\title{
Volume 6 Indexes
}




\section{Compound Pharmacological Activity Index}

This index lists all 3367 normalized pharmacological activity terms appeared in the encyclopedia in alphabetical order and a number code sequence of the related compounds follows the bold term immediately. Authors would like to give some reminder to readers: (1), for normalization of pharmacological terms, please see "Abbreviations and Symbols" and "Cancer Cell Codes in Pharmacological Models" in Volume 1. (2), in the encyclopedia the in vivo anticancer activity data are defined as "antineoplastic" while the in vitro anticancer activity data are defined as "cytotoxic". (3), the inactive experimental data in literatures are also collected. The formatted method of inactive data is putting word "inactive" after the activity term, for example "AChE inhibitor inactive", "Cytotoxic inactive" and so on.

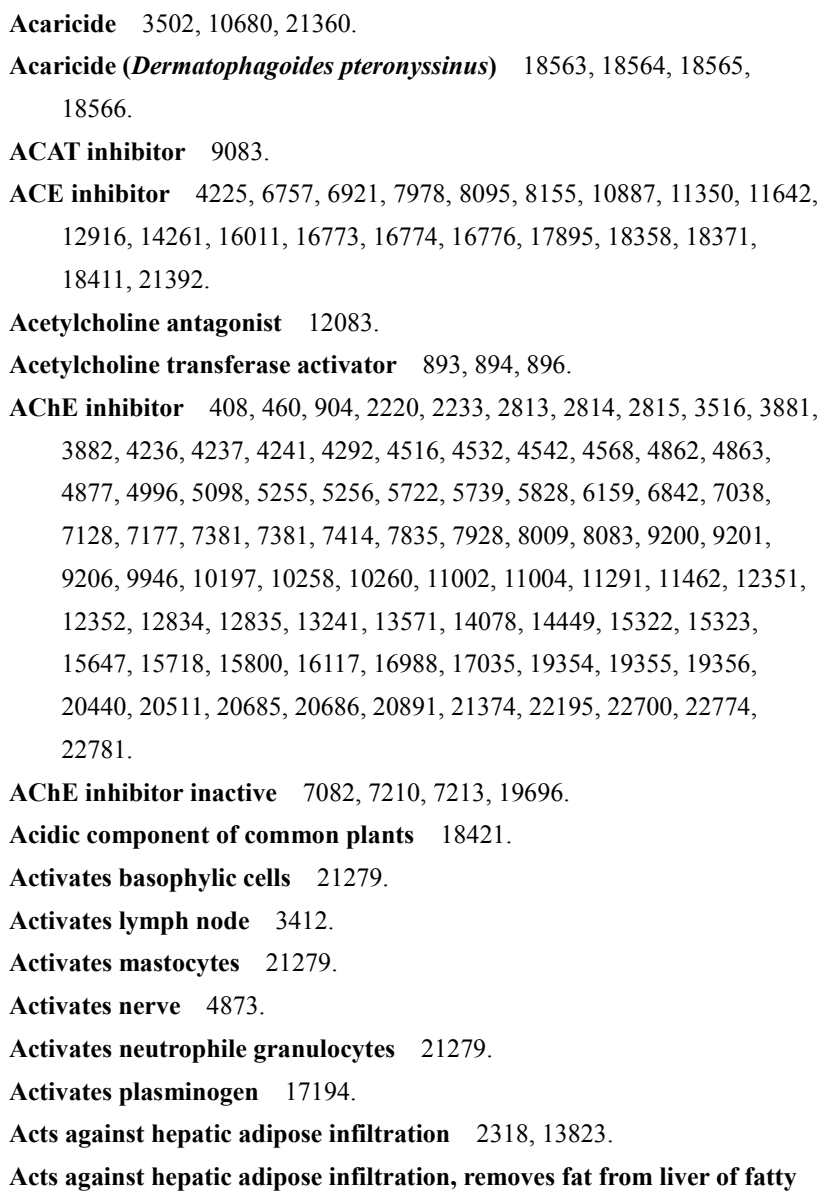


18004, 18005, 18317, 18317, 18345, 18351, 18368, 18378, 18411, 19087, 19087, 19201, 19618, 20102, 21053, 21097, 21362.

Aldose reductase inhibitor inactive 1621, 19425, 19426, 20556. Algicidal (Oscillatoria perornata) 8985, 19077, 19078. Algicidal (Raphidocelis subcapitata) 7049, 7161, 12409, 14405, 14408. Algicidal (Selenastrum capricornutum) 8985, 19077, 19078.

Alkaline phosphatase inhibitor 2208, 3063.

Alkaline phosphatase promoter 2331 .

Allelopathil agent, produced from walnut tree Juglans regia 11903.

Allergen 62, 2890, 3611, 9700, 14971, 16440, 16598, 17828, 17853,

18679, 22104, 22105.

Alluring action (male gamete of Allomyces) 19976.

$\gamma$-Aminobutyric acid antagonist 642 .

AMV-reverse transcriptase inhibitor 11703, 21913.

Amyloglucosidase inhibitor 2792, 7437.

An essential amino acid for children 9569 .

Analgesic $304,554,669,1012,1021,1094,1097,1178,1283,1287,1667$, 2214, 2247, 2303, 2550, 2737, 2738, 2739, 2887, 3141, 3152, 3204, $3218,3498,3689,3693,3712,3885,3907,3935,3937,4080,4103$, 4550, 4645, 4685, 4741, 4745, 4771, 4993, 5108, 5358, 5359, 5417, 6529, 6708, 6767, 7441, 7481, 7665, 7707, 7714, 7800, 7996, 8083, $8150,8151,8156,8170,8257,8273,8276,8307,8428,8965,9187$, $9238,9661,10875,10887,11259,11260,11580,11734,11741,12510$, 12608, 12916, 12916, 13606, 13630, 13774, 13776, 13794, 14184, 14896, 14897, 14981, 15449, 15843, 16451, 16525, 16532, 16555, 16803, 16966, 17042, 17958, 19081, 19184, 19196, 19198, 19200, 19473, 19540, 19922, 19955, 20002, 20141, 20307, 20324, 20444, 20509, 20569, 20578, 21077, 21206, 21234, 21245, 21292, 21366 , 21887, 21955, 22384, 22562, 22602, 22818, 22938, 23004.

Androgen 1169, 21022.

Anesthetic 2113, 2716, 3278,4353, 5199, 6745, 9553, 13774, 13796, 13797, 13798, 14981, 21292, 23003.

Anesthetic antagonist 3079 .

Angiogenesis enhancer 19202.

Angiogenesis inhibitor $2208,5047,5910,11505,13391,16183,17717$, 20900, 20901, 21709.

Angiogenesis inhibitor inactive $6162,9564,10751,12448,21856$. Anorexic 3327, 15789.

Antagonist to body temperature reduction caused by 5-HT 19846. Antagonist to muscle rigidity 20732 .

Antagonizes antibacterial action of paraxin 3118 .

Antemetic 8395, 19846, 21059, 23003.

Anthelmintic $139,759,762,834,936,1319,1475,1654,1658,1779$, $1798,1842,1895,1897,2050,2550,3231,3598,3760,3767,3841$, 4234, 4317, 4329, 5251, 5502, 5648, 6460, 6550, 6767, 7481, 7798, $7809,8312,9288,9704,11203,12221,13559,14728,15131,15485$, 15486,15882 , 16085, 16584, 16770, 16789, 18094, 18299, 18452, $18546,18940,19101,19313,19314,20240,21350,21360,21448$, 22332, 22382.

Anthelmintic (amoeba) 14728.

Anthelmintic (ants) 18293.

Anthelmintic (ascarid) 762, 8312, 11203, 16770, 18452, 21448.

Anthelmintic (Ascaris vermicularis) 18299.
Anthelmintic (Caenorhabditis elegans) 1319, 20240.

Anthelmintic (dwarf tapeworm) 6460.

Anthelmintic (hookworm) 1895, 6460.

Anthelmintic (liver flukes) 16789.

Anthelmintic (tapeworm) 762, 1654, 1895, 6767, 9704, 16789.

Anthelmintic (termites) 5188, 6015, 9857, 14144.

Anthelmintic (Trypanosoma cruzi) 16085.

Antiacetylcholine activity 17007 .

Anti-acne agent 2056.

Anti-addictive 4080 .

Anti-adrenaline $5707,11851,18623,22921$.

Antiallergic $892,893,983,1836,1837,1838,2102,2106,2687,3412$, 4097, 4417, 5415, 5430, 5722, 6105, 6853, 7054, 7924, 8095, 8297, $8841,8846,11095,12683,12766,12767,12891,13137,14995,16268$, 16269, 16525, 18643, 18790, 21206, 21356, 21993, 21994, 22350.

Antiallergic and anti-inflammatory 23, 3602 .

Antiallergic $\boldsymbol{\beta}$-Hexosaminidase inhibitor $2165,2456,2508,5905,9099$, 9100, 9102, 9846, 9848, 13909, 13910, 14154.

Antiamebic $576,774,777,2615,2660,2665,2666,2667,2668,2670$, $2671,2674,2684,3218,3400,5436,6772,8047,8509,11203,12347$, 17862, 18095, 18627, 19414, 19900, 22098, 22146.

Anti-amnesic 4568.

Anti-androgenic $4039,4040,4041,4042,7521,10960,12490,12491$, 12493, 12496, 16358.

Anti-androgenic inactive 16498 .

Antianemic 11882.

Anti-apoptosis $251,580,1181,2547,5314,18192$.

Antiasthmatic $663,957,1162,1628,1840,1903,2797,3235,3237,3689$, $5067,5763,6815,7481,7924,8095,8404,9646,11507,12438,14395$, $15370,17456,17472,17983,18219,18317,19540,20077,20137$, 20254, 20992, 20995, 21312.

Anti-atropine 9224.

Antibacterial inactive summary index $569,1215,1217,1222,1617$, $1635,1636,1637,1646,1647,1764,2590,2624,2945,2990,2991$, 2998, 3004, 3908, 4035, 4128, 4891, 4985, 5925, 5926, 6065, 6809, $6810,7044,7313,7315,7316,8576,8579,8580,9223,9225,9518$, $9857,10629,11071,11078,11079,11086,11258,11485,11570,12310$, $12511,12572,12615,12616,13259,13637,14729,14731,14789$, $14915,15118,15583,15584,15585,15586,15588,15589,15590$, $15591,15592,15916,18673,19308,19349,19350,19428,19429$, 19589, 19591, 19594, 19595, 19999, 20377, 22634, 22777, 22962.

Antibacterial inactive, 13 strains of methicillin-resistant Staphylococcus aureus (MRSA) 7313, 7315, 7316.

Antibacterial inactive, Alteromonas sp. 2624, 12616.

Antibacterial inactive, Azobacter beijerinckii $\quad 11485,12616$.

Antibacterial inactive, Bacillus brevis $\quad 8576,8579,8580$.

Antibacterial inactive, Bacillus megaterium 15518.

Antibacterial inactive, Bacillus subtilis $\quad 1646,1647,1764,3004,8576$, 8579, 8580, 10629, 13259, 19428, 19429.

Antibacterial inactive, Chromobacterium violaceum 11258.

Antibacterial inactive, Clostridium fallax 11570, 12615.

Antibacterial inactive, Clostridium novyi $11570,12615$.

Antibacterial inactive, Clostridium sordellii $11570,12615$. 
Antibacterial inactive, Enterobacter aerogenes $\quad$ 11570, 12615. Antibacterial inactive, Enterobacter cloacae 569, 14789. Antibacterial inactive, Enterobacter dissolvens $\quad 8576,8579,8580$. Antibacterial inactive, Escherichia coli $569,1635,1636,1637,2590$, 2945, 2990, 2991, 2998, 3004, 6065, 9857, 10629, 11071, 11078, $11079,11086,12615,13259,13637,14789,19589,19591,19594$, 19595, 22634.

Antibacterial inactive, gram-negative bacteria $1215,1217,1222$ Antibacterial inactive, gram-positive bacteria $1215,1217,1222$. Antibacterial inactive, Halobacterium sp. 2624, 11485, 12572, 12616. Antibacterial inactive, Halococcus sp. 2624, 11485, 12572, 12616. Antibacterial inactive, Helicobacter pylori NCTC11637 strain 4035. Antibacterial inactive, Helicobacter pylori NCTC11916 strain 4035. Antibacterial inactive, Helicobacter pylori OCO1 strain 4035. Antibacterial inactive, Klebsiella aerogenes 11258.

Antibacterial inactive, Klebsiella pneumoniae 569, 14789, 19589, 19591, 19594, 19595, 22634.

Antibacterial inactive, MDR Staphylococcus aureus RN4220 strain 4985, 9518, 15916

Antibacterial inactive, MDR Staphylococcus aureus SA-1199-B strain 4985, 9518, 15916

Antibacterial inactive, MDR Staphylococcus aureus XU212 strain $4985,9518,15916$

Antibacterial inactive, methicillin-resistant Staphylococcus aureus MRSA 5925, 5926, 12310, 15583, 15584, 15585, 15586, 15588, 15589, 15590, 15591, 15592.

Antibacterial inactive, methicillin-sensitive Staphylococcus aureus MSSA 12310.

Antibacterial inactive, Micrococcus luteus 8576, 19349, 19350, 19589, 19591, 19594, 19595, 22634.

Antibacterial inactive, Mycobacterium smegmatis $\quad$ 1646, 1647.

Antibacterial inactive, Mycobacterium tuberculosis $\quad 6809,6810,14729$, 14731, 14915, 15118, 19999, 20377, 22777.

Antibacterial inactive, Pseudomonas aeruginosa $\quad 569,1635,1636,1637$, 14789.

Antibacterial inactive, Pseudomonas aeruginosa $\quad 569,1635,1636,1637$, 14789.

Antibacterial inactive, Salmonella setubal 19589, 19591, 19594, 19595, 22634.

Antibacterial inactive, Serratia marcescens 14789.

Antibacterial inactive, Shigella flexneri 11570, 12615.

Antibacterial inactive, Staphylococcus aureus $\quad 569,1635,1636,1637$, 1646, 1647, 1764, 3004, 9857, 10629, 11086, 13259, 19349, 19350, 19428, 19429, 19589, 19591, 19594, 19595, 22634.

Antibacterial inactive, Staphylococcus epidermidis $\quad 569,19589,19591$, 19594, 19595, 22634.

Antibacterial inactive, Staphylococcus sangui $\quad$ 1635, 1636, 1637.

Antibacterial inactive, various tested bacteria 9223, 9225 .

Antibacterial inactive, Vibrio anguillarum 2590, 2945, 2990, 2991, 2998, 9857, 11071, 11078, 11079, 11086.

Antibacterial inactive, Vibrio cholerae 11570, 12615.

Antibacterial inactive, Vibrio vulnificus $11570,12615$.

Antibacterial summary index $10,24,50,51,53,99,123,126,143,207$,
$218,370,515,585,589,595,597,598,663,664,675,754,755,756$, $762,783,822,834,847,847,899,901,909,920,930,957,967,1016$, $1080,1135,1159,1178,1179,1244,1250,1252,1277,1293,1348$, 1476, 1643, 1644, 1645, 1648, 1745, 1769, 1842, 1845, 1897, 1904, 1990, 2013, 2034, 2081, 2106, 2168, 2244, 2283, 2284, 2294, 2300, 2303, 2304, 2309, 2331, 2334, 2376, 2380, 2509, 2539, 2550, 2584, 2590, 2591, 2592, 2593, 2594, 2619, 2624, 2678, 2680, 2681, 2682, 2685, 2844, 2887, 2945, 2985, 2990, 2991, 2998, 3004, 3004, 3026, $3029,3052,3055,3057,3058,3094,3096,3122,3148,3153,3157$, $3158,3186,3231,3243,3294,3308,3368,3412,3452$, 3453, 3453, $3463,3472,3473,3474,3475,3485,3490,3498,3502$, 3511, 3551, $3588,3598,3615,3633,3689,3695,3708,3745,3767,3768,3791$, $3799,3940,3941,3962,4005,4029,4048,4055,4135,4140,4200$, 4202, 4203, 4241, 4243, 4292, 4348, 4349, 4362, 4363, 4398, 4422, 4460, 4471, 4536, 4604, 4632, 4633, 4634, 4645, 4832, 4837, 4840, 4923, 4976, 5064, 5100, 5134, 5152, 5161, 5214, 5229, 5235, 5272, $5273,5276,5279,5332,5333,5445,5522,5524,5540,5541,5542$, 5550, 5551, 5572, 5613, 5682, 5694, 5700, 5708, 5722, 5747, 5763, 5789, 5858, 5894, 5926, 5959, 6018, 6018, 6020, 6020, 6032, 6040, $6049,6065,6178,6238,6261,6263,6298,6302,6303,6361,6366$, $6427,6429,6430,6518,6605,6626,6629,6735,6752,6757,6758$, 6776, 6801, 6853, 6901, 6921, 6973, 7015, 7063, 7121, 7154, 7174, 7178, 7181, 7185, 7205, 7221, 7278, 7305, 7306, 7307, 7312, 7314, 7323, 7354, 7355, 7356, 7357, 7358, 7385, 7399, 7413, 7416, 7441, 7481, 7512, 7521, 7703, 7707, 7736, 7756, 7764, 7767, 7768, 7792, 7802, 7809, 7825, 7829, 7830, 7883, 7897, 7897, 7907, 7924, 7926, 7927, 7942, 7944, 7996, 8081, 8081, 8081, 8081, 8085, 8095, 8095, $8136,8140,8218,8218,8219,8221,8272,8275,8278,8306,8307$, $8314,8333,8334,8403,8405,8456,8490,8493,8499,8548,8591$, $8596,8672,8708,8709,8760,8833,8841,8955,8959,8967,8968$, 8982, 9226, 9288, 9321, 9322, 9393, 9441, 9456, 9547, 9557, 9558, 9570, 9596, 9597, 9631, 9631, 9678, 9697, 9702, 9720, 9740, 9788, 9854, 9869, 9877, 9924, 10104, 10126, 10149, 10159, 10208, 10351, 10383, 10484, 10565, 10567, 10652, 10664, 10665, 10732, 10733, 10736, 10747, 10748, 10751, 10819, 10820, 10826, 10883, 10887, 11056, 11071, 11078, 11079, 11092, 11149, 11258, 11259, 11260, $11262,11263,11264,11269,11321,11324,11327,11348,11386$, 11392, 11423, 11434, 11455, 11485, 11526, 11570, 11607, 11642, 11683, 11736, 11736, 11747, 11751, 11777, 11778, 11779, 11811, 11849, 11851, 11866, 11903, 11943, 12018, 12020, 12060, 12163 , $12228,12254,12321,12322,12334,12335,12380,12389,12420$, $12438,12453,12454,12489,12501,12537,12572,12573,12597$, 12615, 12616, 12764, 12766, 12767, 12771, 12843, 12849, 12957 , 12958, 13041, 13088, 13088, 13107, 13109, 13137, 13195, 13196, 13239, 13264, 13281, 13315, 13388, 13398, 13428, 13429, 13469 , 13469, 13481, 13485, 13486, 13487, 13488, 13492, 13493, 13494, 13495, 13606, 13607, 13637, 13638, 13643, 13651, 13652, 13811, 13811, 13874, 13876, 13941, 13979, 14028, 14029, 14083, 14086, $14121,14154,14170,14216,14217,14255,14256,14340,14341$, 14342, 14346, 14454, 14531, 14567, 14592, 14603, 14680, 14705, 14728, 14758, 14789, 14815, 14829, 14840, 14842, 14850, 14967, 14969, 14970, 14971, 15027, 15039, 15042, 15043, 15044, 15056, 15170, 15184, 15221, 15279, 15286, 15297, 15298, 15300, 15301, 
15302, 15303, 15304, 15305, 15306, 15307, 15308, 15309, 15312, $15313,15314,15315,15316,15335,15337,15342,15370,15412$, 15436, 15489, 15506, 15519, 15561, 15587, 15633, 15652, 15715, $15728,15737,15800,15849,15858,15859,15860,15869,15870$, $15872,15881,15885,15888,15910,15939,16050,16079,16080$, $16119,16183,16261,16264,16268,16269,16275,16359,16429$, $16439,16442,16488,16532,16545,16555,16597,16598,16600$, 16666, 16675, 16711, 16741, 16742, 16743, 16744, 16745, 16747, $16747,16748,16748,16749,16754,16770,16799,16800,16802$, 16816, 16912, 16913, 16914, 16915, 16966, 16978, 17009, 17018, $17019,17028,17047,17048,17049,17168,17247,17272,17283$, 17361, 17362, 17363, 17371, 17375, 17403, 17430, 17431, 17432, 17456, 17486, 17487, 17488, 17489, 17491, 17528, 17567, 17568, 17570, 17571, 17602, 17696, 17777, 17859, 17861, 17862, 17870, 17924, 17926, 17983, 18011, 18071, 18086, 18097, 18102, 18212, $18213,18214,18219,18220,18221,18269,18270,18305,18317$, 18376, 18411, 18547, 18643, 18674, 18679, 18759, 18831, 18849, 18857, 18859, 18868, 18972, 19078, 19148, 19174, 19257, 19259, 19299, 19306, 19307, 19312, 19452, 19468, 19469, 19471, 19511, 19533, 19542, 19546, 19547, 19548, 19549, 19550, 19551, 19552, 19553, 19554, 19555, 19556, 19557, 19558, 19559, 19560, 19561, 19574, 19575, 19590, 19592, 19593, 19614, 19707, 19777, 19819, 19827, 19904, 19905, 19906, 19907, 19912, 19933, 19934, 19935, 19985, 20002, 20018, 20019, 20044, 20061, 20093, 20135, 20137, 20150, 20166, 20167, 20307, 20390, 20444, 20489, 20511, 20566, 20575, 20578, 20670, 20679, 20680, 20681, 20685, 20686, 20687, 20697, 20699, 20700, 20701, 20702, 20717, 20732, 20968, 20992, 21018, 21022, 21034, 21052, 21055, 21132, 21206, 21235, 21236, $21237,21239,21251,21253,21257,21260,21261,21266,21268$, $21269,21270,21328,21333,21356,21360,21362,21486,21503$, $21577,21589,21727,21765,21776,21830,21866,22045,22059$, 22080, 22190, 22191, 22195, 22239, 22270, 22270, 22270, 22282, $22321,22332,22380,22420,22496,22498,22499,22592,22593$, 22594, 22596, 22667, 22667, 22700, 22704, 22755, 22759, 22774, 22781, 22815, 22818, 22965, 22970, 22972, 23002, 23014.

Antibacterial, $\alpha$-hemolytic streptococcus 17602.

Antibacterial, 10 strains of methicillin-resistant Staphylococcus aureus (MRSA) 847, 3453, 8140, 15042, 15043, 15044, 15056, 22667.

Antibacterial, 11 kinds of pathogenic bacteria 7924.

Antibacterial, 12 strains of molds 16555.

Antibacterial, 13 strains of methicillin-resistant Staphylococcus aureus (MRSA) 7312, 7314

Antibacterial, 15 strains of dysentery 13606.

Antibacterial, 18 strains of methicillin-resistant Staphylococcus aureus (MRSA) 22593, 5279, 22594, 22592.

Antibacterial, $30 \mathrm{hmn}$ pathogenic bacteria, in which $8 / 30$ effective 16966

Antibacterial, 30 kinds of pathogenic bacteria 20575.

Antibacterial, 40 types of bacteria 22499.

Antibacterial, 40 types of bacteria and fungi 21055 .

Antibacterial, 8 strains of methicillin-resistant Staphylococcus aureus (MRSA) 847, 3453, 8140, 15042, 15043, 15044, 15056, 22667.

Antibacterial, acid-fast bacteria $7323,9631,14454$.
Antibacterial, aflatoxin $\mathbf{B}_{1} 17028$.

Antibacterial, Alcaligenes aquamarinus 2624, 12616.

Antibacterial, Alteromonas sp. 11485, 12572.

Antibacterial, antipyretic 15337.

Antibacterial, Aspergillus niger $\quad$ 1244, 1250, 1252, 2844, 5550, 15298, 15370, 20390.

Antibacterial, Azomonas agilis 2624, 11485, 12572, 12616.

Antibacterial, Azotobacter beijerinckii 2624, 12572.

Antibacterial, Babesia gibsoni 14342, 14340, 14341, 14346.

Antibacterial, Bacillus anthracis 967, 9678, 18759.

Antibacterial, Bacillus brevis 10732.

Antibacterial, Bacillus cereus $\quad$ 143, 207, 218, 589, 755, 756, 2591, 2592, 2593, 3368, 6049, 6065, 7707, 7756, 7767, 9678, 9924, 10159, 10567, $11262,11263,11264,14789,17862,21830,22818$.

Antibacterial, Bacillus cereus ATCC11778 strain 10484, 14592.

Antibacterial, Bacillus coagulans 143, 9924.

Antibacterial, Bacillus coli $\quad$ 51, 123, 515, 589, 663, 957, 1016, 1179, 1244, $1250,1252,2168,2244,2283,2539,2624,2844,3148,3157,3158$, $3186,3452,3962,4140,4203,4241,4243,4645,4840,5161,5235$, $5272,5273,5540,5541,5542,5550,5682,5763,6427,6429,6430$, $6735,6776,6973,7205,7305,7307,7767,7883,8136,8278,8456$, $8968,9596,9788,10104,10159,10567,10733,10819,11259,11260$, $11392,11434,11485,11570,11777,11778,11779,12537,12572$, 12616, 12957, 12958, 13107, 13239, 13398, 13874, 13876, 14255, $14815,15170,15279,15286,15297,15298,15300,15301,15302$, $15303,15304,15305,15306,15307,15308,15309,15312,15313$, $15314,15315,15316,15342,15885,16442,16532,16545,16555$, $17018,17019,17048,17247,17528,18071,18220,18221,18547$, 19590, 19592, 19593, 19707, 19819, 20018, 20019, 20390, 20686, 20687, 20697, 20699, 20700, 20701, 20702, 21251, 21257, 21269, 21270, 22045, 22195, 22270, 22282, 22496, 22667.

Antibacterial, Bacillus coli ATCC11775 strain 10484, 14592.

Antibacterial, Bacillus coli ATCC25922 strain 3689, 4135.

Antibacterial, Bacillus coli NIHJ JC-2 strain 7792, 11811, 12321, 12322.

Antibacterial, Bacillus coli O157:H7 ATCC43894 strain 3689, 4135. Antibacterial, Bacillus diphtheriae $\quad 515,967,1178,1244,1250,1252$, $2844,3157,3158,3186,4832,4840,5272,5273,5540,5541,5542$, $5550,6973,9678,9788,10733,13874,13876,15297,15298,15300$, $15301,15302,15303,15304,15305,15306,15307,15308,15309$, 15312, 15313, 15314, 15315, 15316, 18759, 20390, 22282, 22496.

Antibacterial, Bacillus dysenteriae $\quad 967,6776,7441,7942,8095,8968$, $13137,14971,15170,15279,15286,16555,17247,18759,19574$, 19575, 19819, 20444, 21251, 21269, 21270.

Antibacterial, Bacillus fusiformis 8405.

Antibacterial, Bacillus globisporus 15910, 18097.

Antibacterial, Bacillus influenzae 3615, 6776.

Antibacterial, Lactobacillus spp. 16080.

Antibacterial, Bacillus lactis $\quad 515,1244,1250,1252,2844,3157,3158$, $3186,4840,5272,5273,5540,5541,5542,5550,6973,9788,10733$, $13874,13876,15297,15298,15300,15301,15302,15303,15304$, $15305,15306,15307,15308,15309,15312,15313,15314,15315$, $15316,20390,22496$. 
Antibacterial, Bacillus megaterium 12501, 15519.

Antibacterial, Bacillus mycoides 14829, 15039, 21251.

Antibacterial, Bacillus paratyphosus 967, 6776, 6921, 18759.

Antibacterial, Bacillus paratyphosus A 8095.

Antibacterial, Bacillus pertussis 22282.

Antibacterial, Bacillus pumilus 14789.

Antibacterial, Bacillus pyocyaneus $\quad 3148,4645,5682,5763,6776,8095$, 8334, 10351, 10819, 11736, 13137, 15910, 17247, 17528, 17696, 19148, 19819, 20444, 21251, 21269, 21270, 22045.

Antibacterial, Bacillus pyocyaneus ATCC27853 strain 3689,4135 , 20686, 20687.

Antibacterial, Bacillus septicus $\quad 21251,21269,21270$.

Antibacterial, Bacillus sonne 16770.

Antibacterial, Bacillus sp. $\quad$ 18868, 22059.

Antibacterial, Bacillus sphaericus 99, 6263, 10748, 11258, 16275, 16741, 16742, 16743, 16744, 16745, 18212, 18213.

Antibacterial, Bacillus subtilis $\quad 50,51,53,99,123,143,207,218,515$, $901,957,967,1244,1250,1252,1645,1648,2168,2244,2283,2539$, 2584, 2844, 3004, 3157, 3186, 3463, 3472, 3474, 4048, 4243, 4243, 4422, 4604, 4840, 4923, 5214, 5235, 5272, 5273, 5522, 5540, 5541, $5542,5550,5747,5959,6238,6263,6303,6361,6427,6429,6430$, $6776,6973,7178,7205,7305,7306,7307,7707,7756,7764,7767$, 7883, 8136, 8278, 8456, 8672, 8833, 9288, 9321, 9322, 9788, 9924, 10104, 10732, 10733, 10748, 11056, 11258, 11269, 11321, 11392, $11434,11751,11777,11778,11779,12334,12380,12389,12766$, $12767,12771,13107,13109,13137,13195,13196,13398,13637$, 13874, 13876, 14170, 14680, 14789, 14829, 15039, 15297, 15298, $15300,15301,15302,15303,15304,15305,15306,15307,15308$, $15309,15312,15313,15314,15315,15316,15342$, 15489, 15506, $15561,15939,16275,16359,16429,16532,16545,16741,16742$, $16743,16744,16745,16747,16748,16749,16799,16802,17018$, 17019, 17048, 17371, 17403, 17430, 17431, 17432, 17486, 17487, $17488,17489,17491,18071,18212,18213,18214,18759,19257$, 19259, 19574, 19575, 19707, 20093, 20390, 20697, 20699, 20700, 20701, 20702, 20968, 21022, 21034, 22059, 22195, 22239, 22270, 22282, 22496, 22667

Antibacterial, Bacillus subtilis 6633 strain 3158.

Antibacterial, Bacillus subtilis ATCC1633 strain 13607.

Antibacterial, Bacillus subtilis H17 strain $\quad 15858,15859,15860$

Antibacterial, Bacillus subtilis IFO3134 strain 7792, 11811, 12321, 12322.

Antibacterial, Bacillus subtilis M45 strain $\quad$ 15858, 15859, 15860. Antibacterial, Bacillus termo $\quad 515,1244,1250,1252,2844,3157,3158$, $3186,4840,5272,5273,5540,5541,5542,5550,5763,6973,7521$, $9788,10733,13137,13874,13876,15297,15298,15300,15301$, $15302,15303,15304,15305,15306,15307,15308,15309,15312$, $15313,15314,15315,15316,16770,20390,20444,21251,21269$, 21270, 22282, 22496.

Antibacterial, Bacillus termo I $\quad$ 12537, 20578.

Antibacterial, Bacillus thuringiensis 20992.

Antibacterial, Bacillus typhosus $\quad 3452,3767,3768,6921,8095,13137$, $14971,15170,15279,15286,16770,18270,18759,19819,20444$, 22815 .
Antibacterial, bacteria on skin surface 8081 .

Antibacterial, broad spectrum 4460, 11851, 11903, 16261, 22774.

Antibacterial, broad spectrum and low toxicity 18270 .

Antibacterial, Chromobacterium violaceum 99, 6263, 6735, 10748, $11570,12615,16275,16741,16742,16743,16744,16745,18212$, 18213.

Antibacterial, Citrobacter freundii ATCC8090 strain 3689, 4135.

Antibacterial, Clostridium cellobioparum 6735, 11570, 12615.

Antibacterial, Clostridium fallax 6735.

Antibacterial, Clostridium novyi 6735.

Antibacterial, Clostridium sordellii 6735.

Antibacterial, Coccus catarrhal 3615, 6776, 20444, 13137.

Antibacterial, Coccus spp. 11747.

Antibacterial, Cochliobolus miyabeanus 15370.

Antibacterial, cooperates with berberine to inhibit Staphylococcus aureus 8841.

Antibacterial, Corynebacterium accolens $\quad 3490,4055,6757,6758,8095$, 13428, 13429.

Antibacterial, Corynebacterium acnes 10, 10351.

Antibacterial, Corynebacterium betae 5894, 6032.

Antibacterial, Corynebacterium fascians $\quad 5894,6032,18219$.

Antibacterial, Corynebacterium hoffmanii 16799, 16800, 16802.

Antibacterial, Cryptococcus neoformans 10565.

Antibacterial, Cryptococcus neoformans ATCC90112 strain 10484 , 14592.

Antibacterial, cytochrome C reductase inhibitor 7015 .

Antibacterial, Diplococcus catarrhal 12843.

Antibacterial, Diplococcus pneumoniae $\quad 3158,3615,5763,9678,12334$, $12843,13137,14454,14531,15337,15561,17283,17456,17862$, 20061, 22282.

Antibacterial, Enterobacter cloacae $\quad 5613,7278,8081,8959,13651$, 13652.

Antibacterial, Enterobacter cloacae ATCC23350 strain $3689,4135$.

Antibacterial, Enterococci sp. (VRE) 9393.

Antibacterial, Enterococcus faecalis $\quad 5747,7764$.

Antibacterial, Enterococcus faecalis 11268 strain 13811.

Antibacterial, Enterococcus faecalis 1528(vanA) strain 2680, 2681, 2682.

Antibacterial, Enterococcus faecalis 18292 strain $\quad 6018,6020,13469$, 13811.

Antibacterial, Enterococcus faecalis 19250 strain $\quad 6018,6020,13469$, 13811.

Antibacterial, Enterococcus faecalis ATCC21212 strain 7792, 11811, 12321, 12322.

Antibacterial, Enterococcus faecalis ATCC29212 strain 10484, 14592.

Antibacterial, Enterococcus faecalis ATCC33186 strain $\quad 3689,4135$. Antibacterial, Enterococcus faecalis JCM7783 strain (VSE) 847, 3453, 8140, 15042, 15043, 15044, 15056, 22667.

Antibacterial, Enterococcus faecalis JU1782 (VRE, VanB) 847, 3453, 8140, 15042, 15043, 15044, 15056, 22667.

Antibacterial, Enterococcus faecalis JU1856 (VRE, VanA) 847, 3453, 8140, 15042, 15043, 15044, 15056, 22667. 
Antibacterial, Enterococcus faecium 513811.

Antibacterial, Enterococcus faecium JCM5804 (VSE) 847, 3453, 8140, 15042, 15043, 15044, 15056, 22667.

Antibacterial, Enterococcus faecium JU1777 (VRE,VanB) 847, 3453, 8140, 15042, 15043, 15044, 15056, 22667.

Antibacterial, Enterococcus faecium JU1858 (VRE,VanA) 847, 3453, 8140, 15042, 15043, 15044, 15056, 22667.

Antibacterial, Enterococcus gallinarum CDC-42 strain 3689, 4135. Antibacterial, Enterococcus gallinarum JU2786 (VRE,VanC) 3453. Antibacterial, Enterococcus gallinarum JU2786 strain (VRE,VanC) 847, 8140, 15042, 15043, 15044, 15056, 22667.

Antibacterial, Enterococcus sp. $\quad 18270,22815$.

Antibacterial, Enteromorpha cloacae $15170,15184,18305,18411$, $18679,18857,18859$

Antibacterial, Erwinia amylovora $\quad 2624,11485,12572,12616$.

Antibacterial, Erwinia carotovora $\quad 3490,4055,6757,6758,8095,13428$, 13429.

Antibacterial, Erwinia carotovora var. carotovora 18219.

Antibacterial, Escherichia coli $\quad$ 901, 1178, 4471, 5522, 5700, 5747, 5959, $6238,7521,7764,13195,13196,13651,13652,14170,18219,20093$, $21022,21034$.

Antibacterial, Escherichia coli SG458 strain 2680, 2681, 2682.

Antibacterial, Escherichia sp. 20578.

Antibacterial, Flavobacterium helmiphilum $\quad$ 6735, 11570, 12615.

Antibacterial, Fusobacterium nucleatum 2303, 3055, 3057, 3058, 6366, 9702, 11348, 14567, 19985, 21356.

Antibacterial, gram-negative bacteria $\quad 675,2294,3745,4837,7323$, 9631, 10751, 10883, 14083, 14454, 14728, 16183, 17009, 17489, $17570,17571,17870,22270$

Antibacterial, gram-positive bacteria $\quad 675,2294,3588,3633,3745,4837$, 5789, 7221, 7323, 9570, 9869, 10751, 10883, 11092, 11455, 13109, $14083,14454,14728,15039,15652,16183,17009,17489,17570$, 17571, 17870, 19468, 22239, 22270, 22321, 22596, 22704, 23014.

Antibacterial, gram-positive bacteria (inactive for gram-negative bacteria) 7015 .

Antibacterial, Helicobacter pylori NCTC11637 strain $\quad$ 13643, 16079, 21765.

Antibacterial, Helicobacter pylori NCTC11916 strain $\quad 13643,16079$, 21765.

Antibacterial, Helicobacter pylori OCO1 strain 13643, 16079, 21765.

Antibacterial, hemolytic streptococcus $3452,8967,10736,20686$, 20687, 22282.

Antibacterial, hemolytic $\boldsymbol{\beta}$-Streptococcus $\quad 3158,11259,11260,15337$, 15561.

Antibacterial, Hemophilus influezae 5763.

Antibacterial, hmn Mycobacterium tuberculosis 22282.

Antibacterial, hmn Mycobacterium tuberculosis H37Rv strain 4292, 5722, 6605, 10736.

Antibacterial, enhances antibacterial action of phage by over 100 times 22774.

Antibacterial, inhibits colony formation of $X$. campestris pv. oryzae 7121, 7154, 7174, 7181, 7185.

Antibacterial, Klebsiella aerogenes 99, 6263, 10748, 16275, 16741,
16742, 16743, 16744, 16745, 18212, 18213.

Antibacterial, Klebsiella pneumoniae $\quad 3962,7521,8548,13651,13652$, $14454,14815,15885,16799,16800,17528,19593,20018,20019$, 21251, 21253, 21257, 21269, 21270.

Antibacterial, Klebsiella pneumoniae $\quad 515,1244,1250,1252,2844,3157$, $3158,3186,4840,5272,5273,5540,5541,5542,5550,6973,9788$, $10733,13874,13876,15297,15298,15300,15301,15302,15303$, $15304,15305,15306,15307,15308,15309,15312,15313,15314$, $15315,15316,20390,22496$.

Antibacterial, Klebsiella pneumoniae ATCC13883 strain 3689, 4135. Antibacterial, Klebsiella pneumoniae NCTC9633 strain 10484, 14592. Antibacterial, lactic acid bacteria 19819.

Antibacterial, Listeria monocytogenes 5747, 7764.

Antibacterial, main effective component of Euphorbia humifusa (DI JIN CAO) 22195.

Antibacterial, Micrococcus epidermidis 2081, 4362, 4363.

Antibacterial, Micrococcus kristinae 14789.

Antibacterial, Micrococcus luteus 143, 2081, 3368, 3472, 3473, 3962, $9924,14815,15506,17430,17431,17432,17862,18269,19592$, 19593, 19614, 21830.

Antibacterial, Micrococcus luteus ATCC10240 strain 2680, 2681, 2682.

Antibacterial, Micrococcus lysodeikticus 53, 4243.

Antibacterial, Micrococcus lysodicklycus $\quad$ 16799, 16800.

Antibacterial, Micrococcus sp. 22818.

Antibacterial, Microsporum sp. 2081.

Antibacterial, microzyme $12771,22270$.

Antibacterial, Mucor mucedo 50.

Antibacterial, mutational Streptococcus 8833.

Antibacterial, Mycobacterium intracellulare 19174.

Antibacterial, Mycobacterium leprae $\quad$ 1293, 3485, 8955, 9697.

Antibacterial, Mycobacterium smegmatis 1645, 1648, 3463, 4923, 5100, $5572,6752,8493,8548,9441,9597,11321,11751,12380,12389$, $15881,15885,16439,16816,17049,18102,18831,19174,21235$, 21237, 21251, 21257, 21260, 21261, 21266, 21268, 21269, 21270.

Antibacterial, Mycobacterium smegmatis ATCC607 strain 8490,8499 , 9557, 9558, 10149.

Antibacterial, Mycobacterium sp. $1769,14028,14029,14603,16488$, 17924, 17926, 21239, 21253.

Antibacterial, Mycobacterium tuberculosis $\quad 24,1178,1179,1277,1643$, 1644, 2244, 2300, 2331, 2334, 2334, 2619, 2619, 3412, 3708, 3791, $3799,3940,3941,4048,4837,5064,5134,5926,6298,6518,6629$, $6801,6901,7413,7512,7768,7897,7897,7907,8219,8221,8306$, 8403, 9678, 9854, 10126, 10208, 10383, 10652, 10747, 10820, 11607, $11736,12163,12228,12453,12454,12489,13109,13264,13481$, $13485,13486,13487,13488,13492,13493,13494,13495,13979$, 14216, 14217, 14217, 14758, 14840, 14842, 15027, 15715, 15737, $15800,15881,15939,16050,16439,16912,16913,16914,16915$, 17375, 17568, 17861, 18011, 18086, 19312, 19533, 19777, 19827, 20061, 20511, 20679, 20680, 20681, 20717, 21206, 21236, 21503, 21589, 21776, 21866, 22080, 22270, 22282, 22774.

Antibacterial, Mycobacterium tuberculosis 607 strain 17570.

Antibacterial, Mycobacterium tuberculosis aviun isoniazed-resistant strain 15939. 
Antibacterial, Mycobacterium tuberculosis H37Rv strain 4632, 4633, 4634, 6302, 8085, 13638

Antibacterial, Mycobacterium vaccae IMET10670 strain 2680, 2681, 2682.

Antibacterial, Mycobacterium xenopi 19174.

Antibacterial, Neisseria sp. 8095.

Antibacterial, no explanation of bacterial species 126, 370, 585, 595, $597,598,664,754,762,783,822,834,920,1080,1159,1348,1476$ $1745,1842,1845,1897,1904,1990,2013,2034,2106,2284,2304$, 2309, 2376, 2594, 2678, 2887, 3094, 3231, 3294, 3308, 3498, 3498, $3502,3598,3695,4005,4029,4348,4349,4398,4536,4976,5152$, $5445,5524,5694,5858,6040,6261,6853,7063,7385,7399,7416$, $7481,7703,7768,7802,7809,7926,7944,7996,8272,8275,8307$, $8591,8760,8982,9456,9720,9740,9877,11149,11327,11386$, 11423, 11526, 11866, 11943, 12020, 12438, 12849, 14086, 14121, $14705,14850,15221,15335,15412,15436,15633,15849,15888$, $16268,16269,16675,16711,16978,17047,17168,17567,17859$, 17983, 18317, 18376, 18643, 18849, 19078, 19306, 19307, 19469, 19471, 19511, 19542, 19904, 19905, 19906, 19907, 19912, 19933, 19934, 19935, 20044, 20135, 20166, 20167, 20307, 20566, 20670, $20685,21018,21328,21333,21360,21486,21577,22332,22420$, 22700, 22755, 22781, 22965, 22970, 22972, 23002.

Antibacterial, original hyphomycete of blood red trichophyta 3122 . Antibacterial, pathogenic bacteria 12420, 22498.

Antibacterial, pathogenic bacteria in mouth 13315 .

Antibacterial, Penicillium aureus 675.

Antibacterial, photo-activated antibacterial 7830, 9226, 12254, 20002.

Antibacterial, plant pathogenic bacteria $5894,6032,7924,16666$, 18219.

Antibacterial, Proteus mirabilis $\quad 6735,11570,12615$.

Antibacterial, Proteus mirabilis IFO3849 strain 7792, 11811, 12321, 12322.

Antibacterial, Proteus vulgare 21052.

Antibacterial, Proteus vulgaris $\quad 8456,10104,13398,16442,17489$, 20018, 20019.

Antibacterial, Pseudomonas aeruginosa $\quad 207,218,2244,2283,3472$, 4203, 6065, 7756, 7767, 10565, 10567, 11777, 11778, 11779, 16600.

Antibacterial, Pseudomonas aeruginosa (Bacillus pyocyaneus) 99, 6263, 7707, 10748, 11258, 13651, 13652, 16275, 16799, 18212, 18213.

Antibacterial, Pseudomonas cepacia 18219.

Antibacterial, Pseudomonas flourescens 99.

Antibacterial, Pseudomonas maltophilia $\quad 5613,7278,8081,8959,10887$, $11642,15170,15184,18219,18305,18411,18679,18857,18859$, 20578.

Antibacterial, Pseudomonas sp. 17489.

Antibacterial, Pseudomonas syringae 6361.

Antibacterial, Pyricularia oryzae 15370, 17489, 19257, 19259.

Antibacterial, Ristella melaninogenica 21356.

Antibacterial, S. thermophilus 10351.

Antibacterial, Saccharomyces cerevisiae $15869,15870$.

Antibacterial, Salmonella aertrycke $\quad 3026,7178,13041,17272,20018$, 20019, 21251, 21269, 21270.

Antibacterial, Salmonella aertrycke ATCC14028 strain $3689,4135$.
Antibacterial, Salmonella aertrycke IFO13245 strain 7792, 11811, 12321, 12322.

Antibacterial, Salmonella enteriditis 5747, 7764.

Antibacterial, Salmonella gallinarum 15885, 21251.

Antibacterial, Salmonella paratyphi A $16799,16800$.

Antibacterial, Salmonella setubal 3962, 14815.

Antibacterial, Salmonella sp. $\quad 515,1244,1250,1252,2844,3157,3158$, 3186, 4840, 5272, 5273, 5540, 5541, 5542, 5550, 6973, 9788, 10733 , 13874, 13876, 14829, 15297, 15298, 15300, 15301, 15302, 15303, 15304, 15305, 15306, 15307, 15308, 15309, 15312, 15313, 15314, 15315, 15316, 20390, 20578, 22496.

Antibacterial, Salmonella typhi 16799, 16800.

Antibacterial, Sarcina citrea $\quad 21022,21034$.

Antibacterial, Sarcina lutea 6303, 12537, 20968.

Antibacterial, Sarcina lutea NIHJ strain 5214.

Antibacterial, Sarcina sp. $\quad 8095,15489$.

Antibacterial, Sclerotinia libertiana 50.

Antibacterial, Serratia marcescens 8968.

Antibacterial, Shigella flexneri 6735.

Antibacterial, Shigella flexneri 4645, 6735.

Antibacterial, Shigella shigae $1179,7521$.

Antibacterial, Shigella sonnei 5747, 7764.

Antibacterial, Shigella sp. 16770, 20578.

Antibacterial, six Bacillus 11747.

Antibacterial, Sporothrix sp. 16555.

Antibacterial, Staphylococcus albus 5763.

Antibacterial, Staphylococcus aureus $\quad 10,50,51,53,99,143,207,218$, $515,663,755,756,899,909,1016,1179,1244,1250,1252,1645$, $1648,2081,2081,2081,2168,2244,2283,2380,2509,2539,2590$, 2844, 2945, 2990, 2991, 2998, 3004, 3026, 3096, 3153, 3157, 3186, $3243,3452,3463,3472,3490,3511,3767,3768,3962,4055,4203$, 4241, 4243, 4243, 4292, 4362, 4363, 4471, 4604, 4645, 4840, 4923, $5161,5235,5272,5273,5540,5541,5542,5550,5682,5700,5722$, $5747,5763,5959,6065,6238,6263,6302,6427,6429,6430,6752$, 6757, 6758, 6776, 6921, 6973, 7205, 7305, 7306, 7307, 7413, 7521, 7707, 7736, 7756, 7764, 7767, 7829, 7883, 7927, 8095, 8095, 8136, $8278,8314,8333,8456,8548,8672,8708,8709,8833,8967,9288$, 9441, 9547, 9678, 9788, 9924, 10104, 10351, 10565, 10567, 10664, 10665, 10733, 10736, 10748, 10819, 10826, 11056, 11071, 11078, $11079,11258,11259,11260,11321,11392,11434,11736,11751$, $11777,11778,11779,11849,12228,12335,12380,12389,12537$, 12573, 12764, 12766, 12767, 12771, 12843, 12957, 12958, 13107, 13109, 13137, 13195, 13196, 13398, 13428, 13429, 13637, 13651, 13652, 13874, 13876, 14170, 14255, 14256, 14531, 14680, 14789, $14815,14971,15039,15170,15279,15286,15297,15298,15300$, 15301, 15302, 15303, 15304, 15305, 15306, 15307, 15308, 15309, $15312,15313,15314,15315,15316,15342,15728,15869,15870$, $15872,15881,15885,15910,16119,16183,16264,16275,16429$, $16439,16442,16532,16545,16555,16597,16598,16600,16741$, 16742, 16743, 16744, 16745, 16747, 16748, 16749, 16770, 16799, $16800,16802,17018,17019,17048,17049,17247,17272,17283$, 17361, 17362, 17363, 17371, 17456, 17486, 17487, 17488, 17489, 17491, 17602, 17696, 17777, 17862, 18071, 18097, 18102, 18212, 
$18213,18219,18269,18270,18547,18759,18868,18972,19174$, 19257, 19259, 19574, 19575, 19593, 19614, 19819, 20018, 20019, 20093, 20137, 20390, 20444, 20687, 20697, 20699, 20700, 20701, 20702, 20968, 21052, 21239, 21257, 21266, 21269, 21270, 21362, 22190, 22191, 22239, 22270, 22380, 22496, 22667, 22759, 22815, 22818 .

Antibacterial, Staphylococcus aureus 17380 strain $\quad 6018,6020,13469$, 13811.

Antibacterial, Staphylococcus aureus 17547 strain $\quad$ 6018, 6020, 13469.

Antibacterial, Staphylococcus aureus 17592 strain $\quad 6018,6020,13469$, 13811.

Antibacterial, Staphylococcus aureus 17728 strain $\quad 6018,6020,13469$, 13811.

Antibacterial, Staphylococcus aureus 18110 strain $\quad 6018,6020,13469$, 13811.

Antibacterial, Staphylococcus aureus 18268 strain $\quad 6018,6020,13469$. Antibacterial, Staphylococcus aureus 209P strain 3158, 5214, 6303, 13607, 18011.

Antibacterial, Staphylococcus aureus 3012 strain $\quad 6018,6020,13469$. Antibacterial, Staphylococcus aureus 414 strain $\quad$ 6018, 6020, 13469.

Antibacterial, Staphylococcus aureus ATCC13709 strain 8490, 8493, 8499, 9557, 9558, 10149, 13941.

Antibacterial, Staphylococcus aureus ATCC25923 penicillin-sensitive strain 18674, 22759.

Antibacterial, Staphylococcus aureus ATCC25923 strain 2685, 3029, $3475,3689,4135,4200,4202,5229,6626,7825,8218,8219,8221$, $11324,11683,12018,12060,13088,13088,13281,13492,14969$, 16747, 16748, 16749, 16754, 19299, 19452, 19546, 19547, 19548, $19549,19550,19551,19552,19553,19554,19555,19556,19557$, 19558, 19559, 19560, 19561, 20150, 20686, 20687.

Antibacterial, Staphylococcus aureus ATCC6538 strain 10484, 14592.

Antibacterial, Staphylococcus aureus drug-resistant strain $\quad 4292,8968$, 10736, 12335, 20687.

Antibacterial, Staphylococcus aureus EMRSA-15 strain 16747, 16748, $16749,16754$.

Antibacterial, Staphylococcus aureus H114 strain 2081.

Antibacterial, Staphylococcus aureus methicillin-resistant ATCC33591 strain $5332,5333$.

Antibacterial, Staphylococcus aureus methicillin-resistant JCM2874 strain $847,3453,8140,15042,15043,15044,15056,22667$.

Antibacterial, Staphylococcus aureus methicillin-resistant SK1 strain 2685, 3029, 3475, 4200, 4202, 5229, 6626, 7825, 8218, 8219, 8221, 11324, 11683, 12018, 12060, 13088, 13088, 13281, 13492, 14969, 18674, 19299, 19452, 19546, 19547, 19548, 19549, 19550, 19551, 19552, 19553, 19554, 19555, 19556, 19557, 19558, 19559, 19560, 19561, 20150, 22759.

Antibacterial, Staphylococcus aureus methicillin-resistant strain 1135, 5276, 7354, 7355, 7356, 7357, 7358, 7792, 8218, 9393, 11811, 12321, 12322, 12597, 12766, 14967, 14970, 15587, 21132.

Antibacterial, Staphylococcus aureus methicillin-sansitive strain $\quad 7792$, 11811, 12321, 12322

Antibacterial, Staphylococcus aureus multidrug-resistant ATCC25923 strain 21727
Antibacterial, Staphylococcus aureus multidrug-resistant RN4220 strain 2985, 5551.

Antibacterial, Staphylococcus aureus multidrug-resistant SA-1199-B strain $2985,5551$.

Antibacterial, Staphylococcus aureus multidrug-resistant XU212 strain 2985, 5551.

Antibacterial, Staphylococcus aureus RN4220 strain 15939, 21727.

Antibacterial, Staphylococcus aureus SA-1199B strain 16747, 16748, 16749, 16754, 21727.

Antibacterial, Staphylococcus aureus sansitive strain 8968 .

Antibacterial, Staphylococcus aureus SG511 strain 2680, 2681, 2682.

Antibacterial, Staphylococcus aureus TPR27 strain 5214, 6303.

Antibacterial, Staphylococcus aureus XU-212 strain 16747, 16748, 16749,16754

Antibacterial, Staphylococcus aureus XU212(TetKmecA) strain 21727.

Antibacterial, Staphylococcus epidermidis 589, 909, 2584, 2591, 2592, $2593,3368,3962,6302,8081,10159,10351,11262,11263,11264$, 13651, 13652, 14815, 17272, 17862, 19592, 19593, 21830.

Antibacterial, Staphylococcus epidermidis 2515 strain 6018, 6020, 13469.

Antibacterial, Staphylococcus epidermidis 3112 strain $\quad 6018,6020$, 13469, 13811.

Antibacterial, Staphylococcus epidermidis ATCC12228 strain 3689, 4135.

Antibacterial, Staphylococcus epidermidis ATCC2223 strain 14592, 10484.

Antibacterial, Staphylococcus epidermidis IFO3762 strain 11811, 12322, 12321, 7792.

Antibacterial, Staphylococcus epidermidis TPR25 strain 5214, 6303.

Antibacterial, Staphylococcus hemolyticus flavus 6921.

Antibacterial, Staphylococcus saprophyticus 3010 strain 6018,6020 , 13469.

Antibacterial, Staphylococcus simulans 214 strain $\quad 6018,6020,13469$, 13811.

Antibacterial, Staphylococcus sp. 930, 967, 1178, 3052, 14829, 20489, 20578, 21251, 22080.

Antibacterial, Staphylococcus spp. 20732, 22270.

Antibacterial, Staphylococcus tetragenus $\quad 3511,5161$.

Antibacterial, Streptobacillus sp. $\quad 515,3157,3158,3186,4840,5272$, 5273, 5540, 5541, 5542, 6973, 9788, 10733, 13874, 13876, 15297, $15300,15301,15302,15303,15304,15305,15306,15307,15308$, 15309, 15312, 15313, 15314, 15315, 15316, 22496.

Antibacterial, Streptococcus durans 2313811.

Antibacterial, Streptococcus faecalis $\quad 3472,3474,7829,12380,12389$, 15039, 15910, 16532, 16800, 16802, 20018, 20019, 21251, 21269, 21270.

Antibacterial, Streptococcus mutans $\quad 2303,2509,11348,3057,14567$, 3055, 3058, 6366, 9702, 19985.

Antibacterial, Streptococcus mutans ATCC25175 strain $3689,4135$. Antibacterial, Streptococcus oneumonia 7413, 12228.

Antibacterial, Streptococcus pneumoniae $11259,11260$.

Antibacterial, Streptococcus pyogenes $\quad$ 16799, 16800, 17862.

Antibacterial, Streptococcus pyogenes ATCC19615 strain 3689, 4135. 
Antibacterial, Streptococcus sp. $\quad 515,967,1178,1244,1250,1252,2844$, $3052,3157,3158,3186,3511,4840,5161,5272,5273,5540,5541$, 5542, 5550, 6973, 9596, 9788, 10733, 12771, 13388, 13874, 13876, $14154,15297,15298,15300,15301,15302,15303,15304,15305$, $15306,15307,15308,15309,15312,15313,15314,15315,15316$, 18759, 20390, 22496

Antibacterial, Streptococcus var. 10, 6178, 17696.

Antibacterial, Streptococcus varians 17862.

Antibacterial, Streptococcus viridans $\quad 11259,11260,16799,16800$.

Antibacterial, Streptomyces scabies 207, 218, 7707, 7756, 7767.

Antibacterial, Trichophyton sp. 2081.

Antibacterial, used in treatment of skin diseases 3122 .

Antibacterial, Vibrio cholerae 5161, 6735.

Antibacterial, Vibrio parahaemolyticus $\quad 6735,11570,12615$.

Antibacterial, Vibrio vulnificus 6735.

Antibacterial, Vibrio vulnificus ATCC29307 strain 3689, 4135.

Antibacterial, vinegar organism 19819.

Antibacterial, a-Streptococcus $3615,5763,6776,8095,12843,17456$.

Antibacterial, $\boldsymbol{\beta}$-Streptococcus $\quad 16555,17456,17528$.

Antibiotic 1179, 2063, 14968.

Anti-caducity of plants 22975 .

Anti-cancer-promoted activity 7460 .

Anti-carcinogen, inhibits carcinogenic action of multiring aromatic hydrocarbons 2298 .

Anti-carcinogenic 2890, 18643.

Anti-carcinogenic, inhibits TPA-stimulated ${ }^{32} \mathbf{P}$-incorporation into the phospholipids of HeLa cells 5377, 6424, 6425, 16922.

Anticaries 9631.

Anti-Chagas' disease $184,1141,1145,1147,3258$.

Anticholinergic $1287,1288,2001,2725,2734,2797,6439,7636,8273$, 10872, 11809, 12825, 17546, 18842, 19388, 20488, 21372.

Anticoagulant $126,5858,6040,7063,11414,12766,19469,19471$.

Anticomplement activity $3602,10665,13592,17869,18400,18401$, 18402, 18403, 20422, 20428, 20429.

Anti-complication of diabetes 11505 .

Anticonvulsant $2791,3079,3318,3588,4536,4936,9184,9223,9330$, $11077,15370,16532,17451,18623,19111,19737,19738,20002$, 20509, 22270, 22336, 22346.

Antidepressant 10883, 10886, 11524, 17093, 17955, 22060.

Antidiabetic $12443,12444,22270$.

Antidiabetic 920,1618.

Antidiarrheal 759, 2303, 3308, 4421, 4921, 14933.

Anti-diuretic $6618,13057,22921$.

Antidote $1039,1275,1845,3412,7381,8761,8785,9818,17347,20444$, $22269,22562$.

Antidote (alcohol and venom) 3412 .

Antidote (cobra-poisoning) 9818, 20444.

Antidote (diphacin poisoning) 22562.

Antidote (from poisoning by sulfydryl enzyme) 8785 .

Antidote (poisoning from anticholinergic) 7381 .

Antidote (poisoning from barbital) 17347.

Antidyskinetic 9092 .

anti-early-pregnancy 1191 .
Anti-electroshock 7996, 9330.

Antiemetic 8081, 10186, 10215, 10450, 10630, 12015, 19997.

Anti-endotoxin 9217.

Anti-epilepsy 6708 .

Antiestrogenic 7768, 22937.

Anti-exudation 21282 .

Antifeedant $3087,9013,9456,11843,11844,11845,16652,16653$, 19652, 19653, 19654, 19656, 19657.

Anti-fertility agent $3909,4190,6767,7243,7945,8967,10814,13575$, 15527, 16793, 17337, 17568, 17592, 18033, 19187, 22011, 22815.

Antifibrinolysis 2366, 2367, 5484, 6700, 2183.

Antifibrogenic 2779 .

Antifibrotic 7441 .

Antifungal inactive summary index $504,589,618,1343,1470,2296$, 2590, 2833, 2945, 2990, 2991, 2998, 3048, 3791, 3799, 3856, 3962, 4422, 4550, 4712, 4816, 5134, 5547, 5747, 6263, 6758, 7756, 7764, $8107,8576,8578,8580,8623,8708,8709,8985,9223,9225,9486$, 9597, 9857, 10159, 10565, 10629, 10748, 11001, 11071, 11078, 11079, $11086,11230,11258,11269,11601,12048,13259,13571,13860$, 14109, 14498, 14516, 14698, 14699, 14915, 14957, 14958, 14959, $15118,15152,15518,15737,15881,16247,16248,16439,16555$, 18359, 18784, 19077, 19193, 19589, 19590, 19591, 19592, 19593, 19594, 19595, 19983, 20018, 20019, 20990, 21239, 21266, 21835, $22616,22777$.

Antifungal inactive, Aspergillus flavus KCCM11453 3048, 4550, 20990.

Antifungal inactive, Aspergillus fumigatus $\quad$ 1470, 11230.

Antifungal inactive, Aspergillus niger 7756.

Antifungal inactive, Blastoschyzomyces capitatus KCCM50270 4550, 20990.

Antifungal inactive, Candida albicans $\quad 589,618,2296,3791,3799$, $3962,4422,5134,5547,5747,6758,7764,8623,9486,9597,10159$, $10565,10629,11269,13259,13860,14698,14699,14915,15118$, $15737,15881,16439,16555,19589,19590,19591,19592,19593$, 19594, 19595, 19983, 20018, 20019, 21239, 21266, 21835, 22616, 22777.

Antifungal inactive, Candida albicans $19 \quad 12048,15152,18359$.

Antifungal inactive, Candida albicans 32 12048, 15152, 18359.

Antifungal inactive, Candida albicans ATCC2091 8107, 12048, 15152, 18359.

Antifungal inactive, Candida albicans DSY1024 14109, 14498.

Antifungal inactive, Candida albicans KCCM11282 3048, 4550, 20990.

Antifungal inactive, Candida glabrata $\quad 618,8623,9486,14698,14699$, 19983, 21835.

Antifungal inactive, Candida krusei 5747, 7764, 20018, 20019.

Antifungal inactive, Candida tropicalis $\quad 618,2590,2945,2990,2991$, 2998, 8623, 9486, 9857, 11071, 11078, 11079, 11086, 14698, 14699, $19983,21835$.

Antifungal inactive, Candida utilis KCCM11356 3048, 4550, 20990. Antifungal inactive, Cladosporium cladosporioides 14516.

Antifungal inactive, Cladosporium cucumerinum 1343, 2833, 3856, 4816, 8985, 11001, 11601, 13571, 18784, 19077. 
Antifungal inactive, Cladosporium herbarum 10629.

Antifungal inactive, Cladosporium sphaerospermum 8708, 8709, 14516. Antifungal inactive, Cryptococcus neoformans KCCM0564 4550, 3048, 20990.

Antifungal inactive, Diaporthe nomurai 14958.

Antifungal inactive, Fusarium oxysporum 10629.

Antifungal inactive, Mucor miehei $\quad 8576,8578,8580$.

Antifungal inactive, Nematospora coryli $8576,8580$.

Antifungal inactive, no description on fungi species 9223, 9225.

Antifungal inactive, Paecilomyces notatum $8576,8580$.

Antifungal inactive, Paecilomyces variotii $8576,8580$.

Antifungal inactive, Penicillium notatum 8578.

Antifungal inactive, Penicillium ochrocloron 504.

Antifungal inactive, Pyricularia orizae 16247, 16248.

Antifungal inactive, Rhizopus oryzae 6263, 10748, 11258.

Antifungal inactive, Saccharomyces cerevisiae 3962, 19589, 19590, 19591, 19592, 19593, 19594, 19595.

Antifungal inactive, Sclerotinia sp. 14959.

Antifungal inactive, Stigmina mori 14957.

Antifungal inactive, TLC $2833,3856,8985,10629,11001,11601,13571$, 18784, 19077.

Antifungal inactive, Trichoderma viride 504, 4712, 19193.

Antifungal inactive, Trichosporon mucoides KCCM50570 3048, 4550, 20990.

Antifungal inactive, Ustilago violacea 15518.

Antifungal summary index 51, 140, 206, 207, 218, 233, 234, 242, 318, $319,357,426,432,433,434,436,504,515,660,663,675,676,834$, $844,909,924,1016,1030,1178,1282,1342,1379,1572,1612,1736$, 1764, 1832, 1913, 1914, 2002, 2005, 2029, 2030, 2031, 2032, 2033, 2041, 2166, 2224, 2244, 2283, 2286, 2293, 2317, 2539, 2647, 2648, 2649, 2680, 2681, 2682, 2783, 2887, 2961, 2962, 2988, 3004, 3036, $3037,3048,3140,3145,3157,3158,3186,3231,3318,3464,3490$, $3498,3572,3598,3693,3695,3760,3761,3762,3767,3853,3925$, 3936, 3939, 3983, 4005, 4029, 4055, 4124, 4135, 4140, 4190, 4203, 4241, 4243, 4291, 4301, 4335, 4337, 4347, 4422, 4457, 4536, 4550, 4604, 4712, 4832, 4840, 4873, 4923, 5101, 5104, 5229, 5230, 5231, 5232, 5233, 5234, 5235, 5272, 5273, 5336, 5337, 5366, 5372, 5391, 5392, 5393, 5394, 5395, 5445, 5518, 5524, 5540, 5541, 5542, 5578, 5682, 5694, 5695, 5700, 5763, 5844, 5859, 5860, 5894, 5923, 5923, 6030, 6032, 6041, 6181, 6263, 6290, 6361, 6390, 6391, 6392, 6393, 6402, 6437, 6447, 6496, 6501, 6549, 6757, 6776, 6797, 6973, 7107, 7200, 7206, 7207, 7305, 7306, 7307, 7317, 7382, 7413, 7505, 7506, 7507, 7521, 7703, 7707, 7767, 7768, 7809, 7834, 7883, 7897, 7950, $7973,8034,8079,8080,8095,8136,8218,8278,8312,8330,8389$, $8456,8493,8548,8577,8579,8606,8672,8683,8708,8709,8752$, 8760, 8794, 8805, 8833, 8968, 9200, 9201, 9234, 9321, 9322, 9344, 9387, 9441, 9540, 9546, 9547, 9549, 9596, 9606, 9631, 9644, 9645, 9700, 9759, 9760, 9788, 10041, 10082, 10104, 10126, 10257, 10258, 10260, 10351, 10463, 10464, 10465, 10484, 10600, 10639, 10664, 10665, 10709, 10732, 10733, 10748, 10826, 10831, 10832, 10839, 10840, 10841, 11056, 11092, 11130, 11203, 11246, 11258, 11269 , 11280, 11303, 11322, 11379, 11408, 11434, 11526, 11607, 11673, 11777, 11778, 11779, 11851, 11903, 11943, 11978, 12184, 12228,
$12344,12501,12542,12580,12778,12849,12917,13011,13016$, 13085, 13088, 13105, 13106, 13107, 13137, 13150, 13195, 13196, 13241, 13259, 13281, 13398, 13416, 13428, 13429, 13513, 13637, 13638, 13808, 13820, 13821, 13861, 13874, 13876, 13965, 13990, 13991, 14020, 14029, 14101, 14116, 14182, 14217, 14347, 14446, $14454,14515,14541,14581,14587,14588,14592,14705,14728$, 14789, 14850, 14954, 14955, 14956, 14957, 14958, 14959, 14960 , 15020, 15021, 15027, 15124, 15142, 15204, 15279, 15297, 15300, 15301, 15302, 15303, 15304, 15305, 15306, 15307, 15308, 15309, $15312,15313,15314,15315,15316,15342,15489,15629,15633$, $15635,15741,15855,15872,15885,15998,16130,16168,16169$, $16257,16264,16267,16339,16388,16442,16545,16600,16666$, 16674, 16675, 16934, 17047, 17048, 17049, 17225, 17257, 17258, 17259, 17260, 17261, 17278, 17341, 17376, 17377, 17403, 17420, 17421, 17438, 17439, 17477, 17478, 17481, 17482, 17570, 17602, 17777, 17833, 17841, 17842, 17864, 17865, 17924, 17926, 17931, 18003, 18029, 18031, 18033, 18035, 18036, 18088, 18102, 18103, $18163,18214,18215,18265,18270,18280,18547,18643,18759$, 18780, 18849, 19078, 19086, 19121, 19168, 19172, 19174, 19193, 19316, 19394, 19452, 19542, 19777, 19784, 19909, 19912, 19929 , 20041, 20042, 20060, 20070, 20093, 20134, 20210, 20211, 20382, 20566, 20670, 20901, 20975, 20990, 21119, 21143, 21146, 21147 , $21171,21253,21257,21270,21328,21329,21347,21348,21360$, 21434, 21435, 21488, 21557, 21559, 21737, 21745, 21749, 21790 , 21904, 21908, 22059, 22080, 22104, 22105, 22158, 22190, 22191, 22195, 22213, 22332, 22336, 22347, 22436, 22437, 22438, 22474 , $22496,22505,22526,22638,22667,22700,22741,22742,22755$, 22768, 22903, 22966, 23002, 23014.

Antifungal, 17 kinds of fungi 8095 .

Antifungal, Acrothesium floccosum 17341.

Antifungal, Alternaria alternata $\quad 206,357,2002,3853,4291,6030,9387$, 9759, 9760, 10082, 13416.

Antifungal, Alternaria citri 5694, 17420, 20382.

Antifungal, ash fungi 5763 .

Antifungal, Aspergillus flavus $\quad 206,357,2002,3853,6030,9759,9760$, 10082, 11903, 11978, 13416, 14789, 17478.

Antifungal, Aspergillus flavus $\quad 9200,9201$.

Antifungal, Aspergillus flavus IHEM37.19 2962, 5844, 6181, 10463, 10464, 10831, 10832, 13808, 21790.

Antifungal, Aspergillus flavus KCCM11453 3231, 21360.

Antifungal, Aspergillus fumigatus $\quad 1030,2961,2988,4335,4347,11303$, 11978, 14029, 17478, 21488, 22667.

Antifungal, Aspergillus fumigatus CBS113.26 2962, 5844, 6181, 10463, 10464, 10831, 10832, 13808, 21790.

Antifungal, Aspergillus nidulan 22667.

Antifungal, Aspergillus niger $\quad$ 206, 207, 218, 357, 504, 515, 2002, 3157, $3158,3186,3853,4203,4712,4840,5101,5272,5273,5540,5541$, $5542,6030,6263,6361,6973,7707,7767,8456,8672,8683,9549$, $9759,9760,9788,10082,10104,10733,10748,11246,11258,11778$, $13398,13416,13874,13876,14789,15297,15300,15301,15302$, $15303,15304,15305,15306,15307,15308,15309,15312,15313$, $15314,15315,15316,16257,16442,17602,19193,19784,20060$, $21435,22496$. 
Antifungal, Aspergillus niger IHEM2951 2962, 5844, 6181, 10463, 10464, 10831, 10832, 13808, 21790.

Antifungal, Aspergillus niger KCCM11239 3048, 3231, 4550, 20990, 21360.

Antifungal, Aspergillus ochraceus $\quad$ 206, 357, 504, 2002, 3853, 4712, 6030, 9759, 9760, 10082, 13416, 19193.

Antifungal, Aspergillus parasiticus 22768.

Antifungal, Aspergillus terreus 5029.2000 2962, 5844, 6181, 10463, 10464, 10831, 10832, 13808, 21790.

Antifungal, Aspergillus versicolor $\quad 206,357,2002,3853,6030,9759$, 9760, 10082, 13416.

Antifungal, Bipolaris leersiac 14954.

Antifungal, Blastomyces sp. 15872.

Antifungal, Blastomyces dermatitidis 17049.

Antifungal, Blastoschizomyces capitatus 11978, 17478.

Antifungal, Blastoschyzomyces capitatus KCCM50270 3048, 3231, 21360.

Antifungal, Botrytis cinerea $\quad 1030,5694,5894,6032,10600,11246$, 17420, 20382, 22505.

Antifungal, broad spectrum 11851, 19121.

Antifungal, Candida albicans $\quad 242,675,909,924,1016,2244,2283$, 2680, 2681, 2682, 3318, 3490, 3925, 4055, 4203, 4241, 4832, 4923, 5678, 5682, 5923, 6263, 6437, 6447, 6757, 7107, 7206, 7207, 7897, $8095,8456,8548,8606,8672,8708,8709,9200,9201,9321,9322$, 9441, 9547, 9596, 10041, 10104, 10126, 10257, 10258, 10260, 10639, 10664, 10665, 10748, 10826, 11056, 11258, 11777, 11778, 11779, $11978,12344,13137,13195,13196,13241,13398,13428,13429$, $14217,14446,15027,15124,15489,15885,16339,16442,16600$, 17403, 17478, 17602, 17864, 17865, 18029, 18102, 18214, 18215, 19174, 20093, 20060, 21146, 21147, 21253, 21257, 21270, 21328, 21435, 21559, 22195.

Antifungal, Candida albicans 19 2286, 5518, 13965, 14101.

Antifungal, Candida albicans 32 2286, 5518, 13965, 14101.

Antifungal, Candida albicans ATCC1023 8493.

Antifungal, Candida albicans ATCC10231 6797, 10484, 14592

Antifungal, Candida albicans ATCC2091 2286, 5518, 13965, 14101.

Antifungal, Candida albicans ATCC663.90 2962, 5844, 6181, 10463, 10464, 10831, 10832, 13808, 21790.

Antifungal, Candida albicans KCCM11282 3231, 21360.

Antifungal, Candida glabrata 1572, 4335, 4347, 6437, 6447, 8606, 9200, 9201, 21488.

Antifungal, Candida guilliermondii 8079,8080 .

Antifungal, Candida krusei 19174.

Antifungal, Candida mycoderma 51, 2539, 3004, 3004, 4243, 4604, 5235, 7305, 7306, 7307, 7883, 8136, 8278, 11434, 13107, 13637, 15342, $16545,17048,22667$.

Antifungal, Candida niger ATCC10335 6797.

Antifungal, Candida sp. $3140,8833$.

Antifungal, Candida tropicalis $\quad 6437,6447$.

Antifungal, Cladosporium cladosporioides $\quad 2166,5700,6361,19168$.

Antifungal, Cladosporium cladosporioides 206, 357, 504, 2002, 2293, $3853,4712,5391,5392,5393,5394,5395,5859,5860,6030,6041$, $6501,8330,9759,9760,10082,13416,13820,13821,14116,14515$,
$14587,14588,14789,15279,19172,19193,21119,21737,21745$, 21749.

Antifungal, Cladosporium cucumerinum 426, 432, 433, 434, 436, 2041, 4422, 5336, 5337, 5578, 7973, 9321, 9322, 10465, 10839, 10840, $10841,11269,11408,12344,14182,14541,14789,15204,17833$, 17842, 18280, 19777, 22213, 22505.

Antifungal, Cladosporium cucumerinum 242, 5923, 10257, 10260, 10258.

Antifungal, Cladosporium fulvum 3572, 7200, 10709, 18780.

Antifungal, Cladosporium herbarum 5694, 17420, 20382.

Antifungal, Cladosporium herbarum 12542, 13259.

Antifungal, Cladosporium sp. $\quad$ 17481, 17482.

Antifungal, Cladosporium sp. 17777.

Antifungal, Cladosporium sphaerospermum 1612, 16388.

Antifungal, Cladosporium sphaerospermum 5695, 5859, 5860, 6041, $10664,10665,11280,13820,13821,14020,14116,14515,14587$, 14588, 14789, 15279, 17438, 17439, 19172, 21904, 21908.

Antifungal, Cladosporium sphaerospermum 2293, 5391, 5392, 5393, $5394,5395,6501$.

Antifungal, Claviceps purpurea 16666, 20042.

Antifungal, Colletotrichum acutatum 19078, 19078.

Antifungal, Colletotrichum fragariae 19078, 19078.

Antifungal, Colletotrichum fragariae 233, 234, 16257, 19078, 19078.

Antifungal, Cryptococcus neoformans 924, 1736, 4335, 4347, 8606,

11978, 17403, 17478, 17602, 17924, 17926, 21488, 22667.

Antifungal, Cryptococcus neoformans KCСM0564 3231, 21360.

Antifungal, Cryptococcus neoformans KCCM11356 3231, 21360.

Antifungal, Curvularia lunata 17341.

Antifungal, Curvularia lunata 18103, 19784.

Antifungal, Curvularia sp. 16257.

Antifungal, dermatophyte $3140,3318,4337,5229,5230,5231,5232$,

5233, 5234, 6496, 13088, 13105, 13106, 18759, 21435, 22059, 22966.

Antifungal, Deuterophoma tracheiphila 15635.

Antifungal, Diaporthe nomurai $\quad$ 14954, 14957, 14959, 14960.

Antifungal, Dothichiza populea 21557.

Antifungal, Epidermophyton sp. 11203.

Antifungal, Fusarium 2647, 2648.

Antifungal, Fusarium avenaceum 5694, 17420, 20382.

Antifungal, Fusarium culmorum 20210, 20211.

Antifungal, Fusarium moniliforme 7834.

Antifungal, Fusarium oxysporum 207, 218, 7767, 13259.

Antifungal, Fusarium solani $9201,9200$.

Antifungal, Fusarium tricinctum 504, 4712, 19193.

Antifungal, inhibits spore germination 18780 .

Antifungal, inhibits sporular growth $17258,17259,17260,17261$.

Antifungal, low fungi $11092,23014$.

Antifungal, Magnaparthe grisea $\quad 17257,17258,17259,17260,17261$.

Antifungal, Microsporum audouini 8312, 21435.

Antifungal, Microsporum canis 9201, 9200.

Antifungal, Microsporum sp. 15872.

Antifungal, Microsporum sp. 6776.

Antifungal, Mucor miehei $\quad 8577,8579,10732$.

Antifungal, mycelian Cladosporium cucumerinum 9546. 
Antifungal, mycelian Colletotrichum orbiculare 9546.

Antifungal, mycelian Phytophthora capsici 9546.

Antifungal, mycelian Pythium ultimum 9546.

Antifungal, mycelian Rhizoctonia solani 9546.

Antifungal, Nematospora coryli 8579.

Antifungal, no description on fungi species $140,660,663,676,834,844$,

1178, 1282, 1342, 1764, 1832, 2005, 2031, 2032, 2033, 2224, 2317 ,

2649, 2783, 2887, 3036, 3037, 3145, 3231, 3464, 3498, 3598, 3693,

$3695,3760,3761,3762,3767,3939,3983,4005,4029,4124,4135$,

4140, 4190, 4457, 4536, 4550, 4604, 5104, 5366, 5372, 5445, 5524,

6290, 6402, 6549, 7703, 7768, 7809, 7950, 8034, 8218, 8389, 8752,

$8760,8794,8805,9344,9540,9606,9631,9644,9645,9700,11130$,

$11379,11526,11607,11943,12184,12580,12778,12849,12917$,

13011, 13016, 13150, 13281, 13513, 13861, 14454, 14581, 14705,

$14728,14850,14955,14956,15020,15021,15142,15629,15633$,

15741, 15855, 15998, 16130, 16168, 16169, 16267, 16674, 16675,

16934, 17047, 17225, 17278, 17376, 17377, 17421, 17570, 17841,

17931, 18031, 18033, 18035, 18036, 18088, 18163, 18270, 18547,

18643, 18849, 19086, 19316, 19394, 19542, 19912, 19929, 20041,

20070, 20134, 20566, 20670, 20901, 20975, 21143, 21171, 21329,

21347, 21348, 21434, 22080, 22104, 22105, 22158, 22332, 22336,

22347, 22436, 22437, 22438, 22474, 22638, 22700, 22755, 22903,

23002 .

Antifungal, Oidium porriginis 19909.

Antifungal, Oidium tropioale $8080,22768$.

Antifungal, Paecilomyces notatum 8579.

Antifungal, Paecilomyces variotii 8579.

Antifungal, pathogen fungi 7521 .

Antifungal, pathogen fungi of plants $242,2029,2030,2293,5391,5392$, $5393,5394,5395,5923,6501,10257,10258,10260,17257,17258$,

$17259,17260,17261$.

Antifungal, pathogenic bacteria of celery 3936 .

Antifungal, Penicillium avellaneum 1572, 8606.

Antifungal, Penicillium avellaneum UC-4376 7413, 12228.

Antifungal, Penicillium cirtinum 20093.

Antifungal, Penicillium funiculosum 206, 357, 2002, 3853, 6030, 9759, 9760, 10082, 13416.

Antifungal, Penicillium notatum 8577, 10732.

Antifungal, Penicillium ochrochloron 206, 357, 2002, 3853, 6030, 9759, 9760, 10082, 13416.

Antifungal, Penicillium ochrocloron 4712, 19193.

Antifungal, Penicillium oryzae $1572,8606$.

Antifungal, Penicillium sp. 16257.

Antifungal, Phomopsis helianthi 504, 4712, 19193.

Antifungal, Phytophthora capsici 14789.

Antifungal, plant antitoxin 22741, 22742.

Antifungal, Plasmopara viticola 22505.

Antifungal, Pleurotus ostreatus 17341.

Antifungal, protects heartwood and bark 11673 .

Antifungal, Pyricularia grisea 5694, 17420, 20382.

Antifungal, Pyricularia oryzae $1913,1914,4291,4873,7505,7506,7507$, 13990, 13991.

Antifungal, Pythium spp. 12344.
Antifungal, Pythium ultimus 14347.

Antifungal, Ribes nigrum (Grossulariaceae family) 19174.

Antifungal, Saccharomyces cerevisiae $8833,13195$.

Antifungal, Saccharomyces cerevisiae $\quad 1572,4923,8606,16264,17403$, 18029.

Antifungal, Sclerotinia sp. 2647, 2648, 14957, 14958, 16666, 17477, 20042.

Antifungal, solanum-shape Fusarium 22768.

Antifungal, Sporotrichum pulverulentum 18003.

Antifungal, Stigmina mori $\quad$ 14954, 14958, 14959, 14960.

Antifungal, T. beigelii $1572,8606$.

Antifungal, TLC $\quad 242,1612,4422,5578,5859,5860,5923,6041,6390$, $6391,6392,6393,8330,10258,10260,10465,11269,11322,13259$, $14182,15279,16388,19172,19777,19777,21119,21737,21745$, 21749.

Antifungal, Trichoderma glaucum 1030.

Antifungal, Trichoderma viride $\quad 206,357,2002,3853,6030,9759,9760$, $10082,13416$.

Antifungal, Trichophyton interdigitalis 1379, 6776, 9234.

Antifungal, Trichophyton longifusus 9201, 9200.

Antifungal, Trichophyton mentagrophytes 2244, 2283, 4203, 5229, 5230, $5231,5232,5233,5234,5700,6437,6496,7317,8968,11203,11777$, $11778,11779,13088,13105,13106,13638,15872,19257,19259$, 19452, 21435, 22190, 22191, 22195.

Antifungal, Trichophyton mentagrophytes ATCC28185 318, 319, 6797, 7382.

Antifungal, Trichophyton purpureatum 22195.

Antifungal, Trichophyton rubrum 10351, 15489.

Antifungal, Trichophyton rubrum ATCC28188 318, 319, 6797, 7382.

Antifungal, Trichophyton rubrum ATCC6345 3048, 3231, 4550, 20990, 21360.

Antifungal, Trichophyton sp. $8312,19909$.

Antifungal, Trichophyton spp. 22059.

Antifungal, Trichosporon mucoides KCCM50570 3231, 21360.

Antifungal, Trichothecium roseum 16666, 20042.

Antifungal, Uromyces fabae 13085.

Antifungal, Ustilago violacea 12501.

Antifungal, yeast-like pathogenic bacteria 22966.

Antifungal, YNG-CA 4301.

Antifungal, YNG-CG 4301.

Anti-gastrin 2734.

Anti-gibberellin activity $4320,4323,4324$.

Anti-gonadotrophin 2833, 15170, 16209.

Antigout 3910.

Anti-HBV 19147.

Antihemolysis inactive $12819,12820,12824,12828,13053$.

Anti-hepatitis $4317,4320,8907,8912,8917,22492$.

Antihepatitis (type B) inactive 12004, 19465, 19500, 19501, 19502.

Antihepatotoxin $61,580,892,894,896,924,1020,1162,1372,1928$, $1971,2220,2318,2538,2786,2887,3143,3221,3301,3308,3419$, $3420,3829,3830,3831,4055,4135,4398,4484,4564,4921,4922$, $5101,5178,5213,5359,5763,6193,7768,8388,8426,8846,8906$, $8907,8908,8913,8914,8917,9564,11642,11648,11690,12624$, 
12784, 12916, 13481, 14012, 14732, 15279, 15366, 15623, 16039, $16765,16836,17287,17344,17345,17587,18221,18317,18411$, 18599, 19042, 19142, 19148, 19331, 19377, 19473, 19489, 19490, 19497, 19893, 19895, 19898, 19912, 20502, 20515, 20732, 20910, 22265, 22266, 22656 .

Antihistamine $443,1614,1840,2359,2360,2361,2362,3600,3770$, 3781, 3782, 3973, 4158, 4159, 4681, 4682, 4683, 4684, 5155, 6351, 7802, 8153, 8167, 8273, 9010, 11910, 11913, 14531, 15571, 17278, 19238, 19239, 19846, 19929, 20009, 20447, 21435, 21493, 22282, 22349, 22564, 22720.

Antihistamine inactive 16169 .

Anti-HIV inactive summary index $930,1030,1473,1665,1666,1699$, 1713, 1718, 1725, 1728, 2303, 3096, 3097, 3104, 3105, 3106, 3107, 3108, 3109, 3110, 3111, 3112, 3113, 3159, 3159, 3498, 4189, 4376, 5062, 6072, 6149, 6910, 6911, 7504, 7777, 7788, 9444, 9451, 9885, 10163, 10597, 10688, 11968, 11969, 12861, 12862, 12863, 12864, $12865,12868,12871,12872,12929,12932,12933,13867,13869$, 13871, 14483, 15929, 15966, 16342, 17309, 17983, 18053, 19284, 20407, 20408, 21056.

Anti-HIV inactive, H9 lymphocytes 930, 1665, 1666, 1699, 1713, 1718, 1725, 1728, 2303, 3498, 4189, 5062, 7777, 7788, 17983, 19284, 21056.

Anti-HIV inactive, no explanation of HIV species 3096, 3097, 3104 , 3105, 3106, 3107, 3108, 3109, 3110, 3111, 3112, 3113, 3159, 3159, 9885, 11569, 13867, 13869, 13871, 17309.

Anti-HIV summary index $27,112,144,145,163,483,484,485,1467$, 1483, 1494, 2223, 2527, 3309, 3518, 3600, 3829, 3831, 3861, 4214, 4215, 4216, 4839, 4957, 5269, 5303, 5414, 5515, 5789, 6008, 6090, $6609,6909,7251,7742,7743,7744,7745,7746,7788,8185,8189$, $8246,8551,8554,8804,8822,8833,8844,8846,8914,9449,9535$, 9543, 9546, 9928, 9929, 10473, 11029, 11490, 11525, 11843, 11844, 11845, 11846, 11847, 11992, 11993, 12011, 12019, 12020, 12046, 12093, 12479, 12573, 12766, 12782, 12860, 12930, 12931, 13137, $13315,13369,13431,13518,13865,14004,14027,14201,14206$, $14356,14831,14995,15169,15249,15420,15570,15627,15806$, $15850,15851,15852,15853,15854,16290,16304,16314,16344$, $16352,16587,16652,16653,16836,16999,17000,17001,17187$, $17958,18202,18228,18294,18317,18340,18354,18362,18363$, 18395, 18624, 18662, 19182, 19472, 19479, 19480, 19484, 19487, 19498, 19499, 19629, 19652, 19653, 19654, 19655, 19656, 20088, 20109, 20476, 20477, 20499, 20975, 21233, 21324, 21327, 21381 , 21382, 21493, 21724, 21990, 22208, 22270.

Anti-HIV, a lead candidate 5269 .

Anti-HIV, H9 lymphocytes 27, 112, 2527, 3309, 3829, 3831, 5789, 6609, 7788, 9535, 11029, 13518, 15169, 15249, 19182, 21990, 22208.

Anti-HIV, HIV-INinhibitor 8554.

Anti-HIV, HIV-RT inhibitor and HIV polyase inhibitor 15570.

Anti-HIV, HIV-RTinhibitor 163, 5303, 9543, 13315, 13431, 18202, 18228, 18624, 19182, 20109, 21724.

Anti-HIV, inhibits cell denaturalization affected by HIV 14206. Anti-HIV, inhibits formation of giant-cell without cytotoxicity 12782 . Anti-HIV, inhibits HIV in early stage of its life cycle, inhibits the cell fusion and formation of plasmodia 14831.

Anti-HIV, inhibits HIV replication 27, 2527, 3309, 3600, 3829, 3831,
$5414,5789,7788,9535,11029,12479,13518,15169,15249,19182$, 21493, 21990, 22208.

Anti-HIV, inhibits HIV-induced formation of giant-cells 8844, 12766. Anti-HIV, MT-2 cell infected by HIV-IIIB virus 6909.

Anti-HIV, non-competitively inhibits enzymatic substrates 13369 . Anti-HIV-1 483, 484, 485, 492, 1483, 1494, 3518, 4214, 4215, 4216, 4839, 5414, 6008, 6909, 7251, 8151, 8152, 8156, 8175, 8176, 8182, $8183,8185,8189,8196,8223,8224,8225,8226,8229,8246,9449$, $9952,11843,11844,11845,11846,11847,12019,12020,12046$, 12093, 12573, 12847, 12860, 12930, 12931, 12966, 12967, 12968, 14004, 14027, 14201, 15655, 16304, 16314, 16344, 16587, 16652, 16653, 16999, 17000, 17001, 17187, 17958, 18294, 18317, 18340, 18354, 18362, 18363, 18395, 18662, 18697, 19652, 19653, 19654 , 19655, 19656, 20088, 20389, 20499, 20514, 21324, 21327, 21327 , 21381, 21382.

Anti-HIV-1 inactive $930,1030,1473,1665,1666,1699,1713,1718$, 1725, 1728, 2303, 3498, 4189, 4376, 5062, 6072, 6149, 6910, 6911, 7504, 7777, 7788, 9444, 9451, 10163, 10597, 10688, 11968, 11969, $12861,12862,12863,12864,12865,12868,12871,12872,12929$, 12932, 12933, 14483, 15929, 15966, 16342, 17983, 18053, 19284, 20407, 20408, 21056.

Anti-HIV-1 inactive, H9 lymphocytes $930,1665,1666,1699,1713$, 1718, 1725, 1728, 2303, 3498, 4189, 5062, 7777, 7788, 17983, 19284, 21056.

Anti-HIV-1 inactive, HIV-1 IN inhibitor inactive 9444, 9451, 12861, $12862,12863,12864,12865,12871,12872,14483,15929,16342$, 18053, 20407, 20408, 12868.

Anti-HIV-1 inactive, HOG.R5 cells 1473, 6910, 6911, 7504, 12929, 12932, 12933, 15966.

Anti-HIV-1, , inhibits cytotoxic effect induced by HIV-1 in MT-4 cells 8185,8189 .

Anti-HIV-1, binds to chemokine receptor CCR5 6008, 14004, 14027. Anti-HIV-1, CEM-SS cells infected by HIV-1 17958.

Anti-HIV-1, DDDP inhibitor $\quad 12020,18354,4214,4216,18395,12019$, 20499, 18363, 18317, 12046, 20088, 16587, 16999, 17000, 17001, 18362, 12093, 21324, 21327, 4215.

Anti-HIV-1, H9 lymphocytes 16304, 16314.

Anti-HIV-1, HIV-1 IN inhibitor 12020, 18354, 18395, 12019, 18363 , 18317, 12046, 20088, 16587, 16999, 17000, 17001, 18362, 12093, 21324, 21327, 9449, 16344, 12860, 12573.

Anti-HIV-1, HIV-1 integrase inhibitor 5414, 18340, 1483, 1494, 3518. Anti-HIV-1, HIV-1 protease inhibitor $492,8151,8152,8156,8175$, 8176, 8182, 8183, 8185, 8196, 12966, 12967, 12968, 18697.

Anti-HIV-1, HIV-1 reverse transcriptase assay $8223,8224,8225,8226$. Anti-HIV-1, HIV-1 reverse transcriptase highly selective inhibitor 18662.

Anti-HIV-1, HIV-1IIIB-induced MT-4 cells 1483, 3518, 1494.

Anti-HIV-1, HIV-1-induced cytopathic effect inhibitor 483, 484, 485, 4839, 11843, 11844, 11845, 11846, 11847, 14201, 16652, 16653, 17187, 19652, 19653, 19654, 19655, 19656, 21381, 21382.

Anti-HIV-1, HIV-RT inhibitor $8246,12020,18354,4214,4216,18395$, 12019, 20499, 18363, 18317, 12046, 20088, 16587, 16999, 17000, 17001, 18362, 12093, 21324, 21327, 4215, 20389, 20514. 
Anti-HIV-1, inhibits HIV-1 replication 6909, 12930, 12931, 16304, 16314.

Anti-HIV-1, inhibits produce of viral antigen P24 and plasmodia 17958.

Anti-HIV-1, MT-4 cells 483, 484, 485, 4839, 11843, 11844, 11845, 11846, 11847, 14201, 16652, 16653, 17187, 19652, 19653, 19654, 19655, 19656, 21381, 21382

Anti-HIV-1, RnaseH inhibitor 12020, 4214, 4216, 20499, 4215. Anti-HIV-1, syncytium assay $8229,9952$.

Antihypercholesterolemic $675,891,892,894,896,1845,2102,2379$, $2384,2435,2538,3045,3511,4135,4564,4685,5707,6440,6853$, 7832, 7883, 7978, 8278, 8401, 8423, 8424, 8846, 9184, 9498, 11083, $11648,12624,12891,13137,15419,15420,15421,15528,16599$, 17765, 18001, 18164, 18165, 18317, 18643, 18916, 19070, 19142, 19148, 19316, 19983, 20121, 20122, 20369, 22269, 22270, 22498, 22552, 22718.

Antihypertensive $80,304,554,582,631,651,783,784,824,846,899$, $920,983,1048,1275,1293,1476,1497,1613,1769,1866,1935,1948$, 2039, 2106, 2284, 2300, 2303, 2309, 2318, 2977, 3002, 3057, 3201, 3218, 3296, 3498, 3594, 3633, 3770, 3839, 3864, 3886, 3907, 3915, 3963, 4237, 4319, 4353, 4480, 4685, 4912, 5003, 5004, 5035, 5036, 5100, 5108, 5136, 5248, 5260, 5435, 5550, 5550, 5930, 6691, 6699, 6747, 6776, 6802, 6813, 6814, 7296, 7298, 7303, 7341, 7611, 7714, 7793, 7945, 8010, 8289, 8292, 8297, 8353, 8524, 8846, 9186, 9233 , 9301, 9441, 9597, 9740, 11091, 11202, 11377, 11642, 11680, 11851, 12327, 12385, 12386, 12574, 12646, 12798, 12839, 13089, 13098, 13189, 13237, 13246, 13264, 13373, 13374, 13571, 13631, 13716, 14125, 14184, 14756, 14812, 14889, 14923, 14951, 14952, 15041, $15126,15250,15263,15321,15395,15396,15452,15547,15881$, $15885,15972,16080,16261,16280,16451,16525,16532,16770$, 16966, 17278, 17384, 17412, 17472, 17568, 17869, 17915, 17952, 17995, 18017, 18059, 18165, 18180, 18317, 18552, 18623, 18630, 18632, 18633, 18633, 18652, 18826, 18826, 18833, 19070, 19081, 19195, 19196, 19257, 19259, 19316, 19386, 19388, 19540, 19762, 19846, 19918, 19955, 20076, 20324, 20341, 20434, 20580, 20650, 20732, 21206, 21234, 21235, 21237, 21238, 21239, 21251, 21257 , $21260,21261,21263,21268,21270,21435,21493,21511,21578$, $21887,22195,22277,22349,22386,22395,22427,22433,22489$, 22490, 22502, 22503, 22581, 23033.

Antihypertensive (no influence on heart) 899 .

Antihypophyseal 1613 .

Anti-hypotension 2989, 3000, 5184, 8405, 16730, 21122.

Anti-infective $1845,3202,3203,4995,5189$.

Anti-inflammatory inactive summary index $117,303,371,496,5531$, $5750,8216,8217,8507,8520,9365,10639,11572,14777,15670$, 15928, 16162, 16164, 16165, 17351, 19032, 19871.

Anti-inflammatory inactive, carrageenan-induced rat paw edema 5531.

Anti-inflammatory inactive, inhibiting COX-1 assay 9365, 10639, 11572, 15670, 15928, 19871.

Anti-inflammatory inactive, inhibiting COX-2 assay 11572,15928 , 19871.

Anti-inflammatory inactive, inhibiting NF-кB assay $\quad 16162,16164$,
16165.

Anti-inflammatory inactive, inhibition assay of accumulation of $\mathrm{NO}_{2}^{-}$, LPS/IFN- $\boldsymbol{\gamma}$-induced N9 microglial cells 8216,8217 .

Anti-inflammatory inactive, inhibition assay of accumulation of $\mathrm{NO}_{2}^{-}$, culture media of LPS-induced RAW264.7 cells 8216, 8217.

Anti-inflammatory inactive, inhibition assay of histamine release, rat mast cells stimulated with compound 48/80 8216, 8217.

Anti-inflammatory inactive, inhibition assay of release of $\beta$-glucuronidase, rat mast cells stimulated with compound 48/80 $8216,8217$.

Anti-inflammatory inactive, LPS-activated mouse peritoneal macrophages 303, 371, 496, 14777, 19032.

Anti-inflammatory inactive, mast cells and neutrophils stimulated with various inducers $117,5750,8507,8520$.

Anti-inflammatory inactive, NO production inhibitor assay 16162 , 16164, 16165.

Anti-inflammatory inactive, TNF- $\alpha$ production inhibitor assay, N9 microglial cells stimulated with LPS/INF- $\boldsymbol{\gamma} \quad$ 117, 5750, 8507.

Anti-inflammatory inactive, TNF- $\alpha$ production inhibitor assay, RAW264.7 cells stimulated with LPS $117,5750,8507$.

Anti-inflammatory summary index (not including NO production inhibitor) $27,37,56,118,119,171,173,205,222,304,309,508$, $538,546,547,548,580,660,663,664,668,899,966,977,978,983$, $1476,1492,1573,1614,1768,1790,1791,1802,1828,1854,1928$, 1965, 1971, 2004, 2036, 2038, 2102, 2106, 2303, 2312, 2325, 2414, 2455, 2594, 2687, 2886, 2887, 2890, 2983, 2989, 2990, 3077, 3141, $3221,3269,3270,3272,3273,3307,3362$, 3368, 3462, 3464, 3588, 3595, 3600, 3674, 3689, 3743, 3911, 3923, 3924, 3935, 3937, 3981, 4056, 4097, 4266, 4328, 4398, 4439, 4477, 4565, 4589, 4614, 4645, 4685, 4746, 4876, 4921, 4922, 4963, 5008, 5152, 5161, 5573, 5699, $5763,5930,5939,6183,6211,6288,6428,6454,6487,6491,6643$, 6656, 6767, 6816, 6853, 6923, 6938, 7180, 7195, 7196, 7197, 7239, $7243,7278,7361,7370,7399,7416,7481,7495,7521,7528,7529$, 7530, 7531, 7532, 7586, 7659, 7714, 7793, 7802, 7833, 7860, 7868, 7869, 7870, 7871, 7872, 7873, 7926, 7950, 7951, 8020, 8095, 8214, $8215,8278,8292,8293,8295,8297,8304,8360,8361,8363,8365$, $8388,8401,8405,8423,8424,8520,8521,8522,8664,8679,8788$, $8814,8841,8846,8865,8965,9038,9053,9054,9056,9060,9061$, 9062, 9238, 9276, 9288, 9337, 9419, 9441, 9455, 9456, 9486, 9496, 9705, 10041, 10182, 10363, 10472, 10526, 10639, 10814, 10820, 10875, 10886, 10887, 10967, 11017, 11077, 11121, 11259, 11260, $11339,11416,11417,11505,11521,11522,11530,11572,11642$, $11736,11741,11747,11753,11808,12018,12020,12122,12178$, 12184, 12255, 12420, 12510, 12523, 12608, 12766, 12891, 12907 , 12916, 12982, 12983, 12987, 13088, 13098, 13137, 13145, 13391, 13392, 13435, 13436, 13437, 13474, 13475, 13481, 13492, 13559 , 13591, 13774, 13808, 13809, 13962, 14097, 14106, 14756, 14767 , 14893, 14896, 14897, 14971, 14976, 14977, 14979, 15002, 15038, $15170,15203,15279,15279,15286,15635,15843,15886,15928$, $16025,16031,16050,16080,16163,16166,16167,16216,16221$, 16222, 16362, 16451, 16525, 16532, 16535, 16539, 16540, 16550, $16571,16603,16675,16713,16867,16900,16983,17024,17042$, $17145,17168,17240,17267,17344,17345,17350,17371,17377$, 
$17403,17409,17422,17460,17532,17597,17598,17599,17648$, $17829,17862,17918,17958,18169,18190,18219,18221,18317$, $18376,18408,18409,18411,18440,18513,18643,18644,18645$, 18673, 18685, 18792, 18824, 18859, 19074, 19081, 19087, 19111, 19142, 19143, 19259, 19308, 19312, 19401, 19402, 19403, 19404, 19405, 19406, 19407, 19427, 19450, 19540, 19542, 19545, 19819, 19846, 19871, 19883, 19885, 19894, 19895, 19897, 19898, 19922, 19955, 19983, 20066, 20099, 20168, 20501, 20509, 20569, 20650, 20686, 20710, 20717, 20732, 20900, 20901, 20978, 20992, 21055, $21059,21206,21234,21245,21264,21392,21397,21419,21420$, $21421,21422,21435,21438,21439,21862,21887,21930,21971$, $22023,22059,22080,22123,22124,22151,22270,22274,22282$, $22332,22433,22504,22581,22686,22687,22688,22700,22718$, 22720, 22727, 22728, 22729, 22730, 22731, 22938, 22990.

Anti-inflammatory, 12-LOX inhibitor in hmn platelets, without affecting the levels of cyclooxygenase 2106, 16216, 22718.

Anti-inflammatory, 15-LOX inhibitor $1110,1131,2102,2380,4289$, 13097, 13098, 13137, 19929.

Anti-inflammatory, 5-LOX inhibitor 444, 580, 2101, 2102, 2216, 3188 , 3743, 6491, 7819, 7924, 7926, 8926, 8997, 9202, 9331, 11572, 12936, $15319,15928,16540,16983,17979,18513,19201,19312,19846$, 19871, 22511, 22778

Anti-inflammatory, activity matches with aspirin 12987.

Anti-inflammatory, acute 983, 16451.

Anti-inflammatory, animal model $3595,9276,17344,17350,18859$, 21264.

Anti-inflammatory, antiarthritic $668,3368,7566,8753$.

Anti-inflammatory, anti-edema 118, 119, 2102, 2106, 6211, 10526, 11339, 14767, 14893, 15886, 18408, 19894, 21971.

Anti-inflammatory, anti-inflammatory action in models of atherosclerosis, Alzheimer's disease, arthritis and pancreatitis; proposed mechanisms include macrophage activation inhibitor, lipoxygenase inhibitor, cyclooxygenase 2 inhibitor, and metabolite production via arachidonic acid pathways 4398 .

Anti-inflammatory, antiproliferative, hmn mononuclear cells involving T lymphocytes, B lymphocytes, and macrophages isolated from peripheral blood 8664 .

Anti-inflammatory, apoptosis, K562 cells, via inhibition of both LOX and COX 18643

Anti-inflammatory, appears to be a highly specific antagonist for the CCR1 receptor 19819

Anti-inflammatory, arthritis model, induced by carrageenan, suppresses recruitment of neutrophils 12122 .

Anti-inflammatory, arthritis model, induced by formaldehyde or egg white 8297.

Anti-inflammatory, assay of dimethyl benzene-induced inflammation $660,7361,11416,11417$.

Anti-inflammatory, blocks macrophage inflammatory protein (MIP-1a) banding to hmn monocytes 19819.

Anti-inflammatory, blocks macrophage inflammatory protein (MIP-1a) binding to hmn embryonic kidney (HEK)/293 cells transfected with stable CC chemokine receptor-1 (CCR1) 19819.

Anti-inflammatory, blocks NO production and NOS activity and expression, rat macrophages and pancreatitis tissue 4398 . Anti-inflammatory, blocks regulated upon activation on normal T-cell expressed and secreted (RANTES) banding to hmn monocytes 19819.

Anti-inflammatory, blocks regulated upon activation on normal T-cell expressed and secreted (RANTES) binding to hmn embryonic kidney (HEK)/293 cells transfected with stable CC chemokine receptor-1 (CCR1) 19819.

Anti-inflammatory, blocks TNF- $\alpha$-induced cell-cell adhesion between HUVECs and THP-1 cells 18643.

Anti-inflammatory, blocks VCAM-1 expression induced by IL-4 in endothelial cells 16675 .

Anti-inflammatory, calcium-stimulated, mouse peritoneal macrophages and hmn platelets 3981 .

Anti-inflammatory, caused by formalin 5930 .

Anti-inflammatory, causes a pronounced reduction in the $c$-fos and TGF- $\beta 1$ expression in mouse skin stimulated by phorbol myristate acetate (PMA) 18643.

Anti-inflammatory, chemiluminescence assay 21419, 21420, 21421, 21422.

Anti-inflammatory, CINC-1 formation inhibitor, stimulated with LPS, LPSNRK-52E rat kidney epithelial cells 18673.

Anti-inflammatory, COX inhibitor 16540, 16983, 18376, 19312, 19846, 19895, 19897, 19898.

Anti-inflammatory, COX-1 inhibitor $2890,4056,7180,7860,7868$, 7869, 7870, 7871, 7872, 7873, 8814, 9486, 10041, 11753, 12178, $12891,14106,15203,16550,17371,18317,18513,18643,18644$, 18645,22504

Anti-inflammatory, COX-2 inhibitor $1476,2036,2038,2887,2890$, $3600,4056,6277,6282,7180,7860,7868,7869,7870,7871,7872$, $7873,8020,8814,9486,10041,10438,10639,12122,12891,13137$, $13137,13137,14106,14971,14971,14971,15203,15279,16550$, $17168,17532,18317,18643,18643,18643,18644,18645,19087$, 21924, 22270, 22504, 22718.

Anti-inflammatory, COX-2 inhibitor inactive $330,509,510,511,6278$, 6279, 6280, 11129, 13108, 15134, 19795, 19796.

Anti-inflammatory, COX-2 inhibitor, inhibits expression of COX-2 1476, 2036, 2038, 3600, 8020, 15279, 17168, 18317, 19087, 22718.

Anti-inflammatory, COX-2 inhibitor, inhibits expression of COX-2, through inhibition of NF-кB 2036, 2038.

Anti-inflammatory, COX-2 inhibitor, rat peritoneal macrophages, inhibits PGE2 production 17532.

Anti-inflammatory, COX-2 inhibitor, rat renal medulla $13137,14971$.

Anti-inflammatory, COX-2 inhibitor, selective 16050, 22270.

Anti-inflammatory, cytokine formation inhibitor, hmn peripheral

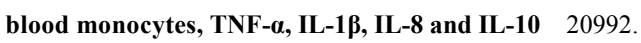

Anti-inflammatory, cytokine formation inhibitor, hmn peripheral blood mononuclear cells, TNF- $\alpha$, IL-4, IL-2 and IFN- $\boldsymbol{\gamma}$ 14976, 14977.

Anti-inflammatory, cytokine formation inhibitor, macrophages, TNF- $\alpha$, IL-6 and IL-12 17240.

Anti-inflammatory, cytokine formation inhibitor, RAW264.7 cells, TNF- $\alpha$ and IL-6 13137, 18317, 8278, 3674. 
Anti-inflammatory, decreases expression of IL-1 $\alpha$, IL-1 $\beta$, TNF- $\alpha$ and IL-6 mRNAs in J774A.1 macrophages 15635.

Anti-inflammatory, diminishes increase in VCAM-1 and MCP-1 levels induced by TNF- $\alpha$ and oxidized LDL in HUVECs 9337.

Anti-inflammatory, ear edema, both PMA and oxazolone-induced 13137.

Anti-inflammatory, ear edema, prevents ear edema formation caused by $\mathrm{PMA}$ and synthesis of LOX products, especially $\mathrm{LTC}_{\mathbf{4}}$ and COX metabolites derived from arachidonic acid 14979, 13591, 10363.

Anti-inflammatory, eczema in mouse ears, repeated administration of TPA 3462, 6938, 11530.

Anti-inflammatory, edema and inflammation, induced by PMA 17958. Anti-inflammatory, experimental colitis 2102.

Anti-inflammatory, free radical scavenger 19087.

Anti-inflammatory, gpg ear edema, induced by benzoic acid 7521 .

Anti-inflammatory, gpg, erythema reaction from ultraviolet irradiation 664.

Anti-inflammatory, ICAM-1 expression inhibitor 4217, 4218.

Anti-inflammatory, ICAM-1 expression inhibitor, PMA-induced 13474, 13475.

Anti-inflammatory, IL-12 production inhibitor 2102, 10886, 17240.

Anti-inflammatory, IL-12 production inhibitor, macrophages,

LPS-activated $10886,17240$.

Anti-inflammatory, IL-12 production inhibitor, macrophages, LPS-activated, 2102.

Anti-inflammatory, IL-1 $\beta$ production inhibitor, hmn monocyte, LPS-stimulated 3368, 17862, 21397.

Anti-inflammatory, IL-1/inhibitor, hmn monocyte 16539.

Anti-inflammatory, IL-2 inhibitor, decreases expression of IL-2 in T-lymphocytes 16675 .

Anti-inflammatory, IL-5inhibitor 1476, 1492, 12020, 13137, 13145, 18409.

Anti-inflammatory, IL-6 and IL-8 blocker, blocking production and expression of IL-6 and IL-8 2102, 22718.

Anti-inflammatory, increases TNF- $\boldsymbol{\alpha}$ level in RAW264.7 cells 2102, 2106, 22718.

Anti-inflammatory, inhibits 5-LOX metabolites especially $\mathrm{LTC}_{4}$ 3981.

Anti-inflammatory, inhibits accumulation of $\mathbf{N O}_{2}^{-1} \quad 8214,8215$.

Anti-inflammatory, inhibits activation of IL-12 gene promoter 10886 .

Anti-inflammatory, inhibits activation of NF- $\mathrm{B}$, PMA- and

TNF- $\alpha$-induced, mechanism not involving antioxidant pathways 10886.

Anti-inflammatory, inhibits binding of several chemokines, such as CXC, $\mathrm{CC}$ to hmn leukocytes or cells transfected with chemokine receptors $2102,2106$.

Anti-inflammatory, inhibits C5a-stimulated release of TNF- $\alpha$ and IL-1 $\beta$ 17460 .

Anti-inflammatory, inhibits C5a-stimulated release of TNF- $\alpha$ and IL-1 $\beta$, RAW264.7 cells 17460.

Anti-inflammatory, inhibits chemotaxis of hmn peripheral blood neutrophils 6428 .
Anti-inflammatory, inhibits COX metabolite PGE $\mathbf{2}_{\mathbf{2}} \mathbf{T X B}_{\mathbf{2}} 3981$.

Anti-inflammatory, inhibits expression and production of of pro-inflammatory cytokines (IL-1 $\beta$, IL-6, TNF- $\alpha$, IFN $\gamma$., MIP-1 $\alpha / \beta)$, hmn peripheral blood mononuclear cells under stimulation with superantigenic staphylococcal exotoxins 2106.

Anti-inflammatory, inhibits expression of iNOS $1790,5669,7982,7985$, $10628,10820,11017,12122,13433,13434,13435,13436,13437$, 16603, 16713, 17409, 18643, 19405, 19406, 19895, 20686.

Anti-inflammatory, inhibits expression of iNOS, TPA-treated hmn monocyte cell line THP-1 16713.

Anti-inflammatory, inhibits induction of VCAM-1, HUVECs, TNF- $\alpha$ and IL-1b-stimulated 3911.

Anti-inflammatory, inhibits inflammation induced by cancer promotor TPA 16221, 16222.

Anti-inflammatory, inhibits lipid peroxidation see "Antioxidant, inhibits lipid peroxidation".

Anti-inflammatory, inhibits lipid peroxidation, cephalopin 8278, 13088, 18685, 19450.

Anti-inflammatory, inhibits LPS-induced DNA binding activity of $\mathrm{NF}-\kappa \mathrm{B}$, associated with decrease of p65 protein levels in nucleus 10820 .

Anti-inflammatory, inhibits $\mathbf{L T B}_{\mathbf{4}}$ biosynthesis 2106 .

Anti-inflammatory, inhibits metabolism of arachidonic acid 7926 , 15038, 19259, 22274.

Anti-inflammatory, inhibits metabolism of arachidonic acid, calcium ionophore-stimulated leukocytes, inhibits $\mathrm{LTB}_{4}$ production 10182, 10472.

Anti-inflammatory, inhibits mRNA expression and production of TNF- $\boldsymbol{\alpha}$ or IL-6 in RAW 264.7 cells $11521,11522$.

Anti-inflammatory, inhibits not only expression of inflammatory NF-кB target genes such as iNOS, COX-2 and TNF- $\alpha$ but also production of PGE 2 and TNF- $\alpha 12122$.

Anti-inflammatory, inhibits over-production of NO and PGE 16532.

Anti-inflammatory, inhibits $\mathrm{PGE}_{2}$ production and inhibits COX-2 production, but not COX-1 17532.

Anti-inflammatory, inhibits production of COX metabolite $\mathrm{PGE}_{2}$, reduces $\mathbf{T X B}_{2}$ level $17145,18792,20569$.

Anti-inflammatory, inhibits production of of pro-inflammatory cytokines (TNF- $\alpha$, IL-1 $\beta$, IL-4, IL-2 and IFN- $\gamma$ ), hmn peripheral mononuclear cells $22686,22687,22688$.

Anti-inflammatory, inhibits production of of pro-inflammatory cytokines (TNF- $\alpha$, IL-1ßand IL-6) 16532.

Anti-inflammatory, inhibits production of of pro-inflammatory cytokines (TNF- $\alpha$ and IL-1/), hmn monocytes and macrophages 3368 .

Anti-inflammatory, inhibits production of $\mathrm{PGE}_{2}$ and proMMP-9 in rabbit synovial fibroblasts 15635 .

Anti-inflammatory, inhibits production of $\mathrm{PGE}_{2}$ in $\mathrm{C6}$ rat glioma cells 2106.

Anti-inflammatory, inhibits protein and mRNA expression levels of iNOS and COX-2 enzymes 10820.

Anti-inflammatory, inhibits RANTES-induced CCR1 cell migration, without interfering with CCR1 cell migration induced by 
epidermal growth factor (EGF) 19819.

Anti-inflammatory, inhibits release and metabolism of arachidonic acid 7802,15170 .

Anti-inflammatory, inhibits release of histamine 8214,8215 .

Anti-inflammatory, inhibits release of $\beta$-glucuronidase, rat mast cells stimulated with compound 48/80 8214,8215 .

Anti-inflammatory, inhibits synthesis of COX-2 transcript 22504. Anti-inflammatory, inhibits synthesis of iNOS transcript 22504. Anti-inflammatory, inhibits synthesis of prostaglandin and leukotriene 22059.

Anti-inflammatory, inhibits thioglycolate-elicited rat peritoneal neutrophil accumulation and LPS-activated nitric oxide production in murine macrophages 20978.

Anti-inflammatory, lead compound to develop new anti-inflammatory drugs $4398,12122,15635,16532$.

Anti-inflammatory, lead compound to treat asthma 13137.

Anti-inflammatory, leukocyte elastase MMP-2/9 inhibitor 6923, 14971.

Anti-inflammatory, $\mathrm{LTC}_{4}$ selective inhibitor, rat resident peritoneal macrophage 2102 .

Anti-inflammatory, lysosome enzyme inhibitor, polymorphonuclear (PMN) leukocytes 8405, 9053, 9054, 9056, 9060, 9061, 9062.

Anti-inflammatory, may be useful for the treatment of various inflammatory diseases 11017 .

Anti-inflammatory, modified Tan and Berridge method 508, 5008, 8788, 12510, 12982, 12983, 18169, 20501.

Anti-inflammatory, modulation of complement 5a-induced chemotaxis and inflammatory cytokines production in macrophages 17460 .

Anti-inflammatory, modulator of cytokine network 2004, 2102, 2106, $2983,3269,3270,3272,3273,3307,3368,3368,3674,3911,4398$, $4565,5699,6923,7278,7714,8278,8423,8424,9276,9337,9456$, $11521,11522,12523,13137,14971,14976,14977,15635,16532$. $16675,17240,17409,17460,17862,18317,18643,18673,19308$, 19427, 19819, 20992, 21206, 21397, 22686, 22687, 22688, 22718 , 22727, 22728, 22729, 22730, 22731.

Anti-inflammatory, most effective component of 10 compounds in Schizonepeta tenuifolia (JING JIE) 17377.

Anti-inflammatory, mus arthritis 4589, 19955, 22023.

Anti-inflammatory, mus ear edema, induced by arachidonic acid $12766,18219$.

Anti-inflammatory, mus ear edema, induced by croton oil 977,978 , 5152, 7521, 8521, 8522, 10814, 11121, 11259, 11260, 17422, 22023

Anti-inflammatory, mus ear edema, induced by croton oil, by down-regulation of COX-2 8401 .

Anti-inflammatory, mus ear edema, induced by phospholipase $\mathrm{A}_{2}$ 19883, 19885.

Anti-inflammatory, mus ear edema, induced by TPA 205, 1971, 2102, 12766, 13098, 16080, 17345, 20710, 21392.

Anti-inflammatory, mus paw edema model 4614, 8965, 22700, 22938. Anti-inflammatory, mus paw edema model, induced by 5-HT 664 , 7243.

Anti-inflammatory, mus paw edema model, induced by carrageenan $663,664,966,1768,1928,2455,2594,2990,3462,3743,4328,5573$.
$6454,6487,7243,7521,7659,8304,8841,9288,9419,9705,11747$, $12122,12916,13481,14896,14897,16050,16080,16525,16532$, $16867,17042,18221,19540,19922,20509,20569,20717,21055$, 21435, 21438, 21439, 22080, 22151, 22433, 22504, 22504

Anti-inflammatory, mus paw edema model, induced by carrageenan, inhibits leucocyte aggregation in inflammatory exudate 22720 .

Anti-inflammatory, mus paw edema model, induced by DMSO 546, 547,548 .

Anti-inflammatory, mus paw edema model, induced by formaldehyde 19111.

Anti-inflammatory, mus paw edema model, induced by glucosan 664 , 16525.

Anti-inflammatory, mus paw edema model, induced by glucosan or acetate acid 16532 .

Anti-inflammatory, mus paw edema model, induced by histamine 663 , 664.

Anti-inflammatory, mus paw edema model, induced by phospholipase A $11530,21392$.

Anti-inflammatory, mus, experimental chronic arthritis $16050,14896$.

Anti-inflammatory, mus, formaldehyde edema model $56,309,8841$, $12510,15286$.

Anti-inflammatory, mus, inflammation caused by TPA 10967,16362 , 4963.

Anti-inflammatory, mus, inhibits phoroplast permeability 899 .

Anti-inflammatory, mus, inhibits vaso-permeability $3588,5161,7495$, $15843,19922,20569$.

Anti-inflammatory, mus, tampon granuloma model $309,3368,4266$, 8841, 8841, 9238, 11505, 11747, 13391, 13481, 13559, 22151, 22938.

Anti-inflammatory, mus, tuberculin reaction model 8841 .

Anti-inflammatory, mus, woolball model 10887, 12018, 12020, 13137, 15279, 19087, 20099, 22270.

Anti-inflammatory, myeloperoxidase inhibitor $2102,13098$.

Anti-inflammatory, NF-кB inhibitor 19401, 19402, 19403, 19404, $13474,13475$.

Anti-inflammatory, NF-кB pathway 2890, 3141, 4398, 6923, 7239, $9288,16031,16713,17862,18317,18643$.

Anti-inflammatory, NF-кB activation inhibitor, LPS-induced, murine RAW264.7 cells $171,173,222,5939,16675$.

Anti-inflammatory, NF-кB inhibitor 1790, 1791, 1802, 2004, 2036, 2038, 3307, 3362, 3368, 4746, 7195, 7196, 7197, 10820, 10886, 14097, $15002,16163,16166,16167,16675,18643,19407,19895,21930$, 2102, 6288 .

Anti-inflammatory, NF-кB inhibitor, hmn monocytes, prevents cytokines (IL-1, IL-6, TNF, IL-8) release and PGE ${ }_{2}$ synthesis 6288 , 21930 .

Anti-inflammatory, NF-кB inhibitor, LPS-induced, RAW264.7 cells 16675, 14097, 4746, 7196, 7197, 15002, 7195, 1802, 1791, 1790.

Anti-inflammatory, no detail information $1828,1965,2325,2414,2687$, 2886, 3221, 3464, 6183, 6816, 7370, 7528, 7529, 7530, 7531, 7532, $7950,9455,8293,8295,12907,13809,8360,8361,8365,8363,12608$, $16025,17267,17648,17918,18440,19074,21245,3935,3937,304$, 538, 580, 1614, 2303, 2312, 2989, 3077, 3600, 3689, 3923, 3924, 4439, $4477,4645,4685,4876,5763,6643,6656,6767,6853,7481,7586$, 
7793, 7951, 9441, 8292, 8095, 11077, 12420, 9038, 9496, 11808, $12255,13392,13492,13808,13962,14756,12184,8846,11642$, $11736,10875,11741,13774,16900,17024,17597,17598,17599$, 17829, 18190, 18411, 19142, 19143, 19542, 19545, 19983, 20168, 20650, 20732, 21059, 21234, 21887, 22282, 22581, 27, 13437, 13435, 13436, 8865, 17403, 16535, 22990, 7833, 18824, 21862, 22332.

Anti-inflammatory, NO production inhibitor see "NO production inhibitor".

Anti-inflammatory, NO, IL-1ß, IL-6 and TNF- $\alpha$ production inhibitor 20686.

Anti-inflammatory, ornithine decarboxylase inhibitor 2102 .

Anti-inflammatory, PGE $_{2}$ production inhibitor $12122,16603$.

Anti-inflammatory, PGE $_{2}$ production inhibitor, LPS-induced, RAW264.7 cells $1573,8020,8679,10820,19081,19405,19406$, 22123, 22124, 22270.

Anti-inflammatory, $\mathrm{PGE}_{2}$ production inhibitor, rat peritoneal macrophages 17532 .

Anti-inflammatory, $\mathrm{PGE}_{2}$ production inhibitor, TPA-stimulated rat peritoneal macrophages 20900, 20901.

Anti-inflammatory, PMA-treated mammary epithelial cells, inhibits COX-2 transcription and COX-2activity, by inhibiting signal transduction through PKC 18643.

Anti-inflammatory, prevents eotaxin production and mRNA eotaxin expression in hmn fibroblasts stimulated with IL-4/TNF- $\boldsymbol{\alpha} 2102$.

Anti-inflammatory, prevents integrin-mediated neutrophil adhesion and fMLP- or leukotriene B4-induced transmigration 7714, 21206.

Anti-inflammatory, prevents production of TNF- $\alpha$, IL-1b and IL-6 in LPS-activated macrophages, possibly via NF-кB inhibition 3307.

Anti-inflammatory, prevents TNF- $\alpha$ and IL-6 production in RBL-2H3 stimulated mast cells, through a mechanism involving the blockade of NF-кB activation 2004.

Anti-inflammatory, prostaglandin biosynthesis inhibitor 4921, 4922, 8388 .

Anti-inflammatory, prostaglandin biosynthesis inhibitor, rbt kidney microsomes 16571 .

Anti-inflammatory, prostanoid inhibitor, LOX pathway 18643.

Anti-inflammatory, rat, experimental gastric ulcer 37 .

Anti-inflammatory, reduces arthritic inflammation in rat adjuvant-induced arthritis as well as abdominal constriction caused by acetic acid 8401 .

Anti-inflammatory, reduces cell surface expression of adhesion molecules, resulting in inhibition of THP-1 monocyte adhesion to TNF- $\boldsymbol{\alpha}$ stimulated HUVECs 9337.

Anti-inflammatory, reduces expression of ICAM-1 and VCAM-1 in THP-1 hmn monocytes 3269, 3270, 3272, 3273.

Anti-inflammatory, reduces expression of VCAM-1, aorta cells of hypercholesterolemic New Zealand rabbits 9337.

Anti-inflammatory, reduces ICAM-1 expression, mouse liver cells, LPS-stimulated 13137.

Anti-inflammatory, reduces IFN- $\gamma$-induced ICAM-1 protein, as well as mRNA expression in hmn keratinocytes 5699.
Anti-inflammatory, reduces leukocyte infiltration, measured as tissue peroxidase activity 6938,11530 .

Anti-inflammatory, reduces permeability of blood capillary and activity of hyaluronidase 20066 .

Anti-inflammatory, reduces permeability of capillary and blood cell walls 4097.

Anti-inflammatory, reduces VCAM-1 expression, HUVECs, TNF-a-induced 9337.

Anti-inflammatory, retardation of macrophage recruitment and suppression of cytokines production might underlie potential usefulness of piperlactam $S$ as an anti-inflammatory agent 17460 .

Anti-inflammatory, specific NF-кB inhibitor of DNA-binding activity of p50 subunit 12122 .

Anti-inflammatory, sulfate of rutin being strong anti-inflammatory 19087.

Anti-inflammatory, suppresses expression of iNOS and COX-2 protein 19405, 19406.

Anti-inflammatory, suppresses expression of iNOS and COX-2, without cytotoxic effect 16603 .

Anti-inflammatory, suppresses expression of NF-кB target genes such as iNOS and COX-2 1790.

Anti-inflammatory, suppresses IL-1 $\beta$-induced production of $\mathrm{PGE}_{2}$ in hmn synovial fibroblasts 15635 .

Anti-inflammatory, suppresses iNOS expression and NO production, cultured cells, by down-regulation of NF- $\mathrm{NB}$ binding activity via blockade of IkB $\boldsymbol{\alpha}$ degradation 18643.

Anti-inflammatory, TNF- $\alpha$ production inhibitor 13137, 14097, 4746, $7196,7197,15002,7195,1802,1791,1790,12122,16675$.

Anti-inflammatory, TNF- $\alpha$ production inhibitor, LPS/IFN- $\gamma$-induced, N9 microglial cell lines 8520 .

Anti-inflammatory, TNF- $\alpha$ production inhibitor, LPS/IFN- $\gamma$-induced, peritoneal macrophages 9337.

Anti-inflammatory, TNF- $\alpha$ production inhibitor, LPS-induced, RAW264.7cells $2983,4565,7278,8423,8424,8520,9276,9456$, 12523, 17409, 18673, 19308, 19427, 22727, 22728, 22729, 22730, 22731.

Anti-inflammatory, TNF- $\alpha$ production inhibitor, LPS-induced, U937cells 8423,8424 .

Anti-inflammatory, TNF-a production inhibitor, sulphydryl (thiol, -SH) compounds abrogated the inhibitory effect 4565 .

Anti-inflammatory, treatment of cervicitis $7399,7416$.

Anti-inflammatory, Ungar method 15635.

Anti-inflammatory, inhibits iNOS $1854,4292,5722,16717,20685$, 20686, 22059.

Antileishmanial $515,580,815,816,1141,1145,1146,1241,1244,1250$, $1252,1937,1939,2004,2229,2346,2683,2833,2844,2920,2924$, $3157,3158,3166,3186,3306,3551,3609,3674,3856,4080,4279$, $4478,4840,5272,5273,5540,5541,5542,5550,5675,6024,6025$, $6776,6973,7646,7650,7651,7925,8325,8985,9750,9788,10310$, $10465,10733,11001,11148,11195,11601,12232,12487,12916$, $13571,13874,13876,14222,15297,15298,15300,15301,15302$, $15303,15304,15305,15306,15307,15308,15309,15312,15313$, 
$15314,15315,15316,15779,15780,16067,16068,16693,16898$, $17135,17940,18221,18784,19077,19234,19235,19542,19568$, 19960, 20369, 20390, 22496, 22551, 22818.

Antileishmanial inactive, Leishmania donovani $4866,15716$.

Antileprotic 3485, 8955, 9697.

Antimalarial inactive summary index $8,472,506,1276,1532,1784$, 2334, 2911, 3097, 3159, 3413, 3852, 3940, 3941, 4068, 4069, 4237, 4866, 4982, 5733, 6034, 6757, 6809, 6810, 7503, 8321, 8335, 8336, 9887, 10159, 10426, 10427, 13241, 13297, 13867, 13869, 13871, $14462,14729,14731,14758,14915,15716,16050,16154,16892$, 16898, 17940, 19533, 19999, 20377, 21475, 21926, 22230.

Antimalarial inactive, Plasmodium 3940, 3941.

Antimalarial inactive, Plasmodium falciparum 506, 580, 815, 816, 1241, 2004, 2334, 2924, 3166, 3306, 3551, 4866, 5675, 6809, 6810, 7925, 9750, 12916, 14222, 14729, 14731, 14758, 15716, 16154, 18221, 19568, 19960.

Antimalarial inactive, Plasmodium falciparum D10 1532.

Antimalarial inactive, Plasmodium falciparum D6 3852.

Antimalarial inactive, Plasmodium falciparum D6 chloroquine-sensitive strain 5733.

Antimalarial inactive, Plasmodium falciparum D6 clone 3097, 3159, 9887, 13867, 13869, 13871, 16892.

Antimalarial inactive, Plasmodium falciparum Dd2 4068, 4069.

Antimalarial inactive, Plasmodium falciparum FAC8 1532.

Antimalarial inactive, Plasmodium falciparum K1 3413, 6034, 10159, 10426, 10427, 14915, 20369.

Antimalarial inactive, Plasmodium falciparum K1 multidrug-resistant strain 2911, 19999, 20377.

Antimalarial inactive, Plasmodium falciparum NF54 4982, 10159, 13297, 22230.

Antimalarial inactive, Plasmodium falciparum poW 1928, 4068, 4069, $5659,7883$.

Antimalarial inactive, Plasmodium falciparum TM91C235 clone 3097, 3159, 9887, 13867, 13869, 13871, 16892.

Antimalarial inactive, Plasmodium falciparum W2 chloroquine-resistant $3852,5733$.

Antimalarial inactive, Plasmodium falciparum W2 clone 3097,3159 , 9887, 13867, 13869, 13871, 16892.

Antimalarial inactive, Plasmodium hmn 11133.

Antimalarial summary index $24,52,52,53,55,261,262,266,580,589$, $602,675,676,777,815,816,919,1141,1145,1147,1241,1364,1460$, $1475,1784,1784,1784,1784,1784,1842,1857,1928,1937,1939$, 2004, 2148, 2191, 2672, 2683, 2870, 2871, 2872, 2873, 2875, 2883, 2884, 2885, 2910, 2914, 2916, 2917, 2920, 2924, 3004, 3005, 3007, 3096, 3166, 3243, 3306, 3551, 3609, 3614, 3674, 3686, 3700, 4048, 4066, 4067, 4104, 4181, 4421, 4478, 4949, 5095, 5146, 5435, 5436, $5659,5675,5833,6018,6020,6409,6413,6433,6464,6776,6896$, 6971, 7304, 7329, 7883, 7925, 8004, 8322, 8509, 9072, 9598, 9738, 9739, 9750, 9857, 9885, 9926, 10310, 10340, 10383, 10398, 10531, $10561,10656,11133,11148,11195,11358,11594,11596,11598$, $11723,11723,11977,11978,11988,12455,12487,12501,12573$, $12573,12751,12751,12766,12916,13241,13811,13918,14029$, $14183,14222,14325,14396,15062,15722,15739,15764,15804$,
$16050,16067,16068,16261,16366,16373,16374,16604,16912$, 16913, 16914, 16915, 16970, 17041, 17309, 17422, 17423, 17478, $17574,17827,17841,17846,17847,17924,17983,18188,18221$, $18252,18253,18425,18425,18515,18862,18867,19234,19235$, 19568, 19753, 19883, 19885, 19886, 19887, 19888, 19891, 19892, 19900, 19960, 20108, 20253, 20333, 20334, 20369, 20414, 20415, $20975,21313,21314,21315,21316,21389,21390,21905,22278$, 22279, 22551, 22818, 23002.

Antimalarial, causes transformation of erythrocytes into stomatocytes 16050.

Antimalarial, cultivated in vitro by Trager and Jensen method 17574 , 20253.

Antimalarial, formerly used to treat malaria 18425 .

Antimalarial, inhibits Plasmodium falciparum. absorbing $\mathrm{H}^{3}$-sarkin in vitro 2672.

Antimalarial, mus Plasmodium sp. 777, 1475, 23002.

Antimalarial, mus, infected by Plasmodium vinckei 16898, 17940.

Antimalarial, no detail information 3686, 8509, 9738, 20108, 21905, 1842, 2191, 4421, 5435, 9739, 9857, 12501, 16261, 17983, 20975.

Antimalarial, Plasmodium $\quad 24,1364,1784,6757,16050,18252,18253$, 20333, 20334, 20414, 21926.

Antimalarial, Plasmodium berghei 18862, 18867.

Antimalarial, Plasmodium berghei NK65 on infected mouse 261, 262, $10531,15062$.

Antimalarial, Plasmodium chloroquine-resistant strain $\quad 3614,11723$, 20415.

Antimalarial, Plasmodium chloroquine-sensitive strain $\quad 3614,11723$, 20415.

Antimalarial, Plasmodium F32 chloroquine-sensitive strain 919.

Antimalarial, Plasmodium falciparum 777, 1784, 1784, 1784, 1937, $1939,2683,2920,3243,3609,3674,5095,6413,6776,7503,8335$, $8336,10383,11148,11195,11977,13918,14183,16067,16068$, $16912,16913,16914,16915,16970,17041,17841,17846,17847$, $18515,19900,21389,22551$.

Antimalarial, Plasmodium falciparum 3D7 675, 10656, 11358, 11594, 11596, 11598, 12455, 12766, 16366, 17422, 17423.

Antimalarial, Plasmodium falciparum D10 8, 472, 1276, 4237, 13241, 21475.

Antimalarial, Plasmodium falciparum D10 chloroquine-sensitive 6018 , $6020,13811$.

Antimalarial, Plasmodium falciparum D2 12573.

Antimalarial, Plasmodium falciparum D6 52, 52, 53, 55, 1460, 1784, 5146, 5833, 6409, 7304, 7329, 8004, 8321, 9072, 9598, 10398, 12751, $12751,14029,14462,16604,17827,17924,18188,18425,19753$, 19883, 19885, 19886, 19887, 19888, 21390.

Antimalarial, Plasmodium falciparum D6 chloroquine-sensitive 2148 , 4949, 6971, 8322, 17309, 22278, 22279, 22818, 4104, 15739, 15764, $15804,1857$.

Antimalarial, Plasmodium falciparum D6 clone 21313, 21314, 21315, 21316.

Antimalarial, Plasmodium falciparum Dd2 676, 1928, 3004, 3005, 3007, 3700, 4066, 4067, 5659, 7883, 12573, 12766.

Antimalarial, Plasmodium falciparum F32 chloroquine-sensitive 
11723.

Antimalarial, Plasmodium falciparum FAC8 472, 1276, 4237, 13241.

Antimalarial, Plasmodium falciparum FCA20 GHANA

chloroquine-sensitive 11723.

Antimalarial, Plasmodium falciparum FcB1 266, 2914, 2916, 2917, 4181, 6464, 9926, 10340, 10561, 16050, 16374.

Antimalarial, Plasmodium falciparum FCB1 COLOMBIA(CQR-) strain 11723.

Antimalarial, Plasmodium falciparum FcM29 10340, 16373, 16374.

Antimalarial, Plasmodium falciparum FCR3 drug-sensitive strain 19891, 19892.

Antimalarial, Plasmodium falciparum FCR-3/A2 clone 2870, 2871, 2872, 2873, 2875, 2883, 2884, 2885, 15722.

Antimalarial, Plasmodium falciparum K1 8, 589, 1141, 1145, 1147, 4048, 6433, 6896, 10310, 11978, 12487, 14325, 14396, 17478, 19234, $19235,21475$.

Antimalarial, Plasmodium falciparum K1 chloroquine-resistant strain 19891, 19892.

Antimalarial, Plasmodium falciparum K1 multidrug-resistant strain 1784, 2910, 11988, 17574, 19533, 20253.

Antimalarial, Plasmodium falciparum NF54 589, 6433, 6896, $13241,14325,14396$.

Antimalarial, Plasmodium falciparum NF54 chloroquine-sensitive strain 11988.

Antimalarial, Plasmodium falciparum PFB(CQR+) strain 11723.

Antimalarial, Plasmodium falciparum PoW 4066, 4067, 12573, 3700, 3005, 676, 3004, 3007.

Antimalarial, Plasmodium falciparum W2 52, 53, 55, 1460, 1784, 5146, 6409, 8004, 8321, 9072, 9598, 12751, 14029, 14462, 16604, 17827, 17924, 18188, 18425, 19753, 19883, 19885, 19886, 19887, 19888, 21390.

Antimalarial, Plasmodium falciparum W2 chloroquine-resistant strain $52,12751,7304,10398,5833,6018,6020,7329,9885,2148,4949$, 6971, 8322, 13811, 17309, 22278, 22279, 22818, 4104, 15739, 15764, $15804,1857$.

Antimalarial, Plasmodium falciparum W2 clone $\quad 3096,21313,21314$, 21315, 21316.

Antimalarial, Plasmodium falciparum W2 INDOCHINA(CQR) strain 11723.

Antimalarial, Plasmodium FcM29-Cameroon chloroquine resistant strain 919.

Antimalarial, Plasmodium moderate chloroquine-resistant strain 3614 , 11723.

Antimalarial, similar action with quinine 602 .

Antimelancholic 10886 .

Anti-metabolism 3059 .

Antimicrobial $933,2057,2303,2628,3079,3499,3600,4032,6606$, $8081,8403,8487,8549,8550,8555,8556,8557,8752,11808,11819$, $12256,12503,12749,13492,15741,15849,16128,16457,16584$, 17695, 17831, 19047, 19257, 19259, 20093, 21597, 22290.

Antimitotic $1372,2890,3909,4547,5074,5093,7768,9740,12732$, 20284, 22408, 22488, 22491.

Antimitotic and antifungal $3458,3459,3461,11319,15361,19890$.
Antimitotic and antifungal inactive 3460 .

Antimutagenic 870, 6193, 7956, 19983, 20355, 20365, 20369.

Antimutagenic 2564, 3026, 3551, 6757, 6853, 8095, 9818, 18545.

Antineoplastic (in vivo) summary index 10, 27, 90, 136, 140, 144, 145, 195, 404, 409, 537, 552, 570, 576, 660, 727, 759, 777, 778, 779, 780, $837,899,909,910,952,962,967,1002,1029,1128,1210,1275,1313$, $1372,1463,1464,1467,1497,1621,1626,1627,1745,1789,1841$, 1866, 1928, 1965, 1971, 1993, 2026, 2073, 2102, 2109, 2119, 2293, $2300,2318,2331,2334,2380,2660,2661,2662,2666,2667,2675$, 2676, 2684, 2687, 2735, 2768, 2892, 2938, 2946, 3053, 3076, 3082, $3094,3095,3181,3188,3197,3198,3209,3218,3300,3308,3402$, 3404,3412 , 3467, 3481, 3498, 3499, 3506, 3529, 3551, 3602, 3637, $3693,3702,3745,3754,3779,3788,3861,3883,3909,3911,3937$, 3964, 3965, 4048, 4053, 4106, 4128, 4140, 4238, 4239, 4289, 4295, 4299, 4300, 4317, 4319, 4320, 4321, 4322, 4323, 4324, 4327, 4352, 4398, 4399, 4400, 4455, 4456, 4457, 4547, 4657, 4739, 4860, 4870, 4872, 4874, 4978, 5063, 5074, 5093, 5098, 5134, 5136, 5168, 5181, 5187, 5236, 5391, 5392, 5393, 5394, 5395, 5414, 5440, 5507, 5537, 5543, 5686, 5796, 6162, 6290, 6303, 6490, 6498, 6501, 6643, 6737, $6741,6750,6751,6759,6762,6767,6772,6776,6804,6889,7030$, 7031, 7167, 7203, 7232, 7236, 7327, 7399, 7416, 7528, 7529, 7530, 7531, 7532, 7550, 7551, 7552, 7553, 7554, 7555, 7559, 7566, 7579, 7580, 7581, 7584, 7585, 7586, 7593, 7594, 7595, 7611, 7618, 7739, 7740, 7768, 7837, 7904, 7909, 7913, 7979, 7994, 7996, 8047, 8095, $8128,8155,8169,8173,8263,8312,8324,8403,8424,8429,8430$, $8436,8458,8509,8524,8563,8569,8570,8841,8846,8848,8906$, $8908,8912,8914,8967,8980,8981,9015,9194,9239,9288,9320$, 9329, 9456, 9463, 9465, 9571, 9572, 9609, 9643, 9671, 9740, 9882, 9928, 9929, 9992, 10067, 10070, 10093, 10148, 10583, 10588, 10710, $10881,10915,11019,11020,11024,11058,11091,11100,11103$, 11104, 11105, 11106, 11179, 11295, 11428, 11449, 11450, 11451, $11525,11601,11747,11748,11773$ 11807, 11848, 11850, 11864, $11903,11970,11992,11993,12011,12012,12145,12146,12220$, $12465,12501,12503,12535,12537,12619,12732,12733,12734$, $12746,12766,12843,12850,12952,13002,13023,13025,13027$, 13029, 13040, 13072, 13092, 13094, 13098, 13100, 13212, 13239, $13241,13430,13514,13606,13618,13619,13620,13621,13622$, $13801,13846,13863,13864,14090,14092,14126,14205,14356$, $14457,14559,14560,14561,14562,14563,14564,14565,14728$, $14739,14821,14822,14826,14828,14830,14835,14836,14837$, 14839, 14896, 14897, 14907, 14923, 14971, 15071, 15073, 15114, $15115,15116,15170,15204,15231,15233,15279,15403,15417$, $15419,15422,15570,15631,15634,15635,15637,15641,15642$, $15737,15741,15776,15806,15990,16019,16050,16082,16083$, $16085,16183,16205,16257,16260,16278,16279,16290,16352$, $16439,16451,16593,16601,16623,16666,16675,16731,16731$, 16732, 16733, 16763, 16793, 16795, 16796, 16797, 16916, 16935, 17009, 17028, 17039, 17042, 17194, 17216, 17217, 17240, 17243, $17278,17337,17341,17342,17409,17411,17413,17417,17557$, 17558, 17591, 17593, 17662, 17664, 17706, 17717, 17722, 17723, $17727,17728,17754,17829,17850,17859,17862,17876,17886$, 17952, 17998, 18011, 18012, 18050, 18067, 18069, 18086, 18091, $18103,18262,18272,18287,18297,18298,18411,18483,18523$, 
18623, 18679, 18759, 18834, 18843, 18882, 18899, 18939, 18940, 19036, 19057, 19142, 19148, 19194, 19201, 19308, 19313, 19316, 19387, 19455, 19472, 19479, 19480, 19484, 19487, 19497, 19498, 19499, 19524, 19540, 19542, 19629, 19697, 19715, 19762, 19784 , 19811, 19819, 19889, 19900, 19983, 20009, 20030, 20044, 20060, 20062, 20063, 20064, 20065, 20069, 20077, 20079, 20098, 20108, 20140, 20160, 20234, 20236, 20387, 20419, 20500, 20576, 20612, 20664, 20670, 20717, 20730, 20745, 20746, 20802, 20809, 20810, 20811, 20814, 20815, 20900, 20901, 20963, 21206, 21234, 21236 , 21239, 21240, 21243, 21250, 21251, 21264, 21320, 21321, 21397 , $21435,21483,21486,21487,21499,21589,21662,21709,21889$, 21913, 21987, 22011, 22072, 22098, 22108, 22146, 22151, 22152, $22153,22194,22195,22231,22232,22268,22270,22282,22368$, 22405, 22407, 22411, 22413, 22414, 22422, 22475, 22497, 22551 , $22581,22596,22615,22700,22704,22718,22733,22768,22777$, $22781,22865,22868,22869,22870,22871,22929,22951,22962$, 22984, 22987, 23033.

Antineoplastic inactive, KB, LNCaP, Lu1 and $\mathbf{P}_{388} 3300$.

Antineoplastic inactive, $\mathbf{P}_{388} \quad 22860,22861,22862,22863,22864,22866$, 22875.

Antineoplastic, induces myelocytic leukemia M1 cell differentiation $3745,8436$.

Antineoplastic, , tubulin assay, ox brain 14739, 20814.

Antineoplastic, 115 Shingi cancer 570 .

Antineoplastic, 3PS leukemia 11848, 17240.

Antineoplastic, 715 adenocarcinoma 19983.

Antineoplastic, 755 adenocarcinoma 9320, 14971, 20140, 22146.

Antineoplastic, 9KB 2768.

Antineoplastic, A2780 2735.

Antineoplastic, A549 11864, 1313, 17342, 17337, 17341.

Antineoplastic, activates glutathione S-transferase in cardiae sinus 952.

Antineoplastic, adenocarcinoma in colon ascendens 22551.

Antineoplastic, antineoplastic and antimetastatic 9992.

Antineoplastic, antitumor promotor $3209,3779,3788,4872,5063,5098$, 5134, 5686, 6498, 6762, 7167, 7203, 7904, 7909, 7913, 8980, 8981, 9456, 9643, 10067, 10710, 11970, 12220, 13002, 14090, 14092, 15737, $16257,16260,17706,18939,19540,19542,19784,21483,21499$, 22194, 22195, 22777, 22781.

Antineoplastic, ARS ascites tumour $\quad 6741,22733$.

Antineoplastic, ascites carcinoma 16439, 17028, 20060, 22270.

Antineoplastic, ascites carcinoma AH130 and AH1974 22282.

Antineoplastic, ascites hepatoma 11179, 13241, 16916, 22232.

Antineoplastic, ascites lymphoma 13241, 21239, 21264.

Antineoplastic, autochthoneous fibrosarcomas induced by methylcholanthrene 6767 .

Antineoplastic, B16 1626, 1627, 1928, 2660, 5414, 6776, 8509, 13621, $15170,15631,17216,17217,17557,18011,18287,22368$.

Antineoplastic, bladder carcinoma 13212.

Antineoplastic, breast cancer 3095, 6776, 16935, 17028, 18759, 20030.

Antineoplastic, breast cancer caused by DMBA 7232 .

Antineoplastic, C1498 marrow-leukemia 570.

Antineoplastic, candidate as antitumor agent $\quad 6162,21709$.
Antineoplastic, cardiae sinus cancer 14126.

Antineoplastic, cardiae sinus cancer induced by benzopyrene 952 .

Antineoplastic, caused tumor cell death selectively, without showing any cytotoxicity to normal fibroblasts 8848 .

Antineoplastic, cervical carcinoma $7399,7416$.

Antineoplastic, chorion cell carcinoma 12734.

Antineoplastic, clinical trial, given orl to 558 patients with cancer of lung and esophagus, or with superficial metastatic cancer during radiotherapy 11179 .

Antineoplastic, Co115 cancer 10881.

Antineoplastic, colorectal cancer $\quad 3095,10915,10915$.

Antineoplastic, deciduoma caused by luteosterone 8967 .

Antineoplastic, dissolves cancer cells 20670.

Antineoplastic, E0771 breast cancer 22700.

Antineoplastic, EAC $\quad 837,2380,3412,3499,4048,4299,4320,4323$, 4323, 4399, 4456, 6741, 6759, 6776, 6804, 8128, 11179, 11747, 12537, $12733,12734,13239,13241,13606,13621,14728,14896,14897$, 16793, 17042, 17593, 18483, 18759, 19142, 19148, 20963, 21206, 21239, 21240, 21251, 22700, 22733.

Antineoplastic, EAC, inhibits cellular proliferation 5440 .

Antineoplastic, EBV-EA activation inhibitor $3209,3937,5796,8155$, $8169,8173,8324,10583,13023,13025,13027,13029,13040,13846$, $14457,14559,14560,14561,14562,14563,14564,14565,15114$, $15115,15116,16082,16083,17409,21913$.

Antineoplastic, EBV-EA activation inhibitor, TPA-induced 409, 537, 778, 779, 780, 1210, 1463, 1464, 1497, 1928, 2331, 3209, 3779, 3788, 4398, 4455, 4860, 4872, 4874, 4978, 5063, 5098, 5134, 5507, 5686, $6498,6762,6889,7167,7203,7528,7529,7530,7531,7532,7611$, $7618,7904,7909,7913,8563,8569,8570,8906,8908,8912,8980$, $8981,9463,9465,9643,10067,10070,10710,11100,11103,11104$, $11105,11106,11451,11970,12012,12220,12619,12952,13002$, 13092, 13094, 13098, 14090, 14092, 15422, 15737, 16257, 16260, 17662, 17664, 17706, 17722, 17723, 17727, 17728, 17829, 18939, 19497, 19540, 19542, 19784, 21483, 21499, 21913, 22108, 22194, 22195, 22777, 22781.

Antineoplastic, Ehrlich ascites carcinoma (EAC) 3964, 3965, 4327, 4400, 11179, 15631, 22232.

Antineoplastic, ESC cancer $1626,1627$.

Antineoplastic, Friend virus leukemia 22768.

Antineoplastic, gastric cancer 20098.

Antineoplastic, glutathione S-transferase activator 9671 .

Antineoplastic, granulation carcinoma 17028.

Antineoplastic, HAC cancer 12537.

Antineoplastic, HCS cancer 22733.

Antineoplastic, HeLa $\quad 837,837,4870,8458,9609,11747,13239,13241$, $17850,20236$.

Antineoplastic, HeLa, inhibits proliferation of cells 11601, 20236. Antineoplastic, HeLa-S 16439.

Antineoplastic, HepA liver cancer 1993.

Antineoplastic, HL-60 8436, 22615, 20670.

Antineoplastic, HL-60, induces leucocyte inhibiting growth of HL-60 leukemia cells 20670 .

Antineoplastic, hollow fiber assay $3300,10588$. 
Antineoplastic, HT29 1313, 11864, 17337, 17341, 17342.

Antineoplastic, hysteromyoma 8967.

Antineoplastic, in clinical trials 22232.

Antineoplastic, in stage of pre-clinic at NCI 17216.

Antineoplastic, in stage of pre-clinic at NCI, shows high antineoplstic activity in NCI's 60 tumor system, the highest activity is of melanotic carcinoma, high activity is of brain tumor, colorectal cancer, lung cancer, kidney cancer 16601.

Antineoplastic, induces cell differentiation 17243.

Antineoplastic, induces normal conformation of cells 18262.

Antineoplastic, inhibits ${ }^{32} \mathrm{P}$ combines with phospholipid in HeLa cells $8458,4870$.

Antineoplastic, inhibits biosynthesis of protein $837,2684,17850$, 18262.

Antineoplastic, inhibits cancer cell mitosis 16793.

Antineoplastic, inhibits carcinogenesis of 3,4-benzopyrene $\quad 552,3602$.

Antineoplastic, inhibits cellular hyperplasia 837 .

Antineoplastic, inhibits DNA synthesis 837, 3412, 14907, 15631, 20815.

Antineoplastic, inhibits formation of melanin $1928,3702,5414,16731$, $16732,18067,18069$

Antineoplastic, inhibits K-ras-NRK 18262.

Antineoplastic, inhibits mus melanoma lung metastasis 8424 .

Antineoplastic, inhibits occurrence of cutaneum carcinoma and duodenum carcinoma 7979 .

Antineoplastic, inhibits RNA synthesis 2684, 14907, 15631.

Antineoplastic, initiative immunity 4400.

Antineoplastic, inoculated tumor 837 .

Antineoplastic, KB $727,3181,3883,8047,10588,13621,15073,21987$, 22146.

Antineoplastic, Kichita sarcoma 13241.

Antineoplastic, $\mathbf{L}_{\mathbf{1 2 1 0}}$ Lymphocytic leukemia 2660, 2687, 2735, 3218 , $6759,9194,9609,11449,13621,13863,14826,14971,15073,15170$, 15279, 15631, 15631, 16085, 16183, 21987, 22011, 22146, 22232.

Antineoplastic, $\mathbf{L}_{1712}$ leukemia 11024 .

Antineoplastic, $\mathbf{L}_{342}$ pulmonary adenocarcinoma $3754,16763$.

Antineoplastic, $\mathbf{L}_{\mathbf{5 1 8 7}}$ Y lymphoma 1965.

Antineoplastic, L $\mathbf{L}_{615}$ leukemia $\quad 5181,9194,9239$.

Antineoplastic, $\mathbf{L}_{7212}$ leukemia 9239.

Antineoplastic, leukemia $727,899,2662,2675,3637,5136,8312,11019$, 13430, 15403, 15806, 15990, 18882, 20108, 22231.

Antineoplastic, leukemia caused by Rauscher leukemia virus 17850 .

Antineoplastic, leukemia, acute 11179.

Antineoplastic, Lewis lung cancer $\quad 2660,3082,7740,8509,9194,13606$, $13621,15631,15635,18011,18287,19983,20901,21236,21239$, $21251,22368$.

Antineoplastic, Lewis lung carcinoma 22232.

Antineoplastic, liver cancer $\quad 6759,13241,12535,20745,20746,20802$, 11179.

Antineoplastic, LLC 13241.

Antineoplastic, llung cancer $12843,15204$.

Antineoplastic, LNCaP 10588.

Antineoplastic, lung cancer, essential or caused by urethan 2892.
Antineoplastic, lymphatic dyscrasia 12734 .

Antineoplastic, lymphatic sarcoma $11179,11179,13241,21234,21435$, 22146.

Antineoplastic, lymphocyte leukemia 9609, 19542.

Antineoplastic, lymphoma 1621.

Antineoplastic, malignant lymphoma 3404.

Antineoplastic, many types of transplanted tumor 17717.

Antineoplastic, maybe useful for the remission of brain tumors 2102 , 22718.

Antineoplastic, MCF7 1313, 11864, 13514, 21320, 21321.

Antineoplastic, melanoma $\quad 8424,16731,16732,17028,18759,22700$.

Antineoplastic, MGc803 adenocarcnoma of stomach 3754 .

Antineoplastic, MQc803 gastric adenocarcinoma 16763, 20236.

Antineoplastic, MS301 breast cancer 1002.

Antineoplastic, MS310 breast cancer 19762.

Antineoplastic, mus pulmonary adenoma caused by nitroso compound 2892.

Antineoplastic, mus tumor due to SV40 virus 3693.

Antineoplastic, nasopharyngeal carcinoma 8524.

Antineoplastic, NK/CY lymphatic carcinoma 21234.

Antineoplastic, no cytotoxic action for normal cells $16731,16732$.

Antineoplastic, no description on tumor types $136,144,145,404,576$, $660,909,1128,1275,1467,1789,1866,1971,2026,2073,2119,2300$, 2318, 2938, 2946, 3053, 3076, 3094, 3197, 3198, 3308, 3467, 3506, $3529,3551,3861,3909,3911,4053,4106,4128,4140,4238,4239$, 4295, 4300, 4317, 4319, 4321, 4322, 4324, 4352, 4457, 4547, 4657, $5093,5187,5236,5543,6262,6290,6490,6737,6741,6772,7030$, $7031,7236,7327,7550,7552,7554,7555,7559,7579,7580,7584$, 7585, 7586, 7593, 7594, 7595, 7739, 7768, 7996, 8403, 8429, 8430, $8846,9015,9329,9571,9572,9740,9882,9928,9929,10881,11295$, $11525,11903,11992,11993,12011,12145,12146,12465,12503$, $12732,13072,13100,13801,14356,14821,14822,14828,14830$, $14835,14836,14839,14923,15417,15419,15570,15634,15637$, $15741,16019,16085,16205,16278,16279,16290,16352,16451$, $16623,16675,16795,16796,16797,17009,17039,17243,17278$, $17411,17413,17417,17558,17859,17862,17952,17998,18012$, 18050, 18086, 18297, 18411, 18623, 18679, 18843, 19057, 19194, 19201, 19308, 19313, 19316, 19455, 19472, 19479, 19480, 19484, 19487, 19498, 19499, 19629, 19697, 19715, 19811, 20160, 20234, 20387, 20419, 20500, 20664, 20730, 21243, 21397, 21486, 21589 , 21889, 22072, 22098, 22151, 22152, 22153, 22268, 22405, 22407, 22413, 22414, 22475, 22497, 22551, 22581, 22718, 22951, 22987, 23033.

Antineoplastic, nude mouse bearing hmn gastric cancer xenografts 22232.

Antineoplastic, Oberling-Guerin transplanting myeloma 8841 . Antineoplastic, 10 of 60 tested flavones show antineoplastic activity, isovitexin was one of the strongest three compounds 11773.

Antineoplastic, P1534 leukemia 12733.

Antineoplastic, $\mathbf{P}_{\mathbf{3 8 8}} \quad 27,90,777,1029,1372,1745,2109,2660,2661$, 2666, 2667, 2676, 2684, 3181, 3402, 3481, 4289, 4739, 5074, 5181, $5537,6750,6751,6759,7553,7581,7740,8509,8967,9194,9288$, 10093, 10148, 10915, 11020, 11058, 11449, 11450, 11748, 11807, 
$11848,11850,12501,12734,12746,13618,13619,13620,13621$, 13622, 13864, 13864, 14826, 14897, 14971, 15071, 15231, 15233, 15631, 15631, 15776, 16601, 16733, 17194, 17216, 17217, 17337, 17341, 17342, 17557, 17591, 18011, 18091, 18298, 18523, 18899 , 19387, 19900, 20576, 20612, 20815, 20963, 21487, 21662, 22011, 22146, 22232, 22596, 22733, 22865, 22868, 22869, 22870, 22871, 22929, 22962, 22984.

Antineoplastic, $\mathbf{P}_{388}$ D1, inhibits growth of cells 22984.

Antineoplastic, p53-deficient hmn head and neck squamous cell carcinoma SQ-20B 3498.

Antineoplastic, papillary epithelioma 12766.

Antineoplastic, PC-1 hepatocellular carcinoma 21234.

Antineoplastic, prevents new vessel formation 8424 .

Antineoplastic, produces cytotoxic activity under exposure of light with special wavelength 18272 .

Antineoplastic, pulmonary adenoma $8095,14126$.

Antineoplastic, QGY7703 liver cancer 22422.

Antineoplastic, radioresistant and chemoresistant 3498 .

Antineoplastic, Raji cells 537, 1210, 1463, 1464, 3779, 3788, 4874, 4978, 5063, 5098, 5134, 5686, 6498, 6889, 7203, 7611, 7618, 7904, 7909, 7913, 8906, 8908, 8912, 8980, 8981, 9463, 9465, 9643, 10070, 10710, $11100,11103,11104,11105,11106,11451,11970,12012,12220$, $12619,13002,14092,15422,15737,16257,16260,17664,17706$, $17722,17723,17727,17728,19497,19540,19542,19784,21499$, 22108, 22194, 22195, 22777, 22781.

Antineoplastic, RNA tumor virus 20717.

Antineoplastic, RS188N(rad+) mutant yeast Saccharomyces cerevisiae 2293, 5391, 5392, 5393, 5394, 5395, 6501.

Antineoplastic, RS321 mutant yeast Saccharomyces cerevisiae 2293, 5391, 5392, 5393, 5394, 5395, 6501.

Antineoplastic, RS52YK(rad52Y) mutant yeast Saccharomyces cerevisiae 2293, 2293, 3053, 5391, 5391, 5392, 5392, 5393, 5393, 5394, 5394, 5395, 5395, 6501, 6501 .

Antineoplastic, $\mathbf{S}_{\mathbf{1 8 0}}$ sarcoma $\quad 10,140,195,759,962,1626,1627,2380$, $3181,3188,3404,3412,3964,3965,4299,4320,4323,6741,6759$, $8128,8263,9194,9239,9320,11747,12850,13241,13606,13621$, $14205,14907,14971,15641,15642,16050,17754,18011,18103$, 19036, 19524, 19819, 20009, 20044, 20069, 20079, 20900, 20901, 21206, 21251, 22700, 22704, 22733.

Antineoplastic, $\mathbf{S}_{\mathbf{1 8 0}}$ vaccinal 11428 .

Antineoplastic, S37 mouse liver cancer 22011.

Antineoplastic, S37 sarcoma 4399, 4400, 13241.

Antineoplastic, S45 sarcoma $12535,18834$.

Antineoplastic, sarcoma $1841,15279$.

Antineoplastic, screened as potential antitumor promoters 1210,8906 , $8908,8912,9463,9465,11100,11103,11104,11105,11106,12012$, $15422,19497$.

Antineoplastic, shows antitumor activity without any cytotoxicity to Raji cells $778,779,780$.

Antineoplastic, skin cancer $409,3209,4455,4872,6762,7167,10067$, 12843, 14090, 16935, 18939, 19889, 21483.

Antineoplastic, skin cancer caused by DMBA 6643 .

Antineoplastic, skin cancer, induced by chemical carcinogen 3095 .
Antineoplastic, SMMC-7721 3754, 16763, 20236.

Antineoplastic, SN36 leukemia 2380 .

Antineoplastic, squamosal carcinoma in skin 14923.

Antineoplastic, stomach tumor 15204.

Antineoplastic, SW620 hmn colorectal cancer $\quad 6162,21709$.

Antineoplastic, SWA16 2331.

Antineoplastic, thyroid carcinoma 7399, 7416.

Antineoplastic, transplant tumor 20077.

Antineoplastic, transplant tumors of six animals 11091 .

Antineoplastic, treatment of breast cancer 20811.

Antineoplastic, treatment of cancer 18940.

Antineoplastic, treatment of cancer of esophagus 16183.

Antineoplastic, treatment of cancer of pancreas $16183,16935$.

Antineoplastic, treatment of cervical cancer 4400.

Antineoplastic, treatment of chronic myelocytic leukemia 15631.

Antineoplastic, treatment of colorectal cancer 9609.

Antineoplastic, treatment of gastric cancer $\quad 8430,21251$.

Antineoplastic, treatment of granulocytic leukemia 11024.

Antineoplastic, treatment of leukemia 967.

Antineoplastic, treatment of liver cancer 16183.

Antineoplastic, treatment of lung cancer 20811.

Antineoplastic, treatment of nasopharyngeal carcinoma 20811.

Antineoplastic, treatment of nonlymphatic leukemia 9609.

Antineoplastic, treatment of ovarian cancer 20811.

Antineoplastic, treatment of skin cancer 5093.

Antineoplastic, tumor caused by TPA $6643,8914,17876,17886$.

Antineoplastic, tumor xenograft $\quad 6162,11179,21709$.

Antineoplastic, U14 cervical carcinoma $1993,4400,11179,11179$, $11179,11179$.

Antineoplastic, U4 mouse cervical carcinoma 4399.

Antineoplastic, unacceptable toxicity profile for clinical use 15631 .

Antineoplastic, V-79 cancer 3181.

Antineoplastic, $\mathbf{W}_{\mathbf{2 5 6}}$ rat Walker sarcoma 910, 2331, 2334, 5168, 6303, $6750,6751,7551,7553,7566,7837,7994,9194,9239,9320,9320$, $11024,12501,12535,13098,14837,14896,14897,15635,16666$, 19983, 20062, 20063, 20064, 20065, 20140, 20809, 20810, 20963, 21236, 21239, 21251, 22011, 22368, 22411.

Antineoplastic, WM cancer 1745 .

Antineoplastic, X5563 phlogocyte myeloma 570 .

Antineoplastic, YAS ascites tumor 6741.

Antineoplastic, yeast bioassay for DNA-modifying agents 2293, 3053, 5391, 5392, 5393, 5394, 5395, 6501.

Antineoplastic, Yoshida sarcoma 16593.

Antinociception $8423,8424,8427,8429$.

Antiosteoporosis 2480 .

Antioxidant inactive summary index $27,113,258,571,572,573,574$, $575,577,580,617,618,959,960,961,1107,1113,1162,1363,1476$, $1513,1935,2073,2107,2136,2311,2491,2539,2805,3004,3175$, $3359,3389,3475,3615,3619,3775,3872,3873,3983,4195,4199$, 4200, 4201, 4202, 4243, 4406, 4604, 4611, 4612, 4613, 4940, 5044, $5096,5276,5652,5864,5865,6060,6365,6622,6626,6628,6629$, $6630,6631,6776,6778,6779,6780,6781,6782,6865,7305,7306$, $7307,7405,7457,7478,7701,7702,7768,7773,7825,7826,7883$, 
7925, 7949, 7974, 8029, 8136, 8210, 8211, 8219, 8221, 8278, 8278, $8423,8426,8427,8504,8546,8547,8869,8871,9243,9347,9423$, 9502, 9551, 9626, 9811, 10127, 10220, 10221, 10222, 10231, 10362, 10376, 10442, 10578, 10751, 10786, 10803, 10883, 10884, 10885, 10889, 10929, 10930, 10931, 10932, 10933, 11324, 11434, 11462, $11527,11658,11669,11683,12053,12070,12082,12178,12420$, $12819,12820,12821,12822,12824,13088,13107,13250,13253$, 13492, 13493, 13572, 13624, 13637, 14723, 15342, 15525, 16159, $16160,16169,16180,16182,16245,16252,16629,16630,16631$, 16632, 16633, 16994, 17012, 17048, 17247, 17251, 17287, 17416, $17511,17517,17518,17548,17559,17588,18086,18317,18368$, $18411,18674,18755,18925,19182,19211,19542,19562,19572$, 19619, 19777, 20389, 20423, 20424, 20425, 20426, 20427, 20569, 20711, 20840, 21357, 21415, 21711, 21763, 21768, 21857, 21996, 22025, 22182, 22252, 22332, 22336, 22609, 22667, 22673, 22775.

Antioxidant inactive, assay on AAPH-induced hemolysis of RBC $12819,12820,12824$.

Antioxidant inactive, Cytochrome-C reduction $1935,11658,11669$, $12053,15525$.

Antioxidant inactive, DCFH method, HL-60cells $1476,4940,5652$, 5864, 5865, 9423, 12070, 21711, 21857.

Antioxidant inactive, DPPH scavenger inactive 27, 258, 577, 580, 617, $618,618,959,1107,1113,1363,1513,1935,2073,2107,2136,2311$, $2491,2805,3175,3359,3389,3475,3615,3615,3619,3619,3775$, 3872, 3873, 3983, 4195, 4199, 4200, 4201, 4202, 4406, 4611, 4612, 4613, 5044, 5096, 5276, 6060, 6365, 6622, 6626, 6628, 6629, 6630, $6631,6776,6776,6778,6779,6865,7457,7825,7826,7925,7949$, 7974, 8210, 8211, 8219, 8221, 8221, 8546, 8547, 9243, 9551, 9626, 9811, 10127, 10231, 10362, 10376, 10578, 10803, 10883, 10885, 10933, 11324, 11462, 11527, 11658, 11669, 11683, 12053, 12082, 13088, 13107, 13492, 13493, 13493, 13572, 13624, 14723, 15525, $16169,16180,16182,16245,16994,17012,17247,17247,17251$, 17416, 17517, 17548, 17559, 17588, 18086, 18411, 18674, 18755, $18925,19182,19542,19562,19619,20389,20423,20424,20425$, 20426, 20427, 20569, 20711, 20840, 21357, 21763, 21768, 21996, 22025, 22182, 22252, 22332, 22336, 22609, 22673, 22775.

Antioxidant inactive, DPPH scavenger inactive, TLC 51, 113, 2539, $3004,4243,4604,7305,7306,7307,7701,7702,7883,8136,8278$, 8278, 11434, 13107, 13637, 15342, 17048, 18317, 19777, 22667.

Antioxidant inactive, ferric thiocyanate method 10751, 19211.

Antioxidant inactive, FMLP-induced and OZ-induced oxidative burst 6780, 6781, 6782, 8029, 10220, 10221, 10222, 10889, 10931, 16629, $16630,16631,16632$.

Antioxidant inactive, $\mathbf{H}_{2} \mathbf{O}_{2}$ /horseradish peroxidase assay 10885 . Antioxidant inactive, hydroxyl radical scavenging assay 1162,7773 , 9347, 10442, 17287.

Antioxidant inactive, inhibits peroxidation of linolenic acid 258 .

Antioxidant inactive, inhibits superoxide anion generation, fMLP/CB method 12178 .

Antioxidant inactive, iron chelating assay $2805,6365,7457,7478$, 18925.

Antioxidant inactive, lipid peroxide inhibitory experiment 8869,8871 , $17518,17559,21415$.
Antioxidant inactive, lipid peroxide inhibitory experiment, rat hepatic homogenate, caused by $\mathrm{FeSO}_{4} \quad 8426,8427$.

Antioxidant inactive, lipid peroxide inhibitory experiment, rat hepatic homogenate, caused by $\mathrm{H}_{2} \mathrm{O}_{2}+\mathrm{FeSO}_{4} 8426$.

Antioxidant inactive, lipid peroxide inhibitory experiment, rat hepatic homogenate, caused by $\mathrm{H}_{2} \mathrm{O}_{2} \quad 8426,8423$.

Antioxidant inactive, lipid peroxide inhibitory experiment, rat liver microsomes 10786, 19542.

Antioxidant inactive, no description on experimental method 571,572 , $573,574,575$.

Antioxidant inactive, PMN cellular chemiluminescence assay 6780 , 6781, 6782, 8029, 10220, 10221, 10222, 10889, 10931, 16629, 16630, $16631,16632$.

Antioxidant inactive, PMN cellular chemiluminescence assay, reduces oxidative burst FMLP-induced 10929, 10932, 10930, 10933, 10885, 10884, 16633.

Antioxidant inactive, rbt, peroxidization of erythrocytic membrane 18368.

Antioxidant inactive, SOD-like activity $617,618,8504,9502$.

Antioxidant inactive, superoxide anion radical scavenging assay 1162 , 7773, 9347, 10442, 10885, 17287, 17511, 19572.

Antioxidant inactive, superoxide anion radical scavenging assay, superoxide dismutase method 7405, 16159, 16160, 22332, 7768.

Antioxidant inactive, superoxide radical scavenging assay 959,960 , $961,2805,6365,7457,7478,9551,12082,12420,13250,13253$, 17548, 17559, 18411, 18925.

Antioxidant inactive, TBARS assay 258 .

Antioxidant summary index $51,113,309,361,398,508,577,580,679$, $680,759,778,779,780,845,856,911,912,959,960,961,962,1074$, $1091,1174,1175,1466,1476,1492,1497,1513,1562,1564,1650$, $1651,1652,1663,1845,1928,1935,1937,1938,1939,1940,1941$, 1942, 1943, 1971, 2073, 2107, 2134, 2135, 2136, 2311, 2312, 2380, $2490,2539,2564,2685,2805,2859,2887,2909,2912,3004,3029$, $3064,3065,3066,3067,3068,3069,3141,3162,3222,3289,3291$, $3301,3303,3308,3370,3389,3475,3490,3493,3517,3544,3551$, $3674,3680,3681,3745,3754,3756,3775,3829,3831,3832,3850$, $3870,3871,3980,3983,3984,4195,4196,4199,4200,4201,4202$, 4225, 4243, 4298, 4385, 4386, 4387, 4398, 4405, 4406, 4407, 4408, 4409, 4410, 4411, 4412, 4413, 4604, 4611, 4612, 4613, 4630, 4631, 4830, 4920, 4930, 4940, 5008, 5227, 5229, 5276, 5279, 5353, 5414, 5484, 5521, 5588, 5725, 5726, 5729, 5730, 5731, 5763, 5791, 5926, 5928, 5929, 5930, 5932, 6002, 6003, 6004, 6074, 6081, 6086, 6105, $6108,6148,6200,6302,6344,6365,6623,6624,6625,6626,6627$, $6700,6701,6718,6767,6819,6853,6854,6860,6861,6863,6865$, 6921, 6923, 6942, 7280, 7305, 7306, 7307, 7405, 7405, 7432, 7457, 7518, 7557, 7591, 7701, 7702, 7746, 7768, 7788, 7825, 7826, 7883, $7924,7925,7926,7974,7991,8051,8095,8136,8210,8211,8218$, $8219,8221,8235,8246,8278,8330,8423,8426,8427,8501,8504$, $8505,8506,8545,8546,8547,8833,8844,8845,8869,8870,8871$, $8872,8873,8914,9003,9053,9054,9055,9056,9057,9060,9061$, 9062, 9063, 9064, 9065, 9066, 9189, 9190, 9240, 9241, 9242, 9243, 9257, 9258, 9275, 9276, 9347, 9376, 9383, 9386, 9423, 9502, 9550, 9551, 9605, 9626, 9631, 9706, 9723, 9757, 9811, 9818, 9857, 9869, 
10038, 10077, 10185, 10231, 10272, 10276, 10314, 10351, 10362, 10376, 10377, 10434, 10442, 10542, 10694, 10695, 10751, 10883, 10884, 10885, 10887, 10893, 10929, 10930, 10932, 10933, 11152, 11154, 11158, 11165, 11171, 11172, 11195, 11266, 11324, 11327, 11434, 11462, 11477, 11514, 11564, 11642, 11648, 11654, 11658, $11669,11672,11683,11694,11703,11704,11732,11735,12018$, $12020,12020,12032,12033,12052,12053,12060,12062,12070$, $12082,12178,12258,12391,12392,12420,12510,12682,12683$, $12684,12685,12686,12687,12688,12689,12690,12691,12695$, 12696, 12697, 12698, 12701, 12702, 12766, 12771, 12817, 12818, 12821, 12822, 12823, 12925, 13054, 13088, 13107, 13212, 13249, 13250, 13253, 13291, 13292, 13296, 13331, 13361, 13370, 13438, 13479, 13481, 13492, 13514, 13572, 13581, 13637, 13872, 13975, 14210, 14261, 14489, 14556, 14711, 14969, 14988, 14997, 15170, $15202,15204,15278,15279,15342,15448,15525,15719,15766$, $15805,15809,16050,16159,16160,16170,16171,16172,16174$, $16175,16179,16180,16182,16246,16252,16253,16258,16399$, $16531,16532,16540,16545,16633,16680,16681,16725,16728$, 16763, 16765, 16836, 16983, 16994, 17012, 17048, 17053, 17173, $17229,17278,17283,17285,17344,17352,17352,17416,17460$, 17486, 17489, 17491, 17507, 17508, 17510, 17511, 17517, 17518, $17519,17548,17553,17554,17869,17876,17876,17884,17884$, 17886, 17895, 18086, 18169, 18306, 18307, 18310, 18316, 18317, 18323, 18330, 18335, 18336, 18361, 18365, 18368, 18376, 18378, 18400, 18401, 18402, 18403, 18411, 18414, 18584, 18643, 18682, 18925, 19056, 19087, 19201, 19203, 19206, 19277, 19299, 19419, 19452, 19531, 19532, 19542, 19562, 19572, 19573, 19587, 19618 , 19619, 19777, 19781, 19819, 19846, 19883, 19885, 19903, 19923, 20121, 20122, 20150, 20310, 20324, 20389, 20424, 20425, 20426, 20451, 20452, 20453, 20454, 20501, 20556, 20566, 20696, 20806, 20840, 20900, 20901, 20910, 21082, 21083, 21105, 21119, 21125 , 21130, 21157, 21320, 21321, 21357, 21360, 21369, 21392, 21396, 21397, 21415, 21417, 21453, 21477, 21478, 21479, 21480, 21500, $21634,21691,21711,21737,21745,21749,21763,21768,21771$, 21808, 21955, 22180, 22181, 22183, 22184, 22185, 22186, 22187 , 22188, 22239, 22306, 22332, 22336, 22416, 22417, 22418, 22505 , $22524,22565,22566,22581,22594,22595,22667,22718,22759$, 22760, 22775.

Antioxidant, ABTS $^{\bullet+}$ radical quenching activity 16531.

Antioxidant, against oxidative toxicity induced by glutamate, PC12 cells 19587.

Antioxidant, amelioration of freeradical-Induced oxidative stress of endothelial cells 17460 .

Antioxidant, antihemolytic $1928,8818,13055,15439,16071,16080$, 16763.

Antioxidant, antihemolytic, AAPH-induced hemolysis of RBC 580, 1492, 1497, 12817, 12818, 12821, 12822, 12823, 16252, 16253.

Antioxidant, antihemolytic, free-radical induced lysis of rat RBC 2887, 3308, 6853, 17876.

Antioxidant, antihemolytic, free-radical induced lysis of RBC 13054. Antioxidant, antihemolytic, $\mathbf{H}_{2} \mathbf{O}_{2}$-induced hemolysis of RBC 8501 , 12766.

Antioxidant, antihemolytic, rat plasma 2887.
Antioxidant, attenuates $\mathrm{Fe}^{2+}$-induced oxidation of cell membrane 17460.

Antioxidant, chemiluminescence method 3551, 2909, 18317, 2887, 19087.

Antioxidant, Cytochrome-C reduction $\quad 11642,18335,18336,18365$, 19087, 11654, 12032.

Antioxidant, DCFH method, HL-60cells $1845,4920,6942,7746,10077$, 21105, 21771.

Antioxidant, DPPH scavenger $508,580,679,680,856,911,912,960$, $961,1091,1091,1466,1476,1562,1564,1650,1651,1652,1845$, $1845,1845,1845,1845,1845,1845,1845,1935,2135,2312,2380$, $2490,2685,2887,2887,2909,3029,3162,3222,3301,3303,3308$, $3308,3370,3475,3517,3544,3551,3551,3551,3674,3850,3870$, 3871, 3980, 4196, 4200, 4202, 4405, 4407, 4408, 4409, 4410, 4411, 4412, 4413, 4930, 5008, 5227, 5229, 5279, 5484, 5521, 5763, 5763, $5791,5926,6002,6003,6004,6074,6081,6108,6623,6624,6625$, $6627,6700,6701,6718,6767,6819,6853,6853,6853,6853,6854$, $6860,6861,6863,6921,6923,7405,7432,7445,7478,7518,7557$, $7768,7768,7991,8051,8095,8218,8218,8219,8235,8246,8278$, $8504,8505,8506,8545,8845,9003,9240,9241,9242,9257,9258$, $9275,9276,9502,9550,9818,9857,10185,10272,10314,10377$, 10434, 10694, 10695, 10887, 11152, 11154, 11158, 11165, 11171, $11172,11477,11514,11564,11642,11642,11642,11642,11654$, 11704, 11732, 12018, 12018, 12020, 12020, 12032, 12033, 12052, 12060, 12062, 12420, 12510, 13088, 13249, 13250, 13253, 13291, 13296, 13361, 13479, 13479, 13481, 13492, 13872, 13975, 14489 , 14556, 14711, 14969, 14988, 15170, 15202, 15448, 15719, 15805, $15809,16159,16160,16174,16179,16246,16258,16399,16680$, $16681,16725,16728,16765,17053,17173,17283,17285,17352$, $17518,17519,17553,17554,17869,17876,17884,17884,17886$, 18169, 18306, 18307, 18310, 18316, 18317, 18317, 18317, 18317, 18317, 18330, 18335, 18336, 18365, 18376, 18378, 18400, 18401, 18402, 18403, 18414, 18584, 18643, 19056, 19087, 19087, 19087, 19087, 19087, 19277, 19299, 19452, 19618, 19618, 19883, 19885, 20150, 20310, 20501, 20556, 20696, 20806, 20910, 21082, 21083, $21125,21396,21397,21415,21415,21417,21453,21500,21634$, $21691,21808,22180,22181,22183,22184,22185,22186,22187$, $22188,22306,22332,22332,22416,22417,22418,22524,22565$, 22566, 22581, 22594, 22595, 22759, 22760.

Antioxidant, DPPH scavenger, TLC $\quad 1845,8095,8330,16545,18317$, 21119, 21415, 21737, 21745, 21749.

Antioxidant, ferric thiocyanate method $5279,10185,22565,22566$, 22594, 22595, 6148, 4830, 22306, 7591, 3745, 580, 11195.

Antioxidant, free radical scavenger, no description on type of free radical $1174,1175,4631,7924,7926,8833,8844,11266,12766$, 13212, 17344, 19203, 19846, 21360.

Antioxidant, $\mathrm{H}_{2} \mathbf{O}_{2}$ scavenger $\quad$ 9257, 9258, 9275, 9276, 20901.

Antioxidant, $\mathrm{H}_{2} \mathbf{O}_{2}$ /horseradish peroxidase assay 10933 .

Antioxidant, hydroxyl radical scavenger $1845,4298,4385,4386,4387$, $6081,6200,6923,7280,9605,9631,13331,16170,16171,16172$, 17510, 17511, 19572, 19573, 20566, 20900, 20901, 21130.

Antioxidant, inhibits formation of active oxygen 1928.

Antioxidant, inhibits formation of superoxide in macrophage 22239 . 
Antioxidant, inhibits formation of MDA, mitochondria of mus heart 17876.

Antioxidant, inhibits $\mathrm{H}_{2} \mathrm{O}_{2}$-induced oxidative stress and DNA oxidative damage, cultured MDCK cells 3303.

Antioxidant, inhibits lipid peroxidation, ADP/Fe ${ }^{2+}$-induced 3141,5588 , $17229,21415$.

Antioxidant, inhibits lipid peroxidation, adriamycin- induced 1937, 1938, 1939, 1940, 1941, 1942, 1943, 20121, 20122.

Antioxidant, inhibits lipid peroxidation, brain tissue, caused by insufficient oxygen and sugar 7788 .

Antioxidant, inhibits lipid peroxidation, caused by $\mathbf{C C l}_{\mathbf{4}} 1971$.

Antioxidant, inhibits lipid peroxidation, cephalopin $\quad 8278,13088$, $18685,19450$.

Antioxidant, inhibits lipid peroxidation, cytoblast and microsome in hepatic cells 5414 .

Antioxidant, inhibits lipid peroxidation, effects on plasma oxidation after incubation with $\mathrm{Fe}^{2+} / \mathrm{H}_{2} \mathrm{O}_{2} 13581$.

Antioxidant, inhibits lipid peroxidation, enzymatic lipid peroxidation 21392.

Antioxidant, inhibits lipid peroxidation, hepatic cell mitochondria in cats 16765 .

Antioxidant, inhibits lipid peroxidation, induced by vitamin Cnicotinamide ADP and $\mathrm{Fe}^{2+}$-cysteine in microsome of murine cerebral, hepatic and renal cells $4631,12420,19201$.

Antioxidant, inhibits lipid peroxidation, linoleic acid emulsion 9257, 9258, 9275, 9276.

Antioxidant, inhibits lipid peroxidation, macrosome of liver cells in rat 962, 3303.

Antioxidant, inhibits lipid peroxidation, microsome $3754,16763$.

Antioxidant, inhibits lipid peroxidation, microsome of hepatocyte in rat 3493, 3831, 3832, 3829, 10038, 13438, 16836.

Antioxidant, inhibits lipid peroxidation, microsome of hepatocyte in rat, ADP+NADPH-induced $17507,17508,17510,21157$.

Antioxidant, inhibits lipid peroxidation, microsome of hepatocyte in rat, $\mathrm{FeSO}_{4}+$ cysteine-induced 1497.

Antioxidant, inhibits lipid peroxidation, microsome of hepatocyte in rat, $\mathbf{C C l}_{4}$-induced 1497.

Antioxidant, inhibits lipid peroxidation, microsome, induced by $\mathrm{Fe}^{2+} / \mathrm{VC}$, $\mathrm{CCl}_{4} / \mathrm{NADPH}$, or $\mathrm{Fe}^{3+} / \mathrm{NADPH} 20324$.

Antioxidant, inhibits lipid peroxidation, microsome, induced by ferrous-cysteine $9063,9064,9065,9066,21415$.

Antioxidant, inhibits lipid peroxidation, microsome, NADPH-dependent lipid peroxidation and autoxidation of linoleic acid 1562,1564 , $6863,8051$.

Antioxidant, inhibits lipid peroxidation, mitochondria 3756.

Antioxidant, inhibits lipid peroxidation, mitochondria of hepatocyte in rat $759,962,3490,16836$.

Antioxidant, inhibits lipid peroxidation, mitochondria of hepatocyte in rat, ADP/NADPH-induced $8914,18643$.

Antioxidant, inhibits lipid peroxidation, mitochondria of hepatocyte in rat, $\mathrm{Fe}^{2+} / \mathrm{VC}$-induced 8914 .

Antioxidant, inhibits lipid peroxidation, mitochondria of hepatocyte in rat, $\mathrm{FeSO}_{4}$-induced 11703 .
Antioxidant, inhibits lipid peroxidation, mus liver 15204.

Antioxidant, inhibits lipid peroxidation, no description on target tissue and method $8870,11648,13250,13253,13514,16983,17278$, $17283,17285,17352,17517,17519,18643,21320,21321,21415$, 22505 .

Antioxidant, inhibits lipid peroxidation, rat brain homogenate 6086, $6105,9189,9190,12682,12683,12684,12685,12686,12687,12688$, 12689, 12690, 12691, 12695, 12696, 12697, 12698, 12701, 12702.

Antioxidant, inhibits lipid peroxidation, rat cytoblast in liver cells 11735 .

Antioxidant, inhibits lipid peroxidation, rat heart and liver mitochondria 9631 .

Antioxidant, inhibits lipid peroxidation, rat hepatic homogenate, caused by $\mathrm{H}_{2} \mathrm{O}_{2} 8427$.

Antioxidant, inhibits lipid peroxidation, rat hepatocyte membrane, effects on $\mathrm{Fe}^{+3} /$ ascorbate-induced lipid peroxidation 13581 .

Antioxidant, inhibits malondialdehyde (MDA) 845, 9053, 9054, 9055, 9056, 9057, 9060, 9061, 9062, 9063, 9064, 9065, 9066, 9869, 12391, 12392, 21415, 21415, 21415.

Antioxidant, inhibits NOR1 (nitric oxide donor) action $778,779,780$.

Antioxidant, inhibits $t$-BuOOH induced luminescence, mus hepatic homogenate 11327 .

Antioxidant, iron chelating assay $9257,9258,9275,9276,12420,14556$.

Antioxidant, iron/ascorbate system for antioxidative potency (AOP) determination 9376, 9383,9386.

Antioxidant, LDL peroxidation inhibitor $\quad 13212,13292,17884,19419$.

Antioxidant, LDL peroxidation inhibitor, $\mathrm{Cu}^{+2}$-induced $\quad 6002,6003$, 6004,11694, 12420, 12925, 17460, 19201, 19206, 21477, 21478, 21479, 21480 .

Antioxidant, $\mathrm{LDL}$ peroxidation inhibitor, $\mathrm{Cu}^{+2}$-induced and AAPH-induced $580,7925,1663,2134,2912$.

Antioxidant, linoleic acid solution $5725,5726,5729,5730,5731$.

Antioxidant, lipid peroxidation assay, enzyme-dependent 3068,3064 , 14210, 3065, 3069, 12420, 3066, 3067.

Antioxidant, lipid peroxidation assay, enzyme-independent 3064 , 3065, 3066, 3067, 3068, 3069, 12420, 14210, 21392.

Antioxidant, minimizes loss of cell viability in endothelial cells 17460 .

Antioxidant, no description on experimental method $309,361,398$, 580, 1074, 2564, 2859, 3368, 3680, 3681, 4225, 4398, 6302, 7521, $10351,11195,11266,12771,13370,14261,14997,15278,15279$, 16532, 16540, 17486, 17489, 17491, 17886, 18643, 19781, 19819, 19903, 20451, 20452, 20453, 20454, 21415, 22718.

Antioxidant, oxygen free radical scavenger $\quad 1938,19201,20901$.

Antioxidant, PEP inhibitor 5791, 9561, 11648, 18682.

Antioxidant, peroxide formed from polymorph 10893 .

Antioxidant, peroxidization of rbt erythrocytic membrane $3303,18323$. Antioxidant, peroxidized anion scavenger 17895.

Antioxidant, PMN cellular chemiluminescence assay 10883,10929 , 10932, 10930, 10933, 10885, 10884, 16633, 18317.

Antioxidant, prevents oxidation of unsaturated fatty acid components to stabilize cell membranes 21415 .

Antioxidant, rat brain, effect on conjugated diene formation of LDL or MDA level 3984, 6344, 19923, 21955. 
Antioxidant, reduces oxidative burst FMLP-induced 18317, 10883. Antioxidant, reduces risk of atherosclerosis 17460 .

Antioxidant, reversed $\mathrm{H}_{2} \mathrm{O}_{2} / \mathrm{FeSO}_{4}$-induced impairment of endothelium in rat aorta 17460 .

Antioxidant, SOD-like activity $1845,3301,3303,5521,7518,8505$, 8506, 16765, 19056, 19277, 20310, 20389, 20910.

Antioxidant, superoxide anion radical scavenger 1845, 1939, 1941, $3289,3291,3308,3754,4385,4386,4387,6081,6200,6923,7280$, $8872,8873,9605,11672,12258,12420,16170,16171,16172,17278$, $17278,17283,17285,17352,17510,18643,18643,19531,19532$, 19573, 20556, 20566, 21369, 21415.

Antioxidant, superoxide anion radical scavenger, cytochrome $\mathrm{C}$ assay 10933.

Antioxidant, superoxide anion radical scavenger, hmn neutrophils, stimulated by fMLP/Cbor PMA 5353, 16050.

Antioxidant, superoxide anion radical scavenger, inhibits superoxide anion generation, fMLP/CB method 5930, 5932, 10542, 9757, 5928, 15766, 9723, 10276, 5929, 9706.

Antioxidant, superoxide anion radical scavenger, protects myocardial ischemia-reperfusion injury in rat myocardium mitochondrial membrane 4630 .

Antioxidant, superoxide anion radical scavenger, superoxide dismutase method 5763, 7432, 12020, 12052, 12062, 13479, 14711, 18317, 18323, 18361, 18376, 19087.

Antioxidant, superoxide radical scavenger 4930, 7445, 9257, 9258, 9275, 9276, 9550, 14489, 14556, 16174, 16175.

Antioxidant, superoxide scavenger $5791,8869,8870,8871,22505$.

Antioxidant, thiobarbituric acid reactive substance (TBARS) assay, inhibits peroxidation of linolenic acid 10377, 10376, 21808.

Antioxidant, up-regulats 50 genes and down-regulats many others 10887.

Antiparasite 16266

Anti-phage 12221.

Antipneumocystis agent 2376 .

Anti-pregnancy 23021.

Antiprotozoal 2303, 3094, 3968, 8304, 8128, 11541, 11851, 14906, $18939,19819,22282$.

Antipyretic 304, 554, 651, 1283, 1520, 1901, 1964, 2106, 2303, 2676, $3220,3588,3689,3693,3809,3907,4421,4614,4876,5108,5436$, $6618,6767,7481,7521,7523,8010,8292,9455,11882,12510,15337$, $16451,16525,16532,16966,19184,19473,20002,20066,20076$, 20444, 20732, 21234, 21508, 21887, 22270, 22384, 22385, 22602, 22938, 23003.

Antipyretic mechanism involves inhibition of PG synthesis in brain 7521.

Antirachitic vitamin 22558 .

Anti-rejection symptom in skin grafting 1191.

Antiretroviral and cytotoxic 9186, 9612, 13241, 22040.

Antirheumatic 8307, 10886, 13391, 16085, 19184, 19427, 22270.

Anti-rheumatoid arthritis 5613, 20481.

Anti-sepsis 14063, 14064.

Anti-sepsis inactive 1184, 1185, 14062, 14065.

Antiseptic 2284, 3237, 3318, 3760, 3761, 3762, 8312, 9703, 12436,
12849, 17089, 17602, 17931, 19187, 21360, 21366.

Anti-seronine 8395.

Anti-sickling of cells $9818,22332$.

Antispasmodic $56,871,904,957,1191,1287,1288,1476,1502,1520$, $1526,1614,1619,1742,1935,1960,2001,2298,2300,2550,2657$, 2803, 3231, 3243, 3394, 3502, 3633, 3695, 3741, 3745, 3794, 3889, $3915,4421,4604,5036,5161,5677,6507,6776,6921,7230,7385$, 7523, 7703, 7802, 7811, 9184, 9419, 9541, 10872, 11002, 11091, $11504,11591,11834,12184,12221,12438,12535,12747,12908$, 13137, 13571, 14531, 14820, 14923, 14971, 15266, 15268, 15279, 15449, 16080, 16525, 16770, 16966, 17013, 17036, 17174, 18165, $19085,19195,19354,19355,19356,19388,19473,19542,19706$, 19712, 19933, 19934, 20140, 20148, 20324, 21060, 21077, 21263, $21362,21511,22195,22269,22282,22314,22350,22530,22552$, 22553, 22581, 22718, 22768, 22774, 22781, 22882.

Antispirochetic 4116, 5152, 6776.

Anti-stimulation 21350 .

Anti-stress 86, 3751 .

Anti-sweetener 2271, 11904, 11905, 11906, 11911, 20037, 23016, 23017, 23018.

Antithrombotic $10,920,921,2102,2266,5440,6700,7978,10684$, $12420,15635,17568,17978,18378,20009,20121,20686,21157$, 22497.

Antitoxin 2106, 3760, 3774.

Anti-Trichomonas vaginalis $\quad 576,2061,2615,7323,8047,9809,9810$, 11203, 14728, 18627, 19414, 22282.

Antitrypanosomal 9235, 12501, 15881, 16555, 21397, 14728, 18094.

Antitrypanosomal inactive (Trypanosoma brucei) 4866.

Antitrypanosomal inactive (Trypanosoma brucei rhodesiense) 10159. Antitrypanosomal inactive (Trypanosoma cruzi) 4866, 10159, 12845, 12846, 13729, 15716, 19983.

Antitrypanosomal (Castellanella gambiense) 7323.

Antitrypanosomal (Trypanosoma brucei rhodesiense) 580, 589, 815, $816,1141,1145,1146,1147,1241,1937,1939,2004,2346,2683$, 2920, 2924, 3166, 3306, 3551, 3609, 3674, 4478, 4982, 5675, 6776, $7925,8325,9750,11148,11195,11978,12916,13297,14222,16067$, 16068, 17478, 18221, 19568, 19960, 22409, 22410, 22551.

Antitrypanosomal (Trypanosoma brucei) 506, 15716.

Antitrypanosomal (Trypanosoma brucei brucei) 585, 3293, 5417, 10310, 12232, 12487, 19234, 19235, 19542, 20369.

Antitrypanosomal (Trypanosoma cruzi) 184, 227, 376, 506, 580, 815, $816,1141,1145,1146,1147,1241,1460,1842,1937,1939,2004$, $2346,2683,2920,2924,3166,3258,3306,3551,3609,3674,3761$, $3762,4088,4204,4205,4478,4891,5477,5675,6348,6759,6776$, $7925,8004,8325,8979,9031,9032,9033,9072,9727,9728,9729$, 9732, 9750, 10296, 10297, 11148, 11195, 11978, 12497, 12842, 12916, $13719,13720,13721,13722,13905,14222,14384,16050,16067$, $16068,16604,16711,17006,17478,17851,18187,18188,18221$, 19568, 19636, 19753, 19960, 21390, 22270, 22551, 22579, 22580, 22963.

Antitrypanosomal (Trypanosoma equiperdum) 7323.

Antitussive $1618,1866,2274,2312,2557,3237,3588,3615,3885,5178$, $6776,7996,8347,9315,9564,10887,11507,12446,13137,15146$, 
$15263,15460,15469,16623,17283,17456,19955,19983,20307$, 22086, 22087.

Antitussive inactive $6839,10188$.

Antitussive, dispels phlegm 10819, 9021, 9617, 10137, 11966, 15614, $17377,17384,17567,17813,19715,663,834,1094,1097,1935,2678$, $3400,4550,6482,6772,7944,9564,12843,13264,14531,15170$, $11524,13574,13610,13611,17376,18095,18317,18816,20254$, $17505,19542,7736,5763,20987,17568$.

Antiulcerative $10,140,195,777,1287,1476,2071,2887,3308,4873$, 4889, 4893, 4941, 5665, 6990, 8297, 8524, 8841, 8965, 11504, 11808, $12908,13388,13492,13678,15705,15882,16085,16505,16525$, $16693,16694,17220,17546,17549,17550,17551,17552,17886$, 19332, 19388, 19422, 19423, 19424, 20082, 20205, 20444, 20711, $20714,22270$.

Anti-venom 2887, 5763.

Antiviral inactive summary index 2994, 2995, 4225, 10681, 11350, 14261, 14847, 14848, 14849, 21509, 21509, 21510, 21510.

Antiviral inactive, HSV-1 virus $\quad 6230,6298,10681,13250,13251,14321$, $16500,21509,21510$.

Antiviral inactive, HSV-2 virus 10681, 21509, 21510.

Antiviral inactive, MDCK cells, Flu-A virus 12828, 13053, 13055, $15439,16071,16080$

Antiviral inactive, Vero cells, HSV-1 virus $12828,13053,13055,15439$, $16071,16080$.

Antiviral inactive, Vero cells, HSV-2 333 virus 4225, 11350, 14261, $14847,14848,14849$

Antiviral inactive, Vero cells, HSV-2 virus 2994, 2995.

Antiviral summary index 26, 736, 740, 784, 1372, 2044, 2811, 2865, 2866, 2867, 2868, 2869, 2887, 2892, 3094, 3197, 3198, 3300, 3308, 3498, 3502, 3551, 3588, 3622, 3623, 3875, 3909, 4048, 4354, 5093, 5161, 5613, 6272, 6402, 6551, 6772, 7323, 7518, 7833, 8095, 8246, $8307,8423,8424,8427,8458,8846,8967,9194,9456,9458,10059$, $10589,10681,10886,11086,11129,11524,11569,11703,11903$, $11978,11980,12070,12420,12733,12828,12849$, 13053, 13055, $13137,13239,13241,13481,13863,14728,14965,14971,15286$, $15439,15462,15849,15881,16003,16071,16080,16268,16498$, $16499,16500,16511,16555,16599,16601,16777,16793,17216$, $17217,17337,17341,17342,17574,17578,17579,17580,17581$, $17582,17592,17593,17896,17952,18028,18050,18251,18272$, $18317,18376,18411,18770,18771,18824,18868,18916,19087$, 19142, 19148, 19211, 19777, 19819, 19900, 20253, 20307, 20369, 20434, 20578, 20670, 21059, 21089, 21328, 21508, 21698, 21862, 22215, 22216, 22721, 22863.

Antiviral, Asia $\boldsymbol{\alpha}$-Influenza virus 16555 .

Antiviral, Bunya virus 16601.

Antiviral, chicken ES4 virus 12849.

Antiviral, chickenpox virus 8846 .

Antiviral, Coxsackie virus 13241.

Antiviral, Cxsackie-B virus 14728.

Antiviral, cytomegalovirus CMV(hmn) $18770,18771$.

Antiviral, cytomegalovirus CMV(mus) $11978,18770,18771$.

Antiviral, EBV virus $\quad 8246,16599$.

Antiviral, EMC virus $=$ Casrdiovirus 18050 .
Antiviral, encephalitis B virus 9194.

Antiviral, encephalitis B virus in mouse 5161 .

Antiviral, encephalitis B virus in mouse, RNA virus 16601.

Antiviral, endomyocarditis virus 13239.

Antiviral, epidemic type-1 poliomyelitis virus-1 $\quad 6402,18376$.

Antiviral, Gesak virus $17216,17217$.

Antiviral, Gesak-B $\mathbf{4}_{4}$ virus $\quad$ 6402, 18376.

Antiviral, H. suis virus 13137.

Antiviral, Hep2 cells infected by Para-3 virus 12828, 13053, 13055, 15439, 16071, 16080.

Antiviral, Hep2cells infected by respiratory syncytial virus RSV 16080.

Antiviral, hepatitis B virus HBV in blood products 3588 .

Antiviral, herpes zoster virus 8846 .

Antiviral, herpesvirus $13863,14971$.

Antiviral, herpesvirus A 13241.

Antiviral, herpetic stomatitis RNA virus 16268.

Antiviral, hmn coronavirus strain 229E (HCoV-229E) 11086, 18251.

Antiviral, hmn T-lymphocytes-phil virus III HTLV-III 3588.

Antiviral, hmn T-lymphocytes-phil virus III HTLV-III 3588.

Antiviral, HSV virus $7518,11524,12420,13481,17216,17217,17593$, 19148.

Antiviral, HSV/CV-1 virus $17337,17341,17342$.

Antiviral, HSV-1 virus $163,422,736,740,1372,3875,5303,5613,6551$, $10059,10589,11129,11569,13098,15462,16499,16511,16793$, 17337, 17592, 17896, 18868, 20253, 21059, 21508, 21724, 22721.

Antiviral, HSV-1, DNA virus 16268.

Antiviral, HSV-2 virus $736,740,6551,10059,16500,10589,15462$, $16499,17574,17900,18272,21059$

Antiviral, influenza virus $3909,8095,9194,11703,15881,19777$.

Antiviral, influenza virus A $661,8967,11903,18411$.

Antiviral, influenza virus $\mathbf{A}_{2} 19142$.

Antiviral, influenza virus B 11903.

Antiviral, influenza virus sialoma inhibitor 19211.

Antiviral, inhibits biosynthesis of RNA 4048, 13239.

Antiviral, inhibits infection from RDV virus in young chicken embryo 16003.

Antiviral, inhibits infection of herpes simplex virus 2 in mus genital 17216, 17217.

Antiviral, inhibits replication of HSV-1 2811, 8423, 8427, 8424, 19537. Antiviral, Japanese encephalitis virus $8967,18050$.

Antiviral, jockos, inactivator to hepatitis B virus HBV 3588.

Antiviral, Mayaro virus 17952.

Antiviral, measles virus $1372,16793,17337,17592,19148$.

Antiviral, Mengo virus 14728 .

Antiviral, meningitis virus 18050 .

Antiviral, Newcastle disease virus 9194.

Antiviral, no explanation of virus species $26,784,2044,2887,2892$, 3094, 3197, 3198, 3300, 3308, 3498, 3502, 3551, 3622, 4354, 5093, $6772,7323,7833,8307,8423,8424,8427,9456,9458,12070,14965$, 15849, 16498, 16777, 18317, 18824, 18916, 19819, 19900, 20307, 20434, 20670, 21328, 21862, 22863.

Antiviral, non-A non-B hepatitis virus NANB 3588 . 
Antiviral, Para-3 virus $\quad 661,2865,2866,2867,2868,2869,20369,22215$, 22216.

Antiviral, poliomyelitis 1 virus 18868.

Antiviral, poliomyelitis virus 11569, 12733, 13241, 17952, 20578.

Antiviral, potato virus 14971.

Antiviral, poxvirus 14728.

Antiviral, PR $_{8}$ virus 8967.

Antiviral, pseudolyssa virus 14728.

Antiviral, respiratory syncytial virus RSV 661, 22215, 22216.

Antiviral, reverse transcriptive virus $10886,18028$.

Antiviral, rhinovirus II 3623.

Antiviral, Sendai virus 19777.

Antiviral, Sindbis virus 11978 .

Antiviral, tobacco masaic virus 8458 .

Antiviral, tobacco mosaic virus TMV 6272.

Antiviral, vaccinia virus 9194, 12733.

Antiviral, Vero cells infected by HSV-1 virus $17578,17579,17580$,

17581, 17582, 21089, 21698.

Antiviral, vesicular stomatitis virus $15286,18411,19087$.

Antiviral, vesicular stomatitis virus VSV 17216, 17217.

Antiviral, vesicular stomatitis virus VSV $11569,11978,11980$.

Antiviral, vesicular stomatitis virus VSV/BHK 17337, 17341, 17342.

Antiviral, yellow fever virus 16601.

Anxiolytic 2016.

Anxiolytic and antidepressant 8524 .

Aphrodisiac 14057.

APN inhibitor inactive $6757,8095,11642,10887,18411,16011,18358$, $18371,21392$.

Apoptosis enhancer 10287.

apoptosis inducer $1307,8526,8527,8528,8530,8531,8532,8533,8535$,

8536, 8541, 8542, 8543, 8544, 16050, 20811.

apoptosis inducer inactive 6689 .

Arachidonic acid 5-lipoxidase selective inhibitor 3743 .

Arachidonic acid oxidase inhibitor 14995.

Aromatase inhibitor 51, 846, 2635, 2647, 5086, 5746, 7485, 8262, 10506, 11490, 14964, 15279, 15934, 20326, 21116, 21151.

Aromatase inhibitor inactive $1764,2172,2629,2630,2648,2650,2651$, 4108, 5894, 6001, 6088, 6102, 6103, 8141, 10445, 12681, 13571, 14956, 14961, 14962, 18643, 21086, 21117, 21128.

Aromatic bitter 3248 .

Aromatic $\boldsymbol{L}$-amino-acid decarboxylase inhibitor 17278 .

Astringent $\quad 660,909,917,1654,7882,8095,17813,21955$.

Attractant for many plant-eating insects 9522 .

Attracts adult male dayfly (Chrysopa septempunctata) 15434.

BACE1 inhibitor 9561 .

BChE inhibitor $2233,2815,4516,4532,4542,4996,5828,6159,7082$, 7128, 7177, 7210, 7381, 7381, 7835, 7928, 8009, 9200, 9201, 11002, $11004,11291,12834,12835,14449,15322,15323,16988,21374$, 22700 .

BChE inhibitor inactive $5739,7213$.

Benzedrine antagonist 16451.

Benzodiazepine receptor agonist 14865 .

Bidirectional action to blood pressure (first increases and then lowers blood pressure, while heart rate slows) $8423,8424,8426,8428$, 8429,8430 .

Bidirectional action to blood pressure (first increases and then lowers blood pressure, while heart rate slows) 20100.

Bidirectional action to blood vessel (dilates at low concentration and contracts at high concentration) 4036 .

Bidirectional action to blood vessel (when dose less than $40 \mathrm{nmol}$ relaxes blood vessel, when dose over $40 \mathrm{nmol}$ contracts blood vessel) 15317.

Bidirectional action to CNS system (central sedation at low dose, central stimulation at moderate dose and paralytic death at high dose) 8297.

Bidirectional action to CNS system (central stimulation at high dose and sedation at low dose) 651.

Bidirectional action to CNS system (first stimulation and then inhibition) 3220, 11202, 19388.

Bidirectional action to CNS system (stimulation in low dose, inhibition in high dose) 20069.

Bidirectional action to drowsiness (excitation in low dose and inhibition in high dose) 15129.

Bidirectional action to heart (first stimulates and then inhibits) 13237.

Bidirectional action to heart (inhibits first and then stimulates) 11851.

Bidirectional action to nerve system (motor depressant in low dose and causes convulsion in high dose) 9234.

Bidirectional action to neuromuscular transmission (in high dose, first enhances and then inhibits) 21593.

Bidirectional action to potassium channel in myocardium membrane 19201.

Bidirectional action to sympathetic nerve (excites sympathetic fibers in low dose and inhibits them in high dose) 22397.

Bile secretion promoter $2887,1742,20734,2538,4135,15279,17983$, 22346, 22397, 5161, 4249

Binding activity to benzodiazepine receptor $1476,3745,8090,9564$, $14085,20577$.

Bioactive in connection with plant photosynthesis and path of respiration 17515 .

Biosynthesis of DNA, promoter 9456.

Biosynthesis of DNA, protein and lipid, promoter $\quad 8423,8424,8426$, 8430.

Biosynthesis of rRNA and mRNA in diabetic rat, promoter 8424 .

Biosynthesis precursor of carthamin 17771 .

Biosynthetic precursor of some drugs 18304.

Bitter principle $\quad 486,1017,1779,3206,4318,15286,15404,15555$, $15652,15655,16080,16551,16650,17215,17705,18299,18425$, 19184, 20913, 21599.

Bitter-sweet taste 8834 .

Blocks nerve 19431.

Blocks permeation of sodium through membranes of nerval fibrocyte 21211.

Blocks self-discipline nerve 20496.

Blocks sympathetic ganglia 20650 .

Bone marrow cell proliferation promotor 3308, 6853, 6864, 6921, 6923.

Bone marrow inhibitor $3404,3911$. 
Bone resorption inhibitor 10896, 14698, 15985, 16408, 16409, 20228.

Bone resorption inhibitor 6456, 6457, 6458, 19778, 20556.

Bradykinin antagonist 20009.

$\mathrm{Ca}^{2+}$-ATPase inhibitor 18668.

Caffeine antagonist 16451 .

Calcitonin gene-related peptide (CGRP) stimulator 7665 .

Calcium antagonist $2041,3935,5135,7512,7721,8423,8424,8426$, $9553,11680,12916,13836,14184,15321,15645,15704,16711$, 20121, 20122, 21077, 21490, 21493, 22144.

Calcium channel blocker 2606, 16263.

Calcium channel receptor inhibitor 2209.

Calmodulin-dependent cAMP phosphodiesterase inhibitor 10564.

CaM interactor 4918, 7838, 14639.

CaM interactor inactive $9480,15936,21038$.

cAMP phosphodiesterase inhibitor 2102, 2613, 2614, 3160, 3602, 3745, 4898, 4918, 4996, 5250, 6254, 7838, 7924, 7926, 8423, 8424, 8426, $8427,8833,8914,8968,9331,9546,13594,9681,9682,12333,12344$, $12355,12358,13145,14639,15038,15455,15456,16867,16989$, 16997, 17020, 17305, 17306, 17409, 18219, 18368, 19072, 19259, 19588, 21493, 22720.

3',5'-cAMP-phosphodiesterase inhibitor 18317.

Cancer cell P-Glycoprotein inhibitor $7596,7597,7598,7599,7600$, 7601, 7602, 7603, 7604, 7605, 7606, 7607.

Cancer cell stimulator, MCF7 and T47D cell proliferation 4418 .

Capillary, enhances capillary permeability $4319,13137$.

Capillary, improves barrier of microcirculation $\quad 4630,10870,18180$.

Capillary, inhibits increase of blood capillary permeability 664,3588 , 4097, 5161, 7495, 8297, 15843, 16050, 19087, 19111, 19922, 20066, 20569.

Capillary, improves osmosis of capillary 11141.

Capillary, prevents brittle rupture of blood capillary 4439,16900 , 17024.

Capillary, reduces blood capillary brittleness $\quad 12255,18317,19087$.

Capillary, reduces effusion of Evan's blue from blood capillary 5152 .

Carboxylation activity during metabolism of protein and carbohydrate 2395.

Carcinogen $1713,1835,3847,4456,11189,12535,12543,16657,17127$, $18098,19121,19731,20527$.

Carcinogen assistant 17187, 18640, 22658.

Carcinogen promotor 13476 .

Carcinogen, causes hepatic cancer 14923.

Cardiac glycoside $9335,9330,16943,17002,19090,20405$.

Cardiotonic $377,1844,2187,2190,2191,2716,2728,3048,3277,3278$, $3729,3732,3942,4005,4029,4036,4192,4544,4547,5104,5523$, $5526,5533,6530,7296,7303,7320,7342,7343,8461,8968,10915$, $10981,11524,16031,16050,16084,18216,18637,18785,19958$, 20056, 20140, 20403, 20671, 20730, 20732, 21325, 21326, 21435 , $22301,22629$.

Cardiovascular activity (against damage in cardiac cells) 19201. Cardiovascular activity (against heart failure) 4108 .

Cardiovascular activity (antiarrhythmic) 618, 783, 930, 983, 1287, $1667,2300,2372,4543,4544,4685,5136,7703,7854,8423,8424$, $8426,8427,9553,9631,9738,11344,11851,12510,12798,13089$,
$13836,15321,15526,15658,15875,15972,16080,16451,16555$, 18180, 18180, 18376, 19955, 20094, 20100, 20732, 21077, 21206, 21263, 21325, 21326, 22404, 22466, 22490.

Cardiovascular activity (anti-arteriosclerosis) 2791, 3909, 11834, 13212, 19738, 18165, 19070, 22270.

Cardiovascular activity (anti-ischemia myocardial) 2300, 2564, 3761, 3762, 4544, 4630, 4645, 9631, 18180, 22172, 22581.

Cardiovascular activity (anti-myocardial infarction) 4630, 9631, 22172 .

Cardiovascular activity (arterial pressure in both types of rats fell substantially, while heart rate of only anaesthetized rats also decreased) 5569.

Cardiovascular activity (assists in treatment of heart failure, coronary heart disease, hypertension and arrhythmia) 22172.

Cardiovascular activity (Cardioprotective) 2606, 3633, 8914.

Cardiovascular activity (causes heart beat to slow and amplitude to increasing in low dose; causes heart paralysis and hypotension in high dose) 17505.

Cardiovascular activity (contracts blood vessels, increases blood pressure and stimulates heart) 15184 .

Cardiovascular activity (electrocardiogram changed) 11020 .

Cardiovascular activity (enhances amplitude of contraction and reduces frequency of heart beat) 15288.

Cardiovascular activity (enhances arterial tension and myocardial contractility) 9419 .

Cardiovascular activity (enhances cardiac motility) 5067.

Cardiovascular activity (enhances collateral circulation and oxygen consumption upon lack of blood in myocardium) 4604.

Cardiovascular activity (enhances contractility of main artery) 20968.

Cardiovascular activity (enhances myocardial contractility and increases blood flow) 6559 .

Cardiovascular activity (enhances myocardial contractility and raises heart rate) 19846.

Cardiovascular activity (enhances myocardial contractility and reduces scope of contraction) 6691 .

Cardiovascular activity (enhances myocardial contractility with peripheral anapetia) 1866 .

Cardiovascular activity (enhances myocardial contractility) 4108, 4353, 7343, 16555, 16756, 16997, 19639, 20094, 21511, 22237.

Cardiovascular activity (improves acute myocardial ischemia) 3633 .

Cardiovascular activity (improves myocardium metabolism and promotes restoration of myocardial function) 4645 .

Cardiovascular activity (improves peripheral circulation and markedly increases amount of urine) 6559 .

Cardiovascular activity (increases atrial and ventricular thresholds, also reduces atrial conduction) 19762.

Cardiovascular activity (increases cerebral blood flow) 19540, 19587.

Cardiovascular activity (increases contractility of atrium); 16451.

Cardiovascular activity (increases coronary flow and cerebral blood flow) 11344 .

Cardiovascular activity (increases coronary flow and slows heart rate) 4609, 20730.

Cardiovascular activity (increases coronary flow) 3633, 4480, 4543, 
4544, 4604, 4631, 4645, 4889, 5414, 6752, 6753, 7303, 8914, 13137, 14796, 22552.

Cardiovascular activity (increases coronary flow, reduces consumption of oxygen in myocardium, increases cerebral blood flow, lowers blood pressure, and slows heart rate) 5750 .

Cardiovascular activity (induces myocardial rhythm) 14796.

Cardiovascular activity (inhibits cardiac contraction, causes a prolongation of the latency time and decrease of contraction force) 4911, 4931, 4932, 4933, 5215, 5869, 7091, 7092, 10071, 10683, 19091, 20162.

Cardiovascular activity (inhibits cardiac fibrillation) 21263.

Cardiovascular activity (inhibits cardiac muscles) $\quad$ 15321, 21239.

Cardiovascular activity (inhibits content of free radicals in myocardial cells) 8424 .

Cardiovascular activity (inhibits contraction of auricular smooth muscle) 2284.

Cardiovascular activity (inhibits contraction of blood vessel and cardiac muscle) 17762 .

Cardiovascular activity (inhibits damage of myocardial cells caused by free radicals) 11266 .

Cardiovascular activity (inhibits frog heart in vitro) $\quad 8289,8297$.

Cardiovascular activity (inhibits heart and relaxes artery) 17568 .

Cardiovascular activity (inhibits heart rate) 18299.

Cardiovascular activity (inhibits heart) $\quad 643,3002,3907,5435,6552$, $16674,22153$.

Cardiovascular activity (inhibits malondialdehyde (MDA) formed by abnormity of calcium concentration in cardiac muscle cells) 8914 .

Cardiovascular activity (inhibits myocardial automatic rhythmicity and contractile power) 13836 .

Cardiovascular activity (inhibits myocardial contractility) 117,304 , 2300 .

Cardiovascular activity (inhibits myocardial contraction and calcium antagonist) 16280 .

Cardiovascular activity (inhibits sino-atrial rate and contraction of atrium papillary musclein dog) 16759.

Cardiovascular activity (makes heart stop in period of relaxation) 3218 .

Cardiovascular activity (Protects cardiac muscle from lack of blood) 20688.

Cardiovascular activity (protects in vitro heart from damage during ischemic re-perfusion) 17762 .

Cardiovascular activity (raises heart rate) 5067 .

Cardiovascular activity (reduces area of myocardial infarction) 16555 , 20094.

Cardiovascular activity (reduces consumption of oxygen in myocardium) 4645, 5763, 14796, 18826.

Cardiovascular activity (reduces myocardial contractility) 15396, 18826.

Cardiovascular activity (reduces resistance of cerebral blood vessels) 19587.

Cardiovascular activity (reduces scleratheroma incidence) 4245 .

Cardiovascular activity (slows heart rate and enhances amount of urine) 1866.
Cardiovascular activity (slows heart rate and enhances myocardial contractility) 9233 .

Cardiovascular activity (slows heart rate) $\quad 554,983,1658,2300,4912$, 7343, 7980, 9232, 11680, 15708, 18165, 18826, 19473, 20061, 20254, 21511.

Cardiovascular activity (slows heart rate, increases output blood pressure, blood flow in aorta and myocardial contractility) 9235 .

Cardiovascular activity (stimulates atrium) 19198.

Cardiovascular activity (stimulates heart) 4108, 5445, 20069, 20077, 20100.

Cardiovascular activity (stimulates heart) 3588 .

Carminative $1186,3237$.

Catechol- $\boldsymbol{O}$-methyltransferase inhibitor 8278 .

Cathartic 136, 6262, 13801, 17411, 17413, 17417.

Causes abortion 3498 .

Causes acute glomerulus necrosis 1713 .

Causes allergic reaction (hmn skin) 19779.

Causes arrhythmia $\quad 304,1738,10875$.

Causes arrhythmia and bradycardia 8353 .

Causes asthma and nasitis 17561 .

Causes asthma, convulsion and breath faintness 22360 .

causes bleeding 4140,5440, 15633.

Causes blistering in cuticle 18545 .

Causes chronic ammonia toxicosis 5351 .

Causes conjunctivitis 18426 .

Causes contact dermatitis $1789,2890,3695,4087,4128,4584,8997$, 16598, 16674, 18257, 18426, 21207, 21974, 22142, 22274.

Causes convulsion and paralysis 12562 .

Causes dermitis 21402 .

Causes epilepsy 4051.

Causes glucopenia and "vomiting sickness" 10898.

Causes goitre 17903.

Causes hmn mammary diseases 21507 .

Causes hypoglycemia $14358,21662$.

Causes involuntary repetitive movement 3692 .

Causes liver injury 4140 .

Causes mental illness $\quad 6418,13795,14583$.

Causes methaemoglobin disease in cattles 14885 .

Causes miosis $1654,1658,7298,17253,17360$.

Causes paralysis, paroxysmal spasm, convulsion and death 7706 .

Causes paralysis $4377,19223$.

Causes paroxysm convulsion and breath inhibition 5035 .

causes Phalaris blind stagger (sheep) 8971.

Causes plant to be infected by Agrobacterium tumefaciens 116.

Causes progressive paralysis of CNS 9233.

Causes pulmonary heart disease 19902.

Causes selenium poisoning 19691 .

Causes slight shrinkage of liver 4329.

Causes St. George disease (ox) 19902.

Causes strong convulsion 3002 .

Causes tachycardia 1134.

Causes tetanic coma 2734 .

Cell cycle inhibitor 2021. 
Cell division arrester 6939, 12130, 12131, 16365.

Cell growth inhibitor 8095,8276 .

Cell growth regulator 3275, 22408 .

Cell viability $8788,12982,12983$.

Central muscle relaxant 2791 .

Choleretic 117, 834, 1742, 1935, 2102, 2106, 2224, 2303, 2538, 2887, $3118,3551,3695,4135,4245,4249,4398,4564,5161,8095,8273$, $9498,9749,11428,13481,15184,15279,16439,16439,16623,16623$, 16770, 17983, 19473, 19473, 19540, 20732, 20732, 20734, 22269 , 22269, 22346, 22397.

Cholineoid action 15126 .

Cholinergic 1658, 5201, 5219.

Cholinesterase activator 1036 .

Cholinesterase inhibitor $6853,7379,7380,8083,9686,9687,10601$, 13236, 13255, 14971, 16555, 17253, 19639, 21434, 22349.

Cholinomimetic 1658.

Chymotrypsin inhibitor 13563, 13564, 13565.

Chymotrypsin inhibitor inactive 9561 .

CNS active $3147,3860,4118,4120,7521,8251,9232,11636,11679$, 12908, 16761, 16762, 18114, 20577, 22145.

CNS depressant $569,669,706,983,1039,1191,1654,1824,2016,2114$, 3502 , 3637, 3907, 3915, 5248, 6656, 6708, 7517, 7523, 8524, 9553, 9631, 10886, 11477, 11869, 13481, 13630, 13795, 14865, 15070, 15126, 16009, 17007, 17065, 17572, 19041, 19057, 19473, 19903, 20002, 20076, 20307, 20444, 21263, 21292, 21372, 21877, 21887, 21991, 22151, 22314, 22490, 22502, 22818.

Coagulant 3615 .

Cockroache killer 2298, 15490, 17332, 17334, 17335, 17336, 18270.

Coenzyme 22554, 22556.

Coenzyme of amino transferase, decarboxylase, racemase and some other amino acids 18261.

Coenzyme of energy transfer in phosphate bonds 626 .

Collagenase inhibitor 7441 .

Component of artificial calculus bovis 2374 .

Component of coconut flavorant 15680 .

component of coenzyme I and II 15526.

Components of plant epicyte 2596,3040 .

Contraceptive 1710, 3457, 6495, 12925, 18841.

Controls dormancy of common yam 2163, 2166.

Controls growth of Hepatica lunularia 13084.

Convulsant $\quad 6552,12562,16746$.

corrosion 7882 .

Curare component in cucurbit 2959 .

Curare main component in cucurbit 4377 .

Curariform action $\quad 2042,6699,7325,7328,7330,12571,13373,13374$, 13716.

Curtials the time of blood clotting and bleeding 3551 .

Cuticle cornification promoter 1853 .

Cycloadenyl mononucleotide phosphodiesterase inhibitor 1621, 9331.

Cyclonucleotide phosphodiesterase inhibitor 710, 4376, 9543, 15278, 18864.

Cyclooxygenase inhibitor $\quad 3188,7819,8081,8395$.

CYP2D6 inhibitor inactive $7105,7106,8395,8397,9664,9668,12024$,
12025, 12026, 12035, 12036, 12082, 19846, 19983, 19987, 21600, 22984, 22985.

CYP3A4 inhibitor $1834,3851,4307,4308,4309,4310,5543,5561$, 5572, 7105, 7106, 7114, 7400, 8395, 8396, 8397, 9664, 9668, 9949, 12024, 12025, 12026, 12035, 12036, 12037, 12044, 12070, 12099, 13261, 13391, 13640, 13898, 14264, 16340, 19846, 19847, 21345, 21392, 21392, 21928, 22984, 22985.

CYP3A4 inhibitor and CYP2D6 inhibitor 1834, 3851, 4307, 4308, 4309, 4310, 5543, 5561, 5572, 7114, 7400, 8396, 9949, 12037, 12044, 12070, 12099, 13391, 13640, 13898, 14264, 16340, 18423, 18423, 19847, 21345, 21928.

CYP3A4 inhibitor inactive 12082, 19983, 19987, 21600.

Cytochrome CyP1A inhibitor 8278, 11152, 11154, 11158, 11165, 11171, 11172.

Cytochrome P450 (CYP3A4) inducer 10883.

Cytochrome P-450inhibitor 17247.

Cytostatic/cytotoxic 5185, 5186 .

Cytotoxic (in vitro) summary index $8,24,51,76,78,84,90,150,159$, $175,225,226,228,240,248,251,265,266,332,349,370,378,463$, $472,492,512,526,530,531,576,577,579,585,589,594,595,597$, $598,607,610,696,777,837,843,846,911,912,913,914,915,919$, 924, 941, 955, 993, 1008, 1010, 1012, 1030, 1136, 1137, 1138, 1177, $1245,1275,1276,1277,1294,1295,1296,1297,1302,1303,1304$, $1305,1306,1308,1310,1311,1312,1313,1319,1320,1321,1322$, $1324,1325,1359,1367,1372,1373,1380,1381,1382,1383,1384$, $1385,1386,1387,1388,1389,1390,1391,1392,1393,1394,1395$, 1396, 1397, 1398, 1399, 1400, 1401, 1402, 1403, 1404, 1405, 1406, $1407,1408,1409,1410,1411,1412,1413,1414,1415,1416,1417$, $1418,1419,1420,1421,1422,1423,1424,1425,1426,1427,1428$, $1429,1430,1431,1432,1433,1434,1435,1436,1437,1438,1439$, $1440,1441,1442,1443,1444,1445,1446,1447,1448,1449,1450$, $1451,1452,1453,1454,1455,1456,1457,1460,1470,1471,1472$, $1492,1508,1552,1570,1571,1575,1576,1578,1581,1583,1617$, $1621,1625,1650,1651,1652,1679,1691,1748,1764,1765,1768$, 1789, 1792, 1845, 1856, 1860, 1863, 1864, 1885, 1913, 1914, 1924, 1925, 1926, 1961, 1971, 1992, 2005, 2006, 2034, 2047, 2073, 2082, $2102,2106,2109,2119,2130,2172,2219,2227,2244,2257,2259$, $2260,2261,2262,2303,2304,2312,2334,2338,2339,2340,2468$, $2492,2493,2495,2498,2499,2500,2527,2528,2529,2590,2601$, $2604,2619,2625,2630,2635,2647,2648,2652,2653,2654,2655$, $2656,2660,2661,2664,2676,2677,2684,2694,2716,2722,2735$, $2736,2769,2810,2811,2824,2826,2830,2856,2857,2946,2948$, 2950, 2952, 2956, 2957, 2958, 2964, 2985, 2990, 2991, 2998, 3012, $3014,3015,3016,3018,3019,3020,3021,3022,3053,3060,3063$, 3071, 3072, 3076, 3087, 3088, 3096, 3097, 3126, 3143, 3181, 3227, $3228,3229,3230,3241,3257,3258,3259,3260,3261,3262,3263$, $3264,3265,3266,3267,3268,3271,3274,3292,3293,3294,3296$, $3300,3303,3308,3324,3325,3368,3388,3402,3405,3406,3412$, 3416, 3429, 3457, 3467, 3476, 3481, 3498, 3499, 3502, 3513, 3514, $3515,3548,3551,3553,3555,3565,3568,3573,3576,3580,3581$, $3582,3583,3600,3602,3626,3667,3668,3708,3710,3724,3729$, $3732,3745,3748,3777,3778,3784,3816,3821,3829,3868,3869$, 3906, 3911, 3920, 3921, 3936, 3940, 3974, 3975, 4046, 4048, 4081, 
4082, 4083, 4084, 4089, 4090, 4091, 4108, 4111, 4112, 4113, 4128, 4135, 4147, 4148, 4151, 4164, 4165, 4166, 4179, 4180, 4190, 4235, 4237, 4268, 4269, 4282, 4284, 4291, 4310, 4317, 4320, 4323, 4324, 4327, 4337, 4338, 4339, 4340, 4341, 4342, 4348, 4349, 4350, 4351, 4353, 4398, 4399, 4414, 4460, 4461, 4471, 4472, 4547, 4565, 4604, 4643, 4680, 4689, 4690, 4701, 4708, 4739, 4740, 4752, 4753, 4754, 4762, 4763, 4775, 4790, 4795, 4820, 4821, 4822, 4825, 4826, 4827, 4828, 4829, 4838, 4880, 4882, 4891, 4893, 4900, 4913, 4930, 4939, 4941, 4986, 5055, 5064, 5074, 5081, 5095, 5136, 5156, 5168, 5175, 5184, 5187, 5196, 5236, 5237, 5238, 5239, 5240, 5274, 5275, 5276, 5284, 5295, 5297, 5314, 5319, 5327, 5343, 5367, 5369, 5375, 5378, 5387, 5401, 5417, 5494, 5522, 5532, 5536, 5537, 5543, 5548, 5551, 5573, 5574, 5580, 5595, 5621, 5622, 5679, 5706, 5746, 5746, 5774, 5775, 5776, 5777, 5778, 5779, 5780, 5781, 5815, 5816, 5817, 5826, 5827, 5832, 5841, 5843, 5851, 5888, 5899, 5900, 5901, 5912, 5913, 5926, 5929, 5941, 5942, 5945, 5973, 6010, 6014, 6027, 6042, 6044, $6045,6049,6055,6064,6075,6103,6107,6125,6128,6129,6130$, $6160,6162,6164,6165,6181,6183,6204,6213,6217,6221,6228$, 6246, 6254, 6261, 6277, 6282, 6284, 6285, 6289, 6309, 6314, 6315, 6322, 6328, 6346, 6367, 6402, 6404, 6405, 6437, 6447, 6459, 6483, $6484,6490,6497,6500,6514,6516,6528,6576,6577,6606,6621$, $6642,6646,6647,6652,6654,6710,6711,6714,6737,6750,6751$, 6756, 6759, 6760, 6761, 6766, 6767, 6776, 6785, 6789, 6790, 6794, 6826, 6827, 6828, 6835, 6844, 6846, 6847, 6848, 6849, 6897, 6906, 6913, 6921, 7016, 7026, 7027, 7030, 7031, 7032, 7033, 7035, 7036, 7041, 7044, 7060, 7062, 7078, 7081, 7083, 7100, 7115, 7125, 7126, 7131, 7132, 7133, 7136, 7139, 7140, 7153, 7182, 7192, 7193, 7204, 7295, 7317, 7323, 7376, 7399, 7436, 7474, 7504, 7515, 7516, 7519, 7535, 7540, 7544, 7545, 7548, 7550, 7551, 7552, 7553, 7554, 7555, 7556, 7558, 7559, 7566, 7568, 7569, 7571, 7579, 7580, 7581, 7583, 7584, 7585, 7586, 7590, 7593, 7594, 7595, 7611, 7613, 7614, 7646, $7650,7655,7665,7670,7674,7675,7677,7681,7682,7683,7684$, 7698, 7704, 7714, 7739, 7740, 7746, 7764, 7768, 7788, 7789, 7815, 7824, 7836, 7911, 7912, 7914, 7951, 8004, 8054, 8065, 8079, 8080, $8095,8121,8128,8148,8149,8159,8160,8161,8167,8174,8186$, $8187,8188,8203,8204,8205,8210,8220,8221,8222,8235,8238$, $8239,8262,8278,8315,8327,8328,8354,8355,8357,8358,8359$, $8360,8361,8363,8364,8365,8379,8436,8452,8483,8489,8508$, $8509,8520,8526,8527,8528,8530,8531,8532,8533,8534,8535$, $8536,8541,8542,8543,8544,8556,8563,8569,8570,8596,8598$, $8599,8653,8654,8655,8656,8661,8662,8669,8670,8671,8687$, $8691,8692,8695,8699,8715,8716,8717,8718,8721,8722,8749$, 8824, 8932, 8933, 8934, 8935, 8936, 8940, 8946, 8947, 8948, 8968, 9011, 9013, 9015, 9035, 9072, 9080, 9089, 9090, 9187, 9194, 9198 , 9226, 9254, 9255, 9256, 9288, 9300, 9304, 9320, 9323, 9330, 9362, 9376, 9381, 9383, 9386, 9388, 9535, 9544, 9546, 9564, 9567, 9570, 9594, 9628, 9655, 9669, 9740, 9747, 9859, 9861, 9869, 9883, 9885, 9902, 9905, 9917, 9918, 9974, 9993, 10047, 10052, 10068, 10077. 10100, 10116, 10126, 10148, 10155, 10206, 10229, 10230, 10235, 10250, 10274, 10277, 10281, 10287, 10297, 10307, 10310, 10317, 10340, 10344, 10396, 10403, 10412, 10430, 10438, 10462, 10468, 10511, 10539, 10558, 10559, 10573, 10588, 10594, 10672, 10673 , 10680, 10692, 10716, 10717, 10719, 10720, 10722, 10725, 10726,
10727, 10741, 10751, 10752, 10769, 10773, 10796, 10815, 10816, 10817, 10827, 10893, 10909, 10915, 11001, 11015, 11023, 11029, 11052, 11056, 11071, 11081, 11179, 11203, 11206, 11230, 11231, 11232, 11248, 11266, 11269, 11278, 11279, 11295, 11316, 11318, $11323,11353,11355,11358,11375,11379,11380,11386,11392$, 11394, 11405, 11423, 11428, 11433, 11490, 11494, 11504, 11541, $11555,11556,11566,11605,11636,11645,11659,11672,11722$, 11723, 11736, 11751, 11783, 11800, 11801, 11802, 11804, 11807, $11842,11850,11854,11855,11856,11858,11860,11861,11862$, 11863, 11864, 11890, 11895, 11949, 11951, 11962, 11977, 11978, 11980, 12106, 12108, 12109, 12110, 12122, 12125, 12138, 12167 , 12171, 12177, 12180, 12188, 12189, 12202, 12206, 12225, 12254 , 12344, 12397, 12398, 12414, 12417, 12423, 12446, 12453, 12454 , 12487, 12501, 12511, 12531, 12532, 12533, 12536, 12537, 12541 , 12587, 12588, 12589, 12595, 12597, 12681, 12746, 12766, 12768 , 12770, 12886, 12887, 12888, 12897, 12916, 12917, 12936, 12944 . 12953, 12954, 12956, 12957, 12958, 12964, 13019, 13020, 13021, 13023, 13038, 13044, 13071, 13072, 13076, 13078, 13083, 13092, 13093, 13098, 13123, 13137, 13153, 13154, 13155, 13157, 13158, 13159, 13160, 13161, 13164, 13165, 13238, 13241, 13294, 13296, 13328, 13403, 13430, 13432, 13464, 13468, 13469, 13470, 13472, 13473, 13484, 13487, 13489, 13490, 13491, 13492, 13493, 13494, $13495,13518,13522,13537,13544,13618,13619,13620,13622$, 13672, 13673, 13685, 13694, 13700, 13701, 13705, 13808, 13845, 13859, 13867, 13869, 13881, 13903, 13943, 14044, 14045, 14074, 14075, 14086, 14121, 14139, 14195, 14227, 14234, 14254, 14304, $14305,14342,14347,14417,14454,14492,14495,14496,14504$, 14572, 14602, 14644, 14645, 14646, 14647, 14698, 14705, 14714, $14719,14775,14813,14821,14822,14828,14830,14835,14836$, 14846, 14896, 14901, 14906, 14931, 14934, 14935, 14936, 14937, 14938, 14939, 14940, 14941, 14942, 14943, 14945, 14961, 14962, $14965,14967,14978,14995,15073,15074,15082,15084,15085$, 15087, 15088, 15089, 15090, 15091, 15092, 15093, 15095, 15097, 15098, 15141, 15169, 15170, 15230, 15231, 15232, 15233, 15234, 15242, 15331, 15335, 15339, 15351, 15352, 15409, 15412, 15417, 15443, 15494, 15495, 15598, 15631, 15634, 15635, 15637, 15645, 15647, 15652, 15714, 15739, 15746, 15750, 15762, 15764, 15776, 15782, 15792, 15796, 15801, 15813, 15857, 15879, 15902, 15903, 15916, 15919, 15920, 15921, 15922, 15923, 15991, 16019, 16020, $16021,16022,16023,16050,16119,16122,16128,16183,16205$, $16216,16221,16222,16229,16230,16231,16232,16261,16262$, $16265,16266,16278,16280,16282,16283,16296,16312,16329$, $16337,16339,16343,16356,16370,16402,16403,16411,16414$, 16427, 16429, 16444, 16495, 16498, 16548, 16549, 16586, 16597 , 16599, 16601, 16604, 16634, 16661, 16662, 16670, 16674, 16675 , 16676, 16677, 16686, 16693, 16694, 16695, 16703, 16733, 16747, 16748, 16749, 16832, 16864, 16891, 16929, 16935, 16966, 16978, 16984, 17008, 17009, 17028, 17037, 17038, 17041, 17042, 17059, 17060, 17061, 17062, 17063, 17064, 17144, 17174, 17200, 17201, 17218, 17239, 17240, 17252, 17265, 17309, 17342, 17343, 17403, 17430, 17431, 17478, 17489, 17517, 17518, 17519, 17528, 17557, 17558, 17564, 17565, 17574, 17588, 17591, 17592, 17594, 17600, 17603, 17613, 17614, 17616, 17619, 17622, 17623, 17643, 17644, 
17645, 17646, 17654, 17665, 17666, 17667, 17668, 17669, 17670, 17671, 17696, 17717, 17722, 17727, 17753, 17763, 17769, 17822, 17830, 17832, 17862, 17872, 17895, 17896, 17928, 17929, 17958, 17973, 17975, 17979, 17980, 17998, 18011, 18033, 18034, 18044, 18054, 18056, 18057, 18091, 18097, 18114, 18170, 18171, 18172, 18188, 18202, 18211, 18214, 18215, 18222, 18224, 18240, 18295, 18296, 18297, 18298, 18444, 18457, 18459, 18461, 18472, 18483, 18484, 18485, 18499, 18515, 18522, 18523, 18545, 18547, 18562, 18567, 18637, 18643, 18644, 18668, 18673, 18679, 18690, 18691, 18692, 18693, 18700, 18701, 18714, 18716, 18733, 18754, 18768, 18769, 18770, 18771, 18772, 18773, 18774, 18775, 18776, 18777 , $18778,18816,18835,18843,18866,18892,18897,18898,18899$, 18931, 18944, 18950, 18951, 18952, 18953, 18994, 19041, 19057, 19061, 19085, 19122, 19128, 19129, 19143, 19146, 19148, 19194, 19200, 19221, 19234, 19235, 19236, 19254, 19257, 19278, 19284, 19296, 19308, 19313, 19315, 19324, 19373, 19376, 19410, 19411, 19412, 19413, 19427, 19458, 19468, 19483, 19485, 19486, 19488, 19511, 19515, 19516, 19517, 19521, 19524, 19525, 19526, 19535, 19536, 19537, 19540, 19542, 19571, 19579, 19618, 19634, 19637, 19638, 19753, 19777, 19790, 19892, 19900, 19904, 19905, 19906, 19907, 19910, 19916, 19929, 19983, 19985, 20002, 20009, 20010, 20051, 20062, 20063, 20068, 20117, 20121, 20122, 20129, 20135, 20137, 20160, 20171, 20221, 20223, 20235, 20240, 20243, 20244, 20248, 20253, 20278, 20283, 20284, 20285, 20326, 20363, 20387, 20389, 20397, 20465, 20481, 20482, 20485, 20495, 20496, 20540, 20542, 20545, 20556, 20575, 20610, 20626, 20629, 20630, 20631, 20632, 20633, 20634, 20635, 20636, 20637, 20638, 20639, 20640, 20641, 20643, 20644, 20664, 20670, 20671, 20679, 20680, 20681, 20700, 20720, 20721, 20722, 20730, 20743, 20771, 20798, 20800, 20803, 20809, 20810, 20811, 20813, 20814, 20833, 20834, 20835, 20836, 20843, 20849, 20850, 20851, 20852, 20853, 20856, 20871, 20872, 20873, 20900, 20901, 20911, 20963, 21080, 21206, 21239, 21240, 21313, 21314, 21315, 21316, 21328, 21337, 21338, 21385, $21387,21390,21410,21435,21440,21447,21448,21483,21490$, 21493 21497, 21498, 21545, 21554, 21559, 21709, 21771, 21797 , 21803, 21830, 21845, 21849, 21887, 21889, 21918, 21924, 21968, 22011, 22013, 22015, 22077, 22103, 22114, 22147, 22148, 22149, 22150, 22208, 22209, 22210, 22211, 22212, 22214, 22232, 22268, 22270, 22272, 22281, 22286, 22290, 22299, 22301, 22405, 22407, $22411,22412,22414,22420,22435,22443,22451,22452,22453$, $22454,22455,22456,22457,22458,22459,22475,22497,22543$, $22548,22549,22551,2258022615,22630,22653,22654,22655$, $22657,22667,22705,22715,22718,22720,22735,22751,22761$, $22768,22775,22777,22778,22781,22784,22792,22793,22794$, 22795, 22797, 22798, 22799, 22802, 22818, 22826, 22828, 22830, $22838,22839,22923,22962,22965,22969,22970,22972,22987$, 22988, 23005, 23013.

Cytotoxic inactive summary index $8,24,26,89,163,174,175,194,215$, $221,223,236,247,248,252,266,330,414,422,465,477,492,509$, $510,511,512,519,579,580,675,815,816,901,984,993,1022,1067$, $1107,1113,1136,1137,1138,1161,1177,1241,1367,1372,1476$, $1505,1506,1507,1509,1532,1580,1581,1583,1584,1585,1586$, $1587,1591,1621,1624,1699,1713,1718,1725,1728,1745,1764$,
$1784,1819,1820,1821,1864,1865,1885,1937,1939,1992,2004$, 2021, 2048, 2091, 2092, 2101, 2102, 2106, 2251, 2254, 2259, 2260, $2286,2309,2331,2346,2384,2415,2473,2591,2592,2593,2618$, 2683, 2716, 2722, 2765, 2766, 2767, 2824, 2826, 2830, 2833, 2856, 2920, 2924, 2945, 3014, 3104, 3105, 3106, 3107, 3108, 3109, 3110, 3111,3112 , 3113, 3125, 3127, 3153, 3159, 3166, 3241, 3266, 3267, $3268,3271,3292,3306,3308,3319,3355,3429,3544,3551,3555$, $3571,3609,3615,3619,3659,3667,3668,3669,3674,3695,3729$, $3732,3778,3829,3831,3908,3932,3940,3941,3980,3982,4046$, 4135, 4169, 4170, 4189, 4281, 4283, 4310, 4416, 4478, 4624, 4648, 4680, 4689, 4690, 4722, 4762, 4763, 4788, 4900, 4913, 4936, 4940, 4957, 4970, 4987, 5042, 5062, 5075, 5150, 5179, 5180, 5194, 5237, $5238,5239,5276,5303,5343,5344,5367,5368,5369,5373,5374$, $5375,5387,5401,5492,5496,5518,5522,5536,5548,5570,5580$, 5607, 5621, 5633, 5648, 5652, 5669, 5675, 5689, 5701, 5703, 5706, $5774,5775,5776,5779,5781,5788,5790,5794,5795,5815,5816$, $5817,5864,5865,5867,5886,5887,5912,5913,5931,5934,5936$, $5944,5975,5976,5982,5983,6017,6042,6066,6067,6090,6114$, $6162,6176,6183,6204,6267,6289,6298,6327,6338,6409,6427$, $6430,6477,6478,6490,6500,6515,6578,6617,6621,6689,6708$, $6741,6760,6761,6776,6778,6779,6785,6791,6792,6942,6954$, 7016, 7025, 7043, 7122, 7125, 7130, 7134, 7135, 7137, 7138, 7190, 7191, 7205, 7366, 7367, 7540, 7571, 7572, 7575, 7582, 7613, 7614, 7657, 7699, 7764, 7777, 7788, 7789, 7897, 7907, 7925, 7951, 7982, $7983,7984,7985,7998,8027,8028,8032,8107,8121,8150,8166$, $8168,8186,8188,8189,8211,8219,8278,8325,8327,8328,8360$, $8361,8363,8364,8365,8439,8528,8569,8576,8577,8578,8579$, $8580,8632,8657,8658,8659,8660,8669,8670,8671,8693,8698$, $8748,8814,8824,8932,8933,8935,8936,8938,8939,8942,8944$, $8948,9035,9081,9082,9199,9406,9423,9452,9474,9475,9488$, 9504, 9507, 9509, 9535, 9546, 9564, 9626, 9627, 9628, 9629, 9698, 9700, 9701, 9750, 9812, 9815, 9857, 9859, 9861, 9905, 9917, 9918, 9944, 9961, 9993, 10020, 10052, 10068, 10089, 10099, 10100, 10126, 10159, 10187, 10206, 10207, 10250, 10279, 10280, 10296, 10306, $10342,10343,10364,10374,10380,10381,10411,10412,10414$, 10446, 10467, 10483, 10485, 10505, 10510, 10512, 10558, 10595, 10672, 10673, 10717, 10718, 10721, 10732, 10747, 10751, 10769, 10815, 10816, 10817, 10871, 10910, 10911, 10912, 11001, 11017, $11079,11129,11148,11191,11192,11195,11201,11234,11262$, $11263,11264,11265,11266,11277,11344,11392,11394,11405$, $11477,11504,11569,11595,11600,11601,11636,11722,11799$, $11843,11844,11845,11846,11847,11853,11857,11859,11890$, $11945,11950,11952,11978,12048,12070,12107,12137,12139$, $12179,12310,12397,12398,12413,12415,12416,12448,12453$, $12481,12489,12512,12536,12541,12587,12588,12589,12590$, 12622, 12639, 12640, 12916, 12917, 12936, 13014, 13015, 13019, 13020, 13023, 13038, 13041, 13058, 13059, 13060, 13067, 13068, 13069, 13070, 13097, 13098, 13108, 13123, 13166, 13167, 13197, 13198, 13199, 13328, 13464, 13467, 13468, 13472, 13484, 13487, 13491, 13537, 13591, 13674, 13693, 13699, 13702, 13703, 13704, $13706,13707,13845,13871,13902,13903,13935,13936,13943$, $13965,14042,14072,14101,14114,14115,14168,14217,14222$, 14226, 14228, 14254, 14300, 14321, 14437, 14438, 14439, 14484, 
$14500,14613,14638,14643,14697,14737,14775,14776,14840$, $14842,14846,14847,14848,14849,14915,14934,14935,14940$, 14942, 14995, 15038, 15040, 15042, 15046, 15054, 15055, 15056, $15057,15082,15118,15134,15152,15242,15249,15279,15351$, 15443, 15483, 15621, 15635, 15719, 15796, 15910, 15931, 15944, 15945, 15946, 15951, 16050, 16067, 16068, 16169, 16177, 16199, 16221, 16222, 16229, 16230, 16231, 16232, 16261, 16296, 16343, $16370,16373,16402,16415,16550,16652,16653,16665,16725$, $16748,16754,16818,16819,16820,16854,16855,16867,16873$, 16906, 16966, 17118, 17196, 17197, 17198, 17199, 17244, 17247, 17574, 17611, 17612, 17615, 17696, 17715, 17727, 17846, 17847, 17852, 17871, 17872, 18088, 18114, 18221, 18222, 18224, 18359 , 18420, 18425, 18499, 18588, 18682, 18697, 18700, 18701, 18916, 18931, 18945, 18946, 18947, 18948, 18949, 18950, 18978, 18998, 19087, 19174, 19220, 19255, 19256, 19257, 19259, 19268, 19349 , 19350, 19522, 19523, 19536, 19540, 19542, 19568, 19571, 19618, 19622, 19652, 19653, 19654, 19655, 19656, 19690, 19795, 19796, 19891, 19960, 19981, 19983, 19985, 19987, 20068, 20070, 20105, 20106, 20117, 20122, 20129, 20237, 20316, 20356, 20362, 20369, 20465, 20493, 20554, 20643, 20697, 20699, 20700, 20701, 20702, 20711, 20712, 20722, 20739, 20765, 20797, 20798, 20803, 20811, 20836, 20840, 20849, 20850, 20851, 20877, 20911, 21027, 21031, $21037,21082,21105,21125,21135,21137,21170,21358,21410$, $21475,21498,21529,21640,21709,21711,21713,21724,21755$, $21758,21798,21801,21804,21805,21806,21807,21845,21856$, $21857,21864,22011,22013,22195,22205,22206,22270,22287$, $22299,22332,22378,22379,22408,22413,22460,22497,22544$, $22545,22546,22547,22551,22630,22667,22736,22748,22774$, 22781, 22792, 22800, 22801, 22803, 22830, 22962, 22988, 23029.

Cytotoxic inactive, 1A9 14934, 14935.

Cytotoxic inactive, 3LL 7998, 11569, 14697.

Cytotoxic inactive, 59 NCI hmn cancer cell lines except HL-60 cell 17727.

Cytotoxic inactive, 9 KB cells 22195 .

Cytotoxic inactive, A2780 cells $1107,1113,3125,3127,4281,7025,7366$, 7367, 7764, 8939, 8942, 8944, 10052, 12107, 13097, 16050, 16415, 19220, 20369, 20711, 20712.

Cytotoxic inactive, $\mathbf{A 3 7 5}$ cells 11799 .

Cytotoxic inactive, A498 cells $1067,3571,4722,4762,9812,9961$, 20722, 20739, 20765, 20803, 22630, 22781.

Cytotoxic inactive, A549 cells $519,984,1067,1367,1699,1713,1718$, $1725,1728,2856,3266,3267,3268,3271,3695,3829,3831,3932$, 4169, 4170, 4189, 4310, 4690, 4722, 4762, 4763, 4957, 5062, 5344, $5367,5368,5369,6708,6761,7572,7575,7777,7788,7788,7788$, $7789,7951,8027,8028,8327,8328,9035,9812,9961,10052,10381$, $10558,12397,12398,12587,12589,13537,13674,13871,13903$, $14072,14437,14438,14439,14484,15082,15249,15621,16343$, $16402,19571,19622,19690,19981,19983,19983,19985,20237$, 20356, 20362, 20554, 20739, 20765, 20803, 22299, 22332, 22630, 22781, 22988.

Cytotoxic inactive, ACHN cells 3355 .

Cytotoxic inactive, AGS cells 2101, 12936.

Cytotoxic inactive, assay to screen for inhibitors of cell division,
Xenopus cells from early Xenopus laevis embryo at blastular stage 12139, 12137.

Cytotoxic inactive, ATCC: CCRF-CEM murine leukemia cells 1136, $1137,1138$.

Cytotoxic inactive, B16 cells $492,6427,6430,7205,9474,9475,16818$, 16819, 16820, 18697, 20697, 20699, 20701, 20702, 21027, 22497.

Cytotoxic inactive, B16(F-10) cells $\quad 984,3932,4169,4170,16402,18945$, 18946, 18947, 18948, 18949.

Cytotoxic inactive, BC cells $14915,17846,17847$.

Cytotoxic inactive, BC-1 cells 7764, 2048, 3292, 3940, 3941, 8219, 10296, 10510, 13484, 13487, 18682, 21358, 5276.

Cytotoxic inactive, BCA-1 cells 13464, 13472, 13467.

Cytotoxic inactive, Bcap37 cells $11394,12536$.

Cytotoxic inactive, Bel7402 cells $465,2254,4987,5344,5867,5975$, $5976,6327,6689,7540,8528,9629,10485,10815,10816,10817$, 14042, 14168, 16050, 21031, 21640, 22011, 22013.

Cytotoxic inactive, Bel7405 cells $8632,11201$.

Cytotoxic inactive, BGC823 cells $6689,8528,13693,13699,13702$, 13703, 13704, 13706, 13707, 20811.

Cytotoxic inactive, BL6 cells 1532.

Cytotoxic inactive, Bowes cells $3695,10381,15621$.

Cytotoxic inactive, Bre04 cells $14847,14848,14849$.

Cytotoxic inactive, BST assay $247,2091,2092,6267,10207,10505,221$, $223,1819,1820,1821,2260,3153,5936,8211,10510,14995,15038$, 20803, 21082, 21125, 22551.

Cytotoxic inactive, BST cells 2346, 8325, 10126.

Cytotoxic inactive, BT474 cells $2251,2618,5944,7122,10306,12413$, $12415,12416,17118$.

Cytotoxic inactive, BT549 cells $11636,22205,22206,22206$.

Cytotoxic inactive, BXPC3 cells 2102, 2106.

Cytotoxic inactive, CA cells 18499.

Cytotoxic inactive, CAKI cells 14934.

Cytotoxic inactive, Calu1 cells $1764,3319,3615,11191,11192,13098$, 16665, 20369, 20711, 21713, 22378, 22379.

Cytotoxic inactive, Capan 1 cells 2101.

Cytotoxic inactive, Capan2 cells 2101, 12936.

Cytotoxic inactive, CaSki cells 6490.

Cytotoxic inactive, CCM2 cells $13023,13038$.

Cytotoxic inactive, CEM cells 16550 .

Cytotoxic inactive, CHAGO cells 248, 2251, 2618, 5944, 7122, 10306, $12413,12415,12416,17118$.

Cytotoxic inactive, Chinese hamster ovarian CHO cells 21475.

Cytotoxic inactive, Col2 cells $175,1864,1865,1885,2048,2259,2765$, $2766,2767,3982,4281,5075,5387,5536,5931,6066,6067,6090$, $6500,9504,9507,9546,10296,10446,10512,10769,11477,12179$, $13464,13467,13468,13845,16415,16854,16855,18682,19220$, $21135,21137,21410,21755,21758,21798,21801,21804,21805$, 21806, 21807.

Cytotoxic inactive, Colon205 cells $\quad 901,2309,2830,3778,5522,6017$, 7699, 10380, 10414, 10467, 11001, 11600, 11601, 13041, 14776, 16261, 16725, 18222, 18998, 19542, 19790, 20465, 22270, 22774.

Cytotoxic inactive, Colon26-L5 cells $3014,3980,4135,4788,4900$, 4936, 5788, 9626, 9627, 9628, 9815, 10099, 10411, 14500, 14613, 
15351, 15443, 16221, 16222, 16230, 16231, 16232, 20840, 20877, 21529 .

Cytotoxic inactive, COS- 7 cells $\quad 10732,8576,8577,8578,8579,8580$.

Cytotoxic inactive, DLD-1 cells 3241 .

Cytotoxic inactive, DU145 cells 5706, 7998, 14697.

Cytotoxic inactive, EAC cells 12622.

Cytotoxic inactive, fibroblasts in normal hmn 3241.

Cytotoxic inactive, FM3A cells 5042, 15719.

Cytotoxic inactive, GLC4 cells 16725.

Cytotoxic inactive, HCC2998 cells 5706.

Cytotoxic inactive, HCT cells 6785 .

Cytotoxic inactive, HCT116 cells 5706, 12310, 15242, 17872.

Cytotoxic inactive, HCT15 cells 3571, 12541, 19622.

Cytotoxic inactive, HCT8 cells 8939, 8942, 8944, 14935.

Cytotoxic inactive, HeLa cells 465, 901, 1022, 1624, 1764, 2021, 2309, 2473, 2833, 3319, 3615, 3908, 4046, 4283, 4987, 5522, 5570, 5607, 5701, 5795, 5867, 5975, 5976, 6017, 6338, 6427, 6430, 6689, 7205, 9406, 9509, 10374, 10380, 10414, 10467, 10483, 10485, 10595, 11001, 11191, 11192, 11265, 11266, 11392, 11595, 11600, 11601, 11799, 12512, 13041, 13098, 13123, 13197, 13198, 13199, 14042, 14737, 14776, 15635, 15910, 16050, 16261, 16665, 16873, 17244, 17611, $17612,17615,18222,18224,18499,18697,18945,18946,18947$, $18948,18949,18950,18998,19174,19540,19542,20369,20465$, 20697, 20699, 20700, 20701, 20702, 20711, 21713, 22378, 22379, 22497, 22774, 22962.

Cytotoxic inactive, HeLa-S3 cells $10732,13166,13167,8579,8580$, $8576,8577,8578,8360,8361,8363,8364,8365$.

Cytotoxic inactive, HEp2 cells $11265,11722$.

Cytotoxic inactive, Hep3B cells $901,2824,2826,3778,5179,5180,5367$, 5368, 5369, 6017, 7043, 8938, 10380, 10414, 10467, 11600, 13041, 14776, 14940, 14942, 18222, 18998, 19542, 19790, 20465, 22270.

Cytotoxic inactive, Hepa cells 12622.

Cytotoxic inactive, Hepa1c1c7 cells 17715 .

Cytotoxic inactive, HEPA59T/VGH cells $11945,11950,11952$.

Cytotoxic inactive, HepG cells $\quad 8669,8670,8671,13328$.

Cytotoxic inactive, HepG2 cells $8,248,2251,2618,3308,3544,3615$, $3619,3908,5179,5180,5368,5570,5607,5701,5795,5944,6776$, $6778,6779,7043,7122,8935,8936,8938,10306,10483,12413$, 12415, 12416, 12512, 16169, 17118, 17247, 21475, 22962.

Cytotoxic inactive, HGF cells $\quad 414,477,512,579,993,1177,1580,1581$, 1583, 1584, 1585, 1586, 1587, 1591, 2309, 3659, 3667, 3668, 3669, 5150, 5774, 5775, 5776, 5781, 5790, 5815, 5816, 5817, 5912, 5913, 5982, 5983, 6176, 7657, 9857, 9905, 10717, 10718, 10721, 14638, 15040, 15042, 15046, 15054, 15055, 15056, 15279, 17696, 18916, 19255, 19256, 19257, 19259, 19268, 20105, 20106, 21170, 21864 , 22667.

Cytotoxic inactive, HL-60 cells $\quad 465,1476,2101,2309,4689,4690,4940$, 4987, 5344, 5652, 5703, 5864, 5865, 5867, 5934, 5975, 5976, 6578, $6689,6760,6761,6942,8528,8576,8577,8578,8579,8580,9423$, $9629,10279,10280,10485,10732,11595,11601,12070,14042$, 16050, 18931, 19349, 19350, 20068, 21105, 21711, 21857, 22287 , 22774.

Cytotoxic inactive, HM02 cells $\quad 6778,3308,3544,3619,6779,17247$,
$3615,6776,16169$.

Cytotoxic inactive, HMEC cells 9199.

Cytotoxic inactive, HO-8910 cells $236,5648,5689,6114,8632,10020$, 11201.

Cytotoxic inactive, HOG.R5 cells 6090 .

Cytotoxic inactive, HONE-1 cells 174, 252, 2415, 4648, 6477, 8948, 9452, 9698, 9700, 9701, 9815, 9944, 11234, 13943, 14254, 15249, 16906, 17871, 18420, 19983, 19987, 20493, 20554, 22195, 23029.

Cytotoxic inactive, Hs578T cells 5580 .

Cytotoxic inactive, Hs742T cells 5343, 5580, 5621, 20122.

Cytotoxic inactive, HSC-2 cells $414,477,993,1505,1506,1507,1509$, $1580,1584,1585,1586,1587,1591,2309,3659,3669,5150,5774$, $5775,5776,5781,5790,5815,5816,5817,5912,5913,5982,5983$, $6176,7130,7134,7135,7137,7138,7657,8657,8658,8659,8660$, $8693,8698,8748,9857,10718,10721,12481,13058,13059,13060$, $14638,15040,15042,15046,15054,15055,15056,15057,15279$, 17696, 18916, 19255, 19256, 19259, 19268, 19522, 19523, 20105, 20106, 21170, 21864, 22667.

Cytotoxic inactive, HSG cells $15040,15042,15046,15054,15055$, 15056, 19255, 19256, 19257, 19259, 19268, 20105, 20106.

Cytotoxic inactive, HT1080 cells $3980,4135,4788,4936,5548,5779$, 5788, 6954, 9626, 9627, 9815, 10099, 10100, 10411, 10412, 11504, $14500,14613,15443,15796,16221,16222,16229,16230,16231$, 19618, 20840, 20877, 21529.

Cytotoxic inactive, HT29 cells $174,252,1067,1621,2856,3266,3267$, $3268,3271,5373,5374,5375,6289,6477,6708,6760,6761,7043$, $7788,8027,8028,8824,9035,10187,10747,13674,14072,14484$, 18588, 19690, 19981, 19983, 19985, 20237, 20356, 20362, 20554, 20643, 22332, 22781.

Cytotoxic inactive, hTERT-RPE1 cells 5931, 6066, 6067, 9504, 9507, $10512,12179,16854,16855,21135,21137,21755,21758,21798$, 21801, 21804, 21805, 21806, 21807.

Cytotoxic inactive, HUVEC cells $\quad 1864,1865,1885,2259,2259,3982$, 5387, 5387, 5401, 5931, 6042, 6066, 6067, 6090, 9504, 9507, 9507, $9546,10512,12179,13845,16854,16855,21135,21137,21755$, 21758, 21798, 21801, 21804, 21805, 21806, 21807.

Cytotoxic inactive, inhibition assay of hmn tumor cell replication 3104 , 3105, 3106, 3107, 3108, 3109, 3110, 3111, 3112, 3113.

Cytotoxic inactive, Jurkat-T cells $2101,2591,2592,2593,11262,11263$, 11264, 12936.

Cytotoxic inactive, K562 cells $492,984,1764,2101,3319,3615,3615$, 3695, 3932, 4169, 4170, 6204, 6776, 6791, 6792, 7998, 9081, 9082, 10068, 10381, 11191, 11192, 11265, 12590, 13067, 13068, 13069, 13070, 13098, 14697, 15621, 16177, 16402, 16665, 18697, 18978, 19983, 20369, 20711, 21713, 21845, 22378, 22379, 22748, 22792, 22800, 22801, 22803.

Cytotoxic inactive, K562/ADM cells $492,18697$.

Cytotoxic inactive, Kato3 cells 248, 2251, 2618, 5944, 7016, 7122, 10306, 12413, 12415, 12416, 17118.

Cytotoxic inactive, KB ATCC CCL17 cells 5794.

Cytotoxic inactive, KB cells $89,174,252,266,675,901,1372,1745$, $1865,2048,2259,2384,2591,2592,2593,2765,2766,2767,2824$, 2826, 2945, 3292, 3429, 3940, 3941, 3982, 4281, 4624, 5075, 5276, 
5387, 5522, 5931, 6017, 6066, 6067, 6090, 6477, 6515, 6689, 6761, 7190, 7191, 7582, 7764, 8219, 8528, 8939, 8942, 8944, 9199, 9504, 9507, 9546, 9564, 10159, 10206, 10296, 10380, 10414, 10446, 10467, 10510, 10512, 10910, 10911, 10912, 11079, 11262, 11263, 11264, 11477, 11600, 11636, 12179, 12453, 13038, 13041, 13467, 13468, 13484, 13487, 13491, 14775, 14776, 14846, 14915, 15118, 16050, $16415,16748,16754,16854,16855,16867,16966,17846,17847$, 17852, 18425, 18682, 18697, 18998, 19220, 19536, 19540, 19542, 20369, 20465, 20797, 20798, 20811, 20836, 20849, 20850, 20851, $21135,21137,21358,21755,21758,21798,21801,21804,21805$, 21806, 21807, 22205, 22206, 22206, 22270, 22413, 22544, 22545, 22546, 22547.

Cytotoxic inactive, KB16 cells $11945,11950,11952$.

Cytotoxic inactive, KB-VI cells 7764.

Cytotoxic inactive, KB-VIN cells 14934.

Cytotoxic inactive, $\mathbf{L}_{\mathbf{1 2 1 0}}$ cells $\quad 6478,6617,7998,8439,10089,10364$, $11569,12639,12640,13014,13015,13591,14697,15483,15931$, 16050, 20797.

Cytotoxic inactive, L-5178Y cells 22408.

Cytotoxic inactive, L6( $=\mathbf{L}-6)$ cells $\quad 580,815,816,1241,1784,1937$, 1939, 2004, 2346, 2683, 2920, 2924, 3166, 3306, 3551, 3609, 3674, $4478,5675,6776,7925,8325,9750,10159,11148,11195,12916$, 14222, 16067, 16068, 18221, 19568, 19960.

Cytotoxic inactive, L-929 cells 11799.

Cytotoxic inactive, leukemia cells 6741 .

Cytotoxic inactive, LLC cells $8150,8166,8168,8186,8188,8189,11277$, 13019.

Cytotoxic inactive, LNCaP cells 266, 1864, 1865, 1885, 2048, 2765, 2766, 2767, 3982, 4281, 4680, 5075, 5931, 6066, 6067, 6090, 7788, 8814, 9504, 9546, 10296, 10446, 10512, 10871, 11477, 12179, 13845, 14114, 14226, 14228, 14300, 14643, 14846, 15944, 15945, 15946, $15951,16415,16854,16855,18682,19087,19220,19983,21037$, $21135,21137,21755,21758,21798,21801,21804,21805,21806$, $21807,22332$.

Cytotoxic inactive, LNCaP-FGC cells $5343,5580,5621,8278,18700$, 18701, 20117, 20122, 20129.

Cytotoxic inactive, LO2 hmn liver cells 5648 .

Cytotoxic inactive, LOX-IMVI cells $\quad 984,3355,3932,4169,4170$, 16402.

Cytotoxic inactive, Lu04 cells $14847,14848,14849$.

Cytotoxic inactive, Lu1 cells $\quad 266,1864,1865,1885,2048,3982,4281$, 5931, 6066, 6067, 6090, 9504, 9507, 9546, 10296, 10512, 12179, $13464,13467,13468,13472,13845,14846,16415,16854,16855$, 18682, 19220, 21135, 21137, 21755, 21758, 21798, 21801, 21804, 21805, 21806, 21807.

Cytotoxic inactive, mammalian cell lines $11636,18114$.

Cytotoxic inactive, McCoy cells 13902.

Cytotoxic inactive, MCF cells $3308,3544,3615,3619,6776,6778,6779$, $16169,17247$.

Cytotoxic inactive, MCF7 cells $1067,1621,1699,1713,1718,1725$, 1728, 3159, 3695, 3829, 3831, 4189, 4416, 4957, 5062, 5179, 5180, 5367, 5368, 5369, 5373, 5374, 5375, 6162, 6490, 6689, 7043, 7777, 7788, 7788, 7998, 8569, 8932, 8933, 8935, 8936, 8938, 9535, 9564,
10187, 10381, 10747, 10751, 11890, 12448, 13871, 13903, 13935, 13936, 13943, 14115, 14697, 15249, 15621, 16050, 18588, 19571, 19983, 21709, 21856, 22830.

Cytotoxic inactive, MCF7/ADM cells 22830.

Cytotoxic inactive, MCF7-ras cells 6490.

Cytotoxic inactive, MDA231 cells 4913.

Cytotoxic inactive, MDA-MB-231 cells 5179, 5180, 5367, 5368, 5369, 6162, 7699, 8935, 8938, 9564, 10751, 12448, 13903, 13943, 21709, 21856.

Cytotoxic inactive, Meth-A cells $8150,8166,8168,8189$.

Cytotoxic inactive, MH-60 cells 2716, 2722, 3729, 3732, 5237, 5238, 5239, 7190, 7191, 8121, 9859, 9861, 9917, 9918, 9993, 10672, 10673, 16296, 20911.

Cytotoxic inactive, MI4 cells 3355 .

Cytotoxic inactive, MK1 cells $18945,18946,18947,18948,18949$.

Cytotoxic inactive, MKN-28 cells 19349, 19350.

Cytotoxic inactive, MRC-5 cells 19891.

Cytotoxic inactive, MT-4 cells 11843, 11844, 11845, 11846, 11847, 16652, 16653, 19652, 19653, 19654, 19655, 19656.

Cytotoxic inactive, MTT antiproliferative assay, B16F10, HeLa, MK1 cells $18945,18946,18947,18948,18949$.

Cytotoxic inactive, myosarcoma cells $2591,2592,2593,11262,11263$, 11264.

Cytotoxic inactive, N04 cells $14847,14848,14849$.

Cytotoxic inactive, NCF-7 cells 5532.

Cytotoxic inactive, NCI-H1417 cells 2101, 12936.

Cytotoxic inactive, NCI-H187 cells $422,422,3941,6183,6298,8032$, $8219,8325,10250,13491,14321,21358$.

Cytotoxic inactive, NCI-H226 cells 4722, 4762, 9812, 9961, 20739, 20765, 20803, 22630.

Cytotoxic inactive, NCI-H23 cells 3355.

Cytotoxic inactive, NCI-H266 cells 5706.

Cytotoxic inactive, NCI-H446 cells $12397,12398,22299,22988$.

Cytotoxic inactive, NCI-H460 cells 7699,8569 .

Cytotoxic inactive, no explanation on cell species $163,1161,1992$, 2286, 3159, 3429, 5303, 5518, 6409, 6490, 6490, 6490, 6490, 8107, $9474,9475,12048,13965,14101,15152,16818,16819,16820,17872$, 18359, 21027, 21724, 16199, 17196, 17198, 17197, 17199.

Cytotoxic inactive, NSCLC-N6 cells 2331, 10342, 11978, 13097, 13098, 16373.

Cytotoxic inactive, NUGC cells $3555,4648,8948,9452,9698,9700$, 9701, 9815, 9944, 10343, 11234, 13943, 14254, 18420, 19983, 19987, 20554, 22195, 22460.

Cytotoxic inactive, NUGC-3 cells $23029,2415,15249,16906,17871$, 20493.

Cytotoxic inactive, NUGC-4 cells 6621, 7016, 11405.

Cytotoxic inactive, OVCAR-3 cells $2101,3908,5570,5607,5701,5795$, 10483, 12512, 12936, 20070.

Cytotoxic inactive, $\mathbf{P}_{388}$ cells $\quad 194,215,492,2309,3292,4281,4689$, 5194, 5344, 5492, 5496, 5886, 5887, 6708, 7571, 7572, 7575, 8439, 10510, 11001, 11601, 11853, 11857, 11859, 13464, 13467, 13472, 13674, 14072, 14437, 14484, 16415, 18697, 19690, 19981, 20237, 20356, 20362, 20554, 22332, 22736, 22774. 
Cytotoxic inactive, $\mathbf{P}_{388} / \mathrm{ADM}$ cells 18697.

Cytotoxic inactive, PACA-2 cells 1067.

Cytotoxic inactive, PANC1 cells 2101, 12936.

Cytotoxic inactive, PBMC cells 9488.

Cytotoxic inactive, PC3 cells 984, 1067, 2101, 3932, 4169, 4170, 4722,

9812, 9961, 12936, 14934, 16402, 20739, 20765, 20803, 22630, 22781.

Cytotoxic inactive, primary culture hmn PBMCs 11001, 16261.

Cytotoxic inactive, PTX10 cells 14935.

Cytotoxic inactive, Raji cells 1764, 3319, 3615, 11191, 11192, 13098, 16665, 20369, 20711, 21713, 22378, 22379.

Cytotoxic inactive, RAW264.7 cells $5633,5669,7982,7983,7984,7985$, 11017.

Cytotoxic inactive, RL33 cells 11978.

Cytotoxic inactive, RXF-393 cells 3355.

Cytotoxic inactive, $\mathbf{S}_{180}$ cells $\quad 8150,8166,8168,8186,8189,13019$, 13020.

Cytotoxic inactive, $\mathbf{S}_{\mathbf{1 8 0}} \mathrm{A}$ cells 12622 .

Cytotoxic inactive, SCL-6 cells 7016 .

Cytotoxic inactive, SF268 cells 7125, 7613, 7614, 8569, 11890.

Cytotoxic inactive, SF539 cells 5706 .

Cytotoxic inactive, SGC7901 cells 19349, 19350.

Cytotoxic inactive, SiHa cells $6490,12541$.

Cytotoxic inactive, SK-MEL cells 11636, 22205, 22206, 22206.

Cytotoxic inactive, SK-MEL-2 cells $19622,984,3932,4169,4170$, 16402.

Cytotoxic inactive, SK-MES-1 cells 12397, 22299, 22988.

Cytotoxic inactive, SK-OV-3 cells 11636, 16370, 19622, 22205, 22206, 22206.

Cytotoxic inactive, SMMC-7721 cells 236, 5648, 5689, 6114, 6427, 6430, 7205, 10020, 20697, 20699, 20700, 20701, 20702, 22497, 22497.

Cytotoxic inactive, SNU-1 cells 18088.

Cytotoxic inactive, SNU-16 cells 18088.

Cytotoxic inactive, SR cells 3355 .

Cytotoxic inactive, SW620 cells $248,2251,2618,5944,7122,10306$, 12413, 12415, 12416, 17118.

Cytotoxic inactive, T24 cells 12588.

Cytotoxic inactive, T24S cells $3695,10381,15621,19571,19983$.

Cytotoxic inactive, T47D cells $2101,6162,8150,8166,8168,8186,8189$, 9564, 10751, 12448, 12936, 13019, 21709, 21856.

Cytotoxic inactive, U251 cells 7998, 14697.

Cytotoxic inactive, U-87-MG cells 14934 .

Cytotoxic inactive, U937 cells 465, 4987, 5867, 5975, 5976, 10485, 14042.

Cytotoxic inactive, Vero cells $\quad 24,26,330,509,510,511,1764,3319$, 3615, 3940, 3941, 7897, 7907, 11129, 11191, 11192, 11636, 12489, $13098,13108,14217,14840,14842,15134,16665,17574,19795$, 19796, 20369, 20711, 21713, 22205, 22206, 22206, 22378, 22379.

Cytotoxic inactive, WI-38 cells $11595,21498$.

Cytotoxic inactive, WiDr cells 2101, 12936.

Cytotoxic inactive, Wish cells $1764,3319,3615,11191,11192,13098$, 16665, 20369, 20711, 21713, 22378, 22379.

Cytotoxic inactive, yeast assay, no selective DNA-damaging, RS321NpRAD52(gal) 4970, 11344, 20316, 12917.
Cytotoxic inactive, yeast assay, no selective DNA-damaging,

RS321NYCp50(gal) 4970, 11344, 20316, 12917.

Cytotoxic, 1,3,8-trihydroxy for anthraquinone plays a significant role in the cytotoxic activity $6776,20010$.

Cytotoxic, 212 cells $1992,6490,8520,11977,17872$.

Cytotoxic, 39 kinds of hmn cancer cell lines 1625.

Cytotoxic, 3LL mus Lewis lung cancer cells 19516, 19517, 19524, $19525,19526,22281$.

Cytotoxic, 780-6 renal cancer cells 3583 .

Cytotoxic, 9KB hmn epidermatoid nasopharyngeal carcinoma cells 2735, 10126, 11978, 16414, 17240.

Cytotoxic, 9L rat glioma cells 2303.

Cytotoxic, 9PS mouse lymphleukemia cells 2735, 16021, 16022, 16023, 17240.

Cytotoxic, a promising lead as potential cancer chemopreventive agents $51,5746,8262,11490,11504,11804,18547$.

Cytotoxic, A2780 hmn ovarian cancer cells $1576,3126,6044,6045$, $8054,8065,8940,9089,9090,9388,11783,11783,12108,12109$, 12110, 17622, 17623, 20809, 21337, 21338, 22761, 22761, 22839.

Cytotoxic, A375 hmn melanoma cells 919, 2492, 2493, 4046, 6437, 11800, 11801, 17654, 18056.

Cytotoxic, A431 hmn epidermic cancer cells 9187, 15739, 15922, 17008, 19284.

Cytotoxic, A498 hmn renal cancer cells $1297,1303,1304,1305,1320$, $1321,1322,3457,3583,4763,5706,6827,6828,10558,11269,14906$, 18211, 20720, 20721, 20811.

Cytotoxic, A549 non-small cell lung cancer cells $150,251,577,843$, $1297,1303,1304,1305,1310,1311,1312,1320,1321,1322,1621$, 1679, 1764, 1924, 1925, 1926, 2006, 2047, 2334, 2338, 2527, 2528, $2652,2653,2654,2655,2656,2677,2735,2736,2857,3053,3096$, $3097,3241,3257,3457,3583,3868,3869,3974,3975,4147,4148$, 4317, 4320, 4323, 4689, 4880, 4986, 5055, 5284, 5295, 5314, 5826, $5832,6044,6045,6075,6162,6204,6277,6282,6289,6314,6315$, $6328,6367,6404,6405,6642,6759,6760,6827,6828,6897,7535$, $7568,7569,7571,7646,7698,7788,8824,8946,9226,9323,9535$, 9544, 9567, 9669, 9747, 9885, 10126, 10438, 11029, 11081, 11269, $11375,11494,11605,12188,12189,12254,12964,13518,13673$, $13685,13867,13869,14254,14492,14495,14496,14504,14644$, $14645,14646,14647,14906,14934,14934,14935,14935,15084$, 15085, 15087, 15088, 15089, 15141, 15169, 15879, 15902, 16021, $16023,16050,16282,16283,16370,16402,16427,16676,16677$, 16693, 17059, 17060, 17061, 17062, 17063, 17064, 17309, 17619, 18011, 18033, 18044, 18211, 18768, 18769, 18770, 18771, 18772, 18773, 18774, 18775, 18776, 18777, 18778, 18892, 19427, 19516, 19517, 19524, 19525, 19526, 20002, 20244, 20363, 20540, 20641, 20643, 20644, 20679, 20680, 20681, 20720, 20721, 20722, 20811, 20811, 20811, 21709, 21924, 22208, 22208, 22209, 22451, 22458, 22923, 23013.

Cytotoxic, ACHN hmn renal cancer cells $\quad 251,2528,4880,5314,5706$, 7698, 20811, 20811.

Cytotoxic, acts on calf thymus DNA 7704, 15631, 19284, 22232.

Cytotoxic, acts on DNA topo II 843 .

Cytotoxic, against a series of tumor cell lines but did not show 
significant effects in clinical studies against solid tumors 2660 . Cytotoxic, AGS gastric adenocarcinoma cells 3498, 17979.

Cytotoxic, animal cap assay to screen for inhibitors of cell division 4820, 4821, 4822, 4825, 4826, 4827, 4828, 4829, 4838, 12138 .

Cytotoxic, animal model $8596,8598,8599$.

Cytotoxic, animal tumor and plant tumor 14705 .

Cytotoxic, antioxidant assay $1400,2635,6103,8095,14454,20481$, 20482.

Cytotoxic, antiproliferation inactive $5528,8665,8677,8678$.

Cytotoxic, antiproliferation inactive, Colon26-L5 15443, 16221, 16222, 16230, 16231, 16232.

Cytotoxic, antiproliferation inactive, HT1080 15443, 15796, 16221, $16222,16229,16230,16231$.

Cytotoxic, antiproliferative $2102,2106,3548,3551,4590,5929,6907$, $7080,9655,10277,10281,10559,12171,12177,12180,12206,14304$, 14305, 15635, 18759, 19458, 19634, 20465, 20670, 20811, 21231, 22213, 22718.

Cytotoxic, antiproliferative $\quad 1389,1390,1439,1440,14901,15494 . ?$

Cytotoxic, antiproliferative inactive, HL-60 20068.

Cytotoxic, antiproliferative, A-2780 11783, 22761.

Cytotoxic, antiproliferative, AGS cells 3498 .

Cytotoxic, antiproliferative, B16(F-10), HeLa, MK1 18944, 18950, 18951, 18952, 18953.

Cytotoxic, antiproliferative, calf pulmonary arterial endothelial cells CPAE 20900, 20901.

Cytotoxic, antiproliferative, Col2 cells 240, 6064, 7204, 20610.

Cytotoxic, antiproliferative, Colon26-L5 15796, 16229.

Cytotoxic, antiproliferative, colorectal cancer cells $\quad 11783,22761$.

Cytotoxic, antiproliferative, cytotoxicity of compounds was measured using the WST-8 proliferation reagent 7376, 21435.

Cytotoxic, antiproliferative, HEK-293 1575, 3821, 8715, 18691, 18693, $18733,22838$.

Cytotoxic, antiproliferative, hmn breast cancer cells $\quad$ 1399, 2635, 11783, 14961, 22761.

Cytotoxic, antiproliferative, HT1080 cells 16232.

Cytotoxic, antiproliferative, HTC cells $8148,8149,8159,8160,8161$, 8174.

Cytotoxic, antiproliferative, J774 macrophages $8662,8717,8718,8749$, 18690, 18692.

Cytotoxic, antiproliferative, J774.A1 1575, 3821, 8715, 8716, 18691, 18693, 18733, 22838

Cytotoxic, antiproliferative, LoVo $3573,6164,6213,19916$.

Cytotoxic, antiproliferative, LoVo/Doxo $3573,6164$.

Cytotoxic, antiproliferative, lymphocytes 22015 .

Cytotoxic, antiproliferative, MCF7 3303, 3498.

Cytotoxic, antiproliferative, MT2 3816.

Cytotoxic, antiproliferative, MTT colorimetric assay 3816 .

Cytotoxic, antiproliferative, PC3 3498.

Cytotoxic, antiproliferative, PMA-induced 17958.

Cytotoxic, antiproliferative, six esophageal cancer cells 2303.

Cytotoxic, antiproliferative, T-lymphoid leukemia cells 2102 .

Cytotoxic, antiproliferative, WEHI-164 1575, 3821, 8715, 8716, 18691, 18693, 22838.
Cytotoxic, APM1840 hmn leukemia cells 17240.

Cytotoxic, aromatase inhibitor 51, 846, 1402, 2635, 2647, 5746, 8262, 11490, 17403.

Cytotoxic, arrests in the G2/M phase of cell cycle 5055 .

Cytotoxic, ascites carcinoma cells $17028,18816$.

Cytotoxic, assay of larva of yellow fever mosquito 18240.

Cytotoxic, B16 mouse melanoma cells 2304, 2811, 6221, 16599, 19143, 19143, 20700.

Cytotoxic, B16(F-10) mouse melanoma cells 2334, 2338, 4147, 4148, 16050, 18944, 18950, 18951, 18952, 18953, 19516, 19517, 19524, 19525, 19526, 23013.

Cytotoxic, Bacillus coli, Bacillus subtilis and nema 21328.

Cytotoxic, BC hmn breast cancer cells 24, 349, 526, 2244, 3416, 3429, $3708,4775,5095,6759,6759,6759,12453,12454,15494,15495$, $18515,20671$.

Cytotoxic, BC-1 hmn breast cancer cells 1277, 2260, 5064, 5327, 6759, $6759,8220,8221,8222,8235,10250,10297,10511,11353,13489$, 13490, 13491, 13492, 13493, 13494, 13495, 15919, 17008, 18866, 20809.

Cytotoxic, BCA-1 hmn breast cancer cells $6759,13468,13469,13470$, 15739.

Cytotoxic, Bcap37 hmn breast cancer cells 843, 11392, 13123, 16183, $17717,18499$.

Cytotoxic, Bel7402 hmn liver cancer cells $\quad 1764,3053,3868,3869,3911$, 5826, 5832, 5888, 6044, 6045, 6075, 6314, 6328, 7436, 7504, 7544, $7545,7548,8526,8527,8530,8531,8532,8533,8534,8535,8536$, $8541,8543,8544,8692,9567,10052,11081,11659,14347,17059$, 17060, 17061, 17062, 17063, 17064, 17928, 19488, 20397, 20811, 20811, 20811.

Cytotoxic, Bel7405 hmn liver cancer cells $1860,8656,9546,14044$, 22828.

Cytotoxic, Bel7420 cells 18545 .

Cytotoxic, BGC823 hmn stomach cancer cells $843,1030,1764,3053$, $3868,3869,4701,5826,5832,6044,6045,6075,6756,8526,8527$, $8530,8531,8532,8533,8534,8535,8536,8541,8543,8544,9567$, $10052,10815,10816,10817,11081,11392,11659,13123,13694$, $13700,13701,13705,16050,17059,17060,17061,17062,17063$, 17064, 17717, 18499, 20397, 20542, 22011, 22013, 22497, 22798.

Cytotoxic, BIU87 bladder cancer cells $16183,17717$.

Cytotoxic, BL6 mouse melanotic carcinoma 472, 1276, 4237, 13241.

Cytotoxic, blocks cell from $\mathbf{G}_{\mathbf{1}}$ to $\mathbf{S}$ phase 9655 .

Cytotoxic, blocks DNA synthesis 19085 .

Cytotoxic, blocks expression of vascular endothelial growth factor (VEGF)mRNA in GI-101A cells 3498 .

Cytotoxic, B-lymphocytes 1359.

Cytotoxic, bone cancer cells 15409.

Cytotoxic, Bowes skin cancer cells $\quad$ 16402, 19571, 19983.

Cytotoxic, breast cancer cells 2601, 11783, 22761.

Cytotoxic, Brine Shrimp Lethality bioassay(Brine Shrimp Test) 1297, 1303, 2082, 3292, 5327, 6309, 8210, 10511, 13296, 17592.

Cytotoxic, BST 1310, 1311, 1312, 1313, 1320, 1321, 1322, 1679, 2736, 7788, 8946, 11375, 11566, 11864, 11978, 14906, 15084, 15085, 15087, 15088, 15089, 15902, 15903, 16676, 16677, 18211, 18214, 18215, 
18240, 18899, 20248.

Cytotoxic, BSY1 breast cancer cells $1625,4880,18714$.

Cytotoxic, BT-20 9869.

Cytotoxic, BT474 hmn galactophore cancer cells 225, 226, 248, 463,

2257, 2261, 2262, 4268, 4269, 5942, 5945, 10307, 12414, 12417,

12423.

Cytotoxic, BT549 hmn galactophore cancer cells $\quad 11636,18114$.

Cytotoxic, BXPC3 pancreas cancer cells 7912, 9859, 9861, 9917, 10672, $10673,16296,16312$.

Cytotoxic, C6 rat glioma cells 9376, 9383, 9386.

Cytotoxic, CA hmn liver cancer cells $11392,13071,13123,16183$, 17717.

Cytotoxic, CaEs-17 hmn esophageal cancer cells 16183.

Cytotoxic, CAKI hmn renal cancer cells 14934.

Cytotoxic, CAKI-1 hmn renal cancer cells 3583.

Cytotoxic, Calu1 hmn lung cancer cells 6776, 10068, 20010.

Cytotoxic, Capan1 pancreas cancer cells 12936, 17979.

Cytotoxic, Capan 2 pancreas cancer cells 17979.

Cytotoxic, CaSki hmn cervical carcinoma cells 1992, 11977.

Cytotoxic, CCM2 8167.

Cytotoxic, cell adhesion inhibitor, adhesion of HL-60 cell to BAEC $3324,3325$.

Cytotoxic, cellular differentiation inducer, HL-60 175, 240, 1438, 1441, 2676, 2684, 6064, 7204, 10769, 15635, 20610, 20670, 21410.

Cytotoxic, cellular differentiation inducer, mus myelocytic leukemia cells $10594,10751,10752,10796$.

Cytotoxic, CEM leukemia cells 6221 .

Cytotoxic, CHAGO hmn undifferentiated lung cancer cells 225, 226, 463, 2257, 2261, 2262, 4268, 4269, 5942, 5945, 10307, 12414, 12417, 12423.

Cytotoxic, changes the shape of Pyricularia oryzae mycelium 17975 , 17980.

Cytotoxic, Chinese hamster ovarian 8 .

Cytotoxic, CNE hmn nasopharyngeal carcinoma $16183,17717$.

Cytotoxic, Col1 hmn colorectal cancer cells 15739.

Cytotoxic, Col2 hmn colorectal cancer cells $\quad 175,240,266,1863,3300$, $5055,5074,5319,5401,6042,6064,6277,6282,6483,6759,6759$, $6759,6759,6759,7078,7204,7764,10297,10396,10438,10769$, $11353,13469,13470,13472,14045,14846,18866,20610,20809$, 20811, 21313, 21314, 21315, 21316, 21410, 21803, 21924.

Cytotoxic, COLO320DM hmn colorectal cancer cells 4913.

Cytotoxic, Colon 205 colorectal cancer cells $\quad 2528,2824,2826,3583$, 3777, 6766, 6906, 7698, 10206, 14775, 18224, 19540, 20811.

Cytotoxic, Colon26-L5 mus colorectal cancer cells $\quad 2468,2498,2499$, 2500, 3012, 3012, 3014, 3015, 3015, 3016, 3016, 3018, 3018, 3019, $3019,3020,3020,3021,3021,4398,4882,5156,5156,5548,5777$, $5778,5779,5780,6217,6844,6844,6846,6846,6847,6848,6848$, $9300,3022,6849,9974,10100,10412,13859,14254,15351,15352$, 15796, 16229, 17174, 17517, 17518, 17519, 19618, 20840, 20877, 21529.

Cytotoxic, colorectal cancer cells 11783, 15409, 16864, 22761.

Cytotoxic, colorimetric method $6759,17574,20253$.

Cytotoxic, COS-7 monkey kidney cells $\quad 8360,8361,8363,8364,8365$.
Cytotoxic, COX-1 inhibitor 1397, 1405, 1417, 1421, 1422, 1423, 1443, $1446,1447,1448,1765,7026,5679,15714,20326,21849,12681$, $11672,1387,1396,1412,1445,1449,1450,2172,2635,3308,4337$, 7768, 18643, 18644, 11248, 14931, 14962, 14961.

Cytotoxic, COX-2 inhibitor $1387,1396,1412,1424,1437,1442,1444$, 1445, 1449, 1450, 2334, 6497, 11490, 14931, 18644, 21440.

Cytotoxic, Crown gall cancer cells 17619.

Cytotoxic, CT-26 mus colorectal cancer cells $3241,9669$.

Cytotoxic, CTV1 hmn leukemia cells 17240.

Cytotoxic, cultural hmn throat epicytoma cells 14896.

Cytotoxic, cultured mouse epidermal 308 cells $1372,1373,1394,1395$, $5543,15412$.

Cytotoxic, CXF94L hmn tumor cells 13241.

Cytotoxic, cytochrome C antioxidant assay 1398.

Cytotoxic, D.mel-II (embryonic tissue of ferment fly, D. melanogaster) 2948, 2950, 2952.

Cytotoxic, DAUDI 17528.

Cytotoxic, decrease in cellular GSH content and increases ROS production 9669 .

Cytotoxic, decrease in thymidine uptake and glutathione levels of the tumor cells 6767 .

Cytotoxic, direct cytocidal effect to arrest carcinoma cells in the G2/M phase, stomach cancer cells 22232 .

Cytotoxic, DLD hmn colorectal adenocarcinoma cells 11949.

Cytotoxic, DLD-1 hmn colorectal adenocarcinoma cells 9669.

Cytotoxic, DMS114 hmn lung cancer cells 4880 .

Cytotoxic, DMS273 hmn lung cancer cells $1625,4880$.

Cytotoxic, DNA-damaging activity $3498,6710,6711,6714,11636$, 12537, 18114, 19061.

Cytotoxic, DU145 prostatic cancer cells $2528,4880,5343,5580,5621$, $6314,7912,8278,9859,9861,9917,10672,10673,16296,16312$, 18700, 18701, 20117, 20122, 20129, 20811, 22281.

Cytotoxic, EAC Ehrlich ascites cancer cells $\quad 3502,3745,3906,6284$, 6285, 6459, 7323, 7566, 11056, 13238, 17717, 18461, 19929, 22778.

Cytotoxic, EBV-EA 7764, 11358, 19236, 20465.

Cytotoxic, EBV-EA inhibitor TPA-induced 7764, 11358, 19236, 20465. Cytotoxic, Ehrlich ascites cancer cells $4471,4472,7399,16183$.

Cytotoxic, EJ-1 hmn bladder cancer cells $\quad 2102,2106,22718$.

Cytotoxic, $\mathbf{E L}_{\mathbf{4}}$ cells 19143.

Cytotoxic, enhances cytotoxicity for drug-resistant strain $\mathbf{P}_{\mathbf{3 8 8}} 8436$.

Cytotoxic, estrogen $\boldsymbol{\alpha}$-receptor-binding assay $\quad 1397,2630,2648,4108$, 14961.

Cytotoxic, estrogen $\boldsymbol{\beta}$-receptor-binding assay $\quad 1397,2630,2648,4108$, 14961.

Cytotoxic, estrone sulfatase assay $\quad 6322,21968$.

Cytotoxic, fibrosarcoma cells 6767 .

Cytotoxic, FM3A mus breast cancer cells $911,912,1650,1651,1652$, 2312, 2380 .

Cytotoxic, gpg horn cells 3096 .

Cytotoxic, GXF251L 13241.

Cytotoxic, H.Ep.-2 hmn cutis cancer cells in throat 7836, 17042.

Cytotoxic, H116 hmn colorectal cancer cells 5284, 5295, 6897, 9747, 14495, 14496, 14504, 14644, 14647. 
Cytotoxic, HBC4 breast cancer cells 4880 .

Cytotoxic, HBC5 breast cancer cells 4880 .

Cytotoxic, HCC2998 hmn colorectal cancer cells 4880 .

Cytotoxic, HCT hmn colorectal cancer cells 2677, 12958, 18931, 22795, 22923.

Cytotoxic, HCT116 hmn colorectal cancer cells 159, 1792, 3300, 3583, 4880, 6160, 6490, 9376, 9383, 9386, 11723, 22077, 22580.

Cytotoxic, HCT15 hmn colorectal cancer cells 1913, 1914, 4880, 5706, 6162, 7032, 11723, 11723, 16370, 18011, 20679, 20680, 20681, 21709, 22208, 22209.

Cytotoxic, HCT8 hmn colorectal cancer cells $1764,2652,2653,2654$, 2655, 2656, 3053, 3868, 3869, 5826, 5832, 6044, 6045, 6075, 6221, $8940,9567,10052,11081,12188,12189,14934,14934,14935,17059$, 17060, 17061, 17062, 17063, 17064, 18044, 20540, 20542, 20545, 22147, 22148, 22149, 22150.

Cytotoxic, HEK-293 hmn epithelial kidney cells $1575,3821,8715$, 18691, 18693, 18733, 22838.

Cytotoxic, HEL normal hmn embryonic lung fibrocytes 5196, 14967. Cytotoxic, HeLa ATCC-17 hmn cervical epithelial cancer cells 1136, 1137, 1138.

Cytotoxic, HeLa culture cervical epithelial cancer cells from Henrietta Lack 492, 585, 696, 1319, 1470, 1471, 1472, 1617, 1625, 2492, 2493, 2811, 3053, 3293, 3412, 3502, 3548, 3553, 3745, 3911, 4128, 4320, 4323, 4324, 4327, 4461, 4565, 4795, 4891, 5196, 5417, 5929, 6437, 6776, 6921, 7044, 7436, 7474, 7586, 7593, 7665, 7714, 8128, 8526, $8527,8528,8530,8531,8532,8533,8534,8535,8536,8541,8543$, 8544, 8968, 9015, 9194, 9254, 9255, 9256, 9288, 9376, 9383, 9386, 9869, 10068, 10206, 10277, 10281, 10559, 10773, 10815, 10816, 10817, 10893, 11179, 11230, 11231, 11232, 11555, 11556, 11645, $11659,11722,11800,11801,11977,12171,12177,12180,12446$, 12511, 13071, 14304, 14305, 14347, 14417, 14775, 14967, 16183, $16599,16670,17265,17613,17614,17616,17643,17644,17645$, 17646, 17654, 17654, 17717, 17862, 18056, 18097, 18484, 18485, 18562, 18673, 18944, 18951, 18952, 18953, 19085, 19278, 19308, 20010, 20135, 20278, 20283, 20284, 20397, 20811, 20811, 20811, 20811, 21206, 21239, 21240, 21385, 21387, 21498, 22011, 22013, 22270, 22286, 22775, 22781, 22784.

Cytotoxic, HeLa-S3 hmn cervical epithelial cancer cells 3412,4752 , 4753, 4754, 4790, 13153, 13154, 13155, 13157, 13158, 13159, 13160, 13161, 13164, 13165, 15409, 17489, 21887.

Cytotoxic, HELF normal hmn embryo lung fibroblasts 20540 . Cytotoxic, Hep2 hmn liver cancer cells 2811, 7586, 11266, 20963. Cytotoxic, Hep2,2,15 transfected with hepatitis B virus hmn liver cancer cells $473,1294,1295,1296,1302,1308,1856,1961,2735,3060$, 4089, 4090, 4091, 4284, 5240, 7515, 7516, 7815, 8167, 12956, 13023, 13038, 15084, 15085, 15090, 15091, 15092, 15093, 15095, 15097, 15098, 15339, 18897, 18898, 19200, 20240, 20243, 22214.

Cytotoxic, Hep3B hmn liver cancer cells $1324,1324,1325,1492,2830$, 3258, 3259, 3260, 3261, 3262, 3263, 3555, 3777, 5297, 5522, 5532, 6490, 8379, 8379, 8932, 8933, 8934, 8935, 8936, 8946, 8947, 8948, 9194, 10206, 11977, 13903, 13943, 14775, 14936, 14936, 14937, 14937, 14938, 14938, 14941, 14942, 14943, 14943, 17872, 18224, 19540, 20626, 20811, 22077.
Cytotoxic, Hepa hmn liver cancer cells 20834, 20835, 20836.

Cytotoxic, Hepa1c1c7 mus liver cancer cells $1380,1381,1382,1383$, 1384, 1386, 1388, 1392, 1393, 1401, 1403, 1404, 1406, 1407, 1408, $1409,1410,1411,1413,1414,1415,1416,1418,1419,1420,1425$, $1427,1428,1429,1430,1431,1432,1433,1434,1435,1436,1451$, $1452,1453,1454,1455,1456,1457,2468,2857,2857,3071,3072$, $3602,4282,6228,6228,6246,6246,7670,7788,8278,9740,10430$, 10827, 11504, 11804, 12953, 14074, 14074, 14075, 14075, 16498, 16935, 17753, 19542, 19910, 21918, 22615, 22715.

Cytotoxic, Hepa59T/VGH hmn liver cancer cells 531, 11951, 20811, 20833, 22630.

Cytotoxic, HepG2 hmn liver cancer cells $\quad 225,226,463,473,1294,1295$, 1296, 1302, 1308, 1324, 1324, 1325, 1617, 1856, 1961, 2102, 2106, 2257, 2261, 2262, 2735, 2948, 2950, 2952, 3060, 3555, 4089, 4090, 4091, 4128, 4268, 4269, 4284, 4891, 5240, 5367, 5369, 5532, 5942, $5945,6490,7044,7515,7516,7815,8167,8379,8379,8932,8933$, 8934, 8946, 8947, 8948, 10307, 11977, 12414, 12417, 12423, 12511, 12597, 12954, 12956, 13023, 13038, 13903, 13943, 14936, 14936, 14937, 14937, 14938, 14938, 14939, 14940, 14940, 14941, 14941, 14942, 14942, 14943, 14943, 15084, 15085, 15090, 15091, 15092, 15093, 15095, 15097, 15098, 15339, 16634, 17872, 17928, 17929 , 18673, 18897, 18898, 19148, 19200, 19308, 19488, 20240, 20243, 22077, 22214.

Cytotoxic, HEPZ hmn epithelial cancer cells 837.

Cytotoxic, HGF normal hmn gingival fibroblast cells $1010,4338,4339$, 4340, 4341, 4342, 5184, 6128, 6129, 6130, 6447, 7133, 7136, 8354, $8355,8357,8358,8359,8653,8654,8655,8687,8721,8722,10716$, 10719, 10720, 10722, 10727, 11206, 11248, 13881, 19254, 19517, 21797.

Cytotoxic, HL-60 leukaemia cells $\quad$ 175, 240, 594, 941, 1570, 1571, 1578, 1691, 1845, 2102, 2106, 2528, 2716, 2722, 3053, 3324, 3325, 3576, $3576,3580,3580,3581,3581,3582,3582,3583,3583,3724,3729$, $3732,3784,3911,4893,5237,5238,5239,5774,5775,5776,5781$, 5815, 5816, 5817, 5899, 5900, 5912, 5913, 5926, 6064, 6107, 6125, 6221, 6328, 6437, 6484, 6528, 6576, 6577, 6785, 7081, 7204, 7436, $7540,7544,7545,7548,7746,7911,7912,8121,8222,8327,8328$, $8360,8361,8363,8364,8365,8526,8527,8530,8531,8532,8533$, $8534,8535,8536,8541,8542,8543,8544,8661,8691,8699,9381$, 9859, 9861, 9917, 9918, 9993, 10077, 10274, 10287, 10287, 10672, 10673, 10725, 10726, 10769, 10773, 10815, 10816, 10817, 11001, 11001, 11323, 11659, 11751, 11962, 12167, 12202, 12595, 12597, 12936, 12958, 13241, 13492, 13493, 13494, 13495, 14347, 14698, $15635,15813,16261,16261,16296,16312,17240,17265,17528$, 17727, 17928, 17929, 17979, 18637, 18714, 18716, 18768, 18769, 18770, 18771, 18772, 18773, 18774, 18775, 18776, 18777, 18778, 18835, 18892, 19122, 19324, 19410, 19410, 19411, 19411, 19412, 19412, 19413, 19413, 19488, 20009, 20068, 20221, 20223, 20397. 20610, 20811, 20811, 20811, 20811, 20911, 21410, 21498, 21771 , 22011, 22013, 22290, 22718, 22720, 22777, 22792, 22793, 22794, 22795, 22826.

Cytotoxic, hmn breast cancer cells $\quad$ 1399, 2635, 14961.

Cytotoxic, hmn cervical carcinoma cells 21497.

Cytotoxic, hmn cervical carcinoma cells 11541 . 
Cytotoxic, hmn colorectal cancer cells $\quad 1389,1390,1439,1440,14901$, 15494.

Cytotoxic, hmn embryo lung cells 11541 .

Cytotoxic, hmn intestinal mucoadenocarcinoma in nude mouse 11179 .

Cytotoxic, hmn keratinocytes 19284

Cytotoxic, hmn liver cancer cells 8128 .

Cytotoxic, hmn lymphocytes 14995.

Cytotoxic, hmn medulloblastoma $1470,1471,1472,11230,11231$, $11232,11645,18562$

Cytotoxic, hmn microvascular endothelial cells 9198.

Cytotoxic, hmn peripheral blood T cells 7519, 11318, 12106, 15331, $16216,19515,22718$

Cytotoxic, hmn throat epicytoma cells 7558 .

Cytotoxic, hmn throat epidermic carcinoma cells H-Ep-2 9288.

Cytotoxic, hmn T-lymphoid leukemia cells 2102.

Cytotoxic, HO-8910 hmn ovarian cancer cells $1860,8656,9546,11203$, 14044, 14828, 16675, 22497, 22828.

Cytotoxic, HOG.R5 green fluorescent protein (GFP)-based reporter cells 1863, 1885, 6759, 9546, 10396, 13845, 14045.

Cytotoxic, HONE-1 hmn nasopharyngeal carcinoma cells 228, 332, 530, 2006, 2338, 3555, 4317, 4320, 4323, 5573, 5574, 8935, 8936, 13294, 16337, 16402, 16891, 20495, 22270, 22272.

Cytotoxic, HOP-62 non-small cell lung cancer cells 3583.

Cytotoxic, Hs578T hmn breast cancer cells $5343,5580,5621,8278$, 18700, 18701, 20117, 20122, 20129.

Cytotoxic, Hs740T hmn stomach cancer cells 5343, 5580, 5621, 8278, 18700, 18701, 20117, 20122, 20129.

Cytotoxic, Hs742T hmn breast cancer cells $5343,5580,5621,8278$, 18700, 18701, 20117, 20129.

Cytotoxic, Hs756T hmn stomach cancer cells 5343, 5580, 5621, 8278, 18700, 18701, 20117, 20122, 20129.

Cytotoxic, HSC-2 hmn oral squamous cell carcinoma cells $\quad 1010,1177$, $1508,1570,1571,1581,1583,4338,4339,4340,4341,4342,5184$, $6128,6129,6130,6447,7083,7131,7132,7139,7140,8354,8355$, $8357,8358,8359,8661,8695,10722,10727,11206,11248,19254$, $19257,19521,21797,22826$.

Cytotoxic, HSC-2 hmn oral squamous cell carcinoma cells, also active for normal hmn gingival fibroblasts HGF $8653,8654,8655,8687,8721$, 8722, 10716, 10719, 10720, 13881.

Cytotoxic, HSC-2 hmn oral squamous cell carcinoma cells, inactive for normal hmn gingival fibroblasts HGF $512,579,3667,3668,9905$, 10717.

Cytotoxic, HSG hmn salivary gland tumor cells 19254.

Cytotoxic, HT sarcoma cells $\quad$ 9187, 15919, 17008, 17009.

Cytotoxic, HT1080 hmn fibrosarcoma cells 2468, 2498, 2499, 2500, 2769, 3012, 3012, 3014, 3014, 3015, 3015, 3016, 3016, 3018, 3018, $3019,3019,3020,3020,3021,3021,3022,4398,4882,4900,5156$, $5156,5777,5778,5780,6217,6277,6282,6759,6844,6844,6846$, 6846, 6847, 6848, 6848, 6849, 9300, 9628, 9974, 10438, 11353, 12766, 12768, 12770, 13098, 13859, 14254, 15351, 15351, 15352, 15352, 15739, 15922, 15923, 16232, 17174, 17343, 18866, 19516, 19517, 19524, 19525, 19526, 21924.
Cytotoxic, HT1197 cells 22208, 22209

Cytotoxic, HT29 hmn colorectal cancer cells 228, 332, 530, 1297, 1303, $1304,1305,1310,1312,1320,1321,1322,1367,1625,1679,1691$, 1924, 1925, 1926, 2005, 2047, 2338, 2528, 2694, 2735, 2736, 2857 , $3257,3457,3583,3974,3975,4164,4165,4166,4310,4880,5841$, $5843,6010,6165,6181,6183,6204,6314,6367,6404,6405,6490$, 6642, 6827, 6828, 7032, 7698, 7788, 7789, 7951, 8203, 8204, 8205, 8556, 8946, 9226, 9323, 9544, 10126, 10462, 11269, 11375, 11494, $11566,11605,12254,12541,12886,12887,12888,13673,13685$, $13808,14254,14492,14572,14645,14646,14906,15082,15084$, $15085,15087,15088,15089,15141,15879,15902,15903,16020$, $16282,16283,16337,16402,16427,16677,16832,17588,17619$, 17872, 18754, 18768, 18769, 18770, 18771, 18772, 18773, 18774, 18775, 18776, 18777, 18778, 18892, 19427, 19516, 19517, 19524, 19525, 19526, 20002, 20244, 20363, 20641, 20644, 20811, 20811, 22077, 22270, 22272, 22451, 22458.

Cytotoxic, HT3 hmn cervicall cancer cells 1992.

Cytotoxic, HTC mus hepatosarcoma cells $8148,8149,8159,8160,8161$, 8174.

Cytotoxic, HTC rat cultural liver cancer cells $\quad$ 18295, 18296.

Cytotoxic, hTERT-RPE1 hmn telomerase reverse transcriptase-retinal pigment epithelial cells $2259,3300,5319,5387,5401,5536,6042$, 6483, 6500, 20811, 21803.

Cytotoxic, Huh7 hmn hepatoma cells 9376, 9383, 9386.

Cytotoxic, HUVEC hmn umbilical vein endothelial cells 1863, 3300, $3300,5319,5319,5401,5536,5536,6483,6483,6500,6500,6759$, 10396, 14045, 20811, 21803.

Cytotoxic, induces apoptosis, B16 19143.

Cytotoxic, induces apoptosis, causes rapidly apoptosis of many radioresistant and chemoresistant hmn squamous cell carcinoma 3498.

Cytotoxic, induces apoptosis, HeLa 7665.

Cytotoxic, induces apoptosis, HL-60 10287, 13492.

Cytotoxic, inhibition assay of ornithine decarboxylase 1373,1394 , 1395.

Cytotoxic, inhibits absorption of thymidine 2664 .

Cytotoxic, inhibits biosynthesis of DNA 17489.

Cytotoxic, inhibits biosynthesis of DNA, RNA and protein 14698, 16183, 21397.

Cytotoxic, inhibits biosynthesis of DNA, RNA, protein and cholesterol 7566 .

Cytotoxic, inhibits $\mathrm{Ca}^{2+}$-induced depolymerization of tubulin to overcome resistance of cancer cells $\quad 20800,20850$.

Cytotoxic, inhibits cellular macromolecular biosyntheses 8360,8361 , $8363,8364,8365$.

Cytotoxic, inhibits DMBA-induced preneoplastic lesion formation 240, 4151, 4179, 4180.

Cytotoxic, inhibits DNA biosynthesis by blocking thymidine to go into HL-60 cells 941, 6484, 22777.

Cytotoxic, inhibits estrogen receptor-positive MCF7 hmn breast cancer cells, the inhibition was not reversible by an addition of estrogen 2102 .

Cytotoxic, inhibits growth of cells $2102,2106,19284,20009,22718$, 
22720.

Cytotoxic, inhibits growth of cells inactive, MCF7, NCIH460, SF268 8569.

Cytotoxic, inhibits growth of cells, A549 5055, 6162, 21709, 22208, 22209.

Cytotoxic, inhibits growth of cells, B16 19143.

Cytotoxic, inhibits growth of cells, Bel7402 5888, 7504, 8692.

Cytotoxic, inhibits growth of cells, breast cancer cells 2601.

Cytotoxic, inhibits growth of cells, Col2 5055 .

Cytotoxic, inhibits growth of cells, $\mathbf{E L}_{\mathbf{4}} 19143$.

Cytotoxic, inhibits growth of cells, GI-101A 3498.

Cytotoxic, inhibits growth of cells, HCT15 6162, 21709, 22208, 22209.

Cytotoxic, inhibits growth of cells, HepG2 2102, 2106.

Cytotoxic, inhibits growth of cells, HL-60 3576, 3580, 3581, 3582, 3583, 14698, 19410, 19411, 19412, 19413.

Cytotoxic, inhibits growth of cells, hmn cancer cells 22210, 22211, 22212.

Cytotoxic, inhibits growth of cells, HT1197 22208, 22209.

Cytotoxic, inhibits growth of cells, KB 13672, 14813, 21447, 21448, 21554.

Cytotoxic, inhibits growth of cells, LOX-IMVI 6162, 21709.

Cytotoxic, inhibits growth of cells, MCF7 8563, 8570, 13092, 13093, 13098, 18170, 18171, 18172, 22208, 22209.

Cytotoxic, inhibits growth of cells, MDA-MB-435 2102.

Cytotoxic, inhibits growth of cells, $\mathbf{M H}_{\mathbf{1}} \mathbf{C}_{\mathbf{1}} 19143$.

Cytotoxic, inhibits growth of cells, NCI-H460 8563, 8570, 13092, 13093, 13098, 18170, 18171, 18172.

Cytotoxic, inhibits growth of cells, PC3 21709, 6162.

Cytotoxic, inhibits growth of cells, rat liver cancer 16935.

Cytotoxic, inhibits growth of cells, SF268 8563, 8570, 13092, 13093, 13098, 18170, 18171, 18172.

Cytotoxic, inhibits growth of cells, SW620 6162, 21709.

Cytotoxic, inhibits growth of cells, ZR-75-1 9564.

Cytotoxic, inhibits invasion of mus MO4 cells into embryonic chick heart fragments 15635, 20670.

Cytotoxic, inhibits phosphorytated action of phospholipid, then inhibits cancer cell's growth and metabolism 17763.

Cytotoxic, inhibits TPA-induced ${ }^{32} \mathrm{P}$ combines with phospholipid in HeLa cells 12766.

Cytotoxic, Ishikawa anti-E2 bioassay $1389,1390,14714$.

Cytotoxic, J774.A1 macrophages $1575,3821,8715,8716,18691,18693$, 18733, 22838.

Cytotoxic, Jurkat-T hmn T-cell leukemia cells $2495,4190,4604,5851$, 5973, 7295, 10047, 11978, 17478, 17528, 17830, 17979.

Cytotoxic, K562 doxorubicin-resistant hmn leukemia cells 5175,8238 , 11433.

Cytotoxic, K562 hmn leukemia cells $607,610,843,1245,1913,1914$, 2334, 2338, 2528, 3548, 3551, 3583, 3920, 3921, 4147, 4148, 4701, 5929, 6756, 6785, 6789, 6790, 6794, 6826, 7062, 8483, 9080, 9381, 10277, 10281, 10559, 11266, 11392, 11394, 11722, 12171, 12177, 12180, 12536, 12587, 12588, 12589, 12936, 12958, 13071, 13076, 13078, 13083, 13123, 13241, 13537, 13544, 13694, 14304, 14305, 15652, 16050, 16183, 16402, 17240, 17717, 17975, 17979, 17980,
18457, 18459, 18499, 18931, 19122, 19458, 19571, 19579, 20010, 20811, 20811, 22281, 22653, 22654, 22655, 22657, 22751, 22793, 22794, 22795, 22797, 22798, 22799, 22802, 23013.

Cytotoxic, Kato3 hmn stomach cancer cells 225, 226, 463, 2257, 2261, $2262,4268,4269,5942,5945,6621,10307,11405,12414,12417$, 12423, 19535, 19537.

Cytotoxic, KB ATCC CCL17 hmn nasopharyngeal carcinoma cells 4941, 5494, 7115, 7182, 10155, 18673.

Cytotoxic, KB drug-resistant cells 18866.

Cytotoxic, KB hmn nasopharyngeal carcinoma cells $24,76,78,84,90$, 228, 265, 332, 349, 492, 526, 530, 531, 577, 589, 777, 837, 913, 914, $915,993,1012,1277,1306,1460,1470,1471,1472,1492,1768,1863$, $1864,1885,2026,2109,2244,2260,2338,2495,2590,2604,2619$, 2652, 2653, 2654, 2655, 2656, 2660, 2661, 2677, 2716, 2722, 2810, 2811, 2830, 2990, 2991, 2998, 3181, 3300, 3368, 3402, 3405, 3406, $3416,3481,3499,3513,3514,3515,3600,3626,3708,3729,3732$, $3748,3777,3778,3829,4048,4164,4165,4166,4190,4320,4323$, 4324, 4327, 4547, 4604, 4708, 4739, 4740, 4775, 4939, 5064, 5074, 5074, 5095, 5237, 5238, 5239, 5274, 5275, 5319, 5327, 5401, 5522, 5536, 5537, 5851, 5941, 5973, 6042, 6049, 6483, 6500, 6514, 6516, $6606,6646,6647,6652,6654,6750,6751,6759,6759,6759,6759$, $6759,6759,6759,6759,6759,6759,6760,6766,6913,7027,7030$, 7031, 7033, 7036, 7041, 7078, 7192, 7193, 7295, 7317, 7550, 7551, 7552, 7553, 7554, 7555, 7556, 7579, 7580, 7581, 7583, 7584, 7585, 7586, 7590, 7593, 7594, 7595, 7650, 7655, 7740, 7912, 7914, 8004, $8079,8080,8121,8220,8221,8222,8235,8278,8327,8328,8489$, $8508,8509,8526,8527,8530,8531,8532,8533,8534,8535,8536$, 8541, 8543, 8544, 8940, 9072, 9187, 9194, 9198, 9320, 9330, 9570, 9859, 9861, 9902, 9917, 9918, 9993, 10047, 10148, 10250, 10297. 10297, 10297, 10310, 10396, 10511, 10588, 10672, 10673, 10680, 10692, 10815, 10816, 10817, 10909, 10915, 11015, 11071, 11230, $11231,11232,11295,11316,11353,11355,11380,11494,11636$, 11645, 11723, 11736, 11807, 11850, 11977, 11978, 11980, 12188, 12189, 12454, 12487, 12746, 12897, 12917, 12944, 12964, 13023, 13430, 13432, 13464, 13469, 13470, 13472, 13489, 13490, 13492, $13493,13494,13495,13618,13619,13620,13622,13672,13845$, 14045, 14195, 14602, 14813, 14906, 14934, 14934, 14935, 14935, 14978, 15170, 15242, 15409, 15417, 15494, 15495, 15598, 15635, 15739, 15746, 15776, 15857, 15991, 16020, 16119, 16266, 16280, 16312, 16329, 16337, 16339, 16356, 16402, 16411, 16429, 16444, 16495, 16604, 16693, 16695, 16733, 16747, 16749, 16864, 16984 , 17008, 17252, 17430, 17431, 17478, 17557, 17591, 17592, 17603, 17830, 17832, 17862, 17928, 17929, 17998, 18044, 18091, 18114, 18188, 18222, 18224, 18298, 18515, 18523, 18562, 18637, 18637 , 18668, 18768, 18769, 18770, 18771, 18772, 18773, 18774, 18775, 18776, 18777, 18866, 18892, 19128, 19129, 19148, 19234, 19235, 19373, 19376, 19511, 19542, 19542, 19637, 19638, 19753, 19790, 19900, 20051, 20062, 20063, 20160, 20284, 20285, 20540, 20575 , 20626, 20629, 20630, 20631, 20632, 20633, 20634, 20635, 20636, 20637, 20638, 20639, 20640, 20671, 20743, 20800, 20803, 20809, 20809, 20810, 20811, 20811, 20813, 20814, 20833, 20834, 20835, 20852, 20853, 20856, 20873, 20911, 20963, 21313, 21314, 21315 , 21316, 21390, 21447, 21448, 21554, 21803, 21830, 22011, 22011, 
22013, 22013, 22103, 22147, 22148, 22149, 22150, 22232, 22270, 22272, 22301, 22405, 22411, 22412, 22414, 22443, 22543, 22548, 22549, 22630, 22667, 22768, 22784.

Cytotoxic, KB15 hmn nasopharyngeal carcinoma cells 15879 .

Cytotoxic, KB16 hmn nasopharyngeal carcinoma cells 11949,11951 , $16282,16343$.

Cytotoxic, KB3 hmn nasopharyngeal carcinoma cells 15920 .

Cytotoxic, KBMRI cells 10126.

Cytotoxic, KB-V cells $\quad 11353,11353,15919,17008,17008,17009$.

Cytotoxic, KB-V1 cells 15739, 20809.

Cytotoxic, KB-VIN vincristine-resistant nasopharyngeal carcinoma cells $1625,14934,14935,14935,16864$.

Cytotoxic, Ketr3 hmn renal cancer cells $\quad 6075,9567,11081,17059$, 17060, 17061, 17062, 17063, 17064.

Cytotoxic, KG-1 hmn leukemia cells 17240.

Cytotoxic, Kichita sarcoma cells $15230,15231,15232,15233,15234$, 17591.

Cytotoxic, KM12 hmn colorectal cancer cells $3583,4880$.

Cytotoxic, KM20L2 hmn colorectal cancer cells 7912, 9859, 9861, 9917, $10672,10673,16296,16312$.

Cytotoxic, KM3 cells 17528.

Cytotoxic, KU-1 hmn bladder cancer cells $\quad 2102,2106,22718$.

Cytotoxic, $\mathbf{L}_{\mathbf{1 2 1 0}}$ lymphocytic leukemia cells $\quad 577,2304,2339,2340,4048$, $4708,7740,8360,8361,8363,8364,8365,8452,15409,15647,16183$, $16411,16429,16586,17557,17600,18523,20009,20240,20743$, 20771, 20798, 20800, 20813, 20814, 20849, 20850, 20851, 20852, 20853, 20871, 20872, 22281.

Cytotoxic, L5178Y lymphosarcoma cells $\quad 2811,13432,17822,21493$.

Cytotoxic, L-6 rat skeletal myoblasts $589,11978,17478,17592,17592$, 22551.

Cytotoxic, L-929 fibrosarcoma cells $\quad 2492,2493,3241,3548,4046,5929$, $6437,9669,10277,10281,10559,11800,11801,12171,12177,12180$, 14304, 14305, 16599, 17654, 18056, 20811.

Cytotoxic, lymphoma cells 21490 .

Cytotoxic, leukemia $\quad 1275,2660,2684,13044,14945,16597,19146$, $19221,22411$.

Cytotoxic, leukemia cells 11227.

Cytotoxic, liver cancer cells 11392, 22270.

Cytotoxic, LLC mouse Lewis lung cancer cells $\quad$ 1792, 3227, 3228, 3229, $3230,4235,5378,8187,10235,10317,10403,10539,11023,11278$, 11279, 13020, 13021, 13241, 17769, 19777, 20556.

Cytotoxic, LMTK mouse fiber cells 5081, 16634.

Cytotoxic, LNCaP hmn prostate cancer cells 1863, 3087, 3088, 5074, 6042, 6759, 6759, 7078, 7764, 9013, 9187, 10297, 10396, 10588, 11353, 14045, 14227, 14234, 15739, 15919, 15922, 17008, 18866, 20809, 20811, 21313, 21314, 21315, 21316, 21803.

Cytotoxic, LO2 hmn liver cells 11203.

Cytotoxic, LoVo hmn colorectal cancer cells $\quad$ 1552, 6164, 6213, 6490, 9376, 9383, 9386, 13403, 16703, 19916, 22077.

Cytotoxic, LoVo/Doxo hmn colorectal cancer cells, drug-resistant subclone 6164 .

Cytotoxic, LOX melanoma cells 4111, 4112, 4113.

Cytotoxic, LOX-IMVI melanoma cells 251, 2334, 2338, 4147, 4148,
4880, 5314, 5706, 6162, 16050, 19516, 19517, 19524, 19525, 19526, $21709,23013$.

Cytotoxic, LS174T colorectal cancer cells 4913.

Cytotoxic, Lu1 hmn lung cancer cells 1863, 2259, 3300, 5319, 5387, $5401,5536,6042,6483,6500,6759,6759,6759,7078,7764,9187$, $10297,10396,10588,11353,13469,13470,14045,15919,17008$, 18866, 20809, 20811, 21313, 21314, 21315, 21316, 21803.

Cytotoxic, LUC-1 15739.

Cytotoxic, lung cancer cells 10229, 10230.

Cytotoxic, LXFL529L hmn large cell lung cancer cells 2102, 2106, 13241, 20009, 22718, 22720.

Cytotoxic, lymphleukemia cells 16864 .

Cytotoxic, lymphocyte sarcoma 2664 .

Cytotoxic, M1 mus myelocytic leukemia 17239.

Cytotoxic, M14 melanoma cells 3583 .

Cytotoxic, M4BEU hmn melanoma cells $3241,9669$.

Cytotoxic, M5076 ovarian sarcoma cells 16601.

Cytotoxic, Ma7373 mus breast cancer cells 11179 .

Cytotoxic, macrophages 1359.

Cytotoxic, MALME-3M melanoma cells 3583 .

Cytotoxic, mammary cancer cells in rat 12957, 12958.

Cytotoxic, many of radioresistant and chemoresistant hmn squamous cell carcinoma 3498 .

Cytotoxic, may serve as a new prototype molecule to develop antitumor agents, the first compound of this type to contain lactone moieties on both sides of the aliphatic chain and to lack either tetrahydrofuran or tetrahydropyran rings 18897 .

Cytotoxic, MBT-2 mus bladder cancer cells $\quad 2102,2106,22718$.

Cytotoxic, MCF7 hmn breast cancer cells $1297,1303,1304,1305,1310$, $1320,1321,1322,1679,1764,2006,2527,2528,2735,2736,2856$, 2857, 2985, 3053, 3096, 3097, 3241, 3303, 3457, 3555, 3583, 3868, $3869,4310,4317,4320,4323,4689,4690,4880,4913,4930,4986$, $5551,5622,5826,5832,6075,6221,6367,6827,6828,7100,7125$, $7126,7376,7613,7614,7646,7698,7788,7912,8526,8527,8528$, $8530,8531,8532,8533,8534,8535,8536,8541,8543,8544,8563$, $8570,8946,8947,8948,9323,9567,9669,9859,9861,9885,9917$, $10672,10673,10815,10816,10817,11029,11081,11269,11566$, 11977, 13092, 13093, 13098, 13518, 13867, 13869, 14934, 14934, $14935,14935,15082,15084,15085,15087,15088,15089,15141$, $15169,15902,15903,15916,16020,16021,16296,16312,16402$, 16676, 16693, 16694, 16695, 16832, 17059, 17060, 17061, 17062, 17063, 17064, 17309, 17619, 17973, 18170, 18171, 18172, 19427, 19516, 19517, 19524, 19525, 19526, 20244, 20641, 20643, 20644, 20811, 20811, 20811, 20811, 20811, 21435, 22011, 22013, 22077, 22208, 22208, 22209, 22281, 22781.

Cytotoxic, MCF7/6 hmn breast cancer cells 12344 .

Cytotoxic, MCF7/ADR-RES hmn breast cancer cells 3583.

Cytotoxic, MCF7-ras hmn breast cancer cells 11977, 22077.

Cytotoxic, MDA-MB-231 hmn breast cancer cells 2528, 3555, 4880, 5532, 7698, 7698, 8932, 8933, 8934, 8936, 8947, 8948, 20811, 20811.

Cytotoxic, MDA-MB-231/ATCC hmn breast cancer cells 3583 .

Cytotoxic, MDA-MB-435 hmn breast cancer cells 2102, 3583, 11659, 20397. 
Cytotoxic, MDA-N hmn breast cancer cells 3583 .

Cytotoxic, mechanism was considered to be an inhibition of oxygen consumption and depletion of glutathione in tumor cells 11179 .

Cytotoxic, mechanism was postulated to be due to covalent binding of oridonin to a specific site of enzymes in tumor cells 16183.

Cytotoxic, Mel-2 cells 15739, 15923, 17008, 20137.

Cytotoxic, MEL-28 hmn melanoma cells 1924, 1925, 1926, 14492, $14645,14646,16427$.

Cytotoxic, melanoma cells 16864 .

Cytotoxic, Meth-A sarcoma cells 1792, 3227, 3228, 3229, 3230, 4235, 5378, 8186, 8187, 8188, 10229, 10230, 10317, 10403, 10539, 13019, $13020,13021,13241,17769,19777,20556$.

Cytotoxic, MG 18461.

Cytotoxic, MGc803 hmn gastric adenocarcinoma cells 16183.

Cytotoxic, $\mathbf{M H}_{1} \mathbf{C}_{1} 19143$.

Cytotoxic, MH-60 mus leukemia cells 7192, 7193, 7912, 16312, 16329, 18637.

Cytotoxic, MI4 melanoma cells 5706.

Cytotoxic, MIA-PaCa-2 hmn pancreas cancer cells $1297,1303,1304$, $1305,6827,6828$.

Cytotoxic, MK1 hmn stomach cancer cells $16599,18944,18950,18951$, 18952, 18953.

Cytotoxic, MKN1 hmn stomach cancer cells 4880 .

Cytotoxic, MKN28 hmn stomach cancer cells 1625, 4880, 6785, 12202, 12958, 18714, 18931, 22793, 22794, 22795.

Cytotoxic, MKN45 hmn stomach cancer cells 4880 .

Cytotoxic, MKN7 hmn stomach cancer cells 4880 .

Cytotoxic, MKN74 hmn stomach cancer cells 4880 .

Cytotoxic, MMOC assay, inhibition of DMBA-induced preneoplastic lesions in vitro $3602,5595,6064,7204,8278,10430,10741,11504$, 20485, 20610, 21410, 21483.

Cytotoxic, Molt4 hmn lymphoma cells 3583, 5081, 13241, 15750, 16634.

Cytotoxic, Mono-Mac-6 mononuclear cells $\quad 2495,4190,4604,5851$, 5973, 7295, 10047, 17830.

Cytotoxic, mouse alveolus non-cancer fibrocytes LMTK 15750 .

Cytotoxic, mouse mammary organ culture model MMOC 240,4151 , $4179,4180$.

Cytotoxic, mouse TPA-induced skin tumor 17763.

Cytotoxic, MRC-5 hmn diploid embryonic cells 19892.

Cytotoxic, MT2 cells 3816 .

Cytotoxic, MT-4 cells 8436 .

Cytotoxic, MTT assay $\quad 2985,4893,5375,5551,12397,12398,15652$, 15916, 19536, 21845, 22299, 22653, 22654, 22655, 22988.

Cytotoxic, mus mammary organ culture assay 1453, 1454, 3568, 4151, 4282, 5679, 6497, 7788, 7824, 10741, 10827, 11504, 11804, 15714, 20326, 21440, 21483, 22705, 22715.

Cytotoxic, mutant yeast strain RAD 52Y 6710, 6711, 6714, 12537, 19061.

Cytotoxic, NCI 60 hmn tumor cell lines screen $\quad 3583,14719,17722$, 19296 .

Cytotoxic, NCI-H1417 hmn small cell lung cancer cells 17979.

Cytotoxic, NCI-H187 hmn small cell lung cancer cells $\quad 349,526,1277$,
$2244,3416,3429,3708,3940,4775,5064,5276,5827,6759,8220$, $8221,8222,8235,8315,13484,13487,13489,13490,13492,13493$, 13494, 13495, 15494, 15495, 20671.

Cytotoxic, NCI-H226 hmn non-small cell lung cancer cells 1625,4763 , 4880, 10558, 20720, 20721, 20722, 20811.

Cytotoxic, NCI-H23 hmn lung cancer cells 3583,4880 .

Cytotoxic, NCI-H460 hmn lung cancer cells 2528, 4880, 7125, 7126, $7613,7614,7698,7912,8563,8570,8669,8670,8671,9859,9861$, $9917,10672,10673,11890,13092,13093,13098,13328,16296$, 16312, 18170, 18171, 18172, 18714, 20811.

Cytotoxic, NCI-H522 hmn lung cancer cells $1625,3583,4880$.

Cytotoxic, NK/LY ascites cancer cells 13137.

Cytotoxic, no explanation on cell species $\quad 370,576,595,597,598,1748$, 1789, 1971, 2034, 2073, 2119, 2130, 2227, 2303, 2529, 2946, 3063, $3076,3294,3388,3467,3565,4348,4349,4350,4351,4353,4399$, $4414,5136,5168,5187,5236,6027,6261,6737,7559,7739,8239$, $9304,9655,11052,11379,11386,11423,11636,12225,12501,12916$, $13072,13473,13522,14086,14121,14821,14822,14830,14835$, $14836,15335,15634,15635,15637,15764,15782,15801,16019$, $16119,16128,16205,16278,16674,16675,16747,16748,16749$, $16929,16978,17037,17038,17041,17200,17201,17558,17594$, $17619,17665,17666,17667,17668,17669,17670,17671,18114$, 18297, 18522, 18567, 18679, 18843, 19194, 19313, 19315, 19468, 19904, 19905, 19906, 19907, 20121, 20171, 20235, 20387, 20496, 20664, 20730, 21559, 21889, 22268, 22407, 22420, 22475, 22818, 22965, 22970, 22972, 22987.

Cytotoxic, no reversal effect of VCR resistance 1625 .

Cytotoxic, no significant differential cellular sensitivities when it was evaluated in the Japanese Foundation for Cancer Research 39 cell line assay 18714.

Cytotoxic, non-parvicellular lung cancer, with strong selectivity 20813.

Cytotoxic, normal EAC-E4cells 7586, 7593.

Cytotoxic, normal hmn cell, fibroblasts 9669 .

Cytotoxic, normal Rkcells 7586, 7593.

Cytotoxic, NSCLC-N6 hmn non-small cell lung cancer cells 10340 , 10573.

Cytotoxic, NSCLCN6-L16 non-small cell lung cancer cells 16495.

Cytotoxic, NUGC hmn stomach cancer cells $955,8935,8936,10344$, 16891, 22114, 22459.

Cytotoxic, NUGC-3 hmn stomach cancer cells 2006, 4317, 4320, 4323, 5573, 5574, 7035, 13294, 17973, 20495.

Cytotoxic, NUGC-4 hmn stomach cancer cells $19535,19537$.

Cytotoxic, ornithine decarboxylase inhibition assay $1372,5543,15412$.

Cytotoxic, OVCAR-2780 ovarian adenocarcinoma cells 12531,12532 , 12533.

Cytotoxic, OVCAR-3 ovarian adenocarcinoma cells $1617,4128,4880$, 4891, 7044, 7698, 12511, 17979, 18673, 18714, 19308, 20811, 22962.

Cytotoxic, OVCAR-4 ovarian adenocarcinoma cells 4880.

Cytotoxic, OVCAR-5 ovarian adenocarcinoma cells 3583,4880 .

Cytotoxic, OVCAR-8 ovarian adenocarcinoma cells $4880,18714$.

Cytotoxic, $\mathbf{P}_{\mathbf{3 8 8}}$ mouse lymphocytic leukemia cells $\quad 378,577,913,914$, 915, 1008, 1306, 1367, 1691, 1924, 1925, 1926, 2005, 2047, 2109, $2219,2260,2303,2304,2677,2694,2811,3181,3257,3266,3267$, 
3268, 3271, 3476, 3602, 3710, 3829, 3936, 3974, 3975, 4135, 4291, 4471, 4680, 4690, 4708, 4986, 5327, 5841, 5843, 5901, 5941, 6010, 6014, 6165, 6181, 6183, 6204, 6204, 6284, 6285, 6289, 6328, 6402, 6404, 6405, 6606, 6642, 6759, 6759, 6760, 6761, 6776, 7568, 7569, 7611, 7674, 7675, 7677, 7681, 7682, 7683, 7684, 7740, 7764, 7788, 7789, 7951, 8167, 8203, 8204, 8205, 8556, 8824, 9035, 9226, 9362 , 9544, 9594, 9883, 10116, 10462, 10468, 10511, 11428, 11494, 11605, $11842,11854,11855,11856,11858,11860,11861,11862,11863$, $11978,12122,12125,12254,12886,12887,12888,12964,13023$, 13038, 13468, 13469, 13470, 13673, 13685, 13808, 14139, 14254, 14492, 14572, 14645, 14646, 14846, 15073, 15074, 15598, 15645, $15739,15762,15792,15879,15919,15920,15921,16122,16261$, $16262,16282,16283,16343,16356,16403,16411,16427,16429$, $16495,16601,16661,16662,16695,16733,16966,17218,17557$, 17591, 17603, 17696, 17973, 18033, 18044, 18054, 18057, 18444, 18472, 18485, 18523, 18754, 18768, 18769, 18770, 18771, 18772 . 18773, 18774, 18775, 18776, 18777, 18778, 18892, 18994, 19041, 19057, 19143, 19148, 19483, 19485, 19486, 19983, 19985, 20002, 20051, 20240, 20244, 20284, 20285, 20363, 20809, 20843, 21080 , 22451, 22452, 22453, 22454, 22455, 22456, 22457, 22458, 22735 , 23005.

Cytotoxic, $\mathbf{P}_{388} / \mathrm{ADM}$ doxorubicin-resistant mouse lymphocytic leukemia cells 492

Cytotoxic, $\mathbf{P}_{\mathbf{3 8 8}} / \mathbf{D O X}$ cells 16495 .

Cytotoxic, $\mathbf{P}_{815} 4135$.

Cytotoxic, PACA-2 hmn pancreas cancer cells 1320, 1321, 1322, 3457, 11269, 14906, 18240, 22781.

Cytotoxic, PANC1 hmn pancreas cancer cells 17979.

Cytotoxic, panel of hmn cancer cell lines, according to established protocols of Likitwitayawuid 1993 and Seo 2001 6346, 14342.

Cytotoxic, partial reversal of doxorubicin resistance 22830 .

Cytotoxic, parvicellular lung cancer, with strong selectivity 20813. Cytotoxic, PBMC peripheral blood mononuclear cells $\quad 11978,17478$. Cytotoxic, PC12 hmn lung cancer cells $159,1792,3300,6160,22580$. Cytotoxic, PC13 2811.

Cytotoxic, PC3 hmn prostatic cancer cells $251,1297,1303,1304,1305$, $1320,1321,1322,2334,2338,2528,3241,3258,3259,3260,3261$, $3262,3263,3264,3265,3274,3457,3498,3583,4147,4148,4762$, $4763,4880,5297,5314,6162,6827,6828,9669,10558,11269,11802$, $12446,14906,14934,14935,14935,16050,17979,18211,18714$, 19516, 19517, 19524, 19525, 19526, 20248, 20720, 20721, 20722, 20811, 20811, 20811, 21709, 23013.

Cytotoxic, PC-3M-1E8 11659, 20397.

Cytotoxic, PC-6 hmn lung cancer cells 17973.

Cytotoxic, PD assay $1310,1679,7788,8946,11864,15084,15085$.

Cytotoxic, platelet aggregation inhibitor 22969.

Cytotoxic, PLC/PRF/5 hmn liver cancer cells 1992, 2102, 2106, 3143, 17252.

Cytotoxic, polymorphism malignancy glioma $16548,16549$.

Cytotoxic, potato culture dish PD test 15902.

Cytotoxic, protein quantification assay 4893 .

Cytotoxic, PSN1 hmn pancreas cancer cells 5284, 5295, 6897, 9747, $14495,14496,14504,14644,14647$.
Cytotoxic, PTX10 ovarian cancer cells with $\boldsymbol{\beta}$-tubulin mutation 14934 , 14935.

Cytotoxic, QGY-7703 hmn liver cancer cells $16686,18483$.

Cytotoxic, quinone reductase induction assay $1380,1381,1382,1383$, $1384,1386,1388,1392,1393,1401,1403,1404,1406,1407,1408$, $1409,1410,1411,1413,1414,1415,1416,1418,1419,1420,1425$, $1427,1428,1429,1430,1431,1432,1433,1434,1435,1436,1451$, $1452,1453,1454,1455,1456,1457,2468,2857,3602,4282,6228$, $6246,7670,7788,8278,10430,10827,11504,11804,12953,14074$, 14075, 16498, 19542, 19910, 21918, 22615, 22715.

Cytotoxic, RAD+ wild type yeast strain $6710,6711,6714,12537$, 19061.

Cytotoxic, radiosensitizing agent 11179 .

Cytotoxic, Raji cells $6254,6776,10068,20010$.

Cytotoxic, RB cells 18545 .

Cytotoxic, RL33 rbt lung cancer cells 11980.

Cytotoxic, Rous sarcoma virus 3481 .

Cytotoxic, RPMI 2677.

Cytotoxic, RPMI-7951 melanoma cells 3296, 16548, 16549, 17144, 17603, 17895, 17896, 18202, 20389, 22435.

Cytotoxic, RPMI-8226 leukemia cells 3583 .

Cytotoxic, RXF-393 renal cancer cells 3583 .

Cytotoxic, RXF-631L renal cancer cells 4880 .

Cytotoxic, $\mathbf{S}_{\mathbf{1 8 0}}$ mouse sarcoma cells $\quad 3502,8187,8188,13021,16414$, 22232.

Cytotoxic, $\mathbf{S - 1 8 0 V}$ cells 11179.

Cytotoxic, Sca7901 hmn gastric adenocarcinoma cells 4460 .

Cytotoxic, SCL hmn stomach cancer cells $\quad 6621,7016,11405,19535$, 19537.

Cytotoxic, SCL-37'6 hmn stomach cancer cells $\quad 6621,7016,11405$, 19535, 19537.

Cytotoxic, SCL-6 hmn stomach cancer cells $\quad 6621,11405,19535,19537$.

Cytotoxic, SCL-9 hmn stomach cancer cells $\quad 6621,7016,11405,19535$, 19537.

Cytotoxic, SDK cells 17528.

Cytotoxic, Selective DNA-damaging activity 1961, 3053.

Cytotoxic, SF268 hmn brain tumor cells $3583,4880,7126,7912,8563$, $8570,8669,8670,8671,9859,9861,9917,10672,10673,13092$, 13093, 13098, 13328, 16296, 16312, 18170, 18171, 18172.

Cytotoxic, SF295 hmn brain tumor cells 3583, 4880, 18714.

Cytotoxic, SF539 hmn brain tumor cells $1625,3583,4111,4112,4113$, 4880.

Cytotoxic, SGC hmn stomach cancer cells 6055 .

Cytotoxic, SGC7901 hmn stomach cancer cells 12202.

Cytotoxic, SiHa hmn cervical carcinomacells $\quad$ 1992, 7032, 11977, 22077.

Cytotoxic, SKBR3 hmn breast cancer cells $5284,5295,6897,9747$, 14495, 14496, 14504, 14644, 14647.

Cytotoxic, SKCO1 colorectal cancer cells 4913.

Cytotoxic, SK-MEL hmn caucasian melanoma cells $\quad 11636,18114$.

Cytotoxic, SK-MEL-2 hmn melanoma cells 2334, 2338, 3583, 4147, 4148, 14934, 14934, 16050, 16370, 18011, 20679, 20680, 20681, 23013.

Cytotoxic, SK-MEL-28 hmn melanoma cells 3583 . 
Cytotoxic, SK-MEL-5 hmn melanoma cells 3583, 5706.

Cytotoxic, SK-MES-1 bronchogenic carcinoma cells 12398.

Cytotoxic, SK-OV-3 ovarian adenocarcinoma cells 4880, 7535, 11636, 18011, 18114, 20679, 20680, 20681.

Cytotoxic, SMMC-7721 hmn liver cancer cells 11203, 14828, 16675, 18034.

Cytotoxic, SNB75 hmn brain tumor cells 4880 .

Cytotoxic, SNB78 hmn brain tumor cells $1625,4880$.

Cytotoxic, SNU638 hmn gastric adenocarcinoma cells 6277, 6282, 6759, 10438, 21924.

Cytotoxic, soft agar transformation assay with JB6 cells 1373, 1385, $1391,1394,1395,1426,1428,1429,1433,3568,10827,11804,17342$, 22715.

Cytotoxic, specific activity for cancer cells 16265 .

Cytotoxic, SR leukemia cells 3583 .

Cytotoxic, St4 stomach cancer cells 4880 .

Cytotoxic, stomach cancer cells 924, 22232.

Cytotoxic, sulforhodamine-B assay 12344 .

Cytotoxic, SW620 hmn colorectal adenocarcinoma cells 225, 226, 251, $463,2257,2261,2262,3583,4268,4269,5314,5942,5945,6162$, 10307, 12414, 12417, 12423, 16599, 17973, 21709.

Cytotoxic, T24 hmn liver cancer cells 1992, 12587, 12589, 13537.

Cytotoxic, T24S hmn bladder cancer cells 16402.

Cytotoxic, T47D hmn breast cancer cells $7032,8187,8188,12541$, 13020, 13021, 17979.

Cytotoxic, T98G hmn caucasian gioblastoma cells $5284,5295,6897$, 9747, 14495, 14496, 14504, 14644, 14647.

Cytotoxic, TE-671 2677.

Cytotoxic, TK10 renal cancer cells 4930 .

Cytotoxic, T-lymphocyte 1359

Cytotoxic, Tmolt3 hmn leukemia cells 15409.

Cytotoxic, topoisomerase I inhibitor $6835,9011$.

Cytotoxic, toxic to topoisomerases I and II 7704 .

Cytotoxic, toxin 12138 .

Cytotoxic, U14 mouse cervical carcinoma cells 11179.

Cytotoxic, U251 brain tumor cells 3583, 4880, 5706, 18714, 22281.

Cytotoxic, U373 caucasian gioblastoma cells $11353,15739,15919$, 15922, 15923, 17008, 18866.

Cytotoxic, U-87-MG caucasian gioblastoma cells $\quad$ 14934, 14935, 14935.

Cytotoxic, U937 hmn monocytic leukemia cells 2956, 2957, 2958, 2964, $3911,7060,7153,7436,8360,8361,8363,8364,8365,11895,13241$, $14347,16675$.

Cytotoxic, UACC62 melanoma cells 3583, 4930.

Cytotoxic, uncoupling of oxidative phosphorylation 3498 .

Cytotoxic, UO-31 renal cancer cells $\quad 2528,3583,20811$.

Cytotoxic, V-79 cells 3181, 4081, 4082, 4083, 4084.

Cytotoxic, Vero green monkey kidney tumour cells $6759,6759,6776$, 10068, 11636, 14965, 16266, 18114, 20010, 20253.

Cytotoxic, W-18Va-2 cells 7586, 9288.

Cytotoxic, $\mathbf{W}_{256}$ rat Walker sarcoma cells $4643,6776,11023$.

Cytotoxic, WEHI-164 mus fibrosarcoma cells $1575,3821,8715,8716$, $18691,18693,22838$.

Cytotoxic, WHCO1 hmn esophageal cancer cells 2619, 2625, 17564,
17565,21545

Cytotoxic, WI-38 hmn lung fibrocyte (normal hmn diploid fibrocyte) 3053, 9288, 10773, 11723, 17265.

Cytotoxic, WiDr colorectal adenocarcinoma cells 4913, 17979.

Cytotoxic, Wish transformed epithelial tumour cells 6776, 10068, 20010.

Cytotoxic, XF-498 hmn tumour cells 18011, 20679, 20680, 20681.

Cytotoxic, yeast assay RS321NpRAD52(glu) 1961, 3053.

Cytotoxic, ZR-75-1 hmn breast cancer cells $\quad 9187,9564,11353,15739$,

15919, 15923, 17008, 17009, 18866.

Damages function of liver and kidney 3692 .

Decolorant 9739 .

Decreases accumulation of reactive oxygen intermediates 2102,2106 .

Decreases some hmn leukocyte functions $18219,18220$.

Deodorant $917,17486,17491$.

Depilatory 14868 .

Dermatitis suppressant $\quad 828,829,830,831,1293,1332,1599,21434$.

Detail study on pharmacokinetics of baicalin 2106 .

Detail study on protective effects of evodiamine on myocardial ischemia-reperfusion injury in rats 7665 .

Detail study on protective effects of rutaecarpine on cardiac anaphylaxis (the protective effects of rutaecarpine on cardiac anaphylactic injury are related to inhibition of TNF- $\alpha$ production by stimulation of CGRP release) 19081 .

Detumescent $3983,5502,12184,19910,21434$.

DGAT inhibitor $7104,7663,12341,12343,12344,12361,12364,13922$.

Diabetogenic 17170 .

Digestive enzymes inhibitor 3968 .

Digestive tract irritant 11024 .

Dihydrocoenzyme I (NADH) oxidase inhibitor 13137.

Disinfectant $4234,4306$.

Dispels phlegm $13137,15146,15221,20137,21360$.

Dissolves oncocytes 22491.

Diuretic $480,664,892,893,899,1048,1476,1618,1866,1904,1935$, 2039, 2102, 2106, 3306, 3307, 3615, 4029, 4036, 4080, 4547, 4589, $6455,6699,6776,7278,7665,7883,7944,8806,9224,9335,9340$, $9604,11141,11524,11642,13504,14057,14796,14971,15170$, $15781,16031,16050,16756,17571,17952,18411,18652,18759$, 18794, 18859, 19540, 20133, 20168, 20169, 20730, 21310, 21312, 21508, 22301, 22498, 22499, 22668, 22718.

DNA cleavage inducer, berberrubine induced 2304 .

DNA isomerase inhibitor $598,2034,2227,4348,4349,11423,14086$, 14121, 15335, 16978, 19468, 19904, 19905, 19906, 19907, 22965, 22969, 22970, 22972.

DNA strand-scission activity 22546 .

DNA strand-scission inactive $22543,22544,22545,22547,22548$, 22549.

DNA topoisomerase I inhibitor 6906.

DNA topoisomerase II inhibitor 2102, 3935, 20389.

DNA topoisomerase inhibitor $370,595,597,6261,19278$.

Dopamine receptor antagonist 20324.

Dopamine receptor antagonist (CNS system) 2734, 20324.

Dopaminergic 763 .

Dormancy factor 13084 . 
Downregulates COX-2 gene expression $13437,13435,13436$.

Downregulates IL-1 $\beta$ gene expression $\quad$ 13437, 13435, 13436.

Downregulates IL-6 gene expression $13437,13435,13436$.

Ecdysteroid agonist 10063, 10064.

Ecdysteroid agonist or antagonist inactive 9326 .

Ecdysteroid antagonist $12240,14890,14891$.

Eclamptogenic $642,1738,2716,3637,4051,5108,5136,7980,10734$, $10877,13001$.

Ectoparasiticide; 17269, 20262.

Effective component in Acacia melanoxylon (HEI MU JIN HE HUAN) known to cause contact dermatitis 62 .

Effective component in Alangium chinense (BA JIAO FENG) 1125.

Effective component in Plantago asiatica (CHE QIAN) 17505.

Effective component in Pueraria lobata (GE GEN) 18180.

Effective component in Schisandra chinensis (WU WEI ZI) 19473.

Effective component in Schizonepeta tenuifolia (JING JIE) to settle pain 13776.

Effective component in seeds of Melia azedarach 2050.

Effective component in total alkaloids of Common Devilpepper, Rauwolfia verticillata (LUO FU MU) 18633.

Effective component in Virgate Wormwood, Artemisia scoparia (HUANG HАО) 19540.

Effective component in Yellow Toadflax, Linaria vulgaris (LIU CHUAN YU) 16756.

Egg laying stimulator $2455,15287$.

Elastase inhibitor $306,580,2887,3695,4163,9238,11195,16536$.

Elastase inhibitor inactive 9561 .

Emetic 3400, 5436, 6772, 13241, 15452, 17995, 18095.

Enhances action of $\boldsymbol{\beta}$-adrenalin 15127.

Enhances action to boost blood pressure caused by adrenalin 6439 .

Enhances activity of esterase 22269 .

Enhances arterial tension and lowers intravenous tension 13137.

Enhances blood pressure increase caused by adrenaline 6686.

Enhances cytotoxic effects of daunomycin and vincaleucoblastine 8423 , 8427.

Enhances effects of cytotoxic drugs against cancer metastasis 8404 , 8405.

Enhances effects of vitamin C 9458.

Enhances fibrinolytic activity 11414 .

Enhances neuromuscular transmission 21593.

Enhances phagocytic function 18114 .

Enhances phagocytosis of granular leukocytes 17568.

Enhances respiration 15288 .

Enhances sedative effects of phenobarbital 582 .

Enhances sex drive 2564, 8633, 8634 .

Enhances tension of intestinal canal of cat, but inhibits the tension of rbt 6691 .

Enhances trypsin activity 3231 .

Enzymatic substrate (arginase, anginine decarboxylase, $L$-aminoacidoxidase) 9798 .

Essential amino acid 17093, 22060.

Essential for growth 18656 .

Estrogenic activity $1290,2384,3911,4080,4190,4604,6440,7387$,
$7883,8282,8372,12344,14883,17028,17834,17838,19540,19618$, 20041, 20685, 22718.

Excitation to the peripheral nervous system 13711.

Extends the period of fertility in rat 1599.

Extremely bitter $4315,15454,15542$.

Farnesyl transferase inhibitor 4302.

Farnesylprenyltransferase (PFTase) inhibitor 6162, 9564, 10751,

12448, 21709, 21856.

Farnesyl-protein transferase inhibitor 9908.

Fatty acid synthetase inhibitor 18317.

Feeding irritant (Plutella maculipennis) 3317, 17903.

Feeding pregnant rat 20670.

Fibrinolytic function $4631,10967$.

Flavorant $2808,2850,3237,3241,3242,3766,3767,3768,4305,6501$, $7463,7734,9110,9601,9669,11183,12569,13779,15498,15500$,

15705, 17913, 17914, 19927, 19928, 22217, 22440, 22441, 22920.

Flavorant, conditioning agent 23010 .

Food additive $832,3139,3142,3209,3350,3354,15705$.

Frees menstruation 1520 .

Fruit protective film 9363.

Funk 19459.

GABA $_{\mathbf{A}}$ receptor antagonist 17347 .

Galactin inhibitor 7236 .

Galactin inhibitor, inhibits release of galactin $3935,6765,7232,7243$, 7234,7240 .

Ganglionic blocker $1134,3633,4459,6686,6699,6734,7325,7328$, $7330,12571,13373,13374,13716,22427$.

Ganglionic stimulant 1658,3070 .

Gastric secretion inhibitor 777, 1599, 20082.

Gastric secretion promotor $2887,4097,8304,16770$.

Gastroprotective 6980, 10364, 19983.

Gastroprotective, ethanol- and indomethacin-induced gastric lesions 2969, 2970, 2971, 2972, 2973, 4961, 9083, 11273, 11281, 14678, 16384, 17439, 17451, 17461, 18658.

Gastroprotective, Inhibits ethanol-induced gastric mucosal lesions 1916, 1917, 21283, 21284, 21287.

Gastroprotective, inhibits gastric contraction 4459 .

Gastroprotective, inhibits gastric injury 4398.

Gastroprotective, inhibits gastric secretion $4889,8273,8297,16084$, 19201, 19473, 20711.

Gastroprotective, inhibits gastric ulcer $8914,13678$.

Genotoxic 967, 18058, 18222.

Germination inhibitor $36,2802,5969,9596,9798,11032,11158,13084$, 13883, 17425, 18020, 19542, 20146, 20147, 21397.

Germination/growth inhibitor/stimulator $7777,7778,7788,14444$, 14445.

Glandular secretion inhibitor 2001 .

Glucagon secretion promotor 832 .

Glucocorticoid (enhances glycogen in liver, reduces glycogen in heart and striated muscles) 22270.

Glucose consumption activity 7505 .

Glucose dehydrogenase inhibitor 1319.

Glucose transferase inhibitor 13315. 
Glucosidase I and $\boldsymbol{\alpha}$-, $\boldsymbol{\beta}$-glucosidase inhibitor 6028 .

Glutaminergic agonist 8308 .

Glycation inhibitor 3605,3606 .

Gonad stimulating principle 823 .

Gonad stimulating principle 12916.

Granular release inhibitor $8278,18685$.

Granulation stimulator 1853 .

Growth factor for animals and microorganisms 11083.

Growth retardant 14134

Growth-Stimulating factor 22305 .

$\mathbf{H}^{+}, \mathbf{K}^{+}$-ATPase inhibitor 19201, 19537, 20082.

Hallucinogen 2726, 3477, 4594, 9235, 13255, 13795, 15204, 21059.

Hematopoietic 1845,7852 .

Hemolytic $660,1554,2029,2030,3939,4005,4029,4457,6755,6756$, $6759,7748,8424,8968,9294,13635,16666,18229,18534,18702$, $18916,19148,19316,19333,19334,19335,19336,19337,19338$, 19339, 19340, 19341, 20060, 20069, 21282.

Hemostatic $759,1058,2048,2304,2887,3308,3551,5101,5105,6757$, 9703, 9719, 12580, 14826, 16756, 17655, 18086, 18411, 19749, 19750, $20254,21955,22562$

Hepatoprotective 4398, 10314, 20806, 21157.

Hepatoprotective, alanine aminopherase inhibitor 16050.

Hepatoprotective, aminotransferase inhibitor 3498, 8907, 10351, $16851,22270$.

Hepatoprotective, biosynthesis of hepatic glycogen, promoter 8906 .

Hepatoprotective, $\mathbf{H}_{2} \mathbf{O}_{2}$-induced toxicity $2039,11897,12082,15184$, $18319,18320,18411$.

Hepatoprotective, hepatic cell recondition and regeneration, promoter 16050 .

Hepatoprotective, hepatotoxin inhibitor, induced by GalN 993, 6290, 7302, 12097, 17493, 17494, 18103, 18398, 22667, 3004, 5235, 5834, $7300,7301,7883,8278,10007,10009,13281,13638,14638,16209$, 21918.

Hepatoprotective, improves appetite and symptoms in hepatitis patients 18261.

Hepatoprotective, inhibits activation of macrophages, inhibits increase in sALT and sAST levels $8423,8425,8426,8427,8428,8430,8435$, 9168, 15836, 15840.

Hepatoprotective, inhibits cellular leakage of LDH and AST, and cell death $9631,13388$.

Hepatoprotective, inhibits GSH depletion 9631, 13388.

Hepatoprotective, Inhibits liver damage $16579,16580,16581,20121$, 20122.

Hepatoprotective, inhibits liver damage induced by GaIN, LPS or $\mathbf{C C l}_{\mathbf{4}}$ 18398.

Hepatoprotective, inhibits SGPT, reduces the raised SGPT due to acute liver injury induced by $\mathbf{C C l}_{\mathbf{4}} 20505$.

Hepatoprotective, inhibits stored triglyceride in primary cultured mouse hepatocytes $15563,15564,15565,15566$.

Hepatoprotective, inhibits tBH-induced lipid peroxidation 9631, 13388.

Hepatoprotective, liver regeneration promoter 7385 .

Hepatoprotective, reduces accumulation of trilaurin in liver 8846 .
Hepatoprotective, reduces GPT 20099.

Hepatoprotective, SGPT inhibitor 19377.

Hepatoprotective, SGPT, SGOT and ALP inhibitor 20503.

Hepatotoxin $2015,7636,9316,9318,10238,11019,11091,13235,17040$, 18842, 18854, 19714, 20488, 21051.

Herbicide $1846,6598,12501,13883,18841$.

Herbicide inactive $15518,15519$.

5-HETE production inhibitor 7924.

12(S)-HETE production inhibitor 4281, 12766, 19983.

12(S)-HETE production inhibitor inactive 9, 3354, 4916, 15705, 20465.

Hexokinase inhibitor 2684.

Histamine antagonist 12083, 16098, 18454, 18455, 22023.

Histamine secretion promotor 15811 .

Histidine decarboxylase inhibitor $8278,15279$.

Hormone of defoliation 36 .

Hormone secreted by medulla of adrenal gland 15708 .

5-HT inhibitor 13255, 15279, 18317.

5-HT receptor antagonist, rbt in vitro aortal contraction induced by 5-HT 1857.

5-HT receptor blocker, gpg uterus 7243.

5-HT receptor inhibitor, D receptor and M receptor 16098.

Hyaluronidase inhibitor $5415,6855,6856,6858,6859,11095,17866$, 17867, 18203, 18368.

11 $\beta$-Hydroxylase inhibitor 1162 .

11/-Hydroxysteroid dehydrogenase inhibitor 8427 .

3 $\alpha$-Hydroxysteroid dehydrogenase inhibitor $9456,16539$.

Hypnotic $115,1048,2113,2303,2734,3220,3368,4119,4120,6193$, $7341,9184,9223,9224,11259,11260,11408,11679,14531,16532$, 21077, 22336, 22346, 22375, 22820.

Hypoglycemic $1972,2303,2969,2970,2971,2972,2973,3321,3907$, $3923,3924,4140,6178,7705,8052,8091,11067,12255,12447$, $12734,13098,13100,14290,15882,16050,16792,16931,18599$, 20341, 20383, 20732, 20896, 20898, 21055, 22269, 22498, 22499.

hypoglycemic and lowers urine sugar 22269 .

Hypotensor and vasodilator 9553 .

Ichthyotoxin $126,136,1990,2402,2403,2697,2976,3541,4976,5858$, 6040, 6262, 7063, 7189, 7404, 7448, 7449, 9691, 9877, 10775, 11077, 11903, 11977, 11978, 13801, 14347, 14348, 16564, 16659, 17411, 17413, 17417, 18165, 19469, 19471, 20986, 21507.

IFN- $\gamma$ inhibitor $2106,14976,14977,22686,22687,22688$.

IL-1 inhibitor, in mus enterocelia macrophages 3368 .

IL-10 inhibitor 20992.

IL-10-like activity $\quad 8095,18317$.

IL-12 inhibitor 2102, 10886, 17240.

IL-1 $\alpha$ inhibitor 15635 .

IL-1 $\boldsymbol{\beta}$ inhibitor $2106,3307,3368,15635,16532,16539,17460,17862$, 20686, 20992, 22686, 22687, 22688, 21397.

IL-2 inhibitor $14976,14977,16675,22686,22687,22688$.

IL-2 inhibitor in mus splenocyte 3368.

IL-2 secretion inhibitor 7519, 11318, 12106, 15331, 16216, 19515, 22718 .

IL-4 inhibitor 5430, 14976, 14977, 22686, 22687, 22688.

IL-4 release inhibitor 5424 . 
IL-4 release inhibitor inactive $5420,5421,5422,5423,5425$.

IL-6 inhibitor 2004, 2102, 2106, 3307, 7714, 11521, 11522, 15635, 16532, 17240, 20686, 21206.

IL-6 release inhibitor 3674, 6288, 8278, , 13137, 18317, 21930.

IL-8 inhibitor 2102, 6288, 20992, 21930, 22718.

IL-8 secretion inhibitor 79, 82, 83, 20486.

Immunoenhancer $266,1275,1626,1627,1713,1936,2300,3906,4837$, 5120, 5122, 5446, 6459, 6961, 7788, 11179, 11680, 12501, 13137, 15221, 17569, 18534, 19143, 19148, 20100, 22938.

Immunoenhancer inactive $11679,18826$.

Immunological adjuvant activity, increases serum IgG level 15818, $15820,15821,15825,15826,15827,15829,15830,15832,18448$, $18449,18451$.

Immunomodulator $85,909,2890,3368,4645,7487,7924,13435,13436$, 13437.

Immunostimulant 11539, 11636, 14889, 18114, 22206, 22207.

Immunosuppressant $899,1359,1544,1545,1546,1547,2208,3368$, $3599,3649,3756,4292,5121,5488,5489,5721,6070,7519,7657$, $8023,8024,9367,10580,10814,11318,11882,12106,12827,15319$, $15331,15538,16216,16410,16649,17592,18221,18221,18549$, 18550, 19515, 19869, 19870, 20094, 20685, 20686, 21521, 21579, 21580, 21581, 21585, 21586, 21587, 21588, 21831, 22664, 22679, $22685,22695,22718$.

Immunosuppressant inactive $1542,1543,10776,18695,18696$. Important medium of inflammation and anaphylaxis 9568 . Important role in normal metabolic processes 8785 .

Improves erythrocyte's ability to change shape $1937,1939$.

Improves hyperplasia of spleen lymphocyte 4384 .

Improves permeability of BBB 19587.

Improves respiration 8289 .

Increases absorption through skin 16066.

Increases activity of choline acetyltransferase in rat cerebrum 11209 , 11211.

Increases amount of lactic acid in zoic blood 1972 .

Increases blood flow through kidney 14796.

Increases blood pressure $\quad 580,2304,2716,2726,3070,3475,3729,3732$, $4477,4547,5229,6418,6815,7825,7980,8257,8423,9459,11507$, $13169,13237,13281,13543,14796,17220,17996,18637,19223$, 19299, 19449, 19451, 19452, 19639, 20077, 22144.

Increases blood pressure and blood flow through the coronary arteries 15708.

Increases blood pressure and enhances myocardial contractility 16261 . Increases blood pressure and raises heart rate 19198.

Increases fertility 8405 .

Increases leucocyte 2300 .

Increases level of arterenol and dopamine 12254

Increases level of blood sugar 8297, 20060, 20066, 20069.

Increases spread and mobility of macrophage 5414 .

Increases the rate and depth of breathing 15708 .

Increases the weight and RNA content of both prostate and testis 6754 .

Increases tolerance to anoxia $9631,1275,2303,3633,4630,4889,11259$, 11260, 15129, 17764, 19196.

Indispensable for cell growth, promotes biosynthesis of protein 18261 .
Induces activity of cytochrome system 15204 .

Induces cell cleavage arrest $2238,4823,4824,6325$.

Induces cell cleavage arrest inactive 12140 .

Induces cell differentiation $\quad 11716,19211,19929,21185,21913,22859$.

Induces distortion of mycelial (mold of rice blast) 6703 .

Induces expression of defense genes in uninfected leaves 9528 .

Induces formation of spore 19534.

Induces gene expression changes (hmn fibroblast) $1853,1854,13337$, 13338.

Induces gene expression of pea nodule bacteria and accrete host Pisum sativum 7278.

Induces geno-defect 22974 .

Induces growth of radial root nodule commensal "Frankia" 6502 .

Induces hyperplasia $20683,20684$.

Induces lipid peroxidization 17168.

Induces mitochondria-mediated apoptosis 317 .

Induces nodulin gene expression of symbion in Rhizobium

leguminosarum and Pisum sativum 15279.

Induces production of estrin synthetase and cruarin 3600 .

Induces quinone reductase $10421,18223$.

Induces sweatiness $1654,2550,7665$.

Induces tissue to produce collagen 1845 .

Inducing cell differentiation activity 17239.

Influences CNS in insects 3059.

Influenza virus sialoma inhibitor 11703, 20515.

Inhibits ${ }^{3} \mathbf{H}$-dobamine absorption by synapse of rat striatum 15797.

Inhibits ${ }^{35}$ S-TBPS specially combines with rat brain meninges 17333 ,

$$
\text { 17334, 17335, } 17336 .
$$

Inhibits absorption of GABA and $\boldsymbol{\beta}$-alanine 9092 .

Inhibits acetylcholine 19388.

Inhibits activation of transcription factor AP-1 18317.

Inhibits activity of cells 17219.

Inhibits adenosine diphosphate transfer to adenosine triphosphate

11141 .

Inhibits adipose peroxidization 8311 .

Inhibits akaryocyte $\mathbf{K}^{+}$effusion caused by lysolecithin 3412 .

Inhibits alcohol in blood 20383, 20384.

Inhibits autonomic movement 11741 .

Inhibits autoxidation of linoleic acid 3559, 19929.

Inhibits basophile to release histamine 3602,7819 .

Inhibits biosynthesis of chlorophyll 11825 .

Inhibits biosynthesis of cholesterol $\quad 675,8190,8191,15419,15420$, $15421,18841$.

Inhibits biosynthesis of DNA in mus embryo 22929.

Inhibits bloom 931.

Inhibits breeding 16808.

Inhibits cancer cell invasion $\quad 2040,3308,6853,6864,6921,6923,8098$, 10944, 12891, 12893, 15947, 15955, 15956, 16066.

Inhibits cancer cell invasion inactive $2892,18411,19087,21310$.

Inhibits carcinogenic action of chemicals 664 .

Inhibits cell proliferation of PMBC $\quad 6923,15180,15184,20394$.

Inhibits CNS 15497.

Inhibits CNS with activity similar to that of morphine 6552. 
Inhibits combination of leucocyte and its receptor 4061 . Inhibits contraction of aorta strip 17762.

Inhibits coronal wart growth in potato flower tray 6776 .

Inhibits cytochrome C and P450 13514, 21320, 21321.

Inhibits degradation of insulin $1618,8095,18265$.

Inhibits degranulation and release of $\beta$-hexosaminidase (did not affect the enzyme activity of $\boldsymbol{\beta}$-hexosaminidase) $\quad 6921,15154,15155$, $15156,15157,15158,15159,15160,15163,15164,15165,15170$, 15184, 18413, 20711, 22272.

Inhibits degranulation of mast cell RBL-2H3 5722. Inhibits ejection of sperm 6302 . Inhibits electron-teransfer and oxidative phosphorylation 11130. Inhibits endotoxin, promotes dissolution of fibrin 1939. Inhibits englobement of $\boldsymbol{\gamma}$-propalanine and $\boldsymbol{\beta}$-alanine 1654 . Inhibits excitation of striatum adenyl cyclase caused by dopamine 2734.

Inhibits expression of tissue factor, hmn hyalin leukocyte, induced by interleukin-1 19929.

Inhibits expression of tissue factor, hum monocyte induced by interleukin $\mathbf{I}$, anti-coagulant 3602 .

Inhibits fatigue $8423,8426,8424,8429,8430,8428$.

Inhibits fatigue and promotes interferon inducing formation 12916 . Inhibits fermentation of indole-3-acetic acid (IAA) 9549.

Inhibits flap and quiver in mus, caused by stress 4080 .

Inhibits formation of indole-3-acetic acid oxidase and ATP 19582. Inhibits formation of peroxide 23 .

Inhibits glandular secretion $10872,10870$.

Inhibits Glu-mediated excitatory signal pathway in hippocampus (probably acts through its anti-calmodulin action) 11909.

Inhibits granulation 7527 .

Inhibits granulocyte and lymphocyte 909 .

Inhibits growth of cell and tissue 21662 .

Inhibits growth of green algae 12564

Inhibits growth of wheat coleoptile 8282 .

Inhibits herpes simplex 16796.

Inhibits hippocampal formation 11909.

Inhibits histamine release $443,1840,2359,2360,2361,2362,3600$, 3781, 3782, 3973, 4158, 4159, 4681, 4682, 4683, 4684, 5155, 6351, $7802,8153,8167,9010,11910,11913,17278,19238,19239,19846$ 19929, 20009, 20447, 21493, 22720.

Inhibits IL-1 $\alpha$-induced proliferation of synovia cells 13391. Inhibits increase of $\mathbf{C a}^{2+}$ concentration caused by ET-1 15321. Inhibits kidney damage in diabetic rat 8424 .

Inhibits lactation hormone 16808 .

Inhibits lactic acid bacteria 6853 .

Inhibits lipolysis $\quad 4055,7518,8311,9456,19143,20389,20910$.

Inhibits L-type calcium current 20148.

Inhibits lymphocyte reproduction caused by sheep red blood cell antibody and concanavalin A 17506 .

Inhibits microzymes 22638 .

Inhibits minimally oxidized LDL-induced cellular toxicity $\quad 580,1663$, 2134, 2912, 7925.

Inhibits mitosis $3502,17595,16796,17590,17592,17593,16795,17699$,
22487.

Inhibits mitosis of plant cells 13241 .

Inhibits molds 17931.

Inhibits neuroaction 7793 .

Inhibits neutrophil cells to release $\boldsymbol{\beta}$-glucuronidase 3602 .

Inhibits OH-free radicals damaging AT-III 7441.

Inhibits onset of senility $\quad 8388,8633,8634,11490,22094$.

Inhibits oxidation of LDL $17869,17886$.

Inhibits oxidative phosphorylation 16602, 23014.

Inhibits phagocytosis of hmn granular cells and stimulates the activity in low dose 3526.

Inhibits plasmin and plasminogen 2266 .

Inhibits present of bilirubin in blood 4245,4249 .

Inhibits production of carcinogen 1845 .

Inhibits production of Trichoderma viride 13635.

Inhibits proliferation of neuroglia 4097.

Inhibits promotor of cancer $266,3625,8968,11320,12165,12166$, $12591,15321,15361,16304,16305,16314,16315,16804,16998$, $17869,22697$.

Inhibits rat neutrophilic cells 23 .

Inhibits rat skin passive allergy 19846.

Inhibits reaction of actomyosin-adenosine triphosphate system 3907 .

Inhibits release of $\beta$-glucuronidase, lysozym and histamine in mastocyte 23 .

Inhibits release of Glu-transmitter $\quad 15370,19737,19738$.

Inhibits respiration of cytoblast $4337,11130$.

Inhibits reuptake of neurotransmitters 10883 .

Inhibits specificly basic fibroblast growth factor (bFGF)-induced proliferation of bovine aorta endothelial cells (BAECs) 6364.

Inhibits sperm movement 6778 .

Inhibits spontaneous movement $3354,4029,12849,16451,18197$, 20076, 20077, 20324, 22336, 22346.

Inhibits spontaneous movement and reflex actions 17065.

Inhibits sympathetic nervous system and relaxes blood vessels 9301 , 17869.

Inhibits synthesis of leukotriene in polymorphonuclear neutrocyte 12766,12767

Inhibits teleocidin 19259.

Inhibits tissue respiration in lung trachea 7736 .

Inhibits transport of active sodium in bladder 9233, 9234.

Inhibits transport of gall 18841 .

Inhibits zoospore motility $8605,8646,8651$.

Inhibits [ $\left.{ }^{125} \mathbf{I}\right]$ sauvagine binding to CRH-1 receptor 10883,10886 , 20012, 20013.

Insect antifeedant $425,524,525,788,789,809,1264,1265,2050,2054$, 2055, 2119, 3037, 3076, 3246, 3307, 3635, 3837, 4087, 4742, 5342, 5991, 6028, 6402, 7031, 7788, 7972, 8201, 8404, 8405, 8971, 9015, 9496, 9540, 9547, 9570, 9604, 9646, 10053, 10130, 10131, 10132, $10762,11052,11203,11256,11386,11430,11903,12254,12336$, $12337,12338,12339,12897,13104,13237,13238,13239,13670$, $14446,14946,15189,15521,15534,15535,15536,15537,15606$, $15607,15608,15609,15655,16119,16572,16674,16973,17047$, $17048,17049,17168,17170,17386,17421,17640,17857,18411$, 
19087, 19168, 19177, 19194, 19313, 19511, 19837, 19846, 20965, $21207,21213,21217,21218,21219,21222,21224,21446,21553$, 21889, 22349, 22405, 22406, 22638, 22780, 22784, 22865.

Insect attractant $8313,11025,17402$.

Insect ecdysone $\quad 794,811,4455,6678,6679,11067,13411,17583,17662$, $17700,18162$.

Insect growth inhibitor $3972,13104,15652,17172,22755$.

Insect juvenile hormone $4935,11987,11988$.

Insect phagostimulant $15355,15458,18411,19087,19463$.

Insect repellent 11143 .

Insecticidal $1124,1348,2598,3356,3761,3762,3864,4550,5035,6437$, 11149, 14581, 15521, 17117, 17444, 18882, 20240, 22677.

Insecticidal (Aedes aegypti larvae) 14284, 17473, 17474.

Insecticidal (Aedes aegypti) 364.

Insecticidal (Cylas formicarius elegantulus adult) 2847, 2855, 5757, 5758, 5784, 9800, 9801, 9802, 9803, 9870, 9872, 9873, 9874, 9875, 9876, 20249.

Insecticidal (Heliothis virescens) 15603

Insecticidal (Mythimna separata larvae) 16817, 21517, 21520, 21525, 21526.

Insecticidal (Mythimna separata) 5300, 5312, 21025.

Insecticidal (Plutella xylostella) 22860, 22861, 22862, 22866, 22871. Insecticidal (Rhyzopertha dominica) 15445, 15446.

Insecticidal (Sitophilus oryzae) 15445, 15446.

Insecticidal (Spodoptera littoralis larvae) 375, 495, 741, 4964, 5463, $5470,10677,10731,14094,14714,16470,16475,16476,16618$,

$16692,17992,18260,18883,18884,18885,18886,18887,18888$, 18889, 18890, 18891, 20296, 20304, 20305.

Insecticidal inactive (Aedes aegypti) 13663.

Insecticidal inactive (Spodoptera littoralis larvae) 10097, 10098, 21274, 21275.

Insulin-like activity $10888,18339$.

Integrin MAC-1 inhibitor 2102, 2106.

Interferes in metabolism of alcohol 4030 .

Intermediate in phytosterol biosynthesis 14352 .

Involves in many plant metabolism processes 10165 .

Involves the metabolism of carbohydrates 22253.

Iodinate thyronine deiodinase inhibitor $2011,3454,3600,7802,12020$, 13137, 14971, 17168, 20481.

Irritant $899,953,1738,1789,2215,2845,2890,3048,3188,3194,3231$, $3361,3593,3695,4087,4128,4584,5188,6482,6521,7616,8827$, $8997,8997,9568,12543,12843,13439,13476,14125,15964,16066$, $16598,16674,17083,17181,17187,17374,17376,17857,18257$, $18426,18639,18640,19846,19909,19935,20550,21207,21974$, 22070, 22142, 22274.

Irritant inactive $9474,9475,16818,16819,16820,21027$.

Irritant of contact-ovipositting (Papilio xuthus) 19087.

Key role in biosynthesis of threonine, isoleucine and methionine 9620. Kibe (frostbite) preventive 9458 .

Kinase inhibitor, cyclin-dependent 11023.

$\boldsymbol{\alpha}$-Glucosidase inhibitor $5198,2102,2792,5206,6054,6089,6203,7437$, $7705,8617,9922,10351,10435,10437,11703,13803,16179,16182$, $16851,18524,18525,20570,20571,21685,21763,21815,21816$,
21838.

$\boldsymbol{\alpha}$-Glucosidase inhibitor inactive $\quad 6052,6053,6094,8616,14989,14990$, 14991, 14992, 14993, 14994, 15812, 20569, 21893.

$\boldsymbol{\beta}$-Galactosidase inhibitor $2792,7437,21838$.

$\boldsymbol{\beta}$-Glucosidase inhibitor $2792,7437,9616,22581$.

$\boldsymbol{\beta}$-Glucosidase inhibitor inactive $6089,6094,6203,10435,10437,20569$, 21893.

$\boldsymbol{\beta}$-Glucuronidase inhibitor $18668,19539$.

$\boldsymbol{\beta}$-Hexosaminidase inhibitor $97,98,100,101,4084,5430,9278,9282$, 15499, 18990, 18996, 18997, 19004, 19005, 19006, 19032.

$\boldsymbol{\beta}$-Hexosaminidase inhibitor inactive $\quad 303,371,496,3308,3399,3831$, $3832,5427,5428,5431,6853,7944,8594,11477,14777,18797$, 18983, 18995, 19001, 19002, 19003, 19007, 19009, 19010, 19011, 19016, 19542, 19618, 20566, 22332, 22332.

$\boldsymbol{\beta}$-Hexosaminidase release inhibitor $4398,5424,11364,11614,11806$, $12403,12520,13986,14775,15705,16462,16463,16464,16465$, 16466, 16468, 19689, 20899.

$\boldsymbol{\beta}$-Hexosaminidase release inhibitor inactive $5420,5421,5422,5423$, 5425.

$\boldsymbol{\beta}$-Hexosaminidase release inhibitor, IgE-induced $9456,9458,11691$, $15279,15286,17705$.

Lacrimation promoter $6550,17912$.

Lactace inhibitor (isomaltose enzyme inhibitor) 6914.

Lactace inhibitor (trehalase inhibitor) $\quad 3010,3011$.

Larvacide $2057,17434,17435,17436,17437,19378$.

Larvacide (Acroepiopsis assectella larvae) 17662.

Larvacide (Eurema hecabe mandarina larvae) 5991.

Larvacide (Heliothis virescens) 3594.

Larvacide (Heliothis zea larvae) 7278, 19582.

Larvacide (insect larvae) 4739, 7621, 21208.

Larvacide (larvae of housefly and apple moth) 15231.

Larvacide (mosquito larvae) 4872, 4898, 10843.

Larvacide (mosquito late third or early fourth-instar larvae of Anopheles gambiae) 5299, 5311, 5316.

Larvacide (order Lepidoptera larvae) 16183.

Larvacide (Spodoptera exempta larvae) 11386.

Larvacide (Stegomyia calopus larvae) 11408, 15204, 18214, 18215, 18899.

Larvacide (toxic to larvae of housefly) $4140,9196,9197$.

Larvacide (toxic to mosquito larvae) 9857.

Laxative $480,967,971,981,1892,2004,2538,2816,3254,3306,3307$, $3598,3599,3692,3695,4319,4477,4531,4543,4716,4717,5093$, $5191,6632,7617,7938,7939,7940,8128,8231,8276,8277,12950$, $15068,15069,16516,16756,17249,17251,17571,18841,19749$, $19750,22397$.

laxative 18759 .

LD $663,2001,2678,3094,3911,4685,4993,6772,9441,11141,12446$, 13621, 14971, 15170, 15527, 16031, 18299, 18859, 18939, 19223, 19431, 20896, 21234, 21263, 21350, 22057.

$\mathbf{L D}_{\mathbf{5 0}} \quad 56,304,554,651,909,924,1161,1191,1283,1376,1520,1526$, 1713, 1844, 1904, 1938, 1972, 2214, 2284, 2294, 2298, 2312, 2380, $2550,2660,2716,2728,2734,2737,2738,2757,2791,2887,2937$, 3002 , 3220, 3277, 3278, 3502, 3511, 3551, 3588, 3633, 3637, 3693, 
$3729,3770,3886,3910,3964,3965,4103,4135,4237,4245,4290$, 4315, 4317, 4319, 4320, 4399, 4399, 4456, 4547, 4550, 4589, 4594, 4645, 4649, 4685, 4693, 5105, 5136, 5152, 5161, 5436, 5502, 5526, 5763, 6454, 6558, 6990, 7272, 7341, 7381, 7385, 7521, 7714, 7736, 7854, 7980, 8297, 8312, 8347, 8430, 8967, 9223, 9330, 9335, 9486, 9541, 9553, 9740, 10351, 10734, 10814, 10875, 11002, 11024, 11091, $11179,11189,11259,11260,11337,11344,11736,12221,12421$, 12422, 12510, 12537, 12571, 12734, 12798, 13001, 13109, 13137, 13236, 13237, 13239, 13241, 13246, 13247, 13373, 13559, 13606, 14796, 14923, 14981, 15126, 15129, 15146, 15266, 15432, 15490, $15561,15658,15781,16084,16169,16183,16261,16439,16505$, $16512,16525,16555,16623,16657,16666,16693,16694,16773$, 16789, 16795, 16796, 16884, 16929, 16958, 16959, 16966, 17028, 17127, 17451, 17456, 17592, 17593, 17708, 17717, 18031, 18033, 18050, 18086, 18256, 18317, 18816, 18826, 18834, 19087, 19111, 19195, 19196, 19227, 19316, 19518, 19528, 19540, 19542, 19587, 19639, 19706, 19749, 19895, 19955, 20002, 20060, 20066, 20076, 20077, 20079, 20137, 20341, 20403, 20405, 20410, 20490, 20650, 20717, 20963, 20995, 21051, 21077, 21251, 21292, 21360, 21435, 21448, 21485, 21511, 21577, 21662, 22011, 22232, 22269, 22270 , 22280, 22282, 22336, 22346, 22395, 22399, 22420, 22421, 22487 , 22489, 22497, 22502, 22552, 22553, 22618, 22774, 22820, 22938.

$\mathbf{L D}_{100} \quad 22384,22385$.

Leads to struma 14868 .

Lettuce cotyledon factor 5564 .

Leucine aminopeptidase inhibitor 7924 .

Leucocyte activation inhibitor 12767.

Leukocyte elastase MMP-2/9 inhibitor 2102, 5011, 16777, 18317.

Leukocytes infiltration effect 118, 119, 14767.

Leukopoietic 617, 1186, 2887, 3094, 3551, 3695, 3844, 7385, 7386.

Leukotriene biosynthesis inhibitor $118,119,230,6211,7663,12766$, 12767, 14394, 14624, 14654, 14771, 14800, 22059.

Leukotriene inhibitor 1628.

Lipase accelerator 20734 .

Lipase inhibitor $4604,8278$.

Liver and nerve protectant 8423 .

Liver sialidase inhibitor 22720, 20515.

Local anesthetic $554,824,2218,2303,3277,3508,3968,4936,4993$, 6439, 6683, 8010, 12184, 12510, 16803, 17909, 19184, 20566, 22938.

Local anticorrosion 4234 .

Local stimulant 3094,7751 .

Lousicide 781,17470 .

Low toxin $195,1618,3431,3907,3912,4036,6679,10887,11067,15337$, 16050, 16080, 17505, 18624, 20016, 20254, 21362.

LOX inhibitor 2102, 2131, 2132, 6921, 8818, 13252, 13261, 15322, $15323,16120,16121,16525,16529,16530,16531,16886,17278$, 19895, 19897, 19898, 20123, 20124, 20125, 20126, 20129.

5-LOX inhibitor 444, 580, 2101, 2102, 2216, 3188, 3743, 6491, 7819, 7924, 7926, 8926, 8997, 9202, 9331, 11572, 12936, 15319, 15928, 16540, 16983, 17979, 18513, 19201, 19312, 19846, 19871, 22511, 22778.

$\Delta^{5}$-LOX inhibitor $7802,12020,14971,15170,16758,18219$.

12-LOX inhibitor inactive 4913.
12(S)-LOX inhibitor inactive 9, 3354, 4916, 15705, 20465.

12-LOX inhibitor 1131, 2102, 5801, 12936, 17979, 21703.

12(S)-LOX inhibitor $4281,19983$.

15-LOX inhibitor 1110, 1131, 2102, 2380, 4289, 13097, 13098, 13137, 19929.

$\mathbf{L T B}_{4}$ inhibitor 7924, 19312.

LTB $_{4}$ production inhibitor $2106,3462,10182,10472,19883,19885$.

LTC $_{4}$ inhibitor 3981.

LTD 4 antagonist $16268,16269,21993,21994$.

Lubricant 1598.

Main active component in Cassave Aerial Parts, Manihot esculenta MU SHU DI SHANG BU FEN 12854.

Main antispasmodic component in Cow-bezoar, Bos taurus domesticus; Bubalus bubalis NIU HUANG 5161.

Main bitter component in beer 9678 .

Main component of breviscarpin in Shortscape Fleabane, Erigeron breviscapus DENG ZHAN XI XIN to cure post-palsy paralysis 19587.

Main component of phenol character acid in Danshen, Salvia miltiorrhiza DAN SHEN. 4631.

Main effective component in Dahurian Rhododendron, Rhododendron dauricum MAN SHAN HONG to treat trachitis 7736.

Main effective component in Orange Daylily, Hemerocallis fulva XUAN CAO GEN 9339.

Main effective component in Tibet Lyonia, Lyonia ovalifolia LI MU 13246, 13247.

Main effective component in Tibet Wormwood, ZANG YIN CHEN 13481.

Main effective component in Yanhusuo, Corydalis yanhusuo YAN HU SUO 4889.

Main odiferous component in cucumber Cucumis sativus HUANG GUA 15676.

Maintains normal vision 18834 .

Maltase inhibitor $2792,7437,21362$.

MAO inhibitor $1007,3440,3441,3442,3443,3444,3445,3446,3447$, $3449,3450,8844,9232,9233,9235,10786,11436,11504,12771$, 12782, 12908, 15204, 19198, 19542, 19783.

MAO inhibitor inactive $3829,3831,3832,10038$.

MAO-A inhibitor 2220, 4292, 5722, 10124, 12889, 12890, 13916, 17278, 17451, 20502, 20685.

MAO-B inhibitor 10124, 12889, 12890, 13916, 17451.

Matrix metalloproteinase-1 (MMP-1) inhibitor 20421.

May has a potential in the treatment of asthma 18376.

M-choline receptor agonist 1658,17360 .

M-cholinergic receptor blocker 10872 .

Medulla vasomotorium inhibitor 19195, 19196.

Melanogenesis inhibitor 17461.

Membrane stabilizer 1769 .

Metabolic intermediate. 8817.

Mild anesthetic 6552 .

MIP-1 $\alpha / \beta$ inhibitor 2106.

Mitochondrial complex I selective inhibitor, NADH oxidase 1317 , 11793, 12452, 14906, 18899, 18939, 22099. 
Mitochondrial respiratory chain complex I inhibitor $1459,1770,13634$, 13715, 15885, 18939, 21258.

Mitochondrial respiratory chain inhibitor, mammalian 241,311, 1008. MLD 1102, 3452, 4029, 8256, 11203, 13089, 21211, 21887.

Molluscacide $1029,2179,2309,2973,2973,3340,4624,6193,8403$, $9288,9589,10881,11379,11903,12621,14279,14541,15253,16044$, $16052,16080,16674,16898,17269,17586,17857,18229,18257$, 18534, 19316, 19391, 19846, 22880.

Molluscacide 19333, 19334, 19335, 19336, 19337, 19338, 19339, 19340, 19341.

Mucin release stimulator 2331, 22270.

Multidrug resistance (MDR) reversing activity $17731,18170,18171$, 18172, 18173, 21026.

Multidrug resistance protein (MRP) inhibitor 19536.

Muscarinic inhibitor 22275.

Muscle relaxant $136,1125,2042,3148,4459,4693,6262,6734,8292$, $9597,11091,11329,13388,13716,13801,17411,17413,17417$, 20732, 21206, 21485, 21887, 22489.

Muscle stimulant 3404 .

Mutagen $701,1713,2220,3847,6011,6684,7994,8081,9320,9882$, $11091,11436,11809,12535,14923,14971,15184,15799,15815$, 18667, 18679, 19706, 19731, 19791, 19805, 19846, 20510, 22011.

Mutagen 1020, 17247.

Mydriatic $1288,2001,3860,6418,9703,10870,10872,15461,16773$, 20496, 22921.

Myotoxic phospholipase A2 (PLA2) inhibitor 15505.

$\mathbf{N a}^{+}, \mathbf{K}^{+}$-ATP inhibitor $10,7156,9233,9234,10285,12178,12180$, 14093, 14543, 14995, 15038, 15705, 19802, 19803, 20222.

NADH dehydrogenase inhibitor 16124.

NADH oxidase inhibitor $241,311,1008,1319,5613,7015,7802,9395$, 9396, 9397, 9398, 9399, 9400, 9401, 15170, 18939.

NADH ubiquinone reductase inhibitor 14906, 16266, 20240.

N-choline receptor agonist 1658 .

Negative chronotropic action 19955.

Negative inotropic action 21206.

Nematocide $2783,3508,4872,4898,6487,10680,14683,17708,19846$, 21018, 21328, 21483.

NEP inhibitor $6757,8095,10887,11642,16011,18358,18371,18411$, 21392.

Nerve growth factor (NGF) enhancer 9436, 22271.

Neurite outgrowth activity $3859,6538,6723,7212,9994,21099,21148$, 21783, 22702, 22708, 22710.

Neurite outgrowth enhancer $1035,1477,1486,8212,8259,8423,8425$, $12934,15822,15839,17500,21168,22318$.

Neurite outgrowth enhancer inactive $7522,21734$.

Neuromuscular blocker $1125,1366,2959,3148,3878,3963,4864,7324$, 7326, 7327, 7331, 7340, 7341, 11337, 14569, 18655, 19198, 21448, 21485 .

Neuromuscular toxicity $905,4926,4927,9077,9078,14024$.

Neuroprotective $1695,2376,2439,2470,2709,2710,2711,2712,2887$, 2960, 3695, 4135, 4185, 4186, 4398, 5045, 7052, 7053, 7768, 8089, $8404,8405,9020,9237,9238,9855,9968,10684,10765,11447$, 12046, 12749, 13290, 13885, 13886, 13888, 13889, 13890, 13891,
13894, 16587, 18354, 18355, 18362, 19777.

Neuroprotective inactive 2466, 2467, 2469.

Neuroprotective, induced by L-glutamate 13895, 14070.

Neurotoxin $833,1057,1058,4452,15490,19223$.

Neurotransmitter 1048, 6559, 15708, 19760.

Neurotrophic 2376.

Neurotrophic $7517,20651$.

Neurotrophic activity $4811,6538,6723,9975,11868$.

Neurotrophic bioassay inactive 4523, 4896, 9954, 13789, 20716.

NF-кB inhibitor 2004, 3307, 3693, 13882.

NFAT transcription inhibitor $2245,2778,3308,4184,5213,5981,8098$, 8910, 8922, 9106, 9107, 9108, 9109, 9382, 10637, 13100, 14530, 15954, 18411, 19377, 19473, 19474, 19497.

NFAT transcription inhibitor inactive 6918, 7951, 20237.

Nicotine antagonist 9238, 12083, 19386, 20496.

Nicotinic acetylcholine receptor competitive inhibitor 14024.

Nitrogen-containing base occurring in DNA and RNA 9070, 14802.

NO production inhibitor inactive $658,1284,2041,2491,4396,4895$, 5577, 6201, 9657, 9818, 11613, 11789, 12016, 12253, 13115, 15805, 15809, 16204, 16231, 17844, 20660, 22978, 22981, 22982, 22983.

NO production inhibitor, accompanied by a decrease in iNOS protein level, did not affect COX-2 protein expression level 16717.

NO production inhibitor, cultured rat aortic smooth muscle cells treated with LPS/IFN- $\boldsymbol{\gamma}$ 11203, 16713.

NO production inhibitor, inhibits iNOS gene expression activated by LPS and recombinant mus IFN- $\gamma$, furthermore, inhibits histamine release from rat peritoneal mast cells $13433,13434$.

NO production inhibitor, LPS-activated macrophage-like $\mathbf{J 7 7 4 . 1}$ cells $4759,4760,4761,5137,6205,6281,6286,6287,9819,10606,10618$, $10621,11146,11147,13850,15443,15444,15795,16221,16222$, $16223,16224,16225,16226,16227,16228,16229,16232,16233$, $16234,16235,16236,16237,16238,16239,16240,16242,16243$, 16244, 19630, 19966, 19967, 19968, 19969, 19970, 20258, 20259, 20260, 20261, 21927.

NO production inhibitor, LPS-activated macrophages RAW264.7 131, 132, 155, 292, 2359, 2360, 2361, 2362, 5155, 52855292.

NO production inhibitor, LPS-activated macrophages RAW264.7, treated simultaneously by $\boldsymbol{\alpha}$-viniferin and LPS 22504.

NO production inhibitor, LPS-activated mus peritoneal macrophages $889,1213,2427,2428,2462,4378,4379,4389,4393,4394,4395$, 4397, 4398, 4400, 4416, 5457, 6875, 7116, 7514, 8014, 8015, 8048, $8049,8347,8348,8525,10147,11001,11359,11364,11412,11462$, $11477,11614,11637,11806,12403,12520,13986,14775,15378$, $15379,15705,16456,16459,16462,16463,16464,16465,16466$, $17875,18282,18983,18990,18995,18996,19001,19002,19003$, 19004, 19005, 19006, 19007, 19009, 19010, 19011, 19016, 19017, 19618, 19689, 20566, 20899, 22124, 22195, 22332, 22775, 22977.

NO production inhibitor, LPS-activated mus peritoneal macrophages, reduces NO production and iNOS gene expression, by inhibiting NFkB 19895.

NO production inhibitor, LPS-induced macrophages RAW264.7, through suppression of NF-кB by inhibiting transactivation activity of RelA subunit 19407. 
NO production inhibitor, LPS-induced mus macrophages RAW264.7 1573, 8679, 10820, 10834, 19405, 19406, 22270, 22984, 22986.

NO production inhibitor, LPS-induced, concentration-dependent manner 3388, 5987, 9765, 12044, 19312.

NO production inhibitor, macrophage-like cell line RAW264.7 activated by $\mathbf{L P S} / \mathbf{I F N} \quad 592,3517,8037,8038,8039,8086,8088,8093,13293$, 13295, 22383, 23030.

NO production inhibitor, macrophages activated by LPS and recombinant mouse IFN- $\gamma \quad 11017,13435,13436,13437,18317$, 19891, 19892.

NO production inhibitor, mus macrophages RAW264.7, activated by LPS/IFN- $\boldsymbol{\gamma} \quad 2359,2360,2361,2362,5155,5633,5669,7982,7983$, $7984,7985,18317$

NO production inhibitor, mus macrophages RAW264.7, activated by LPS/IFN- $\gamma$, without showing cytotoxicity at low (effective) concentrations 5742, 5911, 16199, 22761, 22762, 22764.

NO production inhibitor, mus macrophages, activated by LPS/IFN- $\gamma$ 19536.

NO production inhibitor, neuroprotective, glutamate-injured primary cultures of rat cortical cells 1695 .

NO production inhibitor, no explanation on cell species $171,173,222$, 1159, 1367, 1476, 1790, 1791, 1802, 2036, 2038, 2073, 2075, 2102, $2106,2379,2490,2629,3362,3368,3368,3980,3983,4128,4604$, 4726, 4746, 4788, 4891, 4980, 5939, 6690, 6923, 7195, 7196, 7197, 7239, 8020, 8278, 8401, 8818, 9626, 9627, 9811, 10314, 10894, 10922, $11732,12170,13137,14097,14995,15002,15038,16163,16166$, $16167,16603,16675,16675,17862,19259,19618,20556,20806$, 20840, 20877, 20988, 21280, 22336, 22718, 22876, 22877.

NO production inhibitor, RAW264.7, activated by LPS/IFN- $\gamma$, with strong cytotoxicity $13109,13110,13111,13112,13113,13114$.

NO production inhibitor, summary index $131,132,155,171,173,222$, 292, 592, 889, 1159, 1213, 1367, 1476, 1573, 1695, 1790, 1791, 1802, 2036, 2038, 2073, 2075, 2102, 2106, 2359, 2360, 2361, 2362, 2379, $2427,2428,2462,2490,2629,3362,3368,3388,3517,3980,3983$, $4128,4378,4379,4389,4393,4394,4395,4397,4398,4400,4416$, 4604, 4726, 4746, 4759, 4760, 4761, 4788, 4891, 4980, 5137, 5155, $5285,5292,5457,5633,5669,5742,5911,5939,5987,6205,6281$, $6286,6287,6690,6875,6923,7116,7195,7196,7197,7239,7514$, $7982,7983,7984,7985,8014,8015,8020,8037,8038,8039,8048$, $8049,8086,8088,8093,8278,8347,8348,8401,8525,8679,8818$, $9626,9627,9765,9811,9819,10147,10314,10606,10618,10621$, 10820, 10834, 10894, 10922, 11001, 11017, 11146, 11147, 11203, $11359,11364,11412,11462,11477,11614,11637,11732,11806$, $12044,12170,12403,12520,13109,13110,13111,13112,13113$, $13114,13137,13293,13295,13433,13434,13435,13436,13437$, 13850, 13986, 14097, 14775, 14995, 15002, 15038, 15378, 15379, 15443, 15444, 15705, 15795, 16163, 16166, 16167, 16199, 16221, $16222,16223,16224,16225,16226,16227,16228,16229,16232$, $16233,16234,16235,16236,16237,16238,16239,16240,16242$, 16243, 16244, 16456, 16459, 16462, 16463, 16464, 16465, 16466, 16603, 16675, 16713, 16717, 17196, 17197, 17198, 17199, 17200 17201, 17862, 17875, 18282, 18317, 18983, 18990, 18995, 18996, 19001, 19002, 19003, 19004, 19005, 19006, 19007, 19009, 19010,
19011, 19016, 19017, 19259, 19312, 19405, 19406, 19407, 19536, 19618, 19630, 19689, 19891, 19892, 19895, 19966, 19967, 19968, 19969, 19970, 20258, 20259, 20260, 20261, 20556, 20566, 20806, 20840, 20877, 20899, 20988, 21280, 21927, 22124, 22195, 22270, 22332, 22336, 22383, 22504, 22718, 22761, 22762, 22764, 22775, 22876, 22877, 22977, 22984, 22986, 23030 .

NO production inhibitor, with cytotoxicity $17200,17201$.

NO production inhibitor, without cytotoxicity $17196,17197,17198$, 17199.

Nodulation signal for metabiosis of pea and Rhizobium leguminosarum 1492,8309 .

Nodulation signal for metabiosis of pea and Rhizobium leguminosarum 1476.

Non-addictive antitussive 15266 .

Nonpeptide tachykinin $\mathbf{N K}_{1}$ receptor antagonist 21176.

Nonpeptide tachykinin NK1 receptor antagonist inactive 22035.

Nonpolarizing muscle relaxant 2191 .

Nootropic 21234.

Normalizes the ratio between phosphatide and cholesterol 2379 .

Nourishes nerve $1036,11209,11211,17500$.

Novel $\boldsymbol{\alpha}$-glycerophosphoric acid tetrazole reductase inhibitor 22282 .

Nucleotide diphosphatase inhibitor 1030.

Nutrient 1866, 1886, 12891, 12893.

One of allergens in Hydrangea macrophylla BA XIAN HUA 9700.

One of effective components in danshen, Salvia miltiorrhiza DAN SHEN 10736.

One of effective components in Erycibe obtusifolia DING GONG TENG $19542,19545$.

One of effective components in Hypericum japonicum DI ER CAO for curing hepatitis 11642 .

One of effective components in Red Wing Azalea 491.

One of main components of divasides 6530 .

Opioid agonist $\quad$ 10528, 14886, 14981, 16735, 20138, 20139.

Ornithine decarboxylase inhibitor 4872, 17958, 19255.

Osteoblast inhibitor 2208

Osteoblastic differentiation stimulator 1275 .

Osteoblastic proliferation stimulator 2331, 13990, 13991.

Osteoclastogenesis-inhibitory factor (OCIF) gene expression inhibitor 15376.

Overcomer of dormancy of peppertree fruits 16657.

Oviposition promoter 9458, 17903.

Oxytocic 930, 7243, 16770, 20133.

PAF antagonist $5135,8040,8392,8393,9490,13390,15940,17422$, 19404, 20113, 20114, 21493, 22383.

PAF inhibitor(Anti-PAF) $\quad 1737,4967,5549,8979,11286,12010,12013$, 12611,12612

PAF receptor antagonist $5135,8404,8405,8406,8407,8408,12005$, 12006, 12007, 12008, 13387, 17688, 22528, 22628.

Pancreatic lipase inhibitor 9632, 9633, 19433, 19434, 19435, 19436, 19437, 19438, 19439, 19440, 19441.

Pancreatic lipase inhibitor inactive 16701.

Paralyses CNS 3218.

Paralyses CNS and respiration 983. 
Paralyses motor nerve ending 3988, 23003.

Paralyses muscle $3615,21485$.

Paralyses respiration 2372, 3692, 8256, 21292, 21877.

Paralyses sensory and motor nerve 3502 .

Paralyses striated muscle 6552 .

Paralysis 3968, 13223.

Paralysis of nervous system 16285 .

Paralyzes cardiac and skeletal muscle 21887.

Parasympathetic ganglionic blocker (anti-vagus) 871.

Parasympathomimetic 20017, 22158, 22397.

Passive cutaneous anaphylaxis inhibitor $9456,9458,11691,15279$, 15286, 17705.

PDE isozymes selective inhibitor 18376.

PDE3 selective inhibitor 18376.

Pectinase inhibitor 9616, 22581.

PEP inhibitor 5791, 9561, 11648, 18682.

Peroxidase formation inhibitor 20127.

Peroxidase inhibitor 4190, 8278, 19898.

Perspiration 17360, 18794.

Pesticide 136, 290, 782, 862, 948, 1125, 1319, 1474, 1520, 2298, 2676, $3307,3692,6262,6796,8509,9428,9631,10680,10938,11269$, $13801,14692,15527,15549,16500,16734,17411,17413,17417$, 17451, 17776, 18256, 18262, 18939, 19811, 20485, 21483, 22815.

Pesticide (aphids Schizaphis graminum) 6643, 13022.

Pesticide (curculio, grasshopper, housefly and cockroach) 18270.

Pesticide (dog roundworm larvae) 18202.

Pesticide (Eurema hecabe mandarina) 8083.

Pesticide (housefly) 15232, 15233, 15490.

Pesticide (larvae of mosquito, mustard beetles and houseflies) 20490.

PGE 2 production inhibitor 1573, 2106, 3388, 3981, 4159, 5987, 6288, $6351,8020,8679,8898,9436,9765,10820,11001,11462,12044$, 12122, 15635, 16532, 16603, 17077, 17145, 17532, 18792, 19081, 19201, 19312, 19405, 19406, 20569, 20900, 20901, 21930, 22123, 22124, 22270.

$\mathrm{PGE}_{2}$ production inhibitor inactive 4158, 20447.

$\mathrm{PGE}_{2}$ release inhibitor in rbt synovioblasts 3368 .

$\mathbf{P G E}_{2}$ synthesis stimulator 19143, 19148.

PGI $_{2}$ biosynthesis stimulator 17697.

$\mathbf{P G I}_{2}$ release stimulator $5414,11209$.

P-glucoprotein inhibitor 6604, 17732.

Phagostimulant (Chrysomela vigintipunctata) 3674

Phagostimulant (insect) 11699.

Phagostimulant (order Hemiptera insect) 3199.

Phagostimulant (silkworm) 14971, 11642.

Pheromone of Nasutitermis exitiosus (for tracking) 15360.

Phosphatase inhibitor 4297, 5040.

Phosphodiesterase I inhibitor 2270, 19192, 20528,20547, 20548, 20549.

Phosphodiesterase inhibitor 61, 2369, 16918, 16919, 16920.

Phospholipase $\mathbf{A}_{2}$ inhibitor 8401.

Phospholipase $\mathbf{C} \gamma_{1}$ inhibitor $12280,12355,12358,12364,12365,12366$, 12367.

Phospholipase PLA $\mathbf{P}_{2}$ inhibitor 22055.
Phospholipase PLC $\gamma 1$ inhibitor 22208, 22209, 22210, 22211, 22212.

Photo-activated antibacterial 7830, 9226, 12254, 20002.

photo-activated antifungal 7830, 9226, 12254, 20002.

Photo-activated DNA binding 9226, 12254, 20002.

Photo-activated DNA binding inactive 7830 .

Photosensitive agent $126,1191,2309,5858,6040,7063,10886,18086$, 19469, 19471, 21328, 22774.

Phototoxic 6796, 7592, 9226, 11376, 12221, 12254, 20002, 22553.

Phytoalexin 4135, 4312, 4313, 4966, 7707, 9616, 11773, 13513, 14254, $16168,16196,18643,19122,22581$.

Phyto-estrogen 361, 398.

Phytogrowth inhibitor 421, 1067, 1822, 1833, 2847, 2855, 3457, 3840, 3911, 4938, 4986, 5941, 6312, 6888, 7719, 9304, 9872, 9873, 9875, 9876, 10290, 10564, 11113, 11114, 11115, 11269, 12964, 16601, 16805, $17278,22781$.

Phytohormone 8374, 22122.

Phytohormone active against auxin 22757 .

Phytotoxin 187, 1924, 1925, 1926, 2108, 4918, 5875, 5876, 5956, 5957, 5958, 7079, 7123, 7838, 10087, 10366, 10367, 10368, 10369, 10371, $14492,14639,14645,14646,16427,16805,17605,17607,17742$, 18802, 20332, 21124.

Phytotoxin inactive 9480, 15936, 21038.

Pigment 1013, 1358, 2319, 2320, 2322, 2496, 3146, 3209, 3210, 3215, $3225,3432,4293,4398,4434,4446,4451,5011,7821,8927,8930$, $9290,9421,11018,13243,13456,13460,14879,14880,14881,14882$, 14974, 15477, 17238, 17771, 22520, 22623, 22976.

Pituitary stimulant 1673 .

Piscicide 933, 6490, 11978, 16457, 17478, 18939, 22774.

PKA inhibitor 19284.

PKC activator $\quad 485,14201,17187,21381,21382$

PKC activator inactive $483,484,4839$.

PKC inhibitor $3498,7802,13137,14892,16550,17168,17869,18092$, 18203, 18317, 19255, 19259, 19284, 22504.

Plant growth and germination inhibitor $834,1892,3063,16898,17940$.

Plant growth hormone 11031 .

Plant growth regulator $538,777,1617,2108,3076,3545,4314,4891$, $5236,5938,6643,6750,9234,9291,12227,13238,14705,15229$, $15230,15231,15232,15233,15234,17733,18257,19313,19897$, 19898, 21541, 22411, 22533, 22638, 22975.

Plant growth stimulant 2845 .

Plant growth stimulatory or inhibitory activity $412,2288,5639,5640$, $5641,8675,8676,9590,9840,10953,13640,17409,18000,19226$.

Plasma protein binder 15849 .

Platelet aggregation inhibitor $23,24,54,147,309,322,370,383,424$, $532,595,597,598,664,921,952,955,983,1173,1287,1476,1691$, 2009, 2010, 2018, 2034, 2120, 2121, 2122, 2224, 2227, 2263, 2891, $3244,3408,3412,3434,3519,3520,3600,3613,3633,3694,3723$, $3793,3794,3795,3796,3797,3798,3926,3935,3936,3937,4296$, $4348,4349,4580,4630,4680,4685,4989,5347,5414,5440,5750$, $6204,6216,6261,6266,6302,6466,6745,6921,7441,7521,7714$, $7719,7768,7768,7788,7819,7894,7945,8191,8380,8404,8405$, $8406,8407,8424,8696,9123,9486,9564,9604,9631,9656,9736$, $9742,10236,10237,10545,11000,11414,11421,11423,11547,11648$, 
$11747,12420,12498,12499,12500,12564,12574,12714,12767$, $12917,13098,13330,13331,13332,13387,13987,14037,14086$, $14121,14187,14188,14619,14825,14865,14996,14997,15103$, $15104,15107,15118,15120,15204,15279,15321,15333,15335$, $15470,15635,15645,15647,16050,16072$, 16073, 16094, 16095, $16525,16533,16534,16572,16623,16978,17168,17283,17463$, 17464, 17467, 17508, 17568, 17587, 17688, 17763, 18004, 18005, $18063,18295,18296,18317,18376,18643,18655,18744,18768$, $18769,18770,18772,18773,18774,18775,18776,18778,18901$, $18902,18903,18904,19012,19201,19203,19231,19232,19298$, 19467, 19468, 19470, 19540, 19542, 19587, 19819, 19846, 19904, 19905, 19906, 19907, 19983, 19983, 19993, 20009, 20069, 20178, 20181, 20182, 20183, 20184, 20185, 20186, 20187, 20188, 20194, 20348, 20361, 20369, 20968, 21057, 21206, 21493, 21576, 21887, $22144,22270,22336,22511,22528,22615,22628,22818,22965$, 22969, 22970, 22972.

Platelet aggregation inhibitor inactive $1328,1510,5535,5929,5930$, $5932,10275,12497$.

Platelet aggregation promoter or inhibitor (promoter at low concentration; inhibitor at high concentration) $\quad$ 18995, 19015.

Platelet aggregation selected inhibitor 1326, 5928, 9706, 9723, 9757, $10276,10542,12178$

PNPPase inhibitor 19201.

Polyoma enhancer binding protein 2 $\alpha$ A (PEBP2 $\alpha$ A) inhibitor 15376. Positive inotropic effect $7788,20100$.

Potassium channel activator 2606.

Potato micro-tuber inducer $9979,9980,10315,11825,21311$.

Tumour-promoting agents 17181.

Potential exists for development of new agents for treating stroke 10684.

Potentiates hypnotic effect of barbiturates 18881.

Ppase1 inhibitor 10036, 10035.

Precursor of biosynthesis of vitamin A $\quad 3208,3209,3210,3214,15135$.

Precursor of indoleacetic acid 22058.

Precursor to biosynthesis of 5-HT 10818.

Precursor to biosynthesis of adrenalin 6559 .

Precursor to biosynthesis of arterenol 6558.

Precursor to biosynthesis of chamazulene 13605 .

Precursor to biosynthesis of dopamine 6558 .

Precursor to biosynthesis of ecdysone 19513.

Precursor to biosynthesis of lignin 3981, 19922.

Precursor to biosynthesis of sterol 4473, 12488.

Precursor to essence synthesis; 15203.

Pregnancy terminator 17983, 22937.

Prevention and cure of diabetes syndrome (retinopathy, cataract, nervous system diseases) $16931,16933$.

Prevention and cure of ulcer and gastritis 1020 . prevents action of chemical carcinogens 11320 .

Prevents AIDS 20124, 20125, 20126.

Prevents atherosclerosis 18261.

Prevents atrophy of prostate and spermary 86 .

Prevents cancer $\quad 6812,16888,20478,22779$

Prevents cell division 13239.
Prevents enteritis 2013.

Prevents exudative pulmonary edema, reduces vasa publica permeability 21312 .

Prevents hardening of cardiac muscle and vasa coronaria 4547.

Prevents infection of Schistosoma mansoni 4485.

prevents toxicosis 8906 .

Prevents ulcer 16512.

Proliferation inhibitor 5116, 5118, 11504, 20168, 21156, 22338.

Proliferation stimulator or inhibitor, Jurkat cell lines, stimulator at low concentration, inhibitor at high concentration 8060,8061 .

Proliferation stimulator, B cells $5116,5118,22338$.

Proliferation stimulator, Jurkat cell lines 8059 .

Prolyl endopeptidase inhibitor $5916,5917,6244,8403,9389,10750$, $15215,15217,18917$.

Promoter of biosynthesis of glutathione 13823 .

Promoter of cytotoxic effects of vincaleucoblastine $8423,8427,17475$.

Promoter of cytotoxic effects of vincaleucoblastine, inhibits vinblastine-resistant KB cells 16002.

Promoter of estrogenic activity 8487 .

Promoter of glutathione $\boldsymbol{S}$-transferase 3243.

Promoter of normal growth of female sexual organs and secondary sex characters 7383,7384 .

Promotes aging of leaves 11825 .

Promotes cell division and growth of plants 2598 .

Promotes cell division of plants 22975.

Promotes decomposition of protein to be converted to sugar 4097 .

Promotes germination 19542, 20392.

Promotes growth of bacteria producing butyric and lactic acids 10 .

Promotes hair growth 18655 .

Promotes lipid metabolism 15563, 15564, 15565, 15566.

Promotes lipolysis $\quad 3490,3493,8311,11083,13438$.

Promotes macrophage phagocytic function 4385 .

Promotes metabolism 20341.

Promotes plasma secretion of corticosterone $8423,8426,8427$.

Promotes platelet production in toxin dose 1658 .

Promotes regeneration of muscle tissue 8967.

Promotes resynthesis of heart glycogen 4547 .

Promotes RNA polymerase activity 8424 .

Promotes secretion of digestive juice 19935.

Promotes ulcer healing 9569 .

Promotes wound healing 1853.

Prostaglandin biosynthesis inhibitor $1746,1747,3983,5258,6643$, 7788, 7802, 8386, 10545, 11547, 13962, 19822, 19823.

Prostaglandin synthetase activator 5763,9818 .

Prostaglandin synthetase inhibitor $4921,8388,8403,15705,16001$, 19846, 19910, 22778.

Prostaglandin-like physio-activities $17952,17953$.

Protects cerebrum 1039.

Protects red blood cells from decomposition due to low osmotic pressure 3077.

Protects shock 6452,8297 .

Protein glycation (Maillard reaction) inhibitor 9779 .

Protein opopanax ester transferase (FPT) selective inhibitor 8150 . 
Protein tyrosine phosphatase 1B (PTP1B) inhibitor 2081, 10035, 10036, 18088.

Provides energy and restores body fluid for patients with diabetes and hepatitis 7971 .

PTP1B inhibitor 2975.

Pulmonary fibrosis inhibitor 11491 .

Pyridoxal phosphate enzyme inhibitor 3059 .

$\boldsymbol{\alpha}_{2}$-Macroglobulin inhibitor $17171,17172$.

$\alpha$-Mannosidase inhibitor 13803, 20500.

QR inhibitor 8278, 11152, 11154, 11158, 11165, 11171, 11172.

Quinone reductase inducer 4194, 5657, 5671, 5672, 11804, 21834.

Quinone reductase inducer inactive 2149, 7023, 19586.

Radioprotector 9646, 16943, 22158.

Raises body temperature 7665, 19081.

Raises dopamine metabolite HVA 20094.

Raw material for partial synthesis of steroid hormone 5040,5041 .

Raw material for synthesis $3138,11754$.

Raw material for synthesis of hormonal corticosteroid drugs 21383 .

Raw material for synthesis of ionone and vitamin A 3760 .

Raw material for synthesis of vitamin $\mathbf{D}$ and hormones 3585 .

Raw material of synthesis of pregnane 19390.

Raw material of synthesis of vitamins $\mathbf{K}_{\mathbf{1}}$ and $\mathbf{E} 17265$.

Reagent used in biochemistry research 617, 626, 12802, 13507, 18230.

Reduced coenzyme I (NADH) oxidase inhibitor 2779 .

Reduces activity of LDH 20094.

Reduces ammonia in blood 1673.

Reduces arteriotony 19706.

Reduces consumption of oxygen in blood 1972.

Reduces fertility in insects 22349 .

Reduces G6PD in hematids 22466.

Reduces intestinal vessel tension (gpg, in vitro) 6552.

Reduces intra-ocular pressure in rbt 2303.

Reduces isolation-induced aggression in mouse 12917, 19783.

Reduces learning disorder in mus caused by ischemia-perfusion 19201.

Reduces MDA content in mouse serum 2564.

Reduces muscular twitching 4862, 4863.

Reduces nephrotoxicity of cyclosporine 8405 .

Reduces normal body temperature of mus 22865 .

Reduces plasma's exosmosis 23 .

Reduces serum bilirubin and enhances output of bilirubin in urine $8841,8846$.

Reduces symptom of acute dysemia in myocarditis 4547 .

Reduces symptoms of myocarditis and prevents development of cardiac muscle sclerosis 4036 .

Reduces the level of cholesterol and lecithin in serum 5707, 18165.

Reduces the level of cholesterol and triglyceride in serum 8278,13137 , $18916,22269$.

Reduces the level of cholesterol in serum $891,2384,2379,4685,6440$, $6853,7978,8401,8423,8424,8846,9184,11648,12624,19070$, $20369,22552$.

Reduces toxicity of opium 3361 .

Reduces tube formation of BAECs 6364 . $\mathbf{5} \boldsymbol{\alpha}$-Reductase inhibitor inactive 2071,6923 .

5 $\boldsymbol{\alpha}$-Reductase inhibitor $4398,6185,6488,8330,9038,9044,10191$, $10214,12893,21456$.

Regulates allergic reaction 7802 .

Regulates cAMP level and hydrochloric acid in gastric juice 17220 .

Regulates drug immunological injury 2300 .

Regulates kidney function and inhibits reproduction of glomerulus cells 8427.

Regulates menstrual cycle 19427.

Relaxes aortal contraction induced by $\mathbf{K}^{+}$and $\mathbf{C a}^{2+} 7945$.

Release of CRF and CRF gene express, stimulator 19148.

Releases histamine 19955 .

Releases inhibition of vagus nerve to the heart 2001 .

Relieves headache 7252 .

Relieves itching 13774, 17089.

Respiration promoter 8010 .

Respiratory depressant $\quad 4237,5003,5032,5108,7341,11741,21511$.

Respiratory stimulant $1124,1904,2001,2716,4594,7980,8256,9419$, 10870, 13001, 15263, 15268, 18637, 19639, 22158, 22502.

Restores miocardia force after anoxia $\quad 20679,20680,20681,20688$.

Reverse transcriptase inhibitor 5515, 12503, 15631, 17869.

Reverse transcriptase inhibitorof RNA tumor virus 7806.

Reverses glucopenia and ketosis caused by starvation 832 .

Reverses standing lowblood pressure 17220.

Reversing action to MDR of KBV200 cells $\quad 6709,11001$.

Rodenticide 5440, 19528.

Rodentine antifeedant 10877.

Salivary secretion inhibitor 1288 .

Salivary secretion promotor 582 .

Schistosomacide 762, 1784, 4485, 4624, 7226, 9339, 20492.

Schistosomacide (Bilharzia japonica) 6611.

Schistosomacide (Fasciola hepatica) 834.

Schistosomacide (martensite schistosome) 4128.

Screening ultraviolet $1042,3209$.

Sedative $86,535,651,783,784,917,1094,1097,1178,1502,2106,2113$, $2303,2734,3080,3354,3498,3588,3878,3929,3933,4029,4036$, $4116,4536,4645,5199,5417,5482,6193,6482,6618,7385,9187$, $9223,9224,9335,11741,11851,11903,12446,12843,12849,13392$, $14531,15843,16451,16501,16505,16512,16525,16532,17451$, $17708,17958,17983,18197,18552,18623,18630,18633,18816$, 18826, 19111, 20076, 20205, 20324, 20444, 20509, 20566, 21234 , 21511, 21578, 22195, 22312, 22336, 22346, 22375, 22384, 22433.

Selective DNA-damaging activity 4106.

Selective $\boldsymbol{\alpha}$-mannosidase inhibitor 17506.

Selectively inhibits nucleoside transport across cellular chorion 3652 .

Sensitizer $3551,6482,16622,17083,17561$.

Serotonin (5-HT) antagonist 22921.

Shampoo 16711.

Similar action with aconitine $5032,7706,10875,11867,13794$.

Similar action with arterenol 7240 .

Similar action with atropine $10870,22277$.

Similar action with berberine 2302 .

Similar action with bufotenine 6370 . 
Similar action with codeine 15449 .

Similar action with cokaine 6439 .

Similar action with colchicine 3912.

Similar action with ephedrine 9646.

Similar action with ergometrine 16808 .

Similar action with ergotamine 7243,16808 .

Similar action with linamarin 13003.

Similar action with mesaconitine 10875 .

Similar action with mescaline 14583 .

Similar action with methyllycaconitine 3963 .

Similar action with narceine $4290,7994$.

Similar action with nicotine 3070 .

Similar action with physostigmine 17253.

Similar action with quinine 602 .

Similar action with sanguinarin 5708 .

Similar action with strychnine $\quad 6691,10734$.

Similar action with talatizamine 3201 .

Similar action with vitamin $\mathbf{C}_{2} 6454$.

Similar action with vitamin $\mathbf{P} \quad 56,3308,6454,12018,12714$.

Similar physiological activity with ergot 7234 .

Similar to that of aminoguanidine 3605,3606 .

Skeletal muscle and carotid stimulant 1658 .

Skeletal muscle inhibitor 3502, 3907.

Skeletal muscle relaxant $824,3218,7523,9631,20732,22091$.

Smell of cedar 19303.

Smooth muscle, bladder smooth muscle relaxant $16189,16190,16191$, 20472, 20473, 20474, 20475.

Smooth muscle, bronchial smooth muscle stimulant $1658,2303,9568$, $16773,17055,17056$.

Smooth muscle, bronchodilator $\quad 2284,6815,14395,20254,20995$, 21312, 16773, 18024, 21578.

Smooth muscle, inhibits calcium activation and release (blood vessel smooth muscle) 19540.

Smooth muscle, inhibits ileac tension and contractility 17472.

Smooth muscle, inhibits ileal contraction 893.

Smooth muscle, inhibits intestinal and uterine movement 8289 .

Smooth muscle, inhibits intestinal movement $871,2734,3907,11091$, 13776, 15882, 19101, 19620, 19955.

Smooth muscle, inhibits intestine and other smooth muscle movement 3452 .

Smooth muscle, inhibits $\mathbf{K}^{+}$-induced contraction of colon bands 21493 .

Smooth muscle, inhibits mesenteric venous contraction 19846.

Smooth muscle, inhibits smooth muscle 2284 .

Smooth muscle, inhibits tracheal smooth muscle contraction induced by

TXA2 $_{2} 16268,16269$.

Smooth muscle, paralyses small intestinal smooth muscle 2380 .

Smooth muscle, paralyses uterus (high dose) 6552 .

Smooth muscle, promotes intestinal and uterine motion 17505.

Smooth muscle, promotes intestinal motion $1658,3551,3615,15263$, 15884.

Smooth muscle, promotes small intestinal motion $13223,16929$.

Smooth muscle, reduces duodenum tension 1742 .

Smooth muscle, rises intestinal vessel tension (gpg, in vivo) 6552.
Smooth muscle, smooth muscle contractor (tracheal) 843 .

Smooth muscle, smooth muscle relaxant $126,5035,5858,6040,6402$, 7063, 7802, 9541, 16623, 17983, 18317, 19469, 19471, 19473, 20670, $21362,21589$.

Smooth muscle, smooth muscle relaxant (biliary tract) 1287.

Smooth muscle, smooth muscle relaxant (blood vessel) 7521 .

Smooth muscle, smooth muscle relaxant (blood vessel, intestine, isolated uterus, gpg trachea, gpg ileum, but causes constriction of rat isolated bladder) 7521 .

Smooth muscle, smooth muscle relaxant (colonic) 18826.

Smooth muscle, smooth muscle relaxant (duodenum) 1191, 1287, 11002 .

Smooth muscle, smooth muscle relaxant (ileal) $7521,8846,11002$, 12083, 13836, 16098, 16101, 16102, 16152, 18454, 18455, 19539, 22023.

Smooth muscle, smooth muscle relaxant (ileal) 13137.

Smooth muscle, smooth muscle relaxant (intestinal) 760, 5136, 5286, 7521, 9232, 11580, 15708, 16623, 19706, 21024, 21522.

Smooth muscle, smooth muscle relaxant (small intestine) 4685, 5003, 11002 .

Smooth muscle, smooth muscle relaxant (stomach) 1287.

Smooth muscle, smooth muscle relaxant (tracheal) 7521, 8846, 13137, $15370,17456,21493$.

Smooth muscle, smooth muscle relaxant (uterus) 7521, 11002, 15797, 18190.

Smooth muscle, smooth muscle stimulant 17360, 20060.

Smooth muscle, smooth muscle stimulant (bladder) 2303.

Smooth muscle, smooth muscle stimulant (ileal) 338 .

Smooth muscle, smooth muscle stimulant (intestinal) 2303, 3002, 3318, 11111, 11867, 17592.

Smooth muscle, smooth muscle stimulant (peripheral blood vessel) 7252.

Smooth muscle, smooth muscle stimulant (stomach) 2303.

Smooth muscle, smooth muscle stimulant relaxant (small intestine) $643,13237$.

Smooth muscle, stimulates striated muscles and smooth muscles 22153.

Smooth muscle, uterine relaxant $2797,2803,5003,8423,8426,8428$, $8429,8430,9235,12825,18655$.

Smooth muscle, uterine stimulant $117,642,643,763,1333,1599,2219$, 2303, 2380, 2725, 2726, 2734, 3002, 3114, 3318, 3452, 3551, 3934, 4058, 4103, 4115, 4191, 4192, 4353, 4912, 5108, 5435, 5436, 5445, $6420,6552,6757,7232,7240,7252,7327,8127,8256,8289,9234$, $9646,11091,11202,12646,13089,13223,13237,13241,16664$, $16773,16774,18652,19081,19461,20133,20254,22502,22503$, 22929.

SOD activity enhancer 2564 .

Somatostatin antagonist 18093 .

SP-A gene expression promoter 2106.

Spasm action (picrotoxin-like) 15432 .

Spasmogenic 13246, 21292, 21350.

Special spicery of tea 6923 .

Spermaticidal 924 .

Spermaticidal $107,762,1860,1861,1862,3368,3619,6940,10093$, 
12621, 16044, 16169, 17768, 18229, 19333, 19334, 19335, 19336, 19337, 19338, 19339, 19340, 19341.

Steroid hormone 17901.

Sthenic 136, 6262, 12916, 13801, 17411, 17413, 17417.

Stimulant 2550, 2566, 13796, 13798, 14057, 14125, 15485, 15486.

Stimulates center nerves and cardiac muscles 21312.

Stimulates cerebra 12580 .

Stimulates fibrinolysis 12420 .

Stimulates function of adrenal cortex 5152 .

Stimulates horses 18425 .

Stimulates nerve 3615 .

Stimulates pons, causes spasm and stiffness in limbs 9232.

Stimulates synthesis of NGF 7261, 7262, 7263, 7264.

Stomachic 12229, 14982, 18299.

Strengthens CNS inhibition induced by chlorpromazine 16451.

Stress-induced plant metabolite 22782 .

Striated muscle relaxant 3747 .

Striated muscle stimulant 9232 .

Strongest active component in Chinese Angelica, DANG GUI, Angelica sinensis 12825 .

Succinic oxidase inhibitor $2779,5613,7802,13137,15170$.

Sucrase inhibitor 21362.

Sugar hydrolase inhibitor 16931 .

Sulfonamide antagonist 1042 .

Supercharging for cerebral circulation 4594 .

Supertoxic agent $37,554,631,1021,1134,1849,2372,3063,3075,3979$, 4377, 5093, 6683, 8776, 9067, 9077, 10959, 11020, 12563, 13476, 14821, 17278, 17999, 22070, 22098.

Suppressive effects on ovalbumin (OVA)-induced airway hyperresponsiveness 18376 .

Survival effect on TrkA fibroblasts 12141 .

Sweetener 31, 1596, 4483, 6632, 16930, 17663, 20791, 20919, 20920, 20921, 20922, 20923, 20924, 20925, 20926, 20927, 20928, 20929, 22607.

Sympatholytic $3755,3757,3758$.

Synergist of antineoplastic bleomycin A5 16183.

Synergist of buhach 2298.

Synergist of pesticides $4310,12012,13594,17202$.

Synergist of pyrethrin 1833,6193 .

TACE inhibitor inactive 9561 .

Tanning agent $678,6857,6922,8126,9079,12128,13136,13402,17867$, 18858.

T-Cell Proliferation inhibitor 9419, 11001.

Teratogen 1134, 3979, 3988, 4522, 4594, 9320, 11866, 14868, 15204, 20060, 20066, 20488.

Testosterone 5 $\boldsymbol{\alpha}$-reductase inhibitor $4039,4040,4041,4042,7521$, 10960, 12490, 12491, 12493, 12496, 16358.

Testosterone $\mathbf{5} \boldsymbol{\alpha}$-reductase inhibitor $4610,6275,6276,10394,12559$.

Tetanicum 3293.

TGF- $\beta 1$ antagonist $16050,22270$.

Thrombin inhibitor 18917.

Thrombin inhibitor inactive $5916,5917,6244,6089,6094,6203,10437$, 10435, 10750, 20569, 21893.
Thrombolytic 4055 .

Thymidine phosphorylase (TP) inhibitor 12613, 12614.

Thymidine phosphorylase inhibitor 2270, 19192, 20528, 20547, 20548, 20549.

Thyroid peroxidase inhibitor 16196, 22581.

Tissue factor inhibitor 8621 .

Tissue factor inhibitor inactive 16050, 22270.

Tissue proteinase B inhibitor 1030, 6314, 17588.

TNF inhibitor 22586, 22587.

TNF- $\boldsymbol{\alpha}$ formation enhancer 11977, 22077.

TNF-a inhibitor 5430 .

TNF- $\alpha$ production inhibitor $1071,1790,1791,1802,2004,2106,2469$, 2983, 3307, 3368, 3674, 4565, 4565, 4746, 7195, 7196, 7197, 7278, $8278,8423,8423,8424,8424,8520,8520,9276,9337,9456,11521$, $11522,12122,12122,12523,13137,13137,14097,14976,14977$, $15002,15635,16532,16675,16717,17240,17409,17460,17875$, 18317, 18317, 18376, 18673, 19308, 19427, 20992, 22686, 22687, 22688, 22727, 22728, 22729, 22730, 22731.

TNF- $\alpha$ release inhibitor $4055,4716,5424,6923,8311,8894$.

TNF- $\alpha$ release inhibitor inactive $5420,5421,5422,5423,5425$.

TNF- $\alpha$ secretion inhibitor 79, 82, 83, 18317, 20486.

Tonic 1017, 11882, 19473.

Topical protectant 10,15333 .

Topoisomerase I inhibitor 10691, 15631.

Topoisomerase II inhibitor 2304, 15478, 15479, 15480, 15481, 15869, 17144, 17896, 18202, 18624, 21054.

Topoisomerase inhibitor inactive 19349, 19350.

Topoisomerases I and II inhibitor 22232.

Total cAMP- and cGMP-phosphodiesterase (PDE)inhibitor 18376. Toxic 17732.

Toxic (highly) 18426.

Toxic inactive 14024 .

Toxin $10,126,140,340,551,567,646,655,782,983,1008,1011,1068$, $1125,1242,1250,1283,1352,1353,1364,1366,1461,1842,1887$, $1895,2039,2309,2399,2676,2838,2937,2959,3148,3152,3190$, $3201,3207,3218,3404,3498,3634,3909,3911,3988,4078,4457$, $4741,4745,4771,4857,4988,5004,5283,5440,5523,5858,6040$, $6370,6532,6533,6684,6686,6734,7063,7236,7252,8091,8257$, 8292, 8461, 8756, 8784, 8841, 8906, 8971, 9114, 9183, 9288, 9339, $9467,9596,9877,10238,10818,10898,11012,11130,11189,11690$, 11794, 11867, 12421, 12422, 12854, 12896, 12937, 12951, 13241, $13255,13711,14134,14250,14358,14885,15123,15429,15452$, $15461,15633,15872,15924,16285,16674,17040,17089,17420$, $17863,17903,17904,17963,18000,18017,18023,18785,18814$, 18816, 18840, 18849, 18940, 19041, 19057, 19121, 19374, 19379, 19461, 19469, 19471, 19676, 19698, 19706, 19712, 19889, 20056, 20060, 20061, 20069, 20133, 20341, 20403, 20500, 20963, 21325, $21326,21402,21644,22146,22151,22153,22276,22280,22360$, 22401, 22404, 22465, 22608, 23000.

Transforms into vitamin $\mathbf{D}_{\mathbf{2}}$ under ultraviolet ray 7250 .

Treatment of abdominalgia, cholera in early stage, colitis and dysentery 2013.

Treatment of acne and other pigment diseases 2056 . 
Treatment of acute arrhythmia 17761.

Treatment of acute heart failure with edema of lungs and cardiogenic shock 20403 .

Treatment of acute myocardial ischemia 16525 .

Treatment of AIDS 17958.

Treatment of allergic purpura 6302 .

Treatment of allergy and empyema in nose 13390.

Treatment of amebic dysentery $8513,12347$.

Treatment of amoebic dysentery 19819.

Treatment of angina pectoris 8401 .

Treatment of angina pectoris, asthma, and cardiac edema 21312.

Treatment of angiocardiopathy 8633,8634 .

Treatment of AP 1275.

Treatment of arrhythmia 7703, 18552.

Treatment of arterial blockage in retina 18180 .

Treatment of arteriosclerosis and hyperlipidemia 11083.

Treatment of arteriosclerosis, cerebral wounds and dysemia 20575.

Treatment of arteriosclerosis, hyperlipidemia, thrombus and coronary heart disease 12767 .

Treatment of asthma and allergic disease 7926.

Treatment of asthma and bronchitis 17472, 19540.

Treatment of asthmatic bronchitis 14395, 22552.

Treatment of atherosclerosis 1928.

Treatment of bacillary dysentery 983 .

Treatment of bacillary dysentery 13606.

Treatment of bacillary dysentery and inflammation of upper-respiratory tract 1159 .

Treatment of bacillary dysentery, duodenal ulcer, enteritis, gastrosis and trachitis 3203 .

Treatment of bilious headache (vasomotor headache) 16675 .

Treatment of bilious headache and obstetric process 16808 .

Treatment of blood capillary ailments 19087.

Treatment of bronchitis, scrofula, pulmonary tuberculosis and basal

tuberculosis 13264 .

Treatment of cerebral thrombosis and atherosclerosis 21055 .

Treatment of chronic arrhythmia 5067.

Treatment of chronic bronchitis $8312,9315,13610,13611$.

Treatment of chronic bronchitis 18317.

Treatment of cirrhosis with ascites 19819.

Treatment of coronary heart disease $4889,16525$.

Treatment of dermatitis 21434 .

Treatment of dermatosis and tinea 19187.

Treatment of diabetic cataract 491

Treatment of diarrhea 22190 .

Treatment of diseases in lung and bronchus 7482 .

Treatment of dysentery 17862 .

Treatment of eczema 1599

Treatment of fever, diarrhea and diseases of the urinary system 822 .

Treatment of fig wart 17592 .

Treatment of gastric disorders 22564.

Treatment of gastric hyperacidity 20341.

Treatment of gastric ulcer 19388.

Treatment of gastrosis, ulcer, upper-respiratory tract infection, urethral infection, bronchitis, pneumonia, enteritis, bacillary dysentery and sepsis 3202 .

Treatment of glaucoma 17360, 21059.

Treatment of glaucoma and myoparalysis 7381 .

Treatment of gout 10913 .

Treatment of headache and diseases of nasal cavity 13392.

Treatment of headache, neuralgia, itching, respiratory tract

inflammation, atrophic rhinitis and celostomia (alalia) 13774 .

Treatment of heart failure and auricular arrhythmia 15038 .

Treatment of heart failure and breathing inhibition 18637.

Treatment of hepatic coma and constipation 12440 .

Treatment of hepatic edema 21310.

Treatment of hepatic insufficiency 2538 .

Treatment of hepatitis 13481, 19819, 19895, 19897, 22975.

Treatment of Hodgkin's disease, chorion cancer, lymphatic sarcoma 22487.

Treatment of hypertension and angina pectoris 18180 .

Treatment of hypertension and rheumatalgia 21887 .

Treatment of hypertension and tachycardia caused by smoking 4873 .

Treatment of hypertension, bronchial asthma, ulcer of digestive tract, and chronic enteritis 21511.

Treatment of hypertensive 18826 .

Treatment of hypoprothrombinemia disease 22562 .

Treatment of impotence $12291,12292$.

Treatment of infant bacillary dysentery 7942 .

Treatment of infection from hepatitis virus 18599 .

Treatment of infection of respiratory tract, urethra, digestive tract and wounds 18270 .

Treatment of infectious diseases of respiratory tract and intestinal tract 4876.

Treatment of leprosy $828,829,830,831$.

Treatment of liver coma 1673.

Treatment of maladjustment of blood capillary permeability 13502 .

Treatment of megaloblastic anemia due to lack of folic acid 7852

Treatment of menopathy and female climacteric syndrome 7386 .

Treatment of mental disorder 1039.

Treatment of muscle rigidity and Parkinson's disease 21372.

Treatment of myasthenia gravis 15396 .

Treatment of myocardial infarction 11851.

Treatment of myocardial ischemia and myocardial infarction 20686 .

Treatment of neurasthenic syndrome 1609.

Treatment of neurosis 1608 .

Treatment of neurosis and gastrointestinal diseases 7517 .

Treatment of newbron asphyxia, toxicosis from opium, barbital, carbon monoxide, and respiratory failure induced by pneumonia and diphtheria 12939.

Treatment of pediatric gastrointestinal functional disorder 17886 .

Treatment of pellagrosis, stomatitis and glossitis 15526 .

Treatment of pile 19070.

Treatment of post-palsy paralysis 19587.

Treatment of post-partum uterus bleeding 7240 .

Treatment of primary hypertensionon 20324.

Treatment of psoriasis, rheumatic arthritis and leukemia 22011. 
Treatment of renal insufficiency 12018 .

Treatment of restless extrapyramidal dyscinesia 20324 .

Treatment of rheumatalgia and neuralgia 19542, 19545.

Treatment of rheumatic arthritis 22487.

Treatment of rheumatic arthritis 1928.

Treatment of rheumatic arthritis and asthma 5135 .

Treatment of rheumatic heart disease and coronary heart disease 4543.

Treatment of rheumatism and paralysis 3361 .

Treatment of schistosomiasis 9339.

Treatment of seasickness and carsickness 10870.

Treatment of sexual immaturity and learning disability 3753 .

Treatment of silicosis 21206 .

Treatment of skin disease $1293,1332,13223,17347,17486,17491$.

Treatment of skin disease, hepatitis, and inflammation 7997.

Treatment of some kidney disorders and relieve intracranial pressure in brain injuries 13504.

Treatment of sterilitas virilis 13212.

Treatment of stroke 17978.

Treatment of suppurated wound, burn, and skin infection 22282.

Treatment of terror, anxiety and melancholy 8524 .

Treatment of tinea and sarcoptidosis 13246, 13247.

Treatment of trachitis 21508 .

Treatment of trachitis and dysentery 22151, 22153.

Treatment of trachitis, antitussive 12020.

Treatment of tympanitis, onychia lateralis, viral herpes, burn infection,

suppurative amygdalitis and enteritis 20444.

Treatment of ulcer 11295 .

Treatment of ulcer of digestive tract 17546.

Treatment of urethral infection 834 .

Treatment of vascular headache and bilious headache 20324 .

Treatment of ventricular tachycardia 20133.

Trehalase inhibitor 2792,7437 .

Tremorigenic agent 20016 .

Trypsase inhibitor 20009.

Trypsase inhibitor inactive 9561 .

TXA formation inhibitor 3794.

$\mathbf{T X B}_{\mathbf{2}}$ formation inhibitor $3981,12766,19259$.

Tyrosinase inhibitor $577,618,1091,1618,2380,3567,3829,3831,4249$, $4251,4254,4260,4262,4263,4264,6700,6870,8673,10054,10626$, $10807,11656,12252,12280,12341,12341,12343,12344,12355$, $12358,12361,12365,12366,12367,12510,12847,13097,14452$, $14511,14802,14868,15206,15526,15769,16897,18064,18065$, $18066,18067,18068,18069,18169,19983,20086,21905,21967$, 22237.

Tyrosinase inhibitor inactive $508,571,572,573,574,575,5008,5783$, 20501.

Tyrosine kinase inhibitor 2102, 2106, 3811, 6091, 10638, 17169, 20009, 22718, 22720.

UDP-MurNac synthesis inhibitor $1635,1636,1637$.

Uncoupling action 16024 .

Up-regulation of vascular endothelial growth factor (VEGF) and its receptors VEGF-R1, VEGF-R2 gene 19202.
Urease inhibitor 9740, 15216, 20528.

Uricosuric 2004.

Used to research of $\boldsymbol{\gamma}$-radiation 16929.

Used to resist aridity in agriculture 13084 .

Vanilloid receptor activator 19081.

Vasoconstriction inhibitor $893,894,896,4545,5750,8913,8914$.

Vasoconstriction inhibitor, in artery 15704.

Vasoconstrictor $514,2726,4381,5445,6303,6815,7232,7234,7243$, $9335,15253,15708,15875,17028,17370,17953,18024,19819$, 20100, 22144, 22237.

Vasodilator $1048,1275,1658,2300,2303,2734,3588,4543,4544,4873$, $5707,6677,6691,8010,8290,8423,8424,8426,8427,8430,8808$, $8989,9568,10870,11002,11680,11834,11834,12574,13237,13260$, $13261,13262,14949,14950,15002,15003,15321,15528,15704$, $15705,16263,16439,16867,17283,18651,19081,19540,20275$, 20510, 20575, 21055, 21239, 21877, 22489, 22552.

Vasodilator inactive $1271,1272,8507,14732$.

Vasodilator, cerebral $8989,21055$.

Vasodilator, coronary $784,1275,2298,2796,3633,4108,4630,4889$, $6507,7334,7945,11648,12221,12747,13836,15129,16080,16525$, $16759,17036,17278,17968,18165,18180,18317,19227,19540$, $21310,21312,21511$.

Vasodilator, coronary and cerebral 783 .

Vasodilator, coronary, cerebral and peripheral 5067 .

Vasodilator, peripheral $4543,4544,11002,15528,21055$.

Vasorelaxant $1030,2300,2303,3795,5135,8251,13573,13574,15278$, 19761.

Vasorelaxant, inhibits inward flow of calcium, increase cGMP 5135 .

Vesicant $1179,22146$.

VHR DS-PTPase inhibitor 10035, 10036.

VR1 receptor antagonist 843 .

Vulnerary 21511.

Xanthine oxidase inhibitor $3490,4055,5212,6757,6758,8095,13428$, 13429, 14099, 15451, 15457, 17985, 17986, 17991, 18317, 19347, 19348.

Xanthinoxidase inhibitor $1497,2102,3602,6095,6096,6769,8844$, $11266,12766,12767,12771,12987,13145,15715,17895,18323$, 18361, 18368, 20515, 21054, 21634, 22718. 


\section{Compound Molecular Formula Index}

The Molecular Formula Index lists the molecular formulae of all 23033 isolated compounds from TCM plants and their congeners given in the encyclopedia in Hill convention order. Under a bold formula, main names of all related compounds lists in alphabetical order and the compound code number follows the name immediately.

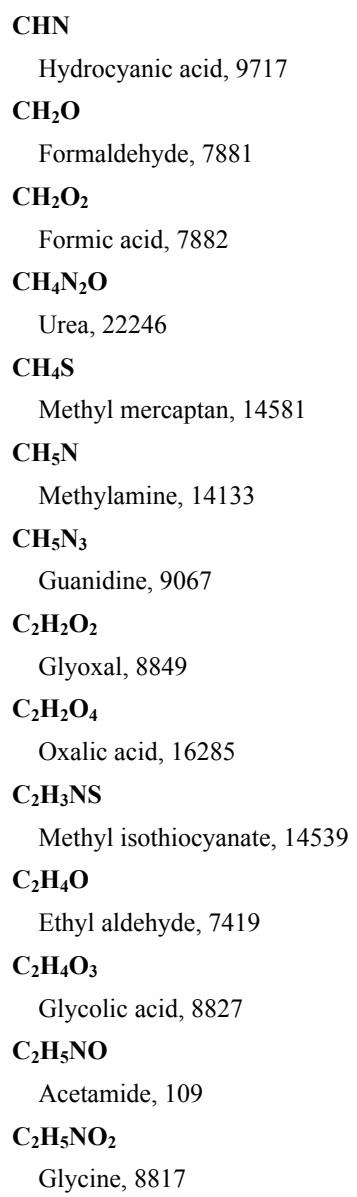

$\mathrm{C}_{2} \mathrm{H}_{6} \mathrm{~N}_{2} \mathrm{O}_{2}$

Methylazoxymethanol, 14151

$\mathrm{C}_{2} \mathrm{H}_{6} \mathrm{O}_{2}$

Glycol, 8826

$\mathrm{C}_{2} \mathrm{H}_{6} \mathrm{O}_{2} \mathrm{~S}$

Dimethyl sulfone, 6408

$\mathrm{C}_{2} \mathrm{H}_{6} \mathrm{~S}$

Dimethyl sulfide, 6407

$\mathrm{C}_{2} \mathrm{H}_{6} \mathrm{~S}_{2}$

Dimethyl disulfide, 6342

$\mathrm{C}_{2} \mathrm{H}_{6} \mathrm{~S}_{3}$

Dimethyl trisulfide, 6417

$\mathrm{C}_{2} \mathrm{H}_{6} \mathrm{~S}_{4}$

Dimethyl tetrasulfide, 6414

$\mathrm{C}_{2} \mathrm{H}_{7} \mathbf{N}$

Ethylamine, 7420

$\mathrm{C}_{2} \mathrm{H}_{7} \mathrm{NO}$

Ethanolamine, 7390

$\mathrm{C}_{2} \mathrm{H}_{7} \mathrm{NO}_{3} \mathrm{~S}$

Taurine, 20732

$\mathrm{C}_{3} \mathrm{H}_{4} \mathrm{O}_{2}$

Acrylic acid, 578

Methylglyoxal, 14475

$\mathrm{C}_{3} \mathrm{H}_{4} \mathrm{O}_{3}$

Pyruvic acid, 18281

$\mathrm{C}_{3} \mathrm{H}_{4} \mathrm{O}_{4}$

Malonic acid, 13439

$\mathrm{C}_{3} \mathrm{H}_{4} \mathrm{~S}_{2}$
Dithiocyclopentene, 6524

\section{$\mathrm{C}_{3} \mathrm{H}_{5} \mathrm{NOS}$}

2-Oxazolidinethione, 16286

$\mathrm{C}_{3} \mathrm{H}_{6} \mathrm{O}$

2-Hydroxy-propylene, 10663

Propionaldehyde, 17930

$\mathrm{C}_{3} \mathrm{H}_{6} \mathrm{OS}$

Propanethial $S$-oxide, 17912

$\mathrm{C}_{3} \mathrm{H}_{6} \mathrm{O}_{2}$

Methylacetate, 14111

Propionic acid, 17931

$\mathrm{C}_{3} \mathrm{H}_{6} \mathrm{O}_{3}$

Dihydroxyacetone, 5748

Lactic acid, 12436

$\mathrm{C}_{3} \mathrm{H}_{6} \mathrm{O}_{4}$

$D$-Glyceric acid, 8806

$\mathrm{C}_{3} \mathrm{H}_{6} \mathrm{~S}_{4}$

5-Methyl-1,2,3,4-tetrathiane, 14755

$\mathrm{C}_{3} \mathrm{H}_{7} \mathrm{NO}_{2}$

L-Alanine, 832

$\beta$-Alanine, 833

Sarcosine, 19360

$\mathrm{C}_{3} \mathrm{H}_{7} \mathrm{NO}_{3}$

(S)-Serine, 19759

$\mathrm{C}_{3} \mathrm{H}_{8} \mathrm{~N}_{2} \mathrm{O}_{2}$

$\alpha, \beta$-Diaminopropionic acid, 5352

$\mathrm{C}_{3} \mathrm{H}_{8} \mathrm{O}$

n-Propanol, 17916 
$\mathrm{C}_{3} \mathrm{H}_{8} \mathrm{O}_{3}$

Glycerol, 8808

\section{$\mathrm{C}_{3} \mathrm{H}_{8} \mathrm{~S}$}

Propane-1-thiol, 17913

Propane-2-thiol, 17914

\section{$\mathrm{C}_{3} \mathrm{H}_{9} \mathrm{~N}$}

Propylamine, 17935

Trimethylamine, 21939

\section{$\mathrm{C}_{3} \mathrm{H}_{9} \mathrm{NO}$}

Trimethylamine oxide, 21940

\section{$\mathrm{C}_{4} \mathbf{H}_{4} \mathbf{N}_{2} \mathbf{O}_{2}$}

Uracil, 22237

\section{$\mathrm{C}_{4} \mathrm{H}_{4} \mathrm{O}_{4}$}

Fumaric acid, 7996

\section{$\mathrm{C}_{4} \mathrm{H}_{4} \mathrm{O}_{5}$}

Oxalacetic acid, 16284

\section{$\mathrm{C}_{4} \mathrm{H}_{5} \mathrm{ClO}_{2}$}

Methyl 2-chloropropenoate, 14225

\section{$\mathrm{C}_{4} \mathrm{H}_{5} \mathrm{NO}_{2}$}

Pyrrolidine-2,5-dione, 18277

\section{$\mathrm{C}_{4} \mathrm{H}_{5} \mathrm{NS}$}

Allyl isothiocyanate, 949

Allylthiocyanate, 956

\section{$\mathrm{C}_{4} \mathrm{H}_{5} \mathrm{O}_{4} \mathrm{~N}_{3}$}

(2,5-Dioxo-4-imidazolidinyl)carbamic acid, 6472

\section{$\mathrm{C}_{4} \mathrm{H}_{6} \mathrm{~N}_{2} \mathrm{O}_{2}$}

\section{$L$ - $\beta$-Cyanoalanine, 4452}

5-Methyl hydantoin, 14494

\section{$\mathrm{C}_{4} \mathrm{H}_{6} \mathrm{~N}_{4} \mathrm{O}_{3}$}

Allantoin, 917

\section{$\mathrm{C}_{4} \mathrm{H}_{6} \mathrm{O}_{2}$}

Crotonic acid, 4271

\section{$\mathrm{C}_{4} \mathrm{H}_{6} \mathrm{O}_{2} \mathrm{~S}$}

Thioacetic anhydride, 21332

\section{$\mathrm{C}_{4} \mathrm{H}_{6} \mathrm{O}_{4}$}

Succinic acid, 20444

Threono-1,4-lactone, 21343

\section{$\mathrm{C}_{4} \mathrm{H}_{6} \mathrm{O}_{5}$}

Malic acid, 13419

\section{$\mathrm{C}_{4} \mathrm{H}_{6} \mathrm{O}_{6}$}

Tartaric acid, 20715

\section{$\mathrm{C}_{4} \mathrm{H}_{6} \mathrm{~S}$}

Divinyl sulfide, 6531

\section{$\mathrm{C}_{4} \mathrm{H}_{6} \mathrm{~S}_{2}$}

3-Methyl-1,2-dithia-3-cyclopentene, 14327

4-Methyl-1,2-dithio-3-cyclopentene, 14328

5-Methyl-1,2-dithio-3-cyclopentene, 14329

\section{$\mathrm{C}_{4} \mathrm{H}_{7} \mathrm{NOS}$}

trans-3-Methylthioacrylamide, 14758

\section{$\mathrm{C}_{4} \mathrm{H}_{7} \mathrm{NO}_{2}$}

Azetidine-2-carboxylic acid, 2057

\section{$\mathrm{C}_{4} \mathrm{H}_{7} \mathrm{NO}_{4}$}

$N$-Acetyl- $N$-hydroxy-2-carbamic acid methyl ester, 413

L-Aspartic acid, 1886

\section{$\mathrm{C}_{4} \mathrm{H}_{7} \mathrm{NO}_{5}$}

erythro- $\beta$-Hydroxy- $L$-aspartic acid, 9804

$\mathrm{C}_{4} \mathrm{H}_{7} \mathrm{NS}$

Isopropyl isothiocyanate, 11625

$\mathrm{C}_{4} \mathrm{H}_{7} \mathrm{~N}_{3} \mathrm{O}$

Creatinine, 4223

1,5-Dihydro-1-methyl-2-amino-imidazol-4-one, 5674

$\mathrm{C}_{4} \mathrm{H}_{8} \mathrm{~N}_{2} \mathrm{O}_{3}$

L-Asparagine, 1866

\section{$\mathrm{C}_{4} \mathrm{H}_{8} \mathrm{~N}_{4} \mathrm{O}_{4}$}

Allantoic acid, 916

\section{$\mathrm{C}_{4} \mathrm{H}_{8} \mathrm{O}$}

Allylcarbinol, 943

n-Butylaldehyde, 2787

Isobutanal, 11267

\section{$\mathrm{C}_{4} \mathrm{H}_{8} \mathrm{O}_{2}$}

Acetoin, 111

Butyric acid, 2808

Ethyl acetate, 7418

Isobutyric acid, 11290

\section{$\mathrm{C}_{4} \mathrm{H}_{8} \mathrm{O}_{2} \mathrm{~S}$}

trans-3-Methylsulfinyl-2-propenol, 14729

\section{$\mathrm{C}_{4} \mathrm{H}_{8} \mathrm{O}_{3} \mathrm{~S}$}

trans-3-Methylsulfonyl-2-propenol, 14731

\section{$\mathrm{C}_{4} \mathrm{H}_{8} \mathrm{O}_{4}$}

(3S),4-Dihydroxybutyric acid, 5783

\section{$\mathrm{C}_{4} \mathrm{H}_{8} \mathrm{O}_{5}$}

(D)-Threonic acid, 21340

(L)-Threonic acid, 21341

\section{$\mathrm{C}_{4} \mathrm{H}_{8} \mathrm{OS}_{2}$}

Methyl allyl thiosulfinate, 14128

Methyl-1-propenyl thiosulfinate, 14690

\section{$\mathrm{C}_{4} \mathrm{H}_{8} \mathrm{~S}$}

Methyl allyl sulfide, 14127

\section{$\mathrm{C}_{4} \mathrm{H}_{8} \mathrm{~S}_{2}$}

Methyl allyl disulfide, 14126

$\mathrm{C}_{4} \mathrm{H}_{8} \mathrm{~S}_{3}$

Allyl methyl trisulfide, 952

4-Methyl-1,2,3-trithiane, 14793

$\mathrm{C}_{4} \mathrm{H}_{8} \mathrm{~S}_{4}$

Allyl methyl tetrasulfide, 951

$\mathrm{C}_{4} \mathrm{H}_{8} \mathrm{~S}_{5}$

Allyl methyl pentasulfide, 950

$\mathrm{C}_{4} \mathrm{H}_{9} \mathrm{~N}$

Pyrrolidine, 18275

$\mathrm{C}_{4} \mathrm{H}_{9} \mathrm{NO}_{2}$

$\alpha$-Aminobutyric acid, 1046

$\beta$-Aminobutyric acid, 1047

$\gamma$-Aminobutyric acid, 1048

Aminoisobutyric acid, 1055

\section{$\mathrm{C}_{4} \mathrm{H}_{9} \mathrm{NO}_{2} \mathrm{~S}$}

$L$-Homocysteine, 9601

$S$-Methyl cysteine, 14277

$\mathrm{C}_{4} \mathrm{H}_{9} \mathrm{NO}_{2} \mathrm{~S}_{2}$

$S$-Methyl mercapto- $L$-cysteine, 14582

$\mathrm{C}_{4} \mathrm{H}_{9} \mathrm{NO}_{2} \mathrm{Se}$

Se-Methyl- $L$-selenocysteine, 19691

\section{$\mathrm{C}_{4} \mathrm{H}_{9} \mathrm{NO}_{3}$}

L-Homoserine, 9620

Threonine, 21342

\section{$\mathrm{C}_{4} \mathrm{H}_{9} \mathrm{NO}_{3} \mathrm{~S}$}

$S$-Methyl- $L$-cysteine sulfoxide, 14278

\section{$\mathrm{C}_{4} \mathrm{H}_{9} \mathrm{NO}_{4}$}

$\alpha$-Hydroxymethylserine, 10521

\section{$\mathrm{C}_{4} \mathrm{H}_{9} \mathrm{~N}_{3} \mathrm{O}_{2}$}

Creatine, 4221

\section{$\mathrm{C}_{4} \mathrm{H}_{9} \mathrm{~N}_{3} \mathrm{O}_{3}$}

L-Albizziine, 862

\section{$\mathrm{C}_{4} \mathrm{H}_{9} \mathrm{O}_{4}$}

Methyl (S)-glycerate, 14473

\section{$\mathrm{C}_{4} \mathrm{H}_{\mathbf{1 0}} \mathbf{N}_{2} \mathrm{O}_{2}$}

2,3-Diaminobutyric acid, 5350

$L$ - $\alpha, \gamma$-Diaminobutyric acid, 5351

3-Methylamino- $L$-alanine, 14134

\section{$\mathrm{C}_{4} \mathrm{H}_{10} \mathrm{~N}_{2} \mathrm{O}_{3}$}

Canaline, 3059

\section{$\mathrm{C}_{4} \mathrm{H}_{10} \mathrm{~N}_{3} \mathrm{O}_{5} \mathrm{P}$}

Creatine phosphoric acid, 4222

\section{$\mathrm{C}_{4} \mathrm{H}_{10} \mathrm{O}$}

Isobutyl alcohol, 11268

\section{$\mathrm{C}_{4} \mathrm{H}_{10} \mathrm{O}_{3}$}

1-Deoxy-L-erythritol, 5171

\section{$\mathrm{C}_{4} \mathrm{H}_{10} \mathrm{O}_{4}$}

Erythritol, 7334

(D)-Threitol, 21339

\section{$\mathrm{C}_{4} \mathrm{H}_{10} \mathrm{~S}$}

Diethyl sulfide, 5503

\section{$\mathrm{C}_{4} \mathrm{H}_{10} \mathrm{~S}_{2}$}

Methyl propyl disulfide, 14691

\section{$\mathrm{C}_{4} \mathrm{H}_{10} \mathrm{~S}_{3}$}

Methyl propyl trisulfide, 14694

$\mathrm{C}_{4} \mathrm{H}_{12} \mathrm{~N}_{2}$

Putrescine, 18230

$\mathrm{C}_{5} \mathrm{H}_{4} \mathrm{Cl}_{2} \mathrm{~N}_{2}$

2,4-Dichloro-6-aminopyridine, 5418

$\mathrm{C}_{5} \mathrm{H}_{4} \mathbf{N}_{2} \mathrm{O}_{4}$

Orotic acid, 16214

$\mathrm{C}_{5} \mathrm{H}_{4} \mathrm{~N}_{4}$

Purine, 18209

$\mathrm{C}_{5} \mathrm{H}_{4} \mathrm{~N}_{4} \mathrm{O}$

Hypoxanthine, 10913

$\mathrm{C}_{5} \mathrm{H}_{4} \mathrm{~N}_{4} \mathrm{O}_{2}$

Xanthine, 22756 


\section{$\mathrm{C}_{5} \mathrm{H}_{4} \mathrm{O}_{2}$}

Anemonol, 1179

2-Furaldehyde, 8011

\section{$\mathrm{C}_{5} \mathrm{H}_{4} \mathrm{O}_{3}$}

2-Furancarboxylic acid, 8012

Pyromeconic acid, 18271

\section{$\mathrm{C}_{5} \mathrm{H}_{5} \mathrm{NO}$}

Pyrrole-2-aldehyde, 18274

$\mathrm{C}_{5} \mathrm{H}_{5} \mathrm{NO}_{2}$

2-Minaline, 14870

$\mathrm{C}_{5} \mathrm{H}_{5} \mathrm{NO}_{3}$

Codopiloic acid, 3887

$\mathrm{C}_{5} \mathrm{H}_{5} \mathrm{~N}_{5}$

Adenine, 617

$\mathrm{C}_{5} \mathrm{H}_{5} \mathrm{~N}_{5} \mathrm{O}$

Guanine (1,7-Dihydro-form), 9070

Isoguanine, 11448

\section{$\mathrm{C}_{5} \mathrm{H}_{6} \mathrm{KN}_{3} \mathrm{O}_{5}$}

Potassium quisqualate, 17746

$\mathrm{C}_{5} \mathrm{H}_{6} \mathrm{~N}_{2} \mathrm{O}_{2}$

1-Imidazolylacetic acid, 10997

5-Methyluracil, 14802

$\mathrm{C}_{5} \mathbf{H}_{6} \mathbf{N}_{2} \mathbf{O}_{4}$

Ibotenic acid, 10938

$\mathrm{C}_{5} \mathrm{H}_{6} \mathrm{~N}_{4} \mathrm{O}_{3}$

Uric acid, 22251

\section{$\mathrm{C}_{5} \mathrm{H}_{6} \mathrm{O}_{2}$}

Furfuryl alcohol, 8025

Tulipalin, 22102

\section{$\mathrm{C}_{5} \mathrm{H}_{6} \mathrm{O}_{3}$}

$\beta$-Hydroxy- $\alpha$-methylene- $\gamma$-butyllactone, 10488

$\mathrm{C}_{5} \mathrm{H}_{6} \mathrm{O}_{4}$

Mesaconic acid, 13793

\section{$\mathrm{C}_{5} \mathrm{H}_{6} \mathrm{~S}_{3}$}

4-Vinyl-1,2,3-trithia-4-cyclohexene, 22518

\section{$\mathrm{C}_{5} \mathrm{H}_{7} \mathrm{NOS}$}

1-Thiocyanato-2-hydroxy-3-butene, 21334

L-5-Vinyl-2-thiooxazolidone, 22517

\section{$\mathrm{C}_{5} \mathrm{H}_{7} \mathrm{NO}_{2}$}

1-Cyano-2-hydroxymethylprop-1-ene-3-ol, 4453

1-Cyano-2-hydroxymethylprop-2-ene-1-ol, 4454

Jatropham, 11842

$\mathrm{C}_{5} \mathrm{H}_{7} \mathrm{NO}_{3}$

Pyroglutamic acid, 18266

\section{$\mathrm{C}_{5} \mathrm{H}_{7} \mathrm{NS}$}

3-Butenyl isothiocyanate, 2782

$\mathrm{C}_{5} \mathrm{H}_{7} \mathbf{N}_{3} \mathrm{O}_{5}$

Quisqualic acid, 18452

$\mathrm{C}_{5} \mathrm{H}_{8} \mathrm{~N}_{2} \mathrm{O}$

2-4'-Imidazolylethanol, 10998

$\mathrm{C}_{5} \mathrm{H}_{8} \mathrm{~N}_{2} \mathrm{O}_{2}$

Squamolone, 20243

$\mathrm{C}_{5} \mathrm{H}_{8} \mathrm{~N}_{2} \mathrm{O}_{4}$
Tricholomic acid, 21562

$\mathbf{C}_{5} \mathbf{H}_{8} \mathbf{N}_{2} \mathbf{O}_{5}$

$L$ - $\alpha$-Amino- $\beta$-oxalylaminopropionic acid, 1058

(S)-Dencichine, 5105

$\mathrm{C}_{5} \mathrm{H}_{8} \mathrm{O}$

3-Methyl-3-butenone, 14177

Tiglaldehyde, 21370

$\mathrm{C}_{5} \mathrm{H}_{8} \mathrm{O}_{2}$

Angelic acid, 1188

Tiglic acid, 21371

$\mathrm{C}_{5} \mathrm{H}_{8} \mathrm{O}_{3}$

Levulinic acid, 12735

$\mathrm{C}_{5} \mathrm{H}_{8} \mathrm{O}_{4}$

Capilliplactone, 3123

2-Deoxy- $D$-ribono-1,4-lactone, 5209

2,3-Dihydroxy-2-methyl-butyolactone, 6027

Glutaric acid, 8784

\section{$\mathrm{C}_{5} \mathrm{H}_{9} \mathrm{NO}$}

4-Pentenamide, 16887

\section{$\mathrm{C}_{5} \mathrm{H}_{9} \mathrm{NOS}_{2}$}

3-Methylsulfinyl propyl isothiocyanate, 14730

$\mathrm{C}_{5} \mathrm{H}_{9} \mathrm{NO}_{2}$

$\gamma$-Amino- $\alpha$-methylene butyric acid, 1056

Proline, 17905

Pterolactam, 18112

Pyrrolidine carboxylic acd, 18276

\section{$\mathrm{C}_{5} \mathrm{H}_{9} \mathrm{NO}_{3}$}

cis-4-Hydroxyproline, 10659

3-Hydroxyproline, 10660

trans-4-Hydroxyproline, 10661

\section{$\mathrm{C}_{5} \mathrm{H}_{9} \mathrm{NO}_{3} \mathrm{~S}$}

1,4-Thiazane-3-carboxylic acid $S$-oxide, 21330

\section{$\mathrm{C}_{5} \mathrm{H}_{9} \mathrm{NO}_{5}$}

3-Amino-2-hydroxy pentanedioic acid, 1052

$\gamma$-Hydroxy glutaminic acid, 10151

\section{$\mathrm{C}_{5} \mathrm{H}_{9} \mathrm{NS}$}

Butyl isothiocyanate, 2798

sec-Butyl isothiocyanate, 2799

$\mathrm{C}_{5} \mathrm{H}_{9} \mathrm{NS}_{2}$

3-Methylthiopropyl isothiocyanate, 14762

\section{$\mathrm{C}_{5} \mathrm{H}_{9} \mathrm{~N}_{3}$}

Histamine, 9568

$\mathrm{C}_{5} \mathrm{H}_{10}$

2-Methyl-2-butene, 14173

$\mathrm{C}_{5} \mathrm{H}_{10} \mathrm{~N}_{2} \mathrm{O}_{2}$

Cucurbitine, 4329

$\mathrm{C}_{5} \mathrm{H}_{10} \mathrm{~N}_{4} \mathrm{O}_{4}$

3-Acetyl-5-carbomethoxy-2H-3,4,5,6-tetrahydro1-oxa-2,3,5,6-tetrazine, 345

\section{$\mathrm{C}_{5} \mathrm{H}_{10} \mathrm{O}$}

Isovaleraldehyde, 11752

3-Methyl butanone, 14163

2-Methylbut-3-en-2-ol, 14176
Pentanal, 16876

1-Penten-3-ol, 16889

\section{$\mathrm{C}_{5} \mathrm{H}_{10} \mathrm{O}_{2}$}

Ethylpropionate, 7475

Isovaleric acid, 11754

2-Methyl butyric acid, 14186

Pentanic acid, 16884

$\mathrm{C}_{5} \mathrm{H}_{10} \mathrm{O}_{3}$

(R)-Artabotriol, 1775

Nilic acid, 15597

\section{$\mathrm{C}_{5} \mathrm{H}_{10} \mathrm{O}_{5}$}

Apiose, 1521

Ribose, 18837

Xylose, 22843

\section{$\mathrm{C}_{5} \mathrm{H}_{11} \mathrm{~N}$}

Piperidine, 17450

\section{$\mathrm{C}_{5} \mathrm{H}_{11} \mathrm{NO}$}

Pentaldehyde oxime, 16862

\section{$\mathrm{C}_{5} \mathrm{H}_{11} \mathrm{NO}_{2}$}

Betaine, 2318

$N, N$-Dimethyl glycine methyl ester, 6353

Norvaline, 15814

2- $N$-Propyl-1,3-dioxolane, 17938

Valine, 22324

\section{$\mathrm{C}_{5} \mathrm{H}_{11} \mathrm{NO}_{2} \mathrm{~S}$}

Methionine, 13823

\section{$\mathrm{C}_{5} \mathrm{H}_{11} \mathrm{NO}_{3}$}

$L$ - $\alpha$-Amino- $\delta$-hydroxyvaleric acid, 1054

1,4-Dideoxy-1,4-imino-arabinitol, 5475

Mesotrihydroxypiperidine, 13803

$\alpha$-Oxyvaline, 16480

$\beta$-Oxyvaline, 16481

$3 \beta, 4 \beta, 5 \alpha$-Trihydroxypiperidine, 21838

\section{$\mathrm{C}_{5} \mathrm{H}_{11} \mathrm{NO}_{3} \mathrm{~S}$}

Methionine sulfoxide, 13824

\section{$\mathrm{C}_{5} \mathrm{H}_{11} \mathrm{NO}_{5} \mathrm{~S}$}

$D$-Rhodoic acid, 18798

\section{$\mathrm{C}_{5} \mathrm{H}_{11} \mathrm{NS}_{2}$}

Nereistoxin, 15490

\section{$\mathrm{C}_{5} \mathrm{H}_{11} \mathrm{~N}_{3} \mathrm{O}_{2}$}

$\gamma$-Guanidinobutyric acid, 9069

\section{$\mathrm{C}_{5} \mathrm{H}_{11} \mathrm{O}_{2} \mathrm{~S}^{+}$}

Dimethyl- $\beta$-propriothetin, 6400

\section{$\mathrm{C}_{5} \mathrm{H}_{12} \mathrm{~N}_{2} \mathrm{O}_{2}$}

Ornithine, 16208

\section{$\mathrm{C}_{5} \mathrm{H}_{12} \mathrm{~N}_{4} \mathrm{O}_{3}$}

Canavanine, 3063

\section{$\mathrm{C}_{5} \mathrm{H}_{12} \mathrm{O}$}

Isoamyl alcohol, 11218

2-Methyl-1-butanol, 14162

Pentanol, 16885

$\mathrm{C}_{5} \mathrm{H}_{12} \mathrm{O}_{2}$

1,5-Pentadiol, 16835 
$\mathrm{C}_{5} \mathrm{H}_{12} \mathrm{O}_{4}$

1-Deoxy- $L$-arabinitol, 5154

1-Deoxy- $D$-lyxitol, 5192

1-Deoxy- $D$-ribitol, 5207

2-Deoxy- $D$-ribitol1, 5208

1-Deoxy- $D$-xylitol, 5220

2-C-Methyl- $D$-erythritol, 14419

\section{$\mathrm{C}_{5} \mathrm{H}_{12} \mathrm{O}_{5}$}

Adonitol, 645

D-Arabitol, 1596

(3R)-2-Hydroxymethylbutane-1,2,3,4-tetrol, 10471

Ribitol, 18832

\section{$\mathrm{C}_{5} \mathrm{H}_{12} \mathrm{~S}$}

Ethylisopropyl sulfide, 7454

\section{$\mathrm{C}_{5} \mathrm{H}_{13} \mathrm{~N}$}

Isoamylamine, 11219

\section{$\mathrm{C}_{5} \mathrm{H}_{13} \mathrm{NO}$}

Neurine, 15516

\section{$\mathrm{C}_{5} \mathrm{H}_{13} \mathrm{~N}_{3} \mathrm{O}$}

4-Guanidino-1-butanol, 9068

$$
\begin{aligned}
& \mathbf{C}_{5} \mathbf{H}_{14} \mathbf{N O}^{+} \\
& \text {Choline, } 3589
\end{aligned}
$$

\section{$\mathrm{C}_{5} \mathrm{H}_{14} \mathrm{~N}_{2}$}

Cadaverine, 2845

\section{$\mathrm{C}_{5} \mathrm{H}_{14} \mathrm{~N}_{4}$}

Agmatine, 749

\section{$\mathrm{C}_{5} \mathbf{H}_{\mathbf{1 4}} \mathbf{O}_{22} \mathbf{P}_{6}$}

3,4-trans-(erythro)-3,5-Bis(tripolyphosphate)-

4-pentanolide, 2494

\section{$\mathrm{C}_{6} \mathrm{H}_{3} \mathrm{~N}_{3} \mathrm{O}_{7}$}

Picric acid, 17328

\section{$\mathrm{C}_{6} \mathrm{H}_{4} \mathrm{O}_{2}$}

Quinone, 18426

\section{$\mathrm{C}_{6} \mathrm{H}_{5} \mathrm{NO}_{2}$}

Nicotinic acid, 15528

\section{$\mathrm{C}_{6} \mathrm{H}_{6} \mathrm{~N}_{2} \mathrm{O}$}

Nicotinamide, 15526

\section{$\mathrm{C}_{6} \mathrm{H}_{6} \mathbf{N}_{2} \mathrm{O}_{2}$}

Urocanic acid, 22260

\section{$\mathrm{C}_{6} \mathrm{H}_{5} \mathrm{~N}_{5} \mathrm{O}$}

2-Amino-6-hydroxypteridine, 1053

\section{$\mathrm{C}_{6} \mathrm{H}_{5} \mathrm{~N}_{5} \mathrm{O}_{2}$}

Isoxanthopterin, 11784

Xanthopterin, 22772

\section{$\mathrm{C}_{6} \mathrm{H}_{6} \mathrm{O}$}

Phenol, 17089

\section{$\mathrm{C}_{6} \mathrm{H}_{6} \mathrm{O}_{2}$}

Catechol, 3318

Hydroquinone, 9740

$\alpha$-Methylfurfural, 14450

Resorcinol, 18641

$\mathrm{C}_{6} \mathrm{H}_{6} \mathrm{O}_{3}$
5-Hydroxymethyl furaldehyde, 10493

4-Hydroxymethyl-2-furaldehyde, 10494

Maltol, 13452

5-Methoxyfuraldehyde, 13931

Phloroglucinol, 17174

Pyrogallol, 18265

$\mathrm{C}_{6} \mathrm{H}_{6} \mathrm{O}_{4}$

5-(Hydroxymethyl)-furan-2-carboxylic acid, 10495

Kojic acid, 12252

\section{$\mathrm{C}_{6} \mathrm{H}_{6} \mathrm{O}_{6}$}

cis-Aconitic acid, 552

trans-Aconitic acid, 553

Dehydroascorbic acid, 4879

\section{$\mathrm{C}_{6} \mathrm{H}_{7} \mathrm{NO}$}

m-Aminophenol, 1059

$o$-Aminophenol, 1060

p-Aminophenol, 1061

3-Hydroxy-2-methylpyridine, 10520

3-Methoxypyridine, 14081

Pyridin-3-yl-methanol, 18259

Pyrroyl- $\alpha$-methyl ketone, 18279

\section{$\mathrm{C}_{6} \mathrm{H}_{7} \mathrm{NO}_{2}$}

5-Hydroxy-2-pyridinemethanol, 10669

\section{$\mathrm{C}_{6} \mathrm{H}_{7} \mathrm{NO}_{3}$}

Gentianaine, 8293

\section{$\mathrm{C}_{6} \mathrm{H}_{7} \mathrm{NO}_{3} \mathrm{~S}$}

Sulfanilic acid, 20467

\section{$\mathrm{C}_{6} \mathrm{H}_{8} \mathrm{~N}_{2} \mathrm{O}_{2}$}

Imidazolylpropionic acid, 10999

\section{$\mathrm{C}_{6} \mathrm{H}_{8} \mathrm{O}_{2}$}

Parasorbic acid, 16657

\section{$\mathrm{C}_{6} \mathrm{H}_{8} \mathrm{O}_{3}$}

$5 R, 6 S$-Osmundalactone, 16255

\section{$\mathrm{C}_{6} \mathrm{H}_{8} \mathrm{O}_{6}$}

Ascorbic acid, 1845

\section{$\mathrm{C}_{6} \mathrm{H}_{8} \mathrm{O}_{7}$}

Citric acid, 3766

Isocitric acid, 11332

Isocitric acid b, 11333

Isocitric acid $c, 11334$

Isocitric acid $\mathrm{d}, 11335$

\section{$\mathrm{C}_{6} \mathrm{H}_{8} \mathrm{OS}_{2}$}

3-Allyldisulfanyl-propenal, 947

2-Vinyl-1,3-dithia-4-cyclohexene-3-oxide, 22512

3-Vinyl-1,2-dithia-4-cyclohexene-2-oxide, 22514

\section{$\mathrm{C}_{6} \mathrm{H}_{8} \mathrm{~S}$}

6-Methyl-1-thio-2,4-cyclohexadiene, 14759

\section{$\mathrm{C}_{6} \mathrm{H}_{8} \mathrm{~S}_{2}$}

2-Vinyl-1,3-dithia-4-cyclohexene, 22511

3-Vinyl-1,2-dithia-4-cyclohexene, 22513

3-Vinyl-1,2-dithia-5-cyclohexene, 22515

$\mathrm{C}_{6} \mathrm{H}_{8} \mathrm{~S}_{2} \mathrm{O}$
3-Vinyl-3,4-dihydro-1,2-dithiin-1-oxide, 22509

$\mathrm{C}_{6} \mathrm{H}_{9} \mathrm{NO}$

trans-3-Ethylidene-2-pyrrolidinone, 7451

$\mathrm{C}_{6} \mathrm{H}_{9} \mathrm{NOS}_{2}$

Raphanin, 18547

$\mathrm{C}_{6} \mathrm{H}_{9} \mathrm{NO}_{2}$

L-Baikiain, 2108

Guvacine, 9092

$\alpha$-(Methylenecyclopropyl) glycine, 14358

4-Methylene- $D L$-proline, 14390

$\mathrm{C}_{6} \mathrm{H}_{9} \mathrm{NO}_{3}$

Methyl pyroglutamate, 14709

\section{$\mathrm{C}_{6} \mathrm{H}_{9} \mathrm{NO}_{4}$}

$\gamma$-Methylene glutamic acid, 14385

\section{$\mathrm{C}_{6} \mathrm{H}_{9} \mathrm{NS}$}

4-Pentenyl isothiocyanate, 16888

\section{$\mathrm{C}_{6} \mathrm{H}_{9} \mathrm{~N}_{3} \mathrm{O}_{2}$}

$\alpha$-Amino- $\beta$-(pyrazolyl- $N$ )propionic acid, 1063 L-Histidine, 9569

\section{$\mathrm{C}_{6} \mathrm{H}_{10}$}

Methylene cyclopentane, 14357

\section{$\mathrm{C}_{6} \mathrm{H}_{10} \mathrm{M}_{\mathrm{g}} \mathrm{O}_{6}$}

Magnesium lactate, 13368

\section{$\mathrm{C}_{6} \mathrm{H}_{10} \mathrm{~N}_{2} \mathrm{O}_{2}$}

Cyclo-(Ala-Ala), 4462

\section{$\mathrm{C}_{6} \mathrm{H}_{10} \mathrm{~N}_{2} \mathrm{O}_{5}$}

$L$ - $\alpha$-Amino- $\gamma$-oxalylaminobutyric acid, 1057

$L$-Glutamic acid- $\gamma$-methylamide, 8769

\section{$\mathrm{C}_{6} \mathrm{H}_{10} \mathrm{O}$}

(E)-2-Hexenal, 9519

Isomesityl oxide, 11535

Mesityl oxide, 13800

2-Methylcyclopentanone, 14275

2-Methyl-2-pentenal, 14662

\section{$\mathrm{C}_{6} \mathrm{H}_{10} \mathrm{OS}_{2}$}

Allicin, 920

1-Propenylallylthiosulfinate, 17919

\section{$\mathrm{C}_{6} \mathrm{H}_{10} \mathrm{O}_{2}$}

trans-2-Hexenoic acid, 9521

\section{$\mathrm{C}_{6} \mathrm{H}_{10} \mathrm{O}_{3}$}

(+)-(3R)-3-Hydroxy-4,4-dimethyl-4-butyrolactone, 10048

\section{$\mathrm{C}_{6} \mathrm{H}_{10} \mathrm{O}_{4}$}

Leucanthemitol, 12713

Methyl $\beta, \gamma$-dihydroxy- $\alpha$-methylene butylate, 14313

Methyl glutarate, 14471

Methyl 5-hydroxy-4-oxopentanoate, 14519

$\mathrm{C}_{6} \mathrm{H}_{10} \mathrm{O}_{5}$

3,6-Anhydrogalactose, 1268

$\mathrm{C}_{6} \mathrm{H}_{10} \mathrm{O}_{7}$

D-Galacturonic acid, 8078

Glucuronic acid, 8761 
D-Mannuronic acid, 13509

$\mathrm{C}_{6} \mathrm{H}_{10} \mathrm{O}_{8}$

Mucic acid, 15007

$\mathrm{C}_{6} \mathrm{H}_{10} \mathrm{~S}$

Allyl monosulfide, 953

$\mathrm{C}_{6} \mathrm{H}_{10} \mathrm{~S}_{2}$

Allyl disulfide, 948

$\mathrm{C}_{6} \mathrm{H}_{10} \mathrm{~S}_{3}$

Allitridin, 924

$\mathrm{C}_{6} \mathrm{H}_{10} \mathrm{~S}_{4}$

Diallyl tetrasulfide, 5349

$\mathrm{C}_{6} \mathrm{H}_{11}$ NOS

Cleomin, 3828

\section{$\mathrm{C}_{6} \mathrm{H}_{11} \mathrm{NOS}_{2}$}

4-Methylsulfinyl butyl isothiocyanate, 14728

Sulforathane, 20478

$\mathrm{C}_{6} \mathrm{H}_{11} \mathrm{NO}_{2}$

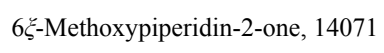

Pipecolic acid, 17425

\section{$\mathrm{C}_{6} \mathrm{H}_{11} \mathrm{NO}_{2} \mathrm{~S}$}

$S$-Allyl- $L$-cystein, 944

Entadamide A, 6809

$S$-(1-Propenyl)-L-cystein, 17921

\section{$\mathrm{C}_{6} \mathrm{H}_{11} \mathrm{NO}_{2} \mathrm{~S}_{2}$}

Erysoline, 7323

\section{$\mathrm{C}_{6} \mathrm{H}_{11} \mathrm{NO}_{3}$}

cis-4-Hydroxymethylproline, 10518

trans-4-Hydroxymethylproline, 10519

Pegaline, 16767

\section{$\mathrm{C}_{6} \mathrm{H}_{11} \mathrm{NO}_{3} \mathrm{~S}$}

Alliin, 921

Entadamide C, 6810

\section{$\mathrm{C}_{6} \mathrm{H}_{11} \mathrm{NO}_{4}$}

$\alpha$-Aminoadipic acid, 1040

\section{$\mathrm{C}_{6} \mathrm{H}_{11} \mathrm{NO}_{4} \mathrm{~S}$}

$S$-(2-Carboxyethyl)- $L$-cysteine, 3171

$\mathrm{C}_{6} \mathrm{H}_{11} \mathrm{~N}_{3} \mathrm{O}_{3}$

$\alpha$-Keto- $\delta$-guanidino-valeric acid, 12200

\section{$\mathrm{C}_{6} \mathrm{H}_{12}$}

1-Hexene, 9520

\section{$\mathrm{C}_{6} \mathrm{H}_{12} \mathrm{~N}_{2} \mathrm{O}$}

$\mathrm{N}$-3-methyl-2-butenyl urea, 14184

\section{$\mathrm{C}_{6} \mathrm{H}_{12} \mathrm{~N}_{2} \mathrm{O}_{3}$}

Aminozide, 1064

n-Butyl allophanate, 2788

\section{$\mathrm{C}_{6} \mathrm{H}_{12} \mathrm{O}$}

Hexanal, 9512

$\beta$-Hexenol, 9522

trans-2-Hexenol, 9523

$\gamma$-Hexenol, 9524

Methyl isobutyl ketone, 14529

$\mathrm{C}_{6} \mathrm{H}_{12} \mathrm{O}_{2}$

Caproic acid, 3139
Ethyl butyrate, 7423

2-Methanol tetrahydropyran, 13822

3-Methyl-1,2-cyclopentanediol, 14274

$\mathrm{C}_{6} \mathrm{H}_{12} \mathrm{O}_{4}$

Digitoxose, 5527

$\mathrm{C}_{6} \mathrm{H}_{12} \mathrm{O}_{5}$

Methyl pentose I, 14665

Methyl pentose II, 14666

1-O-Methyl- $D$-xyloside, 14817

2-O-Methyl- $D$-xyloside, 14818

Polygalitol, 17636

D-Quercitol , 18410

Rhamnose, 18739

Viburnitol, 22461

\section{$\mathrm{C}_{6} \mathrm{H}_{12} \mathrm{O}_{6}$}

Cocositol, 3880

Fructose, 7971

Inositol, 11083

Inositol b, 11084

Inositol $c, 11085$

Mannose, 13507

Mannose-b, 13508

Sorbose, 20104

\section{$\mathrm{C}_{6} \mathrm{H}_{12} \mathrm{O}_{7}$}

Gluconic acid, 8600

\section{$\mathrm{C}_{6} \mathrm{H}_{12} \mathrm{~S}$}

3-[(1-Methylethyl)thio]-1-propene, 14436

\section{$\mathrm{C}_{6} \mathrm{H}_{12} \mathrm{~S}_{2}$}

Allyl propyl disulfide, 954

Propenyl propyl disulfide, 17927

\section{$\mathrm{C}_{6} \mathrm{H}_{13} \mathrm{~N}$}

$D$ - $\alpha$-Pipecoline, 17426

\section{$\mathrm{C}_{6} \mathrm{H}_{13} \mathrm{NO}_{2}$}

Alloisoleucine, 935

\section{$\mathrm{C}_{6} \mathrm{H}_{13} \mathrm{NO}_{2} \mathrm{~S}$}

Homomethionin, 9614

$S$-Propyl-L-cystein, 17937

\section{$\mathrm{C}_{6} \mathrm{H}_{13} \mathrm{NO}_{3}$}

3-Epifagomine, 6914

Fagomine, 7705

$\mathrm{C}_{6} \mathrm{H}_{13} \mathrm{NO}_{4}$

1-Deoxynojirimycin, 5198

2,5-Dihydroxymethyl-3,4-dihydroxypyrrolidine, 6028

\section{$\mathrm{C}_{6} \mathrm{H}_{13} \mathrm{NO}_{5}$}

Galactosamine, 8076

Glucosamine, 8753

$\mathrm{C}_{6} \mathrm{H}_{13} \mathrm{~N}_{3}$

Galegine, 8091

$\mathrm{C}_{6} \mathrm{H}_{13} \mathrm{~N}_{3} \mathrm{O}_{3}$

Citrulline, 3774

$\mathrm{C}_{6} \mathrm{H}_{14} \mathrm{NO}_{2} \mathrm{~S}^{+}$

Vitamin U, 22564
$\mathrm{C}_{6} \mathrm{H}_{14} \mathrm{~N}_{2} \mathrm{O}_{3}$

$\delta$-Hydroxylysine, 10357

$\mathrm{C}_{6} \mathrm{H}_{14} \mathrm{~N}_{4} \mathrm{O}_{2}$

L-Arginine, 1673

$\mathrm{C}_{6} \mathrm{H}_{14} \mathrm{~N}_{4} \mathrm{O}_{3}$

$\gamma$-Hydroxyarginine, 9798

$\mathrm{C}_{6} \mathrm{H}_{14} \mathrm{O}$

Hexanol, 9514

2-Hexanol, 9515

3-Hexanol, 9516

$\mathrm{C}_{6} \mathrm{H}_{14} \mathrm{O}_{2}$

Acetal, 108

$\mathrm{C}_{6} \mathrm{H}_{14} \mathrm{O}_{5}$

1-Deoxy- $D$-glucitol, 5177

L-Fucitol, 7975

D-Quinovitol, 18443

\section{$\mathrm{C}_{6} \mathrm{H}_{14} \mathrm{O}_{6}$}

Dulcitol, 6632

D-Mannitol, 13504

\section{$\mathrm{C}_{6} \mathrm{H}_{14} \mathrm{~S}_{2}$}

Dipropyl disulfide, 6501

Propyl isopropyl disulfide, 17939

\section{$\mathrm{C}_{6} \mathrm{H}_{15} \mathrm{~N}$}

Hexyl amine-1, 9530

$\mathrm{C}_{6} \mathrm{H}_{18} \mathrm{O}_{24} \mathrm{P}_{6}$

Phytic acid, 17256

\section{$\mathrm{C}_{7} \mathrm{H}_{4} \mathrm{OS}$}

3-(2-Thienyl) propargyl aldehyde, 21331

\section{$\mathrm{C}_{7} \mathrm{H}_{4} \mathrm{O}_{6}$}

Chelidonic acid, 3501

\section{$\mathrm{C}_{7} \mathrm{H}_{5} \mathrm{NO}_{2}$}

2-Benzoxazolinone, 2229

\section{$\mathrm{C}_{7} \mathrm{H}_{5} \mathrm{NO}_{4}$}

Pyridine-2,6-dicarboxylic acid, 18258

\section{$\mathrm{C}_{7} \mathrm{H}_{5} \mathrm{NS}$}

Benzothiazole, 2228

Phenyl isothiocyanate, 17125

\section{$\mathrm{C}_{7} \mathrm{H}_{5} \mathrm{~N}_{5} \mathrm{O}_{3}$}

Ranachrome 5, 18530

$\mathrm{C}_{7} \mathrm{H}_{5} \mathrm{~N}_{5} \mathrm{O}_{4}$

Isoxanthopterin-6-carboxylic acid, 11785

\section{$\mathrm{C}_{7} \mathrm{H}_{5} \mathrm{NaO}_{3}$}

Sodium $p$-hydroxybenzoate, 20033

$\mathrm{C}_{7} \mathrm{H}_{5} \mathrm{NaO}_{4}$

Sodium protocatechuate, 20035

$\mathrm{C}_{7} \mathrm{H}_{6} \mathrm{~N}_{4} \mathrm{O}_{3}$

6-Hydroxymethyllumazin, 10500

\section{$\mathrm{C}_{7} \mathrm{H}_{6} \mathrm{O}$}

Benzaldehyde, 2222

\section{$\mathrm{C}_{7} \mathrm{H}_{6} \mathrm{O}_{2}$}

Benzoic acid, 2224

p-Hydroxybenzaldehyde, 9815

$m$-Hydroxybenzaldehyde, 9816 
Salicylaldehyde, 19185

Tropolone, 22052

\section{$\mathrm{C}_{7} \mathrm{H}_{6} \mathrm{O}_{3}$}

$m$-Hydroxybenzoic acid, 9817

p-Hydroxybenzoic acid, 9818

Protocatechuic aldehyde, 17968

Salicylic acid, 19187

Sesamol, 19779

\section{$\mathrm{C}_{7} \mathrm{H}_{6} \mathrm{O}_{4}$}

2,6-Dihydroxybenzoic acid, 5762

3,4-Dihydroxybenzoic acid, 5763

3,5-Dihydroxybenzoic acid, 5764

Gentisic acid, 8307

$o$-Pyrocatechuic acid, 18263

$\beta$-Resorcylic acid, 18642

\section{$\mathrm{C}_{7} \mathrm{H}_{6} \mathrm{O}_{5}$}

Gallic acid, 8095

\section{$\mathrm{C}_{7} \mathrm{H}_{6} \mathrm{O}_{6}$}

Osbeckic acid, 16250

\section{$\mathrm{C}_{7} \mathrm{H}_{7} \mathrm{NO}_{2}$}

p-Aminobenzoic acid, 1042

Trigonelline, 21662

\section{$\mathrm{C}_{7} \mathrm{H}_{7} \mathrm{~N}_{5} \mathrm{O}_{2}$}

Ranachrome 3, 18529

\section{$\mathrm{C}_{7} \mathrm{H}_{7} \mathrm{O}_{2}$}

Pyrolin, 18270

\section{$\mathrm{C}_{7} \mathrm{H}_{8}$}

Methylbenzene, 14155

\section{$\mathrm{C}_{7} \mathrm{H}_{8} \mathrm{~N}_{2} \mathrm{O}$}

$N$ '-Methyl nicotineamide, 14618

\section{$\mathrm{C}_{7} \mathrm{H}_{8} \mathrm{~N}_{4} \mathrm{O}_{2}$}

Theobromine, 21310

Theophylline, 21312

\section{$\mathrm{C}_{7} \mathrm{H}_{8} \mathrm{O}$}

Anisole, 1290

Benzyl alcohol, 2275

$m$-Cresol, 4232

o-Cresol, 4233

p-Cresol, 4234

\section{$\mathrm{C}_{7} \mathrm{H}_{8} \mathrm{O}_{2}$}

Guaiacol, 9021

2-Hydroxybenzyl alcohol, 9832

p-Hydroxybenzyl alcohol, 9833

Orcinol, 16169

Trichodenone A, 21558

\section{$\mathrm{C}_{7} \mathrm{H}_{8} \mathrm{O}_{3}$}

5-Methoxymethyl furfural, 14023

\section{$\mathrm{C}_{7} \mathrm{H}_{8} \mathrm{O}_{4}$}

Doederleinic acid, 6547

Opuntiol, 16159

\section{$\mathrm{C}_{7} \mathrm{H}_{8} \mathrm{O}_{5}$}

Dehydroshikimic acid, 4968

$\mathrm{C}_{7} \mathrm{H}_{8} \mathrm{O}_{7}$
Daucic acid, 4676

$\mathrm{C}_{7} \mathrm{H}_{9} \mathrm{NO}$

4-Hydroxybenzylamine, 9834

Salicylamine, 19186

$\mathrm{C}_{7} \mathrm{H}_{9} \mathrm{NO}_{2}$

Myrothenone B, 15207

$\mathrm{C}_{7} \mathrm{H}_{10} \mathrm{~N}_{5} \mathrm{O}^{+}$

1,3-Dimethylisoguaninium, 6364

$\mathrm{C}_{7} \mathrm{H}_{10} \mathrm{O}$

(E,E)-2,4-Heptadienal, 9392

$\mathrm{C}_{7} \mathrm{H}_{10} \mathrm{O}_{3}$

Theobroxide, 21311

$\mathrm{C}_{7} \mathrm{H}_{10} \mathrm{O}_{5}$

Shikimic acid, 19805

$\mathrm{C}_{7} \mathrm{H}_{11} \mathrm{NO}_{2}$

Arecaidine, 1654

Guvacoline, 9093

L-Hypoglycin, 10898

\section{$\mathrm{C}_{7} \mathrm{H}_{11} \mathrm{NO}_{3}$}

Ethyl pyroglutamate, 7476

$\mathrm{C}_{7} \mathrm{H}_{11} \mathrm{~N}_{3} \mathrm{O}_{2}$

3-Methylhistidine, 14490

\section{$\mathrm{C}_{7} \mathrm{H}_{11} \mathrm{~N}_{5} \mathrm{O}_{2}$}

Stizolamine, 20385

\section{$\mathrm{C}_{7} \mathrm{H}_{12} \mathrm{~N}_{2} \mathrm{O}_{5}$}

$\gamma$-L-Glutamyl-glycine, 8775

\section{$\mathrm{C}_{7} \mathrm{H}_{12} \mathrm{O}$}

$\alpha$-Heptenal, 9413

3-Heptenal, 9414

3-Methylcyclohexanone, 14271

4-Methylcyclohexanone, 14272

\section{$\mathrm{C}_{7} \mathrm{H}_{12} \mathrm{OS}_{3}$}

Z-4,9-Diene-2,3,7-trithiadeca-7-oxide, 5486

\section{$\mathrm{C}_{7} \mathrm{H}_{12} \mathrm{O}_{2}$}

Ethyl-2-methylbut-2-enoate, 7463

$\gamma$-Heptalactone, 9402

2-Heptenic acid, 9415

$\mathrm{C}_{7} \mathrm{H}_{12} \mathrm{O}_{3}$

trans-4-Hydroxycyclohexane-1-carboxylic acid, 9953

$\mathrm{C}_{7} \mathrm{H}_{12} \mathrm{O}_{4}$

Dimelic acid, 6194

$\mathrm{C}_{7} \mathrm{H}_{12} \mathrm{O}_{6}$

Quinic acid, 18421

$\mathrm{C}_{7} \mathrm{H}_{13} \mathrm{NO}$

Nor- $\psi$-tropine, 15812

$\mathrm{C}_{7} \mathrm{H}_{13} \mathrm{NO}_{2}$

$2 \alpha, 3 \beta$-Dihydroxynortropane, 6052

$2 \beta, 3 \beta$-Dihydroxynortropane, 6053

3 $\beta$,6-exo-Dihydroxynortropane, 6054

Erycibelline, 7299

Stachydrine, 20254

$\mathrm{C}_{7} \mathrm{H}_{13} \mathrm{NO}_{3}$
Betonicine, 2325

$2 \alpha, 3 \beta, 4 \alpha$-Trihydroxynortropane, 21815

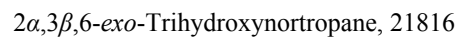

$\mathrm{C}_{7} \mathrm{H}_{13} \mathrm{NO}_{4}$

Calystegine $\mathrm{B}_{2}, 3010$

$\mathrm{C}_{7} \mathrm{H}_{13} \mathrm{NO}_{5}$

Calystegine $\mathrm{C}_{1}, 3011$

1,2,3,4,7-Pentahydroxy-6-nitrobicyclo[3.3.0]octane, 16856

$\mathrm{C}_{7} \mathrm{H}_{14}$

Methylcyclohexane, 14270

\section{$\mathrm{C}_{7} \mathrm{H}_{14} \mathrm{~N}_{2} \mathrm{O}_{3}$}

Theanine, 21281

\section{$\mathrm{C}_{7} \mathrm{H}_{14} \mathrm{~N}_{2} \mathrm{O}_{4} \mathrm{~S}_{2}$}

Cystathionine, 4591

$L$-Djenkolic acid, 6533

\section{$\mathrm{C}_{7} \mathrm{H}_{14} \mathrm{~N}_{2} \mathrm{O}_{4} \mathrm{Se}$}

L-Selenocystathionine, 19676

\section{$\mathrm{C}_{7} \mathrm{H}_{14} \mathrm{O}$}

Heptanal, 9407

$\beta$-Heptenol, 9416

$\gamma$-Heptenol, 9417

Methyl-n-pentyl ketone, 14668

\section{$\mathrm{C}_{7} \mathrm{H}_{14} \mathrm{O}_{2}$}

Amyl acetate, 1103

Enanthic acid, 6795

\section{$\mathrm{C}_{7} \mathrm{H}_{14} \mathrm{O}_{4}$}

Cymarose, 4549

Oleandrose, 16034

Sarmentose, 19375

\section{$\mathrm{C}_{7} \mathrm{H}_{14} \mathrm{O}_{6}$}

$L(+)$-Bornesitol, 2556

Lucidol, 13047

Methyl- $\alpha$ - $D$-fructofuranoside, 14448

Methyl $D$-galactoside, 14453

$\beta$-Methyl- $D$-glucoside, 14469

D-1-O-Methyl-muco-inositol, 14527

Ononitol, 16118

Pinitol, 17391

L-Quebrachitol, 18304

Sequoyitol, 19757

\section{$\mathrm{C}_{7} \mathrm{H}_{14} \mathrm{O}_{7}$}

Coriose, 4057

L-Galactoheptulose, 8050

D-Mannoheptulose, 13505

Sedoheptulose, 19650

$\mathrm{C}_{7} \mathrm{H}_{14} \mathrm{~S}_{3}$

1-(1-Methyl thiopropyl)-1-propenyl disulfide, 14763

$\mathrm{C}_{7} \mathrm{H}_{15} \mathrm{NO}_{4}$

$N$-Methyl-1-deoxynojirimycin, 14290

\section{$\mathrm{C}_{7} \mathrm{H}_{16}$}

Heptane, 9408 
3-Methylhexane, 14488

$\mathrm{C}_{7} \mathrm{H}_{16} \mathrm{NO}_{2}^{+}$

Acetylcholine, 351

$\mathrm{C}_{7} \mathrm{H}_{16} \mathbf{N}_{4} \mathbf{O}_{2}$

L-Homoarginine, 9596

$\mathbf{C}_{7} \mathbf{H}_{16} \mathbf{N}_{4} \mathbf{O}_{3}$

(+)- $\gamma$-Hydroxy- $L$-homoarginine, 10165

$\mathrm{C}_{7} \mathrm{H}_{16} \mathrm{O}$

2-Heptanol, 9409

$\mathrm{C}_{7} \mathrm{H}_{16} \mathrm{O}_{5}$

Xylitol, 22814

$\mathrm{C}_{7} \mathrm{H}_{16} \mathrm{O}_{7}$

Volemitol, 22607

$\mathrm{C}_{7} \mathrm{H}_{19} \mathrm{~N}_{3}$

Spermidine, 20146

$\mathrm{C}_{8} \mathrm{H}_{4} \mathrm{O}_{3}$

Phthalic anhydride, 17204

$\mathrm{C}_{8} \mathrm{H}_{5} \mathrm{NO}_{2}$

Isatin, 11190

$\mathrm{C}_{8} \mathrm{H}_{6} \mathrm{~N}_{2} \mathrm{O}$

4-Quinazolone, 18420

\section{$\mathrm{C}_{8} \mathrm{H}_{6} \mathrm{O}$}

Coumarone, 4143

\section{$\mathrm{C}_{8} \mathrm{H}_{6} \mathrm{O}_{3}$}

$\Delta^{2,4}$-Dihydrophthalic anhydride, 5693

p-Formyl benzoic acid, 7895

Piperonal, 17470

\section{$\mathrm{C}_{8} \mathrm{H}_{6} \mathrm{O}_{5}$}

5-Hydroxyisophthalic acid, 10252

\section{$\mathrm{C}_{8} \mathrm{H}_{7} \mathrm{~N}$}

Indole, 11025

\section{$\mathrm{C}_{8} \mathrm{H}_{7} \mathrm{NO}$}

4-Hydroxybenzyl cyanide, 9835

Mandelonitrile, 13478

\section{$\mathrm{C}_{8} \mathrm{H}_{7} \mathrm{NO}_{2}$}

Gentianadine, 8292

\section{$\mathrm{C}_{8} \mathrm{H}_{7} \mathrm{NO}_{3}$}

Ascosonchine, 1846

Coixol, 3907

\section{$\mathrm{C}_{8} \mathrm{H}_{7} \mathrm{NO}_{4}$}

2,4-Dihydroxy-1,4-benzoxazin-3-one, 5765

\section{$\mathrm{C}_{8} \mathrm{H}_{7} \mathrm{NS}$}

Benzyl isothiocyanate, 2294

Benzyl thiocyanate, 2298

\section{$\mathrm{C}_{8} \mathrm{H}_{7} \mathrm{NaO}_{4}$}

Sodium vanillate, 20039

$\mathrm{C}_{8} \mathrm{H}_{8}$

Styrene, 20430

$\mathrm{C}_{8} \mathrm{H}_{8} \mathrm{~N}_{2} \mathrm{O}_{2}$

Ricinine, 18840

\section{$\mathrm{C}_{8} \mathrm{H}_{8} \mathrm{O}$}

Acetophenone, 115

Phenylacetaldehyde, 17090
$\mathrm{C}_{8} \mathrm{H}_{8} \mathrm{O}_{2}$

p-Anisaldehyde, 1282

Benzyl formate, 2285

p-Hydroxyacetophenone, 9749

$m$-Methoxybenzaldehyde, 13848

4-Methyl salicylaldehyde, 14717

Phenylacetic acid, 17091

\section{$\mathrm{C}_{8} \mathrm{H}_{8} \mathrm{O}_{3}$}

Anisic acid, 1284

2',4'-Dihydroxyacetophenone, 5749

3',4'-Dihydroxyacetophenone, 5750

6-Hydroxy-7,7 $\alpha$-dihydro-2( $6 H)$-benzofuranone, 9997

2-Hydroxy-5-methoxy-benzyldehyde, 10384

2-Hydroxyphenyl acetic acid, 10607

p-Hydroxyphenyl acetic acid, 10608

Isovanillin, 11767

4-Methoxy-1,2-benzodioxole, 13849

4-Methoxysalicylaldehyde, 14088

4-Methyl-2,6-dihydroxy-benzaldehyde, 14297

Methylparaben, 14652

Methyl salicylate, 14718

Vanillin, 22336

\section{$\mathrm{C}_{8} \mathrm{H}_{8} \mathrm{O}_{4}$}

2,6-Dimethoxybenzoquinone, 6204

Homogentisic acid, 9608

3-Hydroxy-4-methoxy benzoic acid, 10381

2-Hydroxy-4-methoxybenzoic acid, 10382

Methyl- $\beta$-resorcylate, 14712

Orsellinic acid, 16218

Protocatechuic acid methyl ester, 17967

Vanillic acid, 22332

\section{$\mathrm{C}_{8} \mathrm{H}_{8} \mathrm{O}_{5}$}

5-(Acetoxymethyl)-furan-2-carboxylic acid, 258

3,5-Dihydroxy-4-methoxybenzoic acid, 5963

Leiocarpic acid, 12607

3-Methoxygallic acid, 13937

Methyl gallate, 14454

$\mathrm{C}_{8} \mathrm{H}_{9} \mathrm{NO}_{2}$

Methylanthranilate, 14142

$\mathrm{C}_{8} \mathrm{H}_{9} \mathrm{NO}_{3}$

$m$-Hydroxyphenylglycine, 10624

2-(4-Hydroxyphenyl)-1-nitroethane, 10629

Myrothenone A, 15206

$\mathrm{C}_{8} \mathrm{H}_{10}$

1,2-Dimethylbenzene, 6318

$\mathrm{C}_{8} \mathrm{H}_{10} \mathrm{~N}_{2} \mathrm{O}$

$\mathrm{N}$-Methylanthranylamide, 14143

3-Methyl-6,7,8-trihydropyrrolo[1,2-a]pyrimidin2-one, 14774

$\mathrm{C}_{8} \mathrm{H}_{10} \mathrm{~N}_{2} \mathrm{O}_{4}$

Mimosine, 14868

$\mathrm{C}_{8} \mathrm{H}_{10} \mathrm{~N}_{4} \mathrm{O}_{2}$
Caffeine, 2892

$\mathrm{C}_{8} \mathrm{H}_{10} \mathrm{~N}_{4} \mathrm{O}_{3}$

Vachellin, 22307

$\mathrm{C}_{8} \mathrm{H}_{10} \mathrm{O}$

Benzyl ethyl alcohol, 2284

2,3-Dicresol, 5442

$m$-Ethylphenol, 7472

p-Ethylphenol, 7473

Methyl benzyl ether, 14156

Methyl phenyl carbinol, 14671

$\mathrm{C}_{8} \mathrm{H}_{10} \mathrm{OS}$

Benzyl hydroxymethyl sulphide, 2293

$\mathrm{C}_{8} \mathrm{H}_{10} \mathrm{O}_{2}$

4-Ethylresorcinol, 7477

4-Hydroxybenzyl methyl ether, 9849

Tyrosol, 22170

Veratrole, 22389

$m$-Xylohydroquinone, 22815

\section{$\mathrm{C}_{8} \mathrm{H}_{10} \mathrm{O}_{2} \mathrm{~S}$}

Foetithiophene A, 7849

\section{$\mathrm{C}_{8} \mathrm{H}_{10} \mathrm{O}_{3}$}

Filicinic acid, 7797

1'-(4-Hydroxyphenyl)ethane-1',2'-diol, 10604

Hydroxytyrosol, 10819

Rengyolone, 18615

Vanillyl alcohol, 22346

\section{$\mathrm{C}_{8} \mathrm{H}_{10} \mathrm{O}_{4}$}

Dictafolin B, 5444

4-Ethoxy-6-hydroxymethyl- $\alpha$-pyrone, 7405

Herierin III, 9438

Herierin IV, 9439

Penicillic acid, 16805

$\mathrm{C}_{8} \mathrm{H}_{10} \mathrm{O}_{5}$

Argutone, 1678

$\mathrm{C}_{8} \mathrm{H}_{11} \mathrm{~N}$

Phenethylamine, 17083

\section{$\mathrm{C}_{8} \mathrm{H}_{11} \mathrm{NO}$}

Tyramine, 22158

\section{$\mathrm{C}_{8} \mathrm{H}_{11} \mathrm{NO}_{2}$}

Dopamine, 6559

\section{$\mathrm{C}_{8} \mathrm{H}_{11} \mathrm{NO}_{3}$}

Noradrenaline, 15708

Pyridoxine, 18261

$\mathrm{C}_{8} \mathrm{H}_{12} \mathrm{~N}_{2}$

Chuanxiongzine, 3633

$\mathrm{C}_{8} \mathrm{H}_{12} \mathrm{~N}_{2} \mathrm{O}_{2}$

Cyclo-(Ala-Pro), 4463

$\mathrm{C}_{8} \mathrm{H}_{12} \mathrm{O}$

Bicyclo[2,2,2]oct-5-en-2-ol, 2356

(E,E)-2,4-Octadienal, 15962

3,5-Octadiene-2-one, 15963

$\mathrm{C}_{8} \mathrm{H}_{12} \mathrm{O}_{3}$

Euscapholide, 7659 


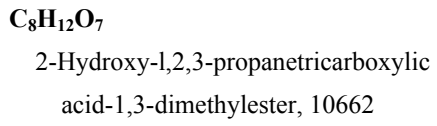

\section{$\mathrm{C}_{8} \mathrm{H}_{13} \mathrm{NO}$}

Tropinone, 22051

\section{$\mathrm{C}_{8} \mathrm{H}_{13} \mathrm{NO}_{2}$}

Arecolidine, 1657

Arecoline, 1658

Heliotridine, 9318

Scopine, 19541

$\mathrm{C}_{8} \mathrm{H}_{13} \mathrm{NO}_{3}$

Desmodilactone, 5266

$\mathrm{C}_{8} \mathrm{H}_{14} \mathrm{~N}_{2} \mathrm{O}_{2}$

Cyclo-(Ala-Val), 4464

$\mathrm{C}_{8} \mathrm{H}_{14} \mathbf{N}_{2} \mathrm{O}_{4}$

Coprine, 4030

\section{$\mathrm{C}_{8} \mathrm{H}_{14} \mathrm{~N}_{2} \mathrm{O}_{5}$}

$\gamma$-Glutamyl-alanine, 8770

$\mathrm{C}_{8} \mathrm{H}_{14} \mathrm{~N}_{2} \mathrm{O}_{6}$

$\gamma$-Glutamyl-serine, 8781

\section{$\mathrm{C}_{8} \mathrm{H}_{14} \mathrm{O}$}

(E)-2-Octenal, 15980

\section{$\mathrm{C}_{8} \mathrm{H}_{14} \mathrm{O}_{2}$}

$\gamma$-Octalactone, 15971

2-Octenic acid, 15982

\section{$\mathrm{C}_{\mathbf{8}} \mathrm{H}_{14} \mathrm{O}_{2} \mathrm{~S}_{2}$}

$\alpha$-Lipoic acid, 12900

\section{$\mathrm{C}_{8} \mathrm{H}_{14} \mathrm{O}_{3}$}

Rengyoxide, 18619

\section{$\mathrm{C}_{8} \mathrm{H}_{14} \mathrm{O}_{3} \mathrm{~S}_{2}$}

$\beta$-Lipoic acid, 12901

\section{$\mathrm{C}_{8} \mathrm{H}_{14} \mathrm{O}_{4}$}

2-(1,4-Dihydroxycyclohexanyl)-acetic acid, 5800

3,7-Dihydroxy-5-octanolide, 6056

Suberic acid, 20439

\section{$\mathrm{C}_{8} \mathrm{H}_{14} \mathrm{~S}_{4}$}

Foetisulfide D, 7848

\section{$\mathrm{C}_{8} \mathrm{H}_{15} \mathrm{~N}$}

$\gamma$-Coniceine, 3979

\section{$\mathrm{C}_{8} \mathrm{H}_{15} \mathrm{NO}$}

Hygrine, 10835

Pelletierine, 16789

Pseudotropine, 18072

Tropine, 22050

$\mathrm{C}_{8} \mathrm{H}_{15} \mathrm{NO}_{2}$

Valerine, 22321

\section{$\mathrm{C}_{8} \mathrm{H}_{15} \mathrm{NO}_{3}$}

Swainsonine, 20500

\section{$\mathrm{C}_{8} \mathrm{H}_{15} \mathrm{NO}_{6}$}

$N$-Acetyl- $D$-glucosamine, 402

\section{$\mathrm{C}_{8} \mathrm{H}_{15} \mathrm{NS}$}

7-Methylthioheptanenitrile, 14760

$\mathrm{C}_{8} \mathrm{H}_{16}$
1,4-Dimethyl-cis-cyclohexane, 6334

1,6-Dimethyl-cis-cyclohexane, 6335

Ethylcyclohexane, 7431

1-Octene, 15981

1,1,3-Trimethylcyclopentane, 21952

\section{$\mathrm{C}_{8} \mathrm{H}_{16} \mathrm{CINO}$}

N-4'-Chlorobutylbutyramide, 3543

\section{$\mathrm{C}_{8} \mathrm{H}_{16} \mathrm{~N}_{2} \mathrm{O}_{2}$}

$N, N, N^{\prime}, N^{\prime}$-Tetramethylsuccinamide, 21203

\section{$\mathrm{C}_{8} \mathrm{H}_{16} \mathrm{~N}_{2} \mathrm{O}_{4}$}

Octopinic acid, 15986

\section{$\mathrm{C}_{8} \mathrm{H}_{16} \mathrm{~N}_{2} \mathrm{O}_{4} \mathrm{~S}_{2}$}

Homocystine, 9602

\section{$\mathrm{C}_{8} \mathrm{H}_{16} \mathrm{~N}_{2} \mathrm{O}_{7}$}

Cycasin, 4456

\section{$\mathrm{C}_{8} \mathrm{H}_{16} \mathrm{O}$}

Isomatsutakeol, 11532

Matsutake alcohol, 13608

Octanal, 15973

3-Octanone, 15979

1-Octen-3-ol, 15983

7-Octen-4-ol, 15984

\section{$\mathrm{C}_{8} \mathrm{H}_{16} \mathrm{OS}_{3}$}

Foetisulfide A, 7845

\section{$\mathrm{C}_{8} \mathrm{H}_{16} \mathrm{O}_{2}$}

Caprylic acid, 3140

\section{$\mathrm{C}_{8} \mathrm{H}_{16} \mathrm{O}_{2} \mathrm{~S}_{3}$}

Foetisulfide B, 7846

Foetisulfide C, 7847

$\mathrm{C}_{8} \mathrm{H}_{16} \mathrm{O}_{3}$

Rengyol, 18614

\section{$\mathrm{C}_{8} \mathrm{H}_{16} \mathrm{O}_{4}$}

erythro-2-Hydroxy-2-(1-hydroxyethyl)-4methylpentanoic acid, 10181

\section{$\mathrm{C}_{8} \mathrm{H}_{16} \mathrm{O}_{6}$}

3,6-Anhydro- $L$-galactose dimethyl acetal, 1269

Dambonitol, 4615

Ethyl- $\alpha$ - $D$-fructoside, 7438

1-Ethyl- $\alpha$ - $D$-galactoside, 7439

1-Ethyl- $\beta$ - $D$-galactoside, 7440

Ethyl $\beta$ - $D$-glucopyranoside, 7443

$\mathrm{C}_{8} \mathrm{H}_{16} \mathrm{O}_{7}$

Ethane-1,2-diol 1-O- $\beta$ - $D$-glucopyranoside, 7389

$\mathrm{C}_{8} \mathrm{H}_{17} \mathrm{~N}$

Coniine, 3988

$\mathrm{C}_{8} \mathrm{H}_{17} \mathrm{NO}_{3}$

$\alpha$-1-C-Ethyl-fagomine, 7437

\section{$\mathrm{C}_{8} \mathrm{H}_{17} \mathrm{O}$}

Pseudoconhydrine, 18023

\section{$\mathrm{C}_{8} \mathrm{H}_{18}$}

2-Methylheptane, 14482

n-Octane, 15974

$\mathrm{C}_{8} \mathrm{H}_{18} \mathrm{O}$
Octanol, 15977

3-Octanol, 15978

$\mathrm{C}_{8} \mathrm{H}_{18} \mathrm{O}_{4}$

$\left(2 S^{*}, 7 S^{*}\right)$-(2)-Octane-1,2,7,8-tetrol, 15976

2,2,2-Triethoxyl-ethanol, 21628

\section{$\mathrm{C}_{8} \mathrm{H}_{18} \mathrm{O}_{5} \mathrm{~S}$}

Musclide $\mathrm{A}_{1}, 15127$

\section{$\mathrm{C}_{8} \mathrm{H}_{20} \mathrm{~N}_{2}$}

Tetramethyl diaminobutane, 21195

$\mathrm{C}_{8} \mathrm{H}_{21} \mathrm{~N}_{3}$

sym-Homospermidine, 9621

\section{$\mathrm{C}_{9} \mathrm{H}_{6} \mathrm{~N}_{2} \mathrm{~S}$}

Brassilexin, 2597

\section{$\mathrm{C}_{9} \mathrm{H}_{6} \mathrm{O}_{2}$}

Coumarin, 4140

\section{$\mathrm{C}_{9} \mathrm{H}_{6} \mathrm{O}_{3}$}

3-Hydroxycoumarin, 9942

4-Hydroxycoumarin, 9943

5-Hydroxycoumarin, 9944

6-Hydroxycoumarin, 9945

Umbelliferone, 22195

\section{$\mathrm{C}_{9} \mathrm{H}_{6} \mathrm{O}_{4}$}

Aesculetin, 663

Daphnetin, 4645

5,7-Dihydroxychromone, 5789

5,7-Dihydroxycoumarin, 5796

\section{$\mathrm{C}_{9} \mathrm{H}_{7} \mathrm{NO}$}

3-Aldehydoindole, 874

\section{$\mathrm{C}_{9} \mathrm{H}_{7} \mathrm{NO}_{2}$}

Indole-3-carboxylic acid, 11029

\section{$\mathrm{C}_{9} \mathrm{H}_{7} \mathrm{NS}$}

5-Phenyl thiazole, 17136

\section{$\mathrm{C}_{9} \mathrm{H}_{7} \mathrm{~N}_{3} \mathrm{O}_{3}$}

Methyl calvatate, 14207

\section{$\mathrm{C}_{9} \mathrm{H}_{8} \mathrm{O}$}

Cinnamaldehyde, 3693

1-Indanone, 11013

\section{$\mathrm{C}_{9} \mathrm{H}_{8} \mathrm{O}_{2}$}

Cinnamic acid, 3695

2'-Hydroxycinnamaldehyde, 9908

\section{$\mathrm{C}_{9} \mathrm{H}_{8} \mathrm{O}_{3}$}

trans-Caffeic aldehyde, 2891

$m$-Coumaric acid, 4133

$o$-Coumaric acid, 4134

p-Coumaric acid, 4135

Coumarinic acid, 4141

Methyl- $p$-formylbenzoate, 14447

Phenyl pyruvic acid, 17134

\section{$\mathrm{C}_{9} \mathrm{H}_{8} \mathrm{O}_{4}$}

Caffeic acid, 2887

$m$-Hydroxyphenylpyruvic acid, 10640 $o$-Hydroxyphenylpyruvic acid, 10641 p-Hydroxyphenylpyruvic acid, 10642 


\section{$\mathrm{C}_{9} \mathrm{H}_{8} \mathrm{O}_{5}$}

2,3-Dihydro-3,6,7-trihydroxy-1- $H$-benzo[b]pyran-4-one, 5728

Haematommic acid, 9188

Meconic acid, 13632

4,5,6-Trihydroxy-7-methylphthalide, 21808

\section{$\mathrm{C}_{9} \mathrm{H}_{9} \mathrm{I}_{2} \mathrm{NO}_{3}$}

Diiodotyrosine, 6187

\section{$\mathrm{C}_{9} \mathrm{H}_{9} \mathrm{~N}$}

6-Methyl indole, 14525

Phenyl propionitrile, 17131

Scatole, 19459

Xylylic acid nitrile, 22855

\section{$\mathrm{C}_{9} \mathrm{H}_{9} \mathrm{NO}$}

trans-Cinnamamide, 3694

\section{$\mathrm{C}_{9} \mathrm{H}_{9} \mathrm{NO}_{2}$}

Gentianidine, 8296

\section{$\mathrm{C}_{9} \mathrm{H}_{9} \mathrm{NO}_{3}$}

Hippuric acid, 9548

\section{$\mathrm{C}_{9} \mathrm{H}_{9} \mathrm{NO}_{4}$}

Peristrophamide, 16964

\section{$\mathrm{C}_{9} \mathrm{H}_{9} \mathrm{NO}_{5}$}

Betalamic acid, 2319

\section{$\mathrm{C}_{9} \mathrm{H}_{9} \mathrm{NS}$}

$\beta$-Phenylethyl isothiocyanate, 17117

\section{$\mathrm{C}_{9} \mathrm{H}_{9} \mathrm{NaO}_{3}$}

(2R)-Sodium 3-phenyllactate, 20034

\section{$\mathrm{C}_{9} \mathrm{H}_{9} \mathrm{NaO}_{5}$}

Sodium syringate, 20031

\section{$\mathrm{C}_{9} \mathrm{H}_{9} \mathrm{O}_{4}{ }^{-}$}

(2S)-(O-Hydroxyphenyl)lactate, 10626

\section{$\mathrm{C}_{9} \mathrm{H}_{9} \mathrm{O}_{8} \mathrm{~S}$}

3,4-Dihydroxy-5-methoxybenzoic acid methyl ester-4-sulfate, 5964

\section{$\mathrm{C}_{9} \mathrm{H}_{10} \mathrm{~N}_{2} \mathrm{O}_{3}$ \\ Armillarisin B, 1743 \\ $\mathrm{C}_{9} \mathrm{H}_{10} \mathrm{O}$}

Chavicol, 3488

Cinnamic alcohol, 3696

p-Methoxystyrene, 14096

$o$-Methylacetophenone, 14112

Phenyl-2-propanone, 17129

2-(2-Propenyl) phenol, 17923

\section{$\mathrm{C}_{9} \mathrm{H}_{10} \mathrm{O}_{2}$}

Allylpyrocatechol, 955

Benzyl acetate, 2273

3,4-Dimethylbenzoic acid, 6320

3,5-Dimethyl-4-hydroxy-benzaldehyde, 6359

Ethyl benzoate, 7421

Hydrocinnamic acid, 9708

p-Hydroxybenzyl acetone, 9831

4-Methoxy-acetophenone, 13827

Methyl phenylacetate, 14669
Phenyl ethyl formate, 17115

$\mathrm{C}_{9} \mathrm{H}_{10} \mathrm{O}_{3}$

Acetovanillone, 117

2,4-Dimethoxybenzyldehyde, 6209

Isopaeonol, 11580

Melilotic acid, 13678

4-Methoxyphenylacetic acid, 14054

Methyl $p$-anisate, 14141

Methylvanillin, 14805

Paeonol, 16532

Phloretinic acid, 17169

Tropic acid, 22049

\section{$\mathrm{C}_{9} \mathrm{H}_{10} \mathrm{O}_{4}$}

2,4'-Dihydroxy-3'-methoxyacetophenone, 5960

3-(3,4-Dihydroxyphenyl)propanoic acid, 6091

2,6-Dimethoxy benzoic acid, 6200

3,4-Dimethoxybenzoic acid, 6201

3,5-Dimethoxybenzoic acid , 6202

Ethyl 3,4-dihydroxybenzoate, 7432

Jacaranone, 11807

Methyl orsellinate, 14639

Methyl vanillate, 14804

Syringaldehyde, 20554

\section{$\mathrm{C}_{9} \mathrm{H}_{10} \mathrm{O}_{5}$}

Danshensu, 4630

1,5-Dimethoxy-3-hydroxybenzoic acid, 6233

3-Ethoxy-4,5-dihydroxy-benzoic acid, 7402

Ethyl gallate, 7441

$\alpha$-Hydroxyhydrocaffeic acid, 10172

Methyl-4-O-methylgallate, 14602

Piscrocin B, 17484

Syringic acid, 20566

2,3,4-Trihydroxy-benzenepropanoic acid, 21689

\section{$\mathrm{C}_{9} \mathrm{H}_{11} \mathrm{NO}$}

D-Cathinone, 3327

Gentialutine, 8291

Isogentialutine, 11435

\section{$\mathrm{C}_{9} \mathrm{H}_{11} \mathrm{NO}_{2}$}

Benzoic acid 2-methyl amino methyl ester, 2225

Gentiatibetine, 8299

Phenylalanine, 17093

\section{$\mathrm{C}_{9} \mathrm{H}_{11} \mathrm{NO}_{3}$}

Lysichitalexin, 13259

(S)-Tyrosine, 22169

\section{$\mathrm{C}_{9} \mathrm{H}_{11} \mathrm{NO}_{4}$}

Dopa, 6558

$\mathrm{C}_{9} \mathrm{H}_{11} \mathrm{~N}_{5} \mathrm{O}_{3}$

Biopterin, 2393

(2R)-Hydroxy-4-(9-adenyl)butyric acid, 9766

\section{$\mathrm{C}_{9} \mathrm{H}_{11} \mathrm{~N}_{5} \mathrm{O}_{4}$}

7-Hydroxybiopterin, 9853

Lentysine, 12624

Trihydroxypropylpterisin, 21841
$\mathrm{C}_{9} \mathrm{H}_{11} \mathrm{O}_{5}$

7-Hydroxy viteoid II, 10825

$\mathrm{C}_{9} \mathrm{H}_{12}$

1-Ethyl-2-methylbenzene, 7462

1,2,3-Trimethylbenzene, 21942

$\mathrm{C}_{9} \mathrm{H}_{12} \mathrm{~N}_{2} \mathrm{O}_{6}$

Uridine, 22252

$\mathrm{C}_{9} \mathrm{H}_{12} \mathrm{O}$

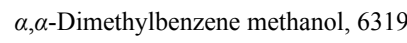

Methyl (phenyl ethyl) ether, 14672

Phenylpropyl alcohol, 17132

$\mathrm{C}_{9} \mathrm{H}_{12} \mathrm{O}_{2}$

3,5-Dimethoxytoluene, 6294

p-Hydroxybenzyl ethyl ether, 9839

$\mathrm{C}_{9} \mathrm{H}_{12} \mathrm{O}_{3}$

3-(3,4-Dihydroxyphenyl)-1-propanol, 6092

Homovanillyl alcohol, 9624

2-Hydroxy-3,5,5-trimethylcyclohex-2-ene-1,4dione, 10807

2-Methoxy-2-(4'-hydroxyphenyl)ethanol, 13966

\section{$\mathrm{C}_{9} \mathrm{H}_{12} \mathrm{O}_{4}$}

Aucubigenin, 2003

Buergerinin G, 2708

(3S,4R)-3-Carboxy-2-methylene-heptan-4-olide, 3179

Chaetoquadrin F, 3445

Decumbic acid, 4861

Genipic acid, 8272

Jiofuran, 11872

Piscrocin A, 17483

\section{$\mathrm{C}_{9} \mathrm{H}_{12} \mathrm{O}_{5}$}

Rehmaglutin C, 18591

\section{$\mathrm{C}_{9} \mathrm{H}_{13} \mathrm{ClO}_{3}$}

Cistachlorin, 3749

\section{$\mathrm{C}_{9} \mathrm{H}_{13} \mathrm{ClO}_{4}$}

Rehmaglutin D, 18592

\section{$\mathrm{C}_{9} \mathrm{H}_{13} \mathrm{ClO}_{5}$}

Rehmaglutin B, 18590

\section{$\mathrm{C}_{9} \mathrm{H}_{13} \mathrm{NO}$}

$\mathrm{N}$-Methyltyramine, 14796

Norephedrine, 15745

$D$-Norpseudoephedrine, 15789

\section{$\mathrm{C}_{9} \mathrm{H}_{13} \mathrm{NO}_{2}$}

Synephrine, 20552

$\mathrm{C}_{9} \mathrm{H}_{13} \mathrm{NO}_{3}$

Adrenaline, 653

$\mathrm{C}_{9} \mathrm{H}_{13} \mathrm{~N}_{2} \mathrm{O}_{9} \mathrm{P}$

Uridinemonophosphate, 22254

Uridylic acid, 22256

$\mathrm{C}_{9} \mathrm{H}_{14}$

Santene, 19309

$\mathrm{C}_{9} \mathrm{H}_{14} \mathrm{~N}_{2} \mathrm{O}_{3}$

2-Methyl-3-(2',3',4'-trihydroxybutyl)pyrazine, 
14780

$\mathrm{C}_{9} \mathrm{H}_{14} \mathrm{~N}_{2} \mathrm{O}_{4}$

2-Methyl-5-(1',2',3',4'-tetrahydroxybutyl)pyrazine, 14752

\section{$\mathrm{C}_{9} \mathrm{H}_{14} \mathrm{~N}_{3} \mathrm{O}_{8} \mathrm{P}$}

Cytidylic acid A, 4592

Cytidylic acid B, 4593

\section{$\mathrm{C}_{9} \mathrm{H}_{14} \mathrm{~N}_{4} \mathrm{O}_{3}$}

Carnosine, 3205

\section{$\mathrm{C}_{9} \mathrm{H}_{14} \mathrm{O}$}

Crocusatin E, 4257

Cryptone, 4288

1(7),8(10)-p-Menthadien-9-ol, 13725

2,4-Nonadienal, 15675

2,6-Nonadienal, 15676

2E,6Z-Nonadienal, 15677

Nopinone, 15706

2-Pentylfuran, 16893

(-)- $\beta$-Pinone, 17408

Santenone, 19310

Spiro[4,4]nonane-2-one, 20204

\section{$\mathrm{C}_{9} \mathrm{H}_{14} \mathrm{OS}_{3}$}

Ajoene, 785

\section{$\mathrm{C}_{9} \mathrm{H}_{14} \mathrm{O}_{2}$}

Boschnialactone, 2566

Crocusatin A, 4253

(2E)-2,6-Dimethyl-2,5-heptadienoic acid, 6354

(4R)-Hydroxy-3,5,5-trimethylcyclohex-2-enone, 10808

(4S)-Hydroxy-3,5,5-trimethylcyclohex-2-enone, 10809

2,4-Nonadienic acid, 15678

3,5,5-Trimethyl-4-hydroxy-1-cyclohexanon-2ene, 21967

\section{$\mathrm{C}_{9} \mathrm{H}_{14} \mathrm{O}_{2} \mathrm{~S}_{2}$}

Foetithiophene B, 7850

\section{$\mathrm{C}_{9} \mathrm{H}_{14} \mathrm{O}_{2} \mathrm{~S}_{3}$}

$E$-1,7,11-Triene-4,5,9-trithiadodeca-9,9-dioxide, 21626

\section{$\mathrm{C}_{9} \mathrm{H}_{14} \mathrm{O}_{3}$}

Boonein, 2546

Buergerinin F, 2707

7-Hydroxy-9-hydroxymethy-3-oxo-bicyclo

$$
\text { [4.3.0]-8-nonene, } 10209
$$

Iridoid-related aglycone, 11148

Ningpogenin, 15615

\section{$\mathrm{C}_{9} \mathrm{H}_{14} \mathrm{O}_{4}$}

Cistanin, 3750

Jioglutolide, 11878

\section{$\mathrm{C}_{9} \mathrm{H}_{14} \mathrm{O}_{5}$}

Buergerinin B, 2703

Rehmaglutin A, 18589

$\mathrm{C}_{9} \mathrm{H}_{14} \mathrm{O}_{6}$
7-Hydroxy eucommic acid, 10107

$\mathrm{C}_{9} \mathrm{H}_{15} \mathrm{NO}$

Pseudopelletierine, 18055

$\mathrm{C}_{9} \mathrm{H}_{15} \mathrm{NO}_{2}$

Homoarecoline, 9595

$\mathrm{C}_{9} \mathrm{H}_{15} \mathrm{NO}_{3}$

n-Butyl pyroglutamate, 2804

Ecgonine, 6683

Isobutyl pyroglutamate, 11285

$\mathrm{C}_{9} \mathrm{H}_{15} \mathrm{~N}_{2} \mathrm{O}_{15} \mathrm{P}_{3}$

Uridine-5'-triphosphatemonophosphate, 22255

$\mathrm{C}_{9} \mathrm{H}_{15} \mathrm{~N}_{3} \mathrm{O}_{2} \mathrm{~S}$

Ergothioneine, 7254

$\mathrm{C}_{9} \mathrm{H}_{16}$

1-Propenyl-cyclohexane, 17920

$\mathrm{C}_{9} \mathrm{H}_{16} \mathrm{~N}_{2} \mathrm{O}_{2}$

Cyclo-(Ile-Ala), 4500

Cyclo-(Leu-Ala), 4505

$\mathrm{C}_{9} \mathrm{H}_{16} \mathrm{~N}_{2} \mathrm{O}_{3}$

Cyclo-(Leu-Ser), 4507

$\mathrm{C}_{9} \mathrm{H}_{16} \mathrm{~N}_{2} \mathrm{O}_{5}$

$\gamma$-L-Glutamyl- $L$ - $\beta$-aminoisobutyric acid, 8771

$\mathrm{C}_{9} \mathrm{H}_{16} \mathrm{O}$

3-Ene-nonanone-2, 6800

2,6-Nonadienol, 15679

(E)-2-Nonenal, 15692

Santenone alcohol, 19311

2,2,6-Trimethyl cyclohexanone, 21949

\section{$\mathrm{C}_{9} \mathrm{H}_{16} \mathrm{O}_{2}$}

$\gamma$-Nonalactone, 15680

2-Nonenoic acid, 15694

8-Nonenoic acid, 15695

2,6,6-Trimethyl-2-hydroxycyclohexanone, 21966

\section{$\mathrm{C}_{9} \mathrm{H}_{16} \mathrm{O}_{2} \mathrm{~S}_{2}$}

Rutadisulfide A, 19080

\section{$\mathrm{C}_{9} \mathrm{H}_{16} \mathrm{O}_{3}$}

1-Deoxyeucommiol, 5173

10-Deoxyeucommiol, 5174

erythro-5-n-Pentyl-4-hydroxytetrahydrofuran2-one, 16895

threo-5-n-Pentyl-4-hydroxy

tetrahydrofuran-2-one, 16896

$\mathrm{C}_{9} \mathrm{H}_{16} \mathrm{O}_{4}$

Azelaic acid, 2056

4,4-Dimethyl-1,7-heptanedioic acid, 6357

Eucommiol, 7488

7-Hydroxy-10-deoxyeucommiol, 9988

3-(2'-Hydroxyethyl)-5-(2"-hydroxypropyl)dihydrofuran-2(3H)-one, 10105

Methyl-5,7-dihydroxy-2(Z)-octenoate, 14314

$\mathrm{C}_{9} \mathrm{H}_{17} \mathrm{~N}$

Pinidine, 17390

$\mathrm{C}_{9} \mathrm{H}_{17} \mathrm{NO}$
Methyl isopelletierine, 14534

$\mathrm{C}_{9} \mathrm{H}_{17} \mathrm{NO}_{5}$

Vitamin $\mathrm{B}_{5}, 22555$

$\mathrm{C}_{9} \mathrm{H}_{17} \mathrm{NO}_{8}$

Miserotoxin, 14885

$\mathrm{C}_{9} \mathrm{H}_{17} \mathrm{NO}_{10} \mathrm{~S}_{2}$

2-Hydroxyethyl glucosinolate, 10103

\section{$\mathrm{C}_{9} \mathrm{H}_{18}$}

1-Nonene, 15693

Propylcyclohexane, 17936

1,2,3-Trimethyl-cyclohexane, 21947

1,3,5-Trimethylcyclohexane, 21948

3,4,4-Trimethyl-2-hexene, 21965

\section{$\mathrm{C}_{9} \mathrm{H}_{18} \mathrm{~N}_{2} \mathrm{O}_{4}$}

Lysopine, 13266

\section{$\mathrm{C}_{9} \mathrm{H}_{18} \mathrm{O}$}

Nonaldehyde, 15681

2-Nonanone, 15687

3-Nonanone, 15688

1-Nonen-3-ol, 15696

\section{$\mathrm{C}_{9} \mathrm{H}_{18} \mathrm{O}_{2}$}

Amyl butyrate, 1104

Isobutylisovalerate, 11276

Methyl caprylate, 14215

Nonanoic acid, 15684

\section{$\mathrm{C}_{9} \mathrm{H}_{18} \mathrm{O}_{6}$}

Isopropyl $\beta$ - $D$-glucopyranoside, 11621

\section{$\mathrm{C}_{9} \mathrm{H}_{18} \mathrm{O}_{7}$}

Glycerol 2- $O$ - $\alpha$ - $L$-fucopyranoside, 8810

(2S)-Propane-1,2-diol 1- $O$ - $\beta$ - $D$-glucopyranoside, 17911

\section{$\mathrm{C}_{9} \mathrm{H}_{18} \mathrm{O}_{8}$}

(2R)-1-O-Glyceryl- $\beta$ - $D$-galactoside, 8813

\section{$\mathrm{C}_{9} \mathrm{H}_{19} \mathrm{~N}$}

(+)-N-Methylconiine, 14250

\section{$\mathrm{C}_{9} \mathrm{H}_{20}$}

2,3-Dimethylheptane, 6356

3-Ethyl-2,3-dimethyl-pentane, 7434

3-Methyl-3-ethylhexane, 14435

2-Methyl octane, 14631

n-Nonane, 15683

2,3,4-Trimethylhexane, 21963

\section{$\mathrm{C}_{9} \mathrm{H}_{20} \mathrm{NO}_{2}^{+}$}

Muscarine I, 15125

Muscarine II, 15126

\section{$\mathrm{C}_{9} \mathrm{H}_{20} \mathrm{~N}_{2} \mathrm{O}_{2}$}

Laminine, 12457

\section{$\mathrm{C}_{9} \mathrm{H}_{20} \mathrm{O}$}

Heptyl ethyl ether, 9418

6-Methyl-1-octanol, 14632

2-Nonanol, 15685

n-Nonanol, 15686

$\mathrm{C}_{10} \mathrm{H}_{6} \mathrm{O}_{2}$ 
1,4-Naphthoquinone, 15255

$\mathrm{C}_{10} \mathrm{H}_{6} \mathrm{O}_{3}$

Juglone, 11903

Lawsone, 12580

\section{$\mathrm{C}_{10} \mathrm{H}_{6} \mathrm{O}_{4}$}

Ayapin, 2048

8-Formyl-7-hydroxycoumarin, 7904

6-Formylumbelliferone, 7913

Naphthazarin, 15253

\section{$\mathrm{C}_{10} \mathrm{H}_{6} \mathrm{O}_{6}$}

Saikochromic acid, 19130

\section{$\mathrm{C}_{10} \mathrm{H}_{7} \mathrm{NO}_{2}$}

Cinchonic acid, 3683

\section{$\mathrm{C}_{10} \mathrm{H}_{7} \mathrm{NO}_{3}$}

6,7-Methylenedioxy-1(2H)-isoquinolinone, 14372

\section{$\mathrm{C}_{10} \mathrm{H}_{7} \mathrm{NO}_{4}$}

Nukagenin, 15867

\section{$\mathrm{C}_{10} \mathrm{H}_{8}$}

Azulene, 2071

Naphthalene, 15252

\section{$\mathrm{C}_{10} \mathrm{H}_{8} \mathrm{~N}_{2}$}

Indole-3-acetonitrile, 11027

\section{$\mathrm{C}_{10} \mathrm{H}_{8} \mathrm{~N}_{2} \mathrm{O}_{2}$}

2,3-Dihydro-4-hydroxy-2-indole-3-acetonitrile, 5637

\section{$\mathrm{C}_{10} \mathrm{H}_{8} \mathrm{~N}_{2} \mathrm{O}_{2} \mathrm{~S}_{2} \mathrm{Zn}$}

Zincpolyanemine, 23002

\section{$\mathrm{C}_{10} \mathrm{H}_{8} \mathrm{O}_{2}$}

1,2-Hydronaphthoquinone, 9724

1,4-Hydronaphthoquinone, 9725

\section{$\mathrm{C}_{10} \mathrm{H}_{8} \mathrm{O}_{2} \mathrm{~S}$}

Methyl trans-5-(2-thienyl)-2-penten-4-yn-1-oate, 14757

\section{$\mathrm{C}_{10} \mathrm{H}_{8} \mathrm{O}_{3}$}

Erythrocentaurin, 7336

Herniarin, 9452

$\alpha$-Hydrojuglone, 9719

$\beta$-Hydrojuglone, 9720

6-Hydroxy-7-methylcoumarin, 10482

\section{$\mathrm{C}_{10} \mathrm{H}_{8} \mathrm{O}_{4}$}

Acamelin, 62

\section{Anemonin, 1178}

Daphnetin-7-methyl ether, 4647

Daphnetin-8-methyl ether, 4648

Erythrocentauric acid, 7335

Isoscopoletin, 11702

$\beta$-Methylaesculetin, 14122

Scopoletin, 19542

\section{$\mathrm{C}_{10} \mathrm{H}_{8} \mathrm{O}_{5}$}

Fraxetin, 7942

\section{$\mathrm{C}_{10} \mathrm{H}_{9} \mathrm{ClO}_{5}$}

Longissiminone B, 12983

\section{$\mathrm{C}_{10} \mathrm{H}_{9} \mathrm{NO}$}

Echinopsine, 6691

Indole-3-acetaldehyde, 11026

\section{$\mathrm{C}_{10} \mathrm{H}_{9} \mathrm{NO}_{2}$}

Gentianine, 8297

3-Indolylacetic acid, 11031

Methyl indole-3-carboxylate, 14526

\section{$\mathrm{C}_{10} \mathrm{H}_{9} \mathrm{NO}_{3}$}

$N$-Demethyldoryphornine, 5077

Noroxyhydrastinine, 15787

\section{$\mathrm{C}_{10} \mathrm{H}_{9} \mathrm{NO}_{4}$}

6-Hydroxy-5-methoxy- $N$-methylphthalimide, 10428

\section{$\mathrm{C}_{10} \mathrm{H}_{9} \mathrm{~N}_{5} \mathrm{O}$}

Kinetin, 12227

\section{$\mathrm{C}_{10} \mathrm{H}_{9} \mathrm{NaO}_{4}$}

Sodium ferulate, 20032

$\mathrm{C}_{10} \mathrm{H}_{10} \mathrm{~N}_{4}$

2-(4'-Aminobenzenamine)-pyrimidine, 1041

\section{$\mathrm{C}_{10} \mathrm{H}_{10} \mathrm{O}$}

2-Phenyl-2-butenal, 17100

(E)-4-Phenyl-3-buten-2-one, 17101

\section{$\mathrm{C}_{10} \mathrm{H}_{10} \mathrm{O}_{2}$}

Cumulene, 4360

(4R)-4-Hydroxy- $\alpha$-tetralone, 10746

(4S)-4-Hydroxy- $\alpha$-tetralone, 10747

Isosafrole, 11690

2-Methoxycinnamaldehyde, 13882

p-Methoxycinnamaldehyde, 13883

5-Methoxy-2-methylbenzofuran, 14003

Methylcinnamate, 14245

Methyl $p$-hydroxycinnamoyl ketone, 14500

1-Phenyl-1,3-butanedion, 17098

Safrole, 19121

\section{$\mathrm{C}_{10} \mathrm{H}_{10} \mathrm{O}_{3}$}

Coniferyl aldehyde, 3983

(E)-4-(3,4-Dihydroxyphenyl)but-3-en-2-one, 6075

(4R)-4,8-Dihydroxy- $\alpha$-tetralone, 6145

3-(4-Hydroxy-3-methoxyphenyl)-2-propenal, 10453

5-Hydroxymethylisochroman-1-one, 10498

p-Hydroxyphenyl crotonic acid, 10615

$m$-Methoxycinnamic acid, 13884

p-Methoxycinnamic acid, 13885

trans-Methyl $p$-coumarate, 14254

$m$-Methyl- $p$-hydroxy-cinnamic acid, 14499

(-)-Regiolone, 18588

\section{$\mathrm{C}_{10} \mathrm{H}_{10} \mathrm{O}_{4}$}

Dimethyl phthalate, 6398

Ferulic acid, 7768

(-)-Gynuraone, 9123

Hesperetic acid, 9455
$3 \xi$-(1 $\xi$-Hydroxyethyl)-7-hydroxy-1-isobenzofuranone, 10104

(-)-(3R,4S)-4-Hydroxymellein, 10376

(-)-(3R)-5-Hydroxymellein, 10377

Kakuol, 12115

Meconine, 13633

Methyl caffeate, 14205

Vanillin acetate, 22337

\section{$\mathrm{C}_{10} \mathrm{H}_{10} \mathrm{O}_{5}$}

Longissiminone A, 12982

Methyl(2,4-dihydroxy-3-formyl-6-methoxy)phenylketone, 14301

\section{$\mathrm{C}_{10} \mathrm{H}_{10} \mathrm{O}_{6}$}

Hemipic acid, 9349

$m$-Hemipic acid, 9350

\section{$\mathrm{C}_{10} \mathrm{H}_{11} \mathrm{NO}$}

Boschniakine, 2565

\section{$\mathrm{C}_{10} \mathrm{H}_{11} \mathrm{NO}_{3}$}

Gentianal, 8294

Gentioflavine, 8303

Northalifoline (tautomeric structure 1), 15802

Northalifoline (tautomeric structure 2), 15803

Tuberosine B, 22082

\section{$\mathrm{C}_{10} \mathrm{H}_{12}$}

$m$-Isopropenyl toluene, 11617

$o$-Isopropenyl toluene, 11618

p-Isopropenyl toluene, 11619

\section{$\mathrm{C}_{10} \mathrm{H}_{12} \mathrm{~N}_{2}$}

Anatabine, 1140

Tryptamine, 22058

\section{$\mathrm{C}_{10} \mathrm{H}_{12} \mathrm{~N}_{2} \mathrm{O}$}

Acanthifoline, 77

Argutine B, 1677

Serotonin, 19760

\section{$\mathrm{C}_{10} \mathrm{H}_{12} \mathrm{~N}_{2} \mathrm{O}_{3}$}

Kynurenine, 12399

\section{$\mathrm{C}_{10} \mathrm{H}_{12} \mathrm{~N}_{2} \mathrm{O}_{4}$}

3-Hydroxykynurenine, 10294

\section{$\mathrm{C}_{10} \mathrm{H}_{12} \mathrm{~N}_{4} \mathrm{O}_{5}$}

Hypoxanthine nucleoside, 10914

Inosine, 11082

6-Isoinosine, 11465

\section{$\mathrm{C}_{10} \mathrm{H}_{12} \mathrm{O}$}

cis-Anethole, 1183

Anethole, 1186

Benzyl acetone, 2274

Cumaldehyde, 4354

Estragole, 7385

Lachnophyllol, 12429

2,4,6-Trimethylbenzaldehyde, 21941

\section{$\mathrm{C}_{10} \mathrm{H}_{12} \mathrm{O}_{2}$}

Anisolacetone, 1289

( \pm )-Car-3-ene-2,5-dione, 3196 
Chavibetol, 3486

Cumic acid, 4356

Egomaketone, 6716

Eugenol, 7521

4-(4-Hydroxyphenyl)-2-butanone, 10614

Isoegomaketone, 11406

Isoeugenol, 11421

trans-4-Methoxycinnamoyl alcohol, 13896

Naginataketone, 15238

Phenylethyl acetate, 17111

$\alpha$-Thujaplicin, 21346

$\beta$-Thujaplicin, 21347

$\gamma$-Thujaplicin, 21348

1,1,5-Trimethyl-2-formyl-cyclohexa-2,5-diene-4one, 21957

\section{$\mathrm{C}_{10} \mathrm{H}_{12} \mathrm{O}_{3}$}

trans-Coniferyl alcohol, 3982

3,5-Dimethyl-4-methoxybenzoic acid, 6368

5,6-Dimethyl-3a,4,7,7a-tetrahydro-1,3-lsoben-

zofurandione, 6412

5-Ethyl-1-methoxy-2,3-methylenedioxybenzene, 7461

2-(1'-Hydroxy-2'-oxopropyl)-5-methylphenol, 10581

p-Methoxydihydrocinnamic acid, 13906

Methyl 4-methoxyphenylacetate, 14589

1,3R,8R-Trihydroxydec-9-en-4,6-yne, 21703

2,4,4-Trimethyl-3-formyl-6-hydroxy-2,5-cyclohexadien-1-one, 21958

\section{$\mathrm{C}_{10} \mathrm{H}_{12} \mathrm{O}_{4}$}

Acetosyringone, 116

Anticancer Benzenoid PMV70P691-58, 1386

Asarylaldehyde, 1840

Cantharidin, 3094

2,4-Dihydroxy-6-methoxy-3-methylacetophenone, 5988

4-Ethoxy-3-methoxybenzoic acid, 7411

3-Hydroxy-1-(4'-hydroxy-3'-methoxyphenyl) propan-1-one, 10192

Methyl- $\beta$-orcinol carboxylate, 14637

Methyl veratrate, 14806

Paeonilactone B, 16527

Rhizonic acid, 18779

Sparassol, 20132

Xanthoxylin, 22778

$\mathrm{C}_{10} \mathrm{H}_{12} \mathrm{O}_{5}$

Antiarolaldehyde, 1379

Methyl 2-hydroxy-3,4-dimethoxy benzoate, 14502

Methyl syringate, 14734

Piscrocin C, 17485

2,4,5-Trimethoxybenzoic acid, 21892

$\mathrm{C}_{10} \mathrm{H}_{12} \mathrm{O}_{6}$
$(5 R, 8 R)-1,6,9,13$-Tetraoxadispiro[4.2.4.2]-

tetradecane-2,10-dione, 21209

$$
\mathrm{C}_{10} \mathrm{H}_{12} \mathrm{O}_{7}
$$

1-O-Galloyl-glycerol, 8110

\section{$\mathrm{C}_{10} \mathrm{H}_{13} \mathrm{BrCl}_{4}$}

8-Bromo-1,3,4,7-tetrachloro-3,7-dimethyl-1E,5Eoctadiene, 2625

\section{$\mathrm{C}_{10} \mathrm{H}_{13} \mathrm{Br}_{2} \mathrm{Cl}_{3}$}

$\left(1 R^{*}, 2 S^{*}, 4 S^{*}, 5 S^{*}\right)-4$-Bromo-5-bromomethyl-1Echlorovinyl-2,5-dichloromethylcyclohexane, 2619

Plocoralide C, 17565

$\mathrm{C}_{10} \mathrm{H}_{13} \mathrm{Br}_{2} \mathrm{NO}_{4}$

1-Acetamide-3,5-dibromo-1-hydroxy-4,4-dimethoxy-2,5-cyclohexadiene, 110

\section{$\mathrm{C}_{10} \mathrm{H}_{13} \mathrm{Br}_{3} \mathrm{Cl}_{2}$}

1,4,8-Tribromo-3,7-dichloro-3,7-dimethyl$1 E, 5 E$-octadiene, 21545

\section{$\mathrm{C}_{10} \mathrm{H}_{13} \mathrm{ClO}_{4}$}

threo-3-Chloro-1-(4-hydroxy-3-methoxyphenyl)propane-1,2-diol, 3559

\section{$\mathrm{C}_{10} \mathrm{H}_{13} \mathrm{~N}$}

Actinidine, 582

5,6,7,8-Tetrahydro-4-methylquinoline, 21075

\section{$\mathrm{C}_{10} \mathrm{H}_{13} \mathrm{NO}_{2}$}

Daechu alkaloid A, 4596

(3 $\alpha, 4 \beta, 5 \alpha)-4,5$-Dihydro-3-(1-pyrryl)-4,5dimethyl-2(3H)-furanone, 5698

$N$-Methylphenylalanine, 14670

(-)-Salsolinol, 19198

\section{$\mathrm{C}_{10} \mathrm{H}_{13} \mathrm{NO}_{3}$}

Damascenine, 4614

$\mathrm{C}_{10} \mathrm{H}_{13} \mathrm{~N}_{5}$

Triacanthine, 21511

$\mathrm{C}_{10} \mathrm{H}_{13} \mathrm{~N}_{5} \mathrm{O}$

Zeatin, 22975

$\mathrm{C}_{10} \mathrm{H}_{13} \mathrm{~N}_{5} \mathrm{O}_{3}$

Cordycepin, 4048

$\mathrm{C}_{10} \mathrm{H}_{13} \mathrm{~N}_{5} \mathrm{O}_{4}$

Adenine nucleoside, 618

$\mathrm{C}_{10} \mathrm{H}_{13} \mathrm{~N}_{5} \mathrm{O}_{5}$

Crotonoside, 4272

Guanosine, 9071

$\mathrm{C}_{10} \mathrm{H}_{14}$

p-Cymene, 4550

3,5-Dimethylene-1,4,4-trimethylcyclopentene, 6350

\section{$\mathrm{C}_{10} \mathrm{H}_{14} \mathrm{Br}_{2} \mathrm{Cl}_{2}$}

Plocoralide A, 17563

Plocoralide B, 17564

$\mathrm{C}_{10} \mathrm{H}_{14} \mathrm{~N}_{2}$

Anabasine, 1124

( \pm )-Anabasine, 1125
(-)-Nicotine, 15527

$\mathrm{C}_{10} \mathrm{H}_{14} \mathrm{~N}_{2} \mathrm{O}_{2}$

$L$-Prolyl- $L$-proline anhydride, 17906

$\mathrm{C}_{10} \mathrm{H}_{14} \mathrm{~N}_{2} \mathrm{O}_{3}$

Chinese bittersweet alkaloid I, 3536

\section{$\mathrm{C}_{10} \mathrm{H}_{14} \mathrm{~N}_{2} \mathrm{O}_{5}$}

Thymidine, 21359

\section{$\mathrm{C}_{10} \mathrm{H}_{14} \mathrm{~N}_{5} \mathrm{O}_{7} \mathrm{P}$}

5'-Adenosine monophosphate, 625

\section{$\mathrm{C}_{10} \mathrm{H}_{14} \mathrm{O}$}

1-Acetyl-4-isopropenyl cyclopentene, 440

1-Acetyl-4-isopropylidene-cyclopentene, 441

Carvacrol, 3231

Carvone, 3237

Chrysanthenone, 3596

Cumic alcohol, 4357

Eucarvone, 7483

Isopiperitenone, 11603

Limonene-10-al, 12842

5-Methylene-2,3,4,4-tetramethylcyclopent-2enone, 14392

Myrtenal, 15219

$\alpha$-Naginatene, 15239

$\beta$-Naginatene, 15240

Perillaldehyde, 16930

(-)-Pinocarvone, 17402

Piperitenone, 17452

Safranal, 19120

Teresantalaldehyde, 20971

Thymol, 21360

3,6,6-Trimethyl-2,4-cycloheptadien-1-one, 21946

Verbenone, 22399

\section{$\mathrm{C}_{10} \mathrm{H}_{14} \mathrm{O}_{2}$}

Actinidialactone, 581

3,7-Dimethyl-2,6-octadien-1,6-olide, 6382

Dolichodial, 6550

Elsholtzia ketone, 6763

Isoactinidialactone, 11196

(-)-Isochaminic acid, 11322

Isoneonepetalactone, 11560

Isonepetalactone, 11562

Myrtenic acid, 15220

Neonepetalactone, 15437

Nepetalactone, 15484

cis-Nepetalactone, 15485

trans-Nepetalactone, 15486

6-n-Pentyl- $\alpha$-pyrone, 16897

Perilla ketone, 16929

Rhododendrol, 18796

(+)-Rhododendrol, 18797

Rotundifolone, 18954

$\alpha$-Teresantalic acid, 20972

3,4,5,5-Tetramethylcyclopenta-1,3-diene- 
carboxylic acid, 21193

Thymolhydroquinone, 21361

\section{$\mathrm{C}_{10} \mathrm{H}_{14} \mathrm{O}_{3}$}

Crocusatin I, 4261

Dihydroconiferyl alcohol, 5564

3-Hydroxy-4(8)-ene- $p$-menthane-3(9)-lactone, 10069

1-(4'-Methoxyphenyl)-(1R,2R)-propanediol, 14062

1-(4'-Methoxyphenyl)-(1R,2S)-propanediol, 14063

1-(4'-Methoxyphenyl)-(1S,2R)-propanediol, 14064

1-(4'-Methoxyphenyl)-(1S,2S)-propanediol, 14065

1,2,3-Trimethoxy-5-methyl benzene, 21919

2,3,5-Trimethoxytoluene, 21935

\section{$\mathrm{C}_{10} \mathrm{H}_{14} \mathrm{O}_{4}$}

Buergerinin C, 2704

2-Carboxymethyl-4-(3'-hydroxybutyl)furan, 3180

Cepharosporolide E, 3414

Cepharosporolide F, 3415

Crocusatin F, 4258

Gardendiol, 8227

1-(4-Hydroxy-3-methoxyphenyl)propan-1,2-diol, 10452

Paeonilactone A, 16526

Suspenolic acid, 20497

3,4,5-Trimethoxy-benzyl alcohol, 21897

\section{$\mathrm{C}_{10} \mathrm{H}_{14} \mathrm{O}_{5}$}

Buergerinin D, 2705

(1R,4R,4aS,7aS)-4,7-Dihydroxymethyl-1-hydroxyl-1,4,4a,7a-tetrahydrocyclopenta-6-ene $[e$ ]pyran-3-one, 6035

4,4-Dimethyl-7 $\alpha, 8 \beta$-dihydroxy-3,5-dioxobicyclo

[4.3.1]dec-1(10)-en-2-one, 6339

erythro-Guaiacylglycerol, 9022

threo-Guaiacylglycerol, 9028

Morindacin, 14972

Non-glycosidic iridoid, 15697

\section{$\mathrm{C}_{10} \mathrm{H}_{14} \mathrm{O}_{5} \mathrm{~S}$}

(S)-4-(4-Hydroxyphenyl)-2-butanol 2-O-sulfate, 10613

\section{$\mathrm{C}_{10} \mathrm{H}_{15} \mathrm{ClO}_{5}$}

Jioglutin A, 11873

Jioglutin B, 11874

$\mathrm{C}_{10} \mathrm{H}_{15} \mathrm{~N}_{5} \mathrm{O}_{10} \mathrm{P}_{2}$

Adenosine diphosphate, 624

\section{$\mathrm{C}_{10} \mathrm{H}_{15} \mathrm{NO}$}

Ephedrine, 6815

Hordenine, 9646

D-Pseudoephedrine, 18024

$\mathrm{C}_{10} \mathrm{H}_{15} \mathrm{NO}_{4}$
$\alpha$-Allokainic acid, 936

$\mathrm{C}_{10} \mathrm{H}_{15} \mathrm{O}_{3}$

GSIR-1, 9019

$\mathrm{C}_{10} \mathrm{H}_{15} \mathrm{O}_{4}$

(5-Butyl-3-oxo-2,3-dihydrofuran-2-yl)-acetic acid, 2802

\section{$\mathrm{C}_{10} \mathrm{H}_{16}$}

Alloocimene, 939

Artemisia triene, 1796

Bornylene, 2559

Camphene, 3045

Carene-3, 3194

Carene-4, 3195

1,1-Dimethyl-2-(3-methyl-1,3-butadiene)cyclo-propane, 6371

Dipentene, 6482

$\alpha$-Fenchene, 7749

$\beta$-Fenchene, 7750

D-Limonene, 12843

L-Limonene, 12844

2,4(8)-p-Menthadiene, 13718

1-Methyl-4-methylethenylcyclohexene, 14599

Myrcene, 15146

Ocimene, 15925

$\beta$-cis-Ocimene, 15926

$\beta$-trans-Ocimene, 15927

$\alpha$-Phellandrene, 17055

$\beta$-Phellandrene, 17056

$\alpha$-Pinene, 17376

$\beta$-Pinene, 17377

Sabinene, 19099

Sylvestrene, 20524

$\alpha$-Terpinene, 20988

$\beta$-Terpinene, 20989

$\gamma$-Terpinene, 20990

Terpinolene, 20998

Thujene, 21349

Tricyclene, 21598

3,6,6-Trimethyl-bicyclo[3.1.1]-2-heptene, 21944

1,3,3-Trimethyltricyclo[2.2.1.0 $\left.0^{2,6}\right]$ heptane, 21975

$\mathrm{C}_{10} \mathrm{H}_{16} \mathrm{~N}_{2} \mathrm{O}_{2}$

Cyclo-(Pro-Val), 4534

$\mathrm{C}_{10} \mathrm{H}_{16} \mathrm{~N}_{2} \mathrm{O}_{3} \mathrm{~S}$

Biotin, 2395

$\mathrm{C}_{10} \mathrm{H}_{16} \mathrm{~N}_{2} \mathrm{O}_{5}$

Pinnatanine, 17392

$\mathrm{C}_{10} \mathrm{H}_{16} \mathrm{~N}_{2} \mathrm{O}_{6}$

Oxypinnatanine, 16469

$\mathrm{C}_{10} \mathrm{H}_{16} \mathbf{N}_{2} \mathrm{O}_{7}$

$\gamma$ - $L$-Glutamyl- $L$-glutamic acid, 8773

$\mathrm{C}_{10} \mathrm{H}_{16} \mathrm{~N}_{4} \mathrm{O}_{3}$

Anserine, 1350

$\mathrm{C}_{10} \mathrm{H}_{16} \mathrm{~N}_{4} \mathrm{O}_{7}$
Vicine, 22466

$\mathrm{C}_{10} \mathrm{H}_{16} \mathrm{~N}_{5} \mathrm{O}_{13} \mathrm{P}_{3}$

Adenosine triphosphate, 626

\section{$\mathrm{C}_{10} \mathrm{H}_{16} \mathrm{O}$}

Artemisia ketone, 1795

Camphor, 3048

cis-Carveol, 3234

trans-Carveol, 3235

Chrysanthemal, 3591

Citral, 3760

(E)-Citral, 3761

(Z)-Citral, 3762

(E,E)-2,4-Decadienal, 4817

3,7-Dimethyl-1,5,7-octatrien-3-ol, 6384

Fenchone, 7751

Isopinocamphone, 11602

2-Isopropenyl-5-methylhexa-trans-3,5-dien-1-ol, 11615

Isopulegone, 11639

Isothujone, 11742

Matatabiether, 13596

1(7),2-p-Menthadien-4-ol, 13723

1(7),2-p-Menthadien-6-ol, 13724

cis-p-2,8-Menthadien-1-ol, 13726

3,8(9)-p-Menthadien-1-ol, 13727

p-Menth-4-en-3-one, 13772

2-Methyl-6-methylene-2,7-octadienol, 14597

Myrtenol, 15221

Perilal, 16926

(R)-Perilla alcohol, 16927

(S)-Perilla alcohol, 16928

Perillyl alcohol, 16935

Phellandral, 17054

L-Pinocamphone, 17400

trans-Pinocarveol, 17401

Piperitone, 17456

Pulegone, 18190

Sabinol, 19101

Teresantalol, 20973

1-Thujone, 21350

Thujylalcohol, 21355

1,3,4-Trimethyl-3-cyclohexene-1-carboxaldehyde, 21950

Verbenol, 22398

3,6,6-Trimethyl norpinan-2-one, 21970

\section{$\mathrm{C}_{10} \mathrm{H}_{16} \mathrm{O}_{2}$}

Aleprestic acid, 883

Allomatatabiol, 937

Ascaridole, 1842

Chrysanthemic acid, 3593

Crocusatin C, 4255

Decadienoic acid, 4819

Dihydronepetalactone, 5680 
Diosphenol, 6455

5-Hydroxymatatabiether, 10365

(+)-Iridodial, 11143

Iridomyrmecin, 11149

Isodihydroepinepetalactone, 11378

Isoiridomyrmecin, 11467

Matatabilactone, 13597

Matatabiol, 13598

(-)-(1R,4S)-p-Mentha-2,8-dien-1-hydroperoxide, 13719

(-)-(1S,4S)-p-Mentha-2,8-dien-1-hydroperoxide, 13720

(-)-(2R,4S)-p-Mentha-1(7),8-dien-2-hydroperoxide, 13721

(-)-(2S,4S)-p-Mentha-1(7),8-dien-2-hydroperoxide, 13722

6 $\alpha$-Methyl-2,6 6 -dihydroxymethylbicyclo-

[3.1.1]-hept-2-ene, 14310

1,10-Oxy- $\alpha$-myrcene hydroxide, 16452

1,10-Oxy- $\beta$-myrcene hydroxide, 16453

Piperitone oxide, 17457

Schizonol, 19509

\section{$\mathrm{C}_{10} \mathrm{H}_{16} \mathrm{O}_{3}$}

Crocusatin B, 4254

Crocusatin D, 4256

Crocusatin J, 4262

Crocusatin K, 4263

Crocusatin L, 4264

(4S,8R)-8,9-Dihydroxy-8,9-dihydrocarvone, 5809

(4S,8S)-8,9-Dihydroxy-8,9-dihydrocarvone, 5810

Elsholtzidiol, 6764

(R)-6-Hydroxy-3-(2-hydroxypropan-2-yl)-6methylcyclohex-2-enone, 10216

(4R)-4-Hydroxymethylboschnialactone, 10470

Nepetalic acid, 15487

Rehmapicrogenin, 18600

Schizonodiol, 19508

\section{$\mathrm{C}_{10} \mathrm{H}_{16} \mathrm{O}_{4}$}

Camphoric acid, 3051

4,7-Dihydroxy-10-methyl-3,4,7,8,9,10-

hexahydro-oxecin-2-one, 6034

$(4 R, 5 R, 7 S, 8 S, 9 S)$-7-Hydroxy-8-hydroxymethyl4-methyl-perhydrocyclopenta[c]pyran-1-one, 10208

Masutakic acid A, 13593

Villosol, 22480

$\mathrm{C}_{10} \mathrm{H}_{16} \mathrm{O}_{5}$

Buergerinin E, 2706

Cepharosporolide C, 3413

$\mathrm{C}_{10} \mathrm{H}_{16} \mathrm{O}_{6}$

Jioglutin C, 11875

Melimessanol A, 13682

$\mathrm{C}_{10} \mathrm{H}_{16} \mathrm{O}_{9}$
6- $O$-malonyl- $\beta$-methyl- $D$-glucopyranoside, 13447

$\mathrm{C}_{10} \mathrm{H}_{17} \mathrm{NO}_{3}$

Erycibe alkaloid II, 7298

Tussilagine, 22143

$\mathrm{C}_{10} \mathrm{H}_{17} \mathrm{NO}_{6}$

Linamarin, 12854

$\mathrm{C}_{10} \mathrm{H}_{17} \mathrm{NO}_{9} \mathrm{~S}_{2}$

Sinigrin, 19935

$\mathrm{C}_{10} \mathrm{H}_{17} \mathrm{~N}_{3} \mathrm{O}_{3}$

Cordycedipeptide A, 4046

\section{$\mathrm{C}_{\mathbf{1 0}} \mathrm{H}_{17} \mathbf{N}_{3} \mathbf{O}_{\mathbf{6}}$}

$\gamma$-L-Glutamyl-glutamine, 8774

Noropthalmic acid, 15784

$\mathrm{C}_{10} \mathrm{H}_{17} \mathrm{~N}_{3} \mathrm{O}_{6} \mathrm{~S}$

Glutathione, 8785

$\mathrm{C}_{10} \mathrm{H}_{17} \mathrm{~N}_{7} \mathbf{O}_{4}$

Saxitoxin, 19431

$\mathrm{C}_{10} \mathrm{H}_{18}$

Butylidenecyclohexane, 2795

$\Delta^{1(7)}$-Menthene, 13742

$\Delta^{3}$-Menthene, 13743

p-1-Menthene, 13744

1-Methyl-4-isoallyl-cyclohexane, 14528

$\mathrm{C}_{10} \mathrm{H}_{18} \mathrm{~N}_{2} \mathrm{O}_{2}$

Slaframine, 20017

$L$-Valyl- $L$-valine anhydride, 22329

\section{$\mathrm{C}_{10} \mathrm{H}_{18} \mathrm{~N}_{2} \mathrm{O}_{3}$}

Cyclo-(Leu-Thr), 4508

\section{$\mathrm{C}_{10} \mathrm{H}_{18} \mathrm{~N}_{2} \mathrm{O}_{5}$}

$\gamma$-Glutamyl-valine, 8783

\section{$\mathrm{C}_{10} \mathrm{H}_{18} \mathrm{O}$}

Artemisia alcohol, 1793

Borneol, 2550

$D$-Borneol, 2553

$L$-Borneol, 2555

2-Caraneol, 3150

3-Caraneol, 3151

1,4-Cineole, 3688

1,8-Cineole, 3689

Citronellal, 3767

Dihydrocarveol, 5555

Dihydroperilla alcohol, 5690

3,7-Dimethyl-7-octenal, 6385

Eucalyptol, 7481

Fenchyl alcohol, 7752

Geraniol, 8312

D-Isoborneol, 11259

L-Isoborneol, 11260

Isomenthone, 11533

Isopulegol, 11638

Lavandulol, 12578

Linalool, 12849
cis-p-2-Menthen-1-ol, 13770

trans-p-2-Menthen-1-ol, 13771

Menthone, 13776

4-Methyl-1-(1-methylethyl)-3-cyclohexene-1-ol, 14600

Myrtanol, 15218

Neoisoisopulegol, 15413

Neoisopulegol, 15415

Nerol, 15498

Piperitol, 17453

$(3 R, 4 S)$-(-)-trans-Piperitol, 17454

$(3 S, 4 R)-(+)$-cis-Piperitol, 17455

Rose oxide, 18920

Sabinene hydrate, 19100

1-Terpinen-5-ol, 20991

Terpinen-4-ol, 20992

cis- $\beta$-Terpineol, 20993

trans- $\beta$-Terpineol, 20994

$\alpha$-Terpineol, 20995

$\delta$-Terpineol, 20997

Thujanol-4, 21344

3,6,6-Trimethyl bicyclo[3.1.1]-2-heptanol, 21943

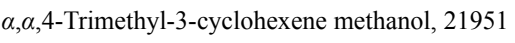

2,2,6-Trimethyl-6-vinyl-tetrahydropyran, 21979

Yomogi alcohol A, 22922

\section{$\mathrm{C}_{10} \mathrm{H}_{18} \mathrm{O}_{2}$}

$\gamma$-Decanolactone, 4835

cis-4-Decenoic acid, 4843

trans-4-Decenoic acid, 4844

1,2-Dihydroxy-8(9)-ene-p-menthane, 5868

$(3 R, 4 R, 6 S)$-3,6-Dihydroxy-1-menthene, 5959

(5E)-2,6-Dimethyl-5,7-octadiene-2,3-diol, 6380

Epoxydihydrolinalool, 7072

7-Hydroxydihydromatatabiether, 10008

4-Hydroxy-2-isopropenyl-5-methylene-hexan1-ol, 10261

trans-5-Hydroxy-2-isopropenyl-5-methylhex-

3-en-1-ol, 10262

4-Hydroxymethyl-3,5,5-trimethylcyclohex-3enol, 10524

Isoneomatatabiol, 11559

Linalyl oxide, 12853

$p$-Mentha-8-en-1,2-diol, 13729

$6 \alpha$-Methyl-2 $\alpha, 6 \beta$-dihydroxymethylbicyclo-

[3.1.1]heptane, 14308

Neomatatabiol, 15434

Sobrerol, 20030

$\mathrm{C}_{10} \mathrm{H}_{18} \mathrm{O}_{3}$

(1S,4S,8S)-8,9-Dihydroxytetrahydrocarvone, 6141

$\omega$-cis-Hydroxy- $\Delta^{2}$-decenoic acid, 9966

$\omega$-trans-Hydroxy- $\Delta^{2}$-decenoic acid, 9967

cis-p-Menth-2-ene-1 $\alpha, 7,8$-triol, 13765 
trans-p-Menth-2-ene-1 $\alpha, 7,8$-triol, 13766

$p$-Menth-3-ene- $1 \beta, 2 \beta, 5 \beta$-triol, 13767

Methyl 4-oxononanoate, 14649

$\mathrm{C}_{10} \mathrm{H}_{18} \mathrm{O}_{4}$

Dibutyl oxalate, 5403

Sebacic acid, 19596

$\mathrm{C}_{10} \mathrm{H}_{18} \mathrm{O}_{10}$

Peltalosa, 16792

$\mathrm{C}_{10} \mathrm{H}_{19} \mathrm{NO}$

Epilupinine, 6955

Lupinine, 13104

$\mathrm{C}_{10} \mathrm{H}_{19} \mathrm{NS}$

9-Methylthiononanenitrile, 14761

\section{$\mathrm{C}_{10} \mathrm{H}_{20}$}

Butyl-cyclohexane, 2790

1-Decene, 4841

\section{$\mathrm{C}_{10} \mathrm{H}_{20} \mathrm{~N}_{2} \mathrm{O}_{3}$}

Azoxyalkene, 2063

$L$-Valine- $L$-valine anhydride, 22325

\section{$\mathrm{C}_{10} \mathrm{H}_{20} \mathrm{O}$}

Carvomenthol, 3236

Citronellol, 3768

Decanal, 4833

Menthol, 13774

Menthol-b, 13775

1-Methyl-3-isopropoxy cyclohexane, 14536

\section{$\mathrm{C}_{10} \mathrm{H}_{20} \mathrm{O}_{2}$}

Capric acid, 3138

Iridodiol, 11145

n-Octyl acetate, 15987

Terpin, 20987

\section{$\mathrm{C}_{10} \mathrm{H}_{20} \mathrm{O}_{3}$}

(3S,6R)-6,7-Dihydroxy-6,7-dihydrolinalool, 5811

(3S,6S)-6,7-Dihydroxy-6,7-dihydrolinalool, 5812

trans-p-Menthane-1 $\alpha, 2 \beta, 8$-triol, 13739

4-p-Menthane-1,7,8-triol, 13740

\section{$\mathrm{C}_{10} \mathrm{H}_{20} \mathrm{O}_{4}$}

3,7-Dimethyloct-3(10)-ene-1,2,6,7-tetrol, 6387

$4 \beta H$-cis- $p$-Menthane- $\alpha \alpha, 6 \alpha, 8,9$-tetrol, 13730

rel-(1R,2R,4R,8S)-p-Menthane-1,2,8,9-tetrol, 13731

$(1 R, 2 S, 4 R, 8 R)$ - $p$-Menthane-1,2,8,9-tetrol, 13732

rel-(1S,2R,4R,8R)-p-Menthane-1,2,8,9-tetrol, 13733

rel-(1S,2R,4R,8S)-p-Menthane-1,2,8,9-tetrol, 13734

(1S,2S,4R,8S)-p-Menthane-1,2,8,9-tetrol, 13735

Thellungianol, 21309

$\mathrm{C}_{10} \mathrm{H}_{20} \mathrm{O}_{6}$

Butan-2-O- $\beta$ - $D$-glucopyranoside, 2778

Isobutyl $\beta$ - $D$-glucopyranoside, 11275

$\beta$ - $n$-Butyl- $D$-tagatopyranoside, 2806
$\mathrm{C}_{10} \mathrm{H}_{20} \mathrm{O}_{7}$

Butane-2,3-diol 2-O- $\beta$ - $D$-glucopyranoside, 2777

$\mathrm{C}_{10} \mathrm{H}_{20} \mathrm{O}_{8}$

1-Deoxy- $L$-erythritol 3- $O$ - $\beta$ - $D$-glucopyranoside, 5172

$\mathrm{C}_{10} \mathrm{H}_{21} \mathrm{NO}_{4}$

6-C-Butyl-DMDP, 2792

$\mathrm{C}_{10} \mathrm{H}_{21} \mathrm{O}_{4}$

(3E)-3,7-Dimethyl-3-octene-1,2,6,7-tetrol, 6386

$\mathrm{C}_{10} \mathrm{H}_{22}$

Decane, 4834

5-Ethyl-2-methylheptane, 7464

3-Methylnonane, 14621

4-Methylnonane, 14622

$\mathrm{C}_{10} \mathrm{H}_{23} \mathrm{~N}$

$N, N$-Dimethylconiine, 6332

$\mathrm{C}_{10} \mathrm{H}_{26} \mathrm{~N}_{4}$

Spermine, 20147

$\mathrm{C}_{11} \mathrm{H}_{6} \mathrm{O}_{3}$

Angelicin, 1191

Psoralen, 18086

$\mathrm{C}_{11} \mathrm{H}_{6} \mathrm{O}_{4}$

Bergaptol, 2310

Xanthotoxol, 22775

$\mathrm{C}_{11} \mathrm{H}_{7} \mathrm{ClO}_{3}$

3-Chloroplumbagin, 3569

$\mathrm{C}_{11} \mathrm{H}_{8}$

Norcapillene, 15724

$\mathrm{C}_{11} \mathrm{H}_{8} \mathrm{~N}_{2}$

Norharman, 15752

$\mathrm{C}_{11} \mathrm{H}_{8} \mathrm{O}$

$\beta$-Naphthaldehyde, 15251

\section{$\mathrm{C}_{11} \mathrm{H}_{8} \mathrm{O}_{2}$}

Dehydromatricaria ester, 4946

trans-Dehydromatricaria ester, 4947

2-Methyl-1,4-naphthoquinone, 14612

$\mathrm{C}_{11} \mathrm{H}_{8} \mathrm{O}_{3}$

7-Methyl juglone, 14541

Plumbagin, 17568

\section{$\mathrm{C}_{11} \mathrm{H}_{8} \mathrm{O}_{4}$}

Angelical, 1189

Droserone, 6605

2,3-Epoxyplumbagin, 7189

8-Formyl-7-methoxycoumarin, 7909

2-Methoxyjuglone, 13978

3-Methoxyjuglone, 13979

$\mathrm{C}_{11} \mathrm{H}_{8} \mathrm{O}_{5}$

5-Carboxy-7-hydroxy-2-methyl-benzopyran- $\gamma$ one, 3173

Hydroxydroserone, 10060

6-Methoxy-7,8-methylenedioxycoumarin, 14013

Yunngnin B, 22954

$\mathrm{C}_{11} \mathrm{H}_{8} \mathrm{O}_{7}$
3,8-Dihydroxy-4-methoxy-2-oxo-2H-1-benzopyran-5-carboxylic acid, 5994

$\mathrm{C}_{11} \mathrm{H}_{9} \mathrm{NO}_{3}$

Doryanine, 6573

$\mathrm{C}_{11} \mathrm{H}_{9} \mathrm{NO}_{4}$

4-Carbomethoxy-6-hydroxy-2-quinolone, 3162

8-Methoxy-4-quinolone-2-caboxylic acid, 14083

$\mathrm{C}_{11} \mathrm{H}_{10}$

1-Methyl naphthalene, 14611

$\mathrm{C}_{11} \mathrm{H}_{10} \mathrm{NO}_{2}{ }^{+}$

6,7-Methylenedioxy- $N$-methylisoquinoline, 14378

$\mathrm{C}_{11} \mathrm{H}_{10} \mathrm{~N}_{2} \mathrm{O}$

Deoxyvasicinone, 5219

1,2,3,4-Tetrahydro-1-oxo- $\beta$-carboline, 21076

$\mathrm{C}_{11} \mathrm{H}_{10} \mathrm{~N}_{2} \mathrm{O}_{2}$

Vasicinone, 22350

$\mathrm{C}_{11} \mathrm{H}_{10} \mathrm{~N}_{6} \mathrm{O}$

Pedatisectine A, 16759

\section{$\mathrm{C}_{11} \mathrm{H}_{10} \mathrm{O}_{2}$}

Matricaria ester, 13603

Methyl(2E,8Z)-decadien-4,6-diynoate, 14283

$\mathrm{C}_{11} \mathrm{H}_{10} \mathrm{O}_{3}$

2,5-Dimethyl-7-hydroxy chromone, 6360

Psilotic acid, 18084

\section{$\mathrm{C}_{11} \mathrm{H}_{10} \mathrm{O}_{4}$}

Citropten, 3770

Dulcinone, 6622

Eugenin, 7519

7-Hydroxy-4-methoxy-5-methylcoumarin, 10419

Isoeugenitol, 11420

Lathodoratin, 12542

(E)-3-Methoxy-4,5-methylenedioxycinnamicaldehyde, 14012

Phellodenol A, 17067

Phellodenol B, 17068

trans-Phenylitaconic acid, 17126

Scoparone, 19540

$\mathrm{C}_{11} \mathrm{H}_{10} \mathrm{O}_{5}$

5,6-Dimethoxy-7-hydroxycoumarin, 6236

Fraxidin, 7943

7-Hydroxy-6,8-dimethoxy coumarin, 10017

Isofraxidin, 11428

Scikochromone A, 19515

$\mathrm{C}_{11} \mathrm{H}_{10} \mathrm{O}_{7}$

(+)-Taraxafolin B, 20696

$\mathrm{C}_{11} \mathrm{H}_{10} \mathrm{O}_{9}$

$L$-Malic acid 2-O-gallate, 13420

\section{$\mathrm{C}_{11} \mathrm{H}_{11} \mathrm{NO}$}

6-Methoxy-4-methylquinoline, 14036

Phlegmariurine N, 17158

$\mathrm{C}_{11} \mathrm{H}_{11} \mathrm{NO}_{2}$

4-Methoxy-1-methyl-2-quinolone, 14037 


\section{$\mathrm{C}_{11} \mathrm{H}_{11} \mathrm{NO}_{3}$}

Doryphornine, 6574

Gentianamine, 8295

Oxyhydrastinine, 16446

\section{$\mathrm{C}_{11} \mathrm{H}_{11} \mathrm{NO}_{4}$}

5,6-Dimethoxy- $N$-methylphthalimide, 6268

\section{$\mathrm{C}_{11} \mathrm{H}_{12} \mathrm{NO}^{+}$}

Echinorine, 6692

\section{$\mathrm{C}_{11} \mathrm{H}_{12} \mathrm{~N}_{2}$ \\ Deoxypeganine, 5201}

\section{$\mathrm{C}_{11} \mathrm{H}_{12} \mathrm{~N}_{2} \mathrm{O}$}

Peganine, 16770

Peganol, 16771

\section{$\mathrm{C}_{11} \mathrm{H}_{12} \mathbf{N}_{2} \mathrm{O}_{2}$}

7-hydroxy vasicine, 10821

Pegamine, 16768

Tryptophan, 22060

Vasicinol, 22349

\section{$\mathrm{C}_{11} \mathrm{H}_{12} \mathrm{~N}_{2} \mathrm{O}_{3}$}

5-Hydroxy-L-tryptophan, 10818

\section{$\mathrm{C}_{11} \mathrm{H}_{12} \mathrm{O}_{2}$}

2-(Butyn-2-ylidene)-- $\Delta^{3}$-dihydrofuran[5-spiro-2']tetrahydrofuran, 2807

Cinnamyl acetate, 3726

Ethylcinnamate, 7430

2-(1'-Methylethenyl)-6-hydroxy-2,3-dihydrobenzo[b]furan, 14429

\section{$\mathrm{C}_{11} \mathrm{H}_{12} \mathrm{O}_{3}$}

Anticancer Benzenoid PMV70P691-57, 1385

Asaricin, 1832

Croweacin, 4276

Dictafolin A, 5443

(-)-5-Hydroxy-4-methoxy-1-tetralone, 10460

(4R)-5-Hydroxy-4-methoxy- $\alpha$-tetralone, 10461

Isomyristicin, 11552

(E)-p-Methoxycinnamic acid methyl ester, 13886

3-(4-Methoxyphenyl)-2-methyl-2-acrylic acid, 14060

Myristicin, 15204

Pedicellanin, 16761

\section{$\mathrm{C}_{11} \mathrm{H}_{12} \mathrm{O}_{4}$}

Caffeic acid dimethyl ether, 2888

(4S)-4,8-Dihydroxy-5-methoxy- $\alpha$-tetralone, 6005

(4R)-5,8-Dihydroxy-4-methoxy- $\alpha$-tetralone, 6006

(4S)-5,8-Dihydroxy-4-methoxy- $\alpha$-tetralone, 6007

6,7-Dimethoxy-2H-1-benzopyran-2-one, 6203

3,4-Dioxymethylene-5-methoxy-1-(1-oxopropyl)benzene, 6479

Ethyl caffeate, 7424

Methyl cis-ferulate, 14442

Methyl trans-ferulate, 14443

3-Methyl-6-methoxy-8-hydroxy-3,4-dihydroisocoumarin, 14586
Sinapaldehyde, 19910

$\mathrm{C}_{11} \mathrm{H}_{12} \mathrm{O}_{5}$

3-Aldehyde-6-methyl-2,4-dihydroxy-ethylbenzoate, 873

Elenolide, 6747

Plumbagic acid, 17567

Sinapic acid, 19912

$\mathrm{C}_{11} \mathrm{H}_{12} \mathrm{O}_{7}$

Piscidic acid, 17479

$\mathrm{C}_{11} \mathrm{H}_{13} \mathrm{NO}_{2}$

$(4 S, 5 R)$ Ephedroxane, 6816

$\mathrm{C}_{11} \mathrm{H}_{13} \mathrm{NO}_{3}$

Cantleyine, 3103

Corydaldine (tautomeric structure 1), 4100

Corydaldine (tautomeric structure 2), 4101

Isocantleyine, 11305

Thalifoline, 21255

$\mathrm{C}_{11} \mathrm{H}_{13} \mathrm{NO}_{4}$

Wilfordic acid, 22676

$\mathrm{C}_{11} \mathrm{H}_{14} \mathrm{~N}_{2}$

Gramine, 8971

\section{$\mathrm{C}_{11} \mathrm{H}_{14} \mathrm{~N}_{2} \mathrm{O}$}

Cytisine, 4594

Gramine $N b$-oxide, 8973

\section{$\mathrm{C}_{11} \mathbf{H}_{14} \mathbf{N}_{4} \mathbf{O}_{6}$}

1-Methylxanthosine, 14816

\section{$\mathrm{C}_{11} \mathrm{H}_{14} \mathrm{O}$}

1-Methoxy-4-(2-methylpropenyl)benzene, 14033

\section{$\mathrm{C}_{11} \mathrm{H}_{14} \mathrm{O}_{2}$}

Actinidiolide, 583

1-Allyl-2,4-dimethoxybenzene, 945

Eugenol methyl ether, 7523

p-Methoxybenzylacetone, 13852

4-(3-Methyl-2-butenyl)-4-cyclohexene-1,3-dione, 14180

cis-Methyl isoeugenol, 14530

trans-Methyl isoeugenol, 14531

\section{$\mathrm{C}_{11} \mathrm{H}_{14} \mathrm{O}_{3}$}

Feroxidin, 7761

Gynunol, 9121

2-Methoxy-4-(3-methoxy-1-propenyl)-phenol, 14001

Nakienone A, 15242

Zingerone, 23003

\section{$\mathrm{C}_{11} \mathrm{H}_{14} \mathrm{O}_{4}$}

Desaspidinol, 5244

Diethylene glycol monobenzoate, 5501

3-(3,4-Dihydroxyphenyl)-2-propen-1-ethanoate, 6094

Robustaol B, 18868

Sinapyl alcohol, 19922

$\mathrm{C}_{11} \mathrm{H}_{14} \mathrm{O}_{5}$

Dehydromorroniaglycone, 4951
Dunnisinin, 6639

Genipin, 8273

3-Hydroxy-1-(4-hydroxy-3,5-dimethoxyphenyl)-1-propanone, 10180

Sarracenin, 19387

Taraxafolin, 20695

$\mathrm{C}_{11} \mathrm{H}_{14} \mathrm{O}_{6}$

2,3-Dihydroxy-1-(4-hydroxy-3,5-dimethoxyphenyl)-1-propanone, 5907

Genipinic acid, 8275

Lamiophlomiol A, 12459

Lamiophlomiol B, 12460

Methyl syramuraldehydate, 14733

$\mathrm{C}_{11} \mathrm{H}_{14} \mathrm{O}_{7}$

Lamiophlomiol C, 12461

$\mathbf{C}_{11} \mathbf{H}_{15} \mathbf{N}$

5,6,7,8-Tetrahydro-2,4-dimethylquinoline, 21069

\section{$\mathrm{C}_{11} \mathrm{H}_{15} \mathrm{NO}$}

3,4-Dimethyl-5-phenyloxazolidine, 6396

\section{$\mathrm{C}_{11} \mathrm{H}_{15} \mathrm{NO}$}

Valerianine, 22319

\section{$\mathrm{C}_{11} \mathrm{H}_{15} \mathrm{NO}_{2}$}

Corypalline, 4121

\section{$\mathrm{C}_{11} \mathrm{H}_{15} \mathrm{NO}_{2}$}

6,7-Dihydroxy-1,1-dimethyl-1,2,3,4-tetrahydroisoquinoline, 5862

\section{$\mathrm{C}_{11} \mathrm{H}_{15} \mathrm{NO}_{2}$}

$N, N$-Dimethylphenylalanine, 6394

\section{$\mathrm{C}_{11} \mathrm{H}_{15} \mathrm{NO}_{2}$}

3-Ethoxymethyl-5,6,7,8-tetrahydro-8indolizinone, 7413

\section{$\mathrm{C}_{11} \mathrm{H}_{15} \mathrm{NO}_{2}$}

Salsoline, 19196

\section{$\mathrm{C}_{11} \mathrm{H}_{15} \mathrm{NO}_{4}$}

Radicamine B, 18525

\section{$\mathrm{C}_{11} \mathrm{H}_{15} \mathrm{NO}_{7}$}

Brachystemoside A, 2583

\section{$\mathrm{C}_{11} \mathrm{H}_{15} \mathrm{~N}_{5} \mathrm{O}$}

Ganoderpurine, 8192

\section{$\mathrm{C}_{11} \mathrm{H}_{16} \mathrm{~N}_{2} \mathrm{O}_{2}$}

Pilocarpine, 17360

\section{$\mathrm{C}_{11} \mathrm{H}_{16} \mathrm{O}$}

2-Isopropyl-5-methylanisole, 11626

Jasmone, 11824

2-Methyl-4-(1,1-dimethylethyl) phenol, 14323

3-Methyl-2-(2-pentenyl)-2-cyclopenten-1-one, 14664

\section{$\mathrm{C}_{11} \mathrm{H}_{16} \mathrm{O}_{2}$}

Jasmololone, 11823

Nortricycloekasantalic acid, 15810

$\mathrm{C}_{11} \mathrm{H}_{16} \mathrm{O}_{3}$

5-Ethyl-1,2,3-trimethoxybenzene, 7479

Isololiolide, 11508 
3-Isopropyl-5-acetoxycyclohexene-2-one-1, 4496 (6S,7 2 R)-Loliolide, 12952

Loliolide isomer, 12953

8-Methoxy-9-hydroxythymol, 13969

$\mathrm{C}_{11} \mathrm{H}_{16} \mathrm{O}_{4}$

Melazolide A, 13660

$\mathrm{C}_{11} \mathrm{H}_{16} \mathbf{O}_{5}$

$(1 R, 4 R, 4 \mathrm{a} S, 7 \mathrm{a} S)-4,7-D i h y d r o x y m e t h y l-1-$ methoxyl-1,4,4a,7a-tetrahydrocyclopenta-6-en e[e]pyran-3-one, 6038

$(1 R, 4 S, 4 \mathrm{a} S, 7 \mathrm{a} S)-4,7-D i h y d r o x y m e t h y l-1-$ methoxyl-1,4,4a,7a-tetrahydrocyclopenta-6-en e[e]pyran-3-one, 6039

\section{$\mathrm{C}_{11} \mathrm{H}_{16} \mathrm{O}_{6}$}

erythro-1-C-Syringylglycerol, 20572

threo-1-C-Syringylglycerol, 20574

\section{$\mathrm{C}_{11} \mathrm{H}_{16} \mathrm{O}_{8}$}

Protoanemonin hydrate glucoside, 17965

Ranunculin, 18545

\section{$\mathrm{C}_{11} \mathrm{H}_{17} \mathrm{NO}$}

$\mathrm{N}$-Methylephedrine, 14395

$D$ - $N$-Methyl-pseudoephedrine, 14701

Tecomanine, 20896

\section{$\mathrm{C}_{11} \mathrm{H}_{17} \mathrm{NO}_{2}$}

Ganoine, 8194

Magnosprengerine, 13393

\section{$\mathrm{C}_{11} \mathrm{H}_{17} \mathrm{NO}_{3}$}

Mescaline, 13795

\section{$\mathrm{C}_{11} \mathrm{H}_{17} \mathrm{NO}_{6}$}

Rhodiocyanoside A, 18790

\section{$\mathrm{C}_{11} \mathrm{H}_{17} \mathrm{NO}_{7}$}

Cardiospermin, 3190

Sarmentosin, 19377

\section{$\mathrm{C}_{11} \mathrm{H}_{17} \mathrm{NO}_{8}$}

Pyroglutamic acid $N$-fructoside, 18267

Sarmentosin epoxide, 19379

\section{$\mathrm{C}_{11} \mathrm{H}_{17} \mathrm{~N}_{3} \mathrm{O}_{8}$}

Tetrodonic acid, 21210

Tetrodotoxin, 21211

\section{$\mathrm{C}_{11} \mathrm{H}_{18} \mathrm{NO}^{+}$}

$\mathrm{N}$-Candicine, 3070

\section{$\mathrm{C}_{11} \mathrm{H}_{18} \mathrm{~N}_{2} \mathrm{O}_{2}$}

$L$-Prolyl- $L$-valine anhydride, 17907

\section{$\mathrm{C}_{11} \mathrm{H}_{18} \mathrm{~N}_{2} \mathrm{O}_{6} \mathrm{~S}$}

$\gamma$-L-Glutamyl-S-(prop-1-enyl)cystein sulfoxide, 8780

\section{$\mathrm{C}_{11} \mathrm{H}_{18} \mathrm{O}_{2}$}

Linalylformate, 12852

$\mathrm{C}_{11} \mathrm{H}_{18} \mathrm{O}_{3}$

Aeginetolide, 657

$\mathrm{C}_{11} \mathrm{H}_{18} \mathrm{O}_{5}$

$(1 R, 4 R, 4 a S, 7 S, 7 a S)$-7-Hydroxyl-4-hydroxymethyl-7-methyl-1-methoxyl-1,4,4a,7a-tetrahy drocyclopenta[e]-pyran-3-one, 10321

$(1 R, 4 S, 4 a S, 7 S, 7 a S)$-7-hydroxyl-4-hydroxymethyl-7-methyl-1-methoxyl-1,4,4a,7a-tetrahy drocyclopenta[e]-pyran-3-one, 10322

$(1 S, 4 R, 4 a S, 7 S, 7 a S)$-7-Hydroxyl-4-hydroxymethyl-7-methyl-1-methoxyl-1,4,4a,7a-tetrahy drocyclopenta[e]-pyran-3-one, 10323

4-Hydroxy-5-methoxy-10-methyl-oxecane-2,7dione $\mathrm{A}, 10426$

4-Hydroxy-5-methoxy-10-methyl-oxecane-2,7dione $\mathrm{B}, 10427$

\section{$\mathrm{C}_{11} \mathrm{H}_{18} \mathrm{O}_{6}$}

Jioglutin D, 11876

\section{$\mathrm{C}_{11} \mathrm{H}_{18} \mathrm{O}_{8}$}

Securiterpenoside, 19643

Tuliposide A, 22104

\section{$\mathrm{C}_{11} \mathrm{H}_{18} \mathrm{O}_{9}$}

Tuliposide B, 22105

6-Tuliposide B, 22106

\section{$\mathrm{C}_{11} \mathrm{H}_{19} \mathrm{NO}_{6}$}

Heterodendrin, 9467

Lotaustralin, 13003

\section{$\mathrm{C}_{11} \mathrm{H}_{19} \mathrm{NO}_{10} \mathrm{~S}_{2}$}

2-Hydroxybut-3-enyl glucosinolate, 9863

Progoitrin, 17903

\section{$\mathrm{C}_{11} \mathrm{H}_{19} \mathrm{~N}_{3} \mathrm{O}_{6}$}

Ophthalmic acid, 16153

\section{$\mathrm{C}_{11} \mathrm{H}_{19} \mathrm{~N}_{3} \mathrm{O}_{6} \mathrm{~S}$}

$S$-Methylglutathione, 14472

\section{$\mathrm{C}_{11} \mathrm{H}_{20} \mathrm{NO}_{10} \mathrm{~S}_{3}{ }^{-}$}

Glucoiberin, 8598

\section{$\mathrm{C}_{11} \mathrm{H}_{20} \mathrm{NO}_{11} \mathrm{~S}_{3}{ }^{-}$}

Glucocheirolin, 8591

\section{$\mathrm{C}_{11} \mathrm{H}_{20} \mathrm{~N}_{2} \mathrm{O}_{2}$}

Cyclo-(Ile-Val), 4501

Cyclo-(Leu-Val), 4510

3-Isopropyl-6-isobutyl-2,5-dioxopiperazine, 11624

3-Isopropyl-6-tert-butyl-2,5-piperazinedione, 11631

$\mathrm{C}_{11} \mathrm{H}_{20} \mathrm{~N}_{2} \mathrm{O}_{6}$

Saccharopine, 19105

$\mathrm{C}_{11} \mathrm{H}_{20} \mathrm{O}_{2}$

3-Acetoxy-1-nonene, 264

$\gamma$-Undecalactone, 22217

10-Undecenoic acid, 22221

$\mathrm{C}_{11} \mathrm{H}_{20} \mathrm{O}_{4}$

Dimethyl azelate, 6317

$\mathrm{C}_{11} \mathrm{H}_{20} \mathrm{O}_{5}$

Jioglutin E, 11877

$\mathrm{C}_{11} \mathrm{H}_{20} \mathrm{O}_{6}$

Crenulatin, 4227
Officinalisin, 16014

\section{$\mathrm{C}_{11} \mathrm{H}_{20} \mathrm{O}_{8}$}

Hymenoside M, 10856

Hymenoside W, 10866

3-(Methoxycarbonyl)propyl- $\beta$ - $D$-glucopyranoside, 13877

\section{$\mathrm{C}_{11} \mathrm{H}_{21} \mathrm{~N}$}

$\beta$-Skytanthine, 20016

\section{$\mathrm{C}_{11} \mathrm{H}_{21} \mathrm{NO}$}

Tecostanine, 20898

\section{$\mathrm{C}_{11} \mathrm{H}_{21} \mathrm{NO}_{2} \mathrm{~S}$}

Rorifone, 18909

$\mathrm{C}_{11} \mathrm{H}_{22}$

Methyl cyclodecane, 14269

Undecene, 22220

\section{$\mathrm{C}_{11} \mathrm{H}_{22} \mathrm{O}$}

Methyl-n-nonylketone, 14623

1-Undecen-3-ol, 22222

\section{$\mathrm{C}_{11} \mathrm{H}_{22} \mathrm{O}_{2}$}

8-Methyl capric acid, 14214

2-Nonyl acetate, 15698

Undecanoic acid, 22218

$\mathrm{C}_{11} \mathrm{H}_{22} \mathrm{O}_{9}$

2-C-Methyl- $D$-erythritol

$1-O-\beta$ - $D$-fructofuranoside, 14420

2-C-Methyl- $D$-erythritol

3-O- $\beta$ - $D$-fructofuranoside, 14421

2-C-Methyl- $D$-erythritol

4-O- $\beta$ - $D$-fructofuranoside, 14422

2-C-Methyl- $D$-erythritol

1-O- $\beta$ - $D$-glucopyranoside, 14423

2-C-Methyl- $D$-erythritol

3-O- $\beta$ - $D$-glucopyranoside, 14424

2-C-Methyl- $D$-erythritol

4- $O$ - $\beta$ - $D$-glucopyranoside, 14425

\section{$\mathrm{C}_{11} \mathrm{H}_{23} \mathrm{NO}_{3} \mathrm{~S}$}

Rorifamide, 18908

\section{$\mathrm{C}_{11} \mathrm{H}_{24}$}

2,4,6-Trimethyl octane, 21972

\section{$\mathrm{C}_{11} \mathrm{H}_{24} \mathrm{O}$}

Nonyl ethyl ether, 15700

Undecan-2-ol, 22219

Undecyl alcohol, 22226

\section{$\mathrm{C}_{12} \mathrm{H}_{6} \mathrm{~S}$}

2-(Buta-1,3-diynyl)-5-(but-3-en-1-ynyl) thiophene, 2775

\section{$\mathrm{C}_{12} \mathrm{H}_{7} \mathrm{ClOS}$}

2-(Buta-1,3-diynyl)-5-(4-chloro-3-hydroxybut-1ynyl) thiophene, 2776

\section{$\mathrm{C}_{12} \mathrm{H}_{8} \mathrm{~N}_{2} \mathrm{O}$}

1-Formyl- $\beta$-carboline, 7898

\section{$\mathrm{C}_{12} \mathrm{H}_{8} \mathrm{~N}_{2} \mathrm{O}_{4}$}

Picrasidine V, 17313 
$\mathrm{C}_{12} \mathrm{H}_{8} \mathrm{O}$

Capillin, 3122

Dibenzofuran, 5380

\section{$\mathrm{C}_{12} \mathrm{H}_{8} \mathrm{OS}$}

cis-1-(2-Furyl)-4-(2-thienyl)-1-buten-3-yne, 8031

\section{$\mathrm{C}_{12} \mathrm{H}_{8} \mathrm{O}_{2} \mathrm{~S}_{2}$}

Arctic acid, 1620

\section{$\mathrm{C}_{12} \mathrm{H}_{8} \mathrm{O}_{4}$}

Bergapten, 2309

Isobergapten, 11249

Sphondin, 20156

Xanthotoxin, 22774

\section{$\mathrm{C}_{12} \mathrm{H}_{8} \mathrm{O}_{5}$}

5-Methoxy-8-hydroxy-psoralen, 13968

\section{$\mathrm{C}_{12} \mathrm{H}_{8} \mathrm{O}_{6}$}

Brevifolin, 2602

\section{$\mathrm{C}_{12} \mathrm{H}_{8} \mathrm{~S}_{2}$}

5-(3-Buten-1-ynyl)-2,2'-bithienyl, 2783

\section{$\mathrm{C}_{12} \mathrm{H}_{8} \mathrm{~S}_{3}$}

$\alpha$-Terthienyl, 21018

\section{$\mathrm{C}_{12} \mathrm{H}_{9} \mathrm{NO}_{2}$}

Dictamnine, 5445

Isodictamnine, 11376

\section{$\mathrm{C}_{12} \mathrm{H}_{9} \mathrm{NO}_{3}$}

Confusameline, 3975

Dictangustine A, 5456

Robustine, 18881

\section{$\mathrm{C}_{12} \mathrm{H}_{9} \mathrm{NO}_{4}$}

Cheliensisamine, 3504

5-Hydroxy-3-amino-2-acetyl-1,4-naphthoquinone, 9778

\section{$\mathrm{C}_{12} \mathrm{H}_{9} \mathrm{~N}_{3} \mathrm{O}$}

Carboline-1-carboxylic acid, amide, 3157

\section{$\mathrm{C}_{12} \mathrm{H}_{10}$}

Capillene, 3121

Neocapillene, 15354

$\mathrm{C}_{12} \mathrm{H}_{10} \mathrm{~N}_{2}$

Harman, 9234

\section{$\mathrm{C}_{12} \mathrm{H}_{10} \mathrm{~N}_{2} \mathrm{O}$}

Harmol, 9236

1-Hydroxymethyl- $\beta$-carboline, 10479

Taraxacine A, 20692

\section{$\mathrm{C}_{12} \mathrm{H}_{10} \mathrm{O}$}

1-Phenyl-2,4-hexadiyne-1-ol, 17123

\section{$\mathrm{C}_{12} \mathrm{H}_{10} \mathrm{O}_{2}$}

Chimaphylin, 3526

$\mathrm{C}_{12} \mathrm{H}_{10} \mathrm{O}_{4}$

5-Acetyl-7-hydroxy-2-methylbenzopyran- $\gamma$-one, 423

Aristolindiquinone, 1710

5-(2-Hydroxyphenoxymethyl)furfural, 10603

Liqcoumarin, 12903

3-Methoxy-7-methyljuglone, 14026
Sappanin, 19346

$\mathrm{C}_{12} \mathrm{H}_{10} \mathrm{O}_{5}$

Armillarisin A, 1742

Bis(5-formylfurfuryl)ether, 2452

2-Methyl-5-carboxymethyl-7-hydroxychromone, 14220

Murraxonin, 15112

$\mathrm{C}_{12} \mathrm{H}_{10} \mathrm{O}_{6}$

Yunngnin A, 22953

$\mathrm{C}_{12} \mathrm{H}_{11} \mathrm{NO}_{3}$

4-Ethoxycarbonyl-2-quinolinone, 7398

$\mathrm{C}_{12} \mathrm{H}_{11} \mathrm{NO}_{4}$

2,3-Methylenedioxy-4,7-dimethoxyquinoline, 14364

$\mathrm{C}_{12} \mathrm{H}_{11} \mathbf{N O}_{5}$

6,7-Dimethoxy- $N$-methyl-3,4-dioxo-1(2H)-isoquinolinone, 6260

$\mathrm{C}_{12} \mathrm{H}_{12}$

Agropyrene, 764

$\mathrm{C}_{12} \mathrm{H}_{12} \mathrm{~N}_{2}$

Dihydroharman, 5625

$\mathrm{C}_{12} \mathrm{H}_{12} \mathrm{~N}_{2} \mathrm{O}$

Harmalol, 9233

\section{$\mathrm{C}_{12} \mathrm{H}_{12} \mathrm{~N}_{2} \mathrm{O}_{2}$}

Cycloanthranilylproline, 4466

Lycoperodine 1, 13213

1,2,3,9-Tetrahydropyrrolo(2,1-b)quinazolin-1carboxylic acid, 21079

\section{$\mathrm{C}_{12} \mathrm{H}_{12} \mathrm{O}$}

Capillon, 3130

\section{$\mathrm{C}_{12} \mathrm{H}_{12} \mathrm{O}_{2}$}

3-Butylidene-phalide, 2797

\section{$\mathrm{C}_{12} \mathrm{H}_{12} \mathrm{O}_{3}$}

Anofinic acid, 1343

3-Butylidene-7-hydroxyphalide, 2796

Fomannoxin acid, 7855

Senkyunolide B, 19732

Senkyunolide C, 19733

Senkyunolide E, 19735

\section{$\mathrm{C}_{12} \mathrm{H}_{12} \mathrm{O}_{4}$}

5,7-Dihydroxy-2,6,8-trimethylchromone, 6167

Eugenitin, 7520

Hispolon, 9567

Isoeugenitin, 11419

Polygonolide, 17648

$\mathrm{C}_{12} \mathrm{H}_{12} \mathrm{O}_{5}$

2-Methyl-5-carboxymethyl-7-hydroxychromanone, 14219

\section{$\mathrm{C}_{12} \mathrm{H}_{12} \mathrm{O}_{5}$}

Schinicoumarin, 19467

6,7,8-Trimethoxy-2H-1-benzopyran-2-one, 21893

5,6,7-Trimethoxycoumarin, 21905
5,7,8-Trimethoxycoumarin, 21906

$\mathrm{C}_{12} \mathrm{H}_{12} \mathrm{O}_{6}$

Apodophyllone, 1531

8-Hydroxy-5,6,7-trimethoxycoumarin, 10786

$\mathrm{C}_{12} \mathrm{H}_{13} \mathrm{~N}$

2-n-Propylquinoline, 17940

$\mathrm{C}_{12} \mathrm{H}_{13} \mathrm{NO}_{2}$

Shihunine, 19803

$\mathrm{C}_{12} \mathrm{H}_{13} \mathrm{NO}_{3}$

Nigellimine $N$-oxide, 15569

Nirurine, 15626

Oleracein E, 16078

Salsoline A, 19197

$\mathrm{C}_{12} \mathrm{H}_{13} \mathrm{NO}_{4}$

Cherianoine, 3512

\section{$\mathrm{C}_{12} \mathrm{H}_{13} \mathrm{NO}_{5}$}

4-[2-(Methoxycarbonyl)anilino]-4-oxobutanoic acid, 13872

$\mathrm{C}_{12} \mathrm{H}_{14} \mathrm{~N}_{2}$

Calligonine, 2977

Eleagnine, 6736

\section{$\mathrm{C}_{12} \mathrm{H}_{14} \mathrm{~N}_{2} \mathrm{O}$}

Dehydrobufotenine, 4884

Shihunidine, 19802

Tetrahydroharmol, 21071

\section{$\mathrm{C}_{12} \mathrm{H}_{14} \mathrm{~N}_{2} \mathrm{O}^{-}$}

Nigeglanine, 15562

\section{$\mathrm{C}_{12} \mathrm{H}_{14} \mathrm{~N}_{2} \mathrm{OS}_{2}$}

Methoxybrassinin, 13861

\section{$\mathrm{C}_{12} \mathrm{H}_{14} \mathrm{~N}_{2} \mathrm{O}_{2}$}

Abrine, 13

Cyclo-(Phe-Ala), 4526

$\mathrm{N}$-Formylcytisine, 7900

\section{$\mathrm{C}_{12} \mathrm{H}_{14} \mathrm{~N}_{2} \mathrm{O}_{3}$}

$L$-Phenylalanyl- $L$-serine anhydride, 17095

\section{$\mathrm{C}_{12} \mathrm{H}_{14} \mathrm{~N}_{2} \mathrm{O}_{4}$}

Cyclo-( $D$-seryl- $L$-tyrosyl), 4535

\section{$\mathrm{C}_{12} \mathrm{H}_{14} \mathrm{~N}_{2} \mathrm{O}_{4} \mathrm{~S}$}

Bufothionine, 2727

\section{$\mathrm{C}_{12} \mathrm{H}_{14} \mathrm{O}$}

Capillanol, 3116

4,7-Dimethyl-1-tetralone, 6413

\section{$\mathrm{C}_{12} \mathrm{H}_{14} \mathrm{O}_{2}$}

Bruguierol A, 2680

3-Butyl-phthalide, 2803

4-(3,4-Dimethoxyphenyl)-but-1,3-diene, 6277

6-Hydroxy-8-methyl-2,2-dimethyl-2H-benzopyran, 10484

Lachnophyllol acetate, 12430

Ligustilide, 12825

2-Methyl-6-(3-methyl-2-butenyl)benzo-1,4quinone, 14592

Neoligustilide, 15427 


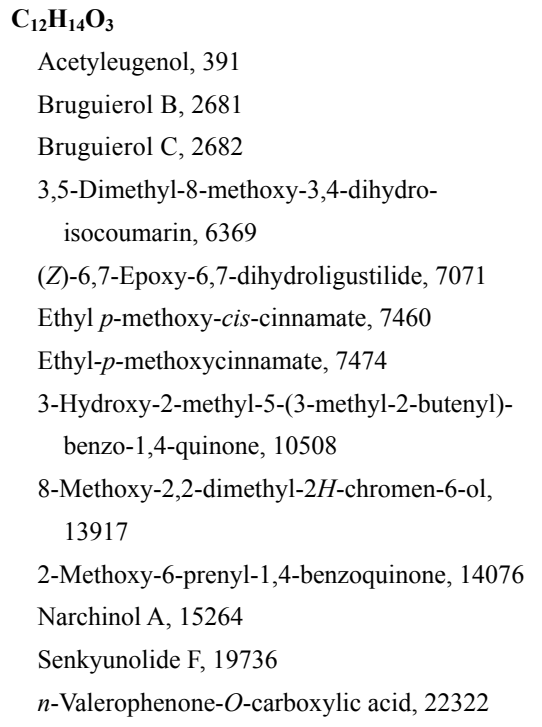

\section{$\mathrm{C}_{12} \mathrm{H}_{14} \mathrm{O}_{4}$}

Apiole, 1520

Diethylphthalate, 5502

2,3-Dihydro-5,7-dihydroxy-2,6,8-trimethyl- $4 \mathrm{H}$ -

1-benzopyran-4-one, 5598

4,7-Dihydroxy-3-butylphthalide, 5782

Dillapiol, 6193

3,5-Dimethyl-8-hydroxy-7-methoxy-3,4dihydroisocoumarin, 6361

Phomapyrone D, 17177

Senkyunolide D, 19734

Tetrahydropiperic acid, 21078

Z-3-(2,4,5-Trimethoxyphenyl)-2-propenal, 21929

\section{$\mathrm{C}_{12} \mathrm{H}_{14} \mathrm{O}_{5}$}

Mono-p-coumaroyl glyceride, 14922

trans-Sinapic acid methylester, 19916

2,4,5-Trimethoxycinnamic acid, 21901

3,4,5-Trimethoxy cinnamic acid, 21902

1-(2,4,5-Trimethoxyphenyl)-1,2-propanedione, 21928

\section{$\mathrm{C}_{12} \mathrm{H}_{14} \mathrm{O}_{6}$}

1-O-Caffeoylglycerol, 2907

\section{$\mathrm{C}_{12} \mathrm{H}_{14} \mathrm{O}_{8}$}

Uralenneoside, 22240

\section{$\mathrm{C}_{12} \mathrm{H}_{15} \mathrm{ClO}_{3}$}

Senkyunolide L, 19741

\section{$\mathrm{C}_{12} \mathrm{H}_{15} \mathrm{NO}_{3}$}

Hydrocotarnine, 9709

$N$-Methylcorydaldine, 14251

Uncinine, 22214

\section{$\mathrm{C}_{12} \mathrm{H}_{15} \mathrm{NO}_{4}$}

(-)-3-Carboxy-1,1-dimethyl-6,7-dihydroxy1,2,3,4-tetrahydroisoquinoline, 3169

(-)-3-Carboxy-1,1-dimethyl-7,8-dihydroxy-

1,2,3,4-tetrahydroisoquinoline, 3170

Cotarnine, 4129
Desmodimine, 5267

\section{$\mathrm{C}_{12} \mathrm{H}_{15} \mathrm{NO}_{8}$}

4-Hydroxypyridyl-3-oic acid 4-O-glucopyranoside, 10670

$\mathrm{C}_{12} \mathrm{H}_{16} \mathrm{~N}_{2}$

3,4-Dihydro-6,7-dimethoxy-2-methylisoquinoline, 5602

$\mathrm{N}, \mathrm{N}$-Dimethyltryptamine, 6418

$\mathrm{C}_{12} \mathrm{H}_{16} \mathrm{NO}_{2}^{+}$

2-Methyl-1,2,3,4-tetrahydro- $\beta$-carboline, 14747

\section{$\mathrm{C}_{12} \mathrm{H}_{16} \mathrm{~N}_{2} \mathrm{O}$}

Bufotenine, 2726

$N, N$-Dimethyltryptamine $N$-oxide, 6420

5-Methoxy- $N$-methyltryptamine, 14038

$N$-Methylcytisine, 14279

\section{$\mathrm{C}_{\mathbf{1 2}} \mathrm{H}_{16} \mathrm{O}_{2}$}

Benzyl D-2-methylbutyrate, 2295

3(S)-3-Butyl-4,5-dihydrophthalide, 2791

Carvacrol acetate, 3232

$\beta$-Phenylethyl isobutanoate, 17116

Thymyl acetate, 21366

7-Trinoreudesm-4(15),8-dien-1 $\beta$-ol-7-one, 21981

\section{$\mathrm{C}_{12} \mathrm{H}_{16} \mathrm{O}_{3}$}

1-Allyl-2,4,5-trimethoxy-benzene, 957

$\alpha$-Asarone, 1834

$\beta$-Asarone, 1835

(E)-4-(3,4-Dimethoxyphenyl)-but-3-en-1-ol, 6279

3,7-Dimethyl-8-hydroxy-6-methoxyisochroman, 6362

Elemicin, 6745

trans-Isoelemicin, 11408

2-Methoxy-6-prenylhydroquinone, 14077

Primin, 17857

Senkyunolide G, 19737

Senkyunolide K, 19740

\section{$\mathrm{C}_{12} \mathrm{H}_{16} \mathrm{O}_{4}$}

Acoramone, 561

Aspidinol, 1897

(Z)-4,5-Dihydro-6,7-trans-dihydroxy-3butylidene phthalide, 5589

(Z)-4,5-Dihydro-6,7-cis-dihydroxy-3-butylidene phthalide, 5590

3,7-Dimethyl-1,8-dihydroxy-6-methoxyisochroman, 6340

cis-1',2'-Epoxyasarone, 7056

Erigerenone A, 7255

Erigerenone C, 7257

Isoacoramone, 11194

Pogostone, 17602

Senkyunolide H, 19738

$\mathrm{C}_{12} \mathrm{H}_{16} \mathrm{O}_{5}$

3,4,5-Trimethoxydihydrocinnamic acid, 21907
$\mathrm{C}_{12} \mathrm{H}_{16} \mathrm{O}_{7}$

Arbutin, 1618

$C$ - $\beta$ - $D$-Glucopyranosyl-2,6-dihydroxyl benzene, 8620

$\mathrm{C}_{12} \mathrm{H}_{16} \mathrm{O}_{8}$

Dianthoside, 5371

Phlorin, 17171

$\mathrm{C}_{\mathbf{1 2}} \mathrm{H}_{17} \mathrm{~N}$

Nigrifactin, 15571

$\mathrm{C}_{12} \mathrm{H}_{17} \mathrm{NO}$

2,3,4-Trimethyl-5-phenyloxazolidine, 21973

\section{$\mathrm{C}_{12} \mathrm{H}_{17} \mathrm{NO}_{2}$}

1-Methyl-corypalline, 14252

$O$-Methyl-corypalline, 14253

Salsolidine, 19195

\section{$\mathrm{C}_{12} \mathrm{H}_{17} \mathrm{NO}_{3}$}

Maokonine, 13543

\section{$\mathrm{C}_{12} \mathrm{H}_{17} \mathrm{NO}_{5}$}

Radicamine A, 18524

\section{$\mathrm{C}_{12} \mathrm{H}_{17} \mathrm{NO}_{6}$}

Deidaclin, 4988

\section{$\mathrm{C}_{12} \mathrm{H}_{17} \mathrm{NO}_{7}$}

Volkenin, 22608

\section{$\mathrm{C}_{12} \mathrm{H}_{17} \mathrm{NO}_{8}$}

Gynocardin, 9114

\section{$\mathrm{C}_{12} \mathrm{H}_{17} \mathrm{~N}_{3} \mathrm{O}_{4}$}

Agaritine, 701

\section{$\mathrm{C}_{12} \mathrm{H}_{17} \mathrm{~N}_{4} \mathrm{OS}^{+}$}

Vitamin $\mathrm{B}_{1}, 22554$

\section{$\mathrm{C}_{12} \mathrm{H}_{18}$}

3,5-Dimethylbutylbenzene, 6329

1-Phenylhexane, 17124

Pregeijerene B, 17780

\section{$\mathrm{C}_{12} \mathrm{H}_{18} \mathrm{NO}_{3}{ }^{+}$}

4-Hydroxybenzoyl choline, 9822

\section{$\mathrm{C}_{12} \mathrm{H}_{18} \mathrm{~N}_{2} \mathrm{O}$}

Gramine methohydroxide, 8972

\section{$\mathrm{C}_{12} \mathrm{H}_{18} \mathrm{~N}_{2} \mathrm{O}_{2}$}

Isokuraramine, 11473

Kuraramine, 12340

\section{$\mathrm{C}_{12} \mathrm{H}_{18} \mathrm{~N}_{2} \mathrm{O}_{5}$}

$L$ - $\gamma$-Glutamyl- $L$-hypoglycin, 8776

$\mathrm{C}_{12} \mathrm{H}_{18} \mathrm{~N}_{\mathbf{2}} \mathrm{O}_{12}$

Cibarian, 3634

Coronarian, 4078

$\mathrm{C}_{\mathbf{1 2}} \mathrm{H}_{\mathbf{1 8}} \mathrm{O}$

(+)-3,4,4 $\alpha R, 7,8,8 \alpha R$-Hexahydro-5, $8 \alpha$ dimethylnaphthalen-2(1H)-one, 9499

\section{$\mathrm{C}_{12} \mathrm{H}_{18} \mathrm{O}_{2}$}

Cnidilide, 3855

Cnidium lactone, 3858

7,7-Dimethyl-2-methylenebicyclo[3.1.1]heptan-6-ol acetate, 6372 
Isocnidilide, 11336

1,8-Menthadien-10-ol acetate, 13728

4-Methylene-1-isopropyl-bicyclo[3.1.0]-

hexan-3-ol acetate, 14386

2-Methyl-6-methylene-2,7-octadienol acetate, 14598

6-Methyl-7-(3-oxobutyl)-bicyclo[4.1.0]heptan3-one, 14642

(+)-Myrtenyl acetate, 15222

Neocnidilide, 15370

\section{$\mathrm{C}_{12} \mathrm{H}_{18} \mathrm{O}_{3}$}

D-8-Acetoxycarvotanacetone, 139

Buergerinin A, 2702

1-(3-Hydroxyphenyl)-hexane-2,5-diol, 10625

(-)-Jasmonic acid, 11825

Oxyphyllenone A, 16464

Oxyphyllenone B, 16465

Sedanonic acid, 19648

\section{$\mathrm{C}_{12} \mathrm{H}_{18} \mathrm{O}_{4}$}

Allixin, 926

Erigerenone B, 7256

2-(1-Ethoxy-2-hydroxy)propyl-4-methoxyphenol, 7406

$(1 R, 2 R)-5$ '-Hydroxyjasmonic acid, 10269

Senkyunolide J, 19739

Senkyunolide N, 19743

\section{$\mathrm{C}_{12} \mathrm{H}_{18} \mathrm{O}_{5}$}

3-n-Butyl-3-hydroxy-4,5,6,7-tetrahydro-6,7dihydroxy phthalide, 2794

Drummondol, 6607

\section{$\mathrm{C}_{12} \mathrm{H}_{18} \mathrm{O}_{8}$}

Opuntiaester, 16157

Osmundalin, 16256

\section{$\mathrm{C}_{12} \mathrm{H}_{19} \mathrm{NO}_{3}$}

$\mathrm{N}$-Methylmescaline, 14583

\section{$\mathrm{C}_{12} \mathrm{H}_{19} \mathrm{~N}_{3} \mathrm{O}$}

Alchorneine, 871

\section{$\mathrm{C}_{12} \mathrm{H}_{20} \mathrm{NO}_{2}^{+}$}

Salicifoline, 19183

\section{$\mathrm{C}_{12} \mathrm{H}_{20} \mathrm{~N}_{2} \mathrm{O}$}

(+)-Ammodendrine, 1068

$N$-Methyltetrahydrocytisine, 14749

\section{$\mathrm{C}_{12} \mathrm{H}_{20} \mathrm{~N}_{2} \mathrm{O}_{2} \mathrm{~S}_{2}$}

Aglaidithioduline, 736

\section{$\mathrm{C}_{12} \mathrm{H}_{20} \mathrm{O}_{2}$}

Acetylborneol, 336

Aleprylic acid, 885

$L$ - $\beta$-Artemisia alcohol acetate, 1794

Bornyl acetate, 2557

Crocusatin G, 4259

Dihydrocarveol acetate, 5556

Ethyl geranate, 7442

Geranyl acetate, 8313
Isobornyl acetate, 11261

Linalyl acetate, 12850

4-Methyl-1-(1-methylethyl)-3-cyclohexen-1ol-acetate, 14601

Neryl acetate, 15512

Terpinyl acetate, 20999

$\mathrm{C}_{12} \mathrm{H}_{20} \mathrm{O}_{3}$

Cucurbic acid, 4314

Gardenone, 8230

Vulgarole, 22620

$\mathrm{C}_{12} \mathrm{H}_{20} \mathrm{O}_{4}$

Crocusatin H, 4260

Dimethyl camphorate, 6330

$(1 R, 4 S)$-1-Hydroperoxy- $p$-menth-2-en-8-ol acetate, 9732

\section{$\mathrm{C}_{12} \mathrm{H}_{20} \mathrm{O}_{7}$}

Clandonensine, 3785

\section{$\mathrm{C}_{12} \mathrm{H}_{20} \mathrm{O}_{8}$}

(3R,5R)-3-( $\beta$ - $D$-Glucopyranosyloxy)-5hexanolide, 8689

Parasorboside, 16658

\section{$\mathrm{C}_{12} \mathrm{H}_{21} \mathrm{NO}$}

$N$-Isobutyl-(2E,4E)-octadienamide, 11283

$N$-Isobutyl-(2Z,4E)-octa-2,4-dienamide, 11284

\section{$\mathrm{C}_{12} \mathrm{H}_{21} \mathrm{NO}_{10} \mathrm{~S}_{2}$}

2-Hydroxypent-4-enylglucosinate, 10598

\section{$\mathrm{C}_{12} \mathrm{H}_{22}$}

1,1'-Bicyclohexyl, 2353

$\mathrm{C}_{12} \mathrm{H}_{22} \mathrm{NO}_{9} \mathrm{~S}_{3}{ }^{-}$

Glucoerucin, 8595

\section{$\mathrm{C}_{12} \mathrm{H}_{22} \mathrm{NO}_{10} \mathrm{~S}_{3}{ }^{-}$}

Glucoraphanin, 8752

$\mathrm{C}_{12} \mathrm{H}_{22} \mathrm{NO}_{11} \mathrm{~S}_{3}{ }^{-}$

Glucoerysolin, 8596

$\mathrm{C}_{12} \mathrm{H}_{22} \mathrm{~N}_{2} \mathrm{O}_{2}$

Cyclo-(Leu-Ile), 4506

\section{$\mathrm{C}_{12} \mathrm{H}_{22} \mathrm{O}$}

Cyclododecanone, 4489

8-Methyl-5-isopropyl-6,8-nonadiene-2-one, 14538

\section{$\mathrm{C}_{12} \mathrm{H}_{22} \mathrm{O}_{2}$}

Citronellyl acetate, 3769

Decanoylacetaldehyde, 4837

$\gamma$-Dodecalactone, 6540

cis-4-Dodecenoic acid, 6543

Dodecenoic acid, 6544

Linderic acid, 12873

Menthyl acetate, 13779

$\mathrm{C}_{12} \mathrm{H}_{22} \mathrm{O}_{4}$

Dimethyl sebacate, 6406

$\mathrm{C}_{12} \mathrm{H}_{22} \mathrm{O}_{5}$

Mioporosidegenin, 14878

$\mathrm{C}_{12} \mathrm{H}_{22} \mathrm{O}_{6}$
(E)-2-Hexenyl- $\beta$ - $D$-glucopyranoside, 9527

(Z)-3-Hexenyl- $\beta$ - $D$-glucopyranoside, 9528

$\mathrm{C}_{12} \mathrm{H}_{22} \mathrm{O}_{10}$

Methyl $\beta$ - $D$-apiofuranosyl- $(1 \rightarrow 6)-\beta$ - $D$-glucopyranoside, 14145

Robinobiose, 18860

\section{$\mathrm{C}_{12} \mathrm{H}_{22} \mathrm{O}_{11}$}

Cellobiose, 3383

Gentiobiose, 8300

Isomaltose, 11523

Lactose, 12440

Maltose, 13454

Sophorose, 20101

Sucrose, 20446

Timobiose, 21393

Trehalose $(\alpha: \alpha), 21504$

Trehalose $(\alpha: \beta), 21505$

Trehalose $(\beta: \beta), 21506$

Turanose, 22118

\section{$\mathrm{C}_{12} \mathrm{H}_{23} \mathrm{NO}_{3}$}

( \pm )-threo- $N$-Isobutyl-4,5-dihydroxy- $2 E$ octaenamide, 11271

\section{$\mathrm{C}_{12} \mathrm{H}_{23} \mathrm{NO}_{9}$}

2-O- $\alpha-D$-Galactopyranosyl-1-deoxynojirimycin, 8052

6-O- $\alpha-D$-Galactopyranosyl-1-deoxynojirimycin, 8053

4-O- $\beta$ - $D$-Glucopyranosyl-1-deoxynojirimycin, 8614

$\mathrm{C}_{12} \mathrm{H}_{24}$

Nonyl cyclopropane, 15699

\section{$\mathrm{C}_{12} \mathrm{H}_{24} \mathrm{O}$}

Lauric aldehyde, 12570

\section{$\mathrm{C}_{12} \mathrm{H}_{24} \mathrm{O}_{2}$}

$n$-Decyl acetate, 4865

Lauric acid, 12569

\section{$\mathrm{C}_{12} \mathrm{H}_{24} \mathrm{O}_{6}$}

$n$-Hexyl- $\beta$ - $D$-glucopyranoside, 9531

\section{$\mathrm{C}_{12} \mathrm{H}_{24} \mathrm{O}_{7}$}

Hexane-1,5-diol-1- $O$ - $\beta$ - $D$-glucopyranoside, 9513

$\mathrm{C}_{12} \mathrm{H}_{26}$

Dodecane, 6541

$\mathrm{C}_{12} \mathrm{H}_{26} \mathrm{O}$

Dodecanol, 6542

\section{$\mathrm{C}_{12} \mathrm{H}_{31} \mathrm{NO}$}

Nominine, 15658

$\mathrm{C}_{13} \mathrm{H}_{6}$

1-Tridecene-3,5,7,9,11-pentyne, 21622

\section{$\mathrm{C}_{13} \mathrm{H}_{8}$}

1,3E-Tridecadiene-5,7,9,11-tetrayne, 21604

1,3Z-Tridecadiene-5,7,9,11-tetrayne, 21605

1,11E-Tridecadiene-3,5,7,9-tetrayne, 21606

1,11Z-Tridecadiene-3,5,7,9-tetrayne, 21607 


\section{$\mathrm{C}_{13} \mathrm{H}_{8} \mathrm{O}_{3}$}

5-Hydroxyxanthone, 10831

7-Hydroxyxanthone, 10832

\section{$\mathrm{C}_{13} \mathrm{H}_{8} \mathrm{O}_{4}$}

2'-Acetylangelicin, 316

1,5-Dihydroxyxanthone, 6181

1,6-Dihydroxyxanthone, 6182

1,7-Dihydroxyxanthone, 6183

\section{$\mathrm{C}_{13} \mathrm{H}_{8} \mathbf{O}_{5}$}

Gentisein, 8306

Mesuaxanthone B, 13809

1,3,5-Trihydroxyxanthone, 21866

\section{$\mathrm{C}_{13} \mathrm{H}_{8} \mathrm{O}_{6}$}

Norathyriol , 15715

Norbellidifodin, 15718

Norswertianin, 15799

Phelligridin A, 17059

\section{$\mathbf{C}_{13} \mathbf{H}_{8} \mathbf{O}_{7}$}

3,4,8,9,10-Pentahydroxydibenzo[b,d]pyran-6-one,

\section{$\mathrm{C}_{13} \mathrm{H}_{8} \mathrm{O}_{8}$}

Brevifolincarboxylic acid, 2603

Phyllanthusiin E, 17221

\section{$\mathrm{C}_{13} \mathrm{H}_{8} \mathrm{~S}$}

2-(Ethenylbutadiynyl)-5-( propinyl)-thiophene, 7391

\section{$\mathrm{C}_{13} \mathrm{H}_{8} \mathrm{~S}_{2}$}

Thiarubrin A, 21328

Thiarubrin B, 21329

\section{$\mathrm{C}_{13} \mathrm{H}_{9} \mathrm{ClO}$}

5-Chloro-2-(octa-2,4,6-triynylidene)-5,6-dihydro$2 H$-pyran, 3564

\section{$\mathrm{C}_{13} \mathrm{H}_{9} \mathrm{ClOS}$}

2-(4-Chloro-3-hydroxybut-1-ynyl)-5-(penta-1,3diynyl) thiophene, 3558

\section{$\mathrm{C}_{13} \mathrm{H}_{9} \mathrm{NO}$}

3-Formylcarbazole, 7897

\section{$\mathrm{C}_{13} \mathrm{H}_{9} \mathrm{NO}_{2}$}

Mukonal, 15027

\section{$\mathrm{C}_{13} \mathrm{H}_{9} \mathrm{NO}_{3}$}

Clausine O, 3802

\section{$\mathrm{C}_{13} \mathrm{H}_{9} \mathrm{NO}_{4}$}

Kokusagine, 12253

1,2,3-Trihydroxyacridone, 21687

\section{$\mathrm{C}_{13} \mathrm{H}_{9} \mathrm{NO}_{5}$}

Megistoquinone I, 13651

$\mathrm{C}_{13} \mathrm{H}_{10}$

(E,E)-1,3,5-Tridecatriene-7,9,11-triyne, 21617

(E,Z)-1,3,5-Tridecatriene-7,9,11-triyne, 21618

(Z,E)-1,3,11-Tridecatriene-5,7,9-triyne, 21619

(Z,Z)-1,3,11-Tridecatriene-5,7,9-triyne, 21620

\section{$\mathrm{C}_{13} \mathrm{H}_{10} \mathrm{~N}_{2} \mathrm{O}$}

1-Acetyl- $\beta$-carboline, 344
$\mathrm{C}_{13} \mathrm{H}_{10} \mathrm{~N}_{2} \mathrm{O}_{2}$

Arenarine D, 1662

$\beta$-Carboline-1-carboxylic acid, methyl ester, 3158

1-Formyl-4-methoxy- $\beta$-carboline, 7908

$\mathrm{C}_{13} \mathrm{H}_{10} \mathrm{~N}_{2} \mathrm{O}_{3}$

Begonanline, 2210

Taraxacine B, 20693

$\mathrm{C}_{13} \mathrm{H}_{10} \mathrm{O}$

Atractylodin, 1969

Ponticaepoxide, 17719

$\mathrm{C}_{13} \mathrm{H}_{10} \mathrm{OS}_{3}$

$\alpha$-Terthienyl methanol, 21019

$\mathrm{C}_{13} \mathrm{H}_{10} \mathrm{O}_{2}$

Annuadiepoxide, 1334

Capillarin, 3117

Dehydrosafynol, 4966

$\mathrm{C}_{13} \mathrm{H}_{10} \mathrm{O}_{3}$

Mycosinol, 15142

$\mathrm{C}_{13} \mathrm{H}_{10} \mathrm{O}_{4}$

Visnagin, 22553

\section{$\mathrm{C}_{13} \mathrm{H}_{10} \mathrm{O}_{5}$}

2,4'-Dihydroxy-5-carboxy-dibenyl ether, 5786

Hispidin, 9561

Iriflophenone, 11153

Isopimpinellin, 11601

Khellol, 12222

Pimpinellin, 17375

2,4,6,3'-Tetrahydroxybenzophenone, 21082

\section{$\mathrm{C}_{13} \mathrm{H}_{10} \mathrm{O}_{6}$}

Maclurin, 13296

\section{$\mathrm{C}_{13} \mathrm{H}_{10} \mathrm{O}_{8}$}

Tricrozarin A, 21597

\section{$\mathrm{C}_{13} \mathrm{H}_{10} \mathrm{O}_{9}$}

4,8-Dimethoxy-7-hydroxy-2-oxo-2H-1-

benzopyran-5,6-dicarboxylic acid, 6249

\section{$\mathrm{C}_{13} \mathrm{H}_{11} \mathrm{~N}$}

3-Methylcarbazole, 14216

\section{$\mathrm{C}_{13} \mathrm{H}_{11} \mathrm{NO}$}

2-Hydroxy-3-methylcarbazole, 10478

$\mathrm{C}_{13} \mathrm{H}_{11} \mathrm{NO}_{3}$

$\gamma$-Fagarine, 7703

Iso- $\gamma$-fagarine, 11424

Melicarpinone, 13674

6-Methoxy dictamnine, 13904

\section{$\mathrm{C}_{13} \mathrm{H}_{11} \mathrm{NO}_{4}$}

Haplopine, 9226

$\mathrm{C}_{13} \mathrm{H}_{12}$

(E,E,E)-1,3,5,11-Tridecatetraene-7,9-diyne, 21613

(E,Z,E)-1,3,5,11-Tridecatetraene-7,9-diyne, 21614

(Z,E,E)-1,3,5,11-Tridecatetraene-7,9-diyne, 21615

\section{$\mathrm{C}_{13} \mathrm{H}_{12} \mathrm{~N}_{2} \mathrm{O}$}

Harmine, 9235

1-Methoxymethyl- $\beta$-carboline, 14007

\section{$\mathrm{C}_{13} \mathrm{H}_{12} \mathrm{~N}_{2} \mathrm{O}_{2}$}

Picrasidine P, 17308

$\mathrm{C}_{13} \mathrm{H}_{12} \mathrm{O}$

Methoxycapillen, 13870

\section{$\mathrm{C}_{13} \mathrm{H}_{12} \mathrm{O}_{2}$}

Atractylodinol, 1970

Dehydrotremetone, 4976

4,4'-Dihydroxydiphenyl methane, 5866

Goniothalamin, 8947

2-(Hexa-2,4-diyn-1-ylidene)-1,6-dioxaspiro[4,4]non-3-ene, 9496

Safynol, 19122

\section{$\mathrm{C}_{13} \mathrm{H}_{12} \mathrm{O}_{3}$}

Euparin, 7578

Garcibiphenyl A, 8204

Goniothalamin epoxide, 8948

$o$-(o-Methoxyphenoxy)phenol, 14053

6-(2-methoxy-Z-vinyl)-7-methyl-pyranocoumarin, 14104

Mukagolactone, 15026

Nepodin, 15489

\section{$\mathrm{C}_{13} \mathrm{H}_{12} \mathrm{O}_{4}$}

Aloesone, 980

Altholactone, 1008

6-(trans-1-Buten-3-oxy)-7-methoxycoumarin, 2780

Cassiachromone, 3279

Goniobutenolide A, 8932

Goniobutenolide B, 8933

Hibicuslide C, 9538

Methyl piperate, 14678

$\mathrm{C}_{13} \mathrm{H}_{12} \mathrm{O}_{5}$

Xanthocerin, 22758

$\mathrm{C}_{13} \mathrm{H}_{12} \mathrm{O}_{6}$

Nigrolineaisoflavone A, 15572

$\mathrm{C}_{13} \mathrm{H}_{12} \mathrm{O}_{8}$

Caffeoyl malic acid, 2912

$\mathrm{C}_{13} \mathrm{H}_{12} \mathrm{O}_{9}$

Monocaffeyltartaric acid, 14920

\section{$\mathrm{C}_{13} \mathrm{H}_{12} \mathrm{O}_{11}$}

Mucic acid 1,4-lactone 2-O-gallate, 15011

Mucic acid 1,4-lactone 3-O-gallate, 15012

Mucic acid 1,4-lactone 5-O-gallate, 15013

$\mathrm{C}_{13} \mathrm{H}_{13} \mathrm{ClO}_{3}$

8-Chlorogoniodiol, 3555

\section{$\mathrm{C}_{13} \mathrm{H}_{13} \mathrm{NO}$}

(E)-3-(3'-Methyl-2'-butenylidene)-2-indolinone, 14181

$N$-(3-Phenylpropanoyl)pyrrole, 17130

$\mathrm{C}_{13} \mathrm{H}_{13} \mathrm{NO}_{2}$ 
Sarmentamide A, 19368

$\mathrm{C}_{13} \mathrm{H}_{13} \mathrm{NO}_{5}$

Glycocitridine, 8824

Melisemine, 13685

\section{$\mathrm{C}_{13} \mathrm{H}_{13} \mathrm{~N}_{2} \mathrm{O}^{+}$}

6-Methoxy-2-methyl- $\beta$-carbolinium (cation), 14008

$\mathrm{C}_{13} \mathrm{H}_{14} \mathrm{~N}_{2} \mathrm{O}$

Harmaline, 9232

$\mathrm{C}_{13} \mathrm{H}_{14} \mathrm{~N}_{2} \mathrm{O}_{3}$

Nigellicine, 15568

$\mathrm{C}_{13} \mathrm{H}_{14} \mathrm{~N}_{2} \mathrm{O}_{8}$

Miraxanthin II, 14880

\section{$\mathrm{C}_{13} \mathrm{H}_{14} \mathrm{O}$}

Carlinoxide, 3200

$\mathrm{C}_{13} \mathrm{H}_{14} \mathrm{O}_{2}$

Tremetone, 21507

\section{$\mathrm{C}_{13} \mathrm{H}_{14} \mathrm{O}_{3}$}

6-Acetyl-2,2-dimethylchroman-4-one, 383

6-Acetyl-5-hydroxy-2-isopropenyl-2,3dihydrobenzofuran, 418

Chuanxiongol, 3631

( \pm )-2,3-Dihydro-2-(1-methylethenyl)-5benzofurancarboxylic acid methyl ester, 5676

5,6-Dimethoxy-2-isopropenylbenzofuran, 6255

2,2-Dimethyl-2H-1-benzopyran-6-carboxylic acid methyl ester, 6321

Eupatoriochromene, 7592

7-Hydroxy-4-isopropyl-6-methyl coumarin, 10263

2-Hydroxy-5-methoxy-6-(3-methylbut-3-en1-ynyl)benzylalcohol, 10418

6-Hydroxytremetone, 10775

Toxol, 21486

\section{$\mathrm{C}_{13} \mathrm{H}_{14} \mathrm{O}_{4}$}

1'-Acetoxychavicol acetate, 140

6-Acetyl-5-hydroxy-2-(1-hydroxy-2-propenyl)-

2,3-dihydrobenzofuran, 416

6-Acetyl-2-hydroxymethyl-2-methylchroman4-one, 424

(+)-9-Deoxygoniopypyrone, 5179

Deoxygoniopypyrone A, 5180

(6R,7R,8R)-Goniodiol, 8934

Goniofupyrone A, 8938

(+)-Gynunone, 9122

7-Hydroxy-2-(2-hydroxy)propyl-5-methylbenzopyran- $\gamma$-one, 10217

(2'S)-7-Hydroxy-2-(2'-hydroxypropyl)-5methylchromone, 10218

Isocorylifonol, 11345

7-Methoxyanofinic acid, 13840

Pedicellin, 16762

$\mathrm{C}_{13} \mathrm{H}_{14} \mathrm{O}_{5}$
$(5 S, 6 R, 7 R, 8 R)$-Goniotriol, 8949

(5S,6R,7S,8S)-Goniotriol, 8950

(2'S)-7-Hydroxy-5-hydroxymethyl-2-(2'hydroxypropyl) chromone, 10205

5-Methanol-7-hydroxy-2,2-dimethyl-2H-1-

chromene-6-carboxylic acid, 13820

\section{$\mathrm{C}_{13} \mathrm{H}_{14} \mathrm{O}_{8}$}

(-)-4-(E)-Caffeoyl- $L$-threonic acid, 2928

\section{$\mathrm{C}_{13} \mathrm{H}_{14} \mathrm{O}_{9}$}

Norbergenin, 15719

$\mathrm{C}_{13} \mathrm{H}_{14} \mathrm{O}_{12}$

Mucic acid 2-O-gallate, 15009

\section{$\mathrm{C}_{13} \mathrm{H}_{15} \mathrm{NO}$}

Argutine A, 1676

(1-Oxo-3-phenyl-2E-propenyl)pyrrolidine, 16406

Zanthonitrile, 22968

\section{$\mathrm{C}_{13} \mathrm{H}_{15} \mathrm{NO}_{2}$}

Allosecurinine, 940

Securinine, 19639

Virosecurinine, 22529

\section{$\mathrm{C}_{13} \mathrm{H}_{15} \mathrm{NO}_{3}$}

ent-Phyllanthidine, 17214

$\mathrm{C}_{13} \mathrm{H}_{15} \mathrm{NO}_{6}$

L-O-Caffeoylhomoserine, 2909

\section{$\mathrm{C}_{13} \mathrm{H}_{15} \mathrm{O}_{8}$}

Demethoxybergenin, 5042

\section{$\mathrm{C}_{13} \mathrm{H}_{15} \mathrm{O}_{13} \mathrm{~S}^{-}$}

Turgorin, 22122

$\mathrm{C}_{13} \mathrm{H}_{16} \mathrm{~N}_{2}$

$N_{b}$-Methyltetrahydroharman, 14750

\section{$\mathrm{C}_{13} \mathrm{H}_{16} \mathrm{~N}_{2} \mathrm{O}$}

2-Methyl-6-methoxy-1,2,3,4-tetrahydro- $\beta$ carboline, 14590

$N_{b}$-Methyltetrahydroharmol, 14751

Tetrahydroharmine, 21070

\section{$\mathrm{C}_{13} \mathrm{H}_{16} \mathrm{~N}_{2} \mathrm{O}_{2}$}

(+)- $N_{b}$-Methyl tryptophan methyl ester $(S), 14795$

\section{$\mathrm{C}_{13} \mathrm{H}_{16} \mathbf{N}_{2} \mathrm{O}_{4}$}

Delamide, 4992

\section{$\mathrm{C}_{13} \mathrm{H}_{16} \mathrm{O}_{2}$}

2-Hexenyl benzoate, 9526

4-Hydroxy-3-(3-methyl-2-butenyl)acetophenone, 10472

Vinyl-2,2-dimethyl-3-phenyl-propionate, 22510

\section{$\mathrm{C}_{13} \mathrm{H}_{16} \mathrm{O}_{3}$}

4-Hydroxy-3-(2-hydroxy-3-isopentenyl)acetophenone, 10182

4-Methoxy-3-(3-methyl-2-butenyl)-benzoic acid, 14004

2-(1-Oxopentyl)-benzoic acid methyl ester, 16404

Precocene II, 17776

4-(2,4,5-Trimethoxyphenyl)-but-1,3-diene, 21924
Wilforonide, 22689

\section{$\mathrm{C}_{13} \mathrm{H}_{16} \mathrm{O}_{4}$}

Asarumin B, 1837

Proglobeflowery acid, 17902

$\mathrm{C}_{13} \mathrm{H}_{16} \mathrm{O}_{5}$

Descurainolide A, 5246

Eugenone, 7524

4-Hydroxy-2-(2,4,5-trimethoxyphenyl)-2Ebutenal, 10801

Methyl 3,4,5-trimethoxycinnamate, 14791

Sinapic acid ethyl ester, 19913

$\mathrm{C}_{13} \mathrm{H}_{16} \mathrm{O}_{6}$

Cardiobutanolide, 3189

\section{$\mathrm{C}_{13} \mathrm{H}_{16} \mathrm{O}_{7}$}

Helicide, 9303

\section{$\mathrm{C}_{13} \mathrm{H}_{16} \mathrm{O}_{8}$}

1-O-(4-Hydroxybenzoyl)- $\beta$-D-glucose, 9824

\section{$\mathrm{C}_{13} \mathrm{H}_{16} \mathrm{O}_{9}$}

3-Carboxy-4-hydroxy-phenoxy glucoside, 3174

1-O-Galloyl- $\alpha$ - $L$-rhamnose, 8118

Protocatechuic acid-3-glucoside, 17966

\section{$\mathbf{C}_{13} \mathbf{H}_{16} \mathbf{O}_{10}$}

1-O-Galloyl-glucose, 8108

6-O-Galloyl-glucose, 8109

\section{$\mathrm{C}_{13} \mathrm{H}_{17} \mathrm{NO}$}

Paniculidine C, 16614

\section{$\mathrm{C}_{13} \mathrm{H}_{17} \mathrm{NO}_{2}$}

Dihydrosecurinine, 5712

14,15-Dihydrovirosecurinine, 5738

\section{$\mathrm{C}_{13} \mathrm{H}_{17} \mathrm{NO}_{3}$}

Lophophorine, 13001

Securinol A, 19640

Securinol B, 19641

Securinol C, 19642

\section{$\mathrm{C}_{13} \mathrm{H}_{17} \mathrm{NO}_{4}$}

2,6-Di- $C$-methyl-nicotinic acid 3,5-diethyl ester, 6378

\section{$\mathrm{C}_{13} \mathrm{H}_{18} \mathrm{~N}_{2} \mathrm{O}$}

Bufotenidine, 2725

$N, N$-Dimethyl-5-methoxy tryptamine, 6370

\section{$\mathrm{C}_{13} \mathrm{H}_{18} \mathrm{~N}_{2} \mathrm{O}_{2}$}

5-Methoxy- $N, N$-dimethyl-tryptamine $N_{b}$-oxide, 13920

$\mathrm{C}_{13} \mathrm{H}_{18} \mathrm{~N}_{2} \mathrm{O}_{4}$

$N^{5}$-(4-Methoxyphenyl)methyl- $L$-glutamine, 14061

\section{$\mathrm{C}_{13} \mathrm{H}_{18} \mathrm{~N}_{2} \mathrm{O}_{7}$}

Justiciamide, 11976

\section{$\mathrm{C}_{13} \mathrm{H}_{18} \mathrm{O}_{2}$}

Arteamisinine I, 1782

3-Keto- $\beta$-ionone, 12203

$\mathrm{C}_{13} \mathrm{H}_{18} \mathrm{O}_{3}$

2-Methoxy-1-O-methyl-6-prenylhydroquinone, 
14031

2-Methoxy-4-O-methyl-6-prenylhydroquinone, 14032

$\mathrm{C}_{13} \mathrm{H}_{18} \mathrm{O}_{4}$

Baeckeol, 2097

$\mathrm{C}_{13} \mathrm{H}_{18} \mathrm{O}_{5}$

Monascusone A, 14915

$\mathrm{C}_{13} \mathrm{H}_{18} \mathrm{O}_{6}$

Benzyl alcohol $O-\beta$ - $D$-glucopyranoside, 2276

$\mathrm{C}_{13} \mathrm{H}_{18} \mathrm{O}_{7}$

Gastrodin, 8237

Homoarbutin, 9594

3-Hydroxybenzyl-1- $O-\beta$ - $D$-glucopyranoside, 9840

4-Hydroxybenzyl- $O-\beta$ - $D$-glucopyranoside, 9841

Isohomoarbutin, 11456

2-Methoxyphenyl $\beta$ - $D$-glucopyranoside, 14059

Orcinol glucoside, 16172

Salicin, 19184

\section{$\mathrm{C}_{13} \mathrm{H}_{18} \mathrm{O}_{8}$}

3,4-Dihydroxybenzyl alcohol-4-glucoside, 5769

4-Hydroxy-3-methoxyphenol

$\beta$-D-glucopyranoside, 10432

Isotachioside, 11726

$\mathrm{C}_{13} \mathrm{H}_{18} \mathrm{O}_{9}$

Opuntioside, 16160

$\mathrm{C}_{13} \mathrm{H}_{19} \mathrm{NO}_{2}$

6,7-Dihydroxy-1,1-dimethyl- $N$-ethyl-1,2,3,4tetrahydroisoquinoline, 5853

Dioscorine, 6439

Kinganone, 12228

Lucidulinone, 13051

Pectenine, 16746

\section{$\mathrm{C}_{13} \mathrm{H}_{19} \mathrm{NO}_{3}$}

$O^{7}$-Angeloylretronecine, 1233

$O^{9}$-Angeloylretronecine, 1234

Rivularine, 18854

\section{$\mathrm{C}_{13} \mathrm{H}_{19} \mathrm{NO}_{4}$}

Tuberostemospironine, 22090

\section{$\mathrm{C}_{13} \mathrm{H}_{20} \mathrm{~N}_{2} \mathrm{O}$}

$N, N$-Dimethyltryptamine-methohydroxide, 6419

\section{$\mathrm{C}_{13} \mathrm{H}_{20} \mathrm{O}$}

$\alpha$-Ionone, 11122

$\beta$-Ionone, 11123

\section{$\mathrm{C}_{13} \mathrm{H}_{20} \mathrm{O}_{2}$}

Actinidol, 584

3-Hydroxy- $\beta$-ionone, 10233

(3R,6R,7E)-3-Hydroxy-4,7-megastigmadien-9one, 10366

(6E,9S)-9-Hydroxy-4,6-megastigmadien-3-one, 10367

(6Z,9S)-9-Hydroxy-4,6-megastigmadien-3-one, 10368
(6R,7E,9R)-9-Hydroxy-4,7-megastigmadien-3one, 10369

(7E,9 $)$-9-Hydroxy-5,7-megastigmadien-4-one, 10370

Theaspirone, 21291

$\mathrm{C}_{13} \mathrm{H}_{20} \mathrm{O}_{3}$

Annuionone D, 1335

Apocynol A, 1527

$(3 S, 5 R, 6 S, 7 E)-5,6$-Epoxy-3-hydroxy-7megastigmen-9-one, 7123

Grasshopperketone, 8983

4'-(4"-Hydroxy-3"-methylbutyloxy)-2phenylethanol, 10474

Icarisidin $\mathrm{B}_{1}, 10956$

cis-Methyl jasmonate, 14540

Norannuic acid, 15709

Vomifoliol, 22615

\section{$\mathrm{C}_{13} \mathrm{H}_{20} \mathrm{O}_{4}$}

Apocynol B, 1528

13-Carboxy-blumenol C, 3165

(1R,2R)-Methyl-5'-hydroxyjasmonate, 14509

\section{$\mathrm{C}_{13} \mathrm{H}_{20} \mathrm{O}_{6}$}

threo-3-(4-Hydroxy-3,5-dimethoxyphenyl)-3ethoxypropane-1,2-diol, 10037

$\mathrm{C}_{13} \mathrm{H}_{21} \mathrm{NO}_{2}$

Tigloidine, 21372

$\mathrm{C}_{13} \mathrm{H}_{21} \mathrm{NO}_{3}$

Macrophylline, 13320

$\mathrm{C}_{13} \mathrm{H}_{21} \mathrm{NO}_{4}$

Meteloidine, 13819

$\mathrm{C}_{13} \mathrm{H}_{22}$

1,4,7-Tridecatriene, 21616

$\mathrm{C}_{13} \mathrm{H}_{22} \mathrm{O}$

Dihydro- $\beta$-ionone, 5646

$\mathrm{C}_{13} \mathrm{H}_{22} \mathrm{O}_{2}$

(3R,6R,7E,9R)-3,9-Dihydroxy-4,7-megastigmadiene, 5956

(3S,7E,9R)-3,9-Dihydroxy-5,7-megastigmadiene, 5957

$(6 R, 9 R)-9$-Hydroxy-4-megastigmen-3-one, 10371

3-Hydroxymegastigm-5-en-9-one, 10372

(S)-9-Hydroxymegastigm-5-en-4-one, 10373

Neryl propionate, 15515

$\mathrm{C}_{13} \mathrm{H}_{22} \mathrm{O}_{3}$

Annuionone E, 1336

Annuionone G, 1338

(2R,6R,9R)-2,9-Dihydroxy-4-megastigmen-3-one, 5958

$(3 S, 5 R, 6 S, 7 E, 9 R)-5,6-E p o x y-3,9-d i h y d r o x y-7-$ megastigmene, 7079

Methyl dihydrojasmonate , 14296

$\mathrm{C}_{13} \mathrm{H}_{22} \mathrm{O}_{4}$

Annuionone F, 1337
$\mathrm{C}_{13} \mathrm{H}_{24} \mathrm{~N}_{2} \mathrm{O}$

Cuscohygrine, 4417

$\mathrm{C}_{13} \mathrm{H}_{24} \mathrm{O}_{2}$

Megastigm-5-ene-3,9-diol, 13646

$\mathrm{C}_{13} \mathrm{H}_{24} \mathrm{O}_{3}$

11-Hydroxy-9-tridecenoic acid, 10784

7-Megastigmene-3,6,9-triol, 13648

\section{$\mathrm{C}_{13} \mathrm{H}_{24} \mathrm{O}_{4}$}

Megastigmenetetrol, 13647

$(3 S, 5 R, 6 R, 7 E, 9 R)-3,5,6,9-T e t r a h y d r o x y-7-$ megastigmene, 21124

\section{$\mathrm{C}_{13} \mathrm{H}_{24} \mathrm{O}_{9}$}

Methyl di- $\alpha$ - $L$-rhamnoside, 14326

Methyl-(3R,5R)-5-hydroxy-3-( $\beta$ - $D$-glucopyranosyloxy)-hexanoate, 14505

Methyl-(3S,5S)-5-hydroxy-3-( $\beta$ - $D$-glucopyranosyloxy) hexanoate, 14506

\section{$\mathrm{C}_{13} \mathrm{H}_{24} \mathrm{O}_{11}$}

Ethane-1,2-diol 1-O- $\beta$ - $D$-apiofuranosyl-( $(\rightarrow 6)$ $\beta$ - $D$-glucopyranoside, 7388

$\mathrm{C}_{13} \mathrm{H}_{26}$

Tridecene, 21621

$\mathrm{C}_{13} \mathrm{H}_{26} \mathrm{O}$

2-Methyl-dodecane-5-one, 14330

2-Tridecanone, 21612

\section{$\mathrm{C}_{13} \mathrm{H}_{26} \mathrm{O}_{2}$}

n-Undecyl acetate, 22224

Tridecanoic acid, 21611

2-Undecyl acetate, 22225

\section{$\mathrm{C}_{13} \mathrm{H}_{26} \mathrm{O}_{4} \mathrm{~S}$}

Undecyl sulfonyl acetic acid, 22228

\section{$\mathrm{C}_{13} \mathrm{H}_{28}$}

2,4-Dimethyl-undecane, 6421

3,6-Dimethyl-undecane, 6422

2-Methyl-5-propyl nonane, 14693

$n$-Tridecane, 21610

2,5,6-Trimethyldecane, 21953

\section{$\mathrm{C}_{13} \mathrm{H}_{28} \mathrm{O}_{2}$}

1,1-Diethoxy- $n$-nonane, 5499

\section{$\mathrm{C}_{13} \mathrm{H}_{28} \mathrm{O}_{4}$}

Homosenkyunolide H, 9618

Homosenkyunolide I, 9619

\section{$\mathrm{C}_{14} \mathrm{H}_{6} \mathrm{O}_{8}$}

Ellagic acid, 6757

\section{$\mathrm{C}_{14} \mathrm{H}_{8} \mathrm{~N}_{2} \mathrm{O}$}

Canthin-6-one, 3096

\section{$\mathrm{C}_{14} \mathrm{H}_{8} \mathrm{~N}_{2} \mathrm{O}_{2}$}

Canthin-6-one 3- $N$-oxide, 3098

1-Hydroxycanthin-6-one, 9884

9-Hydroxycanthin-6-one, 9885

11-Hydroxycanthin-6-one, 9886

\section{$\mathrm{C}_{14} \mathrm{H}_{8} \mathrm{~N}_{2} \mathrm{O}_{3}$}

9-Hydroxycanthin-6-one 3- $N$-oxide, 9887 
Tramesanguin, 21501

$\mathrm{C}_{14} \mathrm{H}_{8} \mathrm{O}_{2}$

Anthraquinone, 1367

$\mathrm{C}_{14} \mathrm{H}_{8} \mathrm{O}_{3}$

$\alpha$-Hydroxyanthraquinone, 9791

2-Hydroxyanthraquinone, 9792

\section{$\mathrm{C}_{14} \mathrm{H}_{8} \mathrm{O}_{4}$}

Alizarin, 899

Chrysazin, 3599

Purpuroxanthin, 18224

\section{$\mathrm{C}_{14} \mathrm{H}_{8} \mathrm{O}_{5}$}

Anthragallol, 1359

Purpurin, 18222

\section{$\mathrm{C}_{14} \mathrm{H}_{8} \mathrm{O}_{6}$}

1,7-Dihydroxy-2,3-methylenedioxyxanthone, 6031

\section{$\mathrm{C}_{14} \mathrm{H}_{8} \mathrm{O}_{9}$}

Luteic acid, 13125

\section{$\mathrm{C}_{14} \mathrm{H}_{9} \mathrm{ClO}_{6}$}

7-Chloro-1,2,3-trihydroxy-6-methoxyxanthone, 3573

\section{$\mathrm{C}_{14} \mathrm{H}_{9} \mathrm{NO}_{2}$}

Trisphaeridine, 22040

\section{$\mathrm{C}_{14} \mathrm{H}_{9} \mathrm{NO}_{3}$}

Crinasiadine, 4238

Dianthalexine, 5366

\section{$\mathrm{C}_{14} \mathrm{H}_{9} \mathrm{O}_{8} \mathrm{~S}^{-}$}

1,3-Dihydroxy-5-methoxyxanthone-4-sulfonate, 6014

\section{$\mathrm{C}_{14} \mathrm{H}_{10} \mathrm{~N}_{2} \mathrm{O}_{2}$}

3-(2'-Hydroxyphenyl)-4-(3H)-quinazolinone, 10643

\section{$\mathrm{C}_{14} \mathrm{H}_{10} \mathrm{O}$}

Anthranol, 1365

\section{$\mathrm{C}_{14} \mathrm{H}_{10} \mathrm{O}_{3}$}

Kvannin, 12395

\section{$\mathrm{C}_{14} \mathrm{H}_{10} \mathrm{O}_{4}$}

Cyperaquinone, 4576

Dengibsin, 5128

2-(2,4-Dihydroxyphenyl)-6-hydroxybenzofuran, 6086

Drypearmoracein B, 6614

5-Hydroxy-1-methoxyxanthone, 10462

6-Hydroxy-7-methoxyxanthone, 10463

7-Hydroxy-8-methoxyxanthone, 10464

4,5-Methylenedioxy-4'-hydroxy-2-aldehyde-

[1,1'-biphenyl], 14369

Moracin M, 14962

\section{$\mathrm{C}_{14} \mathrm{H}_{10} \mathrm{O}_{5}$}

7-Benzoyloxy-6-oxo-2,4E-heptadiene-1,4-olide, 2261

7-Benzoyloxy-6-oxo-2,4Z-heptadiene-1,4-olide, 2262
Buchanaxanthone, 2694

Crataequinone A, 4217

Densiflorolorin, 5133

1,3-Dihydroxy-2-methoxy xanthone, 6009

1,6-Dihydroxy-7-methoxyxanthone, 6010

1,7-Dihydroxy-3-methoxy xanthone, 6011

1,7-Dihydroxy-4-methoxyxanthone, 6012

2,7-Dihydroxy-1-methoxyxanthone, 6013

Isogentisin, 11436

Kigelinone, 12225

Mesuaxanthone A, 13808

Nor-rubrofusarin, 15791

1,3,6-Trihydroxy-8-methylxanthone, 21809

1,5,8-Trihydroxy-3-methylxanthone, 21810

\section{$\mathrm{C}_{14} \mathrm{H}_{10} \mathrm{O}_{6}$}

Bellidifodin, 2220

Crataequinone B, 4218

Lespedezol $\mathrm{H}_{1}, 12704$

Swertianin, 20510

1,3,7-Trihydroxyl-8-methoxyxanthone, 21762

1,3,5-Trihydroxy-2-methoxyxanthone, 21790

1,3,7-Trihydroxy-2-methoxyxanthone, 21791

1,3,8-Trihydroxy-2-methoxyxanthone, 21792

1,3,8-Trihydroxy-5-methoxyxanthone, 21793

\section{$\mathrm{C}_{14} \mathrm{H}_{10} \mathrm{O}_{7}$}

Caloxanthone E, 3000

\section{$\mathrm{C}_{14} \mathrm{H}_{10} \mathrm{O}_{8}$}

Methyl brevifolin carboxylate, 14160

Phyllanthusiin E methyl ester, 17222

\section{$\mathrm{C}_{14} \mathrm{H}_{10} \mathrm{O}_{9}$}

$m$-Digallic acid, 5510

$m$-Digalloyl acid, 5511

p-Digalloyl acid, 5512

\section{$\mathrm{C}_{14} \mathrm{H}_{11} \mathrm{NO}_{2}$}

3-Formyl-6-methoxycarbazole, 7907

1-Hydroxy-10-methylacridone, 10465

Methyl carbazole-3-carboxylate, 14217

Murrayanine, 15118

\section{$\mathrm{C}_{14} \mathrm{H}_{11} \mathrm{NO}_{3}$}

Clausine E, 3795

Clausine I, 3798

Clausine M, 3800

Clausine N, 3801

Clausine Q, 3804

Griffithazanone B, 9005

Isoursuline, 11750

Lansine, 12489

\section{$\mathrm{C}_{14} \mathrm{H}_{11} \mathrm{NO}_{4}$}

Clausine R, 3805

Griffithazanone A, 9004

2-Hydroxy-3-formyl-7-methoxycarbazole, 10126

Indigotiisocoumarin A, 11022

Penduline, 16802
$\mathrm{C}_{14} \mathrm{H}_{11} \mathrm{NO}_{5}$

Melandrin, 13657

\section{$\mathrm{C}_{14} \mathrm{H}_{11} \mathrm{NO}_{6}$}

Dianthramine, 5372

Precatorine, 17772

\section{$\mathrm{C}_{14} \mathrm{H}_{12} \mathrm{~N}_{2} \mathrm{O}$}

1-Vinyl-4-methoxy- $\beta$-carboline, 22516

\section{$\mathrm{C}_{14} \mathrm{H}_{12} \mathrm{~N}_{2} \mathrm{O}_{2}$}

Arenarine A, 1659

Arenarine C, 1661

1-Carboethoxy- $\beta$-carboline, 3156

$\beta$-Carboline-1-propionic acid, 3159

Picrasidine I, 17301

\section{$\mathrm{C}_{14} \mathrm{H}_{12} \mathrm{~N}_{2} \mathrm{O}_{3}$}

Dichotomine A, 5422

7-Hydroxy- $\beta$-carboline-1-propionic acid, 9890

\section{$\mathrm{C}_{14} \mathrm{H}_{12} \mathrm{~N}_{2} \mathrm{O}_{4}$}

Dichotomine B, 5423

Peristrophine, 16965

\section{$\mathrm{C}_{14} \mathrm{H}_{12} \mathrm{O}_{2}$}

Benzyl benzoate, 2280

o-Benzyl benzoic acid, 2281

Pinosylvin, 17420

\section{$\mathrm{C}_{14} \mathrm{H}_{12} \mathrm{O}_{3}$}

Ammirin, 1067

Angenomalin, 1238

Benzyl salicylate, 2297

5,6-Dehydrokawain, 4936

Hibicutaiwanin, 9539

2'-Isopropyl-psoralen, 11630

Resveratrol, 18643

Seselin, 19784

2,4,4'-Trihydroxystilbene, 21849

4,5',8-Trimethyl psoralen, 21974

Xanthyletin, 22781

\section{$\mathrm{C}_{14} \mathrm{H}_{12} \mathrm{O}_{4}$}

Cearoin, 3346

Cedrecoumarin A, 3349

Clematichinenol, 3822

Cudranin, 4337

Dihydrocyperaquinone, 5579

2,6-Dihydroxy-4-methoxybenzophenone, 5965

Eleutherol, 6753

4'-Hydroxy-5,6-dehydrokawain, 9974

5-Hydroxyseselin, 10710

Nakhsmyrin, 15241

Nodachenetin, 15644

Norbraylin, 15721

Obliquin, 15886

Oroselol, 16213

cis-Osthenone, 16259

trans-Osthenone, 16260

E-Piceatannol, 17278 
Suberenone, 20438

Wyerone acid, 22742

$\mathrm{C}_{14} \mathrm{H}_{12} \mathrm{O}_{5}$

Clausenin, 3792

Khellin, 12221

Melodorinol, 13712

2-Methoxy-6-acetyl-7-methyljuglone, 13832

$\mathrm{C}_{14} \mathrm{H}_{12} \mathrm{O}_{6}$

Ammiol, 1066

4,6,3',4'-Tetrahydroxy-2-methoxybenzophenone, 21125

$\mathrm{C}_{14} \mathrm{H}_{12} \mathrm{O}_{7}$

Rheumin, 18767

$\mathrm{C}_{14} \mathrm{H}_{12} \mathrm{O}_{11}$

Chebulic acid, 3492

$\mathrm{C}_{14} \mathrm{H}_{13} \mathrm{NO}$

Murrayafoline A, 15117

$\mathrm{C}_{14} \mathrm{H}_{13} \mathrm{NO}_{2}$

Carbalexin A, 3153

Carbalexin B, 3154

Carbalexin C, 3155

Clausine V, 3808

Flindersine, 7830

$\mathrm{N}$-Salicylidene-salicylamine, 19188

$\mathrm{C}_{14} \mathrm{H}_{13} \mathrm{NO}_{3}$

Isotaifine, 11727

\section{$\mathrm{C}_{14} \mathrm{H}_{13} \mathrm{NO}_{4}$}

Isomaculosidine, 11518

Kokusaginine, 12254

Skimmianine, 20002

\section{$\mathrm{C}_{14} \mathrm{H}_{13} \mathrm{NO}_{6}$}

$E$-Dimethyl rhoifolinate, 6404

Z-Dimethyl rhoifolinate, 6405

Lycoricidine, 13238

Megistoquinone II, 13652

\section{$\mathrm{C}_{14} \mathrm{H}_{13} \mathrm{NO}_{7}$}

Furomegistine II, 8028

Lycoricidinol, 13239

\section{$\mathrm{C}_{14} \mathrm{H}_{14} \mathrm{BrNO}$}

6-Bromo-2-(1,1-dimethyl-2-propenyl)- $1 H$ indole-3-carbaldehyde, 2621

\section{$\mathrm{C}_{14} \mathrm{H}_{14} \mathrm{~N}_{2} \mathrm{O}$}

1-Ethyl-4-methoxy- $\beta$-carboline, 7459

\section{$\mathrm{C}_{14} \mathrm{H}_{14} \mathrm{~N}_{2} \mathrm{O}_{2}$}

Arenarine B, 1660

Picrasidine J, 17302

\section{$\mathrm{C}_{14} \mathrm{H}_{14} \mathrm{O}$}

Tetradec-8,10,12-triyne-6-ene-3-one, 21047

\section{$\mathrm{C}_{14} \mathrm{H}_{14} \mathrm{O}_{2}$}

Dihydropinosylvin, 5694

Ichthyothereol, 10959

Lunularin, 13085

$\mathrm{C}_{14} \mathrm{H}_{14} \mathrm{O}_{3}$
Aucuparin, 2005

Bis(4-hydroxybenzyl)ether, 2458

Bis(2-hydroxyphenyl)methyl ether, 2471

7-Demethylsuberosin, 5098

Dihydroresveratrol, 5700

3',5'-Dimethoxy-biphenyl-4-ol, 6213

4-[(4-hydroxyphenyl)methoxy]benzenemethanol, 10627

Kawain, 12184

Mansonrin C, 13521

Osthenol, 16257

\section{$\mathrm{C}_{14} \mathrm{H}_{14} \mathrm{O}_{4}$}

Columbianetin, 3936

Decursinol, 4863

Demethylauraptenol, 5063

Dihydroosthenon, 5686

(E)-7-Hydroxy-6-(3-hydroxy-methyl-1-butenyl)2H-1-benzopyran-2-one, 10194

(R)-(+)-7-Hydroxy-8-(2-hydroxy-3-methyl-3butenyl)-2H-1-benzopyran-2-one, 10195

7-Hydroxy-6-(2-(R)-hydroxy-3-methylbut-3enyl)coumarin, 10197

(Z)-7-Hydroxy-6-(3-hydroxy-methyl-1-butenyl)2H-1-benzopyran-2-one, 10196

Jatamansinol, 11835

Marmesin, 13571

Methyl-2-(5-acetyl-2,3-dihydrobenzofuran-

2-yl)propenoate, 14117

Nodakenetin, 15645

Phellodenol C, 17069

Tetrahydrocyperaguinone, 21063

Torachrysone, 21452

\section{$\mathrm{C}_{14} \mathrm{H}_{14} \mathrm{O}_{5}$}

Angelidiol, 1194

Celereoin, 3375

3,4-Dihydro-1,2-secomicrominutinin, 5709

1-(3',5'-Dihydroxyphenyl)-2-(4"-hydroxyphenyl)-ethane-1,2-diol, 6087

Khellactone, 12220

Methyl-2-(6-acetyl-5-hydroxy-2,3-dihydrobenzofuran-2-yl)propenoate, 14119

Peucedanone, 17035

$\mathrm{C}_{\mathbf{1 4}} \mathrm{H}_{\mathbf{1 4}} \mathrm{O}_{\mathbf{9}}$

3,5,7-Ttrihydroxylchromone 3-O- $\beta$ - $D$-xylopyranoside, 22071

\section{$\mathrm{C}_{14} \mathrm{H}_{14} \mathrm{O}_{11}$}

Mucic acid 1,4-lactone methyl ester 2-O-gallate, 15014

Mucic acid 1,4-lactone methyl ester 5-O-gallate, 15015

\section{$\mathrm{C}_{14} \mathrm{H}_{14} \mathrm{~S}$}

Dibenzyl sulphide, 5392

$\mathrm{C}_{14} \mathrm{H}_{14} \mathrm{~S}_{2}$
Dibenzyl disulphide, 5391

$\mathrm{C}_{14} \mathrm{H}_{14} \mathrm{~S}_{3}$

Dibenzyl trisulphide, 5394

$\mathrm{C}_{14} \mathrm{H}_{14} \mathrm{~S}_{\mathbf{4}}$

Dibenzyl tetrasulphide, 5393

$\mathrm{C}_{14} \mathrm{H}_{15} \mathrm{NO}_{2}$

Ganodine, 8193

Piperchabamide A, 17440

$\mathrm{C}_{14} \mathrm{H}_{15} \mathrm{NO}_{3}$

Gastrodamine, 8236

1-[1-Oxo-3(3,4-methylenedioxyphenyl)-2Epropenyl]pyrrolidine, 16392

$\mathrm{C}_{14} \mathrm{H}_{15} \mathrm{NO}_{7}$

Isatan $\mathrm{B}, 11188$

$\mathrm{C}_{14} \mathrm{H}_{15} \mathrm{NO}_{8}$

Pancratistatin, 16601

$\mathrm{C}_{14} \mathrm{H}_{16}$

Chamazulene, 3464

$\mathrm{C}_{14} \mathrm{H}_{16} \mathrm{CINO}_{8}$

7-Chloro-(2R)-2-O- $\beta$ - $D$-glucopyranosyl- $2 H$ -

1,4-benzoxazin-3(4H)-one, 3554

\section{$\mathrm{C}_{14} \mathrm{H}_{16} \mathrm{CINO}_{9}$}

7-Cl-DIBOA-Glc, 3815

\section{$\mathrm{C}_{14} \mathrm{H}_{16} \mathrm{~N}_{2} \mathrm{O}_{2}$}

Peganidine, 16769

\section{$\mathrm{C}_{14} \mathrm{H}_{16} \mathrm{~N}_{2} \mathrm{O}_{3}$}

Cyclo-(Pro-Tyr-), 4533

\section{$\mathrm{C}_{14} \mathrm{H}_{16} \mathrm{~N}_{2} \mathrm{O}_{5}$}

Brachystemidine D, 2581

\section{$\mathrm{C}_{14} \mathrm{H}_{16} \mathrm{~N}_{2} \mathrm{O}_{6}$}

Brachystemidine E, 2582

Indicaxanthin, 11018

\section{$\mathrm{C}_{14} \mathrm{H}_{16} \mathbf{N}_{2} \mathrm{O}_{7}$}

Portulaxanthine, 17734

\section{$\mathrm{C}_{14} \mathrm{H}_{16} \mathrm{~N}_{2} \mathrm{O}_{8}$}

Vulgaxanthin II, 22624

\section{$\mathrm{C}_{14} \mathrm{H}_{16} \mathrm{O}$}

Lacinilene A, 12431

\section{$\mathrm{C}_{14} \mathrm{H}_{16} \mathrm{O}_{2}$}

Precolpuchol, 17777

4-(2-Propenyl)-phenyl angelate, 17924

4-(1-Propenyl)-phenyl tiglate, 17926

\section{$\mathrm{C}_{14} \mathrm{H}_{16} \mathrm{O}_{3}$}

Atractylentriol, 1968

Encecalin, 6796

Fraxinellone, 7945

Mansonrin B, 13520

Mexicanin E, 14821

Phomapyrone F, 17179

Pterosin E, 18136

\section{$\mathrm{C}_{14} \mathrm{H}_{16} \mathrm{O}_{4}$}

6-Acetyl-5-hydroxy-2-isopropenyl-3-methoxy2,3-dihydrobenzofuran, 419 
$\left(3 S^{*}, 4 S^{*}, 5 R^{*}\right)-(E)-3,4-$ Dihydroxy-2-(hexa-2,4diynyliden)-1,6-dioxaspiro-(4,5)decane, 5903

Hydroxyachillin, 9765

(6R,7R,8R)-8-Methoxygoniodiol, 13943

Methyl 5-hydroxy-7-methyl-2,2-dimethyl-2H-1chromene-6-carboxylate, 14515

Methyl 7-hydroxy-5-methyl-2,2-dimethyl-2H-1chromene-6-carboxylate, 14516

Prenyl caffeate, 17828

\section{$\mathrm{C}_{14} \mathrm{H}_{16} \mathrm{O}_{5}$}

1'-Acetoxyeugenol acetate, 195

6-Acetyl-5-hydroxy-2-(1-hydroxy-2-propenyl)3-methoxy-2,3-dihydrobenzofuran, 417

Forsythenside B, 7923

5-Methanol-7-methoxy-2,2-dimethyl-2H-1chromene-6-carboxylic acid, 13821

Methyl-2-(6-acetyl-5-hydroxy-2,3-dihydrobenzofuran-2-yl)propanoate, 14118

(R)-Peucedanol, 17032

(S)-Peucedanol, 17033

\section{$\mathrm{C}_{14} \mathrm{H}_{16} \mathrm{O}_{6}$}

Asarumin D, 1839

Caruilignan D, 3230

Zhebeiresinol, 22996

\section{$\mathrm{C}_{14} \mathrm{H}_{16} \mathrm{O}_{6} \mathrm{~S}$}

Petasiformin A, 17012

\section{$\mathrm{C}_{14} \mathrm{H}_{16} \mathrm{O}_{8}$}

1-O-Caffeoyl- $\beta$-xylose, 2930

\section{$\mathrm{C}_{14} \mathrm{H}_{16} \mathrm{O}_{9}$}

Bergenin, 2312

\section{$\mathrm{C}_{14} \mathrm{H}_{16} \mathrm{O}_{10}$}

3-O-Galloyl quinic acid, 8116

4-O-Galloyl quinic acid, 8117

\section{$\mathrm{C}_{14} \mathrm{H}_{16} \mathrm{O}_{12}$}

Mucic acid 1-methyl ester 2-O-gallate, 15016

Mucic acid 6-methyl ester 2-O-gallate, 15017

\section{$\mathrm{C}_{14} \mathrm{H}_{17} \mathrm{Br}_{2} \mathrm{ClO}$}

Dactylyne, 4595

\section{$\mathrm{C}_{14} \mathrm{H}_{17} \mathrm{ClO}$}

Pterosin F, 18137

\section{$\mathrm{C}_{14} \mathrm{H}_{17} \mathrm{ClO}_{2}$}

Pterosin J, 18145

\section{$\mathrm{C}_{14} \mathrm{H}_{17} \mathrm{~N}$}

2-n-Pentylquinoline, 16898

\section{$\mathrm{C}_{14} \mathrm{H}_{17} \mathrm{NO}$}

1-(1-Oxo-3-phenyl-2E-propenyl)piperidine, 16405

$\mathrm{C}_{14} \mathrm{H}_{17} \mathrm{NO}_{2}$

Awaine, 2043

Paniculidine A, 16612

$\mathrm{C}_{14} \mathrm{H}_{17} \mathrm{NO}_{3}$

cis-Fagaramide, 7701

trans-Fagaramide, 7702
6-Hydroxy-2-(3-hydroxy-3-methylbutyl)-4quinolone, 10201

7-Hydroxy-2-(3-hydroxy-3-methylbutyl)-4quinolone, 10202

Securitinine, 19644

\section{$\mathrm{C}_{14} \mathrm{H}_{17} \mathrm{NO}_{6}$}

Indican glucoside, 11014

Prunasin, 18000

Sambunigrin, 19226

\section{$\mathrm{C}_{14} \mathrm{H}_{17} \mathrm{NO}_{7}$}

Dhurrin, 5283

p-Glucosyloxymandelonitrile, 8756

Holocalin, 9590

Taxiphyllin, 20805

Zierin, 23000

\section{$\mathrm{C}_{14} \mathrm{H}_{17} \mathrm{NO}_{9}$}

2,4-Dihydroxy-1,4-benzoxazin-3-one-2- $O-\beta$ - $D$ glucopyranoside, 5766

(2R)-2-O- $\beta$ - $D$-Glucopyranosyl-5-hydroxy-2H1,4-benzoxazin-3(4H)-one, 8666

6-Hydroxy blepharin, 9858

\section{$\mathrm{C}_{14} \mathrm{H}_{17} \mathrm{NO}_{10}$}

Triglochinin, 21644

$\mathrm{C}_{14} \mathrm{H}_{17} \mathrm{~N}_{3} \mathrm{O}_{7}$

Vulgaxanthin I, 22623

$\mathrm{C}_{14} \mathrm{H}_{17} \mathrm{O}_{8}^{-}$

Glochidacuminoside A, 8559

$\mathrm{C}_{14} \mathrm{H}_{18}$

4-Epi-11-nor-aristola-1,9,11-triene, 6979

\section{$\mathrm{C}_{14} \mathrm{H}_{18} \mathrm{BrClO}$}

Srilankenyne, 20251

\section{$\mathrm{C}_{14} \mathrm{H}_{18} \mathrm{~N}_{2} \mathrm{O}_{2}$}

Cyclo-(Phe-Val), 4528

Hypaphorine, 10877

Trimethyl tryptophan, 21978

$\mathrm{C}_{14} \mathrm{H}_{18} \mathrm{~N}_{2} \mathrm{O}_{3}$

Physovenine, 17253

$\mathrm{C}_{14} \mathrm{H}_{18} \mathrm{~N}_{2} \mathrm{O}_{5}$

$\gamma$ - $L$-Glutamyl- $L$-phenylalanine, 8779

$\mathrm{C}_{14} \mathrm{H}_{18} \mathrm{~N}_{2} \mathrm{O}_{6}$

$\gamma$ - $L$-Glutamyl- $L$-tyrosine, 8782

$\mathrm{C}_{14} \mathrm{H}_{18} \mathrm{~N}_{2} \mathrm{O}_{7}$

Humilixanthin, 9662

$\mathrm{C}_{14} \mathrm{H}_{18} \mathrm{~N}_{2} \mathrm{O}_{7} \mathrm{~S}$

Miraxanthin I, 14879

$\mathrm{C}_{14} \mathrm{H}_{18} \mathrm{O}$

3,3-Dimethyl allyl-p-propenyl phenyl ether, 6310

4-Phenylbicyclo[2.2.2] octan-1-ol, 17096

$\mathrm{C}_{14} \mathrm{H}_{18} \mathrm{O}_{2}$

4-(1-Propenyl)-phenyl 2-methylbutanoate, 17925

Pterosin B, 18133

Thellungianin F, 21308

\section{$\mathrm{C}_{14} \mathrm{H}_{18} \mathrm{O}_{3}$}

Encecalinol, 6797

Espeletone, 7382

Lobetyol, 12941

Pterosin C, 18134

Pterosin G, 18138

Pterosin M, 18148

Pterosin N, 18149

Pterosin P, 18151

\section{$\mathrm{C}_{14} \mathrm{H}_{18} \mathrm{O}_{4}$}

(E)-4-(3,4-Dimethoxyphenyl)-but-3-en-1-ol acetate, 6280

Phomapyrone E, 17178

Phomapyrone G, 17180

Pterosin Q, 18152

Pterosin S, 18154

Pterosin T, 18155

\section{$\mathrm{C}_{14} \mathrm{H}_{18} \mathrm{O}_{5}$}

AcronyculatinD, 574

Pterosin U, 18156

\section{$\mathrm{C}_{14} \mathrm{H}_{18} \mathrm{O}_{6}$}

2,3-Dihydroxymethyl-4-(3',4'-dimethoxyphenyl)$\gamma$-butyrolactone, 6029

Drypearmoracein A, 6613

\section{$\mathrm{C}_{14} \mathrm{H}_{18} \mathrm{O}_{7}$}

Picein, 17287

\section{$\mathrm{C}_{14} \mathrm{H}_{18} \mathrm{O}_{8}$}

6-O-Acetylarbutin, 324

Alopecuquinone, 982

Bungeiside A, 2747

Bungeiside B, 2748

2-Carboxylmethylphenol

1-O- $\beta$ - $D$-glucopyranoside, 3176

Cuneataside A, 4362

Cuneataside B, 4363

(2S)-2-O- $\beta$-D-Glucopyranosyl-2-hydroxyphenylacetic acid, 8675

methyl (6-O-p-Hydroxybenzoyl)- $\beta$ - $D$-glucopyranoside, 14497

\section{$\mathrm{C}_{14} \mathrm{H}_{18} \mathrm{O}_{9}$}

1-(3-O- $\beta$-D-Glucopyranosyl-4,5-dihydroxyphenyl)-ethanone, 8622

Maltol-(6- $O$-acetyl)- $\beta$ - $D$-glucopyranoside, 13453 Phaseoloidin, 17050

Vanillic acid 4- $O-\beta-D$-glucopyranoside, 22334 Vanillic acid $\beta$ - $D$-glucopyranosyl ester, 22335

Woodorien, 22721

\section{$\mathrm{C}_{14} \mathrm{H}_{18} \mathrm{O}_{10}$}

4-O- $\beta$ - $D$-Glucopyranosyl methyl gallate, 8682

Methyl-6- $O$-galloyl- $\beta$ - $D$-glucopyranoside, 14455

Polygoacetophenoside, 17639

$\mathrm{C}_{14} \mathrm{H}_{19} \mathrm{NO}_{2}$

3-Methyl-but-2-enoic acid-[2-(4-methoxyphen- 
yl)-ethyl]-amide, 14175

Paniculidine B, 16613

\section{$\mathrm{C}_{14} \mathrm{H}_{19} \mathrm{NO}_{6}$}

Nordomoic acid, 15742

$\mathrm{C}_{14} \mathrm{H}_{19} \mathrm{NO}_{9} \mathrm{~S}_{2}$

Glucotropaeolin, 8760

$\mathrm{C}_{\mathbf{1 4}} \mathrm{H}_{\mathbf{1 9}} \mathrm{NO}_{10} \mathrm{~S}_{\mathbf{2}}$

Sinalbine, 19909

\section{$\mathrm{C}_{\mathbf{1 4}} \mathrm{H}_{\mathbf{2 0}}$}

4-Epi-11-nor-aristola-1(10),11-diene, 6977

4-Epi-11-nor-aristola-9,11-diene, 6978

\section{$\mathrm{C}_{14} \mathrm{H}_{20} \mathrm{~N}_{2} \mathrm{O}_{3}$}

Subaphylline, 20434

\section{$\mathrm{C}_{14} \mathrm{H}_{20} \mathrm{~N}_{2} \mathrm{O}_{9}$}

Mimosine- $O$ - $\beta$ - $D$-glucoside, 14869

\section{$\mathrm{C}_{14} \mathrm{H}_{20} \mathrm{O}$}

2,8-Dimethyl-5-acetyl-bicyclo[5,3,0] decadiene-1,8, 6301

Mayurone, 13625

\section{$\mathrm{C}_{14} \mathbf{H}_{20} \mathbf{O}_{2}$}

2-(p-Cyclohexyl-phenoxy)ethanol, 4499

(+)-1,5-Epoxy-nor-ketoguaia-11-ene, 7179

Glutinosone, 8794

4'-(3"-Methylbut-2"-enyloxy)-3-phenylpropanol, 14182

2-(2-Phenyl cyclohexyloxy) ethanol, 17103

Polygonone, 17649

\section{$\mathrm{C}_{14} \mathrm{H}_{20} \mathrm{O}_{3}$}

Ailanthoidiol, 775

Cuspidiol, 4422

\section{$\mathrm{C}_{14} \mathrm{H}_{20} \mathrm{O}_{4}$}

Fascicularone A, 7737

Norannuic acid formyl ester, 15710

2,2,4,4-Tetramethyl-6-(2-methyl-1-oxopropyl)-

1,3,5-cyclohexanetrione, 21200

\section{$\mathrm{C}_{14} \mathrm{H}_{20} \mathrm{O}_{5}$}

7,8-Dihydroxy-isobutyryl-thymol, 5921

Pectinolide B, 16748

Pectinolide C, 16749

Pectinolide H, 16754

Sphaelactone A, 20149

$\mathrm{C}_{14} \mathrm{H}_{20} \mathrm{O}_{6}$

Phenethyl $\beta$ - $D$-glucopyranoside, 17085

\section{$\mathrm{C}_{14} \mathrm{H}_{20} \mathrm{O}_{7}$}

1-O- $\beta$-D-Glucopyranosyl-2-(3-hydroxyphenyl)ethanol, 8676

Rhodioloside, 18792

Sargencuneside, 19366

Sceptroside, 19462

$\mathrm{C}_{14} \mathrm{H}_{20} \mathrm{O}_{8}$

Cimidahurine, 3653

Cimidahurinine, 3654

3,5-Dihydroxyphenethyl alcohol 3-O- $\beta$-gluco- pyranoside, 6074

3,4-Dihydroxyphenylethyl alcohol glucoside, 6081

Vanilloloside, 22338

$\mathrm{C}_{14} \mathrm{H}_{20} \mathrm{O}_{9}$

2,6-Dimethoxy-4-hydroxyphenol-1- $O-\beta$ - $D$-glucopyranoside, 6250

Jioglutoside A, 11879

Koaburaside, 12238

$\mathrm{C}_{14} \mathrm{H}_{21} \mathrm{ClO}_{11}$

Glutinoside, 8790

$\mathrm{C}_{14} \mathrm{H}_{21} \mathrm{~N}$

2,6-Nonamethylene pyridine, 15682

$\mathrm{C}_{14} \mathrm{H}_{21} \mathrm{NO}$

(2S,2'S)-Sedamine, 19647

$\mathrm{C}_{14} \mathrm{H}_{21} \mathrm{NO}_{4}$

Codonopsine, 3886

6,7-Dihydroxy-1,1-dimethyl- $N$-(2'-glyceryl)1,2,3,4-tetrahydroisoquinoline, 5855

\section{$\mathrm{C}_{14} \mathrm{H}_{21} \mathrm{~N}_{3} \mathrm{O}_{5}$}

Leonurine, 12646

$\mathrm{C}_{14} \mathrm{H}_{22}$

(-)-Norrotundene, 15790

\section{$\mathrm{C}_{14} \mathrm{H}_{22} \mathrm{NO}_{3}{ }^{+}$}

3-(4-Hydroxyphenyl)propionyl choline, 10638

\section{$\mathrm{C}_{14} \mathrm{H}_{22} \mathrm{O}$}

cis- $\alpha$-Irone, 11183

trans- $\alpha$-Irone, 11184

Rudbeckianone, 19046

\section{$\mathrm{C}_{14} \mathrm{H}_{22} \mathrm{O}_{2}$}

2,6-Dibutyl-p-cresol, 5402

4-Hydroxy-14-nor-5-dumorten-7-one, 10540

Isokobusone, 11472

Isopolygonal, 11610

Kobusone, 12242

Norketoagarofuran, 15768

Polygonal, 17642

Rishitin, 18849

\section{$\mathrm{C}_{14} \mathrm{H}_{22} \mathrm{O}_{3}$}

Canusesnol H, 3111

Dihydrocuspidiol, 5578

Oxyphyllenodiol A, 16462

Oxyphyllenodiol B, 16463

$\mathrm{C}_{14} \mathrm{H}_{22} \mathrm{O}_{4}$

1-Oxo-2 $\beta$-[3-butanone]-3 $\alpha$-methyl-6 $\beta$-[2propanoic acid]-cyclohexane, 16297

Russulanorol A, 19075

Russulanorol B, 19076

$\mathrm{C}_{14} \mathrm{H}_{22} \mathrm{O}_{6}$

Bursephenylpropane, 2767

$\mathrm{C}_{14} \mathrm{H}_{23} \mathrm{NO}$

Sarmentine, 19371

$\mathrm{C}_{14} \mathrm{H}_{23} \mathrm{NO}_{4}$
Heliohoustine, 9306

Isoretrohoustine, 11647

Retrohoustine, 18661

$\mathrm{C}_{14} \mathrm{H}_{24}$

Cyprotene, 4587

\section{$\mathrm{C}_{14} \mathrm{H}_{24} \mathrm{O}$}

5-cis-Cyclotetradecen-1-one, 4538

(E)-9-Isopropyl-6-methyl-5,9-decadiene-2-one, 11627

T-Muurolol, 15141

\section{$\mathrm{C}_{14} \mathrm{H}_{24} \mathrm{O}_{2}$}

Alepric acid, 884

Goshuyic acid, 8956

\section{$\mathrm{C}_{14} \mathrm{H}_{24} \mathrm{O}_{3}$}

$(1 R, 5 S, 6 S, 7 S, 10 R)-1 \beta, 6 \alpha$-Dihydroxyeudesman4-one, 5892

15-Nor-10-hydroxy-oplopan-4-oic acid, 15758

\section{$\mathrm{C}_{14} \mathrm{H}_{24} \mathrm{O}_{4} \mathrm{~S}_{2}$}

$t$-Butyl 3-[(1-methylpropyl)dithio]-2-propenyl malonate, 2800

$t$-Butyl 3-[(1-methylthiopropyl)thio]-2-propenyl malonate, 2801

\section{$\mathrm{C}_{14} \mathrm{H}_{24} \mathrm{O}_{8}$}

Rengyoside B, 18617

\section{$\mathrm{C}_{14} \mathrm{H}_{24} \mathrm{O}_{9}$}

Rengynic acid 1'- $O$ - $\beta$ - $D$-glucoside, 18613

\section{$\mathrm{C}_{14} \mathrm{H}_{25} \mathrm{NO}$}

$\mathrm{N}$-Isobutyldeca-trans-2-trans-4-dienamide, 11269

\section{$\mathrm{C}_{14} \mathrm{H}_{25} \mathrm{NO}_{2}$}

$N$-Isobutyl-4,5-epoxy-2E-decaenamide, 11274

\section{$\mathrm{C}_{14} \mathrm{H}_{25} \mathrm{NO}_{3}$}

Pipercycliamide, 17445

\section{$\mathrm{C}_{14} \mathrm{H}_{26} \mathrm{~N}_{2} \mathrm{O}_{12}$}

\section{Neocycasin A, 15380}

Neocycasin B, 15381

Neocycasin E, 15384

\section{$\mathrm{C}_{14} \mathrm{H}_{26} \mathrm{O}$}

Cyclotetradecan-1-one, 4537

3-Methylcyclotridecan-1-one, 14276

\section{$\mathrm{C}_{14} \mathrm{H}_{26} \mathrm{O}_{2}$}

Myristoleic acid, 15205

cis-4-Tetradecenoic acid, 21044

Tetradecenoic acid A, 21045

Tetradecenoic acid B, 21046

$\mathrm{C}_{\mathbf{1 4}} \mathrm{H}_{26} \mathrm{O}_{6}$

(3R)-1-Octan-3-enyl- $\beta$ - $D$-glucopyranoside, 15975

$\mathrm{C}_{14} \mathrm{H}_{26} \mathrm{O}_{8}$

Rengyoside A, 18616

$\mathrm{C}_{14} \mathrm{H}_{26} \mathrm{O}_{9} \mathrm{~S}$

Bacopaside A, 2084

$\mathrm{C}_{14} \mathrm{H}_{26} \mathrm{O}_{10}$ 
Isopropyl $\beta$ - $D$-apiofuranosyl-( $1 \rightarrow 6)-\beta$ - $D$-glucopyranoside, 11620

\section{$\mathrm{C}_{14} \mathrm{H}_{26} \mathbf{O}_{11}$}

Agarobiose dimethylacetal, 702

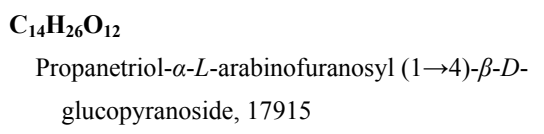

\section{$\mathrm{C}_{14} \mathrm{H}_{27} \mathrm{NO}_{3}$}

$N$-Isobutyl-4,5-dihydroxy-2E-decaenamide, 11270

\section{$\mathrm{C}_{14} \mathrm{H}_{28}$}

1-Tetradecene, 21043

\section{$\mathrm{C}_{14} \mathrm{H}_{28} \mathrm{O}_{2}$}

Ethyllaurate, 7455

Methyl-2,4,6-trimethyl-decanoate, 14792

Myristic acid, 15203

$n$-Dodecyl acetate, 6545

$(-) 2 D, 4 D, 6 D, 8 D$-Tetramethyl decanoic acid, 21194

\section{$\mathrm{C}_{14} \mathrm{H}_{28} \mathrm{O}_{4}$}

Ipurolic acid, 11132

\section{$\mathrm{C}_{14} \mathrm{H}_{30}$}

4,6-Dimethyl dodecane, 6343

Tetradecane, 21040

\section{$\mathrm{C}_{14} \mathrm{H}_{30} \mathrm{O}$}

1-Tetradecanol, 21041

\section{$\mathrm{C}_{15} \mathrm{H}_{8} \mathbf{N}_{2} \mathrm{O}_{2}$}

Tryptanthrine, 22059

\section{$\mathrm{C}_{15} \mathrm{H}_{8} \mathrm{O}_{5}$}

Coumestrol, 4190

4-Hydroxy-2-carboxyanthraquinone, 9891

Nordamnacanthal, 15734

Ophiohayatone C, 16134

\section{$\mathrm{C}_{15} \mathrm{H}_{8} \mathrm{O}_{6}$}

Lucernol, 13018

Munjistin, 15076

Norjuzunal, 15765

Rhein, 18759

\section{$\mathrm{C}_{15} \mathrm{H}_{8} \mathrm{O}_{7}$}

Demethylwedelolactone, 5101

Isodemethylwedelolactone, 11374

Pseudopurpurin, 18058

\section{$\mathrm{C}_{15} \mathrm{H}_{9} \mathrm{ClO}_{2}$}

4'-Chloroaurone, 3542

\section{$\mathrm{C}_{15} \mathrm{H}_{9} \mathrm{NO}_{3}$}

Criasiaticidine A, 4235

\section{$\mathrm{C}_{15} \mathrm{H}_{9} \mathrm{O}_{10} \mathrm{~S}$}

Quercetin-3-sulphate, 18399

\section{$\mathrm{C}_{15} \mathrm{H}_{10} \mathrm{~N}_{2} \mathrm{O}$}

Anticancer Alkaloid PMV70P691-051, 1381

\section{$\mathrm{C}_{15} \mathrm{H}_{10} \mathrm{~N}_{2} \mathrm{O}_{2}$}

Drymaritin, 6609

5-Hydroxymethylcanthin-6-one, 10477
1-Methoxycanthin-6-one, 13865

5-Methoxycanthin-6-one, 13866

9-Methoxycanthin-6-one, 13867

10-Methoxycanthin-6-one, 13868

3-Methyl-canthin-2,6-dione, 14211

Picrasidine L, 17304

\section{$\mathrm{C}_{15} \mathrm{H}_{10} \mathrm{~N}_{2} \mathrm{O}_{3}$}

1-Hydroxy-9-methoxycanthin-6-one, 10390

8-Hydroxy-9-methoxycanthin-6-one, 10391

10-Hydroxy-9-methoxycanthin-6-one, 10392

11-Hydroxy-10-methoxycanthin-6-one, 10393

9-Methoxycanthin-6-one 3- $\mathrm{N}$-oxide, 13869

Nigakinone, 15561

Picrasidine Q, 17309

\section{$\mathrm{C}_{15} \mathrm{H}_{10} \mathrm{O}$}

2,3-Diphenyl-2-cyclopropen-1-one, 6485

\section{$\mathrm{C}_{15} \mathrm{H}_{10} \mathrm{O}_{2}$}

Flavone, 7819

2-Methylanthraquinone, 14144

\section{$\mathrm{C}_{15} \mathrm{H}_{10} \mathrm{O}_{3}$}

Anticancer Flavonoid PMV70P691-94, 1419

Flavonol, 7820

1-Hydroxy-2-methyl-anthraquinone, 10467

2-Hydroxymethylanthraquinone, 10468

2-Isopropenyl naphtho[2,3-b]furan-4,9-quinone, 11616

\section{$\mathrm{C}_{15} \mathrm{H}_{10} \mathrm{O}_{4}$}

Alizarin-1-methylether, 900

Alizarin-2-methylether, 901

Chrysin, 3600

Chrysophanol, 3615

Cypritibetquinone A, 4585

Daidzein, 4604

Digiferrugineol, 5522

4',7-Dihydroxyflavone, 5895

1,4-Dihydroxy-2-methylanthraquinone, 6015

1,8-Dihydroxy-2-methylanthraquinone, 6017

3,9-Dihydroxypterocarp-6a-ene, 6105

2-Hydroxy-3-hydroxymethyl anthraquinone, 10193

1-Hydroxy-3-methoxyanthraquinone, 10380

6-Methylalizarin, 14124

2-(1'-Methylethenyl)-5-hydroxynaphtho[2,3- $b$ ] furan-4,9-dione, 14430

2-(1'-Methylethenyl)-7-hydroxynaphtho[2,3-b] furan-4,9-dione, 14431

Nordalbergin, 15733

Primetin, 17853

Rubiadin, 18998

$\mathrm{C}_{15} \mathrm{H}_{10} \mathrm{O}_{5}$

Aloeemodin, 967

Anthragallol-2-methylether, 1361

Apigenin, 1476
Baicalein, 2102

1,8-Dihydroxy-4-hydroxymethyl anthraquinone, 5914

3,6-Dihydroxy-2-hydroxymethyl-9,10-anthraquinone, 5915

1,6-Dihydroxy-2-methoxyanthraquinone, 5961

Emodin, 6776

Galangin, 8081

Genistein, 8278

Helminthosporin, 9333

5-Hydroxyalizarin-1-methylether, 9771

Islandicin, 11191

Lucidin, 13041

2-Methyl-1,3,6-trihydroxyanthraquinone, 14775

Morindone, 14974

Norobtusifolin, 15782

Norwogonin, 15815

Rubilactone, 19031

Sulfuretin, 20481

Thunberginol A, 21356

Tournefolal, 21477

4,5,6-Trihydroxy-aurone, 21688

5,7,2'-Trihydroxyflavone, 21732

7,3',4'-Trihydroxyflavone, 21733

\section{$\mathrm{C}_{15} \mathrm{H}_{10} \mathrm{O}_{6}$}

Aureusidin, 2011

Catenarin, 3319

Citreorosein, 3765

6-Demethoxycapillarisin, 5043

Fisetin, 7802

6-Hydroxy-1-methoxy-2,3-methylenedioxyxanthone, 10422

7-Hydroxy-1-methoxy-2,3-methylenedioxy xanthone, 10423

3-Hydroxymorindone, 10530

Isosabandin, 11689

Isoscutellarein, 11703

Kaempferol, 12020

Luteolin, 13137

Norartocarpetin, 15714

Orobol, 16209

Scutellarein, 19582

5,7,2',3'-Tetrahydroxyflavone, 21113

5,7,2',6'-Tetrahydroxyflavone, 21114

\section{$\mathrm{C}_{15} \mathrm{H}_{10} \mathbf{O}_{7}$}

6-Demethylcapillarisin, 5065

Herbacetin, 9421

6-Hydroxyluteolin, 10351

Morin, 14971

3,5,7,2',6'-Pentahydroxy flavone, 16848

3,5,6,7,8-Pentahydroxy-2-phenyl-4H-1-benzopyran-4-one, 16859

Quercetin, 18317 
Robinetin, 18857

Robustaquinone F, 18874

\section{$\mathrm{C}_{15} \mathrm{H}_{10} \mathrm{O}_{8}$}

Gossypetin, 8959

Myricetin, 15170

Quercetagetin, 18305

\section{$\mathrm{C}_{15} \mathrm{H}_{10} \mathrm{O}_{9} \mathrm{~S}$}

Kaempferol-3-sulphate, 12098

\section{$\mathrm{C}_{15} \mathrm{H}_{11} \mathrm{ClO}_{3}$}

4'-Chloro-2-hydroxyaurone, 3557

\section{$\mathrm{C}_{15} \mathrm{H}_{11} \mathrm{ClO}_{5}$}

5-Chloro-1,6-dihydroxy-3-methoxy-8-methylxanthone, 3549

\section{$\mathrm{C}_{15} \mathrm{H}_{11} \mathrm{I}_{4} \mathrm{NO}_{4}$}

3,5,3',5'-Tetraiodothyronine, 21175

\section{$\mathrm{C}_{15} \mathrm{H}_{11} \mathrm{~N}$}

$\alpha$-Phenylcinnamic acid nitrile, 17102

2-Phenylquinoline, 17135

\section{$\mathrm{C}_{15} \mathrm{H}_{11} \mathrm{NO}_{5}$}

Marcanine D, 13549

\section{$\mathrm{C}_{15} \mathrm{H}_{11} \mathrm{O}_{5}{ }^{+}$}

Pelargonidin, 16777

\section{$\mathrm{C}_{15} \mathrm{H}_{11} \mathrm{O}_{6}^{+}$}

Cyanidin, 4434

\section{$\mathrm{C}_{15} \mathrm{H}_{11} \mathrm{O}_{7}^{+}$}

Delphinidin, 5011

\section{$\mathrm{C}_{15} \mathrm{H}_{12} \mathrm{I}_{3} \mathrm{NO}_{4}$}

3,3',5'-Triiodothyronine, 21867

3,5,3'-Triiodothyronine, 21868

\section{$\mathrm{C}_{15} \mathrm{H}_{12} \mathrm{O}_{2} \mathrm{~S}_{2}$}

5-(3-Buten-1-ynyl)-2,2'-bithienyl-5'-methylacetate, 2784

\section{$\mathrm{C}_{15} \mathrm{H}_{12} \mathrm{O}_{2} \mathrm{~S}_{3}$}

$\alpha$-Terthienyl methyl acetate, 21020

\section{$\mathrm{C}_{15} \mathrm{H}_{12} \mathrm{O}_{3}$}

Acetyl-atractylodinol, 327

Anticancer Flavonoid PMV70P691-84, 1414

Chrysarobin, 3598

4,4'-Dihydroxychalcone, 5788

Flavidin, 7811

Haginin E, 9190

5-Hydroxyflavanone, 10124

7-Hydroxyflavanone, 10125

Magnoquinone, 13389

Mansonone F, 13516

Moscatin, 14997

Thespone, 21321

Typhaphthalide, 22155

\section{$\mathrm{C}_{15} \mathrm{H}_{12} \mathrm{O}_{4}$}

Bolusanthin IV, 2539

1,7-Dimethoxyxanthone, 6299

Emodin anthrone, 6777

Haginin D, 9189
Hydrangeic acid, 9699

Hydrangenol, 9700

3-Hydroxydehydro-iso- $\alpha$-lapachone, 9973

Isoliquiritigenin, 11504

Lespedezol $\mathrm{F}_{1}, 12702$

Liquiritigenin, 12908

4-Methoxyphenanthrene-2,3,7-triol, 14051

Montroumarin, 14947

Pinocembrin, 17403

2,4,4'-Trihydroxychalcone, 21694

\section{$\mathrm{C}_{15} \mathrm{H}_{12} \mathrm{O}_{5}$}

Butein, 2779

Butin, 2785

Chalconaringenin, 3454

1,6-Dihydroxy-3-methoxy-8-methylxanthone, 5992

1,8-Dihydroxy-5-methoxy-3-methylxanthone, 5993

Garbanzol, 8202

1-Hydroxy-2,4-dimethoxyxanthone, 10044

1-Hydroxy-3,7-dimethoxyxanthone, 10045

2-Hydroxy-1,7-dimethoxyxanthone, 10046

4-Hydroxyhydrangenol, 10171

Isotoralactone, 11744

4-Methoxy-6-(11,12-methylenedioxystyryl)-2pyrone, 14021

4-Methoxyphenanthrene-2,3,6,7-tetrol, 14050

Microminutin, 14841

( \pm -Naringenin, 15278

Naringenin, 15279

Pinobanksin, 17399

Protosappanin A, 17985

Rubrofusarin, 19041

Securixanthone B, 19646

2,4,2',4'-Tetrahydroxychalcone, 21086

Thunberginol C, 21357

Toralactone, 21455

7,3',5'-Trihydroxyflavanone, 21731

\section{$\mathrm{C}_{15} \mathrm{H}_{12} \mathrm{O}_{6}$}

(2R,3R)-Aromadendrin, 1764

(2R,3S)-Aromadendrin, 1765

Carthamidin, 3223

(+)Dihydrofisetin, 5613

(-)Dihydrofisetin, 5614

1,3-Dihydroxy-4,5-dimethoxyxanthone, 5840

1,6-Dihydroxy-3,5-dimethoxyxanthone, 5841

1,6-Dihydroxy-3,7-dimethoxyxanthone, 5842

1,6-Dihydroxy-5,7-dimethoxyxanthone, 5843

1,6-Dihydroxy-7,8-dimethoxyxanthone, 5844

1,7-Dihydroxy-3,4-dimethoxyxanthone, 5845

3,6-Dihydroxy-1,7-dimethoxyxanthone, 5846

Eriodictyol, 7278

Gentiacauleine, 8290
Maesopsin, 13361

2-O-Methylswertianin, 14732

Micromelin, 14839

Okanin, 16024

2',3',4',5',6'-Pentahydroxychalcone, 16842

3,4,5,2',4'-Pentahydroxychalcone, 16843

Steppogenin, 20326

Swerchirin, 20502

$(2 R, 3 R)-2$ ',3,5,7-tetrahydroxyflavanone, 21108

(2S)-5,7,2',5'-Tetrahydroxyflavanone, 21109

Tragopogonic acid, 21500

\section{$\mathrm{C}_{15} \mathrm{H}_{12} \mathrm{O}_{7}$}

Dihydromorin , 5679

Dihydroquercetin, 5699

Dihydrorobinetin, 5702

Epitaxifolin, 7026

2-O-(4-Hydroxybenzoyl)-2,4,6-trihydroxyphenylacetic acid, 9830

Onjixanthone II, 16109

3,5,7,2'6'-Pentahydroxyflavanone, 16846

(+)-3,5,7,3',5'-Pentahydroxyflavanone, 16847

Phelligridin B, 17060

Securixanthone A, 19645

\section{$\mathrm{C}_{15} \mathrm{H}_{12} \mathrm{O}_{8}$}

Ampelopsin, 1074

2-O-(3,4-Dihydroxybenzoyl)-2,4,6-trihydroxyphenylacetic acid, 5768

\section{$\mathrm{C}_{15} \mathrm{H}_{13} \mathrm{ClO}_{4}$}

Chlorosesamone, 3572

\section{$\mathrm{C}_{15} \mathrm{H}_{13} \mathrm{ClO}_{6}$}

6-Chlorocatechin, 3544

(-)-6-Chloroepicatechin, 3550

\section{$\mathrm{C}_{15} \mathrm{H}_{13} \mathrm{I}_{2} \mathrm{NO}_{4}$}

3,3'-Diiodothyronine, 6186

\section{$\mathrm{C}_{15} \mathrm{H}_{13} \mathrm{NO}_{3}$}

Glycozolidal, 8832

Mukonine, 15028

\section{$\mathrm{C}_{15} \mathrm{H}_{13} \mathrm{NO}_{4}$}

Clausine B, 3793

Clausine K, 3799

\section{$\mathrm{C}_{15} \mathrm{H}_{13} \mathrm{NO}_{5}$}

Citrusinine II, 3778

\section{$\mathrm{C}_{15} \mathrm{H}_{14} \mathrm{~N}_{2} \mathrm{O}_{2}$}

Kumujian G, 12335

Methyl-3-( $\beta$-carboline-1-yl) propionate, 14218

4,9-Dimethoxy-1-vinyl- $\beta$-carboline, 6297

\section{$\mathrm{C}_{15} \mathrm{H}_{14} \mathrm{~N}_{2} \mathrm{O}_{3}$}

7-Methoxy- $\beta$-carboline-1-Propionic acid, 13871

\section{$\mathrm{C}_{15} \mathrm{H}_{14} \mathrm{~N}_{2} \mathrm{O}_{4}$}

Dichotomine C, 5424

$\mathrm{C}_{15} \mathrm{H}_{14} \mathrm{~N}_{2} \mathrm{O}_{6}$

Sterculinine II, 20330

$\mathrm{C}_{15} \mathrm{H}_{14} \mathrm{O}$ 
Linderazulene, 12870

$\mathrm{C}_{15} \mathrm{H}_{14} \mathrm{O}_{2}$

Deoxylapachol, 5188

Flavanol, 7807

4'-Methylpinosylvin, 14677

Pinosylvin methyl ether, 17421

$\mathrm{C}_{15} \mathrm{H}_{14} \mathrm{O}_{3}$

Corsifuran B, 4094

7,4'-Dihydroxyflavan, 5894

4,7-Dihydroxy-2-methoxy-9,10-dihydro-

phenanthrene, 5970

1,3-Diphenylpropane-1,2-diol-3-one, 6489

Hircinol, 9549

Lapachol, 12501

$\alpha$-Lapachone, 12502

$\beta$-Lapachone, 12503

Magnotriol A, 13395

Magnotriol B, 13396

Mansonone D, 13514

Mansonone E, 13515

2-Methoxy-9,10-dihydrophenanthrene-4,5-diol, 13909

4-Methoxy-9,10-dihydrophenanthrene-2,7-diol, 13910

Tupichinol C, 22116

\section{$\mathrm{C}_{15} \mathrm{H}_{14} \mathrm{O}_{4}$}

Alloxanthoxyletin, 941

Benzyl 2-hydroxy-6-methoxybenzoate, 2292

Braylin, 2599

Catalpalactone, 3305

Cochinolide, 3876

Davidigenin, 4694

Dehydrogeijerin, 4919

Dehydrooxoperezinone, 4957

1,5-Dihydroxy-3-methylanthraquinone, 6016

Drypemolundein A, 6615

5-Hydroxylapachol, 10313

Isorhapontigenin, 11672

Lunularic acid, 13084

Luvangetin, 13152

Mansonone H, 13518

4-Methoxy-9,10-dihydrophenanthrene-1,2,7-triol, 13912

4-Methoxy-9,10-dihydrophenanthrene-2,3,7-triol, 13913

5-Methoxyseselin, 14092

Micropubescin, 14845

Miliusolide, 14853

Murralongin, 15104

Murrayone, 15122

Naphthoquinone IV, 15259

Peucedanin, 17028

Phebalosin, 17051
Randaiol, 18533

Rhapontigenin, 18748

Rhinacanthin A, 18768

Thespesone, 21320

Wyerone, 22741

Xanthoxyletin, 22777

Yangonin, 22882

$\mathrm{C}_{15} \mathrm{H}_{14} \mathrm{O}_{5}$

5-Acetoxyisogoniothalamin oxide, 241

3-Acetylaltholactone, 311

Afzelechin, 677

Atrochrysone, 1999

Cheliensisin A, 3505

6,3'-Dihydroxy-2,4-dimethoxybenzophenone, 5819

(-)-Epiafzelechin, 6819

2,3-Epoxysesamone, 7200

Fistacacidin, 7804

Hopeyhopin, 9643

Hydroxysesamone, 10709

4-Methoxy-9,10-dihydrophenanthrene-2,3,6,7tetrol, 13911

4-Methoxy-6-(11,12-methylenedioxydihydrostyryl)-2-pyrone, 14014

11-Methoxynoryangonin, 14043

Methysticin, 14820

Naphthoquinone VI, 15261

Panial, 16605

Perforamone D, 16915

Phloretin, 17168

$\alpha$-Pyrufuran, 18280

Umtatin, 22203

Verussustilbene, 22434

\section{$\mathrm{C}_{15} \mathrm{H}_{14} \mathrm{O}_{6}$}

7-Benzoyloxy-4-hydroxy-1-methoxy-2E,4Zheptadien-1,6-dione, 2257

(+)-Catechin, 3308

(-)-Catechin, 3309

(-)-Epicatechin, 6853

Epicatechin, 6854

Isoplumericin, 11607

Leucopelargonidin, 12718

Mikanolide, 14850

Mollisacacidin, 14899

Plumericin, 17570

Ptaeroglycol, 18097

Spirolactone iridoid, 20202

$\mathrm{C}_{15} \mathrm{H}_{14} \mathrm{O}_{7}$

L-Epigallocatechin, 6921

(+)-Gallocatechin, 8098

(-)-Gallocatechin, 8099

Leucocyanidin, 12714

Plagiogyrin A, 17501
Robidandiol, 18856

Sawarachromone, 19428

$\mathrm{C}_{15} \mathrm{H}_{14} \mathrm{O}_{8}$

Dulcisflavan, 6624

Leucodelphinidin, 12717

1-(3,4,5-Trihydroxyphenyl)-3-(2,4,6-trihydroxyphenyl)-2-hydroxy-1-propanone, 21837

$\mathrm{C}_{15} \mathrm{H}_{14} \mathrm{O}_{9}$

2-Acetyl-3-( $p$-coumaroyl)-meso-tartaric acid, 360

$\mathrm{C}_{15} \mathrm{H}_{14} \mathrm{O}_{10}$

7,8-Dihydroxycoumarin-7- $O-\beta$ - $D$-glucuronide, 5797

\section{$\mathrm{C}_{15} \mathrm{H}_{15} \mathrm{NO}$}

$\mathrm{N}$-(2-Phenylethyl)benzamide, 17112

\section{$\mathrm{C}_{15} \mathrm{H}_{15} \mathrm{NO}_{2}$}

Clausine P, 3803

$N$-Methylflindersine, 14446

Onosmin A, 16120

\section{$\mathrm{C}_{15} \mathrm{H}_{15} \mathrm{NO}_{3}$}

Haplophytin A, 9225

\section{$\mathrm{C}_{15} \mathrm{H}_{15} \mathrm{NO}_{5}$}

Acronycidine, 569

Flindersiamine, 7829

\section{$\mathrm{C}_{15} \mathrm{H}_{15} \mathrm{NO}_{6}$}

Melicarpine, 13673

\section{$\mathrm{C}_{15} \mathrm{H}_{15} \mathrm{NO}_{7}$}

Furomegistine I, 8027

$\mathrm{C}_{15} \mathrm{H}_{16} \mathrm{Br}_{2} \mathrm{O}_{2}$

Lembyne B, 12617

$\mathrm{C}_{15} \mathrm{H}_{16} \mathrm{BrClO}_{2}$

Lembyne A, 12615

(12E)-Lembyne-A, 12616

$\mathrm{C}_{15} \mathrm{H}_{16} \mathrm{~N}_{2} \mathrm{O}_{2}$

1-Ethyl-4,8-dimethoxy- $\beta$-carboline, 7433

\section{$\mathrm{C}_{15} \mathrm{H}_{16} \mathrm{O}$}

4-Cumylphenol, 4361

Pyrocurzerenone, 18264

\section{$\mathrm{C}_{15} \mathrm{H}_{16} \mathrm{O}_{2}$}

Dehydrolindestrenolide, 4942

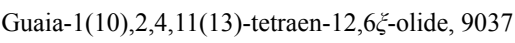

7-Hydroxycadalenal, 9868

3-Hydroxy-4'-methoxybibenzyl, 10389

Lindenenone, 12867

Mansonone C, 13513

Naphthoquinone II, 15257

Shizukanolide B, 19841

Stilbostemin B, 20379

\section{$\mathrm{C}_{15} \mathrm{H}_{16} \mathrm{O}_{3}$}

Batatasin III, 2165

Batatasin IV, 2166

Dehydroleucodin, 4941

1-(2,4-Dihydroxyphenyl)-3-(4-hydroxyphenyl) propane, 6088 


\author{
3-(1,1-Dimethyl allyl) herniarin, 6306 \\ (E)-4-(1,5-Dimethyl-3-oxo-1,4-hexadienyl) \\ benzoic acid, 6390 \\ 4-(4'-Hydroxybenzyloxy)benzyl methyl ether, \\ 9850 \\ Isolinderalactone, 11494 \\ Linderalactone, 12868 \\ Mansonone G, 13517 \\ Naphthoquinone III, 15258 \\ Neolinderalactone, 15428 \\ Osthol, 16261 \\ 2-Oxo-eremophil-1(10),7(11),8(9)-trien-12,8- \\ olide, 16330 \\ (5 $\alpha, 8 \alpha)$-2-Oxo-1(10),3,7(11)-guaiatrien-12,8- \\ olide, 16340 \\ Sinodielide C, 19949 \\ Yomogin, 22923
}

\section{$\mathrm{C}_{15} \mathrm{H}_{16} \mathrm{O}_{4}$}

Breviquinone, 2605

Celerin, 3377

8-Deoxylactucin, 5187

$1 \beta, 10 \beta$-Epoxydehydroleucodin, 7066

$5 \alpha H-3 \beta, 4 \beta$-Epoxy-14-oxo-guaia-1(10),11(13)dien- $6 \alpha, 12$-olide, 7182

Epoxysuberosin, 7203

$8 \beta H$-Eremophil-3,7(11)-diene-12,8 $\alpha(14,6 \alpha)$ diolide, 7228

6-(1-Ethoxyethyl)plumbagin, 7404

Hemigossypol, 9344

7-Hydroxy-6-methoxy-8-(3-methylbut-2-enyl) coumarin, 10417

1-Hydroxy-2-oxoeremophil-1(10),7(11),8(9)-trien-12(8)-olide, 10564

Isomeramazin, 11534

Lindenanolide E, 12862

Linderane, 12869

Meranzin, 13780

7-Methoxy-8-(2'-formyl-2'-methylpropyl) coumarin, 13930

7-Methoxy-5-prenyloxycoumarin, 14078

Murraol, 15111

7-Prenyloxy-6-methoxycoumarin, 17840

Pseudoneolinderane, 18053

Quracol A, 18454

Tristin, 22041

$\mathrm{C}_{15} \mathrm{H}_{16} \mathrm{O}_{5}$

Dihydromethysticin, 5677

3,4-Dihydro-1,2-secomicrominutinin methyl ester, 5711

Goniodiol-7-monoacetate, 8935

Goniodiol-8-monoacetate, 8936

$3^{\prime}(R)-(+)$-Hamaudol, 9204

3'(S)-(-)-Hamaudol, 9205
Isoobtusitin, 11569

Lactucin, 12446

Lophopterol, 13002

5-Methyoxymarmesin, 14819

( \pm )-Murpaniculol, 15101

(S)-Murpanidin , 15102

(+)-Murranganone, 15106

Murrangatin, 15107

erythro-Murrangatin, 15109

threo-Murrangatin, 15110

Notoserolide, 15846

Paniculin, 16615

Peroxyauraptenol, 16969

Peroxymurraol, 16975

Prelunularic acid, 17811

Quracol B, 18455

Vernolepin, 22411

Vernomenin, 22413

Visamminol, 22530

\section{$\mathrm{C}_{15} \mathrm{H}_{16} \mathrm{O}_{6}$}

8-Acetyl goniofufurone, 404

8-O-Acetylgoniotriol, 405

Allamdin, 915

Chaetoquadrin K, 3450

Dihydromikanolide, 5678

cis-Grandmarin, 8980

Norcimifugin, 15726

Picrotoxinin, 17349

\section{$\mathrm{C}_{15} \mathrm{H}_{16} \mathrm{O}_{7}$}

Allamandin, 913

Allamansicin, 914

3-(4-Hydroxyphenyl)-trans-propenoic acid-2,3-dihydroxypropyl ester, 10636

(1R)-2-Oxo-3,4-dehydroneomajucin, 16308

(1S)-2-Oxo-3,4-dehydroneomajucin, 16309

\section{$\mathrm{C}_{15} \mathrm{H}_{16} \mathrm{O}_{8}$}

2-(4-Carboxy-3-methoxystyryl)-2-methoxysuccinic acid, 3178

Plagiogyrin B, 17502

Skimmin, 20003

\section{$\mathrm{C}_{15} \mathrm{H}_{16} \mathrm{O}_{9}$}

Aesculin, 664

Cichoriin, 3635

Daphnetin-8-glucoside, 4646

Daphnin, 4650

Tufulingoside, 22101

$\mathrm{C}_{15} \mathrm{H}_{16} \mathrm{~S}_{6}$

Di(benzyltrithio)methane, 5395

\section{$\mathrm{C}_{15} \mathrm{H}_{17} \mathrm{Br}_{2} \mathrm{ClO}_{2}$}

Obtusallene I, 15893

\section{$\mathrm{C}_{15} \mathrm{H}_{17} \mathrm{NO}_{3}$}

(S)-(+)-Isoplatydesmine, 11605

Isoplatydesmine, 11606
1-[1-Oxo-3(3,4-methylenedioxyphenyl)-2Epropenyl]piperidine, 16390

1-[1-Oxo-3(3,4-methylenedioxyphenyl)-2Zpropenyl]piperidine, 16391

Ribalinine, 18830

\section{$\mathrm{C}_{15} \mathrm{H}_{17} \mathrm{NO}_{4}$}

Dubinidine, 6618

$\mathrm{N}$-[3-(6'-Methoxy-3',4'-methylenedioxyphenyl)-2(Z)-propenoyl]pyrrolidine, 14020

Orixalone D, 16202

Ribalinidine, 18829

Rutalinidine, 19083

$\mathrm{C}_{15} \mathrm{H}_{17} \mathrm{O}_{12} \mathrm{~S}^{-}$

1-Caffeoyl galactose-6-sulphate, 2901

$\mathrm{C}_{15} \mathrm{H}_{18}$

Guaiazulene, 9038

Vetivazulene, 22439

$\mathrm{C}_{15} \mathrm{H}_{18} \mathrm{~N}_{2} \mathrm{O}$

$\Delta^{7}$-Dehydrosophoramine, 4969

Huperzine A, 9686

$\mathrm{C}_{15} \mathrm{H}_{18} \mathrm{~N}_{2} \mathrm{O}_{2}$

6 $\beta$-Hydroxyhuperzine A, 10170

\section{$\mathrm{C}_{15} \mathrm{H}_{18} \mathrm{~N}_{2} \mathrm{O}_{5}$}

Brachystemidine A, 2578

Brachystemidine B, 2579

\section{$\mathrm{C}_{15} \mathrm{H}_{18} \mathrm{~N}_{2} \mathrm{O}_{6}$}

Brachystemidine C, 2580

\section{$\mathrm{C}_{15} \mathrm{H}_{18} \mathrm{O}$}

4,5-Dihydropyrocurzerenone, 5697

Farfugin A, 7717

Farfugin B, 7718

7-Hydroxycadalene, 9869

Lindenene, 12866

Lindestrene, 12874

\section{$\mathrm{C}_{15} \mathrm{H}_{18} \mathrm{O}_{2}$}

Aoifuranone, 1469

Atractylenolide I, 1965

Curzerenone, 4416

Dehydrocostuslactone, 4891

8,9-Dehydrothymol 3-O-tiglate, 4975

Epicurzerenone, 6876

1,8-Epoxy-1(6),2,4,7,10-bisabolapentaen-4-ol, 7059

Eremanthin, 7226

Euryopsin-3-one, 7656

Furanodienone, 8015

$10 \alpha$ - $H$-Furanoligularenone, 8020

13-Hydroxy-lactara-2,6,8-trien-5-oic acid $\gamma$-lactone, 10309

Isodehydrocostus lactone, 11371

Linderene, 12871

Lindestrenolide, 12875

Naphthoquinone I, 15256 
Oleodaphnal, 16069

Oleodaphnone, 16070

Podoandin, 17586

Sinodielide B, 19948

$\mathrm{C}_{15} \mathrm{H}_{18} \mathrm{O}_{3}$

Achillin, 538

Ambrosin, 1029

Arglabin, 1675

Aromaticin, 1768

Asteriscunolide A, 1924

Asteriscunolide C, 1925

Asteriscunolide D, 1926

Curcolone, 4380

Desacetoxymatricarin, 5236

(E)-4-(1,5-Dimethyl-3-oxo-1-hexenyl)benzoic acid, 6391

(R)-4-(1,5-Dimethyl-3-oxo-4-hexenyl)benzoic acid, 6392

3-Epizaluzanin C, 7044

$r e l-1 S, 2 S$-Epoxy-4R-furanogermacr-10(15)-en-6one, 7100

Gmelofuran, 8863

$5 \alpha H$-2 $\beta$-Hydroxyguaia-3(4),10(14),11(13)trien-6 $\alpha, 12$-olide, 10155

Leptene B, 12670

Mansonrin A, 13519

4-Methoxy-2-(1-propenyl)-phenyl angelate, 14079

3-Oxoeudesma-1,4,11(13)-trien-7 $\alpha H$-12-oic acid, 16331

a-Santonin, 19313

$\beta$-Santonin, 19314

Sinodielide D, 19950

Sinodielide G, 19953

Xanthatin, 22755

Xerantholide, 22784

Zaluzanin C, 22962

Zederone, 22977

Zedoarol, 22980

$\mathrm{C}_{15} \mathrm{H}_{18} \mathrm{O}_{4}$

Artemisin, 1798

Asarumin C, 1838

Chinensiolide A, 3533

Chinensiolide C, 3535

Deacetylmatricarin, 4749

Deacylcynaropicrin, 4795

8-Desoxy-3 $\alpha, 4 \alpha$-epoxyrupiculin-A, 5274

8-Desoxy-3 $\alpha, 4 \alpha$-epoxyrupiculin-B, 5275

$5 \alpha H-1 \alpha, 10 \alpha: 3 \alpha, 4 \alpha$-Diepoxyguaia-11(13)-en$6 \alpha, 12$-olide, 5494

(1 $\alpha, 2 \beta, 5 \alpha, 8 \alpha 10 \alpha)$-1,10-Epoxy-2-hydroxy3,7(11)-guaiadien-12,8-olide, 7114

$5 \alpha H-2 \beta, 4 \beta$-Epoxy-3 $\alpha$-hydroxy-guaia-1(10), 11(13)-dien-6 $\alpha$, 12-olide, 7115

8,10-Epoxy-9-hydroxy thymol 3-O-tiglate, 7146 Grossheimin, 9015

Helenalin, 9288

$2 \beta$-Hydroxyepiligustrin, 10078

$10 \alpha$-Hydroxy-1-oxoeremophila-7(11),8(9)dien-12, 8-olide, 10563

(+)-4-Methoxy-2-(3-methyloxiranyl)-phenyl angelate, 14028

(+)-4-Methoxy-2-(3-methyloxiranyl)-phenyl tiglate, 14030

Methyl 5-methoxy-7-methyl-2,2-dimethyl-2H-1chromene-8-carboxylate, 14587

Methyl 7-methoxy-5-methyl-2,2-dimethyl-2H-1chromene-6-carboxylate, 14588

Mexicanin I, 14822

Microhelenin A, 14835

Moroccolide A, 14978

Parthenin, 16674

Psilostachyin B, 18082

Saharanolide A, 19128

Saharanolide B, 19129

Shizukanolide E, 19843

Shizukanolide F, 19844

Sinodielide E, 19951

Sinodielide F, 19952

Sinodielide H, 19954

Stramonin B, 20387

Strychnistenolide, 20412

\section{$\mathrm{C}_{15} \mathrm{H}_{18} \mathrm{O}_{5}$}

AcronyculatinA, 571

Canin, 3076

Charminarone, 3482

Coriamyrtin, 4051

$6 \alpha, 10 \alpha$-Dihydroxy-1-oxoeremophila-7(11),8(9)dien-12,8-olide, 6063

Isohelenol, 11450

Meranzin hydrate, 13781

Paniculide C, 16611

Secotanapartholide A, 19635

Tanparthin- $\alpha$-peroxide, 20677

Ulopterol, 22194

\section{$\mathrm{C}_{15} \mathrm{H}_{18} \mathrm{O}_{6}$}

\section{Aduncin, 654}

Angelitriol, 1196

Apotutin, 1535

Caruilignan C, 3229

Citrusol, 3779

Coriarin, 4054

Tutin, 22145

\section{$\mathrm{C}_{15} \mathrm{H}_{18} \mathrm{O}_{7}$}

1-O-cis-Cinnamoyl- $\beta$ - $D$-glucopyranose, 3704

1-O-trans-Cinnamoyl- $\beta$ - $D$-glucopyranose, 3705
2-Cinnamoyl-glucose, 3706

1-(p-Coumaroyl)- $\alpha$ - $L$-rhamnopyranose, 4184

1,2-Dehydroneomajucin, 4952

2,3-Dehydroneomajucin, 4953

Dendrobiumane E, 5113

(2S)-Hydroxy-3,4-dehydroneomajucin, 9975

Mellitoxin, 13711

3-Oxo-6-deoxyneoanisatin, 16311

Picrotin, 17347

Picrotoxic acid, 17348

\section{$\mathrm{C}_{15} \mathrm{H}_{18} \mathrm{O}_{8}$}

Bilobalide A, 2376

cis-p-Coumaric acid 4- $O-\beta$ - $D$-glucopyranoside, 4137

trans-p-Coumaric acid 4- $O-\beta$ - $D$-glucopyranoside, 4138

$o$-Coumaric acid- $\beta$ - $D$-glucoside, 4139

Coumarinic acid- $\beta$ - $D$-glucoside, 4142

Dihydroxypicrotoxinin, 6097

(Z)-8- $\beta$ - $D$-Glucopyranosyloxycinnamic acid, 8686

2-Oxoneomajucin, 16395

\section{$\mathrm{C}_{15} \mathrm{H}_{18} \mathrm{O}_{9}$}

Caffeic acid-4- $O-\beta$ - $D$-glucopyranoside, 2889

1-Caffeoylglucose, 2906

\section{$\mathrm{C}_{15} \mathrm{H}_{18} \mathrm{O}_{12}$}

Mucic acid dimethyl ester 2-O-gallate, 15008

\section{$\mathrm{C}_{15} \mathrm{H}_{19} \mathrm{Br}_{2} \mathrm{ClO}_{2}$}

Obtusallene II, 15894

\section{$\mathrm{C}_{15} \mathrm{H}_{19} \mathrm{BrO}$}

Isolaurinterol, 11485

Laurinterol, 12572

\section{$\mathrm{C}_{15} \mathrm{H}_{19} \mathrm{ClO}$}

Pterosin H, 18143

\section{$\mathrm{C}_{15} \mathrm{H}_{19} \mathrm{ClO}_{2}$}

Pterosin K, 18146

Pterosin R, 18153

\section{$\mathrm{C}_{15} \mathrm{H}_{19} \mathrm{ClO}_{5}$}

Chlorochrymorin, 3545

Eupalinilide D, 7570

\section{$\mathrm{C}_{15} \mathrm{H}_{19} \mathrm{NO}$}

Neopellitorine A, 15445

\section{$\mathrm{C}_{15} \mathrm{H}_{19} \mathrm{NO}_{3}$}

Cocculolidine, 3864

3',5'-Dimethoxy-cinnamic acid pyrrolidine, 6216

Merresectine A, 13785

1-[1-Oxo-3(3,4-methylenedioxyphenyl)propyl] piperidine, 16393

\section{$\mathrm{C}_{15} \mathrm{H}_{20}$}

Bisabola-1,3,5,7,11-pentaene, 2404

Bisabola-1,3,5,7(14),11-pentaene, 2405

$\alpha$-Calacorene, 2935

$\gamma$-Calacorene, 2936 
$\alpha$-Corocalene, 4076

Dehydro- $\alpha$-curcumene, 4894

Tenuifolene, 20937

\section{$\mathrm{C}_{15} \mathrm{H}_{20} \mathrm{Br}_{2} \mathrm{O}$}

(E)-10,15-Dibromo-9-hydroxy-chamigra-1,3(15), 7(14)-triene, 5399

(Z)-10,15-Dibromo-9-hydroxychamigra-1,3(15), 7(14)-triene, 5400

\section{$\mathrm{C}_{15} \mathrm{H}_{20} \mathrm{BrClO}$}

Bisezakyne A, 2450

Obtusenyne, 15899

\section{$\mathrm{C}_{15} \mathrm{H}_{20} \mathrm{~N}_{2} \mathrm{O}$}

Anagyrine, 1134

Rhombifoline, 18819

Sophoramine, 20094

Thermopsine, 21318

Verruculotoxin, 22421

\section{$\mathrm{C}_{15} \mathrm{H}_{20} \mathrm{~N}_{2} \mathrm{O}_{2}$}

Baptifoline, 2145

$9 \alpha$-Hydroxysophoramine, 10714

\section{$\mathrm{C}_{15} \mathrm{H}_{20} \mathrm{~N}_{2} \mathrm{O}_{3}$}

Cyclo-(Leu-Tyr), 4509

\section{$\mathrm{C}_{15} \mathrm{H}_{20} \mathrm{~N}_{2} \mathrm{O}_{5} \mathrm{~S}$}

$\left(S_{C 2} R_{C 7}\right)$ - $\gamma$-Glutamyl-S-benzylcysteine, 8772

\section{$\mathrm{C}_{15} \mathrm{H}_{20} \mathrm{~N}_{2} \mathrm{O}_{6}$}

1-Ribityl-2,3-diketo-1,2,3,4-tetrahydro-6,7dimethyl-quinoxaline, 18833

\section{$\mathrm{C}_{15} \mathrm{H}_{20} \mathrm{~N}_{2} \mathrm{O}_{6} \mathrm{~S}$}

$\gamma$-L-Glutamyl-petiveriin A, 8777

$\gamma$-L-Glutamyl-petiveriin B, 8778

\section{$\mathrm{C}_{15} \mathrm{H}_{20} \mathrm{~N}_{6} \mathrm{O}_{7}$}

$N^{6}$-[ $\beta$-(Acetylcarbamoyloxy)ethyl $]$ adenosine, 343

\section{$\mathrm{C}_{15} \mathrm{H}_{20} \mathrm{O}$}

Atractylone, 1971

$\alpha$-Cuparenone, 4371

$\beta$-Cuparenone, 4372

Curzerene, 4415

Furanodiene, 8014

(+)-Karanone, 12164

(-)-(1S,5R,6R,7S,10S)-Myli-4(15)-en-3-one, 15144

Nardostachone, 15277

(+)-Plagiochiline X, 17499

(+)-ar-Turumerone, 22124

\section{$\mathrm{C}_{15} \mathrm{H}_{20} \mathrm{O}_{2}$}

Alantolactone, 834

Annulide, 1341

Aristolactone, 1700

Atractylenolide II, 1966

Bilobanone, 2378

Costunolide, 4128

$\alpha$-Cyclocostunolide, 4485
$\beta$-Cyclocostunolide, 4486

Cylindrene, 4545

6,7-Dehydroartemisinic acid, 4878

Dehydropinguisenol, 4960

Epideoxyarteannuin B, 6891

5-Epidilatanolide B, 6895

Eudesma-4(15),11(13)-dien-12,5 $\beta$-olide, 7493

Glechomanolide, 8525

Gloeophyllol A, 8576

(-)-Guaia-1(10),11-dien-15,2-olide, 9034

$3 \beta$-Hydroxyatractylone, 9807

5-Hydroxy-lactara-6,8-dien-13-oic acid $\gamma$-lactone, 10308

Hypacrone, 10876

Indicanone, 11017

Inunolide, 11119

Isoalantolactone, 11203

Isoannulide, 11226

Isovelleral, 11768

Ligularone, 12813

Molli lactone, 14898

Nardonoxide, 15273

Nootkatin, 15703

10,12-Peroxycalamenene, 16970

Pinguisone, 17386

Pterosin O, 18150

Pterosin Z, 18161

Pyrovellerolactone, 18273

Sinodielide A, 19947

Solafuranone, 20043

3,5,11(13)-Trieneudesma-12-oic acid, 21627

Velleral, 22364

Vellerolactone, 22367

Versicolactone A, 22422

\section{$\mathrm{C}_{15} \mathrm{H}_{20} \mathrm{O}_{3}$}

Acutifolone B, 601

Agarol, 705

Artabsin, 1779

Arteannuin B, 1785

Asperilin, 1888

Atractylenolide III, 1967

Carabrone, 3147

Carpesia lactone, 3220

Commiferin, 3943

Confertin, 3972

Curcumenolactone A, 4394

Curcumenolactone B, 4395

Damsin, 4624

Deacetyllaurenobiolide, 4746

Dehydroflourensic acid, 4918

11 $\beta$,13-Dihydro-3-epizaluzanin C, 5607

(R)-4-(1,5-Dimethyl-3-oxohexyl)benzoic acid, 6393
4 $\alpha, 5 \beta$-Epoxy-8-epiinnunolide, 7089

Eupatolide, 7586

Finitin, 7801

Glandulone C, 8503

Gloeophyllol C, 8578

Gloeophyllone, 8580

$1 \beta$-Hydroxy-alantolactone, 9770

9-Hydroxyvelleral, 10822

Inuviscolide, 11120

Isorupestonic acid, 11687

Ivalin, 11794

Ivangustin, 11795

Kanshone C, 12134

Madolin U, 13346

Madolin X, 13348

Magnolialide, 13377

4'-Methoxycinnamoyl isovalerate, 13897

Micheliolide, 14830

Nobilomethylene, 15639

Onitin, 16098

8-Oxo- $\alpha$-humula-6E,9E-dien-12-oic acid, 16346

8-Oxo- $\alpha$-humula-6E,9Z-dien-12-oic acid, 16347

8-Oxo- $\alpha$-humula-6Z,9Z-dien-12-oic acid, 16348

3-Oxoisocostic acid, 16361

Parthenolide, 16675

Pseudoivalin, 18029

Pterosin A, 18132

Pterosin D, 18135

Quadrangolide, 18293

Quadrone, 18297

Reynosin, 18673

Rupestonic acid, 19068

Santamarin, 19308

Saussureal, 19420

Sieversinin, 19882

Suberogorgin, 20440

Tamaulipin A, 20664

Taurin, 20731

Telekin, 20906

Tessaric acid, 21021

Toluccanolide A, 21429

Tomentosin $\ddagger, 21444$

Viscosumic acid, 22535

Xanthalongin, 22746

Xuelianlactone, 22809

$\mathrm{C}_{15} \mathrm{H}_{20} \mathrm{O}_{4}$

Abscisic acid, 36

Amaralin, 1012

Amberboin, 1022

Ambrosic acid, 1028

Baileyin, 2109

Chinensiolide B, 3534

Coronopilin, 4087 
Cumambrin B, 4355

Curcumenolactone C, 4396

$1 \beta, 10 \alpha, 4 \alpha, 5 \beta$-Diepoxyglechoman- $8 \alpha, 12$-olide, 5492

$6 \beta, 10 \beta$-Dihydroxyeremophila-7(11),8(9)-dien12,8-olide, 5883

$1 \beta, 8 \beta$-Dihydroxyeudesman-3,7(11)-dien- $8 \alpha, 12$ olide, 5886

$1 \beta, 8 \beta$-Dihydroxyeudesman-4(15),7(11)-dien$8 \alpha, 12$-olide, 5887

2,4'-Dimethoxy-3'-(2-hydroxy-3-methyl-3butenyl)acetophenone, 6244

Florilenalin, 7836

Granilin, 8982

Hulupinic acid, 9657

8 $\alpha$-Hydroxyarborescin, 9796

$2 \alpha$-Hydroxyeupatolide, 10114

$9 \alpha$-Hydroxy-4 $\beta, 15,11 \beta, 13$-tetrahydro-dehydrozaluzanin C, 10745

Hymenolin, 10843

(-)-Istanbulin A, 11792

Kanshone E, 12136

Lampterol, 12465

Leptol B, 12673

Ligustilone, 12827

Ludovicin A, 13072

Lupulone F, 13115

Melampomagnolide A, 13654

Melampomagnolide B, 13655

(+)-4-Methoxy-2-(3-methyloxiranyl)-phenyl-2methylbutanoate, 14029

Michelenolide, 14828

(S)-Onitisin, 16102

8-Oxo-15-hydroxygermacra-1(10),E,4Z-dien$11 \beta H$-12,6 $\alpha$-olide, 16351

Paniculide A, 16609

Pleniradin, 17557

Plenolin, 17558

Pseudosantonin, 18060

Psilostachyin C, 18083

Pterosin L, 18147

Pterosin W, 18158

Pterosin X, 18159

Ridentin, 18843

Salonitenolide, 19194

Tauremisin A, 20730

Toluccanolide C, 21431

Verlotorin, 22400

Vermeerin, 22401

$\mathrm{C}_{15} \mathrm{H}_{20} \mathrm{O}_{5}$

Artemisitene, 1800

Asarumin A, 1836

Autumnolide, 2026
$1 \beta, 10 \alpha, 4 \alpha, 5 \beta$-Diepoxy- $6 \beta$-hydroxyglechoman$8 \alpha, 12$-olide, 5495

$1 \beta, 10 \alpha, 4 \alpha, 5 \beta$-Diepoxy- $8 \alpha$-hydroxyglechoman$8 \alpha, 12$-olide, 5496

$2 \beta$-Hydroxycoronopilin, 9936

$8 \alpha$-Hydroxy-4-epi-sonchucarpolide, 10082

Hysterone A, 10916

$\left(1 R^{*}\right)$-Minwanenone, 14875

(1S*)-Minwanenone, 14876

Paniculide B, 16610

Peroxyparthenolide, 16977

Phaseic acid, 17045

Psilostachyin, 18081

Pterosin Y, 18160

Zedoalactone B, 22978

\section{$\mathrm{C}_{15} \mathrm{H}_{20} \mathrm{O}_{6}$}

Anislactone A, 1285

Anislactone B, 1286

Chavicol $\beta$-D-glucoside, 3489

Corianin, 4052

Dihydrotutin, 5732

3-Oxopseudoanisatin, 16413

$6 \beta, 8 \beta, 10 \beta$-Trihydroxy-3-oxoeremophilenolide, 21827

Vomitoxin, 22618

Zedoarolide A, 22981

\section{$\mathrm{C}_{15} \mathrm{H}_{20} \mathrm{O}_{7}$}

Anthemolide B, 1355

$1 \beta, 2 \beta$-Epoxy-3 $\beta, 4 \alpha, 8 \beta, 10 \alpha$-tetrahydroxyguaia11(13)-en-12,6 $\alpha$-olide, 7206

$1 \beta, 2 \beta$-Epoxy- $3 \beta, 4 \alpha, 9 \alpha, 10 \alpha$-tetrahydroxyguaia11(13)-en-12,6 $\alpha$-olide, 7207

Eugenylglucoside, 7525

(Z)-4-Hydroxycinnamyl alcohol 4- $O-\beta$ - $D$-glucopyranoside, 9916

Hydroxycoriatin, 9935

Neomajucin, 15432

Nivalenol, 15633

\section{$\mathrm{C}_{15} \mathrm{H}_{20} \mathrm{O}_{8}$}

Androsin, 1162

Anisatin, 1283

Demethyl coniferin, 5068

Dihydromelilotoside, 5665

$1 \beta, 2 \beta$-Epoxy- $10 \alpha$-hydroperoxy- $3 \beta, 4 \alpha, 8 \beta$ -

trihydroxyguaia-11(13)-en-12,6 $\alpha$-olide, 7107

Ethyl (6- $O$ - $p$-hydroxybenzoyl)- $\beta$ - $D$-glucopyranoside, 7446

Icariside $\mathrm{D}_{3}, 10950$

Majucin, 13409

Paeonoside, 16534

\section{$\mathrm{C}_{15} \mathrm{H}_{20} \mathrm{O}_{9}$}

2- $\beta$ - $D$-Glucopyranosyloxy-5-hydroxyphenylacetic acid methyl ester, 8697
Teuhircoside, 21225

\section{$\mathrm{C}_{15} \mathrm{H}_{20} \mathrm{O}_{10}$}

Glucosyringic acid, 8759

$\mathrm{C}_{15} \mathrm{H}_{21} \mathrm{BrO}_{3}$

Laurencin, 12565

$\mathrm{C}_{15} \mathrm{H}_{21} \mathrm{BrO}_{5}$

Obtusallenetriol, 15895

$\mathrm{C}_{15} \mathrm{H}_{21} \mathrm{~N}$

Epiguaipyridine, 6928

Patchoulipyridine, 16712

\section{$\mathrm{C}_{15} \mathrm{H}_{21} \mathrm{NO}$}

Darlinine, 4660

Nupharopumiline, 15871

\section{$\mathrm{C}_{15} \mathrm{H}_{21} \mathrm{NO}_{2}$}

Phlegmariurine A, 17154

Phlegmariurine B, 17155

\section{$\mathrm{C}_{15} \mathrm{H}_{21} \mathrm{NO}_{3}$}

Huperzine R, 9688

$8 \beta$-Hydroxy phlegmariurine $\mathrm{B}, 10644$

\section{$\mathrm{C}_{15} \mathrm{H}_{21} \mathrm{NO}_{6}$}

Isodomoic acid $\mathrm{A}, 11387$

Isodomoic acid B, 11388

Isodomoic acid C, 11389

Isodomoic acid D, 11390

\section{$\mathrm{C}_{15} \mathrm{H}_{21} \mathrm{NO}_{9} \mathrm{~S}_{2}$}

Gluconasturtiin, 8599

\section{$\mathrm{C}_{15} \mathrm{H}_{21} \mathrm{~N}_{2} \mathrm{O}_{2}$}

Methyl ester of $N, N$-dimethyl-tryptophan methocation, 14428

\section{$\mathrm{C}_{15} \mathrm{H}_{21} \mathrm{~N}_{3} \mathrm{O}_{2}$}

Eserine, 7381

\section{$\mathrm{C}_{15} \mathrm{H}_{21} \mathrm{~N}_{3} \mathrm{O}_{3}$}

Eseridine, 7380

\section{$\mathrm{C}_{15} \mathrm{H}_{21} \mathrm{~N}_{5}$}

Trypargine, 22057

\section{$\mathrm{C}_{15} \mathrm{H}_{21} \mathrm{~N}_{5} \mathrm{O}_{5}$}

9-Ribosylzeatin, 18838

\section{$\mathrm{C}_{15} \mathrm{H}_{21} \mathrm{O}_{3}$}

Nazlinin, 15317

$\mathrm{C}_{15} \mathrm{H}_{22}$

(+)-(1R,5S,6S,7S)-Allo-aromadendra-4(15), 10(14)-diene, 927

(-)-Aromadendra-1(10),3-diene, 1754

$(+)-\left(5 S^{*}, 6 S^{*}, 7 S^{*}\right)$-Aromadendra-1(10),4(15)diene, 1755

(1S,6R,7S)-Aromadendra-4,9-diene, 1756

$(+)-(1 S, 6 R, 7 S)$-Aromadendra-4,10(14)-diene, 1757

Bisabola-1,3,5,7-tetraene, 2406

Bisabola-1,3,5,7(14)-tetraene, 2407

cis-Calamenene, 2940

trans-Calamenene, 2941

Copadiene, 4028 
Cuparene, 4367

$\alpha$-Curcumene, 4390

Cypera-2,4-diene, 4573

(-)-Cypera-2,4(15)-diene, 4574

1-(1,5-Dimethyl-4-hexenyl)-4-methyl benzene, 6358

Eudesma-2,4,11-triene, 7498

(-)-Eudesma-2,4(15),11-triene, 7499

Eudesma-3,5,11-triene, 7500

(5S,6S,10S)-Gorgona-1,4(15),11-triene, 8951

(+)-Isozierene, 11791

(-)-(1 $\left.1 R^{*}, 5 S^{*}, 6 R^{*}, 7 S^{*}, 10 S^{*}\right)$-Myli-4(15)-ene, 15143

ar-Tenuifolene, 20938

1,2,9,10-Tetradehydroaristolane, 21048

Thujopsadiene, 21351

Zierene, 22999

\section{$\mathrm{C}_{15} \mathrm{H}_{22} \mathrm{BrClO}$}

Elatol, 6735

\section{$\mathrm{C}_{15} \mathrm{H}_{22} \mathrm{~N}_{2} \mathrm{O}$}

7,11-Dehydromatrine, 4948

(+)-2,3-Dehydro-10-oxo- $\alpha$-isosparteine, 4956

Lehmannine, 12605

Multiflorine, 15070

Sophocarpine, 20077

\section{$\mathrm{C}_{15} \mathrm{H}_{22} \mathbf{N}_{2} \mathbf{O}_{2}$}

(-)-9 $\alpha$-Hydroxylsophocarpine, 10336

(-)-12 $\beta$-Hydroxylsophocarpine, 10337

Mamanine, 13462

$\mathrm{N}$-Oxysophocarpine, 16474

\section{$\mathrm{C}_{15} \mathrm{H}_{22} \mathrm{~N}_{2} \mathrm{O}_{5}$}

(2S)-5-Amino-2-[(3a $\left.R^{*}, 4 S^{*}, 7 R^{*}, 7 \mathrm{a} S^{*}\right)-3 \mathrm{a}, 7 \mathrm{a}-$ dimethyl-1,3-dioxo-4,7-epoxyoctahydroisoind ol-2-yl]-pentanoic acid, 1051

\section{$\mathrm{C}_{15} \mathrm{H}_{22} \mathrm{~N}_{4} \mathrm{O}_{2} \mathrm{~S}_{2}$}

Allithiamine, 923

\section{$\mathrm{C}_{15} \mathrm{H}_{22} \mathrm{O}$}

Amorpha-4,9-dien-14-al, 1072

7,14-Anhydro-amorpha-4,9-diene, 1260

Aristol-1(10)-en-12-al, 1703

(-)-Aristol-1(10)-en-12-al, 1702

1(10)-Aristolen-2-one, 1707

Aristolone, 1723

(+)-Aromadendra-4(15),10(14)-dien-1-ol, 1758

Bisacumol, 2427

Cadina-4,10(15)-dien-3-one, 2847

Cryptomerion, 4287

$\alpha$-Cuparenol, 4368

$\beta$-Cuparenol, 4369

$\gamma$-Cuparenol, 4370

Curcumenether, 4392

$\alpha$-Cyperone, 4580

$\beta$-Cyperone, 4581
Dendrolasin, 5115

Dihydrokaranone, 5660

4,6-Dumortadien-3-one, 6634

(-)-(1 $\left.S^{*}, 5 S^{*}, 6 S^{*}, 7 S^{*}, 10 S^{*}\right)-7-E p i-b o u r b o n-3-e n-$ 5,11-oxide, 6840

$1 \beta, 10 \beta$-Epoxy-nardosin-7,11-diene, 7175

(+)-Eudesma-4(15),7(11)-dien-8-one, 7495

(+)-Eudesma-3,7(11)-dien-8-one, 7496

(+)-Eudesma-3,11-dien-8-one, 7497

Furanoeremophilane, 8016

Germacra-1(10),4,11(13)-trien-12-al, 8338

1-cis,5-cis Germacrone, 8345

1-cis,5-trans Germacrone, 8346

1-trans,5-trans Germacrone, 8347

(-)-Guaia-1(10),11-dien-15-al, 9029

$1 \alpha, 7 \alpha, 10 \alpha H$-Guaia-4,11-dien-3-one, 9035

(+)-Guaia-1(10),11-dien-9-one, 9036

(-)-(1 $\left.S^{*}, 5 S^{*}, 7 S^{*}\right)$-Guai-3,9-dien-5,11-oxide, 9039

(+)-(1 $\left.S^{*}, 5 S^{*}, 7 S^{*}\right)$-Guai-3,10(14)-dien-5,11-oxide, 9040

$\beta$-Herbertenol, 9426

(-)- $\gamma$-Herbertenol, 9427

(+)-8-Hydroxycalamenone, 9877

(+)-Isobicyclogermacrenal, 11253

(-)-Isobicyclogermacrenal, 11254

$\alpha$-Isocuparenol, 11357

Isodaucenal, 11367

Neopetasone, 15447

Nootkatone, 15705

$1,1 \alpha, 4,5,6,7,7 \alpha, 7 \beta$-Octahydro-1,1,7,7 $\alpha$-tetramethyl-2H-cyclopropa $(\alpha)$-naphthalen-2-one, 15968

$1,1 \alpha, 2,4,6,7,7 \alpha, 7 \beta$-Octahydro-1,1,7,7 $\alpha$-tetramethyl-5H-cyclopropa $(\alpha)$-naphthalen-5-one, 15969

ent-9-Oxo- $\alpha$-chamigrene, 16300

3-Oxoishwarane, 16360

Patchoulenone, 16709

(+)-Plagiochiline W, 17498

Rosacorenone, 18913

Rotundone, 18958

Saccogynol, 19106

$\alpha$-Santalal, 19300

$\beta$-Santalal, 19301

(-)-Selina-3,11-dien-14-al, 19677

(+)-Selina-4,11-dien-14-al, 19678

(-)-Selina-3,11-dien-9-one, 19683

Selina-3,7(11)-dien-8-one, 19684

$\alpha$-Sinensal , 19927

$\beta$-Sinensal, 19928

Solavetivone, 20070

Squamulosone, 20249
(-)-(6S,7S,10R)-Taylocyclane, 20886

$(-)-(7 S)-(E)-$ Taylopyran, 20888

$(-)-(6 R, 7 S)-\alpha$-Taylorione, 20889

$\left(1 R^{*}, 4 S^{*}, 5 S^{*}, 6 R^{*}, 7 S^{*}, 9 R^{*}\right)$-Taynudol, 20890

Thujopsenone, 21354

Turmerone, 22123

Valerenone, 22316

$\alpha$-Vetivone, 22440

$\beta$-Vetivone, 22441

Zerumbone, 22984

\section{$\mathrm{C}_{15} \mathrm{H}_{22} \mathrm{O}_{2}$}

Aciphyllic acid, 549

Acoronene, 566

Artemisic acid, 1797

Bakkenolide A, 2119

Bilobanol, 2377

(4S)-2,6,10-Bisabolatrien-4-ol-1-one, 2410

Confertifolin, 3971

Costic acid, 4126

Curcumenol, 4393

Curcumenone, 4397

Debilone, 4815

Dehydrocurdione, 4895

11 $\beta, 13$-Dihydrocostunolide, 5570

Dihydro-deoxyarteannuin B, 5587

Dihydro-epi-deoxyarteannuin B, 5606

Dihydroisoalantolactone, 5648

4-Epicurcumenol, 6875

5-Epidilatanolide A, 6894

$(3 R, 4 R, 6 S)-3,4$-Epoxybisabola-7(14),10-dien-2one, 7058

6,7-Epoxy-4-dumorten-3-one, 7085

1,10-Epoxylepidozenal, 7163

Eremophilenolide, 7230

Fukinanolide, 7986

Furanopinguisanol, 8022

Gajutsulactone A, 8048

Gajutsulactone B, 8049

Germacra-1(10),4,11(13)-trien-12-oic acid, 8339

(+)-Germacrone 4,5-epoxide, 8348

(-)-Guaia-1(10),11-dien-15-carboxylic acid, 9030

(-)-Herbertene-1,12-diol, 9425

(5R)-2,6,9-Humulatrien-5-ol-8-one, 9668

$2 \alpha$-Hydroxyaromadendr-1(10)-en-9-one, 9800

$2 \beta$-Hydroxyaromadendr-1(10)-en-9-one, 9801

13-Hydroxyaromadendr-1(10)-en-9-one, 9802

14-Hydroxyaromadendr-1(10)-en-9-one, 9803

13-Hydroxygermacrone, 10147

Hydroxyisodaucenal, 10245

5-Hydroxyzerumbone, 10834

Isocostic acid, 11349

Isocurcumenol, 11359 
Isodaucenoic acid, 11369

Isopolygodial, 11609

Isoprocurcumenol, 11613

Jatamansic acid, 11833

Kanshone A, 12132

Lactarol, 12433

Lejeuneapinguisenone, 12610

Ligudicin A, 12803

Ligudicin D, 12805

Litseachromolaevane A, 12929

Litseachromolaevane B, 12930

Madolin A, 13339

Madolin R, 13344

Madolin W, 13347

Manshurolide, 13512

Nardoguaianone J, 15270

Nardoguaianone K, 15271

Neocurcumenol, 15378

1,2,2a,3,6,7,8,8a-Octahydro-7-hydroxy-2a,7,8trimethylacenaphthylen-4(4H)-one, 15967

Oxyphyllol B, 16467

Petasalbin, 17010

Polygodial, 17640

Procurcumenol, 17875

$\alpha$-Rotunol, 18959

$\beta$-Rotunol, 18960

$\beta$-Santalic acid, 19304

Santalic acid, 19305

Saussurea lactone, 19421

Schisandronol, 19476

Sclerosporin, 19534

Shizukafuranol, 19840

Tetradymol, 21051

Valerenic acid, 22314

Vellerol, 22366

Zerumbone epoxide, 22985

Zerumboneoxide, 22986

\section{$\mathrm{C}_{15} \mathrm{H}_{22} \mathrm{O}_{3}$}

Aerugidiol, 658

Arbusculin A, 1617

Artemislactone, 1801

Blennin C, 2497

Canusesnol A, 3104

Canusesnol J, 3113

11 $\beta, 13$-Dihydroparthenolide, 5689

11 $\beta, 13$-Dihydroreynosin, 5701

$11 \alpha, 13$-Dihydroxanthalongin, 5741

$2 \beta, 13$-Dihydroxyaromadendr-1(10)-en-9-one, 5757

13,14-Dihydroxyaromadendr-1(10)-en-9-one, 5758

Epoxyarteannuinic acid, 7055

(2R,3R,5R)-2,3-Epoxy-6,9-humuladien-5-ol-8- one, 7105

(2R,3S,5R)-2,3-Epoxy-6,9-humuladien-5-ol-8one, 7106

$7 \alpha$-11 $\alpha$-Epoxy-5 $\beta$-hydroxy-9-guaiaen-8-one, 7116

Furandiol, 8013

Furanoeremophilane-6 $\beta, 10 \beta$-diol, 8017

Furanofukinol, 8018

$1 \beta$-Hydroxycostic acid, 9939

$5 \alpha$-Hydroxycostic acid, 9940

$5 \beta$-Hydroxycostic acid, 9941

$4 \beta$-Hydroxy-4,10 $\alpha$-dimethyl- $7 \alpha H, 8 \alpha H$ eudesman-11-ene-8,12-olide, 10050

$10 \alpha$-Hydroxy-1,2-epoxyaromadendran-9-one, 10083

6-Hydroxyeremophilenolide, 10094

$1 \beta$-Hydroxy- $4 \alpha, 11 \alpha$-eudesma-5-en-12,8 $\beta$-olide, 10111

6-Hydroxy-2-methyl-5-(5'-hydroxy-1'(R),5'dimethylhex-3'-enyl)-phenol, 10497

Illudin I, 10991

Illudin $\mathrm{I}_{2}, 10992$

Illudin J, 10993

Illudin $\mathrm{J}_{2}, 10994$

Ipomeamarone, 11130

Isonardosinone, 11553

Ketosantalic acid, 12207

Madolin B, 13340

Nardofuran, 15269

Nardosinone, 15274

8-Oxo-6,7,9,10-tetrahydrohumulen-1,12-olide, 16427

Piperdial, 17446

Polygonic acid, 17647

Procurcumadiol, 17874

Secocarotanal, 19607

Septuplinolide, 19754

Valdiviolide, 22310

Valerenolic acid, 22315

Versicolactone B, 22423

Warburganal, 22638

4H-Xanthalongin, 22747

$\mathrm{C}_{15} \mathrm{H}_{22} \mathrm{O}_{4}$

Arteamisinine III, 1783

Budlein B, 2699

Canusesnol F, 3109

Damsinic acid, 4625

Dendrobiumane C, 5111

$1 \beta, 4 \beta, 4 \alpha, 5 \beta$-Diepoxy- $10 \alpha, 11 \alpha H$-xantha- $12,8 \beta$ olide, 5498

1 $\beta$-6 $\alpha$-Dihydroxycostic acid, 5794

1,6 $\alpha$-Dihydroxy-4 $\alpha H$-1,10-secoeudesma-5(10), 11(13)-dien-12,8 $\beta$-olide, 6114
$1 \beta, 4 \beta$-Epoxy- $5 \beta$-hydroxy- $10 \alpha H$-xantha-

11(13)-en-12,8 $\beta$-olide, 7147

Fascicularone B, 7738

[4]-Gingerol, 8394

Gloeophyllol D, 8579

Heliannuol L, 9293

Helibisabonol B, 9299

$1 \beta$-Hydroxyarbusculin A, 9797

3-(3-Hydroxybutyl)-1,1-dimethylisochroman-

6,8-diol, 9864

3-O-Isobutyryl-8-methoxy-9-hydroxythymol, 11296

Isoivaxillin, 11468

Isolactarorufin, 11475

Ivaxillin, 11796

Kanshone B, 12133

Kanshone D, 12135

Lactarorufin A, 12435

Lejeuneapinguisanolide, 12609

Magnograndiolide, 13375

8-Methoxy-9-O-isobutyrylthymol, 13973

Mukaadial, 15023

Odoratin I*, 15991

Rugosal A, 19047

Rugosal D, 19048

2,2,4,4-Tetramethyl-6-(2-methyl-1-oxobutyl)-

1,3,5-cyclohexanetrione, 21198

2,2,4,4-Tetramethyl-6-(3-methyl-1-oxobutyl)-

1,3,5-cyclohexanetrione, 21199

Versicolactone C, 22424

Zedoarofuran, 22979

\section{$\mathrm{C}_{15} \mathrm{H}_{22} \mathrm{O}_{5}$}

(+)-Arteannuin, 1784

Arteannuin G, 1786

11-O-Debenzoyltashironin, 4811

3,4-Dehydrofloridanolide, 4917

3-Deoxypseudoanisatin, 5203

6-Deoxypseudoanisatin, 5204

3,6-Dideoxy-10-hydroxypseudoanisatin, 5474

$\left(3 S^{*}, 6 R^{*}\right)-4,7-E p o x y-6$-deoxypseudoanisatin, 7068

2 $\beta$-Hydroxy-3,6-dedioxypseudoanisatin, 9969

Hysterone B, 10917

Hysterone C, 10918

Lactarolide A, 12434

Merrilianone, 13789

Porellapinguisanolide, 17721

Pseudomajucin, 18051

Qinghaosu IV, 18288

Rugosic acid A, 19050

Rugosic acid B, 19051

Rugosic acid D, 19053

$1 \alpha, 2 \beta, 4 \beta$-Trihydroxypseudoguaian-6 $\beta, 12$-olide, 
21842

Zedoarolide B, 22982

\section{$\mathrm{C}_{15} \mathrm{H}_{22} \mathrm{O}_{6}$}

1,2-Dehydrocycloparvifloralone, 4896

$1 \alpha$-Hydroxy-3-deoxypseudoanisatin, 9989

$1 \alpha$-Hydroxy-6-deoxypseudoanisatin, 9990

(2S)-Hydroxy-6-deoxypseudoanisatin, 9991

Parviflorolide, 16679

Pseudoanisatin, 18018

\section{$\mathrm{C}_{15} \mathrm{H}_{22} \mathrm{O}_{6} \mathrm{~S}$}

4-Gingesulfonic acid , 8399

13-Sulfo-dihydroreynosin, 20469

13-Sulfo-dihydrosantamarine, 20470

\section{$\mathrm{C}_{15} \mathrm{H}_{22} \mathrm{O}_{7}$}

$10 \beta$-Hydroxycyclopseudoanisatin, 9955

$8 \alpha$-Hydroxy-10-deoxycyclomerrillianolide, 9987

$10 \beta$-Hydroxypseudoanisatin, 10667

$1 \alpha$-Hydroxypseudoanisatin, 10668

Juniperoside I, 11968

Juniperoside II, 11969

\section{$\mathrm{C}_{15} \mathrm{H}_{22} \mathrm{O}_{8}$}

Bartsioside, 2156

3,4-Dihydroxyphenylpropanol $\beta$ - $D$-glucopyranoside, 6093

Homovanillyl alcohol-4-O-glucoside, 9625

erythro-1'-(4-Hydroxyphenyl)propane-1',2'-diol-

4-O- $\beta$ - $D$-glucopyranoside, 10631

(1'R,2'R)-1'-(4-Hydroxyphenyl)propane-1',2'diol-2'-O- $\beta$ - $D$-glucopyranoside, 10632

(1'R,2'S)-1'-(4-Hydroxyphenyl)propane-1',2'diol-2'-O- $\beta$ - $D$-glucopyranoside, 10633

$\left(1^{\prime} S, 2^{\prime} R\right)-1^{\prime}-(4-H y d r o x y p h e n y l) p r o p a n e-1 ', 2 '-$ diol-2'-O- $\beta$ - $D$-glucopyranoside, 10634

(1'S,2'S)-1'-(4-Hydroxyphenyl)propane-1',2'diol-2'-O- $\beta$ - $D$-glucopyranoside, 10635

\section{$\mathrm{C}_{15} \mathrm{H}_{22} \mathrm{O}_{9}$}

Aucubin, 2004

6-Deoxycatalpol, 5159

8-Epi-grandifloric acid, 6927

Galiridoside, 8094

4-Hydroxy-3,5-dimethoxybenzyl alcohol 4-O- $\beta$ $D$-glucopyranoside, 10014

Pedicularis lactone-1- $O-\beta$ - $D$-glucoside, 16764

\section{$\mathrm{C}_{15} \mathrm{H}_{22} \mathrm{O}_{10}$}

Catalpol, 3306

Clandonoside, 3786

Danmelittoside, 4626

\section{$\mathrm{C}_{15} \mathrm{H}_{23} \mathrm{Br}_{2} \mathrm{ClO}$}

Isoobtusol, 11570

Obtusol, 15910

\section{$\mathrm{C}_{15} \mathrm{H}_{23} \mathrm{BrO}$}

$(6 R, 9 R, 10 S)$-10-Bromo-9-hydroxy-chamigra2,7(14)-diene, 2624
$\mathrm{C}_{15} \mathrm{H}_{23} \mathrm{ClO}_{10}$

Linarioside, 12859

$\mathrm{C}_{15} \mathrm{H}_{23} \mathrm{~N}$

2,6-Decamethylene pyridine, 4831

$\mathrm{C}_{15} \mathrm{H}_{23} \mathrm{NO}$

Cananodine, 3060

Deoxynupharidine, 5199

\section{$\mathrm{C}_{15} \mathrm{H}_{23} \mathrm{NO}_{2}$}

1-(1,6-Dioxo-2E,4E-decadienyl)piperidine, 6465

Nupharidine, 15868

\section{$\mathrm{C}_{15} \mathrm{H}_{23} \mathrm{NO}_{5}$}

$N$-Methyl tyramine- $O$ - $\alpha$ - $L$-rhamnopyranoside, 14797

\section{$\mathrm{C}_{15} \mathrm{H}_{24}$}

$\alpha$-Acoradiene, 555

$\beta$-Acoradiene, 556

$\gamma$-Acoradiene, 557

$\delta$-Acoradiene, 558

Alloaromadendrene, 928

9-Aristolene, 1704

Aromadendrene, 1763

$\alpha$-Bergamotene, 2306

Bicycloelemene, 2351

Bicyclogermacrene, 2352

Bicyclosesquiphellandrene, 2357

Bisabola-2,6,11-triene, 2408

$\alpha$-Bisabolene, 2411

$\beta$-Bisabolene, 2412

$\beta_{2}$-Bisabolene, 2413

$\beta$-Bourbonene, 2570

Brasila-1(6),5(10)-diene, 2587

Brasila-5,10-diene, 2588

Brasila-5(10),6-diene, 2589

Cadina-9,11(12)-diene, 2846

$\alpha$-Cadinene, 2849

$\beta$-Cadinene, 2850

$\gamma$-Cadinene, 2851

$\delta$-Cadinene, 2852

$\varepsilon$-Cadinene, 2853

Calarene, 2943

$\beta$-Caryophyllene, 3242

$\gamma$-Caryophyllene, 3241

$\alpha$-Cedrene, 3350

$\beta$-Cedrene, 3351

$\alpha$-Chamigrene, 3465

$\beta$-Chamigrene, 3466

Clovene, 3848

$\alpha$-Copaene, 4029

$\alpha$-Cubebene, 4305

$\alpha$-Cuprenene, 4374

$\gamma$-Cuprenene, 4375

$\beta$-Curcumene, 4391

Cyperene, 4577
Daucene, 4675

(-)-3,4-Di-epi-3,7-trifara-9,14-diene, 5487

1,8-Dimethyl-4-(1-methylenyl)-spiro(4,5)

dec-7-ene, 6373

(-)-cis- $\beta$-Elemene, 6740

$\beta$-Elemene, 6741

$\gamma$-Elemene, 6742

$\delta$-Elemene, 6743

cis- $\beta$-Elemene diastereomer, 6744

Eremophilene, 7229

(+)-Eudesma-5,7(11)-diene, 7491

$(E, E)-\alpha$-Farnesene, 7728

(Z,Z)- $\alpha$-Farnesene, 7729

$\beta$-Farnesene, 7730

$\alpha$-Funebrene, 8002

(+)-Germacrene $A_{1}, 8342$

Germacrene B, 8343

Germacrene D, 8344

Ginsinsene, 8453

$(+)-(5 R, 6 S, 10 S)$ - $\alpha$-Gorgonene, 8952

$\beta$-Gorgonene, 8953

$\alpha$-Guaiene, 9041

$\delta$-Guaiene, 9042

$\alpha$-Guriunene, 9088

(+)-Helminthogermacrene, 9332

$\alpha$-Humulene, 9669

$\beta$-Humulene, 9670

Isocomene, 11339

Isodaucene, 11368

(-)-(5S,6S,10S)-Iso- $\beta$-elemene, 11407

(+)-(S)-Isogermacrene A, 11437

Iso- $\alpha$-humulene, 11458

(+)- $\beta$-Isolongibornene, 11509

Isolongifolene, 11510

1-Isopropyl-4-methylene-7-methyl-1,2,3,4,4a,5, 6,8a-octahydronaphthalene, 11628

(-)-Isorotundene, 11686

Longifolene, 12955

$\alpha$-Longipinene, 12969

$\beta$-Maaliene, 13284

2-Methyl-5-(1,5-dimethyl-3-hexenyl)-1,3-cyclohexadiene, 14324

Modhephene, 14893

$\alpha$-Muurolene, 15140

$\alpha$-Neoclovene, 15368

$\beta$-Neoclovene, 15369

Panaginsene, 16583

$\beta$-Panasinsene, 16590

$\alpha$-Panasinsene, 16591

$\beta$-Panasinsene, 16592

Panaxene, 16596

$\alpha$-Patchoulene, 16706

$\beta$-Patchoulene, 16707 
$\gamma$-Patchoulene, 16708

Patrinene, 16719

7,10-Pentadecadiynoic acid, 16829

(-)-Perfora-1,7-diene, 16911

Rotundene, 18941

(+)-Sandvicene, 19250

$\alpha$-Santalene, 19302

$\beta$-Santalene, 19303

(-)-Selina-5,7(11)-diene, 19679

3,7(11)-Selinadiene, 19680

Selina-4(15),7(11)-diene, 19681

$\alpha$-Selinene, 19686

$\beta$-Selinene, 19687

$\gamma$-Selinene, 19688

Sesquicarene, 19786

$\beta$-Sesquiphellandrene, 19787

Sesquisabinene, 19788

Seychellene, 19793

Silphinene, 19894

1,1,5,5-Tetramethyl-4-methano-2,3,4,6,7,10-

hexahydronaphthalene, 21197

Thujopsene, 21353

Tricyclovetivene, 21601

(-)-Trifara-9,14-diene, 21629

1,3,5-Triisopropylphene, 21870

cis-4,11,11-Trimethyl-8-methylenebicyclo[7.2.0]-

undeca-4-ene, 21969

$\alpha$-Ylangene, 22920

Zingiberene, 23004

Zonarene, 23022

\section{$\mathrm{C}_{15} \mathrm{H}_{24} \mathrm{BrClO}$}

Glanduliforol, 8502

\section{$\mathrm{C}_{15} \mathrm{H}_{24} \mathrm{Br}_{2} \mathrm{O}$}

Palisadin B, 16542

\section{$\mathrm{C}_{15} \mathrm{H}_{24} \mathrm{~N}_{2}$}

Aloperine, 983

\section{$\mathrm{C}_{15} \mathrm{H}_{24} \mathrm{~N}_{2} \mathrm{O}$}

(+)-Allomatrine, 938

Aphylline, 1474

Isomatrine, 11531

Lupanine, 13089

Matrine, 13606

Sophoridine, 20100

\section{$\mathrm{C}_{15} \mathrm{H}_{24} \mathrm{~N}_{2} \mathrm{O}_{2}$}

(-)-14 $\beta$-Hydroxylsophoridine, 10338

Octalupine, 15972

Oxymatrine, 16451

Sophoranol, 20096

\section{$\mathrm{C}_{15} \mathrm{H}_{24} \mathrm{~N}_{2} \mathrm{O}_{3}$}

$5 \alpha, 9 \alpha$-Dihydroxymatrine, 5955

Sophoranol $N$-oxide, 20097

\section{$\mathrm{C}_{15} \mathrm{H}_{24} \mathrm{~N}_{2} \mathrm{O}_{17} \mathrm{P}_{2}$}

Uridine diphosphate glucose, 22253
$\mathrm{C}_{15} \mathrm{H}_{24} \mathrm{O}$

Acolamone, 550

Acorenone, 564

$1 S^{*}, 4 S^{*}, 5 S^{*}$-Acora-8(15),9-dien-7R*-ol, 559

Acoragermacrone, 560

$\alpha$-Agarofuran, 703

$\beta$-Agarofuran, 704

Alismol, 888

Amorpha-4,9-dien-2-ol, 1073

7,10-Anhydro-11,12-dihydrochiloscypholone, 1266

(-)-Aristol-1(10)-en-12-ol, 1705

9-Aristolen-1- $\alpha$-ol, 1706

9(10)Z, $\alpha$-trans-Bergamotenol, 2307

$\alpha$-Betulol, 2336

$\beta$-Betulol, 2337

$\alpha$-Biotol, 2396

$\beta$-Biotol, 2397

2,(7Z,10Z)-Bisabolatrien-13-ol, 2409

2,6-Bis(1,1-dimethylethyl)-4-methyl phenol, 2446

(4S)-Cadin-10(15)-en-3-one, 2855

Calacone, 2934

Calarenol, 2944

Campherenone, 3047

(6R,7R)-Caryophyllene oxide, 3243

Caryophyllene oxide, 3244

3(15)-Caryophyllen-5-one, 3245

$\alpha$-Cedren- $3 \beta$-ol, 3352

$\alpha$-Cedren-12-ol, 3353

Costol, 4127

Cyperol, 4578

Dehydrojinkoheremol, 4934

$\beta$-Dictyopterol, 5457

11,12-Dihydrochiloscyphone, 5560

2,6-Ditertbutyl-4-methyl phenol, 6522

Epishyobunone, 7019

6,7-Epoxybisabola-2,11-diene, 7057

4,5-Epoxy- $\beta$-caryophyllene, 7061

Epoxycyperene, 7064

(-)-1,10-Epoxy-guaia-11-ene, 7101

Epoxyguaine, 7102

3(15)-Epoxygymnomitrane, 7103

Eremofukinone, 7227

(-)-Eudesma-3,11-dien-5-ol, 7492

Fukinone, 7990

Germacra-1(10),4,7(11)-trien- $9 \alpha$-ol, 8340

Germacra-1(10),4,11(13)-trien-12-ol, 8341

(-)-Guaia-1,11-dien-10 $\alpha$-ol, 9031

(-)-Guaia-1(10),11-dien-15-ol, 9032

(+)-Guaia-10(15),11-dien-1 $\alpha$-ol, 9033

Humuladienone, 9665

Humulene epoxide I, 9671
Humulene epoxide II, 9672

Humulene epoxide III, 9673

Humulenol I, 9675

Humulenol II, 9676

4-Hydroxy- $\beta$-bulnesene, 9862

(4S)-3 $\beta$-Hydroxycadina-10(15),12(13)-diene, 9870

$1 \beta$-Hydroxy-4(15),5-eudesmadiene, 10108

1 $\beta$-Hydroxy-4(15),7-eudesmadiene, 10109

(+)-8 $\alpha$-Hydroxy-eudesma-3,11-diene, 10110

11-Hydroxyguaia-4,6-diene, 10154

14-Hydroxymodhephene, 10529

11-Hydroxy- $\alpha$-santal-9-ene, 10689

$10 \xi$-Hydroxy- $\alpha$-santal-11-ene, 10690

1-Hydroxy-3-sterpurene, 10732

Isoacolamone, 11193

$\beta$-Isobiotol, 11255

Isocyperol, 11364

Isodaucenol, 11370

Isolentideusether, 11487

6-Isopropenyl-4,4a-dimethyl-1,2,3,4,4a,5,6,7-

octahydro-naphthalen-1-ol, 11614

Isoshyobunone, 11713

Lactifloreol, 12438

Lanceoloxide, 12467

Lentideusether, 12623

1-Methyl-4-(5-methyl-1-methylenehex-4-enyl)-

7-oxabicyclo[4.1.0]heptane, 14603

2-Methyl-2-vinyl-3-isopropenyl-5-isopropylidene

cyclohexanol, 14810

Neoacolamone, 15334

Nonylphenol, 15701

Nootkatol, 15704

Oxyphyllol A, 16466

(6Z,9Z,12Z)Pentadecatrien-2-one, 16832

Preisocalamendiol, 17809

Rosacorenol, 18912

Rupestrenol, 19069

$\alpha$-Santalol, 19306

$\beta$-Santalol, 19307

(+)-Selina-3,11-dien-9-ol, 19682

Shizuka-acoradienol, 19839

Shyobunone, 19852

(-)-ent-Spathulenol, 20136

Spathulenol, 20137

6,6,8,9-Tetramethyltricyclo[3.3.3.0]undec-7-en-

2-ol, 21204

Waitziacuminone, 22626

\section{$\mathrm{C}_{15} \mathrm{H}_{24} \mathrm{O}_{2}$}

Acorone, 565

Aphanamol II, 1473

Baimuxinal, 2111

Capsidiol, 3145 
Curcumol, 4399

Curdione, 4400

7,12-Cyclo-6,11-epoxy-4-dumortanol, 4490

(+)-Cyperadione, 4575

Cyperolone, 4579

Dehydrobaimuxinol, 4881

4S-Dihydrocurcumenone, 5577

(-)-Epihernandulcin, 6930

$6 \beta, 14$-Epoxyeudesm-4(15)-en-1 $\beta$-ol, 7097

2-(5-Ethenyl-5-methyl-2-tetrahydrofuranyl)-6methyl-5-hepten-3-one, 7392

(+)-Hernandulcin, 9450

1,2,3,3 $\alpha, 8,8 \alpha$-Hexahydro-2,2,8-trimethyl-5,6azulenedimethanol, 9501

2,9-Humuladien-6-ol-8-one, 9664

$5 \alpha$-Hydroperoxy-eudesma-4(15),11-diene, 9726

$1 \alpha$-Hydroperoxy-guaia-10(15),11-diene, 9727

10 $\alpha$-Hydroperoxy-guaia-1,11-diene, 9728

15 $\alpha$-Hydroperoxy-guaia-1(10),11-diene, 9729

11-Hydroperoxy- $\alpha$-santal-9-ene, 9733

$10 \xi$-Hydroperoxy- $\alpha$-santal-11-ene, 9734

$10 \alpha$-Hydroxycadin-4-en-15-al, 9871

(4S)-12-Hydroxycadin-10(15)-en-3-one, 9875

(4S)-13-Hydroxycadin-10(15)-en-3-one, 9876

$8 \beta$-Hydroxy gymnomitrian-9-one, 10156

11-Hydroxy-12-hydroisodaucenal, 10173

1 $\alpha$-Hydroxyisodauc-4-en-15-al, 10246

10-Hydroxylentideusether, 10318

$7 \alpha$-Hydroxyneoacolamone, 10537

Isocampheren-11-ene-10-one, 11304

Isodrimeninol, 11403

Ligudicin C, 12804

Litseagermacrane, 12931

Litseahumulane A, 12932

Litseahumulane B, 12933

Lubimin, 13016

Muurolan-3-en-9 $\beta$-ol-2-one, 15137

$L$-Myrtenyl isovalerate, 15223

Neocurdione, 15379

Orientalol C, 16191

Orientalol F, 16194

$4 \alpha, 5 \alpha$-Oxidoeudesm-11-en-3 $\alpha$-ol, 16287

9-Oxofarnesol, 16333

9-Oxonerolidol, 16396

Petasitolone, 17017

Sinenofuranal, 19924

Sirenin, 19976

(5S*,7 $\left.S^{*}\right)$-Taylofuran, 20887

Thujopsan-7 $\beta$-ol, 21352

3,7,11-Trimethyldodeca-1,7,10-trien-3-ol-9-one, 21954

Vellerdiol, 22365

$\mathrm{C}_{15} \mathrm{H}_{24} \mathrm{O}_{3}$
Baimuxinfuranic acid, 2112

Baimuxinic acid, 2113

Bisacurone, 2428

Canusesnol E, 3108

Colartin, 3908

Curcarabranol A, 4378

Curcarabranol B, 4379

5-Demethoxyfumagillol, 5047

Epilippidulcine A, 6951

$\alpha$-Epoxy-dihydroartemisinic acid, 7069

Hamanasic acid A, 9203

13-Hydroxycapsidiol, 9889

$3 \alpha$-Hydroxy- $4 \alpha, 5 \alpha$-epoxy-7-oxo- $(8[7 \rightarrow 6]-$

abeoamorphane, 10091

Ilicic acid, 10987

Indicumenone, 11021

Isozedoarondiol, 11789

Lippidulcine A, 12902

Nardosinonediol, 15275

Piperalol, 17433

Sugetriol, 20456

Torilolone, 21457

Zedoarondiol, 22983

\section{$\mathrm{C}_{15} \mathrm{H}_{24} \mathrm{O}_{4}$}

Aeginetic acid, 656

$1 \alpha$-Aldehyde- $2 \beta$-[3-butanone]-3 $\alpha$-methyl-6 $\beta$ -

[2-propanoic acid]-cyclohexane, 872

Canusesnol B, 3105

Canusesnol C, 3106

Dendrobiumane B, 5110

$3 \alpha, 4 \beta$-Dihydroxy- $5 \beta H, 11 \alpha H$-eudesman-6,12olide, 5890

$1 \alpha, 4 \alpha$-Dihydroxyeudesman- $5 \alpha, 6 \beta, 7 \alpha, 11 \beta H$ -

12,6-olide, 5891

$4 \alpha, 5 \alpha$-Epoxy- $6 \alpha$-hydroxy amorphan-12-oic acid, 7108

Helibisabonol A, 9298

$1 \beta$-Hydroxyilicic acid, 10223

$3 \alpha$-Hydroxyilicic acid, 10224

$5 \beta$-Hydroxyilicic acid, 10225

1-(4-Hydroxy-3-methoxyphenyl)-3,5-octane-diol, 10448

1-Oxo-2 $\beta$-[3-butanone]-3 $\alpha$-methyl- $6 \beta$-[2-

propanol formyl ester]-cyclohexane, 16298

3-Oxo-11,12,13-trihydroxy-eudesm-4-ene, 16432

$\mathrm{C}_{15} \mathrm{H}_{24} \mathrm{O}_{4} \mathrm{~S}$

Sulfoorientalol C, 20474

$\mathrm{C}_{15} \mathrm{H}_{24} \mathrm{O}_{5}$

Dendrobiumane D, 5112

sec-Hydroxyaeginetic acid, 9767

Hysterone D, 10919

Minwanensin, 14877

Rugosic acid C, 19052
$\mathrm{C}_{15} \mathrm{H}_{24} \mathrm{O}_{6}$

Cycloparvifloralone, 4523

8-Deoxymerrilliortholactone, 5193

1,6-Dihydroxy-3-deoxyminwanensin, 5805

$\mathrm{C}_{15} \mathrm{H}_{24} \mathrm{O}_{7}$

(2E)-2,6-Dimethyl-2,5-heptadienoic acid $\beta$ - $D$ glucopyranosyl ester, 6355

3 $\alpha$-Hydroxycycloparvifloralone, 9954

(11)7,14-Ortholactone- $3 \alpha$-hydroxyfloridanolide, 16219

\section{$\mathrm{C}_{15} \mathrm{H}_{24} \mathrm{O}_{8}$}

Gluroside, 8768

Ningpogoside A, 15616

Ningpogoside B, 15617

Phyllaemblic acid C, 17206

\section{$\mathrm{C}_{15} \mathrm{H}_{24} \mathrm{O}_{9}$}

Ajugol, 815

Phyllaemblic acid B, 17205

\section{$\mathrm{C}_{15} \mathrm{H}_{24} \mathrm{O}_{10}$}

Dihydrocatalpol, 5557

Harpagide, 9237

Phelypaeside, 17080

\section{$\mathrm{C}_{15} \mathrm{H}_{25} \mathrm{Br}$}

8-Bromo-1-en-Chamigrene, 2623

\section{$\mathrm{C}_{15} \mathrm{H}_{25} \mathrm{NO}$}

Neopellitorine B, 15446

\section{$\mathrm{C}_{15} \mathrm{H}_{25} \mathrm{NO}_{4}$}

Amabiline, 1011

Supinine, 20488

\section{$\mathrm{C}_{15} \mathrm{H}_{25} \mathrm{NO}_{5}$}

Echinatine, 6686

Indicine, 11019

Intermedine, 11107

Lycopsamine, 13235

Rinderine, 18847

\section{$\mathrm{C}_{15} \mathrm{H}_{25} \mathrm{NO}_{6}$}

Echinatine $N$-oxide, 6687

Indicine $N$-oxide, 11020

$\mathrm{C}_{15} \mathrm{H}_{26}$

Patchoulane, 16705

Seychellane, 19792

Tanacetene, 20666

$\mathrm{C}_{15} \mathrm{H}_{26} \mathrm{Br}_{2} \mathrm{O}_{2}$

Obtusenol, 15898

\section{$\mathrm{C}_{15} \mathrm{H}_{26} \mathrm{~N}_{2}$}

Pachycarpine, 16494

Sparteine, 20133

\section{$\mathrm{C}_{15} \mathrm{H}_{26} \mathrm{~N}_{2} \mathrm{O}$}

Retamine, 18652

Thermopsamine, 21317

\section{$\mathrm{C}_{15} \mathrm{H}_{26} \mathrm{O}$}

$\alpha$-Acorenol, 562

$\beta$-Acorenol, 563 
Agarospirol, 706

Allohimachalol, 932

Aristolan-9 $\beta$-ol, 1701

$\beta$-Bisabolol, 2414

Bisabolol, 2415

Blepharostol, 2503

T-Cadinol, 2856

$\alpha$-Cadinol, 2857

$\delta$-Cadinol, 2858

Campherenol, 3046

Carotol, 3216

$\alpha$-Cedrol, 3354

ent-Cubeban-11-ol, 4303

Cubeben camphor, 4304

5-cis-Cyclopentadecen-1-one, 4524

Dihydroagarofuran, 5539

Elema-1,3-dien-7-ol, 6739

Elemol, 6746

(+)-4-Epi- $\alpha$-bisabolol, 6838

(-)-10-Epi- $\gamma$-eudesmol, 6912

(-)-4-Epi-maaliol, 6957

(+)-6,11-Epoxy-eudesmane, 7095

(+)-6,11-Epoxy-isodaucane, 7150

4-Ethenyl-2,2,4-trimethyl-3-(1-methylethenyl)cyclohexane-methanol, 7393

(+)-Eudesm-3-ene-7 $\alpha$-ol, 7508

Eudesm-3-en-7-ol, 7509

Eudesm-4-en- $7 \alpha$-ol, 7510

Eudesm-4(15)-en-7-ol, 7511

$\alpha$-Eudesmol, 7513

$\beta$-Eudesmol, 7514

$\gamma$-Eudesmol, 7515

Farnesol, 7734

1(10)E,5E-Germacradien-4-ol, 8336

Ginsenol, 8409

(-)-(4R,5R,6S,10S)-Gorgon-11-en-4-ol, 8954

Guaiol, 9044

Himachalol, 9541

Hinesol, 9542

1,6-Humuladien-10-ol, 9663

(4R)-3 $\alpha$-Hydroxycadin-10(15)-ene, 9872

(4S)-3 $\alpha$-Hydroxycadin-10(15)-ene, 9873

(4S)-3 $\beta$-Hydroxycadin-10(15)-ene, 9874

(+)-6 $\beta$-Hydroxy-eudesm-11-ene, 10112

(-)-6 $\alpha$-Hydroxy-eudesm-11-ene, 10113

Jinkoheremol, 11869

Jinkohol I, 11870

Jinkohol II, 11871

Junenol, 11961

Juniper camphor, 11966

Kusunol, 12375

Ledol, 12604

Liguloxide, 12814
Maalialcohol, 13283

(+)-Maalioxide, 13285

Nardol, 15272

Neointermedeol, 15411

E-Nerolidol, 15499

Z-Nerolidol, 15500

Panasinsanol A, 16588

Panasinsanol B, 16589

Patchoulic alcohol, 16711

Pogostol, 17601

(-)-6,7-Seco-eudesm-7(11)-en-6-al, 19613

Selin-11-en-4 $\alpha$-ol, 19689

Tamariscol, 20656

Valeranone, 22313

Valerianol, 22320

Widdrol, 22659

\section{$\mathrm{C}_{15} \mathrm{H}_{26} \mathrm{O}_{2}$}

Alismoxide 1, 889

\section{$\mathrm{C}_{15} \mathrm{H}_{26} \mathrm{O}_{2}$}

Alismoxide 2, 890

\section{$\mathrm{C}_{15} \mathrm{H}_{26} \mathrm{O}_{2}$}

Anticancer Sesquiterpene PMV70P691-132, 1437

\section{$\mathrm{C}_{15} \mathrm{H}_{26} \mathrm{O}_{2}$}

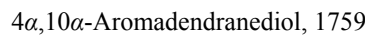

Aromadendrane- $4 \beta, 10 \alpha$-diol, 1760

(+)-4 $\beta, 10 \alpha$-Aromadendranediol, 1761

Aromadendrane- $4 \beta, 10 \beta$-diol, 1762

Baimuxinol, 2114

Bisabolol oxide A, 2416

Bornyl isovalerianate, 2560

Buchariol, 2695

Cadin-10(14)-ene-4 $\beta, 5 \alpha$-diol, 2854

Calamendiol, 2939

Canusesnol I, 3112

Centdarol, 3394

Chrysanthemol, 3595

Curcumadiol, 4388

Daucol, 4679

Debneyol, 4816

11,12-Dihydrochiloscypholone, 5559

$4 \beta, 10 \alpha$-Dihydroxyaromadendrane, 5756

(4R)-3 $\beta, 14$-Dihydroxycadin-10(15)-ene, 5784

$3 \alpha, 15$-Dihydroxy cedrane, 5787

$1 \beta, 4 \alpha$-Dihydroxyeudesman-11-ene, 5888

$1 \beta, 4 \beta$-Dihydroxyeudesman-11-ene, 5889

5-Epi-eudesm-4(15)-ene-1 $\beta, 6 \beta$-diol, 6909

7-Epieudesm-4(15)-ene-1 $\alpha, 6 \alpha$-diol, 6910

7-Epi-eudesm-4(15)-ene-1 $\beta, 6 \beta$-diol, 6911

ent-4(15)-Eudesmene-1 $\beta, 6 \alpha$-diol, 7502

7-Eudesmene-1,4-diol, 7503

Eudesm-4(15)-ene-1 $\beta, 6 \alpha$-diol, 7504

4(15)-Eudesmene-1 $\beta, 5 \alpha$-diol, 7505
4(15)-Eudesmene-1 $\beta, 7 \alpha$-diol, 7506

4(15)-Eudesmene-1 $\beta, 7 \beta$-diol, 7507

Flourensadiol, 7838

$5 E, 10$ (14)-Germacradien-1 $\beta, 4 \beta$-diol, 8335

Hedychiol A, 9281

4-Hydroxydihydroagarofuran, 9996

11-Hydroxy-isocampheren-9-ene, 10242

$10 \xi$-Hydroxy-isocampheren-11-ene, 10243

$4 \alpha$-Hydroxy- $4 \beta$-methyldihydrocostol, 10483

Isobaimuxinol, 11245

Isocalamendiol, 11302

Isocentdarol, 11315

Isokhusinodiol, 11471

Kessyl alcohol, 12194

Khusinodiol, 12223

Liguloxidol, 12815

Lubiminol, 13017

Mucronatone, 15019

(+)-Muurolan-4,7-peroxide, 15139

Neryl pentanoate, 15514

Octahydro-4-hydroxy-3 $\alpha$-methyl-7-methylene- $\alpha$ -

(1-methylethyl)-1H-indene-1-methanol, 15966

Oplopanone, 16154

(7R*)-Opposit-4(15)-ene-1 $\beta, 7$-diol, 16155

Opposit-4(15)-ene-1 $\beta, 11$-diol, 16156

Oxyphyllol C, 16468

Patchoulan-1,12-diol, 16704

1,4-Peroxy-5-muurolene, 16976

(+)-Plagio-4,7-peroxide, 17503

Pterocarpol, 18104

Pterocarpus marsupium sesquiterpene, 18105

Sinenofuranol, 19925

Tricyclohumuladiol, 21600

Verticillatol, 22428

cis-Widdrol $\alpha$-epoxide, 22660

trans-Widdrol $\alpha$-epoxide, 22661

$\mathrm{C}_{15} \mathrm{H}_{26} \mathrm{O}_{3}$

Canusesnol D, 3107

Canusesnol G, 3110

Chrysanthetriol, 3597

Dendrobiumane A, 5109

3,4-Dihydroxydihydroagarofuran, 5807

6,15 $\alpha$-Epoxy-1 $\beta, 4 \beta$-dihydroxyeudesmane, 7077

$4 \alpha, 15$-Epoxyeudesmane-1 $\beta, 6 \alpha$-diol, 7096

$4 \alpha, 5 \alpha$-Epoxy- $6 \alpha$-hydroxy amorphan-12-ol, 7109

Gloeosteretriol, 8581

9-Hydroxy-10,12-pentadecadienoic acid, 10591

Isovaleroyloxylinalool, 11760

Kessoglycol, 12191

Orientalol A, 16189

Orientalol B, 16190

Orientalol E, 16193

Pipelol A, 17427 
$\mathrm{C}_{15} \mathrm{H}_{26} \mathrm{O}_{4}$

Integrifonol A, 11094

\section{$\mathrm{C}_{15} \mathrm{H}_{26} \mathrm{O}_{4} \mathrm{~S}$}

Sulfoorientalol A, 20472

$\mathrm{C}_{15} \mathrm{H}_{26} \mathrm{O}_{5}$

Malkanguniol, 13422

\section{$\mathrm{C}_{15} \mathrm{H}_{26} \mathrm{O}_{5} \mathrm{~S}$}

Sulfoorientalol B, 20473

Sulfoorientalol D, 20475

\section{$\mathrm{C}_{15} \mathrm{H}_{26} \mathrm{O}_{9}$}

EucommiosideI, 7489

Eucommioside-II, 7490

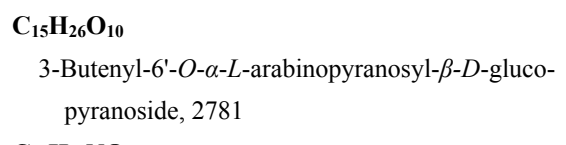

\section{$\mathrm{C}_{15} \mathrm{H}_{27} \mathrm{NO}$}

1-(1-Oxo-2E-decaenyl) piperidine, 16306

\section{$\mathrm{C}_{15} \mathrm{H}_{27} \mathrm{NO}_{3}$}

( \pm )-erythro-1-(1-Oxo-4,5-dihydroxy-2E-decaenyl) piperidine, 16318

( \pm )-threo-1-(1-Oxo-4,5-dihydroxy-2E-decaenyl) piperidine, 16319

\section{$\mathrm{C}_{15} \mathrm{H}_{27} \mathrm{NO}_{5}$}

Floridinine, 7834

\section{$\mathrm{C}_{15} \mathrm{H}_{27} \mathrm{O}_{3}$}

Orientalol E, 16192

\section{$\mathrm{C}_{15} \mathrm{H}_{28}$}

Selinane, 19685

\section{$\mathrm{C}_{15} \mathrm{H}_{28} \mathrm{O}$}

Artemisinol, 1799

Normuscone, 15778

\section{$\mathrm{C}_{15} \mathrm{H}_{28} \mathrm{O}_{2}$}

Cryptomeridiol, 4283

Cyclonerodiol, 4520

Exaltolide, 7672

\section{$\mathrm{C}_{15} \mathrm{H}_{28} \mathrm{O}_{3}$}

Cadinane- $4 \beta, 5 \alpha, 10 \beta$-triol, 2848

Muurolane- $4 \beta, 5 \beta, 10 \beta$-triol, 15138

6,7,10-Trihydroxyisodaucane, 21750

Zingibertriol, 23006

\section{$\mathrm{C}_{15} \mathrm{H}_{28} \mathrm{O}_{4}$}

1 $\beta, 4 \beta, 6 \alpha, 15$-Tetrahydroxyeudesmane, 21107

\section{$\mathrm{C}_{15} \mathrm{H}_{28} \mathrm{O}_{5}$}

$\left(1 R^{*}, 2 R^{*}, 3 R^{*}, 6 R^{*}, 7 R^{*}\right) 1,2,3,6,7-$ Pentahydroxybisabol-10(11)-ene, 16840

\section{$\mathrm{C}_{15} \mathrm{H}_{28} \mathrm{O}_{13}$}

Fuzinoside, 8042

\section{$\mathrm{C}_{15} \mathrm{H}_{30}$}

Humulane, 9667

1-Pentadecene, 16833

$\mathrm{C}_{15} \mathrm{H}_{30} \mathrm{O}_{2}$

Methyl myristate, 14610

12-Methyl tetradecanoic acid, 14742
13-Methyl tetradecanoic acid, 14743

Pentadecanoic acid, 16831

$(-) 2 D, 4 D, 6 D, 8 D$-Tetramethyl undecanoic acid, 21205

$\mathrm{C}_{15} \mathrm{H}_{32}$

$n$-Pentadecane, 16830

$\mathrm{C}_{15} \mathrm{H}_{32} \mathrm{O}$

Dodecyl isopropyl ether, 6546

$\mathrm{C}_{15} \mathrm{H}_{33} \mathrm{~N}_{3}$

1,3,5-Tributylhexahydro-1,3,5-triazine, 21549

$\mathrm{C}_{15} \mathrm{H}_{37} \mathrm{~N}_{5}$

Aminobutyl canavalmine, 1045

$\mathrm{C}_{16} \mathrm{H}_{8} \mathrm{O}_{3}$

Wrightiadione, 22733

\section{$\mathrm{C}_{16} \mathrm{H}_{8} \mathrm{O}_{4}$}

Aristolide B, 1708

Frutinone A, 7973

Methyl- $O$-cedrelopsin, 14223

\section{$\mathrm{C}_{16} \mathrm{H}_{8} \mathrm{O}_{6}$}

Medicagol, 13636

\section{$\mathrm{C}_{16} \mathrm{H}_{8} \mathrm{O}_{9}$}

3,4-Methylenedioxy-3'-O-methyl-5'-hydroxyellagic acid, 14377

\section{$\mathrm{C}_{16} \mathrm{H}_{9} \mathrm{NO}_{3}$}

Aristolactam II, 1699

\section{$\mathrm{C}_{16} \mathrm{H}_{9} \mathrm{NO}_{4}$}

Aristolactam AIIIa, 1693

\section{$\mathrm{C}_{16} \mathrm{H}_{9} \mathrm{NO}_{6}$}

Aristolochic acid II, 1716

\section{$\mathrm{C}_{16} \mathrm{H}_{10} \mathrm{~N}_{2} \mathrm{O}_{2}$}

Indigotin, 11023

Indirubin, 11024

Isoindigo, 11463

\section{$\mathrm{C}_{16} \mathrm{H}_{10} \mathrm{O}_{4}$}

DemethylaristofolinE, 5062

\section{$\mathrm{C}_{16} \mathrm{H}_{10} \mathrm{O}_{5}$}

Corylinal, 4110

Damnacanthal, 4622

Fimbriatone, 7799

12-O-Methylcoumestrol, 14260

Pseudobaptigenin, 18020

Rubiawallin C, 19026

$\mathrm{C}_{16} \mathrm{H}_{10} \mathrm{O}_{6}$

Bowdichione, 2574

3,9-Dihydroxy-4-methoxy-benzo[4,5]furo[3,2-c] chromen-6-one, 5962

Glyzaglabrin, 8862

1-Hydroxy-2-carboxy-3-methoxyanthraquinone, 9892

Irilone, 11158

Isotrifoliol, 11746

Juzunal, 11990

3-Methoxycarbonyl-1,5-dihydroxyanthra- quinone, 13873

Munjistin methyl ether, 15077

Sativol, 19395

\section{$\mathrm{C}_{16} \mathrm{H}_{10} \mathrm{O}_{7}$}

Laccaic acid D, 12427

Wedelolactone, 22656

\section{$\mathrm{C}_{16} \mathrm{H}_{10} \mathrm{O}_{8}$}

Clavorubin, 3814

2,2'-Dimethoxy-3,3'-dihydroxy-5,5'-oxygen-6,6'biphenylformic anhydride, 6220

2,3-Dimethoxyellagic acid, 6224

3,3'-Di-O-methylellagic acid, 6344

\section{$\mathrm{C}_{16} \mathrm{H}_{10} \mathrm{O}_{13} \mathrm{~S}_{2}$}

Quercetin-7-methyl ether-3,3'-disulfate, 18377

\section{$\mathrm{C}_{16} \mathrm{H}_{11} \mathrm{KO}_{10} \mathrm{~S}$}

Persicarin, 16990

\section{$\mathrm{C}_{16} \mathrm{H}_{11} \mathrm{NO}_{2}$}

Lutine, 13151

\section{$\mathrm{C}_{16} \mathrm{H}_{11} \mathrm{NO}_{3}$}

Anhydrolycorin-6-one, 1276

Aristolactam AII, 1692

Piperolactam A, 17463

Pratorimine, 17769

Ungeremine, 22232

\section{$\mathrm{C}_{16} \mathrm{H}_{11} \mathrm{NO}_{4}$}

Buxifoliadine F, 2828

Cheliensisam B, 3503

\section{$\mathrm{C}_{16} \mathrm{H}_{12} \mathrm{~N}_{2} \mathrm{O}$}

Anticancer Alkaloid PMV70P691-050, 1380

Cryptolepinone, 4282

\section{$\mathrm{C}_{16} \mathrm{H}_{12} \mathrm{~N}_{2} \mathrm{O}_{2}$}

Luotonin C, 13086

Nauclefidine, 15303

Perlolyrine, 16968

Tribulusterin, 21548

\section{$\mathrm{C}_{16} \mathrm{H}_{12} \mathrm{~N}_{2} \mathrm{O}_{3}$}

5,9-Dimethoxycanthin-6-one, 6214

9,10-Dimethoxycanthin-6-one, 6215

5-Hydroxymethyl-9-methoxycanthin-6-one, 10503

Kumujian D, 12334

9-Methoxy-3-methylcanthin-5,6-dione, 14006 Picrasidine O, 17307

\section{$\mathrm{C}_{16} \mathrm{H}_{12} \mathrm{O}_{3}$}

Danshenxinkun C, 4634

7-Hydroxy-2-methyl isoflavone, 10499

1-Methoxy-2-methylanthraquinone, 14002

Nordracorhodin, 15743

\section{$\mathrm{C}_{16} \mathrm{H}_{12} \mathrm{O}_{4}$}

Dalbergin, 4609

Formononetin, 7883

7-Hydroxy-3-(4'-hydroxybenzylidene)-chroman4-one, 10177 


\author{
1-Hydroxy-3-methoxy-2-methylanthraquinone, \\ 10414 \\ 1-Hydroxy-5-methoxy-2-methylanthraquinone, \\ 10415 \\ 1-Hydroxy-2-methyl-6-methoxyanthraquinone, \\ 10502 \\ Isodalbergin, 11365 \\ Lespedezol $\mathrm{A}_{1}, 12682$ \\ 7-Methoxy-4'-hydroxyflavone, 13959 \\ Pallidiflorin, 16546 \\ Rubiadin-1-methyl ether, 18999 \\ Rubiawallin B, 19025 \\ Tectochrysin, 20899
}

\section{$\mathrm{C}_{16} \mathrm{H}_{12} \mathrm{O}_{5}$}

Acacetin, 56

Anthragallol-1,3-dimethylether, 1360

Anticancer Flavonoid PMV70P691-85, 1415

Biochanin A, 2384

Calycosin, 3004

2-Carbomethoxy-6-methoxyxanthone, 3163

Cypripedin, 4584

Cypritibetquinone B, 4586

Damnacanthol, 4623

7,6'-Dihydroxy-3'-methoxyisoflavone, 5984

Genkwanin, 8289

Geraldone, 8309

Glycitein, 8818

3-(4-Hydroxybenzylidene)-5,7-dihydroxychroman-4-one, 9842

5-Hydroxy-3, 7-dimethoxy-1,4-phenanthrenequinone, 10035

7-Hydroxy-5,6-dimethoxy-1,4-phenanthrenequinone, 10036

1-Hydroxy-2-hydroxymethyl-3-methoxyanthraquinone, 10206

Islandicin 4-methyl ether, 11192

Izalpinin, 11806

Lespedezol A4, 12685

Lucidin $\omega$-methyl ether, 13044

Maackiain, 13281

Melanettin, 13658

7-Methoxybaicalein, 13847

3'-Methoxydaidzein, 13901

7-Methoxy-4'-hydroxyflavonol, 13960

Morindone-5-methylether, 14975

Obtusifolin, 15900

Ophiohayatone A, 16132

Oroxylin A, 16216

Physcion, 17247

Prunetin, 18001

Questin, 18416

Robustaquinone D, 18872

Rubianthraquinone, 19011
Rubiawallin A, 19024

Stevenin, 20340

Vertia flavone, 22426

Wogonin, 22718

Xenognosin B, 22783

\section{$\mathrm{C}_{16} \mathrm{H}_{12} \mathrm{O}_{6}$}

Anticancer Flavonoid PMV70P691-103, 1407

Cajinin, 2931

Chrysoeriol, 3602

6-Demethoxy-4'-methoxylcapillarisin, 5049

1,6-Dihydroxy-2,4-dimethoxyanthraquinone $\mathrm{V}$, 5818

1,3-Dihydroxy-2-methoxymethylanthraquinone, 5990

Diosmetin, 6452

Evariquinone, 7660

Fallacinol, 7710

Hematein, 9337

Hispidulin, 9564

6-Hydroxy-anthragallol-1,3-dimethylether, 9790

2-Hydroxyemodin 1-methyl ether, 10068

Hydroxygenkwanin, 10137

3'-Hydroxymelanettin, 10375

2-Hydroxy-6-methyl-8-methoxy-9-oxo-9Hxanthene-1-carboxylic acid, 10505

4'-Hydroxywogonin, 10829

Irilin A, 11155

Kaempferide, 12015

Kaempferol 3-methyl ether, 12070

Knoxiadin, 12237

Koparin, 12260

1-Methoxy-3,6-dihydroxy-2-hydroxymethyl9,10-anthraquinone, 13914

5-Methyl kaempferol, 14542

3'-O-Methylorobol, 14638

Parietin, 16665

Pratensein, 17765

Questinol, 18417

Rhamnocitrin, 18682

Robustaquinone C, 18871

Santal, 19299

Scutevulin, 19588

Tectorigenin, 20901

5,2',6'-Trihydroxy-7-methoxyflavone, 21774

5,7,2'-Trihydroxy-6-methoxyflavone, 21775

5,7,3'-Trihydroxy-4'-methoxyflavone, 21776

5,8,2'-Trihydroxy-7-methoxyflavone, 21777

1,3,6-Trihydroxy-2-methoxymethyl-9,10-anthraquinone, 21782

\section{$\mathrm{C}_{16} \mathrm{H}_{12} \mathrm{O}_{7}$}

Azaleatin, 2052

Capillarisin, 3118

Eupafolin, 7556
2-Hydroxy-6-hydroxymethyl-8-methoxy-9-oxo9H-xanthene-1-carboxylic acid, 10207

Irilin D, 11157

Isorhamnetin, 11648

5-Methoxy-3,4-dehydroxanthomegnin, 13902

3-Methoxyherbacetin, 13947

6-Methoxykaempferol, 13980

7-Methoxy-3,3',4',6-tetrahydroxyflavone, 14098

Nodifloretin, 15648

Oryzafuran, 16246

Pedalitin, 16758

Pollenitin, 17617

Quercetin-3-methyl ether, 18376

Rhamnetin, 18679

Robustaquinone E, 18873

Scandione, 19453

Selagin, 19675

Sexangularetin, 19791

Tamarixetin, 20657

Tenuiflorin C, 20936

5,6,7,3'-Tetrahydroxy-4'-methoxyisoflavone,

5,6,7,4'-Tetrahydroxy-8-methoxyisoflavone, 21130

\section{$\mathrm{C}_{16} \mathrm{H}_{12} \mathrm{O}_{8}$}

Annulatin, 1339

Gossypetin-7-methylether, 8964

5-Methylmyricetin, 14609

Patuletin, 16725

5,6,7,3',4'-Pentahydroxy-3-methoxyflavone, 16851

3,5,6,7,8-Pentahydroxy-2-(4-methoxyphenyl)4H-1-benzopyran-4-one, 16852

Vaccaxanthone, 22304

\section{$\mathrm{C}_{16} \mathrm{H}_{12} \mathrm{O}_{10}$}

Rubanthrone C, 18974

$\mathrm{C}_{16} \mathrm{H}_{13} \mathrm{~N}$

Ipomine $\mathrm{A}, 11131$

$N$-Phenyl-2-naphthylamine, 17127

$\mathrm{C}_{16} \mathrm{H}_{13} \mathrm{NO}_{2}$

Reevesianine A, 18568

$\mathrm{C}_{16} \mathrm{H}_{13} \mathrm{NO}_{4}$

Marcanine B, 13547

\section{$\mathrm{C}_{16} \mathrm{H}_{13} \mathrm{NO}_{5}$}

Avenalumin I, 2033

Marcanine C, 13548

$\mathrm{C}_{16} \mathrm{H}_{13} \mathrm{O}_{6}^{+}$

Peonidin, 16900

$\mathrm{C}_{16} \mathrm{H}_{13} \mathrm{O}_{7}^{+}$

Petunidin, 17024

$\mathrm{C}_{16} \mathrm{H}_{14} \mathrm{~N}_{2} \mathrm{O}$

Arborine, 1613

$\mathrm{C}_{16} \mathrm{H}_{14} \mathrm{O}_{2}$ 
Benzyl cinnamate, 2282

$\mathrm{C}_{16} \mathrm{H}_{14} \mathrm{O}_{2}$

Cinnamyl benzoate, 3727

\section{$\mathrm{C}_{16} \mathrm{H}_{14} \mathrm{O}_{3}$}

Chalepensin, 3457

Corsifuran C, 4095

Dalbergenone, 4607

Dalbergichromene, 4608

Gnetin, 8878

2-Hydroxy-3,7-dimethoxyphenanthrene, 10034

(7S,8R)-4-Hydroxy-8',9'-dinor-4',7-epoxy-8,3'neolignan-7'-aldehyde, 10056

4'-Hydroxy-2'-methoxychalcone, 10394

Magnaldehyde D, 13366

Magnaldehyde E, 13367

Medicarpan, 13637

Rhizonone, 18780

\section{$\mathrm{C}_{16} \mathrm{H}_{14} \mathrm{O}_{4}$}

Alloimperatorin, 933

Alloisoimperatorin, 934

Alpinetin, 985

Anhydroalkannin, 1259

Anticancer Flavonoid PMV70P691-101, 1406

Anticancer Flavonoid PMV70P691-95, 1420

Cardamonin, 3187

Demethylfuropinnarin, 5080

2',6'-Dihydroxy-4'-methoxychalcone, 5968

Echinatin, 6685

Imperatorin, 11001

Isoimperatorin, 11462

Isolarrien, 11484

(-)-Medicarpin, 13638

2'-O-Methyl isoliquiritigenin, 14532

8-Methylpinocembrin, 14676

Pallidiflorene, 16545

Physcion anthrone, 17248

Saprionide, 19349

(-)-Strobopinin, 20394

Vignafuran, 22474

\section{$\mathrm{C}_{16} \mathrm{H}_{14} \mathrm{O}_{5}$}

Alpinone, 989

Brasilin, 2594

Coeloginanthrin, 3891

8-Demethylfarrerol, 5079

3-Deoxysappanone B, 5212

Dihydroechioidinin, 5604

Dihydrooroxylin A, 5685

Helichrysetin, 9300

Heraclenin, 9419

Homobutein, 9598

Isogosferol, 11445

Isooxypeucedanin, 11578

Isosakuranetin, 11691
Kushenin, 12353

Lespedezol $\mathrm{D}_{1}, 12693$

Licochalcone B, 12767

2'-Methoxy-3,4,4'-trihydroxychalcone, 14099

5-O-Methylnaringenin, 14613

6-C-Methylnaringenin, 14614

Moracin B, 14954

Moracin F, 14958

(-)-Nissolin, 15629

Oxyimperatorin, 16447

(S)-(-)-Oxypeucedanin, 16456

(+)-Oxypeucedanin, 16457

Pabulenol, 16488

Phyllodulcin, 17225

Quinquangulin, 18444

Sainfuran, 19168

Sakuranetin, 19172

(R)-Sakuranetin, 19173

(S)-Sakuranetin, 19174

7,2',6'-Trihydroxy-5-methoxychalcone, 21769

1,2,8-Trimethoxyxanthone, 21937

Vestitone, 22438

\section{$\mathrm{C}_{16} \mathrm{H}_{14} \mathrm{O}_{6}$}

Acetylmelodorinol, 463

Anticancer Flavonoid PMV70P691-022, 1401

Aromadendrin-7-monomethyl ether, 1767

Baishouwubenzophenone, 2115

Blumeatin, 2521

Carpusin, 3222

Clavilactone D, 3811

2-(3',5'-Dihydroxy-4'-methoxyphenyl)-3methoxy-5-hydroxy benzofuran, 5997

Hesperetin, 9456

Homoeriodictyol, 9604

11b-Hydroxy-11b,1-dihydromaackiain, 10007

1-Hydroxy-2,3,5-trimethoxyxanthone, 10802

1-Hydroxy-3,4,5-trimethoxyxanthone, 10803

1-Hydroxy-3,6,7-trimethoxy xanthone, 10804

6-Hydroxy-1,2,5-trimethoxyxanthone, 10805

7-Hydroxy-1,2,8-trimethoxyxanthone, 10806

Klaivanolide, 12232

Lespedezol D, 12692

4-Methoxy-6-(11,12-methylenedioxy-14methoxystyryl)-2-pyrone, 14019

(3R)-4'-Methoxy-2',3,7-trihydroxyisoflavanone, 14100

7-O-Methylaromadendrin, 14146

7-O-Methyleriodictyol, 14417

(+)-Mopanol, 14949

Onjixanthone I, 16108

Paepalantine, 16535

Perforatic acid, 16916

Protosappanin C, 17988
Sappanone B, 19348

Scuteamoenin, 19580

Suberectin, 20437

2,6,2',4'-Tetrahydroxy-6'-methoxychalcone, 21126

5,7,3',5'-Tetrahydroxy-6-methylflavanone, 21134

5,7,4'-Trihydroxy-6-methoxyflavanone, 21772

7,2'6'-Trihydroxy-5-methoxyflavanone, 21773

\section{$\mathrm{C}_{16} \mathrm{H}_{14} \mathrm{O}_{7}$}

(+)-Dihydroisorhamnetin, 5652

1,3-Dihydroxy-2,4,7-trimethoxyxanthone, 6164

1,6-Dihydroxy-3,5,7-trimethoxyxanthone, 6165

1,8-Dihydroxy-3,4,7-trimethoxyxanthone, 6166

Isolecanoric acid, 11486

Lecanoric acid, 12598

4'-O-Methyltaxifolin, 14737

\section{$\mathrm{C}_{16} \mathrm{H}_{14} \mathrm{O}_{9}$}

Ethyl- $m$-digallate, 7458

Ethyl-p-digallate, 7470

\section{$\mathrm{C}_{16} \mathrm{H}_{15} \mathrm{ClO}_{5}$}

8-(3-Chloro-2-hydroxy-3-methylbutoxy)psoralen, 3560

Saxalin, 19430

\section{$\mathrm{C}_{16} \mathrm{H}_{15} \mathrm{NO}_{3}$}

Apohaemanthamine, 1532

Kalasinamide, 12116

Nantoamide, 15249

\section{$\mathrm{C}_{16} \mathrm{H}_{15} \mathrm{NO}_{4}$}

Arborinine, 1614

Clausine H, 3797

4a, $N$-Dedihydronoraugustamine, 4866

Toddaliopsin A, 21419

\section{$\mathrm{C}_{16} \mathrm{H}_{15} \mathrm{NO}_{5}$}

Citrusinine I, 3777

4-Methoxydianthramide B, 13903

\section{$\mathrm{C}_{16} \mathrm{H}_{15} \mathrm{NO}_{6}$}

Atalafoline B, 1954

Buxifoliadine H, 2830

\section{$\mathrm{C}_{16} \mathrm{H}_{16} \mathrm{~N}_{2} \mathrm{O}_{2}$}

Isolysergic acid, 11516

Lysergic acid, 13256

\section{$\mathrm{C}_{16} \mathrm{H}_{16} \mathrm{O}_{2}$}

(E)-3,4'-Dimethoxystilbene, 6292

3,5-Dimethoxystilbene, 6293

6-Isohexenyl- $\alpha$-naphthoquinone, 11455

Obtustyrene, 15913

$\mathrm{C}_{16} \mathrm{H}_{16} \mathrm{O}_{3}$

Broussin, 2628

Corsifuran A, 4093

7,4'-Dihydroxy-8-methylflavan, 6032

7-Hydroxy-3-(4-hydroxybenzyl)chromane, 10175

4'-Hydroxy-7-methoxyflavan, 10403 
Hydroxyobtustyrene, 10545

3'-(4"-Hydroxyphenyl)-propyl benzoate, 10639

(2S)-5-Methoxy flavan-7-ol, 13928

3,4-Methylenedioxy-3'-methoxybibenzil, 14375

Orchinol, 16168

Pterostilbene, 18163

Xenognosin A, 22782

\section{$\mathrm{C}_{16} \mathrm{H}_{16} \mathrm{O}_{4}$}

Angolensin, 1240

Benzyl 2,6-dimethoxybenzoate, 2283

Densiflorol A, 5132

Deoxyshikonin, 5214

3',4-Dihydroxy-3,5'-dimethoxystilbene, 5839

2',6'-Dihydroxy-4'-methoxydihydrochalcone, 5969

(2S),3',4'-Dihydroxy-7-methoxy flavan, 5978

(2S)-4',7-Dihydroxy-3'-methoxyflavan, 5979

Eleutherin, 6752

4'-Hydroxy-7-methoxyflavan-3-ol, 10404

Isohemigossylic acid lactone-2-methyl ether, 11453

Isohemigossylic acid lactone- 7-methyl ether, 11454

LoureirinC, 13012

5-O-Methylalloptaeroxylin, 14125

Naphthoquinone V, 15260

Phoyunbene C, 17198

Uvangoletin, 22287

(3R)-Vestitol, 22436

(3S)-Vestitol, 22437

\section{$\mathrm{C}_{16} \mathrm{H}_{16} \mathrm{O}_{5}$}

(-)-Alkannin, 909

Angelicone, 1193

Benzyl 2-hydroxy-3,6-dimethoxybenzoate, 2291

Coeloginanthridin, 3890

Columbianetin acetate, 3937

3'-Deoxysappanol, 5211

5,8-Dimethoxyxanthyletin, 6300

11b-Hydroxy-11b,1-dihydromedicarpin, 10009

5-Hydroxymethyl-6-endo-(3'-methoxy-4'-

hydroxyphenyl)-8-oxa-bicyclo[3.2.1]-oct-3-en2-one, 10489

Lespedezol $\mathrm{G}_{1}, 12703$

4-Methoxy-6-(11,12-dimethylstyryl)-2-pyrone, 13919

Nodakenetin acetate, 15646

Shikonin, 19819

Torosachrysone, 21459

4,2',4'-Trihydroxy-6'-methoxydihydrochalcone, 21770

$\mathrm{C}_{16} \mathrm{H}_{16} \mathrm{O}_{6}$

3'(S)-Acetoxy-4'(R)-hydroxy-3',4'-dihydroxanthyletin, 213
Arachidoside, 1600

Aviprin, 2041

(4S,4a $R, 9 \mathrm{a} R)$-4a-Carbomethoxy-1,4,4a,9a-tetrahydro-4,8-dihydroxy-6-methylxanthone, 3164

3',6-Dihydroxy-2,4,4'-trimethoxybenzophenone, 6158

Glochidiolide, 8564

Heraclenol, 9420

Isoglochidiolide, 11442

4-Methoxy-6-(11,12-methylenedioxy-14methoxydihydrostyryl)-2-pyrone, 14018

Oxypeucedanin hydrate, 16458

(S)-(-)-Oxypeucedanin hydrate, 16459

Protosappanin B, 17987

Qianhucoumarin B, 18283

Qianhucoumarin C, 18284

Sappanol, 19347

Ventiloquinone I, 22378

\section{$\mathrm{C}_{16} \mathrm{H}_{16} \mathrm{O}_{7}$}

8-O-Methyl-fusarubin, 14451

Omphalocarpinol, 16094

\section{$\mathrm{C}_{16} \mathrm{H}_{16} \mathrm{O}_{8}$}

3-O-Caffeoylshikimic acid, 2923

5-O-Caffeoylshikimic acid, 2924

\section{$\mathrm{C}_{16} \mathrm{H}_{17} \mathrm{NO}_{2}$}

Onosmin B, 16121

\section{$\mathrm{C}_{16} \mathrm{H}_{17} \mathrm{NO}_{3}$}

Caranine, 3152

Crinine, 4240

(+)-Crinine, 4241

Demethylcoclaurine, 5067

Epivittatine, 7038

Neoacutifolin, 15335

1-[1-Oxo-5(3,4-methylenedioxyphenyl)-2E,4Epentadienyl]pyrrolidine, 16387

1-[1-Oxo-5(3,4-methylenedioxyphenyl)-2E,4Zpentadienyl] pyrrolidine, 16388

1-[1-Oxo-5(3,4-methylenedioxyphenyl)-2Z,4Epentadienyl] pyrrolidine, 16389

Zanthobungeanine, 22966

\section{$\mathrm{C}_{16} \mathrm{H}_{17} \mathrm{NO}_{4}$}

Hamayne, 9206

(+)-11-Hydroxyvittatine, 10826

Lycorine, 13241

Nangustine, 15246

Noraugustamine, 15716

Orixalone C, 16201

(-)-Pancracine, 16600

Pipermethystine, 17462

\section{$\mathrm{C}_{16} \mathrm{H}_{17} \mathrm{NO}_{5}$}

Cenocladamide, 3386

4'-Desmethylpiplartine, 5259

$3 \alpha, 4 \alpha$-Epoxy-5 $\beta$-pipermethystine, 7188

\section{$\mathrm{C}_{16} \mathrm{H}_{17} \mathrm{~N}_{3} \mathrm{O}$}

Lysergamide, 13255

$\mathrm{C}_{16} \mathrm{H}_{17} \mathrm{~N}_{3} \mathrm{O}_{2} \mathrm{~S}$

1-(2'-Pyrrolidinethion-3'-yl)-1,2,3,4-tetrahydro$\beta$-carboline-3-carboxylic acid, 18278

\section{$\mathrm{C}_{16} \mathrm{H}_{18} \mathrm{~N}_{2}$}

Agroclavine, 763

$\mathrm{C}_{16} \mathrm{H}_{18} \mathrm{~N}_{2} \mathrm{O}$

Elymoclavine, 6765

Isosetoclavine, 11712

Lysergol, 13257

Setoclavine, 19789

$\mathrm{C}_{16} \mathrm{H}_{18} \mathrm{~N}_{2} \mathrm{O}_{2}$

Isopenniclavine, 11582

Penniclavine, 16808

$\mathrm{C}_{16} \mathrm{H}_{18} \mathrm{~N}_{2} \mathrm{O}_{3}$

4,8-Dimethoxy-1-(2-methoxyethyl)- $\beta$-carboline, 6257

\section{$\mathrm{C}_{16} \mathrm{H}_{18} \mathrm{~N}_{2} \mathrm{O}_{6}$}

Cappariloside A, 3136

Indole-3-acetonitrile-6- $O-\beta$ - $D$-glucopyranoside, 11028

\section{$\mathrm{C}_{16} \mathrm{H}_{18} \mathrm{~N}_{6} \mathrm{O}_{5}$}

AMG-1, 1039

\section{$\mathrm{C}_{16} \mathrm{H}_{18} \mathrm{O}_{3}$}

Broussonin A, 2647

Broussonin B, 2648

4-Ethoxymethylphenyl-4'-hydroxybenzylether, 7412

3-Methoxy-7-hydroxycadalenal, 13951

3'-O-Methylbatatasin III, 14154

Stilbostemin D, 20380

\section{$\mathrm{C}_{16} \mathrm{H}_{18} \mathrm{O}_{4}$}

Alkannan, 908

Batatasin II, 2164

Bungein A, 2746

Coumurrayin, 4193

Gigantol, 8380

6-Methoxyhemigossypol, 13946

Rutacultin, 19079

Stilbostemin E, 20381

Toddaculine, 21418

\section{$\mathrm{C}_{16} \mathrm{H}_{18} \mathrm{O}_{5}$}

Aculeatin, 588

Albiflorin-2, 859

Albiflorin-3, 860

5,6-Dimethoxy-8-(3'-methyl-2'-oxobutyl) coumarin, 6265

5,7-Dimethoxy-8-(3'-methyl-2'-oxobutyl) coumarin, 6266

11-Methoxy-5,6-dihydroyangonin, 13915

5-O-Methylvisamminol, 14812

Omphamurin, 16095 
Perforamone A, 16912

Perforamone B, 16913

Perforamone C, 16914

\section{$\mathrm{C}_{16} \mathrm{H}_{18} \mathrm{O}_{6}$}

Descurainin, 5245

6-(2',3'-Dihydroxy-3'-methylbutyl)-7-acetoxy2H-1-benzopyran-2-one, 6026

Methyl 5-acetoxymethanol-7-hydroxy-2,2-dimethyl-2H-1-chrome e-6-carboxylate, 14116

1-Naphthol- $\beta$ - $D$-glucopyranoside, 15254

(2S)-Ongokein-4'-one, 16097

\section{$\mathrm{C}_{16} \mathrm{H}_{18} \mathrm{O}_{7}$}

4-Hydroxy-1-naphthalenyl- $\beta$ - $D$-glucopyranoside, 10536

\section{$\mathrm{C}_{\mathbf{1 6}} \mathrm{H}_{18} \mathbf{O}_{\mathbf{8}}$}

5-p-cis-Coumaroylquinic acid, 4182

5-p-trans-Coumaroylquinic acid, 4183

Gerberinside, 8334

$\alpha$-Hydrojuglone glucoside, 9721

Jiadifenin, 11868

1,2,4-Trihydroxynaphthalene-4-glucoside, 21811

\section{$\mathrm{C}_{16} \mathrm{H}_{18} \mathrm{O}_{9}$}

Biflorin, 2369

4-O-Caffeoylquinic acid, 2918

Chlorogenic acid, 3551

8-O- $\beta$ - $D$-Glucopyranosyl-6-hydroxy-2-methyl-

4H-1-benzopyran-4-one, 8673

Isochlorogenic acid, 11327

Neochlorogenic acid, 15363

Scopolin, 19545

Staphylin, 20263

\section{$\mathrm{C}_{16} \mathrm{H}_{18} \mathrm{O}_{10}$}

Fraxin, 7944

\section{$\mathrm{C}_{16} \mathrm{H}_{19} \mathrm{BrCl}_{2} \mathrm{O}_{3}$}

Polyhalogenated homosesquiterpenic fatty acid A, 17650

Polyhalogenated homosesquiterpenic fatty acid B, 17651

\section{$\mathrm{C}_{16} \mathrm{H}_{19} \mathrm{NO}_{3}$}

$\alpha$-Erythroidine, 7340

$\beta$-Erythroidine, 7341

Macowine, 13297

Norgalanthamine, 15750

Norpluviine, 15788

Piperamine, 17438

Piperlonguminine, 17461

\section{$\mathrm{C}_{16} \mathrm{H}_{19} \mathrm{NO}_{4}$}

Acutifolidin, 595

$4 \alpha$-Dehydroxycrinamabine, 4982

( \pm )-8-Methoxyplatydesmine, 14072

1-[1-Oxo-3(3,4-methylenedioxy-5-methoxy-

phenyl)-2Zpropenyl] piperidine, 16382

Pseudolycorine, 18050
$\mathrm{C}_{16} \mathrm{H}_{19} \mathrm{NO}_{5}$

(2S,4R)-2-Carboxy-4-(E)-p-coumaroyloxy-

1,1-dimethylpyrrolidinium inner salt, 3166

Zanthodioline, 22967

$\mathrm{C}_{16} \mathrm{H}_{19} \mathrm{NO}_{6}$

Nor-orixine, 15785

$\mathrm{C}_{16} \mathrm{H}_{19} \mathrm{NO}_{7} \mathrm{~S}$

Chaetoquadrin D, 3443

$\mathrm{C}_{16} \mathrm{H}_{19} \mathrm{NO}_{8}$

6-Acetyl holocalin, 412

$\mathrm{C}_{16} \mathrm{H}_{19} \mathrm{~N}_{3} \mathrm{O}_{3}$

$\alpha$-Dichroine, 5435

$\beta$-Dichroine, 5436

Febrifugine, 7747

$\mathrm{C}_{16} \mathrm{H}_{20} \mathrm{NO}_{3}{ }^{+}$

$\mathrm{N}$-Methylplatydesmin, 14682

$\mathrm{C}_{16} \mathrm{H}_{20} \mathrm{NO}_{4}$

Ribalinium, 18831

$\mathrm{C}_{16} \mathrm{H}_{20} \mathrm{NO}_{4}^{+}$

Pteleatin, 18102

$\mathrm{C}_{\mathbf{1 6}} \mathrm{H}_{\mathbf{2 0}} \mathbf{N}_{\mathbf{2}}$

Costaclavine, 4125

\section{$\mathrm{C}_{16} \mathrm{H}_{20} \mathrm{~N}_{2} \mathrm{O}$}

Chanoclavine, 3477

Fordimine, 7879

Huperzine B, 9687

\section{$\mathrm{C}_{16} \mathrm{H}_{20} \mathrm{~N}_{2} \mathrm{O}_{9} \mathrm{~S}_{2}$}

Glucobrassicin, 8589

$\mathrm{C}_{16} \mathrm{H}_{20} \mathrm{~N}_{2} \mathrm{O}_{10} \mathrm{~S}_{2}$

4-Hydroxy-3-indolyl methyl glucosinolate, 10232

\section{$\mathrm{C}_{16} \mathrm{H}_{20} \mathrm{~N}_{2} \mathrm{O}_{12} \mathrm{~S}_{3}$}

Glucobrassicin-1-Sulfonate, 8590

\section{$\mathrm{C}_{16} \mathrm{H}_{20} \mathrm{O}_{2}$}

Arnebinol, 1746

Isolinderoxide, 11495

\section{$\mathrm{C}_{16} \mathrm{H}_{20} \mathrm{O}_{3}$}

1,4-Dihydroxy-2-(3',7'-dimethyl-1'-oxo-2'-E,6'octadienyl)benzene, 5859

1,4-Dihydroxy-2-(3',7'-dimethyl-1'-oxo-2'-Z,6'octadienyl)benzene, 5860

Lacinilene C 7-methyl ether, 12432

rel-3R-Methoxy-4S-furanogermacra- $1 E, 10(15)$ dien-6-one, 13935

\section{$\mathrm{C}_{16} \mathrm{H}_{20} \mathrm{O}_{4}$}

Acetylpterosin C, 490

2,3-Dihydro-5,7-dihydroxy-2,6-dimethyl-8-(3methyl-2-butenyl)-4H-1-benzopyran-4-one, 5592

2,3-Dihydro-5,7-dihydroxy-2,8-dimethyl-6-(3methyl-2-butenyl)-4H-1-benzopyran-4-one, 5593

5-Hydroxy-6-isobutyryl-7-methoxy-2,2- dimethylbenzopyran, 10239

7-Hydroxy-6-isobutyryl-5-methoxy-2,2dimethylbenzopyran, 10240

\section{$\mathrm{C}_{16} \mathrm{H}_{20} \mathrm{O}_{5}$}

Curvularin, 4414

1,4-Dihydroxy-2-(7'-methyl-3'-methylene-1'-oxo4',7'-peroxide-octyl)benzene, 6041

7-Methoxy-8-(2'-Methoxy-3'-hydroxy-3'-methylbutyl)coumarin, 13996

3-Methoxytanapartholide, 14097

\section{$\mathrm{C}_{\mathbf{1 6}} \mathrm{H}_{\mathbf{2 0}} \mathrm{O}_{\mathbf{6}}$}

(2S)-cis-4'-Hydroxy-ongokein, 10554

(2S)-trans-4'-Hydroxy-ongokein, 10555

Isomexoticin, 11536

Lasiopulide, 12541

Mexoticin, 14825

Toddalolactone, 21423

\section{$\mathrm{C}_{16} \mathrm{H}_{20} \mathrm{O}_{7}$}

Bidensyneoside B, 2361

Buergeriside $\mathrm{C}_{1}, 2712$

(2E,8E)-2,8-Decadiene-4,6-diyne-1,10-diol 1-O$\beta$ - $D$-glucopyranoside, 4818

Juglanoside A, 11898

\section{$\mathrm{C}_{16} \mathrm{H}_{20} \mathrm{O}_{8}$}

Juglanoside B, 11899

Juglanoside C, 11900

Methyl 6- $O$ - $p$-cis-coumaroyl- $\beta$ - $D$-glucopyranoside, 14257

Methyl 6- $O$-p-trans-coumaroyl- $\beta$ - $D$-glucopyranoside, 14258

Veranisatin A, 22384

\section{$\mathrm{C}_{16} \mathrm{H}_{20} \mathrm{O}_{9}$}

6- $O$-Feruloyl- $\beta$ - $D$-glucopyranoside, 7773

1- $O$-Feruloyl- $\beta$-glucose, 7774

Gentiopicroside, 8304

5-Hydroxy-6-methylchromone-7- $O-\beta$ - $D$-glucoside, 10481

Juglanoside D, 11901

Juglanoside E, 11902

Veranisatin B, 22385

\section{$\mathrm{C}_{16} \mathrm{H}_{20} \mathrm{O}_{10}$}

7-Caffeoylsedoheptulose, 2922

\section{$\mathrm{C}_{16} \mathrm{H}_{20} \mathrm{O}_{11}$}

Erinoside, 7266

\section{$\mathrm{C}_{16} \mathrm{H}_{21} \mathrm{BrCl}_{2} \mathrm{O}_{3}$}

Polyhalogenated homosesquiterpenic fatty acid C, 17652

\section{$\mathrm{C}_{16} \mathrm{H}_{21} \mathrm{BrN}_{2}$}

Deformylflustrabromine, 4868

Deformylflustrabromine B, 4869

\section{$\mathrm{C}_{16} \mathrm{H}_{21} \mathrm{NO}_{3}$}

Annotine, 1332

Annotinine, 1333 
Datumetine, 4665

5,6-Dihydropiperlonguminine, 5695

Norhyoscyamine, 15760

3-Oxo-8 $\alpha$-methoxy-10 $\alpha H$-eremophila-1,7(11)-

dien-12,8 $\beta$-lactam, 16378

\section{$\mathrm{C}_{16} \mathrm{H}_{21} \mathrm{NO}_{4}$}

Concneorine, 3960

Edulinine, 6708

Sarmentamide C, 19370

\section{$\mathrm{C}_{16} \mathrm{H}_{21} \mathrm{NO}_{5}$}

Foliosidine, 7854

\section{$\mathrm{C}_{16} \mathrm{H}_{22} \mathrm{Cl}_{2} \mathrm{O}_{3}$}

Polyhalogenated homosesquiterpenic fatty acid D, 17653

\section{$\mathrm{C}_{16} \mathrm{H}_{22} \mathrm{~N}_{2}$}

Lycodine, 13190

6,7-Seco-agroclavine, 19605

\section{$\mathrm{C}_{16} \mathrm{H}_{22} \mathrm{~N}_{2} \mathrm{O}_{2} \mathrm{~S}$}

Aglaithioduline, 740

\section{$\mathrm{C}_{16} \mathrm{H}_{22} \mathrm{~N}_{4} \mathrm{O}_{2}$}

Acanthoine, 81

\section{$\mathrm{C}_{16} \mathrm{H}_{22} \mathrm{~N}_{4} \mathrm{O}_{3}$}

Eseramine, 7379

\section{$\mathrm{C}_{16} \mathrm{H}_{22} \mathrm{NO}_{4}^{+}$}

Rutalinium, 19084

\section{$\mathrm{C}_{16} \mathrm{H}_{22} \mathrm{O}_{2}$}

Gloeophyllol B, 8577

$8 \alpha$-Methoxyfuranodiene, 13932

Pathenolide, 16713

Pterosin I, 18144

\section{$\mathrm{C}_{16} \mathrm{H}_{22} \mathrm{O}_{3}$}

Acutifolone A, 600

$4 \beta$-Methoxycostuslactone, 13899

rel-2R-Methoxy-4R-furanogermacra-1(10)E-en6-one, 13936

Methyl 8- $\alpha$-humula-6Z,9E-dien-12-oate, 14492

Methyl 8-oxo- $\alpha$-humula-6E,9E-dien-12-oate, 14645

Methyl 8-oxo- $\alpha$-humula-6E,9Z-dien-12-oate, 14646

Pterosin V, 18157

\section{$\mathrm{C}_{16} \mathrm{H}_{22} \mathrm{O}_{4}$}

Des- $O$-methyllasiodiplodin, 5258

Dibutyl phthalate, 5404

Ditertbutyl phthalate, 6523

$1 \beta, 10 \beta$-Epoxy- $8 \alpha$-methoxyeremophil-7(11)-en$12,8 \beta$-olide, 7164

$6 \beta$-Hydroxy- $8 \alpha$-methoxyeremophila-1(10),7(11)dien-12,8 $\beta$-olide, 10401

8-Methoxy-9-O-angeloylthymol, 13839

8-Methoxy-9-hydroxythymol 3-O-tiglate, 13970

3-O-(3-Methyl-2-butenoyl)-8-methoxy-9-hydroxythymol, 14179
9'-(3,4-Methylenedioxy-phenyl)-nonanoic acid, 14381

Methyl leptol B, 14551

Senkyunolide M, 19742

Senkyunolide Q, 19744

$\mathrm{C}_{16} \mathrm{H}_{22} \mathrm{O}_{5}$

$(3 R),(5 R)-5$-Hydroxy-de- $O$-methyllasiodiplodin, 9979

$(3 R),(6 R)-6$-Hydroxy-de- $O$-methyllasiodiplodin, 9980

$\mathrm{C}_{16} \mathrm{H}_{22} \mathrm{O}_{6}$

7-Acetoxy-8-hydroxy-9-isobutyryloxythymol, 219

Bidenoside C, 2358

Lindenanolide G, 12864

Pectinolide A, 16747

\section{$\mathrm{C}_{16} \mathrm{H}_{22} \mathrm{O}_{7}$}

Bidensyneoside $\mathrm{A}_{1}, 2359$

Bidensyneoside $\mathrm{A}_{2}, 2360$

3-Deoxybidensyneoside B, 5155

Eugenol- $\beta$ - $D$-glucopyranoside, 7522

Gymnasterkoreaside A, 9104

(E)-3-Hydroxyanethole $\beta$ - $D$-glucopyranoside, 9784

(E)-1'-(2-Hydroxy-5-methoxyphenyl)propene $\beta$ - $D$-glucopyranoside, 10454

Phenylbutanone-glucoside, 17099

\section{$\mathrm{C}_{16} \mathrm{H}_{22} \mathrm{O}_{8}$}

Baihuaqianhuoside, 2107

Bidensyneoside C, 2362

Coniferin, 3981

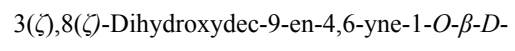
glucopyranoside, 5801

Pectinolide D, 16750

Serratumin A, 19775

\section{$\mathrm{C}_{16} \mathrm{H}_{22} \mathrm{O}_{9}$}

10-Deoxygeniposidic acid, 5176

2,4-Dihydroxy-6-methoxy-3-methylacetophenone-4- $O-\beta$ - $D$-glucopyranoside, 5989

2-O-(2)- $\beta$ - $D$-Glucopyranosyl-4,6-dimethoxy phenylenthanone, 8625

2-O- $\beta$ - $D$-Glucosyloxy-4-methoxybenzenepropanoic acid, 8757

Sweroside, 20503

$\mathrm{C}_{16} \mathrm{H}_{22} \mathrm{O}_{10}$

7,8-Epoxy-8-epi-loganic acid, 7090

Gardoside, 8234

Geniposidic acid, 8277

Methyl syringate 4- $O-\beta$ - $D$-glucopyranoside, 14735

Swertiamarin, 20509

Trimethylgalloylglucose, 21959

$\mathrm{C}_{16} \mathrm{H}_{22} \mathrm{O}_{11}$
Deacetyl asperulosidic acid, 4716

Monotropein, 14933

Scandoside, 19454

Theveside, 21324

\section{$\mathrm{C}_{16} \mathrm{H}_{23} \mathrm{NO}_{2}$}

Acrifoline, 567

Lycophlegmine, 13221

Lycoposerramine H, 13227

Lycoposerramine I, 13228

Lycoposerramine K, 13230

$\mathrm{C}_{16} \mathrm{H}_{23} \mathrm{NO}_{3}$

Neohuperzinine, 15407

\section{$\mathrm{C}_{16} \mathrm{H}_{23} \mathrm{NO}_{5}$}

Assamicadine, 1912

Fulvine, 7994

\section{$\mathrm{C}_{16} \mathrm{H}_{23} \mathrm{NO}_{6}$}

Monocrotaline, 14923

\section{$\mathrm{C}_{16} \mathrm{H}_{23} \mathrm{NO}_{7}$}

6,7-Dihydroxy-1-methyl- $N$-(6'-fructopyranosyl)1,2,3,4-tetrahydroisoquinoline, 6033

\section{$\mathrm{C}_{16} \mathrm{H}_{24} \mathrm{NO}_{5}$}

Sinapine, 19917

$\mathrm{C}_{16} \mathrm{H}_{24} \mathbf{N}_{2}$

Huperzinine C, 9690

\section{$\mathrm{C}_{16} \mathrm{H}_{24} \mathrm{~N}_{2} \mathrm{O}_{2}$}

Carolinianine, 3207

\section{$\mathrm{C}_{16} \mathrm{H}_{24} \mathrm{~N}_{2} \mathrm{O}_{5}$}

(2S)-6-Amino-2-[(3a $\left.R^{*}, 4 S^{*}, 7 R^{*}, 7 \mathrm{a} S^{*}\right)-3 \mathrm{a}, 7 \mathrm{a}-$ dimethyl-1,3-dioxo-4,7-epoxyoctahydroisoind ol-2-yl]-hexanoic Acid, 1050

\section{$\mathrm{C}_{16} \mathrm{H}_{24} \mathrm{~N}_{4} \mathrm{O}_{5}$}

$(2 S)-2-\left[\left(3 \mathrm{a} R^{*}, 4 S^{*}, 7 R^{*}, 7 \mathrm{a} S^{*}\right)-3 \mathrm{a}, 7 \mathrm{a}-\right.$ Dimethyl1,3-dioxo-4,7-epoxy-octahydroisoindol-2-yl]5-guanidino pentanoic acid, 6341

\section{$\mathrm{C}_{16} \mathrm{H}_{24} \mathrm{O}$}

Curcumadione, 4389

\section{$\mathrm{C}_{16} \mathrm{H}_{24} \mathrm{O}_{2}$}

2-Hydroxy-4-methoxycuparene, 10395

Madolin K, 13342

Madolin M, 13343

Madolin S, 13345

Methylarteannuate, 14147

Methyl guaia-1(10),11-dien-15-carboxylate, 14477

(-)-Methyl selina-3,11-dien-14-oate, 14725

(+)-Methyl selina-4,11-dien-14-oate, 14726

Petasalbin methyl ether, 17011

\section{$\mathrm{C}_{16} \mathrm{H}_{24} \mathrm{O}_{3}$}

Actinolide A, 586

Dehydrojuvabione, 4935

$10 \beta$-Hydroxy- $6 \beta$-methoxy-furanoeremophilane, 10406

$\mathrm{C}_{16} \mathrm{H}_{24} \mathrm{O}_{4}$ 
Brefeldin A, 2600

Isotorquatone, 11745

8-Methoxy-9-(2-methylbutyryloxy)thymol, 14005

7-Oxopinguisenol-12-methyl ester, 16407

\section{$\mathrm{C}_{\mathbf{1 6}} \mathrm{H}_{\mathbf{2 4}} \mathrm{O}_{\mathbf{5}}$}

$8 \beta, 10 \beta$-Dihydroxy- $6 \beta$-methoxyeremophil-7(11)-

en-12,8 $\alpha$-olide, 5977

7-O-Methylpseudomajucin, 14704

\section{$\mathrm{C}_{16} \mathrm{H}_{24} \mathrm{O}_{7}$}

Epirhododendrin, 7007

8-Hydroxycuminyl $\beta$ - $D$-glucopyranoside, 9950

(8R)-9-Hydroxycuminyl $\beta$ - $D$-glucopyranoside, 9951

7-Hydroxythymol 3-O- $\beta$ - $D$-glucopyranoside, 10764

Perilloside B, 16932

(2S,3S)-1-Phenyl-2,3-butanediol

3-O- $\beta$ - $D$-glucopyranoside, 17097

Rhododendrin, 18794

Thymoquinol 2- $O-\beta$-glucopyranoside, 21363

Thymoquinol 5- $O-\beta$-glucopyranoside, 21364

\section{$\mathrm{C}_{16} \mathrm{H}_{24} \mathrm{O}_{8}$}

$\left(1^{\prime} R, 2^{\prime} R\right)$-Anethole Glycol 2'-O- $\beta$ - $D$-glucopyranoside, 1184

$(1 ' S, 2$ 'S)-Anethole glycol 2'- $O-\beta$ - $D$-Glucopyranoside, 1185

Boschnaloside, 2564

(8S)-8,9-Dihydroxycuminyl $\beta$ - $D$-glucopyranoside, 5798

1-(4'-Methoxyphenyl)-(1 $R, 2 S)$-propan-1-ol 2-O- $\beta$-D-glucopyranoside, 14066

1-(4'-Methoxyphenyl)-(1S,2R)-propan-1-ol 2-O- $\beta$ - $D$-glucopyranoside, 14067

\section{$\mathrm{C}_{16} \mathrm{H}_{24} \mathrm{O}_{9}$}

Cachineside I, 2839

7-Deoxy-8-epi-loganic acid, 5189

C-8-(S)-7-Deoxyloganic acid, 5190

2,6-Dimethoxy-4-(2-hydroxyethyl)phenol

$1-O-\beta$ - $D$-glucopyranoside, 6239

1'-(4-Hydroxy-2-methoxyphenyl)propane-2',3diol 4-O- $\beta$ - $D$-glucopyranoside, 10433

Ixoroside, 11805

Junipediol A 29-O- $\beta$ - $D$-glucopyranoside, 11963

Junipediol A 4-O- $\beta$ - $D$-glucopyranoside, 11964

Nikoenoside, 15595

Plantarenaloside, 17513

Swertiajaposide B, 20507

\section{$\mathrm{C}_{16} \mathrm{H}_{24} \mathrm{O}_{10}$}

Adoxosidic acid, 652

Cachineside V, 2842

8-O-Debenzoylpaeoniflorin, 4809

Demethylsecologanol, 5097
8-Diebenzoylpaeoniflorin, 5483

6 $\alpha$-Dihydrocornic acid, 5567

$6 \beta$-Dihydrocornic acid, 5568

8-Epiloganic acid, 6952

$D$-threo-Guaiacyl glycerol 8- $\beta$ - $D$-glucopyranoside, 9023

L-threo-Guaiacyl glycerol 8- $\beta$-D-glucopyranoside, 9024

$\left(1^{\prime} R, 2^{\prime} R\right)$-Guaiacyl glycerol 3'- $O-\beta$ - $D$-glucopyranoside, 9025

$\left(1^{\prime} R, 2^{\prime} R\right)$-Guaiacyl glycerol 4- $O-\beta$ - $D$-glucopyranoside, 9026

$\left(1 ' S, 2^{\prime} R\right)$-Guaiacyl glycerol 3'- $O-\beta$ - $D$-glucopyranoside, 9027

8-Hydroxy-10-hydrosweroside, 10174

9-Hydroxysemperoside, 10698

Loganic acid, 12949

6-O-Methyl catalpol, 14222

Mussaenosidic acid, 15134

Tecomoside, 20897

$\mathrm{C}_{16} \mathrm{H}_{24} \mathrm{O}_{11}$

Cachineside IV, 2841

Shanzhiside, 19795

Tuliposide F, 22107

\section{$\mathrm{C}_{16} \mathrm{H}_{25} \mathrm{~N}$}

(E)-3-Isocyanobisabolane-7,10-diene, 11360

Muscopyridine, 15130

\section{$\mathrm{C}_{16} \mathrm{H}_{25} \mathrm{NO}$}

Hydroxymuscopyridine A, 10532

Hydroxymuscopyridine B, 10533

Lycopodine, 13223

Sanshool, 19297

\section{$\mathrm{C}_{16} \mathrm{H}_{25} \mathrm{NO}_{2}$}

Acrifolinol, 568

Annofoline, 1300

Clavatine, 3809

Clavolonine, 3813

Dendrobine, 5108

12-Epilycodoline, 6956

Flabelliformine, 7805

L20, 12400

Lucidioline, 13046

Lycodoline, 13191

Lycofoline, 13194

Lycoposerramine J, 13229

Lycoposerramine L, 13231

Lycoposerramine M, 13232

Pseudoselagine, 18062

$\mathrm{C}_{16} \mathrm{H}_{25} \mathrm{NO}_{3}$

Decoyl vanillylamide, 4858

Dendramine, 5106

6-Hydroxydendrobine, 9982

Lycoposerramine G, 13226
Serrantinine, 19764

Serratine, 19771

Serratinine, 19773

\section{$\mathrm{C}_{16} \mathrm{H}_{25} \mathrm{NO}_{4}$}

Lycoposerramine F, 13225

Serratanidine, 19766

ZP-amide A, 23023

ZP-amide B, 23024

\section{$\mathrm{C}_{16} \mathrm{H}_{25} \mathrm{NO}_{5}$}

Hordenine- $O$ - $\alpha$ - $L$-rhamnopyranoside, 9649

Retusine, 18668

\section{$\mathrm{C}_{16} \mathrm{H}_{25} \mathrm{NO}_{9} \mathrm{~S}$}

Sinapine bisulfate, 19918

\section{$\mathrm{C}_{16} \mathrm{H}_{25} \mathrm{NO}_{10}$}

Proacaciberin, 17863

\section{$\mathrm{C}_{16} \mathrm{H}_{26}$}

3-Phenyldecane, 17104

Valencene, 22311

\section{$\mathrm{C}_{16} \mathrm{H}_{26} \mathrm{~N}_{2} \mathrm{O}$}

Cernuine, 3431

\section{$\mathrm{C}_{16} \mathrm{H}_{26} \mathrm{~N}_{2} \mathrm{O}_{2}$}

Lycocernuine, 13184

$\mathrm{C}_{16} \mathrm{H}_{26} \mathrm{~N}_{4} \mathrm{O}_{2}$

Acanthoidine, 80

\section{$\mathrm{C}_{16} \mathrm{H}_{26} \mathrm{O}$}

7,10,13-Hexadecatrienal, 9491

\section{$\mathrm{C}_{16} \mathrm{H}_{26} \mathrm{O}_{2}$}

1,5-Di-isobutyl-3,3-dimethyl[3,1,0]cyclohexadione, 6188

7Z,10Z,13Z-Hexadecatrienoic acid, 9492

\section{$\mathrm{C}_{16} \mathrm{H}_{26} \mathrm{O}_{3}$}

Bisaborosaol A, 2417

(10R)-Hydroxyhexadeca-7Z,11E,13Z-trienoic acid, 10161

Juvabione, 11987

Juvenile hormone III, 11988

4-Methoxy-5-hydroxybisabola-2,10-diene-9-one, 13950

2-Methoxy-3-nonylresorcinol, 14042

\section{$\mathrm{C}_{16} \mathrm{H}_{26} \mathrm{O}_{4}$}

Bisaborosaol $\mathrm{B}_{1}, 2418$

Bisaborosaol $\mathrm{B}_{2}, 2419$

Bisaborosaol F, 2423

$5 \alpha$-Hydroxy-4-epi-ilicic acid methyl ester, 10072

$7 \alpha$-Hydroxypinguisenol-12-methyl ester, 10650

$7 \beta$-Hydroxypinguisenol-12-methyl ester, 10651

\section{$\mathrm{C}_{16} \mathrm{H}_{26} \mathrm{O}_{5}$}

Bisaborosaol $\mathrm{C}_{1}, 2420$

Bisaborosaol $\mathrm{C}_{2}, 2421$

Bisaborosaol D, 2422

\section{$\mathrm{C}_{16} \mathrm{H}_{26} \mathrm{O}_{6}$}

Limonene-10-ol 10-O- $\beta$ - $D$-glucopyranoside, 12845

Perilloside A, 16931 
Perillylglucopyranoside, 16936

$\mathrm{C}_{16} \mathrm{H}_{26} \mathrm{O}_{7}$

Dissectol A, 6518

$(1 S, 4 R, 6 S)$-6-Hydroxycamphor $\beta$ - $D$-glucopyranoside, 9880

$(1 R, 4 S, 6 S)$-6-Hydroxycamphor $\beta$ - $D$-glucopyranoside, 9881

(4R,6S)-7-Hydroxycarveol 7-O- $\beta$ - $D$-glucopyranoside, 9896

$3 \beta$-Hydroxy- $p$-menth-1-en- $4 \beta, 5 \beta$-oxide 3-O- $\beta$ - $D$ glucopyranoside, 10378

$6 \alpha$-Methyl-2,6 $\beta$-dihydroxymethylbicyclo[3.1.1]hept-2-ene-2 $\beta$-O-glucoside, 14311

Picrocrocin, 17330

Schizonepetoside A, 19503

Schizonepetoside B, 19504

Schizonepetoside C, 19505

Schizonepetoside D, 19506

Thymuside A, 21365

\section{$\mathrm{C}_{16} \mathrm{H}_{26} \mathrm{O}_{8}$}

Bodinierin, 2526

(4S,8S)-8,9-Dihydroxy-8,9-dihydrocarvone 9-O$\beta$ - $D$-glucopyranoside, 5808

(-)-Oleuropeic acid 8- $O-\beta$ - $D$-glucopyranoside, 16079

Picrocrocinic acid $O-\beta$ - $D$-glucopyrinoside, 17331

Rehmapicroside, 18601

Villoside, 22479

\section{$\mathbf{C}_{\mathbf{1 6}} \mathbf{H}_{\mathbf{2 6}} \mathbf{O}_{\mathbf{9}}$}

1-O- $\beta$-D-Glucopyranosylamplexin, 8603

Villosolside, 22481

\section{$\mathrm{C}_{16} \mathrm{H}_{26} \mathrm{O}_{10}$}

3,4-Dihydro-methylcatalpol, 5675

Lamiol, 12458

\section{$\mathrm{C}_{16} \mathrm{H}_{26} \mathrm{O}_{11}$}

3-Methylbut-2-enoyl-1- $O$ - $\beta$ - $D$-glucopyranosyl$\beta$ - $D$-apiofuranoside, 14178

\section{$\mathrm{C}_{16} \mathrm{H}_{27} \mathrm{KO}_{10} \mathrm{~S}$}

(3S,6E)-8-Hydroxylinalool 3-O- $\beta-D-(3-O-$

Potassium sulfo)glucopyranoside, 10326

\section{$\mathrm{C}_{16} \mathrm{H}_{27} \mathrm{NO}$}

Complanatine, 3948

1-(1-Oxo-2E,4E-dodedienyl)pyrrolidine, 16325

\section{$\mathrm{C}_{16} \mathrm{H}_{27} \mathrm{NO}_{2}$}

Deacetyllycoclavine, 4748

\section{$\mathrm{C}_{16} \mathrm{H}_{27} \mathrm{NO}_{4}$}

ZP-amide C, 23025

ZP-amide D, 23026

ZP-amide E, 23027

ZP-amide F, 23028

\section{$\mathrm{C}_{16} \mathrm{H}_{27} \mathrm{NO}_{5}$}

Heliotridine $2 S$-hydroxy-2S-(1S-hydroxyethyl)-4methyl-pentanoyl ester, 9319
Heliotrine, 9320

Retronecine $2 S$-hydroxy-2S-(1S-hydroxyethyl)4-methylpentanoyl ester, 18664

Supinidine $N$-oxide $2 S$-hydroxy- $2 S$-( $1 S$-hydroxyethyl)-4-methylpentanoyl ester, 20487

\section{$\mathrm{C}_{16} \mathrm{H}_{27} \mathrm{NO}_{6}$}

Europine, 7636

Retronecine $N$-oxide $2 S$-hydroxy- $2 S$-( $1 R$-hydroxyethyl)-4-methylpentanoyl ester, 18665

Retronecine $N$-oxide $2 S$-hydroxy-2S-(1S-hydroxyethyl)-4-methylpentanoyl ester, 18666

\section{$\mathrm{C}_{16} \mathrm{H}_{27} \mathrm{NO}_{11}$}

Linustatin, 12896

\section{$\mathrm{C}_{16} \mathrm{H}_{28} \mathrm{O}$}

1-Methoxy-4-cadinene, 13862

\section{$\mathrm{C}_{16} \mathrm{H}_{28} \mathrm{O}_{2}$}

Ambrettolide, 1027

Hydnocarpic acid, 9697

$6 \alpha$-Methoxyeudesm-4(15)-en-1 $\beta$-ol, 13925

11-Methoxyopposit-4(15)-en-1 $\beta$-ol, 14048

\section{$\mathrm{C}_{16} \mathrm{H}_{28} \mathrm{O}_{3}$}

13-Hydroxy-9,11-hexadecadienoic acid, 10160

15-Methoxyisodauc-3-ene-1 $\beta, 5 \alpha$-diol, 13974

\section{$\mathrm{C}_{16} \mathrm{H}_{28} \mathrm{O}_{4}$}

Actinolide B, 587

Methyl $(2 E, 6 E, 10 R)$-10,11-dihydroxy-3,7,11-trimethyl-2,6-dodecadienoate, 14318

\section{$\mathrm{C}_{16} \mathrm{H}_{28} \mathrm{O}_{6}$}

Borneol-2-O- $\beta$ - $D$-glucopyranoside, 2554

Perilloside C, 16933

\section{$\mathbf{C}_{16} \mathbf{H}_{28} \mathbf{O}_{7}$}

$(1 R, 2 S, 4 S, 5 R)$-Angelicoidenol 2- $O-\beta$ - $D$-glucopyranoside, 1192

Betulalbuside A, 2329

(1R,2R,4S,6R)-Bornane-2,6-diol 2-O- $\beta$ - $D$-glucopyranoside, 2548

(1S,2S,4R,6S)-Bornane-2,6-diol 2- $O-\beta$ - $D$-glucopyranoside, 2549

Bucharioside, 2696

(1R,2R,4S)-2-Hydroxy-1,8-cineole $\beta$ - $D$-glucopyranoside, 9907

8-Hydroxygeraniol-1- $\beta$ - $D$-glucopyranoside, 10141

$2 \beta$-Hydroxy- $2 \alpha$-hydroxymethyl-6,6-dimethyl bicyclo[3.1.1] heptane-2 $\alpha$ - $O$-glucoside, 10203

$p$-Menth-1-ene-3,4-diol 4- $O$ - $\beta$-glucopyranoside, 13748

(1R,2R)-p-Menth-4(5)-ene-1,2-diol 1- $O$ - $\beta$ - $D$-glucopyranoside, 13749

$(1 S, 2 R, 4 R)$ - $p$-Menth-5-ene-1,2-diol 1-O- $\beta$ - $D$-glucopyranoside, 13750

$(1 S, 2 R, 4 R)-p$-Menth-5-ene-1,2-diol 2-O- $\beta$ - $D$-glucopyranoside, 13751
$(1 S, 2 R, 4 R)$ - $p$-Menth-8-ene-2,10-diol

2-O- $\beta$-D-glucopyranoside, 13752

$(1 S, 2 S, 4 R)$ - $p$-Menth-8-ene-1,2-diol 1-O- $\beta$ - $D$-glucopyranoside, 13753

(1S,2S,4R)-p-Menth-8-ene-1,2-diol 2-O- $\beta$ - $D$-glucopyranoside, 13754

$(1 R, 2 R)$ - $p$-Menth-3-ene-1,2-diol 2- $O$ - $\beta$ - $D$-glucopyranoside, 13755

$(3 R, 4 R)$ - $p$-Menth-1-ene-3,4-diol 3- $O-\beta$ - $D$-glucopyranoside, 13756

$(3 R, 4 S, 6 R)$ - $p$-Menth-1-ene-3,6-diol 3- $O$ - $\beta$ - $D$-glucopyranoside, 13757

$(3 R, 4 S, 6 R)$ - $p$-Menth-1-ene-3,6-diol 6- $O$ - $\beta$ - $D$-glucopyranoside, 13758

(4R)- $p$-Menth-1-ene-7,8-diol 7- $O-\beta$ - $D$-glucopyranoside, 13759

(4R)- $p$-Menth-1-ene-7,8-diol 8- $O-\beta$ - $D$-glucopyranoside, 13760

$(4 R, 6 S)$ - $p$-Menth-1-ene-4,6-diol 4- $O-\beta$ - $D$-glucopyranoside, 13761

(4S)- $p$-Menth-1-ene-4,7-diol 4- $O$ - $\beta$ - $D$-glucopyranoside, 13762

(4S)- $p$-Menth-1-ene-7,8-diol 8- $O$ - $\beta$ - $D$-glucopyranoside, 13763

(4S,6S)- $p$-Menth-1-ene-4,6-diol 4-O- $\beta$ - $D$-glucopyranoside, 13764

$6 \alpha$-Methyl-2 $\alpha, 6 \beta$-dihydroxymethylbicyclo[3.1.1] heptane-2 $\alpha$-O-glucoside, 14309

$(1 S, 2 R, 4 S, 7 R)$-Vicodiol 2-O- $\beta$ - $D$-glucopyranoside, 22467

$(1 R, 2 S, 4 R, 7 S)$-Vicodiol 9-O- $\beta$ - $D$-glucopyranoside, 22468

\section{$\mathrm{C}_{16} \mathrm{H}_{28} \mathrm{O}_{8}$}

$(1 S, 4 S, 8 S)-8,9-$ Dihydroxytetrahydrocarvone 9-O$\beta$ - $D$-glucopyranoside, 6142

$(3 R, 4 R, 6 R)-p$-Menth-1-ene-3,4,6-triol 3-O- $\beta$ - $D$ glucopyranoside, 13768

$(1 S, 2 R, 4 S)$ - $p$-Menth-5-ene-1,2,4-triol 2-O- $\beta$ - $D$ glucopyranoside, 13769

Schizonepetoside E, 19507

\section{$\mathrm{C}_{16} \mathrm{H}_{28} \mathrm{O}_{10}$}

Isopentenol-1- $O-\beta$ - $D$-apiofuranosyl- $(1 \rightarrow 6)-\beta-D$ glucopyranoside, 11584

\section{$\mathrm{C}_{16} \mathrm{H}_{28} \mathrm{O}_{11}$}

3-Methylbutanoyl-1-O- $\beta$ - $D$-glucopyranosyl- $\beta$ - $D$ apiofuranoside, 14166

\section{$\mathrm{C}_{16} \mathrm{H}_{29} \mathrm{NO}$}

Herculin, 9428

$N$-Isobutyl-2E,4E-dodedienamide, 11272

\section{$\mathrm{C}_{16} \mathrm{H}_{29} \mathrm{NO}_{4}$}

Trachelanthamidine $2 S$-hydroxy-2S-(1S-hydroxyethyl)-4-methylpentanoyl ester, 21489 Viridiflorine, 22527 


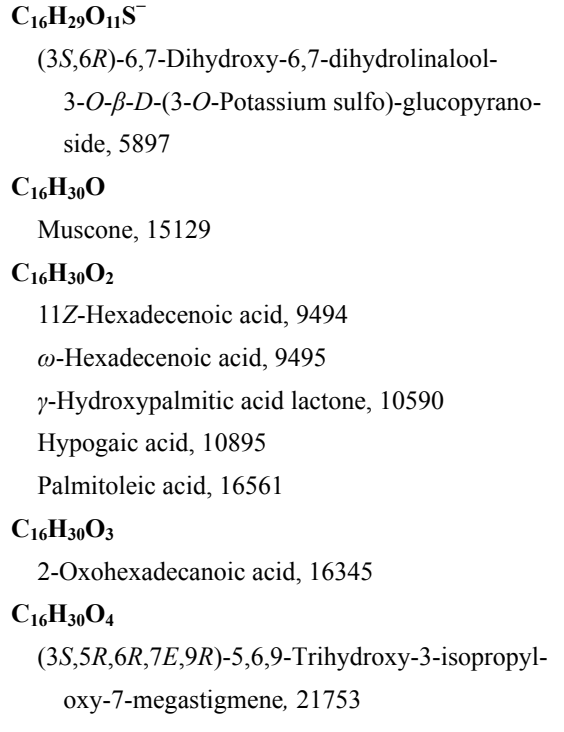

\section{$\mathbf{C}_{16} \mathrm{H}_{30} \mathbf{O}_{7}$}

(3S)-8-Hydroxy-6,7-dihydrolinallol 3-O- $\beta$ - $D$-glucopyranoside, 10005

8-Hydroxy-6,7-dihydrolinalool 8-O-glucopyranoside, 10006

\section{$\mathrm{C}_{16} \mathrm{H}_{30} \mathrm{O}_{8}$}

(3S,6R)-6,7-Dihydroxy-6,7-dihydrolinalool-3-O$\beta$-D-glucopyranoside, 5813

(3S,6S)-6,7-Dihydroxy-6,7-dihydrolinalool-3-O$\beta$ - $D$-glucopyranoside, 5814

Ethyl $O-\beta$ - $D$-oleandropyranosyl-( $1 \rightarrow 4)-O-3-O$ methyl-6-deoxy- $\beta$ - $D$-allopyranoside, 7468

$(1 S, 2 R, 4 R, 8 S)-p$-Menthane-2,8,9-triol 2-O- $\beta-D-$ glucopyranoside, 13741

$(1 S, 2 S, 4 R)$ - $p$-Menth-1,2,8-triol 2-O- $\beta$ - $D$-glucopyranoside, 13777

$(1 R, 2 R, 4 R, 8 R)$ - $p$-Menth-2,8,9-triol 2-O- $\beta$ - $D$-glucopyranoside, 13778

\section{$\mathrm{C}_{16} \mathrm{H}_{30} \mathrm{O}_{9}$}

(2S,6ら)-3,7-Dimethyloct-3(10)-ene-1,2,6,7-tetrol 1-O- $\beta$ - $D$-glucopyranoside, 6388

rel-(1R,2R,4R,8S)-p-Menthane-1,2,8,9-tetrol 9-O- $\beta$ - $D$-glucopyranoside, 13736

$(1 S, 2 S, 4 R, 8 R)-p$-Menthane-1,2,8,9-tetrol 2-O- $\beta$ $D$-glucopyranoside, 13737

(1S,2S,4R,8S)-p-Menthane-1,2,8,9-tetrol 2-O- $\beta$ $D$-glucopyranoside, 13738

$$
\mathrm{C}_{16} \mathrm{H}_{32}
$$

2-Cyclohexyldecane, 4497

1-Hexadecene, 9493

\section{$\mathrm{C}_{16} \mathrm{H}_{32} \mathrm{O}$}

Muscol, 15128

$\mathrm{C}_{16} \mathrm{H}_{32} \mathrm{O}_{2}$

Hexadecanoic acid, 9486

Methyl pentadecanoate, 14655

13-Methyl pentadecanoic acid, 14656
12-Methyl tetradecanoic acid methyl ester, 14744

4,8,12-Trimethyl tridecanoic acid, 21976

$\mathrm{C}_{16} \mathrm{H}_{32} \mathrm{O}_{5}$

Aleuritic acid, 887

$\mathrm{C}_{16} \mathrm{H}_{33} \mathrm{NO}$

Palmitamide, 16559

$\mathrm{C}_{16} \mathrm{H}_{34}$

Hexadecane, 9485

$\mathrm{C}_{17} \mathrm{H}_{9} \mathrm{NO}_{3}$

Liriodenine, 12917

$\mathrm{C}_{17} \mathrm{H}_{10} \mathrm{O}_{6}$

Aristolophenanlactone I, 1724

Flemichapparin C, 7826

$\mathrm{C}_{17} \mathrm{H}_{10} \mathbf{O}_{7}$

Boeravinone F, 2537

Irisoid D, 11169

$\mathrm{C}_{17} \mathrm{H}_{10} \mathrm{O}_{8}$

Irisoid E, 11170

\section{$\mathrm{C}_{17} \mathrm{H}_{11} \mathrm{NO}_{4}$}

Aristolactam I, 1698

4,5-Dioxodehydroasimilobine, 6466

6-Methoxy-aristololactam, 13844

\section{$\mathrm{C}_{17} \mathrm{H}_{11} \mathrm{NO}_{5}$}

Aristoliukine B, 1712

\section{$\mathrm{C}_{17} \mathrm{H}_{11} \mathrm{NO}_{6}$}

Aristolochic acid II methyl ester, 1717

\section{$\mathrm{C}_{17} \mathrm{H}_{11} \mathrm{NO}_{7}$}

Aristolochic acid, 1713

\section{$\mathrm{C}_{17} \mathrm{H}_{11} \mathrm{NO}_{8}$}

Aristolochic acid E, 1715

Aristolochic acid IVa, 1719

7-Hydroxy-aristolochic acid A, 9799

\section{$\mathrm{C}_{17} \mathrm{H}_{11} \mathbf{N}_{3} \mathrm{O}_{2}$}

Bouchardatine, 2569

$\mathrm{C}_{17} \mathrm{H}_{12} \mathrm{~N}_{2} \mathrm{O}_{4}$

Flazin, 7824

$\mathrm{C}_{17} \mathrm{H}_{12} \mathrm{O}_{3}$

Tanshinlactone, 20682

$\mathrm{C}_{17} \mathrm{H}_{12} \mathrm{O}_{4}$

Nortanshinone, 15801

\section{$\mathrm{C}_{17} \mathrm{H}_{12} \mathrm{O}_{5}$}

Flemichapparin B, 7825

3'-Methoxy-4',5'-methylenedioxyflavone, 14016

5-Methoxy-6,7-methylenedioxyflavone, 14017

3',4'-Methylenedioxy-7-methoxyflavone, 14376

\section{$\mathrm{C}_{17} \mathrm{H}_{12} \mathrm{O}_{6}$}

Aflatoxin $\mathrm{B}_{1}, 671$

Boeravinone B, 2533

3,4-Dimethylenedioxypterocarpan, 6349

Leiocarpaquinone, 12606

1-Methoxy-3-hydroxy-2-carbomethoxy-9,10anthraquinone, 13952

5-Methoxy-4'-hydroxy-6,7-methylenedioxyiso- flavone, 13964

Tournefolic acid A, 21478

Tournefolic acid B, 21479

\section{$\mathrm{C}_{17} \mathrm{H}_{12} \mathrm{O}_{7}$}

Aflatoxin $\mathrm{G}_{1}, 673$

Boeravinone E, 2536

Iriflogenin, 11152

Irisoid A, 11166

$\mathrm{C}_{17} \mathrm{H}_{12} \mathrm{O}_{8}$

3,3',4-Tri- $O$-methyl ellagic acid, 21955

\section{$\mathrm{C}_{17} \mathrm{H}_{13} \mathrm{NO}$}

Hypodematine, 10891

\section{$\mathrm{C}_{17} \mathrm{H}_{13} \mathrm{NO}_{3}$}

10-Amino-2,4-dimethoxyphenanthrene-1carboxylic acid lactam, 1049

Aristolactam BII, 1695

Graveoline, 8991

4-Hydroxy-3-methoxy- $N$-methylaristolactam, 10416

Opuntin B, 16158

Sauristolactam, 19417

\section{$\mathrm{C}_{17} \mathrm{H}_{13} \mathrm{NO}_{4}$}

Aristolactam FII, 1696

Gonioffithine, 8937

Goniothalactam, 8945

1-(4-Hydroxybenzoyl)-7-hydroxy-6-methoxyisoquinoline, 9825

Hyoscyamine $6 \beta$-hydroxylase, 10873

Piperlactam S, 17460

Piperolactam B, 17464

Piperolactam D, 17466

\section{$\mathrm{C}_{17} \mathrm{H}_{13} \mathrm{NO}_{5}$}

Aristoliukine A, 1711

\section{$\mathrm{C}_{17} \mathrm{H}_{13} \mathrm{O}_{9} \mathrm{~S}$}

Quercetin-3-methyl-7-methyl ether-4'-sulfate, 18349

\section{$\mathrm{C}_{17} \mathrm{H}_{13} \mathrm{O}_{10} \mathrm{~S}$}

Persicarin-7-methylether, 16991

\section{$\mathrm{C}_{17} \mathrm{H}_{13} \mathrm{O}_{11} \mathrm{~S}^{-}$}

Centradixin, 3398

\section{$\mathrm{C}_{17} \mathrm{H}_{14} \mathrm{~N}_{2}$}

Ellipticine, 6759

Olivacine, 16085

\section{$\mathrm{C}_{17} \mathrm{H}_{14} \mathrm{~N}_{2} \mathrm{O}_{2}$}

Luotonin D, 13087

\section{$\mathrm{C}_{17} \mathrm{H}_{14} \mathrm{~N}_{2} \mathrm{O}_{3}$}

Pedatisectine C, 16760

\section{$\mathrm{C}_{17} \mathrm{H}_{14} \mathrm{O}_{2}$}

2,7-Dihydroxy-1-methyl-5-vinylphenanthrene, 6046

2-(2-Phenylethyl) chromone, 17113

$\mathrm{C}_{17} \mathrm{H}_{14} \mathrm{O}_{3}$

6-Hydroxy-2-(2-phenylethyl) chromone, 10616 
7-Hydroxy-2-(2-phenylethyl)chromone, 10617

7-Methoxy-2-methyl isoflavone, 14025

Sterehirsutinal, 20332

Typharin, 22156

\section{$\mathrm{C}_{17} \mathrm{H}_{14} \mathbf{O}_{4}$}

5,8-Dihydroxy-2-(2-phenylethyl)chromone, 6082

6,8-Dihydroxy-2-(2-phenylethyl)chromone, 6083

2',5'-Dimethoxyflavone, 6225

3',4'-Dimethoxyflavone, 6226

7,4'-Dimethoxyisoflavone, 6254

6-Hydroxy-2-(2-hydroxy-2-phenylethyl)chromone, 10210

6-Hydroxy-2-[2-(2-hydroxyphenyl) ethyl]chromone, 10211

6-Hydroxy-2-[2-(4-hydroxyphenyl) ethyl]chromone, 10212

5-Hydroxy-7-methoxy-6-methylflavone, 10424

5-Hydroxy-7-methoxy-8-methylflavone, 10425

9-Hydroxymicroperfuranone, 10527

Isobonducellin, 11258

$O$-Methyldalbergin, 14280

\section{$\mathrm{C}_{17} \mathrm{H}_{14} \mathrm{O}_{5}$}

Afrormosin, 676

Desmosflavone, 5270

7,4'-Dimethoxy-3'-hydroxyflavone, 6240

7,4'-Dimethoxy-5-hydroxyisoflavone, 6243

3-(4-Hydroxybenzylidene)-5-hydroxy-7methoxychroman-4-one, 9844

3'-Hydroxy-4',5'-dimethoxyflavone, 10023

5-Hydroxy-4',7-dimethoxy-flavone, 10024

5-Hydroxy-6,7-dimethoxyflavone, 10025

5-Hydroxy-7,8-dimethoxyflavone, 10026

7-Hydroxy-3,5-dimethoxyflavone, 10027

7-Hydroxy-5,8-dimethoxyflavone, 10028

1-Hydroxy-2,3-dimethoxy-7-methyl-9,10anthraquinone, 10032

Isoneobavachalcone, 11557

Melimessanol C, 13684

1-Methoxy-2-methoxymethyl-3-hydroxyanthraquinone, 13998

2-Methoxy-obtusifolin, 14046

2'-O-Methylabronisoflavone, 14109

8-o-Methylreyusi, 14713

Neobavachalcone, 15341

Pterocarpin, 18103

Robustaquinone B, 18870

$\mathrm{C}_{17} \mathrm{H}_{14} \mathrm{O}_{6}$

Abrectorin, 11

trans-3-Acetoxy-5,7-dihydroxyflavanone, 165

Aflatoxin $\mathrm{B}_{2}, 672$

Cirsimaritin, 3745

5,3'-Dihydroxy-7,4'-dimethoxyflavone, 5829

5,8-Dihydroxy-6,7-dimethoxyflavone, 5830
2',7-Dihydroxy-4',5'-dimethoxyisoflavone, 5834

3',7-Dihydroxy-4',6-dimethoxyisoflavone, 5835

1,3-Dihydroxy-5,6-dimethoxy-2-methyl-9,10-

anthraquinone, 5836

1,7-Dihydroxy-3,9-dimethoxy pterocarpene, 5837

3-(3,4-Dihydroxyphenyl)-2-propenoic acid (Z,E)-

2-(3,4-dihydroxyphenyl) ethenyl ester, 6095

3-(3,4-Dihydroxyphenyl)-2-propenoic acid $(Z, E)$ -

2-(3,5-dihydroxyphenyl) ethenyl ester, 6096

5,15-Dimethylmorindol, 6376

Gnaphaliin, 8865

3-(4-Hydroxybenzylidene)-5,7-dihydroxy-6methoxychroman-4-one, 9843

5-Hydroxy-7-methoxy-3',4'-methylenedioxy isoflavone, 10420

(-)-3-Hydroxy-4-methoxy-8-9-methylenedioxy pterocarpan, 10421

Irilin B, 11156

Irisolidone, 11171

Kaempferol-3,4-di-O-methyl ether, 12044

Kaempferol-7,4'-dimethyl ether, 12045

Kumatakenin, 12331

Ladanein, 12448

6-C-Methylluteolin 7-methyl ether, 14567

Pectolinarigenin, 16755

Pisatin, 17477

Rhynchotechol, 18828

Salvianolic acid F, 19206

Skullcapflavone I, 20004

\section{$\mathrm{C}_{17} \mathrm{H}_{14} \mathrm{O}_{7}$}

Aflatoxin $\mathrm{G}_{2}, 674$

Anticancer Flavonoid PMV70P691-87, 1416

Aurantioobtusin, 2008

Betuletol, 2330

Calycosin C, 3007

Cirsiliol, 3743

4',7-Dihydroxy-2',5-dimethoxyflavonol, 5832

3,3'-Dimethylquercetin, 6402

Eupalitin, 7577

Hildecarpin, 9540

Irisflavone A, 11160

Irisflavone D, 11163

Laurentinol, 12567

4'-Methylcapillarisin, 14212

7-Methylcapillarisin, 14213

Olibergin A, 16082

Ombuin, 16092

Quercetin-3',4'-dimethyl ether, 18348

Rhamnazin, 18674

Robustaquinone A, 18869

Tricin, 21589

3,5,6-Trihydroxy-7,4'-dimethoxyflavone, 21707
5,2',6'-Trihydroxy-7,8-dimethoxyflavone, 21708

5,6,4'-Trihydroxy-7,3'-dimethoxyflavone, 21709

5,7,2'-Trihydroxy-8,6'-dimethoxyflavone, 21710

5,7,4'-Trihydroxy-3,8-dimethoxyflavone, 21711

5,8,2'-Trihydroxy-6,7-dimethoxyflavone, 21712

1,2,6-Trihydroxy-7,8-dimethoxy-3-methylanthraquinone, 21713

\section{$\mathrm{C}_{17} \mathrm{H}_{14} \mathrm{O}_{\mathbf{8}}$}

Axillarin, 2044

Eupatolitin, 7588

Gossypetin-3,5-dimethyl ether, 8960

Quercetagetin-3,4'-dimethyl ether, 18308

Spinacetin, 20164

Syringetin, 20561

Taxifolin 3-O-acetate, 20791

Tenuiflorin A, 20934

Tenuiflorin B, 20935

$5,7,2$ ',5'-Tetrahydroxy-8,6'-dimethoxy flavone, 21094

$5,7,3^{\prime}, 4^{\prime}$-Tetrahydroxy-6,8-dimethoxy flavone, 21095

$5,7,8,3^{\prime}$-Tetrahydroxy-3,4'-dimethoxy flavone, 21096

5,6,3',4'-Tetrahydroxy-3,7-dimethoxyflavone, 21097

\section{$\mathrm{C}_{17} \mathrm{H}_{14} \mathrm{O}_{9}$}

Rubanthrone B, 18973

\section{$\mathrm{C}_{17} \mathrm{H}_{14} \mathrm{O}_{10}$}

Rubanthrone A, 18972

\section{$\mathrm{C}_{17} \mathrm{H}_{15} \mathrm{NO}_{2}$}

Anonaine, 1348

\section{$\mathrm{C}_{17} \mathrm{H}_{15} \mathrm{NO}_{3}$}

Anolobine, 1344

(-)-Cissaglaberrimine, 3746

Dutadrupine, 6642

Galanthindole, 8084

Juzirine, 11989

Michelalbine, 14827

Reevesianine B, 18569

\section{$\mathrm{C}_{17} \mathrm{H}_{15} \mathrm{NO}_{4}$}

Annocherine A, 1298

(E)-3-(3',5'-Dimethoxy-4'-hydroxybenzylidene)2-indolinone, 6235

Prooxocryprochine, 17910

$\mathrm{C}_{17} \mathrm{H}_{15} \mathrm{O}_{7}$

Malvidin, 13456

$\mathrm{C}_{17} \mathrm{H}_{16} \mathrm{~N}_{2} \mathrm{O}_{3}$

Nauclealine B, 15291

$\mathrm{C}_{17} \mathrm{H}_{16} \mathrm{O}_{2}$

cis-Hinokiresinol, 9546

Thannilignan, 21273

$\mathrm{C}_{17} \mathrm{H}_{16} \mathrm{O}_{3}$ 
Asparenydiol, 1885

Danshenspiroketallactone, 4629

1,3-Di- $p$-hydroxyphenyl-4-penten-1-one, 6090

Epidanshenspiroketallactone, 6877

3-(Hydroxymethyl)-2,5-bis(3-methylbut-3-en-1ynyl)benzene-1,4-diol, 10469

Juncunone, 11959

Obtusilactone, 15902

2,4,7-Trimethoxyphenanthrene, 21920

\section{$\mathrm{C}_{17} \mathrm{H}_{16} \mathrm{O}_{4}$}

Anticancer Flavonoid PMV70P691-93, 1418

Batatasin I, 2163

Caffeic acid phenethyl ester, 2890

Coniferyl benzoate, 3985

Demethoxymatteucinol, 5048

3,9-Dimethoxypterocarpan, 6290

Glypallichalcone, 8850

Isobatatasin I, 11246

Isosativan, 11696

Methyl-(7R,8R)-4-hydroxy-8',9'-dinor-4',7epoxy-8,3'-neolignan-7'-ate, 14503

Mollugin, 14901

Stercurensin, 20331

\section{$\mathrm{C}_{17} \mathrm{H}_{16} \mathrm{O}_{5}$}

2-Carboxymethyl-3-phenyl-2,3-epoxy-1,4-naphthoquinone, 3181

Cnidilin, 3856

Coelogin, 3889

2',3'-Dihydroxy-4',6'-dimethoxychalcone, 5820

2',4'-Dihydroxy-4,6'-dimethoxydihydrochalcone, 5824

7,4'-Dihydroxy-2',5'-dimethoxyisoflav-3-ene, 5833

2,5-Dihydroxy-6-methyl-7-methoxyflavanone, 6036

2,5-Dihydroxy-8-methyl-7-methoxyflavanone, 6037

2,6-Dihydroxy-1,5,7-trimethoxyphenanthrene, 6163

2',6'-Dimethoxy-4,4'-dihydroxychalcone, 6217

Farrerol, 7736

2'-Hydroxy-3',5'-diimethoxyflavanone, 10013

5-Hydroxy-4',7-dimethoxydihydroflavone, 10020

5-Hydroxy-7,2'-dimethoxyflavone, 10022

5-Hydroxy-7-methoxy-3-(4'-hydroxybenzyl)-4chromanone, 10409

6-Isopentenyloxyisobergapten, 11589

Matteucin, 13609

Melilotocarpan A, 13679

3'-O-Methylbrazilin, 14159

Methylnissolin, 14619

Odoriflavene, 16001

Pashanone, 16697
Phellopterin, 17077

Sativanone, 19393

(-)-Sparticarpin, 20134

Tsugafolin, 22067

(-)-Variabilin, 22347

\section{$\mathrm{C}_{17} \mathrm{H}_{16} \mathrm{O}_{6}$}

Aromadendrin-5,7-dimethyl ether, 1766

Byakangelicol, 2834

Cyrtominetin, 4588

Dihydroskullcapflavone I, 5717

(2S)-5,2'-Dihydroxy-7,5'-dimethoxyflavanone, 5828

1,8-Dihydroxy-3-(3'-hydroxy-butoxy) xanthone, 5950

Homoferreirin, 9606

3-(4-Hydroxybenzyl)-5,7-dihydroxy-6-methoxychroman-4-one, 9836

3-(3-Hydroxy-4-methoxybenzyl)-5,7-dihydroxychroman-4-one, 10385

Isobyakangelicol, 11301

Lapathinol, 12504

Melilotocarpan D, 13681

Methoxy-8-(3"-hydroxymethyl-but-2-enyloxy)psoralen, 13963

Neobyakangelicol, 15348

Pendulone, 16804

Persicogenin, 16992

Tamariscina ester A, 20653

1,2,3,7-Tetramethoxyxanthone, 21190

Teucrol, 21216

3,5,7-Trihydroxy-3-(4'-methoxybenzyl)-4-chromanone, 21767

\section{$\mathrm{C}_{17} \mathrm{H}_{16} \mathrm{O}_{7}$}

Americanoic acid methyl ester, 1035

(2R,3S)-(+)-3',5-Dihydroxy-4',7-dimethoxydihydroflavonol, 5825

(2R,3R)-4',7-Dihydroxy-2',5-dimethoxydihydroflavonol, 5826

4',7-Di- $O$-methyldihydroquercetin, 6338

Dulcisxanthone C, 6628

Evernic acid, 7661

1-Hydroxy-2,3,4,5-tetramethoxyxanthone, 10754

1-Hydroxy-2,3,4,7-tetramethoxyxanthone, 10755

6-Hydroxy-1,2,3,7-tetramethoxyxanthone, 10756

6-Hydroxy-1,3,5,7-tetramethoxyxanthone, 10757

Isoamericanoic acid A methyl ester, 11210

Lespedezol E, 12699

Swertiadecoraxanthone, 20504

3,2',4',6'-Tetrahydroxy-4,3'-dimethoxy chalcone, 21093

Ventilagolin, 22377

$\mathrm{C}_{17} \mathrm{H}_{16} \mathrm{O}_{9}$

Bergaptol- $O-\beta$ - $D$-glucopyranoside, 2311
Xanthotoxol 8-O- $\beta$ - $D$-glucopyranoside, 22776

$\mathrm{C}_{17} \mathrm{H}_{16} \mathrm{O}_{10}$

$5-O-\beta-D$-Glucopyranosyl-6-hydroxyangelicin, 8664

6- $O-\beta-D$-Glucopyranosyl-5-hydroxyangelicin, 8665

5-O- $\beta$ - $D$-Glucopyranosyl-8-hydroxypsoralen, 8677

8-O- $\beta$ - $D$-Glucopyranosyl-5-hydroxypsoralen, 8678

$\mathrm{C}_{17} \mathrm{H}_{16} \mathrm{O}_{11}$

Trimethyl ester dehydrochebulic acid, 21956

\section{$\mathrm{C}_{17} \mathrm{H}_{17} \mathrm{ClO}_{5}$}

8-Chloro-2-(2-phenylethyl)-5,6,7-trihydroxy5,6,7,8-tetrahydrochromone, 3566

\section{$\mathrm{C}_{17} \mathrm{H}_{17} \mathrm{ClO}_{6}$}

5-O-(3-Chloro-2-hydroxy-3-methylbutyl)-8methoxypsoralen, 3561

\section{$\mathrm{C}_{17} \mathrm{H}_{17} \mathrm{NO}_{2}$}

Asimilobine, 1857

(-)-Caaverine, 2838

$N$-trans-Cinnamoyltyramine, 3723

\section{$\mathrm{C}_{17} \mathrm{H}_{17} \mathrm{NO}_{3}$}

$N$-p-cis-Coumaroyltyramine, 4188

$\mathrm{N}$-( trans-p-Coumaroyl) tyramine, 4189

Graveolinine, 8992

Norcinnamolaurine, 15727

Semecarpine, 19690

\section{$\mathrm{C}_{17} \mathrm{H}_{17} \mathrm{NO}_{4}$}

Confusadine, 3974

Demethylcephalotaxinone, 5066

Hainanensine, 9191

$N$-[ $\beta$-Hydroxy- $\beta$-(4-hydroxyphenyl)] ethyl-4hydroxy cinnamide, 10213

5,6,8,9-Tetrahydro-1-hydro-4H-cyclopenta[a]$[1,3]$ dioxolo[4,5- $h]$ pyrrolo[2,1- $b][3]$ benzaepin2(3H)-one, 21073

\section{$\mathrm{C}_{17} \mathrm{H}_{17} \mathrm{NO}_{5}$}

Hippeastrine, 9547

\section{$\mathrm{C}_{17} \mathrm{H}_{17} \mathrm{NO}_{6}$}

Atalafoline, 1953

\section{$\mathrm{C}_{17} \mathrm{H}_{17} \mathrm{O}_{4}$}

2,3-Dihydroxy-4-methoxy-6,6,9-trimethyl-6Hdibenzo $[b, d]$ pyran, 6008

\section{$\mathrm{C}_{17} \mathrm{H}_{18}$}

Heptadec-1,7,9-trien-11,13,15-triyne, 9390

\section{$\mathrm{C}_{17} \mathrm{H}_{18} \mathrm{Cl}_{2} \mathrm{O}_{7}$}

Patientoside B, 16715

\section{$\mathrm{C}_{17} \mathrm{H}_{18} \mathrm{~N}_{2} \mathrm{O}_{5}$}

Miraxanthin III, 14881

\section{$\mathrm{C}_{17} \mathrm{H}_{18} \mathrm{~N}_{2} \mathrm{O}_{6}$}

Miraxanthin V, 14882

\section{$\mathrm{C}_{17} \mathrm{H}_{18} \mathrm{O}_{3}$}


Dalbergiphenol, 4610

Daphneolone, 4642

2,5-Dihydroxy-3-isoprenyl-6-(3-methylbut-3-en-

1-ynyl)benzaldehyde, 5927

Isomucronustyrene, 11547

(2S)-5-Methoxy-6-methylflavan-7-ol, 14022

Neocryptotanshinone II, 15375

2,4,7-Trimethoxy-9,10-dihydrophenanthrene, 21910

\section{$\mathrm{C}_{17} \mathrm{H}_{18} \mathrm{O}_{4}$}

7,3'-Dihydroxy-4'-methoxy-8-methylflavan, 5991

6,7-Dihydroxy-2-(2-phenylethyl)-5,6,7,8-tetrahydrochromone, 6084

4-Hydroxy-2',4'-dimethoxydihydrochalcone, 10018

4-Hydroxy-2',6'-dimethoxydihydrochalcone, 10019

6-Hydroxy-7-methoxy-3-(4'-hydroxybenzyl) chroman, 10408

Isoagatharesinol, 11201

Isohemigossylic acid lactone-2, 7-dimethyl ether, 11452

Loureirin A, 13011

Myrigalone $\mathrm{H}, 15192$

Neoboutonin, 15346

Phoyunbene D, 17199

Pygmaeoherin, 18236

Sativin, 19394

Stemanthrene A, 20291

Stemanthrene B, 20292

Stemanthrene D, 20294

Tupichinol A, 22114

\section{$\mathrm{C}_{17} \mathrm{H}_{18} \mathrm{O}_{5}$}

Columbianetin propionate, 3938

3'-Deoxy-4-O-methylsappanol, 5195

5,6-Dihydro-1,7-dihydroxy-3,4-dimethoxy-2methyldibenz[b,f]oxepin, 5591

2',4'-Dihydroxy-4,6'-dimethoxychalcone, 5821

$\alpha$-2'-Dihydroxy-4,4'-dimethoxydihydrochalcone, 5822

2',3'-Dihydroxy-4',6'-dimethoxydihydrochalcone, 5823

Halophilol A, 9198

1-(2-Hydroxy-4-methoxyphenyl)-3-(3,4-methylenedioxyphenyl)propan-2-ol, 10447

Isomucronulatol, 11544

Isomurralonginol acetate, 11549

2-Methoxybenzyl-2,6-dimethoxybenzoate, 13853

(3R)-5'-Methoxyvestitol, 14103

Monascusone B, 14916

Mucronulatol, 15021

Odoratol, 15998

Phellodenol D, 17070
Phoyunbene A, 17196

Phoyunbene B, 17197

Sequirin C, 19755

Shikonine, 19820

Spherosin, 20151

Torosachrysone 8-O-methyl ether, 21460

\section{$\mathrm{C}_{17} \mathrm{H}_{18} \mathrm{O}_{6}$}

3'-O-Acetylhamaudol, 407

Agarotetrol, 707

Albiflorin-1, 858

Clavilactone E, 3812

Evofolin B, 7670

Hainanmurpanin, 9193

Isoagarotetrol, 11199

3'-O-Methyl episappanol, 14402

4-O-Methyl episappanol, 14403

10-O-Methyl protosappanin B, 14700

3'-O-Methyl sappanol, 14720

4-O-Methyl sappanol, 14721

Murrangatin acetate, 15108

Oxypeucedanin methanolate, 16460

Paeoniflorigenone, 16524

Paeonilactone $\mathrm{C}, 16528$

(5R,6R,7S,8R)-2-(2-Phenylethyl)-5e',6a,7e,8e'tetrahydroxy-5,6,7,8-tetrahydrochromone, 17114

Protosappanin A dimethyl acetal, 17986

\section{$\mathrm{C}_{17} \mathrm{H}_{18} \mathrm{O}_{6} \mathrm{~S}$}

(-)-Epiafzelechin-4-(2-hydroxyethyl)thio ether, 6823

\section{$\mathrm{C}_{17} \mathrm{H}_{18} \mathrm{O}_{7}$}

Byakangelicin, 2833

Divaricatol, 6529

4-Methoxy-6(11,12-methylenedioxy-10,14dimethoxydihydrostyryl)-2-pyrone, 14015

$5 \alpha, 6 \beta, 7 \beta, 8 \alpha$-Tetrahydroxy-2-[2-(2'-hydroxy phenyl)ethyl]-5,6,7,8-tetrahydrochromone, 21120

$5 \alpha, 6 \beta, 7 \alpha, 8 \beta$-Tetrahydroxy-2-[2-(2'-hydroxyphenyl)ethyl]-5,6,7,8-tetrahydrochromone, 21121

Ventiloquinone K, 22379

$\mathrm{C}_{17} \mathrm{H}_{18} \mathrm{O}_{7} \mathrm{~S}$

(-)-Epicatechin 4-(2-hydroxyethyl)thio ether, 6866

\section{$\mathrm{C}_{17} \mathrm{H}_{18} \mathrm{O}_{8} \mathrm{~S}$}

(-)-Epigallocatechin 4-(2-hydroxyethyl)thio ether, 6924

\section{$\mathrm{C}_{17} \mathrm{H}_{18} \mathrm{O}_{9}$}

Rubinaphthin A, 19032

\section{$\mathrm{C}_{17} \mathrm{H}_{18} \mathrm{O}_{10}$}

5-Carboxy-7-glucosyloxy-2-methyl-benzopyran$\gamma$-one, 3172

\section{$\mathrm{C}_{17} \mathrm{H}_{19} \mathrm{NO}_{3}$}

Aposcopolamine, 1534

Chavicine, 3487

Cherylline, 3516

$D$-Coclaurine, 3878

Epibuphanisine, 6842

Erysonine, 7324

Erysopine, 7325

Morphine, 14981

Narwedine, 15288

Noryuziphine, 15817

1-[1-Oxo-5(3,4-methylenedioxyphenyl)-2E,4Zpentadienyl]piperidine, 16385

1-[1-Oxo-5(3,4-methylenedioxyphenyl)-2Z,4Epentadienyl] piperidine, 16386

Piperine, 17451

\section{$\mathrm{C}_{17} \mathrm{H}_{19} \mathrm{NO}_{4}$}

(-)-Amarbellisine, 1016

Crinamine, 4237

9-Demethylhomolycorine, 5081

Haemanthamine, 9186

Orixalone B, 16200

Powelline, 17751

\section{$\mathrm{C}_{17} \mathrm{H}_{19} \mathrm{NO}_{5}$}

Crinamidine, 4236

Haemanthidine, 9187

6-Hydroxycrinamine, 9946

Isoptelefolidine, 11633

Orixinone, 16204

Piplartine, 17472

Preorixine, 17844

Sarmentamide B, 19369

$8(Z)-N$-(12,13,14-Trimethoxycinnamoyl)-

$\Delta^{3}$-pyridin-2-one, 21904

\section{$\mathrm{C}_{17} \mathrm{H}_{19} \mathrm{~N}_{3} \mathrm{O}_{3}$}

Hydrachine A, 9698

\section{$\mathrm{C}_{17} \mathrm{H}_{19} \mathrm{~N}_{3} \mathrm{O}_{4}$}

Polanrazine F, 17608

$\mathrm{C}_{17} \mathrm{H}_{20}$

Heptadeca-1,7,9,15-tetraen-11,13-diyne*, 9381

\section{$\mathrm{C}_{17} \mathrm{H}_{20} \mathrm{~N}_{2} \mathrm{O}_{3}$}

Phlegmariurine M, 17157

Salacin, 19176

\section{$\mathrm{C}_{17} \mathrm{H}_{20} \mathrm{~N}_{4} \mathrm{O}_{\mathbf{6}}$}

Riboflavine, 18834

$\mathrm{C}_{17} \mathrm{H}_{20} \mathrm{O}$

Dehydrofalcarinone, 4915

$\mathrm{C}_{17} \mathrm{H}_{20} \mathrm{O}_{2}$

Bupleuronol, 2752

\section{$\mathrm{C}_{17} \mathrm{H}_{20} \mathrm{O}_{3}$}

Linderene acetate, 12872

3,4,3'-Trimethoxybibenzil, 21899

3,5,3'-Trimethoxybibenzil, 21900 


\section{$\mathrm{C}_{17} \mathrm{H}_{20} \mathbf{O}_{4}$}

9-Acetoxy-8,10-dehydrothymol 3-O-tiglate, 153

Amoenylin, 1070

Batatasin V, 2167

Broussonin E, 2650

Broussonin F, 2651

Celaphanol A, 3362

2-(2-Hydroxy-1-methyl-2-phenylethyl)-4,5-dimethoxyphenol, 10514

Isoamoenylin, 11216

Lindenanolide H, 12865

Stilbostemin G, 20382

Zaluzanin D, 22963

\section{$\mathrm{C}_{17} \mathrm{H}_{20} \mathrm{O}_{5}$}

8 $\alpha$-Acetoxyarglabin, 124

Arteglasin A, 1789

Matricarin, 13604

3-Methoxy-4-acetoxycinnamyl angelate, 13828

Moscatilin, 14996

Murpanicin, 15100

1-Oxo-4 $\alpha$-acetoxyeudesma-2,1(13)-dien-12,8 $\beta$ olide, 16288

1-Oxo-4 $\beta$-acetoxyeudesma-2,1(13)-dien-12,8 $\beta$ olide, 16289

Shizukanolide D, 19842

Strychinstenolide 6- $O$-acetate A, 20407

Strychinstenolide 6-O-acetate B, 20408

Strychnistenolide 6-O-acetate, 20413

\section{$\mathrm{C}_{17} \mathrm{H}_{20} \mathrm{O}_{6}$}

9-Acetoxy-8,10-epoxy-6-hydroxythymol 3-O-angelate, 186

3-O-Deacetyl-9-O-acetylsalograviolide A, 4712

Murrayanone, 15120

PR toxin, 17999

Salograviolide A, 19193

\section{$\mathrm{C}_{17} \mathrm{H}_{20} \mathbf{O}_{7}$}

Casearinone, 3271

\section{$\mathrm{C}_{17} \mathrm{H}_{20} \mathrm{O}_{8}$}

3-O-Acetyl-2-O-(p-hydroxycinnamoyl)- $\alpha-L-$ rhamnose, 415

Ningposide C, 15620

Plumbaside A, 17569

Psilotin, 18085

Rossoliside, 18930

\section{$\mathrm{C}_{17} \mathrm{H}_{20} \mathrm{O}_{9}$}

3-O-Caffeoylquinic acid methyl ester, 2920

4-O-Caffeoylquinic acid methyl ester, 2921

Cnidioside A, 3857

3-O-Feruloylquinic acid, 7786

6-Methoxy-7-O- $\beta-D-(4$ '-methoxy) glucopyranosyl coumarin, 13995

Methyl chlorogenate, 14224

Phellodenol E, 17071
$\mathrm{C}_{17} \mathrm{H}_{20} \mathrm{O}_{10}$

Eleutheroside $\mathrm{B}_{1}, 6754$

Isofraxidin glucoside, 11429

Saikochromoside A, 19131

Tomenin, 21436

$\mathrm{C}_{17} \mathrm{H}_{21} \mathrm{BrN}_{2} \mathrm{O}$

Flustrabromine, 7842

$\mathrm{C}_{17} \mathrm{H}_{21} \mathrm{NO}_{2}$

Apoatropine, 1526

trans-3 $\beta$-Cinnamoyloxytropane, 3718

Cocculine, 3863

Erythrozeylanine B, 7348

\section{$\mathrm{C}_{17} \mathrm{H}_{21} \mathrm{NO}_{3}$}

2-Epigalanthamine, 6920

Galanthamine, 8083

Maritidine, 13568

Mesembrenone, 13796

Piperanine, 17439

Pluviine, 17573

\section{$\mathrm{C}_{17} \mathrm{H}_{21} \mathrm{NO}_{4}$}

Acutifolin, 597

Carinatine, 3198

Cocaine, 3860

Dasycarpamine, 4663

Hyoscine, 10870

Orixalone A, 16199

Preskimmianine, 17849

\section{$\mathrm{C}_{17} \mathrm{H}_{21} \mathrm{NO}_{5}$}

Anisodine, 1288

(S)-(-)-7,8-Dimethoxyplatydesmine, 6289

1-Epideacetylbowdensine, 6882

1-Epideacetylbowdensineł, 6883

$N$-(12,13,14-Trimethoxydihydrocinnamoyl)- $\Delta^{3}$ pyridin-2-one, 21908

Zascanol epoxide, 22972

$\mathrm{C}_{17} \mathrm{H}_{21} \mathrm{NO}_{6}$

Orixine, 16203

$\mathrm{C}_{17} \mathrm{H}_{21} \mathrm{~N}_{3} \mathrm{O}_{3} \mathrm{~S}$

Polanrazine C, 17605

Polanrazine D, 17606

$\mathrm{C}_{17} \mathrm{H}_{21} \mathbf{N}_{3} \mathrm{O}_{4}$

Polanrazine E, 17607

$\mathrm{C}_{17} \mathrm{H}_{21} \mathrm{~N}_{4} \mathrm{O}_{9} \mathrm{P}$

Flavin mononucleotide, 7816

\section{$\mathrm{C}_{17} \mathrm{H}_{22} \mathrm{BrClO}_{3}$}

2-Bromo-3-chloro-5-acetoxy-chamigra-7(14),9dien-8-one, 2620

\section{$\mathrm{C}_{17} \mathrm{H}_{22} \mathrm{NO}_{4}{ }^{+}$}

$O$-Methylbalfourodinium (salt), 14153

\section{$\mathrm{C}_{17} \mathrm{H}_{22} \mathrm{~N}_{2} \mathrm{O}$}

Huperzinine, 9689

$N$-Methyl huperzine B, 14493

$\mathrm{C}_{17} \mathrm{H}_{22} \mathrm{~N}_{2} \mathrm{O}_{2}$
Anticancer Amide PMV70P691-053, 1383

Piriferine, 17475

\section{$\mathrm{C}_{17} \mathrm{H}_{22} \mathrm{~N}_{2} \mathrm{O}_{3}$}

13-O-Acetylbaptifoline, 329

\section{$\mathrm{C}_{\mathbf{1 7}} \mathrm{H}_{22} \mathbf{N}_{\mathbf{2}} \mathbf{O}_{10} \mathbf{S}_{\mathbf{2}}$}

Neoglucobrassicin, 15400

\section{$\mathrm{C}_{17} \mathrm{H}_{22} \mathrm{O}$}

Bupleurynol, 2758

Cicutol, 3636

Dehydrofalcarinol, 4914

2(E), $9(Z), 16$-Heptadecatriene-4,6-diyne-8-ol, 9382

\section{$\mathrm{C}_{17} \mathrm{H}_{22} \mathrm{O}_{2}$}

Bupleurotoxin, 2757

Cicutoxin, 3637

$3 R, 8 R$-Dehydrofalcarindiol, 4913

Ginsenoyne A, 8448

Ginsenoyne E, 8452

Gymnasterkoreayne B, 9106

Gymnasterkoreayne F, 9108

Linalyl benzoate, 12851

\section{$\mathrm{C}_{17} \mathrm{H}_{22} \mathrm{O}_{3}$}

$3 \beta$-Acetoxy-atractylon, 125

8-Methoxy-2-methyl-2-(4-methyl-3-pentenyl)2H-1-benzopyran-6-ol, 14027

Nervogenic acid, 15506

\section{$\mathrm{C}_{17} \mathrm{H}_{22} \mathrm{O}_{4}$}

$1 \beta$-Acetoxyeudesman-4(15),7(11)-dien- $8 \alpha, 12$ olide, 194

9-Acetoxythymo 13-O-tiglate, 289

Acetyl cannabispirol, 342

6-Dehydrogingerdione, 4921

Epitulipinolide, 7030

Eupatoranolide, 7589

Germacranolide, 8337

6-Isobutyryl-5,7-dimethoxy-2,2-dimethylbenzopyran, 11294

Kushenquinone A, 12371

Lappalone, 12512

Laurenobiolide, 12566

$1 \beta, 13,14$-Trihydroxy-8,11,13-podocarpatrien-7one, 21839

Tulipinolide, 22103

\section{$\mathrm{C}_{17} \mathrm{H}_{22} \mathrm{O}_{5}$}

$6 \beta$-Acetoxyglechomafuran, 199

$1 \beta$-Acetoxy- $8 \beta$-hydroxyeudesman-4(15),7(11)dien- $8 \alpha, 12$-olide, 215

15-O-Acetylamphoricarpolide, 314

Arnicolide A, 1748

11,13-Dehydrolanuginolide, 4939

Ergolide, 7239

Eupaformonin, 7558

Gaillardin, 8047 
Heleniamarin, 9289

Hiyodorilactone C, 9572

Inuchinenolide A, 11108

Inuchinenolide B, 11109

Isotenulin, 11734

Lipiferolide, 12897

Matricin, 13605

7-Methoxy-8-(2'-ethoxy-3'-hydroxy-3'-methylbutyl)coumarin, 13923

7-Methoxy-8-(2'-hydroxy-3'-ethoxy-3'-methylbutyl)coumarin, 13958

Ovatifolin, 16278

Pyrethrosin, 18257

Sieversin, 19881

Tenulin, 20963

Viscidulin B, 22533

Xanthinin, 22757

Xanthumin, 22780

$\mathrm{C}_{17} \mathrm{H}_{22} \mathrm{O}_{6}$

Angelol J, 1199

Anthemolide A, 1354

Anthemolide C, 1356

Chaetoquadrin E, 3444

8,10-Dihydroxy-9-acetoxythymol 3-O-tiglate, 5751

Epitulipinolide diepoxide, 7031

(E)-1 $\alpha, 10 \beta$-Epoxy- $3 \beta$-acetoxy- $6 \alpha$-hydroxygermacra-4,11(13)-dien-12,8 $\alpha$-olide, 7048 8-Hydroxy-10-acetoxythymol 9-O-angelate, 9761

2-Methyl-4-[2',4',6'-trihydroxy-3'-(2-methylpropanoyl)phenyl]but-2-enyl acetate, 14789

Tetraneurin A, 21207

\section{$\mathrm{C}_{17} \mathrm{H}_{22} \mathrm{O}_{7}$}

7-O-Acetylanislactone $\mathrm{B}, 320$

9-O-Deacetylanthemolide $\mathrm{D}, 4715$

8-Deoxy-9-O-acetylanthemolide B, 5148

Murragleinin, 15103

1-Peroxyferolide, 16973

\section{$\mathrm{C}_{17} \mathrm{H}_{22} \mathrm{O}_{8}$}

1,2-Methylenedioxy-4-methoxy-5-allyl-phen-3-yl $\beta$ - $D$-glucopyranoside, 14374

\section{$\mathrm{C}_{17} \mathrm{H}_{22} \mathrm{O}_{9}$}

Perilloside E, 16934

Sinapaldehyde glucoside, 19911

\section{$\mathrm{C}_{17} \mathrm{H}_{22} \mathrm{O}_{10}$}

3'-O- $\beta$-Glucopyranosyl plumbagic acid, 8712

cis-Sinapic acid glucoside, 19914

trans-Sinapic acid glucoside, 19915

\section{$\mathrm{C}_{17} \mathrm{H}_{23} \mathrm{BrCl}_{2} \mathrm{O}_{3}$}

Bisezakyne B, 2451

\section{$\mathrm{C}_{17} \mathrm{H}_{23} \mathrm{BrN}_{2} \mathrm{O}_{2} \mathrm{~S}$}

$N$-(2-[6-Bromo-2-(1,1-dimethyl-2-propenyl)$1 H$-indol-3-yl] ethyl)- $N$-methylmethanesulfo- namide, 2622

$\mathrm{C}_{17} \mathrm{H}_{23} \mathrm{ClO}_{2}$

Ginsenoyne B, 8449

$\mathrm{C}_{17} \mathrm{H}_{23} \mathrm{ClO}_{6}$

Eupaglehnin E, 7564

$\mathrm{C}_{17} \mathrm{H}_{23} \mathrm{NO}$

Schinifoline, 19468

$\mathrm{C}_{17} \mathrm{H}_{23} \mathrm{NO}_{3}$

Atropine, 2001

Hyoscyamine, 10872

Lycoramine, 13236

Mesembrine, 13797

$\mathrm{C}_{17} \mathrm{H}_{23} \mathrm{NO}_{4}$

Anisodamine, 1287

$\mathrm{C}_{17} \mathrm{H}_{23} \mathrm{NO}_{5}$

Nilgirine, 15596

$\mathrm{C}_{17} \mathrm{H}_{23} \mathrm{NO}_{5} \mathrm{~S}$

Sakambullin, 19169

$\mathrm{C}_{17} \mathrm{H}_{24} \mathrm{~N}_{2}$

$\mathrm{N}$-Methyllycodine, 14571

\section{$\mathrm{C}_{17} \mathrm{H}_{24} \mathrm{~N}_{2} \mathrm{O}$}

$\beta$-Obscurine, 15890

\section{$\mathrm{C}_{17} \mathrm{H}_{24} \mathrm{O}$}

Falcarinol, 7709

Panaxynol, 16598

\section{$\mathrm{C}_{17} \mathrm{H}_{24} \mathrm{O}_{2}$}

1 $\beta, 13$-Dihydroxy-8,11,13-podocarpatriene, 6099

(9R,10S)-Epoxyheptadecan-4,6-diyn-3-one, 7104

cis-Falcarindiol, 7707

trans-Falcarindiol, 7708

1,8-Heptadecadiene-4,6-diyne-3,10-diol, 9375

Panaxydol, 16597

\section{$\mathrm{C}_{17} \mathrm{H}_{24} \mathrm{O}_{3}$}

Buddledin A, 2697

Ginsenoyne C, 8450

Gymnasterkoreayne E, 9107

Gymnasterkoreayne G, 9109

Madolin H, 13341

Moxartenone, 15003

PQ-2, 17752

6-Shogaol, 19846

Valeriana-sesquiterpene CPB2006542, 22318

$\mathrm{C}_{17} \mathrm{H}_{24} \mathrm{O}_{4}$

9-Acetoxyfukinanolide, 198

6-Acetylfuranofukinol, 395

Ethylleptol B, 7456

[6]-Gingerdione (enol form), 8386

[6]-Gingerdione (keto form), 8387

3-Oxo- $8 \alpha, 12 \alpha$-dimethoxy-8,12-dihydro-10 $\alpha H$ furanoeremophil-1-ene, 16324

Toluccanolide B, 21430

Trichodermin, 21559

$\mathrm{C}_{17} \mathrm{H}_{24} \mathrm{O}_{5}$
$1 \beta$-Acetoxy-7-drimen-11 $\alpha$-ol-12,11-lactone, 176

4 -Acetoxy-1 $\beta, 5 \beta$-epoxy-10 $\alpha H$-xantha-11(13)-en-

$12,8 \beta$-olide, 191

$8 \alpha$-Acetoxy- $1 \beta$-hydroxyeudesm-3-en- $5 \alpha, 6 \beta, 7 \alpha$,

11 $\beta H$-12,6-olide, 217

1-Acetoxy- $6 \alpha$-hydroxy- $4 \alpha H$-1,10-secoeudesma5(10),11(13)-dien-12,8 $\beta$-olide, 236

6- $\alpha$-Acetyl-4- $O$-oxobedfordiaic acid, 474

(3R),(4S)-4-Hydroxylasiodiplodin, 10315

Inulicin, 11111

\section{$\mathrm{C}_{17} \mathrm{H}_{24} \mathrm{O}_{6}$}

Blumealactone C, 2520

Borenolide, 2547

Strychnilactone, 20409

Tetraneurin E, 21208

$\mathrm{C}_{17} \mathrm{H}_{24} \mathrm{O}_{7}$

8- $\beta$-Acetoxyhysterone C, 239

\section{$\mathrm{C}_{17} \mathrm{H}_{24} \mathrm{O}_{8}$}

Feroxin A, 7762

Methylconiferin, 14249

\section{$\mathrm{C}_{17} \mathrm{H}_{24} \mathrm{O}_{9}$}

Lysidiside A, 13261

Methyl

2-O- $\beta$ - $D$-glucosyloxy-4-methoxyben

zenepropanoate, 14470

Multifidol glucoside, 15063

1-[(Pentanoyl)phloroglucinyl]- $\beta$ - $D$-glucopyranoside, 16886

Syringenone, 20560

Syringin, 20569

Tangshenoside II, 20673

\section{$\mathrm{C}_{17} \mathrm{H}_{24} \mathrm{O}_{10}$}

Cornin, 4060

Dehydrologanin, 4943

8-Epiapodantheroside, 6829

Epivogeloside, 7040

Fissistigmoside, 7803

Geniposide, 8276

3-O-( $\beta$ - $D$-Glucopyranosyl)-1-(3',5'-dimethoxy-

4'-hydroxyphenyl)-1-propanone, 8624

Majoroside, 13406

Secologanin, 19623

Swertiajaposide A, 20506

Verbenalin, 22397

Vogeloside, 22606

$\mathrm{C}_{17} \mathrm{H}_{24} \mathrm{O}_{11}$

Acetylcatalpol, 347

8-O-Acetylclandonoside, 356

Deacetyl asperulosidic acid methyl ester, 4717

7,8-Dehydropenstemoside, 4959

$7 \beta, 8 \beta$-Epoxy- $8 \alpha$-dihydrogeniposide, 7070

Gardenoside, 8231

Hastatoside, 9244 
Kingiside, 12229

6-Methoxygeniposidic acid, 13939

6-Methoxyginiposidic acid, 13940

Scandoside methyl ester, 19455

Secologanoside 7-methyl ester, 19626

Secoxyloganin, 19636

Theviridoside, 21327

\section{$\mathrm{C}_{17} \mathrm{H}_{24} \mathrm{O}_{12}$}

Sesamoside, 19782

\section{$\mathrm{C}_{17} \mathrm{H}_{24} \mathrm{O}_{14}$}

Nuezhenidic acid, 15866

\section{$\mathrm{C}_{17} \mathrm{H}_{25} \mathrm{ClO}_{3}$}

10-Chloro-1-heptadecene-4,6-diyne-3,8,9-triol, 3556

Chloropanaxydiol, 3565

\section{$\mathrm{C}_{17} \mathrm{H}_{25} \mathrm{NO}$}

$\gamma$-Sanshool, 19298

\section{$\mathrm{C}_{17} \mathrm{H}_{25} \mathrm{NO}_{2}$}

Hydroxy- $\gamma$-Sanshool, 9742

Lycoflexine, 13193

(-)-Sedinine, 19649

\section{$\mathrm{C}_{17} \mathrm{H}_{25} \mathrm{NO}_{3}$}

Annopodine, 1323

Dendroxine, 5127

Lyconnotine, 13200

Mesembrinol, 13798

\section{$\mathrm{C}_{17} \mathrm{H}_{25} \mathrm{NO}_{4}$}

4-Hydroxydendroxine, 9983

6-Hydroxydendroxine, 9984

Stemonine, 20307

\section{$\mathrm{C}_{17} \mathrm{H}_{25} \mathrm{NO}_{7}$}

6,7-Dihydroxy-1,1-dimethyl- $N$-(6'-fructopyra-

nosyl)-1,2,3,4-tetrahydroisoquinoline, 5854

\section{$\mathrm{C}_{17} \mathrm{H}_{26} \mathrm{~N}_{2} \mathrm{O}$}

$\alpha$-Obscurine, 15889

\section{$\mathrm{C}_{17} \mathrm{H}_{26} \mathrm{O}_{2}$}

14-Acetoxymodhephene, 260

Ginsenoyne D, 8451

Heptadeca-1-en-4,6-diyne-3,9-diol, 9377

$\alpha$-Humulenol acetate, 9674

Methyl 9-hydroxyselina-4,11-dien-14-oate,

$$
14522
$$

Patchouleny acetate, 16710

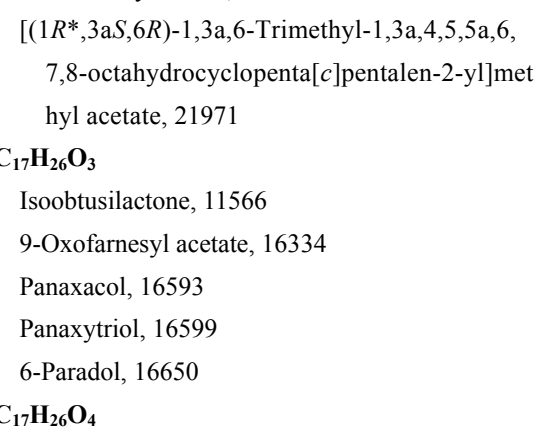

$1 \beta, 6 \alpha$-Dihydroxycostic acid ethyl ester, 5795

Embelin, 6767

[6]-Gingerol, 8395

14,15,16-Trisnor-8(17)-labdene-13,19-dioic acid, 22039

$\mathrm{C}_{17} \mathrm{H}_{26} \mathrm{O}_{5}$

$1 \beta$-Acetoxy-11,12-epoxy-6-drimen- $8 \alpha, 11 \alpha$-diol, 182

$6 \beta, 8 \beta$-Dimethoxy-10 $\beta$-hydroxyeremophil-7(11)en-12,8 $\alpha$-olide, 6238

2-(1-Ethoxy-2-hydroxy)propyl-4-methoxyphenol-2-methyl-butyrate, 7407

\section{$\mathrm{C}_{17} \mathrm{H}_{26} \mathrm{O}_{6} \mathrm{~S}$}

6-Gingesulfonic acid, 8400

\section{$\mathrm{C}_{17} \mathrm{H}_{26} \mathrm{O}_{7}$}

(Z)-5'-Hydroxyjasmone 5'- $O$ - $\beta$ - $D$-glucopyranoside, 10268

\section{$\mathrm{C}_{17} \mathrm{H}_{26} \mathrm{O}_{9}$}

Deoxyloganin, 5191

(1'R)-1'-(4-Hydroxy-3,5-dimethoxyphenyl) propan-1'-ol 4-O- $\beta$ - $D$-glucopyranoside, 10040

\section{$\mathrm{C}_{17} \mathrm{H}_{26} \mathrm{O}_{10}$}

6-O-Acetylajugol, 308

Ajugoside, 816

3,4-Dihydroverbenalin, 5735

8-Epiloganin, 6953

1'-(3-Hydroxy-4,5-dimethoxyphenyl)-propane2',3'-diol 3'-O- $\beta$ - $D$-glucopyranoside, 10039 loganin, 12950

$\left(1^{\prime} R, 2^{\prime} R\right)-4-O$-Methylguaiacyl glycerol 3'- $O-\beta-D$ glucopyranoside, 14476

Mussaenoside, 15133

Reptoside, 18628

\section{$\mathrm{C}_{17} \mathrm{H}_{26} \mathrm{O}_{11}$}

8-Acetylharpagide, 409

Caryoptoside, 3247

6-Epi-8-O-acetylharpagide, 6818

Ipolamiide, 11128

Morroniside, 14982

Shanzhiside methyl ester, 19796

(7S,8S)-Syringoylglycerol

9-O- $\beta$ - $D$-glucopyranoside, 20571

4-(1,2,3-Trihydroxypropyl)-2,6-dimethoxyphenyl-1- $O-\beta$ - $D$-glucopyranoside, 21840

\section{$\mathrm{C}_{17} \mathrm{H}_{26} \mathrm{O}_{12}$}

Clandonoside II, 3787

Genameside A, 8266

Genameside B, 8267

Lamiide, 12456

Yopaaoside C, 22928

$\mathrm{C}_{17} \mathrm{H}_{26} \mathrm{O}_{13}$

7-Epiphlomiol, 6997

$\mathrm{C}_{17} \mathrm{H}_{27} \mathrm{NO}_{2}$
Neostenine, 15460

Stenine, 20309

\section{$\mathrm{C}_{17} \mathrm{H}_{27} \mathrm{NO}_{3}$}

Nobilonine, 15640

Nonyl vanillylamide, 15702

Nordihydrocapsacine, 15740

\section{$\mathrm{C}_{17} \mathrm{H}_{27} \mathrm{NO}_{6}$}

Acetylindicine, 437

\section{$\mathrm{C}_{17} \mathrm{H}_{28}$}

Aplotaxene, 1525

3-Phenylundecane, 17088

4-Phenylundecane, 17138

6-Phenylundecane, 17139

\section{$\mathrm{C}_{17} \mathrm{H}_{28} \mathrm{NO}_{2}{ }^{+}$}

$N$-Methyldendrobium, 14288

\section{$\mathrm{C}_{17} \mathrm{H}_{28} \mathrm{O}_{2}$}

Farnesyl acetate, 7735

Isoambreinolide, 11208

Scleropyric acid, 19533

\section{$\mathrm{C}_{17} \mathrm{H}_{28} \mathrm{O}_{3}$}

7-Acetoxy-elema-1,3-dien-8-ol, 177

9-Acetoxy-10-hydroxyaromadendrane, 209

$1 \alpha$-Acetoxy-ent-junenol, 246

15-Acetoxy-T-muurolol, 263

$3 \alpha, 7 \alpha$-Dihydroxy amorph-4-ene 3-acetate, 5755

(-)-Eudesm-3-ene-6 $\alpha$-acetoxy-7 $\alpha$-ol, 7501

5-Hydroxy-6-methyl-3-(undec-10-enyl)-5,6dihydropyran-2-one, 10525

Kessyl acetate, 12193

Liguloxidol acetate, 12816

Polygodial acetal, 17641

\section{$\mathrm{C}_{17} \mathrm{H}_{28} \mathrm{O}_{4}$}

3-Acetoxy-7,11-dihydroxy-farnesa-1,5,9-triene, 164

[6]-Gingediol, 8383

\section{$\mathrm{C}_{17} \mathrm{H}_{28} \mathrm{O}_{6}$}

$3 \beta$-Acetoxy, $5 \alpha, 11,12,13$-tetrahydroxy-eudesm4(15)-ene, 287

Griffonianone C, 9009

\section{$\mathrm{C}_{17} \mathrm{H}_{29} \mathrm{NO}_{11}$}

Neolinustatin, 15429

\section{$\mathrm{C}_{17} \mathrm{H}_{30} \mathrm{O}$}

Civetone, 3780

(Z,Z)-8,11-Heptadecadienal, 9374

\section{$\mathrm{C}_{17} \mathrm{H}_{30} \mathrm{O}_{6}$}

$\left(1 R^{*}, 2 R^{*}, 3 R^{*}, 6 R^{*}, 7 R^{*}\right) 1,2,3,6,7-$ Pentahydroxy1 -acetoxy-bisabol-10(11)-ene, 16837

$\left(1 R^{*}, 2 R^{*}, 3 R^{*}, 6 R^{*}, 7 R^{*}\right) 1,2,3,6,7-P e n t a h y d r o x y-$ 2-acetoxy-bisabol-10(11)-ene, 16838

\section{$\mathrm{C}_{17} \mathrm{H}_{30} \mathrm{O}_{10}$}

(E)-2-Hexenyl- $\alpha$ - $L$-arabinopyranosyl-( $1 \rightarrow 2)$ $\beta$ - $D$-glucopyranoside, 9525

$\mathrm{C}_{17} \mathrm{H}_{30} \mathrm{O}_{12}$ 


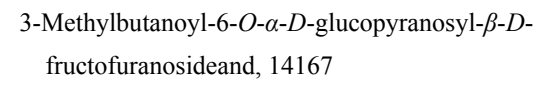

\section{$\mathrm{C}_{18} \mathrm{H}_{8} \mathrm{O}_{6}$}

Erosnine, 7289

\section{$\mathrm{C}_{18} \mathrm{H}_{10} \mathrm{~N}_{2} \mathrm{O}_{4}$}

Melanin, 13659

\section{$\mathrm{C}_{18} \mathrm{H}_{10} \mathrm{O}_{5}$}

Pongaglabrone, 17707

\section{$\mathrm{C}_{18} \mathrm{H}_{10} \mathrm{O}_{8}$}

Euphorbetin, 7615

Isoeuphorbetin, 11422

Torreyaflavone, 21462

\section{$\mathrm{C}_{18} \mathrm{H}_{10} \mathrm{O}_{9}$}

Eckstolonol, 6701

\section{$\mathrm{C}_{18} \mathrm{H}_{11} \mathrm{NO}_{4}$}

Atherospermidine, 1961

Bianfugedine, 2344

Cepharadione A, 3408

Lanuginosine, 12497

\section{$\mathrm{C}_{18} \mathrm{H}_{11} \mathrm{NO}_{5}$}

7-Oxohernangerine, 16344

\section{$\mathrm{C}_{18} \mathrm{H}_{11} \mathrm{~N}_{3} \mathrm{O}$}

(E)-2-[(3'-Indole)cyanomethylene]-3-indolinone, 11030

$$
\mathrm{C}_{18} \mathrm{H}_{12}
$$

(Z)-1,1'-Biindenyliden, 2370

\section{$\mathrm{C}_{18} \mathrm{H}_{12} \mathrm{O}_{3}$}

Isotanshinone I, 11729

Isotanshinone II, 11730

Tanshinone I, 20685

$\mathrm{C}_{18} \mathrm{H}_{12} \mathrm{O}_{4}$
Cauliflorin A, 3334

Karanjin, 12163

Pinnatin, 17398

Przewaquinone B, 18012

Tanshinol A, 20683

\section{$\mathrm{C}_{18} \mathrm{H}_{12} \mathrm{O}_{5}$}

Cauliflorin B, 3335

6-Hydroxy,3-methoxy furo[8,7:4",5"]flavone, 10407

3',4'-Methylenedioxy-(2",3":7,8)-furanoflavanone, 14368

$\mathrm{C}_{18} \mathrm{H}_{12} \mathrm{O}_{6}$

Atromentin, 2000

$\mathrm{C}_{18} \mathrm{H}_{12} \mathrm{O}_{7}$

Salvianolic acid G, 19207

\section{$\mathrm{C}_{18} \mathrm{H}_{12} \mathrm{O}_{8}$}

Usararotenoid A, 22278

\section{$\mathrm{C}_{18} \mathrm{H}_{12} \mathrm{O}_{9}$}

Eckol, 6700

3,4-Methylenedioxy-3',4'-O-dimethyl-5'-methoxyellagic acid, 14366

Norstictic acid, 15798

\section{$\mathrm{C}_{18} \mathrm{H}_{12} \mathrm{O}_{10}$}

Parmatic acid, 16673

\section{$\mathrm{C}_{18} \mathrm{H}_{13} \mathrm{NO}_{3}$}

Bianfugecine, 2343

Lysicamine, 13258

\section{$\mathrm{C}_{18} \mathrm{H}_{13} \mathrm{NO}_{4}$}

Artabonatine D, 1772

Daurioxoisoporphine D, 4692

Norcepharadione B, 15725

Pareirubrine B, 16662

Piperadione, 17429

\section{$\mathrm{C}_{18} \mathrm{H}_{13} \mathrm{NO}_{5}$}

9-Methoxyaristolactam I, 13842

\section{$\mathrm{C}_{18} \mathrm{H}_{13} \mathrm{NO}_{7}$}

Aristolochic acid I methyl ester, 1718

Debilic acid, 4814

\section{$\mathrm{C}_{18} \mathrm{H}_{13} \mathrm{NO}_{8}$}

Aristolochic acid D methyl ether, 1714

Aristolochic acid IVa methyl ester, 1720

7-Methoxy-aristolochiac acid, 13843

\section{$\mathrm{C}_{18} \mathrm{H}_{13} \mathrm{~N}_{3} \mathrm{O}$}

Rutaecarpine, 19081

\section{$\mathrm{C}_{18} \mathrm{H}_{13} \mathrm{~N}_{3} \mathrm{O}_{3}$}

1,2-Dihydroxyrutaecarpine, 6112

\section{$\mathrm{C}_{18} \mathrm{H}_{14} \mathrm{O}_{3}$}

Dihydroisotanshinone I, 5653

Dihydroisotanshinone II, 5654

15,17-Dihydrotanshinone I, 5721

Dihydrotanshinone I, 5722

1,2-Dihydrotanshiquinone, 5723

Methylene tanshinquinone, 14391

\section{$\mathrm{C}_{18} \mathrm{H}_{14} \mathrm{O}_{4}$}

7-Acetoxy-2-methylisoflavone, 259

3- $\beta$-Hydroxymethylenetanshiquinone, 10492

3-Methoxy-(2",3":7,8)-furanoflavanone, 13933

(-)-(2S)-6-Methoxy-[2",3":7,8]-furanoflavanone, 13934

Pongamol, 17708

\section{$\mathrm{C}_{18} \mathrm{H}_{14} \mathrm{O}_{5}$}

6-Deoxyisojacareubin, 5182

6-Deoxyjacareubin, 5184

Nigrolineaxanthone F, 15579

Nigrolineaxanthone H, 15581

Rheediachromenoxanthone, 18754

Tovoxanthone, 21482

\section{$\mathrm{C}_{18} \mathrm{H}_{14} \mathrm{O}_{6}$}

Boeravinone A, 2532

7,8-Dimethoxy-3',4'-methylenedioxyflavone, 6264

Globulixanthone C, 8555

Jacareubin, 11808

3',4'-Methylenedioxy-6,7-dimethoxyflavone, 14363

Millettocalyxin A, 14856

Morusignin C, 14984

Ophiopogonone A, 16148

Tlatancuayin, 21411

1,3,5-Trihydroxy-13,13-dimethyl-2H-pyran[6,7b]-xanthen-9-one, 21723

\section{$\mathrm{C}_{18} \mathrm{H}_{14} \mathrm{O}_{7}$}

Boeravinone D, 2535

4',5-Dimethoxy-3-hydroxy-6,7-methylenedioxyisoflavone, 6248

Iriskashmirianin, 11165

Irisoid B, 11167

Irisoid C, 11168

9-O-Methyl-4-hydroxyboeravinone, 14498

Sagittin, 19127

5,7,2'-Trihydroxy-6-methyl-3-(3',4'-methylenedioxybenzyl) chromone, 21800

\section{$\mathrm{C}_{18} \mathrm{H}_{14} \mathrm{O}_{8}$}

Calycosin B, 3006

Dichotomitin, 5426

12-Dihydrousararotenoid A, 5733

5,3'-Dihydroxy-4'-methoxy-7-methoxycarbonylflavonol, 5987

3,8-Dimethoxy-5,7-dihydroxy-3',4'-methylenedioxyflavone, 6218

Epiphyllic acid, 6999

Psoromic acid, 18090

Robustaquinone G, 18875

Virensic acid, 22523

$\mathrm{C}_{18} \mathrm{H}_{14} \mathrm{O}_{9}$

Protocetraric acid, 17970 


\section{$\mathrm{C}_{18} \mathrm{H}_{15} \mathrm{KO}_{8}$}

Potassium rosmarinate, 17747

$\mathrm{C}_{18} \mathrm{H}_{15} \mathrm{NO}_{2}$

Murrayacine, 15113

$\mathrm{C}_{18} \mathrm{H}_{15} \mathrm{NO}_{3}$

$N$-Demethylnoracronycine, 5089

$N$-Formylanonaine, 7894

\section{$\mathrm{C}_{18} \mathrm{H}_{15} \mathrm{NO}_{4}$}

Atalaphyllidine, 1958

(S)-Cryptodorine, 4279

(-)- $N$-Demethylcrychine, 5069

Norrufescine, 15792

Oriciacridone C, 16179

Oriciacridone D, 16180

Piperolactam C, 17465

Piperolactam E, 17467

\section{$\mathrm{C}_{18} \mathrm{H}_{15} \mathrm{NO}_{5}$}

Lindechunine B, 12861

\section{$\mathrm{C}_{18} \mathrm{H}_{15} \mathrm{NO}_{6}$}

Ariskanin A, 1691

$\mathrm{C}_{18} \mathrm{H}_{15} \mathrm{~N}_{3} \mathrm{O}_{2}$

Naucledine, 15301

$\mathrm{C}_{18} \mathrm{H}_{15} \mathrm{~N}_{3} \mathrm{O}_{4}$

Dichotomide II, 5421

$\mathrm{C}_{18} \mathrm{H}_{15} \mathrm{NaO}_{8}$

Sodium rosmarinate, 20036

\section{$\mathrm{C}_{18} \mathrm{H}_{15} \mathrm{O}_{8}{ }^{-}$}

Calcium rosmarinate, 2955

\section{$\mathrm{C}_{18} \mathrm{H}_{15} \mathrm{O}_{10} \mathrm{~S}$}

Quercetin-3',4',7-trimethyl ether-3-sulfate, 18406

\section{$\mathrm{C}_{18} \mathrm{H}_{16} \mathrm{~N}_{2} \mathrm{O}_{5}$}

Picrasidine E, 17298

\section{$\mathrm{C}_{18} \mathrm{H}_{16} \mathrm{~N}_{2} \mathrm{O}_{8}$}

Betanidin, 2320

Isobetanidin, 11250

\section{$\mathrm{C}_{18} \mathrm{H}_{16} \mathrm{O}_{2}$}

Cinnamyl cinnamate, 3728

12-Deoxydanshenxinkun B, 5165

2,7-Dihydroxy-1,6-dimethylpyrene, 5861

2-Isopropyl-8-methylphenanthrene-3,4-dione

$$
\text { ( } \left.\mathrm{R}_{0}-090680\right), 11629
$$

Purpureamethide, 18218

Styracin, 20417

\section{$\mathrm{C}_{18} \mathrm{H}_{16} \mathrm{O}_{3}$}

Danshenxinkun B, 4633

2-Hydroxy-7-carboxy-1-methyl-5-ethenyl-9,10dihydrophenanthrene, 9893

2-Hydroxy-8-carboxy-1-methyl-5-ethenyl-9,10dihydrophenanthrene, 9894

Magnaldehyde B, 13364

4-[5-(4-Methoxyphenoxy)-3-penten-1-ynyl]phenol, 14052

2-[2-(4'-Methoxyphenyl) ethyl] chromone, 14057
6-Methoxy-2-(2-phenylethyl) chromone, 14058

Randainal, 18532

Styracin epoxide, 20418

1,2,15,16-Tetrahydrotanshinone I, 21080

\section{$\mathrm{C}_{18} \mathrm{H}_{16} \mathrm{O}_{4}$}

Calophyllin B, 2989

Danshenxinkun A, 4632

(E)-4,4'-Dihydroxy-7,7'-dioxolign-8(8')-ene, 5864

(Z)-4,4'-Dihydroxy-7,7'-dioxolign-8(8')-ene, 5865

5-Hydroxy-6-methoxy-2-(2-phenylethyl) chromone, 10439

6-Hydroxy-2-[2-(4'-methoxyphenyl)ethyl] chromone, 10440

6-Hydroxy-7-methoxy-2-(2-phenylethyl) chromone, 10441

3"-Methoxyasparenydiol, 13845

4-[(2S,3R)-3-Methyl-7-(1-propynyl)-2,3-dihydro1,4-benzodioxin-2-yl]-1,3-benzenediol, 14695

4-[(2S,3S)-3-Methyl-7-(1-propynyl)-2,3-dihydro1,4-benzodioxin-2-yl]-1,3-benzenediol, 14696

Tanshinol B, 20684

Tanshinone VI, 20688

\section{$\mathrm{C}_{18} \mathrm{H}_{16} \mathrm{O}_{5}$}

Bangangxanthone $\mathrm{B}, 2136$

5,8-Dihydroxy-2-[2-(4'-methoxyphenyl) ethyl]chromone, 5996

(2S)-5,7-Dimethoxy-8-formylflavanone, 6228

3'-Formyl-4',6'-dihydroxy-2'-methoxy-5'-methylchalcone, 7903

(2S)-8-Formyl-5-hydroxy-7-methoxy-6-methylflavanone, 7905

Globuxanthone, 8558

12b-Hydroxy-des- $D$-ring-garcigerrin A, 9994

3,4-Methylenedioxy-2',4'-dimethoxychalcone, 14361

Przewaquinone F, 18013

Tanshindiol A, 20679

Tanshindiol B, 20680

Tanshindiol C, 20681

1,3,7-Trihydroxy-2-(3-methylbut-2-enyl)

xanthone, 21797

5,7,2'-Trimethoxyflavone, 21912

5,7,4'-Trimethoxyflavone, 21913

7,2',4'-Trimethoxyflavone, 21914

7,2',5'-Trimethoxyflavone, 21915

7,3',4'-Trimethoxyisoflavone, 21918

\section{$\mathrm{C}_{18} \mathrm{H}_{16} \mathrm{O}_{6}$}

Americanin A, 1034

Andrographin, 1157

Betagarin, 2317

Cudraxanthone S, 4347

Desmosdumotin D, 5269
5,7-Dihydroxy-3',4'-dimethoxyspiro $\{2 H$-1-benzopyran-7'-bicyclo[4.2.0]octa[1,3,5]-trien\}-4one, 5838

(2S)-5,7-Dimethoxy-3',4'-methylenedioxyflavanone, 6263

2,8-Dimethoxy-6-methyl-9-oxo-9H-xanthene-

1-carboxylic acid methyl ester, 6267

6,8-Di-C-methylluteolin 7-methyl ether, 6366

Erycibenin C, 7302

3-Hydroxy-5,7,4'-trimethoxyflavone, 10790

5-Hydroxy-3,7,4'-trimethoxyflavone, 10791

5-Hydroxy-7,3',4'-trimethoxyflavone, 10792

5-Hydroxy-7,2',3'-trimethoxyflavone, 10793

5-Hydroxy-7,2',5'-trimethoxyflavone, 10794

5-Hydroxy-7,2',6'-trimethoxyflavone, 10795

8-Hydroxy-5,7,4'-trimethoxyflavone, 10796

2'-Hydroxy-5,6,7-trimethoxyisoflavonoid, 10799

3-Hydroxy-1,5,6-trimethoxy-2-methyl-9,10-

anthraquinone, 10800

Isoamericanin A, 11209

Isoophiopogonone $\mathrm{A}, 11577$

Melimessanol B, 13683

3-(4-Methoxybenzylidene)-5,7-dihydroxy-6-methoxychroman-4-one, 13856

Milletenone, 14855

Morusignin A, 14983

Ophiopogonanone A, 16136

Salvigenin, 19211

Symphoxanthone, 20526

1,3,5,6-Tetrahydroxy-2-isoprenylxanthone, 21122

1,3,6,7-Tetrahydroxy-8-(3-methyl-2-butenyl)-9Hxanthen-9-one, 21133

1,3,5,6-Tetrahydroxy-4-prenylxanthone, 21152

Toxyloxanthone C, 21488

\section{$\mathrm{C}_{18} \mathrm{H}_{16} \mathrm{O}_{7}$}

Ayanin, 2047

Boeravinone C, 2534

Calycosin A, 3005

Cirsilineol, 3741

5,2'-Dihydroxy-6,7,8-trimethoxyflavone, 6161

5,6-Dihydroxy-7,3',4'-trimethoxyflavone, 6162

Eupatilin, 7582

Eupatorin, 7591

Feralolide, 7755

Herbacetin 3,8,4'-trimethyl ether, 9423

Irisflavone $\mathrm{B}, 11161$

Lysionotin, 13264

Obtusin, 15908

Pachypodol, 16498

Penduletin, 16801

Rivularin, 18853

Robustaquinone H, 18876 
Santin, 19312

Tambulin, 20665

1,3,5-Trihydroxy-2-ethoxymethyl-6-methoxylanthraquinone, 21730

(-)-Usnic acid, 22281

Usnic acid, 22282

Vavain, 22358

Xanthomicrol, 22768

\section{$\mathrm{C}_{18} \mathrm{H}_{16} \mathrm{O}_{8}$}

Acerosin, 107

Areapillin, 1653

Centaureidin, 3388

Chrysograyanin, 3611

Chrysosplenol, 3623

Chrysosplenol D, 3624

Eupatin, 7583

Irigenin, 11154

Irisflavone C, 11162

Isoarcapillin, 11235

Labiatenic acid, 12420

Myricetin-3,5,3'-trimethyl ether, 15186

Oxyayanin B, 16440

Sideritiflavone, 19872

Thymonin, 21362

3,5,6-Trihydroxy-7,3',4'-trimethoxyflavone, 21854

5,2',5'-Trihydroxy-6,7,8-trimethoxyflavone, 21855

5,7,3'-Trihydroxy-6,4',5'-trimethoxyflavone, 21856

5,7,4'-Trihydroxy-3,8,3'-trimethoxyflavone, 21857

5,7,4'-Trihydroxy-6,8,3'-trimethoxy flavone, 21858

6,3',4'-Trimethoxy-7,8,5'-trihydroxyisoflavone, 21936

\section{$\mathrm{C}_{18} \mathrm{H}_{16} \mathrm{O}_{9}$}

Limocitrol, 12840

Vermicularin, 22402

\section{$\mathrm{C}_{18} \mathrm{H}_{16} \mathrm{O}_{10}$}

2-C- $\beta$ - $D$-Xylopyranosyl-1,3,6,7-tetrahydroxyxanthone, 22841

\section{$\mathrm{C}_{18} \mathrm{H}_{17} \mathrm{ClO}_{6}$}

2-Chlorosamaderine A, 3571

\section{$\mathrm{C}_{18} \mathrm{H}_{17} \mathrm{NO}$}

Girinimbine, 8456

$\mathrm{C}_{18} \mathrm{H}_{17} \mathrm{NO}_{2}$

Clausine D, 3794

Cycloclausenamide, 4484

Glycoborinine, 8822

Heptaphylline, 9410

Koenine, 12250

Micromeline, 14840
Roemerine, 18896

$\mathrm{C}_{18} \mathrm{H}_{17} \mathrm{NO}_{3}$

Clausine S, 3806

Fugapavine, 7980

Japonine, 11821

L-Mecambroline, 13631

Micheline A, 14829

Norstephalagine, 15797

PC-2000-53-503-15, 16738

$N$ - $\beta$-Phenethyl-3-(3,4-methylenedioxy phenyl) propenamide, 17086

Xylopine, 22818

\section{$\mathrm{C}_{18} \mathrm{H}_{17} \mathrm{NO}_{4}$}

Actinodaphnine, 585

Annocherine B, 1299

Cularicine, 4350

Hernangerine, 9451

Launobine, 12564

7-Methoxyoxoassoanine, 14049

Norannuradhapurine, 15711

1,3,5-Trihydroxyl-4-prenylacridone, 21763

\section{$\mathrm{C}_{18} \mathrm{H}_{17} \mathrm{NO}_{5}$}

Terrestriamide, 21004

\section{$\mathrm{C}_{18} \mathrm{H}_{17} \mathrm{NO}_{6}$}

Toddaliopsin C, 21421

\section{$\mathrm{C}_{18} \mathrm{H}_{17} \mathrm{NO}_{8} \mathrm{~S}$}

2-Hydroxy-7-[(4-hydroxy-3-methoxyphenyl) methyl]-3-methoxy-8-quinolyl sulfate, 10190

\section{$\mathrm{C}_{18} \mathrm{H}_{17} \mathrm{~N}_{3} \mathrm{O}_{2}$}

Nauclexine, 15314

$\mathrm{C}_{18} \mathrm{H}_{17} \mathrm{~N}_{3} \mathrm{O}_{4}$

Dichotomide I, 5420

$\mathrm{C}_{18} \mathrm{H}_{17} \mathrm{O}_{7}^{+}$

3-O-Methyl malvidin, 14575

$\mathrm{C}_{18} \mathrm{H}_{18} \mathrm{~N}_{2} \mathrm{O}_{3}$

Cyclo-(Phe-Tyr), 4527

\section{$\mathrm{C}_{18} \mathrm{H}_{18} \mathrm{~N}_{2} \mathrm{O}_{4}$}

3,6-Di(4-hydroxy) benzyl-2,5-dioxopiperazine, 5770

Isaindigodione, 11186

\section{$\mathrm{C}_{18} \mathrm{H}_{18} \mathrm{O}$}

Juncunol, 11958

$\mathrm{C}_{18} \mathrm{H}_{18} \mathrm{O}_{2}$

Honokiol, 9631

(7S,8R)-4-Hydroxy-4',7-epoxy-8,3'-neolignan(7E)-ene, 10088

Isomagnolol, 11519

Juncusol, 11960

Magnolol, 13388

(-)-4'-O-Methyl-nyasol, 14627

Monomethyl-cis-hinokiresinol, 14926

Phenylpropyl cinnamate, 17133

$\mathrm{C}_{18} \mathrm{H}_{18} \mathrm{O}_{3}$
3'-Hydroxy-4'-methoxy-4'-dehydroxynyasol, 10396

3'-Methoxynyasin, 14044

3"-Methoxynyasol, 14045

Obovatol, 15888

Termilignan, 20975

\section{$\mathrm{C}_{18} \mathrm{H}_{18} \mathrm{O}_{4}$}

Champanone B, 3473

Champanone C, 3474

Combretastatin $\mathrm{D}_{4}, 3941$

3,4-Dehydrolarreatricin, 4940

4',6'-Dihydroxy-3',5'-dimethyl-2'-methoxychalcone, 5857

Enterolactone, 6812

7-Hydroxy-5-methoxy-6,8-dimethylflavanone, 10399

Magnolignan E, 13382

4-[(2S,3R)-3-Methyl-7-((E)-1-propenyl)-2,3-dihydro-1,4-benzodioxin-2-yl]-1,3-benzenediol, 14688

4-[(2S,3S)-3-Methyl-7-((E)-1-propenyl)-2,3-dihydro-1,4-benzodioxin-2-yl]-1,3-benzenediol, 14689

\section{$\mathrm{C}_{18} \mathrm{H}_{18} \mathrm{O}_{5}$}

Agrimonolide, 760

Chrysotoxene, 3627

4,7-Dihydroxy-5-methoxyl-6-methyl-8-formylflavan, 5985

5,7-Dimethoxy-3-(4-hydroxybenzyl)chroman-4one, 6234

Flavokawain A, 7818

Helilandin B, 9305

(4-Hydroxyphenyl)ethyl trans-ferulate, 10619

2'-Hydroxy-2,4',6'-trimethoxychalcone, 10785

Iso-mucromatol, 11543

Magnaldehyde C, 13365

Matteucinol, 13611

(2S)-3',4'-Methylenedioxy-5,7-dimethoxyflavane, 14362

Naringenin trimethyl ether, 15285

Ophiopogonanone B, 16137

\section{$\mathrm{C}_{18} \mathrm{H}_{18} \mathrm{O}_{6}$}

Acetylalkannin, 309

Acetylshikonin, 514

Americanol, 1036

( \pm )-3',3"-Bisdemethylpinoresinol, 2440

5,7-Dihydroxy-6-methoxy-3-(4-methoxybenzyl)

chroman-4-one, 5986

3-(3,4-Dimethoxybenzyl)-5,7-dihydroxychroman-4-one, 6210

5-Hydroxy-6,7-dimethoxy-3-(4'-hydroxybenzyl)

-4-chromanone, 10030

3-(4-Hydroxy-3-methoxybenzyl)-5-hydroxy-7- 
methoxychroman-4-one, 10387

5-Hydroxy-7-methoxy-3-(3-hydroxy-4-methoxy-

benzyl)-chroman-4-one, 10410

(2R)-5-Hydroxy-7,2',3'-trimethoxyflavanone, 10789

Isoamericanol A, 11211

Melilotocarpan C, 13680

Methoxymatteucin, 13993

3'-O-Methylviolanone, 14811

Nigrolineaxanthone D, 15577

Odoricarpan, 16000

Samaderine A, 19221

2(S)-5,7,3'-Trihydroxy-6,8-dimethyl-5'-methoxyflavanone, 21721

1,3,5-Trihydroxy-4-(3-hydroxy-3-methylbutyl) xanthone, 21747

1,3,7-Trihydroxy-2-(3-hydroxy-3-methylbutyl) xanthone, 21748

(2R,3R)-(+)-4',5,7-Trimethoxydihydroflavonol, 21909

\section{$\mathrm{C}_{18} \mathrm{H}_{18} \mathrm{O}_{7}$}

(R)-2,3-Dihydro-7-demethylrobustigenin, 5586

5,7-Dihydroxy-3-(3-hydroxy-4-methoxy-benzyl)6-methoxychroman-4-one, 5910

(2S)-5,2'-Dihydroxy-7,4',5'-trimethoxyflavanone, 6159

5,3'-Dihydroxy-6,7,4'-trimethoxyflavanone, 6160

Inumakilactone B, 11115

Laurentiquinone, 12568

3-Methyl betuletol, 14157

1,2,3,6,7-Pentamethoxyxanthone, 16874

Qianhucoumarin D, 18285

Ramalic acid, 18527

\section{$\mathrm{C}_{18} \mathrm{H}_{18} \mathrm{O}_{8}$}

2-Caffeoyloxy-3-hydroxy-3-(3,4-dihydroxyphenyl)propyl alcohol, 2915

2,3-Dihydroirigenin, 5647

Futoxide, 8041

\section{$\mathrm{C}_{18} \mathrm{H}_{18} \mathrm{O}_{9}$}

Vaccihein A, 22306

\section{$\mathrm{C}_{18} \mathrm{H}_{19} \mathrm{ClO}_{5}$}

Eurycolactone B, 7640

\section{$\mathrm{C}_{18} \mathrm{H}_{19} \mathrm{NO}_{2}$}

Lirinidine, 12915

$N$-Methylasimilobine, 14148

(S)-Nornuciferine, 15780

$\mathrm{N}$-Nornuciferine, 15781

\section{$\mathrm{C}_{18} \mathrm{H}_{19} \mathrm{NO}_{3}$}

Clausenamide, 3789

Erythraline, 7330

Glaziovine, 8524

(-)-Isopiline, 11593

Neoclausenamide, 15366
1-[1-Oxo-7(3,4-methylenedioxyphenyl)-2E,4E, $6 E$-heptatrienyl]pyrrolidine, 16383

Stepharine, 20322

Tuduranine, 22100

$\mathrm{C}_{18} \mathrm{H}_{19} \mathrm{NO}_{4}$

Bellamarine, 2219

Cephalotaxinone, 3407

7'-(3',4'-Dihydroxyphenyl)- $N$-[(4-methoxyphenyl) ethyl]propenamide, 6089

Eryphrinine, 7308

$N$-trans-Feruloyltyramine, 7788

$\mathrm{N}$-cis-Feruloyltyramine, 7789

Haplopine-3,3'-dimethylallylether, 9227

Hernovine, 9454

Isocephalotaxinone, 11317

Krepowine, 12296

Laurolitsine, 12573

Nobiline, 15638

Perfamine, 16910

Pessoine, 17006

Tecleabine, 20892

Tecleanatalensine B, 20894

\section{$\mathrm{C}_{18} \mathrm{H}_{19} \mathrm{NO}_{5}$}

3-O-Acetylhamayne, 408

1-O-Acetyllycorine, 460

2-O-Acetyllycorine, 461

Anhydroevoxine, 1267

Bueropyridinium A, 2713

Cephalotaxinamide, 3403

Clausine U, 3807

Epibueropyridinium A, 6841

$N$-cis-Feruloyloctopamine, 7781

$N$-trans-Feruloyloctopamine, 7782

Hernandonine, 9449

Isotecleoxine, 11733

Tecleanatalensine A, 20893

Toddaliopsin D, 21422

\section{$\mathrm{C}_{18} \mathrm{H}_{19} \mathrm{NO}_{6}$}

3'-O-Acetylisopteleflorine, 442

$\mathrm{C}_{18} \mathrm{H}_{19} \mathrm{NO}_{7}$

Glyfoline, 8848

$\mathrm{C}_{18} \mathrm{H}_{19} \mathrm{NO}_{11}$

Ebracteolatinoside A, 6671

$\mathrm{C}_{18} \mathrm{H}_{19} \mathrm{~N}_{3} \mathrm{O}$

$\mathrm{N}$-(2-Methylaminobenzoyl)tryptamine, 14135

\section{$\mathrm{C}_{18} \mathrm{H}_{20} \mathrm{CINO}_{5}$}

Chlorodesnkolbisine, 3547

\section{$\mathrm{C}_{18} \mathrm{H}_{20} \mathrm{CIN}_{3} \mathrm{O}_{6} \mathrm{~S}_{2}$}

Sporidesmin, 20234

$\mathrm{C}_{18} \mathrm{H}_{20} \mathrm{~N}_{2}$

Tabernoschizine, 20578

$\mathrm{C}_{18} \mathrm{H}_{20} \mathrm{~N}_{2} \mathrm{O}_{4}$

Dichotomine D, 5425
$\mathrm{C}_{18} \mathrm{H}_{20} \mathrm{~N}_{2} \mathrm{O}_{6}$

Sterculinine I, 20329

\section{$\mathrm{C}_{18} \mathrm{H}_{20} \mathrm{O}_{2}$}

FB3, 7744

Salvinone, 19218

\section{$\mathrm{C}_{18} \mathrm{H}_{20} \mathrm{O}_{3}$}

Allogibberic acid, 931

1,3-Di-O-[2',2'-di-( $p$-phenylene) isopropylidene] glycerol, 5481

4-Epi-larreatricin, 6942

FB5, 7746

Garcibenzopyran, 8203

Garcibiphenyl B, 8205

5-(1-Hydroxyethyl)-2,6-dihydroxy-1,7-dimethyl9,10-dihydrophenanthrene, 10101

5-(1-Hydroxyethyl)-2,8-dihydroxy-1,7-dimethyl9,10-dihydrophenanthrene, 10102

threo-(7S,8R)-1-(4-Hydroxyphenyl)-2-[4-(E)-propenylphenoxy]-propan-1-ol, 10637

\section{$\mathrm{C}_{18} \mathrm{H}_{20} \mathrm{O}_{4}$}

Hibicuslide A, 9536

Hibicuslide B, 9537

3"-Hydroxy-4-epi-larreatricin, 10077

Magnolignan A, 13378

Magnolignan C, 13380

Stemanthrene C, 20293

Toxyl angelate, 21487

\section{$\mathrm{C}_{18} \mathrm{H}_{20} \mathrm{O}_{5}$}

Arnebifuranone, 1744

Cnidiadin, 3854

Gobicusin A, 8903

3'-(4"-Hydroxy-3",5'-dimethoxyphenyl)-propyl benzoate, 10041

2'-Hydroxy-4,4',6'-trimethoxydihydrochalcone, 10787

Laurycolactone B, 12576

Magnolignan B, 13379

2'-Methoxymollugin, 14040

7-O-Methyl isomucronulatol, 14533

Shikonofuran A, 19821

meso-(rel-7S,8S,7'R,8'R)-3,4,3',4'-Tetrahydroxy7,7'-epoxylignan, 21105

2,3,4,4'-Tetrahydroxy-5-methoxy-6-prenyl-biphenyl, 21132

Tupichinol B, 22115

\section{$\mathrm{C}_{18} \mathrm{H}_{20} \mathrm{O}_{6}$}

trans-4,5-Bis(4-hydroxy-3-methoxyphenyl)-1,3dioxacyclohexane, 2463

6-O-cis-p-Coumaroyl-7-deoxyrehmaglutin A, 4152

6-O-trans-p-Coumaroyl-7-deoxyrehmaglutin A, 4153

5 $\beta, 6$-Dihydrosamaderine A, 5705 
Eurycolactone C, 7641

Isoduartin, 11404

2-Methoxybenzyl-2,3,6-trimethoxybenzoate, 13857

1'-Methoxy-2'-hydroxydihydromollugin, 13954

Nagilactone D, 15232

$5 \alpha, 6 \beta, 7 \beta$-Trihydroxy- $8 \alpha$-methoxy-2-(2-phenylethyl)-5,6,7,8-tetrahydro chromone, 21785

\section{$\mathrm{C}_{18} \mathrm{H}_{20} \mathrm{O}_{7}$}

tert-O-Methyl byakangelicin, 14204

Protosappanin C dimethyl acetal, 17989

$5 \alpha, 6 \beta, 7 \beta, 8 \alpha$-Tetrahydroxy-2-[2-(4'-methoxyphenyl)ethyl]-5,6,7,8-tetrahydrochromone, 21131

\section{$\mathrm{C}_{18} \mathrm{H}_{20} \mathrm{O}_{8}$}

Inumakilactone $\mathrm{A}, 11113$

Uvaribonol F, 22295

Uvaribonol G, 22296

\section{$\mathrm{C}_{18} \mathrm{H}_{21} \mathrm{NO}_{3}$}

Codeine, 3885

(+)-8,9-Dihydrostepharine, 5719

Erysodine, 7322

Erysovine, 7328

Magnococline, 13372

$D$-N-Methyl coclaurine, 14247

Neopine, 15449

$\mathrm{N}$-Norarmepavine, 15713

Sarmentosine, 19378

Yuziphine, 22958

\section{$\mathrm{C}_{18} \mathrm{H}_{21} \mathrm{NO}_{4}$}

1-O-Acetylnorpluviine, 472

3-O-Acetylsanguinine, 506

Buphanidrine, 2751

Cephalotaxine, 3404

Homolycorine, 9612

Isocephalotaxine, 11316

\section{$\mathrm{C}_{18} \mathrm{H}_{21} \mathrm{NO}_{5}$}

Ambelline, 1021

Cephalotaxine $\alpha$ - $N$-oxide, 3405

Cephalotaxine $\beta$ - $N$-oxide, 3406

Drupacine, 6608

4-Hydroxycephalotaxine, 9900

11-Hydroxycephalotaxine, 9901

Pretazettine, 17850

Stephabyssine, 20311

Tazettine, 20891

(+)-Undulatine, 22229

(-)-Undulatine, 22230

\section{$\mathrm{C}_{18} \mathrm{H}_{21} \mathrm{NO}_{6}$}

Haploperine, 9223

11- $\beta$-Hydroxycephalotaxine $\beta$ - $N$-oxide, 9902

\section{$\mathrm{C}_{18} \mathrm{H}_{22} \mathrm{CINO}_{6}$}

Acutumidine, 602
$\mathrm{C}_{18} \mathrm{H}_{22} \mathrm{NO}^{+}$

$N$-( $p$-Hydroxyphenethyl)actinidine, 10601

$\mathrm{C}_{18} \mathrm{H}_{22} \mathrm{NO}_{2}$

Valerianae alkaloid B, 22317

$\mathrm{C}_{18} \mathrm{H}_{22} \mathrm{NO}_{4}^{+}$

$O$-Methylptelefolonium, 14705

\section{$\mathrm{C}_{18} \mathrm{H}_{22} \mathrm{~N}_{2} \mathrm{O}$}

(+)-Manilamine, 13501

$\mathrm{C}_{18} \mathrm{H}_{22} \mathrm{~N}_{2} \mathrm{O}_{2}$

Dehydroodorine, 4955

$\mathrm{C}_{18} \mathrm{H}_{22} \mathrm{O}$

2,2-Dimethyl-8-prenyl-6-vinylchromene, 6399

\section{$\mathrm{C}_{18} \mathrm{H}_{22} \mathrm{O}_{2}$}

Deoxodehydrocyclopiloselloidone, 5142

Estrone, 7387

1-(7-Hydroxy-2,6-dimethyl-1-naphthyl)-4-methyl-3-pentanone, 10052

Tetrahydromagnolol, 21074

\section{$\mathrm{C}_{18} \mathrm{H}_{22} \mathrm{O}_{3}$}

Cyclopiloselloidone, 4530

3 $\beta, 12$-Dihydroxy-13-methyl-5,8,11,13-podocarpatetraen-7-one, 6045

FB4, 7745

Isocrotonoylpterosin $\mathrm{B}, 11351$

\section{$\mathrm{C}_{18} \mathrm{H}_{22} \mathrm{O}_{4}$}

Arnebinone, 1747

$\left(1 \mathrm{a} S^{*}, 1 \mathrm{~b} S^{*}, 7 \mathrm{a} S^{*}, 8 \mathrm{a} S^{*}\right)-4,5-D i m e t h o x y-1 \mathrm{a}, 7 \mathrm{a}-$ dimethyl-1,1a,1b,2,7,7a,8,8a-octahydrocyclo propa $[3,4]$ cyclopenta[1,2-b]naphthalene-3,6dione, 6221

Heliotropinone A, 9321

Heliotropinone B, 9322

13-Methoxy-8,12-podocarpadiene-2,11,14-trione, 14073

Nordihydroguaiaretic acid, 15741

\section{$\mathrm{C}_{18} \mathrm{H}_{22} \mathrm{O}_{5}$}

5-Acetyl-3 $\beta$-angeloyloxy-2 $\beta$-(1-hydroxyisopropyl)-2,3-dihydrobenzofuran, 318

Celaphanol B, 3363

Chrysotoxin, 3628

1 $\beta, 14$-Dihydroxy-13-methoxy-8,11,13-podocar-

$$
\text { patriene-2,7-dione, } 5999
$$

Eurycolactone D, 7642

Laurycolactone A, 12575

Zearalenone, 22974

$\mathrm{C}_{18} \mathrm{H}_{22} \mathrm{O}_{6}$

Arteanomalactone, 1788

2,5-Dimethyl-3-O- $\beta$ - $D$-glucopyranosylnaphthol, 6352

$\mathrm{C}_{18} \mathrm{H}_{22} \mathrm{O}_{7}$

Nagilactone J, 15235

$\mathrm{C}_{18} \mathrm{H}_{22} \mathrm{O}_{8}$

Buergeriside $\mathrm{B}_{1}, 2710$
Buergeriside $\mathrm{B}_{2}, 2711$

Ningposide D, 15621

Pectinolide G, 16753

\section{$\mathrm{C}_{18} \mathrm{H}_{22} \mathrm{O}_{9}$}

Ethyl chlorogenate, 7425

6 $\beta$-C-Glucopyranosyl-5,7-dihydroxy-2-isopropylchromone, 8618

$8 \beta$-C-Glucopyranosyl-5,7-dihydroxy-2-isopropylchromone, 8619

Inumakilactone $\mathrm{C}, 11116$

Methylcnidioside A, 14246

Ningposide A, 15618

Ningposide B, 15619

Uncinoside A, 22215

\section{$\mathrm{C}_{18} \mathrm{H}_{22} \mathrm{O}_{10}$}

Picraquassioside A, 17297

\section{$\mathrm{C}_{18} \mathrm{H}_{22} \mathrm{O}_{11}$}

Asperuloside, 1892

$\mathrm{C}_{18} \mathrm{H}_{22} \mathrm{O}_{11} \mathrm{~S}$

Paederoside, 16516

\section{$\mathrm{C}_{18} \mathrm{H}_{23} \mathrm{NO}$}

(E)-3-(3-Hydroxymethyl-2-butenyl)-7-(3-methyl2-butenyl)- $1 H$-indole, 10473

$N$-Isobutyl-2E,4E,12E-tetradecatrien-8,10-diynamide, 11288

$N$-Isobutyl-2E,4E,12Z-tetradecatrien-8,10-diynamide, 11289

\section{$\mathrm{C}_{18} \mathrm{H}_{23} \mathrm{NO}_{2}$}

Cocculidine, 3862

3,3-Dimethylallyl-4-methoxy-2-quinolone, 6308 Isococculidine, 11337

2-(Nonan-8-one)-(1H)-4-quinolone, 15691

\section{$\mathrm{C}_{18} \mathrm{H}_{23} \mathrm{NO}_{3}$}

Coccutrine, 3865

Futoamide, 8035

1-Hydroxycryprochine, 9948

(3Z,5Z)- $N$-Isobutyl-8-(3',4'-methylenedioxyphenyl)-heptadienamide, 11280

$O$-Methylmaritidine, 14578

Piperamide C 7:1(6E), 17434

\section{$\mathrm{C}_{18} \mathrm{H}_{23} \mathrm{NO}_{4}$}

Haplophyllidine, 9224

Isostemonamine, 11719

Lycorenine, 13237

$O$-Methylacutifolin, 14121

Papyramine, 16634

\section{$\mathrm{C}_{18} \mathrm{H}_{23} \mathrm{NO}_{5}$}

(E)-Seneciphylline, 19712

\section{$\mathrm{C}_{18} \mathrm{H}_{23} \mathrm{NO}_{6}$}

Dechloroacutumidine, 4846

Riddelline, 18842

\section{$\mathrm{C}_{18} \mathrm{H}_{23} \mathrm{~N}_{3} \mathrm{O}_{2}$}

Picrasidine K, 17303 


\section{$\mathrm{C}_{18} \mathrm{H}_{23} \mathrm{~N}_{3} \mathrm{O}_{2} \mathrm{~S}_{2}$}

Polanrazine B, 17604

\section{$\mathrm{C}_{18} \mathrm{H}_{24} \mathrm{NO}_{5}{ }^{+}$}

Veprisinium, 22380

\section{$\mathrm{C}_{18} \mathrm{H}_{24} \mathrm{~N}_{2} \mathrm{O}_{2}$}

Anticancer Amide PMV70P691-052, 1382

Grandiamide B, 8975

Odorine, 16002

\section{$\mathrm{C}_{18} \mathrm{H}_{24} \mathrm{~N}_{2} \mathrm{O}_{3}$}

Elliptinol, 6761

Grandiamide C, 8976

(+)-Odorinol, 16003

\section{$\mathrm{C}_{18} \mathrm{H}_{24} \mathrm{O}$}

Backuchiol, 2081

2,6-Diprenyl-4-vinylphenol, 6499

\section{$\mathrm{C}_{\mathbf{1 8}} \mathrm{H}_{\mathbf{2 4}} \mathbf{O}_{\mathbf{2}}$}

3 $\beta, 12$-Dihydroxy-13-methyl-6,8,11,13-podocarpatetraen, 6044

$\alpha$-Estradiol, 7383

$\beta$-Estradiol, 7384

Octadeca-8,10,12-triynoic acid, 15955

Przewalskin, 18008

\section{$\mathrm{C}_{18} \mathrm{H}_{24} \mathrm{O}_{3}$}

3-(3',7'-Dimethyl-2',6'-octadienyl)-4-methoxybenzoic acid, 6383

Estriol, 7386

Piloselloidone, 17367

\section{$\mathrm{C}_{18} \mathrm{H}_{24} \mathrm{O}_{4}$}

$3 \beta, 14$-Dihydroxy-13-methoxy-8,11,13-podocarpatrien-7-one, 6000

Hydroxyisopiloselloidone, 10254

$3 \beta$-Hydroxy-13-methoxy-8,12-podocarpadiene-

11,14-dione, 10455

18-Hydroxy-13-methoxy-8,12-podocarpadiene11,14-dione, 10456

Hydroxypiloselloidone, 10646

Ialibinone E, 10933

Miliusol, 14852

Tanzawaic acid F, 20691

\section{$\mathrm{C}_{18} \mathrm{H}_{24} \mathrm{O}_{5}$}

$r e l-2 R$-Methyl-5S-acetoxy- $4 R$-furanogermacr1(10)Z-en-6-one, 14115

\section{$\mathrm{C}_{18} \mathrm{H}_{24} \mathrm{O}_{6}$}

8-Acetoxy-2-methoxy-10-hydroxy-3,11(13)guaiadien-12,6-olide, 251

\section{$\mathrm{C}_{18} \mathrm{H}_{24} \mathrm{O}_{7}$}

(2S)-4',4'-Dimethoxy-ongokein, 6271 Iridoid CPB-53-710-1, 11146

Iridoid CPB-53-710-2, 11147

Renifolin, 18620

\section{$\mathrm{C}_{18} \mathrm{H}_{24} \mathrm{O}_{8}$}

Celephtalide A, 3372

$\mathrm{C}_{18} \mathrm{H}_{24} \mathrm{O}_{9}$
Tenuifoliside D, 20958

$\mathrm{C}_{18} \mathrm{H}_{24} \mathrm{O}_{10}$

Dracunculifoside E, 6584

3'-O- $\beta$-Glucopyranosyl plumbagic acid methyl ester, 8713

Regaloside A, 18571

Regaloside D, 18573

$\mathrm{C}_{18} \mathrm{H}_{24} \mathbf{O}_{12}$

10-O-Acetylmonotropein, 470

6-O-Acetylscandoside, 507

Asperulosidic acid, 1894

6-O-Epiacetylscandoside, 6869

\section{$\mathrm{C}_{18} \mathrm{H}_{24} \mathrm{O}_{12} \mathrm{~S}$}

Paederosidic acid, 16517

\section{$\mathrm{C}_{18} \mathrm{H}_{25} \mathrm{NO}$}

Dehydro- $\gamma$-sanshool, 4967

$N$-Isobutyl-2E,4E,10E,12Z-tetradecatetraen-8ynamide, 11287

\section{$\mathrm{C}_{18} \mathrm{H}_{25} \mathrm{NO}_{2}$}

Isolobinine, 11507

\section{$\mathrm{C}_{18} \mathrm{H}_{25} \mathrm{NO}_{5}$}

Integerrimine, 11091

Isostemotinine, 11720

Senecionine, 19706

Senecivernine, 19714

Stemotinine, 20308

$3 \alpha-\left(3^{\prime}, 4^{\prime}, 5^{\prime}\right.$-Trimethoxybenzoyloxy)tropane, 21896

\section{$\mathrm{C}_{18} \mathrm{H}_{25} \mathrm{NO}_{5} \mathrm{~S}$}

$O$-Methylsakambullin, 14715

\section{$\mathrm{C}_{18} \mathrm{H}_{25} \mathrm{NO}_{6}$}

Angularine, 1242

Crotalaburnine, 4266

Jacobine, 11809

Mucronatinine, 15018

Retrorsine, 18667

Usaramine, 22277

\section{$\mathrm{C}_{18} \mathrm{H}_{25} \mathrm{NO}_{7}$}

Isatidine, 11189

Spectabiline, 20140

\section{$\mathrm{C}_{18} \mathrm{H}_{26} \mathrm{O}_{2}$}

3 $\beta, 12$-Dihydroxy-13-methyl-8,10,13-podocarpanetriene, 6043

(Z)-Octadec-12-ene-8,10-diynoic acid, 15956

\section{$\mathrm{C}_{18} \mathrm{H}_{26} \mathrm{O}_{3}$}

15,16-Bisnor-13-oxo-8(17),11E-labdadien-19-oic acid, 2488

14,18-Dihydroxy-13-methoxy-8,11,13-podocarpatriene, 5998

8 $\beta$-Ethoxy atractylenolide III, 7396

1-Methoxy-(9R,10S)-epoxyheptadecan-4,6diyn-3-one, 13922

Tanzawaic acid E, 20690

\section{$\mathrm{C}_{18} \mathrm{H}_{26} \mathrm{O}_{4}$}

Aurantiacone, 2007

$n$-Butyl-2-ethylbutylphthalate, 2793

2-(1' $\beta$-Geranyl-5' $\beta$-hydroxy-2'-oxocyclohex-3'enyl)acetic acid, 8321

3-Teracrylmelazolide B, 20967

\section{$\mathbf{C}_{18} \mathbf{H}_{26} \mathbf{O}_{5}$}

6- $\alpha$-Acetyl-4- $O$-oxobedfordiaic methyl ester, 475

$1 \beta$-Hydroxy- $8 \beta$-acetoxycostic acid methyl ester, 9754

$1 \beta$-Hydroxy- $8 \beta$-acetoxyisocostic acid methyl ester, 9756

3-Teracrylmelazolide A, 20966

\section{$\mathrm{C}_{18} \mathrm{H}_{26} \mathrm{O}_{\mathbf{8}}$}

(E)-4-(3,4-Dimethoxyphenyl)but-3-en-1-O- $\beta$ - $D$ glucopyranoside, 6278

4-Hydroxy-2-[(E)-4-hydroxy-3-methyl-2butenyl]-5-methylphenyl $\beta$ - $D$-glucopyranoside, 10198

(S)-4-(4-Hydroxyphenyl)-2-butanol 2-O-(6-O-acetyl)- $\beta$ - $D$-glucopyranoside, 10610

\section{$\mathrm{C}_{18} \mathrm{H}_{26} \mathrm{O}_{9}$}

Icariside $\mathrm{H}_{1}, 10954$

\section{$\mathrm{C}_{18} \mathrm{H}_{26} \mathrm{O}_{10}$}

Benzyl alcohol $O-\beta-D$-primveroside, 2278

Benzyl alcohol $\beta$ - $D$-(2'-O- $\beta$-xylopyranosyl) glucopyranoside, 2279

Icariside $\mathrm{F}_{2}, 10953$

\section{$\mathrm{C}_{18} \mathrm{H}_{26} \mathbf{O}_{11}$}

3'-O-Acetylloganic acid, 454

4'-O-Acetylloganic acid, 455

6'-O-Acetylloganic acid, 456

7-O-Acetylloganic acid, 457

6-O-Methyldeacetylasperulosidic acid methyl ester, 14282

2-C-Methyl- $D$-erythritol 1- $O-\beta-D$-(6-O-4-hydroxybenzoyl)glucopyranoside, 14426 8-O-Methylmonotropein methyl ester, 14605 6-O-Methylscandoside methyl ester, 14722 Oleoside dimethyl ester, 16071

Orcinol-1- $O-\beta$ - $D$-apiofuranosyl- $(1 \rightarrow 6)-\beta$ - $D$ glucopyranoside, 16170

Secologanoside dimethyl ester, 19625

\section{$\mathrm{C}_{18} \mathrm{H}_{26} \mathrm{O}_{12}$}

8-O-Acetylshanzhiside, 509

Canthoside C, 3101

Canthoside D, 3102

Digupigan A, 5534

4-Hydroxyphenyl- $\beta$-gentiobioside, 10623

\section{$\mathrm{C}_{18} \mathrm{H}_{27} \mathrm{NO}$}

$(2 E, 4 E, 8 E, 10 E, 12 E)-N$-Isobutyl-2, $4,8,10,12-$ tetradecapentaenamide, 11286

\section{$\mathrm{C}_{18} \mathrm{H}_{27} \mathrm{NO}_{3}$}


Capsaicin, 3141

Lycophlegmarine, 13220

\section{$\mathrm{C}_{18} \mathrm{H}_{27} \mathrm{NO}_{4}$}

$L-3 \alpha, 6 \beta$-Ditigloyloxytropane, 6525

$\omega$-Hydroxycapsaicin, 9888

Lycofawcine, 13192

Lycoposerramine N, 13233

\section{$\mathrm{C}_{18} \mathrm{H}_{27} \mathrm{NO}_{5}$}

7-Hydroxy-3,6-bis(tigloyloxy)tropane, 9856

Platyphylline, 17546

Sarracine, 19388

\section{$\mathrm{C}_{18} \mathrm{H}_{27} \mathrm{NO}_{6}$}

Rosmarinine, 18926

Trichodesmine, 21560

\section{$\mathrm{C}_{18} \mathrm{H}_{27} \mathrm{NO}_{7}$}

Sceleratine, 19461

Syneilesine, 20551

\section{$\mathrm{C}_{18} \mathrm{H}_{28} \mathrm{~N}_{2} \mathrm{O}_{2}$}

Serratinidine, 19772

\section{$\mathrm{C}_{18} \mathrm{H}_{28} \mathrm{O}_{2}$}

Octadeca-8,10-diynoic acid, 15947

Parinaric acid, 16667

1-Undecylenyl-3,4-methylenedioxybenzene, 22227

\section{$\mathrm{C}_{18} \mathrm{H}_{28} \mathrm{O}_{3}$}

15,16-Bisnor-13-oxo-8(17)-labden-19-oic acid, 2489

2-Dehydroxy-5-O-methylembelin, 4987

Licanic acid, 12748

Panaquinquecol 1, 16586

\section{$\mathrm{C}_{18} \mathrm{H}_{28} \mathrm{O}_{4}$}

5-O-Methylembelin, 14347

\section{$\mathrm{C}_{18} \mathrm{H}_{28} \mathrm{O}_{5}$}

$3 \alpha$-Acetoxydiversifolol, 175

$3 \alpha$-Acetoxy-4 $\alpha$-hydroxy-11(13)-eudesmen-12oic acid methyl ester, 216

\section{$\mathrm{C}_{18} \mathrm{H}_{28} \mathrm{O}_{8}$}

Celephtalide C, 3374

4-Hydroxy-2-[3-hydroxy-3-methylbutyl]-5methylphenyl $\beta$ - $D$-glucopyranoside, 10200

\section{$\mathrm{C}_{18} \mathrm{H}_{28} \mathrm{O}_{9}$}

$(1 R, 2 R)-5$ '-Hydroxyjasmonic acid 5'- $O-\beta$ - $D$-glucopyranoside, 10270

Nuezhengalaside, 15865

Qingjueine I, 18289

Tuberonic acid glucoside, 22078

\section{$\mathrm{C}_{18} \mathrm{H}_{28} \mathrm{O}_{11}$}

3-Epiphlomurin, 6998

Lamioside, 12464

7-O-Methyl morroniside, 14607

Phlomurin, 17167

$\mathrm{C}_{18} \mathrm{H}_{28} \mathrm{O}_{12}$

Repenoside, 18626
$\mathrm{C}_{18} \mathrm{H}_{29} \mathrm{NO}$

Lanyuamide III, 12500

$\mathrm{C}_{18} \mathrm{H}_{29} \mathrm{NO}_{2}$

O-Acetyl-dihydrolycopodine, 379

Bungeanool, 2745

(2E,4E,8Z,11E)-2'-Hydroxy- $N$-isobutyl-2,4,8,11tetradecatetraenamide, 10236

(2E,4E,8Z,11Z)-2'-Hydroxy- $N$-isobutyl-2,4,8,11tetradecatetraenamide, 10237

5-Hydroxy-2-methyl-6-(11'-oxododecyl)pyridine, 10510

Lanyuamide II, 12499

$\mathrm{C}_{18} \mathrm{H}_{29} \mathrm{NO}_{3}$

Dihydrocapsaicin, 5553

Fawcettiine, 7741

5-Hydroxy-2-methyl-6-(11'-oxododecyl)pyridine $N$-oxide, 10511

Lycoclavine, 13187

$\mathrm{C}_{18} \mathrm{H}_{29} \mathrm{NO}_{7}$

Croalbidine, 4244

\section{$\mathrm{C}_{18} \mathrm{H}_{30}$}

2-Phenyldodecane, 17105

3-Phenyldodecane, 17106

4-Phenyldodecane, 17107

5-Phenyldodecane, 17108

6-Phenyldodecane, 17109

\section{$\mathrm{C}_{18} \mathrm{H}_{30} \mathrm{~N}_{2} \mathrm{O}_{3}$}

Chinese bittersweet alkaloid II, 3537

\section{$\mathrm{C}_{18} \mathrm{H}_{30} \mathrm{O}$}

2,4,6-Tri-t-butyl phenol, 21550

\section{$\mathrm{C}_{18} \mathrm{H}_{30} \mathrm{O}_{2}$}

$\alpha$-Eleostearic acid, 6748

Gorlic acid, 8955

Linolenic acid, 12893

(Z)-7-Octadecen-9-ynoic acid, 15958

Trichosanic acid, 21576

\section{$\mathrm{C}_{18} \mathrm{H}_{30} \mathrm{O}_{3}$}

13(R)-Hydroxy-octadeca-(9Z,11E,15Z)-trien-oic acid, 10546

Hygrophorone $\mathrm{F}^{12}, 10841$

Hygrophorone $\mathrm{G}^{12}, 10842$

\section{$\mathrm{C}_{18} \mathrm{H}_{30} \mathrm{O}_{4}$}

Auxin B, 2028

Hygrophorone $\mathrm{C}^{12}, 10839$

Hygrophorone $\mathrm{D}^{12}, 10840$

6-Methylgingediol, 14464

$\mathrm{C}_{18} \mathrm{H}_{30} \mathrm{O}_{5}$

1-O-Acetyl hygrophorone $\mathrm{E}^{10}, 435$

\section{$\mathrm{C}_{18} \mathrm{H}_{30} \mathrm{O}_{8}$}

Dictamnoside N, 5455

$(1 R, 2 R)$ - $p$-Menth-4(5)-ene-1,2-diol

$1-O-\beta-D-(2-O$-acetyl)glucopyranoside, 13745

$(1 R, 2 R)$-p-Menth-4(5)-ene-1,2-diol
1-O- $\beta$ - $D$-(6- $O$-acetyl)glucopyranoside, 13746

$\mathrm{C}_{18} \mathrm{H}_{31} \mathrm{NO}$

$N$-Methyl-6 $\beta$-(deca-l',3',5'-trienyl)-3 $\beta$-methoxy-

$2 \beta$-methylpiperidine, 14284

\section{$\mathrm{C}_{18} \mathrm{H}_{31} \mathrm{NO}_{2}$}

Dihydrobungeanool, 5549

Lanyuamide I, 12498

$\mathrm{C}_{18} \mathrm{H}_{31} \mathrm{NO}_{4}$

Broussonetine W, 2645

$\mathrm{C}_{18} \mathrm{H}_{31} \mathrm{NO}_{6}$

Broussonetine R, 2639

$\mathrm{C}_{18} \mathrm{H}_{31} \mathrm{~N}_{3} \mathrm{O}_{3}$

Palustridine, 16573

\section{$\mathrm{C}_{18} \mathrm{H}_{32} \mathrm{O}$}

9,12-Octadecadienal, 15938

\section{$\mathrm{C}_{18} \mathrm{H}_{32} \mathrm{O}_{2}$}

Chaulmoogric acid, 3485

Isolinolic acid, 11503

cis-9,cis-12-Linoleic acid, 12891

trans-9,trans-12-Linoleic acid, 12892

Malvic acid, 13455

Micromolide, 14842

10,13-Octadecadienoic acid, 15941

\section{$\mathrm{C}_{18} \mathrm{H}_{32} \mathrm{O}_{3}$}

(S)-Coriolic acid, 4056

Coronaric acid, 4079

$( \pm)$-12,13-Epoxyoleic acid, 7180

\section{$\mathrm{C}_{18} \mathrm{H}_{32} \mathrm{O}_{4}$}

Hygrophorone $\mathrm{A}^{12}, 10836$

\section{$\mathrm{C}_{18} \mathrm{H}_{32} \mathrm{O}_{5}$}

Auxin A, 2027

\section{$\mathrm{C}_{18} \mathrm{H}_{32} \mathrm{O}_{11}$}

(E)-2-Hexenyl- $\beta$ - $D$-glucopyranosyl- $(1 \rightarrow 2)$ $\beta$ - $D$-glucopyranoside, 9529

\section{$\mathrm{C}_{18} \mathrm{H}_{32} \mathrm{O}_{16}$}

Gentianose, 8298

1-Kestose, 12195

Manninotriose, 13503

Neokestose, 15424

Nigellamose, 15567

Panose, 16619

Panose B, 16620

Panose C, 16621

Planteose, 17514

Raffinose, 18526

\section{$\mathrm{C}_{18} \mathrm{H}_{33} \mathrm{NO}_{2}$}

Tetrahydrobungeanool, 21057

\section{$\mathrm{C}_{18} \mathrm{H}_{33} \mathrm{NO}_{5}$}

Broussonetine U, 2642

Broussonetine $\mathrm{U}_{1}, 2643$

Broussonetine V, 2644

$\mathrm{C}_{18} \mathrm{H}_{34} \mathrm{~N}_{4} \mathrm{O}_{12}$

Argininyl-fructosyl-glucose, 1674 
$\mathrm{C}_{18} \mathrm{H}_{34} \mathrm{O}_{2}$

Elaidic acid, 6731

Isooleic acid, 11574

Oleic acid, 16066

Petroselaidic acid, 17021

Petroselinic acid, 17022

Vaccenic acid, 22305

$\mathrm{C}_{18} \mathrm{H}_{34} \mathrm{O}_{3}$

9-D-Hydroxy-cis-12-octadecenoic acid, 10547

Ricinoleic acid, 18841

$\mathrm{C}_{18} \mathrm{H}_{34} \mathrm{O}_{5}$

Sanleng acid, 19295

Tianshic acid, 21368

\section{$\mathrm{C}_{18} \mathrm{H}_{34} \mathrm{O}_{11}$}

Hexyl- $\beta$ - $D$-glucopyranosyl-( $1 \rightarrow 2)-\beta$ - $D$-glucopyranoside, 9532

Hexyl- $\beta$ - $D$-glucopyranosyl-( $1 \rightarrow 6)-\beta$ - $D$-glucopyranoside, 9533

\section{$\mathrm{C}_{18} \mathrm{H}_{35} \mathrm{NO}_{2}$}

(-)-Cassine, 3292

\section{$\mathrm{C}_{18} \mathrm{H}_{35} \mathrm{NO}_{4}$}

Morusimic acid B, 14990

Morusimic acid D, 14992

Morusimic acid F, 14994

\section{$\mathrm{C}_{18} \mathrm{H}_{35} \mathrm{NO}_{7}$}

Broussonetine T, 2641

\section{$\mathrm{C}_{18} \mathrm{H}_{36}$}

3-Cyclohexyldodecane, 4498

\section{$\mathrm{C}_{18} \mathrm{H}_{36} \mathrm{~N}_{2} \mathrm{O}_{5}$}

Broussonetine $\mathrm{J}_{2}, 2637$

\section{$\mathrm{C}_{18} \mathrm{H}_{36} \mathrm{O}$}

Hexahydrofarnesyl acetone, 9500

9(Z)-Octadecen-1-ol, 15957

\section{$\mathrm{C}_{18} \mathrm{H}_{36} \mathrm{O}_{2}$}

Ethylpalmitate, 7469

4-Methyl heptadecanoic acid, 14481

14-Methyl hexadecanoic acid methyl ester, 14486

Stearic acid, 20280

\section{$\mathrm{C}_{18} \mathrm{H}_{36} \mathrm{O}_{4}$}

9,10-Dihydroxystearic acid, 6132

\section{$\mathrm{C}_{18} \mathrm{H}_{37} \mathrm{NO}$}

Stearamide, 20279

\section{$\mathrm{C}_{18} \mathrm{H}_{37} \mathrm{NO}_{5}$}

Broussonetine $\mathrm{M}_{1}, 2638$

\section{$\mathrm{C}_{18} \mathrm{H}_{37} \mathrm{NO}_{6}$}

Broussonetine S, 2640

\section{$\mathrm{C}_{18} \mathrm{H}_{38}$}

n-Octadecane, 15948

\section{$\mathrm{C}_{18} \mathrm{H}_{38} \mathrm{O}$}

Octadecanol, 15950

\section{$\mathrm{C}_{18} \mathrm{H}_{38} \mathrm{O}_{2}$}

1,1-Diethoxy- $n$-tetradecane, 5500

erythro-2,3-Octadecane-diol, 15949
$\mathrm{C}_{18} \mathrm{H}_{39} \mathrm{NO}_{3}$

Phytosphingosine, 17273

$\mathrm{C}_{19} \mathrm{H}_{11} \mathrm{NO}_{4}$

Lettowianthine, 12707

Norsanguinarine, 15793

$\mathrm{C}_{19} \mathrm{H}_{11} \mathrm{NO}_{6}$

Hypecoumine, 10880

Stephadione, 20313

$\mathrm{C}_{19} \mathrm{H}_{12} \mathrm{NO}_{5}$

8-Oxocoptisine, 16302

$\mathrm{C}_{19} \mathrm{H}_{12} \mathrm{O}_{3}$

Anticancer Phenylphenalone PMV70P691-130, 1435

$\mathrm{C}_{19} \mathrm{H}_{12} \mathrm{O}_{6}$

Bhubaneswin, 2342

Dehydroneotenone, 4954

Dicoumarin, 5440

Dolineone, 6551

Gamatin, 8124

Pachyrrhizin, 16499

Pongapin, 17714

$\mathbf{C}_{19} \mathbf{H}_{12} \mathbf{O}_{7}$

Daphnoretin, 4657

5,8-Dihydroxy-7-(4-hydroxy-5-methyl-coumarin3)-coumarin, 5918

12 $\alpha$-Hydroxydolineone, 10059

Isodaphnoretin, 11366

\section{$\mathrm{C}_{19} \mathrm{H}_{12} \mathrm{O}_{8}$}

Boennin, 2531

$\mathrm{C}_{19} \mathrm{H}_{13} \mathrm{NO}_{3}$

Evodianinine, 7666

$\mathrm{C}_{19} \mathrm{H}_{13} \mathrm{NO}_{4}$

Zanthoxyline, 22970

$\mathrm{C}_{19} \mathrm{H}_{13} \mathrm{NO}_{5}$

Nandazurine, 15243

$\mathrm{C}_{19} \mathrm{H}_{13} \mathrm{NO}_{6}$

Lindechunine A, 12860

$\mathrm{C}_{19} \mathrm{H}_{14} \mathrm{NO}_{4}{ }^{+}$

Coptisine, 4032

$\mathrm{C}_{19} \mathrm{H}_{14} \mathrm{~N}_{2} \mathrm{O}$

Nauclefine, 15305

$\mathrm{C}_{19} \mathrm{H}_{14} \mathrm{~N}_{4}$

Bilatriene, 2373

$\mathrm{C}_{19} \mathrm{H}_{14} \mathrm{O}_{2}$

Sterequinone A, 20335

$\mathrm{C}_{19} \mathrm{H}_{14} \mathrm{O}_{5}$

Millettocalyxin C, 14858

Pachycarin D, 16492

Vulpinic acid, 22625

$\mathrm{C}_{19} \mathrm{H}_{14} \mathrm{O}_{6}$

9-Ethoxy-aristololactone, 7395

3'-Hydroxy,3,5'-dimethoxy furo[8,7:4",5"]flavone, 10029
2-O-Methylatromentin, 14150

Neotenone, 15462

$\mathrm{C}_{19} \mathrm{H}_{14} \mathrm{O}_{7}$

6-Aldehydo-isoophiopogone A, 875

Anadanthoflavone, 1131

Ophiopogonone C, 16149

$\mathrm{C}_{19} \mathrm{H}_{14} \mathrm{O}_{9}$

Stictic acid, 20342

$\mathrm{C}_{19} \mathrm{H}_{14} \mathrm{O}_{10}$

3,4-Methylenedioxy-3',4'-O-dimethyl-5,5'-dimethoxyellagic acid, 14365

\section{$\mathrm{C}_{19} \mathrm{H}_{14} \mathrm{O}_{12}$}

Ellagic acid-4- $O-\beta$ - $D$-xylopyranoside, 6758

\section{$\mathrm{C}_{19} \mathrm{H}_{15} \mathrm{NO}_{3}$}

$\mathrm{N}$-Acetyldehydroanonaine, 370

\section{$\mathrm{C}_{19} \mathrm{H}_{15} \mathrm{NO}_{4}$}

Artabonatine C, 1771

Berberrubine, 2304

Cepharadione B, 3409

Griffinin, 9002

Griffithdione, 9006

Menisporphine, 13717

O-Methylmoschatoline, 14608

\section{$\mathrm{C}_{19} \mathrm{H}_{15} \mathrm{NO}_{5}$}

Artabonatine E, 1773

9-Ethoxy-aristololactam, 7394

7-Oxohernagine, 16342

\section{$\mathrm{C}_{19} \mathrm{H}_{15} \mathrm{NO}_{6}$}

Integriamide, 11093

\section{$\mathrm{C}_{19} \mathrm{H}_{15} \mathrm{~N}_{3} \mathrm{O}$}

Angustidine, 1244

Dehydroevodiamine, 4912

\section{$\mathrm{C}_{19} \mathrm{H}_{15} \mathrm{~N}_{3} \mathrm{O}_{2}$}

14-Formyldihydrorutaecarpine, 7902

1-Methoxyrutaecarpine, 14086

\section{$\mathrm{C}_{19} \mathrm{H}_{16} \mathrm{NO}_{4}^{+}$}

Groenlandicin, 9011

Thalifendine, 21254

\section{$\mathrm{C}_{19} \mathrm{H}_{16} \mathrm{~N}_{2}$}

Sempervirine II, 19697

\section{$\mathrm{C}_{19} \mathrm{H}_{16} \mathrm{~N}_{2} \mathrm{O}_{3}$}

Alangimarine, 831

Daurioxoisoporphine C, 4691

Nauclefoline, 15306

$\mathrm{C}_{19} \mathrm{H}_{16} \mathrm{~N}_{2} \mathrm{O}_{4}$

Daurioxoisoporphine B, 4690

$\mathrm{C}_{19} \mathrm{H}_{16} \mathrm{O}_{3}$

1,7-Bis(4-hydroxyphenyl)-1,4,6-heptatrien-

3-one, 2469

$\Delta^{1}$-Dehydrotanshinone, 4974

Sterequinone C, 20337

$\mathrm{C}_{19} \mathrm{H}_{16} \mathrm{O}_{4}$

Anthrakunthone, 1364 
Bisdemethoxycurcumin, 2439

Bis(4-hydroxycinnamoyl)methane, 2462

2-(3,4-Methylenedioxyphenyl)-3-methyl-5-(2oxopropyl)benzofuran, 14380

$O$-Methylpongamol, 14683

Moracin D, 14956

Moracin E, 14957

Moracin G, 14959

Sterequinone E, 20339

Tanshinaldehyde, 20678

\section{$\mathrm{C}_{19} \mathrm{H}_{16} \mathrm{O}_{5}$}

Combretastatin $\mathrm{D}_{3}, 3940$

Linixanthone B, 12887

Sarcomeginal, 19359

Vitrofolal E, 22594

\section{$\mathrm{C}_{19} \mathrm{H}_{16} \mathrm{O}_{6}$}

6-Aldehydo-isoophiopogone B, 876

Caledonixanthone E, 2962

Psorospermin, 18091

Tournefolic acid B ethyl ester, 21480

Vitrofolal F, 22595

\section{$\mathrm{C}_{19} \mathrm{H}_{16} \mathrm{O}_{7}$}

Hemerocallone, 9340

2'-Hydroxymethylophiopogonone A, 10509

Ophiopogonanone C, 16138

\section{$\mathrm{C}_{19} \mathrm{H}_{16} \mathrm{O}_{9}$}

9'-(O-Methyl)protocetraric acid, 14697

3,6,8-Trimethoxy-5,7-dihydroxy-3',4'-methylenedioxyflavone, 21911

\section{$\mathrm{C}_{19} \mathrm{H}_{16} \mathrm{O}_{11}$}

Thamnolic acid, 21271

\section{$\mathrm{C}_{19} \mathrm{H}_{17} \mathrm{NO}_{3}$}

$N$-Acetylanonaine, 322

Cusparine, 4421

Dehydrostephalagine, 4970

$\mathrm{N}$-Demethyl-acronycine, 5057

Noracronycine, 15707

Rutacridone, 19077

\section{$\mathrm{C}_{19} \mathrm{H}_{17} \mathrm{NO}_{4}$}

Crychine, 4277

$\mathrm{N}$-Methyl ovigerine, 14640

Neolitsine, 15431

Reframidine, 18570

Romucosine, 18900

Rutacridone epoxide, 19078

Stylopine, 20416

(-)-Tetrahydrocoptisine, 21061

\section{$\mathrm{C}_{19} \mathrm{H}_{17} \mathrm{NO}_{5}$}

Cassythidine, 3295

(-)-12-Hydroxycrychine, 9947

Norimelutein, 15762

Pendulamine B, 16800

$\mathrm{C}_{19} \mathrm{H}_{17} \mathrm{NO}_{5}^{+}$
Thalidastine, 21252

$\mathrm{C}_{19} \mathrm{H}_{17} \mathrm{~N}_{3}$

Naufoline, 15315

$\mathrm{C}_{19} \mathrm{H}_{17} \mathrm{~N}_{3} \mathrm{O}$

Evodiamine, 7665

$\mathrm{C}_{19} \mathrm{H}_{17} \mathrm{~N}_{3} \mathrm{O}_{2}$

GoshuyuamideII, 8958

Hydroxyevodiamine, 10117

$\mathrm{C}_{19} \mathrm{H}_{17} \mathrm{O}_{13} \mathrm{~S}$

1,3-Dihydroxy-5-O- $\beta$ - $D$-glucopyranosyl-

xanthone-4-sulfonate, 5901

\section{$\mathrm{C}_{19} \mathrm{H}_{18} \mathrm{CINO}_{4}$}

Gravacridonechlorine, 8984

Romucosine B, 18902

\section{$\mathrm{C}_{19} \mathrm{H}_{18} \mathrm{CINO}_{5}$}

Gravacridonolchlorine, 8987

\section{$\mathrm{C}_{19} \mathrm{H}_{18} \mathrm{NO}_{4}{ }^{+}$}

Dehydrocoreximine (perchlorate), 4886

Stepharanine, 20321

\section{$\mathrm{C}_{19} \mathrm{H}_{18} \mathrm{~N}_{2} \mathrm{O}_{4}$}

Alamarine, 828

\section{$\mathrm{C}_{19} \mathrm{H}_{18} \mathrm{O}$}

Alnustone, 966

\section{$\mathrm{C}_{19} \mathrm{H}_{18} \mathrm{O}_{3}$}

Alnusone, 963

1,7-Bis(4-hydroxyphenyl)hepta-4E,6E-dien-3one, 2468

Isotanshinone IIA, 11731

(7R,8R)-3,4-Methylenedioxy-4',7-epoxy-8,3'neolignan-7'E-ene, 14367

Tanshinone IIa, 20686

\section{$\mathrm{C}_{19} \mathrm{H}_{18} \mathrm{O}_{4}$}

Alnusoxide, 965

Anticancer Benzofuran PMV70P691-005, 1387

1,7-Bis(4-hydroxyphenyl)-1-heptene-3,5-dione, 2470

Coniferyl cinnamate, 3986

Demethylmoracin I, 5086

1,2-Dihydrobis(de- $O$-methyl)-curcumin, 5548

(2,3-trans-3,4-trans)-3,4-Dimethoxy-(2",3":7,8)furanoflavan, 6229

6,7-Dimethoxy-2-(2-phenylethyl) chromone, 6283

Gobicusin B, 8904

Hainanolide, 9194

$3 \alpha$-Hydroxytanshinone IIA, 10736

$3 \beta$-Hydroxytanshinone IIA, 10737

1-Keto-isocryptotanshinone, 12204

4-Methoxy-2,2-dimethyl-6-(2-(2,4-dihydroxy) phenyl-trans-ethenyl)chromene, 13918

6-Methoxy-2-[2-(3'-methoxyphenyl) ethyl] chromone, 13999

6-Methoxy-2-[2-(4'-methoxyphenyl) ethyl] chro- mone, 14000

Moracin C, 14955

Moracin N, 14964

Przewaquinone A, 18011

Tanshinone IIb, 20687

\section{$\mathrm{C}_{19} \mathrm{H}_{18} \mathrm{O}_{5}$}

Ailanthoidol, 776

Anticancer Benzofuran PMV70P691-64, 1388

1,5-Bis(4-hydroxy-3-methoxyphenyl)-1,4-pen-

tadien-3-one, 2467

1,5-Dihydroxy-2-isoprenyl-3-methoxyxanthone, 5925

1,7-Dihydroxy-2-isoprenyl-3-methoxyxanthone, 5926

2,5-Dimethoxy-4-hydroxy-[2",3":7,8]-furano-

flavan, 6242

Eucalyptin, 7480

Globulixanthone D, 8556

5-(3"-Hydroxypropyl)-7-methoxy-2-(3',4'methylenedioxyphenyl)benzofuran, 10665

6-Methoxy-2-[2-(3-methoxy-4-hydroxyphenyl) ethyl]chromone, 13997

3-Methoxy-4-[(2S,3R)-3-methyl-7-(1-propynyl)2,3-dihydro-1,4-benzodioxin-2-yl]-1,2-ben-zen ediol, 14035

1-O-Methylglobuxanthone, 14467

Teysmannic acid, 21232

\section{$\mathrm{C}_{19} \mathrm{H}_{18} \mathrm{O}_{6}$}

Afzeliixanthone A, 679

Celebixanthone, 3370

2',3'-Epoxyisocapnolactone, 7149

5-Hydroxy-3',4',7-trimethoxyspiro $\{2 H$-1-benzopyran-7'-bicyclo[4.2.0]octa[1,3,5]-trien\}-4-one, 10676

Luzonidial A, 13155

Luzonidial B, 13156

Methyl ophiopogonanone A, 14635

Qianhucoumarin E, 18286

Scriblitifolic acid, 19567

5,7,2',3'-Tetramethoxyflavone, 21183

$5,7,3^{\prime}, 4^{\prime}$-Tetramethoxyflavone, 21184

5,7,8,4'-Tetramethoxyflavone, 21185

7,2',3',4'-Tetramethoxyflavone, 21186

Tetramethylscutellarein, 21202

1,2,6-Trihydroxy-5-methoxy-7-(3-methylbut-2enyl)xanthone, 21783

Turgeniifolin A, 22119

\section{$\mathrm{C}_{19} \mathrm{H}_{18} \mathrm{O}_{7}$}

Anticancer Flavonoid PMV70P691-114, 1411

Chrysoobtusin, 3612

Cimiracemate A, 3662

Cimiracemate B, 3663

3'-Hydroxy-5,7,8,4'-tetramethoxyflavone, 10749 
5-Hydroxy-3,6,7,4'-tetramethoxyflavone, 10750

5-Hydroxy-6,7,3',4'-tetramethoxyflavone, 10751

7-Hydroxy-5,6,8,4'-tetramethoxyflavone, 10752

6-Hydroxy-7,2',4',5'-tetramethoxyisoflavone, 10753

Hyperxanthone, 10890

Mono- $O$-methylwightin, 14928

Polygalolide A, 17637

2,5,7-Trihydroxy-6,8-dimethyl-3-(3',4'-methylenedioxybenzyl)-chroman-4-one, 21722

\section{$\mathrm{C}_{19} \mathrm{H}_{18} \mathrm{O}_{8}$}

Arteanoflavone, 1787

Atranorin, 1990

Baeomycesic acid, 2101

Casticin, 3300

Chrysosplenetin, 3622

Chrysosplenol E, 3625

Chrysosplenol G, 3626

3,5-Dihydroxy-6,7,3',4'-tetramethoxyflavone, 6146

5,3'-Dihydroxy-3,7,4',5'-tetramethoxyflavone, 6147

5,3'-Dihydroxy-6,7,4',5'-tetramethoxyflavone, 6148

5,7-Dihydroxy-2',3',4',5'-tetramethoxy-flavone, 6149

5,7-Dihydroxy-6,8,2',3'-tetramethoxyflavone, 6150

Eupatoretin, 7590

Gephyromycin, 8308

5,6,7,8,3',4'-Hexamethoxyflavone, 9510

6-Methoxyboeravinone C, 13860

Rosmarinic acid methyl ester, 18925

Skullcapflavone II, 20009

Ternatin, 20978

\section{$\mathrm{C}_{19} \mathrm{H}_{18} \mathrm{O}_{9}$}

Squamatic acid, 20238

\section{$\mathrm{C}_{19} \mathrm{H}_{18} \mathrm{O}_{10}$}

1,5-Dihydroxyxanthone-6- $O-\beta$ - $D$-glucoside, 6184

Lancerin, 12468

\section{$\mathrm{C}_{19} \mathrm{H}_{18} \mathrm{O}_{11}$}

5-O- $\beta$ - $D$-Glucopyranosyl-1,3,8-trihydroxyanthone, 8740

Isomangiferin, 11524

Mangiferin, 13481

Norswertianolin, 15800

$1,3,7,8$-Tetrahydroxyxanthone-1- $O$ - $\beta$ - $D$-glucopyranoside, 21172

$1,3,7,8$-Tetrahydroxyxanthone-8- $O-\beta$ - $D$-glucopyranoside, 21173

Tripteroside, 21991

$\mathrm{C}_{19} \mathrm{H}_{19} \mathrm{NO}_{2}$
Drazepinone, 6598

Koenimbine, 12249

Stephanthrine, 20320

$\mathrm{C}_{19} \mathrm{H}_{19} \mathrm{NO}_{3}$

Clausine F, 3796

Koenigine, 12248

Stephalagine, 20316

(-)-Stephalagine, 20317

Stephanine, 20319

\section{$\mathrm{C}_{19} \mathrm{H}_{19} \mathrm{NO}_{4}$}

Amurensine, 1094

Amurine, 1097

Armatamide, 1737

(-)-Ayuthianine, 2049

Bulbocapnine, 2734

Buxifoliadine C, 2825

(-)-Caryachine, 3238

dl-Caryachine, 3239

(+)-Cassythicine, 3294

Cheilanthifoline, 3494

Domesticine, 6552

(+)-Isocaryachine, 11308

(-)-Isocaryachine, 11309

$\mathrm{N}$-Methylhernangerine, 14483

Nandinine, 15245

Neocaryachine, 15359

(+)-Nordicentrine, 15738

(-)-Nordicentrine, 15739

$\mathrm{N}$-Nornantenine, 15779

Oriciacridone E, 16181

Pachystaudine, 16511

Palaudine, 16538

(-)-Phanostenine, 17041

Romucosine A, 18901

\section{$\mathrm{C}_{19} \mathrm{H}_{19} \mathrm{NO}_{5}$}

(-)-Caryachine- $N$-oxide, 3240

Cassyfiline, 3293

Gravacridonediol, 8985

Hernandine, 9444

(+)-Isocaryachine- $N$-oxide, 11310

(-)-Isocaryachine- $N$-oxide, 11311

(-)-Isocaryachine- $N$-oxide $\mathrm{B}, 11312$

Ledecorine, 12602

Pendulamine A, 16799

\section{$\mathrm{C}_{19} \mathrm{H}_{19} \mathrm{NO}_{6}$}

Epiporphyroxine, 7002

Toddaliopsin B, 21420

\section{$\mathrm{C}_{19} \mathrm{H}_{19} \mathrm{~N}_{3} \mathrm{O}$}

Decarbomethoxy naucl echine, 4840

Goshuyuamide I, 8957

Naucleonine, 15309

Vasicolinone, 22352

$\mathrm{C}_{19} \mathrm{H}_{19} \mathrm{~N}_{3} \mathrm{O}_{2}$
Nauclederine, 15300

$\mathrm{C}_{19} \mathrm{H}_{19} \mathrm{~N}_{7} \mathrm{O}_{6}$

Folic acid, 7852

$\mathrm{C}_{19} \mathrm{H}_{20} \mathrm{~N}_{2} \mathrm{O}$

Vellosimine, 22368

$\mathrm{C}_{19} \mathrm{H}_{20} \mathrm{~N}_{2} \mathrm{O}_{4}$

19-Oxo-gelsenicine, 16338

$\mathrm{C}_{19} \mathrm{H}_{20} \mathrm{~N}_{2} \mathrm{O}_{6}$

Pycnosanguin, 18231

\section{$\mathrm{C}_{19} \mathrm{H}_{20} \mathrm{O}$}

7,8-Dimethyl-2-(1-methylethyl)phenanthren3-ol, 6374

1,7-Diphenylhept-4-en-3-one, 6488

\section{$\mathrm{C}_{19} \mathrm{H}_{20} \mathrm{O}_{2}$}

Dehydromiltirone, 4950

6'-O-Methylhonokiol, 14491

Sibiriquinone A, 19869

\section{$\mathrm{C}_{19} \mathrm{H}_{20} \mathrm{O}_{3}$}

Acerogenin C, 99

Acerogenin E, 100

Anticancer Stilbenoid PMV70P691-040, 1444 Cryptotanshinone, 4292

(3S,7R)-5,6-Dehydro-1,7-bis(4-hydroxyphenyl)4 "-de- $O$-methylcentrolobine, 4882

(3S,7S)-5,6-Dehydro-4"-de- $O$-methylcentrolobine, 4900

(2R,3R)-2,3-Dihydro-2-(4-hydroxyphenyl)-5methoxy-3-methyl-7-propenylbenzofuran, 5644

3-(1,1-Dimethylallyl)-xanthyletin, 6312

Diospongin A, 6456

Diospongin B, 6457

Isocryptotanshinone, 11354

Methoxy-3-(1,1'-dimethylallyl)-6a,10a-dihydrobenzo(1,2-c)chroman-6-one, 13916

Parakmerin A, 16651

Perrottetin D, 16983

4-Prenylresveratrol, 17842

\section{$\mathrm{C}_{19} \mathrm{H}_{20} \mathrm{O}_{4}$}

Alnusonol, 964

Anticancer Stilbenoid PMV70P691-039, 1443

Champanone A, 3472

Engelhardione, 6801

Hainanolidol, 9195

trans-4-(3-Methyl-E-but-1-enyl)-3,5,2',4'-tetrahydroxystilbene, 14183

Miltionone I, 14862

Miltionone II, 14863

Nordentatin, 15737

4'-Prenyloxyresveratrol, 17841

Trachyphyllin, 21499

\section{$\mathrm{C}_{19} \mathrm{H}_{20} \mathrm{O}_{5}$}

Clausenidin, 3791 
Columbianadin, 3935

Cudranone, 4338

Decursin, 4862

Dehydrohirsutanonol, 4930

Deltoin, 5039

3",4"-Dihydrocapnolactone, 5552

5,7-Dimethoxy-3-(4-methoxybenzyl)chroman-

4-one, 6256

(1S,2R,5S,6R)-2-(4-Hydroxyphenyl)-6-(3-meth-

oxy-4-hydroxyphenyl)-3,7-dioxabicyclo[3.3.0]

octane, 10628

Jatamansin, 11834

Kadsurenin M, 12009

Libanorin, 12747

3-Methoxy-4-[(2S,3R)-3-methyl-7-(E)-1-propenyl-2,3-dihydro-1,4-benzodioxin-2-yl]-1,2-benz enediol, 14034

Methyl ophiopogonanone B, 14636

Teucvin, 21223

\section{$\mathrm{C}_{19} \mathrm{H}_{20} \mathrm{O}_{6}$}

3'-Angeloyl-cis-khellactone, 1213

Calaxin, 2946

Chaetoquadrin I, 3448

Daucoidin A, 4677

Daucoidin B, 4678

Decumbensol, 4859

Deoxyelephantopin, 5168

5,7-Dihydroxy-6,8-dimethyl-3-(4'-hydroxy-3'methoxybenzyl)chroman-4-one, 5856

Diosbulbin B, 6435

( $2 " R, 3 " R)$-Epoxyangeloyldecursinol, 7052

(2"S,3"S)-Epoxyangeloyldecursinol, 7053

3'(S)-Hydroxy-4' $(R)$-angeloyloxy-3',4'-dihydroxanthyletin, 9786

3-(4-Hydroxybenzyl)-5,6,7-trimethoxychroman4-one, 9851

4"-Hydroxydecursin, 9968

(3'S)-Hydroxydeltoin, 9976

(+)-(2'S,3'R)-3'-Hydroxyprantschimgin, 10658

2'-Hydroxy-2,3,4'6'-tetramethoxychalcone, 10748

4"-Hydroxytigloyldecursinol, 10765

D-Laserpitin, 12527

3-(4-Methoxybenzyl)-6-hydroxy-5,7-dimethoxychroman-4-one, 13854

3-(4-Methoxybenzyl)-8-hydroxy-5,7-dimethoxychroman-4-one, 13855

1-Methyl-acetylshikonin, 14120

9'-O-Methylamericanol A, 14132

Nigrolineaxanthone A, 15574

Odoratin III*, 15993

Peguangxienin, 16772

Propionylshikonin, 17933

Qianhucoumarin A, 18282
(+)-2"-Senecioyloxymarmesin, 19709

(-)-(2'S,3'R)-3'-Senecioyloxymarmesin, 19710

(2S)-5,7,2',3'-Tetramethoxyflavanone, 21180

$5,7,2^{\prime}, 5^{\prime}$-Tetramethoxyflavanone, 21181

7,2',3',4'-Tetramethoxyflavanone, 21182

2,5,7-Trihydroxy-6,8-dimethyl-3-(4'-methoxy-

benzyl)chroman-4-one, 21720

5,7,8-Trimethoxy-3-(4'-hydroxybenzyl)-4chromanone, 21917

Turgeniifolin $\mathrm{C}, 22121$

\section{$\mathrm{C}_{19} \mathrm{H}_{20} \mathrm{O}_{7}$}

Abruquinone A, 23

Barbatic acid, 2146

(-)-3'-O-Demethyl-5-hydroxymatairesinol, 5082

3,4-Dihydroxy-6,7,3',4'-tetramethoxyflavonol, 6151

Elephantopin, 6751

Heliangolide 17,18-dehydro-viguiepinin, 9292

5-Hydroxy-7,8-dimethoxy-6-methyl-3-(3',4'dihydroxybenzyl) chroman-4-one, 10033

3-(4-Hydroxy-3-methoxybenzyl)-5-hydroxy-6,7dimethoxychroman-4-one, 10386

Luzonial A, 13153

Luzonial B, 13154

Murrayacoumarin C, 15116

Nigrolineaxanthone C, 15576

Ophiopogonanone E, 16140

$E$-Resveratrol 3-O- $\beta$ - $D$-xylopyranoside, 18651

1,5,6-Trihydroxy-3-methoxy-4-(3-hydroxyl-3methylbutyl)xanthone, 21780

Typhic acid, 22157

Vernodalin, 22405

\section{$\mathrm{C}_{19} \mathrm{H}_{20} \mathrm{O}_{8}$}

Abruquinone C, 25

1,1'-Bis(2,6-dihydroxy-3-acetyl-4-methoxyphenyl)methane, 2442

Eximine, 7695

8-Hydroxyisocapnolactone-2',3'-diol, 10244

Piceatannol 3'- $O-\beta$ - $D$-xylopyranoside, 17282

Sheganone, 19797

Trideca-2 $\beta$ - $D$-glucopyranosyl-1,13-dihydroxy11(E)-en-3,5,7,9-tetrayne, 21609

$\mathrm{C}_{19} \mathrm{H}_{20} \mathrm{O}_{9}$

Garcimangosone D, 8209

\section{$\mathrm{C}_{19} \mathrm{H}_{20} \mathrm{O}_{10}$}

Annulatophenonoside, 1340

4-O- $\beta$ - $D$-Glucopyranosyl-2,6,4'-trihydroxybenzophenone, 8741

$\mathrm{C}_{19} \mathrm{H}_{20} \mathrm{O}_{11}$

2,3,4',5,6-Pentahydroxybenzophenone-4-Cglucoside, 16839

\section{$\mathrm{C}_{19} \mathrm{H}_{21} \mathrm{ClO}_{8}$}

Patientoside A, 16714

\section{$\mathrm{C}_{19} \mathrm{H}_{21} \mathrm{NO}_{2}$}

Glybomine B, 8804

Nuciferine, 15856

\section{$\mathrm{C}_{19} \mathrm{H}_{21} \mathrm{NO}_{3}$}

Homodihydrocapsaicin, 9603

Isothebaine, 11741

Lemairamin, 12612

(-)- $N$-Methylisopiline, 14535

(-)- $O$-Methyl- $N$-norlirinine, 14626

$N$ - $\beta$-Phenethyl-3-(3,4-dimethoxy phenyl) propenamide, 17084

Piperettine, 17449

Pronuciferine, 17909

Thebaine, 21292

\section{$\mathrm{C}_{19} \mathrm{H}_{21} \mathrm{NO}_{4}$}

Amurinine, 1098

Amurinol I, 1099

Artacinatine, 1780

Bisnorargemonine, 2486

Boldine, 2538

Comosimine, 3947

Coreximine, 4050

Corytuberine, 4123

Cularidine, 4351

Cularimine, 4352

(-)-10-O-Demethyldiscretine, 5076

$N$-Demethylphyllocryptine, 5090

(+)-Flavinantine, 7815

Floripavine, 7837

Isoboldine, 11256

Laurotetanine, 12574

(-)-Munitagine, 15075

Norisocorydine, 15763

Pallidine, 16547

(-)-Salutaridine, 19200

Scoulerine, 19566

Stepholidine, 20324

\section{$\mathrm{C}_{19} \mathrm{H}_{21} \mathrm{NO}_{5}$}

11-O-Acetyl haemanthamine, 406

$\mathrm{N}$-trans-Feruloylmethoxytyramine, 7777

$N$-trans-Feruloyl-4'-O-methyldopamine, 7778

Isoboldine- $\beta$ - $N$-oxide, 11257

Prostephanaberrine, 17957

Tuberosine A, 22081

\section{$\mathrm{C}_{19} \mathrm{H}_{21} \mathrm{NO}_{7} \mathrm{~S}$}

Erysothiopine, 7326

$\mathrm{C}_{19} \mathrm{H}_{21} \mathrm{~N}_{3}$

Vasicoline, 22351

\section{$\mathrm{C}_{19} \mathrm{H}_{21} \mathrm{~N}_{3} \mathrm{O}$}

Evodiamide, 7664

\section{$\mathrm{C}_{19} \mathrm{H}_{22} \mathrm{~N}_{2} \mathrm{O}$}

Cinchonicine, 3684

Cinchonidine, 3685 
Cinchonine, 3686

Eburnamonine, 6677

Koumidine, 12288

Normacusine B, 15775

\section{$\mathrm{C}_{19} \mathrm{H}_{22} \mathrm{~N}_{2} \mathrm{O}_{2}$}

Cupreine, 4373

$N$-Desmethoxyrankinidine, 5254

16-Episarpagine, 7012

n-Pentyl $\beta$-carboline-1-propionate, 16892

Peraksine, 16909

Sarpagine, 19386

\section{$\mathrm{C}_{19} \mathrm{H}_{22} \mathrm{~N}_{2} \mathrm{O}_{3}$}

Caribine, 3197

Humantenmine, 9661

\section{$\mathrm{C}_{19} \mathrm{H}_{22} \mathrm{~N}_{2} \mathrm{O}_{4}$}

Humantenidine, 9658

\section{$\mathrm{C}_{19} \mathrm{H}_{22} \mathrm{~N}_{2} \mathrm{O}_{5}$}

Gelsemoxonine, 8260

\section{$\mathrm{C}_{19} \mathrm{H}_{22} \mathrm{~N}_{2} \mathrm{O}_{7}$}

Ruine, 19062

\section{$\mathrm{C}_{19} \mathrm{H}_{22} \mathrm{O}$}

9,10-Dihydro-7,8-dimethyl-2-(1-methylethyl) phenanthren-3-ol, 5603

trans-1,7-Diphenyl-1-hepten-5-ol, 6487

\section{$\mathrm{C}_{19} \mathrm{H}_{22} \mathrm{O}_{2}$}

Dihydroyashabushiketol, 6185

Miltirone, 14865

4-Prenyldihgdropinosylvin, 17831

Sibiriquinone B, 19870

\section{$\mathrm{C}_{19} \mathrm{H}_{22} \mathrm{O}_{3}$}

Acerogenin A, 97

Acerogenin B, 98

Acerogenin K, 101

Auraptene, 2010

Bibenzyl CPB-2002-50-1390-3, 2348

Bibenzyl CPB-2002-50-1390-4, 2349

$\alpha, \alpha^{\prime}$-Dihydro-3,5,4'-trihydroxy-5'-isopentenylstilbene, 5730

6,8-Diprenylumbelliferone, 6498

8-Geranyl-7-hydroxycoumarin, 8320

Glepidotin C, 8550

Gravelliferone, 8988

5R-Hydroxy-7-(4"-hydroxyphenyl)-1-phenyl-3heptanone, 10214

5-Hydroxy-7-(4"-hydroxyphenyl)-1-phenyl-3heptanone, 10215

5-(1-Methoxyethyl)-2,6-dihydroxy-1,7-dimethyl9,10-dihydrophenanthrene, 13924

Ostruthin, 16264

Perrottetin A, 16980

\section{$\mathrm{C}_{19} \mathrm{H}_{22} \mathrm{O}_{4}$}

Alnusdiol, 958

Centaur X2, 3391
cis-Dehydrocrotonin, 4892

trans-Dehydrocrotonin, 4893

$\alpha, \alpha^{\prime}$-Dihydro-3,5,3',4'-tetrahydroxy-5'-isopentenyl stilbene, 5726

Ferulagol A, 7765

Ferulagol B, 7766

5-Geranyloxy-7-hydroxycoumarin, 8324

Heliettin, 9304

3-Isobutyl-4-[4-(3-methyl-2-butenyloxy)phenyl] furan-2,5-dione, 11277

Neocryptotanshinone, 15374

Odoratin II*, 15992

Perrottetin B, 16981

Platyphyllonol, 17547

\section{$\mathrm{C}_{19} \mathrm{H}_{22} \mathrm{O}_{5}$}

Anticancer Stilbenoid PMV70P691-038, 1442

2',3'-Dihydro-jatamansin, 5658

(3S,5R,6S,7R)-5,6-Dihydroxy-1,7-bis(4-hydroxyphenyl)-de- $O$-methylcentrolobine, 5777

(3S,5S,6R,7R)-5,6-Dihydroxy-1,7-bis(4-hydroxyphenyl)-de- $O$-methylcentrolobine, 5778

(3S,5S,6S,7R)-5,6-Dihydroxy-1,7-bis(4-hydroxyphenyl)-4"-de- $O$-methylcentrolobine, 5779

$(5 S, 6 S)-5,6$-Dihydroxy-4"-de- $O$-methylcentrolobine, 5804

7-(5',6'-Dihydroxy-3',7'-dimethylocta-2',7'-dienyloxy)-coumarin, 5858

12-Epi-methyl-barbascoate, 6967

Gibberellin $\mathrm{A}_{5}, 8373$

Hericenone A, 9429

$5 \beta$-Hydroxy-cis-dehydrocrotonin, 9972

3-Hydroxy-4-(3,7-dimethyl-5-oxo-2,6-octadienyl)-5-methoxy-benzo[1,2-c]furan-2-one, 10055

Imperanene, 11000

Isoponcimarin, 11611

$O$-Isovalerylcolum bianetin, 11761

Lactiflorasyne, 12437

Magnolignan D, 13381

Muricarpone B, 15081

Podolide, 17591

Poncimarin, 17704

( $\left.7 S, 8 S, 7^{\prime} S, 8^{\prime} S\right)$-3,3',4'-Trihydroxy-4-methoxy-

$$
\text { 7,7'-epoxylignan, } 21771
$$

\section{$\mathrm{C}_{19} \mathrm{H}_{22} \mathrm{O}_{6}$}

Cajucarinolide, 2933

Canavalia gibberellin II, 3062

Cynaropicrin, 4565

5,6-Dihydroeurycomalactone, 5610

(7S,8R)-Dihydro-3'-hydroxy-8-hydroxy-methyl-

7-(4-hydroxy-3-methoxyphenyl)-1'-benzofuran propanol, 5636

(2R-trans)-2,3-Dihydro-2-(4-hydroxy-3-me- thoxyphenyl)-3-(hydroxymethyl)-7-hydroxy-

5-benzofuran-propanol, 5639

$8 \alpha$-Epoxymethylacrylyloxyambrosin, 7170

Gibberellin $\mathrm{A}_{3}, 8372$

Hallactone A, 9196

Hirsutanonol, 9550

Inumakilactone D, 11117

Isotaxiresinol, 11732

Junosmarin, 11970

Molephantin, 14896

Ponalactone A, 17699

Saupirin, 19414

Strigol, 20392

Tagitinin C, 20610

Taxiresinol, 20806

Turgeniifolin B, 22120

\section{$\mathrm{C}_{19} \mathrm{H}_{22} \mathrm{O}_{7}$}

9-O-Acetylsalograviolide A, 504

Canavalia gibberellin I, 3061

6-O-cis- $p$-Coumaroyl-3 $\alpha$-O-methyl-7-deoxyrehmaglutin $\mathrm{A}, 4171$

6-O-cis-p-Coumaroyl-3 $\beta$ - $O$-methyl-7-deoxyrehmaglutin A, 4172

6-O-trans- $p$-Coumaroyl-3 $\alpha$ - $O$-methyl-7-deoxyrehmaglutin $\mathrm{A}, 4173$

6-O-trans- $p$-Coumaroyl-3 $\beta$ - $O$-methyl-7-deoxyrehmaglutin A, 4174

Diosbulbin C, 6436

$8 \alpha$-Epoxymethylacrylyloxyparthenin, 7172

6-Hydroxy-5,6-dihydroeurycomalactone, 9999

Nagilactone C, 15231

Repin, 18627

Vernolide, 22412

\section{$\mathrm{C}_{19} \mathrm{H}_{22} \mathrm{O}_{8}$}

8-Hydroxy-3",4"-dihydrocapnolactone-2',3'-diol, 9998

Musizin-8-O- $\beta$ - $D$-glucoside, 15132

Trideca-2 $\beta$ - $D$-glucopyranosyl-1,13-dihydroxy3(E),11(E)-dien-5,7,9-triyne, 21608

\section{$\mathrm{C}_{19} \mathrm{H}_{22} \mathrm{O}_{8} \mathrm{~S}$}

Shogasulfonic acid C, 19850

\section{$\mathrm{C}_{19} \mathrm{H}_{22} \mathrm{O}_{9}$}

Aloesin, 979

Breynioside A, 2611

Eurylactone B, 7654

6-Hydroxymusizin-8-O- $\beta$ - $D$-glucoside, 10534

Podolactone B, 17590

\section{$\mathrm{C}_{19} \mathrm{H}_{22} \mathrm{O}_{10}$}

Aloenin, 972

Isoaloesin, 11205

\section{$\mathrm{C}_{19} \mathrm{H}_{23} \mathrm{ClO}_{7}$}

Acroptilin, 576

$\mathrm{C}_{19} \mathrm{H}_{23} \mathrm{NO}_{3}$ 
Amuronine, 1101

Armepavine, 1738

3-Epischelhammericine, 7014

Erysotrine, 7327

Isoamuronine, 11217

3-Isobutyl-4-[4-(3-methyl-2-butenyloxy)phenyl]-

$1 H$-pyrrole-2,5-dione, 11278

6-Methylcodeine, 14248

4'-Methyl- $N$-methylcoclaurine, 14593

\section{$\mathrm{C}_{19} \mathrm{H}_{23} \mathrm{NO}_{4}$}

trans-6 $\beta$-Acetoxy-3 $\alpha$-(cinnamoyloxy)tropane, 142

Cepharamine, 3410

Erythrozeylanine C, 7349

Haplotubinone, 9229

3-Isobutyl-4-[4-(3-methyl-2-butenyloxy)phenyl]-

$1 H$-pyrrol-1-ol-2,5-dione, 11279

Isosinomenine, 11717

Reticuline, 18655

Sinomenine, 19955

\section{$\mathrm{C}_{19} \mathrm{H}_{23} \mathrm{NO}_{5}$}

Aknadicine, 822

$N$-Desmethyl- $8 \alpha$-ethoxy pretazettine, 5255

$N$-Desmethyl- $8 \beta$-ethoxy pretazettine, 5256

Metaphanine, 13812

6-O-Methylpretazettine, 14687

Prostephabyssine, 17956

\section{$\mathrm{C}_{19} \mathrm{H}_{23} \mathrm{NO}_{6}$}

Methylnkolbisine, 14620

\section{$\mathrm{C}_{19} \mathrm{H}_{23} \mathrm{~N}_{3} \mathrm{O}_{2}$}

Ergometrine, 7240

Ergometrinine, 7241

\section{$\mathrm{C}_{19} \mathrm{H}_{24} \mathrm{ClNO}_{5}$}

Acutuminine, 604

\section{$\mathrm{C}_{19} \mathrm{H}_{24} \mathrm{CINO}_{6}$}

Acutumine, 603

\section{$\mathrm{C}_{19} \mathrm{H}_{24} \mathrm{Cl}_{2} \mathrm{O}_{7}$}

Centaurepensin, 3390

\section{$\mathrm{C}_{19} \mathrm{H}_{24} \mathrm{NO}_{3}$}

Lotusine, 13010

\section{$\mathrm{C}_{19} \mathrm{H}_{24} \mathrm{NO}_{3}{ }^{+}$}

Magnocurarine, 13373

\section{$\mathrm{C}_{19} \mathrm{H}_{24} \mathrm{~N}_{2} \mathrm{O}$}

Cinchamidine, 3675

Cinchonamine, 3682

Cinchotine, 3687

20-Epi-19,20-dihydro-decarbomethoxy vobasine, 6893

\section{$\mathrm{C}_{19} \mathrm{H}_{24} \mathrm{~N}_{2} \mathrm{O}_{2}$}

Conquinamine, 4004

\section{$\mathrm{C}_{19} \mathrm{H}_{24} \mathrm{~N}_{2} \mathrm{O}_{3}$}

Gelsedine, 8253

$\mathrm{C}_{19} \mathrm{H}_{24} \mathrm{~N}_{2} \mathrm{O}_{4}$
14-Hydroxygelsedine, 10136

Stephadiamine, 20312

$\mathrm{C}_{19} \mathrm{H}_{24} \mathrm{O}_{2}$

Androst-4,6-diene-3,17-dione, 1168

$\mathrm{C}_{19} \mathrm{H}_{24} \mathrm{O}_{3}$

Acetyl-bupleurotoxin, 340

Artepillin C, 1808

Biondinin C, 2389

Biondinin D, 2390

(-)-Centrolobol, 3399

Dichroanone, 5434

Epi-6-methylcryptoacetalide, 6968

(3R,5R)-1-(4-Hydroxyphenyl)-7-phenyl-3,5heptanediol, 10630

6-Methylcryptoacetalide, 14262

Miltipolone, 14864

\section{$\mathrm{C}_{19} \mathrm{H}_{24} \mathrm{O}_{4}$}

AcronyculatinE, 575

Artepillin A, 1807

Capillartemisin A, 3119

Capillartemisin B, 3120

(3S,5S)-3,5-Dihydroxy-1,7-bis(4-hydroxyphenyl) heptane, 5780

Diospongin C, 6458

FB1, 7742

FB2, 7743

Gibberellin A9, 8375

Hannokinol, 9219

Hyperguinone A, 10884

(4E,6E,12E)-3-Isovaleryloxy-tetradeca-4,6,12triene-8,10-diyne-1,14-diol, 11763

$(3 S, 4 E, 6 E, 12 E)-1-$ Isovaleryloxy-tetradeca-4,6,

12-triene-8,10-diyne-3,14-diol, 11764

14( $\alpha$-Methyl butyryl)-2E, $8 E, 10 E$-atractylentriol, 14191

$14(\alpha$-Methyl butyryl)-2E,8Z,10E-atractylentriol, 14192

Nagilactone F, 15234

Perrottetin C, 16982

Strictifolione, 20388

\section{$\mathrm{C}_{19} \mathrm{H}_{24} \mathrm{O}_{5}$}

3,5-Dihydroxy-1-(3,4-dihydroxyphenyl)-7-(4hydroxyphenyl)heptane, 5815

4,6-Dihydroxy-3-[3'-methyl-2'-butenyl]-5-[4"hydroxy-3"-methyl-2"-butenyl]-cinnamic acid, 6023

Erinacerin B, 7260

Gibberellin $\mathrm{A}_{20}, 8377$

Glabratolide, 8489

Trichothecin, 21577

\section{$\mathrm{C}_{19} \mathrm{H}_{24} \mathrm{O}_{6}$}

Acantholide, 84

$2 \alpha$-Acetoxyepitulipinolide, 181
5-Acetyl-3 $\beta$-angeloyloxy-2 $\beta$-(1-hydroxyisopropyl)-6-methoxy-2,3-dihydrobenzofuran, 319

Anticancer Benzenoid PMV70P691-004, 1384

Anticancer Sesquiterpene PMV70P691-136, 1440

erythro-2,3-Bis(4-hydroxy-3-methoxyphenyl)-3ethoxypropan-1-ol, 2464

threo-2,3-Bis(4-hydroxy-3-methoxyphenyl)-3ethoxypropan-1-ol, 2465

Chamissonin diacetate, 3467

(3R,5R)-3,5-Dihydroxy-1,7-bis-(3,4-dihydroxyphenyl)heptane, 5774

Erioflorin, 7286

Eurycomalactone, 7646

Eurycomalide B, 7648

Gibberellin $\mathrm{A}_{1}, 8371$

9-Hydroxyglabratolide, 10148

Murrayacoumarin A, 15114

Nagilactone A, 15229

Nagilactone E, 15233

Onopordopicrin, 16119

Plagiochiline T, 17496

Radiatin, 18523

Tagitinin F, 20612

Xanthanthusin E, 22748

\section{$\mathrm{C}_{19} \mathrm{H}_{24} \mathrm{O}_{7}$}

$9 \alpha$-Acetoxycumambrin A, 148

Ailanquassin, 773

Anticancer Sesquiterpene PMV70P691-135, 1439

Cedronin, 3355

3,15-Di- $O$-acetyl- $9 \beta$-hydroxyamphoricarpolide, 5335

$8 \alpha$-Epoxymethylacrylyloxy-11,13-dihydroparthenin, 7171

$1 \beta, 2 \alpha$-Epoxytagitinin C, 7204

Gibberellin $\mathrm{A}_{8}, 8374$

$6 \alpha$-Hydroxyeurycomalactone, 10115

Inumakilactone $\mathrm{E}, 11118$

Nagilactone B, 15230

Vernomygdin, 22414

Zexbrevin B, 22987

\section{$\mathrm{C}_{19} \mathrm{H}_{24} \mathrm{O}_{8}$}

$1 \alpha, 3 \alpha, 15$-Trihydroxy-3,10-epoxy- $8 \beta$ - $O$-methacryloyl-4,11-germacradiene, $6 \alpha, 12$-olide, 21726

\section{$\mathrm{C}_{19} \mathrm{H}_{24} \mathrm{O}_{9}$}

$\left(2 R^{*}, 3 S^{*}\right)-1-(2-[1-($ Hydroxymethyl)vinyl]-3$[\beta$ - $D$-glucosyloxy]-2,3-dihydrobenzo $[b]$ furan -5-yl)-1-ethanone, 10526

Macrophylloside C, 13323

2-Methyl-5-(2'-hydroxypropyl)-7-hydroxy- 
chromone-2'-O- $\beta$ - $D$-glucopyranoside, 14521

\section{$\mathrm{C}_{19} \mathrm{H}_{24} \mathrm{O}_{10}$}

Dracunculifoside J, 6589

Methylpicraquassioside A, 14675

\section{$\mathrm{C}_{19} \mathrm{H}_{25} \mathrm{ClO}_{7}$}

Eupaglehnin F, 7565

\section{$\mathrm{C}_{19} \mathrm{H}_{25} \mathrm{Cl}_{3} \mathrm{O}_{11}$}

Curculigin C, 4383

\section{$\mathrm{C}_{19} \mathrm{H}_{25} \mathrm{NO}_{2}$}

2-(Nonan-8-one)-4-methoxy-quinoline, 15689

2-(Nonan-8-one)- $N$-methyl-4-quinolone, 15690

\section{$\mathrm{C}_{19} \mathrm{H}_{25} \mathrm{NO}_{3}$}

Amuroline, 1100

Cryprochine, 4278

Isocryprochine, 11352

Piperolein A, 17468

Taxodine, 20808

\section{$\mathrm{C}_{19} \mathrm{H}_{25} \mathrm{NO}_{4}$}

Erythratidine, 7331

$3 R *, 4 R^{*}$-1-Hydroxy-3-isobutyl-4-[4-(3-methyl2-butenyloxy)phenyl]pyrrolidine-2,5-dione, 10234

$3 R *, 4 S^{*}$-1-Hydroxy-3-isobutyl-4-[4-(3-methyl2-butenyloxy)phenyl]pyrrolidine-2,5-dione, 10235

\section{$\mathrm{C}_{19} \mathrm{H}_{25} \mathrm{NO}_{5}$}

Acanthamolide, 76

Nerinine, 15496

Pyridostemin, 18260

\section{$\mathrm{C}_{19} \mathrm{H}_{25} \mathrm{NO}_{6}$}

Dechlorodauricumine, 4847

1-Epidechloroacutumine, 6886

Hernandine A, 9445

Hernandine B, 9446

\section{$\mathrm{C}_{19} \mathrm{H}_{25} \mathrm{NO}_{10}$}

Vicianin, 22465

\section{$\mathrm{C}_{19} \mathrm{H}_{25} \mathrm{NO}_{12}$}

1-(2-Hydroxyphenylamino)-1-deoxy- $\beta$-gentiobioside 1,2-carbamate, 10609

\section{$\mathrm{C}_{19} \mathrm{H}_{26}$}

19-Nordehydroabiet-4(18)-ene, 15736

\section{$\mathrm{C}_{19} \mathrm{H}_{26} \mathrm{Cl}_{2} \mathrm{O}_{11}$}

Curculigin B, 4382

\section{$\mathrm{C}_{19} \mathrm{H}_{26} \mathrm{~N}_{2}$}

Kamassine, 12120

\section{$\mathrm{C}_{19} \mathrm{H}_{26} \mathrm{~N}_{2} \mathrm{O}$}

Pareirine, 16660

\section{$\mathrm{C}_{19} \mathrm{H}_{26} \mathrm{~N}_{2} \mathrm{O}_{3}$}

Alstonoxine B, 1004

\section{$\mathrm{C}_{19} \mathrm{H}_{26} \mathrm{~N}_{2} \mathrm{O}_{5}$} Saussureamine C, 19424

\section{$\mathrm{C}_{19} \mathrm{H}_{26} \mathrm{O}_{2}$}

Androst-4-ene-3,17-dione, 1169
$\mathrm{C}_{19} \mathrm{H}_{26} \mathrm{O}_{3}$

8-Acetoxyfalcarinol, 196

Acetyl panaxydol, 479

3,15-Dihydroxy-18-norabieta-3,8,11,13-tetraene, 6049

4-Hydroxy-[2-trans-3',7'-dimethyl-octa-2',6'dienyl]-6-methoxy-acetophenone, 10774

$\mathrm{C}_{19} \mathrm{H}_{26} \mathrm{O}_{3} \mathrm{~S}$

Spetasin, 20148

$\mathrm{C}_{19} \mathrm{H}_{26} \mathrm{O}_{4}$

1-[2',4'-Dihydroxy-3',5'-di-(3"-methylbut-2"enyl)-6'-methoxy]phenylethanone, 5852

\section{$\mathrm{C}_{19} \mathrm{H}_{26} \mathrm{O}_{5}$}

AcronyculatinB, 572

AcronyculatinC, 573

$1 R, 5 R$-Diacetoxycyclomyltaylan-10-one, 5290

Rubrosterone, 19043

\section{$\mathrm{C}_{19} \mathrm{H}_{26} \mathrm{O}_{6}$}

Alatolide, 837

6-Dehydroxylongilactone, 4986

3,4-Dihydroeurycomalactone, 5609

Eurycolactone E, 7643

Eurycomalide A, 7647

7 $\alpha$-Hydroxyeurycomalactone, 10116

Millefin, 14854

Sipaucin B, 19962

\section{$\mathrm{C}_{19} \mathrm{H}_{26} \mathrm{O}_{7}$}

Anticancer Sesquiterpene PMV70P691-134, 1438

Britanin, 2615

3,4 $\beta$-Dihydrosamaderine $\mathrm{C}, 5706$

$4 \alpha, 10 \alpha$-Dihydroxy-3-oxo- $8 \beta$-isobutyryloxyguaia-

11(13)-en-12,6 $\alpha$-olide, 6064

Eurycolactone G, 7645

Inuchinenolide C, 11110

Longilactone, 12964

Orizabin, 16205

Sipaucin A, 19961

Sipaucin C, 19963

$\mathrm{C}_{19} \mathrm{H}_{26} \mathrm{O}_{8}$

Eurylactone A, 7653

\section{$\mathrm{C}_{19} \mathrm{H}_{26} \mathrm{O}_{9}$}

Hymenoside S, 10862

Hymenoside T, 10863

\section{$\mathrm{C}_{19} \mathrm{H}_{26} \mathrm{O}_{10}$}

Hymenoside K, 10854

Hymenoside Q, 10860

Hymenoside R, 10861

Ptelatoside A, 18099

Ptelatoside C, 18101

\section{$\mathrm{C}_{19} \mathrm{H}_{26} \mathrm{O}_{11}$}

10-O-Acetylgeniposide, 397

Bungeiside C, 2749
4-Hydroxyacetophenone 4-O-(6-O- $\beta$ - $D$-apiofuranosyl)- $\beta$ - $D$-glucopyranoside, 9750

Hymenoside L, 10855

Regaloside F, 18575

\section{$\mathrm{C}_{19} \mathrm{H}_{26} \mathrm{O}_{12}$}

10-Acetoxymajoroside, 250

Bungeiside D, 2750

Canthoside A, 3099

Daphylloside, 4659

Violutoside, 22521

\section{$\mathrm{C}_{19} \mathrm{H}_{26} \mathrm{O}_{12} \mathrm{~S}$}

Paederia glucoside $1^{*}, 16514$

Paederia glucoside 3*, 16515

\section{$\mathrm{C}_{19} \mathrm{H}_{26} \mathrm{O}_{13}$}

Sibiricose $\mathrm{A}_{3}, 19864$

\section{$\mathrm{C}_{\mathbf{1 9}} \mathrm{H}_{\mathbf{2 6}} \mathbf{O}_{15}$}

1 -Galloyl $\beta-D$-glucopyranosyl-( $1 \rightarrow 4)-\beta$ - $D$ galactopyranoside, 8107

\section{$\mathrm{C}_{19} \mathrm{H}_{27} \mathrm{NO}$}

1-Methyl-2-nonyl-4(1H)-quinolone, 14624

\section{$\mathrm{C}_{19} \mathrm{H}_{27} \mathrm{NO}_{3}$}

Nudicaulonol, 15862

\section{$\mathrm{C}_{19} \mathrm{H}_{27} \mathrm{NO}_{4}$}

Stemonamine, 20306

\section{$\mathrm{C}_{19} \mathrm{H}_{27} \mathrm{NO}_{6}$}

Senkirkine, 19731

\section{$\mathrm{C}_{19} \mathrm{H}_{27} \mathrm{NO}_{7}$}

Petasitenine, 17015

\section{$\mathrm{C}_{19} \mathrm{H}_{27} \mathrm{NO}_{12}$}

6'-O- $\beta$-D-Apiofuranosylthalictoside, 1518

6'-O- $\alpha$ - $L$-Arabinofuranosylthalictoside, 1558

Proteacin, 17963

\section{$\mathrm{C}_{19} \mathrm{H}_{28} \mathrm{NO}_{2}$}

Delavayine A, 4995

\section{$\mathrm{C}_{19} \mathrm{H}_{28} \mathrm{O}$}

18-Nordehydroabietan- $4 \alpha$-ol, 15735

\section{$\mathrm{C}_{19} \mathrm{H}_{28} \mathrm{O}_{2}$}

5 $\alpha$-Androstan-3,17-dione, 1165

$5 \beta$-Androstan-3,17-dione, 1166

$3 \alpha$-Hydroxy-androst-4-ene-17-one, 9782

$3 \beta$-Hydroxy-androst-5-ene-17-one, 9783

16 $\beta H, 17$-Hydroxy-3-oxo-19-nor-ent-kaur-4-ene, 10567

Pipataline, 17424

Testosterone, 21022

\section{$\mathrm{C}_{19} \mathrm{H}_{28} \mathrm{O}_{3}$}

Agallochin I, 692

10, 19 -Dihydroxy-15,16-epoxy-8(17),13(16),14nor-ent-labdatriene, 5876

16 $\alpha$,17-Dihydroxy-3-oxo-19-nor-ent-kaur-4-ene, 6065

ent-15,16-Epoxy-3-oxa-kauran-2-one, 7181

$\mathrm{C}_{19} \mathrm{H}_{28} \mathrm{O}_{3} \mathrm{~S}$ 
$S$-Japonine, 11822

$\mathrm{C}_{19} \mathrm{H}_{28} \mathrm{O}_{4}$

$10 \beta$-Hydroxy- $6 \beta$-isobutyryl furanoeremophilane, 10238

Methyl 2-(1' $\beta$-geranyl-5' $\beta$-hydroxy-2'-oxocyclohex-3'-enyl)acetate, 14462

$13 \alpha, 16 \alpha, 17-T r i h y d r o x y-9 \alpha$-methyl-19,20-dinor-kauran-4-en-15-one, 21798

\section{$\mathrm{C}_{19} \mathrm{H}_{28} \mathrm{O}_{5}$}

6-Acetyl gingerol, 399

Deisobutyryl bakkenolide H, 4989

\section{$\mathrm{C}_{19} \mathrm{H}_{28} \mathrm{O}_{6}$}

2-O-n-Butyrylpseudomajucin, 2812

1-(4-Hydroxy-3-methoxyphenyl)-3,5-diacetoxyoctane, 10436

Tagitinin D, 20611

\section{$\mathrm{C}_{19} \mathrm{H}_{28} \mathrm{O}_{7}$}

Eurycolactone F, 7644

2 $\alpha$-Hydroxytirotundin, 10769

Murrayacoumarin B, 15115

Tagitinin A, 20609

\section{$\mathrm{C}_{19} \mathrm{H}_{28} \mathrm{O}_{8}$}

Excoecarioside B, 7694

\section{$\mathrm{C}_{19} \mathrm{H}_{28} \mathrm{O}_{9}$}

Corchoionoside B, 4034

Icariside $\mathrm{B}_{2}, 10944$

\section{$\mathrm{C}_{19} \mathrm{H}_{28} \mathrm{O}_{10}$}

Benzyl 6- $O-\alpha$ - $L$-rhamnopyranosyl- $(1 \rightarrow 6) \beta-D$ glucopyranoside, 2296

Icariside $\mathrm{D}_{1}, 10949$

Sayaendoside, 19432

\section{$\mathrm{C}_{19} \mathrm{H}_{28} \mathrm{O}_{11}$}

Cuchiloside, 4311

2-C-Methyl- $D$-erythritol

1-O- $\beta$ - $D$-(6-O-4-methoxybenzoyl)glucopyrano side, 14427

Osmanthuside H, 16254

Phenyl ethanol 4-O- $\beta$ - $D$-xylopyranosyl- $(1 \rightarrow 6)-\beta$ $D$-glucopyranoside, 17110

Zizybeoside I, 23011

\section{$\mathrm{C}_{19} \mathrm{H}_{28} \mathrm{O}_{12}$}

6-O-Acetylshanzhiside methyl ester, 510

8-O-Acetylshanzhiside methyl ester, 511

Cuneataside C, 4364

Cuneataside D, 4365

3,4-Dihydroxyphenylethanol-8- $O$-[ $\beta$ - $D$-apiofuranosyl $(1 \rightarrow 2)]-\beta-D$-glucopyranoside, 6076

3,4-Dihydroxyphenylethanol-8- $O$-[ $\beta$ - $D$-apiofuranosyl $(1 \rightarrow 3)]$ - $\beta$ - $D$-glucopyranoside, 6077

3,4-Dimethoxyphenol $\beta$ - $D$-apiofuranosyl $(1 \rightarrow 6)$ $\beta$ - $D$-glucopyranoside, 6273

2'-O- $\beta$ - $D$-Glucopyranosylsalicin, 8731

(1'S)-1'-(4-Hydroxyphenyl)ethane-1',2'-diol
2'-O- $\beta$ - $D$-Apiofuranosyl-( $1 \rightarrow 6)-\beta$ - $D$-glucopyra noside, 10605

Ipolamiidoside, 11129

Orcinol-1- $O-\beta$ - $D$-glucopyranosyl- $(1 \rightarrow 6)-\beta$ - $D$ -

glucopyranoside, 16171

\section{$\mathrm{C}_{19} \mathrm{H}_{28} \mathrm{O}_{13}$}

Canthoside B, 3100

Diderroside, 5477

$\mathrm{C}_{19} \mathrm{H}_{29} \mathrm{NO}_{3}$

Homocapsaicin, 9599

$\mathrm{C}_{19} \mathrm{H}_{29} \mathrm{NO}_{4}$

Ankorine, 1293

Dendrine, 5107

\section{$\mathrm{C}_{19} \mathrm{H}_{30} \mathrm{O}$}

ent-17-Norkauran-16-one, 15767

\section{$\mathrm{C}_{19} \mathrm{H}_{30} \mathrm{O}_{2}$}

Androsterone, 1170

Ardisinol II, 1644

CPB5212-1492-1, 4204

$3 \alpha$-Hydroxy-5 $\beta$-androstan-17-one, 9780

$3 \beta$-Hydroxy- $5 \alpha$-androstan-17-one, 9781

\section{$\mathrm{C}_{19} \mathrm{H}_{30} \mathrm{O}_{3}$}

Agallochin E, 688

$4 \alpha$-Hydroxy-19-nor-ent-kauran-17-oic acid, 10542

Tagalsin H, 20607

\section{$\mathrm{C}_{19} \mathrm{H}_{30} \mathrm{O}_{4}$}

5-O-Ethylembelin, 7436

[8]-Gingerol, 8396

9'-Oxopodopyrone, 16408

10'-Oxopodopyrone, 16409

Rapanone, 18546

Vitedoin B, 22567

Vitexifolin D, 22578

\section{$\mathrm{C}_{19} \mathrm{H}_{30} \mathrm{O}_{5}$}

Hedychiol B 8,9-diacetate, 9282

Kessoglycol diacetate, 12192

Shiromodiol diacetate, 19837

\section{$\mathrm{C}_{19} \mathrm{H}_{30} \mathrm{O}_{7}$}

14-O-n-Butyrylfloridanolide, 2809

(6S,9R)-Deoxyroseoside, 5210

\section{$\mathrm{C}_{19} \mathrm{H}_{30} \mathrm{O}_{8}$}

Apocynoside I, 1529

Austroside B, 2023

Cannabiside E, 3086

Citroside A, 3771

Citroside B, 3772

Corchoionoside C, 4035

Glochidionionoside C, 8567

Glochidionionoside D, 8568

Icariside $\mathrm{B}_{1}, 10943$

Junipeionoloside, 11965

(6S,9R)-Roseoside, 18918
Roseoside II, 18919

Saussureoside B, 19426

Staphylionoside A, 20264

Staphylionoside D, 20267

\section{$\mathrm{C}_{19} \mathrm{H}_{30} \mathrm{O}_{9}$}

Apocynoside II, 1530

Austroside A, 2022

Cannabiside D, 3085

Glochidionionoside A, 8565

6-Hydroxy-junipeionoloside, 10271

$(1 R, 2 R)$-Methyl-5'-hydroxyjasmonate 5'-O- $\beta$ - $D$ glucopyranoside, 14510

Sauroposide, 19418

Saussureoside A, 19425

Spionoside A, 20173

Spionoside B, 20174

$(1 R, 6 R, 9 S)-6,9,11$-Trihydroxy-4,7-megastigmadien-3-one 11-O- $\beta$ - $D$-glucopyranoside, 21765

\section{$\mathrm{C}_{19} \mathrm{H}_{30} \mathrm{O}_{10}$}

2-[4-(3-Hydroxypropyl)-2-methoxyphenoxy]1,3-propanediol 1-O-glucoside, 10666 Qingjueine II, 18290

\section{$\mathrm{C}_{19} \mathrm{H}_{30} \mathrm{O}_{11}$}

Secologanin dimethyl acetal, 19624

\section{$\mathrm{C}_{19} \mathrm{H}_{31} \mathrm{NO}_{2}$}

Samandarine, 19223

\section{$\mathrm{C}_{19} \mathrm{H}_{32}$}

4-Phenyltridecane, 17128

5-Phenyltridecane, 17137

\section{$\mathrm{C}_{19} \mathrm{H}_{32} \mathrm{O}_{2}$}

$5 \beta$-Androstan- $3 \alpha, 17 \alpha$-diol, 1163

$5 \beta$-Androstan-3 $\alpha, 17 \beta$-diol, 1164

$5 \alpha$-Androstane- $3 \beta, 17 \alpha$-diol, 1167

Annosquamosin C, 1327

Grevillol, 8997

Methyllinolenate, 14555

\section{$\mathrm{C}_{19} \mathrm{H}_{32} \mathrm{O}_{3}$}

Annosquamosin B, 1326

Isoobtusilactone A, 11567

19-Nor-ent-kaurane-4 $\alpha, 16 \beta, 17$-triol, 15766

ObtusilactoneA, 15903

\section{$\mathrm{C}_{19} \mathrm{H}_{32} \mathrm{O}_{4}$}

Annosquamosin G, 1331

[8]-Gingediol, 8384

Protolichesterinic acid, 17979

\section{$\mathrm{C}_{19} \mathrm{H}_{32} \mathrm{O}_{7}$}

Blumenol C glucoside, 2522

Byzantionoside B, 2835

Icariside $\mathrm{B}_{6}, 10946$

Icariside $\mathrm{B}_{9}, 10947$

\section{$\mathrm{C}_{19} \mathrm{H}_{32} \mathrm{O}_{8}$}

Breyniaionoside D, 2610

Corchoionoside A, 4033 
Excoecarioside A, 7693

Glochidionionoside B, 8566

$(3 S, 5 S, 6 R, 9 R)$-3-Hydroxy-5,6-epoxy- $\beta$-ionol-

9- $O$ - $\beta$-glucopyranoside, 10085

Lasianthionoside B, 12529

Lasianthionoside C, 12530

Officinoside A, 16015

Officinoside B, 16016

Rehmaionoside C, 18595

Sammangaoside A, 19228

Staphylionoside E, 20268

Staphylionoside F, 20269

Staphylionoside H, 20271

Turpinionoside E, 22129

\section{$\mathrm{C}_{19} \mathrm{H}_{32} \mathrm{O}_{9}$}

Breyniaionoside A, 2607

Lasianthionoside A, 12528

Pisumionoside, 17495

Staphylionoside C, 20266

\section{$\mathrm{C}_{19} \mathrm{H}_{33} \mathrm{NO}_{2}$}

3-O-Tetradecanoyl-1-cyano-2-methyl-1-propene, 21042

\section{$\mathrm{C}_{19} \mathrm{H}_{34} \mathrm{O}_{2}$}

Anticancer Fatty acid PMV70P691-75, 1396

Methyl linoleate, 14554

Methyl octadeca-8,11-dienoate, 14628

Sterculic acid, 20328

\section{$\mathrm{C}_{19} \mathrm{H}_{34} \mathrm{O}_{4}$}

1-Acetoxy-2,4-dihydroxy- $N$-heptadeca-16-yne, 167

\section{$\mathrm{C}_{19} \mathrm{H}_{34} \mathrm{O}_{7}$}

Alatoside E, 842

Linarionoside A, 12856

Linarionoside B, 12857

$(3 R, 9 S)$-Megastigman-5-en-3,9-diol-3-O- $\beta-D$ glucopyranoside, 13643

Myrsinionoside A, 15210

Platanionoside J, 17527

\section{$\mathrm{C}_{19} \mathrm{H}_{34} \mathrm{O}_{8}$}

Myrsinionoside B, 15211

Rehmaionoside A, 18593

Rehmaionoside B, 18594

Sammangaoside B, 19229

Turpinionoside A, 22125

Turpinionoside B, 22126

Turpinionoside D, 22128

\section{$\mathrm{C}_{19} \mathrm{H}_{34} \mathrm{O}_{9}$}

Breyniaionoside B, 2608

Breyniaionoside C, 2609

(3S,5R,6R,7E,9S)-Megastigman-7-ene-3,5,6,9tetrol-3-O- $\beta$ - $D$-glucopyranoside, 13644

$(3 S, 5 R, 6 R, 7 E, 9 S)$-Megastigman-7-ene-3,5,6,9tetrol-9-O- $\beta$ - $D$-glucopyranoside, 13645
Staphylionoside B, 20265

Staphylionoside J, 20273

$\mathrm{C}_{19} \mathrm{H}_{34} \mathrm{O}_{11}$

Ebracteatoside D, 6666

$\mathrm{C}_{19} \mathrm{H}_{34} \mathrm{O}_{12}$

3- $O$ - $\alpha$ - $L$-Arabinopyranosyl- $(1 \rightarrow 6)-\beta$ - $D$-glucopyranoside of butyl (3S)-hydroxybutanoate, 1563

3-O- $\alpha$ - $L$-Arabinopyranosyl-( $1 \rightarrow 6)-\beta$ - $D$-glucopyranoside of butyl (3R)-hydroxybutanoate, 1567

$\mathrm{C}_{19} \mathrm{H}_{36} \mathrm{O}_{2}$

Methyl 9-octadecenoate, 14630

$\mathrm{C}_{19} \mathrm{H}_{36} \mathrm{O}_{4}$

1-Acetoxy-2,4-dihydroxy- $N$-heptadeca-16-ene, 166

9,12-Dihydroxy-15-nonadecenoic acid, 6047

$\mathrm{C}_{19} \mathrm{H}_{36} \mathrm{O}_{5}$

2,3-Dihydroxy-2-(1-hydroxytridecyl)-4methoxycyclopentanone, 5920

\section{$\mathrm{C}_{19} \mathrm{H}_{36} \mathrm{O}_{7}$}

Myrsinionoside C, 15212

Myrsinionoside D, 15213

\section{$\mathrm{C}_{19} \mathrm{H}_{36} \mathrm{O}_{8}$}

Meliaionoside A, 13664

Meliaionoside B, 13665

Turpinionoside C, 22127

\section{$\mathrm{C}_{19} \mathrm{H}_{36} \mathrm{O}_{10}$}

Rhodiooctanoside, 18793

\section{$\mathrm{C}_{19} \mathrm{H}_{38} \mathrm{O}_{2}$}

Ethyl heptadecanoate, 7444

Ethylisoheptadecanoate, 7452

Methyl octadecanoate, 14629

Nonadecanoic acid, 15672

\section{$\mathrm{C}_{19} \mathrm{H}_{38} \mathrm{O}_{4}$}

$L$-(-)- $\alpha$-Monopalmitin, 14931

$\mathrm{C}_{19} \mathrm{H}_{40}$

n-Nonadecane, 15671

\section{$\mathrm{C}_{19} \mathrm{H}_{40} \mathrm{O}$}

Nonacasyl alcohol-10, 15659

$\mathrm{C}_{20} \mathrm{H}_{11} \mathrm{~N}_{3} \mathrm{O}_{2}$

Arcyriaflavin A, 1624

$\mathrm{C}_{20} \mathrm{H}_{11} \mathrm{~N}_{3} \mathrm{O}_{3}$

Arcyriaflavin B, 1625

$\mathrm{C}_{20} \mathrm{H}_{12}$

3,4-Benzopyrene, 2226

$\mathrm{C}_{20} \mathrm{H}_{12} \mathrm{~N}_{2} \mathrm{O}_{2}$

20-Formylbenzo[6,7]indolizino[1,2-b]quinolin-

$11(13 H)$-one, 7896

$\mathrm{C}_{20} \mathrm{H}_{12} \mathrm{O}_{4}$

Palmarumycin $\mathrm{CP}_{1}, 16552$

$\mathrm{C}_{20} \mathrm{H}_{12} \mathrm{O}_{6}$

Helioxanthin, 9323
Justicidine E, 11981

Taiwanin C, 20643

\section{$\mathrm{C}_{20} \mathrm{H}_{12} \mathrm{O}_{7}$}

Jusmicranthin, 11972

Justicidone, 11982

Phelligridin C, 17061

Taiwanin E, 20644

\section{$\mathrm{C}_{20} \mathrm{H}_{12} \mathrm{O}_{8}$}

Phelligridin D, 17062

\section{$\mathrm{C}_{20} \mathrm{H}_{13} \mathrm{NO}_{5}$}

11-Methoxylettowianthine, 13988

Oxysanguinarine, 16473

\section{$\mathrm{C}_{20} \mathrm{H}_{13} \mathrm{~N}_{3} \mathrm{O}$}

Naulafine, 15316

Staurosporinone, 20278

\section{$\mathrm{C}_{20} \mathrm{H}_{13} \mathrm{~N}_{3} \mathrm{O}_{2}$}

Bisindolylpyrrole CPB-53-594-6, 2476

\section{$\mathrm{C}_{20} \mathrm{H}_{13} \mathrm{~N}_{3} \mathrm{O}_{8}$}

Xanthommatin, 22769

\section{$\mathrm{C}_{20} \mathrm{H}_{14} \mathrm{NO}_{4}$}

Avicine, 2037

Sanguinarine, 19284

\section{$\mathrm{C}_{20} \mathrm{H}_{14} \mathrm{~N}_{2} \mathrm{O}_{2}$}

Naucleficine, 15302

\section{$\mathrm{C}_{20} \mathrm{H}_{14} \mathrm{~N}_{2} \mathrm{O}_{3}$}

Nauclealine A, 15290

Nauclequiniine, 15312

\section{$\mathrm{C}_{20} \mathrm{H}_{14} \mathrm{~N}_{4}$}

Porphyrin, 17730

\section{$\mathrm{C}_{20} \mathrm{H}_{14} \mathrm{O}_{4}$}

4,5,4',5'-Tetrahydroxy-1:1'-binaphthyl, 21083

\section{$\mathrm{C}_{20} \mathrm{H}_{14} \mathrm{O}_{5}$}

Palmarumycin $\mathrm{JC}_{1}, 16553$

Palmarumycin $\mathrm{JC}_{2}, 16554$

Sophoracoumestan A, 20081

\section{$\mathrm{C}_{20} \mathrm{H}_{14} \mathrm{O}_{6}$}

Jayantinin, 11865

Matsukaze lactone, 13607

Pachycarin E, 16493

Taiwanin A, 20641

\section{$\mathrm{C}_{20} \mathrm{H}_{14} \mathrm{O}_{7}$}

Daphnoretin methyl ether, 4658

3'-Methoxypongapin, 14074

Pachyrrhizone, 16500

\section{$\mathrm{C}_{20} \mathrm{H}_{14} \mathrm{O}_{8}$}

(-)-Diasesamin-di- $\gamma$-lactone, 5376

12 $\alpha$-Hydroxypachyrrhizone, 10589

\section{$\mathrm{C}_{20} \mathrm{H}_{14} \mathrm{O}_{9}$}

Fuscoporine, 8033

\section{$\mathrm{C}_{20} \mathrm{H}_{15} \mathrm{NO}_{4}$}

Dihydrosanguinarine, 5708

7,9-Dimethoxy-2,3-methylendioxybenzophenanthridine, 6261 
$\mathrm{C}_{20} \mathrm{H}_{15} \mathrm{NO}_{5}$

Oxyterihanine, 16477

$\mathrm{C}_{20} \mathrm{H}_{15} \mathrm{NO}_{6}$

6,7-Methylenedioxy-2-(6-acetyl-2,3-methylenedioxybenzyl)-1(2H)-isoquinolinone, 14360

Thalicminine, 21242

\section{$\mathrm{C}_{20} \mathrm{H}_{15} \mathrm{~N}_{3} \mathrm{O}$}

Angustine, 1250

Evodiaxinine, 7667

\section{$\mathrm{C}_{20} \mathrm{H}_{15} \mathrm{~N}_{3} \mathrm{O}_{2}$}

10-Hydroxyangustine, 9788

Naucletine, 15313

\section{$\mathrm{C}_{20} \mathrm{H}_{16} \mathrm{NO}_{4}^{+}$}

Isofagaridine, 11423

Worenine, 22732

\section{$\mathrm{C}_{20} \mathrm{H}_{16} \mathrm{~N}_{2} \mathrm{O}_{3}$}

Deoxycamptothecin, 5157

\section{$\mathrm{C}_{20} \mathrm{H}_{16} \mathrm{~N}_{2} \mathrm{O}_{4}$}

Camptothecin, 3053

\section{$\mathrm{C}_{20} \mathrm{H}_{16} \mathrm{~N}_{2} \mathrm{O}_{5}$}

10-Hydroxycamptothecin, 9882

18-Hydroxycamptothecin, 9883

\section{$\mathrm{C}_{20} \mathrm{H}_{16} \mathrm{O}_{2}$}

(E)-2-(4-Methylpenta-1,3-dienyl)anthraquinone, 14661

\section{$\mathrm{C}_{20} \mathrm{H}_{16} \mathrm{O}_{3}$}

Sterequinone D, 20338

\section{$\mathrm{C}_{20} \mathrm{H}_{16} \mathrm{O}_{4}$ \\ Bidwillon C, 2365 \\ Corylin, 4109}

\section{$\mathrm{C}_{20} \mathrm{H}_{16} \mathrm{O}_{5}$}

Alpinumisoflavone, 993

Atalantoflavone, 1956

Carpachromene, 3217

Derrone, 5235

Erylatissin B, 7306

Glabrocoumarin, 8496

Glabrone, 8501

Isopsoralidin, 11632

Kanzonol W, 12153

Licoagroisoflavone, 12756

Phaseol, 17046

Psoralidin, 18088

Psorothamnone A, 18092

Sojagol, 20041

Vogelin J, 22605

Yinyanghuo C, 22910

\section{$\mathrm{C}_{20} \mathrm{H}_{16} \mathrm{O}_{6}$}

Bavacoumestan A, 2176

Bavacoumestan B, 2177

Detetrahydroconidendrin, 5279

Elliptone, 6762

Erosone, 7290
(-)-(R,E)-Hibalactone, 9534

I-23, 10928

Licoisoflavone B, 12779

Negundin A, 15322

Pachycarin A, 16489

Pachycarin B, 16490

Picropolygamain, 17343

Psoralidin-2',3'-oxide, 18089

Savinin, 19427

Semilicoisoflavone B, 19695

3,3',5'-Trimethoxy furo[8,7:4",5"]flavone, 21916

Yinyanghuo E, 22912

\section{$\mathrm{C}_{20} \mathrm{H}_{16} \mathrm{O}_{7}$}

(-)-Aptosimon, 1537

Corylidin, 4107

Genkdaphin, 8286

6a $\alpha, 12 \mathrm{a} \alpha-12 \mathrm{a}-H y d r o x y e l l i p t o n e, 10067$

(+)-Malaccol, 13415

\section{$\mathrm{C}_{20} \mathrm{H}_{16} \mathrm{O}_{12}$}

Ducheside A, 6619

Ducheside B, 6620

4-( $\alpha$-Rhamnopyranosyl)ellagic acid, 18697

\section{$\mathrm{C}_{20} \mathrm{H}_{16} \mathrm{O}_{15}$}

Mucic acid 1,4-lactone 3,5-di-O-gallate, 15010

\section{$\mathrm{C}_{20} \mathrm{H}_{17} \mathrm{ClO}_{4}$}

Anthrasesamone C, 1370

\section{$\mathrm{C}_{20} \mathrm{H}_{17} \mathrm{ClO}_{7}$}

5-Chloropropacin, 3570

\section{$\mathrm{C}_{20} \mathrm{H}_{17} \mathrm{NO}_{5}$}

Bianfugenine, 2345

Oxoglaucine, 16339

Oxyberberine, 16441

Vitedoamine A, 22565

\section{$\mathrm{C}_{20} \mathrm{H}_{17} \mathrm{NO}_{6}$}

Adlumidine, 642

Bicuculline, 2350

Hypecorinine, 10879

Leptopidinine, 12675

Pareirubrine A, 16661

1,2,3,10-Tetramethoxy-9-hydroxy-4,5,6,6 $\alpha$ dehydro-7-aporhhinone, 21187

$\mathrm{C}_{20} \mathrm{H}_{17} \mathrm{NO}_{7}$

Procumbine, 17873

\section{$\mathrm{C}_{20} \mathrm{H}_{17} \mathrm{~N}_{3} \mathrm{O}$}

3,14-Dihydroangustine, 5540

18,19-Dihydroangustine, 5541

$\mathrm{C}_{20} \mathrm{H}_{17} \mathrm{~N}_{3} \mathrm{O}_{2}$

Angustoline, 1252

$\mathrm{C}_{20} \mathrm{H}_{18} \mathrm{NO}_{4}$

Berberine, 2303

Epiberberine, 6835

$\mathrm{C}_{20} \mathrm{H}_{18} \mathrm{NO}_{5}{ }^{+}$

Berberastine, 2302
$\mathrm{C}_{20} \mathrm{H}_{18} \mathrm{NO}_{6}^{+}$

Leptopine, 12676

$\mathrm{C}_{20} \mathrm{H}_{18} \mathrm{~N}_{2} \mathrm{O}_{2}$

Kopsorinine, 12269

$\mathrm{C}_{20} \mathrm{H}_{18} \mathrm{~N}_{2} \mathrm{O}_{4}$

Corydamine, 4105

\section{$\mathrm{C}_{20} \mathrm{H}_{18} \mathrm{~N}_{2} \mathrm{O}_{7}$}

Canthin-6-one 9- $O$ - $\beta$-glucopyranoside, 3097

$\mathrm{C}_{20} \mathrm{H}_{18} \mathrm{O}_{2}$

1,4-Bis-benzyloxy-benzene, 2436

2-(4-Methylpent-3-enyl)anthraquinone, 14663

$\mathrm{C}_{20} \mathrm{H}_{18} \mathrm{O}_{3}$

Anthrasesamone A, 1368

\section{$\mathrm{C}_{20} \mathrm{H}_{18} \mathrm{O}_{4}$}

$(2 R)$-Abyssinone I, 50

Anthrasesamone B, 1369

Anticancer Phenylphenalone PMV70P691-129, 1434

Bavachromene, 2175

Capitellataquinone D, 3135

Erypoegin E, 7311

Erypoegin H, 7314

Glabrene, 8490

4-Hydroxyonchocarpin, 10335

Inflacoumarin A, 11037

Kanzonol B, 12149

Licoflavone A, 12774

Neobavaisoflavone, 15342

Obovatin, 15887

Phaseollin, 17048

8-Prenyldaidzein, 17830

Sterekunthal B, 20334

\section{$\mathrm{C}_{20} \mathrm{H}_{18} \mathrm{O}_{5}$}

Capitellataquinone C, 3134

Demethoxycurcumin, 5045

8-(1,1-Dimethylallyl)genistein, 6305

Eurycarpin A, 7637

Glabroisoflavanone A, 8497

Glepidotin A, 8548

(2S)-5-Hydroxy-7-methoxy-8-[(E)-3-oxo-1butenyl]flavanone, 10430

Hydroxyphaseollin, 10600

Isodispar B, 11380

Licoflavone C, 12776

Lupiwighteone, 13107

Methyl tanshinonate, 14736

Moracin H, 14960

6-Prenylapigenin, 17823

Psoralenol, 18087

Sterekunthal A, 20333

Tuberosin, 22080

Vismiaguianin A, 22543

Vitrofolal A, 22590 
Wighteone, 22667

Yinyanghuo D, 22911

\section{$\mathrm{C}_{20} \mathrm{H}_{18} \mathrm{O}_{6}$}

Asarinin, 1833

Capitellataquinone A, 3132

Cubebinolide, 4310

Desmosdumotin A, 5268

Erythrinin C, 7333

Gancaonin C, 8136

Glyasperin F, 8801

Glycyrrhisoflavone, 8844

(+)-Hinokinin, 9544

2-(p-Hydroxyphenoxy)-5,7-dihydroxy-6isopentenylchromone, 10602

Isogancaonin C, 11434

Isolicoflavonol, 11490

8-Isopentenyl-kaempferol, 11588

8-( $\Delta^{2}$-Isopentenyl)-5,7,3',4'-tetrahydroxyflavone, 11590

Licoflavonal, 12773

Licoisoflavone, 12778

Luteone, 13150

4-Oxoobovatachromene, 16399

Phellodensin A, 17072

Sanggenon F, 19261

Sanggenon H, 19263

Sesamin, 19777

5,7,4'-Trihydroxy-6-(3,3-dimethylallyloxiranylmethyl)isoflavone, 21716

Ugonin F, 22179

Vitrofolal B, 22591

\section{$\mathrm{C}_{20} \mathrm{H}_{18} \mathrm{O}_{7}$}

Calocedrin, 2983

Capitellataquinone B, 3133

Gancaonin P, 8141

Glycyrrhiza-flavonol A, 8845

7-Hydroxyhinokinin, 10164

Isopaulownin, 11581

Meridinol, 13783

Neosesamin, 15459

Neouralenol, 15471

Ophiopogonanone D, 16139

Parabenzlactone, 16649

Paulownin, 16734

Sesaminone, 19778

Sesamolin, 19780

Styraxin, 20419

Uralenol, 22241

\section{$\mathrm{C}_{20} \mathrm{H}_{18} \mathrm{O}_{8}$}

Cleomiscosin A, 3829

Cleomiscosin B, 3830

Daphneticin, 4643

8'-Epi-cleomiscosin A, 6870
Irisflorentin, 11164

5,6,7,5'-Tetramethoxy-3',4'-methylenedioxy-

flavone, 21188

\section{$\mathrm{C}_{20} \mathrm{H}_{18} \mathrm{O}_{9}$}

Frangulin B, 7940

5,3',4',5'-Tetramethoxy-6,7-methylenedioxyisoflavone, 21189

Xanthopurpurin-3- $O-\beta$ - $D$-glucoside, 22773

$\mathrm{C}_{20} \mathrm{H}_{18} \mathrm{O}_{10}$

Juglanin, 11897

Salvianolic acid D, 19204

$\mathrm{C}_{20} \mathrm{H}_{18} \mathrm{O}_{11}$

Avicularin, 2039

Fukinolic acid, 7987

Guaijaverin, 9043

Quercetin-3- $\beta-D$-xylopyranoside, 18407

$\mathrm{C}_{20} \mathrm{H}_{18} \mathrm{O}_{12}$

(-)-2-Galloyl-4-(E)-caffeoyl- $L$-threonic acid, 8103

Melicitrin, 13675

$\mathrm{C}_{20} \mathrm{H}_{18} \mathrm{O}_{14}$

2,3-O-(S)-Hexahydroxydiphenoyl- $D$-glucopyranose, 9502

\section{$\mathrm{C}_{20} \mathrm{H}_{19} \mathrm{NO}_{2}$}

Benzosimuline, 2227

$\mathrm{C}_{20} \mathrm{H}_{19} \mathrm{NO}_{3}$

Acronycine, 570

2-[4(3,4-Methylenedioxyphenyl)butyl]-4quinolone, 14379

\section{$\mathrm{C}_{20} \mathrm{H}_{19} \mathrm{NO}_{4}$}

(-)- $N$-Acetylnorstephalagine, 473

$\mathrm{N}$-Acetylxylopine, 532

Annuloline, 1342

Tetrahydrocorysamine, 21062

\section{$\mathrm{C}_{20} \mathrm{H}_{19} \mathrm{NO}_{5}$}

Chelidonine, 3502

2,3-Dihydrodauriporphine, 5581

Hypecorine, 10878

Isocorynoline, 11346

Parfumine, 16663

Protopine, 17983

Pseudoprotopine, 18057

Xanthaline, 22745

$\mathrm{C}_{20} \mathrm{H}_{19} \mathrm{NO}_{6}$

10-Hydroxychelidonine, 9903

Ledeborine, 12600

Papaverrubine A, 16624

Papaverrubine E, 16628

Rhoeagenine, 18817

Taspine, 20717

$\mathrm{C}_{20} \mathrm{H}_{19} \mathrm{NO}_{7}$

Rhodesiacridone, 18784

$\mathrm{C}_{20} \mathrm{H}_{19} \mathrm{~N}_{3} \mathrm{O}_{2}$
3,14-Dihydroangustoline, 5542

$\mathrm{C}_{20} \mathrm{H}_{19} \mathrm{~N}_{3} \mathrm{O}_{3}$

Anisotine, 1291

$\mathrm{C}_{20} \mathrm{H}_{19} \mathrm{O}_{3}$

Decussine, 4864

$\mathrm{C}_{20} \mathrm{H}_{19} \mathrm{O}_{10} \mathrm{~S}^{-}$

Torvanol A, 21468

$\mathrm{C}_{20} \mathrm{H}_{19} \mathrm{O}_{11}{ }^{+}$

Delphinidin-3-arabinoside, 5012

\section{$\mathrm{C}_{20} \mathrm{H}_{20} \mathrm{NO}_{4}{ }^{+}$}

Columbamine, 3934

Dehydrocorydalmine, 4890

Jatrorrhizine, 11851

$\mathrm{C}_{20} \mathrm{H}_{20} \mathrm{NO}_{6}{ }^{+}$

Leptopidine, 12674

\section{$\mathrm{C}_{20} \mathrm{H}_{20} \mathrm{~N}_{2} \mathrm{O}_{2}$}

10,22-Dioxokopsan, 6473

Nauclefiline, 15304

\section{$\mathrm{C}_{20} \mathrm{H}_{20} \mathrm{~N}_{2} \mathrm{O}_{3}$}

Centcyamine, 3392

cis-Centcyamine, 3393

17-Epinaucleidinal, 6973

Naucleamide C, 15294

Naucleamide D, 15295

Naucleidinal, 15307

\section{$\mathrm{C}_{20} \mathrm{H}_{20} \mathrm{~N}_{2} \mathrm{O}_{4}$}

cis-Moschamine, 14998

trans-Moschamine, 14999

\section{$\mathrm{C}_{20} \mathrm{H}_{20} \mathrm{~N}_{2} \mathrm{O}_{5}$}

Aquiledine, 1541

Cheliensisine, 3506

Isoaquiledine, 11233

\section{$\mathrm{C}_{20} \mathrm{H}_{20} \mathrm{O}_{2}$}

2,7-Dimethoxy-1,6-dimethyl-5-vinylphenanthrene, 6223

Salvilenone, 19217

\section{$\mathrm{C}_{20} \mathrm{H}_{20} \mathrm{O}_{3}$}

Derricidin, 5221

Ovalifoliolatin B, 16276

\section{$\mathrm{C}_{20} \mathrm{H}_{20} \mathbf{O}_{4}$}

(2R)-Abyssinone, 49

(2S)-Abyssinone II, 51

Ajuforrestine A, 786

Anticancer Diarylheptanoid PMV70P691-010, 1393

Avicennin, 2035

Bavachin, 2172

Broussochalcone B, 2630

Burttinol D, 2773

Corylifolinin, 4108

Difengpin, 5504

3,9-Dihydroxy-4-(3,3-dimethylallyl)-[6a $R$, $11 \mathrm{a} R]$-pterocarpane, 5851 
Dorsmanin A, 6561

Glabranin, 8487

Glabridin, 8493

Lespedezol $\mathrm{D}_{2}, 12694$

Licarin B, 12750

Licoagrochalcone A, 12751

Licoagropin, 12758

Moracin I, 14961

Otobain, 16267

Ovalifoliolatin A, 16275

(-)-Phaseollidin, 17047

Phaseollinisoflavan, 17049

Pygmaeocine E, 18235

Pyranokunthone A, 18252

Pyranokunthone B, 18253

Spathelia bischromene, 20135

\section{$\mathrm{C}_{20} \mathrm{H}_{20} \mathrm{O}_{5}$}

Bakuchalcone, 2123

Bavachromanol, 2174

Blepharolide B, 2502

Broussochalcone A, 2629

Burchellin, 2760

Dehydroxycubebin, 4983

Desmethylxanthohumol, 5261

1,10-Didehydrosalviarin, 5469

6,7-Dimethoxy-2-[2-(4'-methoxyphenyl)ethyl]chromone, 6258

(2S)-Euchrenone $\mathrm{A}_{7}, 7485$

Flavanone 4',5-dihydroxy-7-prenyloxyflavanone, 7808

Futoenone, 8036

(+)-Galbacin, 8085

(-)-Galbacin, 8086

Glepidotin B, 8549

Hancinone, 9209

Isobavachin, 11248

Kosamol S, 12283

Lespedezol $\mathrm{D}_{3}, 12695$

Licocoumarone, 12771

Licoflavanone, 12772

Linearolactone, 12879

Linixanthone C, 12888

PM-2004-70-452-3, 17575

5-Prenylbutein, 17827

5'-Prenyllicodione, 17836

6-Prenylnaringenin, 17839

Salvifarcin, 19210

2,4,2',4'-Tetrahydroxy-3'-prenylchalcone, 21151

Teuvincenone E, 21231

Ugonin D, 22177

Ugonin E, 22178

(-)-Zuonin A, 23030

$\mathrm{C}_{20} \mathrm{H}_{20} \mathrm{O}_{6}$
Afzeliixanthone B, 680

Balanophonin, 2125

Blepharolide A, 2501

$\alpha$-Conidendrin, 3980

Cowagarcinone B, 4196

Cowagarcinone C, 4197

Cubebin, 4306

(-)-Cubebin, 4307

Cudraflavanone B, 4335

Desmethylxanthohumol B, 5262

Desmethylxanthohumol J, 5263

5,6-Didehydropygmaeocin A, 5468

(2S)-2',4'-Dihydroxy-2"-(1-hydroxy-1-methylethyl)dihydrofuro[2,3-h]-flavanone, 5746

5,4'-Dihydroxy-[2"-(1-hydroxy-1-methylethyl)dihydrofurano]-(7,8:5",4")flavanone, 5919

6-Hydroxy-4-(4-hydroxy-3-methoxyphenyl)-3hydroxymethyl-7-methoxy-3,4-dihydro-2-naph thaldehyde, 10185

Leachianone G, 12597

Melianoninol, 13670

Neolignan in Magnolia denudata, 15426

Neophellamuretin, 15448

Phellamuretin, 17052

Plaunol B, 17549

Polystachyne E, 17687

6-Prenylated eriodictyol, 17825

8-Prenylated eriodictyol, 17826

Sauchinone, 19407

Teuperinin A, 21228

Tomentosanol D, 21440

Uralenin, 22239

Vismiaguianin B, 22544

Vitedoin A, 22566

Xanthoxylol, 22779

$\mathrm{C}_{20} \mathrm{H}_{20} \mathrm{O}_{7}$

Anticancer Lignan PMV70P691-124, 1425

Artocarpesin, 1809

Cimicifuge acid, 3650

Erycibenin A, 7300

Erycibenin B, 7301

Isosinensetin, 11716

Licoleafol, 12780

3,5,7,3',4'-Pentamethoxyflavone, 16867

5,6,2',3',6'-Pentamethoxyflavone, 16868

5,7,2',3',4'-Pentamethoxyflavone, 16869

5,7,2',4',6'-Pentamethoxyflavone, 16870

5,7,3',4',5'-Pentamethoxyflavone, 16871

6,7,8,3',4'-Pentamethoxyflavone, 16872

Phellodensin C, 17073

Plaunol C, 17550

Praeruptorin C, 17762
Sesamolinol, 19781

Sinensetin, 19929

Tangeretin, 20670

\section{$\mathrm{C}_{20} \mathrm{H}_{20} \mathrm{O}_{8}$}

Artemisetin, 1792

Cimiracemate C, 3664

Cimiracemate D, 3665

Demethylnobiletin, 5088

Dimethyl ester of

(1 $\alpha, 2 \alpha, 3 \alpha, 4 \alpha)$-2,4-bis(3,4-dihydroxyphenyl)-1,

3-cyclobutanedicarboxylic acid, 6351

Ethyl rosmarinate, 7478

$(8 S, 8 ' S)-(+)-8$-Hydroxy-oxomatairesinol, 10574

3'-Hydroxy-5,6,7,8,4'-pentamethoxyflavone, 10594

4'-Hydroxy-5,6,7,3',5'-pentamethoxyflavone, 10595

5-Hydroxy-6,7,3',4',5'-pentamethoxyflavone, 10596

5-Hydroxy-7,2',3',4',5'-pentamethoxyflavone, 10597

Polygalolide B, 17638

3,4,6-Trihydroxyphenanthrene-3- $O-\beta$ - $D$-glucopyranoside, 21835

\section{$\mathrm{C}_{20} \mathrm{H}_{20} \mathrm{O}_{9}$}

5,7-Dihydroxy-2',3',4',5',6'-pentamethoxyflavone, 6072

Moracin M 3'-O- $\beta$ - $D$-glucopyranoside, 14963

3,6,8,3',4'-Pentamethoxy-5,7-dihydroxyflavone, 16863

\section{$\mathrm{C}_{20} \mathrm{H}_{20} \mathrm{O}_{10}$}

Cassiaside, 3284

1,6-Di- $O$ - $p$-hydroxybenzoyl- $\beta$ - $D$-glucopyranoside, 5767

$5,7,4$ '-Trihydroxy dihydroflavonol-3- $O-\alpha-L$ arabinofuranoside, 21706

\section{$\mathrm{C}_{20} \mathrm{H}_{20} \mathrm{O}_{11}$}

6'-O-Caffeoylerigeroside, 2900

4- $C$ - $\beta$ - $D$-Glucopyranosyl-1,3,6-trihydroxy-7methoxyxanthone, 8743

1-(2'- $\gamma$-Pyranone)-6-caffeoyl- $\alpha-D$-pyranoglucose, 18254

Swertianolin, 20511

\section{$\mathrm{C}_{20} \mathrm{H}_{20} \mathrm{O}_{12}$}

Homomangiferin, 9613

\section{$\mathrm{C}_{20} \mathrm{H}_{20} \mathrm{O}_{13}$}

Aceritannin, 95

Neocretanin, 15372

$\mathrm{C}_{20} \mathrm{H}_{20} \mathrm{O}_{14}$

1,3-Di- $O$-galloyl- $\beta$ - $D$-glucopyranose, 5516

1,4-Di-O-galloylglucose, 5517

1,6-Di- $O$-galloyl- $\beta$-glucose, 5518

3,6-Di- $O$-galloylglucose, 5519 
2,3-Di- $O$-galloyl- $D$-glucose, 5520

Gallic acid-3-O-(6'-O-galloyl)glucoside, 8096

Gallic acid-4-O-(6'-O-galloyl)-glucoside, 8097

Hamamelitannin, 9202

$\mathrm{C}_{20} \mathrm{H}_{21} \mathrm{NO}_{3}$

Koenigicine, 12247

\section{$\mathrm{C}_{20} \mathrm{H}_{21} \mathrm{NO}_{4}$}

Amurensinine, 1096

Canadine, 3057

(-)-Crebanine, 4224

(-)-Dicentrine, 5417

Glycocitrine I, 8825

$\mathrm{N}$-Methoxylcarbonyl-nornuciferine, 13987

$O$-Methylbulbocapnine, 14161

$O$-Methyl domesticine, 14332

Papaverine, 16623

Podocarpamide, 17587

Sinactine, 19908

(-)-Tetrahydroberberine, 21056

\section{$\mathrm{C}_{20} \mathrm{H}_{21} \mathrm{NO}_{5}$}

(+)-Eschscholtzidine- $N$-oxide, 7360

Gravacridonediol monomethyl ether, 8986

(-)-12-Hydroxy-O-methylcaryachine, 10480

Ocokryptine, 15929

Ophiocarpine, 16128

Prenylcitpressine, 17829

Romucosine C, 18903

Thalictricine, 21249

\section{$\mathrm{C}_{20} \mathrm{H}_{21} \mathrm{NO}_{6}$}

Ledeboridine, 12599

$\mathrm{N}$-Methyl-14-O-desmethyl-epiporphyroxine, 14291

Ophiocarpine $N$-oxide, 16129

16-Oxodelavaine, 16310

Papaverrubine C, 16626

Papaverrubine D, 16627

\section{$\mathrm{C}_{20} \mathrm{H}_{21} \mathrm{~N}_{3} \mathrm{O}_{2}$}

Adhatodine, 627

\section{$\mathrm{C}_{20} \mathrm{H}_{22} \mathrm{NO}_{6}{ }^{+}$}

Leptopinine, 12677

\section{$\mathrm{C}_{20} \mathrm{H}_{22} \mathrm{~N}_{2} \mathrm{O}$}

Demethoxypurpeline, 5054

Kopsanone, 12261

Koumine, 12289

\section{$\mathrm{C}_{20} \mathrm{H}_{22} \mathrm{~N}_{2} \mathrm{O}_{2}$}

Akuammicine, 823

Alstolactone, 995

$N(4)$-Demethylalstonerinal, 5058

N(4)-Demethylalstonerine, 5059

Gelsemine, 8257

Koumine $N$-oxide, 12290

12-Methoxyvellosimine, 14102

Pericyclivine, 16921
Pleiocarpamine, 17555

Quinidinone, 18424

$\mathrm{C}_{20} \mathrm{H}_{22} \mathrm{~N}_{2} \mathrm{O}_{3}$

Alstomaline, 996

Alstonal, 998

Alstonisine, 1003

Fluorocarpamine, 7839

Gelsemine $N$-oxide, 8258

Gomaline, 8905

Naucleamide E, 15296

Perivine, 16966

Picrinine, 17329

Vinervine, 22503

\section{$\mathrm{C}_{20} \mathrm{H}_{22} \mathrm{~N}_{2} \mathrm{O}_{4}$}

Akuammigine pseudoindoxyl, 826

Angustilobine B N4-oxide, 1249

Fluorocarpamine- $N$-oxide, 7840

16-Hydroxyalstonal, 9776

16-Hydroxyalstonisine, 9777

Isomitraphyllic acid, 11537

Isopteropodic acid, 11635

3-Isorauniticine pseudoindoxyl, 11644

Lagumicine, 12451

Mitraphyllic acid, 14887

Pteropodic acid, 18113

Rauniticine pseudoindoxyl, 18555

\section{$\mathrm{C}_{20} \mathrm{H}_{22} \mathrm{~N}_{2} \mathrm{O}_{6}$}

GS-1, 9016

\section{$\mathrm{C}_{20} \mathrm{H}_{22} \mathrm{~N}_{2} \mathrm{O}_{9}$}

Glucodichotomine B, 8594

\section{$\mathrm{C}_{20} \mathrm{H}_{22} \mathrm{O}_{3}$}

Anticancer Flavonoid PMV70P691-015, 1399

Brosimine B, 2627

(2S)-7,4'-Dihydroxy-3'-prenylflavan, 6102

6(1,1-Dimethylallyl)-7,4'-dihydroxylflavan, 6304

7-(4"-Hydroxy-3"-methoxyphenyl)-1-phenyl-

hept-4-en-3-one, 10451

6-Methylcryptotanshinone

[14,16-epoxy-6-methyl-5(10),6,8,13-abietatetr aene-11,12-dione], 14263

Myricarborin, 15167

Picealactone A, 17275

Pygmaeocin B, 18233

Sinensol G, 19930

Yakuchinone B, 22877

$\mathrm{C}_{20} \mathrm{H}_{22} \mathrm{O}_{4}$

Ajuforrestine B, 787

Anticancer Flavonoid PMV70P691-013, 1397

3,3'-Bis(6-methoxychroman), 2481

Brosimine A, 2626

Dehydrodieugenol, 4906

Demethylracemosol, 5095

Dentatin, 5134
Dipetaline, 6484

Gingerenone C, 8391

7-Hydroxy-12-methoxy-20-nor-abieta-

1,5(10),7,9,12-pentaen-6,14-dione, 10429

Licarin A, 12749

Machilin A, 13290

(3S)-Methoxy-1,7-bis(4-hydroxyphenyl)-6E-

hepten-5-one, 13859

Neomillinol, 15436

Picealactone B, 17276

Picealactone C, 17277

Piloselloidal, 17366

Preracemosol B, 17847

Teuflin, 21224

Triptonolide, 22015

Uncinatone, 22213

\section{$\mathrm{C}_{20} \mathrm{H}_{22} \mathrm{O}_{5}$}

(-)-Chicanine, 3517

Diarylheptanoid CPB-51-262-1, 5373

5-(3"-Hydroxypropyl)-7-methoxy-2-(3',4'dimethoxyphenyl)benzofuran, 10664

Hypopurin B, 10910

Hypopurin C, 10911

Kadsurenin B, 12005

Kadsurenin K, 12007

Machilin F, 13293

Maoecrystal P, 13537

Moxartenolide, 15002

Neoclerodan-5,10-en-19,6 $\beta ; 20,12$-diolide, 15367

Odoratisol D, 15997

Polystachyne A, 17682

Talaumidin, 20651

Wulignan $\mathrm{A}_{1}, 22737$

Wulignan $A_{2}, 22738$

\section{$\mathrm{C}_{20} \mathrm{H}_{22} \mathrm{O}_{6}$}

3-O-Angeloylhamaudol , 1211

Baenzigeride A, 2098

Chaetoquadrin G, 3446

Chaetoquadrin $\mathrm{H}, 3447$

Clemaphenol A, 3818

Columbin, 3939

Dihydroconiferyl ferulate, 5565

Dihydrocubebin, 5572

(+)-Epipinoresinol, 7000

Eupachinilide D, 7543

2-Hydroxy-3,2'-dimethoxy-4'-(2,3-epoxy-1hydroxypropyl)-5-(3-hydroxy-1-propenyl) biphenyl, 10021

6 $\beta$-Hydroxyteuscordin, 10762

Isobutyryl shikonin, 11300

Isocolumbin, 11338

Isomurranganone senecioate, 11550

Jamesoniellide I, 11813 
Matairesinol, 13594

Miroestrol, 14883

Multiradiatin, 15073

Multisatin, 15074

Negundin B, 15323

(+)-Pinoresinol, 17409

Polystachyne B, 17683

Polystachyne C, 17684

Polystachyne D, 17685

4aH-Polystachyne D, 17686

Pygmaeocin A, 18232

Saururin A, 19419

Sigmoidin B, 19884

Spiraformin A, 20189

4,6,4'-Trihydroxy-2,3'-dimethoxy-3-prenylbenzophenone, 21715

Triptonide, 22013

Triptotin A, 22019

\section{$\mathrm{C}_{20} \mathrm{H}_{22} \mathrm{O}_{7}$}

Angeliticin A, 1195

$3 \beta$-Angeloyloxy-10 $\beta$-hydroxyeremophil8(9),7(11)-diene-12,8(14 $\beta, 6 \alpha)$-diolide, 1224

\section{Budlein A, 2698}

Chasmanthin, 3484

(-)-3'-O-Demethyl-5-methoxymatairesinol, 5085

Diffractaic acid, 5507

(2R,3S)-Dihydro-2-(3',5'-dimethoxy-4'hydroxyphenyl)-7-methoxy-5-acetyl-benzofuran, 5601

Elephantin, 6750

Eupachinilide G, 7546

(+)-1-Hydroxy-2,6-bis-epi-pinoresinol, 9854

2'-Hydroxy-3,4,3',4',6'-pentamethoxychalcone, 10593

(+)-1-Hydroxypinoresinol, 10652

$5 \alpha$-Hydroxytriptonide, 10815

16-Hydroxytriptonide, 10816

17-Hydroxytriptonide, 10817

3'-Isobutyryloxy-O-acetyl-2',3'-dihydro-oroselol, 11297

Ledebouriellol, 12601

(+)-Nortrachelogenin, 15805

(-)-Nortrachelogenin, 15806

Ophiopogonanone F, 16141

Palmarin, 16551

(2S)-5,6,7,3',4'-Pentamethoxyflavanone, 16865

(2S)-5,6,7,8,4'-Pentamethoxyflavanone, 16866

Plaunol D, 17551

Pumilin, 18198

$\mathrm{C}_{20} \mathrm{H}_{22} \mathrm{O}_{8}$

Abruquinone B, 24

Anticancer Stilbenoid PMV70P691-142, 1446 Anticancer Stilbenoid PMV70P691-146, 1447
CPB-53-641-1, 4208

4,8-Dihydroxyepipinoresinol, 5871

Jamesoniellide K, 11814

Jamesoniellide L, 11815

Piceid, 17283

Populin, 17720

Prinsepiol, 17861

Resveratrol-10- $C$ - $\beta$-glucopyranoside, 18649

Resveratrol-4'- $O$ - $\beta$ - $D$-glucoside, 18650

Samaderolactone A, 19222

Spiramongolin, 20196

Tremuloidin, 21510

$\mathrm{C}_{20} \mathrm{H}_{22} \mathrm{O}_{9}$

Astringin, 1951

Benzyl 2-O- $\beta$ - $D$-glucopyranosyl-2,6-dihydroxybenzoate, 2288

2-Carboxyl-3,4'-dihydroxy-5- $\beta$ - $D$-xylopyranosyloxybibenzyl, 3175

Isoeleutherol glucoside, 11409

Oxyresveratrol 2-O- $\beta$ - $D$-glucopyranoside, 16471

Oxyresveratrol 3'-O- $\beta$ - $D$-glucopyranoside, 16472

Piceatannol 4'- $O$ - $\beta$ - $D$-glucopyranoside, 17281

Salireposide, 19192

2,3,5,4'-Tetrahydroxystilbene-2- $O$ - $\beta$ - $D$-glucoside, 21157

3,4,3',5'-Tetrahydroxystilbene-3-glucoside, 21158

Trichocarpin, 21557

\section{$\mathrm{C}_{20} \mathrm{H}_{22} \mathrm{O}_{10}$}

Benzyl 6'-O-galloyl- $\beta$ - $D$-glucopyranoside, 2286

Catechin 7-O- $\beta$ - $D$-xyloside, 3317

p-Hydroxybenzoyl calleryanin, 9820

6-Methoxy-2-acetyl-3-methyl-1,4-naphthoquinone-8-O- $\beta$ - $D$-glucopyranoside, 13833

Plicatic acid, 17561

Rumexoside, 19066

2,4',6-Trihydroxy-4-methoxybenzophenone-2$O$-glucoside, 21766

\section{$\mathrm{C}_{20} \mathrm{H}_{22} \mathrm{O}_{11}$}

3-Hydroxy-5-methylphenol

1-O- $\beta$ - $D$-(6'-galloyl)glucopyranoside, 10513

Protocatechuoyl calleryanin, 17969

$\mathrm{C}_{20} \mathrm{H}_{23} \mathrm{ClO}$

Fritillaziebinol, 7958

$\mathrm{C}_{20} \mathrm{H}_{23} \mathrm{NO}_{2}$

4-(3'-Methyl-but-2'-ene)oxy, $N$-benzoyl phenethyl amine, 14174

Zanthosimuline, 22969

$\mathrm{C}_{20} \mathrm{H}_{23} \mathrm{NO}_{3}$

Huajiaosimuline, 9656

Piperamide C 9:3(2E,4E,8E), 17437

Simulenoline, 19907

$\mathrm{C}_{20} \mathrm{H}_{23} \mathrm{NO}_{4}$

Corydalmine, 4104
Corydine, 4106

Corypalmine, 4122

Cularine, 4353

Isocorydine, 11344

Isocorypalmine, 11348

Lirioferine, 12918

(+)- $N$-Methyl laurotetanine, 14550

Norglaucine, 15751

Peroxysimulenoline, 16978

(+)-Sebiferine, 19598

Thaliporphine, 21259

\section{$\mathrm{C}_{20} \mathrm{H}_{23} \mathrm{NO}_{5}$}

Acetylcephalotaxine, 348

Capaurimine, 3115

Delavaine, 4994

C-3-Epiwilsonione, 7043

$N$-trans-4-O-Methylferuloyl

4'-O-methyldopamine, 14445

\section{$\mathrm{C}_{20} \mathrm{H}_{23} \mathrm{NO}_{6}$}

Bujeine, 2733

16-Oxoprometaphanine, 16412

\section{$\mathrm{C}_{20} \mathrm{H}_{23} \mathrm{NO}_{7}$}

Senaetnine, 19698

\section{$\mathrm{C}_{20} \mathrm{H}_{23} \mathrm{NO}_{9}$}

Nigrumin-5-p-coumarate, 15593

\section{$\mathrm{C}_{20} \mathrm{H}_{23} \mathrm{~N}_{3} \mathrm{O}$}

3,3'-Bis(indolylmethyl)dimethyl ammonium hydroxide, 2472

\section{$\mathrm{C}_{20} \mathrm{H}_{23} \mathrm{O}_{9} \mathrm{~S}$}

(+)-Isolariciresinol-2a-sulfate, 11483

\section{$\mathrm{C}_{20} \mathrm{H}_{24} \mathrm{NO}_{4}$}

Magnoflorine, 13374

Steponine, 20325

\section{$\mathrm{C}_{20} \mathrm{H}_{24} \mathrm{NO}_{4}{ }^{+}$}

Cissamine, 3747

Cyclanoline, 4459

2,3-Dimethoxy-9,10-dihydroxy- $N$-methyltetrahydroprotoberberine quaternary salt, 6219

Laurifoline, 12571

Phellodendrine, 17065

Trilobinine, 21888

(R)-Zizyphusine, 23015

\section{$\mathrm{C}_{20} \mathrm{H}_{24} \mathrm{~N}_{2} \mathrm{O}$}

Affinisine, 669

Dihydrokoumine, 5661

19-(Z)-Taberpsychine, 20579

\section{$\mathrm{C}_{20} \mathrm{H}_{24} \mathrm{~N}_{2} \mathrm{O}_{2}$}

Affinisine oxindole, 670

Aglaiduline, 737

Akagerine, 817

Cyclocapitelline, 4479

$N$-Desmethoxyhumantenine, 5253

Epiquinidine, 7003 
Epiquinine, 7004

19-(R)-Hydroxydihydrokoumine, 10003

19-(S)-Hydroxydihydrokoumine, 10004

Isocyclocapitelline, 11363

Lochnerine, 12946

Quinicine, 18422

Quinidine, 18423

Quinine, 18425

20(S)-Tubotaiwine, 22092

\section{$\mathrm{C}_{20} \mathrm{H}_{24} \mathrm{~N}_{2} \mathrm{O}_{3}$}

Echitamidine, 6698

Gelsemamide, 8255

19-(R)-Hydroxydihydrogelsemine, 10000

Lochneridine, 12945

Naucleamide A, 15292

Naucleamide B, 15293

Rankinidine, 18543

19,20-(E)-Vallesamine, 22327

\section{$\mathrm{C}_{20} \mathbf{H}_{24} \mathbf{N}_{2} \mathbf{O}_{4}$}

Amsonic acid, 1088

11-Hydroxyrankinidine, 10671

6,7-Seco-19,20-epoxyangustilobine B, 19612

\section{$\mathrm{C}_{20} \mathrm{H}_{\mathbf{2 4}} \mathbf{N}_{\mathbf{2}} \mathrm{O}_{5}$}

GS-2, 9017

\section{$\mathrm{C}_{20} \mathbf{H}_{24} \mathbf{N}_{2} \mathrm{O}_{6}$}

GS-3, 9018

\section{$\mathrm{C}_{20} \mathrm{H}_{24} \mathrm{O}_{2}$}

5-Methoxy-1,7-diphenyl-3-heptanone, 13921

Saprirearine, 19350

\section{$\mathrm{C}_{20} \mathrm{H}_{24} \mathrm{O}_{3}$}

3-Acetoxy-17-oxo-estra-1,3,5(10)-triene, 273

Broussonin C, 2649

1-(2,4-Dihydroxy-3-prenylphenyl)-3-(4-hydroxyphenyl)-propane, 6103

15,16-Epoxy-neo-clerodan-1,3,13(16),14-tetraen18,19-olide, 7176

15,16-Epoxy-5,10-seco-clerodan-1(10),2,4, 13(16),14-pentaen-18,19-olide, 7199

Gravelliferone methyl ether, 8989

(3S,5R)-3-Hydroxy-5-methoxy-1-(4-hydroxyphenyl)-7-phenyl-6E-heptene, 10411

(3S,5S)-3-Hydroxy-5-methoxy-1-(4-hydroxyphenyl)-7-phenyl- $6 E$-heptene, 10412

Hypolide, 10899

Jatrophone, 11850

3-Oxomicrostegiol, 16394

1-Oxo-salvibretol, 16415

3-Oxosaprorthoquinone, 16417

Pygmaeocin C, 18234

Yakuchinone A, 22876

$\mathrm{C}_{20} \mathrm{H}_{24} \mathrm{O}_{4}$

Anwulignan, 1466

Collinin, 3926
$\alpha$-Crocetin, 4245

Dehydrokerlin, 4937

Diarylheptanoid CPB-51-262-2, 5374

$\alpha, \alpha^{\prime}$-Dihydro-3,5,3'-trihydroxy-4'-methoxy-5'isopentenylstilbene, 5731

15,16-Epoxy-12-oxo-8(17),13(16),14-entlabdatrien-20,19-olide, 7183

15,16-Epoxy-12-oxo-8(17),13(16),14-entlabdatrien-19,20-olide, 7184

(+)-Guaiacin, 9020

5-Hydroxy-7-(4"-hydroxy-3"-methoxy-phenyl)1-phenyl-3-heptanone, 10191

Hypopurin A, 10909

(-)-Isoguaiacin, 11447

3-Keto-4-hydroxysaprorthoquinone, 12202

6-Methoxy aurapten, 13846

Obtusanal B, 15897

Ovatodiolide, 16280

3-Oxoisotaxodione, 16363

3-Oxosapriparaquinone, 16416

Taiwaniaquinone D, 20636

Vernoflexin, 22407

\section{$\mathrm{C}_{20} \mathrm{H}_{24} \mathrm{O}_{5}$}

$3 \beta$-Angeloyloxyeremophil-7,11-dien-14 $\beta, 6 \alpha$ olide, 1220

Candelabroquinone, 3065

Coleon U quinone, 3921

Coniferyl diangelate, 3987

Cornutin L, 4075

Epi-eriocalyxin A, 6908

Epoxycollinin, 7063

Eriocalysin A, 7267

Eriocalysin B, 7268

Fercomin, 7756

Galdosol, 8090

5R-Hydroxy-7-(4-hydroxy-3-methoxyphenyl)1-(4-hydroxyphenyl)-3-heptanone, 10186

$5 S$-Hydroxy-7-(4-hydroxy-3-methoxyphenyl)1-(4-hydroxyphenyl)-3-heptanone, 10187

5R-Hydroxy-1-(4-hydroxy-3-methoxyphenyl)-

7-(4-hydroxyphenyl)-3-heptanone, 10188

5 $\xi$-Hydroxy-1-(4-hydroxy-3-methoxyphenyl)-

7-(4-hydroxyphenyl)-3-heptanone, 10189

3-Hydroxy-4-methyl-2-(7'-piperonyl- $n$-heptyl)butenolide, 10515

Laxiflorin B, 12584

Laxiflorin D, 12586

Laxiflorin J, 12587

Longirabdolactone, 12971

Machilin C, 13291

Machilin D, 13292

Macrocalyxoformin B, 13312

Nectandrin B, 15319
Odoratisol B, 15995

Odoratisol C, 15996

Ravidin A, 18559

Ravidin B, 18560

Schinilenol, 19469

Schininallylol, 19470

Sculponeatin J, 19579

Shikonofuran D, 19824

\section{$\mathrm{C}_{20} \mathrm{H}_{24} \mathrm{O}_{6}$}

$3 \beta$-Angeloyloxy- $8 \beta H$-eremophil-7(11)-ene-

$12,8 \alpha(14 \beta, 6 \alpha)$-diolide, 1221

Bacchariol, 2080

Burselignan, 2765

Candesalvolactone, 3066

Chaetoquadrin A, 3440

Chaetoquadrin B, 3441

Chaetoquadrin C, 3442

Cordato-oblongic acid, 4038

Dihydrodehydrodiconiferyl alcohol, 5582

( $\left.2 S, 3 S, 1^{\prime} S, 2^{\prime} R\right)$-and $\left(2 S, 3 S, 1^{\prime} R, 2^{\prime} R\right)$-2,3-Dihydro5-(1',2'-dihydroxypropyl)-2-(4-hydroxy-3-methoxyphenyl)-7-methoxy-3-methylbenzofuran, 5597

(2R-trans)-2,3-Dihydro-2-(4-hydroxy-3methoxyphenyl)-3-(hydroxymethyl)-7methoxy-5-benzofuran-propanol, 5640

(7,8-cis-8,8'-trans)-2',4'-Dihydroxyl-3,5dimethoxyl-lariciresinol, 5948

3-( $\alpha, 4$-Dihydroxy-3-methoxybenzyl)-4-(hydroxy-

3-methoxybenzyl) tetrahydrofuran, 5967

7-(2',6'-Dihydroxy-7'-methyl-3'-methyleneocta-

7'-en-1-oxy)-8-methoxycoumarin, 6040

$8 \alpha, 9 \alpha$-Epoxycoleon U-quinone, 7062

12(S)-15,16-Epoxy-19-hydroxy-neo-cleroda-

13(16),14-dien-18,6 $\alpha: 20,12$-diolide, 7124

3 $\alpha, 4 \alpha$-Epoxyrupicolin C, 7195

Eupalinilide B, 7568

Euponin, 7621

Fastigilin C, 7740

Helivypolide D, 9324

5-Hydroxy-1-(4-hydroxy-3-methoxyphenyl)-7-

(3,4-dihydroxyphenyl)heptan-3-one, 10184

$8 \beta$-(4'-Hydroxytigloyloxy)-2 $\beta$-hydroxy- $1 \alpha H, 5 \alpha H$, $6 \beta H, 7 \alpha H$-guai-3,10(14),11(13)-trien-6,12-olide, 10767

Isocordatooblongic acid, 11341

Isolariciresinol, 11477

(+)-Lariciresinol, 12520

(-)-Lariciresinol, 12521

Ludongnin A, 13066

Maoecrystal N, 13535

5-O-Methylhirsutanonol, 14489

Minumicrolin, 14874 
Molephantinin, 14897

Multigilin, 15071

Muricarpone A, 15080

1-Oxo-6 $\beta$-senecioyloxy- $8 \alpha$-hydroxyeremophil-

7(11),9-(10)-dien-8 $\beta$ (12)-olide, 16419

Paniculatin, 16606

Paniculonol isovalerate, 16617

(2S)-7,8,3',4',5'-Pentamethoxyflavan, 16864

Sculponeatin A, 19574

Sculponeatin C, 19576

Teucrin $\mathrm{H}_{2}, 21213$

Tripdiotolnide, 21988

Triptolide, 22011

\section{$\mathrm{C}_{20} \mathrm{H}_{24} \mathrm{O}_{7}$}

Ailanthone, 777

Angelol, 1197

Angelol D, 1198

Angelol K, 1200

$3 \beta$-Angeloyloxy- $8 \beta$-hydroxyeremophil-7(11)-

ene-12,8 $\alpha(14 \beta, 6 \alpha)$-diolide, 1226

(+)-Cycloolivil, 4521

Deacetylpseudolaric acid $\mathrm{C}_{2}, 4770$

eythro-2,2'-Dimethoxy-4-(3-hydroxy-1-

propenyl)-4'-(1,2,3-trihydroxypropyl) diphenyl

ether, 6252

threo-2,2'-Dimethoxy-4-(3-hydroxy-1-

propenyl)-4'-(1,2,3-trihydroxypropyl) diphenyl

ether, 6253

Diosbulbin A, 6434

2-Epitripdiolide, 7029

Eupachinilide B, 7541

Eupalinilide C, 7569

Euparotin, 7579

Eupatundin, 7595

trans-Grandmarin isovalerate, 8981

Helivypolide E, 9325

(7'R)-7'-Hydroxylariciresinol, 10314

16-Hydroxytriptolide, 10814

Isoangelol, 11221

Neoolivil, 15440

(-)-Olivil, 16088

8-Prenylated coumarin microfalcatin isovalerate, 17824

Shinjudilactone, 19826

Tripdiolide, 21987

Triptolidenol, 22012

$\mathrm{C}_{20} \mathrm{H}_{24} \mathrm{O}_{8}$

Bis(4-hydroxybenzyl)ether mono- $\beta$ - $D$-glucopyranoside, 2459

6-O-cis- $p$-Coumaroyl-1 $\beta$ - $O$-methylovatofuranic acid methyl ester, 4175

6-O-trans-p-Coumaroyl-1 $\beta$-O-methylovatofuranic acid methyl ester, 4176
erythro-Dihydroxydehydrodiconiferyl alcohol, 5802

threo-Dihydroxydehydrodiconiferyl alcohol, 5803

10-Epieupatoroxin, 6913

Epivernodalol, 7032

Eupalinilide G, 7573

Eupatoroxin, 7594

1-Keto-3,10-epoxy-11 $\alpha$-methoxymethyl- $8 \beta$ - $O$ methacryloyl-15-hydroxy-2,4-germacradiene, 6 $\alpha, 12$-olide, 12199

Massoniresinol, 13588

Peroxyeupahakonin A, 16971

Peroxyeupahakonin B, 16972

$8 \beta$-Tigloyloxy-2,3-seco- $6 \beta H, 7 \alpha H$-helianga-

$4 Z, 11$ (13)-diene-3,10 $\beta ; 6,12$-diolid-2-oic acid, 21380

Vernodalol, 22406

\section{$\mathrm{C}_{20} \mathrm{H}_{24} \mathrm{O}_{8} \mathrm{~S}$}

Shogasulfonic acid B, 19849

\section{$\mathrm{C}_{20} \mathrm{H}_{24} \mathrm{O}_{9}$}

Abruquinone G, 26

6-O-(trans-Cinnamoyl)-1-O-(4"-hydroxy-3"methylfuran-2"-one)- $\beta$ - $D$-glucopyranose, 3707

\section{Ginkgolide A, 8404}

6-O-(4'-Hydroxy-2'-methylene-butyryl)-1-Ocis-cinnamoyl- $\beta$ - $D$-glucopyranose, 10490

6-O-(4'-Hydroxy-2'-methylene-butyryl)-1-Otrans-cinnamoyl- $\beta$ - $D$-glucopyranose, 10491

Marmesinin, 13572

7-O-Methylaloeresin, 14129

Nodakenin, 15647

Pasakbumin A, 16693

Praeroside IV, 17759

Praeroside V, 17760

Torachrysone-8- $O$ - $\beta$ - $D$-glucoside, 21453

Uvaribonol E, 22294

\section{$\mathrm{C}_{20} \mathrm{H}_{24} \mathrm{O}_{9} \mathrm{~S}$}

Hallactone B, 9197

\section{$\mathrm{C}_{20} \mathrm{H}_{24} \mathrm{O}_{10}$}

Apterin, 1536

Celereoside, 3376

3,4-Dihydro-1,2-secomicrominutinin-9-Oglucoside, 5710

Ginkgolide B, 8405

Ginkgolide J, 8407

Ginkgolide M, 8408

(3R')-Hydroxymarmesin 4'- $O$ - $\beta$ - $D$-glucopyranoside, 10362

Isorutarin, 11688

Oxymarmesinin 5'- $O-\beta$ - $D$-glucopyranoside, 16450

Pasakbumin B, 16694
Picrodendrin B, 17333

Praeroside II, 17757

Praeroside III, 17758

Rutarin, 19086

Uncinoside B, 22216

Yunngnoside B, 22956

$\mathrm{C}_{20} \mathrm{H}_{24} \mathrm{O}_{11}$

Ginkgolide C, 8406

$\mathrm{C}_{20} \mathrm{H}_{24} \mathrm{O}_{12}$

6'-Acetyl asperuloside, 325

Apiosylskimmin, 1523

$O$ - $[\beta$ - $D$-Xylopyranosyl $(1 \rightarrow 6) \beta$ - $D$-glucopyranosyl] 7-hydroxycoumarin, 22829

\section{$\mathrm{C}_{20} \mathrm{H}_{25} \mathrm{ClO}_{6}$}

Eupalinilide E, 7571

Tripchlorolide, 21986

\section{$\mathrm{C}_{20} \mathrm{H}_{25} \mathrm{ClO}_{7}$}

Eupachinilide C, 7542

Eupalinilide A, 7567

Eupalinilide H, 7574

\section{$\mathrm{C}_{20} \mathrm{H}_{25} \mathrm{ClO}_{8}$}

Eupachinilide E, 7544

Eupachloroxin, 7552

\section{$\mathrm{C}_{20} \mathrm{H}_{25} \mathrm{NO}$}

Spirasine IV, 20197

Spirasine IX, 20198

\section{$\mathrm{C}_{20} \mathrm{H}_{25} \mathrm{NO}_{2}$}

Spiradine A, 20175

\section{$\mathrm{C}_{20} \mathrm{H}_{25} \mathrm{NO}_{3}$}

3-Epifortuneine, 6916

Fortuneine, 7929

Hetisinone, 9472

Lemairamide, 12611

Leonticine, 12641

Piperamide C 9:2(2E,8E), 17436

Retrofractamide A, 18657

Simulansine, 19906

\section{$\mathrm{C}_{20} \mathrm{H}_{25} \mathrm{NO}_{4}$}

Codamine, 3884

Epiwilsonine, 7041

C-3-Epiwilsonine, 7042

Erythroculine, 7337

Fuzitine, 8043

$d l$-Laudanidine, 12561

Laudanidine, 12562

Wilsonine, 22696

\section{$\mathrm{C}_{20} \mathrm{H}_{25} \mathrm{NO}_{5}$}

$8 \alpha$-Ethoxyprecriwelline, 7414

Hernandoline, 9447

Homostephanoline, 9622

Prometaphanine, 17908

\section{$\mathrm{C}_{20} \mathrm{H}_{25} \mathrm{NO}_{6}$}

Seneciphyllinine, 19713 
$\mathrm{C}_{20} \mathrm{H}_{25} \mathrm{NO}_{7}$

Eupachlorin, 7550

\section{$\mathrm{C}_{20} \mathrm{H}_{25} \mathrm{~N}_{2} \mathrm{O}$}

C-Alkaloid O, 905

Macusine B, 13335

$\mathrm{C}_{20} \mathrm{H}_{25} \mathrm{~N}_{2} \mathrm{O}^{+}$

Mavacurine, 13615

$\mathrm{C}_{20} \mathrm{H}_{25} \mathrm{~N}_{2} \mathrm{O}_{3}$

Verticillatine, 22427

$\mathrm{C}_{20} \mathrm{H}_{26} \mathrm{NO}_{3}^{+}$

Petaline, 17007

$\mathrm{C}_{20} \mathrm{H}_{26} \mathrm{NO}_{4}^{+}$

Xylopinidine, 22819

$\mathrm{C}_{20} \mathrm{H}_{26} \mathrm{~N}_{2} \mathrm{O}$

Ibogaine, 10937

Tabernanthine, 20577

\section{$\mathrm{C}_{20} \mathrm{H}_{26} \mathrm{~N}_{2} \mathrm{O}_{2}$}

Ajmaline, 784

Alstomicine, 997

Hydroquinidine, 9738

Hydroquinine, 9739

Isoajmaline, 11202

\section{$\mathrm{C}_{20} \mathrm{H}_{26} \mathbf{N}_{\mathbf{2}} \mathbf{O}_{\mathbf{4}}$}

Gelselegine, 8254

Gelsemicine, 8256

20-Hydroxydihydrorankinidine, 10010

\section{$\mathrm{C}_{20} \mathrm{H}_{26} \mathrm{O}_{2}$}

8,11,13,15-Abietatetraen-19-oic acid, 5

Hedychenone, 9277

ent-9(11),16-Kauradiene-12,15-dione, 12167

Microstegiol, 14846

Przewalskin D, 18010

\section{$\mathrm{C}_{20} \mathrm{H}_{26} \mathrm{O}_{3}$}

Cryptoquinone, 4291

6,7-Dehydroroyleanone, 4965

Dichroanal B, 5433

6,12-Dihydroxy-5,8,11,13-abietetraen-7-one, 5747

Fukujusonorone, 7993

Helioscopinolide E, 9314

ent-2-Hydroxyatis-1,16(17)-dien-3,14-dione, 9805

2-Hydroxy-1,15-beyeradiene-3,12-dione, 9852

7-Hydroxyhedychenone, 10157

4-Hydroxysaprorthoquinone, 10691

Jatrophatrione, 11848

Mandarone A, 13477

Neonootkatol, 15438

7-Oxodehydroabietic acid, 16307

Oxyphyllacinol, 16461

Potamogetonin, 17742

Senecioyloxyeuryopsin, 19708

Taiwaniaquinone H, 20640
Taxodione, 20809

Triptinin B, 21994

Xanthoperol, 22770

Yunnancoronarin D, 22948

$\mathrm{C}_{20} \mathrm{H}_{26} \mathrm{O}_{4}$

(-)-Bonyl ferulate, 2545

Carnosol, 3206

Caudicifolin, 3330

Cornutin I, 4072

Crotohalimoneic acid, 4269

Cynajapogenin A, 4551

2,19;15,16-Diepoxy-neo-clerodan-3,13(16),14-

trien-18-oic acid, 5497

(-)-Dihydroguaiaretic acid, 5624

Forrestiin A, 7914

Helioscopinolide C, 9313

Heteronone A, 9468

12(S)-Hydroxy-15,16-epoxy-8(17),13(16),14-entlabdatrien-20,19-olide, 10087

1-(4-Hydroxy-3-methoxyphenyl)-7-phenyl-3,5heptanediol, 10450

13-Hydroxy-15-oxozoapatlin, 10588

Hyperguinone B, 10885

Hypopurin D, 10912

Liangshanin A, 12739

Neocaesalpin I, 15350

Taiwaniaquinone A, 20635

Torreyayunnin, 21465

\section{$\mathrm{C}_{20} \mathrm{H}_{26} \mathrm{O}_{5}$}

6 $\beta$-Angeloyloxy- $8 \alpha$-hydroxyeremophil1(10),7(11)-dien-8 $\beta(12)$-olide, 1225

\section{Caesalmin G, 2869}

Candelabrone, 3064

Coleon T, 3918

Coleon U, 3919

Cornutin E, 4068

Deacetylpseudolaric acid A, 4769

Eupaglehnin C, 7562

Guidongnin B, 9081

Inflexusin B, 11056

Isodocarpin, 11382

Laxiflorin A, 12583

Laxiflorin C, 12585

Laxiflorin L, 12589

Leonotinin, 12640

Longirabdacetal, 12970

Macrocalyxoformin A, 13311

Maoecrystal C, 13525

Maoyecrystal I, 13544

3 $\beta$-O-(2-Methylbutyryl)moroccolide A, 14195

Microhelenin C, 14836

Myristargenol B, 15202

Nobilin, 15637
4,7-Oxycycloanisomelic acid, 16444

Rabdoserrin A, 18484

Rosmadial, 18921

Taiwaniaquinol F, 20634

Trichorabdal A, 21563

Xerophilusin B, 22787

\section{$\mathrm{C}_{20} \mathrm{H}_{26} \mathrm{O}_{6}$}

Acanthospermolide, 90

Ailantinol F, 779

$3 \beta$-Angeloyloxy- $6 \beta, 10 \beta$-dihydroxyeremophila-

7(11),8(9)-dien-8,12-olide, 1215

Candesalvone B, 3067

Deacetyleupaserrin, 4739

11-Dehydroklaineanone, 4938

Ememodin, 6770

Enmein, 6804

12-Epi-11-dehydroklaineanone, 6888

Epinodosin, 6975

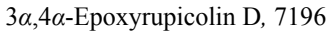

$3 \alpha, 4 \alpha$-Epoxyrupicolin E, 7197

Fastigilin B, 7739

Guidongnin, 9080

Guidongnin C, 9082

Heliangin, 9291

1,10-Hydrobahia, 9707

13-Hydroxy ballonigrinolide, 9814

Laxiflorin K, 12588

Leptocarpin, 12671

Macrocalin B, 13304

Macrocalyxin E, 13308

$3 \beta$-(2'-Methylbutanoyloxy)-8 $\beta H$-eremophil-

7(11)-ene-12,8 $\alpha$-(14,6 $\alpha)$-diolide, 14169

(-)-Methyl 16-hydroxy-19-nor-2-oxo-cis-

cleroda-3,13-dien-15,16-olide-20-oate, 14517

Mollisorin B, 14900

Montanin D, 14946

Murrayatin, 15121

Nodosin, 15652

Parvifoline B, 16685

Phyllostachysin A, 17226

Ponicidin, 17717

Rubescensin D, 18976

Sauriol A, 19415

Schinindiol, 19471

Sculponeatin B, 19575

(-)-Secoisolariciresinol, 19618

$3 \beta$-Senecioyloxy- $1 \beta, 10 \beta$-epoxy- $8 \alpha$-hydroxyeremophil-7(11)-en-8 $\beta(12)$-olide, 19707

Shikodonin, 19811

$7 \beta, 11 \beta, 14 \beta, 20$-Tetrahydroxy-ent-kaur-16-en6,15-dione, 21123

$8 \beta$-Tigloyloxy-3 $\beta, 14$-dihydroxy- $6 \beta H, 7 \alpha H$ germacra-1(10)Z,4E,11(13)-trien-6,12-olide, 
21367

Trichorabdal F, 21568

Triptotin B, 22020

Xerophilusin D, 22789

$\mathrm{C}_{20} \mathrm{H}_{26} \mathrm{O}_{7}$

$8 \alpha$-Angeloxy- $1 \beta, 2 \beta: 4 \beta, 5 \beta$-diepoxy- $10 \beta$-hydroxy$6 \beta H, 7 \alpha H, 11 \beta H-12,6 \alpha$-guaianolide, 1201

$8 \alpha$-Angeloxy- $4 \alpha, 10 \beta$-dihydroxy-2-oxo$6 \beta H, 7 \alpha H, 11 \beta H$-1(5)-guaien-12,6 $\alpha$-olide, 1202

$6 \beta$-Angeloyloxy- $8 \beta, 10 \beta$-dihydroxy-3-oxoeremophilenolide, 1218

Anpubesol, 1349

Chaparrinone, 3481

Cnicin, 3853

3-Deacetyleupalinin A, 4738

8,10-Diacetoxy-2-methoxy-3,11(13)guaiadiene-12,6-olide, 5314

$8 \alpha$-(3,4-Dihydroxy-2-methylene-butanoyloxy)dehydro-melitensin, 6030

Ememogin, 6771

13,14-Epoxide 9,11,12-trihydroxytriptolide, 7045

Eupachinilide A, 7540

Eupaformosanin, 7559

Eupahyssopin, 7566

Eupatoriopicrin, 7593

1-(4-Hydroxy-3-methoxyphenyl-2-[4- $(\omega-$ hydroxypropyl)-2-methoxyphenoxy]propane1,3-diol, 10449

$8 \beta$-(4'-Hydroxytigloyloxy)-3 $\beta, 14$-dihydroxy$6 \beta H, 7 \alpha H$-germacra-1(10)Z,4E,-11(13)-trien-6, 12-olide, 10766

Longirabdolide C, 12972

Niveusin C, 15634

Plagiochiline U, 17497

Teulolin A, 21226

Teulolin B, 21227

Triptriolide, 22027

\section{$\mathrm{C}_{20} \mathrm{H}_{26} \mathrm{O}_{8}$}

Anticancer Simaroubolide PMV70P691-137, 1441

Bruceene, 2663

Bruceine G, 2671

Eupalinilide F, 7572

Glaucarubolone, 8510

Malacitanolide, 13416

8 $\alpha$-Propionyloxyanthemolide C, 17932

Triptotetraolide, 22018

\section{$\mathrm{C}_{20} \mathrm{H}_{26} \mathrm{O}_{9}$}

Bruceine D, 2667

Butyl chlorogenate, 2789

5-O-Caffeoyl quinic acid butyl ester, 2919

Eupalinilide I, 7575

Eurycomanol, 7650
Iandonone, 10934

Pasakbumin C, 16695

Yadanziolide C, 22859

$\mathrm{C}_{20} \mathrm{H}_{26} \mathrm{O}_{10}$

Bruceine H, 2672

Hymenoside V, 10865

Pasakbumin D, 16696

Pectinolide E, 16751

(R)-Peucedanol 7-O- $\beta$ - $D$-glucopyranoside, 17034

Yadanziolide A, 22857

$\mathrm{C}_{20} \mathrm{H}_{26} \mathrm{O}_{11}$

Hymenoside N, 10857

Hymenoside O, 10858

Hymenoside P, 10859

Hymenoside U, 10864

Regaloside B, 18572

Yadanziolide B, 22858

\section{$\mathrm{C}_{20} \mathrm{H}_{26} \mathrm{O}_{12}$}

(Z)-4-Coumaric acid 4- $O-\beta$ - $D$-apiofuranosyl$\left(1^{\prime \prime} \rightarrow 2^{\prime}\right)-O-\beta$ - $D$-glucopyranoside, 4136

Regaloside E, 18574

\section{$\mathrm{C}_{20} \mathrm{H}_{26} \mathrm{O}_{13}$}

5-Methoxyphthalide 7-O- $\beta$-xylopyranosyl$(1 \rightarrow 6)$ - $\beta$-glucopyranoside, 14068

\section{$\mathrm{C}_{20} \mathrm{H}_{27} \mathrm{NO}$}

Spirasine XI, 20199

\section{$\mathrm{C}_{20} \mathrm{H}_{27} \mathrm{NO}_{2}$}

2-(Decan-9-one)- $N$-methyl-4-quinolone, 4836

Spiradine B, 20176

Tangutimine, 20676

Tongolinine, 21445

$\mathrm{C}_{20} \mathrm{H}_{27} \mathrm{NO}_{3}$

3-Epimethylschelhammericine B, 6969

Kusnesoline, 12372

Piperamide C 9:1(8E), 17435

Retrofractamide C, 18659

Tanwusine, 20689

Tatsirine, 20729

$\mathrm{C}_{20} \mathrm{H}_{27} \mathrm{NO}_{4}$

7-Deoxycephalofortuneine, 5160

Guan-fu aminealcohol, 9045

Guan-fu base K, 9049

Isorosmaricine, 11684

Rosmaricine, 18924

Saussureamine B, 19423

$\mathrm{C}_{20} \mathrm{H}_{27} \mathrm{NO}_{5}$

Cephalofortuneine, 3401

Hernandolinol, 9448

$\mathrm{C}_{20} \mathrm{H}_{27} \mathrm{NO}_{6}$

Stephasunoline, 20323

$\mathrm{C}_{20} \mathrm{H}_{27} \mathrm{NO}_{7}$

Erythrozeylanine A, 7347

$\mathrm{C}_{20} \mathrm{H}_{27} \mathrm{NO}_{11}$
Amygdalin, 1102

$\mathrm{C}_{20} \mathrm{H}_{27} \mathrm{~N}_{2} \mathrm{O}_{3}$

$C$-profluorocurine, 4209

$\mathrm{C}_{20} \mathrm{H}_{28}$

(+)-8,11,13,15-Abietatetraene, 4

\section{$\mathrm{C}_{20} \mathrm{H}_{28} \mathrm{~N}_{2} \mathrm{O}_{6}$}

Broussonetine $\mathrm{J}_{1}, 2636$

\section{$\mathrm{C}_{20} \mathrm{H}_{28} \mathrm{O}$}

Abieta-8,11,13-trien-7-one, 9

Coronarin E, 4086

$\Delta^{6}$-Dehydroferruginol, 4916

5,6-Didehydroferruginol, 5467

Pisiferin, 17490

Vitexifolin C, 22577

\section{$\mathrm{C}_{20} \mathrm{H}_{28} \mathrm{O}_{2}$}

Abieta-8,12-dien-11,14-dione, 3

Aritasone, 1731

Barbatusol, 2147

Bornyl cinnamate, 2558

Coronarin A, 4081

Dehydroabietic acid, 4873

3,11-Dioxo-labda-8(17),13(16),14-triene, 6474

4-Epidehydroabietic acid, 6887

12,15-Epoxylabda-8(17),12,14-trien-16-al, 7160

Hispanone, 9560

$7 \beta$-Hydroxyabieta-8,11,13-trien-19-al, 9745

11-Hydroxyabieta-8,11,13-trien-7-one, 9746

ent-1 $\beta$-Hydroxy-9(11),16-kauradien-15-one,

10274

Jolkinolide E, 11893

Jungermannenone A, 11962

Labda-8(17),11,13-trien-15(16)-olide, 12418

Pisiferal, 17486

5,15-Rosadiene-3,11-dione, 18914

Spruceanol, 20235

Sugikurojin A, 20457

Sugiol, 20465

Tagalsin C, 20602

Triptonoterpene, 22016

Velamone, 22363

Yunnancoronarin A, 22946

\section{$\mathrm{C}_{20} \mathrm{H}_{28} \mathrm{O}_{3}$}

Albopetasin, 864

Aldovibsanin B, 881

Anticancer Diterpenoid PMV70P691-011, 1394

Anticancer Diterpenoid PMV70P691-74, 1395

Cafestol, 2886

16-Carboxytotarol, 3185

Crotohalimaneic acid, 4268

$13 \beta, 14 \beta$-Epoxyabiet-7-en-19,6 $\beta$-olide, 7047 ent-15,16-Epoxy-kauran-2,3-dione, 7154

15,16-Epoxy-8(17),13(16),14-ent-labdatrien19-oic acid, 7161 
Furanojaponin, 8019

(+)-Hardwickiic acid, 9230

(-)-Hardwickiic acid, 9231

Helioscopinolide A, 9311

Helioscopinolide B, 9312

12-Hydroxy-6,7-secoabieta-8,11,13-triene-6,7dial, 10696

Isopetasin, 11591

(12R)-Judrpxucascainone, 11896

ent-Kaur-16-en-15-one 18-oic acid, 12183

7-Keto-L-pimara-8(14),15-dien-19-oic acid, 12205

Lambertianic acid, 12455

Leoheteronin A, 12627

Leoheteronin B, 12628

3-Methylbutyryloxyeuryopsin, 14197

Petasin, 17013

Phytocassane A, 17257

Phytocassane D, 17260

Phytocassane E, 17261

Pisiferic acid, 17489

Portlanquinol, 17732

Rabdoumbrosanin, 18509

Rosenonolactone, 18917

Royleanone, 18971

Sarcodonin A, 19358

7,8-Seco-para-ferruginone, 19614

Tagalsin A, 20600

Tagalsin B, 20601

Taiwaniaquinone G, 20639

Taxodone, 20810

Velamolone, 22361

(+)-Vouacapenic acid, 22619

Yunnancoronarin B, 22947

Yunnancoronarin E, 22949

\section{$\mathrm{C}_{20} \mathrm{H}_{28} \mathrm{O}_{4}$}

Aldovibsanin C, 882

6-Angeloylfuranofukinol, 1205

Callicarpone, 2976

(+)-3,13-Clerodadien-16,15-olid-18-oic acid, 3834

Cyrtophyllone B, 4590

Dehydroandrographolide, 4876

3-Dehydrodeoxyandrographolide, 4901

14-Deoxy-11,12-didehydroandrographolide, 5167

Dichroanal A, 5432

8,12-Dihydroxydielmentha-5,9-diene-7,11-dione, 5806

$7 \beta, 12 \alpha$-Dihydroxykaurenolide, 5938

$10 \alpha, 13 \alpha$-Dihydroxy- $9 \alpha$-methyl-15-oxo-20-nor-

kauran-19-oic acid $\gamma$-lactone, 6042

3,12-Dioxo-15,16-epoxy-4-hydroxy-cleroda- 13(16),14-diene, 6467

Ebracteolatanolide A, 6669

Floridiolide A, 7835

Glaucocalyxin A, 8515

Hautriwaic acid, 9249

Hedychilactone C, 9280

Horminone, 9650

Inflexarabdonin I, 11049

8(17),13-ent-Labdadien-15 $\rightarrow$ 16-lactone-19-oic acid, 12409

8(17),13-Labdadien-12,15-olid-19-oic acid, 12411

Leoheteronin C, 12629

Liangshanin D, 12742

Marrubiin, 13574

9,17-Octadecadiene-12,14-diyne-1,11,16-triol, 1-acetate, 15939

Petasitin, 17016

Phlogacantholide B, 17159

Phlogacantholide C, 17160

Pinusolidic acid, 17423

Premarrubiin, 17813

Pseudolaric acid E, 18038

Rabdolatifolin, 18476

ent-8,9-Seco-7 $\alpha, 11$ b-dihydroxykaura-14(14),16dien-9,15-dione, 19611

Taiwaniaquinol B, 20630

Taiwaniaquinol E, 20633

11,12,14-Trihydroxyabieta-8,11,13-trien-7-one, 21679

Umbrosin B, 22202

\section{$\mathrm{C}_{20} \mathrm{H}_{28} \mathrm{O}_{5}$}

Amethystonal, 1038

Coetsoidin A(Huang), 3898

Compound 9 (Isodon umbrosa), 3954

Deacetylfruticolone, 4742

14-Deoxy-11-oxoandrographolide, 5200

Dihydroisodocarpin, 5650

(-)-12,16-Dihydroxy-cis-cleroda-3,13-dien-15oic acid-15,16-olide, 5793

$3 \alpha, 4 \beta$-Dihydroxy-15,16-epoxy-12-oxo-cleroda13(16),14-dien-9-oal, 5878

Effusanin A, 6710

4-Epihenryine, 6929

ent-8S,12S-Epoxy-7R,16-dihydroxyhalima5(10),13-dien-15,16-olide, 7078

Excisanin H, 7681

(-)-6 $\beta$-Hydroxy-5 $\beta, 8 \beta, 9 \beta, 10 \alpha$-cleroda-3,13-dien16,15-olid-18-oic acid, 9922

16-Hydroxy-8(17),13-ent-labdadien-15,16-olid19-oic acid, 10298

$15 \xi$-Hydroxypinusolidic acid, 10656 Ingenol, 11057
Isolongirabdiol, 11511

Leonotin, 12639

Longikaurin A, 12957

Longirabdolide E, 12974

Macrocalyxin D, 13307

Macrocalyxoformin D, 13313

Macrocalyxoformin E, 13314

Megathyrin A, 13649

Neocaesalpin H, 15349

16-Oxo-8(17),13-labdadiene-15,19-dioic acid, 16366

Rabdocoetsin A, 18456

Rabdosichuanin B, 18491

Sibiricinone B, 19859

Taiwaninal, 20642

$1 \alpha, 10 \alpha, 13 \alpha$-Trihydroxy- $9 \alpha$-methyl-15-oxo-

20-nor-kauran-19-oic acid $\gamma$-lactone, 21801

$6 \beta, 10 \alpha, 13 \alpha$-Trihydroxy- $9 \alpha$-methyl-15-oxo-

20-nor-kauran-19-oic acid (19,10)-lactone, 21802

$10 \alpha, 13 \alpha, 17$-Trihydroxy- $9 \alpha$-methyl-15-oxo-

20-nor-kauran-19-oic acid $\gamma$-lactone, 21803

$10 \alpha, 16 \alpha, 17$-Trihydroxy- $9 \alpha$-methyl-15-oxo-

20-nor-kauran-19-oic acid $\gamma$-lactone, 21804

Wikstroemioidin A, 22669

Xerophilusin I, 22793

Xindongnin I, 22805

Xindongnin J, 22806

\section{$\mathrm{C}_{20} \mathrm{H}_{28} \mathrm{O}_{6}$}

Amarolide, 1018

Amethystoic acid, 1037

Anhydrocinnzeylanone, 1265

Blumealactone A, 2518

Blumealactone B, 2519

Coetsoidin F, 3904

Coetsoidin G, 3905

Dihydroenmein, 5605

Effusanin E, 6714

Epinodosinol, 6976

Excisanin F, 7679

Junceellolide H, 11947

Langduin B, 12480

Lasiocarpanin, 12534

Lasiodonin, 12536

Longikaurin G, 12963

LS-1(furanoditerpenelactone), 13014

Oridonin, 16183

13-Oxyingenol, 16448

Phorbol, 17181

Rabdoinflexin A, 18464

Rabdoloxin A, 18478

Rabdonervosin A, 18480

Rabdosichuanin C, 18492 
Rabdoternin A, 18501

Resiniferonol, 18640

Rosthorin A, 18931

$2 \alpha, 10 \alpha, 13 \alpha, 17-T$ etrahydroxy- $9 \alpha$-methyl-15oxo-20-nor-kauran-19-oic acid $(19,10)$ -

lactone, 21136

$10 \alpha, 13 \alpha, 16 \alpha, 17-$ Tetrahydroxy- $9 \alpha$-methyl-15oxo-20-nor-kauran-19-oic acid $\gamma$-lactone, 21137

Xerophilusin C, 22788

$\mathrm{C}_{20} \mathrm{H}_{28} \mathrm{O}_{7}$

$8 \alpha$-Angeloxy- $2 \alpha, 4 \alpha, 10 \beta$-trihydroxy- $6 \beta H, 7 \alpha H$, 11 $\beta H$-1(5)-guaien-12,6 $\alpha$-olide, 1203

2,3-Didehydrocinnzeylanone, 5465

Eupachinilide H, 7547

Gibberellin $\mathrm{A}_{13}, 8376$

15 $\beta$-Hydroxyklaineanone, 10290

$2 \alpha, 10 \alpha, 13 \alpha, 16 \alpha, 17-P e n t a h y d r o x y-9 \alpha$-methyl-

15-oxo-20-nor-kauran-19-oic acid $(19,10)$ lactone, 16854

$3 \alpha, 10 \alpha, 13 \alpha, 16 \alpha, 17-P e n t a h y d r o x y-9 \alpha$-methyl-

15-oxo-20-nor-kaur-an-19-oic acid $\gamma$-lactone, 16855

(2S)-Pteroside B, 18117

Pteroside B, 18118

Rabdoternin B, 18502

$1 \alpha, 7 \beta, 10 \alpha, 13 \alpha$-Tetrahydroxy- $9 \alpha$-methyl-15-

oxo-20-nor-kauran-19-oic acid $\gamma$-lactone,

21135

Trichorabdonin, 21573

\section{$\mathrm{C}_{20} \mathrm{H}_{28} \mathrm{O}_{8}$}

$14 \beta, 15 \beta$-Dihydroxyklaineanone, 5941

$2 \alpha, 3 \alpha, 10 \alpha, 13 \alpha, 16 \alpha, 17-H e x a h y d r o x y-9 \alpha$-methyl-

15-oxo-20-nor-kauran-19-oic acid $(19,10)$ -

lactone, 9504

Leopersin I, 12656

Lobetyolin, 12942

Picrodendrin O, 17335

(2S,3R)-Pteroside C, 18119

Pteroside C, 18120

Pteroside M, 18123

Pteroside P, 18124

Pterosin C-3-O-glucoside, 18139

$2 S, 3 S$-Pterosin C-3-O- $\beta$ - $D$-glucoside, 18142

Sculponeatin D, 19577

\section{$\mathrm{C}_{20} \mathrm{H}_{28} \mathrm{O}_{9}$}

Bruceine E, 2668

13 $\beta, 18$-Dihydroeurycomanol, 5611

Pteroside Q, 18125

Pteroside S, 18126

Pteroside T, 18127

$5 \alpha, 14 \beta, 15 \beta$-Trihydroxyklaineanone, 21759

$\mathrm{C}_{20} \mathrm{H}_{28} \mathrm{O}_{10}$
Bruceine F, 2670

trans-p-Ferulylalcohol-4-O-[6-(2-methyl-3-

hydroxypropionyl)] glucopyranoside, 7790

Hyuganoside II, 10923

Hyuganoside V, 10927

Ptelatoside B, 18100

Pteroside U, 18128

$p$ - $\beta$-Rutinosyloxy styrene, 19088

$\mathrm{C}_{20} \mathrm{H}_{28} \mathrm{O}_{11}$

Adinoside A, 636

13 $\beta, 21$-Dihydroxyeurycomanol, 5893

Hyuganoside IV, 10926

Rossicasin A, 18928

Rossicasin B, 18929

Sibiricaphenone, 19855

$\mathrm{C}_{20} \mathrm{H}_{28} \mathrm{O}_{12}$

Aplopaeonoside, 1524

2-Methoxy-4-acetylphenyl-1- $O-\beta$ - $D$-apiofuranosyl-(1" $\left.\rightarrow 6^{\prime}\right)$ - $\beta$-glucopyranoside, 13834

Paeonolide, 16533

\section{$\mathrm{C}_{20} \mathrm{H}_{28} \mathrm{O}_{13}$}

Erythroxyloside A, 7345

\section{$\mathrm{C}_{20} \mathrm{H}_{28} \mathrm{O}_{14}$}

2,4,6-Trihydroxyacetophenone-2,4-di- $O-\beta$ - $D$ glucopyranoside, 21682

\section{$\mathrm{C}_{20} \mathrm{H}_{29} \mathrm{NO}_{3}$}

O-Methyltaxodine, 14738

\section{$\mathrm{C}_{20} \mathrm{H}_{29} \mathrm{NO}_{4}$}

Saussureamine A, 19422

\section{$\mathrm{C}_{20} \mathrm{H}_{30} \mathrm{~N}_{2} \mathrm{O}_{2}$}

$3 \alpha$-Ureido-androst-4-en-17-one, 22248

\section{$\mathrm{C}_{20} \mathrm{H}_{30} \mathrm{O}$}

Abietatriene- $3 \beta$-ol, 6

Abieta-8,11,13-trien-7 $\beta$-ol, 7

(+)-8,11,13-Abietatrien-12-ol, 8

Casearimene B, 3268

Ferruginol, 7764

$5 \beta$-Hydro-8, 11,13-abietatrien-6 $\alpha$-ol, 9705

ent-16-Kauren-15-one, 12182

Labda-7,12(E),14-triene-17-al, 12414

3-Oxo-labda-8(17),13(16),14-triene, 16369

Retinol, 18656

Stemod-12-en-2-one, 20298

Tagalsin E, 20604

Totarol, 21476

ent-Trachyloban-3-one, 21498

$\mathrm{C}_{20} \mathrm{H}_{30} \mathrm{O}_{2}$

Abietic acid, 10

Acanthoic acid, 79

Agallochin D, 687

Agallochin G, 690

Azorellolide, 2062

Casearimene A, 3267
Cassa-13(14),15-dien-19-oic acid, 3276

Ceriopsin G, 3428

Cryptopimaric acid, 4289

Dolabeserpenoic acid A, 6549

4-Epi-abietic acid, 6817

4-Epi-sandaracopimaric acid, 7010

ent-16,17-Epoxykauran-15-one, 7158

Hinokiol, 9545

$8 \beta$-Hydroxy-9(11),13-abietadien-12-one, 9744

12-Hydroxydehydroabietinol, 9970

$\left(1 S^{*}, 6 S^{*}, 11 S^{*}\right)-6$-Hydroxydolabella-3E,7E,12trien-14-one, 10058

16-Hydroxy ferruginol, 10122

$11 \beta$-Hydroxy-8,15-isopimaradiene-3-one, 10256

$7 \beta$-Hydroxyisopimara-8(14),15-dien-1-one, 10257

$7 \beta$-Hydroxyisopimara-8,15-dien-14-one, 10258

$9 \alpha$-Hydroxyisopimara-8(14),15-dien-7-one, 10259

$14 \alpha$-Hydroxyisopimara-7,15-dien-1-one, 10260

17-Hydroxy-16 $\beta$-ent-kauran-19-al, 10275

13-Hydroxy-16-ent-kauren-19-al, 10281

ent-3 $\alpha$-Hydroxy-16-kauren-15-one, 10286

ent-11 $\alpha$-Hydroxy-16-kauren-15-one, 10287

ent-14 $\alpha$-Hydroxy-16-kauren-15-one, 10288

$3 \alpha$-Hydroxy-11-oxo-labda-8(17),13(16),14-triene, 10568

$3 \beta$-Hydroxy-11-oxo-labda-8(17),13(16),14-triene, 10569

11-Hydroxy-3-oxo-labda-8(17),13(16),14-triene, 10570

$11 \beta$-Hydroxy-7-oxo-rosa-5,15-diene, 10582

$7 \beta$-Hydroxypimara-8,15-dien-14-one, 10649

ent-18-Hydroxy-trachyloban-3-one, 10773

Isopachydictyolal, 11579

Isopimaric acid, 11600

L-Kaur-16-en-19-oic acid, 12178

ent-Kaur-16-en-19-oic acid, 12179

(E)-Labda-8(17),12-diene-15,16-dial, 12403

Labda-8(17),13(14)-dien-15,16-olide, 12410

Labdatriene, 12412

Labda-7,12(E),14-triene-17-oic acid, 12415

Labta-7,13(E)-diene-17,12-olide, 12423

Mulin-11,13-dien-20-oic acid, 15059

Neoabietic acid, 15333

Neodictyolactone, 15391

Neoisodextropimaric acid, 15412

Obtunone, 15891

Obtusadione, 15892

Pachylactone, 16495

Palustric acid, 16572

Perrottetianal, 16979

L-Pimara-8(14),15-dien-19-oic acid, 17371 
Pisiferanol, 17487

Pisiferol, 17491

Salviol, 19219

Sandaracopimaric acid, 19236

3,4-Secoisopi-mara-4(18),7,15-trien-3-oic acid, 19620

Tagalsin F, 20605

Tagalsin G, 20606

Totaradiol, 21474

(+)-8,11,13-Totaratriene-12,13-diol, 21475

Trachyloban-18-oic acid, 21495

Trachyloban-19-oic acid, 21496

\section{$\mathrm{C}_{20} \mathrm{H}_{30} \mathrm{O}_{3}$}

Aframodial, 675

Agallochin F, 689

Agallochin H, 691

Andrograpanin, 1149

Antriptolactone, 1465

Caesalpinin MI, 2879

Coronarin C, 4083

Coronarin D, 4084

Dihydrosolidagenone, 5718

ent-3 $\beta,(13 S)$-Dihydroxyatis-16-en-14-one, 5760

19,20-Dihydroxy-15,16-epoxy-8(17),13(16),14ent-labdatriene, 5875

3 $\beta, 19$-Dihydroxy-8(9),15-isopimaradien-7-one, 5924

16 $\alpha, 17$-Dihydroxy-ent-9(11)-kauren-19-al, 5933

$7 \alpha, 14 \beta$-Dihydroxykaur-16-en-15-one, 5939

ent-7 $\alpha, 18$-Dihydroxykaur-16-en-3-one, 5940

7 $\beta, 17$-Dihydroxystemod-12-en-2-one, 6136

$\left(1 S^{*}, 3 R^{*}, 4 R^{*}, 6 S^{*}, 11 S^{*}\right)$-3,4-Epoxy-6-hydroxy-

dolabella-7E,12-dien-14-one, 7110

ent-15,16-Epoxy-3 $\beta$-hydroxy-kauran-2-one, 7121

(-)-16ק,17-Epoxykauran-19-oic acid, 7156

12,15-Epoxy-8(17),13-labdadien-18-oic acid, 7159

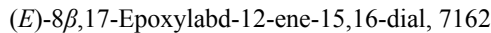

Galanal A, 8079

Galanal B, 8080

Galanolactone, 8082

Hedychilactone A, 9278

Hedychilactone B, 9279

Hispanolone, 9559

16 $\alpha$-Hydro-19-al-ent-kauran-17-oic acid, 9706

(12R)-12-Hydroxy cascarill one, 9897

2- $\alpha$-Hydroxy-cis-cleroda-3,13(Z),8(17)-trien15-oic acid, 9924

(-)-17-Hydroxy-kaur-15-en-19-oic acid, 10285 ent-12R-Hydroxylabda-7,13-dien-15,16-olide, 10296

ent-16-Hydroxylabda-7,13-dien-15,16-olide, 10297
(13R)-13-Hydroxy-8(17),11E, 14-labdatrien-18oic acid, 10303

13-Hydroxylabta-7,14-diene-17,12-olide, 10306

15-Hydroxylabta-7,13(E)-diene-17,12-olide, 10307

$(4 R, 5 S, 8 R, 9 R, 10 S, 13 S)$-ent-17-Hydroxy-16oxobeyeran-19-al, 10559

$7 \alpha$-Hydroxy- $L$-pimara-8(14),15-dien-19-oic acid, 10647

$7 \beta$-Hydroxy- $L$-pimara-8(14),15-dien-19-oic acid, 10648

$3 \beta$-Hydroxysandara copimaric acid, 10688

Hypodiolide A, 10892

Ineketone, 11032

Inflexarabdonin J, 11050

Isocoronarin $\mathrm{D}, 11343$

Leucothol A, 12727

8-Methylandrograpanin, 14137

Mulin-12-ene-14-one-20-oic acid, 15061

15-Oxolabda-8(17),13E-dien-19-oic acid, 16367

15-Oxolabda-8(17),13Z-dien-19-oic acid, 16368

8-Oxo-8,14-seco-abiet-12-en-14,19-dial, 16418

Phytocassane C, 17259

Pisiferdiol, 17488

Polyalthialdoic acid, 17619

Prehispanolone, 17807

Pseudolaric acid D, 18037

Solidagonal acid, 20071

Torreyagrandate, 21464

Tripterfordin, 21990

Vibsanin V, 22457

\section{$\mathrm{C}_{20} \mathrm{H}_{30} \mathrm{O}_{4}$}

Acanthokoreoic acid A, 82

Ballotenic acid, 2132

Bis(2-ethylbutyl)phthalate, 2448

Coronarin B, 4082

Deoxyandrographolide, 5152

$3 \alpha, 4 \beta$-Dihydroxy-15,16-epoxy-12-oxo-cleroda -13(16),14-dien, 5877

16 $\alpha$,17-Dihydroxy-ent-9(11)-kauren-19-oic acid, 5937

ent-2,7-Dioxo-3-cleroden-15-oic acid, 6464

8,19-Dioxo-8,14-seco-chinan-14,11-olide, 6476

Enanderianin P, 6794

Epoxyeleganolactone, 7086

Excisanin C, 7676

Excoecarin $\mathrm{V}_{1}, 7690$

Excoecarin $\mathrm{V}_{2}, 7691$

Fumotoshidin C, 8001

Glaucocalyxin C, 8517

Glaucocalyxin E, 8519

Glutinic acid, 8787
Haplopappic acid, 9222

16 $\alpha$-Hydro-ent-kauran-17,19-dioic acid, 9722

16 $\beta$-Hydro-ent-kauran-17,19-dioic acid, 9723

$5 \beta$-Hydroxy-10 $\alpha$ - $O$-angeloyl-3-oxodauc- 8 -ene, 9785

$10 \beta$-Hydroxyfuranoeremophilan- $6 \beta$-yl-2' $\xi$ methylbutanoate, 10133

$13 S$-Hydroxy-9-oxo-9,10-seco-abiet-8(14)en-18,10 $\alpha$-olide, 10583

Isodopharicin D, 11397

Kamebanin, 12125

Lathyrol, 12543

Leoheterin, 12626

Leoheteronin E, 12631

Leopersin G, 12654

Mebadonin, 13629

13'-(3,4-Methylene-dioxyphenyl)-tridecanoic acid, 14383

Phytocassane B, 17258

Preleoheterin, 17810

Pseurata A, 18075

Rabdokunmin B, 18471

Rabdosinatol, 18498

Sawaradienone, 19429

Umbrosin A, 22201

Wanghzaozin A, 22635

\section{$\mathrm{C}_{20} \mathrm{H}_{30} \mathrm{O}_{5}$}

2-O-Acetyl-5- $O$-methylembelin, 465

Andrographolide, 1159

Ballotenol, 2133

Coetsoidin B, 3900

8-Deacetylleopersin A, 4747

16 $\alpha$,17-Dihydroxy-15-oxo-ent-kaur-19-oic acid, 6066

Ebracteolatanolide B, 6670

Enmelol, 6806

Excisanin A, 7674

Excisanin I, 7682

Excisanin J, 7683

Excisanin K, 7684

Kamebakaurin, 12123

Kamebakaurinin, 12124

Leopersin D, 12650

Leopersin L, 12658

Megathyrin B, 13650

Oreskaurin C, 16178

7-Oxo-12 $\alpha, 13 \beta$-dihydroxyabie-8(14)-en-18-oic acid, 16316

Parvifoline A, 16684

Portulenone, 17737

Pseurata B, 18076

Rabdoinflexin B, 18465

Rabdokunmin C, 18472 


\author{
Rabdokunmin D, 18473 \\ Rabdoloxin B, 18479 \\ Rabdoserrin D, 18486 \\ 13,16 $\alpha, 17$-Trihydroxy-ent-9(11)-kauren-19-oic \\ acid, 21757 \\ Umbrosianin, 22200

\section{$\mathrm{C}_{20} \mathrm{H}_{30} \mathrm{O}_{6}$} \\ Chiromodine, 3538 \\ Coetsanoic acid, 3895 \\ Cornutin C, 4066 \\ Enmenol, 6808 \\ 19-Hydroxygaleopsin, 10135 \\ Ingol, 11063 \\ Leopersin H, 12655 \\ Leopersin P, 12661 \\ Maoecrystal K, 13532 \\ Nervosanin B, 15509 \\ Rabdokunmin E, 18474 \\ Rabdoserrin B, 18485 \\ Rubescensin C, 18975 \\ Sibiricinone A, 19858

\section{$\mathrm{C}_{20} \mathrm{H}_{30} \mathrm{O}_{7}$} \\ Anhydrospiganthol, 1281 \\ 15- $O$-Isovaleroyl-3 $\beta$ - $O$-acetyl-2 $\alpha$-hydroxy- \\ amphoricarpolide, 11756 \\ Parvifolin, 16683
}

\section{$\mathrm{C}_{20} \mathrm{H}_{30} \mathrm{O}_{7} \mathrm{~S}$}

14-Deoxyandrographolide-3-O-sulfate, 5153

\section{$\mathrm{C}_{20} \mathrm{H}_{30} \mathrm{O}_{8}$}

Lushanrubescensin G, 13122

Ptaquiloside, 18098

\section{$\mathrm{C}_{20} \mathrm{H}_{30} \mathrm{O}_{8} \mathrm{~S}$}

Andrographolide-3- $O$-sulfate, 1160

Isoandrographolide-3-O-sulfate, 11220

\section{$\mathrm{C}_{20} \mathrm{H}_{30} \mathrm{O}_{9}$}

Dichotomoside E, 5431

\section{$\mathrm{C}_{20} \mathrm{H}_{30} \mathrm{O}_{10}$}

Phenethyl rutinoside, 17087

\section{$\mathrm{C}_{20} \mathrm{H}_{30} \mathrm{O}_{11}$}

Cistanoside G, 3757

Echipuroside A, 6697

Hedyoside, 9283

1-(1-Hydroxyethyl)-4 $\beta$-rutinosyloxybenzene, 10106

4-Hydroxyphenylethyl 4- $O-\beta$ - $D$-glucopyranosyl$(1 \rightarrow 3)-O-\alpha$ - $L$-rhamnopyranoside, 10620

Phenethylalcohol 8-O- $\beta$ - $D$-glucopyranosyl$(1 \rightarrow 2)-\beta$ - $D$-glucopyranoside, 17082

\section{$\mathrm{C}_{20} \mathrm{H}_{30} \mathrm{O}_{12}$}

Decaffeoylacteoside, 4830

\section{$\mathrm{C}_{20} \mathrm{H}_{30} \mathrm{O}_{13}$}

Forsythoside D, 7927

7-Methoxydiderroside, 13905
Scroside D, 19573

2,4,6-Trimethoxyphenyl 1- $O-\beta-D$-apiofuranosyl$(1 \rightarrow 6)-\beta$ - $D$-glucopyranoside, 21922

$3,4,5$-Trimethoxyphenyl 1-O- $\beta$ - $D$-apiofuranosyl $(1 \rightarrow 6)-\beta$ - $D$-glucopyranoside, 21923

\section{$\mathrm{C}_{20} \mathrm{H}_{30} \mathrm{O}_{15}$}

3'-O- $\beta$-Glucopyranosyl-stilbericoside, 8733

\section{$\mathrm{C}_{20} \mathrm{H}_{31} \mathrm{ClO}_{3}$}

17-Chloro-13,16 $\beta$-dihydroxy-ent-kauran-19-al, 3548

$\mathrm{C}_{20} \mathrm{H}_{31} \mathrm{NO}$

( $E, E, E)-N$-(2-Methylpropyl)-hexadeca-2,6,8trien-10-ynamide, 14692

\section{$\mathrm{C}_{20} \mathrm{H}_{31} \mathrm{NO}_{4}$}

Acetyllycoclavine, 459

4'-Dihydroconsabatine, 5566

\section{$\mathrm{C}_{20} \mathrm{H}_{31} \mathrm{NO}_{6}$}

Myoscorpine, 15145

Symlandine, 20525

Symphytine, 20527

\section{$\mathrm{C}_{20} \mathrm{H}_{31} \mathrm{NO}_{7}$}

Echimidine, 6684

Heliosupine, 9316

Hydroxymyoscorpine, 10535

\section{$\mathrm{C}_{20} \mathrm{H}_{31} \mathrm{NO}_{8}$}

Heliosupine $N$-oxide, 9317

\section{$\mathrm{C}_{20} \mathrm{H}_{32}$}

(+)-Axinyssene, 2045

$\alpha$-Camphorene, 3049

$\gamma$-Camphorene, 3050

Cembrene, 3385

Cosbene, 4124

(-)-Dolabella-3,7,18-triene, 6548

Kaurene, 12176

Labda-7,12(E),14-triene, 12413

Neocembrene, 15360

Stemod-12-ene, 20297

Viscida-3,9,14-triene, 22531

Viscida-3,11(18),14-triene, 22532

$\mathrm{C}_{20} \mathrm{H}_{32} \mathrm{~N}_{2} \mathrm{O}_{2}$

$3 \alpha$-Ureido-androst-4-en-17 $\beta$-ol, 22247

\section{$\mathrm{C}_{20} \mathrm{H}_{32} \mathrm{O}$}

Acanthol, 83

ent-(16S)-Atisan-13,17-oxide, 1963

trans-Communol, 3946

12,13-Dehydrogeranylgeraniol, 4920

ent-16 $\beta, 17$-Epoxy-kaurane, 7155

$6 \alpha$-Hydroxy-3,12E,14-clerodatriene, 9923

(2R)-ent-2-Hydroxyisopimara-8(14),15-diene, 10255

ent-18-Hydroxykaur-16-ene, 10282

ent-11 $\alpha$-Hydroxy-16-kaurene, 10283

ent-15 $\alpha$-Hydroxy-16-kaurene, 10284
3 $\beta$-Hydroxy-labda-8(17),13(16),14-triene, 10302

$11 \beta$-Hydroxy-rosa-5,15-diene, 10678

$2 \alpha$-Hydroxystemod-12-ene, 10729

$2 \beta$-Hydroxystemod-12-ene, 10730

Isopimara-7,15-dien-3 $\beta$-ol, 11595

8(9),15-Isopimaradien-3 $\beta$-ol, 11596

(16R)-ent-Kauran-15-one, 12174

(-)-Kaur-16-en-19-ol, 12180

ent-Kaur-15-en-17-ol, 12181

Labda-7,12(E),14-triene-17-ol, 12417

Marginatol, 13560

L-Pimara-8(14),15-dien-19-ol, 17372

(3R)-ent-1(10),15-Rosadien-3-ol, 18915

Sandaracopimarinol, 19237

ent-Trachyloban-3 $\beta$-ol, 21497

ent-Verticilla-4,9,13-trien-2 $\alpha$-ol, 22429

ent-Verticilla-4(18),9,13-trien-12 $\alpha$-ol, 22430

\section{$\mathrm{C}_{20} \mathrm{H}_{32} \mathrm{O}_{2}$}

Arachidonic acid, 1599

Ardisinol I, 1643

Caesalpinin ML, 2882

Dihydroazorellolide, 5545

(1R,2R)-ent-1,2-Dihydroxyisopimara-8(14),15diene, 5922

$1 \beta, 14 \alpha$-Dihydroxyisopimara-7,15-diene, 5923

ent-11 $\alpha, 15 \alpha$-Dihydroxy-16-kaurene, 5934

ent-14 $\alpha, 15 \alpha$-Dihydroxy-16-kaurene, 5935

ent-15 $\alpha, 18$-Dihydroxy-16-kaurene, 5936

2,3-Dihydroxy-labda-8(17),12(E),14-triene, 5945

$3 \alpha, 19$-Dihydroxy trachylobane, 6156

Eicosanetetraenoic acid, 6721

7-Epicandicandiol, 6851

13-Epi-2-oxo-kolavelool, 6988

$5 \beta, 20$-Epoxy-20-hydroxy-ros-15-ene, 7141

ent-15 $\beta, 16$-Epoxy-kauran-17-ol, 7157

$(3 R, 15 R)$-ent-15,16-Epoxy-1(10)-rosen-3-ol, 7194

Flavidusin B, 7813

13 $\beta$-Hydroxyabiet-8(14)-en-7-one, 9747

$2 \beta$-Hydroxy-9-epi-ent-labda-8(17),13(E)-dien15-al, 10073

$2 \beta$-Hydroxy-9-epi-ent-labda-8(17),13(Z)-dien15-al, 10074

18-Hydroxy-7 $\alpha, 8 \alpha$-epoxy-9-epi-ent-pimara15-ene, 10084

16-Hydroxy-19,20-epoxy-kaurane, 10086 cis-(S)-12-Hydroxygeranylgeranial, 10142 trans-(S)-12-Hydroxygeranylgeranial, 10143 $8 \alpha$-Hydroxy-11E,13Z-labdadien-15-al, 10295 ent-15-Hydroxylabda-8(17),13E-dien-3-one, 10299

8(14),15-Isopimaradien-3 $\beta, 19$-diol, 11594

ent-Kauran-16 $\beta$-ol-3-one, 12173 
16-ent-Kaurene-13,19-diol, 12177

Kolavenic acid, 12257

Liangshanin G, 12745

Lophanthin A, 12991

Lophanthin B, 12992

Oriediterpenol, 16184

Oryzalexin E, 16247

Oryzalexin F, 16248

ent-3-Oxo-13-epi-manoyl oxide, 16326

ent-8(14),15-Sandaracopimaradiene-2 $\alpha, 18$-diol, 19234

ent-8(14),15-Sandaracopimaradiene-2 $\beta, 18$-diol, 19235

Stemodinone, 20301

Tagalsin D, 20603

Trachyloban-7 $\beta, 18$-diol, 21494

Vitetrifolin G, 22574

\section{$\mathrm{C}_{20} \mathrm{H}_{32} \mathrm{O}_{3}$}

Alepterolic acid, 886

1 $\alpha$-Angeloyloxycarotol, 1214

Calliterpenone, 2979

$(13 R, 14 S)$-ent-8 $\alpha, 13 ; 14,15$-Diepoxy-13-epi-

labdan-3-one, 5491

ent-16 $\alpha, 17$-Dihydroxyatisan-3-one, 5759

6 $\beta, 18$-Dihydroxy-7 $\alpha, 8 \alpha$-epoxy-9-epi-ent-

pimara-15-ene, 5872

9 $\beta, 18$-Dihydroxy-7 $\alpha, 8 \alpha$-epoxy-9-epi-ent-

pimara-15-ene, 5873

16ק,17-Dihydroxy-ent-kauran-19-al, 5928

16 $\alpha$,17-Dihydroxy-ent-kauran-19-al, 5929

ent-8 $\alpha, 9 \beta$-Dihydroxylabda-13(16),14-dien-1-one, 5943

ent-16,18-Dihydroxy-8(14)-pimaren-15-one, 6098

6 $\alpha, 13(S)$-Dihydroxystemodan-2-one, 6135

Dunniana acid B, 6638

$9 \alpha, 13 \alpha$-Epi-dioxiabiet-8(14)-en-18-ol, 6897

Excoecarin $\mathrm{V}_{3}, 7692$

(+)-15-Hydroxy-cis-cleroda-3,13-dien-18-oic acid, 9919

2 $\beta$-Hydroxy-9-epi-ent-labda-8(17),13(E)-dien15-oic acid, 10075

(S)-12-Hydroxygeranylgeranic acid, 10144

17-Hydroxy-16 $\beta$-ent-kauran-19-oic acid, 10276

(-)-17-Hydroxy-16 $\beta$-kauran-19-oic acid, 10277

ent-16-Hydroxy-3-oxo-13-epi-manoyl oxide, 10562

14-Hydroxyvibsanin F, 10823

Isoanomallotusin, 11227

Isocupressic acid, 11358

Isodoglutinosin B, 11385

Isonerylgeraniol-18-oic acid, 11563

Lophanic acid, 12990
Mulinolic acid, 15062

Nerylgeraniol-18-oic acid, 15513

Onychiol C, 16123

Otostegindiol, 16273

Pilosanone A, 17364

Preotostegindiol, 17845

Pterokaurane $\mathrm{P}_{1}, 18107$

Sideroxol, 19873

Suaveolic acid, 20431

$6 \beta, 14 \alpha, 18$-Trihydroxy-9-epi-ent-pimara-7,15diene, 21725

$3 \alpha, 18,19$-Trihydroxy trachylobane, 21851

Vitetrifolin A, 22568

\section{$\mathrm{C}_{20} \mathrm{H}_{32} \mathrm{O}_{4}$}

$4 \alpha$-Acetyldictyodial, 374

16,17-Dihydrorostronol F, 5703

(ent-2 $\alpha, 7 \beta, 13 E)$ 2,7-Dihydroxy-3,13-clerodadien-

15-oic acid, 5792

(-)-16,17-Dihydroxy-16 $\beta$-kauran-19-oic acid, 5930

16 $\alpha, 17$-Dihydroxy-ent-kauran-19-oic acid, 5931

16 $\beta$,17-Dihydroxy-ent-kauran-19-oic acid, 5932

Dunniana acid A, 6637

15-Epileoheteronone B, 6943

15-Epileoheteronone D, 6944

ent-12 $\alpha, 16$-Epoxy-2 $\beta, 15 \alpha, 19$-trihydroxypimar-

8 -ene, 7215

ent-12 $\alpha, 16$-Epoxy-2 $\beta, 15 \alpha, 19$-trihydroxypimar-

8(14)-ene, 7216

Fumotoshidin A, 7999

Fumotoshidin B, 8000

Grayanotoxin XVIII, 8996

$14(R)$-Hydroxy-7 $\beta$-isovaleryloxyoplop-8(10)-en2-one, 10265

ent-7 $\beta$-Hydroxy-2-oxo-3-cleroden-15-oic acid, 10561

Leoheteronone B, 12633

Leoheteronone D, 12635

Marrubenol, 13573

ent-2-Oxo-15,16,19-trihydroxypimar-8(14)-ene, 16434

ent-15-Oxo-2 $\beta, 16,19$-trihydroxypimar-8(14)-ene, 16435

Pilosanone B, 17365

Portulal, 17733

Premarrubenol, 17812

Prostaglandin $\mathrm{A}_{1}, 17952$

Prostaglandin $\mathrm{B}_{1}, 17953$

Pterokaurane $\mathrm{P}_{2}, 18109$

Pterokaurane $\mathrm{P}_{3}, 18110$

Pterokaurane $\mathrm{P}_{4}, 18111$

Siegesbeckic acid, 19875

Tetrahydroxytaxadiene, 21160
$1 \alpha, 5 \beta, 11 \beta$-Trihydroxy-7-oxo-ros-15-ene, 21829

$\mathrm{C}_{20} \mathrm{H}_{32} \mathrm{O}_{\mathbf{5}}$

4-O-Acetyl hygrophorone $\mathrm{C}^{12}, 432$

4-O-Acetyl hygrophorone $\mathrm{D}^{12}, 433$

15-Epileopersin C, 6947

15-Epi-leopersin J, 6948

Grayanotoxin II, 8995

Leopersin C, 12649

Leopersin J, 12657

Methyl 2-(1' $\beta$-geranyl-5' $\beta$-hydroxy-4' $\beta$-methoxy-

2'-oxocyclohexyl)acetate, 14461

Portulene, 17735

Portulenol, 17736

Portulic acid, 17738

Portulic lactone, 17739

Rhodomollein II, 18805

3 $\beta, 15 x i, 16$-Trihydroxy-isopimaric acid, 21752

Wulingzhic acid, 22739

\section{$\mathrm{C}_{20} \mathrm{H}_{32} \mathrm{O}_{\mathbf{6}}$}

1,4-Di-O-acetyl hygrophorone $\mathrm{E}^{10}, 5339$

15-Epi-leopersin O, 6949

15-Epi-leopersin Q, 6950

Excisanin G, 7680

Excoecarin S, 7687

Excoecarin $\mathrm{T}_{1}, 7688$

Excoecarin $\mathrm{T}_{2}, 7689$

Hexahydroxytaxadiene, 9505

Leopersin O, 12660

Leopersin Q, 12662

Lyoniol B, 13247

Rhodojaponin III, 18801

Rhodomollein I, 18804

Rhodomollein IX, 18806

Rhodomollein X, 18807

$\mathrm{C}_{20} \mathrm{H}_{32} \mathrm{O}_{7}$

Icariside $\mathrm{B}_{2}, 10945$

$\mathrm{C}_{20} \mathrm{H}_{32} \mathrm{O}_{8}$

Spiganthol, 20163

$\mathrm{C}_{20} \mathrm{H}_{33} \mathrm{ClO}_{3}$

Agallochin A, 684

Agallochin B, 685

$\mathrm{C}_{20} \mathrm{H}_{33} \mathrm{~N}_{3}$

Panamine, 16585

$\mathrm{C}_{20} \mathrm{H}_{34}$

Kaurane, 12170

\section{$\mathrm{C}_{20} \mathrm{H}_{34} \mathrm{O}$}

4,6-Bis(4-methylpent-3-en-1-yl)-6-methylcyclohexa-1,3-diene-carbaldehyde, 2484

4-Epiisocembrol, 6937

Geranylgeraniol, 8316

9-Geranyl-terpineol, 8329

13 $\alpha$-Hydroxyazorellane, 9809

13 $\beta$-Hydroxyazorellane, 9810 
$5 \beta$-Hydroxy-ros-15-ene, 10679

13S-Hydroxystemodane, 10728

Isocembrol, 11314

ent-Labda-13(16),14-diene-8 $\alpha$-ol, 12407

Manoyloxide, 13511

cis-Neoabienol, 15332

Vitexifolin A, 22575

(+)-(1 $\left.R^{*}, 4 S^{*}, 5 S^{*}, 7 S^{*}, 10 S^{*}\right)$-Viticulol, 22583

\section{$\mathrm{C}_{20} \mathrm{H}_{34} \mathrm{O}_{2}$}

Agathodienediol, 711

Crotonadiol, 4270

Dihydrogrindelaldehyde, 5622

12,17-Dihydroxylabda-7,13(E)-diene, 5942

$5 \beta, 11 \beta$-Dihydroxy-ros-15-ene, 6109

$5 \beta, 12 \beta$-Dihydroxy-ros-15-ene, 6110

$2 \alpha, 13(R)$-Dihydroxystemodane, 6133

$2 \beta, 13(S)$-Dihydroxystemodane, 6134

$\beta$-4,8,13-Duvatriene-1,3-diol, 6643

$\beta$-3,8,13-Duvatriene-1,5-diol, 6644

12,17-Epoxy-17-hydroxylabda-13(E)-ene, 7122

(9S,10S)-ent-9,10-Epoxyverticillol, 7218

Flavidusin A, 7812

Fusicoserpenol A, 8034

Glutinosin, 8791

Glutinosin A, 8792

Glutinosin B, 8793

ent-3 $\beta$-Hydroxy-13-epi-manoyl oxide, 10080

(S)-12-Hydroxygeranylgeraniol, 10146

(+)-2 $\alpha$-Hydroxykolavelool, 10291

(-)-2 $\beta$-Hydroxykolavelool, 10292

$7 \beta$-Hydroxymanoyl oxide, 10360

(E,Z,E)-7-Hydroxymethyl-3,11,15-trimethyl-

2,6,10,14-hexadecatetraen-1-ol, 10523

16-Hydroxy-2,6,10,14-tetramethyl-6,10,14-

hexadecatrien-4-one, 10758

Insecticidea diterpene, 11087

ent-Isopimara-15-en- $\alpha, \alpha$-diol, 11597

15-Isopimaren-3 $\beta, 8 \beta$-diol, 11598

ent-Kauran-16 $\alpha, 17$-diol, 12168

ent-Kauran-16 $\beta, 17$-diol, 12169

16 $\alpha H$-17,19-ent-Kauranediol, 12171

16ßH-17,19-ent-Kauranediol, 12172

Labdadiene, 12402

Labda-8(17),13E-diene-3 $\beta, 15$-diol, 12404

ent-Labda-13(16),14-diene-8 $\alpha, 9 \beta$-diol, 12405

ent-Labda-13(16),14-diene-1 $\beta, 8 \alpha$-diol, 12406

ent-Pimara-15-ene-3 $\alpha, 8 \alpha$-diol, 17373

Sagittariol, 19124

Stemarin, 20295

Stemodin, 20299

Suaveolol, 20432

$\mathrm{C}_{20} \mathrm{H}_{34} \mathrm{O}_{3}$

$(+)-(4 \rightarrow 2)$-Abeo-kolavelool-3-oic acid, 2
ent-Atisane-3 $\beta, 16 \alpha, 17$-triol, 1962

Austroinulin, 2021

Ceriopsin C, 3425

Ceriopsin D, 3426

Darutigenol, 4661

Dihydrogrindelic acid, 5623

(2E,6E,10E,14Z)-17,20-Dihydroxygeranylnerol, 5898

$12 \beta, 19$-Dihydroxymanoyl oxide, 5954

(-)-2 $\beta$-Hydroperoxykolavelool, 9731

ent-18-Hydroxy-3-cleroden-15-oic acid, 9925

(13S)-ent-7 $\beta$-Hydroxy-3-cleroden-15-oic acid, 9926

ent-15-Hydroxy-8(17)-labden-19-oic acid, 10304

ent-18-Hydroxy-8(17)-labden-15-oic acid, 10305

Imbricatolic acid, 10996

ent-Isopimar-15-en-6 $\alpha, 7 \alpha, 8 \alpha$-triol, 11599

ent-Kauran-16 $\beta, 17,18$-triol, 12175

ent-Labda-13(16),14-diene-1 $\beta, 8 \alpha, 9 \beta$-triol, 12408

Siegesbeckiol, 19876

8,10,18-Trihydroxy-2,6-dolabelladiene, 21724

$1 \beta, 16 \alpha, 17$-Trihydroxy-ent-kaurane, 21755

$2 \beta, 7 \beta, 13(S)$-Trihydroxystemodane, 21847

\section{$\mathrm{C}_{20} \mathrm{H}_{34} \mathrm{O}_{4}$}

Ballodiolic acid, 2131

Kirenol, 12230

Portulol, 17740

ent-2 $\alpha, 15,16,19$-Tetrahydroxypimar-8(14)-ene, 21149

$2 \alpha, 3 \beta, 13(S), 16 \alpha$-Tetrahydroxystemodane, 21153

Zoapatanol, 23021

\section{$\mathrm{C}_{20} \mathrm{H}_{34} \mathrm{O}_{5}$}

4-O-Acetyl hygrophorone $\mathrm{A}^{12}, 426$

6-O-Acetyl hygrophorone $\mathrm{A}^{12}, 427$

1-O-Acetyl hygrophorone $\mathrm{E}^{12}, 436$

12-Hydroxykirenol, 10289

ent-2 $\alpha, 14 \alpha, 15 \beta, 16 S, 17-P e n t a h y d r o x y$ kaurane, 16849

Prostaglandin $\mathrm{E}_{1}, 17954$

$\mathrm{C}_{20} \mathrm{H}_{34} \mathrm{O}_{6}$

Grayanotoxin III, 8994

Kalmanol, 12117

$\mathrm{C}_{20} \mathrm{H}_{34} \mathrm{O}_{7}$

Rhodojaponin VI, 18803

Rhodomollein XIV, 18811

$\mathrm{C}_{20} \mathrm{H}_{34} \mathrm{O}_{12}$

$1 \alpha-O$-[2'-(2'-Methyl-5'-isopropyl,3'-en-bihydrofuryl)]- $\beta$ - $D$-lactose, 14537

\section{$\mathrm{C}_{20} \mathrm{H}_{35} \mathrm{ClO}_{3}$}

Agallochin C, 686

$\mathrm{C}_{20} \mathrm{H}_{35} \mathrm{~N}_{3}$

Ormosanine, 16206

$\mathrm{C}_{20} \mathrm{H}_{36} \mathrm{~N}_{2} \mathrm{O}_{17}$
Neocycasin C, 15382

Neocycasin F, 15385

Neocycasin G, 15386

$\mathrm{C}_{20} \mathrm{H}_{36} \mathrm{O}_{2}$

12,17-Dihydroxylabda-13(E)-ene, 5944

13-Epi-sclareol, 7015

Leoheteronin D, 12630

Linoleyl acetate, 12894

10,13-Octadecadienoic acid ethyl ester, 15942

\section{$\mathrm{C}_{20} \mathrm{H}_{36} \mathrm{O}_{3}$}

Clerod-14-ene- $3 \alpha, 4 \beta, 13 \xi$-triol, 3840

Vitexifolin B, 22576

\section{$\mathrm{C}_{20} \mathrm{H}_{36} \mathrm{O}_{4}$}

Hygrophorone $\mathrm{B}^{14}, 10837$

1-Monolinolein, 14925

\section{$\mathrm{C}_{20} \mathrm{H}_{36} \mathrm{O}_{9}$}

Staphylionoside I, 20272

\section{$\mathrm{C}_{20} \mathrm{H}_{36} \mathrm{O}_{10}$}

$O-\beta$ - $D$-Oleandropyranosyl- $(1 \rightarrow 4)-O-\beta$ - $D$-digitoxopyranosyl-( $1 \rightarrow 4)-\beta$ - $D$-oleandropyranose, 16033

\section{$\mathrm{C}_{20} \mathrm{H}_{36} \mathrm{O}_{11}$}

(R)-Oct-1-en-3-yl $O$ - $\alpha$ - $L$-arabinopyranosyl-

$(1 \rightarrow 6)-\beta$ - $D$-glucopyranoside, 15985

\section{$\mathrm{C}_{20} \mathrm{H}_{36} \mathrm{O}_{12}$}

Ebracteatoside C, 6665

\section{$\mathrm{C}_{20} \mathrm{H}_{38}$}

trans-1,3-Phytodiene, 17262

\section{$\mathrm{C}_{20} \mathrm{H}_{38} \mathrm{O}_{2}$}

11-Eicosenoic acid, 6725

Gadoleic acid, 8045

\section{$\mathrm{C}_{20} \mathrm{H}_{38} \mathrm{O}_{4}$}

Eicosandioic acid, 6719

\section{$\mathrm{C}_{20} \mathrm{H}_{39} \mathrm{NO}_{2}$}

(-)-Spectaline, 20141

\section{$\mathrm{C}_{20} \mathrm{H}_{40} \mathrm{~N}_{2} \mathrm{O}_{8}$}

Vitamin $\mathrm{B}_{15}, 22557$

\section{$\mathrm{C}_{20} \mathrm{H}_{40} \mathrm{O}$}

Phytol, 17265

\section{$\mathrm{C}_{20} \mathrm{H}_{40} \mathrm{O}_{2}$}

Arachidic acid, 1598

Ethylisooctadecanoate, 7453

Ethyloctadecanoate, 7467

(4R)-4-Hydroxyisophytol, 10253

Phytene-1,2-diol, 17254

\section{$\mathrm{C}_{20} \mathrm{H}_{40} \mathrm{O}_{3}$}

2Z-Phytene-1,15,20-triol, 17255

$\mathrm{C}_{20} \mathrm{H}_{42}$

Eicosane, 6720

$\mathrm{C}_{20} \mathrm{H}_{42} \mathrm{O}$

Eicosanol, 6722

$\mathrm{C}_{20} \mathrm{H}_{50} \mathrm{O}_{5}$

ent-16 $\beta, 17,18$-Trihydroxy-kauran-19-oic acid, 
21756

$\mathrm{C}_{21} \mathrm{H}_{7} \mathrm{NO}_{3}$

Retrofractamide D, 18660

$\mathrm{C}_{21} \mathrm{H}_{10} \mathrm{O}_{13}$

Sanguisobic acid dilactone, 19285

$\mathrm{C}_{21} \mathrm{H}_{14} \mathrm{O}_{4}$

2-Benzylxanthopurpurin, 2299

$\mathrm{C}_{21} \mathrm{H}_{14} \mathrm{O}_{6}$

Tephcalostan, 20964

$\mathrm{C}_{21} \mathrm{H}_{14} \mathrm{O}_{7}$

Jusmicranthin methyl ether, 11973

Justicidin D, 11980

Taiwanin E methyl ether, 20645

\section{$\mathrm{C}_{21} \mathrm{H}_{14} \mathrm{O}_{8}$}

Prostalidin A, 17955

$\mathrm{C}_{21} \mathrm{H}_{16} \mathrm{NO}_{5}^{+}$

Chelirubine, 3508

\section{$\mathrm{C}_{21} \mathrm{H}_{16} \mathrm{O}_{6}$}

Cassigarol B, 3286

Chinensin, 3530

Gancaonin F, 8139

Gerberinol I, 8333

Gnetal, 8877

Justicidin B, 11978

Retrojusticidin B, 18662

Scandenal, 19449

Vitrofolal D, 22593

$\mathrm{C}_{21} \mathrm{H}_{16} \mathrm{O}_{7}$

Chinensinaphthol, 3531

Diphyllin, 6490

Parviflorol, 16678

Piscatorin, 17478

\section{$\mathrm{C}_{21} \mathrm{H}_{16} \mathrm{O}_{8}$}

4'-Demethyldehydropodophyllotoxin, 5070

\section{$\mathrm{C}_{21} \mathrm{H}_{16} \mathrm{O}_{9}$}

Anthraxin, 1371

\section{$\mathrm{C}_{21} \mathrm{H}_{16} \mathrm{O}_{10}$}

3-Carboxy-6,7-dihydroxy-1-(3',4'-dihydroxyphenyl)-naphthalene-9,2"-O-malic acid ester, 3167

$\mathrm{C}_{21} \mathrm{H}_{16} \mathrm{O}_{18} \mathrm{~S}_{2}{ }^{2-}$

Isoscutellarein 8-O- $\beta$ - $D$-glucuronide $2 ", 4 "$ disulfate, 11706

\section{$\mathrm{C}_{21} \mathrm{H}_{17} \mathrm{NO}_{5}$}

Oxychelerythrine, 16443

Oxynitidine, 16454

\section{$\mathrm{C}_{21} \mathrm{H}_{18} \mathrm{CINO}_{4}$}

Nitidine chloride, 15632

\section{$\mathrm{C}_{21} \mathrm{H}_{18} \mathrm{NO}_{4}^{+}$}

Chelerythrine, 3498

Nitidine, 15631

\section{$\mathrm{C}_{21} \mathrm{H}_{18} \mathrm{~N}_{2} \mathrm{O}_{4}$}

Zanthobisquinolone, 22965
$\mathrm{C}_{21} \mathrm{H}_{18} \mathrm{~N}_{2} \mathrm{O}_{5}$

$N$-Formylcorydamine, 7899

10-Methoxycamptothecin, 13863

9-Methoxycamptothecin, 13864

$\mathrm{C}_{21} \mathrm{H}_{18} \mathrm{O}_{4}$

Candidin, 3071

\section{$\mathrm{C}_{21} \mathrm{H}_{18} \mathrm{O}_{5}$}

[6",6"-Dimethylpyrano-(2",3":7,8)]-4'-methoxy-

3-arylcoumarin, 6401

5-O-Methylalpinumisoflavone, 14131

3',4'-Methylenedioxy-7-hydroxy-6-isopentenyl flavone, 14371

\section{$\mathrm{C}_{21} \mathrm{H}_{18} \mathrm{O}_{6}$}

Detetrahydroconidendrin B, 5280

Detetrahydroconidendrin C, 5281

Glycyrol, 8839

3'-Hydroxy-4'-O-methylalpinumisoflavone, 10466

Isoglycyrol, 11444

Piscisoflavone $\mathrm{B}, 17482$

\section{$\mathrm{C}_{21} \mathrm{H}_{18} \mathrm{O}_{7}$}

Artoindonesianin T, 1818

Burseranin, 2769

4,4'-Dimethoxyvulpinic acid, 6298

Pachycarin C, 16491

\section{$\mathrm{C}_{21} \mathrm{H}_{18} \mathrm{O}_{8}$}

Lignan from Justicia heterocarpa, 12799

$\mathrm{C}_{21} \mathrm{H}_{18} \mathrm{O}_{9}$

Pinnatifinoside A, 17394

$\mathrm{C}_{21} \mathrm{H}_{18} \mathrm{O}_{10}$

Chrysin 7-O- $\beta$-galactopyranuronoside, 3601

$\mathrm{C}_{21} \mathrm{H}_{18} \mathrm{O}_{11}$

Apigenin-4'-O-glucuronide, 1493

Apigenin-7- $O-\beta$ - $D$-glucuronide, 1494

Baicalein-6-glucuronide, 2104

Baicalin, 2106

Rhein-8-O- $\beta$ - $D$-glucopyranoside, 18761

$\mathrm{C}_{21} \mathrm{H}_{18} \mathrm{O}_{12}$

Breviscapine, 2606

Demethylwedelolactone-7-glucoside, 5102

Kaempferol-3- $\beta$ - $D$-glucuronide, 12065

Luteolin-3'-O-glucuronide, 13146

Luteolin-7-O- $\beta$ - $D$-glucuronide, 13147

3-O-methylellagic acid

3'-O- $\alpha$ - $L$-rhamnopyranoside, 14343

3-O-Methylellagic acid

4-O- $\alpha$ - $L$-rhamnopyranoside, 14344

3-O-Methylellagic acid

4'-O- $\alpha$ - $L$-rhamnopyranoside, 14345

4-O-Methylellagic acid 3'- $\alpha$-rhamnoside, 14346 Scutellarin, 19587

$5,7,2^{\prime}, 5^{\prime}$-Tetrahydroxyflavone

7-O- $\beta$ - $D$-glucuronopyranoside, 21115
5,6,4'-Trihydroxyflavone-7- $O-\beta$ - $D$-galactonic acid, 21735

\section{$\mathrm{C}_{21} \mathrm{H}_{18} \mathrm{O}_{13}$}

Mingjinianuronide A, 14872

Quercetin-3-O-glucuronide, 18371

Querciturone, 18414

\section{$\mathrm{C}_{21} \mathrm{H}_{19} \mathrm{NO}_{4}$}

Buxifoliadine G, 2829

Dihydrochelerythrine, 5558

\section{$\mathrm{C}_{21} \mathrm{H}_{19} \mathrm{NO}_{5}$}

Corynoloxine, 4117

Thaliglucinone, 21257

\section{$\mathrm{C}_{21} \mathrm{H}_{19} \mathrm{NO}_{6}$}

Isoarnottianamide, 11237

6-Oxocorynoline, 16303

Oxopurpureine, 16414

\section{$\mathrm{C}_{21} \mathrm{H}_{19} \mathrm{NO}_{7}$}

Lederine, 12603

\section{$\mathrm{C}_{21} \mathrm{H}_{19} \mathrm{~N}_{3} \mathrm{O}_{2}$}

16-Methoxycarbonyl naufoline, 13876

19-O-Methylangustoline, 14139

\section{$\mathrm{C}_{21} \mathrm{H}_{19} \mathrm{~N}_{3} \mathrm{O}_{4}$}

16-Methoxycarbonyl-18,19-dihydroxynaufoline, 13874

\section{$\mathrm{C}_{21} \mathrm{H}_{19} \mathrm{O}_{15} \mathrm{~S}^{-}$}

Centabractein, 3387

\section{$\mathrm{C}_{21} \mathrm{H}_{20} \mathrm{NO}_{4}$}

Fagaronine, 7704

$\mathrm{C}_{21} \mathrm{H}_{20} \mathrm{NO}_{13}$

Isoaffinetin, 11198

\section{$\mathrm{C}_{21} \mathrm{H}_{20} \mathrm{~N}_{2} \mathrm{O}_{3}$}

Alstonine, 1002

Serpentine, 19762

\section{$\mathrm{C}_{21} \mathrm{H}_{20} \mathrm{O}_{4}$}

Anhydronotoptol, 1278

Burttinol B, 2771

Crassichalcone, 4212

Danshenol A, 4627

Danshenxinkun D, 4635

3-Hydroxy-9-methoxy-10-(3,3-dimethylallyl) pterocarpene, 10398

Licoagrochalcone B, 12752

\section{$\mathrm{C}_{21} \mathrm{H}_{20} \mathrm{O}_{5}$}

Anhydronotoptoloxide, 1279

Anticancer Flavonoid PMV70P691-026, 1404

1,7-Bis(4-hydroxy-3-methoxyphenyl)-1,4,6-

heptatrien-3-one, 2466

Erylatissin A, 7305

Erypoegin F, 7312

Gancaonin A, 8134

Glabroisoflavanone B, 8498

Glyurallin A, 8854

5-Hydroxy-4'-methoxy-2",2"-dimethylpyrano- 
(7,8:6",5")flavanone, 10400

Pervilline, 17004

Pervillinine, 17005

Sigmoidin C, 19887

Sterequinone B, 20336

Xanthohumol C, 22763

\section{$\mathrm{C}_{21} \mathrm{H}_{20} \mathrm{O}_{6}$}

Anhydroicaritin, 1273

Bursehemin, 2764

Chisulactone, 3540

Curcumin, 4398

4',5"-Dihydroxy-6"-methoxy-4",4"-dimethyl-

4",5"-dihydro-6" $H$-pyrano[2",3":7,8]-

isoflavone, 5973

Gancaonin B, 8135

Glicoricone, 8552

Glyasperin M, 8803

Glycycoumarin, 8833

Glycyrrhisoflavanone, 8843

Isoanhydroicaritin, 11222

Isoglycycoumarin, 11443

Isokaerophyllin, 11470

Kaerophyllin, 12106

Licoarylcoumarin, 12763

7-O-Methylluteone, 14568

Piscisoflavone A, 17481

Sophoflavescenol, 20078

Suchilactone, 20445

Topazolin, 21451

Ugonin C, 22176

\section{$\mathrm{C}_{21} \mathrm{H}_{20} \mathrm{O}_{7}$}

Anticancer Glycerol Ester PMV70P691-117, 1422

Calebin A, 2960

(+)-5'-Demethoxyepiexcelsin, 5046

4'-Demethyldeoxypodophyllotoxin, 5074

$O$-[3-(2,2-Dimethyl-3-oxo-2H-furan-5-yl)-3hydroxybutyl]bergaptol, 6389

Gancaonin D, 8137

Gancaonin P-3'-methylether, 8142

Glyasperin E, 8800

2-O-[2-(5-Hydroxy-2,6,6-trimethyl-3-oxo- $2 \mathrm{H}$ pyran-2-yl)ethyl]bergaptol, 10812

Kweichowenol B, 12398

Licofuranocoumarin, 12777

Licopyranocoumarin, 12782

Piperenol A, 17447

Piperenol B, 17448

Podoverin A, 17597

Uralene, 22238

Uralenol-3-methylether, 22242

Uvaribonol D, 22293

Uvarigranol G, 22299
Zeylenol, 22988

(+)-Zeylenol, 22989

\section{$\mathrm{C}_{21} \mathrm{H}_{20} \mathrm{O}_{8}$}

(-)-Altissinone, 1009

Coniselin, 3990

4-Demethyl-picropodophyllotoxin, 5091

4-Demethyl-podophyllotoxin, 5093

2-(4-Hydroxy-3,5-dimethoxy-phenyl)-10methoxy-3-methyl-2,3-dihydro-1,4,5-trioxaphenanthren-6-one, 10038

$\alpha$-Peltatin, 16793

$\mathrm{C}_{21} \mathrm{H}_{20} \mathrm{O}_{9}$

Apigenin-5-rhamnoside, 1498

Chrysophanol-8- $O-\beta$ - $D$-glucopyranoside, 3619

Chrysophanol-1- $O-\beta$ - $D$-glucoside, 3620

Cleomiscosin C, 3831

Cleomiscosin D, 3832

Daidzin, 4606

Frangulin A, 7939

5,6,7,8,5'-Pentamethoxy-3',4'-methylenedioxy-

flavone, 16873

Puerarin, 18180

Sophoraflavone B, 20087

\section{$\mathrm{C}_{21} \mathrm{H}_{20} \mathrm{O}_{10}$}

Aloeemodin- $\omega$ - $O-\beta$ - $D$-glucopyranoside, 970

Aloeemodin-8-monoglucoside, 971

Anthraglycoside B, 1363

Apigenin-4'-O- $\beta$ - $D$-glucopyranoside, 1491

Apigenin-7-O-glucoside, 1492

Baicalein-7-O- $\beta$ - $D$-glucopyranoside, 2103

Emodin-1-O- $\beta$ - $D$-glucopyranoside, 6778

Emodin-3-O- $\beta$ - $D$-glucopyranoside, 6779

Genistein 7-glucoside, 8282

Genistein 8- $C$-glucoside, 8283

Isovitexin, 11773

Kaempferol-3-rhamnoside, 12082

Kaempferol-7-rhamnoside, 12083

Sophoricoside, 20099

Sorbarin, 20102

Sulfuretin glucoside, 20482

Tetuin, 21212

$5,7,2^{\prime}$-Trihydroxyflavone

2'-O- $\beta$ - $D$-glucopyranoside, 21736

Vitexin, 22581

$\mathrm{C}_{21} \mathrm{H}_{20} \mathrm{O}_{11}$

Amoenin $\mathrm{A}_{3}, 1069$

Astragalin, 1935

Aureusidin-6-glucoside, 2012

Carthamone, 3225

Cernuoside, 3432

Cinaroside, 3674

Dihydrobaicalin, 5546

Galuteolin, 8120
Homoorientin, 9616

Isorhamnetin-3- $\alpha-L$-arabofuranoside, 11652

Isoscutellarein 7-O- $\beta$ - $D$-glucopyranoside, 11705

Kaempferol 3-O- $\beta$ - $D$-glucopyranoside, 12050

Kaempferol-7- $O$-glucoside, 12062

Luteolin 7-O- $\beta$ - $D$-galactoside, 13143

Luteolin-4'-O-glucoside, 13145

Mollupentin, 14905

Orientin, 16196

Oroboside, 16211

Plantaginin, 17505

Quercetin-3- $\alpha$ - $L$-rhamnofuranoside, 18379

Quercitrin, 18411

Rhodiolatuntoside, 18791

Scutellarein-5-galactoside, 19584

Trifolin, 21634

Vincetoxicoside B, 22492

\section{$\mathrm{C}_{21} \mathrm{H}_{20} \mathrm{O}_{12}$}

(2R)-Eriodictyol-7- $O-\beta$ - $D$-glucopyranosiduronic acid, 7283

(2S)-Eriodictyol-7- $O-\beta$ - $D$-glucopyranosiduronic acid, 7284

Herbacin, 9424

6-Hydroxykaempferol-7-O-glucoside, 10273

8-Hydroxyluteolin-8- $\beta$ - $D$-glucopranoside, 10354

6-Hydroxyluteolin-7-O-glucoside, 10355

Hyperin, 10887

Isoarticulatin, 11238

Isohyperoside, 11461

Isoquercitrin, 11642

Myricetin-3-O- $\alpha$ - $L$-rhamnoside, 15184

Quercetin-4'-glucoside, 18368

Quercetin-5-O- $\beta$ - $D$-glucoside, 18369

Quercimeritrin, 18409

(2S)-5,7,2',5'-Tetrahydroxyflavanone

7-O- $\beta$ - $D$-glucuronopyranoside, 21111

\section{$\mathrm{C}_{21} \mathrm{H}_{20} \mathrm{O}_{13}$}

Cannabiscitrin, 3084

Gossypin, 8965

Gossypitrin, 8966

Isomyricitrin, 11551

Quercetagetin-3-galactoside, 18309

Quercetagitrin, 18316

Tagetiin, 20608

\section{$\mathrm{C}_{21} \mathrm{H}_{20} \mathbf{O}_{13} \mathrm{~S}$}

Sulfemodin-8-O- $\beta$ - $D$-glucoside, 20468

\section{$\mathrm{C}_{21} \mathrm{H}_{21} \mathrm{NO}_{4}$}

Isoochotensine, 11571

Ochotensine, 15918

\section{$\mathrm{C}_{21} \mathrm{H}_{21} \mathrm{NO}_{5}$}

Corycavine, 4099

Corynoline, 4116

(+)-14-Epicorynoline, 6874 


\section{$\mathrm{C}_{21} \mathrm{H}_{21} \mathrm{NO}_{6}$}

Adlumine, 643

Corlumine, 4058

Cornigerine, 4059

( $1 R, 9 R)$-Hydrastine, 9703

$D$-(1S,9R)-Hydrastine, 9704

12-Hydroxycorynoline, 9938

Isorhoeadine, 11677

Methoxychelidonine, 13880

Rhoeadine, 18816

Zijinlongine, 23001

$\mathrm{C}_{21} \mathrm{H}_{21} \mathrm{NO}_{7}$

Narcotoline, 15268

\section{$\mathrm{C}_{21} \mathrm{H}_{21} \mathrm{NO}_{10}$ \\ 6'-O-Galloylprunasin, 8114}

\section{$\mathrm{C}_{21} \mathrm{H}_{21} \mathrm{NO}_{11}$}

Glochidacuminoside D, 8562

\section{$\mathrm{C}_{21} \mathrm{H}_{21} \mathrm{~N}_{3} \mathrm{O}_{3}$}

Nauclechine, 15297

Naucleonidine, 15308

\section{$\mathrm{C}_{21} \mathrm{H}_{21} \mathrm{O}_{10}$}

Pelargonidin-3-galactoside, 16785

\section{$\mathrm{C}_{21} \mathrm{H}_{21} \mathrm{O}_{10}{ }^{+}$}

Callistephin, 2978

Luteolinidin-5-glucoside, 13148

\section{$\mathrm{C}_{21} \mathrm{H}_{21} \mathrm{O}_{11}$}

Petunidin-3-arabinoside, 17025

\section{$\mathrm{C}_{21} \mathrm{H}_{21} \mathrm{O}_{11}{ }^{+}$}

Chrysanthemin, 3594

Cyanidin-3- $O-\beta$ - $D$-galactoside, 4439

Cyanidin-4'-O- $\beta$ - $D$-glucopyranoside, 4442

\section{$\mathrm{C}_{21} \mathrm{H}_{21} \mathrm{O}_{12}{ }^{+}$}

Delphinidin-3-O- $\beta$ - $D$-galactopyranoside, 5022

Delphinidin-3-glucoside, 5027

\section{$\mathrm{C}_{21} \mathrm{H}_{22} \mathrm{NO}_{4}^{+}$}

Dehydrocorybulbine, 4888

Palmatine, 16555

Thalphenine, 21266

\section{$\mathrm{C}_{21} \mathrm{H}_{22} \mathrm{~N}_{2} \mathrm{O}_{2}$}

Isostrychnine, 11721

Kopsifoline D, 12265

Strychnine, 20410

\section{$\mathrm{C}_{21} \mathrm{H}_{22} \mathrm{~N}_{2} \mathrm{O}_{3}$}

4-Hydroxystrychnine, 10734

Isostrychnine $N$-oxide, 11722

Pseudostrychnine, 18070

Strychnine $N$-oxide, 20411

Vallesiachotamine, 22328

\section{$\mathrm{C}_{21} \mathrm{H}_{22} \mathrm{~N}_{2} \mathrm{O}_{4}$}

Picralinal, 17296

$\mathrm{C}_{21} \mathrm{H}_{22} \mathrm{O}_{2}$

$5,7,3$ ', 5'-Tetrahydroxyflavanonol-3- $O-\beta-D$ glucoside, 21112
$\mathrm{C}_{21} \mathrm{H}_{22} \mathrm{O}_{3}$

Benzoylpterosin B, 2267

$\mathrm{C}_{21} \mathrm{H}_{22} \mathrm{O}_{4}$

Bavachalcone, 2171

Bavachinin, 2173

Bergamotin, 2308

Burttinol A, 2770

Burttinol C, 2772

Gancaonin X, 8143

Gancaonin Y, 8144

8-Geranyloxy psoralen, 8326

4-Hydroxyderricin, 9992

Licochalcone A, 12766

Licochalcone C, 12768

Licochalcone E, 12770

3-O-Methylcalopocarpin, 14206

7-O-Methylglabranin, 14465

4'-O-Methylglabridin, 14466

Sandwicensin, 19251

Taxamairin A, 20745

\section{$\mathrm{C}_{21} \mathrm{H}_{22} \mathrm{O}_{5}$}

(-)-Aristotetralone, 1730

Cristacarpin, 4243

Dehydroglyasperin C, 4924

5-Deoxyabyssinin II, 5146

1",2"-Dihydroxanthohumol C, 5743

Erylatissin C, 7307

Futoquinol, 8040

Gancaonin I, 8140

Glyceollin IV, 8805

5-(6-Hydroxy-3,7-dimethylocta-2,7-dienyloxy) psoralen, 10053

Isoxanthohumol, 11783

Lespedezol $\mathrm{D}_{4}, 12696$

Licoagrochalcone C, 12753

Licoagrochalcone D, 12754

Licobenzofuran, 12764

Licochalcone D, 12769

3'-Methoxyglabridin, 13941

(-)-4'-O-Methylenshicine, 14393

Notopterol, 15843

Notoptol, 15844

Sigmoidin B, 19885

1,2,5-Tri-O-methylglobuxanthone, 21960

Xanthohumol, 22761

$\mathrm{C}_{21} \mathrm{H}_{22} \mathrm{O}_{6}$

Alkannin angelate, 910

Arnebin, 1745

Dextrobursehernin, 5282

$\beta, \beta$-Dimethylacrylalkannin, 6302

$\beta, \beta$-Dimethylacrylshikonin, 6303

12,16-Epoxy-11,14-dihydroxy-6-methoxy-17

(15-16)-abeo-abieta-5,8,11,13,15-pentaene- 3,7-dione, 7080

Erypoegin C, 7309

Erypoegin I, 7315

Fargesin, 7719

Glyasperin B, 8797

trans-Hydroxyxanthohumol, 10830

Isoocobullenone, 11572

Kobusin, 12241

Kosamol V, 12286

Kosamol W, 12287

Kusunokinin, 12374

Lysisteisoflavanone, 13265

$\alpha$-O-Methylcubebin, 14264

$\beta$-O-Methylcubebin, 14265

Morelensin, 14966

Ocobullenone, 15928

L-Planinin, 17504

5'-Prenylhomoeriodictyol, 17833

Sibyllenone, 19871

Sophoraisoflavanone A, 20093

Spiraformin B, 20190

XanthohumolB, 22762

XanthohumolD, 22764

Xanthohumol I, 22767

\section{$\mathrm{C}_{21} \mathrm{H}_{22} \mathrm{O}_{7}$}

$( \pm)-4$ '-O-Acetyl-3'-O-angeloyl-cis-khellactone, 317

(-)-3'-(S)-Acetyloxy-4'-(S)-angeloyloxy-3',4'dihydroseselin, 476

Crotozambefuran C, 4275

Edultin, 6709

Floranol, 7831

$1 \xi$-Hydroxy-1,7-bis(4-hydroxy-3-methoxyphenyl)-6-heptene-3,5-dione, 9855

(2S,3S)-2-(4-Hydroxy-3,5-dimethoxybenzyl)-3(3,4-methylenedioxybenzyl)butyrolactone, 10016

(2S,3S)-2-(4-Hydroxy-3-methoxybenzyl)-3(5-methoxy-3,4-methylenedioxybenzyl)butyrol actone, 10388

Isopteryxin, 11637

Omphamurrayin, 16096

Ostruhol, 16263

Peucedanocoumarin II, 17030

Peucedanocoumarin III, 17031

Peucenidin, 17036

Praeruptorin A, 17761

Pteryxin, 18165

Samidin, 19227

7,8-Seco-holostylone B, 19616

Sen-byakangelicol, 19704

(+)-Senecioylprangol, 19711

$\mathrm{C}_{21} \mathrm{H}_{22} \mathrm{O}_{8}$ 
Bannamurpanisin, 2137

Descurainolide B, 5247

$\left(3^{\prime} R, 4^{\prime} R\right)-3^{\prime}$-Epoxyangeloyloxy-4'-acetoxy-3',4'dihydroseselin, 7054

Gossypetin hexamethyl ether, 8963

3,5,6,7,3',4'-Hexamethoxyflavone, 9507

$5,6,2^{\prime}, 3^{\prime}, 5^{\prime}, 6^{\prime}$-Hexamethoxyflavone, 9508

5,6,7,3',4',5'-Hexamethoxyflavone, 9509

5,6,8,3',4',5'-Hexamethoxyflavone, 9511

Isoepoxypteryxin, 11412

Nobiletin, 15635

Pteroside, 18115

\section{$\mathrm{C}_{21} \mathrm{H}_{22} \mathrm{O}_{9}$}

Aloin, 981

Cassialoin, 3280

Dihydrodaidzin, 5580

1,3-Dihydroxy-6,7-dimethylxanthone-1-O- $\beta$ - $D$ glucoside, 5863

Gardenin, 8228

4'-Hydroxy-3,5,6,7,3',5'-hexamethoxyflavone, 10162

5-Hydroxy-7, 2',3',4',5',6'-hexamethoxyflavone, 10163

Isoliquiritin, 11505

Isorobustaside A, 11682

Liquiritin, 12913

Neoisoliquiritin, 15414

Neoliquiritin, 15430

Pleuropyrone A, 17559

Robustaside A, 18877

\section{$\mathrm{C}_{21} \mathrm{H}_{22} \mathrm{O}_{10}$}

2-O-Caffeoyl arbutin, 2893

Choerospondin, 3574

Dihydrogenistin, 5621

Dihydrokaempferol-3- $O-\alpha-L$-rhamnopyranoside, 5659

5-Hydroxyaloin A, 9772

7-Hydroxyaloin A, 9773

Isoengelitin, 11411

Isosalipurposide, 11693

Malto-3-O-[6'-O-(4"-hydroxy-trans-cinnamoyl)]$\beta$ - $D$-glucopyranoside, 13451

Marsuposide, 13579

Prunin, 18003

Robustaflavone 4'-O-methyl ether, 18865

Robustaside B, 18878

Robustaside D, 18880

Salipurposide, 19191

Schoenoside, 19512

Tadehaginoside, 20599

$1 \alpha, 3,2^{\prime}, 4^{\prime}$-Tetrahydroxy-2'- $O-\beta$ - $D$-glucopyranosylchalcone, 21118

Vijayoside, 22476
$\mathrm{C}_{21} \mathrm{H}_{22} \mathrm{O}_{11}$

Acetylannulatophenonoside, 321

Astilbin, 1928

Bockioside A, 2524

Bockioside B, 2525

Carthamin, 3224

1,3-Dihydroxy-4,5-dimethoxyxanthone-1-O- $\beta$ - $D$ glucopyranoside, 5847

1,3-Dihydroxy-4,5-dimethoxyxanthone-3- $O-\beta$ - $D$ glucopyranoside, 5848

Eriodictyol-7-glucoside, 7285

Isoastilbin B, 11241

Isookanin-7-O- $\beta$ - $D$-glucoside, 11573

Kaempferol 3-O- $\beta$ - $D$-galactopyranoside, 12047

Miscanthoside, 14884

Neocarthamin, 15358

Smitilbin, 20029

Steppogenin 4'- $O$ - $\beta$ - $D$-glucoside, 20327

(2S)-5,7,2',5'-Tetrahydroxy-flavanone

7-O- $\beta$ - $D$-glucopyranoside, 21110

2,4,6-Trihydroxyacetophenone

3-C- $\beta$-(2'-O-p-hydroxybenzoyl)-glucopyranosi

de, 21683

\section{$\mathrm{C}_{21} \mathrm{H}_{22} \mathrm{O}_{12}$}

Plantagoside, 17506

Quercetin-3-O- $\beta$ - $D$-galactopyranoside, 18356

$(2 S, 3 S)-(-)$-Taxifolin-3-O- $\beta$ - $D$-glucopyra-noside, 20792

5-O- $\beta$ - $D$-(6- $O$-Vanilloyl glucopyranosyl) gentisic acid, 22342

$\mathrm{C}_{21} \mathrm{H}_{22} \mathrm{O}_{13}$

Myrciaphenone B, 15152

Myricetin-3-O- $\beta$ - $D$-galactopyranoside, 15176

\section{$\mathrm{C}_{21} \mathrm{H}_{22} \mathrm{O}_{14}$}

Methyl 4,6-di- $O$-galloyl- $\beta$ - $D$-glucopyranoside, 14293

Methyl 6- $O$-digalloyl- $\beta$ - $D$-glucopyranoside I, 14294

Methyl 6- $O$-digalloyl- $\beta$ - $D$-glucopyranoside II, 14295

$\mathrm{C}_{21} \mathrm{H}_{23} \mathrm{NO}_{2}$

Isolobelanine, 11506

$\mathrm{C}_{21} \mathrm{H}_{23} \mathrm{NO}_{3}$

(+)-Eschscholtzidine, 7359

\section{$\mathrm{C}_{21} \mathrm{H}_{23} \mathrm{NO}_{4}$}

Dehydroglaucine, 4923

Thalictrifoline, 21250

$\mathrm{C}_{21} \mathrm{H}_{23} \mathrm{NO}_{5}$

Allocryptopine, 930

Argemexicaine A, 1665

Argemexicaine B, 1666

Canadaline, 3055

Cryptopine, 4290
Fumaricine, 7997

Homochelidonine, 9600

Romucosine D, 18904

Tenellin, 20932

Thalicmine, 21241

\section{$\mathrm{C}_{21} \mathrm{H}_{23} \mathrm{NO}_{6}$}

Canadinic acid, 3058

Colchiceine, 3910

$N$-Formyl- $N$-deacetylcolchicine, 7901

$\beta$-Hydrastine, 9702

Papaverrubine B, 16625

Raddeanine, 18519

Romucosine H, 18907

\section{$\mathrm{C}_{21} \mathrm{H}_{24} \mathrm{NO}_{4}$}

$\mathrm{N}$-Methyl canadine, 14209

Takatonine, 20649

\section{$\mathrm{C}_{21} \mathrm{H}_{24} \mathrm{~N}_{2} \mathrm{O}_{2}$}

Alstonerinal II*, 1000

Alstonerine, 1001

Catharanthine, 3321

12-Methoxy- $N_{a}$-methyl-vellosimine, 14039

Purpeline, 18210

Tabersonine, 20580

Venalstonine, 22374

Vindolinine, 22499

\section{$\mathrm{C}_{21} \mathrm{H}_{24} \mathrm{~N}_{2} \mathrm{O}_{3}$}

Ajmalicine, 783

19-(Z)-Akuammidine, 824

16-Epideacetylakuammiline, 6878

Gelseverine, 8261

16-Hydroxymethylpleiocarpamine, 10517

Lochnericine, 12944

Rauniticine, 18553

Rhazine, 18753

Tetrahydroalstonine, 21055

Vincarin, 22490

Vincoridine, 22494

Vindolinine $N$-oxide, 22500

Vobasine, 22602

\section{$\mathrm{C}_{21} \mathrm{H}_{24} \mathrm{~N}_{2} \mathrm{O}_{4}$}

Akuammidine $N$-oxide, 825

$N(4)$-Demethylalstophyllal oxindole, 5060

$N(4)$-Demethylalstophylline oxindole, 5061

16-Epideacetylakuammiline $N(4)$-oxide, 6879

16-Epi-voacarpine, 7039

11-Hydroxy-14,15 $\alpha$-epoxytabersonine, 10093

$16 \beta$-Hydroxy-19S-vindolinine $N$-oxide, 10824

Isoformosanine, 11426

Isomitraphylline, 11539

Isopteropodine, 11636

Mitraphylline, 14889

Pteropodine, 18114

Rauniticine oxindole A, 18554 
Uncarine B, 22204

Uncarine C, 22205

Uncarine D, 22206

Uncarine F, 22207

Vincoline, 22493

\section{$\mathrm{C}_{21} \mathrm{H}_{24} \mathrm{~N}_{2} \mathrm{O}_{5}$}

16-Hydroxy- $N(4)$-demethylalstophyllal oxindole, 9977

16-Hydroxy- $N$ (4)-demethylalstophylline oxindole, 9978

\section{$\mathrm{C}_{21} \mathrm{H}_{24} \mathrm{O}_{4}$}

Acuminatin, 591

(+)-Acuminatin, 592

(2S)-2',4'-Dihydroxy-7-methoxy-8-prenylflavan, 6001

2',4'-Dihydroxy-7-methoxy-8-prenylflavan, 6002

2',7-Dihydroxy-4'-methoxy-8-prenylflavan, 6003

Gancaonin Z, 8145

Myricanene A, 15155

Myricanene B, 15157

Racemosol\$, 18515

$\mathrm{C}_{21} \mathrm{H}_{24} \mathrm{O}_{5}$

Acetoxyauraptene, 126

(-)-Denudatin B, 5135

Dihydroxanthohumol, 5742

(-)8,8'R-Epi-holostylone, 6932

(-)8,8'S-Epi-holostylone, 6933

Fragransol C, 7933

Futokadsurin B, 8038

Futokadsurin C, 8039

Gingerenone A, 8389

Glyasperin C, 8798

Hancinone B, 9210

(+)-Holostylone, 9592

Kadsurenone, 12010

(-)-Machilusin, 13295

Myricanone, 15165

Odoratisol A, 15994

Rutamarin, 19085

Schisandrone, 19475

Shikonofuran E, 19825

\section{$\mathrm{C}_{21} \mathrm{H}_{24} \mathrm{O}_{6}$}

L-Arctigenin, 1621

Fargesone A, 7721

Fargesone B, 7722

Isoarctigenin, 11236

Isovalerylshikonin, 11765

Kadsurin A, 12013

$\alpha$-Methyl- $n$-butyrylshikonin, 14202

Phillygenin, 17143

(+)-Pinoresinol monomethyl ether, 17418

7,8-Seco-holostylone A, 19615

Theraphin D, 21316
Xanthohumol H, 22766

$\mathrm{C}_{21} \mathrm{H}_{24} \mathrm{O}_{7}$

Ailantinol E, 778

Dihydrosamidin, 5707

Dihydrosinapyl ferulate, 5713

2,3,4,2',4',6'-Hexamethoxychalcone, 9506

$\beta$-Hydroxyisovalerylshikonin, 10266

2-Hydroxy-3,2',6'-trimethoxy-4'-(2,3-epoxy-1hydroxypropyl)-5-(3-hydroxy-1-propenyl) biphenyl, 10788

Lithospermidin A, 12927

Lithospermidin B, 12928

(+)-Medioresinol, 13640

$(2 R, 3 R)-5$ '-Methoxyguayarol, 13945

(2S,3S)-2-(5-Methoxy-3,4-methylenedioxybenzyl)-3-(3,4-methylenedioxybenzyl)butane1,4-diol, 14010

Peucedanocoumarin I, 17029

Trachelogenin, 21490

Visnadine, 22552

Xanthohumol G, 22765

$\mathrm{C}_{21} \mathrm{H}_{24} \mathrm{O}_{8}$

Albaspidin AA, 848

Deoxyrhaponticin, 5206

Mallotophenone, 13432

7S,8S-Nitidanin, 15630

Orientalide, 16186

\section{$\mathrm{C}_{21} \mathrm{H}_{24} \mathrm{O}_{9}$}

Anticancer Flavonoid PMV70P691-77, 1412

Cochinolide $\beta$-glucopyranoside, 3877

Glycyphyllin, 8834

Isorhapontin, 11673

Monnieraside I, 14917

Nodifloridin B, 15650

Rhaponticin, 18744

Rhapontigenin 3'-O- $\beta$ - $D$-glucopyranoside, 18749

cis-3,5,3'-Trihydroxy-4'-methoxystilbene-3-O-

$$
\beta \text { - } D \text {-glucopyranoside, } 21788
$$

\section{$\mathbf{C}_{21} \mathbf{H}_{24} \mathbf{O}_{10}$}

1-Keto-3,10-epoxy-8 $\beta$ - $O$-methacryloyl-4,15dihydroxy-5-acetoxy-2,11-germacradiene, $6 \alpha$, 12-olide, 12198

Licoagroside F, 12762

Monnieraside III, 14919

Phloridzin, 17170

Pterosupin, 18164

Rocymosin B, 18895

Trilobatin, 21878

Triptephenoside, 21989

$\mathrm{C}_{21} \mathrm{H}_{24} \mathrm{O}_{11}$

(+)-Catechin-5-O-glucoside, 3315

Curculigoside B, 4386

Epicatechin-8-C- $\beta$ - $D$-galactopyranoside, 6863
3-Hydroxyphlorizin, 10645

Vanilloyl calleryanin, 22340

$\mathrm{C}_{21} \mathrm{H}_{24} \mathrm{O}_{12}$

2-Methoxy-5-hydroxymethyl-phenyl-1-O-

(6"-galloyl)- $\beta$ - $D$-glucopyranoside, 13965

Rocymosin A, 18894

\section{$\mathrm{C}_{21} \mathrm{H}_{24} \mathrm{O}_{13}$}

3,5-Dimethoxy-4-hydroxyphenol

1-O- $\beta$ - $D$-(6'-O-galloyl)glucopyranoside, 6251

Palustroside, 16577

\section{$\mathrm{C}_{21} \mathrm{H}_{25} \mathrm{NO}_{3}$}

Carduchorone, 3191

Dehydrodeacetylheterophylloidine, 4897

Dehydropipernonaline, 4961

\section{$\mathrm{C}_{21} \mathrm{H}_{25} \mathrm{NO}_{4}$}

(-)-Argemonine, 1667

(+)-Corybulbine, 4098

Glaucine, 8513

Lienkonine, 12797

Tetrahydropalmatine, 21077

Thalicthuberine, 21247

Xylopinine, 22820

Yuanhunine, 22934

\section{$\mathrm{C}_{21} \mathrm{H}_{25} \mathrm{NO}_{5}$}

Acutifolidine, 596

Capauridine, 3114

Colchamine, 3909

$\mathrm{N}$-trans-4-O-Methylferuloyl

3',4'-O-dimethyldopamine, 14444

\section{$\mathrm{C}_{21} \mathrm{H}_{25} \mathrm{NO}_{6}$}

16-Oxohasubanonine, 16341

\section{$\mathrm{C}_{21} \mathrm{H}_{25} \mathrm{NO}_{7}$}

Oxoepistephamiersine, 16328

Oxostephamiersine, 16424

\section{$\mathrm{C}_{21} \mathrm{H}_{25} \mathrm{NO}_{10}$}

Nigrumin-5-ferulate, 15594

\section{$\mathrm{C}_{21} \mathrm{H}_{25} \mathrm{~N}_{2} \mathrm{O}_{3}$}

N4-Methyl angustilobine B, 14138

\section{$\mathrm{C}_{21} \mathrm{H}_{25} \mathbf{N}_{7} \mathbf{O}_{7}$}

Folinic acid, 7853

\section{$\mathrm{C}_{21} \mathrm{H}_{26} \mathrm{NO}_{4}$}

(S)-trans- $N$-Methyltetrahydrocolumbamine, 14748

\section{$\mathrm{C}_{21} \mathrm{H}_{26} \mathrm{NO}_{4}{ }^{+}$}

Menisperine, 13716

Xanthoplanine, 22771

\section{$\mathrm{C}_{21} \mathrm{H}_{26} \mathrm{~N}_{2} \mathrm{O}_{2}$}

Aspidospermatine, 1903

Chrysotricine, 3629

Coronaridine, 4080

Dihydrovindolinine, 5737

16-Epikopsinine, 6941

Isochrysotricine, 11330 
Kopsinine, 12268

10-Methoxyaffinisine, 13837

12-Methoxyaffinisine, 13838

17-O-Methylakagerine, 14123

\section{$\mathrm{C}_{21} \mathrm{H}_{26} \mathrm{~N}_{2} \mathrm{O}_{3}$}

Alloyohimbine, 942

Corynanthine, 4115

Humantenine, 9659

15 $\alpha$-Hydroxykopsinine, 10293

Isositsirikine, 11718

Protostrychnine, 17994

Pseudoyohimbine, 18074

Rhazimanine, 18752

Sitsirikine, 20000

Tabernaemontanine, 20575

Vincamine, 22489

Yohimbine, 22921

\section{$\mathrm{C}_{21} \mathrm{H}_{26} \mathrm{~N}_{2} \mathrm{O}_{4}$}

Amsosinine, 1089

Aspidodasycarpine, 1901

Humantenirine, 9660

19-(R)-Hydroxydihydrogelsevirine, 10001

19-(S)-Hydroxydihydrogelsevirine, 10002

11-Hydroxyhumantenine, 10168

15-Hydroxyhumantenine, 10169

Isorhynchophyllic acid, 11678

Isorhynchophylline, 11679

Lonicerine, 12988

11-Methoxygelsemamide, 13938

Rhynchophyllic acid, 18825

\section{$\mathrm{C}_{21} \mathrm{H}_{26} \mathrm{O}_{2}$}

Cannabinol, 3082

\section{$\mathrm{C}_{21} \mathrm{H}_{26} \mathrm{O}_{3}$}

5-(1-Ethoxy-ethyl)-2-hydroxy-7-methoxy-1,8dimethyl-9,10-dihydrophenanthrene, 7403

(3S,5R)-3-Hydroxy-5-ethoxy-1-(4-hydroxyphenyl)-7-phenyl-6E-heptene, 10099

(3S,5S)-3-Hydroxy-5-ethoxy-1-(4-hydroxyphenyl)-7-phenyl-6E-heptene, 10100

1-(4-Hydroxy-2-methoxyphenyl)-3-(4-hydroxy-

3-prenylphenyl)propane, 10445

$11 \alpha$-Hydroxy 18,20-oxido-3-oxo-pregna-

1,4,17(20)-triene, 10557

Hypolide methyl ether, 10900

5-Methoxy-7-(4"-hydroxyphenyl)-1-phenyl-3heptanone, 13967

Neridienone A, 15493

Octabenzone, 15932

S-I, 19853

\section{$\mathrm{C}_{21} \mathrm{H}_{26} \mathrm{O}_{4}$}

Crocetin monomethyl ester, 4248

Dehydroagastol, 4875

Isoneotriptophenolide, 11561 5-methoxy-7-(4"-hydroxy-3"-methoxy-

phenyl)-1-phenyl-3-heptanone, 13962

Neotriptophenolide, 15468

Preracemosol A, 17846

Serpendione, 19761

Terresterone A, 21002

$\mathrm{C}_{21} \mathrm{H}_{26} \mathrm{O}_{5}$

Diarylheptanoid CPB-51-262-4, 5375

Futokadsurin A, 8037

Kadsurenin C, 12006

4-Methoxymachilin D, 13992

12-( $\alpha$-Methyl butyryl)-14-acetyl-2E, $8 E, 10 E$ atractylentriol, 14189

12-( $\alpha$-Methyl butyryl)-14-acetyl-2E,8Z,10Eatractylentriol, 14190

(+)-S-Myricanol, 15159

Myricanol, 15160

(-)-Nectandrin A, 15318

PC-66-633-3, 16744

Rosmaquinone A, 18922

Rosmaquinone B, 18923

Shikonofuran B, 19822

Shikonofuran C, 19823

Tashironin, 20716

Terresterone B, 21003

\section{$\mathrm{C}_{21} \mathrm{H}_{26} \mathrm{O}_{6}$}

Biondinin A, 2387

Candesalvoquinone, 3069

Cascarinin B, 3252

Daphneligin, 4641

$\left(2 S, 3 S, 1^{\prime} S, 2^{\prime} R\right)$-and $\left(2 S, 3 S, 1^{\prime} R, 2^{\prime} R\right)-2,3$-Dihydro5-(1',2'-dihydroxypropyl)-2-(3,4-dimethoxyphe nyl)-7-methoxy-3-methylbenzofuran, 5596

(E)-3-(3,4-Dimethoxyphenyl)-2-propen-1-yl (Z)-2-[(Z)-2-methyl-2-butenoyloxymethyl] butenoate, 6288

5-O-Ethyl-hirsutanonol, 7445

Hexahydrocurcumin, 9498

erythro-1-(4-Hydroxy-3-methoxyphenyl)-2(4-allyl-2,6-dimethoxyphenoxy) propan-1-ol, 10434

Phantomolin, 17042

Scaphopetalone, 19456

Theraphin A, 21313

$\mathrm{C}_{21} \mathrm{H}_{26} \mathrm{O}_{7}$

Acetylerioflorin, 390

$3 \beta$-Angeloyloxy- $8 \beta$-methoxyeremophil-7(11)ene-12, $8 \alpha(14 \beta, 6 \alpha)$-diolide, 1230

Aspidinin, 1896

Eurycolactone A, 7639

5-Hydroxy-1-(3,4-dihydroxy-5-methoxyphenyl)7-(4-hydroxy-3-methoxyphenyl)heptan-3-one, 10011
5-Hydroxy-1-(4-hydroxy-3-methoxyphenyl)7-(3,4-dihydroxy-5-methoxyphenyl)heptan-3one, 10183

5-Methoxy-trans-dihydrodehydrodiconiferyl alcohol, 13907

(-)-8-Methoxyisolariciresinol, 13975

(-)-5'-Methoxyisolariciresinol, 13976

5'-Methoxylariciresinol, 13986

Norcaesalpinin F, 15722

Nudaphantin, 15857

Pseudolaric acid C, 18035

\section{$\mathrm{C}_{21} \mathrm{H}_{26} \mathrm{O}_{8}$}

Calealactone C, 2958

Calein D, 2964

15 -Hydroxy- $9 \alpha$-acetoxy- $8 \beta$-isobutyryloxy-14oxo-melampolide, 9755

Icariol $\mathrm{A}_{1}, 10940$

Torosaflavone A, 21461

\section{$\mathrm{C}_{21} \mathrm{H}_{26} \mathrm{O}_{8} \mathrm{~S}$}

Shogasulfonic acid A, 19848

\section{$\mathrm{C}_{21} \mathrm{H}_{26} \mathrm{O}_{9}$}

Calealactone B, 2957

\section{$\mathrm{C}_{21} \mathrm{H}_{26} \mathrm{O}_{10}$}

Bruceolide, 2674

$\mathrm{C}_{21} \mathrm{H}_{26} \mathrm{O}_{10} \mathrm{~S}$

Shogasulfonic acid D, 19851

$\mathrm{C}_{21} \mathrm{H}_{26} \mathrm{O}_{12}$

Plumieride, 17571

\section{$\mathrm{C}_{21} \mathrm{H}_{26} \mathrm{O}_{13}$}

Scopoletin $\beta$ - $D$-xylopyranosyl-( $1 \rightarrow 6)-\beta$ - $D$ glucopyranoside, 19544

\section{$\mathrm{C}_{21} \mathrm{H}_{27} \mathrm{NO}_{2}$}

Holonamine, 9591

Variegatine, 22348

\section{$\mathrm{C}_{21} \mathrm{H}_{27} \mathrm{NO}_{3}$}

Delcarduchol, 5002

3,18-Dioxo-11 $\alpha$-hydroxycona-1,4-diene, 6469

1-[1-Oxo-9(3,4-methylenedioxyphenyl)-2E, $8 E$ nonadienyl] piperidine, 16384

\section{$\mathrm{C}_{21} \mathrm{H}_{27} \mathrm{NO}_{4}$}

Laudanosine, 12563

Protostephanine, 17993

\section{$\mathrm{C}_{21} \mathrm{H}_{27} \mathrm{NO}_{5}$}

Hasubanonine, 9245

Runanine, 19067

\section{$\mathrm{C}_{21} \mathrm{H}_{27} \mathrm{NO}_{6}$}

Stephamiersine, 20318

\section{$\mathrm{C}_{21} \mathrm{H}_{27} \mathrm{NO}_{7}$}

Clivorine, 3847

Trachelogenin amide, 21491

\section{$\mathrm{C}_{21} \mathrm{H}_{27} \mathrm{~N}_{2} \mathrm{O}_{2}$}

Fluorocurine, 7841

$\mathrm{C}_{21} \mathrm{H}_{27} \mathrm{~N}_{3} \mathrm{O}_{3}$ 
Celafurine, 3358

$\mathrm{C}_{21} \mathrm{H}_{27} \mathrm{~N}_{3} \mathrm{O}_{6}$

Casimiroedine, 3275

$\mathbf{C}_{\mathbf{2 1}} \mathrm{H}_{\mathbf{2 7}} \mathrm{N}_{7} \mathbf{O}_{\mathbf{1 4}} \mathbf{P}_{\mathbf{2}}$

Coenzyme I, 3893

$\mathbf{C}_{21} \mathbf{H}_{27} \mathbf{N}_{7} \mathbf{O}_{17} \mathbf{P}_{3}$

Coenzyme II, 3894

$\mathrm{C}_{21} \mathrm{H}_{28} \mathrm{~N}_{2} \mathrm{O}_{2}$

Macrocarpine A, 13316

$\mathrm{C}_{21} \mathrm{H}_{28} \mathrm{~N}_{2} \mathrm{O}_{3}$

Alstohentine, 994

Dihydrositsirikine, 5716

\section{$\mathrm{C}_{21} \mathrm{H}_{28} \mathrm{~N}_{2} \mathrm{O}_{6}$}

11-Methoxy-19-(R)-hydroxygelselegine, 13961

\section{$\mathrm{C}_{21} \mathrm{H}_{28} \mathrm{O}_{3}$}

Pyrethrin I, 18255

Salvibracteone, 19208

Triptinin A, 21993

\section{$\mathrm{C}_{21} \mathrm{H}_{28} \mathrm{O}_{4}$}

$3 \beta$-Acetoxy-12-methoxy-13-methyl-podocarpa-

8,11,13-trien-7-one., 254

Adonilide, 644

Anhydrohirundigenin, 1271

Bractealine, 2584

$t$-Cajucarin B, 2932

11-Dehydrocorticosterone, 4887

Erectquione A, 7223

Geranyloxy sinapyl aldehyde, 8328

Ialibinone A, 10929

Ialibinone B, 10930

Methyl 15,16-epoxy-12-oxo-8(17),13(16),14ent-labdatrien-19-oate, 14408

Neotussilagolactone, 15470

Stizophyllin, 20386

Taiwaniaquinol A, 20629

Taiwaniaquinol D, 20632

Taiwaniaquinone F, 20638

Triptobenzene H, 21995

Triptonoditerpenic acid, 22014

\section{$\mathrm{C}_{21} \mathrm{H}_{28} \mathrm{O}_{5}$}

Aldosterone, 880

Cascarinin C, 3253

CinerinII, 3692

Limbatolide A, 12834

Ludongnin J, 13071

7-Methoxyrosmanol, 14085

1-Methylene-2,4a-dimethyl-6,8-dihydroxy-5methoxy-7-(1,1-dimethyl hydroxymethyl)-

1,2,3,4,9,10,10a-heptahydro-9-phenanthrone, 14359

9 $\beta$-(3-Methyl-pentoyl-3-ene)-parthenolide, 14667

Taiwaniaquinone E, 20637

Tricyclodehydroisohumulone, 21599
$\mathrm{C}_{21} \mathrm{H}_{28} \mathrm{O}_{6}$

$3 \beta$-Angeloyloxy-10 $\beta$-hydroxy- $6 \beta$-methoxy-

eremophila-7(11),8(9)-dien-8,12-olide, 1227

Angustifolin, 1245

Candesalvone B methyl ester, 3068

6-Epiangustifolin, 6826

Epi-maoecrystal P, 6958

Eupaglehnin D, 7563

Macrocalyxin B, 13306

Maoecrystal Q, 13538

12-O-Methylcandesalvone B, 14210

Nervosin, 15510

Nigakilactone I, 15554

Octahydrocurcumin, 15965

Rabdosin A, 18496

Sauriol B, 19416

\section{$\mathrm{C}_{21} \mathrm{H}_{28} \mathrm{O}_{6} \mathrm{~S}$}

Bakkenolide D, 2120

S-Fukinolide, 7988

\section{$\mathrm{C}_{21} \mathrm{H}_{28} \mathrm{O}_{7}$}

$3 \beta$-Acetoxy- $8 \beta$-isobutyryloxyreynosin, 240

$3 \beta$-Angeloyloxy-8,12-epoxy-12 $\alpha$-hydroxy- $8 \beta$ methoxyeremophil-7(11)-en-14 $\beta, 6 \alpha$-olide, 1219

Hancogenin B, 9212

8-O-Isobutyryl-9 $\alpha$-acetoxycumambrin B, 11292

3'-Methoxysecoisolariciresinol, 14089

Picrasin G, 17317

Stauntonine, 20275

Viguiestin, 22475

\section{$\mathrm{C}_{21} \mathrm{H}_{28} \mathrm{O}_{8}$}

1-(4'-Hydroxy-3'-methoxyphenyl)-2-[4"-(3hydroxypropyl)-2",6"-dimethoxyphenoxy]propane-1,3-diol, 10446

$8 \alpha$-Isobutyryloxyanthemolide A, 11298

$8 \alpha$-Isobutyryloxyanthemolide C, 11299

Ixerisoside, 11797

Picrodendrin Q, 17336

Vernoflexuoside, 22408

\section{$\mathrm{C}_{21} \mathrm{H}_{28} \mathrm{O}_{9}$}

Anthemolide F, 1357

Deacetylmatricarin 8-O- $\beta$-glucopyronoside, 4750

$2 \alpha$-Hydroperoxy-8-O-isobutyryl- $9 \alpha$-acetoxycumambrin B, 9730

11 $\beta$-Hydroxyleukodin 11-O- $\beta$-glucopyranoside, 10319

8-O-Isobutyryl-9- $O$-acetylanthemolide B, 11293

Picrodendrin M, 17334

Taraxinic acid-1'- $O-\beta$ - $D$-glucopyranoside, 20714

\section{$\mathrm{C}_{21} \mathrm{H}_{28} \mathrm{O}_{10}$}

Picrodendrin A, 17332

Taraxafolide, 20694

$\mathrm{C}_{21} \mathrm{H}_{28} \mathrm{O}_{12}$
Cistanoside I, 3759

Sibirioside A, 19867

\section{$\mathrm{C}_{21} \mathrm{H}_{28} \mathrm{O}_{13}$}

Cistanoside F, 3756

(6- $O$ - $(E)$ - $p$-Coumaroyl)- $\beta$ - $D$-fructofuranosyl$(2 \rightarrow 1)$ - $\alpha$ - $D$-glucopyranoside, 4159

Swertiamacroside, 20508

\section{$\mathrm{C}_{21} \mathrm{H}_{28} \mathrm{O}_{14}$}

1-O-(E)-Caffeoyl- $\beta$-gentiobiose, 2905

1-O-(3'-O- $\beta$ - $D$-Glucopyranosyl)-(E)-caffeoyl$\beta$ - $D$-glucopyranose, 8612

\section{$\mathrm{C}_{21} \mathrm{H}_{29} \mathrm{BrN}_{2}$}

Flustramine A, 7843

Flustramine B, 7844

\section{$\mathrm{C}_{21} \mathrm{H}_{29} \mathrm{ClO}_{7}$}

(2S)-Pteroside K, 18122

\section{$\mathrm{C}_{21} \mathrm{H}_{29} \mathrm{NO}$}

1-Methyl-2-[(Z)-5-undecenyl]-4(1H)-quinolone, 14799

1-Methyl-2-[(Z)-6-undecenyl]-4(1H)-quinolone, 14800

\section{$\mathrm{C}_{21} \mathrm{H}_{29} \mathrm{NO}_{2}$}

Regholarrhenine B, 18586

\section{$\mathrm{C}_{21} \mathrm{H}_{29} \mathrm{NO}_{3}$}

Piperolein B, 17469

Spiradine G, 20180

\section{$\mathrm{C}_{21} \mathrm{H}_{29} \mathrm{NO}_{4}$}

Incarvine $\mathrm{C}, 11011$

\section{$\mathrm{C}_{21} \mathrm{H}_{29} \mathrm{~N}_{2} \mathrm{O}_{2}$}

9-Methoxy- $N_{b}$-methylgeissoschizol, 14024

\section{$\mathrm{C}_{21} \mathrm{H}_{29} \mathrm{O}_{6}$}

Eupaglehnin A, 7560

\section{$\mathrm{C}_{21} \mathrm{H}_{30} \mathrm{O}$}

6-Dehydrohinokiol, 4929

\section{$\mathrm{C}_{21} \mathrm{H}_{30} \mathrm{O}_{2}$}

Cannabichromene, 3077

Cannabidiol, 3079

Dehydroabietic acid methyl ester, 4874

12-Methoxy-6,8,11,13-abietatraen-11-ol, 13825

4-Nerolidylcatechol, 15505

Progesterone, 17901

$\Delta^{8}$-Tetrahydrocannabinol , 21058

$\Delta^{9}$-Tetrahydrocannabinol, 21059

\section{$\mathrm{C}_{21} \mathrm{H}_{30} \mathrm{O}_{3}$}

Cryptojaponol, 4281

Deoxycorticosterone, 5163

3 $\beta$-Hydroxy-1-oxo-13- $O$-methyltotarol, 10575

8,11,13-Icetexantrien-10,11,12-triol, 10957

Methyl dehydro-15-hydroxy-abietan-18-oate, 14285

Methyl lambertianate, 14548

(+)-Methyl vouacapenate, 14814

Przewalskin C, 18009 
Sugikurojin F, 20462

Tupipregnenolone, 22117

Turricolol E, 22142

\section{$\mathrm{C}_{21} \mathrm{H}_{30} \mathrm{O}_{4}$}

Bornyl-2-methoxy-4-hydroxycinnamate, 2562

Corticosterone, 4096

10-Dehydrogingerdione, 4922

Geranyloxy sinapyl alcohol, 8327

Glaucopine A, 8521

Glaucopine B, 8522

$3 \beta$-Hydroxy-cis-dehydrocrotonin, 9971

17-Hydroxy-11-deoxy-corticosterone, 9986

Otogirin, 16268

PC-66-633-5, 16741

Pinusolide, 17422

Triptonoterpenol, 22017

\section{$\mathrm{C}_{21} \mathrm{H}_{30} \mathrm{O}_{5}$}

Adhumulone, 628

Coetsoidin C, 3901

4-O-[(2E,5E)-3,7-Dimethyl-2,5-octadiene-7-ol]sinapyl alcohol, 6381

Enaimeone B, 6781

Humulone, 9678

17-Hydroxycorticosterone, 9937

1'-Hydroxyialibinone A, 10220

1'-Hydroxyialibinone B, 10221

Isohumulone A, 11459

Isohumulone B, 11460

Kamebacetal A, 12121

Kamebacetal B, 12122

Limbatolide B, 12835

Ludongnin F, 13067

Ludongnin G, 13068

Ludongnin H, 13069

Ludongnin I, 13070

(+)-15-Methoxyfloridiolide A, 13929

(10R)-15-Methoxypinusolidic acid, 14069

(10S)-15-Methoxypinusolidic acid, 14070

Nelumol B, 15326

PC-66-633-1, 16742

Poststerone, 17741

4-Pregnene-17 $\alpha, 20 \beta, 21$-triol-3,11-dione, 17804

Taiwaniaquinol C, 20631

1,3,5-Trihydroxy-6-[2"',3"'-epoxy-3"'-methylbutyl]-2-[2"-methyl-butanoyl]-4-[3'-methyl-2'butenyl]-benzene, 21727

\section{$\mathrm{C}_{21} \mathrm{H}_{30} \mathrm{O}_{6}$}

$6 \beta$-Angeloyloxy-10 $\beta$-hydroxy- $8 \alpha$-methoxyeremophil-7(11)-en-12,8 $\beta$-olide, 1228

$6 \beta$-Angeloyloxy-10 $\beta$-hydroxy- $8 \beta$-methoxyeremophil-7(11)-en-12,8 $\alpha$-olide, 1229

Coetsoidin D, 3902

Humulinone, 9677
Irroratin A, 11185

Nigakilactone A, 15547

PC-66-633-2, 16743

PC-66-633-4, 16745

Rabdonervosin B, 18481

Rubescensin W, 18978

Syringenin diisovalerate, 20559

$\mathrm{C}_{21} \mathrm{H}_{30} \mathrm{O}_{7}$

$3 \beta$-Angeloyloxy- $8 \beta, 10 \beta$-dihydroxy- $6 \beta$ methoxyeremophilenolide, 1217

Isodotricin, 11402

Nigakihemiacetal E, 15544

Nigakilactone M, 15558

Nigakilactone N, 15559

Pteroside Z, 18131

Rabdoternin E, 18505

Rabdoternin F, 18506

Rabdoternin G, 18507

Rubescensin H, 18977

Taibaijaponicain A, 20620

\section{$\mathrm{C}_{21} \mathrm{H}_{30} \mathrm{O}_{8}$}

Cuminoside A, 4358

Eurycolactone, 7638

Methyl $8 \alpha$-(3,4-dihydroxy-2-methylenebutanoyloxy)- $6 \alpha, 15$-dihydroxyelema1,3,11(13)-trien-12-oate, 14312

Onitin-2'-O- $\beta$ - $D$-alloside, 16099

Onitin-2'- $O-\beta$ - $D$-glucoside, 16100

Onitinoside, 16101

Pteroside A, 18116

Pteroside D, 18121

Tithofolinolide, 21410

$3 \beta, 6 \beta, 8 \alpha$-Triacetoxy-4 $\beta, 5 \alpha$-epoxy-1-oxogermacr-10(14)-ene, 21519

\section{$\mathrm{C}_{21} \mathrm{H}_{30} \mathrm{O}_{9}$}

$11 \beta, 13$-Dihydro-taraxinic acid-1'- $O-\beta$ - $D$ glucopyranoside, 5724

10 $\beta, 14$-Dihydroxy-10(14),11 $\beta(13)$-tetrahydro8,9-didehydro-3-deoxyzaluzanin C 10-O- $\beta$ - $D$-glucopyranoside, 6143

(3S)-3-Hydroxyatractylenolide III

3-O- $\beta$-D-glucopyranoside, 9806

$9 \alpha$-Hydroxy-11 $\beta, 13$-dihydrozaluzanin C

3-O- $\beta$-allopyranoside, 10012

Napiferoside, 15262

Prenantheside A, 17822

Pteroside W, 18129

Pteroside X, 18130

$2 R, 3 R$-Pterosin L-2'- $O$ - $\beta$ - $D$-glucoside, 18140

$2 S, 3 R$-Pterosin L-2'- $O$ - $\beta$ - $D$-glucoside, 18141

\section{$\mathrm{C}_{21} \mathrm{H}_{30} \mathrm{O}_{10}$}

15-O- $\beta$ - $D$-Glucopyranosyl-11 $\beta, 13$-dihydrourospermal A, 8615
(1'S,6'R)-8'-Hydroxyabscisic acid $\beta$ - $D$-glucoside, 9748

$\mathrm{C}_{21} \mathrm{H}_{30} \mathrm{O}_{11}$

(2E)-2-Decene-4,6-diyne-1,8-diol 8- $O-\beta$ - $D$-apiofuranosyl- $(1 \rightarrow 6)-\beta$ - $D$-glucopyranoside, 4842

3,4-Dihydroxyallylbenzene

4- $O$ - $[\alpha$ - $L$-rhamnopyranosyl- $(1 \rightarrow 6)]-\beta$ - $D$-gluco pyranoside, 5754

Gein, 8250

Gymnasterkoreaside B, 9105

Pectinolide F, 16752

4-Propenyl-2-methoxyphenyl 6-O- $\beta$ - $D$-apiofuranosyl

$(1 \rightarrow 6)-\beta$ - $D$-glucopyranoside, 17922

Rhododendroketoside, 18795

\section{$\mathrm{C}_{21} \mathrm{H}_{30} \mathrm{O}_{12}$}

4- $[\beta$ - $D$-Apiofuranosyl-( $1 \rightarrow 6)-\beta$ - $D$-glucopyranosyloxy]-3-methoxypropiophenone, 1513

Coelovirin A, 3892

Coniferyl 9-O- $[\beta-D$-apiofuranosyl $(1 \rightarrow 6)]-O-\beta$ - $D$ glucopyranoside, 3984

\section{$\mathrm{C}_{21} \mathrm{H}_{30} \mathrm{O}_{13}$}

Acetylbarlerin, 330

6'-O- $\beta$-Apiofuranosylsweroside, 1517

Ebractelatinoside C, 6668

Erythroxyloside B, 7346

Everninic acid methyl ester 2- $O-\beta$-xylopyranosyl-( $1 \rightarrow 6)$ - $\beta$-glucopyranoside, 7662

Tectoruside, 20904

\section{$\mathrm{C}_{21} \mathrm{H}_{30} \mathrm{O}_{14}$}

6'-O-Acetyldiderroside, 376

6'-O- $\alpha$ - $L$-Arabinopyranosylswertiamarin, 1593

Chironiside, 3539

Syringic acid methyl ester-4- $O-\beta$ - $D$-apiofuranosyl-( $1 \rightarrow 2)$ - $\beta$ - $D$-glucopyranoside, 20567

\section{$\mathrm{C}_{21} \mathrm{H}_{31} \mathrm{NO}$}

1-Methyl-2-undecyl-4(1H)-quinolilone, 14801

\section{$\mathrm{C}_{21} \mathrm{H}_{31} \mathrm{NO}_{4}$}

$N$-Deethyl- $N$-19-didehydrosachaconitine, 4867

Liconosine A, 12781

\section{$\mathrm{C}_{21} \mathrm{H}_{32} \mathrm{~N}_{2}$}

Conkurchine, 3991

\section{$\mathrm{C}_{21} \mathrm{H}_{32} \mathrm{O}_{2}$}

Cannabigerol, 3081

trans-Communic acid methyl ester, 3945

1,4-Epoxy-16-hydroxyheneicos-1,3,12,14,18pentaene, 7117

Labda-7,12(E),14-triene-17-oic acid methyl ester, 12416

1-(3,4-Methylenedioxyphenyl)-1E-tetradecene, 14382

(-)-Methyl kaur-16-en-19-oate, 14543

12-O-Methylpisiferanol, 14679 
Pregnenolone, 17805

Urushiol III, 22274

\section{$\mathrm{C}_{21} \mathrm{H}_{32} \mathrm{O}_{3}$}

$3 \beta$-Acetoxy-17 $\beta$-hydroxy-androst-5-ene, 205

Agallochin O, 695

4(18),13-Clerodadien-3-oxo-15-oic acid methyl ester, 3835

Coronarin D methyl ether, 4085

10-Deoxy-4,18-epoxy-12-methoxy-4,5-secopisiferan-19-ol, 5170

$15 \alpha, 20 \beta$-Dihydroxy- $\Delta^{4}$-pregnen-3-one, 6101

$1 \beta$-Hydroxypisiferanol, 10657

12-Methoxy-8,11,13-abietatriene-7 $\beta, 11$-diol, 13826

Preclavulone A methyl ester, 17773

Preclavulone A methyl ester 12-isomer, 17775

Salvicanol, 19209

trans-10-Shogaol, 19847

\section{$\mathrm{C}_{21} \mathrm{H}_{32} \mathrm{O}_{4}$}

Ceriopsin A, 3423

Ceriopsin F, 3427

CPB5212-1492-2, 4205

17 $\alpha, 20 R$-Dihydroxypregnan-3,16-dione, 6100

Fukujusone, 7992

[10]-Gingerdione, 8388

Isoramanone, 11643

Methyl 16 $\alpha, 17$-dihydroxy-ent-9(11)-kauren-19oate, 14305

Methyl $7 \alpha, 12 \beta$-dihydroxysandaracopimarate, 14317

Methyl 12-oxo-8 $\alpha$-hydroxyabiet-13-en-19-oate, 14647

Ramanone, 18528

\section{$\mathrm{C}_{21} \mathrm{H}_{32} \mathrm{O}_{5}$}

Agallochin N, 694

1-[2',4'-Dihydroxy-3'-(3"-methylbut-2"-enyl)-5'(1"'-ethoxy-3"'-methylbutyl)-6'-methoxy]phenylethanone, 6022

16 $\alpha, 17$-Dihydroxy-15-oxo-ent-kaur-19-oic acid methyl ester, 6067

Isolineolone, 11497

Lineolone, 12880

Methyl 15-hydroxy- $8 \alpha, 12 \alpha$-epidioxiabiet-13-en19-oate, 14504

Methyl 12-oxo-8 $\alpha, 15$-dihydroxyabiet-13-en-19oate, 14644

Nepetalic anhydride, 15488

Pergularin, 16917

$\mathrm{C}_{21} \mathrm{H}_{32} \mathrm{O}_{6}$

Ajugalide D, 798

Anhydrocinnzeylanol, 1264

Deacylmetaplexigenin, 4796

[6]-Gingediacetate, 8382
$15 \beta$-Hydroxyisolineolon, 10248

$15 \beta$-Hydroxylineolon, 10327

6 $\beta$-(2'-Methylbutanoyloxy)-10 $\beta$-hydroxy- $8 \alpha$ methoxyeremophil-7(11)-en-12,8 $\beta$-olide, 14170

$6 \beta$-(2'-Methylbutanoyloxy)-10 $\beta$-hydroxy- $8 \beta$ methoxyeremophil-7(11)-en-12,8 $\alpha$-olide, 14171

Methyl 15-hydroperoxy- $8 \alpha, 14 \alpha, 12 \alpha, 13 \alpha$ diepoxiabietan-13-en-19-oate, 14495

Methyl 15-hydroperoxy- $8 \alpha, 12 \alpha$-epidioxiabiet13-en-19-oate, 14496

Nardostachin, 15276

Nervosanin A, 15508

Nigakihemiacetal C, 15543

Sibiricinone C, 19860

$10 \alpha, 16 \alpha, 17-T r i h y d r o x y-9 \alpha$-methyl-15-oxo-20nor-kauran-19-oic acid methyl ester, 21807

Xindongnin K, 22807

\section{$\mathrm{C}_{21} \mathrm{H}_{32} \mathrm{O}_{7}$}

Furonewguinone B, 8029

Lushanrubescensin F, 13121

$3 \beta, 6 \beta, 8 \alpha$-Triacetoxy- $4 \beta, 5 \alpha$-epoxygermacr$1(10) E$-ene, 21518

\section{$\mathrm{C}_{21} \mathrm{H}_{32} \mathrm{O}_{8}$}

Alatoside C, 840

Alatoside D, 841

Mukaadial 6- $O-\alpha-L$-rhamnopyranoside, 15025

Sonchuside A, 20073

$3 \beta, 6 \beta, 8 \alpha$-Triacetoxy- $4 \beta, 5 \alpha: 1 \alpha, 10 \beta$-diepoxygermacrane, 21514

\section{$\mathrm{C}_{21} \mathrm{H}_{32} \mathrm{O}_{9}$}

Cuminoside B, 4359

Mukaadial 6-O- $\beta$ - $D$-glucopyranoside, 15024

\section{$\mathrm{C}_{21} \mathrm{H}_{32} \mathrm{O}_{10}$}

Dendromoniliside C, 5118

Dihydrophaseic acid 4'-O- $\beta$ - $D$-glucopyranoside, 5691

\section{$\mathrm{C}_{21} \mathrm{H}_{32} \mathrm{O}_{11}$}

Adinoside B, 637

Adinoside C, 638

Apiosylepirhododendrin, 1522

Dendromoniliside B, 5117

Loniceracetalide A, 12985

Loniceracetalide B, 12986

$\mathrm{C}_{21} \mathrm{H}_{32} \mathrm{O}_{12}$

Cistanoside E, 3755

4'-Deoxykanokoside A, 5185

\section{$\mathrm{C}_{21} \mathrm{H}_{32} \mathrm{O}_{13}$}

Diospyrososide, 6463

2-(3-Hydroxy-4-methoxyphenyl)-ethyl- $O-\beta$ - $D$ glucopyranosyl $(1 \rightarrow 3)-\beta-D$-glucopyranoside, 10442
1 -[ $\alpha$ - $L$-Rhamnosyl-( $1 \rightarrow 6)$ - $\beta$ - $D$-glucopyranosyl]3,4,5-trimethoxybenzene, 18740

Sinuatol, 19960

$\mathrm{C}_{21} \mathrm{H}_{32} \mathrm{O}_{14}$

6- $O-\beta$ - $D$-Apiofuranosyl-mussaenosidic acid, 1516

6-O- $\alpha$ - $L$-Rhamnopyranosylcatalpol, 18694

Ulmoside, 22193

\section{$\mathbf{C}_{\mathbf{2 1}} \mathbf{H}_{\mathbf{3 2}} \mathbf{O}_{\mathbf{1 5}}$}

3'-O- $\beta$ - $D$-Glucopyranosyl-catalpol, 8613

Melittoside, 13710

Rehmannioside A, 18596

Rehmannioside B, 18597

\section{$\mathrm{C}_{21} \mathrm{H}_{33} \mathrm{NO}_{3}$}

Dictysine, 5458

\section{$\mathrm{C}_{21} \mathrm{H}_{33} \mathrm{NO}_{4}$}

16 $\beta$-Hydroxycardiopetaline, 9895

\section{$\mathrm{C}_{21} \mathrm{H}_{33} \mathrm{NO}_{7}$}

Lasiocarpine, 12535

\section{$\mathrm{C}_{21} \mathrm{H}_{33} \mathrm{O}_{3}$}

$O$-Methylpisiferic acid, 14680

\section{$\mathrm{C}_{21} \mathrm{H}_{34} \mathrm{NO}_{2}^{+}$}

$\mathrm{N}$-Isopentenyl dendrobine, 11585

\section{$\mathrm{C}_{21} \mathrm{H}_{34} \mathrm{~N}_{2}$}

Wrightiamine A, 22735

\section{$\mathrm{C}_{21} \mathrm{H}_{34} \mathrm{O}$}

Cardanol, 3188

\section{$\mathrm{C}_{21} \mathrm{H}_{34} \mathrm{O}_{2}$}

Bilobol, 2380

1,4-Epoxy-16-hydroxyheneicos-1,3,12,14tetraene, 7118

5 $\beta, 20$-Epoxy-20-methoxy-ros-15-ene, 7165

3-(Pantadec-10-enyl)-catechol, 16622

5-Pregnene-3 $\beta, 20 \alpha$-diol, 17801

\section{$\mathrm{C}_{21} \mathrm{H}_{34} \mathrm{O}_{3}$}

Hydroginkgolinic acid, 9718

$2 \alpha$-Hydroxy-3,13-clerodadien-15-oic acid methyl ester, 9920

2 $\beta$-Hydroxy-3,13-clerodadien-15-oic acid methyl ester, 9921

$2 \beta$-Hydroxy-9-epi-ent-labda-8(17),13(Z)-dien15-oic acid, 10076

(S)-12-Hydroxygeranylgeranic acid methyl ester, 10145

Isoobtusilactone B, 11568

Obtusilactone B, 15904

5-Pregnene-3 $\beta, 17 \alpha, 20 \alpha$-triol, 17803

Siegesmethyletheric acid, 19880

Toosendansterol A, 21449

Toosendansterol B, 21450

\section{$\mathrm{C}_{21} \mathrm{H}_{34} \mathrm{O}_{4}$}

Annosquamosin D, 1328

Ceriopsin B, 3424 
[10]-Gingerol, 8397

Methyl 16 $\alpha, 17$-dihydroxy-ent-kauran-19-oate, 14304

\section{$\mathrm{C}_{21} \mathrm{H}_{34} \mathrm{O}_{5}$}

Annosquamosin F, 1330

15-Epi-sibiricinone D, 7020

15-Epi-sibiricinone E, 7021

Sibiricinone D, 19861

Sibiricinone E, 19862

Utendin, 22285

\section{$\mathrm{C}_{21} \mathrm{H}_{34} \mathrm{O}_{6}$}

Deniagenin, 5129

Sarcostin, 19361

\section{$\mathrm{C}_{21} \mathrm{H}_{34} \mathrm{O}_{8}$}

Dendromoniliside A, 5116

Dictamnoside L, 5453

\section{$\mathrm{C}_{21} \mathrm{H}_{34} \mathrm{O}_{9}$}

Alatoside B, 839

Celerioside A, 3378

Dendroside F, 5125

\section{$\mathrm{C}_{21} \mathrm{H}_{34} \mathrm{O}_{10}$}

Dendromoniliside D, 5119

Dendroside G, 5126

(Z)-(IS,5R)- $\beta$-Pinen-10-yl- $\beta$-vicianoside, 17378

Sacranoside A, 19109

$(1 S, 5 R, 7 R, 10 R)$-Secoatractylolactone

11-O- $\beta$ - $D$-glucopyranoside, 19606

\section{$\mathrm{C}_{21} \mathrm{H}_{34} \mathrm{O}_{11}$}

$(1 R, 4 S, 6 S)$-6-Hydroxycamphor $\beta$ - $D$-apiofuranosyl-(1 $\rightarrow 6)-\beta-D$-glucopyranoside, 9879

Patrinoside, 16720

Valerosidatum, 22323

\section{$\mathrm{C}_{21} \mathrm{H}_{34} \mathrm{O}_{13}$}

Phyllaemblicin D, 17207

\section{$\mathrm{C}_{21} \mathrm{H}_{34} \mathrm{O}_{14}$}

Rehmannioside C, 18598

\section{$\mathrm{C}_{21} \mathrm{H}_{35} \mathrm{NO}$}

Funtumine, 8010

Wrightiamine B, 22736

\section{$\mathrm{C}_{21} \mathrm{H}_{35} \mathrm{O}_{7}$}

$(5 R, 7 R, 10 S)$-Isopterocarpolon $\beta$ - $D$-gluco-

$$
\text { pyranoside, } 11634
$$

\section{$\mathrm{C}_{21} \mathrm{H}_{36} \mathrm{~N}_{2} \mathrm{O}$}

Holarrhidine, 9586

Holarrhimine, 9587

\section{$\mathrm{C}_{21} \mathrm{H}_{36} \mathrm{O}_{2}$}

Alkylresorcinol A, 911

Casealactone, 3257

ent-16 $\alpha$-Methoxy-kauran-17-ol, 13984

Methyl labd-13E-en-15-oate, 14546

$5 \alpha$-Pregnane-3 $\beta, 20 \beta$-diol, 17784

\section{$\mathrm{C}_{21} \mathrm{H}_{36} \mathrm{O}_{4}$}

[10]-Gingediol, 8385
$\mathrm{C}_{21} \mathrm{H}_{36} \mathrm{O}_{5}$

Agallochin M, 693

Desacylkondurangogenin C , 5242

$\gamma$-Eudesmol 11- $\alpha$ - $L$-rhamnoside, 7516

Tomentogenin, 21437

\section{$\mathrm{C}_{21} \mathrm{H}_{36} \mathrm{O}_{7}$}

Atractyloside C, 1976

$1 \beta$ - $D$-Glucopyranosyloxy- $6 \alpha$-hydroxyeudesman-4(15)-ene, 8692

Liriopeoside A, 12919

\section{$\mathrm{C}_{21} \mathrm{H}_{36} \mathrm{O}_{8}$}

Alatoside A, 838

Atractyloside G, 1979

Celerioside B, 3379

Celerioside C, 3380

Celerioside D, 3381

Dendroside A, 5122

Dendroside E, 5124

Dictamnoside H, 5449

(1S,4S,5R,7R,10R)-11,14-Dihydroxyguai-3-one

11-O- $\beta$ - $D$-glucopyranoside, 5902

Integrifoside A, 11095

Integrifoside B, 11096

\section{$\mathrm{C}_{21} \mathrm{H}_{36} \mathrm{O}_{9}$}

Dictamnoside A, 5446

Dictamnoside B, 5447

Integrifoside C, 11097

Integrifoside D, 11098

(1S,4S,5S,7R,10R)-10,11,14-Trihydroxyguai-

3-one 11-O- $\beta$ - $D$-glucopyranoside, 21739

$(1 S, 4 S, 5 S, 7 R, 10 S)-10,11,14$-Trihydroxyguai-

3-one 11-O- $\beta$ - $D$-glucopyranoside, 21740

\section{$\mathrm{C}_{21} \mathrm{H}_{36} \mathrm{O}_{10}$}

Atractyloside A, 1973

Borneol-2- $O-\beta$ - $D$-apiofuranosyl $(1 \rightarrow 6)-\beta$ - $D$ -

glucopyranoside, 2551

Borneol-2- $O-\alpha-L$-arabinofuranosyl $(1 \rightarrow 6)-\beta$ - $D$ glucopyranoside, 2552

10-Epiatractyloside A, 6833

2-(8-Methyl-2,8-dihydroxy-9-oxo-2hydroxymethylbicyclo[5.3.0]decan-7-yl) isopropanol glucoside, 14316

Neohancoside A, 15401

Shionoside A, 19831

$(4 S)-\alpha$-Terpineol $O-\beta$ - $D$-Apiofuranosyl- $(1 \rightarrow 6)$ $\beta$ - $D$-glucopyranoside, 20996

$\mathrm{C}_{21} \mathrm{H}_{36} \mathrm{O}_{11}$

$(1 S, 2 S, 4 R)-p$-Menth-8-ene-1,2-diol 2-O- $\beta$ - $D$ apiofuranosyl-( $1 \rightarrow 6)-\beta$ - $D$-glucopyranoside, 13747

Neohancoside B, 15402

$\mathrm{C}_{21} \mathrm{H}_{37} \mathrm{~N}_{3} \mathrm{O}_{2}$

Anhydrocannabisativine, 1262
$\mathrm{C}_{21} \mathrm{H}_{38} \mathrm{O}_{4}$

Glyceryl linolenate I, 8814

$\mathrm{C}_{21} \mathrm{H}_{38} \mathrm{O}_{6}$

Cryptomeridiol 11- $\alpha$ - $L$-rhamnoside, 4284

$\mathrm{C}_{21} \mathrm{H}_{38} \mathrm{O}_{8}$

Celerioside E, 3382

Dendronobiloside B, 5121

Icariside $\mathrm{C}_{3}, 10948$

Ophiopogonoside A, 16150

\section{$\mathrm{C}_{21} \mathrm{H}_{38} \mathrm{O}_{9}$}

Dictamnoside I, 5450

Dictamnoside J, 5451

\section{$\mathrm{C}_{21} \mathrm{H}_{38} \mathrm{O}_{10}$}

Atractyloside B, 1975

Dictamnoside K, 5452

2-(8-Methyl-2,8,9-trihydroxy-2-hydroxymethylbicyclo[5.3.0]decan-7-yl)isopropanolglucoside, 14782

$O-\beta-D$-Oleandropyranosyl- $(1 \rightarrow 4)-O-\beta-D$ cymaropyranosyl-( $(1 \rightarrow 4)-\beta$ - $D$-oleandropyranose, 16032

\section{$\mathrm{C}_{21} \mathrm{H}_{39} \mathrm{~N}_{7} \mathrm{O}_{13}$}

Reticulin, 18654

\section{$\mathrm{C}_{21} \mathrm{H}_{39} \mathrm{O}_{5}$}

Enaimeone A, 6780

\section{$\mathrm{C}_{21} \mathrm{H}_{40} \mathrm{O}_{2}$}

Methyl 11-eicosenoate, 14336

\section{$\mathrm{C}_{21} \mathrm{H}_{40} \mathrm{O}_{4}$}

$\alpha$-Monoolein, 14929

$\beta$-Monoolein, 14930

\section{$\mathrm{C}_{21} \mathrm{H}_{40} \mathrm{O}_{5}$}

Monoricinolein, 14932

\section{$\mathrm{C}_{21} \mathrm{H}_{41} \mathrm{NO}$}

1-(14-Methylhexadecanoyl)pyrrolidine, 14487

\section{$\mathrm{C}_{21} \mathrm{H}_{42} \mathrm{O}_{2}$}

Heneicosanoic acid, 9360

Methyl eicosanoate, 14335

\section{$\mathrm{C}_{21} \mathrm{H}_{42} \mathrm{O}_{4}$}

1,2-O-[2'-Hydroxyoctadecyl]-glycerol, 10548

1-O-Octadecanoyl glycerol, 15951

\section{$\mathrm{C}_{21} \mathrm{H}_{44}$}

Heneicosane, 9359

3-Methyl eicosane, 14334

\section{$\mathrm{C}_{22} \mathrm{H}_{14} \mathrm{O}_{6}$}

3,3'-Biplumbagin, 2402

3,8'-Biplumbagin, 2403

Chitranone, 3541

Diospyrin, 6459

Isodiospyrin, 11379

Isozeylanone, 11790

Mamegakinone, 13463

Neodiospyrin, 15392

$\mathrm{C}_{22} \mathrm{H}_{15} \mathrm{~N}_{3} \mathrm{O}_{4}$ 
Lycogaric acid A, 13197

$\mathrm{C}_{22} \mathrm{H}_{16} \mathrm{O}_{5}$

Latinone, 12559

$\mathrm{C}_{22} \mathrm{H}_{16} \mathrm{O}_{6}$

$6 \alpha, 12 \alpha$-Dehydromillettone, 4949

$\mathrm{C}_{22} \mathrm{H}_{16} \mathrm{O}_{7}$

8-C-p-Hydroxybenzylkaempferol, 9845

$\mathrm{C}_{22} \mathrm{H}_{16} \mathbf{O}_{10}$

Amurenisin, 1090

$\mathrm{C}_{22} \mathrm{H}_{16} \mathrm{O}_{12}$

Fumarprotocetraric acid, 7998

$\mathrm{C}_{22} \mathrm{H}_{16} \mathrm{O}_{15} \mathrm{~S}$

Myricatin, 15168

$\mathrm{C}_{22} \mathrm{H}_{17} \mathrm{NO}_{4}$

Crinasiatine, 4239

\section{$\mathrm{C}_{22} \mathrm{H}_{17} \mathrm{~N}_{3} \mathrm{O}_{3}$}

Cinereapyrrole B, 3691

\section{$\mathrm{C}_{22} \mathrm{H}_{17} \mathrm{~N}_{3} \mathrm{O}_{4}$}

Cinereapyrrole A, 3690

\section{$\mathrm{C}_{22} \mathrm{H}_{18} \mathrm{~N}_{2} \mathrm{O}_{5}$}

20-O-Acetylcamptothecin, 341

\section{$\mathrm{C}_{22} \mathrm{H}_{18} \mathrm{O}_{4}$}

Diospyrol, 6460

1-p-Hydroxybenzyl-4-methoxyphenanthrene2,7-diol, 9848

Ovalifolin, 16274

\section{$\mathrm{C}_{22} \mathrm{H}_{18} \mathrm{O}_{5}$}

Chamanetin, 3463

Howiinin A, 9654

Isochamanetin, 11321

\section{$\mathrm{C}_{22} \mathrm{H}_{18} \mathrm{O}_{6}$}

Vitrofolal C, 22592

\section{$\mathrm{C}_{22} \mathrm{H}_{18} \mathrm{O}_{7}$}

Chaihunaphthone, 3451

Chinensinaphthol methyl ether, 3532

12a-Epimillettosin, 6971

Griffonianone B, 9008

Justicidin A, 11977

Justicidin C, 11979

\section{$\mathrm{C}_{22} \mathrm{H}_{18} \mathrm{O}_{8}$}

Cleistanone, 3816

Dehydropodophyllotoxin, 4962

\section{$\mathrm{C}_{22} \mathrm{H}_{18} \mathrm{O}_{9}$}

4',5',7-Triacetoxy-2'-methoxyisoflavone, 21528

Triacetylhispidulin, 21534

\section{$\mathrm{C}_{22} \mathrm{H}_{18} \mathrm{O}_{10}$}

(-)-Epicatechin-3-O-gallate, 6864

\section{$\mathrm{C}_{22} \mathrm{H}_{18} \mathrm{O}_{11}$}

Epigallocatechin 3-gallate (EGCG), 6923

(+)-Leucocyanidin gallate, 12715

\section{$\mathrm{C}_{22} \mathrm{H}_{18} \mathrm{O}_{12}$}

Chicoric acid, 3518

$\mathrm{C}_{22} \mathrm{H}_{18} \mathrm{O}_{13}$
4-(4"-O-Acetyl- $\alpha$-rhamnopyranosyl)ellagic acid, 492

Nyssoside, 15880

$\mathrm{C}_{22} \mathrm{H}_{18} \mathrm{O}_{18} \mathrm{~S}_{2}{ }^{2-}$

Isoscutellarein 4 '-methyl ether $8-O-\beta$ - $D$ glucuronide 2",4"-disulfate, 11709

$\mathrm{C}_{22} \mathrm{H}_{19} \mathrm{NO}_{5}$

Ethoxysanguinarine, 7416

$\mathrm{C}_{22} \mathrm{H}_{19} \mathrm{NO}_{6}$

Bungeanine, 2744

$\mathrm{C}_{22} \mathrm{H}_{19} \mathrm{NO}_{9}$

3,4-Methylenedioxy-10-hydroxy aristololactam- $N$ - $\beta$ - $D$-glucoside, 14370

\section{$\mathrm{C}_{22} \mathrm{H}_{19} \mathrm{NO}_{10}$}

Aristolactam AIIIa $N$ - $\beta$ - $D$-glucoside, 1694

$\mathrm{C}_{22} \mathrm{H}_{19} \mathrm{O}_{15} \mathrm{~S}^{-}$

Isoscutellarein 4'-methyl ether

8-O- $\beta$ - $D$-glucuronide $2 "$-sulfate, 11710

\section{$\mathrm{C}_{22} \mathrm{H}_{20} \mathrm{NO}_{5}^{+}$}

Chelilutine, 3507

$\mathrm{C}_{22} \mathrm{H}_{20} \mathrm{NO}_{6}{ }^{+}$

Leptocarpinine, 12672

\section{$\mathrm{C}_{22} \mathrm{H}_{20} \mathrm{O}_{4}$}

Gymconopin A, 9099

Gymconopin B, 9100

1-(p-Hydroxybenzyl)-4-methoxy-9,10-dihydrophenanthrene-2,7-diol, 9846

3-( $p$-Hydroxybenzyl)-4-methoxy-9,10-dihydrophenanthrene-2,7-diol, 9847

\section{$\mathrm{C}_{22} \mathrm{H}_{20} \mathrm{O}_{5}$}

3,5-Dimethoxy-2",2"-dimethylpyrano-(5",6":8,7)flavone, 6222

Dimethylalpinumisoflavone, 6313

Gnetupendin A, 8897

Rhinacanthin M, 18776

\section{$\mathrm{C}_{22} \mathrm{H}_{20} \mathrm{O}_{6}$}

Gnetupendin B, 8898

Howiinol A, 9655

Indicanine E, 11016

Millettocalyxin B, 14857

\section{$\mathrm{C}_{22} \mathrm{H}_{20} \mathrm{O}_{7}$}

$\beta$-Apopicropodophyllin, 1533

Artoindonesianin S, 1817

Methyl 4,4'-dimethoxyvulpinate, 14321

\section{$\mathrm{C}_{22} \mathrm{H}_{20} \mathrm{O}_{8}$}

Isopicropodophyllone, 11592

(cis-head-to-head)-Limettin dimer, 12836

(cis-head-to-tail)-Limettin dimer, 12837

Peperomin E, 16906

Picropodophyllone, 17341

Podophyllotoxone, 17594

$\mathrm{C}_{22} \mathrm{H}_{20} \mathrm{O}_{9}$

3,5-Diacetyltambulin, 5347
Hyosgerin, 10874

Venkatasin, 22376

$\mathrm{C}_{22} \mathrm{H}_{20} \mathrm{O}_{10}$

Licoagroside C, 12759

$\mathrm{C}_{22} \mathrm{H}_{20} \mathrm{O}_{11}$

Baicalein 6-methylether-7-O- $\beta$-galactopyranuronoside, 2105

Irilone 4'- $O-\beta$ - $D$-glucoside, 11159

Kaempferol-3- $O-\alpha-L-3$ "-acetyl-arabinofuranoside, 12021

Kaempferol-3- $O-\alpha-L-5$ "-acetyl-arabinofuranoside, 12022

Oroxylin A 7-O-glucuronide, 16217

Palustrinoside, 16575

Wogonoside, 22720

\section{$\mathrm{C}_{22} \mathrm{H}_{20} \mathrm{O}_{12}$}

Bracteoside, 2585

Clerodendroside A, 3839

3,4 '- $O$-Dimethylellagic acid 4- $O-\alpha-L$-rhamnopyranoside, 6347

Hispidulin-7-O-glucuronide, 9566

Isoscutellarein 4'-methyl ether 8-O- $\beta-D$ - glucuronide, 11707

Quercetin-3-O- $\alpha-L-(3$ "- $O$-acetyl)-arabinofuranoside, 18319

Quercetin-3-O- $\alpha-L-(5$ "- $O$-acetyl)-arabinofuranoside, 18320

3,5,4'-Trihydroxy-6,7-methylenedioxyflavone3-O- $\beta$ - $D$-glucopyranoside, 21799

\section{$\mathrm{C}_{22} \mathrm{H}_{21} \mathrm{NO}_{5}$}

Bocconoline, 2523

Chelerythrine methanolate, 3499

\section{$\mathrm{C}_{22} \mathrm{H}_{21} \mathrm{~N}_{3} \mathrm{O}_{2}$}

Acetonylevodiamine, 114

\section{$\mathrm{C}_{22} \mathrm{H}_{22} \mathrm{NO}_{6}$}

Alborine, 866

$\mathrm{C}_{22} \mathrm{H}_{22} \mathrm{~N}_{2} \mathrm{O}_{4}$

Venacarpine A, 22372

\section{$\mathrm{C}_{22} \mathrm{H}_{22} \mathrm{O}_{4}$}

3,3'-Dihydroxy-2-(4-hydroxybenzyl)-5methoxybibenzyl, 5905

Microphyllone, 14844

\section{$\mathrm{C}_{22} \mathrm{H}_{22} \mathrm{O}_{5}$}

Anticancer Flavonoid PMV70P691-107, 1410

Ponganone I, 17713

Praecansone B, 17753

$\mathrm{C}_{22} \mathrm{H}_{22} \mathrm{O}_{6}$

Erypoegin G, 7313

Glycyrin, 8838

Licoricone, 12794

\section{$\mathrm{C}_{22} \mathrm{H}_{22} \mathrm{O}_{7}$}

Angelafolone, 1187

Anhydropodorhizol, 1280 
Anthricin, 1372

Anthricin isomer, 1373

Artoindonesianin Q, 1815

Artoindonesianin R, 1816

Baohuosu, 2144

Dulxanthone E, 6633

Isoanthricin, 11228

Isochaihulactone, 11318

Nemerosin, 15331

Pyramidatin H, 18248

\section{$\mathrm{C}_{22} \mathrm{H}_{22} \mathrm{O}_{8}$}

Anticancer Glycerol Ester PMV70P691-118, 1423

(2S,3S)-2,3-Bis(5-methoxy-3,4-methylenedioxybenzyl)-butyrolactone, 2483

7-Hydroxyanhydropodorhizol, 9789

$\beta$-Peltatin, 16794

Picropodophyllin, 17337

Podophyllotoxin, 17592

\section{$\mathrm{C}_{22} \mathrm{H}_{22} \mathrm{O}_{9}$}

Chrysoeriol 6- $C$ - $\beta$ - $L$-boivinopyranoside, 3605

Formononetin-7-glucoside, 7885

Isoononin, 11576

Kaplanin, 12157

8-Methoxy-5-O-glucoside flavone, 13942

4'-O-Methylpuerarin, 14706

Nantenoside A, 15247

Nantenoside B, 15248

Strobilanthin, 20393

\section{$\mathrm{C}_{22} \mathrm{H}_{22} \mathrm{O}_{10}$}

Acacetin-7-O- $\beta$ - $D$-galactopyranoside, 57

Anthraglycoside A, 1362

Derriscandenoside A, 5222

Echioidinin-2'-O- $\beta$ - $D$-glucopyranoside, 6695

Echioidinin-5-O- $\beta$ - $D$-glucopyranoside, 6696

2-( $\beta$ - $D$-Glucopyranosyloxy)-8-hydroxy-1methoxy-3-methyl-9,10-anthraquinone, 8696

Glycitin, 8819

3'-Hydroxy-4'-methoxyisoflavone-7- $O-\beta-D$ glucoside, 10413

Isoswertisin, 11724

Lasianthuoside A, 12531

$\alpha$-Maackiain- $\beta$ - $D$-glucoside, 13282

7-Methoxykaempferol 3-O- $\alpha-L$-rhamnopyranoside, 13983

3'-Methoxypuerarin, 14080

Physcion-8-O- $\beta$ - $D$-glucopyranoside, 17251

Prunetin 4'-O- $\beta$ - $D$-glucopyranoside, 18002

Pteroisoauroside, 18106

Sophojaponicin, 20079

Swertisin, 20515

Tilianin, 21391

Wogonin 5- $\beta$ - $D$-glucoside, 22719
$\mathrm{C}_{22} \mathrm{H}_{22} \mathrm{O}_{11}$

Azalein, 2053

Chrysoeriol 4'-O- $\beta$ - $D$-glucopyranoside, 3608

Chrysoeriol 7- $O-\beta$ - $D$-glucopyranoside, 3609

2-O-Cinnamoyl-glucogallin, 3703

(2S)-7,2'-Dihydroxy-5-methoxyflavanone

7- $O$ - $\beta$ - $D$-glucuronopyranoside, 5980

Diosmetin 7-O- $\beta$ - $D$-glucopyranoside, 6453

3-O- $\beta$ - $D$-Glucopyranosyl-5,9,4'-trihydroxy-8methoxyflavone, 8744

Homoplataginin, 9617

Isorhamnetin-3- $\alpha$ - $L$-rhamnofuranoside, 11663

Isorhamnetin 3-O-rhamnoside, 11666

Isoscoparin, 11699

Leptosin, 12678

Nodososide, 15654

Oroboside-3'-methylether, 16212

Putorinoside B, 18227

Rhamnocitrin-3-O- $\beta$ - $D$-glucoside, 18684

$7 \alpha$-L-Rhamnosyl-6-methoxyluteolin, 18743

Stachannin A, 20252

Swertiajaponin, 20505

Tectoridin, 20900

$5,7,2^{\prime}$-Trihydroxy-8-methoxyflavone 2'- $O-\beta$ - $D$ glucopyranoside, 21778

$5,7,2^{\prime}$-Trihydroxy-8-methoxyflavone $7-O-\beta-D$ glucopyranoside, 21779

\section{$\mathrm{C}_{22} \mathrm{H}_{22} \mathrm{O}_{12}$}

Alliumoside A, 925

Cacticin, 2843

Dracunculifoside B, 6581

Eriodictyl 7- $O-\beta-D-(6$ '-methylester)-glucuronopyranoside, 7277

Eupafolin-7-glucoside, 7557

4-O-(6'-O-Galloyl- $\beta$ - $D$-glucopyranosyl)-cis- $p$ coumaric acid, 8104

2-(3-Hydroxy-4-methoxyphenyl)-3,5-dihydroxy7-O- $\beta$ - $D$-glucopyranoside-4H-1-benzopyran-4one, 10437

Isorhamnetin-3-O-glucoside, 11659

Isorhamnetin-5-O-glucoside, 11660

Mearnsitrin, 13628

6-Methoxykaempferol 3-O-glycoside, 13982

3-O-Methyl quercetin 7-O- $\beta$ - $D$-glucopyranoside, 14711

Ordoritin-glucoside, 16173

Pedaliin, 16757

Putorinoside A, 18226

Rhamnetin-3-galactoside, 18680

$\mathrm{C}_{22} \mathrm{H}_{22} \mathrm{O}_{13}$

1-O-(E)-Caffeoyl-3- $O$-galloyl- $\beta$ - $D$-glucopyranose, 2902

3-O-(E)-Caffeoyl-4-O-galloyl- $\beta$ - $D$-glucopyra- nose, 2903

Myricomplanoside, 15188

Patuletin-3-O- $\beta$ - $D$-galactopyranoside, 16726

Patuletin 3-O- $\beta$ - $D$-glycopyranoside, 16727

Patuletin-7-O- $\beta$ - $D$-glucopyranoside, 16728

Quercetagetin 7-methylether-3- $O-\beta$ - $D$-glucopyranoside, 18313

Quercetagetin 7-methylether-4'-O- $\beta$ - $D$-glucopyranoside, 18314

$5,7,3^{\prime}, 4^{\prime}$-Tetrahydroxy-8-methoxyflavonol-

3-O- $\beta$ - $D$-galactoside, 21127

\section{$\mathrm{C}_{22} \mathrm{H}_{23} \mathrm{NO}_{4}$}

Ochotensimine, 15917

Tylophoridicine D, 22148

Tylophorinidine, 22152

\section{$\mathrm{C}_{22} \mathrm{H}_{23} \mathrm{NO}_{5}$}

$3 \alpha, 7 \beta$-Dibenzoyloxy- $6 \beta$-hydroxy-tropane, 5385

Tylophoridicine E, 22149

Tylophoridicine F, 22150

\section{$\mathrm{C}_{22} \mathrm{H}_{23} \mathrm{NO}_{6}$}

Corydalic acid methyl ester, 4102

Simulansamide, 19905

\section{$\mathrm{C}_{22} \mathrm{H}_{23} \mathrm{NO}_{7}$}

$\alpha$-Narcotine, 15266

$\beta$-Narcotine, 15267

\section{$\mathrm{C}_{22} \mathrm{H}_{23} \mathrm{NO}_{8}$}

Acuminaminoside, 590

\section{$\mathrm{C}_{22} \mathrm{H}_{23} \mathrm{O}_{11}{ }^{+}$}

Malvidin-3-arabinoside, 13457

Peonidin-3-glucoside, 16901

\section{$\mathrm{C}_{22} \mathrm{H}_{23} \mathrm{O}_{12}$}

Petunidin-3-glucoside, 17027

\section{$\mathrm{C}_{22} \mathrm{H}_{24} \mathrm{CINO}_{6}$}

Romucosine F, 18905

\section{$\mathrm{C}_{22} \mathrm{H}_{24} \mathrm{NO}_{4}{ }^{+}$}

Dehydrocorydaline, 4889

\section{$\mathrm{C}_{22} \mathrm{H}_{24} \mathrm{~N}_{2} \mathrm{O}_{3}$}

$\alpha$-Colubrine, 3930

$\beta$-Colubrine, 3931

Icajine, 10939

Kopsifoline E, 12266

16-Methoxystrychnine, 14095

Venacarpine B, 22373

\section{$\mathrm{C}_{22} \mathrm{H}_{24} \mathrm{~N}_{2} \mathrm{O}_{4}$}

11-Demethoxymyrtoidine, 5050

16-Hydroxy- $\alpha$-colubrine, 9930

16-Hydroxy- $\beta$-colubrine, 9931

2-Hydroxy-3-methoxystrychnine, 10457

4-Hydroxy-3-methoxystrychnine, 10459

6-Oxoalstophyllal, 16292

6-Oxoalstophylline, 16293

3-Oxo-11-methoxytabersonine, 16379

Vomicine, 22614 


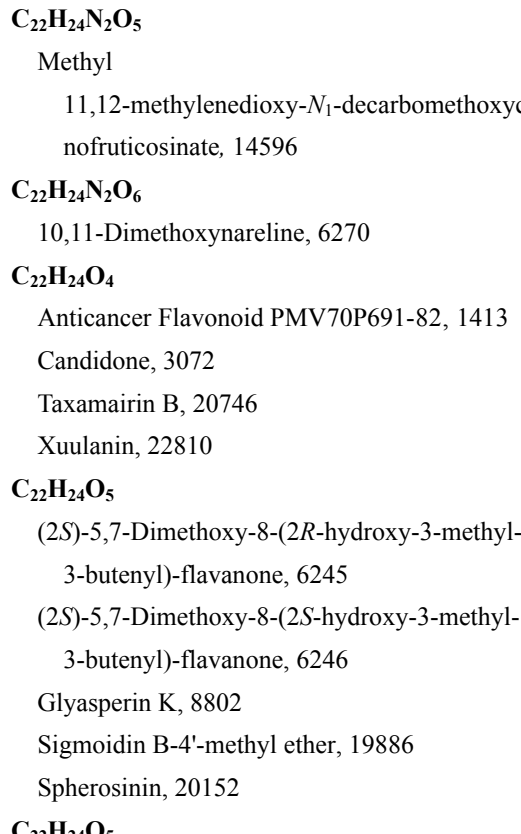

$\mathrm{C}_{22} \mathrm{H}_{24} \mathrm{O}_{5}$

Tomentolide B, 21439

Erypoegin D, 7310

Schizandrin C, 19497

Urinatetralin, 22258

Xanthanthusin K, 22754

$\mathrm{C}_{22} \mathrm{H}_{24} \mathbf{O}_{7}$

$6 \beta$-Acetylteuscordin, 525

Aschantin, 1843

Crotozambefuran A, 4273

Dihydroanhydropodorhizol, 5543

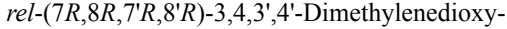

5,5'-dimethoxy-7,7'-epoxylignan, 6348

Echinoisoflavanone, 6690

Epiaschantin, 6832

Gomisin R, 8924

Hemiariensin, 9343

Heteroclitin G, 9466

Melafolone, 13653

Nodifloridin A, 15649

Pyramidatin F, 18246

Urinaligran, 22257

Valafolone, 22309

\section{$\mathrm{C}_{22} \mathrm{H}_{24} \mathrm{O}_{8}$}

trans-4,5-Bis(4-acetoxy-3-methoxyphenyl)-1,3dioxacyclohexane, 2424

Butyl rosmarinate, 2805

$\left(8 R, 8^{\prime} R\right)$-4-Hydroxycubebinone, 9949

(2S,3S)-2-(4-Hydroxy-3,5-dimethoxybenzyl)-3(5-methoxy-3,4-methylenedioxybenzyl)butyrol actone, 10015

7-Hydroxyyatein, 10833

Nymphone, 15879

Plaunol E, 17552
$\mathrm{C}_{22} \mathrm{H}_{24} \mathrm{O}_{9}$

9,9-Bisacetylneoolivil, 2426

$3,5,6,7,3^{\prime}, 4^{\prime}, 5^{\prime}$-Heptamethoxyflavone, 9403

$3,5,6,8,3^{\prime}, 4^{\prime}, 5^{\prime}$-Heptamethoxy flavone, 9404

3,5,7,8,3',4',5'--Heptamethoxyflavone, 9405

5,6,7,8,3',4',5'-Heptamethoxy flavone, 9406

Homonataloin, 9615

Medicarpin-3-O-glucoside, 13639

$\mathrm{C}_{22} \mathrm{H}_{24} \mathrm{O}_{10}$

Biochanin-7-glucoside, 2386

$4 "$-Hydroxyimperatorin 4"-O- $\beta$ - $D$-glucopyranoside, 10231

Licoagroside D, 12760

Licoagroside E, 12761

Scorzocreticoside I, 19562

2,3,5,4'-Tetrahydroxystilbene-2-O-(6"-O-acetyl)-

$$
\beta \text { - } D \text {-glucopyranoside, } 21154
$$

\section{$\mathrm{C}_{22} \mathrm{H}_{24} \mathrm{O}_{11}$}

Caffeoyl calleryanin, 2894

Hesperetin-5-glucoside, 9457

Lanceolin, 12466

3'-Methyl eriodictyol-7-O- $\beta$ - $D$-glucoside, 14418

7-O-Methyl luteolin-6-C- $\beta$ - $D$-glucoside, 14566

Mopanolside, 14950

Protogenkwanin-4'-glucoside, 17974

Scuteamoenoside, 19581

\section{$\mathrm{C}_{22} \mathrm{H}_{24} \mathrm{O}_{12}$}

Torachrysone-8- $O-\beta-D-(6 '$-oxayl)-glucoside, 21454

4-O- $\beta-D-(6-O$-Vanilloyl glucopyranosyl) vanillic acid, 22343

\section{$\mathrm{C}_{22} \mathrm{H}_{25} \mathrm{NO}$}

Exozoline, 7697

\section{$\mathrm{C}_{22} \mathrm{H}_{25} \mathrm{NO}_{2}$}

Lobelanine, 12938

Mahanimbinine, 13399

$\mathrm{C}_{22} \mathrm{H}_{25} \mathrm{NO}_{6}$

Colchicine, 3911

$N$-Ethoxycarbonyllaurotetanine, 7397

Glaudine, 8523

$\beta$-Lumicolchicine, 13073

Mecambridine, 13630

$\mathrm{C}_{22} \mathrm{H}_{25} \mathbf{N}_{2} \mathbf{O}_{7}$

Pseudopalmatine methyl nitrate, 18054

\section{$\mathrm{C}_{22} \mathrm{H}_{26} \mathrm{Br}_{3} \mathrm{~N}_{3} \mathrm{O}_{4}$}

Purpuramine L, 18213

$\mathrm{C}_{22} \mathrm{H}_{26} \mathrm{NO}_{5}{ }^{+}$

$\mathrm{N}$-Methylphoebine, 14674

\section{$\mathrm{C}_{22} \mathrm{H}_{26} \mathrm{~N}_{2} \mathrm{O}_{2}$}

Alstonerinal, 999

\section{$\mathrm{C}_{22} \mathrm{H}_{26} \mathrm{~N}_{2} \mathrm{O}_{3}$}

Alstophyllal, 1005

Alstophylline, 1006
Corynantheine, 4114

Geissoschizine methyl ether, 8251

Hirsuteine, 9552

Kopsifoline F, 12267

\section{$\mathrm{C}_{22} \mathrm{H}_{26} \mathrm{~N}_{2} \mathrm{O}_{4}$}

Aricine, 1680

Corynoxeine, 4118

Gambireine, 8125

14- $\beta$-Hydroxy-3-isorauniticine, 10264

Isocorynoxeine, 11347

Kopsifoline A, 12262

Lochnerinine, 12947

Methyl 12-methoxy- $N_{I}$-decarbomethoxychanofruticosinate, 14585

Raumitorine, 18551

Reserpinine 1, 18634

\section{$\mathrm{C}_{22} \mathrm{H}_{26} \mathrm{~N}_{2} \mathrm{O}_{5}$}

Vineridine, 22502

Volkensine, 22610

\section{$\mathrm{C}_{22} \mathrm{H}_{26} \mathrm{~N}_{2} \mathrm{O}_{6}$}

Alkaloid US-7, 906

Alkaloid US-8, 907

11,12-Methylenedioxykopsinaline $N(4)$-oxide, 14373

\section{$\mathrm{C}_{22} \mathrm{H}_{26} \mathrm{~N}_{4}$}

Calycanthine, 3002

Chimonanthine, 3527

\section{$\mathrm{C}_{22} \mathrm{H}_{26} \mathrm{O}_{4}$}

(+)-Bornyl piperate, 2563

Cannabinolic acid, 3083

Danshenol B, 4628

\section{$\mathrm{C}_{22} \mathrm{H}_{26} \mathrm{O}_{5}$}

6-Allyl-7-(3,4-dimethoxyphenyl)-2,3-dimethoxy8-methyl-tricyclo[4.2.0.0 $0^{2,8}$ ]oct-3-en-5-one, 946

(-)-Aristoligone, 1709

3,4-Dimethoxy-3',4'-methylenedioxy-7,9'-epoxylignan-9-ol, 6262

(+)-8,8'-Epi-aristoligone, 6830

(-)-8,8'-Epi-aristoligone, 6831

Glyasperin D, 8799

Mammearin A, 13472

Wallichinine, 22628

\section{$\mathrm{C}_{22} \mathrm{H}_{26} \mathrm{O}_{6}$}

(-)-Acetoxycollinin, 147

Burcellin, 2759

Burseran, 2768

Coetsoidin A(Wang), 3899

Dimethylmatairesinol, 6367

Eriocalysin E, 7271

Eudesmin, 7512

Gingerenone B, 8390

(-)-Gomisin $\mathrm{L}_{1}, 8918$ 
(-)-Gomisin $\mathrm{L}_{2}, 8919$

$( \pm)$-Gomisin $\mathrm{M}_{1}, 8920$

(+)-Gomisin $\mathrm{M}_{2}, 8921$

Isogingerenone $\mathrm{B}, 11438$

Orthosilignin, 16220

Pinoresinol dimethyl ether, 17414

Pseudolaric acid H, 18041

Schisanhenol B, 19478

\section{$\mathrm{C}_{22} \mathrm{H}_{26} \mathrm{O}_{7}$}

Binankadsurin A, 2381

Caesalpinin I, 2871

(-)-Clusin, 3851

Crotocorylifuran, 4267

2-Deoxo-8- $O$-acetyl pumilin, 5141

2-Deoxo-5-deoxy-8-O-acetyl-17,18-epoxy pumilin, 5143

(-)-Dihydroclusin, 5561

(2R-trans)

2,3-Dihydro-2-(4-hydroxy-3-methoxyphenyl)-

3-(hydroxymethyl)-7-methoxy-5-benzofuranpr opanol acetate, 5641

1-[2,4-Dihydroxy-3-(3-hydroxy-2-methoxy-3methylbutyl)-6-methoxyphenyl]-3-(4-hydroxy phenyl)propenone, 5911

Enanderianin F, 6786

Glaucocalactone, 8514

(+)-4-Hydroxy-2,6-di(3,4-dimethoxy)phenyl3,7-dioxabicyclo[3.3.0]octane, 9995

Neociwujiaphenol, 15365

Pseudolaric acid F, 18039

Teuquadrin B, 21229

Woorenogenin, 22726

\section{$\mathrm{C}_{22} \mathrm{H}_{26} \mathrm{O}_{8}$}

Albaspidin AP, 850

Euparotin acetate, 7580

Liatrin, 12746

Magnostellin B, 13394

Pseudolaric acid $\mathrm{C}_{2}, 18036$

(+)-Syringaresinol, 20556

2,6,2',6'-Tetramethoxy-4,4'-bis(2,3-epoxy-1hydroxypropyl)biphenyl, 21178

Teucrolivin H, 21220

Xerophilusin E, 22790

\section{$\mathrm{C}_{22} \mathrm{H}_{26} \mathrm{O}_{9}$}

Ciwujiatone, 3783

Daldinin C, 4611

Eleganin, 6737

\section{$\mathrm{C}_{22} \mathrm{H}_{26} \mathrm{O}_{10}$}

Asebotin, 1847

Auriculoside, 2016

Benzyl 2 $\beta$-O-D-glucopyranosyl-3,6-dimethoxybenzoate, 2289

Forsythenside A, 7922
Melampyroside, 13656

Trifochalcanoloside I, 21631

\section{$\mathrm{C}_{22} \mathrm{H}_{26} \mathrm{O}_{11}$}

15-Acetyl-13 $\alpha(21)$-epoxyeurycomanone, 389

Curculigoside, 4385

1'-O- $\beta$ - $D$-(3,4-Dihydroxyphenyl)-ethyl-6'-Ovanilloyl-glucopyranoside, 6085

7-O-Methyl leucopelargonidin-3-mono-glucofuranoside, 14552

4'-O-Methyl leucopelargonidin-3-mono-glucofuranoside, 14553

Trifochalcanoloside II, 21632

Veronicoside, 22415

Yunngnoside A, 22955

$\mathrm{C}_{22} \mathrm{H}_{26} \mathrm{O}_{12}$

Catalposide, 3307

Curculigoside C, 4387

Rubinaphthin C, 19034

\section{$\mathrm{C}_{22} \mathrm{H}_{26} \mathrm{O}_{13}$}

Seguinoside H, 19671

3,4,5-Trimethoxyphenyl (6'-O-galloyl)- $\beta$ - $D$ glucopyranoside, 21926

Verproside, 22419

\section{$\mathrm{C}_{22} \mathrm{H}_{27} \mathrm{ClO}_{8}$}

Eupachifolin D, 7539

Eupachlorin acetate, 7551

\section{$\mathrm{C}_{22} \mathrm{H}_{27} \mathrm{ClO}_{9}$}

Eupachinilide F, 7545

$\mathrm{C}_{22} \mathrm{H}_{27} \mathrm{NO}_{2}$

Lobeline, 12939

\section{$\mathrm{C}_{22} \mathrm{H}_{27} \mathrm{NO}_{3}$}

Cyclostachine A, 4536

2,3-Dimethoxy-6-(3-oxo-butyl)-7,9,10,11,11a,12hexahydrobenzo[f]pyrrolo[ $[1,2-b]$ isoquinoline, 6272

7'-(4'-Hydroxy,3'-methoxyphenyl)- $N$-[(4-butylphenyl)ethyl]propenamide, 10435

\section{$\mathrm{C}_{22} \mathrm{H}_{27} \mathrm{NO}_{4}$}

(+)-Corydaline, 4103

Thalicsessine, 21245

$\mathrm{C}_{22} \mathrm{H}_{27} \mathrm{NO}_{5}$

Didehydrostemofoline, 5470

Guanfu base Q, 9051

Muramine, 15079

Thalicsimidine, 21246

$\mathrm{C}_{22} \mathrm{H}_{27} \mathrm{O}_{11} \mathrm{~S}$

(-)-Lyoniresinol-2a-sulfate, 13254

$\mathrm{C}_{22} \mathrm{H}_{28} \mathrm{~N}_{2} \mathrm{O}_{2}$

$N(4)$-Methyl-N(4),21-seco-talpinine, 14724

Talcarpine, 20652

$\mathrm{C}_{22} \mathrm{H}_{28} \mathrm{~N}_{2} \mathrm{O}_{3}$

Dihydrocorynantheine, 5569

Hirsutine, 9553
10-Methoxycathafoline, 13878

Vincorine, 22495

Voacangine, 22597

\section{$\mathrm{C}_{22} \mathrm{H}_{28} \mathrm{~N}_{2} \mathrm{O}_{4}$}

Alstovenine, 1007

Catharosine, 3323

Corynoxine, 4119

Corynoxine B, 4120

Diangoutengjian I, 5357

Gambirine, 8127

Isogambirine, 11432

Isorhyncophylline, 11680

10-Methoxycathafoline N(4)-oxide, 13879

11-Methoxyhumantenine, 13948

9-Methoxy-3-epi- $\alpha$-yohimbine, 14108

Rhynchophylline, 18826

Venenatine, 22375

\section{$\mathrm{C}_{22} \mathrm{H}_{28} \mathrm{~N}_{2} \mathrm{O}_{5}$}

Anti-isorhynchophylline $N$-oxide, 1458

Kopsifoline B, 12263

Rhynchophylline $N$-oxide, 18827

Rotundifoline, 18943

\section{$\mathrm{C}_{22} \mathrm{H}_{28} \mathrm{~N}_{2} \mathrm{O}_{6}$}

Kopsifoline C, 12264

3-Oxo-7-hydroxy-3,7-secorhynchophylline, 16353

\section{$\mathrm{C}_{22} \mathrm{H}_{28} \mathrm{~N}_{2} \mathrm{O}_{11}$}

Cappariloside B, 3137

\section{$\mathrm{C}_{22} \mathrm{H}_{28} \mathrm{O}_{3}$}

Dehydroguggulsterone M, 4925

\section{$\mathrm{C}_{22} \mathrm{H}_{28} \mathrm{O}_{4}$}

2-Acetoxy-1,15-beyeradiene-3,12-dione, 134

Crocetin dimethyl ester, 4246

\section{$\mathrm{C}_{22} \mathrm{H}_{28} \mathrm{O}_{5}$}

17-Acetoxyjolkinolide A, 245

15-Acetoxy-7-oxo-dehydroabietic acid, 271

Di- $O$-methyltetrahydrofuriguaiacin B, 6410

(-)-Galbelgin, 8088

(+)-Galbelgin, 8089

$( \pm)$-Galgravin, 8093

Hancinol, 9208

$5 \alpha$ - $p$-Hydroxybenzoyloxydauc-2-ene-1-one, 9829

Isorecedensolide, 11645

Kuhistanicaol B, 12312

Liangshanin B, 12740

Liangshanin C, 12741

Pyrethrin II, 18256

Recedensolide, 18562

Saucernetin, 19404

Veraguensin, 22383

\section{$\mathrm{C}_{22} \mathrm{H}_{28} \mathrm{O}_{6}$}

Apetalic acid, 1470

Caesalmin B, 2864 
Calanolide $\mathrm{E}_{2}, 2942$

Calolongic acid, 2988

Cascarillin B, 3249

Coleon U 11-acetate, 3920

Cornutin G, 4070

Cornutin H, 4071

Disparpropylinol B, 6516

Effusin, 6715

Gomisin J, 8914

Isoapetalic acid, 11230

Isocalolongic acid, 11303

Isodoacetal, 11381

LS-2(furanoditerpenelactone), 13015

Macrophyllin B, 13319

Maoecrystal A, 13523

Maoecrystal B, 13524

Maoecrystal U, 13542

Nigakilactone D, 15549

Pellucidin A, 16791

Picrasmin, 17327

Pseudolaric acid A, 18031

Quassin, 18299

Surinamensin, 20492

Theraphin B, 21314

Theraphin C, 21315

\section{$\mathrm{C}_{22} \mathrm{H}_{28} \mathrm{O}_{7}$}

16-O-Acetylcoleon C, 358

Acetylleptocarpin, 447

$3 \beta$-Angeloyloxy- $8 \beta$-ethoxyeremophil-7(11)-ene-

$12,8 \alpha(14 \beta, 6 \alpha)$-diolide, 1223

Biondinin B, 2388

Caesalpinin H, 2870

Caesalpinin O, 2884

Enmein-3-acetate, 6805

1,5-Epoxy-3-hydroxy-1-(4-hydroxy-3,5-

dimethoxyphenyl)-7-(4-hydroxy-3-methoxy-

phenyl)heptane, 7119

Eriocalysin C, 7269

4-[1-Ethoxyl-1-(4'-hydroxy-3'-methoxy)benzyl] methyl-2-(4-hydroxy-3-methoxy)benzyl-3hydroxymethyl-tetrahydro-furan, 7409

Eupachinilide I, 7548

Eupacunin, 7553

Eupaglehnin B, 7561

Eupaserrin, 7581

Eupatocunin, 7584

(-)-Fargesol, 7720

Hiyodorilactone B, 9571

5 $\xi$-Hydroxy-1-(4-hydroxy-3,5-dimethoxy-

phenyl)-7-(4-hydroxy-3-methoxyphenyl)-3heptanone, 10178

5 $\xi$-Hydroxy-7-(4-hydroxy-3,5-dimethoxyphenyl)-1-(4-hydroxy-3-methoxyphenyl)-3- heptanone, 10179

Isodonal, 11392

Laxiflorin M, 12590

(+)-Leosibiricin, 12663

Leosibiricin, 12664

$8 \alpha$-(Z-2-Methyl-4-acetoxybut-2-enoyloxy)-

15-hydroxygermacra-1(10),E,4Z,11(13)-trien-

12,6 $\alpha$-olide, 14113

Nepetaefuran, 15483

Orthosiphol Z, 16241

Pseudolaric acid G, 18040

Rugosanin, 19049

Trichorabdal B, 21564

Trichorabdal C, 21565

Trichorabdal $\mathrm{F}$ acetate, 21569

Trichorabdal G, 21570

Trichorabdal H, 21572

1-(3,4,5-Trimethoxyphenyl)-2-propenyl 2-(2methyl-2Z-butenoyloxymethyl)-2Z-butenoate, 21930

Ursiniolide A, 22268

Xerophilusin A, 22786

Xerophilusin F, 22791

\section{$\mathrm{C}_{22} \mathrm{H}_{28} \mathrm{O}_{8}$}

$3 \beta$-Acetoxy- $9 \beta$-angeloyloxy- $1 \beta, 10 \beta$-epoxy- $8 \alpha$ hydroxyeremophil-7(11)-en- $8 \beta(12)$-olide, 123

$8 \alpha$-[(4-Acetoxy-5-hydroxy)-angelate]salonitenolide, 206

4'-Acetylcnicin, 357

Caesalmin A, 2863

Carpalasionin, 3219

6-Deacetyl-teucrolivin A, 4789

Eupachinilide J, 7549

Eupacunolin, 7554

Eupacunoxin, 7555

Eupatocunoxin, 7585

Hiyodorilactone A, 9570

8 $\alpha$-(3-Hydroxy-4-acetoxy-2-methylene-butanoyloxy)-dehydro-melitensin, 9759

Isodonoic acid, 11393

(+)-Lyoniresinol, 13249

Oreskaurin A, 16176

Teuctosin, 21222

8 $\alpha$-Tigloyloxyanthemolide C, 21379

Trichorabdal D, 21566

Trichorabdal E, 21567

$\mathrm{C}_{22} \mathrm{H}_{28} \mathrm{O}_{9}$

8-O-Angeloyl-9-O-acetylanthemolide B, 1204

Bruceine I, 2673

$8 \alpha$-(3'-Hydroxy-4'-acetoxy-2'-methylene-butanoyloxy)4-epi-sonchucarpolide, 9760

8-O-Tigloyl-9-O-acetylanthemolide B, 21373

$\mathrm{C}_{22} \mathrm{H}_{28} \mathrm{O}_{10}$
12-Acetyl-13,21-dihydroeurycomanone, 378

Davisioside, 4698

Eupalinilide J, 7576

Glochidacuminoside B, 8560

4'- $O$ - $\beta$-Glucopyranosyl-5- $O$-methylvisamminol, 8684

Ovatolactone 7-O-(6'- $O-p$-hydroxybenzoyl)- $\beta-D$ glucopyranoside, 16281

Tschimganic ester A, 22064

\section{$\mathrm{C}_{22} \mathrm{H}_{28} \mathrm{O}_{11}$}

6-O-(4-Hydroxybenzoyl)-ajugol, 9819

Prim- $O$-glucosylcimifugin, 17856

\section{$\mathrm{C}_{22} \mathrm{H}_{28} \mathrm{O}_{12}$}

7-Methyl-1,4,5-trihydroxy-naphthalene-4- $O$ -

(6'- $O$ - $\beta$-xylopyranosyl)- $\beta$-glucopyranoside, 14790

Rubinaphthin B, 19033

\section{$\mathrm{C}_{22} \mathrm{H}_{28} \mathrm{O}_{13}$}

5-Methylcoumarin-4-cellobioside, 14255

5-Methylcoumarin-4-gentiobioside, 14256

\section{$\mathrm{C}_{22} \mathrm{H}_{28} \mathrm{O}_{14}$}

Racemosic acid, 18513

Scopoletin 7- $O-\beta$ - $D$-sophoroside, 19543

\section{$\mathrm{C}_{22} \mathrm{H}_{29} \mathrm{NO}_{2}$}

Lobelanidine, 12937

Spiradine D, 20178

Spirafine IV, 20184

\section{$\mathrm{C}_{22} \mathrm{H}_{29} \mathrm{NO}_{3}$}

Laetispicine, 12449

Pipercide, 17444

Retrofractamide B, 18658

Songoramine, 20075

Spiradine C, 20177

\section{$\mathrm{C}_{22} \mathrm{H}_{29} \mathrm{NO}_{4}$}

Didehydrotuberostemonine, 5471

Epi-bisdehydrotuberostemonine J, 6839

\section{$\mathrm{C}_{22} \mathrm{H}_{29} \mathrm{NO}_{5}$}

Guan-fu base I, 9048

Stemofoline, 20304

Tuberostemoenone, 22084

\section{$\mathrm{C}_{22} \mathrm{H}_{29} \mathrm{NO}_{6}$}

2'-Hydroxystemofoline, 10731

\section{$\mathrm{C}_{22} \mathrm{H}_{29} \mathrm{NO}_{6} \mathrm{~S}$}

$\beta$-Hydroxygerambullal, 10138

\section{$\mathrm{C}_{22} \mathrm{H}_{29} \mathrm{~N}_{2} \mathrm{O}_{4}$}

Echitamine, 6699

$\mathrm{C}_{22} \mathrm{H}_{30} \mathrm{NO}_{4}^{+}$

$N, N$-Dimethylanomurine, 6316

\section{$\mathrm{C}_{22} \mathrm{H}_{30} \mathrm{~N}_{2} \mathrm{O}_{2}$}

Aspidospermine, 1904

\section{$\mathrm{C}_{22} \mathrm{H}_{30} \mathrm{~N}_{2} \mathrm{O}_{3}$}

Aspidocarpine, 1900

Malagashanol, 13417 


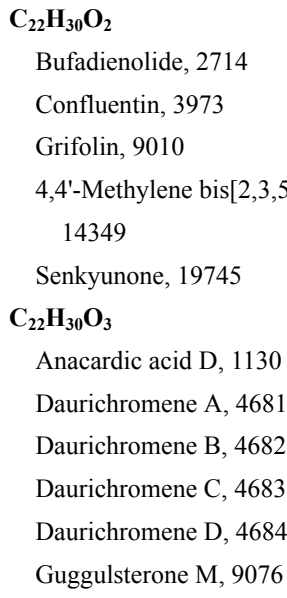

\section{$\mathrm{C}_{22} \mathrm{H}_{30} \mathrm{O}_{4}$}

19-Acetoxy-15,16-epoxy-8(17),13(16),14-entlabdatrien-20-al, 187

Cannabichromenic acid, 3078

Cannabidiolic acid, 3080

CPB-2004-52-608-2, 3510

Ferutinin, 7792

Gesneroidin B, 8367

4-Hydroxy-2,6,8-trimethyl-6-(3,7-dimethyl-2,6octadienyl)-2H-1-benzopyran-5,7(3H,6H)dione, 10810

5-Hydroxy-2,6,8-trimethyl-8-(3,7-dimethyl-2,6octadienyl)-2H-1-benzopyran-4,7(3H,8H)dione, 10811

Ialibinone C, 10931

Ialibinone D, 10932

Jatrophenone, 11849

Kuhistanol D, 12322

Kuhistanol E, 12323

Kuhistanol F, 12324

Kuhistanol G, 12325

Palbinone, 16539

ent-8,9-Seco-7 $\alpha$-acetoxykaura-8(14),16-dien9,15-dione, 19604

$\Delta^{1}$-Tetrahydrocannabinolic acid A, 21060

Velamolone acetate, 22362

\section{$\mathrm{C}_{22} \mathrm{H}_{30} \mathrm{O}_{5}$}

$7 \alpha$-Acetoxyroyleanone, 277

15-O-Acetyl-15-epi-(4E)-jatrogrossidentadione, 385

14-Acetylumbrosin B, 528

3 $\beta$-Angeloyloxy-8-oxoeremophila-6,9-dien-12oic acid ethyl ester, 1231

Cascarillin C, 3250

Cascarillin D, 3251

Cornutin K, 4074

Cortisone, 4097

15,16-Epoxy-12(R)-acetoxy-8(17),13(16),14-entlabdatrien-19-oic acid, 7049
8,9-Epoxy-ferutinin, 7098

Glaucocalyxin B, 8516

Glaucocalyxin D, 8518

Inflexarabdonin C, 11043

Kuhistanicaol G, 12317

Lancerotriol $9 \alpha$-( $p$-hydroxybenzoate), 12470

ent-8,9-Seco-7 $\alpha$-acetoxy-11 $\beta$-hydroxykaura-

(14),16-dien-9,15-dione, 19603

ent-8,9-Seco-7 $\alpha$-hydroxy-11-acetoxykaura-

8(14),16-dien-9,15-dione, 19617

Shikoccin, 19807

\section{$\mathrm{C}_{22} \mathrm{H}_{30} \mathrm{O}_{6}$}

Caesalpinin N, 2883

Coleon S, 3917

Dubiin, 6617

Effusanin B, 6711

Enanderianin L, 6790

Enmedol, 6803

Epoxyshikoccin, 7201

$3 \beta$-Ethoxy-leptocarpin, 7408

Fruticolone, 7972

Fukinolide, 7989

Ganervosin B, 8147

Inflexarabdonin E, 11045

Isodoglutinosin A, 11384

Kuhistaferone, 12310

Kuhistanicaol H, 12318

Kuhistanicaol J, 12320

Kuhistanol H, 12326

Liangshanin E, 12743

Liangshanin F, 12744

Longikaurin C, 12959

Longikaurin E, 12961

Longirabdolide F, 12975

Longirabdolide G, 12976

Lushanrubescensin H, 13123

Maoecrystal L, 13533

Maoecrystal S, 13540

Neoquassin, 15454

Oreskaurin B, 16177

Picrasin D, 17314

Pregomisin, 17806

Preschisanthrin, 17848

Prostratin, 17958

Pseurata C, 18077

Rabdocoetsin B, 18457

Rabdocoetsin D, 18459

Rabdosianin C, 18489

Rabdoternin H, 18508

Xindongnin H, 22804

$\mathrm{C}_{22} \mathrm{H}_{30} \mathrm{O}_{7}$

$(1 R, 3 R, 4 R, 5 S, 6 S)$-1-Acetoxy-8-angeloyloxy3,4-epoxy-5-hydroxybisabola-7(14),10-dien-2- one, 122

Acetylamarolide, 312

Baiyecrystal B, 2117

Dihydrorugosanin, 5704

(3R,5S)-3,5-Dihydroxy-1-(4-hydroxy-3,5dimethoxyphenyl)-7-(4-hydroxy-3-methoxyph enyl)heptane, 5906

Effusanin C, 6712

Enanderianin C, 6785

Enanderianin K, 6789

$7 \beta$-Hydroxyfruticolone, 10130

$8 \beta$-Hydroxyfruticolone, 10131

11-Hydroxyfruticolone, 10132

Isodonoiol, 11394

15 - $O$-Isovaleroyl-3 $\beta$ - $O$-acetyl- $9 \beta$-hydroxyamphoricarpolide, 11757

Lasiokaurin, 12537

Lasiokaurin (Lasiodin), 12539

Longikaurin B, 12958

Longikaurin D, 12960

Lophanthoidin C, 12995

Lophanthoidin E, 12997

Lushanrubescensin I, 13124

Maoecrystal O, 13536

Nigakilactone K, 15556

Nigakilactone L, 15557

Phyllostachysin B, 17227

Phyllostachysin C, 17228

Picrasin E, 17315

Pseurata F, 18080

Rabdokaurin D, 18469

Rabdolasional, 18475

Rabdosichuanin A, 18490

Rabdosin C, 18500

Sculponeatin E, 19578

Trichoranin, 21574

Wikstroemioidin C, 22671

Xerophilusin K, 22795

\section{$\mathrm{C}_{22} \mathrm{H}_{30} \mathrm{O}_{8}$}

Adenolin B, 620

Dihydrocarpalasionin, 5554

Enanderianin B, 6784

Isovaltrate, 11766

Leocardin, 12625

Maoecrystal I, 13530

Picrasin F, 17316

Teucrolivin C, 21219

Valepotriate, 22312

Xeroferin, 22785

Xerophilusin G, 22792

Xerophilusin J, 22794

$\mathrm{C}_{22} \mathrm{H}_{30} \mathrm{O}_{9}$

$6 \alpha$-Acetyl-15 $\beta$-hydroxyklaineanone, 420 
15 $\beta$-O-Acetyl-14-hydroxyklaineanone, 421

Burseneolignan, 2766

\section{$\mathrm{C}_{22} \mathrm{H}_{30} \mathrm{O}_{10}$}

$6 \alpha$-Acetyl-14 $\beta, 15 \beta$-dihydroxyklaineanone, 381

\section{$\mathrm{C}_{22} \mathrm{H}_{30} \mathrm{O}_{12}$}

Seguinoside G, 19670

\section{$\mathrm{C}_{22} \mathrm{H}_{30} \mathrm{O}_{13}$}

Sibirioside B, 19868

\section{$\mathrm{C}_{22} \mathrm{H}_{30} \mathrm{O}_{14}$}

Arillanin B, 1682

Arillatose B, 1685

4'- $O$ - $\beta$ - $D$-Glucopyranosylgentiopicroside, 8635

6'-O- $\beta$ - $D$-Glucopyranosylgentiopicroside, 8636

Olivieroside, 16087

\section{$\mathrm{C}_{22} \mathrm{H}_{31} \mathrm{NO}_{2}$}

Geranyl $N$-Dimethyllallylanthranilate, 8314

Regholarrhenine A, 18585

Spirafine II, 20181

Spirafine III, 20182

\section{$\mathrm{C}_{22} \mathrm{H}_{31} \mathrm{NO}_{3}$}

Bullatine G, 2742

Piperchabamide D, 17443

Songorine, 20076

Spirafine V, 20185

Spirafine VI, 20187

Spiramine $\mathrm{N}_{6}, 20194$

Yuzurimic acid B, 22959

\section{$\mathrm{C}_{22} \mathrm{H}_{31} \mathrm{NO}_{4}$}

Equisetin, 7221

\section{$\mathrm{C}_{22} \mathrm{H}_{31} \mathrm{NO}_{5}$}

Oxotuberostemonine, 16437

Oxotuberostemonine II, 16438

Stemocochinin, 20296

Stemokerrin, 20305

Tuberostemonol, 22088

\section{$\mathrm{C}_{22} \mathrm{H}_{31} \mathrm{NO}_{5} \mathrm{~S}$}

Gerambullol, 8310

$\beta$-Hydroxygerambullin, 10139

\section{$\mathrm{C}_{22} \mathrm{H}_{31} \mathrm{NO}_{6}$}

Oxystemokerrin, 16475

Tuberostemonone, 22089

\section{$\mathrm{C}_{22} \mathrm{H}_{31} \mathrm{NO}_{6} \mathrm{~S}$}

$\beta$-Hydroxygerambullol, 10140

Sakerinol A, 19170

Sakerinol B, 19171

\section{$\mathrm{C}_{22} \mathrm{H}_{31} \mathrm{NO}_{7}$}

Oxystemokerrin- $N$-oxide, 16476

$\mathrm{C}_{22} \mathrm{H}_{32} \mathrm{O}_{2}$

2,4-Dimethyl-6-(3'-methyl-isobuten-5'-isopropyl)-phenyl-3,5-hexanedione, 6375 (16R)-ent-3 $\alpha$-Hydroxykauran-15-one, 10278 (16R)-ent-7 $\beta$-Hydroxykauran-15-one, 10279 (16R)-ent-11 $\alpha$-Hydroxykauran-15-one, 10280
$\mathrm{C}_{22} \mathrm{H}_{32} \mathrm{O}_{3}$

$7 \beta$-Acetoxyisopimara-8(14),15-dien-1-one, 242

Acetylsanadaol, 505

Anacardic acid C, 1129

3-Hydroxyneogrifolin, 10538

Micranoic acid A, 14833

Micranoic acid B, 14834

\section{$\mathrm{C}_{22} \mathrm{H}_{32} \mathrm{O}_{4}$}

2- $\alpha$-Acetoxy-cis-cleroda-3,13(Z),8(17)-trien-15oic acid, 143

ent-20-Acetoxy-11 $\alpha$-hydroxy-16-kauren-15-one, 201

ent-7 $\beta$-Acetoxy-11 $\alpha$-hydroxykaur-16-en-15-one, 221

18-Acetoxy-7 $\alpha$-hydroxykaur-16-en-15-one, 222 ent-18-Acetoxy-11 $\alpha$-hydroxykaur-16-en-15-one, 223

ent-18-Acetoxy-14 $\alpha$-hydroxykaur-16-en-15-one, 224

ent-11 $\alpha$-Acetoxykaur-16-en-18-oic acid, 247

17-Acetoxymulin-11,13-dien-20-oic acid, 261

Caesaldekarin A, 2861

Gesneroidin A, 8366

Juniperexcelsic acid, 11967

Melissoidesin L, 13698

PC-1999-52-1447-7b, 16736

Rabdohakusin, 18463

Sugikurojin E, 20461

Vitetrifolin C, 22570

\section{$\mathrm{C}_{22} \mathrm{H}_{32} \mathrm{O}_{5}$}

ent-3 $\beta$-Acetoxy-7 $\alpha, 18$-dihydroxykaur-15-en-17al, 168

ent-18-Acetoxy-3 $\beta, 7 \alpha$-dihydroxykaur-15-en-17al, 169

ent-1 $\alpha$-Acetoxy-7 $\beta, 14 \alpha$-dihydroxy-kaur-16-en15-one, 170

$1 \beta$-Acetoxy-7 $\alpha, 14 \beta$-dihydroxykaur-16-en-15-one, 171

ent-11 $\alpha$-Acetoxy-7 $\beta, 14 \alpha$-dihydroxykaur-16-en-

$$
\text { 15-one, } 172
$$

18-Acetoxy-7 $\alpha, 14 \beta$-dihydroxykaur-16-en-15-one, 173

\section{Caesalpinin K, 2873}

\section{Coetsoidin E, 3903}

Compound 1 (Isodon umbrosa), 3950

Compound 2 (Isodon umbrosa), 3951

Enaimeone C, 6782

Galeopsin, 8092

1'-Hydroxyialibinone D, 10222

Hypoestoxide, 10894

Inflexanin A, 11039

Inflexarabdonin K, 11051

Isodopharicin A, 11395
Kuhistanol A, 12321

Leucamenin E, 12712

Lungshengenin D, 13080

Maoecrystal G, 13529

Melissoidesin N, 13700

Otostegin A, 16272

Pregaleopsin, 17779

Prehumulone, 17808

Reniformin C, 18622

Rosthornin A (Isodopharicin C), 18932

Torilin, 21456

\section{$\mathrm{C}_{22} \mathrm{H}_{32} \mathrm{O}_{6}$}

17-Acetoxymulinic acid, 262

(12 $\alpha$ )-2 $\alpha$-Acetoxy-5 $\alpha, 9 \alpha, 10 \beta$-trihydroxy-3,11cyclotax-4(20)-en-13-one, 293

Adenanthin L, 616

Albopilosin A, 865

Bakkenolide G, 2121

Compound 3 (Isodon umbrosa), 3952

Compound 4 (Isodon umbrosa), 3953

Dihydrofukinolide, 5617

Enanderianin M, 6791

Enanderianin N, 6792

Excisanin B, 7675

Excisanin D, 7677

Flexicaulin A, 7828

Glabcensin J, 8471

Henryin, 9362

Inflexanin B, 11040

Isodomedin, 11386

Isodoternifolin B, 11401

Isofruticolone, 11430

Leopersin A, 12647

Leucamenin A, 12708

Lophanthoidin D, 12996

Lushanrubescensin D, 13119

Maoecrystal E, 13527

Nigakilactone B, 15548

Picrasinol B, 17319

Rabdokunmin A, 18470

Rabdonervosin C, 18482

Reniformin B, 18621

Rosthornin C, 18934

Rosthornin D, 18935

Rostronol F, 18938

Shikoccidin, 19806

Suimiyain A, 20466

Taxezopidine C, 20782

Taxezopidine D, 20783

$4 \beta, 8 \beta, 9 \alpha$-Trihydroxy- $6 \alpha$ - $p$-hydroxybenzoyloxydaucane, 21744

Xindongnin B, 22798

Xindongnin F, 22802 


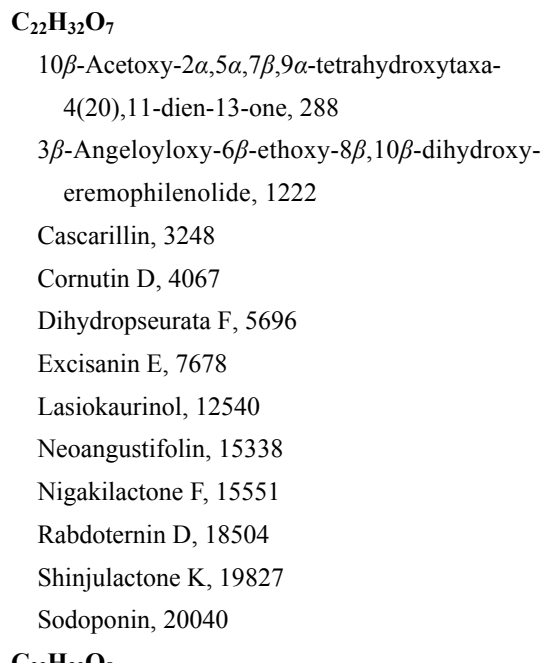

$\mathrm{C}_{22} \mathrm{H}_{32} \mathrm{O}_{8}$

Didrovaltratum, 5482

Dihydrovalepotriate, 5734

Nigakilactone $\mathrm{H}, 15553$

\section{$\mathrm{C}_{22} \mathrm{H}_{32} \mathrm{O}_{9}$}

Garajonone, 8201

\section{$\mathrm{C}_{22} \mathrm{H}_{32} \mathrm{O}_{10}$}

4-Deacetyltaxagifine III, 4778

Glochidacuminoside C, 8561

Rengyoside C, 18618

\section{$\mathrm{C}_{22} \mathrm{H}_{32} \mathrm{O}_{11}$}

Eugenyl- $\beta$-rutinoside, 7526

\section{$\mathrm{C}_{22} \mathrm{H}_{32} \mathrm{O}_{13}$}

Cistanoside H, 3758

2-(2-Methylbutyryl)phloroglucinol 1-O-(6"-O$\beta$ - $D$-apiofuranosyl)- $\beta$ - $D$-glucopyranoside, 14200

Sinapyl 9- $O-[\beta-D$-apiofuranosyl $(1 \rightarrow 6)]-O-\beta-D$ glucopyranoside, 19923

8-O-Tigloyldiderroside, 21376

Tinosinen, 21399

\section{$\mathrm{C}_{22} \mathrm{H}_{32} \mathrm{O}_{14}$}

Swertiapunimarin, 20512

\section{$\mathbf{C}_{22} \mathbf{H}_{32} \mathbf{O}_{15}$}

6'-O- $\beta$ - $D$-Fructofuranosylswertiamarin, 7970

3'-O- $\beta$ - $D$-Galactopyranosylswertiamarin, 8069

6'-O- $\alpha$ - $D$-Galactopyranosylswertiamarin, 8070

$3^{\prime}-O-\beta$ - $D$-Glucopyranosylswertiamarin, 8734

$4^{\prime}-O-\beta$ - $D$-Glucopyranosylswertiamarin, 8735

6'-O- $\beta$-D-Glucopyranosylswertiamarin, 8736

6'-O- $\alpha$ - $D$-Mannopyranosylswertiamarin, 13506

Segetoside A, 19658

\section{$\mathrm{C}_{22} \mathrm{H}_{33} \mathrm{NO}$}

1-Methyl-2-dodecyl-4(1H)-quinolone, 14331

2-Tridecyl-4(1H)-quinolone, 21625

\section{$\mathrm{C}_{22} \mathrm{H}_{33} \mathrm{NO}_{2}$}

Atisine, 1964

Bullatine A, 2737

\section{Denudatine, 5136}

Paravallarine, 16659

Spirafine III A, 20183

Veatchine, 22360

\section{$\mathrm{C}_{22} \mathrm{H}_{33} \mathrm{NO}_{3}$}

Ajaconine, 782

8-Deoxy-14-dehydro-aconosine, 5166

Lepenine, 12668

Napelline, 15250

Spirafine VA, 20186

Spirafine VIA, 20188

Spiratine A, 20200

\section{$\mathrm{C}_{22} \mathrm{H}_{33} \mathrm{NO}_{4}$}

Isotuberostemonine, 11749

Karakanine, 12162

Neotuberostemonine, 15469

Spiramine W, 20195

Tuberostemonine, 22085

Tuberostemonine H, 22086

Tuberostemonine J, 22087

\section{$\mathrm{C}_{22} \mathrm{H}_{33} \mathrm{NO}_{5}$}

Heteratisine, 9459

Parvistemonine, 16692

\section{$\mathrm{C}_{22} \mathrm{H}_{33} \mathrm{NO}_{6}$}

Excelsine, 7673

Glabrephinine, 8491

\section{$\mathrm{C}_{22} \mathrm{H}_{34} \mathrm{NO}_{3}$}

$\mathrm{N}$-Isopentenyl dendroxine, 11586

\section{$\mathrm{C}_{22} \mathrm{H}_{34} \mathrm{NO}_{4}^{+}$}

$\mathrm{N}$-Isopentenyl-6-hydroxydendroxine, 11587

\section{$\mathrm{C}_{22} \mathrm{H}_{34} \mathrm{~N}_{2}$}

Conessidine, 3966

Regholarrhenine C, 18587

\section{$\mathrm{C}_{22} \mathrm{H}_{34} \mathrm{O}_{2}$}

17-Acetoxylabda-7,12(E),14-triene, 248

Clupanodonic acid, 3849

$15 \alpha$-Hydroxymansumbinone, 10361

20-Hydroxymulin-11,13-dienyl acetate, 10531

2-Methylcardol, 14221

\section{$\mathrm{C}_{22} \mathrm{H}_{34} \mathrm{O}_{3}$}

2-Acetoxy-3-hydroxy-labda-8(17),12(E),14triene, 225

3-Acetoxy-2-hydroxy-labda-8(17),12(E),14triene, 226

18-Acetoxy-7-oxo-9-epi-ent-pimara-15-ene, 272 Albaconol, 843

7 $\beta, 12$-Dimethoxy-8,11,13-abietatrien-11-ol, 6198 Ginkgolic acid, 8403

Rhusone, 18823

\section{$\mathrm{C}_{22} \mathrm{H}_{34} \mathrm{O}_{4}$}

16-Acetoxy-12(R), 15 -epoxy-15 $\beta$-hydroxylabda8(17),13(16)-diene, 185

ent-(16S)-18-Acetoxy-7 $\beta$-hydroxykauran-15-one,
220

ent-18-Acetoxy-16-hydroxy-8(14)-pimaren-15one, 235

7 $\beta$-Acetoxytrachyloban-18-oic acid, 291

Aglatomin B, 743

Calliterpenone monoacetate, 2980

Compound 2 (Periploca sepium), 3957

Fritillebic acid, 7960

2-Hydroxy-5-methoxy-3-pentadecenyl benzoquinone, 10431

Linearol, 12878

Rotundifuran, 18955

Sidol, 19874

Taxuyunnanine J, 20882

Vitetrifolin B, 22569

\section{$\mathrm{C}_{22} \mathrm{H}_{34} \mathrm{O}_{5}$}

6-Acetoxy-9-hydroxy-13(14)-labden-16,15-olide, 227

ent-3 $\beta$-Acetoxy-7 $\alpha, 17,18$-trihydroxykaur-15-ene, 298

ent-18-Acetoxy-3 $\beta, 7 \alpha, 17$-trihydroxykaur-15-ene, 299

Annosquamosin E, 1329

Compound 1a (Periploca sepium), 3956

Cornudentanone, 4061

Epoxyisolinearol, 7151

$16 \beta$-Hydroxy-17-acetoxy-ent-kauran-19-oic acid, 9757

2-Keto-16-acetyl-kirenol, 12196

Melissoidesin M, 13699

21-O-Methyl-5,14-pregndiene-3 $\beta, 14 \beta, 17 \beta, 21$ tetrol, 14684

21-O-Methyl-5-pregnene-3 $\beta, 17 \beta, 21$-tetrol-20one, 14686

Mulin-11-ene-13 $\alpha, 14 \alpha$-dihydroxy-20-oic acid, 15060

Previtexilactone, 17851

$2 \alpha, 10 \beta, 14 \beta$-Trihydroxy-5 $\alpha$-acetoxytaxa-4(20),11diene, 21686

Vitexilactone, 22580

$\mathrm{C}_{22} \mathrm{H}_{34} \mathrm{O}_{6}$

ent-3 $\beta$-Acetoxy-7 $\alpha, 17,18$-trihydroxy-15 $\beta, 16 \beta$ epoxykaurane, 296

ent-18-Acetoxy-3 $\beta, 7 \alpha, 17$-trihydroxy-15 $\beta, 16 \beta$ epoxykaurane, 297

7-Debenzoyloxy-10-deacetyl-brevifoliol, 4808 Enanderianin O, 6793

15-Epileoheteronone E, 6945

15-Epileopersin B, 6946

Inflexarabdonin F, 11046

Leoheteronone E, 12636

Leopersin B, 12648

Melissoidesin I, 13695 
Melissoidesin P, 13702

6-Methylgingediacetate, 14463

Nigakihemiacetal F, 15545

\section{$\mathrm{C}_{22} \mathrm{H}_{34} \mathbf{O}_{7}$}

Coleonol, 3915

Lyoniol A, 13246

Nigakihemiacetal A, 15542

Rhodojaponin II, 18800

Rhodomollein XII, 18809

\section{$\mathrm{C}_{22} \mathrm{H}_{34} \mathrm{O}_{8}$}

Cornutin J, 4073

Macrocalyxin G, 13309

Macrocalyxin H, 13310

Patridoid I, 16716

Patridoid II, 16717

Patridoid IIA, 16718

\section{$\mathrm{C}_{22} \mathrm{H}_{34} \mathrm{O}_{10}$}

Marioside, 13567

\section{$\mathrm{C}_{22} \mathrm{H}_{34} \mathrm{O}_{11}$}

Carvacrol 2- $O-\beta$-glucopyranosyl-( $1 \rightarrow 2)-\beta$-glucopyranoside, 3233

\section{$\mathrm{C}_{22} \mathrm{H}_{34} \mathrm{O}_{12}$}

6-Hydroxythymol 3,6-di- $O-\beta$ - $D$-glucopyranoside, 10763

\section{$\mathrm{C}_{22} \mathrm{H}_{34} \mathbf{O}_{15}$}

Gmephiloside, 8864

\section{$\mathrm{C}_{22} \mathrm{H}_{35} \mathrm{NO}$}

Holadysamine, 9576

Holadysine, 9577

\section{$\mathrm{C}_{22} \mathrm{H}_{35} \mathrm{NO}_{2}$}

Kurchiphyllamine, 12349

\section{$\mathrm{C}_{22} \mathrm{H}_{35} \mathrm{NO}_{3}$}

17-Hydroxyhomodaphniphyllic acid, 10166

\section{$\mathrm{C}_{22} \mathrm{H}_{35} \mathrm{NO}_{4}$}

Carmichaeline, 3201

Genicunine A, 8271

Karacoline, 12161

\section{$\mathrm{C}_{22} \mathrm{H}_{35} \mathrm{NO}_{5}$}

Karacolidine, 12160

\section{$\mathrm{C}_{22} \mathrm{H}_{35} \mathrm{NO}_{6}$}

Tatsinine, 20728

\section{$\mathrm{C}_{22} \mathrm{H}_{35} \mathrm{NO}_{7}$}

Sinomontanine E, 19957

\section{$\mathrm{C}_{22} \mathrm{H}_{35} \mathrm{NO}_{8}$}

Sinomontanine D, 19956

\section{$\mathrm{C}_{22} \mathrm{H}_{36} \mathrm{~N}_{2}$}

Conamine, 3959

Conimine, 3989

3-Epiconamine, 6873

\section{$\mathrm{C}_{22} \mathrm{H}_{36} \mathrm{O}_{2}$}

Belamcandol B, 2217

Garcinielliptone N, 8216

$\mathrm{C}_{22} \mathrm{H}_{36} \mathrm{O}_{3}$ ent-3 $\beta$-Acetoxyisopimar-15-en-8 $\beta$-ol, 243

Aglatomin A, 742

Anacardic acid A, 1128

Azorellanol, 2061

5-Pregnene-3 $\beta, 16 \alpha, 20 \alpha$-triol, 17802

Siegesetheric acid II, 19879

\section{$\mathrm{C}_{22} \mathrm{H}_{36} \mathrm{O}_{4}$}

10-Acetoxy-8,18-dihydroxy-2,6-dolabelladiene, 163

ent-18-Acetoxy-8(14)-pimarene-15S,16-diol, 276

2,5-Dimethoxy-6-methyl-3-tridecyl-1,4-benzoquinone, 6269

Fritillebeinol, 7959

Vitetrifolin E, 22572

Vitetrifolin F, 22573

Vitexifolin F, 22579

\section{$\mathrm{C}_{22} \mathrm{H}_{36} \mathrm{O}_{5}$}

$\left(1 R^{*}, 3 S^{*}, 4 R^{*}, 6 S^{*}\right)-9-($ Acetoxy)-4-hydroxy-1-

[(2Z)-2-methylbut-2-enoyloxy]bisabol-

10(11)-ene, 230

4-O-Acetyl hygrophorone $\mathrm{D}^{14}, 434$

16-Acetylkirenol, 445

Compound 1 (Periploca sepium), 3955

21-O-Methyl-5-pregnene-3 $\beta, 14 \beta, 17 \beta, 20,21$ pentol, 14685

Orientalin A, 16187

Orientalin B, 16188

\section{$\mathrm{C}_{22} \mathrm{H}_{36} \mathrm{O}_{6}$}

4,6-Di- $O$-acetyl hygrophorone $\mathrm{A}^{12}, 5336$

1,4-Di-O-acetyl hygrophorone $\mathrm{E}^{12}, 5340$

Rollicosin, 18897

\section{$\mathrm{C}_{22} \mathrm{H}_{36} \mathrm{O}_{7}$}

Andromedotoxin, 1161

Grayanotoxin I, 8993

Rhodomollein XIII, 18810

\section{$\mathrm{C}_{22} \mathrm{H}_{36} \mathrm{O}_{8}$}

Lyoniol D, 13248

Rhodomollein XI, 18808

\section{$\mathrm{C}_{22} \mathrm{H}_{36} \mathrm{O}_{11}$}

Limonene-10-ol 10- $O-\beta$ - $D$-glucopyranosyl$(1 \rightarrow 2)-\beta$ - $D$-glucopyranoside, 12846

\section{$\mathrm{C}_{22} \mathrm{H}_{36} \mathrm{O}_{12}$}

Iridodialo- $\beta$ - $D$-gentiobioside, 11144

$\mathrm{C}_{22} \mathrm{H}_{37} \mathrm{NO}$

$\mathrm{N}$-Methylfuntumine, 14449

$\mathrm{C}_{22} \mathrm{H}_{38} \mathrm{~N}_{2}$

Kurchamine, 12346

$\mathrm{C}_{22} \mathrm{H}_{38} \mathrm{O}_{5}$

4-O-Acetyl hygrophorone $\mathrm{A}^{14}, 428$

6-O-Acetyl hygrophorone $\mathrm{A}^{14}, 429$

4-O-Acetyl hygrophorone $\mathrm{B}^{14}, 430$

6-O-Acetyl hygrophorone $\mathrm{B}^{14}, 431$

\section{$\mathrm{C}_{22} \mathrm{H}_{38} \mathrm{O}_{7}$}

Leopersin E, 12651

7-epi-Leopersin F, 12652

Leopersin F, 12653

$\mathrm{C}_{22} \mathrm{H}_{38} \mathrm{O}_{10}$

Shionoside B, 19832

\section{$\mathrm{C}_{22} \mathrm{H}_{39} \mathrm{NO}$}

$N$-Isobutyl-2E,4E,12Z-octadecatrienamide, 11282

$\mathrm{C}_{22} \mathrm{H}_{39} \mathrm{NO}_{4}$

N,O-Diacetylcassine, 5327

$\mathrm{C}_{22} \mathrm{H}_{40} \mathrm{~N}_{6} \mathrm{O}_{4}{ }^{+2}$

Chaksine, 3452

$\mathrm{C}_{22} \mathrm{H}_{40} \mathrm{O}_{4}$

Hygrophorone $\mathrm{B}^{16}, 10838$

$\mathrm{C}_{22} \mathrm{H}_{40} \mathrm{O}_{7}$

Agaricic acid, 700

\section{$\mathrm{C}_{22} \mathrm{H}_{40} \mathrm{O}_{11}$}

(E)-3,7-Dimethyl-1-O-[ $\alpha$ - $L$-rhamnopyranosyl$(1 \rightarrow 6)-\beta$ - $D$-glucopyranosyl-oct-2-en-7-ol, 6403

\section{$\mathrm{C}_{22} \mathrm{H}_{40} \mathrm{O}_{13}$}

3-O- $\beta$ - $D$-Glucopyranosyl-( $1 \rightarrow 6)-\beta$ - $D$-glucopyranoside of ethyl 3-hydroxyoctanoate, 8637

\section{$\mathrm{C}_{22} \mathrm{H}_{41} \mathrm{NO}$}

$N$-Isobutyl-(2E,4E)-octadecadienamide, 11281

\section{$\mathrm{C}_{22} \mathrm{H}_{42} \mathrm{O}_{2}$}

Cyclodocosalactone, 4488

Erucic acid, 7291

\section{$\mathrm{C}_{22} \mathrm{H}_{42} \mathrm{O}_{4}$}

Docosanedioic acid, 6535

\section{$\mathrm{C}_{22} \mathrm{H}_{44} \mathrm{O}_{2}$}

Docosanoic acid, 6537

$\mathrm{C}_{22} \mathrm{H}_{46}$

Docosane, 6534

\section{$\mathrm{C}_{22} \mathrm{H}_{46} \mathrm{O}_{2}$}

1,22-Docosanediol, 6536

\section{$\mathrm{C}_{23} \mathrm{H}_{13} \mathrm{~N}_{3} \mathrm{O}_{2}$}

Qingdainone, 18287

$\mathrm{C}_{23} \mathrm{H}_{16} \mathrm{O}_{5}$

Ohioensin A, 16019

Ohioensin C, 16021

\section{$\mathrm{C}_{23} \mathrm{H}_{16} \mathrm{O}_{6}$}

Methylene-3,3'-biplumbagin, 14348

\section{$\mathrm{C}_{23} \mathrm{H}_{17} \mathrm{~N}_{3} \mathrm{O}_{2}$}

Scarlet808, 19458

\section{$\mathrm{C}_{23} \mathrm{H}_{17} \mathrm{~N}_{3} \mathrm{O}_{4}$}

Bisindolylpyrrole CPB-53-594-3, 2473

\section{$\mathrm{C}_{23} \mathrm{H}_{17} \mathrm{~N}_{3} \mathrm{O}_{5}$}

Bisindolylpyrrole CPB-53-594-4, 2474

Bisindolylpyrrole CPB-53-594-5, 2475

\section{$\mathrm{C}_{23} \mathrm{H}_{18} \mathrm{O}_{3}$}

Pallidisetin A, 16548

Pallidisetin B, 16549 


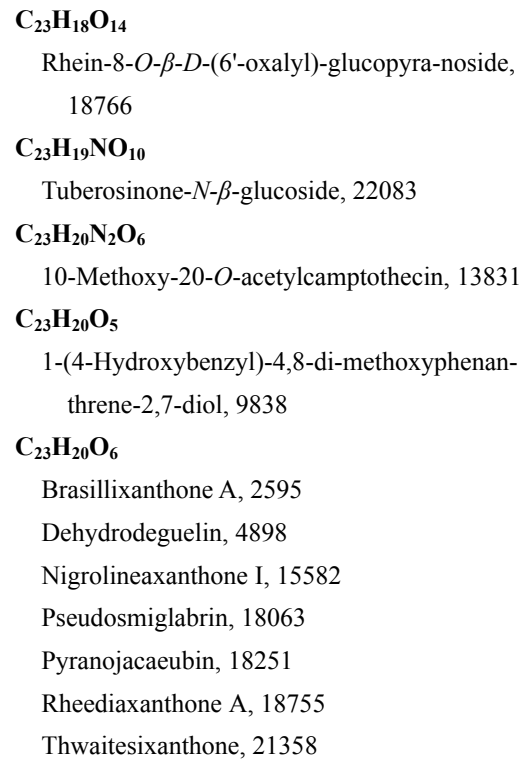

\section{$\mathrm{C}_{23} \mathrm{H}_{20} \mathrm{O}_{7}$}

Rhinacanthin D, 18771

\section{$\mathrm{C}_{23} \mathrm{H}_{20} \mathrm{O}_{9}$}

Pongamoside A, 17709

\section{$\mathrm{C}_{23} \mathrm{H}_{20} \mathrm{O}_{10}$}

Pinnatifin I, 17393

Pinnatifinoside B, 17395

Pinnatifinoside C, 17396

Pinnatifinoside D, 17397

\section{$\mathrm{C}_{23} \mathrm{H}_{20} \mathrm{O}_{13}$}

3,3'-Di- $O$-methylellagic acid

4-(5"-acetyl)- $\alpha$ - $L$-arabinofuranoside, 6345

3-O-Methylellagic acid

3'-O- $\alpha-2$--O-acetylrhamnopyranoside, 14337

3-O-Methylellagic acid

3'-O- $\alpha-3$ "-O-acetylrhamnopyranoside, 14338

3-O-Methylellagic acid

3'-O- $\alpha-4$ "-O-acetylrhamnopyranoside, 14339

4-O-Methylellagic acid

3 '-(3"--O-acetyl)- $\alpha$-rhamnoside, 14340

4-O-Methylellagic acid

3'-(4"--O-acetyl)- $\alpha$-rhamnoside, 14341

\section{$\mathrm{C}_{23} \mathrm{H}_{21} \mathrm{NO}_{10}$}

Aristolactam- $N$ - $\beta$ - $D$-glucoside, 1697

\section{$\mathrm{C}_{23} \mathrm{H}_{21} \mathrm{NO}_{13}$}

Aristoloside, 1725

$\mathrm{C}_{23} \mathrm{H}_{22} \mathrm{NO}_{3}{ }^{+}$

Tylophoridicine C, 22147

\section{$\mathrm{C}_{23} \mathrm{H}_{22} \mathrm{~N}_{2} \mathrm{O}_{5}$}

Isaindigotidione, 11187

\section{$\mathrm{C}_{23} \mathrm{H}_{22} \mathrm{O}_{4}$}

8-( $\alpha, \beta$-Dimethylallyl)-pongamol, 6309

1-(4-Hydroxybenzyl)-4,7-dimethoxy-9,10dihydrophenanthrene-2-ol, 9837

$\mathrm{C}_{23} \mathrm{H}_{22} \mathrm{O}_{5}$
Ananixanthone, 1135

Cudraxanthone Q, 4345

Demethylcalabaxanthone, 5064

Inoxanthone, 11086

Isouvaretin, 11751

Merguenone, 13782

Nigrolineaxanthone K, 15584

Trapezifolixanthone, 21503

Uvaretin, 22290

\section{$\mathrm{C}_{23} \mathrm{H}_{22} \mathrm{O}_{6}$}

Bangangxanthone A, 2135

Barbigerone, 2148

Caledonixanthone M, 2963

Caloxanthone A, 2998

Deguelin, 4872

Garcinone B, 8219

Gerontoxanthone A, 8354

Gerontoxanthone B, 8355

3-Hydroxyblancoxanthone, 9857

Latisxanthone D, 12560

Morusignin I, 14987

Morusignin J, 14988

Nigrolineaxanthone G, 15580

(+)-Purpurin 2, 18223

Rotenone, 18939

Smeathxanthone B, 20019

Subelliptenone H, 20436

\section{$\mathrm{C}_{23} \mathrm{H}_{22} \mathrm{O}_{7}$}

Anticancer Flavonoid PMV70P691-105, 1408

3,4 $\beta$-Dihydro-15-dehydrolactucopicrin, 5585

$12 \alpha$-Hydroxyrotenone, 10680

12a-Hydroxyrotenone, 10681

Lactucopicrin, 12447

Pongapinone A, 17715

Sumatrol, 20485

(-)-Tephrosin, 20965

$\alpha$-Toxicarol, 21483

$\beta$-Toxicarol, 21484

Usararotenoid C, 22279

$\mathrm{C}_{23} \mathrm{H}_{22} \mathrm{O}_{8}$

Chaetoquadrin J, 3449

11-Hydroxytephrosin, 10741

$\mathrm{C}_{23} \mathrm{H}_{22} \mathrm{O}_{10}$

6"-O-Acetyldaidzin, 361

8-O- $\beta$-D-(6'-O-Acetyl)glucopyranosylchrysophanol, 401

5,3'-Diacetoxy-3,6,7,4'-tetramethoxyflavone, 5319

Phrymarolin II, 17203

$\mathrm{C}_{23} \mathrm{H}_{22} \mathbf{O}_{11}$

6"-O-Acetylgenistin, 398

Germanaism B, 8350

Kaempferol-3-O-(2-O-acetyl- $\alpha-L$-rhamnopyra- noside), 12024

Kaempferol-3- $O$-(3- $O$-acetyl- $\alpha-L$-rhamnopyranoside), 12025

Kaempferol-3- $O$-(4- $O$-acetyl- $\alpha-L$-rhamnopyranoside), 12026

Pongamoside D, 17712

1,3,6-Trihydroxy-2-methyl-9,10-anthra-quinone3-O-(6'- $O$-acetyl)- $\beta$ - $D$-glucoside, 21794

$\mathrm{C}_{23} \mathrm{H}_{22} \mathrm{O}_{12}$

2"-O-Acetylquercitrin, 491

\section{$\mathrm{C}_{23} \mathrm{H}_{22} \mathrm{O}_{13}$}

Myricetin-3-O-(2"- $O$-acetyl- $\alpha$-rhamnopyranoside), 15173

3,3',4-O-Trimethyl-4'-O- $\beta$ - $D$-glucopyranosylellagic acid, 21961

\section{$\mathrm{C}_{23} \mathrm{H}_{22} \mathrm{O}_{15} \mathrm{~S}$}

Tamarixetin 3-glucoside-7-sulphate, 20659

\section{$\mathrm{C}_{23} \mathrm{H}_{23} \mathrm{NO}_{3}$}

Buxifoliadine D, 2826

Severifoline, 19790

\section{$\mathrm{C}_{23} \mathrm{H}_{23} \mathrm{NO}_{4}$}

Atalaphyllinine, 1959

\section{$\mathrm{C}_{23} \mathrm{H}_{23} \mathrm{NO}_{5}$}

Ethoxychelerythrine, 7399

Waltherione A, 22634

\section{$\mathrm{C}_{23} \mathrm{H}_{23} \mathrm{NO}_{6}$}

Acetylcorynoline, 359

Acetylisocorynoline, 438

$\mathrm{C}_{23} \mathrm{H}_{23} \mathrm{NO}_{10}$

Nandinin, 15244

\section{$\mathrm{C}_{23} \mathrm{H}_{24} \mathrm{O}_{4}$}

Cudraphenone A, 4339

Gymconopin D, 9102

5-Hydroxy-4-( $p$-hydroxybenzyl)-3',3-dimethoxybibenzyl, 10176

\section{$\mathrm{C}_{23} \mathrm{H}_{24} \mathrm{O}_{5}$}

Caloxanthone L, 3001

Cudraphenone C, 4341

8-Desoxygartanin, 5276

7-Geranyloxy-1,3-dihydroxyxanthone, 8323

Gerontoxanthone H, 8358

Mangostinone, 13495

7-O-Methoxypraecansone B, 14075

Nigrolineaxanthone J, 15583

Nigrolineaxanthone Q, 15590

Subelliptenone B, 20435

1,3,7-Trihydroxy-2,4-bis(3-methyl-2-butenyl)xanthone, 21691

\section{$\mathrm{C}_{23} \mathrm{H}_{24} \mathrm{O}_{6}$}

Alvaxanthone, 1010

Anticancer Flavonoid PMV70P691-106, 1409 BR-Xanthone A, 2685

Cudraxanthone J, 4343 
Cudraxanthone P, 4344

Demethoxy-cochinchinone D, 5044

Gartanin, 8235

Gerontoxanthone G, 8357

Gerontoxanthone I, 8359

Isoalvaxanthone, 11206

Isonormangostin, 11564

Ixerochinolide, 11802

Mangostenone D, 13490

$\gamma$-Mangostin, 13494

Mangoxanthone, 13496

Montrouxanthone, 14948

Morusignin H, 14986

Nigrolineaxanthone M, 15586

Patulone, 16730

Pongapinone B, 17716

Smeathxanthone A, 20018

1,4,5,6-Tetrahydroxy-7,8-di(3-methylbut-2-enyl) xanthone, 21099

\section{$\mathrm{C}_{23} \mathrm{H}_{24} \mathrm{O}_{7}$}

Cudraxanthone R, 4346

(2S)-5,7-Dimethoxy-8-(2S-hydroxy-3-methyl-3butenyl)-3',4'-methylenedioxyflavanone, 6247

Garcimangosone C, 8208

Morusignin E, 14985

(R)-Saclenone, 19108

\section{$\mathrm{C}_{23} \mathrm{H}_{24} \mathrm{O}_{8}$}

3,7-Dihydroxy-3'-(2-hydroxy-3-methyl-3-

butenyl)-5,6,4'-trimethoxyflavone, 5916

5,7-Dihydroxy-3'-(2-hydroxy-3-methyl-3-

butenyl)-3,6,4'-trimethoxyflavone, 5917

Hernandin, 9443

$\beta$-Peltatin A methyl ether, 16797

\section{$\mathrm{C}_{23} \mathrm{H}_{24} \mathrm{O}_{10}$}

Andrographidine C, 1153

Lasianthuoside B, 12532

2,4,6-Trihydroxyacetophenone 3-C- $\beta$-(2'-O-Ecinnamoyl)-glucopyranoside, 21680

\section{$\mathrm{C}_{23} \mathrm{H}_{24} \mathrm{O}_{11}$}

Abrusin, 28

Camaroside, 3027

5,7-Dihydroxy-8,2'-dimethoxyflavone-7-O- $\beta-D$ glucopyranoside, 5831

6,4'-dimethoxy-5-hydroxyflavone 7-glucoside, 6241

5-Hydroxyaloin A 6'-O-acetate, 9774

Irisolidone-7-O- $\alpha$ - $D$-glucoside, 11172

4'-Methyltectorigenin 7-glucoside, 14740

Orobol 5,3'-di-O-methyl-8- $C$-glucoside, 16210

Skullcapflavone I 2'-O- $\beta$ - $D$-glucopyranoside, 20008

2,4,6-Trihydroxyacetophenone 3-C- $\beta$-(2'-O-Ecoumaroyl)-glucopyranoside, 21681
$\mathrm{C}_{23} \mathrm{H}_{24} \mathrm{O}_{12}$

Andrographidine B, 1152

Aurantioobtusin $\beta$ - $D$-glucoside, 2009

Burkinabin A, 2761

Burkinabin B, 2762

Burkinabin C, 2763

Cirsiliol-4'-monoglucoside, 3744

3',4'-Dimethoxy-5, 7,5'-trihydroxyl-flavone 3-O$\alpha$ - $L$-rhamnopyranoside, 6296

Eriodictyl 7- $O-\beta$ - $D$-(6'-ethyl ester)-glucuronopyranoside, 7276

Eupatolin, 7587

Iristectorin A, 11181

Iristectorin $\mathrm{B}, 11182$

Kaempferol 3-O-(6"-acetyl)- $\beta$ - $D$-galactopyranoside, 12023

Rhamnazin-3-O- $\beta$ - $D$-glucoside, 18675

Tricin-7-O- $\beta$ - $D$-glucopyranoside, 21590

\section{$\mathrm{C}_{23} \mathrm{H}_{24} \mathrm{O}_{13}$}

7,4'-Dimethoxy-8,3',5'-trihydroxy-6- $O-\beta-D$ glucopyranosylisoflavone, 6295

Gossypetin-3,8-dimethyl ether-5- $O-\beta$-glucoside, 8961

Limocitrin- $\beta$ - $D$-glucoside, 12839

Quercetin-3-O-(6"-acetyl)- $\beta$ - $D$-galactopyranoside, 18321

Syringetin-3-O- $\beta$ - $D$-galactopyranoside, 20562

Syringetin-3- $O-\beta$ - $D$-glucoside, 20563

Viscidulin III-6'-O- $\beta$ - $D$-glucopyranoside, 22534

$\mathrm{C}_{23} \mathrm{H}_{24} \mathrm{O}_{14}$

Myricetin-3-O-(6"-acetyl)- $\beta$ - $D$-galactopyranoside, 15171

\section{$\mathrm{C}_{23} \mathrm{H}_{25} \mathrm{NO}$}

Bicyclomahanimbicine, 2354

Bicyclomahanimbine, 2355

Cyclomahanimbine, 4515

Isomahanimbine, 11520

Mahanimbidine, 13397

(+)-Mahanimbine, 13398

$\mathrm{C}_{23} \mathrm{H}_{25} \mathrm{NO}_{2}$

Clausenatine A, 3790

\section{$\mathrm{C}_{23} \mathrm{H}_{25} \mathrm{NO}_{3}$}

Boehmeriasin B, 2529

7-Demethoxytylophorine, 5055

1-Epi-dioncophylline B, 6896

3,6,7-Trimethoxyphenanthroindolizidine, 21921

\section{$\mathrm{C}_{23} \mathrm{H}_{25} \mathrm{NO}_{4}$}

Dioncophyllinol B, 6433

Tylophorinine, 22153

$\mathrm{C}_{23} \mathrm{H}_{25} \mathrm{NO}_{5}$

Bassianin, 2161

Buxifoliadine E, 2827

$\mathrm{C}_{23} \mathrm{H}_{25} \mathrm{NO}_{6}$
Gnoscopine, 8902

$\mathrm{C}_{23} \mathrm{H}_{25} \mathrm{O}_{12}{ }^{+}$

Oenin, 16010

$\mathrm{C}_{23} \mathrm{H}_{26} \mathrm{~N}_{2} \mathrm{O}_{3}$

16-Ethoxystrychnine, 7417

$\mathrm{C}_{23} \mathrm{H}_{26} \mathrm{~N}_{2} \mathrm{O}_{4}$

Brucine, 2678

Isobrucine, 11265

3-Methoxyicajine, 13972

\section{$\mathrm{C}_{23} \mathrm{H}_{26} \mathrm{~N}_{2} \mathrm{O}_{5}$}

Akuammiline N(4)-oxide, 827

Brucine $N$-oxide, 2679

Isobrucine $N$-oxide, 11266

Myrtoidine, 15226

Pseudobrucine, 18021

\section{$\mathrm{C}_{23} \mathrm{H}_{26} \mathrm{~N}_{4} \mathrm{O}_{3}$}

Argentine, 1672

\section{$\mathrm{C}_{23} \mathrm{H}_{26} \mathrm{O}_{4}$}

Clusiaphenone B, 3850

Cudraphenone B, 4340

$4 \beta$-Demethylxuulanin- $4 \beta$-ethyl ether, 5103

( \pm -trans-3-(4-Hydroxy-3-methoxyphenyl)-4-

[(E)-3,4-dimethoxystyryl]cyclohex-1-ene, 10438

\section{$\mathrm{C}_{23} \mathrm{H}_{26} \mathrm{O}_{5}$}

Cudraphenone D, 4342

2,6-Dihydroxy-4-[(E)-5-hydroxy-3,7-dimethylocta-2,7-dienyloxy] benzophenone, 5908

Ethylnotopterol, 7466

$3 \beta$-Methoxyxuulanin, 14105

Vismiaguianone A, 22545

Vismiaguianone B, 22546

Vismiaguianone C, 22547

\section{$\mathrm{C}_{23} \mathrm{H}_{26} \mathrm{O}_{6}$}

Nigrolineaxanthone L, 15585

Nigrolineaxanthone N, 15587

Nigrolineaxanthone R, 15591

Spiraformin C, 20191

3,4-Teracrylshikonin, 20968

\section{$\mathrm{C}_{23} \mathrm{H}_{26} \mathrm{O}_{7}$}

Garcinone C, 8220

Neoisostegane, 15417

Neokadsuranin, 15422

Neokadsuranin, 15423

Pyramidatin G, 18247

\section{$\mathrm{C}_{23} \mathrm{H}_{26} \mathrm{O}_{8}$}

$\alpha$-Acetoxyisovalerylalkannin, 244

Caruilignan B, 3228

5'-Methoxyyatein, 14106

(2S,3S)-(+)-5'-Methoxyyatein, 14107

Sesartemin, 19783

Sikkimotoxin, 19889

$\mathrm{C}_{23} \mathrm{H}_{26} \mathrm{O}_{10}$ 
Andrographidine A, 1151

Exoticin, 7696

Icariside $\mathrm{A}_{7}, 10942$

Lactiflorin, 12439

6-O-Methylscorzocreticoside I, 14723

\section{$\mathrm{C}_{23} \mathrm{H}_{26} \mathrm{O}_{11}$}

Aquaticoside C, 1540

Decentapicrin A, 4845

Desrhamnosylacteoside, 5277

Desrhamnosylisoacteoside, 5278

Isolindleyin, 11496

Lindleyin, 12876

Myrciacitrin I, 15147

Myrciacitrin III, 15149

Nyasicoside, 15875

Persicoside, 16993

Pilosidine, 17370

Plantainoside A, 17507

Plantainoside B, 17508

Tschimganic ester B, 22065

Tschimganic ester C, 22066

\section{$\mathrm{C}_{23} \mathrm{H}_{26} \mathrm{O}_{12}$}

2'-(o,m-Dihydroxybenzyl) sweroside, 5772

Peruvianoside I, 16999

Peruvianoside II, 17000

Polygalaxanthone VI, 17634

\section{$\mathrm{C}_{23} \mathrm{H}_{26} \mathrm{O}_{13}$}

Mallophenol A, 13424

1-O-Vanillic acid-6-O-(3",5"-dimethoxy-galloyl)$\beta$ - $D$-glycoside, 22333

\section{$\mathbf{C}_{23} \mathbf{H}_{26} \mathbf{O}_{15}$}

5,6-O- $\beta$-D-Diglucopyranosylangelicin, 5528

\section{$\mathrm{C}_{23} \mathrm{H}_{27} \mathrm{NO}_{3}$}

Hispidine, 9562

\section{$\mathrm{C}_{23} \mathrm{H}_{27} \mathrm{NO}_{6}$}

Ambinine, 1023

\section{$\mathrm{C}_{23} \mathrm{H}_{27} \mathrm{NO}_{7}$}

Methoxymecambridine, 13994

Romucosine G, 18906

\section{$\mathrm{C}_{23} \mathrm{H}_{27} \mathrm{NO}_{8}$}

Narceine, 15263

\section{$\mathrm{C}_{23} \mathrm{H}_{28} \mathrm{~N}_{2} \mathrm{O}_{4}$}

Paynantheine, 16735

Vellosine, 22369

\section{$\mathrm{C}_{23} \mathrm{H}_{28} \mathrm{~N}_{2} \mathrm{O}_{5}$}

19-(R)-Acetoxydihydrogelsevirine, 161

22-O-Acetyl- $N_{b}$-demethyl-echitamine, 372

Quaternine, 18302

Quaternine, 18303

Rauvanine, 18556

Reserpiline, 18632

\section{$\mathrm{C}_{23} \mathrm{H}_{28} \mathrm{~N}_{2} \mathrm{O}_{6}$}

Rauvoxine, 18558

\author{
$\mathrm{C}_{23} \mathrm{H}_{28} \mathrm{O}_{5}$ \\ 2,6-Dihydroxy-4-[(E)-7-hydroxy-3,7-dimethyl- \\ octa-2-enyloxy]benzophenone, 5909 \\ rel-(5S,6S,7R,10R,12S,13R)-7-Hydroxyapiana- \\ 8,14-diene-11,16-dion-(22,6)-olide, 9793 \\ rel-(5S,6S,7S,10R,12R,13S)-7-Hydroxyapiana- \\ 8,14-diene-11,16-dion-(22,6)-olide, 9794 \\ rel-(5S,6S,7S,10R,12S,13R)-7-Hydroxyapiana- \\ 8,14-diene-11,16-dion-(22,6)-olide, 9795

\section{$\mathrm{C}_{23} \mathrm{H}_{28} \mathrm{O}_{6}$} \\ Deoxygomisin A, 5178 \\ Gomisin N, 8922 \\ Hancinone C, 9211 \\ Isokadsuranin, 11469 \\ Kadsuranin, 12003 \\ Kadsurenin L, 12008 \\ Moslolignan A, 15000 \\ Pseudo- $\gamma$-schisandrin, 18061

\section{$\mathrm{C}_{23} \mathrm{H}_{28} \mathrm{O}_{7}$}

(5S)-5-Acetoxy-1,7-bis(4-hydroxy-3-methoxyphenyl)heptan-3-one, 135

Crotozambefuran B, 4274

Epigomisin O, 6926

Gomisin A, 8906

Gomisin O, 8923

Kadsulignan A, 11996

Korberin B, 12271

Leonubiastrin, 12645

Magnolin, 13387

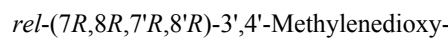

3,4,5,5'-tetramethoxy-7,7'-epoxylignan, 14384

Pyramidatin B, 18242

Pyramidatin C, 18243

Pyramidatin D, 18244

Pyramidatin E, 18245

Schisandrol B, 19474

Schisanlignone B, 19486

(-)-Thujaplicatin trimethyl ether, 21345

\section{$\mathrm{C}_{23} \mathrm{H}_{28} \mathrm{O}_{8}$}

Albaspidin AB, 849

erythro-2,3-Bis(4-acetoxy-3-methoxyphenyl)-

3-ethoxypropan-1-ol acetate, 2425

Flavaspidinin, 7810

(-)-Hernolactone, 9453

Isoaspidin $\mathrm{AB}, 11239$

Juanislamin, 11895

Korberin A, 12270

(8R,8'R,9'S)-5-Methoxyclusin, 13898

Norcaesalpinin MD, 15723

Paludosic acid, 16571

Pseudolaric acid B, 18033

$\mathrm{C}_{23} \mathrm{H}_{28} \mathrm{O}_{9}$

2,3-Epoxyjuanislamin, 7153

\section{$\mathrm{C}_{23} \mathrm{H}_{28} \mathrm{O}_{10}$}

9,10-Dimethoxy-pterocarpane-3- $O-\beta$ - $D$-glucoside, 6291

Enhydrin, 6802

Isomucronulatol-7-O-glucoside, 11546

Methyl-9 $\beta$-(epoxyangeloyloxy)-5 $\alpha, 6 \alpha$-dihydroxy-2-oxo-3,4-dehydro- $\delta$-guaien-12-oate, 14406

$3 S$-(-)-Mucronulatol-7-D-glucopyranoside, 15022

\section{$\mathrm{C}_{23} \mathrm{H}_{28} \mathrm{O}_{11}$}

Albiflorin, 857

Albiflorin $\mathrm{R}_{1}, 861$

Aquaticoside A, 1538

Bruceine B, 2665

Glochiflavanoside A, 8571

Glochiflavanoside D, 8574

(S)-4-(4-Hydroxyphenyl)-2-butanol 2-O-(6-O-galloyl)- $\beta$ - $D$-glucopyranoside, 10612

Paeoniflorin, 16525

Paeonin B, 16530

\section{$\mathrm{C}_{23} \mathrm{H}_{28} \mathrm{O}_{12}$}

Ampelopsisin, 1079

Aquaticoside B, 1539

Curculigin, 4381

Negundoside, 15324

Oxypaeoniflorin, 16455

Paeonin C, 16531

Swertiaside A, 20513

Tetracentronside A, 21035

\section{$\mathrm{C}_{23} \mathrm{H}_{28} \mathrm{O}_{13}$}

Amphicoside, 1082

Kutkoside, 12376

Picroside II, 17345

6'-O-Vanilloyl catalpol, 22341

\section{$\mathrm{C}_{23} \mathrm{H}_{28} \mathrm{O}_{14}$}

2-Methoxy-5-(1',2',3'-trihydroxypropyl)phenyl-1- $O$-(6"-galloyl)- $\beta$ - $D$-glucopyrano -side, 14101

\section{$\mathrm{C}_{23} \mathrm{H}_{29} \mathrm{NO}_{3}$}

Piperundecalidine, 17471

\section{$\mathrm{C}_{23} \mathrm{H}_{29} \mathrm{NO}_{6}$}

Dehydroprotostemonine, 4964

Haplotubine, 9228

$\mathrm{C}_{23} \mathrm{H}_{29} \mathrm{NO}_{7}$

Oxyprotostemonine, 16470

\section{$\mathrm{C}_{23} \mathrm{H}_{29} \mathrm{~N}_{3} \mathrm{O}_{2}$}

Celabenzine, 3356

$\mathrm{C}_{23} \mathrm{H}_{30} \mathrm{NO}_{5}^{+}$

$\mathrm{N}$-Methylpurpuerine, 14708

$\mathrm{C}_{23} \mathrm{H}_{30} \mathrm{~N}_{2} \mathrm{O}_{4}$

Acetyl-alstohentine, 310 
Mitragynine, 14886

Speciociliatine, 20138

Speciogynine, 20139

\section{$\mathrm{C}_{23} \mathrm{H}_{30} \mathrm{~N}_{2} \mathrm{O}_{5}$}

12-Hydroxy-19-epi-malagashanine, 10079

7-Hydroxymitragynine, 10528

\section{$\mathrm{C}_{23} \mathrm{H}_{30} \mathrm{O}_{4}$}

2-(4',8'-Dimethylnona-3',7'-dienyl)-8-hydroxy-

2-methyl-2H-chromene-6-carboxylic methyl ester, 6379

\section{$\mathrm{C}_{23} \mathrm{H}_{30} \mathrm{O}_{5}$}

Ardisinone E, 1649

Euglobal $\mathrm{Ia}_{1}, 7527$

Euglobal Ib, 7528

Euglobal Ic, 7529

Euglobal Iia, 7530

PM04701085-02, 17574

Robustadial A, 18862

Robustadial B, 18863

Stachybotrystrydial (Mer-NF5003F), 20253

\section{$\mathrm{C}_{23} \mathrm{H}_{30} \mathrm{O}_{6}$}

Apetalic acid methyl ester, 1472

Ardisinone D, 1648

16-Dehydrostrophanthidin, 4972

5-Demethoxyniranthin, 5052

(-)-Gomisin $\mathrm{K}_{1}, 8915$

(+)-Gomisin $\mathrm{K}_{2}, 8916$

(+)-Gomisin $\mathrm{K}_{3}, 8917$

Isoapetalic methyl ester, 11232

Lapidol vanillate, 12509

Methyl pseudolarate A, 14702

$( \pm)$-Virolongin, 22528

\section{$\mathrm{C}_{23} \mathrm{H}_{30} \mathrm{O}_{7}$}

Acanthoglabrolide, 78

(3R,5S)-3-Acetoxy-5-hydroxy-1,7-bis(4-hydroxy-3-methoxyphenyl)heptane, 210

Albaspidin PP, 854

Biondinin E, 2391

Gomisin H, 8913

Gomisin S, 8925

Gomisin T, 8926

Kuhistanicaol I, 12319

Polysyphorin, 17688

\section{$\mathrm{C}_{23} \mathrm{H}_{30} \mathrm{O}_{8}$}

Acanthospermal A, 89

Calealactone A, 2956

Coetsin A, 3896

\section{$\mathrm{C}_{23} \mathrm{H}_{30} \mathrm{O}_{9}$}

2,3-Epoxycalealactone A, 7060

\section{$\mathrm{C}_{23} \mathrm{H}_{30} \mathrm{O}_{11}$}

Aglycone of yadanzioside D, 748

Broceaketolic acid, 2616

(1S,2R)-1-(4'-O- $\beta$-D-Glucopyranosyl-3'- methoxyphenyl)-2-(4"-hydroxy-3"-methoxy-

phenyl)-1,3-propanediol, 8681

6-O-(4-Methoxybenzoyl)-ajugol, 13850

$\mathrm{C}_{23} \mathrm{H}_{30} \mathrm{O}_{12}$

Diospyrosonaphthoside, 6461

Nishindaside, 15628

Trifochalcanoloside III, 21633

6-O-Vanilloylajugol, 22339

\section{$\mathrm{C}_{23} \mathrm{H}_{31} \mathrm{NO}$}

1-Methyl-2-(4Z,7Z)-4,7-tridecadienyl-4(1H)quinolinone, 14771

\section{$\mathrm{C}_{23} \mathrm{H}_{31} \mathrm{NO}_{3}$}

Piperchabamide B, 17441

\section{$\mathrm{C}_{23} \mathrm{H}_{31} \mathrm{NO}_{4}$}

Spiradine F, 20179

\section{$\mathrm{C}_{23} \mathrm{H}_{31} \mathrm{NO}_{6}$}

Protostemonine, 17992

\section{$\mathrm{C}_{23} \mathrm{H}_{31} \mathrm{NO}_{8}$}

Thesinine-4'- $O-\beta$ - $D$-glucoside, 21319

\section{$\mathrm{C}_{23} \mathrm{H}_{31} \mathrm{NO}_{9}$}

Ligularine, 12812

$\mathrm{C}_{23} \mathrm{H}_{32} \mathrm{O}_{3}$

1-Formylneogrifolin, 7910

$\mathrm{C}_{23} \mathrm{H}_{32} \mathrm{O}_{4}$

14-Deoxy-15-isopropylidene-11,12-didehydroandrographolide, 5183

Ferutidin, 7791

1-Formyl-3-hydroxyneogrifolin, 7906

Xysmalogenin, 22856

\section{$\mathrm{C}_{23} \mathrm{H}_{32} \mathrm{O}_{5}$}

Corotoxigenin, 4092

2,3-Epoxy-jaeschkeanadiol $p$-methoxybenzoate, 7152

Jaeschkeanadiol vanillate, 11811

Kuhistanicaol C, 12313

Kuhistanicaol F, 12316

Methyl-15,16-epoxy-12(R)-acetoxy-8(17),

13(16),14-ent-labdatrien-19-oate, 14405

$o$-Methylshikoccin, 14727

Periforgenin A, 16923

\section{$\mathrm{C}_{23} \mathrm{H}_{32} \mathrm{O}_{6}$}

16-Acetoxy-7 $\alpha$-methoxyroyleanone, 256

Calotropagenin, 2996

16-Dehydrostrophanthidol, 4973

$4 \beta, 8 \alpha$-Dihydroxy- $6 \alpha$-vanilloyloxydauc-9-ene, 6179

4 $\beta, 8 \beta$-Dihydroxy- $6 \alpha$-vanilloyloxydauc-9-ene, 6180

Kuhistanicaol D, 12314

Kuhistanicaol E, 12315

Lancerotriol 6-vanillate, 12471

$o$-Methylepoxyshikoccin, 14409

Sarmutogenin, 19380
Strophanthidin, 20397

$5 \alpha$-Vanillate of 2,3-epoxy-jaeschkeanadiol, 22331

\section{$\mathrm{C}_{23} \mathrm{H}_{32} \mathrm{O}_{7}$}

Antiarigenin, 1375

Lophanthoidin A, 12993

Nodosinin, 15653

Strophadogenin, 20395

\section{$\mathrm{C}_{23} \mathrm{H}_{32} \mathrm{O}_{8}$}

Dihydroacanthospermal A, 5537

Leopersin N, 12659

15 $\beta$-Methoxyfaciculatin, 13926

15 $\alpha$-Methoxyfaciculatin B, 13927

Methoxynepetaefolin, 14041

\section{$\mathrm{C}_{23} \mathrm{H}_{32} \mathrm{O}_{9}$}

Methyl

$8 \alpha$-(Z-2-hydroxymethyl-4-acetoxybut-2-enoylo xy)-6 $\alpha, 15$-dihydroxyelema-1,3,11(13)-trien-12 -oate, 14514

Taibaijaponicain B, 20621

\section{$\mathrm{C}_{23} \mathrm{H}_{32} \mathrm{O}_{10}$}

Atticin, 2002

2'-Hydroxy-3',4'-dimethoxy-isoflavane-7-O- $\beta-D$ glucoside, 10031

Paucin, 16733

\section{$\mathrm{C}_{23} \mathrm{H}_{32} \mathrm{O}_{11}$}

CPB-2001-49-1359-1, 4203

2,7-Dimethyl-1,4-dihydronaphthalene-5,8-diol 5 - $O$ - $\beta$ - $D$-xylopyranosyl $(1 \rightarrow 6)$ - $\beta$ - $D$-glucopyran oside, 6336

Ovatic acid methyl ester 7-O-(6'-O-p-hydroxybenzoyl)- $\beta$ - $D$-glucopyranoside, 16277

\section{$\mathrm{C}_{23} \mathrm{H}_{32} \mathrm{O}_{12}$}

Celephtalide B, 3373

Seguinoside J, 19673

\section{$\mathrm{C}_{23} \mathrm{H}_{32} \mathrm{O}_{13}$}

Dracunculifoside G, 6586

\section{$\mathrm{C}_{23} \mathrm{H}_{32} \mathrm{O}_{15}$}

Arillanin C, 1683

Sibiricose $\mathrm{A}_{6}, 19866$

\section{$\mathrm{C}_{23} \mathrm{H}_{33} \mathrm{NO}$}

Evocarpine, 7663

1-Methyl-2-[(Z)-7-tridecenyl]-4(1H)-quinolone, 14741

\section{$\mathrm{C}_{23} \mathrm{H}_{33} \mathrm{NO}_{3}$}

2-(12-Oxo-tridecanyl)-3-methoxy-4-quinolone, 16431

\section{$\mathrm{C}_{23} \mathrm{H}_{33} \mathrm{NO}_{6}$}

Methoxystemokerrin- $N$-oxide, 14094

\section{$\mathrm{C}_{23} \mathrm{H}_{33} \mathrm{NO}_{6} \mathrm{~S}$}

O-Methylsakerinol A, 14716

$\mathrm{C}_{23} \mathrm{H}_{34} \mathrm{O}_{2}$

$10^{\prime}(Z), 13^{\prime}(E), 15^{\prime}(E)$-Heptadecatrienylhydro- 
quinone, 9383

$$
\begin{gathered}
\mathbf{C}_{\mathbf{2 3}} \mathbf{H}_{\mathbf{3 4}} \mathbf{O}_{\mathbf{4}} \\
\text { Digitoxigenin, } 5525 \\
\text { Proceragenin, } 17870 \\
\text { Uzarigenin, } 22301
\end{gathered}
$$

$\mathrm{C}_{23} \mathrm{H}_{34} \mathrm{O}_{5}$

7-Acetoxy-3-oxo-4(3 $\rightarrow 2$ )-abeo-2(4),13-clerodadien-15-oic acid methyl ester, 268

7-Acetoxy-2-oxo-3,13-clerodadien-15-oic acid methyl ester, 269

Bryophollenone, 2688

Coroglaucigenin, 4077

Farfaratin, 7716

Gitoxigenin, 8460

$(2 S, 3 R, 4 S)$-3-Hydroxy-4-methyl-2-(11'piperonyl- $n$-undecyl)-butanolide, 10516

Nelumol C, 15327

Otogirone, 16269

Periplogenin, 16959

Rabdocoetsin C, 18458

Sarmentogenin, 19372

Sugikurojin D, 20460

Tussilagone, 22144

\section{$\mathrm{C}_{23} \mathrm{H}_{34} \mathrm{O}_{6}$}

12-O-Acetyllineolone, 448

Bakkenolide H, 2122

8-Hydroxy-periplogenin, 10599

Nelumol D, 15328

Nelumol E, 15329

Strophanthidol, 20401

Wikstroemioidin B, 22670

\section{$\mathrm{C}_{23} \mathrm{H}_{34} \mathrm{O}_{7}$}

Adenolin D, 622

Anhydrocinnzeylanine, 1263

Enanderianin H, 6788

Metaplexigenin, 13813

Nigakilactone J, 15555

Xindongnin L, 22808

\section{$\mathrm{C}_{23} \mathrm{H}_{34} \mathrm{O}_{8}$}

Adenolin C, 621

Adenolin E, 623

Enanderianin G, 6787

Lasiokaurinin, 12538

Pirolatin, 17476

Wikstroemioidin D, 22672

\section{$\mathrm{C}_{23} \mathrm{H}_{34} \mathrm{O}_{9}$}

11-Homohydroxyldidrovaltrate, 9610 Hypoloside A, 10901

\section{$\mathrm{C}_{23} \mathrm{H}_{34} \mathrm{O}_{12}$}

1,5-Bis $(\beta$ - $D$-glucopyranosyloxy-2-(3',3'dimethylallyl) benzene, 2454

\section{$\mathrm{C}_{23} \mathrm{H}_{34} \mathrm{O}_{13}$}

Jioglutoside B, 11880
$\mathrm{C}_{23} \mathrm{H}_{34} \mathrm{O}_{14}$

Lysidiside B, 13262

$\mathrm{C}_{23} \mathrm{H}_{34} \mathrm{O}_{15}$

Genameside C, 8268

Genameside D, 8269

Genipingentiobioside, 8274

$\mathrm{C}_{23} \mathrm{H}_{35} \mathrm{ClO}_{5}$

Guisinol, 9084

$\mathrm{C}_{23} \mathrm{H}_{35} \mathrm{NO}$

2-Tridecyl-1-methyl-4(1H)-quinolone, 21624

$\mathrm{C}_{23} \mathrm{H}_{35} \mathrm{NO}_{3}$

2-(10-Hydroxy-10-methyldodecanyl)-3-methoxy4-quinolone, 10486

2-(11-Hydroxy-11-methyldodecanyl)-3-methoxy4-quinolone, 10487

2-(12-Hydroxytridecanyl)-3-methoxy-4-quinolone, 10783

Lepedine, 12667

$\mathrm{C}_{23} \mathrm{H}_{35} \mathrm{NO}_{6}$

Gadesine, 8044

Tatsidine, 20726

$\mathrm{C}_{23} \mathrm{H}_{35} \mathrm{O}_{16}$

6'-O- $\beta$ - $D$-Glucopyranosylmorroniside, 8685

\section{$\mathrm{C}_{23} \mathrm{H}_{36} \mathrm{O}_{2}$}

Alkylresorcinol C, 912

$10^{\prime}(Z), 13^{\prime}(E)$-Heptadecadienylhydroquinone, 9376

\section{$\mathrm{C}_{23} \mathrm{H}_{36} \mathrm{O}_{3}$}

$7 \beta$-Ethoxy-12-methoxy-8,11,13-abietatrien-11-ol, 7410

S-20, 19095

\section{$\mathrm{C}_{23} \mathrm{H}_{36} \mathrm{O}_{4}$}

Ardisiphenol B, 1651

\section{$\mathrm{C}_{23} \mathrm{H}_{36} \mathrm{O}_{5}$}

2-Acetoxy-5-methoxy-6-methyl-3-tridecyl-1,4benzoquinone, 255

$2 \beta$-Hydroxy-7-acetoxy-3,13-clerodadien-15-oic acid methyl ester, 9751

$3 \alpha$-Hydroxy-7-acetoxy-4(18),13-clerodadien15-oic acid methyl ester, 9752

$3 \beta$-Hydroxy-7-acetoxy-4(18),13-clerodadien15-oic acid methyl ester, 9753

2-Methoxy-5-acetoxy-6-methyl-3-tridecyl-1,4benzoquinone, 13830

\section{$\mathrm{C}_{23} \mathrm{H}_{36} \mathrm{O}_{6}$}

Asebotoxin II, 1849

Leoheteronone A, 12632

Leoheteronone C, 12634

$\mathrm{C}_{23} \mathrm{H}_{36} \mathrm{O}_{8}$

Asebotoxin III, 1850

$\mathrm{C}_{23} \mathrm{H}_{36} \mathrm{O}_{14}$

Capillipnin, 3124

Pheloside, 17079
$\mathrm{C}_{23} \mathrm{H}_{37} \mathrm{NO}_{2}$

Kurchaline, 12345

Kurchiline, 12348

Kurchiphylline, 12350

$\mathrm{C}_{23} \mathrm{H}_{37} \mathrm{NO}_{4}$

Sachaconitine, 19107

\section{$\mathrm{C}_{23} \mathrm{H}_{37} \mathrm{NO}_{5}$}

Crispulidine, 4242

Isotalatizidine, 11728

\section{$\mathrm{C}_{23} \mathrm{H}_{37} \mathrm{NO}_{6}$}

Dihydrogadesine, 5618

Senbusine A, 19702

Senbusine B, 19703

Umbrofine, 22199

\section{$\mathrm{C}_{23} \mathrm{H}_{37} \mathrm{NO}_{7}$}

Takaosamine, 20648

\section{$\mathrm{C}_{23} \mathrm{H}_{37} \mathrm{O}_{5}$}

Norerythrostachaldine, 15746

\section{$\mathrm{C}_{23} \mathrm{H}_{38} \mathrm{~N}_{2}$}

Conessimine, 3967

Isoconessimine, 11340

\section{$\mathrm{C}_{23} \mathrm{H}_{38} \mathrm{O}_{2}$}

$10^{\prime}(Z)$-Heptadecenylhydroquinone, 9386

5-(Heptadec-12E-enyl)resorcinol, 9387

5-(Heptadec-12Z-enyl)resorcinol, 9388

$\mathrm{C}_{23} \mathrm{H}_{38} \mathrm{O}_{3}$

Belamcandol A, 2216

\section{$\mathrm{C}_{23} \mathrm{H}_{38} \mathrm{O}_{4}$}

$(E, Z, Z)$-1-Acetoxy-2-hydroxy-4-oxo-heneicosa-

5,12,15-triene, 234

Ardisiphenol A, 1650

[12]-Gingerol, 8398

Irisoquin A, 11173

Isopropyl idenekirenol, 11623

\section{$\mathrm{C}_{23} \mathrm{H}_{38} \mathrm{O}_{7}$}

Asebotoxin I, 1848

$\mathrm{C}_{23} \mathrm{H}_{39} \mathrm{NO}$

Funtumafrine C, 8009

\section{$\mathrm{C}_{23} \mathrm{H}_{39} \mathrm{NO}_{9} \mathrm{~S}_{2}$}

14-Deoxy-12-(cysteine-S-yl)-andrographolide-3-O-sulfate, 5164

\section{$\mathrm{C}_{23} \mathrm{H}_{40} \mathrm{O}_{2}$}

Laccol, 12428

\section{$\mathrm{C}_{23} \mathrm{H}_{40} \mathrm{O}_{3}$}

Zuihoenalide, 23029

$\mathrm{C}_{23} \mathrm{H}_{40} \mathrm{O}_{4}$

(Z,Z)-1-Acetoxy-2-hydroxy-4-oxo-heneicosa-

12,15-diene, 233

\section{$\mathrm{C}_{23} \mathrm{H}_{41} \mathrm{NO}_{2}$}

Terminaline, 20976

\section{$\mathrm{C}_{23} \mathrm{H}_{41} \mathrm{NO}_{4}$}

8-Ethoxysachaconitine, 7415

$\mathrm{C}_{23} \mathrm{H}_{42} \mathrm{~N}_{2}$ 
Epipachysamine F, 6995

$\mathrm{C}_{23} \mathrm{H}_{42} \mathrm{O}_{20}$

Chalcononaringenin 2'-O- $\beta$ - $D$-glucoside-4'-O- $\beta$ gentiobioside, 3456

$\mathrm{C}_{23} \mathrm{H}_{46} \mathrm{NO}_{3}$

1,3-Dihydroxy-2-hexanoylamino-(4E)-heptadecene, 5904

$\mathrm{C}_{23} \mathrm{H}_{46} \mathrm{O}_{2}$

$n$-Tricosanoic acid, 21595

$\mathrm{C}_{23} \mathrm{H}_{46} \mathrm{O}_{4}$

Glyceryl-1-eicosanoate, 8812

$\mathrm{C}_{23} \mathrm{H}_{47} \mathrm{NO}_{8}$

Germine, 8353

$\mathrm{C}_{23} \mathrm{H}_{48}$

n-Tricosane, 21594

$\mathrm{C}_{23} \mathrm{H}_{48} \mathrm{O}$

12-Tricosanol, 21596

\section{$\mathrm{C}_{24} \mathrm{H}_{16} \mathrm{O}_{12}$}

Laccaic acid B, 12425

2-O-Phloroeckol, 17172

\section{$\mathrm{C}_{24} \mathrm{H}_{18} \mathrm{~N}_{2} \mathrm{O}_{4}$}

Caulerpin, 3333

\section{$\mathrm{C}_{24} \mathrm{H}_{18} \mathrm{O}_{5}$}

Ohioensin B, 16020

\section{$\mathrm{C}_{24} \mathrm{H}_{18} \mathrm{O}_{6}$}

Ethylidene-3,3'-biplumbagin, 7448

Ethylidene-3,6'-biplumbagin, 7449

Ohioensin D, 16022

\section{$\mathrm{C}_{24} \mathrm{H}_{19} \mathrm{~N}_{3} \mathrm{O}_{4}$}

Lycogarubin C, 13199

\section{$\mathrm{C}_{24} \mathrm{H}_{19} \mathrm{~N}_{3} \mathrm{O}_{5}$}

Lycogarubi B, 13198

\section{$\mathrm{C}_{24} \mathrm{H}_{20} \mathrm{O}_{6}$}

Diosindigo A, 6450

\section{$\mathrm{C}_{24} \mathrm{H}_{20} \mathrm{O}_{9}$}

Cinchonain Ia, 3680

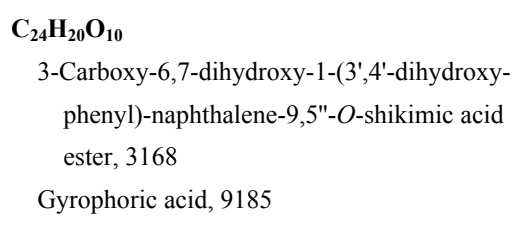

\section{$\mathrm{C}_{24} \mathrm{H}_{21} \mathbf{N}_{3} \mathrm{O}_{6}$}

Hyperectine, 10882

\section{$\mathrm{C}_{24} \mathrm{H}_{21} \mathrm{O}_{13}$}

5-Carboxypyranocyanidin-3-O- $\beta$-glucopyranoside, 3182

$\mathrm{C}_{24} \mathrm{H}_{22} \mathrm{O}_{5}$

Mesuagin, 13805

$\mathrm{C}_{24} \mathrm{H}_{22} \mathrm{O}_{6}$

Eurycomalin A, 7649

$\mathrm{C}_{24} \mathrm{H}_{22} \mathrm{O}_{7}$

JSPC0305368-18, 11894

$\mathrm{C}_{24} \mathrm{H}_{22} \mathrm{O}_{8}$
Curtisian O, 4411

Thonningine C, 21336

\section{$\mathrm{C}_{24} \mathrm{H}_{22} \mathrm{O}_{9}$}

Curtisian L, 4408

Tetraacetylbrazilin, 21033

$\mathrm{C}_{24} \mathrm{H}_{22} \mathrm{O}_{10}$

Liquidamboside, 12905

Lysidicichin, 13260

Pongamoside B, 17710

Pongamoside C, 17711

$\mathrm{C}_{24} \mathrm{H}_{22} \mathrm{O}_{12}$

Chrysophanol 8-O- $\beta$ - $D$-(6'-O-malonyl) glucopyranoside, 3621

\section{$\mathrm{C}_{24} \mathrm{H}_{22} \mathrm{O}_{13}$}

Quercetin-3-O- $\alpha-L-3$ ",5"-diacetyl-arabinofuranoside, 18337

\section{$\mathrm{C}_{24} \mathrm{H}_{22} \mathrm{O}_{14}$}

Quercetin-3-O-(4"-O-malonyl)- $\alpha$ - $L$-rhamnopyranoside, 18375

\section{$\mathrm{C}_{24} \mathrm{H}_{22} \mathbf{O}_{15}$}

Myricetin-3-O-(4"-O-malonyl)- $\alpha-L$-rhamnopyranoside, 15183

Quercetin-3-O-(6"-malonyl)- $D$-galactoside, 18373

Quercetin-3-O-malonyl- $\beta$ - $D$-glucoside, 18374

\section{$\mathrm{C}_{24} \mathrm{H}_{23} \mathrm{NO}_{5}$}

( \pm )-6-Acetonyldihydrochelerythrine, 112

6-Acetonyldihydrochelerythrine, 113

\section{$\mathrm{C}_{24} \mathrm{H}_{23} \mathrm{O}_{14}{ }^{+}$}

Cyanidin-3-O-(6"-O-malonyl- $\beta$-glucopyranoside), 4445

\section{$\mathrm{C}_{24} \mathrm{H}_{23} \mathrm{O}_{15}$}

Delphinidin-3-neohesperidoside, 5029

\section{$\mathrm{C}_{24} \mathrm{H}_{24} \mathrm{~N}_{2} \mathrm{O}_{13}$}

Neobetanin, 15343

\section{$\mathrm{C}_{24} \mathrm{H}_{24} \mathrm{O}_{4}$}

Inophynone, 11080

Isoinophynone, 11464

$\mathrm{C}_{24} \mathrm{H}_{24} \mathrm{O}_{5}$

Mammea A/BD, 13470

Mesuol, 13811

\section{$\mathrm{C}_{24} \mathrm{H}_{24} \mathrm{O}_{6}$}

Anhydromangostanol*, 1277

1,5-Dihydroxy-3-methoxy-6',6'-dimethyl-

2H-pyrano(2',3':6,7)-4-(3-methylbut-2enyl)xanthone, 5974

Disparinol D, 6515

Dulcisxanthone F, 6631

Garcimangosone B, 8207

Mammea A/AC cyclo F, 13471

10-O-Methylmacluraxanthone, 14572

Racemosol, 18514

$\mathrm{C}_{24} \mathrm{H}_{24} \mathrm{O}_{7}$
Anticancer Flavonoid PMV70P691-025, 1403

Kweichowenol A, 12397

Linixanthone A, 12886

$\mathrm{C}_{24} \mathrm{H}_{24} \mathrm{O}_{8}$

3",4"--DihydrothonningineC, 5727

$\mathrm{C}_{24} \mathrm{H}_{24} \mathrm{O}_{9}$

4,5-Dihydro-5' $\alpha$-hydroxy-4' $\alpha$-methoxy- $6 \alpha, 12 \alpha$ dehydro- $\alpha$-toxicarol, 5638

Picropodophyllotoxin acetate, 17342

Steganacin, 20284

\section{$\mathrm{C}_{24} \mathrm{H}_{24} \mathrm{O}_{10}$}

3-Carboxy-6-methoxy-1-(3',4'-dihydroxyphenyl)naphthalene-7-O- $\alpha-L$-rhamnopyranoside, 3177

\section{$\mathrm{C}_{24} \mathrm{H}_{24} \mathrm{O}_{11}$}

Agastachoside, 709

2-O-(E)-Caffeoyl-1-O-p-(E)-coumaroyl- $\beta-D$-glucopyranose, 2895

Isoagastachoside, 11200

Phrymarolin I, 17202

Quercitrin derivative CPB-50-208-18, 18413

\section{$\mathrm{C}_{24} \mathrm{H}_{24} \mathrm{O}_{12}$}

Chrysoeriol 4'- $O$-(6"- $O$-acetyl)- $\beta$ - $D$-glucopyranoside, 3603

1,2-Di- $O$ - $(E)$-caffeoyl- $\beta$ - $D$-glucopyranose, 5407

1,3-Di- $O$ - $(E)$-caffeoyl- $\beta$ - $D$-glucopyranose, 5408

3,6-Di- $O$-caffeoyl- $(\alpha / \beta)$-glucose, 5409

Germanaism A, 8349

7-(6-O-Malonyl- $\beta$ - $D$-glucopyransyloxy)-3-(4-

hydroxyphenyl)-4H-1-benzopyran-4-one, 13445

Neocomplanoside, 15371

\section{$\mathrm{C}_{24} \mathrm{H}_{24} \mathrm{O}_{13}$}

Mearnsetin 3-O-(4"-O-acetyl)- $\alpha-L-{ }^{1} C_{4}$-rhamnopyranoside, 13626

\section{$\mathrm{C}_{24} \mathrm{H}_{25} \mathrm{NO}_{4}$}

Ancistroheynine A, 1144

Ancistroheynine B, 1145

Ancistrolikokine D, 1146

\section{$\mathrm{C}_{24} \mathrm{H}_{25} \mathrm{NO}_{5}$}

$O$-Methyl-waltherione A, 14815

\section{$\mathrm{C}_{24} \mathrm{H}_{25} \mathrm{NO}_{11}$}

Oleracein A, 16074

\section{$\mathrm{C}_{24} \mathrm{H}_{26} \mathrm{~N}_{2} \mathrm{O}_{8}$}

Pauciflorine A, 16731

\section{$\mathrm{C}_{24} \mathrm{H}_{26} \mathrm{~N}_{2} \mathrm{O}_{13}$}

Betanin, 2322

Gomphrenin I, 8927

Gomphrenin II, 8928

Isobetanin, 11252

$\mathrm{C}_{24} \mathrm{H}_{26} \mathrm{~N}_{2} \mathrm{O}_{16} \mathrm{~S}$

Prebetanin, 17770

$\mathrm{C}_{24} \mathrm{H}_{26} \mathrm{O}_{4}$

Albafuran A, 844

Mulberrofuran L, 15046 
Mulberrofuran V, 15053

Myrtiaphenone B, 15225

\section{$\mathrm{C}_{24} \mathrm{H}_{26} \mathrm{O}_{5}$}

2,3-Dihydro-7-hydroxy-2 $R^{*}, 3 R^{*}$-dimethyl-2-[4methyl-5-(4-methyl-2-furyl)-3(E),7-pentenyl]furo[2,3- $b]$ chromone, 5630

2,3-Dihydro-7-hydroxy-2 $S^{*}, 3 R^{*}$-dimethyl-2-[4methyl-5-(4-methyl-2-furyl)-3(E),7-pentenyl] furo[2,3- $b]$ chromone, 5631

2,3-Dihydro-7-hydroxy-2 $R^{*}, 3 R^{*}$-dimethyl-2-[4methyl-5-(4-methyl-2-furyl)-3(E)-pentenyl]furo[3,2-c]coumarin, 5632

2,3-Dihydro-7-hydroxy-2 $S^{*}, 3 R^{*}$-dimethyl-2-[4methyl-5-(4-methyl-2-furyl)-3(E)-pentenyl]furo[3,2-c] coumarin, 5633

\section{$\mathrm{C}_{24} \mathrm{H}_{26} \mathrm{O}_{6}$}

Caloxanthone B, 2999

Cochinchinone C, 3872

Cowaxanthone, 4202

Cudrafrutixanthone, 4336

Dulcisxanthone B, 6627

Gerontoxanthone E, 8356

1-Isomangostin, 11526

3-Isomangostin, 11527

Mangostin, 13492

4-[3"-(1c-Methylbutanoyloxy)propyl]-2-methoxy-(3',4'-methylenedioxyphenyl)-1a,5b-dihydrobenzo-[3,4]-cyclobutaoxirene, 14172 Nigrolineaxanthone E, 15578

1,3,6-Trihydroxy-7-methoxy-2,5-bis(3-methyl2-butenyl)-xanthone, 21768

\section{$\mathrm{C}_{24} \mathrm{H}_{26} \mathrm{O}_{7}$}

(+)-Anomalin, 1346

Anomalin, 1347

Archangelicin, 1619

Cochinchinone D, 3873

Disenecionyl cis-khellactone, 6507

11-Hydroxy-1-isomangostin, 10250

Mangostanin A, 13485

Mangostanol, 13486

Mangostenol, 13487

Nigrolineaxanthone B, 15575

Praeruptorin D, 17763

Xanthalin, 22744

\section{$\mathrm{C}_{24} \mathrm{H}_{26} \mathrm{O}_{8}$}

Catalpafurxin, 3304

Interiorin C, 11101

Isomallotochromene, 11522

Mallotochromene, 13430

Mangostenone C, 13489

Picropodophyllin-1-ethyl ether, 17338

6-Prenylisocaviunin, 17834

1",2",3",4"-TetrahydrothonningineC, 21081
$\mathrm{C}_{24} \mathrm{H}_{26} \mathrm{O}_{10}$

Anticancer Rotenoid PMV70P691-036, 1436

4',5'-Dihydro-11,5'-dihydroxy-4'-methoxytephrosin, 5595

Scrophuloside A, 19569

Scrophuloside B, 19570

$\mathrm{C}_{24} \mathrm{H}_{26} \mathrm{O}_{11}$

Andrographidine E, 1155

Leptostachyol, 12679

Spiraformin D, 20192

$\mathrm{C}_{24} \mathrm{H}_{26} \mathrm{O}_{12}$

Cirsilineol-4'-monoglucoside, 3742

Cyrtophylin, 4589

Dracunculifoside C, 6582

5-Hydroxy-6,7,3'-trimethoxyflavone-8- $O-\beta$ - $D$ glucoside, 10797

Vavain 3'- $O$ - $\beta$ - $D$-glucopyranoside, 22359

Viscumneoside VI, 22541

\section{$\mathrm{C}_{24} \mathrm{H}_{26} \mathrm{O}_{13}$}

Benzenepropanoic acid,

8-\{[7'-(3',4'-dihydroxy-phenyl)-9'-oxo-7'-prope nyl]oxy $\}-3-(1 "-O-\beta$ - $D$-glucopyranosyl)-4-hydr oxy- $[R-(E)], 2223$

Centaurein, 3389

Iridin, 11141

\section{$\mathrm{C}_{24} \mathrm{H}_{26} \mathrm{O}_{14}$}

Isolimocitrol-3- $\beta$ - $D$-glucoside, 11492

Limocitrol- $\beta$ - $D$-glucoside, 12841

Sibiricaxanthone A, 19856

Sibiricaxanthone B, 19857

\section{$\mathrm{C}_{24} \mathrm{H}_{27} \mathrm{NO}_{3}$}

Boehmeriasin A, 2528

8-O-Methyl-1-epi-dioncophylline B, 14396

\section{$\mathrm{C}_{24} \mathrm{H}_{27} \mathrm{NO}_{4}$}

Buxifoliadine B, 2824

$N$-Methylatalaphylline, 14149

8-O-Methyldioncophyllinol B, 14325

Tylocrebrine, 22146

Tylophorine, 22151

$\mathrm{C}_{24} \mathrm{H}_{27} \mathrm{NO}_{6}$

Merredissine, 13784

$\mathrm{C}_{24} \mathrm{H}_{28} \mathrm{Br}_{3} \mathrm{~N}_{3} \mathrm{O}_{6}$

Purpuramine K, 18212

$\mathrm{C}_{24} \mathrm{H}_{28} \mathrm{~N}_{2} \mathrm{O}_{3}$

16-Propoxystrychnine, 17934

$\mathrm{C}_{24} \mathrm{H}_{28} \mathrm{~N}_{2} \mathrm{O}_{5}$

Novacine, 15847

$\mathrm{C}_{24} \mathrm{H}_{28} \mathrm{~N}_{2} \mathrm{O}_{6}$

Methyl 12-methoxychanofruticosinate, 14584

Terrestribisamide, 21005

$\mathrm{C}_{24} \mathrm{H}_{28} \mathrm{~N}_{4} \mathrm{O}_{4}$

Ergosecalinine, 7242

$\mathrm{C}_{24} \mathrm{H}_{28} \mathrm{O}_{3}$
Ugonstilbene A, 22186

Ugonstilbene B, 22187

Ugonstilbene C, 22188

\section{$\mathrm{C}_{24} \mathrm{H}_{28} \mathrm{O}_{4}$}

Angelicide, 1190

Chlorophorin, 3567

Clausarin, 3788

Conferone, 3970

$(Z, Z)$-Diligustilide, 6192

$( \pm)$-trans-3-(3,4-Dimethoxyphenyl)-4-[(E)-3,4dimethoxystyryl]cyclohex-1-ene, 6282

Ligustilide dimer, 12826

Norbixin, 15720

Ponfolin, 17706

Vismiaphenone C, 22550

\section{$\mathrm{C}_{24} \mathrm{H}_{28} \mathrm{O}_{5}$}

2,3-Dihydro-7-hydroxy-2 $S^{*}, 3 R^{*}$-dimethyl-2-

[4,8-dimethyl-3(E),7-nonadien-6-onyl]-furo

$[3,2-c]$ coumarin, 5626

Pseudoguttiaphenone-A, 18027

Shijiaocao lactone, 19804

$Z-3^{\prime}, 8^{\prime}, 3^{\prime} \alpha, 7^{\prime} \alpha$-Tetrahydro-6,3',7,7' $\alpha$-diligustilide8 -one, 21068

\section{$\mathrm{C}_{24} \mathrm{H}_{28} \mathrm{O}_{6}$}

Nigrolineaxanthone O, 15588

\section{$\mathrm{C}_{24} \mathrm{H}_{28} \mathrm{O}_{7}$}

3'(S)-Angeroyloxy-4'(R)-isovaleryloxy-3',4'-dihydroxanthyletin, 1239

Cratoxylone, 4220

Garcinone D, 8221

Hyuganin A, 10922

(+)-Praeruptorin E, 17764

$\psi$-Rhodomyrtoxin, 18814

\section{$\mathrm{C}_{24} \mathrm{H}_{28} \mathrm{O}_{8}$}

Acetylbinankadsurin A, 333

Mangostenone E, 13491

Schisantherin O, 19496

Xanthanthusin H, 22751

Xanthanthusin I, 22752

Xanthanthusin J, 22753

\section{$\mathrm{C}_{24} \mathrm{H}_{28} \mathrm{O}_{9}$}

6-Acetylpicropoline, 486

Anticancer Lignan PMV70P691-126, 1426

(2S,3S)-2,3-Bis(5-methoxy-3,4-methylenedioxybenzyl)-butane-1,4-diol monoacetate, 2482

Daldinin E, 4612

Daldinin F, 4613

Isomallotochromanol, 11521

\section{$\mathrm{C}_{24} \mathrm{H}_{28} \mathrm{O}_{10}$}

Agrimonolide-6-O- $\beta$ - $D$-glucopyranoside, 761

\section{$\mathrm{C}_{24} \mathrm{H}_{28} \mathrm{O}_{11}$}

Bacopaside B, 2085

10-O-trans-Coumaroyl-eranthemoside, 4154 
(-)-7-O-Methyleucomol 5-O- $\beta$ - $D$-glucopyranoside, 14437

Monnieraside II, 14918

Myrciacitrin II, 15148

Pagoside, 16536

Picroside I, 17344

\section{$\mathrm{C}_{24} \mathrm{H}_{28} \mathrm{O}_{12}$}

Breynioside B, 2612

Cucurbitoside E, 4334

12,15-Diacetyl-13 $\alpha(21)$-epoxyeurycomanone, 5334

Javanicin, 11852

Odontoside, 15989

\section{$\mathrm{C}_{24} \mathrm{H}_{28} \mathrm{O}_{13}$}

Gentiopicroside tetraacetate, 8305

Verminoside, 22403

\section{$\mathrm{C}_{24} \mathrm{H}_{29} \mathrm{ClO}_{7}$}

Armillarinin, 1740

\section{$\mathrm{C}_{24} \mathrm{H}_{30} \mathrm{~N}_{2} \mathrm{O}_{5}$}

Vindorosine, 22501

\section{$\mathbf{C}_{24} \mathrm{H}_{30} \mathbf{O}_{3}$}

$\alpha, \alpha^{\prime}$-Dihydro-3,5,4'-trihydroxy-4,5'-diisopentenylstilbene , 5729

Ferulenol, 7767

Glepidotin D, 8551

14 $\beta$-Hydroxybufa-3,5,20,22-tetraenolide, 9860

Umbelliprenin, 22196

\section{$\mathrm{C}_{24} \mathrm{H}_{30} \mathrm{O}_{4}$}

Assafoetidin, 1909

Badrakemin, 2096

Conferol, 3969

(Z')-3,8-Dihydro-6,6'; 7,3' $\alpha$-diligustilide, 5599

2,3-Dihydro-7-hydroxy-2 $S^{*}, 3 R^{*}$-dimethyl-2-

[4,8-dimethyl-3(E),7-nonadienyl]-furo[2,3- $b]$ chromone, 5627

2,3-Dihydro-7-hydroxy-2 $R^{*}, 3 R^{*}$-dimethyl-2-

[4,8-dimethyl-3(E),7-nonadienyl]-furo[3,2-c] coumarin, 5628

2,3-Dihydro-7-hydroxy-2 $S^{*}, 3 R^{*}$-dimethyl-2-

[4,8-dimethyl-3(E),7-nonadienyl]-furo[3,2-c] coumarin, 5629

$\alpha, \alpha^{\prime}$-Dihydro-3,5,3',4'-tetrahydroxy-4,5'-diisopentenylstilbene, 5725

Farnesiferol A, 7731

Farnesiferol B, 7732

Farnesiferol C, 7733

Gummosin, 9085

Uralstilbene, 22245

\section{$\mathrm{C}_{24} \mathrm{H}_{30} \mathrm{O}_{5}$}

Assafoetidnol A, 1910

Galbanic acid, 8087

4-[(2"E)-7"-Hydroxy-3",7"-dimethyloct-2"enyl]-2',3,4',5-tetrahydroxy-trans-stilbene,
10054

Kamolonol, 12127

Neveskone, 15517

3-Oxo-20S,21-epoxyresibufogenin, 16329

3-Oxo-11 $\alpha$-hydroxy-12-dehydroxy-scilliphaeosidin, 16349

Resibufagin, 18636

\section{$\mathrm{C}_{24} \mathrm{H}_{30} \mathrm{O}_{6}$}

Armillaripin, 1741

Bufotalinin, 2723

Caesaldekarin E, 2862

Magnoshinin, 13392

Obtusanal A, 15896

19-Oxodesacetylcinobufagin, 16312

\section{$\mathrm{C}_{24} \mathrm{H}_{30} \mathrm{O}_{7}$}

Apetalic acid 5-O-acetate, 1471

Armillarilin, 1739

Athamantin, 1960

Ethoxyclusin, 7400

Isoapetalic acid 5-O-acetate, 11231

Mallotojaponin, 13431

Moslolignan B, 15001

Nirtetralin, 15624

Novelrabdosin, 15848

Odonicin, 15988

Pyramidatin A, 18241

Schisanlignone A, 19485

\section{$\mathrm{C}_{24} \mathrm{H}_{30} \mathrm{O}_{8}$}

16-Acetoxycoleon U 11-acetate, 146

9'-O-Acetyl-(7R,8S,7R,8S)-(-)-fargesol, 394

6-Acetyl-teucjaponin B, 524

Albaspidin PB, 853

Cornutin F, 4069

(-)-Cubebininolide, 4309

Diacetyl montanin D, 5342

Diayangambin, 5378

Flavaspidic acid BB, 7809

Lirioresinol B dimethyl ether, 12920

Margaspidin, 13559

Methyl pseudolarate B, 14703

Platyphyllonol-5-O- $\beta$ - $D$-xylopyranoside, 17548

Rugosinin, 19061

Salvigresin, 19216

Saroaspidin A, 19382

Trichorabdal G acetate, 21571

$\mathrm{C}_{24} \mathrm{H}_{30} \mathrm{O}_{9}$

6-Acetyl-10-hydroxyteucjaponin B, 425

Alnuside A, 959

Alnuside B, 960

Coetsin B, 3897

1,1'-Dibenzene-6', 8',9'-trihydroxy-3-allyl-4- $O-\beta$ D-glucopyranoside, 5379

Loxothyrin A, 13013
Luzonoid A, 13157

Luzonoid B, 13158

Luzonoid C, 13159

Luzonoid D, 13160

Macrocalyxin A (Macrocalin A), 13305

Teucrolivin A, 21217

Xanthanthusin F, 22749

\section{$\mathrm{C}_{24} \mathrm{H}_{30} \mathrm{O}_{10}$}

8-Cinnamoylmyoporoside, 3711

Icariside $A_{1}, 10941$

Oregonin, 16174

\section{$\mathrm{C}_{24} \mathrm{H}_{30} \mathrm{O}_{11}$}

6-O-p-Coumaroylajugol, 4144

Harpagoside, 9238

\section{$\mathrm{C}_{24} \mathrm{H}_{30} \mathrm{O}_{12}$}

8-p-Coumaroylharpagide, 4163

Demethylligstroside, 5084

Glochiflavanoside B, 8572

Glochiflavanoside C, 8573

8-O-(2-Hydroxycinnamoyl)harpagide, 9912

\section{$\mathrm{C}_{24} \mathrm{H}_{30} \mathrm{O}_{13}$}

2-Acetyl-1,5-dihydroxy-3-methyl-8- $O(\beta$-xylopyranosyl-( $(1 \rightarrow 6)-O-(\beta$-glucopyranosyl $))$ naphthalene, 382

Inumakilactone A glucoside, 11114

6-O-Veratroyl-catalpol, 22392

\section{$\mathrm{C}_{24} \mathrm{H}_{32} \mathrm{O}_{4}$}

Gerronemin B, 8361

Resibufogenin, 18637

\section{$\mathrm{C}_{24} \mathrm{H}_{32} \mathrm{O}_{5}$}

Bufagin, 2715

Desacetylcinobufagin, 5238

20R,21-Epoxyresibufogenin, 7192

20S,21-Epoxyresibufogenin, 7193

Gamabufotalininol, 8122

12 $\beta$-Hydroxyresibufogenin, 10672

19-Hydroxyresibufogenin, 10673

Lucidone B, 13049

19-Oxobufalin, 16296

Samarcandin, 19224

3- $\alpha, 12 \alpha-$ Scilliphaeosidin, 19527

\section{$\mathrm{C}_{24} \mathrm{H}_{32} \mathrm{O}_{5} \mathrm{~S}$}

$S$-Furanopetasitin, 8021

\section{$\mathrm{C}_{24} \mathrm{H}_{32} \mathrm{O}_{6}$}

Andamanicin, 1148

Ardisinone C, 1647

Arenobufagin, 1664

Atranone A, 1983

Atranone C, 1985

Bisasaricin, 2435

Bufarenogin, 2718

Bufotalidin, 2721

Caesalpinin MJ, 2880 
Caesalpinin MK, 2881

Caesalpinin P, 2885

Deoxyschizandrin, 5213

Desacetylcinobufotalin, 5239

Heterotropan, 9471

19-Hydroxydesacetylcinobufagin, 9993

Lancerotriol 9-acetate-6- $p$-hydroxybenzoate, 12469

Magnosalin, 13391

Phyltetralin, 17231

\section{$\mathrm{C}_{24} \mathrm{H}_{32} \mathrm{O}_{7}$}

Atranone F, 1988

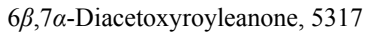

Gerardianin A, 8331

Gerardianin B, 8332

Glabcensin X, 8485

Grandisin, 8979

Inflexarabdonin $\mathrm{H}, 11048$

Inflexin, 11052

Inflexin II, 11053

Isoschizandrin, 11698

Leucophyllin F, 12724

Magnosalicin, 13390

Malkangunin, 13421

Maoecrystal D, 13526

Niranthin, 15622

Rabdokaurin A, 18466

Schisandrol A, 19473

Shikodomedin, 19810

Xindongnin A, 22797

\section{$\mathrm{C}_{24} \mathrm{H}_{32} \mathrm{O}_{8}$}

Asatone, 1841

Calcicolin B, 2949

(-)-Cubebinin, 4308

Effusanin D, 6713

Ganervosin A, 8146

Leosibirin, 12665

Leucophyllin B, 12720

Longikaurin F, 12962

Longirabdolide D, 12973

Lophanthoidin B, 12994

Maoecrystal J, 13531

Maoecrystal R, 13539

Nirphyllin, 15623

Orthosiphol Y, 16240

Pseurata D, 18078

Rabdokaurin B, 18467

Rabdosin B, 18499

Shikokiamedin, 19812

Shikokianin, 19815

$\mathrm{C}_{24} \mathrm{H}_{32} \mathrm{O}_{9}$

Caesalpinin MH, 2878

$(1 R, 2 R, 5 Z, 7 R, 8 S, 9 R, 10 R, 12 R, 14 R, 17 S)-2,14-D i-$ acetoxy-8,17-epoxy-9,12-dihydroxybriara-5,11

(20)-dien-19-one, 5296

Enanderianin A, 6783

Shikokianoic acid, 19816

Teucrolivin B, 21218

\section{$\mathrm{C}_{\mathbf{2 4}} \mathbf{H}_{32} \mathbf{O}_{\mathbf{1 0}}$}

Acevaltrate, 534

Acevaltratum, 535

Ailantinol G, 780

8 $\beta$-Hydroxy-teucrolivin B, 10761

\section{$\mathrm{C}_{24} \mathrm{H}_{32} \mathbf{O}_{12}$}

6-O-(3,4-Dimethoxybenzoyl)-ajugol, 6205

\section{$\mathrm{C}_{24} \mathrm{H}_{33} \mathrm{NO}_{3}$}

Guineensine, 9083

Pipyahyine, 17474

Sylvatine, 20523

\section{$\mathrm{C}_{24} \mathrm{H}_{33} \mathrm{NO}_{4}$}

11-Acetyl-1,19-epoxydenudatine, 388

\section{$\mathrm{C}_{24} \mathrm{H}_{33} \mathrm{NO}_{5}$}

Guan-fu base Z, 9052

Spiratine B, 20201

\section{$\mathrm{C}_{24} \mathrm{H}_{34} \mathbf{N}_{6} \mathrm{O}_{7}$}

Stellarria cyclopeptide, 20286

\section{$\mathrm{C}_{24} \mathrm{H}_{34} \mathrm{O}_{4}$}

Atranone D, 1986

Atranone E, 1987

Bufalin, 2716

Gerronemin A, 8360

\section{$\mathrm{C}_{24} \mathrm{H}_{34} \mathrm{O}_{5}$}

2-Acetyl-3,5-dihydroxy-1-geranoxy-6-methyl-4(2-methyl)butyryl-benzene, 380

Desacetylbufotalin, 5237

Gamabufogenin, 8121

1 $\beta$-Hydroxybufalin, 9859

Lucidone A, 13048

Taxuyunnanine D, 20876

Telocinobufagin, 20911

\section{$\mathrm{C}_{24} \mathrm{H}_{34} \mathrm{O}_{6}$}

16-Acetoxy-7 $\alpha$-ethoxyroyleanone, 193

ent-1 $\alpha, 7 \beta$-Diacetoxy-14 $\alpha$-hydroxykaur-16-en-15one, 5305

ent-1 $\alpha, 14 \alpha$-Diacetoxy-7 $\beta$-hydroxykaur-16-en-15one, 5306

ent-11 $\alpha, 18$-Diacetoxy-7 $\beta$-hydroxykaur-16-en-15-

$$
\text { one, } 5307
$$

Inflexarabdonin G, 11047

Isodopharicin B, 11396

Melissoidesin O, 13701

Phyllanthin, 17215

$\mathrm{C}_{24} \mathrm{H}_{34} \mathrm{O}_{7}$

Adenanthin F, 610

Adenanthin G, 611

Ajugarin I, 809
Caesalpinin L, 2874

Calcicolin C, 2951

Clerodin, 3841

Eriocalysin D, 7270

Glabcensin V, 8483

Glabcensin Y, 8486

Inflexarabdonin B, 11042

Inflexinol, 11054

Leucamenin B, 12709

Leucophyllin E, 12723

Lophanthoidin F, 12998

Lushanrubescensin E, 13120

Maoecrystal F, 13528

Melissoidesin G, 13694

MelissoidesinS, 13705

Nigakilactone C, 15546

Rabdoternin C, 18503

Rosthornin B, 18933

Taibairubescensin A, 20622

Taibairubescensin B, 20623

Taxuspine G, 20855

Trichokaurin, 21561

Xindongnin C, 22799

\section{$\mathrm{C}_{24} \mathrm{H}_{34} \mathrm{O}_{8}$}

Adenanthin I, 613

Ajugalide C, 797

Deaminoacyltaxine A, 4798

$2 \alpha, 7 \beta$-Diacetoxy- $5 \alpha, 10 \beta, 13 \alpha$-trihydroxy-2(3 $\rightarrow 20)$

abeotaxane-9-one, 5320

$7 \beta, 13 \alpha$-Diacetoxy- $2 \alpha, 5 \alpha, 10 \beta$-trihydroxy-2(3 $\rightarrow 20)$

abeotaxane-9-one, 5321

$7 \beta, 9 \alpha$-Diacetoxy- $5 \alpha, 13 \alpha, 14 \beta$-trihydroxy-10-oxotaxa-4(20),11-diene, 5322

(3E,7E)-2 $\alpha, 10 \beta$-Diacetoxy- $5 \alpha, 13 \alpha, 20$-trihydroxy-

3,8-secotaxa-3,7,11-trien-9-one, 5323

Diacetyldecinnamoyltaxicin I, 5331

Glabcensin H, 8469

Glabcensin I, 8470

Isoleosibirin, 11488

Jiuhuanin A, 11889

Leosibirin, 12666

Leucophyllin C, 12721

Melissoidesin A, 13688

Meso-2,3-bis(3,4,5-trimethoxybenzyl)-1,4butanediol, 13801

Nigakilactone E, 15550

Pseurata E, 18079

Rabdokaurin C, 18468

Rabdolongin A, 18477

Rabdosichuanin D, 18493

Taxacustone, 20740

Ternifolin, 20979

$\mathrm{C}_{24} \mathrm{H}_{34} \mathrm{O}_{9}$ 
Ajugamarin $\mathrm{C}_{1}, 804$

T2 Toxin, 22070

$\mathrm{C}_{24} \mathrm{H}_{34} \mathrm{O}_{11}$

Taxagifin III, 20744

$\mathrm{C}_{24} \mathrm{H}_{34} \mathrm{O}_{13}$

Dracunculifoside F, 6585

$\mathrm{C}_{24} \mathrm{H}_{34} \mathrm{O}_{15}$

Glomeratose A, 8582

Sibiricose $\mathrm{A}_{2}, 19863$

\section{$\mathrm{C}_{24} \mathrm{H}_{35} \mathrm{NO}$}

1-Methyl-2-(Z)-8-tetradecenyl-4(1H)-quinolone, 14745

$\mathrm{C}_{24} \mathrm{H}_{35} \mathrm{NO}_{3}$

Ovatine, 16279

$\mathrm{C}_{24} \mathrm{H}_{35} \mathrm{NO}_{4}$

Lepetine, 12669

Lucidusculine, 13057

\section{$\mathrm{C}_{24} \mathrm{H}_{35} \mathrm{NO}_{7}$}

8-Acetylexcelsine, 392

\section{$\mathrm{C}_{24} \mathrm{H}_{36} \mathrm{O}_{4}$}

18-Acetoxy-15 $\alpha$-hydroxymansumbinone, 229

Thouvenol B, 21338

2-n-Tridecyl-5,7-dihydroxy-6,8-dimethyl chromone, 21623

\section{$\mathrm{C}_{24} \mathrm{H}_{36} \mathrm{O}_{5}$}

Hexanorcucurbitacin F, 9517

Lucidone C, 13050

\section{$\mathrm{C}_{24} \mathrm{H}_{36} \mathrm{O}_{6}$}

$\left(1 R^{*}, 3 S^{*}, 4 R^{*}, 6 S^{*}\right)-9$-(Acetoxy)-4-acetoxy-1-

[(2Z)-2-methylbut-2-enoyloxy]bisabol-2(3),

$$
\text { 10(11)-diene, } 118
$$

$9 \alpha, 10 \beta$-Diacetoxy-5 $\alpha, 13 \alpha$-dihydroxy-4(20),11-

taxadiene, 5294

$7 \alpha, 18$-Diacetoxy,9 $\beta, 13 \beta$-epi-dioxiabiet-8(14)-ene, 5295

Melissoidesin F, 13693

Taxuyunnanine G, 20879

\section{$\mathrm{C}_{24} \mathrm{H}_{36} \mathrm{O}_{7}$}

Adenanthin E, 609

Adenanthin H, 612

CPB-53-1114-4, 4206

Inflexarabdonin A, 11041

Inflexarabdonin D, 11044

Lihsienin A, 12831

Melissoidesin E, 13692

Melissoidesin J, 13696

MelissoidesinT, 13706

Picrasinol A, 17318

$2 \alpha, 5 \alpha, 9 \alpha$-Trihydroxy-10 $\beta, 13 \alpha$-diacetoxytaxa4(20),11-diene, 21705

Xindongnin E, 22801

$\mathrm{C}_{24} \mathrm{H}_{36} \mathrm{O}_{8}$

Diacetoxytetrahydroxytaxadiene, 5318
Forrestin B, 7916

Glabcensin K, 8472

Melissoidesin C, 13690

Melissoidesin K, 13697

Rhodojaponin I, 18799

$2 \alpha, 5 \alpha, 9 \alpha$-Trihydroxy- $10 \beta, 13 \alpha$-diacetoxy- $4 \beta, 20$ epoxytaxa-11-ene, 21704

\section{$\mathrm{C}_{24} \mathrm{H}_{36} \mathrm{O}_{9}$}

Junceellolide I, 11948

Maoyerabdosin, 13545

Rabdophyllin H, 18483

\section{$\mathrm{C}_{24} \mathrm{H}_{36} \mathrm{O}_{10}$}

Taxumairol Q, 20833

Taxumairol X, 20837

\section{$\mathrm{C}_{24} \mathrm{H}_{36} \mathrm{O}_{15}$}

Benzyl alcohol $\beta$ - $D$-glucopyranosyl-( $1 \rightarrow 2)$ - $[\beta$ - $D$ xylopyranosyl-( $(1 \rightarrow 6)]-\beta$ - $D$-glucopyranoside, 2277

Benzyl

$\beta$ - $D$-glucopyranosyl-( $(1 \rightarrow 4)$ - $[\beta$ - $D$-apiofurano

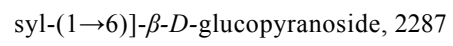

$\mathrm{C}_{24} \mathbf{H}_{36} \mathbf{O}_{16}$

2'-O- $\beta$ - $D$-Glucopyranosyl-6'- $O-\beta$ - $D$-xylopyranosylsalicin, 8751

\section{$\mathrm{C}_{24} \mathrm{H}_{37} \mathrm{NO}_{2}$}

Buxtauine, 2832

\section{$\mathrm{C}_{24} \mathrm{H}_{37} \mathrm{NO}_{5}$}

14-Debenzoylfranchetine, 4806

\section{$\mathrm{C}_{24} \mathrm{H}_{37} \mathrm{NO}_{7}$}

18-Hydroxy-14-O-methylgadesine, 10496

\section{$\mathrm{C}_{24} \mathrm{H}_{37} \mathrm{O}_{7}$}

MelissoidesinQ, 13703

$\mathrm{C}_{24} \mathrm{H}_{38} \mathrm{O}_{3}$

6-(10'Z-Heptadecenyl)salicylic acid, 9389

Irisquinone A, 11179

Merulinic acid C, 13792

Suranone, 20491

\section{$\mathrm{C}_{24} \mathrm{H}_{38} \mathrm{O}_{4}$}

Bis(2-ethyl-hexyl)-phthalate, 2449

Dicapryl phthalate, 5416

Diisocapryl phthalate, 6189

Garcinielliptone O, 8217

$3 \alpha$-Hydroxy-6-oxo- $5 \alpha$-cholanic acid, 10560

Merulinic acid A, 13790

Merulinic acid B, 13791

Thouvenol A, 21337

\section{$\mathrm{C}_{24} \mathrm{H}_{38} \mathrm{O}_{5}$}

Ardisianone, 1628

7 $\alpha, 18$-Diacetoxyabiet-8(14)-en-13 $\beta$-ol, 5284

10,18-Diacetoxy-8-hydroxy-2,6-dolabelladiene, 5303

6 $\beta, 7 \beta$-Diacetoxy-13-hydroxy-labda-8,14-diene, 5308
Vitetrifolin D, 22571

$\mathrm{C}_{24} \mathrm{H}_{38} \mathrm{O}_{6}$

$\left(1 R^{*}, 3 S^{*}, 4 R^{*}, 6 S^{*}\right)-9$-(Acetoxy)-4-acetoxy-1-

[(2Z)-2-methylbut-2-enoyloxy]bisabol-10

(11)-ene, 119

7-Acetoxy-4-hydroxy-3-oxo-4( $3 \rightarrow 2)$-abeo-13clerodaen-15-oic acid methyl ester, 232

$\mathrm{C}_{24} \mathrm{H}_{38} \mathrm{O}_{7}$

4,5,6-Tri- $O$-acetyl hygrophorone $\mathrm{A}^{12}, 21535$

$\mathrm{C}_{24} \mathrm{H}_{38} \mathrm{O}_{8}$

Rhodojaponin IV, 18802

$\mathrm{C}_{24} \mathrm{H}_{38} \mathrm{O}_{10}$

Taxuyunnanine P, 20883

\section{$\mathrm{C}_{24} \mathrm{H}_{38} \mathrm{O}_{11}$}

Eriojaposide A, 7287

Platanionoside F, 17523

\section{$\mathrm{C}_{24} \mathbf{H}_{38} \mathbf{O}_{12}$}

$(6 S, 9 R)$-Vomifoliol-9-O- $\beta$-apiofuranosyl-

$\left(1^{\prime \prime} \rightarrow 6\right.$ ')- $O-\beta$-glucopyranoside, 22616

$(6 S, 9 R)$-Vomifoliol-9- $O-\beta$-xylopyranosyl-

$\left(1^{\prime \prime} \rightarrow 6\right.$ ')- $O-\beta$-glucopyranoside, 22617

\section{$\mathrm{C}_{24} \mathrm{H}_{39} \mathrm{NO}_{4}$}

Cassaine, 3278

\section{$\mathrm{C}_{24} \mathrm{H}_{39} \mathrm{NO}_{5}$}

Talatisamine, 20650

\section{$\mathrm{C}_{24} \mathrm{H}_{39} \mathrm{NO}_{6}$}

Bullatine B, 2738

6-Epiforesticine, 6915

Foresticine, 7880

6-Methylumbrofine, 14798

\section{$\mathrm{C}_{24} \mathrm{H}_{39} \mathrm{NO}_{7}$}

Bullatine F, 2741

Circinadine B, 3736

Delcosine, 5004

Delectinine, 5006

6-Demethyldelsoline, 5073

Gigaconitine, 8378

10-Hydroxynudicaulidine, 10543

Nagarine, 15228

Potanine, 17745

Senbusine C, 19701

\section{$\mathrm{C}_{24} \mathrm{H}_{40} \mathrm{~N}_{2}$}

Concuressine, 3961

Conessine, 3968

\section{$\mathrm{C}_{24} \mathrm{H}_{40} \mathrm{~N}_{2} \mathrm{O}$}

Holarrhenine, 9584

$7 \alpha$-Hydroxyconessine, 9932

$\mathrm{C}_{24} \mathrm{H}_{40} \mathrm{O}_{2}$

$5 \beta$-Cholanic acid, 3575

\section{$\mathrm{C}_{24} \mathrm{H}_{40} \mathrm{O}_{3}$}

Bufodihydroxycholanic acid, 2719

Lithocholic acid, 12923

Pallasone B, 16543 


\section{$\mathrm{C}_{24} \mathrm{H}_{40} \mathrm{O}_{4}$}

Allochenodeoxycholic acid, 929

Chenodeoxycholic acid, 3511

Deoxycholic acid, 5161

$\alpha$-Hyodeoxycholic acid, 10868

$\beta$-Hyodeoxycholic acid, 10869

Irisoquin B, 11174

Proctorione C, 17871

Ursodeoxycholic acid, 22269

$\mathrm{C}_{24} \mathrm{H}_{40} \mathrm{O}_{5}$

Cholic acid, 3588

Hyocholic acid, 10867

\section{$\mathbf{C}_{24} \mathbf{H}_{40} \mathbf{O}_{6}$}

(rel-5S,6R,8R,9R,10S,13S,15R,16R)-6-Acetoxy-

9,13;15,16-diepoxy-15,16-dimethoxylabdane, 159

4,5-Di- $O$-acetyl hygrophorone $\mathrm{A}^{14}, 5337$

4,6-Di- $O$-acetyl hygrophorone $\mathrm{B}^{14}, 5338$

1,4-Di-O-acetyl hygrophorone $\mathrm{E}^{14}, 5341$

\section{$\mathrm{C}_{24} \mathrm{H}_{40} \mathrm{O}_{10}$}

Shionoside C, 19833

\section{$\mathrm{C}_{24} \mathrm{H}_{40} \mathrm{O}_{11}$}

Cuneataside E, 4366

Platanionoside E, 17522

Platanionoside G, 17524

\section{$\mathrm{C}_{24} \mathrm{H}_{40} \mathrm{O}_{12}$}

Ebracteatoside A, 6663

$(6 R, 9 R)$-9-Hydroxy-4-megastigmen-3-one 9- $O-\beta$ -

$D$-glucopyranosyl-(1 $\rightarrow 6)-\beta$ - $D$-glucopyranoside, 10374

\section{$\mathrm{C}_{24} \mathrm{H}_{41} \mathrm{NO}$}

Alkaloid C, 904

2-Noryl-5-decanoylpyridine, 15816

\section{$\mathrm{C}_{24} \mathrm{H}_{41} \mathrm{NO}_{4}$}

Cassaidine, 3277

\section{$\mathrm{C}_{24} \mathrm{H}_{41} \mathrm{NO}_{8}$}

Retronecine $2 S$-hydroxy-2S-(1S-hydroxyethyl) $2 S$-[(1'S-hydroxyethyl)-4-methylpentanoyl]4-methylpentanoyl ester, 18663

\section{$\mathrm{C}_{24} \mathrm{H}_{41} \mathrm{NO}_{9}$}

Broussonetine X, 2646

\section{$\mathrm{C}_{24} \mathrm{H}_{42} \mathrm{~N}_{2}$}

Croomionidine, 4265

Dihydroconcuressine, 5562

Dihydroconessine, 5563

\section{$\mathrm{C}_{24} \mathrm{H}_{42} \mathrm{O}_{3}$}

3-(1-Methoxyoctadecyl)-5-methylene-5 $H$-furan2-one, 14047

\section{$\mathrm{C}_{24} \mathrm{H}_{42} \mathrm{O}_{11}$}

$(3 R, 9 S)$-Megastigman-5-en-3,9-diol-3- $O-[\alpha-L$ arabinofuranosyl-( $1 \rightarrow 6)]-\beta$ - $D$-glucopyranoside, 13642

Myrsinionoside E, 15214
Platanionoside H, 17525

$\mathrm{C}_{24} \mathrm{H}_{42} \mathrm{O}_{12}$

Platanionoside I, 17526

$\mathrm{C}_{24} \mathrm{H}_{42} \mathrm{O}_{21}$

Isolychnose, 11515

Lychnose, 13178

Stachyose, 20255

\section{$\mathrm{C}_{24} \mathrm{H}_{43} \mathrm{NO}$}

$N$-Isobutyl-(2E,4E,14Z)-eicosatrienamide, 11273

Pipnoohine, 17473

$\mathrm{C}_{24} \mathrm{H}_{44} \mathrm{~N}_{2}$

Pachysamine A, 16501

$\mathrm{C}_{24} \mathrm{H}_{44} \mathrm{~N}_{2} \mathrm{O}$

Pachysandrine C, 16507

$\mathrm{C}_{\mathbf{2 4}} \mathrm{H}_{\mathbf{4 4}} \mathrm{O}_{\mathbf{4}}$

Secomahubanolide, 19628

\section{$\mathrm{C}_{24} \mathrm{H}_{45} \mathrm{NO}_{9}$}

Morusimic acid A, 14989

Morusimic acid C, 14991

\section{$\mathrm{C}_{24} \mathrm{H}_{45} \mathrm{NO}_{10}$}

Morusimic acid E, 14993

\section{$\mathrm{C}_{24} \mathrm{H}_{46} \mathrm{O}_{2}$}

Tetracosan-4-olide, 21038

\section{$\mathrm{C}_{24} \mathrm{H}_{48} \mathrm{O}_{2}$}

Lignoceric acid, 12800

21-Methyl tricosanoic acid, 14770

\section{$\mathrm{C}_{25} \mathrm{H}_{14} \mathrm{O}_{5}$}

Newbouldiaquinone, 15519

\section{$\mathrm{C}_{25} \mathrm{H}_{14} \mathrm{O}_{10}$}

Phelligridin E, 17063

\section{$\mathrm{C}_{25} \mathrm{H}_{17} \mathrm{NO}_{13}$}

Laccaic acid C, 12426

\section{$\mathrm{C}_{25} \mathrm{H}_{18} \mathrm{O}_{5}$}

Blespirol, 2504

\section{$\mathrm{C}_{25} \mathrm{H}_{18} \mathrm{O}_{9}$}

Inoscavin A, 11081

$\mathrm{C}_{25} \mathrm{H}_{20} \mathrm{O}_{6}$

Ohioensin E, 16023

\section{$\mathrm{C}_{25} \mathrm{H}_{20} \mathrm{O}_{9}$}

Neohydnocarpin, 15409

$\mathrm{C}_{25} \mathrm{H}_{22} \mathrm{O}_{5}$

Inophyllolide, 11077

Isorobustone, 11683

Tomentolide A, 21438

$\mathrm{C}_{25} \mathrm{H}_{22} \mathrm{O}_{6}$

Cyclomorusin, 4517

Kraussianone 1, 12291

$\mathrm{C}_{25} \mathrm{H}_{22} \mathrm{O}_{7}$

Formosanatin D, 7892

Gnetumontanin C, 8895

Kraussianone 4, 12294

$\mathrm{C}_{25} \mathrm{H}_{22} \mathrm{O}_{9}$

Silandrin, 19893
Silymonin, 19899

\section{$\mathrm{C}_{25} \mathrm{H}_{22} \mathrm{O}_{10}$}

Isosilybin A, 11714

Isosilybin B, 11715

Silybin, 19895

Silybin B, 19896

Silychristin, 19897

Silydianin, 19898

\section{$\mathrm{C}_{25} \mathrm{H}_{22} \mathrm{O}_{13}$}

6,7-Dihydroxy-3-methoxy-8-[2-oxo-2 $H$-1-benzopyran-7-(O- $\beta$ - $D$-glucopyranosyl)-8-yl]-2H-1-b enzopyran-2-one, 5995

\section{$\mathrm{C}_{25} \mathrm{H}_{22} \mathrm{O}_{14}$}

4'-O-Methylellagic acid 3-(2",3"-di- $O$-acetyl)- $\alpha$ $L$-rhamnoside, 14342

\section{$\mathrm{C}_{25} \mathrm{H}_{24} \mathrm{O}_{4}$}

Xambioona, 22743

\section{$\mathrm{C}_{25} \mathrm{H}_{24} \mathrm{O}_{5}$}

Calaustralin, 2945

Chandalone, 3475

8,9-Dihydro-5-hydroxy-6-(2-methylbutanoyl)-4phenyl-8-(prop-1-en-2-yl)furo[2,3- $h$ ]chromen2-one, 5642

8,9-Dihydro-5-hydroxy-6-(3-methylbutanoyl)4-phenyl-8-(prop-1-en-2-yl)furo[2,3- $h]$ chrome n-2-one, 5643

Glycyrdione D, 8837

Inophyllum C, 11078

Inophyllum E, 11079

Isochandalone, 11324

Lespedezol A 3,12684

Mammea A/AB cyclo D, 13464

Mammea A/A cyclo D, 13467

Mesuarin, 13807

Osajin, 16249

Ponnalide, 17718

Puerarol, 18184

Scanderone, 19451

Soulattrolide, 20109

Ulexone A, 22189

Warangalone, 22637

\section{$\mathrm{C}_{25} \mathrm{H}_{24} \mathrm{O}_{6}$}

Acetyl blancoxanthone, 335

Auriculatin, 2014

Cyclomulberrin, 4518

5,4'-Dihydroxy-8-(3,3-dimethylallyl)-2"-hydroxymethyl-2"-methylpyrano[5,6:6,7]iso-

flavone, 5849

Eryvarin G, 7351

Kanzonol T, 12152

Kraussianone 2, 12292

Kuwanon A, 12380

Kuwanon B, 12381 
Lespedezol $\mathrm{A}_{6}, 12687$

Lespedezol $\mathrm{C}_{1}, 12691$

Morusin, 14995

Pomiferin, 17695

5,7,3'-Trihydroxy-2'-(3-methylbut-2-enyl)-4',5'-

(3,3-dimethylpyrano)isoflavone, 21796

Yinyanghuo A, 22908

$\mathrm{C}_{25} \mathrm{H}_{24} \mathrm{O}_{7}$

Broussonol A, 2652

Broussonol B, 2653

Kraussianone 5, 12295

Macrophyllin, 13318

Mammea A/AB dioxalanocyclo F, 13466

Petalopurpurenol, 17008

Sanggenon A, 19255

Sanggenon M, 19268

\section{$\mathrm{C}_{25} \mathrm{H}_{24} \mathrm{O}_{8}$}

Anticancer Stilbenolignan PMV70P691-042, 1448

Kompasinol A, 12258

5-Methoxy-(3",4"-dihydro-3",4"-diacetoxy)-2", 2"-dimethylpyrano-(7,8:5",6")-flavone, 13908

\section{$\mathrm{C}_{25} \mathrm{H}_{24} \mathrm{O}_{10}$}

Anticancer Flavonoid PMV70P691-014, 1398

\section{$\mathrm{C}_{25} \mathrm{H}_{24} \mathrm{O}_{11}$}

(+)-Catechin-pentaacetate, 3316

(-)-Epicatechin-pentaacetate, 6867

\section{$\mathrm{C}_{25} \mathrm{H}_{24} \mathrm{O}_{12}$}

Cynarin, 4564

1,4-Di-O-caffeoylquinic acid, 5413

3,4-Di- $O$-caffeoylquinic acid, 5414

Isochlorogenic acid A, 11328

Kaempferol-3-O-(2,3-di- $O$-acetyl- $\alpha-L$-rhamnopyranoside), 12035

Kaempferol-3-O-(2,4-di- $O$-acetyl- $\alpha$ - $L$-rhamnopyranoside), 12036

Kaempferol-3-O-(3,4-di- $O$-acetyl- $\alpha$ - $L$-rhamnopyranoside), 12037

\section{$\mathrm{C}_{25} \mathrm{H}_{24} \mathrm{O}_{13}$}

Trifolirhizin-6"-O-malonate, 21638

Trilobatin D, 21879

\section{$\mathrm{C}_{25} \mathrm{H}_{24} \mathrm{O}_{15}$}

Mearnsetin 3-O-(4"-O-malonyl)- $\alpha-L$-rhamnopyranoside, 13627

\section{$\mathrm{C}_{25} \mathrm{H}_{25} \mathrm{O}_{13}{ }^{+}$}

Peonidin-3-O-(6"- $O$-malonyl- $\beta$-glucopyranoside), 16902

\section{$\mathrm{C}_{25} \mathrm{H}_{26} \mathrm{BrN}_{5} \mathrm{O}_{13}$}

Surugatoxin, 20496

$\mathrm{C}_{25} \mathrm{H}_{26} \mathrm{~N}_{2} \mathrm{O}_{9}$

Nauclecosidine, 15299

$\mathrm{C}_{25} \mathrm{H}_{26} \mathrm{O}_{3}$

8,8-Dimethyl-2-phenyl-10-prenyl-2,3-dihydro-
$8 H$-pyrano[3,2-g]chroman-4-one, 6397

$$
\mathrm{C}_{25} \mathrm{H}_{26} \mathrm{O}_{4}
$$

Erycristagallin, 7304

Euchrenone, 7484

Hispaglabridin B, 9558

3-Hydroxy-4-(3,3-dimethylallyl)-4",5"-dehydropyrano[8,9:2",3"][6aR,11aR]-pterocarpane, 10047

Licoflavone B, 12775

\section{$\mathrm{C}_{25} \mathrm{H}_{26} \mathrm{O}_{5}$}

Calopolyanolide C, 2994

Calopolyanolide D, 2995

(E)-1-[2,4-Dihydroxy-3-(3-methyl-2-butenyl) phenyl]-3-(2,2-dimethyl-8-hydroxy-2H-benzopyran-6-yl)-2-propen-1-one, 6024

6,8-Diprenylgenistein, 6496

Dulcisisoflavone, 6625

2-Geranylemodin, 8315

3-Geranyloxy-6-methyl-1,8-dihydroxyanthraquinone, 8325

Glycyrdione B, 8836

Isolupalbigenin, 11514

Kanzonol Z, 12156

Lespedezol A 2,12683

Lespedezol $\mathrm{E}_{1}, 12700$

Lupalbigenin, 13088

Mammea A/BA, 13468

Mammea A/BB, 13469

Mammeisin, 13473

\section{$\mathrm{C}_{25} \mathrm{H}_{26} \mathrm{O}_{6}$}

Anticancer Flavonoid PMV70P691-024, 1402

Anticancer Flavonoid PMV70P691-100, 1405

Broussoflavonol F, 2635

Calopolyanolide A, 2992

Calopolyanolide B, 2993

Derrisisoflavone B, 5230

Derrisisoflavone F, 5234

2,3-Dihydroauriculatin, 5544

Disparinol B, 6514

Dorsmanin I, 6571

Erysenegalensein E, 7317

Eryvarin F, 7350

Glyarallin B, 8795

Glyasperin A, 8796

Isoerysenegalensein E, 11415

Isosenegalensin, 11711

Kushenol F, 12359

Kuwanol C, 12378

Kuwanon D, 12382

Kuwanon F, 12384

Lespedezol E2, 12701

LupinisoflavoneG, 13105

Lupinisol A, 13106
Mammea A/AB cyclo E, 13465

Mulberrin, 15038

8-Prenylerythrinin, 17832

8-Prenylluteone, 17837

Sanggenol L, 19253

Sanggenon I, 19264

Sanggenon N, 19269

5,7,3',4'-Tetrahydroxy-2',5'-di(3-methylbut-2enyl)isoflavone, 21098

5,7,2',4'-Tetrahydroxy-3-geranylflavone, 21116

2',3,5-Trihydroxy-6,7-(2",2"-dimethylchromene)-

8-(3"',3"'-dimethylallyl)-flavanone, 21719

Ugonin A, 22174

Ugonin G, 22180

Ugonin J, 22183

Vogelin H, 22603

Yinyanghuo B, 22909

\section{$\mathrm{C}_{25} \mathrm{H}_{26} \mathrm{O}_{7}$}

Breviflavone B, 2601

Broussonol C, 2654

Broussonol D, 2655

Broussonol E, 2656

Erysenegalensein N, 7318

Erysenegalensein O, 7319

Kraussianone 3, 12293

Kushenol C, 12356

Oxydihydromorusin, 16445

5,7,3',4'-Tetrahydroxy-6-geranylflavonol , 21117

Vogelin I, 22604

\section{$\mathrm{C}_{25} \mathrm{H}_{26} \mathrm{O}_{9}$}

Eryvarinol A, 7352

\section{$\mathrm{C}_{25} \mathrm{H}_{26} \mathrm{O}_{10}$}

$(5 S, 6 S, 7 R)-2-[2-(2-$ Acetoxyphenyl)ethyl]-5 $\alpha, 6 \beta$, $7 \alpha$-triacetoxy-5,6,7,8-tetrahydrochro- mone (AH9), 275

5,3'-Dipropanoyloxy-3,6,7,4'-tetramethoxy-flavone, 6500

\section{$\mathrm{C}_{25} \mathrm{H}_{26} \mathrm{O}_{11}$}

Apigenin-7- $O-\beta$ - $D$-glucuronide butyl ester, 1495 4',6"-Diacetyl puerarin, 5345

\section{$\mathrm{C}_{25} \mathrm{H}_{26} \mathrm{O}_{13}$}

Apigenin-6- $C$ - $\alpha$ - $L$-arabinopyranosyl- $8-C-\beta-L$ arabinopyranoside, 1480

Apigenin-6- $C$ - $\alpha$ - $L$-arabinopyranosyl-8- $C-\beta-D$ xylopyranoside, 1481

Apigenin-6- $C$ - $\beta$ - $D$-xylopyranosyl-8- $C$ - $\alpha$ - $L$-arabinopyranoside, 1501

6-C- $\beta$ - $L$-Arabinopyranosyl-8- $C$ - $\alpha-L$-arabinopyranosylapigenin, 1560

6- $\beta$ - $C$-(2'-Galloylglucopyranosyl)-5,7-dihydroxy2-isopropylchromone, 8105

8 - $\beta$-C-(2'-Galloylglucopyranosyl)-5,7-dihydroxy2-isopropylchromone, 8106 
Viscumneoside II, 22537

$\mathrm{C}_{25} \mathrm{H}_{27} \mathrm{NO}_{4}$

Ancistrocladidine, 1141

$\mathrm{C}_{25} \mathrm{H}_{27} \mathrm{NO}_{5}$

Ancistrocladisine, 1143

$\mathrm{C}_{25} \mathrm{H}_{27} \mathrm{NO}_{12}$

Oleracein B, 16075

$\mathrm{C}_{25} \mathrm{H}_{28} \mathrm{O}_{4}$

Abyssinone IV, 52

Abyssinone VI, 54

(E)-1-[2,4-Dihydroxy-3-(3-methyl-2-butenyl) phenyl]-3-(4-hydroxy-3-[3-methyl-2-butenyl)p henyl]-2-propen-1-one, 6025

Erybraedin C, 7295

Erythrabssin II, 7329

Eryzerin D, 7357

4'-O-Geranylisoliquiritigenin, 8322

Glabrol, 8499

Hispaglabridin A, 9557

Isograbrol, 11446

Kazinol B, 12187

Mulberrofuran A, 15039

Mulberrofuran B, 15040

Mulberrofuran N, 15048

Perrottetinenic acid, 16985

Spiniavanone-B, 20170

2',4,4'-Trihydroxy-3'-geranylchalcone, 21737

\section{$\mathrm{C}_{25} \mathrm{H}_{28} \mathrm{O}_{5}$}

Abyssinone V, 53

Bartericin A, 2153

Bartericin B, 2154

Bartericin C, 2155

2,3-Dihydro-7-methoxy-2S*,3R*-dimethyl-2-[4methyl-5-(4-methyl-2-furyl)-3(E)-pentenyl]-

furo[3,2-c] coumarin, 5669

6,8-Diprenylnaringenin, 6497

Dorsmanin B, 6562

Eryzerin A, 7354

(2S)-Euchrenone $\mathrm{A}_{16}, 7486$

Fukanefuromarin E, 7982

Fukanefuromarin F, 7983

Fukanefuromarin G, 7984

Fukanemarin B, 7985

3'-Geranyl-2',3,4,4'-tetrahydroxychalcone, 8330

Glycyrdione A, 8835

3-Hydroxyglabrol, 10149

Isoemericellin, 11410

Kushenol A, 12354

Kuwanol D, 12379

Lespedezol B 12688

Mulberrofuran Y, 15056

Rhinacanthin B, 18769

2',4,4'-Trihydroxy-3'-[6-hydroxy-3,7-dimethyl-
2(E),7-octadienyl]chalcone, 21745

2',4',4-Trihydroxy-3'-[2-hydroxy-7-methyl-3-

methylene-6-octaenyl]chalcone, 21749

$\mathrm{C}_{25} \mathrm{H}_{28} \mathrm{O}_{6}$

1,3-Dihydroxy-6,7-dimethoxy-2,8-diprenylxanthone, 5827

3'-( $\gamma, \gamma$-Dimethylallyl)-kievitone, 6307

Dorsmanin E, 6565

Dorsmanin J, 6572

Gancaonin E, 8138

Glisoflavanone, 8553

Kushenol E, 12358

Kuwanon E, 12383

(2R,3R)-Lespedezaflavanone C, 12681

Lespedezol $D_{5}, 12697$

Lespedezol $\mathrm{D}_{6}, 12698$

Lespedol A, 12705

Lespedol B, 12706

$\beta$-Mangostin, 13493

Norkurarinone, 15770

Pluviatilol- $\gamma, \gamma$-dimethylallyl ether, 17572

Sophoraflavanone G, 20086

2',3,4,4'-Tetrahydroxy-3'-[6-hydroxy-3,7-di-

methyl-2(E),7-octadienyl]chalcone, 21119

\section{$\mathrm{C}_{25} \mathrm{H}_{28} \mathrm{O}_{7}$}

Cedkathryn A, 3347

Cedkathryn B, 3348

Dispardiol B, 6513

Dorsmanin Fa, 6566

Dorsmanin Fb, 6567

Dorsmanin Ga, 6568

Dorsmanin Gb, 6569

Dorsmanin H, 6570

Kushenol L, 12365

Kushenol X, 12370

Mangostanin, 13484

Norkurarinol, 15769

$\mathrm{C}_{25} \mathrm{H}_{28} \mathrm{O}_{8}$

Kushenol G, 12360

Lobaric acid, 12936

$\mathrm{C}_{25} \mathrm{H}_{28} \mathrm{O}_{10}$

5 -[3"- $\beta-D$-Glucopyranosyloxy)propyl]-7-methoxy-2-(3',4'-methylenedioxyphenyl)benzofuran, 8709

$\mathrm{C}_{25} \mathrm{H}_{28} \mathrm{O}_{11}$

Dracunculifoside K, 6590

6-Hydroxy-5-methyl-3',4',5'-trimethoxyaurone 4- $O$ - $\alpha$ - $L$-rhamnopyranoside, 10522

Shakuchirin, 19794

Umbilicaxanthoside A, 22197

$\mathrm{C}_{25} \mathrm{H}_{28} \mathrm{O}_{12}$

Andrographidine D, 1154

Chrysoobtusin glucoside, 3613

\section{$\mathrm{C}_{25} \mathrm{H}_{28} \mathrm{O}_{13}$}

Andrographidine F, 1156

Polycaudoside A, 17621

$\mathrm{C}_{25} \mathrm{H}_{28} \mathrm{O}_{14}$

2-O-[ $\alpha$ - $L$-Rhamnopyranosyl-( $1 \rightarrow 2)-\beta$ - $D$-xylopyranosyl]-1,8-dihydroxy-6-methoxyxanthone, 18736

\section{$\mathrm{C}_{25} \mathrm{H}_{28} \mathrm{O}_{15}$}

Polygalaxanthone III, 17631

8-O-Primeverosylbellidifolin, 17854

1-O-Primeverosyl-3,8-dihydroxy-5-methoxyxanthone, 17855

\section{$\mathrm{C}_{25} \mathrm{H}_{29} \mathrm{NO}_{4}$}

Ancistrocladine, 1142

Ancistrotanzanine C, 1147

Buxifoliadine A, 2823

\section{$\mathrm{C}_{25} \mathrm{H}_{29} \mathrm{NO}_{5}$}

Decodine, 4856

Dihydroverticillatine, 5736

\section{$\mathrm{C}_{25} \mathrm{H}_{30}$}

Undecenyl phenanthrene, 22223

\section{$\mathrm{C}_{25} \mathrm{H}_{30} \mathrm{~N}_{2} \mathrm{O}_{7}$}

Methyl 11,12-dimethoxychanofruticosinate, 14319

\section{$\mathrm{C}_{25} \mathrm{H}_{30} \mathrm{~N}_{2} \mathrm{O}_{8}$}

Pauciflorine B, 16732

\section{$\mathrm{C}_{25} \mathrm{H}_{30} \mathrm{O}_{4}$}

Bixin, 2496

Eryzerin C, 7356

Kanzonol X, 12154

Kazinol A, 12186

Myrtiaphenone A, 15224

\section{$\mathrm{C}_{25} \mathrm{H}_{30} \mathrm{O}_{5}$}

2,3-Dihydro-7-methoxy-2 $S^{*}, 3 R^{*}$-dimethyl-2-

[4,8-dimethyl-3(E),7-nonadien-6-onyl]-furo

[3,2-c] coumarin, 5666

Kanzonol Y, 12155

Rhinacanthin C, 18770

Vismione D, 22551

\section{$\mathrm{C}_{25} \mathrm{H}_{30} \mathrm{O}_{6}$}

(+)-3,4-(6",6"-Dimethyldihydropyrano)-4',5'-[2"'(1-hydroxy-1-methylethyl)-dihydrofurano]-

2',3"'-dihydroxydihydrochalcone, 6337

Kosamol T, 12284

Notoptolide, 15845

(+)-Pinoresinol-3,3-dimethylallyl ether, 17413

Rhinacanthin G, 18772

Rhinacanthin H, 18773

Rhinacanthin I, 18774

$\mathrm{C}_{25} \mathrm{H}_{30} \mathrm{O}_{7}$

Kosamol Q, 12281

Rhinacanthin K, 18775

$\mathrm{C}_{25} \mathrm{H}_{30} \mathrm{O}_{8}$ 
Kadsurin, 12012

Longipedunin B, 12967

Rubschisantherin, 19044

\section{$\mathrm{C}_{25} \mathrm{H}_{30} \mathrm{O}_{9}$}

Gaultherin A, 8240

Kadsulignan B, 11997

Robustaol A, 18867

\section{$\mathrm{C}_{25} \mathrm{H}_{30} \mathrm{O}_{11}$}

5-Hydroxycampenoside, 9878

Yemuoside $\mathrm{YM}_{1}, 22884$

\section{$\mathrm{C}_{25} \mathrm{H}_{30} \mathrm{O}_{12}$}

Cucurbitoside C, 4332

6-p-Methoxycinnamoyl catalpol, 13887

\section{$\mathrm{C}_{25} \mathrm{H}_{30} \mathrm{O}_{13}$}

Alboside IV, 870

Cachineside III, 2840

Cucurbitoside D, 4333

Ebractelatinoside B, 6667

Fraxamoside, 7941

Grandifloroside, 8977

Minecoside, 14871

Picroside III, 17346

\section{$\mathrm{C}_{25} \mathrm{H}_{30} \mathrm{O}_{14}$}

Ligustrosidic acid, 12830

\section{$\mathrm{C}_{25} \mathrm{H}_{30} \mathrm{O}_{15}$}

Oleuropeinic acid, 16081

Tenuiphenone B, 20960

\section{$\mathrm{C}_{25} \mathrm{H}_{31} \mathrm{NO}_{10}$}

Isocimicifugamide, 11331

\section{$\mathrm{C}_{25} \mathrm{H}_{31} \mathrm{~N}_{3} \mathrm{O}_{2}$}

Celacinnine, 3357

Celallocinnine, 3360

\section{$\mathrm{C}_{25} \mathrm{H}_{32} \mathrm{CIN}_{5} \mathrm{O}_{6}$}

Astin D, 1932

\section{$\mathrm{C}_{25} \mathrm{H}_{32} \mathrm{CIN}_{5} \mathrm{O}_{7}$}

Astin E, 1933

\section{$\mathrm{C}_{25} \mathrm{H}_{32} \mathrm{~N}_{2} \mathrm{O}$}

Vindoline, 22498

\section{$\mathrm{C}_{25} \mathrm{H}_{32} \mathrm{O}_{4}$}

Artemisolide, 1802

2,3-Dihydro-7-methoxy-2 $R^{*}, 3 R^{*}$-dimethyl-2-

[4,8-dimethyl-3(E),7-nonadienyl]-furo[3,2-c] coumarin, 5667

2,3-Dihydro-7-methoxy-2 $S^{*}, 3 R^{*}$-dimethyl-2-

[4,8-dimethyl-3(E),7-nonadienyl]-furo[3,2-c]

coumarin, 5668

Kazinol F, 12188

\section{$\mathrm{C}_{25} \mathrm{H}_{32} \mathrm{O}_{5}$}

3 $\beta$-Formyloxyresibufogenin, 7912

Wallichilide, 22627

\section{$\mathrm{C}_{25} \mathrm{H}_{32} \mathrm{O}_{6}$}

20R,21-Epoxyresibufogenin 3-formate, 7190 20S,21-Epoxyresibufogenin 3-formate, 7191
$\mathrm{C}_{25} \mathrm{H}_{32} \mathrm{O}_{7}$

Ardisinone A, 1645

Ichangensin, 10958

Nigrolineaxanthone P, 15589

Perlatolic acid, 16967

Quassinoid PC03-579A, 18300

Schisanhenol acetate, 19477

\section{$\mathrm{C}_{25} \mathrm{H}_{32} \mathrm{O}_{8}$}

Aceroside $\mathrm{B}_{1}, 103$

Aceroside $\mathrm{B}_{2}, 104$

Albaspidin BB, 851

Albaspidin $\mathrm{BBiB}, 852$

Aspidin, 1895

(3S,5S)-3,5-Diacetoxy-1,7-bis(4-hydroxy-3methoxyphenyl)heptane, 5288

Isoaspidin BB, 11240

Meso-3,5-diacetoxy-1,7-bis-(4-hydroxy-3methoxyphenyl) heptane, 13802

Paraaspidin BB, 16648

Pseudoaspidin, 18019

Saroaspidin B, 19383

$\mathrm{C}_{25} \mathrm{H}_{32} \mathrm{O}_{9}$

Caesalpinin J, 2872

Caruilignan A, 3227

Luzonoid E, 13161

Luzonoid F, 13162

Luzonoid G, 13163

Sudachinoid B, 20449

\section{$\mathrm{C}_{25} \mathrm{H}_{32} \mathrm{O}_{10}$}

(-)-Isolariciresinol-2 $\alpha-O-\beta$ - $D$-xylopyranoside, 11479

Massonianoside A, 13583

Massonianoside B, 13584

Massonianoside E, 13587

\section{$\mathrm{C}_{25} \mathrm{H}_{32} \mathrm{O}_{11}$}

(2R,3S)-2,3-Dihydro-7-hydroxy-2-(4'-hydroxy-

3'-methoxyphenyl)-3-hydroxymethyl-5-benzo-

furanpropanol 4'- $O-\beta$ - $D$-glucopyranoside, 5634

(2S,3R)-2,3-Dihydro-7-hydroxy-2-(4'-hydroxy-

3'-methoxyphenyl)-3-hydroxymethyl-5-benzo-

furanpropanol 4'- $O-\beta$ - $D$-glucopyranoside, 5635

5,4'-Dihydroxy-3- $\alpha$ - $L$-rhamnosyl-( $1^{\prime \prime} \rightarrow 3$ ')- $\beta$ - $D$ -

xylopyranosyloxybibenzyl, 6108

Hirsutanonol-5-O- $\beta$ - $D$-glucopyranoside, 9551

(-)-Isolariciresinol-3 $\alpha-O-\beta$ - $D$-glucopyranoside, 11480

Neoglabrescin A tetraacetate, 15398

Syringafghanoside, 20553

\section{$\mathrm{C}_{25} \mathrm{H}_{32} \mathrm{O}_{12}$}

6-O-E-Feruloylajugol, 7769

6-O-Z-Feruloylajugol, 7770

Gelsemiol 6'-trans-caffeoyl-1-glucoside, 8259

(8E)-Ligustroside, 12828
(8Z)-Ligustroside, 12829

6'-O-E-p-Methoxycinnamoylharpagide, 13888

6'-O-Z-p-Methoxycinnamoylharpagide, 13889

8-O-E-p-Methoxycinnamoylharpagide, 13890

8-O-Z-p-Methoxycinnamoylharpagide, 13891

8-(O-Methyl- $p$-coumaroyl)harpagide, 14259

\section{$\mathrm{C}_{25} \mathrm{H}_{32} \mathrm{O}_{13}$}

8-O-Feruloylharpagide, 7776

10-Hydroxyligustroside, 10324

Oleuropein, 16080

Orientaloside, 16195

\section{$\mathrm{C}_{25} \mathrm{H}_{32} \mathrm{O}_{14}$}

(2"R)-2"-Hydroxyoleuropein, 10552

(2"S)-2"-Hydroxyoleuropein, 10553

\section{$\mathrm{C}_{25} \mathrm{H}_{33} \mathrm{Cl}_{2} \mathrm{~N}_{5} \mathrm{O}_{6}$}

Astin C, 1931

\section{$\mathrm{C}_{25} \mathrm{H}_{33} \mathrm{Cl}_{2} \mathrm{~N}_{5} \mathrm{O}_{7}$}

Astin A, 1929

Astin B, 1930

\section{$\mathrm{C}_{25} \mathrm{H}_{33} \mathrm{NO}_{3}$}

Piperchabamide C, 17442

$\mathrm{C}_{25} \mathrm{H}_{33} \mathrm{NO}_{9}$

8,9-Dehydro-10-epi-ryanodine, 4911

\section{$\mathrm{C}_{25} \mathrm{H}_{33} \mathrm{NO}_{10}$}

(13S)-8,9-Dehydro-18-hydroxy-10-epi-ryanodine, 4931

8,9-Dehydro-20-hydroxy-10-epi-ryanodine, 4932

8,9-Dehydro-21-hydroxy-10-epi-ryanodine, 4933

6-Deoxy- $6 \beta, 9 \beta$-epoxy- $8 \alpha$-hydroxy-10-epi-ryanodine, 5169

$8 \alpha, 9 \alpha$-Epoxy-10-epi-ryanodine, 7091

$8 \beta, 9 \beta$-Epoxy-10-epi-ryanodine, 7092

20-Norspiganthine-5-carboxylic acid, 15794

\section{$\mathrm{C}_{25} \mathrm{H}_{33} \mathrm{NO}_{11}$}

(13S)-8,9-Dehydro-18,21-dihydroxy-10-epiryanodine, 4907

8,9-Dehydro-20,21-dihydroxy-10-epi-ryanodine, 4908

(13S)-8 $\alpha, 9 \alpha$-Epoxy-18-hydroxy-10-epi-ryanodine, 7111

$8 \alpha, 9 \alpha$-Epoxy-20-hydroxy-10-epi-ryanodine, 7112

$8 \alpha, 9 \alpha$-Epoxy-21-hydroxy-10-epi-ryanodine, 7113

\section{$\mathrm{C}_{25} \mathrm{H}_{33} \mathrm{~N}_{5} \mathrm{O}_{8}$}

Asterin A, 1922

\section{$\mathrm{C}_{25} \mathrm{H}_{34} \mathrm{O}_{4}$}

Lupulone B, 13111

Neovibsanin G, 15474

14-epi-Neovibsanin G, 15475

\section{$\mathrm{C}_{25} \mathrm{H}_{34} \mathrm{O}_{5}$}

3-Epi-O-Methyl-scilliphaeosidin, 6970

\section{$\mathrm{C}_{25} \mathrm{H}_{34} \mathrm{O}_{6}$}

Erectquione C, 7225

Homofukinolide, 9607 
Rouyolide B, 18963

$\mathrm{C}_{25} \mathrm{H}_{34} \mathrm{O}_{7}$

Atranone B, 1984

Rouyolide A, 18962

Rouyolide F, 18967

Vibsanin M, 22450

\section{$\mathrm{C}_{25} \mathrm{H}_{34} \mathrm{O}_{8}$}

7-Acetoxybonducellpin C, 137

16 $\beta$-Acetoxystrophanthidin, 281

Atranone G, 1989

Caesalpinin MF, 2876

Kuhistanicaol A, 12311

Rouyolide D, 18965

Rouyolide E, 18966

\section{$\mathrm{C}_{25} \mathrm{H}_{34} \mathrm{O}_{9}$}

Ailanthinone, 774

Caesalpinin M, 2875

(3R,5R)-3,5-Dihydroxy-1,7-bis(4-hydroxyphenyl)

heptane 3-O- $\beta$ - $D$-glucopyranoside, 5781

Kusulactone, 12373

Schkuhrin II, 19511

Simalikalactone D, 19900

\section{$\mathrm{C}_{25} \mathrm{H}_{34} \mathrm{O}_{10}$}

Amritoside D, 1087

(3R,5R)-3,5-Dihydroxy-1-(3,4-dihydroxyphenyl)-

7-(4-hydroxyphenyl)heptane 3- $O-\beta$ - $D$-gluco-

pyranoside, 5816

(3R,5R)-3,5-Dihydroxy-1-(3,4-dihydroxy-

phenyl)-7-(4-hydroxyphenyl)heptane

5-O- $\beta$ - $D$-glucopyranoside, 5817

$(2 E, 6 R)-2,6$-Dimethyl-8-hydroxy-2-octenoic acid

8-O-[6'- $O-(E)$ - $p$-coumaroyl]- $\beta$ - $D$-glucopyra-

noside, 6363

Glaucarubinone, 8509

1-Homoacevaltrate, 9593

1-Homoisoacevaltrate, 9611

Soularbinone, 20108

\section{$\mathbf{C}_{25} \mathbf{H}_{34} \mathbf{O}_{11}$}

$(3 R, 5 R)-3,5$-Dihydroxy-1,7-bis(3,4-dihydroxyphenyl)heptane 3-O- $\beta$ - $D$-glucopyranoside, 5775

Nagilactoside A, 15236

Nagilactoside B, 15237

\section{$\mathrm{C}_{25} \mathrm{H}_{34} \mathrm{O}_{12}$}

Lucidumoside A, 13053

\section{$\mathrm{C}_{25} \mathrm{H}_{34} \mathrm{O}_{13}$}

Lucidumoside B, 13054

6-O-(3,4,5-Trimethoxybenzoyl)-ajugol, 21894

\section{$\mathrm{C}_{25} \mathrm{H}_{34} \mathrm{O}_{14}$}

Macrophylloside D, 13324

\section{$\mathrm{C}_{25} \mathrm{H}_{35} \mathrm{NO}$}

1-Methyl-2-[(6Z,9Z)-6,9-pentadecadienyl]4(1H)-quinolone, 14654
$\mathrm{C}_{25} \mathrm{H}_{35} \mathrm{NO}_{3}$

Merresectine C, 13787

$\mathrm{C}_{25} \mathrm{H}_{35} \mathrm{NO}_{5}$

Yuzurimine E, 22960

$\mathrm{C}_{25} \mathrm{H}_{35} \mathrm{NO}_{8}$

20-Deoxyspiganthine, 5215

$\mathrm{C}_{25} \mathrm{H}_{35} \mathrm{NO}_{9}$

Spiganthine, 20162

$\mathrm{C}_{25} \mathrm{H}_{35} \mathrm{NO}_{10}$

9-Hydroxy-9-epi-10-epi-ryanodine, 10071

9-Hydroxy-10-epi-ryanodine, 10081

20-Hydroxyryanodine, 10683

$8 \alpha$-Hydroxyspiganthine, 10715

Ryanodine, 19091

\section{$\mathrm{C}_{25} \mathrm{H}_{35} \mathrm{NO}_{11}$}

(13S)-9,18-Dihydroxy-9-epi-10-epi-ryanodine, 5869

9,20-Dihydroxy-9-epi-10-epi-ryanodine, 5870

\section{$\mathrm{C}_{25} \mathrm{H}_{35} \mathrm{~N}_{5} \mathrm{O}_{6}$}

Pseudostellarin A, 18064

\section{$\mathrm{C}_{25} \mathrm{H}_{36} \mathrm{O}_{2}$}

Abridin, 12

$\mathrm{C}_{25} \mathrm{H}_{36} \mathrm{O}_{3}$

Surinone B, 20494

\section{$\mathrm{C}_{25} \mathrm{H}_{36} \mathrm{O}_{4}$}

Vibsanin R, 22453

\section{$\mathrm{C}_{25} \mathrm{H}_{36} \mathrm{O}_{5}$}

Caseamembrin F, 3263

5-Epi-vibsanin C, 7033

5-Epi-vibsanin E, 7034

Lupulone E, 13114

Vibsanin B, 22442

Vibsanin C, 22443

Vibsanin E, 22444

Vibsanin T, 22455

\section{$\mathrm{C}_{25} \mathrm{H}_{36} \mathrm{O}_{6}$}

16-Acetylgitoxigenin, 400

Ajugacumbin B, 789

ent-15 $\beta$-Angeloyloxy- $7 \alpha, 9 \alpha$-dihydroxy-kaur-

16-en-19-oic acid, 1216

5-Epivibsanin G, 7035

5-Epi-vibsanin H, 7036

$14 R^{*}, 15$-Epoxyvibsanin C, 7219

$14 S^{*}, 15$-Epoxyvibsanin C, 7220

Erinacine A, 7261

Erinacine B, 7262

Erinacine E, 7264

Vibsanin G, 22445

Vibsanin H, 22446

Vibsanin L, 22449

Vibsanol A, 22459

Vibsanol B, 22460

$\mathrm{C}_{25} \mathrm{H}_{36} \mathrm{O}_{7}$
5-Epi-vibsanin K, 7037

Preclavulone A methyl ester derivative CPB51-909-3, 17774

Trichurusin F, 21583

Trichurusin G, 21584

Vibsanin I, 22447

Vibsanin K, 22448

\section{$\mathrm{C}_{25} \mathrm{H}_{36} \mathrm{O}_{8}$}

Erinacine G, 7265

\section{$\mathrm{C}_{25} \mathrm{H}_{36} \mathrm{O}_{9}$}

Adenolin A, 619

2,5-Epoxy-5,10-dihydroxy-6-angeloyloxy-9-(2methylbutyryloxy)-germacran-8,12-olide, 7074

2,5-Epoxy-5,10-dihydroxy-6-angeloyloxy-9-(2Rmethylbutyryloxy)-germacran-8,12-olide, 7075

2,5-Epoxy-5,10-dihydroxy-6-angeloyloxy-9-(3methylbutyryloxy)-germacran-8,12-olide, 7076

\section{$\mathrm{C}_{25} \mathrm{H}_{36} \mathrm{O}_{10}$}

10-Acetoxy-1-homovaltrate hydrin, 200

\section{$\mathrm{C}_{25} \mathrm{H}_{36} \mathrm{O}_{13}$}

Dracunculifoside Q, 6596

\section{$\mathrm{C}_{25} \mathrm{H}_{37} \mathrm{NO}$}

1-Methyl-2-[(Z)-6-pentadecenyl]-4(1H)quinolone, 14657

1-Methyl-2-[(Z)-9-pentadecenyl]-4(1H)quinolone, 14658

1-Methyl-2-[(Z)-10-pentadecenyl]-4(1H)quinolone, 14659

\section{$\mathrm{C}_{25} \mathrm{H}_{38} \mathrm{O}_{4}$}

Ardisiphenol C, 1652

12,25-Dihydroxy-16-scalaren-24,25-olide, 6113

\section{$\mathrm{C}_{25} \mathrm{H}_{38} \mathrm{O}_{5}$}

2-Acetoxy-5-methoxy-6-methyl-3-[(Z-10'pentadecenyl)-1,4-benzoquinone], 253

\section{$\mathrm{C}_{25} \mathrm{H}_{38} \mathrm{O}_{5}$}

Caesaldecan, 2860

2-Methoxy-5-acetoxy-6-methyl-3-[(z)-10'pentadecenyl]-1,4-benzoquinone, 13829

Vibsanin P, 22451

Vibsanin S, 22454

\section{$\mathrm{C}_{25} \mathrm{H}_{38} \mathrm{O}_{6}$}

Erinacine C, 7263

Philadelphinone, 17142

Rasfonin, 18549

Taxuyunnanine H, 20880

\section{$\mathrm{C}_{25} \mathrm{H}_{38} \mathrm{O}_{7}$}

Ajugacumbin F, 793

ent-(16S)-1 $\alpha, 14 \alpha$-Diacetoxy-7 $\beta$-hydroxy-17methoxykauran-15-one, 5309

Trichurusin C, 21580

Trichurusin D, 21581

Trichurusin E, 21582

Trichurusin H, 21585 
Trichurusin I, 21586

Vibsanin U, 22456

Vibsanin W, 22458

$\mathrm{C}_{25} \mathrm{H}_{38} \mathrm{O}_{8}$

Xindongnin G, 22803

$\mathrm{C}_{25} \mathrm{H}_{38} \mathrm{O}_{12}$

Eurycomaoside, 7652

$\mathrm{C}_{25} \mathrm{H}_{38} \mathrm{O}_{15}$

Phenethylalcohol 8-O- $\beta$ - $D$-glucopyranosyl$(1 \rightarrow 2)-O-\beta$ - $D$-apiofuranosyl- $(1 \rightarrow 6)-\beta-D$ glucopyranoside, 17081

$\mathrm{C}_{25} \mathrm{H}_{38} \mathrm{O}_{16}$

Lupulinoside, 13108

Zizybeoside II, 23012

\section{$\mathrm{C}_{25} \mathrm{H}_{38} \mathrm{O}_{17}$}

3,4-Dihydroxyphenylethanol-8- $O-[\beta-D$-apiofu-

$\operatorname{ranosyl}(1 \rightarrow 3)-\beta$ - $D$-glucopyranosyl $(1 \rightarrow 6)]-\beta$ -

$D$-glucopyranoside, 6078

\section{$\mathrm{C}_{25} \mathrm{H}_{39} \mathrm{NO}$}

1-Methyl-2-pentadecyl-4(1H)-quinolone, 14660

\section{$\mathrm{C}_{25} \mathrm{H}_{39} \mathrm{NO}_{2}$}

Buxpiine, 2831

\section{$\mathrm{C}_{25} \mathrm{H}_{39} \mathrm{NO}_{3}$}

3(R)-Benzoyloxy-2(R)-methyl-6(R)-(11'-oxododecyl)-piperidine, 2260

\section{$\mathrm{C}_{25} \mathrm{H}_{39} \mathrm{NO}_{6}$}

14-Acetylgenicunine B, 396

Condelphine, 3963

Erythrophlamine, 7342

Erythrophleguine, 7343

Erythrosuamine, 7344

\section{$\mathrm{C}_{25} \mathrm{H}_{39} \mathrm{NO}_{7}$}

Dehydrodeltatsine, 4899

Delbrunine, 5000

Deltamine, 5037

18-Demethoxypubescenine, 5053

\section{$\mathrm{C}_{25} \mathrm{H}_{39} \mathrm{NO}_{8}$}

14-O-Acetyltakaosamine, 518

\section{$\mathrm{C}_{25} \mathrm{H}_{40} \mathrm{~N}_{2} \mathrm{O}_{5}$}

Arboreumine, 1612

\section{$\mathrm{C}_{25} \mathrm{H}_{40} \mathrm{O}_{4}$}

2,5-Dihydroxy-3-(nonadec-14-enyl)-benzoquinone, 6048

16 $\alpha H, 17-$ Isovalerate-ent-kauran-19-oic acid, 11753

$\mathrm{C}_{25} \mathrm{H}_{40} \mathrm{O}_{5}$

$7 \alpha, 18$-Diacetoxy-13 $\beta$-methoxyabiet-8(14)-ene, 5313

$\mathrm{C}_{25} \mathrm{H}_{40} \mathrm{O}_{6}$

Erigerol, 7258

Oriediterpenoside, 16185

Trichurusin B, 21579

$\mathrm{C}_{25} \mathrm{H}_{40} \mathrm{O}_{11}$
Eriojaposide B, 7288

$\mathrm{C}_{25} \mathrm{H}_{40} \mathrm{O}_{12}$

Zizyvoside I, 23019

$\mathrm{C}_{25} \mathrm{H}_{41} \mathrm{NO}_{2}$

3,5-Didecanoylpyridine, 5462

$\mathrm{C}_{25} \mathrm{H}_{41} \mathrm{NO}_{6}$

Chasmanine, 3483

$\mathrm{C}_{25} \mathrm{H}_{41} \mathrm{NO}_{7}$

Browniine, 2657

Delsoline, 5035

Deltatsine, 5038

Ezochasmanine, 7700

Lycoctonine, 13189

$\mathrm{C}_{25} \mathrm{H}_{41} \mathrm{NO}_{8}$

Pseudoaconine, 18016

Swatinine, 20501

$\mathrm{C}_{25} \mathrm{H}_{42} \mathrm{~N}_{2} \mathrm{O}$

Cyclobuxine D, 4477

$\mathrm{C}_{25} \mathrm{H}_{42} \mathrm{~N}_{2} \mathrm{O}_{7}$

Hemsleyatine, 9356

\section{$\mathrm{C}_{25} \mathrm{H}_{42} \mathrm{O}_{6}$}

Stemodin- $\alpha$ - $L$-arabinofuranoside, 20300

Stemodinoside A, 20302

$\mathrm{C}_{25} \mathrm{H}_{42} \mathrm{O}_{12}$

Staphylionoside G, 20270

$\mathrm{C}_{25} \mathrm{H}_{42} \mathrm{O}_{17}$

Ilicifolioside C, 10990

$\mathrm{C}_{25} \mathrm{H}_{43} \mathrm{NO}_{2}$

3-O-14,15-Eicosylenoyl-1-cyano-2-methyl-

$$
\text { 1,2-propene, } 6727
$$

\section{$\mathrm{C}_{25} \mathrm{H}_{44} \mathrm{~N}_{2}$}

Epiheteroconessine, 6931

Kurchessine, 12347

\section{$\mathrm{C}_{25} \mathrm{H}_{44} \mathrm{~N}_{2} \mathrm{O}$}

Saracorine, 19356

$N, N, N^{\prime}, N^{\prime}$-Tetramethyl-holarrhimine, 21196

\section{$\mathrm{C}_{25} \mathrm{H}_{44} \mathrm{O}_{12}$}

Linarionoside C, 12858

Staphylionoside K, 20274

\section{$\mathrm{C}_{25} \mathrm{H}_{44} \mathrm{O}_{15}$}

Asystoside, 1952

Ebracteatoside B, 6664

Lunaroside, 13075

$\mathrm{C}_{25} \mathrm{H}_{44} \mathrm{O}_{16}$

Ilicifolioside B, 10989

$\mathrm{C}_{25} \mathrm{H}_{46} \mathrm{~N}_{2}$

Epipachysamine C, 6992

$N$-Methyl pachysamine A, 14650

$\mathrm{C}_{25} \mathrm{H}_{46} \mathrm{~N}_{2} \mathrm{O}$

$N, O$-Dideacyl- $N$-methylpachysandrine A, 5461

$\mathrm{C}_{25} \mathrm{H}_{46} \mathrm{O}_{12}$

Platanionoside D, 17521

$\mathrm{C}_{25} \mathrm{H}_{47} \mathrm{O}_{11} \mathrm{~S}$
1'-O-Palmitoyl-3'-O-(6-sulfo- $O$ - $\alpha-D$-quino-

vopyranosyl)glycerol, 16568

$\mathrm{C}_{25} \mathrm{H}_{48} \mathrm{O}_{2}$

Pentacosan-4-olide, 16824

$\mathrm{C}_{25} \mathrm{H}_{50} \mathrm{O}$

11-Pentacosanone, 16825

$\mathrm{C}_{25} \mathrm{H}_{50} \mathrm{O}_{2}$

n-Pentacosanoic acid, 16823

$\mathrm{C}_{25} \mathrm{H}_{52}$

Pentacosane, 16822

$\mathrm{C}_{26} \mathrm{H}_{18} \mathrm{O}_{7}$

Thelephantin I, 21301

$\mathrm{C}_{26} \mathrm{H}_{19} \mathrm{NO}_{12}$

Laccaic acid A, 12424

\section{$\mathrm{C}_{26} \mathrm{H}_{20} \mathrm{O}_{10}$}

Globoidnan A, 8554

Isosalvianolic acid C, 11694

Moellendorffiline, 14894

Salvianolic acid C, 19203

\section{$\mathrm{C}_{26} \mathrm{H}_{22} \mathrm{~N}_{2} \mathrm{O}_{4}$}

Daurioxoisoporphine A, 4689

\section{$\mathrm{C}_{26} \mathrm{H}_{22} \mathrm{O}_{6}$}

5-(3"-Benzoyloxypropyl)-7-methoxy-2-(3',4'methylenedioxyphenyl)-benzofuran, 2264

Hemerocallin, 9339

\section{$\mathrm{C}_{26} \mathrm{H}_{22} \mathrm{O}_{9}$}

3'-Benzoyloxy-5-hydroxy-3,6,7,4'-tetramethoxyflavone, 2259

Phelligridin F, 17064

\section{$\mathrm{C}_{26} \mathrm{H}_{22} \mathrm{O}_{10}$}

Salvianolic acid A, 19201

\section{$\mathrm{C}_{26} \mathrm{H}_{22} \mathrm{O}_{18}$}

Pelargoniin B, 16787

\section{$\mathrm{C}_{26} \mathrm{H}_{24} \mathrm{O}_{5}$}

Calophyllolide, 2990

\section{$\mathrm{C}_{26} \mathrm{H}_{24} \mathrm{O}_{6}$}

6-Methyltetrapterol A, 14754

\section{$\mathrm{C}_{26} \mathrm{H}_{24} \mathrm{O}_{7}$}

Goniolactone A, 8939

Rocaglamide derivative 7, 18887

\section{$\mathrm{C}_{26} \mathrm{H}_{24} \mathrm{O}_{10}$}

Curtisian N, 4410

\section{$\mathrm{C}_{26} \mathrm{H}_{24} \mathrm{O}_{11}$}

Tuberculatin, 22077

\section{$\mathrm{C}_{26} \mathrm{H}_{24} \mathrm{O}_{12}$}

Boehmerin, 2530

Justalakonin, 11975

$\mathrm{C}_{26} \mathrm{H}_{24} \mathrm{O}_{14}$

4,4'-O-Dimethylellagic acid

3-(2",3"-di- $O$-acetyl)- $\alpha$ - $L$-rhamnoside, 6346

\section{$\mathrm{C}_{26} \mathrm{H}_{24} \mathrm{O}_{18}$}

Pelargoniin C, 16788

$\mathrm{C}_{26} \mathrm{H}_{26} \mathrm{~N}_{2}$ 


\author{
Caulindole A, 3336 \\ Caulindole B, 3337 \\ Yuehchukene, 22937
$\mathbf{C}_{26} \mathrm{H}_{26} \mathbf{N}_{2} \mathbf{O}_{10}$
20-O- $\beta$-Glucopyranosyl 18-hydroxycampto- thecin, 8667

\section{$\mathrm{C}_{26} \mathrm{H}_{26} \mathrm{O}_{5}$} \\ Scandinone, 19452 \\ $\mathrm{C}_{26} \mathrm{H}_{26} \mathrm{O}_{6}$ \\ Artocommunol CE, 1814 \\ 5,4'-Dihydroxy-8-(3,3-dimethylallyl)-2"-me- \\ thoxyisopropylfurano[4,5:6,7]isoflavone, 5850 \\ Flemiphilippinin C, 7827 \\ (+)-(10S)-Ligulacephalin C, 12810 \\ (-)-(10S)-Ligulacephalin C, 12811 \\ Rocaglamide derivative 1, 18883 \\ Scandenin, 19450
}

\section{$\mathrm{C}_{26} \mathrm{H}_{26} \mathrm{O}_{7}$}

Broussoflavonol A, 2631

Digoniodiol, 5532

Ugonin I, 22182

\section{$\mathrm{C}_{26} \mathrm{H}_{26} \mathrm{O}_{8}$}

$\left(2 R^{*}, 3 S^{*}\right)$-3-Hydroxymethyl-9-methoxy-2-

(4'-hydroxy-3',5'-dimethoxyphenyl)-2,3,6,7-tet rahydrophenanthro[4,3-b]furan-5,11-diol, 10504

Interiotherin D, 11106

Terrestrin B, 21007

\section{$\mathrm{C}_{26} \mathrm{H}_{26} \mathrm{O}_{11}$ \\ 2",6"-O-Diacetyloninin, 5343}

\section{$\mathrm{C}_{26} \mathrm{H}_{26} \mathrm{O}_{12}$}

3,4-Di- $O$-caffeoylquinic acid methyl ester, 5415

Juspurpurin, 11974

Macranthoin F, 13300

Macranthoin G, 13301

\section{$\mathrm{C}_{26} \mathrm{H}_{26} \mathrm{O}_{14}$}

Mingjinianuronide B, 14873

\section{$\mathrm{C}_{26} \mathrm{H}_{26} \mathrm{O}_{18}$}

Amritoside, 1083

$\mathrm{C}_{26} \mathrm{H}_{27} \mathrm{NO}_{7}$

Squamosamide, 20245

\section{$\mathrm{C}_{26} \mathrm{H}_{28} \mathrm{~N}_{2} \mathrm{O}_{8}$}

(3R)-Deoxypumiloside, 5205

\section{$\mathrm{C}_{26} \mathrm{H}_{28} \mathrm{~N}_{2} \mathrm{O}_{9}$}

Nauclecoside, 15298

Naucleoside A, 15310

Naucleoside B, 15311

Ophiorine A, 16151

Ophiorine B, 16152

Pumiloside, 18199

\section{$\mathrm{C}_{26} \mathrm{H}_{28} \mathrm{O}_{4}$}

Monachosorin A, 14911

Monachosorin C, 14913
Sigmoidin E, 19888

$\mathrm{C}_{26} \mathrm{H}_{28} \mathrm{O}_{5}$

Derrisisoflavone A, 5229

Erypoegin J, 7316

Lyfoline, 13244

Olibergin B, 16083

\section{$\mathrm{C}_{26} \mathrm{H}_{28} \mathrm{O}_{6}$}

Burttinonedehydrate, 2774

Derrisisoflavone C, 5231

Derrisisoflavone D, 5232

Derrisisoflavone E, 5233

Desmodianone D, 5264

Desmodianone E, 5265

5,4'-Dihydroxy-2'-methoxy-8-(3,3-di-methylallyl)-2",2"-dimethylpyrano[5,6:6,7] iso-

flavanone, 5971

5,2'-Dihydroxy-3-methoxy-6,7-(2",2"-dimethylchromene)-8-(3"',3"'-dimethylallyl)flavanone, 5972

Kanzonol K, 12150

(+)-(10S,10'S)-Ligulacephalin A, 12806

(-)-(10R,10'R)-Ligulacephalin A, 12807

Ugonin B, 22175

Ugonin K, 22184

Ugonin L, 22185

\section{$\mathrm{C}_{26} \mathrm{H}_{28} \mathrm{O}_{7}$}

Artocommunol CC, 1812

Broussoflavonol B, 2632

Dorsmanin D, 6564

(+)-(10R,10'R)-Ligulacephalin B, 12808

(-)-(10S,10'S)-Ligulacephalin B, 12809

5-O-Methyl kushenol C, 14545

5,7,3',4'-Tetrahydroxy-3-methoxy-6-geranyl-

flavone , 21128

Ugonin H, 22181

$\mathrm{C}_{26} \mathrm{H}_{28} \mathrm{O}_{8}$

Jangomolide, 11816

$\mathrm{C}_{26} \mathrm{H}_{28} \mathrm{O}_{9}$

Evodol, 7669

Sudachinoid C, 20450

$\mathrm{C}_{26} \mathrm{H}_{28} \mathrm{O}_{10}$

Baohuoside II, 2139

$12 \alpha$-Hydroxyevodol, 10118

\section{$\mathrm{C}_{26} \mathrm{H}_{28} \mathrm{O}_{11}$}

Epimedoside C, 6964

2"-O-(2"'-Methylbutyryl)vitexin, 14203

$5 \alpha, 6 \beta, 7 \beta, 8 \alpha$-Tetraacetoxy-2-[2-(4'-methoxy-

phenyl)ethyl]-5,6,7,8-tetrahydro-chromone, 21030

\section{$\mathrm{C}_{26} \mathrm{H}_{28} \mathrm{O}_{12}$}

Isoscutellarein 4 '-methyl ether 8 - $O$ - $\beta$ - $D$-glucuronide 6"-n-butyl ester, 11708

Leptostachyol acetate, 12680
2"-O-(2"'-Methylbutyryl)orientin, 14196

Styraxlignolide B, 20423

\section{$\mathrm{C}_{26} \mathrm{H}_{28} \mathrm{O}_{13}$}

Apigenin-bioside, 1482

6-C-Arabinopyranosyl-8-C-glucopyranosyl-

5,7-dihydroxyflavone, 1568

8-C-Arabinopyranosyl-6- $C$-glucopyranosyl-

5,7-dihydroxyflavone, 1569

Daphneticin-4"- $O-\alpha$ - $D$-glucopyranoside, 4644

Puerarin-xyloside I, 18182

Puerarin xyloside II, 18183

Rubiadin primeveroside, 19000

\section{$\mathrm{C}_{26} \mathrm{H}_{28} \mathrm{O}_{14}$}

Apigenin-4'- $O-\beta$ - $D$-xylofuranosyl $(1 \rightarrow 4)-O-\beta$ - $D$ glucopyranoside, 1500

Apiin, 1502

6"- $\beta$-D-Arabinose-genistin, 1594

6- $C$-Arabinosyl-8- $C$-glucosyl apigenin, 1595

Artabotryside B, 1778

Isoschaftoside, 11697

Kaempferol-3- $O-\alpha-L$-rhamnosyl $(1 \rightarrow 2)-\beta-D$ xyloside, 12090

Kaempferol-3-rhamno-4'-xyloside, 12091

Lucidin primeveroside, 13045

Morindin, 14973

Neoschaftoside, 15458

Schaftoside, 19463

Sorbifolin, 20103

5,7,4'-Trihydroxy-6- $C$-glucoside- 8 - $C$-arabinoside flavone, 21738

Vicenin 1, 22464

2 "-O- $\beta$-D-Xylopyranosylisovitexin, 22836

$6 "-\beta-D$-Xylose-genistin, 22844

$O$-D-Xylosylvitexin, 22854

Yopaaoside B, 22927

\section{$\mathrm{C}_{26} \mathrm{H}_{28} \mathrm{O}_{15}$}

Artabotryside A, 1777

Carlinoside, 3199

Graveobioside A, 8990

Isocarlinoside, 11306

Kaempferol 3-O- $\alpha$-arabinopyranosyl $(1 " ' \rightarrow 6 ")-\beta$ glucopyranoside, 12028

Neocarlinoside, 15355

Phlomisflavoside B, 17166

Quercetin-3- $O-\beta$ - $D$-xylose-( $1 \rightarrow 4)-\alpha-L$-rhamnoside, 18408

1,3,6-Trihydroxy-2-hydroxymethyl-9,10-anthraquinone 3-O- $\beta$-Primeveroside, 21746

\section{$\mathrm{C}_{26} \mathrm{H}_{28} \mathrm{O}_{16}$}

Phlomisflavoside A, 17165

Quercetin-3- $L$-arabino-7- $D$-glucoside, 18322

Quercetin-3-O- $\alpha$ - $L$-arabinopyranosyl-( $(\rightarrow 2)$ -

$\beta$ - $D$-galactopyranoside, 18326 
Quercetin-3-O- $\alpha$-arabinopyranosyl(1"' $\rightarrow 6$ ")-

$\beta$-glucopyranoside, 18327

\section{$\mathrm{C}_{26} \mathrm{H}_{29} \mathrm{NO}_{5}$}

Vertine, 22433

\section{$\mathrm{C}_{26} \mathrm{H}_{29} \mathrm{O}_{15}{ }^{+}$}

Cyanidin-3-xylosyl-glucoside, 4450

Lycoricyanin' 13240

\section{$\mathrm{C}_{26} \mathrm{H}_{30} \mathrm{~N}_{2} \mathrm{O}_{8}$}

Strictosamide, 20390

Vincosamide, 22496

\section{$\mathrm{C}_{26} \mathrm{H}_{30} \mathrm{~N}_{2} \mathrm{O}_{9}$}

10-Hydroxystrictosamide, 10733

Lyalosidic acid, 13168

\section{$\mathrm{C}_{26} \mathrm{H}_{30} \mathrm{O}_{4}$}

Lakoochin A, 12453

Monachosorin B, 14912

Panduratin A, 16603

Tunicatachalcone, 22110

\section{$\mathrm{C}_{26} \mathrm{H}_{30} \mathrm{O}_{5}$}

Abyssinone V-4'-methyl ether, 55

(5Ar,6R,9R,9Ar)-4-Cinnamoyl-3,6-dihydroxy-1methoxy-6-methyl-9-(1-methylethyl)-5a,6,7,8, 9a-hexahydro-dibenzofuran, 3702

Eryzerin B, 7355

Eryzerin E, 7358

Kosamol R, 12282

Kosamol U, 12285

5'-Prenylxanthohumol, 17843

Sigmoidin A, 19883

\section{$\mathrm{C}_{26} \mathrm{H}_{30} \mathrm{O}_{6}$}

Amoradicin, 1071

Fuscaxanthone C, 8032

Isokurarinone, 11474

Kuraridin, 12341

Kurarinone, 12344

Leachianone A, 12595

\section{$\mathrm{C}_{26} \mathrm{H}_{30} \mathrm{O}_{7}$}

1-Hydroxy-8-(2-hydroxy-3-methylbut-3-enyl)3,6,7-trimethoxy-2-(3-methylbut-2-enyl)-xanthone, 10199

Kurarinol, 12343

Kushenol I, 12362

Kushenol N, 12367

6-O-Methylmangostanin, 14577

Obacunone, 15884

(+)-Pinoresinol-3-hydroxy-4-methyl-4-pentenyl ether, 17417

\section{$\mathrm{C}_{26} \mathrm{H}_{30} \mathrm{O}_{8}$}

Butyrylma Ilotochromene, 2810

Drummondin A, 6606

Isobutyrylmallotochromene, 11295

Limonin, 12847

Mallotophilippen B, 13434
Obaculactone, 15882

11-Oxo-7 $\alpha$-obacunol, 16397

Zapoterin, 22971

\section{$\mathrm{C}_{26} \mathrm{H}_{30} \mathrm{O}_{9}$}

Egonol glucoside, 6717

Evodinone, 7668

$12 \alpha$-Hydroxylimonin, 10325

Kihadanin B, 12226

\section{$\mathrm{C}_{26} \mathrm{H}_{30} \mathrm{O}_{10}$}

Glaucin A, 8512

Massonianoid A, 13582

(2R)-Phellodensin F, 17074

Shihulimonin A, 19801

\section{$\mathrm{C}_{26} \mathrm{H}_{30} \mathrm{O}_{11}$}

Balanophonin-4- $O-\beta$ - $D$-glucopyranoside, 2126

Dracunculifoside D, 6583

Phellamurin, 17053

Phellodendroside, 17066

Simplexoside, 19903

\section{$\mathrm{C}_{26} \mathrm{H}_{30} \mathrm{O}_{12}$}

Amurensin, 1091

6- $\gamma, \gamma$-Dimethylallyltaxifolin 7-O- $\beta$ - $D$-glucoside, 6311

Noricariside, 15761

Phellatin, 17057

\section{$\mathrm{C}_{26} \mathrm{H}_{30} \mathrm{O}_{13}$}

Angustiamarin, 1243

6-O-E-p-Coumaroyl scandoside methyl ester, 4185

6-O-Z-p-Coumaroyl scandoside methyl ester, 4186

Dunnisinoside, 6640

Licuroside, 12796

Liquiritigenin-7- $O-\beta$ - $D$-apiofuranosyl)-4' $-O-\beta$ $D$-glucopyranoside, 12910

Liquiritigenin

4'-O- $\beta$ - $D$-apio- $D$-furanosyl $(1 \rightarrow 2)-\beta$ - $D$-glucopy ranoside, 12911

(2S)-Pinocembrin

$$
\text { 7-O-[ } \beta \text { - } D \text {-apiosyl }(1 \rightarrow 2)]-\beta \text { - } D \text {-glucoside, }
$$
17405

Pinocembrin-7-O- $\alpha$-arabinopyranosyl-( $(1 \rightarrow 2)$ $\beta$-glucopyranoside, 17406

Styraxjaponoside A, 20420

\section{$\mathrm{C}_{26} \mathrm{H}_{30} \mathrm{O}_{14}$}

Angustioside, 1251

2'-O-[ $\beta$ - $D$-Apiofuranosyl $(1 \rightarrow 2)-\beta$ - $D$-glucopyranosyl]isoliquiritigenin, 1511

6-[( $\alpha$-Apiofuranosyl-( $1 \rightarrow 6)-O-\beta$ - $D$-glucopyranosyl)oxyrubrofusarin], 1514

6'-O-E-Feruloylmonotropein, 7779

10-O-E-Feruloylmonotropein, 7780

$\mathrm{C}_{26} \mathrm{H}_{30} \mathrm{O}_{15}$
Asperuloside tetraacetate, 1893

Polygalaxanthone $\mathrm{V}, 17633$

$\mathrm{C}_{26} \mathrm{H}_{30} \mathrm{O}_{16}$

Vijayosine, 22477

$\mathrm{C}_{26} \mathrm{H}_{31} \mathrm{NO}_{5}$

Decamine, 4832

Decinine, 4848

Lagerstremine, 12450

Methyl lagerine, 14547

\section{$\mathrm{C}_{26} \mathrm{H}_{32} \mathrm{~N}_{2} \mathrm{O}_{9}$}

Isomitraphyllic acid (16 $\rightarrow 1)-\beta$ - $D$-glucopyranosyl ester, 11538

Mitraphyllic acid ( $16 \rightarrow 1)-\beta$ - $D$-gluco-pyranosyl ester, 14888

Strictosidinic acid, 20391

\section{$\mathrm{C}_{26} \mathrm{H}_{32} \mathrm{O}_{4}$}

Nimbocinol, 15603

\section{$\mathrm{C}_{26} \mathrm{H}_{32} \mathrm{O}_{5}$}

Licoricidin, 12793

Polyanthinin, 17620

Tecleanin, 20895

\section{$\mathrm{C}_{26} \mathrm{H}_{32} \mathrm{O}_{6}$}

Kleinioxanthrone 1, 12233

( \pm -trans-3-(2,4,5-Trimethoxyphenyl)-4-[(E)2,4,5-trimethoxystyryl]-cyclohexene, 21931

1,2-bis(2,4,5-Trimethoxy-Z-styryl)cyclobutane, 21932

cis-1-(2,4,5-Trimethoxy-E-styryl)-2-(2,4,5-trimethoxy-Z-styryl)cyclobutane, 21933

trans-1-(2,4,5-Trimethoxy-E-styryl)-2-(2,4,5-trimethoxy-Z-styryl)cyclobutane, 21934

\section{$\mathrm{C}_{26} \mathrm{H}_{32} \mathrm{O}_{7}$}

Assafoetidnol B, 1911

Kuraridinol, 12342

Kushenol P, 12369

Quassinoid PC03-579B, 18301

\section{$\mathrm{C}_{26} \mathrm{H}_{32} \mathrm{O}_{8}$}

2-Acetoxycaesaldekarin E, 138

$6 \beta$-Acetoxy- $3 \beta, 8 \beta, 14 \beta$-trihydroxy-12-oxobufa4,20,22-trienolide, 300

Bryophyllin A, 2689

Butyrylmallotojaponin, 2811

Deacetylnomilin, 4757

Kushenol H, 12361

Kushenol K, 12364

Longirabdosin, 12977

Obacunoic acid, 15883

\section{$\mathrm{C}_{26} \mathrm{H}_{32} \mathrm{O}_{9}$}

Isolimonic acid $(16 \rightarrow 17)$ lactone, 11493

Limonoic acid A ring lactone, 12848

\section{$\mathrm{C}_{26} \mathrm{H}_{32} \mathrm{O}_{10}$}

Gaultherin B, 8241

5-[3"-( $\beta$ - $D$-Glucopyranosyloxy)propyl]-7-me- 


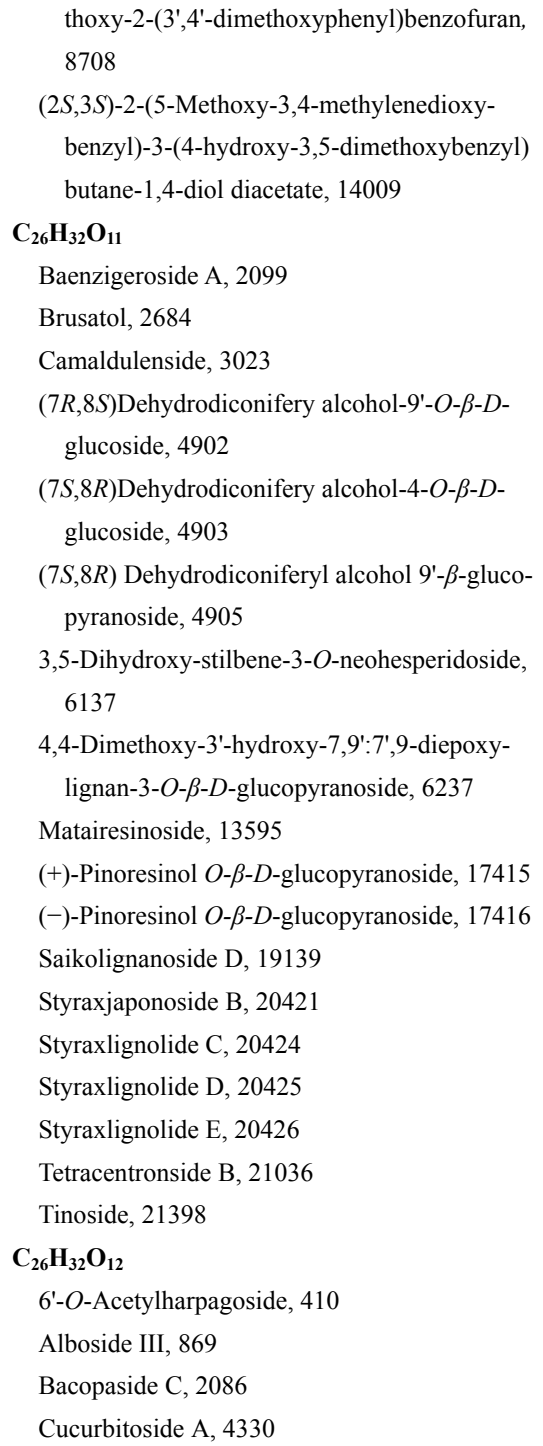

\section{$\mathbf{C}_{26} \mathrm{H}_{32} \mathbf{O}_{12}$}

6'-O-Acetylharpagoside, 410

Alboside III, 869

Bacopaside C, 2086

Cucurbitoside A, 4330

(+)-1-Hydroxypinoresinol-4'-O- $\beta$ - $D$-glucopyra noside, 10654

(+)-1-Hydroxypinoresinol-4"- $O-\beta$ - $D$-glucopyra noside, 10655

Luzonoside C, 13166

Luzonoside D, 13167

Nortrachelogenin-5'- $C$ - $\beta$ - $D$-glucopyranoside, 15807

Nortrachelogenin-8'- $O-\beta$ - $D$-glucopyranoside, 15808

\section{Nortracheloside, 15809}

Phellavin, 17058

(7S,8S)-Syringoylglycerol 9-O-(6'-O-cinnamoyl)- $\beta$ - $D$-glucopyranoside, 20570

\section{$\mathrm{C}_{26} \mathrm{H}_{32} \mathrm{O}_{13}$}

Alboside I, 867

Cucurbitoside B, 4331
Dichotomoside A, 5427

Glehlinoside C, 8547

Grandifloroside 11-methyl ester, 8978

2-(4-Hydroxyphenyl)ethyl-1- $O-\beta-D-[5-O-(4-$ hydroxybenzoyl)]-apiofuranosyl-( $(1 \rightarrow 6)-\beta-D$ glucopyranoside, 10621

Lactucaside, 12444

Resveratrol 3,4'-O, $O^{\prime}$-di- $\beta$ - $D$-glucopyranoside, 18647

Tenuiphenone A, 20959

\section{$\mathbf{C}_{26} \mathbf{H}_{32} \mathbf{O}_{14}$}

3,4-Dimethoxyphenyl $1-O-\beta$ - $D$-[5-O-(4-hydroxybenzoyl)]-apiofuranosyl-(1 $\rightarrow 6)-\beta$ - $D$-glucopyranoside, 6286

Mulberroside A, 15058

2,3,5,4'-Tetrahydroxystilbene-2-O-(6"-O- $\alpha-D$ glucopyranosyl)- $\beta$ - $D$-glucopyranoside, 21156

$2,3,5,4$ '-Tetrahydroxystilbene-2,3-O- $\beta$ - $D$-diglucoside, 21159

\section{$\mathrm{C}_{26} \mathrm{H}_{32} \mathrm{O}_{15}$}

Seguinoside F, 19669

\section{$\mathrm{C}_{26} \mathrm{H}_{32} \mathrm{O}_{16}$}

Leucocyanidin-3-O- $\alpha-D$-glucopyranosyl-( $1 \rightarrow 4)$ $O-\beta$ - $D$-arabinopyranoside, 12716

\section{$\mathrm{C}_{26} \mathrm{H}_{33} \mathrm{ClO}_{10}$}

Junceellolide C, 11945

$\mathrm{C}_{26} \mathrm{H}_{33} \mathrm{ClO}_{12}$

Juncenolide G, 11954

$\mathrm{C}_{26} \mathrm{H}_{33} \mathrm{NO}_{7}$

Guan-fu base G, 9047

\section{$\mathrm{C}_{26} \mathrm{H}_{34} \mathrm{O}_{4}$}

Gerronemin E, 8364

Kazinol J, 12189

\section{$\mathrm{C}_{26} \mathrm{H}_{34} \mathrm{O}_{5}$}

Driportlandin, 6604

\section{$\mathrm{C}_{26} \mathrm{H}_{34} \mathrm{O}_{6}$}

Cinobufagin, 3729

Samarcandin acetate, 19225

\section{$\mathrm{C}_{26} \mathrm{H}_{34} \mathrm{O}_{7}$}

Ardisinone B, 1646

Cinobufaginol, 3731

Cinobufotalin, 3732

$1 \alpha, 8 \beta$-Diacetoxy- $9 \beta$-benzoyloxydihydro- $\beta$ agarofuran, 5286

Forrestin G, 7921

Hellebrigenin 3-acetate, 9329

$6 \alpha$-Hydroxycinobufagin, 9917

$12 \beta$-Hydroxycinobufagin, 9918

1-O-Methylichangensin, 14524

\section{$\mathrm{C}_{26} \mathrm{H}_{34} \mathrm{O}_{8}$}

Agrimophol, 762

Caesalmin C, 2865
12 $\beta$-Hydroxyscillirosidin, 10693

Nigakilactone G, 15552

Saroaspidin C, 19384

\section{$\mathrm{C}_{26} \mathrm{H}_{34} \mathrm{O}_{9}$}

Acetylexidonin, 393

Adenanthin, 605

Bryophyllin B, 2690

Crocetin mono( $\beta$ - $D$-glucosyl) ester, 4247

3,5-Diacetoxy-1-(4-hydroxy-3,5-dimethoxyphenyl)-7-(4-hydroxy-3-methoxyphenyl)

heptane, 5302

Glabcensin Q, 8478

Leucophyllin A, 12719

Lungshengenin G, 13083

Rabdoepigibberellolide, 18460

Shikokianal acetate, 19813

Shikokianidin, 19814

Sudachinoid A, 20448

11-O- $\beta$ - $D$-Xylopyranosylmyricanol, 22837

\section{$\mathrm{C}_{26} \mathrm{H}_{34} \mathrm{O}_{10}$}

Aviculin, 2040

Baiyecrystal A, 2116

2,3-Dihydrobenzofuran-2-(4'-hydroxy-3'-methoxyphenyl)-3- $\alpha-L$-rhamnopyranosyloxymet hyl-7-methoxy-5-propanol, 5547

2',3'-Dihydroxy-1'-propoxypseudolarate B, 6104 16,17-Epoxyshikokianal acetate, 7202

(+)-Isolariciresinol-9'-O- $\alpha$ - $L$-rhamnoside, 11482 Massonianoside C, 13585

Massonianoside D, 13586

$1 \alpha, 8 \beta, 14$-Triacetoxy- $9 \beta$-furoyloxydihydro- $\beta$ agarofuran, 21522

\section{$\mathrm{C}_{26} \mathrm{H}_{34} \mathrm{O}_{11}$}

Bruceine A, 2664

Dihydrodehydrodiconiferyl alcohol 4'- $O-\beta-D-$ glucoside, 5583

(-)-Isolariciresinol-4- $O-\beta$ - $D$-glucopyranoside, 11481

(+)-1-Lariciresinol-4'- $\beta$ - $D$-glucopyranoside, 12522

(+)-Lariciresinol-4- $\beta$ - $D$-glucopyranoside, 12523

Lariciresinol-9- $O-\beta$ - $D$-glucoside, 12525

(-)-5'-Methoxyisolariciresinol-2 $\alpha-O-\beta-D$-xylopyranoside, 13977

Neoglabrescin B triacetate, 15399

Saikolignanoside A, 19138

\section{$\mathrm{C}_{26} \mathrm{H}_{34} \mathrm{O}_{12}$}

Berchemol-4'-O- $\beta$ - $D$-glucoside, 2305

Citrusin A, 3775

Hyuganoside IIIa, 10924

Hyuganoside IIIb, 10925

Mallophenol B, 13425

$7 R, 7^{\prime} R, 8 S, 8^{\prime} S$-(+)-Neo-olivil-4- $O-\beta$ - $D$-glucopyra- 
noside, 15441

Neoolivil-4- $O-\beta$ - $D$-glucoside, 15442

(-)-Olivil-4"-O- $\beta$ - $D$-glucopyranoside, 16090

(-)-Olivil-4'-O- $\beta$ - $D$-glucopyranoside, 16091

Sinenoside, 19926

Tamariscinoside C, 20655

Tanegoside, 20669

\section{$\mathrm{C}_{26} \mathrm{H}_{34} \mathrm{O}_{13}$}

Juncin Q, 11957

(-)-Massoniresinol 4'- $O$ - $\beta$ - $D$-glucopyranoside, 13589

(-)-Massoniresinol 4"- $O$ - $\beta$ - $D$-glucopyranoside, 13590

Osthenol-7- $O$ - $\beta$-gentiobioside, 16258

\section{$\mathrm{C}_{26} \mathrm{H}_{34} \mathrm{O}_{14}$}

Pyrolaside A, 18268

\section{$\mathrm{C}_{26} \mathrm{H}_{35} \mathrm{NO}_{4}$}

Lythranidine, 13279

\section{$\mathrm{C}_{26} \mathrm{H}_{35} \mathrm{NO}_{6}$}

Guan-fu base F, 9046

Spiramide, 20193

\section{$\mathrm{C}_{26} \mathrm{H}_{35} \mathrm{~N}_{5} \mathrm{O}_{8}$}

Asterin B, 1923

\section{$\mathrm{C}_{26} \mathrm{H}_{36} \mathrm{NO}_{11}$}

L-Phenylalaninosecologanin, 17094

\section{$\mathrm{C}_{26} \mathrm{H}_{36} \mathrm{O}_{4}$}

Gerronemin D, 8363

4-Hydroxy-2,6-dimethyl-6-(3,7-dimethyl-2,6octadienyl)-8-(3-methyl-2-butenyl)-2H-1-benzopyran-5,7(3H,6H)-dione, 10049

5-Hydroxy-2,8-dimethyl-6-(3-methyl-2-butenyl)8-(3,7-dimethyl-2,6-octadienyl)-2H-1-benzopyran-4,7(3H,8H)-dione, 10051

Lupulone A, 13110

Papuaforin A, 16629

\section{$\mathrm{C}_{26} \mathrm{H}_{36} \mathrm{O}_{6}$}

Bufotalin, 2722

15-O-Methyl-18-oxoneovibsanin F, 14648

\section{$\mathrm{C}_{26} \mathrm{H}_{36} \mathrm{O}_{7}$}

$5 \beta$-Hydroxybufotalin, 9861

$9 \alpha$-Hydroxy- $2 \alpha, 10 \beta, 13 \alpha$-triacetoxytaxa-4(20), 5(6),11(12)-triene, 10781

$\left(2 R^{*}, 3 S^{*}, 4 R^{*}, 5 R^{*}\right.$,

$\left.9 S^{*}, 11 S^{*}, 15 R^{*}\right)-3,5,15$-Triacetoxy-14-oxolathyra-6(17), $12 E$-diene, 21530

\section{$\mathrm{C}_{26} \mathrm{H}_{36} \mathrm{O}_{8}$}

Adenanthin C, 607

Casearinone A, 3272

Casearinone B, 3273

Dawoensin A, 4701

$3 \beta, 6 \beta$-Diangeloyloxy-10 $\alpha$-hydroxy- $8 \alpha$-methoxyeremophilenolide, 5356

Enmenin monoacetate, 6807
Isodoternifolin A, 11400

Leucamenin C, 12710

Leucamenin D, 12711

Lungshengenin C, 13078

Lungshengenin C, 13079

Lungshengenin F, 13082

Rabyuennane B, 18511

Shikodokaurin A, 19808

Shikodokaurin B, 19809

Taxinine A, 20798

Taxuspinanane K, 20848

Trillenogenin, 21872

\section{$\mathrm{C}_{26} \mathrm{H}_{36} \mathrm{O}_{9}$}

4-Acetoxy-2,3-bis(3,4,5-trimethoxybenzyl)-1butanol, 136

Adenanthin J, 614

Adenanthin K, 615

Caesalmin D, 2866

Caesalmin E, 2867

Caryoptin, 3246

2-Deacetyltaxine B, 4781

14-Deoxo-14-O-acetylorthosiphol Y, 5137

Glabcensin C, 8464

Glabcensin D, 8465

Glabcensin E, 8466

Glabcensin F, 8467

Glabcensin G, 8468

Glabcensin U, 8482

1-Hydroxytaxinine A, 10739

Leucophyllin D, 12722

Lungshengenin A, 13076

Lushanrubescensin B, 13117

Melissoidesin B, 13689

Phlogacanthoside B, 17162

Rabdosianin A, 18487

Taxezopidine A, 20780

Taxinine A 11,12-epoxide, 20799

Taxuspine W, 20870

$2 \alpha, 7 \beta, 13 \alpha$-Triacetoxy- $5 \alpha, 9 \alpha$-dihydroxy-2(3 $\rightarrow 20)$ abeotaxa-4(20),11-dien-10-one, 21515

(3E,7E)-2 $\alpha, 10 \beta, 13 \alpha$-Triacetoxy-5 $\alpha, 20$-dihydroxy-3,8-seco-taxa-3,7,11-trien-9-one, 21516

Weisiensin A, 22657

\section{$\mathrm{C}_{26} \mathrm{H}_{36} \mathrm{O}_{10}$}

Ajugalide A, 795

Ajugalide B, 796

(3R,5R)-3,5-Dihydroxy-1-(4-hydroxy-3-methoxyphenyl)-7-(4-hydroxyphenyl)heptane 3-O- $\beta$-D-glucopyranoside, 5913

Nortrilobolide, 15811

Taxumairol J, 20830

Taxuspine O, 20862

$\mathrm{C}_{26} \mathrm{H}_{36} \mathrm{O}_{11}$
$2 \alpha$-Deacetyl-5 $\alpha$-decinnamoyltaxagifine, 4736

(3R,5R)-3,5-Dihydroxy-1-(4-hydroxy-3-methoxyphenyl)-7-(3,4-dihydroxyphenyl)heptane 3-O- $\beta$ - $D$-glucopyranoside, 5912

Javanicolide C, 11853

(-)-Secoisolariciresinol

4-O- $\beta$ - $D$-glucopyranoside, 19619

\section{$\mathrm{C}_{26} \mathrm{H}_{36} \mathrm{O}_{12}$}

10-Acetoxy-1-acevaltrate hydrin, 121

Foliamenthin, 7851

Menthiafolin, 13773

Tamariscinoside B, 20654

7,9,9'-Trihydroxy-3,3'-dimethoxy-8-O-4'-neolignan-4- $O-\beta$ - $D$-glucopyranoside, 21714

\section{$\mathrm{C}_{26} \mathrm{H}_{36} \mathrm{O}_{13}$}

Amritoside A, 1084

Dracunculifoside P, 6595

\section{$\mathrm{C}_{26} \mathrm{H}_{36} \mathrm{O}_{14}$}

Eurycomanol-2- $O$ - $\beta$-glucopyranoside, 7651

\section{$\mathrm{C}_{26} \mathrm{H}_{36} \mathrm{O}_{15}$}

Seguinoside K, 19674

\section{$\mathrm{C}_{26} \mathrm{H}_{37} \mathrm{NO}_{3}$}

Brachystamide C, 2575

\section{$\mathrm{C}_{26} \mathrm{H}_{37} \mathrm{NO}_{8}$}

(-)-(trans-3'-Methoxy-4'- $\alpha-L$-rhamnosyloxy cinnamoyl)epilupinine, 14084

\section{$\mathrm{C}_{26} \mathrm{H}_{37} \mathrm{~N}_{3} \mathrm{O}_{4}$}

Discarine M, 6505

\section{$\mathrm{C}_{26} \mathrm{H}_{38} \mathrm{O}_{3}$}

Papuaforin B, 16630

\section{$\mathrm{C}_{26} \mathrm{H}_{38} \mathrm{O}_{4}$}

Gerronemin C, 8362

Hyperpapuanone, 10889

Lupulone, 13109

\section{$\mathrm{C}_{26} \mathrm{H}_{38} \mathrm{O}_{5}$}

Lupulone C, 13112

Lupulone D, 13113

Methyl butyric acid 3,14-dehydro-Z-tussilagin ester, 14187

15-O-Methyl-14-epi-neovibsanin F, 14399

15-O-Methylneovibsanin F, 14615

2-O-Methylneovibsanin H, 14616

2-O-Methylneovibsanin I, 14617

\section{$\mathrm{C}_{26} \mathrm{H}_{38} \mathrm{O}_{6}$}

Methyl ent-7 $\alpha, 9 \alpha$-dihydroxy-15 $\beta-[(2 Z)-2-$ methyl-but-2-enoyloxy]kaur-16-en-19-oate, 14394

18-O-Methylvibsanin G, 14807

\section{$\mathrm{C}_{26} \mathrm{H}_{38} \mathrm{O}_{7}$}

10-Deacetyltaxuyunnanine C, 4788

$2 \alpha$-Hydroxy- $5 \alpha, 10 \beta, 14 \beta$-triacetoxytaxa-4(20), 11-diene, 10779

Hydroxytriacetoxytaxadiene, 10780 
18-O-Methyl-5-epi-vibsanin K, 14404

18-O-Methylvibsanin K, 14808

Neovibsanin D, 15472

7-epi-Neovibsanin D, 15473

Pierisformoside C, 17354

\section{$\mathrm{C}_{26} \mathrm{H}_{38} \mathrm{O}_{8}$}

Adenanthin B, 606

2-Deacetyldecinnamoyltaxinine E, 4737

Melissoidesin R, 13704

Melissoidesin U, 13707

3-Methyl-1-\{2-[(1R*,2 $\left.S^{*}, 5 R^{*}, 6 R^{*}\right)$-2,5,6-tri (acetyloxy)-4-methyl-3-cyclohexenyl]-propyl\}-2-butenyl (Z)-2-methyl-2-butenoate, 14767

Xindongnin D, 22800

\section{$\mathrm{C}_{26} \mathrm{H}_{38} \mathrm{O}_{9}$}

Baiyecrystal C, 2118

Epi-baiyecrystal C, 6834

Forrestin C, 7917

Forrestin E, 7919

Glabcensin L, 8473

Glabcensin M, 8474

$\beta$-D-Glucopyranosyl-8(17),13-ent-labdadien16,15-olid-18-oate, 8680

Phlogacanthoside A, 17161

Taxane 2, 20749

Taxawallin F, 20755

Taxawallin G, 20756

Taxawallin H, 20757

$7 \beta, 9 \alpha, 10 \beta$-Triacetoxy- $2 \alpha, 5 \alpha, 13 \alpha$-trihydroxy4(20),11-taxadiene, 21532

\section{$\mathrm{C}_{26} \mathrm{H}_{38} \mathrm{O}_{10}$}

10-Debenzoyl-2 $\alpha$-acetoxy-brevifoliol, 4801

Taxezopidine B, 20781

$5 \alpha, 7 \beta, 10 \beta, 13 \alpha$-Tetrahydroxy- $2 \alpha, 9 \alpha, 15$-triacetoxy-11(15 $\rightarrow 1)$-abeo-taxa-4(20),11-diene, 21161

\section{$\mathrm{C}_{26} \mathrm{H}_{38} \mathrm{O}_{11}$}

1-Hydroxy-2,7,9-trideacetylbaccatin I, 10782

Tasumatrol B, 20719

Taxumairol Y, 20838

\section{$\mathrm{C}_{26} \mathrm{H}_{38} \mathrm{O}_{12}$}

Agnucastoside A, 750

Dihydrofoliamenthin, 5615

Jasnudifloside I, 11829

Jasnudifloside J, 11830

Pratialin A, 17766

$\mathrm{C}_{26} \mathrm{H}_{38} \mathrm{O}_{13}$

Casteloside B, 3299

Iandonoside A, 10935

Lobetyolinin, 12943

$\mathrm{C}_{26} \mathrm{H}_{38} \mathrm{O}_{14}$

Bruceine E 2- $\beta$ - $D$-glucopyranoside, 2669
Iandonoside B, 10936

$\mathrm{C}_{26} \mathrm{H}_{39} \mathrm{NO}_{6}$

8-Acetyldolaconine, 384

$\mathrm{C}_{26} \mathrm{H}_{39} \mathrm{NO}_{8}$

$3 \beta$-Acetoxynorerythrosuamine, 265

$\mathrm{C}_{26} \mathrm{H}_{40} \mathrm{O}_{4}$

2-n-Pentadecyl-5,7-dihydroxy-6,8-dimethyl chromone, 16834

\section{$\mathrm{C}_{26} \mathrm{H}_{40} \mathrm{O}_{5}$}

Vibsanin Q, 22452

$\mathrm{C}_{26} \mathrm{H}_{40} \mathrm{O}_{6}$

Siegesetheric acid I, 19878

$\mathrm{C}_{26} \mathrm{H}_{40} \mathrm{O}_{6}$

Taxuyunnanine I, 20881

$\mathrm{C}_{26} \mathrm{H}_{40} \mathrm{O}_{\mathbf{8}}$

3,4-Dihydro-excelsin, 5612

\section{$\mathrm{C}_{26} \mathrm{H}_{40} \mathrm{O}_{8}$}

18- $\beta$-D-Glucopyranosyloxy-8(17),13-ent-labdadien-16,15-olide, 8700

\section{$\mathrm{C}_{26} \mathrm{H}_{40} \mathrm{O}_{8}$}

Neoandrographolide, 15337

\section{$\mathrm{C}_{26} \mathrm{H}_{40} \mathrm{O}_{8}$}

Pierisformoside F, 17357

\section{$\mathrm{C}_{26} \mathrm{H}_{40} \mathrm{O}_{9}$}

Cussoracoside E, 4427

\section{$\mathrm{C}_{26} \mathrm{H}_{40} \mathrm{O}_{9}$}

Melissoidesin D, 13691

$\mathrm{C}_{26} \mathrm{H}_{40} \mathrm{O}_{9}$

Pierisformoside D, 17355

$\mathrm{C}_{26} \mathrm{H}_{40} \mathrm{O}_{9}$

Pierisformoside E, 17356

\section{$\mathrm{C}_{26} \mathrm{H}_{40} \mathrm{O}_{10}$}

Andrographiside, 1158

(3S)-3-O-(3',4'-Diangeloyl- $\beta$ - $D$-glucopyranosyloxy)-6-hydroperoxy-3,7-dimethylocta-1,7-diene, 5354

(3S)-3-O-(3',4'-Diangeloyl- $\beta$ - $D$-glucopyranosyloxy)-7-hydroperoxy-3,7-dimethylocta-1,5-dien e, 5355

Isodoforrestin, 11383

Shikokiaside B, 19818

$\mathrm{C}_{26} \mathrm{H}_{40} \mathrm{O}_{11}$

Parvifoliside, 16687

Rabdoside 1, 18494

Shikokiaside A, 19817

Taxuyunnanine Q, 20884

$\mathrm{C}_{26} \mathrm{H}_{40} \mathrm{O}_{12}$

Agnucastoside B, 751

Rabdoside 2, 18495

$\mathrm{C}_{26} \mathrm{H}_{40} \mathrm{O}_{15}$

LigurobustosideM, 12822

$\mathrm{C}_{26} \mathrm{H}_{41} \mathrm{NO}$

Buxbodine C, 2820
$\mathrm{C}_{26} \mathrm{H}_{41} \mathrm{NO}_{2}$

Buxbodine B, 2819

$\mathrm{C}_{26} \mathrm{H}_{41} \mathrm{NO}_{7}$

Akirane, 821

Bullatine C, 2739

Delbruline, 4999

Delcorine, 5003

$\mathrm{C}_{26} \mathrm{H}_{41} \mathrm{NO}_{8}$

Pubescenine, 18174

$\mathrm{C}_{26} \mathrm{H}_{42} \mathrm{O}_{4}$

Aculeatin D, 589

Maesaquinone, 13350

Pallasone C, 16544

$\mathrm{C}_{26} \mathrm{H}_{42} \mathrm{O}_{6}$

Acetylcholic acid, 350

$\mathbf{C}_{\mathbf{2 6}} \mathrm{H}_{\mathbf{4 2}} \mathrm{O}_{7}$

4,5,6-Tri- $O$-acetyl hygrophorone $\mathrm{B}^{14}, 21536$

\section{$\mathrm{C}_{26} \mathrm{H}_{42} \mathrm{O}_{8}$}

ent-2-Oxo-15,16-dihydroxypimar-8(14)-en-16-

$O$ - $\beta$-glucopyranoside, 16320

Pierisformoside B, 17353

Pterokaurane $\mathrm{P}_{1}$-2-O- $\beta$ - $D$-glucoside, 18108

\section{$\mathrm{C}_{26} \mathrm{H}_{42} \mathrm{O}_{9}$}

Cussoracoside A, 4423

Isodopharicin $\mathrm{F}, 11399$

ent-2-Oxo-3 $\beta, 15,16$-trihydroxypimar-8(14)-en-

3-O- $\beta$-glucopyranoside, 16436

Pubeside C, 18177

Pubeside D, 18178

Rhodomoside A, 18812

Sumogaside, 20486

\section{$\mathrm{C}_{26} \mathrm{H}_{42} \mathrm{O}_{10}$}

Rhodomoside B, 18813

\section{$\mathrm{C}_{26} \mathrm{H}_{43} \mathrm{NO}_{6}$}

6,14-Dimethoxyforesticine, 6227

Glycocholic acid, 8823

\section{$\mathrm{C}_{26} \mathrm{H}_{43} \mathrm{NO}_{7}$}

Delphatine, 5008

8-Methyllycoctonine, 14570

\section{$\mathrm{C}_{26} \mathrm{H}_{43} \mathrm{NO}_{8}$}

18-O-Methyldelterine, 14286

8-Methyl-10-hydroxylycoctonine, 14513

\section{$\mathrm{C}_{26} \mathrm{H}_{44} \mathrm{NNaO}_{7} \mathrm{~S}$}

Sodium tauropythocholate, 20038

\section{$\mathrm{C}_{26} \mathrm{H}_{44} \mathrm{~N}_{2}$}

Buxamine E, 2816

Buxbodine E, 2822

\section{$\mathrm{C}_{26} \mathrm{H}_{44} \mathrm{~N}_{2} \mathrm{O}$}

Buxaminol E, 2817

Saracocine, 19354

\section{$\mathrm{C}_{26} \mathrm{H}_{44} \mathrm{O}_{4}$}

Irisoquin C, 11175

Surinone C, 20495 
$\mathrm{C}_{26} \mathrm{H}_{44} \mathrm{O}_{6}$

Dresigenin B, 6600

$\mathrm{C}_{26} \mathrm{H}_{44} \mathrm{O}_{7}$

Stemodinoside B, 20303

$\mathrm{C}_{26} \mathrm{H}_{44} \mathrm{O}_{8}$

Darutoside, 4662

Pubeside A, 18175

Pubeside B, 18176

Salvigreside A, 19212

Siegesbeckioside, 19877

$1 \beta, 16 \alpha, 17$-Trihydroxy-ent-kaur-17-O- $\beta$ - $D$ glucopyranoside, 21758

\section{$\mathrm{C}_{26} \mathrm{H}_{44} \mathrm{O}_{9}$}

ent-2 $\beta, 15,16,19$-Tetrahydroxypimar-8(14)-en-

19-O- $\beta$-glucopyranoside, 21150

\section{$\mathbf{C}_{26} \mathbf{H}_{44} \mathbf{O}_{10}$}

2,6-Diacetyl-3,4-diisobutyryl-1- $O$-octylglucopyranoside, 5332

Eriocaside A, 7275

\section{$\mathrm{C}_{26} \mathrm{H}_{45} \mathrm{~N}$}

Buxbodine A, 2818

\section{$\mathrm{C}_{26} \mathrm{H}_{45} \mathrm{NO}_{6} \mathrm{~S}$}

Taurochenodeoxycholic acid, 20733

Tauroursodeoxycholic acid, 20735

\section{$\mathrm{C}_{26} \mathrm{H}_{45} \mathrm{NO}_{7} \mathrm{~S}$}

Taurocholic acid, 20734

\section{$\mathrm{C}_{26} \mathrm{H}_{46} \mathrm{~N}_{2} \mathrm{O}$}

Cyclovirobuxine D, 4544

Epipachysamine A, 6989

Epipachysamine AII, 6990

Saracodine, 19355

\section{$\mathrm{C}_{26} \mathrm{H}_{46} \mathrm{~N}_{\mathbf{2}} \mathrm{O}_{22}$}

Neocycasin D, 15383

\section{$\mathrm{C}_{26} \mathrm{H}_{46} \mathrm{O}_{5}$}

Ranol, 18544

\section{$\mathrm{C}_{26} \mathrm{H}_{50} \mathrm{O}_{2}$}

Hexacosan-4-olide, 9480

5-Hydroxy-hexacos-1-en-3-one, 10159

\section{$\mathrm{C}_{26} \mathrm{H}_{52} \mathrm{O}_{2}$}

Cerotic acid, 3434

$$
\mathrm{C}_{26} \mathrm{H}_{54}
$$

n-Hexacosane, 9479

\section{$\mathrm{C}_{26} \mathrm{H}_{54} \mathrm{~N}_{4} \mathrm{O}$}

Budmunchiamine $\mathrm{L}_{4}, 2700$

\section{$\mathrm{C}_{26} \mathrm{H}_{54} \mathrm{O}$}

Ceryl alcohol, 3435

\section{$\mathrm{C}_{27} \mathrm{H}_{12} \mathbf{O}_{18}$}

Isocorilagin, 11342

\section{$\mathrm{C}_{27} \mathrm{H}_{20} \mathrm{MgO}_{12}$}

Magnesium lithospermate, 13369

$\mathrm{C}_{27} \mathrm{H}_{20} \mathrm{O}_{6}$

Microphyllaquinone, 14843

$\mathrm{C}_{27} \mathrm{H}_{20} \mathbf{O}_{7}$
Mulberrofuran R, 15052

$\mathrm{C}_{27} \mathrm{H}_{20} \mathrm{O}_{12}$

Sagecoumarin, 19123

$\mathrm{C}_{27} \mathrm{H}_{20} \mathrm{O}_{17}$

Phyllanemblinin A, 17208

$\mathrm{C}_{27} \mathrm{H}_{22} \mathrm{~N}_{4} \mathbf{O}_{3}$

1-( $\beta$-Carbolin-1-yl)-3-(4,8-dimethoxy- $\beta$-carbolin1-yl)propan-1-one, 3161

$\mathrm{C}_{27} \mathrm{H}_{22} \mathrm{O}_{12}$

Lithospermic acid, 12925

Melitric acid A, 13709

$\mathrm{C}_{27} \mathrm{H}_{22} \mathrm{O}_{15}$

Quercetin-3-O- $\alpha$-arabinopyranoside-2"-gallate, 18323

$\mathrm{C}_{27} \mathrm{H}_{22} \mathrm{O}_{16}$

(-)-2,3-Digalloyl-4-(E)-caffeoyl- $L$-threonic acid, 5513

\section{$\mathrm{C}_{27} \mathrm{H}_{22} \mathrm{O}_{18}$}

Corilagin, 4055

Helioscopinin B, 9310

Phyllanemblinin B, 17209

Punicacortein A, 18200

Punicacortein B, 18201

Sanguiin $\mathrm{H}_{4}, 19276$

Sanguiin $\mathrm{H}_{5}, 19277$

Strictinin, 20389

\section{$\mathrm{C}_{27} \mathrm{H}_{22} \mathrm{O}_{19}$}

Furosin, 8030

\section{$\mathrm{C}_{27} \mathrm{H}_{23} \mathrm{O}_{16}$}

5-Carboxypyranocyanidin-3-O-(6"-O-malonyl$\beta$-glucopyranoside), 3183

\section{$\mathrm{C}_{27} \mathrm{H}_{24} \mathrm{~N}_{2} \mathrm{O}_{3}$}

Melicobisquinolinone B, 13677

\section{$\mathrm{C}_{27} \mathrm{H}_{24} \mathrm{~N}_{2} \mathrm{O}_{5}$}

Tyraminoporphine, 22159

\section{$\mathrm{C}_{27} \mathrm{H}_{24} \mathrm{O}_{5}$}

Vismiaguianone D, 22548

Vismiaguianone E, 22549

$\mathrm{C}_{27} \mathrm{H}_{24} \mathrm{O}_{7}$

Rhinacanthin N, 18777

\section{$\mathrm{C}_{27} \mathrm{H}_{24} \mathrm{O}_{18}$}

1,2,3-Tri- $O$-galloyl- $\beta$ - $D$-glucose, 21641

1,2,6-Tri- $O$-galloyl- $\beta$ - $D$-glucose, 21642

1,3,6-Trigalloyl- $\beta$ - $D$-glucose, 21643

\section{$\mathrm{C}_{27} \mathrm{H}_{24} \mathbf{O}_{19}$}

Amlaic acid, 1065

Chebulanin, 3491

$\mathrm{C}_{27} \mathrm{H}_{26} \mathrm{O}_{7}$

Bletilol B, 2516

Bletilol C, 2517

$\mathrm{C}_{27} \mathrm{H}_{26} \mathrm{O}_{8}$

Rocagloic acid, 18892

$\mathrm{C}_{27} \mathrm{H}_{26} \mathrm{O}_{9}$
Artabotrycinol, 1776

Isoamericanol $\mathrm{B}_{1}, 11212$

Isoamericanol $\mathrm{B}_{2}, 11213$

Isoamericanol $\mathrm{C}_{1}, 11214$

Isoamericanol $\mathrm{C}_{2}, 11215$

Isoprincepin, 11612

Princepin, 17860

\section{$\mathrm{C}_{27} \mathrm{H}_{26} \mathbf{O}_{10}$}

Benzoylsalireposide, 2270

Piceid 2'-O-p-hydroxybenzoate, 17286

Symplososide, 20547

\section{$\mathrm{C}_{27} \mathrm{H}_{26} \mathrm{O}_{11}$}

Symponoside, 20549

Torreyaflavonoside, 21463

\section{$\mathrm{C}_{27} \mathrm{H}_{26} \mathrm{O}_{12}$}

Cleistanthin B, 3817

Piceid-2"-O-gallate, 17285

Resveratrol-4'-O-(6"-- $O$-galloyl)- $\beta$ - $D$-glucopyranoside, 18648

Symplocomoside, 20528

\section{$\mathrm{C}_{27} \mathrm{H}_{26} \mathrm{O}_{13}$}

$(+)$-Gallocatechin-hexacetate, 8102

Kaempferol-3-O-(2,3,4-tri- $O$-acetyl- $\alpha$ - $L$-rhamnopyranoside), 12099

Piceatannol 3'-O- $\beta$ - $D$-(6"-O-galloyl)glucopyranoside, 17279

Piceatannol

4'-O-(6"- $O$-galloyl) $\beta$ - $D$-glucopyranoside, 17280

(E)-3,5,3',4'-Tetrahydroxystilbene 3-O- $\beta-D-(6-O-$ galloyl)glucopyranoside, 21155

\section{$\mathrm{C}_{27} \mathrm{H}_{26} \mathrm{O}_{17}$}

Apigenin-7-O-diglucuronide, 1489

\section{$\mathrm{C}_{27} \mathrm{H}_{26} \mathrm{O}_{18}$}

Isochesnatin, 11325

Luteolin-7-O-diglucuronide, 13142

Scutellarein-7-O-diglucuronide, 19583

\section{$\mathrm{C}_{27} \mathrm{H}_{26} \mathrm{O}_{19}$}

Quercetin-3,7-diglucuronide, 18347

\section{$\mathrm{C}_{27} \mathrm{H}_{26} \mathrm{O}_{20}$}

Phyllanemblinin D, 17211

Phyllanemblinin E, 17212

Phyllanemblinin F, 17213

\section{$\mathrm{C}_{27} \mathrm{H}_{28} \mathrm{~N}_{2} \mathrm{O}_{4}$}

Anomalamide, 1345

Patriscabratine, 16724

Trichosanatine, 21575

$\mathrm{C}_{27} \mathrm{H}_{28} \mathrm{~N}_{2} \mathrm{O}_{11}$

Desoxycordifolinic acid, 5273

$\mathrm{C}_{27} \mathrm{H}_{28} \mathrm{O}_{7}$

Lespedezol A 5,12686

$\mathrm{C}_{27} \mathrm{H}_{28} \mathrm{O}_{9}$

Angeloylpodophyllotoxin, 1232 
Steganagin, 20285

$$
\begin{aligned}
& \mathbf{C}_{\mathbf{2 7}} \mathbf{H}_{\mathbf{2 8}} \mathbf{O}_{\mathbf{1 0}} \\
& \text { Acuminatin II*, } 593 \\
& \mathbf{C}_{\mathbf{2 7}} \mathbf{H}_{\mathbf{2 8}} \mathbf{O}_{\mathbf{1 1}} \\
& \text { Cochinchiside A, } 3874 \\
& \text { Tremulacin, 21508 }
\end{aligned}
$$

\section{$\mathrm{C}_{27} \mathrm{H}_{28} \mathrm{O}_{12}$}

Cochinchiside B, 3875

Hirsutissimiside A, 9554

1-O-Methyl-3,5-O-dicaffeoyl quinic acid methyl ester, 14292

$$
\begin{aligned}
& \mathbf{C}_{27} \mathbf{H}_{28} \mathbf{O}_{13} \\
& \text { 3-O-Caffeoyl-4-O-sinapoylquinic acid, } 2925 \\
& \text { Cassiaoccidentalin A, } 3281
\end{aligned}
$$

\section{$\mathrm{C}_{27} \mathrm{H}_{28} \mathrm{O}_{14}$}

Cassiaoccidentalin B, 3282

\section{$\mathrm{C}_{27} \mathrm{H}_{28} \mathrm{O}_{15}$}

1,3-Dihydroxy-2-carbomethoxy-9,10-anthraquinone 3- $O$ - $\beta$-primeveroside, 5785

3',5'-O-Dimethylmyricetin

3-O- $\beta$ - $D$-2",3"-diacetylglucopyranoside, 6377

Yopaaoside A, 22926

\section{$\mathrm{C}_{27} \mathrm{H}_{28} \mathrm{O}_{16}$}

Kaempferol 3-O-(2"- $\alpha$-rhamnopyranosyl)- $\beta$ glucuronopyranoside, 12078

Rhein diglucoside, 18760

\section{$\mathrm{C}_{27} \mathrm{H}_{28} \mathrm{O}_{17}$}

Quercetin-3- $O$-(2"-O-rhamnopyranosyl)- $\beta$ glucuronopyranoside, 18330

\section{$\mathrm{C}_{27} \mathrm{H}_{28} \mathrm{O}_{18}$}

Nelumboside, 15325

Quercetin-7-O-glucuronoglucoside, 18372

\section{$\mathrm{C}_{27} \mathrm{H}_{29} \mathrm{NO}_{6}$}

(+)-Plicamine, 17560

(-)-Secoplicamine, 19633

\section{$\mathrm{C}_{27} \mathrm{H}_{29} \mathrm{NO}_{13}$}

Cartormin, 3226

\section{$\mathrm{C}_{27} \mathrm{H}_{30} \mathrm{~N}_{2} \mathrm{O}_{10}$}

$3 \alpha, 3 \beta$-Carboxyvincoside lactam, 3186

\section{$\mathrm{C}_{27} \mathrm{H}_{30} \mathrm{O}_{4}$}

Isovouacapenol D, 11779

\section{$\mathrm{C}_{27} \mathrm{H}_{30} \mathrm{O}_{6}$}

Parvifloron E, 16680

Parvifloron F, 16681

Plectranthol A, 17553

$\mathrm{C}_{27} \mathrm{H}_{30} \mathrm{O}_{7}$

Nimbolide, 15604

\section{$\mathrm{C}_{27} \mathrm{H}_{30} \mathrm{O}_{8}$}

Angeloylgomisin R, 1210

Daphnetoxin, 4649

Heteroclitin D, 9463

Interiorin A, 11099

Interiorin B, 11100
Schiarisanrin E, 19465

$\mathrm{C}_{27} \mathrm{H}_{30} \mathrm{O}_{9}$

Heteroclitin E, 9464

Interiotherin B, 11104

Schisantherin L, 19493

\section{$\mathrm{C}_{27} \mathrm{H}_{30} \mathrm{O}_{10}$}

Baohuoside I, 2138

5,3'-Dibutanoyloxy-3,6,7,4'-tetramethoxyflavone, 5401

Heteroclitin F, 9465

$\mathrm{C}_{27} \mathrm{H}_{30} \mathrm{O}_{11}$

Icariside I, 10955

2"-O-(2"'-Methylbutyryl)isoswertisin, 14193

3"-O-(2"'-Methylbutyryl)isoswertisin, 14194

Neoicariin, 15410

Tremulacinol, 21509

Wushanicariin, 22740

\section{$\mathrm{C}_{27} \mathrm{H}_{30} \mathrm{O}_{12}$}

4'-Demethyldesoxypodophyllotoxin-4- $O-\beta$ - $D$ glucoside, 5075

\section{$\mathrm{C}_{27} \mathrm{H}_{30} \mathrm{O}_{13}$}

4-Demethylepipodophyllotoxin 7'- $O-\beta$ - $D$-glucopyranoside, 5078

4-Demethyl-picropodophyllotoxin 7'- $O-\beta-D$ glucopyranoside, 5092

4-Demethyl-podophyllotoxin 7'- $O$ - $\beta$ - $D$-glucopyranoside, 5094

Formononetin-7-O- $\beta$ - $D$-apiofuranosyl- $(1 \rightarrow 6)$ $\beta$ - $D$-glucopyranoside, 7884

Glycyroside, 8840

Kushenol O, 12368

$\alpha$-Peltatin glucoside, 16795

Rhamnellaflavoside A, 18676

Rhamnellaflavoside B, 18677

Rhamnellaflavoside C, 18678

\section{$\mathrm{C}_{27} \mathrm{H}_{30} \mathrm{O}_{14}$}

Apigenin-7-O-neohesperidoside, 1497

Apigenin-7-O-rutinoside, 1499

Camellianin A, 3038

Chrysophanol-1- $\beta$-gentiobioside, 3617

Chrysophanol-8-O-gentiobioside, 3618

Daidzein 4',7-diglucoside, 4605

6,8-Di- $C$ - $\beta$-glucosylchrysin, 5531

6,4'-Dihydroxy aurone 4- $O$-rutinoside, 5761

Dulcinoside, 6623

Isoviolanthin, 11772

Kaempferitrin, 12018

2-Methyl-1,3,6-trihydroxyanthraquinone

3-O- $\alpha$ - $L$-rhamnosyl-( $1 \rightarrow 2)$ - $\beta$ - $D$-glucoside, 14778

Ophiohayatone B, 16133

Puerarin-4'- $O$ - $D$-glucoside, 18181

7-O- $\alpha$-Rhamno( $1 \rightarrow 6)-\beta$-glucosylgenistein,
18685

2"-O- $\alpha-L$-Rhamnopyranosylisovitexin, 18729

Sophorabioside, 20080

4',6,7-Trihydroxyisoflavone-6-methylether-7-

$O$ - $\beta$ - $D$-xylopyranosyl-( $1 \rightarrow 6)-\beta$ - $D$-glucopyrano side, 21751

Violanthin, 22519

\section{$\mathrm{C}_{27} \mathrm{H}_{30} \mathrm{O}_{15}$}

Aloeemodin diglucoside, 969

Apigenin-7-O-gentiobioside, 1490

6,8-Bis( $C$ - $\beta$-glucosyl)-apigenin, 2455

Chrysoeriol-7-apio-glucoside, 3604

$2 "-O-\beta$ - $L$-Galactopyranosylvitexin, 8072

Genistein 7- $O-\beta$ - $D$-glucopyranoside-4'- $O-\beta-D$ glucopyranoside, 8279

Isorhamnetin-3-arabino-7-rhamnoside, 11651

Isosaponarin, 11695

Kaempferide-3- $O$ - $\beta$-xylosyl $(1 \rightarrow 2)-\beta$-glucoside, 12017

Kaempferol 3-O- $\beta$ - $D$-glucopyranosyl-(1-2)$\alpha$ - $L$-rhamnopyranoside, 12053

Kaempferol-3- $\beta$ - $D$-gluco-7- $\alpha-L$-rhamnoside, 12060

Kaempferol-3- $\beta$ - $D$-gluco-7- $\beta$ - $L$-rhamnoside, 12061

Kaempferol-3- $O$-neohesperidoside, 12071

Kaempferol 3-O- $\alpha$ - $L$-rhamnopyranosyl $(1 \rightarrow 6)$ $\beta$ - $D$-galactopyranoside, 12074

Lonicerin, 12987

Luteolin-7-rutinoside, 13149

Multiflorin B, 15069

Nicotiflorin, 15525

Oroxin B, 16215

Palustroside,+ 16578

Quercetin-3,4'-di- $O$ - $\alpha$ - $L$-rhamnopyranoside, 18350

Quercetin-3,7- $\alpha$ - $L$-dirhamnoside, 18352

Rheinoside C, 18764

Rheinoside D, 18765

Safflower yellow A, 19111

Saponarin, 19331

Scutellarein-7-rutinoside, 19585

Tectorigenin-7- $O$ - $[\beta$ - $D$-apiofuranosyl- $(1 \rightarrow 6)$ $\beta$ - $D$-glucopyranoside], 20902

4',5,7-Trihydroxy-6-methoxyisoflavone7-O$\beta$ - $D$-xylopyranosyl-( $(\rightarrow 6)$ - $\beta$ - $D$-glucopyranoside, 21781

Vitexin-7-glucoside, 22582

Yuankanin, 22935

\section{$\mathrm{C}_{27} \mathrm{H}_{30} \mathrm{O}_{16}$}

Bioquercetin, 2394

Equisetrin, 7222

2 "-O- $\beta$-L-Galactopyranosylorientin, 8066 
Isorhamnetin-3-arabinoglucoside, 11650

Kaempferol-3,7-diglucoside, 12043

Kaempferol 7- $O-\beta$ - $D$-glucopyranosyl-( $1 \rightarrow 4)$ -

$\beta$ - $D$-glucopyranoside, 12052

Luteolin-7-O-digalactoside, 13140

Luteolin-6,8-C-diglucoside, 13141

Luteolin-7-O-gentiobioside, 13144

Multinoside A, 15072

Neoisorutin, 15416

Panasenoside, 16587

Quercetin-3- $O-\beta$ - $D$-glucopyranosyl- $(1 \rightarrow 2)-\alpha-L$ rhamnopyranoside, 18365

Quercetin-3- $\beta$ - $D$-gluco-7- $\alpha-L$-rhamnoside, 18367

Quercetin-3- $O$-neohesperidoside, 18378

Quercetin-3-rhamnoside-7-glucoside, 18387

Quercetin-3-robinobioside, 18390

Rheinoside A, 18762

Rheinoside B, 18763

Rutin, 19087

Sophoraflavonoloside, 20088

\section{$\mathrm{C}_{27} \mathrm{H}_{30} \mathrm{O}_{17}$}

Heliosin, 9315

6-Hydroxyluteolin-7-diglucoside, 10353

Myricetin-3-rutinoside, 15185

Quercetin-3,5-di- $D$-galactoside, 18338

Quercetin-5,4'-di- $O$ - $\beta$ - $D$-glucopyranoside, 18341

Quercetin-3-diglucoside, 18342

Quercetin-3,4'-diglucoside, 18343

Quercetin-3,5-diglucoside, 18344

Quercetin-3,7-diglucoside, 18345

Quercetin-7,4'-diglucoside, 18346

Quercetin-3- $O-\beta$ - $D$-galactoside-7- $O$ - $\beta$-glucoside, 18357

Quercetin-3-O-[ $\beta$ - $D$-glucopyranosyl-( $1 \rightarrow 2)-\beta$ $D$-galactopyranoside], 18362

Quercetin-3-O-[ $\beta$ - $D$-glucopyranosyl- $(1 \rightarrow 2)-\beta$ $D$-glucopyranoside], 18363

Quercetin-7-O- $\beta$ - $D$-glucopyranosyl $(1 \rightarrow 6)-\beta-D$ glucopyranoside, 18364

\section{$\mathrm{C}_{27} \mathrm{H}_{31} \mathrm{NO}_{4}$}

Erinacerin A, 7259

Hericenone B, 9430

\section{$\mathrm{C}_{27} \mathrm{H}_{31} \mathrm{NO}_{5}$}

Ignavine, 10962

\section{$\mathrm{C}_{27} \mathrm{H}_{31} \mathrm{~N}_{2} \mathrm{O}_{9}{ }^{+}$}

3,4,5,6-Tetradehydropalicoside, 21050

\section{$\mathrm{C}_{27} \mathrm{H}_{31} \mathrm{O}_{15}{ }^{+}$}

Cyanidin-3-rutinoside, 4446

Pelargonidin-3,5-diglucoside, 16781

$\mathrm{C}_{27} \mathrm{H}_{31} \mathrm{O}_{16}{ }^{+}$

Cyanidin-3,4'-di- $O$ - $\beta$-glucopyranoside, 4438

Cyanidin-3-gentiobioside, 4441

Cyanin, 4451
$\mathrm{C}_{27} \mathrm{H}_{31} \mathrm{O}_{17}{ }^{+}$

Delphin, 5010

Delphinidin-3-diglucoside, 5020

$\mathrm{C}_{27} \mathrm{H}_{32} \mathbf{N}_{2} \mathbf{O}_{10}$

Cadambine, 2844

$\mathrm{C}_{27} \mathrm{H}_{32} \mathrm{O}_{4}$

Bitucarpin A, 2495

Isovouacapenol A, 11777

$\mathrm{C}_{27} \mathrm{H}_{32} \mathrm{O}_{5}$

12-Benzoyloxycrotohalimaneic acid, 2251

$\mathrm{C}_{27} \mathrm{H}_{32} \mathrm{O}_{6}$

Kushenol D, 12357

2'-Methoxykurarinone, 13985

$\mathrm{C}_{27} \mathrm{H}_{32} \mathrm{O}_{7}$

Kadsutherin, 12014

$\mathrm{C}_{27} \mathrm{H}_{32} \mathrm{O}_{8}$

Atalantolide, 1957

PC-1999-52-1525-6, 16737

$\mathrm{C}_{27} \mathrm{H}_{32} \mathrm{O}_{9}$

Atalantin, 1955

Orbiculin D, 16163

$\mathrm{C}_{27} \mathrm{H}_{32} \mathrm{O}_{10}$

Spicatin, 20160

\section{$\mathrm{C}_{27} \mathrm{H}_{32} \mathrm{O}_{11}$}

Hemislienoside, 9352

Hymenoside A, 10844

Hymenoside J, 10853

\section{$\mathrm{C}_{27} \mathrm{H}_{32} \mathrm{O}_{12}$}

Hymenoside C, 10846

Hymenoside D, 10847

\section{$\mathrm{C}_{27} \mathrm{H}_{32} \mathrm{O}_{13}$}

Cascaroside C, 3255

Cascaroside D, 3256

10R-Chrysaloin 1-O- $\beta$ - $D$-glucopyranoside, 3590

Dracunculifoside H, 6587

Hymenoside B, 10845

Hymenoside E, 10848

6-O-Z-p-Methoxycinnamoyl scandoside methyl ester, 13894

5-O-p-Methoxy cinnamoyl scandoside methyl ester, 13895

Naringenin-7- $O-\alpha$ - $L$-rhamnosyl $(1 \rightarrow 4)$-rhamnoside, 15284

Onychin, 16122

$\mathrm{C}_{27} \mathrm{H}_{32} \mathrm{O}_{14}$

Cascaroside A, 3254

Glypentoside A, 8851

10-Hydroxycascaroside C, 9898

10-Hydroxycascaroside D, 9899

Kushenol J, 12363

Liquiritigenin-7,4'-diglucoside, 12912

Naringin, 15286

Narirutin, 15287
Nobiletin-3- $O-\beta$ - $D$-glucoside, 15636

\section{$\mathrm{C}_{27} \mathrm{H}_{32} \mathbf{O}_{15}$}

Butrin, 2786

Chalcononaringenin 2',4'-di- $O-\beta$ - $D$-glucoside, 3455

9-[( $\beta$ - $D$-Glucopyranosyl- $(1 \rightarrow 6)-O-\beta$ - $D$-glucopyranosyl)oxy]-10-hydroxy-7-methoxy-3-

methyl-1H-naphthol[2,3-c]pyran-1-one, 8649

Neoeriocitrin, 15393

Polygalaxanthone IV, 17632

Robustaside C, 18879

Rubrofusarin-6- $\beta$-gentiobioside, 19042

Veratriloside D, 22388

Viscumneoside I, 22536

Viscumneoside III, 22538

\section{$\mathrm{C}_{27} \mathrm{H}_{32} \mathrm{O}_{16}$}

(2R)-Eriodictyol-7,4'-di- $O$ - $\beta$ - $D$-glucopyranoside, 7280

1-O-Gentiobiosyl-3,7-dimethoxy-8-hydroxyxanthone, 8301

Polygalaxanthone VII, 17635

Safflomin A, 19110

Suffruticoside A, 20451

Suffruticoside B, 20452

Suffruticoside C, 20453

Suffruticoside D, 20454

\section{$\mathrm{C}_{27} \mathrm{H}_{32} \mathrm{O}_{17}$}

Eriodictyol-7,3-diglucoside, 7281

\section{$\mathrm{C}_{27} \mathrm{H}_{33} \mathrm{NO}_{3}$}

Hericerin, 9437

$\mathrm{C}_{27} \mathrm{H}_{33} \mathrm{NO}_{11}$

Colchicoside, 3912

$\mathrm{C}_{27} \mathrm{H}_{33} \mathrm{~N}_{2} \mathrm{O}_{9}{ }^{+}$

3,4-Dehydropalicoside, 4958

\section{$\mathrm{C}_{27} \mathrm{H}_{34} \mathrm{~N}_{2} \mathrm{O}_{4}$}

Pleiocarpine, 17556

\section{$\mathrm{C}_{27} \mathrm{H}_{34} \mathrm{~N}_{2} \mathrm{O}_{9}$}

Glabratine, 8488

Palicoside, 16541

\section{$\mathrm{C}_{27} \mathrm{H}_{34} \mathrm{~N}_{2} \mathrm{O}_{10}$}

$3 \alpha$-Dihydrocadambine, 5550

3 $\beta$-Isodihydrocadambine, 11377

\section{$\mathrm{C}_{27} \mathrm{H}_{34} \mathrm{O}_{5}$}

$6 \beta$-Benzoyl-7 $\beta$-hydroxyvouacapen-5 $\alpha$-ol, 2244

4-Episcopadulcic acid B, 7016

Isovouacapenol B, 11778

Licorisoflavan A, 12795

\section{$\mathrm{C}_{27} \mathrm{H}_{34} \mathrm{O}_{6}$}

Anticancer Norwithanolide PMV70P691-029, 1427

Jolkinol A', 11891

\section{$\mathrm{C}_{27} \mathrm{H}_{34} \mathrm{O}_{7}$}

Anticancer Norwithanolide PMV70P691-030, 
1428

[(2S,3R,4R)-4-(3,4-Dimethoxybenzyl)-2-(3,4dimethoxyphenyl)-tetrahydrofuran-3-yl]methyl (2Z)-2-methylbut-2-en-oate, 6211

Lucidenic acid $\mathrm{D}_{1}, 13026$

Neokurarinol, 15425

$\mathrm{C}_{27} \mathrm{H}_{34} \mathrm{O}_{8}$

Deacetylsecomahoganin, 4772

Kleinioxanthrone 4, 12236

(+)-4-(3-Methylbutanoyl)-2,6-di(3,4-dimethoxy)phenyl-3,7-dioxabicyclo[3.3.0]octane, 14165

\section{$\mathrm{C}_{27} \mathrm{H}_{34} \mathrm{O}_{9}$}

Anticancer Norwithanolide PMV70P691-035, 1433

Verrucarin A, 22420

\section{$\mathrm{C}_{27} \mathrm{H}_{34} \mathrm{O}_{10}$}

Isoveprisonic acid, 11769

(2S,3S)-2-(5-Methoxy-3,4-methylenedioxybenzyl)-3-(3,4,5-trimethoxybenzyl)butane1,4-diol diacetate, 14011

Provincialin, 17998

Veprisonic acid, 22381

\section{$\mathrm{C}_{27} \mathrm{H}_{34} \mathrm{O}_{11}$}

Arctiin, 1623

CPB-2001-49-282-32, 3509

3,4,1-O-3,4-Dimethoxy-phenylethyl-6- $O$ cinnamoyl- $\beta$ - $D$-glucopyranose, 6284

3-Methoxyarctii-4"-O- $\beta$ - $D$-xyloside, 13841

Phillyrin, 17145

(+)-Pinoresinol monomethyl ether

$O-\beta$ - $D$-glucopyranoside, 17419

Styraxlignolide F, 20427

Tetra- $O$-methylpilosidine, 21201

Undulatone, 22231

\section{$\mathrm{C}_{27} \mathrm{H}_{34} \mathrm{O}_{12}$}

Eucommin A, 7487

Tracheloside, 21493

Woorenoside I, 22727

\section{$\mathrm{C}_{27} \mathrm{H}_{34} \mathrm{O}_{13}$}

Alboside II, 868

Brunneogaleatoside, 2683

2-(4-Hydroxyphenyl)ethyl-1-O- $\beta-D-[5-O-(4-$ methoxybenzoyl)]-apiofuranosyl-( $1 \rightarrow 6)-\beta$ - $D$ glucopyranoside, 10606

$\mathrm{C}_{27} \mathrm{H}_{34} \mathrm{O}_{14}$

3,4-Dimethoxyphenyl

1-O- $\beta$ - $D$-[5-O-(4-methoxybenzoyl)]-apiofuranosyl-(1 $\rightarrow 6)-\beta$ - $D$-glucopyranoside, 6287

4-Methoxyphenyl

1-O- $\beta$ - $D$-[5-O-(3,4-dimethoxybenzoyl)]-apiofuranosyl-( $1 \rightarrow 6)-\beta$ - $D$-glucopyranoside, 14055 Phlomisethanoside, 17164

\section{$\mathrm{C}_{27} \mathrm{H}_{34} \mathbf{O}_{15}$}

13- $O$ - $[\beta$ - $D$-Apiofuranosyl( $1 \rightarrow 6)-\beta$ - $D$-glucopyranosyl]-(12R)-heraclenol, 1510

3,4-Dihydroxyphenylethanol-8- $O-[(5-O-$ vanilloyl) $-\beta$ - $D$-apiofuranosyl $(1 \rightarrow 2)]-\beta-D$ glucopyranoside, 6080

\section{$\mathrm{C}_{27} \mathrm{H}_{34} \mathrm{O}_{16}$}

Albibrissinoside B, 856

Glypentoside B, 8852

Hexaacetyl catalpol, 9476

Leiocarposide, 12608

\section{$\mathrm{C}_{27} \mathrm{H}_{35} \mathrm{BrO}_{2}$}

4-Bromobenzyl-labda-7,12(E),14-triene-17-oate, 2618

$\mathrm{C}_{27} \mathrm{H}_{35} \mathrm{NO}_{4}$

Lythrancepine I, 13269

\section{$\mathrm{C}_{27} \mathrm{H}_{35} \mathrm{NO}_{5}$}

Hokbusine B, 9575

Lythracine I, 13267

Lythrancine I, 13272

Nicotinoylisoramanone, 15530

$\mathrm{C}_{27} \mathrm{H}_{35} \mathrm{NO}_{6}$

12-O-Nicotinoylisolineolone, 15529

\section{$\mathrm{C}_{27} \mathrm{H}_{35} \mathrm{NO}_{7}$}

Rostratamine, 18936

$\mathrm{C}_{27} \mathrm{H}_{35} \mathrm{~N}_{9} \mathrm{O}_{15} \mathrm{P}_{2}$

Flavinadenine dinucleotide, 7814

\section{$\mathrm{C}_{27} \mathrm{H}_{36} \mathrm{O}_{4}$}

Isodulcinol, 11405

Scopadulciol, 19537

Scopanolal, 19538

\section{$\mathrm{C}_{27} \mathrm{H}_{36} \mathrm{O}_{5}$}

Scopadulcic acid C, 19536

Scoparic acid A, 19539

Surangin A, 20489

$\mathrm{C}_{27} \mathrm{H}_{36} \mathrm{O}_{6}$

Anticancer Norwithanolide PMV70P691-031, 1429

Ganolactone, 8195

Lucidenic acid F, 13030

$\mathrm{C}_{27} \mathrm{H}_{36} \mathrm{O}_{7}$

Anticancer Norwithanolide PMV70P691-032, 1430

Lanciaquinone, 12472

Lucidenic acid K, 13035

$\mathrm{C}_{27} \mathrm{H}_{36} \mathrm{O}_{8}$

12-O-Acetylphorbol-13-tigliate, 485

\section{$\mathrm{C}_{27} \mathrm{H}_{36} \mathbf{O}_{9}$}

Crocin 4, 4252

Xanthanthusin G, 22750

\section{$\mathrm{C}_{27} \mathrm{H}_{36} \mathrm{O}_{10}$}

Caesalpinin MG, 2877

Dihydrodehydrodiconiferyl alcohol
9'-O-glucoside, 5584

$R$-Myricanol 5-O- $\beta$ - $D$-glucopyranoside, 15163

(+)-S-Myricanol 5-O- $\beta$ - $D$-glucopyranoside, 15164

$\mathrm{C}_{27} \mathrm{H}_{36} \mathrm{O}_{11}$

Quassimarin, 18298

\section{$\mathrm{C}_{27} \mathrm{H}_{36} \mathrm{O}_{12}$}

Gaultheroside A, 8242

Nudiposide, 15864

\section{$\mathrm{C}_{27} \mathrm{H}_{36} \mathrm{O}_{13}$}

Amritoside C, 1086

Citrusin B, 3776

Lucidumoside D, 13056

\section{$\mathrm{C}_{27} \mathrm{H}_{36} \mathrm{O}_{14}$}

Lucidumoside C, 13055

\section{$\mathrm{C}_{27} \mathrm{H}_{38} \mathrm{O}_{3}$}

Nigrolineaquinone A, 15573

\section{$\mathrm{C}_{27} \mathrm{H}_{38} \mathrm{O}_{4}$}

Dulcidiol, 6621

Lygodinolide, 13245

Papuaforin C, 16631

Plastoquinone $\mathrm{C}_{1}, 17516$

Scopadiol, 19535

25R-Spirost-4-en-3,12-dione, 20218

\section{$\mathrm{C}_{27} \mathrm{H}_{38} \mathrm{O}_{6}$}

Caledonic acid, 2961

Lucidenic acid A, 13023

\section{$\mathrm{C}_{27} \mathrm{H}_{38} \mathrm{O}_{7}$}

Ajugacumbin A, 788

Diterpenoid EF-D, 6521

Lucidenic acid B, 13024

Lucidenic acid $\mathrm{E}_{1}, 13028$

Lucidenic acid I, 13033

Lucidenic acid L, 13036

\section{$\mathrm{C}_{27} \mathrm{H}_{38} \mathrm{O}_{8}$}

Ajugacumbin D, 791

Lucidenic acid J, 13034

Maoecrystal T, 13541

\section{$\mathrm{C}_{27} \mathrm{H}_{38} \mathrm{O}_{9}$}

Caesalmin F, 2868

\section{$\mathrm{C}_{27} \mathrm{H}_{38} \mathrm{O}_{10}$}

2-Debenzoyl-2-tigloyl-10-deacetylbaccatin III, 4812

Taxuyunnanine R, 20885

Trilobolide, 21889

\section{$\mathrm{C}_{27} \mathrm{H}_{38} \mathrm{O}_{11}$}

(3R,5R)-3,5-Dihydroxy-1,7-bis(4-hydroxy-3methoxyphenyl)heptane

3-O- $\beta$-D-glucopyranoside, 5776

Picrasinoside A, 17320

Tinosposide A, 21400

$\mathrm{C}_{27} \mathrm{H}_{38} \mathrm{O}_{12}$

Baenzigeroside B, 2100 
Icariside $\mathrm{E}_{6}, 10951$

Tinosposide B, 21401

\section{$\mathrm{C}_{27} \mathrm{H}_{38} \mathrm{O}_{13}$}

Seguinoside I, 19672

\section{$\mathrm{C}_{27} \mathrm{H}_{38} \mathrm{O}_{15}$ \\ 2"-Epiframeroside, 6917 \\ Frameroside, 7934 \\ Rubricauloside, 19040}

\section{$\mathrm{C}_{27} \mathrm{H}_{39} \mathrm{NO}_{2}$}

Veratramine, 22386

\section{$\mathrm{C}_{27} \mathrm{H}_{39} \mathrm{NO}_{3}$}

Brachystamide D, 2576

Fetisinine, 7794

Jervine, 11866

\section{$\mathrm{C}_{27} \mathrm{H}_{39} \mathrm{NO}_{7}$}

Tatsiensine, 20727

\section{$\mathrm{C}_{27} \mathrm{H}_{39} \mathrm{NO}_{8}$}

Siwanine A, 20001

\section{$\mathrm{C}_{27} \mathrm{H}_{39} \mathrm{NO}_{9}$}

Merresectine D $\beta$ - $D$-glucoside, 13788

\section{$\mathrm{C}_{27} \mathrm{H}_{40} \mathrm{~N}_{2} \mathrm{O}_{3}$}

Epoxysarcovagenine D, 7198

\section{$\mathrm{C}_{27} \mathrm{H}_{40} \mathrm{O}_{2}$}

Caseamemin, 3266

$\Delta^{3,5}$-Deoxyneotigogenin, 5197

$\Delta^{3,5}$-Deoxytigogenin, 5216

$25 \alpha$-Spirosta-3,5-diene, 20207

$25 \beta$-Spirosta-3,5-diene, 20208

$\delta$-Tocotrienol, 21417

\section{$\mathrm{C}_{27} \mathrm{H}_{40} \mathrm{O}_{3}$}

Walsurol, 22631

\section{$\mathrm{C}_{27} \mathrm{H}_{40} \mathrm{O}_{4}$}

9(11)-Dehydrohecogenin, 4928

3-Epineoruscogenin, 6974

Plastoquinone $\mathrm{C}_{2}, 17517$

Schisanterpene B, 19488

Sisalagenone, 19978

\section{$\mathrm{C}_{27} \mathrm{H}_{40} \mathrm{O}_{5}$}

Asparacosin A, 1864

9(11)-Dehydromanogenin, 4945

Kammogenin, 12126

Proliferin, 17904

\section{$\mathrm{C}_{27} \mathrm{H}_{40} \mathrm{O}_{6}$}

Lucidenic acid $\mathrm{LW}_{1}, 13038$

2,3-Seco-porrigenin, 19634

\section{$\mathrm{C}_{27} \mathrm{H}_{40} \mathrm{O}_{7}$}

Hongdoushan B, 9627

Lucidenic acid C, 13025

Lucidenic acid H, 13032

Trichurusin J, 21587

Trichurusin K, 21588

\section{$\mathrm{C}_{27} \mathrm{H}_{40} \mathrm{O}_{10}$}

14,15-Dihydro-15 $\beta$-methoxy-3-epicaryoptin,
5670

Glabcensin P, 8477

$\mathrm{C}_{27} \mathrm{H}_{40} \mathrm{O}_{11}$

Isovaleroxy-hydroxy dihydrovaltrate, 11755

$\mathrm{C}_{27} \mathrm{H}_{40} \mathrm{O}_{14}$

Amritoside B, 1085

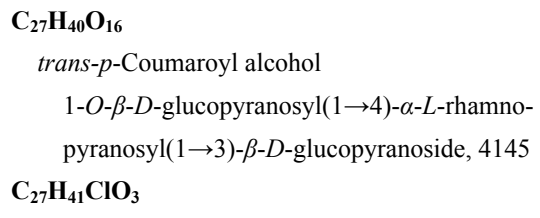

\section{$\mathrm{C}_{27} \mathrm{H}_{41} \mathrm{ClO}_{3}$}

26-Chloro-26-deoxycryptogenin, 3546

$\mathrm{C}_{27} \mathrm{H}_{41} \mathrm{NO}_{2}$

Cyclopamine, 4522

Ebeinone, 6661

\section{$\mathrm{C}_{27} \mathrm{H}_{41} \mathrm{NO}_{3}$}

Brachystamide E, 2577

Ebeiensine, 6658

Hupehenisine, 9684

Korsevinine, 12278

Peimisine, 16776

Petisidine, 17020

Songbeisine, 20074

\section{$\mathrm{C}_{27} \mathrm{H}_{41} \mathrm{NO}_{4}$}

Yibeissine, 22907

\section{$\mathrm{C}_{27} \mathrm{H}_{41} \mathrm{NO}_{8}$}

Deltaline, 5036

\section{$\mathrm{C}_{27} \mathrm{H}_{41} \mathrm{~N}_{3} \mathrm{O}_{5}$}

Cordycepeptide A, 4047

\section{$\mathrm{C}_{27} \mathrm{H}_{42} \mathrm{~N}_{4} \mathrm{O}_{4}$}

Hovenine A, 9653

\section{$\mathrm{C}_{27} \mathrm{H}_{42} \mathrm{O}_{2}$}

Calicoferol E, 2975

(22E)-25,26,27-Trinor-3 $\beta$-hydroxycycloart-22en-24-al, 21982

\section{$\mathrm{C}_{27} \mathrm{H}_{42} \mathrm{O}_{3}$}

22-Cyclopentyloxil-22-deisopenty-3 $\beta$-hydroxylfurostanol, 4525

Diosgenin, 6440

Neotigogenone, 15467

Sarsasapogenone, 19392

Smilagenone, 20028

Tigogenone, 21388

Trigonegenin A, 21659

Trigonegenin B, 21660

Yamogenin, 22878

\section{$\mathrm{C}_{27} \mathrm{H}_{42} \mathrm{O}_{4}$}

Cryptogenin, 4280

3-Epiruscogenin, 7009

Gloriogenin, 8588

Hecogenin, 9253

Heloniogenin, 9334

Isonarthogenin, 11554
Laxogenin, 12591

Lilagenin, 12832

Nuatigenin, 15855

Pennogenin, 16809

Plastoquinone from Sargassum micracanthum, 17520

Ruscogenin, 19070

Sisalagenin, 19977

Torvogenin, 21469

Yuccagenin, 22936

\section{$\mathrm{C}_{27} \mathrm{H}_{42} \mathrm{O}_{5}$}

12-Ketoporrigenin, 12206

Manogenin, 13510

Mexogenin, 14823

Neomanogenin, 15433

Neomexogenin, 15435

Tupichigenin E, 22112

\section{$\mathrm{C}_{27} \mathrm{H}_{42} \mathrm{O}_{6}$}

$2 \alpha$-Acetoxy-14 $\beta$ - $[(S)$-2-methyl-butyryloxy $]$ 4(20),11-taxadiene, 257

Hongdoushan C, 9628

Lucidenic acid M, 13039

Podecdysone B, 17583

Tupichigenin D, 22111

\section{$\mathrm{C}_{27} \mathrm{H}_{42} \mathrm{O}_{7}$}

22-Oxo-ajugasterone C, 16291

22-Oxo-20-hydroxyecdysone, 16350

425(27)-Pentrogenin, 16891

\section{$\mathrm{C}_{27} \mathrm{H}_{42} \mathrm{O}_{7} \mathrm{~S}$}

Ruscogenin 1-O-sulfate, 19073

\section{$\mathrm{C}_{27} \mathrm{H}_{42} \mathrm{O}_{9}$}

Spirost-25(27)-ene-1,2,3,4,5,6,7-heptol, 20220

\section{$\mathrm{C}_{27} \mathrm{H}_{42} \mathrm{O}_{13}$}

Jasnudifloside F, 11826

Jasnudifloside G, 11827

$\mathrm{C}_{27} \mathrm{H}_{42} \mathrm{O}_{13}$

Nudifloside D, 15863

\section{$\mathrm{C}_{27} \mathrm{H}_{42} \mathrm{O}_{17}$}

4'-Deoxykanokoside C, 5186

\section{$\mathrm{C}_{27} \mathrm{H}_{42} \mathrm{O}_{20}$}

Rehmannioside D, 18599

\section{$\mathrm{C}_{27} \mathrm{H}_{43} \mathrm{NO}$}

Shinonomenine, 19828

Solanidine, 20056

\section{$\mathrm{C}_{27} \mathrm{H}_{43} \mathrm{NO}_{2}$}

Chuanbeinone, 3630

Delavinone, 4997

Ebeienine, 6657

Fritillarizine, 7957

Hupehenirine, 9683

Hupehenizine, 9685

Korseverinine, 12277

Rubijervine, 19030 
Solasodine, 20066

Tomatidenol, 21433

Zhebeirine, 22997

Ziebeimine, 22998

$\mathrm{C}_{27} \mathrm{H}_{43} \mathrm{NO}_{3}$

Ebeietinone, 6659

15 $\alpha$-Hydroxysolasodine, 10713

15 $\alpha$-Hydroxytomatidenol, 10771

Imperialine, 11002

Impericine, 11004

Korsine, 12279

Peiminine, 16774

Taipaienine, 20624

Zhebeinone, 22995

\section{$\mathrm{C}_{27} \mathrm{H}_{43} \mathrm{NO}_{4}$}

Imperialine $N$-oxide, 11003

Verticinone- $\mathrm{N}$-oxide, 22432

\section{$\mathrm{C}_{27} \mathrm{H}_{43} \mathrm{NO}_{6}$}

Pingbeimine C, 17380

\section{$\mathrm{C}_{27} \mathrm{H}_{43} \mathrm{NO}_{7}$}

Bullatine E, 2740

Delbrusine, 5001

Zygadenine, 23033

\section{$\mathrm{C}_{27} \mathrm{H}_{43} \mathrm{NO}_{8}$}

Acetylbrowniine, 338

Delbonine, 4998

Tricornine, 21593

Veracevine, 22382

\section{$\mathrm{C}_{27} \mathrm{H}_{43} \mathrm{NO}_{9}$}

Protoverine, 17996

\section{$\mathrm{C}_{27} \mathrm{H}_{44} \mathrm{O}$}

Cholest-4-ene-3-one, 3578

7-Dehydrocholesterol, 4885

\section{$\mathrm{C}_{27} \mathrm{H}_{44} \mathrm{O}_{2}$}

$(2 R, 3 R, 4 S, 6 S)-3-M e t h y l-4,6-\operatorname{di}(3-m e t h y l-2-$ butenyl)-2-(2-methyl-1-oxopropyl)-3-(4-methyl-3-pentenyl)-cyclohexanone, 14322

Wrightial, 22734

\section{$\mathrm{C}_{27} \mathrm{H}_{44} \mathrm{O}_{3}$}

Epi-sarsasapogenin, 7013

Epismilagenin, 7022

Neotigogenin, 15463

Octadecyl (E)-p-coumarate, 15959

Octadecyl (Z)-p-coumarate, 15960

Sarsasapogenin, 19390

Smilagenin, 20025

Tigogenin, 21383

\section{$\mathrm{C}_{27} \mathrm{H}_{44} \mathrm{O}_{4}$}

Barbourgenin, 2150

Chlorogenin, 3552

Cordylagenin, 4049

12-Epirockogenin, 7008

Gitogenin, 8457
Isorhodeasapogenin, 11674

Markogenin, 13569

Neochlorogenin, 15364

Neogitogenin, 15397

Octadecanyl caffeate, 15953

Rhodeasapogenin, 18781

Rockogenin, 18893

Ruizgenin, 19065

Samogenin, 19230

Yonogenin, 22924

\section{$\mathrm{C}_{27} \mathrm{H}_{44} \mathrm{O}_{5}$}

Agavogenin, 726

Hainangenin, 9192

Hongguanggenin, 9630

Igagenin, 10961

Isocarneagenin, 11307

Isoreineckiagenin, 11646

Paniculogenin, 16616

Reineckiagenin, 18602

$5 \alpha$-Spirostan-3 $\beta, 12 \beta, 15 \alpha$-triol, 20215

Tokorogenin, 21426

$\Delta^{23}-3 \alpha, 7 \alpha, 12 \alpha$-Trihydroxy coprostenic acid,

$$
21696
$$

Trihydroxy-isosterocholenic acid, 21754

\section{$\mathrm{C}_{27} \mathrm{H}_{44} \mathrm{O}_{6}$}

Ecdysone, 6678

Glycoside E, 8828

Kitigenin, 12231

Kogagenin, 12251

Periplocoside N, 16956

Ponasterone A, 17700

Taxisterone, 20807

Tupichigenin F, 22113

\section{$\mathrm{C}_{27} \mathrm{H}_{44} \mathrm{O}_{7}$}

Ajugasterone C, 811

Commisterone, 3944

Ecdysterone, 6679

20-Hydroxyecdysone, 10061

24-Hydroxyecdysone, 10062

Inokosterone, 11067

(25R)-Inokosterone, 11068

(25S)-Inokosterone, 11069

Locin, 12948

Pentologenin, 16890

Ponasterone C, 17702

Pterosterone, 18162

$\mathrm{C}_{27} \mathrm{H}_{44} \mathrm{O}_{8}$

$1 \alpha, 20 R$-Hydroxyecdysone, 10063

$5 \beta$-Hydroxyecdysterone, 10066

Polypodine B, 17662

Rhapontisterone, 18750

$\mathrm{C}_{27} \mathrm{H}_{44} \mathrm{O}_{10} \mathrm{~S}$

Aspidistrogenin A, 1899

\section{$\mathrm{C}_{27} \mathrm{H}_{44} \mathrm{O}_{13}$}

Atractyloside I, 1981

cis-Atractyloside I, 1982

$\mathrm{C}_{27} \mathrm{H}_{44} \mathrm{O}_{14}$

Dendroside D, 5123

\section{$\mathrm{C}_{27} \mathrm{H}_{45} \mathrm{NO}_{2}$}

Delavine, 4996

Ebeinine, 6660

Forticine, 7928

Hupehenine, 9681

17 $\alpha H$-Persicanidine A, 16988

Soladulcidine, 20042

Tomatidine, 21434

Tortifoline, 21466

\section{$\mathrm{C}_{27} \mathrm{H}_{45} \mathrm{NO}_{3}$}

Baimonidine, 2110

Delafrine, 4990

Hupehenidine, 9680

$15 \alpha$-Hydroxysoladulcidine, 10711

$15 \beta$-Hydroxysoladulcidine, 10712

15 $\alpha$-Hydroxytomatidine, 10772

Isobaimonidine, 11244

Isoverticine, 11770

Korseveramine, 12275

Korseveriline, 12276

Peimine, 16773

Wanpeinine A, 22636

Zhebeinine, 22993

\section{$\mathrm{C}_{27} \mathrm{H}_{45} \mathrm{NO}_{4}$}

Esculeogenin B, 7375

Isoesculeogenin A, 11418

Isoverticine- $\beta-\mathrm{N}$-oxide, 11771

Verticine- $N$-oxide, 22431

\section{$\mathrm{C}_{27} \mathrm{H}_{45} \mathrm{NO}_{5}$}

Pingpeimine A, 17384

\section{$\mathrm{C}_{27} \mathrm{H}_{45} \mathrm{NO}_{6}$}

Pingpeimine B, 17385

\section{$\mathrm{C}_{27} \mathrm{H}_{46} \mathrm{~N}_{2} \mathrm{O}_{2}$}

Solanocapsine, 20061

\section{$\mathrm{C}_{27} \mathrm{H}_{46} \mathrm{O}$}

Cholesterol, 3585

\section{$\mathrm{C}_{27} \mathrm{H}_{46} \mathrm{O}_{2}$}

$7 \alpha$-Hydroxycholesterol, 9904

$\delta$-Tocopherol, 21412

Vitamin $\mathrm{E}_{\delta}, 22560$

$\mathrm{C}_{27} \mathrm{H}_{46} \mathrm{O}_{4}$

Guggulsterol Y, 9075

Irisoquin D, 11176

$\mathrm{C}_{27} \mathrm{H}_{46} \mathrm{O}_{5}$

Coprocholic acid, 4031

Ponasterone B, 17701

$\mathrm{C}_{27} \mathrm{H}_{46} \mathrm{O}_{8}$

Microlepin, 14838 


\section{$\mathrm{C}_{27} \mathrm{H}_{46} \mathrm{O}_{10}$}

Crenulatoside A, 4228

Nerolidol-3- $O$ - $\alpha$ - $L$-rhamnopyranosyl $(1 \rightarrow 2)-\beta-D$ glucopyranoside, 15501

$\mathrm{C}_{27} \mathrm{H}_{46} \mathrm{O}_{11}$

Officinoside C, 16017

Officinoside D, 16018

$\mathrm{C}_{27} \mathrm{H}_{46} \mathrm{O}_{12}$

Atractyloside D, 1977

$\mathbf{C}_{27} \mathbf{H}_{46} \mathbf{O}_{13}$

$(2 R, 3 R, 5 R, 7 R, 10 S)$-Atractyloside G 2-O- $\beta$ - $D$ glucopyranoside, 1980

\section{$\mathrm{C}_{27} \mathrm{H}_{46} \mathbf{O}_{14}$}

Dictamnoside G, 5448

\section{$\mathrm{C}_{27} \mathrm{H}_{46} \mathbf{O}_{15}$}

Atractyloside A 14- $O-\beta$ - $D$-fructofuranoside, 1974

$\mathrm{C}_{27} \mathrm{H}_{47} \mathrm{NO}_{2}$

Hapepunine, 9220

$\mathrm{C}_{27} \mathrm{H}_{47} \mathrm{O}_{7}$

Marsin, 13578

$\mathrm{C}_{27} \mathrm{H}_{48} \mathrm{~N}_{2}$

Cycloprotobuxine C, 4531

$\mathrm{C}_{27} \mathrm{H}_{48} \mathrm{~N}_{2} \mathrm{O}$

Cyclovirobuxine, 4543

$\mathrm{C}_{27} \mathrm{H}_{48} \mathrm{O}_{4}$

Agavegenin D, 725

Tetrahydroxynorbufostane, 21138

\section{$\mathrm{C}_{27} \mathrm{H}_{48} \mathrm{O}_{5}$}

$5 \beta$-Bufol sulfate, 2720

$5 \alpha$-Cyprinol, 4582

$5 \beta$-Cyprinol, 4583

\section{$\mathrm{C}_{27} \mathrm{H}_{48} \mathrm{O}_{7}$}

$5 \alpha$-Cholest-3 $\beta, 6 \alpha, 7 \alpha, 8 \beta, 15 \alpha, 16 \beta, 26$-sevol, 3587

\section{$\mathrm{C}_{27} \mathrm{H}_{48} \mathrm{O}_{9}$}

(2S)-1-O-(9Z,12Z-Octadeca-dien-noyl)-3-O- $\beta$ - $D$ galactopyranosyl-glycerol, 15940

\section{$\mathrm{C}_{27} \mathrm{H}_{48} \mathrm{O}_{12}$}

Dendronobiloside A, 5120

\section{$\mathrm{C}_{27} \mathrm{H}_{48} \mathrm{O}_{14}$}

Dictamnoside M, 5454

\section{$\mathrm{C}_{27} \mathrm{H}_{49} \mathrm{NO}_{2}$}

Haloxyline A, 9200

\section{$\mathrm{C}_{27} \mathrm{H}_{49} \mathrm{NO}_{3}$}

Haloxyline B, 9201

\section{$\mathrm{C}_{27} \mathrm{H}_{51} \mathrm{O}_{11} \mathrm{~S}^{-}$}

(2S)-1-Stearoyl-3-O-(6-sulpho- $\alpha$ - $D$-quinovopyranosyl)-glycerol, 20282

$\mathrm{C}_{27} \mathrm{H}_{52} \mathrm{O}_{2}$

Heptacosan-4-olide, 9371

Pentacosyl vinyl ester, 16827

$\mathrm{C}_{27} \mathrm{H}_{52} \mathrm{O}_{4}$

Bifloride A, 2368
$\mathrm{C}_{27} \mathrm{H}_{56}$

n-Heptacosane, 9368

$\mathrm{C}_{27} \mathrm{H}_{56} \mathrm{O}$

14-Heptacosanol, 9369

Heptacosanol, 9370

$\mathrm{C}_{28} \mathrm{H}_{10} \mathrm{O}_{16}$

Tetrameric gallic acid, 21177

$\mathrm{C}_{28} \mathrm{H}_{14} \mathrm{Cl}_{8} \mathrm{O}_{4}$

Bazzanin R, 2186

$\mathrm{C}_{28} \mathrm{H}_{16} \mathrm{Cl}_{6} \mathrm{O}_{4}$

Bazzanin Q, 2185

$\mathrm{C}_{28} \mathrm{H}_{17} \mathrm{Cl}_{5} \mathrm{O}_{4}$

Bazzanin P, 2184

$\mathrm{C}_{28} \mathrm{H}_{17} \mathrm{O}_{7}$

Hopeafuran, 9636

$\mathrm{C}_{28} \mathrm{H}_{18} \mathrm{Cl}_{4} \mathrm{O}_{4}$

Bazzanin N, 2182

$\mathrm{C}_{28} \mathrm{H}_{18} \mathrm{O}_{10}$

Ustilaginoidin A, 22283

$\mathrm{C}_{28} \mathrm{H}_{19} \mathrm{Cl}_{3} \mathrm{O}_{4}$

Bazzanin M, 2181

$\mathrm{C}_{28} \mathrm{H}_{\mathbf{2 0}} \mathrm{Cl}_{\mathbf{6}} \mathbf{O}_{\mathbf{4}}$

6,6',10,10',12,12'-Hexachloroisoperrottetin A, 9478

$\mathrm{C}_{28} \mathrm{H}_{20} \mathrm{O}_{8}$

Cassigarol D, 3288

Cassigarol G, 3291

$\mathrm{C}_{28} \mathrm{H}_{22} \mathrm{O}_{4}$

Isoplagiochin C, 11604

$\mathrm{C}_{28} \mathrm{H}_{22} \mathrm{O}_{6}$

Ampelopsin B, 1077

Ampelopsin D, 1078

Gnetin C, 8879

Marchantin G, 13555

Maximol A, 13616

Maximol B, 13617

Pallidol, 16550

Resveratrol $E$-dehydrodimer, 18644

$\varepsilon$-Viniferin, 22505

(+)-६-Viniferin, 22506

$(-)-\varepsilon$-Viniferin, 22507

$\varepsilon$-iso-Viniferin, 22508

$\mathrm{C}_{28} \mathrm{H}_{22} \mathrm{O}_{7}$

(+)-Ampelopsin A, 1075

(-)-Ampelopsin A, 1076

(+)-Balanocarpol, 2124

Cassigarol C, 3287

Gnetuhainin A, 8882

Gnetuhainin C, 8883

2b-Hydroxyampelopsin F, 9779

Parvifolol A, 16688

Parvifolol B, 16689

Scirpusin A, 19531

\section{$\mathrm{C}_{28} \mathrm{H}_{22} \mathrm{O}_{8}$}

Cassigarol E, 3289

Cassigarol F, 3290

Gnetumontanin A, 8893

Parvifolol C, 16690

Scirpusin B, 19532

Terrestrin D, 21009

Tibeticanol, 21369

\section{$\mathrm{C}_{28} \mathrm{H}_{23} \mathrm{NO}_{8}$}

Oxohernandaline, 16343

\section{$\mathrm{C}_{28} \mathrm{H}_{24} \mathbf{N}_{2} \mathbf{O}_{3}$}

3,3'-[Oxybis(methylene)]bis(9-methoxy-9Hcarbazole), 16442

\section{$\mathrm{C}_{28} \mathrm{H}_{24} \mathrm{~N}_{4} \mathrm{O}_{4}$}

Picrasidine H, 17300

\section{$\mathrm{C}_{28} \mathrm{H}_{24} \mathrm{O}_{4}$}

Isomarchantin $\mathrm{C}, 11528$

Isoriccardin C, 11681

Marchantin B, 13551

Riccardin C, 18839

\section{$\mathrm{C}_{28} \mathrm{H}_{24} \mathrm{O}_{5}$}

Marchantin A, 13550

$\mathrm{C}_{28} \mathrm{H}_{24} \mathrm{O}_{6}$

Gnetin F, 8881

Marchantin C, 13552

Marchantin D, 13553

Marchantin L, 13558

\section{$\mathrm{C}_{28} \mathrm{H}_{24} \mathrm{O}_{7}$}

Goniolactone B, 8940

Goniolactone C, 8941

Goniolactone E, 8943

\section{$\mathrm{C}_{28} \mathrm{H}_{24} \mathrm{O}_{8}$}

Alboctalol, 863

Cassigarol A, 3285

Goniolactone D, 8942

\section{$\mathrm{C}_{28} \mathrm{H}_{24} \mathrm{O}_{12}$}

Methyl melitrate A, 14580

Monomethyl lithospermate, 14927

\section{$\mathrm{C}_{28} \mathrm{H}_{24} \mathrm{O}_{13}$}

Chrysophanol-8- $O-\beta-D-(6 '-O$-galloyl)-glucopyranoside, 3616

2"-O-p-Hydroxybenzoylorientin, 9827

\section{$\mathrm{C}_{28} \mathrm{H}_{24} \mathrm{O}_{14}$}

2"-O-Galloylisovitexin, 8112

2"-O-Galloylvitexin, 8119

\section{$\mathrm{C}_{28} \mathrm{H}_{24} \mathrm{O}_{15}$}

2"-O-Galloylisoorientin, 8111

2"-O-Galloylorientin, 8113

3'-Hydroxyscutellarein 7-O-(6"-O-protocatechuoyl)- $\beta$-glucopyranoside, 10695

Kaempferol 3-O-(6"-galloyl)- $\beta$ - $D$-glucopyranoside, 12048

Kaempferol-3-O-(2"-O-galloyl)- $\beta$ - $D$-glucoside, 
12049

Quercitrin-2"-gallate, 18412

\section{$\mathrm{C}_{28} \mathrm{H}_{24} \mathrm{O}_{16}$}

Desmanthin 2, 5252

Hyperin-2"- $O$-gallate, 10888

Myricetin-3-O-(2"-O-galloyl)- $\alpha$-rhamnopyranoside, 15180

Myricetin-3- $O$-(3"-O-galloyl)- $\alpha$-rhamnopyranoside, 15181

Quercetin-3-O-(6"-galloyl)-galactoside, 18358

Quercetin-3-O-(6"-galloyl)- $\beta$ - $D$-glucopyranoside, 18359

Quercetin-3-O-(2"-O-galloyl)- $\beta$ - $D$-glucoside, 18360

Quercetin-4'- $O-\beta$ - $D$-glucopyranoside-6"- gallate, 18361

\section{$\mathrm{C}_{28} \mathrm{H}_{24} \mathrm{O}_{17}$}

Myricetin-3- $O-\beta-D-(6 "-O$-galloyl)-galactopyranoside, 15177

Myricetin-3- $O$-(2"-O-galloyl)- $\beta$ - $D$-glucopyranoside, 15178

Myricetin-3- $O-\beta-D$-(6"- $O$-galloyl)-glucopyranoside, 15179

Quercetagetin-7- $O$-(6- $O$-galloyl- $\beta$ - $D$-glucopyranoside), 18310

\section{$\mathrm{C}_{28} \mathrm{H}_{25} \mathrm{~N}_{4} \mathrm{O}_{2}^{+}$}

Kumujansine A, 12332

\section{$\mathrm{C}_{28} \mathrm{H}_{25} \mathrm{~N}_{4} \mathrm{O}_{4}$}

Picrasidine T, 17311

\section{$\mathrm{C}_{28} \mathrm{H}_{25} \mathrm{O}_{16}{ }^{+}$}

Delphinidin-3'- $O$-(2"- $O$-galloyl- $\beta$-galactopyranoside), 5024

\section{$\mathrm{C}_{28} \mathrm{H}_{26} \mathrm{~N}_{2} \mathrm{O}_{10}$}

Ophiocordin, 16130

\section{$\mathrm{C}_{28} \mathrm{H}_{26} \mathrm{O}_{4}$}

Perrottetin E, 16984

$\mathrm{C}_{28} \mathrm{H}_{26} \mathrm{O}_{5}$

Perrottetin F, 16986

\section{$\mathrm{C}_{28} \mathrm{H}_{26} \mathrm{O}_{7}$}

Neocalyxin A, 15351

Neocalyxin B, 15352

Rhinacanthin Q, 18778

\section{$\mathrm{C}_{28} \mathrm{H}_{26} \mathrm{O}_{9}$}

Guangsangon L, 9064

Pannellin, 16618

Rocaglamide derivative 8,18888

\section{$\mathrm{C}_{28} \mathrm{H}_{26} \mathrm{O}_{12}$}

Diphyllin acetylapioside, 6491

\section{$\mathrm{C}_{28} \mathrm{H}_{26} \mathrm{O}_{14}$}

Naringenin 7-O-(6"-O-galloyl)-glucoside, 15281

\section{$\mathrm{C}_{28} \mathrm{H}_{26} \mathrm{O}_{15}$}

$(S)$-Eriodictyol-7-O-(6"-O-galloyl)- $\beta$ - $D$-glucopyranoside, 7282
$\mathrm{C}_{28} \mathrm{H}_{26} \mathrm{O}_{18}$

Methyl 2,3,6-tri- $O$-galloyl- $\beta$ - $D$-glucopyranoside, 14772

Methyl 3,4,6-tri- $O$-galloyl- $\beta$ - $D$-glucopyranoside, 14773

$\mathrm{C}_{28} \mathrm{H}_{28} \mathrm{O}_{6}$

Caloxanthone, 2997

Dulcisxanthone D, 6629

Garcimangosone A, 8206

Mangostenone A, 13488

\section{$\mathrm{C}_{28} \mathrm{H}_{28} \mathrm{O}_{8}$}

Bletilol A, 2515

Methyl rocaglate, 14714

$\mathrm{C}_{28} \mathrm{H}_{28} \mathrm{O}_{9}$

3'-Hydroxyrocaglate, 10677

\section{$\mathrm{C}_{28} \mathrm{H}_{28} \mathrm{O}_{11}$}

Aloeresin A, 973

6'-O-p-Coumaroylaloesin, 4146

Curtisian I, 4405

Isoaloeresin A, 11204

\section{$\mathrm{C}_{28} \mathrm{H}_{28} \mathrm{O}_{12}$}

(-)-Epicatechin-5-O- $\beta$ - $D$-glucopyranosyl-3benzoate, 6865

\section{$\mathrm{C}_{28} \mathrm{H}_{28} \mathrm{O}_{13}$}

Rhaponticin 2"-O-gallate, 18746

Rhaponticin 6"-O-gallate, 18747

cis-3,5,3'-Trihydroxy-4'-methoxystilbene-3- $O-\beta$ -

$D$-(2"-O-galloyl) glucopyranoside, 21786

cis-3,5,3'-Trihydroxy-4'-methoxystilbene-3-O- $\beta$ -

$D$-(6"-O-galloyl) glucopyranoside, 21787

\section{$\mathrm{C}_{28} \mathrm{H}_{28} \mathrm{O}_{15}$}

Isorhamnetin 3-O- $\beta-D-2 ", 3 ", 4 "$-triacetylglucopyranoside, 11670

\section{$\mathrm{C}_{28} \mathrm{H}_{28} \mathrm{O}_{17}$}

Acacetin-7-glucurono-( $1 \rightarrow 2)$-glucuronide, 58

$\mathrm{C}_{28} \mathrm{H}_{29} \mathrm{NO}_{7}$

Grossamide K, 9014

$\mathrm{C}_{28} \mathrm{H}_{29} \mathrm{NO}_{8}$

3'-Hydroxy- $N$-demethylrocaglamide, 9981

$\mathrm{C}_{28} \mathrm{H}_{30} \mathrm{~N}_{2} \mathrm{O}_{11}$

Desoxycordifoline, 5272

\section{$\mathrm{C}_{28} \mathrm{H}_{30} \mathrm{O}_{5}$}

Dulcisxanthone A, 6626

Nigrolineaxanthone S, 15592

\section{$\mathrm{C}_{28} \mathrm{H}_{30} \mathrm{O}_{6}$}

Allanxanthone B, 918

Cowagarcinone D, 4198

Tovophyllin A, 21481

Virgataxanthone B, 22525

\section{$\mathrm{C}_{28} \mathrm{H}_{30} \mathrm{O}_{9}$}

Physalin B, 17240

Physalin C, 17241

$\mathrm{C}_{28} \mathrm{H}_{30} \mathrm{O}_{10}$
Buergeriside $A_{1}, 2709$

$5 \beta, 6 \beta$-Epoxyphysalin B, 7187

Physalin A, 17239

Pilosanol C, 17363

$\mathrm{C}_{28} \mathrm{H}_{30} \mathrm{O}_{12}$

6"'-O-Acetylamurensin, 315

\section{$\mathrm{C}_{28} \mathrm{H}_{30} \mathrm{O}_{13}$}

Dracunculifoside L, 6591

Pabularin A, 16485

Pabularin B, 16486

Pabularin C, 16487

Praeroside I, 17756

\section{$\mathrm{C}_{28} \mathrm{H}_{30} \mathrm{O}_{14}$}

Cassiaoccidentalin C, 3283

\section{$\mathrm{C}_{28} \mathrm{H}_{30} \mathrm{O}_{15}$}

1-Methoxy-3-hydroxy-2-carbomethoxy-9,10anthraquinone 3-O- $\beta$-primeveroside, 13953

2-Methyl-1,3,6-trihydroxy-9,10-anthraquinone3 - $O-\beta$ - $D$-xylosyl- $(1 \rightarrow 2)-\beta$ - $D$-(6'- $O$-acetyl $)$ glucoside, 14779

\section{$\mathrm{C}_{28} \mathrm{H}_{30} \mathrm{O}_{17}$}

Quercetin-7-O- $\alpha-L$-Arabinosyl-3- $O-\beta-D-6$ "-acetyl glucopyranoside, 18329

\section{$\mathrm{C}_{28} \mathrm{H}_{31} \mathrm{NO}_{8}$}

erythro-Canabisine H, 3054

\section{$\mathrm{C}_{28} \mathrm{H}_{32} \mathrm{~N}_{2} \mathrm{O}_{9}$}

6-O-Acetylstritosamide, 515

\section{$\mathrm{C}_{28} \mathrm{H}_{32} \mathrm{O}_{5}$}

Cochinchinone A, 3870

\section{$\mathrm{C}_{28} \mathrm{H}_{32} \mathrm{O}_{6}$}

Allanxanthone C, 919

Cochinchinone B, 3871

Dulcisxanthone E, 6630

GarcinianoneA, 8210

GarcinianoneB, 8211

Garciniaxanthone E, 8212

Garcinone E, 8222

Norcowanin, 15728

1,3,5,6-Tetrahydroxy-4,7,8-tri(3-methyl-2-butenyl)xanthone, 21168

Virgataxanthone A, 22524

\section{$\mathrm{C}_{28} \mathrm{H}_{32} \mathrm{O}_{8}$}

Griffonianone A, 9007

\section{$\mathrm{C}_{28} \mathrm{H}_{32} \mathrm{O}_{9}$}

11-Oxo-7 $\alpha$-obacunyl acetate, 16398

Physalin M, 17244

\section{$\mathrm{C}_{28} \mathrm{H}_{32} \mathrm{O}_{10}$}

Physalin L, 17243

Pilosanol B, 17362

Rutaevin acetate, 19082

$\mathrm{C}_{28} \mathrm{H}_{32} \mathrm{O}_{11}$

Physalin D, 17242

$\mathrm{C}_{28} \mathrm{H}_{32} \mathrm{O}_{12}$ 
6"'-O-Acetylphellamurin, 481

Hymenoside I, 10852

\section{$\mathrm{C}_{28} \mathrm{H}_{32} \mathrm{O}_{13}$}

Derriscanoside A, 5227

$\beta$-Peltatin glucoside, 16796

$L$-Picropodophyllin 7'- $O$ - $\beta$ - $D$-glucopyranoside, 17339

Podophyllotoxin 7'- $O$ - $\beta$ - $D$-glucopyranoside, 17593

\section{$\mathrm{C}_{28} \mathrm{H}_{32} \mathrm{O}_{14}$}

Acaciin, 61

Chrysoeriol 6- $C$ - $\beta$-boivinopyranosyl-7- $O-\beta$ glucopyranoside, 3606

Derriscandenoside B, 5223

1,2-Di- $O$ - $E$-sinapoyl- $\beta$-glucopyranose, 6509

1,6-Di- $O$-sinapoylglucose, 6510

Fortunellin, 7930

Hirsutissimiside B, 9555

Lasianthuoside C, 12533

Liquiritigenin-7-O- $\beta$ - $D$-(3- $O$-acetyl)-apiofuranosyl-4'- $O-\beta$ - $D$-glucopyranoside, 12909

Physcion-8-O-rhamnosyl-( $1 \rightarrow 2$ )-glucoside, 17252

Saikoisoflavonoside A, 19137

\section{$\mathrm{C}_{28} \mathrm{H}_{32} \mathrm{O}_{15}$}

Abrusin-2"- $O$-apioside, 29

Chrysoeriol 7-O-rutinoside, 3610

5,4'-Dihydroxyl-6,7-dimethoxyl-8- $C$-[ $\beta$ - $D$-xylocopyranosyl-( $(1 \rightarrow 2)]-\beta$ - $D$-glucopyranosyl flavone, 5949

Diosmin, 6454

Flavocommelin, 7817

Hirsutissimiside C, 9556

Kaempferide-3-O-neohesperidoside, 12016

Physciondiglucoside, 17249

Physcion-8- $O-\beta$ - $D$-gentiobioside, 17250

Rhamnetin-3-O-rhamnosyl

(1 $\rightarrow 4$ )-rhamnopyranoside, 18681

Spinosin, 20172

Wikstroemin, 22668

Zivulgarin, 23007

\section{$\mathrm{C}_{28} \mathrm{H}_{32} \mathrm{O}_{16}$}

Complanatuside, 3949

Isorhamnetin 3-O- $\beta$-D-glucopyranosyl-(1-2)$\alpha$-L-rhamnopyranoside, 11658

Isorhamnetin-3-O-glucosyl-7- $O$-rhamnoside, 11661

Isorhamnetin-3-O-neohesperidoside, 11662

Isorhamnetin-3-O-robinobioside, 11668

Isorhamnetin-3-O-rutinoside, 11669

Keioside, 12190

Narcissin, 15265

Tamarixetin 3-O-neohesperidoside, 20660
Tectorigenin-4'-glucosyl $(1 \rightarrow 6)$ glucoside, 20903

5,3',4'-Trihydroxyl-6,7-dimethoxyl-8- $C$ - $[\beta-D$ xylocopyranosyl- $(1 \rightarrow 2)]$ - $\beta$ - $D$-glucopyranosyl flavone, 21761

$\mathrm{C}_{28} \mathrm{H}_{32} \mathrm{O}_{17}$

Isorhamnetin 3,7-O-di- $\beta$ - $D$-glucopyranoside, 11655

Isorhamnetin-3,4'-diglucoside, 11656

Isorhamnetin-7- $\beta-\mathrm{D}-$ gentiobioside, 11657

Patuletin-3-O- $\beta$ - $D$-robinabioside, 16729

Quercetagetin 7-methylether 3- $O$-neohesperidoside, 18315

Tamarixetin 3-O- $\beta$ - $D$-glucopyranoside

7-O- $\beta$ - $D$-glucopyranoside, 20658

\section{$\mathrm{C}_{28} \mathrm{H}_{32} \mathrm{O}_{18}$}

Quercetagetin 7-methylether 3- $O$-cellobioside, 18312

\section{$\mathrm{C}_{28} \mathrm{H}_{33} \mathrm{~N}_{2} \mathrm{O}_{9}^{+}$}

3,4,5,6-Tetradehydrodolichantoside, 21049

\section{$\mathrm{C}_{28} \mathrm{H}_{33} \mathrm{O}_{15}{ }^{+}$}

Peonidin-3-O-(6"- $O$ - $\alpha$-rhamnopyranosyl- $\beta$-glucopyranoside), 16904

$\mathrm{C}_{28} \mathrm{H}_{33} \mathrm{O}_{16}{ }^{+}$

Peonin, 16905

$\mathrm{C}_{28} \mathrm{H}_{33} \mathrm{O}_{17}{ }^{+}$

Petudin-3,7-di- $O$-( $\beta$ - $D$-glucopyranoside), 17023

$\mathrm{C}_{28} \mathrm{H}_{34} \mathrm{~N}_{2} \mathrm{O}_{11}$

(5S)-5-Carboxystrictosidine, 3184

\section{$\mathrm{C}_{28} \mathrm{H}_{34} \mathrm{O}_{2}$}

Bornylmagnolol, 2561

Piperitylmagnolol, 17458

Piperitylhonokiol, 17459

$\mathrm{C}_{28} \mathrm{H}_{34} \mathrm{O}_{5}$

Peperovulcanone B, 16908

$\mathrm{C}_{28} \mathrm{H}_{34} \mathrm{O}_{6}$

$6 \alpha$-Hydroxyazadiradione, 9808

Nicandrenone II, 15521

Nimbinin, 15602

Withaphysalin A, 22716

$\mathrm{C}_{28} \mathrm{H}_{34} \mathrm{O}_{7}$

Gedunin, 8249

\section{$\mathrm{C}_{28} \mathrm{H}_{34} \mathrm{O}_{8}$}

Angeloyl gomisin O, 1207

Angeloylisogomisin O, 1212

Heteroclitin B, 9461

Heteroclitin C, 9462

Mallotophilippen A, 13433

Uliginosin B, 22191

\section{$\mathrm{C}_{28} \mathrm{H}_{34} \mathrm{O}_{9}$}

Ananosin A, 1139

Angeloyl gomisin P, 1208

Gomisin B, 8907

Gomisin E, 8910
Gomisin F, 8911

$11 \beta$-Hydroxy-7 $\alpha$-obacunyl acetate, 10544

Nomilin, 15655

Schisantherin C, 19489

Tigloylgomisin P, 21377

3,7,4'-Trihydroxy-3'-(8"-acetoxy-7"-methyloctyl)-5,6-dimethoxyflavone, 21685

\section{$\mathrm{C}_{28} \mathrm{H}_{34} \mathrm{O}_{10}$}

Gomisin D, 8909

Taiwanschirin D, 20646

\section{$\mathrm{C}_{28} \mathrm{H}_{34} \mathrm{O}_{13}$}

Dracunculifoside O, 6594

Podorhizol $\beta$ - $D$-glucoside, 17595

\section{$\mathrm{C}_{28} \mathrm{H}_{34} \mathrm{O}_{14}$}

Isosakuranetin-7-rutinoside, 11692

Poncirin, 17705

\section{$\mathrm{C}_{28} \mathrm{H}_{34} \mathrm{O}_{15}$}

Cusianoside A, 4419

Cusianoside B, 4420

Hesperidin, 9458

Hydroxysafflor yellow A, 10684

Mesuein, 13810

Neohesperidin, 15404

Paederotoside, 16518

\section{$\mathrm{C}_{28} \mathrm{H}_{34} \mathrm{O}_{16}$}

(2S)-Homoeriodictyol

7,4'-di- $O$ - $\beta$ - $D$-glucopyranoside, 9605

$2,3,4,7$-Tetramethoxyxanthone-1- $O-\beta$ - $D$-xylopyranosyl-( $(1 \rightarrow 6)-\beta$ - $D$-glucopyranoside, 21191

Veratriloside C, 22387

\section{$\mathrm{C}_{28} \mathrm{H}_{35} \mathrm{ClO}_{11}$}

Junceellin, 11944

$\mathrm{C}_{28} \mathrm{H}_{35} \mathrm{ClO}_{12}$

Praelolide, 17755

$\mathrm{C}_{28} \mathrm{H}_{35} \mathrm{ClO}_{13}$

Juncin P, 11956

\section{$\mathrm{C}_{28} \mathrm{H}_{35} \mathrm{NO}_{8}$}

Anhydroharringtonine, 1270

\section{$\mathrm{C}_{28} \mathrm{H}_{36} \mathrm{~N}_{2} \mathrm{O}_{4}$}

Psychotrine, 18095

$\mathrm{C}_{28} \mathrm{H}_{36} \mathrm{~N}_{2} \mathrm{O}_{5}$

Alangicine, 829

$\mathrm{C}_{28} \mathrm{H}_{36} \mathrm{O}_{3}$

6-Oxo-iguesterol, 16359

Tingenone, 21397

\section{$\mathrm{C}_{28} \mathrm{H}_{36} \mathrm{O}_{4}$}

Daturilin, 4673

Grevirobstol C, 9000

6-Oxotingenol, 16429

Tingenin B, 21396

\section{$\mathrm{C}_{28} \mathrm{H}_{36} \mathrm{O}_{5}$}

$6 \alpha$-O-Acetyl-7-deacetylnimocinol, 364

Benzoylramanone, 2268 
$5 \beta, 6 \beta$-Epoxy-4 $\beta$-hydroxy-1-oxowitha-2,14,24trienolide, 7128

$5 \beta, 6 \beta$-Epoxy- $4 \beta$-hydroxy-1-oxo-witha-2,16,24trienolide, 7129

$17 \beta$-Hydroxy-14 $\alpha, 20 \alpha$-epoxy-1-oxo-(22R)-witha3,5,24-trienolide, 10092

Salaquinone B, 19178

Withacoagulin, 22699

\section{$\mathrm{C}_{28} \mathrm{H}_{36} \mathrm{O}_{6}$}

Azadironolide, 2051

12-O-Benzoylisolineolone, 2246

Withanicandrin, 22701

Withanone, 22705

Withaphysalin B, 22717

$\mathrm{C}_{28} \mathrm{H}_{36} \mathrm{O}_{7}$

Turraparvin A, 22138

Turraparvin B, 22139

\section{$\mathrm{C}_{28} \mathrm{H}_{36} \mathrm{O}_{8}$}

Angeloyl gomisin H, 1206

[(2S,3R,4R)-4-(3,4-Dimethoxybenzyl)-2-

(3,4,5-trimethoxyphenyl)-tetrahydrofuran-3-

yl]-methyl (2Z)-2-methylbut-2-en-oate, 6212

Heteroclitin A, 9460

Propinquanin F, 17929

Qingyangshengenin, 18291

Uliginosin A, 22190

\section{$\mathrm{C}_{28} \mathrm{H}_{36} \mathrm{O}_{9}$}

Forrestin F, 7920

Kupitengester 4, 12339

\section{$\mathrm{C}_{28} \mathrm{H}_{36} \mathrm{O}_{10}$}

Celahin C, 3359

Nomilinic acid, 15656

\section{$\mathrm{C}_{28} \mathrm{H}_{36} \mathrm{O}_{11}$}

Bruceantin, 2660

Glabcensin R, 8479

\section{$\mathrm{C}_{28} \mathrm{H}_{36} \mathrm{O}_{12}$}

Bruceine C, 2666

\section{$\mathrm{C}_{28} \mathrm{H}_{36} \mathrm{O}_{13}$}

Acanthoside B, 85

JuncenolideB, 11950

\section{$\mathrm{C}_{28} \mathrm{H}_{36} \mathrm{O}_{14}$}

2-(4-Hydroxyphenyl)ethyl 1-O- $\beta$ - $D$-[5-O-(3,4-dimethoxybenzoyl)]-apiofuranosyl-( $1 \rightarrow 6)-\beta-D$ glucopyranoside, 10618

Magnolenin C, 13376

Tyrolobibenzyl E, 22160

\section{$\mathrm{C}_{28} \mathrm{H}_{36} \mathrm{O}_{15}$}

2,4-Dimethoxyphenol 1-O- $\beta$ - $D$-[5-O-(3,4-dimethoxybenzoyl)]-apiofuranosyl- $(1 \rightarrow 6)$ $\beta$ - $D$-glucopyranoside, 6274

3,4-Dimethoxyphenyl 1-O- $\beta$ - $D$-[5- $O$-(3,4-dimethoxybenzoyl)]-apiofuranosyl-( $1 \rightarrow 6)$ $\beta$ - $D$-glucopyranoside, 6281

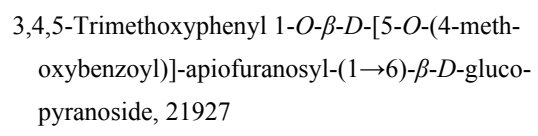

3,4,5-Trimethoxyphenyl 1-O- $\beta$ - $D$-[5-O-(4-methoxybenzoyl)]-apiofuranosyl-( $1 \rightarrow 6)-\beta$ - $D$-glucopyranoside, 21927

$\mathrm{C}_{28} \mathrm{H}_{36} \mathrm{O}_{16}$

Piloside A, 17368

$\mathrm{C}_{28} \mathrm{H}_{36} \mathrm{O}_{17}$

Piloside B, 17369

Rubinaphthin D, 19035

$\mathrm{C}_{28} \mathrm{H}_{37} \mathrm{NO}_{5}$

Lythranine, 13280

Turraparvin D, 22141

$\mathrm{C}_{28} \mathrm{H}_{37} \mathrm{NO}_{7}$

Guan-fu base P, 9050

$\mathrm{C}_{28} \mathrm{H}_{37} \mathrm{NO}_{8}$

Deoxyharringtonine, 5181

$\mathrm{C}_{28} \mathrm{H}_{37} \mathrm{NO}_{9}$

Harringtonine, 9239

Isoharringtonine, 11449

$\mathrm{C}_{28} \mathrm{H}_{38} \mathrm{~N}_{2} \mathrm{O}_{4}$

Cephaeline, 3400

$\mathrm{C}_{28} \mathrm{H}_{38} \mathrm{~N}_{8} \mathrm{O}_{5}$

Hordatine A, 9644

\section{$\mathrm{C}_{28} \mathrm{H}_{38} \mathrm{O}_{4}$}

Gerronemin F, 8365

Grevirobstol A, 8998

20 $\beta$-Hydroxy-1-oxo-(22R)-witha-2,5,24-trienolide, 9743

27-Hydroxy-3-oxo-witha-1,4,24-trienolide, 10587

\section{$\mathrm{C}_{28} \mathrm{H}_{38} \mathrm{O}_{5}$}

Daturilinol, 4674

Euglobal III, 7531

Euglobal V, 7532

Lycium substance B, 13181

Meldenin, 13661

Withacoagin, 22698

Withanolide B, 22703

\section{$\mathrm{C}_{28} \mathrm{H}_{38} \mathrm{O}_{6}$}

Ajugin, 812

(20S,22R)-5 $\alpha, 27-D i h y d r o x y-6 \alpha, 7 \alpha$-epoxy-1-oxowitha-2,24-dienolide, 5880

$6 \alpha, 7 \alpha$-Epoxy-5 $\alpha, 20 \beta$-dihydroxy-1-oxowitha- 2 ,

24-dienolide, 7082

Methyl lucidenate F, 14562

Nicandrin B, 15522

Withaferin A, 22700

Withanolide A, 22702

Withanolide D, 22704

\section{$\mathrm{C}_{28} \mathrm{H}_{38} \mathrm{O}_{7}$}

Ajugin E, 813

$5 \beta, 6 \beta$-Epoxy- $4 \beta, 17 \alpha, 27$-trihydroxy-1-oxowitha-2, 24-dienolide, 7210

(20S,22R)-5 $\beta, 6 \beta$-epoxy- $4 \beta, 14 \beta, 15 \alpha$-trihydroxy-1- oxowith-2,24-dienolide, 7214

18-Hydroxywithanolide D, 10827

27-Hydroxy withanone, 10828

\section{$\mathrm{C}_{28} \mathrm{H}_{38} \mathrm{O}_{8}$}

Anticancer Withanolide PMV70P691-046, 1452

Penisimplicin B, 16807

\section{$\mathrm{C}_{28} \mathrm{H}_{38} \mathrm{O}_{8} \mathrm{~S}$}

Daturametelin F, 4670

\section{$\mathrm{C}_{28} \mathrm{H}_{38} \mathrm{O}_{9}$}

Gesneroidin D, 8369

Glabcensin W, 8484

Lungshengenin E, 13081

Lushanrubescensin C, 13118

Rabyuennane A, 18510

Taxinine H, 20801

\section{$\mathrm{C}_{28} \mathrm{H}_{38} \mathrm{O}_{10}$}

Bulleyanin, 2743

Calcicolin A, 2947

Gesneroidin C, 8368

Glabcensin A, 8462

Glabcensin B, 8463

Inflexusin, 11055

Lungshengenin B , 13077

Lushanrubescensin A, 13116

Nervosanin, 15507

Rabdoforrestin A, 18461

Rabdoforrestin A', 18462

Rabdosianin B, 18488

Rabdosinate, 18497

Taxine B', 20796

Taxuspine F, 20854

$2 \alpha, 5 \alpha, 7 \beta, 13 \alpha$-Tetraacetoxy-10 $\beta$-hydroxy-2

$(3 \rightarrow 20)$ abeotaxan-9-one, 21029

\section{$\mathrm{C}_{28} \mathrm{H}_{38} \mathrm{O}_{11}$}

Ajugapantin A, 808

Decinnamoyltaxinine B 11,12-oxide, 4855

Junceellolide D, 11946

Pseudolaric acid A- $O-\beta$ - $D$-glucopyranoside, 18032

Tasumatrol N, 20724

Taxumairol C, 20823

Taxumairol I, 20829

\section{$\mathrm{C}_{28} \mathrm{H}_{38} \mathrm{O}_{12}$}

$5 \alpha$-Decinnamoyltaxagifine, 4853

Javanicolide D, 11854

\section{$\mathrm{C}_{28} \mathrm{H}_{38} \mathrm{O}_{13}$}

(+)-5,5'-Dimethoxy-9-O- $\beta$ - $D$-glucopyranosyl lariciresinol, 6230

(+)-Lyoniresinol-2 $\alpha$ - $O$ - $\beta$ - $D$-glucopyranoside, 13250

(-)-Lyoniresinol-3 $\alpha$ - $O-\beta$ - $D$-glucopyranoside, 13253

$\mathrm{C}_{28} \mathrm{H}_{39} \mathrm{ClO}_{10}$ 
Juncenolide A, 11949

$$
\begin{aligned}
\mathbf{C}_{28} \mathbf{H}_{39} \mathbf{N O} \\
N \text {-[ } \\
\quad \text { 17-amide, } 17118
\end{aligned}
$$

\section{$\mathrm{C}_{28} \mathrm{H}_{39} \mathrm{NO}_{6}$}

Lycoposerramine O, 13234

\section{$\mathrm{C}_{28} \mathrm{H}_{40} \mathrm{O}$}

Ergosta-4,6,8(14),22-tetraen-3-one, 7247

\section{$\mathrm{C}_{28} \mathrm{H}_{40} \mathrm{O}_{3}$}

9(11)-Dehydroaxinysterol, 4880

2,8-Dimethyl-2-[(3E,7E)-4,8,12-trimethyltrideca3,7,11-trienyl]-5-formyl-chroman-6-ol, 6415

2,8-Dimethyl-2-[(3E,7E)-4,8,12-trimethyltrideca-

3,7,11-trienyl]-7-formyl-chroman-6-ol, 6416

\section{$\mathrm{C}_{28} \mathrm{H}_{40} \mathrm{O}_{4}$}

Grevirobstol B, 8999

Regeol A, 18584

Siraitic acid C, 19973

\section{$\mathrm{C}_{28} \mathrm{H}_{40} \mathrm{O}_{5}$}

Siraitic acid D, 19974

Siraitic acid E, 19975

\section{$\mathrm{C}_{28} \mathrm{H}_{40} \mathrm{O}_{6}$}

Ajugin F, 814

Anticancer Withanolide PMV70P691-049, 1455

Anticancer Withanolide PMV70P691-149, 1457

4,20-Dideoxyphorbol 12,13-bis(isobutyrate), 5476

2,3-Dihydrowithaferin A, 5739

24,25-Dihydrowithanolide A, 5740

Macrocarpal A, 13315

Methyl lucidenate A, 14559

Surinone A, 20493

$4 \beta, 7 \beta, 20 R$-Trihydroxy-1-oxowitha-2,5-dien-

22,26-olide, 21834

\section{$\mathrm{C}_{28} \mathrm{H}_{40} \mathrm{O}_{7}$}

Anticancer Withanolide PMV70P691-047, 1453

4-Deoxyphorbol 12,13-bis(isobutyrate), 5202

4-Epi-4-deoxyphorbol 12,13-bis(isobutyrate), 6892

$6 \alpha, 7 \alpha$-Epoxy-3 $\beta, 5 \alpha, 17 \alpha$-trihydroxy-1-oxo-witha24-enolide, 7211

(20S,22R)-3 $\alpha, 6 \alpha$-Epoxy-4 $\beta, 5 \beta, 27-$ trihydroxy-1oxowitha-24-enolide, 7212

$6 \alpha, 7 \alpha$-Epoxy-3 $\beta, 5 \alpha, 20 \beta$-trihydroxy-1-oxowitha-24-enolide, 7213

Methyl lucidenate L, 14563

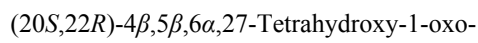
witha-2,24-dienolide, 21148

Withaphysacarpin, 22715

\section{$\mathrm{C}_{28} \mathrm{H}_{40} \mathrm{O}_{8}$}

Anhydrohirundigenin monothevetoside, 1272 Anticancer Withanolide PMV70P691-045, 1451 Anticancer Withanolide PMV70P691-048, 1454
2,3-Dihydroixocarpalactone B, 5657

Ixocarpalactone $\mathrm{A}, 11804$

$3 \beta$-Oxo-formyl-7 $\beta, 12 \beta$-dihydroxy-4,4,14 $\alpha$-trimethyl-5 $\alpha$-chol-11,15-dioxo-8-en $(E)$-24-oic acid, 16336

Taxusin, 20840

Taxuyunnanine C, 20875

\section{$\mathrm{C}_{28} \mathrm{H}_{40} \mathrm{O}_{9}$}

Adenanthin D, 608

2-Deacetoxy-5-decinnamoyl taxinine J, 4704

Decinnamoyltaxinine E, 4854

Glaocogenin $C$ mono- $D$-thevetoside, 8507

14 $\beta$-Hydroxytaxusin, 10740

$7 \beta$-Hydroxy- $2 \alpha, 5 \alpha, 10 \beta, 14 \beta$-tetraacetoxytaxa4(20),11-diene, 10743

Rabyuennane C, 18512

Taxa-4(20),11-diene-5 $\alpha$-hydroxy- $1 \beta, 7 \beta, 9 \alpha, 10 \beta$ tetraacetate, 20741

$5 \alpha, 9 \alpha, 10 \beta, 13 \alpha$-Tetraacetoxy-15-hydroxy11(15 $\rightarrow 1$ )-abeo-taxa-4(20),11-diene, 21028

\section{$\mathrm{C}_{28} \mathrm{H}_{40} \mathrm{O}_{10}$}

Calcicolin D, 2953

Calcicolin E, 2954

$2 \alpha, 5 \alpha$-Dihydroxy-7 $\beta, 9 \alpha, 10 \beta, 13 \alpha$-tetraacetoxy4(20),11-taxadiene, 6140

Forrestin D, 7918

Neocynapanogenin $\mathrm{C}$

3-O- $\beta$ - $D$-oleandropyranoside, 15388

Taxezopidine F, 20785

Teixidol, 20905

\section{$\mathrm{C}_{28} \mathrm{H}_{40} \mathrm{O}_{10} \mathrm{~S}$}

$5 \alpha, 17 \alpha$-Dihydroxy- $6 \alpha, 7 \alpha$-epoxy-1-oxo- $3 \beta-O$ sulfate-witha-24-enolide, 5879

\section{$\mathrm{C}_{28} \mathrm{H}_{40} \mathrm{O}_{11}$}

7-Deacetylcanadensene, 4734

13-Deacetylcanadensene, 4735

5-Deacetyltaxachitriene B, 4777

Picrasinoside B, 17321

Tasumatrol M, 20723

Taxchinin G, 20774

Taxuspine U, 20868

$\mathrm{C}_{28} \mathrm{H}_{40} \mathrm{O}_{12}$

7,9-Deacetylbaccatin IV, 4723

$1 \beta$-Hydroxy- $2 \alpha, 7 \beta$-deacetylbaccatin I, 9960

Taxacustin, 20739

Taxumairol B, 20822

Taxumairol W, 20836

\section{$\mathrm{C}_{28} \mathrm{H}_{40} \mathrm{O}_{13}$}

(+)-5,5'-Dimethoxy-9-O- $\beta$ - $D$-glucopyranosyl secoisolariciresinol, 6231

\section{$\mathrm{C}_{28} \mathrm{H}_{40} \mathrm{O}_{18}$}

Arillatose C, 1686

Arillatose E, 1688
$\mathrm{C}_{28} \mathrm{H}_{41} \mathrm{O}_{11}$

Dracunculifoside A, 6580

$\mathrm{C}_{28} \mathrm{H}_{42} \mathrm{Cl}_{2} \mathrm{O}_{4}$

Abamagenin, 1

$\mathrm{C}_{28} \mathrm{H}_{42} \mathrm{~N}_{4} \mathrm{O}_{6}$

Kukoamine A, 12327

$\mathrm{C}_{28} \mathrm{H}_{42} \mathrm{O}$

Isoergosterone, 11413

$\mathrm{C}_{28} \mathrm{H}_{42} \mathrm{O}_{3}$

Axinysterol, 2046

$5 \alpha, 8 \alpha$-Epidioxyergosta-6,9(11),22-trien-3 $\alpha$-ol, 6903

$5 \alpha, 8 \alpha$-Epidioxyergosta-6,9(11),22-trien-3 $\beta$-ol, 6904

Peperovulcanone A, 16907

\section{$\mathrm{C}_{28} \mathrm{H}_{42} \mathrm{O}_{4}$}

$5 \alpha, 9 \alpha$-Epidioxy- $8 \alpha, 14 \alpha$-epoxy-(22E)-ergosta6,22-dien-3 $\beta$-ol, 6898

$5 \alpha, 9 \alpha$-Epidioxy-3 $\beta$-hydroxy-(22E)-ergosta7,22-dien-6-one, 6905

Triptocalline A, 21996

\section{$\mathrm{C}_{28} \mathrm{H}_{42} \mathrm{O}_{6}$}

Lucidenic acid $\mathrm{LM}_{1}, 13037$

Methyl lucidenate Q, 14565

Periplocagenin, 16942

$3 \beta, 6 \beta, 19 \alpha$-Trihydroxy-23-oxo-urs-12-en-28-oic acid, 21833

\section{$\mathrm{C}_{28} \mathrm{H}_{42} \mathrm{O}_{7}$}

Caseamembrin B, 3259

Caudatin, 3329

Lucidenic acid G, 13031

Methyl butyric acid tussilagin ester, 14188

\section{$\mathrm{C}_{28} \mathrm{H}_{42} \mathrm{O}_{8}$}

(20R,22R)-5 $\alpha, 6 \beta, 14 \alpha, 20,27-P e n t a h y d r o x y-1-$ oxowitha-24-enolide, 16858

Salvigreside D, 19215

$\mathrm{C}_{28} \mathrm{H}_{42} \mathrm{O}_{11}$

Picrasinoside C, 17322

Taxane 5, 20752

$\mathrm{C}_{28} \mathrm{H}_{42} \mathrm{O}_{12}$

Taxayuntin G, 20765

Taxumairol V, 20835

\section{$\mathrm{C}_{28} \mathrm{H}_{43} \mathrm{~N}_{5} \mathrm{O}_{12} \mathrm{~S}$}

Ustiloxin, 22284

\section{$\mathrm{C}_{28} \mathrm{H}_{44} \mathrm{~N}_{2} \mathrm{O}_{2}$}

Sarcovagine D, 19365

\section{$\mathrm{C}_{28} \mathrm{H}_{44} \mathrm{~N}_{4} \mathrm{O}_{4}$}

Adouetine X, 648

Frangulanine, 7938

$\mathrm{C}_{28} \mathrm{H}_{44} \mathrm{O}$

Ergosterol, 7250

Vitamin $\mathrm{D}_{2}, 22558$

$\mathrm{C}_{28} \mathrm{H}_{44} \mathrm{O}_{2}$ 


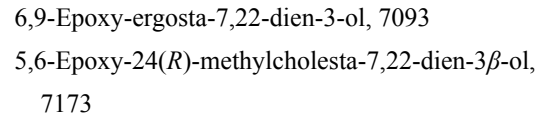

$5 \alpha, 8 \alpha$-Epidioxy-24(R)-methylcholesta-6,22-diene-3 $\beta$-ol, 6906

Ergosterol peroxide, 7251

24-Methyl-7-oxocholesta-5,24(28)-diene-3 $\beta, 19$ diol, 14643

\section{$\mathrm{C}_{28} \mathrm{H}_{44} \mathrm{O}_{4}$}

Bethogenin, 2324

$5 \alpha, 9 \alpha$-Epidioxy-(22E)-ergosta-7,22-diene-3 $\beta, 6 \alpha$ diol, 6899

$5 \alpha, 9 \alpha$-Epidioxy-(22E)-ergosta-7,22-diene-3 $\beta, 6 \beta$ diol, 6900

2-n-Heptadecy-5,7-dihydroxy-6,8-dimethyl chromone, 9391

$3 \beta, 5 \alpha, 9 \alpha$-Trihydroxy-(22E)-ergosta-7,22-dien-6one, 21729

\section{$\mathrm{C}_{28} \mathrm{H}_{44} \mathrm{O}_{5}$}

Polyporusterone D, 17668

Polyporusterone E, 17669

Polyporusterone G, 17671

$3 \beta, 5 \alpha, 9 \alpha, 14 \beta$-Tetrahydroxy-(22E)-ergosta-7,22dien-6-one, 21106

\section{$\mathrm{C}_{28} \mathrm{H}_{44} \mathrm{O}_{6}$}

Polyporusterone B, 17666

Polyporusterone C, 17667

\section{$\mathrm{C}_{28} \mathrm{H}_{44} \mathrm{O}_{8}$}

Lemnabourside D, 12622

\section{$\mathrm{C}_{28} \mathrm{H}_{44} \mathrm{O}_{8} \mathrm{~S}_{2}{ }^{2-}$}

Lembehsterol B, 12614

\section{$\mathrm{C}_{28} \mathrm{H}_{44} \mathrm{O}_{9} \mathrm{~S}$}

Cilistol Y, 3648

\section{$\mathrm{C}_{28} \mathrm{H}_{44} \mathrm{O}_{12}$}

Picrasinoside G, 17325

\section{$\mathrm{C}_{28} \mathrm{H}_{45} \mathrm{NO}_{2}$}

Impranine, 11005

\section{$\mathrm{C}_{28} \mathrm{H}_{45} \mathrm{NO}_{5}$}

Holantosine B, 9580

Holantosine D, 9582

\section{$\mathrm{C}_{28} \mathrm{H}_{45} \mathrm{NO}_{6}$}

Coumingidine, 4191

$\mathrm{C}_{28} \mathrm{H}_{45} \mathrm{O}_{12} \mathrm{~S}_{3}{ }^{3-}$

Lembehsterol A, 12613

\section{$\mathrm{C}_{28} \mathrm{H}_{46} \mathrm{~N}_{2} \mathrm{O}$}

Buxbodine D, 2821

\section{$\mathrm{C}_{28} \mathrm{H}_{46} \mathrm{O}$}

Brassicasterol, 2596

$\Delta^{7}$-Campesterol, 3039

5,6-Dihydroergosterol, 5608
Ergost-7-en-3-one, 7249

24-Methylcholesta-5,7-dien-3 $\beta$-ol, 14229

24-Methylcholesta-5,24-dien-3 $\beta$-ol, 14230

24-Methylcholesta-7,22-dien-3 $\beta$-ol, 14231

24-Methylene cholesterol, 14350

\section{$\mathrm{C}_{28} \mathrm{H}_{46} \mathrm{O}_{2}$}

24-Methylcholesta-5,24(28)-diene-3 $\beta, 19$-diol, 14226

$\mathrm{C}_{28} \mathrm{H}_{46} \mathrm{O}_{3}$

(22Z,24S)-Cerevisterol, 3422

$3 \beta, 5 \alpha$-Dihydroxyergost-7-en-6-one, 5885

24-Methylcholesta-5,24(28)-diene-3 $\beta, 7 \beta, 19$-triol, 14227

24-Methylcholesta-9(11),24(28)-diene-3 $\beta, 12 \alpha$, 19-triol, 14228

\section{$\mathrm{C}_{28} \mathrm{H}_{46} \mathrm{O}_{4}$}

26,27-Bisnor-8,14-dioxo- $\alpha$-onocerin, 2487

$22 E, 24 R$-Ergosta-7,22-diene-3 $\beta, 5 \alpha, 6 \beta, 9 \alpha$-tetraol, 7245

Octadecanyl-3-methoxy-4-hydroxy benzeneacrylate, 15954

Octadecyl (Z)-ferulate, 15961

\section{$\mathrm{C}_{28} \mathrm{H}_{46} \mathrm{O}_{5}$}

Polyporusterone F, 17670

Trihydroxybufosterocholenic acid, 21693

$\mathrm{C}_{28} \mathrm{H}_{46} \mathrm{O}_{6}$

Polyporusterone A, 17665

\section{$\mathrm{C}_{28} \mathrm{H}_{46} \mathrm{O}_{7}$}

Makisterone A, 13410

Makisterone B, 13411

Paristerone, 16671

Periplocoside L, 16954

\section{$\mathrm{C}_{28} \mathrm{H}_{46} \mathrm{O}_{8}$}

20-Hydroxy 24-hydroxymethyl ecdysone, 10204

\section{$\mathrm{C}_{28} \mathrm{H}_{46} \mathrm{O}_{9}$}

Hythiemoside A, 10920

Hythiemoside B, 10921

Salvigreside B, 19213

$\mathrm{C}_{28} \mathrm{H}_{46} \mathrm{O}_{10}$

Salvigreside C, 19214

$\mathrm{C}_{28} \mathrm{H}_{47} \mathrm{NO}_{2}$

Dihydroimpranine, 5645

Ningpeisine, 15613

Puqietinone, 18208

$\mathrm{C}_{28} \mathrm{H}_{47} \mathrm{NO}_{3}$

Pingbeinine, 17381

Valivine, 22326

$\mathrm{C}_{28} \mathrm{H}_{47} \mathrm{NO}_{6}$

Holantosine A, 9579

Holantosine C, 9581

$\mathrm{C}_{28} \mathrm{H}_{47} \mathrm{~N}_{7} \mathrm{O}_{7}$

Glaucacyclopeptide A, 8508

$\mathrm{C}_{28} \mathrm{H}_{48} \mathrm{~N}_{2}$
Cycloprotobuxine $\mathrm{C}_{1}, 4532$

$\mathrm{C}_{28} \mathrm{H}_{48} \mathrm{~N}_{2} \mathrm{O}$

Cyclovirobuxeine A, 4542

Epipachysamine E, 6994

$\mathrm{C}_{28} \mathrm{H}_{48} \mathrm{~N}_{2} \mathrm{O}_{2}$

Cyclomicrophylline A, 4516

\section{$\mathrm{C}_{28} \mathrm{H}_{48} \mathrm{~N}_{2} \mathrm{O}_{3}$}

Sarcovagine A, 19362

\section{$\mathrm{C}_{28} \mathrm{H}_{48} \mathrm{O}$}

Campesterol, 3040

Fungisterol, 8003

$4 \alpha$-Methyl-cholest-7-en-3 $\beta$-ol, 14235

$4 \alpha$-Methyl-cholest-8-en-3 $\beta$-ol, 14236

(24S)-Methylcholest-7-en-3 $\beta$-ol, 14237

(24R)-Methyl cholest-8(14)-enol, 14238

(24S)-Methyl cholest-8(14)-enol, 14239

Pollinastanol, 17618

Spongesterol, 20225

\section{$\mathrm{C}_{28} \mathrm{H}_{48} \mathrm{O}_{2}$}

(24S)-Ergost-5-en-3 $\beta, 7 \beta$-diol, 7248

$\beta$-Tocopherol, 21413

$\gamma$-Tocopherol, 21414

Vitamin $\mathrm{E}_{\beta}, 22559$

Vitamin $\mathrm{E}_{\gamma}, 22561$

\section{$\mathrm{C}_{28} \mathrm{H}_{48} \mathrm{O}_{4}$}

Irisoquin $\mathrm{E}, 11177$

24-Methylcholest-24(28)-ene-3 $\beta, 5 \alpha, 6 \beta, 19$-tetraol, 14234

\section{$\mathrm{C}_{28} \mathrm{H}_{48} \mathrm{O}_{5}$}

24-Epicastasterone, 6852

Trihydroxybufosterocholanic acid, 21692

\section{$\mathrm{C}_{28} \mathrm{H}_{48} \mathrm{O}_{6}$}

Brassinolide, 2598

\section{$\mathrm{C}_{28} \mathrm{H}_{48} \mathrm{O}_{10}$}

2,6-Diacetyl-3,4-dimethylbutyryl-1-O-octylglucopyranoside, 5333

\section{$\mathrm{C}_{28} \mathrm{H}_{48} \mathrm{O}_{14}$}

Periplocae oligosaccharide $\mathrm{F}_{1}, 16940$

\section{$\mathrm{C}_{28} \mathrm{H}_{50} \mathrm{~N}_{2} \mathrm{O}$}

Cyclokoreanine B, 4502

\section{$\mathrm{C}_{28} \mathrm{H}_{50} \mathrm{~N}_{2} \mathrm{O}_{4}$}

Carpaine, 3218

Pseudocarpaine, 18022

\section{$\mathrm{C}_{28} \mathrm{H}_{50} \mathrm{O}$}

(24R)-Methyl cholestanol, 14232

(24S)-Methyl cholestanol, 14233

$\mathrm{C}_{28} \mathrm{H}_{50} \mathrm{O}_{4}$

Clavatol, 3810

$\mathrm{C}_{28} \mathrm{H}_{50} \mathrm{O}_{5}$

Pentahydroxybufostane, 16841

\section{$\mathrm{C}_{28} \mathrm{H}_{52} \mathrm{O}_{11}$}

Muricatin B, 15086

$\mathrm{C}_{28} \mathrm{H}_{54} \mathrm{O}_{2}$ 
Octacosan-4-olide, 15936

$\mathrm{C}_{28} \mathrm{H}_{54} \mathrm{O}_{4}$

Octacosanedioic acid, 15933

$\mathrm{C}_{28} \mathrm{H}_{56} \mathrm{O}_{2}$

Montanic acid, 14944

$\mathrm{C}_{28} \mathrm{H}_{57} \mathrm{~N}_{3} \mathrm{O}$

Solapalmitenine, 20062

$\mathrm{C}_{28} \mathrm{H}_{58} \mathrm{~N}_{4} \mathrm{O}$

Budmunchiamine $\mathrm{L}_{5}, 2701$

$\mathrm{C}_{28} \mathrm{H}_{58} \mathrm{O}$

14-Octacosanol, 15934

1-Octacosanol, 15935

\section{$\mathrm{C}_{28} \mathrm{H}_{59} \mathrm{~N}_{3} \mathrm{O}$}

Solapalmitine, 20063

\section{$\mathrm{C}_{29} \mathrm{H}_{19} \mathrm{Cl}_{5} \mathrm{O}_{4}$}

Bazzanin O, 2183

\section{$\mathrm{C}_{29} \mathrm{H}_{21} \mathrm{Cl}_{3} \mathrm{O}_{4}$}

Bazzanin L, 2180

\section{$\mathrm{C}_{29} \mathrm{H}_{22} \mathrm{~N}_{4} \mathrm{O}_{4}$}

Picrasidine M, 17305

Picrasidine N, 17306

\section{$\mathrm{C}_{29} \mathrm{H}_{22} \mathrm{O}_{6}$}

Acumitin, 594

\section{$\mathrm{C}_{29} \mathrm{H}_{22} \mathrm{O}_{16}$}

Balanophotannin B, 2128

\section{$\mathrm{C}_{29} \mathrm{H}_{24} \mathrm{O}_{5}$}

1,8-Bis(4-hydroxybenzyl)-4-methoxyphenanthrene-2,7-diol, 2461

Isochamuvaritin, 11323

\section{$\mathrm{C}_{29} \mathrm{H}_{24} \mathrm{O}_{7}$}

Gnetuhainin Q, 8891

\section{$\mathrm{C}_{29} \mathrm{H}_{24} \mathrm{O}_{8}$}

Curtisian P, 4412

Gnetuhainin J, 8888

Gnetuhainin K, 8889

\section{$\mathrm{C}_{29} \mathrm{H}_{24} \mathrm{O}_{9}$}

Thelephantin A, 21293

\section{$\mathrm{C}_{29} \mathrm{H}_{24} \mathrm{O}_{12}$}

Isotheaflavin, 11740

\section{$\mathrm{C}_{29} \mathrm{H}_{24} \mathrm{O}_{16}$ \\ 1-O-p-(E)-Coumaroyl-4,6-(S)-HHDP- $\beta$ - $D$-gluco- pyranose, 4167}

$\mathrm{C}_{29} \mathrm{H}_{24} \mathrm{O}_{17}$

1-O-(E)-Caffeoyl-4,6-(S)-HHDP- $\beta$ - $D$-glucopyranose, 2908

\section{$\mathrm{C}_{29} \mathrm{H}_{26} \mathrm{~N}_{4} \mathrm{O}_{4}$}

1-( $\beta$-Carbolin-1-yl)-4-(4,8-dimethoxy- $\beta$-carbolin1-yl)-2-methoxy-butan-1-one, 3160

\section{$\mathrm{C}_{29} \mathrm{H}_{26} \mathrm{O}_{4}$}

5-Methoxy-3-(2-phenyl-E-ethenyl)-2,4-bis(4hydroxybenzyl) phenol, 14056

$\mathrm{C}_{29} \mathrm{H}_{26} \mathrm{O}_{5}$

1,6-Bis(4-hydroxybenzyl)-4-methoxy-9,10-di- hydrophenanthrene-2,7-diol, 2460

1,3-Di(4-hydroxybenzyl)-4-methoxy-9,10-dihydrophenanthrene-2,7-diol, 5771

\section{$\mathrm{C}_{29} \mathrm{H}_{26} \mathrm{O}_{6}$}

Marchantin E, 13554

Plagiochin A, 17500

\section{$\mathrm{C}_{29} \mathrm{H}_{26} \mathrm{O}_{7}$}

Anticancer Flavonoid PMV70P691-91, 1417

Anticancer Flavonoid PMV70P691-97, 1421

Marchantin K, 13557

\section{$\mathrm{C}_{29} \mathrm{H}_{26} \mathrm{O}_{8}$}

3'-[ $\gamma$-Hydroxymethyl- $(E)-\gamma$-methylallyl $]-2,4,2^{\prime}, 4^{\prime}-$ tetrahydroxychalcone 11'-O-coumarate, 10506

\section{$\mathrm{C}_{29} \mathrm{H}_{26} \mathrm{O}_{10}$}

Rocaglamide derivative 9, 18889

$\mathrm{C}_{29} \mathrm{H}_{26} \mathrm{O}_{12}$

Dimethyl lithospermate, 6365

\section{$\mathrm{C}_{29} \mathrm{H}_{26} \mathrm{O}_{13}$}

2"-O-Vanilloylvitexin, 22345

\section{$\mathrm{C}_{29} \mathrm{H}_{26} \mathrm{O}_{15}$}

1,6-Digalloyl-2-cinnamoyl-glucose, 5514

$\mathrm{C}_{29} \mathrm{H}_{26} \mathrm{O}_{17}$

1-O-(E)-Caffeoyl-3,4-di- $O$-galloyl- $\beta$ - $D$-glucopyranose, 2897

1-O-(E)-Caffeoyl-4,6-di- $O$-galloyl- $\beta$ - $D$-glucopyranose, 2898

3-O-(E)-Caffeoyl-1,4-di- $O$-galloyl- $\beta$ - $D$-glucopyranose, 2899

\section{$\mathrm{C}_{29} \mathrm{H}_{27} \mathrm{~N}_{4} \mathrm{O}_{3}{ }^{+}$}

Picrasidine F, 17299

\section{$\mathrm{C}_{29} \mathrm{H}_{28} \mathrm{~N}_{2} \mathrm{O}_{7}$}

Ipobscurine $\mathrm{C}, 11126$

Ipobscurine D, 11127

$\mathrm{C}_{29} \mathrm{H}_{28} \mathrm{~N}_{4}$

Usambarensine, 22275

$\mathrm{C}_{29} \mathrm{H}_{28} \mathrm{O}_{5}$

2',6'-Bis(p-hydroxybenzyl)-3,3'-dihydroxy-5methoxybibenzyl, 2456

Perrottetin G, 16987

\section{$\mathrm{C}_{29} \mathrm{H}_{28} \mathrm{O}_{8}$}

Interiorin D, 11102

Interiotherin A, 11103

$\mathrm{C}_{29} \mathrm{H}_{28} \mathrm{O}_{9}$

Angustifolin C, 1248

Rocaglamide derivative 5, 18885

Schisantherin D, 19490

$\mathrm{C}_{29} \mathrm{H}_{28} \mathrm{O}_{10}$

Pieceid-2"-O-coumarate, 17352

Rocaglamide derivative 6, 18886

$\mathrm{C}_{29} \mathrm{H}_{28} \mathrm{O}_{11}$

Cucumerin A, 4312

Cucumerin B, 4313

$\mathrm{C}_{29} \mathrm{H}_{28} \mathrm{O}_{13}$
Amaronitidin, 1019

$\mathrm{C}_{29} \mathrm{H}_{29} \mathrm{NO}_{8}$

1-O-Acetyl- $N, N$-didemethylrocaglamide, 375

Aglaroxin A, 741

$\mathrm{C}_{29} \mathrm{H}_{30} \mathrm{~N}_{2} \mathrm{O}_{7}$

Ipobscurine B, 11125

\section{$\mathrm{C}_{29} \mathrm{H}_{30} \mathrm{O}_{5}$}

5-Hydroxy-6-isobutyryl-8-methyl-8-(4-methylpent-3-enyl)-4-phenyl-2 $H$-pyrano[2,3- $h]$ chromen-2-one, 10241

\section{$\mathrm{C}_{29} \mathrm{H}_{30} \mathrm{O}_{7}$}

Piperaduncin A, 17430

\section{$\mathrm{C}_{29} \mathrm{H}_{30} \mathrm{O}_{8}$}

Longipedunin C, 12968

Piperaduncin B, 17431

\section{$\mathrm{C}_{29} \mathrm{H}_{30} \mathrm{O}_{9}$}

5-Hydroxy-7-methoxy-3',4'-diacetoxy-6-(6,6dimethyl-2-oxo-cyclohexylmethyl)flavone, 10397

Rocaglamide derivative 4, 18884

\section{$\mathrm{C}_{29} \mathrm{H}_{30} \mathrm{O}_{10}$}

Aloeresin G, 976

5,3'-Dipent-4-enoyloxy-3,6,7,4'-tetramethoxyflavone, 6483

\section{$\mathrm{C}_{29} \mathrm{H}_{30} \mathrm{O}_{11}$}

7-O-Methylaloeresin A, 14130

\section{$\mathrm{C}_{29} \mathrm{H}_{30} \mathrm{O}_{12}$}

(E)-3,5,4'-Trihydroxystilbene 3-O- $\beta$ - $D$-(6-Ogalloyl)glucopyranoside, 21850

\section{$\mathrm{C}_{29} \mathrm{H}_{30} \mathrm{O}_{13}$}

Amarogentin, 1017

Phyllanthostatin A, 17219

\section{$\mathrm{C}_{29} \mathrm{H}_{30} \mathrm{O}_{14}$}

Amaroswerin, 1020

6'-O-p-Hydroxybenzoylcatalposide, 9821

\section{$\mathrm{C}_{29} \mathrm{H}_{30} \mathrm{O}_{15}$}

Oraposide, 16161

\section{$\mathrm{C}_{29} \mathrm{H}_{30} \mathrm{O}_{17}$}

Luteolin 7-O-[2"-O-(4"'- $O$-acetyl- $\alpha$ - $L$-rhamnopyranosyl)]- $\beta$ - $D$-glucuronopyranoside, 13138

\section{$\mathrm{C}_{29} \mathrm{H}_{31} \mathrm{NO}_{7}$}

Hernandaline, 9440

Rocaglamide, 18882

\section{$\mathrm{C}_{29} \mathrm{H}_{31} \mathrm{O}_{15}$}

Sutchuenoside A, 20499

$\mathrm{C}_{29} \mathrm{H}_{32} \mathrm{O}_{4}$

Artoindonesianin Y, 1820

$\mathrm{C}_{29} \mathrm{H}_{32} \mathrm{O}_{6}$

Fucaxanthone A, 7974

$\mathrm{C}_{29} \mathrm{H}_{32} \mathrm{O}_{10}$

Bruceantarin, 2659

Pilosanol A, 17361

Symploveroside, 20548 
Yuanhuafin, 22931

$\mathrm{C}_{29} \mathrm{H}_{32} \mathrm{O}_{11}$

Aloeresin D, 975

Kadsulignan F, 12001

$\mathrm{C}_{29} \mathrm{H}_{32} \mathrm{O}_{12}$

2"-O-Feruloylaloesin, 7771

\section{$\mathrm{C}_{29} \mathrm{H}_{32} \mathrm{O}_{15}$}

2"'-O-Acetyl-2"- $O-\alpha$ - $L$-rhamnopyranosylisovitexin, 493

4"'-O-Acetyl-2"-O- $\alpha-L$-rhamnopyranosylisovitexin, 494

Apigenin-7-O- $\alpha-L-3-O$-acetylrhamnopyranosyl$(1 \rightarrow 6)-\beta$ - $D$-glucopyranoside, 1477

Camellianin A, 3035

2-Methyl-1,3,6-trihydroxy-9,10-anthraquinone 3-O- $(\alpha$ - $L$-rhamnopyranosyl- $(1 \rightarrow 2)(6 '$-acetyl)$\beta$ - $D$-glucopyranoside), 14777

1,3,6-Trihydroxy-2-methylanthraquinone-3-O$\alpha$-rhamnosyl $(1 \rightarrow 2)$-3'- $O$-acetylglucoside, 21795

\section{$\mathrm{C}_{29} \mathrm{H}_{32} \mathrm{O}_{16}$}

2-Methyl-1,3,6-trihydroxyanthraquinone 3-O-(6'- $O$-acetyl)- $\alpha$ - $L$-rhamnosyl-( $1 \rightarrow 2)$ $\beta$ - $D$-glucoside, 14776

Multiflorin A, 15068

Periginatorine, 16925

Viscumneoside IV, 22539

\section{$\mathrm{C}_{29} \mathrm{H}_{32} \mathrm{O}_{17}$}

2"-O-Acetylrutin, 497

Isoscutellarein 7-O-(6"'-O-acetyl)- $\beta$-allopyranosyl(1"' $\rightarrow 2 ")-\beta$-glucopyranoside, 11704

Kaempferol-3-O- $\beta$ - $D$-glucopyranosyl $(1 \rightarrow 2)-\beta$ $D$-6-acetylglucopyranoside, 12051

\section{$\mathrm{C}_{29} \mathrm{H}_{33} \mathrm{NO}_{9}$}

Hernandifoline, 9442

\section{$\mathrm{C}_{29} \mathrm{H}_{34} \mathrm{~N}_{4}$}

Ochrolifuanine A, 15924

\section{$\mathrm{C}_{29} \mathrm{H}_{34} \mathrm{O}_{4}$}

Artoindonesianin X, 1819

Lakoochin B, 12454

Mulberrofuran D, 15042

Mulberrofuran W, 15054

$\mathrm{C}_{29} \mathrm{H}_{34} \mathrm{O}_{5}$

Mulberrofuran X, 15055

\section{$\mathrm{C}_{29} \mathrm{H}_{34} \mathrm{O}_{6}$}

Cowanin, 4200

Kansuiphorin C, 12147

Kansuiphorin D, 12148

Triptofordin $\mathrm{B}_{1}, 21999$

$\mathrm{C}_{29} \mathrm{H}_{34} \mathrm{O}_{7}$

Cowanol, 4201

Microlenin, 14837

Scortechinone D, 19549
Scortechinone E, 19550

$\mathrm{C}_{29} \mathrm{H}_{34} \mathrm{O}_{9}$

10,15-Epoxy-11(15 $\rightarrow 1)$-abeo-10-deacetylbaccatin III, 7046

2-Hydroxyangustidienolide, 9787

Lancifodilactone D, 12475

\section{$\mathrm{C}_{29} \mathrm{H}_{34} \mathrm{O}_{10}$}

15-Benzoyl-10-deacetyl-2-debenzoyl-10-dehydro-abeo-baccatin III, 2236

10-Deacetyl-13-oxobaccatin III, 4762

10-Deacetyl-10-oxobaccatin III, 4763

10-Deacetyl-10-oxobaccatin V, 4764

Wallifoliol, 22630

Yuanhuapin, 22932

\section{$\mathrm{C}_{29} \mathrm{H}_{34} \mathrm{O}_{11}$}

Diandraflavone, 5353

Ixerisoside A, 11798

Lancifodilactone B, 12473

Orbiculin H, 16166

Schizanrin G, 19501

Tasumatrol A, 20718

\section{$\mathrm{C}_{29} \mathrm{H}_{34} \mathrm{O}_{12}$}

7-O-p-Hydroxybenzoylovatol 1-O-(6'-O-phydroxybenzoyl)- $\beta$ - $D$-glucopyranoside, 9828

\section{$\mathrm{C}_{29} \mathrm{H}_{34} \mathrm{O}_{14}$}

Derriscandenoside C, 5224

Derriscandenoside D, 5225

Derriscanoside B, 5228

Embinin, 6769

Pueroside A, 18185

\section{$\mathrm{C}_{29} \mathrm{H}_{34} \mathrm{O}_{15}$}

Crenatoside, 4225

Derriscandenoside E, 5226

Dicliripariside C, 5439

Isocrenatoside, 11350

Pectolinarin, 16756

Reiniose B, 18604

Reiniose C, 18605

Tyrolobibenzyl F, 22161

$\mathrm{C}_{29} \mathrm{H}_{34} \mathrm{O}_{16}$

5-Hydroxy-7,4',5'-trimethoxyisoflavone 3'-O- $\alpha$ $L$-arabinofuranosyl-( $1 \rightarrow 6)-\beta$ - $D$-glucopyranoside, 10798

Ombuoside, 16093

$\mathrm{C}_{29} \mathrm{H}_{34} \mathrm{O}_{17}$

Iristectorigenin B 7-glucosyl $(1 \rightarrow 6)$ glucoside, 11180

Syringetin-3- $O$-robinobioside, 20564

$\mathrm{C}_{29} \mathrm{H}_{35} \mathrm{~N}_{3} \mathrm{O}_{4}$

Sanjoinenine, 19289

$\mathrm{C}_{29} \mathrm{H}_{35} \mathrm{O}_{16}$

Isolugrandoside, 11513

$\mathrm{C}_{29} \mathrm{H}_{35} \mathrm{O}_{17}{ }^{+}$
Malvidin-3,5-diglucoside, 13460

\section{$\mathrm{C}_{29} \mathrm{H}_{36} \mathrm{~N}_{2} \mathrm{O}_{6}$}

Elegansamine, 6738

Gelsamydine, 8252

$\mathrm{C}_{29} \mathrm{H}_{36} \mathrm{O}_{2}$

Machillene, 13294

\section{$\mathrm{C}_{29} \mathrm{H}_{36} \mathrm{O}_{5}$}

6 $\beta$-Cinnamoyl-7 $\beta$-hydroxyvouacapen- $5 \alpha$-ol, 3708

Jolkinol B, 11892

\section{$\mathrm{C}_{29} \mathrm{H}_{36} \mathrm{O}_{6}$}

5-Cinnamoylphototaxicin II, 3719

Jolkinol A, 11890

\section{$\mathrm{C}_{29} \mathrm{H}_{36} \mathrm{O}_{7}$}

Turraflorin B, 22131

Turraflorin G, 22135

\section{$\mathrm{C}_{29} \mathrm{H}_{36} \mathrm{O}_{8}$}

Turraflorin F, 22134

\section{$\mathrm{C}_{29} \mathrm{H}_{36} \mathrm{O}_{9}$}

$2 \alpha$-Acetoxy- $9 \alpha$-benzoyloxy- $5 \alpha, 7 \beta, 10 \beta, 15$-tetrahydroxy-11(15 $\rightarrow 1)$-abeotaxa-4(20),11-dien13-one, 133

3-O-Deacetylorthosiphol I, 4760

Orthosiphonone C, 16243

\section{$\mathrm{C}_{29} \mathrm{H}_{36} \mathrm{O}_{10}$}

10-Deacetyl baccatin III, 4722

13-Epi-10-deacetylbaccatin III, 6880

Lancifodilactone C, 12474

Taxuspinanane C, 20843

\section{$\mathrm{C}_{29} \mathrm{H}_{36} \mathrm{O}_{11}$}

2-Debenzoyl-14 $\beta$-benzoyloxy-10-deacetylbaccatin III, 4803

19-Hydroxy-10-deacetylbaccatin III, 9961

14 $\beta$-Hydroxy-10-deacetylbaccatin III, 9962

Labriformidin, 12421

Lancifodilactone E, 12476

\section{$\mathrm{C}_{29} \mathrm{H}_{36} \mathrm{O}_{12}$}

Micrandilactone A, 14832

\section{$\mathrm{C}_{29} \mathrm{H}_{36} \mathrm{O}_{13}$}

Jionoside C, 11884

Osmanthuside B, 16252

Osmanthuside $\mathrm{B}_{6}, 16253$

Tabularin, 20586

Woorenoside II, 22728

\section{$\mathrm{C}_{29} \mathrm{H}_{36} \mathrm{O}_{14}$}

7-O- $\beta$ - $D$-Apiofuranosyl-( $1 \rightarrow 6)-\beta$ - $D$-glucopyranosylmatteucinol, 1512

Clerodendronoside, 3838

Isosyringalide 3'- $\alpha$ - $L$-rhamnopyranoside, 11725 Syringalide 3 '- $\alpha$ - $L$-rhamnopyranoside, 20555

\section{$\mathrm{C}_{29} \mathrm{H}_{36} \mathrm{O}_{15}$}

Acteroside, 580

Forsythoside A, 7924 
Isoacteoside, 11195

\section{$\mathrm{C}_{29} \mathrm{H}_{36} \mathrm{O}_{16}$}

Forsythoside C, 7926

Plantainoside D, 17510

Purpureaside A, 18219

$\mathrm{C}_{29} \mathrm{H}_{36} \mathrm{O}_{17}$

Hellicoside, 9331

Prunose II, 18005

\section{$\mathrm{C}_{29} \mathrm{H}_{37} \mathrm{NO}_{5}$}

Lythramine, 13268

Lythrancepine II, 13270

$\mathrm{C}_{29} \mathrm{H}_{37} \mathrm{NO}_{6}$

Benzoylheteratisine, 2243

Lythrancine II, 13273

\section{$\mathrm{C}_{29} \mathrm{H}_{37} \mathrm{~N}_{3} \mathrm{O}_{3}$}

Alangimarckine, 830

Tubulosine, 22098

\section{$\mathrm{C}_{29} \mathrm{H}_{38} \mathrm{~N}_{4} \mathrm{O}_{4}$}

Mucronine A, 15020

\section{$\mathrm{C}_{29} \mathrm{H}_{38} \mathrm{~N}_{4} \mathrm{O}_{5}$}

Ephedradine B, 6813

\section{$\mathrm{C}_{29} \mathrm{H}_{38} \mathrm{O}_{4}$}

Celastrol, 3368

\section{$\mathrm{C}_{29} \mathrm{H}_{38} \mathrm{O}_{5}$}

Kleinioxanthrone 2, 12234

\section{$\mathrm{C}_{29} \mathrm{H}_{38} \mathrm{O}_{7}$}

$2 \alpha$-Acetoxy- $4 \beta$-hydroxy- $6 \alpha$-angeloyloxy- $10 \beta$ benzoyloxy-dauc-8-ene, 207

Anticancer Norwithanolide PMV70P691-034, 1432

Surangin B, 20490

\section{$\mathrm{C}_{29} \mathrm{H}_{38} \mathrm{O}_{8}$}

9-Deacetyl-9-benzoyl-10-debenzoylbrevifoliol, 4729

Lucidenic acid $\mathrm{D}_{2}, 13027$

\section{$\mathrm{C}_{29} \mathrm{H}_{38} \mathrm{O}_{9}$}

Angeloylgomisin Q, 1209

[(2S,3R,4R)-4-(3,4,5-Trimethoxybenzyl)-2-(3, 4,5-trimethoxyphenyl)-tetrahydrofuran-3-yl] methyl (2Z)-2-methylbut-2-en-oate, 21898

Uscharidin, 22280

\section{$\mathrm{C}_{29} \mathrm{H}_{38} \mathrm{O}_{10}$}

7,13-Dideaceyl-9,10-debenzoyltaxchinin C, 5460

\section{$\mathrm{C}_{29} \mathrm{H}_{38} \mathrm{O}_{11}$}

Alnuside C, 961

Eriocarpin, 7272

\section{$\mathrm{C}_{29} \mathrm{H}_{38} \mathrm{O}_{13}$}

6'- $O$-Acetylpseudolaric acid B- $O-\beta$ - $D$-glucopyranoside, 489

Icariside $\mathrm{E}_{7}, 10952$

Pseudolaric acid B- $O-\beta$ - $D$-glucopyranoside, 18034

$\mathrm{C}_{29} \mathrm{H}_{38} \mathrm{O}_{15}$

$$
\begin{aligned}
& \text { 2-(4-Hydroxyphenyl)ethyl 1-O- } \beta \text { - } D-[5-O-(3,4,5- \\
& \text { trimethoxybenzoyl)]-apiofuranosyl- }(1 \rightarrow 6)-\beta \text { - } \\
& \text { D-glucopyranoside, } 10622
\end{aligned}
$$

Isomucronulatol-7,2'-di- $O$-glucoside, 11545

$\mathrm{C}_{29} \mathrm{H}_{38} \mathrm{O}_{16}$

5'-Hydroxy-isomucronulatol-2',5'-di- $O$-glucoside, 10251

3,4,5-Trimethoxyphenyl $1-O-\beta-D-[5-O-(3,4-$ dimethoxybenzoyl $)]$-apiofuranosyl-( $1 \rightarrow 6)-\beta$ - $D$-glucopyranoside, 21925

Yadanzioside F, 22865

Yadanzioside I, 22868

$\mathrm{C}_{29} \mathrm{H}_{38} \mathrm{O}_{17}$

Albibrissinoside A, 855

$\mathrm{C}_{29} \mathrm{H}_{39} \mathrm{NO}_{6}$

Delavaconitine, 4993

\section{$\mathrm{C}_{29} \mathrm{H}_{39} \mathrm{NO}_{9}$}

Homoharringtonine, 9609

$\mathrm{C}_{29} \mathrm{H}_{40} \mathrm{NO}_{6}^{+}$

Nudicaulin, 15861

$\mathrm{C}_{29} \mathrm{H}_{40} \mathrm{~N}_{2} \mathrm{O}_{4}$

Emetine, 6772

$\mathrm{C}_{29} \mathrm{H}_{40} \mathrm{~N}_{8} \mathrm{O}_{5}$

Hordatine B, 9645

$\mathrm{C}_{29} \mathrm{H}_{40} \mathrm{O}_{2}$

Papyriogenin H, 16641

\section{$\mathrm{C}_{29} \mathrm{H}_{40} \mathrm{O}_{4}$}

$2 \alpha, 3 \beta$-Dihydroxy-28-norurs-12,17,19(20),21-tetraen-23-oic cid, 6055

$8 \alpha$-Hydroxyabda-13(16),14-dien-19-yl-(Z)-4-hydroxycinnamate, 10300

\section{$\mathrm{C}_{29} \mathrm{H}_{40} \mathrm{O}_{5}$}

(13S)-ent-18-(E)-Coumaroyloxy-8(17)-labden15-oic acid, 4181

Datumetelin, 4664

Daturametelin C, 4668

Zhankuic acid A, 22990

\section{$\mathrm{C}_{29} \mathrm{H}_{40} \mathrm{O}_{6}$}

ent-15-(E)-Caffeoyloxy-8(17)-labden-18-oic acid, 2916

ent-18-(E)-Caffeoyloxy-8(17)-labden-15-oic acid, 2917

$16 \beta, 22 R ; 21,23 S$-Diepoxy-21S,24-dihydroxy-5 $\alpha$ stigmasta-8,14-diene-3,28-dione, 5490

Erectquione B, 7224

Zafaral, 22961

$\mathrm{C}_{29} \mathrm{H}_{40} \mathrm{O}_{7}$

Ardisiaquinone E, 1633

ent-18-(E)-Caffeoyloxy-7 $\beta$-hydroxy-3-cleroden15-oic acid, 2914

Neocucurbitacin B, 15377

\section{$\mathrm{C}_{29} \mathrm{H}_{40} \mathrm{O}_{8}$}

Ajugalactone, 794
Lucidenic acid $\mathrm{E}_{2}, 13029$

Phorbol-12-tiglate-13-butyrate, 17192

\section{$\mathrm{C}_{29} \mathrm{H}_{40} \mathrm{O}_{9}$}

Calactin, 2937

Calcicolin A $\$ 2948$

\section{$\mathrm{C}_{29} \mathrm{H}_{40} \mathrm{O}_{10}$}

Ajugamarin, 801

\section{$\mathrm{C}_{29} \mathrm{H}_{40} \mathrm{O}_{11}$}

Hellebortin A, 9326

Hellebortin C, 9328

$1 \alpha$-( $\alpha$-Methyl)-butanoyl-2 $\alpha, 15$-diacetoxy- $4 \beta$-hydroxy-9 $\beta$ - $(\beta$-)furoyloxy- $\beta$-dihydroagarofuran, 14164

Neocynanversicoside, 15387

\section{$\mathrm{C}_{29} \mathrm{H}_{40} \mathrm{O}_{15}$}

$1 \beta, 2 \beta, 3 \alpha, 5 \alpha, 7 \beta, 8 \beta, 11$-Heptaacetoxy-dihydroagarofuran, 9367

\section{$\mathrm{C}_{29} \mathrm{H}_{40} \mathrm{O}_{16}$}

6-O-(4"- $O-\alpha-L$-Rhamnopyranosyl) vanilloylajugol, 18735

Yadanzioside D, 22863

\section{$\mathrm{C}_{29} \mathrm{H}_{42} \mathrm{O}_{2}$}

Papyriogenin I, 16642

$\mathrm{C}_{29} \mathrm{H}_{42} \mathrm{O}_{3}$

Bryophyllone, 2692

$\mathrm{C}_{29} \mathrm{H}_{42} \mathrm{O}_{4}$

17 $\beta$-Hydroxy-3,11,16-trioxo-28-norolean-12-ene, 10813

Tripterygone, 21992

\section{$\mathrm{C}_{29} \mathrm{H}_{42} \mathrm{O}_{5}$}

Siraitic acid B, 19972

Zhankuic acid B, 22991

\section{$\mathrm{C}_{29} \mathrm{H}_{42} \mathrm{O}_{6}$}

Zhankuic acid C, 22992

\section{$\mathrm{C}_{29} \mathrm{H}_{42} \mathrm{O}_{8}$}

Caseamembrin E, 3262

Caseamembrol A, 3264

Casearinol A, 3269

Casearlucin A, 3274

rel-(2S,5R,6R,8S,9S,10R,18S,19R)-diacetoxy-18, 19-epoxy-6-hydroxy-2-(2-methylbutanoyloxy) cleroda-3,13(16),14-triene, 5297

Lucidenic acid P, 13040

Rasfonin diacetate, 18550

$2 \alpha, 5 \alpha, 10 \beta$-Triacetoxy- $14 \beta$-propionyloxytaxa4(20),11-diene, 21531

\section{$\mathrm{C}_{29} \mathrm{H}_{42} \mathrm{O}_{9}$}

Ajugamarin $\mathrm{F}_{4}, 805$

Caseamembrol B, 3265

Corchoroside A, 4036

2,3-Dihydro-3 $\beta$-methoxyixocarpalactone B, 5672 Helveticoside, 9335

Malayoside, 13418 
Peruvoside, 17002

Rhapontisterone $\mathrm{R}_{1}, 18751$

\section{$\mathrm{C}_{29} \mathrm{H}_{42} \mathrm{O} 9 \mathrm{~S}$}

Daturametelin E, 4669

\section{$\mathrm{C}_{29} \mathrm{H}_{42} \mathrm{O}_{10}$}

Adonitoxin, 646

Aspecioside, 1887

Convallatoxin, 4012

Deglucocheirotoxin, 4871

Securigenin-3 $\beta$ - $O$ - $\beta$-6-deoxyguloside, 19638

Strophalloside, 20396

\section{$\mathrm{C}_{29} \mathrm{H}_{42} \mathrm{O}_{11}$}

$\alpha$-Antiarin, 1376

$\beta$-Antiarin, 1377

Glabcensin O, 8476

Sarmentosigenin-3 $\beta$-O- $\beta$-6-deoxyguloside, 19376

Strophanthidin-glucoside, 20399

\section{$\mathrm{C}_{29} \mathrm{H}_{42} \mathrm{O}_{12}$}

Antialloside, 1374

\section{$\mathrm{C}_{29} \mathrm{H}_{42} \mathrm{O}_{18}$}

Tangshenoside I, 20672

\section{$\mathrm{C}_{29} \mathrm{H}_{42} \mathrm{O}_{19}$}

Arillatose D, 1687

Arillatose F, 1689

\section{$\mathrm{C}_{29} \mathrm{H}_{44} \mathrm{NO}$ \\ (22E,24R)-3 $\alpha$-Ureido-ergosta-4,6,8(14),22-tetra- ene, 22249}

\section{$\mathrm{C}_{29} \mathrm{H}_{44} \mathrm{O}$}

(22E,24S)-Stigmasta-1,4,22-trien-3-one, 20361

\section{$\mathrm{C}_{29} \mathrm{H}_{44} \mathrm{O}_{2}$}

Koelpinin C, 12245

Maragenin II, 13546

\section{$\mathrm{C}_{29} \mathrm{H}_{44} \mathrm{O}_{3}$}

17 $\beta$-Hydroxy-3,16-dioxo-28-norolean-12-ene, 10057

Larreagenin A, 12526

3-Oxobetulinic acid, 16295

Pfaffic acid, 17037

\section{$\mathrm{C}_{29} \mathrm{H}_{44} \mathrm{O}_{4}$}

$2 \alpha, 3 \alpha$-Dihydroxy-30-noroleana-12,20(29)-dien28-oic acid, 6050

$2 \alpha, 3 \beta$-Dihydroxy-30-noroleana-12,20(29)-dien28-oic acid, 6051

Diosgenin acetate, 6441

Ilekudinol B, 10969

Quinatic acid, 18419

Yamogenin acetate, 22879

\section{$\mathrm{C}_{29} \mathrm{H}_{44} \mathrm{O}_{5}$}

Siraitic acid A, 19971

$2 \alpha, 3 \beta, 23$-Trihydroxy-30-noroleana-12,20(21)dien-28-oic acid, 21812

$2 \alpha, 3 \beta, 23$-Trihydroxy-30-noroleana-12,20(29)dien-28-oic acid, 21813

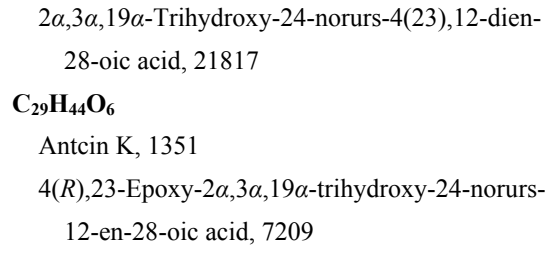

$2 \alpha, 3 \alpha, 19 \alpha$-Trihydroxy-24-norurs-4(23),12-dien-

28-oic acid, 21817

$\mathrm{C}_{29} \mathrm{H}_{44} \mathrm{O}_{6}$

Antcin K, 1351

$4(R), 23$-Epoxy-2 $\alpha, 3 \alpha, 19 \alpha$-trihydroxy-24-norurs12-en-28-oic acid, 7209

\section{$\mathrm{C}_{29} \mathrm{H}_{44} \mathrm{O}_{7}$}

Capitasterone, 3131

Hongdoushan A, 9626

$\mathrm{C}_{29} \mathrm{H}_{44} \mathrm{O}_{8}$

Casearinol B, 3270

Cyasterone, 4455

10-Deacetylyunnanaxane, 4793

Isocyasterone, 11361

Precyasterone, 17778

\section{$\mathrm{C}_{29} \mathrm{H}_{44} \mathrm{O}_{9}$}

Corchorosol A, 4037

Diginatin, 5523

2,3-Dihydro-3 $\beta$-methoxyixocarpalactone A, 5671

Helveticosol, 9336

Peripalloside, 16937

Periplogenin-3-O- $\alpha$ - $L$-rhamnopyranoside, 16960

Rhodexin A, 18785

Rhodexin B, 18786

Sengosterone, 19730

\section{$\mathrm{C}_{29} \mathrm{H}_{44} \mathrm{O}_{10}$}

Alliside, 922

Antioside, 1461

$\alpha$-Antioside, 1462

Convallatoxol, 4013

19-Hydroxy-sarmentogenin-3 $\beta$ - $O$ - $\beta$-6-deoxyguloside, 10692

Lokundjoside, 12951

$3 \beta, 5 \beta, 11 \alpha, 14 \beta$-Tetrahydroxy-5 $\beta$-card-20(22)eno-

lide-3 $\alpha$ - $L$-rhamnoside, 21084

\section{$\mathrm{C}_{29} \mathrm{H}_{44} \mathrm{O}_{11}$}

Bipindogenin-3-O- $\beta$ - $D$-allopyranoside, 2400

Hativene A, 9246

Hativene B, 9247

Hativene C, 9248

Sarmentoloside, 19374

$\mathrm{C}_{29} \mathrm{H}_{44} \mathrm{O}_{12}$

Strophanthin G, 20403

$\mathrm{C}_{29} \mathrm{H}_{44} \mathrm{O}_{13}$

4-Hydroxy-2-[(2E,6Z)-8- $\beta$ - $D$-glucopyranosyloxy-3,7-dimethylocta-2,6-dien-1-yl]-5-methyl

phenyl $\beta$ - $D$-glucopyranoside, 10150

\section{$\mathrm{C}_{29} \mathrm{H}_{45} \mathrm{NO}_{8}$}

Zygacine, 23031

\section{$\mathrm{C}_{29} \mathrm{H}_{45} \mathrm{~N}_{3} \mathrm{O}$}

Epipachysamine B, 6991

$\mathrm{C}_{29} \mathrm{H}_{46}$

24-Nor-4(23),9(11)-fernadiene, 15748
$\mathrm{C}_{29} \mathrm{H}_{46} \mathrm{~N}_{2} \mathrm{O}_{2}$

Holafrine, 9578

\section{$\mathrm{C}_{29} \mathrm{H}_{46} \mathrm{O}$}

7-Dehydroporiferasterol, 4963

(24S)-Ethylcholesta-5,22,25-trien-3 $\beta$-ol, 7428

$24 \beta$-Ethylcholesta-5,9(11),22-trien-3 $\beta$-ol, 7429

24-Methylenepollinastanone, 14389

(22E,24R)-Stigmasta-1,4-dien-3-one, 20348

(22E,20S,24S)-Stigmasta-7,22-dien-3-one, 20349

Stigmasta-4,22-dien-3-one, 20350

Stigmasta-5,22-dien-3-one, 20351

$5 \alpha$-Stigmasta-7,22-dien-3-one, 20352

7,22,25-Stigmastatrienol, 20360

\section{$\mathrm{C}_{29} \mathrm{H}_{46} \mathrm{O}_{2}$}

22-Dihydrostigmast-4-en-3,6-dione, 5720

Koelpinin A, 12243

Lactucasterol, 12445

Myricaolal*, 15166

(23E)-27-Nor-3 $\beta$-hydroxycycloart-23-en-25-one, 15757

Stigmasta-5,22-dien-3 $\beta$-ol-7-one, 20347

Stigmast-4-ene-1,3-dione, 20364

Stigmast-4-ene-3,6-dione, 20365

\section{$\mathrm{C}_{29} \mathrm{H}_{46} \mathrm{O}_{4}$}

24-Hydroxy-11-deoxoglycyrrhetic acid, 9985

$(2 R, 3 R, 4 S, 6 R)-6-M e t h o x y c a r b o n y l-3-m e t h y l-4,6-$ di(3-methyl-2-butenyl)-2-(2-methyl-1-oxoprop yl)-3-(4-methyl-3-pentenyl)cyclohexanone, 13875

Trisnorcycloartenoloic acid acetate, 22038

\section{$\mathrm{C}_{29} \mathrm{H}_{46} \mathrm{O}_{6}$}

Asparacosin B, 1865

Isothankunic acid, 11738

Pogosterol, 17600

\section{$\mathrm{C}_{29} \mathrm{H}_{46} \mathrm{O}_{7}$}

Ajugasterone B, 810

Decumbesterone A, 4860

\section{$\mathrm{C}_{29} \mathrm{H}_{46} \mathrm{O}_{9}$}

Pubeside E, 18179

\section{$\mathrm{C}_{29} \mathrm{H}_{47} \mathrm{NO}_{6}$}

Coumingine, 4192

\section{$\mathrm{C}_{29} \mathrm{H}_{48}$}

4-Methyl-7-ergosta-8,24(28)-diene, 14414

\section{$\mathrm{C}_{29} \mathrm{H}_{48} \mathrm{~N}_{2} \mathrm{O}_{2}$}

Pachysantermine A, 16509

Pachystermine A, 16512

\section{$\mathrm{C}_{29} \mathrm{H}_{48} \mathrm{~N}_{8} \mathrm{O}_{9}$}

Cherimolacyclopeptide D, 3513

\section{$\mathrm{C}_{29} \mathrm{H}_{48} \mathrm{O}$}

Adiantone, 632

Adiantulupanone, 634

Bessisterol, 2316

Clerosterol, 3844 
Fucosterol, 7978

Isoadiantone, 11197

Isofucosterol, 11431

24-Lophenolmethylene, 12999

24-Methylenelophenol, 14388

$14 \alpha$-Methyl-5 $\alpha$-ergosta-9(11),24(28)-dien-3 $\beta$-ol, 14415

Poriferasterol, 17729

5,25-Sitost-dienol, 19980

$\beta$-Sitostenone, 19981

$\beta$-Sitosterone, 19993

$\alpha$-Spinasterol, 20168

7,24-Stigmastadienol, 20344

7,25-Stigmastadienol, 20345

$5 \alpha$-Stigmasta-22-en-3-one, 20354

7-Stigmastenone-3, 20368

Stigmasterol, 20369

\section{$\mathrm{C}_{29} \mathrm{H}_{48} \mathrm{O}_{2}$}

Adiantuoleanone, 635

Adipedatol, 641

$22,29 \xi$-Epoxy-30-norhopane-13 $\beta$-ol, 7178

$3 \beta$-Hydroxy-29-norcycloart-24-one, 10539

21-Hydroxy-30-norhopan-22-one, 10541

27-nor-3 $\beta$-Hydroxy-25-oxocycloartane, 15759

30-Nor-lupan-3 $\beta$-ol-20-one, 15774

28-Nor-urs-12-ene-3 $\beta$-, 17 $\beta$-diol, 15813

Saringosterol, 19367

Stigmasta-4,25-dien-3 $\beta, 6 \beta$-diol, 20343

Stigmasta-5-en-3 $\beta$-ol-7-one, 20353

$5 \alpha$-Stigmastan-3,6-dione, 20355

\section{$\mathrm{C}_{29} \mathrm{H}_{48} \mathrm{O}_{3}$}

(24R)-7 $\alpha$-Hydroperoxystigmasta-5,22-dien-3 $\beta$ ol, 9735

(22E,24S)-7 $\alpha$-Hydroperoxystigmasta-5,22-dien3 $\beta$-ol, 9736

$4 \alpha$-Methyl-3 $\beta, 14 \beta$-dihydroxy-5 $\alpha$-ergost-24(28)en-23-one, 14300

26-Nor-8-oxo- $\alpha$-onocerin, 15786

1-Oxo-7 $\alpha$-hydroxysitosterol, 16356

$2 \alpha, 3 \alpha, 19 \alpha$-Trihydroxy-28-norurs-12-ene, 21818

$2 \alpha, 3 \beta, 19 \alpha$-Trihydroxy-28-norurs-12-ene, 21819

\section{$\mathrm{C}_{29} \mathrm{H}_{48} \mathrm{O}_{4}$}

Eicosyl caffeate, 6726

\section{$\mathrm{C}_{29} \mathrm{H}_{48} \mathrm{O}_{5}$}

Gloeophyllic acid A dimethylacetal, 8575

\section{$\mathrm{C}_{29} \mathrm{H}_{48} \mathrm{O}_{7}$}

Amarasterone A, 1014

Lemmasterone, 12620

Makisterone D, 13412

$\mathrm{C}_{29} \mathrm{H}_{48} \mathrm{O}_{9}$

6'-O-Acetylmicrolepin, 468

17-O-Acetylmicrolepin, 469

$\mathrm{C}_{29} \mathrm{H}_{48} \mathrm{O}_{10} \mathrm{~S}$
Cilistol W, 3647

$\mathrm{C}_{29} \mathrm{H}_{48} \mathrm{O}_{11}$

Crenulatoside B, 4229

Crenulatoside C, 4230

$\mathrm{C}_{29} \mathrm{H}_{49} \mathrm{NO}_{2}$

24-Hydroxyimino-29-norcycloart-3-ol, 10230

$\mathrm{C}_{29} \mathrm{H}_{50} \mathrm{~N}_{2} \mathrm{O}$

Pachysamine B, 16502

$\mathrm{C}_{29} \mathrm{H}_{50} \mathrm{~N}_{2} \mathrm{O}_{2}$

$O$-Deacetylpachysandrine B, 4767

Pachysandrine D, 16508

Pachystermine B, 16513

$N^{3}, N^{20}, N^{20}$-Trimethyl- $N^{3}$-(3-methyl-2-butenoyl)-

3,20-diaminopregnan-12-ol, 21968

\section{$\mathrm{C}_{29} \mathrm{H}_{50} \mathrm{O}$}

7-Dehydrostigmasterol, 4971

$\beta$-Dihydrofucosterol, 5616

24,24-Dimethyl-5 $\alpha$-cholesta-8-en-3 $\beta$-ol, 6331

$14 \alpha$-Methyl-5 $\alpha$-ergosta-9(11)-en-3 $\beta$-ol, 14416

24-Methyllophenol, 14558

29-Norcycloartan-3 $\beta$-ol, 15729

29-Nor-22-hopanol, 15756

29-Norlanost-8-enol, 15771

29-Norlanost-9(11)-enol, 15772

29-Norlanosterol, 15773

Schottenol, 19513

$\beta$-Sitosterol, 19983

$\gamma$-Sitosterol, 19984

$5 \alpha$-Stigmast-7-en-3 $\beta$-ol, 20366

22-Stigmasterol, 20370

\section{$\mathrm{C}_{29} \mathrm{H}_{50} \mathrm{O}_{2}$}

Ikshusterol, 10967

Stigmast-5-ene-3 $\beta, 7 \alpha$-diol, 20362

Stigmast-5-ene-3 $\beta, 7 \beta$-diol, 20363

$\alpha$-Tocopherol, 21415

\section{$\mathrm{C}_{29} \mathrm{H}_{50} \mathrm{O}_{4}$}

Bryophyllol, 2691

Irisoquin F, 11178

(-)- $\alpha$-Tocospirone, 21416

\section{$\mathrm{C}_{29} \mathrm{H}_{52} \mathrm{O}$}

$24 \alpha$-Ethyl-5 $\alpha$-cholestan-3 $\beta$-ol, 7427

Stigmastanol, 20357

$\mathrm{C}_{29} \mathrm{H}_{52} \mathrm{O}_{3}$

Stigmastane- $3 \beta, 5 \alpha, 6 \beta$-triol, 20356

$3 \beta, 5 \alpha, 6 \beta$-Trihydroxystigmastane, 21848

$\mathrm{C}_{29} \mathrm{H}_{53} \mathrm{O}_{3}$

$7 \alpha, 22 S$-Dihydroxysitosterol, 6123

$\mathrm{C}_{29} \mathrm{H}_{54} \mathrm{O}_{9}$

1-O-Gluco-2-O-gadoleic-glyceride, 8597

$\mathrm{C}_{29} \mathrm{H}_{56} \mathrm{O}_{2}$

Nonacosan-4-olide, 15666

$\mathrm{C}_{29} \mathrm{H}_{58} \mathrm{O}$

6-Nonacosanone, 15667
10-Nonacosanone, 15668

2-Nonacosanone, 15669

$\mathrm{C}_{29} \mathrm{H}_{58} \mathrm{O}_{2}$

Methyl montanate, 14606

$\mathrm{C}_{29} \mathrm{H}_{60}$

Nonacosane, 15660

$\mathrm{C}_{29} \mathrm{H}_{60} \mathrm{O}$

10-Nonacosanol, 15664

Nonacosanol, 15665

$\mathrm{C}_{29} \mathrm{H}_{60} \mathrm{O}_{2}$

Nonacosanediol-6,21, 15661

Nonacosanediol-6,8, 15662

Nonacosanediol-6,10, 15663

$\mathrm{C}_{30} \mathrm{H}_{16} \mathrm{O}_{8}$

Hypericin, 10886

$\mathrm{C}_{30} \mathrm{H}_{16} \mathrm{O}_{9}$

Pseudohypericin, 18028

$\mathrm{C}_{30} \mathrm{H}_{16} \mathrm{O}_{12}$

Anhydrobartramiaflovone, 1261

$\mathrm{C}_{30} \mathrm{H}_{18} \mathrm{CaO}_{14}$

Cochinchinol B, 3869

\section{$\mathrm{C}_{30} \mathrm{H}_{18} \mathrm{O}_{8}$}

2,2'-Bis[(1,8-dihydroxy-3-methyl)anthraquinone], 2443

Protohypericin, 17976

\section{$\mathrm{C}_{30} \mathrm{H}_{18} \mathrm{O}_{10}$}

Agathisflavone, 710

Amentoflavone, 1030

Anticancer Flavonoid PMV70P691-018, 1400

3,8"-Biapigenin, 2347

Cupressiflavone, 4376

Hinokiflavone, 9543

Loniflavone, 12989

Robustaflavone, 18864

Sennidin A, 19746

Sennidin B, 19747

Skyrin, 20010

Taiwaniaflavone, 20628

\section{$\mathrm{C}_{30} \mathrm{H}_{18} \mathrm{O}_{11}$}

Ridiculuflavone A, 18844

\section{$\mathrm{C}_{30} \mathrm{H}_{18} \mathrm{O}_{12}$}

Philonotisflavone, 17147

$\mathrm{C}_{30} \mathrm{H}_{18} \mathrm{O}_{14}$

Phlorofucofuroeckol A, 17173

\section{$\mathrm{C}_{30} \mathrm{H}_{20} \mathrm{~N}_{4} \mathrm{O}_{6}$}

Trichotomine, 21578

\section{$\mathrm{C}_{30} \mathrm{H}_{20} \mathrm{O}_{8}$}

Lespedezol $\mathrm{B}_{2}, 12689$

Rheidin B, 18757

\section{$\mathrm{C}_{30} \mathrm{H}_{20} \mathrm{O}_{9}$}

Calodenin B, 2985

Flavumone B, 7823

Rheidin A, 18756 
Sennidin C, 19748

$\mathrm{C}_{30} \mathrm{H}_{20} \mathrm{O}_{10}$

2",3"-Dihydroochnaflavone, 5683

Flavumone A, 7822

Rhusflavone, 18822

Volkensiflavone, 22609

\section{$\mathrm{C}_{30} \mathrm{H}_{20} \mathrm{O}_{11}$}

Hegoflavone A, 9286

(I-2S)-I-5,II-5,I-7,II-7,I-2',II-2',II-5'-Heptahy-

droxy-[I-6,II-6']-flavanonylflavone, 9394

Morelloflavone, 14969

\section{$\mathrm{C}_{30} \mathrm{H}_{20} \mathrm{O}_{12}$}

2,3-Dihydrophilonotisflavone, 5692

Hegoflavone B, 9287

(I-2S)-I-5,II-5,I-7,II-7,I-2',II-2',I-5',II-5'-Octahydroxy-[I-6,II-6']-flavanonylflavone, 15970

\section{$\mathrm{C}_{30} \mathrm{H}_{22} \mathrm{O}_{6}$}

Blestriarene C, 2510

$\mathrm{C}_{30} \mathrm{H}_{22} \mathrm{O}_{7}$

Palmidin B, 16557

Palmidin C, 16558

\section{$\mathrm{C}_{30} \mathrm{H}_{22} \mathrm{O}_{8}$}

Aloeemodin bianthrone, 968

Isolophirone C, 11512

Lophirone C, 13000

Palmidin A, 16556

\section{$\mathrm{C}_{30} \mathrm{H}_{22} \mathrm{O}_{9}$}

Afzelone B, 682

Afzelone C, 683

Calodenin A, 2984

Dihydrocalodenin B, 5551

Gnetuhainin G, 8885

\section{$\mathrm{C}_{30} \mathrm{H}_{22} \mathrm{O}_{10}$}

3,8"-Binaringenin, 2382

(+)-Chamaejasmin, 3462

Daphnodorin B, 4651

GB1a, 8244

Isochamaejasmin, 11320

Isoneochamaejasmin A, 11558

Neochamaejasmenin A, 15361

Rhusflavanone, 18821

Tetrahydrohinokiflavone, 21072

\section{$\mathrm{C}_{30} \mathrm{H}_{22} \mathrm{O}_{11}$}

Daphnodorin G, 4652

Daphnodorin H, 4654

GB1, 8243

GB2a, 8246

3",4',4"',5,5",7,7"-Heptahydroxy-3,8"-biflavanone, 9393

$\mathrm{C}_{30} \mathrm{H}_{22} \mathrm{O}_{12}$

GB2, 8245

Vitisinol, 22589

$\mathrm{C}_{30} \mathrm{H}_{22} \mathrm{O}_{13}$
Manniflavanone, 13502

$\mathrm{C}_{30} \mathrm{H}_{24} \mathrm{~N}_{4} \mathrm{O}_{5}$

Picrasidine U, 17312

$\mathrm{C}_{30} \mathrm{H}_{24} \mathrm{O}_{6}$

Blestriarene B, 2509

Blestrin C, 2513

3-3"Linked-(2'-hydroxy-4-hydroxychalcone)-

(2"'-hydroxy-4"-hydroxy dihydrochalcone), 12889

\section{$\mathrm{C}_{30} \mathrm{H}_{24} \mathrm{O}_{8}$}

Dihydrolophirone C, 5662

Dimeric 4,2',4'-trihydroxydihydrochalcone, 6195

\section{$\mathrm{C}_{30} \mathrm{H}_{24} \mathrm{O}_{9}$}

Cordigol, 4045

Gnetuhainin H, 8886

Mahuangnin B, 13400

Mahuangnin D, 13402

Ochnone, 15916

Shiraiachrome C, 19836

\section{$\mathrm{C}_{30} \mathrm{H}_{24} \mathrm{O}_{10}$}

ent-Epiafzelechin- $(2 \alpha \rightarrow O \rightarrow 7,4 \alpha \rightarrow 8)-(+)$-afzelechin, 6820

ent-Epiafzelechin-( $2 \alpha \rightarrow O \rightarrow 7,4 \alpha \rightarrow 8)$-(-)-afzelechin, 6821

ent-Epiafzelechin-( $2 \alpha \rightarrow O \rightarrow 7,4 \alpha \rightarrow 8)$-epi-afzelechin, 6822

Mahuangnin C, 13401

\section{$\mathrm{C}_{30} \mathrm{H}_{24} \mathrm{O}_{12}$}

Epicatechin- $(2 \beta \rightarrow O \rightarrow 7,4 \beta \rightarrow 6)$-catechin, 6855

Epicatechin- $(2 \beta \rightarrow O \rightarrow 7,4 \beta \rightarrow 6)$-ent-catechin, 6856

Epicatechin- $(2 \beta \rightarrow O \rightarrow 7,4 \beta \rightarrow 8)$-ent-epicatechin, 6858

Epicatechin- $(4 \beta \rightarrow 6,2 \beta \rightarrow O \rightarrow 7)$-ent-epicatechin, 6859

Proanthocyanidin $\mathrm{A}_{1}, 17866$

Proanthocyanidin $\mathrm{A}_{2}, 17867$

Proanthocyanidin $\mathrm{A}_{6}, 17868$

$\mathrm{C}_{30} \mathrm{H}_{24} \mathrm{O}_{14}$

Apigenin-7-O- $\beta$ - $D$-(4"-caffeoyl)glucuronide, 1483

\section{$\mathrm{C}_{30} \mathrm{H}_{26} \mathrm{O}_{6}$}

Blestrianol A, 2505

Blestriarene A, 2508

Blestrin A, 2511

Blestrin B, 2512

Blestrin D, 2514

Gymconopin C, 9101

Phoyunnanin B, 17201

\section{$\mathrm{C}_{30} \mathrm{H}_{26} \mathrm{O}_{8}$}

Gnemonol M, 8874

Gnetuhainin F, 8884

Gnetulin, 8892
Goniolactone F, 8944

Littorachalcone, 12934

Parvifolol D, 16691

Terrestrin C, 21008

Thelephantin D, 21296

\section{$\mathrm{C}_{30} \mathrm{H}_{26} \mathrm{O}_{10}$}

Afzelechin- $(4 \alpha \rightarrow 8)$-afzelechin, 678

Guibourtinidol-( $4 \alpha \rightarrow 6)$-catechin, 9079

Shiraiachrome A, 19834

Shiraiachrome B, 19835

\section{$\mathrm{C}_{30} \mathrm{H}_{26} \mathrm{O}_{11}$}

Gambiriin C, 8126

\section{$\mathrm{C}_{30} \mathrm{H}_{26} \mathrm{O}_{12}$}

Apigenin-7- $O$-(6"-(E)-p-coumaroyl)- $\beta$ - $D$ galactopyranoside, 1484

Apigenin-7- $O-\beta$ - $D$-(6"-p-courmaroyl)-glucoside, 1485

Apigenin-7- $O-\beta-D$-(6'- $p$-hydroxy-cinnamoyloxy)-mannoside, 1496

4-O-8',5'-5"-Dehydrotriferulic acid, 4977

Epioritin-4 $\alpha$-ol-(6 $\rightarrow 6)$-epioritin-4 $\beta$-ol, 6984

Peroxyhypocrellin, 16974

Proanthocyanidin $\mathrm{B}_{2}, 17869$

Procyanidin $\mathrm{B}_{1}, 17876$

Procyanidin $\mathrm{B}_{3}, 17884$

Procyanidin $\mathrm{B}_{4}, 17886$

Procyanidin $\mathrm{B}_{5}, 17888$

Procyanidin $\mathrm{B}_{7}, 17890$

Procyanidin C, 17892

\section{$\mathrm{C}_{30} \mathrm{H}_{26} \mathrm{O}_{13}$}

Biondnoid I, 2392

Castanoside A, 3297

Gallocatechin- $(4 \alpha \rightarrow 8)$ epicatechin, 8100

Tiliroside, 21392

\section{$\mathrm{C}_{30} \mathrm{H}_{26} \mathrm{O}_{14}$}

Ergoflavine, 7237

Gallocatechin-( $4 \alpha \rightarrow 8)$-epigallocatechin, 8101

Helichrysoside, 9301

Prodelphinidin $\mathrm{B}_{1}, 17898$

Prodelphinidin $\mathrm{B}_{2}, 17899$

\section{$\mathrm{C}_{30} \mathrm{H}_{26} \mathrm{O}_{15}$}

6-Hydroxykaempferol-7-O-(6-O-caffeoyl- $\beta-D$ glucopyranoside), 10272

6-Hydroxyluteolin

7-O-(6"-O-(E)-caffeoyl)- $\beta$-glucopyranoside, 10352

Myricetin-3'-O-(6"-p-coumaroyl) glucoside, 15174

Nympholide A, 15877

Nympholide B, 15878

Quercetagetin-7-O-(6- $O$ - $p$-coumaroyl- $\beta$ - $D$-glucopyranoside), 18307

Quercetin-3-O- $\beta-D$-(6"-caffeoyl galactoside), 
18332

$\mathrm{C}_{30} \mathrm{H}_{26} \mathrm{O}_{16}$

Quercetagetin-7- $O$-(6- $O$-caffeoyl- $\beta$ - $D$-glucopyranoside), 18306

\section{$\mathrm{C}_{30} \mathrm{H}_{26} \mathrm{O}_{17}$}

Myricetin-3-O-(4"-O-acetyl-2"-O-galloyl)- $\alpha-L$ -

${ }^{1} C_{4}$-rhamnopyranoside, 15172

\section{$\mathrm{C}_{30} \mathrm{H}_{27} \mathrm{O}_{13}{ }^{+}$}

Hyacinthin, 9696

\section{$\mathrm{C}_{30} \mathrm{H}_{27} \mathrm{O}_{14}{ }^{+}$}

Delphinidin-3- $O-\beta$ - $D$-(6-(E)-p-coumaryl) galactopyranoside, 5018

\section{$\mathrm{C}_{30} \mathrm{H}_{27} \mathrm{O}_{17}{ }^{+}$}

Delphinidin-3'-O-(2"-O -galloyl-6"- $O$-acetyl- $\beta$ galactopyranoside), 5023

\section{$\mathrm{C}_{30} \mathrm{H}_{28} \mathrm{O}_{6}$}

Dibothrioclinin I, 5396

Dibothrioclinin II, 5397

Diuvaretin, 6528

Marchantin J, 13556

Phoyunnanin A, 17200

\section{$\mathrm{C}_{30} \mathrm{H}_{28} \mathrm{O}_{8}$}

Isorottlerin, 11685

Rottlerin, 18940

\section{$\mathrm{C}_{30} \mathrm{H}_{28} \mathrm{O}_{9}$}

Gemichalcone C, 8262

Gnetuhainin I, 8887

4-Hydroxyrottlerin, 10682

\section{$\mathrm{C}_{30} \mathrm{H}_{28} \mathrm{O}_{10}$}

Hypomycin C, 10904

Hypomycin D, 10905

Rocaglamide derivative 10, 18890

\section{$\mathrm{C}_{30} \mathrm{H}_{28} \mathrm{O}_{11}$}

Crenatoside, 4226

\section{$\mathrm{C}_{30} \mathrm{H}_{28} \mathrm{O}_{12}$}

3'-O-trans-Cinnamoyl-astilbin, 3700

Ethyl lithospermate, 7457

Naringenin 7-O-(6"-O-trans-p-coumaroyl)glucoside, 15280

\section{$\mathrm{C}_{30} \mathrm{H}_{28} \mathrm{O}_{13}$}

2"-O-(3"',4"'-Dimethoxybenzoyl)vitexin, 6208

$(S)$-Eriodictyol-7-O-(6"-O-trans-p-coumaroyl)$\beta$-D-glucopyranoside, 7279

\section{$\mathrm{C}_{30} \mathrm{H}_{28} \mathrm{O}_{14}$}

2"-O-(3"',4"'-Dimethoxybenzoyl)orientin, 6207

$\mathrm{C}_{30} \mathrm{H}_{29} \mathrm{~N}_{4} \mathrm{O}_{4}^{+}$

Kumujansine B, 12333

\section{$\mathrm{C}_{30} \mathrm{H}_{30} \mathrm{~N}_{2} \mathrm{O}_{4}$}

Melicobisquinolinone A, 13676

$\mathrm{C}_{30} \mathrm{H}_{30} \mathrm{O}_{5}$

2,6-Bis( $p$-hydroxybenzyl)-3',5-dimethoxy-3hydroxybibenzyl, 2457

$\mathrm{C}_{30} \mathrm{H}_{30} \mathrm{O}_{7}$
Artonin A, 1821

Cycloheterophyllin, 4495

Formosanatin C, 7891

Paleatin B, 16540

p-Tolyl-methyl carbinol diferuloyl methane,

21432

$\mathrm{C}_{30} \mathrm{H}_{30} \mathrm{O}_{8}$

Gossypol, 8967

$\mathrm{C}_{30} \mathrm{H}_{30} \mathrm{O}_{9}$

1-O-Acetyl-rocaglatic acid methyl ester, 495

Bismurrangatin, 2485

\section{$\mathrm{C}_{30} \mathrm{H}_{30} \mathrm{O}_{10}$}

rel-5-Hydroxy-7,4'-dimethoxy-2"S-(2,4,5-trimethoxy-E-styryl)-tetrahydrofuro[4" $R, 5 " R: 2,3]$

flavanonol, 10042

rel-5-Hydroxy-7,4'-dimethoxy-3"S-(2,4,5-trimethoxy- $E$-styryl)tetrahydrofuro[4"R,5"R:2,3]

flavanonol, 10043

\section{$\mathrm{C}_{30} \mathrm{H}_{30} \mathrm{O}_{11}$}

Piceid 2'-O-E-ferulate, 17284

Rhaponticin 2"-O-p-coumarate, 18745

$\mathrm{C}_{30} \mathrm{H}_{30} \mathrm{O}_{12}$

(+)-Catechin-3- $O-\beta$ - $D$-gluco(2-cinnamoyl)pyranoside, 3311

(+)-Catechin-3-O- $\beta$ - $D$-gluco(6-cinnamoyl)pyranoside, 3312

Curtisian M, 4409

\section{$\mathrm{C}_{30} \mathrm{H}_{30} \mathrm{O}_{13}$}

Myrciacitrin V, 15151

\section{$\mathrm{C}_{30} \mathrm{H}_{31} \mathrm{NO}_{7}$}

Murrayanine II*, 15119

\section{$\mathrm{C}_{30} \mathrm{H}_{32} \mathrm{O}_{5}$}

5-Hydroxy-8-methyl-6-(2-methylbutanoyl)-8-(4methylpent-3-enyl)-4-phenyl-2H-pyrano[2,3h] chromen-2-one, 10507

\section{$\mathrm{C}_{30} \mathrm{H}_{32} \mathrm{O}_{6}$}

BF-4, 2339

BF-5, 2340

Kanzonol L, 12151

Sanggenon J, 19265

Sanggenon K, 19266

$\mathrm{C}_{30} \mathrm{H}_{32} \mathrm{O}_{7}$

Broussoflavonol D, 2634

Dorsmanin C, 6563

Lactucain A, 12441

Sanggenon L, 19267

Sorocein D, 20105

\section{$\mathrm{C}_{30} \mathrm{H}_{32} \mathrm{O}_{8}$}

Benzoylisogomisin O, 2245

Formosanatin A, 7889

\section{$\mathrm{C}_{30} \mathrm{H}_{32} \mathrm{O}_{9}$}

Benzoylgomisin P, 2241

Formosanatin B, 7890
Gomisin C, 8908

Gomisin G, 8912

Lactucain B, 12442

Lappaol A, 12513

\section{$\mathrm{C}_{30} \mathrm{H}_{32} \mathrm{O}_{10}$}

Dumosaol, 6635

\section{$\mathrm{C}_{30} \mathrm{H}_{32} \mathrm{O}_{12}$}

Benzoylpaeoniflorin, 2266

Paeonin A, 16529

\section{$\mathrm{C}_{30} \mathrm{H}_{32} \mathrm{O}_{13}$}

Benzoyl-oxypaeoniflorin, 2263

\section{$\mathrm{C}_{30} \mathrm{H}_{32} \mathrm{O}_{18}$}

Kaempferol-3-O-(2"-O- $\alpha$-rhamnosyl-6"- $O$ malonyl)- $\beta$-glucoside, 12088

\section{$\mathrm{C}_{30} \mathrm{H}_{32} \mathrm{O}_{19}$}

Quercetin-3-O-(2"-O- $\alpha$-rhamnosyl-6"-Omalonyl)- $\beta$-glucoside, 18389

\section{$\mathrm{C}_{30} \mathrm{H}_{33} \mathrm{NO}_{8}$}

3'-Hydroxy-8b-ethyl ether-rocaglatic acid methylamide, 10098

Neoharringtonine, 15403

Thaliadine, 21238

\section{$\mathrm{C}_{30} \mathrm{H}_{33} \mathrm{O}_{19}$}

Delphinidin-3-O-(2"- $O$ - $\alpha$-rhamnosyl-6"-Omalonyl)- $\beta$-glucoside, 5030

\section{$\mathrm{C}_{30} \mathrm{H}_{33} \mathrm{O}_{20}{ }^{+}$}

Delphinidin-3-O-( $\beta$ - $D$-glucopyranoside $)-5-O$ (6- $O$-malonyl-( $\beta$ - $D$-glucopyranoside, 5025

Delphinidin-3- $O$-(6- $O$-malonyl- $\beta$ - $D$-glucoside)5- $O$ - $\beta$ - $D$-glucoside, 5028

\section{$\mathrm{C}_{30} \mathrm{H}_{34} \mathrm{BrN}_{5} \mathrm{O}_{15}$}

Neosurugatoxin, 15461

\section{$\mathrm{C}_{30} \mathrm{H}_{34} \mathrm{~N}_{2} \mathrm{O}_{5}$}

Rauvomitine, 18557

\section{$\mathrm{C}_{30} \mathrm{H}_{34} \mathrm{~N}_{2} \mathrm{O}_{19}$}

Amaranthin, 1013

Isoamaranthin, 11207

\section{$\mathrm{C}_{30} \mathrm{H}_{34} \mathrm{~N}_{4}$}

Usambarine, 22276

\section{$\mathrm{C}_{30} \mathrm{H}_{34} \mathrm{O}_{4}$}

Harunmadagascarin A, 9242

Sophoradochromene, 20085

Sophoranochromene, 20095

\section{$\mathrm{C}_{30} \mathrm{H}_{34} \mathrm{O}_{5}$}

5,7-Dihydroxy-6-(2-methylbutanoyl)-8-[(E)-3,7dimethylocta-2,6-dienyl]-4-phenyl- $2 H$-chrome n-2-one, 6018

5,7-Dihydroxy-6-(3-methylbutanoyl)-8-[(E)-3,7dimethylocta-2,6-dienyl]-4-phenyl- $2 H$-chrome n-2-one, 6019

5,7-Dihydroxy-8-(2-methylbutanoyl)-6-[(E)-3,7dimethylocta-2,6-dienyl]-4-phenyl- $2 H$-chrome n-2-one, 6020 


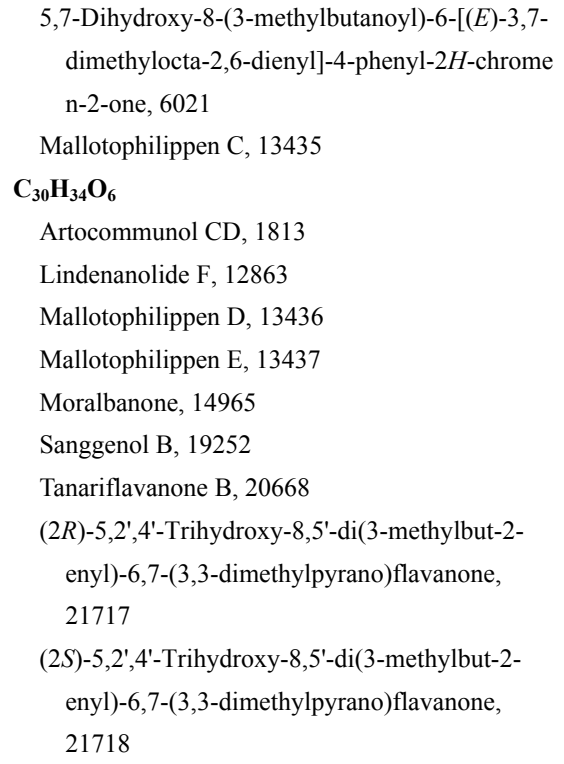

\section{$\mathrm{C}_{30} \mathrm{H}_{34} \mathrm{O}_{7}$}

Broussoflavonol C, 2633

Sorocein F, 20106

\section{$\mathrm{C}_{30} \mathrm{H}_{34} \mathrm{O}_{8}$}

Benzoylgomisin $\mathrm{H}, 2240$

Saucerneol E, 19402

\section{$\mathrm{C}_{30} \mathrm{H}_{34} \mathrm{O}_{9}$}

Eryvarinol B, 7353

Schisantherin E, 19491

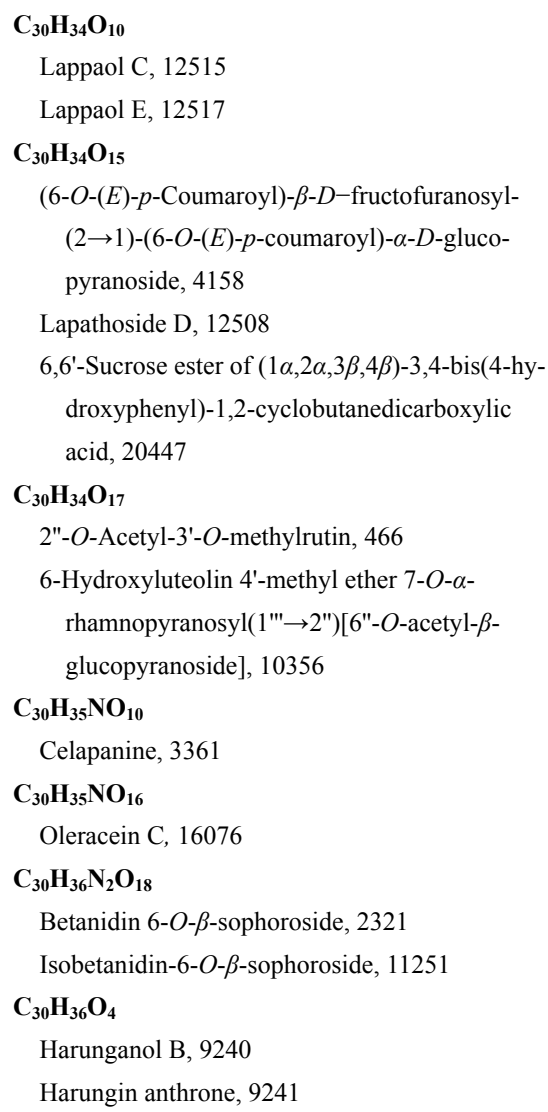

\section{$\mathrm{C}_{30} \mathrm{H}_{34} \mathrm{O}_{17}$}

2"-O-Acetyl-3'-O-methylrutin, 466

6-Hydroxyluteolin 4'-methyl ether 7-O- $\alpha$ rhamnopyranosyl $(1 " ' \rightarrow 2 ")[6 "-O$-acetyl- $\beta$ glucopyranoside], 10356

\section{$\mathrm{C}_{30} \mathrm{H}_{35} \mathrm{NO}_{10}$}

Celapanine, 3361

\section{$\mathrm{C}_{30} \mathrm{H}_{35} \mathrm{NO}_{16}$}

Oleracein C, 16076

\section{$\mathrm{C}_{30} \mathrm{H}_{36} \mathrm{~N}_{2} \mathrm{O}_{18}$}

Betanidin 6-O- $\beta$-sophoroside, 2321

Isobetanidin-6- $O-\beta$-sophoroside, 11251

\section{$\mathrm{C}_{30} \mathrm{H}_{36} \mathrm{O}_{4}$}

Harunganol B, 9240

Harungin anthrone, 9241

Sophoradin, 20082

Sophoranone, 20098

Vielanin B, 22470

$\mathrm{C}_{30} \mathrm{H}_{36} \mathrm{O}_{5}$

Mulberrofuran Z, 15057

$\mathrm{C}_{30} \mathrm{H}_{36} \mathrm{O}_{6}$

Artemyriantholide A, 1803

Artemyriantholide B, 1804

Artemyriantholide D, 1806

Kushenol B, 12355

(-)-Nymphaeol C, 15876

$\mathrm{C}_{30} \mathrm{H}_{36} \mathrm{O}_{7}$

Cynaphylloside, 4555

Kushenol M, 12366

Petalostemumol, 17009

Tanariflavanone A, 20667

\section{$\mathrm{C}_{30} \mathrm{H}_{36} \mathrm{O}_{\mathbf{8}}$}

$11 \beta, 12 \alpha$-Diacetoxyneotecleanin, 5316

Saucerneol C, 19400

Vielanin C, 22471

\section{$\mathrm{C}_{30} \mathrm{H}_{36} \mathrm{O}_{9}$}

$11 \beta, 12 \alpha$-Diacetoxy-14 $\beta, 15 \beta$-epoxyneotecleanin, 5299

Nimbin, 15601

Norstaminone A, 15796

\section{$\mathrm{C}_{30} \mathrm{H}_{36} \mathrm{O}_{10}$}

$11 \beta, 12 \alpha$-Diacetoxy-1-deoxo-14 $\beta, 15 \beta$-epoxy-3 $\beta$ hydroxy-2-oxo-neotecleanin, 5291

Interiotherin C, 11105

Toosendanal, 21447

\section{$\mathrm{C}_{30} \mathrm{H}_{36} \mathrm{O}_{11}$}

$2 \beta$-( $\beta$ - $D$-glucopyranosyloxy)-8 $\beta$-(4"-methoxyphenylacetoxy)-guaia-4(15),10(14),11(13)-trien- $1 \alpha, 5 \alpha, 6 \beta, 7 \alpha H$-12,6-olide, 8706

Kadsurarin, 12004

Nigakilactone $\mathrm{O}, 15560$

11-Oxocneorin G, 16301

\section{$\mathrm{C}_{30} \mathrm{H}_{36} \mathrm{O}_{12}$}

14-O-Methylacetal-15-O-[6'-( $p$-hydroxyphenylacetyl)]- $\beta$ - $D$-glucopyranosylurospermal A, 14110

$\mathrm{C}_{30} \mathrm{H}_{36} \mathrm{O}_{13}$

Oregonin peracetate, 16175

$\mathrm{C}_{30} \mathrm{H}_{36} \mathrm{O}_{14}$

Dracunculifoside N, 6593

7-O- $\alpha$-D-Glucopyranosyl-3,4'-dihydroxy-3'-(4"acetoxyl-3"-methylbutyl)-5,6-dimethoxyflavone, 8616

7-O- $\alpha-D$-Glucopyranosyl-3,5-dihydroxy-3'-(4"acetoxyl-3"-methylbutyl)-6,4'-dimethoxyflavone, 8617

Masutakeside I, 13592

$\mathrm{C}_{30} \mathrm{H}_{36} \mathrm{O}_{15}$
Dracunculifoside I, 6588

3"'-O-Methylcrenatoside, 14261

Pueroside B, 18186

\section{$\mathrm{C}_{30} \mathrm{H}_{36} \mathrm{O}_{16}$}

$\beta$-D-[5-O-(3,4-Dimethoxybenzoyl)]-apiofuranosyl-( $1 \rightarrow 6)-\beta$ - $D$-glucopyranosyl, 6206

Syringetin-3-rutinoside, 20565

\section{$\mathrm{C}_{30} \mathrm{H}_{36} \mathbf{O}_{17}$}

Tenuifoliside B, 20956

\section{$\mathrm{C}_{30} \mathrm{H}_{37} \mathrm{NO}_{7}$}

Lathyrol-3,15-diacetate-5-nicotinate, 12545

\section{$\mathrm{C}_{30} \mathrm{H}_{37} \mathrm{NO}_{9}$}

Nicotaxine, 15524

\section{$\mathrm{C}_{30} \mathrm{H}_{37} \mathrm{NO}_{11}$}

$3 \alpha-(3,4,5$-Trimethoxy-cinnamoyloxy)-7 $\beta$-(3,4,5trimethoxybenzoyloxy)- $6 \alpha$-hydroxy-tropane, 21903

\section{$\mathrm{C}_{30} \mathrm{H}_{37} \mathrm{~N}_{5} \mathrm{O}_{5}$}

Dianthin F, 5370

Ergosine, 7243

Ergosinine, 7244

\section{$\mathrm{C}_{30} \mathrm{H}_{38} \mathrm{O}_{4}$}

Bis-[8-eudesma-4(15),7(11)-dien-12,8 $\alpha$-olide], 7494

Lancilactone B, 12478

\section{$\mathrm{C}_{30} \mathrm{H}_{38} \mathrm{O}_{6}$}

Artemyriantholide C, 1805

Dysobinin, 6656

Lappadilactone, 12511

(+)-Pinoresinol-di-3,3-dimethylallyl ether, 17411

\section{$\mathrm{C}_{30} \mathrm{H}_{38} \mathrm{O}_{7}$}

Kidjolanin, 12224

\section{$\mathrm{C}_{30} \mathrm{H}_{38} \mathrm{O}_{8}$}

$12 \alpha$-Acetoxyazadironolide, 128

$7 \alpha, 12 \alpha$-Diacetoxy-11 $\beta$-hydroxyneotecleanin, 5311

Ganosporeric acid A, 8200

Kosamol A, 12280

Turraparvin C, 22140

\section{$\mathrm{C}_{30} \mathrm{H}_{38} \mathrm{O}_{9}$}

$6 \alpha$-Acetoxy-5-epilimonin, 179

$6 \beta$-Acetoxy-5-epilimonin, 180

$7 \alpha, 12 \alpha$-Diacetoxy-14 $\beta, 15 \beta$-epoxy-11 $\beta$-hydroxyneotecleanin, 5298

Sapxanthone, 19353

\section{$\mathrm{C}_{30} \mathrm{H}_{38} \mathbf{O}_{10}$}

Secoorthosiphol B, 19630

\section{$\mathrm{C}_{30} \mathrm{H}_{38} \mathrm{O}_{11}$}

11 $\beta$-Hydroxycneorin G, 9927

Isotoosendanin, 11743

Kupitengester 3, 12338

$1 \alpha, 6 \beta, 8 \beta, 14$-Tetraacetoxy-9 $\beta$-benzoyloxydihydro- $\beta$-agarofuran, 21024 
Toosendanin, 21448

$\mathrm{C}_{30} \mathrm{H}_{38} \mathrm{O}_{12}$

Aceroketoside, 102

$2 \alpha, 9 \beta$-Di-( $\beta$-furancarbonyloxy)- $4 \beta, 6 \beta, 15$-trihydroxy-1 $\alpha$-(2)-methylbutanoyloxy-dihydro- $\beta$-ag arofuran, 5508

$1 \alpha, 6 \beta, 8 \alpha, 13$-Tetraacetoxy-9 $\alpha$-benzoyloxy- $2 \alpha$ hydroxy- $\beta$-dihydroagarofuran, 21025

$\mathrm{C}_{30} \mathrm{H}_{38} \mathrm{O}_{13}$

Bruceantinol, 2661

\section{$\mathrm{C}_{30} \mathrm{H}_{38} \mathrm{O}_{15}$}

Cistanoside C, 3753

Eukovoside, 7533

$1 \beta$-Furanoyl-2 $\beta, 3 \alpha, 7 \alpha, 8 \beta, 11$-pentaacetoxy-5 $\alpha$ hydroxy-dihydroagarofuran, 8024

Jionoside D, 11885

JuncenolideC, 11951

Leucosceptoside A, 12725

Matteucinin, 13610

(-)-7- $O$-Methyleucomol 5- $O-\beta$-neohesperidoside, 14438

(-)-7-O-Methyleucomol 5- $O$ - $\beta$-rutinoside, 14439

Plantainoside C, 17509

\section{$\mathrm{C}_{30} \mathrm{H}_{38} \mathrm{O}_{16}$}

Campneoside I, 3052

$1 \beta$-Furanoyl- $2 \beta, 3 \alpha, 7 \alpha, 8 \beta, 11$-pentaacetoxy- $4 \alpha, 5 \alpha$ dihydroxy-dihydroagarofuran, 8023

Saccatoside, 19104

\section{$\mathrm{C}_{30} \mathrm{H}_{39} \mathrm{ClO}_{14}$}

Gemmacolide A, 8264

\section{$\mathrm{C}_{30} \mathrm{H}_{39} \mathrm{O}_{3}$}

$2 \alpha, 7 \beta, 13(S)$-Trihydroxystemodane, 21846

\section{$\mathrm{C}_{30} \mathrm{H}_{40} \mathrm{~N}_{4} \mathrm{O}_{4}$}

Sanjoinine B, 19290

\section{$\mathrm{C}_{30} \mathrm{H}_{40} \mathrm{~N}_{4} \mathrm{O}_{5}$}

Ephedradine C, 6814

\section{$\mathrm{C}_{30} \mathrm{H}_{40} \mathrm{O}_{2}$}

$\alpha$-Citraurin, 3763

$\beta$-Citraurin, 3764

\section{$\mathrm{C}_{30} \mathrm{H}_{40} \mathrm{O}_{3}$}

Versicolactone D, 22425

\section{$\mathrm{C}_{30} \mathrm{H}_{40} \mathrm{O}_{4}$}

Lancilactone C, 12479

Pristimerin, 17862

Schisanlactone A, 19479

\section{$\mathrm{C}_{30} \mathrm{H}_{40} \mathrm{O}_{5}$}

Lancilactone A, 12477

6-Oxopristimerol, 16411

Schisanlactone C, 19481

$\mathrm{C}_{30} \mathrm{H}_{40} \mathrm{O}_{6}$

Absinthin, 37

Anabsinthin, 1127

2,3,7-Trihydroxy-6-oxo-1,3,5(10),7-tetraene- 24-nor-friedelane-29-oic acid methylester,

\section{0}

\section{$\mathrm{C}_{30} \mathrm{H}_{40} \mathrm{O}_{7}$}

Anticancer Norwithanolide PMV70P691-033, 1431

Ganoderenic acid D, 8162

Ganoderenic acid H, 8163

Ganoderic acid E, 8169

Ganosporelactone A, 8198

12-O-2Z,4E-Octadienoyl-4-deoxyphorbol-13acetate, 15964

Penupogenin, 16899

Seemarin, 19651

\section{$\mathrm{C}_{30} \mathrm{H}_{40} \mathrm{O}_{8}$}

$7 \beta$-Acetoxywithanolide $\mathrm{D}, 302$

Ardisiaquinone A, 1629

Ardisiaquinone B, 1630

Methyl lucidenate $\mathrm{D}_{2}, 14560$

Physachenolide D, 17235

Terpenoid EA-1, 20986

\section{$\mathrm{C}_{30} \mathrm{H}_{40} \mathrm{O}_{9}$}

Anticancer Withanolide PMV70P691-148, 1456

Calcicolin B, 2950

Physachenolide E, 17236

\section{$\mathrm{C}_{30} \mathrm{H}_{40} \mathrm{O}_{10}$}

Penisimplicin A, 16806

Strophantojavoside, 20404

\section{$\mathrm{C}_{30} \mathrm{H}_{40} \mathrm{O}_{11}$}

7-Acetyllushanrubescensin A, 458

Hellebortin B, 9327

\section{$\mathrm{C}_{30} \mathrm{H}_{40} \mathrm{O}_{12}$}

Aceroside III, 105

Glabcensin S, 8480

$3 \beta, 5 \alpha, 7 \beta, 8 \alpha, 15 \beta$-Pentaacetoxyjatropha-6(17),

$11 E$-dien-9,14-dione, 16820

\section{$\mathrm{C}_{30} \mathrm{H}_{40} \mathrm{O}_{13}$}

5 $\alpha$-Acetyl-5 $\alpha$-decinnamoyltaxagifine, 368

Dichotomoside B, 5428

Dichotomoside C, 5429

\section{$\mathrm{C}_{30} \mathrm{H}_{40} \mathrm{O}_{14}$}

19-Debenzoyl-19-acetyltaxinine M, 4802

5-Decinnamoyl-11-acetyl-19-hydroxyl taxagifine, 4850

Luzonoside A, 13164

Luzonoside B, 13165

$\mathrm{C}_{30} \mathrm{H}_{40} \mathrm{O}_{16}$

6-O- $\alpha-D$-Galactopyranosylharpagoside, 8064

6'-O- $\alpha-D$-Galactopyranosylsyringopicroside, 8071

3'-O- $\beta$ - $D$-Glucopyranosylsyringopicroside, 8737

4'-O- $\beta$ - $D$-Glucopyranosylsyringopicroside, 8738

6'-O- $\alpha$ - $D$-Glucopyranosylsyringopicroside, 8739

$\mathrm{C}_{30} \mathrm{H}_{40} \mathrm{O}_{17}$
Safghanoside C, 19114

$\mathrm{C}_{30} \mathrm{H}_{40} \mathrm{O}_{18}$

7-O-(4- $\beta$ - $D$-Glucopyranosyloxy-3-methoxybenzoyl) secologanolic acid, 8701

$\mathrm{C}_{30} \mathrm{H}_{41} \mathrm{NO}_{6}$

15-Veratroyldictizine, 22393

$\mathrm{C}_{30} \mathrm{H}_{42} \mathrm{~N}_{2} \mathrm{O}_{2} \mathrm{~S}$

Thiobinupharidine, 21333

$\mathrm{C}_{30} \mathrm{H}_{42} \mathrm{~N}_{2} \mathrm{O}_{4} \mathrm{~S}$

Nuphleine, 15872

\section{$\mathrm{C}_{30} \mathrm{H}_{42} \mathrm{~N}_{2} \mathrm{O}_{6}$}

Delphicrispuline, 5009

\section{$\mathrm{C}_{30} \mathrm{H}_{42} \mathrm{~N}_{2} \mathrm{O}_{7}$}

$\mathrm{N}$-Deacetyllappaconitine, 4745

Puberanidine, 18168

\section{$\mathrm{C}_{30} \mathrm{H}_{42} \mathrm{~N}_{2} \mathrm{O}_{8}$}

$N$-Deacetylranaconitine, 4771

Jiufengtine, 11888

\section{$\mathrm{C}_{30} \mathrm{H}_{42} \mathrm{~N}_{2} \mathrm{O}_{9}$}

$N$-Deacetylfinaconitine, 4741

\section{$\mathrm{C}_{30} \mathrm{H}_{42} \mathrm{~N}_{4} \mathrm{O}_{5}$}

Sanjoinine $\mathrm{G}_{2}, 19294$

\section{$\mathrm{C}_{30} \mathrm{H}_{42} \mathrm{O}_{3}$}

Argentatin F, 1669

Tyromycic acid F, 22167

\section{$\mathrm{C}_{30} \mathrm{H}_{42} \mathrm{O}_{4}$}

Papuaforin E, 16633

Papyriogenin A, 16635

Papyriogenin B, 16636

Pseudolarifuroic acid, 18042

Pseudolarolide B, 18044

Schisanlactone B, 19480

\section{$\mathrm{C}_{30} \mathrm{H}_{42} \mathrm{O}_{5}$}

3-O-(2E,4E-Decadienoyl)-20-deoxyingenol, 4823

3-O-(2E,4Z-Decadienoyl)-20-deoxyingenol, 4824

$8 \alpha$-Hydroxyabda-13(16),14-dien-19-yl- $(E)-4-$ hydroxy-3-methoxycinnamate, 10301

Kadsulactone A, 11993

Kadsulactone A', 11994

\section{$\mathrm{C}_{30} \mathrm{H}_{42} \mathrm{O}_{6}$}

3-O-(2'E,4'E-Decadienoyl)ingenol, 4825

3-O-(2'E,4'Z-Decadienoyl)ingenol, 4826

5-O-(2'E,4'E-Decadienoyl)ingenol, 4827

20-O-(2'E,4'E-Decadienoyl)ingenol, 4828

20-O-(2'E,4'Z-Decadienoyl)ingenol, 4829

Picfeltarraegenin V, 17292

Pseudolarolide E, 18047

\section{$\mathrm{C}_{30} \mathrm{H}_{42} \mathrm{O}_{7}$}

Ardisiaquinone F, 1634

Cucurbitacin I, 4323

$7 \beta, 23 \xi$-Dihydroxy-3,11,15-trioxolanosta-8,20E(22)-dien-26-oic acid, 6168 
Diketouncaric acid, 6191

Drevogenin II, 6603

Ganode-8-en-ric acid $\mathrm{C}_{1}, 8152$

Ganode-8-en-ric acid $\mathrm{LM}_{2}, 8157$

Ganoderenic acid I, 8164

Ganoderic acid $\mathrm{AM}_{1}, 8165$

Ganoderic acid $\mathrm{C}_{1}, 8168$

Ganoderic acid $\zeta, 8180$

Ganosporelactone B, 8199

Pseudolarolide H, 18048

\section{$\mathrm{C}_{30} \mathrm{H}_{42} \mathrm{O}_{8}$}

12-Deacetylganoderic acid $\mathrm{H}, 4743$

Ganode-8-en-ric acid D, 8153

Ganoderic acid N, 8172

Ganoderic acid $\theta, 8181$

Methyl lucidenate $\mathrm{E}_{2}, 14561$

Proscillaridin A, 17951

12-O-Tigloylphorbol-13-(2-methylbutyrate), 21382

\section{$\mathrm{C}_{30} \mathrm{H}_{42} \mathrm{O}_{9}$}

17-Acetoxy-4-deoxyphorbol 12,13-bis (isobutyrate), 154

Calcicolin C $\$, 2952$

Decoside, 4857

\section{$\mathrm{C}_{30} \mathrm{H}_{42} \mathbf{O}_{\mathbf{1 0}}$}

Fugaxin, 7981

Physachenolide A, 17232

Rouyolide C, 18964

\section{$\mathrm{C}_{30} \mathrm{H}_{42} \mathrm{O}_{11}$}

Decinnamol taxinine J, 4849

Forrestin A, 7915

Gesneroidin E, 8370

Glabcensin N, 8475

Physachenolide B, 17233

5,10,13-Triacetyl-10- debenzoyl brevifoliol, 21533

\section{$\mathrm{C}_{30} \mathrm{H}_{42} \mathrm{O}_{12}$}

Aceroside VIII, 106

Canadensene, 3056

$5 \alpha$-DeacetylbaccatinI, 4721

2-Deacetyltaxachitriene A, 4776

Decinnamoyl-1-hydroxy-taxinine J, 4852

$1 \beta$-Dehydroxy- $4 \alpha$-deacetylbaccatin IV, 4984

5-Epicanadense, 6850

Glabcensin T, 8481

Taxane 1, 20748

\section{$\mathrm{C}_{30} \mathrm{H}_{42} \mathrm{O}_{13}$}

4-Deacetylbaccatin IV, 4724

$1 \beta, 7 \beta$-Dihydroxy- $4 \beta, 20$-epoxy- $2 \alpha, 5 \alpha, 9 \alpha, 10 \beta$, $13 \alpha$-pentaacetoxytax-11-ene, 5881

$1 \beta, 9 \alpha$-Dihydroxy-4 $\beta, 20$-epoxy- $2 \alpha, 5 \alpha, 7 \beta, 10 \beta$,

$13 \alpha$-penta-acetoxytax-11-ene, 5882

$1 \beta$-Hydroxy- $7 \beta$-deacetyoxy- $7 \alpha$-hydroxybaccatin

$$
\text { I, } 9965
$$

Taxane 4, 20751

Taxayuntin H, 20766

Taxumairol D, 20824

Taxumairol F, 20826

Taxuspine K, 20858

Taxuspine V, 20869

Taxuyunnanine F, 20878

\section{$\mathrm{C}_{30} \mathrm{H}_{42} \mathrm{O}_{14}$}

(-)-Sakuraresinoside, 19175

$$
\begin{aligned}
& \mathbf{C}_{30} \mathbf{H}_{44} \mathbf{N}_{2} \mathbf{O}_{2} \\
& N^{20}, N^{20} \text {-Dimethyl- } N^{3} \text {-benzoyl-3,20-diamino- } \\
& \quad \text { pregn-2-en-4-ol, } 6322
\end{aligned}
$$

\section{$\mathrm{C}_{30} \mathrm{H}_{44} \mathrm{~N}_{6} \mathrm{O}_{7}$}

Dianthin E, 5369

\section{$\mathrm{C}_{30} \mathrm{H}_{44} \mathrm{O}_{3}$}

Ananosic acid C, 1138

Kadsulactone, 11992

Kulactone, 12328

Lucialdehyde B, 13020

Neokadsuranic acid A, 15419

Neokadsuranic acid B, 15420

Ochraceolide A, 15919

Ochraceolide C, 15921

3-Oxo-11,13(18)-oleanadien-28-oic acid, 16400

Papyriogenin J, 16643

Schisanlactone D, 19482

Tyromycic acid, 22162

Tyromycic acid E, 22166

Wilforlide B, 22683

Zizyberenalic acid, 23013

\section{$\mathrm{C}_{30} \mathrm{H}_{44} \mathrm{O}_{4}$}

Changnanic acid, 3476

3,7-Dioxo-8,24Z-tirucalladien-26-oic acid, 6478

Ganode-8-en-ric acid DM, 8154

Glabrolide, 8500

6 $\beta$-Hydroxy-3-oxo-11,13(18)-oleanadien-28-oic acid, 10576

24-Hydroxy-3-oxo-11,13(18)-oleanadien-28-oic acid, 10577

Isoglabrolide, 11441

Kadsudilactone, 11991

Kadsulactone acid, 11995

$\alpha$-Kudinlactone, 12297

Liquidambaric lactone, 12904

Ochraceolide B, 15920

Ochraceolide E, 15923

Papyriogenin C, 16637

Papyriogenin D, 16638

Papyriogenin G, 16640

Pseudolarolide A, 18043

Schisanlactone E, 19483

Schisanlactone F, 19484
Seco-neokadsuranic acid A, 19629

Semialactone, 19692

Tyromycic acid D, 22165

\section{$\mathrm{C}_{30} \mathrm{H}_{44} \mathrm{O}_{5}$}

Cangoronine, 3075

Chiisanogenin, 3519

7,11-Dioxo-3 $\alpha$-hydroxy-8,24Z-tirucalladien-26oic acid, 6471

$14 \beta, 15 \beta$-Epoxy-3 $\beta$-hydroxy-9-oxo-11(10 $\rightarrow 8$ )abeolanosta-22-cis,24-trans-dien-26-oic acid, 7125

Garcinielliptone L, 8214

Garcinielliptone M, 8215

Glyyunnansapogenin F, 8861

$21 \alpha$-Hydroxyisoglabrolide, 10247

Isomasticadienonalic acid, 11530

Liquoric acid, 12914

3-Oxo-30-carbomethoxy-23-norolean-12-en-28oic acid, 16299

Picfeltarraegenin I, 17288

Poricoic acid B, 17723

Propapyriogenin $\mathrm{A}_{2}, 17918$

Pseudolarolide D, 18046

\section{$\mathrm{C}_{30} \mathrm{H}_{44} \mathbf{O}_{6}$}

$3 \beta, 19 \alpha$-Dihydroxy-6-oxo-urs-12-en-23-al-28-oic acid, 6069

$2 \alpha, 3 \beta$-Dihydroxyurs-12,19-dien-23,28-oic acid, 6170

Ganoderic acid $\beta, 8176$

Ganolucidic acid A, 8196

$6 \alpha$-Hydroxylup-20(29)-en-3-oxo-27,28-dioic acid, 10343

$6 \beta$-Hydroxylup-20(29)-en-3-oxo-27,28-dioic acid, 10344

Isodonadenanthin, 11391

Officinalic acid, 16013

7-Oxo-3 $\beta$-hydroxyurs-12-en-27,28-dioic acid, 16357

Picfeltarraegenin III, 17290

Picfeltarraegenin VI, 17293

Saponaceolide G, 19330

\section{$\mathrm{C}_{30} \mathrm{H}_{44} \mathrm{O}_{7}$}

Adynerin, 655

Cucurbitacin D, 4319

Cucurbitacin L, 4327

$8 \beta, 9 \alpha$-Dihydroganoderic acid J, 5619

Ganode-8-en-ric acid A, 8150

Ganode-8-en-ric acid B, 8151

Ganoderic acid $\mathrm{B}_{8}, 8166$

Ganoderic acid $\gamma, 8177$

Ganoderic acid $\delta, 8178$

Ganoderic acid $\varepsilon, 8179$

Isocucurbitacin D, 11355 
Neriantin, 15491

Saponaceolide F, 19329

\section{$\mathrm{C}_{30} \mathrm{H}_{44} \mathrm{O}_{8}$}

Cerleaside A, 3429

Cucurbitacin J, 4324

Ganode-8-en-ric acid G, 8170

Ganoderic acid I, 8171

Methyl lucidenate P, 14564

15-Oxo-cucurbitacin F, 16304

Pseudolarolide I, 18049

Simplexin, 19902

$2 \alpha, 5 \alpha, 10 \beta$-Triacetoxy- $14 \beta$-iso-butyryloxytaxa4(20),11-diene, 21527

$7 \beta, 20,23 \xi$-Trihydroxy-3,11,15-trioxolanosta-8en-26-oic acid, 21859

\section{$\mathrm{C}_{30} \mathrm{H}_{44} \mathrm{O}_{9}$}

Anticancer Cardiac Glycoside PMV70P691-007, 1389

Anticancer Cardiac Glycoside PMV70P691-008, 1390

Caudoside, 3331

Caudostroside, 3332

Cymarin, 4547

Deacetyltanghinin, 4775

20-Hydroxylganoderic acid G, 10320

Pubescene B, 18171

Sarmutoside, 19381

Sinoside, 19958

Sinostroside, 19959

\section{$\mathrm{C}_{30} \mathrm{H}_{44} \mathrm{O}_{10}$}

3,17-Bisdeoxo-3,17-dihydroxypenisimplicin A, 2441

Musaroside, 15123

Perusitin, 16996

Strophanthidin- $\beta$ - $D$-digitaloside, 20398

Vernadigin, 22404

\section{$\mathrm{C}_{30} \mathrm{H}_{44} \mathrm{O}_{11}$}

Antiarojavoside, 1378

Dracunculifoside M, 6592

\section{$\mathrm{C}_{30} \mathrm{H}_{44} \mathrm{O}_{12}$}

7-Deacetyltaxuspine L, 4786

Taxumairol E, 20825

\section{$\mathrm{C}_{30} \mathrm{H}_{44} \mathrm{O}_{13}$}

Picrasinoside H, 17326

Taxayuntin J, 20767

Taxumain A, 20819

Taxumairol U, 20834

\section{$\mathrm{C}_{30} \mathrm{H}_{46} \mathrm{~N}_{2} \mathrm{O}$}

Epipachysamine D, 6993

\section{$\mathrm{C}_{30} \mathrm{H}_{46} \mathrm{~N}_{2} \mathrm{O}_{2}$}

Epipachysandrine A, 6996

$\mathrm{C}_{30} \mathrm{H}_{46} \mathrm{O}$

Glochidone, 8569
3-Oxo-olean-9(11),12-diene, 16401

$\mathrm{C}_{30} \mathrm{H}_{46} \mathrm{O}_{2}$

Bryophynol, 2693

3,11-Dioxo-olean-12-ene, 6475

3,22-Dioxo-20-taraxastene, 6477

Dymacrin D, 6648

Ganodermanonol, 8188

23-Hydroxy-5 $\alpha$-lanosta-7,9(11),24-triene-3-one, 10310

11-Keto- $\alpha$-amyrenone, 12197

3-Oxolup-20(29)-en-30-al, 16373

Schisandraflorin, 19472

\section{$\mathrm{C}_{30} \mathrm{H}_{46} \mathrm{O}_{3}$}

Abruslactone A, 30

Ananosic acid A, 1136

Anwuweizonic acid, 1468

Betulonic acid, 2338

Coccinic acid, 3861

Cyclocaric acid A, 4480

Dymacrin B, 6646

Dymacrin C, 6647

Dymacrin E, 6649

Ebelin lactone, 6662

Embelinone, 6768

Esculentoic acid B, 7367

Friedelan-1,3-dion-24-al, 7946

Ganode-7,9-dien-ric acid Y, 8149

Ganoderiol F, 8185

Garciosaterpene C, 8226

(24E)-3 $\alpha$-Hydroxy-17,14-friedo-lanostan-8,14,

24-trien-26-oic acid, 10128

$3 \beta$-Hydroxylanosta-7,9(11),24-trien-21-oic acid, 10311

28-Hydroxy-3-oxo-lup-20-(29)-en-30-al, 10573

(24Z)-26-Hydroxy-3-oxo-7,24-tirucalladien-21-al, 10584

Kulolactone, 12330

Liquidambronovic acid, 12906

Lucialdehyde C, 13021

Machaerinic acid lactone, 13288

Mangiferonic acid, 13483

Masticadienonic acid, 13591

Moronic acid, 14980

3-Oxo-24-cycloarten-21-oic acid, 16305

3-Oxo-olean-12-en-28-oic acid, 16402

3-Oxo-olean-12-en-29-oic acid, 16403

Pinicolic acid A, 17387

Pinicolol C, 17389

Regelide, 18576

Sanguisorbigenin, 19286

Schisanol, 19487

Schizandronic acid, 19499

Triptotriterpenoidel lactone A, 22026
Ursolic acid lactone, 22271

Ursonic acid, 22272

\section{$\mathrm{C}_{30} \mathrm{H}_{46} \mathrm{O}_{4}$}

Acacic acid lactone, 59

Coleonolic acid, 3916

Colubrinic acid, 3932

Cordianal B, 4042

Demethylregelin, 5096

3,24-Dioxo-friedelan-29-oic acid, 6468

Dymacrin A, 6645

Ganoderiol B, 8183

Gardenolic acid A, 8229

Glycyrrhetinic acid, 8841

Gypsogenin, 9183

Hederagenic acid, 9259

$3 \beta$-Hydroxy-29(or 30)-al-olean-12-en-28-oic acid, 9775

$12 \alpha$-Hydroxycoccinic acid, 9928

$12 \beta$-Hydroxycoccinic acid, 9929

Hydroxymangiferonic acid, 10359

3-Hydroxy-2-oxo-3-friedelen-20 $\alpha$-carboxylic acid, 10566

(25R)-3 $\alpha$-Hydroxy-23-oxo-9,16-lanostadien-26oic-acid, 10571

(25R)-3 $\beta$-Hydroxy-23-oxo-9,16-lanostadien-26oic acid, 10572

22 $\alpha$-Hydroxy-3-oxoolean-12-en-29-oic acid, 10578

$3 \alpha$-Hydroxy-6-oxo-7,24Z-tirucalladien-26-oic acid, 10585

$3 \alpha$-Hydroxy-7-oxo-8,24Z-tirucalladien-26-oic acid, 10586

Ilekudinol A, 10968

Julibrotriterpenoidal lactone A, 11942

Kadsuric acid, 12011

Lantanolic acid, 12493

Lantic acid, 12496

Liquiritic acid, 12907

Manwuweizic acid, 13522

Maytenfolone A, 13623

Melianone, 13669

Momordic acid, 14909

Neokadsuranic acid C, 15421

Nigranoic acid, 15570

3-Oxo-23-dihydroxycycloart-24-en-26-oic acid, 16317

16-Oxo-3 $\alpha, 21 \beta$-dihydroxyserrat-14-en-24-al, 16321

(24Z)-3-Oxo-12 $\alpha$-hydroxylanosta-8,24-dien-26oic acid, 16352

3-Oxo-19 $\alpha$-hydroxyurs-12-en-28-oic acid, 16358

11-Oxo-kansenonol, 16365

Papyriogenin E, 16639 
Pinicolic acid E, 17388

Pulsatillic acid, 18191

Rubianol G, 19006

Rubiarbonone E, 19020

Rutundanonic acid, 19089

Sabialactone, 19097

(24E)-3,4-Secodammara-4(28),20,24-trien-3,26dioic acid, 19609

24(E)-3,4-Seco-9 $\beta H$-lanosta-4(28),7,24-triene3,26-dioic acid, 19622

Semialatic acid, 19693

4,4,8-Trimethyl-3 $\beta, 7 \alpha, 23$-trihydroxy-chola-14, 24-dien-21-oic acid-21,23-lactone, 21977

\section{$\mathrm{C}_{30} \mathrm{H}_{46} \mathrm{O}_{5}$}

Acerionol, 94

Alisol C, 895

Ceanothic acid, 3345

Cimigenol-3-one, 3660

Cincholic acid, 3676

Coussaric acid, 4194

Cyclocaric acid B, 4481

$2 \alpha, 3 \alpha$-Dihydroxy-19-oxo-18,19-seco-urs-11,

13(18)-dien-28-oic acid, 6068

19,24-Dihydroxyurs-12-en-3-one-28-oic acid, 6177

3,8-Dioxo-7 $\beta$-hydroxy-7,9-cyclo-7,8-seco-24Ztirucalladien-26-oic acid, 6470

Dymacrin H, 6652

3-Epi-isomasticadienolalic acid, 6938

$14 \beta, 15 \beta$-Epoxy-3 $\beta$-hydroxy-9-oxo-11(10 $\rightarrow 8)$ -

abeolanosta-24-trans-en-26-oic acid, 7126

$14 \beta, 15 \beta$-Epoxy-3 $\alpha$-hydroxy-9-oxo-11(10 $\rightarrow 8)$ abeolanost-24-trans-en-26-oic acid, 7127

Floridic acid methyl ester, 7833

Glyyunnansapogenin A, 8857

$18 \alpha$-Hydroxy glycyrrhetic acid, 10152

24-Hydroxyglycyrrhetic acid, 10153

Iridotectoral A, 11150

Iridotectoral B, 11151

Isoceanothic acid, 11313

$\beta$-Kudinlactone, 12298

Lucyin, 13061

Ochraceolide D, 15922

16-Oxo-3 $\alpha, 21 \beta$-dihydroxyserrat-14-en-24-oic acid, 16322

3-Oxo-6 $\beta, 19 \alpha$-dihydroxyurs-12-en-28-oic acid, 16323

2-Oxopomolic acid, 16410

3-Oxo-skimmiarepin, 16423

Poricoic acid G, 17727

Quillaic acid, 18418

Quinovic acid, 18427

Rubiarbonone F, 19019
Serratagenic acid, 19765

$2 \alpha, 3 \beta, 19 \alpha$-Trihydroxy-olean-11,13(18)-dien-28oic acid, 21822

$\mathrm{C}_{30} \mathrm{H}_{46} \mathrm{O}_{6}$

20-O-(Decanoyl)ingenol, 4838

$2 \alpha, 19 \alpha$-Dihydroxy-3-oxo-12-ursen-28-oic acid, 6070

$3 \beta, 19 \alpha$-Dihydroxy-6-oxo-urs-12-en-23-ol-28-oic acid, 6071

$3 \beta, 19 \alpha$-Dihydroxyurs-12-en-24,28-dioic acid, 6175

Ganolucidic acid B, 8197

$1 \alpha$-Hydroxy-2-oxopomolic acid, 10580

23-Nor-24-esomethylene-3,6,19-thihydroxyurs12-en-28 oic acid, 15747

Picfeltarraegenin II, 17289

$3 \beta, 19 \alpha, 23$-Trihydroxy-6-oxo-olean-12-en-28-oic acid, 21828

$1 \beta, 2 \alpha, 19 \alpha$-Trihydroxy-3-oxo-12-ursen-28-oic acid, 21831

$2 \alpha, 3 \alpha, 19 \alpha$-Trihydroxy-11-oxo-urs-12-en-28-oic acid, 21832

\section{$\mathrm{C}_{30} \mathrm{H}_{46} \mathrm{O}_{7}$}

Beaumontoside, 2187

Cucurbitacin F, 4321

Cucurbitacin R, 4328

Ganoderic acid C, 8167

Jaligonic acid, 11812

Odoroside A, 16005

Odoroside B, 16006

Perulactone, 16995

Saponaceolide A, 19327

Saponaceolide E, 19328

Somalin, 20072

Taxa-4(20),11-diene-10 $\beta$-methoxy- $2 \alpha, 5 \alpha$-diacetoxy-14 $\beta$-( $\alpha$-methyl)butyrate, 20742

Wallichoside, 22629

\section{$\mathrm{C}_{30} \mathrm{H}_{46} \mathrm{O}_{8}$}

Cucurbitacin H, 4322

Deacetyloleandrin, 4758

Divaricoside, 6530

Divostroside, 6532

Neriaside, 15492

$17 \beta$-Neriifolin, 15494

$17 \alpha$-Neriifolin, 15495

Odoroside H, 16009

15-Oxo-23,24-dihydrocucurbitacin F, 16314

Periplocymarin, 16958

Platycogenic acid A, 17535

Platycogenic acid B, 17536

$3 \beta, 7 \beta, 20,23 \xi$-Tetrahydroxy-11,15-dioxolanosta8-en-26-oic acid, 21100

Thevefoline, 21323

\section{$\mathrm{C}_{30} \mathrm{H}_{46} \mathrm{O}_{9}$}

Cymarol, 4548

Ruvoside, 19090

Strospeside, 20406

$\mathrm{C}_{30} \mathrm{H}_{46} \mathrm{O}_{10}$

Bipindaloside, 2399

\section{$\mathrm{C}_{30} \mathrm{H}_{46} \mathrm{O}_{11}$}

$9 \alpha, 10 \beta$-Diacetoxy-13 $\alpha$-hydroxy-5 $\alpha-O-(\beta-D$-glucopyranosyl)taxa-4(20),11-diene, 5304

\section{$\mathrm{C}_{30} \mathrm{H}_{46} \mathrm{O}_{12}$}

Picrasinoside D, 17323

\section{$\mathrm{C}_{30} \mathrm{H}_{46} \mathrm{O}_{13}$}

Picrasinoside E, 17324

\section{$\mathrm{C}_{30} \mathrm{H}_{46} \mathrm{O}_{16} \mathrm{~S}_{2}$}

Atractyloside, 1972

\section{$\mathrm{C}_{30} \mathrm{H}_{46} \mathrm{O}_{20}$}

Benzyl $\beta$ - $D$-glucopyranosyl- $(1 \rightarrow 4)$ - $\beta$ - $D$-glucopyranosyl- $(1 \rightarrow 4)-[\beta$ - $D$-apiofuranosyl$(1 \rightarrow 6)]$ - $\beta$ - $D$-glucopyranoside, 2290

\section{$\mathrm{C}_{30} \mathrm{H}_{47} \mathrm{NO}_{6}$}

Holarosine A, 9583

\section{$\mathrm{C}_{30} \mathrm{H}_{48}$}

11,13(18)-Neohopadiene, 15405

Olean-11,13(18)-diene, 16029

\section{$\mathrm{C}_{30} \mathrm{H}_{48} \mathrm{~N}_{2} \mathrm{O}_{2}$}

Holarrhetine, 9585

\section{$\mathrm{C}_{30} \mathrm{H}_{48} \mathrm{O}$}

$\alpha$-Amyrenone, 1106

$\beta$-Amyrenone, 1107

$\delta$-Amyrenone, 1108

Antiquol C, 1464

Arborinone, 1616

Champalin A, 3468

Cimicifugenol, 3651

Filicenal, 7795

1-Friedelen-3-one, 7953

D:C-Friedo-urs-7-en-3-one, 7956

Glutinone, 8789

Hancolupenone, 9216

$19 \alpha$-Hydroxyferna-7,9(11)-diene, 10119

$3 \beta$-Hydroxy-olean-9(11),12-diene, 10549

Lupenone, 13097

Olean-12-en-3-one, 16042

Olean-18-en-3-one, 16043

16-Oxotaraxer-14-ene, 16426

Taraxerone, 20712

\section{$\mathrm{C}_{30} \mathrm{H}_{48} \mathrm{O}_{2}$}

Abrisapogenol F, 19

$\beta$-Amyrenonol, 1109

Betulinaldehyde, 2333

Champalinone, 3471

Drypemolundein B, 6616

Epi-kansenone, 6939 
20R,24R-Epoxy-25-dammaren-3-one, 7065

Friedelane-3,7-dione, 7948

Ganodermadiol, 8186

Glochidonol, 8570

16 $\beta$-Hydroxy-dammara-20(22),25-dien-3-one, 9956

26-Hydroxy-dammara-20,24-dien-3-one, 9957

28-Hydroxy- $D$-friedo-olean-14-en-3-one, 10129

$3 \beta$-Hydroxy-olean-12-en-28-al, 10334

$3 \beta$-Hydroxy-lup-20(29)-en-30-al, 10340

28-Hydroxy-lup-20-(29)-en-3-one, 10342

$3 \beta$-Hydroxy-urs-12-en-28-al, 10350

$21 \beta$-Hydroxyserrat-14-en-3-one, 10702

$3 \beta$-Hydroxyserrat-14-en-21-one, 10703

(24Z)-27-Hydroxy-7,24-tirucalladien-3-one, 10770

Kansenol, 12129

Kansenone, 12130

Karounidiol, 12165

Kulinone, 12329

$5 \alpha$-Lanosta-7,9(11),24-triene-3 $\alpha, 23$-diol, 12487

14-Methylmangiferolic aldehyde, 14576

Mollugogenol B, 14903

Mupinensisone, 15078

Myricolal, 15187

Oleanolic aldehyde, 16064

7-Oxoisomultiflorenol, 16362

24(E)-3-Oxo-9 $\beta H$-lanosta-7,24-dien-26-ol, 16370

3-Oxolupenol, 16374

Roburic acid, 18861

Sabianone, 19098

Sebiferic acid, 19597

Soyasapogenol C, 20118

Squasapogenol, 20250

Tamarixone, 20663

Tirucall-7-en-3,24-dione, 21408

\section{$\mathrm{C}_{30} \mathrm{H}_{48} \mathrm{O}_{3}$}

Abrisapogenol J, 21

Anticancer Triterpene PMV70P691-044, 1450

Anwuweizic acid, 1467

Betulinic acid, 2334

$\alpha$-Boswellic acid, 2567

$\beta$-Boswellic acid, 2568

Bryonolic acid, 2687

Celasdin C, 3366

Cladocalol, 3784

Clerodolone, 3842

CTHD0384-2, 4301

20(R)-21,24-Cyclo-3 $\beta, 25$-dihydroxyldammar23(24)-en-21-one, 4487

11-Deoxoglycyrrhetinic acid, 5144

$3 \beta, 15 \alpha$-Dihydroxy-olean-12-en-16-one, 6061

$3 \beta, 16 \beta$-Dihydroxy-olean-12-en-15-one, 6062
$3 \beta, 21 \beta$-Dihydroxyserrat-14-en-16-one, 6122

(24Z)-3 $\beta, 27-D i h y d r o x y-7,24-$ tirucalladien-21-al, 6152

$3 \beta, 22 S$-Dihydroxy-tirucalla-7,24-dien-23-one, 6154

23,26-Dihydroxy-tirucalla-7,24-dien-3-one, 6155

Dymacrin G, 6651

Dymacrin K, 6655

3-Epikatonic acid, 6940

3-Epioleanolic acid, 6980

$14 \beta, 15 \beta$-Epoxy-21 $\beta$-hydroxyserratan-3-one, 7145

Esculentoic acid A, 7366

Friedelan-1,3-dion-7 $\alpha$-ol, 7947

Ganoderic acid Z, 8174

Ganodermanondiol, 8187

Garciosaterpene B, 8225

Glycyrrhetol, 8842

$8 \alpha$-Hydroxyfernan-25,7 $\beta$-olide, 10120 $3 \beta$-Hydroxylupane-13 $\beta$,28-lactone, 10339 $3 \alpha$-Hydroxy-lup-20(29)-en-24-oic acid, 10341 3 $\beta$-Hydroxy-masticadienolic acid, 10363 3 $\alpha$-Hydroxymasticadienonic acid, 10364

Isoanwuweizic acid, 11229

Isomangiferolic acid, 11525

Kansenonol, 12131

Mangiferolic acid, 13482

Maytenoic acid, 13624

Morolic acid, 14979

Niloticin, 15598

Oleanolic acid, 16050

16-Oxodiepiserratenediol, 16313

7-Oxodihydro karounidiol, 16315

3-Oxofriedelan-28-oic acid, 16337

16-Oxo-3 $\alpha$-hydroxyserrat-14-en-21 $\beta$-ol, 16354

16-Oxoserratenediol, 16420

Palmitoylpterosin B, 16566

$\beta$-Peltoboykinolic acid, 16798

Petatrichol A, 17018

Petatrichol B, 17019

Rosacea acid B, 18911

Rubiarbonone D, 19018

Saikogenin E, 19133

Schimperinone, 19466

Schizandrolic acid, 19498

2,3-seco-3-Oxoolean-12-en-2-oic acid, 19631

2,3-Seco-3-oxours-12-en-2-oic acid, 19632

Soyasapogenol E, 20120

Thailandiol, 21233

Trametenolic acid, 21502

Ursolic acid, 22270

Woodwardic acid, 22722

$\mathrm{C}_{30} \mathrm{H}_{48} \mathrm{O}_{4}$

Abrusgenic acid, 27
$6 \beta$-Acetoxy-(22E)-ergosta-7,22-diene-3 $\beta, 5 \alpha$-diol, 192

Alisol B, 893

Alphitolic acid, 984

Anagalligenone B, 1133

Anemosapogenin, 1180

Bourjotinolone A, 2571

Cochalic acid, 3866

Cordianal C, 4043

Corosolic acid, 4088

Crategolic acid, 4219

CTHD0384-3, 4302

Deacetylturraeanthin, 4791

3-Deoxycimigenol, 5162

$3 \beta, 23(R$ or $S$ )-dihydroxycycloart-24-en-26-oic acid, 5799

$2 \alpha, 3 \alpha$-Dihydroxylup-20(29)-en-28-oic acid, 5953

$2 \alpha, 3 \alpha$-Dihydroxyolean-12-en-28-oic acid, 6058

$2 \alpha, 3 \beta$-Dihydroxyolean-12-en-28-oic acid, 6059

$3 \beta, 22 \beta$-Dihydroxyolean-12-en-29-oic acid, 6060

$3 \alpha, 21 \alpha$-Dihydroxyserrat-14-en-24-oic acid, 6117

Dymacrin I, 6653

Dymacrin J, 6654

Echinocystic acid, 6689

23-Epimeric

3ß,23-dihydroxycycloart-24-en-26-oic acid, 6966

3-Epipomolic acid, 7001

Epoxydihydro-oleanolic acid, 7073

Eriocarpin B, 7273

Ganode-8-en-ric acid U, 8159

Ganodermanontriol, 8189

Hederagenin, 9260

Hispidone, 9563

Hydroxymangiferolic acid, 10358

$2 \beta$-Hydroxyoleanolic acid, 10550

$3 \alpha$-Hydroxy-2-oxofriedelane-20 $\alpha$-carboxylic acid, 10565

23-Hydroxyursolic acid, 10820

Imberbic acid, 10995

Jujubogenin, 11907

Lucidumol A, 13052

Lycernuic acid A, 13170

Lycernuic ketone C, 13177

Machaerinic acid, 13287

$(2 \beta, 3 \beta)$-Maslinic acid, 13581

Melianol, 13668

Mesembryanthemoidigenic acid, 13799

24-Methyl-7 $\beta$-acetoxycholesta-5,24(28)-diene-

3 $\beta, 19$-diol, 14114

2-n-Nonadecyl-5,7-dihydroxy-6,8-dimethyl

chromone, 15674

16-Oxo-21-episerratriol, 16327 
16-Oxolycoclavanol, 16377

16-Oxoserratriol, 16421

Palmitoylpterosin C, 16567

Palustrolide, 16576

Phlegmaric acid, 17153

Pomolic acid, 17696

Queretaroic acid, 18415

Rosacea acid A, 18910

Rubiarbonone B, 19016

Rubifolic acid, 19029

Saikogenin D, 19132

Saikogenin F, 19134

Saikogenin G, 19135

Salaspermic acid, 19182

Saponaceoic acid I, 19321

Saponaceoic acid II, 19322

Saponaceoic acid III, 19323

Scutellaric acid, 19586

Siaresinolic acid, 19854

Sumaresinolic acid, 20484

Syringic aldehyde, 20568

(24R)-7 $\beta, 24,25$-Trihydroxy-9,19-cycloart-1-en-

3-one, 21760

Triptotin C, 22021

Triptotriterpenic acid A, 22022

Triptotriterpenic acid A', 22023

Triptotriterpenic acid B, 22024

Triptotriterpenic acid C, 22025

Wilfolic acid C, 22673

Wistariasapogenol A, 22697

\section{$\mathrm{C}_{30} \mathrm{H}_{48} \mathrm{O}_{5}$}

Aescigenin, 659

Anticancer Triterpene PMV70P691-043, 1449

Arjunic acid, 1734

Arjunolic acid, 1736

Asiatic acid, 1853

Barbinervic acid, 2149

Bayogenin acid, 2178

Bemeuxin, 2221

Camelliagenin B, 3031

Cimigenol, 3658

Collinsogenin, 3927

$2 \alpha, 6 \alpha$-Dihydroxybetulinic acid, 5773

$2 \alpha, 19 \alpha$-Dihydroxyursolic acid, 6178

Entagenic acid, 6811

3-epi-spathodic acid, 7023

Euscaphic acid, 7657

Fomitopinic acid A, 7860

Ganoderiol D, 8184

Glyyunnansapogenin B, 8858

Glyyunnansapogenin $\mathrm{B}_{1}, 8859$

Hovenic acid, 9652

Lycernuic acid B, 13171
Lycoclavanin, 13185

Madasiatic acid, 13336

Maesagenin A, 13349

Melianodiol, 13667

Neoalisol, 15336

Orthosphenic acid, 16245

Rotundic acid, 18942

Rubianol A, 19001

Saikogenin Q, 19136

Terminoic acid, 20977

$2 \alpha, 3 \alpha, 19 \alpha$-Trihydroxy-12-oleanen-28-oic acid, 21824

$3 \beta, 6 \beta, 18 \beta$-Trihydroxyurs-12-en-28-oic acid, 21862

$3 \beta, 6 \beta, 19 \alpha$-Trihydroxyurs-12-en-28-oic acid, 21863

\section{$\mathrm{C}_{30} \mathrm{H}_{48} \mathrm{O}_{6}$}

Arjungenin, 1732

Brahmic acid, 2586

Camelliagenin D, 3033

Camelliagenin E, 3034

(22R)-22-Hydroxycimigenol, 9906

Madecassic acid, 13337

Myrianthic acid, 15153

16-Oxolyclanitin, 16375

Platycogenic acid C, 17537

Polygalacic acid, 17628

Roxburic acid, 18970

Sublateriol A, 20441

$1 \beta, 2 \alpha, 3 \alpha, 24$-Tetrahydroxyolean-12-en-28-oic acid, 21143

$2 \alpha, 3 \alpha, 23,29$-Tetrahydroxyolean-12-en-28-oic acid, 21144

$2 \alpha, 3 \alpha, 11 \alpha, 19 \alpha$-Tetrahydroxy-urs-12-en-28-oic acid, 21169

$1 \beta, 2 \alpha, 3 \alpha, 19 \alpha$-Tetrahydroxyurs-12-en-28-oic acid, 21170

$1 \beta, 2 \alpha, 3 \alpha, 24$-Tetrahydroxyurs-12-en-28-oic acid, 21171

$\mathrm{C}_{30} \mathrm{H}_{48} \mathrm{O}_{7}$

Dihydrocucurbitacin F, 5575

Gamboukokoensein A, 8129

20-Hydroxyecdysone-20,22-monoacetonide, 10065

Isocucurbitacin R, 11356

Paristerone 20,22-monoacetonide, 16672

Platycodigenin, 17529

$\mathrm{C}_{30} \mathrm{H}_{48} \mathrm{O}_{10}$

Marginatoside A, 13561

$\mathrm{C}_{30} \mathrm{H}_{49} \mathrm{ClO}_{2}$

25-Chloro-24-hydroxytirucall-7-en-3-one, 3562

$\mathrm{C}_{30} \mathrm{H}_{49} \mathrm{NO}_{8}$

Glaucedine, 8511

\section{$\mathrm{C}_{30} \mathrm{H}_{49} \mathrm{~N}_{3} \mathrm{O}$}

Lucidine A, 13042

Lucidine B, 13043

\section{$\mathrm{C}_{30} \mathrm{H}_{49} \mathrm{~N}_{3} \mathrm{O}_{2}$}

Oxolucidine A, 16371

Oxolucidine B, 16372

Spirolucidine, 20203

\section{$\mathrm{C}_{30} \mathrm{H}_{50}$}

Adianene, 629

Bacchara-12,21-diene, 2079

7-Fernene, 7757

9(11)-Fernene, 7758

Filicene, 7796

Hop-16-ene, 9637

Hop-17(21)-ene, 9638

Hop-21-ene, 9639

21 $\alpha H$-22(29)-Hopene, 9640

21BH-22(29)-Hopene, 9641

Hopene II, 9642

Isofernene, 11425

Isohop-22-(29)-ene, 11457

Lemmaphylla-7,21-diene, 12618

$13 \alpha H$-Malabaricatriene, 13413

$13 \beta H$-Malabaricatriene, 13414

21-Methyl bacchara-12,22(29)-diene, 14152

Multiflor-7-ene, 15064

Multiflor-8-ene, 15065

Multiflor-9(11)-ene, 15066

12-Neohopene, 15406

Olean-12-ene, 16037

Olean-18-ene, 16038

$\alpha$-Onoceradiene, 16110

$\beta$-Onoceradiene, 16111

Onocera-7,13-diene, 16112

Onocera-7,14-diene, 16113

Onocera-7,14(27)-diene, 16114

Onocera-8,14(27)-diene, 16115

7,17,21-Podiodatriene, 17584

8,17,21-Podiodatriene, 17585

$\alpha$-Polypodatetraene, 17660

Serratene, 19767

Shiona-3,21-diene, 19829

Squalene, 20237

$\psi$-Taraxastene, 20698

Taraxer-14-ene, 20709

Tirucalla-7,24-diene, 21404

Tirucalla-8,24-diene, 21405

\section{$\mathrm{C}_{30} \mathrm{H}_{50} \mathrm{~N}_{2} \mathrm{O}_{3}$}

Sarcovagine C, 19364

$\mathrm{C}_{30} \mathrm{H}_{50} \mathrm{~N}_{\mathbf{2}} \mathrm{O}_{\mathbf{4}}$

Sarcovagine B, 19363

\section{$\mathrm{C}_{30} \mathrm{H}_{50} \mathrm{O}$}

Achilleol A, 536 
Achilleol C, 537

$\delta$-Amyrenol, 1105

$\alpha$-Amyrin, 1110

$\beta$-Amyrin, 1113

Antiquol B, 1463

Arborinol, 1615

Bauerenol, 2169

Champalin B, 3469

Citrostadienol, 3773

Clerodone, 3843

Cycloartenol, 4473

Dammara-20,24-dien-3 $\beta$-ol, 4616

Epi- $\alpha$-amyrin, 6825

3-Epilupeol, 6954

Epitaraxerol, 7025

(20R)-3 $\alpha, 10 \alpha$-Epoxy-9-epi-cucurbita-24-ene, 7087

(20S)-3 $\alpha, 10 \alpha$-Epoxy-9-epi-cucurbita-24-ene, 7088

$3 \alpha, 4 \alpha$-Epoxyfilicane, 7099

24-Ethylidene lophenol, 7450

Euphol, 7611

$\alpha$-Euphol, 7612

Fernenol, 7759

Fern-9(11)-en-12 $\beta$-ol, 7760

Friedelan-3-one, 7951

Germanicol, 8351

$\alpha$-Glutenol, 8786

$\beta$-Glutinol, 8788

Hancokinol, 9213

Hancolupenol, 9214

Hemistriterpene ether, 9353

$12 \alpha$-Hydroxyfern-9(11)-ene, 10121

Isoarborinol, 11234

Lanosterol, 12488

Lemmaphylla-7,21-dien-3 $\beta$-ol, 12619

Lupeol, 13098

$4 \alpha$-Methyl-24 $\beta$-ethyl-5 $\alpha$-cholesta-14,25-dien$3 \beta$-ol, 14432

4 $\alpha$-Methyl-24-ethylcholesta-7,24-dienol, 14433

Nepehinol, 15482

31-Norcyclolaudenol, 15731

Obtusifoliol, 15901

(2E,6E,12E,16E)-3,7,13,17,21-Pentamethyl-

10-(1-methylethenyl)-2,6,12,16,20-docosapentaen-1-ol, 16875

(13E,17E)-Polypoda-7,13,17,21-tetraen-3 $\beta$-ol, 17661

Pseudotaraxasterol, 18071

Rhoiptelenol, 18818

Shionone, 19830

Simiarenol, 19901

$\alpha_{1}$-Sitosterol, 19982
Taraxast-20-ene-3 $\beta$-ol, 20701

Taraxast-20(30)-ene-3 $\beta$-ol, 20702

Taraxasterol, 20704

$\psi$-Taraxasterol, 20705

Taraxer-14-en-3 $\beta$-ol, 20710

Taraxerol, 20711

Tirucallol, 21409

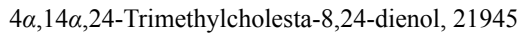

Ulmoprenol, 22192

\section{$\mathrm{C}_{30} \mathrm{H}_{50} \mathrm{O}_{2}$}

Abrisapogenol G, 20

Aglaiol, 739

Arnidiol, 1749

Bauer-7-ene-3 $\beta, 16 \alpha$-diol, 2168

Betulin, 2331

Champalinol, 3470

9,19-Cycloart-23-ene-3 $\beta, 25$-diol, 4471

9,19-Cycloart-25-ene-3 $\beta, 24$-diol, 4472

$3 \beta, 11 \alpha$-Dihydroxy-olean-12-ene, 6057

$3 \beta, 25$-Dihydroxy-tirucalla-7,23-diene, 6153

Dipterocarpol, 6502

21-Episerratenediol, 7017

Erythrodiol, 7338

Faradiol, 7715

Friedelane-3-one-29-ol, 7949

Glochidiol, 8563

Glyyunnansapogenin $\mathrm{B}_{2}, 8860$

$4 \alpha$-Hydroxyfilican-3-one, 10123

$15 \alpha$-Hydroxyfriedelane-3-one, 10127

3 $\beta$-Hydroxylcycloart-24-one, 10317

$21 \alpha$-Hydroxyserrat-14-en-3 $\beta$-ol, 10699

$21 \beta$-Hydroxyserrat-14-en-3 $\alpha$-ol, 10700

$21 \beta$-Hydroxyserrat-14-en-3 $\beta$-ol, 10701

Lup-20(29)-ene-1 $\beta, 3 \beta$-diol, 13092

Lup-20(29)-ene-3 $\alpha, 23$-diol, 13093

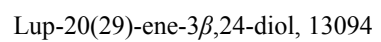

Myricadiol, 15154

Obtusol, 15911

Olean-12-en-3 $\beta, 28$-diol, 16036

Olean-12-ene-3 $\alpha, 16 \beta$-diol, 16039

Olean-12-ene-3 $\beta, 16 \alpha$-diol, 16040

Olean-12-ene-3 $\beta, 16 \beta$-diol, 16041

$\alpha$-Onocerin, 16117

3-Oxo taraxastan-20( $R$ or $S)$-ol, 16425

Pachysonol, 16510

3,4-Seco-20(29)-lupen-3-oic acid, 19627

Serratenediol, 19768

Sophoradiol, 20083

Tamarixol, 20662

Taraxast-20(30)-ene-3 $\beta, 21 \alpha$-diol, 20699

Taraxast-20-ene-3 $\beta, 30$-diol, 20700

Tirucalla-7,24-diene-3 $\beta, 23$-diol, 21406

(24Z)-7,24-Tirucalladien-3 $\beta, 26$-diol, 21407
Urs-12-en-3 $\beta, 28$-diol, 22264

Urs-12-ene-3 $\alpha, 16 \beta$-diol, 22265

Urs-12-ene-3 $\beta, 16 \beta$-diol, 22266

Uvaol, 22288

\section{$\mathrm{C}_{30} \mathrm{H}_{50} \mathrm{O}_{3}$}

Abrisapogenol A, 14

Abrisapogenol D, 17

Adian-5-ene ozonide, 630

Celasdin A, 3364

Celasdin B, 3365

Dammar-20(21),24-diene-3 $\beta, 6 \alpha, 12 \beta$-triol, 4617

Dihydroniloticin, 5681

$3 \beta, 9 \alpha$-Dihydroxylanost-24-en-26-ol, 5947

$3 \beta, 21 \alpha$-Dihydroxyserrat-14-en-24-ol, 6118

$3 \alpha, 21 \beta$-Dihydroxyserrat-14-en-24-ol, 6119

$3 \beta, 21 \beta$-Dihydroxyserrat-14-en-24-ol, 6120

$3 \beta, 21 \beta$-Dihydroxyserrat-14-en-29-ol, 6121

Dryobalanone, 6610

Dymacrin F, 6650

21-Episerratriol, 7018

24,25-Epoxy-3 $\beta, 23$-dihydroxy-7-tirucallene, 7084

$14 \beta, 15 \beta$-Epoxy-3 $\beta$-hydroxyserratan- $21 \alpha$-ol, 7143

$14 \beta, 15 \beta$-Epoxy-3 $\beta$-hydroxy-serratan- $21 \beta$-ol, 7144

$20 \alpha, 21 \alpha$-Epoxy-taraxastane-3 $\beta, 22 \alpha$-diol, 7205

Fouquierone, 7931

22 $\alpha$-Hydroxyerythrodiol, 10096

Ilekudinol C, 10970

Iridal, 11133

Kapurone, 12159

Kudzusapogenol C, 12309

Longispinogenin, 12980

Lycoclavanol, 13186

Myrrhanone A, 15209

Ocotillone, 15931

Phlegmanol E, 17151

Primulagenin A, 17858

Rubiarbonol B, 19012

Rubiatriol, 19023

Salasone D, 19180

Salasone E, 19181

Salvistamineol, 19220

Serratriol, 19774

Soyasapogenol B, 20116

\section{$\mathrm{C}_{30} \mathrm{H}_{50} \mathrm{O}_{4}$}

Abrisapogenol B, 15

Abrisapogenol C, 16

Abrisapogenol E, 18

Aglinin D, 747

Anagalligenin B, 1132

Camelliagenin A, 3030 
11-Deoxyalisol A, 5151

16-Desoxybarringtogenol C, 5271

$3 \alpha, 21 \beta$-Dihydroxyserrat-14-ene-24,29-diol, 6116

$3 \beta, 19 \alpha$-Dihydroxyursan-28-oic acid, 6169

Eicosyl ferulate, 6729

3-Episapeline A, 7011

22,23-Epoxy-10-deoxy-21-hydroxyiridal, 7067

22,23-Epoxyiridal, 7148

22,23-Epoxy-tirucalla-7-ene-3 $\beta, 24,25$-triol, 7208

Ganoderiol A, 8182

Iridobelamal A, 11142

Isofouquierone peroxide, 11427

Isoiridogermanal, 11466

Lyclaninol, 13182

Lycocryptol, 13188

Melialactone, 13666

Piscidinol A, 17480

Rubiarbonol A, 18995

Sapelin A, 19315

Soyasapogenol A, 20115

\section{$\mathrm{C}_{30} \mathrm{H}_{50} \mathrm{O}_{5}$}

Aglinin A, 744

Alisol A, 891

Barrigenol $\mathrm{A}_{1}, 2151$

Barringtogenol C, 2152

Camelliagenin C, 3032

Cycloastragenol, 4476

22,23-Epoxy-21-hydroxyiridal, 7120

Kudzusapogenol A, 12307

Lycernuic acid E, 13174

Lyclanitin, 13183

Melianotriol, 13671

Rubiarbonol F, 18996

(23E,20S)-20,25,26-Trihydroxy-3,4-seco-

dammara-4(28),23-dien-3-oic acid, 21843

(23E,12R,20S)-12,20,25-Trihydroxy-3,4-seco-

dammara-4(28),23-dien-3-oic acid, 21844

\section{$\mathrm{C}_{30} \mathrm{H}_{50} \mathrm{O}_{6}$}

$13 \beta, 17 \beta$-Epoxyalisol A, 7050

Lycernuic acid D, 13173

2,3,16,20,25-Pentahydroxy-9-methyl-19-nor-

lanost-5-en-22-one, 16853

Protoaescigenin, 17964

Sublateriol B, 20442

Sublateriol C, 20443

$\mathrm{C}_{30} \mathrm{H}_{50} \mathrm{O}_{7}$

Amarasterone B, 1015

Lycernuic acid C, 13172

$\mathrm{C}_{30} \mathrm{H}_{50} \mathrm{O}_{15}$

Periplocae oligosaccharide $\mathrm{C}_{1}, 16938$

$\mathrm{C}_{30} \mathrm{H}_{51} \mathrm{NO}_{2}$

24-Hydroxyiminocycloart-3-ol, 10229

$\mathrm{C}_{30} \mathrm{H}_{51} \mathrm{~N}_{3} \mathrm{O}$
Dihydrolucidine A, 5663

Dihydrolucidine B, 5664

\section{$\mathrm{C}_{30} \mathrm{H}_{51} \mathrm{~N}_{3} \mathrm{O}_{2}$}

Dihydrooxolucidine A, 5687

Dihydrooxolucidine B, 5688

$\mathrm{C}_{30} \mathrm{H}_{52} \mathrm{~N}_{2} \mathrm{O}_{2}$

$\mathrm{N}$-Isobutyroylbuxahyrcanine, 11291

\section{$\mathrm{C}_{30} \mathrm{H}_{52} \mathrm{O}$}

Adiantulanosterol, 633

Ambrein, 1026

Epifriedelanol, 6918

Friedelan-3 $\alpha$-ol, 7950

29-Hopanol, 9635

Hydroxyhopane, 10167

Isohelianol, 11451

24-Methyl-31-norlanost-9(11)-enol, 14625

Onoceranoxide, 16116

Tetrahymanol, 21174

\section{$\mathrm{C}_{30} \mathrm{H}_{52} \mathrm{O}_{2}$}

Argentatin E, 1668

Dammar-24-ene-3 $\beta, 20$-diol I, 4618

6 $\alpha, 22$-Hopanediol, 9634

Pachysandiol A, 16503

Pachysandiol B, 16504

Sitostanyl formate, 19979

Taraxastane-3 $\beta, 20 \alpha$-diol, 20697

Ursan-3 $\beta, 5 \alpha$-diol, 22263

\section{$\mathrm{C}_{30} \mathrm{H}_{52} \mathrm{O}_{3}$}

Betulafolientriol, 2328

Kapurol, 12158

Myrrhanol A, 15208

Ocotillol II, 15930

Panaxadiol, 16594

Protopanaxadiol, 17981

Tohogenol, 21425

\section{$\mathrm{C}_{30} \mathrm{H}_{52} \mathrm{O}_{4}$}

Aglinin C, 746

Betulafolienetetrol, 2326

Betulafolientetraol A, 2327

20(S)-Dammar-25(26)-ene-3 $\beta, 6 \alpha, 12 \beta, 20$-tetrol, 4621

Mollugogenol A, 14902

Panaxatriol, 16595

Phlegmanol F, 17152

Protopanaxatriol, 17982

$3 \beta, 16 \beta, 20(S), 25$-Tetrahydroxydammar-23-ene, 21090

Tohogeninol, 21424

$\mathrm{C}_{30} \mathrm{H}_{52} \mathrm{O}_{5}$

20(S),24(S)-Dammar-25(26)-ene-3 $\beta, 6 \alpha, 12 \beta, 20$,

24-pentanol, 4620

Hosenkol A, 9651

(20S)-3 $\beta, 12 \beta, 16 \beta, 25$-Pentahydroxydammar-23- ene, 16844

Polacamdrin, 17603

Teurilene, 21230

Trilocularol A, 21890

\section{$\mathrm{C}_{30} \mathrm{H}_{52} \mathrm{O}_{6}$}

Aglinin B, 745

20(S)-Dammar-23-ene-25-hydroperoxyl-3 $\beta, 6 \alpha$,

12 $\beta, 20$-tetrol, 4619

\section{$\mathrm{C}_{30} \mathrm{H}_{52} \mathrm{O}_{8}$}

$15 \beta, 16 \beta$-Isopropylidenedioxy- $5 \alpha$-cholest- $3 \beta, 4 \beta$,

$6 \alpha, 7 \alpha, 8 \beta, 26$-hexaol, 11622

Longilene peroxide, 12965

\section{$\mathrm{C}_{30} \mathrm{H}_{52} \mathrm{O}_{26}$}

Lantanose A, 12494

Verbascose, 22396

$\mathrm{C}_{30} \mathrm{H}_{53}$

$18 \alpha$-Oleanane, 16028

\section{$\mathrm{C}_{30} \mathrm{H}_{53} \mathrm{~N}_{3}$}

Tetrahydrodeoxylucidine A, 21064

Tetrahydrodeoxylucidine B, 21065

\section{$\mathrm{C}_{30} \mathrm{H}_{53} \mathrm{~N}_{3} \mathrm{O}$}

Tetrahydrodeoxyoxolucidine A, 21066

Tetrahydrodeoxyoxolucidine B, 21067

\section{$\mathrm{C}_{30} \mathrm{H}_{54} \mathrm{O}_{2}$}

Tamarixinol, 20661

\section{$\mathrm{C}_{30} \mathrm{H}_{58} \mathrm{O}_{2}$}

Triacontan-4-olide, 21542

\section{$\mathrm{C}_{30} \mathrm{H}_{58} \mathrm{O}_{3}$}

11-Oxotriacontanoic acid, 16430

\section{$\mathrm{C}_{30} \mathrm{H}_{58} \mathrm{O}_{4}$}

Triacontanedioic acid, 21538

\section{$\mathrm{C}_{30} \mathrm{H}_{60} \mathrm{O}_{2}$}

Melissic acid, 13687

\section{$\mathrm{C}_{30} \mathrm{H}_{60} \mathrm{O}_{4}$}

(2S)-1-O-Heptacosanoyl glycerol, 9411

\section{$\mathrm{C}_{30} \mathrm{H}_{62}$}

Melissane, 13686

Triacontane, 21537

\section{$\mathrm{C}_{30} \mathrm{H}_{62} \mathrm{O}$}

16-Triacontanol, 21540

$n$-Triacontanol, 21541

\section{$\mathrm{C}_{30} \mathrm{H}_{62} \mathrm{O}_{10}$}

Chaetochromin A, 3439

\section{$\mathrm{C}_{31} \mathrm{H}_{20} \mathrm{O}_{9}$}

Sesguoiaflavone, 19785

\section{$\mathrm{C}_{31} \mathrm{H}_{20} \mathrm{O}_{10}$}

Bilobetin, 2379

Cryptomerin A, 4285

Isocryptomerin, 11353

3'-O-Methyl loniflavone, 14557

Neocryptomerin, 15373

Podocarpusflavone A, 17588

Robustaflavone-7"-methyl ether, 18866 
Sequoiaflavone, 19756

Sotetsuflavone, 20107

\section{$\mathrm{C}_{31} \mathrm{H}_{20} \mathrm{O}_{11}$}

Ridiculuflavone B, 18845

\section{$\mathrm{C}_{31} \mathrm{H}_{21} \mathrm{NO}_{8}$}

Thelephantin N, 21306

$\mathrm{C}_{31} \mathrm{H}_{22} \mathrm{O}_{5}$

Nordracorubin, 15744

$\mathrm{C}_{31} \mathrm{H}_{22} \mathrm{O}_{9}$

Rheidin C, 18758

\section{$\mathrm{C}_{31} \mathrm{H}_{22} \mathrm{O}_{10}$}

2,3-Dihydro-4"'- $O$-methyl amentoflavone, 5673

2",3"-Dihydroochnflavone 7"- $O$-methyl ether, 5684

$\mathrm{C}_{31} \mathrm{H}_{24} \mathrm{O}_{9}$

Afzelone A, 681

$\mathrm{C}_{31} \mathrm{H}_{24} \mathrm{O}_{10}$

Arurasperone D, 1824

7-Methoxylneochaejasmin A, 13989

Rubioncolin B, 19036

Sikokianin A, 19890

Sikokianin B, 19891

Sikokianin C, 19892

\section{$\mathrm{C}_{31} \mathrm{H}_{24} \mathrm{O}_{11}$}

Daphnodorin G-3"--methyl ether, 4653

Daphnodorin H 3-methyl ether, 4655

Daphnodorin H 3"-methyl ether, 4656

\section{$\mathrm{C}_{31} \mathrm{H}_{24} \mathrm{O}_{12}$}

Kolaflavanone, 12255

\section{$\mathrm{C}_{31} \mathrm{H}_{28} \mathrm{~N}_{2} \mathrm{O}_{6}$}

Pyrimidinone, 18262

\section{$\mathrm{C}_{31} \mathrm{H}_{28} \mathrm{~N}_{2} \mathrm{O}_{7}$}

Didehydro-3'-hydroxyaglaiastatin, 5463

\section{$\mathrm{C}_{31} \mathrm{H}_{28} \mathrm{~N}_{4} \mathrm{O}_{5}$}

Picrasidine R, 17310

\section{$\mathrm{C}_{31} \mathrm{H}_{28} \mathrm{O}_{9}$}

Curtisian K, 4407

Thelephantin B, 21294

\section{$\mathrm{C}_{31} \mathrm{H}_{28} \mathrm{O}_{13}$}

Hispidulin 7-(6-E-p-coumaroyl- $\beta$ - $D$-glucopyranoside), 9565

\section{$\mathrm{C}_{31} \mathrm{H}_{28} \mathrm{O}_{14}$}

Ergochrysin, 7231

2"-O-Feruloylorientin, 7783

6-Methoxykaempferol-3-O- $\beta-D-6$ "( $p$-coumaroyl) glucopyranoside, 13981

\section{$\mathrm{C}_{31} \mathrm{H}_{28} \mathrm{O}_{15}$}

3'-Hydroxyscutellarein

7-O-(6"-O-trans-feruloyl)- $\beta$-glucopyranoside, 10694

6-Methoxyquercetin-3- $O-\beta-D-6$ "( $p$-coumaroyl) glucopyranoside, 14082

$\mathrm{C}_{31} \mathrm{H}_{28} \mathrm{O}_{16}$
1,3-Di- $O$ - $(E)$-caffeoyl-4- $O$-galloyl- $\beta$ - $D$-glucopyranose, 5406

Quercetin-3-O-(6"-feruloyl)- $\beta$ - $D$-galactopyranoside, 18353

$\mathrm{C}_{31} \mathrm{H}_{30} \mathrm{O}_{7}$

Cochinchinenin, 3867

$\mathrm{C}_{31} \mathrm{H}_{30} \mathrm{O}_{9}$

3,4-Dihydroxyrottlerin, 6111

$\mathrm{C}_{31} \mathrm{H}_{30} \mathbf{O}_{10}$

Angustifolin B, 1247

$\mathrm{C}_{31} \mathrm{H}_{30} \mathrm{O}_{11}$

Kadsulignan C, 11998

Kadsulignan E, 12000

$\mathrm{C}_{31} \mathrm{H}_{30} \mathrm{O}_{15}$

6"-O-p-Hydroxybenzoyliridin, 9826

$\mathrm{C}_{31} \mathrm{H}_{32} \mathrm{~N}_{4} \mathrm{O}_{2}$

Roxburghine, 18968

Roxburghine X, 18969

$\mathrm{C}_{31} \mathrm{H}_{32} \mathrm{O}_{6}$

Artocommunol CA, 1810

$\mathrm{C}_{31} \mathrm{H}_{32} \mathrm{O}_{8}$

Longipedunin A, 12966

6-Methoxygossypol, 13944

\section{$\mathrm{C}_{31} \mathrm{H}_{32} \mathrm{O}_{12}$}

Gnetumontanin D, 8896

$\mathrm{C}_{31} \mathrm{H}_{32} \mathrm{O}_{14}$

Cypellogin C, 4572

$\mathrm{C}_{31} \mathrm{H}_{32} \mathrm{O}_{15}$

Majidine, 13403

Procumbenoside A, 17872

\section{$\mathrm{C}_{31} \mathrm{H}_{32} \mathrm{O}_{16}$}

3,4-Di- $O$-caffeoyl-5-O-(3-hydroxy-3-methyl) glutaroyl quinic acid, 5411

3,5-Di- $O$-caffeoyl-4- $O$-(3-hydroxy-3-methyl) glutaroylquinic acid, 5412

\section{$\mathrm{C}_{31} \mathrm{H}_{33} \mathrm{O}_{18}{ }^{+}$}

3,5-Di- $O$-( $\beta$-glucopyranosyl)pelargonidin

6"-O-4,6"'-O-1-cyclic malate, 5530

$\mathrm{C}_{31} \mathrm{H}_{33} \mathrm{O}_{19}{ }^{+}$

3,5-Di- $O$-( $\beta$-glucopyranosyl)cyanidin

$6 "-O-4,6 " '-O-1$-cyclic malate, 5529

\section{$\mathrm{C}_{31} \mathrm{H}_{34} \mathrm{~N}_{2} \mathrm{O}_{8}$}

Deacetylpicraline-3,4,5-trimethoxybenzoat, 4768

$\mathrm{C}_{31} \mathrm{H}_{34} \mathrm{O}_{6}$

Shizukaol A, 19845

$\mathrm{C}_{31} \mathrm{H}_{34} \mathrm{O}_{8}$

Japonicin C, 11819

$\mathrm{C}_{31} \mathrm{H}_{34} \mathrm{O}_{9}$

Lappaol B, 12514

$\mathrm{C}_{31} \mathrm{H}_{34} \mathrm{O}_{14}$

Cypellogin A, 4570

Cypellogin B, 4571

$\mathrm{C}_{31} \mathrm{H}_{34} \mathrm{O}_{16}$
Apigenin-7- $O-\alpha-L-2,3-$ di- $O$-acetylrhamnopyranosyl-( $(1 \rightarrow 6)-\beta$ - $D$-glucopyranoside, 1486

Crassirhizomoside A, 4214

Crassirhizomoside B, 4215

Crassirhizomoside C, 4216

\section{$\mathrm{C}_{31} \mathrm{H}_{34} \mathbf{O}_{17}$}

5,6,4'-Trihydroxyflavone 7- $O-\alpha-L-2,3-\mathrm{di}-O$ acetylrhamnopyranosyl-( $1 \rightarrow 6)-\beta$ - $D$-glucopyranoside, 21734

\section{$\mathrm{C}_{31} \mathrm{H}_{35} \mathrm{NO}_{8}$}

3'-Hydroxy-8b-ethyl ether-rocaglatic acid dimethylamide, 10097

\section{$\mathrm{C}_{31} \mathrm{H}_{35} \mathrm{O}_{18}{ }^{+}$}

Peonidin-3- $O$-(6"- $O$-malonyl- $\beta$-glucopyranoside)-5- $O$ - $\beta$-glucopyranoside, 16903

\section{$\mathrm{C}_{31} \mathrm{H}_{36} \mathrm{~N}_{2} \mathrm{O}_{8}$}

Raunescine, 18552

$\mathrm{C}_{31} \mathrm{H}_{36} \mathrm{~N}_{2} \mathrm{O}_{11}$

Novobiocin, 15849

$\mathrm{C}_{31} \mathrm{H}_{36} \mathrm{O}_{6}$

Triptofordin A, 21998

$\mathrm{C}_{31} \mathrm{H}_{36} \mathrm{O}_{8}$

$6 \alpha$-Acetoxy- $1 \beta, 8 \beta$-dibenzoyloxy- $9 \beta$-hydroxy- $\beta$ dihydroagarofuran, 155

$2 \alpha$-Acetoxy- $4 \beta$-hydroxy- $6 \alpha$ - $p$-hydroxybenzoyloxy-10 $\beta$-benzoyloxy-dauc-8-ene, 218

Cowagarcinone E, 4199

Saucerneol B, 19399

Saucerneol D, 19401

\section{$\mathrm{C}_{31} \mathrm{H}_{36} \mathrm{O}_{9}$}

Benzoylgomisin Q, 2242

7-Isovaleroylcycloepiatalantin, 11758

Orthosiphol D, 16223

\section{$\mathrm{C}_{31} \mathrm{H}_{36} \mathrm{O}_{10}$}

Lappaol D, 12516

Orbiculin E, 16164

Staminol D, 20261

$\mathrm{C}_{31} \mathrm{H}_{36} \mathrm{O}_{11}$

13-O-Acetylwallifoliol, 531

Propinquanin E, 17928

\section{$\mathrm{C}_{31} \mathrm{H}_{36} \mathrm{O}_{12}$}

19-Hydroxy-13-oxobaccatin III, 10558

\section{$\mathrm{C}_{31} \mathrm{H}_{36} \mathrm{O}_{14}$}

11,12-Diacetoxyharrisonin, 5301

Ikarisoside F, 10964

\section{$\mathrm{C}_{31} \mathrm{H}_{36} \mathrm{O}_{16}$}

Acetylpectolinarin, 480

Dicliripariside B, 5438

\section{$\mathrm{C}_{31} \mathrm{H}_{37} \mathrm{NO}_{17}$}

Oleracein D, 16077

\section{$\mathrm{C}_{31} \mathrm{H}_{38} \mathrm{O}_{6}$}

7-Deacetyl-7-angeloyl- $6 \alpha$-hydroxyazadiradione, 4714 
$\mathrm{C}_{31} \mathrm{H}_{38} \mathrm{O}_{7}$

5-Cinnamoyl-10-aceyltaxicin II, 3699

$\left(2 R^{*}, 3 S^{*}, 4 R^{*}, 5 R^{*}, 9 S^{*}, 11 S^{*}, 15 R^{*}\right)-5,15-$

Diacetoxy-3-benzoyloxy-14-oxolathyra6(17),12E-diene, 5287

Lathyrol-3,15-diacetate-5-benzoate, 12544

\section{$\mathrm{C}_{31} \mathrm{H}_{38} \mathrm{O}_{8}$}

2-O-Acetyl-5-O-cinnamoyltaxicin I, 355

5-Cinnamoyl-9-O-acetylphototaxicin I, 3697

5-Cinnamoyl-10-aceyltaxicin I, 3698

$O$-Cinnamoyltaxicin I, 3720

Saucerneol, 19397

Taxezopidine E, 20784

Turraflorin A, 22130

\section{$\mathrm{C}_{31} \mathrm{H}_{38} \mathrm{O}_{9}$}

3,12-Diacetyl-8-benzoylingol, 5325

7-Isovaleroylcycloseverinolide, 11759

Taxuspine Y, 20872

Toonacilin, 21446

\section{$\mathrm{C}_{31} \mathrm{H}_{38} \mathrm{O}_{10}$}

2-O-Deacetylorthosiphol J, 4761

1-Dehydroxybaccatin III, 4979

5,3'-Dihexanoyloxy-3,6,7,4'-tetramethoxyflavone, 5536

Meliacinanhydride, 13662

Orthosiphol I, 16227

Orthosiphol M, 16231

Turraflorin D, 22132

Turraflorin E, 22133

\section{$\mathrm{C}_{31} \mathrm{H}_{38} \mathrm{O}_{11}$}

Acernikol, 96

1-Acetyl-10-deacetylbaccatin III, 362

Baccatin III, 2073

Baccatin V, 2076

6-Deoxy- $6 \alpha$-acetoxyatalantin acetate, 5147

11-Epi-21-hydroxytoonacilide, 6935

11-Epi-23-hydroxytoonacilide, 6936

Taxuspinanane D, 20844

\section{$\mathrm{C}_{31} \mathrm{H}_{38} \mathrm{O}_{12}$}

7-Epi-19-hydroxybaccatin III, 6934

19-Hydroxybaccatin III, 9812

Tasumatrol O, 20725

\section{$\mathrm{C}_{31} \mathrm{H}_{38} \mathrm{O}_{13}$}

Woorenoside V, 22731

\section{$\mathrm{C}_{31} \mathrm{H}_{38} \mathrm{O}_{15}$}

Tubuloside E, 22097

\section{$\mathrm{C}_{31} \mathrm{H}_{38} \mathrm{O}_{16}$}

2'-Acetylacteoside, 305

6'-O-Acetylacteoside, 306

3,4-Dihydroxyphenethoxy- $O$ - $\alpha$ - $L$-rhamnopyranosyl-( $1 \rightarrow 3)-\beta-D$-(2-O-acetyl-4- $O$-caffeoyl)galactopyranoside, 6073

Tubuloside B, 22094
$\mathrm{C}_{31} \mathrm{H}_{38} \mathrm{O}_{17}$

Pinocembrin 7-O-apiosyl( $1 \rightarrow 5$ )apiosyl $(1 \rightarrow 2)$ $\beta$-D-glucopyranoside, 17404

Tenuifoliside A, 20955

$\beta$ - $D$-[5-O-(3,4,5-Trimethoxybenzoyl)]-apiofuranosyl-( $1 \rightarrow 6)-\beta$ - $D$-glucopyranosyl, 21895

\section{$\mathrm{C}_{31} \mathrm{H}_{38} \mathrm{O}_{18}$}

Prunose I, 18004

$\mathrm{C}_{31} \mathrm{H}_{39} \mathrm{NO}_{6}$

Lythrancepine III, 13271

$\mathrm{C}_{31} \mathrm{H}_{39} \mathrm{NO}_{7}$

Lythrancine III, 13274

Lythrancine VI, 13277

Lythrancine VII, 13278

$\mathrm{C}_{31} \mathrm{H}_{39} \mathrm{NO}_{10} \mathrm{~S}$

Labriformine, 12422

$\mathrm{C}_{31} \mathrm{H}_{39} \mathrm{~N}_{5} \mathrm{O}_{5}$

Ergocornine, 7232

\section{$\mathrm{C}_{31} \mathrm{H}_{40} \mathrm{O}_{7}$}

$2 \alpha$-Acetoxy- $4 \beta$-hydroxy- $6 \alpha$-angeloyloxy- $10 \beta$ cinnamoyloxy-dauc-8-ene, 208

\section{$\mathrm{C}_{31} \mathrm{H}_{40} \mathrm{O}_{8}$}

Pubescene C, 18172

Pubescene D, 18173

\section{$\mathrm{C}_{31} \mathrm{H}_{40} \mathrm{O}_{9}$}

$2 \alpha$-Benzoyloxy- $9 \alpha, 10 \beta$-diacetoxy- $1 \beta, 5 \alpha, 13 \alpha$ trihydroxy-4(20),11-taxadiene, 2255

Brevifoliol, 2604

Turraflorin H, 22136

\section{$\mathrm{C}_{31} \mathbf{H}_{40} \mathbf{O}_{10}$}

Ardisiaquinone G, 1635

9-Deacetyl-9-benzoyl-10-debenzoyltaxchinin A, 4730

Orthosiphol G, 16225

Orthosiphol V, 16237

Orthosiphol W, 16238

\section{$\mathrm{C}_{31} \mathrm{H}_{40} \mathrm{O}_{11}$}

7,9,10-Deacetylbaccatin VI, 4725

7,9-Diacetyltaxayuntin, 5348

7-Epi-9,10-deacetylbaccatin VI, 6881

Taxane 3, 20750

Taxayuntin B, 20760

Taxumairol K, 20831

Taxuspinanane F, 20845

Taxuspine E, 20853

7,9,10-Trideacetyl-abeo-baccatin VI, 21602

7,9,13-Trideacetylbaccatin VI, 21603

\section{$\mathrm{C}_{31} \mathrm{H}_{40} \mathrm{O}_{12}$}

13-O-Deacetyltaxumairol Z, 4785

9( $\beta H)$-9-Dihydro-19-acetoxy-10-deacetylbaccatin III, 5538

\section{$\mathrm{C}_{31} \mathrm{H}_{40} \mathrm{O}_{13}$}

Hydrangenoside A, 9701
$\mathrm{C}_{31} \mathrm{H}_{40} \mathrm{O}_{14}$

Styraxlignolide A, 20422

\section{$\mathrm{C}_{31} \mathrm{H}_{40} \mathrm{O}_{15}$}

Cistanoside D, 3754

Epimeredinoside A, 6965

2-(3-Hydroxy-4-methoxyphenyl) ethyl $O-\alpha-L$ rhamnopyranosyl-( $1 \rightarrow 3)-(4-O$-cis-feruloyl)- $\beta$ -

$D$-glucopyranoside, 10443

2-(3-Hydroxy-4-methoxyphenyl)-ethyl-1- $O-\alpha-L$ rhamnosyl-( $1 \rightarrow 3)-\beta$ - $D$-(4-feruloyl)-glucoside, 10444

JuncenolideD, 11952

Martynoside, 13580

Isomartynoside, 11529

\section{$\mathrm{C}_{31} \mathrm{H}_{40} \mathrm{O}_{16}$}

Hemiphroside A, 9347

Hemiphroside C, 9348

Ilicifolioside A, 10988

6-O- $\alpha$ - $L$-(2"-O-trans- $p$-Methoxycinnamoyl) rhamnopyranosylcatalpol, 13892

6-O- $\alpha$ - L-(3"-O-trans- $p$-Methoxycinnamoyl) rhamnopyranosylcatalpol, 13893

Plantainoside E, 17511

Plantainoside F, 17512

\section{$\mathrm{C}_{31} \mathrm{H}_{41} \mathrm{ClO}_{11}$}

Taxuchin B, 20818

\section{$\mathrm{C}_{31} \mathrm{H}_{41} \mathrm{NO}_{6}$}

Franchetine, 7935

Franchitine, 7936

\section{$\mathrm{C}_{31} \mathrm{H}_{41} \mathrm{NO}_{10}$}

Polyschistine C, 17674

\section{$\mathbf{C}_{31} \mathbf{H}_{41} \mathrm{NO}_{11}$}

(E)-Hordenine-(6-O-cinnamoyl- $\beta$ - $D$-glucopyranosyl)-( $1 \rightarrow 3)-\alpha$ - $L$-rhamnopyranoside, 9647

\section{$\mathrm{C}_{31} \mathrm{H}_{41} \mathrm{NO}_{12}$}

(E)-Hordenine-[6-O-(4-hydroxycinnamoyl)- $\beta$ - $D$ glucopyranosyl]-(1 $\rightarrow 3)-\alpha-L$-rhamnopyranoside, 9648

\section{$\mathrm{C}_{31} \mathrm{H}_{41} \mathrm{NO}_{15}$}

Aristomanoside, 1728

\section{$\mathrm{C}_{31} \mathrm{H}_{41} \mathrm{~N}_{5} \mathrm{O}_{5}$}

Ergocorninine, 7233

\section{$\mathrm{C}_{31} \mathrm{H}_{42} \mathrm{~N}_{4} \mathrm{O}_{4}$}

Adouetine $\mathrm{Y}^{\prime}, 650$

Frangufoline, 7937

Scutianine C, 19591

Waltherine A, 22632

\section{$\mathrm{C}_{31} \mathrm{H}_{42} \mathrm{O}_{5}$}

Kleinioxanthrone 3, 12235

\section{$\mathrm{C}_{31} \mathrm{H}_{42} \mathrm{O}_{7}$}

$7 \beta$-Hydroxy-3,11,15,23-tetraoxolanosta-8,20E

(22)-dien-26-oic acid methyl ester, 10759

Methyl ganoderate H, 14458 
$\mathrm{C}_{31} \mathrm{H}_{42} \mathrm{O}_{8}$

Neocucurbitacin A, 15376

$\mathrm{C}_{31} \mathrm{H}_{42} \mathrm{O}_{9}$

Ardisiaquinone D, 1632

$\mathrm{C}_{31} \mathbf{H}_{42} \mathbf{O}_{10}$

Asclepin, 1844

Physachenolide C, 17234

$\mathrm{C}_{31} \mathrm{H}_{42} \mathrm{O}_{11}$

Ajugacumbin C, 790

Ajugamarin $\mathrm{A}_{2}, 802$

$\mathrm{C}_{31} \mathrm{H}_{42} \mathrm{O}_{12}$

Ajugacumbin E, 792

Clerodendrin A, 3837

$\mathrm{C}_{31} \mathrm{H}_{42} \mathrm{O}_{13}$

LigurobustosideO, 12824

\section{$\mathrm{C}_{31} \mathrm{H}_{42} \mathrm{O}_{15}$}

(S)-4-(4-Hydroxyphenyl)-2-butanol 2-O-[6-O(3,5-dimethoxy-4-O- $\alpha$ - $L$-rhamnopyranosylgalloyl)- $\beta$ - $D$-glucopyranoside], 10611

(-)-Isolariciresinol-3 $\alpha-O-\beta$-apiofuranosyl-

$(1 \rightarrow 2)$ - $O$ - $\beta$-glucopyranoside, 11478

Symplolignanoside A, 20546

\section{$\mathrm{C}_{31} \mathrm{H}_{42} \mathrm{O}_{16}$}

Yemuoside $\mathrm{YM}_{2}, 22885$

Yemuoside $\mathrm{YM}_{6}, 22886$

\section{$\mathrm{C}_{31} \mathrm{H}_{42} \mathrm{O}_{17}$}

Isonuezhenide, 11565

(8E)-Nüzhenide, 15873

(8Z)-Nüzhenide, 15874

\section{$\mathrm{C}_{31} \mathrm{H}_{42} \mathrm{O}_{18}$}

Neonuezhenide, 15439

\section{$\mathrm{C}_{31} \mathrm{H}_{43} \mathrm{NO}_{7}$}

$N$-Ethyl-1 $\alpha$-hydroxy-17-veratroyldictizine, 7447

\section{$\mathrm{C}_{31} \mathrm{H}_{43} \mathrm{NO}_{10}$}

Benzoylmesaconine, 2247

\section{$\mathrm{C}_{31} \mathbf{H}_{44} \mathbf{N}_{2} \mathbf{O}_{8}$}

Delectine, 5005

Isodelectine, 11372

\section{$\mathrm{C}_{31} \mathrm{H}_{44} \mathbf{N}_{4} \mathbf{O}_{5}$}

Pandamine, 16602

Sanjoinine F, 19292

Sanjoinine $\mathrm{G}_{1}, 19293$

$\mathrm{C}_{31} \mathrm{H}_{44} \mathrm{O}_{4}$

Papuaforin D, 16632

\section{$\mathrm{C}_{31} \mathrm{H}_{44} \mathrm{O}_{6}$}

Brasiliensic acid, 2590

Glyuranolide, 8855

$\mathrm{C}_{31} \mathrm{H}_{44} \mathrm{O}_{7}$

Methyl ganoderate I, 14459

$\mathrm{C}_{31} \mathrm{H}_{44} \mathrm{O}_{9}$

Methyl ganoderate AP, 14456

$3 \beta$-Oxo-formyl-7 $\beta, 12 \beta$-dihydroxy- $5 \alpha$-lanost-

11,15,23-trioxo-8-en $(E)$-26-0ic acid, 16335
$\mathrm{C}_{31} \mathrm{H}_{44} \mathrm{O}_{11}$

Ajugamacrin B, 800

Ajugamarin $\mathrm{B}_{2}, 803$

$1 \alpha$-( $\alpha$-Methyl)-butanoyl-2 $\alpha$-( $\alpha$-methyl)-propynoyloxy- $4 \beta$-hydroxy- $9 \beta$ - $(\beta$-)furoyloxy-15acetoxy- $\beta$-dihydroagarofuran, 14168

\section{$\mathbf{C}_{31} \mathbf{H}_{44} \mathbf{O}_{12}$}

$16 \beta$-Acetoxy-strophanthidin-3- $\beta$ - $D$ - $O$-rhamnoside, 282

LigurobustosideC, 12819

LigurobustosideE, 12820

$\mathrm{C}_{31} \mathrm{H}_{44} \mathbf{O}_{13}$

Ligurobustoside I, 12821

$\mathrm{C}_{31} \mathbf{H}_{44} \mathbf{O}_{16}$

$2^{\prime}, 3^{\prime}, 4^{\prime}, 3^{\prime \prime}$-Tetramethoxy-1,3-diphenylpropane 5',4"-di- $O-\beta$ - $D$-glucopyranoside, 21179

\section{$\mathrm{C}_{31} \mathrm{H}_{45} \mathrm{NO}_{8}$}

Auriculine, 2015

Merresectine B, 13786

$\mathrm{C}_{31} \mathrm{H}_{46} \mathrm{~N}_{2} \mathrm{O}$

Spiropachysine, 20205

\section{$\mathrm{C}_{31} \mathrm{H}_{46} \mathrm{O}_{2}$}

Lucialdehyde A, 13019

Vitamin $\mathrm{K}_{1}, 22562$

\section{$\mathrm{C}_{31} \mathrm{H}_{46} \mathrm{O}_{3}$}

Dehydroeburiconic acid, 4910

\section{$\mathrm{C}_{31} \mathrm{H}_{46} \mathrm{O}_{4}$}

26,27-Dihydroxy-lanosta-7,9(11),24-trien-3,16dione, 5946

Fomlactone C, 7878

Methyl

(24E)-3 $\alpha, 16 \alpha, 23 \alpha(=16 R, 23 R)$-trihydroxy-epox y-17,14-friedolan-8,14,24-trien-26-oate, 14407 Polyporenic acid C, 17664

Poricoic acid C, 17724

\section{$\mathrm{C}_{31} \mathrm{H}_{46} \mathrm{O}_{5}$}

Poricoic acid A, 17722

Propapyriogenin $\mathrm{A}_{1}, 17917$

\section{$\mathrm{C}_{31} \mathrm{H}_{46} \mathrm{O}_{6}$}

$3 \beta$-Methoxy-16 $\alpha$-hydroxyursa-12,19(29)-dien27,28-dioic acid, 13971

Methyl (24E)-9 $\alpha, 23 \alpha$-dihydroxy-3,15-dioxo17,15-friedo-lanostan-8(14),24-dien-26-oate, 14298

Poricoic acid D, 17725

$\mathrm{C}_{31} \mathrm{H}_{46} \mathrm{O}_{7}$

Acinospesigenin B, 547

$8 \beta, 9 \alpha$-Dihydroganoderic acid J methyl ester, 5620

Methyl-7-epiganoderate, 14397

\section{$\mathrm{C}_{\mathbf{3 1}} \mathrm{H}_{\mathbf{4}} \mathrm{O}_{\mathbf{8}}$}

rel-(2S,5R,6R,8S,9S,10R,18S,19R)-19-Acetoxy18,19-epoxy-6-hydroxy-18-butanoyloxy-2- (2-methylbutanoyloxy)cleroda-3,13 (16),14-

triene, 184

Acinospesigenin C, 548

Caseamembrin A, 3258

$2 \alpha$-( $\alpha$-Methylbutyryl)-oxy- $5 \alpha, 7 \beta, 10 \beta$-triacetoxy4(20),11-taxadiene, 14199

Methyl ganoderate I, 14460

$2 \alpha, 5 \alpha, 10 \beta$-Triacetoxy-14 $\beta$-((S)2-methyl)butyryloxytaxa-4(20),11-diene, 21529

\section{$\mathrm{C}_{31} \mathrm{H}_{46} \mathrm{O}_{9}$}

22-Acetylcasterone, 346

Ajugalide E, 799

Caseamembrin C, 3260

$5 \alpha$-Hydroxy- $\alpha \alpha$-( $\alpha$-methylbutyryl)-oxy-7 $\beta, 9 \alpha$, $10 \beta$-triacetoxy-4(20),11-taxadiene, 10475

$9 \alpha$-Hydroxy-14 $\beta$-(2-methylbutyryl)-oxy- $2 \alpha, 5 \alpha$, $10 \beta$-tri acetoxytaxa-4(20),11-diene, 10476

Taiwanxan, 20647

Yunnanxane, 22951

\section{$\mathrm{C}_{31} \mathrm{H}_{48} \mathbf{N}_{2} \mathrm{O}_{6}$}

Epoxynepapakistamine A, 7177

\section{$\mathrm{C}_{31} \mathrm{H}_{48} \mathrm{O}$}

24-Methylenecycloartenone, 14356

\section{$\mathrm{C}_{31} \mathrm{H}_{48} \mathrm{O}_{3}$}

Ambonic acid, 1025

Dehydroeburicoic acid, 4909

Fomefficinic acid A, 7856

$3 \alpha$-Hydroxy-11 $\alpha, 12 \alpha$-epoxy-oleanane- $28,13 \beta$ olide, 10089

$3 \beta$-Hydroxy-11 $\alpha, 12 \alpha$-epoxy-oleanane-28,13 $\beta$ olide, 10090

Koelpinin B, 12244

\section{$\mathrm{C}_{31} \mathrm{H}_{48} \mathrm{O}_{4}$}

Dehydrotumulosic acid, 4978

3-Epidehydrotumulosic acid, 6889

Fomefficinic acid B, 7857

Fomefficinic acid D, 7858

Fomlactone B, 7877

$6 \alpha$-Hydroxylup-20(29)-en-3-oxo-28-oic acid, 10345

Macedonic acid, 13286

Methyl (24E)-3 $\alpha, 23 \alpha(=R)$-dihydroxy-17,14friedo-lanostan-8,14,24-trien-26-oate, 14302

Methyl (24E)-3 $\alpha, 23$-dihydroxy-17,14-friedolanstan-8,14,24-trien-26-oate, 14303

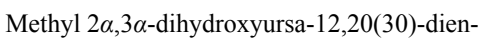
28-oate, 14306

Methylglycyrrhetate, 14474

Methyl (25R)-3 $\beta$-hydroxy-23-oxo-9, 16-lanostadien-26-oate, 14518

Methyl(24Z)-26-hydroxy-3-oxo-7,24-tirucalladienoate, 14520

Methyl kulonate, 14544 
Quadrangularic acid E, 18294

Regelin, 18578

Regelin D, 18580

24(E)-3,4-Secodammara-4(28),20,24-trien-3,26dioic acid-3-methylester, 19610

\section{$\mathrm{C}_{31} \mathrm{H}_{48} \mathrm{O}_{5}$}

25-Hydroxy-3-epidehydrotumulosic acid, 10070

Methyl (24E)-3 $\alpha, 23 \alpha$-dihydroxy-15-oxo-17,14friedo-lanostan-8(14),24-dien-26-oate, 14315

Methyl 16-epiquillate, 14401

Methyl 18 $\alpha$-hydroxyglycyrrhetate, 14507

Methyl-24-hydroxyglycyrrhetate, 14508

Methyl $(13 S, 14 R) 2 \alpha, 3 \alpha, 24$-trihydroxy-13,14cyclo-oleana-11-en-28-oate, 14783

Methyl $(12 R, 13 S) 2 \alpha, 3 \alpha, 24$-trihydroxy-12,13cyclo-taraxer-14-en-28-oate, 14784

Methyl $2 \alpha, 3 \alpha, 24$-trihydroxyoleana-11,13(18)dien-28-oate, 14785

Methyl $2 \alpha, 3 \alpha, 24$-trihydroxyursa-12,20(30)-dien28-oate, 14788

Poricoic acid H, 17728

Regelinol, 18583

\section{$\mathrm{C}_{31} \mathrm{H}_{48} \mathrm{O}_{6}$}

Lycernuic ketone A, 13175

Lycernuic ketone B, 13176

Methyl (24E)-3 $\alpha, 23 \alpha$-dihydroxy- $8 \alpha, 9 \alpha$-epoxy15-oxo-17,14-friedo-lanostan-24-en-26-oate, 14299

Methyl 11-oxoasiatate, 14641

Phytolaccagenic acid, 17266

Pseudolarolide C, 18045

\section{$\mathrm{C}_{31} \mathrm{H}_{48} \mathrm{O}_{7}$}

Phytolaccagenin, 17267

\section{$\mathrm{C}_{31} \mathrm{H}_{48} \mathrm{O}_{12}$}

Cussoracoside B, 4424

Cussoracoside D, 4426

\section{$\mathrm{C}_{31} \mathrm{H}_{49} \mathrm{~N}_{3} \mathrm{O}_{5}$}

Beauverilide A, 2189

\section{$\mathrm{C}_{31} \mathrm{H}_{50} \mathrm{O}$}

Agrostophyllinone, 767

24-Methylene cycloartanone, 14354

3-Oxo-24-methylenecycloartane, 16380

\section{$\mathrm{C}_{31} \mathrm{H}_{50} \mathrm{O}_{2}$}

Eburical, 6672

$3 \beta$-Hydroxy-lanost-9(11),24(25)-dien-26-oic acid, 10312

21 $\alpha$-Methoxyserrat-13-en-3-one, 14091

12-Oxoarundoin, 16294

25(R)-3-Oxo-24-methylenecycloartan-26-ol, 16381

$\mathrm{C}_{31} \mathrm{H}_{50} \mathrm{O}_{3}$

Ambolic acid, 1024

Eburicoic acid, 6674
$13 \alpha, 14 \alpha$-Epoxy-21 $\alpha$-methoxyserratan-3-one, 7169

$3 \beta$-Hydroxy-5 $\alpha$-cycloart-24(31)-en-28-oic acid, 9952

Lithocarpolone, 12922

Marianine, 13563

Methylbetulinate, 14158

Methyl oleanolate, 14634

Methyl trametenolate, 14766

Methyl ursolate, 14803

\section{$\mathrm{C}_{31} \mathrm{H}_{50} \mathrm{O}_{4}$}

Hederagenin mathyl ester, 9267

Machaerinic acid methyl ester, 13289

Methyl $2 \alpha, 3 \alpha$-dihydroxyursa-12-en-28-oate, 14307

Methyl 3-epimaslinate, 14398

Methyl-24-hydroxy-11-deoxoglycyrrhetate, 14501

Methyl $2 \alpha$-hydroxyursa-28-oate, 14512

Methyl maslinate, 14579

Palmitoylpterosin A, 16565

Regelindiol A, 18581

Regelindiol B, 18582

$3 \alpha$-Sulfurenic acid, 20480

3 $\beta$-Sulphurenic acid, 20483

Triptodihydroxy acid methyl ester, 21997

Tumulosic acid, 22108

\section{$\mathrm{C}_{31} \mathrm{H}_{50} \mathrm{O}_{5}$}

15-O-Methylcimigenol, 14242

25-O-Methylcimigenol, 14243

Methyl euscaphate, 14440

Methyl tormentate, 14765

Methyl (24E)-3 $\alpha, 9,23$-trihydroxy-17,14-friedo-

lanostan-14,24-dien-26-oate, 14781

Methyl $2 \alpha, 3 \alpha, 23$-trihydroxyolean-12-en-28-oate, 14786

Methyl $2 \alpha, 3 \alpha, 24$-trihydroxyolean-12-en-28-oate, 14787

$2 \alpha, 3 \alpha, 24$-Trihydroxyursa-12-en-28-oate, 21764

$\mathrm{C}_{31} \mathrm{H}_{50} \mathrm{O}_{6}$

Kudzusapogenol B methyl ester, 12308

Methyl $11 \alpha$-hydroxytormentate, 14523

$2 \alpha, 3 \beta, 7 \beta$-Trihydroxy-11 $\alpha$-methoxyurs-12-en-28oic acid, 21789

\section{$\mathrm{C}_{31} \mathrm{H}_{50} \mathrm{O}_{8}$}

5-Ene-methyl-7,12-didehydroxy-cholate-3- $O-\beta$ $D$-glucopyranoside, 6798

\section{$\mathrm{C}_{31} \mathrm{H}_{50} \mathrm{O}_{11}$}

Cussoracoside F, 4428

$\mathrm{C}_{31} \mathrm{H}_{50} \mathrm{O}_{12}$

Crenulatoside D, 4231

$\mathrm{C}_{31} \mathrm{H}_{50} \mathrm{O}_{18}$

Zizyvoside II, 23020
$\mathrm{C}_{31} \mathrm{H}_{52}$

21-Methyldammara-18(28),22(29)-diene, 14281

$\mathrm{C}_{31} \mathrm{H}_{52} \mathrm{~N}_{2} \mathrm{O}_{2}$

$\mathrm{N}$-Tigloylbuxahyrcanine, 21374

$\mathrm{C}_{31} \mathrm{H}_{52} \mathrm{~N}_{2} \mathrm{O}_{3}$

Pachysandrine B, 16506

\section{$\mathrm{C}_{31} \mathrm{H}_{52} \mathrm{O}$}

Agrostophyllinol, 766

Arundoin, 1823

Cycloartenone, 4474

Cyclolaudenol, 4503

Cyclopholidonol, 4529

Cylindrin, 4546

Eburicol, 6675

Euphorbol, 7618

24-Methylene cycloartan-3 $\beta$-ol, 14352

24-Methylene lanost-8-enol, 14387

Miliacin, 14851

Sinetirucallol, 19932

\section{$\mathrm{C}_{31} \mathrm{H}_{52} \mathrm{O}_{2}$}

Dryocrassyl formate, 6612

Eburicodiol, 6673

$3 \beta$-Methoxyserrat-13-en-21 $\beta$-ol, 14090

24-Methylene cycloartan-3 $\beta, 21$-diol, 14351

14-Methyl-24-methylene-dihydromangiferodiol, 14594

31-Norcycloartanyl acetate, 15730

$\beta$-Sitosteryl acetate, 19994

\section{$\mathrm{C}_{31} \mathrm{H}_{52} \mathrm{O}_{3}$}

$13 \alpha, 14 \alpha$-Epoxy-3 $\beta$-methoxyserratan- $21 \beta$-ol, 7166

$13 \beta, 14 \beta$-Epoxy-3 $\beta$-methoxyserratan- $21 \beta$-ol, 7167

$14 \beta, 15 \beta$-Epoxy-3 $\beta$-methoxyserratan- $21 \beta$-ol, 7168

4'-Hydroxy-cis-cinnamic acid docosyl ester, 9909

Lithocarpdiol, 12921

$3 \beta$-Methoxy- $\beta, 19$-cyclolanost-23(E)-en-25,26diol, 13900

Soyasapogenol D, 20119

\section{$\mathrm{C}_{31} \mathrm{H}_{52} \mathrm{O}_{4}$}

(20S)-3 $\beta$-Acetoxy-20-hydroperoxy-30-norlupane, 202

Docosyl caffeate, 6539

\section{$\mathrm{C}_{31} \mathrm{H}_{52} \mathrm{O}_{6}$}

21-O-Methyl toosendanpentol, 14764

\section{$\mathrm{C}_{31} \mathrm{H}_{52} \mathrm{O}_{8}$}

Drevogenin I, 6602

$\mathrm{C}_{31} \mathrm{H}_{52} \mathrm{O}_{17}$

Pipeloside A, 17428

$\mathrm{C}_{31} \mathrm{H}_{53} \mathrm{NO}_{3}$

Ethyl $N$-docosanoylanthranilate, 7435

$\mathrm{C}_{31} \mathrm{H}_{54} \mathrm{O}$ 
Cycloeucalenol, 4491

$\mathrm{C}_{31} \mathrm{H}_{54} \mathrm{O}_{2}$

3 $\alpha$-Hydroxy-4-methoxyfilicane, 10402

$\mathrm{C}_{31} \mathrm{H}_{54} \mathrm{O}_{5}$

Foveolin B, 7932

$\mathrm{C}_{31} \mathrm{H}_{54} \mathrm{O}_{12}$

Cuscutic resinoside A, 4418

$\mathrm{C}_{31} \mathrm{H}_{56} \mathrm{O}$

Cycloartanol, 4468

$\mathrm{C}_{31} \mathrm{H}_{56} \mathrm{O}_{3}$

Thamnolin, 21272

$\mathrm{C}_{31} \mathrm{H}_{58} \mathrm{O}_{14}$

1'- $O$-Palmitoyl-3'- $O$-(6- $O-\alpha-D$-galactopyranosyl-

$\beta$ - $D$-galactopyranosyl)glycerol, 16563

\section{$\mathrm{C}_{31} \mathrm{H}_{60} \mathrm{O}$}

(2E)-2-Ethyl-2-nonacosenal, 7465

$\mathrm{C}_{31} \mathrm{H}_{62} \mathrm{O}$

7-Methyl-4-triacontanone, 14769

Palmitone, 16562

\section{$\mathrm{C}_{31} \mathrm{H}_{62} \mathrm{O}_{2}$}

Hentriacontic acid, 9366

n-Hexacosanyl isovalerate, 9483

Methyl triacontanate, 14768

$\mathrm{C}_{31} \mathrm{H}_{64}$

Hentriacontane, 9363

$\mathrm{C}_{31} \mathrm{H}_{64} \mathrm{O}$

Hentriacontanol-6, 9364

\section{$\mathrm{C}_{32} \mathrm{H}_{20} \mathrm{O}_{9}$}

Thelephantin M, 21305

\section{$\mathrm{C}_{32} \mathrm{H}_{22} \mathrm{MgO}_{14}$}

Cochinchinol A, 3868

\section{$\mathrm{C}_{32} \mathrm{H}_{22} \mathrm{O}_{8}$}

Thelephantin J, 21302

\section{$\mathrm{C}_{32} \mathrm{H}_{22} \mathrm{O}_{10}$}

Cryptomerin B, 4286

7,7"-Dimethoxyamentoflavone, 6199

4',7-Dimethylamentoflavone, 6314

7,7"-Di-O-methylcupressu-flavone, 6333

Ginkgetin, 8401

Isoginkgetin, 11439

Podocarpusflavone B, 17589

Thelephantin G, 21299

$\mathrm{C}_{32} \mathrm{H}_{22} \mathrm{O}_{11}$

5'-Methoxybilobetin, 13858

\section{$\mathrm{C}_{32} \mathrm{H}_{24} \mathrm{O}_{5}$}

Dracorubin, 6579

\section{$\mathrm{C}_{32} \mathrm{H}_{24} \mathrm{O}_{10}$}

2,3-Dihydro-7,7"-dimethoxyamentoflavone, 5600

Taiwanhomoflavone B, 20626

$\mathrm{C}_{32} \mathrm{H}_{26} \mathrm{O}_{7}$

Neocandenatone, 15353

$\mathrm{C}_{32} \mathrm{H}_{26} \mathrm{O}_{8}$

Rhuschalcone 1, 18820
$\mathrm{C}_{32} \mathrm{H}_{26} \mathrm{O}_{10}$

Biphenanthrene, 2398

Chamaejasmenin A, 3458

Chamaejasmenin B, 3459

Chamaejasmenin D, 3461

(2S,2"S)-7,7"-Di-O-methyltetrahydroamento-

flavone, 6409

7,7"-Di-O-methyltetrahydrohinokiflavone, 6411

Isochamaejasmenin B, 11319

Licobichalcone, 12765

Neoprotosappanin, 15451

$\mathrm{C}_{32} \mathrm{H}_{26} \mathrm{O}_{11}$

Protosappanin $\mathrm{E}_{1}, 17990$

Protosappanin $\mathrm{E}_{2}, 17991$

$\mathrm{C}_{32} \mathrm{H}_{28} \mathrm{O}_{10}$

Dahuribirin D, 4600

\section{$\mathrm{C}_{32} \mathrm{H}_{28} \mathrm{O}_{11}$}

Neosappanone A, 15457

\section{$\mathrm{C}_{32} \mathrm{H}_{28} \mathrm{O}_{15} \mathrm{~S}$}

Prodelphinidin $\mathrm{A}_{2}$ 4'-(2-hydroxyethyl)thio ether, 17897

\section{$\mathrm{C}_{32} \mathrm{H}_{30} \mathrm{~N}_{2} \mathrm{O}_{4}$}

Anabellamide, 1126

(-)-Auranamide, 2006

\section{$\mathrm{C}_{32} \mathrm{H}_{30} \mathrm{O}_{9}$}

Thelephantin C, 21295

$\mathrm{C}_{32} \mathrm{H}_{30} \mathrm{O}_{10}$

Occidentalol II, 15915

\section{$\mathrm{C}_{32} \mathrm{H}_{30} \mathrm{O}_{11}$}

Dahuribirin E, 4601

(12R,12"R)-Diheraclenol, 5535

\section{$\mathrm{C}_{32} \mathrm{H}_{30} \mathrm{O}_{12}$}

SkullcapflavoneI 2'- $O-\beta-D$-(2"- $E$-cinnamoyl)glucopyranoside, 20005

Skullcapflavone I 2'-O- $\beta$ - $D$-(3"-E-cinnamoyl)glucopyranoside, 20006

Skullcapflavone I 2'-O- $\beta$ - $D$-(4"-E-cinnamyl)glucopyranoside, 20007

\section{$\mathrm{C}_{32} \mathrm{H}_{30} \mathrm{O}_{14}$}

Secalonic acid A, 19599

Secalonic acid B, 19600

Secalonic acid C, 19601

Secalonic acid D, 19602

$\mathrm{C}_{32} \mathrm{H}_{30} \mathrm{O}_{15}$

Limocitrin-3-O-(6"-- $O$ - $p$-coumaryl)- $\beta$ - $D$-glucopyranoside, 12838

$\mathrm{C}_{32} \mathrm{H}_{31} \mathrm{NO}_{8}$

Aristoloterpenate, 1726

Aristoloterpenate III, 1727

Aristophyllide A, 1729

$\mathrm{C}_{32} \mathrm{H}_{32} \mathrm{O}_{8}$

Mexolide, 14824

$\mathrm{C}_{32} \mathrm{H}_{32} \mathrm{O}_{13}$
Myrciacitrin IV, 15150

$\mathrm{C}_{32} \mathrm{H}_{32} \mathrm{O}_{16}$

Shegansu A, 19798

6"-O-Vanilloyliridin, 22344

$\mathrm{C}_{32} \mathrm{H}_{33} \mathrm{~N}_{3} \mathrm{O}_{4}$

Scutianene D, 19589

\section{$\mathrm{C}_{32} \mathrm{H}_{34} \mathrm{~N}_{2} \mathrm{O}_{7}$}

Discarine N, 6506

$O$-Methyl punjabine, 14707

\section{$\mathrm{C}_{32} \mathrm{H}_{34} \mathrm{O}_{8}$}

6,6'-Dimethoxygossypol, 6232

\section{$\mathrm{C}_{32} \mathrm{H}_{34} \mathrm{O}_{10}$}

Murramarin A, 15105

\section{$\mathrm{C}_{32} \mathrm{H}_{34} \mathrm{O}_{11}$}

Schizanrin F, 19500

$\mathrm{C}_{32} \mathrm{H}_{34} \mathrm{O}_{12}$

Orbiculin I, 16167

\section{$\mathrm{C}_{32} \mathrm{H}_{34} \mathrm{O}_{13}$}

$1 \alpha, 2 \alpha, 9 \beta$-Tri- $(\beta$-)furoyloxy- $4 \beta$-hydroxy- 15 acetoxy- $\beta$-dihydroagarofuran, 21640

\section{$\mathrm{C}_{32} \mathrm{H}_{34} \mathrm{O}_{16}$}

Arabelline, 1552

Neesiinoside A, 15320

\section{$\mathrm{C}_{32} \mathrm{H}_{36} \mathrm{O}_{8}$}

Artanomaloide, 1781

\section{$\mathrm{C}_{32} \mathrm{H}_{36} \mathrm{O}_{10}$}

Schisantherin M, 19494

Schisantherin N, 19495

\section{$\mathrm{C}_{32} \mathrm{H}_{36} \mathrm{O}_{11}$}

Kadsulignan D, 11999

Kadsulignan G, 12002

\section{$\mathrm{C}_{32} \mathrm{H}_{36} \mathrm{O}_{16}$}

3,6-Di- $O$ - $p$-coumaroyl- $\beta$ - $D$-fructofuranosyl 6- $O$-acetyl- $\alpha$ - $D$-glucopyranoside, 5441

\section{$\mathrm{C}_{32} \mathrm{H}_{37} \mathrm{NO}_{9}$}

Kupitengester 2, 12337

\section{$\mathrm{C}_{32} \mathrm{H}_{37} \mathrm{NO}_{13}$}

$1 \alpha$-Nicotinoyloxy- $2 \alpha$-acetoxy- $6 \beta$-acetoxy- $9 \beta$ furoyloxy-11-acetoxy- $4 \beta$-hydroxydihydro- $\beta$ agarofuran, 15535

\section{$\mathrm{C}_{32} \mathrm{H}_{38} \mathrm{~N}_{2} \mathrm{O}_{5}$}

Nigellamine C, 15566

\section{$\mathrm{C}_{32} \mathrm{H}_{38} \mathrm{~N}_{2} \mathrm{O}_{8}$}

Deserpidine, 5248

\section{$\mathrm{C}_{32} \mathrm{H}_{38} \mathrm{~N}_{2} \mathrm{O}_{9}$}

Pseudoreserpine 16,17-stereoisomer, 18059

\section{$\mathrm{C}_{32} \mathrm{H}_{38} \mathrm{O}_{14}$}

Baohuoside III, 2140

\section{$\mathrm{C}_{32} \mathrm{H}_{38} \mathrm{O}_{14}$}

Baohuoside IV, 2141

2"-O-Rhamnosylikarisoside A, 18742

Safghanoside D, 19115

$\mathrm{C}_{32} \mathrm{H}_{38} \mathrm{O}_{15}$ 


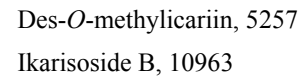

\section{$\mathrm{C}_{32} \mathrm{H}_{38} \mathrm{O}_{17}$}

Glomeratose B, 8583

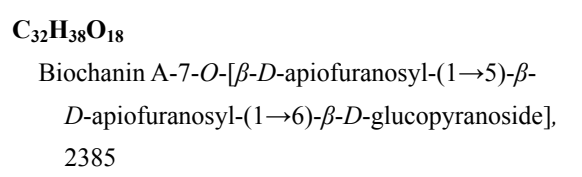

Kaempferol-3-rhamnoside-7-xylosyl $(1 \rightarrow 2)$ rhamnoside, 12086

Sagittatin A, 19125

Vaccarin, 22302

\section{$\mathrm{C}_{32} \mathrm{H}_{38} \mathrm{O}_{19}$}

$A$ pigenin-7- $O$ - $\beta$-apiofuranosyl-6,8-di- $C$ - $\beta$ glucopyranoside, 1478

Kaempferol 3- $O-\beta$ - $D$-apiosyl- $(1 \rightarrow 2)$ - $[\alpha-L$ rhamnosyl $(1 \rightarrow 6)]-\beta$ - $D$-glucoside, 12027

\section{$\mathrm{C}_{32} \mathrm{H}_{38} \mathrm{O}_{20}$}

Kaempferol 3-O- $\alpha$-arabinopyranosyl( 1 "' $\rightarrow 6$ ")- $\beta$ glucopyranoside 7- $O$ - $\beta$-glucopyranoside, 12029

Saluenin, 19199

\section{$\mathrm{C}_{32} \mathrm{H}_{38} \mathrm{O}_{21}$}

Quercetin-3- $O$ - $\alpha$-arabinopyranosyl( $1 " ' \rightarrow 6 ")$ - $\beta$ glucopyranoside 7-O- $\beta$-glucopyranoside, 18328

\section{$\mathrm{C}_{32} \mathrm{H}_{39} \mathrm{NO}_{11}$}

Myrsinol-type diterpene ester CPB51-719-2, 15216

\section{$\mathrm{C}_{32} \mathrm{H}_{39} \mathrm{NO}_{15}$}

1,6-Bis-deacetyl evonine, 2438

\section{$\mathrm{C}_{32} \mathrm{H}_{40} \mathrm{~N}_{2} \mathrm{O}_{13}$}

$N, \beta$ - $D$-Glucopyranosyl vincosamide, 8746

\section{$\mathrm{C}_{32} \mathrm{H}_{40} \mathrm{O}_{7}$}

Plectranthol B, 17554

\section{$\mathrm{C}_{32} \mathrm{H}_{40} \mathrm{O}_{8}$}

Euphorbiasteroid, 7617

Saucerneol A, 19398

(-)-Saucerneol methyl ether, 19403

\section{$\mathrm{C}_{32} \mathrm{H}_{40} \mathrm{O}_{9}$}

17-Epi-12-dehydroxyheudebolin, 6890

\section{$\mathrm{C}_{32} \mathrm{H}_{40} \mathrm{O}_{10}$}

2-Hydroxy-3-O-tigloylswietenolide, 10768

\section{$\mathrm{C}_{32} \mathrm{H}_{40} \mathrm{O}_{11}$}

Hypoloside B, 10902

Hypoloside C, 10903

Myrsinol-type diterpene ester CPB51-719-3, 15217

\section{$\mathrm{C}_{32} \mathrm{H}_{40} \mathrm{O}_{13}$}

15-Acetoxy- $2 \alpha, 9 \beta$-di-( $\beta$-furancarbonyloxy)$4 \beta, 6 \beta$-dihydroxy-1 $\alpha$-(2-methylbutanoyloxy)dihydro- $\beta$-agarofuran, 160

$\mathrm{C}_{32} \mathrm{H}_{40} \mathrm{O}_{14}$

$$
\begin{gathered}
1 \alpha, 2 \alpha, 6 \beta, 8 \beta, 13 \text {-Pentaacetoxy-9 } \beta \text {-benzoyloxy- } \\
4 \beta \text {-hydroxy- } \beta \text {-dihydroagarofuran, } 16817
\end{gathered}
$$

\section{$\mathrm{C}_{32} \mathrm{H}_{40} \mathrm{O}_{15}$}

Nirurin, 15625

$\mathrm{C}_{32} \mathrm{H}_{40} \mathrm{O}_{16}$

Javanicoside C, 11856

Phellodensin G, 17075

$\mathrm{C}_{32} \mathrm{H}_{40} \mathrm{O}_{17}$

6"-O-p-Coumaroylgenipingentiobioside, 4160

Phelloside, 17078

Safghanoside A, 19112

Safghanoside B, 19113

\section{$\mathrm{C}_{32} \mathrm{H}_{40} \mathrm{O}_{18}$}

Telephiose C, 20909

\section{$\mathrm{C}_{32} \mathrm{H}_{40} \mathrm{O}_{19}$}

Viscumneoside V, 22540

\section{$\mathrm{C}_{32} \mathrm{H}_{41} \mathrm{NO}_{2}$}

$N$-(1',4'-Dihydroxy-1',2',3',4'-tetrahydronaphthyl)-propyl- $N$-diphenylmethyl- $N$-3,3-dimethylbutylamine, 6144

\section{$\mathrm{C}_{32} \mathrm{H}_{41} \mathrm{NO}_{8}$}

15-Veratroyl-17-acetyl-19-oxodictizine, 22391

\section{$\mathrm{C}_{32} \mathrm{H}_{41} \mathrm{~N}_{5} \mathrm{O}_{5}$}

Ergocryptine, 7236

$\alpha$-Ergokryptinine, 7238

Mauritine A, 13613

$O-12$ '-Methyl ergocornine, 14410

$O$-12'-Methyl ergocorninine, 14411

\section{$\mathrm{C}_{32} \mathrm{H}_{42} \mathrm{~N}_{2} \mathrm{O}_{6}$}

Rescinnaminol, 18631

\section{$\mathrm{C}_{32} \mathrm{H}_{42} \mathrm{O}_{5}$}

Vielanin A, 22469

\section{$\mathrm{C}_{32} \mathrm{H}_{42} \mathrm{O}_{7}$}

Euphorbia factor $\mathrm{Ti}_{2}, 7616$

Vielanin D, 22472

Vielanin E, 22473

\section{$\mathrm{C}_{32} \mathrm{H}_{42} \mathrm{O}_{8}$}

Acrovestone, 577

Cipadesin, 3734

Meliacinol, 13663

\section{$\mathrm{C}_{32} \mathrm{H}_{42} \mathrm{O}_{9}$}

Ardisiaquinone C, 1631

Ganode-8-en-ric acid F, 8155

\section{$\mathrm{C}_{32} \mathrm{H}_{42} \mathrm{O}_{10}$}

Ardisiaquinone H, 1636

Azedarachin C, 2055

Yuanhuadin, 22930

\section{$\mathrm{C}_{32} \mathrm{H}_{42} \mathrm{O}_{11}$}

Taccalonolide G, 20589

\section{$\mathrm{C}_{32} \mathrm{H}_{42} \mathrm{O}_{12}$}

1-Deoxy-3-methacrylyl-11-methoxymeliacarpinin, 5194

Taccalonolide M, 20595

\section{$\mathrm{C}_{32} \mathrm{H}_{42} \mathrm{O}_{13}$}

Myricanene A 5-O- $\alpha$ - $L$-arabinofuranosyl $(1 \rightarrow 6)$ $\beta$ - $D$-glucopyranoside, 15156

Myricanene B 5-O- $\alpha$ - $L$-arabinofuranosyl $(1 \rightarrow 6)$ $\beta$ - $D$-glucopyranoside, 15158

$1 \beta, 2 \beta, 9 \alpha$-Triacetoxy- $8 \alpha$-(2-hydroxy-isobutyryoxy)-15-benzoyloxy- $4 \alpha$-hydroxy- $\beta$-dihydoagarofuran, 21524

\section{$\mathrm{C}_{32} \mathrm{H}_{42} \mathrm{O}_{15}$}

$1 \beta, 7 \beta, 8 \alpha$-Triacetoxy- $2 \beta$-furanoyl- $4 \alpha$-hydroxy11-isobutyryloxy-dihydroagarofuran, 21521

\section{$\mathrm{C}_{32} \mathrm{H}_{42} \mathrm{O}_{16}$}

Bruceoside A, 2675

Bruceoside B, 2676

Bruceoside C, 2677

Dehydrodiconiferyl alcohol 4, $\gamma^{\prime}$-di- $O-\beta$ - $D$-glucopyranoside, 4904

Javanicoside I, 11860

(+)-Pinoresinol-di- $O-\beta$ - $D$-glucoside, 17412

Scrolepidoside, 19568

\section{$\mathrm{C}_{32} \mathrm{H}_{42} \mathrm{O}_{17}$}

(+)-1-Hydroxypinoresinol-4',4"-di- $O$ - $\beta$ - $D$-glucopyranoside, 10653

\section{$\mathrm{C}_{32} \mathrm{H}_{42} \mathrm{O}_{21}$}

Saprosmoside G, 19351

\section{$\mathrm{C}_{32} \mathrm{H}_{43} \mathrm{NO}_{7}$}

15-Veratroyl-17-acetyldictizine, 22390

\section{$\mathrm{C}_{32} \mathrm{H}_{44} \mathrm{~N}_{2} \mathrm{O}_{4}$}

Phlegmariurine $\mathrm{C}, 17156$

\section{$\mathrm{C}_{32} \mathrm{H}_{44} \mathrm{~N}_{2} \mathrm{O}_{8}$}

Isolappaconitine, 11476

Lappaconitine, 12510

\section{$\mathrm{C}_{32} \mathrm{H}_{44} \mathrm{~N}_{2} \mathrm{O}_{9}$}

$\mathrm{N}$-Acetylsepaconitine, 508

Puberanine, 18169

Ranaconitine, 18531

\section{$\mathrm{C}_{32} \mathbf{H}_{44} \mathbf{N}_{2} \mathbf{O}_{10}$}

Finaconitine, 7800

\section{$\mathrm{C}_{32} \mathrm{H}_{44} \mathrm{O}_{6}$}

Bisacutifolone C, 2434

\section{$\mathrm{C}_{32} \mathrm{H}_{44} \mathrm{O}_{7}$}

Bisacutifolone A, 2429

Bisacutifolone B, 2432

3-O-(2'E,4'E-Decadienoyl)-20-O-acetylingenol, 4820

3-O-(2'E,4'Z-Decadienoyl)-5-O-acetylingenol, 4821

3-O-(2'E,4'Z-Decadienoyl)-20-O-acetylingenol, 4822

Resibufogenin 3-hydrogen suberate, 18638

\section{$\mathrm{C}_{32} \mathrm{H}_{44} \mathrm{O}_{8}$}

Cucurbitacin E, 4320

Elaterin, 6733 
$\mathrm{C}_{32} \mathrm{H}_{44} \mathrm{O}_{9}$

Ganode-8-en-ric acid H, 8156

$\mathrm{C}_{32} \mathrm{H}_{44} \mathrm{O}_{11}$

$6 \beta$-Acetoxy scillarenin 3-O- $\beta$ - $D$-glucopyranoside, 278

$\mathrm{C}_{32} \mathrm{H}_{44} \mathrm{O}_{12}$

Jatrophane 9, 11845

Scilliroside, 19528

$\mathrm{C}_{32} \mathrm{H}_{44} \mathrm{O}_{13}$

Baccatin I, 2072

$1 \beta$-Dehydroxybaccatin IV, 4980

Taxachitriene A, 20736

Taxachitriene B, 20737

\section{$\mathrm{C}_{32} \mathrm{H}_{44} \mathrm{O}_{14}$}

$1 \beta$-Acetoxy- $5 \alpha$-deacetyl-baccatin I, 151

Baccatin IV, 2075

Crocin 3, 4251

$1 \beta$-Hydroxybaccatin I, 9811

Taxuchin A, 20817

\section{$\mathrm{C}_{32} \mathrm{H}_{44} \mathrm{O}_{15}$}

10-Deacetyl-10-glycolylbaccatin IV, 4744

$1 \beta$-Hydroxy-10-deacetyl-10-glycolylbaccatin I, 9963

\section{$\mathrm{C}_{32} \mathrm{H}_{44} \mathrm{O}_{16}$}

Javanicoside B, 11855

(+)-Lariciresinol-4'- $O-\beta$ - $D$-glucopyranosyl$(1 \rightarrow 3)-\beta$ - $D$-glucopyranoside, 12524

Yadanzioside A, 22860

Yadanzioside B, 22861

Yadanzioside E, 22864

\section{$\mathrm{C}_{32} \mathrm{H}_{44} \mathrm{O}_{17}$}

(-)-Olivil-4',4"-di- $O-\beta$ - $D$-glucopyranoside, 16089

Yadanzioside J, 22869

\section{$\mathrm{C}_{32} \mathrm{H}_{45} \mathrm{NO}_{8}$}

14-O-Anisoylneoline, 1292

\section{$\mathrm{C}_{32} \mathrm{H}_{45} \mathrm{NO}_{9}$}

Circinadine A, 3735

Ludaconitine, 13065

\section{$\mathrm{C}_{32} \mathrm{H}_{45} \mathrm{NO}_{10}$}

Benzoylaconine, 2231

Hokbusine A, 9574

\section{$\mathrm{C}_{32} \mathrm{H}_{46} \mathrm{~N}_{2} \mathrm{O}_{3}$}

(-)-Culantraramine $\mathrm{N}$-oxide, 4348

\section{$\mathrm{C}_{32} \mathrm{H}_{46} \mathrm{~N}_{2} \mathrm{O}_{8}$}

Anthranoyllycoctonine, 1366 Inuline, 11112

\section{$\mathrm{C}_{32} \mathrm{H}_{46} \mathrm{~N}_{4} \mathrm{O}_{5}$}

Sanjoinine D, 19291

\section{$\mathrm{C}_{32} \mathrm{H}_{46} \mathrm{O}_{5}$}

Ganoderic acid T-Q, 8173

Inophylloidic acid $A_{2}, 11073$

Tyromycic acid G, 22168

\section{$\mathrm{C}_{32} \mathrm{H}_{46} \mathrm{O}_{6}$}

Inocalophyllin B, 11065

Inophylloidic acid, 11070

Inophylloidic acid $A_{1}, 11072$

Inophylloidic acid $\mathrm{A}_{3}, 11074$

Inophylloidic acid $\mathrm{B}_{1}, 11075$

Inophylloidic acid $\mathrm{B}_{2}, 11076$

Lanosta-7,9(11),24-trien-3 $\alpha$-acetoxy-15 $\alpha$-hydroxy-23-oxo-26-oic acid, 12483

Lanosta-7,9(11),24-trien-15 $\alpha$-acetoxy-3 $\alpha$-hydroxy-23-oxo-26-oic acid, 12484

Picfeltarraegenin IV, 17291

$\mathrm{C}_{32} \mathrm{H}_{46} \mathrm{O}_{7}$

Bufalin-3-hydrogen suberate, 2717

$\mathrm{C}_{32} \mathrm{H}_{46} \mathrm{O}_{8}$

Cucurbitacin B, 4317

Dihydrocucurbitacin E, 5574

Gamabufotalin-3-suberate, 8123

\section{$\mathrm{C}_{32} \mathrm{H}_{46} \mathrm{O}_{9}$}

2'-O-Acetyl cerleaside A, 349

$3 \beta$-O-(2'-O-Acetyl- $\alpha$ - $L$-thevetosyl)-14 $\beta$-hydroxy-7-en-5 $\beta$-card-20(22)-enolide, 526

Cucurbitacin A, 4315

Ganoderic acid $\alpha, 8175$

\section{$\mathrm{C}_{32} \mathrm{H}_{46} \mathrm{O}_{10}$}

Peruvoside-2'-monoacetate, 17003

Tanghinin, 20671

\section{$\mathrm{C}_{32} \mathrm{H}_{46} \mathrm{O}_{11}$}

$1 \alpha, 2 \alpha$-Di-(a-methyl)-butanoyl-4 $\beta$-hydroxy- $9 \beta$ -

$(\beta$-)furoyloxy-15-acetoxy- $\beta$-dihydroagarofuran, 6327

\section{$\mathrm{C}_{32} \mathrm{H}_{46} \mathrm{O}_{12}$}

Euphopubescene, 7613

\section{$\mathrm{C}_{32} \mathrm{H}_{46} \mathrm{O}_{13}$}

Taxchin A, 20768

Taxuspine L, 20859

\section{$\mathrm{C}_{32} \mathrm{H}_{46} \mathrm{O}_{14}$}

$\beta$ - $D$-Glucopyranosyl-( $1 \rightarrow 2)-\beta$ - $D$-glucopyranosyl-15,16-epoxy-12-oxo-8(17),13(16)-14-entlabdatrien-19-oate, 8641

Taxumain B, 20820

Taxumairol L, 20832

Taxuspine R, 20865

$\mathrm{C}_{32} \mathrm{H}_{46} \mathrm{O}_{16}$

19-Hydroxydeacetylnomilinic acid-17- $\beta$ - $D$-glucopyranoside, 9964

Javanicoside L, 11863

Yadanzioside H, 22867

$\mathrm{C}_{32} \mathrm{H}_{47} \mathrm{NO}_{8}$

Zygadenilic acid $\delta$-lactone-16-angelate, 23032

$\mathrm{C}_{32} \mathrm{H}_{48} \mathrm{~N}_{2} \mathrm{O}_{3}$

Avicennamine, 2034

$\mathrm{C}_{32} \mathrm{H}_{48} \mathrm{~N}_{2} \mathrm{O}_{4}$
(-)-Culantraraminol $\mathrm{N}$-oxide, 4349

$\mathrm{C}_{32} \mathrm{H}_{48} \mathrm{O}_{4}$

3 $\beta$-Acetoxy-12,19-dioxo-13(18)-oleanene, 174

$3 \beta$-Acetoxy-11 $\alpha, 12 \alpha$-epoxy-16-oxo-14-taraxerene, 188

$3 \beta$-Acetoxy-11-ursen-13 $\alpha, 30$-olide, 301

Ananosic acid B, 1137

Lanosta-7,9(11),24-trien-3 $\alpha$-acetoxy-26-oic acid, 12485

\section{$\mathrm{C}_{32} \mathrm{H}_{48} \mathrm{O}_{5}$}

$12 \alpha$-Acetoxycoccinic acid, 144

$12 \beta$-Acetoxycoccinic acid, 145

(25R)-3 $\beta$-Acetoxy-23-oxo-9, 16-lanostandien-

26-oic acid, 274

3-O-Acetyl-glycyrrhetinic acid, 403

Acetyl-11-keto- $\beta$-boswellic acid, 444

Cordianal A, 4041

Ganode-7,9-dien-ric acid X, 8148

$3 \beta$-Hydroxy-16 $\alpha$-acetoxy-lanosta-7,9(11),24trien-21-oic acid, 9758

Lanosta-7,9(11),24-trien-3 $\alpha$-acetoxy-15 $\alpha, 22 \beta$ dihydroxy-26-oic acid, 12482

(24Z)-3-Oxo-12 $\alpha$-acetoxylanosta-8,24-dien-26oic acid, 16290

Sendanone acetate, 19705

Tyromycic acid C, 22164

\section{$\mathrm{C}_{32} \mathrm{H}_{48} \mathrm{O}_{6}$}

Alisol C monoacetate, 896

Belamcandal, 2215

Camarinic acid, 3026

Cordiaketal A, 4039

Ganode-8-en-ric acid V, 8160

Poricoic acid DM, 17726

\section{$\mathrm{C}_{32} \mathrm{H}_{48} \mathrm{O}_{7}$}

Acantrifoic acid A, 91

12-O-Acetylphorbla-13-decanoate, 482

Dendrocyin, 5114

\section{$\mathrm{C}_{32} \mathrm{H}_{48} \mathrm{O}_{8}$}

12-O-Acetylphorbol-13-decanoate, 483

Acinospesigenin, 545

Cucurbitacin F 25-acetate, 4316

Cucurbitacin C, 4318

Dihydrocucurbitacin B, 5573

Dihydroisocucurbitacin B, 5649

Montanin, 14945

Phorbol-12-caprate-13-acetate, 17185

\section{$\mathrm{C}_{32} \mathrm{H}_{48} \mathrm{O}_{9}$}

Beauwalloside, 2190

Cerberin, 3416

Oleandrin, 16031

\section{$\mathrm{C}_{32} \mathrm{H}_{48} \mathrm{O}_{10}$}

Neritaloside, 15497

$\mathrm{C}_{32} \mathrm{H}_{48} \mathrm{O}_{12}$ 


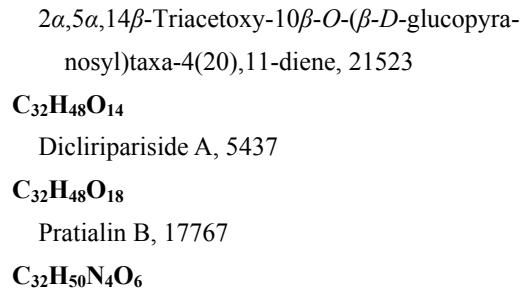

\section{$\mathrm{C}_{32} \mathrm{H}_{50} \mathrm{O}_{4}$}

$3 \beta$-Acetoxy-1 $\beta, 11 \alpha$-epidioxy-12-ursene, 178

$3 \beta$-Acetoxyolean-12-en-28-oic acid, 266

Acetylbetulinic acid, 332

(20S,22S,23E)-22-O-Acetyl-25-hydroxylanosta8,23(E)-dien-3-one, 422

$3 \beta$-O-Acetyl-mangiferolic acid, 462

3-O-Acetylursolic acid, 530

3,7-Dihydroxy-2,5-diundecylnaphthoquinone, 5867

3-Epi-betulinic acid acetate, 6836

$17 \beta$-Formyloxy-3 $\beta$-acetyloxy-28-nor-urs-12-ene, 7911

Garciosaterpene A, 8224

Lup-20(29)-ene-3 $\alpha$-acetoxy-24-oic acid, 13091

Niloticin acetate, 15599

16-Oxo-21 $\beta$-hydroxyserrat-14-en- $3 \alpha$-yl acetate, 16355

Tsugaric acid A, 22068

\section{$\mathrm{C}_{32} \mathrm{H}_{50} \mathrm{O}_{5}$}

$3 \beta$-Acetoxy-18 $\alpha$-hydroperoxy-12-oleanen-11-one, 203

$3 \alpha$-Acetoxy-25-hydroxyolean-12-en-28-oic acid, 231

Alisol B monoacetate, 894

25-Anhydroalisol A 11-acetate, 1257

25-Anhydroalisol A 24-acetate, 1258

Radermasinin, 18522

Rubiarbonone C', 18997

Rubiarbonone A, 19015

$\mathrm{C}_{32} \mathrm{H}_{50} \mathrm{O}_{6}$

25-O-Acetylcimigenol, 353

21-O-Acetyl toosendantriol, 527
Acinospesigenin A, 546

Alizexol A, 902

Cordiaketal B, 4040

Cordianone, 4044

Guavanoic acid, 9074

Rubianol B, 19002

$\mathrm{C}_{32} \mathrm{H}_{50} \mathrm{O}_{7}$

Alizexol B, 903

$\mathrm{C}_{32} \mathrm{H}_{50} \mathrm{O}_{8}$

Baliospermin, 2130

Dihydrocucurbitacin F-25-acetate, 5576

$\mathrm{C}_{32} \mathrm{H}_{50} \mathrm{O}_{\mathbf{9}}$

Hemslecin G, 9355

$\mathrm{C}_{32} \mathrm{H}_{50} \mathrm{O}_{11}$

Marginatoside B, 13562

$\mathrm{C}_{32} \mathrm{H}_{50} \mathrm{O}_{13}$

Cussoracoside C, 4425

$\mathrm{C}_{32} \mathrm{H}_{52} \mathrm{~N}_{2} \mathrm{O}_{4}$

Incarvine A, 11010

\section{$\mathrm{C}_{32} \mathrm{H}_{52} \mathrm{O}_{2}$}

$3 \beta$-Acetoxy-dammara-20,24-diene, 149

$\alpha$-Amyrin acetate, 1111

$\beta$-Amyrin acetate, 1112

Bauerenyl acetate, 2170

$D: C$-Friedomadeir-7-en-3 $\beta$-yl acetate, 7954

$D$-Friedomadeir-14-en-3 $\beta$-yl acetate, 7955

Isobauerenyl acetate, 11247

Isomultiflorenyl acetate, 11548

Lupeol acetate, 13100

(24R)-4 $\alpha$-Methyl-24-ethylcholesta-7,25-dien-3 $\beta$ -

yl acetate, 14434

Multiflor-7-ene-3 $\beta$-yl acetate, 15067

Nematocyphol acetate, 15330

31-Norcyclolaudenyl acetate, 15732

Olean-13(18)-en-3-acetate, 16035

Sablacaurin B, 19103

$\psi$-Taraxasteryl acetate, 20706

Taraxasteryl acetate, 20707

Taraxeryl acetate, 20713

\section{$\mathrm{C}_{32} \mathrm{H}_{52} \mathrm{O}_{3}$}

$3 \beta$-Acetoxy-25-hydroxydammara-20,23-diene, 212

$3 \beta$-Acetoxy-25-hydroxylanosta-8,23-diene, 228

$3 \beta$-Acetoxy-19(29)-taraxasten-20 $\alpha$-ol, 283

Acetylmyricadiol, 471

Betulin-3-acetate, 2332

$3 \beta$-Hydroxyserrat-14-en-21 $\alpha$-yl acetate, 10704

$21 \alpha$-Hydroxyserrat-14-en-3 $\beta$-yl acetate, 10705

$21 \beta$-Hydroxyserrat-14-en-3 $\beta$-yl acetate, 10706

Methyl eburicoate, 14333

Serratenediol-21-acetate, 19769

Serratenediol-3-acetate, 19770

Sophoradiol-22-O-acetate, 20084
Taraxaster-20-en-3 $\beta, 16 \alpha$-diol-3-acetate, 20703

$\mathrm{C}_{32} \mathrm{H}_{52} \mathrm{O}_{4}$

$3 \beta$-Acetoxy-21 $\alpha, 22 \alpha$-epoxytaraxastan-20 $\alpha$-ol, 189

(20S)-3 $\beta$-Acetoxylupan-29-oic acid, 249

$14 \beta, 15 \beta$-Epoxy- $3 \beta$-hydroxyserratan- $21 \alpha$-ol- $3 \beta$ -

$O$-acetate, 7142

2-n-Heneicosyl-5,7-dihydroxy-6,8-dimethyl chromone, 9361

Methyl 2 $\alpha$-methoxyursolate, 14591

Phlegmanol D, 17150

Rubiprasin B, 19038

Tumulosic acid methyl ester, 22109

\section{$\mathrm{C}_{32} \mathrm{H}_{52} \mathrm{O}_{5}$}

$3 \beta$-Acetoxy-12 $\beta, 13 \beta$-epoxy-11 $\alpha$-hydroperoxyursane, 183

$3 \beta$-Acetoxy-11 $\alpha$-hydroperoxy-13 $\alpha H$-ursan-12one, 204

16-O-Acetyl isoiridogermanal, 439

$21 R, 23 R$-Epoxy,21 $\alpha$-ethoxy, 24S,25-dihydroxyapotirucalla-7-en-3-one, 7094

Rubianol C, 19003

Rubiarbonol D, 19013

Rubiarbonol G, 19014

Rubiprasin A, 19037

\section{$\mathrm{C}_{32} \mathrm{H}_{52} \mathrm{O}_{6}$}

Alisol A monoacetate, 892

Alisol E 23-acetate, 897

Alisol E 24-acetate, 898

Fomitopinic acid B, 7861

Rubianol D, 19004

$7 \alpha, 21 S, 25$-Trihydroxy-3 $\beta$-acetoxy- $21 S, 23 R$ epoxy-9(11)-en-dammarane, 21684

\section{$\mathrm{C}_{32} \mathrm{H}_{52} \mathrm{O}_{7}$}

$13 \beta, 17 \beta$-Epoxyalisol A 24-acetate, 7051

\section{$\mathrm{C}_{32} \mathrm{H}_{52} \mathrm{O}_{8}$}

Yononin, 22925

$\mathrm{C}_{32} \mathrm{H}_{52} \mathrm{O}_{9}$

Convallasaponin A, 4008

\section{$\mathrm{C}_{32} \mathrm{H}_{52} \mathrm{O}_{10}$}

Convallasaponin B, 4009

\section{$\mathrm{C}_{32} \mathrm{H}_{52} \mathrm{O}_{11}$}

Wattoside G, 22653

\section{$\mathrm{C}_{32} \mathrm{H}_{52} \mathrm{O}_{14}$}

Acantrifoside D, 93

\section{$\mathrm{C}_{32} \mathrm{H}_{54} \mathrm{~N}_{2} \mathrm{O}_{3}$}

(-)-Buxahejramine, 2813

(-)-Buxakarachiamine, 2814

\section{$\mathrm{C}_{32} \mathrm{H}_{54} \mathrm{O}$}

Cyclolaudenone, 4504

Cycloneolitsol, 4519

(24S)-24-Methyl-25,32-cyclo-cycloartane-3 $\beta$-ol, 14268 
(24S)-24-Methyl-25,32-cyclo-5 $\alpha$-lanosta9(11)-en-3 $\beta$-ol, 14273

\section{$\mathrm{C}_{32} \mathrm{H}_{54} \mathrm{O}_{2}$}

Epifriedelinol acetate, 6919

Friedelan-3 $\alpha$-yl acetate, 7952

\section{$\mathrm{C}_{32} \mathrm{H}_{54} \mathrm{O}_{4}$}

$24 R$-Acetoxy-3 $\beta, 25$-dihydroxycycloartane, 162

$3 \beta$-Acetyl-20,25-epoxydammarane- $24 \alpha$-ol, 386

$3 \beta$-Acetyl-20,25-epoxydammarane-24 $\beta$-ol, 387

$\mathrm{C}_{32} \mathrm{H}_{54} \mathrm{O}_{5}$

$3 \beta$-Acetoxy-12 $\beta, 16 \beta, 20 S$-trihydroxydammar-

24-ene, 294

$3 \beta$-Acetoxy-16 $\beta, 20 S, 25$-trihydroxydammar-

23-ene, 295

\section{$\mathrm{C}_{32} \mathrm{H}_{54} \mathrm{O}_{6}$}

(20S)-3 $\beta$-Acetoxy-12 $\beta, 16 \beta, 25$-tetrahydroxy-

dammar-23-ene, 286

\section{$\mathrm{C}_{32} \mathrm{H}_{54} \mathrm{O}_{13}$}

Neodarutoside, 15390

\section{$\mathrm{C}_{32} \mathrm{H}_{54} \mathrm{O}_{14}$}

Pyishiauoside $\mathrm{II}_{\mathrm{b}}, 18238$

\section{$\mathrm{C}_{32} \mathrm{H}_{54} \mathrm{O}_{16}$}

Atractyloside E, 1978

\section{$\mathrm{C}_{32} \mathrm{H}_{55} \mathrm{O}_{5}$}

RubiarbononeC, 19017

\section{$\mathrm{C}_{32} \mathrm{H}_{56} \mathrm{O}_{4}$}

Mollugogenol D, 14904

\section{$\mathrm{C}_{32} \mathrm{H}_{56} \mathrm{O}_{7}$}

14-Deacetyleurylene, 4740

\section{$\mathrm{C}_{32} \mathrm{H}_{57} \mathrm{NO}_{2}$}

Violyedoenamide, 22522

\section{$\mathrm{C}_{32} \mathrm{H}_{60} \mathrm{O}_{4}$}

Nodolidate, 15651

Triacontanedioic acid dimethyl ester, 21539

\section{$\mathrm{C}_{32} \mathrm{H}_{63} \mathrm{NO}_{3}$}

(4E,2S,3R)-2-N-Octadecanoyl-4-tetradecasphingenine, 15952

\section{$\mathrm{C}_{32} \mathrm{H}_{64} \mathrm{O}_{2}$}

Dotriacontanic acid, 6575

Triacontanyl acetate, 21543

\section{$\mathrm{C}_{33} \mathrm{H}_{22} \mathrm{O}_{10}$}

Thelephantin H, 21300

\section{$\mathrm{C}_{33} \mathrm{H}_{24} \mathrm{O}_{8}$}

Thelephantin E, 21297

\section{$\mathrm{C}_{33} \mathrm{H}_{24} \mathrm{O}_{9}$}

Thelephorin A, 21307

\section{$\mathrm{C}_{33} \mathrm{H}_{24} \mathrm{O}_{10}$}

Heveaflavone, 9473

Kayaflavone, 12185

Sciadopitysin, 19514

Stephaflavone B, 20315

Taiwanhomoflavone A, 20625

7,4',7"-Tri-O-methyl amentoflavone, 21938

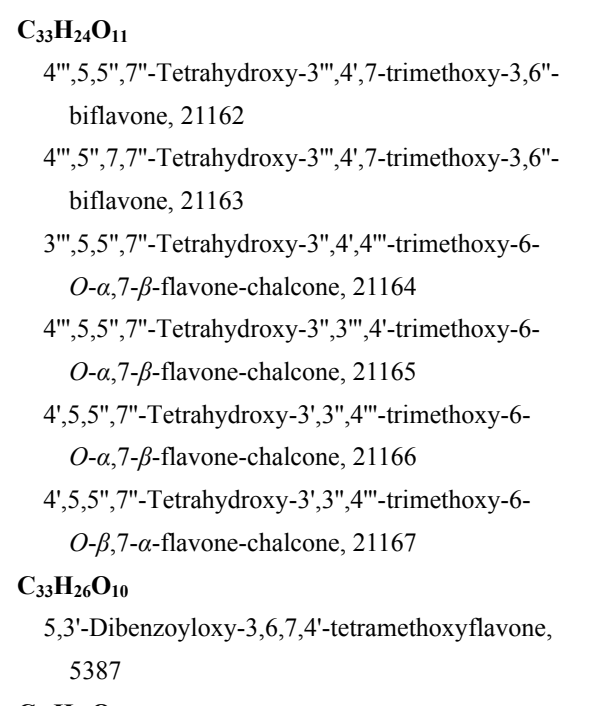

$\mathrm{C}_{33} \mathrm{H}_{26} \mathrm{O}_{17}$

Swertipunicoside, 20514

\section{$\mathrm{C}_{33} \mathrm{H}_{28} \mathrm{~N}_{2} \mathrm{O}_{6}$}

Kurramine-2'- $\alpha$ - $N$-oxide, 12351

Kurramine-2'- $\beta$ - $N$-oxide, 12352

\section{$\mathrm{C}_{33} \mathrm{H}_{28} \mathrm{O}_{9}$}

Dehydroxyhexaspermone C, 4985

\section{$\mathrm{C}_{33} \mathrm{H}_{28} \mathrm{O}_{10}$}

Chamaejasmenin C, 3460

Hexaspermone C, 9518

Ruixianglangdusu A, 19063

Ruixianglangdusu B, 19064

\section{$\mathrm{C}_{33} \mathrm{H}_{28} \mathrm{O}_{24}$ \\ Mallonin, 13423 \\ $\mathrm{C}_{33} \mathrm{H}_{30} \mathrm{O}_{6}$}

BF-6, 2341

2-[4,5-Dimethoxy-5-(3-phenyl-trans-allyl)cyclohexa-3,6-dien-2-on-1-ylmethyl]-5-hydroxy-6methoxy-3-phenylbenzofuran, 6275

2-[4,5-Dimethoxy-2-(3-phenyl-trans-allyloxy) benzyl]-5-hydroxy-6-methoxy-3-phenylbenzofuran, 6276

Magnolignan I, 13386

\section{$\mathrm{C}_{33} \mathrm{H}_{30} \mathbf{O}_{8}$}

rel-1 $\beta$-(4,6-Dihydroxy-2-methoxy)-benzoyl-rel$2 \alpha$-(2,6-dimethoxy-4-hydroxy)-benzoyl-rel-

(3 $\beta, 4 \alpha)$-diphenylcyclobutane, 5966

\section{$\mathrm{C}_{33} \mathrm{H}_{30} \mathrm{O}_{9}$}

Sanggenon B, 19256

$\mathrm{C}_{33} \mathrm{H}_{30} \mathbf{O}_{10}$

Curtisian J, 4406

Dahuribirin A, 4597

$\mathrm{C}_{33} \mathrm{H}_{30} \mathrm{O}_{11}$

Dahuribirin C, 4599

\section{$\mathrm{C}_{33} \mathrm{H}_{30} \mathrm{O}_{15}$}

1,2,6-Tri- $O$ - $(E)$-caffeoyl- $\beta$ - $D$-glucopyranose, 21551

\section{$\mathrm{C}_{33} \mathrm{H}_{32} \mathbf{N}_{2} \mathbf{O}_{15}$}

Gomphrenin III, 8929

\section{$\mathrm{C}_{33} \mathrm{H}_{32} \mathrm{O}_{8}$}

Piperaduncin C, 17432

Thelephantin F, 21298

$\mathrm{C}_{33} \mathrm{H}_{32} \mathrm{O}_{9}$

Occidentalol I, 15914

\section{$\mathrm{C}_{33} \mathrm{H}_{32} \mathrm{O}_{10}$}

Tricolorin A, 21591

\section{$\mathrm{C}_{33} \mathrm{H}_{34} \mathrm{~N}_{4} \mathrm{O}_{3}$}

Pyropheophorbide a, 18272

\section{$\mathrm{C}_{33} \mathrm{H}_{34} \mathrm{~N}_{4} \mathrm{O}_{6}$}

Biliverdin, 2375

\section{$\mathrm{C}_{33} \mathrm{H}_{35} \mathrm{~N}_{5} \mathrm{O}_{5}$}

Ergotamine, 7252

Ergotaminine, 7253

$\mathrm{C}_{33} \mathrm{H}_{35} \mathrm{O}_{23}{ }^{+}$

Delphinidin-3,5-di- $O$-(6- $O$-malonyl- $\beta$ - $D$-glucoside), 5021

\section{$\mathrm{C}_{33} \mathrm{H}_{36} \mathrm{~N}_{4} \mathrm{O}_{6}$}

Bilirubin, 2374

\section{$\mathrm{C}_{33} \mathrm{H}_{36} \mathrm{O}_{5}$}

Humuladien-10-yl-(3-Hydroxy-5-oxo-4-phenyl$5 H$-furan-2-ylidene)-phenylacetic acid-ester, 9666

\section{$\mathrm{C}_{33} \mathrm{H}_{36} \mathrm{O}_{6}$}

(3-Hydroxy-5-oxo-4-phenyl-5H-furan-2-ylidene)-phenylacetic Acid 6-hydroxy-1,7(11)humuladienyl-10-yl ester, 10579

\section{$\mathrm{C}_{33} \mathrm{H}_{36} \mathrm{O}_{7}$}

Isomorellin, 11541

Morellin, 14968

\section{$\mathrm{C}_{33} \mathrm{H}_{36} \mathrm{O}_{8}$}

Isomorellic acid, 11540

Morellic acid, 14967

\section{$\mathrm{C}_{33} \mathrm{H}_{36} \mathrm{O}_{11}$}

Triptofordin $\mathrm{B}_{2}, 22000$

\section{$\mathrm{C}_{33} \mathrm{H}_{36} \mathrm{O}_{19}$}

Quercetin-3-O-[(2,3,4-triacetyl- $\alpha$-rhamnopyranosyl)-( $1 \rightarrow 6)]$ - $\beta$-galactopyranoside, 18403

\section{$\mathrm{C}_{33} \mathrm{H}_{38} \mathrm{~N}_{4} \mathrm{O}_{4}$}

Condaline A, 3962

Scutianine M, 19595

\section{$\mathrm{C}_{33} \mathrm{H}_{38} \mathrm{O}_{6}$}

Deoxymorellin, 5196

$\mathrm{C}_{33} \mathrm{H}_{38} \mathrm{O}_{7}$

Dihydroisomorellin, 5651

Gaudichaudione A, 8239

$\mathrm{C}_{33} \mathrm{H}_{38} \mathrm{O}_{8}$

$5 \alpha$-Acetoxy- $1 \beta$-benzoyl- $8 \alpha$-cinnamoyl- $4 \alpha$-hydroxy-dihydroagarofuran, 130

Gaudichaudic acid, 8238 
$\mathrm{C}_{33} \mathrm{H}_{38} \mathrm{O}_{9}$

$1 \beta, 6 \alpha$-Diacetoxy- $8 \beta, 9 \beta$-dibenzoyloxy- $\beta$-dihydroagarofuran, 5292

Orbiculin A, 16162

\section{$\mathrm{C}_{33} \mathrm{H}_{38} \mathrm{O}_{10}$}

Salasol B, 19179

\section{$\mathrm{C}_{33} \mathrm{H}_{38} \mathrm{O}_{11}$}

$1 S, 13$-Diacetyloxy-4S-hydroxy-6R-(3-)furancarbonyloxy-9S-cinnamoyloxy- $\beta$-dihydroagarofuran, 5344

Schizanrin H, 19502

Triptofordin $\mathrm{C}_{2}, 22001$

Triptofordin E, 22004

\section{$\mathrm{C}_{33} \mathrm{H}_{38} \mathrm{O}_{17}$ \\ 3-O-p-Coumaroyl-6- $O$-feruloyl- $\beta$ - $D$-fructo- furanosyl 6- $O$-acetyl- $\alpha-D$-glucopyranoside, 4157}

\section{$\mathrm{C}_{33} \mathrm{H}_{38} \mathrm{O}_{21}$}

Kaempferol 3-O- $\beta$-(2"-O- $\alpha$-rhamnopyranosyl3 "- $O$ - $\beta$-glucopyranosyl)- $\beta$-glucuronopyranoside, 12077

\section{$\mathrm{C}_{33} \mathrm{H}_{40} \mathrm{~N}_{2} \mathrm{O}_{9}$}

Renoxidine, 18623

Reserpine, 18633

\section{$\mathrm{C}_{33} \mathrm{H}_{40} \mathrm{O}_{6}$}

Deoxygaudichaudione A, 5175

\section{$\mathrm{C}_{33} \mathrm{H}_{40} \mathrm{O}_{8}$}

2,9-Diacetyl-5-cinnamoylphototaxicin II, 5329

2,10-Diacetyl-5-cinnamoylphototaxicin II, 5330

Morinol A, 14976

Morinol B, 14977

\section{$\mathrm{C}_{33} \mathrm{H}_{40} \mathrm{O}_{9}$}

5-Cinnamoyl-9,10-diacetyltaxicin I, 3701

2,10-Diacetyl-5-cinnamoyl-7 $\beta$-hydroxy

phototaxicin II, 5328

Taxuspinanane $\mathrm{H}, 20847$

\section{$\mathrm{C}_{33} \mathrm{H}_{40} \mathbf{O}_{\mathbf{1 0}}$}

$\left(2 R^{*}, 3 S^{*}, 4 R^{*}, 5 R^{*}, 8 R^{*}, 13 S^{*}, 15 R^{*}\right)-5,8,15$-Triacetoxy-3-benzoyloxy-9,14-dioxojatropha6(17),11E-diene, 21513

\section{$\mathrm{C}_{33} \mathrm{H}_{40} \mathrm{O}_{11}$}

Acetoxytoonacilin, 290

Euphopubescenol, 7614

Orthosiphol J, 16228

Taxuspinanane B, 20842

\section{$\mathrm{C}_{33} \mathrm{H}_{40} \mathrm{O}_{14}$}

Anhydroicaritin-3- $O-\alpha-L$-rhamnopyranosyl$(1 \rightarrow 2)-\alpha$ - $L$-rhamnopyranoside, 1274

Linarin isovalerate, 12855

2"-O-Rhamnosyl icariside II, 18741

Sandrapin A, 19240

\section{$\mathrm{C}_{33} \mathrm{H}_{40} \mathrm{O}_{15}$}

Anhydroicaritin-3- $O-\alpha-L$-rhamnosyl-7- $O-\beta-D-$ glucopyranoside, 1275

Baohuoside VII, 2143

$\mathrm{C}_{33} \mathrm{H}_{40} \mathrm{O}_{16}$

Tabulalide C, 20583

$\mathrm{C}_{33} \mathrm{H}_{40} \mathrm{O}_{17}$

Glomeratose C, 8584

\section{$\mathrm{C}_{33} \mathrm{H}_{40} \mathrm{O}_{18}$}

Arillanin A, 1681

Genistein 7- $O-\alpha-L$-rhamnopyranoside-4'- $O$ $[(\alpha-L$-rhamnopyranosyl)-( $1 \rightarrow 2)-\beta$ - $D$-glucopyranoside], 8285

Kaempferol-3-O-rhamnosyl-7-rhamnosyl$(1 \rightarrow 3)$-rhamnoside, 12089

3'-Sinapoyl-6-feruloylsucrose, 19919

\section{$\mathrm{C}_{33} \mathrm{H}_{40} \mathrm{O}_{19}$}

Clitorin, 3846

5,4'-Dihydroxyflavone-6- $C$ - $\beta$ - $D$-glycosylrhamnoside-7-O-glycoside, 5896

4',5-Dihydroxy-7-methoxyflavonol 3-O- $\beta-D-$ xylopyranosyl-( $(1 \rightarrow 2)-O-[\alpha-L$-rhamnopyranos-

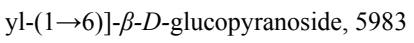

Genistein 7-O- $\beta$ - $D$-glucopyranoside-4' $O-[(\alpha-L-$ rhamnopyranosyl)-(1 $\rightarrow 2)-\beta$ - $D$-glucopyranoside], 8281

Genistein 7-O- $\alpha-L$-rhamnopyranoside-4'- $O$-[ $(\beta-$ $D$-glucopyranosyl)-(1 $\rightarrow 2)$ - $\beta$ - $D$-glucopyranoside], 8284

1-[( $\beta$ - $D$-Glucopyranosyl-( $1 \rightarrow 3)-O-\beta$ - $D$-glucopyranosyl-(1 $\rightarrow 6)-O-\beta$ - $D$-glucopyranosyl)oxy]8-hydroxy-3-methyl-9,10-anthraquinone, 8645

Kaempferol 3- $O-\alpha-L$-rhamnopyranosyl- $(1 \rightarrow 2)-\beta$ $D$-galactopyranosyl-7- $O-\alpha-L$-rhamnopyranoside, 12075

Kaempferol-3- $O$-[ $\alpha$-rhamnopyranosyl- $(1 \rightarrow 4)$ rhamnopyranosyl-( $1 \rightarrow 6)-\beta$-galactopyranoside], 12080

Oxytroside, 16479

Robinin, 18859

\section{$\mathrm{C}_{33} \mathrm{H}_{40} \mathrm{O}_{20}$}

Genistein 7- $O-\beta$ - $D$-glucopyranoside-4'- $O$-[( $\beta$ $D$-glucopyranosyl)-( $1 \rightarrow 2)-\beta$ - $D$-glucopyranoside], 8280

Isorhamnetin 3- $O-\beta-D$-apiofuranosyl-(1"' $\rightarrow 2$ ") $[\alpha-$ $L$-rhamnopyranosyl-(1"' $\rightarrow 6$ ")]- $\beta$ - $D$-galactopyranoside, 11649

Isorhamnetin 3- $O-\beta-D$-xylopyranosyl-(1"' $\rightarrow 3$ "')$\alpha$ - $L$-rhamnopyranosyl-(1"' $\rightarrow 6$ ")- $\beta$ - $D$-galactopyranoside, 11671

Kaempferol 3- $O$ - $\{\beta$ - $D$-glucopyranosyl-( $(1 \rightarrow 2)-[\alpha$ $L$-rhamonopyranosyl-( $(1 \rightarrow 6)]-\beta$ - $D$-galactopyranoside\}, 12019

Kaempferol 3-O- $\alpha$-(2,3-di-O- $\beta$ - $D$-glucopyranosyl) rhamnopyranoside, 12041
Kaempferol 3-O- $\alpha-L-(2-O-\beta-D$-glucopyranosyl) rhamnopyranoside-7- $O-\beta$ - $D$-glucopyranoside, 12055

Kaempferol 3- $O$ - $\alpha$-rhamnoside-7,4'-di- $O$ - $\beta$ galactoside, 12084

Kaempferol-3-rhamnosyl glucoside-7-glucoside, 12087

Manghaslin, 13479

Quercetin-3-O-(2",6"- $\alpha$ - $L$-dirhamnopyranosyl)$\beta$-D-galactopyranoside, 18351

Quercetin-3- $O$ - $[\alpha$-rhamnopyranosyl- $(1 \rightarrow 4)$ rhamnopyranosyl-( $(\rightarrow 6)]$ - $\beta$-galactopyranoside, 18385

Quercetin-3-O-[ $\alpha$-rhamnopyranosyl- $(1 \rightarrow 4)-\alpha$ rhamnopyranosyl-( $1 \rightarrow 6)$ - $\beta$-glucopyranoside], 18386

Quercetin-3-rutinoside-7-rhamnoside, 18392

\section{$\mathrm{C}_{33} \mathrm{H}_{40} \mathrm{O}_{21}$}

Aescuflavoside A, 662

Kaempferol-3-diglucose-7-glucoside, 12042

Kaempferol 3- $O-\beta$ - $D$-glucosyl $(1 \rightarrow 2)-\beta$ - $D$-galactoside 7-O- $\beta$ - $D$-glucoside, 12064

Kaempferol-3- $O$-sophoroside-7- $O$ - $\beta$-glucopyranoside, 12095

Kaempferol-3-sophorotrioside, 12097

Kaempferol-3,7,4'-tri- $O$ - $\beta$-glucoside, 12100

Myricetin-3- $O$-(2",6"-di- $O$ - $\alpha$-rhamnosyl)- $\beta$ glucoside, 15175

Peruvianoside III, 17001

Quercetin-3- $O-\alpha-L-(2-O-\beta-D$-glucopyranosyl) rhamnopyranoside-7- $O-\beta$ - $D$-glucopyranoside, 18366

Quercetin-3-rutinoside-7-glucoside, 18391

Quercetin-3-rutinosyl-7-galactoside, 18393

\section{$\mathrm{C}_{33} \mathrm{H}_{40} \mathrm{O}_{22}$}

Herbacetin-3- $\beta$ - $D$-(2- $O-\beta$ - $D$-glucopyranosylglucopyranoside)-8- $\beta$ - $D$-glucopyranoside, 9422

Moracetin, 14953

Myricetin-3-O- $\alpha-L-(2-O-\beta$ - $D$-glucopyranosyl) rhamnopyranoside-7- $O-\beta$ - $D$-glucopyranoside, 15182

Quercetin-3- $O-\beta$ - $D$-glucosyl $(1 \rightarrow 2)-\beta$ - $D$-galactoside 7-O- $\beta$ - $D$-glucoside, 18370

Quercetin-3-sophorotrioside, 18398

Quercetin-3,3',4'-tri- $O-\beta$ - $D$-glucopyranoside, 18404

Quercetin-5,7,4'-tri- $O-\beta$ - $D$-glucopyranoside, 18405

\section{$\mathrm{C}_{33} \mathrm{H}_{40} \mathrm{O}_{23}$}

Gossypetin-3- $\beta$ - $D$-(2-O- $\beta$ - $D$-glucopyranosylglucopyranoside)-8- $\beta$ - $D$-glucopyranoside, 8962 


\section{$\mathrm{C}_{33} \mathrm{H}_{41} \mathrm{NO}_{8}$}

Lythrancine IV, 13275

Lythrancine V, 13276

\section{$\mathrm{C}_{33} \mathrm{H}_{41} \mathrm{O}_{20}{ }^{+}$}

Cyanidin-3-rutinoside-5-glucoside, 4447

Cyanidin-3- $O$ - $\beta$-rutinoside-7- $O-\beta$-glucoside, 4448

Raphanusin, 18548

$\mathrm{C}_{33} \mathrm{H}_{42} \mathrm{~N}_{4}$

Auricularine, 2013

$\mathrm{C}_{33} \mathrm{H}_{42} \mathrm{~N}_{4} \mathrm{O}_{6}$

Urobilin, 22259

\section{$\mathrm{C}_{33} \mathbf{H}_{42} \mathbf{N}_{6} \mathbf{O}_{7}$}

Cherimolacyclopeptide E, 3514

\section{$\mathrm{C}_{33} \mathrm{H}_{42} \mathrm{O}_{4}$}

Kolanone, 12256

\section{$\mathrm{C}_{33} \mathrm{H}_{42} \mathrm{O}_{5}$}

Garcinielliptone K, 8213

Hypercalin B, 10881

\section{$\mathrm{C}_{33} \mathrm{H}_{42} \mathrm{O}_{6}$}

Garciosaphenone A, 8223

\section{$\mathrm{C}_{33} \mathrm{H}_{42} \mathrm{O}_{7}$}

Dantaxusin B, 4637

$9 \alpha, 10 \beta$-Diacetoxy- $5 \alpha$-cinnamoyloxytaxa4(20),11-dien-13 $\alpha$-ol, 5289

\section{$\mathrm{C}_{33} \mathrm{H}_{42} \mathrm{O}_{8}$}

$5 \alpha$-Cinnamoyloxy- $2 \alpha, 13 \alpha$-dihydroxy- $9 \alpha, 10 \beta$ diacetoxy-4(20),11-taxadiene, 3714

2-Deacetoxy-7,9-dideacetyltaxinine J, 4705 Japonicin B, 11818

\section{$\mathrm{C}_{33} \mathrm{H}_{42} \mathrm{O}_{9}$}

10 $\beta$-Deacetylspicatine, 4773

Euphoscopin B, 7619

Pubescene A, 18170

Quivisianthone, 18453

\section{$\mathrm{C}_{33} \mathrm{H}_{42} \mathrm{O}_{10}$}

13-Acetylbrevifoliol, 337

$2 \alpha$-Benzoyloxy- $9 \alpha, 10 \beta, 13 \alpha$-triacetoxy- $1 \beta, 5 \alpha$ dihydroxy-4(20),11-taxadiene, 2265

$7 \beta, 16 \alpha$-Diacetoxy withanolide D, 5324

Euphoheliosnoid C, 7610

Paraliane 13, 16653

Taxawallin D, 20754

\section{$\mathrm{C}_{33} \mathrm{H}_{42} \mathrm{O}_{11}$}

13-Acetyl-9-deacetyl-9-benzoyl-10-debenzoyltaxchinin A, 363

Orthosiphol U, 16236

Taxchinin A, 20770

\section{$\mathrm{C}_{33} \mathrm{H}_{42} \mathrm{O}_{12}$}

15-Benzoyl-2-debenzoyl-7,9-dideacetyl-abeobaccatin VI, 2237

7,9-Deacetylbaccatin VI, 4726

13-Decinnamoyl-9-deacetyltaxchinin B, 4851
10-( $\beta$-Hydroxybutyryl)-10-deacetylbaccatin I, 9865

Tasumatrol G, 20722

Taxayuntin E, 20763

Taxayuntin F, 20764

Taxumairol H, 20828

Taxuyunnanine E, 20877

$\mathrm{C}_{33} \mathrm{H}_{42} \mathrm{O}_{13}$

Taxumairol Z, 20839

$\mathrm{C}_{33} \mathrm{H}_{42} \mathrm{O}_{14}$

Woorenoside III, 22729

$\mathrm{C}_{33} \mathrm{H}_{42} \mathrm{O}_{16}$

Wiedemannioside A, 22662

$\mathrm{C}_{33} \mathrm{H}_{42} \mathrm{O}_{19}$

Naringenin-4'-glucoside-7-neohesperidoside, 15282

Naringenin-4'-glucoside-7-rutinoside, 15283

$\mathrm{C}_{33} \mathrm{H}_{43} \mathrm{ClO}_{14}$

Juncin O, 11955

$\mathrm{C}_{33} \mathrm{H}_{43} \mathrm{NO}_{11}$

Aldohypaconitine, 877

$\mathrm{C}_{33} \mathrm{H}_{43} \mathrm{~N}_{5} \mathrm{O}_{4}$

Amphibine A, 1080

Waltherine B, 22633

\section{$\mathrm{C}_{33} \mathrm{H}_{43} \mathrm{~N}_{5} \mathrm{O}_{5}$}

$O$-12'-Methyl- $\alpha$-ergokryptine, 14412

$O$-12'-Methyl- $\alpha$-ergokryptinine, 14413

\section{$\mathrm{C}_{33} \mathrm{H}_{44} \mathrm{O}_{4}$}

Eudesobovatol A, 7517

$\mathrm{C}_{33} \mathrm{H}_{44} \mathrm{O}_{6}$

rel-Labd-12-en-15(16)-olid-7-one-8R-spiro-1'-

[2S-(2,4,5-trimethoxyphenyl)-3-cyclohexene], 12419

\section{$\mathrm{C}_{33} \mathrm{H}_{44} \mathrm{O}_{8}$}

Sarothralen A, 19385

\section{$\mathrm{C}_{33} \mathrm{H}_{44} \mathrm{O}_{9}$}

1-Deacetylnimbolinin B, 4756

Methyl ganoderate F, 14457

\section{$\mathrm{C}_{33} \mathrm{H}_{44} \mathrm{O}_{10}$}

Ardisiaquinone I, 1637

$\mathrm{C}_{33} \mathrm{H}_{44} \mathrm{O}_{12}$

Tasumatrol F, 20721

$\mathrm{C}_{33} \mathrm{H}_{44} \mathrm{O}_{13}$

Tasumatrol E, 20720

\section{$\mathrm{C}_{33} \mathrm{H}_{44} \mathrm{O}_{14}$}

2',7-Dihydroxy-4'-methoxy-8-prenylflavan

2',7-di- $O-\beta$ - $D$-glucopyranoside, 6004

\section{$\mathrm{C}_{33} \mathrm{H}_{44} \mathbf{O}_{15}$}

$1 \alpha, 2 \alpha, 6 \beta, 15$-Tetraacetoxy-3 $\alpha$-( $\alpha$-methyl)-butanoyl-4 $\beta$-hydroxy- $9 \beta$-( $\beta$-)furoyloxy- $\beta$-dihydroagarofuran, 21031

\section{$\mathrm{C}_{33} \mathrm{H}_{44} \mathrm{O}_{16}$}

Arctigenin 4'-gentiobioside, 1622
Javanicoside F, 11859

\section{$\mathrm{C}_{33} \mathrm{H}_{44} \mathbf{O}_{17}$}

(+)-Medioresinol di- $O$ - $\beta$ - $D$-glucopyranoside, 13641

Syringaresinol-4- $O-\beta$ - $D$-apiofuranosyl-( $(1 \rightarrow 2)$ $\beta$ - $D$-glucopyranoside, 20557

Trachelogenin 4'-O- $\beta$-gentiobioside, 21492

\section{$\mathrm{C}_{33} \mathrm{H}_{44} \mathbf{O}_{20}$}

Adinoside D, 639

Adinoside E, 640

Epigeoside, 6925

$\mathrm{C}_{33} \mathrm{H}_{45} \mathrm{ClO}_{14}$

Gemmacolide B, 8265

\section{$\mathrm{C}_{33} \mathrm{H}_{45} \mathrm{NO}_{6}$}

Leueantine $\mathrm{C}, 12730$

\section{$\mathrm{C}_{33} \mathrm{H}_{45} \mathrm{NO}_{7}$}

8-O-Cinnamoylneoline, 3712

14-O-Cinnamoylneoline, 3713

2-Deacetyl-9-acetoxytaxine B, 4711

2-Deacetyl-10-acetyltaxine B, 4713

Kongboendine, 12259

Leueantine D, 12731

\section{$\mathrm{C}_{33} \mathrm{H}_{45} \mathrm{NO}_{8}$}

Taxine B, 20795

\section{$\mathrm{C}_{33} \mathrm{H}_{45} \mathrm{NO}_{9}$}

2-Deacetyltaxine A, 4780

Delphinine, 5032

Isodelphinine, 11373

\section{$\mathrm{C}_{33} \mathrm{H}_{45} \mathrm{NO}_{10}$}

Hypaconitine, 10875

\section{$\mathrm{C}_{33} \mathrm{H}_{45} \mathrm{NO}_{11}$}

Mesaconitine, 13794

\section{$\mathrm{C}_{33} \mathrm{H}_{45} \mathrm{NO}_{12}$}

Beiwutine, 2214

\section{$\mathrm{C}_{33} \mathrm{H}_{46} \mathrm{~N}_{8} \mathrm{O}_{8}$}

Pseudostellarin B, 18065

\section{$\mathrm{C}_{33} \mathrm{H}_{46} \mathrm{O}_{8}$}

Phorbol-12-tiglate-13-caprylenate, 17193

\section{$\mathrm{C}_{33} \mathrm{H}_{46} \mathrm{O}_{10}$}

Ostodin, 16262

\section{$\mathrm{C}_{33} \mathrm{H}_{46} \mathrm{O}_{13}$}

Taxuspine Q, 20864

\section{$\mathrm{C}_{33} \mathrm{H}_{46} \mathrm{O}_{15}$}

Myricanol gentiobioside, 15162

\section{$\mathrm{C}_{33} \mathrm{H}_{46} \mathrm{O}_{17}$}

(+)-Lyoniresinol-3 $\alpha-O-\beta$ - $D$-apiofuranosyl$(1 \rightarrow 2)-\beta$ - $D$-glucopyranoside, 13251

\section{$\mathrm{C}_{33} \mathrm{H}_{46} \mathbf{O}_{19}$}

6'-O-(7 $\alpha$-Hydroxyswerosyloxy)loganin, 10735

\section{$\mathrm{C}_{33} \mathrm{H}_{46} \mathbf{O}_{20}$}

Randinoside, 18542

$\mathrm{C}_{33} \mathrm{H}_{47} \mathrm{ClO}_{12}$

Juncenolide F, 11953 


\section{$\mathrm{C}_{33} \mathrm{H}_{47} \mathrm{NO}_{2}$ \\ Emindole PA, 6773 \\ Emindole PB, 6774 \\ Emindole PC, 6775 \\ $\mathrm{C}_{33} \mathrm{H}_{47} \mathrm{NO}_{9}$}

8-Deacetylsungpanconitine, 4774

Neojiangyouaconitine, 15418

14-O-Veratroylneoline, 22394

\section{$\mathrm{C}_{33} \mathrm{H}_{47} \mathrm{NO}_{10}$}

14-Benzoyl-8-O-methyl-aconine I, 2248

8-Deacetylyunaconitine, 4792

\section{$\mathrm{C}_{33} \mathrm{H}_{48} \mathrm{O}_{\mathbf{5}}$}

3-O-Acetyl-methyl-(24E)-3 $\alpha, 16 \alpha, 23 \alpha(=16 R$,

$23 R$ )-trihydroxy-epoxy-17,14-friedolan-8,14,

24-trien-26-oate, 467

Fomefficinic acid E, 7859

Fomitopsic acid B, 7862

\section{$\mathrm{C}_{33} \mathrm{H}_{48} \mathrm{O}_{6}$}

Inocalophyllin B methyl ester, 11066

\section{$\mathrm{C}_{33} \mathrm{H}_{48} \mathrm{O}_{7}$}

Revandchinone 4, 18672

\section{$\mathrm{C}_{33} \mathrm{H}_{48} \mathrm{O}_{8}$}

Phorbol-12- $\alpha$-methylbutyrate-13-caprylenate, 17189

\section{$\mathrm{C}_{33} \mathrm{H}_{48} \mathrm{O}_{9}$}

$12 \beta$-Acetoxy-3 $\beta$-hydroxy-24,25,26,27-tetranorcycloart-7-en-23,16 $\beta$-olide 3-O- $\beta$ - $D$-xylopyranoside, 238

\section{$\mathrm{C}_{33} \mathrm{H}_{48} \mathrm{O}_{10}$}

Caseamembrin D, 3261

$2 \alpha$-( $\alpha$-Methylbutyryl)-oxy- $5 \alpha, 7 \beta, 9 \alpha, 10 \beta$-tetraacetoxy-4(20),11-taxadiene, 14198

Taxuyunnanine B, 20874

\section{$\mathrm{C}_{33} \mathrm{H}_{49} \mathrm{NO}_{8}$}

Pseudojervine, 18030

\section{$\mathrm{C}_{33} \mathrm{H}_{49} \mathrm{~N}_{5} \mathrm{O}_{6}$}

Zizyphine A, 23014

\section{$\mathrm{C}_{33} \mathrm{H}_{50} \mathrm{~N}_{2} \mathrm{O}_{2}$}

$N$-Benzoylbuxahyrcanine, 2233

\section{$\mathrm{C}_{33} \mathrm{H}_{50} \mathrm{~N}_{2} \mathrm{O}_{3}$}

Pachysandrine A, 16505

\section{$\mathrm{C}_{33} \mathrm{H}_{50} \mathrm{O}_{5}$}

Fomlactone A, 7876

\section{$\mathrm{C}_{33} \mathrm{H}_{50} \mathrm{O}_{6}$}

Methylcamaralate, 14208

Regelin C, 18579

\section{$\mathrm{C}_{33} \mathrm{H}_{50} \mathrm{O}_{9}$}

$12 \beta$-Acetoxy-3 $\beta$-hydroxy-24,25,26,27-tetranorcycloartan-23,16 $\beta$-olide 3-O- $\beta$ - $D$-xylopyranoside, 237

\section{$\mathrm{C}_{33} \mathrm{H}_{50} \mathrm{O}_{11}$}

Spongipregnoloside A, 20230

Spongipregnoloside B, 20231
$\mathrm{C}_{33} \mathrm{H}_{52} \mathrm{~N}_{4} \mathrm{O}_{6}$

$\mathrm{N}$-Methylsansalvamide, 14719

$\mathrm{C}_{33} \mathrm{H}_{52} \mathrm{O}_{5}$

$3 \beta$-Acetoxy-12 $\alpha$-formyloxy-13,27-cycloursan$11 \alpha$-ol, 197

Euscaphic acid 2,3-monoacetonide, 7658

$21 \alpha$-Hydroxyserrat-14-en-3 $\beta$-yl propanedioic acid monoester, 10708

Pachymic acid, 16496

Tsugaric acid B, 22069

$\mathrm{C}_{33} \mathrm{H}_{52} \mathrm{O}_{8}$

Collettinside I, 3922

Diosgenin-3- $O-\beta$ - $D$-glucopyranoside, 6443

$\mathrm{C}_{33} \mathrm{H}_{52} \mathrm{O}_{9}$

Agavoside A, 727

Convallamarogen-3- $O-\beta$ - $D$-glucopyranoside, 4006

\section{$\mathrm{C}_{33} \mathrm{H}_{52} \mathrm{O}_{10}$}

22-Hydroxy-25( $R, S)$-furost-5-en-12-on-3 $\beta, 22,26$ triol 26- $O-\beta$ - $D$-glucopyranoside, 10134

\section{$\mathrm{C}_{33} \mathrm{H}_{52} \mathrm{O}_{12}$}

Aspidoside A, 1902

$\mathrm{C}_{33} \mathrm{H}_{52} \mathbf{O}_{15}$

Wattoside $\mathrm{H}, 22654$

\section{$\mathrm{C}_{33} \mathrm{H}_{52} \mathrm{O}_{17}$}

10-Isovaleryl kanokoside C, 11762

\section{$\mathrm{C}_{33} \mathrm{H}_{52} \mathrm{O}_{18}$}

Jasnudifloside $\mathrm{K}, 11831$

$\mathrm{C}_{33} \mathrm{H}_{53} \mathrm{NO}_{6}$

Solaplumbinine, 20065

$\mathrm{C}_{33} \mathrm{H}_{53} \mathrm{NO}_{7}$

Yibeinoside A, 22904

Yibeinoside B, 22905

\section{$\mathrm{C}_{33} \mathrm{H}_{53} \mathrm{NO}_{8}$}

Hupehemonoside, 9679

\section{$\mathrm{C}_{33} \mathrm{H}_{54} \mathrm{O}_{2}$}

Eburicyl acetate, 6676

24-Methylenecycloartanyl acetate, 14355

Sablacaurin A, 19102

$\mathrm{C}_{33} \mathrm{H}_{54} \mathrm{O}_{3}$

$3 \beta$-Acetoxy-25-methoxy-lanosta-8,23-diene, 252

\section{$\mathrm{C}_{33} \mathrm{H}_{54} \mathrm{O}_{8}$}

Asparagoside A, 1867

(22S)-Cholesta-5,24-diene-3 $\beta, 11 \alpha, 16 \beta, 22$-tetrol 16-O- $\alpha$ - $L$-rhamnopyranoside, 3577

Timosaponin $\mathrm{A}^{1}, 21394$

\section{$\mathrm{C}_{33} \mathrm{H}_{54} \mathrm{O}_{9}$}

22-Epirhodeasapogenin-3- $O-\beta$ - $D$-glucopyranoside, 7005

$16 \beta$-[( $\beta$-D-Glucopyranosyl)-oxy]-3 $\beta, 7 \beta$-dihydroxycholest-5-en-23-one, 8687

(25R)-3 $\beta$-Hydroxy- $5 \alpha$-spirostan- $6 \alpha$-yl $O-\beta$ - $D$ glucopyranoside, 10717
Isorhodeasapogenin-3- $O-\beta$ - $D$-glucopyranoside, 11675

Rhodeasapogenin-3- $O-\beta$ - $D$-glucopyranoside, 18782

Tokoronin, 21428

\section{$\mathrm{C}_{33} \mathrm{H}_{54} \mathrm{O}_{10}$}

Agamenoside I, 698

Agamenoside J, 699

(25R)-5 $\alpha$-Spirostan- $3 \beta, 6 \alpha, 23 \alpha$-triol-6- $O-\beta-D-$ glucopyranoside, 20216

Tokorogenin- $L$ - $O-\beta$ - $D$-glucopyranoside, 21427

\section{$\mathrm{C}_{33} \mathrm{H}_{54} \mathrm{O}_{11}$}

Ponasteroside A, 17703

(25S)-1 $\beta, 3 \beta, 4 \beta$-Trihydroxyspirostan-5 $\beta$-yl- $O-\beta$ $D$-glucopyranoside, 21845

\section{$\mathrm{C}_{33} \mathrm{H}_{54} \mathrm{O}_{12}$}

$20 \beta$-Ecdysterone 2- $O-\beta$ - $D$-glucopyranoside, 6680

Ecdysterone-3- $O-\beta-D$-glucopyranoside, 6681

Ecdysterone-22- $O-\beta-D$-glucopyranoside, 6682

\section{$\mathrm{C}_{33} \mathrm{H}_{54} \mathrm{O}_{13}$}

20-Hydroxyecdysone 3-O- $\beta$ - $D$-glucoside, 10064

\section{$\mathrm{C}_{33} \mathrm{H}_{55} \mathrm{NO}_{5}$}

Acutifolin palmitate, 598

\section{$\mathrm{C}_{33} \mathrm{H}_{55} \mathrm{NO}_{7}$}

Capsicastrine, 3143

Hupeheninoside, 9682

Persicanidine B-3- $O$ - $\beta$ - $D$-glucoside, 16989

\section{$\mathrm{C}_{33} \mathrm{H}_{55} \mathrm{NO}_{8}$}

Peiminoside, 16775

Zhebeininoside, 22994

\section{$\mathrm{C}_{33} \mathrm{H}_{56} \mathrm{O}_{4}$}

Tetracosyl caffeate, 21039

\section{$\mathrm{C}_{33} \mathrm{H}_{56} \mathrm{O}_{8}$}

(22S)-Cholest-5-ene-3 $\alpha, 11 \alpha, 16 \beta, 22$-tetrol 16$O$ - $\alpha$ - $L$-rhamnopyranoside, 3583

\section{$\mathrm{C}_{33} \mathrm{H}_{56} \mathrm{O}_{12}$}

6'-O- $\alpha$ - $L$-Rhamnopyranosyl-4-epimicrolepin, 18698

\section{$\mathrm{C}_{33} \mathrm{H}_{56} \mathrm{O}_{13}$}

Wattoside D, 22651

\section{$\mathrm{C}_{33} \mathrm{H}_{56} \mathrm{O}_{14}$}

Denin, 5131

$\alpha, \beta$-Digalactosyl- $\alpha$-linolenic-glyceride, 5509

Nerolidol-3- $O-\alpha$ - $L$-rhamnopyranosyl-( $1 \rightarrow 4)-\alpha$ $L$-rhamnopyranosyl-( $1 \rightarrow 2)-\beta$ - $D$-glucopyranoside, 15502

Nerolidol-3- $O$ - $\alpha$ - $L$-rhamnopyranosyl-( $(1 \rightarrow 4)-\alpha$ $L$-rhamnopyranosyl-( $1 \rightarrow 6)-\beta$ - $D$-glucopyranoside, 15503

Pyishiauoside $\mathrm{I}_{\mathrm{b}}, 18237$

\section{$\mathrm{C}_{33} \mathrm{H}_{56} \mathrm{O}_{17}$}

Periplocae oligosaccharide $\mathrm{F}_{2}, 16941$

$\mathrm{C}_{33} \mathrm{H}_{57} \mathrm{NO}_{3}$ 
Qinjiaoamide, 18292

$\mathrm{C}_{33} \mathrm{H}_{57} \mathrm{NO}_{8}$

Jurubine, 11971

$\mathrm{C}_{33} \mathrm{H}_{58} \mathrm{O}_{2}$

Cycloartanol acetate, 4469

$\mathrm{C}_{33} \mathrm{H}_{58} \mathrm{O}_{14}$

Gingerglycolipid B, 8392

2,3,4-Tri(5-methylhexanoyl)- $\alpha$ - $D$-glucopyranosyl- $\beta$ - $D$-fructofuranoside, 21964

$\mathrm{C}_{33} \mathrm{H}_{60} \mathrm{O}_{6}$

Montalicin A, 14936

$\mathrm{C}_{33} \mathrm{H}_{60} \mathrm{O}_{7}$

Muricin D, 15093

Muricin E, 15094

$\mathrm{C}_{33} \mathrm{H}_{60} \mathrm{O}_{14}$

Gingerglycolipid C, 8393

$\mathrm{C}_{33} \mathrm{H}_{63} \mathrm{NO}_{4}$

$\left(2 S, 2^{\prime} R, 3 R, 4 E, 8 E\right)-N-2^{\prime}$-Hydroxytetradecanoyl-

2-amino-9-methyl-4,8-octadecadiene-1,3-diol, 10744

\section{$\mathrm{C}_{33} \mathrm{H}_{64} \mathrm{O}_{2}$}

Eucalyptus wax, 7482

$\mathrm{C}_{33} \mathrm{H}_{66} \mathrm{O}_{2}$

Psyllic acid, 18096

\section{$\mathrm{C}_{33} \mathrm{H}_{67} \mathrm{NO}_{5}$}

Trufflesphingolipid C, 22055

\section{$\mathrm{C}_{33} \mathrm{H}_{68}$}

$n$-Tritriacontane, 22042

\section{$\mathrm{C}_{33} \mathrm{H}_{68} \mathrm{O}$}

6-Tritriacontanol, 22043

Tritriacontanol, 22044

\section{$\mathrm{C}_{34} \mathrm{H}_{22} \mathrm{O}_{8}$}

( \pm )-Albanol B, 847

\section{$\mathrm{C}_{34} \mathrm{H}_{22} \mathrm{O}_{9}$}

Mulberrofuran P, 15050

\section{$\mathrm{C}_{34} \mathrm{H}_{22} \mathrm{O}_{10}$}

Mulberrofuran M, 15047

\section{$\mathrm{C}_{34} \mathrm{H}_{22} \mathrm{O}_{22}$}

Punicalin, 18205

\section{$\mathrm{C}_{34} \mathrm{H}_{24} \mathrm{O}_{10}$}

Mulberrofuran Q, 15051

\section{$\mathrm{C}_{34} \mathrm{H}_{24} \mathrm{O}_{21}$}

Balanophotannin A, 2127

\section{$\mathrm{C}_{34} \mathrm{H}_{24} \mathrm{O}_{22}$}

Casuariin, 3302

5-Desgalloylstachyurin, 5249

Pedunculagin, 16765

Tomentosin, 21443

\section{$\mathrm{C}_{34} \mathrm{H}_{26} \mathrm{O}_{6}$}

2,2'-Di-(2-phenylethyl)-8,6'-dihydroxy-5,5'-bichromone (AH11), 6486

$\mathrm{C}_{34} \mathrm{H}_{26} \mathrm{O}_{8}$

Albanol A, 846
Curtisian Q, 4413

Ganbajunin C, 8132

Ganbajunin E, 8133

Mulberrofuran G, 15044

Terrestrin A, 21006

$\mathrm{C}_{34} \mathrm{H}_{26} \mathrm{O}_{10}$

Kuwanon Z, 12394

Stephaflavone A, 20314

7,4',7",4"'-Tetra- $O$-methylamentoflavone, 21192

\section{$\mathrm{C}_{34} \mathrm{H}_{26} \mathrm{O}_{22}$}

1,2-Di- $O$-galloyl-4,6-O-(S)-hexahydroxydiphenoyl- $\beta$ - $D$-glucopyranose, 5521

Mallorepanin, 13428

Mallotinic acid, 13429

Sanguiin $\mathrm{H}_{1}, 19273$

Tellimagrandin I, 20910

Tercatain, 20969

\section{$\mathrm{C}_{34} \mathrm{H}_{26} \mathrm{O}_{23}$}

Punigluconin, 18206

Sanguiin $\mathrm{H}_{7}, 19279$

\section{$\mathrm{C}_{34} \mathrm{H}_{28} \mathrm{O}_{7}$}

Kuwanol A, 12377

$\mathrm{C}_{34} \mathrm{H}_{28} \mathrm{O}_{8}$

$\mathrm{AH} 21,772$

$\mathrm{C}_{34} \mathrm{H}_{28} \mathrm{O}_{9}$

Albafuran C, 845

Mulberrofuran C, 15041

\section{$\mathrm{C}_{34} \mathrm{H}_{28} \mathrm{O}_{22}$}

1,2,3,4-Tetragalloyl- $\alpha$ - $D$-glucose, 21052

1,2,3,6-Tetra- $O$-galloyl- $\beta$ - $D$-glucose, 21053

1,2,4,6-Tetra- $O$-galloyl- $\beta$ - $D$-glucose, 21054

\section{$\mathrm{C}_{34} \mathrm{H}_{30} \mathrm{~N}_{2} \mathrm{O}_{5}$}

1,2-Dehydroapateline, 4877

\section{$\mathrm{C}_{34} \mathrm{H}_{30} \mathrm{~N}_{2} \mathrm{O}_{6}$}

Puertogaline A, 18187

\section{$\mathrm{C}_{34} \mathrm{H}_{30} \mathrm{O}_{8}$}

Guangsangon B, 9054

(5S,6R,7R,8S)-2-(2-Phenylethyl)-5,6,7-tri-hydroxy-5,6,7,8-tetrahydro-8-[2-(2-phenylethyl) chromonyl-6-oxy]chromone (AH13), 17119

(5S,6S,7S,8R)-2-(2-Phenylethyl)-6,7,8-trihydroxy-5,6,7,8-tetrahydro-5-[2-(2-phenylethyl) chromonyl-6-oxy]chromone (AH14), 17120

\section{$\mathrm{C}_{34} \mathrm{H}_{30} \mathrm{O}_{9}$}

Kuwanon P, 12390

Kuwanon X, 12391

Kuwanon Y, 12392

Kuwanon Y, 12393

(5S,6S,7R,8S)-2-(2-Phenylethyl)-6,7,8-trihydroxy-5,6,7,8-tetrahydro-5-[2-(2-phenyl-ethyl) -7-hydroxy-chromonyl-6-oxy]-chromone (AH15), 17122

$\mathrm{C}_{34} \mathrm{H}_{30} \mathrm{O}_{15}$
1,3,5-Tri-O-caffeoyl quinic acid, 21552

$\mathrm{C}_{34} \mathrm{H}_{30} \mathrm{O}_{18}$

Trifuhalol A octaacetate, 21639

Triisofuhalol octaacetate, 21869

$\mathrm{C}_{34} \mathrm{H}_{32} \mathrm{FeN}_{4} \mathrm{O}_{4}{ }^{+}$

Hemin, 9345

$\mathrm{C}_{34} \mathrm{H}_{32} \mathrm{~N}_{2} \mathrm{O}_{5}$

Cocsoline, 3881

$\mathrm{C}_{34} \mathrm{H}_{32} \mathrm{~N}_{2} \mathrm{O}_{6}$

Pangkorimine, 16604

$\mathrm{C}_{34} \mathrm{H}_{32} \mathrm{O}_{8}$

rel-(1 $\alpha, 2 \beta)$-Di-(2,6-dimethoxy-4-hydroxy)benzoyl-rel-( $(3 \alpha, 4 \beta)$-diphenylcyclobutane, 5480

$\mathrm{C}_{34} \mathrm{H}_{32} \mathrm{O}_{9}$

6"-Demethoxyneocalycopterone, 5051

\section{$\mathrm{C}_{34} \mathrm{H}_{32} \mathrm{O}_{10}$}

(rel)-1 $\beta, 2 \alpha$-Di-(2,4-dihydroxy-6-methoxybenzoyl)-3 $\beta, 4 \alpha$-di-(4-methoxyphenyl)-cyclobutane, 5479

\section{$\mathrm{C}_{34} \mathrm{H}_{32} \mathrm{O}_{11}$}

Anticancer Stilbenoid PMV70P691-041, 1445

Resveratrol (E)-dehydrodimer 11-O- $\beta$ - $D$-glucopyranoside, 18645

Resveratrol (E)-dehydrodimer 11'-O- $\beta$ - $D$-glucopyranoside, 18646

\section{$\mathrm{C}_{34} \mathrm{H}_{32} \mathrm{O}_{12}$}

Dahuribirin F, 4602

\section{$\mathrm{C}_{34} \mathrm{H}_{33} \mathrm{FeN}_{4} \mathrm{O}_{5}$}

Hematin, 9338

\section{$\mathrm{C}_{34} \mathrm{H}_{34} \mathrm{~N}_{2} \mathbf{O}_{16}$}

Gomphrenin V, 8930

Gomphrenin VI, 8931

\section{$\mathrm{C}_{34} \mathrm{H}_{34} \mathrm{~N}_{4} \mathrm{O}_{4}$}

Protoporphyrin, 17984

\section{$\mathrm{C}_{34} \mathrm{H}_{34} \mathrm{~N}_{4} \mathrm{O}_{5}$}

Phellophyll a, 17076

\section{$\mathrm{C}_{34} \mathrm{H}_{34} \mathrm{O}_{10}$}

Austrocolorin $\mathrm{A}_{1}, 2019$

Austrocolorin $\mathrm{B}_{1}, 2020$

Calyflorenone D, 3009

Genkwadaphnin, 8287

Singueanol I, 19933

Singueanol II, 19934

\section{$\mathrm{C}_{34} \mathrm{H}_{34} \mathrm{O}_{13}$}

Dahuribirin B, 4598

Dahuribirin G, 4603

\section{$\mathrm{C}_{34} \mathrm{H}_{35} \mathrm{O}_{19}$}

Cyanidin-3-O-(2"-O-galloyl-6"-O- $\alpha$-rhamnopyranosyl- $\beta$-galactopyranoside), 4440

\section{$\mathrm{C}_{34} \mathrm{H}_{36} \mathrm{~N}_{2} \mathrm{O}_{6}$}

Lindoldhamine, 12877

Pseudomorphine, 18052 


\section{$\mathrm{C}_{34} \mathrm{H}_{36} \mathrm{O}_{6}$}

Isovouacapenol E, 11780

$\mathrm{C}_{34} \mathrm{H}_{36} \mathrm{O}_{7}$

Ingenol-3,20-dibenzoate, 11058

$\mathrm{C}_{34} \mathrm{H}_{36} \mathrm{O}_{8}$

Phorbol-12-benzoate-13-benzoate, 17183

$\mathrm{C}_{34} \mathrm{H}_{36} \mathrm{O}_{10}$

Yuanhuatin, 22933

$\mathrm{C}_{34} \mathrm{H}_{36} \mathrm{O}_{11}$

Orbiculin F, 16165

$\mathrm{C}_{34} \mathrm{H}_{36} \mathrm{O}_{15}$

Agnucastoside $\mathrm{C}, 752$

$\mathrm{C}_{34} \mathrm{H}_{37} \mathrm{~N}_{5} \mathrm{O}_{6}$

Longicalycinin A, 12954

$\mathrm{C}_{34} \mathrm{H}_{38} \mathrm{O}_{7}$

Sarbronine B, 19357

\section{$\mathrm{C}_{34} \mathrm{H}_{38} \mathrm{O}_{10}$}

Triptofordin $\mathrm{D}_{1}, 22002$

\section{$\mathrm{C}_{34} \mathrm{H}_{38} \mathrm{O}_{16}$}

Aloeresin C, 974

Pentaacetyl-6'-cinnamoyl catalpol, 16821

Senburiside I, 19699

\section{$\mathrm{C}_{34} \mathbf{H}_{38} \mathbf{O}_{19}$}

Kaempferol-3-O-lysimachiatrioside, 12066

\section{$\mathrm{C}_{34} \mathrm{H}_{39} \mathrm{NO}_{11}$}

Kupitengester 1, 12336

\section{$\mathrm{C}_{34} \mathrm{H}_{39} \mathrm{NO}_{12}$}

$1 \alpha$-Nicotinoyloxy- $2 \alpha$-acetoxy- $6 \beta$-acetoxy- $9 \beta$ benzoyloxy-11-acetoxy-4 $\beta$-hydroxydihydro$\beta$-agarofuran, 15534

$1 \beta, 5 \alpha, 11$-Triacetoxy-7 $\beta$-benzoyl- $4 \alpha$-hydroxy- $8 \beta$ nicotinoyl-dihydroagarofuran, 21512

\section{$\mathrm{C}_{34} \mathrm{H}_{40} \mathbf{N}_{2} \mathbf{O}_{10}$}

10,11-Dimethoxy-1-methyldeacetylpicraline-3', 4',5'-trimethoxy-benzoate, 6259

\section{$\mathrm{C}_{34} \mathrm{H}_{40} \mathrm{~N}_{4} \mathrm{O}_{4}$}

Adouetine Y, 649

Scutianine B, 19590

\section{$\mathrm{C}_{34} \mathrm{H}_{40} \mathrm{~N}_{4} \mathrm{O}_{5}$}

Scutianine D, 19592

Scutianine E, 19593

\section{$\mathrm{C}_{34} \mathrm{H}_{40} \mathrm{O}_{8}$}

Isomoreollin B, 11542

Scortechinone H, 19553

\section{$\mathrm{C}_{34} \mathrm{H}_{40} \mathrm{O}_{9}$}

Moreollic acid, 14970

Scortechinone B, 19547

Scortechinone F, 19551

\section{$\mathrm{C}_{34} \mathrm{H}_{40} \mathrm{O}_{10}$}

Scortechinone C, 19548

Scortechinone K, 19556

Scortechinone M, 19558

$\mathrm{C}_{34} \mathrm{H}_{40} \mathrm{O}_{12}$
Agrimol F, 755

Swietephragmin G, 20522

$\mathrm{C}_{34} \mathrm{H}_{40} \mathrm{O}_{14}$

Myricanol galloyl glucoside, 15161

$\mathrm{C}_{34} \mathrm{H}_{40} \mathrm{O}_{16}$

Javanicoside J, 11861

Yadanzioside M, 22872

$\mathrm{C}_{34} \mathrm{H}_{40} \mathrm{O}_{17}$

Tabulalide A, 20581

$\mathrm{C}_{34} \mathrm{H}_{40} \mathrm{O}_{18}$

Sagittatin B, 19126

$\mathrm{C}_{34} \mathrm{H}_{40} \mathrm{O}_{19}$

Arillatose A, 1684

Glomeratose E, 8586

\section{$\mathrm{C}_{34} \mathrm{H}_{40} \mathrm{O}_{20}$}

Viscumneoside VII, 22542

\section{$\mathrm{C}_{34} \mathrm{H}_{41} \mathrm{NO}_{13}$}

$1 \alpha$-Nicotinoyloxy- $2 \alpha$-acetoxy- $6 \beta$-acetoxy- $9 \beta$ furoyloxy-11-isobutyryloxy- $4 \beta$-hydroxydihydro- $\beta$-agarofuran, 15536

\section{$\mathrm{C}_{34} \mathrm{H}_{41} \mathrm{NO}_{17}$}

Anthemis glycoside B, 1353

\section{$\mathrm{C}_{34} \mathrm{H}_{42} \mathrm{~N}_{2} \mathrm{O}_{4}$}

Belladonnine, 2218

\section{$\mathrm{C}_{34} \mathrm{H}_{42} \mathrm{O}_{6}$}

Cowagarcinone A, 4195

\section{$\mathrm{C}_{34} \mathrm{H}_{42} \mathrm{O}_{7}$}

Scortechinone A, 19546

Scortechinone J, 19555

Scortechinone L, 19557

\section{$\mathrm{C}_{34} \mathrm{H}_{42} \mathrm{O}_{10}$}

Handelin, 9218

Scortechinone N, 19559

Scortechinone P, 19561

\section{$\mathrm{C}_{34} \mathrm{H}_{42} \mathrm{O}_{11}$}

Hanburinone, 9207

\section{$\mathrm{C}_{34} \mathrm{H}_{42} \mathrm{O}_{13}$ \\ PC-2004-65-2003-18, 16739 \\ $\mathrm{C}_{34} \mathrm{H}_{42} \mathrm{O}_{18}$}

Isopodophyllotoxin 7'- $O$ - $\beta$ - $D$-glucopyranosyl$(1 \rightarrow 6)-\beta$ - $D$-glucopyranoside, 11608

Neobudofficide, 15347

$L$-Picropodophyllin 7'-O-( $\beta$ - $D$-glucopyranosyl$(1 \rightarrow 6)-\beta$ - $D$-glucopyranoside), 17340

Reiniose A, 18603

\section{$\mathrm{C}_{34} \mathrm{H}_{42} \mathrm{O}_{19}$}

5,4'-Dihydroxy-7-methoxyflavone-3-O- $[\alpha-L$ rhamnopyranosyl $(1 \rightarrow 3)-O-\alpha$ - $L$-rhamnopyra$\operatorname{nosyl}(1 \rightarrow 6)-O-\beta$ - $D$-glucopyranoside], 5981

1,2-Di- $O$ - $E$-sinapoyl- $\beta$-gentiobiose, 6508

3',6-Disinapoylsucrose, 6511

Sibiricose $\mathrm{A}_{4}, 19865$

$\mathrm{C}_{34} \mathrm{H}_{42} \mathrm{O}_{20}$
Isorhamnetin 3-O- $\alpha-L$-rhamnopyranosyl$(1 " ' \rightarrow 2 ")[a-L$-rhamnopyranosyl-(1"' $\rightarrow 6 ")]-$ $\beta$ - $D$-galactopyranoside, 11664

Isorhamnetin 3-O-[ $\alpha$-rhamnopyranosyl- $(1 \rightarrow 4)$ $\alpha$-rhamnopyranosyl-( $1 \rightarrow 6)$ - $\beta$-glucopyranoside], 11665

Isorhamnetin $3-O-2^{\mathrm{G}}$-rhamnosylrutinoside, 11667

Typhaneoside, 22154

\section{$\mathrm{C}_{34} \mathrm{H}_{42} \mathrm{O}_{21}$}

Saprosmoside H, 19352

\section{$\mathrm{C}_{34} \mathrm{H}_{42} \mathrm{O}_{22}$}

3-O-Methylquercetin-7- $O$-diglucoside-4'- $O$ glucoside, 14710

\section{$\mathrm{C}_{34} \mathrm{H}_{43} \mathrm{NO}_{11}$}

Euphocharacin J, 7605

3,5,7,15-Tetraacetoxy-9-nicotinoyloxy-14oxojatropha-6(17),11-diene, 21032

\section{$\mathrm{C}_{34} \mathrm{H}_{43} \mathrm{NO}_{12}$}

Kansuinin G, 12143

\section{$\mathrm{C}_{34} \mathrm{H}_{43} \mathrm{O}_{14}$}

Glehlinoside A, 8545

\section{$\mathrm{C}_{34} \mathrm{H}_{43} \mathrm{O}_{20}{ }^{+}$}

Cyanidin-3-sophoroside-5-glucoside, 4449

\section{$\mathrm{C}_{34} \mathrm{H}_{44} \mathrm{O}_{9}$}

Neobonaspectin B, 15345

Salannin, 19177

\section{$\mathrm{C}_{34} \mathrm{H}_{44} \mathrm{O}_{12}$}

Taccalonolide E, 20587

\section{$\mathrm{C}_{34} \mathrm{H}_{44} \mathrm{O}_{13}$}

12-O-Deacetyltrichilin $\mathrm{H}, 4790$

Taccalonolide F, 20588

Taccalonolide I, 20591

\section{$\mathrm{C}_{34} \mathrm{H}_{44} \mathrm{O}_{14}$}

Taccalonolide K, 20593

7-( $\beta$-Xylosyl)-10-deacetylbaccatin III, 22846

\section{$\mathrm{C}_{34} \mathrm{H}_{44} \mathrm{O}_{16}$}

Viburtinoside B, 22462

Viburtioside A, 22463

\section{$\mathrm{C}_{34} \mathrm{H}_{44} \mathrm{O}_{19}$}

Arenarioside, 1663

Ehrenoside, 6718

Forsythoside B, 7925

Lavansulifoliside, 12579

Myricoside, 15189

Pedicularioside A, 16763

\section{$\mathrm{C}_{34} \mathrm{H}_{44} \mathrm{O}_{20}$}

Aragoside, 1602

3,4-Dihydroxyphenylethanol-8-O-[4-O-transcaffeoyl- $\beta$ - $D$-apiofuranosyl $(1 \rightarrow 3)]$ - $\beta$ - $D$-glucopyranosyl- $(1 \rightarrow 6)]-\beta$ - $D$-glucopyranoside, 6079

Lavandulifolioside, 12577

$\mathrm{C}_{34} \mathrm{H}_{45} \mathrm{ClN}_{2} \mathrm{O}_{11}$ 
Maytanbutacine, 13618

\section{$\mathrm{C}_{34} \mathrm{H}_{45} \mathrm{NO}_{10}$}

Euphocharacin G, 7602

Euphocharacin K, 7606

\section{$\mathrm{C}_{34} \mathrm{H}_{46} \mathrm{CIN}_{3} \mathrm{O}_{10}$}

Maytansine, 13621

$\mathrm{C}_{34} \mathrm{H}_{46} \mathrm{O}_{6}$

Revandchinone 1, 18669

$\mathrm{C}_{34} \mathrm{H}_{46} \mathrm{O}_{8}$

Bisacutifolone A mono acetate, 2431

\section{$\mathrm{C}_{34} \mathrm{H}_{46} \mathrm{O}_{9}$}

Cinobufagin-3-hydrogen suberate, 3730

12-O-Methylvolkensin, 14813

\section{$\mathrm{C}_{34} \mathrm{H}_{46} \mathrm{O}_{13}$}

cis-Cleroda-3,13(14)-dien-15,16-olide-18-O-

[ $\beta$ - $D$-galactopyranosyl]-peracetylester, 3833

\section{$\mathrm{C}_{34} \mathrm{H}_{46} \mathrm{O}_{14}$}

Jatrophane 7, 11843

\section{$\mathrm{C}_{34} \mathrm{H}_{46} \mathrm{O}_{15}$}

1-Acetoxy-baccatin I, 129

$1 \beta$-Acetylbaccatin IV, 328

$4 \alpha$-Hydroxy- $\beta, 2 \beta, 5 \alpha$-triacetoxy- $7 \beta, 11$-diiso-

butyryloxy- $8 \alpha$-furanoyl-dihydroagarofuran, 10776

\section{$\mathrm{C}_{34} \mathrm{H}_{46} \mathrm{O}_{16}$}

Bruceantinoside A, 2662

Yadanzioside N, 22873

Yadanzioside P, 22875

\section{$\mathrm{C}_{34} \mathrm{H}_{46} \mathrm{O}_{17}$}

Bis[4-( $\beta$ - $D$-glucopyranosyloxy) benzyl $](S)$-2butylmalate, 2453

Tangshenoside III, 20674

Yadanzioside C, 22862

Yadanzioside L, 22871

\section{$\mathrm{C}_{34} \mathrm{H}_{46} \mathrm{O}_{18}$}

Acanthoside D, 86

Liriodendrin, 12916

\section{$\mathrm{C}_{34} \mathrm{H}_{46} \mathrm{O}_{19}$}

(E)-Aldosecologanin, 878

(Z)-Aldosecologanin, 879

\section{$\mathrm{C}_{34} \mathrm{H}_{47} \mathrm{NO}_{8}$}

8-Acetyl-14-benzoylchasmanine, 331

Crassicaudine, 4210

13-Deoxo-13 $\alpha$-acetyloxy-1-deoxynortaxine B, 5138

Gymnaconitine, 9103

\section{$\mathrm{C}_{34} \mathrm{H}_{47} \mathrm{NO}_{9}$}

13,15-Dideoxyaconitine, 5473

Penduline, 16803

\section{$\mathrm{C}_{34} \mathrm{H}_{47} \mathrm{NO}_{10}$}

Deoxyaconitine, 5149

Falaconitine, 7706

Indaconitine, 11012
$\mathrm{C}_{34} \mathrm{H}_{47} \mathrm{NO}_{11}$

Aconitine, 554

Geniculine, 8270

Polyschistine B, 17673

Polyschistine D, 17675

$\mathrm{C}_{34} \mathrm{H}_{47} \mathrm{NO}_{12}$

Aconifine, 551

$\mathrm{C}_{34} \mathrm{H}_{48} \mathrm{~N}_{2} \mathrm{O}_{9}$

Ajacine, 781

$\mathrm{C}_{34} \mathrm{H}_{48} \mathrm{O}_{7}$

Fomitopsin B, 7864

Lanosta-7,9(11),24-trien-3 $\alpha, 15 \alpha$-diacetoxy-23oxo-26-oic acid, 12486

\section{$\mathrm{C}_{34} \mathrm{H}_{48} \mathrm{O}_{8}$}

Corymbulosin A, 4111

Huratoxin, 9691

\section{$\mathrm{C}_{34} \mathrm{H}_{48} \mathrm{O}_{9}$}

Daturametelin A, 4666

$\mathrm{C}_{34} \mathrm{H}_{48} \mathrm{O}_{10}$

Daturametelin B, 4667

\section{$\mathrm{C}_{34} \mathrm{H}_{48} \mathrm{O}_{11}$}

Ajugamarin $\mathrm{G}_{1}, 806$

Ajugamarin $\mathrm{H}_{1}, 807$

\section{$\mathrm{C}_{34} \mathrm{H}_{48} \mathrm{O}_{13}$}

Dichotomoside D, 5430

$\mathrm{C}_{34} \mathrm{H}_{48} \mathrm{O}_{14}$

Hyrcanoside, 10915

\section{$\mathrm{C}_{34} \mathrm{H}_{48} \mathrm{O}_{16}$}

Nomilinic acid glucoside, 15657

$\mathrm{C}_{34} \mathrm{H}_{48} \mathrm{O}_{17}$

Javanicoside K, 11862

\section{$\mathrm{C}_{34} \mathrm{H}_{48} \mathrm{O}_{18}$}

(+)-Lyoniresinol-4,4'-bis- $O$ - $\beta$ - $D$-glucopyranoside, 13252

\section{$\mathrm{C}_{34} \mathrm{H}_{49} \mathrm{~N}_{5} \mathrm{O}_{6}$}

Apicidin, 1475

$\mathrm{C}_{34} \mathrm{H}_{50} \mathrm{O}_{4}$

Inophylloidic acid, 11071

$\mathrm{C}_{34} \mathrm{H}_{50} \mathrm{O}_{5}$

Argentatin G, 1670

$\mathrm{C}_{34} \mathrm{H}_{50} \mathrm{O}_{6}$

Ganodermic acid R, 8190

Ganodermic acid S, 8191

Muscanone, 15124

$\mathrm{C}_{34} \mathrm{H}_{50} \mathrm{O}_{7}$

Fomitopsin C, 7865

Tyromycic acid B, 22163

\section{$\mathrm{C}_{34} \mathrm{H}_{50} \mathrm{O}_{8}$}

Fomitopsin A, 7863

$\mathrm{C}_{34} \mathrm{H}_{50} \mathrm{O}_{12}$

Thapsigargin, 21279

$\mathrm{C}_{34} \mathrm{H}_{50} \mathrm{O}_{13}$

Erychroside, 7296

\section{$\mathrm{C}_{34} \mathrm{H}_{50} \mathrm{O}_{20}$}

Cornuside, 4062

$\mathrm{C}_{34} \mathrm{H}_{52} \mathrm{O}_{5}$

Argentatin H, 1671

$\mathrm{C}_{34} \mathrm{H}_{52} \mathrm{O}_{6}$

$\mathrm{C}_{17}$-Obtusilactone dimer, 2836

\section{$\mathrm{C}_{34} \mathrm{H}_{52} \mathrm{O}_{7}$}

Ganode-8-en-ric acid Ma, 8158

Ganode-8-en-ric acid W, 8161

\section{$\mathrm{C}_{34} \mathrm{H}_{52} \mathrm{O}_{8}$}

Corymbulosin B, 4112

Corymbulosin C, 4113

(22E,24R)-5 $\alpha, 8 \alpha$-Epidioxyergosta-6,9,22-triene3 $\beta$-ol 3-O- $\beta$ - $D$-glucopyranoside, 6902

30-Norhederagenin-3-O- $\alpha-L$-arabinopyranoside, 15753

Periploside B, 16962

Phorbol-12-acetate-13-laurate, 17182

Phorbol-12-laurate-13-acetate, 17186

\section{$\mathrm{C}_{34} \mathrm{H}_{52} \mathrm{O}_{9}$}

Periplocoside M, 16955

\section{$\mathrm{C}_{34} \mathrm{H}_{52} \mathrm{O}_{10}$}

Coagulin Q, 3859

$16 \alpha, 24 \alpha$-Dihydroxy-12 $\beta$-acetoxy-25,26,27-trinor16,24-cycloartan-23-one $3 \beta-O-\alpha$ - $L$-arabinopyranoside, 5752

24-Hydroxy-12 $\beta$-acetoxy-25,26,27-trinorcycloartan-16,23-dione 3 $\beta$ - $O-\alpha-L$-arabinopyranoside, 9762

Physagulin D, 17237

\section{$\mathrm{C}_{34} \mathrm{H}_{52} \mathrm{O}_{11}$}

Cilistol U, 3645

Withanoside XI, 22714

\section{$\mathrm{C}_{34} \mathrm{H}_{52} \mathrm{O}_{12}$}

Withanoside III, 22707

\section{$\mathrm{C}_{34} \mathrm{H}_{52} \mathrm{O}_{13}$}

Erychrosol, 7297

\section{$\mathrm{C}_{34} \mathrm{H}_{52} \mathrm{O}_{15}$}

Bipindogenin-3-O- $\beta$ - $D$-xylopyranosyl $(1 \rightarrow 4)-\beta$ $D$-allopyranoside, 2401

\section{$\mathrm{C}_{34} \mathrm{H}_{54} \mathrm{O}_{6}$}

Ingenol-3-myristinate, 11061

Ingenol-20-myristinate, 11062

\section{$\mathrm{C}_{34} \mathrm{H}_{54} \mathrm{O}_{7}$}

Rubianol E, 19005

\section{$\mathrm{C}_{34} \mathrm{H}_{54} \mathrm{O}_{8}$}

$5 \alpha, 8 \alpha$-Epidioxy-24(R)-methylcholesta-6,22dien-3 $\beta$ - $D$-glucopyranoside, 6907

\section{$\mathrm{C}_{34} \mathrm{H}_{54} \mathrm{O}_{10}$}

Cilistol T, 3644

\section{$\mathrm{C}_{34} \mathrm{H}_{54} \mathrm{O}_{12}$}

Cilistol p, 3641

$\mathrm{C}_{34} \mathrm{H}_{54} \mathrm{O}_{13}$ 
Styraxoside A, 20428

$\mathrm{C}_{34} \mathrm{H}_{56} \mathrm{O}_{4}$

ent-15,16-Epoxy-3 $\beta$-myristoyloxy-kauran-2-one, 7174

Pisosterol, 17492

\section{$\mathrm{C}_{34} \mathrm{H}_{56} \mathrm{O}_{9}$}

Cilistol V, 3646

Heminine, 9346

$\mathrm{C}_{34} \mathrm{H}_{56} \mathrm{O}_{11}$

Cilistol J, 3640

$\mathrm{C}_{34} \mathrm{H}_{57} \mathrm{NO}_{7}$

Ningpeisinoside, 15614

$\mathrm{C}_{34} \mathrm{H}_{57} \mathrm{NO}_{8}$

Pingbeininoside, 17382

$\mathrm{C}_{34} \mathrm{H}_{57} \mathrm{NO}_{9}$

Pingbeidinoside, 17379

\section{$\mathrm{C}_{34} \mathrm{H}_{58} \mathrm{O}_{4}$}

Lignoceryl ferulate, 12801

\section{$\mathrm{C}_{34} \mathrm{H}_{58} \mathrm{O}_{5}$}

$\beta$-Sitosterol-3-O- $\beta$ - $D$-xylopyranoside, 19992

\section{$\mathrm{C}_{34} \mathrm{H}_{58} \mathrm{O}_{6}$}

Campesteryl- $D$-glucoside, 3042

24-Methylcholest-5-enyl-3 $\beta$ - $O$-glucopyranoside, 14240

\section{$\mathrm{C}_{34} \mathrm{H}_{58} \mathrm{O}_{8}$}

Eurylene, 7655

\section{$\mathrm{C}_{34} \mathrm{H}_{58} \mathrm{O}_{12}$}

Wattoside B, 22649

\section{$\mathrm{C}_{34} \mathrm{H}_{65} \mathrm{NO}_{3}$}

(4E,6E,2S,3R)-2- $N$-Eicosanoyl-4,6-tetradecasphingadienine, 6723

$(2 S, 3 R, 4 E, 8 E)$-2-Hexadecanoylamino-4,8octadecadien-1,3-diol, 9488

\section{$\mathrm{C}_{34} \mathrm{H}_{65} \mathrm{NO}_{4}$}

$\left(2 S, 2^{\prime} R, 3 R, 4 E, 8 E\right)-N-2^{\prime}$-Hydroxypentadecanoyl-2-amino-9-methyl-4,8-octadecadiene-1,3diol, 10592

\section{$\mathrm{C}_{34} \mathrm{H}_{66} \mathrm{O}_{3}$}

Methyl-2 $\beta(2 S)$-hydroxyl-7(E)-tritriacontenoate, 14511

\section{$\mathrm{C}_{34} \mathrm{H}_{67} \mathrm{NO}_{3}$}

(4E,2S,3R)-2- $N$-Eicosanoyl-4-tetradecasphingenine, 6724

\section{$\mathrm{C}_{34} \mathrm{H}_{68} \mathrm{O}_{2}$}

4-Acetyl-2-methoxy-5-methyltriacontane, 464

\section{$\mathrm{C}_{34} \mathrm{H}_{69} \mathrm{NO}_{5}$}

Trufflesphingolipid D, 22056

\section{$\mathrm{C}_{34} \mathbf{H}_{70}$}

6-Methyl tritriacontane, 14794

\section{$\mathrm{C}_{35} \mathrm{H}_{18} \mathrm{O}_{15}$}

Norbadione A, 15717

$\mathrm{C}_{35} \mathrm{H}_{26} \mathrm{O}_{8}$

Upunaphenol E, 22236
$\mathrm{C}_{35} \mathrm{H}_{26} \mathrm{O}_{14}$

$S$-(+)-Skyrin-6- $O$ - $\alpha$-arabinofuranoside, 20011

$R$-(-)-Skyrin-6-O- $\beta$ - $D$-xylopyranoside, 20014

$S$-(+)-Skyrin-6- $O$ - $\beta$ - $D$-xylopyranoside, 20015

$\mathrm{C}_{35} \mathrm{H}_{28} \mathrm{O}_{10}$

Guangsangon G, 9059

Oliveriflavone, 16086

$\mathrm{C}_{35} \mathrm{H}_{28} \mathrm{O}_{11}$

Guangsangon I, 9061

$\mathrm{C}_{35} \mathrm{H}_{28} \mathrm{O}_{17}$

Pelliatin, 16790

Trilobatin K, 21886

\section{$\mathrm{C}_{35} \mathrm{H}_{28} \mathrm{O}_{20}$}

Quercetin-3-O-(2",3"'-digalloyl)- $\beta$ - $D$-galactopyranoside, 18339

Quercetin-3-O-(2",6"-digalloyl)- $\beta$ - $D$-galactopyranoside, 18340

\section{$\mathrm{C}_{35} \mathrm{H}_{28} \mathrm{O}_{22}$}

3,5-Di- $O$-galloyl-4- $O$-digalloylquinic acid, 5515

\section{$\mathrm{C}_{35} \mathrm{H}_{30} \mathrm{O}_{6}$}

Sinensol H, 19931

$\mathrm{C}_{35} \mathrm{H}_{30} \mathrm{O}_{10}$

Guangsangon C, 9055

Guangsangon D, 9056

Guangsangon M, 9065

Guangsangon N, 9066

\section{$\mathrm{C}_{35} \mathrm{H}_{30} \mathrm{O}_{11}$}

Guangsangon K, 9063

Kuwanon L, 12389

\section{$\mathrm{C}_{35} \mathrm{H}_{30} \mathrm{O}_{22}$}

Methyl 2,3,4,6-tetra- $O$-galloyl- $\beta$ - $D$-glucopyranoside, 14746

\section{$\mathrm{C}_{35} \mathrm{H}_{32} \mathrm{~N}_{2} \mathrm{O}_{6}$}

Normenisarine, 15777

Philogaline, 17146

Puertogaline B, 18188

$\mathrm{C}_{35} \mathrm{H}_{32} \mathrm{~N}_{2} \mathrm{O}_{7}$

Punjabine, 18207

\section{$\mathrm{C}_{35} \mathrm{H}_{32} \mathrm{O}_{9}$}

(5S,6S,7R,8S)-2-(2-Phenylethyl)-5,6,7-trihydroxy-5,6,7,8-tetrahydro-8-[2-(2-phenylethyl)-7-methoxychromonyl-6-oxy]chromone (AH12), 17121

$\mathrm{C}_{35} \mathrm{H}_{32} \mathrm{O}_{16}$

3T-O-Arabinopyranosyl-ent-epicatechin- $(2 \alpha \rightarrow 7$, $4 \alpha \rightarrow 8)$-catechin, 1564

\section{$\mathrm{C}_{35} \mathrm{H}_{34} \mathrm{MgN}_{4} \mathrm{O}_{5}$}

Chlorophyllide a, 3568

\section{$\mathrm{C}_{35} \mathrm{H}_{34} \mathrm{~N}_{2} \mathrm{O}_{5}$}

Cocsuline, 3882

Trilobine, 21887

$\mathrm{C}_{35} \mathrm{H}_{34} \mathrm{~N}_{2} \mathrm{O}_{6}$

Cocsulinine, 3883
(+)-Guatteboline, 9072

\section{$\mathrm{C}_{35} \mathrm{H}_{34} \mathrm{O}_{6}$}

Alpinnanin A, 986

Alpinnanin B, 987

Alpinnanin C, 988

Magnolignan H, 13385

$\mathrm{C}_{35} \mathrm{H}_{34} \mathrm{O}_{7}$

Calyxin H, 3017

Epicalyxin H, 6845

\section{$\mathrm{C}_{35} \mathrm{H}_{34} \mathrm{O}_{8}$}

Calyxin B, 3013

Calyxin E, 3014

Calyxin F, 3015

Calyxin G, 3016

Calyxin K, 3020

Calyxin L, 3021

Calyxin M, 3022

Deoxycalyxin A, 5156

Epicalyxin B, 6843

Epicalyxin F, 6844

Epicalyxin K, 6848

Epicalyxin M, 6849

$\mathrm{C}_{35} \mathrm{H}_{34} \mathrm{O}_{9}$

Calyxin A, 3012

\section{$\mathrm{C}_{35} \mathrm{H}_{34} \mathrm{O}_{10}$}

Calycopterone, 3003

\section{$\mathrm{C}_{35} \mathrm{H}_{34} \mathrm{O}_{11}$}

Tricolorin A acetate, 21592

\section{$\mathrm{C}_{35} \mathrm{H}_{34} \mathrm{O}_{18}$}

Quercetin-3-O- $\alpha$ - $L$-arabinopyranosyl- $(1 \rightarrow 6)-[2 "-$ $O$-(E)-p-coumaroyl]- $\beta$ - $D$-galactopyranoside, 18324

Quercetin-3-O- $\alpha$ - $L$-arabinopyranosyl-( $1 \rightarrow 6)$-[2"$O$-(E)-p-coumaroyl]- $\beta$ - $D$-glucopyranoside, 18325

\section{$\mathrm{C}_{35} \mathrm{H}_{34} \mathrm{O}_{19}$}

Quercetin-3-O-(2-E-caffeoyl)- $\alpha$ - $L$-arabinopyranosyl-( $1 \rightarrow 2)-\beta$ - $D$-galactopyranoside, 18318

\section{$\mathrm{C}_{35} \mathrm{H}_{36} \mathrm{~N}_{2} \mathrm{O}_{6}$}

Trilobamine, 21877

\section{$\mathrm{C}_{35} \mathrm{H}_{36} \mathrm{~N}_{2} \mathrm{O}_{9}$}

Rocaglamide derivative 11, 18891

Thapsakone A, 21277

Thapsakone B, 21278

\section{$\mathrm{C}_{35} \mathrm{H}_{36} \mathrm{O}_{11}$}

Calyflorenone C, 3008

6"-Epi-calyflorenone C, 6872

cis-p-Hydroxycinnamoylrutaevin, 9913

trans-p-Hydroxycinnamoylrutaevin, 9914

$\mathrm{C}_{35} \mathrm{H}_{36} \mathrm{O}_{12}$

Feroxin B, 7763

$\mathrm{C}_{35} \mathrm{H}_{36} \mathrm{O}_{14}$

$(2 S)$-Pinocembrin 7-O-[cinnamoyl $(1 \rightarrow 5)-\beta$ - $D$ - 
apiosyl $(1 \rightarrow 2)]$ - $\beta$ - $D$-glucoside, 17407

$\mathrm{C}_{35} \mathrm{H}_{37} \mathrm{NO}_{8}$

Regelidine, 18577

$\mathrm{C}_{35} \mathrm{H}_{38} \mathrm{~N}_{2} \mathrm{O}_{6}$

Berbamunine, 2301

$\mathrm{C}_{35} \mathrm{H}_{38} \mathrm{~N}_{2} \mathrm{O}_{9}$

Isothapsakin B, 11739

Thapsakin B, 21276

$\mathrm{C}_{35} \mathrm{H}_{38} \mathrm{~N}_{5} \mathrm{O}_{2}$

Chrysopentamine, 3614

\section{$\mathrm{C}_{35} \mathrm{H}_{38} \mathrm{O}_{14}$}

Pinoresinol $O$-[6- $O$ - $(E)$-caffeoyl]- $\beta$ - $D$-glucopyranoside, 17410

\section{$\mathrm{C}_{35} \mathrm{H}_{38} \mathrm{O}_{15}$}

Hymenoside F, 10849

\section{$\mathrm{C}_{35} \mathrm{H}_{38} \mathrm{O}_{17}$}

Hymenoside G, 10850

Hymenoside H, 10851

\section{$\mathrm{C}_{35} \mathrm{H}_{38} \mathrm{O}_{20}$}

Quercetin-3-O-[(2,3,4-triacetyl- $\alpha$-rhamnopyranosyl)-( $1 \rightarrow 6)]$-3-acetyl- $\beta$-galactopyranoside, 18400

Quercetin-3-O-[(2,3,4-triacetyl- $\alpha$-rhamnopyranosyl)-( $1 \rightarrow 6)]$-4-acetyl- $\beta$-galactopyranoside, 18401

\section{$\mathrm{C}_{35} \mathrm{H}_{39} \mathrm{~N}_{5} \mathrm{O}_{4}$}

Integerrine, 11092

\section{$\mathrm{C}_{35} \mathrm{H}_{39} \mathrm{~N}_{5} \mathrm{O}_{5}$}

Ergocristine, 7234

Ergocristinine, 7235

\section{$\mathrm{C}_{35} \mathrm{H}_{40} \mathrm{O}_{6}$}

Artocommunol CB, 1811

\section{$\mathrm{C}_{35} \mathrm{H}_{40} \mathrm{O}_{7}$}

Celastrine B, 3367

\section{$\mathrm{C}_{35} \mathrm{H}_{40} \mathrm{O}_{8}$}

$5 \alpha$-Acetyl-1 $\beta, 8 \alpha$-bis-cinnamoyl- $4 \alpha$-hydroxydihydroagarofuran, 334

Arteminolide B, 1790

Arteminolide D, 1791

\section{$\mathrm{C}_{35} \mathrm{H}_{40} \mathrm{O}_{9}$}

$1 \beta, 2 \beta$-Diacetoxy- $6 \alpha$-benzoyloxy- $9 \alpha$-cinnamoyloxy- $\beta$-dihydroagarofuran, 5285

\section{$\mathrm{C}_{35} \mathrm{H}_{40} \mathrm{O}_{10}$}

$(1 R, 2 S, 4 S, 5 R, 7 R, 9 S, 10 R)-1 \alpha$-Benzoyloxy-2 $\alpha, 15$ diacetoxy- $4 \beta$-hydroxy- $9 \beta$-cinnamoyloxy- $\beta$-dihydroagarofuran, 2254

Dantaxusin A, 4636

\section{$\mathrm{C}_{35} \mathrm{H}_{40} \mathrm{O}_{11}$}

Schisantherin J, 19492

\section{$\mathrm{C}_{35} \mathrm{H}_{40} \mathrm{O}_{12}$}

Triptofordin $\mathrm{F}_{4}, 22008$

\section{$\mathrm{C}_{35} \mathrm{H}_{40} \mathrm{O}_{13}$}

Triptofordin $\mathrm{F}_{2}, 22006$

\section{$\mathrm{C}_{35} \mathrm{H}_{40} \mathrm{O}_{16}$}

6-O- $\alpha-L-(2 "-O-, 3$ "- $O$-Dibenzoyl)rhamnopyranosylcatalpol, 5388

6-O- $\alpha-L-(2 "-O-, 4$ "-O-Dibenzoyl)rhamnopyranosylcatalpol, 5389

6-O- $\alpha-L-(3 "-O-, 4$ "-O-Dibenzoyl)rhamnopyranosylcatalpol, 5390

$\mathrm{C}_{35} \mathrm{H}_{40} \mathrm{O}_{17}$

2-Methoxy-4-[(4-hydroxybenzoyl)phenol] 1- $O-\beta$ $D$-[5-O-(3,4-dimethoxybenzoyl)]-apiofuranosyl-( $1 \rightarrow 6)-\beta$ - $D$-glucopyranoside, 13949

$\mathrm{C}_{35} \mathrm{H}_{40} \mathrm{O}_{18}$

5"-O- $\beta$-D-Glucopyranosylamarogentin, 8601

$\mathrm{C}_{35} \mathrm{H}_{40} \mathrm{O}_{19}$

5"-O- $\beta$-D-Glucopyranosylamaroswerin, 8602

Hexaacetyl-6-vaniloyl catalpol, 9477

Kaempferol-3-O-lysimachiatrioside, 12067

\section{$\mathrm{C}_{35} \mathrm{H}_{40} \mathrm{O}_{23}$}

Luteolin 7-O-[2-( $\beta$ - $D$-apiofuranosyl)-4-( $\beta$ - $D$-glucopyranosyl)-6-malonyl]- $\beta$ - $D$-glucopyranoside, 13139

\section{$\mathrm{C}_{35} \mathrm{H}_{42} \mathrm{~N}_{2} \mathrm{O}_{9}$}

Rescinnamine, 18630

Reserpinine 2, 18635

\section{$\mathrm{C}_{35} \mathrm{H}_{42} \mathrm{O}_{4}$}

Harunmadagascarin B, 9243

$\mathrm{C}_{35} \mathrm{H}_{42} \mathrm{O}_{7}$

Plocigenin, 17562

\section{$\mathrm{C}_{35} \mathrm{H}_{42} \mathrm{O}_{8}$}

Ethoxydihydroisomoreollin, 7401

Trichilinin E, 21556

\section{$\mathrm{C}_{35} \mathrm{H}_{42} \mathrm{O}_{9}$}

2-Deacetoxytaxinine B, 4707

1-Deacetylnimbolinin A, 4755

Dibenzoylgagaimol, 5384

Scortechinone G, 19552

Taxinine, 20797

Taxuspine C, 20851

\section{$\mathrm{C}_{35} \mathrm{H}_{42} \mathrm{O}_{10}$}

O-Cinnamoyltaxicin I triacetate, 3721

10-Deacetyl taxinine B, 4782

Taxezopidine J, 20788

Taxinine N,N-4, 20804

Taxuspine B, 20850

$\mathrm{C}_{35} \mathrm{H}_{42} \mathrm{O}_{12}$

13-Deacetoxy-13,15-epoxy-11(15 $\rightarrow 1)$-abeo-13epi-baccatin VI, 4706

Swietephragmin F, 20521

$3 \beta, 5 \alpha, 8 \alpha, 15 \beta$-Tetraacetoxy-7 $\beta$-benzoyloxyjatropha-6(17),11E-dien-9,14-dione, 21027

Trisdesaspidin BBB, 22032

Trisflavaspidic acid, 22033

$\mathrm{C}_{35} \mathrm{H}_{42} \mathrm{O}_{14}$
$1 \alpha, 2 \alpha$-Diacetoxy- $8 \beta$-( $\beta$-furancarbonyloxy)- $9 \alpha$ benzoyloxy-13-isobutanoyloxy- $4 \beta, 6 \beta$-dihydroxy- $\beta$-dihydroagarofuran, 5300

Sandrapin E, 19244

Taxinine M, 20803

\section{$\mathrm{C}_{35} \mathrm{H}_{42} \mathrm{O}_{17}$}

Tabulalide B, 20582

Tabulalide D, 20584

\section{$\mathrm{C}_{35} \mathrm{H}_{42} \mathbf{O}_{20}$}

Trifloroside, 21630

\section{$\mathrm{C}_{35} \mathrm{H}_{42} \mathrm{O}_{21}$}

Kaempferol

3-O- $\alpha-L-(2-O-\beta-D$-glucopyranosyl)rhamnopyranoside-7- $O-\beta$ - $D$-(6- $O$-acetyl)glucopyranoside, 12054

Rindoside, 18848

\section{$\mathrm{C}_{35} \mathrm{H}_{42} \mathrm{O}_{22}$}

Kaempferol 3- $O-\beta$ - $D$-glucosyl $(1 \rightarrow 2)-(6 "-O$-acetyl)- $\beta$ - $D$-galactoside 7- $O-\beta$ - $D$-glucoside, 12063

\section{$\mathrm{C}_{35} \mathrm{H}_{43} \mathrm{NO}_{13}$}

$1 \alpha$-Nicotinoyloxy- $2 \alpha$-acetoxy- $6 \beta$-acetoxy- $9 \beta$ furoyloxy-11-(2-methyl)butyryloxy-4 $\beta$-hydroxydihydro- $\beta$-agarofuran, 15537

\section{$\mathrm{C}_{35} \mathrm{H}_{43} \mathrm{~N}_{5} \mathrm{O}$}

Isostrychnopentamine, 11723

Strychnopentamine, 20415

\section{$\mathrm{C}_{35} \mathrm{H}_{44} \mathrm{~N}_{2} \mathrm{O}_{9}$}

Rescinnamidine, 18629

\section{$\mathrm{C}_{35} \mathrm{H}_{44} \mathrm{O}_{6}$}

Brasiliensophyllic acid A, 2591

Brasiliensophyllic acid B, 2592

Calophynic acid, 2991

Isobrasiliensophyllic acid A, 11262

Isobrasiliensophyllic acid B, 11263

\section{$\mathrm{C}_{35} \mathrm{H}_{44} \mathrm{O}_{8}$}

$5 \alpha$-Cinnamoyloxy- $9 \alpha, 10 \beta, 13 \alpha$-triacetoxytaxa4(20),11-diene, 3717

\section{$\mathrm{C}_{35} \mathrm{H}_{44} \mathrm{O}_{9}$}

Candletoxin A, 3074

$5 \alpha$-Cinnamoyloxy-7 $\beta$-hydroxy- $9 \alpha, 10 \beta, 13 \alpha$-triacetoxytaxa-4(20),11-diene, 3715

$5 \alpha$-Cinnamoyloxy-10 $\beta$-hydroxy- $2 \alpha, 9 \alpha, 13 \alpha$-triacetoxytaxa-4(20),11-diene, 3716

Taxezopidine G, 20786

Taxezopidine H, 20787

\section{$\mathrm{C}_{35} \mathrm{H}_{44} \mathrm{O}_{10}$}

$9 \alpha$-Acetyl-10 $\beta$-deacetyl-spicatine, 366

Scortechinone I, 19554

Spicatine, 20161

Taxuspine M, 20860

\section{$\mathrm{C}_{35} \mathrm{H}_{44} \mathrm{O}_{11}$}

Myrsinoltype diterpene ester CPB51-719-1, 15215 
$\mathrm{C}_{35} \mathrm{H}_{44} \mathrm{O}_{12}$

Paraliane 12, 16652

Portlandicine, 17731

Taxchinin D, 20772

$1 \beta, 5 \alpha, 14 \alpha, 17 \alpha$-Tetraacetoxy-3 $\beta$-benzoyloxy- $15 \beta$ -

hydroxy-9-oxoparaliane, 21026

\section{$\mathrm{C}_{35} \mathrm{H}_{44} \mathrm{O}_{13}$}

4-Deacetyl-11(15 $\rightarrow 1)$-abeo-baccatin VI, 4710

10-Deacetylbaccatin VI, 4727

9-Deacetylbaccatin VI, 4728

Segetene 6, 19655

Taxayunnansin A, 20758

Taxchinin M, 20779

\section{$\mathrm{C}_{35} \mathrm{H}_{44} \mathrm{O}_{14}$}

PC-2004-65-2003-19, 16740

Sandrapin C, 19242

\section{$\mathrm{C}_{35} \mathrm{H}_{44} \mathrm{O}_{15}$}

Glehlinoside B, 8546

Woorenoside IV, 22730

\section{$\mathrm{C}_{35} \mathrm{H}_{44} \mathrm{O}_{16}$}

Azadirachtin, 2050

\section{$\mathrm{C}_{35} \mathrm{H}_{44} \mathbf{O}_{18}$}

$\alpha-D$-(6-O-4-Methyl-3,5-dimethoxycinnamoyl)glucopyranosyl-(1 $\rightarrow 2)-\beta$ - $D$-(3-O-sinapoyl)fructofuranose, 14320

Tabulalide E, 20585

\section{$\mathrm{C}_{35} \mathrm{H}_{44} \mathrm{O}_{19}$}

Tenuifoliside C, 20957

\section{$\mathrm{C}_{35} \mathrm{H}_{45} \mathrm{NO}_{11}$}

Euphocharacin I, 7604

\section{$\mathrm{C}_{35} \mathrm{H}_{46} \mathrm{O}_{6}$}

Mesuaferrol, 13804

\section{$\mathrm{C}_{35} \mathrm{H}_{46} \mathrm{O}_{8}$}

12-O-Cinnamoyl-20-O-tigloyl sarcostin, 3722

\section{$\mathrm{C}_{35} \mathrm{H}_{46} \mathrm{O}_{10}$}

Bonaspectin A, 2541

Bonaspectin B, 2542

Nimbolinin B, 15612

\section{$\mathrm{C}_{35} \mathrm{H}_{46} \mathrm{O}_{11}$}

Scortechinone O, 19560

Spirosendan, 20206

Turraflorin I, 22137

\section{$\mathrm{C}_{35} \mathrm{H}_{46} \mathrm{O}_{12}$}

Meliatoxin $\mathrm{B}_{1}, 13672$

\section{$\mathrm{C}_{35} \mathrm{H}_{46} \mathrm{O}_{13}$}

Phlogacanthoside C, 17163

Trichilin A, 21553

\section{$\mathrm{C}_{35} \mathrm{H}_{46} \mathrm{O}_{14}$}

Jatrophane 8, 11844

Yunantaxusin A, 22939

\section{$\mathrm{C}_{35} \mathrm{H}_{46} \mathrm{O}_{17}$}

Ligupurpuroside B, 12818

$\mathrm{C}_{35} \mathrm{H}_{46} \mathrm{O}_{18}$
LigurobustosideN, 12823

Yadanzioside K, 22870

\section{$\mathbf{C}_{35} \mathbf{H}_{46} \mathbf{O}_{19}$}

6'- $\beta$ - $D$-Apiofuranosylcistanoside C, 1504

Jionoside E, 11886

Leonoside A, 12637

Ligupurpuroside A, 12817

Methyl epipodophyllate 7'- $O-\beta$ - $D$-Glucopyranosyl-(1 $\rightarrow 6)-\beta$ - $D$-glucopyranoside, 14400

Teucrioside-3'"'-O-methylether, 21215

Teucroside, 21221

Verpectoside A, 22416

\section{$\mathrm{C}_{35} \mathrm{H}_{46} \mathrm{O}_{\mathbf{2 0}}$}

Caerulescenoside, 2859

Purpureaside B, 18220

Purpureaside C, 18221

Verpectoside B, 22417

\section{$\mathrm{C}_{35} \mathrm{H}_{46} \mathrm{O}_{21}$}

Persicoside, 16994

\section{$\mathrm{C}_{35} \mathrm{H}_{47} \mathrm{NO}_{9}$}

9-Acetoxytaxine B, 284

10-Acetoxytaxine B, 285

\section{$\mathrm{C}_{35} \mathrm{H}_{47} \mathrm{NO}_{10}$}

Liaconitine A, 12736

Taxine A, 20793

$2^{\prime} \beta, 13 \alpha, 14 \beta$-Trisdeacetylaustrotaxine, 22031

\section{$\mathrm{C}_{35} \mathrm{H}_{47} \mathrm{~N}_{5} \mathrm{O}_{5}$}

Mauritine B, 13614

$\mathrm{C}_{35} \mathrm{H}_{48} \mathrm{ClN}_{3} \mathrm{O}_{10}$

Maytanprine, 13620

$\mathrm{C}_{35} \mathrm{H}_{48} \mathrm{~N}_{8} \mathrm{O}_{11} \mathrm{~S}$

Phalloidin, 17040

\section{$\mathrm{C}_{35} \mathrm{H}_{48} \mathrm{O}_{6}$}

Mesuanic acid, 13806

\section{$\mathrm{C}_{35} \mathrm{H}_{48} \mathrm{O}_{13}$}

$1 \alpha, 2 \alpha$-Diacetoxy- $8 \beta$-isobutanoyloxy- $9 \alpha$-benzoyloxy-13-( $\alpha$-methyl)butanoyloxy- $4 \beta, 6 \beta$-dihydro xy- $\beta$-dihydroagarofuran, 5312

\section{$\mathrm{C}_{35} \mathrm{H}_{48} \mathrm{O}_{14}$}

Jatrophane 10, 11846

$\mathrm{C}_{35} \mathrm{H}_{48} \mathrm{O}_{15}$

$1 \alpha, 2 \alpha, 6 \beta$-Triacetoxy- $8 \beta$-isobutanoyloxy- $9 \beta-(\beta$ furancarbonyloxy)-13-( $\alpha$-methyl)butanoyloxy$4 \beta$-hydroxy- $\beta$-dihydroagarofuran, 21526

\section{$\mathrm{C}_{35} \mathrm{H}_{48} \mathrm{O}_{17}$}

Javanicoside D, 11857

$\mathrm{C}_{35} \mathrm{H}_{49} \mathrm{NO}_{8}$

13-Deoxo-13 $\alpha$-acetyloxy-1-deoxytaxine B, 5139

Methyl gymnaconitine, 14478

$\mathrm{C}_{35} \mathrm{H}_{49} \mathrm{NO}_{9}$

13-Deoxo-3 $\alpha$-acetyloxytaxine B, 5140

Liaconitine C, 12738

$2^{\prime} \beta, 7 \beta, 9 \alpha$-Trisdeacetylaustrospicatine, 22030
Vilmorrianine C, 22482

\section{$\mathrm{C}_{35} \mathrm{H}_{49} \mathrm{NO}_{10}$}

Crassicauline A, 4211

\section{$\mathrm{C}_{35} \mathrm{H}_{49} \mathrm{NO}_{11}$}

Yunaconitine, 22938

\section{$\mathrm{C}_{35} \mathrm{H}_{49} \mathrm{NO}_{12}$}

Jesaconitine, 11867

\section{$\mathrm{C}_{35} \mathrm{H}_{50} \mathrm{~N}_{2} \mathrm{O}_{9}$}

Trifoliolasine A, 21635

\section{$\mathrm{C}_{35} \mathrm{H}_{50} \mathrm{O}_{4}$}

Pyrano-[7,28-b]hyperforin, 18250

$\mathrm{C}_{35} \mathrm{H}_{50} \mathrm{O}_{7}$

Camangeloyl acid, 3024

\section{$\mathrm{C}_{35} \mathrm{H}_{52} \mathrm{~N}_{2} \mathrm{O}_{4}$}

Semperviraminol, 19696

$\mathrm{C}_{35} \mathrm{H}_{52} \mathrm{O}_{4}$

Hyperforin, 10883

\section{$\mathrm{C}_{35} \mathrm{H}_{52} \mathrm{O}_{5}$}

Furohyperforin, 8026

8-Hydroxyhyperforin-8,1-hemiacetal, 10219

Lantadene A, 12490

Lantadene B, 12491

\section{$\mathrm{C}_{35} \mathrm{H}_{52} \mathrm{O}_{6}$}

Camaric acid, 3025

2,7-Dihydroxy-8-methoxy-3,6-diundecyldibenzofuran-1,4-dione, 5975

2,8-Dihydroxy-7-methoxy-3,9-diundecyldibenzofuran-1,4-dione, 5976

Icterogenin, 10960

\section{$\mathrm{C}_{35} \mathrm{H}_{52} \mathrm{O}_{7}$}

10-Hydroxy-4-O-methyl-2,11-diundecylgomphilactone, 10485

\section{$\mathrm{C}_{35} \mathrm{H}_{52} \mathrm{O}_{8}$}

Kudinoside J, 12306

Phorbol 12-tiglate 13-decanonate, 17194

Vilangin, 22478

\section{$\mathrm{C}_{35} \mathrm{H}_{52} \mathrm{O}_{9}$}

Pentadecadienoic acid, 16828

\section{$\mathrm{C}_{35} \mathrm{H}_{52} \mathrm{O}_{11}$}

$16 \beta, 22 R ; 21,23 S$-Diepoxy-3 $\beta$ - $O-\beta$ - $D$-glucopyranosyloxy-21S,24-dihydroxy- $5 \alpha$-stigmasta- 8 , 14-dien-28-one, 5493

Vernoguinosterol, 22410

\section{$\mathrm{C}_{35} \mathrm{H}_{52} \mathrm{O}_{12}$}

Atratoglaucoside A, 1991

3 - $O-\beta$-D-Glucuronopyranosyl-2 $\beta, 3 \beta, 16 \beta$-trihydroxy-28-norolean-12-en-15-on-23-oic acid, 8767

\section{$\mathrm{C}_{35} \mathrm{H}_{52} \mathrm{O}_{13}$}

$(2 R, 3 R, 4 R, 5 R, 7 S, 8 S, 9 S, 11 E, 13 S, 15 R)-2,3,5,7,8$, 9,15-Heptahydroxyjatropha-6(17),11-diene-14one-2,8,9-triacetate-7-isobutyrate-5-(2-methyl butyrate), 9399 


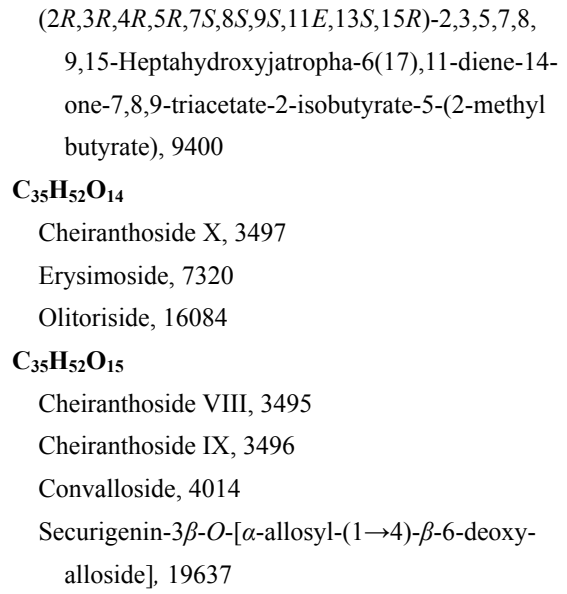

\section{$\mathrm{C}_{35} \mathrm{H}_{54} \mathrm{O}_{7}$}

22-O-Angeloyl theasapogenol E, 1237

$3 \beta$-[( $\alpha$ - $L$-Arabinopyranosyl)oxy]urs-12,18-dien28-oic acid, 1587

$3 \beta$-[( $\alpha$ - $L$-Arabinopyranosyl)oxy]-urs-12,19-dien28-oic acid, 1588

$3 \beta$-[( $\alpha$-L-Arabinopyranosyl)oxy]-urs-12,19(29)dien-28-oic acid, 1589

Fomitoside C, 7868

Ilexolide A, 10981

\section{$\mathrm{C}_{35} \mathrm{H}_{54} \mathrm{O}_{8}$}

$3 \beta$-[( $\alpha$ - $L$-Arabinopyranosyl)oxy]-19 $\beta$-hydroxyurs-12,20(30)-dien-28-oic acid, 1582

Cimiside E, 3672

12-O-Decanoylphorbol-13-(2-methylbutyrate), 4839

Fomitoside A, 7866

Fomitoside B, 7867

Phorbol-12- $\alpha$-methylbutyrate-13-caprate, 17188

$\mathrm{C}_{35} \mathrm{H}_{54} \mathrm{O}_{9}$

7,8-Didehydrocimigenol 3- $O-\beta$ - $D$-xylopyranoside, 5464

Gamboukokoenside B, 8131

\section{$\mathrm{C}_{35} \mathrm{H}_{54} \mathrm{O}_{10}$}

Bugbanoside F, 2732

24-Hydroxy-15,16-seco-cycloart-7-en 3-O-xyloside, 10697

3-O- $\beta$ - $D$-Xylopyranosyl-esculentic acid, 22822

\section{$\mathrm{C}_{35} \mathrm{H}_{54} \mathrm{O}_{11}$}

Esculentoside B, 7369
Esculentoside E, 7372

\section{$\mathrm{C}_{35} \mathrm{H}_{54} \mathrm{O}_{12}$}

$2 \alpha, 5 \alpha$-Diacetoxy-14 $\beta$-2 $2^{\prime} \alpha$-methylbutanoate- $10 \beta$ $O$-( $\beta$ - $D$-glucopyranosyl)taxa-4(20),11-diene, 5315

\section{$\mathrm{C}_{35} \mathrm{H}_{54} \mathrm{O}_{13}$}

$2 \alpha, 5 \alpha$-Diacetoxy-14 $\beta$-(2'S,3' $R)$-3'-hydroxy-2' $\alpha$ methylbutanoate-10 $\beta$ - $O-(\beta-D$-glucopyranosyl) taxa-4(20),11-diene, 5310

\section{$\mathrm{C}_{35} \mathrm{H}_{54} \mathrm{O}_{14}$}

Erycordine, 7303

Erysimosol, 7321

Euonymoside A, 7535

Rhodexin C, 18787

Rhodexoside, 18789

Sarmentogenin-3 $\beta$-O- $[\alpha$-allosyl- $(1 \rightarrow 4)-\beta-6$ deoxyalloside], 19373

\section{$\mathrm{C}_{35} \mathrm{H}_{56} \mathrm{O}_{5}$}

Lantaiursolic acid, 12492

\section{$\mathrm{C}_{35} \mathrm{H}_{56} \mathrm{O}_{6}$}

22-O-Angeloyl theasapogenol $\mathrm{B}, 1236$

\section{$\mathrm{C}_{35} \mathrm{H}_{56} \mathrm{O}_{7}$}

$O$-Acetylpachymic acid-25-ol, 478

22-O-Angeloyl theasapogenol A, 1235

3-O- $\alpha$ - $L$-Arabinopyranosyloleanolic acid, 1576

Inermiside II, 11036

Sanguisorbin B, 19287

3-O- $\beta$ - $D$-Xylopyranosyloleanolic acid, 22839

\section{$\mathrm{C}_{35} \mathrm{H}_{56} \mathrm{O}_{8}$}

3-O- $\alpha$-L-Arabinopyranosyl-23-hydroxyursolic acid, 1573

3- $\beta$ - $O-\alpha$ - $L$-Arabinopyranosyl jujubogenin, 1574

$3 \beta$-[( $\alpha$-L-Arabinopyranosyl)oxy]-19 $\alpha$-hydroxyolean-12-en-28-oic acid, 1579

$3 \beta$-[( $\alpha$-L-Arabinopyranosyl)oxy $]-19 \alpha$-hydroxyurs-12-en-28-oic acid, 1584

Cauloside A, 3340

Leontoside A, 12642

Prosapogenin $\mathrm{CP}_{0}, 17943$

Prostratoside J, 17962

Pulsatilloside A, 18192

Ziyu glycoside II, 23009

\section{$\mathrm{C}_{35} \mathrm{H}_{56} \mathrm{O}_{9}$}

Cauloside B, 3341

Cimiaceroside B, 3649

Cimigenol 3- $O-\alpha-L$-arabinopyranoside, 3659

Cimigenoside, 3661

Shengmanol xyloside, 19800

\section{$\mathrm{C}_{35} \mathrm{H}_{56} \mathrm{O}_{10}$}

Cimiside A, 3670

Eupteleasaponin VIII, 7631

Gamboukokoenside A, 8130

Pictoside B, 17351
$\mathrm{C}_{35} \mathrm{H}_{56} \mathrm{O}_{11}$

12 $\beta, 21$-Dihydroxycimigenol

3- $O-\alpha$ - $L$-arabinopyranoside, 5790

\section{$\mathrm{C}_{35} \mathrm{H}_{56} \mathrm{O}_{12}$}

Cilistol pl, 3642

Cilistol pm, 3643

Deacetylmetaplexigenin 3- $O-\beta$ - $D$-oleandropyranosyl-( $1 \rightarrow 4)-\alpha-D$-oleandropyranoside, 4751

\section{$\mathrm{C}_{35} \mathrm{H}_{58} \mathrm{O}_{6}$}

$\alpha$-Spinasterol- $\beta$ - $D$-glucoside, 20169

5,25-Stigmastadien-3 $\beta$-ol- $\beta$ - $D$-glucoside, 20346

Stigmasterol- $\beta$ - $D$-glucoside, 20372

$\mathrm{C}_{35} \mathrm{H}_{58} \mathrm{O}_{7}$

7-Oxositosteryl- $\beta$ - $O$-glucopyranoside, 16422

\section{$\mathrm{C}_{35} \mathrm{H}_{58} \mathrm{O}_{9}$}

Astramembrannin II, 1946

Beesioside A, 2192

Cycloalpioside C, 4465

\section{$\mathrm{C}_{35} \mathrm{H}_{58} \mathrm{O}_{10}$}

Beesioside B, 2193

Beesioside C, 2194

Beesioside E, 2196

Beesioside N, 2207

Denicunine, 5130

$12 \beta$-Hydroxycimigenol 3-O- $\alpha$ - $L$-arabinopyranoside, 9905

\section{$\mathrm{C}_{35} \mathrm{H}_{58} \mathrm{O}_{11}$}

Cilistol I, 3639

\section{$\mathrm{C}_{35} \mathrm{H}_{58} \mathrm{O}_{11} \mathrm{~S}$}

Spergulin A, 20144

\section{$\mathrm{C}_{35} \mathrm{H}_{58} \mathrm{O}_{18}$}

Periplocae oligosaccharide $\mathrm{D}_{2}, 16939$

\section{$\mathrm{C}_{35} \mathrm{H}_{60} \mathrm{O}_{3}$}

4'-Hydroxy-cis-cinnamic acid hexacosyl ester, 9910

\section{$\mathrm{C}_{35} \mathrm{H}_{60} \mathrm{O}_{4}$}

Hexacosanyl caffeate, 9481

\section{$\mathrm{C}_{35} \mathrm{H}_{60} \mathrm{O}_{6}$}

Bacosterol-3-O- $\beta$ - $D$-glucopyranoside, 2095

Daucosterol, 4680

3-O- $\beta$ - $D$-Glucopyranosyl- $\beta$-sitosterol, 8732

$\beta$-Sitosterol-3-O- $\beta$ - $D$-glucoside, 19985

$\beta$-Sitosterol- $\alpha$ - $D$-glucoside, 19986

$\beta$-Sitosterol- $\beta$ - $D$-glucoside, 19987

7-Stigmastenol-3-O- $\beta$ - $D$-glucoside, 20367

\section{$\mathrm{C}_{35} \mathrm{H}_{62} \mathrm{O}_{3}$}

Sabadelin, 19096

\section{$\mathrm{C}_{35} \mathrm{H}_{62} \mathrm{O}_{6}$}

Annocatacin A, 1294

Annocatacin B, 1295

Corossolone, 4090

cis-Corossolone, 4091 
$\mathrm{C}_{35} \mathrm{H}_{62} \mathrm{O}_{7}$

Annomolon A, 1304

Annonacin-10-one, 1315

cis-Annonacin-10-one, 1316

34-Epiannomolon A, 6827

22-Epimolvizarin, 6972

Molvizarin, 14906

Muricin F, 15095

Muricin G, 15096

Parviflorin, 16677

Reticulacinone, 18653

Squamone, 20244

$\mathrm{C}_{35} \mathrm{H}_{62} \mathrm{O}_{8}$

Annomolon B, 1305

34-Epiannomolon B, 6828

Montacin, 14934

cis-Montacin, 14935

Parvifloracin, 16676

\section{$\mathrm{C}_{35} \mathrm{H}_{64} \mathrm{O}_{5}$}

Solamin, 20051

\section{$\mathrm{C}_{35} \mathrm{H}_{64} \mathrm{O}_{6}$}

Corossoline, 4089

Gigantriocin, 8381

Longifolicin, 12956

Montalicin B, 14937

Muricin H, 15097

Murisolin, 15099

\section{$\mathrm{C}_{35} \mathrm{H}_{64} \mathrm{O}_{7}$}

Annocatalin, 1296

Annomolin, 1303

cis-Annomuricin, 1310

Annonacin, 1313

cis-Annonacin, 1314

Annoreticuin, 1324

cis-Annoreticuin, 1325

Arianacin, 1679

Asitrilobin B, 1859

cis-Goniothalamicin, 8946

Itrabin, 11793

Javoricin, 11864

Montalicin C, 14938

Montalicin D, 14939

Montalicin F, 14941

Muricatetrocin A, 15084

Muricatetrocin B, 15085

Muricin A, 15090

Muricin B, 15091

Muricin C, 15092

Pyragonicin, 18240

Pyranicin, 18249

Xylopianan, 22817

$\mathrm{C}_{35} \mathrm{H}_{64} \mathrm{O}_{8}$

Annomonicin, 1306
Annomuricin A, 1311

Annomuricin B, 1312

Annopentocin A, 1320

Annopentocin B, 1321

Annopentocin C, 1322

Muricatalicin, 15083

Muricatocin A, 15087

Muricatocin B, 15088

Muricatocin C, 15089

$\mathrm{C}_{35} \mathrm{H}_{66} \mathrm{O}_{6}$

Gardnerilin B, 8233

$\mathrm{C}_{35} \mathrm{H}_{66} \mathrm{O}_{8}$

Gardnerilin A, 8232

$\mathrm{C}_{35} \mathrm{H}_{67} \mathrm{NO}_{3}$

(2S,3R,4E,8E)- $N$-Hexadecanoyl-2-amino-9-methyl-4,8-octadecadiene-1,3-diol, 9487

\section{$\mathrm{C}_{35} \mathrm{H}_{67} \mathrm{NO}_{4}$}

(2S,3R,4E,8E)-[(2'R)-2'-Hydroxyheptadecanoylamino]-4,8-octadecadiene-1,3-diol, 10158

\section{$\mathrm{C}_{36} \mathrm{H}_{22} \mathrm{O}_{18}$}

6,6'-Bieckol, 2366

8,8'-Bieckol, 2367

Dieckol, 5484

\section{$\mathrm{C}_{36} \mathrm{H}_{26} \mathrm{O}_{10}$}

Terrestrin F, 21011

$\mathrm{C}_{36} \mathrm{H}_{26} \mathrm{O}_{20}$

Balanophotannin C, 2129

$\mathrm{C}_{36} \mathrm{H}_{28} \mathrm{~K}_{2} \mathrm{O}_{16}$

Dipotassium rabdosiin, 6495

$\mathrm{C}_{36} \mathrm{H}_{28} \mathrm{KNaO}_{16}$

NP02140176-41-KNa, 15853

$\mathrm{C}_{36} \mathrm{H}_{28} \mathrm{MgO}_{16}$

Magnesium lithospermate B, 13370

$\mathrm{C}_{36} \mathrm{H}_{28} \mathrm{~N}_{2} \mathrm{O}_{6}$

Artabonatine F, 1774

$\mathrm{C}_{36} \mathrm{H}_{28} \mathrm{O}_{6}$

Neo-przewaquinone A, 15453

$\mathrm{C}_{36} \mathrm{H}_{28} \mathrm{O}_{12}$

Griffipavixanthone, 9003

$\mathrm{C}_{36} \mathrm{H}_{28} \mathrm{O}_{13}$

Bijaponicaxanthone, 2371

$\mathrm{C}_{36} \mathrm{H}_{28} \mathrm{O}_{15}$

$R$-(-)-Skyrin-6-O- $\beta$-glucopyranoside, 20012

$S$-(+)-Skyrin-6- $O$ - $\beta$-glucopyranoside, 20013

$\mathrm{C}_{36} \mathrm{H}_{28} \mathrm{O}_{16}$

Schizotenuin A, 19510

$\mathrm{C}_{36} \mathrm{H}_{28} \mathrm{O}_{21}$

1-O-(E)-Caffeoyl-3- $O$-galloyl-4,6-(S)-HHDP- $\beta$ -

$D$-glucopyranose, 2904

$\mathrm{C}_{36} \mathrm{H}_{29} \mathrm{KO}_{16}$

NP02140176-38-K, 15850

NP02140176-42-K, 15854

$\mathrm{C}_{36} \mathrm{H}_{29} \mathrm{NaO}_{16}$
NP02140176-39-Na, 15851

$\mathrm{C}_{36} \mathrm{H}_{30} \mathrm{MgO}_{16}$

Lithospermate B, 12924

Magnesium rosmarinate, 13371

$\mathrm{C}_{36} \mathrm{H}_{30} \mathrm{O}_{4}$

Dineolignan, 6423

$\mathrm{C}_{36} \mathrm{H}_{30} \mathrm{O}_{14}$

Podoverin C, 17599

$\mathrm{C}_{36} \mathrm{H}_{30} \mathrm{O}_{15}$

Podoverin B, 17598

Spicataside, 20158

$\mathrm{C}_{36} \mathrm{H}_{30} \mathrm{O}_{16}$

1,3-Bis-[2-(3,4-dihydroxyphenyl)-1-carboxy] ethoxycarbonyl-2-(3,4-dihydroxyphenyl)-7,8di-hydroxy-1,2-dihydronaphthalene, 2444

Danshensuan B, 4631

Fukugiside, 7991

Lithospermic acid B, 12926

NP02140176-40, 15852

Salvianolic acid E, 19205

$\mathrm{C}_{36} \mathrm{H}_{30} \mathrm{O}_{21}$

6-O-(E)-Caffeoyl-1,3,4-tri- $O$-galloyl- $\beta$ - $D$-glucopyranose, 2929

\section{$\mathrm{C}_{36} \mathrm{H}_{32} \mathrm{~N}_{2} \mathrm{O}_{4}$}

Kwangsine, 12396

\section{$\mathrm{C}_{36} \mathrm{H}_{32} \mathrm{~N}_{2} \mathrm{O}_{8}$}

Oriciacridone F, 16182

\section{$\mathrm{C}_{36} \mathrm{H}_{32} \mathrm{O}_{6}$}

1,3,6-Tri(4-hydroxybenzyl)-4-methoxydihydrophenanthrene-2,7-diol, 21690

\section{$\mathrm{C}_{36} \mathrm{H}_{32} \mathrm{O}_{10}$}

Angustifolin A, 1246

\section{$\mathrm{C}_{36} \mathrm{H}_{32} \mathrm{O}_{15}$}

3,8"-Binaringenin-7"- $O$ - $\beta$-glucoside, 2383

\section{$\mathrm{C}_{36} \mathrm{H}_{32} \mathrm{O}_{16}$}

Xanthochymusside, 22760

\section{$\mathrm{C}_{36} \mathrm{H}_{33} \mathrm{O}_{16}$}

Salvianolic Acid B, 19202

\section{$\mathrm{C}_{36} \mathrm{H}_{34} \mathrm{~N}_{2} \mathrm{O}_{6}$}

Menisarine, 13713

Stebisimine, 20283

\section{$\mathrm{C}_{36} \mathrm{H}_{34} \mathrm{~N}_{2} \mathrm{O}_{9}$}

Tribulusamide B, 21547

\section{$\mathrm{C}_{36} \mathrm{H}_{36} \mathrm{~N}_{2} \mathrm{O}_{5}$}

Isotrilobine, 11747

Tiliacorine, 21389

\section{$\mathrm{C}_{36} \mathrm{H}_{36} \mathrm{~N}_{2} \mathrm{O}_{6}$}

Cepharanoline, 3411

Hypoepistephanine, 10893

Isotrilobine-2- $N$-oxide, 11748

$O$-Methyldeoxopunjabine, 14289

\section{$\mathrm{C}_{36} \mathrm{H}_{36} \mathrm{~N}_{2} \mathrm{O}_{8}$}

Cannabisin D, 3087 
Cannabisin G, 3088

Grossamide, 9013

Hyoscyamide, 10871

Tribulusamide A, 21546

$\mathrm{C}_{36} \mathrm{H}_{36} \mathrm{O}_{6}$

Magnolignan F, 13383

$\mathrm{C}_{36} \mathrm{H}_{36} \mathrm{O}_{8}$

Magnolignan G, 13384

$\mathrm{C}_{36} \mathrm{H}_{36} \mathrm{O}_{11}$

3'-Prenyl-4'-methoxy-isoflavone-7-O'- $\beta-D-(2 "-O$ p-coumaroyl)glycopyranoside, 17838

\section{$\mathrm{C}_{36} \mathrm{H}_{36} \mathrm{O}_{16}$}

Luteoliflavan-( $4 \beta \rightarrow 8)$-eriodictyol-5-glucoside, 13136

\section{$\mathrm{C}_{36} \mathrm{H}_{36} \mathrm{O}_{17}$}

Isovitexin 2"-O-(6"'-(E)-p-coumaroyl)glucoside, 11774

Kaempferol 3-O- $\alpha-L$-[6"'-p-coumaroyl- $(\beta-D)$-glucopyranosyl-(1,2)-rhamnopyranoside], 12032

Kaempferol 3-O- $\alpha$ - $L$-rhamnopyranosyl $(1 \rightarrow 6)$ (4-O-trans- $p$-coumaroyl)- $\beta$ - $D$-galactopyranoside, 12072

Procyanidin $\mathrm{B}_{1}-6-C-\beta$ - $D$-glucopyranoside, 17878 Procyanidin $\mathrm{B}_{1}-8-C-\beta$ - $D$-glucopyranoside, 17879 Procyanidin $\mathrm{B}_{2}-3$ "- $O-\beta$ - $D$-allopyranoside, 17880 Procyanidin $\mathrm{B}_{2}-6-C$-glucopyranoside, 17882

Procyanidin $\mathrm{B}_{2}-8-C$ - $\beta$ - $D$-glucopyranoside, 17883

Procyanidin $\mathrm{B}_{3}$-7-O- $\beta$ - $D$-glucopyranoside, 17885

\section{$\mathrm{C}_{36} \mathrm{H}_{36} \mathrm{O}_{18}$}

Kaempferol 3-O- $\beta$-(6"-E-p-coumaroylglucopyranoside)-7- $O$ - $\beta$-glucopyranoside, 12031

Quercetin-3-O- $\alpha-L$-[6"'- $p$-coumaroyl- $(\beta-D)$-glucopyranosyl-(1,2)-rhamnopyranoside], 18335

Quercetin-3-O- $\alpha$ - $L$-rhamnopyranosyl $(1 \rightarrow 6)$-(3-

$O$-trans- $p$-coumaroyl)- $\beta$ - $D$-galactopyranoside, 18381

Quercetin-3-O- $\alpha$ - $L$-rhamnopyranosyl $(1 \rightarrow 6)$-(4$O$-trans- $p$-coumaroyl)- $\beta$ - $D$-galactopyranoside, 18382

\section{$\mathrm{C}_{36} \mathrm{H}_{36} \mathrm{O}_{19}$}

Quercetin-3-O- $\beta$-(6"- $E$ - $p$-coumaroylglucopyranoside)-7-O- $\beta$-glucopyranoside, 18334

\section{$\mathrm{C}_{36} \mathrm{H}_{37} \mathrm{O}_{18}{ }^{+}$}

Shisonin, 19838

$\mathrm{C}_{36} \mathrm{H}_{37} \mathrm{O}_{19}{ }^{+}$

Caffeoylcyanin, 2896

\section{$\mathrm{C}_{36} \mathrm{H}_{38} \mathrm{~N}_{2} \mathrm{O}_{6}$}

Aromoline, 1769

(+)-Aromoline, 1770

L-Bebeerine, 2191

Hayatine, 9251

Isochondrodendrin, 11329

(+)-2-Northalrugosine, 15804
(+)-Obamegine, 15885

Sepeerine, 19753

Thalicrine, 21244

$\mathrm{C}_{36} \mathrm{H}_{38} \mathrm{~N}_{2} \mathrm{O}_{8}$

Wilforcidine, 22674

$\mathrm{C}_{36} \mathrm{H}_{38} \mathrm{~N}_{2} \mathrm{O}_{9}$

Heterophylline, 9470

$\mathrm{C}_{36} \mathrm{H}_{38} \mathrm{~N}_{2} \mathrm{O}_{10}$

Thapoxepine A, 21274

$\mathrm{C}_{36} \mathrm{H}_{38} \mathrm{~N}_{2} \mathrm{O}_{11}$

Rhynchophine, 18824

$\mathrm{C}_{36} \mathrm{H}_{38} \mathrm{~N}_{4} \mathrm{O}_{5}$

Methyl pheophorbide a, 14673

$\mathrm{C}_{36} \mathrm{H}_{38} \mathrm{O}_{8}$

Tinyatoxin, 21402

$\mathrm{C}_{36} \mathrm{H}_{38} \mathrm{O}_{11}$

6"-Epi-calyflorenone B, 6871

Voamatin D, 22601

$\mathrm{C}_{36} \mathrm{H}_{38} \mathrm{O}_{15}$

Glycerol sinapate, 8811

$\mathrm{C}_{36} \mathrm{H}_{40} \mathrm{~N}_{2} \mathrm{O}_{6}$

Dauricoline, 4687

$\mathrm{C}_{36} \mathrm{H}_{40} \mathrm{~N}_{2} \mathrm{O}_{8}$

Elliptifoline, 6760

$\mathrm{C}_{36} \mathrm{H}_{40} \mathrm{~N}_{2} \mathrm{O}_{9}$

Homothapsakin A, 9623

\section{$\mathrm{C}_{36} \mathrm{H}_{40} \mathrm{O}_{10}$}

Orthosiphol N, 16232

Orthosiphonone D, 16244

\section{$\mathrm{C}_{36} \mathrm{H}_{40} \mathrm{O}_{11}$}

Voamatin A, 22598

Voamatin B, 22599

\section{$\mathrm{C}_{36} \mathrm{H}_{40} \mathrm{O}_{12}$}

1-O-3,4-Dimethoxy-phenylethyl-4-O-3,4-dimethoxy cinnamoyl-6- $O$-cinnamoyl- $\beta$ - $D$-glucopyranose, 6285

\section{$\mathrm{C}_{36} \mathrm{H}_{40} \mathrm{O}_{19}$}

Ciliatoside A, 3638

Patavine, 16703

$\mathrm{C}_{36} \mathrm{H}_{41} \mathrm{NO}_{7}$

Aristoloin II, 1722

$\mathrm{C}_{36} \mathrm{H}_{41} \mathrm{NO}_{14}$

$1 \beta, 2 \beta, 5 \alpha, 11$-Tetraacetoxy- $8 \alpha$-benzoyl- $4 \alpha$-hydrox-

y-7 $\beta$-nicotinoyl-dihydroagarofuran, 21023

$\mathrm{C}_{36} \mathrm{H}_{42} \mathrm{~N}_{2}$

Caulindole C, 3338

Caulindole D, 3339

$\mathrm{C}_{36} \mathrm{H}_{42} \mathrm{~N}_{2} \mathrm{O}_{9}$

3'-Hydroxyaglaine B, 9768

3'-Hydroxyaglaine C, 9769

$\mathrm{C}_{36} \mathrm{H}_{42} \mathrm{~N}_{2} \mathrm{O}_{10}$

3',19-Dihydroxyaglaine C, 5753

$\mathrm{C}_{36} \mathrm{H}_{42} \mathrm{O}_{10}$
7-O-Deacetylorthosiphol B, 4759

Orthosiphol K, 16229

Orthosiphol T, 16235

Orthosiphol X, 16239

\section{$\mathrm{C}_{36} \mathrm{H}_{42} \mathrm{O}_{11}$}

Taxayuntin A, 20759

Triptofordin $\mathrm{D}_{2}, 22003$

$\mathrm{C}_{36} \mathrm{H}_{42} \mathrm{O}_{19}$

3"'-O-Glucosylsenburiside II, 8758

\section{$\mathrm{C}_{36} \mathrm{H}_{42} \mathrm{O}_{23}$}

Kaempferol 3-O- $\alpha-L-(2-O-\beta-D$-glucopyranosyl)rhamnopyranoside-7- $O-\beta$ - $D$-(6- $O$-malonyl)glucopyrano- side, 12056

\section{$\mathrm{C}_{36} \mathrm{H}_{43} \mathrm{NO}_{8}$}

Gagaminine, 8046

\section{$\mathrm{C}_{36} \mathrm{H}_{43} \mathrm{NO}_{15}$}

$1 \beta$-Nicotinoyl- $2 \beta, 5 \alpha, 7 \beta$-triacetoxy- $4 \alpha$-hydroxy11-isobutyryloxy- $8 \alpha$-furanoyl-dihydroagarofuran, 15538

\section{$\mathrm{C}_{36} \mathrm{H}_{43} \mathrm{NO}_{17}$}

Evonine, 7671

\section{$\mathrm{C}_{36} \mathrm{H}_{43} \mathrm{O}_{24}{ }^{+}$}

Cyanidin-3-O-(3"-O- $\beta$-glucopyranosyl-6"-Omalonyl- $\beta$-glucopyranoside)-4'- $O-\beta$-glucopyranoside, 4443

Cyanidin-7-O-(3"-O- $\beta$-glucopyranosyl-6"- $O$ malonyl- $\beta$-glucopyranoside)-4'- $O$ - $\beta$-glucopyranoside, 4444

\section{$\mathrm{C}_{36} \mathrm{H}_{44} \mathrm{~N}_{2} \mathrm{O}_{7}$}

Nigellamine $\mathrm{A}_{4}, 15564$

$\mathrm{C}_{36} \mathrm{H}_{44} \mathrm{~N}_{2} \mathrm{O}_{11}$

14-Demethyl-14-acetylanhweidelphinine, 5056

\section{$\mathrm{C}_{36} \mathrm{H}_{44} \mathrm{O}_{12}$}

Agrimol C, 754

Agrimol G, 756

Filixic acid BBB, 7798

Japonicin D, 11820

Swietephragmin D, 20519

Trisaspidin, 22029

\section{$\mathrm{C}_{36} \mathrm{H}_{44} \mathrm{O}_{14}$}

Sandrapin D, 19243

Taccalonolide H, 20590

\section{$\mathrm{C}_{36} \mathrm{H}_{44} \mathrm{O}_{15}$}

Dracunculifoside R, 6597

\section{$\mathrm{C}_{36} \mathrm{H}_{44} \mathrm{O}_{16}$}

Epimedokoreanoside II, 6963

\section{$\mathrm{C}_{36} \mathrm{H}_{44} \mathrm{O}_{20}$}

5-Hydroxy-4-methoxy-flavone-7- $O-\alpha$ - $L$-rhamnopyranosyl- $(1 \rightarrow 6)[2-O$-acetyl- $\beta$ - $D$-glucopyranosyl-( $1 \rightarrow 2)]-\beta$ - $D$-glucopyranoside, 10405

Telephiose A, 20907

Telephiose B, 20908

$\mathrm{C}_{36} \mathrm{H}_{44} \mathrm{O}_{22}$ 


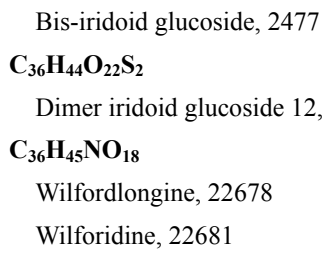

\section{$\mathrm{C}_{36} \mathrm{H}_{45} \mathrm{NO}_{18}$}

Wilfordlongine, 22678

Wilforidine, 22681

\section{$\mathrm{C}_{36} \mathrm{H}_{46} \mathrm{O}_{6}$}

Brasiliensophyllic acid C, 2593

Inocalophyllin A methyl ester, 11064

Isobrasiliensophyllic acid C, 11264

\section{$\mathrm{C}_{36} \mathrm{H}_{46} \mathrm{O}_{11}$}

Neobonaspectin A, 15344

\section{$\mathrm{C}_{36} \mathrm{H}_{46} \mathrm{O}_{14}$}

Sandrapin B, 19241

Taccalonolide J, 20592

Trichilin H, 21554

\section{$\mathrm{C}_{36} \mathrm{H}_{46} \mathrm{O}_{15}$}

Taccalonolide L, 20594

\section{$\mathrm{C}_{36} \mathrm{H}_{46} \mathrm{O}_{16}$}

Umbilicaxanthoside B, 22198

\section{$\mathrm{C}_{36} \mathrm{H}_{46} \mathrm{O}_{19}$}

Glomeratose D, 8585

\section{$\mathrm{C}_{36} \mathrm{H}_{47} \mathrm{NO}_{11}$}

Euphocharacin $\mathrm{H}, 7603$

\section{$\mathrm{C}_{36} \mathrm{H}_{48} \mathrm{CIN}_{3} \mathrm{O}_{10}$}

Normaytancyprine, 15776

\section{$\mathrm{C}_{36} \mathrm{H}_{48} \mathrm{~N}_{2} \mathrm{O}_{10}$}

Lycaconitine, 13169

\section{$\mathrm{C}_{36} \mathrm{H}_{48} \mathrm{~N}_{6} \mathrm{O}_{7}$}

Dianthin C, 5367

\section{$\mathrm{C}_{36} \mathrm{H}_{48} \mathrm{O}_{5}$}

Oleoyl danshenxinkun A, 16072

\section{$\mathrm{C}_{36} \mathrm{H}_{48} \mathrm{O}_{9}$}

( \pm )-Schefflone, 19464

\section{$\mathrm{C}_{36} \mathrm{H}_{48} \mathrm{O}_{11}$}

15-O-Deacetylnimbolidin B, 4754

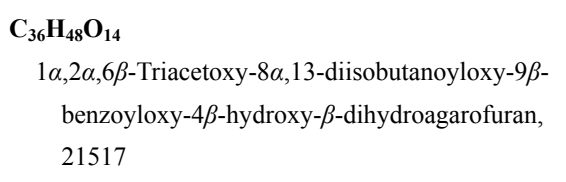

\section{$\mathrm{C}_{36} \mathrm{H}_{48} \mathrm{O}_{16}$}

Phyllanthostatin 6, 17218

\section{$\mathrm{C}_{36} \mathrm{H}_{48} \mathrm{O}_{18}$}

Yadanzioside G, 22866

\section{$\mathrm{C}_{36} \mathrm{H}_{48} \mathrm{O}_{19}$}

Angoroside C, 1241

Lamiophlomioside A, 12462

cis-Lamiophlomiside A, 12463

Leonoside B, 12638

Leucosceptoside B, 12726

Teucrioside-3"'",4"'-O-dimethylether, 21214

\section{$\mathrm{C}_{36} \mathrm{H}_{48} \mathrm{O}_{20}$}

Cistanoside A, 3751
Jionoside $A_{2}, 11881$

Verpectoside C, 22418

Wiedemannioside C, 22664

$\mathrm{C}_{36} \mathrm{H}_{48} \mathrm{O}_{21}$

Scroside A, 19572

$\mathrm{C}_{36} \mathrm{H}_{49} \mathrm{NO}_{8}$

Leueantine B, 12729

$\mathrm{C}_{36} \mathrm{H}_{49} \mathrm{NO}_{9}$

Leueantine A, 12728

$\mathrm{C}_{36} \mathrm{H}_{49} \mathrm{NO}_{12}$

3-Acetylaconitine, 304

$\mathrm{C}_{36} \mathrm{H}_{49} \mathrm{~N}_{5} \mathrm{O}_{5}$

Amphibine D, 1081

$\mathrm{C}_{36} \mathrm{H}_{49} \mathrm{~N}_{7} \mathrm{O}_{10}$

Glabrin D, 8495

$\mathrm{C}_{36} \mathrm{H}_{50} \mathrm{ClN}_{3} \mathrm{O}_{10}$

Maytanbutine, 13619

$\mathrm{C}_{36} \mathrm{H}_{50} \mathrm{~N}_{2} \mathrm{O}_{11}$

Puberaconitine, 18167

$\mathrm{C}_{36} \mathrm{H}_{50} \mathrm{O}_{4}$

Physanol A, 17245

$\mathrm{C}_{36} \mathrm{H}_{50} \mathrm{O}_{18}$

Javanicoside E, 11858

$\mathrm{C}_{36} \mathrm{H}_{51} \mathrm{NO}_{10}$

Veratroyl zygadenine, 22395

$\mathrm{C}_{36} \mathrm{H}_{51} \mathrm{NO}_{11}$

Bikhaconitine, 2372

Polyschistine A, 17672

$\mathrm{C}_{36} \mathrm{H}_{51} \mathrm{NO}_{12}$

Pseudoaconitine, 18017

\section{$\mathrm{C}_{36} \mathrm{H}_{51} \mathbf{N}_{3} \mathbf{O}_{10}$}

Awadcharidine, 2042

Trifoliolasine B, 21636

\section{$\mathrm{C}_{36} \mathrm{H}_{52} \mathrm{~N}_{2} \mathrm{O}_{9}$}

Jiufengdine, 11887

$\mathrm{C}_{36} \mathrm{H}_{52} \mathbf{N}_{4} \mathbf{O}_{10}$

Bufotalin 3-succinoylarginine ester, 2724

\section{$\mathrm{C}_{36} \mathrm{H}_{52} \mathrm{O}_{4}$}

Physanol B, 17246

\section{$\mathrm{C}_{36} \mathrm{H}_{52} \mathrm{O}_{6}$}

Hericenone H, 9436

$\mathrm{C}_{36} \mathrm{H}_{52} \mathrm{O}_{7}$

Methylanhydrovilangin, 14140

$\mathrm{C}_{36} \mathrm{H}_{52} \mathrm{O}_{8}$

Mancinellin, 13476

$\mathrm{C}_{36} \mathrm{H}_{52} \mathrm{O}_{10}$

Khekadaengoside G, 12214

Khekadaengoside H, 12215

$\mathrm{C}_{36} \mathrm{H}_{52} \mathrm{O}_{11}$

Khekadaengoside C, 12210

Khekadaengoside I, 12216

3,11,22-Trioxo-16 $\alpha$-hydroxy-(20S,24)-epoxycucurbit-5,23-diene-2 $\beta$ - $O-\beta$ - $D$-glucopyrano- side, 21985

$\mathrm{C}_{36} \mathrm{H}_{52} \mathrm{O}_{13}$

Scillaren A, 19518

Scillirubrosidin 3-O- $\alpha-L$-rhamnopyranosyl-

$(1 \rightarrow 4)-\alpha$ - $L$-rhamnopyranoside, 19530

$\mathrm{C}_{36} \mathrm{H}_{52} \mathrm{O}_{14}$

Baccatin VII, 2078

$\mathrm{C}_{36} \mathrm{H}_{52} \mathrm{O}_{15}$

Hellebrin, 9330

$\mathrm{C}_{36} \mathrm{H}_{53} \mathrm{NO}_{12}$

Nervosine, 15511

$\mathrm{C}_{36} \mathrm{H}_{53} \mathrm{~N}_{7} \mathrm{O}_{8}$

Dianthin D, 5368

$\mathrm{C}_{36} \mathbf{H}_{54} \mathbf{N}_{2} \mathbf{O}_{6}$

6",7"-Dihydro-5',5"'-dicapsaicin, 5588

$\mathrm{C}_{36} \mathrm{H}_{54} \mathrm{O}_{6}$

Camaryolic acid, 3028

\section{$\mathrm{C}_{36} \mathrm{H}_{54} \mathrm{O}_{8}$}

Methylvilangin, 14809

Synaptolepis factor $\mathrm{K}_{1}, 20550$

\section{$\mathrm{C}_{36} \mathrm{H}_{54} \mathrm{O}_{10}$}

Abrusoside A, 31

3-O- $\beta$ - $D$-Glucuronopyranosyl gypsogenin, 8765 Vaccaroside, 22303

\section{$\mathrm{C}_{36} \mathrm{H}_{54} \mathrm{O}_{12}$}

Arvenin III, 1827

Bryoamaride, 2686

Khekadaengoside D, 12211

Khekadaengoside E, 12212

\section{$\mathrm{C}_{36} \mathrm{H}_{54} \mathrm{O}_{13}$}

Cucurbitacin J 2-O- $\beta$-glucopyranoside, 4325

Cucurbitacin K 2-O- $\beta$-glucopyranoside, 4326

$(2 R, 3 R, 4 R, 5 R, 7 S, 8 S, 9 S, 11 E, 13 S, 15 R)-2,3,5,7$, $8,9,15$-Heptahydroxyjatropha-6(17),11-diene14-one-7,8,9-triacetate-2,5-bis(2-methylbutyrate), 9398

Khekadaengoside F, 12213

Periforoside I, 16924

\section{$\mathrm{C}_{36} \mathrm{H}_{54} \mathrm{O}_{15}$}

Strophanthidin- $\beta$ - $D$-glucosyl-( $1 \rightarrow 4)-\beta$ - $D$-digitaloside, 20400

Strophanthin, 20402

\section{$\mathrm{C}_{36} \mathrm{H}_{55} \mathrm{NO}_{11}$}

Neogermitrine, 15396

\section{$\mathrm{C}_{36} \mathrm{H}_{55} \mathrm{~N}_{7} \mathrm{O}_{8}$}

Pseudostellarin D, 18067

$\mathrm{C}_{36} \mathrm{H}_{56} \mathrm{~N}_{2} \mathrm{O}_{5}$

(-)-Buxakashmiramine, 2815

\section{$\mathrm{C}_{36} \mathrm{H}_{56} \mathrm{O}_{7}$}

Fomitoside D, 7869

\section{$\mathrm{C}_{36} \mathrm{H}_{56} \mathrm{O}_{8}$}

Phorbol-12-butyrate-13-laurate, 17184

Phorbol-4-methoxy-12-myristate-13-acetate, 
17187

$\mathrm{C}_{36} \mathrm{H}_{56} \mathrm{O}_{9}$

Calendula officinalis Glycoside F, 2973

Cimifoetiside III, 3657

Eriocarpin C, 7274

Quinovic acid $3 \beta-O-\beta$ - $D$-quinovopyranoside, 18439

Quinovic acid $3 \beta-O-\alpha$ - $L$-rhamnopyranoside, 18442

\section{$\mathrm{C}_{36} \mathrm{H}_{56} \mathrm{O}_{10}$}

Dianoside H, 5365

Hederagenin-3- $O$ - $\beta$-glucuronopyranoside, 9265

$12 \beta$-Hydroxylcimigenol

3-O- $\alpha$ - $L$-arabinopyranoside, 10316

Myrioside B, 15199

Periplocoside O, 16957

Phytolaccoside A, 17268

Quinovic acid 27- $O$ - $\beta$ - $D$-glucopyranosyl ester, 18430

$2 \alpha, 3 \beta, 24$-Trihydroxyurs-12,18-dien-28-oic acid 28-O- $\beta$ - $D$-glucopyranosyl ester, 21860

\section{$\mathrm{C}_{36} \mathrm{H}_{56} \mathrm{O}_{11}$}

Dianoside F, 5363

28-O- $\beta$ - $D$-Glucopyranosyl-2 $\alpha$-3 $\beta$-dihydroxy-

olean-12-ene-24,28-dioic acid, 8621

Phytolaccoside B, 17269

\section{$\mathrm{C}_{36} \mathrm{H}_{56} \mathrm{O}_{12}$}

Odoroside D, 16007

Suavissimoside $\mathrm{R}_{1}, 20433$

Tenuifolin, 20939

\section{$\mathrm{C}_{36} \mathrm{H}_{56} \mathrm{O}_{13}$}

Odorobioside G, 16004

Odoroside F, 16008

Periplocin, 16943

Thevebioside, 21322

Tirlocularoside A, 21403

$2 \alpha, 3 \alpha, 23$-Trihydroxyurs-12-en-24,28-dioic acid 28- $\beta$ - $D$-glucopyranosylester, 21861

\section{$\mathrm{C}_{36} \mathrm{H}_{58} \mathrm{O}_{6}$}

20-Hexadecanoylingenol, 9489

Ingenol-20-hexadecanoate, 11059

Ingenol-3-hexadecanoate, 11060

$\mathrm{C}_{36} \mathrm{H}_{58} \mathrm{O}_{7}$

$\alpha$-L-Rhamnopyranosyl-3 $\beta$-hydroxy-lup-20(29)en-28-oic acid, 18728

\section{$\mathrm{C}_{36} \mathrm{H}_{58} \mathrm{O}_{8}$}

Fomitoside I, 7874

3-O- $\beta$ - $D$-Galactopyranosyloleanolic acid, 8065

Ilexoside B methyl ester, 10982

Oleanolic acid-28- $O-\beta$ - $D$-glucopyranoside, 16052

Prosaikogenin F, 17941

Prosaikogenin G, 17942
$\mathrm{C}_{36} \mathrm{H}_{58} \mathrm{O}_{9}$

$3 \beta, 19 \alpha$-Dihydroxyurs-12-en-28-oic acid 28- $\beta$ - $D$ glucopyranosyl ester, 6176

Ecliptasaponin A, 6705

Ecliptasaponin D, 6707

3-O- $\beta$ - $D$-Glucopyranosyl-23-hydroxyursolic acid, 8679

$16 \beta$-Hydroxy-18 $\beta H$-oleanolic acid-28- $O-\beta$ - $D$ glucopyranoside, 10551

Longispinogenin 3-O- $\beta$ - $D$-glucuronopyranoside, 12981

Lucyoside Q, 13064

25-O-Methoxycimigenol 3- $O-\alpha-L$-arabinopyranoside, 13881

25-O-Methylcimigenoside, 14244

Pomolic acid-28-O- $\beta$ - $D$-glucopyranoside, 17698

Prosapogenin $\mathrm{CP}_{2 \mathrm{a}}, 17944$

Soyasapogenol B monoglucuronide, 20117

\section{$\mathrm{C}_{36} \mathrm{H}_{58} \mathrm{O}_{10}$}

Arjunic acid-28-O-glucoside, 1735

Cimiside F, 3673

21-O- $\beta$-D-Glucopyranosyl-3 $\beta, 21 \alpha, 30$-trihydroxyolean-13(18)-en-24-oic acid, 8745

Lucyoside N, 13063

Pedunculoside, 16766

Rosamultin, 18916

$2 \alpha, 3 \alpha, 19 \alpha$-Trihydroxyurs-12-en-28-oic acid 28- $\beta$ - $D$-glucopyranosyl ester, 21864

$2 \alpha, 3 \alpha, 24$-Trihydroxyurs-12-en-28-oic acid-28-

$O-\beta$ - $D$-glucopyranosyl ester, 21865

Vulgarsaponin, 22621

\section{$\mathrm{C}_{36} \mathrm{H}_{58} \mathrm{O}_{11}$}

Arjunglucoside I, 1733

Lucyoside R, 13062

Niga-ichigoside $\mathrm{F}_{1}, 15539$

Niga-ichigoside $\mathrm{F}_{2}, 15540$

Pruvuloside B, 18007

Sericoside, 19758

$2 \alpha, 3 \beta, 23,29$-Tetrahydroxyolean-12-en-28-oic acid 29- $O-\beta$ - $D$-glucopyranoside, 21145

\section{$\mathrm{C}_{36} \mathrm{H}_{58} \mathrm{O}_{12}$}

3-O- $\beta$-D-Glucopyranosyl platycodigenin, 8711

$\mathrm{C}_{36} \mathrm{H}_{60} \mathrm{O}_{2}$

Cynanester A, 4554

\section{$\mathrm{C}_{36} \mathrm{H}_{60} \mathrm{O}_{4}$}

ent-15,16-Epoxy-2 $\beta$-palmitoyloxy-kauran-2-one, 7185

ent-15,16-Epoxy-3 $\alpha$-palmitoyloxy-kauran-2-one, 7186

2-n-Pentacosyl-5,7-dihydroxy-6,8-dimethyl chromone, 16826

\section{$\mathrm{C}_{36} \mathrm{H}_{60} \mathrm{O}_{8}$}

Ginsenoside $\mathrm{Rh}_{4}, 8439$
Rubianoside II, 19008

\section{$\mathrm{C}_{36} \mathrm{H}_{60} \mathrm{O}_{9}$}

Ginsenoside $\mathrm{Rh}_{5}, 8440$

Ginsenoside $\mathrm{Rh}_{7}, 8442$

Ginsenoside $\mathrm{Rh}_{8}, 8443$

Ginsenoside $\mathrm{Rh}_{9}, 8444$

Kahiricoside II, 12107

Rubianoside III, 19009

\section{$\mathrm{C}_{36} \mathrm{H}_{60} \mathrm{O}_{10}$}

Beesioside G, 2198

Rubianoside IV, 19010

\section{$\mathrm{C}_{36} \mathrm{H}_{62} \mathrm{O}_{4}$}

Hexacosanyl ferulate, 9482

Tenuiphenone C, 20961

\section{$\mathrm{C}_{36} \mathrm{H}_{62} \mathrm{O}_{8}$}

20(R)-Ginsenoside- $\mathrm{Rh}_{2}, 8436$

20(S)-Ginsenoside $\mathrm{Rh}_{2}, 8437$

\section{$\mathrm{C}_{36} \mathrm{H}_{62} \mathrm{O}_{9}$}

Ginsenoside $F_{1}, 8410$

20(R)-Ginsenoside $\mathrm{Rh}_{1}, 8434$

20(S)-Ginsenoside $\mathrm{Rh}_{1}, 8435$

Gynosaponin $\mathrm{TN}_{1}, 9115$

Gypenoside LXXVI, 9178

Sanchinoside $\mathrm{B}_{1}, 19233$

(20S)-12 $\beta, 16 \beta$-Trihydroxydammar-24-ene-3 $\beta$ - $O$ $\beta$-glucopyranoside, 21699

\section{$\mathrm{C}_{36} \mathrm{H}_{62} \mathrm{O}_{10}$}

Gycomoside III, 9096

Momorcharaside B, 14908

(24S)-Pseudoginsenoside $\mathrm{RT}_{4}, 18026$

Trilocularol A 3-glucoside, 21891

$\mathrm{C}_{36} \mathrm{H}_{62} \mathrm{O}_{11}$

Ginsenoside $\mathrm{Rh}_{6}, 8441$

$\mathrm{C}_{36} \mathrm{H}_{62} \mathrm{O}_{31}$

Lantanose B, 12495

Lycopose, 13224

\section{$\mathrm{C}_{36} \mathrm{H}_{64} \mathrm{O}_{14}$}

2,3,4-Tri(6-methylheptanoyl)- $\alpha$ - $D$-glucopyranosyl- $\beta$ - $D$-fructofuranoside, 21962

\section{$\mathrm{C}_{36} \mathrm{H}_{68} \mathrm{~N}_{2} \mathrm{O}_{3}$}

Secojuliprosopinal, 19621

\section{$\mathrm{C}_{36} \mathrm{H}_{69} \mathrm{NO}_{3}$}

(4E,6E,2S,3R)-2-N-Docosanoyl-4,6-tetradecasphingadienine, 6538

\section{$\mathrm{C}_{36} \mathrm{H}_{70} \mathrm{O}_{2}$}

3-Methoxy-5-acetyl-31-tritriacontene, 13835

\section{$\mathrm{C}_{36} \mathrm{H}_{72} \mathrm{O}_{2}$}

Pentyl hentriacontanoate, 16894

\section{$\mathrm{C}_{36} \mathrm{H}_{73} \mathrm{NO}_{4}$}

$(2 S, 3 S, 4 R)-N$-[2-(1,3,4-Trihydroxy-octadecanyl)]-octadecamamide, 21820

\section{$\mathrm{C}_{37} \mathrm{H}_{30} \mathrm{~N}_{2} \mathrm{O}_{9}$}

(+)-Ovihernangerine, 16282 


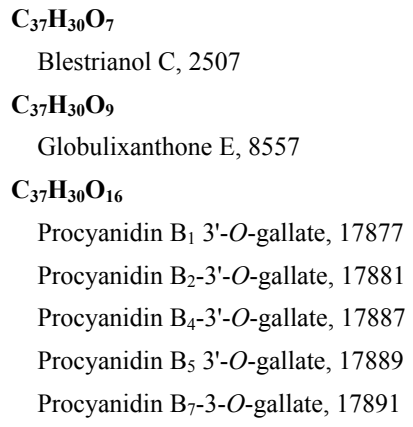

\section{$\mathrm{C}_{37} \mathrm{H}_{30} \mathrm{O}_{17}$}

Epigallocatechin-( $4 \beta \rightarrow 8)$-epicatechin-3- $O$ gallate ester, 6922

\section{$\mathrm{C}_{37} \mathrm{H}_{32} \mathrm{O}_{7}$}

Blestrianol B, 2506

\section{$\mathrm{C}_{37} \mathbf{H}_{34} \mathbf{N}_{2} \mathbf{O}_{7}$}

Oxofangchirine, 16332

Simulanoquinoline, 19904

\section{$\mathrm{C}_{37} \mathrm{H}_{34} \mathbf{O}_{20}$ \\ 4'-O-[2'-O-E-Feruloyl- $O-\beta$ - $D$-glucuronopyranos- $\mathrm{yl}(1 \rightarrow 2)-O-\beta$ - $D$-glucuronopyranoside]apigenin, 7775}

\section{$\mathbf{C}_{37} \mathbf{H}_{36} \mathbf{N}_{2} \mathbf{O}_{7}$}

Isothalmidine, 11737

\section{$\mathrm{C}_{37} \mathrm{H}_{36} \mathbf{N}_{2} \mathrm{O}_{8}$}

Secocepharanthine, 19608

\section{$\mathrm{C}_{37} \mathrm{H}_{36} \mathrm{O}_{12}$}

Curtisian E, 4401

\section{$\mathrm{C}_{37} \mathrm{H}_{36} \mathrm{O}_{22}$}

Quercetagetin 7-methylether-3-O-[2-O-caffeoyl$\beta$ - $D$-glucopyranosyl $(1 \rightarrow 2)-O-\beta$ - $D$-glucuronopyranoside], 18311

\section{$\mathrm{C}_{37} \mathrm{H}_{37} \mathrm{~N}_{2} \mathrm{O}_{6}{ }^{+}$}

Fenfangjine D, 7754

\section{$\mathrm{C}_{37} \mathrm{H}_{38} \mathrm{~N}_{2} \mathrm{O}_{6}$}

Cepharanthine, 3412

Cissampareine, 3748

Coclobine, 3879

Epistephanine, 7024

(-)-Medelline, 13634

\section{$\mathrm{C}_{37} \mathrm{H}_{38} \mathrm{~N}_{4} \mathrm{O}_{7}$}

Petasiphyll A, 17014

\section{$\mathrm{C}_{37} \mathrm{H}_{38} \mathrm{O}_{17}$}

6"'-p-Coumaroylspinosin, 4187

\section{$\mathrm{C}_{37} \mathrm{H}_{38} \mathrm{O}_{18}$}

Isorhamnetin 3-O- $\alpha-L$ - $[6$ "'- $p$-coumaroyl- $\beta$ - $D$-glucopyranosyl-(1,2)-rhamnopyranoside], 11654

Isoscoparin 2"-O-(6"'-(E)-p-coumaroyl)glucoside, 11700

\section{$\mathrm{C}_{37} \mathrm{H}_{38} \mathrm{O}_{19}$}

Isorhamnetin 3-O- $\beta$-(6"-E-p-coumaroyglucopyranoside)-7-O- $\beta$-glucopyranoside, 11653 Kaempferol 3-O-[(6- $O$-feruloyl)- $\beta$ - $D$-glucopy- ranosyl-( $1 \rightarrow 2)-\beta$ - $D$-galactopyranoside], 12046

Kaempferol 3-O-[2-O-(trans-3-methoxy-4-hydroxycinnamoyl)]- $\beta$ - $D$-galactopyranosyl-

$(1 \rightarrow 4)-O-\beta$ - $D$-glucopyranoside, 12068

Kaempferol 3-O-[2-O-(trans-3-methoxy-4-hydroxycinnamoyl)]- $\beta$ - $D$-glucopyranosyl-( $1 \rightarrow 6)$ $O$ - $\beta$ - $D$-glucopyranoside, 12069

Ozturkoside B, 16483

\section{$\mathrm{C}_{37} \mathrm{H}_{38} \mathrm{O}_{20}$}

Quercetin-3- $O$-[(6- $O$-feruloyl)- $\beta$ - $D$-glucopyranosyl- $(1 \rightarrow 2)-\beta$ - $D$-galactopyranoside], 18354

Quercetin-3-O-[2-O-(6-O-E-feruloyl)- $\beta$ - $D$-glucopyranosyl]- $\beta$ - $D$-glucopyranoside, 18355

\section{$\mathrm{C}_{37} \mathrm{H}_{40} \mathrm{~N}_{2} \mathrm{O}_{6}$}

(+)-Antioquine, 1459

(-)-Antioquine, 1460

Berbamine, 2300

Fangchinoline, 7714

Hayatidine, 9250

Hayatinine, 9252

Homoaromoline, 9597

Menisidine, 13714

(+)-4"-O-Methylcurine, 14266

(+)-2-Norisotetrandrine, 15764

Oxyacanthine, 16439

Thalicberine, 21240

Thalictine, 21248

Thalifortine, 21256

Thalmine, 21264

Thalrugosine, 21270

Tiliageine, 21390

\section{$\mathrm{C}_{37} \mathrm{H}_{40} \mathrm{~N}_{2} \mathrm{O}_{7}$}

$\mathrm{N}$-Desmethylthalidezine, 5260

\section{$\mathrm{C}_{37} \mathrm{H}_{40} \mathbf{N}_{2} \mathbf{O}_{10}$}

Thapsakin A 10-O-acetate, 21275

\section{$\mathrm{C}_{37} \mathrm{H}_{40} \mathrm{O}_{9}$}

Resiniferatoxin, 18639

\section{$\mathrm{C}_{37} \mathrm{H}_{40} \mathrm{O}_{13}$}

Ixerochinoside, 11803

$\mathrm{C}_{37} \mathrm{H}_{40} \mathrm{O}_{21}$

Quercetin-3-O-[(2,3,4-triacetyl- $\alpha$-rhamnopyranosyl)-(1 $\rightarrow 6)]$-3,4-diacetyl- $\beta$-galactopyranoside, 18402

\section{$\mathrm{C}_{37} \mathrm{H}_{41} \mathrm{NO}_{10}$}

3,12-Diacetyl-7-benzoyl-8-nicotinoylingol, 5326

\section{$\mathrm{C}_{37} \mathrm{H}_{42} \mathrm{~N}_{2} \mathrm{O}_{6}$}

Dauricinoline, 4686

Daurinoline, 4688

Daurisoline, 4693

Isoliensinine, 11491

Liensinine, 12798

(+)-Thaligrisine, 21258

$\mathrm{C}_{37} \mathrm{H}_{42} \mathrm{~N}_{2} \mathbf{O}_{6}{ }^{2+}$
(+)-Tubocurarine, 22091

$\mathrm{C}_{37} \mathrm{H}_{42} \mathrm{O}_{10}$

Odoratrin, 15999

\section{$\mathrm{C}_{37} \mathrm{H}_{42} \mathrm{O}_{12}$}

Norstaminol A, 15795

\section{$\mathrm{C}_{37} \mathrm{H}_{42} \mathrm{O}_{13}$}

Triptofordin $\mathrm{F}_{1}, 22005$

\section{$\mathrm{C}_{37} \mathrm{H}_{42} \mathrm{O}_{14}$}

Triptofordin $\mathrm{F}_{3}, 22007$

\section{$\mathbf{C}_{37} \mathbf{H}_{42} \mathbf{O}_{17}$}

6-O- $\alpha-L$-(2"-O-Benzoyl,3"-O-trans-p-coumaroyl) rhamnopyranosylcatalpol, 2234

\section{$\mathrm{C}_{37} \mathrm{H}_{43} \mathrm{NO}_{8}$}

Aristoloin I, 1721

\section{$\mathrm{C}_{37} \mathrm{H}_{43} \mathrm{NO}_{9}$}

Euphoheliosnoid A, 7608

Euphoheliosnoid B, 7609

\section{$\mathrm{C}_{37} \mathrm{H}_{43} \mathrm{NO}_{10}$}

Euphocharacin E, 7600

\section{$\mathrm{C}_{37} \mathrm{H}_{43} \mathrm{NO}_{11}$}

Euphocharacin B, 7597

Euphocharacin L, 7607

\section{$\mathbf{C}_{37} \mathbf{H}_{44} \mathbf{N}_{2} \mathbf{O}_{10}$}

19-Hydroxy-3'-methoxyaglaine C, 10379

\section{$\mathbf{C}_{37} \mathbf{H}_{44} \mathbf{N}_{4} \mathbf{O}_{6}$}

$N 1, N 5, N 10$-Tris[3-(4-hydroxyphenyl)-2-propenoyl]-1,5,10,14-tetraazatetradecane, 22035

\section{$\mathrm{C}_{37} \mathrm{H}_{44} \mathrm{O}_{8}$}

Trichilinin D, 21555

\section{$\mathrm{C}_{37} \mathrm{H}_{44} \mathbf{O}_{9}$}

$6 \alpha$-Acetoxy- $9 \beta$-benzoyloxy- $1 \beta$-cinnamoyloxy$8 \beta$-butanoyloxy- $\beta$-dihydroagarofuran, 131

\section{$\mathrm{C}_{37} \mathrm{H}_{44} \mathrm{O}_{10}$}

Gnidilatidin, 8900

Odoracin, 15990

Yuanhuacin, 22929

\section{$\mathrm{C}_{37} \mathrm{H}_{44} \mathrm{O}_{11}$}

Taxinine B, 20800

\section{$\mathrm{C}_{37} \mathrm{H}_{44} \mathrm{O}_{12}$}

Dantaxusin C, 4638

\section{$\mathrm{C}_{37} \mathrm{H}_{44} \mathrm{O}_{13}$}

1-Cinnamoyl -11-methoxymeliacarpinin, 3710 Taxagifin, 20743

\section{$\mathrm{C}_{37} \mathrm{H}_{44} \mathrm{O}_{14}$}

Jatrophane 11,11847

$3 \beta, 5 \alpha, 7 \beta, 8 \alpha, 15 \beta$-Pentaacetoxy-2 $\alpha$-benzoyloxyjatropha-6(17),11E-dien-9,14-dione, 16818

Segetene A, 19656

Segetene B, 19657

Taxuspine S, 20866

Taxuspine T, 20867

\section{$\mathrm{C}_{37} \mathrm{H}_{44} \mathbf{O}_{15}$}

$1 \alpha, 2 \alpha, 6 \beta$-Triacetoxy- $8 \alpha$-( $\beta$-furancarbonyloxy)- 
9 $\beta$-benzoyloxy-13-isobutanoyloxy- $4 \beta$-hy-

droxy- $\beta$-dihydroagarofuran, 21520

\section{$\mathrm{C}_{37} \mathrm{H}_{44} \mathrm{O}_{17}$}

Korepimedoside A, 12272

$\mathrm{C}_{37} \mathrm{H}_{46} \mathbf{O}_{10}$

2-Deacetoxytaxinine J, 4708

$\mathrm{C}_{37} \mathrm{H}_{46} \mathrm{O}_{11}$

Dantaxusin D, 4639

Taxamedin A, 20747

Taxawallin A, 20753

Taxuspinanane G, 20846

Taxuspine J, 20857

$\mathrm{C}_{37} \mathrm{H}_{46} \mathbf{O}_{12}$

(R)-(-)-Agrimol B, 753

Swietephragmin C, 20518

Taxezopidine K, 20789

\section{$\mathbf{C}_{37} \mathrm{H}_{46} \mathbf{O}_{13}$}

$1 \beta$-Dehydroxybaccatin VI, 4981

$3 \beta, 5 \alpha, 8 \alpha, 9 \alpha,-15 \beta$-Pentaacetoxy-7 $\beta$-benzoyloxyjatropha-6(17),11E-dien-14-one, 16819

Swietephragmin E, 20520

\section{$\mathrm{C}_{37} \mathrm{H}_{46} \mathrm{O}_{14}$}

13-Acetyl-13-decinnamoyltaxchinin B, 369

Baccatin VI, 2077

Segetene 3, 19652

Segetene 4, 19653

Taxumairol G, 20827

\section{$\mathrm{C}_{37} \mathrm{H}_{46} \mathrm{O}_{15}$}

14 $\beta$-Benzoyloxy-13-deacetylbaccatin IV, 2252

14 $\beta$-Benzoyloxy-2-deacetylbaccatin VI, 2253

10-Hydroxyacetylbaccatin VI, 9763

14 $\beta$-Hydroxy-baccatin VI, 9813

Kansuinin A, 12137

\section{$\mathrm{C}_{37} \mathrm{H}_{46} \mathrm{O}_{16}$}

Kansuinin H, 12144

\section{$\mathrm{C}_{37} \mathrm{H}_{46} \mathrm{O}_{17}$}

Acanfolioside, 63

\section{$\mathrm{C}_{37} \mathrm{H}_{46} \mathrm{O}_{18}$}

Wiedemannioside B, 22663

\section{$\mathrm{C}_{37} \mathrm{H}_{48} \mathrm{~N}_{2} \mathrm{O}_{11}$}

Potanidine B, 17744

\section{$\mathrm{C}_{37} \mathrm{H}_{48} \mathrm{O}_{6}$}

3,6-Epoxy-5,3',4'-trihydroxy-12',13',20'-trinor$\beta, \beta$-caroten-19,11-olide, 7217

\section{$\mathrm{C}_{37} \mathrm{H}_{48} \mathrm{O}_{10}$}

Cynaphylloside A, 4556

\section{$\mathrm{C}_{37} \mathrm{H}_{48} \mathrm{O}_{14}$}

$5 \alpha-O-(\beta$ - $D$-Glucopyranosyl $)-10 \beta$-benzoyltaxacustone, 8609

Taxumairol A, 20821

\section{$\mathrm{C}_{37} \mathrm{H}_{48} \mathrm{O}_{21}$}

Tubuloside A, 22093

$\mathrm{C}_{37} \mathrm{H}_{48} \mathrm{O}_{23} \mathrm{~S}_{2}$
Dimer iridoid glucoside 10, 6196

$\mathrm{C}_{37} \mathrm{H}_{49} \mathrm{NO}_{9}$

Taxuspine H, 20856

$\mathrm{C}_{37} \mathrm{H}_{49} \mathrm{NO}_{10}$

Comptonine, 3958

2'-Hydroxytaxine II, 10738

Spicaledonine, 20157

Taxine A', 20794

$\mathrm{C}_{37} \mathrm{H}_{49} \mathrm{NO}_{11}$

7-O-Acetyltaxine A, 519

$\mathrm{C}_{37} \mathrm{H}_{49} \mathrm{NO}_{12}$

Euphocharacin D, 7599

$\mathrm{C}_{37} \mathrm{H}_{49} \mathrm{NO}_{13}$

$(2 R, 3 R, 4 R, 5 R, 7 S, 8 S, 9 S, 11 E, 13 S, 15 R)-2,3,5,7,8$,

9,15-Heptahydroxyjatropha-6(17),11-diene-14one-7,8,9-triacetate-2-nicotinate-5-(2-methylbutyrate), 9401

\section{$\mathrm{C}_{37} \mathrm{H}_{50} \mathbf{N}_{2} \mathbf{O}_{10}$}

Methyllycaconitine, 14569

\section{$\mathrm{C}_{37} \mathrm{H}_{50} \mathbf{N}_{2} \mathrm{O}_{11}$}

10-Hydroxy-methyllycaconitine, 10501

\section{$\mathrm{C}_{37} \mathrm{H}_{50} \mathrm{O}_{8}$}

12-O-Cinnamoyl-20-O-ikemaoyl sarcostin, 3709

\section{$\mathrm{C}_{37} \mathrm{H}_{50} \mathrm{O}_{9}$}

Pimelea factor $\mathrm{P}_{2}, 17374$

$\mathrm{C}_{37} \mathrm{H}_{50} \mathbf{O}_{11}$

15-O-Deacetyl-15-O-methylnimbolidin B, 4753

\section{$\mathrm{C}_{37} \mathrm{H}_{50} \mathrm{O}_{12}$}

Nimbolidin C, 15607

\section{$\mathrm{C}_{37} \mathrm{H}_{50} \mathrm{O}_{14}$}

$1 \alpha, 2 \alpha, 6 \beta$-Triacetoxy- $8 \alpha$-isobutanoyloxy- $9 \beta$-benzoyloxy-13-( $\alpha$-methyl)butanoyloxy-4 $\beta$-hydroxy- $\beta$-dihydroagarofuran, 21525

\section{$\mathrm{C}_{37} \mathrm{H}_{50} \mathrm{O}_{18}$}

Yadanzioside O, 22874

\section{$\mathrm{C}_{37} \mathrm{H}_{50} \mathbf{O}_{20}$}

Cistanoside B, 3752

Jionoside $\mathrm{B}_{1}, 11882$

Jionoside $\mathrm{B}_{2}, 11883$

$\mathrm{C}_{37} \mathrm{H}_{50} \mathbf{O}_{21}$

Rossicaside F, 18927

$\mathrm{C}_{37} \mathrm{H}_{51} \mathrm{NO}_{8}$

7,2'-Didesacetoxy austrospicatine, 5478

\section{$\mathrm{C}_{37} \mathrm{H}_{51} \mathrm{NO}_{9}$}

10 $\beta$-Hydroxy- $2 \alpha, 9 \alpha, 13 \alpha$-triacetoxy- $5 \alpha$-(3'-(dimethylamino)-3'-phenyl)butanoatetaxa-4(20), 11-diene, 10777

$9 \alpha$-Hydroxy- $2 \alpha, 10 \beta, 13 \alpha$-triacetoxy-5 $\alpha-\left(3^{\prime}-N, N-\right.$ dimethylamino-3'-phenyl)-propionyloxytaxa4(20),11-diene, 10778

Taxuspine Z, 20873

\section{$\mathrm{C}_{37} \mathrm{H}_{51} \mathrm{NO}_{10}$}

$9 \alpha$-Acetyl-10 $\beta$-deacetyl-spicataxine, 365
$7 \beta, 9 \alpha$-Bisdeacetylaustrospicatine, 2437

$2 \alpha 17$-Dihydroxy- $9 \alpha, 10 \beta, 13 \alpha$-triacetoxy- $5 \alpha$-(3'$N, N$-dimethylamino-3'-phenyl)-propionyloxytaxa-4(20),11-diene, 6157

Spicataxine, 20159

$\mathrm{C}_{37} \mathrm{H}_{52} \mathrm{CIN}_{\mathbf{3}} \mathrm{O}_{10}$

Maytanvaline, 13622

$\mathrm{C}_{37} \mathrm{H}_{52} \mathrm{~N}_{2} \mathrm{O}_{11}$

Demethyldelavaine A, 5071

Demethyldelavaine B, 5072

Puberaconitidine, 18166

$\mathrm{C}_{37} \mathrm{H}_{52} \mathrm{O}_{3}$

Karounidiol 3-benzoate, 12166

\section{$\mathrm{C}_{37} \mathrm{H}_{53} \mathrm{~N}_{3} \mathrm{O}_{10}$}

Delsemine A, 5033

Delsemine B, 5034

\section{$\mathrm{C}_{37} \mathrm{H}_{54} \mathrm{~N}_{2} \mathrm{O}_{9}$}

Delajacine, 4991

\section{$\mathrm{C}_{37} \mathrm{H}_{54} \mathrm{O}_{4}$}

Carpesterol, 3221

Cholesteryl ferulate, 3586

\section{$\mathrm{C}_{37} \mathrm{H}_{54} \mathrm{O}_{5}$}

Oleoyl neocryptotanshinone, 16073

Revandchinone 3, 18671

\section{$\mathrm{C}_{37} \mathrm{H}_{54} \mathrm{O}_{6}$}

Hericenone E, 9433

\section{$\mathrm{C}_{37} \mathrm{H}_{54} \mathrm{O}_{10}$}

Bugbanoside E, 2731

7,8-Didehydro-27-deoxyactein, 5466

\section{$\mathrm{C}_{37} \mathrm{H}_{54} \mathrm{O}_{11}$}

Bugbanoside D, 2730

Cimicifugoside, 3652

\section{$\mathrm{C}_{37} \mathbf{H}_{54} \mathrm{O}_{16}$}

(3S)-O- $\alpha$ - $L$-Rhamnopyranosyl-( $1 \rightarrow 3)$-[4- $O-(E)-$ coumaroyl]- $\alpha$ - $L$-rhamnopyranosyl- $(1 \rightarrow 6)-\beta-D$ glucopyranosyl-linalool, 18695

(3S)-O- $\alpha$ - $L$-Rhamnopyranosyl-( $1 \rightarrow 3)$-[4-O-(Z)coumaroyl]- $\alpha-L$-rhamnopyranosyl-( $1 \rightarrow 6)-\beta-D$ glucopyranosyl-linalool, 18696

Trillenoside C, 21875

\section{$\mathrm{C}_{37} \mathrm{H}_{56} \mathrm{O}_{8}$}

Phorbol-12-tiglate-13-laurate, 17195

\section{$\mathrm{C}_{37} \mathrm{H}_{56} \mathrm{O}_{10}$}

Acetylcimifugoside, 352

3-O- $\alpha$-(2"-O-Acetyl)- $D$-xylopyranosyl-3 $\beta$-hydroxyolean-12-ene-28,29-dioic acid, 533

\section{$\mathrm{C}_{37} \mathrm{H}_{56} \mathrm{O}_{11}$}

Cimiracemoside F, 3667

Cimiracemoside G, 3668

27-Deoxyactein, 5150

\section{$\mathrm{C}_{37} \mathrm{H}_{56} \mathrm{O}_{12}$}

24-Acetoxy-15,16-seco-cycloart-7-en 3-O-xyloside, 280 
Actein, 579

Bugbanoside C, 2729

Cimidahuside D, 3656

$\mathrm{C}_{37} \mathrm{H}_{56} \mathrm{O}_{15}$

Wattoside E, 22652

$\mathrm{C}_{37} \mathbf{H}_{56} \mathbf{O}_{16}$

Rhodexin D, 18788

$\mathrm{C}_{37} \mathrm{H}_{58} \mathrm{O}_{6}$

Hericenone D, 9432

Hericenone G, 9435

\section{$\mathrm{C}_{37} \mathrm{H}_{58} \mathrm{O}_{8}$}

Fomitoside F, 7871

12-O-(2-Methylbutyryl)phorbol-13-dodecanoate 14201

Phorbol-12- $\alpha$-methylbutyrate-13-laurate, 17190

\section{$\mathrm{C}_{37} \mathrm{H}_{58} \mathrm{O}_{9}$}

Fomitoside E, 7870

Oleanolic acid-3- $O-\beta-D$-(6'- $O$-methyl)-glucuronoside, 16048

\section{$\mathrm{C}_{37} \mathrm{H}_{58} \mathrm{O}_{10}$}

25-O-Acetylcimigenoside, 354

23-O-Acetylshengmanol 3-O- $\alpha-L$-arabinopyranoside, 512

Acetyl shengmanol xyloside, 513

Beesioside L, 2205

Beesioside O, 2208

(22S)-Cholesta-5,24-diene-3 $\beta, 11 \alpha, 16 \beta, 22$-tetrol

16- $O$-(2,3-di- $O$-acetyl- $\alpha$ - $L$-rhamnopyranoside), 3576

Cimiracemoside E, 3666

Eclalbasaponin XIII, 6704

Saponin 1, 19333

Soulieoside C, 20112

\section{$\mathrm{C}_{37} \mathrm{H}_{58} \mathrm{O}_{11}$}

$12 \beta$-Acetoxycimigenol-3- $O-\beta$ - $D$-xylopyranoside, 141

25-O-Acetyl-12 $\beta$-hydroxycimigenol 3-O- $\alpha-L$ arabinopyranoside, 414

$(22 R, 23 R, 24 R)-12 \beta$-Acetyloxy-16 $\beta, 23: 22,25$-diepoxy-23,24-dihydroxy-9,19-cyclolanostan$3 \beta$-yl $\alpha$ - $L$-arabinopyranoside, 477

Beesioside K, 2204

Cimiracemoside H, 3669

\section{$\mathrm{C}_{37} \mathrm{H}_{58} \mathrm{O}_{12}$}

Cimidahuside C, 3655

Esculentoside D, 7371

$\mathrm{C}_{37} \mathrm{H}_{58} \mathrm{O}_{13}$

5-Ene-methyl-7,12-didehydroxy-cholate-3-O-

$\alpha$ - $L$-rhamnopyranosyl-( $1 \rightarrow 4)-\beta$ - $D$-glucuronopyranoside, 6799

$\mathrm{C}_{37} \mathrm{H}_{58} \mathrm{O}_{19}$

Prostratoside I, 17961

$\mathrm{C}_{37} \mathrm{H}_{59} \mathrm{NO}_{11}$
Germerine, 8352

$\mathrm{C}_{37} \mathrm{H}_{59} \mathrm{NO}_{12}$

Neogermbudine, 15395

$\mathrm{C}_{37} \mathrm{H}_{60} \mathrm{O}_{8}$

Fomitoside J, 7875

$\mathrm{C}_{37} \mathrm{H}_{60} \mathrm{O}_{10}$

Beesioside F, 2197

Beesioside M, 2206

(22S)-Cholest-5-ene-3 $\beta, 11 \alpha, 16 \beta, 22$-tetrol 16-O-

(2,3-di- $O$-acetyl- $\alpha$ - $L$-rhamnopyranoside), 3582

Tormentic acid-6-methoxy $\beta$ - $D$-glucopyranosyl ester, 21458

\section{$\mathrm{C}_{37} \mathrm{H}_{60} \mathrm{O}_{11}$}

Beesioside D, 2195

Beesioside III, 2202

Methyl-3-O- $\beta$ - $D$-glucopyranosyl polygalacate, 14468

\section{$\mathrm{C}_{37} \mathrm{H}_{60} \mathrm{O}_{12}$}

(22R)-22-Hydroxy-24-O-acetylhydroshengmanol

3-O- $\beta$ - $D$-xylopyranoside, 9764

\section{$\mathrm{C}_{37} \mathrm{H}_{62} \mathrm{O}_{6}$}

Marianoside B, 13565

\section{$\mathrm{C}_{37} \mathrm{H}_{62} \mathrm{O}_{7}$}

Ginsenoside $\mathrm{Rh}_{3}, 8438$

Marianoside A, 13564

\section{$\mathrm{C}_{37} \mathrm{H}_{62} \mathrm{O}_{11}$}

Beesioside P, 2209

\section{$\mathrm{C}_{37} \mathrm{H}_{62} \mathrm{O}_{16}$}

$1^{\prime}, 3,3$ ', 4',6'-Pentakis- $O$-(3-methylbutanoyl)- $\beta$ - $D$ fructofuranosyl $\alpha$ - $D$-glucopyranoside, 16861

\section{$\mathrm{C}_{37} \mathrm{H}_{64} \mathrm{O}_{3}$}

4'-Hydroxy-cis-cinnamic acid octacosyl ester, 9911

$\mathrm{C}_{37} \mathrm{H}_{64} \mathrm{O}_{4}$

Erythrinassinate B, 7332

\section{$\mathrm{C}_{37} \mathrm{H}_{64} \mathrm{O}_{7}$}

Panacon, 16582

\section{$\mathrm{C}_{37} \mathrm{H}_{66} \mathrm{O}_{5}$}

Squamocenin, 20239

$\mathrm{C}_{37} \mathrm{H}_{66} \mathrm{O}_{6}$

Desacetyluvaricin, 5240

Isodesacetyluvaricin, 11375

Muricin I, 15098

Neoannonin, 15339

\section{$\mathrm{C}_{37} \mathrm{H}_{66} \mathrm{O}_{7}$}

Annocherimolin, 1297

Annonareticin, 1317

Annonin VI, 1319

Asimicin, 1856

Bullatacin, 2735

Gigantetronenin, 8379

Isoannonareticin, 11223

2,4-cis-Isoannonareticin, 11224
2,4-trans-Isoannonareticin, 11225

Montalicin E, 14940

Rolliniastatin 1, 18899

Squamocin, 20240

Squamostatin D, 20247

Squamotacin, 20248

Uvaribonianin, 22291

Uvarigrandin A, 22298

\section{$\mathrm{C}_{37} \mathrm{H}_{66} \mathrm{O}_{8}$}

Annoglaucin, 1302

Bullatanocin, 2736

Panalicin, 16584

Purpuracenin, 18211

Purpureacin 1, 18214

Purpureacin 2, 18215

Rollimusin, 18898

Squamocin $\mathrm{O}_{1}, 20241$

Squamocin $\mathrm{O}_{2}, 20242$

Squamostatin B, 20246

\section{$\mathrm{C}_{37} \mathrm{H}_{67} \mathrm{NO}_{3}$}

(2S,3R,4E,8E,9'Z,12'Z)-N-9',12'-Octadecadienoyl-2-amino-9-methyl-4,8-octadecadiene-1,3diol, 15943

\section{$\mathrm{C}_{37} \mathrm{H}_{68} \mathrm{O}_{6}$}

Uvarigrin, 22300

\section{$\mathrm{C}_{37} \mathrm{H}_{68} \mathrm{O}_{7}$}

Annomontacin, 1307

cis-Annomontacin, 1308

Asitrilobin A, 1858

Laherradurin, 12452

Montalicin I, 14942

Montalicin J, 14943

Tucumanin, 22099

Xylomaticin, 22816

\section{$\mathrm{C}_{37} \mathrm{H}_{68} \mathrm{O}_{8}$}

Otivarin, 16266

\section{$\mathrm{C}_{37} \mathrm{H}_{75} \mathrm{NO}_{4}$}

$(2 S, 3 S, 4 R)-2-$ Nonadecanoylamino-octadecane1,3,4-triol, 15673

Trufflesphingolipid B, 22054

\section{$\mathrm{C}_{38} \mathrm{H}_{30} \mathrm{O}_{20}$}

1,3-Di- $O$-(E)-caffeoyl-4,6-(S)-HHDP- $\beta$ - $D$-glucopyranose, 5410

\section{$\mathrm{C}_{38} \mathrm{H}_{32} \mathrm{~N}_{2} \mathrm{O}_{9}$}

Glycobismine F, 8820

Glycobismine G, 8821

\section{$\mathrm{C}_{38} \mathrm{H}_{32} \mathrm{O}_{15}$}

Ginkgetin 7"- $O$ - $\beta$ - $D$-glucopyranoside, 8402

Isoginkgetin-7-O- $\beta$ - $D$-glucopyranoside, 11440

$\mathrm{C}_{38} \mathrm{H}_{34} \mathrm{~N}_{2} \mathrm{O}_{9}$

(+)-Oviisocorydine, 16283

\section{$\mathrm{C}_{38} \mathrm{H}_{34} \mathrm{O}_{10}$}

$(3 S)-6-(3-P h e n y l-5-a c e t o x y-6-m e t h o x y b e n z o[b]$ 
furan-2-ylmethyl)-vestitol-triacetate, 17092

$\mathrm{C}_{38} \mathrm{H}_{34} \mathrm{O}_{16}$

1,3-Bis-[2-(3,4-dihydroxyphenyl)-1-methoxycarbonyl] ethoxycarbonyl-2-(3,4-dihydroxyphenyl)-7,8-dihydroxy-1,2-dihydronaphthalene, 2445

Methyl lithospermate B, 14556

\section{$\mathrm{C}_{38} \mathbf{H}_{36} \mathbf{N}_{2} \mathbf{O}_{6}$}

8,8"-Biskoenigine, 2480

\section{$\mathrm{C}_{38} \mathrm{H}_{36} \mathrm{~N}_{2} \mathrm{O}_{8}$}

Thalphine, 21267

$\mathrm{C}_{38} \mathrm{H}_{38} \mathrm{O}_{10}$

Mezerein, 14826

$\mathrm{C}_{38} \mathrm{H}_{40} \mathrm{~N}_{2} \mathrm{O}_{11}$

Catheduline $\mathrm{E}_{2}, 3326$

$\mathrm{C}_{38} \mathrm{H}_{40} \mathrm{~N}_{2} \mathrm{O}_{6}$

Insularine, 11088

\section{$\mathrm{C}_{38} \mathrm{H}_{40} \mathrm{~N}_{2} \mathrm{O}_{7}$}

Calafatimine, 2938

Thalcimine, 21234

\section{$\mathrm{C}_{38} \mathrm{H}_{40} \mathrm{~N}_{4}$}

Dihydronortoxiferine I, 5682

\section{$\mathrm{C}_{38} \mathrm{H}_{40} \mathrm{~N}_{4} \mathrm{O}_{2}$}

Caracurine V, 3148

\section{$\mathrm{C}_{38} \mathrm{H}_{40} \mathrm{O}_{6}$}

Blepharocalyxin D, 2499

\section{$\mathrm{C}_{38} \mathrm{H}_{40} \mathrm{O}_{9}$}

$6 \alpha$-Acetoxy- $1 \beta, 8 \beta, 9 \beta$-tribenzoyloxy- $\beta$-dihydroagarofuran, 292

\section{$\mathrm{C}_{38} \mathrm{H}_{40} \mathrm{O}_{18}$ \\ 6"'-Feruloylspinosin, 7787}

\section{$\mathrm{C}_{38} \mathrm{H}_{40} \mathrm{O}_{20}$ \\ Kaempferol-3-O-[(6- $O$-sinapoyl)- $\beta$ - $D$-glucopyra- nosyl-(1 $\rightarrow 2)-\beta$ - $D$-galactopyranoside], 12093}

\section{$\mathrm{C}_{38} \mathrm{H}_{40} \mathrm{O}_{21}$}

Quercetin-3-O-[(6-O-sinapoyl)- $\beta$ - $D$-glucopyranosyl-(1 $\rightarrow 2)-\beta$ - $D$-galactopyranoside], 18395

Quercetin-3-O-[2-O-(6-O-E-sinapoyl)- $\beta$ - $D$-glucopyranosyl]- $\beta$ - $D$-glucopyranoside, 18396

\section{$\mathrm{C}_{38} \mathrm{H}_{41} \mathrm{O}_{20}{ }^{+}$}

Alatanin 1,835

$\mathrm{C}_{38} \mathrm{H}_{42} \mathrm{Br}_{2} \mathrm{O}_{9}$

CPB-53-1114-4 6,7-di-p-bromobenzoate, 4207

\section{$\mathrm{C}_{38} \mathrm{H}_{42} \mathrm{~N}_{2} \mathrm{O}_{6}$}

Cycleaneonine, 4460

Cycleanine, 4461

Funiferine, 8004

Isocycleanine, 11362

Isotetrandrine, 11736

Menisine, 13715

(+)-O-Methylthalicberine, 14756

Obaberine, 15881

Pakistanamine, 16537
Sutchuenensine, 20498

Tetrandrine, 21206

$\mathrm{C}_{38} \mathrm{H}_{42} \mathrm{~N}_{2} \mathrm{O}_{7}$

Fenfangjine A, 7753

Thalfoetidine, 21236

Thalidezine, 21253

Thalisamine, 21262

Thalisopine, 21263

Thalrugosidine, 21269

$\mathrm{C}_{38} \mathrm{H}_{42} \mathrm{O}_{7}$

Blepharocalyxin C, 2498

$\mathrm{C}_{38} \mathrm{H}_{42} \mathrm{O}_{11}$

Orthosiphonone A, 16242

\section{$\mathrm{C}_{38} \mathrm{H}_{42} \mathrm{O}_{14}$}

Kansuinin B, 12138

Kansuinin C, 12139

$\mathrm{C}_{38} \mathrm{H}_{42} \mathrm{O}_{16}$

Curtisian F, 4402

Labadoside, 12401

\section{$\mathrm{C}_{38} \mathrm{H}_{42} \mathrm{O}_{17}$}

Aloeresin H, 977

Niruriside, 15627

\section{$\mathrm{C}_{38} \mathrm{H}_{43} \mathrm{~N}_{2} \mathrm{O}_{6}$}

(+)-2- $N$-Methylfangchinoline, 14441

\section{$\mathrm{C}_{38} \mathrm{H}_{44} \mathbf{N}_{2} \mathrm{O}_{6}$}

Dauricine, 4685

Neferine, 15321

\section{$\mathrm{C}_{38} \mathrm{H}_{44} \mathrm{~N}_{2} \mathrm{O}_{8}$}

Disinomenine, 6512

\section{$\mathrm{C}_{38} \mathrm{H}_{44} \mathrm{~N}_{2} \mathrm{O}_{9}$}

10-O-Acetylaglaine B, 307

Aglaine A, 738

4-Epiaglain A, 6824

Grandiamide A, 8974

\section{$\mathrm{C}_{38} \mathrm{H}_{44} \mathrm{O}_{8}$}

Gambogic acid, 8128

\section{$\mathrm{C}_{38} \mathrm{H}_{44} \mathrm{O}_{9}$}

Euphoscopin C, 7620

\section{$\mathrm{C}_{38} \mathrm{H}_{44} \mathrm{O}_{11}$}

Euphocharacin C, 7598

Orthosiphol A, 16221

Orthosiphol B, 16222

Orthosiphol F, 16224

Orthosiphol O, 16233

\section{$\mathrm{C}_{38} \mathrm{H}_{44} \mathrm{O}_{12}$}

$4 \alpha, 7 \beta$-Diacetoxy-2 $\alpha, 9 \alpha$-dibenzoyloxy- $5 \beta, 20$ epoxy-10 $\beta, 13 \alpha, 15$-trihydroxy-11(15 $\rightarrow 1)$-abeotaxene, 5293

\section{$\mathrm{C}_{38} \mathrm{H}_{44} \mathrm{O}_{12}$}

6-Hydroxyorthosiphol B, 10556

Neoorthosiphol A, 15443

Neoorthosiphol B, 15444

Orthosiphol L, 16230
Orthosiphol R, 16234

Siphonol B, 19967

Siphonol C, 19968

Staminol B, 20259

Staminol C, 20260

\section{$\mathrm{C}_{38} \mathrm{H}_{44} \mathrm{O}_{18}$}

Glypentoside C, 8853

$\mathrm{C}_{38} \mathrm{H}_{44} \mathrm{O}_{20}$

Smiglaside C, 20022

\section{$\mathrm{C}_{38} \mathrm{H}_{45} \mathrm{~N}_{5} \mathrm{O}_{5}$}

Scutianine F, 19594

$\mathrm{C}_{38} \mathrm{H}_{46} \mathrm{~N}_{2} \mathrm{O}_{17}$

Emarginatine, 6766

\section{$\mathrm{C}_{38} \mathrm{H}_{46} \mathrm{O}_{8}$}

Isogambogenic acid, 11433

\section{$\mathrm{C}_{38} \mathrm{H}_{46} \mathrm{O}_{9}$}

$6 \alpha$-Acetoxy-9 $\beta$-benzoyloxy-1 $\beta$-cinnamoyloxy$8 \beta$-(2-methylbutanoyloxy)- $\beta$-dihydroagarofuran, 132

Neogambogic acid, 15394

\section{$\mathrm{C}_{38} \mathrm{H}_{46} \mathrm{O}_{13}$}

Swietephragmin A, 20516

\section{$\mathrm{C}_{38} \mathrm{H}_{46} \mathrm{O}_{18}$}

Insularoside-3'-O- $\beta$ - $D$-glucoside, 11089

\section{$\mathrm{C}_{38} \mathrm{H}_{47} \mathrm{NO}_{18}$}

Euonymine, 7534

Wilformine, 22684

\section{$\mathrm{C}_{38} \mathrm{H}_{48} \mathrm{~N}_{2} \mathrm{O}_{7}$}

Nigellamine $\mathrm{A}_{3}, 15563$

\section{$\mathrm{C}_{38} \mathrm{H}_{48} \mathrm{~N}_{2} \mathrm{O}_{11}$}

14-Demethyl-14-isobutyrylanhweidelphinine, 5083

\section{$\mathrm{C}_{38} \mathrm{H}_{48} \mathrm{O}_{18}$}

8-Prenylkaempferol-4'-methoxy-3-[xylosyl $(1 \rightarrow 4)$ rhamnoside]-7-glucoside, 17835

Safghanoside F, 19117

\section{$\mathrm{C}_{38} \mathrm{H}_{48} \mathrm{O}_{19}$}

Baohuoside V, 2142

Diphylloside B, 6493

Epimedin B, 6960

Safghanoside E, 19116

\section{$\mathrm{C}_{38} \mathrm{H}_{48} \mathrm{O}_{20}$}

Diphylloside A, 6492

Rouhuoside, 18961

\section{$\mathrm{C}_{38} \mathrm{H}_{48} \mathrm{O}_{23}$}

Kaempferol-3-O- $\{[\beta-D$-xylopyranosyl $(1 \rightarrow 3)-\alpha-$ $L$-rhamnopyranosyl $(1 \rightarrow 6)][\alpha-L$-rhamnopyra$\operatorname{nosyl}(1 \rightarrow 2)]\}-\beta$ - $D$-galactopyranoside, 12105

Rhamnocitrin 3-O-apiosyl $(1 \rightarrow 5)$-apiosyl $(1 \rightarrow 2)$ $[\alpha$ - $L$-rhamnopyranosyl $(1 \rightarrow 6)]$ - $\beta$ - $D$-glucopyranoside, 18683

\section{$\mathrm{C}_{38} \mathrm{H}_{48} \mathrm{O}_{25}$}

Aescuflavoside, 661 


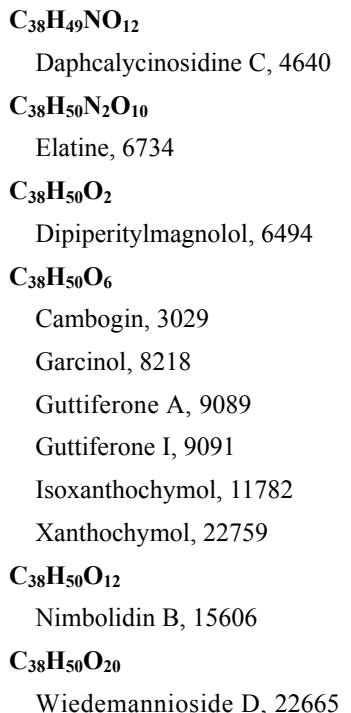

\section{$\mathrm{C}_{38} \mathrm{H}_{51} \mathrm{NO}_{18}$}

4-Acetylaminoethylphenyl-1-O-[6-O-(Z)-p-methoxycinnamoyl- $\beta$ - $D$-glucopyranosyl $(1 \rightarrow 2)]-[\beta$ $D$-glucopyranosyl $(1 \rightarrow 3)]$ - $\alpha$ - $L$-rhamnopyranoside, 313

\section{$\mathrm{C}_{38} \mathrm{H}_{52} \mathrm{O}_{6}$}

3 $\beta$-p-Hydroxybenzoyldehydrotumulosic acid, 9823

\section{$\mathrm{C}_{38} \mathrm{H}_{52} \mathrm{O}_{8}$}

3-O-(4-Hydroxy-3-methoxybenzoyl)ceanothic acid, 10383

\section{$\mathrm{C}_{38} \mathrm{H}_{52} \mathrm{O}_{16}$}

Chantriolide A, 3478

\section{$\mathrm{C}_{38} \mathrm{H}_{54} \mathrm{O}_{13}$ \\ Marinobufagin 3-suberoyl- $L$-glutamine ester, 13566}

\section{$\mathrm{C}_{38} \mathrm{H}_{54} \mathrm{O}_{14}$}

Scillarenin 3-O- $\beta$ - $D$-glucopyranosyl-( $1 \rightarrow 4)-2$ '- $O$ acetyl- $\alpha$ - $L$-rhamnopyranoside, 19519

Scillarenin 3-O- $\beta$ - $D$-glucopyranosyl-(1 $\rightarrow 4)-3$ '- $O$ acetyl- $\alpha$ - $L$-rhamnopyranoside, 19520

\section{$\mathrm{C}_{38} \mathrm{H}_{54} \mathrm{O}_{15}$}

$6 \beta$-Acetoxy scillarenin 3-O- $\beta$ - $D$-glucopyranosyl$(1 \rightarrow 4)$ - $\alpha$ - $L$-rhamnopyranoside, 279

cis-Cleroda-15,16-dihydroxy-3,13(Z)-dien-18-O[ $\beta$ - $D$-galactopyranosyl]-peracetylester, 3836

\section{$\mathrm{C}_{38} \mathrm{H}_{54} \mathrm{O}_{16}$}

Chantriolide B, 3479

Scillirosidin 3-O- $\beta$-D-glucopyranosyl-( $1 \rightarrow 4)$ $\alpha$-L-rhamnopyranoside, 19529

\section{$\mathrm{C}_{38} \mathrm{H}_{54} \mathrm{O}_{19}$}

Crocin 2, 4250

$\mathrm{C}_{38} \mathrm{H}_{54} \mathrm{O}_{22}$

4'- $O-\beta-D$-Glucosyl-9- $O$-(6"-deoxysaccharosyl) olivil, 8754

$\mathrm{C}_{38} \mathrm{H}_{56} \mathrm{~N}_{8} \mathrm{O}_{10}$
Pseudostellarin F, 18068

$$
\mathrm{C}_{38} \mathrm{H}_{56} \mathrm{O}_{4}
$$

Campesteryl ferulate, 3041

$\mathrm{C}_{38} \mathrm{H}_{56} \mathrm{O}_{8}$

Colchiside A, 3913

$\mathrm{C}_{38} \mathrm{H}_{56} \mathrm{O}_{11}$

Methylcimicifugoside, 14241

$\mathrm{C}_{38} \mathrm{H}_{56} \mathrm{O}_{13}$

25-O-Acetylbryoamaride, 339

Arvenin I, 1825

Opercurin A, 16126

Opercurin B, 16127

\section{$\mathrm{C}_{38} \mathrm{H}_{58} \mathrm{O}_{13}$}

2-O-Acetylsuavissimoside $\mathrm{F}_{1}, 516$

Arvenin II, 1826

$(2 R, 3 R, 4 R, 5 R, 7 S, 8 S, 9 S, 11 E, 13 S, 15 R)-2,3,5,7,8$,

9,15-Heptahydroxyjatropha-6(17),11-diene-14-

one-8,9-diacetate-7-isobutyrate-2,5-bis(2-meth

ylbutyrate), 9396

$(2 R, 3 R, 4 R, 5 R, 7 S, 8 S, 9 S, 11 E, 13 S, 15 R)-2,3,5,7,8$,

9,15-Heptahydroxyjatropha-6(17),11-diene-14one-8,9-diacetate-7-isobutyrate-2,3-bis(2-meth ylbutyrate), 9397

\section{$\mathrm{C}_{38} \mathrm{H}_{58} \mathrm{O}_{14}$}

Funingenoside C, 8007

\section{$\mathrm{C}_{38} \mathrm{H}_{60} \mathrm{O}_{7}$}

3-O-(2,3-Dimethylbutanoyl)-13-O-dodecanoyl20-deoxyingenol, 6324

\section{$\mathrm{C}_{38} \mathrm{H}_{60} \mathrm{O}_{8}$}

3-O-(2,3-Dimethylbutanoyl)-13-O-dodecanoylingenol, 6325

20-O-(2,3-Dimethylbutanoyl)-13-O-dodecanoylingenol, 6326

Fomitoside G, 7872

13-Oxyingenol-13-dodecanoate-20-hexanoate, 16449

Phorbol-12-palmitate-13-acetate, 17191

Welensalifactor $\mathrm{F}_{1}, 22658$

\section{$\mathrm{C}_{38} \mathrm{H}_{60} \mathrm{O}_{9}$}

12-O-Palmitoyl-16-hydroxyphorbol-13-acetate, 16564

\section{$\mathrm{C}_{38} \mathrm{H}_{60} \mathrm{O}_{10}$}

$1 \alpha, 3 \beta$-Hydroxyimberbic acid-23-O- $\alpha-L$-4-acetylrhamnopyranoside, 10226

Rubianoside I, 19007

\section{$\mathrm{C}_{38} \mathrm{H}_{60} \mathrm{O}_{11}$}

Lycogalinoside A, 13195

Lycogalinoside B, 13196

$\mathrm{C}_{38} \mathrm{H}_{60} \mathrm{O}_{12}$

Convallamarogen-1-O- $\alpha$ - $L$-rhamnopyranosyl $(1 \rightarrow 2)-\beta$ - $D$-xylopyranoside, 4007

Niga-ichigoside $F_{3}, 15541$

Polyspirostanol $\mathrm{PO}_{5}, 17679$
25(S)-Ruscogenin 1-O- $\alpha$ - $L$-rhamnopy-ranosyl$(1 \rightarrow 2)-\beta$ - $D$-xylopyranoside, 19071

\section{$\mathrm{C}_{38} \mathrm{H}_{60} \mathrm{O}_{13}$}

Laxogenin 3-O-[O- $\alpha$ - $L$-arabinopyranosyl-( $1 \rightarrow 6)$ $\beta$ - $D$-glucopyranoside], 12593

Pennogenin-3- $O-\alpha$ - $L$-arabinofuranosyl $(1 \rightarrow 4)-\beta$ $D$-glucopyranoside, 16810

$\mathrm{SQD}_{4}, 20236$

$\mathrm{C}_{38} \mathrm{H}_{60} \mathrm{O}_{14}$

26-O- $\beta$ - $D$-Glucopyranosyl-furostan-5,25(27)-diene- $1 \beta, 3 \beta, 22 \beta, 26$-tetrahydroxy-1- $O-\alpha-L$-arabinopyranoside, 8630

\section{$\mathrm{C}_{38} \mathrm{H}_{60} \mathrm{O}_{18}$}

Stevioside, 20341

\section{$\mathrm{C}_{38} \mathrm{H}_{61} \mathrm{NO}_{11}$}

Obtusolactam-20(R)-O-[ $\beta$-thevetopyranosyl-

$(1 \rightarrow 4)$ - $\beta$-cymaropyranoside], 15912

\section{$\mathrm{C}_{38} \mathrm{H}_{62}$}

Annoglabayin, 1301

\section{$\mathrm{C}_{38} \mathrm{H}_{62} \mathrm{O}_{9}$}

26- $O$-(3'-Isopentanoyl)- $\beta$ - $D$-glucopyranosyl-5 $\alpha$ furost-20(22)-ene-3 $\beta, 26$-diol, 11583

\section{$\mathrm{C}_{38} \mathrm{H}_{62} \mathrm{O}_{10}$}

2-O-Acetyl-28-dehydroxy-rubianoside IV, 371

Kahiricoside III, 12108

Kahiricoside IV, 12109

Rubiarboside A, 19021

\section{$\mathrm{C}_{38} \mathrm{H}_{62} \mathrm{O}_{11}$}

2-O-Acetyl-rubianoside IV, 496

\section{$\mathrm{C}_{38} \mathrm{H}_{62} \mathrm{O}_{12}$}

Aspafilioside A, 1860

Asparanin $\mathrm{B}_{3}, 1871$

Asparanin $\mathrm{B}_{4}, 1872$

22-Epirhodeasapogenin-1-O- $\alpha$ - $L$-rhamnopyra$\operatorname{nosyl}(1 \rightarrow 2)-\beta$ - $D$-xylopyranoside, 7006

Isorhodeasapogenin-1- $O-\alpha$ - $L$-rhamnopyranosyl $(1 \rightarrow 2)-\beta$ - $D$-xylopyranoside, 11676

Rhodeasapogenin-1-O- $\alpha$ - $L$-rhamnopyranosyl$(1 \rightarrow 2)-\beta$ - $D$-xylopyranoside, 18783

\section{$\mathrm{C}_{38} \mathrm{H}_{62} \mathrm{O}_{13}$}

$16 \beta$-[( $\alpha$-L-Arabinopyranosyl)oxy $]-3 \beta$-[( $\beta$ - $D$-glucopyranosyl)oxy]-17 $\alpha$-hydroxycholest-5-en22-one, 1578

(25R)-3 $\beta$-Hydroxy- $5 \alpha$-spirostan- $6 \alpha$-yl $O-\beta-D$ xylopyranosyl-( $1 \rightarrow 2)-\beta$ - $D$-glucopyranoside, 10721

Pentandroside A, 16877

\section{$\mathrm{C}_{38} \mathrm{H}_{62} \mathrm{O}_{14}$}

Glucoconvallasaponin A, 8592

Pentandroside C, 16879

\section{$\mathrm{C}_{38} \mathrm{H}_{62} \mathrm{O}_{15}$}

Glucoconvallasaponin B, 8593

Hemeroside A, 9341 


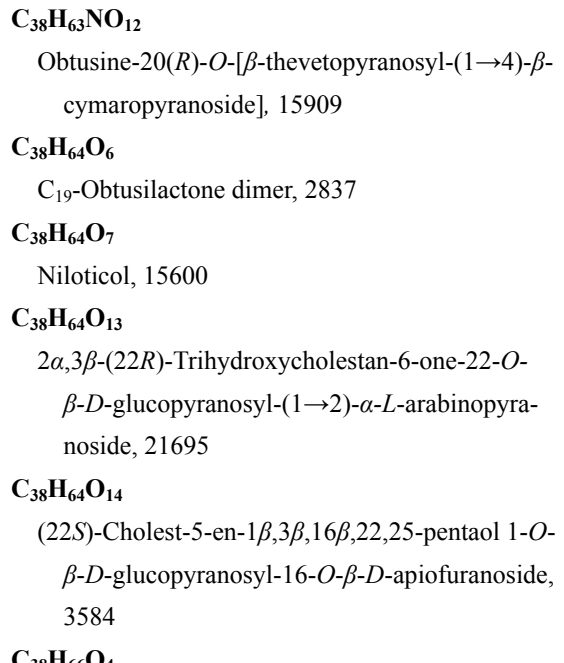

$\mathrm{C}_{38} \mathrm{H}_{66} \mathrm{O}_{4}$

Cluytyl ferulate, 3852

Nonacosanyl caffeate, 15670

Tenuiphenone D, 20962

\section{$\mathrm{C}_{38} \mathrm{H}_{70} \mathrm{O}_{4}$}

Coixenolide, 3906

\section{$\mathrm{C}_{39} \mathrm{H}_{26} \mathrm{O}_{9}$}

Thelephantin K, 21303

\section{$\mathrm{C}_{39} \mathrm{H}_{32} \mathrm{O}_{8}$}

Mulberrofuran K, 15045

\section{$\mathrm{C}_{39} \mathrm{H}_{32} \mathrm{O}_{14}$}

Apigenin-7-O-(3",6"-di-(E)-p-coumaroyl)- $\beta$ - $D$ galactopyranoside, 1487

Apigenin-4'- $O$-(2',6"-di- $O-p$-coumaroyl)- $\beta$ - $D$ glucoside, 1488

Cernoside, 3430

Kaempferol 3-O- $\alpha-L-\left(2^{\prime}, 4^{\prime}\right.$-di-Z-p-coumaroyl)rhamnoside, 12039

Platanoside, 17528

\section{$\mathrm{C}_{39} \mathrm{H}_{32} \mathrm{O}_{15}$}

Castanoside B, 3298

Cinchonain Ib, 3681

Kaempferol-3-O-(2",6"-di- $O$-(E)-p-coumaroyl$\beta$ - $D$-glucopyranoside), 12038

Kandelin $\mathrm{A}_{1}, 12128$

\section{$\mathrm{C}_{39} \mathrm{H}_{34} \mathrm{O}_{8}$}

Mulberrofuran F, 15043

\section{$\mathrm{C}_{39} \mathrm{H}_{34} \mathrm{O}_{9}$}

Mulberrofuran O, 15049

\section{$\mathrm{C}_{39} \mathrm{H}_{36} \mathrm{O}_{9}$}

Chalcomoracin, 3453

Guangsangon E, 9057

Guangsangon J, 9062

\section{$\mathrm{C}_{39} \mathrm{H}_{36} \mathrm{O}_{13}$}

(+)-Catechin-3- $O-\beta$ - $D$-gluco(2,6-bis-cinnamoyl)pyranoside, 3310

$\mathrm{C}_{39} \mathrm{H}_{38} \mathrm{O}_{9}$

Leachianone C, 12596
$\mathrm{C}_{39} \mathrm{H}_{38} \mathrm{O}_{10}$

Guangsangon A, 9053

$\mathrm{C}_{39} \mathrm{H}_{39} \mathrm{O}_{21}{ }^{+}$

Malonyl shisonin, 13450

$\mathrm{C}_{39} \mathrm{H}_{39} \mathrm{O}_{22}{ }^{+}$

Caffeoyl malonyl cyanin, 2913

Malonylawobanin, 13440

$\mathrm{C}_{39} \mathrm{H}_{40} \mathrm{~N}_{2} \mathrm{O}_{6}{ }^{2+}$

Phaeantharine, 17039

$\mathrm{C}_{39} \mathrm{H}_{40} \mathrm{O}_{13}$

Curtisian G, 4403

$\mathrm{C}_{39} \mathrm{H}_{40} \mathrm{O}_{17}$

Hydropiperoside, 9737

$\mathrm{C}_{\mathbf{3 9}} \mathrm{H}_{40} \mathrm{O}_{19}$

Ozturkoside C, 16484

$\mathrm{C}_{39} \mathrm{H}_{40} \mathrm{O}_{20}$

Ozturkoside A, 16482

$\mathrm{C}_{39} \mathrm{H}_{42} \mathrm{~N}_{2} \mathrm{O}_{8}$

Thalfinine, 21235

$\mathrm{C}_{39} \mathrm{H}_{43} \mathrm{O}_{20}{ }^{+}$

Alatanin 2, 836

$\mathrm{C}_{39} \mathbf{H}_{44} \mathbf{N}_{2} \mathbf{O}_{7}$

Hernandezine, 9441

Thalidasine, 21251

Thaliracebine, 21261

Thalrugosaminine, 21268

$\mathrm{C}_{39} \mathrm{H}_{44} \mathrm{O}_{14}$

Siphonol E, 19970

$\mathrm{C}_{39} \mathrm{H}_{44} \mathrm{O}_{19}$

6"'-Sinapoylspinosin, 19921

$\mathrm{C}_{39} \mathrm{H}_{45} \mathrm{NO}_{11}$

Euphocharacin F, 7601

$\mathrm{C}_{39} \mathrm{H}_{45} \mathrm{NO}_{12}$

Euphocharacin A, 7596

$\mathrm{C}_{39} \mathrm{H}_{45} \mathrm{NO}_{19}$

Wilfordconine, 22675

$\mathrm{C}_{39} \mathrm{H}_{45} \mathrm{~N}_{2} \mathrm{O}_{6}{ }^{+}$

(+)-2- $N$-Methyltetrandrine, 14753

\section{$\mathbf{C}_{39} \mathbf{H}_{46} \mathbf{N}_{2} \mathbf{O}_{10}$}

$5 \alpha-O-(3$ '-Amino-3'-phenylpropionyl)nicotaxine, 1062

$\mathrm{C}_{39} \mathrm{H}_{46} \mathrm{O}_{8}$

Nimbolin A, 15610

$\mathrm{C}_{39} \mathrm{H}_{46} \mathrm{O}_{10}$

Nimbolin B, 15611

$\mathrm{C}_{39} \mathrm{H}_{46} \mathrm{O}_{15}$

Taxezopidine L, 20790

$\mathrm{C}_{39} \mathrm{H}_{46} \mathrm{O}_{18}$

Korepimedoside B, 12273

\section{$\mathrm{C}_{39} \mathrm{H}_{47} \mathrm{BrO}_{8}$}

Bisacutifolone A $p$-bromobenzoate, 2430

Bisacutifolone B $p$-bromobenzoate, 2433

$\mathrm{C}_{39} \mathrm{H}_{48} \mathrm{O}_{11}$
15-O-Deacetyl-15-O-methylnimbolidin A, 4752

$\mathrm{C}_{39} \mathrm{H}_{48} \mathrm{O}_{12}$

Taxinine J, 20802

$\mathrm{C}_{39} \mathrm{H}_{48} \mathrm{O}_{13}$

Swietephragmin B, 20517

Taxuspine D, 20852

$\mathrm{C}_{\mathbf{3 9}} \mathrm{H}_{\mathbf{4 8}} \mathbf{O}_{\mathbf{1 5}}$

$2 \alpha, 3 \beta, 5 \alpha, 8 \alpha, 9 \alpha, 15 \beta$-Hexaacetoxy-7 $\beta$-benzoyloxyjatropha-6(17),11E-dien-14-one, 9474

$3 \beta, 5 \alpha, 7 \beta, 8 \alpha, 9 \alpha, 15 \beta$-Hexaacetoxy-2 $\alpha$-benzoyloxyjatropha-6(17),11E-dien-14-one, 9475

\section{$\mathrm{C}_{39} \mathrm{H}_{48} \mathrm{O}_{16}$}

14 $\beta$-Benzoyloxybaccatin IV, 2249

Segetene 5, 19654

\section{$\mathrm{C}_{39} \mathrm{H}_{49} \mathrm{NO}_{21}$}

Anthemis glycoside A, 1352

\section{$\mathrm{C}_{39} \mathrm{H}_{50} \mathrm{O}_{7}$}

Peridinin, 16922

\section{$\mathrm{C}_{39} \mathrm{H}_{50} \mathrm{O}_{13}$}

$1 S, 6 R$-Di(2-)methylbutanoyloxy-4S-hydroxy$8 S$-benzoyloxy-9R-(3-)furancarbonyloxy-13acetyloxy- $\beta$-dihydroagarofuran, 6328

\section{$\mathrm{C}_{39} \mathrm{H}_{50} \mathrm{O}_{19}$}

Epimedin C, 6961

\section{$\mathrm{C}_{39} \mathrm{H}_{50} \mathrm{O}_{20}$}

Epimedin A, 6959

\section{$\mathrm{C}_{39} \mathrm{H}_{50} \mathrm{O}_{21}$}

Pyrolaside B, 18269

\section{$\mathrm{C}_{39} \mathrm{H}_{50} \mathrm{O}_{23}$}

Astrasikokioside I, 1950

Reiniose E, 18607

\section{$\mathrm{C}_{39} \mathrm{H}_{50} \mathrm{O}_{24}$}

1-[( $\beta$ - $D$-Glucopyranosyl-( $(1 \rightarrow 6)-O-\beta-D$-glucopyranosyl-(1 $\rightarrow 3)-O-\beta$ - $D$-glucopyranosyl$(1 \rightarrow 6)-O-\beta$ - $D$-glucopyranosyl)oxy]-8-hydroxy-3-methyl-9,10-anthraquinone, 8644

Kaempferol 3-O-[2"-O- $\alpha$-rhamnopyranosyl-3"$O$-(6"'--O- $\alpha$-rhamnopyranosyl- $\beta$-glucopyranosyl)]- $\beta$-glucopyranoside, 12081

\section{$\mathrm{C}_{39} \mathrm{H}_{50} \mathrm{O}_{25}$}

Quercetin-3-O-[ $\alpha$-rhamnosyl $(1 \rightarrow 6)]$ [ $\beta$-glucosyl $(1 \rightarrow 2)]$ - $\beta$-glucoside-7- $O$ - $\alpha$-rhamnoside, 18388

\section{$\mathrm{C}_{39} \mathrm{H}_{51} \mathrm{NO}_{11}$}

2'-Deacetoxyaustrotaxine, 4703

\section{$\mathrm{C}_{39} \mathrm{H}_{51} \mathrm{NO}_{12}$}

2'-Deacetylaustrotaxine, 4720

\section{$\mathrm{C}_{39} \mathrm{H}_{52} \mathrm{O}_{23}$}

Ballotetroside, 2134

Lunariifolioside, 13074

$\mathrm{C}_{39} \mathrm{H}_{52} \mathrm{O}_{24}$

Kaempferol 3-O- $\alpha-L$-rhamnopyranosyl $(1 \rightarrow 6)-\beta$ $D$-glucopyranosyl $(1 \rightarrow 2)-\beta$ - $D$-glucopyranoside-7-O- $\alpha$ - $L$-rhamnopyranoside, 12076 


\section{$\mathrm{C}_{39} \mathrm{H}_{53} \mathrm{NO}_{10}$}

2 $\alpha$-Acetoxy-2',7-dideacetoxy austrospicatine, 156

2' $\beta$-Deacetoxyaustrospicatine, 4702

\section{$\mathrm{C}_{39} \mathrm{H}_{53} \mathrm{NO}_{11}$}

(-)-2 $\alpha$-Acetoxy-2',7-dideacetoxy-1-hydroxy-11 $(15 \rightarrow 1)$-abeo-austrospicatine, 157

(+)-2 $\alpha$-Acetoxy-2',7-dideacetoxy-1-hydroxyaustrospicatine, 158

2'-Deacetylaustrospicatine, 4718

7-Deacetylaustrospicatine, 4719

$2 \alpha$-Hydroxy-2' $\beta$-Deacetylaustrospicatine, 9959

\section{$\mathrm{C}_{39} \mathrm{H}_{53} \mathrm{NO}_{12}$}

$2 \alpha$-Hydroxy- $7 \beta, 9 \alpha, 10 \beta, 13 \alpha$-tetraacetoxy- $5 \alpha-(2$ hydroxy-3'- $N, N$-dimethylamino-3'-phenyl)propionyloxytaxa-4(20),11-diene, 10742

\section{$\mathrm{C}_{39} \mathrm{H}_{53} \mathrm{~N}_{7} \mathrm{O}_{11}$}

Cyclo-(Gly-Asp-Leu-Thr-Val-Tyr-Phe), 4493

\section{$\mathrm{C}_{39} \mathrm{H}_{54} \mathrm{O}_{5}$}

Chuanxiongterpene, 3632

3-O-(E)-Coumaroyloleanolic acid, 4177

3-O-(Z)-Coumaroyloleanolic acid, 4178

Lup-20(29)en-28-al-3 $\beta$-yl-caffeate, 13090

$3 \beta$-[( $m$-Methoxybenzoyl)oxyl]urs-12-en-28-oic acid, 13851

Olean-28-al-3 $\beta$-yl-caffeate, 16027

\section{$\mathrm{C}_{39} \mathrm{H}_{54} \mathrm{O}_{6}$}

3-O-(cis-p-Coumaroyl)-alphitolic acid, 4147

3-O-(trans-p-Coumaroyl)-alphitolic acid, 4148

(3Z)-Coumaroylhederagenin, 4164

(23E)-Coumaroylhederagenin, 4165

(23Z)-Coumaroylhederagenin, 4166

3-O-(cis-p-Coumaroyl)-maslinic acid, 4169

3-O-(trans- $p$-Coumaroyl)-maslinic acid, 4170

$3 \alpha$-(3",4"-Dihydroxy-trans-cinnamoyloxy)- $D$ -

friedo-olean-14-en-28-oic acid, 5791

Diospyrosooleanolide, 6462

3-O-p-Hydroxy-trans-cinnamoylmaslinic acid, 9915

Jacoumaric acid, 11810

Lup-20(29)-en-28-oic-3 $\beta$-yl caffeate, 13095

Olean-28-oic-3 $\beta$-yl caffeate, 16045

Rubicoumaric acid, 19028

Uncarinic acid C, 22210

Zamanic acid, 22964

\section{$\mathrm{C}_{39} \mathrm{H}_{54} \mathrm{O}_{7}$}

(3E)-Coumaroylarjunolic acid, 4151

$3 \beta$-cis-p-Coumaroyloxy-2 $\alpha, 23$-dihydroxyolean12-en-28-oic acid, 4179

23-trans- $p$-Coumaroyloxy- $2 \alpha, 3 \beta$-dihydroxyolean-12-en-28-oic acid, 4180

Guavacoumaric acid, 9073

$\mathrm{C}_{39} \mathrm{H}_{54} \mathrm{O}_{8}$

3-O-Benzoyl-13-O-dodecanoateingenol, 2238
20-O-Benzoyl-13-O-dodecanoateingenol, 2239

16-Oxolyclanitin 30-(4-hydroxycinnamoyl), 16376

$\mathrm{C}_{39} \mathrm{H}_{54} \mathrm{O}_{22}$

Syringaresinol-4- $O-\beta$ - $D$-apiofuranosyl- $(1 \rightarrow 2)$ $\beta$ - $D$-glucopyranosyl-4'- $O-\beta$ - $D$-glucopyranoside, 20558

$\mathrm{C}_{39} \mathrm{H}_{56} \mathrm{O}_{2}$

$\alpha$-Amyrin cinnamate, 1114

Lupeol cinnamate, 13101

$\mathrm{C}_{39} \mathrm{H}_{56} \mathrm{O}_{3}$

cis-Careaborin, 3192

trans-Careaborin, 3193

3- $O$-(E)-Coumaroyl- $\beta$-amyrin, 4149

3-O-(Z)-Coumaroyl- $\beta$-amyrin, 4150

3-(Z)-Coumaroyllupeol, 4168

Dioslupecin A, 6451

\section{$\mathrm{C}_{39} \mathrm{H}_{56} \mathrm{O}_{4}$}

3-(E)-Caffeoyllupeol, 2910

3-(Z)-Caffeoyllupeol, 2911

$3 \beta$-(E)-Caffeoyltaraxerol, 2926

$3 \beta$-(Z)-Caffeoyltaraxerol, 2927

3-O-(E)-Coumaroylerythrodiol, 4155

3-O-(Z)-Coumaroylerythrodiol, 4156

Stigmasteryl ferulate, 20376

\section{$\mathrm{C}_{39} \mathrm{H}_{56} \mathrm{O}_{5}$}

Lup-20(29)-en-28-ol-3 $\beta$-yl caffeate, 13096

\section{$\mathrm{C}_{39} \mathrm{H}_{56} \mathrm{O}_{12}$}

Spinoside A, 20171

\section{$\mathrm{C}_{39} \mathrm{H}_{58} \mathrm{O}_{2}$}

Lupeol $\beta$-phenyl propionate, 13102

\section{$\mathrm{C}_{39} \mathrm{H}_{58} \mathrm{O}_{3}$}

3-O-Dihydrocoumaroyl- $\beta$-amyrin, 5571

\section{$\mathrm{C}_{39} \mathrm{H}_{58} \mathrm{O}_{4}$}

$21 \alpha$-Hydroxyserrat-14-en-3 $\beta$-yl $p$-dihydrocoumarate, 10707

$\beta$-Sitosteryl ferulate, 19995

\section{$\mathrm{C}_{39} \mathrm{H}_{58} \mathrm{O}_{5}$}

Phlegmanol A, 17148

\section{$\mathrm{C}_{39} \mathrm{H}_{60} \mathrm{O}_{3}$}

5-(1-Phytoxy-ethyl)-2-hydroxy-7-methoxy-1,8dimethyl-9,10-dihydrophenanthrene, 17274

\section{$\mathrm{C}_{39} \mathrm{H}_{60} \mathrm{O}_{4}$}

Dihydro- $\beta$-sitosteryl ferulate, 5714

Dihydro- $\gamma$-sitosteryl ferulate, 5715

\section{$\mathrm{C}_{39} \mathrm{H}_{60} \mathbf{O}_{9}$}

Quinovic acid-3 $\beta$-O-(3',4'-O-isopropylidene)$\beta$ - $D$-fucopyranoside, 18437

Quinovic acid-3 $\beta$-(2',3'-O-isopropylidene) $-\alpha-L$ rhamnopyranoside, 18438

\section{$\mathrm{C}_{39} \mathrm{H}_{60} \mathrm{O}_{11}$}

30-Noroleanolic acid-3-O- $\beta$ - $D$-xylosyl( $1 \rightarrow 2)-\alpha$ $L$-arabinopyranoside, 15783
Soulieoside A, 20110

Soulieoside B, 20111

\section{$\mathrm{C}_{39} \mathrm{H}_{60} \mathrm{O}_{12}$}

Beesioside I, 2200

30 -Norhederagenin-3- $O-\beta$ - $D$-xylosyl $(1 \rightarrow 2)-\alpha$ $L$-arabinopyranoside, 15755

\section{$\mathrm{C}_{39} \mathrm{H}_{60} \mathrm{O}_{13}$}

3-O- $\alpha$ - $L$-Rhamnopyranosyl $(1 \rightarrow 2)-\beta$ - $D$-glucuronopyranosyl-3 $\beta$-hydroxy-25R-spirost-5-ene, 18724

3-O- $\alpha$ - $L$-Rhamnopyranosyl $(1 \rightarrow 2)-\beta$ - $D$-glucuronopyranosyl-3 $\beta$-hydroxy- $25 S$-spirost-5-ene, 18725

Saundersioside D, 19409

\section{$\mathrm{C}_{39} \mathrm{H}_{60} \mathbf{O}_{14}$}

$1 \beta, 2 \alpha$-Dihydroxyspirosta-5,25(27)-dien-3 $\beta$-yl $O$ - $\alpha$ - $D$-rhamnopyranosyl-( $(1 \rightarrow 2)-\beta$ - $L$-galactopyranoside, 6124

\section{$\mathrm{C}_{39} \mathrm{H}_{60} \mathrm{O}_{15}$}

Polygonatoside $\mathrm{C}, 17645$

Pregna-5,16-dien-3 $\beta$-ol-20-one 3- $O-\beta$-chacotrioside, 17781

\section{$\mathrm{C}_{39} \mathrm{H}_{60} \mathrm{O}_{16}$}

Pregnadienolone-3- $O$ - $\beta$-gracillimatriose, 17782

\section{$\mathrm{C}_{39} \mathrm{H}_{62} \mathrm{O}_{12}$}

Beesioside II, 2201

Beesioside J, 2203

Collettinside II, 3923

Ophiopogonin B, 16144

Ophiopogonin C', 16145

Polyphyllin C, 17654

\section{$\mathrm{C}_{39} \mathrm{H}_{62} \mathrm{O}_{13}$}

Deacylbrownioside, 4794

Diosgenin-3-di- $\beta$ - $O$-glucopyranoside, 6442

$(22 S, 23 R)-16 \beta, 23$-Epoxy-18,23-dihydroxy-22(2-methyl-1-propenyl)-24-norchol-5-en-3 $\beta$-yl $O$ - $\alpha$ - $L$-rhamnopyranosyl-( $(1 \rightarrow 2)-\beta$ - $D$-glucopyr anoside, 7081

Pennogenin-3- $O-\alpha-L$-rhamnopyranosyl $(1 \rightarrow 2)-\beta$ $D$-glucopyranoside, 16813

Pingbeisaponin, 17383

Trillarin, 21871

\section{$\mathrm{C}_{39} \mathrm{H}_{62} \mathrm{O}_{14}$}

Cantalanin A, 3089

(25R)-1 $\beta, 2 \alpha$-Dihydroxyspirost-5-en-3 $\beta$-yl $O-\alpha-L$ rhamnopyranosyl-( $(1 \rightarrow 2)-\beta$ - $D$-galactopyranoside, 6127

$1 \beta, 2 \alpha$-Dihydroxy-5 $\alpha$-spirost-25(27)-en- $3 \beta$-yl $O$ - $\alpha$ - $L$-rhamnopyranosyl-( $(1 \rightarrow 2)-\beta$ - $D$-galactopyranoside, 6131

$(24 S, 25 R)-1 \beta-[(\beta-D$-Fucopyranosyl)oxy]-6 $\beta$ hydroxy- $3 \alpha, 5 \alpha$-cyclospirostan-24-yl $\beta$ - $D$ glucopyranoside, 7976 
Ophiogenin-3-O- $\alpha$ - $L$-rhamnopyranosyl $(1 \rightarrow 2)$ $\beta$ - $D$-glucopyranoside, 16131

Polyspirostanol $\mathrm{PO}_{1}, 17676$

$(23 S, 25 R)$-Spirost-5-ene-3 $\beta, 23$-diol 23-O-[O- $\beta$ $D$-glucopyranosyl-( $1 \rightarrow 6)-\beta$ - $D$-glucopyranoside], 20219

Terreside B, 21001

\section{$\mathbf{C}_{39} \mathbf{H}_{62} \mathbf{O}_{15}$}

Agave americana Compound 3, 714

$(24 S, 25 R)-1 \beta-[(\beta-D$-Glucopyranosyl) oxy $]-6 \beta$ hydroxy-3 $\alpha, 5 \alpha$-cyclospirostan-24-yl $\beta$ - $D$-glucopyranoside, 8690

\section{$\mathrm{C}_{39} \mathrm{H}_{63} \mathrm{NO}_{10}$}

Solanidine-3- $O-\alpha$ - $L$-rhamnopyranosyl-( $(1 \rightarrow 2)$ $\beta$ - $D$-glucopyranoside, 20058

\section{$\mathrm{C}_{39} \mathrm{H}_{63} \mathrm{NO}_{11}$}

$\beta$-Solamargine, 20045

$\gamma_{1}$-Solamarine, 20048

$\gamma_{2}$-Solamarine, 20049

Solaplumbine, 20064

\section{$\mathrm{C}_{39} \mathrm{H}_{63} \mathrm{NO}_{12}$}

$\delta$-Solamarine, 20050

\section{$\mathrm{C}_{39} \mathrm{H}_{64} \mathrm{O}_{11}$}

Spergulin B, 20145

\section{$\mathrm{C}_{39} \mathrm{H}_{64} \mathrm{O}_{12}$}

Asparanin $\mathrm{B}_{6}, 1874$

Neotigogenin-3-O- $\alpha$ - $L$-rhamnopyranosyl $(1 \rightarrow 6)$ $\beta$ - $D$-glucopyranoside, 15466

\section{$\mathrm{C}_{39} \mathrm{H}_{64} \mathrm{O}_{13}$}

Asparanin A, 1868

(22S)-3 $\beta, 22-$ Dihydroxy-1 $\beta$ - $[(\alpha-L$-rhamnopyranosyl)oxy]cholest-5,24-dien-16 $\beta$-yl $\beta$-D-glucopyranoside, 6107

3-O- $[\beta$ - $D$-Glucopyranosyl $(1 \rightarrow 2)-\beta$ - $D$-glucopyranosyl]-(25S)-5 $\beta$-spirostan-3 $\beta$-ol, 8656 Gurillin G, 9086

Pentandroside D, 16880

Smilagenin-3- $O$-[ $\beta$ - $D$-glucopyranosyl- $(1 \rightarrow 2)]$ $\beta$-D-mannopyranoside, 20027

Timosaponin $\mathrm{A}_{3}, 21395$

Torvoside J, 21471

Torvoside K, 21472

Torvoside L, 21473

\section{$\mathrm{C}_{39} \mathrm{H}_{64} \mathbf{O}_{14}$}

Agave cantala Compound 1, 721

Agave cantala Saponin 1, 723

(25R)-1 $\beta, 2 \alpha$-Dihydroxy-5 $\alpha$-spirostan- $3 \beta$-yl

$O$ - $\alpha$ - $L$-rhamnopyranosyl-( $1 \rightarrow 2)-\beta$ - $D$-galactopyranoside, 6126

(25R)-3 $\beta$-Hydroxy-5 $\alpha$-spirostan- $6 \alpha$-yl $O-\beta-D$ glucopyranosyl-( $1 \rightarrow 2)$ - $\beta$ - $D$-glucopyranoside, 10718

Markogenin3- $O-\beta$ - $D$-glucopyranosyl-( $(1 \rightarrow 2)-\beta$ -
$D$-galactopyranoside, 13570

(25R)-Samogenin 3-O- $\beta$ - $D$-glucopyranosyl

$(1 \rightarrow 2)$ - $\beta$ - $D$-galactopyranoside, 19231

(25S)-Samogenin 3-O- $\beta$ - $D$-glucopyranosyl

$(1 \rightarrow 2)$ - $\beta$ - $D$-galactopyranoside, 19232

(5 $\beta, 25 S)$-Spirostan-3 $\beta, 15 \alpha, 23 \alpha$-diol-3- $O$ - $D$-glucopyranosyl-( $1 \rightarrow 2)-\beta$ - $D$-galactopyranoside, 20209

\section{$\mathrm{C}_{39} \mathrm{H}_{64} \mathrm{O}_{15}$}

Cantalasaponin 1, 3090

Proampeloside $\mathrm{Bf}_{2}, 17865$

(5 $\beta, 25 S)$-Spirostan-3 $\beta, 15 \alpha, 23 \alpha$-triol-3- $O$ - $D$-glucopyranosyl-( $1 \rightarrow 2)-\beta$ - $D$-galactopyranoside, 20217

Wattoside I, 22655

\section{$\mathrm{C}_{39} \mathrm{H}_{64} \mathrm{O}_{16}$}

Agamenoside H, 697

$\mathrm{C}_{39} \mathrm{H}_{64} \mathrm{O}_{17}$

S-10, 19094

$\mathrm{C}_{39} \mathrm{H}_{65} \mathrm{NO}_{12}$

Yibeinoside C, 22906

$\mathrm{C}_{39} \mathrm{H}_{66} \mathrm{O}_{13}$

(22S)-Cholest-5-ene-1 $\beta, 3 \beta, 16 \beta, 22$-tetraol-1- $O$ $\alpha$ - $L$-rhamnopyranosyl-16- $O-\beta$ - $D$-glucopyranoside, 3579

\section{$\mathrm{C}_{39} \mathrm{H}_{66} \mathrm{O}_{14}$}

Anemarrhenasaponin I, 1171

26- $O-\beta$ - $D$-Glucopyranosylfurostane-3 $\beta, 26$-diol3-O- $\beta$ - $D$-glucopyranoside, 8631

\section{$\mathrm{C}_{39} \mathrm{H}_{66} \mathrm{O}_{18}$}

Nerolidol-3-O- $\{\alpha-L$-rhamnopyranosyl- $(1 \rightarrow 4)-\alpha$ $L$-rhamnopyranosyl-( $1 \rightarrow 2)$-[ $\alpha$ - $L$-rhamnopyranosyl- $(1 \rightarrow 6)]-\beta$ - $D$-glucopyranoside\}, 15504

\section{$\mathrm{C}_{39} \mathrm{H}_{68} \mathrm{O}_{5}$}

Glyceryl linolenate II, 8815

1-O-(9Z,12Z-Octadecadienoyl)-2-O-(9Z,12Zoctadecadienoyl) glycerol, 15945

\section{$\mathrm{C}_{39} \mathrm{H}_{68} \mathrm{O}_{8}$}

Uvaribonone, 22297

\section{$\mathrm{C}_{39} \mathrm{H}_{70} \mathrm{O}_{5}$}

1-O-(9Z,12Z-Octadecadienoyl)-3-O-(9Z-octadecenoyl) glycerol, 15946

\section{$\mathrm{C}_{39} \mathrm{H}_{70} \mathrm{O}_{8}$}

Uvaribonin, 22292

$\mathrm{C}_{39} \mathrm{H}_{72} \mathrm{O}_{5}$

Anticancer Glycerol Ester PMV70P691-119, 1424

$\alpha: \alpha$-Diolein, 6431

$\alpha: \beta$-Diolein, 6432

$\mathrm{C}_{39} \mathrm{H}_{79} \mathrm{NO}_{5}$

Spongiamine A, 20226

$\mathrm{C}_{40} \mathrm{H}_{26} \mathrm{O}_{25}$

Glansrin B, 8505

\section{$\mathrm{C}_{40} \mathrm{H}_{28} \mathrm{O}_{26}$}

Phyllanthusiin U, 17224

\section{$\mathrm{C}_{40} \mathrm{H}_{30} \mathrm{O}_{24}$}

Diellagic acid rhamnoside $(1 \rightarrow 4)$ glucopyranoside, 5485

\section{$\mathrm{C}_{40} \mathrm{H}_{30} \mathrm{O}_{26}$}

Pelargoniin A, 16786

\section{$\mathrm{C}_{40} \mathrm{H}_{34} \mathrm{O}_{15}$}

Chrysoeriol 7-O-(3",6"-Di- $O-E$ - $p$-coumaroyl)$\beta$ - $D$-glucopyranoside, 3607

\section{$\mathrm{C}_{40} \mathrm{H}_{36} \mathrm{O}_{9}$}

Lespedezol B 3,12690

\section{$\mathrm{C}_{40} \mathrm{H}_{36} \mathrm{O}_{10}$}

Guangsangon F, 9058

\section{$\mathrm{C}_{40} \mathrm{H}_{36} \mathrm{O}_{11}$}

Kuwanon G, 12385

Kuwanon K, 12388

\section{$\mathrm{C}_{40} \mathrm{H}_{36} \mathrm{O}_{12}$}

Cathayanon A, 3324

Cathayanon B, 3325

Sanggenon C, 19257

Sanggenon $\mathrm{C}_{1}, 19258$

Sanggenon D, 19259

Sanggenon O, 19270

Sanggenon Q, 19272

\section{$\mathrm{C}_{40} \mathrm{H}_{38} \mathrm{O}_{10}$}

Guangsangon H, 9060

Kuwanon I, 12387

\section{$\mathrm{C}_{40} \mathrm{H}_{38} \mathrm{O}_{11}$}

Sanggenon G, 19262

\section{$\mathrm{C}_{40} \mathrm{H}_{38} \mathrm{O}_{12}$}

Moracenin D, 14952

$\mathrm{C}_{40} \mathrm{H}_{40} \mathrm{~N}_{4} \mathrm{O}_{2}^{+2}$

5',6'-Dehydroguiaflavine, 4927

\section{$\mathrm{C}_{40} \mathrm{H}_{40} \mathrm{O}_{6}$}

3-3"Linked-(2'-hydroxy-4-O-isoprenylchalcone)(2"'-hydroxy-4"-O-isoprenyl dihydrochalcone), 12890

\section{$\mathrm{C}_{40} \mathrm{H}_{40} \mathrm{O}_{12}$}

Boehmenan, 2527

\section{$\mathrm{C}_{40} \mathrm{H}_{42} \mathrm{~N}_{4} \mathrm{O}_{2}$}

Guiaflavine, 9078

$\mathrm{C}_{40} \mathrm{H}_{42} \mathrm{~N}_{4} \mathrm{O}_{3}^{+2}$

5',6'-Dehydroguiachrysine, 4926

\section{$\mathrm{C}_{40} \mathrm{H}_{42} \mathrm{O}_{9}$}

PM-2004-70-452-5, 17577

\section{$\mathrm{C}_{40} \mathrm{H}_{42} \mathrm{O}_{12}$}

Lappaol F, 12518

\section{$\mathrm{C}_{40} \mathrm{H}_{42} \mathrm{O}_{14}$}

Bis-5,5-nortrachelogenin, 2490

Bis-5,5'-nortrachelogenin, 2491

\section{$\mathrm{C}_{40} \mathrm{H}_{42} \mathrm{O}_{16}$}

Gnemonoside E, 8875 
$\mathrm{C}_{40} \mathrm{H}_{42} \mathrm{O}_{18}$

Lapathoside C, 12507

$\mathrm{C}_{40} \mathrm{H}_{42} \mathrm{O}_{26}$

Isochestanin, 11326

$\mathrm{C}_{40} \mathrm{H}_{44} \mathrm{~N}_{\mathbf{2}} \mathrm{O}_{7}$

Nigellamine $\mathrm{A}_{5}, 15565$

$\mathrm{C}_{40} \mathrm{H}_{44} \mathrm{~N}_{4} \mathrm{O}^{2+}$

C-Curarine, 4377

$\mathrm{C}_{40} \mathrm{H}_{44} \mathrm{~N}_{4} \mathrm{O}_{3}$

De-O-methyltenuicausine, 5099

Guiachrysine, 9077

\section{$\mathrm{C}_{40} \mathrm{H}_{44} \mathrm{O}_{12}$}

Baccatin III 13-cinnamate, 2074

\section{$\mathrm{C}_{40} \mathrm{H}_{44} \mathrm{O}_{20}$}

Scabroside, 19448

\section{$\mathrm{C}_{40} \mathrm{H}_{44} \mathrm{O}_{22}$}

Isomacrophylloside, 11517

Macrophylloside A, 13321

\section{$\mathrm{C}_{40} \mathrm{H}_{44} \mathrm{O}_{23}$}

Macrophylloside B, 13322

\section{$\mathrm{C}_{40} \mathrm{H}_{45} \mathrm{~N}_{4} \mathrm{O}_{3}$}

Macrospegatrine, 13325

\section{$\mathrm{C}_{40} \mathrm{H}_{46} \mathrm{Cl}_{2} \mathrm{~N}_{2} \mathrm{O}_{6}{ }^{2+}$}

2',2'-N,N-Dichloromethyltetrandrine, 5419

\section{$\mathrm{C}_{40} \mathrm{H}_{46} \mathrm{~N}_{2} \mathrm{O}_{8}$}

Fetidine, 7793

Pennsylvanine, 16816

\section{$\mathrm{C}_{40} \mathrm{H}_{46} \mathrm{~N}_{4} \mathrm{O}_{2}{ }^{2+}$ \\ Toxiferine I, 21485}

\section{$\mathrm{C}_{40} \mathrm{H}_{46} \mathrm{O}_{10}$ \\ $2 \alpha$-Benzoyloxy-5 $\alpha$-cinnamoyloxy- $9 \alpha, 10 \beta$-di- acetoxy-1 $\beta, 13 \alpha$-dihydroxy-4(20),11-taxadiene, 2250}

Taxchinin H, 20775

\section{$\mathrm{C}_{40} \mathrm{H}_{46} \mathrm{O}_{12}$}

Orthosiphol H, 16226

\section{$\mathrm{C}_{40} \mathrm{H}_{46} \mathrm{O}_{13}$}

2,7-Dideacetyl-2,7-dibenzoyl-taxayunnanine F, 5459

Siphonol A, 19966

Siphonol D, 19969

Staminol A, 20258

Taxchinin I, 20776

\section{$\mathrm{C}_{40} \mathrm{H}_{46} \mathrm{O}_{14}$}

Lappaol H, 12519

Yunnanxol, 22952

\section{$\mathrm{C}_{40} \mathrm{H}_{46} \mathrm{O}_{20}$}

Longitin, 12984

$\mathrm{C}_{40} \mathrm{H}_{47} \mathrm{~N}_{2} \mathrm{O}_{8}{ }^{+}$

Thalirabine, 21260

\section{$\mathrm{C}_{40} \mathrm{H}_{48} \mathrm{~N}_{2} \mathrm{O}_{8}$}

$\mathrm{N}$-Demethylthalistyline, 5100

$\mathrm{C}_{40} \mathrm{H}_{48} \mathrm{~N}_{2} \mathrm{O}_{10}$

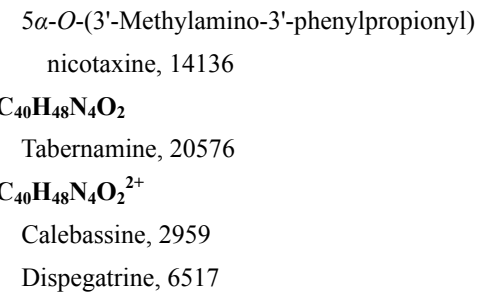

$\mathrm{C}_{40} \mathrm{H}_{48} \mathrm{~N}_{6} \mathrm{O}_{9}$

Rubia akane RA-II, 18980

Rubia akane RA-V, 18983

$\mathrm{C}_{40} \mathrm{H}_{48} \mathrm{~N}_{6} \mathrm{O}_{10}$

Rubia akane RA-I, 18979

$\mathrm{C}_{40} \mathrm{H}_{48} \mathrm{O}_{12}$

Nimbolidin A, 15605

$\mathrm{C}_{40} \mathrm{H}_{50} \mathrm{O}_{2}$

Rhodoxanthin, 18815

$\mathrm{C}_{40} \mathrm{H}_{50} \mathrm{O}_{5}$

Hongencaotone, 9629

$\mathrm{C}_{40} \mathrm{H}_{50} \mathrm{O}_{7}$

PM-2004-70-452-4, 17576

$\mathrm{C}_{40} \mathrm{H}_{52} \mathrm{O}$

3,4-Dehydrolycopen-16-al, 4944

\section{$\mathrm{C}_{40} \mathrm{H}_{52} \mathrm{O}_{2}$}

Canthaxanthin, 3095

Cynthiaxanthin, 4569

$\mathrm{C}_{40} \mathrm{H}_{52} \mathrm{O}_{3}$

4,4'-Diketo-3-hydroxy- $\beta$-carotene, 6190

\section{$\mathrm{C}_{40} \mathrm{H}_{52} \mathrm{O}_{4}$}

Astaxanthin, 1921

\section{$\mathrm{C}_{40} \mathrm{H}_{52} \mathrm{O}_{17}$}

Phyllanthoside, 17216

Phyllanthostatin 1, 17217

$\mathrm{C}_{40} \mathrm{H}_{52} \mathrm{O}_{21}$

Wiedemannioside E, 22666

\section{$\mathrm{C}_{40} \mathrm{H}_{52} \mathrm{O}_{24}$}

Reiniose F, 18608

$\mathrm{C}_{40} \mathrm{H}_{54}$

Torulene, 21467

$\mathrm{C}_{40} \mathrm{H}_{54} \mathrm{~N}_{2} \mathrm{O}_{6}$

Incarvillateine C, 11007

\section{$\mathrm{C}_{40} \mathrm{H}_{54} \mathrm{O}$}

Celaxanthin, 3369

3,4-Didehydroxy-3'-deoxycapsanthin, 5472

Echinenone, 6688

$\mathrm{C}_{40} \mathrm{H}_{54} \mathrm{O}_{2}$

Diatoxanthin, 5377

4-Keto-4'-hydroxy- $\beta$-carotene, 12201

$\mathrm{C}_{40} \mathrm{H}_{54} \mathrm{O}_{3}$

Adonixanthin, 647

Doradexanthin, 6560

$\mathrm{C}_{40} \mathrm{H}_{54} \mathrm{O}_{6}$

Macrophyllic acid, 13317

$\mathrm{C}_{40} \mathrm{H}_{54} \mathrm{O}_{11}$
$10 \alpha, 13 \alpha, 16 \alpha$-Trihydroxy-9 $\alpha$-methyl-15-oxo-20nor-kauran-19-oic acid $\gamma$-lactone-17-yl-14' $\alpha$, $16 ' \alpha, 17$ '-trihydroxy-15'-oxo-ent-kaur-11'-en19 '-oate, 21805

$\mathrm{C}_{40} \mathrm{H}_{54} \mathrm{O}_{12}$

Nimbolidin E, 15609

$\mathrm{C}_{40} \mathrm{H}_{54} \mathrm{O}_{15}$

Bonaspectin C 4"- $\beta$-glucoside, 2543

$\mathrm{C}_{40} \mathrm{H}_{54} \mathrm{O}_{16}$

Bonaspectin D 4"- $\beta$-glucoside, 2544

\section{$\mathrm{C}_{40} \mathrm{H}_{54} \mathrm{O}_{24}$}

Velutinoside I, 22370

\section{$\mathrm{C}_{40} \mathrm{H}_{56}$}

$\alpha$-Carotene, 3208

$\beta$-Carotene, 3209

$\gamma$-Carotene, 3210

$\delta$-Carotene, 3211

$\varepsilon$-Carotene, 3212

Lycopene, 13212

Neo- $\beta$-carotene B, 15356

Neo- $\beta$-carotene U, 15357

\section{$\mathrm{C}_{40} \mathrm{H}_{56} \mathrm{O}$}

$\alpha$-Carotene-5,6-epoxide, 3213

$\beta$-Carotene-5,6-epoxide, 3214

$\beta$-Cryptoxanthin, 4293

Lycoxanthin, 13243

Mutatochrome, 15135

Rubixanthin, 19039

\section{$\mathrm{C}_{40} \mathrm{H}_{56} \mathrm{O}_{2}$}

Cryptoxanthin epoxide, 4294

3'-Deoxycapsanthin, 5158

Lutein, 13126

Lycophyll, 13222

Mutatoxanthin, 15136

Rubichrome, 19027

Semi- $\alpha$-carotenone, 19694

Zeaxanthin, 22976

\section{$\mathrm{C}_{40} \mathrm{H}_{56} \mathrm{O}_{3}$}

Antheraxanthin, 1358

Capsanthin, 3142

Chrysanthemaxanthin, 3592

Flavoxanthin, 7821

Lutein epoxide, 13127

(9Z,9'Z)-Lutein-5,6-epoxide, 13128

(9Z,13Z)-Lutein-5,6-epoxide, 13129

(9Z,13'Z)-Lutein-5,6-epoxide, 13130

(9'Z,13'Z)-Lutein-5,6-epoxide, 13131

(13Z,9'Z)-Lutein-5,6-epoxide, 13132

\section{$\mathrm{C}_{40} \mathrm{H}_{56} \mathrm{O}_{4}$}

Auroxanthin, 2017

Capsorubin, 3146

Formosaninol, 7893

Neoxanthin, 15477 
Siphonaxanthin, 19964

Sugikurojin C, 20459

Violaxanthin, 22520

\section{$\mathrm{C}_{40} \mathrm{H}_{56} \mathrm{O}_{5}$}

3,4-Dihydroxy-3',6'-epoxy-1',2',5',6'7',8'-hexahydro-6'-methyl-16'-nor- $\beta, \varphi$-carotene-1',8'dione, 5874

3,4,3',8'-Tetrahydroxy- $\beta, \kappa$-caroten-6'-one, 21085

\section{$\mathrm{C}_{40} \mathrm{H}_{56} \mathrm{O}_{6}$}

Lawsonic acid, 12581

Methyl helicterate, 14479

Methyl helicterilate, 14480

Scaphopetalumate, 19457

\section{$\mathrm{C}_{40} \mathrm{H}_{56} \mathrm{O}_{7}$}

$3 \alpha$-trans-Feruloyloxy- $2 \alpha$-hydroxyurs-12-en-28oic acid, 7785

Uncarinic acid A, 22208

Uncarinic acid B, 22209

Uncarinic acid D, 22211

Uncarinic acid E, 22212

\section{$\mathrm{C}_{40} \mathrm{H}_{56} \mathrm{O}_{8}$}

3-O-trans-Feruloyleuscaphic acid, 7772

\section{$\mathrm{C}_{40} \mathrm{H}_{56} \mathrm{O}_{10}$}

$10 \alpha, 13 \alpha, 16 \alpha$-Trihydroxy-9 $\alpha$-methyl-15-oxo-20nor-kauran-19-oic acid $\gamma$-lactone-17-yl-16' $\alpha$, 17'-dihydroxy-15'-oxo-ent-kaur-19'-oate, 21806

\section{$\mathrm{C}_{40} \mathrm{H}_{57} \mathrm{~N}_{3} \mathrm{O}_{11}$}

Giraldine G, 8454

\section{$\mathrm{C}_{40} \mathrm{H}_{58}$}

$\beta$-Zeacarotene, 22973

\section{$\mathrm{C}_{40} \mathrm{H}_{58} \mathrm{~N}_{3} \mathrm{O}_{11}$}

Trifoliolasine C, 21637

\section{$\mathrm{C}_{40} \mathrm{H}_{58} \mathrm{~N}_{4} \mathrm{O}_{10}$}

Cinobufotoxin, 3733

\section{$\mathrm{C}_{40} \mathrm{H}_{58} \mathrm{O}_{2}$}

Parasiloxanthin, 16656

Podototarin, 17596

\section{$\mathrm{C}_{40} \mathrm{H}_{58} \mathrm{O}_{3}$}

Pholidotanin, 17175

Pholidotin, 17176

\section{$\mathrm{C}_{40} \mathrm{H}_{58} \mathrm{O}_{4}$}

(3S,5S,6R,3'R,6'R)-5,6-Dihydro-5,6-dihydroxylutein, 5594

\section{$\mathrm{C}_{40} \mathrm{H}_{58} \mathrm{O}_{5}$}

$3 \beta$-trans-Feruloyloxy-16 $\beta$-hydroxylup-20(29)ene, 7784

Lawsonin, 12582

Phlegmanol B, 17149

$\mathrm{C}_{40} \mathrm{H}_{58} \mathrm{O}_{15}$

Stauntoside B, 20277

$\mathbf{C}_{40} \mathbf{H}_{60}$

$\zeta$-Carotene, 3215
$\mathrm{C}_{40} \mathrm{H}_{60} \mathrm{~N}_{4} \mathrm{O}_{10}$

Bufotoxin, 2728

$\mathrm{C}_{40} \mathrm{H}_{60} \mathrm{~N}_{8} \mathrm{O}_{10}$

Pseudostellarin C, 18066

$\mathrm{C}_{40} \mathrm{H}_{60} \mathrm{O}_{5}$

Annomosin A, 1309

$\mathrm{C}_{40} \mathrm{H}_{60} \mathrm{O}_{7}$

Acacigenin B, 60

$\mathrm{C}_{40} \mathrm{H}_{60} \mathrm{O}_{8}$

13-O-Acetylphorbol-20-linoleate, 484

$\mathrm{C}_{40} \mathrm{H}_{60} \mathrm{O}_{13}$

Atratoside D, 1996

Cynascyroside D, 4566

Pfaffoside A, 17038

$\mathrm{C}_{40} \mathrm{H}_{60} \mathrm{O}_{15}$

DraconinC, 6578

$\mathrm{C}_{40} \mathrm{H}_{62}$

Phytofluene, 17264

$\mathrm{C}_{40} \mathrm{H}_{62} \mathrm{O}_{4}$

Annonebinide B, 1318

\section{$\mathrm{C}_{40} \mathrm{H}_{62} \mathrm{O}_{9}$}

3-O-(2,3-Dimethylbutanoyl)-13-O-dodecanoyl20-acetylingenol, 6323

\section{$\mathrm{C}_{40} \mathrm{H}_{62} \mathrm{O}_{11}$}

$1 \alpha, 3 \beta$-Hydroxyimberbic acid-23-O- $\alpha-L-3,4-$ diacetylrhamnopyranoside, 9741

\section{$\mathrm{C}_{40} \mathrm{H}_{62} \mathrm{O}_{12}$}

Prieurianoside, 17852

\section{$\mathrm{C}_{40} \mathrm{H}_{62} \mathrm{O}_{13}$}

Bidentatoside II, 2364

30 -Norhederagenin-3-O- $\beta$ - $D$-glucosyl $(1 \rightarrow 3)$ $\alpha$ - $L$-arabinopyranoside, 15754

\section{$\mathrm{C}_{40} \mathrm{H}_{62} \mathrm{O}_{14}$}

Laxogenin 3-O-[O-(2-O-acetyl- $\alpha$ - $L$-arabinopyranosyl$(1 \rightarrow 6)-\beta$-D-glucopyranoside)], 12592

Withanoside V, 22709

\section{$\mathrm{C}_{40} \mathrm{H}_{62} \mathrm{O}_{15}$}

Withanoside IV, 22708

Withanoside VI, 22710

Withanoside X, 22713

\section{$\mathbf{C}_{40} \mathbf{H}_{62} \mathbf{O}_{16}$}

Dumoside, 6636

21-Methoxyl-3 $\beta$-[( $O-\alpha-L$-rhamnopyranosyl$(1 \rightarrow 2)-O$ - $[\alpha-L$-rhamnopyranosyl- $(1 \rightarrow 4)]-\beta-D$ glucopyranosyl)oxy]pregn-5,16-en-20-one, 13991

Withanoside II, 22706

\section{$\mathrm{C}_{40} \mathrm{H}_{64}$}

Phytoene, 17263

$\mathrm{C}_{40} \mathrm{H}_{64} \mathrm{O}_{2}$

Fritillebinide A, 7965

$\mathrm{C}_{40} \mathrm{H}_{64} \mathrm{O}_{3}$
Fritillebin C, 7963

Fritillebin D, 7964

\section{$\mathrm{C}_{40} \mathrm{H}_{64} \mathrm{O}_{11}$}

Excoecarin $\mathrm{R}_{1}, 7685$

Excoecarin $\mathrm{R}_{2}, 7686$

Oleanolic acid 3-O- $\beta$ - $D$-xylopyranosyl $(1 \rightarrow 2)$ $\alpha$ - $L$-arabinopyranoside, 16061

Rubusside A, 19045

Vulgarsaponin B, 22622

\section{$\mathrm{C}_{40} \mathrm{H}_{64} \mathrm{O}_{12}$}

2-O-Acetyl-28-O-acetyl-rubianoside IV, 303

Bacopasaponin G, 2083

Hederagenin-3-O- $\beta$ - $D$-xyl $(1 \rightarrow 3)-\alpha-L$-arabinopyranoside, 9274

3-O- $\alpha$ - $L$-Rhamnopyranosyl $(1 \rightarrow 2)-\alpha$ - $L$-arabinopyranosyl-3 $\beta, 23$-dihydroxylup- $\Delta^{20(29)}$-en-28oic-acid, 18686

Yamogenin 3-O-neohesperidoside, 22880

\section{$\mathrm{C}_{40} \mathrm{H}_{64} \mathrm{O}_{13}$}

Brisbagenin-1-O-[O- $\alpha$ - $L$-rhamnopyranosyl$(1 \rightarrow 3)-4-O$-acetyl- $\alpha$ - $L$-arabinopyranoside], 2613

Cimiside B, 3671

Fargoside A, 7723

\section{$\mathrm{C}_{40} \mathrm{H}_{64} \mathrm{O}_{14}$}

Pentandroside B, 16878

\section{$\mathrm{C}_{40} \mathrm{H}_{64} \mathrm{O}_{16}$}

$16 \alpha$-Methoxyl-3 $\beta$-[(O- $\alpha$ - $L$-rhamnopyranosyl$(1 \rightarrow 2)-O-[\alpha-L$-rhamnopyranosyl- $(1 \rightarrow 4)]-\beta-D$ glucopyranosyl)oxy]pregn-5-en-20-one, 13990 Spongipregnoloside D, 20233

\section{$\mathrm{C}_{40} \mathrm{H}_{66} \mathrm{~N}_{6}$}

Ormosinine, 16207

\section{$\mathrm{C}_{40} \mathrm{H}_{66} \mathrm{O}_{12}$}

Gypenoside XXIX, 9141

Smilagenin-3- $O$ - $\beta$ - $D$-glucopyranosyl $(1 \rightarrow 2)-\beta$ - $D$ galactopyranoside, 20026

\section{$\mathrm{C}_{40} \mathrm{H}_{66} \mathrm{O}_{14}$}

(25S)-1 $\beta$-[( $\beta$ - $D$-Fucopyranosyl)oxy]-6 $\beta$-hydroxy$22 \alpha$-methoxy-3 $\alpha, 5 \alpha$-cyclofurostan-26-yl $\beta$ - $D$-glucopyranoside, 7977

\section{$\mathrm{C}_{40} \mathrm{H}_{66} \mathrm{O}_{15}$}

(25S)-1 $\beta$-[( $\beta$ - $D$-Glucopyranosyl)oxy]-6 $\beta$-hydroxy-22 $\alpha$-methoxy-3 $\alpha, 5 \alpha$-cyclofurostan-26-yl $\beta$ - $D$-glucopyranoside, 8694

\section{$\mathrm{C}_{40} \mathrm{H}_{66} \mathrm{O}_{16}$}

Glycoside K (Periplocae), 8831

\section{$\mathrm{C}_{40} \mathrm{H}_{67} \mathrm{NO}_{11}$}

Hapepunine 3-O- $\alpha$ - $L$-rhamnosyl- $(1 \rightarrow 2)-\beta$ - $D$ glucopyranoside, 9221

\section{$\mathrm{C}_{40} \mathrm{H}_{68} \mathrm{O}_{2}$}

(E)-Phytol(5Z,8Z,11Z,14Z,17Z)-eicosapentaenoate, 17272 


\section{$\mathrm{C}_{40} \mathrm{H}_{68} \mathrm{O}_{12}$}

Gypenoside LXXVII, 9179

Lotoideside D, 13007

Lotoideside E, 13008

$\mathrm{C}_{40} \mathrm{H}_{68} \mathrm{O}_{13}$

24S-Cycloartane-3 $\beta, 16 \beta, 24,25,30$-pentaol-3- $O$ -

(2-O- $\beta$ - $D$-xylosyl) $-\beta$ - $D$-xyloside, 4467

\section{$\mathrm{C}_{40} \mathrm{H}_{68} \mathrm{O}_{14}$}

Anemarrhenasaponin Ia, 1172

$\mathrm{C}_{40} \mathrm{H}_{70} \mathrm{O}_{4}$

Hentriacontanyl caffeate, 9365

$\mathrm{C}_{40} \mathrm{H}_{73} \mathrm{~N}_{3} \mathrm{O}_{3}$

3"'-Oxo-juliprosopine, 16364

\section{$\mathbf{C}_{40} \mathbf{H}_{74} \mathbf{O}_{5}$}

1-O-(9Z,12Z-Octadecadienoyl)-3-O-nonadecanoyl glycerol, 15944

\section{$\mathrm{C}_{40} \mathrm{H}_{74} \mathrm{O}_{12}$}

Azralidoside, 2064

\section{$\mathbf{C}_{40} \mathbf{H}_{74} \mathbf{O}_{19}$}

1-O-[ $\alpha$ - $L$-Rhamnopyranosyl-( $(1 \rightarrow 2)-\beta$ - $D$-glucopyranosyl-( $1 \rightarrow 3)-\alpha-L$-rhamnopyranosyl$(1 \rightarrow 6)-\beta$ - $D$-glucopyranosyl]hexadecanol, 18718

\section{$\mathrm{C}_{40} \mathrm{H}_{75} \mathrm{NO}_{9}$}

Celebroside, 3371

1-O- $\beta$-D-Glucopyranosyl-( $2 S, 3 R, 4 E, 11 E)-2$ ( 2 'R-hydroxyhexadecenoylamino)-4,11-octadecadiene-1,3-diol, 8672

Soyacerebroside I, 20113

Soyacerebroside II, 20114

\section{$\mathrm{C}_{40} \mathrm{H}_{75} \mathrm{~N}_{3} \mathrm{O}_{2}$}

Juliflorine, 11943

\section{$\mathrm{C}_{40} \mathrm{H}_{77} \mathrm{NO}_{10}$}

Bonaroside, 2540

\section{$\mathrm{C}_{40} \mathrm{H}_{78}$}

Lycopadiene, 13201

\section{$\mathrm{C}_{40} \mathrm{H}_{79} \mathrm{NO}_{5}$}

Urtica ceramide, 22273

\section{$\mathrm{C}_{40} \mathrm{H}_{81} \mathrm{NO}_{4}$}

Trufflesphingolipid A, 22053

\section{$\mathrm{C}_{40} \mathrm{H}_{82}$}

Andrographan, 1150

\section{$\mathrm{C}_{41} \mathrm{H}_{26} \mathrm{O}_{25}$}

Mauotusinin, 13612

\section{$\mathrm{C}_{41} \mathrm{H}_{26} \mathrm{O}_{26}$}

Alnusiin, 962

Castalagin, 3296

Glansrin C, 8506

Vescalagin, 22435

\section{$\mathrm{C}_{41} \mathrm{H}_{28} \mathrm{O}_{10}$}

Thelephantin L, 21304

\section{$\mathrm{C}_{41} \mathrm{H}_{28} \mathrm{O}_{11}$}

Terrestrin G, 21012

\section{$\mathrm{C}_{41} \mathrm{H}_{28} \mathrm{O}_{26}$}

Casuarictin, 3301

Casuarinin, 3303

Potentillin, 17750

Stachyurin, 20257

$\mathrm{C}_{41} \mathrm{H}_{28} \mathrm{O}_{27}$

Geraniin, 8311

Helioscopinin A, 9309

2,3-O-Hexahydroxydiphenoyl-4,6- $O$-sanguisorboyl- $(\alpha / \beta)$-glucose, 9503

Praecoxin A, 17754

\section{$\mathrm{C}_{41} \mathrm{H}_{30} \mathrm{O}_{26}$}

Cercidinin A, 3418

Eugeniin, 7518

Nupharin $\mathrm{A}(S), 15869$

Nupharin $\mathrm{B}(R), 15870$

Punicafolin, 18203

\section{$\mathrm{C}_{41} \mathrm{H}_{30} \mathrm{O}_{27}$}

Chebulagic acid, 3490

Isoterchebin, 11735

Rugosin B, 19055

\section{$\mathrm{C}_{41} \mathrm{H}_{30} \mathrm{O}_{28}$}

Phyllanemblinin C, 17210

Phyllanthusiin G, 17223

Repandusinic acid A, 18624

\section{$\mathrm{C}_{41} \mathrm{H}_{32} \mathrm{O}_{\mathbf{2 6}}$}

1,2,3,4,6-Pentagalloylglucose, 16836

\section{$\mathrm{C}_{41} \mathrm{H}_{32} \mathbf{O}_{27}$}

Chebulinic acid, 3493

\section{$\mathrm{C}_{41} \mathrm{H}_{32} \mathrm{O}_{28}$}

Neochebulagic acid, 15362

\section{$\mathrm{C}_{41} \mathrm{H}_{37} \mathrm{O}_{16}$}

Kaempferol 3-O- $\alpha-L-(2 ", 4 "-d i-E$-feruloyl)rhamnoside, 12040

\section{$\mathrm{C}_{41} \mathrm{H}_{41} \mathrm{O}_{23}{ }^{+}$}

Delphinidin-3-O-[6- $O$-( $p$-coumaroyl)- $\beta$ - $D$-glucopyranoside]-5-O-[4- $O$-acetyl-6- $O$-malonyl$\beta$ - $D$-glucopyranoside], 5017

\section{$\mathrm{C}_{41} \mathrm{H}_{42} \mathrm{O}_{14}$}

Curtisian H, 4404

\section{$\mathrm{C}_{41} \mathrm{H}_{43} \mathrm{NO}_{12}$}

Delgrandine, 5007

Triptofordinine $\mathrm{A}_{1}, 22009$

Triptofordinine $\mathrm{A}_{2}, 22010$

$\mathrm{C}_{41} \mathrm{H}_{44} \mathrm{O}_{21}$

Myriophylloside D, 15195

Sphaerobioside acetate, 20150

$\mathrm{C}_{41} \mathrm{H}_{44} \mathrm{O}_{22}$

Myriophylloside B, 15193

$\mathrm{C}_{41} \mathrm{H}_{44} \mathrm{O}_{23}$

Myriophylloside C, 15194

\section{$\mathrm{C}_{41} \mathrm{H}_{44} \mathrm{O}_{24}$}

Quercetin-3-O-(2-E-caffeoyl)- $\alpha-L$-arabinopyra- nosyl-(1 $\rightarrow 2)-\beta$ - $D$-galactopyranoside-7- $O-\beta$ - $D$ glucopyranoside, 18331

\section{$\mathrm{C}_{41} \mathrm{H}_{46} \mathrm{~N}_{4} \mathrm{O}_{3}$}

Tenuicausine, 20933

$\mathrm{C}_{41} \mathrm{H}_{46} \mathrm{O}_{13}$

3-Acetyl-5 $\beta, 8 \alpha$-dibenzylformyl-14-propanoyl myrsinoltype diterpene with C9-C10 cyclized to form an additional lactone ring, 373

\section{$\mathrm{C}_{41} \mathrm{H}_{47} \mathrm{NO}_{14}$}

Kansuinin E, 12141

$\mathrm{C}_{41} \mathrm{H}_{47} \mathrm{NO}_{15}$

Kansuinin D, 12140

\section{$\mathrm{C}_{41} \mathrm{H}_{47} \mathrm{NO}_{17}$}

Wilforzine, 22690

\section{$\mathrm{C}_{41} \mathrm{H}_{47} \mathrm{NO}_{19}$}

Hyponine F, 10908

Wilforgine, 22680

\section{$\mathrm{C}_{41} \mathrm{H}_{47} \mathrm{NO}_{20}$}

Hypoglaunine, 10897

Isowilfortrine, 11781

Wilfotrine, 22695

\section{$\mathrm{C}_{41} \mathrm{H}_{48} \mathrm{~N}_{2} \mathrm{O}_{8}$}

Thalicarpine, 21239

\section{$\mathrm{C}_{41} \mathrm{H}_{48} \mathrm{~N}_{2} \mathrm{O}_{9}$}

Thaliadanine, 21237

\section{$\mathrm{C}_{41} \mathrm{H}_{48} \mathrm{O}_{11}$}

Manassantin B, 13475

Saucernetin 7, 19405

\section{$\mathrm{C}_{41} \mathrm{H}_{49} \mathrm{NO}_{13}$}

$N$-Debenzoyl- $N$-propanoyl-10-deacetyl paclitaxel, 4810

\section{$\mathrm{C}_{41} \mathrm{H}_{50} \mathrm{~N}_{6} \mathrm{O}_{9}$}

Rubia akane RA-VII, 18985

\section{$\mathrm{C}_{41} \mathrm{H}_{50} \mathrm{~N}_{6} \mathrm{O}_{10}$}

Rubia akane RA-III, 18981

Rubia akane RA-IV, 18982

Rubia akane RA-VI, 18984

\section{$\mathrm{C}_{41} \mathrm{H}_{50} \mathrm{O}_{14}$}

Taxuspine X, 20871

\section{$\mathrm{C}_{41} \mathrm{H}_{51} \mathrm{NO}_{11}$}

Liaconitine B, 12737

\section{$\mathrm{C}_{41} \mathrm{H}_{52} \mathrm{O}_{14}$}

Taxchin B, 20769

\section{$\mathrm{C}_{41} \mathrm{H}_{52} \mathrm{O}_{21}$}

Korepimedoside C, 12274

\section{$\mathrm{C}_{41} \mathrm{H}_{53} \mathrm{NO}_{11}$}

Liwaconitine, 12935

\section{$\mathrm{C}_{41} \mathrm{H}_{53} \mathrm{NO}_{13}$}

Austrotaxine, 2025

$\mathbf{C}_{41} \mathbf{H}_{54} \mathbf{N}_{4} \mathbf{O}_{3}$

20-Epi-16'-decarbomethoxy-conoduramine, 6885

\section{$\mathrm{C}_{41} \mathrm{H}_{54} \mathrm{O}_{12}$}

Nimbolidin D, 15608 


\section{$\mathrm{C}_{41} \mathrm{H}_{55} \mathrm{NO}_{12}$}

Austrospicatine, 2024

\section{$\mathrm{C}_{41} \mathrm{H}_{55} \mathrm{NO}_{13}$}

$7 \beta$-Acetoxy-9-acetylspicataxine, 120

$2 \alpha$-Acetoxy-2' $\beta$-deacetylaustrospicatine, 150

Taxuspine P, 20863

\section{$\mathrm{C}_{41} \mathrm{H}_{55} \mathrm{NO}_{14}$}

$2 \alpha$-Acetoxy-2' $\beta$-deacetyl-1-hydroxyaustrospicatine, 152

\section{$\mathbf{C}_{41} \mathbf{H}_{56} \mathbf{N}_{2} \mathbf{O}_{7}$}

Incarvillateine $\mathrm{D}, 11008$

\section{$\mathbf{C}_{41} \mathbf{H}_{56} \mathbf{N}_{\mathbf{2}} \mathbf{O}_{11}$}

Elanine, 6732

\section{$\mathrm{C}_{41} \mathrm{H}_{56} \mathrm{O}_{2}$}

Vitamin $\mathrm{K}_{2}, 22563$

\section{$\mathrm{C}_{41} \mathrm{H}_{56} \mathrm{O}_{4}$}

3-Methoxy-3'-hydroxy-5', 8'-epoxy-5', 8'-dihydro5,6-seco-4,6-cyclo- $\beta, \beta$-caroten-5-one, 13955

(all-E)-3-Methoxy-3'-hydroxy-5',6'-epoxy-5',6'dihydro-5,6-seco-4,6-cyclo- $\beta, \beta$-caroten-5-one, 13956

(9'Z)-3-Methoxy-3'-hydroxy-5',6'-epoxy-5',6'-

dihydro-5,6-seco-4,6-cyclo- $\beta, \beta$-caroten-5-one, 13957

\section{$\mathrm{C}_{41} \mathrm{H}_{56} \mathrm{O}_{6}$}

Fucoxanthin, 7979

\section{$\mathrm{C}_{41} \mathrm{H}_{56} \mathrm{O}_{13}$}

$(2 R, 3 R, 4 R, 5 R, 7 S, 8 S, 9 S, 11 E, 13 S, 15 R)-2,3,5,7,8$, 9,15-Heptahydroxyjatropha-6(17),11-diene-14one-7,9-diacetate-8-benzoate-2,3-bis(2-methylbutyrate), 9395

\section{$\mathrm{C}_{41} \mathrm{H}_{56} \mathrm{O}_{24}$}

Velutinoside II, 22371

\section{$\mathrm{C}_{41} \mathrm{H}_{58} \mathrm{O}_{3}$}

Formosadimer A, 7886

Sugikurojin B, 20458

\section{$\mathrm{C}_{41} \mathrm{H}_{58} \mathrm{O}_{10}$}

$10 \alpha$-Hydroxy-9 $\alpha$-methyl-15-oxo-20-nor-kauran19-oic acid methyl ester- $16 \alpha$-yl-14' $\alpha, 16^{\prime} \alpha, 17^{\prime}$ trihydroxy-15'-oxo-ent-kaur-11'-en-19'-oate, 10512

\section{$\mathrm{C}_{41} \mathrm{H}_{59} \mathrm{~N}_{3} \mathrm{O}_{11}$} Giraldine H, 8455

\section{$\mathrm{C}_{41} \mathrm{H}_{60} \mathrm{~N}_{2} \mathrm{O}_{11}$} Potanidine A, 17743

\section{$\mathrm{C}_{41} \mathrm{H}_{60} \mathrm{O}_{4}$}

24-Methylene cycloartanol ferulate, 14353

\section{$\mathrm{C}_{41} \mathrm{H}_{60} \mathrm{O}_{6}$}

3 $\beta$-trans-Sinapoyloxylup-20(29)-en-28-ol, 19920

\section{$\mathbf{C}_{41} \mathbf{H}_{60} \mathbf{O}_{7}$}

Myxoxanthophyll, 15227

\section{$\mathrm{C}_{41} \mathrm{H}_{60} \mathrm{O}_{16}$}

Neocynapanoside A, 15389

\section{$\mathrm{C}_{41} \mathrm{H}_{62} \mathrm{O}_{4}$}

Cycloartenyl ferulate, 4475

24-Methylcycloartanol ferulate, 14267

$\mathrm{C}_{41} \mathrm{H}_{62} \mathrm{O}_{13}$

Picfeltarraenin IA, 17294

$\mathrm{C}_{41} \mathrm{H}_{62} \mathrm{O}_{14}$

Apioglycyrrhizin, 1519

Araboglycyrrhizin, 1597

Fargoside D, 7726

Hollow alternanthera saponin D, 9589

$\mathrm{C}_{41} \mathrm{H}_{62} \mathrm{O}_{15}$

Achyranthoside II, 544

$\mathrm{C}_{41} \mathrm{H}_{62} \mathrm{O}_{16}$

Ageratoside $\mathrm{C}_{1}, 735$

$\mathrm{C}_{41} \mathrm{H}_{63} \mathrm{NO}_{14}$

Protoveratrine A, 17995

$\mathrm{C}_{41} \mathrm{H}_{63} \mathrm{NO}_{15}$

Neoprotoveratrine, 15452

$\mathrm{C}_{41} \mathrm{H}_{64} \mathbf{N}_{8} \mathbf{O}_{9}$

Glabrin C, 8494

\section{$\mathrm{C}_{41} \mathrm{H}_{64} \mathrm{O}_{4}$}

Cycloartanol ferulate, 4470

\section{$\mathrm{C}_{41} \mathrm{H}_{64} \mathrm{O}_{8}$}

Julibrogenin A, 11916

\section{$\mathbf{C}_{41} \mathbf{H}_{64} \mathbf{O}_{12}$}

$3 \beta$-[( $\alpha$-L-Arabinopyranosyl)oxy]-urs-11,13(18)dien-28-oic acid $\beta$ - $D$-glucopyranosyl ester, 1590

$3 \beta$-[( $\alpha$-L-Arabinopyranosyl)oxy]urs-12,19(29)dien-28-oic acid 28- $\beta$ - $D$-glucopyranosyl ester, 1591

Dehydrobryogenin glycoside, 4883

\section{$\mathrm{C}_{41} \mathrm{H}_{64} \mathrm{O}_{13}$}

$3 \beta$-[( $\alpha$-L-Arabinopyranosyl)oxy]-23-hydroxyurs-12,19(29)-dien-28-oic acid 28- $\beta$ - $D$-glucopyranosyl ester, 1583

Digitoxin, 5526

$2 \alpha, 3 \beta$-Dihydroxyurs-12,20(30)-dien-28-oic acid $3-O-\{[O-\beta-D$-xylopyranosyl-( $(1 \rightarrow 3)]-D$-glucopyranoside $\}, 6171$

Oleanolic acid-3- $\alpha$ - $L$-arabinofuranosyl $(1 \rightarrow 4)$ $\beta$ - $D$-glucuronopyrannoside, 16046

Ophiopogonin A, 16143

Styraxoside B, 20429

\section{$\mathrm{C}_{41} \mathrm{H}_{64} \mathrm{O}_{14}$}

Aquilegioside B, 1543

Digoxin, 5533

Gitoxin, 8461

Ilekudinoside I, 10979

Randiasaponin I, 18535

$\mathrm{C}_{41} \mathrm{H}_{64} \mathrm{O}_{16}$

Esculentoside F, 7373

$\mathrm{C}_{41} \mathrm{H}_{65} \mathrm{O}_{16} \mathrm{~S}^{-}$
Bacopaside III, 2088

$\mathrm{C}_{41} \mathrm{H}_{66} \mathrm{O}_{11}$

$\alpha$ - $L$-Arabinofuranosyl-( $(1 \rightarrow 4)-O-\beta$ - $D$-glucuronopyranosyl-( $1 \rightarrow 3$ )3 $\beta$-hydroxy-lup-20(29)-ene, 1557

Eleutheroside I, 6755

Eleutheroside K, 6756

Obtusilobin, 15906

\section{$\mathrm{C}_{41} \mathrm{H}_{66} \mathrm{O}_{12}$}

Anemoside $\mathrm{A}_{3}, 1181$

3-O- $\alpha$ - $L$-Arabinopyranosyl hederagenin 28-O$\alpha$ - $L$-rhamnopyranosyl ester, 1572

$3 \beta$-D-O-( $\alpha$ - L-Arabinopyranosyl)-lup-20(29)ene-28-O- $\beta$ - $D$-glucopyranosyl ester, 1575

Flaccidin B, 7806

3 - $O$ - $\beta$ - $D$-Glucopyranosyl $(1 \rightarrow 3)-\alpha-L$-arabinopyranosyl oleanolic acid, 8607

$\alpha$-Hederin, 9276

Oleanolic acid-3- $O-\beta$ - $D$-glucopyranosyl $(1 \rightarrow 2)$ $\alpha$ - $L$-arabinopyranoside, 16053

Polyfoliolide A, 17622

Polyfoliolide B, 17623

Raddeanoside, 18521

Sapindoside A, 19316

Saponin $\mathrm{E}_{3}, 19342$

Scabioside B, 19443

\section{$\mathbf{C}_{41} \mathbf{H}_{66} \mathbf{O}_{13}$}

$3 \beta$-[( $\alpha$-L-Arabinopyranosyl)oxy]-19 $\alpha$-hydroxyolean-12-en-28-oic acid 28- $\beta$ - $D$-glucopyranosyl ester, 1580

$3 \beta$-[( $\alpha$-L-Arabinopyranosyl)oxy]-23-hydroxyolean-12-en-28-oic acid 28- $\beta$ - $D$-glucopyranosyl ester, 1581

$3 \beta-[(\alpha-L$-Arabinopyranosyl)oxy $]-19 \alpha$-hydroxyurs-12-en-28-oic acid 28- $\beta$ - $D$-glucopyranosyl ester, 1586

Atriplicosaponin A, 1997

Bacopaside IIIb*, 2089

Bacopaside IV, 2090

Bacopaside V, 2093

Candidoside A, 3073

Cauloside C, 3342

Leontoside B, 12643

Pictoside A, 17350

Ziyu glycoside I, 23008

\section{$\mathrm{C}_{41} \mathbf{H}_{66} \mathbf{O}_{14}$}

3-O- $\alpha$ - $L$-Arabinopyranosylcimigenol $15-O-\beta-D$ glucopyranoside, 1561

Congmuyanoside A, 3976

Conyzasaponin G, 4018

Ilekudinoside C, 10973

Ilekudinoside D, 10974

Ilexoside XXVII, 10983 
Randiasaponin II, 18536

Randiasaponin VI, 18540

Scorzoneroside C, 19565

\section{$\mathrm{C}_{41} \mathrm{H}_{68} \mathrm{O}_{10}$}

Lupeol-3-O- $\beta$ - $D$-xylopyranosyl $(1 \rightarrow 4)-O-\beta-D$ glucopyranoside, 13103

\section{$\mathrm{C}_{41} \mathrm{H}_{68} \mathrm{O}_{12}$}

Spergulacin, 20142

Spergulacin A, 20143

\section{$\mathrm{C}_{41} \mathrm{H}_{68} \mathrm{O}_{13}$}

Astrachrysoside, 1934

$3 \beta, 23$-Dihydroxy-lup-20(29)-ene-28- $O-\beta$ - $D$ glucopyranosyl-( $1 \rightarrow 6)-\beta$ - $D$-glucopyranoside, 5951

Gynoside E, 9120

Gypenoside LIII, 9158

Lotoideside F, 13009

\section{$\mathrm{C}_{41} \mathrm{H}_{68} \mathrm{O}_{14}$}

Astragaloside III, 1938

Astragaloside IV, 1939

Astramembrannin I, 1945

Bupleuroside VI, 2754

Bupleuroside XIII, 2755

3-O- $\beta$ - $D$-Xylopyranosyl-25- $O-\beta$ - $D$-glucopyranosyl cycloastragenol, 22824

\section{$\mathrm{C}_{41} \mathrm{H}_{70} \mathrm{O}_{12}$}

Cyclocarioside I, 4483

Gypenoside XIII, 9133

Gypenoside LXXI, 9173

$20-O-\beta$ - $D$-Xylopyranosyl $(1 \rightarrow 6)-\beta$ - $D$-glucopyranosyl-20(S)-protopanaxadiol, 22830

\section{$\mathrm{C}_{41} \mathrm{H}_{70} \mathrm{O}_{13}$}

Gynoside A, 9116

Gynoside C, 9118

Gypenoside XXI, 9138

Gypenoside XXX, 9142

Gypenoside LIV, 9159

Gypenoside LXV, 9169

Gypenoside LXXVIII, 9180

Notoginsenoside $\mathrm{R}_{2}, 15837$

$7 \beta, 20,26$-Trihydroxy-(20S)-dammar-24E-en-3-

$O$ - $\alpha$ - $L$-arabinopyranosyl-( $1 \rightarrow 2)-\beta$ - $D$-glucopyranoside, 21698

\section{$\mathrm{C}_{41} \mathrm{H}_{70} \mathrm{O}_{14}$}

Cyclocanthoside E, 4478

Gynoside D, 9119

Gypenoside LIX, 9164

Gypenoside LX, 9165

Majonoside $\mathrm{R}_{2}, 13405$

$7 \beta, 18,20,26$-Tetrahydroxy-(20S)-dammar-24Een-3- $O$ - $\alpha$ - $L$-arabinopyranosyl- $(1 \rightarrow 2)-\beta$ - $D$-glucopyranoside, 21089

$3 \beta, 12 \beta, 23 S, 24 R$-Tetrahydroxy-20S,25-epoxy-

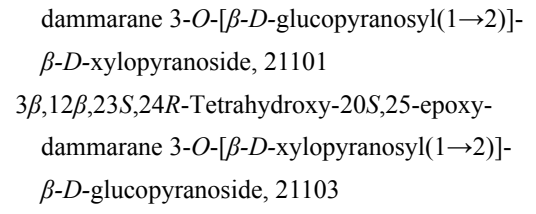

\section{$\mathrm{C}_{41} \mathrm{H}_{78} \mathrm{O}_{12} \mathrm{~S}$}

(2S)-1,2-Di- $O$-palmitoyl-3- $O$-(6-sulpho- $\alpha$ - $D$ quinovopyranosyl) glycerol, 6481

$\mathrm{C}_{41} \mathrm{H}_{78} \mathrm{O}_{8}$

Methyl-2 $\beta(2 S)$ - $O$ - $\beta$-D-galactopyranosyl-7(E)tetratriacontenoate, 14452

\section{$\mathrm{C}_{41} \mathrm{H}_{81} \mathrm{NO}_{3}$}

Sertularamide, 19776

\section{$\mathrm{C}_{42} \mathrm{H}_{26} \mathrm{O}_{21}$ \\ 2-O-(2,4,6-Trihydroxyphenyl)-6,6'-bieckol, 21836}

\section{$\mathrm{C}_{42} \mathrm{H}_{30} \mathrm{O}_{9}$}

Amurensin D, 1093

Caragaphenol A, 3149

$\alpha$-Viniferin, 22504

\section{$\mathrm{C}_{42} \mathrm{H}_{30} \mathrm{O}_{11}$}

Terrestrin E, 21010

\section{$\mathrm{C}_{42} \mathrm{H}_{30} \mathrm{O}_{26}$}

Paeonianiin E, 16519

\section{$\mathrm{C}_{42} \mathrm{H}_{32} \mathrm{O}_{9}$}

Amurensin B, 1092

Amurensin G, 1095

Davidiol A, 4695

cis-Diptoindonesin B, 6503

trans-Diptoindonesin B, 6504

Gnemonol K, 8872

Gnemonol L, 8873

Gnetin E, 8880

cis-Miyabenol C, 14891

Miyabenol C, 14892

Suffruticosol A, 20455

Vaticanol A, 22354

\section{$\mathrm{C}_{42} \mathrm{H}_{32} \mathrm{O}_{10}$}

Gnemonol A, 8866

Gnemonol D, 8869

Gnemonol E, 8870

Gnemonol F, 8871

Latifolol, 12546

\section{$\mathrm{C}_{42} \mathrm{H}_{32} \mathrm{O}_{11}$}

Gnetuhainin M, 8890

Gnetumontanin B, 8894

\section{$\mathrm{C}_{42} \mathrm{H}_{34} \mathrm{O}_{10}$}

Davidiol B, 4696

Nepalensinol D, 15478
$\mathrm{C}_{42} \mathrm{H}_{38} \mathrm{O}_{9}$

Calyxin I, 3018

Calyxin J, 3019

Epicalyxin I, 6846

Epicalyxin J, 6847

\section{$\mathrm{C}_{42} \mathrm{H}_{38} \mathrm{O}_{20}$}

Amentoflavone-4',4"'-di- $O$ - $\beta$ - $D$-glucopyranoside, 1031

Amentoflavone-7,4"'-di- $O$ - $\beta$ - $D$-glucopyranoside, 1032

Sennoside A, 19749

Sennoside B, 19750

\section{$\mathrm{C}_{42} \mathrm{H}_{40} \mathrm{O}_{19}$}

Sennoside C, 19751

Sennoside D, 19752

\section{$\mathrm{C}_{42} \mathrm{H}_{40} \mathrm{O}_{25}$}

7-O- $\beta$ - $D$-Glucuronopyranosyl-4'-O-[2'-O-p-Ecoumaroyl- $O-\beta$ - $D$-glucuronopyranosyl $(1 \rightarrow 2)$ $O-\beta$ - $D$-glucuronopyranoside]apigenin, 8762

\section{$\mathrm{C}_{42} \mathrm{H}_{41} \mathrm{O}_{23}{ }^{+}$}

Monardaein, 14914

\section{$\mathrm{C}_{42} \mathrm{H}_{42} \mathrm{~N}_{4} \mathrm{O}_{3}$}

Strychnogucine C, 20414

$\mathrm{C}_{42} \mathrm{H}_{42} \mathrm{O}_{7}$

Crassifolin, 4213

\section{$\mathrm{C}_{42} \mathrm{H}_{42} \mathrm{O}_{23}$}

Trilobatin H, 21883

\section{$\mathrm{C}_{42} \mathrm{H}_{44} \mathrm{~N}_{4} \mathrm{O}_{5}$}

Serpentinine, 19763

\section{$\mathrm{C}_{42} \mathrm{H}_{44} \mathrm{O}_{17}$}

6-O- $\alpha$ - $L-(2 "-O-, 3$ "- $O-, 4$ "- $O$-Tribenzoyl)rhamnopyranosylcatalpol, 21544

\section{$\mathrm{C}_{42} \mathrm{H}_{45} \mathrm{~N}_{5} \mathrm{O}_{5}$}

Adouetine Z, 651

\section{$\mathrm{C}_{42} \mathrm{H}_{46} \mathrm{O}_{12}$}

Neoarctin B, 15340

\section{$\mathrm{C}_{42} \mathrm{H}_{46} \mathrm{O}_{22}$}

Apigenin-7- $O-\beta$-apiofuranosyl-6- $C$ - $\beta$-glucopyranosyl-8- $C$-(6"'-- $O$ - $E$-feruloyl)- $\beta$-glucopyranoside, 1479

Isovitexin 2"-O-(6"'-(E)-p-coumaroyl)glucoside4'- $O$-glucoside, 11775

Kaempferol 3-O- $\alpha-L$-[6"'-p-coumaroyl- $(\beta-D)$-glucopyranosyl-(1,2)-rhamnopyranoside]-7- $O-\beta$ $D$-glucopyranoside, 12033

Kaempferol 7-O-(6-trans- $p$-coumaroyl)- $\beta$-glucopyranosyl- $(1 \rightarrow 3)$ - $\alpha$-rhamnopyranoside-3-O- $\beta$ glucopyranoside, 12034

\section{$\mathrm{C}_{42} \mathrm{H}_{46} \mathrm{O}_{23}$}

Kaempferol 7-O-(6-trans-caffeoyl)- $\beta$-glucopyranosyl-( $1 \rightarrow 3)$ - $\alpha$-rhamnopyranoside-3- $O$ - $\beta$-glucopyranoside, 12030

Myriophylloside E, 15196 
Myriophylloside F, 15197

Quercetin-3- $O-\alpha-L$-[6"'-p-coumaroyl- $(\beta-D)$-glucopyranosyl-(1,2)-rhamnopyranoside]-7- $O-\beta$ D-glucopyranoside, 18336

\section{$\mathrm{C}_{42} \mathrm{H}_{46} \mathrm{O}_{24}$}

Pisumflavonoside I, 17493

Quercetin-7-O-(6-trans-caffeoyl)- $\beta$-glucopyranosyl-( $1 \rightarrow 3)$ - $\alpha$-rhamnopyranoside-3- $O$ - $\beta$-glucopyranoside, 18333

\section{$\mathrm{C}_{42} \mathrm{H}_{47} \mathrm{O}_{23}$}

Nasunin, 15289

$\mathrm{C}_{42} \mathrm{H}_{47} \mathrm{O}_{24}{ }^{+}$

Cayratinin, 3344

\section{$\mathrm{C}_{42} \mathrm{H}_{48} \mathrm{~N}_{2} \mathrm{O}_{18}$}

Wilfornine, 22685

\section{$\mathrm{C}_{42} \mathrm{H}_{48} \mathrm{O}_{11}$}

Taxuspine A, 20849

\section{$\mathrm{C}_{42} \mathrm{H}_{48} \mathrm{O}_{12}$}

Taxchinin E, 20773

\section{$\mathrm{C}_{42} \mathrm{H}_{48} \mathrm{O}_{13}$}

2-Deacetyl-2 $\alpha$-benzoyl-5,13-diacetyltaxchinin A, 4731

Taxchinin J, 20777

\section{$\mathrm{C}_{42} \mathrm{H}_{48} \mathrm{O}_{14}$}

9-O-Benzoyl-9-de- $O$-acetyl-11(15 $\rightarrow 1)$-abeobaccatin VI, 2235

$2 \alpha, 7 \beta$-Dibenzoyl- $5 \beta, 20$-epoxy- $1 \beta$-hydroxy- $4 \alpha$, $9 \alpha, 10 \beta, 13 \alpha$-tetraacetoxytax-11-ene, 5383

Kansuinin F, 12142

Taxayuntin C, 20761

Taxchinin K, 20778

\section{$\mathrm{C}_{42} \mathrm{H}_{48} \mathrm{O}_{18}$}

Scrophuloside $\mathrm{B}_{4}, 19571$

\section{$\mathrm{C}_{42} \mathrm{H}_{48} \mathrm{O}_{20}$}

Reiniose D, 18606

\section{$\mathrm{C}_{42} \mathrm{H}_{48} \mathrm{O}_{22}$}

Segetoside J, 19667

\section{$\mathrm{C}_{42} \mathrm{H}_{49} \mathrm{NO}_{14}$}

$N$-Acetyl- $N$-debenzoyltaxol, 367

\section{$\mathrm{C}_{42} \mathrm{H}_{50} \mathrm{~N}_{2} \mathrm{O}_{9}$}

Adiantifoline, 631

\section{$\mathrm{C}_{42} \mathrm{H}_{50} \mathrm{~N}_{4} \mathrm{O}_{4}$}

Ervahanine A, 7294

\section{$\mathrm{C}_{42} \mathrm{H}_{50} \mathrm{~N}_{4} \mathrm{O}_{5}$}

Conodutarine B, 4001

19'(S)-Hydroxyervahanine A, 10095

\section{$\mathrm{C}_{42} \mathrm{H}_{50} \mathrm{~N}_{4} \mathrm{O}_{6}$}

Conodurinine, 3999

\section{$\mathrm{C}_{42} \mathrm{H}_{50} \mathrm{~N}_{6} \mathrm{O}_{11}$}

Rubia akane RA-XI, 18989

\section{$\mathrm{C}_{42} \mathrm{H}_{50} \mathrm{O}_{16}$}

Phyllostadimer A, 17229

Phyllostadimer B, 17230
$\mathrm{C}_{42} \mathrm{H}_{51} \mathrm{NO}_{13}$

$N$-Debenzoyl- $N$-butanoyl-10-deacetylpaclitaxel, 4804

\section{$\mathrm{C}_{42} \mathrm{H}_{52} \mathrm{~N}_{2} \mathrm{O}_{11}$}

$N$-Demethylnicaustrine, 5087

$\mathrm{C}_{42} \mathrm{H}_{52} \mathrm{~N}_{4} \mathrm{O}_{5}$

Conodutarine A, 4000

\section{$\mathrm{C}_{42} \mathrm{H}_{52} \mathrm{~N}_{6} \mathrm{O}_{10}$}

Rubia akane RA-VIII, 18986

\section{$\mathrm{C}_{42} \mathrm{H}_{52} \mathrm{O}_{11}$}

Manassantin A, 13474

Saucernetin 8, 19406

\section{$\mathrm{C}_{42} \mathrm{H}_{56} \mathrm{~N}_{8} \mathrm{O}_{9}$}

Pseudostellarin G, 18069

\section{$\mathrm{C}_{42} \mathrm{H}_{56} \mathrm{O}_{14}$}

Daturametelin G-AC, 4671

\section{$\mathrm{C}_{42} \mathrm{H}_{58} \mathrm{~N}_{2} \mathrm{O}$}

Staphidine, 20262

\section{$\mathrm{C}_{42} \mathrm{H}_{58} \mathrm{~N}_{2} \mathrm{O}_{8}$}

Incarvillateine, 11006

\section{$\mathrm{C}_{42} \mathrm{H}_{58} \mathrm{O}_{5}$}

Dinochrome A, 6424

Dinochrome B, 6425

\section{$\mathrm{C}_{42} \mathrm{H}_{60} \mathrm{O}_{13}$}

Diterp-Complex-RA, 6520

\section{$\mathrm{C}_{42} \mathrm{H}_{60} \mathrm{O}_{16}$}

Licoricesaponin $\mathrm{E}_{2}, 12787$

Yunganoside $\mathrm{E}_{2}, 22944$

\section{$\mathrm{C}_{42} \mathrm{H}_{60} \mathrm{O}_{20}$}

Premnaodoroside G, 17821

\section{$\mathrm{C}_{42} \mathrm{H}_{62} \mathrm{O}_{8}$}

(1E,22Z)-1,22-Diferuloyloxydocosane, 5505

\section{$\mathrm{C}_{42} \mathrm{H}_{62} \mathrm{O}_{15}$}

Licoricesaponin $\mathrm{C}_{2}, 12785$

\section{$\mathrm{C}_{42} \mathrm{H}_{62} \mathrm{O}_{16}$}

Abrusoside D, 34

Abrusoside E, 35

Atratoglaucoside B, 1992

DraconinB, 6577

Glycyrrhizic acid, 8846

Glyeurysaponin, 8847

Glyyunnanprosapogenin D, 8856

Licoricesaponin $\mathrm{H}_{2}, 12790$

Licoricesaponin $\mathrm{K}_{2}, 12792$

Uralsaponin A, 22243

Uralsaponin B, 22244

Yunganoside $\mathrm{F}_{2}, 22945$

\section{$\mathrm{C}_{42} \mathrm{H}_{62} \mathrm{O}_{17}$}

Licoricesaponin $\mathrm{G}_{2}, 12789$

Urginin, 22250

$\mathrm{C}_{42} \mathrm{H}_{62} \mathrm{O}_{19}$

Deoxytrillenoside B, 5218

$\mathrm{C}_{42} \mathrm{H}_{62} \mathrm{O}_{20}$
Premnaodoroside E, 17818

Trillenoside B, 21874

\section{$\mathrm{C}_{42} \mathrm{H}_{63} \mathrm{~N}_{9} \mathrm{O}_{8}$}

Cyclo-(Gly-Leu-Pro-Trp-Leu-Ile-Ala-Ala), 4494

\section{$\mathrm{C}_{42} \mathrm{H}_{64} \mathrm{O}_{13}$}

Atratoside A, 1993

\section{$\mathrm{C}_{42} \mathrm{H}_{64} \mathrm{O}_{14}$}

Picfeltarraenin IB, 17295

\section{$\mathrm{C}_{42} \mathrm{H}_{64} \mathrm{O}_{15}$}

Abrusoside C, 33

3- $O$ - $\beta$ - $D$-Galactopyranosyl-( $1 \rightarrow 2)-\beta$ - $D$-glucuronopyranosyl-gypsogenin, 8057

Glaucogenin C 3-O- $\beta$ - $D$-cymaropyranosyl$(1 \rightarrow 4)$ - $\alpha$ - $L$-diginopyranosyl-( $1 \rightarrow 4)-\beta$ - $D$ thevetopyranoside, 8520

Licoricesaponin $\mathrm{B}_{2}, 12784$

\section{$\mathrm{C}_{42} \mathrm{H}_{64} \mathrm{O}_{16}$}

Khekadaengoside A, 12208

Khekadaengoside J, 12217

Licoricesaponin $\mathrm{J}_{2}, 12791$

\section{$\mathrm{C}_{42} \mathrm{H}_{64} \mathrm{O}_{17}$}

Khekadaengoside B, 12209

\section{$\mathrm{C}_{42} \mathrm{H}_{64} \mathrm{O}_{19}$}

k-Strophantylside, 20405

Thevetin A, 21325

\section{$\mathrm{C}_{42} \mathrm{H}_{64} \mathrm{O}_{20}$}

Premnaodoroside C, 17816

Premnaodoroside $\mathrm{F}_{1}, 17819$

Premnaodoroside $\mathrm{F}_{2}, 17820$

\section{$\mathrm{C}_{42} \mathrm{H}_{66} \mathrm{O}_{4}$}

Fritillebinide B, 7966

Fritillebinide C, 7967

\section{$\mathrm{C}_{42} \mathrm{H}_{66} \mathrm{O}_{5}$}

Fritillebin A, 7961

\section{$\mathrm{C}_{42} \mathrm{H}_{66} \mathrm{O}_{11}$}

Fomitoside H, 7873

\section{$\mathrm{C}_{42} \mathrm{H}_{66} \mathrm{O}_{13}$}

Putranoside A, 18229

3-O- $\alpha$ - $L$-Rhamnopyranosyl $(1 \rightarrow 3)-\beta$ - $D$-glucuronopyranosyl-3 $\beta$-hydroxyolean-12-en-28-oate, 18723

3-O-[ $\alpha$ - $L$-Rhamnopyranosyl-( $1 \rightarrow 4)-\beta$ - $D$-glucuronopyranosyl]oleanolic acid, 18726

\section{$\mathrm{C}_{42} \mathrm{H}_{66} \mathrm{O}_{14}$}

Calendula officinalis Glycoside D, 2972

Chikusetsu saponin Iva, 3524

Cincholic acid

$3 \beta$ - $O-\beta$ - $D$-fucopyranosyl-28- $O-\beta$ - $D$-glucopyranosyl ester, 3677

Cincholic acid

$3 \beta$ - $O$ - $\beta$ - $D$-glucopyranosyl-( $1 \rightarrow 4)-\beta$ - $D$-fucopyranoside, 3678

Eupteleasaponin X, 7633 
Fargoside E, 7727

3 - $O$-[ $\beta$ - $D$-Glucopyranosyl-( $1 \rightarrow 3)-O-\beta$ - $D$-glucuronopyranosyl]-15- $\alpha$-hydroxyolean-12-en16-one, 8663

3- $O$ - $\beta$ - $D$-glucopyranosyl-( $1 \rightarrow 2)-\beta$ - $D$-quinovopyranosyl quinovic acid, 8714

Hollow alternanthera saponin A, 9588

Palustroside I, 16579

Quinovic acid $3 \beta-O-\beta-D$-fucopyranosyl-(27-1)$\beta$ - $D$-glucopyranosyl ester, 18428

Quinovic acid 3-O- $\beta$ - $D$-galactopyranosyl-( $(\rightarrow 3)$ $\beta$-D-quinovopyranoside, 18429

Quinovic acid 3 $\beta$ - $O-\beta$ - $D$-glucopyranosyl-( $(1 \rightarrow 3)$ $\beta$ - $D$-fucopyranoside, 18431

Quinovic acid 3- $O-\beta$ - $D$-glucopyranosyl-( $1 \rightarrow 3$ )$\beta$-D-quinovopyranoside, 18434

Quinovic acid 3- $O-\beta$ - $D$-glucopyranosyl-( $(1 \rightarrow 3)$ $\alpha$ - $L$-rhamnopyranoside, 18435

Quinovic acid 3-O- $\beta$ - $D$-quinovopyranoside 27$O$ - $\beta$ - $D$-glucopyranosyl ester, 18440

Quinovic acid 3-O- $\beta$ - $D$-quinovopyranoside 28$O$ - $\beta$ - $D$-glucopyranosyl ester, 18441

3-O-[ $\alpha$ - $L$-Rhamnopyranosyl-( $1 \rightarrow 4)-\beta$ - $D$-glucuronopyranosyl]echinocystic acid, 18721

Spinasaponin A, 20166

\section{$\mathrm{C}_{42} \mathrm{H}_{66} \mathrm{O}_{15}$}

Azukisaponin III, 2067

Dianoside A, 5358

Esculentoside C, 7370

3- $O$ - $[\beta$ - $D$-Glucopyranosyl- $(1 \rightarrow 2)-\beta$ - $D$-xylopyranosyl]phytolaccinic Acid, 8750

Hederagenin-28-O- $\beta$ - $D$-glucuronopyranosyl $(1 \rightarrow 4)$ - $\beta$ - $D$-glucopyranoside, 9266

Ilekudinoside B, 10972

Ilekudinoside J, 10980

Myrioside C, 15200

Phytolaccoside $\mathrm{D}_{2}, 17270$

Rivaloside E, 18852

Saikosaponin X, 19167

Spinasaponin B, 20167

\section{$\mathrm{C}_{42} \mathrm{H}_{66} \mathrm{O}_{16}$}

Dianoside C, 5360

Dianoside E, 5362

Esculentoside A, 7368

Metaplexigenin-3- $O-\beta$ - $D$-oleandropyranosyl$(1 \rightarrow 4)-\beta$ - $D$-digitoxopyranosyl-( $(1 \rightarrow 4)-\beta$ - $D$ digitoxopyranoside, 13818

\section{$\mathrm{C}_{42} \mathrm{H}_{66} \mathrm{O}_{17}$}

Stelmatotriterpenoside H, 20290

\section{$\mathrm{C}_{42} \mathrm{H}_{66} \mathrm{O}_{18}$}

Cerberoside, 3417

Thevetin B, 21326

$\mathrm{C}_{42} \mathrm{H}_{66} \mathrm{O}_{20}$
Premnaodoroside A, 17814

Premnaodoroside B, 17815

Premnaodoroside D, 17817

\section{$\mathrm{C}_{42} \mathrm{H}_{67} \mathrm{KO}_{14}$}

Potassium salt of longispinogenin 3- $O-\beta$ - $D$-glucopyranosyl $(1 \rightarrow 3)-\beta$ - $D$-glucuronopyranoside, 17749

\section{$\mathrm{C}_{42} \mathrm{H}_{67} \mathrm{KO}_{15}$}

Potassium salt of 29-hydroxylongispinogenin 3-O- $\beta$ - $D$-glucopyranosyl $(1 \rightarrow 3)-\beta$ - $D$-glucuronopyranoside, 17748

\section{$\mathrm{C}_{42} \mathrm{H}_{67} \mathrm{NaO}_{18} \mathrm{~S}$}

$2 \alpha, 23$-Dihydroxy-3 $\beta$-sulfoxyolean-12-en-28-oic acid $O-\beta$ - $D$-glucopyranosyl-( $1 \rightarrow 6)-\beta$ - $D$-glucopyranosyl ester sodium salt, 6138

\section{$\mathrm{C}_{42} \mathrm{H}_{68} \mathrm{O}_{12}$}

Oleanolic acid-3- $\beta$ - $D$-galactopyranosyl $(1 \rightarrow 2)$ $\beta$ - $D$-fucopyranoside, 16047

Saikosaponin E, 19149

Saikosaponin M, 19152

\section{$\mathrm{C}_{42} \mathrm{H}_{68} \mathrm{O}_{13}$}

Arvensoside A, 1829

Azukisaponin I, 2065

Carnosifloside I, 3202

3- $O-\beta$ - $D$-Galactopyranosyl-( $1 \rightarrow 4)-\beta$ - $D$-galactopyranosyloleanolic acid, 8054

\section{Hemslonin A, 9357}

Ilexside I methyl ester, 10986

Kaikasaponin I, 12111

Randianin, 18534

Saikosaponin A, 19142

Saikosaponin $\mathrm{B}_{2}, 19143$

Saikosaponin D, 19148

Saponin $\mathrm{E}_{6}, 19343$

\section{$\mathrm{C}_{42} \mathrm{H}_{68} \mathrm{O}_{14}$}

Azukisaponin II, 2066

Bacopaside $\mathrm{N}_{1}, 2091$

Bacopaside $\mathrm{N}_{2}, 2092$

Bourneioside A, 2572

Congmuyanoside C, 3978

Hederagenin-28-O- $\beta$ - $D$-glucopyranosyl $(1 \rightarrow 6)$ $\beta$ - $D$-glucopyranoside, 9264

Jujubasaponin VI, 11906

Pulsatilloside B, 18193

Saikosaponin L, 19151

Soyasaponin III, 20128

\section{$\mathrm{C}_{42} \mathrm{H}_{68} \mathrm{O}_{15}$}

Aquilegioside G, 1548

Bayogenin 3-O-cellobioside, 2179

Ilexoside XXXVII, 10984

Palustroside II, 16580

Pruvuloside A, 18006

Randiasaponin VII, 18541
Rivaloside D, 18851

\section{$\mathrm{C}_{42} \mathrm{H}_{68} \mathrm{O}_{16}$}

Centellasaponin B, 3395

\section{$\mathrm{C}_{42} \mathrm{H}_{68} \mathrm{O}_{17}$}

Platycoside K, 17544

Platycoside L, 17545

\section{$\mathrm{C}_{42} \mathrm{H}_{68} \mathrm{O}_{17} \mathrm{~S}$}

Sulfopatrinoside I, 20476

Sulfopatrinoside II, 20477

\section{$\mathrm{C}_{42} \mathrm{H}_{70} \mathrm{O}_{12}$}

Acanthoside $\mathrm{K}_{3}, 88$

Ginsenoside $\mathrm{F}_{4}, 8411$

Ginsenoside Ic, 8415

\section{$\mathrm{C}_{42} \mathrm{H}_{70} \mathrm{O}_{13}$}

Curculigosaponin G, 4384

Ginsenoside La, 8418

\section{$\mathrm{C}_{42} \mathrm{H}_{70} \mathrm{O}_{14}$}

23-O-Acetyl-3 $\beta, 12 \beta, 23 S, 24 R$-tetrahydroxy-20S, 25 -epoxydammarane 3- $O$ - $[\beta$ - $D$-xylopyranosyl $(1 \rightarrow 2)]-\beta$ - $D$-xylopyranoside, 521

Gypenoside XL, 9149

Hydroxysaikosaponin A, 10685

Hydroxysaikosaponin D, 10687

Justicioside A, 11983

Rubiarboside G, 19022

Thalicoside A, 21243

\section{$\mathrm{C}_{42} \mathrm{H}_{70} \mathrm{O}_{14} \mathrm{~S}$}

Atriplicosaponin B, 1998

\section{$\mathrm{C}_{42} \mathrm{H}_{70} \mathrm{O}_{15}$}

Beesioside H, 2199

\section{$\mathrm{C}_{42} \mathrm{H}_{70} \mathrm{O}_{16}$}

Iridalglycoside 5b, 11135

Iridalglycoside 6c, 11138

\section{$\mathrm{C}_{42} \mathrm{H}_{72} \mathrm{O}_{2}$}

$\alpha$-Amyrin laurate, 1115

$\beta$-Amyrin laurate, 1116

\section{$\mathrm{C}_{42} \mathrm{H}_{72} \mathrm{O}_{13}$}

Ginsenoside $\mathrm{Rg}_{2}, 8431$

20(S)-Ginsenoside $\mathrm{Rg}_{3}, 8432$

Gypenoside XII, 9132

Gypenoside XXXVIII, 9147

Gypenoside XLI, 9150

Gypenoside LXXV, 9177

Gypenoside LXXIX, 9181

\section{$\mathrm{C}_{42} \mathrm{H}_{72} \mathrm{O}_{14}$}

Ginsenoside Ia, 8413

Ginsenoside Ib, 8414

Ginsenoside Rf, 8429

Ginsenoside $\operatorname{Rg}_{1}, 8430$

Ginsenoside $\operatorname{Rg}_{7}, 8433$

Gynoside B, 9117

Gypenoside XXXIX, 9148

Gypenoside LI, 9156 
Gypenoside LXXIV, 9176

$2 \alpha, 3 \beta, 12 \beta, 20(S)$-3-Hydroxydammar-24-en-20$O$ - $\beta$ - $D$-glucopyranoside, 9958

Pseudoginsenoside $\mathrm{F}_{11}, 18025$

$24 S, 3 \beta, 11 \alpha, 16 \beta, 24$-Tetrahydroxycycloartanol3- $O$ - $\beta$ - $D$-glucopyranosyl-( $1 \rightarrow 2)-\beta$ - $D$-glucopyranoside, 21088

Yixinoside B, 22914

\section{$\mathrm{C}_{42} \mathrm{H}_{72} \mathrm{O}_{15}$}

6- $O$ - $\beta$ - $D$-Glucopyranosyl-20- $O-\beta$ - $D$-glucopyranosyl-3 $\beta, 6 \alpha, 12 \beta, 20(S), 25$-pentahydroxydammar-23-ene, 8651

Gycomoside I, 9094

Majonoside $\mathrm{R}_{1}, 13404$

Momorcharaside A, 14907

$\mathrm{C}_{42} \mathrm{H}_{72} \mathrm{O}_{16}$

Dresioside I, 6601

Iridalglycoside 7, 11139

Iridalglycoside 8, 11140

\section{$\mathrm{C}_{42} \mathrm{H}_{74} \mathrm{O}_{15}$}

Quinquenoside $\mathrm{F}_{1}, 18445$

\section{$\mathrm{C}_{42} \mathrm{H}_{74} \mathrm{O}_{16}$}

Notoginsenoside J, 15828

\section{$\mathrm{C}_{42} \mathrm{H}_{76} \mathrm{O}_{3}$}

Parvifolinoic acid, 16686

\section{$\mathrm{C}_{42} \mathrm{H}_{79} \mathrm{NO}_{9}$}

Cerebroside 5, 3420

Sphingolipid Lipids01-521, 20154

\section{$\mathrm{C}_{42} \mathrm{H}_{81} \mathrm{NO}_{4}$}

1,3,5-Trihydroxy-2-hexadecanoylamino-(6E, 9E)-heptacosdiene, 21741

\section{$\mathrm{C}_{42} \mathrm{H}_{83} \mathrm{NO}_{5}$}

Anticancer Ceramide PMV70P691-009, 1391

\section{$\mathrm{C}_{42} \mathrm{H}_{85} \mathrm{NO}_{5}$}

Anticancer Ceramide PMV70P691-69, 1392

Hemisceramide, 9351

Newbouldiamide, 15518

Soyasphingosine B, 20131

\section{$\mathrm{C}_{43} \mathrm{H}_{32} \mathrm{~N}_{2} \mathrm{O}_{9}$}

meso-Chelidimerine, 3500

\section{$\mathrm{C}_{43} \mathrm{H}_{32} \mathrm{O}_{20}$}

Theaflavin 3,3'-digallate, 21280

\section{$\mathrm{C}_{43} \mathrm{H}_{33} \mathrm{O}_{12}$}

Mohsenone, 14895

\section{$\mathrm{C}_{43} \mathrm{H}_{34} \mathrm{O}_{22}$}

Trilobatin G, 21882

\section{$\mathrm{C}_{43} \mathrm{H}_{34} \mathrm{O}_{23}$}

Trilobatin F, 21881

\section{$\mathrm{C}_{43} \mathrm{H}_{36} \mathrm{O}_{18}$}

1,3,4,5-Tetracaffeoylquinic acid, 21034

\section{$\mathrm{C}_{43} \mathrm{H}_{36} \mathrm{O}_{21}$}

Trilobatin E, 21880

$\mathrm{C}_{43} \mathrm{H}_{42} \mathrm{O}_{23}$
Trilobatin I, 21884

$\mathrm{C}_{43} \mathrm{H}_{42} \mathrm{O}_{26}$

7-O- $\beta$ - $D$-Glucuronopyranosyl-4'-O-[2'-O-E-

feruloyl- $O-\beta$ - $D$-glucuronopyranosyl $(1 \rightarrow 2)$ -

$O$ - $\beta$ - $D$-glucuronopyranoside]apigenin, 8763

\section{$\mathrm{C}_{43} \mathrm{H}_{44} \mathrm{O}_{6}$}

GBB A, 8247

GBB B, 8248

\section{$\mathrm{C}_{43} \mathrm{H}_{46} \mathrm{O}_{15}$}

Epioritin- $4 \alpha$-ol- $(6 \rightarrow 6)$-epioritin- $4 \beta$-ol hepta- $O$ methylether triacetate, 6985

\section{$\mathrm{C}_{43} \mathrm{H}_{47} \mathrm{~N}_{3} \mathrm{O}_{9}$}

Milliamine B, 14860

Milliamine C, 14861

$\mathrm{C}_{43} \mathrm{H}_{48} \mathrm{~N}_{2} \mathrm{O}_{10}$

Isopythaldine, 11641

$\mathrm{C}_{43} \mathrm{H}_{48} \mathrm{O}_{16}$

Dryocrassin, 6611

Norflavaspidic acid, 15749

\section{$\mathrm{C}_{43} \mathrm{H}_{48} \mathrm{O}_{22}$}

Kaempferol-3-rhamnoside-7-O-[6-feruloylglucosyl $(1 \rightarrow 3)$-rhamnoside], 12085

\section{$\mathrm{C}_{43} \mathrm{H}_{48} \mathrm{O}_{23}$}

Isoscoparin 2"-O-(6"'-(E)-coumaroyl)glucoside4'-O-glucoside, 11701

Isovitexin 2"-O-(6"'-(E)-feruloyl)glucoside-4'$O$-glucoside, 11776

\section{$\mathrm{C}_{43} \mathrm{H}_{49} \mathrm{NO}_{17}$}

Neowilforine, 15476

\section{$\mathrm{C}_{43} \mathrm{H}_{49} \mathrm{NO}_{18}$}

Euoverrine B, 7538

Wilforine, 22682

\section{$\mathrm{C}_{43} \mathrm{H}_{49} \mathrm{NO}_{19}$}

Wilfordine, 22677

Wilfordside, 22679

Wilfornine B, 22687

\section{$\mathrm{C}_{43} \mathrm{H}_{49} \mathrm{O}_{23}{ }^{+}$}

Pelargonidin-3-O-[6-O-(E)-feruloyl-2- $O-\beta-D-$ glucopyranosyl]-( $1 \rightarrow 2)-\beta$ - $D$-glucopyranoside (-5-O- $\beta$ - $D$-glucopyranoside), 16784

\section{$\mathrm{C}_{43} \mathrm{H}_{50} \mathrm{~N}_{6} \mathrm{O}_{10}$}

Rubia akane RA-IX, 18987

$\mathrm{C}_{43} \mathrm{H}_{51} \mathrm{NO}_{13}$

10-Deacetyltaxol B, 4784

\section{$\mathrm{C}_{43} \mathrm{H}_{52} \mathrm{~N}_{2} \mathrm{O}_{10}$}

Methoxyadiantifoline, 13836

$\mathrm{C}_{43} \mathrm{H}_{52} \mathrm{~N}_{4} \mathrm{O}_{5}$

Conodurine, 3998

Voacamine, 22596

$\mathrm{C}_{43} \mathrm{H}_{52} \mathrm{~N}_{4} \mathrm{O}_{6}$

Cononitarine B, 4003

19'(S)-Hydroxyconoduramine, 9933

19'(S)-Hydroxyconodurine, 9934

\section{$\mathrm{C}_{43} \mathrm{H}_{52} \mathrm{~N}_{4} \mathrm{O}_{7}$}

Callophylline A, 2981

Conodiparine E, 3996

Conodiparine F, 3997

Cononitarine A, 4002

\section{$\mathrm{C}_{43} \mathrm{H}_{52} \mathrm{~N}_{4} \mathrm{O}_{8}$}

Callophylline B, 2982

$\mathrm{C}_{43} \mathrm{H}_{52} \mathrm{~N}_{6} \mathrm{O}_{11}$

Rubia akane RA-X, 18988

\section{$\mathrm{C}_{43} \mathrm{H}_{53} \mathrm{NO}_{11}$}

$10 \beta$-Benzoyloxy-1 $\beta$-hydroxy-5 $\alpha$-(3'-methylamino-3'-phenyl)propanoxy-7 $\beta, 9 \alpha, 13 \alpha$-triacetoxy-11(15 $\rightarrow$ 1)-abeo-taxa-4(20),11-diene, 2258

\section{$\mathrm{C}_{43} \mathrm{H}_{53} \mathrm{~N}_{9} \mathrm{O}_{12}$}

Lyciumin A methylate, 13179

\section{$\mathrm{C}_{43} \mathrm{H}_{54} \mathrm{~N}_{2} \mathrm{O}_{11}$}

Nicaustrine, 15523

\section{$\mathrm{C}_{43} \mathrm{H}_{54} \mathrm{O}_{4}$} 3,29-O-Dibenzoyloxykarounidiol, 5386

\section{$\mathrm{C}_{43} \mathrm{H}_{54} \mathrm{O}_{23}$}

Tubuloside D, 22096

\section{$\mathrm{C}_{43} \mathrm{H}_{54} \mathrm{O}_{24}$}

Tubuloside C, 22095

\section{$\mathrm{C}_{43} \mathrm{H}_{56} \mathrm{~N}_{4} \mathrm{O}_{5}$}

Ervadivaricatine A, 7292

Ervadivaricatine B, 7293

\section{$\mathrm{C}_{43} \mathrm{H}_{56} \mathrm{O}_{13}$}

Rediocide E, 18565

\section{$\mathrm{C}_{43} \mathrm{H}_{56} \mathrm{O}_{21}$}

Hedyotol C 4,4"-di- $O$ - $\beta$ - $D$-glucopyranoside, 9284

\section{$\mathrm{C}_{43} \mathrm{H}_{57} \mathrm{NO}_{14}$}

$2 \alpha$-Acetoxyaustrospicatine, 127

\section{$\mathrm{C}_{43} \mathrm{H}_{57} \mathrm{NO}_{15}$}

(2'S,3'R)-5-( $N, N$-Dimethyl-3'-phenylisoseryl)taxachitriene A, 6395

\section{$\mathrm{C}_{43} \mathrm{H}_{58} \mathrm{O}_{6}$}

Guttiferone G, 9090

\section{$\mathrm{C}_{43} \mathrm{H}_{58} \mathrm{O}_{15}$}

Cynaphylloside B, 4557

\section{$\mathrm{C}_{43} \mathrm{H}_{60} \mathrm{O}_{4}$}

6-Geranylgeranyl 8'-methyl-6,8'-diapocaroten6,8'-dioate, 8317

\section{$\mathrm{C}_{43} \mathrm{H}_{60} \mathrm{O}_{20}$}

Azecin 1, 2054

\section{$\mathrm{C}_{43} \mathrm{H}_{62} \mathrm{~N}_{8} \mathrm{O}_{8}$}

Grifficyclocin A, 9001

\section{$\mathrm{C}_{43} \mathrm{H}_{62} \mathrm{O}_{11}$}

$21 \beta$-Benzoylsitakisogenin-3- $O-\beta$ - $D$-glucuronopyranoside, 2272

Voamatin C, 22600

$\mathrm{C}_{43} \mathrm{H}_{62} \mathrm{O}_{17}$ 


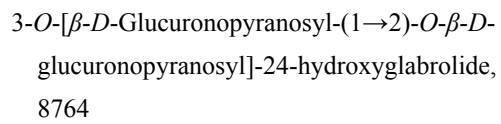

$\mathrm{C}_{43} \mathrm{H}_{62} \mathrm{O}_{23}$

Jasnudifloside H, 11828

$\mathrm{C}_{43} \mathrm{H}_{64} \mathrm{O}_{8}$

13-O-Tigloylphorbol-20-linoleate, 21381

\section{$\mathrm{C}_{43} \mathrm{H}_{64} \mathrm{O}_{11}$}

(22S)-Cholest-5-ene-3 $\beta, 11 \alpha, 16 \beta, 22$-tetrol 16-O$\{2-O$-acetyl-3- $O$-( $p$-methoxybenzoyl)- $\alpha-L$ rhamnopyranoside\}, 3580

\section{$\mathrm{C}_{43} \mathrm{H}_{64} \mathrm{O}_{16}$}

Abrusoside B, 32

$\mathrm{C}_{43} \mathrm{H}_{66} \mathbf{N}_{12} \mathrm{O}_{12} \mathrm{~S}_{2}$

Oxytocin, 16478

\section{$\mathrm{C}_{43} \mathrm{H}_{66} \mathrm{O}_{5}$}

Revandchinone 2, 18670

\section{$\mathrm{C}_{43} \mathrm{H}_{66} \mathrm{O}_{14}$}

$\alpha$-Acetyldigitoxin, 377

Gymnemic acid I, 9110

\section{$\mathbf{C}_{43} \mathbf{H}_{66} \mathbf{O}_{16}$}

Neoruscogenin 1-O-\{O- $\alpha-L$-rhamnopyranosyl$(1 \rightarrow 2)-O-[\beta$ - $D$-xylopyranosyl- $(1 \rightarrow 3)]-\alpha-L$ arabinopyranoside\}, 15455

\section{$\mathrm{C}_{43} \mathrm{H}_{68} \mathrm{O}_{13}$}

Sanguisorbin E, 19288

\section{$\mathrm{C}_{43} \mathrm{H}_{68} \mathrm{O}_{14}$}

Pomolic acid-3 $\beta-O-\alpha-L-2$-acetoxyarabinopyranosyl-28- $O-\beta$ - $D$-glucopyranoside, 17697

\section{$\mathrm{C}_{43} \mathrm{H}_{68} \mathrm{O}_{15}$}

Eupteleasaponin IX, 7632

3-O-[ $\beta$ - $D$-Glucopyranosyl $(1 \rightarrow 3)-\beta-D$-6- $O$-methyl-glucoronopyranosyl]-3 $\beta, 15 \alpha, 23$-trihydroxyolean-12-en-16-one, 8683

Saponin 2, 19334

Saponin 3, 19335

Yiamoloside B, 22903

\section{$\mathrm{C}_{43} \mathrm{H}_{68} \mathrm{O}_{16}$}

Aferoside A, 668

\section{$\mathrm{C}_{43} \mathrm{H}_{68} \mathrm{O}_{17}$}

Laxogenin 3-O- $\{O-\beta$ - $D$-xylopyranosyl- $(1 \rightarrow 4)-$ $O$-[ $\alpha$ - $L$-arabinopyranosyl-( $1 \rightarrow 6)-\beta$ - $D$-glucopyranoside]\}, 12594

\section{$\mathrm{C}_{43} \mathrm{H}_{68} \mathrm{O}_{22}$}

Rebaudioside F, 18561

\section{$\mathbf{C}_{43} \mathrm{H}_{70} \mathbf{O}_{13}$}

3-O-[ $\beta$ - $D$-Galactopyranosyl-( $1 \rightarrow 2)-\beta$ - $D$-glucuronopyranosyl]-sophoradiol methyl ester, 8063 16 $\alpha, 23,28,30$-Tetrahydroxyolean-11,13(18)dien-3 $\beta$-yl- $\beta$ - $D$-glucopyranosyl-( $1 \rightarrow 3)-\beta$ - $D$ fucopyranoside, 21139

\section{$\mathrm{C}_{43} \mathrm{H}_{70} \mathrm{O}_{15}$}

Astragaloside II, 1937
Davuricoside D, 4699

Isoastragaloside II, 11243

PM-2004-70-458-3b, 17578

\section{$\mathrm{C}_{43} \mathrm{H}_{70} \mathrm{O}_{16}$}

Aquilegioside F, 1547

Aspafilioside B, 1861

Asparanin C, 1878

Methyl 3-O- $\beta$-laminaribiosyl polygalacate, 14549

3 - $O$ - $\{[\beta$ - $D$-Xylopyranosyl $(1 \rightarrow 4)][\alpha-L$-arabinopyranosyl $(1 \rightarrow 6)]-\beta$ - $D$-glucopyranosyl $\}-(25 S)$ $5 \beta$-spirostan- $3 \beta$-ol, 22821

\section{$\mathrm{C}_{43} \mathrm{H}_{72} \mathrm{O}_{4}$}

Diosgenin palmitate, 6445

Yamogenin palmitate, 22881

$\mathrm{C}_{43} \mathrm{H}_{72} \mathrm{O}_{11}$

Acanthoside $\mathrm{K}_{2}, 87$

$\mathrm{C}_{43} \mathrm{H}_{72} \mathrm{O}_{13}$

Bupleuroside IX, 2756

Cyclocarioside A, 4482

Saikosaponin T, 19162

\section{$\mathrm{C}_{43} \mathrm{H}_{72} \mathrm{O}_{14}$}

Bupleuroside III, 2753

Justicioside C, 11985

Saikosaponin $\mathrm{B}_{3}, 19144$

Saikosaponin $\mathrm{B}_{4}, 19145$

\section{$\mathrm{C}_{43} \mathrm{H}_{72} \mathrm{O}_{15}$}

23- $O$-Acetyl-3 $\beta, 12 \beta, 23 S, 24 R$-tetrahydroxy- $20 S$, 25 -epoxydammarane 3- $O$ - $[\beta$ - $D$-xylopyranosyl $(1 \rightarrow 2)]-\beta$ - $D$-glucopyranoside, 520

Vina-ginsenoside $\mathrm{R}_{2}, 22484$

\section{$\mathrm{C}_{43} \mathrm{H}_{72} \mathrm{O}_{16}$}

Cyclotricuspidoside A, 4539

\section{$\mathrm{C}_{43} \mathrm{H}_{72} \mathbf{O}_{17}$}

Cyclotricuspidoside B, 4540

Cyclotricuspidoside C, 4541

\section{$\mathrm{C}_{43} \mathrm{H}_{73} \mathrm{NO}_{13}$}

Abutiloside K, 44

\section{$\mathrm{C}_{43} \mathrm{H}_{74} \mathrm{O}_{2}$}

Ergosta-7,22-dien-3 $\beta$-yl pentadecanoate, 7246

\section{$\mathrm{C}_{43} \mathrm{H}_{74} \mathrm{O}_{4}$}

2-Tritriacontyl-5-hydroxy-7-methoxychromone, 22045

$\mathrm{C}_{43} \mathrm{H}_{77} \mathrm{NO}_{4}$

3-O-14,15-Eicosylenoyl-4- $O$-stearoyl-1-cyano-

$$
\text { 2-oxymethyl-1,2-propene, } 6728
$$

\section{$\mathrm{C}_{43} \mathrm{H}_{80} \mathrm{O}_{10}$}

Arisaema glyceride 3, 1690

\section{$\mathrm{C}_{43} \mathrm{H}_{87} \mathrm{NO}_{5}$}

Soyasphingosine A, 20130

Sponge sphingolipid, 20224

\section{$\mathrm{C}_{44} \mathrm{H}_{26} \mathrm{O}_{12}$}

Bisisodiospyrin, 2478

$\mathrm{C}_{44} \mathrm{H}_{26} \mathrm{O}_{14}$
6",8'-Bisdiosquinone, 2447

\section{$\mathrm{C}_{44} \mathrm{H}_{32} \mathrm{O}_{27}$}

Phyllanthusiin D, 17220

\section{$\mathrm{C}_{44} \mathrm{H}_{34} \mathbf{O}_{20}$}

Procyanidin $\mathrm{B}_{2}$ 3,3'-di-O-gallate, 17896

\section{$\mathrm{C}_{44} \mathrm{H}_{34} \mathbf{O}_{22}$}

Prodelphinidin B 23,3'-di-O-gallate, 17900

Theasinensin A, 21290

\section{$\mathrm{C}_{44} \mathbf{H}_{44} \mathbf{O}_{24}$}

Precarthamin, 17771

\section{$\mathrm{C}_{44} \mathbf{H}_{46} \mathbf{O}_{\mathbf{1 8}}$}

6-O- $\alpha$-L-(2"-O-,3"-O-Dibenzoyl,4"-O-cis-pcoumaroyl)rhamnopyranosylcatalpol, 5381 6-O- $\alpha-L-(2 "-O-, 3 "-O-D i b e n z o y 1,4 "-O-$ transp-coumaroyl)rhamnopyranosylcatalpol, 5382

\section{$\mathrm{C}_{44} \mathbf{H}_{48} \mathbf{O}_{15}$}

Taxacin, 20738

\section{$\mathrm{C}_{44} \mathrm{H}_{48} \mathrm{O}_{21}$}

Shegansu C, 19799

\section{$\mathrm{C}_{44} \mathrm{H}_{50} \mathrm{~N}_{8}$}

Psycholeine, 18093

Quadrigemine A, 18295

Quadrigemine B, 18296

\section{$\mathrm{C}_{44} \mathrm{H}_{50} \mathrm{O}_{14}$}

Taxchinin B, 20771

\section{$\mathrm{C}_{44} \mathrm{H}_{50} \mathrm{O}_{25}$}

Kaempferol-3- $\beta$ - $D$-glucopyranosyl-7-O-[(2$O$-trans-sinnapoyl)- $\beta$ - $D$-glucopyranosyl $(1 \rightarrow 6)]-\beta$ - $D$-glucopyranoside, 12059

Kaempferol-4'-(6-O-E-sinapoyl- $\beta$-glucopyranoside)-3,7-di- $O-\beta$-glucopyranoside, 12092

Kaempferol-3-(2"-O-E-sinapoylsophoroside)7-O- $\beta$-glucopyranoside, 12094

Kaempferol-3-O-sophoroside-7-O-(2-O-Esinapoyl- $\beta$-glucopyranoside), 12096

\section{$\mathrm{C}_{44} \mathrm{H}_{50} \mathrm{O}_{26}$}

Quercetin-3'-(6-sinapoyl- $O-\beta$ - $D$-glucopyranosyl)3,4'-di-O- $\beta$ - $D$-glucopyranoside, 18394

\section{$\mathbf{C}_{44} \mathbf{H}_{52} \mathbf{N}_{4} \mathbf{O}_{7}$}

Conodiparine C, 3994

\section{$\mathrm{C}_{44} \mathrm{H}_{52} \mathrm{~N}_{2} \mathrm{O}_{10}$}

Isopyruthaldine, 11640

\section{$\mathrm{C}_{44} \mathrm{H}_{52} \mathrm{~N}_{4} \mathrm{O}_{7}$}

Conodiparine D, 3995

\section{$\mathrm{C}_{44} \mathrm{H}_{53} \mathrm{NO}_{13}$}

10-Deacetyl-10-oxo-7-epitaxuyunnanine A, 4766

\section{$\mathrm{C}_{44} \mathrm{H}_{53} \mathrm{NO}_{14}$}

Taxol D, 20814

\section{$\mathrm{C}_{44} \mathrm{H}_{54} \mathbf{N}_{4} \mathbf{O}_{7}$}

Conodiparine A, 3992

Conodiparine B, 3993

\section{$\mathrm{C}_{44} \mathrm{H}_{54} \mathrm{O}_{8}$}

Jatropha factor $\mathrm{C}_{1}, 11836$ 


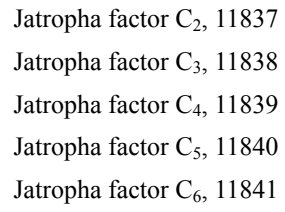

Jatropha factor $\mathrm{C}_{2}, 11837$

Jatropha factor $\mathrm{C}_{3}, 11838$

Jatropha factor $\mathrm{C}_{4}, 11839$

Jatropha factor $\mathrm{C}_{5}, 11840$

Jatropha factor $\mathrm{C}_{6}, 11841$

\section{$\mathrm{C}_{44} \mathrm{H}_{55} \mathrm{NO}_{11}$}

10 $\beta$-Benzoyloxy-5 $\alpha$-(3'-dimethylamino-3'phenyl)propanoxy-1 $\beta$-hydroxy- $7 \beta, 9 \alpha, 13 \alpha$-triacetoxy-11(15 $\rightarrow 1)$-abeo-taxa-4(20),11-dien, 2256

\section{$\mathrm{C}_{44} \mathrm{H}_{55} \mathrm{NO}_{13}$}

10-Deacetyltaxuyunnanine $\mathrm{A}, 4787$

7-Epi-10-deacetyltaxuyunnanine A, 6884

\section{$\mathrm{C}_{44} \mathrm{H}_{56} \mathbf{N}_{4} \mathbf{O}_{7}$}

Deacetoxyvinblastine, 4709

\section{$\mathrm{C}_{44} \mathrm{H}_{56} \mathrm{~N}_{4} \mathrm{O}_{8}$}

Pseudovincaleukoblastine diol, 18073

\section{$\mathrm{C}_{44} \mathrm{H}_{56} \mathrm{O}_{22}$}

Epimedokoreanoside I, 6962

\section{$\mathrm{C}_{44} \mathrm{H}_{56} \mathrm{O}_{23}$}

Insularoside-6"'- $O-\beta$-glucosi-(3' $\rightarrow 1)-\beta$ - $D$-glucoside, 11090

\section{$\mathrm{C}_{44} \mathrm{H}_{57} \mathrm{NO}_{4}$}

3-O-p-Aminobenzoyl-29-O-benzoylmultiflora7,9(11)-diene-3 $\alpha, 29$-diol, 1043

\section{$\mathrm{C}_{44} \mathrm{H}_{58} \mathrm{O}_{13}$}

Rediocide A, 18563

\section{$\mathrm{C}_{44} \mathrm{H}_{58} \mathrm{O}_{21}$}

Hedysalignan A, 9285

\section{$\mathrm{C}_{44} \mathrm{H}_{59} \mathrm{Br}_{2} \mathrm{~N}_{3} \mathrm{O}_{4}$}

Di- $p$-bromobenzoate of tetrahydrodeoxyoxolucidine B, 5398

\section{$\mathrm{C}_{44} \mathrm{H}_{59} \mathrm{NO}_{5}$}

3-O-p-Aminobenzoyl-29-O-benzoylmultiflora8-ene-3 $\alpha, 7 \beta, 29$-triol, 1044

\section{$\mathrm{C}_{44} \mathrm{H}_{60} \mathrm{O}_{24}$}

Gymnoside VIII, 9111

\section{$\mathrm{C}_{44} \mathrm{H}_{62} \mathrm{O}_{18}$}

Hancoside A, 9217

\section{$\mathrm{C}_{44} \mathrm{H}_{64} \mathrm{~N}_{2} \mathrm{O}_{3}$}

Pukeensine, 18189

\section{$\mathrm{C}_{44} \mathrm{H}_{64} \mathbf{O}_{10}$}

Isodopharicin E, 11398

\section{$\mathrm{C}_{44} \mathrm{H}_{64} \mathrm{O}_{17}$}

DraconinA, 6576

\section{$\mathrm{C}_{44} \mathrm{H}_{64} \mathrm{O}_{24}$} Crocin, 4249

\section{$\mathrm{C}_{44} \mathrm{H}_{66} \mathrm{O}_{8}$}

(1E,24Z)-1,-24-Diferuloyloxytetracosane, 5506 1,24-Tetracosanediol diferulate, 21037

\section{$\mathrm{C}_{44} \mathrm{H}_{66} \mathrm{O}_{19}$}

Funingenoside A, 8005

$\mathrm{C}_{44} \mathrm{H}_{68} \mathrm{O}_{6}$
Fritillebinide D, 7968

Fritillebinide E, 7969

\section{$\mathrm{C}_{44} \mathrm{H}_{68} \mathrm{O}_{7}$}

Fritillebin B, 7962

\section{$\mathrm{C}_{44} \mathrm{H}_{68} \mathrm{O}_{16}$}

Neoruscogenin 1-O-\{O- $\alpha-L$-rhamnopyranosyl$(1 \rightarrow 2)-O$ - $[\beta$ - $D$-xylopyranosyl-( $(1 \rightarrow 3)]-\beta-D$ fucopyranoside\}, 15456

\section{$\mathrm{C}_{44} \mathrm{H}_{68} \mathrm{O}_{19}$}

Funingenoside B, 8006

\section{$\mathrm{C}_{44} \mathrm{H}_{70} \mathrm{O}_{\mathbf{1 4}}$}

3"-O-Acetylsaikosaponin A, 498

2"-O-Acetylsaikosaponin D, 500

3"-O-Acetylsaikosaponin D, 501

4"-O-Acetylsaikosaponin D, 502

6"-O-Acetylsaikosaponin D, 503

$1 \alpha, 3 \beta$-Hydroxyimberbic acid-23-O- $\alpha$ - $[L-4$-acetylrhamnopyranosyl]-29- $O-\alpha$-rhamnopyranoside, 10227

\section{$\mathrm{C}_{44} \mathrm{H}_{70} \mathrm{O}_{16}$}

Diosgenin-3- $O-\alpha-L$-rhamnopyranosyl- $(1 \rightarrow 2)[\alpha-$ $L$-arabinofuranosyl-(1 $\rightarrow 4)]$ - $\beta$ - $D$-glucopyranoside, 6446

Lililancifoloside A, 12833

Ophiopogonin D, 16146

Ophiopogonin D', 16147

Pariphyllin, 16668

Polyphyllin D, 17655

Rivaloside C, 18850

25(S)-Ruscogenin 1-O-[ $\alpha-L$-rhamnopyranosyl$(1 \rightarrow 2)][\beta$ - $D$-xylopyra-nosyl- $(1 \rightarrow 3)]-\beta$ - $D$ fucopyranoside, 19072

\section{$\mathrm{C}_{44} \mathrm{H}_{70} \mathrm{O}_{17}$}

Caudaside A, 3328

Pennogenin-3-O- $\alpha$ - $L$-arabinofuranosyl $(1 \rightarrow 4)$ $[\alpha$ - $L$-rhamnopyranosyl $(1 \rightarrow 2)]-\beta$ - $D$-glucopyranoside, 16811

\section{$\mathrm{C}_{44} \mathrm{H}_{70} \mathrm{O}_{18}$}

CTHD0233276-4, 4299

Ophiopogon A, 16135

$\mathrm{C}_{44} \mathrm{H}_{70} \mathrm{O}_{19}$

Chinenoside VI, 3528

\section{$\mathrm{C}_{44} \mathrm{H}_{70} \mathrm{O}_{21}$}

3-O- $\beta$-Lycotetraosyl $3 \beta$-hydroxy-5 $\alpha$-pregn-16en-20-one, 13242

\section{$\mathbf{C}_{44} \mathbf{H}_{72} \mathbf{O}_{13}$}

3- $O$-[ $\beta$ - $D$-Galactopyranosyl-( $(1 \rightarrow 2)-\beta$ - $D$-glucuronopyranosyl]-sophoradiol ethyl ester, 8062

\section{$\mathrm{C}_{44} \mathrm{H}_{72} \mathrm{O}_{15}$} 6"-O-Acetylsaikosaponin $\mathrm{B}_{3}, 499$

Kahiricoside V, 12110

$\mathrm{C}_{44} \mathrm{H}_{72} \mathrm{O}_{16}$
Asparanin $\mathrm{B}_{2}, 1870$

Convallasaponin C, 4010

\section{$\mathrm{C}_{44} \mathbf{H}_{72} \mathbf{O}_{17}$}

AS-1 A, 1830

3-O-[ $\beta$ - $D$-Xylopyranosyl $(1 \rightarrow 4)-\beta$ - $D$-glucopy$\operatorname{ranosyl}(1 \rightarrow 2)-\beta$ - $D$-glucopyranosyl]-(25S)- $5 \beta$ spirostan-3 $\beta$-ol, 22828

\section{$\mathrm{C}_{44} \mathrm{H}_{72} \mathbf{O}_{18}$}

Trigoneoside VIII, 21673

\section{$\mathrm{C}_{44} \mathrm{H}_{74} \mathbf{O}_{15}$}

Vina-ginsenoside $\mathrm{R}_{1}, 22483$

Yesanchinoside D, 22896

\section{$\mathrm{C}_{44} \mathbf{H}_{74} \mathbf{O}_{16}$}

Yesanchinoside A, 22893

\section{$\mathrm{C}_{44} \mathrm{H}_{74} \mathbf{O}_{18}$}

26-O- $\beta$ - $D$-Glucopyranosylfurostane-3 $\beta, 26$-diol3 - $O$ - $\beta$ - $D$-xylopyranosyl $(1 \rightarrow 4)-\beta$ - $D$-glucopyranoside, 8632

Trigoneoside IIa, 21667

Trigoneoside IIb, 21668

\section{$\mathrm{C}_{44} \mathrm{H}_{74} \mathrm{O}_{19}$}

Trigoneoside Ia, 21665

Trigoneoside Ib, 21666

Trigoneoside XIb, 21676

\section{$\mathrm{C}_{44} \mathrm{H}_{74} \mathrm{O}_{24}$}

Mukurozioside $\mathrm{I}_{\mathrm{b}}, 15030$

\section{$\mathrm{C}_{44} \mathrm{H}_{75} \mathrm{NO}_{13}$}

Abutiloside I, 42

\section{$\mathrm{C}_{44} \mathrm{H}_{76} \mathrm{O}_{2}$}

$\alpha$-Amyrin myristate, 1119

$\beta$-Amyrin myristate, 1120

\section{$\mathrm{C}_{44} \mathrm{H}_{76} \mathrm{O}_{3}$}

Arnidiol 3-O-myristate, 1751

16 $\beta$-Hydroxylupeol 3-O-myristate, 10347

Maniladiol 3-O-myristate, 13498

\section{$\mathrm{C}_{44} \mathrm{H}_{76} \mathrm{O}_{18}$}

Pentandroside E, 16881

\section{$\mathrm{C}_{44} \mathrm{H}_{76} \mathrm{O}_{24}$}

Mukurozioside $\mathrm{I}_{\mathrm{a}}, 15029$

\section{$\mathrm{C}_{44} \mathrm{H}_{78} \mathrm{O}_{2}$}

Campesteryl palmitate, 3044

\section{$\mathrm{C}_{44} \mathrm{H}_{78} \mathrm{O}_{5}$}

34-Hydroxytetratriacontanylferulate, 10760

\section{$\mathrm{C}_{44} \mathrm{H}_{78} \mathrm{O}_{26}$}

Pharbitic acid C, 17043

\section{$\mathrm{C}_{44} \mathrm{H}_{83} \mathrm{NO}_{9}$}

Cerebroside 1, 3419

1-O-( $\beta$ - $D$-Glucopyranosyl)-(2S,3R,4E,8E)-2[(2'R)-2'-hydroxynonadecanoylamino]-9methyl-4,8-octadecadiene-1,3-diol, 8674

\section{$\mathrm{C}_{44} \mathrm{H}_{87} \mathrm{NO}_{4}$}

Spongiamine B, 20227

$\mathrm{C}_{44} \mathrm{H}_{88} \mathrm{O}_{2}$ 
Hexacosyl stearate, 9484

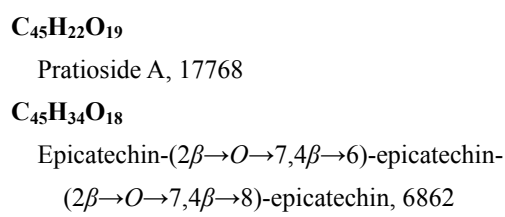

\section{$\mathrm{C}_{45} \mathrm{H}_{36} \mathrm{O}_{18}$}

Aesculitannin B, 666

Cinnamtannin $\mathrm{B}_{1}, 3725$

Epicatechin- $(2 \beta \rightarrow O \rightarrow 7,4 \beta \rightarrow 6)$-[epicatechin$(4 \beta \rightarrow 8)]$-catechin, 6860

\section{$\mathrm{C}_{45} \mathrm{H}_{36} \mathrm{O}_{22}$}

Oolonghomobisflavan A, 16124

Oolonghomobisflavan B, 16125

\section{$\mathrm{C}_{45} \mathrm{H}_{38} \mathrm{O}_{13}$}

Caloflavan A, 2986

Caloflavan B, 2987

\section{$\mathrm{C}_{45} \mathrm{H}_{38} \mathrm{O}_{18}$}

Arecatannin $\mathrm{A}_{1}, 1655$

Procyanidin $\mathrm{C}_{1}, 17893$

Robinetinidol- $(4 \alpha \rightarrow 8)$-catechin- $(6 \rightarrow 4 \alpha)$ robinetinidol, 18858

\section{$\mathrm{C}_{45} \mathrm{H}_{42} \mathrm{O}_{10}$}

Licoagrone, 12757

\section{$\mathrm{C}_{45} \mathrm{H}_{44} \mathrm{O}_{9}$}

Licoagrodin, 12755

\section{$\mathrm{C}_{45} \mathrm{H}_{44} \mathrm{O}_{11}$}

Kuwanon H, 12386

Moracenin C, 14951

\section{$\mathrm{C}_{45} \mathrm{H}_{44} \mathrm{O}_{12}$}

Sanggenon E, 19260

Sanggenon P, 19271

\section{$\mathrm{C}_{45} \mathrm{H}_{44} \mathrm{O}_{16}$}

Epioritin- $(4 \beta \rightarrow 6)$-epioritin- $(4 \beta \rightarrow 6)$-epioritin$4 \alpha$-ol, 6982

Epioritin-( $4 \beta \rightarrow 6)$-oritin-( $4 \alpha \rightarrow 6)$-epioritin- $4 \alpha$-ol, 6986

Oritin-( $4 \beta \rightarrow 6)$-oritin-( $4 \alpha \rightarrow 6)$-epioritin- $4 \alpha$-ol, 16197

\section{$\mathrm{C}_{45} \mathrm{H}_{46} \mathrm{O}_{11}$}

Sanggenol M, 19254

\section{$\mathrm{C}_{45} \mathrm{H}_{47} \mathrm{NO}_{13}$}

10-Deacetyl-10-oxo-7-epitaxol, 4765

\section{$\mathrm{C}_{45} \mathrm{H}_{48} \mathrm{~N}_{2} \mathrm{O}_{19}$}

Hyponine E, 10907

\section{$\mathrm{C}_{45} \mathrm{H}_{48} \mathrm{O}_{13}$}

7-Deacetyl-7-benzoyltaxchinin I, 4733

7-Deacetyltaxayuntin D, 4779

\section{$\mathrm{C}_{45} \mathrm{H}_{48} \mathrm{O}_{21}$}

Smiglaside E, 20024

\section{$\mathrm{C}_{45} \mathrm{H}_{49} \mathrm{NO}_{13}$}

10-Deacetyltaxol, 4783

$\mathrm{C}_{45} \mathrm{H}_{49} \mathrm{~N}_{3} \mathrm{O}_{10}$
Milliamine A, 14859

$\mathrm{C}_{45} \mathrm{H}_{50} \mathrm{O}_{19}$

Mangicrocin, 13480

$\mathrm{C}_{45} \mathrm{H}_{51} \mathrm{NO}_{20}$

Wilfornine A, 22686

$\mathrm{C}_{45} \mathrm{H}_{52} \mathrm{~N}_{2} \mathrm{O}_{10}$

Thalmineline, 21265

$\mathrm{C}_{45} \mathrm{H}_{52} \mathrm{O}_{13}$

Rediocide F, 18566

$\mathrm{C}_{45} \mathrm{H}_{52} \mathrm{O}_{23}$

1,2,2'-Tri- $O$ - $E$-sinapoyl- $\beta$-gentiobiose, 22036

1,2,6'-Tri- $O$ - $E$-sinapoyl- $\beta$-gentiobiose, 22037

\section{$\mathrm{C}_{45} \mathrm{H}_{52} \mathrm{O}_{24}$}

4',5-Dihydroxy-7-methoxyflavonol 3-O-[6-O-

(E)-3,5-dimethoxy-4-hydroxycinnamoyl- $\beta$ - $D$ glucopyranosyl]-( $1 \rightarrow 2)-O$-[ $\alpha$ - $L$-rhamnopyranosyl-(1-6)]- $\beta$ - $D$-glucopyranoside, 5982

\section{$\mathrm{C}_{45} \mathrm{H}_{53} \mathrm{NO}_{14}$}

Cephalomannine, 3402

2-Debenzoyl-2-tigloyltaxol, 4813

7-Epicephalomannine, 6868

Taxol B, 20812

\section{$\mathrm{C}_{45} \mathrm{H}_{55} \mathrm{NO}_{14}$}

$N$-Debenzoyl- $N$-(2-methylbutyryl)taxol, 4807

Taxoline, 20815

\section{$\mathrm{C}_{45} \mathrm{H}_{56} \mathrm{~N}_{8} \mathrm{O}_{8}$}

Microtoenin B, 14848

\section{$\mathrm{C}_{45} \mathrm{H}_{56} \mathrm{O}_{23}$}

Chaohuoside A, 3480

\section{$\mathrm{C}_{45} \mathrm{H}_{56} \mathrm{O}_{25}$}

Tris-[4-( $\beta$ - $D$-glucopyranosyloxy)benzyl]citrate, 22034

$\mathrm{C}_{45} \mathrm{H}_{57} \mathrm{~N}_{3} \mathrm{O}_{9}$

Beauvericin, 2188

\section{$\mathrm{C}_{45} \mathrm{H}_{60} \mathrm{O}_{23}$}

Syringylglycerol- $\beta$-syringaresinol ether-4",4"'-di$O$ - $\beta$ - $D$-glucopyranoside, 20573

\section{$\mathrm{C}_{45} \mathrm{H}_{62} \mathrm{~N}_{8} \mathrm{O}_{9}$}

Psammosilenin B, 18015

\section{$\mathrm{C}_{45} \mathrm{H}_{62} \mathrm{O}_{4}$}

6-Geranylgeranyl 6'-methyl-(9'E)-6,6'-diapocaroten-6,6'-dioate, 8318

6-Geranylgeranyl 6'-methyl-(9'Z)-6,6'-diapocaroten-6,6'-dioate, 8319

\section{$\mathrm{C}_{45} \mathrm{H}_{62} \mathrm{O}_{16}$}

Vernoguinoside, 22409

\section{$\mathrm{C}_{45} \mathrm{H}_{68} \mathrm{O}_{18}$}

Fargoside C, 7725

$\mathrm{C}_{45} \mathrm{H}_{69} \mathrm{~N}_{9} \mathrm{O}_{10} \mathrm{~S}_{2}$

Cherimolacyclopeptide F, 3515

\section{$\mathrm{C}_{45} \mathrm{H}_{69} \mathrm{O}_{13}$}

(22S)-Cholest-5-ene-3 $\beta, 11 \alpha, 16 \beta, 22$-tetrol 16-O$\{2-O$-acetyl-3-O-(3,4,5-trimethoxybenzoyl)- $\alpha$ - $L$-rhamnopyranoside $\}, 3581$

$\mathrm{C}_{45} \mathrm{H}_{70} \mathrm{O}_{14}$

Cynatroside B, 4568

\section{$\mathrm{C}_{45} \mathrm{H}_{70} \mathrm{O}_{16}$}

Malonylsaikosaponin A, 13448

Malonylsaikosaponin D, 13449

\section{$\mathrm{C}_{45} \mathrm{H}_{70} \mathrm{O}_{17}$}

Brownioside, 2658

\section{$\mathrm{C}_{45} \mathrm{H}_{70} \mathrm{O}_{18}$}

Saundersioside C, 19408

\section{$\mathrm{C}_{45} \mathrm{H}_{70} \mathrm{O}_{19}$}

$1 \beta, 2 \alpha$-Dihydroxyspirosta-5,25(27)-dien-3 $\beta$-yl

$O$ - $\alpha$ - $L$-rhamnopyranosyl- $(1 \rightarrow 2)-O$ - $[\beta$ - $D$-glucopyranosyl- $(1 \rightarrow 4)]-\beta$ - $D$-galactopyranoside, 6125

Polygonatoside A, 17643

Pregna-5,16-dien-3 $\beta$-ol-20-one-3 $\beta-O-\alpha-L$ rhamnopyranosyl $(1 \rightarrow 2)$ - $[\alpha-L$-rhamnopyranosyl $(1 \rightarrow 4)-\alpha$ - $L$-rhamnopyranosyl $(1 \rightarrow 4)]-\beta$ - $D$-glucopyranoside-, 17783

\section{$\mathrm{C}_{45} \mathrm{H}_{70} \mathrm{O}_{20}$}

Polygonatoside B, 17644

$3 \beta$ - $[(O-\alpha-L$-Rhamnopyranosyl- $(1 \rightarrow 2)-O-[O-$ $\beta$ - $D$-glucopyranosyl-( $1 \rightarrow 4)-\alpha$ - $L$-rhamnopyranosyl-( $1 \rightarrow 3)]-\beta$ - $D$-glucopyranosyl)oxy] pregna-5,16-dien-20-one, 18719

Spongipregnoloside C, 20232

\section{$\mathrm{C}_{45} \mathrm{H}_{72} \mathrm{O}_{16}$}

Astragaloside I, 1936

Collettiside III, 3925

Desglucomusennin, 5251

Dioscin, 6437

Isoastragaloside I, 11242

Protohypoglaucine A, 17977

\section{$\mathrm{C}_{45} \mathrm{H}_{72} \mathrm{O}_{17}$}

3- $O$-[Bis- $\alpha$ - $L$-rhamnopyranosyl- $(1 \rightarrow 2$ and $1 \rightarrow 4)$ - $\beta$ - $D$-glucopyranosyl]-22R,25R-spirost5-ene-3 $\beta, 20 \alpha$-diol, 2493

\section{$\mathrm{C}_{45} \mathrm{H}_{72} \mathrm{O}_{17}$}

Collettinside IV, 3924

Deltonin, 5040

Gracillin, 8968

Graecunin G, 8970

(24S,25R)-24-Hydroxyspirost-5-en-3 $\beta$-yl $O-\alpha-L$ rhamnopyranosyl-( $1 \rightarrow 2)-O$ - $[\alpha-L$-rhamnopyranosyl-(1 $\rightarrow 3)]-\beta$ - $D$-glucopyranoside, 10726

Isonarthogenin-3-O- $\alpha$ - $L$-rhamnopyranosyl$(1 \rightarrow 2)-O$ - $[\alpha-L$-rhamnopyranosyl- $(1 \rightarrow 4)]-\beta$ $D$-glucopyranoside, 11556

Mubenoside A, 15006

Pennogenin-3- $O-\alpha$ - $L$-rhamnopyranosyl $(1 \rightarrow 2)$ $[\alpha$ - $L$-rhamnopyranosyl $(1 \rightarrow 4)]-\beta$ - $D$-glucopyranoside, 16814 
Polypodoside A, 17663

3 - $O$ - $[\alpha$ - $L$-Rhamnopyranosyl-( $1 \rightarrow 4)-\beta$ - $D$-glucopyranosyl]-26- $O$-( $\beta$ - $D$-glucopyranosyl)(25R)-furosta-5,20-dien-3 $\beta$, 26-diol, 18711

(25S)-Spirost-5-en-3 $\beta$-yl $O-\beta$ - $D$-glucopyranosyl$(1 \rightarrow 4)-O$ - $\alpha$ - $L$-rhamnopyranosyl- $(1 \rightarrow 3)-\beta$ - $D$ glucopyranoside, 20221

(25R)-Spirost-5-en-3 $\beta$-yl- $O-\alpha$ - $L$-rhamnopyranosyl- $(1 \rightarrow 2)-O-[\beta$ - $D$-glucopyranosyl- $(1 \rightarrow 6)]$ $\beta$ - $D$-glucopyranoside, 20222

Trigonella-glucoside A, 21661

Zingiberoside $\mathrm{A}_{3}, 23005$

\section{$\mathbf{C}_{45} \mathrm{H}_{72} \mathbf{O}_{18}$}

CTHD0233276-9, 4300

$26-O-\beta$ - $D$-Glucopyranosyl-3 $\beta, 26$-dihydroxy- $\Delta^{5}$ choleslen-16,22-dioxo-3-O- $\alpha$ - $L$-rhamnopyranosyl-( $(1 \rightarrow 2)-\beta$ - $D$-glucopyranoside, 8610

$3 \beta$-[( $\beta$-D -Glucopyranosyl)oxy]-17 $\alpha$-hydroxy$16 \beta$-[(O- $\beta$ - $D$-xylopyranosyl-( $(1 \rightarrow 2)-2-O$-acetyl- $\alpha$ - $L$-arabinopyranosyl)oxy]cholest-5-en-22one, 8699

(23S,25R)-23-Hydroxyspirost-5-en-3 $\beta$-yl- $O-\alpha$ $L$-rhamnopyranosyl-( $1 \rightarrow 2)$ - $O$-[ $\beta$ - $D$-glucopyranosyl-( $1 \rightarrow 6)]-\beta$ - $D$-glucopyranoside, 10723

(25S)-27-Hydroxyspirost-5-en-3 $\beta$-yl-O- $\alpha$-Lrhamnopyranosyl-( $1 \rightarrow 2)$-O-[ $\beta$-D-glucopyranosyl-(1 $\rightarrow 6)]$ - $\beta$-D-glucopyranoside, 10724

Polygonatoside D, 17646

Trilloside B, 21876

\section{$\mathrm{C}_{45} \mathrm{H}_{72} \mathbf{O}_{19}$}

Agave cantala Substance 1, 724

CTHD0233276-15, 4296

26- $O-\beta$ - $D$-Glucopyranosyl $(25 R, S)$-5 $\alpha$-furostane-12-one-20(22)-en-3 $\beta$,26-diol-3-O- $\beta$ - $D$ glucopyranosyl-( $1 \rightarrow 4)-\beta$ - $D$-galactopyranoside, 8634

Hecogenin 3-O- $\beta$ - $D$-glucopyranosyl-( $(1 \rightarrow 2)$ $\beta$ - $D$-glucopyranosyl-( $1 \rightarrow 4)-\beta$ - $D$-galactopyranoside, 9254

Isonarthogenin 3-O- $\beta$ - $D$-glucopyranosyl- $(1 \rightarrow 2)$ $\beta$ - $D$-glucopyranosyl-( $1 \rightarrow 4)-\beta$ - $D$-galactopyranoside, 11555

Terreside A, 21000

Terrestrosin C, 21015

Terrestrosin E, 21017

\section{$\mathrm{C}_{45} \mathrm{H}_{73} \mathrm{NO}_{15}$}

Solamargine, 20044

$\beta$-Solamarine, 20047

Solanidine-3- $O-\alpha-L$-rhamnopyranosyl-( $(1 \rightarrow 2)$ $\beta$ - $D$-glucopyranosyl-(1 $\rightarrow 4)$ - $\beta$ - $D$-glucopyranoside, 20057

Solanidine 3- $O-\alpha$ - $L$-rhamnopyranosyl $(1 \rightarrow 2)-[\beta$ $D$-glucopyranosyl $(1 \rightarrow 4)]-\beta$ - $D$-glucopyrano- side, 20059

Solanine, 20060

\section{$\mathrm{C}_{45} \mathrm{H}_{73} \mathrm{NO}_{16}$}

$\alpha$-Solamarine, 20046

Solasodine 3-O- $\alpha$ - $L$-rhamnopyranosyl $(1 \rightarrow 2)-O$ [ $\beta$ - $D$-glucopyranosyl $(1 \rightarrow 4)]-\beta$ - $D$-glucopyranoside, 20067

Solasonine, 20069

\section{$\mathrm{C}_{45} \mathrm{H}_{73} \mathrm{NO}_{17}$}

(25) $)$-Solanid-5-en-3 $\beta, 23 \beta$-dihydroxy 3-O- $\beta$ - $D$ glucopyranosyl $(1 \rightarrow 2)-\beta-D$-glucopyranosyl $(1 \rightarrow 4)$ - $\beta$ - $D$-galactopyranoside, 20054

\section{$\mathrm{C}_{45} \mathrm{H}_{74} \mathrm{O}$}

Malloprenol, 13426

$\mathrm{C}_{45} \mathrm{H}_{74} \mathrm{O}_{5}$

Plastoquinone $\mathrm{C}_{4}, 17519$

\section{$\mathbf{C}_{45} \mathbf{H}_{74} \mathbf{O}_{10}$}

(2S)-1,2-Di-O-[(9Z,12Z,15Z)-octadeca-9,12,15trienoyl]-3-O- $\beta-D$-galactopyranosyl glycerol, 6428

Monogalactosyldiglyceride, 14924

\section{$\mathrm{C}_{45} \mathrm{H}_{74} \mathrm{O}_{17}$}

AS-1 B, 1831

Asparanin $\mathrm{B}_{1}, 1869$

Asparanin $\mathrm{B}_{7}, 1875$

3- $O$-[Bis- $\alpha$ - $L$-rhamnopyranosyl-( $1 \rightarrow 2$ and $1 \rightarrow 4)$ - $\beta$ - $D$-glucopyranosyl]-25R-furost-5ene-3 $\beta, 22 \alpha, 26$-triol, 2492

Fenbaqia saponin, 7748

Gurillin H, 9087

Neotigogenin-3- $O-\beta$ - $D$-glucopyranosyl $(1 \rightarrow 4)$ $O$-[ $\alpha$ - $L$-rhamnopyranosyl $(1 \rightarrow 6)]-\beta$ - $D$-galactopyranoside, 15465

Osladin, 16251

Sarsasapogenin 3-O-4 ${ }^{\mathrm{G}}$-rhamnosyl-sophoroside, 19391

Terrestrosin B, 21014

Tubeimoside D, 22075

\section{$\mathrm{C}_{45} \mathrm{H}_{74} \mathrm{O}_{18}$}

Anemarsaponin B, 1173

Anemarsaponin C, 1174

CTHD0233276-21, 4298

Macrostemonoside F, 13331

Neotigogenin-3- $O-\beta$ - $D$-glucopyranosyl $(1 \rightarrow 2)$ $\beta$ - $D$-glucopyranosyl $(1 \rightarrow 4)$ - $\beta$ - $D$-galactopyranoside, 15464

Terrestrosin A, 21013

Tigogenin-3-O- $\beta$ - $D$-glucopyranosyl $(1 \rightarrow 2)-\beta-D$ glucopyranosyl( $(1 \rightarrow 4)-\beta-D$-galactopyranoside, 21384

Torvoside H, 21470

Trigofoenoside A, 21645

Trigoneoside XIIa, 21677
Trigoneoside XIIb, 21678

Tubeimoside E, 22076

Tuberoside A, 22079

Xilingsaponin B, 22796

\section{$\mathrm{C}_{45} \mathrm{H}_{74} \mathbf{O}_{19}$}

Macrostemonoside L, 13334

$(25 R, S)-5 \alpha$-Spirostane- $2 \alpha, 3 \beta$-diol 3-O-[O- $\beta$ - $D$ glucopyranosyl-( $(1 \rightarrow 2)-O-\beta$ - $D$-glucopyranosyl$(1 \rightarrow 4)-\beta$ - $D$-galactopyranoside], 20212

\section{$\mathrm{C}_{45} \mathrm{H}_{74} \mathbf{O}_{20}$}

$26-O-\beta$ - $D$-Glucopyranosyl $(25 R)-5 \alpha$-furostane12-one-3 $\beta, 22 \alpha, 26$-triol-3- $O-\beta$ - $D$-glucopyranosyl- $(1 \rightarrow 2)-\beta$ - $D$-galactopyranoside, 8633

Macrostemonoside G, 13332

Proampeloside $\mathrm{Bf}_{1}, 17864$

\section{$\mathrm{C}_{45} \mathrm{H}_{75} \mathrm{NO}_{9}$}

1,2-Di- $O$-(9Z,12Z,15Z-octadecatrienoyl)-3-O-(6amino-6-deoxy- $\alpha$ - $D$-glucosyl)-glycerol, 6427

\section{$\mathrm{C}_{45} \mathrm{H}_{75} \mathrm{NO}_{17}$}

(25))-Solanidan-3 $\beta, 23 \beta$-dihydroxy $3-O-\beta$ - $D$-glucopyranosyl $(1 \rightarrow 2)-\beta$ - $D$-glucopyranosyl $(1 \rightarrow 4)$ - $\beta$ - $D$-galactopyranoside, 20052

\section{$\mathrm{C}_{45} \mathrm{H}_{76} \mathrm{O}_{5}$}

Plastoquinone $\mathrm{C}_{3}, 17518$

\section{$\mathrm{C}_{45} \mathrm{H}_{76} \mathrm{O}_{13}$}

Dregeoside B, 6599

\section{$\mathrm{C}_{45} \mathrm{H}_{76} \mathrm{O}_{16}$}

Lotoideside C, 13006

\section{$\mathrm{C}_{45} \mathrm{H}_{76} \mathrm{O}_{17}$}

Oleifolioside A, 16067

\section{$\mathrm{C}_{45} \mathrm{H}_{76} \mathrm{O}_{18}$}

Abutiloside F, 40

Asp-IV, 1905

Asp-V, 1906

Trigoneoside IIIa, 21669

Trigoneoside IIIb, 21670

\section{$\mathbf{C}_{45} \mathbf{H}_{76} \mathbf{O}_{19}$}

(25R)-26-O- $\beta$ - $D$-Glucopyranosyl-22-hydroxy$5 \beta$-furostane-3 $\beta$,26-diol 3- $O-\beta$ - $D$-glucopyranosyl-(1 $\rightarrow 2)-O-\beta$ - $D$-galactopyranoside, 8669 (25S)-26- $O-\beta$ - $D$-Glucopyranosyl-22-hydroxy$5 \beta$-furostane-3 $\beta, 26$-diol 3-O- $\beta$ - $D$-glucopyranosyl-(1 $\rightarrow 2)-O-\beta$ - $D$-galactopyranoside, 8670

Trigofoenoside B, 21647

Trigoneoside Xa, 21674

Trigoneoside $\mathrm{Xb}, 21675$

\section{$\mathrm{C}_{45} \mathrm{H}_{76} \mathrm{O}_{20}$}

26- $O-\beta$ - $D$-Glucopyranosyl-(25S)-3 $\beta, 5 \beta, 6 \alpha, 22 \xi$, 26-pentahydroxyl-5 3 -furostane 3-O- $\alpha-L$ rhamnopyranosyl-(1 $\rightarrow 4)-\beta$ - $D$-glucopyranoside, 8710

Macrostemonoside J, 13333 


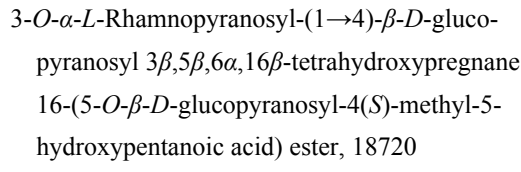

24-Ethylcholesta-5,22-dien-3 $\beta$-ol palmitic acid ester, 7426

\section{$\mathrm{C}_{45} \mathrm{H}_{80} \mathrm{O}_{2}$}

$\beta$-Sitosteryl palmitate, 19998

\section{$\mathbf{C}_{45} \mathrm{H}_{82} \mathbf{O}_{23}$}

1-O-[[ $\alpha-L$-Arabinopyranosyl- $(1 \rightarrow 3)]-\alpha-L-$ rhamnopyranosyl-( $1 \rightarrow 2)-\beta$ - $D$-glucopyranosyl$(1 \rightarrow 3)$ - $\alpha$ - $L$-rhamnopyranosyl-( $1 \rightarrow 6)-\beta$ - $D$-glucopyranosyl] hexadecanol, 1592

\section{$\mathrm{C}_{45} \mathrm{H}_{85} \mathrm{O}_{12} \mathrm{~S}^{-}$}

(2S)-1,2-Distearoyl-3- $O$-(6-sulpho- $\alpha$ - $D$-quinovopyranosyl)-glycerol, 6519

\section{$\mathrm{C}_{45} \mathrm{H}_{86} \mathrm{O}_{6}$}

Trimyristin, 21980

\section{$\mathrm{C}_{45} \mathrm{H}_{91} \mathrm{NO}_{5}$}

Sphingolipid Lipids01-175, 20153

\section{$\mathrm{C}_{46} \mathrm{H}_{36} \mathrm{O}_{31}$}

Putranjivain A, 18228

\section{$\mathrm{C}_{46} \mathrm{H}_{44} \mathrm{O}_{13}$}

Lactucain C, 12443

\section{$\mathrm{C}_{46} \mathrm{H}_{48} \mathrm{~N}_{2} \mathrm{O}_{4}$}

Bisisomahanine, 2479

\section{$\mathrm{C}_{46} \mathrm{H}_{48} \mathrm{~N}_{2} \mathrm{O}_{8}$}

Michellamine B, 14831

\section{$\mathrm{C}_{46} \mathrm{H}_{50} \mathbf{N}_{4} \mathbf{O}_{8}$}

$N 1, N 5, N 10, N 14$-Tetrakis[3-(4-hydroxyphenyl)2-propenoyl]-1,5,10,14-tetraazatetradecane, 21176

\section{$\mathrm{C}_{46} \mathrm{H}_{50} \mathrm{O}_{22}$}

Smiglaside B, 20021

\section{$\mathrm{C}_{46} \mathrm{H}_{54} \mathrm{~N}_{4} \mathrm{O}_{10}$}

Catharine, 3322

\section{$\mathrm{C}_{46} \mathrm{H}_{54} \mathrm{O}_{13}$}

Rediocide C, 18564

Rediocide G, 18567

\section{$\mathrm{C}_{46} \mathrm{H}_{55} \mathrm{NO}_{15}$}

$5 \alpha-O-(3$ '-Dimethylamino-3'-phenylpropionyl) taxinine M, 6315

\section{$\mathrm{C}_{46} \mathrm{H}_{56} \mathrm{~N}_{4} \mathrm{O}_{9}$}

Catharanthamine, 3320

Leurosine, 12734

Vincathicine, 22491

\section{$\mathrm{C}_{46} \mathrm{H}_{56} \mathrm{~N}_{4} \mathrm{O}_{10}$}

Vinamidine, 22486

Vincristine, 22497

\section{$\mathrm{C}_{46} \mathrm{H}_{57} \mathrm{NO}_{14}$}

7-Epitaxuyunnanine A, 7028

Taxol C, 20813
Taxuspine N, 20861

$\mathrm{C}_{46} \mathrm{H}_{58} \mathrm{~N}_{4} \mathrm{O}_{8}$

Isoleurosine, 11489

$\mathrm{C}_{46} \mathrm{H}_{58} \mathrm{~N}_{4} \mathrm{O}_{9}$

Leurosidine, 12733

Vinblastine, 22487

\section{$\mathrm{C}_{46} \mathrm{H}_{58} \mathrm{~N}_{4} \mathrm{O}_{10}$}

Leurocolumbine, 12732

Vincadioline, 22488

$\mathrm{C}_{46} \mathrm{H}_{58} \mathrm{~N}_{6} \mathrm{O}_{14}$

Rubia akane RA-XII, 18990

$\mathrm{C}_{46} \mathrm{H}_{58} \mathrm{O}_{25}$

Isooleoacteoside, 11575

$\mathrm{C}_{46} \mathrm{H}_{64} \mathbf{O}_{15}$

Saundersioside E, 19410

$\mathrm{C}_{46} \mathrm{H}_{64} \mathrm{O}_{26}$

Tangshenoside IV, 20675

$\mathrm{C}_{46} \mathrm{H}_{65} \mathbf{N}_{15} \mathrm{O}_{12} \mathrm{~S}_{2}$

Vasopressin, 22353

$\mathrm{C}_{46} \mathrm{H}_{66} \mathrm{O}_{15}$

Saundersioside G, 19412

$\mathrm{C}_{46} \mathrm{H}_{68} \mathrm{O}_{4}$

Formosadimer B, 7887

$\mathrm{C}_{46} \mathrm{H}_{69} \mathrm{NO}_{11}$

Saponaceol A, 19324

$\mathrm{C}_{46} \mathrm{H}_{69} \mathrm{NO}_{12}$

Saponaceol B, 19325

\section{$\mathrm{C}_{46} \mathrm{H}_{70} \mathrm{O}_{17}$}

Eupteleasaponin V, 7626

\section{$\mathrm{C}_{46} \mathrm{H}_{70} \mathbf{O}_{18}$}

Cynascyroside E, 4567

\section{$\mathrm{C}_{46} \mathrm{H}_{70} \mathrm{O}_{19}$}

Spinacoside C, 20165

\section{$\mathbf{C}_{46} \mathbf{H}_{72} \mathbf{O}_{\mathbf{1 5}}$}

3",6"-O,O-diacetylsaikosaponin $\mathrm{b}_{2}, 5346$

$1 \alpha, 3 \beta$-Hydroxyimberbic acid-23- $\alpha-L$-[3,4-diacetyl-rhamnopyranosyl]-29-O- $\alpha$-rhamnopyranoside, 10228

\section{$\mathrm{C}_{46} \mathrm{H}_{72} \mathrm{O}_{17}$}

3-O- $\beta$ - $D$-Apiofuranosyl-( $1 \rightarrow 4)$ - $[\alpha-L$-arabinopyranosyl-(1 $\rightarrow 2)] \beta$ - $D$-glucuronopyranosyl oleanolic acid, 1503

$2 \alpha, 3 \beta$-Dihydroxyurs-12,20(30)-dien-28-oic acid $3-O-\{O-\alpha-L$-arabinopyranosyl- $(1 \rightarrow 2)-O-[\beta-D-$

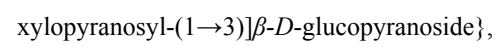
6172

Nudicaucin A, 15858

Yemuoside $\mathrm{YM}_{11}, 22890$

$\mathrm{C}_{46} \mathrm{H}_{72} \mathrm{O}_{19}$

Abutiloside O, 48

$\mathrm{C}_{46} \mathrm{H}_{72} \mathrm{O}_{20}$

Withanoside VIII, 22711

$\mathrm{C}_{46} \mathrm{H}_{73} \mathrm{O}_{20} \mathrm{~S}^{-}$
3-O-[6-O-Sulfonyl- $\beta$ - $D$-glucopyranosyl- $(1 \rightarrow 3)]$ $[\alpha-L$-arabinopyranosyl-( $(1 \rightarrow 2)]-\alpha-L$-arabinopyranosyl-pseudojujubogenin, 20471

\section{$\mathrm{C}_{46} \mathrm{H}_{74} \mathrm{O}_{2}$}

Stigmasteryl-3-O-scleropyrate, 20377

$\mathrm{C}_{46} \mathrm{H}_{74} \mathrm{O}_{10}$

Longiside A, 12978

$\mathrm{C}_{46} \mathrm{H}_{74} \mathrm{O}_{14}$

Taibaienoside IV, 20616

\section{$\mathrm{C}_{46} \mathrm{H}_{74} \mathrm{O}_{15}$}

Huzhangoside A, 9692

Oleanolic acid 3-O- $\beta$ - $D$-ribopyranosyl $(1 \rightarrow 3)-\alpha$ $L$-rhamnopyranosyl $(1 \rightarrow 2)-\alpha$ - $L$-arabinopyranoside, 16060

Oleanolic acid 3-O- $\beta$ - $D$-xylopyranosyl $(1 \rightarrow 3)-\alpha-$ $L$-rhamnopyranosyl $(1 \rightarrow 2)$ - $\alpha$ - $L$-arabinopyranoside, 16063

$3 \beta-[(O-\beta$ - $D$-Ribopyranosyl-( $(1 \rightarrow 3)-O-\alpha-L-$ rhamnopyranosyl-( $(1 \rightarrow 2)-\alpha$ - $L$-arabinopyranosyl)oxy]olean-12-en-28-oic acid, 18835

\section{$\mathrm{C}_{46} \mathrm{H}_{74} \mathrm{O}_{16}$}

Araloside D, 1611

Pometia ridleyi saponin 6, 17692

Prosapogenin $\mathrm{CP}_{5}, 17945$

Prosapogenin $\mathrm{CP}_{6}, 17946$

Sapindoside B, 19317

Scabioside D, 19444

\section{$\mathrm{C}_{46} \mathrm{H}_{74} \mathrm{O}_{17}$}

Bacopasaponin C, 2082

Brisbagenin 1-O-[O- $\alpha-L$-rhamnopyranosyl$(1 \rightarrow 2)-O$-[ $\alpha-L$-rhamnopyranosyl-( $(1 \rightarrow 3)]-4-$ $O$-acetyl- $\alpha$ - $L$-arabinopyranoside], 2614

3 - $O$-[ $\beta$ - $D$-Glucopyranosyl- $(1 \rightarrow 3)][\alpha-L$-arabinopyranosyl-( $(1 \rightarrow 2)]-\alpha-L$-arabinopyranosylpseudojujubogenin, 8604

Isolineolon 3-O- $\beta$ - $D$-oleandropyranosyl- $(1 \rightarrow 4)$ $\beta$-D-digitoxopyranosyl-( $(1 \rightarrow 4)-\beta$ - $D$-digitoxopyranosyl-( $1 \rightarrow 4)-\beta$ - $D$-digitoxopyranoside, 11502

Jujubogenin 3-O- $\alpha$ - $L$-arabinofuranosyl-( $(1 \rightarrow 2)$ $[\beta$ - $D$-glucopyranosyl $(1 \rightarrow 3)]-\alpha-L$-arabinopyranoside, 11908

Lineolon-3- $O-\beta$ - $D$-oleandropyranosyl-( $(1 \rightarrow 4)$ $\beta$ - $D$-digitoxopyranosyl-( $(1 \rightarrow 4)-\beta$ - $D$-digitoxopyranosyl-( $1 \rightarrow 4)-\beta$ - $D$-digitoxopyranoside, 12885

\section{$\mathrm{C}_{46} \mathrm{H}_{74} \mathrm{O}_{18}$}

Deacylmetaplexigenin 3-O- $\beta$ - $D$-oleandropyranosyl-( $1 \rightarrow 4)-\beta$ - $D$-digitoxopyranosyl-( $1 \rightarrow 4)$ $\beta$-D-digitoxopyranosyl-( $(1 \rightarrow 4)-\beta$ - $D$-digitoxopyranoside, 4797

Fargoside B, 7724

$15-\beta$-Hydroxylineolon 3-O- $\beta$ - $D$-oleandropyra- 


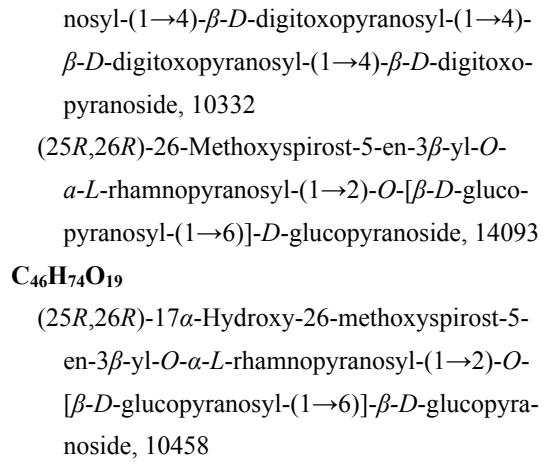

\section{$\mathrm{C}_{46} \mathrm{H}_{74} \mathrm{O}_{19}$}

$(25 R, 26 R)-17 \alpha$-Hydroxy-26-methoxyspirost-5en-3 $\beta$-yl- $O-\alpha-L$-rhamnopyranosyl- $(1 \rightarrow 2)-O$ [ $\beta$ - $D$-glucopyranosyl-( $1 \rightarrow 6)]$ - $\beta$ - $D$-glucopyranoside, 10458

\section{$\mathrm{C}_{46} \mathrm{H}_{74} \mathrm{O}_{20} \mathrm{~S}$}

Bacopaside I, 2087

\section{$\mathrm{C}_{46} \mathrm{H}_{75} \mathrm{NO}_{17}$}

Abutiloside H, 41

\section{$\mathrm{C}_{46} \mathrm{H}_{76} \mathrm{O}_{2}$}

$\beta$-Sitosteryl-3- $O$-scleropyrate, 19999

\section{$\mathrm{C}_{46} \mathrm{H}_{76} \mathrm{O}_{16}$ \\ Gylongiposide I, 9098}

\section{$\mathrm{C}_{46} \mathrm{H}_{76} \mathrm{O}_{17}$}

Astrasieversianin XV, 1949

CTHD0233276-2, 4297

Gypenoside XXVI, 9140

\section{$\mathrm{C}_{46} \mathrm{H}_{76} \mathrm{O}_{18}$}

Marsdekoiside C, 13575

Trigofoenoside $\mathrm{A}_{1}, 21646$

\section{$\mathrm{C}_{46} \mathrm{H}_{76} \mathrm{O}_{19}$}

Polyfuroside $\mathrm{PO}_{8}, 17627$

\section{$\mathrm{C}_{46} \mathrm{H}_{76} \mathrm{O}_{20}$}

Polyfuroside $\mathrm{PO}_{6}, 17625$

Wattoside C, 22650

\section{$\mathrm{C}_{46} \mathrm{H}_{77} \mathrm{NO}_{17}$}

Abutiloside B, 39

\section{$\mathrm{C}_{46} \mathrm{H}_{78} \mathrm{O}_{2}$}

9(11),12-Ursadien-3 $\beta$-ol 3-O-palmitate, 22261

\section{$\mathbf{C}_{46} \mathrm{H}_{78} \mathbf{O}_{4}$}

$5 \alpha, 8 \alpha$-Epidioxyergosta-6,22-dien- $3 \beta$-yl stearate, 6901

\section{$\mathrm{C}_{46} \mathrm{H}_{78} \mathrm{O}_{18}$}

Myrioside A, 15198

Oleifolioside B, 16068

$3 \beta, 12 \beta, 23 S, 24 R$-Tetrahydroxy-20S,25-epoxy-

dammarane 3- $O-[\beta$ - $D$-xylopyranosyl $(1 \rightarrow 2)]$

[ $\beta$ - $D$-xylopyranosyl-( $1 \rightarrow 6)]$ - $\beta$ - $D$-glucopyranoside, 21104

\section{$\mathrm{C}_{46} \mathrm{H}_{78} \mathbf{O}_{19}$}

Anemarsaponin E, 1175

Trigofoenoside $\mathrm{B}_{1}, 21648$

\section{$\mathrm{C}_{46} \mathrm{H}_{80} \mathrm{O}_{2}$}

$\alpha$-Amyrin palmitate, 1121

$\beta$-Amyrin palmitate, 1122

Lupeol palmitate, 13099

Taraxasteryl palmitate, 20708
$\mathrm{C}_{46} \mathrm{H}_{80} \mathrm{O}_{3}$

Arnidiol 3-O-palmitate, 1752

Betulin 3-O-palmitate, 2335

Erythrodiol 3-O-palmitate, 7339

16 $\beta$-Hydroxylupeol 3- $O$-palmitate, 10348

Maniladiol 3-O-palmitate, 13499

12-Ursene-3 $\beta, 11 \alpha$-diol 3- $O$-palmitate, 22267

Uvaol 3-O-palmitate, 22289

$\mathrm{C}_{46} \mathrm{H}_{80} \mathrm{O}_{6}$

34-O-Acetyltetratriacontanylferulate, 523

$\mathrm{C}_{46} \mathrm{H}_{90} \mathrm{O}_{2}$

Myricyl hypogaeate, 15190

$\mathrm{C}_{46} \mathrm{H}_{92} \mathrm{O}_{2}$

Myricyl palmitate, 15191

$\mathrm{C}_{47} \mathrm{H}_{34} \mathrm{O}_{32}$

Elaeocarpusin, 6730

Helioscopin A, 9307

$\mathrm{C}_{47} \mathrm{H}_{36} \mathrm{O}_{32}$

Helioscopin B, 9308

\section{$\mathrm{C}_{47} \mathrm{H}_{44} \mathrm{O}_{22}$}

Agastachin, 708

$\mathrm{C}_{47} \mathrm{H}_{48} \mathrm{O}_{19}$

Aloeresin I, 978

$\mathrm{C}_{47} \mathrm{H}_{50} \mathrm{~N}_{2} \mathrm{O}_{18}$

Hyponine D, 10906

$\mathrm{C}_{47} \mathrm{H}_{50} \mathrm{O}_{14}$

7-Deacetyl-7-benzoyltaxayuntin C, 4732

Taxayuntin D, 20762

\section{$\mathrm{C}_{47} \mathrm{H}_{50} \mathrm{O}_{22}$}

Smiglaside D, 20023

\section{$\mathrm{C}_{47} \mathrm{H}_{51} \mathrm{NO}_{14}$}

7-Epitaxol, 7027

Taxol, 20811

\section{$\mathrm{C}_{47} \mathrm{H}_{51} \mathrm{NO}_{17}$}

Euophelline, 7536

\section{$\mathrm{C}_{47} \mathrm{H}_{52} \mathrm{O}_{23}$}

Senburiside III, 19700

\section{$\mathrm{C}_{47} \mathrm{H}_{53} \mathrm{NO}_{14}$}

9-Deoxo-9 $\alpha$-hydroxytaxol, 5145

Yunnanxamine, 22950

\section{$\mathrm{C}_{47} \mathrm{H}_{54} \mathrm{O}_{25}$}

Kaempferol-3-O- $\{[\beta-D$-xylopyranosyl $(1 \rightarrow 3)-\alpha-$ $L$-rhamnopyranosyl $(1 \rightarrow 6)][\alpha-L$-rhamnopyra$\operatorname{nosyl}(1 \rightarrow 2)]\}$ - $\beta$ - $D$-3-trans- $p$-coumaroylgalactopyranoside, 12101

Kaempferol-3- $O$ - $\{[\beta-D$-xylopyranosyl $(1 \rightarrow 3)-\alpha$ $L$-rhamnopyranosyl $(1 \rightarrow 6)][\alpha-L$-rhamnopyra$\operatorname{nosyl}(1 \rightarrow 2)]\}$ - $\beta$ - $D$-4-trans- $p$-coumaroylgalactopyranoside, 12102

\section{$\mathrm{C}_{47} \mathrm{H}_{57} \mathrm{NO}_{15}$}

10-( $\beta$-Hydroxybutyryl)-10-deacetylcophalomannine, 9866

$\mathrm{C}_{47} \mathrm{H}_{59} \mathrm{NO}_{14}$
N-Methyltaxol C, 14739

Taxuspinanane A, 20841

$\mathrm{C}_{47} \mathrm{H}_{59} \mathrm{NO}_{17}$

7-( $\beta$-xylosyl)-10-deacetyltaxol D, 22849

$\mathrm{C}_{47} \mathrm{H}_{66} \mathrm{~N}_{8} \mathrm{O}_{9}$

Microtoenin A, 14847

$\mathrm{C}_{47} \mathrm{H}_{66} \mathrm{O}_{15}$

Saundersioside F, 19411

$\mathrm{C}_{\mathbf{4 7}} \mathrm{H}_{68} \mathrm{O}_{\mathbf{1 5}}$

OSW-1, 16265

Saundersioside H, 19413

\section{$\mathrm{C}_{47} \mathrm{H}_{68} \mathrm{O}_{21}$}

Basellasaponin B, 2158

\section{$\mathrm{C}_{47} \mathrm{H}_{68} \mathrm{O}_{22}$}

Basellasaponin C, 2159

Basellasaponin D, 2160

\section{$\mathrm{C}_{47} \mathrm{H}_{70} \mathrm{O}_{20}$}

Betavulgaroside I, 2323

Bidentatoside I, 2363

\section{$\mathrm{C}_{47} \mathrm{H}_{70} \mathrm{O}_{21}$}

Basellasaponin A, 2157

$\mathrm{C}_{47} \mathrm{H}_{70} \mathrm{O}_{23}$

Deoxytrillenoside A, 5217

\section{$\mathrm{C}_{47} \mathrm{H}_{70} \mathrm{O}_{24}$}

Trillenoside A, 21873

\section{$\mathrm{C}_{47} \mathrm{H}_{72} \mathrm{O}_{16}$}

Inflasaponin I, 11038

Methyl- $n$-butyl-uralsaponin A esters, 14185

\section{$\mathrm{C}_{47} \mathrm{H}_{72} \mathrm{O}_{17}$}

Kudinoside D, 12302

\section{$\mathrm{C}_{47} \mathrm{H}_{72} \mathrm{O}_{18}$}

Acanjaposide B, 65

Eupteleasaponin VI, 7628

Methyl oleanate-(3)-[ $\alpha$ - $L$-arabinofuranosyl$(1 \rightarrow 4)]$-[( $\beta$ - $D$-galactopyranosyl- $(1 \rightarrow 2))$-methyl-( $\beta$ - $D$-glucopyranoside) uronate], 14633

\section{$\mathrm{C}_{47} \mathrm{H}_{72} \mathbf{O}_{19}$}

Acanjaposide A, 64

3-O- $\beta$ - $D$-Galactopyranosyl-( $1 \rightarrow 2)$-[ $\beta$ - $D$-xylopyranosyl $(1 \rightarrow 3)]-\beta$ - $D$-glucuronopyranosylgypsogenin, 8074

Oldhamianoside, 16026

\section{$\mathrm{C}_{47} \mathrm{H}_{73} \mathrm{NO}_{10}$}

Lipohypaconitine, 12899

\section{$\mathbf{C}_{47} \mathrm{H}_{74} \mathbf{O}_{17}$}

Acetyl astragaloside I, 326

Ardisimamilloside $\mathrm{H}, 1642$

$3 \beta$-[(O- $\beta$ - $D$-Glucuronopyranosyl-( $(1 \rightarrow 3)-O-[\alpha-L$ rhamnopyranosyl-( $(1 \rightarrow 2)]-\alpha$ - $L$-arabinopyranosyl)oxy]olean-12-en-28-oic acid, 8766

Periandradulcin B, 16919

3-O- $\alpha$ - $L$-Rhamnopyranosyl-( $1 \rightarrow 4)$ - $[\alpha-L$-arabinopyranosyl-(1 $\rightarrow 2)-] \beta$ - $D$-glucuronopyranosyl 
oleanolic acid, 18687

Trojanoside I, 22046

\section{$\mathrm{C}_{47} \mathrm{H}_{74} \mathrm{O}_{18}$}

3- $O$ - $[\beta$ - $D$-Apiofuranosyl- $(1 \rightarrow 4)-\beta$ - $D$-glucuronopyranosyl]oleanolic acid $28-O-\beta$ - $D$-glucopyranosyl ester, 1515

Aquilegioside I, 1550

Araloside A, 1608

Chikusetsu saponin Ib, 3521

Chikusetsusaponin IV, 3523

Kudinoside A, 12299

Kudinoside F, 12304

Momordin IIc, 14910

Purpurea glycoside A, 18216

Purpurea glycoside B, 18217

Saikosaponin $\mathrm{S}_{1}, 19161$

Stipuleanoside $\mathrm{R}_{1}, 20383$

Symplocos glomerata saponin 1, 20529

Symplocos glomerata saponin 5, 20533

\section{$\mathrm{C}_{47} \mathrm{H}_{74} \mathrm{O}_{19}$}

Acanjaposide C, 66

Aquilegioside A, 1542

Aquilegioside J, 1551

Hydrocotyloside I, 9710

Kudinoside B, 12300

Myrioside D, 15201

Norarjunolic acid-28-O- $\alpha$ - $L$-rhammnosyl $(1 \rightarrow 4)$ $\beta$-D-glucosyl $(1 \rightarrow 6)$ - $\beta$ - $D$-glucopyranoside, 15712

$2 \alpha, 3 \beta, 23$-Trihydroxy-30-norolean-12-en-28-oic acid $O-\alpha$ - $L$-rhamnopyranosyl- $(1 \rightarrow 4)-O-\beta-D$ glucopyranosyl-( $1 \rightarrow 6)-\beta$ - $D$-glucopyranosyl ester, 21814

Yunganoside $\mathrm{B}_{1}, 22941$

\section{$\mathrm{C}_{47} \mathrm{H}_{74} \mathrm{O}_{20}$}

$3 \beta$-O-( $\beta$-Glucopyranosyl- $(1 \rightarrow 3)$ - $\beta$-xylopyranosyl)-16 $\alpha$-hydroxyolean-12-ene-28,30-dioic acid 28-O-( $\beta$-galactopyranosyl) ester, 8749

\section{$\mathrm{C}_{47} \mathrm{H}_{76} \mathrm{O}_{16}$}

3-O- $\alpha$ - $L$-Glucopyranosyl-( $1 \rightarrow 3)$ - $\alpha$ - $L$-rhamnopyranosyl-( $1 \rightarrow 2)-\alpha-L$-arabinopyranosyloleanclic acid, 8719

3-O- $\beta$ - $D$-Glucopyranosyl-( $1 \rightarrow 3)-\alpha$ - $L$-rhamnopyranosyl-( $1 \rightarrow 2)-\beta$ - $D$-xylopyranosyl oleanolic acid, 8726

Obtusilobinin, 15907

Oleanolic acid-3-O- $\alpha-L$-rhamnopyranosyl$(1 \rightarrow 2)$-[ $\beta$ - $D$-glucopyranosyl- $(1 \rightarrow 4)]-\alpha-L$ arabinopyranoside, 16059

Patrinoside $\mathrm{C}_{1}, 16722$

Raddeanin A, 18516

Raddeanin E, 18520

$3 \beta-D-O-(\alpha-L$-Rhamnopyranosyl- $(1 \rightarrow 2)-\alpha-L-$ arabinopyranosyl)-lup-20(29)-ene-28- $O-\beta$ -

$D$-glucopyranosyl ester, 18691

3-O-[ $\alpha$ - $L$-Rhamnopyranosyl $(1 \rightarrow 2)]-[\beta$ - $D$-glucopyranosyl $(1 \rightarrow 3)]-\alpha$ - $L$-arabinopyranosyl

oleano-lic acid, 18709

3-O-[ $\alpha$ - $L$-Rhamnopyranosyl $(1 \rightarrow 2)]-[\beta$ - $D$-glucopyranosyl $(1 \rightarrow 3)]-\alpha$ - $L$-arabinopyranosyl ursolic acid, 18710

Rotundifolioside I, 18952

Rotundioside G, 18957

\section{$\mathrm{C}_{47} \mathrm{H}_{76} \mathrm{O}_{17}$}

3-O- $\alpha-L$-Arabinopyranosyl oleanolic acid 28-O$\beta$ - $D$-glucopyranosyl-( $1 \rightarrow 6)-\beta$ - $D$-glucopyranoside, 1577

Astragaloside VIII, 1943

Cussosaponin A, 4429

Cussosaponin D, 4432

$3 \beta$ - $O-\beta$ - $D$-Galactopyranosyl- $(1 \rightarrow 3)-\alpha-L$-rhamnopyranosyl- $(1 \rightarrow 2)$ - $\alpha$ - $L$-arabinopyranosyl-23-hydroxyolean-12-en-28-oic acid, 8067

$3 \beta$ - $O$ - $\beta$ - $D$-Glucopyranosyl- $(1 \rightarrow 4)-\alpha-L$-arabinopyranosyl hederagenin 28- $O$ - $\alpha$ - $L$-rhamnopyranosyl ester, 8606

$3 \beta$-[ $(O-\beta$ - $D$-Glucopyranosyl- $(1 \rightarrow 2)-O-[\beta-D$ glucopyranosyl-( $1 \rightarrow 3)]-\alpha$ - $L$-arabinopyranosyl) oxy] olean-12-en-28-oic acid, 8640

Hederagenin-3-O- $\alpha$ - $L$-rhamnopyranosyl- $(1 \rightarrow 2)$ $\alpha$ - $L$-arabinopyranosyl-28- $O$ - $\beta$ - $D$-glucopyranoside, 9270

Inermiside I, 11035

Isolineolon 3-O- $\beta$ - $D$-oleandropyranosyl-( $(\rightarrow 4)$ $\beta$ - $D$-digitoxopyranosyl- $(1 \rightarrow 4)-\beta$ - $D$-digitoxopyranosyl-( $1 \rightarrow 4)-\beta$ - $D$-cymaropyranoside, 11501

Kalopanax saponin H, 12119

Latifoloside A, 12547

Latifoloside B, 12548

Latifoloside D, 12550

Lineolon-3- $O-\beta$ - $D$-oleandropyranosyl-( $1 \rightarrow 4)-\beta$ $D$-digitoxopyranosyl-( $1 \rightarrow 4)-\beta$ - $D$-digitoxopyranosyl-( $1 \rightarrow 4)-\beta$ - $D$-cymaropyranoside, 12884

Macranthoside A, 13302

Nudicaucin B, 15859

Oleanolic acid 3-O- $\beta$ - $D$-xylopyranosyl $(1 \rightarrow 6)-\beta$ $D$-glucopyranosyl $(1 \rightarrow 6)$ - $\beta$ - $D$-glucopyranoside, 16062

Pometia ridleyi saponin 2, 17689

Pometia ridleyi saponin 7, 17693

Randiasaponin IV, 18538

Randiasaponin V, 18539

Rotundifolioside A, 18944

Rotundifolioside D, 18947

Rotundifolioside E, 18948
Rotundifolioside H, 18951

Saikosaponin K, 19150

Saponin $\mathrm{E}_{7}, 19344$

Tomentoside A, 21441

Tomentoside B, 21442

2 "-O- $\beta$-D-Xylopyranosylsaikosaponin $\mathrm{b}_{2}, 22840$

Zizyphussaponin I, 23016

Zizyphussaponin II, 23017

\section{$\mathrm{C}_{47} \mathrm{H}_{76} \mathrm{O}_{18}$}

Akebia saponin D, 818

Bacoside $\mathrm{A}_{3}, 2094$

Cyclaminorin, 4458

Durupcoside C, 6641

$3 \beta$ - $O$ - $[\beta$ - $D$-Glucopyranosyl- $(1 \rightarrow 3)]$ - $[\beta$ - $D$-glucopyranosyl-( $(1 \rightarrow 2)]-\alpha-L$-arabinopyranosyl echinocystic acid, 8638

Hederagenin-3- $O-\alpha-L$-arabinopyranosyl-28- $O-\beta$ $D$-glucopyranosyl $(1 \rightarrow 6)$ - $\beta$ - $D$-glucopyranoside, 9262

$15-\beta$-Hydroxyisolineolon 3- $O-\beta-D$-oleandropyranosyl- $(1 \rightarrow 4)-\beta$ - $D$-digitoxopyranosyl- $(1 \rightarrow 4)-\beta$ $D$-digitoxopyranosyl-( $1 \rightarrow 4)-\beta$ - $D$-cymaropyranoside, 10249

15- $\beta$-Hydroxylineolon 3- $O-\beta$ - $D$-oleandropyranosyl-( $1 \rightarrow 4)-\beta$ - $D$-digitoxopyranosyl-( $(1 \rightarrow 4)$ $\beta$-D-digitoxopyranosyl-( $1 \rightarrow 4)-\beta$ - $D$-cymaropyranoside, 10331

Ilekudinoside E, 10975

Ilexside II, 10985

Ixerissaponin A, 11799

Pulsatiloside A, 18195

Randiasaponin III, 18537

Rotundifolioside G, 18950

\section{$\mathrm{C}_{47} \mathrm{H}_{76} \mathrm{O}_{19}$}

$20 S, 22 R, 23 S, 24 R-16 \beta, 23 ; 22,25$-Diepoxycycloartane-3 $\beta, 23,24$-triol 3-O- $\beta$ - $D$-glucopyranosyl-( $1 \rightarrow 2)-\beta$ - $D$-glucopyranosyl- $(1 \rightarrow 2)-\beta$ - $D$-xylopyranoside, 5489

Saikosaponin $\mathrm{V}_{1}, 19165$

Scorzoneroside B, 19564

\section{$\mathrm{C}_{47} \mathrm{H}_{76} \mathrm{O}_{20}$}

Platycoside F, 17540

\section{$\mathrm{C}_{47} \mathbf{H}_{78} \mathbf{O}_{17}$}

3 $\beta$,23-Dihydroxy-lup-20(29)-ene-28- $O-\alpha-L$ rhamnopyranosyl-( $1 \rightarrow 4)-\beta$ - $D$-glucopyranos-

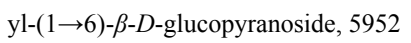

Rotundifolioside B, 18945

\section{$\mathrm{C}_{47} \mathrm{H}_{78} \mathrm{O}_{18}$}

Asernestioside A, 1851

Astrasieversianin XIV, 1948

Gypenoside LII, 9157

\section{$\mathrm{C}_{47} \mathrm{H}_{78} \mathrm{O}_{19}$}

Astragaloside V, 1940 
Astragaloside VI, 1941

Astragaloside VII, 1942

Trojanoside K, 22048

\section{$\mathrm{C}_{47} \mathrm{H}_{78} \mathrm{O}_{20}$}

Volubiloside B, 22612

\section{$\mathrm{C}_{47} \mathrm{H}_{80} \mathrm{O}_{17}$}

Chikusetsusaponin III, 3522

$3 \beta$ - $O$ - $\beta$ - $D$-Glucopyranosyl-20- $O$ - $[\alpha-L$-arabinopyranosyl $(1 \rightarrow 2)-\beta$ - $D$-glucopyranosyl] $3 \beta, 12 \beta$,

20(S)-trihydroxydammar-24-ene, 8605

Gypenoside IX, 9130

Gypenoside XLV, 9154

Gypenoside LV, 9160

Lotoideside B, 13005

Notoginsenoside Fe, 15824

\section{$\mathrm{C}_{47} \mathrm{H}_{80} \mathrm{O}_{18}$}

Cyclofoetoside B, 4492

Gypenoside XLIV, 9153

Gypenoside XLVI, 9155

Gypenoside LVII, 9162

Gypenoside LXIV, 9168

Notoginsenoside $\mathrm{R}_{1}, 15836$

\section{$\mathrm{C}_{47} \mathrm{H}_{80} \mathrm{O}_{19}$}

Notoginsenoside H, 15826

$3 \beta, 12 \beta, 23 S, 24 R$-Tetrahydroxy-20S,25-epoxy-

dammarane 3-O-[ $\beta$ - $D$-glucopyranosyl $(1 \rightarrow 2)]$

$[\beta$ - $D$-xylopyranosyl- $(1 \rightarrow 6)]-\beta$ - $D$-glucopyranoside, 21102

Vina-ginsenoside $\mathrm{R}_{6}, 22485$

Yesanchinoside C, 22895

\section{$\mathrm{C}_{47} \mathrm{H}_{88} \mathrm{O}_{15}$}

1 -O- $\beta$ - $D$-Galactosyl $(6 \rightarrow 1)-\alpha-D$-galactosyl-2,3$O$-dihexadecanoyl-glycerol, 8077

\section{$\mathrm{C}_{48} \mathrm{H}_{28} \mathrm{O}_{30}$}

Punicacortein C, 18202

Punicalagin, 18204

Terchebulin, 20970

\section{$\mathrm{C}_{48} \mathrm{H}_{30} \mathrm{O}_{30}$}

Cornusiin B, 4064

Terflavin A, 20974

\section{$\mathrm{C}_{48} \mathrm{H}_{32} \mathrm{O}_{31}$}

Agrimonic acid A, 757

Agrimonic acid B, 758

Glansrin A, 8504

Rugosin C, 19056

Sanguiin $\mathrm{H}_{2}, 19274$

\section{$\mathrm{C}_{48} \mathrm{H}_{32} \mathrm{O}_{32}$}

Mallotusinic acid, 13438

\section{$\mathrm{C}_{48} \mathrm{H}_{34} \mathrm{O}_{31}$}

Rugosin A, 19054

\section{$\mathrm{C}_{48} \mathrm{H}_{34} \mathrm{O}_{33}$}

Repandusinic acid B, 18625

$\mathrm{C}_{48} \mathrm{H}_{42} \mathrm{O}_{14}$
Cotyleloside A, 4131

Cotyleloside B, 4132

\section{$\mathrm{C}_{48} \mathrm{H}_{48} \mathrm{O}_{9}$}

3,12,21-Trihydroxy-1,10,19-tri(4-hydroxyphenyl)-5,14,23-trimethoxy[3.3.3]metacyclophane, 21852

\section{$\mathrm{C}_{48} \mathrm{H}_{48} \mathrm{O}_{25}$}

Amentoflavone-7,4',4"'-tri- $O-\beta$ - $D$-glucopyranoside, 1033

\section{$\mathrm{C}_{48} \mathrm{H}_{51} \mathrm{NO}_{18}$}

Euoverrine A, 7537

$\mathrm{C}_{48} \mathrm{H}_{52} \mathrm{O}_{23}$

Smiglaside A, 20020

$\mathrm{C}_{48} \mathrm{H}_{53} \mathrm{NO}_{14}$

$N$-Methylpaclitaxel, 14651

\section{$\mathrm{C}_{48} \mathrm{H}_{56} \mathrm{O}_{26}$}

Kaempferol-3-O- $\{[\beta-D$-xylopyranosyl $(1 \rightarrow 3)-\alpha-$ $L$-rhamnopyranosyl $(1 \rightarrow 6)][\alpha$ - $L$-rhamnopyra$\operatorname{nosyl}(1 \rightarrow 2)]\}-\beta$ - $D$-3-trans-feruloylgalactopyranoside, 12103

Kaempferol-3-O- $\{[\beta-D$-xylopyranosyl $(1 \rightarrow 3)-\alpha$ $L$-rhamnopyranosyl $(1 \rightarrow 6)][\alpha-L$-rhamnopyra$\operatorname{nosyl}(1 \rightarrow 2)]\}-\beta$ - $D$-4-trans-feruloylgalactopyranoside, 12104

\section{$\mathrm{C}_{48} \mathrm{H}_{56} \mathrm{O}_{29}$}

Pisumflavonoside II, 17494

\section{$\mathrm{C}_{48} \mathrm{H}_{57} \mathrm{O}_{28}{ }^{+}$}

Pigment 26, 17359

$\mathrm{C}_{48} \mathrm{H}_{58} \mathrm{~N}_{6} \mathrm{O}_{15}$

Rubia akane RA-XIV, 18992

\section{$\mathrm{C}_{48} \mathrm{H}_{60} \mathrm{~N}_{6} \mathrm{O}_{15}$}

Rubia akane RA-XV, 18993

\section{$\mathrm{C}_{48} \mathrm{H}_{60} \mathrm{~N}_{6} \mathrm{O}_{16}$}

Rubia akane RA-XIII, 18991

Rubia akane RA-XVI, 18994

\section{$\mathrm{C}_{48} \mathrm{H}_{60} \mathrm{O}_{9}$}

Hibicusin, 9535

\section{$\mathrm{C}_{48} \mathrm{H}_{60} \mathrm{O}_{10}$}

Myriceric acid C, 15169

$\mathrm{C}_{48} \mathrm{H}_{64} \mathrm{O}_{16}$

Maoecrystal M, 13534

$\mathrm{C}_{48} \mathrm{H}_{69} \mathrm{NO}_{12}$

Saponaceol C, 19326

$\mathrm{C}_{48} \mathrm{H}_{70} \mathrm{O}_{5}$

Formosadimer C, 7888

$\mathrm{C}_{48} \mathrm{H}_{70} \mathrm{O}_{17}$

$3 \beta$-[( $\alpha$-L-Arabinopyranosyl)oxy $]-19 \alpha$-hydroxyurs-12-en-28-oic acid 28-(6-O-galloyl- $\beta$ - $D$ glucopyranosyl)ester, 1585

\section{$\mathrm{C}_{48} \mathrm{H}_{72} \mathrm{O}_{18}$}

Atratoside C, 1995

Eupteleasaponin V acetate, 7627

$\mathrm{C}_{48} \mathrm{H}_{72} \mathrm{O}_{19}$
Licoricesaponin $\mathrm{F}_{3}, 12788$

Stauntoside A, 20276

\section{$\mathrm{C}_{48} \mathrm{H}_{72} \mathrm{O}_{21}$}

Licoricesaponin $\mathrm{A}_{3}, 12783$

\section{$\mathrm{C}_{48} \mathrm{H}_{74} \mathrm{O}_{16}$}

Ethyl- $n$-buthy-uralsaponin A esters, 7422

\section{$\mathrm{C}_{48} \mathrm{H}_{74} \mathrm{O}_{18}$}

Atratoside B, 1994

\section{$\mathrm{C}_{48} \mathrm{H}_{74} \mathrm{O}_{19}$}

Chiisanoside, 3520

(1R)-1,11- $\alpha$-Dihydroxy-3,4-seco-lupa-4(23), 20(29)-diene-3,28-dioic acid 3,11-lactone 28 - $O$ - $\alpha$ - $L$-rhamnopyranosyl- $(1 \rightarrow 4)-\beta$ - $D$-glucopyranosyl $(1 \rightarrow 6)-\beta$ - $D$-glucopyranoside, 6115

Papyrioside L-IIc, 16646

Yunganoside $\mathrm{D}_{1}, 22943$

\section{$\mathrm{C}_{48} \mathrm{H}_{74} \mathrm{O}_{20}$}

Abrisaponin I, 22

Acanjaposide I, 72

3 - $O-\beta$ - $D$-Galactopyranosyl $(1 \rightarrow 2)$ - $[\beta$ - $D$-xylopyranosyl $(1 \rightarrow 3)]-\beta-D$-6- $O$-methylglucoronopyranosyl-quillaic acid, 8075

Sinocrassuloside IV, 19939

\section{$\mathrm{C}_{48} \mathrm{H}_{75} \mathrm{O}_{23} \mathrm{~S}^{-}$}

Sandrosaponin III, 19246

\section{$\mathrm{C}_{48} \mathrm{H}_{75} \mathrm{O}_{24} \mathrm{~S}^{-}$}

Sandrosaponin IV, 19247

\section{$\mathrm{C}_{48} \mathrm{H}_{76} \mathrm{O}_{16}$}

Oleanolic acid 3-O-(4-O-acetyl- $\beta$ - $D$-xylopyranosyl)-( $1 \rightarrow 3)-\alpha$ - $L$-rhamnopyranosyl-( $1 \rightarrow 2)$ $\alpha$ - $L$-arabinopyranoside, 16051

\section{$\mathrm{C}_{48} \mathrm{H}_{76} \mathrm{O}_{17}$}

Hederagenin 3-O-(2- $O$-acetyl- $\beta$ - $D$-xylopyranosyl)-( $1 \rightarrow 3)-\alpha-L$-rhamnopyranosyl- $(1 \rightarrow 2)$ $\alpha$ - $L$-arabinopyranoside, 9261

Mukurozisaponin $\mathrm{E}_{1}, 15033$

\section{$\mathrm{C}_{48} \mathrm{H}_{76} \mathrm{O}_{18}$}

12-O-Acetyllineolon-3- $O-\beta$ - $D$-oleandropyranosyl-( $1 \rightarrow 4)-\beta$ - $D$-digitoxopyranosyl- $(1 \rightarrow 4)-\beta$ - $D$ digitoxopyranosyl-( $1 \rightarrow 4)-\beta$ - $D$-digitoxopyranoside, 453

Achyranthes saponin A, 539

Achyranthes saponin C, 541

Colubrin, 3929

Olaxoside, 16025

Periandradulcin C, 16920

3- $O$ - $\alpha$ - $L$-Rhamnopyranosyl-( $1 \rightarrow 4)$ - $[\beta$ - $D$-galactopyranosyl- $(1 \rightarrow 2)]-\beta$ - $D$-glucuronopyranosyl oleanolic acid, 18704

3- $O$ - $[\alpha$ - $L$-Rhamnopyranosyl $(1 \rightarrow 2)-\beta$ - $D$-galactopyranosyl $(1 \rightarrow 2)-\beta$ - $D$-glucuronopyranosyl] soyasapogenol E, 18706

3- $O$-[ $\alpha$ - $L$-Rhamnopyranosyl-( $1 \rightarrow 3)-\beta$ - $D$-glucu- 
ronopyranosyl]-28- $O-(\beta$ - $D$-glucopyranosyl)-

$3 \beta$-hydroxyolean-12-en-28-oate, 18722

3 - $O-\alpha-L$-Rhamnopyranosyl $(1 \rightarrow 2)-\beta$ - $D$-xylopyranosyl $(1 \rightarrow 2)$-6- $O$-methyl- $\beta$ - $D$-glucuronopyranosyl-3 $\beta, 22 \beta, 24$-trihydroxy-11-oxo-olean12-ene, 18738

\section{$\mathrm{C}_{48} \mathrm{H}_{76} \mathrm{O}_{19}$}

Acanjaposide G, 70

Acanjaposide H, 71

Acankoreoside D, 74

Calendula officinalis Glycoside B, 2970

Calendula officinalis Glycoside C, 2971

Cincholic acid 3 $\beta-O-\beta$ - $D$-glucopyranosyl$(1 \rightarrow 4)-\beta$ - $D$-fucopyranosyl-28- $O$ - $\beta$ - $D$-glucopyranosyl ester, 3679

Cirenshenoside V, 3740

Ginsenoside Ro, 8445

Hemsgiganoside B, 9354

Hydrocotyloside II, 9711

Metaplexigenin-3- $O-\beta$ - $D$-oleandropyranosyl$(1 \rightarrow 4)-\beta$ - $D$-digitoxopyranosyl-( $(1 \rightarrow 4)-\beta$ - $D$ digitoxopyranosyl-( $1 \rightarrow 4)-\beta$ - $D$-digitoxopyranoside, 13817

Papyrioside L-IId, 16647

Phytolaccoside F, 17271

Quinovic acid 3 $\beta$-O- $\beta$ - $D$-glucopyranosyl-( $1 \rightarrow 3$ )$\beta$-D-fucopyranosyl-(27-1)- $\beta$ - $D$-glucopyranosyl ester, 18432

Quinovic acid $3 \beta$ - $O-\beta$ - $D$-glucopyranosyl-( $1 \rightarrow 3)$ $\beta$ - $D$-fucopyranosyl-(28-1)- $\beta$ - $D$-glucopyranosyl ester, 18433

Quinovic acid 3- $O$ - $\beta$ - $D$-glucopyranosyl-(1 $\rightarrow 3)$ $\alpha$ - $L$-rhamnopyranoside 27- $O$ - $\beta$ - $D$-glucopyranosyl ester, 18436

Sandosaponin A, 19238

Sandosaponin B, 19239

Saponin 6, 19338

Yunganoside $\mathrm{A}_{1}, 22940$

Yunganoside $\mathrm{C}_{1}, 22942$

\section{$\mathrm{C}_{48} \mathrm{H}_{76} \mathrm{O}_{20}$}

Acanjaposide D, 67

Acanjaposide E, 68

Acanjaposide F, 69

Acankoreoside E, 75

Azukisaponin IV, 2068

Calendasaponin B, 2966

Dianoside G, 5364

Palustroside III, 16581

Sophoraflavoside II, 20090

$3 \beta, 16 \alpha, 23,28$-Tetrahydroxyolean-11,13(18)dien-30-oic acid 3-O- $\beta$ - $D$-glucopyranosyl$(1 \rightarrow 2)-\beta$ - $D$-glucopyranosyl-( $1 \rightarrow 3)-\beta-D$ fucopyranoside, 21141

\section{$\mathrm{C}_{48} \mathrm{H}_{76} \mathrm{O}_{21}$}

Ageratoside $\mathrm{B}_{1}, 733$

Dianoside D, 5361

Esculentoside H, 7374

Medicagenic acid 3-O-triglucoside, 13635

Sinocrassuloside I, 19936

\section{$\mathrm{C}_{48} \mathrm{H}_{76} \mathrm{O}_{23}$}

Lucilianoside C, 13058

\section{$\mathrm{C}_{48} \mathrm{H}_{77} \mathrm{NaO}_{22} \mathrm{~S}$}

$2 \alpha, 23$-Dihydroxy-3 $\beta$-sulfoxyolean-12-en-28-oic acid $O-\alpha-L$-rhamnopyranosyl-( $1 \rightarrow 4)-O-\beta-D$ glucopyranosyl-( $1 \rightarrow 6)-\beta$ - $D$-glucopyranosyl ester sodium salt, 6139

\section{$\mathrm{C}_{48} \mathrm{H}_{77} \mathrm{O}_{22} \mathrm{~S}^{-}$}

Sandrosaponin II, 19245

Sandrosaponin V, 19248

Sandrosaponin VI, 19249

\section{$\mathrm{C}_{48} \mathrm{H}_{78} \mathrm{O}_{9}$}

Glabrescin, 8492

\section{$\mathrm{C}_{48} \mathrm{H}_{78} \mathrm{O}_{16}$}

Rotundifolioside J, 18953

Rotundioside F, 18956

\section{$\mathrm{C}_{48} \mathrm{H}_{78} \mathrm{O}_{17}$}

Astragaloside VIII methylester, 1944

3-Epibetulinic acid 28-O- $\alpha$ - $L$-rhamnopyranosyl$(1 \rightarrow 4)-\beta$ - $D$-glucopyranosyl $(1 \rightarrow 6)-\beta$ - $D$-glucopyranoside, 6837

3-Epi-oleanolic acid-28- $O-\alpha-L$-rhamnopyranosyl-(1 $\rightarrow 4)-\beta$ - $D$-glucopyranosyl- $(1 \rightarrow 6)-\beta$ - $D$ glucopyranoside, 6981

Isolineolon 3- $O-\beta$ - $D$-oleandropyranosyl- $(1 \rightarrow 4)$ $\beta$-D-digitoxopyranosyl-(1 $\rightarrow 4)-\beta$ - $D$-cymaropyranosyl-( $(1 \rightarrow 4)-\beta$ - $D$-cymaropyranoside, 11500

Kaikasaponin II, 12112

Kaikasaponin III, 12113

Lineolon-3- $O-\beta$ - $D$-oleandropyranosyl- $(1 \rightarrow 4)-\beta$ $D$-digitoxopyranosyl-( $(1 \rightarrow 4)-\beta$ - $D$-cymaropyranosyl-(1 $\rightarrow 4)-\beta$ - $D$-cymaropyranoside, 12883

Rotundifolioside F, 18949

Saikosaponin $\mathrm{BK}_{1}, 19146$

Saikosaponin C, 19147

$3 \beta, 16 \alpha, 28$-Trihydroxyolean-11,13(18)-dien-3 $\beta$ yl- $\beta$ - $D$-glucopyranosyl-( $(1 \rightarrow 6)-\beta$ - $D$-[ $\alpha$ - $L$-rhamnopyranosyl-( $1 \rightarrow 4)]-\beta$ - $D$-glucopyranoside, 21821

\section{$\mathrm{C}_{48} \mathrm{H}_{78} \mathrm{O}_{18}$}

ArvenosideA, 1828

Astrasieversianin IX, 1947

Azukisaponin V, 2069

Carnosifloside III , 3203

3-O- $\beta$ - $D$-Glucopyranosyl betulinic acid-28-O- $\beta$ $D$-glucopyranosyl-(1 $\rightarrow 6)-\beta$ - $D$-glucopyranoside, 8611
3-O- $\beta$ - $D$-Glucopyranosyl-( $1 \rightarrow 6)-\beta$ - $D$-glucopyranosyl oleanolic acid 28- $O-\beta$ - $D$-glucopyranosyl ester, 8647

2 "- $O-\beta$ - $D$-glucopyranosyl saikosaponin $\mathrm{b}_{2}, 8730$

Hederagenin 28-O- $\alpha$-L-rhamnopyranosyl- $(1 \rightarrow 4)$ $\beta$ - $D$-glucopyranosyl $(1 \rightarrow 6)-\beta$ - $D$-glucopyranoside, 9273

HN Saponin H, 9573

Jujubasaponin IV, 11904

Jujubasaponin V, 11905

Latifoloside J, 12556

Lemmatoxin, 12621

Oleanoglycotoxin A, 16044

Pulsatilloside C, 18194

Pulsatiloside C, 18197

Saikosaponin N, 19153

Saikosaponin S, 19160

Saponin $E_{8}, 19345$

Soyasaponin I, 20127

$3 \beta, 16 \alpha, 28,30$-Tetrahydroxyolean-11,13(18)-dien$3 \beta$-yl- $\beta$ - $D$-glucopyranosyl- $(1 \rightarrow 6)-\beta-D$ - $[\alpha-L$ -

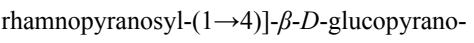
side, 21140

$3 \beta, 16 \beta, 23$-Trihydroxy-13,28-epoxyolean-11-en$3 \beta$-yl-[ $\beta$ - $D$-glucopyranosyl-( $1 \rightarrow 2)]$-[ $\beta$ - $D$-glucopyranosyl- $(1 \rightarrow 3)]-\beta$ - $D$-fucopyranoside, 21728

Udosaponin B, 22173

\section{$\mathrm{C}_{48} \mathrm{H}_{78} \mathrm{O}_{19}$}

Asiaticoside, 1854

Bourneioside B, 2573

Centellasaponin C, 3396

Centellasaponin D, 3397

Clinodiside A, 3845

Congmuyanoside B, 3977

Davuricoside J, 4700

Ecliptasaponin B, 6706

$3 \beta, 16 \alpha, 23,28,30$-Pentahydroxyolean-11,13(18)diene 3-O- $\beta$ - $D$-glucopyranosyl-(1 $\rightarrow 2)-\beta$ - $D$ glucopyranosyl- $(1 \rightarrow 3)-\beta$ - $D$-fucopyranoside, 16857

Saikosaponin P, 19155

Saikosaponin Q, 19156

Saikosaponin $Q_{1}, 19157$

Saikosaponin $Q_{2}, 19158$

Saikosaponin R, 19159

Spartium junceum saponin, 19332

Sceffoleoside A, 19460

Soyasaponin $\mathrm{A}_{3}, 20123$

Soyasaponin V, 20129

$2 \alpha, 3 \beta, 23$-Trihydroxyolean-12-en-28-oic acid $O$ - $\alpha$ - $L$-rhamnopyranosyl-( $1 \rightarrow 4)$ - $O-\beta$ - $D$-glucopyranosyl-( $1 \rightarrow 6)-\beta$ - $D$-glucopyranosyl ester, 
21825

$\mathrm{C}_{48} \mathrm{H}_{78} \mathrm{O}_{20}$

Aquilegioside H, 1549

Asiaticoside B, 1855

Madecassoside, 13338

Stelmatotriterpenoside E, 20287

Stelmatotriterpenoside F, 20288

Stelmatotriterpenoside G, 20289

Volubiloside C, 22613

\section{$\mathrm{C}_{48} \mathrm{H}_{78} \mathrm{O}_{22}$}

Aesculuside B, 667

$\mathrm{C}_{48} \mathrm{H}_{78} \mathrm{O}_{22} \mathrm{~S}$

Eclalbasaponin XII, 6703

\section{$\mathrm{C}_{48} \mathrm{H}_{80} \mathrm{O}_{2}$}

$\alpha$-Amyrin linoleate, 1117

$\beta$-Amyrin linoleate, 1118

\section{$\mathrm{C}_{48} \mathrm{H}_{80} \mathrm{O}_{16}$}

Telosmoside $\mathrm{A}_{6}, 20917$

\section{$\mathrm{C}_{48} \mathrm{H}_{80} \mathrm{O}_{17}$}

$3 \beta$ - $O$ - $\beta$ - $D$-Glucopyranosyl-( $1 \rightarrow 4)-\beta$ - $D$-fucopyranosyl $(22 S, 24 Z)$-cycloart-24-en-3 $\beta, 22$, 26 -triol 26- $O$ - $\beta$ - $D$-glucopyranoside, 8627

Latifoloside I, 12555

Rotundifolioside C, 18946

\section{$\mathrm{C}_{48} \mathrm{H}_{80} \mathrm{O}_{18}$}

Carnosifloside VI, 3204

Hydroxysaikosaponin C, 10686

Khekadaengoside N, 12219

Saikosaponin 15, 19140

\section{$\mathrm{C}_{48} \mathrm{H}_{80} \mathrm{O}_{19}$}

23-O-Acetyl-3 $\beta, 12 \beta, 23 S, 24 R$-tetrahydroxy$20 S, 25$-epoxydammarane 3-O-[ $\beta$ - $D$-xylopyra$\operatorname{nosyl}(1 \rightarrow 2)][\beta-D$-xylopyranosyl $(1 \rightarrow 6)]-\beta-D$ glucopyranoside, 522

Ginsenoside III, 8417

Justicioside B, 11984

Notoginsenoside G, 15825

$11 \alpha, 16 \beta, 23,28$-Tetrahydroxyolean-12-en-3 $\beta$-yl$[\beta$ - $D$-glucopyranosyl- $(1 \rightarrow 2)]$-[ $\beta$ - $D$-glucopyranosyl-(1 $\rightarrow 3)]-\beta$ - $D$-fucopyranoside, 21142

\section{$\mathrm{C}_{48} \mathrm{H}_{80} \mathrm{O}_{20}$}

Volubiloside A, 22611

\section{$\mathrm{C}_{48} \mathrm{H}_{80} \mathrm{O}_{21}$}

Iridalglycoside 5a, 11134

Iridalglycoside 6a, 11136

Iridalglycoside 6b, 11137

\section{$\mathrm{C}_{48} \mathrm{H}_{81} \mathrm{NO}_{17}$}

Abutiloside J, 43

\section{$\mathrm{C}_{48} \mathrm{H}_{82} \mathrm{O}_{2}$}

Stigmasta-7,22,25-triene-3-nonadecanoic acid ester, 20358

\section{$\mathrm{C}_{48} \mathrm{H}_{82} \mathrm{O}_{17}$}

Gypenoside XI, 9131
Gypenoside LXXIII, 9175

$\mathrm{C}_{48} \mathrm{H}_{82} \mathrm{O}_{18}$

Ginsenoside Rd, 8427

Ginsenoside Re, 8428

3- $O$ - $[\beta$ - $D$-Glucopyranosyl $(1 \rightarrow 6)-\beta$ - $D$-glucopyranosyl]-20- $O-\beta$ - $D$-glucopyranosyl-3 $\beta, 12 \beta$,

20(S)-trihydroxydammar-24-ene, 8646

Gypenoside VIII, 9129

Gypenoside XVII, 9134

Gypenoside LXXII, 9174

Khekadaengoside M, 12218

\section{$\mathrm{C}_{48} \mathrm{H}_{82} \mathrm{O}_{19}$}

20-Glucosylginsenoside Rf, 8755

Gycomoside II, 9095

Majoroside $\mathrm{F}_{5}, 13407$

Majoroside $\mathrm{F}_{6}, 13408$

Notoginsenoside M, 15831

Notoginsenoside N, 15832

Notoginsenoside $\mathrm{R}_{3}, 15838$

Notoginsenoside $\mathrm{R}_{6}, 15840$

\section{$\mathrm{C}_{48} \mathrm{H}_{82} \mathrm{O}_{20}$}

Ginsenoside I, 8412

Ginsenoside II, 8416

Gycomoside IV, 9097

Yesanchinoside B, 22894

\section{$\mathrm{C}_{48} \mathrm{H}_{84} \mathrm{~N}_{4} \mathrm{O}_{12}$}

Bassianolide, 2162

\section{$\mathrm{C}_{48} \mathrm{H}_{84} \mathrm{O}_{2}$}

$\alpha$-Amyrin stearate, 1123

\section{$\mathrm{C}_{48} \mathrm{H}_{84} \mathrm{O}_{3}$}

Arnidiol 3-O-stearate, 1753

16 $\beta$-Hydroxylupeol 3-O-stearate, 10349

Maniladiol 3- $O$-stearate, 13500

\section{$\mathrm{C}_{48} \mathrm{H}_{91} \mathrm{NO}_{9}$}

1-O- $\beta$ - $D$-Glucopyranosyl-(2S,3R,4E,8Z)-2- $N$-(2'hydroxydocosanoyl) eicosasphinga-4,8-dienine, 8668

1,3,5-Trihydroxy-2-hexadecanoylamino-( $6 E, 9 E)$ heptacosdiene-1-O-glucopyranoside, 21742

$\mathrm{C}_{48} \mathrm{H}_{93} \mathrm{NO}_{8}$

N-Lignoceryl sphingosyl glucose, 12802

\section{$\mathrm{C}_{48} \mathrm{H}_{93} \mathrm{NO}_{10}$}

Helicia cerebroside A, 9302

Iotroridoside B, 11124

Linum cerebroside, 12895

$\mathrm{C}_{49} \mathrm{H}_{38} \mathrm{O}_{28}$

Stachyuranin B, 20256

$\mathrm{C}_{49} \mathrm{H}_{48} \mathrm{O}_{20}$

Vanicoside B, 22330

$\mathrm{C}_{49} \mathrm{H}_{50} \mathrm{O}_{11}$

Australone B, 2018

$\mathrm{C}_{49} \mathrm{H}_{53} \mathrm{NO}_{14}$

$N$-Debenzoyl- $N$-cinnamoyltaxol, 4805
$\mathrm{C}_{49} \mathrm{H}_{55} \mathrm{NO}_{15}$

10-( $\beta$-Hydroxybutyryl)-10-deacetyltaxol, 9867

\section{$\mathrm{C}_{49} \mathrm{H}_{58} \mathrm{O}_{27}$}

Watterose D, 22642

Watterose I, 22647

\section{$\mathrm{C}_{49} \mathrm{H}_{60} \mathrm{O}_{23}$}

Safghanoside G, 19118

Safghanoside H, 19119

$\mathrm{C}_{49} \mathrm{H}_{61} \mathrm{NO}_{18}$

7-( $\beta$-Xylosyl)taxol D, 22853

$\mathrm{C}_{49} \mathrm{H}_{63} \mathrm{NO}_{17}$

7-( $\beta$-Xylosyl)-10-deacetyltaxol C, 22848

$\mathrm{C}_{49} \mathrm{H}_{67} \mathrm{~N}_{9} \mathrm{O}_{10}$

Microtoenin C, 14849

$\mathrm{C}_{49} \mathrm{H}_{72} \mathrm{O}_{16}$

$21 \beta$ - $O$-Benzoylsitakisogenin 3- $O-\beta$ - $D$-glucopyra$\operatorname{nosyl}(1 \rightarrow 3)-\beta$ - $D$-glucuronopyranoside, 2271

\section{$\mathrm{C}_{49} \mathrm{H}_{72} \mathrm{O}_{17}$}

Otophylloside A, 16270

\section{$\mathrm{C}_{49} \mathrm{H}_{74} \mathrm{O}_{4}$}

Ubiquinone 8, 22171

\section{$\mathrm{C}_{49} \mathrm{H}_{74} \mathrm{O}_{19}$}

Eupteleasaponin VI acetate, 7629

\section{$\mathrm{C}_{49} \mathrm{H}_{76} \mathrm{O}_{16}$}

Periplocoside C, 16946

\section{$\mathrm{C}_{49} \mathrm{H}_{76} \mathrm{O}_{19}$}

Papyrioside L-IIa, 16644

Symplocos glomerata saponin 2, 20530

Symplocos glomerata saponin 3, 20531

Symplocos glomerata saponin 6, 20534

Symplocos glomerata saponin 11, 20539

\section{$\mathrm{C}_{49} \mathrm{H}_{76} \mathrm{O}_{20}$}

3-O- $\beta$ - $D$-Galactopyranosyl $(1 \rightarrow 2)$ - $[\beta$ - $D$-xylo$\operatorname{pyranosyl}(1 \rightarrow 3)]-\beta-D$-6- $O$-ethylglucoronopyranosyl-quillaic acid, 8073

\section{$\mathbf{C}_{49} \mathbf{H}_{77} \mathrm{NO}_{10}$}

Lipo-14-O-anisoylbikhaconine, 12898

\section{$\mathrm{C}_{49} \mathrm{H}_{78} \mathrm{O}_{16}$}

Otophylloside B, 16271

WilfosideC ${ }_{3}$ N, 22693

\section{$\mathrm{C}_{49} \mathrm{H}_{78} \mathrm{O}_{18}$}

12-O-Acetyllineolon-3- $O-\beta$ - $D$-oleandropyranosyl-( $1 \rightarrow 4)-\beta$ - $D$-digitoxopyranosyl- $(1 \rightarrow 4)-\beta$ - $D$ digitoxopyranosyl-( $1 \rightarrow 4)$ - $\beta$ - $D$-cymaropyranoside, 452

3-O- $\alpha$ - $L$-Rhamnopyranosyl $(1 \rightarrow 2)-\beta$ - $D$-galactopyranosyl $(1 \rightarrow 2)$-6- $O$-methyl- $\beta$ - $D$-glucuronopyranosyl-3 $\beta, 22 \beta, 24$-trihudroxy-11-oxo-olean12-ene, 18708

Taibaienoside II, 20614

\section{$\mathbf{C}_{49} \mathbf{H}_{78} \mathbf{O}_{19}$}

3-O-(4-O-Acetyl)- $\alpha-L$-arabinopyranosyl-hederagenin $28-O-\beta$ - $D$-glucopyranosyl- $(1 \rightarrow 6)-\beta-D$ - 
glucopyranoside, 323

Chikusetsu saponin V methyl ester, 3525

Metaplexigenin-3- $O-\beta$ - $D$-oleandropyranosyl$(1 \rightarrow 4)-\beta$ - $D$-digitoxopyranosyl-( $1 \rightarrow 4)-\beta$ - $D$ digitoxopyranosyl-( $1 \rightarrow 4)-\beta$ - $D$-cymaropyranoside, 13816

Papyrioside L-IIb, 16645

Saponin 5, 19337

\section{$\mathrm{C}_{49} \mathrm{H}_{78} \mathrm{O}_{20}$}

Acetyl-subproside II, 517

Peruvianoside A, 16997

Saponin 4, 19336

Saponin 7, 19339

Saponin 8, 19340

\section{$\mathrm{C}_{49} \mathrm{H}_{78} \mathrm{O}_{21}$}

Extensumside A, 7698

3- $O$ - $[\beta$ - $D$-Glucopyranosyl- $(1 \rightarrow 3)-\beta$ - $D$-glucopyranosyl] phytolaccagenic acid $28-O-\beta-D-$ glucopyranosyl ester, 8652

Peruvianoside B, 16998

\section{$\mathrm{C}_{49} \mathrm{H}_{78} \mathrm{O}_{22}$}

Chinenoside II, 3529

\section{$\mathrm{C}_{49} \mathrm{H}_{79} \mathrm{O}_{21}$}

Capilliposide J, 3129

\section{$\mathrm{C}_{49} \mathrm{H}_{80} \mathrm{O}_{17}$}

Kaikasaponin III methyl ester, 12114

\section{$\mathrm{C}_{49} \mathrm{H}_{80} \mathrm{O}_{18}$}

15- $\beta$-Hydroxylineolon 3- $O-\beta$ - $D$-oleandropyranosyl-( $1 \rightarrow 4)-\beta$ - $D$-oleandropyranosyl- $(1 \rightarrow 4)$ $\beta$ - $D$-cymaropyranosyl-( $1 \rightarrow 4)-\beta$ - $D$-cymaropyranoside, 10333

\section{$\mathrm{C}_{49} \mathrm{H}_{80} \mathrm{O}_{19}$}

Asernestioside B, 1852

\section{$\mathrm{C}_{49} \mathrm{H}_{80} \mathrm{O}_{20}$}

Aquilegioside C, 1544

Aquilegioside D, 1545

\section{$\mathrm{C}_{49} \mathrm{H}_{80} \mathrm{O}_{21}$}

Aquilegioside E, 1546

Asparacoside, 1863

\section{$\mathrm{C}_{49} \mathrm{H}_{80} \mathrm{O}_{22}$}

Asparanin D, 1879

\section{$\mathrm{C}_{49} \mathrm{H}_{82} \mathrm{O}_{16}$}

Telosmoside $\mathrm{A}_{4}, 20915$

\section{$\mathrm{C}_{49} \mathrm{H}_{82} \mathrm{O}_{17}$}

Telosmoside $\mathrm{A}_{1}, 20912$

\section{$\mathrm{C}_{49} \mathrm{H}_{82} \mathrm{O}_{18}$}

$11 \alpha$-Methoxysaikosaponin f, 14087

\section{$\mathrm{C}_{49} \mathrm{H}_{82} \mathrm{O}_{19}$}

Justicioside D, 11986

Saikosaponin 16, 19141

$16 \alpha, 23,28$-Trihydroxy-11 $\alpha$-methoxyolean-12-en$3 \beta$-yl-[ $\beta$ - $D$-glucopyranosyl- $(1 \rightarrow 2)]$ - $[\beta$ - $D$-glucopyranosyl-( $(\rightarrow 3)]-\beta$ - $D$-fucopyranoside,
21784

$\mathrm{C}_{49} \mathrm{H}_{82} \mathrm{O}_{22}$

Aspafilioside C, 1862

$\mathrm{C}_{49} \mathrm{H}_{82} \mathrm{O}_{28}$

Pyishiauoside $\mathrm{IV}_{\mathrm{b}}, 18239$

$\mathrm{C}_{49} \mathrm{H}_{83} \mathrm{NO}_{17}$

Abutiloside A, 38

$\mathrm{C}_{49} \mathrm{H}_{86} \mathrm{O}_{2}$

Stigmasterol arachidate, 20371

\section{$\mathrm{C}_{49} \mathrm{H}_{88} \mathrm{O}_{15}$}

1-O-Hexadecanoyl-2-O-(9Z,12Z-octadecadienoly)-3-O-[ $\alpha$ - $D$-galactopyranosyl-(1"'-6')- $O-\beta-D$ galactopyranosyl]-glycerol, 9490

\section{$\mathrm{C}_{50} \mathrm{H}_{44} \mathrm{O}_{22}$}

$3 \mathrm{~T}-O-\alpha-L$-Arabinopyranosylcinnamtannin $\mathrm{B}_{1}$, 1562

$\mathrm{C}_{50} \mathrm{H}_{50} \mathrm{O}_{21}$

Lapathoside A, 12505

$\mathrm{C}_{50} \mathrm{H}_{53} \mathrm{NO}_{20}$

Wilfornine C, 22688

\section{$\mathrm{C}_{50} \mathrm{H}_{54} \mathrm{O}_{8}$}

Bianthrone $\mathrm{A}_{1}, 2346$

$\mathrm{C}_{50} \mathrm{H}_{57} \mathrm{NO}_{17}$

7-( $\beta$-Xylosyl)-10-deacetyltaxol, 22847

$\mathrm{C}_{50} \mathrm{H}_{59} \mathrm{~N}_{9} \mathrm{O}_{12}$

Lyciumin C methylate, 13180

$\mathrm{C}_{50} \mathrm{H}_{61} \mathrm{NO}_{18}$

7-( $\beta$-Xylosyl)cephalomannine, 22845

$\mathrm{C}_{50} \mathrm{H}_{64} \mathbf{O}_{11}$

Genkwadaphnin-20-palmitate, 8288

$\mathrm{C}_{50} \mathrm{H}_{70} \mathrm{O}_{17}$

Cynaphylloside E, 4560

$\mathrm{C}_{50} \mathrm{H}_{70} \mathrm{O}_{18}$

Cynaphylloside C, 4558

$\mathrm{C}_{50} \mathrm{H}_{74} \mathrm{O}_{28}$

Jasnudifloside L, 11832

$\mathbf{C}_{50} \mathbf{H}_{76} \mathbf{O}_{21}$

Licoricesaponin $\mathrm{D}_{3}, 12786$

Acantrifoside C, 92

$\mathrm{C}_{50} \mathrm{H}_{78} \mathrm{O}_{16}$

Dibutyl uralsaponin A ester, 5405

\section{$\mathrm{C}_{50} \mathrm{H}_{78} \mathrm{O}_{18}$}

23-O-Acetylhederagenin 3-O-(4-O-acetyl- $\beta$ - $D$ xylopyranosyl)-( $1 \rightarrow 3)-\alpha$ - $L$-rhamnopyranosyl-

$(1 \rightarrow 2)-\alpha$ - $L$-arabinopyranoside, 411

Mukurozisaponin G, 15034

$\mathrm{C}_{50} \mathrm{H}_{78} \mathrm{O}_{19}$

Colubrinoside, 3933

$\mathrm{C}_{50} \mathrm{H}_{78} \mathrm{O}_{24}$

Funingenoside D, 8008

\section{$\mathrm{C}_{50} \mathrm{H}_{78} \mathbf{O}_{27}$}

$3 \beta$-[(O- $\beta$ - $D$-Glucopyranosyl- $(1 \rightarrow 3)-O-\beta-D$ glucopyranosyl-( $1 \rightarrow 2)-O$-[ $\beta$ - $D$-xylopyranos- yl-( $1 \rightarrow 3)]-O-\beta$ - $D$-glucopyranosyl- $(1 \rightarrow 4)-\beta$ -

$D$-galactopyranosyl)oxy]-2 $\alpha$-hydroxypregna-

5,16-dien-20-one, 8660

\section{$\mathrm{C}_{50} \mathrm{H}_{79} \mathrm{NaO}_{20}$}

Sodium salt of alternoside II, 20037

\section{$\mathrm{C}_{50} \mathrm{H}_{80} \mathrm{O}_{18}$}

12- $O$-Acetyllineolon-3- $O-\beta$ - $D$-oleandropyranosyl-( $1 \rightarrow 4)-\beta$ - $D$-digitoxopyranosyl- $(1 \rightarrow 4)-\beta$ - $D$ cymaropyranosyl- $(1 \rightarrow 4)-\beta$ - $D$-cymaropyranoside, 451

\section{$\mathrm{C}_{50} \mathrm{H}_{80} \mathrm{O}_{19}$}

23-Acetoxy-16 $\alpha$-hydroxy-13,28-epoxyolean-11en-3 $\beta$-yl- $[\beta$ - $D$-glucopyranosyl $(1 \rightarrow 2)]$ - $[\beta-D$ glucopyranosyl-( $(1 \rightarrow 3)]-\beta$ - $D$-fucopyranoside, 214

Trojanoside J, 22047

\section{$\mathrm{C}_{50} \mathrm{H}_{80} \mathrm{O}_{21}$}

Pariphyllin B, 16669

Parissaponin $\mathrm{Pb}, 16670$

\section{$\mathrm{C}_{50} \mathrm{H}_{80} \mathrm{O}_{22}$}

Aspidistrin, 1898

CTHD0233276-10, 4295

Polyspirostanol $\mathrm{PO}_{3}, 17678$

Polyspirostanol $\mathrm{PO}_{\mathrm{e}}, 17681$

\section{$\mathrm{C}_{50} \mathrm{H}_{80} \mathrm{O}_{23}$}

Agave americana Compound 4, 715

Anemarsaponin G, 1177

Cantalasaponin 2, 3091

Hecogenin 3-O- $\beta$ - $D$-glucopyranosyl- $(1 \rightarrow 2)-[\beta$ $D$-xylopyranosyl-(1 $\rightarrow 3)]-\beta$ - $D$-glucopyranosyl$(1 \rightarrow 4)$ - $\beta$ - $D$-galactopyranoside, 9255

Neoprazerigenin A 3-O- $\beta$ - $D$-lycotetraoside, 15450

Ophiopogon B, 16142

Polyspirostanol $\mathrm{PO}_{2}, 17677$

Polyspirostanol $\mathrm{PO}_{6}, 17680$

Ruscoside, 19074

Terrestrosin D, 21016

\section{$\mathrm{C}_{50} \mathrm{H}_{80} \mathrm{O}_{24}$}

(25R)- $2 \alpha, 17 \alpha$-Dihydroxyspirost-5-en-3 $\beta$-yl $O-\beta$ $D$-glucopyranosyl-( $1 \rightarrow 2)-O$ - $[\beta$ - $D$-xylopyranosyl- $(1 \rightarrow 3)]-O-\beta$ - $D$-glucopyranosyl-( $(\rightarrow 4)$ $\beta$-D-galactopyranoside, 6130

\section{$\mathrm{C}_{50} \mathrm{H}_{80} \mathrm{O}_{27}$}

Woodwardinoside, 22723

\section{$\mathrm{C}_{50} \mathrm{H}_{81} \mathrm{NO}_{19}$}

Arudonine, 1822

\section{$\mathrm{C}_{50} \mathrm{H}_{81} \mathrm{NO}_{21}$}

(25))-Solanid-5-en-3 $\beta, 22 \beta$-dihydroxy 3-O- $\beta$ - $D$ glucopyranosyl $(1 \rightarrow 2)$ - $[\beta$ - $D$-xylopyranosyl $(1 \rightarrow 3)]-\beta$ - $D$-glucopyranosyl $(1 \rightarrow 4)-\beta$ - $D$-galactopyranoside, 20055

$\mathrm{C}_{50} \mathrm{H}_{82} \mathrm{O}_{19}$ 


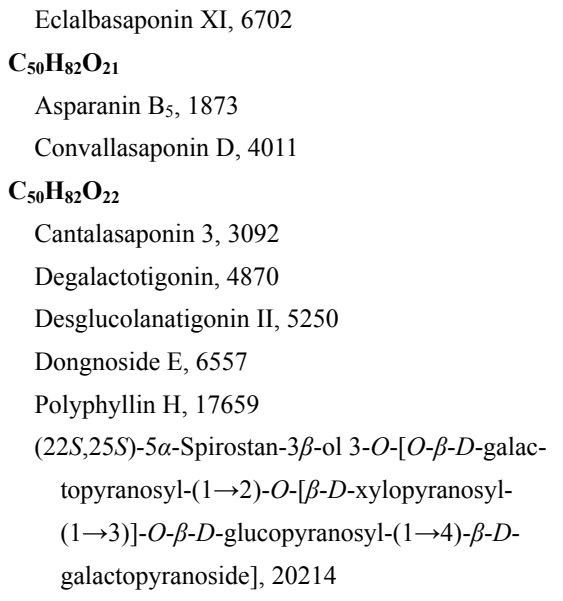

Tigogenin 3-O- $\beta$ - $D$-glucopyranosyl-( $1 \rightarrow 2)$ - $[\beta-D-$ xylopyranosyl-( $(1 \rightarrow 3)]-\beta$ - $D$-glucopyranosyl$(1 \rightarrow 4)-\beta$ - $D$-galactopyranoside, 21385

Trigofoenoside E, 21653

\section{$\mathrm{C}_{50} \mathrm{H}_{82} \mathrm{O}_{23}$}

Anemarsaponin F, 1176

F-Gitonin, 8458

Gitonin, 8459

Hemeroside B, 9342

(25R)-5 $\alpha$-Spirostan-3 $\beta, 6 \beta$-diol 3-O- $\{O-\beta-D$-glucopyranosyl-(1 $\rightarrow 2)-O-[\beta$ - $D$-xylopyranosyl$(1 \rightarrow 3)]-O-\beta$ - $D$-glucopyranosyl-( $1 \rightarrow 4)-\beta$ - $D$ galactopyranoside\}, 20211

\section{$\mathrm{C}_{50} \mathrm{H}_{83} \mathrm{NO}_{20}$}

Demissine, 5104

\section{$\mathrm{C}_{50} \mathrm{H}_{83} \mathrm{NO}_{21}$}

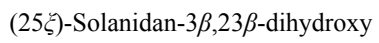
3-O- $\beta$ - $D$-glucopyranosyl $(1 \rightarrow 2)$-[ $\beta$ - $D$-xylopyranosyl $(1 \rightarrow 3)]-\beta$ - $D$-glucopyranosyl $(1 \rightarrow 4)$ $\beta$ - $D$-galactopyranoside, 20053

Tomatine, 21435

\section{$\mathrm{C}_{50} \mathrm{H}_{83} \mathrm{NO}_{22}$}

Lycoperoside H, 13219

\section{$\mathrm{C}_{50} \mathrm{H}_{84} \mathrm{O}_{19}$}

Quinquenoside III, 18448

\section{$\mathrm{C}_{50} \mathrm{H}_{84} \mathrm{O}_{23}$}

Asparanin $\mathrm{B}_{9}, 1877$

Asparaside $\mathrm{B}_{1}, 1881$

Asparaside $\mathrm{B}_{2}, 1882$

\section{$\mathrm{C}_{50} \mathrm{H}_{84} \mathrm{O}_{28}$}

Mukurozioside $\mathrm{II}_{\mathrm{b}}, 15032$

\section{$\mathrm{C}_{50} \mathrm{H}_{86} \mathrm{O}_{28}$}

Mukurozioside $\mathrm{II}_{\mathrm{a}}, 15031$

$\mathrm{C}_{50} \mathrm{H}_{88} \mathrm{O}_{3}$

Arnidiol 3-O-eicosanoate, 1750

16 $\beta$-Hydroxylupeol 3-O-eicosanoate, 10346

Maniladiol 3-O-eicosanoate, 13497

$\mathrm{C}_{50} \mathrm{H}_{88} \mathrm{O}_{7}$

Campesteryl- $D$-glucoside-6'-palmitate, 3043
$\mathrm{C}_{50} \mathrm{H}_{88} \mathrm{O}_{30}$

Pharbitic acid D, 17044

$\mathrm{C}_{50} \mathrm{H}_{\mathbf{1 0 0}} \mathrm{O}_{2}$

Ceryl lignocerate, 3437

$\mathrm{C}_{\mathbf{5 1}} \mathbf{H}_{\mathbf{4 6}} \mathbf{O}_{\mathbf{1 4}}$

AH18, 768

AH19a, 769

AH19b, 770

AH20, 771

$\mathrm{C}_{51} \mathrm{H}_{46} \mathrm{O}_{23}$

3T- $O-\beta$ - $D$-Galactopyranosylcinnamtannin $\mathrm{B}_{1}$, 8051

$\mathrm{C}_{51} \mathrm{H}_{52} \mathrm{O}_{22}$

Lapathoside B, 12506

\section{$\mathrm{C}_{51} \mathrm{H}_{52} \mathrm{O}_{23}$}

Catechin-3- $O-\beta-D$-glucopyranosyl-( $4 \alpha \rightarrow 8)$ catechin-3-O- $\beta$ - $D$-gluco(2-cinnamoyl)pyranoside, 3313

Catechin-3- $O-\beta$ - $D$-glucopyranosyl-( $4 \alpha \rightarrow 8)$-epicatechin-3-O- $\beta$ - $D$-gluco(6-cinnamoyl)pyranoside, 3314

Kaempferol 3-O- $\alpha$ - $L$-rhamnopyranosyl $(1 \rightarrow 6)$ [(4-O-trans-p-coumaroyl)- $\alpha$ - $L$-rhamnopyra$\operatorname{nosyl}(1 \rightarrow 2)]$-(4-O-trans- $p$-coumaroyl)- $\beta$ - $D$ galactopyranoside, 12073

\section{$\mathrm{C}_{51} \mathrm{H}_{52} \mathrm{O}_{24}$}

Quercetin-3-O- $\alpha$ - L-rhamnopyranosyl $(1 \rightarrow 6)-[(4-$ $O$-trans- $p$-coumaroyl)- $\alpha-L$-rhamnopyranosyl $(1 \rightarrow 2)]$-(3-O-trans- $p$-coumaroyl)- $\beta$ - $D$-galactopyranoside, 18383

Quercetin-3-O- $\alpha-L$-rhamnopyranosyl $(1 \rightarrow 6)$ [(4-O-trans- $p$-coumaroyl)- $\alpha$ - $L$-rhamnopyra$\operatorname{nosyl}(1 \rightarrow 2)]-(4-O-$ trans- $p$-coumaroyl) $-\beta$ - $D$ galactopyranoside, 18384

\section{$\mathrm{C}_{51} \mathrm{H}_{52} \mathrm{O}_{25}$}

Quercetin-3-O- $\alpha$ - $L$-rhamnopyranosyl $(1 \rightarrow 6)$ [(4-O-trans-caffeoyl)- $\alpha$ - $L$-rhamnopyranosyl $(1 \rightarrow 2)]$-(3-O-trans- $p$-coumaroyl)- $\beta$ - $D$-galactopyranoside, 18380

\section{$\mathrm{C}_{51} \mathrm{H}_{53} \mathrm{O}_{25}{ }^{+}$}

Pelargonidin-3-O-[6-O-(E)-p-coumaroyl-2-O-(6(E)-caffeoyl- $\beta$ - $D$-glucopyranosyl)-(1 $\rightarrow 2)-\beta$ - $D$ glucopyranoside]-5- $O$-( $\beta$ - $D$-glucopyranoside), 16779

\section{$\mathrm{C}_{51} \mathrm{H}_{53} \mathrm{O}_{28}$}

Gentiodelphin, 8302

\section{$\mathrm{C}_{51} \mathrm{H}_{60} \mathrm{Br}_{3} \mathrm{~N}_{3} \mathrm{O}_{5}$}

$p$-Bromobenzoyl derivative of tetrahydrodeoxyoxolucidine A, 2617

\section{$\mathrm{C}_{51} \mathrm{H}_{60} \mathrm{O}_{27}$}

Fallaxose C, 7711

Watterose H, 22646

$\mathrm{C}_{51} \mathrm{H}_{60} \mathrm{O}_{28}$
Watterose C, 22641

$\mathrm{C}_{51} \mathrm{H}_{60} \mathrm{O}_{29}$

Watterose J, 22648

$\mathrm{C}_{51} \mathrm{H}_{64} \mathrm{~N}_{8} \mathrm{O}_{8}$

Psammosilenin A, 18014

$\mathrm{C}_{51} \mathrm{H}_{64} \mathrm{O}_{24}$

Gymnoside IX, 9112

Gymnoside X, 9113

$\mathrm{C}_{51} \mathrm{H}_{65} \mathrm{NO}_{18}$

7-( $\beta$-Xylosyl)taxol C, 22852

$\mathbf{C}_{51} \mathbf{H}_{72} \mathbf{O}_{17}$

Cynaphylloside D, 4559

$\mathrm{C}_{51} \mathrm{H}_{74} \mathrm{O}_{19}$

Pennogenin-hexaacetyl-3- $O-\alpha-L$-rhamnopyranosyl $(1 \rightarrow 2)-\beta$ - $D$-glucopyranoside, 16812

\section{$\mathrm{C}_{51} \mathrm{H}_{78} \mathrm{O}_{7}$}

Indicanine $\mathrm{D}, 11015$

\section{$\mathrm{C}_{51} \mathrm{H}_{78} \mathrm{O}_{17}$}

Marsdekoiside E, 13576

\section{$\mathrm{C}_{51} \mathrm{H}_{78} \mathrm{O}_{18}$}

3-O- $\alpha$ - $L$-Arabinofuranosyl-( $(1 \rightarrow 2)-\beta-D$-glucuronopyranosyl-21 $\beta, 22 \alpha$-di- $O$-angeloylprotoaescigenin, 1556

3- $O$ - $\beta$ - $D$-Xylopyranosyl-( $1 \rightarrow 2)-\beta$ - $D$-glucuronopyranosyl-21 $\beta, 22 \alpha$-di- $O$-angeloylprotoaescigenin, 22835

\section{$\mathrm{C}_{51} \mathrm{H}_{78} \mathrm{O}_{20}$}

Symplocos glomerata saponin 4, 20532

\section{$\mathrm{C}_{51} \mathbf{H}_{80} \mathbf{O}_{15}$}

(2S)-1,2-O-6,9,12,15-Dioctadecatetraenoyl-3-O$[\alpha-D$-galactopyranosyl-(1'"' $\rightarrow 6$ "')- $O-\beta$ - $D$-galactopyranosyl]-glycerol, 6426

\section{$\mathrm{C}_{51} \mathrm{H}_{80} \mathrm{O}_{18}$}

Ziziphin, 23010

\section{$\mathrm{C}_{51} \mathrm{H}_{80} \mathrm{O}_{20}$}

$\beta$-D-Xylopyranosyl-( $1 \rightarrow 3)-\beta$ - $D$-xylopyranosyl$(1 \rightarrow 4)-\alpha$ - $L$-rhamnopyranosyl-( $1 \rightarrow 2)-\beta$ - $D$-xylopyranosyl gypsogenin, 22842

\section{$\mathrm{C}_{51} \mathrm{H}_{80} \mathrm{O}_{21}$}

Yemuoside $\mathrm{YM}_{13}, 22891$

\section{$\mathrm{C}_{51} \mathrm{H}_{80} \mathrm{O}_{23}$}

$(23 R)-3 \beta-[(O-\beta-D$-Apiofuranosyl- $(1 \rightarrow 2)-O-\beta-D-$ glucopyranosyl-( $1 \rightarrow 2)-O-\alpha$ - $L$-arabinopyranosyl-(1 $\rightarrow 6)-\beta$ - $D$-glucopyranosyl)oxy]-17 $\alpha, 23-$ epoxy-29-hydroxy-27-norlanost-8-ene-15,24dione, 1507

$(23 S)-3 \beta-[(O-\beta-D-$ Apiofuranosyl-( $(1 \rightarrow 2)-O-\beta-D-$ glucopyranosyl-( $(1 \rightarrow 2)-O-\alpha-L$-arabinopyranosyl-(1 $\rightarrow 6)-\beta$ - $D$-glucopyranosyl)oxy]-17 $\alpha, 23-$ epoxy-29-hydroxy-27-norlanost-8-ene-15,24dione, 1508

\section{$\mathrm{C}_{51} \mathrm{H}_{80} \mathbf{O}_{28}$}

$3 \beta$-[(O- $\beta$ - $D$-Glucopyranosyl-( $(1 \rightarrow 3)-O-\beta$ - $D$-glu- 
copyranosyl-( $(1 \rightarrow 2)-O-[\beta$ - $D$-xylopyranosyl$(1 \rightarrow 3)]-O-\beta$ - $D$-glucopyranosyl-( $1 \rightarrow 4)-\beta$ - $D$-galactopyranosyl)-oxy]-2 $\alpha, 16 \beta$-dihydroxypregn5-ene-20-carboxylic acid $\gamma$-lactone, 8657

\section{$\mathrm{C}_{51} \mathrm{H}_{82} \mathrm{O}_{18}$}

Hemslonin B, 9358

Taibaienoside I, 20613

\section{$\mathbf{C}_{51} \mathbf{H}_{82} \mathbf{O}_{20}$}

Asperin, 1889

Diosgenin 3-O- $\alpha$ - $L$-rhamnopyranosyl-( $(1 \rightarrow 4)-\alpha-$ $L$-rhamnopyranosyl-( $1 \rightarrow 4)$-[ $\alpha$ - $L$-rhamnopyranosyl-(1 $\rightarrow 2)]-\beta$ - $D$-glucopyranoside, 6447

Diosgenin 3-O-[ $\alpha-L$-rhamnopyranosyl $(1 \rightarrow 3)$ $\alpha$-L-rhamnopyranosyl $(1 \rightarrow 4)$ - $\alpha$ - $L$-rhamnopyranosyl $(1 \rightarrow 4)]$ - $\beta$ - $D$-glucopyranoside, 6448

Polyphyllin E, 17656

Polyphyllin F, 17657

\section{$\mathrm{C}_{51} \mathrm{H}_{82} \mathrm{O}_{21}$}

Diosgenin -3- $O-\alpha-L$-rhamnopyranosyl $(1 \rightarrow 3)$ $\alpha$ - $L$-rhamnopyranosyl $(1 \rightarrow 4)$ - $\beta$ - $D$-glucopyranosyl $(1 \rightarrow 4)-\beta$ - $D$-glucopyranoside, 6449 (25R)-2 $\alpha$-Hydroxyspirost-5-en-3 $\beta$-yl $O-\alpha-L$ rhamnopyranosyl-( $(1 \rightarrow 2)-O-[O-\alpha-L$-rhamnopyranosyl-( $1 \rightarrow 4)-\alpha-L$-rhamnopyranosyl$(1 \rightarrow 4)]$ - $\beta$ - $D$-glucopyranoside, 10727

Musennin, 15131

Pennogenin rhamnosyl chacotrioside, 16815

Pseudoprotodioscin, 18056

Solasodoside A, 20068

(25S)-Spirost-5-en-3 $\beta$-yl $O-\alpha$ - $L$-rhamnopyranosyl-( $(1 \rightarrow 2)-O-[O-\beta$ - $D$-glucopyranosyl$(1 \rightarrow 4)-\alpha-L$-rhamnopyranosyl-( $1 \rightarrow 3)]-\beta-D$ glucopyranoside, 20223

\section{$\mathrm{C}_{51} \mathrm{H}_{82} \mathrm{O}_{22}$}

Diosgenin $-3-O-\beta$ - $D$-glucopyranosyl $(1 \rightarrow 4)$ - $\alpha$ - $L$-rhamnopyranosyl $(1 \rightarrow 4)-\beta$ - $D$-glucopyranosyl $(1 \rightarrow 4)-\beta$ - $D$-glucopyranoside, 6444 Graecunin E, 8969

(24S,25R)-24-Hydroxyspirost-5-en-3 $\beta$-yl $O-\alpha-L$ rhamnopyranosyl-( $1 \rightarrow 2)-O$-[O- $\beta$ - $D$-glucopyranosyl-( $(1 \rightarrow 4)-\alpha-L$-rhamnopyranosyl- $(1 \rightarrow 3)]$ $\beta$ - $D$-glucopyranoside, 10725

Soyasaponin $\mathrm{A}_{6}, 20126$

woodwardinoside B, 22724

\section{$\mathrm{C}_{51} \mathrm{H}_{82} \mathrm{O}_{23}$}

Abutiloside L, 45

(23S)-3 $\beta$ - $[(O-\beta$ - $D$-Apiofuranosyl- $(1 \rightarrow 2)-O-\beta-D-$ glucopyranosyl-( $(1 \rightarrow 2)-\alpha$ - $L$-arabinopyranosyl( $1 \rightarrow 6)-\beta$ - $D$-glucopyranosyl)oxy]-17 $\alpha, 23$ epoxy-28,29-dihydroxy-27-norlanost-8-en24-one, 1506

Avenacoside A, 2031

Deltoside, 5041
Hypoglaucin G, 10896

Luciamin, 13022

\section{$\mathrm{C}_{51} \mathrm{H}_{82} \mathrm{O}_{24}$}

Abutiloside N, 47

Protozingiberensisaponin, 17997

$\mathrm{C}_{51} \mathrm{H}_{84} \mathrm{O}_{13}$

Glycerol- $\alpha, \beta$-dilinolenate- $\alpha^{\prime}$-rhamno-rhamnoside, 8809

$\mathrm{C}_{51} \mathrm{H}_{84} \mathrm{O}_{15}$

1,2-Di- $O$-(9Z,12Z,15Z-octadecatrienoyl)-3- $O$-[ $\alpha$ -

$D$-glucose( $1 \rightarrow 6)-\beta$ - $D$-allose]-glycerol, 6429

\section{$\mathrm{C}_{51} \mathrm{H}_{84} \mathrm{O}_{21}$}

Malonylginsenoside Rd, 13443

\section{$\mathrm{C}_{51} \mathrm{H}_{84} \mathrm{O}_{22}$}

Parillin, 16666

Polyphyllin G, 17658

Protodioscin, 17973

Protoneodioscin, 17980

Trigofoenoside $\mathrm{E}_{1}, 21654$

Trigonelloside C, 21663

\section{$\mathrm{C}_{51} \mathrm{H}_{84} \mathrm{O}_{23}$}

Macrostemonoside A, 13326

Protogracillin, 17975

Trigofoenoside D, 21651

Trigofoenoside F, 21655

\section{$\mathrm{C}_{51} \mathrm{H}_{84} \mathrm{O}_{24}$}

Isoerubioside $\mathrm{B}, 11414$

(25R,S)-5 $\alpha$-Spirostane- $2 \alpha, 3 \beta$-diol 3-O-[O- $\beta$ - $D$ glucopyranosyl-( $(1 \rightarrow 2)]-O$-[ $\beta$ - $D$-glucopyranosyl-( $(1 \rightarrow 3)]-O-\beta$ - $D$-glucopyranosyl-( $(1 \rightarrow 4)-\beta$ - $D$ galactopyranoside], 20213

\section{$\mathrm{C}_{51} \mathrm{H}_{85} \mathrm{NO}_{21}$}

Commersonine, 3942

\section{$\mathrm{C}_{51} \mathrm{H}_{86} \mathrm{O}_{7}$}

Stigmasta-7,22,25-triene-3- $O-\beta$ - $D$-(6'-palmitoyl) glucopyranoside, 20359

\section{$\mathrm{C}_{51} \mathrm{H}_{86} \mathrm{O}_{21}$}

Spongioside A, 20228

\section{$\mathrm{C}_{51} \mathrm{H}_{86} \mathrm{O}_{22}$}

Asp-VI, 1907

26-O- $\beta$ - $D$-Glucopyranosyl-(25R)-3 $\beta, 22 \xi, 26$-trihydroxyl- $5 \alpha$-furostane $3-O-\beta$-chacotrioside, 8742

\section{$\mathrm{C}_{51} \mathrm{H}_{86} \mathrm{O}_{23}$}

Asparanin $\mathrm{B}_{8}, 1876$

Asparaside A, 1880

Trigofoenoside C, 21649

Trigoneoside 1, 21664

\section{$\mathrm{C}_{51} \mathrm{H}_{88} \mathrm{O}_{7}$}

6'-Palmityl- $\alpha$-spinasteryl- $\beta$ - $D$-glucoside, 16569

$\beta$-Sitosterol-3-(6-palmitoleoyl)glucopyranoside, 19990

Stigmast-3-O- $\beta$ - $D$-glucopyanosyl-6-hexa- decanoate, 20378

\section{$\mathrm{C}_{51} \mathrm{H}_{90} \mathrm{O}_{7}$}

6'-Palmityl- $\Delta^{7}$-spinasteryl- $\beta$ - $D$-glucoside, 16570

$\beta$-Sitosteryl- $D$-glucoside-6'-palmitate, 19997

\section{$\mathrm{C}_{51} \mathrm{H}_{98} \mathrm{O}_{6}$}

Palmitin, 16560

\section{$\mathrm{C}_{52} \mathrm{H}_{46} \mathrm{O}_{26}$}

Trilobatin J, 21885

\section{$\mathrm{C}_{52} \mathrm{H}_{55} \mathrm{O}_{25}{ }^{+}$}

Pelargonidin-3-O-[6-O-(E)-p-coumaroyl-2-O-(6$(E)$-feruloyl- $\beta$ - $D$-glucopyranosyl)-( $1 \rightarrow 2)-\beta$ - $D$ glucopyranoside]-5- $O$-( $\beta$ - $D$-glucopyranoside), 16780

$\mathrm{C}_{52} \mathrm{H}_{55} \mathrm{O}_{26}{ }^{+}$

Pelargonidin-3-O-[6-O-(E)-caffeoyl-2-O-(6-(E)feruloyl- $\beta$ - $D$-glucopyranosyl)-( $1 \rightarrow 2)-\beta$ - $D$-glucopyranoside]-5-O-( $\beta$ - $D$-glucopyranoside $)$, 16778

Pelargonidin-3-O-[6-O-(E)-feruloyl-2- $O-(6-(E)-$ caffeoyl- $\beta$ - $D$-glucopyranosyl)-( $1 \rightarrow 2)-\beta$ - $D$-glucopyranoside]-5- $O$-( $\beta$ - $D$-glucopyranoside $)$, 16782

\section{$\mathrm{C}_{52} \mathrm{H}_{59} \mathrm{NO}_{18}$}

Taxol C-7-xylose, 20816

7-( $\beta$-Xylosyl)taxol, 22851

\section{$\mathrm{C}_{52} \mathrm{H}_{62} \mathrm{O}_{28}$}

Fallaxose D, 7712

Watterose A, 22639

\section{$\mathrm{C}_{52} \mathrm{H}_{62} \mathrm{O}_{29}$}

Watterose F, 22644

\section{$\mathrm{C}_{52} \mathrm{H}_{64} \mathrm{O}_{26}$}

Coelobillardin, 3888

\section{$\mathrm{C}_{52} \mathrm{H}_{66} \mathrm{O}_{11}$}

Gnidicin-20-palmitate, 8899

\section{$\mathrm{C}_{52} \mathrm{H}_{74} \mathrm{O}_{19}$}

Inerme A, 11033

\section{$\mathrm{C}_{52} \mathrm{H}_{76} \mathrm{O}_{5}$}

Siphonein, 19965

\section{$\mathrm{C}_{52} \mathrm{H}_{77} \mathrm{NO}_{18}$}

12-O-Nicotinoyllineolon 3-O- $\beta$ - $D$-oleandropyranosyl-(1 $\rightarrow 4)-\beta$ - $D$-digitoxopyranosyl-( $1 \rightarrow 4)-\beta$ $D$-digitoxopyranosyl-(1 $\rightarrow 4)-\beta$ - $D$-digitoxopyranoside, 15533

\section{$\mathrm{C}_{52} \mathrm{H}_{77} \mathrm{NO}_{19}$}

Rostratamine 3-O- $\beta$ - $D$-oleandropyra-nosyl$(1 \rightarrow 4)-\beta$ - $D$-digitoxopyranosyl-( $(1 \rightarrow 4)-\beta$ - $D$ digitoxopyranosyl-(1 $\rightarrow 4)-\beta$ - $D$-digitoxopyranoside, 18937

\section{$\mathrm{C}_{52} \mathrm{H}_{80} \mathrm{O}_{\mathbf{1 8}}$}

3 - $O$ - $\beta$ - $D$-Galactopyranosyl-( $1 \rightarrow 2)-\beta$ - $D$-glucuronopyranosyl-21 $\beta, 22 \alpha$-di- $O$-angeloylbarringtogenol C, 8055

$\mathrm{C}_{52} \mathrm{H}_{82} \mathrm{O}_{21}$ 
Begoniifolide B, 2212

Begoniifolide C, 2213

Ciwujianoside $\mathrm{C}_{1}, 3781$

$2 \alpha, 3 \beta$-Dihydroxyurs-12,20(30)-dien-28-oic acid $3-O$ - $\{O-\beta$ - $D$-quinovopyranosyl- $(1 \rightarrow 2)-O-\alpha-L-$ arabinopyranosyl-( $(1 \rightarrow 2)-O$-[ $\beta$ - $D$-xylopyranosyl-( $1 \rightarrow 3)] \beta$ - $D$-glucopyranoside $\}, 6174$

Eupteleasaponin I, 7622

Eupteleasaponin XII, 7635

3-O-methyl malonylhederagenin $28-O-\alpha-L-$ rhamnopyranosyl-( $(\rightarrow 4)-\beta$ - $D$-glucopyranosyl$(1 \rightarrow 6)$ - $\beta$ - $D$-glucopyranoside, 14573

23-O-Methyl malonylhederagenin 28-O- $\alpha-L-$ rhamnopyranosyl-( $(\rightarrow 4)-\beta$ - $D$-glucopyranosyl$(1 \rightarrow 6)-\beta$ - $D$-glucopyranoside, 14574

Yemuoside $\mathrm{YM}_{14}, 22892$

\section{$\mathrm{C}_{52} \mathrm{H}_{82} \mathrm{O}_{22}$}

Araloside B, 1609

$2 \alpha, 3 \beta$-Dihydroxyurs-12,20(30)-dien-28-oic acid 3-O-\{O- $\beta$ - $D$-glucopyranosyl-( $(1 \rightarrow 2)-O-\alpha-L$ arabinopyranosyl-( $(1 \rightarrow 2)-O$ - $[\beta$ - $D$-xylopyranosyl-(1 $\rightarrow 3)] \beta$ - $D$-glucopyranoside\}, 6173

Symplocos glomerata saponin 7, 20535

3-O-[(2'-O-Xylosyl)-(3'-O-rarbinosyl)]-glucuronyloleanolic acid-28-O- $\beta$ - $D$-glucopyranoside, 22850

\section{$\mathrm{C}_{52} \mathrm{H}_{82} \mathrm{O}_{23}$}

(23R)-17 $\alpha, 23$-Epoxy-29-hydroxy-3 $\beta$-[(O- $\alpha-L-$ rhamnopyranosyl-( $1 \rightarrow 2)-O-\beta-D$-glucopyranosyl-( $1 \rightarrow 2)-O-\alpha$ - $L$-arabinopyranosyl- $(1 \rightarrow 6)$ $\beta$ - $D$-glucopyranosyl)oxy]-27-norlanost-8-ene15,24-dione, 7130

(23S)-17 $\alpha, 23$-Epoxy-29-hydroxy-3 $\beta$-[(O- $\alpha-L$ rhamnopyranosyl-(1 $\rightarrow 2)-O-\beta-D$-glucopyranosyl-( $(1 \rightarrow 2)-O-\alpha$ - $L$-arabinopyranosyl- $(1 \rightarrow 6)$ $\beta$ - $D$-glucopyranosyl)oxy]-27-norlanost-8-ene15,24-dione, 7131

\section{$\mathrm{C}_{52} \mathrm{H}_{82} \mathrm{O}_{24}$}

Aesculiside A, 665

$(23 S, 25 R)-3 \beta-[(O-\beta-D-$ Apiofuranosyl- $(1 \rightarrow 2)-O-$ $\beta$ - $D$-glucopyranosyl-( $(\rightarrow 2)-\alpha$ - $L$-arabinopyranosyl-( $1 \rightarrow 6)-\beta$ - $D$-glucopyranosyl)oxy]-17 $\alpha$, 23-epoxy-28,29-dihydroxylanost-8-en-23,26olide, 1505

\section{$\mathrm{C}_{52} \mathrm{H}_{82} \mathrm{O}_{25}$}

Desacyl-theasaponin E, 5243

Withanoside IX, 22712

\section{$\mathrm{C}_{52} \mathrm{H}_{84} \mathrm{O}_{20}$}

Clematis prosapogenin, Cp7a, 3823

Obtusilobicinin, 15905

Oleanolic acid 3-O- $\beta$ - $D$-glucopyranosyl $(1 \rightarrow 4)$ $\beta$-D-ribopyranosyl( $(1 \rightarrow 3)-\alpha$ - $L$-rhamnopyranosyl $(1 \rightarrow 2)-\alpha-L$-arabinopyranoside, 16057
Oleanolic acid-3-O- $\beta$ - $D$-glucopyranosyl $(1 \rightarrow 4)$ $\beta$ - $D$-xylopyranosyl $(1 \rightarrow 3)$ - $\alpha$ - $L$-rhamnopyranosyl $(1 \rightarrow 2)-\alpha-L$-arabinopyranoside, 16058

Scabioside E, 19445

\section{$\mathrm{C}_{52} \mathrm{H}_{84} \mathrm{O}_{21}$}

Hederagenin-3-O- $\alpha-L$-rhamnopyranosyl-( $(1 \rightarrow 2)$ $\alpha$ - $L$-arabinopyranosyl-28- $O-\beta-D$-xylopyranosyl-( $1 \rightarrow 6)-\beta$ - $D$-glucopyranoside, 9272

Jujuboside B, 11911

Jujuboside $\mathrm{B}_{1}, 11912$

Pometia ridleyi saponin 3, 17690

Pometia ridleyi saponin 8, 17694

Prosapogenin $\mathrm{CP}_{8}, 17947$

Prosapogenin $\mathrm{CP}_{8 \mathrm{a}}, 17948$

Sapindoside C, 19318

Yuzhizioside, 22957

Zizyphussaponin III, 23018

\section{$\mathrm{C}_{52} \mathrm{H}_{84} \mathrm{O}_{22}$}

Dioscoreside C, 6438

Echinosophoroside B, 6694

(23S)-17 $\alpha, 23$-Epoxy-29-hydroxy-3 $\beta$-[(O- $\alpha-L-$ rhamnopyranosyl-( $(1 \rightarrow 2)-O-\beta$ - $D$-glucopyranosyl-( $(1 \rightarrow 2)-O-\alpha$ - $L$-arabinopyranosyl-( $(1 \rightarrow 6)$ $\beta$ - $D$-glucopyranosyl)oxy]-27-norlanost-8-en24-one, 7132

Isolineolon 3-O- $\beta$ - $D$-glucopyranosyl-(1 $\rightarrow 4)-\beta-D$ oleandropyranosyl-(1 $\rightarrow 4)-\beta$ - $D$-digitoxopyranosyl-(1 $\rightarrow 4)-\beta$ - $D$-digitoxopyranosyl-( $1 \rightarrow 4)$ $\beta$ - $D$-digitoxopyranoside, 11499

Lineolon-3- $O-\beta$ - $D$-glucopyranosyl-( $1 \rightarrow 4)-\beta$ - $D$ oleandropyranosyl-(1 $\rightarrow 4)-\beta$ - $D$-digitoxopyranosyl-( $1 \rightarrow 4)-\beta$ - $D$-digitoxopyranosyl-( $1 \rightarrow 4)-\beta$ $D$-digitoxopyranoside, 12882

Prostratoside D, 17959

Prostratoside E, 17960

\section{$\mathrm{C}_{52} \mathrm{H}_{84} \mathrm{O}_{23}$}

Abutiloside M, 46

Echinosophoroside $\mathrm{A}_{1}, 6693$

(23S)-17 $\alpha, 23$-Epoxy-28,29-dihydroxy-3 $\beta-[(O-\alpha-$ $L$-rhamnopyranosyl-(1 $\rightarrow 2)-O-\beta$ - $D$-glucopyranosyl-( $(1 \rightarrow 2)-\alpha-L$-arabinopyranosyl- $(1 \rightarrow 6)-\beta-$ $D$-glucopyranosyl)oxy]-27-norlanost-8-en-24one, 7083

15- $\beta$-Hydroxylineolon 3- $O-\beta$ - $D$-allopyranosyl$(1 \rightarrow 4)-\beta$ - $D$-oleandropyranosyl-(1 $\rightarrow 4)-\beta-D$ digitoxopyranosyl-( $1 \rightarrow 4)-\beta$ - $D$-digitoxopyrano-

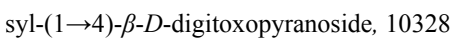

15- $\beta$-Hydroxylineolon 3-O- $\beta$ - $D$-glucopyranosyl$(1 \rightarrow 4)-\beta$ - $D$-oleandropyranosyl-( $(1 \rightarrow 4)-\beta-D$ digitoxopyranosyl-( $1 \rightarrow 4)-\beta$ - $D$-digitoxopyranosyl-(1 $\rightarrow 4)-\beta$ - $D$-digitoxopyranoside, 10330

Platycoside J, 17543

Soyasaponin $\mathrm{A}_{5}, 20125$

\section{$\mathrm{C}_{52} \mathrm{H}_{84} \mathrm{O}_{24}$}

Deapio platycodin D, 4799

\section{$\mathrm{C}_{52} \mathrm{H}_{85} \mathrm{NO}_{23}$}

Lycoperoside A, 13214

Lycoperoside B, 13215

Lycoperoside C, 13216

\section{$\mathrm{C}_{52} \mathrm{H}_{86} \mathrm{O}_{14}$}

Taibaienoside V, 20617

\section{$\mathrm{C}_{52} \mathrm{H}_{86} \mathrm{O}_{19}$}

Quinquenoside I, 18446

\section{$\mathrm{C}_{52} \mathrm{H}_{86} \mathrm{O}_{22}$}

Gypenoside XXXV, 9144

22-O-Methylprotodioscin, 14698

19-Oxo-3 $\beta, 20 S, 21,24 S$-tetrahydroxydammar-25-

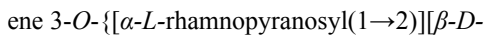
xylopyranosyl $(1 \rightarrow 3)]-\alpha$ - $L$-arabinopyranosyl $\}$ 21-O- $\beta$ - $D$-glucopyranoside, 16428

$\mathrm{S}-5,19093$

\section{$\mathrm{C}_{52} \mathrm{H}_{86} \mathrm{O}_{23}$}

Capilliposide A, 3125

(25R)-26-( $\beta$ - $D$-Glucopyranosyloxy)-22-methoxyfurost-5-en-3 $\beta$-yl- $O-\alpha$ - $L$-rhamnopyranosyl$(1 \rightarrow 2)-O$-[ $\beta$ - $D$-glucopyranosyl-( $(1 \rightarrow 6)]-\beta$ - $D$ glucopyranoside, 8705

Methyl deltoside, 14287

19-Oxo-3 $\beta, 20 S, 21$-trihydroxy-25-hydroperoxydammar-23-ene 3-O-\{[$\alpha-L$-rhamnopyranosyl $(1 \rightarrow 2)][\beta$ - $D$-xylopyranosyl $(1 \rightarrow 3)]-\alpha-L$-arabinopyranosyl\}-21-O- $\beta$ - $D$-glucopyranoside, 16433

(25R)-26-[( $\alpha$-L-Rhamnopyranosyl)oxy]-22 $\alpha$ methoxyfurost-5-en-3 $\beta$-yl- $O-\beta$ - $D$-glucopyranosyl-(1 $\rightarrow 3)-O-\beta$ - $D$-glucopyranosyl-( $1 \rightarrow 3$ )$O-\beta$ - $D$-glucopyranoside, 18731

Trigofoenoside $\mathrm{D}_{1}, 21652$

Trigofoenoside $\mathrm{F}_{1}, 21656$

\section{$\mathrm{C}_{52} \mathrm{H}_{88} \mathrm{O}_{21}$}

Gypenoside LVIII, 9163

Lotoideside A, 13004

Notoginsenoside O, 15833

Notoginsenoside P, 15834

$3 \beta, 19,20 S, 21$-Tetrahydroxydammar-24-ene 3-O$\{[\alpha-L$-rhamnopyranosyl $(1 \rightarrow 2)][\beta$ - $D$-xylopyra$\operatorname{nosyl}(1 \rightarrow 3)]-\alpha-L$-arabinopyranosyl $\}-21-O-\beta$ $D$-glucopyranoside, 21091

\section{$\mathrm{C}_{52} \mathrm{H}_{88} \mathrm{O}_{22}$}

Gypenoside XXII, 9139

\section{$\mathrm{C}_{52} \mathrm{H}_{88} \mathrm{O}_{23}$}

Trigofoenoside $\mathrm{C}_{1}, 21650$

\section{$\mathrm{C}_{52} \mathrm{H}_{92} \mathrm{O}_{7}$}

$\beta$-Sitosteryl glucoside 3'- $O$-heptadecoicate, 19996

$\mathrm{C}_{52} \mathrm{H}_{104} \mathrm{O}_{2}$ 
Ceryl cerotate, 3436

Octacosyl lignocerate, 15937

$$
\begin{aligned}
& \mathrm{C}_{53} \mathrm{H}_{57} \mathrm{O}_{26}{ }^{+} \\
& \text {Pelargonidin-3-O-[6-O- }(E) \text {-feruloyl-2-O- }(2-(E)- \\
& \quad \text { feruloyl- } \beta-D \text {-glucopyranosyl })-(1 \rightarrow 2)-\beta-D \text {-glu- } \\
& \quad \text { copyranoside]-5-O- }(\beta \text { - } D \text {-glucopyranoside }), \\
& 16783
\end{aligned}
$$

\section{$\mathrm{C}_{53} \mathrm{H}_{62} \mathrm{O}_{28}$}

Reiniose G, 18609

\section{$\mathrm{C}_{53} \mathrm{H}_{62} \mathrm{O}_{29}$}

Watterose B, 22640

\section{$\mathrm{C}_{53} \mathrm{H}_{62} \mathrm{O}_{30}$}

Watterose G, 22645

\section{$\mathrm{C}_{53} \mathrm{H}_{64} \mathrm{O}_{29}$}

Senegose G, 19722

Senegose H, 19723

\section{$\mathrm{C}_{53} \mathrm{H}_{74} \mathrm{O}_{11}$}

Gnidilatidin 20-palmitate, 8901

\section{$\mathrm{C}_{53} \mathrm{H}_{76} \mathrm{O}_{20}$}

Inerme B, 11034

\section{$\mathrm{C}_{53} \mathrm{H}_{79} \mathrm{NO}_{18}$}

12-O-Nicotinoyllineolon 3- $O-\beta-D$-oleandropyranosyl-( $1 \rightarrow 4)-\beta$ - $D$-digitoxopyranosyl- $(1 \rightarrow 4)-\beta$ $D$-digitoxopyranosyl-( $1 \rightarrow 4)-\beta$ - $D$-cymaropyranoside, 15532

\section{$\mathrm{C}_{53} \mathrm{H}_{80} \mathrm{O}_{2}$}

Plastoquinone, 17515

\section{$\mathrm{C}_{53} \mathrm{H}_{80} \mathrm{O}_{20}$}

TR-saponin A, 22061

\section{$\mathrm{C}_{53} \mathrm{H}_{81} \mathrm{~N}_{3} \mathrm{O}_{8}$}

Incarvillateine E, 11009

\section{$\mathrm{C}_{53} \mathrm{H}_{82} \mathrm{O}_{20}$}

TR-saponin B, 22062

\section{$\mathrm{C}_{53} \mathrm{H}_{82} \mathrm{O}_{22}$}

Eupteleasaponin VII, 7630

Kudinoside E, 12303

\section{$\mathrm{C}_{53} \mathrm{H}_{82} \mathrm{O}_{23}$}

Koelreuteriasaponin B, 12246

\section{$\mathrm{C}_{53} \mathrm{H}_{82} \mathrm{O}_{25}$}

Achyranthoside I, 543

\section{$\mathrm{C}_{53} \mathrm{H}_{84} \mathrm{O}_{21}$}

3-O- $\beta$ - $D$-Glucopyranosyl- $(1 \rightarrow 2)$ - $[\alpha-L$-rhamnopyranosyl-(1 $\rightarrow 4)]-\beta$ - $D$-glucuronopyranosyl-22$O$ - $\beta, \beta$-dimethylacryloyl-barrigenol $\mathrm{A}_{1}, 8725$

Latifoloside K, 12557

\section{$\mathrm{C}_{53} \mathrm{H}_{84} \mathrm{O}_{22}$}

Ardisimamilloside B, 1640

3-O- $\beta$ - $D$-Glucopyranosyl-( $1 \rightarrow 2)$ - $[\alpha-L$-rhamnopyranosyl-( $1 \rightarrow 4)]-\beta$ - $D$-glucuronopyranosyl-22$O$-angeloyl-barrigenol $\mathrm{R}_{1}, 8724$

Ilekudinoside G, 10977

3-O- $\alpha$ - $L$-Rhamnopyranosyl- $(1 \rightarrow 4)$ - $[\alpha$ - $L$-arabino-

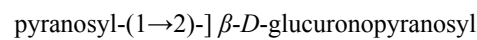

oleanolic acid 28-O- $\beta$ - $D$-glucopyranosyl ester, 18688

3-O-[ $\alpha$ - $L$-Rhamnopyranosyl- $(1 \rightarrow 3)-\beta$ - $D$-glucuronopyranosyl $]-28-O-[\beta-D$-xylopyranosyl $(1 \rightarrow 2)$ - $\beta$ - $D$-glucopyranosyl]-3 $\beta$-hydroxyolean12-en-28-oate, 18727

Rivularinin, 18855

\section{$\mathrm{C}_{53} \mathrm{H}_{84} \mathrm{O}_{22} \mathrm{~S}$}

Extensumside B, 7699

\section{$\mathrm{C}_{53} \mathrm{H}_{84} \mathrm{O}_{23}$}

Araloside C, 1610

Escin IVg, 7363

Escin IVh, 7364

Ilekudinoside H, 10978

Kudinoside C, 12301

Lanostane glycoside, 12481

Stipuleanoside $\mathrm{R}_{2}, 20384$

Yiyeliangwenoside XI, 22919

\section{$\mathrm{C}_{53} \mathrm{H}_{84} \mathrm{O}_{24}$}

Camellidin II, 3037

Sophoraflavoside III, 20091

\section{$\mathrm{C}_{53} \mathrm{H}_{84} \mathrm{O}_{25}$}

$3 \beta$ - $O$ - $(\beta$-Glucopyranosyl- $(1 \rightarrow 2)-\beta$-glucopyra$\operatorname{nosyl}(1 \rightarrow 3)-\beta$-xylopyranosyl)-16 $\alpha$-hydroxyolean-12-en-28- $O$-( $\beta$-galactopyranosyl $)$ ester-30-oic acid, 8662

\section{$\mathrm{C}_{53} \mathrm{H}_{86} \mathrm{O}_{20}$}

Eupteleasaponin XI, 7634

Raddeanin C, 18517

\section{$\mathrm{C}_{53} \mathrm{H}_{86} \mathrm{O}_{21}$}

Anhuienoside C, 1253

Anhuienoside D, 1254

Helianthoside A, 9294

Ilekudinoside A, 10971

Matesaponin 2, 13599

Mubenin A, 15004

Nudicaucin C, 15860

Oleanolic acid 3-O- $\alpha$ - $L$-rhamnopyranosyl $(1 \rightarrow 2)$ $\alpha$ - $L$-arabinopyransyl-28- $O-\beta$ - $D$-glucopyranosyl $(1 \rightarrow 6)-\beta$ - $D$-glucopyranoside, 16049

\section{Pastuchoside E, 16702}

$3 \beta$-D- $O$ - $(\alpha-L$-Rhamnopyranosyl- $(1 \rightarrow 2)-\alpha-L-$ arabinopyranosyl)-olean-12-ene-28- $O-(\beta-D$ glucopyranosyl-( $1 \rightarrow 4)-\beta$ - $D$-glucopyranosyl) ester, 18693

3- $O$ - $\alpha$ - $L$-Rhamnopyranosyl-( $1 \rightarrow 4)-\beta$ - $D$-quinovopyranosyl pyrocincholic acid $28-O-\beta$ - $D$-glucopyranosyl-( $1 \rightarrow 6)-\beta$ - $D$-glucopyranosyl ester, 18732

$3 \beta-D-O-(\beta-D-X y l o p y r a n o s y 1)-o l e a n-12$-ene-28$O$ - $(\alpha$ - $L$-rhamnopyranosyl- $(1 \rightarrow 4)-\beta$ - $D$-glucopyranosyl-( $1 \rightarrow 4)-\beta$ - $D$-glucopyranosyl) ester, 22838

\section{$\mathrm{C}_{53} \mathrm{H}_{86} \mathrm{O}_{22}$}

Ardipusilloside I, 1626

Ardisicrispin B, 1638

Ardisimamilloside G, 1641

Cauloside D, 3343

Cernuoside C, 3433

Clematibetoside C, 3821

$3 \beta$ - $O$ - $(\beta$ - $D$-Glucopyranosyl- $(1 \rightarrow 3)-\alpha-L$-rhamnopyranosyl-( $1 \rightarrow 2)$ - $\alpha$ - $L$-arabinopyranosyl)hederagenin-28- $O-\beta$ - $D$-glucopyranosyl ester, 8715

Hederagenin-3- $O-\alpha$ - $L$-rhamnopyranosyl-( $1 \rightarrow 2)$ $\alpha$ - $L$-arabinopyranosyl-28- $O-\beta$ - $D$-glucopyranosyl-(1 $\rightarrow 6)-\beta$ - $D$-glucopyranoside, 9271

Ioniceroside $\mathrm{C}, 11121$

Isolineolon 3- $O-\beta$ - $D$-glucopyranosyl- $(1 \rightarrow 4)-\beta$ - $D$ oleandropyranosyl-( $1 \rightarrow 4)-\beta$ - $D$-digitoxopyranosyl-( $1 \rightarrow 4)-\beta$ - $D$-digitoxopyranosyl-( $1 \rightarrow 4)-\beta$ $D$-cymaropyranoside, 11498

Kudinoside G, 12305

Latifoloside C, 12549

Latifoloside E, 12551

Latifoloside L, 12558

Lineolon-3- $O-\beta$ - $D$-glucopyranosyl-( $1 \rightarrow 4)-\beta$ - $D$ oleandropyranosyl-( $1 \rightarrow 4)-\beta$ - $D$-digitoxopyranosyl-( $1 \rightarrow 4)-\beta$ - $D$-digitoxopyranosyl- $(1 \rightarrow 4)$ $\beta$ - $D$-cymaropyranoside, 12881

Macranthoside B, 13303

Matesaponin 3, 13600

Mubenin C, 15005

Mukurozisaponin X, 15035

Oleanolic acid-3-O- $\beta$ - $D$-glucopyranosyl $(1 \rightarrow 2)$ $\alpha$ - $L$-arabinopyranosyl-28- $O-\beta$ - $D$-glucopyra$\operatorname{nosyl}(1 \rightarrow 6)$ - $\beta$ - $D$-glucopyranoside, 16054

Pometia ridleyi saponin 5, 17691

$3 \beta$ - $O$ - $(\alpha$ - $L$-Rhamnopyranosyl $(1 \rightarrow 2)-\alpha$ - $L$-arabinopyranosyl)-hederagenin-28- $O-\beta$ - $D$-glucopyra$\operatorname{nosyl}(1 \rightarrow 4)-\beta$ - $D$-glucopyranosyl)ester, 18689

$3-O$ - $\beta$ - $D$-Xylopyranosyl-( $1 \rightarrow 6)-\beta$ - $D$-glucopyranosyl-(1 $\rightarrow 6)-\beta$ - $D$-glucopyranosyl oleanolic acid 28-O- $\beta$ - $D$-glucopyranosyl ester, 22827

\section{$\mathrm{C}_{53} \mathrm{H}_{86} \mathrm{O}_{23}$}

Aralia-saponin IX, 1607

Ardisimamilloside A, 1639

Hederagenin-3-O- $\beta$ - $D$-glucopyranosyl $(1 \rightarrow 2)$ - $\alpha$ $L$-arabinopyranosyl-28- $O-\beta$ - $D$-glucopyranosyl $(1 \rightarrow 6)-\beta$ - $D$-glucopyranoside, 9263

Ixerissaponin B, 11800

Spongioside B, 20229

$2 \alpha, 3 \beta, 23$-Trihydroxyolean-12-en-28-oic acid $O$ $\beta$ - $D$-xylopyranosyl-( $1 \rightarrow 3)-O$ - $\alpha$ - $L$-rhamnopyranosyl-( $1 \rightarrow 4)-O$ - $\beta$ - $D$-glucopyranosyl- $(1 \rightarrow 6)$ $\beta$ - $D$-glucopyranosyl ester, 21826 


\section{$\mathrm{C}_{53} \mathrm{H}_{86} \mathrm{O}_{24}$}

Ixerissaponin C, 11801

Macrostemonoside D, 13329

Saikosaponin V, 19164

Scorzoneroside A, 19563

Soyasaponin $\mathrm{A}_{2}, 20122$

\section{$\mathrm{C}_{53} \mathrm{H}_{88} \mathbf{O}_{21}$}

3- $O$ - $\beta$ - $D$-Glucopyranosyl-( $1 \rightarrow 4)-\beta$ - $D$-fucopyranosyl (22S,24Z)-cycloart-24-en-3 $\beta, 22,26$-triol $26-O-\alpha$ - $L$-arabinopyranosyl-( $(1 \rightarrow 6)-\beta$ - $D$-glucopyranoside, 8626

3 - $O$ - $\beta$ - $D$-Glucopyranosyl-( $1 \rightarrow 4)-\beta$ - $D$-fucopyranosyl (22S,24Z)-cycloart-24-en-3 $\beta, 22,26$-triol $26-O-\beta$ - $D$-xylopyranosyl-( $(1 \rightarrow 6)-\beta$ - $D$-glucopyranoside, 8629

\section{$\mathrm{C}_{53} \mathrm{H}_{88} \mathrm{O}_{22}$}

Gypenoside XXXIV, 9143

Gypenoside XXXVII, 9146

\section{$\mathrm{C}_{53} \mathrm{H}_{88} \mathrm{O}_{23}$}

$3 \beta, 11 \alpha, 16 \beta$-Trihydroxycycloartane-24-one-3-O-

$[\beta$ - $D$-glucopyranosyl $(1 \rightarrow 3)$ - $\beta$ - $D$-glucopyra$\operatorname{nosyl}(1 \rightarrow 2)-\beta$ - $D$-glucopyranosyl]-16- $O-\alpha-L-$ arabinopyranoside, 21697

Yesanchinoside G, 22899

\section{$\mathrm{C}_{53} \mathrm{H}_{88} \mathrm{O}_{7}$}

Stigmasterol-3-(6-linoleoyl)glucopyranoside, 20373

\section{$\mathrm{C}_{53} \mathrm{H}_{90} \mathrm{O}_{7}$}

$\beta$-Sitosterol-3-(6-linoleoyl)glucopyranoside, 19989

Stigmasterol-3-(6-oleoyl)glucopyranoside, 20374

\section{$\mathrm{C}_{53} \mathrm{H}_{90} \mathrm{O}_{21}$}

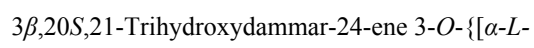
rhamnopyranosyl $(1 \rightarrow 2)][\beta$ - $D$-xylopyranosyl $(1 \rightarrow 3)]-\beta$ - $D$-glucopyranosyl\}-21-O- $\beta$ - $D$-glucopyranoside, 21702

\section{$\mathrm{C}_{53} \mathrm{H}_{90} \mathrm{O}_{22}$}

Ginsenoside $\mathrm{Rb}_{2}, 8424$

Ginsenoside Rc, 8426

Gypenoside XLIII, 9152

Gypenoside LVI, 9161

Gypenoside LXIII, 9167

Notoginsenoside L, 15830

$3 \beta, 19,20 S, 21-T e t r a h y d r o x y d a m m a r-24-e n e ~ 3-O-$ $\{[\alpha-L$-rhamnopyranosyl $(1 \rightarrow 2)][\beta$ - $D$-xylopyra$\operatorname{nosyl}(1 \rightarrow 3)]-\beta-D$-glucopyranosyl $\}-21-O-\beta-D$ glucopyranoside, 21092

\section{$\mathrm{C}_{53} \mathrm{H}_{90} \mathrm{O}_{23}$}

Ginsenoside $\mathrm{Rb}_{3}, 8425$ Gypenoside XLII, 9151 Gypenoside LXII, 9166 Gypenoside LXVII, 9170 Gypenoside LXVIII, 9171
Gypenoside LXX, 9172

Yesanchinoside H, 22900

\section{$\mathrm{C}_{\mathbf{5 3}} \mathrm{H}_{90} \mathrm{O}_{\mathbf{2 4}}$}

$3 \beta, 6 \alpha, 12 \beta, 20 S, 25$-Pentahydroxyl-dammar-23ene-6- $O-\beta-D$-glucopyranoside-20- $O-\beta-D$-glucopyranosyl-3-O- $\beta$ - $D$-xylopyranosyl $(1 \rightarrow 6)-D$ glucopyranoside, 16850

$3 \beta, 12,20 S$-Trihydroxy-25-hydroperoxydammar23 -ene 3- $O$ - $\{[\beta$ - $D$-glucopyranosyl $(1 \rightarrow 2)-\beta$ - $D$ glucopyranosyl $\}-20-O-\{[\beta$ - $D$-xylopyranosyl $(1 \rightarrow 6)]$ - $\beta$ - $D$-glucopyranoside\}, 21743

\section{$\mathrm{C}_{53} \mathrm{H}_{92} \mathrm{O}_{7}$}

Stigmasterol-3-(6-stearoyl)glucopyranoside, 20375

$\mathrm{C}_{53} \mathrm{H}_{94} \mathrm{O}_{7}$

$\beta$-Sitosterol-3-(6-stearoyl)glucopyranoside, 19991

\section{$\mathrm{C}_{53} \mathrm{H}_{102} \mathrm{O}_{6}$}

(2S)-1,3-Di-( $O$-palmitoyl)-2-O-octadecanoyl glycerol, 6480

\section{$\mathrm{C}_{54} \mathrm{H}_{42} \mathrm{O}_{36}$}

Sanguiin $\mathrm{H}_{8}, 19280$

Sanguiin $\mathrm{H}_{9}, 19281$

\section{$\mathrm{C}_{54} \mathrm{H}_{46} \mathrm{O}_{28}$}

Pentaisofuhalol dodecaacetate, 16860

\section{$\mathrm{C}_{54} \mathrm{H}_{54} \mathrm{O}_{11}$}

Blepharocalyxin E, 2500

\section{$\mathrm{C}_{54} \mathrm{H}_{64} \mathbf{O}_{29}$}

Glomeratose F, 8587

Reiniose H, 18610

\section{$\mathrm{C}_{54} \mathrm{H}_{64} \mathrm{O}_{30}$}

Watterose E, 22643

\section{$\mathrm{C}_{54} \mathrm{H}_{66} \mathrm{O}_{31}$}

Paniculatonoid A, 16607

\section{$\mathrm{C}_{54} \mathrm{H}_{78} \mathbf{O}_{19}$}

$16 \beta-[(O-(2-O-(E)$-Cinnamoyl- $\beta$-D-xylopyranosyl)-(1 $\rightarrow 2)-2-O$-acetyl- $\alpha$-L-arabinopyranosyl) oxy]-3 $\beta$-[( $\beta$-D-glucopyranosyl)oxy]-17 $\alpha$-hydroxycholest-5-en-22-one, 3724

\section{$\mathrm{C}_{54} \mathrm{H}_{80} \mathrm{O}_{\mathbf{2 0}}$}

Avenacin $\mathrm{B}_{2}, 2030$

\section{$\mathrm{C}_{54} \mathrm{H}_{80} \mathrm{O}_{21}$}

$3 \beta, 17 \alpha$-Dihydroxy-16 $\beta$-[ $(O-\beta$ - $D$-glucopyranosyl$(1 \rightarrow 4)-O$-(2-O-3,4-dimethoxybenzoyl- $\beta$ - $D$-xylopyranosyl)-( $(\rightarrow 3)-2-O$-acetyl- $\alpha-L$-arabinopyranosyl)oxy]cholest-5-en-22-one, 5899

$3 \beta$-[( $\beta$-D-Glucopyranosyl)oxy $]-17 \alpha$-hydroxy$16 \beta$-[( $O$-(2- $O-3,4-$ dimethoxybenzoyl- $\beta$ - $D$-xylopyranosyl)-( $(\rightarrow 2)-2-O$-acetyl- $\alpha$ - $L$-arabinopyranosyl)oxy]cholest-5-en-22-one, 8691

\section{$\mathrm{C}_{54} \mathrm{H}_{82} \mathrm{O}_{22}$ \\ Az III, 2059 \\ $\mathrm{C}_{54} \mathrm{H}_{82} \mathrm{O}_{23}$}

Az II, 2058

$\mathbf{C}_{54} \mathbf{H}_{83} \mathbf{N O}_{\mathbf{1 1}}$

1,2-Di-O-(9Z,12Z,15Z-octadecatrienoyl)-3-O-(6$p$-hydroxy-phenyl-propionamido-6-deoxy- $\alpha$ $D$-glucosyl)-glycerol, 6430

$\mathrm{C}_{54} \mathrm{H}_{84} \mathrm{O}_{21}$

Capilliposide I, 3128

$\mathrm{C}_{54} \mathrm{H}_{84} \mathrm{O}_{22}$

Az IV, 2060

$\mathrm{C}_{54} \mathrm{H}_{84} \mathrm{O}_{23}$

Symplocos glomerata saponin 8, 20536

\section{$\mathrm{C}_{54} \mathrm{H}_{84} \mathbf{O}_{25}$}

Ageratoside $\mathrm{A}_{4}, 731$

Ageratoside $A_{5}, 732$

Sinocrassuloside II, 19937

Sinocrassuloside V, 19940

$\mathrm{C}_{54} \mathrm{H}_{84} \mathrm{O}_{27}$

Lucilianoside D, 13059

$\mathrm{C}_{54} \mathrm{H}_{86} \mathrm{O}_{19}$

12-O-Tigloyllineolon, 21378

\section{$\mathrm{C}_{54} \mathrm{H}_{86} \mathrm{O}_{20}$}

12-O-Tigloyldeacylmetaplexigenin, 21375

\section{$\mathrm{C}_{54} \mathrm{H}_{86} \mathrm{O}_{21}$}

Cynanauriculoside B, 4553

\section{$\mathrm{C}_{54} \mathrm{H}_{86} \mathrm{O}_{22}$}

Acetyljujuboside B, 443

Cirenshenoside U, 3739

Hederagenin-3-O- $\alpha$ - $L$-rhamnopyranosyl-( $(1 \rightarrow 2)$ $\alpha$ - $L$-arabinopyranosyl-28- $O$-3-acetyl $\beta-D$-xylopyranosyl-( $1 \rightarrow 6)-\beta$ - $D$-glucopyranoside, 9269

\section{$\mathrm{C}_{54} \mathrm{H}_{86} \mathrm{O}_{23}$}

12-O-Acetyllineolon 3- $O-\beta$ - $D$-glucopyranosyl$(1 \rightarrow 4)-\beta$ - $D$-oleandropyranosyl-( $(1 \rightarrow 4)-\beta$ - $D$ digitoxopyranosyl-(1 $\rightarrow 4)-\beta$ - $D$-digitoxopyranosyl-( $1 \rightarrow 4)$ - $\beta$ - $D$-digitoxopyranoside, 450 Achyranthes saponin D, 542

3- $O-\alpha$ - $L$-Rhamnopyranosyl-( $1 \rightarrow 4)$-[ $[\beta$ - $D$-galactopyranosyl-( $1 \rightarrow 2)]-\beta$ - $D$-glucuronopyranosyl oleanolic acid 28- $O-\beta$ - $D$-Glucopyranosylester, 18705

\section{$\mathrm{C}_{54} \mathrm{H}_{86} \mathrm{O}_{24}$}

Berneuxia saponin C, 2315

Calendasaponin A, 2965

Calendula officinalis Glycoside A, 2969

Hydrocotyloside III, 9712

Hydrocotyloside IV, 9713

Metaplexigenin-3- $O-\beta$ - $D$-glucopyranosyl-( $(1 \rightarrow 4)$ $\beta$ - $D$-oleandropyranosyl-(1 $\rightarrow 4)$ - $\beta$ - $D$-digitoxopyranosyl-(1 $\rightarrow 4)-\beta$ - $D$-digitoxopyranosyl$(1 \rightarrow 4)$ - $\beta$ - $D$-digitoxopyranoside, 13815

\section{$\mathrm{C}_{54} \mathrm{H}_{86} \mathrm{O}_{25}$}

Azukisaponin VI, 2070

Calendasaponin D, 2968 
Dianoside B, 5359

\section{$\mathrm{C}_{54} \mathrm{H}_{86} \mathrm{O}_{26}$}

Segetoside K, 19668

\section{$\mathrm{C}_{54} \mathrm{H}_{88} \mathrm{O}_{21}$}

Gypentonoside A, 9182

Mimengoside A, 14866

\section{$\mathrm{C}_{54} \mathrm{H}_{88} \mathrm{O}_{22}$}

Achyranthes saponin B, 540

3-O- $\alpha$ - $L$-Rhamnopyranosyl $(1 \rightarrow 2)-\beta$ - $D$-xylopyranosyl $(1 \rightarrow 2)-6-O$-methyl- $\beta$ - $D$-glucuronopyranosyl-soyasapogenol B 22- $O-\beta$ - $D$-glucopyranoside, 18737

$3 \beta, 16 \alpha, 28$-Trihydroxyolean-11,13(18)-dien-3 $\beta$ yl- $\beta$ - $D$-glucopyranosyl-( $1 \rightarrow 6)$ - $[\alpha-L$-rhamnopyranosyl- $(1 \rightarrow 4)]-\beta$ - $D$-glucopyranoside-28- $O-\beta$ $D$-glucopyranoside, 21823

\section{$\mathrm{C}_{54} \mathrm{H}_{88} \mathrm{O}_{23}$}

Acankoreoside C, 73

Aralia-saponin V, 1603

Calenduloside D, 2974

Cirenshenoside S, 3737

Cirenshenoside T, 3738

3-O- $\beta$ - $D$-Glucopyranosyl-( $(1 \rightarrow 6)-\beta$ - $D$-glucopyranosyl oleanolic acid 28- $\beta$ - $D$-glucopyranosyl-( $1 \rightarrow 6)-\beta$ - $D$-glucopyranosyl ester, 8648

15- $\beta$-Hydroxylineolon 3-O- $\beta$ - $D$-glucopyranosyl$(1 \rightarrow 4)-\beta$ - $D$-oleandropyranosyl- $(1 \rightarrow 4)-\beta$ - $D$ digitoxopyranosyl-( $1 \rightarrow 4)-\beta$ - $D$-cymaropyranosyl-( $1 \rightarrow 4)-\beta$ - $D$-cymaropyranoside, 10329

Paridiformoside, 16664

3-O- $\{\alpha-L$-Rhamnopyranosyl-( $1 \rightarrow 2)-\beta$ - $D$-galactopyranosyl- $(1 \rightarrow 3)$ - $[\beta$ - $D$-glucopyranosyl$(1 \rightarrow 2)]$ - $\beta$ - $D$-glucuronopyranosyl $\}$-primulagenin A, 18699

3-O-[ $\alpha$ - $L$-Rhamnopyranosyl $(1 \rightarrow 2)-\beta$ - $D$-galactopyranosyl $(1 \rightarrow 2)-\beta$ - $D$-glucuronopyranosyl]22-O- $\beta$ - $D$-glucopyranosylsoyasapogenol $\mathrm{B}$, 18703

$3 \beta, 21 \beta, 22 \beta, 24$-Tetrahydroxyolean-12-en-3- $O-\alpha$ $L$-rhamnopyranosyl-( $1 \rightarrow 3)$ - $[\alpha-L$-rhamnopyranosyl-( $1 \rightarrow 2)]$ - $\beta$ - $D$-galactopyranosyl- $(1 \rightarrow 2)$ $\beta$ - $D$-glucuronopyranoside, 21146

\section{$\mathrm{C}_{54} \mathrm{H}_{88} \mathrm{O}_{24}$}

Aralia-saponin VI, 1604

Aralia-saponin VII, 1605

(25R)-26-[( $\alpha$ - $L$-Rhamnopyranosyl)oxy]-22 $\alpha$ methoxyfurost-5-en-3 $\beta$-yl- $O-\beta$ - $D$-glucopyranosyl-( $1 \rightarrow 3)-O$-[6-acetyl- $\beta$ - $D$-glucopyranosyl$(1 \rightarrow 3)]-O$ - $\beta$ - $D$-glucopyranoside, 18730

Saikosaponin O, 19154

Ternstroemiaside A, 20980

Ternstroemiaside C, 20982

$\mathrm{C}_{54} \mathrm{H}_{88} \mathrm{O}_{25}$
Desacyl-boninsaponin A, 5241

Glycoside $\mathrm{H}_{2}, 8830$

S-4a, 19092

$\mathrm{C}_{54} \mathrm{H}_{90} \mathrm{O}_{9}$

Kansuiphorin A, 12145

$\mathrm{C}_{54} \mathrm{H}_{90} \mathrm{O}_{10}$

Kansuiphorin B, 12146

$\mathrm{C}_{54} \mathrm{H}_{90} \mathrm{O}_{21}$

3-O- $\beta$ - $D$-Glucopyranosyl-( $1 \rightarrow 4)-\beta$ - $D$-fucopyranosyl $(22 S, 24 Z)$-cycloart-24-en-3 $\beta, 22,26$-triol $26-O$ - $\beta$ - $D$-quinovopyranosyl-( $1 \rightarrow 6)-\beta$ - $D$-glucopyranoside, 8628

Telosmoside $\mathrm{A}_{8}, 20919$

\section{$\mathrm{C}_{54} \mathrm{H}_{90} \mathrm{O}_{22}$}

Gypenoside XXXVI, 9145

Primulasaponin, 17859

Telosmoside A9, 20920

$3 \beta, 20 S, 21-T r i h y d r o x y d a m m a r-24-e n e ~ 3-O$ $\{[\alpha$ - $L$-rhamnopyranosyl $(1 \rightarrow 2)][\beta$ - $D$-glucopy$\operatorname{ranosyl}(1 \rightarrow 3)]-\beta$ - $D$-glucopyranosyl $\}-21-O-\beta$ -

$D$-glucopyranoside, 21700

\section{$\mathrm{C}_{54} \mathrm{H}_{90} \mathrm{O}_{24}$}

Notoginsenoside B, 15819

Quinquenoside IV, 18449

\section{$\mathrm{C}_{54} \mathrm{H}_{92} \mathrm{O}_{21}$}

Gypenoside VII, 9128

\section{$\mathrm{C}_{54} \mathrm{H}_{92} \mathrm{O}_{22}$}

Gypenoside V, 9126

Gypenoside VI, 9127

Gypenoside XVIII, 9135

Notoginsenoside I, 15827

\section{$\mathrm{C}_{54} \mathrm{H}_{92} \mathrm{O}_{23}$}

Ginsenoside $\mathrm{Rb}_{1}, 8423$

Gypenoside XIX, 9136

Yesanchinoside E, 22897

Yixinoside A, 22913

\section{$\mathrm{C}_{54} \mathrm{H}_{92} \mathrm{O}_{24}$}

Grosmomoside I, 9012

Notoginsenoside A, 15818

(24S)-3 $\beta, 11 \alpha, 16 \beta, 24-$ Tetrahydroxycycloartane-3$O$-[ $\beta$ - $D$-glucopyranosyl $(1 \rightarrow 3)-\beta$ - $D$-glucopyra$\operatorname{nosyl}(1 \rightarrow 2)-\beta$ - $D$-glucopyranosyl]-24-O- $\beta$ - $D$ glucopyranoside, 21087

\section{$\mathrm{C}_{54} \mathrm{H}_{92} \mathrm{O}_{25}$}

Notoginsenoside C, 15820

Notoginsenoside K, 15829

$\mathrm{C}_{54} \mathrm{H}_{108} \mathrm{O}_{2}$

Ceryl montanate, 3438

Heptacosyl heptacosanate, 9372

Melissyl lignocerate, 13708

\section{$\mathrm{C}_{55} \mathrm{H}_{60} \mathrm{O}_{30}$}

Quercetin-3-(2-sinapoyl- $O$ - $\beta$ - $D$-glucopyranosyl)3'-(6-sinapoyl- $O-\beta$ - $D$-glucopyranosyl)-4'- $O-\beta$ -
D-glucopyranoside, 18397

$\mathrm{C}_{55} \mathrm{H}_{62} \mathrm{~N}_{10}$

Psychotridine, 18094

$\mathrm{C}_{55} \mathrm{H}_{66} \mathrm{O}_{30}$

Senegose F, 19721

$\mathrm{C}_{55} \mathrm{H}_{69} \mathrm{~N}_{11} \mathrm{O}_{13}$

Celogenamide A, 3384

$\mathrm{C}_{55} \mathrm{H}_{72} \mathrm{~N}_{4} \mathrm{O}_{6}$

Pheophytin b, 17141

\section{$\mathrm{C}_{55} \mathrm{H}_{73} \mathrm{~N}_{9} \mathrm{O}_{9} \mathrm{~S}_{2}$}

Cyclolinopeptide I, 4514

$\mathrm{C}_{55} \mathrm{H}_{73} \mathrm{~N}_{\mathbf{9}} \mathrm{O}_{10} \mathrm{~S}_{2}$

Cyclolinopeptide F, 4511

\section{$\mathrm{C}_{55} \mathrm{H}_{74} \mathrm{~N}_{4} \mathrm{O}_{5}$}

Pheophytin a, 17140

\section{$\mathrm{C}_{55} \mathrm{H}_{80} \mathrm{O}_{18}$}

Ikemagenin 3-O- $\beta$ - $D$-oleandropyranosyl- $(1 \rightarrow 4)$ $\beta$ - $D$-digitoxopyranosyl-( $1 \rightarrow 4)-\beta$ - $D$-digitoxopyranosyl-( $1 \rightarrow 4)-\beta$ - $D$-digitoxopyranoside, 10966

\section{$\mathrm{C}_{55} \mathrm{H}_{82} \mathrm{O}_{22}$}

$3 \beta,-17 \alpha$-Dihydroxy-16 $\beta$-[( $O-\beta$ - $D$-glucopyranosyl-(1 $\rightarrow 4)-O-(2-O-3,4,5$-trimethoxybenzoyl- $\beta$ $D$-xylopyranosyl)-( $1 \rightarrow 3)-2-O$-acetyl- $\alpha$ - $L$-arabinopyranosyl)oxy]cholest-5-en-22-one, 5900

\section{$\mathrm{C}_{55} \mathrm{H}_{83} \mathrm{NO}_{20}$}

Pregnane glycoside HI, 17793

\section{$\mathrm{C}_{55} \mathrm{H}_{83} \mathrm{NO}_{21}$}

Avenacin $\mathrm{A}_{1}, 2029$

\section{$\mathrm{C}_{55} \mathrm{H}_{84} \mathrm{O}_{21}$}

TR-saponin C, 22063

\section{$\mathrm{C}_{55} \mathrm{H}_{86} \mathrm{O}_{22}$}

Tenacissoside K, 20931

\section{$\mathrm{C}_{55} \mathrm{H}_{86} \mathrm{O}_{23}$}

Escin IIIa, 7362

\section{$\mathrm{C}_{55} \mathrm{H}_{86} \mathrm{O}_{24}$}

Aescin, 660

Escin Ia, 7361

Escin VIb, 7365

Isoescin Ia, 11416

Isoescin Ib, 11417

Symplocos glomerata saponin 9, 20537

Symplocos glomerata saponin 10, 20538

Yiyeliangwenoside IX, 22917

Yiyeliangwenoside X, 22918

\section{$\mathrm{C}_{55} \mathrm{H}_{86} \mathrm{O}_{25}$}

Camellidin I, 3036

Sinocrassuloside III, 19938

\section{$\mathrm{C}_{55} \mathrm{H}_{88} \mathrm{O}_{21}$}

Cynanauriculoside A, 4552

\section{$\mathrm{C}_{55} \mathrm{H}_{88} \mathrm{O}_{22}$}

Ciwujianoside $\mathrm{D}_{1}, 3782$

$\mathrm{C}_{55} \mathrm{H}_{88} \mathrm{O}_{23}$ 


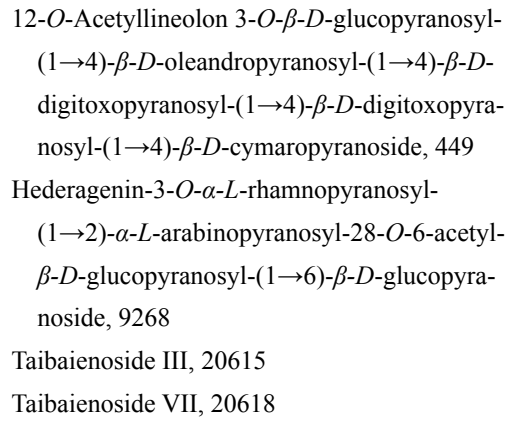

Taibaienoside III, 20615

Taibaienoside VII, 20618

\section{$\mathrm{C}_{55} \mathrm{H}_{88} \mathrm{O}_{24}$}

Metaplexigenin-3- $O-\beta$ - $D$-glucopyranosyl-( $(1 \rightarrow 4)$ $\beta$ - $D$-oleandropyranosyl-( $1 \rightarrow 4)-\beta$ - $D$-digitoxopyranosyl-( $1 \rightarrow 4)-\beta$ - $D$-digitoxopyranosyl$(1 \rightarrow 4)$ - $\beta$ - $D$-cymaropyranoside, 13814

Saponin 9, 19341

\section{$\mathrm{C}_{55} \mathrm{H}_{88} \mathrm{O}_{25}$}

Calendasaponin C, 2967

3 - $O$ - $[\alpha$ - $L$-Rhamnopyranosyl- $(1 \rightarrow 2)-\beta$ - $D$-glucopyranosyl-( $(1 \rightarrow 2)-\beta$ - $D$-glucopyranosyl] phytolaccagenic acid 28- $O-\beta$ - $D$-glucopyranosyl ester, 18713

\section{$\mathrm{C}_{55} \mathrm{H}_{88} \mathrm{O}_{27}$}

$(25 R)-3 \beta-[(O-\alpha-L$-Arabinopyranosyl-( $(1 \rightarrow 3)-\beta-D-$ glucopyranosyl-( $1 \rightarrow 2)-O$ - $[\beta$ - $D$-xylopyranosyl$(1 \rightarrow 3)]-O-\beta$ - $D$-glucopyranosyl-( $1 \rightarrow 4)-\beta$ - $D$-galactopyranosyl)oxy]-5 $\alpha$-spirostan-12-one, 1571

\section{Cantalasaponin 4, 3093}

Hecogenin 3-O- $\beta$ - $D$-xylopyranosyl- $(1 \rightarrow 3)-\beta-D$ glucopyranosyl-( $(1 \rightarrow 2)$-[ $\beta$ - $D$-xylopyranosyl$(1 \rightarrow 3)]-\beta$ - $D$-glucopyranosyl-( $1 \rightarrow 4)-\beta$ - $D$-galactopyranoside, 9256

\section{$\mathrm{C}_{55} \mathrm{H}_{90} \mathrm{O}_{23}$}

3-O- $\alpha$ - $L$-Rhamnopyranosyl $(1 \rightarrow 2)-\beta$ - $D$-galactopyranosyl $(1 \rightarrow 2)-6-O$-methyl- $\beta$ - $D$-glucuronopyranosyl-soyasapogenol B-22- $O-\beta$ - $D$-glucopyranoside, 18707

\section{$\mathrm{C}_{55} \mathrm{H}_{90} \mathrm{O}_{26}$}

Agave americana Glycoside 1, 716

Agave cantala Agaveside B, 718

Agave cantala Compound 1', 722

Dongnoside D, 6556

Tigogenin 3-O- $\beta-D$-xylopyranosyl-( $(1 \rightarrow 3)-\beta-D-$ glucopyranosyl-( $(1 \rightarrow 2)$-[ $\beta$ - $D$-xylopyranosyl$(1 \rightarrow 3)]-\beta$ - $D$-glucopyranosyl-( $(1 \rightarrow 4)-\beta$ - $D$ galactopyranoside, 21387

\section{$\mathrm{C}_{55} \mathrm{H}_{90} \mathrm{O}_{27}$}

Chlorogenin-3- $O-\beta-D$-xylopyranosyl-( $(1 \rightarrow 3)-\beta$ $D$-glucopyranosyl-( $(1 \rightarrow 2)$-[ $\beta$ - $D$-xylopyranosyl$(1 \rightarrow 3)]-\beta$ - $D$-glucopyranosyl-( $1 \rightarrow 4)-\beta$ - $D$-galactopyranoside, 3553

\section{$\mathrm{C}_{55} \mathrm{H}_{92} \mathrm{O}_{19}$}

Telosmoside $\mathrm{A}_{7}, 20918$
$\mathrm{C}_{55} \mathrm{H}_{92} \mathrm{O}_{22}$

Mimengoside B, 14867

Telosmoside $\mathrm{A}_{2}, 20913$

$3 \beta, 20 S, 21$-Trihydroxydammar-24-ene 3-O- $\{[\alpha-L-$ rhamnopyranosyl $(1 \rightarrow 2)][\beta$ - $D$-xylopyranosyl $(1 \rightarrow 3)]-\beta-D$-[6-O-acetylglucopyranosyl]\}-21$O-\beta$ - $D$-glucopyranoside, 21701

\section{$\mathrm{C}_{55} \mathrm{H}_{92} \mathrm{O}_{23}$}

Ginsenoside $\mathrm{Rs}_{1}, 8446$

Ginsenoside $\mathrm{Rs}_{2}, 8447$

\section{$\mathrm{C}_{55} \mathrm{H}_{92} \mathrm{O}_{26}$}

Asparaside C, 1883

$\mathrm{C}_{55} \mathrm{H}_{92} \mathrm{O}_{27}$

Asparaside D, 1884

$\mathrm{C}_{55} \mathrm{H}_{98} \mathrm{O}_{7}$

$\beta$-Sitosterol-3- $O-\beta$ - $D$-glucoside-6'- $O$-eicosanate, 19988

\section{$\mathrm{C}_{55} \mathrm{H}_{99} \mathrm{~N}_{3} \mathrm{O}_{18}$}

Polaramycin A, 17609

$\mathrm{C}_{56} \mathrm{H}_{38} \mathrm{O}_{12}$

Upunaphenol B, 22233

$\mathrm{C}_{56} \mathrm{H}_{40} \mathrm{O}_{12}$

Davidiol C, 4697

Kobophenol B, 12240

\section{$\mathrm{C}_{56} \mathrm{H}_{40} \mathrm{O}_{13}$}

Nepalensinol G, 15481

\section{$\mathrm{C}_{56} \mathrm{H}_{40} \mathrm{O}_{31}$}

Stenophyllanin A, 20310

\section{$\mathrm{C}_{56} \mathrm{H}_{42} \mathrm{O}_{12}$}

Cotylelophenol C, 4130

Gnemonol B, 8867

Gnemonol C, 8868

Halophilol B, 9199

cis-Miyabenol A, 14890

Nepalensinol F, 15480

Upunaphenol D, 22235

Vaticanol B, 22355

Vaticanol C, 22356

(+)-Vitisin A, 22584

(+)-cis-Vitisin A, 22585

Vitisin B, 22586

Vitisin C, 22587

(+)-Vitisin D, 22588

\section{$\mathrm{C}_{56} \mathrm{H}_{42} \mathrm{O}_{13}$}

Upunaphenol C, 22234

$\mathrm{C}_{56} \mathrm{H}_{44} \mathbf{O}_{13}$

Kobophenol A, 12239

Nepalensinol E, 15479

\section{$\mathrm{C}_{56} \mathrm{H}_{46} \mathrm{O}_{8}$}

Pusilatin C, 18225

$\mathrm{C}_{56} \mathrm{H}_{72} \mathrm{O}_{6}$

Xuxuasin A, 22812

$\mathrm{C}_{56} \mathrm{H}_{72} \mathrm{O}_{7}$
7', 8'-Dihydroxuxuarine A $\alpha, 5744$

Xuxuasin B, 22813

$\mathrm{C}_{56} \mathrm{H}_{72} \mathrm{O}_{8}$

7', 8'-Dihydroxuxuarine $\mathrm{D} \beta, 5745$

\section{$\mathrm{C}_{56} \mathrm{H}_{75} \mathrm{~N}_{9} \mathrm{O}_{9} \mathrm{~S}_{2}$}

Cyclolinopeptide $\mathrm{H}, 4513$

$\mathrm{C}_{56} \mathrm{H}_{75} \mathrm{~N}_{\mathbf{9}} \mathrm{O}_{10} \mathrm{~S}_{\mathbf{2}}$

Cyclolinopeptide G, 4512

$\mathrm{C}_{56} \mathrm{H}_{80} \mathrm{O}_{22}$

Cynaphylloside G, 4562

\section{$\mathrm{C}_{56} \mathrm{H}_{82} \mathrm{O}_{18}$}

Ikemagenin 3-O- $\beta$ - $D$-oleandropyranosyl-( $(1 \rightarrow 4)$ $\beta$ - $D$-digitoxopyranosyl-( $1 \rightarrow 4)$ - $\beta$ - $D$-digitoxopyranosyl-( $1 \rightarrow 4)-\beta$ - $D$-cymaropyranoside, 10965

\section{$\mathrm{C}_{56} \mathrm{H}_{82} \mathrm{O}_{22}$}

Periandradulcin A, 16918

\section{$\mathrm{C}_{56} \mathrm{H}_{84} \mathrm{O}_{19}$}

Pregnane glycoside AI, 17785

Pregnane glycoside BI, 17786

\section{$\mathrm{C}_{56} \mathrm{H}_{84} \mathrm{O}_{20}$}

Pregnane glycoside CI, 17787

Pregnane glycoside CII, 17788

Pregnane glycoside WI, 17800

12-O-Salicyloyllineolon, 19190

\section{$\mathrm{C}_{56} \mathrm{H}_{84} \mathrm{O}_{21}$}

Pregnane glycoside UI, 17798

Pregnane glycoside VI, 17799

12-O-Salicyloyldeacylmetaplexigenin, 19189

\section{$\mathrm{C}_{56} \mathrm{H}_{86} \mathrm{O}_{3}$}

Lutein-3-palmitate, 13135

\section{$\mathrm{C}_{56} \mathrm{H}_{86} \mathrm{O}_{19}$}

Pregnane glycoside OI, 17795

\section{$\mathrm{C}_{56} \mathrm{H}_{86} \mathrm{O}_{22}$}

3-O- $\alpha$ - $L$-Arabinofuranosyl-( $(1 \rightarrow 3)-[\alpha-L$-arabinofuranosyl-( $1 \rightarrow 2)]$ - $\beta$ - $D$-glucuronopyranosyl$21 \beta, 22 \alpha$-di- $O$-angeloylprotoaescigenin, 1553

3- $O-\alpha-L$-Arabinofuranosyl-(1 $\rightarrow 3)$-[ $\beta$ - $D$-xylopyranosyl-( $1 \rightarrow 2)]-\beta$ - $D$-glucuronopyranosyl-21 $\beta$, $22 \alpha$-di- $O$-angeloylprotoaescigenin, 1559

\section{$\mathrm{C}_{56} \mathrm{H}_{88} \mathrm{O}_{19}$}

Periplocoside B, 16945

\section{$\mathrm{C}_{56} \mathrm{H}_{88} \mathrm{O}_{26}$}

Segetoside C, 19660

\section{$\mathrm{C}_{56} \mathrm{H}_{88} \mathrm{O}_{29}$}

$(25 R)-3 \beta-[(O-\beta$ - $D$-Glucopyranosyl- $(1 \rightarrow 2)-O-[\beta$ -

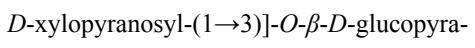
nosyl-(1 $\rightarrow 4)-\beta$ - $D$-galactopyranosyl)oxy]-26[( $\beta$ - $D$-glucopyranosyl)oxy]-2 $\alpha$-hydroxycholesta-5,17-diene-16,22-dione, 8748

\section{$\mathrm{C}_{56} \mathrm{H}_{90} \mathrm{O}_{19}$}

Pregnane glycoside FI, 17791

WilfosideC $\mathrm{C}_{1} \mathrm{~N}, 22692$ 


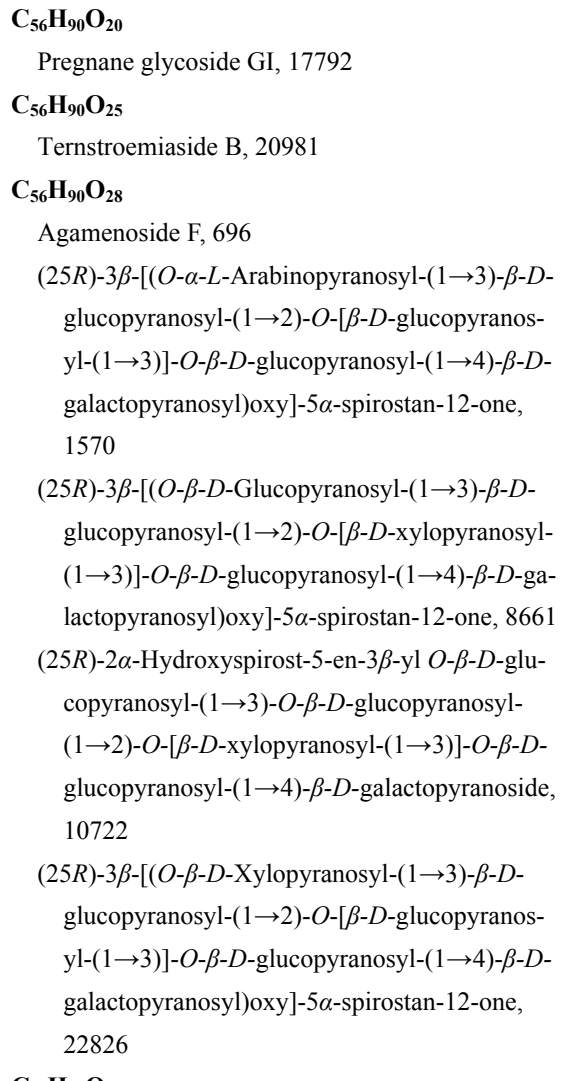

$\mathrm{C}_{56} \mathrm{H}_{90} \mathrm{O}_{29}$

(25R)-2 $\alpha, 17 \alpha$-Dihydroxyspirost-5-en- $3 \beta$-yl $O-\beta$ -

$D$-glucopyranosyl-(1 $\rightarrow 3)-O-\beta$ - $D$-glucopyranosyl-( $1 \rightarrow 2)-O$-[ $\beta$ - $D$-xylopyranosyl- $(1 \rightarrow 3)]-$

$O-\beta$ - $D$-glucopyranosyl-( $1 \rightarrow 4)-\beta$ - $D$-galactopyranoside, 6128

(25R)-2 $\alpha, 15 \beta$-Dihydroxyspirost-5-en-3 $\beta$-yl

$O-\beta$ - $D$-glucopyranosyl-( $1 \rightarrow 3)-O-\beta$ - $D$-glucopyranosyl-( $1 \rightarrow 2)-O-[\beta$ - $D$-xylopyranosyl- $(1 \rightarrow 3)]$ $O$ - $\beta$ - $D$-glucopyranosyl-( $1 \rightarrow 4)-\beta$ - $D$-galactopyranoside, 6129

\section{$\mathrm{C}_{56} \mathrm{H}_{92} \mathrm{O}_{22}$}

Lysimachoside, 13263

\section{$\mathrm{C}_{56} \mathrm{H}_{92} \mathrm{O}_{24}$}

Glycoside $\mathrm{H}_{1}, 8829$

Periplocoside $\mathrm{H}_{1}, 16950$

\section{$\mathrm{C}_{56} \mathrm{H}_{92} \mathrm{O}_{25}$}

Malonylginsenoside $\mathrm{Rb}_{2}, 13441$

Malonylginsenoside Rc, 13442

Periplocoside $\mathrm{H}_{2}, 16951$

Plocoside B, 17566

\section{$\mathrm{C}_{56} \mathrm{H}_{92} \mathrm{O}_{26}$}

Dongnoside C, 6555

\section{$\mathrm{C}_{56} \mathrm{H}_{92} \mathrm{O}_{27}$}

Polianthoside B, 17611

Trigofoenoside G, 21657

\section{$\mathrm{C}_{56} \mathrm{H}_{92} \mathrm{O}_{28}$}

(25R)-5 $\alpha$-Spirostan-3 $\beta, 6 \beta$-diol 3-O-\{O- $\beta-D$-glu- copyranosyl-( $(1 \rightarrow 3)-O-\beta$ - $D$-glucopyranosyl$(1 \rightarrow 2)-O$-[ $\beta$ - $D$-xylopyranosyl- $(1 \rightarrow 3)]-O-\beta$ -

$D$-glucopyranosyl-( $1 \rightarrow 4)-\beta$ - $D$-galactopyranoside $\}, 20210$

\section{$\mathrm{C}_{56} \mathrm{H}_{92} \mathrm{O}_{29}$}

Digitonin, 5524

Polianthoside D, 17613

\section{$\mathrm{C}_{56} \mathrm{H}_{94} \mathrm{O}_{24}$}

Quinquenoside $\mathrm{R}_{1}, 18450$

Yesanchinoside F, 22898

\section{$\mathrm{C}_{56} \mathrm{H}_{94} \mathrm{O}_{28}$}

Protodesgalactotigonin, 17972

Uttroside B, 22286

\section{$\mathrm{C}_{56} \mathrm{H}_{101} \mathrm{~N}_{3} \mathrm{O}_{18}$}

Polaramycin B, 17610

\section{$\mathrm{C}_{57} \mathrm{H}_{61} \mathrm{O}_{34}{ }^{+}$}

Delphinidin-3-O-[2-O-(2-O-(trans-caffeoyl)- $\beta-D$ glucopyranosyl)- $\beta$ - $D$-galactopyranoside]-7-O[6-O-(trans-caffeoyl)- $\beta$ - $D$-glucopyranoside]3'- $O$-[ $\beta$ - $D$-glucuronopyranoside], 5013

\section{$\mathrm{C}_{57} \mathrm{H}_{63} \mathrm{O}_{31}{ }^{+}$}

Delphinidin-3,7-di- $O-\beta$-glucopyranoside-3',5'-di$O$-(6- $O$ - $p$-coumaroyl- $\beta$-glucopyranoside), 5019

Pigment 25, 17358

\section{$\mathrm{C}_{57} \mathrm{H}_{70} \mathrm{O}_{32}$}

Tenuifoliose K, 20949

\section{$\mathrm{C}_{57} \mathrm{H}_{72} \mathrm{O}_{33}$}

Senegose D, 19719

\section{$\mathrm{C}_{57} \mathrm{H}_{82} \mathrm{O}_{22}$}

Cynaphylloside F, 4561

\section{$\mathrm{C}_{57} \mathrm{H}_{84} \mathrm{O}_{22}$}

Alpinoside B, 991

$20 S, 22 R, 23 S, 24 R-16 \beta, 23 ; 22,25$-Diepoxy-cycloartane-3 $\beta, 23,24-$ triol 3-O-(6-O-trans-isoferuloyl- $\beta$ - $D$-glucopyranosyl)-( $1 \rightarrow 2)-\beta$ - $D$-glucopyranosyl-(1 $\rightarrow 2)-\beta$ - $D$-xylopyranoside, 5488

\section{$\mathrm{C}_{57} \mathrm{H}_{88} \mathrm{O}_{22}$}

3-O- $\alpha-L$-Arabinofuranosyl-( $(1 \rightarrow 3)-[\beta$ - $D$-galactopyranosyl-( $(1 \rightarrow 2)]-\beta$ - $D$-glucuronopyranosyl21 $\beta, 22 \alpha$-di- $O$-angeloylbarringtogenol C, 1554 Marsdeoreophiside B, 13577

\section{$\mathrm{C}_{57} \mathrm{H}_{88} \mathrm{O}_{23}$}

3- $O-\alpha-L$-Arabinofuranosyl-( $(1 \rightarrow 3)-[\beta$ - $D$-glucopyranosyl-(1 $\rightarrow 2)]-\beta$ - $D$-glucuronopyranosyl$21 \beta, 22 \alpha$-di- $O$-angeloylprotoaescigenin, 1555

\section{$\mathrm{C}_{57} \mathrm{H}_{88} \mathrm{O}_{25}$}

Assamsaponin A, 1915

Yiyeliangwanoside I, 22915

Yiyeliangwanoside III, 22916

\section{$\mathrm{C}_{57} \mathrm{H}_{88} \mathrm{O}_{26}$}

Theasaponin $\mathrm{E}_{3}, 21285$

Theasaponin $\mathrm{E}_{6}, 21288$

\section{$\mathrm{C}_{57} \mathrm{H}_{88} \mathrm{O}_{28}$}

Platyconic acid A lactone-28-[ $\beta$ - $D$-apiofuranosyl $(1 \rightarrow 3)-\beta$ - $D$-xylopyranosyl $(1 \rightarrow 4)-\alpha$ - $L$-rhamnopyranosyl $(1 \rightarrow 2)$ - $L$-arabinopyranosyl] 3-O$\beta$ - $D$-glucopyranoside, 17538

\section{$\mathrm{C}_{57} \mathrm{H}_{90} \mathrm{O}_{24}$}

Eupteleasaponin IV, 7625

\section{$\mathrm{C}_{57} \mathrm{H}_{90} \mathrm{O}_{25}$}

Yemuoside I, 22883

Yemuoside $\mathrm{YM}_{7}, 22887$

\section{$\mathrm{C}_{57} \mathrm{H}_{90} \mathrm{O}_{27}$}

Ageratoside $\mathrm{B}_{2}, 734$

$\mathrm{C}_{57} \mathrm{H}_{92} \mathrm{O}_{6}$

$\alpha$-Eleostearin, 6749

\section{$\mathrm{C}_{57} \mathrm{H}_{92} \mathrm{O}_{23}$}

Taibaienoside VIII, 20619

\section{$\mathrm{C}_{57} \mathrm{H}_{92} \mathrm{O}_{24}$}

Clematoside A', 3825

Scabioside F, 19446

\section{$\mathrm{C}_{57} \mathrm{H}_{92} \mathrm{O}_{26}$}

Astersaponin G, 1927

(25S)-26-[( $\beta$ - $D$-Glucopyranosyl)oxy]furosta-5, 20(22)-dien-3 $\beta$-yl $O-\alpha$ - $L$-rhamnopyranosyl$(1 \rightarrow 2)-O-[O-\beta$ - $D$-glucopyranosyl-( $1 \rightarrow 4)-\alpha-L$ rhamnopyranosyl-( $(1 \rightarrow 3)]-\beta$ - $D$-glucopyranoside, 8688

Hydrocotyloside VII, 9716

\section{$\mathrm{C}_{57} \mathrm{H}_{\mathbf{9}} \mathrm{O}_{27}$}

26- $O-\beta-D$-Glucopyranosyl-3 $\beta, 26$-dihydroxy20,22 -seco-25(R)-furost-5-en-20,22-dione-3$O$ - $\alpha$ - $L$-rhamnopyranosyl-( $1 \rightarrow 4)-\alpha$ - $L$-rhamnopyranosyl-( $1 \rightarrow 4)$-[ $\alpha$ - $L$-rhamnopyranosyl$(1 \rightarrow 2)]-\beta$ - $D$-glucopyranoside, 8623

Polygalacin D, 17629

Scillascilloside $\mathrm{E}_{2}, 19525$

\section{$\mathrm{C}_{57} \mathrm{H}_{92} \mathrm{O}_{28}$}

(23S)-3 $\beta$-[( $O-\beta$ - $D$-Apiofuranosyl-( $(1 \rightarrow 2)-O-[\beta-D-$ glucopyranosyl-( $(1 \rightarrow 3)]$ - $O-\beta$ - $D$-glucopyranosyl-( $(1 \rightarrow 2)-\alpha$ - $L$-arabinopyranosyl- $(1 \rightarrow 6)-\beta$ - $D$ glucopyranosyl)oxy]-17 $\alpha, 23$-epoxy-28,29-dihydroxy-27-norlanost-8-en-24-one, 1509

Avenacoside B, 2032

$16 \beta$-[[(4S)-5-( $\beta$ - $D$-Glucopyranosyloxy)-4-methyl-1-oxopentyl $]$ oxy $]-3 \beta$-[( $O-\alpha-L$-rhamnopyranosyl-( $1 \rightarrow 2)-O-[O-\beta-D$-glucopyranosyl$(1 \rightarrow 4)-\alpha-L$-rhamnopyranosyl-( $1 \rightarrow 3)]-\beta$ - $D$ glucopyranosyl)oxy]pregn-5-en-20-one, 8707 $(25 R)-3 \beta-[(O-\beta-D-$ Glucopyranosyl- $(1 \rightarrow 2)-O-[O-$ $\alpha$ - $L$-rhamnopyranosyl-( $1 \rightarrow 4)-\beta$ - $D$-glucopyranosyl-( $(1 \rightarrow 3)]-O-\beta$ - $D$-glucopyranosyl-( $(1 \rightarrow 4)$ $\beta$ - $D$-galactopyranosyl)oxy]-5 $\alpha$-spirostan-12one, 8721

Platycodin D, 17532 


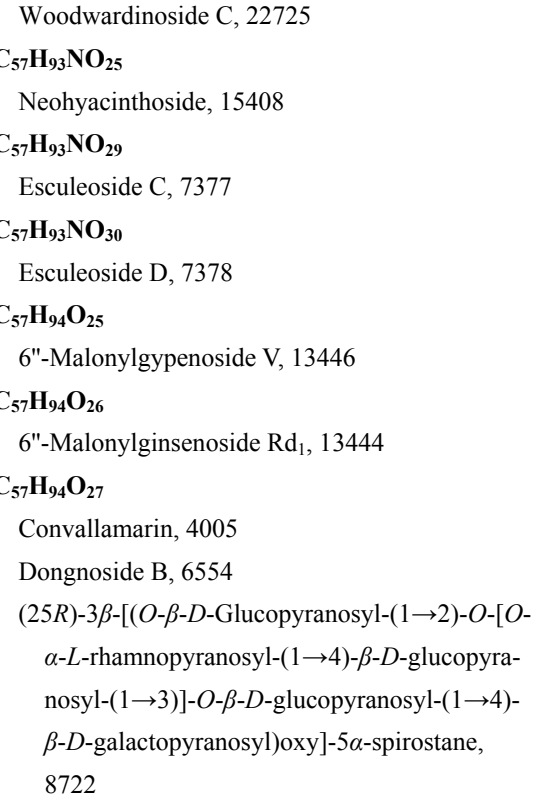

Tigogenin-3- $O-\alpha-L$-rhamnopyranosyl-( $(1 \rightarrow 3)-\beta$ $D$-glucopyranosyl $(1 \rightarrow 2)$-[ $\beta$ - $D$-glucopyranosyl $(1 \rightarrow 3)]-\beta$ - $D$-glucopyranosyl $(1 \rightarrow 4)-\beta$ - $D$-galactopyranoside, 21386

Trigofoenoside $\mathrm{G}_{1}, 21658$

\section{$\mathrm{C}_{57} \mathrm{H}_{94} \mathrm{O}_{28}$}

(25R)-15 $\alpha$-Hydroxy- $5 \alpha$-spirostan- $3 \beta$-yl $O-\beta$ - $D$ glucopyranosyl-( $(1 \rightarrow 2)-O-[O-\alpha-L$-rhamnopyranosyl-( $(1 \rightarrow 4)-\beta$ - $D$-glucopyranosyl-( $(1 \rightarrow 3)]-$ $O$ - $\beta$ - $D$-glucopyranosyl-( $1 \rightarrow 4)$ - $\beta$ - $D$-galactopyranoside, 10716

Macrostemonoside E, 13330

Parvifloside, 16682

Polianthoside C, 17612

\section{$\mathrm{C}_{57} \mathrm{H}_{94} \mathrm{O}_{29}$}

(25R)-26- $O-\beta$ - $D$-Glucopyranosyl-22-hydroxyfurost-5(6)-ene-3 $\beta, 26$-diol-3- $O-\beta$ - $D$-glucopyra$\operatorname{nosyl}(1 \rightarrow 2)[\beta$ - $D$-glucopyranosyl $(1 \rightarrow 3)]-\beta$ - $D$ glucopyranosyl $(1 \rightarrow 4)-\beta$ - $D$-galactopyranoside, 8671

Polyfuroside, 17624

\section{$\mathrm{C}_{57} \mathrm{H}_{96} \mathrm{O}_{10}$}

Longiside B, 12979

$\mathrm{C}_{57} \mathrm{H}_{96} \mathbf{O}_{27}$

Asp-VII, 1908

\section{$\mathrm{C}_{57} \mathrm{H}_{96} \mathrm{O}_{28}$}

Sarsaparilloside, 19389

\section{$\mathrm{C}_{57} \mathrm{H}_{96} \mathbf{O}_{29}$}

Macrostemonoside B, 13327

\section{$\mathrm{C}_{57} \mathrm{H}_{96} \mathrm{O}_{30}$}

Pentandroside F, 16882

Proto-iso-erubioside B, 17978

$\mathrm{C}_{57} \mathrm{H}_{98} \mathrm{O}_{6}$

Glyceryl linolenate III, 8816
$\mathrm{C}_{57} \mathrm{H}_{104} \mathrm{O}_{6}$

Triolein, 21984

$\mathrm{C}_{\mathbf{5 7}} \mathrm{H}_{\mathbf{1 0 4}} \mathrm{O}_{\mathbf{9}}$

Triricinolein, 22028

$\mathrm{C}_{57} \mathrm{H}_{110} \mathrm{O}_{6}$

Stearin, 20281

$\mathrm{C}_{57} \mathrm{H}_{114} \mathrm{O}_{2}$

Heptacosyl melissate, 9373

Heptatriacontanyl eicosanoate, 9412

$\mathrm{C}_{57} \mathrm{H}_{117} \mathbf{N}_{2} \mathrm{O}_{7} \mathrm{P}$

Sphingomyelin, 20155

$\mathrm{C}_{58} \mathrm{H}_{59} \mathrm{O}_{36}{ }^{+}$

Delphinidin-3-O-[2-O-(2-O-(trans-caffeoyl) $-\beta-D-$ glucopyranosyl)-6-O-(2-O-(tartaryl)malonyl)$\beta$ - $D$-galactopyranoside] -7-O-[6-O-(transcaffeoyl) $-\beta-D$-glucopyranoside], 5015

\section{$\mathrm{C}_{58} \mathrm{H}_{65} \mathrm{O}_{31}{ }^{+}$}

Petunidin 3-O-(6-O-(4-O-(4-O-(6-O-feruloyl- $\beta$ $D$-glucopyranosyl)- $E$ - $p$-coumaroyl)- $\alpha$-rhamnosyl)- $\beta$ - $D$-glucopyranoside)-5- $\beta$ - $D$-glucopyranoside, 17026

\section{$\mathrm{C}_{58} \mathrm{H}_{72} \mathrm{O}_{33}$}

Senegose K, 19725

Senegose M, 19727

Tenuifoliose C, 20942

Tenuifoliose E, 20944

\section{$\mathrm{C}_{58} \mathrm{H}_{74} \mathrm{O}_{8}$}

Isoxuxuarine $\mathrm{F} \alpha, 11787$

Isoxuxuarine $\mathrm{G} \beta, 11788$

Xuxuarine $F \alpha, 22811$

\section{$\mathrm{C}_{58} \mathrm{H}_{76} \mathrm{O}_{8}$}

7,8-Dihydroisoxuxuarine $\mathrm{F} \alpha, 5655$

7,8-Dihydroisoxuxuarine $\mathrm{G}_{\alpha}, 5656$

\section{$\mathrm{C}_{58} \mathrm{H}_{84} \mathrm{O}_{3}$}

Lutein-3-linolenate, 13133

\section{$\mathrm{C}_{58} \mathrm{H}_{86} \mathrm{O}_{19}$}

Pregnane glycoside DI, 17789

WilfosideK $\mathrm{K}_{1}$, 22694

\section{$\mathrm{C}_{58} \mathrm{H}_{86} \mathrm{O}_{20}$}

Pregnane glycoside EI, 17790

\section{$\mathrm{C}_{58} \mathrm{H}_{88} \mathrm{O}_{3}$}

Lutein oleic acid ester, 13134

\section{$\mathrm{C}_{58} \mathrm{H}_{88} \mathrm{O}_{20}$}

Pregnane glycoside NI, 17794

\section{$\mathrm{C}_{58} \mathrm{H}_{88} \mathrm{O}_{21}$}

12-O-Benzoyl-20- $O$-acetylsarcostin-3- $O-\beta$ - $D$ thevetopyranosyl-(1 $\rightarrow 4)-\beta$ - $D$-cymaropyranosyl-( $(1 \rightarrow 4)-\beta$ - $D$-cymaropyranosyl-( $(1 \rightarrow 4)-\beta$ - $D$ cymaropyranoside, 2230

\section{$\mathrm{C}_{58} \mathrm{H}_{90} \mathrm{O}_{22}$}

3-O- $\alpha$ - $L$-Rhamnopyranosyl-( $1 \rightarrow 3)$-[ $[\beta$ - $D$-galactopyranosyl-(1 $\rightarrow 2)]-\beta$ - $D$-glucuronopyranosyl$21 \beta, 22 \alpha$-di- $O$-angeloylbarringtogenol C,
18702

$\mathrm{C}_{58} \mathrm{H}_{92} \mathrm{O}_{25}$

Eupteleasaponin II, 7623

$\mathrm{C}_{58} \mathrm{H}_{92} \mathrm{O}_{26}$

Yemuoside $\mathrm{YM}_{8}, 22888$

$\mathrm{C}_{58} \mathrm{H}_{92} \mathrm{O}_{27}$

Scillasaponin E, 19521

$\mathrm{C}_{58} \mathrm{H}_{92} \mathrm{O}_{29}$

Methyl platyconate A, 14681

\section{$\mathrm{C}_{58} \mathrm{H}_{94} \mathbf{O}_{25}$}

$3 \beta$-[ $(O-\beta$ - $D$-Glucopyranosyl-( $1 \rightarrow 4)-O-\beta-D$-ribopyranosyl-( $1 \rightarrow 3)-O-\alpha$ - $L$-rhamnopyranosyl-

$(1 \rightarrow 2)$ - $\alpha$ - $L$-arabinopyranosyl)oxy]olean-12-en-

28 -oic acid $O$ - $\beta$ - $D$-glucopyranosyl ester, 8728

Helianthoside B, 9295

Oleanolic acid-3- $O-\beta$ - $D$-glucopyranosyl $(1 \rightarrow 4)$ $\beta$ - $D$-glucopyranosyl $(1 \rightarrow 4)$ - $\beta$ - $D$-ribopyranosyl $(1 \rightarrow 3)$ - $\alpha$ - $L$-rhamnopyranosyl $(1 \rightarrow 2)-\alpha$ - $L$-arabinopyranoside, 16055

Oleanolic acid-3- $O-\beta$ - $D$-glucopyranosyl $(1 \rightarrow 4)-\beta$ $D$-glucopyranosyl $(1 \rightarrow 4)-\beta$ - $D$-xylopyranosyl $(1 \rightarrow 3)$ - $\alpha$ - $L$-rhamnopyranosyl $(1 \rightarrow 2)-\alpha$ - $L$-arabinopyranoside, 16056

Scabiosaponin E, 19436

\section{$\mathrm{C}_{58} \mathrm{H}_{94} \mathrm{O}_{26}$}

Fulvotomentoside A, 7995

Jujuboside A, 11909

Jujuboside $A_{1}, 11910$

Mukurozisaponin $Y_{1}, 15036$

Mukurozisaponin $\mathrm{Y}_{2}, 15037$

Prosapogenin $\mathrm{CP}_{10}, 17949$

Prosapogenin $\mathrm{CP}_{10 \mathrm{a}}, 17950$

Scillascilloside $\mathrm{E}_{1}, 19524$

\section{$\mathrm{C}_{58} \mathrm{H}_{94} \mathrm{O}_{27}$}

Ardipusilloside II, 1627

Cyclamin, 4457

(23S)-17 $\alpha, 23-$-Epoxy-29-hydroxy-3 $\beta-[(O-\alpha-L-$ rhamnopyranosyl-( $1 \rightarrow 2)-O-[\beta$ - $D$-glucopyranosyl- $(1 \rightarrow 3)]-O-\beta$ - $D$-glucopyranosyl- $(1 \rightarrow 2)$ $\alpha$ - $L$-arabinopyranosyl-( $1 \rightarrow 6)-\beta$ - $D$-glucopyranosyl)oxy]-27-norlanost-8-en-24-one, 7134

\section{$\mathrm{C}_{58} \mathrm{H}_{94} \mathrm{O}_{28}$}

Platycoside H, 17541

Scillanoside $\mathrm{L}_{1}, 19516$

Soyasaponin $\mathrm{A}_{4}, 20124$

\section{$\mathrm{C}_{58} \mathrm{H}_{94} \mathrm{O}_{29}$}

Deapio platycodin $\mathrm{D}_{3}, 4800$

Platycoside A, 17539

\section{$\mathrm{C}_{58} \mathrm{H}_{95} \mathrm{NO}_{29}$}

Esculeoside A, 7376

Lycoperoside F, 13217

Lycoperoside G, 13218

$\mathrm{C}_{58} \mathrm{H}_{96} \mathrm{O}_{24}$ 
Capilliposide B, 3126

$$
\begin{aligned}
& \mathbf{C}_{58} \mathbf{H}_{\mathbf{9 6}} \mathbf{O}_{\mathbf{2 6}} \\
& \text { Methyl protodiosgenin tetraglycoside, } 14699 \\
& \mathbf{C}_{\mathbf{5 8}} \mathbf{H}_{\mathbf{9 6}} \mathbf{O}_{27} \\
& (25 S)-26 \text { - }[(\beta-D \text {-Glucopyranosyl)oxy }]-22 \alpha \text {-meth- } \\
& \quad \text { oxyfurost-5-en-3 } \beta \text {-yl } O \text { - } \alpha \text { - } L \text {-rhamnopyranosyl- } \\
& \quad(1 \rightarrow 2)-O \text { - }[O-\beta \text { - } D \text {-glucopyranosyl- }(1 \rightarrow 4)-\alpha-L \text { - } \\
& \text { rhamnopyranosyl- }(1 \rightarrow 3)] \text { - } \beta \text { - } D \text {-glucopyrano- } \\
& \text { side, } 8704
\end{aligned}
$$

\section{$\mathrm{C}_{58} \mathrm{H}_{96} \mathrm{O}_{28}$}

Capilliposide C, 3127

Methyl parvifloside, 14653

Taccasteroside C, 20598

\section{$\mathrm{C}_{58} \mathrm{H}_{96} \mathrm{O}_{29}$}

Polyfuroside $\mathrm{PO}_{7}, 17626$

\section{$\mathrm{C}_{58} \mathrm{H}_{98} \mathrm{O}_{26}$}

Ginsenoside $\mathrm{Ra}_{1}, 8420$

Ginsenoside $\mathrm{Ra}_{2}, 8421$

Notoginsenoside Fc, 15823

\section{$\mathrm{C}_{58} \mathrm{H}_{98} \mathrm{O}_{29}$}

Macrostemonoside C, 13328

\section{$\mathrm{C}_{58} \mathrm{H}_{102} \mathrm{O}_{3}$}

Ursa-12-ene-11-one-3-ol octocosate, 22262

\section{$\mathrm{C}_{58} \mathrm{H}_{104} \mathrm{O}_{2}$}

Hancolupenol octacosanate, 9215

\section{$\mathrm{C}_{59} \mathrm{H}_{42} \mathrm{O}_{34}$}

5-O-Galloylpunicacortein D, 8115

\section{$\mathrm{C}_{59} \mathrm{H}_{46} \mathrm{O}_{26}$}

Procyanidin $\mathrm{C}_{1}$-3',3"-di-O-gallate, 17894

\section{$\mathrm{C}_{59} \mathrm{H}_{67} \mathrm{O}_{30}{ }^{+}$}

Malvidin 3-O-(6-O-(4-O-(4-O-(6-O-E- $p$ coumaroyl- $\beta$ - $D$-glucopyranosyl)- $E$ - $p$-coumaroyl)- $\alpha$-rhamnosyl)- $\beta$ - $D$-glucopyranoside) $-5-\beta$ $D$-glucopyranoside, 13459

\section{$\mathrm{C}_{59} \mathrm{H}_{67} \mathrm{O}_{31}{ }^{+}$}

Malvidin 3-O-(6-O-(4-O-(4- $O$-(6- $O$-caffeoyl- $\beta$ $D$-glucopyranosyl)- $E$ - $p$-coumaroyl)- $\alpha$-rhamnosyl)- $\beta$ - $D$-glucopyranoside)-5- $O-\beta$ - $D$-glucopyranoside, 13458

\section{$\mathrm{C}_{59} \mathrm{H}_{72} \mathrm{O}_{33}$}

Tenuifoliose I, 20947

Tenuifoliose J, 20948

\section{$\mathrm{C}_{59} \mathrm{H}_{74} \mathrm{O}_{34}$}

Fallaxose E, 7713

Senegose B, 19717

Senegose C, 19718

Tenuifoliose P, 20954

\section{$\mathrm{C}_{59} \mathrm{H}_{88} \mathrm{O}_{22}$}

Condurangoglycoside $\mathrm{A}_{0}, 3964$

\section{$\mathrm{C}_{59} \mathrm{H}_{89} \mathrm{NO}_{23}$}

12-O-Nicotinoyllineolon 3- $O-\beta-D$-glucopyranosyl- $(1 \rightarrow 4)-\beta$ - $D$-oleandropyranosyl- $(1 \rightarrow 4)-\beta$ - $D$ digitoxopyranosyl-( $1 \rightarrow 4)-\beta$ - $D$-digitoxopyra- nosyl-(1 $\rightarrow 4)-\beta$ - $D$-cymaropyranoside, 15531

\section{$\mathrm{C}_{59} \mathrm{H}_{90} \mathrm{O}_{4}$}

Ubiquinone 10, 22172

$\mathrm{C}_{59} \mathrm{H}_{90} \mathrm{O}_{22}$

Condurangoglycoside $\mathrm{C}_{0}, 3965$

\section{$\mathrm{C}_{59} \mathrm{H}_{90} \mathrm{O}_{27}$}

Assamsaponin C, 1917

Theasaponin $\mathrm{E}_{1}, 21283$

Theasaponin $\mathrm{E}_{2}, 21284$

Theasaponin $\mathrm{E}_{4}, 21286$

Theasaponin $\mathrm{E}_{7}, 21289$

$\mathrm{C}_{59} \mathrm{H}_{92} \mathrm{O}_{26}$

Floratheasaponin A, 7832

\section{$\mathrm{C}_{59} \mathrm{H}_{92} \mathrm{O}_{27}$}

Assamsaponin D, 1918

Theasaponin, 21282

\section{$\mathrm{C}_{59} \mathrm{H}_{92} \mathrm{O}_{29}$}

Ageratoside $A_{1}, 728$

Ageratoside $A_{3}, 730$

$\mathrm{C}_{59} \mathrm{H}_{92} \mathrm{O}_{31}$

Lucilianoside E, 13060

\section{$\mathrm{C}_{59} \mathrm{H}_{92} \mathrm{O}_{32} \mathrm{~S}_{2}$}

Colochiroside A, 3928

\section{$\mathrm{C}_{59} \mathrm{H}_{94} \mathrm{O}_{25}$}

Maetenoside B, 13363

\section{$\mathrm{C}_{59} \mathrm{H}_{94} \mathrm{O}_{26}$}

Berneuxia saponin A, 2313

Berneuxia saponin B, 2314

Clematibetoside B, 3820

\section{$\mathrm{C}_{59} \mathrm{H}_{94} \mathrm{O}_{27}$}

Assamicin IV, 1914

(25S)-26-[( $\beta$-D-Glucopyranosyl)oxy]-22 $\alpha$-methoxyfurosta-5,20(22)-dien-3 $\beta$-yl $O-\alpha-L$-rhamnopyranosyl- $(1 \rightarrow 2)-O$-[O- $\beta$ - $D$-glucopyranosyl$(1 \rightarrow 4)-\alpha-L$-rhamnopyranosyl-( $1 \rightarrow 3)]-6-O$ acetyl- $\beta$ - $D$-glucopyranoside, 8702

Maesasaponin I, 13351

\section{$\mathrm{C}_{59} \mathrm{H}_{94} \mathrm{O}_{28}$}

Scillanoside $\mathrm{L}_{2}, 19517$

Scillascilloside $\mathrm{E}_{3}, 19526$

\section{$\mathrm{C}_{59} \mathrm{H}_{94} \mathrm{O}_{29}$}

Methyl 2-O-methyl platycogenate A, 14604

Platycodin A, 17530

Platycodin C, 17531

Sophoraflavoside IV, 20092

\section{$\mathrm{C}_{59} \mathrm{H}_{96} \mathrm{O}_{25}$}

Akeboside $\mathrm{st}_{\mathrm{h}}, 819$

Cussosaponin C, 4431

Raddeanin D, 18518

$3 \beta$ - $O$ - $(\alpha$-Rhamnopyranosyl- $(1 \rightarrow 2)$ - $\alpha$-arabinopyranosyl)-lup-12-en-28- $O$-( $\alpha$-rhamnopyranosyl-( $1 \rightarrow 4)$ - $\beta$-glucopyranosyl- $(1 \rightarrow 6)$ - $\beta$-galactopyranosyl) ester, 18692
$3 \beta-O-(\alpha-L$-Rhamnopyranosyl- $(1 \rightarrow 3)-\alpha-L-$ rhamnopyranosyl- $(1 \rightarrow 2)-\alpha-L$-arabinopyranosyl)-olean-12-ene-28- $O$ - $(\beta$ - $D$-glucopyranosyl-( $1 \rightarrow 4)-\beta$ - $D$-glucopyranosyl) ester, 18733

\section{$\mathrm{C}_{59} \mathrm{H}_{96} \mathrm{O}_{26}$}

Anemoside $\mathrm{B}_{4}, 1182$

Begoniifolide A, 2211

Cussosaponin B, 4430

Cussosaponin E, 4433

3-O-[ $\beta$ - $D$-Glucopyranosyl- $(1 \rightarrow 4)][\alpha$ - $L$-rhamnopyranosyl- $(1 \rightarrow 3)]-\beta$ - $D$-glucopyranosyl-

$(1 \rightarrow 3)$ - $\alpha$ - $L$-rhamnopyranosyl- $(1 \rightarrow 2)$ - $\alpha$-arabinoyranosyl-hederagenin, 8723

Hederasaponin C, 9275

Japondipsaponin $\mathrm{E}_{1}, 11817$

Jujuboside D, 11914

Kalopanax saponin C, 12118

Latifoloside F, 12552

Latifoloside G, 12553

Latifoloside H, 12554

Matesaponin 4, 13601

Pastuchoside D, 16701

$3 \beta$ - $O$ - $(\alpha$-Rhamnopyranosyl- $(1 \rightarrow 2)$ - $\alpha$-arabinopyranosyl)-23-hydroxylup-12-en-28- $O$ - $(\alpha$ rhamnopyranosyl-( $1 \rightarrow 4)$ - $\beta$-glucopyranosyl$(1 \rightarrow 6)$ - $\beta$-galactopyranosyl)ester, 18690

3-O- $\{[\alpha-L$-Rhamnopyranosyl-( $1 \rightarrow 2)-\beta$ - $D$-galactopyranosyl- $(1 \rightarrow 2)]-\beta$ - $D$-glucuronopyranosyl $\}$ -22- $O$ - $[\alpha$ - $L$-rhamnopyranosyl- $(1 \rightarrow 2)$ - $\alpha$ - $L$-arabinopyranosyl]-3 $\beta, 22 \beta, 24$-trihydroxyolean-12ene, 18700

Scabiosaponin F, 19437

\section{$\mathrm{C}_{59} \mathrm{H}_{96} \mathrm{O}_{27}$}

Aradecoside D, 1601

$3 \beta$-[(O- $\beta$ - $D$-Glucopyranosyl- $(1 \rightarrow 3)-\alpha-L$-arabinopyranosyl)oxy]-23-hydroxyolean-12-en-28oic acid $O-\alpha$ - $L$-rhamnopyranosyl-( $1 \rightarrow 4)-O-\beta$ $D$-glucopyranosyl-( $1 \rightarrow 6)-\beta$ - $D$-glucopyranosyl ester, 8608

$3 \beta$ - $O$ - $(\beta$ - $D$-Glucopyranosyl- $(1 \rightarrow 3)-\alpha-L$-rhamnopyranosyl-( $1 \rightarrow 2)-\alpha-L$-arabinopyranosyl)hederagenin-28-O-( $\beta$ - $D$-glucopyranosyl$(1 \rightarrow 4)$ - $\beta$ - $D$-glucopyranosyl)ester, 8716

Hydrocotyloside V, 9714

Jujuboside C, 11913

Leontoside D, 12644

Macranthoidin A, 13298

Pastuchoside C, 16700

Pulsatiloside B, 18196

Scabiosaponin I, 19440

Scabiosaponin J, 19441

Virgaureasaponin 1, 22526 


\section{$\mathrm{C}_{59} \mathrm{H}_{96} \mathrm{O}_{29}$}

Saikosaponin U, 19163

Saikosaponin $\mathrm{V}_{2}, 19166$

Soyasaponin $\mathrm{A}_{1}, 20121$

\section{$\mathrm{C}_{59} \mathrm{H}_{98} \mathrm{O}_{26}$}

SophoraflavosideI, 20089

\section{$\mathrm{C}_{59} \mathrm{H}_{98} \mathrm{O}_{27}$}

20,23-Dihydroxy-3 $\beta$-[(O- $\alpha-L$-rhamnopyranosyl$(1 \rightarrow 2)-\alpha$ - $L$-arabinopyranosyl)oxy]lupan-28-oic acid 28- $O-\alpha$ - $L$-rhamnopyranosyl-( $1 \rightarrow 4)-O-\beta$ -

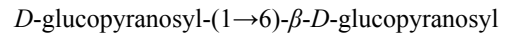
ester, 6106

$\mathrm{C}_{59} \mathrm{H}_{100} \mathrm{O}_{26}$

Yesanchinoside I, 22901

\section{$\mathrm{C}_{59} \mathrm{H}_{100} \mathrm{O}_{27}$}

Ginsenoside $\mathrm{Ra}_{3}, 8422$

Notoginsenoside Fa, 15822

Notoginsenoside $\mathrm{R}_{4}, 15839$

\section{$\mathrm{C}_{60} \mathrm{H}_{46} \mathrm{O}_{24}$}

Parameritannin $\mathrm{A}_{2}, 16655$

\section{$\mathrm{C}_{60} \mathrm{H}_{48} \mathrm{O}_{24}$}

Epicatechin- $(2 \beta \rightarrow O \rightarrow 7,4 \beta \rightarrow 8)$ epicatechin( $4 \alpha \rightarrow 8)$-catechin-( $4 \alpha \rightarrow 8)$-epicatechin, 6861 Parameritannin $\mathrm{A}_{1}, 16654$

\section{$\mathrm{C}_{60} \mathrm{H}_{50} \mathrm{O}_{24}$}

Arecatannin $\mathrm{A}_{2}, 1656$

\section{$\mathrm{C}_{60} \mathrm{H}_{52} \mathrm{O}_{30}$}

Monochaetin, 14921

\section{$\mathrm{C}_{60} \mathrm{H}_{62} \mathrm{O}_{24}$}

Gnemonoside K, 8876

\section{$\mathrm{C}_{60} \mathrm{H}_{63} \mathrm{O}_{37}{ }^{+}$}

Delphinidin-3-O-[2-O-(2-O-(trans-caffeoyl) $-\beta-D-$ glucopyranosyl)-6- $O$-(malonyl)- $\beta$ - $D$-galactopyranoside]-7- $O-[6-O-($ trans - caffeoyl) $-\beta-D-$ glucopyranoside]-3'- $O$-[ $\beta$ - $D$-glucuronopyranoside], 5014

\section{$\mathrm{C}_{60} \mathrm{H}_{69} \mathrm{O}_{31}{ }^{+}$}

Malvidin 3-O-(6-O-(4-O-(4-O-(6-O-feruloyl- $\beta$ $D$-glucopyranosyl)-E-p-coumaroyl)- $\alpha$-rhamnosyl)- $\beta$ - $D$-glucopyranoside)-5- $\beta$ - $D$-glucopyranoside, 13461

\section{$\mathrm{C}_{60} \mathrm{H}_{74} \mathrm{O}_{34}$}

Reiniose J, 18612

Senegose J, 19724

Senegose L, 19726

Senegose N, 19728

Senegose O, 19729

Tenuifoliose B, 20941

Tenuifoliose D, 20943

$\mathrm{C}_{60} \mathrm{H}_{78} \mathrm{O}_{9}$

Isoxuxuarine $\mathrm{E} \beta, 11786$

\section{$\mathrm{C}_{60} \mathrm{H}_{80} \mathrm{O}_{10}$}

$7 \alpha$-Hydroxyisoxuxuarine $\mathrm{E} \alpha, 10267$
$\mathrm{C}_{60} \mathrm{H}_{92} \mathrm{O}_{28}$

Assamsaponin I, 1920

$\mathrm{C}_{60} \mathrm{H}_{94} \mathrm{O}_{30}$

Ageratoside $\mathrm{A}_{2}, 729$

$\mathrm{C}_{60} \mathrm{H}_{96} \mathrm{O}_{27}$

$\alpha$ - $L$-Rhamnopyranosyl-( $(1 \rightarrow 4)-\beta$ - $D$-glucopyranosyl-( $1 \rightarrow 6)-\beta$ - $D$-glucopyranosyl-( $(1 \rightarrow 6)-\beta$ -

$D$-glucopyranosyloleanate-3- $\alpha$ - $L$-arabinofuran osyl ( $1 \rightarrow 4)$-methyl-( $\beta$ - $D$-glucuronopyranoside) uronate, 18712

\section{$\mathrm{C}_{60} \mathrm{H}_{98} \mathrm{O}_{26}$}

Anhuienoside E, 1255

Colchiside B, 3914

$3 \beta$ - $\{O-\alpha-L$-Rhamnopyranosyl-( $(1 \rightarrow 4)-O-\alpha-L-$ rhamnopyranosyl-( $1 \rightarrow 4)$-[ $O-\alpha$ - $L$-rhamnopyranosyl-( $(1 \rightarrow 2)-O-\beta$ - $D$-glucopyranosyl $(1 \rightarrow 4)-O-$ $\beta$ - $D$-glucuronopyranosyl] $\}$-16 $\alpha$-hydroxy-13 $\beta$, 28-epoxyoleanane, 18734

\section{$\mathrm{C}_{60} \mathrm{H}_{98} \mathrm{O}_{27}$}

Hydrocotyloside VI, 9715

3-O- $\{[\alpha-L$-Rhamnopyranosyl-( $(1 \rightarrow 2)-\beta$ - $D$-galactopyranosyl- $(1 \rightarrow 2)]-\beta$ - $D$-glucuronopyranosyl $\}-22-O-[\alpha-L$-rhamnopyranosyl-( $(1 \rightarrow 2)-\beta$ - $D$ glucopyranosyl]-3 $\beta, 22 \beta, 24$-trihydroxyolean12-ene, 18701

$3 \beta, 21 \beta, 22 \beta, 24-T e t r a h y d r o x y o l e a n-12-e n-3-O-\alpha$ $L$-rhamnopyranosyl-( $1 \rightarrow 3)$-[ $\alpha$ - $L$-rhamnopyranosyl-(1 $\rightarrow 2)]-\beta$ - $D$-galactopyranosyl-( $1 \rightarrow 2)-\beta$ $D$-glucuronopyranosyl-21-O- $\alpha$ - $L$-rhamnopyranoside, 21147

\section{$\mathrm{C}_{60} \mathrm{H}_{98} \mathrm{O}_{28}$}

(25S)-26-[( $\beta$ - $D$-Glucopyranosyl)oxy]-22 $\alpha$-methoxyfurost-5-en-3 $\beta$-yl $O-\alpha$ - $L$-rhamnopyranosyl$(1 \rightarrow 2)-O-[O-\beta$ - $D$-glucopyranosyl-( $1 \rightarrow 4)-\alpha-L-$ rhamnopyranosyl-( $1 \rightarrow 3)]-6$ - $O$-acetyl- $\beta$ - $D$-glucopyranoside, 8703

\section{$\mathrm{C}_{60} \mathrm{H}_{98} \mathrm{O}_{29}$}

Ternstroemiaside D, 20983

\section{$\mathrm{C}_{60} \mathrm{H}_{98} \mathrm{O}_{30}$}

Agave cantala Agaveside A, 717

Aralia-saponin VIII, 1606

$\mathrm{C}_{60} \mathrm{H}_{102} \mathrm{O}_{26}$

Gypenoside II, 9125

$\mathrm{C}_{60} \mathrm{H}_{102} \mathrm{O}_{27}$

Gypenoside I, 9124

$\mathrm{C}_{60} \mathrm{H}_{102} \mathrm{O}_{28}$

Ginsenoside $\mathrm{Ra}_{0}, 8419$

Gypenoside XX, 9137

Quinquenoside $\mathrm{V}, 18451$

$\mathrm{C}_{61} \mathrm{H}_{74} \mathbf{O}_{34}$

Tenuifoliose H, 20946

$\mathrm{C}_{61} \mathrm{H}_{76} \mathrm{O}_{35}$

Reiniose I, 18611
Senegose A, 19716

Senegose E, 19720

Tenuifoliose O, 20953

\section{$\mathrm{C}_{61} \mathrm{H}_{92} \mathrm{O}_{28}$}

Assamsaponin B, 1916

Theasaponin $\mathrm{E}_{5}, 21287$

\section{$\mathrm{C}_{61} \mathrm{H}_{96} \mathrm{O}_{27}$}

Tenacissoside J, 20930

\section{$\mathrm{C}_{61} \mathrm{H}_{96} \mathrm{O}_{28}$}

Assamicin III, 1913

Maesasaponin II, 13352

Maesasaponin III, 13353

\section{$\mathrm{C}_{61} \mathrm{H}_{98} \mathrm{O}_{21}$}

Symplocososide F, 20545

\section{$\mathrm{C}_{61} \mathrm{H}_{100} \mathrm{O}_{23}$}

Periplocoside J, 16952

\section{$\mathrm{C}_{61} \mathrm{H}_{100} \mathrm{O}_{32}$}

Chloromaloside E, 3563

Pentandroside G, 16883

\section{$\mathrm{C}_{61} \mathrm{H}_{100} \mathrm{O}_{33}$}

Polianthoside E, 17614

\section{$\mathrm{C}_{61} \mathrm{H}_{102} \mathrm{O}_{23}$}

Telosmoside $\mathrm{A}_{13}, 20924$

\section{$\mathrm{C}_{61} \mathrm{H}_{102} \mathrm{O}_{24}$}

Telosmoside $\mathrm{A}_{12}, 20923$

\section{$\mathrm{C}_{61} \mathrm{H}_{102} \mathrm{O}_{26}$}

Telosmoside $\mathrm{A}_{5}, 20916$

\section{$\mathrm{C}_{61} \mathrm{H}_{102} \mathrm{O}_{27}$}

Telosmoside $\mathrm{A}_{3}, 20914$

\section{$\mathrm{C}_{61} \mathrm{H}_{102} \mathrm{O}_{28}$}

Yesanchinoside J, 22902

\section{$\mathrm{C}_{61} \mathrm{H}_{102} \mathrm{O}_{32}$}

Polianthoside F, 17615

\section{$\mathrm{C}_{62} \mathrm{H}_{64} \mathrm{O}_{20}$}

Epioritin-( $4 \beta \rightarrow 6$ )-epioritin- $(4 \beta \rightarrow 6)$-epioritin- $4 \alpha$ ol nona- $O$-methylether tetra-acetate, 6983

Epioritin-( $4 \beta \rightarrow 6$ )-oritin- $(4 \alpha \rightarrow 6$ )-epioritin- $4 \alpha$-ol nona- $O$-methylether tetra-acetate, 6987 Oritin- $(4 \beta \rightarrow 6)$-oritin-( $4 \alpha \rightarrow 6$ )-epioritin- $4 \alpha$-ol nona- $O$-methylether tetra-acetate, 16198

\section{$\mathrm{C}_{62} \mathrm{H}_{76} \mathrm{O}_{35}$}

Tenuifoliose A, 20940

\section{$\mathrm{C}_{62} \mathrm{H}_{90} \mathrm{O}_{27}$}

Cynaphylloside H, 4563

\section{$\mathrm{C}_{62} \mathrm{H}_{94} \mathrm{O}_{29}$}

Assamsaponin F, 1919

\section{$\mathrm{C}_{62} \mathrm{H}_{98} \mathrm{O}_{4}$}

(14-Methyl-24-methylene-dihydroman-giferodiol)-14-methyl-24-methylene dihydromangiferonate, 14595

\section{$\mathrm{C}_{62} \mathrm{H}_{98} \mathrm{O}_{28}$}

Maesasaponin $\mathrm{IV}_{3}, 13355$

$\mathrm{C}_{62} \mathrm{H}_{98} \mathrm{O}_{31}$ 


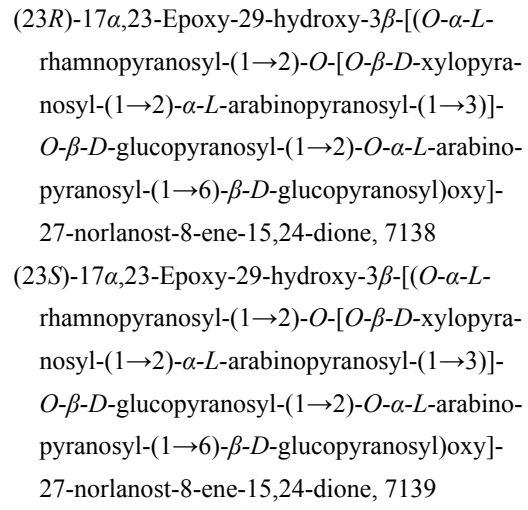

\section{$\mathrm{C}_{62} \mathrm{H}_{100} \mathrm{O}_{24}$}

WilfosideC $\mathrm{C}_{1} \mathrm{G}, 22691$

\section{$\mathrm{C}_{62} \mathrm{H}_{100} \mathrm{O}_{29}$}

Ternstroemiaside E, 20984

\section{$\mathrm{C}_{62} \mathrm{H}_{100} \mathrm{O}_{30}$}

Conyzasaponin A, 4015

Conyzasaponin N, 4024

Conyzasaponin P, 4026

(23S)-17 $\alpha, 23-$-Epoxy-29-hydroxy-3 $\beta-[(O-\alpha-L-$

rhamnopyranosyl-( $(1 \rightarrow 2)-O-[O-\beta$ - $D$-xylopyranosyl-( $(1 \rightarrow 2)-\alpha-L$-arabinopyranosyl- $(1 \rightarrow 3)]-O$ $\beta$ - $D$-glucopyranosyl-( $1 \rightarrow 2)-O-\alpha-L$-arabinopyranosyl-(1 $\rightarrow 6)-\beta$ - $D$-glucopyranosyl)oxy]-27norlanost-8-en-24-one, 7140

\section{$\mathrm{C}_{62} \mathrm{H}_{100} \mathrm{O}_{31}$}

Agavasaponin E, 712

\section{$\mathrm{C}_{62} \mathrm{H}_{100} \mathrm{O}_{33}$}

Diuranthoside F, 6526

Diuranthoside G, 6527

(25R)-26-[( $\beta$ - $D$-Glucopyranosyl)oxy]-2 $\alpha$-hydroxyfurosta-5,20(22)-dien-3 $\beta$-yl $O-\beta$ - $D$-glucopyranosyl-(1 $\rightarrow 3)-O-\beta-D$-glucopyranosyl$(1 \rightarrow 2)-O$-[ $\beta$ - $D$-xylopyranosyl- $(1 \rightarrow 3)]-O-\beta-D$ glucopyranosyl-( $(1 \rightarrow 4)-\beta$ - $D$-galactopyranoside, 8693

\section{$\mathrm{C}_{62} \mathrm{H}_{100} \mathrm{O}_{34}$}

(24S,25S)-24-[( $\beta$ - $D$-Glucopyranosyl)oxy]-2 $\alpha$ hydroxyspirost-5-en-3 $\beta$-yl $O-\beta$ - $D$-glucopyranosyl-( $1 \rightarrow 3)-O-\beta$ - $D$-glucopyranosyl-( $(1 \rightarrow 2)-$ $O$-[ $\beta$ - $D$-xylopyranosyl-( $1 \rightarrow 3)]-O-\beta$ - $D$-glucopyranosyl-(1 $\rightarrow 4)-\beta$ - $D$-galactopyranoside, 8698

\section{$\mathbf{C}_{62} \mathbf{H}_{100} \mathbf{O}_{35}$}

$3 \beta$-[( $O-\beta$ - $D$-Glucopyranosyl-( $(1 \rightarrow 3)-O-\beta$ - $D$-glucopyranosyl-( $1 \rightarrow 2)-O-[\beta$ - $D$-xylopyranosyl-
$(1 \rightarrow 3)]-O-\beta$ - $D$-glucopyranosyl-( $1 \rightarrow 4)-\beta-D-$ galactopyranosyl)oxy]-16 $\beta$-[[(4R)-5-( $\beta$ - $D$-glucopyranosyloxy)-4-methyl-1-oxopentyl]oxy]$2 \alpha$-hydroxypregn-5-en-20-one, 8659

\section{$\mathbf{C}_{\mathbf{6 2}} \mathrm{H}_{\mathbf{1 0 2}} \mathrm{O}_{\mathbf{3 0}}$}

Agave cantala Agaveside C, 719

Agave cantala Agaveside D, 720

$\mathrm{C}_{62} \mathrm{H}_{102} \mathrm{O}_{31}$

Dongnoside A, 6553

$\mathrm{C}_{62} \mathrm{H}_{104} \mathrm{O}_{22}$

Telosmoside $\mathrm{A}_{10}, 20921$

$\mathrm{C}_{62} \mathrm{H}_{104} \mathrm{O}_{23}$

Telosmoside $\mathrm{A}_{11}, 20922$

$\mathrm{C}_{62} \mathrm{H}_{104} \mathrm{O}_{24}$

Quinquenoside II, 18447

$\mathrm{C}_{62} \mathrm{H}_{104} \mathrm{O}_{33}$

Polianthoside G, 17616

$\mathrm{C}_{63} \mathrm{H}_{72} \mathrm{O}_{33}$

Paniculatonoid B, 16608

$\mathrm{C}_{63} \mathrm{H}_{78} \mathrm{O}_{36}$

Tenuifoliose N, 20952

$\mathrm{C}_{63} \mathrm{H}_{88} \mathrm{CoN}_{14} \mathrm{O}_{14} \mathrm{P}$

Vitamin $\mathrm{B}_{12}, 22556$

\section{$\mathrm{C}_{63} \mathrm{H}_{88} \mathrm{O}_{22}$}

12-O-Benzoyl-19-benzoyloxydeacylmetaplexigenin-3- $O-\beta$ - $D$-thevetopyranosyl-( $1 \rightarrow 4)-\beta$ - $D$ cymaropyranosyl-(1 $\rightarrow 4)-\beta$ - $D$-cymaropyranosyl-(1 $\rightarrow 4)$ - $\beta$ - $D$-cymaropyranoside, 2232

\section{$\mathrm{C}_{63} \mathrm{H}_{94} \mathrm{O}_{26}$}

Alpinoside C, 992

\section{$\mathrm{C}_{63} \mathrm{H}_{94} \mathrm{O}_{27}$}

Alpinoside A, 990

\section{$\mathrm{C}_{63} \mathrm{H}_{98} \mathrm{O}_{29}$}

Maesasaponin $\mathrm{IV}_{2}, 13354$

PM-2004-70-458-4, 17579

Tubeimoside A, 22072

\section{$\mathrm{C}_{63} \mathrm{H}_{98} \mathrm{O}_{30}$}

PM-2004-70-458-5, 17581

Tubeimoside B, 22073

$\mathrm{C}_{63} \mathrm{H}_{98} \mathrm{O}_{31}$

Thladioside $\mathrm{H}_{1}, 21335$

$\mathrm{C}_{63} \mathrm{H}_{100} \mathrm{O}_{23}$

Symplocososide A, 20540

Symplocososide B, 20541

$\mathrm{C}_{63} \mathrm{H}_{100} \mathrm{O}_{28}$

Maesasaponin $\mathrm{V}_{3}, 13357$

$\mathrm{C}_{63} \mathrm{H}_{100} \mathrm{O}_{29}$

Eupteleasaponin III, 7624

$\mathrm{C}_{63} \mathrm{H}_{100} \mathrm{O}_{31}$

Scillasaponin F, 19522

$\mathrm{C}_{63} \mathrm{H}_{100} \mathrm{O}_{32}$

Scillasaponin G, 19523

$\mathrm{C}_{63} \mathrm{H}_{102} \mathrm{O}_{2}$
Malloprenyl linolenate, 13427

$\mathrm{C}_{63} \mathrm{H}_{\mathbf{1 0 2}} \mathrm{O}_{\mathbf{1 1}}$

(2S)-1,2,6'-Tri- $O$-[(9Z,12Z,15Z)-octadeca-9,12, 15 -trienoyl]-3- $O-\beta$ - $D$-galactopyranosyl glycerol, 21983

\section{$\mathrm{C}_{63} \mathrm{H}_{102} \mathrm{O}_{29}$}

Scabioside G, 19447

\section{$\mathrm{C}_{63} \mathrm{H}_{102} \mathrm{O}_{30}$}

Conyzasaponin I, 4019

\section{$\mathrm{C}_{63} \mathrm{H}_{102} \mathrm{O}_{31}$}

Conyzasaponin J, 4020

\section{$\mathrm{C}_{63} \mathrm{H}_{102} \mathrm{O}_{32}$}

Polygalacin $\mathrm{D}_{2}, 17630$

\section{$\mathrm{C}_{63} \mathrm{H}_{102} \mathrm{O}_{33}$}

$(25 R)-3 \beta-[(O-\beta-D$-Glucopyranosyl- $(1 \rightarrow 3)-O-\beta$ $D$-glucopyranosyl-( $1 \rightarrow 2)-O$ - $[O-\alpha-L$-rhamnopyranosyl-(1 $\rightarrow 4)-\beta$ - $D$-glucopyranosyl$(1 \rightarrow 3)]-O-\beta$ - $D$-glucopyranosyl-( $1 \rightarrow 4)-\beta$ - $D$-galactopyranosyl)oxy]-5 $\alpha$-spirostan-12-one, 8653

Platycodin $\mathrm{D}_{2}, 17533$

Platycodin $\mathrm{D}_{3}, 17534$

\section{$\mathrm{C}_{63} \mathrm{H}_{102} \mathbf{O}_{\mathbf{3 4}}$}

$(25 R)-3 \beta-[(O-\beta-D-$ Glucopyranosyl- $(1 \rightarrow 3)-O-\beta-$ $D$-glucopyranosyl-( $(1 \rightarrow 2)-O$-[ $O-\alpha-L$-rhamnopyranosyl-(1 $\rightarrow 4)-\beta$ - $D$-glucopyranosyl$(1 \rightarrow 3)]-O-\beta$ - $D$-glucopyranosyl-( $1 \rightarrow 4)$ - $\beta$ - $D$-galactopyranosyl)oxy]-15 $\alpha$-hydroxy- $5 \alpha$-spirostan-12-one, 8654

\section{$\mathrm{C}_{63} \mathrm{H}_{104} \mathrm{O}_{23}$}

Periplocoside F, 16949

\section{$\mathrm{C}_{63} \mathrm{H}_{104} \mathrm{O}_{33}$}

(25R)-12 $\beta$-Hydroxy- $5 \alpha$-spirostan- $3 \beta$-yl $O-\beta$ - $D$-glucopyranosyl-( $(1 \rightarrow 3)-O-\beta$ - $D$-glucopyr anosyl-(1 $\rightarrow 2)-O-[O-\alpha-L$-rhamnopyranosyl-(1 $\rightarrow 4)$ - $\beta$ - $D$-glucopyranosyl-( $1 \rightarrow 3)]$ - $O$ - $\beta$ - $D$-gluc opyranosyl-( $1 \rightarrow 4)-\beta$ - $D$-galactopyranoside, 10719

(25R)-15 $\alpha$-Hydroxy- $5 \alpha$-spirostan-3 $\beta$-yl $O$ - $\beta$ - $D$-glucopyranosyl-( $1 \rightarrow 3)-O-\beta$ - $D$-glucopyr anosyl-( $1 \rightarrow 2)-O-[O-\alpha-L$-rhamnopyranosyl-(1 $\rightarrow 4)$ - $\beta$ - $D$-glucopyranosyl-( $(1 \rightarrow 3)]-O-\beta$ - $D$-gluc opyranosyl-( $(1 \rightarrow 4)-\beta-D$-galactopyranoside, 10720

\section{$\mathrm{C}_{63} \mathrm{H}_{104} \mathrm{O}_{34}$}

(25R)-26-[( $\beta$ - $D$-Glucopyranosyl)oxy]-2 $\alpha$-hydroxy-22 $\alpha$-methoxyfurost-5-en-3 $\beta$-yl- $O-\beta$ - $D$-glucopyranosyl-(1 $\rightarrow 3)-O-\beta$ - $D$-glucopyranosyl$(1 \rightarrow 2)-O$-[ $\beta$ - $D$-xylopyranosyl-( $(1 \rightarrow 3)]-O-\beta-D-$ glucopyranosyl-( $1 \rightarrow 4)-\beta$ - $D$-galactopyranoside, 8695

\section{$\mathrm{C}_{63} \mathrm{H}_{106} \mathrm{O}_{30}$}

Notoginsenoside Q, 15835

Notoginsenoside S, 15841 


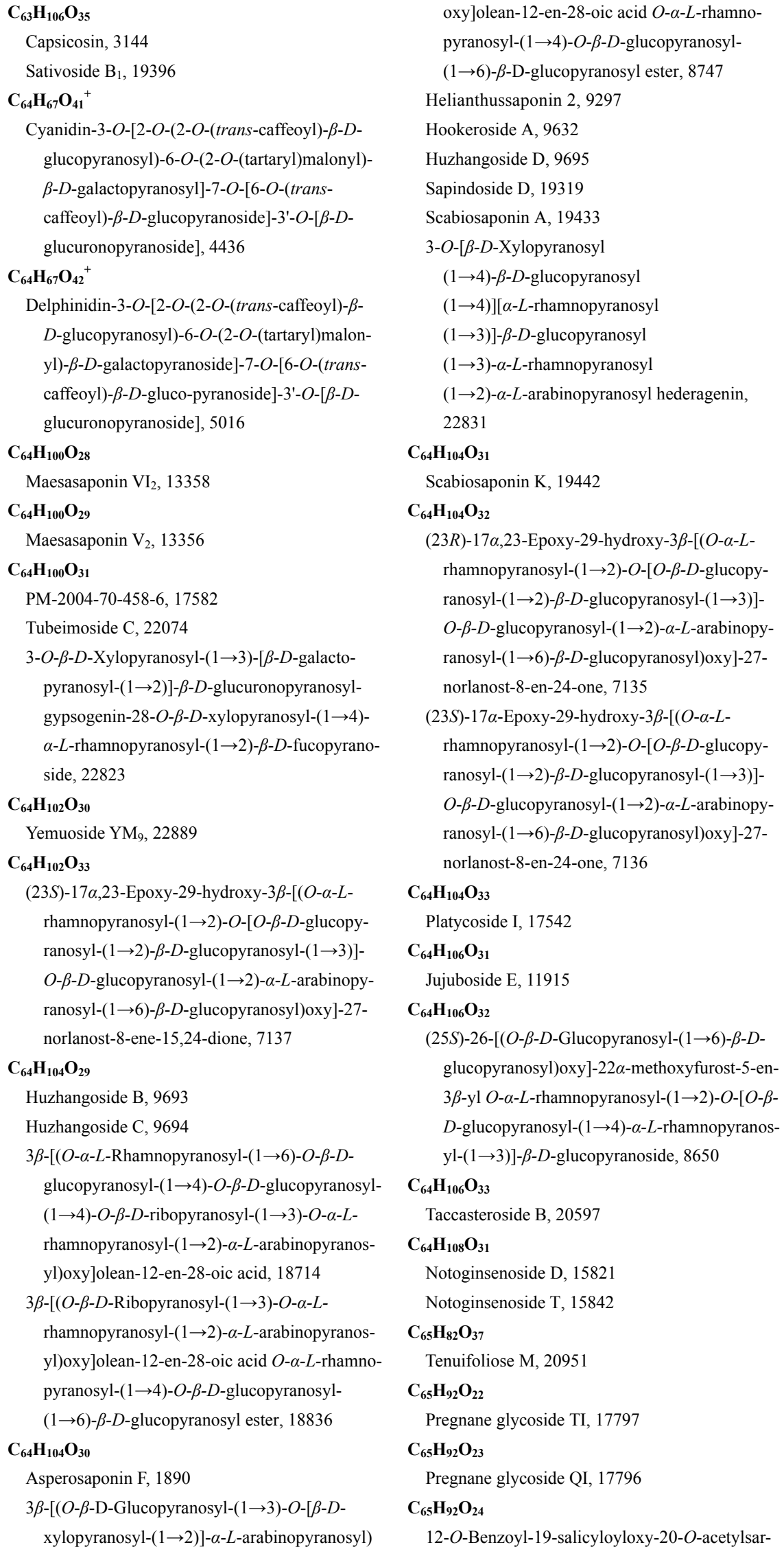

costin 3- $O-\beta$ - $D$-thevetopyranosyl- $(1 \rightarrow 4)-\beta-D$ cymaropyranosyl-( $1 \rightarrow 4)-\beta$ - $D$-cymaropyranosyl-( $(1 \rightarrow 4)-\beta$ - $D$-cymaropyranoside, 2269

\section{$\mathrm{C}_{65} \mathrm{H}_{98} \mathrm{O}_{23}$}

Symplocososide E, 20544

$\mathrm{C}_{65} \mathrm{H}_{102} \mathrm{O}_{29}$

Maesasaponin $\mathrm{VI}_{3}, 13359$

$\mathrm{C}_{65} \mathrm{H}_{104} \mathrm{O}_{30}$

Maetenoside A, 13362

$\mathrm{C}_{65} \mathrm{H}_{104} \mathrm{O}_{31}$

Ilekudinoside F, 10976

$\mathbf{C}_{65} \mathbf{H}_{104} \mathbf{O}_{33}$

$(23 S, 25 R)-17 \alpha, 23$-Epoxy-29-hydroxy-3 $\beta$-[( $O-\alpha$ $L$-rhamnopyranosyl-( $1 \rightarrow 2)-O-[O-\beta$ - $D$-glucopyranosyl-( $(1 \rightarrow 2)-\beta$ - $D$-glucopyranosyl$(1 \rightarrow 3)]-O-\beta$ - $D$-glucopyranosyl-( $(1 \rightarrow 2)-\alpha-L-$ arabinopyranosyl-( $1 \rightarrow 6)-\beta$ - $D$-glucopyranosyl) oxy]lanost-8-en-23,26-olide, 7133

\section{$\mathrm{C}_{65} \mathrm{H}_{104} \mathrm{O}_{34}$}

2"-O-Acetylplatycodin $\mathrm{D}_{2}, 487$

3"-O-Acetylplatycodin $\mathrm{D}_{2}, 488$

\section{$\mathrm{C}_{65} \mathrm{H}_{\mathbf{1 0 6}} \mathrm{O}_{24}$}

Periplocoside E, 16948

\section{$\mathrm{C}_{65} \mathrm{H}_{106} \mathrm{O}_{30}$}

Akeboside st $\mathrm{j}, 820$

Anhuienoside F, 1256

$3 \beta$ - $O$ - $(\beta$-Glucopyranosyl-( $(1 \rightarrow 3)-\alpha$-rhamnopyranosyl-(1 $\rightarrow 2)$ - $\alpha$-arabinopyranosyl)-lup12 -en-28- $O$ - $(\alpha$-rhamnopyranosyl- $(1 \rightarrow 4)-\beta$ glucopyranosyl-( $1 \rightarrow 6)-\beta$-galactopyranosyl) ester, 8718

$3 \beta-[(O-\beta$ - $D$-Glucopyranosyl- $(1 \rightarrow 3)-O-[\alpha-L-$ rhamnopyranosyl- $(1 \rightarrow 2)]-\alpha-L$-arabinopyranosyl)oxy]olean-12-en-28-oic acid $O-\alpha-L$ rhamnopyranosyl-( $1 \rightarrow 4)-O-\beta$ - $D$-glucopyranosyl-( $1 \rightarrow 6)-\beta$ - $D$-glucopyranosyl ester, 8720

Hederacolchiside E, 9257

\section{$\mathrm{C}_{65} \mathrm{H}_{106} \mathrm{O}_{31}$}

$3 \beta$-O- $\beta$ - $D$-Galactopyranosyl- $(1 \rightarrow 3)-\alpha$ - $L$-rhamnopyranosyl-( $(1 \rightarrow 2)-\alpha$ - $L$-arabinopyranosyl-23-hydroxyolean-12-en-28-oic acid 28-O- $\alpha-L$ rhamnopyranosyl-(1 $\rightarrow 4)-\beta$ - $D$-glucopyranosyl$(1 \rightarrow 6)-\beta$ - $D$-glucopyranosylester, 8068

$3 \beta$ - $O$ - $(\beta$-Glucopyranosyl- $(1 \rightarrow 3)-\alpha$-rhamnopyranosyl-( $1 \rightarrow 2)-\alpha$-arabinopyranosyl)-23hydroxylup-12-en-28-O-( $\alpha$-rhamnopyranosyl-( $(\rightarrow 4)-\beta$-glucopyranosyl-( $1 \rightarrow 6)-\beta$-galactopyranosyl) ester, 8717

Hederacolchiside F, 9258

23 -Hydroxy-3 $\beta$-[(O- $\alpha-L$-rhamnopyranosyl$(1 \rightarrow 2)-O$-[ $\beta$ - $D$-glucopyranosyl- $(1 \rightarrow 4)]-\alpha-L$ arabinopyranosyl)oxy]lup-20(29)-en-28-oic acid 28-O- $\alpha$ - $L$-rhamnopyranosyl-( $1 \rightarrow 4)-O$ - 
$\beta$ - $D$-glucopyranosyl-( $1 \rightarrow 6)-\beta$ - $D$-glucopyranosyl ester, 10674

Matesaponin 5, 13602

Patrinoside C, 16721

3-O-2-L-Rhamnopyranosyl-( $1 \rightarrow 3)-\beta$ - $D$-glucopyranosyl-( $1 \rightarrow 3)-\alpha$ - $L$-rhamnopyranosyl- $(1 \rightarrow 2)$ $\alpha$ - $L$-arabinopyranosyl hederagenin $28-O-\beta$ - $D$ gluco-pyra-nosyl-(1 $\rightarrow 6$ )- $\beta$ - $D$-glucopyra-nosyl ester, 18717

Scabiosaponin G, 19438

\section{$\mathrm{C}_{65} \mathrm{H}_{\mathbf{1 0 6}} \mathrm{O}_{\mathbf{3 2}}$}

3-O-[ $\beta$ - $D$-Glucopyranosyl- $(1 \rightarrow 3)]$-[ $\beta$ - $D$-glucopyranosyl- $(1 \rightarrow 2)]-\alpha-L$-arabinopyranosyl echinocystic acid 28-O-[ $\alpha-L$-rhamnopyranosyl$(1 \rightarrow 4)$ - $\beta$ - $D$-glucopyranosyl-( $1 \rightarrow 6)-\beta$ - $D$-glucopyranosyl] ester, 8639

Macranthoidin B, 13299

New triterpennoid glycoside, 15520

Scabiosaponin H, 19439

Ternstroemiaside F, 20985

\section{$\mathrm{C}_{66} \mathrm{H}_{46} \mathrm{O}_{21}$}

RidiculuflavonylchalconeA, 18846

(4"',5",7-Trihydroxy-4',5,7"-trimethoxy-3,6"-biflavone)-3"'-O-4"'-(5,5",7"-trihydroxy-3',3",4'trimethoxy-6- $O-\beta, 7-\alpha$-flavone-chalcone), 21853

\section{$\mathrm{C}_{66} \mathrm{H}_{50} \mathrm{O}_{20}$}

Taiwanhomoflavone C, 20627

\section{$\mathrm{C}_{66} \mathrm{H}_{50} \mathrm{O}_{30}$}

Procyanidin $\mathrm{C}_{1}-3,3^{\prime}, 3^{\prime \prime}$-tri- $O$-gallate, 17895

\section{$\mathrm{C}_{66} \mathrm{H}_{69} \mathrm{O}_{33}{ }^{+}$}

Cyanidin-3-O-[2-O-(6-O-E- $p$-coumaroyl- $\beta-D$ glucopyranosyl)]-\{6-O-[4-O-(6-O-E-p-coumaroyl- $\beta$ - $D$-glucopyranosyl)- $E$-caffeoyl $]-\beta$ $D$-glucopyranosyl $\}-5-O-\beta$ - $D$-glucopyranoside, 4437

Delphinidin-3- $O-\beta-D$-glucopyranoside-7,3',5'-tri$O$-(6-O-p-coumaroyl- $\beta$-glucopyranoside), 5026

\section{$\mathrm{C}_{66} \mathrm{H}_{69} \mathrm{O}_{35}{ }^{+}$}

Cyanidin-3-O-[2-O-(6-O-E-caffeoyl- $\beta$ - $D$-glucopyranosyl)]-\{6-O-[4-O-(6-O-E-3,5-dihydroxycinnamoyl- $\beta$ - $D$-glucopyranosyl)- $E$-caffeoyl]$\beta$ - $D$-glucopyranosyl $\}-5-O-\beta$ - $D$-glucopyranoside, 4435

\section{$\mathrm{C}_{66} \mathrm{H}_{94} \mathrm{O}_{27}$}

3- $O$ - $[\beta$ - $D$-Galactopyranosyl-( $(1 \rightarrow 2)-\beta$ - $D$-glucuronopyranosyl]quillaic acid-28- $O-\alpha-L$-rhamnopyranosyl-( $1 \rightarrow 2)$-3-O-acetyl-4-O-cis- $p$-methoxycinnamoyl $\beta$ - $D$-fucopyranoside, 8060

3- $O$-[ $\beta$ - $D$-Galactopyranosyl-( $(1 \rightarrow 2)-\beta$ - $D$-glucuronopyranosyl]quillaic acid-28- $O-\alpha-L$-rhamnopyranosyl-( $(1 \rightarrow 2)-3-O$-acetyl-4-O-trans- $p$ - methoxycinnamoyl $\beta$ - $D$-fucopyranoside, 8061

\section{$\mathrm{C}_{66} \mathrm{H}_{102} \mathrm{O}_{29}$}

Maesasaponin $\mathrm{VII}_{1}, 13360$

$\mathrm{C}_{66} \mathrm{H}_{106} \mathrm{O}_{23}$

Symplocososide C, 20542

Symplocososide D, 20543

$\mathrm{C}_{66} \mathrm{H}_{108} \mathrm{O}_{24}$

Periploside A, 16961

$\mathrm{C}_{67} \mathrm{H}_{84} \mathrm{O}_{39}$

Tenuifoliose L, 20950

$\mathrm{C}_{67} \mathrm{H}_{104} \mathrm{O}_{32}$

Segetoside F, 19663

$\mathrm{C}_{67} \mathrm{H}_{104} \mathrm{O}_{33}$

3- $O-\beta$ - $D$-Galactopyranosyl-( $1 \rightarrow 2)-\beta$ - $D$-glucuronopyranosyl-gypsogenin-28- $O-\beta-D$-xylopyra$\operatorname{nosyl}(1 \rightarrow 4)$-[ $\beta$ - $D$-6- $O$-acetylglucopyranosyl $(1 \rightarrow 3)]-\alpha$ - $L$-rhamnopyranosyl $(1 \rightarrow 2)-\beta$ - $D$ fucopyranoside, 8058

\section{$\mathrm{C}_{67} \mathrm{H}_{108} \mathrm{O}_{34}$}

Conyzasaponin B, 4016

Conyzasaponin M, 4023

Conyzasaponin Q, 4027

\section{$\mathrm{C}_{67} \mathrm{H}_{112} \mathrm{O}_{29}$}

Telosmoside $\mathrm{A}_{16}, 20927$

$\mathrm{C}_{67} \mathrm{H}_{112} \mathrm{O}_{30}$

Telosmoside A 18,20929

\section{$\mathrm{C}_{68} \mathrm{H}_{48} \mathrm{O}_{44}$}

Oenothein B, 16011

Sanguiin $\mathrm{H}_{3}, 19275$

Sanguiin $\mathrm{H}_{10}, 19282$

\section{$\mathrm{C}_{68} \mathrm{H}_{50} \mathrm{O}_{44}$}

Nobotanin R, 15643

\section{$\mathrm{C}_{68} \mathrm{H}_{56} \mathrm{O}_{44}$}

Cornusiin A, 4063

\section{$\mathrm{C}_{68} \mathrm{H}_{86} \mathrm{O}_{39}$}

Tenuifoliose F, 20945

\section{$\mathrm{C}_{68} \mathrm{H}_{104} \mathrm{O}_{33}$}

Segetoside H, 19665

$\mathrm{C}_{68} \mathrm{H}_{104} \mathrm{O}_{34}$

Segetoside I, 19666

$\mathrm{C}_{68} \mathrm{H}_{108} \mathrm{O}_{26}$

Periplocoside K, 16953

$\mathrm{C}_{68} \mathrm{H}_{110} \mathrm{O}_{33}$

Gleditsioside I, 8538

$\mathrm{C}_{68} \mathrm{H}_{110} \mathrm{O}_{34}$

Conyzasaponin C, 4017

$\mathrm{C}_{68} \mathrm{H}_{110} \mathrm{O}_{35}$

Conyzasaponin K, 4021

Conyzasaponin O, 4025

$\mathrm{C}_{68} \mathrm{H}_{112} \mathrm{O}_{37}$

Agavasaponin $\mathrm{H}, 713$

$\mathrm{C}_{68} \mathrm{H}_{114} \mathrm{O}_{27}$

Telosmoside $\mathrm{A}_{14}, 20925$

\section{$\mathrm{C}_{68} \mathrm{H}_{114} \mathrm{O}_{28}$}

Telosmoside $\mathrm{A}_{15}, 20926$

Telosmoside $\mathrm{A}_{17}, 20928$

\section{$\mathrm{C}_{68} \mathrm{H}_{114} \mathrm{O}_{38}$}

Trigoneoside Va, 21671

Trigoneoside $\mathrm{Vb}, 21672$

$\mathrm{C}_{68} \mathrm{H}_{134} \mathrm{O}_{2}$

Lycopanerol $\mathrm{C}_{1}, 13205$

\section{$\mathrm{C}_{69} \mathrm{H}_{82} \mathrm{O}_{35}$}

Kaempferol-3-O-[a-L-rhamnopyranosyl $(1 \rightarrow 6)$ $\{\alpha$ - $L$-rhamnopyranosyl $(1 \rightarrow 3)\}$-(2-O-trans- $p$ coumaroyl) $]-\beta$ - $D$-glucopyranoside-7- $O-[\alpha-L$ rhamnopyranosyl $(1 \rightarrow 3)-\alpha-L$-rhamnopyranosyl $(1 \rightarrow 3)$-(4-O-trans- $p$-coumaroyl)]- $\alpha$ - $L$-rhamnopyranoside, 12079

\section{$\mathrm{C}_{69} \mathrm{H}_{82} \mathrm{O}_{36}$}

Kaempferol-3- $O$-[ $\beta$ - $D$-glucopyranosyl $(1 \rightarrow 6)$ $\{\alpha$-L-rhamnopyranosyl $(1 \rightarrow 3)\}$-(2-O-trans- $p$ coumaroyl) $]-\beta-D$-glucopyranoside-7- $O-[\alpha-L-$ rhamnopyranosyl $(1 \rightarrow 3)-\alpha-L$-rhamnopyranosyl $(1 \rightarrow 3)-(4-O-c i s-p$-coumaroyl)]- $\alpha$ - $L$-rhamnopyranoside, 12057

Kaempferol-3- $O$-[ $\beta$ - $D$-glucopyranosyl $(1 \rightarrow 6)$ $\{\alpha$-L-rhamnopyranosyl $(1 \rightarrow 3)\}$-(2-O-trans- $p$ coumaroyl) $]-\beta$ - $D$-glucopyranoside-7- $O-[\alpha-L$ rhamnopyranosyl $(1 \rightarrow 3)-\alpha$ - $L$-rhamnopyranosyl $(1 \rightarrow 3)$-(4-O-trans- $p$-coumaroyl)]- $\alpha$ - $L$-rhamnopyranoside, 12058

\section{$\mathrm{C}_{69} \mathrm{H}_{100} \mathrm{O}_{30}$}

Sinocrassuloside X, 19945

\section{$\mathrm{C}_{69} \mathrm{H}_{106} \mathrm{O}_{33}$}

Segetoside B, 19659

\section{$\mathrm{C}_{69} \mathrm{H}_{106} \mathrm{O}_{34}$}

Segetoside D, 19661

\section{$\mathrm{C}_{69} \mathrm{H}_{108} \mathrm{O}_{35}$}

3-O- $\alpha$ - $L$-Arabinopyranosyl- $(1 \rightarrow 2)-[\beta$ - $D$-galactopyranosyl-( $(1 \rightarrow 3)]-\beta$ - $D$-glucuronopyranosylgypsogenin-28- $O-\beta-D$-xylopyranosyl-( $(1 \rightarrow 3)$ $\beta$-D-xylopyranosyl-( $1 \rightarrow 4)$ - $\alpha$ - $L$-rhamnopyranosyl-( $1 \rightarrow 2)-\beta$ - $D$-fucopyranoside, 1566

\section{$\mathrm{C}_{69} \mathrm{H}_{112} \mathrm{O}_{34}$}

Gleditsiasaponin E', 8529

Hookeroside B, 9633

Scabiosaponin B, 19434

\section{$\mathrm{C}_{69} \mathrm{H}_{112} \mathrm{O}_{36}$}

Conyzasaponin L, 4022

\section{$\mathrm{C}_{70} \mathrm{H}_{104} \mathrm{O}_{32}$}

Onjisaponin G, 16107

Senegin II, 19715

$\mathrm{C}_{70} \mathrm{H}_{110} \mathrm{O}_{32}$

Segetoside G, 19664

\section{$\mathbf{C}_{70} \mathbf{H}_{110} \mathbf{O}_{36}$}

3-O- $\alpha$ - $L$-Arabinopyranosyl- $(1 \rightarrow 2)-[\beta$ - $D$-galacto- 


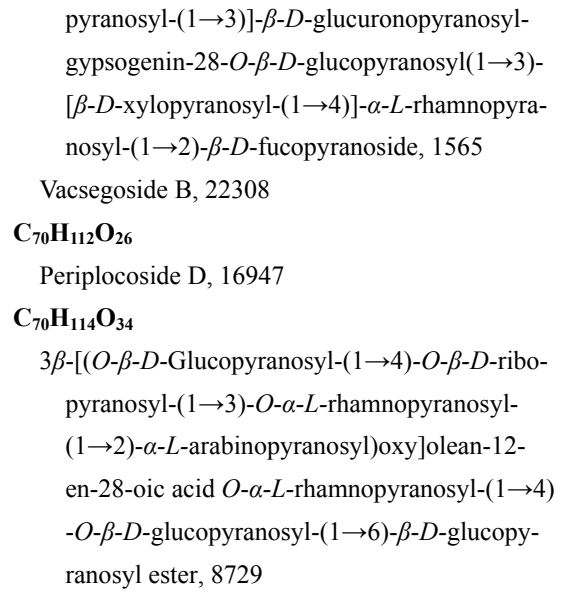

\section{$\mathbf{C}_{70} \mathbf{H}_{114} \mathbf{O}_{35}$}

$3 \beta-[(O-\beta-D$-Glucopyranosyl-( $(1 \rightarrow 4)-O-\beta$ - $D$-ribopyranosyl-( $1 \rightarrow 3)-O-\alpha$ - $L$-rhamnopyranosyl$(1 \rightarrow 2)-\alpha-L$-arabinopyranosyl)oxy]-21 $\alpha$-hydroxyolean-12-en-28-oic acid $O-\alpha$ - $L$-rhamnopyranosyl-( $1 \rightarrow 4)-O-\beta$ - $D$-glucopyranosyl-

$(1 \rightarrow 6)-\beta$ - $D$-glucopyranosyl ester, 8727

Patrinoside D, 16723

Scabiosaponin C, 19435

3 - $O$ - $[\beta$ - $D$-Xylopyranosyl $(1 \rightarrow 4)$ - $\beta$ - $D$-glucopyra$\operatorname{nosyl}(1 \rightarrow 4)][\alpha-L$-rhamnopyranosyl $(1 \rightarrow 3)]-\beta$ $D$-glucopyranosyl $(1 \rightarrow 3)-\alpha-L$-rhamnopyranosyl $(1 \rightarrow 2)-\alpha-L$-arabinopyranosyl hederagenin-

28-O- $\beta$ - $D$-glucopyranoside, 22832

\section{$\mathrm{C}_{70} \mathrm{H}_{116} \mathbf{O}_{38}$}

Taccasteroside A, 20596

\section{$\mathrm{C}_{70} \mathrm{H}_{118} \mathrm{O}_{38}$}

$(25 R)-3 \beta-[(O-\beta-D$-Glucopyranosyl- $(1 \rightarrow 3)-O-\beta-$

$D$-glucopyranosyl-( $1 \rightarrow 2)-O$-[O- $\alpha$ - $L$-rhamnopyranosyl- $(1 \rightarrow 4)-\beta$ - $D$-glucopyranosyl-

$(1 \rightarrow 3)]-O-\beta$ - $D$-glucopyranosyl-( $1 \rightarrow 4)-\beta$ - $D$ galactopyranosyl)oxy]-22 $\xi$-methoxy-5 $\alpha$ furostan-26-yl $\beta$ - $D$-glucopyranoside, 8655

\section{$\mathrm{C}_{70} \mathrm{H}_{138} \mathrm{O}_{2}$}

Lycopanerol $\mathrm{C}_{2}, 13206$

\section{$\mathrm{C}_{71} \mathrm{H}_{102} \mathrm{O}_{31}$}

Sinocrassuloside VI, 19941

Sinocrassuloside VII, 19942

$\mathbf{C}_{71} \mathbf{H}_{106} \mathbf{O}_{33}$

Onjisaponin E, 16105

$\mathrm{C}_{71} \mathrm{H}_{116} \mathrm{O}_{34}$

Pastuchoside B, 16699
$\mathrm{C}_{71} \mathrm{H}_{116} \mathrm{O}_{35}$

Pastuchoside A, 16698

$\mathrm{C}_{71} \mathrm{H}_{116} \mathrm{O}_{36}$

28 - $O-\beta$ - $D$-Glucopyranosyl-( $1 \rightarrow 6)-\beta$ - $D$-glucopyranosyl ester of 3-O-[ $\beta$ - $D$-glucopyranosyl$(1 \rightarrow 4)][\alpha-L$-rhamnopyranosyl- $(1 \rightarrow 3)]-\beta-D$ glucopyranosyl-( $1 \rightarrow 3)-\alpha-L$-rhamnopyranosyl$(1 \rightarrow 2)$ - $\alpha$-arabinopyranosyl-hederagenin, 8642 23 -Hydroxy-3 $\beta-[(O-\alpha-L$-rhamnopyranosyl$(1 \rightarrow 2)-O-[O-\beta-D$-glucopyranosyl-( $(1 \rightarrow 4)-\beta$ - $D$ glucopyranosyl-(1 $\rightarrow 4)]$ - $\alpha$ - $L$-arabinopyranosyl) oxy]lup-20(29)-en-28-oic acid 28-O- $\alpha-L$ rhamnopyranosyl-( $1 \rightarrow 4)$ - $O-\beta$ - $D$-glucopyranosyl-(1 $\rightarrow 6)-\beta$ - $D$-glucopyranosyl ester, 10675

\section{$\mathrm{C}_{72} \mathrm{H}_{104} \mathrm{O}_{31}$}

Sinocrassuloside VIII, 19943

Sinocrassuloside IX, 19944

\section{$\mathrm{C}_{72} \mathbf{H}_{106} \mathrm{O}_{31}$}

3-O-[ $\beta$ - $D$-Galactopyranosyl-( $1 \rightarrow 2)-\beta$ - $D$-glucuronopyranosyl]-28- $O$-[ $\beta$ - $D$-glucopyranosyl-

$(1 \rightarrow 2)-\alpha-L$-rhamnopyranosyl-( $1 \rightarrow 2)-\beta-D-4-O-$ trans-p-methoxycinnamoyl-fucopyranosyl] quillaic acid, 8056

\section{$\mathrm{C}_{72} \mathrm{H}_{112} \mathrm{O}_{34}$}

Segetoside E, 19662

\section{$\mathrm{C}_{72} \mathrm{H}_{112} \mathrm{O}_{37}$}

Agrostemmasaponin 1, 765

$\mathrm{C}_{72} \mathrm{H}_{112} \mathrm{O}_{38}$

Sinocrassuloside XI, 19946

$\mathrm{C}_{72} \mathrm{H}_{114} \mathrm{O}_{27}$

Periplocoside A, 16944

\section{$\mathrm{C}_{72} \mathrm{H}_{116} \mathrm{O}_{4}$}

Helenien, 9290

Physalien, 17238

\section{$\mathrm{C}_{72} \mathrm{H}_{142} \mathrm{O}_{2}$}

Lycopanerol $\mathrm{C}_{3}, 13207$

$\mathrm{C}_{73} \mathrm{H}_{116} \mathbf{O}_{27}$

Periploside C, 16963

\section{$\mathrm{C}_{73} \mathrm{H}_{118} \mathrm{O}_{38}$}

Gleditsioside K, 8540

\section{$\mathrm{C}_{74} \mathrm{H}_{112} \mathrm{O}_{34}$}

3-O-[ $\beta$-D-3-O-cis-p-Coumaroyl-glucopyranosyl$(1 \rightarrow 3)]$-[ $\beta$ - $D$-glucopyranosyl- $(1 \rightarrow 2)]$ - $\alpha$ - $L$-arabinopyranosyl echinocystic acid 28- $O-[\alpha-L-$ rhamnopyranosyl-( $1 \rightarrow 4)-\beta$ - $D$-glucopyranosyl$(1 \rightarrow 6)-\beta-D$-glucopyranosyl] ester, 4161

3-O-[ $\beta$-D-3-O-trans- $p$-Coumaroyl-glucopyranosyl-( $(1 \rightarrow 3)]$-[ $\beta$ - $D$-glucopyranosyl- $(1 \rightarrow 2)]-\alpha$ $L$-arabinopyranosyl echinocystic acid 28-O$[\alpha$ - $L$-rhamnopyranosyl-( $1 \rightarrow 4)$ - $\beta$ - $D$-glucopyranosyl-(1 $\rightarrow 6)-\beta$ - $D$-glucopyranosyl] ester, 4162

$\mathrm{C}_{74} \mathrm{H}_{120} \mathrm{O}_{37}$

Gleditsioside H, 8537

\section{$\mathrm{C}_{74} \mathrm{H}_{120} \mathrm{O}_{38}$}

Gleditsiasaponin $\mathrm{C}^{\prime}, 8528$

\section{$\mathbf{C}_{74} \mathbf{H}_{120} \mathbf{O}_{39}$}

Gleditsioside J, 8539

\section{$\mathrm{C}_{75} \mathrm{H}_{52} \mathrm{O}_{48}$}

Nobotanin A, 15641

\section{$\mathrm{C}_{75} \mathrm{H}_{54} \mathrm{O}_{47}$}

Paeonianin A, 16520

Paeonianin B, 16521

Paeonianin C, 16522

Paeonianin D, 16523

\section{$\mathrm{C}_{75} \mathrm{H}_{54} \mathrm{O}_{48}$}

Rugosin E, 19058

\section{$\mathrm{C}_{75} \mathrm{H}_{56} \mathrm{O}_{48}$}

Cornusiin G, 4065

\section{$\mathrm{C}_{75} \mathrm{H}_{75} \mathrm{O}_{35}$}

Delphinidin-3,7,3',5'-tetra-O-(6-O-p-coumaroyl$\beta$-glucopyranoside), 5031

\section{$\mathrm{C}_{75} \mathrm{H}_{81} \mathrm{O}_{42}{ }^{+}$}

Lobelinin A, 12940

\section{$\mathrm{C}_{75} \mathrm{H}_{112} \mathrm{O}_{35}$}

Onjisaponin B, 16104

\section{$\mathrm{C}_{75} \mathrm{H}_{112} \mathrm{O}_{36}$}

Onjisaponin F, 16106

\section{$\mathrm{C}_{76} \mathrm{H}_{116} \mathbf{O}_{34}$}

Protocrocin, 17971

\section{$\mathrm{C}_{76} \mathrm{H}_{124} \mathrm{O}_{39}$}

3- $O$ - $[\beta$ - $D$-Xylopyranosyl $(1 \rightarrow 4)-\beta$ - $D$-glucopyra$\operatorname{nosyl}(1 \rightarrow 4)][\alpha-L$-rhamnopyranosyl $(1 \rightarrow 3)]-\beta$ $D$-glucopyranosyl $(1 \rightarrow 3)]-\alpha$ - $L$-rhamnopyranosyl $(1 \rightarrow 2)-\alpha-L$-arabinopyranosyl oleanolic acid-28- $O-\beta$ - $D$-glucopyranosyl $(1 \rightarrow 6)-\beta$ - $D$ glucopyranoside, 22833

\section{$\mathrm{C}_{76} \mathrm{H}_{124} \mathrm{O}_{40}$}

Asperosaponin $\mathrm{H}_{1}, 1891$

28-O- $\beta$ - $D$-Glucopyranosyl-(1 $\rightarrow 6)-\beta$ - $D$-glucopyranosyl ester of 3- $O$-[ $\beta$ - $D$-xylopyranosyl$(1 \rightarrow 4)-\beta$ - $D$-glucopyranosyl-( $1 \rightarrow 4)][\alpha-L$-rhamnopyranosyl-( $(1 \rightarrow 3)]$ - $\beta$ - $D$-glucopyranosyl$(1 \rightarrow 3)$ - $\alpha$ - $L$-rhamnopyranosyl-( $(1 \rightarrow 2)$ - $\alpha$-arabinopyranosyl-hederagenin, 8643

3- $O$-[ $\beta$ - $D$-Xylopyranosyl $(1 \rightarrow 4)-\beta$ - $D$-glucopyra$\operatorname{nosyl}(1 \rightarrow 4)][\alpha-L$-rhamnopyranosyl $(1 \rightarrow 3)]-\beta$ $D$-glucopyranosyl $(1 \rightarrow 3)$ - $\alpha$ - $L$-rhamnopyranosyl $(1 \rightarrow 2)-\alpha-L$-arabinopyranosyl hederagenin28 - $O$ - $\beta$ - $D$-glucopyranosyl $(1 \rightarrow 6)-\beta$ - $D$-glucopyranoside, 22834

\section{$\mathrm{C}_{78} \mathrm{H}_{124} \mathrm{O}_{35}$}

Gleditsioside A, 8530

\section{$\mathrm{C}_{78} \mathrm{H}_{124} \mathrm{O}_{36}$}

Gleditsioside B, 8531

\section{$\mathrm{C}_{78} \mathrm{H}_{124} \mathrm{O}_{37}$}

Gleditsioside Q, 8544 


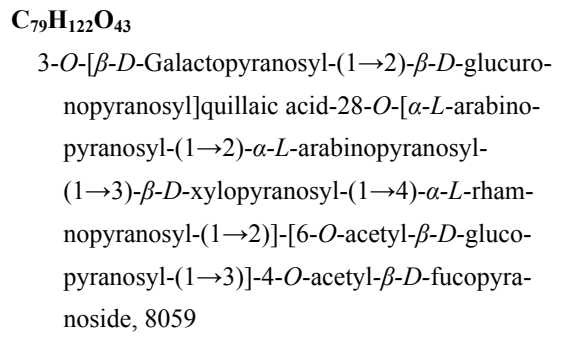

$\mathrm{C}_{79} \mathrm{H}_{128} \mathrm{O}_{30}$

PM-2004-70-458-4a, 17580

\section{$\mathrm{C}_{80} \mathrm{H}_{120} \mathrm{O}_{39}$}

Onjisaponin A, 16103

\section{$\mathrm{C}_{\mathbf{8 0}} \mathrm{H}_{\mathbf{1 2 6}} \mathbf{O}_{44}$}

Gypsoside, 9184

\section{$\mathrm{C}_{80} \mathrm{H}_{130} \mathrm{O}_{42}$}

Sapindoside E, 19320

\section{$\mathrm{C}_{80} \mathrm{H}_{158} \mathrm{O}_{5}$}

Lycopanerol D, 13208

\section{$\mathrm{C}_{81} \mathrm{H}_{132} \mathrm{O}_{43}$}

Clematoside A, 3824

\section{$\mathrm{C}_{82} \mathrm{H}_{54} \mathrm{O}_{52}$}

Agrimoniin, 759

Sanguiin $\mathrm{H}_{6}, 19278$

\section{$\mathrm{C}_{82} \mathrm{H}_{56} \mathrm{O}_{52}$}

Gemin A, 8263

Heterophylliin B, 9469

Nobotanin F, 15642

Rugosin F, 19059

\section{$\mathrm{C}_{82} \mathrm{H}_{58} \mathrm{O}_{52}$}

Coriariin A, 4053

Rugosin D, 19057

\section{$\mathrm{C}_{82} \mathrm{H}_{60} \mathrm{O}_{52}$}

Phillyraeoidin A, 17144

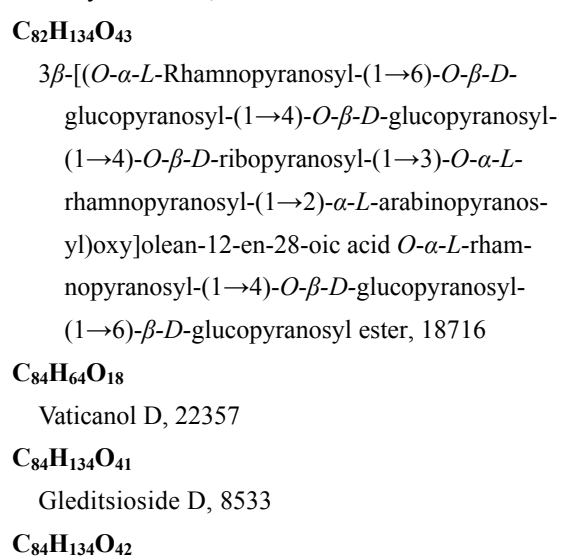

Gleditsioside $\mathrm{C}, 8532$

$\mathrm{C}_{84} \mathrm{H}_{134} \mathrm{O}_{43}$

Julibroside $\mathrm{J}_{20}, 11934$

$\mathrm{C}_{85} \mathrm{H}_{130} \mathrm{O}_{43}$

Clematibetoside A, 3819

$\mathrm{C}_{85} \mathrm{H}_{136} \mathrm{O}_{42}$

Julibroside $\mathrm{J}_{26}, 11940$

$\mathrm{C}_{85} \mathrm{H}_{136} \mathrm{O}_{43}$

Julibroside $\mathrm{J}_{25}, 11939$

$\mathrm{C}_{87} \mathrm{H}_{139} \mathrm{NO}_{43}$

Julibroside $\mathrm{J}_{22}, 11936$

$\mathrm{C}_{87} \mathrm{H}_{142} \mathrm{O}_{48}$

Clematoside B, 3826

$\mathrm{C}_{88} \mathrm{H}_{138} \mathrm{O}_{37}$

Gleditsioside O, 8542

$\mathrm{C}_{88} \mathrm{H}_{138} \mathrm{O}_{38}$

Gleditsioside N, 8541

$\mathrm{C}_{88} \mathrm{H}_{140} \mathrm{O}_{51}$

Acutifoliside, 599

$\mathrm{C}_{88} \mathrm{H}_{144} \mathrm{O}_{48}$

Clematoside C, 3827

\section{$\mathrm{C}_{90} \mathrm{H}_{74} \mathrm{O}_{36}$}

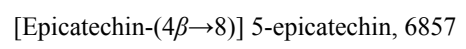

\section{$\mathrm{C}_{94} \mathrm{H}_{148} \mathrm{O}_{42}$}

Gleditsioside F, 8535

Gleditsioside G, 8536

\section{$\mathrm{C}_{94} \mathrm{H}_{148} \mathrm{O}_{43}$}

Gleditsiasaponin C, 8527

Gleditsioside E, 8534

\section{$\mathrm{C}_{94} \mathrm{H}_{148} \mathrm{O}_{44}$}

Gleditsiasaponin B, 8526

\section{$\mathrm{C}_{94} \mathrm{H}_{148} \mathrm{O}_{46}$}

Julibroside $\mathrm{J}_{24}, 11938$

$\mathrm{C}_{95} \mathrm{H}_{150} \mathrm{O}_{46}$

Julibroside $\mathrm{J}_{2}, 11918$

Julibroside $\mathrm{J}_{7}, 11922$

$\mathrm{C}_{96} \mathrm{H}_{152} \mathrm{O}_{46}$

Julibroside $\mathrm{J}_{27}, 11941$

$\mathrm{C}_{97} \mathrm{H}_{153} \mathrm{NO}_{46}$

Julibroside $\mathrm{J}_{6}, 11921$

$\mathrm{C}_{98} \mathrm{H}_{155} \mathrm{NO}_{46}$

Avicin G, 2038

Julibroside $\mathrm{J}_{18}, 11933$

$\mathrm{C}_{98} \mathrm{H}_{155} \mathrm{NO}_{47}$

Avicin D, 2036

$\mathrm{C}_{100} \mathrm{H}_{158} \mathrm{O}_{49}$
Julibroside $\mathrm{J}_{21}, 11935$

$\mathrm{C}_{\mathbf{1 0 1}} \mathrm{H}_{\mathbf{1 6 0}} \mathrm{O}_{\mathbf{4 8}}$

Julibroside $\mathrm{J}_{14}, 11929$

Julibroside $\mathrm{J}_{15}, 11930$

$\mathrm{C}_{\mathbf{1 0 1}} \mathrm{H}_{\mathbf{1 6 0}} \mathrm{O}_{\mathbf{4 9}}$

Julibroside, 11917

Julibroside $\mathrm{J}_{5}, 11920$

Julibroside $\mathrm{J}_{9}, 11924$

Julibroside $\mathrm{J}_{10}, 11925$

Julibroside $\mathrm{J}_{11}, 11926$

Julibroside $\mathrm{J}_{17}, 11932$

Julibroside $\mathrm{J}_{23}, 11937$

$\mathrm{C}_{102} \mathrm{H}_{72} \mathrm{O}_{67}$

Oenotherin $\mathrm{T}_{1}, 16012$

$\mathrm{C}_{102} \mathrm{H}_{162} \mathrm{O}_{48}$

Julibroside $\mathrm{J}_{16}, 11931$

\section{$\mathrm{C}_{\mathbf{1 0 2}} \mathrm{H}_{162} \mathrm{O}_{49}$}

Julibroside $\mathrm{J}_{8}, 11923$

\section{$\mathrm{C}_{\mathbf{1 0 3}} \mathrm{H}_{160} \mathrm{O}_{43}$}

Gleditsioside P, 8543

$\mathrm{C}_{103} \mathrm{H}_{163} \mathrm{NO}_{49}$

Julibroside $\mathrm{J}_{3}, 11919$

\section{$\mathrm{C}_{104} \mathrm{H}_{165} \mathrm{NO}_{49}$}

Julibroside $\mathrm{J}_{12}, 11927$

Julibroside $\mathrm{J}_{13}, 11928$

$\mathrm{C}_{108} \mathrm{H}_{212} \mathrm{O}_{6}$

Lycopanerol $\mathrm{B}_{1}, 13202$

\section{$\mathrm{C}_{110} \mathrm{H}_{\mathbf{2 1 6}} \mathrm{O}_{6}$}

Lycopanerol $\mathrm{B}_{2}, 13203$

\section{$\mathrm{C}_{112} \mathrm{H}_{220} \mathrm{O}_{6}$}

Lycopanerol $\mathrm{B}_{3}, 13204$

\section{$\mathrm{C}_{\mathbf{1 2 0}} \mathrm{H}_{236} \mathrm{O}_{7}$}

Lycopanerol F, 13210

Lycopanerol G, 13211

$\mathrm{C}_{122} \mathrm{H}_{232} \mathrm{O}_{7}$

Lycopanerol E, 13209

\section{$\mathrm{C}_{123} \mathrm{H}_{86} \mathrm{O}_{78}$}

Rugosin G, 19060

\section{$\mathrm{C}_{\mathbf{1 6 4}} \mathrm{H}_{\mathbf{1 0 6}} \mathrm{O}_{\mathbf{1 0 4}}$}

Sanguiin $\mathrm{H}_{11}, 19283$

$\mathrm{O}_{2} \mathrm{~S}$

Sulfur dioxide, 20479 


\section{Compound Name Synonym Index}

This index lists in alphabetical order all compound's synonym names contained in the bodies of compound entries. A equal sign (=) and compound code number (from 1 to 23033) follow the synonym name immediately for locating the compound in the encyclopedia. Following symbols in prefix are ineffective in ordering: $D-, L-, d l, R-, S-, E-, Z-, O-$, $N_{-}, C_{-}, H_{-}$, cis-, trans-, ent-, meso-, rel-, erythro-, threo-, sec-, chiro-, para-, exo-, $m-, o-, p-, n-, \alpha-, \beta-, \gamma-, \delta-, \varepsilon_{-}, \kappa_{-}, \xi-$, $\psi-, \omega-,(+),(-),( \pm)$ etc., and: $0,1,2,3,4,5,6,7,8,9,\{\},,[],,(),,,, ;,,^{*}, ',{ }^{\prime},{ }^{\prime},, \rightarrow$, etc.

\footnotetext{
A

$($ rel-3R,5R,10S)-19(4 $\rightarrow 3)$-Abeo-4(18),8,11,13-abietatetraen-3-ol = 22577

$(9 S, 13 S, 15 S)$-20-(10 $\rightarrow 9)$-Abeo-12 $\alpha$-acetoxy-7-hydroxy-13,16-cycloabieta-

$1,5(10), 7$-trien-6,11,14-trione $=22754$

$(9 S, 13 S, 15 S)$-20-(10 $\rightarrow 9)$-Abeo-2 $\alpha, 12 \alpha$-diacetoxy-7-hydroxy-13,16-cyclo-

abieta-5(10),7-dien-6,11,14-trione $=22753$

$17-(15 \rightarrow 6)$-Abeo-2 $\alpha, 11$-diacetoxy-16- $O$-isopropyl-6,12,14-trihydroxyabieta-

$5,8,11,13$-tetraen-7-one $=22750$

17-(15 $\rightarrow 16)$-Abeo-2 $\alpha, 11$-diacetoxy-6,12,14,16-tetrahydroxyabieta-5,8,11,13-

tetraen-7-one $=22749$

19(4 $\rightarrow$ 3)-Abeo-11,14-dihydroxy-12-methoxy-abieta-8,11,13,15-tetraen-7-one = 4875

$19(10 \rightarrow 9)$ Abeo- $8 \alpha, 9 \beta, 10 \alpha$-eupha-5,24-dien-3 $\beta$-ol = 1463

$26(14 \rightarrow 8)$ Abeo- $D: B$-friedo-ursane-3 $\beta, 16 \alpha$-dihydroxy-7 $\alpha, 14 \alpha$-epoxy-5(10)-

ene $=17019$

$18-(13 \rightarrow 12 \beta)$-Abeo-lanostene triterpenoid acid $=1136$

$20(10 \rightarrow 5)$-Abeo-4,5-seco-5(10),6,8,11,13-podocarpapentaen-3-one $=10052$

Abieforrestin $=11383$

8,13-Abietadien-18-oic acid $=16572$

8(14),13(15)-Abietadien-18-oic acid $=15333$

7,13-Abietadien-18-oil acid $=10$

8,11,13-Abietatrien-18-oic acid $=4873$

8,11,13-Abietatrien-12-ol $=7764$

Acacetin-7-O-glucoside $=21391$

Acacetin 7-O-(6"- $\alpha$ - $L$-rhamnopyranosyl) $-\beta-D$-glucopyranoside $=61$

Acetaldehyde $=7419$

3-Acetamino-6-isobutyl-2,5-dioxopiperazine $=4046$

$7 \beta$-Acetate- $O$-taxinine $\mathrm{A}=20800$

Acetic acid amide $=109$

Acetic acid methyl ester $=14111$

Acetonylgeranin $\mathrm{A}=17220$

2-Acetonyl-6-C- $\beta$ - $D$-glucopyranosyl-7-hydroxy-5-methyl-chromone $=11205$

$12-\alpha$-Acetoxyamoorastatin $=21448$
}

$8 \beta$-(4'-Acetoxyangelyloxy)-2 $\alpha, 3 \beta$-dihydroxy- $6 \beta H, 7 \alpha H$-germacra-

$1(10) E, 4 E, 11(13)$-triene-6,12-olide $=7549$

$8 \beta$-(4'-Acetoxyangelyloxy)-2 $\alpha$-hydroxy- $6 \beta H, 7 \alpha H$-germacra-

1(10)- $E, 4 E, 11$ (13)-triene-6,12-olide $=7548$

$1 \beta$-Acetoxy- $9 \alpha$-benzoyloxy- $2 \beta, 6 \alpha$-dinicotinoyloxy- $\beta$-dihydroagarofuran $=9470$

4-Acetoxy-2,3-bis(3,4-methylene-dioxybenzyl)-butan-1-ol = 9343

2-Acetoxy-3-bornanol $=22620$

$2 \alpha$-Acetoxybrevifoliol $=20770$

rel-(2S,5R,6S,7R,8S,9S,10R,18S,19R)-19-Acetoxy-18-butanoyloxy-18,19-epoxy-6,

7-dihydroxy-2-(2-methylbutanoyloxy)cleroda-3,13(16),14-triene $=3260$

rel-(2S,5R,6R,8S,9S,10R,18S,19R)-19-Acetoxy-18-butanoyloxy-18,19-epoxy-6-hy

droxy-2-(2-methylbutanoyloxy)cleroda-3,13(16),14-triene $=3258$

12-Acetoxy-7 $\alpha$-butoxyethoxyabieta- $6 \alpha$-yl 6,7-dehydroabieta-8,11,13-trien-

12 -yl ether $=7888$

cis- $6 \beta$-Acetoxy- $3 \alpha$-(cinnamoyloxy)tropane $=7349$

$7 \alpha$-Acetoxy-3,4,15,16-diepoxy-cleroda-13(16),14-dien-20-al = 3251

(20S,24S)-16 $\beta$-Acetoxy-18,24;20,24-diepoxy-9,19-cyclanostane-3 $\beta, 25$-diol-3-

$O-\beta$ - $D$-xylopyranoside $=2205$

$(20 S, 24 S)$-16 $\beta$-Acetoxy-18,24;20,24-diepoxy-9,19-cyclanostane-3 $\beta, 15 \beta, 25$ -

triol-3- $O-\beta$ - $D$-xylopyranoside $=2204$

$7 \alpha$-Acetoxy-15,16,12,20-diepoxy-20-hydroxy-cleroda-3,4,13(16),14-triene $=3250$

$7 \alpha$-Acetoxy-3,4,15,16-diepoxy-12-oxo-cleroda-13(16),14-dien-20-al = 3249

$3 \beta$-Acetoxy-11 $\beta, 17$-dihydroxy-ent-abieta-6(7),8(14),15(16)-triene = 13698

$6 \alpha$-Acetoxy-7 $\beta, 12$-dihydroxy- $8,11,13$-abietatriene $=20461$

$15 \beta$-Acetoxy- $6 \beta, 13 \alpha$-dihydroxy-3 $\alpha, 20$-epoxy-ent-kaur-16-en-1,7-dione $=12590$

$7 \beta$-Acetoxy- $4 \beta, 20 R$-dihydroxy- $5 \beta, 6 \beta$-epoxy-1-oxo-witha-2,24-dienolide $=302$

$(1 R, 3 S, 4 S)$-3-Acetoxy-1,4-dihydroxy-8-isobutyloxyeudesm-11(13)-en-6,12olide $=21410$

$6 \alpha$-Acetoxy-7 $\beta, 11$-dihydroxy-12-methoxy-8,11,13-abietatriene $=20460$

$3 \beta$-Acetoxy-6 $\beta, 11 \alpha$-dihydroxy-16 $\alpha$-methoxymethyl-6,20-epoxy-6,7-seco-entkaur-15-one-1,7-olide $=20621$

$2 \alpha$-Acetoxy-3 $\alpha, 19 \alpha$-dihydroxy-olean-12-en-28-oic acid 28-O- $\beta$ - $D$-glucopyranosyl- $(1 \rightarrow 6)-\beta$ - $D$-glucopyranoside $=18850$ 
$3 \beta$-Acetoxy-11 $\alpha, 23$-dihydroxytaraxer-14-en-28-oic acid $=546$

$20 \beta$-Acetoxy- $2 \alpha, 3 \beta$-dihydroxyurs-12-en-28-oic acid $=9074$

$11 \alpha$-Acetoxyeffusanin $\mathrm{D}=2116$

$\left(20 S^{*}, 24 R^{*}\right)$-16 $\beta$-Acetoxy-20,24-epoxy-9,19-cyclolanostane-3 $\beta, 12 \alpha, 18,25$ tetraol-3- $O-\beta-D$-xylopyranoside $=2195$

$\left(20 S^{*}, 24 R^{*}\right)$-16 $\beta$-Acetoxy-20,24-epoxy-9,19-cyclolanostane-3 $\beta, 12 \beta, 25$-triol$3-O-\beta$ - $D$-xylopyranoside $=2197$

$12 \beta$-Acetoxy-24R,25-epoxy-3 $\beta, 15 \alpha$-dihydroxy-16,23-dione-cycloart-7-ene 3- $O-\alpha-L$-arabinopyranoside $=2730$

$12 \beta$-Acetoxy-24R,25-epoxy-3 $\beta$-hydroxy-16,23-dione-cycloart-7-ene 3- $O-\alpha-L$ arabinopyranoside $=2731$

rel-(2S,5R,6R,8S,9S,10R,18S,19R)-19-Acetoxy-18,19-epoxy-6-hydroxy-18methoxy-2-(2-methylbutanoyloxy)cleroda-3,13(16),14-triene $=3259$

$(8 R)-4 \beta$-Acetoxy-3 $\beta$-[( $O-\beta$ - $D$-glucopyranosyl-( $1 \rightarrow 4)-\alpha$ - $L$-cymaropyranosyl) oxy]-14-oxo-5 $\alpha$-15-(14 $\rightarrow 8)$-abeo-card-20(22)-dihydroenolide $=8007$

$(8 R)-4 \beta$-Acetoxy-3 $\beta$-[( $O-\beta$-D-glucopyranosyl-( $1 \rightarrow 6)-O-\beta$ - $D$-glucopyranosyl$(1 \rightarrow 4)$ - $\alpha$ - $L$-cymaropyranosyl)-oxy]-14-oxo- $5 \alpha-15(14 \rightarrow 8)$-abeo-card20(22)-dihydroenolide $=8006$

$(8 R)-4 \beta$-Acetoxy-3 $\beta$-[( $O-\beta$ - $D$-glucopyranosyl-( $1 \rightarrow 6)-O-\beta$ - $D$-glucopyranosyl$(1 \rightarrow 6)-O-\beta$ - $D$-glucopyranosyl-( $1 \rightarrow 4)-\alpha-L$-cymaopyranosyl)oxy]-14-oxo$5 \alpha-15(14 \rightarrow 8)$-abeo-card-20(22)-dihydroenolide $=8008$

$5 \alpha$-Acetoxy-goniothalamin oxide $=3505$

$($ rel $6 S, 7 R, 8 S, 9 R)$-6-Acetoxy-5(10),14-halimadien-7,13-diol $=22572$

$($ rel $6 S, 7 R, 8 S, 9 R)$-7-Acetoxy-5(10),14-halimadien-6,13-diol $=22573$

4,5-cis-4-Acetoxy-5-hydroxy-5-(1-acetoxypentadecyl)-2-cyclopenten-1-one $=5338$ 4,5-trans-4-Acetoxy-5-hydroxy-5-(1-acetoxypentadecyl)-2-cyclopenten-1-one = 5337 3'(S)-Acetoxy-4' $(S)$-hydroxy-3',4'-dihydroseselin $=18283$

$3 \alpha$-Acetoxy-4 $\alpha$-hydroxy-4 $\beta, 10 \beta$-dimethyl-7 $\beta$-(methyl-1E-propenoate)-transdecalin $=175$

4,5-cis-4-Acetoxy-5-hydroxy-5-(1-hydroxypentadecyl)-2-cyclopenten-1-one $=430$ 4,5-trans-4-Acetoxy-5-hydroxy-5-(1-hydroxytridecyl)-2-cyclopenten-1-one $=5336$ $3 \alpha$-Acetoxy-30-hydroxylup-20(29)-ene-23,28-dioic Acid = 91

$3 \alpha$-Acetoxy-30-hydroxylup-20(29)-ene-23,28-dioic Acid 28-O- $\alpha$ - $L$-rhamno-pyranosyl-( $1 \rightarrow 4)-\beta$ - $D$-glucopyranosyl-( $(1 \rightarrow 6)-\beta$ - $D$-glucopyranosyl ester $=92$

$16 \beta$-Acetoxy-17-hydroxy-19-nor-ent-kauran- $4 \alpha$-ol $=1328$

$21 \beta$-Acetoxy-22 $\beta$-hydroxy-3-oxours-12-en-28-al $=4041$

11-(2-Acetoxy-6-hydroxyphenyl)-1-(2,6-dihydroxy-4-methoxyphenyl) undecan-1-one $=1646$

11-(2-Acetoxy-6-hydroxyphenyl)-1-(2,4,6-trihydroxyphenyl)-undecan-1-one = 1645 ent-17-Acetoxy-18-isobutyryloxy-16( $\alpha$ )-kauran-19-oic acid $=19878$ ent-3 $\beta$-Acetoxy-kauran-16 $\beta, 17$-diol $=7959$

$3 \alpha$-Acetoxylabda-8(17),13(16),14-trien-19-oic acid $=11967$

$3 \alpha$-Acetoxylanosta-8,24(31)-dien-21-oic acid 21- $O-\beta$ - $D$-xylopyranoside $=7872$

(24Z)-12 $\beta$-Acetoxylanosta-7,9(11),24-trien-26-oic acid $=22168$

$22 \beta$-Acetoxylantic acid $=3026$

$3 \beta$-Acetoxyl-9,19-cyclolanost-24(E)-en-26-oic acid $=462$

1-(2-Acetoxyl-1,5-dihydroxy-cyclopent-3-enyl)-tridecan-1-one $=436$

1-(2-Acetoxyl-1,5-dihydroxy-cyclopent-3-enyl)-undecan-1-one $=435$

4,5-trans-4-Acetoxyl-5-hydroxy-5-(1-hydroxypentadecyl)-2-cyclopenten-1-one $=428$ 4,5-trans-4-Acetoxyl-5-hydroxy-5-(1-hydroxytridecyl)-2-cyclopenten-1-one $=426$ trans-4-Acetoxyl-5-hydroxy-5-pentadecanoyl-2-cyclopenten-1-one $=434$ cis-4-Acetoxyl-5-hydroxy-5-tridecanoyl-2-cyclopenten-1-one $=432$ trans-4-Acetoxyl-5-hydroxy-5-tridecanoyl-2-cyclopenten-1-one $=433$
$6 \beta$-Acetoxyl-2 $\alpha, 3 \beta, 5 \beta, 14 \beta, 16 \alpha$-pentahydroxygrayanane $=18808$ $6 \beta$-Acetoxyl-2 $\alpha, 3 \beta, 5 \beta, 14 \beta, 16 \alpha$-pentahydroxygrayan-10(20)-ene $=18809$ $1 \beta$-Acetoxyl-2 $\beta, 6 \alpha, 9 \alpha$-trifuroyloxydihydro- $\beta$-agarofuran $=16167$

5-Acetoxyl-2,6,8-trimethylchromone 7- $O-\beta$ - $D$-glucopyranoside $=22216$

$3 \beta$-Acetoxy-28-nor-lup-12,17-dien-16 $\alpha$-ol $=12244$

15 $\alpha$-Acetoxy-3-oxo-24-methylenelanosta-7,9(11)-dien-21-oic acid = 7859

19-Acetoxytaxagifine $=20790$

$6 \beta$-Acetoxy-3 $\beta, 8 \beta, 12 \beta, 14 \beta$-tetrahydroxybufa- $4,20,22$-trienolide $=10693$

$12 \beta$-Acetoxy-3 $\beta, 15 \alpha,-24 R, 25$-tetrahydroxy-16,23-dione-cycloart-7-ene

$3-O-\alpha$ - $L$-arabinopyranoside $=2729$

$23 R$-Acetoxytomatine $=13214$

11-Acetoxy-6,12,14-trihydroxyabieta-5,8,11,13-tetraen-7-one $=3920$

$11 \alpha$-Acetoxy- $6 \beta, 7 \beta, 15 \beta$-trihydroxy-7 $\alpha, 20$-epoxy-entkaur-16-ene $=11401$

$3 \beta$-Acetoxy- $6 \alpha, 15 \beta, 17$-trihydroxy-11 $\beta, 16 \beta$-epoxy-ent-kaurane $=13695$

28 -Acetoxy- $6 \beta, 21 \beta, 22 \beta$-trihydroxy-3-oxours-12-ene $=4044$

$1 R, 3 R, 5 S, 6 R$-6-Acetoxy-3-(3',4',5'-trimethoxybenzoyloxy)tropane $=7347$

$(\mathrm{rel}-3 S, 5 S, 8 R, 9 R, 10 S)$-3-Acetoxy-14,15,16-trinor-13,9-labdanolide $=22567$

2-Acetylamino-2-deoxy- $D$-glucose $=402$

26 -Acetylamino-3 $\beta, 16 \alpha$-dihydroxy-cholest-5-en-22-one 3-O- $\beta$ - $D$-xylopyranosyl- $(1 \rightarrow 2)-\alpha-L$-rhamnopyranosyl- $(1 \rightarrow 4)-\beta-D$-glucopyranoside $=41$

28-O-Acetyl-21- $O$-(4- $O$-angeloyl)-6-deoxy- $\beta$-glucopyranosyl-3- $O$-[ $\beta$-glucopyranosyl $(1 \rightarrow 2)-O$-[ $\beta$-glucopyranosyl $(1 \rightarrow 4)]$ - $\beta$-glucuronopyranosyl] protoaescigenin $=1913$

16-O-Acetyl-21-O-angeloyltheasapogenol E 3-O- $\beta$ - $D$-galactopyranosyl $(1 \rightarrow 2)$ $[\beta$ - $D$-xylopyranosyl $(1 \rightarrow 2)-\alpha$ - $L$-arabinopyranosyl $(1 \rightarrow 3)]-\beta$ - $D$-glucopyranosiduronic acid $=21286$

2 -Acetyl-4-chloro-1,8-dihydroxy-3-methylnaphthalene-8- $O-\beta$ - $D$-glucopyranoside $=16714$

$O$-Acetylcholine $=351$

$25-O$-Acetylcimigenol-3- $O-\beta-D$-(2-acetyl)xylopyranoside $=20110$

6-O-(2"-O-Acetyl-3"-O-cinnamoyl-4"--O-p-methoxycinnamoyl- $\alpha-L$-rhamnopy ranosyl)catalpol $=19571$

$O$-Acetyl columbianetin $=3937$

10-Acetyl-2-deacetoxy-10-debenzoyltaxchinin A $=20757$

13-Acetyl-2-deacetoxy-10-debenzoyltaxchinin $\mathrm{A}=20755$

5,10,13-Acetyl-10-debenzoyl brevifoliol $=21533$

1-O-Acetyl-9- $O$-demethylpluviine $=472$

25 - $O$-Acetyl-7,8-didehydrocimigenol 3-O- $\beta$ - $D$-xylopyranoside $=352$

2-Acetyl-1,8-dihydroxy-3-methyl naphthalene $=15489$

2-O-Acetyl-3,4-di- $O$ - $(E)-p$-methoxycinnamoyl- $\alpha-L$-rhamnopyranoside $=2709$

3-O-Acetyl-2- $O$-feruloyl- $\alpha$ - $L$-rhamnopyranose $=15618$

4- $O$-Acetyl-2- $O$-feruloyl- $\alpha-L$-rhamnopyranose $=15619$

6 -Acetyl- $\beta$ - $D$-glucopyranosyldiderroside $=376$

$6 \alpha-O$-(6-O-Acetyl- $\beta-D$-glucopyranosyl)-15,16-dihydroxycleroda-3,13(14)$\operatorname{dien}=19213$

6 $\alpha$-O-(6-O-Acetyl- $\beta$ - $D$-glucopyranosyl)-15,16-epoxycleroda-3,13(16),14-trien = 19215 6-O-[6-O-Acetyl- $\beta$ - $D$-glucopyranosyl]-20- $O$-( $\beta$ - $D$-glucopyranosyl)-20(S)-protopanaxatriol $=22896$

(22S)-16 $\beta$-[(6-O-Acetyl- $\beta$ - $D$-glucopyranosyl)oxy]-22-hydroxy-3 $\beta-[(O-\alpha-L$ rhamnopyranosyl-( $(1 \rightarrow 2)-O-[\alpha-L$-rhamnopyranosyl- $(1 \rightarrow 4)]-\beta$ - $D$-glucopyranosyl)oxy]cholest-5-en-12-one $=20229$

$6 \alpha-O$-(6-O-Acetyl- $\beta$ - $D$-glucopyranosyl)-15,16,18-trihydroxy-cleroda3,13(14)-dien $=19214$ 
Acetylgomisin $\mathrm{O}=19044$

8-O-Acetylharpagide $=409$

8- $O$-Acetylharpagide-aglucone-1- $O-\beta$ - $D$-ribohexo-3-ulopyranoside $=356$

6-Acetyl-7-hydroxy-2,2-dimethyl-2H-1-benzopyran $=7592$

6-Acetyl-5-hydroxy-4-methyl coumarin $=12903$

$24-O$-Acetyl-isodahurinol-3-O- $\beta-D$-(2-acetyl)xylopyranoside $=20111$

$24-O$-Acetylisodahurinol-3- $O-\beta-D$-xylopyranoside $=3666$

7-Acetyl kamebakaurin $=3953$

14-Acetyl kamebakaurin $=3952$

7-Acetyl kamebanin $=3951$

14-Acetyl kamebanin $=3950$

6'- $O$-Acetylmartynoside $=22662$

3-O-Acetyl-2- $O-p$-methoxycinnamoyl $-\alpha(\beta)-L$-rhamnopyranose $=15621$

2-O-Acetyl-3-O-(E)-p-methoxycinnamoyl- $\alpha-L$-rhamnopyranoside $=2710$

$2-O$-Acetyl-3-O-(Z)- $p$-methoxycinnamoyl- $\alpha-L$-rhamnopyranoside $=2711$

2 -Acetyl-3-methyl-6-carboxy-1,8-dihydroxynaphthalene-8- $O-\beta$ - $D$-glucopyranoside $=19066$

2-Acetyl-3-methyl-1,8-dihydroxynaphthalene-8-O- $\beta$ - $D$-glucopyranosyl $(1 \rightarrow 3)$ $\beta$ - $D$-glucopyranoside $=16195$

3- $O$-[2-Acetyl-3- $O$-methyl- $\beta$ - $D$-fucopyranosyl-( $1 \rightarrow 4$ )-2,6-dideoxy-3-O-methyl- $\beta$ - $D$-ribo-hexopyranoside] $20-O$-[ $\beta$ - $D$-glucopyranosyl- $(1 \rightarrow 6)-\beta$ - $D$-glucopyranosyl- $(1 \rightarrow 2)-3-O$-methyl $-\beta-D$-fucopyranoside $]=17566$

3-O-[4- $O$-Acetyl-3- $O$-methyl- $\beta$ - $D$-fucopyranosyl-( $1 \rightarrow 4$ )-2,6-dideoxy-3- $O$-me thyl- $\beta$ - $D$-ribo-hexopyranoside] $20-O$ - $[\beta$ - $D$-glucopyranosyl- $(1 \rightarrow 6)-\beta$ - $D$ glucopyranosyl- $(1 \rightarrow 2)-3-O$-methyl $-\beta$ - $D$-fucopyranoside $]=8829$

14- $O$-Acetylneoline $=2739$

$3 \beta$-Acetyloleanolic acid $=266$

7-(Acetyloxy)- $O^{2}$-furanoyl- $O^{2}$-deacetyl-7-deoxo-evonine $=10908$

7-(Acetyloxy)- $O^{2}$-nicotinoyl- $O^{2}$-deacetyl- $O^{5}$-benzoyl- $O^{5}$-deacetyl-7-deoxoevonine $=10906$

$6 \beta$-Acetyloxy-3 $\beta, 5 \beta, 10 \alpha, 14 \beta, 16 \alpha$-pentahydroxygrayanane $=18810$

13-O-Acetylphorbol-20-(9Z,12Z-octadecadienoate $)=484$

2"-O-Acetyl platycodin $\mathrm{D}=17530$

3"-O-Acetyl platycodin $\mathrm{D}=17531$

Acetylrabdosin $\mathrm{B}=393$

1-O-[2-O-Acetyl- $\alpha$ - $L$-rhamnopyranosyl-( $1 \rightarrow 6)-\beta$ - $D$-glucopyranosyl]-(2E,6E)farnesol $=4230$

1-O-[3-O-Acetyl- $\alpha$ - $L$-rhamnopyranosyl-( $1 \rightarrow 6)-\beta$ - $D$-glucopyranosyl]-(2E,6E)farnesol $=4229$

$O$-Acetylrutaevin $=19082$

2'-O-Acetylsalsoloside $\mathrm{C}=20531$

3'- $O$-Acetylsalsoloside $\mathrm{C}=20530$

1-[6-(3-Acetyl-2,4,6-trihydroxy-5-methyl-benzyl)-5,7-dihydroxy-2,2-dimethyl-2H-chromen-8-yl]-2-methyl-butan-1-one $=13434$

Aconitane-13,14,15-trihydroxyl,20-ethyl-1,6,8,16-tetramethoxy-4-(methoxymethyl)-14-benzoate $(1 \alpha, 6 \alpha, 14 \alpha, 15 \alpha, 16 \beta)=15418$

Acorodin $=2435$

$3 \beta$-Acyloxytropane $=3960$

Adaptinol $=9290$

Adenosine $=618$

Adenosine, $N$-[(5-hydroxy-2-pyridinyl $)$ methyl $]=1039$

Aesculetin dimethylether $=19540$

Aethalic acid $=9486$
Afaxin $=18656$

Afromosin 7-O-[ $\alpha$-rhamnopyranosyl- $(1 \rightarrow 6)]-\beta$-glucopyranoside $=5225$

Aglafolin $=14714$

$\mathrm{AH} 1=707$

$\mathrm{AH} 1 \mathrm{a}=21030$

$\mathrm{AH} 2=11199$

$\mathrm{AH} 2 \mathrm{a}=21131$

$\mathrm{AH} 2 \mathrm{~b}=21121$

$\mathrm{AH} 16=17114$

$\mathrm{AH} 23=21120$

$\mathrm{AHb}_{1}=13999$

Akebiasaponin $\mathrm{P}_{\mathrm{D}}=19316$

Akebiasaponin $\mathrm{P}_{\mathrm{G}}=19317$

Aknadinine $=9447$

$\beta$-Alanyl- $L$-histidine $=3205$

Albanin $\mathrm{F}=12385$

Albanin $\mathrm{G}=12386$

Albanol $=15044$

Alcapton $=9608$

6-Aldehydo-isoophiopogonone $\mathrm{A}=875$

$(+)$-Alismol $=888$

Alisol F 24-acetate $=902$

Alkylresorcinol B $=2380$

Alleoside $\mathrm{A}=9335$

$L$-Alloisoleucine $=935$

26-O- $\beta$ - $D$-Allopyranosyl-(16S,20S,22S)-16 $\beta, 22$-epoxy-16 $\alpha$-methoxy-3 $\beta, 26$ dihydroxy-cycloartan-24-one-3- $O-\beta$ - $D$-glucopyranosyl-( $1 \rightarrow 2)-\beta$ - $D$-glucopyranoside $=1544$

4-Allylcatechol $=955$

rel-(7S,8S,1'R,3'S,4'R)-1'-Allyl-7-(3,4-dimethoxyphenyl)-4'-hydroxy-5'-methoxy-8-methyl-2'-oxobicyclo[3.2.1]oct-5'-ene $=9208$

4-Allyl-2,6-dimethoxyphenyol cinnamate $=5504$

4-Allyl-2-methoxyphenol $=7521$

4-Allylphenol $=3488$

4-Allylpyrocatechol $=955$

Allyl sulphide $=953$

5-Allyl-2,2',5'-trihydroxybiphenyl $=18533$

Alspopicralamine $=18303$

Amarin $=4317$

Amarylline $=13241$

Amethystoidin A $=6929$

$N$-Amidinosarcasine $=4221$

4-Aminobenzenesulfonic acid $=20467$

10-Amino-2,4-dimethoxyphenanthrene-1-carboxylic acid lactam $=8937$

$\alpha$-Amino-3,5-dioxo-1,2,4-oxadiazolidine-2-propanoic acid $=18452$

Aminoethane $=7420$

Aminoethanoic acid $=8817$

2-Aminoethanol $=7390$

3-Amino-5-ethenyl-5-hydroxy-2-cyclopenten-1-one $=15207$

3 - $(2$-Aminoethyl $)$ indole $=22058$

4-(2-Aminoethyl)pyrocatechol $=6559$

(R)-4-(2-Amino-1-hydroxyethyl)-1,2-benzenediol $=15708$

2-Amino-4-hydroxy-pteridine-6-carboxylic acid $=18530$ 
2-Amino-3-(3-indolyl)propanoic acid $=22060$

2-Amino-2-methylpropionic acid $=1055$

2-Amino-4-(methylthio)butanoic acid $=13823$

2-Amino-3-phenylpropanoic acid $=17093$

3-Aminopregna-5,17(20)-diene-2,16-diol = 12345

2-Amino-4,7-pteridinediol $=11784$

6-Amino-4,5,9-trimethoxyoxoisoaporphine $=4690$

$L$-(-)-2-Amino-3-ureidopropionic acid $=862$

2-Amino-5-ureidovaleric acid $=3774$

Amyl ethyl ketone $=15979$

$\delta$-Amyrone $=1108$

Anacarol $=3188$

Anacrotine $=4266$

Anaprel $=18635$

Anchoic acid $=2056$

Andrographoside $=1158$

Anethol 2-methyl-butyrate $=17925$

16-Angeloyl-21-acetylprotoaescigenin 3- $O$-[ $\beta$ - $D$-glucopyranosyl $(1 \rightarrow 2)]$

$[\beta$ - $D$-glucopyranosyl $(1 \rightarrow 4)]-\beta$ - $D$-glucopyranosiduronic acid $=7365$

21-O-Angeloyl-22-Oacetylprotoaescigenin-3- $O$ - $[\beta$ - $D$-glucopyranosyl $(1 \rightarrow 2)]$

$[\beta$ - $D$-glucopyranosyl $(1 \rightarrow 4)]-\beta$ - $D$-glucopyranosiduronic acid $=660$

21-O-Angeloyl-28-O-acetylprotoaescigenin-3-O-[ $\beta$ - $D$-glucopyranosyl $(1 \rightarrow 2)]$

$[\beta$ - $D$-glucopyranosyl $(1 \rightarrow 4)]-\beta$ - $D$-glucopyranosiduronic acid $=11417$

21-O-(4-O-Angeloyl)-6-deoxy- $\beta$-glucopyranosyl-3- $O$-[ $\beta$-glucopyranosyl $(1 \rightarrow 2)$ -

$O$-[ $\beta$-glucopyranosyl $(1 \rightarrow 4)]$ - $\beta$-glucuronopyranosyl $]$ protoaescigenin $=1914$

8-Angeloyloxy- $1 \alpha$-hydroxy-3 $\alpha, 4 \alpha$-epoxy-5 $\alpha, 7 \alpha H$-10(14),11(13)-guaiadien-

$12,6 \alpha$-olide $=7195$

22-Angeloylprotoaescigenin 3- $O$-[ $\beta$ - $D$-glucopyranosyl $(1 \rightarrow 2)][\beta$ - $D$-glucopy-

ranosyl $(1 \rightarrow 4)]$ - $\beta$ - $D$-glucopyranosiduronic acid $=7364$

21-O-Angeloyltheasa-21- $O$-angeloyltheasapogenol E 3-O- $\beta$ - $D$-galactopyra-

$\operatorname{nosyl}(1 \rightarrow 2)[\beta$ - $D$-xylopyranosyl $(1 \rightarrow 2)-\alpha$ - $L$-arabinopyranosyl $(1 \rightarrow 3)]$ -

$\beta$ - $D$-glucopyranosiduronic acid $=21285$

$8 \beta$-Angelyloxy-4 $\alpha$-hydroxy-14-oxo-5 $\alpha H, 6 \beta H, 7 \alpha H$-guai-2,10(14),11(13)-

triene-6,12-olide $=7543$

$8 \beta$-Angelyloxy-3 $\beta, 4 \beta, 14$-trihydroxy- $\alpha \alpha H, 6 \beta H, 7 \alpha H$-guai-1(10),11(13)-diene-

6,12 -olide $=7540$

Anhydrobyakangelicin $=11301$

25 -Anhydrocimicigenol-3- $O-\beta$ - $D$-xylopyranoside- $(23 R, 24 S)=3672$

Anhydroicaritin 3-O- $\beta$ - $D$-(6-acetyl) glucopyranosyl-(1 $\rightarrow 3)-\alpha-L$-(4-acetyl)

rhamnopyranoside $=12272$

Anhydroicaritin 3-O- $\beta$ - $D$-(2,6-diacetyl)glucopyranosyl-( $1 \rightarrow 3)-\alpha-L$-(4-acet-

yl)rhamnopyranoside-7- $O-\beta$ - $D$-glucopyranosyl $=12273$

Anhydroicaritin-3-O- $\alpha$-rhamnoside $=2138$

Anhydromarmesin $=11630$

2-Anilino-1,4-naphthoquinone $=13151$

Anisic ketone $=1289$

Annonacinone $=1315$

cis-Annonacinone $=1316$

Annonin I = 20240

Anol tiglate $=17926$

$(-)$-Anonaine $=1348$

9,10-Anthracenedione $=1367$

9-Anthracenol $=1365$
Anticancer Alkaloid PMV70P691-001 = 6322

Anticancer Alkaloid PMV70P691-002 = 21968

Anticancer Benzenoid PMV70P691-003 = 6103

Anticancer Benzofuran PMV70P691-006 = 5086

Anticancer Benzofuran PMV70P691-65 = 14961

Anticancer Benzofuran PMV70P691-66 = 14962

Anticancer Benzofuran PMV70P691-67 = 14964

Anticancer Cyclopenta[b] Benzofuran PMV70P691-71 = 14714

Anticancer Diarylheptanoid PMV70P691-72 = 2468

Anticancer Flavonoid PMV70P691-016 $=6228$

Anticancer Flavonoid PMV70P691-017 = 6246

Anticancer Flavonoid PMV70P691-019 = 10430

Anticancer Flavonoid PMV70P691-020 = 8262

Anticancer Flavonoid PMV70P691-021 = 10506

Anticancer Flavonoid PMV70P691-023 = 14075

Anticancer Flavonoid PMV70P691-102 = 14074

Anticancer Flavonoid PMV70P691-112 = 1402

Anticancer Flavonoid PMV70P691-113 = 21151

Anticancer Flavonoid PMV70P691-115 = 21918

Anticancer Flavonoid PMV70P691-79 = 2630

Anticancer Flavonoid PMV70P691-80 = 2635

Anticancer Flavonoid PMV70P691-81 = 3071

Anticancer Flavonoid PMV70P691-90 = 7485

Anticancer Flavonoid PMV70P691-99 = 12681

Anticancer Lignan PMV70P691-027 = 17342

Anticancer Lignan PMV70P691-125 = 14106

Anticancer Monoterpene PMV70P691-127 = 12953

Anticancer Sesquiterpene Lactone PMV70P691-037 = 21410

Anticancer Sesquiterpene PMV70P691-133 = 240

Anticancer Stilbenoid PMV70P691-140 = 11672

Anticancer Stilbenoid PMV70P691-144 = 18644

Antofine $=5055$

Aoibaclyin $=12214$

Aphanamixis grandifolia $=1473$

ent-Apigeniflavan- $(2 \alpha \rightarrow 7,4 \alpha \rightarrow 8)$-epiafzelechin $=13402$

Apigenin-4',7-dimethyl ether $=10024$

Apigenin-6-C- $\beta$ - $D$-glucopyranoside $=11773$

Apigenin-8- $C$ - $\beta$ - $D$-glucopyranoside $=22581$

Apigenin-6- $C$-[(6- $O$ - $p$-hydroxybenzoyl)- $\beta$ - $D$-glucopyranosyl $(1 \rightarrow 2)-\beta$ - $D$-glucopyranoside $=4187$

Apigenin-5- $O$ - $\alpha$ - $L$-rhamnosyl- $(1 \rightarrow 4)-6$ "-acetyl- $\beta$ - $D$-glucoside $=3035$

Apigenin trimethyl ether $=21913$

Apigenside $=1492$

3-O- $\beta$ - $D$-Apiofuranosyl-( $1 \rightarrow 3)-\alpha$ - $L$-arabinopyranosyl-( $1 \rightarrow 3)$-[ $\beta$ - $D$-glucopyranosyl-( $1 \rightarrow 2)]-\beta$ - $D$-glucopyranosyl-oleanolic acid $=17690$

3-O- $\beta$ - $D$-Apiofuranosyl-( $1 \rightarrow 3)-\beta$ - $D$-galactopyranosyl- $(1 \rightarrow 3)-[\beta-D$ glucopyranosyl- $(1 \rightarrow 2)]-\alpha-L$-arabinopyranosyl-oleanolic acid $=$ 17694

3- $O$ - $\beta$ - $D$-Apiofuranosyl-( $1 \rightarrow 3)-\beta$ - $D$-galactopyranosyl-( $1 \rightarrow 3)$ - $[\beta$ - $D$-glucopyra nosyl- $(1 \rightarrow 2)]-\beta-D$-glucopyranosyl-oleanolic acid $=17691$

$3 \beta$ - $[(O-\beta$ - $D$-Apiofuranosyl-( $1 \rightarrow 2)-O-\beta$ - $D$-glucopyranosyl- $(1 \rightarrow 2)-O-\alpha-L$ arabinopyranosyl-( $1 \rightarrow 6)-\beta$ - $D$-glucopyranosyl)oxy]-28,29-dihydroxy$24,25,26,27$-tetranorlanost-8-en-17 $\alpha, 23$-olide $=13058$ 
3- $O$ - $\beta$ - $D$-Apiofuranosyl-( $(1 \rightarrow 3)$-[ $\beta$ - $D$-glucopyranosyl-( $(1 \rightarrow 2)]-\beta$ - $D$-glucopyranosyl-oleanolic acid $=17689$

4-[4 $\beta$-O- $\beta$-D-Apiofuranosyl-(1" $\rightarrow 6$ ')- $\beta$ - $D$-glucopyranosyl-2,6,6-trimethyl-1cyclohexen-1-yl]-butan-2-one $=4366$

25 - $O-\beta$ - $D$-Apiofuranosyl $(1 \rightarrow 4)-\beta$ - $D$-xylopyranosyl $(1 \rightarrow 4)-\beta$ - $D$-glucopyranosyl $1 \alpha, 3 \beta, 7 \beta, 24(S), 25$-pentahydroxycycloartane $=15198$

Apocynamarin $=20397$

Aptinol $=9290$

Aquilide A $=18098$

Aquillochin $=3831$

$D$-Arabinitol $=1596$

3-O-[ $\alpha$ - $L$-Arabinofuranosyl- $(1 \rightarrow 2)]-\alpha-L$-arabinopyranosyl-jujubogenin $=2083$

$(23 S, 24 S, 25 R)-3 \beta-[(O-\alpha-L$-Arabinofuranosyl-( $1 \rightarrow 2)-O-\alpha-L$-arabinopyranosyl$(1 \rightarrow 3)-O$-[ $\alpha$ - $L$-rhamnopyranosyl-( $1 \rightarrow 2)]-O-\beta$ - $D$-glucopyranosyl-( $(1 \rightarrow 2)$ $O$ - $\alpha$ - $L$-arabinopyranosyl-( $1 \rightarrow 6)-\beta$ - $D$-glucopyranosyl)oxy]-17 $\alpha, 23$-epoxy-24,29-dihydroxylanost-8-en-23,26-olide $=19523$

$(23 S, 25 R)-3 \beta-[(O-\alpha-L$-Arabinofuranosyl-( $1 \rightarrow 2)-O-\alpha-L$-arabinopyranosyl- $(1 \rightarrow$ $3)-O$-[ $\alpha$ - $L$-rhamnopyranosyl-( $1 \rightarrow 2)]-O-\beta$ - $D$-glucopyranosyl-( $(1 \rightarrow 2)-O-\alpha$ $L$-arabinopyranosyl-( $(1 \rightarrow 6)-\beta$ - $D$-glucopyranosyl)oxy]-17 $\alpha, 23$-epoxy-29hydroxylanost-8-en-23,26-olide $=19522$

3- $O$-[ $\alpha$ - $L$-Arabinofuranosyl $(1 \rightarrow 4)-6$ '- $O-n$-butyl- $\beta$ - $D$-glucuronopyranosyl $]$ oleanolic acid-28- $O-\beta$ - $D$-glucopyranoside $=20613$

3-O-[ $\alpha$ - $L$-Arabinofuranosyl-( $1 \rightarrow 4)-6$ '- $O$-ethyl- $\beta$ - $D$-glucuronopyranosyl]oleanolic acid-28- $O-\beta-D$-glucopyranoside $=20614$

$3 \beta-[(O-\alpha-L$-Arabinofuranosyl- $(1 \rightarrow 2)-O-\alpha-1$-arabinopyranosyl- $(1 \rightarrow 3)-O-[\alpha-L-$ rhamnopyranosyl-( $(1 \rightarrow 2)]-O-\beta$ - $D$-glucopyranosyl-( $(1 \rightarrow 2)-O-\alpha-L$-arabinopyranosyl-( $1 \rightarrow 6)$ - $\beta$ - $D$-glucopyranosyl)oxy]-29-hydroxy-15-oxo24,25,26,27-tetranorlanost-8-en-17 $\alpha, 23$-olide $=13060$

3 - $O$-[ $\alpha$ - $L$-Arabinopyranosyl-( $(1 \rightarrow 4)$-[2- $O$-acetyl]- $\beta$ - $D$-glucuronopyranosyl]28 - $O$-[ $\beta$ - $D$-glucopyranosyl]-oleanolic acid $=20534$

4- $O-\alpha$ - $L$-Arabinopyranosyl-(1"' $\rightarrow 2 ")-\beta$ - $D$-apiofuranosyldiphyllin $=17872$

3-O- $\alpha$ - $L$-Arabinopyranosyl-3 $\beta, 23$-dihydroxy-lup-20(29)-en-28-oic acid $=18192$

3 - $O-\alpha$ - $L$-Arabinopyranosyl-( $1 \rightarrow 3)$-[ $\beta$ - $D$-glucopyranosyl-( $(1 \rightarrow 2)]-\alpha-L$-arabino pyranosyl-oleanolic acid $=17692$

3- $O$-[ $\alpha$ - $L$-Arabinopyranosyl- $(1 \rightarrow 6)]$-[ $\beta$ - $D$-glucopyranosyl- $(1 \rightarrow 4)]-\beta$ - $D$-gluco pyranosyl-( $1 \rightarrow 2)-\beta$ - $D$-glucopyranosyl-21- $O$-acetyl-22- $O$-acetyl-3 $\beta, 16 \alpha$, $21 \beta, 22 \alpha, 28$-pentahydroxyolean-12-ene $=9716$

3 - $O-\alpha$-[L-Arabinopyranosyl-( $(1 \rightarrow 6)]$-[ $\beta$ - $D$-glucopyranosyl- $(1 \rightarrow 4)]-\beta-D$-glucopyranosyl-(1 $\rightarrow 2)-\beta$ - $D$-glucopyranosyl-22-O-acetyl-21- $O$-(2-methylbuta-noyl)$3 \beta, 15 \alpha, 16 \alpha, 21 \beta, 22 \alpha, 28$-hexahydroxyolean-12-ene $=9715$

3-O-[ $\alpha$ - $L$-Arabinopyranosyl- $(1 \rightarrow 6)]$-[ $\beta$ - $D$-glucopyranosyl-( $(1 \rightarrow 4)]-\beta$ - $D$-gluco pyranosyl-( $1 \rightarrow 2)-\beta$ - $D$-glucopyranosyl-22- $O$-acetyl-21- $O$-(2-methylpropa noyl)-3 $\beta, 15 \alpha, 16 \alpha, 21 \beta, 22 \alpha, 28$-hexahydroxyolean-12-ene $=9714$

3 - $O$-[ $\alpha$ - $L$-Arabinopyranosyl-( $1 \rightarrow 4)-\beta$ - $D$-glucuronopyranosyl]-28- $O$-[ $\beta$ - $D$-glucopyranosyl]-oleanolic acid $=20533$

3- $O$ - $\alpha$ - $L$-Arabinopyranosyl hederagenin $28-O-\beta$ - $D$-glucopyranosyl $(1 \rightarrow 6)-\beta$ $D$-glucopyranoside $=818$

3-O- $\alpha$-L-Arabinopyranosyl-16 $\alpha$-hydroxy-22 $\alpha$-acetoxy-saikogenin $\mathrm{E}=17961$ $3-O-\alpha-L$-Arabinopyranosyl ilexosapogenin A 28- $\beta$ - $D$-glucopyranosyl ester $=18540$ $(23 S, 25 R)-3 \beta-[(O-\alpha-L$-Arabinopyranosyl-( $(1 \rightarrow 3)-O-[\alpha-L$-rhamnopyranosyl$(1 \rightarrow 2)]-O-\beta$ - $D$-glucopyranosyl-( $(\rightarrow 2)-O-\alpha-L$-arabinopyranosyl- $(1 \rightarrow 6)$ $\beta$-D-glucopyranosyl)oxy]-17 $\alpha, 23$-epoxy-29-hydroxylanost-8-en-23,26olide $=19521$
$3 \beta-[(O-\alpha-L$-Arabinopyranosyl-( $(1 \rightarrow 3)-O-[\alpha-L$-rhamnopyranosyl- $(1 \rightarrow 2)]-O-\beta-$ $D$-glucopyranosyl-( $1 \rightarrow 2)-O-\alpha$ - $L$-arabinopyranosyl-( $1 \rightarrow 6)-\beta$ - $D$-glucopyranosyl)oxy]-29-hydroxy-15-oxo-24,25,26,27-tetranorlanost-8-en$17 \alpha, 23$-olide $=13059$

3-O- $\alpha$ - $L$-Arabinopyranosyl saikogenin $\mathrm{F}=17962$

3-O- $\alpha$ - $L$-Arabinopyranosyl-3 $\beta, 19 \alpha, 23$-trihydroxyursa-12,20(30)-dien-28-oic acid 28- $\beta$ - $D$-glucopyranosyl ester $=18535$

3 - $\alpha$-O- $L$-Arabonipyranosyl $(1 \rightarrow 3)$-(6'-butyl ester)- $\beta$ - $D$-glucopyranosyloleanolic acid-28- $O-\beta-D$-glucopyranoside $=9358$

Araregai toxin $=21211$

Ardisin $=1643$

Aristolactam C $N$-glucoside $=1694$

Aristolene $=1704$

1(10)-Aristolene $=2943$

Aristolochic acid BII methyl ester $=1691$

Aristolodione $=17429$

Aristololactam $=1698$

Arnidenediol $=1749$

Arolisic acid B $=2312$

Aromadendr-1(10)-en-9-one $=20249$

Aromadendrin-4',7-dimethyl ether $=8202$

Aromadendrol $=1764$

Arteamisinine IV $=18288$

Artemisinin $\mathrm{G}=1786$

Artilesin A $=13604$

$\gamma$-Asarone $=957$

Asebotoside $=1847$

Asimicin $=1317$

$(-)$-Asimilobine $=1857$

Asparagamine $\mathrm{A}=5470$

Astragalin $=12050$

Astrantiagenin $\mathrm{E}=9260$

Astrasieversianin VIII $=1937$

Atractylin $=1969$

Atractyloxide $=1971$

$\beta$-Daucosterol $=4680$

Aurantiin $=15286$

Ausraptin $=11462$

Azacyclohexane $=17450$

1-Aza-9,10-dimethoxy-4-methyl-2-oxo-1,2-dihydroanthracene $=12116$

Azedarachin $=21448$

Baicalein-7-glucuronide $=2106$

Bakuchicin $=1191$

Bakuchiol $=2081$

Balanitin $6=23005$

Balanophorin $\mathrm{A}=1122$

Balanophorin $\mathrm{B}=13099$

Banisterine $=9235$

Baogongteng B $=19542$

Baphinitone $=6290$

Barlerin $=511$ 
Batatacin III $=2165$

Bayogenin 3- $O-\beta$ - $D$-xylopyranosyl- $(1 \rightarrow 3)-\beta$ - $D$-glucopyranoside $=4018$

Beauverician $=2188$

Beetroot red $=2322$

Belamarine $=2219$

Belamcandaphenol $=2216$

Bellidifolin 8- $O$ - $\beta$-glucopyranoside $=20511$

Bellidin 8- $O$ - $\beta$-glucopyranoside $=15800$

1,2-Benzenediol $=3318$

1,4-Benzenediol $=9740$

1,3-Benzodioxol-5-ol = 19779

4-(1,3-Benzodioxol-5-yl)-6-methoxynaphtho[2,3-c]furan-1,5,8(3H)-trione $=11982$

$(2 E, 4 E, 11 E)-12-(B e n z o[1,3]$ dioxol-5-yl)- $N$-(3-methylbutyl)dodeca-2,4,11-trie n-amide $=17474$

1,3-Benzodioxol-5-yl-1-oxo-2,4-pentadienylpiperine $=17451$

(-)-2-(15-Benzo[1,3]dioxol-5-yl-pentadecanoyl)-3,6-dihydroxy-cyclohex-2-

enone $=20493$

2,3-Benzofuran $=4143$

4H-1-Benzopyran-4-one,3,5,7-trihydroxy-2-phenyl $=8081$

6,7-Benzopyrene $=2226$

1,2-Benzopyrone $=4140$

2,3-Benzopyrrole $=11025$

$1,4-$ Benzoquinone $=18426$

10 - $O$-Benzoyl-6'- $O$ - $\alpha$-arabino $(1 \rightarrow 6)-\beta$-glucopyranosyl arborescosidic acid $=16518$

9-Benzoyl-2-deacetoxy-9-deacetyl-10-debenzoyl-10,13-diacetyltaxchinin A = 20754

3-O-Benzoyl-7-O-deacetylorthosiphol $\mathrm{M}=16232$

9- $O$-Benzoyl-9,10-dide- $O$-acetyl-11(15 $\rightarrow 1)$-abeo-baccatin $\mathrm{VI}=20776$

$2 \alpha$-Benzoyl-9,9-dimethyl-6 $\alpha, 8 \beta$-di- $(\gamma, \gamma$-dimethylallyl)-3,4-[2 $\alpha$-(2'-hydroxyiso propyl)-2,3-dihydrofuran]-8 $\alpha$ - $H$-cis-bicyclo[3.3.1]nona-3-ene-1,5-dione $=8213$

6'-O-Benzoyl-8-epiloganic acid $=1538$

6'- $O$-Benzoyl-gardoside $=1540$

12-O-Benzoyllineolon 3-O- $\beta$ - $D$-thevetopyranosyl-( $1 \rightarrow 4)-\beta$ - $D$-cymaropyranosyl-

$(1 \rightarrow 4)-\beta$ - $D$-cymaropyranosyl-( $1 \rightarrow 4)-\beta$ - $D$-cymaropyranoside $=17785$

1-Benzoylmethyl-5-hydroxyphenyl- $\beta$ - $D$-(3'-benzoyl) glucopyranoside $=2270$

1-Benzoylmethyl-5-hydroxyphenyl- $\alpha$ - $D$-glucopyranoside $=19192$

$10 \beta$-Benzoyloxy-2 $\alpha, 4 \alpha$-diacetoxy-5 $\beta, 20$-epoxy- $1 \beta, 7 \beta, 9 \alpha, 13 \alpha$-tetrahydrox-11

$(15 \rightarrow 1)$-abeo-taxene $=5348$

$9 \alpha$-(Benzoyloxy)-2 $\alpha, 4 \alpha$-diacetoxy- $5 \beta, 20$-epoxy- $1 \beta, 7 \beta, 10 \beta, 13 \alpha$-tetrahydroxy-

$11(15 \rightarrow 1)$-abeo-taxene $=20831$

6'-Benzoylseguinoside $\mathrm{A}=2612$

Benzyl alcohol xylopyranosyl $(1 \rightarrow 6)$ glucopyranoside $=2278$

Benzyl $\beta$ - $D$-apiofuranosyl- $(1 \rightarrow 6)-\beta$ - $D$-glucopyranoside $=10953$

2-Benzyl-1,3-dihydroxy-anthraquinone $=2299$

Berbericinine $=16555$

9-Berberoline $=2304$

Bergamottin $=2308$

Betuletol 3-methyl ether $=19312$

Betulic acid $=2334$

Betulinaldehyde- $3 \beta$-yl-caffeate $=13090$

Betulinol $=2331$

Bicoloirin $=664$

Bicuculline $=642$
Bidenoside $\mathrm{D}=2362$

Biflorine $=17983$

4,4"-Binaphthalene-8,8"-O,O-di- $\beta$ - $D$-glucopyranoside $=12401$

$D(+)$-Biotin $=2395$

Bipindogenin 3-O- $\beta$ - $D$-digitaloside $=2399$

$3,6^{\prime}$-Biplumbagin $=3541$

$L$-Bisabolene $=2412$

$\alpha$-Bisabolol $=2414$

Bischroman $=2481$

3,3'-Bis(3,4-dihydro-6-methoxy-2H-1-benzopyran) $=2481$

1,2-Bis(2,4-dihydroxy,3-C-glucopyranosyl)-ethanedione $=22477$

1,7-Bis-(3,4-dihydroxyphenyl)-5-hydroxy-3-heptanone-5-O-[2-(2-methylbute noyl) $]-\beta$ - $D$-xylopyranoside $=961$

1,7-Bis-(3,4-dihydroxyphenyl)-5-methoxy-3-heptanone $=14489$

3,10-Bis(1,1-dimethyl-2-propenyl)-5-hydroxy-8,8-dimethyl-2 $H, 8 H$-benzo [1,2-b:5,4-b']dipyran-2-one $=3788$

3 - $O$ - $\{$ Bis $[\beta$ - $D$-glucopyra-nosyl $(1 \rightarrow 2$ and $1 \rightarrow 3)$ - $\alpha$ - $L$-arabinopyranosyl $]\}$ echinocystic acid 28- $O-\beta$ - $D$-glucopyranosyl ester $=11800$

$(16 \rightarrow 16)$-Bis-16 $\beta$-hydro-ent-kaurane $=1301$

1,7-Bis(4-hydroxy-3-methoxyphenyl)-1,6-heptadiene-3,5-dione $=4398$

1,7-Bis(4-hydroxyphenyl)-3R,5S-heptanediol $=9219$

1,7-Bis(4-hydroxyphenyl)-5S-hydroxy-3-heptanone $=17547$

3-[Bis(3,4-methylenedioxyphenyl)methyl]-4-( $\beta$ - $D$-glucopyranosyloxymethyl)-2(5H)-furanone $=11974$

Bis(3-methylthio-2E-propenyl) disulfide $=7848$

$\beta$-Bitter acid $=13109$

$\alpha$-Bixin $=2496$

Blancoxanthone $=11086$

Blumenol $\mathrm{A}=22615$

Bogoroside $=4014$

6- $C-\beta-D$-Boivinopyranosyl-4'- $O-\beta-D$-glucopyranosylapigenin $=18677$

Bolusanthin III = 16545

2-Bornanone $=3048$

$(+)-(1 R, 2 S)$-Borneol $=2553$

$(-)-(1 S, 2 R)$-Borneol $=2555$

Brachyamide $\mathrm{B}=17436$

Brazilin $=2594$

Britannin $=2615$

Bruceantinoside $\mathrm{B}=22875$

Brucein $A=2664$

Brucein $\mathrm{B}=2665$

Brucein $\mathrm{C}=2666$

Brucein D $=2667$

Brucein $\mathrm{E}=2668$

Brucein $\mathrm{F}=2670$

Brucein $\mathrm{G}=2671$

Brucein $\mathrm{H}=2672$

Brucein I = 2673

Brucineoxide $=2679$

bryodulcosigenin 3-O- $\beta$-glucopyranosyl-26- $O$ - $\alpha$-rhamnopyranosyl-( $(1 \rightarrow 2)$ -

$O$ - $\beta$-glucopyranoside $=12219$

Buddledin $\mathrm{E}=3245$

Bufarenogenin $=2718$ 
Bufogenin $=18637$

Bulbispermine $=9206$

Bullatine $\mathrm{F}=15228$

Butanoic acid $=2808$

(E)-2-Butenedioic acid $=7996$

Butenoic acid $=4271$

3-Buten-1-ol = 943

$7 \alpha$-Butoxyethoxy-12-hydroxyabieta-6 $\alpha$-yl 6,7-dehydroabieta-8,11,13-trien-

12 -yl ether $=7887$

6- $C$-Butyl derivative of $2 R, 5 R$-bis(hydroxymethyl)- $3 R, 4 R$-dihydroxy-

pyrrolidine $=2792$

$3 S, 6 S, 7 S-3-$ Butyl-4,5-dihydro-6,7-dihydroxy phthalide $=19743$

4- $n$-Butyl-4-hydroxybutyric acid lactone $=15971$

$n$-Butylidene-phthalide $=2797$

$n$-Butyl-phthalide $=2803$

1-Butyl-3,4,5-trihydroxy-cyclohexanol $=21309$

Buxpiine $\mathrm{K}=2831$

Buxtauine $\mathrm{M}=2832$

\section{C}

Cadabine $=20254$

4-Cadinene- $2 \alpha, 10 \alpha$-diol $=12223$

Caesalpinine $\mathrm{C}=3360$

trans-Caffeic acid-1-O-rutinose ester $=20508$

1-O-(E)-Caffeoyl-3-O-galloyl-4,6-[1',1"-(3',3",4',4"-tetrahydroxydibenzofuran dicarboxyl)]- $\beta$ - $D$-glucopyranose $=2129$

3-O-(2-O-Caffeoyl)- $\beta$ - $D$-glucopyranosyl-( $1 \rightarrow 4)$ - $\beta$ - $D$-glucopyranosyl-( $1 \rightarrow 4)$ -

$\beta$ - $D$-ribopyranosyl- $(1 \rightarrow 3)$ - $\alpha$ - $L$-rhamnopyranosyl- $(1 \rightarrow 2)-\alpha$ - $L$-arabinopyra nosyl hederagenin 28- $O$ - $\alpha$ - $L$-rhamnopyranosyl-( $1 \rightarrow 4)-\beta$ - $D$-glucopyranosyl- $(1 \rightarrow 6)-\beta$ - $D$-glucopyranoside $=3819$

5- $O$-Caffeoylquinic acid $=15363$

5-O-Caffeoylquinic acid methyl ester $=14224$

3-Caffeoylquinid acid $=3551$

1-O-(E)-Caffeoyl-4,6-[1',1"-(3',3",4',4"-tetrahydroxydibenzofurandicarboxyl)]- $\beta$ - $D$-glucopyranose $=2128$

Calendula officinalis Glycoside $\mathrm{D}_{2}=9588$

Calogenin 3- $O-\beta$ - $D$-cymaropyranosyl- $(1 \rightarrow 4)-O-\beta-D$-digitoxopyranoside $=9346$

Calogenin 3-O-3-O-methyl- $\beta$ - $D$-fucopyranosyl-( $1 \rightarrow 4)-O-\beta$ - $D$-oleandropyranoside

$$
=5130
$$

Calophyn $=1466$

Calotropin $=2937$

Camboginol $=8218$

Camelliol $\mathrm{C}=537$

Campestrinoside $=17757$

11-Campherennene-4,10-diol $=10243$

Camptothecine $=3053$

Cangorosin $\mathrm{B}=5655$

Cannabiscetin $=15170$

Capaurine $=3114$

Capric aldehyde $=4833$

Caprylic aldehyde $=15973$

Capsicosin $\mathrm{D}_{1}=8459$

Carbamoylamine $=22246$
3-Carboxyacetyloxy-24-exomethylene-12 $\beta$-hydroxy-23-oxo-lanost-8-en-26oic acid $=7863$

3-(3'-Carboxybutyl)-4-methoxyxanthone $=21232$

28-Carboxy-7 $\beta, 19 \alpha$-dihydroxyarbon-9(11)-en-3-one $=19019$

6-Carboxyethyl-7-methoxy-5-hydroxybenzofuran-5- $O$ - $\beta$ - $D$-glucopyranoside $=17297$

19-Carboxy 8(17)-13(16)-14-labdatriene $=12412$

2-Carboxyl-3-(3'-carboxyl-2'-hydroxy)-butyl-1,4-naphthohydroquinone-4- $O$ $\beta$ - $D$-glucopyranoside $=19034$

2-Carboxyl-3-(3'-carboxyl-2'-hydroxy)-butyl-1,4-naphthohydroquinone-4-O$\beta$ - $D$-glucopyranosyl- $(1 \rightarrow 6)-\beta$ - $D$-glucopyranoside $=19035$

5-Carboxyl-3,4-dihydrogen-1H-2-benzopyran-1-one $=7335$

2-Carboxyl-1,4-naphthohydroquinone-4- $O-\beta$ - $D$-glucopyranoside $=19032$

5-(4-Carboxy-3-methoxyphenyl)-3-methoxy-3-carboxy-4-pentenoic acid $=3178$

$\alpha$-Carboxy- $N, N, N$-trimethyl- $1 H$-indole-3-ethanaminium hydroxide inner salt $=10877$

$(17 \beta)$-Card-20(22)-enolide $=5525$

Cardol monoene $=2380$

trans $-\beta$-Carotene $=3209$

$\beta, \beta$-Carotene-4,4'-dione $=3095$

$\psi$-Carpaine $=18022$

Carpoxidine $=16129$

Caryolane- $5 \beta, 9 \beta$-diol $=20137$

(-)-trans-Caryophyllene $=3242$

$\alpha$-Caryophyllene $=9669$

$\beta$-Caryophyllene epoxide $=3243$

Casealutine $=11348$

Caseanine $=21077$

Cassiaside $\mathrm{C}=8649$

Cassic acid $=18759$

Cassythine $=3293$

Castanin $=676$

Catalpin $=3307$

Catalpinoside $=3306$

Catechinic acid $=3308$

Catechin-3- $O$ - $\alpha$ - $L$-rhamnopyranosyl-( $1 \rightarrow 4)-\beta$ - $D$-glucopyranosyl-( $1 \rightarrow 6)-\beta$ - $D$ glucopyranoside $=6925$

Catechol dimethyl ether $=22389$

Catechuic acid $=3308$

$(+)$-Catharanthine $=3321$

Cathedulin $\mathrm{E}_{2}=3326$

$D$-Cathine $=15789$

Cauda-3- $O$ - $\beta$ - $D$-glucopyranosyl-( $1 \rightarrow 4)-\beta$ - $D$-cymaropynanosyl-( $1 \rightarrow 4)-\beta$ - $D$ oleandropyranosyl-( $(1 \rightarrow 4)-\beta$ - $D$-cymaropynanoside $=4552$

Cauda-3- $O$ - $\beta$ - $D$-glucopyranosyl- $(1 \rightarrow 4)-\beta$ - $D$-oleandropyranosyl-( $1 \rightarrow 4)-\beta$ - $D$ digitoxopyranosyl-( $1 \rightarrow 4)-\beta$ - $D$-cymaropynanoside $=4553$

Caulophyllin $=14279$

Caulophyllogenin 3-O- $\alpha-L$-rhamnopyranosyl $(1 \rightarrow 2)-\alpha-L$-arabinopyranoside $=$ 17350

Caulosapogenin $=9260$

Celidoniol $=15659$

$(1 S, 2 E, 4 R, 6 R, 7 E, 11 E)-2,7,11$-Cembratriene-4,6-diol = 6643

Cembrene $\mathrm{A}=15360$ 
Centauridin $=19312$

Centellasaponin $\mathrm{A}=1854$

Cephalomannine $=20812$

Cepharanone $\mathrm{B}=1695$

Cetane $=9485$

Cetin 3- $O-\beta$ - $D$-xylopyranosyl-( $1 \rightarrow 2)-\alpha-L$-rhamnopyranosyl-( $1 \rightarrow 6)-\beta-D$ glucopyranoside $=19199$

Cetylic acid $=9486$

$5 \alpha, 14 \alpha$-Cevanine-13,17-dehydro- $3 \alpha, 6 \beta$-diol $=22998$

Cevaninedihydroxyone $=22995$

$(20 S, 22 S, 25 S)-5 \alpha$-Cevanine- $3 \beta, 6 \beta$-diol $=7928$

$5 \alpha, 17 \beta, 22 \alpha$-Cevanine- $3 \beta, 6 \alpha, 12 \alpha, 14 \alpha, 16 \beta, 20 \beta$-hexol $=17385$

$5 \alpha, 14 \alpha$-Cevanine- $6 \alpha$-hydroxyl-3 $\alpha-\beta$ - $D$-glucoside $=9682$

$(20 R, 22 S, 25 S)-5 \alpha$-Cevanin-23-ene-3 $\beta, 6 \beta, 16 \beta$-triol $=11004$

$5 \alpha, 14 \alpha, 22 \alpha$-Cevanine- $3 \beta, 6 \alpha, 20 \beta$-triol $=22993$

$5 \alpha, 14 \alpha, 22 \beta$-Cevanine- $3 \beta, 6 \alpha, 20 \beta$-triol $=22636$

3- $O-\beta$ - $D$-Chacotriosyl-3 $\beta, 16 \beta$-dihydroxy-pregn-5-en-20-one 16- $O$-(2,5-

epoxy-2-methoxy-4-methyl-pentanolic acid)-ester $=48$

Chaerophyllin $=12106$

Chalepin $=9304$

Chalepin acetate $=19085$

$\psi$-Cheierythrine $=19284$

Cheiranthidin 3-O- $\beta$ - $D$-glucopyranosyl- $(1 \rightarrow 4)-\beta$ - $D$-boiviopyranoside $=3496$

Cheiranthidin 3- $O-\alpha$ - $L$-rhamnopyranosyl- $(1 \rightarrow 4)-\beta$ - $D$-digitoxopyranoside $=3497$

Chikusetsusaponin IVc $=8428$

Chimonin $=13481$

ent-15-Chloro-13,14-dihydroxylabd-8(9)-en-3-one $=685$

2-Chloro-1,4-dihydroxy-3-(4-methylpent-3-enyl)anthraquinone $=1370$

7-Chloro-(2R)-2-O- $\beta$ - $D$-glucopyranosyl-4-hydroxy-2 $H$-1,4-benzoxazin-

$3(4 H)$-one $=3815$

$(6 R, 7 R, 8 R)-8-$ Chlorogoniodiol $=3555$

3-Chloro-2-hydroxy-4,5,6a,7-tetrahydro-dibenzo[de,g]quinoline-6-carboxylic acidmethyl ester $=18902$

ent-15-Chloro-labd-8(9)ene-3 $\alpha, 13,14$-triol $=686$

14-Chloro-2 $\beta, 8 \beta,-10 \alpha$-trihydroxy- $1 \alpha H, 5 \alpha H, 6 \beta H, 7 \alpha H$-guai-3,11(13)-dien-6,

$$
\text { 12-olide }=7570
$$

Cholaic acid $=20734$

Cholestane-3,7,12,26-tetrol $=21138$

$(22 S, 25 S)-5 \alpha$-Cholestane-3 $\beta, 16 \beta, 22,26$-tetrol $=725$

5-Cholesten-3 $\beta$-ol $=3585$

$5 \beta$-Cholic acid $=3588$

$N$-Choloyl-taurine $=20734$

Cholyltaurine $=20734$

Chrysanthelide $=9218$

Chrysanthemyl alcohol $=3595$

Chrysartemin A = 3076

Chrysatroic acid $=19542$

Chrysoeriol 7-O-[2"'- $O$-caffeoyl-6"'- $O$-acetyl- $\beta$ - $D$-glucopyranosyle-( $1 \rightarrow 2)$ $\beta$ - $D$-glucopyranoside $]=16482$

Chrysoeriol 7-O-[2'"- $O$-caffeoyl- $\beta$ - $D$-glucopyranosyl-( $1 \rightarrow 2)-\beta$ - $D$-glucopyranoside $]=16483$

Chrysoeriol 7-O-[2'"- $O$-p-coumaroyl-6"'- $\beta$ - $O$-acetyl- $D$-glucopyranosy-( $1 \rightarrow 2)$ $\beta$ - $D$-glucopyranoside $]=16484$
Chrysophanein $=3620$

Chrysophanol-1- $O-\beta$ - $D$-glucopyranoside $=3620$

Chrysosplenol $\mathrm{C}=3623$

Chuanliansu $=21448$

Chuan-Wu-base B = 3201

Cichoric acid $=3518$

Cichorigenin $=663$

$(8 \alpha, 9 R)$-Cinchonan-9-ol $=3685$

Cinnamic aldehyde $=3693$

6'-Cinnamoylcatalpol $=17344$

Cinnamoyl cinnamate $=20417$

$\mathrm{N}$-Cinnamoyl-trans-3,4-diacetoxypyrrolidine $=19369$

1-Cinnamoyl-3-hydroxy-11-methoxymeliacarpinin $=3710$

12(R)-O-Cinnamoyloxy-3 $\beta, 5 \beta$-dihydroxy- 8,14 -seco- $17 \alpha$-pregn-6-ene- 8,14 , 20 -trione $=4562$

12(R)-O-Cinnamoyloxy-3 $\beta, 5 \beta$-dihydroxy- 8,14 -seco- $17 \beta$-pregn-6-ene- 8,14 , 20 -trione $=4555$

$5 \alpha$-Cinnamoyloxy-9 $\alpha$-hydroxy-10 $\beta, 13 \alpha$-diacetoxytaxa-4(20), 11-diene $=4637$

$5 \alpha$-Cinnamoyloxy-2 $\alpha, 7 \beta, 13 \alpha$-triacetoxy-2(3 $\rightarrow 20)$ abeo-taxa-4(20),11-diene-

9,10 -dione $=4636$

cis-3 $\beta$-(Cinnamoyloxy)tropane $=7348$

$O$-Cinnamoyltaxicin II triacetate $=20797$

12-O-Cinnamyldihydrosarcostin-3- $O-\beta$ - $D$-glucopyranosyl- $(1 \rightarrow 4)-O-3-O$ methyl-6-deoxy- $\beta$ - $D$-allopyranosyl- $(1 \rightarrow 4)-O-\beta$ - $D$-oleandropyranosyl$(1 \rightarrow 4)-O-\beta$ - $D$-cymaropyranoside $=13577$

Cinobufotenine $=2726$

Cirantin $=9458$

Cirensenoside $\mathrm{P}=8611$

Cirontin $=9458$

Cirsiumaldehyde $=2452$

Citroside $\mathrm{A}=4716$

Citrus-hesperidin $=9458$

cis-Civetone $=3780$

Clauszoline $\mathrm{C}=3797$

Clauszoline $\mathrm{I}=3795$

Clauszoline $\mathrm{J}=3799$

Clemaphenol A $=3818$

Cleosandrin $=3829$

(rel-3S,4S,5R,8R,9R,10S)-14-Clerodene-3,4,13-triol $=22576$

Cnidimine $=6709$

Coccellinic acid $=18779$

$(+)$-Coclaurine $=3878$

Coculine $=19955$

Codonolactone $=1967$

Con-5-enin- $3 \beta$-amine $=3959$

Coniferaldehyde $=10453$

Conioselin $=3990$

Convallagenin B 5-O- $\beta$ - $D$-glucopyranoside $=21845$

Convallaton $=4012$

Corbisterol $=4963$

Cornustannin $2=7518$

Corydalactam $=7451$

Corydalis $=21077$ 
Corylifolin $=2172$

Corysamine $=22732$

Cosmosiin $=1492$

Cotoin $=5965$

$10-O-(E)-p$-Coumaroyladoxoside $=13166$

$10-O-(Z)-p$-Coumaroyladoxoside $=13167$

$2^{\prime}-O-p$-Coumaroylaloesin $=973$

7-O-trans- $p$-Coumaroyl-6'-O-trans-caffeoyl-8-epiloganic acid $=752$

7-O-p-Coumaroylhydroxymaltol 3-O- $\beta-D$-glucopyranoside $=2525$

(2-trans- $p$-coumaroyloxymethyl-4- $\beta$ - $D$-glucopyranosyloxy-2(E)-butenenitrile) $=15593$

6-O- $\alpha$-L-(2"-O-trans- $p$-Coumaroyl)rhamnopyranosylcatalpol $=19104$

7-O-(E)-p-Coumaroylsuspensolide $\mathrm{F}=13164$

7-O-(Z)-p-Coumaroylsuspensolide $\mathrm{F}=13165$

trans-p-Coumaryl-(6'-O- $\alpha-L$-arabinopyranosyl) $-O-\beta$ - $D$-glucopyranoside $=18929$

3-O-(6-O- $p$-coumaryl- $\beta$ - $D$-glucopyranosyl)-5- $O$-(6- $O$-malonyl- $\beta$ - $D$-glucopyra nosyl)cyanidin $=13450$

trans- $p$-Coumaryl-(6'- $O-\beta$ - $D$-xylopyranosyl)- $O-\beta$ - $D$-glucopyranoside $=18928$

Couroupitine $\mathrm{B}=11024$

CQ-1 $=3440$

$\mathrm{CQ}-2=3441$

$\mathrm{CQ}-3=3442$

CQ-4 $=3443$

$\mathrm{CQ}-5=3444$

Crassicaulisine $=15228$

Crategolic acid $=6059$

Crocin-1 $=4249$

Crotaline $=14923$

Crotepoxide $=8041$

Cryptocavine $=4290$

Cryptopalmatine $=15079$

Cryptopimaric acid $=19236$

Crysoeriol $=3602$

Cubebol $=4304$

Cucurbitacin $=6733$

Cucurbitacin L 2-O- $\alpha$-glucopyranosyl-( $1 \rightarrow 4)$ - $\beta$-glucopyranoside $=12209$

Cucurbitacin L 2- $O$ - $\alpha$-rhamnopyranosyl- $(1 \rightarrow 2)-\beta$-glucopyranoside $=12208$

Cunabic acid $=12178$

Cupressuflavone $=4376$

Curculigine $=4381$

$L$-Curine $=2191$

Cyanidin-3-O- $\beta$ - $D-(6-O-p$-coumaroyl $)$-glucoside $=9696$

Cyanidin diglucoside $=4451$

Cyanidin 3-O-glucoside $=3594$

Cyanidin rhamnoglucoside $=4446$

Cyanidin 3-O-(4"-O-sinapoyl gentiobioside $)=835$

Cyanidol $=3308$

(S)-trans-Cyclanoline $=3747$

Cyclene $=21598$

Cycloart-23-ene- $3 \beta, 25$-diol $=4471$

$9 \beta, 19$-Cyclolanost-24E-ene-3 $\beta, 6 \alpha, 16 \beta, 27$-tetraol-3-O-(2'-O-acetyl)- $\beta$ - $D$-gluco pyranoside $=12108$

9 $\beta, 19$-Cyclolanost-24E-ene-3 $\beta, 6 \alpha, 16 \beta, 27$-tetraol-3-O-(6'- $O$-acetyl)- $\beta$ - $D$-gluco pyranoside $=12109$

$9 \beta, 19$-Cyclolanost-24E-ene-3 $\beta, 6 \alpha, 16 \beta, 27$-tetraol-3- $O-\beta$ - $D$-glucopyranoside $=12107$

$9 \beta, 19$-Cyclolanost-24E-ene-3 $\beta, 6 \alpha, 16 \beta, 27$-tetraol-3- $O-\beta-D$-glucopyranosyl-27

$-O-\beta$ - $D$-glucopyranoside $=12110$

$9 \beta, 19$-Cyclo-24-lanosten- $3 \beta$-ol $=4473$

Cyclomulberrochromene $=4517$

13-(2-Cyclopenten-1-yl)tridecanoic acid $=3485$

Cyclo-(Pro-Ala) $=4463$

Cyclo-(prolyl-glycyl-tyrosyl-valy-leucyl-alanyl-leucyl-valyl) $=8494$

Cyclo-(prolyl-prolyl-valyl-tyrosyl-glycyl-prolyl-glutamyl) $=8495$

Cyclo-(Val-Pro) $=4534$

Cymaroside $=3674$

2- $p$-Cymenol $=3231$

Cynajapogenin A 3- $O-\alpha-L$-cymaropyranosyl-( $(1 \rightarrow 4)-\beta$ - $D$-digitoxopyranosyl$(1 \rightarrow 4)-\beta$ - $L$-cymaropyranoside $=4566$

Cynajapogenin A 3-O- $\beta$ - $D$-glucopyranosyl- $(1 \rightarrow 4)-\alpha-L$-diginopyranosyl-

$$
(1 \rightarrow 4)-\beta \text { - } L \text {-cymaropyranosyl- }(1 \rightarrow 4)-\beta \text { - } D \text {-digitoxopyranoside }=4567
$$

\section{D}

Daidzein 7-O- $\beta$ - $D-(6 "-O$-acetylglucopyranoside $)=361$

Daidzoside $=4606$

Damma-12,20(22)Z-dien-3 $\beta$-ol $=3469$

Damma-12,20(22)Z-dien-3-one $=3468$

Dammardienyl acetate $=149$

Dammar-25-ene-20,24-diol-3-one $=7931$

Dammar-22(32)-ene-3 $\beta, 6 \alpha, 12 \beta, 20 S, 24 \xi$-pentaol-(20-O- $\beta$ - $D$-glucopyranosyl6- $O$ - $\alpha$ - $L$-rhamnopyranosyl- $(1 \rightarrow 2)-\beta-D$-glucopyranoside $)=13407$

Dammar-23(24)-ene-3 $\beta, 6 \alpha, 12 \beta, 20(S), 25$-pentaol-(20- $O-\beta$ - $D$-glucopyranosyl6- $O$ - $\alpha$ - $L$-rhamnopyranosyl- $(1 \rightarrow 2)-\beta$ - $D$-glucopyranoside $)=13408$

Dammar-24-ene-3,12,17,20-tetrol $=2326$

Dammar-24-ene-3,12,20-triol $=2328$

Daniellic acid $=7161$

Daphnetin-7-glucoside $=4650$

Darutin $=4662$

4,8-Daucadiene $=4675$

Dauriporphine $=2345$

10,13-Deacetyl-abeo-baccatin IV $=20739$

7-Deacetyl-7-angeloyl-16-ketokihadalactone $\mathrm{A}=18453$

$2 \alpha$-Deacetyl- $2 \alpha$-benzoyl-13 $\alpha$-acetyltaxayuntin $=20761$

10-Deacetylcephalomannine $=4784$

$9 \alpha$-Deacetyl- $9 \alpha$-debenzoyl taxayuntin $=20762$

Deacetyldecinnamoyltaxinine $\mathrm{E}=4737$

2-Deacetyl-7,10-diacetyl-5-deaminoacyl taxine $\mathrm{A}=4781$

2-O-Deacetylorthosiphol A $=16229$

3- $O$-Deacetylorthosiphol $\mathrm{B}=16229$

3- $O$-Deacetylorthosiphol $\mathrm{J}=16231$

9-Deacetyltaxayuntin $\mathrm{E}=20845$

2-Deacetyltaxinine $\mathrm{A}=20855$

$2 \alpha$-Deacetyltaxinine $\mathrm{J}=20846$

10-Deacetyltaxol C $=4787$

Deacylcynanchogenin $=12880$

Deaminoacylcinnamoyltaxine A $=20847$

Deaminoacylcinnamoyltaxol $=2074$ 
$2 \alpha$-Debenzoyl-2 $\alpha$-acetyl taxayuntin $=20760$

$N$-Debenzoyl- $N$-butanoyltaxol $=20814$

$N$-Debenzoyl- $N$-hexanoyltaxol $=20813$

Deca-2E,4Z-dienoic acid piperidide $=15446$

Deca-8(E)-en-4,6-diyne-1,3,10-triol 1- $O$ - $\beta$ - $D$-glucopyranoside $=2362$

$8 Z$-Decaene-4,6-diyn-1- $O-\beta$ - $D$-glucopyranoside $=2358$

Decaffeoyl forsythoside $\mathrm{C}=7927$

Decaffeoylverbascoside $=4830$

[1S-(1 $\alpha, 3 \beta, 4 \alpha, 8 \beta)]$-Decahydro-9-methylene-4,8,8-trimethyl-1,4-methanoazulene $=12955$

(-)-(2R,4aS,8aR)-Decahydro-4a-methyl-8-methylene-2-(1-methylethyl)-2naphthalenol $=7511$

$n$-Decane $=4834$

Decanedioic acid $=19596$

Decanoic acid $=3138$

9'-Decarboxyrosmarinic acid 4'- $O$ - $\alpha$-rhamnosyl-(1"'' $\rightarrow 6$ "')- $O$ - $\beta$-galactosyl$\left(1^{\prime \prime \prime} \rightarrow 4 "\right)-O-\alpha$-rhamnoside $=21221$

3(R)-Deca-4,6,8-triyne-1,3-diol 1- $O$ - $\beta$ - $D$-glucopyranoside $=2361$

$(3 R)$-8-Decene-4,6-diyne-1,3-diol 1- $O-\beta$ - $D$-apiofuranosyl-( $1 \rightarrow 6)$ - $\beta$ - $D$-glucopyraside $=9105$

$3(R), 8(E)$-8-Decene-4,6-diyne-1,3-diol 1-O- $\beta$ - $D$-glucopyranoside $=2359$

(E)-8-Decene-4,6-diyne-1,10-diol 1-O- $\beta$ - $D$-glucopyranoside $=5155$

(3R)-8-Decene-4,6-diyne-1,3-diol 1- $O-\beta$ - $D$-glucopyraside $=9104$

Degueline $=4872$

6,7-Dehydroabieta-8,11,13-trien-12-yl 7 $\alpha$-hydroxy-12-methoxyabieta-

$8,11,13$-trien- $6 \alpha$-yl ether $=7886$

13-Dehydro- $1 \beta$-acetyl- $2 \alpha, 6 \beta$-dihydroxyhetisine $=9051$

7,8-Dehydrocerberin $=526$

$6 \alpha, 12 \alpha$-Dehydrodeguelin $=4898$

9(11)-Dehydroergosterol peroxide $=6904$

11-Dehydro-17-hydroxycorticosterone $=4097$

Dehydrolarreatricin $=4940$

6-Dehydrolongilactone $=4986$

$\Delta^{1}$-Dehydromiltirone $=4950$

Dehydroxypodorhizol $=5543$

Dehydroxytetrahydrocurcumin $=8389$

1-O-Deisovaeroyl-1-O-3-methylvaleroylluzonoid $\mathrm{A}=13161$

Delphamine $=5004$

Delphanin $=15289$

Delphinidin 3-O-[6-O-(4-O-(4-O-(6-O-(trans-caffeoyl)- $\beta$ - $D$-glucopyranosyl)trans- $p$-coumaroyl)- $\alpha$ - $L$-rhamnopyranosyl)- $\beta$ - $D$-glucopyranoside]-5- $O$ -

$[\beta$ - $D$-glucopyranoside $]=17358$

Delphinidin-3,5-diglucoside $=5010$

Delphinidin 3-O-[6-O-(4-O-(4-O-( $\beta$ - $D$-glucopyranosyl)-trans- $p$-coumaroyl)$\alpha$ - $L$-rhamnopyranosyl)- $\beta$ - $D$-glucopyranoside]-5- $O$-[ $\beta$ - $D$-glucopyranoside $]$ $=17359$

Delphinidol $=5011$

Demecolcine $=3909$

Demethoxycurcumin $=2439$

3'-Demethoxy-6-O-demethylisoguaicin $=7746$

4-Demethoxytyraminoporphine $=4689$

4-Demethylantiarol 4-O-[(3-methoxy-4-hydroxy-benzoyl)- $\beta$ - $D$-apiofuranosyl$(1 \rightarrow 2)-\beta$ - $D$-glucopyranoside $=8852$
Demethylcrinamine $=9206$

4-Demethyl-deoxypodophyllotoxin $=5074$

$N$-Demethylfrangulanine $=9653$

$\left(8 R, 8^{\prime} R\right)-3^{\prime}-O$-Demethyl-5-hydroxymatairesinol $=5082$

$\left(8 R, 8^{\prime} R\right)-3$ '- $O$-Demethyl-5-methoxymatairesinol $=5085$

Demethylpedalitin $=10351$

4'-Demethylpodophyllotoxin $=5093$

Demethyltetrandrine $=7714$

Dendramine $=9982$

Densiflorol $\mathrm{B}=4585$

15-Deoxo-30-hydroxyeucosterol 3- $O-\alpha-L$-rhamnopyranosyl-( $1 \rightarrow 2)-[(\beta-D$ glucopyranosyl-( $(1 \rightarrow 3))-\beta$ - $D$-glucopyranosyl-( $(\rightarrow 2)-\alpha$ - $L$-arabinopyranosyl- $(1 \rightarrow 6)-\beta$ - $D$-glucopyranoside $=19516$

9-Deoxo-9 $\alpha$-hydroxytaxol $=22950$

11-Deoxojervine $=4522$

4-Deoxyasimicin $=11375$

Deoxycapillartemisin $=1808$

7-Deoxycholic acid $=5161$

3-Deoxycrassicaulidine $=15228$

2-Deoxy- $D$-ribono-3,5-bis(tripolyphosphate)-1,4-lactone $=2494$

22-Deoxyecdysterone $=20807$

6-Deoxy- $\beta$ - $D$-glucopyranosyl-[3- $O-\beta$ - $D$-glucopyranosyl-( $1 \rightarrow 6)-\beta$ - $D$-glucopyranosyl]-pyrocincholate $=11035$

6-Deoxy- $\beta$ - $D$-glucopyranosyl-pyrocincholate $=11036$

11-Deoxyglycyrrhetinic acid $=5144$

Deoxyisoartemisinin $\mathrm{B}=6891$

3-[[2-O-(6-Deoxy- $\alpha$ - $L$-mannopyranosyl)- $\beta$ - $D$-glucopyrano-syl]oxy]-5,7-dihy-

droxy-2-(3-hydroxy-4-methoxyphenyl)-4H-1-benzopyran-4-one $=20660$

3-[[2-O-(6-Deoxy- $\alpha-L$-man-nopyranosyl)- $\beta$ - $D$-glucopyranosyl]oxy]-5,7-dihydroxy-2-(4-methoxyphenyl)-4H-1-benzopyran-4-one $=12016$

6-Deoxy-L-mannose $=18739$

1-Deoxymorusignin $\mathrm{J}=1135$

Deoxypodophyllotoxin $=1372$

7-Deoxypseudoanisatin $=5204$

12-Deoxyroyleanone $=3$

9-Deoxysanaconitine $=11476$

Desacetylcinobufaginol $=9993$

Desacetyleupaserrin $=4739$

Desacetyl pseudolaric acid B $=18035$

Desacylescin I = 667

Desacylkondurangogenin $C-3-O-\alpha$ - $D$-glucopyranosyl-(1 $\rightarrow 4)-O-\alpha-L$-fucopyranoside $=5131$

1-Desgalloyleugeniin $=20910$

5-Desgalloyl stachyurin $=3302$

Desglucosyrioside $=7272$

Desmanthin $1=15181$

Desoxodehydrocyclopiloselloidone $=5142$

21-Desoxy-iridogermanal $=11133$

Desoxymorellin $=5196$

7-Desoxyneocynapanogenin A 3-O- $\beta$ - $D$-cymaropyranosyl-( $1 \rightarrow 4)$ - $\alpha$ - $L$-diginopyranosyl- $(1 \rightarrow 4)-\beta$ - $D$-thevetopyranoside $=1992$

4,5-cis-4,5-Diacetoxy-(1-acetoxypentadecyl)-2-cyclopenten-1-one $=21536$ $3 \beta, 9 \alpha$-Diacetoxy-7 $\beta$-benzoyloxy-15 $\beta$-hydroxy-14-oxojatropha-5E, $12 E$ - 
diene $=18172$

rel-(2S,5R,6S,7R,8S,9S,10R,18S,19R)-7,19-Diacetoxy-18-butanoyloxy-18,19-ep oxy-6-hydroxy-2-(2-methylbutanoyloxy)cleroda-3,13(16),14-triene = 3261 (22R)-1 $\alpha, 12 \alpha$-Diacetoxy-2 $\alpha, 3 \alpha ; 6 \alpha, 7 \alpha$-diepoxy-27-[( $\beta$ - $D$-glucopyranosyl)oxy] $-5 \alpha,-16 \beta$-dihydroxywith-24-enolide $=3479$

(22R)-1 $\alpha, 12 \alpha$-Diacetoxy-2 $\alpha, 3 \alpha ; 6 \alpha, 7 \alpha$-diepoxy-27-[( $\beta$ - $D$-glucopyranosyl)oxy]

-5 $\alpha$-hydroxy-16-oxowith-24-enolide $=3478$

$3^{\prime}(S), 4^{\prime}(S)$-Diacetoxy-3',4'-dihydroseselin $=18285$

$3 \beta, 6 \alpha$-Diacetoxy-15 $\beta, 17$-dihydroxy- $11 \beta, 16 \beta$-epoxy-ent-kaurane $=13696$

$7 \beta, 16 \alpha$-Diacetoxy-4 $\beta, 20 R$-dihydroxy- $5 \beta, 6 \beta$-epoxy-1-oxowitha-2,24-dienolide $=5324$

$2 \beta, 3 \beta$-Diacetoxy-11 $\beta, 13 \alpha$-dihydroxy-ent-kaur-16-en-15-one $=20622$

$3 \beta, 11 \beta$-Diacetoxy-2 $\beta, 6 \alpha$-dihydroxy-ent-kaur-16-en-15-one $=20623$

Diacetoxydihydroxytaxadiene $=5294$

(20S,24R)-15 $\alpha, 16 \beta$-Diacetoxy-20,24-epoxy-9,19-cyclolanostane-3 $\beta, 18,25$-triol-3- $O-\beta$ - $D$-xylopyranoside $=2203$

rel-(2R,5R,6R,8S,9S,10R,18S,19R)-18,19-Diacetoxy-18,19-epoxy-6-hydroxy-

2-(2-methylbutanoyloxy)cleroda-3,13(16),14-triene $=3262$

$2 \alpha, 15$-Diacetoxy-2,3-epoxy-2,3-seco-( $1 \alpha, 5 \alpha, 6 \beta, 7 \beta)$-aromadendra-3,10(14)-

dien-13-al $=17496$

12 $\beta, 21$-Diacetoxy-29- $\beta$ - $D$-glucopyranosyloxy-23 $\xi$-hydroxytirucalla-7,24-

dien-3-one $=17852$

(rel 6S,7R,8S,9R)-6,7-Diacetoxy-5(10),14-halimadien-13-ol = 22571

12,16-Diacetoxy-6-hydroxyabieta-5,8,12-trien-7,11,14-trione $=22751$

(13S,15S)-2 $\alpha, 12 \alpha$-Diacetoxy-6-hydroxy-13,16-cycloabieta-5,8-dien-7,11,14-

trione $=22752$

1-(2,5-Diacetoxy-1-hydroxy-cyclopent-3-enyl)-pentadecan-1-one $=5341$

1-(2,5-Diacetoxy-1-hydroxy-cyclopent-3-enyl)-tridecan-1-one $=5340$

1-(2,5-Diacetoxy-1-hydroxy-cyclopent-3-enyl)-undecan-1-one $=5339$

(12S)-6 $\alpha, 19$-Diacetoxy-12-hydroxy-4,18-epoxyneoclerod-13(14)-en-15,16-

olide $=797$

$6 S$-[3S,6S-(Diacetoxy)-5R-hydroxy-1Z-heptenyl]-5S-hydroxy-5,6-dihydro-

$2 H$-pyran-2-one $=16750$

4,5-trans-4,5-Diacetoxy-5-(1-hydroxytridecyl)-2-cyclopenten-1-one $=21535$

$1 \beta, 8 \beta$-Diacetoxyl- $6 \alpha, 9 \alpha$-difuroyloxydihydro- $\beta$-agarofuran $=16166$

$2 \alpha, 9 \alpha$-Diacetoxy-1 $\beta, 5 \alpha, 10 \beta, 13 \alpha$-tetrahydroxytaxa-4(20),11-diene $=5318$

11,16-Diacetoxy-6,12,14-trihydroxyabieta-5,8,11,13-tetraen-7-one $=146$

$3 \beta, 6 \alpha$-Diacetoxy-7 $\beta, 15 \beta, 17$-trihydroxy-11 $\beta, 16 \beta$-epoxy-ent-kaurane $=13697$

3- $O$ - $\alpha(2$ ', 4'- $O$-Diacetyl)- $L$-arabinopyranosyl-3 $\beta$-hydroxyolean-12-ene-28,29-

dioicacid-28- $O$ - $[\alpha-L$-rhamnopyranosyl-( $1 \rightarrow 4)-\beta$ - $D$-glucopyranosyl-

$(1 \rightarrow 6)-\beta$-D-glucopyranosyl] ester $=22916$

2,10-Di- $O$-acetyl-5-decinnamoyl-taxicin I $=5331$

3,3'-Diacetyl-4,4'-dimethoxy-2,2',6,6'-tetrahydroxy diphenyl methane $=2442$

3,3'-Diacetyl-4,4'-dimethoxy-2,2',6,6'-tetrahydroxy diphenyl methane-6'- $O$ - $\beta$ -

$D$-glusopyranoside $=6667$

5,10-Diacetyl-6,20-epoxy-3-phenyl-acetyllathyrol $=7617$

11,13-Diacetyl-14-hydroxy-2-isobutyryl hetisine $=9050$

21,28-Di- $O$-acetylprotoaescigenin-3- $O$ - $[\beta$ - $D$-glucopyranosyl $(1 \rightarrow 2)][\beta$ - $D$-glucopyranosyl $(1 \rightarrow 4)]-\beta$ - $D$-glucopyranosiduronic acid $=665$

1-O-[2,3-Di- $O$-acetyl- $\alpha$ - $L$-rhamnopyranosyl-(1 $\rightarrow 6)-\beta$ - $D$-glucopyranosyl]-(2E, $6 E)$-farnesol $=4231$

2',3'-O-Diacetylsalsoloside $\mathrm{C}=20532$

Diacetyltaxine $\mathrm{B}=20794$

3-O- $\beta$-(2',3'-Di- $O$-acetyl)- $D$-xylopyranosyl-6- $O-\beta-D$-glucopyranosyl-16- $O$ -
acetoxy-20(R),24(S)-epoxycycloartane-3 $\beta, 6 \alpha, 16 \beta, 25$-tetrol $=22046$

Diallyldisulfide $=948$

Diallyl trisulfide $=924$

1,9-Diamino-5-azanonane $=9621$

1,4-Diaminobutane $=18230$

4,4'-Diaminobutylamine $=9621$

2,4-Diaminobutyric acid $=5351$

2,6-Diamino-4,5-dihydroxy pyrimidine-5- $\beta$-glucoside $=22466$

1,5-Diaminopentane $=2845$

2,5-Diaminopentanoic acid $=16208$

3'(S),4'(S)-Diangeloyloxy-3',4'-dihydroseselin = 17763

Dibenzocyclooctadiene lignan $=1139$

Di[benzoic acid]2,7,8-trihydroxy-3(-4-hydroxyphenyl)dibenzofuran-1,4-diyl $=21305$

4,6-Dibromo-1,1-dichloro-3,7-dimethyl-2E,7-octadiene $=17564$

4,8-Dibromo-1,1-dichloro-3,7-dimethyl-2E,6E-octadiene $=17563$

4,8-Dibromo-1,1,7-trichloro-3,7-dimethyl-2E,5Z-octadiene $=17565$

1,5 -Di- $O$-caffeoylquinic acid $=4564$

2,4-Dichloro-1,8-dihydroxy-3-methylnaphthalene-8- $O$ - $\beta$ - $D$-glucopyranoside $=16715$

Dicoumarol $=5440$

Dictamnolactone $=15882$

2,10-Dideacetyltaxin $\mathrm{B}^{\prime}=5321$

$(5 ' R)$-3,4-Didehydro- $\beta, \kappa$-caroten-6'-one $=5472$

1,2-Didehydro-6,7-epoxy-3 $\alpha, 16,17$-trimethoxyerythrinan-8-one $=7043$

Diderroside methyl ester $=13905$

rel-(1 $\beta, 2 \alpha)$-Di-(2,4-dihydroxybenzoyl)-rel-(3 $\beta, 4 \alpha)$-di-(4-hydroxyphenyl)-

cyclobutane $=6195$

1,7-Di-(3',4'-dihydroxyphenyl)-4-hepten-3-one $=4930$

3',3'-Di-( $\gamma, \gamma$-dimethylallyl)-2',4'-di-oxo-enolchalcone $=22110$

$3,3^{\prime}$-Di-( $\gamma, \gamma$-dimethylallyl)-2',4,4'-trihydroxychalcone $=6025$

4,9-Dien-eudesmine-13,15-dicarboxylic acid-15- $\beta$ - $D$-glucopyranoside $=15262$

$3 \beta, 20: 15 R, 16 S$-Diepoxy-3 $\alpha$-beyeranol $=691$

$16 \beta, 22 R ; 21,23 S$-Diepoxy-3 $\beta$ - $O$ - $\beta$ - $D$-glucopyranosyloxy-21S,24-dihydroxy-

stigmasta-8,14-dien-28-one $=22409$

$(12 R, 20 S, 24 S)-20,24 ; 20,12-D i e p o x y-25$-hydroxydammaran-3 $\beta$-yl

$O-\beta$ - $D$-glucopyranosyl- $(1 \rightarrow 2)-\beta$ - $D$-xylopyranoside $=9120$

9(13),15(16)-Diepoxy-3 $\alpha$-hydroxy-16-dihydrolabda-14-ene $=17845$

9,13:15,16-Diepoxy-7-hydroxy-14-labden-6-one $=17810$

rel-(7 $\left.\alpha, 7^{\prime} \alpha, 8 \alpha, 8^{\prime} \alpha\right)-7,9^{\prime}: 7^{\prime}, 9-$ Diepoxylignan-3,3',4,4'-tetraol $)=2440$

$16,23 R$ : $16,24 S$-Diepoxy-3 $\beta, 12 \beta, 15 \alpha, 25$-tetrahydroxy-cycloart-7-ene 3-O- $\alpha-L$ arabinopyranoside $=2732$

$20(S), 22(R), 23(S), 24(R)-16 \beta: 23 ; 22: 25$-Diepoxy-3 $\beta, 23,24$-trihydroxy-9,19cyclolanostane-3-O- $\beta$ - $D$-(4-acetyl)xylopyranoside $=20112$

$(22 R, 24 S, 25 R, 26 S)-24,25 ; 22,26$-diepoxy-1 $\alpha, 3 \delta, 26$-trihydroxyergost-5-ene $26-O-\beta-D$-glucopyranoside $=3644$

$16 \beta, 22 R ; 21,23 S$-Diepoxy-3 $\beta, 21 S, 24$-trihydroxystigmasta-8,14-dien-28-one = 22410

2,17-Diethenyl-1,10,19,22,23,24-hexahydro-3,7,13,17-tetramethyl-1,19-dioxo -21H-biline-8,12-dipropanoic acid $=2374$

1,1-Diethoxyethane $=108$

Di-(2-ethylbutyl)phthalate $=2448$

1,3-O-Di-galloyl-4,6-[1',1"-(3',3",4',4"-tetrahydroxydibenzofurandicarboxyl)]- 
$\beta$ - $D$-glucopyranose $=2127$

3- $O$-Digilanidobioside $=7321$

Digiprolactone $=12952$

Digitoxigenin 3- $O$-oleandroside $=2187$

28,31 -Di- $O$ - $\beta$ - $D$-glucopyranosides of $1 \alpha, 3 \beta, 24 \xi, 31$-tetrahydroxy- $24 \xi$-methylcycloartan-28-oic acid $=4539$

6,8-Di- $\beta$-glucopyranosyl-5,7-dihydroxy-2-phenyl-4H-1-benzopyran-4-one $=5531$

3,5 -Di- $C$ - $\beta$-glucopyranosyl-2,4,6,3'-tetrahydroxybenzophenone $=20960$

$3 \beta, 22 \alpha$-Digydroxy-11-oxo- $\Delta^{12}$-olean-ene-27 $\alpha$-methoxy carbonyl-29-oic acid

$(29,22 \alpha)$ lactone $=8855$

3,4-Dihdroxy-trans-cinnamic acid hentriacontanylester $=9365$

3,4-Dihdroxy-trans-cinnamic acid nonacosanylester $=15670$

9-Dihydro-13-acetylbaccatin III $=4726$

2,3-Dihydroambrosin $=4624$

10,11-Dihydro-5,10[1',2']benzeno-5H-dibenzo[a,d]cycloheptene-2,4,7,8,15,

17 -hexol $=3286$

2,3-Dihydro-6,6"'-biluteolin $=9287$

$3 \alpha-19-(S)$-Dihydrocadambine $=5550$

Dihydrocherimolin $=16266$

(-)-Dihydrocubebin $=5572$

23,24-Dihydrocucurbitacin B $=5573$

$(-)$-Dihydrodaidzin $=5580$

9,10-Dihydro-5,9-dihydroxy-8,8-dimethyl-6-(2-methyl-1-oxobutyl)-4-phenyl-

$2 H, 8 H$-benzo[1,2-b:5,6- $b$ ] -dipyran-2-one $=13465$

(2E)-1-[2,3-Dihydro-4,6-dihydroxy-2-(1-hydroxy-1-methylethyl)-7-benzofuranyl]-3-(4-hydroxyphenyl)-2-propen-1-one $=5263$

2,3-Dihydro-5,7-dihydroxy-6-methyl-3-(1a,2,3,3a,8b,8c-hexahydro-6-hydroxy-1,1,3a-trimethyl-1H-4-oxabenzo[f]cyclobut [c,d]inden-7-yl)-4H-1-benzopyran-4-one $=5264$

2,3-Dihydro-5,7-dihydroxy-6-methyl-3-(3-hydroxy-6,6,9-trimethyl-6H-dibenzo[b,d]pyran-2-yl) $4 H$-1-benzopyran-4-one $=14754$

2,3-Dihydro-5,7-dihydroxy-6-methyl-3-(6a,7,8,10a-tetrahydro-3-hydroxy-6,6,

9-trimethyl-6H-dibenzo[ $[b, d]$ pyran-2-yl)-4H-1-benzopyran-4-one $=5265$

22,23-Dihydroergosterol $=3039=14229$

$13 \beta, 21$-Dihydroeuycomanone $=16695$

$\alpha$ - $D$-(6-O-Dihydroferuloyl)-glucuronosyl $(1 \rightarrow 2)-\beta$ - $D$-fructofuranoside $=19658$

6'-O-(6,7-Dihydrofoliamenthoyl)mussaenosidic acid $=751$

$(-)$-Dihydrogenistin $=5621$

2,3-Dihydrogeraniol $=3768$

Dihydroharmine $=9232$

Dihydrohetisine $=9472$

2,3-Dihydro-3"'-hydroxy-6,6"'-biapigenin = 9286

(7S,8R)-7,8-Dihydro-7-(4-hydroxy-3,5-dimethoxyphenyl)-8-hydroxymethyl-

[1'-(7'-hydroxyethyl)-5'-methoxyl] benzofuran-4- $O-\beta$ - $D$-glucopyranoside $=20655$

7a,10a-Dihydro-5-hydroxy-8,8-dimethyl-6-(2-methyl-1-oxobutyl)-4-phenyl-2 $H$-8H-[1,2]-dioxolano[4",5":4',5']furo[2',3':5,6]benzo[1,2-b]pyran-2-one $=13466$

(2E)-1-[2,3-Cihydro-4-hydroxy-2-(1-hydroxy-1-methylethyl)-6-methoxy-5-be nzofuranyl]-3-(4-hydroxyphenyl)-2-propen-1-one $=22767$

Dihydro-4-hydroxy-5-hydroxymethyl-2 $(3 H)$-furanone $=5209$

(2E)-1-(3,4-Dihydro-5-hydroxy-7-methoxy-2,2-dimethyl-2H-1-benzopyran-6- yl)-3-(4-hydroxyphenyl)-2-propen-1-one $=5743$

1-[(9,10-Dihydro-4-hydroxy-2-methoxy-7-phenanthrenyl)oxy]-4,7-dihydroxy-2-methoxy-9,10-dihydrophenanthrene $=17201$

(2R,3S)-2,3-Dihydro-2-(4'-hydroxy-3'-methoxyphenyl)-3-hydroxymethyl-7methoxy-5-benzofuranpropanol 4'- $O-\beta$ - $D$-glucopyranoside $=5583$

2,3-Dihydro-2 $\alpha$-(4-hydroxy-3-methoxyphenyl)-3 $\beta$-methyl-5E-propenylbenzofuran $=16651$

(R)-2,3-Dihydro-3-hydroxy-pyrrolo[2,1- $b]$ quinazolin- $9(1 H)$-one $=22350$

2,3-Dihydro-5-hydroxy-6,8,8-trimethyl-2-phenyl-4H-1-benzopyran-4,7(8H)dione $=3474$

Dihydroirisquinone $=16543$

$11 \alpha, 13$-Dihydroisoalantolactone $=5648$

Dihydrokaempferol $=1764$

2,3-Dihydro-7-methoxy-2 $S^{*}, 3 R^{*}$-dimethyl-3-[4-methyl-5-(4-methyl-2-furyl)$3(E)$-pentenyl]-furo[3,2-c] coumarin $=7982$

2,3-Dihydro-7-methoxy-2 $R^{*}, 3 R^{*}$-dimethyl-3-[4-methyl-5-(4-methyl-2-furyl)$3(E)$-pentenyl]-furo[3,2-c] coumarin $=7983$

2,3-Dihydro-7-methoxy-2R*,3R*-dimethyl-2-[4-methyl-5-(4-methyl-2-furyl)$3(E)$-pentenyl]-furo[3,2-c] coumarin $=7984$

4,9-Dihydro-7-methoxy-1-methyl-3H-pyrido[3,4- $b$ indole $=9232$

Dihydromorelloflavone $=8246$

Dihydromyricetin $=1074$

Dihydronepetalactol $=15434$

Dihydrooroselol $=3936$

Dihydrooroxylin $=5685$

3,7-Dihydropurine-2,6-dione $=22756$

Dihydrosarcostin 3-O- $\beta$ - $D$-thevetopyranosyl-(1 $\rightarrow 4)-\beta$ - $D$-oleandropyranosyl-

$$
(1 \rightarrow 4)-\beta \text { - } D \text {-cymaropyranoside }=6601
$$

1,2-Dihydrotanshinone $=5723$

15,16-Dihydrotanshinone $\mathrm{I}=5722$

15,17-Dihydrotanshinone IIa $=4292$

10,11-Dihydro-2,4,7,8-tetrahydroxy-10-(3,4-dihydroxyphenyl)-5-[(3,5-dihydroxyphenyl)methyl]-5H-dibenzo[a,d]cycloheptene $=3285$

( \pm )-\{(2E)-1-(3,4-Dihydro-3,5,7-trihydroxy-2,2-dimethyl-2H-1-benzopyran-6yl)-3-(4-hydroxyphenyl)-2-propen-1-one $\}=5262$

4",5"-Dihydro-3,5,4'-trihydroxy-4",4",5"-trimethylfurano[2",3":7,8]flavone = 22179 2,3-Dihydro-3,6,9-trimethyl naphtho[1,8-bc]pyran-7-oxa-8-one $=13521$

Dihydrovaltrate $=5482$

14 $\alpha, 19$-Dihydroxyabieta-8,13(15)-dien-16,12-olide $=17160$

14 $\beta, 19$-Dihydroxyabieta- 8,13 (15)-dien-16,12-olide $=17159$

$14 \beta, 19$-Dihydroxyabieta-8,13(15)-dien-16,12-olide 19- $O-\beta$ - $D$-glucopyranoside $=17161$

14 $\beta$,19-Dihydroxyabieta-8,13(15)-dien-16,12-olide 19-O-[2-(4-hydroxy-3,5dimethoxybenzoyl)]- $\beta$ - $D$-glucopyranoside $=17163$

6,12-Dihydroxyabieta-5,8,11,13-tetraen-7-one $=5747$

$7 \beta, 15 \beta$-Dihydroxy-6 $\beta$-acetoxy-7 $\alpha, 20$-epoxy-11 $\beta, 12 \beta$-epoxy-ent-kaur-16-ene $=16177$ $7 \beta, 13 \alpha$-Dihydroxy- $1 \alpha$-acetoxy-7 $\alpha, 20$-epoxy-ent-kaur-16-en-15-one $=6790$ $3 \beta, 11 \beta$-Dihydroxy- $6 \alpha$-acetoxy-ent-kaur-16-en- 15 -one $=13700$

24,25-Dihydroxy-3 $\alpha$-acetoxylanost-8-en-21-oic acid $=7861$

4,5-cis-4,5-Dihydroxy-5-(1-acetoxypentadecyl)-2-cyclopenten-1-one $=431$ 4,5-trans-4,5-Dihydroxy-5-(1-acetoxypentadecyl)-2-cyclopenten-1-one $=429$ $1 \beta, 11 \alpha$-Dihydroxy-6 $\beta$-acetoxy-6,7-seco-6,19-epoxy-7,20-olide-ent-kaur-16en-15-one $=16176$ 
4,5-trans-4,5-Dihydroxy-5-(1-acetoxytridecyl)-2-cyclopenten-1-one $=427$

5,7-Dihydroxy-2-(1-acetyl-2-methoxycarbonylethyl)-chromone $=19428$

3,4-Dihydroxy-allylbenzene-4- $O-\beta$ - $D$-glucopyranoside $=7525$

$3 \alpha, 17 \alpha$-Dihydroxy- $5 \beta$-androstane $=1163$

$3 \alpha, 17 \beta$-Dihydroxy- $5 \beta$-androstane $=1164$

$3 \beta, 17 \alpha$-Dihydroxy- $5 \alpha$-androstane $=1167$

$3 \beta, 22 \alpha$-Dihydroxy-16 $\alpha$-angeloyloxy-28 $\rightarrow 13$-lactone-oleanane-3- $O$ - $[\beta-D$-glucopyranosyl-( $(1 \rightarrow 2)-\alpha-L$-Arabinpyraosyl]-22-O-(6-acetyl)- $\beta$ - $D$-glucopyranoside $=3128$

1,3-Dihydroxy-9,10-anthraquinone $=18224$

1,8 -Dihydroxy-anthraquinone $=3599$

1,3-Dihydroxy-9,10-anthraquinone 3- $O-\beta$ - $D$-glucoside $=22773$

$7 \beta, 19 \alpha$-Dihydroxyarbor-9(11)-en-3-one $=19018$

19-O-(3,4-Dihydroxybenzoyl)-11,12-dihydroxy-20(10 $\rightarrow 5$ )-abeo-abieta-1(10), $6,8,11,13$-tetraene $=17553$

rel-4 $\alpha$-(2,4-Dihydroxybenzoyl)-3 $\beta$-(4-hydroxybenzoyl)-2 $\alpha$-(2,4-dihydroxyphenyl)-5 $\alpha$-(4-hydroxyphenyl)tetrahydrofuran $=15916$

3-(2,4-Dihydroxy-benzoyl)-4-hydroxy-2,7-bis-(4-hydroxy-phenyl)-7,8-dihydro-furo[2,3-f]chromen-9-on $=7823$

4,4"-Dihydroxy-2'-benzoyloxy-5'-methoxy [1,1':4',1"-terphenyl]-3',6'-dione = 21301

1-[3-(2,4-Dihydroxy-benzoyl)-4,5,6-trihydroxy-2-(4-hydroxy-phenyl)-benzofuran-7-yl] -3-(4-hydroxy-phenyl) -propenone $=7822$

3,4-Dihydroxybenzyl aldehyde $=17968$

4,4'-Dihydroxybenzyl ether $=2458$

10-(3,4-Dihydroxybenzyl)-isorhapontigenin $=8898$

8,9-Dihydroxy-4,4-bis-(3,3-dimethylallyl)-6-methyl-2,3-(2,2-dimethylpyrano) anthrone $=9242$

3,3'-Dihydroxy-2,6-bis(4-hydroxybenzyl)-5-methoxybibenzyl = 2456

1,3-Dihydroxy-2,6-bis(3-methyl-2-butenyl)-2,2-dimethylchromeno(5"',6"':8,7) xanthone $=6626$

2,5-Dihydroxy-3,6-bis(3-methylbut-3-en-1-ynyl)benzaldehyde $=20332$

$7,7^{\prime}$-Dihydroxybursehernin $4-\beta$ - $D$-glucoside $=20420$

2,3-Dihydroxybutanedioic acid $=20715$

3,3'-Dihydroxy- $\beta$-carotene-4,4'-dione $=1921$

3,9-Dihydroxy-10-c,c-dimethylallylpterocarpan $=17048$

$3 \alpha, 7 \alpha$-Dihydroxy- $5 \beta$-cholanic acid $=3511$

(25S)-12 $\alpha, 16 \beta$-Dihydroxycholest-4-en-3-one 16- $O-\beta$ - $D$-glucopyranosyl-

$(1 \rightarrow 3)-\beta$ - $D$-glucopyranoside $=16880$

5,7-Dihydroxy-chromone-3- $\alpha$ - $L$-rhamnopyranoside $=22101$

trans-3,4-Dihydroxycinnamic acid $=2887$

6'-(3",4"-Dihydroxycinnamoyl)arbutin $=18878$

$2 \alpha, 12$-Dihydroxycopacamphan-15-one $2-O-\beta$ - $D$-glucopyranoside $=5116$

16,24-Dihydroxycycloart-20,25-dien-3-one diacetate $=1670$

$22 S$-3 $\beta, 16 \alpha$-Dihydroxy-cycloart-24-en-26,22-olide 3- $O-\beta$ - $D$-glucopyranosyl-

$(1 \rightarrow 6)-\beta$ - $D$-glucopyranosyl- $(1 \rightarrow 2)-\alpha$ - $L$-arabinopyranoside $=1550$

$22 S$-3 $\beta, 16 \alpha$-Dihydroxy-cycloart-24-en-26,22-olide-3-O- $\beta$ - $D$-glucopyranosyl$(1 \rightarrow 4)-\beta$ - $D$-glucopyranosyl- $(1 \rightarrow 2)-\alpha$ - $L$-arabinopyranoside $=1551$

16,24-Dihydroxycycloart-25-en-3-one $=1671$

$3 \beta, 12 \beta$-Dihydroxy-dammar-20(22),24-diene-3- $O-\beta$ - $D$-glucopyranoside $=8438$

[20S]-3 $\beta, 20$-Dihydroxy-24-dammaren-12,23-dione-3- $O$-[ $\alpha$ - $L$-rhamnopyranosyl-( $1 \rightarrow 2)$ - $[\alpha-L$-rhamnopyra-nosyl- $(1 \rightarrow 3)]-\alpha-L$-rhamnopyranosyl-

$(1 \rightarrow 6)]-\beta$ - $D$-glucopyranoside $=9182$

20,21-Dihydroxydammar-24-en-3-one $=6610$
$1 \alpha, 3 \beta$-Dihydroxy-7 $\beta, 11 \beta$-diacetoxy-ent-kaur-16-en-15-one $=611$

$1 \alpha, 11 \beta$-Dihydroxy-3 $\beta, 6 \alpha$-diacetoxy-ent-kaur-16-en-15-one $=13705$

$1 \alpha, 11 \beta$-Dihydroxy-3 $\beta, 7 \beta$-diacetoxy-ent-kaur-16-en-15-one $=610$

$2 \alpha, 9 \alpha$-Dihydroxy-10 $\beta, 13 \alpha$-diacetoxy-5 $\alpha$-(3'- $N$-methylamino-3'-phenyl)-propionyloxytaxa-4(20),11-diene $=5138$

$(4 S, 5 R, 6 S)$-5,6-Dihydroxy-5,6-dihydro-4H-cyclopenta-[ $c]$ furan-4-acetic acid $=17484$

$(4 S, 5 R, 6 S)-5,6$-Dihydroxy-5,6-dihydro- $4 H$-cyclopenta- $[c]$ furan-4-acetic acid methyl ester $=17485$

$(4 S, 5 R, 6 S)-5,6$-Dihydroxy-5,6-dihydro-4H-cyclopenta $[c]$ furan-4-ethanol = 17483

$(3 R, 5 R, 7 R)-3,5$-Dihydroxy-5,6-dihydro-6,7-dehydro- $\beta$-ionol $=1338$

7,4'-Dihydroxy-8-[(2"' $\xi, 3$ "'-dihydroxy-3"'-methyl)butyl]-2",2"-dimethyl-3",4"dehydropyrano[1",4":5,6]isoflavone $=22604$

$\pm\{(2 E)$-1-[2,4-Dihydroxy-3-(2,3-dihydroxy-3-methylbutyl)-6-methoxyphenyl]-3-(4-hydroxyphenyl)-2-propen-1-one $\}=22765$

5,7-Dihydroxy-8-(2,3-dihydroxy-3-methylbutyl)-6-(2-methyl-1-oxobutyl)-4phenyl-2H-[1] benzopyran-2-one $=6513$

1,2-Dihydroxy-3-[12-(2,3-dihydroxyphenyl)-(Z)-dodec-4-enyl]benzene $=8361$

1,2-Dihydroxy-3-[12-(2,3-dihydroxyphenyl)dodecyl]benzene $=8360$

1,2-Dihydroxy-3-[16-(2,3-dihydroxyphenyl)-(Z,Z)-hexadeca-5,8-dienyl]benzene $=8365$

1,2-Dihydroxy-3-[14-(2,3-dihydroxyphenyl)-(Z,Z)-tetradeca-3,6-dienyl]benzene $=8364$

1,2-Dihydroxy-3-[14-(2,3-dihydroxyphenyl)-(Z)-tetradec-6-enyl]benzene $=8363$

1,2-Dihydroxy-3-[14-(2,3-dihydroxyphenyl)tetradecyl]benzene $=8362$

2,8-Dihydroxy-1,3-dimethoxyanthraquinone $=18871$

1,8-Dihydroxy-9,10-dimethoxy-[1]benzopyrano-[3,2-c][2]-benzopyran$7(5 H)$-one $=11167$

1,10-Dihydroxy-8,9-dimethoxy-[1]benzopyrano-[3,2-c][2]-benzopyran$7(5 H)$-one $=11168$

2,7-Dihydroxy-4,8-dimethoxy-1,3-dimethyl-9,10-dihydrophenanthrene = 20293

3',7-Dihydroxy-4',6-dimethoxyflavone $=11$

4',5-Dihydroxy-3,7-dimethoxyflavone $=12331$

5,6-Dihydroxy-7,4'-dimethoxyflavone $=12448$

4',5-Dihydroxy-3,7-dimethoxyflavone-4'- $O-\beta$ - $D$-glucopyranoside $=3027$

5,7-Dihydroxy-6,4'-dimethoxyisoflavone $=11171$

5,7-Dihydroxy-6,4'-dimethoxyisoflavone-7- $O-\alpha$ - $D$-glucopyranoside $=11172$

1,6-Dihydroxy-7,8-dimethoxy-2-methyl-9,10-anthraquinone $=18828$

1,6-Dihydroxy-3,5-dimethoxy-2-(3-methylbut-2-enyl)xanthone $=4197$

1,6-Dihydroxy-3,7-dimethoxy-2-(3-methylbut-2-enyl)-xanthone $=4196$

2,5-Dihydroxy-1,7-dimethoxy-6-methyl-9,10-dihydrophenanthrene $=20292$

2,7-Dihydroxy-1,5-dimethoxy-6-methyl-9,10-dihydrophenanthrene $=20291$

$(2 R, 3 R)-3,4$ '-Dihydroxy-5,7-dimethoxy-8-methylflavan $=22115$

1,4-Dihydroxy-7,8-dimethoxy-2-methyl-5,6-methylenedioxyanthraquinone $=18875$ trans-3,3'-Dihydroxy-2',5-dimethoxystilbene $=17198$

\{5,5'-Dihydroxy-8-( $\gamma, \gamma$-dimethylallyl)-[6",6"-dimethyl-4",5"-dihydropyrano(2",3":7,6)]-[6"'",6"''-dimethylpyrano-(2"'",3"'":4',3')]\}-coumaronochromone $=7891$

5,7-Dihydroxy-8-( $\gamma, \gamma$-dimethylallyl)-[6"',6"'-dimethyl-4"',5"'-dihydropyrano$\left.\left(2^{\prime \prime \prime}, 3^{\prime \prime \prime}: 4^{\prime}, 3^{\prime}\right)\right]$-flavanone $=7486$

2',4'-Dihydroxy-8- $\gamma, \gamma$-dimethylallyl-2",2"-dimethylpyrano-[5,6:6,7]isoflavan = 7357 
4',7-Dihydroxy-8-(3,3-dimethylallyl)flavan $=2627$

7,4'-Dihydroxy-8-( $\gamma, \gamma$-dimethylallyl)-2" $\xi$-(4"-hydroxyisopropyl)dihydrofurano

$[1 ", 3 ": 5,6]$ isoflavone $=22603$

5,7-Dihydroxy-6( $\gamma, \gamma$-dimethylallyl)-8-(2"'-hydroxy-3"'-methylbut-3"'-enyl)-4'-

(1"-hydroxymethylpenta cosanyl)isoflavone $=11015$

7,4 '-Dihydroxy-3'- $\gamma, \gamma$-dimethylallyl isoflavone $=15342$

5,7-Dihydroxy-6,8-di(3-methylbut-2-enyl)flavanone $=20170$

6,8-Dihydroxy-2,7-dimethyl-4H-chromen-4-one $=6622$

7,4'-Dihydroxy-2",2"-dimethyl-3",4"-dehydropyrano[1",4":5,6]flavone = 22605

7,3'-Dihydroxy-6",6"-dimethyl-4",5"-dehydropyrano [2",3":4',5']isoflavone = 7306

$\left\{5,5^{\prime}\right.$-Dihydroxy-8-[6",6"-dimethyl-4",5"-dihydropyrano-(2",3":7,8)]-[6"',6"''-

dimethylpyrano-(2"', $\left.\left.\left.3^{\prime \prime \prime}: 4^{\prime}, 3^{\prime}\right)\right]\right\}$-coumaronochromone $=7892$

1,5-Dihydroxy-6',6'-dimethyldihydropyrano(2',3':3,2)-6",6"'-dimethylpyrano-

$\left(2^{\prime \prime}, 3 ": 6,7\right)$ xanthone $=15580$

$N$-(2,4-Dihydroxy-3,3-dimethyl-1-oxobutyl)- $\beta$-alanine $=22555$

$5,2^{\prime}$-Dihydroxy-[(6",6"-dimethylpyrano(2",3":4',5')][(6"',6"'-dimethylpyrano

$\left.\left(2^{\prime \prime \prime}, 3 " ': 7,6\right)\right]$-isoflavone $=12291$

1,7-Dihydroxy-6',6'-dimethylpyrano(2',3':3,2)-6",6"-dimethylpyrano

$(2 ", 3 ": 6,5)$ xanthone $=15582$

7,2'-Dihydroxy-6",6"-dimethylpyrano-(2",3":4',3')isoflavone = 8501

1,5-Dihydroxy-6', $6^{\prime}$-dimethylpyrano(2',3':3,2)xanthone $=5184$

1,7-Dihydroxy-6',6'-dimethylpyrano( $\left(2,33^{\prime}: 3,4\right)$ xanthone $=15579$

1,7-Dihydroxy-6',6'-dimethylpyrano(2',3':6,5)xanthone $=15581$

1-[5,7-Dihydroxy-2,2-dimethyl-6-(2,4,6-trihydroxy-3-isobutyryl-5-methyl-

benzyl)-2H-chromen-8-yl]-2-methyl-butan-1-one $=13433$

(7S,8R)-4,9'-Dihydroxy-3,3'-dimethyoxyl-7,8-dihydrobenzofunan-1'-propanol

neolignan-9-O- $\alpha-L$-rhamnopyranoside $=13586$

$1 \alpha, 20 R$-Dihydroxyecdysone $=10063$

$5 \beta, 20 R$-Dihydroxyecdysone $=10066$

$1 \alpha, 20 R$-Dihydroxyecdysone 3-O- $\beta$ - $D$-glucoside $=10064$

$2,20(S)$-dihydroxy-16 $\alpha, 23(R)$-epoxycucurbita-5,24-diene-3,11-dione 2- $O-\beta$ glucopyranoside $=12214$

2,20(S)-dihydroxy-16 $\alpha, 23(S)$-epoxycucurbita-5,24-diene-3,11-dione 2- $O-\beta$ glucopyranoside $=12215$

$\left(1 R^{*}, 4 S^{*}, 5 R^{*}, 6 S^{*}, 7 R^{*}, 10 R^{*}\right)-4,6$-Dihydroxy-7,10-epoxy-1,5-trans-guaiane = 16192

(2R)-Dihydroxy-3,10-epoxy-8-isobutyloxygermacra-11-(13)-en-6,12-olide = 10769

16(S)-6 $\beta, 17$-Dihydroxy-3 $\alpha, 20$-epoxy-ent-kaur-1,7,15-trione $=12588$

$3 \beta, 17 \beta$-Dihydroxy-14,20-epoxy-1-oxo-22 $R$-witha-5,24-dienolide $=812$

$1 \beta, 6 \alpha$-Dihydroxy-eudesman-4(15)-ene $=7504$

$1 \beta, 5 \alpha$-Dihydroxyeudesman-4(15)-ene $=7505$

$1 \beta, 7 \alpha$-Dihydroxyeudesman-4(15)-ene $=7506$

$(1 R, 4 S, 5 R, 7 R, 10 S)$-1,11-Dihydroxy-eudesman-14,4-olide 11-O- $\beta$ - $D$-glucopyranoside $=3378$

$1 \beta, 11$-Dihydroxy-eudesman-4,14-oxide $11-O-\beta$ - $D$-glucopyranoside $=3379$

$1 \beta, 6 \beta$-Dihydroxy-cis-eudesm-3-ene-6- $O-\beta$ - $D$-glucopyranoside $=12919$

$(1 R, 5 R, 6 S, 7 S, 9 S, 10 S, 11 R)$-1,9-Dihydroxyeudesm-3-en-12,6-olide 9-O- $\beta$ - $D$ glucopyranoside $]=4359$

$(2 S)-7,4^{\prime}$-Dihydroxyflavan $=5894$

4,7-Dihydroxyflavanone $=12908$

5,7-Dihydroxyflavone $=3600$

$4 \alpha, 12 \alpha$-Dihydroxy-8(17)-fusicoccene $=8034$
9,14-dihydroxy-1(10),4-germacratrien-12,8-olide[1(10)E,4E,8 $\alpha, 9 \beta]=20149$

2,4-Dihydroxy-10(14),11(13)-guaiadien-12,8-olide $=7836$

$4 \beta, 5 \beta$-Dihydroxy-7(11),9-guaiadien- 8 -one $=17874$

$2 \alpha, 21 \beta$-Dihydroxyhederagenin-3-O- $\beta$ - $D$-glucopyranoside $=13062$

11,13-Dihydroxyhetisan-2-one $=9472$

4,5-cis-4,5-Dihydroxy-5-(1-hydroxyheptadecyl)-2-cyclopenten-1-one $=10838$

5,7-Dihydroxy-2-(3-hydroxy-4-methoxyphenoxy)chromone $=20936$

5,7-Dihydroxy-2-(3-hydroxy-4-methoxyphenoxy)-6-methoxychromone $=20934$

5,7-Dihydroxy-2-(4-hydroxy-3-methoxyphenoxy)-6-methoxychromone $=20935$

1-[2',4'-Dihydroxy-5'-(2"-hydroxy-3"-methyl-3"-butenyl)-6'-methoxy]acetophenone $=574$

1-[2,4-Dihydroxy-3-(4-hydroxy-3-methyl-2-butenyl)-6-methoxyphenyl]-3-(4hydroxyphenyl)-2-propen-1-one $=10830$

5,7-Dihydroxy-8-(2-hydroxy-3-methylbut-3-enyl)-6-(2-methyl-1-oxobutyl)-4propyl-2H-[1]benzopyran-2-one $=6516$

5,7-Dihydroxy-8-(2-hydroxy-3-methylbut-3-enyl)-6-(2-methyl-1-oxopropyl)4-phenyl-2 $H$-[1]benzopyran-2-one $=6515$

(2E)-1-[2,4-Dihydroxy-3-(3-hydroxy-3-methylbutyl)-6-methoxyphenyl]-3-(4hydroxyphenyl)-2-propen-1-one $=22766$

$(2 R)$-2-\{(1S,2S)-1,2-Dihydroxy-8-[(2R,3R,4R,5R)-5-(2-hydroxymethyl-3,4dihydroxy-1-acetylpyrrolidinyl)]octyl $\}$ piperidine $=2636$

$(2 R)$-2-\{(1S,2S)-1,2-Dihydroxy-8-[(2R,3R,4R,5R)-5-(2-hydroxymethyl-3,4dihydroxypyrrolidinyl $)]$ octyl $\}$ piperidine $=2637$

1,8-Dihydroxy-3-hydroxymethyl-6-methoxyanthraquinone $=7710$

(10RS,11RS)-(2E,6Z,8E)-10,11-Dihydroxy- $N$-(2-hydroxy-2-methylpropyl)2,6,8-dodecatrienamide $=23026$

(6RS,11RS)-(2E,7E,9E)-6,11-Dihydroxy- $N$-(2-hydroxy-2-methylpropyl)2,7,9-dodecatrienamides $=23027=23028$

4,5-cis-4,5-Dihydroxy-5-(1-hydroxypentadecyl)-2-cyclopenten-1-one $=10837$ 6,7-Dihydroxy-3-(2-hydroxy-propyl)-7-methyl-1,5,6,7-tetrahydro-isochro-

men-8-one $=14915$

4,5-trans-4,5-Dihydroxy-5-(1-hydroxytridecyl)-2-cyclopenten-1-one $=10836$

(Z)-(-)-3,6-Dihydroxy-2-icos-14-enoyl-cyclohex-2-enone $=20495$

22,23 -Dihydroxy-iridal-3, 16 -di- $\beta$ - $D$-glucopyranoside $=11140$

4',7-Dihydroxyisoflavone $=4604$

22,23-Dihydroxy-isoiridal-3,16-di- $\beta$ - $D$-glucopyranoside $=11139$

$16 \alpha, 17$-Dihydroxy-ent-kauran-19-oic acid 16-O- $\beta$-D-glucopyranoside $19-O$ -

$\beta$ - $D$-glucopyranosyl ester $=93$

3 $\beta, 25$-Dihydroxylanosta-8,23Edien-21-oic acid $=19321$

3,24-Dihydroxylanosta-8,25-dien-21-oic acid $=19322$

$3 \beta, 24 R$-Dihydroxylanosta-8,25-dien-21-oic acid $=19323$

(7S,8R)9,9'-Dihydroxyl-3,3'-dimethyoxyl-7,8-dihydrobenzofunan-1'-propanol neoligan-4- $O-\alpha-L$-rhamnopyranoside $=13585$

$(2 R)-7,4$ '-Dihydroxylflavan $=22116$

2,6-Dihydroxyl-3-geranyl-5-isoprenyl-2,5-dihexadiene-1,4-dione $=7223$

15,19-Dihydroxyl 8(17)-13(E)-labdatriene $=12402$

$(8 R)-3 \beta, 4 \beta$-Dihydroxyl-14-oxo- $5 \alpha$-15(14 $\rightarrow 8)$-abeo-card-20(22)-enolide $=8005$

1-(2',3'-Dihydroxylphenyl)-2- $n$-heptyl-1-nonene-3-one $=18823$

$3 \alpha, 11 \alpha$-Dihydroxylup-23-al-20(29)-en-28-oic acid 28-O- $\alpha$ - L-rhamnopyra-

nosyl- $(1 \rightarrow 4)-\beta$ - $D$-glucopyranosyl- $(1 \rightarrow 6)-\beta$ - $D$-gluco-pyranosyl ester $=74$

$2 \alpha, 3 \beta$-Dihydroxylup-20(29)-en-28-oic acid $=984$

3,19-Dihydroxy-20(29)-lupen-12-one $=3842$

$(3 S, 5 R, 6 S, 9 R)-3,9$-Dihydroxymegastigmane di- $O-\beta-D$-glucopyranoside $=17521$ 
$(2 R, 9 R)-2,9-$ Dihydroxymegastigman-5-ene 9- $O-\beta$ - $D$-glucopyranoside $=17527$ $(3 S, 9 R)$-3,9-Dihydroxymegastigman-5-ene 3-O-primeveroside $=17525$

$(3 S, 4 S, 6 S, 7 E)$-3,4-Dihydroxymegastigman-7-en-9-one-4- $O-\beta$ - $D$-glucopyranoside $=12530$

$(2 S, 9 R)$-2,9-Dihydroxymegastigman-5-en-4-one 2-O- $\beta$ - $D$-glucopyranoside $=22129$ 6,6'-Dihydroxy-4'-methoxy-2-arylbenzofuran $=2539$

2,5-Dihydroxy-4-methoxybenzophenone $=3346$

2,6-Dihydroxy-4-methoxy-3,5-bis(3-methyl-2-butenyl)benzophenone $=22550$

(3R)-7,4'-Dihydroxy-2'-methoxy-6,8-di $(\gamma, \gamma$-dimethylallyl)isoflavanone $=7355$

(6aS,11aS)-3,6a-Dihydroxy-9-methoxy-4,10-di( $\gamma, \gamma$-dimethylallyl)pterocarpan $=7358$

1,5-Dihydroxy-3-methoxy-4-(2,3-dihydroxy-3-methylbutyl) $x$ anthone $=15576$

(-)-7,3'-Dihydroxy-4'-methoxy-5'-( $\gamma, \gamma$-dimethylallyl)flavanone $=7307$

7,3'-Dihydroxy-4'-methoxy-5'-( $\gamma, \gamma$-dimethylallyl)isoflavone $=7305$

3,6a-Dihydroxy-9-methoxy-10- $\gamma, \gamma$-dimethylallylpterocarpan $=4243$

5,7-Dihydroxy-4'-methoxyflavone $=56$

4',5-Dihydroxy-7-methoxy flavone $=8289$

5,4'-Dihydroxy-7-methoxyflavone 8-C-glucopyranoside $=11724$

5,7-Dihydroxy-4'-methoxyflavone-7- $O-\alpha-L$-rhamno-pyranosyl-( $1 \rightarrow 2)-[\alpha-L$ -

rhamnopyranosyl- $(1 \rightarrow 6)]-\beta$ - $D$-glucopyranoside $=15347$

1,5-Dihydroxy-3-methoxy-4-(3-hydroxy-3-methylbuty1)-6',6'-dimethylpyrano- $\left(2^{\prime}, 3^{\prime}: 6,7\right)$ xanthone $=15575$

1,5-Dihydroxy-3-methoxy-4-(3-hydroxy-3-methylbutyl)xanthone = 15574

7,2'-Dihydroxy-4'-methoxyisoflav-3-ene $=16545$

5,7-Dihydroxy-4'-methoxyisoflavone $=2384$

7,3'-Dihydroxy-4'-methoxyisoflavone $=3004$

2',7-Dihydroxy-4'-methoxyisoflavone $=22783$

7,8-Dihydroxy-4'-methoxyisoflavone8- $O-\beta$-glucopyranoside $=5222$

7,8-Dihydroxy-4'-methoxyisoflavone 7-O-[ $\alpha$-rhamnopyranosyl-( $(1 \rightarrow 6)]-\beta$ glucopyranoside $=5223$

2,4-Dihydroxy-6-methoxy-3-methyl acetophenone 4-O- $\beta$ - $D$-xylopyranosyl-

$(1 \rightarrow 6)-\beta$ - $D$-glucopyranoside $=6668$

2,8-Dihydroxy-1-methoxy-3-methylanthraquinone $=15900$

1,7-Dihydroxy-6-methoxy-2-methylanthraquinone $=18872$

5,7-Dihydroxy-4'-methoxy-3'-(3-methylbutadienyl)-5'-(3-methylbut-2-enyl)avanone $=2774$

1-[2',4'-Dihydroxy-6'-methoxy-3'-(3"'-methylbutanoyl)-5'-(3"-methylbut-2"enyl) $]$ acetophenone $=573$

1,6-Dihydroxy-7-methoxy-8-(3-methylbut-2-enyl)6',6'-dimethylpyrano(2',3':3, 2) $x$ anthone $=1277$

1,5-Dihydroxy-3-methoxy-2-(3-methylbut-2-enyl)-xanthone $=5925$

1,7-Dihydroxy-3-methoxy-2-(3-methylbut-2-enyl)xanthone $=5926$

1,6-Dihydroxy-5-methoxy-7-(3-methylbut-2-enyl)xanthone $=8556$

2',2"-Dihydroxy-4'-methoxy-4",5"-methylenedioxybenzil = 19453

5,4'-Dihydroxy-3'-methoxy-6,7-methylenedioxyisoflavone $=11152$

9,10-Dihydroxy-8-methoxy-3,4-methylenedioxy-phenanthrene-1-carboxylic acid lactone $=1724$

$6 \beta, 11 \alpha$-Dihydroxy-16 $\alpha$-methoxymethyl-6,20-epoxy-6,7-seco-ent-kaur-15-one $-1,7$-olide $=20620$

$(2 R, 3 R)-3,4$ '-Dihydroxy-7-methoxy-8-methylflavan $=22114$

5,7-Dihydroxy-8-methoxy-6-methyl-3-(2'-hydroxy-4'-methoxybenzyl)chroman-4-one $=16140$

3,5-Dihydroxy-7-methoxy-2-phenyl-4H-1-benzopyran-4-one $=11806$

1-(2,6-Dihydroxy-4-methoxyphenyl)-11-(2,6-dihydroxyphenyl)undecan-1-one
$=1647$

2-[3',4'-Dihydroxy-2'-methoxyphenyl]-4-hydroxy-6-methoxy-benzofuran-3-ca rbaldehyde $=3007$

4',7-Dihydroxy-3'-methoxy-5'-prenylflavanone $=5146$

1,6-Dihydroxy-5-methoxyxanthone $=2694$

$8 \beta$-(2',3'-Dihydroxy-2'-methybutanoxy)-2 $\alpha$-hydroxy- $6 \beta H, 7 \alpha H$-germacra1(10)- $E, 4 E, 11(13)$-triene-6,12-olide $=7547$

5,7-Dihydroxy-8-methyl-6-aldehydo-3-(4'-methoxybenzyl) chromone $=876$

1,8-Dihydroxy-3-methyl-9 $(10 H)$-anthracenone-10-oxyhexadecanoate $=12235$

1,8-Dihydroxy-3-methyl-9 $(10 H)$-anthracenone-10-oxytetradecanoate $=12234$

1,8-Dihydroxy-3-methyl-9-anthrone $=3598$

1,6-Dihydroxy-5-(3-methyl-2-butenyl)-2',2'-dimethylchromeno(5',6':2,3)-2"',

2"'-dimethylchromeno $\left(5^{\prime \prime}, 66^{\prime \prime}: 8,7\right)$ xanthone $=6629$

1,7-Dihydroxy-8-(3-methylbut-2-enyl)-6',6'-dimethylpyrano(2',3':3,2)-xanthone = 5064

5,4'-Dihydroxy-6-(3'"-methylbut-2"'-enyl)-2"-hydroxyisopropyl dihydrofurano[4",5":8,7]isoflavone $=11711$

1,6-Dihydroxy-2-(3-methyl-2-butenyl)-3-methoxy-2",2"-dimethylchromeno $(5 ", 6 ": 8,7)$-xanthone $=6631$

6-((S)-3,4-Dihydroxy-2-methylenebutanoate $)-D$-glucopyranose $=22106$

1,8-Dihydroxy-9,10-methylenedioxy-[1]benzopyrano-[3,2-c][2]-benzopyran$7(5 H)$-one $=11169$

5,4'-Dihydroxy-6,7-methylenedioxyisoflavone $=11158$

$3 \alpha, 15 \alpha$-Dihydroxy-24-methylene-lanosta-7,9(11)-dien-21-oic acid $=7857$

$3 \alpha, 15 \alpha$-Dihydroxy-24-methylene-lanost- 8 -en-21-oic acid $=20480$

1,8-Dihydroxy-3-methyl-6-methoxy-9 $(10 H)$-anthracenone-10-oxydecanoate = 12233

1-[5,7-Dihydroxy-2-methyl-6-(3-methyl-but-2-enyl)-2-(4-methyl-pent-3enyl)-2 $H$-chromen-8-yl]-3-(3,4-dihydroxy-phenyl)-propenone $=13437$

3,5-Dihydroxy-2-methyl-1,4-naphthoquinone $=6605$

5,7-Dihydroxy-8-(2-methyl-1-oxobutyl)-4-phenyl-2H-[1]benzopyran-2-one $=11380$

1,4-Dihydroxy-2-(4-methylpent-3-enyl)-anthraquinone $=1369$

( $r e l)-4,6$-Dihydroxy-5-[3-methyl-(E)-propenoic acid-3-yl]-7 $\beta$-glucopyranosyl$\{2 \alpha, 3 \beta$-dihydrobenzofuran $\}$-(3,2:b)-[4 $\alpha, 5 \beta$-dihydroxy-6a-hydroxymethyl tetrahydropyran] $=18513$

5,8-Dihydroxy-1,4-naphthoquinone $=15253$

16 $\beta, 17$-Dihydroxy-18-nor-ent-kauran- $4 \beta$-hydroperoxide $=1331$

3,24-Dihydroxy-30-nor-12,20(29)-oleanadien-28-oic acid = 18419

$3 \beta, 23$-Dihydroxy-30-norolean-2,20(29)-dien-28-oic acid 3- $O-\alpha-L$-arabinopyranosyl-( $1 \rightarrow 2)$-[ $\beta$ - $D$-glucopyranosyluronic acid- $(1 \rightarrow 3)]-\alpha-L$-arabinopyranoside $=7725$

3 $\beta$,23-Dihydroxy-30-norolean-12,20(29)-dien-28-oic acid 3- $O$-methyl $\beta$ - $D$ glucopyranosyluronate- $(1 \rightarrow 3)-\alpha-L$-arabinopyranoside $=7726$

$3 \beta, 20 \alpha$-Dihydroxy-29-norolean-12-en-28-oic acid 3-O- $\beta$ - $D$-xylopyranosyl$(1 \rightarrow 2)-\beta$ - $D$-glucopyranoside $=7723$

$3 \beta, 17 \beta$-Dihydroxy-28-norolean-12-en-16-one 3- $O$-[ $\alpha-L$-rhamopyranoside$(1 \rightarrow 2)-\beta$ - $D$-glucuronopyranoside $]=20429$

$2 \beta, 7 \beta$-Dihydroxynortropane $=7299$

$3 \beta, 16 \alpha$-Dihydroxy-olean-12-en-28-al-3-O- $\beta$ - $D$-glucopyranosyl-23-O- $\alpha-D$ ribofuranoside $=3073$

$3 \beta, 16 \alpha$-Dihydroxyolean-12-en-23,28-dioic acid 28- $O$-[ $\beta$ - $D$-glucopyranosyl $(1 \rightarrow 3)][\beta$ - $D$-glucopyranosyl $(1 \rightarrow 6)]-\beta$ - $D$-glucopyranosyl ester $=19936$

$3 \beta, 16 \alpha$-Dihydroxyolean-12-en-23,28-dioic acid 28- $O$-[ $\beta$ - $D$-glucopyranosyl $(1 \rightarrow 3)][\beta-D-6-O$-(3-hydroxy-5-methoxy-3-methyl-5-oxopentanoyl)gluco 
pyranosyl $(1 \rightarrow 6)]-\beta$ - $D$-glucopyranosyl ester $=19938$

$3 \beta, 16 \alpha$-Dihydroxyolean-12-en-23,28-dioic acid 28- $O$-[ $\beta$ - $D$-glucopyranosyl

$(1 \rightarrow 3)][\beta-D-6-O-((3 R)-3$-hydroxy-3-methylglutaryl)glucopyranosyl

$(1 \rightarrow 6)]-\beta$ - $D$-glucopyranosyl ester $=19937$

$3 \beta, 30$-Dihydroxy-olean-12-en-23,28-dioic acid 28- $O$ - $\alpha$ - $L$-rhamnopyranosyl-

$(1 \rightarrow 4)-\beta$ - $D$-glucopyranosyl- $(1 \rightarrow 6)]-\beta$ - $D$-glucopyranoside $=68$

$3 \beta, 23$-Dihydroxy-olean-12-ene-28,29-dioic acid 28- $O-\alpha$ - $L$-rhamnopyranosyl-

$(1 \rightarrow 4)-\beta$ - $D$-glucopyranosyl-( $1 \rightarrow 6)]-\beta$ - $D$-glucopyranoside $=67$

$3 \beta, 16 \alpha$-Dihydroxyolean-12-ene-28-oic acid 3- $O-\beta$ - $D$-glucopyranoside $=6705$

$3 \beta, 22 \alpha$-Dihydroxyolean-12-en-29-oic acid $=27$

2,3-Dihydroxy-12-oleanen-28-oic acid $=4219$

$3 \beta, 29$-Dihydroxyolean-12-en-28-oic acid $=13799$

$3 \beta, 23$-Dihydroxy-olean-12-en-28-oic acid 3- $O$-methyl $\beta$ - $D$-glucopyranosyl-

uronate- $(1 \rightarrow 3)-\alpha$ - $L$-arabinopyranoside $=7727$

$3 \beta, 15 \alpha$-Dihydroxy-olean-12(13)-en-16-one $=6061$

$16 \alpha$-28-dihydroxyolean-12-en-21-one-3- $O-\alpha$ - $L$-rhamnopyranosyl-( $1 \rightarrow 2)-\beta-D$ -

galactopyranosyl- $(1 \rightarrow 3)[\beta$ - $D$-glucopyranosyl- $(1 \rightarrow 2)-\beta$ - $D$-glucuronopyra noside $]=2315$

$8 \beta, 15$-Dihydroxy-2-oxo-guaia-1(10),3,11(13)-trien-5 $\alpha, 6 \beta, 7 \alpha H$-12,6-olide $=12446$

24S,25-Dihydroxy-3-oxolanost-8-en-21-oic acid $=7860$

$3 \beta, 24 \alpha$-Dihydroxy-16-oxo-oleana-11,13(18)-dien-30-oic acid $=8861$

$3 \beta, 28$-dihydroxy-16-oxo-12-oleanene $=19466$

$3 \beta, 24$-Dihydroxy-16-oxo-olean-12-en-29-oic acid $=8857$

3,16-Dihydroxy-23-oxo-12-oleanen-28-oic acid $=18418$

$6 \beta, 28$-Dihydroxy-3-oxoolean-12-en-23-oic acid 23- $O$ - $\alpha$ - $L$-arabinopyranosyl

ester $=8131$

$21 \beta, 22 \beta$-Dihydroxy-3-oxours-12-en-28-al $=4042$

$3 \alpha, 19 \alpha$-Dihydroxy-2-oxo-12-ursen-28-oic acid $=16410$

20 $\beta, 27$-Dihydroxy-1-oxo-(22R)-witha-2,5,24-tetraenolide $=22699$

$3 \beta, 7 \beta$-Dihydroxy-4 $\alpha-4 \beta, 8 \beta, 10 \beta, 14 \alpha$-pentamethyl-5 $\alpha$-gon-16-en-2-one 3- $O$ - $[\beta$ -

$D$-glucopyranoside- $(1 \rightarrow 2)-\beta$ - $D$-glucopyranoside $]=20428$

3,4-Dihydroxy- $\beta$-phenethanol-3- $O-\beta$ - $D$-galactopyranoside $=3653$

3,4-Dihydroxy- $\beta$-phenethanol-3- $O-\beta$ - $D$-glucopyranoside $=3654$

3 ,4-Dihydroxy- $\beta$-phenethyl- $O$ - $\beta$ - $D$-glucopyranosyl-( $1 \rightarrow 3)-4-O$-caffeoyl- $\beta$ - $D$ -

glucopyranoside $=18219$

3,4-Dihydroxy- $\beta$-phenethyl- $O$ - $\beta$ - $D$-gluco-pyranosyl-( $1 \rightarrow 3)-O-\alpha$ - $L$-rhmnopyra

nosyl-( $1 \rightarrow 6)-4-O$-caffeoyl- $\beta$ - $D$-glucopyranoside $=18220$

2-(3,4-Dihydroxyphenethyl)- $O-\alpha$ - $L$-lyxopyranosyl-( $1 \rightarrow 2)-\alpha-L$-rhamnopyra-

nosyl-(1 $\rightarrow 3)$-4-O-3,4-dimethoxy-trans-cinnamoyl- $\beta$ - $D$-glucopyranoside

$=21214$

2-(3,4-Dihydroxyphenethyl)- $O$ - $\alpha$ - $L$-lyxopyranosyl-( $1 \rightarrow 2)-\alpha-L$-rhamnopyrano syl- $(1 \rightarrow 3)-4-O$-trans-feruloyl- $\beta$ - $D$-glucopyranoside $=21215$

3-(2,4-dihydroxyphenoxy)-8-(3,3-dimethylallyl)-2,2-dimethylpyrano[5,6:6,7] chromen-4-one $=7351$

3-(2,4-Dihydroxyphenoxy)-7-hydroxy-6,8-di(3,3-dimethylallyl)chromen-4one $=7350$

(2,5-Dihydroxyphenyl)acetic acid $=9608$

2-(3,4-Dihydroxyphenylacetyl)- $\beta$ - $D$-glucopyranosyl (E)-2-methyl-but-2-en-

$4-\mathrm{ol}=10854$

$L-3,4-$ Dihydroxyphenylalanine $=6558$

2-(3,4-Dihydroxyphenyl)-4,6-dihydroxybenzofuran-3-carboxylic acid methyl ester $=16246$

3-(3,4-Dihydroxy-phenyl)-1-[6-(3,7-dimethyl-octa-2,6-dienyl)-5,7-dihydroxy- 2,2-dimethyl-2 $H$-chromen-8-yl]-propenone $=13436$

1,2-O-[2S-(3,4-Dihydroxyphenyl)-1,2-ethanediyl]-3- $O-\alpha$ - $L$-rhamnopyranosyl4- $O$-feruloyl- $\beta$ - $D$-glucopyranoside $=14261$

$7 \beta$-(3,4-Dihydroxyphenyl)-ethane 7,8-(2',1'-O- $\beta$ - $D$-glucopyranosyl)-7,8-diol $=4362$

$7 \alpha$-(3,4-Dihydroxyphenyl)-ethane 7,8-(2',1'-O- $\beta$ - $D$-glucopyranosyl)-7,8-diol $=4363$

2 -(3,4-Dihydroxy) phenyl ethanol $(1 \rightarrow 1)(2 \rightarrow 2)$-[(1 $\rightarrow 3)$-rhamnopyranosyl-

4-O-caffeoyl] glucoside $=16161$

2-(3,4-Dihydroxyphenyl)- $R, S$-2-ethoxy-ethyl- $O-\beta$ - $D$-glucopyranosyl $(1 \rightarrow 4)-\alpha-L$ rhamnopyranosyl $(1 \rightarrow 3)(4-O$-trans-caffeoyl $)-\beta$ - $D$-glucopyranoside $=18927$

3,4-Dihydroxyphenylethyl alcohol (2-O-feruloyl)- $\beta$ - $D$-glucopyranoside $=2085$

2-(3,4-Dihydroxyphenyl)ethyl $O$ - $\beta$-apiofuranosyl- $(1 \rightarrow 6)-O$-[ $O-\beta$-apiofuranosyl-( $1 \rightarrow 4)-\alpha$-rhamnopyranosyl- $(1 \rightarrow 3)]-4-O-(E)$-caffeoyl- $\beta$-glucopyranoside $=13074$

2-(3,4-Dihydroxyphenyl) ethyl- $O-\beta$ - $D$-apiofuranosyl-(1" $\left.\rightarrow 6^{\prime}\right)-\beta$ - $D$-glucopyranoside $=4364$

2-(3,4-Dihydroxyphenyl)ethyl- $O$ - $\alpha$ - $L$-arabinopyranosyl-( $1 \rightarrow 2)$-[ $\alpha$ - $L$-rhamnopyranosyl-( $1 \rightarrow 3)]$-(4-O-trans-feruloyl)- $\beta$ - $D$-glucopyranoside $=22416$

3,4 -Dihydroxy- $\beta$-phenylethyl caffeate, 9'-Decarboxyrosmarinic acid $=21216$

[2-(3,4-Dihydroxyphenylethyl)]-3- $O-\alpha-D$-apiofuranosyl-( $1 \rightarrow 4)-(4-O$-caffeoyl)- $\beta$ - $D$-glucopyranoside $=4419$

2-(3,4-Dihydroxy-phenyl)-ethyl- $O-\beta$ - $D$-glucopyranoside $=6081$

2-(3,4-dihydroxyphenyl)-ethyl- $O$ - $\beta$ - $D$-glucopyranosyl-( $1 \rightarrow 6)$-3-O-transcaffeoyl- $\beta$ - $D$-glucopyranoside $=11513$

2-(3,4-Dihydroxyphenyl)ethyl- $O-\beta$ - $D$-glucopyranosyl-( $1 \rightarrow 2)$ - $[\alpha-L$-rhamnopyranosyl- $(1 \rightarrow 3)]$-(4-O-trans-caffeoyl)- $\beta$ - $D$-glucopyranoside $=22417$

2 -(3,4-Dihydroxyphenyl)ethyl- $O-\beta$ - $D$-glucopyranosyl-( $1 \rightarrow 2)$ - $[\alpha-L$-rhamnopyranosyl-( $1 \rightarrow 3)]$-(4-O-trans-feruloyl)- $\beta$ - $D$-glucopyranoside $=22418$

[2-(3,4-Dihydroxyphenylethyl)]-3-O- $\beta$ - $D$-xylopyranosyl-( $1 \rightarrow 3)-(4-O$ caffeoyl) $\beta-D$-glucopyranoside $=4420$

7-(3,4-Dihydroxyphenyl)-5-hydroxy-1-(4-hydroxyphenyl)-3-heptanone-5-O$\beta$ - $D$-xylopyranoside $=959$

1-(3,4-Dihydroxyphenyl)-5-hydroxy-7-(4-hydroxyphenyl)-3-heptanone-5-O$\beta$ - $D$-xylopyranoside $=960$

3-[1-[[3-Di(4-hydroxyphenyl)methyl]2,4,6-trihydroxyphenyl]3-di(4-hydroxyphenyl)1-propanone-2-yl]5,7-dihydroxy-4H-1-benzopyran-4-one = 14895

1-(2,6-Dihydroxyphenyl)-octadec-8-en-1-one $=20491$

13(2',3'-Dihydroxy-3'-phenyl)propionyl baccatin III = 22952

10,12-Dihydroxypicrotoxane 10,12 -di- $O-\beta$ - $D$-glucopyranoside $=5120$

3,4-Dihydroxy-2-piperidinemethanol $=7705$

$1 \beta, 3 \alpha$-Dihydroxypregna-5,16-dien-20-one $=22117$

$2 \beta, 3 \alpha$-Dihydroxypregna-4,7,16-trien-12,20-dione $=21002$

(20S)-3 $\beta, 16 \beta$-dihydroxy pregn-5-ene-22-carboxylic acid $(22,16)$-lactone-3-O$\beta$-chacotrioside $=6636$

$11 \beta, 21$-Dihydroxypregn-4-ene-3,20-dione $=4096$

2-[(17,20-Dihydroxypregn-5-en-3-yl)oxy]-4-methoxy-6-methyl-2H-pyran$3(6 H)$-one $=16942$

4',5-Dihydroxy-3'-prenyl-2",2"-dimethylchromeno-[7,8:6",5"]isoflavone = 19451

4',7-Dihydroxy-3'-prenylflavanone $=51$

3,16 $\alpha$-Dihydroxy-3,4-seco-olean-4(24),12-dien-23,28-dioic acid 28-O$[\beta$-D-glucopyranosyl $(1 \rightarrow 3)]\{\beta$ - D-6-O-[(3R)-3-hydroxy-3-methylglutaryl]- glucopyranosyl $(1 \rightarrow 6)\}-\beta$ - $D$-glucopyranosyl ester $=$ 
19940

3,16 $\alpha$-Dihydroxy-3,4-seco-olean-4(24),12-dien-23,28-dioic acid 28- $O$ - $[\beta$ - $D$ -

6-O-(3-hydroxy-3-methylglutaryl)-glucopyranosyl( $1 \rightarrow 6)]-\beta$ - $D$-glucopyranosyl ester $=19939$

$3 \beta$-21 $\alpha$-Dihydroxy-14-sekogammasera-8(26),14(27)-diene $=16117$

$(24 S, 25 S)-1 \beta, 3 \beta$-Dihydroxy-5 $\beta$-spirostan-24-yl- $O$ - $\beta$ - $D$-glucopyranosyl-

$(1 \rightarrow 6)-\beta$ - $D$-glucopyranoside $=22655$

$(25 R)-12 \beta, 17 \alpha$-Dihydroxyspirost-4-en-3-one $=1864$

(25S)-3 $\beta, 27$-Dihydroxyspirost-5-en-12-one 27- $O$ - $\beta$ - $D$-glucopyranosyl-3- $O-\beta$ -

$D$-glucopyranosyl- $(1 \rightarrow 4)-\beta$ - $D$-fucopyranoside $=17643$

(25S)-3 $\beta, 27$-Dihydroxyspirost-5-en-12-one 27- $O$ - $\beta$ - $D$-glucopyranosyl-3- $O-\beta$ -

$D$-glucopyranosyl- $(1 \rightarrow 4)-\beta$ - $D$-galactopyranoside $=17644$

5,2 '-Dihydroxy-6,7,8,6'-tetamethoxyflavone $=20009$

2,3-Dihydroxy-9,10-tetrahydroanthra-1,4-quinone $=6614$

$12 \alpha, 14 \beta$-Dihydroxy-2 $\alpha, 3 \beta$-(tetrahydro-3',5'-dihydroxy-4'-methoxy-6'-methyl-

$2 H$-pyran-2',4'-diylbisoxy)-card-4,20-dienolide $=7981$

8,9-Dihydroxy-1,5,6,10b-tetrahydro-2 $H$-pyrrolo[2,1- $a$ ]-isoquinolin-3-one $=16078$

(4S)-4,8-Dihydroxy- $\alpha$-tetralone $=18588$

(4S)-4,5-Dihydroxy- $\alpha$-tetralone 4- $O-\beta-D$-glucopyranoside $=11899$

(4S)-4,6-Dihydroxy- $\alpha$-tetralone 4- $O-\beta$ - $D$-glucopyranoside $=11900$

5,5"-Dihydroxy-7,4',7",4"'-tetramethoxy-[3 $\rightarrow 6$ "]-biflavone $=20314$

3,3'-Dihydroxy-4',5,6,7-tetramethoxyflavone $=7590$

4',5-Dihydroxy-3,3',7,8-tetramethoxyflavone $=20978$

9,9"-Dihydroxy-3,3",8,8"-tetramethyl-3,3"-bis-(4-methyl-3-pentenyl)-3,3",

11,11"-tetrahydro-10,10"-(bipyrano[3,2-a]carbazole) $=2479$

5b,7-Dihydroxy-2,2,10,10-tetramethyl-5b,13a-dihydro- $2 H, 6 H, 10 H$-chromeno

$\left[6^{\prime}, 7^{\prime}: 4,5\right]$ furo[2,3-b]pyrano[3,2-g]chromene-6-one $=12294$

$7 \beta, 20$-Dihydroxy-3,11,15,23-tetraoxolanost-8-en-26-oic acid $=8172$

2,5-Dihydroxytoluene $=18270$

$11 \beta, 15 \beta$-Dihydroxy-3 $\beta, 6 \alpha, 7 \beta$-triacetoxy-ent-kaur-16-ene $=13704$

$11 \beta, 15 \beta$-Dihydroxy-1 $\alpha, 3 \beta, 6 \alpha$-triacetoxy-ent-kaur-16-ene $=13707$

$3 \beta, 15 \beta$-Dihydroxy- $1 \alpha, 7 \beta, 11 \beta$-triacetoxy-ent-kaur-16-en-6-one $=614$

$3 \beta, 7 \beta$-Dihydroxy-1 $\alpha, 11 \beta, 15 \beta$-triacetoxy-ent-kaur-16-en-6-one $=615$

4,4"-Dihydroxy-2',3',5'-tribenzoyloxy-6'-acetyloxy[1,1':4',1"-terphenyl] = 21304

cis-4,5-Dihydroxy-5-tridecanoyl-2-cyclopenten-1-one $=10839$

trans-4,5-Dihydroxy-5-tridecanoyl-2-cyclopenten-1-one $=10840$

5,4'-Dihydroxy-6,7,3'-trimethoxyflavone $=3741$

5,7-Dihydroxy-3',4',6-trimethoxyflavone $=7582$

3',5-Dihydroxy-4',6,7-trimethoxyflavone $=7591$

2',5-Dihydroxy-3,6,7-trimethoxyflavone $=11161$

5,7-Dihydroxy-3,6,4'-trimethoxyflavone $=19312$

(R)-5,7-Dihydroxy-2',4',5'-trimethoxyisoflavanone $=5586$

1,7-Dihydroxy-2,3,8-trimethoxy-6-methylanthraquinone $=15908$

trans-3,3'-Dihydroxy-2',4',5-trimethoxystilbene $=17196$

trans-3,4'-Dihydroxy-2',3',5-trimethoxystilbene $=17197$

3,8-Dihydroxy-2,4,6-trimethoxyxanthone $=6165$

$3 \beta, 7 \beta$-Dihydroxy-4,4,14 $\alpha$-trimethyl-11,15-dioxo-5 $\alpha$-chol-8-en-24-oic acid $=13038$

3,23-Dihydroxy-7,11,15-trioxolanosta-8,24-dien-26-oic acid = 8180

8,9-Dihydroxy-4,4,5-tris-(3,3-dimethylallyl)-6-methyl-2,3-(2,2-dimethylpyra-

no)anthrone $=9243$

2,5-Dihydroxy-3-undecyl-2,5-cyclohexadiene-1,4-dione $=6767$

$3 \beta, 19 \alpha$-Dihydroxyurs-12-en-28-oic acid $=17696$

1,7-Dihydroxyxanthone-7- $O$ - $\alpha$ - $L$-rhamnopyrano-syl-( $1 \rightarrow 2)-\beta$ - $D$-glucopyra- noside $=17621$

$2 \alpha, 3 \beta$-Dihydroxy-24- $p$-z-coumaroyloxyurs-12-en-28-oic acid $=9073$

2,(3,4-Dihyroxyphenyl)-3,5,7-trihydroxy-4H-1-benzopyran-4-one = 18317

2,6-Diisobutyryloxy-3-geranyl-5-isoprenyl-2,5-dihexadiene-1,4-dione $=7224$

$12,20$-Di- $O$-isovaleryl-tomentogenin-3- $O-\alpha$ - $L$-oleandropyranosyl-( $1 \rightarrow 4)-O-\alpha$ -

$L$-oleandropyrano-side $=6599$

3',6'-Diketo-7-hydroxy-8,2',4'-trimethoxyisoflavan $=12568$

3,20-Diketo-11 $\beta, 18$-oxido-4-pregnene-14,21-diol $=880$

Dilinolenin $=8815$

Dillapiole $=6193$

2,6-Dimethoxy- $p$-benzoquinone $=6204$

$3 \alpha, 6 \beta$-Di-(4-methoxybenzoyloxy)tropane $=13784$

$(2 S, 3 S)-2 \alpha-(3 ", 4 "-$ Dimethoxybenzyl)-3 $\beta$-(4'-hydroxy-3'-methoxybenzyl)- $\gamma$ -

butyrolactone $4 '-O-(\beta$ - $D$-glucopyranoside $)=20427$

7,7'-Dimethoxy-8,8'-bicoumarin $=11865$

4,5-Dimethoxycanthin-6-one $=12334$

2,6-Dimethoxy-9H-Carbazole-3-carboxaldehyde $=8832$

3,4-Dimethoxy-cinnamic acid $=2888$

6-O-[4"-O-trans-(3,4-Dimethoxycinnamoyl)- $\alpha$ - $L$-rhamnopyranosyl] $]$ aucubin $=19568$

5,7-Dimethoxycoumarin $=3770$

6,7-Dimethoxycoumarin $=19540$

Dimethoxydaidzein $=6254$

5,9-Dimethoxy-7H-dibenzo(de,h)quinolin-7-one $=2343$

5,7-Dimethoxy-8-(2,3-dihydroxyisopentyl) coumarin $=14825$

3',4'-Dimethoxy-5',5-dihydroxy-6,7-methylenedioxyisoflavone $=5426$

4 $\beta, 5$-Dimethoxy-6",6"-dimethyl-2H-pyrano-(2",3":7,6)-flavan = 22810

2,6-Dimethoxy-3-formylcarbazole $=8832$

5,8-Dimethoxy-6,7-furanocoumarin $=11601$

3,3'-Dimethoxyfurano[4",5":8,7]foavone $=16492$

2,3-Dimethoxyfurano[4',5':11,10]-7-oxo-[2] benzopyrano[4',3-b][1]benzopy-

$\operatorname{ran}=16493$

11,12-Di-methoxy-3,4-furo-1,2-naphthoquinone $=4217$

4,8-Dimethoxyfuro[2,3-b]quinoline $=7703$

1,3-Dimethoxy-2-hydroxyanthraquinone $=1360$

6,8-Dimethoxy-7-hydroxycoumarin $=11428$

5,7-Dimethoxy-4'-hydroxyflavone-4'- $O$-apioside $=20393$

11,12-Dimetho-xy-5-hydroxy-3,4-furo-1,2-naphthoquinone $=4218$

5,7-Dimethoxy-8-[2'-hydroxy-3'-methyl, 3'- $O-\beta$ - $D$-apiofuranosyl-(1 $\rightarrow 6)-\beta-D$ glucopyranosylbutyl ]-coumaring $=19040$

2-(3',5'-Dimethoxy-4'-hydroxyphenyl)-3,7-dioxabicyclo[3.3.0]octan-6-one $=22996$

2-[2',4'-Dimethoxy-3'-hydroxyphenyl]-4-hydroxy-6-methoxy-benzofuran-3carbaldehyde $=3005$

2-[2',4'-Dimethoxy-3'-hydroxyphenyl]-4-hydroxy-5,6-methylendioxybenzofuran-3-carbaldehyde $=3006$

(25R)-3,3-Dimethoxy-17 $\alpha$-hydroxyspirostan-3-al-12-one $=1865$

3,7-Dimethoxy-4-hydroxyxanthone $=19646$

5,6-Dimethoxyl-9-hydroxyoxoisoaporphine $=4692$

4,7-Dimethoxy-6-[(3-methyl-2-butenyl)oxy]furo-[2,3-b]quinoline $=20894$

$\left(7 R, 8 R, 7^{\prime} R, 8^{\prime} S\right)$-3,4-Dimethoxy-3',4'-methylenedioxy-7,7'-epoxylignan $=8038$ 6,7-Dimethoxy-3',4'-methylenedioxyflavone $=14363$

(7'R,8'S)-3,4-Dimethoxy-3',4'-methylenedioxy-7,8-seco-7,7'-epoxylignan-7,8dione $=19616$

2,3-Dimethoxy-8a-methyl-5-methylene-5,6,7,8,8a,9,10,10a-octahydroanthrac 
ene-1,4-dione $=9322$

rel-4',7-Dimethoxy-4-oxo-2,3-trans-isoflavanyl-(2 $\rightarrow 2 ")-4 ", 5$ "-dihydroxy-7"methoxy-2",3"-trans-isoflavanone $=4985$

5,7-Dimethoxy-8-(1-oxo-2-senecioyl-3-methyl-3-butenyl)-2H-1-benzopyran2-one $=16096$

(1R,2R,5R,6S)-2-(3',4'-Dimethoxy-phenyl)-6-(3",4"-methylene dioxyphenyl)-

3,7-dioxabicyclo $(3,3,0)$ octane $=17504$

4,6-Dimethoxy-8-prenyloxyfuroquinoline $=20892$

1,2-Dimethoxy-4-(1-cis-propenyl)-benzene $=14530$

3,3'-Dimethoxyquercetin $=6402$

2,3-Dimethoxy-4,6, $\alpha, \beta$-tetrahydroxy- $\alpha$-chalcanol-4- $O$ - $\beta$ - $D$-glucopyranoside $=21633$

6,4'-Dimethoxy-3,5,7-trihydroxyflavone $=2330$

6,7-Dimethoxy-3,5,4'-trihydroxyflavone $=7577$

3',4'-Dimethoxy-5,7,5'-trihydroxyl-flavone 3-O- $\alpha$ - $L$-rhamnopyranoside $=6296$

3,6-Dimethoy kaempferide $=14157$

Dimethulene $=3464$

cis-2,3-Dimethylacrylic acid $=1188$

(-)-3-(3,3-Dimethylallyl)-5'-(2-hydroxy-3-methylbut-3-enyl)-4,2',4'-trihy-

droxychalcone $=2153$

(+)-3-(3,3-Dimethylallyl)-4',5'-[2'"-(1-hydroxy-1-methylethyl)-dihydrofura-

no]-4,2'-dihydroxychalcone $=2154$

3'-(3,3-Dimethylallyl)-kievitone $=6307$

$3 \beta$-Dimethylamino-20 $\alpha$-acetoxylamino- $9 \beta, 19$-cyclo- $4,4,14 \alpha$-trimethy-5 $\alpha$ -

pregn-6(7)-ene $=2821$

3(-Dimethylamino-20 $\alpha$-amino-9 $\beta, 19$-cyclo-4,4,14 $\alpha$-trimethy-5 $\alpha$-pregn-6(7)-

ene $=2822$

$20 \alpha$-Dimethy-lamino- $9 \beta, 19$-cyclo-4,4,14 $\alpha$-trimethy- $5 \alpha$-pregnane $=2818$

$3 \beta$-Dimethylamino-9 $\beta, 19$-cyclo-4,4,14 $\alpha$-trimethy-5 $\alpha$-pregn-6(7),17(20)-dien16 -one $=2820$

(20S)-20-Dimethylamino-4',6'-dimethoxy-5'-hydroxybenzoylamino-3 $\beta$-methyl buxan-31-ol $=2815$

(20S)-20-Dimethylamino-2'-hydroxy-3 $\beta$-methyl-3'-methyl-butanoylamino9,10-seco-buxa-9(11),10(19)-dien-31-ol = 2814

(20S)-20-Dimethylamino-20-hydroxy-3 $\beta$-methyl-3'-methyl-pentanoylamino9,10-seco-buxa-9(11),10(19)-dien-31-ol = 2813

$3 \beta$-Dimethylamino-2 $\alpha$-hydroxypregn-5-en-20-one $=12348$

(20S)-20-( $N, N$-dimethylamino)-5 $\alpha$-pregna-3-one $=8009$

5,6-Dimethylbenzimidazolyl cyanocobamide $=22556$

2,2-Dimethyl-8-benzoyl-3,7-dihydroxy-5-methoxy-6-(3-methyl-2-butenyl)3,4-dihydrobenzopyran $=18027$

2,2-Dimethyl-8-benzoyl-7-hydroxy-5-methoxy-6-(3-methyl-2-butenyl)benzopyran $=15225$

rel-10 $\beta H$-trans-1 $\beta, 12 \xi$-Di(2-methylbut-2(E)-enoyl)-6 $\alpha, 13 \xi$-dihydroxyclero-

dan-4(20),8(18)-dien-7,15-dione-15,16-oxide $=2950$

4,24-Dimethyl cholest-7-en-3-ol $=14558$

2,2-Dimethyl-2H-1-chromene-6-carboxylicacid $=1343$

Di-O-Methylcrenatin $=10014$

$(E, E, E)$-1,7-Dimethylcyclodeca-1,4,7-triene $=17780$

4-(1,3-Dimethylcyclohexenyl)-1-methylbenzene $=20938$

4-(1,3-Dimethylcyclohexenyl)-1-methyl-1,4-cyclohexadiene $=20937$

9,9-Dimethyl-6 $\alpha, 8 \beta$-di( $\gamma, \gamma$-dimethylallyl)-3,4-[2 $\beta$-(2'-hydroxyisopropyl)-2,3dihydrofuran]-2 $\alpha$-(1-oxo-2-methylpropyl)- $8 \alpha-H$-cis-bicyclo[3.3.1]nona-
1,5 -dione $=8214$

9,9-Dimethyl- $6 \alpha, 8 \beta$-di( $\gamma, \gamma$-dimethylallyl)-3,4-[2 $\beta$-(2'-hydroxyisopropyl)-2,3dihydrofuran]-2 $\alpha$-(1-oxo-2-methylpropyl)-8 $\alpha$-H-cis-bicyclo[3.3.1]nona1,5 -dione $=8215$

3,3-Dimethyl-4 $\alpha, 6 \alpha$-di $(\gamma, \gamma$-dimethylallyl)-2 $\alpha$-(2-methyl-1-oxopropyl)cyclohexanone $=8216$

3,4-(6",6"-Dimethyldihydropyrano)-4',5'-[2"',-(1-hydroxy-1-methylethyl)dihydrofurano]-2'-hydroxychalcone $=2155$

6,7-(2,2-Dimethyldihydropyrano)-8-prenyl-5,3',4'-trihydroxyflavanone $=6572$

2,5-Dimethyl-8,10-dihydroxynaphthopyrone 10-O- $\beta$ - $D$-glucopyranoside $=17559$

$1 \beta^{*}, 2 \beta^{*}, 3 \alpha^{*}, 4 \alpha^{*}$-1,2-dimethyl-3-(2,5-dimethoxy-3,4-methylenedioxyphenyl)4-(2,4,5-trimethoxyphenyl)cyclobutane $=15001$

$\left(7^{\prime} R, 8 S, 8^{\prime} R\right)-8,8^{\prime}$-Dimethyl-4,5-dimethoxy-3',4'-methylenodioxy-2,7'-cyclolignan-7-one $=1730$

( 7'R, 8S, 8'S)-8,8'-Dimethyl-3',4'-dimethoxy-4,5-methylenodioxy-2,7'-cyclolignan-7-one $=14393$

(+)-1,1-Dimethyl-4,7-dimethylene-decahydro-cyclopropa[ $e$ azulen-4a-ol = 1758

$(+)-(1 S, 4 \mathrm{a} R, 7 \mathrm{a} S, 8 S)$-1,1-Dimethyl-4,7-dimethylenedecahydro-cyclopropa[ $e]$ azulene $=927$

7,8-Dimethyl-10- $d$-ribityl-isoalloxazine $=18834$

6,7-Dimethyl-9- $D$-ribitylisoalloxazine $=18834$

$3,4-\mathrm{Di}-O$-methylellagic acid $=6224$

5-[(2E)-3,7-Dimethyl-5,6-epoxy-2,7-octadienyloxy $]$ psoralen $=1279$

5 - $\{(2 E, 5 E)-3,7-$ Dimethyl-7-[(1-ethoxy)ethoxy-2,5-octadienyloxy $]\}$ psoralen $=7466$

4-O-[(2E,5E)-3,7-Dimethyl-5-ethoxy-2,5-octadiene-7-ol]-sinapyl alcohol = 15328

4-O-[(2E)-3,7-Dimethyl-6-ethoxy-2,7-octadiene]-sinapyl alcohol = 15327

4-O-[(2E,4E)-3,7-Dimethyl-5-ethoxy-2,4-octadien-7-ol]-sinapyl alcohol = 15329

5-(2E)-3,7-dimethyl-5-ethoxy-2,6-octadienyloxy psoralen $=15845$

Dimethyl (2R)-2-[(4-ethoxy-4-oxobutanoyl)oxy]succinate $=16157$

4-(1,5-Dimethyl-1,4-hexadienyl)-1-methyl-cyclohexene $=2411$

(1-(1,5-Dimethylhex-4-enyl)-4,8-dimethylspiro[4.5]deca-1,7-diene $)=22531$

(4-(1,5-Dimethylhex-5-enylidene)-1-methylcyclohexene $)=2408$

$\left[S-\left(R^{*}, S^{*}\right)\right]-5-(1,5-$ Dimethyl-4-hexenyl)-2-methyl-1,3-cyclohexadiene $=23004$

(-)-(3aS,5R,7aR)-4,4-Dimethyl-7a-hydroxymethyl-5-hydroxy-2,3,3a,4,5,7ahexahydrobenzo[b]furan-2-one $=13660$

(-)-(3a $, 5 R, 7 \mathrm{a} R)-4,4-D i m e t h y l-7 \mathrm{a}-h y d r o x y m e t h y l-2-o x o-2,3,3 \mathrm{a}, 4,5,7 \mathrm{a}-$ hexa- hydrobenzo[b]furan-5-yl 3,4-dimethyl-3-pentenoate $=$ 20966

( 7'R, 8S, 8'R)-8, '-'Dimethyl-4-hydroxy-3',4',5-trimethoxy-2,7'-cyclolignan-7one $=6932$

(7' $\left.R, 8 S, 8^{\prime} S\right)-8,8^{\prime}$-Dimethyl-4-hydroxy-3',4',5-trimethoxy-2,7'-cyclolignan-7one $=6933$

(7'R,8R,8'S)-8, 8'-Dimethyl-4-hydroxy-3',4',5-trimethoxy-2,7'-cyclolignan-7one $=9592$

1,6-Dimethyl-4-isopropyl-7,8-dihydro-naphthalene $=4076$

3,8-Dimethyl-5-isopropyl-6-hydroxycoumarin $=13520$

3,8-Dimethyl-5-isopropyl-6-methoxycoumarin $=13519$

1,6-Dimethyl-4-keto-tetrahydronaphthalene $=6413$

$N, N$-Dimethylmethanamine $N$-oxide $=21940$

2,2-Dimethyl-6-methoxycarbonyl-2 $H$-benzopyran $=6321$

$1 \beta^{*}, 2 \beta^{*}, 3 \alpha^{*}, 4 \alpha^{*}$-1,2-dimethyl-3-(3-methoxy-4,5-methylene-dioxyphenyl)-4$(2,4,5$-trimethoxyphenyl)-cyclobutane $=15000$

4-[2,2-Dimethyl-3-(5-methylcyclopenta-1,4-dien-1-yl)-cyclopropyl]-butan-2- 
one $=20889$

2,8-Dimethyl-5-methylene-2a,3,4,5,5a,6,7,8,8a,8b-decahydro-cyclobuta[ $e$ ] azulen-4-ol $=20890$

2,2-Dimethyl-3-methylenebicyclo[2,2,1]heptane $=3045$

1,4-Dimethyl-8-methylene-2-oxabicyclo[3.2.1] octane $=13596$

$(+)-(S)-1,5-D i m e t h y l-7-(1-m e t h y l e t h e n y l)-c y c l o d e c a-1 E, 5 E$-diene $=11437$

(-)-(1R,4aS,8S,9R)-1,4a-Dimethyl-8-(1-methylethenyl)-decahydro-naphthalen-1-ol $=8954$

4,8-Dimethyl-2-(1-methylethyl)-azulene $=22439$

1,5-Dimethyl-1-(4-methylhexenyl)-4-cycloheptenylether $=12467$

6,6-Dimethyl-12 $\alpha$-(2 $\alpha, 3 \alpha-H)$-12 $\alpha$-(2-methyl-3-hydroxybutanoyl)-8b-hydroxy4-phenyl-pyranodihydrocoumarin $=2992$

6,6-Dimethyl-12 $\alpha$-(2 $\alpha, 3 \beta-H)$-12 $\alpha$-(2-methyl-3-hydroxybutanoyl)-8b-hydroxy4-phenyl-pyranodihydrocoumarin $=2993$

(+)-(1 $\left.S^{*}, 3 \mathrm{a} R^{*}, 4 S^{*}, 7 S^{*}, 8 \mathrm{a} S^{*}\right)-1,4-$ Dimethyl-7-(5-methyl-1-methylene-hex-4enyl)-decahydroazulen-4-ol $=22583$

(1,8-Dimethyl-4-(5-methyl-1-methylenehex-4-enyl)spiro[4.5]dec-7-ene) $=22532$

2,6-Dimethyl-6-(4-methyl-3-pentenyl)biscyclo[3,1,1]hept-2-ene $=2306$

(2,6-Dimethyl-2-(4-methylpent-4-enyl)-1-oxaspiro[2.5] oct-5-ene) $=7057$

(+)-(1S,4aS,8aR)-4a,8-Dimethyl-1-(1-methylvinyl)-1,2,3,4,4a,5,6,8a-octahydro-naphthalene $=8952$

3,7-Dimethylocta-1,6-dien-3,8-diol 8-O- $\beta$ - $D$-glucopyranosiode $=2329$

(Z)-3,7-Dimethyl-2,6-octadien-1-ol = 15498

4-O-[(2E)-3,7-Dimethyl-2,7-octadien-5-ol] sinapyl alcohol $=15326$

1-[6-(3,7-Dimethyl-octa-2,6-dienyl)-5,7-dihydroxy-2,2-dimethyl-2 $H$-chromen-8-yl]-3-(4-hydroxy-phenyl)-propenone $=13435$

2-(3,7-Dimethyl-2,6-octadienyl)-1,3,5,8-tetrahydroxyxanthone $=20018$

2,6-Dimethyl-2,4,6-octatriene $=939$

3,7-Dimethyl-6-octen-1-al $=3767$

3,7-Dimethyl-6-octen-1-yl acetate $=3769$

(+)-(1R,5S,6S,7S)-5,6-Dimethyl-9-oxo-8-isopropylidene-tricyclo[5.2.2.0 $\left.{ }^{1,6}\right]$ undecane $=1266$

(3,4-trans)-3-[3'-(1"',1"'-Dimethyl-2"'-propenyl)-5'-indolyl]-1,4-dimethyl-4$\{2-[3 "-(1 " ',, 1$ "'--dimethyl-2"'"-propenyl)-5"-indolyl]-ethyl-2-enyl $\}$-cyclohex-1-ene $=3338$

(3,4-cis)-3-[3'-(1"',1"'-Dimethyl-2"'-propenyl)-5'-indolyl]-1,4-dimethyl-4-\{2[3"-(1"', 1 "''-dimethyl-2"'--propenyl)-5"-indolyl]-ethyl-2-enyl $\}$-cyclohex1 -ene $=3339$

6",6"-Dimethylpyrano(2",3":4,5)-3'- $\gamma, \gamma$-dimethylallyl-2',3,4'-trihydroxychalcone $=6024$

6,7-(2,2-Dimethylpyrano)-8-prenyl-5,3',4'-trihydroxyflavanone $=6571$

( $\left.7^{\prime} R, 8 S, 8^{\prime} R\right)-8,8^{\prime}$-Dimethyl-3',4',4,5-tetramethoxy-2,7'-cyclolignan-7-one = 1709

$\left(7^{\prime} R, 8 R, 8^{\prime} S\right)-8,8^{\prime}-$ Dimethyl-3',4',4,5-tetramethoxy-2,7'-cyclolignan-7-one $=6830$

$\left(7^{\prime} R, 8 S, 8^{\prime} S\right)-8,8^{\prime}$-Dimethyl-3',4',4,5-tetramethoxy-2,7'-cyclolignan-7-one $=6831$

$N, N$-Dimethyltyramine $=9646$

Dimidin $=5707$

Dinatin $=9564$

18,20-Dinor-1,3,5(10),6,8,12-abietahexaene-11,14-dione $=5165$

25,26-Dinor-9,13-dimethyllup-5-en-3-ol = 9213

26,27-Dinor-3,8,14,21-onoceranetetrol $=3810$

16,28-Di-Oacetyl-21- $O$-angeloyltheasapogenol E 3-O- $\beta$ - $D$-galactopyranosyl $(1 \rightarrow 2)[\beta$ - $D$-xylopyranosyl $(1 \rightarrow 2)$ - $\alpha$ - $L$-arabinopyranosyl $(1 \rightarrow 3)]-\beta$ - $D$-glucopyranosiduronic acid $=21287$
Dioctyl 1,2-benzenedicarboxylate $=2449$

3-O- $\alpha$-(3',4'-O-Dioic-acetyl)- $L$-arabino-pyranosyl-3 $\beta$-hydroxylean-12-ene-28, 29-dioic acid-28- $O$-[ $\alpha$ - $L$-rhamnopyranosyl-( $1 \rightarrow 6)-\beta$ - $D$-glycopyranosyl$(1 \rightarrow 4)-\beta$ - $D$-glycopyranosyl] ester $=22915$

Dioscorea sapogenin $=6440$

Diosgenin-dioglucoside $=6442$

Diosgenin-3- $O$ - $[\alpha-L$-Rhamnopyranosyl- $(1 \rightarrow 2)$ - $[\alpha-L$-arabinopyranosyl- $(1 \rightarrow$ 4)]- $\beta$ - $D$-glucopyranoside $=17655$

Diosgenin-3- $\alpha$ - $L$-rhamnopyranosyl $(1 \rightarrow 3)-\beta$ - $D$-glucopyranoside $=17654$

Diosgenin-3- $O$ - $\alpha$ - $L$-rhamnopyranosyl $(1 \rightarrow 4)-\beta$ - $D$-glucopyranosyl $(1 \rightarrow 4)-\beta$ - $D$ glucopyranoside $=21661$

Diosgenin 3-O-[ $\alpha$ - $L$-rhamnopyranosyl- $(1 \rightarrow 3)-\alpha$ - $L$-rhamnopyranosyl- $(1 \rightarrow 4)$ $[\beta$ - $D$-glucopyranosyl- $(1 \rightarrow 2)]-\alpha$ - $L$-rhamnopyranoside $]=17657$

Diosgenin-3-O- $\alpha$ - $L$-rhamnopyranosyl $(1 \rightarrow 2)$ - $\alpha$ - $L$-rhamnopyranosyl $(1 \rightarrow 4)$ $[\alpha$ - $L$-rhamnopyranosyl $(1 \rightarrow 3)]$ - $\beta$ - $D$-glucopyranoside $=17656$

Diosgenin tetraglycoside $=6447$

11,24-Dioxo-5,21-dien-cucuebit-3 $\alpha$ - $O-\beta$ - $D$-xylopyranosyl-16 $\alpha$ - $O$ - $\alpha$ - $L$-rhamno pyranoside $=4883$

(24E)-3,7-Dioxo-5 $\alpha$-lanosta-8,24-dien-26-al = 13020

3,16-dioxo-13 $\beta$ 17-methyleneoxyoleanane $=6768$

2,6-Dioxo-1,2,3,6-tetrahydro-4-pyrimidinecarboxylic acid $=16214$

trans,trans-1,7-Diphenyl-1,3-heptadien-5-one $=966$

1,7-Diphenyl-5-hydroxy-3-heptanone $=6185$

trans-1,7-Diphenyl-5-hydroxy-1-heptene $=6487$

Diphyline $=3502$

Diploptene $=9641$

Diplopterol $=10167$

1,6-Di- $O$ - $E$-sinapoyl- $\beta$-glucopyranose $=6510$

Distylin $=5699$

Diterpenoid SP II $=5930$

2,3-Dithiabutane $=6342$

4,5-Dithiaoctane $=6501$

Di(5,7,4"'-trihydroxy-2,3-dihydro-4',7"-dimethoxy-6"-methyl-3',3"'-biflavanyl)-6,6-tetraflavone $=20627$

3,4-O-Divanilloylquinic acid $=2761$

3,5-O-Divanilloylquinic acid $=2762$

4,5-O-Divanilloylquinic acid $=2763$

$D$-(+)-Maltose $=13454$

$D$-Maltose $=13454$

$\mathrm{DMX}-\mathrm{B}=5262$

$\mathrm{DMX}-\mathrm{J}=5263$

Docosapentenoic acid $=3849$

cis-13-Docosenoic acid $=7291$

$(4 \mathrm{a} \alpha, 5 \beta, 6 \mathrm{a} \beta, 7 \beta, 11 \mathrm{a} \alpha, 11 \mathrm{~b} \beta)-1,2,3,4,4 \mathrm{a}, 5,6,6 \mathrm{a}, 7,11,11 \mathrm{a}, 11 \mathrm{~b}-$ Dodecahydro-4,4,7, 11b-tetramethylphenanthro[3,2-b]furan-4a,5,7-triol-5-benzoate $=11778$

$(4 \mathrm{a} \alpha, 5 \beta, 6 \mathrm{a} \beta, 11 \mathrm{a} \alpha, 11 \mathrm{~b} \beta)-1,2,3,4,4 \mathrm{a}, 5,6,6 \mathrm{a}, 7,11,11 \mathrm{a}, 11 \mathrm{~b}-$ Dodecahydro-4,4,11btrimethyl-7-methylenephenanthro[3,2- $b]$ furan-4a,5-diol-5-benzoate $=$ 11777

Dodecanal $=12570$

(3E,7Z)-Dollabella-3,7,12-trien-17-oic acid $=6549$

Donaxine $=8971$

Dotorioside I $=1733$

(9S)-Drummondol-9- $O-\beta$ - $D$-glucopyranoside $)=20174$ 
Dulcinol $=19537$

\section{E}

Eburicoic acid 21-O- $\beta$ - $D$-glucopyranoside $=7875$

$\alpha$-Ecdysone $=6678$

$\beta$-Ecdysone $=6679$

Echinaystic acid $=6689$

Echinocystic acid 3-O- $\beta$ - $D$-glucopyranosyl( $1 \rightarrow 3)-\beta$ - $D$-glucopyra-nosyl $(1 \rightarrow 3)$ - $\alpha$ - $L$-arabinopyranoside $=11799$

Echinocystic acid 3- $O$-(6'- $O$-methyl) $-\beta$ - $D$-glucuronopyranoside $=6704$

Eemanin $=12044$

Egonol $=10665$

$n$-Eicosane $=6720$

1 -Eicosanol $=6722$

Elatericin $=4319$

Elatoside $\mathrm{J}=3976$

Eleutheroside $\mathrm{C}=7439$

Eleutheroside $\mathrm{E}=86$

Eleutheroside $\mathrm{M}=819$

Emodin-8- $O-\beta$ - $D$-glucopyranoside $=1363$

Emodin 8- $O-\beta$ - $D$-glucopyranosyl-6- $O$-sulfate $=20468$

Emodin-3-monomethyl ether $=17247$

Emodin-1-monomethyl ether $=18416$

Empetrin $=5022$

Encecalol $=6797$

Encordin $=17002$

Engeletin $=5659$

Engelitin $=5659$

Enmenin $=21561$

$\mathrm{EP}-1=7113$

Ephedine $=6815$

Epialisol A $=891$

Epicatechin- $(2 \beta \rightarrow O \rightarrow 7,4 \beta \rightarrow 8)$-[epicatechin- $(4 \beta \rightarrow 6)]$-epicatechin- $(4 \beta \rightarrow 8)$ -

$$
\text { epicatechin }=16654
$$

Epicatechin- $(2 \beta \rightarrow O \rightarrow 5,4 \beta \rightarrow 6)$-[epicatechin- $(2 \beta \rightarrow O \rightarrow 7,4 \beta \rightarrow 8)]$-epicatechin-

$$
(4 \beta \rightarrow 8) \text {-epicatechin }=16655
$$

Epicatechol $=6854$

2-Epicephalofortuneine $=3401$

7-Epi-10-deacetyltaxol $=6884$

$\left(1^{\prime} R, 3^{\prime} R, 5^{\prime} R, 8^{\prime} S\right)$-Epi-dihydrophaseic acid $\beta$ - $D$-glucoside $=5691$

$5 \alpha, 8 \alpha$-Epidioxyergosta- 6,22 -dien- $3 \beta$-ol $=7251$

$5 \alpha, 8 \alpha$-Epi-dioxyergost-6-en- $3 \beta$-ol $=7249$

2 "-Epidorsmanin $\mathrm{Fa}=6566$

2"-Epidorsmanin $\mathrm{Fb}=6567$

2 "-Epidorsmanin $\mathrm{Ga}=6568$

2 "-Epidorsmanin $\mathrm{Gb}=6569$

20-Epieuphol $=21409$

15 -Epifuziline $=15228$

$(-)$-Epigallocatechin $=6921$

$(-)$-Epigallocatechin-3-O-gallate $=6923$

3-Epihydroxyetioallocholan-17-one $=1170$

8 '-Epi-larreatricin $=6942$

1-O-(8-Epi-loganoyl)- $\beta$ - $D$-glucopyranose $=8864$
Epilupinyl rhamnosylferulate $=14084$

Epimedoside $\mathrm{A}=5257$

22,26-Epiminocholest-6-one-3- $O$ - $\beta$ - $D$-glucopyranosyl-( $1 \rightarrow 4)-\beta$ - $D$-galactopyranoside $=22906$

$(5 \alpha, 22 S, 23 R, 25 S)$-22,26-Epimino-16 $\beta, 23$-epoxy-3 $\beta, 23,27$-trihydroxycholestane = 7375

$L$-Epinephrine $=653$

6-C- $\beta$ - $D$-4-Epioliosyl-4'- $O-\beta$ - $D$-glucopyranosylapigenin $=18678$

Epiquinamine $=4004$

13-Epi-roseosta-chenol $=10291$

15-Episenbusine $\mathrm{C}=15228$

$20 \xi_{1}, 24 \xi_{2}$-Epoxy-15R-acetoxy-9,19-cyclolanostane-3 $\beta, 16 \beta, 25$-triol-3- $O-\beta$ - $D$ xylopyranoside $=2206$

$3 \beta, 25$-Epoxy-21 $\beta$-acetoxy-3 $\alpha, 22 \beta$-dihydroxyurs-12-en-28-al $=4039$

$3 \beta, 25$-Epoxy-28-acetoxy-3 $\alpha, 21 \beta, 22 \beta$-trihydroxyurs-12-ene $=4040$

Epoxyactinidionoside $=20271$

4,5-Epoxy-8(14)-caryophyllene $=3243$

15,16-Epoxy-1,3,13(16),14-clerodatetraen-20,12-olide-18,19-dioic acid dimethylester $=4273$

15,16-Epoxy-1,3,13(16),14-clerodatetraen-18,19,20-trioic acid trimethylester $=4274$

15,16-Epoxy-3,13(16),14-clerodatrien-19,1 $\alpha: 20,12$-diolide-18-oic acid methylester $=4275$

$20 \xi_{1}, 24 \xi_{2}$-Epoxy-9,19-cyclolanostane- $3 \beta, 12 \alpha, 15 \alpha, 16 \beta, 25$-pentaol-3- $O-\beta-D$ xylopyranoside $=2207$

$\left(20 S^{*}, 24 R^{*}\right)$-Epoxy-9,19-cyclolanostane-3 $\beta, 12 \beta, 16 \beta, 18,25$-pentaol-3- $O-\beta-D$ xylopyranoside $=2193$

$\left(20 S^{*}, 24 R^{*}\right)$-Epoxy-9,19-cyclolanostane-3 $\beta, 12 \alpha, 16 \beta, 18,25$-pentaol-3- $O-\beta-D$ xylopyranoside $=2194$

$\left(20 S^{*}, 24 R^{*}\right)$-Epoxy-9,19-cyclolanostane-3 $\beta, 15 \alpha, 16 \beta, 18,25$-pentaol-3- $O-\beta-D$ xylopyranoside $=2196$

$20 \xi_{1}, 24 \xi_{2}$-Epoxy-9,19-cyclolanostane-3 $\beta, 16 \beta, 18,25$-tetraol-3- $O$ - $\beta$ - $D$-glucopyranoside $=2198$

$20 \xi_{1}, 24 \xi_{2}$-Epoxy-9,19-cyclolanostane- $3 \beta, 16 \beta, 18,25$-tetraol-3- $O$-[ $\beta$ - $D$-glucopyranosyl- $(1 \rightarrow 6)]-\beta$ - $D$-glucopyranoside $=2199$

$\left(20 S^{*}, 24 R^{*}\right)$-Epoxy-9,19-cyclolanostane-3 $\beta, 16 \beta, 18,-25$-tetraol-3- $O-\beta$ - $D$ xylopyranoside $=2192$

$\left(20 S^{*}, 24 R^{*}\right)$-Epoxy-9,19-cyclolanostane-3 $\beta, 12 \beta, 16 \beta, 25$-tetraol-3-O- $\beta$ - $D$ xylopyranoside $=4465$

20,24-Epoxy-dammarane-( $3 \beta, 12 \beta, 20 S, 24 R)$-12-O- $\beta$ - $D$-quinovopyranosyl-25hydroxy-3- $O-\alpha-L$-arabinofuranoside $=4483$

$\left(3 S, 5 R, 6 R, 3^{\prime} S, 5^{\prime} R, 8^{\prime} R\right)$-Epoxy-6,7-didehydro-5,6,5', $8^{\prime}$-tetrahydro- $\beta, \beta$-carotene$3,5,3^{\prime}$-triol 3-O-acetate $=6424$

$\left(3 S, 5 R, 6 R, 3^{\prime} S, 5^{\prime} R, 8^{\prime} S\right)-5^{\prime}, 8^{\prime}$-Epoxy-6,7-didehydro-5,6,5',8'-tetrahydro- $\beta, \beta$-carot ene-3,5,3'-triol 3-O-acetate $=6425$

5,8-Epoxy-5,8-dihydro- $\beta$,eplsilon-carotene-3,3'-diol $=7821$

12,16-Epoxy-11,14-dihydroxy-3,5,8,11,13,15-abietahexaene-7-one $=786$

12,16-Epoxy-11,14-dihydroxy-3,5,8,11,13-abietapentaene-7-one $=787$

$16 \beta, 23 \beta$-Epoxy-11 $\beta, 25$-dihydroxy-24( $R$ )-acetoxy-protost-13(17)-en-3-one = 902

(20S,24S)-20,24-Epoxy-12,25-dihydroxydammaran-3-yl $O$ - $\beta$ - $D$-glucopyranosyl- $(1 \rightarrow 2)-\beta$ - $D$-glucopyranoside $=9117$

$(20 S, 24 S)-20,-24-E p o x y-12,25$-dihydroxydammaran-3-yl $O-\beta$ - $D$-glucopyra- 
nosyl- $(1 \rightarrow 2)-\beta$ - $D$-xylopyranoside $=9116$

(20S,24R)-20,24-Epoxy-12,25-dihydroxydammaran-3-yl $O-\beta$ - $D$-glucopyranosyl- $(1 \rightarrow 2)-\beta$ - $D$-xylopyranoside $=9118$

1,4-trans-7 $\beta, 10 \beta$-Epoxy-4 $\alpha, 6 \alpha$-dihydroxyguaiane $=16193$

ent-3 $\beta, 20$-Epoxy-3 $\alpha, 6 \alpha$-dihydroxykaur-16-ene $=689$

15,16-Epoxy-3 $\alpha, 9 \alpha$-dihydroxy-labda-13(16),14-diene $=16273$

$3 \beta, 20$-Epoxy-3 $\alpha, 6 \alpha$-dihydroxy-18-nor-beyer- 15 -ene $=692$

$13 \beta, 28$-Epoxy-16 $\alpha, 21 \beta$-dihydroxyurs-11-en- $3 \beta$-yl $\alpha$ - $L$-rhamnopyranosyl$(1 \rightarrow 2)-\beta$ - $D$-glucopyranosyl- $(1 \rightarrow 2)-\beta$ - $D$-fucopyranoside $=18949$

$13 \beta, 28$-Epoxy-16 $\alpha, 23$-dihydroxyurs-11-en-3 $\beta$-yl $\beta$ - $D$-xylopyranosyl-

$(1 \rightarrow 2)-\beta$ - $D$-glucopyranosyl $-(1 \rightarrow 2)-\beta$ - $D$-fucopyranoside $=18944$

$15 \alpha, 16 \alpha$-Epoxy-11 $\alpha, 28$-dihydroxyurs-12-en-3 $\beta$-yl $\beta$ - $D$-xylopyranosyl-

$(1 \rightarrow 2)-\beta$ - $D$-glucopyranosyl- $(1 \rightarrow 2)-\beta$ - $D$-fucopyranoside $=18947$

$13 \beta, 28$-Epoxy-16 $\alpha, 21 \beta$-dihydroxyurs-11-en-3 $\beta$-yl $\beta$ - $D$-xylopyranosyl-

$(1 \rightarrow 2)-\beta$ - $D$-glucopyranosyl- $(1 \rightarrow 2)-\beta$ - $D$-fucopyranoside $=18948$

$13 \beta, 28$-Epoxy-16 $\alpha, 23$-dihydroxyurs-11-en-3 $\beta$-yl $\beta$ - $D$-xylopyranosyl-

$(1 \rightarrow 2)-\beta$ - $D$-glucopyranosyl- $(1 \rightarrow 2)-\beta$ - $D$-glucopyranoside $=18950$

$13 \alpha(21)$-Epoxyeurycomanone $=16694$

(22S,23S,25R,26S)-23,26-Epoxy-5 $\alpha$-furostane-3 $\beta, 22,26$-triol

$26-O-\beta$ - $D$-glucopyranoside $=699$

6,7-Epoxy-10(14)-guaien-4-ol = 16191

2,3-Epoxy-6,9-humuladiene $=9671$

6,7-Epoxy-2,9-humuladiene $=9672$

9,10-Epoxy-2,6-humuladiene $=9673$

2,3-Epoxy-6,9-humuladien-8-one $=22985$

$3 \beta, 20$-Epoxy-3 $\alpha$-hydroxybeyer-15-ene $=690$

16,24-Epoxy-25-hydroxycycloart-1,11,22-trien-3-one $=1669$

$5 \alpha H$-2 $\beta, 4$-Epoxy-3 $\beta$-hydroxyguaia-1(10),11(13)-dien-6 $\beta,-12$-olide $=14978$

$5 \alpha H$-2,4 $\beta$-Epoxy-1-hydroxyguaia-9(10),11(13)-dien- $6 \beta,-12$-olide $=19128$

$5 \alpha H$-2,4 $\beta$-Epoxy-1-hydroxyguaia-10(14),11(13)-dien-6 $\beta,-12$-olide $=19129$

4,10 -Epoxy- $6 \alpha$-hydroxyguaiane $=2695$

16,24-Epoxy-3 $\alpha$-hydroxylanost-8-ene $=1668$

3,25-epoxy-3 $\alpha$-hydroxy-22 $\beta$-[(Z)-29-methyl-29-butenoyloxy]-11-oxoolean-12 -en-28-oic acid $=3024$

1,2-Epoxy-13-hydroxy-6,10,14-phytatrien-20,1-olide $=7086$

(23S,25R)-17 $\alpha, 23$-Epoxy-29-hydroxy-3 $\beta$-[( $O-\alpha-L$-rhamnopyranosyl-(1 $\rightarrow 2)-O$

-[ $\beta$-D-glucopyranosyl- $(1 \rightarrow 3)]-O-\beta$ - $D$-glucopyranosyl- $(1 \rightarrow 2)-\alpha-L$-arabin opyranosyl-( $(1 \rightarrow 6)-\beta$ - $D$-glucopyranosyl)oxy]lanost- 8 -en-23,26-olide $=$ 19517

13 $\beta, 28$-epoxy-16 $\alpha$-hydroxyurs-11-en-3 $\beta$-yl $\alpha$ - $L$-rhamnopyranosyl-( $1 \rightarrow 2)$ -

$\beta$ - $D$-glucopyranosyl- $(1 \rightarrow 2)-\beta$ - $D$-fucopyranoside $=18953$

$13 \beta, 28$-epoxy-16 $\alpha$-hydroxyurs-11-en-3 $\beta$-yl $\beta$ - $D$-xylopyranosyl-( $(1 \rightarrow 2)$ -

$\beta$ - $D$-glucopyranosyl- $(1 \rightarrow 2)-\beta$ - $D$-fucopyranoside $=18952$

$13 \beta, 28$-epoxy-16 $\alpha$-hydroxyurs-11-en-3 $\beta$-yl $\beta$ - $D$-xylopyranosyl- $(1 \rightarrow 2)$ -

$\beta$ - $D$-glucopyranosyl- $(1 \rightarrow 2)-\beta$ - $D$-glucopyranoside $=18951$

Epoxyimperatorin $=9419=16447$

15,16-Epoxy-8(17),13(16),14-ent-labdatrien-20,19-olide $=17742$

8,13 -Epoxy-14-labdene $=5954$

$(E)$-8 $8(17)$-Epoxylabd-12-ene-15,16-dial $=675$

Epoxylinalool $=12853$

(3S,5R,8S,9R)-5,8-Epoxy-6-megastigmene-3,9-diol 3-O- $\beta$ - $D$-glucopyranoside $=16015$

(3S,5R,8R,9R)-5,8-Epoxy-6-megastigmene-3,9-diol 3-O- $\beta$ - $D$-glucopyranoside
$=16016$

3,25-Epoxy-3 $\alpha$-methoxy-22 $\beta$-[ $\beta, \beta$-dimethylacryloyloxy]-urs-12-en-28-oic acid $=3028$

$(16 S, 20 S, 22 S)$-16 $\beta, 22$-Epoxy-16 $\alpha$-methoxy-3 $\beta, 25,26$-trihydroxy-cycloartan24-one 3- $O-\beta$ - $D$-glucopyranosyl- $(1 \rightarrow 2)-\beta$ - $D$-glucopyranoside $=1547$

14,16-Epoxy-6-methyl-5(10),6,8,13-abietatetraene-11,12-dione $=14263$

$8 \beta$-(2',3'-Epoxy-2'-methylbutanoxy)-4 $\alpha$-hydroxy-14-oxo-5 $\alpha H, 6 \beta H, 7 \alpha H$-guai1(10),2,11(13)-triene-6,12-olide $=7546$

6-[(2,3-Epoxy-3-methylbutyl)oxy]-4,7-dimethoxyfuro[2,3-b]quinoline $=20893$

$5 \alpha H$-2 $\beta, 4-E p o x y-3 \beta$-(2-methylbutyryloxy)guaia-1(10),-11(13)-dien-6 $\beta, 12$ olide $=14195$

$(22 R, 24 R, 25 R, 26 S)$-22,26-Epoxy-24- $O$-methyl- $1 \alpha, 3 \beta, 24,25,26$-pentahydroxyergost-5-ene $26-O-\beta$ - $D$-glucopyranoside $=3639$

8,13-Epoxy-3-nor-2,3-seco-14-epilabden-2,4-olide $=688$

$3,23-\beta$-Epoxy-olean-12-en-28-oic acid $=4480$

$6 S$-(1S,2R-Epoxyphenethyl-5S-cinnamyloxy)-5,6-dihydro-2-pyrone $=9654$

Epoxypseudoisoeugenyl 2-mehylbutyrate $=14029$

cis-Epoxy-pseudoisoeugenyl tiglate $=14028$

trans-Epoxy-pseudoisoeugenyl tiglate $=14030$

15,16-Epoxy-6 $6,7 \beta, 18,19$-tetrahydroxy-neo-cleroda-3(4),13(16),14-trien$20,12(S)$-olide $=21226$

15,16-Epoxy-3 $\alpha, 6 \beta, 7 \beta, 18,19$-tetrahydroxy-neo-cleroda-4(18),13(16),14-trien$20,12(S)$-olide $=21227$

13,28-Epoxy-3 $\beta, 16 \beta, 23,29$-tetrahydroxyolean-11-en-3b-yl 30-oic acid $\beta$ - $D$ glucopyrano-syl-( $1 \rightarrow 2)$-[4-O-sulfo- $\beta$ - $D$-glucopyranosyl- $(1 \rightarrow 3)-] \beta$ - $D$ fucopyranoside $=19247$

13,28-Epoxy-3 $\beta, 16 \beta, 23,29$-tetrahydroxyolean-11-en-3- $\beta$-yl $\beta$ - $D$-glucopyranosyl-(1 $\rightarrow 2)$-[4- $O$-sulfo- $\beta$ - $D$-glucopyranosyl-( $(\rightarrow 3)] \beta$ - $D$-fucopyranoside $=19245$

13,28-Epoxy-3 $\beta, 16 \beta, 23,30$-tetrahydroxyolean-11-en-3 $\beta$-yl $\beta$ - $D$-glucopyranosyl-( $1 \rightarrow 2)$-[4- $O$-sulfo- $\beta$ - $D$-glucopyranosyl-( $1 \rightarrow 3)$ - $] \beta$ - $D$-fucopyranoside $=19248$

5,6-Epoxy-4,16,20,22-tetrahydroxy-1-oxoergost-2-eno-26,23-lactone $=11804$ (20S,22R,24S,25S)-22,25-Epoxy-3 $\beta, 24,27$-trihydroxy-cycloartan-16-one 3- $O-\beta$ - $D$-glucopyranosyl- $(1 \rightarrow 2)-\beta$ - $D$-glucopyranoside $=1548$

$(20 S, 24 S)$-20,24-Epoxy-12,23 $\beta, 25$-trihydroxydammaran-3 $\beta$-yl $O$ - $\beta$ - $D$-glucopyranosyl- $(1 \rightarrow 2)-\beta$ - $D$-xylopyranoside $=9119$

$14 \alpha, 20$-Epoxy- $1 \alpha, 7 \alpha, 14 \beta$-trihydroxy-ent-kaur-16-en-15-one $=7681$

13,28 -Epoxy-3 $\beta, 16 \beta, 23$-trihydroxyolean-11-en-3 $\beta$-yl-30-oic acid $\beta$ - $D$-glucopyranosyl-( $(1 \rightarrow 2)$-[4- $O$-sulfo- $\beta$ - $D$-glucopyranosyl- $(1 \rightarrow 3)] \beta$ - $D$-fucopyranoside $=19246$

$\mathrm{EQ}-7=3164$

$\mathrm{EQ}-8=10527$

1(10),11-Eremophiladien-2-one $=15705$

Ergobasine $=7240$

Ergocalciferol $=22558$

Ergokryptine $=7236$

Ergosta-7,22-dien-3 $\beta$-ol $=5608$

Ergosta-5,7-dien-3-ol = 14229

(22E)-Ergosta-5,7,22-trien-3 3 -ol $=7250$

Ergost-7-en-3-ol $=8003$

Ergosterol peroxide $=6906$

Erinacin A $=7261$ 


$$
\begin{aligned}
& \text { Erinacin } \mathrm{B}=7262 \\
& \text { Erinacin } \mathrm{C}=7263 \\
& \text { Erinacin } \mathrm{E}=7264 \\
& \text { Erinacin } \mathrm{G}=7265 \\
& \text { Eriocarpin } \mathrm{A}=6061 \\
& \text { Eriodictyonone }=9604 \\
& \text { Ervine }=783 \\
& \text { Erypoegin } \mathrm{A}=2772 \\
& \text { Erypoegin } \mathrm{B}=2771 \\
& \text { Erysimin }=9335 \\
& \text { Erysimotoxin }=9335 \\
& \text { Escholine }=13374 \\
& \text { Esculetin }=663 \\
& \text { Esculin }=664 \\
& \text { Estratriol }=7386
\end{aligned}
$$

1,2-Ethanedicarboxylic acid $=20444$

Ethanediol $=8826$

4,5-Etheno-9,10-dihydroxy-6-phenanthridone $=4235$

$5(R)$-5-Ethenyl-3-formamido-5-hydroxy-2-cyclopenten-1-one $=15206$

(+)-(1R,2R,4R,5R)-4-Ethenyl-2-hydroxy-4-methyl-5-(1-methylethenyl)-1-(1methylethyl)-cyclohexylacetate $=177$

(-)-(1S,2S,3S)-1-Ethenyl-1-methyl-2,3-di(1-methylethenyl)-cyclohexane = 11407

$\left(1 R^{*}, 2 S^{*}, 5 S^{*}\right)$-2-Ethenyl-1(1-methylethenyl)-2,6,6-trimethylbicyclo[3.2.0] heptane $=16596$

(+)-(1R,3R,4R)-4-Ethenyl-4-methyl-3-(1-methylethenyl)-1-(1-methylethyl)cyclohexanol $=6739$

9-Ethoxy-aristololide $=7395$

$24 \beta$-Ethoxy-20-25-epoxy-3 $\alpha, 16 \alpha$-dihydroxy-9-methyl-19-norlanost-5(6)ene-

$$
2,11,22 \text {-trione }=5114
$$

ent-17-Ethoxy-16( $\alpha$ )-kauran-19-oic acid $=19879$

$4 \beta$-Ethoxy-5-methoxy-6",6"-dimethyl-2H-pyrano-(2",3":7,6)-flavan = 5103

2-Ethoxymethylene-3,5-dihydroxy- $\gamma$-pyrone $=1678$

20-Ethyl-8-acetoxy-14-( $p$-hydroxybenzoyloxy)-1 $\alpha$-,6 $\alpha, 16 \beta, 18$-tetramethoxy-

aconitane- $3 \alpha, 13 \beta$-diol $=8270$

Ethylamylcarbinol $=15978$

Ethyl benzenecarboxylate $=7421$

Ethyl butanoate $=7423$

$24 \alpha$-ethyl-5 $\alpha$-cholesta-7-trans, 22 -dien-3 $\beta$-ol $=20168$

trans-Ethyl cinnamate $=7430$

Ethyl dodecanoate $=7455$

Ethyl ethanoate $=7418$

Ethyl hexadecanoate $=7469$

Ethyl margarate $=7444$

25-Ethyl,23-methyl-19-nor-24-methylene-3,4-seco-4(28)-lanosten-10,3-olide $=19102$

24-Ethyl,24-methyl-19-nor-3,4-seco-4(28),25(26)-lanostadiene-10,3-olide = 19103 Ethyl nonyl ether $=15700$

Ethyl trans-3-phenylpropenoate $=7430$

Ethyl stearate $=7467$

Ethyl sulfide $=5503$

$N$-Ethyl-1 $\alpha, 6 \alpha, 16 \beta, 18$-tetramethoxy-8-ethoxy-13 $\beta$-ol-2,3-dehydroaconitane14 -anisoylate $=12738$

$N$-Ethyl-1 $\alpha, 6 \alpha, 16 \beta, 18$-tetramethoxy-13 $\beta$-ol-2,3-dehydroaconitane- 8 -acetate- 14-anisoylate $=12736$

$N$-Ethyl-1 $\alpha, 6 \alpha, 16 \beta, 18$-tetramethoxy-13 $\beta$-ol-2,3-dehydroaconitane-8,14-dianisoylate $=12737$

Eucannabinolide $=9570$

$5 \alpha H$-Eudesma-4(15),11(13)-dien-12,8 $\beta$-olide $=11203$

Eudesmane-4(15)-ene-1 $\beta, 11,14$-triol 11- $O-\beta$ - $D$-glucopyranoside $=3380$

Eudesmane-4(15)-ene-1 $\beta, 2 \alpha, 11$-triol 11- $O-\beta$ - $D$-glucopyranoside $=3381$

Eudesmane-1 $\beta, 4 \alpha, 11$-triol 11-O- $\beta$ - $D$-glucopyranoside $=3382$

$1 \alpha, 5 \beta, 6 \beta, 7 \alpha, 10 \alpha-4(15)$-Eudesmen-6-ol-1-yl-acetate $=246$

4-Eudesmen-6-one $=15334$

Eugenyl acetate $=391$

Eupassopin $=7566$

(24Z)-7,24-Euphadien-3 $\beta, 26$-diol = 21407

Eupha-7,24-diene $=21404$

Eupha-8,24-diene $=21405$

(23E)-Eupha-8,23-diene-3 $\beta, 25$-diol-7,11-dione $=16365$

(23E)-Eupha-8,23-diene-3 $\beta, 25$-diol-7-one $=12131$

Eupha-8,24-diene-3 $\beta$-ol-7-one $=12130$

Euphadienol $=7611$

Eupha-8,24-dien-3 $\beta$-ol $=7611$

Eupha-7-en-3,24-dione $=21408$

Eupha-7,9(11),24-trien-3 $\beta$-ol = 1464

Euphorbia factor $\mathrm{L}_{3}=5287$

$\alpha$-Euphorbol $=7618$

Eupteleogenin 3-O-[ $\alpha$ - $L$-rhamnopyranosyl $(1 \rightarrow 2)][\beta$ - $D$-galactopyranosyl $(1 \rightarrow 3)][\alpha-L$-rhamnopyranosyl $(1 \rightarrow 4)]-\beta$ - $D$-glucopyranoside $=7630$

Eupteleogenin 3-O-[ $\alpha$ - $L$-rhamnopyranosyl $(1 \rightarrow 2)][\beta$ - $D$-glucopyranosyl

$(1 \rightarrow 3)]-6$ '- $O$-acetyl- $\beta$ - $D$-glucopyranoside $=7629$

Eupteleogenin 3-O- $\alpha$ - $L$-rhamnopyranosyl $(1 \rightarrow 2)$ - $[\beta$ - $D$-glucopyranosyl $(1 \rightarrow 3)]$ -

$$
\beta \text { - } D \text {-glucopyranoside }=7628
$$

Eurycarpin $\mathrm{B}=8501$

Eurycomanone $=16693$

Euxanthogen $=13481$

Euxanthone $=6183$

Evoxine $=9223$

Excoecaria factor $\mathrm{A}_{3}=20986$

Excoecaria factor $\mathrm{B}_{3}=20986$

Excoecariatoxin $=20986$

Exidonin $=18499$

\section{F}

Fabacei II $=4317$

Fanchinin $=21206$

Farcarindiol $=7708$

Farnoquinone $=22563$

Febrivugine $=5436$

Feloside $=17079$

$(-)$-Feroxidin $=7761$

Ferrugin $=18892$

$N$-trans-Feruloyl-3-methyldopamine $=7777$

$N$-cis-Feruloyl-3-methyldopamine $=22081$

$3 \beta-O-E$-Feruloyl oleanolic acid $=19457$

(2-trans-Feruloyloxymethyl-4- $\beta$ - $D$-glucopyranosyloxy-2(E)-butenenitrile) $=15594$ 
$3 \alpha$-E-Ferulyloxy-lup-20(29)-en-28-oic acid $=12581$

$3 \alpha$ - $E$-Ferulyloxy-urs-11-en-13 $\beta$-ol $=12582$

Ficusin $=18086$

Finetose $=13454$

Flaconitine $=304$

Flavan-4-ol $=7807$

Flavesone $=21200$

Flemiphilippinin $D=12358$

Foeniculin $=9043$

6'-O-Foliamenthoylmussaenosidic acid $=750$

Foresaconitine $=22482$

Formonetin $=7883$

Formononetin-7-O-(2",6"-O-diacetyl)glucopyranoside $=5343$

Formononetin 7-O- $\beta-D-(6 "$-ethylmalonyl $)$-glucopyranoside $=9554$

Formosanine $=22204$

3'-Formyl-4',5-dihydroxy-2",2"-dimethylchromeno-[6,7:5",6"]isoflavone = 19449

1-[3'-Formyl-2',4'-dihydroxy-6'-methoxy-5'-(3"-methylbut-2"-enyl)]acetophenone $=571$

rel-(4aS,9R,9aS)-8-Formyl-1,2,3,4,4a,9a-hexahydro-5,6,9-trihydroxy-7-isopropyl-1,1,4a-trimethylfluorene $=5432$

8-Formyl-7-hydroxy-5,6-dimethoxy coumarin $=22953$

3 -Formyl indole $=874$

2-Formyl-5-methoxyfuran $=13931$

$17 \beta$-Formyloxy-28-nor-urs-12-en-3 $\beta$-ol $=3784$

$4 \mathrm{a} S^{*}$-8-Formyl-2,3,4,4a-tetrahydro-5,6-dihydroxy-7-isopropyl-1,1,4a-trimethyl- $1 H$-fluorene $=5433$

Forskolin $=3915$

Forsythiaside $=7924$

Forsythin $=17145$

Friedelan-3 $\beta$-ol $=6918$

Friedelin $=7951$

Friedelinol $=6918$

$D: C$-Friedolean-7-ene $=15064$

$D: B$-Friedoursane-3 $\alpha, 16 \alpha$-dihydroxy- $7 \alpha, 8 \alpha$-epoxy-5(10)-ene $=17018$

$12-O-\beta$-D-Fucopyranosyl flourensadiol 10- $O-\beta-D$-glucopyranoside $=16018$

3-O- $\beta$ - $D$-Fucopyranosyl saikogenin $\mathrm{F}=17941$

$12-O-\beta$ - $D$-Fucopyranosyl selin-4(15)-en-3 $\beta, 11$-diol 3-O- $\beta$ - $D$-glucopyranoside $=16017$

Fugu poison $=21211$

Fujic acid $=8376$

Fukinanolide $=2119$

Fumarilicine $=16663$

Furan-2-carboxaldehyde $=8011$

$13 \beta$-Furanolabda-8(17),11-dien-6 $\beta, 7 \alpha$-diol $=22949$

Furanoligularenone $=8020$

6-Furfurylaminopurine $=12227$

$7 H$-Furo[3,2,g][1] benzopyran-7-one $=18086$

$(+)$-Fustin $=5613$

$(-)$-Fustin $=5614$

Fuziline $=19701$

Fysaproliferin $=17904$

\section{G}

Galactaric acid $=15007$
Galactitol $=6632$

3-O- $\alpha$ - $D$-Galactopyranosyl $(1 \rightarrow 2)-\alpha$ - $L$-arabinopyranosyl betulinic acid $28-O-$ $\alpha$ - $L$-rhamnopyranosyl $(1 \rightarrow 4)-\beta$ - $D$-glucopyranosyl $(1 \rightarrow 6)-\beta$ - $D$-glucopyranosyl ester $=4430$

3- $O-\beta$ - $D$-Galactopyranosyl-(1 $\rightarrow 4)-\alpha-L$-arabinopyranosyloleanolic acid $=17623$

3-O- $\beta$ - $D$-Galactopyranosyl $(1 \rightarrow 2) \alpha$ - $L$-arabinopyranosyl ursolic acid 28- $O$ $\beta$ - $D$-glucopyranosyl ester $=4432$

3-O- $\alpha-D$-Galactopyranosyl-( $(1 \rightarrow 2)-\alpha-L$-arabinopyranosyl ursolic acid 28-O$\alpha$ - $L$-rhamnopyranosyl $(1 \rightarrow 4)-\beta$ - $D$-glucopyranosyl $(1 \rightarrow 6)$ - $\beta$ - $D$-glucopyranosyl ester $=4433$

3-O- $\beta$ - $D$-Galactopyranosyl-( $1 \rightarrow 3)$-[ $\beta$ - $D$-glucopyranosyl-( $1 \rightarrow 2)]-\alpha-L$-arabinopyranosyl-oleanolic acid $=17693$

$(3 \beta, 5 \alpha)-3-[(O-\beta$ - $D$-Galactopyranosyl-( $1 \rightarrow 2)-O-\beta$ - $D$-glucopyranosyl-( $1 \rightarrow 4)$ $\beta$ - $D$-galactopyranosyl)oxy]-spirostan-12-one $=21015$

3 - $O$ - $(\beta$ - $D$-Galactopyranosyl-( $(1 \rightarrow 3)-\beta$ - $D$-glucopyranosyl)serjanic acid $=19334$

3-O-( $\beta$ - $D$-Galactopyranosyl-(1 $\rightarrow 3)-\beta$ - $D$-glucopyranosyl)serjanic acid 28-O$\beta$ - $D$-glucopyranoside $=19339$

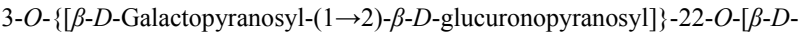
glucopyranosyl $(1 \rightarrow 3)-\alpha-L$-arabinopyranosyl] soyasapogenol $\mathrm{A}=20122$

3-O-[ $\beta$ - $D$-Galactopyranosyl-( $1 \rightarrow 3)-\beta-D$-glucuronopyranosyl] $-28-O-\beta-D$ glucopyranosylolean-12-en-28-oic acid $=1828$

3-O-[ $\beta$ - $D$-Galactopyranosyl-(1 $\rightarrow 2)-\beta-D$-glucuronopyranosyl] $-28-O-\beta-D$ glucopyranosylolean-12-en-28-oic acid $=22173$

$(3 \beta, 5 \alpha, 25 R)-3-[(O-\beta$ - $D$-Galactopyranosyl- $(1 \rightarrow 2)-O-[\beta$ - $D$-xylopyranosyl$(1 \rightarrow 3)]$ - $O-\beta$ - $D$-glucopyranosyl-( $1 \rightarrow 4)-\beta$ - $D$-galactopyranosyl)oxy]spirostan-12-one $=21016$

3 - $O$-[ $\beta$ - $D$-Galactopyranosyl $(1 \rightarrow 2)][\beta$ - $D$-xylopyranosyl $(1 \rightarrow 3)]-\beta$ - $D$-glucuronopyranosylquillaic acid 28-O-[ $\alpha-L$-rhamnopyranosyl $(1 \rightarrow 2)]-3-O-$ acetyl-4-O-(E)-para-methoxycinnamoyl- $\beta$ - $D$-fucopyranosyl ester $=$ 19941

3 - $O$ - $[\beta$ - $D$-Galactopyranosyl $(1 \rightarrow 2)][\beta$ - $D$-xylopyranosyl $(1 \rightarrow 3)]$ - $\beta$ - $D$-glucuronopyranosyl quillaic acid 28-O-[ $\alpha$ - $L$-rhamnopyranosyl $(1 \rightarrow 2)]-$ 3-O-acetyl-4-O-(Z)-para-methoxycinnamoyl- $\beta$ - $D$-fucopyranosyl ester $=19942$

3 - $O$-[ $\beta$ - $D$-Galactopyranosyl $(1 \rightarrow 2)][\beta$ - $D$-xylopyranosyl $(1 \rightarrow 3)]-\beta$ - $D$-glucuronopyranosyl quillaic acid $28-O-[\alpha-L$-rhamnopyranosyl $(1 \rightarrow 2)]-4-O-(E)-$ para-methoxyciunamoyl $-\beta$ - $D$-fucopyranosyl ester $=19945$

3 - $O$-[ $\beta$-D-Galactopyranosyl $(1 \rightarrow 2)][\beta$ - $D$-xylopyranosyl $(1 \rightarrow 3)]-\beta$ - $D$-glucurono pyranosyl quillaic acid $28-O-\{[\beta-D$-xylopyranosyl $(1 \rightarrow 4)]-\alpha-L$-rhamnopyranosyl $(1 \rightarrow 2)\}[\beta$ - $D$-glucopyranosyl $(1 \rightarrow 3)]-4-O$-acetyl- $\beta$ - $D$-fucopyranosyl ester $=19946$

3- $O$-[ $\beta$ - $D$-Galactopyranosyl $(1 \rightarrow 2)][\beta$ - $D$-xylopyranosyl $(1 \rightarrow 3)]$-[6-O-methyl$\beta$ - $D$-glucuronopyranosyl $]$ quillaic acid $28-O-[\alpha-L$-rhamnopyranosyl $(1 \rightarrow 2)]$-[3-O-acetyl-4- $O-(E)$-para-methoxycinnamoyl- $\beta$ - $D$-fucopyranosyl] ester $=19943$

3- $O$-[ $\beta$-D-Galactopyranosyl $(1 \rightarrow 2)][\beta$ - $D$-xylopyranosyl $(1 \rightarrow 3)]$-[6- $O$-methyl$\beta$ - $D$-glucuronopyranosyl] quillaic acid 28-O-[ $\alpha-L$-rhamnopyranosyl $(1 \rightarrow 2)]$-3-O-acetyl-4-O-(Z)-paramethoxyciunamoyl- $\beta$ - $D$-fucopyranosyl ester $=19944$

3-O- $\beta$ - $D$-Galactopyranosyl- $(1 \rightarrow 4)-\beta$ - $D$-xylopyranosyloleanolic acid $=17622$ $\alpha$ - $D$-Galactosyl- $\alpha$ - $D$-galactosyl- $\alpha$ - $D$-glucosyl $-\beta$ - $D$-fructose $=20255$

4,6-(S,S)-Gallagyl- $D$-glucose $=18205$

Gallic acid ethyl ester $=7441$ 
1-Galloyl-2-cinnamoyl-glucose $=3703$

Galloylepicatechin $=6864$

6-Galloyl-2,3-(S)-hexahydroxydiphenoyl- $D$-glucose $=18201$

1-O-Galloyl-3,6- $O$-HHDP-2,4- $O$-dehydroxymethyl-chebuloyl- $\beta$ - $D$-glucopyra $\operatorname{nos}=17224$

1-O-Galloyl-2,4- $(R)$-HHDP- $\beta$ - $D$-glucose $=17209$

$1-O$-Galloylpedunculagin $=3301$

1-O-Galloyl-2,4-tetrahydroxydibenzofurandicarboxyl- $\beta$ - $D$-glucose $=17208$

Gamabufotalin $=8121$

Ganoderic acid $\mathrm{A}=8150$

Ganoderic acid $\mathrm{B}=8151$

Ganoderic acid $\mathrm{C}_{1}=8152$

Ganoderic acid $D=8153$

Ganoderic acid $\mathrm{DM}=8154$

Ganoderic acid $\mathrm{F}=8155$

Ganoderic acid $\mathrm{G}=8170$

Ganoderic acid $\mathrm{H}=8156$

Ganoderic acid $\mathrm{LM}_{2}=8157$

Ganoderic acid $\mathrm{Ma}=8158$

Ganoderic acid $U=8159$

Ganoderic acid V $=8160$

Ganoderic acid $\mathrm{W}=8161$

Ganoderic acid $\mathrm{X}=8148$

Ganoderic acid $\mathrm{Y}=8149$

Ganwuweizic acid $=19499$

Gelidoside $=18848$

Gelsenicine $=9661$

Geneserine $=7380$

Genipin $\beta$-cellobioside $=8268$

Genistein 7-O- $\beta-D-(6 "-O$-acetylglucopyranoside $)=398$

Genistin $=8282$

Genkwanin 8-C-glucoside $=11724$

Genkwanin-5- $O$-xylosylglucoside $=22935$

Gentiocrucine $=8293$

Gentiopicrin $=8304$

Gentisin $=6011$

Geoside $=8250$

Geranial $=3761$

Geranyl alcohol $=8312$

3-Geranyl-4-hydroxy-6-(2-hydroxypropyl)-2-pyrone = 2007

7-Geranyloxy-8-methoxycoumarin $=3926$

7-Geranyloxy-6-methoxycoumarin $=13846$

4-Geranyl-2',3,4',5-tetrahydroxy-trans-stilbene $=3567$

2-Geranyl-1,3,6-trihydroxy-2',2'-dimethyl[5',6':7,8] xanthone $=918$

1(10),4(15)-Germacradien-6-one $=17809$

$1(10) E, 4 E$-Germacrone $=8347$

Gertiopicrin $=8304$

Gesneroidin $\mathrm{F}=8483$

6 - $O$ - $\alpha$ - $L$-Ghamnopyranosyl-( $1 \rightarrow 3)-\beta$ - $D$-quinovopyranosyl-(22R,23S,25S)-

$3 \beta, 6 \alpha, 23$-trihydroxy-5 $\alpha$-spirostane $=21471$

6- $O$ - $\alpha$ - $L$-Ghamnopyranosyl-( $1 \rightarrow 3)-\beta$ - $D$-quinovopyranosyl $(22 R, 23 S, 25 R)$ -

$3 \beta, 6 \alpha, 23$-trihydroxy-5 $\alpha$-spirostane $=21472$

Gibberellic acid $=8372$
Ginkgoic acid $=8403$

Ginsenoside $\mathrm{A}=18025$

Ginsenoside $\mathrm{A}_{2}=8430$

Ginsenoside $\mathrm{B}_{2}=8428$

Ginsenoside $\mathrm{C}=8431$

Ginsenoside $\mathrm{Rh}_{1}=8435$

Gitogenin $\beta$-lycotetraoside $=8458$

Glabralactone $=1193$

Glaucogenin C 3- $O$ - $\alpha$ - $L$-diginopyranosyl-(1 $\rightarrow 4)-\beta$ - $D$-thevetopyranoside $=1991$

Glaucogenin C 3-O- $\beta$ - $D$-thevetopyranoside $=8507$

Glucogallin $=8108$

1- $\beta$-Glucogeniposide $=8276$

$D$-Gluconic acid $=8600$

10-O-Glucopyranoside aristolochic acid $\mathrm{D}=1725$

6- $O$ - $\beta$ - $D$-Glucopyranosyl-( $1 \rightarrow 2)$-6- $O$-acetyl- $\beta$ - $D$-glucopyranosyl $20(S), 24(S)$ epoxydammane- $3 \beta, 6 \alpha, 12 \beta,-25$-tetrol $=22893$

3-O-[ $\beta$ - $D$-Glucopyranosyl-( $1 \rightarrow 2)$-6- $O$-acetyl- $\beta$ - $D$-glucopyranosyl]-20- $O$ - $[\beta$ -

$D$-xylopyranosyl-( $1 \rightarrow 6)-\beta$ - $D$-glucopyranosyl-( $1 \rightarrow 6)-\beta$ - $D$-glucopyranosyl-3 $\beta,-12 \beta, 20(S)$-trihydroxydamm-24-ene $=22902$

8 - $O$ - $\beta$-D-Glucopyranosylakebonoic acid 3- $O-\alpha$ - $L$-arabinopyranosyl $(1 \rightarrow 3)$ $[\alpha$ - $L$-rhamnopyranosyl $(1 \rightarrow 4)]-\beta-D$-glucopyranoside $=7635$

7-O- $\beta$ - $D$-Glucopyranosyl-anhydroicaritin-3- $O-\beta$ - $D$-(3,6- $O$-diacetyl)-glucopyranosyl-( $1 \rightarrow 3)-\alpha-L-(4-O$-acetyl)-rhamnopyranoside $=3480$

4'-O-Glucopyranosyl apigenin-6- $C$ - $\alpha$ - $L$-arabinopyranosyl $(1 \rightarrow 2)-\beta-D-(6-O$ dihydroferuloyl)-glucopyranoside $=19667$

3-O- $\{[\beta-D$-Glucopyranosyl $(1 \rightarrow 2)][\alpha-L$-arabinofuranosyl- $(1 \rightarrow 4)]-[3-O$-acetyl]- $\beta-D$ glucuronopyranosyl $\}-28-O-[\beta-D$-glucopyranosyl]-oleanolic acid $=20538$

3- $O$ - $[\beta$ - $D$-Glucopyranosyl- $(1 \rightarrow 3)[\alpha-L$-arabinofuranosyl- $(1 \rightarrow 4)]$-6'- $O$-ethyl- $\beta$ - $D$ glucuronopyranosyl]-oleanolic acid-28- $O-\beta$ - $D$-glucopyranoside $=20615$

3-O-[ $\beta$ - $D$-Glucopyranosyl- $(1 \rightarrow 3)-O-\{\alpha-L$-arabinofuranosyl- $(1 \rightarrow 2)\}-O-(\beta-D-$ glucopyranosyl)]jujubogenin $=2094$

$\left(4^{\prime} R\right)-4$ '- $O$-[ $\beta$ - $D$-Glucopyranosyl-( $\left.1 \rightarrow 4\right)-\beta$ - $D$-arabinofuranosyl-( $\left.1 \rightarrow 3\right)-\beta$ - $D$-glu copyranosyl- $(1 \rightarrow 4)]$-[ $\beta$ - $D$-xylopyranosyl-( $(1 \rightarrow 2)]$ - $\beta$ - $D$-glucopyranosyl-14-woodwardine $=22723$

$3-O-[\beta-D$-Glucopyranosyl- $(1 \rightarrow 3)]-\alpha-L$-arabinopyranosyl $]$ caulophyllogenin $=3976$ $3-O-\beta$ - $D$-Glucopyranosyl $(1 \rightarrow 2)-\alpha$ - $L$-arabinopyranosyl $\beta$-kudinlactone $=10979$

$3-O-\beta$ - $D$-Glucopyranosyl $(1 \rightarrow 3)-\alpha$ - $L$-arabinopyranosyl oleanolic acid 28- $O$ - $\alpha$ - $L$-rhamnopyranosyl $(1 \rightarrow 4)-\beta$ - $D$-glucopyranosyl $(1 \rightarrow 6)-\beta$ - $D$-glucopyranoside $=2211$

3-O- $\beta$ - $D$-Glucopyranosyl-( $1 \rightarrow 3)$ - $\alpha$ - $L$-arabinopyranosyl pomolic acid $28-\beta-D$ glucopyranosyl ester $=18537$

$3-O-\beta$ - $D$-Glucopyranosyl- $(1 \rightarrow 3)-\alpha-L$-arabinopyranosyl rotundic acid $=18536$

3-O- $\beta$ - $D$-Glucopyranosylbayogenin $28-O-\beta$ - $D$-apiofuranosyl- $(1 \rightarrow 3)-\beta$ - $D$ xylopyranosyl-( $1 \rightarrow 4)$-[ $\beta$ - $D$-apiofuranosyl- $(1 \rightarrow 3)]$ - $\alpha$ - $L$-rhamnopyranosyl- $(1 \rightarrow 2)-\alpha-L$-arabinopyranosyl ester $=4024$

3-O- $\beta$ - $D$-Glucopyranosyl betulinic acid $28-O-\alpha$ - $L$-arabinopyranosyl $(1 \rightarrow 6)$ $\beta$ - $D$-glucopyranosyl ester $=4429$

2-O- $(\beta-D$-Glucopyranosyl $)$ bruceantinol $=22866$

3- $O$ - $(\beta-D$-Glucopyranosyl $)$ brucein $\mathrm{A}=22861$

2-O- $(\beta-D-$ Glucopyranosyl $)$ brucein $\mathrm{C}=22862$

2-exo- $\beta$ - $D$-Glucopyranosyl-1,8-cineol $=2696$

$28-O-\beta$ - $D$-Glucopyranosyl cochalic acid 3-O- $\beta$ - $D$-galactopyranosyl $(1 \rightarrow 3)-\beta$ -

$D$-glucopyranosiduronic acid $=2966$

$28-O-\beta-D$-Glucopyranosyl cochalic acid $3-O-\beta-D$-glucopyranosyl $(1 \rightarrow 2)-[\beta$ - 
$D$-galactopyranosyl $(1 \rightarrow 3)]-\beta$ - $D$-glucopyranosiduronic acid $=2967$ $\left(8 R, 8^{\prime} R\right)$ 9- $\beta$ - $D$-Glucopyranosyl dihydrocubebin $=21036$ $6 \alpha-O-(\beta-D$-Glucopyranosyl)-15,16-dihydroxycleroda-3,13(14)-dien = 19212 $(2 R)-5-O-\beta$ - $D$-Glucopyranosyl-7,4'-dihydroxy-3',5'-dimethoxyflavanone = 16999 (2S)-5-O- $\beta$ - $D$-Glucopyranosyl-7,4'-dihydroxy-3',5'-dimethoxyflavanone $=17000$ 3-O- $\beta$ - $D$-Glucopyranosyl $3 \alpha, 11 \alpha$-dihydroxylup-20(29)-en-28-oic acid 28-O-

$\alpha$ - $L$-rhamnopyranosyl-( $1 \rightarrow 4)$ - $\beta$ - $D$-glucopyranosyl-( $1 \rightarrow 6)-\beta$ - $D$-glucopyranosyl ester $=73$

3-O- $\beta$ - $D$-Glucopyranosyl 3 $\beta, 23$-dihydroxylup-20(29)-en-28-oic acid 28- $O$ -

$\alpha$ - $L$-rhamnopyranosyl $(1 \rightarrow 4)$ - $\beta$ - $D$-glucopyranosyl $(1 \rightarrow 6)-\beta$ - $D$-glucopyranoside $=3737$

$6 \beta$-C-Glucopyranosyl-5,7-dihydroxy-2-methylchromone $=2369$

3-O- $\beta$ - $D$-Glucopyranosyl-2 $\beta, 3 \beta$-dihydroxyolean-12-ene-23,28-dioic acid (medicagenic acid) $28-O-\beta$ - $D$-glucopyranosyl- $(1 \rightarrow 6)-O-\beta$ - $D$-glucopyranosyl ester $=733$

(25S)-24- $O-\beta$ - $D$-Glucopyranosyl-3 $\beta, 24 \beta$-dihydroxy- $5 \alpha$-spirost-3- $O$ - $\alpha$-arabinopyranosyl-( $(1 \rightarrow 6)-\beta$ - $D$-glucopyranoside $=3528$

(25S)-26- $O-\beta$ - $D$-Glucopyranosyl-6 $\alpha$-26-dihydroxy-5 $\alpha$-spirosten-3-one 6- $O$ $[\alpha-L$-rhamnopyranosyl-( $1 \rightarrow 3)-\beta$ - $D$-quinovopyranoside $]=21470$

$(22 S, 25 S)$ 26- $O-\beta$ - $D$-Glucopyranosyl-22,25-epoxy-furost-5-ene-3 $\beta, 7 \beta, 26$-triol 3- $O$ - $\beta$-chacotrioside $=45$

$(22 S, 25 S)$ 26- $O-\beta$ - $D$-Glucopyranosyl-22,25-epoxy-furost-5-ene-3 $\beta, 7 \beta, 26$-triol 3- $O$ - $\beta$-solatrioside $=47$

26-O- $\beta$ - $D$-Glucopyranosyl-(16S,20S,22S)-16 $\beta, 22$-epoxy-16 $\alpha$-methoxy-3 $\beta, 26$ dihydroxy-cycloartan-24-one-3- $O-\beta$ - $D$-glucopyranosyl-( $1 \rightarrow 2)-\beta$ - $D$-gluco pyranoside $=1545$

(22S,25S) 26- $O-\beta$ - $D$-Glucopyranosyl-22,25-epoxy-7 $\beta$-methoxy-furost-5-ene$3 \beta, 26$-diol 3- $O-\beta$-chacotrioside $=46$

$26-O-\beta$ - $D$-Glucopyranosyl-(16S,20S,22S)-16 $\beta, 22$-epoxy-16 $\alpha$-methoxy-3 $\beta, 25$, 26 -trihydroxy-cycloartan-24-one-3- $O-\beta$ - $D$-glucopyranosyl- $(1 \rightarrow 2)-\beta$ - $D$ glucopyranoside $=1546$

$27-O-\beta$ - $D$-Glucopyranosyl-(20S,22R,24S,25S)-22,25-epoxy-3 $\beta, 24,27$-trihydroxy-cycl oartan-16-one-3- $O-\beta$ - $D$-glucopyranosyl- $(1 \rightarrow 2)-\beta$ - $D$-glucopyranoside $=1549$

3- $O-\alpha-D$-Glucopyranosyl- $\beta-D$-fructopyranose $=22118$

3-O-[ $\beta$-D-glucopyranosyl( $1 \rightarrow 3)$ - $\beta$ - $D$-fucopyranosyl]-3 $\beta, 16 \alpha, 23$,28-tetrahydroxy-olean-11,13(18)-dien-30-oic acid 30- $O$-[pentito( $1 \rightarrow 1)]-\beta$ - $D$-glucopyranosyl-6-ester $=19164$

3'-O- $\beta$ - $D$-Glucopyranosyl[2",3":7,8] furanoflavone $=17709$

26-O- $\beta$ - $D$-Glucopyranosyl-25(R)-5 $\alpha$-furostan-20(22)-en-2 $\alpha, 3 \beta, 26$-triol-3- $O-\beta$ $D$-xylopyranosyl $(1 \rightarrow 6)-\beta$ - $D$-glucopyranoside $=21673$

(25S)-26- $O-\beta$ - $D$-Glucopyranosyl-5 $\alpha$-furostan-2 $\alpha, 3 \beta, 22 \alpha, 26$-tetraol 3-O- $\{\beta-D$ glucopyranosyl-( $1 \rightarrow 2)-O$-[ $\beta$ - $D$-glucopyranosyl- $(1 \rightarrow 3)]-O$ - $\beta$ - $D$-glucopyranosyl- $(1 \rightarrow 4)-\beta$ - $D$-galactopyranoside $=16882$

(25S)- $O$ - $\beta$ - $D$-Glucopyranosyl-5 $\beta$-furost-20(22)-ene-3 $\beta, 26$-diol-3- $O-\beta$ - $D$-glucopyranosyl- $(1 \rightarrow 2)-\beta$ - $D$-glucopyranoside $=1174$

26-O- $\beta$ - $D$-Glucopyranosyl-(25R)-5 $\alpha$-furost-3 $\beta, 22 \alpha, 26$-triol 3- $O-\beta$ - $D$-glucopyranosyl- $(1 \rightarrow 2)$ - $[\beta$ - $D$-xylopyranosyl- $(1 \rightarrow 3)]$ - $\beta$ - $D$-glucopyranosyl- $(1 \rightarrow 4)$ $\beta$-D-galactopyranoside $=22286$

26-O- $\beta$ - $D$-Glucopyranosyl-(25R)-5 $\alpha$-furost-3 $\beta, 22 \alpha,-26$-triol-12-one3- $O-\beta$ - $D$ xylopyranosyl- $(1 \rightarrow 3)-\beta$ - $D$-glucopyranosyl- $(1 \rightarrow 2)$ - $[\beta$ - $D$-xylopyranosyl$(1 \rightarrow 3)]$ - $\beta$ - $D$-glucopyranosyl-( $1 \rightarrow 4)-\beta$ - $D$-galactopyranoside $=17614$

26- $O-\beta$ - $D$-Glucopyranosyl-(25R)-5 $\alpha$-furost-3 $\beta, 22 \alpha, 26$-triol-12-one 3- $O-\beta$ - $D$ glucopyranosyl-( $1 \rightarrow 2)$-[ $\beta$ - $D$-xylopyranosyl- $(1 \rightarrow 3)]-\beta$ - $D$-glucopyranos- yl- $(1 \rightarrow 4)-\beta$ - $D$-galactopyranoside $=17613$

$26-O-\beta$ - $D$-Glucopyranosyl-(25R)-5 $\alpha$-furost-3 $\beta, 22 \alpha, 26$-triol 3- $O-\beta$ - $D$-xylopyranosyl-( $1 \rightarrow 3)-\beta$ - $D$-glucopyranosyl- $(1 \rightarrow 2)$ - $[\beta$ - $D$-glucopyranosyl$(1 \rightarrow 3)]$ - $\beta$ - $D$-glucopyranosyl-( $1 \rightarrow 4)$ - $\beta$ - $D$-galactopyranoside $=17616$ 26- $O$ - $\beta$ - $D$-Glucopyranosyl-(25R)-5 $\alpha$-furost-3 $\beta, 22 \alpha, 26$-triol 3- $O-\beta$ - $D$-xylopyranosyl- $(1 \rightarrow 3)-\beta$ - $D$-glucopyranosyl- $(1 \rightarrow 2)$ - $[\beta$ - $D$-xylopyranosyl$(1 \rightarrow 3)]$ - $\beta$ - $D$-glucopyranosyl-( $1 \rightarrow 4)-\beta$ - $D$-galactopyranoside $=17615$

3 - $O$ - $[\beta$ - $D$-Glucopyranosyl-( $1 \rightarrow 2)$ - $\beta$ - $D$-galactopyranosyl $(1 \rightarrow 2)-\beta$ - $D$-glucuronopyranosyl]soyasapogenol $\mathrm{B}=20129$

$10-O-\beta-D$-Glucopyranosyl geniposide $=8269$

3 - $O$ - $[\beta$ - $D$-Glucopyrano-syl $(1 \rightarrow 3)-\beta$ - $D$-glucopyranosyl $(1 \rightarrow 3)-\alpha$ - $L$-arabino-pyranosyl ]-16 $\alpha$,23-dihydroxyolean-12-ene 28-O- $\beta$ - $D$-glucopyranosyl ester $=11801$

3 - $O$-[ $\beta$ - $D$-Glucopyranosyl- $(1 \rightarrow 2)$ - $[\beta$ - $D$-glucopyranosyl- $(1 \rightarrow 3)]-\alpha$ - $L$-arabinopyranosyl]hederagenin 28- $O-\beta$ - $D$-glucopyranosyl ester $=1607$

3 - $O$ - $\beta$ - $D$-Glucopyranosyl-( $1 \rightarrow 2)$ - $\beta$ - $D$-glucopyranosyl- $(1 \rightarrow 3)$ - $\alpha$ - $L$-arabinopyranosyl- $\beta$-kudinlactone $=12300$

3-O- $\beta$-D-Glucopyranosyl-( $1 \rightarrow 3$ )- $\beta$ - $D$-glucopyranosylbayogenin $28-O-\beta$ - $D$ apiofuranosyl-( $(1 \rightarrow 3)-\beta$ - $D$-xylopyranosyl- $(1 \rightarrow 4)$ - $[\beta$ - $D$-apiofuranosyl$(1 \rightarrow 3)]-\alpha-L$-rhamnopyranosyl- $(1 \rightarrow 2)-\alpha$ - $L$-arabinopyranosyl ester $=4025$ 3-O- $\beta$ - $D$-Glucopyranosyl $(1 \rightarrow 4)-\beta$ - $D$-glucopyranosyl echinocystic acid $=3978$

3 - $O$-[ $\beta$-D-Glucopyranosyl $(1 \rightarrow 2)$ - $\beta$ - $D$-glucopyranosyl]-16 $\alpha$-ethoxy-olean-12ene-28-oic acid 28- $O-\beta$ - $D$-glucopyranoside $=6702$

3 - $O$-[ $\beta$ - $D$-Glucopyranosyl- $(1 \rightarrow 2)-\beta$ - $D$-glucopyranosyl- $(1 \rightarrow 3)-\beta$ - $D$-fucopyranosyl]-3 $\beta, 16 \alpha, 23,28$-tetrahydroxy-olean-11,13(18)-dien-30-oic acid-30$O$-[pentito $(1 \rightarrow 1)]$ - $\beta$ - $D$-glucopyranosyl-6-ester $=19163$

9- $O$ - $\beta$ - $D$-Glucopyranosyl-( $1 \rightarrow 3)$ - $[\beta$ - $D$-glucopyranosyl- $(1 \rightarrow 6)]-\beta$ - $D$-Glucopyranoside $=22724$

3 - $O$-[ $\beta$ - $D$-Glucopyranosyl- $(1 \rightarrow 2)$ - $[\beta$ - $D$-glucopyranosyl- $(1 \rightarrow 3)]-\beta$ - $D$-glucopyranosyl] echinocystic acid 28-O- $\beta$ - $D$-glucopyranosyl ester $=1604$

3-O-[ $\beta$ - $D$-Glucopyranosyl- $(1 \rightarrow 2)]$ - $[\beta$ - $D$-glucopyranosyl- $(1 \rightarrow 3)] \beta$ - $D$-glucopyranosyl echinostic acid $=3977$

6- $O$-[ $\alpha$ - $D$-Glucopyranosyl-( $1 \rightarrow 6)]-\beta$ - $D$-glucopyranosyl-( $1 \rightarrow 2)-\beta$ - $D$-glucopyra nosyl 20(S),24(S)-epoxydammane-3 $\beta, 6 \alpha, 12 \beta, 25$-tetrol $=22894$

10 '- $O$ - $[\beta$ - $D$-Glucopyranosyl- $(1 \rightarrow 6)]-\beta$ - $D$-glucopyranosyl- $(1 \rightarrow 3)-\beta$ - $D$-glucopy ranosyl- $(1 \rightarrow 3)-\beta$ - $D$-glucopyranoside $=22725$

3- $O$ - $[\beta$ - $D$-Glucopyranosyl-( $1 \rightarrow 3)$ - $\beta$ - $D$-glucopyranosyl-( $1 \rightarrow 3)$ - $[\beta$ - $D$-glucopyranosyl-( $1 \rightarrow 2)]-\beta$ - $D$-glucopyranosyl]caulophyllogenin $28-O-\beta$ - $D$-glucopyranosyl ester $=1606$

3-O- $\beta$ - $D$-Glucopyranosyl-( $1 \rightarrow 2)$ - $[\beta$ - $D$-glucopyranosyl-( $1 \rightarrow 3)]-\beta$ - $D$-glucopyranosyl]hederagenin 28-O- $\beta$ - $D$-glucopyranosyl ester $=1605$

3 - $O$ - $\beta$ - $D$-Glucopyranosyl- $(1 \rightarrow 6)$ - $[\beta$ - $D$-glucopyranosyl $(1 \rightarrow 4)](\beta$ - $D$-glucopyranosyl-olean-11,13(18)-diene-3 $\beta, 16 \beta, 23,28$-tetrol $=3845$

3 - $O$-[ $\beta$ - $D$-Glucopyranosyl- $(1 \rightarrow 2)$ - $[\beta$ - $D$-glucopyranosyl-( $1 \rightarrow 3)]-\beta$ - $D$-glucopyranosyl]oleanolic acid 28-O- $\beta$ - $D$-glucopyranosyl ester $=1603$

3 - $O$ - $(\beta$ - $D$-Glucopyranosyl-( $1 \rightarrow 3)-\beta$ - $D$-glucopyranosyl-( $1 \rightarrow 3)-\beta$ - $D$-glucopyranosyl)serjanic acid $=19336$

3-O- $\beta$ - $D$-Glucopyranosyl-( $1 \rightarrow 6)-\beta$ - $D$-glucopyranosyl-( $1 \rightarrow 6)-\beta$ - $D$-glucopyra-nosyl $-2 \beta, 3 \beta, 16 \alpha, 23$-tetrahydroxyolean-12-en-28-oic acid 28-O- $\beta$ - $D$-xylopyranosyl$(1 \rightarrow 4)$ - $\alpha$ - $L$-rhamnopyranosyl- $(1 \rightarrow 2)-\alpha-L$-arabinopyranoside $=17542$

$3 \beta$ - $O-\beta$ - $D$-Glucopyranosyl-( $1 \rightarrow 2)-O-\beta$ - $D$-glucopyranosyl-20- $O-\beta$ - $D$-glucopyranos yl-3 $\beta, 12 \beta, 20(S)$-trihydroxy-24-hydrogenperoxide-dammar-25-ene $=8416$

3-O-[ $\beta$ - $D$-Glucopyranosyl-( $1 \rightarrow 3)]-\beta$ - $D$-glucopyranosyl- $(1 \rightarrow 2)-\beta$ - $D$-glucuronopyranosyl-22-O-acetyl-21-O-(2-methylpropanoyl)-3 $\beta, 15 \alpha, 16 \alpha, 21 \beta$, 
$22 \alpha, 28$-hexahydroxyolean-12-ene $=9712$

3 - $O$ - $\beta$ - $D$-Glucopyranosyl-( $1 \rightarrow 2)-\beta$ - $D$-glucopyranosyl- $(1 \rightarrow 2)-\beta$ - $D$-glucuronopyranosyl-22- $O$-acetyl-21- $O$-(2-methylpropanoyl)-3 $\beta, 15 \alpha, 16 \alpha, 21 \beta, 22 \alpha$, 28-hexahydroxyolean-12-ene $=9713$

$3 \beta$ - $O$ - $(\beta$ - $D$-Glucopyranosyl-( $1 \rightarrow 4)-\beta$ - $D$-glucopyranosyl-16 $\alpha$-hydroxyolean-

12-ene-28-oic acid-28- $O-\beta$ - $D$-glucopyranoside $=6706$

$3-O$ - $[\beta$ - $D$-Glucopyranosyl- $(1 \rightarrow 3)-\beta$ - $D$-glucopyranosyl]jujubogenin $=2091$

3 - $O$ - $\beta$ - $D$-Glucopyranosyl $(1 \rightarrow 2)-\beta$ - $D$-glucopyranosyl $\beta$-kudinlactone $=10980$

3-O- $\beta$ - $D$-Glucopyranosyl-( $1 \rightarrow 6)-\beta$ - $D$-glucopyranosyl $2 \beta, 3 \beta, 16 \alpha, 23,24$-penta-

hydroxyolean-12-ene-28-oic acid 28- $O-\beta$ - $D$-xylopyranosyl-( $1 \rightarrow 4)-\alpha-L$ -

rhamnopyranosyl- $(1 \rightarrow 2)-\alpha-L$-ara- binopyranoside $=4800$

3-O- $\beta$ - $D$-Glucopyranosyl-( $\rightarrow 3)$ - $\beta$ - $D$-glucopyranosyl $2 \beta, 3 \beta, 16 \alpha, 23,24$-penta-

hydroxyolean-12-ene-28-oic acid 28- $O-\beta$ - $D$-xylopyranosyl-(1 $\rightarrow 4)-\alpha-L$ -

rhamnopyranosyl-( $1 \rightarrow 2)-\alpha-L$-arabinopyranoside $=17539$

3-O- $\beta$ - $D$-Glucopyranosyl-( $1 \rightarrow 3$ )- $\beta$ - $D$-glucopyranosyl-2 $\beta, 3 \beta, 16 \alpha, 23,24$-penta-

hydroxyolean-12-en-28-oic acid $=17544$

3-O- $\beta$ - $D$-Glucopyranosyl-( $1 \rightarrow 6)$ - $\beta$ - $D$-glucopyranosyl-2 $\beta, 3 \beta, 16 \alpha, 23,24$-penta-

hydroxyolean-12-en-28-oic acid $=17545$

$3-O$ - $[\beta$ - $D$-Glucopyranosyl-( $1 \rightarrow 3)-\beta$ - $D$-glucopyranosyl $]$ pseudojujubogenin $=2092$

$27-O$ - $\beta$ - $D$-Glucopyranosyl $(1 \rightarrow 6)-\beta$ - $D$-glucopyranosylpubesenolide $3-O-\beta$ - $D$ -

glucopyranosyl $(1 \rightarrow 6)-\beta$ - $D$-glucopyranoside $=22712$

3-O- $\beta$ - $D$-Glucopyranosyl $(1 \rightarrow 2)-\beta$ - $D$-glucopyranosyl- $[\alpha$ - $L$-rhamnopyranosyl

$(1 \rightarrow 2)]$ - $\alpha$ - $L$-arabinopyranosyl $3 \beta, 19 \alpha$-dihydroxy-urs-12-en-28,20 $\beta$-olide

$=10977$

$3-O-\beta$ - $D$-Glucopyranosyl-( $1 \rightarrow 2)-\beta$ - $D$-glucopyranosyl-( $1 \rightarrow 3)$ - $[\alpha-L$-rhamnopyranos

yl-(1 $\rightarrow 2)]-\alpha$ - $L$-arabinopyranosyl $3 \beta, 19 \alpha$-dihydroxyursolic acid $=12558$

3 - $O$ - $\beta$ - $D$-Glucopyranosyl-( $1 \rightarrow 4)-\beta$ - $D$-glucopyranosyl-( $1 \rightarrow 3)-\alpha$ - $L$-rhamnopy-

ranosyl- $(1 \rightarrow 2)$ - $\alpha$ - $L$-arabinopyranosyl-hederagenin-28- $O-\beta$ - $D$-glucopyra-

nosyl- $(1 \rightarrow 6)-\beta$ - $D$-glucopyranosyl ester $=13299$

3-O- $\beta$ - $D$-Glucopyranosyl $(1 \rightarrow 2)-\beta$ - $D$-glucopyranosyl- $[\alpha$ - $L$-rhamnopyranosyl

$(1 \rightarrow 2)]-\alpha$ - $L$-arabinopyranosyl $\gamma$-kudinlactone $=10978$

3- $O$ - $\beta$ - $D$-Glucopyranosyl-( $1 \rightarrow 2)-\beta$ - $D$-glucopyranosyl-( $1 \rightarrow 3)$ - $[\alpha$ - $L$-rhamnopyranosyl- $(1 \rightarrow 2)]-\alpha-L$-arabinopyranosyl- $\beta$-kudinlactone $=12301$

3 - $O$ - $\beta$ - $D$-Glucopyranosyl-( $\rightarrow 4)-\beta$ - $D$-glucopyranosyl-( $1 \rightarrow 3)$ - $\alpha$ - $L$-rhamnopyranosyl- $(1 \rightarrow 2)-\alpha$ - $L$-arabinopyranosylpomolic acid $28-O-\beta$ - $D$-glucopyranosyl- $(1 \rightarrow 6)-\beta$ - $D$-glucopyranoside $=19439$

3-O- $\beta$ - $D$-Glucopyranosyl-( $1 \rightarrow 4)-\beta$ - $D$-glucopyranosyl-( $1 \rightarrow 3)$ - $\alpha$ - $L$-rhamnopyranosyl-( $1 \rightarrow 2)-\beta$ - $D$-xylopyranosyloleanolic acid $28-O-\beta$ - $D$-glucopyranosyl- $(1 \rightarrow 6)-\beta$ - $D$-glucopyranoside $=19438$

3- $O$ - $(\beta-D$-Glucopyrano-syl- $(1 \rightarrow 2)-\beta$ - $D$-glucopyranosyl $)$ serjanic acid $=19335$

3-O-( $\beta$ - $D$-Glucopyranosyl-( $1 \rightarrow 2)-\beta$ - $D$-glucopyranosyl)serjanic acid $28-O-\beta$ -

$D$-glucopyranoside $=19340$

3-O- $\beta$ - $D$-Glucopyranosyl-( $1 \rightarrow 6)-\beta$ - $D$-glucopyranosyl- $2 \beta, 3 \beta, 16 \alpha, 23$-tetrahy-

droxyolean-12-en-28-oic acid 28-O- $\beta$ - $D$-xylopyranosyl- $(1 \rightarrow 4)-\alpha-L$ -

rhamnopyranosyl- $(1 \rightarrow 2)-\alpha$ - $L$-arabinopyranoside $=17541$

3- $O$-[ $\beta$ - $D$-Glucopyranosyl-( $1 \rightarrow 2)-\beta$ - $D$-glucopyranosyl]-20- $O$-[ $\beta$ - $D$-xylopyranosyl-(1 $\rightarrow 6)-\beta$-D-glucopyranosyl-3 $\beta, 12 \beta, 20(S), 25$-tetrahydroxydammar23-ene $=22900$

3-O-[ $\beta$ - $D$-Glucopyranosyl-( $1 \rightarrow 2)-\beta$ - $D$-glucopyranosyl $]-20-O$ - $[\beta-D$-xylopyranosyl-( $1 \rightarrow 6)-\beta$ - $D$-glucopyranosyl-( $1 \rightarrow 6)-\beta$ - $D$-glucopyranosyl-3 $\beta, 20(S)$ dihydroxy dammar-24-ene $=22901$

3-O-[ $\beta$ - $D$-Glucopyranosyl-( $1 \rightarrow 2)-\beta$ - $D$-glucopyranosyl]-20- $O$-[ $\beta$ - $D$-xylopyra-

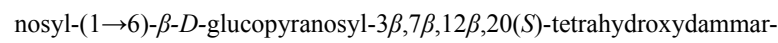

5,24 -diene $=22899$

3-O-[O- $\beta$ - $D$-Glucopyranosyl-( $1 \rightarrow 4)-\beta$ - $D$-glucopyranosyl] zanhic acid 28- $O$ -

$\beta$ - $D$-apiofuranosyl-( $1 \rightarrow 3$ )- $O$-(4- $O$-acetyl)- $\alpha$ - $L$-rhamnopyranosyl-( $1 \rightarrow 2)$ -

$O-\alpha$ - $L$-arabinopyranosyl ester $=729$

4- $O-\alpha$ - $D$-Glucopyranosyl- $D$-glucose $=13454$

4- $O-\beta$ - $D$-Glucopyranosyl- $D$-glucose $=3383$

3- $O$ - $\beta$ - $D$-Glucopyranosyl-( $1 \rightarrow 2)-\beta$ - $D$-glucuronopyranosyl-22- $O$-acetyl-21- $O$ - $(2-\mathrm{m}$ ethylpropanoyl)-3 $\beta, 15 \alpha, 16 \alpha, 21 \beta, 22 \alpha, 28$-hexahydroxyolean-12-ene $=9711$

3-O- $\beta$ - $D$-Glucopyranosyl-( $1 \rightarrow 2)-\beta$ - $D$-glucuronopyranosyl-22- $O$-acetyl-21-Opropanoyl-3 $\beta, 15 \beta, 16 \alpha, 21 \beta, 22 \alpha, 28$-hexahydroxyolean-12-ene $=9710$

3- $O$ - $\beta$ - $D$-Glucopyranosyl hederagenin $28-O-\alpha$ - $L$-rhamnopyranosyl $(1 \rightarrow 4)-\beta$ -

$D$-glucopyranosyl $(1 \rightarrow 6)-\beta$ - $D$-glucopyranoside $=3738$

3-O- $\beta$ - $D$-Glucopyranosyl hederagenin $28-O-\alpha$ - $L$-rhamnopyranosyl $(1 \rightarrow 2)$ -

$[\beta$ - $D$-xylopyranosyl $(1 \rightarrow 6)]-\beta$ - $D$-glucopyranosyl ester $=11121$

$2-O-\beta$ - $D$-Glucopyranosyl-6-hydroxy-2H-1,4-benzoxazin-3(4H)-one $=9858$

3-O-[2'- $\beta$ - $D$-Glucopyranosyl-3'-O-(2"-hydroxy-1"-carboxyethoxycarboxy-

propyl)]- $\beta$ - $D$-glucopyranosyl oleanolic acid $28-O-\beta$ - $D$-glucopyranoside $=543$

6'-(4"-O- $\beta$-Glucopyranosyl-3"-hydroxycinnamoyl)arbutin = 18879

$26-O-\beta$ - $D$-Glucopyranosyl-22-hydroxy-25(S)-5 $\alpha$-furostan-12-oxo-3 $\beta$,26-diol-

3 - $O$ - $\beta$ - $D$-xylopyranosyl $(1 \rightarrow 3)[\alpha$ - $L$-arabinopyranosyl $(1 \rightarrow 2)]$ - $\beta$-D-gluco-

pyranosyl $(1 \rightarrow 4)$-[ $\alpha-L$-rhamnopyranosyl $(1 \rightarrow 2)]-\beta$ - $D$-galactopyranoside

$=3563$

(25R)-26- $O-\beta$ - $D$-glucopyranosyl-22-hydroxy-5 $\beta$-furost- $2 \beta, 3 \beta, 26$-triol-3- $O-\beta$ -

$D$-glucopyranosyl-( $(1 \rightarrow 2)-\beta$ - $D$-galactopyranoside $=13333$

3-O- $\beta$ - $D$-Glucopyranosyl-21- $\beta$-hydroxyhederangenin $=13063$

7-O- $\beta$ - $D$-Glucopyranosyl-4'-hydroxyisoflavanone $=5580$

3-O- $\beta$ - $D$-Glucopyranosyl 3 $\beta$-hydroxylean-9(11),12-dien-28-oic acid 28-O-

$\alpha$ - $L$-rhamnopyranosyl $(1 \rightarrow 4)$ - $\beta$ - $D$-glucopyranosyl $(1 \rightarrow 6)-\beta$ - $D$-glucopyranoside $=3739$

1- $\beta$ - $D$-Glucopyranosyl-(2S,3S,4R,8Z)-2-[(2'R)-2'-hydroxylignocenoyl-amino]-

8-octadecene-1,3,4-triol $=9302$

3-O- $\beta$ - $D$-Glucopyranosyl-23-hydroxy-lup-20(29)-en-28-oic acid-28- $O-\beta$ - $D$ glucopyranosyl ester $=2572$

3-O- $\beta$ - $D$-Glucopyranosyl-23-hydroxy-lup-20(29)-en-28-oic acid-28- $O$-[ $\beta$ - $D$ glucopyranosyl- $(1 \rightarrow 6)-\beta$ - $D$-glucopyranosyl $]$ ester $=2573$

$3-O-\beta$ - $D$-Glucopyranosyl ilexosapogenin A $28-\beta$ - $D$-glucopyranosyl ester $=$ 18541

(+)-9-O- $\beta-D$-Glucopyranosyl lyoniresinol $=13250$

$28-O-\beta$ - $D$-Glucopyranosyl machaerinic acid 3- $O-\beta$ - $D$-glucopyranosyl $(1 \rightarrow 2)$ $[\beta$ - $D$-galactopyranosyl $(1 \rightarrow 3)]-\beta$ - $D$-glucopyranosiduronic acid $=2968$

26-O- $\beta$ - $D$-Glucopyranosyl-22-methoxy-3 $\beta, 26$-dihydroxy-25( $R$-furost-5-en3-O- $\alpha$ - $L$-rhamnopyranosyl-( $1 \rightarrow 4)-\alpha$ - $L$-rhamnopyranosyl- $(1 \rightarrow 4)-[\alpha-L$ rhamnopyranosyl- $(1 \rightarrow 2)]-\beta$ - $D$-glucopyranoside $=14699$

(25R)-26-O- $\beta$ - $D$-Glucopyranosyl-22-methoxy-5 $\beta$-furost-2 $\beta, 3 \beta, 26$-triol-3- $O$ $\beta$ - $D$-glucopyranosyl- $(1 \rightarrow 2)-\beta$ - $D$-galactopyranoside $=13334$

$28-O-\beta$ - $D$-Glucopyranosyl moronic acid 3-O- $\beta$ - $D$-glucopyranosyl $(1 \rightarrow 2)$ -

$[\beta$-D-galactopyranosyl $(1 \rightarrow 3)]-\beta$ - $D$-glucopyranosiduronic acid $=2965$

2- $\beta$ - $D$-Glucopyranosyloxy-4,6-dihydroxyisovalerophenone $=13261$

(-)-(6S,9S)-9-O- $\beta$ - $D$-Glucopyranosyloxy-6,13-dihydroxy-3-oxo- $\alpha$-ionol = 20173

5-[ $\beta$ - $D$-Glucopyranosyloxy]-3,4-epoxy-10,14-dihydroxy-19-nor-bufa-20,22dienolide $=9328$

5-[ $\beta$ - $D$-Glucopyranosyloxy]-3,4-epoxy-14-hydroxy-19-oxo-bufa-20,22- 
dienolide $=9327$

$(24 R, 25 S)-3 \beta$-[( $\beta$ - $D$-Glucopyranosyl)-oxy]ergost-5-en-26-oic acid $O-\beta-D$ glucopyranosyl-( $1 \rightarrow 2)-O$ - $[O-\beta$ - $D$-glucopyranosyl-( $1 \rightarrow 4)-\beta$ - $D$-glucopyranosyl- $(1 \rightarrow 6)]-\beta$ - $D$-glucopyranosyl ester $=20598$

$(24 R, 25 S)-3 \beta$-[ $(\beta$ - $D$-Glucopyranosyl)oxy]ergost-5-en-26-oic acid $O-\beta$ - $D$-glucopyranosyl-( $(1 \rightarrow 4)-O-\beta$ - $D$-glucopyranosyl-( $1 \rightarrow 2)-O$ - $[O-\beta$ - $D$-glucopyranosyl$(1 \rightarrow 4)-\beta$ - $D$-glucopyranosyl- $(1 \rightarrow 6)]-\beta$ - $D$-glucopyranosyl ester $=20597$

$(24 R, 25 S)-3 \beta$-[( $\beta$ - $D$-Glucopyranosyl)oxy]ergost-5-en-26-oic acid $O-\beta$ - $D$-glucopyranosyl-( $1 \rightarrow 4)$ - $O$ - $\beta$ - $D$-glucopyranosyl-( $1 \rightarrow 4)-O-\beta$ - $D$-glucopyranosyl-( $1 \rightarrow 2)-O$ - $[O-\beta$ - $D$-glucopyranosyl-( $1 \rightarrow 4)-\beta$ - $D$-glucopyranosyl$(1 \rightarrow 6)]-\beta$ - $D$-glucopyranosyl ester $=20596$

$(24 S, 25 S)$-24-[( $\beta$-D-Glucopyranosyl)oxy]-1 $\beta, 2 \beta, 3 \beta, 4 \beta, 5 \beta, 7 \beta$-hexahydroxyspirostan-6-one $=22654$

(22S)-16 $\beta$-[( $\beta$ - $D$-Glucopyranosyl)oxy]-22-hydroxycholest-5-en-3 $\beta$-yl $O-\alpha-L$ rhamnopyranosyl-( $1 \rightarrow 2)$ - $O$-[ $\alpha$ - $L$-rhamnopyranosyl-( $(1 \rightarrow 4)]-\beta$ - $D$-glucopyranoside $=20228$

$(E)-4-(\beta$ - $D$-Glucopyranosyloxy)-2-(hydroxymethyl)-2-butenenitrile $=19377$ $1 \alpha$-O-( $\beta$ - $D$-Glucopyranosyloxyl)-7-epi-eudesma-11-en-2b,4 $\alpha$-diol $=838$ $5 \beta$ - $O$-( $\beta$ - $D$-Glucopyranosyloxyl)-eudesma-4(15),11(13)-dien-12-oic-acid $=840$ $5 \alpha$ - $O$-( $\beta$ - $D$-Glucopyranosyloxyl)-eudesma-3,11(13)-dien-12-oic acid $=841$ $2 \beta$-O-( $\beta$ - $D$-Glucopyranosyloxyl)-eudesma-4 $\alpha$-hydroxyl-11(13)-en-12-oic-acid $=839$ $3 \beta$ - $O$ - $(\beta$ - $D$-Glucopyranosyloxyl $)$-megastigma-9-one $=842$

$3 \alpha$-[4-( $\beta$ - $D$-Glucopyranosyloxy)-3-methoxy-5-(3-methyl-2-butenyl)benzoyloxy] tropane $=13788$

(S)-2-( $\beta$ - $D$-Glucopyranosyloxy)-3-methylbutanenitrile $=9467$

(Z)-4-( $\beta$-D-Glucopyranosyloxy)-2-methyl-2-butenenitrile $=18790$

(4S)-4-(1- $\beta$-D-Glucopyranosyloxy-1-methyl)ethyl-1-cyclohexene-1-carboxylic acid $=16079$

$16 \beta$-[(4'S)-5'-( $\beta$ - $D$-Glucopyranosyloxy)-4'-methylpentanoyloxy]-3 $\beta$-hydroxy$5 \alpha$-pregnan-20-one 3- $O$ - $\{\alpha-L$-rhamnopyranosyl-( $(1 \rightarrow 2)-O\}-\beta$ - $D$-xylopyranosyl-(1 $\rightarrow 2)-O$-[ $\beta$ - $D$-xylopyranosyl-( $(1 \rightarrow 3)]-O-\beta$ - $D$-glucopyranosyl$(1 \rightarrow 4)]-\beta$ - $D$-galactopyranoside $\}=16883$

(22S,23S,24R,25S)-24-[( $\beta$-D-Glucopyranosyl) oxy]-5 $\alpha$-spirostane-3 $\beta, 6 \alpha, 23$ triol 6-O- $\beta$ - $D$-glucopyranoside $=697$

$3 \beta$-D-Glucopyranosyloxy- $6 \alpha, 16 \alpha, 20(S), 27$-tetrahydroxydammar-24(Z)-ene $=21891$

5 -[ $\beta$-D-Glucopyranosyloxy]-10,14,16-trihydroxy-19-nor- $\{5 \beta, 10 \beta, 14 \beta, 16 \beta\}$ bufa-3,20,22-trienolide $=9326$

3 - $O-\beta$ - $D$-Glucopyranosyl-2 $\beta, 3 \beta, 16 \alpha, 23,24$-pentahydroxyolean-12-ene-28-oic acid $=8711$

3-O- $\beta$ - $D$-Glucopyranosyl-2 $\beta, 3 \beta, 16 \alpha, 23,24$-pentahydroxyolean-12-ene-28-oic acid 28- $O-\alpha-L$-rhamnopyranosyl- $(1 \rightarrow 2)-\alpha-L$-arabinopyranoside $=17540$

3-O- $\beta$-D-Glucopyranosyl-2 $\beta, 3 \beta, 16 \alpha, 23,24$-pentahydroxyolean-12-ene-28-oic acid 28-O- $\beta$ - $D$-xylopyranosyl-( $1 \rightarrow 4)-\alpha-L$-rhamnopyranosyl-( $1 \rightarrow 2)-\alpha-L$ arabinopyranoside $=4799$

3-O- $\beta$ - $D$-Glucopyranosyl-20(S)-protopanaxadiol 20-O- $\beta$ - $D$-xylopyranosyl $(1 \rightarrow 3)-\beta$ - $D$-xylopyranosyl $(1 \rightarrow 6)-\beta$ - $D$-glucopyranoside $=15833$

3-O- $\beta$-D-Glucopyranosyl-20(S)-protopanaxadiol 20-O- $\beta$ - $D$-xylopyranosyl $(1 \rightarrow 4)-\beta$ - $D$-xylopyranosyl $(1 \rightarrow 6)-\beta$ - $D$-glucopyranoside $=15834$

27- $O-\beta$ - $D$-Glucopyranosylpubesenolide 3-O- $\beta$ - $D$-glucopyranoside $=22713$

$27-O-\beta$ - $D$-Glucopyranosylpubesenolide 3-O- $\beta$ - $D$-glucopyranosyl $(1 \rightarrow 6) \beta$ - $D$ glucopyranoside $=22711$

1 '- $\beta$-D-Glucopyranosyl-2-pyrrole-carboxylate $=2583$

7-O- $\beta$ - $D$-Glucopyranosyl-quercetin 3-O-(6-O-trans- $p$-coumaroyl)- $\beta$ - $D$-glucopyra- $\operatorname{nosyl}(1 \rightarrow 2)-\beta$ - $D$-glucopyranosyl $(1 \rightarrow 2)-\beta$ - $D$-glucopyranoside $=17494$

3 - $O-\beta$ - $D$-Glucopyranosyl $(1 \rightarrow 3)-\alpha-L$-rhamnopyranosyl $(1 \rightarrow 2)-\alpha$ - $L$-arabinopyranosyl hederagenin 28 - $O-\beta$ - $D$-glucopyranosyl $(1 \rightarrow 6)-\beta$ - $D$-glucopyranos$\mathrm{yl}$ ester $=13298$

3 - $O$ - $\beta$ - $D$-Glucopyranosyl $(1 \rightarrow 3)-\alpha-L$-rhamnopyranosyl-( $(1 \rightarrow 2)-\alpha$ - $L$-arabinopyranosyl-hederagenin-28- $O-\beta$ - $D$-glucopyranosyl $(1 \rightarrow 6)-\beta$ - $D$-glucopyranosyl ester $=15520$

3 - $O$ - $\beta$ - $D$-Glucopyranosyl-( $1 \rightarrow 3)$-[ $\alpha$ - $L$-rhamnopyranosyl-( $(1 \rightarrow 2)]-\alpha-L$-arabino pyranosyl $3 \beta$-hydroxy-urs-12,18-dien-28-oic acid $28-O-\beta$ - $D$-glucopyranosyl ester $=12557$

3 - $O$ - $\beta$ - $D$-Glucopyranosyl-( $(1 \rightarrow 3)$-[ $[\alpha-L$-rhamnopyranosyl-( $1 \rightarrow 2)]-\alpha-L$-arabinopyranosyl- $\beta$-kudinlactone $=12299$

3 - $O$ - $\beta$ - $D$-Glucopyranosyl-( $1 \rightarrow 3)-\alpha-L$-rhamnopyranosyl-( $1 \rightarrow 2)-\alpha-L$-arabinopyra-nosylpomolic acid 28- $O-\beta$ - $D$-glucopyranosyl-( $(1 \rightarrow 6)-\beta$ - $D$-glucopyranoside $=19440$

3 - $O-\beta$ - $D$-Glucopyranosyl-( $1 \rightarrow 3)-\alpha$ - $L$-rhamnopyranosyl-( $1 \rightarrow 2)-\alpha-L$-arabinopyranosylsiaresinolic acid 28- $O-\beta$ - $D$-glucopyranosyl- $(1 \rightarrow 6)-\beta$ - $D$-glucopyranoside $=19441$

3-O-[ $\beta$ - $D$-Glucopyranosyl-( $(1 \rightarrow 2)-\alpha$ - $L$-rhamnopyranosyl-( $(1 \rightarrow 2)-\beta$ - $D$-galactopyranosyl-( $(1 \rightarrow 3)][\beta$ - $D$-glucopyranosyl- $(1 \rightarrow 2)]-\beta$ - $D$-glucuronopyranosyl camelliagenin A 22-O-angelate $=13362$

$3 \beta$-[(O- $\beta$ - $D$-Glucopyranosyl- $(1 \rightarrow 3)-O-[\alpha-L$-rhamnopyranosyl- $(1 \rightarrow 2)]-O-[\alpha-$ $L$-rhamnopyranosyl-(1 $\rightarrow 4)]$ - $\beta$ - $D$-glucopyranosyl)oxy]pregna-5,16-dien20 -one $=20232$

3 - $O$ - $\beta$ - $D$-Glucopyranosyl-( $(1 \rightarrow 3)-\alpha-L$-rhamnopyranosyl-( $(1 \rightarrow 2)-\beta-D$-xylopyranosyloleanolic acid 28-O- $\beta$ - $D$-glucopyranosyl-( $1 \rightarrow 6)-\beta$ - $D$-glucopyranoside $=19437$

3-O- $\beta$ - $D$-Glucopyranosyl-( $(1 \rightarrow 3)-\alpha$ - $L$-rhamnopyranosyl-( $(1 \rightarrow 2)-\beta$ - $D$-xylopyranosyl oleanolic acid 28-O- $\alpha$ - $L$-rhamnopyranosyl-( $(1 \rightarrow 4)-\beta$ - $D$-glucopyranosyl- $(1 \rightarrow 6)-\beta$ - $D$-glucopyranosyl ester $=1256$

3-O-( $\beta$ - $D$-Glucopyranosyl-( $1 \rightarrow 3)-O-[\alpha-L$-rhamno-pyransyl-( $1 \rightarrow 2)-\beta-D$-gluco pyranosyl $(1 \rightarrow 2)]-\beta$ - $D$-glucopyranosyl)cyclamiretin $\mathrm{A}=16664$

3-O- $\beta$ - $D$-Glucopyranosylserjanic acid $=19333$

6'- $O-\beta$ - $D$-Glucopyranosylsweroside $=20512$

$2 "-O-\beta$ - $D$-Glucopyranosylswertisin $=20172$

$4 "-\beta$-O-Glucopyranosyl swertisin $=23007$

3-O- $\beta$-D-Glucopyranosyl $3 \beta, 12 \beta, 20(S), 24(R)$-tetrahydroxy-dammar-25-ene $20-O-\beta$ - $D$-glucopyranoside $=8433$

3-O- $\beta$-D-Glucopyranosyl-2 $\beta, 3 \beta, 16 \alpha, 23$-tetrahydroxyolean-12-en-28-oic acid $28-O-\beta$ - $D$-xylopyranosyl- $(1 \rightarrow 4)-\alpha-L$-rhamnopyranosyl-( $(1 \rightarrow 2)-\alpha-L$-arabinopyranoside $=17543$

8 - $(C-\beta$ - $D$-Glucopyranosyl $)-7,3^{\prime}, 4$ '-trihydroxyflavone $=22476$

7-O- $\beta$ - $D$-Glucopyranosyl-5,7,4'-trihydroxyisoflavanone $=5621$

3 - $O$ - $\{[\beta$ - $D$-Glucopyranosyl- $(1 \rightarrow 2)]$-[ $\beta$ - $D$-xylopyranosyl-( $(1 \rightarrow 4)]$-[3- $O$-acetyl] - $\beta$ - $D$-glucuronopyranosyl $\}-28-O-[\beta-D$-glucopyranosyl] - oleanolic acid $=$ 20537

3-O-[ $\beta$ - $D$-Glucopyranosyl-( $(1 \rightarrow 6)$-[ $[\beta$ - $D$-xylopyranosyl-( $(1 \rightarrow 2)]-\beta$ - $D$-glucopyranosyl- $(1 \rightarrow 3)-[\alpha-D$-fucopyranosyl- $(1 \rightarrow 2)]-\alpha-L$-arabinopyranoside $]=11910$

3 - $O$-[ $\beta$ - $D$-Glucopyranosyl-( $(1 \rightarrow 3)-[\beta$ - $D$-xylopyranosyl-( $(1 \rightarrow 2)]-\beta$ - $D$-glucopyranosyl- $(1 \rightarrow 4)-\beta$ - $D$-galactopyranoside $]=6557$

$3-O-[\alpha-D$-Glucopyranosyl- $(1 \rightarrow 2)-\beta-D$-xylopyranosyl $]$-hederagenin $=1997$

3 - $O$-[ $\beta$ - $D$-Glucopyranosyl-( $(1 \rightarrow 4)-\beta$ - $D$-xylopyranosyl-( $(1 \rightarrow 3)-\alpha$ - $L$-rhamnopyranosy-( $(1 \rightarrow 2)][\beta$ - $D$-glucopyranosyl- $(1 \rightarrow 4)]-\alpha$ - $L$-arabinopyranosylolea- 
nolic acid 28- $O-\beta$ - $D$-glucopyranosyl-( $(\rightarrow 6)-\beta$ - $D$-glucopyranoside $=19435$ 3 - $O$ - $\beta$ - $D$-Glucopyranosyl-( $1 \rightarrow 4)-\beta$ - $D$-xylopyranosyl-( $(1 \rightarrow 3)-\alpha$ - $L$-rhamnopyranosyl-(1 $\rightarrow 2)-\alpha$ - $L$-arabinopyranosyloleanolic acid 28-O- $\beta$ - $D$-glucopyranosyl- $(1 \rightarrow 6)-\beta$ - $D$-glucopyranoside $=19433$

3 - $O$ - $\beta$ - $D$-Glucopyranosyl-( $1 \rightarrow 4)-\beta-D$-xylopyranosyl-( $1 \rightarrow 3)$ - $\alpha$ - $L$-rhamnopyranosyl-(1 $\rightarrow 2)-\alpha-L$-arabinopyranosylsiaresinolic acid 28-O- $\beta$ - $D$-glucopyranosyl- $(1 \rightarrow 6)-\beta$ - $D$-glucopyranoside $=19442$

3 - $O$-[ $\beta$ - $D$-Glucopyranosyl-( $1 \rightarrow 3)$-[ $\beta$ - $D$-xylopyranosyl-( $1 \rightarrow 3)-\beta$ - $D$-xylopyranosyl-( $1 \rightarrow 2)]-\beta$ - $D$-glucopyranosyl-( $(1 \rightarrow 4)-\beta-D$-galactopyranoside $]=6556$ 3 - $O$ - $\beta$ - $D$-Glucopyranosyl zanhic acid 28-O- $\beta$ - $D$-apiofuranosyl-( $(1 \rightarrow 3)-O-(4-$

$O$-acetyl)- $\alpha$ - $L$-rhamnopyranosyl-( $(1 \rightarrow 2)-O-\alpha-L$-arabinopyranosyl ester $=732$ 3-O- $\beta$ - $D$-Glucopyranosyl zanhic acid 28-O- $\alpha-L$-arabinopyranosyl-(1 $\rightarrow 3)$ -

$O$-(4-O-acetyl)- $\alpha$ - $L$-rhamnopyranosyl-( $1 \rightarrow 2)-O-\alpha$ - $L$-arabinopyranosyl ester $=731$

2- $O-(\beta-D$-Glucosyl $)$ brucein $\mathrm{A}=22860$

$2-O-(\beta-D$-Glucosyl $)$ brucein $\mathrm{B}=22865$

$3-O-(\beta-D$-Glucosyl $)$ brucein $\mathrm{B}=22868$

3- $O-(\beta-D$-Glucosyl $)$ brucein $\mathrm{C}=22871$

8 - $C$-Glucosylgenkwanin $=11724$

1-(3'- $\beta$ - $D$-Glucosyloxy-4'-hydroxyphenyl)-3-hydroxymethyl-4-methoxy-6,7methylenedioxy-2-naphthoic acid lactone $=11975$

$3-(2-O-\beta-D$-Glucosylphenyl)propanoic acid $=5665$

2 - $\beta$ - $D$-Glucosyl-1,3,6,7-tetrahydroxyxanthone $=13481$

3- $O-\beta$ - $D$-Glucuronopyranosyl azukisapogenol $=15199$

$3-O-\beta$ - $D$-Glucuronopyranosyl azukisapogenol 29-O- $\beta$ - $D$-glucopyranosyl ester $=15200$

3-O- $\beta$ - $D$-Glucuronopyranosyl-24-hydroxy-olean-12-en-28-oic acid 28- $O-\beta$ - $D$ glucopyranoside $=18852$

3-O-[ $\beta$ - $D$-Glucuronopyranosyl] soyasapogenol $\mathrm{B}=20117$

$\gamma$ - $L$-Glutamyl- $L$ - $\alpha$-aminobutyryl glycine $=16153$

$\left(S_{C 2} R_{C 7} R_{S}\right)$ - $\gamma$-Glutamyl-S-benzylcysteine sulfoxide $=8777$

$\left(S_{C 2} R_{C 7} S_{S}\right)-\gamma$-Glutamyl-S-benzylcysteine sulfoxide $=8778$

$\gamma$ - $L$-Glutamyl- $L$-cysteinylglycine $=8785$

Glut-5-en-3 $\alpha$-ol $=8786$

Glutin-5-en- $3 \beta$-ol $=8788$

Glycerol-2-O- $\beta$ - $D$-galactofuranosyl $(1 \rightarrow 3)$-galactofuranoside $=8042$

Glycitein-7- $O-\beta$ - $D$-glucoside $=8819$

3-O- $\beta$-[29-(20-O-Glycolyl)-glyoxylyl]-oleanolic acid 28- $O-\beta$ - $D$-glucopyranoside $=2364$

3-O- $\beta$ - $D$-Glycopyranosylsitosterol $=19987$

Glycuronic acid $=8761$

Glycyrrhetic acid-3-O- $\beta-D-6$ "- $n$-butyl-glucuronopyranosyl-( $1 \rightarrow 2)-\beta-D-6$ '- $n$ butyl-glucuronopyranoside $=5405$

Glycyrrhetic acid-3- $O-\beta-D-6$ "- $n$-methyl-glucuronopyranosyl-( $1 \rightarrow 2)-\beta-D-6$ - $n$ butyl-glucuronopyranoside $=11038$

Glycyrrhetinic acid acetate $=403$

Glycyrrhetinic acid glycyside $=8846$

Glycyrrhizin $=8846$

Glycyrrhizinic acid $=8846$

Gniditrin $=15999$

Gomisin T-ol = 8926

Goniothalenol $=1008$

$(+)-(5 S, 6 S, 10 S)-\beta$-Gorgonene $=8953$
Goshuynic acid $=8956$

Gracilline $=8968$

Graveobioside B = 3604

Greenhartin $=12501$

Grosheimin $=9015$

$5 \alpha, 6 \beta, 7 \beta$ - $H$-1(10),3-Guaiadien-12,6 $\alpha$-olide $=19947$

1(10),4,11-guaiatrien-3,9-dione $=16070$

4,10,11-guaiatriene-3-one-15-al = 16069

$5 \alpha, 6 \beta$ - $H$-1(10),3,7(11)-Guaiatrien-12,6 $\alpha$-olide $=19948$

1(5)-Guaien-11-ol = 9044

$(-)$-Guaiol $=9044$

Guaipyridine $=6928$

Guanandin $=2989$

4-Guanidino-butyric acid $=9069$

Guatambuinine $=16085$

Guidongnin A $=9080$

Gulsamanin $=5995$

$\beta$-Guriunene $=2943$

$\beta$-Guttiferin $=8128$

$\alpha_{2}$-Guttiferin $=14967$

Guttiferone E = 8218

Gynosaponin E $=9126$

Gynosaponin $\mathrm{G}=9128$

Gynosaponin $\mathrm{K}=9131$

Gynosaponin $\mathrm{M}=9133$

Gynosaponin S = 9134

Gypenoside III $=8423$

Gypenoside VIII $=8427$

Gypsogenic acid-28- $O$ - $\beta$ - $D$-(6- $O$-acetyl)-glucopyranosyl( $1 \rightarrow 2)-\beta$ - $D$-glucopyranosyl-( $1 \rightarrow 6)-[\beta$ - $D$-glucopyranosyl $(1 \rightarrow 3)]-\beta$ - $D$-glucopyranoside $=$ 19660

Gypsogenic acid 28- $O$ - $\alpha$ - $L$-rhamnopyranosyl-( $1 \rightarrow 4)-\beta$ - $D$-glucopyranosyl$(1 \rightarrow 6)]-\beta$ - $D$-glucopyranoside $=70$

Gypsogenin-3- $\beta$ - $D$-glucecronoside $=22303$

\section{$\mathbf{H}$}

Haematin $=9338$

Haemin $=9345$

Haitinosporine $=6219$

$($ rel 7S,8S,9R)-1(10),5,14-Halimatrien-7,13-diol = 22574

Halleridone $=18615$

Hancinone $\mathrm{D}=8040$

Hancoside $=9217$

Hanfangchin A = 21206

Hannokinin $=17547$

Haplophytin B = 9223

Harmidine $=9232$

Harpagide-aglucone-1- $O-3$ ', 4'-seco-glycopyranoside $=3787$

Harpagide-aglucone-1- $O-\beta$ - $D$-ribohexo-3-ulopyranoside $=3786$

Harringtonolide $=9194$

Hederacolchiside $\mathrm{C}=819$

Hederacoside $\mathrm{B}=819$

Hederagenin-23- $O-\alpha$ - $L$-arabinopyranoside $=17943$ 
Hederagenin 3- $O$-arabinoside $=3340$

Hederagenin-23- $O-\beta$ - $D$-glucopyranoside $=17944$

Hederagenin-3- $O-\beta$ - $D$-glucopyranosyl $(1 \rightarrow 2)$ - $\alpha$ - $L$-arabinopyranoside $=3342$

Hederagenin 3-O- $\beta$ - $D$-glucopyranosyl $(1 \rightarrow 3)-\beta$ - $D$-glucopyranosyl $(1 \rightarrow 3)-\alpha-L$ arabinopyranoside $=6641$

Hederagenin-3- $O-\beta$ - $D$-glucopyranosyl $(1 \rightarrow 4)-\beta$ - $D$-glucopyranosyl $(1 \rightarrow 4)-\beta$ $D$-ribopyranosyl-( $1 \rightarrow 3)-\alpha$ - $L$-rhamnopyranosyl-( $1 \rightarrow 2)-\alpha$ - $L$-arabinopyranoside $=17949$

Hederagenin-3- $O$ - $\beta$ - $D$-glucopyranosyl-( $1 \rightarrow 4)-\beta$ - $D$-glucopyranosyl-( $1 \rightarrow 4)$ $\beta$ - $D$-xylopyranosyl- $(1 \rightarrow 3)$ - $\alpha$ - $L$-rhamnopyranosyl- $(1 \rightarrow 2)$ - $\alpha$ - $L$-arabinopyranoside $=17950$

Hederagenin-3- $O$ - $\beta$ - $D$-glucopyranosyl- $(1 \rightarrow 4)-\beta$ - $D$-ribopyranosyl- $(1 \rightarrow 3)-\alpha$ $L$-rhamnopyranosyl-( $1 \rightarrow 2)-\alpha$ - $L$-arabinopyranoside $=17947$

Hederagenin-3- $O$ - $\beta$ - $D$-glucopyranosyl-( $1 \rightarrow 4)-\beta$ - $D$-xylopyranosyl-( $(1 \rightarrow 3)-\alpha$ $L$-rhamnopyranosyl-( $1 \rightarrow 2)-\alpha$ - $L$-arabinopyranoside $=17948$

Hederagenin-3- $O-\alpha$ - $L$-rhamnopyranosyl- $(1 \rightarrow 2)-\alpha-L$-arabinopyranoside $=9276$

Hederagenin-3- $O-\beta$ - $D$-ribopyranosyl-( $1 \rightarrow 3$ )- $\alpha$ - $L$-rhamnopyranosyl-( $1 \rightarrow 2)$ $\alpha$ - $L$-arabinopyranoside $=17946$

Hederagenin-3- $O-\beta-D$-xylopyranosyl- $(1 \rightarrow 3)-\alpha$ - $L$-rhamnopyranosyl- $(1 \rightarrow 2)$ $\alpha$ - $L$-arabinopyranoside $=17945$

Hederasaponin $\mathrm{B}=819$

Hederidin $=9260$

$\beta$-Hederin $=6756$

Heliotridine viridiflorate $N$-oxide $=6687$

Hellebrigenin $=2721$

Hellebrigenin glucorhamnoside $=9330$

Hemanthamine $=9186$

Hemipinic acid $=9349$

$m$-Hemipinic acid $=9350$

Hendecanoic acid $=22218$

Henryine A $=6929$

Heptacosane $=9368$

1-Heptacosanol $=9370$

1,9E-Heptadecadiene-4,6-diyne-3R,8S-diol $=7708$

1,9Z-Heptadecadiene-4,6-diyne-3R,8S-diol $=7707$

6-(8'Z,11'Z-Heptadecadienyl)-1,2,4-tetrahydroxybenzene-1- $O$-acetate $=1652$

$Z$-14-Heptadecene $=9497$

3"',4',4"',5,5",7,7"-Heptahydroxy-3,6"-biflavone = 18844

$(20 R, 22 R, 24 S)-2 \beta, 3 \beta, 11 \alpha, 14 \alpha, 20,22,24-H e p t a-h y d r o x y-5 \beta$-cholest-7-en-6-one $=18750$

$3,5,6,7,8,3^{\prime}, 4^{\prime}$-Heptahydroxyflavan $=6624$

$2 \alpha, 3 \beta, 5 \beta, 6 \beta, 8 \alpha, 10 \alpha, 16 \alpha$-Heptahydroxykalmane $=18811$

Heptanedioic acid $=6194$

Heptanoic acid $=6795$

5-Heptyldihydrofuranone $=22217$

Heraclenin $=16447$

Heramandiol $=1194$

Herbacitrin $=11238$

Hernandine $=9445$

Hesperetinic acid $=9455$

Hesperitin $=9456$

Heteroauxin $=11031$

Hetisan-2,3,9,15-tetrol 2-benzoate $=10962$
$3 \beta, 7 \beta, 8 \beta, 9 \alpha, 14 \alpha, 15 \beta$-Hexaacetoxy- $2 \beta H$-jatropha- $5 E, 11 E$-diene $=7613$

$2,10,12,6$ ', 10',14'-Hexachloroisoplagiochin $\mathrm{C}=2185$

Hexacosanoic acid $=3434$

Hexacosanol-1 $=3435$

Hexadecanamide $=16559$

cis-9-Hexadecenoic acid $=16561$

3,14,15,16,17,20-Hexadehydro-16-ethenyloxayohimban-19,21-dione $=15290$

(2E,4E)-Hexa-2,4-dienoic acid,2-methyl-7S-(acetyloxy)-3',4,4',5',6,6',7,8octahydro-3'-hydroxy-6',7-dimethyl-6,8-dioxospiro[3H-2-benzopyran$3,2^{\prime}-[2 H]$ pyran $]-4-y l$ ester $=4612$

$(2 E, 4 E)$-Hexa-2,4-dienoic acid,4-methyl-7S-(acetyloxy)-3',4,4',5',6,6',7,8octahydro-3'-hydroxy-6',7-dimethyl-6,8-dioxospiro[3H-2-benzopyran$3,2^{\prime}-[2 H]$ pyran $]-4-y l$ ester $=4613$

4"a,5",6",7",8",8"a-Hexahydro-3',4'-dihydroxy-7-methoxy-5",5",8"a-trimethyl-4H-chromeno[2",3":5,6]flavone $=22185$

$4,4 \alpha, 5,6,7,8$-Hexahydro-4,4 $\alpha$-dimethyl-6-(1-methylethenyl)-2(3H)-naphthalene $=15705$

(+)-(1S,4aS,5R,8aS)-1,2,4a,5,6,8a-Hexahydro-3,8-dimethyl-5-(1-methylethyl)-naphthalenol $=1073$

(+)-(1S,7S,7aS)-2,3,5,6,7,7a-Hexahydro-7,7a-dimethyl-1-(2-methylpropanonyl)- $1 H$-indene $=5560$

Hexahydrodoluene $=14270$

$7,7^{\prime}, 8,8^{\prime}, 11,12-$ Hexahydrolycopene $=17264$

(1R)-1,2,3,4,5,6-Hexahydro-3-methyl-1,5-methano-8H-pyrido[1,2-a][1,5] diazocin-8-one $=14279$

(+)-(4R,4aR,8aS)-3,4,4a,7,8,8a-Hexahydro-6-methyl-4-(1-methylethyl)naphthalene-1-carbaldehyde $=1072$

$(2 S, 4 R)$-(-)-1,3,4,5,6,7-Hexahydro-1,1,5,5-tetramethyl-2H-2,4a-methanonaphthalene $=11510$

$5,7,4^{\prime}, 5$ ", 7",4"'-Hexahydroxy-(3,8")-biflavone $=2347$

2,3,14,20,22,24-Hexahydroxycholest-7-en-6-one $=18162$

$5 \alpha, 6 \beta, 7 \alpha, 10 \alpha, 11 \beta, 12 \alpha$-Hexahydroxycyclodeca-1,4-benzoquinone $=982$

$3,3^{\prime}, 4,4^{\prime}, 5^{\prime}, 7-$ Hexahydroxyflavan $=18856$

$3,3^{\prime}, 4^{\prime}, 5,5^{\prime}, 7-$ Hexahydroxyflavone $=15170$

$2 \alpha, 3 \beta, 5 \beta, 6 \beta, 10 \alpha, 14 \beta$-Hexahydroxygrayan-16-ene $=18806$

$2 \alpha, 3 \beta, 5 \beta, 6 \beta, 10 \alpha, 14 \beta$-Hexahydroxygrayan-15-ene $=18807$

3"',4',4"',5",7,7"-Hexahydroxy-5-methoxy-3,6"-biflavone = 18845

$3 \beta, 11 \alpha, 12 \beta, 14 \beta, 17 \beta, 20$-Hexahydroxy pregn-5-ene = 5129

$1 \beta, 2 \alpha, 5 \alpha, 9 \alpha, 10 \beta, 13 \alpha$-Hexahydroxy-4(20),11-taxadiene $=9505$

3',4,4',5,9,9'-Hexamethoxy-2,7'-cyclolignan $=17231$

$5,7,8,3^{\prime}, 4^{\prime}, 5^{\prime}-$ Hexamethoxyflavone $=2137$

2,6,10,15,19,23-Hexamethyl-2,6,10,14,18,22-tetracosahexaene $=20237$

1,6-Hexanedicarboxylic acid $=20439$

$n$-Hexanoic acid $=3139$

trans-3-Hexen-1-ol = 9524

Hexylvinylcarbinol $=15696$

Hibiscetin-heptamethylether $=9405$

Higenamine $=5067$

(10RS,11RS)-(2E,6Z,8E)-10,11-Hihydroxy- $N$-(2-hydroxy-2-methylpropyl)2,6,8-dodecatrienamide $=23025$

Hinokiflavone 4"',7"-dimethylether $=4286$

Hinokiflavone 4"'-methylether $=4285$

$(-)$-Hinokinin $=4310$ 
Hippacine $=4235$

$(+)$-Hippeastrine $=9547$

Hirsutrin $=11642$

Hispiduloside $=9617$

Holarrhesine $=9578$

Hollow alternanthera saponin $B=2973$

Homoaromoline $=9597$

$\alpha$-Homochelidonine $=9600$

24,31-Homocyclotirucall-9(11)-ene-3 $\beta$-ol = 19932

Homoeriodictyol-7- $O-\beta-D$-(6"- $O$-acetyl)-glucopyranoside $=22541$

Homoeriodictyol-7- $O-\beta$ - $D$-apiosyl- $(1 \rightarrow 5)-\beta$ - $D$-apiosyl- $(1 \rightarrow 2)-\beta$ - $D$-glucopyranoside $=22540$

Homoeriodictyol-7- $O-\beta$ - $D$-apiosyl- $(1 \rightarrow 2)-\beta$ - $D$-glucopyranoside $=22538$

Homopterocarpin $=6290$

4-Homopterocarpinol $=13679$

Homothalicrine $=9597$

Homotrilobine $=11747$

Homovitexin $=11773$

Hopene-B $=9641$

Houttuynin $=4837$

HSYA $=10684$

2,9-Humuladien-6-one $=9665$

2,7(14),9-Humulatriene $=9670$

2,6,9-Humulatrien-8-one $=22984$

Hydrangetin $=4648$

Hydrocinchonidine $=3675$

Hydrocoumaric acid $=13678$

Hydroginkgolic acid $=1128$

$16 \alpha$-Hydro-17-hydroxy-19-nor-ent-kauran- $4 \alpha$-ol = 1327

Hydromagnolol $=11519$

(24Z)-3 $\beta$-Hydroxy-14(13 $\rightarrow 12)$ abeo-lanosta-7,9(11),13(18),24-tetraen-26-oic acid $=22166$

16-Hydroxy-abieta-8,12-diene-11,14-dione $=17732$

$(5 R, 10 S)$-12-Hydroxy-8,11,13-abietatriene-3,7-dione $=13477$

14-Hydroxy-abieta-8,11,13-trien-3-one $=22016$

Hydroxyacetic acid $=8827$

(4S,5R,6S,7S,8R)-6-Hydroxy-7-( $\alpha$-acetoxybenzyl)-tetrahydrofuro[3,2- $b]$ furan2 -one $=404$

$3^{\prime}(S)$-Hydroxy-4' $(S)$-acetoxy-3',4'-dihydroseselin $=18284$

$3 \beta$-Hydroxy-12 $\beta$-acetoxylanosta-7,9(11),24(Z)-trien-26-oic acid $=22164$

25 -Hydroxy-3 $\alpha$-acetoxylanost-8-en-21-oic acid 21- $O-\beta$ - $D$-xylopyranoside $=7870$

$16 \beta$-Hydroxy-17-acetoxy-19-nor-ent-kauran-4 $\alpha$-formate $=1329$

$16 \beta$-Hydroxy-17-acetoxy-18-nor-ent-kauran- $4 \beta$-hydroperoxide $=1330$

2-Hydroxy-6-aminopurine-9- $\beta$ - $D$-ribofuranoside $=4272$

$17 \beta$-Hydroxyandros-4-en-3-one $=21022$

3-Hydroxy- $p$-anisaldehyde $=11767$

8-Hydroxyapigenin $=11703$

9-Hydroxy- $\Delta^{1(10)}$-aristolen-2-one $=4815$

Hydroxybenzene $=17089$

5-Hydroxy-1,3-benzenedicarboxylic acid $=10252$

4-Hydroxybenzoic acid methyl ester $=14652$

6'-O-p-Hydroxybenzoyl-8-epiloganic acid $=1539$

$\alpha-O-[2-O-(4-H y d r o x y b e n z o y l)-\beta-D$-glucopyranosyl]-3,4-dihydroxyphenyl- ethanol $=14919$

$\alpha$-O-[2-O-(4-Hydroxybenzoyl)- $\beta$ - $D$-glucopyranosyl]-4-hydroxyphenylethanol $=14917$

$N$ - $p$-Hydroxybenzoyl-5-hydroxy anthranilic acid $=13657$

4-Hydroxybenzyl alcohol 4- $O-\beta$ - $D$-glucopyranoside $=8237$

10 -(4-Hydroxybenzyl)-isorhapontigenin $=8897$

7-Hydroxy-3-benzylphthalide $=22155$

p-Hydroxybenzyltartaric acid $=17479$

Hydroxybrazilein $=9337$

$14 \beta$-Hydroxybufa-4,20,22-trienolide $3 \beta$ - $O$ - $\{\alpha$ - $L$-rhamnopyranosyl-[( $1 \rightarrow 4)$ -

$\beta$ - $D$-glucopyranosyl]-( $1 \rightarrow 3)-\alpha$ - $L$-rhamnopyranoside $\}=22250$

14-Hydroxy-bupleurynol $=2757$

Hydroxybutanedioic acid $=13419$

3-Hydroxy-2-butanone $=111$

7-Hydroxy-3-butylidene-phthalide $=2796$

(Z)-5-Hydroxy-3-butylidene-phthalide $=19733$

(3'S)-3'-Hydroxy-3-butyl phthalide $\beta$ - $D$-apiofuranosyl-(1 $\rightarrow 6)-\beta$ - $D$-glucopyranoside $=3373$

(3'S)-3'-Hydroxy-3-butyl phthalide $\beta$ - $D$-glucopyranoside $=3372$

7-Hydroxycadalin $=9869$

$(+)-\left(1 R^{*}, 6 S^{*}, 7 S^{*}, 10 S^{*}\right)$-12-hydroxy-4,11(13)-cadinadiene $=19069$

$\left(3 R, 5^{\prime} R\right)$-3-Hydroxy- $\beta, \kappa$-caroten-6'-one $=5158$

$3 \beta$-Hydroxy $\gamma$-cerane $=21174$

$6 R$-(7R-Hydroxy-8R-chloro-8-phenyl)-5,6-dihydro-2-pyrone $=3555$

$3 \alpha$-Hydroxy- $5 \beta$-cholan-24-oic acid $=12923$

Hydroxychromolaenide $=9570$

Hydroxycinchonine $=4373$

$m$-Hydroxycinnamic acid $=4133$

trans-o-Hydroxycinnamic acid $=4134$

4-Hydroxycinnamic acid $=4135$

6'-(4"-Hydroxycinnamoyl) arbutin = 18877

(E) $N$-(4-Hydroxycinnamoyl)-5-methoxytryptamine $=3392$

(Z)- $N$-(4-Hydroxycinnamoyl)-5-methoxytryptamine $=3393$

$6 S$-(1R-Hydroxy-2R-cinnamyloxyphenethyl)-5,6-dihydro-5S-hydroxy-2-

pyrone $=9655$

$12 \beta$-Hydroxyconessine $=9584$

5-Hydroxy-1- $p$-coumaric acyl-2,3-dihydro- $1 H$-indole-2-carboxylic acid-6$O-\beta$ - $D$-glucopyranoside $=16074$

5-Hydroxy-1-( $p$-coumaric acyl-7'- $O-\beta$ - $D$-glucopyranose)-2,3-dihydro- $1 H$ indole-2-carboxylic acid-6- $O-\beta$ - $D$-glucopyranoside $=16076$

7-Hydroxycoumarin $=22195$

7-Hydroxycoumarin farnesyl ether $=22196$

$2 \alpha$-Hydroxy-3 $\beta$-trans- $p$-coumaroyl-28,19 $\beta$-oleanolide $=6462$

2-Hydroxy-2,4,6-cycloheptatrien-1-one $=22052$

1-Hydroxy-2-deacetoxytaxinine $\mathrm{J}=20753$

$1 \beta$-Hydroxy-10-deacetylbaccatin $\mathrm{I}=20824$

$1 \beta$-Hydroxy-5 $\alpha$-deacetylbaccatin I $=20869$

$1 \alpha$-Hydroxy-3-dehydroxypseudoanisatin $=9989$

$11 \beta$-Hydroxy-3 $\beta, 6 \alpha$-diacetoxy-ent-kaur-16-en-15-one $=13701$

$1 \xi$-Hydroxydihydrocurcumin $=9855$

$\alpha$-Hydroxy-6,7-dihydrogardenoside $=8266$

(2S)-5-Hydroxy-2-(cis-1',4'-dihydroxycyclohexyl)-7-methoxychroman-4-one = 10554

(2S)-5-Hydroxy-2-(trans-1',4'-dihydroxycyclohexyl)-7-methoxychroman-4- 
one $=10555$

7-Hydroxy-2-(3,4-dihydroxyphenyl)benzofuran-4-al = 21477

7-Hydroxy-2-(3,4-dihydroxyphenyl)-4-(1E-propenoyl-3-oic acid)benzofuran $=21478$

10-Hydroxy-1,2-dimethoxy-4,5,6a,7-tetrahydro-dibenzo[de,g]quinoline-6carboxylic acidmethyl ester $=18903$

1-Hydroxy-2,3-dimethoxy-10-acetoxymethylacridone $=21421$

4-Hydroxy-3,5-dimethoxybenzoic acid $=20566$

trans-3-(4-Hydroxy-3,5-dimethoxy-benzylidene)-5-(4-hydroxy-3,5-dimethoxy-phenyl)-dihydrofuran-2-one $=5247$

6-Hydroxy-2,4-dimethoxy-3,5-bis(3-methyl-2-butenyl)benzophenone $=15224$

4-Hydroxy-2,4'-dimethoxychalcone $=8850$

$N$-(4'-Hydroxy-3',5'-dimethoxycinnamoyl)- $\Delta^{2}$-pyridin-4-one $=3386$

$N$-(4'-Hydroxy-3',5'-dimethoxycinnamoyl)- $\Delta^{3}$-pyridin-2-one $=5259$

7-Hydroxy-6,8-dimethoxycoumarin glucoside $=6754$

5-Hydroxy-6,7-dimethoxy-coumarin-5- $O$-glucoside $=21436$

5-Hydroxy-4,9-dimethoxy-6-(3,4-dimethoxyphenyl)-2-(1-methylethenyl)-7Hfurano[3,2-g]chromen-7-one $=21336$

2'-Hydroxy-5,4'-dimethoxy-2"-2"-dimethylpyran-[5"-6":6,7]isoflavone = 11016

7-Hydroxy-5,4'-dimethoxyflavanone $=22067$

7-Hydroxy-3',4'-dimethoxyisoflavanquinone $=16804$

8 -Hydroxy-4',7-dimethoxyisoflavone 8- $O$-[ $\alpha$-rhamnopyranosyl- $(1 \rightarrow 6)]$ - $\beta$-glucopyranoside $=5224$

1-Hydroxy-6,7-dimethoxy-2-methylanthraquinone $=18870$

5-Hydroxy-6,7-dimethoxy-3',4'-methylene-dioxy-flavone $=19127$

4'-Hydroxy-5,3'-dimethoxy-6,7-methylenedioxyisoflavone $=11165$

7-Hydroxy-5,8-dimethoxy-6-methyl-3-(2'-hydroxy-4'-methoxybenzyl)chroman-4-one $=16141$

7-Hydroxy-2,10-dimethoxy-1,4-phenanthraquinone $=4586$

3-(4-Hydroxy-3,5-dimethoxy-phenyl)-acrylic acid methyl ester $=19916$

$1 R^{*}$-(4-Hydroxy-3,5-dimethoxy-phenyl)-2 $R^{*}$-[4-(3-hydroxy-propyl)-2,6dimethoxy-phenoxy]-propane-1,3-diol $=2766$

4-(4-Hydroxy-3,5-dimethoxy-phenyl)-5-methyl-dihydro-furan-2-one $=5246$

3-(4-Hydroxy-3,5-dimethoxyphenyl)-prop-2-enol = 19922

5-Hydroxy-8-(1',1'-dimethylallyl) psoralen $=934$

$16 \beta$-Hydroxy-20 $\alpha$-dimethylamino- $9 \beta, 19$-cyclo-4,4,14 $\alpha$-trimethy-5 $\alpha$-pregn $=2819$

$2 \alpha$-Hydroxy-3 $\beta$-dimethylaminopregn-5-en-16-one $=12350$

$O^{7}$-(2S-2-Hydroxy-2,3-dimethyl-butanoyl $)=11647$

$O^{9}-(2 S-2 \alpha$-Hydroxy-2,3-dimethyl-butanoyl $)=9306$

$O^{9}-(2 S-2 \beta$-Hydroxy-2,3-dimethyl-butanoyl $)=18661$

7-Hydroxy-2",2"-dimethylchromano[5,6:6",5"]-2"',2"'-dimethylchromano $\left[3^{\prime}, 4^{\prime}: 5^{\prime \prime}, 6\right.$ "']isoflavone $=6625$

(1'E)-(1 $\left.R^{*}, 5 R^{*}, 9 S^{*}\right)-9$-Hydroxy-1-(2,6-dimethylhepta-1,5-dienyl)-3,6-dioxo-2-oxa-spiro[4.5]dec-7-ene $=14852$

(1'S,6'R,2Z,4Z)-5-[(1'-Hydroxy-2',6'-dimethyl-6'-hydroxymethyl-4'-oxo-8'- $\beta-D$ glucosyl)-cyclohex-2'-en-1'-yl]-3-methyl-penta-2,4-dienoic acid $=9748$

3-\{rel-(2R,3R)-4-Hydroxy-2,3-dimethyl-6-(3-methylbut-2-enyl)-6-[5-methyl-2-(2-methylpropenyl)hex-5-enyl]-5,7-dioxo-3,5,6,7-tetrahydro-2 $H$ chromen-8-yl $\}-3$-phenylpropionic acid $=2593$

3-\{rel-(2S,3R)-4-Hydroxy-2,3-dimethyl-6-(3-methylbut-2-enyl)-6-[5-methyl-2-(2-methylpropenyl)hex-5-enyl]-5,7-dioxo-3,5,6,7-tetrahydro-2 $H$ chromen-8-yl $\}-3$-phenylpropionic acid $=11264$

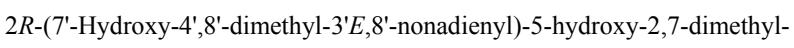

$2 H$-chromene $=4681$

$2 R$-(8'-Hydroxy-4',8'-dimethyl-3' $E, 6^{\prime} Z$-nonadienyl)-5-hydroxy-2,7-dimethyl$2 \mathrm{H}$-chromene $=4683$

$2 R$-(9'-Hydroxy-4',8'-dimethyl-3' $E, 7^{\prime} E$-nonadienyl)-5-hydroxy-2,7-dimethyl$2 \mathrm{H}$-chromene $=4684$

$7-[(E)-3 '-H y d r o x y-3 ', 7 '$-dimethyl-4',6'-octadienyloxy $]$ coumarin $=7765$

7 -[(3'Z,5' E)-7'-Hydroxy-3',7'-dimethyl-3',5'-octadienyloxy $]$ coumarin $=7766$

3-Hydroxy-2,24-dioxo-3-friedelen-29-oic acid $=3075$

2-Hydroxy-3-docosanyl-5-methoxy-1,4-benzoquinone $=11178$

2-Hydroxy-3-eicosanyl-5-methoxy-1,4-benzoquinone $=11176$

$\omega$-Hydroxyemodin $=3765$

3-Hydroxy-8-epi-larreatricin $=10077$

$(3 S, 5 R, 6 S, 9 R)-3$-Hydroxy-5,6-epoxy- $\beta$-dihydroionyl-9- $O$ - $\beta$-glucopyranoside $=19229$

$6 \beta$-Hydroxy-7 $\alpha, 10 \alpha$-epoxyguaiane-4,5-ene $=16194$

$(3 S, 5 R, 6 S, 7 E, 9 S)$-3-Hydroxy-5,6-epoxy- $\beta$-ionyl-9- $O$ - $\beta$-glucopyranoside $=19228$

$6 \beta$-Hydroxy-3 $\alpha, 20$-epoxy-ent-kaur-16-en-1,7,15-trione $=12587$

2-Hydroxyesculentic acid $=11812$

3 -Hydroxyestragole- $\beta$ - $D$-glucopyranoside $=7522$

3-Hydroxy-1,3,5(10)-estratrien-17-one $=7387$

$6 \beta$-Hydroxy-7- $\alpha$-ethoxy-16-acetoxy royleanone $=12998$

(24Z)-26-Hydroxy-7,24-euphadien-3-one $=10770$

4'-Hydroxyeximine $=2611$

5-Hydroxy-1-ferulic acyl-2,3-dihydro- $1 H$-indole-2-carboxylic acid-6- $O-\beta$ - $D$ glucopyranoside $=16075$

5-Hydroxy-1-(ferulic acyl-7'- $O$ - $\beta$ - $D$-glucopyranose)-2,3-dihydro- $1 H$-indole-2carboxylic acid-6- $O-\beta$ - $D$-glucopyranoside $=16077$

2-Hydroxy-3-formylcarbazole $=15027$

6-Hydroxy-3-(3- $O-\beta$ - $D$-glucopyranosyl-but-1-enyl)-2,4,4-trimethyl-cyclohex2-enone $=3086$

9' $\alpha$-Hydroxy-9 $\alpha$ - $O-\beta$ - $D$-glucopyranosylpinoresinol $=12444$

$(1 S, 5 S, 6 S, 10 S)$-10-Hydroxyguaia-3,7(11)-dien-12,6-olide $\beta$ - $D$-glucopyranoside $=4358$

$(4 R, 10 R)$-10-Hydroxyguaia-1(5),6-dien-2-one $)=15270$

$(4 R, 10 S)$-10-Hydroxyguaia-1(5),6-dien-2-one $=15271$

$21 \beta$-Hydroxy-gypsogenin $=13061$

7-Hydroxyharman $=9236$

2-Hydroxy-3-heptadecyl-5-methoxy, 1,4-benzoquinone $=11174$

2-Hydroxy-3-hexadecyl-5-methoxy-1,4-benzoquinone $=11173$

5-Hydroxy-6,7,8,3',4',5'-hexamethoxy flavone $=8228$

4-Hydroxyhomopterocarpin $=13679$

5-Hydroxy-2E,6E,9E-humulatrien-8-one $=10834$

$3 \beta$-Hydroxy-18-hydroperoxy-15,20 $\alpha$ :18,20 $\beta$-diepoxy-13,14:14,15-disecopregna-5,12-dien-14-oic acid 16-oxy-lactone $=20275$

6-Hydroxy-2-(4-hydroxybenzyl)-benzofuran-7-C- $\beta$ - $D$-glucopyranoside = 18115

2-Hydroxy-2- $p$-hydroxybenzyl-3(2H)-6-hydroxybenzofuranone-7- $C$ - $\beta$ - $D$ glucopyranoside $=13579$

(2S)-5-Hydroxy-2-(1'-hydroxy-4',4'-dimethoxycyclohexyl)-7-methoxychroman-4-one $=6271$

(3R)-3-Hydroxy-12-\{(1S,4S)-4-[(1S)-1-hydroxyethyl]-pyrrolidin-1-yl $\}$ dodecanoic acid $=14990$

(3R)-3-Hydroxy-12-\{(1S,4S)-4-[(1S)-1-hydroxyethyl]-pyrrolidin-1-yl $\}$ - 
dodecanoic acid-3- $O-\beta$ - $D$-glucopyranoside $=14989$

(3R)-3-Hydroxy-12-[(1R,4R,5S)-4-hydroxy-5-hydroxymethyl-piperidin-1-yl]dodecanoic acid-3- $O-\beta$ - $D$-glucopyranoside $=14993$

4'-Hydroxy-7,8-[2-(2-hydroxyisopropyl)dihydrofuran]flavan $=2626$

1-Hydroxy-2-[2-hydroxy-3-methoxy-5-(1-hydroxyethyl)-phenyl]-3-(4-hydroxy-3,5-dimethoxy)-propane-1-O- $\beta$ - $D$-glucuronopyranoside $=20654$

6-Hydroxy-4 $\beta$-(4-hydroxy-3-methoxyphenyl)-3 $\alpha$-hydroxylmethyl-5-methoxy-3,4-dihydro-2-naphthaldehyde $=22566$

6-Hydroxy-4-(4-hydroxy-3-methoxyphenyl)-7-methoxy-3-nicoticmethyl-2naphthoic acid- $\gamma$-lactam $=22565$

1-Hydroxy-2-hydroxymethyl anthraquinone $=5522$

(S)-(-)-7-Hydroxy-8-(2-hydroxy-3-methyl-3-butenyl)-2H-1-benzopyran-2one $=5063$

7-Hydroxy-6-(3-hydroxy-3-methyl-2-oxobutyl)-coumarin = 17035

(3R)-3-Hydroxy-12-[(1R,4R,5S)-4-hydroxy-5-methyl-piperidin-1-yl]dodecanoic acid $=14992$

(3R)-3-Hydroxy-12-[(1R,4S,5S)-4-hydroxy-5-methyl-piperidin-1-yl]dodecanoic acid $=14994$

(3R)-3-Hydroxy-12-[(1R,4R,5S)-4-hydroxy-5-methyl-piperidin-1-yl]dodecanoic acid-3-O- $\beta$ - $D$-glucopyranoside $=14991$

$(6 R S)-(2 E, 7 E, 9 E)-6-H y d r o x y-N$-(2-hydroxy-2-methylpropyl)-11-oxo-2,7,9dodecatrienamide $=23023$

(11RS)-(2E,7E,9E)-11-Hydroxy- $N$-(2-hydroxy-2-methyl-propyl)-6-oxo-2,7,9dodecatrienamide $=23024$

(2S)-5-Hydroxy-2-(1'-hydroxy-4'-oxocyclohexyl)-7-methoxychroman-4-one = 16097

5-Hydroxy-3-(4-hydroxyphenyl)-8,8-dimethyl-4H,8H-benzo[1,2-b:3,4-b'] dipyran-4-one $=5235$

$(6 S, 9 S)$-6-Hydroxyinamoside $=20173$

$15 \beta$-Hydroxyisolineolone $=10248$

ent-11 $\beta$-Hydroxy-8(14),15-isopimaradien-3-one $=687$

1-[2'-Hydroxy-3',4'-(2"'-isopropanoyldihydrofuran)-6'-methoxy-5'-(3"-methylbut-2"-enyl)] acetophenone $=572$

3-[rel-(2R,3R)-4-hydroxy-6-(3 $\alpha$-isopropenyl-2,2-dimethylcyclobutyl- $\beta$-methyl)-2,3-dimethyl-6-(3-methylbut-2-enyl)-5,7-dioxo-3,5,6,7-tetrahydro$2 H$-chromen-8-yl]-3-phenylpropionic acid $=2591$

3-[rel-(2R,3R)-4-hydroxy-6-(3 $\alpha$-isopropenyl-2,2-dimethylcyclobutyl- $\beta$-methyl)-2,3-dimethyl-6-(3-methylbut-2-enyl)-5,7-dioxo-3,5,6,7-tetrahydro$2 H$-chromen-8-yl]-3-phenylpropionic acid $=2592$

3-[rel-(2S,3R)-4-hydroxy-6-(3 $\alpha$-isopropenyl-2,2-dimethylcyclobutyl- $\beta$-methyl)-2,3-dimethyl-6-(3-methylbut-2-enyl)-5,7-dioxo-3,5,6,7-tetrahydro-

$2 H$-chromen-8-yl]-3-phenylpropionic acid $=11262$

3-[rel-(2S,3R)-5-hydroxy-6-( $3 \alpha$-isopropenyl-2,2-dimethylcyclobutyl- $\beta$-methyl)-2,3-dimethyl-6-(3-methylbut-2-enyl)-4,7-dioxo-3,4,6,7-tetrahydro-

$2 H$-chromen-8-yl]-3-phenylpropionic acid $=11263$

6-Hydroxykaempferol 3,6,4'-trimethylether $=19312$

$16 \alpha H$-17-Hydroxy-ent-kauran-19-oic acid $=10277$

16 $\alpha$-Hydroxy-ent-17-kauranyl aristolochate $\mathrm{I}=1721$

16 $\alpha$-Hydroxy-ent-17-kauranyl aristolochate II $=1722$

$6 \beta$-Hydroxy-labdane-15,16-epoxy-14,13(16),8(9)-trien-3,7-dione $=9468$

(24E)-3 $\beta$-Hydroxy-5 $\alpha$-lanosta-7,9(11),24-trien-26-al = 13019

(3R,20S)-3-Hydroxyl-20-(5'-hydroxy-3'-methylpyridin-6'-yl)-5 $\alpha$-pregnan-6one) $=7794$
6-Hydroxy-limocitrin $=12840$

8-Hydroxy-linalool-3- $O$ - $\beta$ - $D$-xylpyranosyl $(1 \rightarrow 6)-\beta$ - $D$-glucopyranoside $=15402$

$15 \beta$-Hydroxylineolone $=10327$

(6R)-6-Hydroxyl-7-octenoic acid 6- $O-\beta$ - $D$-xylopyranosyl-(1"'- $\rightarrow 6 ")-O-\beta-D-$ glucopyranosyl-(1" $\rightarrow 2 ')-O-\beta$ - $D$-glucopyranoside $=10990$

$3 \beta$-Hydroxyl-olean-12-en-28,29-dioic acid 28- $O-\alpha$ - $L$-rhamnopyranosyl$(1 \rightarrow 4)$ - $\beta$ - $D$-glucopyranosyl- $(1 \rightarrow 6)]-\beta$ - $D$-glucopyranoside $=72$

$21 \beta$-Hydroxyloleanoic acid-28- $O-\beta$ - $D$-glucopyranoside $=13064$

$3 \beta$-Hydroxyl-23-oxo-olean-12-en-28-oic acid 28- $O-\alpha$ - $L$-rhamnopyranosyl$(1 \rightarrow 4)$ - $\beta$ - $D$-glucopyranosyl- $(1 \rightarrow 6)]$ - $\beta$ - $D$-glucopyranoside $=71$

13-Hydroxylupanine $=15972$

$3 \beta$-Hydroxy-18 $\beta, 19 \alpha H$-lup-20(29)-ene $=15482$

30-Hydroxylup-20(29)-en-3-one $=16374$

6-Hydroxyluteolol $=10351$

Hydroxymaltol 3- $O$-(6- $O$ - $p$-coumaryl $)-\beta$ - $D$-glucopyranoside $=2524$

$p$-Hydroxymandelonitril-glucoside $=5283$

5-Hydroxymatrine $=20096$

$(5 R, 6 R, 7 E)$-9-Hydroxymegastigmen-7-en-3-one $O$-primeveroside $=17522$

$(9 R, 7 E)$-9-Hydroxymengastigmane-5,7-dien-4-one $O$-primeveroside $=17523$

(9R)-9-Hydroxymengastigman-5-en-4-one $O$-primeveroside $=17524$

2-Hydroxy-1-methoxy-4,5,6a,7-tetrahydro-dibenzo[de,g]quinoline-6-carboxylic acidmethyl ester $=18901$

2'-Hydroxy-4'-methoxyacetophenone $=16532$

2-Hydroxy-1-methoxyanthraquinone $=900$

1-Hydroxy-2-methoxyanthraquinone $=901$

2(S)-(3'-Hydroxy-5'-methoxy)-benz-3(S)-ethoxycarbonyl-6-trans-ethyl acrylate-8-methoxy-benzofuran $=3304$

$p$-Hydroxy- $m$-methoxy-benzonic acid $=22332$

4-Hydroxy-3-methoxybenzyl alcohol $=22346$

$(2 S, 3 S)-2 \alpha-(3 "-H y d r o x y-4 "-m e t h o x y b e n z y l)-3 \beta$-(4'-hydroxy-3'-methoxybenzyl)- $\gamma$-butyrolactone $4 '-O-(\beta$ - $D$-glucopyranoside $)=20424$

$(2 S, 3 S)-2 \alpha-(4 "-H y d r o x y-3 "-m e t h o x y b e n z y l)-3 \beta$-(4'-hydroxy-3'-methoxybenzyl)- $\gamma$-butyrolactone $4^{\prime}-O-(\beta$ - $D$-glucopyranoside $)=20425$

$(2 S, 3 S)-2 \alpha-(4 "-H y d r o x y-3 "$--methoxybenzyl)-3 $\beta$-(4'-hydroxy-3'-methoxybenzyl)- $\gamma$-butyrolactone $4 "-O-(\beta$ - $D$-glucopyranoside $)=20426$

3-(4-Hydroxy-3-methoxy-benzyl)-5-[2-(4-hydroxy-3-methoxy-phenyl)-3-hydroxymethyl-7-methoxy-2,3-dihydro-benzofuran-5-yl]-4-hydroxymetyldihydro-furan-2-one $=6635$

4-Hydroxy-3-methoxy-trans-cinnamaldehyde $=3983$

4-Hydroxy-3-methoxycinnamic acid $=7768$

2-Hydroxy-1-methoxy-4H-dibenzo[de,g]quinoline-4,5-(6H)-dione $=6466$

5-Hydroxy-7-methoxy-6,8-dimethyflavone $=5270$

7-Hydroxy-8-methoxyflavanone $=11484$

5-Hydroxy-7-methoxyflavone $=20899$

5-Hydroxy-7-methoxy-8-formyl-3-benzoyl-2,6-dimethyl-2S,3R-dihydrochromone $=5268$

3'-Hydroxy,3-methoxy furo[8,7:4",5"]flavone $=3335$

5 -Hydroxy-7-methoxy-8- $C$ - $\beta$-glucosylflavone $=12157$

3-Hydroxy-1-methoxy-2-hydroxymethyl-9, 10-anthraquinone-3- $O$ - $\beta$ - $D$-glucop yranoside $=12531$

1-[2'-Hydroxy-6'-methoxy-5'-(2"-hydroxy-3"-methyl-3"-butenyl)-3',4'-(3"',3"'dimethyl-1"'-pyrenyl)] acetophenone $=575$

7-Hydroxy-4'-methoxyisoflavone $=7883$ 
5-Hydroxy-4'-methoxyisoflavone $=16546$

7-Hydroxy-6-methoxy-1 $(2 H)$-isoquinolinone $=5077$

3 -Hydroxy-1-methoxy-2-methoxymethylanthraquinone-3- $O-\beta$ - $D$-glucopyranoside $=12532$

3-Hydroxy-1-methoxy-2-methoxymethylanthraquinone-3- $O-\beta$ - $D$-primeveroside $=12533$

5-Hydroxy-6-methoxy-7-(3-methyl-but-2-enyloxy)-2H-1-benzopyran-2-one = 11569

2-Hydroxy-5-methoxy-3-methylcarbazole $=3153$

2-Hydroxy-8-methoxy-3-methylcarbazole $=3154$

2-Hydroxy-6-methoxy-3-methylcarbazole $=3155$

(7S,7'S,8S,8'S)-4-Hydroxy-3-methoxy-3',4'-methylenedioxy-7,7'-epoxilignan = 20651

5-Hydroxy-7-methoxy-2-methyl-8-(2,3-epoxy-3-methylbutyl)chromone = 16912

5-Hydroxy-7-methoxy-2-methyl-8-(1-hydroxy-3-methyl-3-butenyl)chromone = 16913

5-Hydroxy-7-methoxy-2-methyl-8-(2-hydroxy-3-methyl-3-butenyl)chromone = 16914

9-Hydroxy-3-methoxy-2-methyl-9-(2-methoxyphenyl)-14-oxa-biciclo

[3.2.1]-octa-[f]quinolinone $=22634$

1-(3-Hydroxy-5-methoxy-4-methylphenyl)-2-(3-hydroxy-2-methoxyphenyl)ethane $=20382$

1-Hydroxy-2-methoxy noraporphine $=2838$

14-Hydroxy-13-methoxy-8-oxocyatha-3,11-diene-12-carbaldehyde $=8521$

14-Hydroxy-11-methoxy-8-oxocyatha-3,12-diene-12-carbaldehyde $=8522$

7-Hydroxy-2-methoxy-1,4-phenanthraquinone $=4585$

$8 \alpha$-(4-Hydroxy-3-methoxy-phenyl)-6 $\beta, 7 \alpha$-bis-hydroxymethyl-3-methoxy-

5,6,7,8-tetrahydro-naphthalen-2-ol $=2765$

6-Hydroxy-7-Methoxy-4-phenylcoumarin $=4609$

5-Hydroxy-2-(4-methoxyphenyl)-2,3-dihydrobenzofuran $=4094$

$6 R$-(7R-Hydroxy-8R-methoxy-8-phenyl)-5,6-dihydro-2-pyrone $=13943$

1-(4-Hydroxy-3-methoxyphenyl)-ethanone $=117$

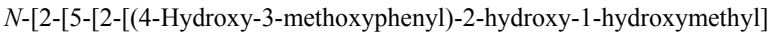
ethoxy]indol-3-yl]ethyl]-4-hydroxycinnamoyl amide $=11125$

2-(4-Hydroxy-3-methoxyphenyl)-3-hydroxymethyl-5-[ $N$-2-(4-hydroxyphenyl) ethyl]carbamoylethenyl-7-methoxybenzodihydrofuran $=9014$

erythro-1-(4-Hydroxy-3-methoxyphenyl)-2-\{4-\{2-[N-2-(4-hydroxyphenyl) ethyl]carbamoylethenyl-2-methoxyphenoxyl $\}\}$-1,3-propanodiol $=3054$

2-(4-Hydroxy-3-methoxyphenyl)-3-[N-2-(4-hydroxyphenyl)ethyl]carbamoyl-5-[N-2-(4-hydroxyphenyl)ethyl]carbamoylethenyl-7-methoxybenzodihydrofuran $=9013$

1-(4-Hydroxy-3-methoxyphenyl)-7-(4-hydroxyphenyl)-1,6-heptadiene-3,5dione $=5045$

$2 \alpha$-(4'-Hydroxy-3'-methoxyphenyl)-6 $\alpha$-(3",4"-methylenedioxyphenyl)-8oxo-3,7-dioxabicyclo[3.3.0]octane 4'-O-( $\beta$ - $D$-glucopyranoside $)=20423$

1-(4'-Hydroxy-3'-methoxyphenyl)-7-phenyl-3-heptanol $=16461$

1-4-(Hydroxy-3-methoxyphenyl)-7-phenyl-1-hepten-3-one $=22877$

4-Hydroxy-3-methoxyphenylpropanol $=5564$

3-(4-Hydroxy-3-methoxyphenyl)-prop-2-enol = 3982

4-Hydroxy-3-methoxyphenyl-1-O- $\beta$ - $D$-(5- $O$-syringoyl)-apiofuranosyl-( $1 \rightarrow 2)$ $\beta$ - $D$-glucopyranoside $=856$

4-Hydroxy-3-methoxyphenyl- $\beta$ - $D$-xylopyranosyl $(1 \rightarrow 6)-\beta$ - $D$-glucopyranoside $=5534$

4 '-Hydroxy-7-methoxy-6-prenylflavanone $=2173$

3 -Hydroxy-9-methoxypterocarpan $=13637$

$L$-3-Hydroxy-9-methoxypterocarpan $=13638$

$(4 S)-5$-Hydroxy-4-methoxy- $\alpha$-tetralone $=10460$

4-Hydroxy-7-methoxy-3-[1,2,6-trimethyl-7-(4-methyl-2-furyl)-hepta-2(E),
5(E)-dienyl]-coumarin $=7985$

2-Hydroxymethylalloptaeroxylin $=16915$

$2 \alpha$-Hydroxy-3 $\beta$-methylaminopregn-5-en-16-one $=12349$

rel-(2S,5R,6R,8S,9S,10R,18S,19R)-6-Hydroxy-2-(2-methylbutanoyloxy)

cleroda-3,13(16),14-triene-18,19-dicarboxaldehyde $=3263$

1-Hydroxy-4-(3-methyl-2-butenyl)-9H-carbazole-3-carboxaldehyde $=3794$

2-Hydroxy-1-(3-methyl-2-butenyl)-carbazole-3-carboxaldehyde $=9410$

5 -[2" $(R)$-Hydroxy-3"-methyl-3"-butenyloxy]furocoumarin $=16488$

2-(3'-Hydroxy-3'-methylbutyl)-4-hydroxy-3,6-dimethoxyphenol-1-O- $\beta$ - $D$ glucopyranoside $=18290$

2-(3'-Hydroxy-3'-methylbutyl)-4-hydroxy-5-methoxyphenol-1- $O-\beta$ - $D$-glucopyranoside $=18289$

$(2 R, 3 R, 4 R, 5 R)$-2-Hydroxymethyl-3,4-dihydroxy-5-[7-(cyclohexy-2-on-1(6)enyl)heptyl]pyrrolidine $=2645$

$(2 R, 3 S, 4 R, 5 R)$-2-Hydroxymethyl-3,4-dihydroxy-5-[7-(cyclohexy-2-on-1(6)enyl)heptyl]pyrrolidine-4- $O-\beta$ - $D$-glucopyranoside $=2646$

$(2 R, 3 R, 4 R, 5 R)$-2-Hydroxymethyl-3,4-dihydroxy-5-[( $9 R)-9,13$-dihydroxytridecyl]pyrrolidine $=2638$

$(2 R, 3 R, 4 R, 5 R)-2$-Hydroxymethyl-3,4-dihydroxy-5-\{(1R)-1-hydroxy-3-[6-(4hydroxybutyl)-cyclohexy-2-on-1(6)-enyl]propyl $\}$ pyrrolidine $=2639$

$(2 S, 3 S, 4 S, 5 S)$-2-Hydroxymethyl-3,4-dihydroxy-5-(3-hydroxy-4-methoxyphenyl)-pyrrolidine $=18524$

(2S,3S,4S,5S)-2-Hydroxymethyl-3,4-dihydroxy-5-(4-hydroxyphenyl)-pyrrolidine $=18525$

(2R,3S,4R,5R)-2-Hydroxymethyl-3,4-dihydroxy-5-[(E)-9-oxo-13-hydroxy-3tridecenyl] pyrrolidine $=2644$

(2S,3S,4S)-2-Hydroxymethyl-3,4-dihydroxy-5-(9-oxo-13-hydroxytridecyl)5 -pyrroline $=2642$

(2S,3S,4S)-2-Hydroxymethyl-3,4-dihydroxy-5-(10-oxo-13-hydroxytridecyl)5 -pyrroline $=2643$

$(2 R, 3 R, 4 R, 5 R)$-2-Hydroxymethyl-3,4-dihydroxy-5-[(1R,5S)-1,5,13-trihydroxy-10-oxo-tridecyl] pyrrolidine $=2641$

$(2 R, 3 R, 4 R, 5 R)$-2-Hydroxymethyl-3,4-dihydroxy-5-[(1R,10S)-1,10,13-trihydroxytridecyl] pyrrolidine $=2640$

1-(4-Hydroxy-2-methylenebutanoate)-6-((S)-3,4-dihydroxy-2-methylenebutanoate) $-\beta$ - $D$-glucopyranose $=22107$

$3 \beta$-Hydroxy-24-methylene-lanosta-7,9(11)-dien-21-oic acid $=4909$

(4S,3Z)-4-Hydroxy-5-methylene-3-octadecylidene-di-hydro-furan-2-one = 23029

2-Hydroxymethylfuran $=8025$

(-)-1R-3-Hydroxymethyl-1-(4'-hydroxy-3',5'-dimethoxyphenyl)-7-hydroxy-6, 8-dimethoxy-1,2,3,4-tetrahydro-2-naphthalenylmethanol sulfate $=13254$

5-Hydroxymethyl-6-(4-hydroxy-3,5-dimethoxyphenyl)-8-oxa-bicyclo[3,2,1] oct-3-en-2-one $=5245$

2-Hydroxymethyl-3,4-[1'-hydroxy-2'-(1-hydroxy-1-methylethyl)-dihydrofurano]-8-hydroxyanthraquinone $=3133$

2-Hydroxymethyl-3,4-[2'-1-hydroxy-1-methylethyl]-dihydrofurano]anthraquinone $=3134$

2-Hydroxymethyl-3,4-[2'-(1-hydroxy-1-methylethyl)-dihydrofurano]-8-hydroxyanthraquinone $=3132$

2-Hydroxymethyl-1-methoxy-3,6-dihydroxyanthraquinone 3-O- $\beta$-glucopyranoside $=18227$

2-Hydroxymethyl-1-methoxy-3,5,6-trihydroxyanthraquinone 3-O- $\beta$-glucopy- 
ranoside $=18226$

3'-[ $\gamma$-Hydroxymethyl $(E)$ - $\gamma$-methylallyl $]-2,4,2^{\prime}, 4^{\prime}$-tetrahydroxychalcone 11 '- $O$ coumarate $=8262$

$2 R$-(3'-Hydroxy-8'-methyl-4'-methyliden-7'-nonaenyl)-5-hydroxy-2,7-dimethyl-2H-chromene $=4682$

21-O-(2-Hydroxymethyl-6-methyl-6-methoxy-2,7-octadienoyl)acacic acid $=11916$

5-Hydroxy-2-methyl-1,4-naphthoquinone $=17568$

1-Hydroxy-2-(4-methylpent-3-enyl)anthraquinone $=1368$

2-(Hydroxymethyl)phenyl- $\beta$ - $D$-glucopyranoside $=19184$

(-)-4-Hydroxymethyl-2-(1',2',2'-trimethylcyclopentyl) phenol = 9425

10-Hydroxymontanin $\mathrm{C}=425$

$9 \beta$-Hydroxy-monticamine $=7673$

13-Hydroxymulin-11-en-20-oic acid = 15062

2-Hydroxy-1,4-naphthalenedione $=12580$

5-Hydroxy-1,4-naphthoquinone $=11903$

$24 S$-Hydroxy-neotokorogenin $1-O-\alpha$ - $L$-arabinopyranosyl $24-O-\beta$ - $D$-glucopyranoside $=9341$

2-Hydroxy-1,2,3-nonadecanetricarboxylic acid $=700$

2-Hydroxy-3-nonadecyl-5-methoxy-1,4-benzoquinone $=11175$

3-Hydroxy-25-norfriedel-3,1(10)-dien-2-one-30-oic acid = 21992

$11-\beta$-Hydroxyobacunone $=22971$

$(12 R)$-Hydroxy-cis-9-octadecenoic acid $=18841$

3-Hydroxy-1-octene $=15984$

7-Hydroxy-2-octen-5-olide $=7659$

$3 \beta, 29$-Hydroxy-olean-12-en-23,28-dioic acid 28- $O$ - $\alpha$ - $L$-rhamnopyranosyl-

$(1 \rightarrow 4)$ - $\beta$ - $D$-glucopyranosyl-( $(1 \rightarrow 6)]-\beta$ - $D$-glucopyranoside $=69$

$3 \alpha$-Hydroxyolean-12-en-23,28-dioic acid 28- $O-\alpha$ - $L$-rhamnopyranosyl $(1 \rightarrow 4)$ - $\beta$ - $D$-glucopyranosyl $(1 \rightarrow 6)-\beta$ - $D$-glucopyranoside $=3740$

$3 \alpha$-Hydroxy-12-oleanen-24-oic acid $=2567$

3-Hydroxy-12-oleanen-28-oic acid $=16050$

$27-H y d r o x y o l e a n-12-e n-28$-oic-acid-3- $O-\alpha-L$-rhamnopyranosyl-( $1 \rightarrow 2)-\alpha-L$ arabinopyranoside $=18521$

$3 \beta$-Hydroxyolean-12-en-28-oic acid $O-\alpha$ - $L$-rhamnopyranosyl- $(1 \rightarrow 4)-O-\beta-D$ glucopyranosyl- $(1 \rightarrow 6)-\beta$ - $D$-glucopyranosyl ester $=6981$

27-Hydroxyolean-12-en-3-one $=3471$

29-Hydroxy oleanic acid $=13799$

12-Hydroxyorthosiphol $\mathrm{A}=16230$

19-Hydroxy-14-oxoabieta-8,13(15)-dien-16,12-olide 19-O- $\beta$ - $D$-glucopyranoside $=17162$

21-Hydroxy-23-oxo-20-en-limonin $=19801$

(24Z)-26-Hydroxy-3-oxo-7,24-euphadien-21-al = 10584

(24E)-3 $\beta$-Hydroxy-7-oxo-5 $\alpha$-lanosta-8,24-dien-26-al = 13021

$16 \alpha$-Hydroxy-3-oxolanosta-8,24-dien-21-oic acid $=17388$

(20S)-3 $\alpha$-Hydroxy-30-oxolupan-23,28-dioic acid 28- $O$ - $[\alpha-L$-rhamnopyranosyl-( $(1 \rightarrow 4)-\beta$ - $D$-glucopyranosyl-( $(1 \rightarrow 6)-\beta$ - $D$-glucopyranosyl $]$-ester $=75$

23-Hydroxy-3-oxo-20(29)-lupen-28-oic acid = 18191

$15 \alpha$-Hydroxy-3-oxo-24-methylenelanost-8-en-21-oic acid $=7858$

$15 \alpha$-Hydroxy-16-oxo-olean-12(13)-en-3- $O$ - $\beta$-glucuronopyranoside $=7274$

$3 \beta$-Hydroxy-11-oxo-olean-12-en-30-oic acid-3- $O-\beta$ - $D$ - $(n$-butyl-glucuronopyranosyl ester)- $(1 \rightarrow 2)-\beta$ - $D$-(ethyl-glucuronopyranosyl ester $=7422$

$3 \beta$-Hydroxy-11-oxo-olean-12-en-30-oic acid-3- $O-\beta-D$-( $n$-butyl-glucuronopyranosyl ester)-( $1 \rightarrow 2)-\beta$ - $D$-(methyl-glucuronopyranosyl ester $=14185$

$3 \beta$-Hydroxy-11-oxo-olean-12-en-30-oic acid-3- $O-\beta$ - $D$-glucoronopyranosyl-
$(1 \rightarrow 4)-\beta$ - $D$-glucuronopyranoside $=8847$

$3 \beta$-Hydroxy-11-oxo-olean-12-en-30-oic acid-3- $O-\beta$ - $D$-glucuronopyranosyl$(1 \rightarrow 2)-\beta$ - $D$-glucuronopyranoside $=22243$

$3 \beta$-Hydroxy-11-oxo-olean-12-en-30-oic acid-3- $O-\beta$ - $D$-glucuronopyranosyl$(1 \rightarrow 3)-\beta$ - $D$-glucuronopyranoside $=22244$

(2Z)-2-[(1R)-1-Hydroxy-2-oxo-propyl]-icos-2-enoic acid methyl ester $=19628$

3-Hydroxy-4-oxo-4H-pyran-2,6-dicarboxylic acid = 13632

3 -[9 $\beta$-Hydroxy-2-oxo-4 $\alpha$-quinolizidyl]-4-quinazolinone $=9698$

$12 \alpha$-Hydroxypachyrhizone $=10589$

$5 \alpha$-Hydroxy-2 $\alpha, 7 \beta, 9 \alpha, 10 \beta, 13 \alpha$-Pentaacetoxy-4(20),11-taxadiene $=4849$

5-Hydroxy-3,6,7,3',4'-pentamethoxy-flavone $=1792$

5-Hydroxy-6,7,8,3',4'-pentamethoxyflavone $=5088$

threo- $\Delta^{7 '}-7$-Hydroxy-3,4,5,3', $5^{\prime}$-pentamethoxy-8-O-4'-neoligna $=17688$

Hydroxypeucedanin hydrate $=2041$

$6 \alpha$-Hydroxyphaseollin $=10600$

4-Hydroxyphenethyl alcohol $=22170$

$(2,3)$ trans $-N$ - $(p$-Hydroxyphenethyl)ferulamide $=7788$

$p$-Hydroxyphenethyl- $\beta$ - $D$-glucoside $=18792$

7-[2-(3-Hydroxyphenethyl)-4-hydroxy-6-methoxyphenoxy]-4-hydroxy-2-met hoxy-9,10-dihydrophenanthrene $=17200$

1-[4-Hydroxyphenoxy]-3-[3-hydroxy-4-methoxyphenyl]penta-1,4-diene = 10396

1-[4-Hydroxyphenoxy]-5-[3-methoxy-4-hydroxyphenyl]pent-2-en-3-yne = 13845

2-Hydroxy-2-phenylacetonitrile $=13478$

2-Hydroxy-3-(phenylaminocarbonyl)naphthalene-1-azobenzene $=19458$

$\beta$-(4-Hydroxyphenyl)ethyl- $O$ - $\beta$ - $D$-apiofuranosyl-( $1 \rightarrow 2)$-4-O-trans-feruloyl-

$$
\beta \text { - } D \text {-glucopyranoside }=3838
$$

2-(4-Hydroxyphenyl)ethyl- $O-\beta$ - $D$-apiofuranosyl-(1" $\left.\rightarrow 6^{\prime}\right)-\beta$ - $D$-glucopyranosid

$$
\mathrm{e}=16254
$$

4-(4'-Hydroxy-phenyl)-6-prenyl-7-hydroxy-coumarin $=11037$

3-(2-Hydroxyphenyl)propanoic acid $=13678$

3-(4-Hydroxyphenyl)propanoic acid $=17169$

3-Hydroxy-2-(13-phenyltridecanoyl)-cyclohex-2-enone $=20494$

$(2 E, 6 E, 10 Z)-1$-Hydroxy-2,6,10,14-phytatetraen-18-oic Acid = 11563

8 '-Hydroxypinoresinol $=10652$

8 '-Hydroxypinoresinol-4'- $O-\beta$ - $D$-glucoside $=10654$

8-Hydroxypinoresinol-4'- $O-\beta$ - $D$-glucoside $=10655$

21-Hydroxypregn-4-ene-3,20-dione $=5163$

2-Hydroxy-1,2,3-propanetricarboxylic acid $=3766$

2-Hydroxypropanoic acid $=12436$

2-[4-(3-Hydroxy-propyl)-2,6-dimethoxyphenoxy]-propane-1,3-diol = 2767

5-(3"-Hydroxypropyl)-7-methoxy-2-(3',4'-dimethoxyphenyl)-benzofuran

$3 "-O$ - $[\beta$ - $D$-xylopyranoside- $(1 \rightarrow 6)-\beta$ - $D$-glucopyranoside $=20422$

(S)-4-Hydroxypterosin $\mathrm{A}=16102$

3-Hydroxy-4H-pyran-4-one $=18271$

5-Hydroxy-2-pyridylmethyl-adenine $=16759$

(Z)-2-Hydroxy-3-(4-pyridyl)-2-propenoic acid = 1846

4-Hydroxy-2-pyrrolidinecarboxylic acid $=10660$

Hydroxysanguiarine $=16473$

$16 \alpha$-Hydroxy-3,4-seco-lanosta-4(28),8,24-triene-3,21-dioic acid = 17727

$16 \alpha$-Hydroxy-3,4-seco-24-methyllanosta-4(28),8,24(24 $\left.{ }^{1}\right)$-triene-3,21-dioic acid $=17728$

(3S)-3'-Hydroxysedanolide $\beta$ - $D$-glucopyranoside $=3374$

12-Hydroxysenecionan-11,16-dione $=19706$ 
$3 \beta$-Hydroxy- $5 \alpha, 25 D$-spirostan-12-one $=9253$

(25R)-3 $\beta$-Hydroxy-5 $\alpha$-spirostan-6-one 3- $O$ - $[O-\alpha$ - $L$-arabinopyranosyl-( $1 \rightarrow 6$ )-

$\beta$ - $D$-glucopyranoside $]=12593$

$(2 \alpha, 3 \beta, 5 \alpha)$-Hydroxyspirostan-3-yl $O-\beta$ - $D$-galactopyranosyl-( $1 \rightarrow 2)-O-\beta-D$ -

glucopyranosyl- $(1 \rightarrow 4)-\beta$ - $D$-galactopyranoside $=21017$

Hydroxysuccinic acid $=13419$

$3 \beta$-Hydroxy- $1 \alpha, 7 \beta, 11 \beta, 15 \beta$-tetraacetoxy-ent-kaur-16-ene $=608$

$N$-(2'-Hydroxy- $n$-tetracosanoyl)- $n$-eicosasphinga-(4E)-ene $=20227$

$N$-(2'-Hydroxy- $n$-tetracosanoyl)-1,3,4-trihydroxy- $n$-pentadeca-sphingosine $=20226$

(5E)-5-(2-Hydroxytetradexylidene)-furan-2 $(5 H)$-one $=10841$

(5Z)-5-(2-Hydroxytetradexylidene)-furan-2 $(5 H)$-one $=10842$

$(-)$-4-Hydroxy-1-tetralone $=10747$

$(4 S)$-4-Hydroxy- $\alpha$-tetralone 4- $O$ - $\beta$ - $D$-glucopyranoside $=11898$

5-Hydroxy-1,2,3,4-tetramethoxyanthraquinone $=18876$

4-Hydroxy-3,5,3',4'-tetramethoxybibenzyl $=3628$

6'-Hydroxy-2',3',4,4'-tetramethoxychalcon $=15993$

2-Hydroxy-1,6,7,8-tetramethoxy-3-methyl-9,10-anthracenedione $=3612$

2-Hydroxy-1,5,6,7-tetramethoxyphenanthrene $=3627$

1-Hydroxy-4,5,6,7-tetramethoxy-9H-xanthen-9-one $=20504$

1-Hydroxy-2,3,4,6-tetramethoxyxanthone $=6628$

(E)-7 $\beta$-Hydroxy-3,11,15,23-tetraoxolanosta-8,20(22)-dien-26-oic acid = 8162

$8 \beta$-(4'-Hydroxytigloyloxy)-14-chloro- $\beta \beta, 10 \beta$-dihydroxy- $\alpha H, 5 \alpha H, 6 \beta H, 7 \alpha H$ -

guai-2,11(13)-dien-6,12-olide $=7567$

$8 \beta$-(4'-Hydroxytigloyloxy)-3 $\alpha, 4 \alpha: 10 \alpha, 14$-diepoxy-2 $\beta$-hydroxy- $1 \alpha H, 5 \alpha H, 6 \beta H$,

$7 \alpha H$-guai-11(13)-en-6,12-olide $=7573$

$3^{\prime}(R)$-Hydroxy-4' $(R)$-tigloyloxy-3',4'-dihydroseslin $=18282$

$8 \beta$-(4'-Hydroxytigloyloxy)-3 $\alpha, 4 \alpha$-epoxy- $2 \beta$-acetoxy- $10 \alpha, 14$-dihydroxy- $1 \alpha H$,

$5 \alpha H, 6 \beta H, 7 \alpha H$-guai-11(13)-en-6,12-olide $=7576$

$8 \beta$-(4'-Hydroxytigloyloxy)-2 $\beta, 14$-epoxy-10 $\alpha$-hydroxy- $\alpha H, 5 \alpha H, 6 \beta H, 7 \alpha H$ -

guai-3,11(13)-dien-6,12-olide $=7569$

$8 \beta$-(4'-Hydroxytigloyloxy)-3 $\alpha, 4 \alpha$-epoxy- $2 \beta, 10 \alpha, 14$-trihydroxy- $\alpha H, 5 \alpha H, 6 \beta H$,

$7 \alpha H$-guai-11(13)-en-6,12-olide $=7575$

$8 \beta$-(4'-Hydroxytigloyloxy)-4 $\alpha$-hydroxy- $\alpha H, 5 \alpha H, 6 \beta H, 7 \alpha H$-guai-2,10-(14),

11(13)-trien-6,12-olide $=7568$

$8 \beta$-(4'-Hydroxytigloyloxy)-2 $\beta, 10 \alpha, 14$-trihydroxy- $\alpha H, 5 \alpha H, 6 \beta H, 7 \alpha H$-guai-

3,11(13)-dien-6,12-olide $=7572$

$8 \beta$-(4'-Hydroxytiglyloxy)-2 $\beta$-acetoxy-14-chlorine- $3 \alpha, 4 \alpha$-epoxy-10 $\alpha$-hydrox-

$\mathrm{y}-1 \alpha H, 5 \alpha H, 6 \beta H, 7 \alpha H$-guai-11(13)-ene-6,12-olide $=7545$

$8 \beta$-(4'-Hydroxytiglyloxy)-14-chrorine-3 $\alpha, 4 \alpha$-epoxy- $2 \beta, 10 \alpha$-dihydroxy- $\alpha H$, $5 \alpha H, 6 \beta H, 7 \alpha H$-guai-11(13)-ene-6,12-olide $=7544$

$8 \beta$-(4'-Hydroxytiglyloxy)-3 $\alpha, 4 \alpha$-epoxy-2 $\beta$-hydroxy- $\alpha H, 5 \alpha H, 6 \beta H, 7 \alpha H$-guai1(10),11(13)-diene-6,12-olide $=7541$

$5 \alpha$-Hydroxy-2 $\alpha, 9 \alpha, 10 \beta$-triacetoxy-11,12-epoxy-taxa-4(20)-en-13-one = 20799

$3 \beta$-Hydroxy-1 $\alpha, 7 \beta, 1 \beta$-triacetoxy-ent-kaur-16-en-15-one $=607$

$5 \alpha$-Hydroxy- $9 \alpha, 10 \beta, 13 \alpha$-triacetoxytaxa-4(20),11-diene $=10780$

rel-(2R,4S,6S)-4-Hydroxy-2-tridecyl-1,7-dioxa-dispiro[5.1.5.2]pentadeca-

9,12-dien-11-one $=589$

2'-Hydroxy-4,4',6'-trimethoxychalcone $=7818$

(R)-5-Hydroxy-2',4',5'-trimethoxy-2",2"-dimethylpyrano[5",6":6,7]isoflavanone $=19108$

$\left(7^{\prime} S, 8^{\prime} R\right)$-4-Hydroxy-3',4',5-trimethoxy-7,8-seco-2,7'-cyclolignan-7,8-dione $=19615$ trans-3-Hydroxy-2',3',5-trimethoxystilbene(6) = 17199

7-Hydroxy-1,2,3-trimethoxyxanthone $=16108$
5-Hydroxy-2,6,8-trimethylchromone 7- $O$ - $\beta$ - $D$-glucopyranoside $=22215$

4-Hydroxy-3,5,5-trimethyl-2-cyclohexen-1-one $=21967$

1-(2-Hydroxy-2,6,6-trimethyl-4- $\beta$ - $D$-glucosyloxyclohexylidene)-butane-2,3dione $=3085$

3-Hydroxy-3,7,11-trimethyl-9-oxododeca-1,10-diene $=15019$

3-Hydroxy-12-ursene-27,28-dioic acid $=18427$

$27 \alpha$-Hydroxyurs-12-ene-3- $O$ - $[\beta$ - $D$-glucopyranosyl $(1 \rightarrow 4)(2$ - $O$-sulpho $)-\beta$ - $D$ quinovopyranoside $=1998$

$2 \alpha$-Hydroxyursolic acid $=4088$

$19 \alpha$-Hydroxyursolic acid $=17696$

$\alpha$-Hydroxyvaline $=16480$

$\beta$-Hydroxyvaline $=16481$

14'-Hydroxyvincaleukoblastine $=12732$

$15^{\prime} \alpha$-Hydroxyvincaleukoblastine $=22488$

17-Hydroxy withaferin $=7210$

Hymenoratin $=15991$

Hyndarine $=21077$

Hyperoside $=10887$

Hypolepin A = 18143

Hypolepin $\mathrm{C}=18144$

Icariin $=1275$

Icaritin $=1273$

Ichthyopterin $=9853$

Idaein $=4439$

$\alpha, \beta$-Dihydroxanthohumol $=5742$

Ikarisoside $\mathrm{A}=2139$

Ikarisoside $\mathrm{C}=6492$

Ilexgenin $A=6175$

Ilexol $=2169$

Ilexolide $=10981$

$7 H$-Imadazo[4,5-d]pyrimidine $=18209$

4-Imidazoleacrylic acid $=22260$

22,26-Imino-17,23-oxidojerv-12-en-6-oxo-3 $\beta, 11 \alpha$-diol $=22907$

Imperatorin oxide $=16447$

$(17 R, 20 S, 22 R)-5 \alpha$-Impra-15,16-ene-3 $\beta, 6 \beta$-diol $=5645$

$3 S, 17 R, 20 S, 22 R)$-5 $\alpha$-Impra-15,16-ene-6-one $=11005$

Inapine glucosinalbate $=19909$

Indan-1-one $=11013$

$\alpha$-Indanone $=11013$

Indicine $=6686$

Indigo $=11023$

$1 H$-Indole-3-acetonitrile 4- $O$ - $\beta$-glucopyranoside $=3136$

$1 H$-Indole-3-acetonitrile 4- $O-\beta$ - $(6$ - $O-\beta$-glucopyranosyl $)$-glucopyranoside $=3137$

2,3-Indolinedione $=11190$

Indolo[2,1b]-quinazoline-6,12-dione $=18287$

Indolo[2,3- $a$ ]quinolizine-2-(1-hydoxyethyl)-3-hydroxy-4,6,7,12-tetrahydro-4one $=15291$

(3,4-trans)-3-(5'-Indolyl)-1,4-dimethyl-4-[ethyl-2-(5"-indolyl)enyl]-cyclohex1 -ene $=3336$

(3,4-cis)-3-(5'-Indolyl)-1,4-dimethyl-4-[ethyl-2-(5"-indolyl)enyl]-cyclohex-1ene $=3337$ 
2- $\{4-[1-(1 H$-Indol-2-yl)-vinyl]-1-methyl-piperidin-3-ylidene $\}$ ethanol $=13501$

Ineupatolide $=7075$

Inflasaponin IV $=5405$

Inflatine $=12939$

Infractine $=14218$

Ingenol-3-(2,4-decadienoate)-20-acetate $=4820$

Inocalophyllin A $=2991$

Chiro-Inositol $=11083$

Inrosidine $=12733$

Insularoside-3'- $O-\beta$ - $D$-glucoside $=11089$

Irigenin-5- $O$-(6"'- $O$-vanillin acid) $-\beta$ - $D$-glucoside $=19798$

Iriskashmirianin 4' $-O-\beta$ - $D$-glucoside $=8349$

Irisquinone $=11179$

Iryelliptin B $=10515$

Isoacoradinene $=6373$

Isoamyl-3-furyl ketone $=16929$

Isoartemisia ketone $=1795$

Isoasarone $=957$

Isobarbaloin $=981$

Isobutanol $=11268$

$N$-Isobutyl-4-hexanoyl-4-hydroxypyrrolidin-1-one $=17445$

$N$-Isobutyl-3,4-methylenedioxybenzethenylamide $=7702$

4-Isobutyl-6-methyl-5-oxo-3a,4,5,7a-tetrahydro-1 $\mathrm{H}$-inden-13-oic acid $=22535$

Isocaboxine $\mathrm{A}=22502$

Iso-4-cadinene- $2 \alpha, 10 \beta$-diol $=11471$

Isocarthamin $=15358$

Isocaryophyllene $=3241$

Isocephalomannine $=4813$

Isochamic acid $=11322$

Isochlorogenic acid B $=5414$

$(+)$-Isocorydine $=11344$

Isocucurbitacin $=5114$

Isodextropimaric acid $=11600$

Isodihydronepetalactol $=11559$

Isodonol $=16183$

trans-Isoelemicine $=11408$

Isofebrifugine $=5435$

Isoferulic acid $=9455$

Isoformononetin-4'-glucoside $=11576$

28-Isofucosterol $=11431$

Isofuranodiene $=8014$

Isofuranodienone $=8015$

Isofuranogermacrene $=4415$

Isogemichalcone $\mathrm{C}=8262$

Isohenin $=11203$

Isokaempferide 7-O- $\beta-D$-glucopyranouronide $=2585$

Isokempferide $=12070$

$(+)$-Isolariciresinol $=11477$

Isoliquiritigenin $=21694$

Isoliquiritigenin-4- $\beta$-glucoside $=15414$

$(-)$-Isolongifolene $=11510$

Isolupinine $=6955$

Isomammeisin $=13469$
Isomesuol $=13470$

$L$-Isomexoticin $=14825$

Isoorientin $=9616$

Isopelletierine $=16789$

Isopentanal $=11752$

Isopentanol $=11218$

8-Isopentenyllimettin $=4193$

trans-4-Isopentenyl-3,5,2',4'-tetrahydroxystilbene $=17841$

$8(14), 15$-Isopimaradi-ene- $6 \alpha$-ol $=13560$

2-Isopropenyl-2,3-dihydrobenzofuran-5-carboxylic acid $=7855$

7-Isopropenyl-2,3-dimethoxy-6-methyl-6-vinyl-5,6,7,8-tetrahydronaphthalene-1,4-dione $=9321$

$p$-Isopropyl-benzaldehyde $=4354$

4-Isopropylbenzyl alcohol $=4357$

4-Isopropyltoluene $=4550$

Isopsoralen $=1191$

Isoquassin $=17327$

Isoquercetin $=11642$

Isoquercetrin $=11642$

Isorhamnetin-3-O- $\beta$ - $D$-galactopyranoside $=2843$

Isorhamnetin 3-O-(6"-O- $\alpha-L$-rhamnopyranosyl) $-\beta-D$-glucopyranoside $=11669$

Isorhamnetin-3-O-(2 $2^{\mathrm{G}}-\alpha-L$-rhamnopyranosyl) $\alpha-L$-rhamnopyranosyl-( $(1 \rightarrow 6)$ $\beta$ - $D$-glucopyranoside $=22154$

Isorhodeasapogenin 3- $O-\beta$ - $D$-glucopyranosyl-( $1 \rightarrow 3)$-[ $\beta$ - $D$-xylopyranosyl-

$(1 \rightarrow 2)]-\beta$ - $D$-glucopyranosyl- $(1 \rightarrow 4)-\beta$ - $D$-galactopyranoside $=9342$

7-Isorhyncophylline $=11680$

Isosarsapogenin $=20025$

Isosarsapogenin-3- $O$ - $[\beta$ - $D$-glucopyranosyl- $(1 \rightarrow 2)]-\beta$ - $D$-mannopyranoside $=20027$

Isoschizandrolic acid $=11525$

Isoscutellarein-8-methyl ether $=10829$

Isostrictinin $=19277$

Isotadeonal $=11609$

Isotazettine $=17850$

Isoterpinene $=20998$

$(+)$-Isotetrandrine $=13715$

Isothiocyanato-benzene $=17125$

2-Isothiocyanato-butane $=2799$

3-Isothiocyanato-1-propene $=949$

Isovaleral $=11752$

Isovanillic acid $=10381$

Isoverbascoside $=11195$

Isovitexin 2"- $O$-(6"'-(E)-feruloyl)- $\beta$-glucopyranoside-4'- $O-\beta$-glucopyranoside = 11776

Isovitexin 7- $O-\beta-D$-glucopyranoside $=19331$

Isovouacapenol $\mathrm{C}=2244$

Isozaluzanin $\mathrm{C}=7044$

Jaeschkeanadiol $p$-hydroxybenzoate $=7792$

Jaeschkeanadiol $p$-methoxybenzoate $=7791$

Japonicine A $=852$

Jatamansone $=22313$

Jatrophalactam $=11842$

Jayacanol $=21719$ 
Jayacanol 3-methyl ether $=5972$

Jikon $=8083$

Jionoside $\mathrm{A}_{1}=22664$

Judaicin $=20730$

Juruenolide $\mathrm{E}=10516$

Justicidin $E=11981$

Juziphine $=22958$

\section{K}

Kadsulignan $\mathrm{K}=9466$

Kaemferol 3,7-di- $O$ - $\alpha$-rhamnopyranoside $=12018$

Kaempferol 3- $\alpha-L-(4-O$-acetyl)rhamnopyranoside-7- $\alpha-L$-rhamnopyranoside $=$ 20499

Kaempferol 3-O- $\alpha$ - $L$-arabinofuranoside $=11897$

Kaempferol-3-O-(6"-coumaroyl)-glucoside $=21392$

Kaempferol-3-O-[6"-(E)-p-coumaroyl]- $\alpha-D$-mannopyranoside $=3297$

Kaempferol 3- $\alpha-L-(2,4-$ di- $O$-acetyl)rhamnopyranoside-7- $\alpha$ - $L$-rhamnopyranoside $=4214$

Kaempferol 3- $\alpha-L$-(3,4-di- $O$-acetyl)rhamnopyranoside-7- $\alpha-L$-rhamnopyranoside $=4215$

Kaempferol 3- $\alpha$ - $L$-(2,3-di- $O$-acetyl)rhamnopyranosside-7- $\alpha-L$-rhamnopyranoside $=4216$

Kaempferol-3-O-[6",4"-di-(E)-p-coumaroyl]- $\alpha$ - $D$-mannopyranoside $=3298$

Kaempferol 3-(2,3-di- $E$ - $p$-coumaroyl- $\alpha-L$-rhamnopyranoside) $=17528$

Kaempferol 3- $O$-(2",6"-di- $O-\alpha$ - $L$-rhamnopyranosyl) $-\beta$ - $D$-glucopyra- noside $=3846$

Kaempferol 3-O- $\beta$ - $D$-galactopyranoside $=21634$

Kaempferol-3-O- $\beta$ - $D$-glucopyranoside $=1935$

Kaempferol-3- $O$ - $[\beta$ - $D$-glucopyranosyl- $(1 \rightarrow 2)-\beta$ - $D$-galactopyranoside $]=16587$

Kaempferol-3- $O$ - $\beta$ - $D$-glucopyranosyl $(1 \rightarrow 2)-\beta$ - $D$-glucopyranoside $=20088$

Kaempferol-3-glucose-7-diglucoside $=12042$

Kaempferol-7- $O-\beta-D$-(6"- $O$ - $p$-hydrocinnamoyl)- $D$-glucoside $=2392$

Kaempferol 3- $\beta$ - $D$-mannoside $=1069$

Kaempferol-4'-methylether $=12015$

Kaempferol-7-methylether $=18682$

Kaempferol 3-O- $\alpha-L$-rhamnopyranoside $=12082$

Kaempferol-3-O- $\alpha$ - $L$-rhamnopyranosyl-( $1 \rightarrow 6)-\beta$ - $D$-galactopyranosyl-7- $O-\alpha$ $L$-rhamnopyranoside $=18859$

Kaempferol 3-O-(2"-O- $\alpha$-rhamnopyranosyl)- $\beta$-glucopyranoside $=12071$

Kaempferol-7- $O-\alpha-L$-rhamnopyranosyl-3- $O$-(6"- $P$-coumaroyl)- $\beta$ - $D$-glucopyranosyl- $(1 \rightarrow 2)-\beta$ - $D$-xylopyranoside $=15195$

Kaempferol-3- $O-\alpha$ - $L$-rhamnopyranosyl $(1 \rightarrow 2)-\alpha$ - $L$-rarbinofuranoside $=1778$

Kaempferol-3-O- $\alpha-L$-rhamnopyranosyl-( $1 \rightarrow 6)$-[ $\alpha$ - $L$-rhamnopyranosyl$(1 \rightarrow 2)]-\beta$ - $D$-galactopyranosyl-7- $O-\alpha-L$-rhamnopyranoside $=1950$

Kaempferol-7- $O-\alpha$ - $L$-rhamnoside $=12083$

Kaempferol 3-O-(6"-O- $\alpha$-rhamonpyranosyl)- $\beta$-glucopyranoside $=15525$

Kaempferol-3- $O-\beta$-rutinoside $=15525$

Kaempferol-3-rutinoside-7-glucoside $=12087$

Kainic acid $=936$

Kajiichigoside F1 = 21864

Kakkonein $=22080$

Kalopanasaponin A $=9276$

Kalopanaxsaponin G $=18197$

Kalopanax septemlobus asponin A $=9276$
Kalosapogenin $=9260$

Kanzuiol $=21409$

Karounidiol 3- $O$-benzoate $=12166$

Kashmirine $=11002$

ent-Kauran-16 $\beta, 17$-acetal ent-16 $\beta$-kauran-17(S)-aldehyde $=7965$

ent-Kauran-16 $\beta$-hydroxy-17-chloride $=7958$

Kaurenoic acid $=12178$

16-ent-Kauren-19-ol = 12180

Kelampayoside A = 21923

Keracyanin $=4446$

Ketocalogenin-3-O- $\alpha$ - $L$-fucopyranoside $=13578$

Ketologanin $=4943$

4-Ketolutein $=6560$

Kidjolanin 3- $O$ - $\beta$-D-glucopyranosyl-( $1 \rightarrow 4)-\beta$ - $D$-glucopyranosyl-( $1 \rightarrow 4)$ $\beta$ - $D$-oleandropyranosyl $-(1 \rightarrow 4)-\beta$ - $D$-cymaropyranosyl- $(1 \rightarrow 4)-\beta$ - $D$ cymaropyranoside $=992$

Kidjolanin 3-O- $\beta$ - $D$-glucopyranosyl-( $1 \rightarrow 4)-\beta$-D-glucopyranosyl-( $1 \rightarrow 4)$ $\beta$ - $D$-thevetopyranosyl- $(1 \rightarrow 4)-\beta$ - $D$-cymaropyranosyl-( $(1 \rightarrow 4)-\beta$ - $D$ cymaropyranoside $=990$

Kidjolanin 3-O- $\beta$ - $D$-glucopyranosyl-( $1 \rightarrow 4)-\beta$ - $D$-thevetopyranosyl-( $1 \rightarrow 4)$ $\beta$ - $D$-cymaropyranosyl- $(1 \rightarrow 4)-\beta$ - $D$-cymaropyranoside $=991$

Kikemanine $=4104$

Kiuminine $=8257$

Kizutasaponin $\mathrm{K}_{10}=3343$

Klinit $=22814$

Koelreuteriasaponin A = 3340

Koenidine $=12247$

Komalin $=9420$

$(-)$ Koumine $=12289$

Kumujancine $=7908$

Kumujian A = 3156

Kumujian B $=3158$

$3 \alpha$-Kurameroyloxytropane $=13786$

Kuwanon $\mathrm{C}=15038$

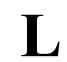

8(17),13-Labdadiene- $6 \alpha, 15$-diol $=4270$

Lagerstroemine $=12450$

Lanceolatin $\mathrm{C}=17708$

(24R)-Lanosta-7,9(11)-diene-2 $\alpha, 3 \beta, 12 \alpha, 21,24,25$-hexaol = 20443

Lanosta-8,24-dien-3 $\beta$-ol $=12488$

Lanost-20(22)-ene-30-ol $=633$

$\left(7^{\prime} S, 8 R, 8^{\prime} R\right)-(+)$-Lariciresinol $=12520$

Larixinic acid $=13452$

Laserpitin $=18282$

Laudanosoline 4',6-dimethyl ethe $=18655$

Laurencenone $\mathrm{C}=16300$

Lauric acid $=12569$

Lecobetaine $=22232$

Ledebouridine $=12599$

Lederbourine $=12600$

Leonuride $=815$

Leptospermol $=21199$ 
Leptospermone $=21199$

Leucaenol $=14868$

Leucuharmine $=9235$

Leukamenin $\mathrm{F}=8515$

Leuroristine $=22497$

Licoagrochalcone $=12751$

Licoagroside $=12759$

Licoflavone $=12774$

Licoisoflavone $A=12778$

Liconeolignan $=12764$

Limbatolide $\mathrm{C}=7835$

Limettin $=3770$

$(R)-(+)$-Limonene $=12843$

$(S)-(-)$-Limonene $=12844$

Limonianin $=1956$

Limonindiosphenol $=7669$

Limonoid $=5147$

Linalool acetate $=12850$

Lindeneol $=12871$

Lindeneyl acetate $=12872$

Linderic acid $=6543$

Linearoside $=15133$

trans, trans-Linoleic acid $=12892$

Linolenic acid methyl ester $=14555$

Liquidambronic acid $=2338$

Lirioresinol B $=20556$

Lirioresinol dimethyl ether $=5378$

$\alpha$-Lobeline $=12939$

$\beta$-Lofoline $=7741$

Loganoside $=12950$

Lomatin $=11835$

Lonchocarpol A = 6497

Loniceroside $=19623$

Lophenol $=14235$

Lucidenic acid D = 13027

Lucidenolactone $=8195$

Ludongnin B $=9081$

lupanidine $=13606$

12-Lupanone $=3843$

Lupenyl acetate $=13100$

Lupeol caffeate $=2910$

Luteolin-7-apio-glucoside $=8990$

Luteolin 6- $C-\alpha-L$-arabinopyranosyl-8-C- $\beta$ - $D$-glucopyranoside $=11306$

Luteolin-6- $C$ - $\beta$ - $D$-glucopyranoside $=9616$

Luteolin-8- $C-\beta-D$-glucopyranoside $=16196$

Luteolin 7-O- $\beta$ - $D$-glucopyranosiduronic acid $=13147$

Luteolin-7- $O$-glucoside $=3674$

Luteolin-7- $O$ - $\alpha$ - $L$-rhamnopyranosyl $(1 \rightarrow 2)-\beta$ - $D$-glucopyranoside $=12987$

Luteolin 7-O-rutinoside $=13149$

Lyciumamide $=1345$

Lyclavatol $=3810$

Lycobergine $=13193$

Lycopersicin $=21435$
Lycopersidin $=21435$

Lycoremine $=8083$

3-O- $\beta$-Lycotetraosyl-(23S,24R)-23-acetoxy-24- $O-\beta$ - $D$-glucopyranosylsoladulc idine-24-ol = 13218

3-O- $\beta$-Lycotetraosyl-23(R)-23-acetoxy-27-hydroxy-27-O- $\beta$ - $D$-glucopyranosyl tomatidine $=13217$

3-O- $\beta$-Lycotetraosyl $3 \beta, 16 \beta$-dihydroxy-5 $\alpha$-pregn-20-one 16-O-[(4S)-2,5-epimino-2-methoxy-4-( $\beta$ - $D$-glucopyranosyloxy) methyl-pentanoic acid]ester $=7378$

3-O- $\beta$-Lycotetraosyl(5S,25S)-22,26-epimino-16 $\beta, 23$-epoxy-23 $\alpha$-methoxy-22( $N$ )ene-3 $\beta, 20 \alpha, 27$-trihydroxycholestane $27-O-\beta$ - $D$-glucopyranoside $=7377$

$3-O-\beta$-Lycotetraosyl-22-isopimpifolidine $=13219$

$(+)$-Lyoniresinol-2 $\alpha-O-\alpha$ - $L$-arabinopyranoside $\left(\mathrm{D}_{1}\right)=8242$

$(+)$-Lyoniresinol-9,9'-diacetate $=8241$

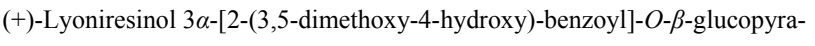
noside $=63$

Lyonotin $=1847$

\section{M}

Maackolin $=12258$

Maaliol $=13283$

$\left(8 S, 8^{\prime} R\right)$-Macelignan $=1466$

Macluraxanthone $=9857$

Macrocalyxin $\mathrm{C}=1038$

Macrocalyxoformin $\mathrm{C}=11056$

Maculotoxin $=21211$

Madasiatic acid 28-O- $\alpha$ - $L$-rhamnopyranosyl( $1 \rightarrow 4)-\beta$ - $D$-glucopyranosyl

$$
(1 \rightarrow 6)-\beta \text { - } D \text {-glucopyranoside }=3396
$$

Madecassic acid $=2586$

Madecassic acid 28-O- $\beta$ - $D$-glucopyranosyl $(1 \rightarrow 6)-\beta$ - $D$-glucopyranoside $=3395$

Maesaponin $=13358$

Magnolenin $=20569$

Mahanimbicine $=11520$

Mahuangnin $\mathrm{A}=6822$

Makisterone $\mathrm{C}=12620$

Malonylginsenoside $\mathrm{Rb}_{1}=13444$

Maltobiose $=13454$

Maltodiose $=13454$

Maltol-3- $O-\beta$ - $D$-glucopyranoside $=5371$

Malvalic acid $=13455$

Malvin $=13460$

Mammeigin $=13467$

$\alpha$-Mangostin $=13492$

Maniladiol $=16041$

Margaric acid $=4672$

Margaspidin $\mathrm{BB}=13559$

Margetine $=13238$

$(+)$-Marmesinin $=15647$

Marsupsin $=3222$

$(-)$-Maslinic acid $=6059$

Masticadienolic acid $=10363$

cis-Matrine $=13606$

Matrine $N$-oxide $=16451$ 
Matsutake alcohol $=15983$

Matsutakeol $=13608$

Maytenfolic acid $=27$

Mearnsetin-3- $O$ - $\alpha$-rhamnopyranoside $=13628$

Mecambrine $=7980$

Medioresinol $=13640$

(+)-Medioresinol monoglucoside $=7487$

(3S,9S,5Z,7E)-Megastigma-5,7-diene-3,9-dihydroxy-4-one 9- $O$ - $\beta$ - $D$-glucopyranoside $=20264$

$(3 S, 5 R, 6 S, 9 S)$-Megastigman-3,9-diol 3- $O-\beta$ - $D$-glucopyranoside $=15212$

$(3 S, 9 S)$-Megastigman-5-ene-3,9-diol 3,9-di- $O$ - $\beta$ - $D$-glucopyranoside $=20274$

$(1 S, 3 S, 5 R, 6 R, 9 R)$-Megastigman-7-ene-3,9-diol-5,12-epoxide 9- $O$ - $\beta$ - $D$-glucopyranoside $=2610$

$(3 S, 4 S, 5 R, 6 S, 9 S, 7 E)$-Megastigman-7-ene-5,6-epoxy-3,4,9-triol 9- $O$ - $\beta$ - $D$-glucopyranoside $=20266$

$(3 R, 4 R, 5 R, 6 R, 9 S, 7 E)$-Megastigman-7-ene-3,4,5,9-tetraol 9- $O-\beta$ - $D$-glucopyranoside $=20273$

$(3 R, 5 R, 6 R, 9 S, 7 E)$-Megastigman-7-ene-3,5,6,9-tetrol 9- $O-\beta$ - $D$-glucopyranoside $=20265$

$\left(3 S^{*}, 5 R^{*}, 6 S^{*}, 9 \xi\right)$-Megastigman-7-ene-3,6,9,10-tetrol 9- $O-\beta$ - $D$-glucopyranoside $=2608$

$\left(3 S^{*}, 5 R^{*}, 6 S^{*}, 9 \xi\right)$-Megastigman-7-ene-3,6,9,10-tetrol 10- $O$ - $\beta$ - $D$-glucopyranoside $=2609$

$(5 R, 6 S, 9 \xi)$-Megastigman-7-ene-6,9,10-triol-3-one 9- $O-\beta$ - $D$-glucopyranoside $=2607$

$(3 S, 5 R, 6 R, 9 S, 7 E)$-Megastigman-5,6-epoxy-7-ene-3,9-diol 9- $O-\beta$ - $D$-glucopyranoside $=20271$

$(5 R, 6 R, 9 R)$-Megastigman-3-on-6,9-diol 9- $O-\beta$ - $D$-glucopyranoside $=15211$

$(6 R, 7 E, 9 S)$-Megastigman-3-one-4,7-diene-9,13-diol 13- $O-\beta-D$-glucopyranoside $=8567$

$(6 S, 7 E, 9 S)$-Megastigman-3-one-4,7-diene-6,9,13-triol 13- $O$ - $\beta$ - $D$-glucopyranoside $=8565$

$(6 R, 9 S)$-Megastigman-3-on-4-ene-9,13-diol = 8566

$(6 S, 9 R)$-Megastigman-3-on-4-en-9-ol 9- $O-\beta$ - $D$-glucopyranoside $=2522$

$(5 R, 6 S, 9 R)$-Megastigma-3-on-9-ol 9- $O-\beta$ - $D$-glucopyranoside $=15210$

Melanthigenin $=9260$

Meliotocarpan A $=13679$

Melissic acid $\mathrm{B}=9366$

Melittoside 3"- $O$ - $\beta$-glucopyranoside $=18599$

Menisporohine $=13717$

$1,8-p$-Menthadiene $=6482$

$(R)-(+)-m$-Mentha-6,8-diene $=20524$

$p$-Mentha-1,4(8)-diene $=20998$

$p$-3,8(9)-Menthadien-1-ol $=13727$

$p$ - $(R)$-Mentha-1,8-dien-7-ol $=16927$

$p$-(S)-Mentha-1,8-dien-7-ol $=16928$

Meratin $=18342$

Mesatlantin E $=4941$

Methanal $=7881$

Methanoic acid $=7882$

$7 \alpha$-Methoxy-8,13-abietadien-11,12-dione $=20462$

$7 \alpha$-Methoxyabieta-8,13-diene-11,12-dione-(20,6 $\beta)$-olide $=18923$

$7 \beta$-Methoxyabieta-8,13-diene-11,12-dione- $(20,6 \beta)$-olide $=18922$

4-Methoxyallylbenzene $=7385$

4-Methoxybenzoic acid $=1284$
$3 \alpha$-(4-Methoxybenzoyloxy)nortropane $=13785$

3-(4-Methoxybenzyl)-5,7-dimethoxychroman-4-one $=6256$

3-Methoxycarbonylcarbazole $=14217$

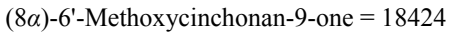

$p$-Methoxycinnamic acid ethyl ether $=7474$

4-O-(E)-p-Methoxycinnamoyl- $\alpha$ - $L$-rhamnopyranoside $=2712$

6-O-E-p-Methoxycinnamoyl scandoside methyl ester $=13895$

5'-Methoxy-cleomiscosin $\mathrm{A}=3831$

7-Methoxycoumarin $=9452$

7-Methoxydaphnoritin $=4658$

6-Methoxy-5,6-dihydrochelerythrine $=3499$

1- $\beta$-Methoxy-3,4-dihydro-3 $\alpha$-hydroxy-8- $O$-acetylharpagide aglucone $=3785$

2-Methoxy-4,6-dihydroxy- $\alpha$-chalcanol- $\alpha, \beta$-epoxide-4- $O-\beta$ - $D$-glucopyrano-

side $=21631$

2-Methoxy-4,7-dihydroxy-6-isopentenyl-9,10-dihydrophenanthrene $=19930$

7-Methoxy-8-(2',3'-dihydroxy-3'-methylbutyl)coumarin = 13781

3'-Methoxy-4',5-dihydroxy-6,7-methylenedioxyisoflavone $=11152$

7-Methoxy-2,2-dimethyl-2H-1-benzopyran-6-carboxylic acid $=13840$

5-Methoxy- $N, N$-dimethyl-tryptamine $=6370$

$\left(5^{\prime} \beta, 9^{\prime} \alpha, 10^{\prime} \alpha\right)-7-O-\left(3 \alpha-\right.$ Methoxy-8' $\left(12^{\prime}\right)$-drimen-11'-yl $)$-scopoletin $=6604$

3-Methoxy-6- $O-\beta$ - $D$-glucopyranosyl[2",3":7,8]furanoflavone $=17711$

6-Methoxy-3'-O- $\beta$ - $D$-glucopyranosyl[2",3":7,8]furanoflavone $=17710$

4-Methoxy-6-( $\beta$ - $D$-glucopyranoxymethyl)-2H-pyran-2-one $=16160$

3-Methoxy-4- $O-\beta$ - $D$-glycopyranosylpropiophenone $=2107$

$(2 R, 3 R)-5$ '-Methoxyguayarol [(2R,3R)-3-(3,4-dihydroxybenzyl)-2-(3,4,5trimethoxybenzyl)-butyrolactone] $=13945$

10-Methoxyheptadeca-1-ene-4,6-diyne-3,9-diol = 16586

3-( $\alpha$-Methoxy-4-hydroxybenzylidene)-6-hydroxybenzo-2(3H)-furanone-7- $C$ $\beta$ - $D$-glucopyranoside $=18106$

4-Methoxy-5-hydroxycanthin-6-one $=15561$

$\alpha$ - $O$-[2-O-(3-Methoxy-4-hydroxycinnamoyl)- $\beta$ - $D$-glucopyranosyl]-3,4-dihydroxyphenylethanol $=14918$

(E)-N-(3-Methoxy-4-hydroxycinnamoyl)-5-hydroxytryptamine $=14999$

(Z)- $N$-(3-Methoxy-4-hydroxycinnamoyl)-5-hydroxytryptamine $=14998$

6-Methoxy-7-hydroxycoumarin $=19542$

4'-Methoxy-5-hydroxy-8-3,3-dimethylallyl-flavone-3-glucosyl-(1 $\rightarrow 2)$ rhamnoside-7-glucoside $=6959$

4'-Methoxy-5-hydroxy-8-3,3-dimethyl allylflavone-3-rhamnosyl-(1 $\rightarrow 2)$ rhamnoside-7-glucoside $=6961$

4'-Methoxy-5-hydroxy-8-3,3-dimethyl allylflavone-3-xyloxyl-( $\rightarrow 2)$ rhamnoside-7-glucoside $=6960$

5-Methoxy-12-hydroxy-11,14-dioxo-abieta-8,12,15-triene $=2584$

3-Methoxy-4-hydroxyl-5-(3'-methyl-2') butylenyl benzoic acid = 17902

3 -Methoxy-4-hydroxylphenyl-1- $O-\alpha-L$-rhamnopyranosyl-(1" $\left.\rightarrow 6^{\prime}\right)-\beta$ - $D$-glucopyranoside $=4365$

5-Methoxy-8-(3"-hydroxymethyl-but-2"-enyloxy)-psoralen = 13963

$N$-(3'-Methoxy-4'-hydroxyphenethyl)-4- $O-\beta$ - $D$-galactopyranosyl-isoferulamide $=11331$

3-Methoxy-4-hydroxy-phenethyl- $O$ - $[\alpha-L$-rhamno-pyranosyl- $(1 \rightarrow 3)]-O-[\beta-D-$

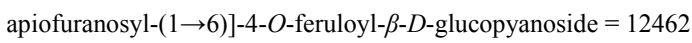

2-(3-Methoxy-4-hydroxy) phenyl ethanol 1- $O-\alpha-L-[(1 \rightarrow 3)$-rhamnopyranosyl-6- $O$-feruloyl] glucoside $=6965$

4"-(3"'-Methoxy-4"'-hydroxyphenyl)-2"-oxo-3"-enebutanyl 3-(3'-methoxy-4'- 
hydroxyphenyl)propenoate $=2960$

5-Methoxy-(+)-isolariciresinol-9,9'-diacetate $=8240$

3'-Methoxyisovitexin 2"- $O$-(6"'-(E)- $p$-coumaroyl $)-\beta$-glucopyranoside $=11700$

3'-Methoxyisovitexin 2"- $O$-(6"'-(E)- $p$-coumaroyl)- $\beta$-glucopyranoside-4'- $O$ - $\beta$ -

glucopyranoside $=11701$

(10R)-15-Methoxy-8(17),13-labdadien-16,15-olide-19-oic acid = 14069

(10S)-15-Methoxy-8(17),13-labdadien-16,15-olide-19-oic acid = 14070

6-Methoxylcarbonyl-3,3-dimethyl-4 $\alpha, 6 \alpha$-di $(\gamma, \gamma$-dimethylallyl)-2 $\alpha$-(2-methyl-

1-oxo-propyl)cyclohexanone $=8217$

4'-Methoxylquercetin-3- $O-\beta$ - $D$-xylopyranosyl $(1 \rightarrow 2)-\beta$ - $D$-glucopyranoside-3'-

$O-\beta$ - $D$-glucopyranoside $=662$

3-Methoxyl-9'- $\alpha$ - $L$-rhamnopyranosyl-4':7,5':8-diepoxyneoligan-4,9-diol = 13587

6-Methoxyluteolin $=7556$

6-Methoxyluteolin-7-glucoside $=7557$

7-Methoxy-8-(1'-methoxy-2'-hydroxy-3'-methyl-3'-butenyl)coumarin = 859

5-Methoxy-2-(4-methoxyphenyl)-benzofuran $=4095$

5-Methoxy-2-(4-methoxyphenyl)-2,3-dihydrobenzofuran $=4093$

7-Methoxy-8-(3-methyl-2-butenyl)-2H-1-benzopyran-2-one = 16261

2-Methoxy-3-methyl-4,6-dihydroxy-5(3'-hydroxyl)cinnamoylbenzaldehyde = 5269

7-Methoxy-3',4'-methylenedioxyflavone $=14376$

3-Methoxy-3',4'-methylenedioxy-7-O- $\beta$ - $D$-glucopyranosyl flavone $=17712$

2-(3-Methoxy-4,5-methylenedioxyphenyl)-4H-1-benzopyran-4-one = 14016

4-Methoxy-3-methyl-5-[(2Z,11aS)-3at,11t-epoxy-8t-((1R)-1-hydroxypropyl)-

1c-methyl-(11ar,11bc)-dodecahydro-furo[3,2-c]pyrido[1,2-a]azepin-2-

ylidene]-5H-furan-2-one $=16475$

4-Methoxy-3-methyl-5-[(2Z,11aS)-3at,11t-epoxy-8t-((1R)-1-hydroxypropyl)-

$1 c$-methyl-(11ar,11bc)-dodecahydro-furo[3,2-c]pyrido[1,2-a]azepin-2-

ylidene]-5H-furan-2-one- $N$-oxide $=16476$

4-Methoxy-3-methyl-5-[(2Z,11aS)-3at,11t-epoxy-1c-methyl-(11ar,11bc)-

dodecahydrofuro[3,2-c]pyrido[1,2- $a$ ]azepin-2-ylidene $]-5 H$-furan-2-one $=$ 18260

4-Methoxy-3-methyl-5-[(2Z,11aS)-8t-((1R)-1-hydroxypropyl)-1c-methyl-

(11ar,11bc)-1,2,5,6,8,9,10,11,11a,11b-decahydro-furo[3,2-c]pyrido

[1,2-a]azepin-2-ylidene $]-5 H$-furan-2-one $=20305$

4-Methoxy-3-methyl-5-[(2Z,11aS)-8t-((1R)-1-methoxypropyl)-1c-methyl-

(11ar,11bc)-1,2,5,6,8,9,10,11,11a,11b-decahydrofuro[3,2-c]pyrido

[1,2-a]azepin-2-ylidene]-5H-furan-2-one- $N$-oxide $=14094$

5 '-Methoxynobiletin $=9406$

$11 \alpha$-Methoxy-olean-12-ene-1 $\beta, 3 \beta, 28$-triol 28 - $O$ - $\beta$ - $D$-glucopyranosyl- $(1 \rightarrow 2)$ -

$\beta$ - $D$-glucopyranoside $=11985$

$11 \alpha$-Methoxy-olean-12-ene-1 $\beta, 3 \beta, 28$-triol 28 - $O$ - $\beta$ - $D$-glucopyranosyl- $(1 \rightarrow 2)$ -

$\beta$ - $D$-glucopyranosyl- $(1 \rightarrow 2)-\beta$ - $D$-glucopyranoside $=11986$

$1 \beta$-Methoxy- $2 \beta, 3 \alpha, 4 \beta, 5 \alpha, 6 \beta$-pentahydroxycyclohexane $=13047$

$o$-Methoxy phenol $=9021$

7-Methoxy-4-phenyl-2H-1-benzopyran-6-ol = 4608

2-(4-Methoxyphenyl)-1-nitroethane $=13259$

1-(4'-Methoxyphenyl)-(1R,2R)-propan-1-ol 2- $O-\beta$ - $D$-glucopyranoside $=1184$

[1-(4'-Methoxyphenyl)]-(1S,2S)-propan-1-ol 2- $O-\beta$ - $D$-glucopyranoside $=1185$

5 '-Methoxypongapin $=14074$

4-Methoxy-9-prenyl-2,6,10-trihydroxybenzophenone $=4338$

1-Methoxy-4-(1-propenyl)benzene $=1186$

2-Methoxy-4-propenyl phenol $=11421$
5-Methoxypsoralen $=2309$

8-Methoxypsoralen $=22774$

Methoxyquinol 4- $O$-[(5-O-trans- $p$-coumaroyl)- $\beta$ - $D$-apiofuranosyl- $(1 \rightarrow 2)-\beta$ -

$D$-glucopyranoside $=8851$

$16 \beta$-Methoxy-3 $\beta$-[( $O-\alpha-L$-rhamnopyranosyl- $(1 \rightarrow 2)-O$ - $[\alpha-L$-rhamnopyranosyl$(1 \rightarrow 4)]$ - $\beta$ - $D$-glucopyranosyl)oxy]pregna-5,16-dien-20-one $=20233$

$6 \alpha$-Methoxy-6,7-seco-6,19-epoxy-7,20-olide-ent-kaur-16(17)-en-15-one = 13071

(16R)-6 $\beta$-Methoxy-6,7-seco-6,19-epoxy-7,20-olide-entkaur-15-one $=13070$

(16R)-6 $\alpha$-Methoxy-6,7-seco-6,19-epoxy-7,20-olide-ent-kaur-15-one = 13067

(16S)-6 $\alpha$-Methoxy-6,7-seco-6,19-epoxy-7,20-olide-ent-kaur-15-one = 13068

(16S)-6 $\beta$-Methoxy-6,7-seco-6,19-epoxy-7,20-olide-ent-kaur-15-one = 13069

(25R,26R)-26-Methoxyspirost-5-en-3 $\beta$-ol 3- $O$ - $\{O-\alpha-L$-rhamnopyranosyl-

$(1 \rightarrow 2)-O$-[ $\beta$ - $D$-xylopyranosyl-( $1 \rightarrow 2)-O-\alpha-L$-rhamnopyranosyl-( $1 \rightarrow 4)]$ -

$\beta$ - $D$-glucopyranoside $\}=20068$

trans-3-Methoxy-2,3',4,5'-tetrahydroxystilbene $=22434$

2-Methoxy-3,4,6-trihydroxy- $\alpha$ '-chalcanol- $\alpha, \beta$-epoxide-4- $O-\beta$ - $D$-glucopyranoside $=21632$

3'-Methoxy-4',5,7-trihydroxyflavone $=3602$

$8 \alpha$-Methybutyryloxy- $1 \alpha$-hydroxy-3 $\alpha, 4 \alpha$-epoxy-5 $\alpha, 7 \alpha H$-10(14),11(13)-guaiadien-12,6 $\alpha$-olide $=7196$

Methylabrusgenate $=18582$

2-Methyl-5-acetonyl-7-hydroxychromone $=3279$

Methyl 22 $\beta$-acetoxy-3,25-epoxy-3 $\alpha$-hydroxy-urs-12-en-28-oate $=14208$

$O$-Methylaknadinine $=9245$

Methyl allyl pentasulfide $=950$

Methylallyltetrasulfide $=951$

6-Methylamino-5,9-dimethoxyoxoisoaporphine $=4691$

$3 \alpha$-(Methylamino)pregn-5-en-16-one $=9577$

(20S)-20-( $N$-Methylamino)-3 $\beta$-(tigloylamino)-5 $\alpha$-pregna-2-en-16 $\alpha, 17 \alpha$ epoxy-4-one $=7198$

(20S)-20-( $N$-Methylamino)-3 $\beta$-(tigloylamino)-5 $\alpha$-pregna- $16 \alpha, 17 \alpha$-epoxy$2 \beta, 4 \beta$-di- $O$-acetate $=7177$

$O$-Methylanolobine $=22818$

$O$-Methylaromoline $=9597$

$O$-Methylatheroline $=16339$

Methyl azelate $=6317$

5-Methyl-1,3-benzenediol $=16169$

Methyl betulinate $=14158$

3-Methylbutanoic acid $=11754$

1-[(3-Methylbutanoyl)phloroglucinyl]- $\beta$ - $D$-glucopyranoside $=13261$

(E)-2-Methyl-2-butenal $=21370$

trans-2-Methyl-2-butenal $=21370$

(E)-2-Methylbut-2-en-1-al = 21370

(E)-2-Methyl-2-butenoic acid $=21371$

trans-2-Methyl-2-butenoic acid $=21371$

2-Methyl-3-buten-2-ol $\beta$ - $D$-glucopyranoside $=4227$

12-O-(3-Methyl-2-butenoyl)-19-O-(3,4-dihydroxybenzoyl)-11-hydroxyabieta-8,11,13-triene $=17554$

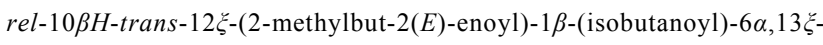
di- hydroxyclerodan-4(20),8(18)-dien-7,15-dione-15,16-oxide $=$ 2948

rel-10 $\beta H$-trans-12 $\xi$-(2-Methylbut-2(E)-enoyl)-1 $\beta$-(2-methylbutanoyl)$6 \alpha, 13 \xi$-dihydroxyclerodan-4(20),8(18)-dien-7,15-dione-15,16-oxide $=$ 
2952

12-(3-Methylbut-2-enoyloxy)pregn-5-en-20-one 3-O-[ $\beta$-cymaropyranosyl-

$(1 \rightarrow 4)$ - $\beta$-cymaropyranosyl- $(1 \rightarrow 4)-\beta$-thevetopyranosyl- $(1 \rightarrow 4)-(6-O$ -

sulfo- $\beta$-glucopyranoside) $]=7699$

6-(3-Methyl-2-butenyl)-1,5-dihydroxyxanthone $=2989$

2-(3-Methyl-2-butenyl)-3-O-(3-methyl-2-butenyl)-5-[2-(3-hydroxyphenyl)

ethyl]-1,3-benzenediol $=8551$

2-(2-Methylbutyryl)-phloroglucinol 1-O- $\beta$ - $D$-glucopyranoside $=15063$

$20-O$-(2-Methylbutyryl)tomentogenin $=6600$

3-Methyl-9H-carbazole $=14216$

3-Methylcarbazol-2-ol $=10478$

$2 \alpha, 3 \beta-7-O$-Methylcedrusin $=5582$

24-Methylcholesta-5,22-dien-3 $\beta$-ol $=2596$

(24S)-Methylcholest-5-en-3 $\beta$-ol $=3040$

3-Methylchrysazin $=3615$

3'-O-Methylconiferin $=14249$

3'-Methyl crenatoside $=14261$

(E)-2-Methyl-crotonaldehyde $=21370$

$\alpha$-Methylcrtonaldehyde $=21370$

Methyl-1-cyclohexen-1-yl-1,3,5,7,9,11,13-tetradecaheptaene $=13189$

$(-)$ - $N$-Methylcytisine $=14279$

$\mathrm{N}$-Methyldaphnandrine $=9597$

Methyldeacetylasperulosidate $=4717$

Methyl $(2 Z, 8 Z)$-decadien-4,6-diynoate $=13603$

Methyl-trans-2-decene-4,6,8-triynoate $=4947$

Methyl-cis-2-decen-4,6,8-triynate $=4946$

$N$-Methyldendrobine $=14288$

Methyl-2 $\alpha, 15$-diacetoxy-2,3-epoxy-2,3-seco-( $1 \alpha, 5 \alpha, 6 \beta, 7 \beta)$-aromadendra-

3,10(14)-dien-13-oate $=17497$

21 $\beta$-Methyl-20,22-dideoxy-25-hydroxymicrandilactone $\mathrm{A}=12476$

Methyl-2,4-dihydroxy-benzoate $=14712$

Methyl 2,3-dihydroxy-2-(5-hydroxy-11-methyl-6-oxo-1,2,6,11-tetrahydrofuro[2,3-c $]$ acridin-2-yl) propanoate $=18784$

(12S)-Methyl 6 $\alpha, 12$-dihydroxy-4 $\alpha$-methoxycarbonyl-18-norneo-clerod-

13(14)-en-15,16-olide $=798$

6-Methyl-2,5-dihydroxymethyl- $\gamma$-pyranone III $=9438$

Methyl-3 $\beta, 22 \alpha$-dihydroxy-olean-12-ene-29-oate $=18582$

Methyl-3 $\beta, 22 \alpha$-dihydroxy-urs-12-ene-30-oate $=18581$

(+)-1-Methyl-4-(5,9-dimethyl-1-methylene-deca-4,8-dienyl)cyclohexene = 2045

Methyl eicos-11-enoate $=14336$

$3^{\prime}-O$-Methyl-ellagic acid 4- $O-\alpha-L$-arabinofuranoside $=6620$

4-O-Methylellagic acid 3'-(2",3'-di- $O$-acetyl)-3'- $\alpha$-rhamnoside $=14342$

$3^{\prime}-O$-Methyl-ellagic acid-4- $O-\beta$ - $D$-xylopyranoside $=6619$

3,4-Methylendioxy -5-methoxy-1-(1-oxopropyl)benzene $=6479$

25-Methylenecycloartanyl- $p$-hydroxy-trans-cinnamate $=17175$

1,2-Methylenedioxy-4-allyl-benzene $=19121$

$6,7-$ Methylenedioxycoumarin $=2048$

$\left(7 R, 8 R, 7^{\prime} S, 8^{\prime} S\right)$-3,4-Methylenedioxy-3',4'-dimethoxy-7,7'-epoxylignan = 8039

3,4-Methylenedioxy-10-hydroxy aristololactam $=1693$

3,4-Methylenedioxy-3'-methoxybibenzyl $=14375$

5,6-Methylenedioxy-9-methoxy-7H-dibenzo(de,h)quinoli-7-one $=2344$

(6aR,12aS)-2,3-Methylenedioxy-9-methoxy-8-(3,3-dimethylallyl)-12ahydroxy rotenoid $=22279$
3,4-Methylenedioxyphenanthrene-1-carboxylic acid $=5062$

$N$-[10-(13,14-Methylenedioxyphenyl)-7(E),9(Z)-pentadienoyl]-pyrrolidine $=$ 16388

$(6 E, 8 E, 12 E)$-3-Methylene-( $4 R)$-hydroxy-7,11-dimethyl-(10R*,11R*)-dichloro-13-bromo-trideca-6,8,12-trienoic acid $=17652$

$(6 E, 8 E, 12 E)$-3-Methylene-( $4 R)$-hydroxy-7,11-dimethyl-( $\left.10 S^{*}, 11 R^{*}\right)$-dichloro-13-bromo-trideca-6,8,12-trienoic acid oil $=17653$

24-Methylene-lanosta-9(11)-en-3 $\beta$-ol $=766$

24-Methylene-lanosta-9(11)-en-3-one $=767$

4-Methylene-1-(1-methylethyl)bicyclo[3.1.0]hexane $=19099$

3-Methylene-6-(1-methylethyl)cyclohexene $=17056$

$(6 E, 8 E, 12 E)$-3-Methylene-4-oxo-7,11-dimethyl-( $\left.10 S^{*}, 11 R^{*}\right)$-dichloro-13bromo-trideca-6,8,12-trienoic acid $=17650$

$(6 E, 8 E, 12 E)$-3-Methylene-4-oxo-7,11-dimethyl-(10R*,11 $\left.R^{*}\right)$-dichloro-13bromo-trideca-6,8,12-trienoic acid $=17651$

24-Methylene-3-oxo-lanost-8-en-21-oic acid $=7856$

Methyl ent-13,17-epoxy-16-hydroxykauran-19-oate $=3427$

Methyl $(2 E, 6 E, 10 R)$-10,11-epoxy-3,7,11-trimethyl-2,6-dodecadien-oate $=11988$

3'- $O$-Methyl eriodictyol $=9604$

7-O-Methylesculetin $=11702$

1-Methyl ether of 10,12,10"-trichloroisoplagiochin C $=2180$

1-Methyl-3-ethylacrolein $=14662$

4-(1-Methylethyl)benzoic acid $=4356$

8-Methyleugenitol $=6167$

Methyl eugenol $=7523$

22 - $O$-Methyl-25(S)-furost-1 $\beta, 2 \beta, 3 \beta, 4 \beta, 5 \beta, 22 \xi, 26 \beta$-heptol-26- $O-\beta$ - $D$-glucopyranoside $=22651$

22-O-Methyl-25(R,S)-furost-1 $\beta, 3 \beta, 4 \beta, 5 \beta, 22 \xi, 26 \beta$-hexol-26- $O-\beta$ - $D$-glucopyranoside $=22649$

$5-O$-Methyl genistein 7-O- $\alpha$ - $L$-rhamnopyranosyl-( $(1 \rightarrow 6)-\beta$ - $D$-glucopyranoside $=9555$

22-O-Methyl-26-O- $\beta$ - $D$-glycopyranosyl-25(S)-furost-5-en-1 $\beta, 3 \beta, 22 \xi, 26 \beta$-tetrol$3-O-[O-\beta$ - $D$-glucopyranosyl $(1 \rightarrow 4)]-\beta$ - $D$-galactopyranoside $=22650$

Methylheptyl-ketone $=15687$

$N$-methyl-2-heptyl-4(1H)-quinolone $=19468$

$N$-Methylhernandine $=15929$

$N$-Methyl hernovine $=14640$

Methyl hexadecanoate $=14484$

Methyl 3'-Hydroxyaglafolin $=10677$

Methyl- $p$-hydroxycinnamate $=14254$

2-Methyl-3,4-[2'-(1-hydroxy-1-methylethyl)-dihydrofurano]anthraquinone $=3135$

Methyl(24Z)-26-hydroxy-3-oxo-7,24-euphadienoate $=14520$

$N$-Methyl-3 $\beta$-hydroxy- $5 \alpha$-veratranine-6-one $=15613$

1-O-Methyl-myo-inositol $=2556$

$O$-Methyllansine $=8832$

24-Methyl lathosterol $=14237$

5-O-Methyl licoricidin $=12795$

23-O-Methyl malonyl hederagenin 28-O- $\alpha-L$-rhamnopyranosyl $(1 \rightarrow 4)-\beta-D$ glucopyranosyl $(1 \rightarrow 6)-\beta$ - $D$-glucopyranoside $=2212$

3-O-Methyl malonyl hederagenin 28- $O-\alpha-L$-rhamnopyranosyl $(1 \rightarrow 4)-\beta$ - $D$ glucopyranosyl $(1 \rightarrow 6)-\beta-D$-glucopyranoside $=2213$

$(3 R, 4 R, 6 R)-4-[(2 R, 3 R)-2-M e t h y l-3-(3-m e t h y l-b u t-2-e n y l)-o x i r a n y l]-1-o x a-$ spiro[2,5] octan-6-ol $=5047$ 
(+)-4-Methyl-9-methylene-bicyclo[8.2.1] trideca-1(13),4-diene $=11791$

(4S,4aS,8aS)-8a-Methyl-5-methylene-4-(1-methylvinyl)-1,2,3,4,4a,5,6,8aoctahydro-naphthalene $=8951$

2-Methyl-5-(1-methylethenyl)-2-cyclohexen-1-one $=3237$

$(1 \alpha, 2 \beta, 5 \alpha)$-2-Methyl-5-(1-methylethyl)cyclohexanol = 3236

3-Methyl-6-(1-methylethyl)-2-cyclohexen-1-one = 17456

$(+)-(2 S, 7 R, 8 S)-5-M e t h y l-8-(1-m e t h y l e t h y l)-9-o x a-t r i c y c l o\left[6.2 .2 .0^{2,7}\right]$ dodeca-

$1(11), 5$-diene $=1260$

3'- $O$-Methyl-3,4- $O, O$-methylideneellagic acid-4'- $O-\beta$ - $D$-glucopyranoside $=15880$

3-Methyl-5-[(2Z,3aR)-1t-methyl-8t-((2S)-4c-methyl-5-oxo-tetrahydrofuran-

$2 r$-yl)-(3ar,10at,10bt)-decahydro-2H-furo[3,2-c]pyrrolo[1,2-a]azepin-

2-yl]-5H-furan-2-one $=20296$

(2-Methyl-6-(4-methylphenyl)-1,5-heptadiene $)=2404$

(2-Methyl-6-(4-methylphenyl)-1,6-heptadiene) $=2405$

$N$-Methyl nandigerine $=14483$

7-O-Methylnaringenin $=19174$

Methylnigakinone $=12334$

$(-)$-Methylnissolin $=14619$

Methyl octadecanoate $=14629$

Methyl oleate $=14630$

Methyl 3-oxo-22 $\alpha$-acetoxy-23-hydroxy-urs-12-ene-30-oate $=18579$

Methyl 3-oxo-22 $\alpha$-hydroxy-olean-12-ene-29-oate $=18580$

Methyl palmitate $=14484$

7-O-Methylpectolinarigenin $=19211$

4-Methyl-4-penten-2-one $=11535$

$(2 E, 4 E, 12 Z)-N$-(4-Methylpentyl)octadeca-2,4,12-trienamide $=17473$

2-Methylphenol $=4233$

3-Methylphenol $=4232$

4-Methylphenol $=4234$

2-Methyl-3-phytyl-1,4-naphthoquinone $=22562$

4'-O-Methyl piceid $=5206$

6-Methylpinocembrin $=20394$

2-Methylpropanoic acid $=11290$

2-Methyl-1-propanol $=11268$

2-Methyl-6-(prop-2-enyl)piperidine $=17390$

Methyl 2-propenyl trisulfide $=952$

3-(1-Methyl-2-pyrrolidinyl)pyridine $=15528$

3 - $O$-Methylquercetin $=18376$

3'- $O$-Methylquercetin 3- $O$ - $\alpha$ - $L$-rhamnopyranosyl $(1 \rightarrow 6)-2$ "- $O$-acetyl- $\beta$ - $D$ -

glucopyranoside $=466$

Methyl rosmarinate $=18925$

$E$-3-Methylsulfinyloxy-2-propenyl sec-butyl disulfide $=7847$

Z-3-Methylsulfinyloxy-2-propenyl sec-butyl disulfide $=7846$

$E$-3-Methylsulfinyl-2-propenyl sec-butyl disulfide $=7845$

(E)-3-(Methylsulfonyl)-propenoic acid (E)-2-[4-(3,7-dimethyl-2,6-octadienyloxy)-phenyl]-2-hydroxyethyl amide $=10139$

(E)-3-(Methylsulfonyl)-propenoic acid (2E,6E)-2-[4-(3,7-dimethyl-8-oxo2,6-octadienyloxy)-phenyl]-2-hydroxyethyl amide $=10138$

(E)-3-(Methylsulfonyl)-propenoic acid (2E,6E)-4-(8-hydroxy-3,7-dimethyl2,6-octadienyloxy)-3-methoxyphenethyl amide $=14716$

(E)-3-(Methylsulfonyl)-propenoic acid $(2 E, 6 E)$-4-(8-hydroxy-3,7-dimethyl2,6-octadienyloxy)-phenethyl amide $=8310$

(E)-3-(Methylsulfonyl)-propenoic acid $(2 E, 6 E)$-2-[4-(8-hydroxy-3,7-di- methyl-2,6-octadienyloxy)-phenyl]-2-hydroxyethyl amide $=10140$

(E)-3-(Methylsulfonyl)-propenoic acid (2E,6E)-3-hydroxy-4-(8-hydroxy-

3,7-dimethyl-2,6-octadienyloxy)-phenethyl amide $=19170$

(E)-3-(Methylsulfonyl)-propenoic acid (2E,6Z)-3-hydroxy-4-(8-hydroxy-

3,7-dimethyl-2,6-octadienyloxy)-phenethyl amide $=19171$

(E)-3-(Methylsulfonyl)-propenoic acid 3-hydroxy-4-(3-methyl-2-butenyloxy)-phenethyl amide $=19169$

(E)-3-(Methylsulfonyl)-propenoic acid 4-(3-methyl-2-butenyloxy)-3-methoxyphenethyl amide $=14715$

$N$-Methyltaxol $=14651$

Methyl tetradecanoate $=14610$

3-Methyl-4-tetradecanoyloxy-2-butenenitrile $=21042$

2-Methyl-3-thiapentane $=7454$

$N$-[N'-(E)-(3-Methylthio-2-propenoyl)-4-aminobu-tyl]-(E)-3-methylthiopropenamide $=736$

$N$-[N'-(E)-(3-Methylthio-2-propenoyl)-4-aminobutyl $]$ phenylacetamide $=740$

5-Methyl thymol ether $=11626$

$\mathrm{N}$-Methyl-1,4,5-trihydroxy-3,6-dimethoxyacridine-9-one $=1954$

(-)-5-Methyl-3-(1',2',2'-trimethylcyclopentyl) phenol = 9427

$N$-Methyl- $L$-tryptophan $=13$

(1-Methylundecyl) benzene $=17104$

$N$-methyl-5 $\alpha$-veratranine-6-oxo-3 $\beta$ - $O-\beta$ - $D$-glucoside $=15614$

Methylwarifteine $=3748$

7-O-Methylwogonin $=10026$

Micromarin B $=17824$

Micropinic acid $=11600$

Mimoside $=14869$

Minorine $=22489$

Minumicroline $=14874$

Mitrinermine $=18826$

Miyoshianine $\mathrm{A}=13225$

Miyoshianine $\mathrm{B}=13229$

Mogro-3-O- $\beta$ - $D$-glucopyranoside-24- $O\{[\beta$ - $D$-glucopyranosyl $(2 \rightarrow 1)]-[\beta-D$ glucopyranosyl $(6 \rightarrow 1)]-\beta$ - $D$-glucopyranoside $\}=9012$

Mogrol 3-O- $\beta$-glucopyranosyl-26- $O$ - $\alpha$-rhamnopyranosyl $(1 \rightarrow 2)-O$ - $\beta$-glucopyranoside $=12218$

Mollis lactone $=14898$

Momordin $\mathrm{IIb}=9588$

Monardein $=14914$

Mono(2,2-dimethylhydrazide) butanedioic acid $=1064$

Monomelittoside $=4626$

7"-Monomethylhinoliflavone $=11353$

Montanin $\mathrm{C}=524$

Moracenin $\mathrm{A}=12386$

Moracenin $\mathrm{B}=12385$

Moranoline $=5198$

Morinobufagin $=2715$

Morphine monomethyl ether $=3885$

Morusinol $=16445$

Moupinamide $=7788$

$3-\mathrm{MQ}=18376$

Mubenin B $=6755$

Mukoenine $\mathrm{B}=3790$ 
Mukurosigenin $=9260$

Mulberrochromene $=14995$

Multifidin $=18790$

Murraxocin $=15100$

Murrayazoline $=13397$

Musizin $=15489$

Mutumol $=17492$

Myoinositol $=11085$

Myo-inositol hexaphosphate $=17256$

Myricardiol $=15154$

Myricetin-3-glucoside $=11551$

Myricetin-3- $O$-methyl ether $=1339$

Myricetin-5-methyl ether $=14609$

Myricetin-4'- $O$-methyl ether-3- $O-\alpha-L$-rhamnopyranoside $=13628$

Myricetin 3- $O$-(6"- $O$ - $\alpha$ - $L$-rhamnopyranosyl) $\beta$ - $D$-glucopyranoside $=15185$

Myricetol $=15170$

Myricitrin $=15184$

Myristic alcohol $=21041$

Myrkolal $=15166$

\section{N}

Nagarine $=274$

Nandigerine $=9451$

$(+)$-Nantenine $=14332$

Napellonine $=2742$

1,4-Naphthohydroquinone-1,4-di- $O-\beta$ - $D$-glucopyranoside $=19033$

Narciclasine $=13239$

Narcipoetine $=9612$

(2S)-Naringenin $=15279$

NDGA $=15741$

Nehipetol $=4380$

Neoaristolactone $=22422$

Neocerotic acid $=16823$

Neochamaejasmin A $=15361$

Neofinaconitine $=5009$

Neoglycyrol $=8839$

Neohecogenin-3- $O-\beta$ - $D$-glucopyranosyl $(1 \rightarrow 4)-\beta$ - $D$-galactopyranoside $=21001$

Neohecogenin-3-O- $\beta$ - $D$-glucopyranosyl $(1 \rightarrow 2)$ - $\beta$ - $D$-glucopyranosyl $(1 \rightarrow 4)$ -

$$
\beta \text { - } D \text {-galactopyranoside }=21000
$$

13(18)-Neohopene $=9642$

Neojusticin A = 11980

Neojusticin B = 11979

Neolinderalactone $=12868$

Neoline $=2738$

Neorautenone $=15462$

Neosamogenin $=13569$

Neotanshinone $\mathrm{A}=4632$

Neotanshinone $\mathrm{B}=4633$

Neotanshinone $\mathrm{C}=4634$

Nepitrin $=7557$

Neral $=3762$

Neriifolin $=15494$

Neriifoliol $=9635$
Neriine $=3968$

$(+)$-Nerolidol $=15499$

$3 \alpha$-Nervogenoyloxytropane $=13787$

Nevadensin $=13264$

Niacin $=15526$

Nicocitin $=5520$

7-(Nicotinoyloxy)- $O^{5}$-furanoyl- $O^{5}$-deacetyl-7-deoxo-evonine $=10907$

Nigakihemiacetal B $=15454$

Nigakilactone $=15546$

Nigricin 4'- $O-\beta-D$-glucoside $=8350$

Ningposide $\mathrm{C}=415$

Nivalenone $=15633$

6,10-Nonacosanediol $=15663$

Nonadecane $=15671$

Nonadecylic acid $=15672$

Nonane $=15683$

Nonyl alcohol $=15686$

Nonylaldehyde $=15681$

4-Nonylphenol = 15701

18-Nor-4(19),8,11,13-abietatetraene $=15736$

Noranhydroicaritin $=11588$

Noraporphine $=16339$

Norarmepavine $=15713$

$(+)$-Norboldine $=12573$

29-Norcycloartanyl acetate $=15730$

Nordihydrocapsaicin $=15740$

Noreugenin-7- $O-\beta-D$-glucoside $=20263$

$C$-Nor- $D$-homosteroid alkaloid $=17380$

$(+)$-Norisocorydine $=15763$

19-Nor-ent-kaurane-4 $\alpha, 16 \beta, 17$-triol = 1326

Norlobelanine $=11506$

28-Nor-lup-12,17-dien-3 $\beta, 16 \alpha$-diol, = 12243

28 -Nor-lup-12,17-dien-3 $\beta$-ol-16-one $=12245$

30 -Normethyl lupane-20-one $=634$

30 -Normethyl olean-3-on-30 $\beta$-ol $=635$

$(S)$-Nornantenine $=15779$

$O$-Nornuciferine $=14148$

Noroxylin $=2102$

$(-)$-Norstephalagine $=15797$

Nortropanoline $=3010$

(-)-Norushinsunine $=14827$

Norwedelolactone $=5101$

Notoginsenoside $\mathrm{B}_{1}=19233$

Nubiletin $=15635$

Nudaurine $=1099$

Nuezhenide $=15873$

Nyasol $=9546$

\section{O}

Obacunonic acid $=15883$

Obtusifolin-2- $O-\beta$ - $D$-glucoside $=8696$

Obtusilic acid $=4843$

$\alpha$-Ocimene $=15925$ 
Ocoteine $=21241$

2,10,12,6', 7', 8',10',14'-Octachloroisoplagiochin C = 2186

Octacosanic acid $=14944$

Octacosyl ferulate $=3852$

9,11-Octadecadienoic acid $=11503$

cis-9, cis-12-Octadecadienoic acid $=12891$

$1-O-(9 Z, 12 Z$-Octadecadienoyl) glycerol $=8814$

Octadecane $=15948$

Octadecanoic acid $=20280$

Octadecanoic acid-2,3-dihydroxypropyl ester $=15951$

Octadecanyl 3-(4-hydroxy-3-methoxy-phenyl)-acrylate ester $=15954$

$(Z, Z, Z)-9,12,15$-Octadecatrienoic acid $=12893$

$(Z, Z, Z)-9,12,15$-Octadecatrienoic acid methyl ester $=14555$

$(-)$-Z-9-Octadecene-4-olide $=14842$

trans-9-Octadecenoic acid $=6731$

10-Octadecenoic acid $=11574$

(-)-1,2,3,4,4a,5,6,7-Octahydro-4a,8-dimethyl-2-(1-methylethyl)-naphthalen-

2 -ol $=7510$

(+)-(2R,4aS,8aR)-1,2,3,4,4a,5,6,8a-Octahydro-4a,8-dimethyl-2-(1-methyl-

ethyl)-2-naphthalenol $=7509$

(+)-(1S,3aR,7S,7aS)-2,3,3a,4,5,6,7,7a-Octahydro-3a-hydroxyl-7,7a-dimeth-

yl-1-(2-methylpropanonyl)- $1 H$-indene $=5559$

$7,7^{\prime}, 8,8^{\prime}, 11,11^{\prime}, 12,12^{\prime}$-Octahydrolycopene $=17263$

$(4 \mathrm{a} \alpha, 5 \beta, 11 \mathrm{~b} \beta)-1,2,3,4,4 \mathrm{a}, 5,6,11 \mathrm{~b}$-Octahydro-4,4,7,11b-tetramethyl-phenanthro

$[3,2-b]$ furan-4a,5-diol-5-benzoate $=11779$

$3,5,6,7,8,3^{\prime}, 4^{\prime}, 5^{\prime}-$ Octamethoxyflavone $=7696$

Octanoic acid $=3140$

Octanoic acid methyl ester $=14215$

$1-$ Octanol $=15977$

(3R)-1-Octan-3-yl-(6- $O$-sulfonyl)- $\beta$ - $D$-glucopyranoside $=2084$

(6R)-7-Octene-1,6-diol 6- $O$ - $\beta$ - $D$-glucopyranosyl-( $1 \rightarrow 2)-O-\beta$ - $D$-glucopyranoside $=6665$

(6R)-7-Octene-1,6-diol 6-O- $\beta$ - $D$-xylopyranosyl-( $1 \rightarrow 6)-O-\beta$ - $D$-glucopyranoside $=6666$

1-Octen-3-ol $=13608$

(E)-2-Octen-1-ol = 11532

(3R)-1-Octen-3-ol-3-O- $\beta$ - $D$-xylopyranosyl- $(1 \rightarrow 6)-\beta$ - $D$-glucopyranosyl$(1 \rightarrow 2)-\beta$ - $D$-glucopyranoside $=1952$

(3R)-1-Octen-3-ol-3-O- $\beta$ - $D$-xylopyranosyl-(1'"' $\left.\rightarrow 6^{\prime}\right)-O$-[ $\beta$ - $D$-glucopyranosyl$\left.\left(1^{\prime \prime} \rightarrow 2^{\prime}\right)\right]-O-\beta-D$-glucopyranoside $=6664$

1-Octen-3-yl $O$ - $\beta$-apiofuranosyl- $(1 \rightarrow 6)-O$-[ $\beta$-glucopyranosyl- $(1 \rightarrow 2)]-\beta$-glucopyranoside $=13075$

Octyl 6- $O-\alpha-L$-arabinopyranosyl- $\beta$ - $D$-glucopyranoside $=18793$

$17 \beta$-Oestradiol $=7384$

Oleana-11,13(18)-dien-29-oic acid 3 $\beta, 21 \alpha$-di- $O-\beta$ - $D$-glucuronopyranoside $=8856$

Olean-11,13(18)-diene-3 $\beta, 22 \beta$-diol $=20250$

Olean-11,13(18)-diene-3 $\beta, 16 \beta, 23,28,30$-pentol = 19136

Oleandrigenin $=400$

Oleandrigenin 3-O- $L$-cymaroside $=2190$

Oleandrigenin-3-O-[O- $\beta$ - $D$-glucopyranosyl $(1 \rightarrow 2)]-\alpha-L$-rhamnopyranoside $=22652$

Olean-12-en-23-al-2 $\beta, 3 \beta$-dihydroxy-30-methoxycarbonyl-28-oic acid $=547$

Olean-12-en-23-al-2 $\beta, 3 \beta, 11 \alpha$-trihydroxy-30-methoxycarbonyl-28-oic acid $=548$
Olean-12-en-3 $\beta, 27$-diol $=3470$

Olean-12-en-3,28-diol $=7338$

Olean-12-ene-23 $\alpha, 28 \beta$-dioic acid-3 $\beta$-,16 $\alpha$-dihydroxy-28- $O-\beta$ - $D$-glucopyra$\operatorname{nosyl}(1 \rightarrow 2)-\beta$ - $D$-glucopyranosyl- $(1 \rightarrow 6)$ - $[\beta$ - $D$-glucopyranosyl $(1 \rightarrow 3)]$ $\beta$ - $D$-glucopyranoside $=19668$

12-Oleanene-3,15,16,22,28-pentol $=2151$

Olean-12-ene-1 $\beta, 3 \beta, 11 \alpha, 28$-tetraol 28-O- $\beta$ - $D$-glucopyranosyl- $(1 \rightarrow 2)-\beta$ - $D$ glucopyranoside $=11983$

Olean-12-ene-1 $\beta, 3 \beta, 11 \alpha, 28$-tetraol 28- $O$ - $\beta$ - $D$-glucopyranosyl-( $1 \rightarrow 2)-\beta$ - $D$ glucopyranosyl- $(1 \rightarrow 2)-\beta$ - $D$-glucopyranoside $=11984$

12-Oleanene-3,22,28-triol $=10096$

Oleanolic acid-3- $O-\alpha$ - $L$-arabinopyranosyl-28- $O-\beta$ - $D$-glucopyranosyl $(1 \rightarrow 6)$ $\beta$ - $D$-glucopyranoside $=1577$

Oleanolic acid 28- $O-\beta$ - $D$-glucopyranosyl- $(1 \rightarrow 6)-\beta$ - $D$-glucopyranoside $=9357$

Oleanolic acid 3-O-[ $\beta$ - $D$-glucopyranosyl- $(1 \rightarrow 2)][\beta$ - $D$-glucopyranosyl$(1 \rightarrow 3)]-\alpha$ - $L$-arabinopyranosyl-28- $\beta$ - $D$-glucopyranosyl- $(1 \rightarrow 6)-\beta-D$ glucopyranoside $=1601$

Oleanolic acid 3- $O$-glucuronide $=2973$

Oleanolic acid 3-O-[3'-O-(2"-hydroxy-1"-carboxyethoxycarboxypropyl)]$\beta$-D-glucopyranoside $=544$

Oleanolic acid 3-O- $\alpha-L$-rhamnopyranosyl $(1 \rightarrow 2)-\alpha-L$-arabinopyranoside $=6756$

Oleanolic acid 3-O- $\{\alpha-L$-rhamnopyranosyl $(1 \rightarrow 2)$ - $[\alpha-L$-rhamnopyranosyl $(1 \rightarrow 4)]$ - $\beta$ - $D$-glucopyranosyl $(1 \rightarrow 3)\}-\beta$ - $D$-xylopyranoside $=7634$

Oleanonic acid $=16402$

Oleanoside $\mathrm{A}=1610$

Oleanoside $\mathrm{C}=1609$

Oleanoside $\mathrm{E}=1608$

cis-Oleic acid $=16066$

Oleoeuropeine $=16080$

Oleovitamin $\mathrm{A}=18656$

Oleyl alcohol $=15957$

6-C- $\beta$ - $D$-Oliopyranosyl-4'-O- $\beta$ - $D$-glucopyranosylapigenin $=18676$

Olitorin $=16084$

Olmelin $=2384$

$8(26), 14(27)$-Onoceradiene $=16110$

Ononin $=7885$

Ononoside $=7885$

Ophiopogenin-3-O- $\alpha$ - $L$-rhamnopyranosyl $(1 \rightarrow 2)[\beta$ - $D$-xylopyranosyl $(1 \rightarrow 3)]-$ $\beta$ - $D$-giucopyranoside $=16135$

Ophiopogenin-3-O- $\alpha$ - $L$-rhamnopyranosyl $(1 \rightarrow 2)[\beta$ - $D$-xylopyranosyl $(1 \rightarrow 3]$ $[\beta$ - $D$-giucopyranosyl $(1 \rightarrow 4)]$ - $\beta$ - $D$-giucopyranoside $=16142$

Opuntioside $\mathrm{I}=16160$

Oraposide $=4225$

Orobanchoside $=4225=16161$

Oroselone $=12395$

Oroxin $\mathrm{A}=2103$

Oroxylin $=16216$

Oryzadione $=7154$

Oryzanol A $=4475$

Osage orange $=14971$

Osthenon $=16260$

Ouabain $=20403$

$7(\beta)$-Oxa-bicyclo-[4,1,0]-hept-3-ene-3-carboxylic acid-5 $(\beta)$-hydroxy $=6547$ 
Oxacyclohexadecan-2-one $=7672$

$\beta$ - $N$-Oxalyl- $L$ - $\alpha, \beta$-diaminopropionic acid $=1058$

Oxedrine $=20552$

(24Z)-3-Oxo-14(13 $\rightarrow$ 12)abeo-lanosta-7,9(11),13(18),24-tetraen-26-oic acid = 22167

Oxoagarospirol $=2111$

ent-16-Oxobeyeran-19-al $=3428$

13-Oxo-7,9-bis-deacetylbaccatin VI $=20844$

2-Oxobutanedioic acid $=16284$

1-(1-Oxo-2E,4E-decadienyl)pyrrolidine $=19371$

(24Z)-3-Oxo-11 $R, 12 S$-diacetoxylanosta-8,24-dien-26-oic acid $=22163$

$(22 R, 24 S, 25 R, 26 R)-1-O x o-22,26 ; 24,25$-diepoxy-3 $\beta, 17 \alpha, 26$-trihydroxyergost-

5-ene 3- $O$-sulfate $=3648$

2-[(2-Oxo-1,2-dihydro-quinoline-4-carbonyl)-amino]-succinic acid 4- $n$-butyl ester $=20329$

2-[(2-Oxo-1,2-dihydro-quinoline-4-carbonyl)-amino]-succinic acid 4-methyl ester $=20330$

$(1 R, 5 R, 6 S, 9 R)$-3-Oxo-6,13-dihydroxy-5,6-dihydro- $\beta$-ionol $=1337$

3-Oxo-2 $\alpha, 23$-dihydroxyolean-12-en-28-oic acid $=4481$

3-Oxo-ent-13epi-8(13)-epoxy-15-chloro-14-hydroxylabdane $=684$

16-Oxo-21-episerratenediol $=6122$

(22R,24R,25R,26S)-1-Oxо-22,26-epoxy-3 $\alpha, 5 \alpha$-cycloergostane-6 $\beta, 17 \alpha, 24,25,2$ 6-pentaol 26- $O-\beta$-Dglucopyranoside $=3641$

(22R,24R,25R,26S)-1-Oxo-22,26-epoxy-3 $\alpha, 5 \alpha$-cycloergostane-6 $\beta, 17 \alpha, 24,25$,

26-pentaol 26- $O-\beta$-Dglucopyranoside $24-O$-methyl ether $=3642$

$(22 R, 24 R, 25 R, 26 S)$-1-Oxo-22,26-epoxy-3 $\alpha, 5 \alpha$-cycloergostane- $6 \beta, 17 \alpha, 24,25$,

26-pentaol 26- $O-\beta$ - $D$-glucopyranoside 6- $O$-methyl ether $=3643$

$(22 R, 24 R, 25 R, 26 \Phi)$-1-oxo-22,26-epoxy-24- $O$-methyl-3 $\beta, 17 \alpha, 24,25,26$-pentah

ydroxyergost-5-ene 3-O-sulfate $=3647$

3-Oxo-1(10),4,11-guaiatrien-14-al = 16069

19-Oxo-humantenmine $=16338$

21-Oxo-23 $\xi$-hydroxy-21,23-dihydroveprisone $=11769$

23-Oxo-21 $\xi$-hydroxy-21,23-dihydroveprisone $=22381$

22-Oxo-23-hydroxy-iridal-3,16-di- $\beta$ - $D$-glucopyranoside $=11135$

22-Oxo-23-hydroxyiridal-3-[ $\beta$ - $D$-glucopyranosyl-( $1 \rightarrow 6)-\beta$ - $D$-glucopyranosid

e]-16- $\beta$ - $D$-glucopyranoside $=11134$

22-Oxo-23-hydroxy-isoiridal-3,16-di- $\beta$ - $D$-glucopyranoside $=11138$

3-Oxo-12 $\beta$-hydroxylanosta-7,9(11),24(Z)-trien-26-oic acid $=22165$

11-Oxo-3 $\beta$-hydroxy-olean-12-ene $=1109$

$(6 R, 9 R)$-3-Oxo- $\alpha$-ionyl-9- $O-\alpha$-rhamnopyranosyl- $\left(1^{\prime \prime} \rightarrow 6^{\prime}\right)-\beta$-glucopyranoside $=7288$

$(6 R, 9 R)-3-O x o-\alpha$-ionyl-9- $O-\beta$-xylopyranosyl- $\left(1^{\prime \prime} \rightarrow 6^{\prime}\right)-\beta$-glucopyranoside $=7287$

22-Oxo-isoiridal-3,16,23-tri- $\beta$ - $D$-glucopyranoside $=11136$

3-Oxolanosta-8,24-dien-26-oic acid $=1467$

3-Oxolanosta-9(11),24-dien-26-oic acid = 3861

3-Oxolanosta-8,24-dien-21-oic acid 21- $O-\beta-D$-xylopyranoside $=7868$

3-Oxolanosta-8,24(31)-dien-21-oic acid 21- $O-\beta-D$-xylopyranoside $=7869$

3-Oxolanosta-7,9(11),24-trien-15 $\alpha, 21$-diol = 17389

1-Oxo-12-methoxy-7,20-epoxyabieta-8,11,13-trienestructureforsalvibractone $=19208$

1-[1-Oxo-7(3,4-methylenedioxyphenyl)-2E,6E-heptadienyl]pyrrolidine $=19378$ 1-[1-Oxo-9(3,4-methylenedioxyphenyl)- $8 E$-nonenyl $])$ piperidine $=17469$ 1-[1-Oxo-5(3,4-methylenedioxyphenyl)-2E-pentenyl $]$ piperidine $=17439$ 1-[1-Oxo-5(3,4-methylene dioxyphenyl)-2E-pentenyl $]$ pyrrolidine $=17438$ 2-Oxopalmitic acid $=16345$
3-Oxopanaxydol $=8452$

4-Oxopentanoic acid $=12735$

5-Oxoproline $=18266$

1-Oxo-1,2,3,4-tetrahydro- $\beta$-carboline $=21076$

12-Oxotigogenin $=9253$

Oxoushinsunine $=12917$

Oxyayanin $\mathrm{A}=3611$

Oxy\{bis[5"(4',5,7"-trihydroxy-3',3",4"'-trimethoxy-7-O- $\alpha$ :6- $\beta$-flavone-chalcone) $]\}=18846$

Oxycanthine $=16439$

Oxycoccicyanin $=16901$

17-Oxycorticosterone $=9937$

5,5'-Oxydimethylene-bis-(2-furaldehyde) $=2452$

11,16-Oxy-18,20-dinor-1,3,5(10),6,8,11,15-abietaheptaene-13,14-dione $=11730$

11,16-Oxy-18,20-dinor-1,3,5(10),6,8,11-abietahexaene-13,14-dione $=5654$

$(R)-(+)$-Oxypeucedanin hydrate $=2041$

Oxyresveratrol $=4337$

\section{$\mathbf{P}$}

$6 \beta, 7 \alpha, 9 \alpha, 11 \alpha$-Pachycarpine $=20133$

Paclitaxel $=20811$

Paederosidic acid methyl ester $=16515$

Paeonidin $=16900$

Paeonin $=16905$

Pallasone $\mathrm{A}=11179$

Palmatoside $\mathrm{D}=21398$

Palmitic acid $=9486$

(2S)-1-O-Palmitoyl glycerol $=14931$

6'-O-Palmityl-sitosteryl-3-O- $\beta$ - $D$-glucoside $=19997$

3(15)-Panasinsanene $=16592$

10-Panasinsanene $=16591$

Pancratine $=9187$

Panicolin $=20004$

Paniculol $=16614$

Papaveraldine $=22745$

Patrinia-glycoside B-II = 18709

Patulitrin $=16728$

Pd-Ia $=17762$

Pd-II $=17763$

Pedatisectine $\mathrm{D}=14780$

Pedatisectine $\mathrm{E}=14752$

Pelargonic acid $=15684$

Pellitorine $=11269$

Pennogenin-3-O- $\alpha$ - $L$-rhamnopyranosyl( $1 \rightarrow 4)-\alpha$ - $L$-rhamnopyranosyl

$(1 \rightarrow 4)$ - $[\alpha$ - $L$-rhamnopyranosyl $(1 \rightarrow 2)]-\beta$ - $D$-glucopyranoside $=16815$

$5 \alpha, 7 \beta, 9 \alpha, 10 \beta, 13 \alpha$-Pentaacetoxy-20(benzolyoxy)-2 $\alpha, 4 \alpha$-dihydroxytax-11-ene $=20821$

$5 \alpha, 6,11 \alpha, 14 \beta, 17(R)$-Pentaacetoxy-3 $\beta$-benzoyloxy-15 $\beta$-hydroxyseget-8(12)en-9-one $=19656$

$5 \alpha, 7 \beta, 9 \alpha, 10 \beta, 13 \alpha$-Pentaacetoxy-2 $\alpha, 20$-dihydroxytax-11-ene $=20825$

$2 \alpha, 7 \beta, 9 \alpha, 10 \beta, 13$-Pentaacetoxy-11 $\beta$-hydroxy-5 $\alpha$-(3'- $N, N$-dimethylamino-3'phenyl)-propionyloxytaxa-4(20),12-diene $=20863$

$5 \alpha, 7 \beta, 9 \alpha, 13 \alpha, 20$-Pentaacetoxy-2 $\alpha, 10 \beta, 15$-trihydroxy-11(15 $\rightarrow 1)$-abeo-taxene 
$=20834$

1,4,3',4',6'-Penta- $O$-acetyl-6- $O$ - $p$-coumaroylsucrose $=18004$

2,7,9,10,13-Pentaacetyl-4(20),12-taxadiene-2,5,7,9,10,11,13-heptol = 20748

2,10,12,6',10'-Pentachloroisoplagiochin $\mathrm{C}=2184$

$10,12,6$ ', 10',14'-Pentachloroisoplagiochin $\mathrm{C}=2183$

$N$-Pentacosyl-2-carboxy-benzoyl amide $=18292$

Pentadecane $=16830$

Pentadecanoic acid methyl ester $=14655$

6-(8'Z-Pentadecenyl)-1,2,4-trihydroxybenzene-1- $O$-acetate $=1651$

Pentadecylic acid $=16831$

6-Pentadecyl-1,2,4-trihydroxybenzene-1- $O$-acetate $=1650$

1,3-Pentadiynylbenzene $=15724$

2,3',4,4',6-Pentahydroxy benzophenone $=13296$

$5,5 ", 7,7 ", 3$ '-Pentahydroxy 4',4"'-biflavonyl ether = 12989

$\left\{5,7,5^{\prime}, 44^{\prime \prime \prime}, 5\right.$ "'-Pentahydroxy-6,8-bis-( $\gamma, \gamma$-dimethylallyl)-[6"'',6"'-dimethyl-

4"'",5"'-dihydropyrano-(2'"',3"'::4',3')]\}-coumaronochromone $=7890$

$2 \beta, 3 \beta, 11 \beta, 14 \beta, 20$-Pentahydroxy-cholest-7-en-6,22-dione $=16291$

$2 \beta, 3 \beta, 14 \alpha, 20,25$-Pentahydroxy-cholest-7-en-6,22-dione $=16350$

$3 \beta, 6 \alpha, 16 \alpha, 20(S), 27-P e n t a h y d r o x y d a m m a r-24(Z)$-ene $=21890$

$1 \beta, 3 \beta, 12 \beta, 20(S), 26$-Pentahydroxy-dammer-24(25)-en-20(S)- $O$ - $\beta$ - $D$-glucopy-

ranoside $=9096$

$1 \beta, 3 \beta, 12 \beta, 20(S), 26$-Pentahydroxy-dammer-24(25)-en-20(S)- $O$ - $\beta$ - $D$-glucopyranosyl- $(1 \rightarrow 6)-\beta$ - $D$-gluco-pyranoside $=9094$

$1 \beta, 3 \beta, 12 \beta, 20(S), 26$-Pentahydroxy-dammer-24(25)-en-3- $O-\beta$ - $D$-glucopyra-nosyl-

20(S)-O- $\beta$ - $D$-glucopyranosyl-( $(1 \rightarrow 6)-\beta$ - $D$-glucopyranoside $=9097$

rel-(7" $E)-\left(7 \alpha, 8 \beta, 7^{\prime} \alpha, 8^{\prime} \beta\right)-3,4,9,9^{\prime}, 9^{\prime \prime}-$ pentahydroxy-3',7:3",7'-diepoxy-8,4':8',4"-

bisoxysesquineolign-7"-ene $=11212$

rel-(7"E)-(7 $\left.\alpha, 8 \beta, 7^{\prime} \alpha, 8^{\prime} \beta\right)-3,4,9,9^{\prime}, 9^{\prime \prime}-$ pentahydroxy-4',7:3",7'-diepoxy-8,3':8',4"-

bisoxysesquineolign-7"-ene $=11214$

rel-(7"E)-(7 $\left.\alpha, 8 \beta, 7^{\prime} \beta, 8^{\prime} \alpha\right)-3,4,9,9^{\prime}, 9^{\prime \prime}$-pentahydroxy-3',7:3",7'-diepoxy-8,4':8',4"-

bisoxysesquineolign-7"-ene) $=11213$

rel-(7" $E)-\left(7 \alpha, 8 \beta, 7^{\prime} \beta, 8^{\prime} \alpha\right)-3,4,9,9^{\prime}, 9^{\prime \prime}-$ pentahydroxy-4',7:3",7'-diepoxy-8,3':8',4"-

bisoxysesquineolign-7"-ene $=11215$

$3,5,6,7,8$-Pentahydroxy flavone $=16859$

$3,5,7,2^{\prime}, 4^{\prime}$-Pentahydroxyflavone $=14971$

$5,6,7,3^{\prime}, 4^{\prime}$-Pentahydroxyflavone $=10351$

$5,7,3^{\prime}, 4^{\prime}, 5^{\prime}$-Pentahydroxyflavone-6- $C$-glucoside $=11198$

$3,5,7,8,4$ '-Pentahydroxyflavone-7- $O-\beta$ - $D$-glucuronopyranoside $=14872$

$3,5,7,8,4$ '-Pentahydroxyflavone-8- $O-\beta-D-2$ "-O-(2-methylbutanoyl) glucuro-

nide $=14873$

$3,5,7,3^{\prime}, 4^{\prime}$-Pentahydroxy-5'-isoprenylflavone $=22241$

$3,6,7,3$ ', 4 '-Pentahydroxy-2'-isoprenylflavone $=15471$

(24R)-2,12 $\alpha, 21,24,25$-Pentahydroxylanosta-1,8-dien-3-one $=20441$

(24R)-3 $\beta, 12 \alpha, 21,24,25$-Pentahydroxylanost-8-en-2-one $=20442$

$3,5,6,7,8$-Pentahydroxy-4'-methoxy flavone $=16852$

$1 \alpha, 3 \beta, 16 \alpha, 24 \xi, 31$-Pentahydroxy-24 $\xi$-methylcycloartan-28-oic $=4541$

$1 \alpha, 3 \beta, 16 \beta, 24 \xi, 31$-Pentahydroxy-24 $\xi$-methylcycloartan-28-oic acid $=4540$

$3 \beta, 16 \beta, 23,28,30$-Pentahydroxyoleana-11,13,(18)-diene-3 $\beta$ - $D$-glucopyranosyl-

$(1 \rightarrow 6)$ - $[\alpha$ - $L$-rhamno-pyranosyl- $(1 \rightarrow 4)]$ - $\beta$ - $D$-glucopyranoside $=19156$

$3 \beta, 16 \alpha, 23,28,30$-Pentahydroxyoleana-11,13(18)-dien-3-O- $\beta$ - $D$-glucopyranosyl- $(1 \rightarrow 3)-\beta-D$-fucopyranoside $=19151$

$3 \beta, 16 \alpha, 23,28,29$-Pentahydroxy-11,13(18)-oleanedien-3 $\beta$-yl $\beta$ - $D$-glucopyranosyl-( $1 \rightarrow 2)$-[4- $O$-sulfo- $\beta$ - $D$-glucopyranosyl-( $1 \rightarrow 3)$ - $] \beta$ - $D$-fucopyrano- side $=19249$

$1 \beta, 1 \beta, 3 \beta, 4 \beta, 5 \beta$-Pentahydroxy-spirost-25(27)-ene5- $O-\beta$ - $D$-glucopyranoside $=1902$

2',4',3",2"',4"'-Pentahydroxy-4-O-4"-tetrahydrobichalcone = 12934

$4 \beta, 9 \alpha, 12 \beta, 13 \alpha, 20$-Pentahydroxy-1,6-tigliadien-3-one $=17181$

$1 \alpha, 2 \alpha, 3 \beta, 19 \alpha, 23$-Pentahydroxyurs-12-en-28-oic acid $=8129$

$5,6,7,3^{\prime}, 4^{\prime}$-Pentamethoxyflavone $=19929$

$5,6,7,8,4^{\prime}$-Pentamethoxyflavone $=20670$

$5,7,8,3^{\prime}, 4^{\prime}$-Pentamethoxyflavone $=11716$

Pentandrin $=22358$

Pentandrin glucoside $=22359$

Pentanedioic acid $=8784$

1,2,3,4-Pentanepentol $=645$

Pentyl butanoate $=1104$

Pentyl ethanoate $=1103$

Peonidin-3,5-diglucoside $=16905$

Peonidin 3-O-(4"-O-sinapoyl gentiobioside $)=836$

Perconval $=4013$

Perforatin A = 14125

Pericalline $=20578$

Pericarsaponin $\mathrm{Pk}=9275$

Periforoside 3-O-(2,6-dideoxy-4- $O-\beta$ - $D$-glucopyranosidyl-3- $O$-methyl- $\beta$ - $D$ ribo-hexopyranoside) $=16924$

Perilla alcohol $=16935$

Perillylaldehyde $=16930$

Periplocoside $=16943$

Peroxycostunolide $=22400$

Persicanidine A $=9681$

PG-3 = 14080

$\mathrm{PGE}_{1}=17954$

Phellandrene $=17055$

Phellizide $=17078$

Phenethyl 1-O- $\beta$ - $D$-apiofuranosyl $(1 \rightarrow 2)-\beta-D$-glucopyranoside $=19432$

Phenethyl caffeate $=2890$

Phenethyl $\alpha$ - $L$-rhamnopyranosyl $(1 \rightarrow 6)-\beta-D$-glucopyranoside $=17087$

Phenol-2-carboxylic acid $=19187$

Phenylacetone $=17129$

$N$-[N'-(Phenylacetyl)-4-aminobutyl $]$ phenylacetamide $=737$

$3 \beta$-(6"-Phenylacetyloxyb- $D$-glucopyranosyloxy)- $8 \beta$-( $p$-hydroxyphenylacetyloxy)-guaia-4(15),10(14),11(13)-trien- $\alpha, 5 \alpha, 6 \beta, 7 \alpha H$-12,6-olide $=11803$

3-Phenylacrylic acid $=3695$

Phenylallyl acetate $=3726$

4-Phenylbutan-2-one $=2274$

2-Phenylchromone $=7819$

Phenylethanone $=115$

$\beta$-Phenylethyl alcohol $=2284$

Phenylethyl alcohol [5- $O$ - $p$-hydroxybenzoyl- $\beta$ - $D$-apiofuranosyl- $(1 \rightarrow 2)]-\beta-D$ glucopyranoside $=2086$

Phenylethylene $=20430$

2-Phenylethyl glucosinolate $=8599$

3-[(1-Phenyl)ethylidene]amino-2-hydroxy-propyl $\alpha$-(benzoyl amino)benzenepropanoate $=21575$

Phenylformic acid $=2224$

1-Phenyl-1,3-hexadiyne $=15354$ 
Phenylmethanal $=2222$

Phenylmethyl glucopyranoside $=2276$

$N$-(Phenylpropanoyl)- $\Delta^{3}$-2-pyrrolidone $=19368$

2-Phenyl-2-propen-1-ol = 3696

Phlegmanol C $=19770$

Phloroacetophenone $=22778$

2-O-Phloro-6,6'-bieckol $=21836$

Phorbol-12-tiglate-13-caprate $=17194$

Phosphocreatine $=4222$

$\alpha$-Photosantalol A $=10689$

$\alpha$-Photosantolol B diastereoisomer $=10690$

1,3-Phthalandione $=17204$

Phyllanthin $=20805$

Phyllanthoside $=20805$

Physcion-8-O- $\beta$ - $D$-glucopyranoside $=1362$

Physeteric acid $=21046$

Physostigmine $=7381$

(E) -Phytol $=17265$

Phytolaccasaponin B $=7374$

Phytolaccasaponin $\mathrm{G}=17269$

Phytolaccinic acid $=17266$

Phytolaccoside $\mathrm{D}=7370$

Phytolaccoside $\mathrm{E}=7368$

Piceatannol 3- $O-\beta-D$-glucopyranoside $=1951$

Piceatannol 3'-O- $\beta$ - $D$-glucopyranoside $=21158$

Piceoside $=17287$

Picrasidine $\mathrm{C}=3160$

Picrasidine $\mathrm{S}=12333$

Picrasin $A=15552$

Picrasin $\mathrm{B}=15554$

Picrasin $\mathrm{C}=15555$

Picropodophyllotoxin $=17337$

Pictogenin (3 $\beta, 6 \beta, 16 \alpha, 23$-tetrahydroxyolean-12-ene-28-oic acid) 3-O- $\alpha-L$-ara-

binopyranoside $=17351$

ent-Pimara-8(14),15-dien-19-oic acid $=17371$

2-Pinen-4-ol $=22398$

2-Pinen-4-one $=22399$

$(-)$-2(10)-Pinen-3-one $=17402$

Pingbeimine $\mathrm{A}=17384$

Pingpeisaponin $=17383$

$(S)$-Pinocembrin $=17403$

Pinoresinol-4,4'-di- $O-\beta$ - $D$-glucoside $=17412$

Pinosolide acid $=17423$

Pinusolic acid $=17423$

$d l$-Pipecolinic acid $=17425$

Piperamide A 7:3 $(2 E, 4 E, 6 E)=17449$

Piperamide A 9:1 $(8 E)=17469$

Piperamide B 9: $3(2 E, 4 E, 8 E)=18657$

Piperamide-C 5:2(E,E) $=16387$

Piperamide C 5:1 $(2 E)=17438$

Piperamide $\mathrm{C} 7: 2(2 E, 6 E)=19378$

Piperitenone oxide $=18954$

Pipernonaline $=16384$
Piperponylaldehyde $=17470$

Piperyline $=16387$

Piptanthine $=16206$

Plantagoside $\mathrm{A}=17507$

Plantamajoside $=18219$

Platynecine $N$-oxide $2 S$-hydroxy- $2 S$-( $1 S$-hydroxyethyl)-4-methyl-pentanoyl ester $=18666$

Podocarprene $=12176$

Polargonin $=16781$

Polyanthin $=17620$

Polydatin $=17283$

Polystachoside $=2039$

Pomonic acid $=16358$

Ponciretin $=11691$

Poncitrin $=5134$

Pongol methyl ether $=3334$

Pontigenin $=18748$

Populnin $=12062$

Porphyroxine $=16627$

Portulaxanthin $\mathrm{I}=17734$

Potengriffioside $\mathrm{A}=21392$

PQ-1 = 16586

$\mathrm{PQ}-3=8452$

Praeruptorin III $=1239$

Prangeferol $=15645$

Prangenin $=16447$

Prangenin hydrate $=9420$

Pratol $=7883$

Pregn-4-ene-3,20-dione $=17901$

Pregn-5-ene-3,17,20-triol 20- $O$-[2- $O$-acetyl- $\beta$ - $D$-digitalopyranosyl-( $1 \rightarrow 4)-\beta$ -

$D$-cymaropyranosyl- $(1 \rightarrow 4)-\beta$ - $D$-cymaropyranosyl- $(1 \rightarrow 4)-\beta$ - $D$-cymaropyranosyl-(1 $\rightarrow 5)$-3,7-dideoxy-4- $O$-methyl- $\alpha$ - $D$-gluco-2-heptulopyranosyl- $(2 \rightarrow 4)$-dioxy- $(1 \rightarrow 3)-\beta$ - $D$-canaropyranoside $]=16948$

8-Prenylnaringenin $=11248$

Proanthocyanidin $\mathrm{B}_{5}=17888$

Prochamazulene $=13605$

Procyanidin $\mathrm{B}_{2}=17869$

Progoitrin $=9863$

Propanal $=17930$

Propanecarbaldehyde $=2787$

Propanedioic acid $=13439$

Propanoic acid $=17931$

trans-1-Propene-1,2,3-tricarboxylic acid $=553$

Propenoic acid $=578$

cis-4-(1-Propenyl)anisole $=1183$

2-[E]-Propenyl-7-methoxy-8-C- $\beta-D-[2 '-(E)-p$-coumaroyl]-glucopyranosyl-5metylchromone $=976$

1-Propenyl methyl thiosulfinate $=14690$

2-Propenyl propyl disulfide $=954$

$n$-Propylamine $=17935$

Prosapogenin $\mathrm{CP}_{2 \mathrm{~b}}=16061$

Prosapogenin $\mathrm{CP}_{3}=16063$

Prosapogenin $\mathrm{CP}_{3 \mathrm{~b}}=9276$ 


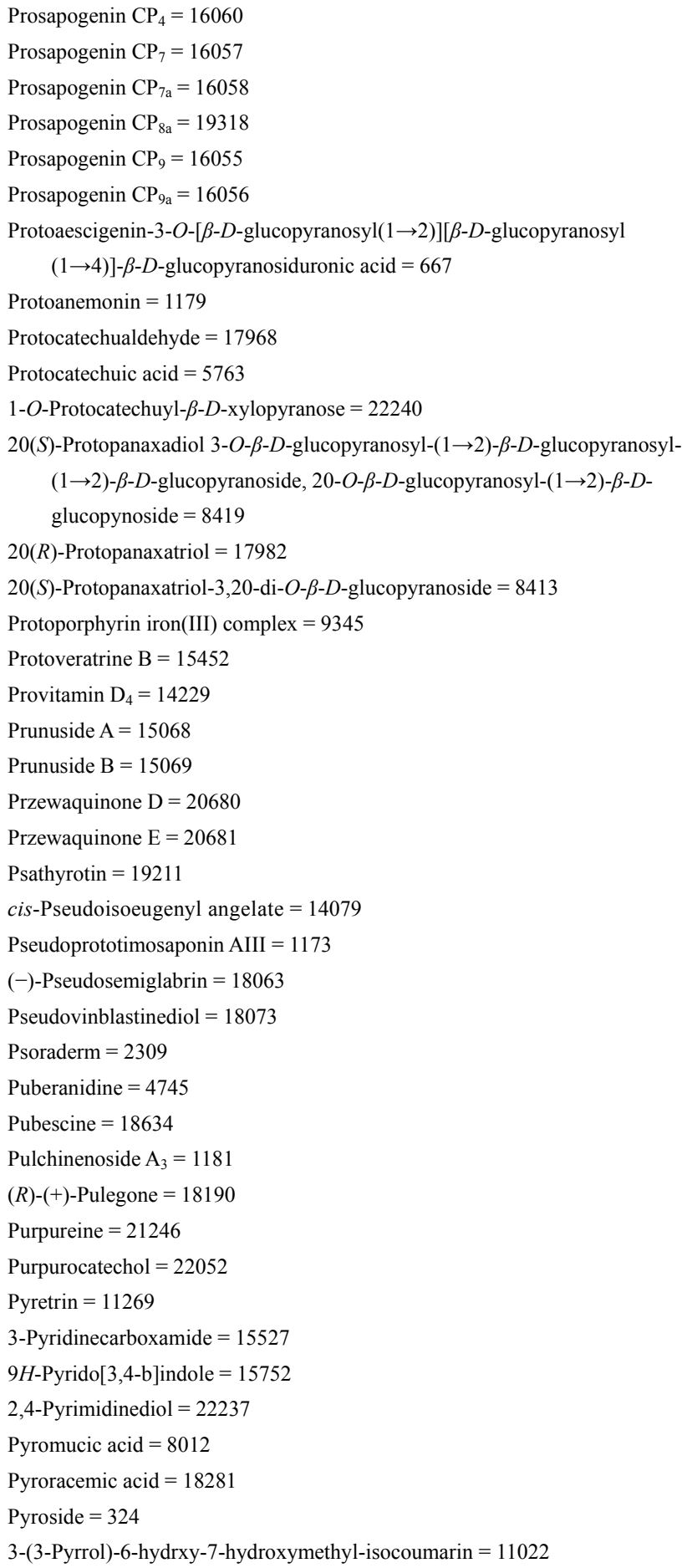

\section{Q}

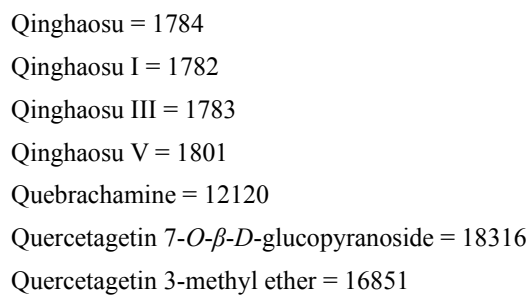

Quercetagetin-6,7,3', 4'-tetramethyl ether $=6146$

Quercetin-3- $O$-(2"-O-acetyl- $\alpha$-rhamnopyranoside $)=491$

Quercetin-3-arabinoside $=7844$

Quercetin-3-O-arabinoside $=9043$

Quercetin-3-O-(6-O-cis- $p$-coumaroyl)- $\beta$ - $D$-glucopyranosyl ( $1 \rightarrow 2)-\beta$ - $D$-glucopyranosyl $(1 \rightarrow 2)-\beta$ - $D$-glucopyranoside $=17493$

Quercetin-3,7-di- $O-\beta$ - $D$-glucopyranoside $=18345$

Quercetin-3-O- $\beta$ - $D$-galactoside $=10887$

Quercetin-3-O-(2"-galloyl)- $\beta$ - $D$-galactopyranoside $=10888$

Quercetin-3-O- $\beta$ - $D$-glucopyranoside $=11642$

Quercetin 3-O- $\beta$ - $D$-glucopyranoside 3'-sulphate $=3387$

Quercetin-3-O- $\{\beta-D$-glucopyranosyl-( $1 \rightarrow 2)$-[ $\alpha-L$-rhamnopyranosyl-( $1 \rightarrow 6)]$ $\beta$ - $D$-galactopyranoside $\}=17001$

Quercetin-3- $\beta$ - $D$-glucoside-2"-gallate $=18360$

Quercetin-3-O- $\beta$-glucuronopyranoside $=18414$

Quercetin 3'-methoxy-4'- $O-\beta$ - $D$-glucopyranoside $=925$

Quercetin 3'-methyl ether $=11648$

Quercetin-5-methyl ether $=2052$

Quercetin-3-O- $\alpha-L$-rhamnopyranoside $=18411$

Quercetin 3- $O-\alpha-L$-rhamnopyranosyl $(1 \rightarrow 6)-2$ "- $O$-acetyl $\beta-D$-glucopyranoside $=497$ Quercetin-7- $O-\alpha-L$-rhamnopyranosyl-3- $O$-(6"-caffeoyl)- $\beta$ - $D$-glucopyranosyl$(1 \rightarrow 2)-\beta$ - $D$-xylopyranoside $=15194$

Quercetin-7-O- $\alpha-L$-rhamnopyranosyl-3- $O$-(6"-feruloyl)- $\beta$ - $D$-glucopyranosyl$(1 \rightarrow 2)-\beta$ - $D$-xylopyranoside $=15196$

Quercetin-3- $O$ - $[\alpha-L$-rhamnopyranosyl $(1 \rightarrow 6)]-\beta$ - $D$-galactofuranoside $=2394$

Quercetin-3-O- $\alpha$ - $L$-rhamnopyranosyl $(1 \rightarrow 6)-\beta$ - $D$-galactopyranoside $=18390$

Quercetin-3-O-(2"-O- $\alpha$-rhamnopyranosyl) $\beta$-glucopyranoside $=18378$

Quercetin-3-O-(6"-O- $\alpha$-rhamnopyranosyl) $-\beta$-glucopyranoside $=19087$

Quercetin-3-O-(6"-O- $\alpha-L$-rhamnopyranosyl)- $\beta$ - $D$-glucopyranoside-7- $O-\beta-D-$ glucopyranoside $=18391$

Quercetin-7-O- $\alpha-L$-rhamnopyranos-yl-3- $O$-(6"- $P$-coumaroyl)- $\beta$ - $D$-glucopyranosyl- $(1 \rightarrow 2)-\beta-D$-glucopyranoside $=15197$

Quercetin-7-O- $\alpha-L$-rhamnopyranosyl-3- $O-(6 "-P$-coumaroyl)- $\beta-D$-glucopyranosyl- $(1 \rightarrow 2)-\beta$ - $D$-xylopyranoside $=15193$

Quercetin-3-O- $\alpha-L$-rhamnopyranosyl $(1 \rightarrow 2)-\alpha-L$-rarbinofuranoside $=1777$

Quercetin-3-O- $\alpha$ - $L$-rhamnopyranosyl $(1 \rightarrow 2)-[\alpha-L$-rhamnopyranosyl $(1 \rightarrow 6)]$ $\beta$ - $D$-glucopyranoside $=13479$

Quercetin-7-O-rhamnoside $=22492$

Quercetin-3- $O-[\beta$ - $D$-xylopyranosyl $(1 \rightarrow 2)]-[\alpha-L$-rhamnopyranosyl $(1 \rightarrow 6)]-\beta$ $D$-glucopyranoside-3'- $O-\beta$ - $D$-glucopyranoside $=661$

$\beta$-Quinine $=18423$

$p$-Quinol $=9740$

\section{$\mathbf{R}$}

\author{
Rabdolongin $\mathrm{B}=13526$ \\ Rabdophyllin $\mathrm{G}=11394$ \\ Rabdosianone $=7268$ \\ Rabdosianone II = 13524 \\ Rabdosin C = 11394 \\ Racemosin $=6300$ \\ Raddeanin $\mathrm{B}=6755$ \\ Raddeanine $=11002$ \\ $D$-Raffinose pentahydrate $=18526$
}


Ranachrome $1=2393$

Ranachrome $4=11784$

Reidin A $=18756$

Reidin B $=18757$

Reidin C $=18758$

Reniformin A = 9362

Resibufaginol $=10673$

Resveraltrol 3- $O-\beta$ - $D$-glucopyranoside $=17283$

Resveratroloside $=18650$

Retrofractamide B = 17444

Retronecine $\dagger=9318$

Retronecine $\dagger=14923$

Retusin 7,8-di- $O-\beta$ - $D$-glucopyranoside $=9556$

Reynoutrin $=18407$

Rhamnazin-3- $O-\beta$ - $D$-apisoyl- $(1 \rightarrow 2)$-[6"- $O$-(3-hydroxy-3-methylglutarate)] glucoside $=22542$

Rhamnazin- $O-\beta$ - $D$ - $(6$ "- $\beta$-hydroxy- $\beta$-methylglutaryl $)$ glucoside $=22539$

Rhamnocitrin-3,4'-O- $\beta$ - $D$-diglucoside $=3949$

6- $O$-[ $\alpha$ - $L$-Rhamnopyranosyl-( $1 \rightarrow 2)$-6- $O$-acetyl- $\beta$ - $D$-glucopyranosyl]-20- $O$ -

[ $\beta$ - $D$-glucopyranosyl-( $1 \rightarrow 6)-\beta$ - $D$-glucopyranosyl]-20(S)-protopanaxatriol $=22898$

3- $O$ - $\alpha$ - $L$-Rhamnopyranosyl $(1 \rightarrow 2)-\alpha-L$-arabinopyranosyl-( $1 \rightarrow 2)-\beta$ - $D$-glucuronopyranosyl abrisapogenol C 22-O- $\alpha-L$-arabinopyranoside $=6694$

3 - $O$ - $\alpha$ - $L$-Rhamnopyranosyl $(1 \rightarrow 2)-\alpha$ - $L$-arabinopyranosyl $(1 \rightarrow 2)-\beta$ - $D$-glucuronopyranosyl kudzusapogenol A 22- $O$-acetate $=517$

3 - $O$ - $\alpha$ - $L$-Rhamnopyranosyl $(1 \rightarrow 2)-\alpha$ - $L$-arabinopyranosyl $(1 \rightarrow 2)-\beta$ - $D$-glucuronopyranosyl kudzusapogenol A 22- $O-\alpha-L$-arabinopyranoside $=6693$

3-O- $\alpha$ - $L$-Rhamnopyranosyl- $(1 \rightarrow 2)-\alpha$ - $L$-arabinopyranosyl gypsogenin $28-O$ $\alpha$ - $L$-rhamnopyranosyl-( $1 \rightarrow 4)-\beta$ - $D$-glucopyranosyl-( $1 \rightarrow 6)-\beta$ - $D$-glucopyranoside $=3820$

3-O-[ $\alpha$ - $L$-Rhamnopyranosyl-( $1 \rightarrow 2)-\alpha$ - $L$-arabinopyranosyl]-hedragenin-28$[\alpha$-L-rhamnopyranosyl-( $1 \rightarrow 2)-\beta$ - $D$-glucopyranosyl $(1 \rightarrow 6)-\beta$ - $D$-glucopyranosyl] ester $=12118$

$3 \beta$-[(O- $\alpha$ - $L$-Rhamnopyranosyl-( $1 \rightarrow 2)-\alpha$ - $L$-arabinopyranosyl)oxy $]$ lup-20-(29)en-28-oic acid 28- $O$ - $\alpha$ - $L$-rhamnopyranosyl- $(1 \rightarrow 4)-O-\beta$ - $D$-glucopyranosyl- $(1 \rightarrow 6)-\beta$ - $D$-glucopyranosyl ester $=4431$

6- $O$-[ $\alpha$ - $L$-Rhamnopyranosyl-( $\rightarrow 2)-\beta$ - $D$-glucopyranosyl]-20- $O$-[ $\beta$ - $D$-glucopyranosyl-(1 $\rightarrow 6)-\beta$ - $D$-glucopyranosyl]-20(S)-protopanaxatriol $=22897$

$3 \beta$-[(O- $\alpha$ - $L$-Rhamnopyranosyl $(1 \rightarrow 2)$ - $\alpha$ - $L$-arabinopyranosyl)oxy]olean-12-en28 -oic acid $O$ - $\alpha$ - $L$-rhamnopyranosyl $(1 \rightarrow 4)$ - $O$ - $\beta$ - $D$-glucopyranosyl $(1 \rightarrow 6)-\beta$ - $D$-glucopyranosyl ester $=819$

$3-O-\alpha$ - $L$-Rhamnopyranosyl-( $1 \rightarrow 2)-\alpha$ - $L$-arabinopyranosyl pomolic acid $28-\beta$ - $D$-glucopyranosyl ester $=18538$

3-O- $\alpha$ - $L$-Rhamnopyranosyl-( $1 \rightarrow 2)-\alpha-L$-arabinopyranosyl siaresinolic acid $28-\beta$ - $D$-glucopyranosyl ester $=18539$

6- $O$ - $\alpha$ - $L$-Rhamnopyranosyl-aucubin $=19960$

3- $O$-[ $\alpha-L$-Rhamnopyranosyl-( $1 \rightarrow 2)-\beta-\left(3^{\prime}, 4\right.$ '-di- $O$-acetyl)- $D$-xylopyranosyl]-6- $O-\beta-D$ -xylopyranosyl-20(R),24(S)-epoxycycloartane-3 $\beta, 6 \alpha, 16 \beta, 25$-tetrol $=22047$

$3 \beta-O-\{[\alpha-L$-Rhamnopyranosyl- $(1 \rightarrow 2)-\beta$ - $D$-galactopyranosyl- $(1 \rightarrow 3)]-[\beta-D$ galactopyranosyl-( $1 \rightarrow 2)]$ - $\beta$ - $D$-glucuronopyranosyl $\}-21 \beta, 22 \alpha$-diangeloyloxy-13 $\beta, 28$-oxidoolean- $16 \alpha, 28 \alpha$-diol $=13358$

$3 \beta-O-\{[\alpha-L$-Rhamnopyranosyl- $(1 \rightarrow 2)-\beta$ - $D$-galactopyranosyl- $(1 \rightarrow 3)]-[\beta-D$ -

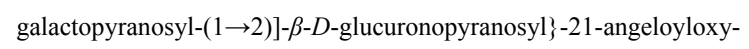

13,28-oxidoolean- $16 \alpha, 22 \alpha, 28 \alpha$-triol $=13351$

$3 \beta-O$ - $\{[\alpha-L$-Rhamnopyranosyl-( $1 \rightarrow 2)-\beta$ - $D$-galactopyranosyl- $(1 \rightarrow 3)]-[\beta-D$ galactopyranosyl- $(1 \rightarrow 2)]$ - $\beta$ - $D$-glucuronopyranosyl $\}-16 \alpha$-acetoxy-21 $\beta$ angeloyloxy-13 $\beta, 28$-oxidoolean-22 $\alpha, 28 \alpha$-diol $=13352$

$3 \beta$ - $O$ - $\{[\alpha-L$-Rhamnopyranosyl-( $1 \rightarrow 2)-\beta$ - $D$-galactopyranosyl- $(1 \rightarrow 3)]-[\beta-D$ galactopyranosyl- $(1 \rightarrow 2)]$ - $\beta$ - $D$-glucuronopyranosyl $\}-22 \alpha$-acetoxy-21 $\beta$ angeloyloxy)-13 $\beta, 28$-oxidoolean-16 $\alpha, 28 \alpha$-diol = 13353

$3 \beta-O$ - $\{[\alpha-L$-Rhamnopyranosyl-( $1 \rightarrow 2)-\beta$ - $D$-galactopyranosyl- $(1 \rightarrow 3)]-[\beta-D$ galactopyranosyl-(1 $\rightarrow 2)]$ - $\beta$ - $D$-glucuronopyranosyl $\}-16 \alpha, 22 \alpha$-diacetoxy$21 \beta$-angeloyloxy)-13 $\beta, 28$-oxidoolean-28 $\alpha$-ol = 13354

$3 \beta$ - $O$ - $\{[\alpha-L$-Rhamnopyranosyl-( $1 \rightarrow 2)-\beta$ - $D$-galactopyranosyl- $(1 \rightarrow 3)]-[\beta-D$ galactopyranosyl- $(1 \rightarrow 2)]$ - $\beta$ - $D$-glucuronopyranosyl $\}-21 \beta$-angeloyloxy$22 \alpha$-propanoyloxy-13 $\beta, 28$-oxidoolean- $16 \alpha, 28 \alpha$-diol $=13355$

$3 \beta$ - $O$ - $\{[\alpha-L$-Rhamnopyranosyl-( $1 \rightarrow 2)-\beta$ - $D$-galactopyranosyl- $(1 \rightarrow 3)]-[\beta-D$ galactopyranosyl- $(1 \rightarrow 2)]$ - $\beta$ - $D$-glucuronopyranosyl $\}-21 \beta$-angeloyloxy$22 \alpha$-butanoyloxy-13 $\beta, 28$-oxidoolean- $16 \alpha, 28 \alpha$-diol $=13356$

$3 \beta-O$ - $\{[\alpha-L$-Rhamnopyranosyl-( $1 \rightarrow 2)-\beta$ - $D$-galactopyranosyl- $(1 \rightarrow 3)]-[\beta-D$ galactopyranosyl- $(1 \rightarrow 2)]$ - $\beta$ - $D$-glucuronopyranosyl $\}-16 \alpha$-acetoxy-21 $\beta$ angeloyloxy-22 $\alpha$-propanoyloxy-13 $\beta, 28$-oxidoolean-28 $\alpha$-ol $=13357$

$3 \beta-O$ - $\{[\alpha-L$-Rhamnopyranosyl-( $1 \rightarrow 2)-\beta$ - $D$-galactopyranosyl- $(1 \rightarrow 3)]-[\beta-D$ galactopyranosyl- $(1 \rightarrow 2)]$ - $\beta$ - $D$-glucuronopyranosyl $\}-16 \alpha$-acetoxy-21 $\beta$ angeloyloxy-22 $\alpha$-butanoyloxy-13 $\beta, 28$-oxidoolean-28 $\alpha$-ol = 13359

$3 \beta$ - $O$ - $\{[\alpha-L$-Rhamnopyranosyl-( $1 \rightarrow 2)-\beta$ - $D$-galactopyranosyl- $(1 \rightarrow 3)]-[\beta-D$ galactopyranosyl- $(1 \rightarrow 2)]$ - $\beta$ - $D$-glucuronopyranosyl $\}-16 \alpha$-acetoxy$21 \beta, 22 \alpha$-diangeloyloxy-13 $\beta, 28$-oxidoolean-28 $\alpha$-ol = 13360

3 - $O$ - $[\alpha$ - $L$-Rhamnopyranosyl- $(1 \rightarrow 2)-\beta$ - $D$-galactopyranosyl- $(1 \rightarrow 3)][\beta$ - $D$-glucopyranosyl- $(1 \rightarrow 2)]$ - $\beta$ - $D$-glucuronopyranosyl camelliagenin A 22-Oangelate $=13363$

$3 \beta$ - $O$ - $\{\alpha$ - $L$-Rhamnopyranosyl- $(1 \rightarrow 2)$ - $[\beta$ - $D$-glucopyranosyl- $(1 \rightarrow 4)]-\alpha-L$-arabinopyranosyl $\}-28-O$-[ $\beta$ - $D$-glucopyranosyl-( $1 \rightarrow 6)-\beta$ - $D$-glucopyranosyl]hederagenin $=16700$

$3 \beta$ - $O$ - $\{\alpha$ - $L$-Rhamnopyranosyl- $(1 \rightarrow 2)$ - $[\beta$ - $D$-glucopyranosyl- $(1 \rightarrow 4)]-\alpha$ - $L$-arabinopyranosyl $\}-28-O$-[ $\beta$ - $D$-glucopyranosyl-( $1 \rightarrow 6)-\beta$ - $D$-glucopyranosyl]oleanolate $=16701$

$3 \beta$ - $O$ - $\{\alpha$ - $L$-Rhamnopyranosyl-( $1 \rightarrow 2)$-[ $\beta$ - $D$-glucopyranosyl- $(1 \rightarrow 4)]-\alpha$ - $L$-arabinopyranosyl $\}-28-O-\beta$ - $D$-glucopyranosyl-oleanolate $=16702$

3- $O$ - $\alpha$ - $L$-Rhamnopyranosyl-( $1 \rightarrow 2)[\beta$ - $D$-glucopyranosyl $(1 \rightarrow 4)]-\alpha-L$-arabinopyranosyl hederagenin 28- $O$ - $D$-glucopyranosyl ester $=3433$

$3-O$ - $\{\alpha$ - $L$-Rhamnopyranosyl-( $1 \rightarrow 2)-\beta$ - $D$-glucopyranosyl-( $1 \rightarrow 4)-\alpha$ - $L$-arabinopyranosyl $\}$-3 $\beta$-hydroxy-13 $\beta, 28$-epoxy-16-oxo-oleanan-30-al = 1642

3-O- $\alpha$ - $L$-Rhamnopyranosyl-( $1 \rightarrow 2$ )- $\beta$ - $D$-glucopyranosyl-3 $\beta, 21 \alpha$-dihydroxyursolic acid 21-O- $\beta$ - $D$-glucopyranoside $=12556$

$1-O$ - $[\alpha$ - $L$-Rhamnopyranosyl- $(1 \rightarrow 6)-\beta$ - $D$-glucopyranosyl $]-(2 E, 6 E)$-farnesol $=4228$

3-O-[ $\alpha$ - $L$-Rhamnopyranosyl- $(1 \rightarrow 2)-\beta$ - $D$-gluco-pyranosyl- $(1 \rightarrow 3)][\beta$ - $D$-glucopyranosyl- $(1 \rightarrow 2)]-\alpha-L$-arabinopyranosyl cyclamiretin $A=1626$

3-O- $\{\alpha$ - $L$-Rhamnopyranosyl-( $1 \rightarrow 2)-\beta$ - $D$-glucopyranosyl-( $1 \rightarrow 4)$ - $[\beta$ - $D$-glucopyranosyl- $(1 \rightarrow 2)]$ - $\alpha$ - $L$-arabinopyranosyl $\}-13 \beta, 28$-epoxy-16-oxooleanan-3 $\beta, 30$-diol $=1641$

3- $O$ - $\{\alpha$ - $L$-Rhamnopyranosyl-( $1 \rightarrow 2)-\beta$ - $D$-glucopyranosyl-( $1 \rightarrow 4)$ - $[\beta$ - $D$-glucopyranosyl- $(1 \rightarrow 2)]$ - $\alpha$ - $L$-arabinopyranosyl $\}-3 \beta$-hydroxy-13 $\beta, 28$-epoxyoleanan-16-oxo-30-al $=1640$

3-O- $\{\alpha$ - $L$-Rhamnopyranosyl-( $1 \rightarrow 2)-\beta$ - $D$-glucopyranosyl-( $1 \rightarrow 4)$ - $[\beta$ - $D$-glucopyranosyl- $(1 \rightarrow 2)]-\alpha-L$-arabinopyranosyl $\}-3 \beta, 16 \alpha, 28 \alpha$-trihydroxy- 
$13 \beta, 28$-epoxy-oleanan-30-al $=1639$

3-O-[ $\alpha$ - $L$-Rhamnopyranosyl-( $1 \rightarrow 4)$ - $\beta$ - $D$-glucopyranosyl- $(1 \rightarrow 2)$ - $[\beta$ - $D$-glucopy ranosyl-( $1 \rightarrow 3)]$ - $\beta$ - $D$-glucopyranosyl- $(1 \rightarrow 4)-\beta$ - $D$-galactopyranoside $]=$ 6554

3-O-( $\alpha$ - $L$-Rhamnopyranosyl- $(\rightarrow)-\beta$ - $D$-glucopyranosyl-( $(\rightarrow 2)-\beta$ - $D$-glucopyranosyl)serjanic acid $=19337$

3- $O$ - $(\alpha$ - $L$-Rhamnopyranosyl-( $1 \rightarrow 2)-\beta$ - $D$-glucopyranosyl-( $(1 \rightarrow 2)-\beta$ - $D$-glucopyranosyl)serjanic acid 28- $O-\beta$ - $D$-glucopyranoside $=19341$

3 - $O$ - $(\alpha$ - $L$-Rhamnopyranosyl- $(1 \rightarrow 2)-\beta$ - $D$-glucopyranosyl- $(1 \rightarrow 2)-\beta$ - $D$-glucopyranosyl)spergulagenic acid $=19338$

3- $O$ - $[\alpha$ - $L$-Rhamnopyranosyl- $(1 \rightarrow 2)-O-\beta$ - $D$-glucopyranosyl- $(1 \rightarrow 2)-\beta$ - $D$-glucuronopyranosyl]-3 $\beta, 16 \beta, 22 \beta, 24$-tetrahydroxy-olean-12-ene $=19332$

4-O-[ $\alpha$ - $L$-Rhamnopyranosyl-( $1 \rightarrow 2)-\beta$ - $D$-glucopyranosyl]-2-hydroxyl-6methoxybenzophenone $=20959$

3- $O$ - $\alpha$ - $L$-Rhamnopyranosyl- $(1 \rightarrow 2)-\beta$ - $D$-glucopyranosyl oleanolic acid 28 - $O$ - $\alpha$ - $L$-rhamnopyranosyl- $(1 \rightarrow 4)-\beta$ - $D$-glucopyranosyl- $(1 \rightarrow 6)-\beta$ - $D$ glucopyranosyl ester $=1255$

$3 \beta$-[(O- $\alpha$ - $L$-Rhamnopyranosyl-( $1 \rightarrow 2)-\beta$ - $D$-glucopyranosyl)oxy $]$-pregna5,16-dien-20-one $=20230$

$3 \beta$-[( $O-\alpha-L$-Rhamnopyranosyl-( $1 \rightarrow 4)-\beta$ - $D$-glucopyranosyl)oxy $]$ pregna5,16-dien-20-one $=20231$

3-O- $\alpha$ - $L$-Rhamnopyranosyl-( $1 \rightarrow 3)-\beta$ - $D$-glucopyranosyl-( $1 \rightarrow 3)-\alpha$ - $L$-rhamnopyranosyl- $(1 \rightarrow 2)-\alpha$ - $L$-arabinopyranosyl-hederagenin $28-O-\beta$ - $D$-glucopyranoside $=11817$

3-O- $\alpha$ - $L$-Rhamnopyranosyl-( $1 \rightarrow 2)$ - $\beta$ - $D$-glucopyranosyl-3 $\beta, 21 \alpha, 28$-trihydroxy-urs-12-ene 21- $O-\beta$ - $D$-glucopyranoside $=12555$

3-O-[ $\alpha$ - $L$-Rhamnopyranosyl- $(1 \rightarrow 2)$ - $[\beta$ - $D$-glucopyranosyl- $(1 \rightarrow 6)-[\beta$ - $D$-xylopyranosyl-( $(1 \rightarrow 2)]-\beta$ - $D$-glucopyranosyl-( $(1 \rightarrow 3)]-\alpha$ - $L$-arabinopyranoside] $=11909$

3- $O$ - $[\alpha$ - $L$-Rhamnopyranosyl-( $1 \rightarrow 4)-\beta$ - $D$-glucopyranosyl-( $(1 \rightarrow 2)$ - $[\beta$ - $D$-xylopyranosyl-( $1 \rightarrow 3)-\beta$ - $D$-glucopyranosyl-( $1 \rightarrow 3)]-\beta$ - $D$-glucopyranosyl$(1 \rightarrow 4)-\beta$ - $D$-galactopyranoside $]=6553$

3- $O$-[- $\alpha$ - $L$-Rhamnopyranosyl-( $1 \rightarrow 3)-\beta$ - $D$-glucuronopyranosyl] $-28-O-[-\alpha-L$ rhamnopyranosyl-( $1 \rightarrow 4)-\beta$ - $D$-glucopyranosyl-( $1 \rightarrow 6)-\beta$ - $D$-glucopyranosyl]-oleanolate $=3914$

3- $O$-[ $\alpha$ - $L$-Rhamnopyranosyl-( $1 \rightarrow 2)-\beta$-(3'- $O$-november acetyl)- $D$-xylopyranosyl]-6- $O-\beta$ - $D$-xylopyranosyl-20(R),24(S)-epoxy-3 $\beta, 6 \alpha, 16 \beta, 25$-tetrahydroxycycloartane $=1947$

6- $O$ - $\alpha$ - $L$-Rhamnopyranosyl-( $1 \rightarrow 3)-\beta$ - $D$-quinovopyranosyl $(22 R, 23 R, 25 S)$ $3 \beta, 6 \alpha, 23$-trihydroxy-5 $\alpha$-spirostane $=21473$

$3 \beta$-O- $\{\alpha-L$-Rhamnopyranosyl- $(1 \rightarrow 2)$ - $[\alpha-L$-rhamnopyranosyl- $(1 \rightarrow 4)-\beta$ - $D$ glucopyranosyl-( $(1 \rightarrow 4)]-\alpha-L$-arabinopyranosyl $\}-28-O$-[ $\alpha$ - $L$-rhamnopyranosyl-( $1 \rightarrow 4)-\beta$ - $D$-glucopyranosyl-( $1 \rightarrow 6)-\beta$ - $D$-glucopyranosyl]-hederagenin $=16698$

$3 \beta$ - $O$ - $\{\alpha-L$-Rhamnopyranosyl- $(1 \rightarrow 2)$ - $[\alpha-L$-rhamnopyranosyl- $(1 \rightarrow 4)-\beta$ - $D$ glucopyranosyl-( $1 \rightarrow 4)]-\alpha-L$-arabinopyranosyl $\}-28-O-[\alpha-L$-rhamnopyranosyl-( $1 \rightarrow 4)-\beta$ - $D$-glucopyranosyl-( $1 \rightarrow 6)-\beta$ - $D$-glucopyranosyl]-oleanolate $=16699$

3-O-[ $\alpha$ - $L$-Rhamnopyranosyl-( $1 \rightarrow 4)-\beta$ - $D$-xylopyranosyl-( $1 \rightarrow 2)$ - $[\beta$ - $D$-glucopyranosyl- $(1 \rightarrow 3)]-\beta$ - $D$-glucopyranosyl- $(1 \rightarrow 4)-\beta$ - $D$-galactopyranoside $]=$ 6555

3-O- $\alpha$ - $L$-Rhamnopyranosyl-( $1 \rightarrow 2)-\beta$ - $D$-xylopyranosyl oleanolic acid 28- $O$ $\beta$-D-glucopyranosyl- $(1 \rightarrow 6)-\beta$ - $D$-glucopyranosyl ester $=1253$
3- $O$-[ $\alpha$-Rhamnopyranosyl $(1 \rightarrow 2)-\beta$ - $D$-xylopyranosyl $]$-spergulatriol $=20145$

3 - $O$-[ $\alpha$ - $L$-Rhamnopyranosyl- $(1 \rightarrow 2)-\beta$ - $D$-xylopyranosyl]-6- $O-\beta$ - $D$-xylopyra$\operatorname{nosyl}-20(R), 24(S)$-epoxy-3 $\beta, 6 \alpha, 16 \beta, 25$-tetrahydroxycycloartane $=1949$

$4[\alpha$ - $L$-Rhamnosyl $(1 \rightarrow 2)$ - $\beta$ - $D$-glucopyranosyloxy $]$-2-hydroxymethylene,5hydroxy naphthalene $=6461$

$1[\alpha$ - $L$-Rhamnosyl $(1 \rightarrow 2)$-( $\beta$ - $D$-glucopyranosyloxy $)]-3,4,5$-trimethoxy benzene $=6463$

Rhapontigenin 3-O- $\beta$ - $D$-glucopyronoside $=18744$

Rhein-8-monoglucoside $=18761$

Rhetsinine $=10117$

Rhizopin $=11031$

Rhoifolin $=1497$

Rhoifoloside $=1497$

Ribaline $=19083$

9- $\beta$-Ribofuranosylguanine $=9071$

9- $\beta$ - $D$-Ribofuranosyl-9H-purin-6-amine $=618$

3-O- $\beta$ - $D$-Ribopyranosyl hederagenin $28-O-\alpha-L$-rhamnopyranosyl- $(1 \rightarrow 4)-\beta-D$ glucopyranosyl- $(1 \rightarrow 6)-\beta$ - $D$-glucopyranoside $=3821$

Ricinolic acid $=18841$

rosmanol $=8090$

Rosmarinic acid $=12420$

Rotenolone $=10680$

Rotundine $=21077$

Roupelliol $=1644$

Roxburghilin $=16002$

Roxburghiline $=16002$

Royleose $=16032$

Rubescensin $\mathrm{A}=16183$

Rubescensine $=16183$

Rubescensin $\mathrm{E}=18503$

Rubescensine B = 17717

Rubiarboside $\mathrm{F}=19010$

Rubrobrassicin $=18548$

Rubrofusarin gentiobioside $=19042$

Rubschisandrin $=12003$

Rugosal $=19047$

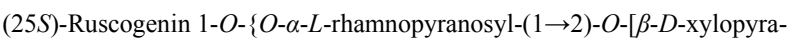
$\operatorname{nosyl}-(1 \rightarrow 3)]-\beta-D$-fucopyranoside $\}=19072$

Rutaevin $=7668$

Rutaevin $(E)$-p-hydroxycinnamate $=9914$

Rutaevin $(Z)$ - $p$-hydroxycinnamate $=9913$

Rutamarin alcohol $=9304$

\section{S}

$(+)-\left(1 R^{*}, 3 S^{*}, 5 R^{*}, 6 S^{*}\right)-$ Saccogynol $=19106$

Safflor yellow A = 19111

Salazinic acid $=16673$

$m$-Salicylic acid $=9817$

$p$-Salicylic acid $=9818$

Salidroside $=18792$

Salsoloside C $=20529$

Salsoloside E $=20535$

$(+)$-Salutaridine $=7837$ 
Salvianolic acid $\mathrm{B}=4631$

Sanchinoside $\mathrm{E}_{1}=8423$

Sanggenone $\mathrm{G}=19262$

Sanjoinine $A=7937$

$\beta$-Santala $(3,15), 10$-diene $=19303$

Santamarine $=19308$

Santol $=16209$

Sapogenin ST-I = 10096

Saponaretin $=11773$

Saponin C $=16025$

Sappanchalcone $=14099$

Sarisan $=1832$

Sarmentogenin 3-O- $\alpha-L$-diginoside $=6532$

Sarmentogenin 3-O- $\alpha$-oleandroside $=6530$

Sarotanoside $=16122$

Sarothralen B $=11818$

Sarothralin $=11819$

Sarsasapogenin-3-O- $\beta$ - $D$-glucopyranosyl-( $1 \rightarrow 3)-\beta$ - $D$-glucopyranosyl-( $1 \rightarrow 2)$ -

$$
\beta \text { - } D \text {-galactopyranoside }=22796
$$

Sativan $=19394$

$(+)$-Saucernetin $=19404$

Saurolactam $=19417$

Saussurine $=19427$

Saxifragin $=18369$

Scabioside $A=12642$

Scabioside C $=12643$

Scandenone $=22637$

Schinol $=10364$

$\gamma$-Schisandrin $=5178$

Schisandrin $\mathrm{B}=5178$

Schisandrin C $=19497$

Schisandronic acid $=19499$

Schisanhenol $=8917$

Schisanhenrin $=8907$

Schisantherin $A=8908$

Schisantherin $\mathrm{B}=8907$

Schizandrin A = 5213

Schizandrin B $=5178$

Schizanhenol $=8917$

Schizantherin B $=8907$

Schizantherin D = 19490

Schkuhrin I = 9570

Scopolamine $=10870$

Scroside B $=17511$

Scutellarein 4'- $O$-methylether 7- $O$ - $\beta$-glucopyranoside $=20252$

1,10-Seco-dihydroisoparthenin-1,10-dione $=3482$

ent-3,4-Seco-16 $\alpha$-hydroxyatis-4(19)-en-3-oic acid $=7692$

$\left(8 R, 8^{\prime} R\right)-(-)-$ Secoisolariciresinol $=19618$

ent-2,3-Secokaur-16-en-2,3-dioic acid $=7691$

Selagine $=9686$

Selina-4(14),7(11)-diene $=19681$

Selina-4(14),7(11)-dien-8-one $=7495$

Selina-4(15),7(11)-dien-8-one $=7495$
Selinan $=19685$

Selinidin $=11834$

Sempervine $=19697$

Sempervirine $=19697$

Senburiside IV $=8758$

Senkyunolide A $=2791$

Senkyunolide $\mathrm{I}=5590$

$(L)$-Serine $=19759$

Serratagenic acid 3- $O-\alpha$ - $L$-rhamnopyranosyl $(1 \rightarrow 4)-\beta$ - $D$-glucopyranoside $=7633$

Serratanetetrol $=21424$

Serratendiol $=7017$

Serrat-14-en-3 $\beta, 21 \alpha$-diol $=19768$

Serrat-14-ene $=19767$

$(+)$-Sesamin $=19777$

Shashenoside I = 20569

Shegansu B = 16691

Shogaol $=19846$

trans-6-Shogaol $=19846$

Sibiricose $\mathrm{A}_{1}=1683$

Sibiricose $A_{5}=1682$

Silerin $=21889$

Silibinin $=19895$

Silybin A = 19895

Simalikalactone $\mathrm{A}=15548$

Sinapyl alcohol diisovalerate $=20559$

Sinapyl alcohol 4'-O- $\beta$-glucopyranoside $=20569$

Sinoacutine $=19200$

Sinpeinine-3- $O$ - $\beta$-glucoside $=22904$

Sipeimine $\dagger=11002$

Sipeimine $\uparrow=18519$

$\triangle^{5}$-Sitosterol-3-one $=19993$

$\beta$-Sitosteryl 3-O- $\beta$ - $D$-glucoside $=19985$

Skatole $=19459$

Sobrepin $=20030$

Solandulcidine $=20042$

Solanidine-3- $O$ - $\alpha$ - $L$-rhamnopyranosyl- $(1 \rightarrow 2)$ - $[\beta$ - $D$-glucopyranosyl- $(1 \rightarrow 6)-\beta$ $D$-glucopyranosyl-( $1 \rightarrow 3)$ - $\beta$ - $D$-glucopyranosyl- $(1 \rightarrow 4)] \beta$ - $D$-glucopyranoside $=15408$

$\alpha$-Solanine $=20060$

Solasodine $O$ - $\alpha$ - $L$-rhamnopyranosyl- $(1 \rightarrow 2)-\{[\beta-D$-xylopyranosyl- $(1 \rightarrow 3)]$, $[\alpha$ - $L$-rhamnopyranosyl- $(1 \rightarrow 4)]\}-\beta$ - $D$-glucopyranoside $=1822$

Sophoraflavanone B $=11248$

(-)-Sophoramide $=20094$

$8 \alpha$-Sovaleryloxy-1 $\alpha$-hydroxy-3 $\alpha, 4 \alpha$-epoxy- $5 \alpha, 7 \alpha H$-10(14),11(13)-guaiadien-

$12,6 \alpha$-olide $=7197$

Soyasaponin $\mathrm{Bb}=20127$

Soyasaponin Bd $=19238$

Speciophylline $=22206$

Specneuzhenide $=15873$

Spermatheridine $=12917$

Sphaerophysone A = 21760

Spheroidine $=21211$

$\alpha$-Spinasterol glucoside $=20169$ 
Spiperlactam S $=17460$

Spiraeoside $=18368$

$(+)$-Spiropachysine $=20205$

$25 R$-Spirosta-3,5-diene $=20207$

$25 S$-Spirosta-3,5-diene $=20208$

$(23 S, 24 S)$-Spirosta-5,25(27)-diene-1 $\beta, 3 \beta, 23,24-$ tetrol 1-O- $\{O$-(2-O-acetyl-

$\alpha$ - $L$-rhamnopyranosyl)-( $1 \rightarrow 2)-\alpha-L$-arabinopyranosyl $\}=6578$

$(23 S, 24 S)$-Spirosta-5,25(27)-diene-1 $\beta, 3 \beta, 23,24$-tetrol 1-O- $\{O$-(2,3-di- $O$ -

acetyl- $\alpha$ - $L$-rhamnopyranosyl)-( $(1 \rightarrow 2)-\alpha-L$-arabinopyranosyl $\}=6577$

$(23 S, 24 S)$-Spirosta-5,25(27)-diene-1 $\beta, 3 \beta, 23,24$-tetrol-O- $\{O-(2,3,4$-tri- $O$ -

acetyl- $\alpha$ - $L$-rhamnopyranosyl)-( $(1 \rightarrow 2)-\alpha$ - $L$-arabinopyranosyl $\}=6576$

Spirostane-3,27-diol $=2150$

Spirostane-1,2,3,5-tetrol $=12251$

$(22 S, 23 S, 24 R, 25 S)-5 \alpha$-Spirostane-3 $\beta, 23,24-$ triol 24-O- $\beta$ - $D$-glucopyranoside $=698$

$(25 S)-5 \beta$-Spirostan- $3 \beta$-ol $=19390$

(25S)-5 $\beta$-Spirostan-3 $\beta$-ol-3- $O-\alpha-L$-arabinopyranosyl- $(1 \rightarrow 6)-[\alpha-L$-arabino pyranosyl- $(1 \rightarrow 4)]$ - $[\beta$ - $D$-glucopyranosyl- $(1 \rightarrow 2)]-\beta$ - $D$-glucopyranoside $=1863$

(3 $\beta, 5 \alpha)$-Spirostan-3-yl $O$-6-deoxy- $\alpha$ - $L$-mannopyranosyl-( $1 \rightarrow 2)-O$-[ $\beta$ - $D$-glucopyranosyl- $(1 \rightarrow 4)]-\beta$ - $D$-galactopyranoside $=21014$

$(3 \beta, 5 \alpha)$-Spirostan-3-yl $O-\beta$ - $D$-galactopyranosyl-( $1 \rightarrow 2)-O-\beta$ - $D$-glucopyranosyl- $(1 \rightarrow 4)-\beta$ - $D$-galactopyranoside $=21013$

Spirost-5,25(27)-dien-1 $\beta, 3 \mathrm{r}$-diol $=6974$

$(25 R)$-Spirost-5-en- $1 \beta, 3 \alpha$-diol $=7009$

(22R,25S)-Spirost-5-en-3 $\beta, 15 \alpha$-diol 3-O- $\{\beta$ - $D$-glucopyranosyl $(1 \rightarrow 2)-\beta$ - $D$ glucopyranosyl-( $(1 \rightarrow 4)$-[ $\alpha$ - $L$-rhamnopyranosyl-( $(1 \rightarrow 2)]-\beta$ - $D$-galactopyranoside $\}=13022$

Spirost-5-ene-3,17-diol $=16809$

(25S)-Spirost-5-ene-3 $\beta, 27-$ diol 27-O- $\beta$ - $D$-glucopyranosyl-3- $O$ - $[\alpha-L$-rhamnopyranosyl- $(1 \rightarrow 4)]-\beta$ - $D$-glucopyranoside $=17646$

Spirost-25(27)-en- $1 \beta, 3 \alpha, 4 \beta, 5 \beta$-tetraol $=22111$

Spirost-25(27)-en- $1 \beta, 3 \beta, 5 \beta$-triol $=22112$

$(25 S)$-Spirost- $1 \beta, 2 \beta, 3 \beta, 4 \beta, 5 \beta$-pentol 2 -sulfate $=1899$

$(25 S)$-Spirost- $1 \beta, 2 \beta, 3 \beta, 5 \beta$-tetraol $=22113$

1(10)-Spirovetiven-11-ol = 9542

Squalidine $=11091$

Squamostatin $\mathrm{C}=2736$

Stauntogenin 3-O-[ $\alpha$ - $L$-Cymaropyranosyl-( $(1 \rightarrow 4)-\beta$ - $D$-digitoxopyranosyl$(1 \rightarrow 4)-\beta$ - $D$-3-demethyl-2-deoxy-thevetopyranoside $]=20277$

Stauntogenin 3-O-[ $\alpha-L$-Cymaropyranosyl- $(1 \rightarrow 4)-\beta-D$-digitoxopyranosyl$(1 \rightarrow 4)$-2,6-dideoxy- $\beta$-D-ribo-hexopyranoside $]=20277$

Stauntogenin 3- $O$ - $[\alpha-L$-diginopyranosyl-( $1 \rightarrow 4)-\beta$ - $L$-cymaropyranosyl- $(1 \rightarrow 4)$ $\beta$-D-digitoxopyranosyl- $(1 \rightarrow 4)-\beta$ - $D$-thevetopyranoside $]=20276$

Stearyl alcohol $=15950$

Stemodin- $\alpha$ - $L$-arabinopyranoside $=20302$

Stemodin- $\beta$ - $D$-glucopyranoside $=20303$

Stemodinol $=20295$

Stephaniaflavone A $=20314$

Stephaniaflavone B $=20315$

Stephenanthrine $=20320$

Stevein $=20340$

Stevenine $=20340$

Stigmasta-5,28-diene-3,24-diol $=19367$
$\Delta^{5,25}$-Stigmastadienol $=3844$

Stigmasta-5,24(28)E-dien-3-ol = 7978

(22E)-Stigmasta-5,22-dien-3 $\beta$-ol = 20369

Stigmast-5-ene- $3 \beta, 7 \alpha$-diol $=10967$

$(3 \beta, 24 R)$ Stigmast-5-en-3-ol $=19983$

$(3 \beta, 24 S)$ Stigmast-5-en-3-ol $=19984$

Stigmast-4-en-3-one $=19981$

Stigmast-5-en-3-one $=19993$

Stigmasterol 3- $O-\beta-D$-glucoside $=20372$

Stigmasteryl palmitate $=7426$

Stoindoside $\mathrm{I}=19997$

Strophanthidin 3-O- $\beta$ - $D$-glucopyranosyl- $(1 \rightarrow 4)-\beta-D$-antiaropyranoside $=3495$

$(-)$-Strychnine $=20410$

Stypandrol $=9339$

Styptysat $=3141$

Suavissimoside $\mathrm{F}_{1}=20433$

1,2,3,9,10-Substituted aporphine alkaloid $=14708$

2,3,10,11-Substituted pseudoprotoberberine alkaloid $=4886$

$D$-(+)-Sucrose $=20446$

Sudachiflavone $=21858$

Sulfapatrinoside I $=20476$

Sulfapatrinoside II = 20477

5-Sulfonyl -1,7-bis(4,5-dihydroxy-3-methoxyphenyl) -heptan-3-one = 19851

5-Sulfonyl-1,7-bis(3,4-dihydroxyphenyl)-heptan-3-one $=19850$

5-Sulfonyl-1,7-bis(4-hydroxy-3-methoxyphenyl)-heptan-3-one = 19848

Sulfonyl bis-methane $=6408$

3-O-[6-O-Sulfonyl- $\beta$ - $D$-glucopyranosyl-( $1 \rightarrow 3)]-\alpha$ - $L$-arabinopyranosyl-pseudo jujubogenin $=2088$

5-Sulfonyl-1-(4-hydroxy-3-methoxyphenyl)-7-(3,4-dihydroxyphenyl)-heptan3-one $=19849$

Sulforaphane $=14728$

3-O-[(2-O-Sulfuryl- $\beta$ - $D$-glucopyranosyl) $(1 \rightarrow 2)-\beta$ - $D$-glucopyranosyl]-echinocystic acid 28- $O-\beta$ - $D$-glucopyranoside $=6703$

Surgatoxin $=20496$

Suspensaside $=7926$

Swertiaperennin $=14732$

Sylvic acid $=10$

$(+)$-Syringaresinol-di- $O-\beta$ - $D$-glucoside $=12916$

Syringaresinol-4' $-O-\beta$ - $D$-glucopyranoside $=85$

(+)-Syringaresinol $O-\beta$ - $D$-glucopyranoside $=85$

Syringetin-3-O- $\alpha$ - $L$-rhamnopyranosyl- $(1 \rightarrow 6)-\beta$ - $D$-galactopyranoside $=20564$

\section{$\mathbf{T}$}

Tachioside $=10432$

Tadeonal $=17640$

Tanetin $=19312$

Tanshinaldehyde II $=20678$

(+)-Tanshindiol A = 20679

Tanshiquinone $\mathrm{A}=4632$

Tanshiquinone $\mathrm{B}=4633$

Tanshiquinone $\mathrm{C}=4634$

Taraxanthin $=13127$

20-Taraxastene $=20698$ 
20-Taraxastene-3,16-diol $=7715$

Tarichatoxin $=21211$

Tatasaponin $\mathrm{I}=20383$

Tauremisin $=20730$

Tauroside $\mathrm{G}_{2}=819$

Taxa-4(20), 11 -diene- $2 \alpha, 5 \alpha, 10 \beta, 14 \beta$-tetraol- $2 \alpha, 5 \alpha, 10 \beta$-triacetate- $14 \beta$ - $\alpha$ methyl- $\beta$-hydroxyl butyrate $=22951$

Taxawallin $\mathrm{C}=20775$

Taxayuntin $=20758$

Taxayuntin $\mathrm{H}=20751$

Taxchinin $\mathrm{C}=20762$

Taxchinin $\mathrm{L}=20764$

Taxcultine $=20814$

Taxifolin $=5699$

Taxifolin-3- $O-\alpha-L$-rhamnoside $=1928$

Taxine $\mathrm{C}=4780$

Taxine $\mathrm{I}=20795$

Taxol B $=3402$

Taxuspinanane $\mathrm{E}=6884$

Taxuspinanane $\mathrm{F}=20750$

Taxuspinanane $\mathrm{I}=14651$

Taxuspinanane $\mathrm{J}=2074$

Taxuyunnanine $\mathrm{A}=20813$

Tecleoxine $=20893$

Tectoquinone $=14144$

Tectorigin $=20900$

Telepathine $=9235$

Tellimagrandin II $=7518$

Telosmogenin I 3-O- $\beta$ - $D$-glucopyranosyl-( $1 \rightarrow 4)-\beta$ - $D$-thevetopyranosyl-

$(1 \rightarrow 4)-\beta$ - $D$-oleandropyranosyl- $(1 \rightarrow 4)-\beta$ - $D$-cymaropyranoside $=20913$

Telosmogenin I 3-O- $\beta$ - $D$-glucopyranosyl-( $1 \rightarrow 4)-\beta$ - $D$-thevetopyranosyl-

$(1 \rightarrow 4)-\beta$ - $D$-oleandropyranosyl- $(1 \rightarrow 4)-\beta$ - $D$-digitoxopyranoside $=20920$

Telosmogenin I 3-O- $\beta$ - $D$-oleandropyranosyl-(1 $\rightarrow 4)-\beta$ - $D$-cymaropyranosyl-

$(1 \rightarrow 4)-\beta$ - $D$-cymaropyranoside $=20915$

Telosmogenin I 3-O- $\beta$ - $D$-oleandropyranosyl-( $1 \rightarrow 4)-\beta$ - $D$-cymaropyranosyl$(1 \rightarrow 4)-\beta$ - $D$-digitoxopyranoside $=20917$

Telosmogenin I 3-O- $\beta$ - $D$-thevetopyranosyl-( $1 \rightarrow 4)-\beta$ - $D$-oleandropyranosyl$(1 \rightarrow 4)-\beta$ - $D$-cymaropyranoside $=20912$

Teracrylshikonin $=20968$

Teresantalaldehyde $=20971$

Teresantanane $=21598$

Terpeneol acetate $=20999$

Terpinen-4-ol = 20992

$2 \alpha, 5 \alpha, 14 \alpha, 17 \alpha$-Tetraacetoxy-3 $\beta$-benzoyloxy-15 $\beta$-hydroxy-9-oxoparaliane = 17731

$2 \alpha, 4 \alpha, 7 \beta, 10 \beta$-Tetraacetoxy-5 $\beta, 20$-epoxy- $9 \alpha, 13 \alpha, 15$-trihydroxy-11(15 $\rightarrow 1)$ abeo-taxene $=20836$

$2 \alpha, 5 \alpha, 10 \beta, 14 \beta$-Tetraacetoxytaxa-4(20),11-diene $=20875$

$5 \alpha, 9 \alpha, 10 \beta, 13 \alpha$-Tetraacetoxytaxa-4(20),11-diene $=20840$

$5 \alpha, 9 \alpha, 10 \beta, 13 \alpha$-Tetraacetoxytaxa-4(20),11-dien-14 $\beta$-ol $=10740$

$5 \alpha, 7 \beta, 9 \alpha, 20$-Tetraacetoxy-2 $\alpha, 10 \beta, 13 \alpha, 15$-tetrahydroxy-11-(15 $\rightarrow 1)$-abeotaxene $=20835$

$1,3^{\prime}, 4^{\prime}, 6^{\prime}$-Tetra- $O$-acetyl-6- $O$ - $p$-coumaroylsucrose $=18005$
2,5,10,13-Tetraacetyl-11-taxene-2,5,7,9,10,13,20-heptol $=20752$

$2,10,12,10^{\prime}$-Tetrachloroisoplagiochin $\mathrm{C}=2182$

$n$-Tetracosanoic acid $=12800$

Tetracosanoyl $-p$-hydroxy phenethylamine $=22522$

Tetradecanoic acid $=15203$

12-Tetradecanoylphorbol 13-acetate $=17187$

5-Tetradecenoic acid $=21046$

cis-9-Tetradecenoic acid $=15205$

Tetradecenoic acid $\mathrm{C}=15205$

3,14,19,20-Tetradehydro-16-ethenyl-17-( $\beta$ - $D$-glucopyranosyloxy)-19-hydroxy- $(15 \beta, 16 \alpha, 17 \beta)$-oxayohimban-21-one $=15310$

$14,15,19,20$-Tetradehydro-16-ethenyl-17-( $\beta$ - $D$-glucopyranosyloxy)-19-hydroxy- $(3 \alpha, 16 \alpha, 17 \beta)$-oxayohimban-21-one $=15311$

(-)-(2R,3S,4R,5R,10S,2'S)-1-[2,3,4,5-Tetrahydro-10-acetyloxy-5-hydroxy-2(4-methoxyphenyl)-6-methoxy-7,8-methylenedioxy-3-phenyl-2,5methano-1-benzoxepin-4-carbonyl]-2-(2-methylpropanoylamino)pyrrolidine $=21275$

( $3 \alpha)-3,4,5,6$-Tetrahydroalstonine $=21055$

Tetrahydrobenzylisoquinoline alkaloid $=6316$

$\Delta^{2}$-Tetrahydrocannabinolic acid $=21060$

(-)-Tetrahydrocolumbamine $=11348$

Tetrahydrodesoxycordifoline $=3184$

(-)-(1R,2R,3S,3aR)-16,7,8,8a-Tetrahydro-8,8a-dihydroxy-9-methoxy-5a-(4methoxyphenyl)-6-phenyl-5a $H$-cyclopenta[4,5]furo[2,3-f]-1,3-benzodioxole-7- $N, N$-dimethyl amide $=741$

(-)-(2R,3S,4R,5R,10S,2'S)-1-[2,3,4,5-Tetrahydro-5,10-dihydroxy-2-(4-methoxyphenyl)-6-methoxy-7,8-methylenedioxy-3-phenyl-2,5-methano-1benzoxepin-4-carbonyl]-2-(2-methylbutanoylamino)-pyrrolidine $=9623$

(-)-(2R,3R,4S,5R,10R,2S)-1-[2,3,4,5-Tetrahydro-5,10-dihydroxy-2-(4-methoxyphenyl)-6-methoxy-7,8-methylenedioxy-3-phenyl-2,5-methano-1-ben zoxepin-4-carbonyl]-2-(2-methylpropanoylamino)-pyrrolidine $=11739$

(+)-(2R,3R,4S,5R,10S,2'RS)-1-[2,3,4,5-Tetrahydro-5,10-dihydroxy-2-(4-methoxyphenyl)-6-methoxy-7,8-methylenedioxy-3-phenyl-2,5-methano-1-ben zoxepin-4-carbonyl]-2-(2-methylpropanoylamino)-pyrrolidine $=21276$

$(S)-5,8,13,13 a-T e t r a h y d r o-3,10$-dimethoxy-6H-dibenzo[ $a, g]$ quinolizine-2,9diol $=19566$

1,2,3,4 Tetrahydro-6,7-dimethoxy-2-methylisoquinoline $=14253$

Tetrahydroharman $=6736$

$3,3 \alpha, 7,7 \alpha$-Tetrahydro-3 $\alpha$-hydroxy-6(2H)-benzofuranone $=18615$

4a $S^{*}-2,3,4,4 a-T e t r a h y d r o-6-h y d r o x y-7-i s o p r o p y l-1,1,4 a-t r i m e t h y l-5,8(1 H)$ fluorene-dione $=5434$

(+)-(2R,3S,4R,5R,2'S)-1-[2,3,4,5-Tetrahydro-5-hydroxy-2-(4-methoxyphenyl)6-methoxy-7,8-methylenedioxy-10-oxo-3-phenyl-2,5-methano-1-benzoxepin-4-carbonyl]-2-(2-methylpropanoylamino)-pyrrolidine $=21277$

(+)-(2R,3R,4S,5R,2'S)-1-[2,3,4,5-Tetrahydro-5-hydroxy-2-(4-methoxyphenyl)-6-methoxy-7,8-methylenedioxy-10-oxo-3-phenyl-2,5-methano-1-ben zoxepin-4-carbonyl]-2-(2-methylpropanoylamino)-pyrrolidine $=21278$

1,2,3,4-Tetrahydro-4-hydroxy-4-quinolin carboxylic acid $=22082$

Tetrahydrojatrorrhizine $=4122$

(+)-(2R,3S,4R,2'RS)-1-[2,3,4,5-Tetrahydro-2-methoxycarbonyl-2-(4-methoxyphenyl)-6-methoxy-7,8-methylenedioxy-5-oxo-3-phenyl-1-benzoxepin4-carbonyl]-2-(2-methylpropanoylamino)-pyrrolidine $=21274$

1,2,3,4-Tetrahydro-6-methoxy-7-hydroxy-1,2-dimethylisoquinoline $=14252$ 
1,2,3,4-Tetrahydro-6-methoxy-7-hydroxy-2-methylisoquinoline $=4121$

$8,13,13 b, 14$-Tetrahydro-14-methylindolo[2',3':3,4]pyrido[2,1b]quinazolin-

$5(7 H)$-one $=7665$

$(6 R, 8 R)-5,6,7,8$-Tetrahydro-8-methyl-1,3,6-naphthalenetriol $=7761$

Tetrahydropiperinic acid $=21078$

Tetrahydropyrrole $=18275$

$1,2,15,16$-Tetrahydrotanshiquinone $=21080$

4"a,5",6",8"a-Tetrahydro-5,7,4'-trihydroxy-3-methoxy-5",5",8"a-trimethyl-

$4 H$-chromeno[2",3": $\left.3^{\prime}, 2^{\prime}\right]$ flavone $=22182$

$1 \beta, 6 \beta, 7 \beta, 14 \beta$-Tetrahydroxy-19-acetoxy-7 $\alpha, 20$-epoxy-ent-kaur-16-en-15-one = 22792

$3 \beta, 7 \beta, 11 \beta, 15 \beta$-Tetrahydroxy- $6 \alpha$-acetoxyent -kaur-16-ene $=13702$

2,3,7,8-Tetrahydroxy[1] benzopyrano[5,4,3-cde][1] benzopyran-5,7-dione $=6757$

3',4,4",6'-Tetrahydroxy-2'-benzoyloxy-5'-(3-pyridinecarboxyl)[1,1':4', 1'-terphe nyl $]=21306$

(E)-4,5,6,7-Tetrahydroxy-2-benzylhept-2-enoic acid $=6613$

$2,4,3^{\prime}, 4^{\prime}$-Tetrahydroxybiphenyl $=19346$

$3,4,2^{\prime}, 4^{\prime}$-Tetrahydroxychalcone $=2779$

(22S,25S)-11 $\alpha, 16 \beta, 22,26$-Tetrahydroxycholest-4-en-3-one 16- $O-\beta$ - $D$-gluco-

pyranosyl- $(1 \rightarrow 3)-\beta$ - $D$-xylopyranoside $=16879$

$(1 \alpha, 3 \alpha, 4 \alpha, 5 \beta)-1,3,4,5$-Tetrahydroxy-cyclohexanecarboxylic acid $=18421$

$3 \beta, 6 \alpha, 12 \beta, 24 \xi$-Tetrahydroxy-dammar-20(22),25-diene 6- $O-\beta$ - $D$-glucopyranoside $=8440$

$3 \beta, 7 \beta, 12 \beta, 20(S)$-Tetrahydroxy-dammar-5,24-diene $20-O-\beta$ - $D$-glucopyranoside $=8442$

$1 \beta, 3 \beta, 12 \beta, 20(S)$-Tetrahydroxy-dammer-24(25)-en-3-O- $\beta$ - $D$-glucopyranosyl-

$20(S)$ - $O-\beta$ - $D$-glucopyranosyl- $(1 \rightarrow 6)-\beta-D$-glucopyranoside $=9095$

3',4,4',6'-Tetrahydroxy-2',5'-dibenzoyloxy [1,1':4',1"-terphenyl $]=21302$

$3,5,3^{\prime}, 4^{\prime}$-Tetrahydroxy-6,7-dimethoxyflavone $=7588$

5,7,3',4'-Tetrahydroxy-6-(6,6-dimethyl-2-methylene-cyclohexylmethyl)flavone $=22183$

1,3,5,6-Tetrahydroxy-4-[(2E)-3,7-dimethylocta-2,6-dienyl]-8-(3-methylbut2 -enyl) xanthone $=22524$

1,3,6,7-Tetrahydroxy-2-(3,7-dimethyl-2,6-octadienyl)-5-(3-methyl-2-butenyl) xanthone $=6630$

1,3,5,6-Tetrahydroxy-2-(1,1-dimethyl-2-propenyl)xanthone $=4347$

$(22 R, 24 Z)-1 \alpha, 3 \beta, 22,26$-tetrahydroxyergost-5,24-diene $26-O-\beta$ - $D$-glucopyranoside $=3646$

3',4',5,7-Tetrahydroxyflavanol $=6853$

$(2 S)-5,7,2^{\prime}, 4^{\prime}-$ Tetrahydroxyflavanone $=20326$

$3,5,7,4^{\prime}$-Tetrahydroxyflavone $=12020$

$3,7,3^{\prime}, 4^{\prime}$-Tetrahydroxyflavone $=7802$

$5,7,3^{\prime}, 4^{\prime}$-Tetrahydroxyflavone $=13137$

$5,7,8,4^{\prime}$-Tetrahydroxyflavone $=11703$

$3,4^{\prime}, 5,7$-Tetrahydroxyflavone-3- $L$-rhamnoside $=12082$

3',4',5,7-Tetrahydroxyflavonol-3- $\beta$ - $D$-galactoside $=10887$

$6 \beta, 7 \beta, 14 \beta, 15 R$-Tetrahydroxy-11 $\beta$-O-formyl-7 $\alpha, 20$-epoxy-ent-kaur-16-ene $=18977$

$2^{\prime}, 3,4,4^{\prime}$-Tetrahydroxy-3'-geranylchalcone $=8330$

$3 \beta, 12 \beta, 20(S), 24 \xi$-Tetrahydroxy-20-O- $\beta$ - $D$-glucopyranosyl-3- $O-\beta$ - $D$-glucopyranoside $=8414$

$3 \beta, 6 \alpha, 12 \beta, 20(S)$-Tetrahydroxy-25-hydroperoxy-dammar-23-ene $20-O-\beta$ - $D$ glucopyranoside $=8441$

5,7,2',4'-Tetrahydroxy-6-(2"-hydroxy-3"-methylbut-3"-enyl)-8-( $\gamma, \gamma$-dimethyl- allyl) isoflavone $=7318$

$5,7,3^{\prime}, 4^{\prime}-$ Tetrahydroxyisoflavone $=16209$

cis-5 $\alpha$ - $H, 3 \beta, 8 \beta, 14 \beta, 17 \beta$-Tetrahydroxy-12 $\beta$ - $O$-isovaleryl-20-O-isovaleryl-

pregnane $=6602$

$1 \alpha, 7 \alpha, 12 \alpha, 14 \beta$-Tetrahydroxy-ent-kaur-16-en-15-one $=7674$

$1 \alpha, 7 \alpha, 14 \beta, 18$-Tetrahydroxy-ent-kaur-16-en-15-one $=7684$

$7 \alpha, 12 \alpha, 14 \beta, 18$-Tetrahydroxy-ent-kaur-16-en-15-one $=18472$

$7 \alpha, 12 \alpha, 14 \beta, 20$-Tetrahydroxy-ent-kaur-16-en-15-one $=7682$

$7 \alpha, 14 \beta, 18,20$-Tetrahydroxy-ent-kaur-16-en-15-one $=7683$

3,5,7,8-Tetrahydroxyl flavone $4 '-O-\alpha-L$-rhamnopyranoside $=18791$

1,3,5,8-Tetrahydroxy-2-methoxyanthraquinone $=18874$

5,5 ", 7,7"-Tetrahydroxy 3'-methoxy 4',4"'-biflavonyl ether = 14557

$3,5,7,3^{\prime}$-Tetrahydroxy-4'-methoxy flavone $=20657$

$3,5,8,4^{\prime}$-Tetrahydroxy-7-methoxy flavone $=17617$

$5,6,3^{\prime}, 4^{\prime}$-Tetrahydroxy-7-methoxyflavone $=16758$

$5,7,3^{\prime}, 4^{\prime}$-Tetrahydroxy-6-methoxyflavone $=7556$

3',4',5,7-Tetrahydroxy-6-methoxyisoflavone $=11157$

3,5,7,4'-Tetrahydroxy-3'-methoxy-6-isoprenyl flavone $=8142$

$5,6,3^{\prime}, 4^{\prime}$-Tetrahydroxy-3-methoxy-6'-isoprenyl flavone $=22238$

$5,7,3^{\prime}, 4^{\prime}$-Tetrahydroxy-3-methoxy-5'-isoprenyl flavone $=22242$

5,7,3',4'-Tetrahydroxy-3-methoxy-2'-(2,6,6-trimethyl-2-cyclohexenylmethyl) flavone $=22181$

$3 \beta, 16 \beta, 23,28$-Tetrahydroxyoleana-11,13(18)-dien-3-O- $\beta$ - $D$-glucopyranosyl-

$(1 \rightarrow 6)$-[ $\alpha-L$-rhamnopyranosyl- $(1 \rightarrow 4)]-\beta-D$-glucopyranoside $=19153$

$3 \beta, 16 \beta, 23,28$-Tetrahydroxyoleana-11,13(18)-dien-3-O- $\beta$ - $D$-xylopyranosyl-

$(1 \rightarrow 2)$ - $\beta$ - $D$-glucopyranosyl-( $(1 \rightarrow 3)-\beta$ - $D$-fucopyranoside $=19150$

$2 \beta, 3 \beta, 6 \beta, 28$-Tetrahydroxyolean-12-en-23-oic acid $23-O-\alpha$ - $L$-arabinopyranosyl ester $=8130$

$2 \alpha, 3 \alpha, 11,12$-Tetrahydroxypicrotoxan-3(15 $\alpha$ )-olide $11-O-\beta$ - $D$-glucopyranoside $=5119$

$2^{\prime}, 3,4,4^{\prime}$-Tetrahydroxy-5-prenylchalcone $=17827$

2',4',5,7-Tetrahydroxy-6-prenylflavanone $=4335$

$5,7,3^{\prime}, 4^{\prime}$-Tetrahydroxy-5'-prenylflavoanone $=22239$

$3,5,7,4$ '-Tetrahydroxy-8-prenylflavone-3,7-O- $\alpha$ - $L$-dirhamnopyranoside $=2141$

$3,5,7,4$ '-Tetrahydroxy-8-prenylflavone-3- $O-\alpha-L$-rhamnopyranoside $=2139$

$3,5,7,4^{\prime}$-Tetrahydroxy-8-prenylflavone-3- $O-\alpha-L$-rhamnopyranosyl-(1 $\left.\rightarrow 4\right)-\alpha-L$ -

rhamnopyranoside $=2140$

3,5,7,4'-Tetrahydroxy-8-prenylflavone-3- $O-\alpha-L$-rhamnopyranosyl-(1 $\rightarrow 4)-\alpha-L$ -

rhamnopyranosyl-7- $O-\beta$ - $D$-glucopyranoside $=2142$

$(25 R)-1 \beta, 2 \beta, 3 \beta, 5 \beta$-Tetrahydroxyspirostan- $4 \beta$-yl- $O-\beta$ - $D$-xylopyranoside $=22653$

Tetrahydroxystilbene $=4337$

$3,5,3^{\prime}, 4^{\prime}$-Tetrahydroxystilbene $=17278$

$5 \alpha, 9 \alpha, 10 \beta, 13 \alpha$-Tetrahydroxy-4(20),11-taxadiene $=21160$

3,5,7,4'-Tetrahydroxy-6-(2,6,6-trimethyl-2-cyclohexenylmethyl)flavone $=22180$

$2 \alpha, 3 \beta, 19 \alpha, 30$-Tetrahydroxyurs-12-en-24,28-dioic acid $28-O-\beta$ - $D$-glucopyra-

nosyl ester $=21403$

$2 \beta, 3 \beta, 7 \beta, 19 \alpha$-Tetrahydroxyurs-12-en-28-oic acid $=18970$

$2 \alpha, 3 \alpha, 19 \alpha, 24$-Tetrahydroxyurs-12-en-28-oic acid 28-O- $\beta$ - $D$-glucopyranoside $=18007$

$(20 R, 22 R)-1 \alpha, 3 \mathrm{~b}, 20,27-T e t r a h y d r o x y w i t h a-5,24$-dienolide 3-O- $\beta$ - $D$-glucopyranoside $=22714$

$1,3,6,7$-Tetrahydroxyxanthone $=15715$

$1,3,5,8$-Tetrahydroxyxanthone $=15718$ 
4,4',6,6'-Tetramethoxy-[1,1'-biphenanthrene]-2,2',3,3',7,7'-hexol = 2398

$5,9,10,12$-Tetramethoxy-2,2-dimethyl-2 $H$-pyrano[5,6- $b]$ xanthen-6-one $=6633$

$3,3^{\prime}, 4^{\prime}, 5^{\prime}$-Tetramethoxyfurano[4",5":8,7]foavone $=16491$

$2,3,4,5$-Tetramethoxy-xanthone-1- $O-\beta$ - $D$-glucopyranosyl-( $1 \rightarrow 6)-\beta$ - $D$-xylopyranoside $=22387$

1,1,4,8-Tetramethylcycloundecane $=9667$

1,3,3,8-Tetramethylcycloundeca-1,4,8-triene $=11458$

$(1 S, 8 S)-4 \alpha, 7 \beta, 10,10$-Tetramethyl-3 $\beta, 6 \beta$-dihydroxytricyclo[5,3,0,0 2,5$]$ decan-

$$
2 \alpha, 6 \text {-olide }=7738
$$

1,1,4,7-Tetramethyl-1a,2,4a,5,6,7b-hexahydro- $1 H$-cyclopropa $[e]$ azulene $=1756$

2,2,5,7-Tetramethyl-4-hydroxy-6-(2-hydroxyethyl)-indanone $=16098$

Tetra- $O$-methylisoscutellarein $=21186$

2,2,4,4-Tetramethyl-6-(1-oxo-3-phenylprop-2-enyl)cyclohexane-1,3,5-trione $=3472$

2,3,5,6-Tetramethylpyrazine $=3633$

2,2,6,9-Tetramethyl-3,4,6a,7-tetrahydro-2 $H$-3,9a-methanocyclopent $[b]$ oxocine $=9039$

$\left(1 R^{*}, 6 R^{*}, 7 R^{*}\right)-3,7,10,10$-Tetramethyltricyclo[4.3.2. $\left.0^{2,6}\right]$ undec-2-ene $=8453$

$\left(1 S^{*}, 8 S^{*}, 11 R^{*}\right)-4,7,7,11$-Tetramethyl-tricyclo[6.3.0.0 $\left.0^{1,5}\right]$-undec-4-ene $=16583$

$24,25,26,27$-Tetra-norapotirucalla-(apoeupha)- $6 \alpha$-acetoxy- $\alpha \alpha$-hydroxy-

1,14,20,22-tetraen-21,23-epoxy-3-one $=364$

[24,25,26,27-Tetranorapotirucalla-(apoeupha)-6 $\alpha$-hydroxy, $11 \alpha$-methoxy-

$7 \alpha, 12 \alpha$-diacetoxy,1,14,20(22)-trien-3-one] $=13662$

$[24,25,26,27-T e t r a n o r a p o t i r u c a l l a-(a p o e u p h a)-6 \alpha$-methoxy-7 $\alpha$-acetoxy-1,14dien-3,16-dione-21-al] $=22961$

24,25,26,27-Tetranorapotirucalla-(apoeupha)-1 $\alpha$-trimethylacryloxy-21,23-

$6 \alpha, 28$-diepoxy-16-oxo-17-oxa-14,20,22-trien-3 $\alpha, 7 \alpha$-diol $=13663$

$6,7,3^{\prime}, 4^{\prime}-$ Tetrasubstituted tetrahydrobenzylisoquinoline alkaloid $=22819$

Tetrodontoxin $=21211$

Teucrol-4'- $O$ - $\alpha$-rhamnopyranosyl-(1"'" $\rightarrow 6$ "')- $\beta$ - $O$-galacto-pyranosyl-(1"' $\rightarrow 4 ")$ $\alpha$-O-rhamnopyranoside $=21221$

Texogenin $=13569$

Thalicsimine $=9441$

Thalictiin $=1492$

Thalictrine $=13374$

Thalictrisine $=21249$

Thaliximine $=9441$

Thalphinine $=21235$

Thalrugosamine $=9597$

Thalsimine $=21234$

Thaspine $=20717$

$\Delta^{2}-\mathrm{THC}=21060$

$\Delta^{8}-\mathrm{THC}=21058$

$\Delta^{9}-\mathrm{THC}=21059$

Theasapogenol $\mathrm{B}=2152$

Theasapogenol $\mathrm{C}=3032$

Theasapogenol $\mathrm{D}=3030$

Theasapogenol E = 3034

Thiamine $=22554$

3-Thiapentane $=5503$

Threitol $=7334$

Thunbergene $=3385$

Thymine $=14802$

Thymoquinol 2,5-O- $\beta$-diglucopyranoside $=10763$
Thyrosine $=21175$

trans-Tiglaldehyde $=21370$

Tiglic aldehyde $=21370$

21-O-Tigloyl-28- $O$-acetylprotoaescigenin-3- $O$-[ $\beta$ - $D$-glucopyranosyl $(1 \rightarrow 2)][\beta$ - $D$-glucopyranosyl $(1 \rightarrow 4)]-\beta$ - $D$-glucopyranosiduronic acid $=11416$

21-O-Tigloyl-22- $O$-acetylprotoaescigenin-3- $O$ - $[\beta$ - $D$-glucopyranosyl $(1 \rightarrow 2)]$ $[\beta$ - $D$-glucopyranosyl $(1 \rightarrow 4)]$ - $\beta$ - $D$-glucopyranosiduronic acid $=7361$

21-O-Tigloyl-22- $O$-acetyltheasapogenol E 3- $O-\beta$ - $D$-galactopyranosyl $(1 \rightarrow 2)$ $[\beta$ - $D$-xylopyranosyl $(1 \rightarrow 2)$ - $\alpha$ - $L$-arabinopyranosyl $(1 \rightarrow 3)]-\beta$ - $D$-glucopyranosiduronic acid $=21289$

21-Tigloylbarringtogenol C $3 \beta-O-\alpha$ - $L$-rhamnopyranosyl-( $1 \rightarrow 2)-\beta$ - $D$-galactopyranosyl- $(1 \rightarrow 3)[\beta$ - $D$-glucopyranosyl-( $(1 \rightarrow 2)-\beta$ - $D$-glucuronopyranoside $]$ $=2313$

28-Tigloylbarringtogenol C $3 \beta$ - $O-\alpha$ - $L$-rhamnopyranosyl-( $1 \rightarrow 2)-\beta$ - $D$-galactopyranosyl- $(1 \rightarrow 3)[\beta$ - $D$-glucopyranosyl- $(1 \rightarrow 2)-\beta$ - $D$-glucuronopyranoside $]$ $=2314$

$8 \beta$-Tigloyloxy-14-chloro- $2 \beta, 10 \alpha$-dihydroxy- $\alpha H, 5 \alpha H, 6 \beta H, 7 \alpha H$-guai3,11(13)-dien-6,12-olide $=7571$

$8 \beta$-Tigloyloxy-14-chloro-3 $\alpha, 4 \alpha$-epoxy- $2 \beta, 10$-dihydroxy- $\alpha H, 5 \alpha H, 6 \beta H, 7 \alpha H$ guai-11(13)-en-6,12-olide $=7574$

3'(R)-Tigloyloxy-4'-keto-3',4'-dihydroseselin $=18286$

$3 \alpha$-Tigloyloxytropane $=21372$

13-O-Tigloylphorbol-20-(9Z,12Z-octadecadienoate $)=21381$

22-Tigloylprotoaescigenin 3- $O$ - $[\beta$ - $D$-glucopyranosyl $(1 \rightarrow 2)][\beta$ - $D$-glucopyranosyl $(1 \rightarrow 4)]-\beta$ - $D$-glucopyranosiduronic acid $=7363$

21 - $O$-Tigloyltheasapogenol E 3-O- $\beta$ - $D$-galactopyranosyl $(1 \rightarrow 2)[\beta$ - $D$-xylopy$\operatorname{ranosyl}(1 \rightarrow 2)$ - $\alpha$ - $L$-arabinopyranosyl $(1 \rightarrow 3)]-\beta$ - $D$-glucopyranosiduronic acid $=21288$

Tigogenin-3-O- $\beta$ - $D$-glucopyranosyl- $(1 \rightarrow 2)[\beta$ - $D$-glucopyranosyl- $(1 \rightarrow 3)(6-O$ acetyl- $\beta$ - $D$-glucopyranosyl)]-( $1 \rightarrow 4)-\beta$ - $D$-galactopyranoside $=13329$

Tigogenin-3- $O-\beta$ - $D$-glucopyranosyl- $(1 \rightarrow 2)[\beta$ - $D$-glucopyranosyl- $(1 \rightarrow 3)]-\beta$ - $D$ glucopyranosyl- $(1 \rightarrow 4)-\beta$ - $D$-galactopyranoside $=13326$

Tigogenin 3- $O$ - $\beta$ - $D$-Glucopyranosyl-( $1 \rightarrow 3)-\beta$ - $D$-glucopyranosyl-( $1 \rightarrow 2)-[\beta-D$ glucopyranosyl-( $(1 \rightarrow 3)]$ - $\beta$ - $D$-glucopyranosyl- $(1 \rightarrow 4)-\beta$ - $D$-galactopyranoside $=17612$

Tigogenin 3-O- $\beta$ - $D$-Xylopyranosyl-( $1 \rightarrow 3)-\beta$ - $D$-glucopyranosyl-( $1 \rightarrow 2)-[\beta-D$ glucopyranosy- $(1 \rightarrow 3)]$ - $\beta$ - $D$-glucopyranosyl-( $(1 \rightarrow 4)-\beta$ - $D$-galactopyranoside $=17611$

$6 \beta, 15 \beta$-Dihydoxy-3 $\alpha, 20$-epoxy-ent-kaur-16-en-1,7-dione $=12589$

Timosaponia $\mathrm{F}=20217$

Timosaponia $\mathrm{G}=20209$

Timosaponin $\mathrm{B}_{1}=1175$

Timosaponin $\mathrm{F}=1176$

Timosaponin $\mathrm{G}=1177$

Tinosporin $=3939$

Tirotundin $=20611$

Tirucalla-8,24-diene-3 $\beta$-ol-7-one $=6939$

$\beta$-Tocopherol $=22559$

$\gamma$-Tocopherol $=22561$

$\delta$-Tocopherol $=22560$

Toddaline $=3498$

Toddasin $=14824$ 
Toluene $=14155$

Toluol $=14155$

Tomatin $=21435$

$\alpha$-Tomatine $=21435$

Toralactone 9-gentiobioside $=8649$

Tormentic acid $=6178$

Tormentic acid $\beta$ - $D$-glucopyranosyl ester $=18916$

2-(2-[3-Tormylindolyl])-(3H)-quinazolin-4-one $=2569$

Torquatone in Eucalyptus torquata var. grandiflora $=11745$

Torreyol $=2858$

Toruogenin $=21469$

Tovophyllin B $=6629$

Toxyloxanthone $\mathrm{A}=21503$

Trametenolic acid $\mathrm{B}=21502$

Trametenolic acid 21- $O-\beta-D$-glucopyranoside $=7874$

Triacanthin $=21511$

$6,14 \alpha, 17(R)$-Triacetoxy-5 $\alpha$-(2-acetoxyacetoxy)-3 $\beta$-benzoyloxy- $15 \beta$-hydroxyseget-8(12)-en-9-one $=19657$

$5 \alpha, 8 \alpha, 15 \beta$-Triacetoxy-3 $\alpha$-benzoyloxy- $4 \alpha$-hydroxy-9,14-dioxo-13 $\beta H$-jatropha6(17), $11 E$-diene $=7614$

$3 \beta, 9 \alpha, 15 \beta$-Triacetoxy-7 $\beta$-benzoyloxy-14-oxojatropha- $5 E, 12 E$-diene $=18170$

$3 \beta, 9 \alpha, 15 \beta$-Triacetoxy-7 $\beta$-butyroyloxy-14-oxojatropha- $5 E, 12 E$-diene $=18171$

$2 \alpha, 7 \beta, 13 \alpha$-Triacetoxy- $5 \alpha, 10 \beta$-dihydroxy-9-keto-2(3 $\rightarrow 20)$-abeo-taxane $=$ 20870

$6 S$-[3S,5R,6S-(Triacetoxy)-1Z-heptenyl]-5S-acetoxy-5,6-dihydro-2H-pyran-2one $=16751$

$6 S$-[3S,5R,6S-(triacetoxy)-1Z-heptenyl]-5S-acetoxy- $4 R$-methoxy-3,4,5,6-tetra hydro-4Hpyran-2-one $=16752$

$\beta, 11 \alpha, 15 \beta$-Triacetoxy-7 $\beta$-hydroxy-7 $\alpha, 20$-epoxy-entkaur-16-ene $=11400$

(12S)-6 $\alpha, 12,19$-Triacetoxy-1 $\beta$-hydroxy-4,18-epoxyneoclerod-13(14)-en15,16 -olide $=795$

(12S)-1 $\beta, 6 \alpha, 19$-Triacetoxy-12-hydroxy-4,18-epoxyneoclerod-13(14)-en15,16 -olide $=796$

$\left[2^{\prime} Z, 5\left(1^{\prime}\right) Z\right] 5-\left(4^{\prime} S, 6^{\prime} R, 7^{\prime} S\right.$-triacetoxy-2-octenylidene)-2(5H)-furanone = 16753

Triacetyl-5-decinnamoyltaxicin $\mathrm{I}=10739$

$2 \alpha, 7 \beta, 9 \alpha$-Triacetyl- $2 \alpha, 7 \beta$-dibenzoyl-10 $\beta$-debenzoyltaxayuntin $=20759$

2,10,13-Triacetyl-4(20),11-taxadiene-2,5,7,9,10,13-hexol $=20749$

$n$-Triacontanoic acid $=13687$

10,12,10'-Trichloroisoplagiochin $\mathrm{C}=2181$

Trichodonin $=21572$

Tricholein $=17435$

$7 \beta, 9 \alpha, 10 \beta$-Trideacetyl-1 $\beta$-hydroxybaccatin $\mathrm{I}=20823$

Tridecane $=21610$

5-Tridecyl-1,3-benzenediol $=8997$

1,3,4-Tridehydrofangchinolium $=7754$

rel-(7 $\left.\alpha, 7^{\prime} \alpha, 8 \alpha, 8^{\prime} \alpha, 7^{\prime \prime} \alpha, 8^{\prime \prime} \beta\right)-3^{\prime}, 7^{\prime \prime}: 7,9^{\prime}: 7^{\prime}, 9-T r i e p o x y-4^{\prime} 8^{\prime \prime}$-oxy- $8,8^{\prime}$-sesquineo-

lignan-3, 3",4,4",9"-pentaol and rel-(7 $\left.\alpha, 7^{\prime} \alpha, 8 \alpha, 8^{\prime} \alpha, 7^{\prime \prime} \beta, 8^{\prime \prime} \alpha\right)-3^{\prime}, 7^{\prime \prime}: 7,9^{\prime}: 7^{\prime}, 9-$

Triepoxy-4'8"oxy-8,8'-sesqui- neolignan-3,3",4,4",9"-pentaol) = 11612

rel-((7 $\left.\alpha, 7^{\prime} \alpha, 8 \alpha, 8^{\prime} \alpha, 7^{\prime \prime} \alpha, 8^{\prime \prime} \beta\right)-4^{\prime}, 7^{\prime \prime}: 7,9^{\prime}: 7^{\prime}, 9$-Triepoxy-3',8"-oxy-8, $8^{\prime}$-sesquineolignan-3,3",4,4",9"-pentaol and rel-(7 $\left.\alpha, 7^{\prime} \alpha, 8 \alpha, 8^{\prime} \alpha, 7^{\prime \prime} \beta, 8^{\prime \prime} \alpha\right)-4^{\prime}, 7^{\prime \prime}: 7,9^{\prime}: 7^{\prime}, 9-$ Triepoxy-3',8"-oxy-8,8'-sesquineolignan-3,3",4,4",9"-pentaol) = 17860

Trifolirhizin $=13282$

1,2,6-Tri-O-galloyl-3,4-(R)-hexahydroxydiphenoyl- $\beta$ - $D$-glucose $=3418$
3-(4,7,8-Trihydoxydibenzo-[ $b, f]$ oxepin-1-yl)acrylic acid $=21479$

rel-(8R,10R,20S)-8,10,20-Trihydroxy-9(10 $\rightarrow 20)$-abeo-abieta-9,13-dien-12one $=19429$

$1 \alpha, 11 \beta, 16$-Trihydroxy-3 $\beta$-acetoxy-ent-abieta-7,15(17)-dien-6-one $=616$

$7 \beta, 14 \beta, 15 \beta$-Trihydroxy- $1 \alpha$-acetoxy- $7 \alpha, 20$-epoxy-ent-kaurane $=6793$

$7 \beta, 13 \alpha, 15 \beta$-Trihydroxy- $1 \alpha$-acetoxy-7 $\alpha, 20$-epoxy-ent-kaur-16-ene $=6791$

$7 \beta, 14 \beta, 15 \beta$-Trihydroxy- $1 \alpha$-acetoxy-7 $\alpha, 20$-epoxy-ent-kaur-16-ene $=6792$

$1 \alpha, 7 \beta, 13 \alpha$-Trihydroxy-11 $\beta$-acetoxy-7 $\alpha, 20$-epoxy-ent-kaur-16-en-15-one $=6789$

$3 \beta, 11 \beta, 15 \beta$-Trihydroxy- $6 \alpha$-acetoxy-ent-kaur-16-ene $=13699$

$1 \alpha, 7 \alpha, 14 \beta$-Trihydroxy-12 $\alpha$-acetoxy-ent-kaur-16-en-15-one $=7675$

$1 \alpha, 7 \alpha, 14 \beta$-Trihydroxy-20-acetoxy-ent-kaur-16-en-15-one $=9362$

cis-5 $\alpha-H, 3 \beta, 14 \beta, 17 \beta$-Trihydroxy-12 $\beta$ - $O$-acetyl-20- $O$-benzoyl-pregnane $=6603$

$3 \beta, 13 \beta, 22 \alpha$-Trihydroxy-16 $\alpha$-acetyloxy-oleanane-28-oic acid 3- $O$-[ $\beta$ - $D$-gluco-

pyranosyl- $(1 \rightarrow 2)-\alpha-L$-arabinpyraosyl]-22- $O-\beta$ - $D$-glucopyranoside $=3129$

$3 \alpha, 14 \beta, 17$-Trihydroxy-18-alent-abieta-7(8),15(16)-diene $=6794$

$10 \beta, 12,14$-Trihydroxyalloaromadendrane $14-O-\beta$ - $D$-glucopyranoside $=5122$

$5,7,4^{4}$-Trihydroxy-6- $C$-arabinoside- 8 - $C$-glucoside flavone $=11697$

1,3,5-Trihydroxybenzene $=17174$

1,3,5-Trihydroxybenzene 1- $O-\beta$ - $D$-glucoside $=17171$

3,4,5-Trihydroxybenzoic acid $=8095$

4,5,7-Trihydroxy-1,8-bis(4-hydroxybenzyl)-3-methoxy-6-phenyl-9,10-dihydr

ophenanthrene $=19931$

$1 \alpha, 6 \beta, 15 \beta$-Trihydroxy-6,7-B-seco-ent-kaur-16-en-6,20-epoxy-7,20- $\delta$-olide $=18480$

$4,2^{\prime}, 4^{\prime}$-Trihydroxychalcone $=11504$

$(22 S, 25 S)$-16 $\beta, 22,26$-Trihydroxycholest-4-en-3-one 16-O-[6- $O$-acetyl- $\beta$ - $D$ -

glucopyranosyl]-(1 $\rightarrow 3)-\beta$ - $D$-xylopyranoside $]=16878$

$(22 S, 25 S)$-16 $\beta, 22,26$-Trihydroxycholest-4-en-3-one 16- $O-\beta$ - $D$-glucopyra-

nosyl- $(1 \rightarrow 3)-\beta$ - $D$-xylopyranoside $=16877$

$3 \alpha, 7 \alpha, 12 \alpha$-Trihydroxy coprostanic acid $=4031$

$3,4,5$-Trihydroxy-1-cyclohexene-1-carboxylic acid $=19805$

$3 \beta, 6 \alpha, 12 \beta$-Trihydroxy-20(22),24-dammardiene-6- $O-\alpha-L$-rhamnopyranosyl

$(1 \rightarrow 2)$ - $O-\beta$ - $D$-glucopyranoside $=8411$

$3 \beta, 6 \alpha, 12 \beta$-Trihydroxy-20(22),24-dammar-20(H),24-diene-6- $O-\alpha-L$-rhamno-

pyranosyl $(1 \rightarrow 2)-O-\beta$ - $D$-glucopyranoside $=8415$

$3 \beta, 6 \alpha, 20(S)$-Trihydroxy-dammar-24-ene-12-one 20-O- $\beta$ - $D$-glucopyranoside $=8443$

$1 \alpha, 11 \beta, 15 \beta$-Trihydroxy-3 $\beta, 6 \alpha$-diacetoxy-ent-kaur-16-ene $=13706$

$1 \alpha, 11 \beta, 15 \beta$-Trihydroxy-3 $\beta, 7 \beta$-diacetoxy-ent-kaur-16-ene $=609$

$3 \beta, 11 \beta, 15 \beta$-Trihydroxy- $1 \alpha, 7 \beta$-diacetoxy-ent-kaur-16-ene $=612$

$3 \beta, 11 \beta, 15 \beta$-Trihydroxy- $6 \alpha, 7 \beta$-diacetoxy-ent-kaur-16-ene $=13703$

$1 \alpha, 6 \alpha, 11 \beta$-Trihydroxy-3 $\beta, 7 \beta$-diacetoxy-ent-kaur-16-en-15-one $=613$

Trihydroxydiacetoxytaxadiene $=21705$

$(3 R)-7,2^{\prime}, 4^{\prime}$-Trihydroxy-6,8-di( $\gamma, \gamma$-dimethylallyl)isoflavan $=7356$

( \pm - $-7,2^{\prime}, 4^{\prime}$-Trihydroxy-8,3'-di( $\gamma, \gamma$-dimethylallyl $)$ isoflavanone $=7354$

2,5,8-Trihydroxy-1,3-dimethoxyanthraquinone $=18873$

2',4",2"'-Trihydroxy-4',4"'-dimethoxy-4-O-5"'-bichalcone = 18820

3,5,7-Trihydroxy-1,2-dimethoxy-9,10-dihydrophenanthrene $=3890$

$5,3,3^{\prime}$-Trihydroxy-7,4'-dimethoxyflavanone $=6338$

3,5,3'-Trihydroxy-7,2'-dimethoxyflavone $=11163$

3,5,3'-Trihydroxy-7,4'-dimethoxyflavone $=16092$

$3,7,4^{\prime}$-Trihydroxy-3',5'-dimethoxyflavone $=12567$

$5,7,2^{\prime}$-Trihydroxy-3,6-dimethoxyflavone $=11160$

$5,7,4$ '-Trihydroxy-3',5'-dimethoxyl-8-prenylflavone $=2144$

2,6,7-Trihydroxy-1,8-dimethoxy-3-methyl-9(10H)-anthracenone-10-oxyde- 
canoate $=12236$

1,4,7-Trihydroxy-6,8-dimethoxy-1-methylanthraquinone $=18869$

3,5,7-Trihydroxy-1,2-dimethoxyphenanthrene $=3891$

1,3,6-Trihydroxy-2,7-dimethoxyxanthone $=16109$

1,3,7-Trihydroxy-2,8-dimethoxyxanthone $=19645$

$7,2^{\prime}, 4^{\prime}$-Trihydroxy-(3,3-dimethylally)isoflavone $=7637$

1,3,5-Trihydroxy-4- $(\gamma, \gamma$-dimethylallyl)acridone $=21763$

5,7,2'-Trihydroxy-6-(3,3-dimethylallyl)-[(6",6"-dimethylpyrano(2",3":4',5')]isoflavone $=12292$

5,7,4'-Trihydroxy-8-(1,1-dimethylallyl)flavanone $=22178$

5,2 ',4'-Trihydroxy-6-( $\gamma, \gamma$-dimethylallyl)-3"'-hydroxy-2"',2"'-dimethyldihydropyrano[5"',6"';8,7]isoflavone $=7319$

5b,7,9-Trihydroxy-2,2-dimethyl-8-(3-methyl-2-butenyl)-5b,11a-dihydro-

$2 H, 6 H$-chromeno[ $\left.6^{\prime}, 7^{\prime}: 4,5\right]$ furo[2,3b]chromen-6-one $=12295$

1,3,6-Trihydroxydimethylpyrano-4-[(2E)-3,7-dimethylocta-2,6-dienyl]xanthone $=22525$

1,7,8-Trihydroxy-2,2-dimethylpyrano[5',6': 3,4] xanthone $=8555$

$(7 \beta, 15 \alpha, 23 S, 24 E)-7,15,23$-Trihydroxy-3,11-dioxolanosta-8,24-dien-26-oic acid $=8177$

3,7,23-Trihydroxy-11,15-dioxolanosta-8,24-dien-26-oic acid $=8179$

$5,7,4$ '-Trihydroxy-6,8-diprenylisoflavone $=6496$

5,7,4'-Trihydroxy-6,5'-diprenylisoflavone $=13088$

$11 \beta, 13 \alpha, 15 \alpha$-Trihydroxy-entkaur-16-en-3 $\alpha$ - $\beta$ - $D$-glucoside $=11399$

$3 \alpha, 11 \beta, 13 \alpha$-Trihydroxy-entkaur-16-en-15-one $=11397$

$2 \alpha, 3 \alpha, 18$-Trihydroxy-3 $\beta, 20$-epoxybeyer-15-ene $=7690$

2,20(S),22-Trihydroxy-16 $\alpha, 23(S)$-epoxycucurbita-5,24-diene-3,11-dione

2-O- $\beta$-glucopyranoside $=12216$

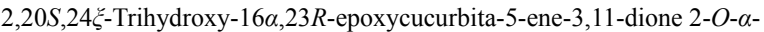
rhamnopyranosyl- $(1 \rightarrow 2)-\beta$-glucopyranoside $=12217$

$3 \beta, 6 \alpha, 20(S)$-Trihydroxy-12 $\beta, 23$-epoxy-dammar-24-ene $20-O-\beta$ - $D$-glucopyranoside $=8444$

$3 \beta, 20,23$-Trihydroxy-16,30-epoxy-dammar-24-ene-23-O- $\beta$ - $D$-glucopyranosyl-3$O$ - $\beta$-D-xylopyranosyl $(1 \rightarrow 2)$-[ $\beta$ - $D$-glucopyranosyl $(1 \rightarrow 6)]-\beta$ - $D$-glucopyra-

$\operatorname{nosyl}(1 \rightarrow 3)-[\alpha-L$-rhamnopyranosyl $(1 \rightarrow 2)]-\alpha-L$-arabinopyranoside $=11915$

$6 \beta, 12 \alpha, 15 \beta$-Trihydroxy-7 $\alpha, 20$-epoxy-ent-kaur-16-ene $=16178$

$1 \beta, 6 \beta, 7 \beta$-Trihydroxy- $7 \alpha, 20$-epoxy-ent-kaur-16-en- 15 -one $=22793$

$3 \beta, 16 \alpha, 29$-Trihydroxy-13,28-epoxy-oleanane-3-O- $\beta$ - $D$-glucopyranosyl-

$(1 \rightarrow 2)$-(6-methyl ester)- $\beta$ - $D$-glucuronopyranoside $=4699$

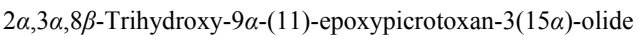

8 - $O-\beta$ - $D$-glucopyranoside $=5118$

$(3 \alpha, 5 \beta, 7 \alpha, 12 \alpha, 24 \xi)-3,7,12$-Trihydroxy-ergost-22-en-28-oic acid $=21693$

$1 \beta, 4 \beta, 6 \beta$-Trihydroxy-cis-eudesmane-6-O- $\beta$ - $D$-glucopyranoside $=16150$

$5,7,4^{\prime}$-Trihydroxyflavanone $=15279$

$7,3^{\prime}, 4^{\prime}$-Trihydroxyflavanone $=2785$

5,7,4'-Trihydroxyflavone 6 - $C$-[ $\alpha$-rhamnopyranosyl-( $(1 \rightarrow 6)]-\beta$-glucopyranoside $=6623$

5,7,4'-Trihydroxy-6-[1-hydroxy-2-methylbuten-2-yl]isoflavone $=11434$

$\left\{5,7,5^{\prime}\right.$-Trihydroxy-6-(3-hydroxy-3-methylbutyl)-8- $(\gamma, \gamma$-dimethylallyl)-

[6"'",6"''-dimethylpyrano-(2'"',3"'":4',3')]\}-coumaronochromone = 7889

5,7,2'-Trihydroxy-6-(3-hydroxy-3-methylbutyl)-[(6",6"-dimethylpyrano

$\left.\left(2^{\prime \prime}, 3^{\prime \prime}: 4^{\prime}, 5^{\prime}\right)\right]$-isoflavone $=12293$

1,3,7-Trihydroxy-8-(3-hydroxy-3-methylbutyl)xanthone $=15577$

$2^{\prime}, 4$ ', 7-Trihydroxyisoflavene $=9189$

$3 \beta, 15 \xi, 16$-trihydroxy isopimaric acid $=22739$
$1 \alpha, 7 \alpha, 14 \beta$-Trihydroxy-ent-kaur-16-en-15-one $=12125$

(7S,8R)-3,9,9'-Trihydroxyl-3-methoxyl-7,8-dihydrobenzofunan-1'-propanolneoligan-4-O- $\alpha$ - $L$-rhamnopyranoside $=13584$

( $7 S, 8 R) 3,4,9$ '-Trihydroxyl-3-methyoxyl-7,8-dihydrobenzofunan-1'-propanolneoligan-9-O- $\alpha-L$-rhamnopyranoside $=13583$

$(1 S, 3 S, 5 R, 6 S, 9 R)-3,9,12$-Trihydroxymegastigmane 3- $O-\beta-D$-glucopyranoside $=22127$ $(3 S, 5 R, 6 S, 9 S)-3,6,9$-Trihydroxymegastigman-7-ene 3-O- $\beta$ - $D$-glucopyranoside $=22125$ $(3 S, 4 R, 9 R)-3,4,6$-Trihydroxymegastigman-5-ene 3-O- $\beta$ - $D$-glucopyranoside $=22128$ $(3 S, 5 R, 6 S, 9 S)-3,6,9$-Trihydroxymegastigman-7-ene 9-O- $\beta$ - $D$-glucopyranoside $=22126$ $(3 S, 4 R, 9 \xi)-3,4,9$-Trihydroxymegastigman-5-ene 3-O-primeveroside $=17526$

1,3,6-Trihydroxy-7-methoxy-2-(4-acetoxy-3-methyl-2-butenyl)-8-(3,7-dimeth yl-2,6-octadienyl) $x a n t h o n e=4199$

1,8,10-Trihydroxy-9-methoxy-[1]benzopyrano-[3,2-c][2]-benzopyran-7(5H)one $=11166$

1,3,6-Trihydroxy-7-methoxy-2,5-bis(3-methyl-2-butenyl)-8-(3,7-dimethyl2,6 -octadienyl) xanthone $=4195$

1,6,7-Trihydroxy-3-methoxy-2,8-bis(3-methyl-2-butenyl)xanthone $=6627$

$5,3^{\prime}, 5^{\prime}$-Trihydroxy-7-methoxy dihydroflavone $=2521$

5,3',4'-Trihydroxy-7-methoxy-6-(6,6-dimethyl-2-methylene-cyclohexylmethyl)flavone $=22184$

(2S)-2',5,6'-Trihydroxy-7-methoxyflavanone $=19580$

(2S)-2',5,6'-trihydroxy-7-methoxyflavanone-2'- $O-\beta-D$-glucopyranoside $=19581$

$5,7,2^{\prime}$-Trihydroxy-8-methoxyflavone $=19588$

$5,7,3^{\prime}$-Trihydroxy-4'-methoxyflavone $=6452$

$5,7,4^{\prime}$-Trihydroxy-8-methoxyflavone $=10829$

$5,7,4^{\prime}$-Trihydroxy-3'-methoxyisoflavone $=14638$

3,4',5-Trihydroxy-7-methoxy-8-isopentenylflavone $=11222$

3,5,7-Trihydroxy-4'-methoxyl-8-prenylflavone-3-O- $\alpha$ - $L$-rhamnopyranosyl-

$(1 \rightarrow 4)-\beta-D$-glucopyranoside $=2143$

1,5,6-Trihydroxy-3-methoxy-2-(3-methyl-2-butenyl)-4-(1,1-dimethylallyl) xanthone $=15578$

5,7,2'-Trihydroxy-4'-methoxy-5'-(3"-methylbut-2"-enyl)isoflavanone $=13265$ $5,2^{\prime}, 4$ '-Trihydroxy-7-methoxy-6-(3-methylbut-2-enyl)isoflavone $=14568$

$3 \beta, 16 \beta, 28$-Trihydroxy-11- $\alpha-\beta$-methoxy-olean-12-ene-3-O- $\beta$ - $D$-glucosyl-

$(1 \rightarrow 3)-\beta-D$-fucoside $=19162$

4',5,7-Trihydroxy-3-methoxy-6-prenylflavone $=21451$

(7S,8R)-3',4,9-Trihydroxy-4-methoxy-9-O-shikimoyl-7,8-dihydrobenzofuran-

$1^{\prime}$-propylneolignan $=13582$

1,2,8-Trihydroxy-6-methoxyxanthone $=20510$

$1,3,8$-Trihydroxy-6-methylanthraquinone $=6776$

5,7,4'-Trihydroxy-8-(3"'-methylbut-2"'-enyl)-6-(2"-hydroxy-3"-methylbut3 "enyl) isoflavone $=11415$

6,8,12-Trihydroxy-7-(3-methyl-2-butenyl)-2-methyl-2-(4-methyl-3-pentenyl) pyrano- $\left(2^{\prime}, 3^{\prime}: 7,8\right)$ xanthone $=4198$

2,5,8-Trihydroxy-3-(3-methyl-2-butenyl)-1,4-naphthoquinone $=10709$

1,8,11-Trihydroxy-9,10-methylenedioxy-[1]benzopyrano-[3,2-c][2]-benzopyran-7(5H)-one $=11170$

$3 \alpha, 4 \beta, 7 \beta$-Trihydroxy-4 $\alpha$-methylergosta-8,24(28)-dien-11-on-26-oic acid = 1351

1,2,3-Trihydroxy-6-methyl-8-methoxyanthraquinone $=7660$

1,3,5-Trihydroxy-2-methyl-6-methoxyanthraquinone $=12237$

5,7,10-Trihydroxy-2-methyl-2-(4-methylpent-3-enyl)[2H,6H]pyrano[3,2-b]

xanthen-6-one $=20019$

1,5,8-Trihydroxy-6'-methyl-6'-(4-methylpent-3-enyl)-pyrano[2',3':3,4]xanth- 
one $=2135$

$3 \beta, 20 \alpha, 24$-Trihydroxy-29-norolean-12-en-28-oic acid 23- $O-\beta$ - $D$-fucopyranosyl-

$(1 \rightarrow 2)$ - $[\alpha$ - $L$-arabinopyranosyl- $(1 \rightarrow 3)]-\beta$ - $D$-glucopyranoside $=7724$

$3 \beta, 20 \alpha, 24$-Trihydroxy-29-norolean-12-en-28-oic acid 24- $O$ - $\beta$ - $D$-glucopyranoside $=7631$

$3 \beta, 20 \alpha, 24$-Trihydroxy-29-norolean-12-en-28-oic acid 24- $O$ - $[\alpha-L$-rhamnopyra$\operatorname{nosyl}(1 \rightarrow 4)]-6$ '- $O$-acetyl- $\beta$ - $D$-glucopyranoside $=7632$

$3 \beta, 23,28$-Trihydroxyoleana-11,13(18)-dien-3- $O-\beta$ - $D$-glucopyranosyl-( $1 \rightarrow 3$ )$\beta$ - $D$-fucopyranoside $=19152$

$3 \beta, 16 \alpha, 24$-Trihydroxyoleana-12-en-28-oic acid-3- $O$-(6'-butyryl)- $\beta$ - $D$-glucopyranoside $=22622$

2,3,27-Trihydroxy-12-oleanene-23,28-dioic acid 3- $O-\beta$ - $D$-glucopyranoside, $28-O$ - $[\beta$ - $D$-galactopyranosyl- $(1 \rightarrow 4)-\beta$ - $D$-xylopyranosyl- $(1 \rightarrow 4)-\alpha-L$ rhamnopyranosyl-( $1 \rightarrow 2)$-[3,4-dimethoxycinnamoyl-( $1 \rightarrow 4)]-\alpha-L$-fucopyra- nosyl] ester $=19715$

$2 \alpha, 3 \alpha, 24$-Trihydroxy-olean-12-ene-28-oic acid-28- $O-\beta$ - $D$-glucopyranosyl ester $=22621$

$3 \beta, 16 \alpha, 28$-Trihydroxy-olean-12-en-3- $O$ - $[\beta$ - $D$-glucopyranosyl-( $(1 \rightarrow 2)-\beta$ - $D$ glucuronopyranosyl]-28- $O-\beta$ - $D$-glucuronopyranoside $=4700$

$1 \alpha, 3 \beta, 23$-Trihydroxy-olean-12-en-29-oate-23-O- $\alpha-L-3,4$-acetyl-29-dirhamnopyranoside $=10228$

$1 \alpha, 3 \beta, 23$-Trihydroxy-olean-12-en-29-oate-23- $O$ - $\alpha$ - $L$-4-acetylrhamnopyranoside $=10226$

$1 \alpha, 3 \beta, 23$-Trihydroxy-olean-12-en-29-oate-23-O- $\alpha$ - $L$-3,4-diacetylrhamnopyranoside $=9741$

$1 \alpha, 3 \beta, 23$-Trihydroxy-olean-12-en-29-oate-23-O- $\alpha$-1-4-acetyl-29-dirhamnopyranoside $=10227$

$2 \alpha, 3 \beta, 19 \alpha$-Trihydroxy-12-oleanen-28-oic acid $=1734$

$3 \beta, 21 \alpha, 24$-Trihydroxy-olean-12-en-30-oic acid $=8858$

$2 \alpha, 3 \alpha, 19 \alpha$-Trihydroxy-olean-12-en-28-oic acid 28-O- $\beta$ - $D$-glucopyranosyl- $(1 \rightarrow 6)-\beta-D$-glucopyranoside $=18851$

$2 \alpha, 3 \beta, 23$-Trihydroxyolean-12-en-28-oic acid-3- $O-\beta$ - $D$-glucopyranosyl-28-

$O-\beta$ - $D$-glucopyranosyl- $(1 \rightarrow 6)-\beta$ - $D$-glucopyranosyl ester $=20287$

$3 \beta, 6 \beta, 23$-Trihydroxyolean-12-en-28-oic acid 28- $O$ - $\alpha$ - $L$-rhamnopyranosyl

$(1 \rightarrow 4)-\beta$ - $D$-glucopyranosyl- $(1 \rightarrow 6)-\beta$ - $D$-glucopyranoside $=3397$

$3 \beta, 15 \alpha, 23$-Trihydroxy-olean-12-en-16-one $=7273$

1-(2,4,6-Trihydroxyphenyl)-11-(2,6-dihydroxyphenyl)undecan-1-one $=1648$

2',4',6'-Trihydroxyphenyl-(26Z)-dotriacontene-1-one $=20962$

1-(2,4,6-Trihydroxyphenyl)-11-(2-hydroxyphenyl)undecan-1-one = 1649

2',4',6'-Trihydroxyphenyl-(24Z)-triacontene-1-one $=20961$

$6 \alpha, 10,12$-Trihydroxypicrotoxane $10-O-\beta$ - $D$-glucopyranoside $=5121$

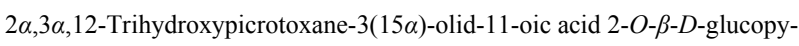

ranoside $=5117$

ent-(15R),16,19-Trihydroxypimar-8(14)-ene 19- $O-\beta$ - $D$-glucopyranoside $=18176$ $1 \alpha, 2 \beta, 3 \alpha$-Trihydroxypregna-4,7,16-trien-12,20-dione $=21003$

$2 \beta, 3 \alpha, 12 \beta$-Trihydroxypregna-4,7,16-trien-20-one $=20386$

$3 \beta, 8 \beta, 14 \beta$-Trihydroxypregn-5-en-20-one $=7992$

$2 ', 4,4^{\prime}$-Trihydroxy-3-prenylchalcone $=12751$

$5,7,4^{\prime}$-Trihydroxy-6-prenyl-isoflavone $=22667$

$5,7,4^{\prime}$-Trihydroxy-8-prenylisoflavone $=13107$

1,4,8-Trihydroxy-2-prenylxanthone $=2136$

2,6,8-Trihydroxy-purine $=22251$

$3 \alpha, 21 \beta, 24$-Trihydroxyserrat-14-en-16-one $=13177$
$(5 \alpha, 22 R, 23 R, 25 S)-3 \beta, 23,27-$ Trihydroxyspirosolane $=11418$

$(2 \alpha, 3 \beta, 5 \alpha, 25 S)-2,3,27-T r i h y d r o x y s p i r o s t a n e ~ 3-O-\alpha$ - $L$-rhamnopyranoyl-

$(1 \rightarrow 2)-O$ - $[\alpha-L$-rhamnopyranoyl- $(1 \rightarrow 4)]-\beta$ - $D$-glucopyranoside $=22079$

$(23 S, 25 S)-3 \beta, 23,27$-Trihydroxyspirost-5-en-12-one 3- $O$ - $\beta$ - $D$-glucopyranosyl-

$(1 \rightarrow 4)-\beta$ - $D$-fucopyranoside $=17645$

$3,5,4^{\prime}$-Trihydroxystilbene $=18643$

3,5,4'-Trihydroxystilbene-4'-(6"-galloyl)-glucoside $=18648$

$3,5,4$ '-Trihydroxystilene-4'-glucoside $=18650$

3,6,7-Trihydroxy-4,5,6,7-tetrahydro-3-butyl-phthalide $=2794$

(4S)-4,5,8-Trihydroxy- $\alpha$-tetralone $4-O-\beta$ - $D$-glucopyranoside $=11901$

(4S)-4,5,8-Trihydroxy- $\alpha$-tetralone 5-O- $\beta$ - $D$-glucopyranoside $=11902$

$1 \beta, 7 \beta, 9 \alpha$-Trihydroxy-5 $\alpha, 10 \beta, 13 \alpha$-triacetoxytaxa-4(20),11-diene $=20756$

$4,4^{\prime \prime}, 6^{\prime}$-Trihydroxy-2',3',5'-tribenzoyloxy [1,1':4',1"-terphenyl $]=21303$

9,4', $9^{\prime}$-Trihydroxy 4,5,3'-trimethoxy aryltetralin lignan $=19456$

5,4',5"-Trihydroxy-7,7",4"'-trimethoxy-[3 $\rightarrow 6$ "]-biflavone = 20315

$3,5,3^{\prime}$-Trihydroxy-6,7,4'-trimethoxy flavone $=7583$

$5,2^{\prime}, 4^{\prime}$-Trihydroxy-6,7,5'-trimethoxy flavone $=1653$

$5,2^{\prime}, 6^{\prime}$-Trihydroxy-3,6,7-trimethoxyflavone $=11162$

$5,6,3$ '-Trihydroxy-3,7,4'-trimethoxyflavone $=16440$

$5,7,3$ '-Trihydroxy-6,4',5'-trimethoxyisoflavone $=11154$

1,3,8-Trihydroxy-2,4,5-tris-(3,3-dimethylallyl)-6-methylanthrone $=9241$

1,3,8-Trihydroxy-4,5,7-tris-(3,3-dimethylallyl)-6-methyl-anthrone $=9240$

$3 \beta, 21 \beta, 22 \beta$-Trihydroxyurs-12-en-28-al $=4043$

$2 \beta, 3 \beta, 19 \alpha$-Trihydroxy-urs-12-en-24,28-dioic acid-24- $O$ - $\beta$ - $D$-glucopy-

ranosyl-28- $O-\beta$ - $D$-glucopyranosyl diester $=20290$

$3 \beta, 11 \alpha, 21 \alpha$-Trihydroxyurs-12-ene $=19220$

$2 \alpha, 3 \alpha, 19 \alpha$-Trihydroxyurs-12-en-28-oic acid $=7657$

$2 \alpha, 3 \beta, 19 \alpha$-Trihydroxyurs-12-en-28-oic acid 28- $\beta$ - $D$-glucopyranosyl ester $=18916$

$2 \alpha, 3 \beta, 19 \alpha$-Trihydroxy-urs-12-en-28-oic acid-3- $O-\beta$ - $D$-glucopyranosyl-

28 - $O$ - $\beta$ - $D$-glucopyranosyl- $(1 \rightarrow 2)-\beta$ - $D$-glucopyranosyl ester $=20289$

$2 \alpha, 3 \beta, 23$-Trihydroxy-urs-12-en-28-oic acid-3-O- $\beta$ - $D$-glucopyranosyl-

28 - $O$ - $\beta$ - $D$-glucopyranosyl- $(1 \rightarrow 6)-\beta$ - $D$-glucopyranosylester $=20288$

$2 \alpha, 3 \beta, 23$-Trihydroxyurs-12-en-28-oic acid $O$ - $\alpha$ - $L$-rhamnopyranosyl-( $1 \rightarrow 4)$ -

$O$ - $\beta$ - $D$-glucopyranosyl- $(1 \rightarrow 6)-\beta$ - $D$-glucopyranosyl ester $=1854$

$11 \alpha, 16 \alpha, 28$-trihydroxyurs-12-en-3 $\beta$-yl $\alpha$ - $L$-rhamnopyranosyl-( $1 \rightarrow 2)-\beta$ - $D$-glucopyranosyl- $(1 \rightarrow 2)-\beta$ - $D$-fucopyranoside $=18946$

$11 \alpha, 16 \alpha, 28$-trihydroxyurs-12-en-3 $\beta$-yl $\beta$ - $D$-xylopyranosyl-( $1 \rightarrow 2)$ - $\beta$ - $D$-gluco-

pyranosyl- $(1 \rightarrow 2)-\beta$ - $D$-fucopyranoside $=18945$

$1,3,7$-Trihydroxyxanthone $=8306$

1,5,6-Trihydroxyxanthone $=13809$

Trilinoleyl glyceride $=8816$

Trillin $=6443$

Trilloside $\mathrm{A}=5040$

1,2,3-Trimethoxy-10-acetoxymethylacridone $=21420$

1,2,3-Trimethoxyacridone $=21419$

$5,7,4^{\prime}$-Trimethoxyapigenin $=21913$

$N$-(3',4',5'-Trimethoxycinnamoyl)- $\Delta^{3}$-pyridin-2-one $=17472$

$N$-(2,4,5-Trimethoxycinnamoyl)pyrrolidine $=19370$

$3,7,8$-Trimethoxycoumarin $=19467$

6,6',7-Trimethoxy-2,2'-dimethyloxyacanthan-12'-ol = 16439

7,2',6'-Trimethoxy-6",6"-dimethylpyrano-(3',4':2",3")-chalcone = 14075

$3 \beta, 4 \beta, 5$-Trimethoxy-6",6"-dimethyl-2H-pyrano-(2",3":7,6)-flavan = 14105

$5,7,4^{\prime}$-Trimethoxyflavanone $=15285$ 
$\left(7 S, 8 S, 7^{\prime} S, 8^{\prime} R\right)-3,4,3^{\prime}-$ Trimethoxy-4'-hydroxy-7,7'-epoxylignan $=8037$

1-2,3-Trimethoxy-10-methoxymethylacridone $=21422$

1,2,4-Trimethoxy-5-(E-3'-methyloxiranyl) benzene $=7056$

1,2,4-Trimethoxyphenyl-5-aldehyde $=1840$

3,4,5-Trimethoxyphenyl-1- $O-\beta$ - $D$-(5- $O$-syringoyl)-apiofuranosyl-( $1 \rightarrow 2)$ -

$\beta$ - $D$-glucopyranoside $=855$

3,4,5-Trimethoxyphenyl- $O$-6' $-O$-vanilloyl- $\beta$ - $D$-glucopyranoside $=21035$

$2,4,5$-Trimethoxypropenyl benzene $=1835$

$3,4,5$-Trimethoxytoluene $=21919$

$3,4,5$-Trimethoxy-xanthone-1- $O-\beta$ - $D$-glucopyranosyl-( $1 \rightarrow 6)-\beta$ - $D$-xylopyranoside $=22388$

1,7,7-Trimethyl-acetate-endobicyclo[2.2.1] heptan-2-ol $=336$

7,4',4'"-Tri- $O$-methyl amentoflavone $=19514$

$N$-Trimethylamine $=21939$

1,3,3-Trimethylbicyclo[2.2.1] heptan-2-one $=7751$

cis-1,2,2-Trimethyl-1,3-cyclopentanedicarboxylic acid $=3051$

1,3,7-Trimethyl-2,6-dioxopurine $=2892$

2,6,10-Trimethyl-2,6,9,11-dodecatetraenal $=19927$

3,7,11-Trimethyl-1,6,10-dodecatrien-3-ol $=15500$

3,7,11-Trimethyl-2,6,10-dodecatrien-1-ol $=7734$

2,6,11-Trimethyl-dodesa-2,6,10-triene $=20666$

$3,3^{\prime}, 4^{\prime}$-Tri- $O$-methylellagic acid $=21955$

1,7,7-Trimethyl endo-bicyclo[2.2.1] heptan-2-ol $=2550$

$3-O-(1 ", 8 ", 14$ "-Trimethylhexadecanyl)naringenin $=15124$

3,5,5-Trimethyl-2-hydroxy-1,4-cyclohexadion-2-ene $=10807$

6- $N$-Trimethyl- $L$-lysine betaine $=12457$

1,3,3-Trimethyl-7-(5-methylcyclopenta-1,4-dien-1-yl)-2-oxabicyclo[2,2,1] heptane $=20886$

2,2,6-Trimethyl-3-[(E)(2-methylcyclopenta-2-en-1-ylidene)methyl]-3,4-dihydro- $2 H$-pyran $=20888$

1,1,3a-Trimethyl-6-methylene-1,1a,2,3,3a,3b,4,5,6,6b-decahydrocyclopenta $[2,3]$ cyclopropa-[1,2-a]cyclopropa $[c]$-benzene $=15143$

2,2,9-Trimethyl-6-methylene-3,4,5,6,6a,7-hexahydro-2H-3,9a-methanocyclopent $[b]$ oxocine $=9040$

1,1,4-Trimethyl-7-methylene-1a,2,3,5,6,7,7a,7b-octahydro- $1 H$-cyclopropa $[e]$ azulene $=1755$

1,1,7-Trimethyl-4-methylene-1a,2,3,4,4a,5,6,7b-octahydro- $1 H$-cyclopropa[e] azulene $=1757$

1,1,3a-Trimethyl-6-methylene-5-oxo-1,1a,2,3,3a,3b,4,-5,6,6b-decahydrocyclopenta[2,3]cyclopropa[1,2- $a]$ cyclopropa $[c]$ benzene $=15144$

3,4,5-Trimethyl-2-(methylsulfinyloxymethyl) thiophene $=7850$

1,3,3-Trimethyl-2-norbornanol $=7752$

1,3,3-Trimethyl-2-oxabicyclo[2.2.2] octane $=3689$

4-(2,2,6-Trimethyl-1-oxaspiro[4,4]non-6-en-3-yl)-butan-2-one $=20887$

$4,4,14 \alpha$-Trimethyl-24-oxo-5 $\alpha$-chol-8-en-21-oic acid dimethylacetal $=8575$

(3a $S, 5 R, 7 \mathrm{a} R)-4,4,7 \mathrm{a}-$ Trimethyl-2-oxo-2,3,3a,4,5,7a-hexahydrobenzo[b] furan-5-yl 3,4-dimethyl-3-pentenoate $=20967$

2,2,4-Trimethyl-6-(1-oxo-3-phenylprop-2-enyl)cyclohexane-1,3,5-trione $=3473$

6,10,14-Trimethyl-pentadecan-2-one $=9500$

$3,6,7$-Trimethylquercetagenin $=3624$

$3,6,4^{\prime}$-Tri- $O$-methylquercetagetin-7- $O-\beta-D$-glucopyranoside $=3389$

3,5,12a-Trimethyl-2,5,5a,12a-tetrahydro- $1 H$-naphtho[2',3':4,5]furo[2,3- $b$ ] azepin-2-one $=6598$

3,4,5-Trimethyl-2-thiophenecarboxylic acid $=7849$

$(1 S, 9 S)-4 \alpha, 11,11$-Trimethyl-2 $\alpha, 5 \alpha, 6 \beta$-trihydroxytricyclo[ $\left[5,4,0,0^{2,5}\right]$ undec-3en-7-one $=7737$

(rel-5S,6S,8R,9R,10S)-14,15,16-Trinor-13,9-labdanolide $=22578$

Triox $=21940$

Tripterifordine $=21990$

Tripterine $=3368$

Tripterygic acid $\mathrm{A}=22025$

Triptoditerpenic acid B $=21993$

Triptophenolide $=10899$

Triptophenolide methyl ether $=10900$

Trisdesaspidin $=22032$

Trisphaerine $=9547$

Trispherine $=9547$

Tropaeolin $=2298$

3-Tropanol $=22050$

Tsuzuic aicd $=21045$

Tubeimoside $\mathrm{I}=22072$

Tubeimoside II $=22073$

Tubeimoside III $=22074$

Tulipalin $\mathrm{A}=22102$

$\beta$-Turmerone $=22123$

Tussilagin $=22144$

\section{U}

Ubiquitous 1,2-dilinolenoyl-3-galactopyranosylglycerol = 14924

Umbellatine $=2303$

Umbelliferone 7-O- $\beta$ - $D$-glucopyranoside $=20003$

Uncarine $\mathrm{A}=11426$

Uncarine $\mathrm{C}=18114$

Uncarine $\mathrm{E}=11636$

Undeca-2E,4Z-dien-7,9-diynoic acid isobutylamide $=15445$

Undecanol $=22226$

Undecylenic acid $=22221$

Untriacontane $=9363$

(2S)-6-(1,4-Ureylenebutyl)-5,7-dihydroxyflavanone $=1541$

(2S)-8-(1,4-Ureylenebutyl)-5,7-dihydroxyflavanone $=11233$

5 -Uridylic acid $=22254$

Ursa-12-ene-11-one-3-ol octocosate $=22262$

12-Ursene-3 $\beta, 27$-diol $=15911$

Urs-12-en- $3 \beta$-ol $=1110$

$\beta$-Ursolic acid $=22270$

Usaramine $=15018$

Ushinsunine $=14829$

Usninic acid $=22282$

$17 \beta$-Uzarigenin-3- $O$ - $\beta$-glucopyranosyl- $(1 \rightarrow 6)$ - $\beta$-glucopyranosyl- $(1 \rightarrow 4)$ $\beta$-theveto-pyranosyl- $(1 \rightarrow 4)$ - $\beta$-cymaropyranoside $=7698$

\section{V}

Valerianae alkaloid $\mathrm{A}=10601$

Valerianol $=12375$ 
Valeric acid $=16884$

Valeric aldehyde $=16876$

Valtrate $=22312$

Valtratum $=22312$

Vanillosmin $=7226$

6-Vanilloylcatalpol $=17345$

3- $O$-Vanillylceanothic acid $=10383$

Vasicine $=16770$

$L$-Vasicinone $=22350$

$\mathrm{VCR}=22497$

Veneniferin $=3416$

Venoterpine $=8291$

$(-)$-Veraguensin $=22383$

Veratric acid $=6201$

Verbascoside $=580$

Verbenalin $=4060$

Verticine $=16773$

Verticinone $=16774$

Vestitol[6 $\rightarrow 9 " ; 7 O \rightarrow 7$ "] obtusaquinone $=15353$

Vicenin $2=2455$

$\beta$-Vicianosyl-3-quercetin $=18327$

Viguiestenin $=22475$

Vilmorrianine D = 19107

Vincaleukoblastine $=22487$

Vincoside lactam $=22496$

Vindolidine $=22501$

$7 \alpha, 8 \alpha-c i s-\varepsilon$-Viniferin $=22505$

Vinleurosine $=12734$

Vinrosidine $=12733$

1-Vinyl-4,8-dimethoxy- $\beta$-carboline $=12335$

Vinylformic acid $=578$

Vinyl sulfide $=6531$

Violanin $=15289$

Virolongin $=22528$

Viscidone $=416$

Viscidulin $\mathrm{I}=16848$

Visnamine $=22552$

Vitamin A = 18656

Vitamin B $=9458$

Vitamin $\mathrm{B}_{2}=18834$

Vitamin $B_{6}=18261$

Vitamin $B_{7}=2395$

Vitamin C $=1845$

Vitamin $\mathrm{E}=21415$

Vitamin $G=18834$

Vitexicarpin $=3300$

Vitexin 7-methyl ether $=11724$

$(+)$-Vittatine $=4241$

Vladinol A $=13588$

$(6 S, 7 E, 9 R)$-Vomifoliol-9- $O-\beta$ - $D$-xylopyranosyl- $(1 \rightarrow 6)-O-\beta$ - $D$-glucopyranoside $=22617$

Vulgarin $=20730$

Vulpic acid $=22625$

Wallichiene $=9642$

Wallichienene $=15405$

Wallichinin $=22628$

Warabisterone $=17703$

Weedone $=4832$

$(+)$-Wikstromol $=15805$

$(-)$-Wikstromol $=15806$

Wikstrotoxin $=19902$

Withametelin $=4673$

Wogonin-7-O-glucoside $=22720$

Wuweizi alcohol B = 8906

Wuweizichun A = 19473

Wuweizi ester $\mathrm{A}=8908$

Wuweizi ester $\mathrm{B}=8907$

Wuweizisu $\mathrm{A}=5213$

Wuweizisu $\mathrm{B}=5178$

Wuweizisu C $=19497$

Xanthalongin $=21444$

Xanthopurpurin $=18224$

Xanthoxylin $\mathrm{N}=22777$

Xantofyl palmitate $=9290$

Xerophilusin $\mathrm{H}=6785$

$\mathrm{XH}-\mathrm{I}=22767$

$3 \beta$ - $O$-[ $\beta$ - $D$-Xylopyranosyl $(1 \rightarrow 4)$-[2- $O$-acetyl]- $\beta$ - $D$-glucuronopyranosyl $]-28$ $O$ - $[\beta$ - $D$-glucopyranosyl $]$-morolic acid $=20539$

3-O-[ $\beta$ - $D$-Xylopyranosyl-( $1 \rightarrow 4)$-[2- $O$-acetyl]- $\beta$ - $D$-glucuronopyranosyl]-28-

$O$-[ $\beta$ - $D$-glucopyranosyl $]$-oleanolic acid $=20531$

3-O-[ $\beta$ - $D$-Xylopyranosyl-( $1 \rightarrow 4)$-[3- $O$-acetyl]- $\beta$ - $D$-glucuronopyranosyl]-28-

$O$-[ $\beta$ - $D$-glucopyranosyl $]$-oleanolic acid $=20530$

3-O-[ $\beta$ - $D$-Xylopyranosyl-( $1 \rightarrow 2)$ - $\alpha$ - $L$-arabinopyranosyl-( $1 \rightarrow 6)$-2-acetamido-

2-deoxy- $\beta$ - $D$-glucopyranosyl] -21-O-\{(6S)-2-trans-2-hydroxymethyl-6-

methyl-6-O-[4-O-((6S)-2-trans-2,6-dimethyl-6- $O$-(6-deoxy- $\beta$ - $D$-glucopy

ranosyl)-2,7-octadienoyl)-6-deoxy- $\beta$ - $D$-glucopyranosyl]-2,-7-octadieno-

yl $\}$-acacic acid $28-O-\beta$-glucopyransyl-( $1 \rightarrow 3)$ - $\alpha$ - $L$-arabinofuranosyl-

$(1 \rightarrow 4)]$ - $\alpha$ - $L$-rhamnnopyransyl- $(1 \rightarrow 2)-\beta$ - $D$-glucopyranosyl ester $=11919$

3-O-[ $\beta$ - $D$-Xylopyranosyl $(1 \rightarrow 2)-\alpha$ - $L$-arabinopyranosyl $(1 \rightarrow 6)-\beta-D$-2-deoxy-2acetamido-glucopyranosyl]-21-O-\{(6S)-2-trans-2-hydroxymethyl-6-met hyl-6-O-(4-O-[(6S)-2-trans-2-hydroxymethyl-6-methyl-6-hydroxy-2,7octadienoyl)]- $\beta$ - $D$-quinovopyranosyl-2,7-octadienoyl $\}$-acacic acid-28- $O$ $\beta$ - $D$-glucopyranosyl $(1 \rightarrow 3)$ - $[\alpha-L$-arabinofuranosyl $(1 \rightarrow 4)]$ - $\alpha$ - $L$-rhamnopyranosyl $(1 \rightarrow 2)-\beta$ - $D$-glucopyranosyl ester $=11921$

3-O- $\beta$ - $D$-Xylopyranosyl-16 $\beta-O-\alpha-L$-arabinopyranosyl- $6 \alpha, 22 \beta$-dihydroxyhopane $=13007$

$3-O$ - $[\beta$ - $D$-Xylopyranosyl- $(1 \rightarrow 2)-\alpha-L$-arabinopyranosyl- $(1 \rightarrow 6)-\beta$ - $D$-glucopyranosyl]-21-O-\{(6S)-2-trans-2-hydroxymethyl-6-methyl-6-O-[4-O-((6S)2-trans-2,6-dimethyl-6- $O$-(6-deoxy- $\beta$ - $D$-glucopyranosyl)-2,7-octadienoyl)-6-deoxy- $\beta$ - $D$-glucopyranosyl]-2,7-octadienoyl $\}$-acacic acid $28-O-\beta$ glucopyransyl-( $1 \rightarrow 3)-\alpha$ - $L$-arabinofuranosyl-( $1 \rightarrow 4)]-\alpha$ - $L$-rhamnnopyranosyl-( $1 \rightarrow 2)-\beta$ - $D$-glucopyranosyl ester $=11917$ 
3 - $O$ - $[\beta$ - $D$-Xylopyranosyl $(1 \rightarrow 2)-\alpha-L$-arabinopyranosyl $(1 \rightarrow 6)-\beta$ - $D$-glucopyranosyl]-21-O-\{(6S)-2-trans-2-hydroxymethyl-6-methyl-6-O-(2-O-[(6S)-2trans-2,6-dimethyl-6- $O-\beta$ - $D$-quinovopyranosyl-2,7-octadienoyl]- $\beta$ - $D$ quinovopyranosyl-2,7-octadienoyl)-acacic acid-28-O- $\beta$ - $D$-glucopyranosyl $(1 \rightarrow 3)$ - $[\alpha-L$-arabinofuranosyl $(1 \rightarrow 4)]-\alpha-L$-rhamnopyranosyl $(1 \rightarrow 2)-\beta$ $D$-glucopyranosyl ester $=11926$

3 - $O$-[ $\beta$ - $D$-Xylopyranosyl-( $(1 \rightarrow 2)-\alpha-L$-arabinopyranosyl-( $(1 \rightarrow 6)-\beta$ - $D$-glucopyranosyl]-21-O-\{(6S)-2-trans-2-hydroxymethyl-6-methyl-6-O-[4-O-((6S)-2-trans-2hydroxymethyl-6-methyl-6-hydroxyl-2,7-octadienoyl)-6-deoxy- $\beta$ - $D$-glucopy-r anosyl]-2,-7-octadienoyl $\}$-acacic acid $28-O-\beta$-glucopyransyl-( $1 \rightarrow 3$ )- $\alpha$ - $L$-arabinofuranosyl-( $(1 \rightarrow 4)]$ - $\alpha$ - $L$-rhamnnopyransyl- $(1 \rightarrow 2)-\beta$ - $D$-glucopyranosyl ester $=11918$

3-O-[ $\beta$-Xylopyranosyl-( $1 \rightarrow 2)$ - $\alpha$-arabinopyranosyl]-6- $O-\beta$-glucopyranosyl$3 \beta, 6 \alpha, 16 \beta, 24(S), 25$-pentahydroxycycloartane $=16068$

3 - $O$ - $\beta$ - $D$-Xylopyranosyl $(1 \rightarrow 2)$ - $\alpha$ - $L$-arabinopyranosyl $(1 \rightarrow 6)-\beta$ - $D$-glucopyranosyl-28- $O-\beta$ - $D$-Xylopyranosyl $(1 \rightarrow 3)-\beta$ - $D$-Xylopyranosyl $(1 \rightarrow 4)-\alpha-L$ rhamnopyranosyl $(1 \rightarrow 2)$-[ $\alpha$ - $L$-rhamnopyranosyl $(1 \rightarrow 6)]-\beta$ - $D$-glucopyranosyl echinocystic acid $=8528$

3 - $O-\beta$ - $D$-xylopyranosyl-( $(1 \rightarrow 2)-\alpha-L$-arabinopy-ranosyl oleanolic acid $28-O-$ $\beta$ - $D$-glucopyranosyl- $(1 \rightarrow 6)-\beta$ - $D$-glucopyranoside $=22957$

3-O-[ $\beta$-Xylopyranosyl-( $1 \rightarrow 2)$ - $\alpha$-arabinopyranosyl]-6- $O-\beta$-xylopyranosyl$3 \beta, 6 \alpha, 16 \beta, 24(S), 25$-pentahydroxycycloartane $=16067$

(22S,25S)-16-O- $\beta$ - $D$-Xylopyranosyl-5 $\alpha$-cholestan-3 $\beta, 16 \beta, 22,26$-tetraol

3- $O$ - $\beta$ - $D$-glucopyranosyl- $(1 \rightarrow 4)-\beta$ - $D$-galactopyranoside $=16881$

3 - $O$-[ $\beta$ - $D$-Xylopyranosyl-( $1 \rightarrow 4)$-[2,3- $O$-diacetyl]- $\beta$ - $D$-glucuronopyranosyl]28-O-[ $\beta$ - $D$-glucopyranosyl]-oleanolic acid $=20532$

3-O- $\beta$ - $D$-Xylopyranosyl-6,16-di- $O-\beta$ - $D$-glucopyranosyl-20(R),24(S)-epoxycycloartane- $3 \beta, 6 \alpha, 16 \beta, 25$-tetrol $=22048$

3 - $O$-[ $\beta$ - $D$-Xylopyranosyl $(1 \rightarrow 2)-\alpha$ - $L$-fucopyranosyl $(1 \rightarrow 6)-\beta$ - $D$-glucopyranosyl]21-O-\{(6S)-2-trans-2-hydroxymethyl-6-methyl-6-O-(4-O-[(6R)-2-trans-2,6-dimethyl-6- $O$-( $\beta$ - $D$-quinovopyranosyl)-2,7-octadienoyl]- $\beta$ - $D$-quinovopyranosyl2,7-octadienoyl\}-acacic acid-28- $O$ - $\beta$ - $D$-glucopy- ranosyl $(1 \rightarrow 3)$ - $[\alpha-L$-arabinofuranosyl $(1 \rightarrow 4)]-\alpha-L$-rhamnopyranosyl $(1 \rightarrow 2)-\beta$ - $D$-glucopyranosyl ester $=$ 11920

3 - $O$ - $\beta$ - $D$-Xylopyranosyl-( $(1 \rightarrow 3)$-[ $\beta$ - $D$-galactopyranosyl-( $(1 \rightarrow 2)]-\beta$ - $D$-glucopyranosyl gypsogenin $=16026$

6- $C$-Xylopyranosyl-8- $C$-glucopyranosylapigenin $=22464$

3-O- $\beta$ - $D$-Xylopyranosyl- $(1 \rightarrow 3)-\beta$ - $D$-glucopyranosylbayogenin $28-O$ $\beta$-D-apiofuranosyl-( $(1 \rightarrow 3)-\beta$ - $D$-xylopyranosyl-( $1 \rightarrow 4)$-[ $\beta$ - $D$-apiofuranosyl-( $1 \rightarrow 3)]-\alpha-L$-rhamnopyranosyl-( $(\rightarrow 2)-\alpha-L$-arabinopyranosyl ester $=4023$

3-O- $\beta$ - $D$-Xylopyranosyl-( $(1 \rightarrow 3)-\beta$ - $D$-glucopyranosyl bayogenin $28-O-\beta$ - $D$ apiofuranosyl- $(\rightarrow 3)-\beta$ - $D$-xylopyranosyl- $(1 \rightarrow 4)$-[ $\alpha$ - $L$-arabinopyranosyl$(1 \rightarrow 3)]-\alpha$ - $L$-rhamnopyranosyl- $(1 \rightarrow 2)-\alpha$ - $L$-arabinopyranosylester $=4016$

$3-O-\beta$ - $D$-Xylopyranosyl- $(1 \rightarrow 3)-\beta$ - $D$-glucopyranosyl bayogenin $28-O-\beta$ - $D$ apiofuranosyl-( $1 \rightarrow 3)-\beta$ - $D$-xylopyranosyl-( $(1 \rightarrow 4)-\alpha-L$-rhamnopyranosyl$(1 \rightarrow 2)-\alpha$ - $L$-arabinopyranosylester $=4015$

3-O- $\beta$ - $D$-Xylopyranosyl-( $(1 \rightarrow 3)-\beta$ - $D$-glucopyranosylbayogenin $28-O-\beta-D$ apiofuranosyl-( $(1 \rightarrow 3)$-[ $\beta$ - $D$-xylopyranosyl- $(1 \rightarrow 4)]-\alpha$ - $L$-rhamnopyranosyl- $(1 \rightarrow 2)-\alpha-L$-arabinopyranosyl ester $=4026$

3-O- $\beta$ - $D$-Xylopyranosyl-( $(1 \rightarrow 3)-\beta$ - $D$-glucopyranosyl bayogenin $28-O-\alpha-L$-rhamnopyranosyl-( $1 \rightarrow 3)-\beta$ - $D$-xylopyra-nosyl-( $1 \rightarrow 4)$-[ $\beta$ - $D$-apiofuranosyl-( $(1 \rightarrow 3)]$ $\alpha$ - $L$-rhamnopyranosyl- $(1 \rightarrow 2)-\alpha-L$-arabinopyranosyl ester $=4017$
3- $O-\beta$ - $D$-Xylopyranosyl-( $1 \rightarrow 3)-\beta$ - $D$-glucopyranosylbayogenin $28-O-\alpha-L$ rhamnopyranosyl-( $(1 \rightarrow 3)-\beta$ - $D$-xylopyranosyl-( $1 \rightarrow 4)-\alpha$ - $L$-rhamnopyranosyl- $(1 \rightarrow 2)-\alpha-L$-arabinopyranosyl ester $=4019$

3 - $O-\beta$ - $D$-Xylopyranosyl-( $1 \rightarrow 3)-\beta$ - $D$-glucopyranosylbayogenin $28-O-\beta$ - $D$ xylopyranosyl-( $(1 \rightarrow 3)-\beta$ - $D$-xylopyranosyl- $(1 \rightarrow 4)$-[ $\beta$ - $D$-apiofuranosyl$(1 \rightarrow 3)]-\alpha$ - $L$-rhamnopyranosyl- $(1 \rightarrow 2)-\alpha$ - $L$-arabinopyranosyl ester $=4027$

3 - $O$-[ $\beta$ - $D$-Xylopyranosyl- $(1 \rightarrow 2)[\beta$ - $D$-glucopyranosyl- $(1 \rightarrow 3)]-6$ - $O$-butyl $-\beta$ - $D$ glucuronopyranosyl]oleano-lic acid-28- $O-\beta-D$-glucopyranoside $=20619$

3 - $O$-[ $\beta$ - $D$-Xylopyranosyl- $(1 \rightarrow 2)[\beta$ - $D$-glucopyranosyl- $(1 \rightarrow 3)]-6$ - $O$-ethyl $-\beta$ - $D$ glucuronopyranosyl] oleanolic acid-28- $O-\beta$ - $D$-glucopyranoside $=20618$

3 - $O$ - $\{\beta$ - $D$-Xylopyranosyl-( $1 \rightarrow 2)-\beta$ - $D$-glucopyranosyl- $(1 \rightarrow 4)$-[ $\beta$ - $D$-glucopyranosyl- $(1 \rightarrow 2)]-\alpha-L$-arabinopyranoside $\}$-saikogenin $=17959$

3 - $O$ - $\{\beta$ - $D$-Xylopyranosyl-( $1 \rightarrow 2)-\beta$ - $D$-glucopyranosyl-( $1 \rightarrow 4)$-[ $\beta$ - $D$-glucopyranosyl- $(1 \rightarrow 2)]-\alpha-L$-arabinopyranoside $\}$-saikogenin $\mathrm{G}=17960$

6 - $O$ - $\beta$ - $D$-Xylopyranosyl-( $1 \rightarrow 2)-\beta$ - $D$-glucopyranosyl- $(1 \rightarrow 2)-\beta$ - $D$-glucopyranosyl 20(S),24(S)-epoxydammane-3 $\beta, 6 \alpha, 12 \beta, 25$-tetrol $=22895$

3-O-[ $\alpha$ - $L$-Xylopyranosyl-( $(1 \rightarrow 2)-\beta$ - $D$-glucopyranosyl- $(1 \rightarrow 4)][\beta$ - $D$-glucopyra nosyl-(1 $\rightarrow 2)-\beta$ - $D$-glucopyranosyl-( $(1 \rightarrow 2)]-\alpha-L$-rhamnopyranosyl cyclamiretin $\mathrm{A}=1627$

3 - $O-\beta$ - $D$-Xylopyranosyl-( $(\rightarrow 2)-\beta$ - $D$-glucopyranosyl $(1 \rightarrow 2)-\beta$ - $D$-glucopyranosyl-20(S)-protopanaxadiol 20-O- $\beta$ - $D$-xylopyranosyl $(1 \rightarrow 4)-\beta$ - $D$-xylopyranosyl $(1 \rightarrow 6)-\beta$ - $D$-glucopyranoside $=15835$

3 - $O-\beta$ - $D$-Xylopyranosyl $(1 \rightarrow 2)-\beta$ - $D$-glucopyranosyl $(1 \rightarrow 2)-\beta$ - $D$-glucopyranosyl-20(S)-protopanaxadiol 20-O- $\beta-D$-xylopyranosyl $(1 \rightarrow 5)-\alpha-L-$ arabino-furanosyl $(1 \rightarrow 6)-\beta$ - $D$-glucopyranoside $=15841$

$3-O-\beta$ - $D$-Xylopyranosyl $(1 \rightarrow 2)-\beta$ - $D$-glucopyranosyl $(1 \rightarrow 2)-\beta$ - $D$-glucopyranosyl-20-(S)-protopanaxadiol 20- $O-\beta-D$-xylopyranosyl $(1 \rightarrow 3)-\beta$ - $D$-glucopyranosyl $(1 \rightarrow 6)-\beta$ - $D$-glucopyranoside $=15842$

3-O- $\beta$ - $D$-Xylopyranosyl-22 $\beta$ - $O-\beta$ - $D$-glucopyranosyl-16 $\beta$-hydroxyhopan-6-one $=13009$

$3-O-[O-\beta$ - $D$-Xylopyranosyl-( $1 \rightarrow 4)-\beta$ - $D$-glucopyranosyl] medicagenic acid $28-O-\beta$ - $D$-xylopyranosyl-( $1 \rightarrow 3)-O-\alpha-L$-rhamnopyranosyl-( $1 \rightarrow 2)-O-\alpha-L$ arabinopyranosyl ester $=734$

3-O- $\beta$ - $D$-Xylopyranosyl-(1 $\rightarrow 3)-\beta$ - $D$-glucopyranosylpolygalacic acid $28-O-\beta$ - $D$-galactopyranosyl-( $(1 \rightarrow 2)-\alpha-L$-rhamnopyranosyl-( $1 \rightarrow 3)-\beta$ - $D$-xylopyranosyl$(1 \rightarrow 4)-\alpha$ - $L$-rhamnopyranosyl- $(1 \rightarrow 2)-\alpha-L$-arabinopyranosyl ester $=4022$

3-O- $\beta$ - $D$-Xylopyranosyl-(1 $\rightarrow 3)-\beta$ - $D$-glucopyranosylpolygalacic acid 28- $O$ $\alpha$ - $L$-rhamnopyranosyl-( $(1 \rightarrow 3)-\beta$ - $D$-xylopyranosyl-( $(1 \rightarrow 4)-\alpha$ - $L$-rhamnopyranosyl- $(1 \rightarrow 2)-\alpha$ - $L$-arabinopyranosyl ester $=4020$

3-O- $\beta$ - $D$-Xylopyranosyl-( $(1 \rightarrow 3)-\beta$ - $D$-glucopyranosylpolygalacic acid 28- $O-\alpha-L$ rhamnopyranosyl-( $(1 \rightarrow 3)-\beta$ - $D$-xylopyranosyl-( $1 \rightarrow 4)$-[ $\beta$ - $D$-apiofuranos-yl$(1 \rightarrow 3)]$ - $\alpha$ - $L$-rhamnopyranosyl- $(1 \rightarrow 2)-\alpha-L$-arabinopyranosyl ester $=4021$

3-O-[ $\beta$-D-Xylopyranosyl $(1 \rightarrow 4)$-[ $[\beta$ - $D$-glucopyranosyl]-(25S)-5 $\beta$-spirostan-3 $\beta$-ol $=1860$ 3 - $O$-[O- $\beta$ - $D$-Xylopyranosyl-( $(1 \rightarrow 4)-\beta$ - $D$-glucopyranosyl] $2 \beta, 3 \beta, 16 \alpha, 21 \beta$-tetrahydroxyolean-12-ene-23,28-dioic acid 21,28-lactone $=735$

3 - $O$-[O- $\beta$ - $D$-Xylopyranosyl-( $1 \rightarrow 4)-\beta$ - $D$-glucopyranosyl] $2 \beta, 3 \beta, 16 \alpha$-trihydroxyolean12-ene-23,28-dioic acid (zanhic acid)28- $O-\beta-D$-apiofuranosyl-( $1 \rightarrow 3$ )-O-(4-Oacetyl)- $\alpha$ - $L$-rhamnopyranosyl- $(1 \rightarrow 2)-O-\alpha-L$-arabino- pyranosyl ester $=728$

$3-O-\beta$ - $D$-Xylopyranosyl-( $(1 \rightarrow 4)-\beta$ - $D$-glucopyranosyl-( $1 \rightarrow 4)-\beta$ - $D$-xylopyranosyl-( $1 \rightarrow 3)-\alpha$ - $L$-rhamnopyranosyl-( $1 \rightarrow 2)-\alpha-L$-arabinopyranosyloleanolic acid 28-O- $\beta$ - $D$-glucopyranosyl- $(1 \rightarrow 6)-\beta-D$-glucopyranoside $=19434$

3-O-[O- $\beta$ - $D$-Xylopyranosyl-(1 $\rightarrow 4)-\beta$ - $D$-glucopyranosyl] zanhic acid $28-O-\alpha$ $L$-arabinopyranosyl-(1 $\rightarrow 3)-O-(4-O$-acetyl)- $\alpha-L$-rhamnopyranosyl-( $1 \rightarrow 2)$ $O-\alpha-L$-arabinopyranosyl ester $=730$ 
3-O-[ $\beta$ - $D$-Xylopyranosyl-( $(1 \rightarrow 4)-\beta$ - $D$-glucopyranoyl-( $(1 \rightarrow 4)][\alpha-L$-rhamnopyranosyl$(1 \rightarrow 3)-\beta$ - $D$-galactopyranosyl-( $(1 \rightarrow 3)-\alpha-L$-rhamnopyranosyl $(1 \rightarrow 2)-\alpha-L$-rabinopyra nosyl-hederagenin-28-O- $\beta$ - $D$-glucopyranosyl $(1 \rightarrow 6)-\beta$ - $D$-glucopyranoside $=1891$

3 - $O$ - $\beta$ - $D$-Xylopyranosyl $(1 \rightarrow 2)-\beta$ - $D$-glucuronopyranosyl azukisapogenol $29-O-\beta-D$-glucopyranoside ester $=15201$

3 - $O$-[ $\beta$ - $D$-Xylopyranosyl-( $1 \rightarrow 4)-\beta$ - $D$-glucuronopyranosyl]-28- $O$ - $[\beta$ - $D$-glucopyranosyl]-oleanolic acid $=20529$

$3-O-(\beta-D$-Xylopyranosyl $)$-hederagenin $=3913$

3 - $O-\beta$ - $D$-Xylopyranosyl oleanolic acid $28-O-\alpha-L$-rhamnopyranosyl- $(1 \rightarrow 4)$ -

$\beta$ - $D$-glucopyranosyl- $(1 \rightarrow 6)-\beta$ - $D$-glucopyranosyl ester $=1254$

$3 \beta-O-(\beta-D$-Xylopyranosyl $)$ pomolic acid methyl ester $=10982$

28 - $O$ - $\beta$ - $D$-Xylopyranosyl-( $(1 \rightarrow 4)-\alpha$ - $L$-rhamnopyranosyl- $(1 \rightarrow 2)$-[ $\alpha-L$-(5-O-acetyl)-arabinofuranosyl $(1 \rightarrow 3)]-\beta$ - $D$-(4- $O$-acetyl)-fucopyranosyl-gypsogenin-3-O- $\beta$ - $D$-galactopyranosyl $(1 \rightarrow 2)-\beta-D$-(6- $O$-methyl ester)-glucuronopyranoside $=19659$

$28-O-\beta$ - $D$-Xylopyranosyl- $(1 \rightarrow 4)-\alpha-L$-rhamnopyranosyl- $(1 \rightarrow 2)-[\alpha-L-(5-O-$ acetyl)-arabinofuranosyl $(1 \rightarrow 3)]-\beta$ - $D$-(4- $O$-acetyl)-fucopyranosyl-quillaic acid-3-O- $\beta$ - $D$-galactopyranosyl $(1 \rightarrow 2)-\beta$ - $D$-(6- $O$-methylester)-glucuronopyranoside $=19661$

28- $O-\beta$ - $D$-Xylopyranosyl-( $(1 \rightarrow 4)-\alpha-L$-rhamnopyranosyl- $(1 \rightarrow 2)-[\alpha-L-(5-O-$ acetyl)-arabinofuranosyl $(1 \rightarrow 3)]-\beta$ - $D$-(4- $O$-acetyl)-fucopyranosyl-quillaic acid-3-O- $\beta$ - $D$-galactopyranosyl $(1 \rightarrow 2)-\beta$ - $D$-(6-O-n-butyl ester)-glucuronopyranoside $=19662$

28 - $O$ - $\beta$ - $D$-Xylopyranosyl- $(1 \rightarrow 4)-\alpha-L$-rhamnopyranosyl-( $(1 \rightarrow 2)-[\alpha-L-(5-O$-ace tyl)-arabinofuranosyl $(1 \rightarrow 3)]-\beta$ - $D$-(4-O-acetyl)-fucopyranosyl-gypsogenin-3- $O-\beta$ - $D$-galactopyranosyl $(1 \rightarrow 2)-\beta$ - $D$-glucuronopyranoside $=19665$

28 -O- $\beta$ - $D$-Xylopyranosyl-( $(1 \rightarrow 4)-\alpha-L$-rhamnopyranosyl- $(1 \rightarrow 2)-[\alpha-L-(5-O-$ acetyl)-arabinofuranosyl $(1 \rightarrow 3)]-\beta-D$-(4-O-acetyl)-fucopyranosyl-quillaic acid-3- $O-\beta$ - $D$-galactopyranosyl $(1 \rightarrow 2)-\beta$ - $D$-glucuronopyranoside $=19666$

$28-O$-[ $\beta$ - $D$-Xylopyranosyl-( $(1 \rightarrow 4)-\alpha-L$-rhamnopyranosyl- $(1 \rightarrow 2)]-\alpha-L$-arabinofura$\operatorname{nosyl}(1 \rightarrow 3)-\beta-D-(4-O$-acetyl)-fucopyranosyl-gypsogenin-3-O- $\beta$ - $D$-galactopyranosyl $(1 \rightarrow 2)-\beta-D-(6-O$-methyl ester $)$-glucuronopyranoside $=19663$

$28-O-\beta$ - $D$-Xylopyranosyl- $(1 \rightarrow 4)-\alpha$ - $L$-rhamnopyranosyl- $(1 \rightarrow 2)$ - $[\alpha-L$-arabino-fura$\operatorname{nosyl}(1 \rightarrow 3)]-\beta-D-(4-O$-acetyl)-fucopyranosyl-gypsogenin-3-O- $\beta$ - $D$-galactopyranosyl $(1 \rightarrow 2)-\beta$ - $D$-(6-O- $n$-butyl ester)-glucuronopyranoside $=19664$

3 - $O$-[ $\beta$ - $D$-Xylopyranosyl- $(1 \rightarrow 4)-\beta$ - $D-(1 \rightarrow 4)][\alpha-L$-rhamnopyranosyl-( $(1 \rightarrow 3)-$ $\beta$ - $D$-galactopyranosyl-( $(1 \rightarrow 3)-\alpha-L$-rhamnopyranosyl-( $(1 \rightarrow 2)-\alpha$ - $L$-arabinopyranosyl-hederagenin $=1890$

3-O- $\beta$ - $D$-Xylopyranosyl $(1 \rightarrow 2)-\alpha-L$-rhamnopyranosyl-22 $\beta$ - $O-\beta$ - $D$-glucopyranosyl-6 $\alpha, 16 \beta$-dihydroxyhopane $=13005$

3-O- $\beta$ - $D$-Xylopyranosyl $(1 \rightarrow 2)-\alpha$ - $L$-rhamnopyranosyl- $6 \alpha-O-\beta$ - $D$-xylopyranosyl-22-O- $\beta$ - $D$-glucopyranosyl-16 $\beta$-hydroxy hopane $=13004$

3-O- $\beta$ - $D$-Xylopyranosyl-( $(1 \rightarrow 3)-\alpha-L$-rhamnopyranosyl-( $(1 \rightarrow 2)-\beta$ - $D$-xylopyranosyloleanolic acid 28-O- $\beta$ - $D$-glucopyranosyl-( $1 \rightarrow 6)-\beta$ - $D$-glucopyranoside $=19436$

3-O-( $\beta$ - $D$-Xylopyranosyl 4-sulphate)-spergulagenin $\mathrm{A}=20144$

$6 '-O-\beta-D-X y l o p y r a n o s y l s w e r t i a m a r i n=3539$

3 - $O$ - $\{[\beta-D$-Xylopyranosyl- $(1 \rightarrow 2)]-[\beta-D$-xylopyranosyl- $(1 \rightarrow 4)]-[3-O$-acetyl] $\beta-D-D-$ glucuronopyranosyl $\}$-28- $O$-[ $\beta$ - $D$-glucopyranosyl]-oleanolic acid $=20536$

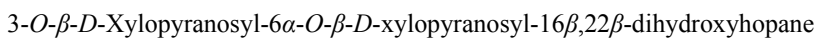
$=13008$

3 - $O$ - $\{[\beta$ - $D$-Xylopyranosyl $(1 \rightarrow 2)]-[\beta$ - $D$-xylopyranosyl $(1 \rightarrow 4)]-\beta$ - $D$-glucuronopyranosyl $\}-28-O-[\beta-D$-glucopyranosyl]-oleanolic acid $=20535$

3-O- $\beta$ - $D$-Xylopyranosyl-6 $\alpha-O-\beta-D$-xylopyranosyl-16 $\beta-O-\beta-D$-xylopyranosyl$22 \beta$-hydroxyhopane $=13006$
$D$-Xylose $=22843$

3,4 -Xylylic acid $=6320$

Yadanziolide A $=2672$

Yageine $=9235$

Yangambin $=12920$

Yatansin $=2684$

$(-)$-Yatein $=5543$

Yatrorizine $=11851$

Yemuoside YM14 = 3781

(-)-Yiyeliangwanoside XI = 22919

Yuanhuacin A $=22929$

Yuanhuadin $B=22930$

Yuglon $=11903$

Yuheinoside $=17513$

Zeaxanthin dipalmitate $=17238$

Zedoarone $=4416$

Zeorin $=9634$

$(+)-\left(1 R^{*}, 5 R^{*}, 6 S^{*}\right)$-Zierene $=22999$

Zingiberenin $\mathrm{B}=3924$ 


\section{TCM Plant English Name Index}

TCM Plant English Name

Abbreviate Balanophora*

Abelmusk

Abrams Cypress*

Absus Senna*

Abura Mitragyna

Abutilon Nightshade*

Abyssinia Coralbean*

Abyssinia Harrisonia*

Acacia Terminalia*

Acanthoid Pricklyash*

Acanthus-leaved Mahonia

Acicular Thorowax

Acid Gambirplant*

Aconite

Actino-spiny Barberry

Aculeate Amomum*

Aculeate Nightshade*

Aculeate Poisonnut*

Aculeate Ruffle Palm

Acuminate Aussiepoplar*

Acuminate Banana

Acuminate Colanut

Acuminate Glochidion*

Acuminate Lasianthus

Acuminate Swallowwort

Acuminate Tobacco*

Acuminate Uvaria*

Acuminatum Epimedium

Acutangular Scopolia

Acute Antirhea*

Acute Common Perilla

Acute Common Perilla Leaf

Acute Rush*

Acute Sida

Acute Spiraea
TCM Plant Code and Latin Name

T0857 Balanophora abbreviata

T4303 Moschus moschiferus; Moschus berezovskii; Moschus sifanicus

T1894 Cupressus abramsiana

T1228 Cassia absus

T4257 Mitragyna macrophylla

T5988 Solanum abutiloides

T2457 Erythrina abyssinica

T3108 Harrisonia abyssinica

T6349 Terminalia stuhlmannii

T6858 Zanthoxylum acanthopodium

T4053 Mahonia acanthifolia

T1057 Bupleurum bicaule

T6604 Uncaria acida

T0118 Aconitum napellus

T0896 Berberis actinacantha

T0415 Amomum aculeatum

T5989 Solanum aculeatissimum

T6163 Strychnos aculeata

T0259 Aiphanes aculeata

T3273 Homalanthus acuminatus

T4329 Musa acuminata

T1615 Cola acuminata

T2987 Glochidion acuminatum

T3696 Lasianthus acuminatissimus

T1950 Cynanchum ascyrifolium

T4424 Nicotiana acuminata

T6657 Uvaria acuminata

T2389 Epimedium acuminatum

T5819 Scopolia acutangula [Syn. Anisodus acutangulus]

T0536 Antirhea acutata

T4717 Perilla frutescens var. acuta [Syn. Perilla frutescens var.

purpurascens]

T4718 Perilla frutescens var. acuta [Syn. Perilla frutescens var.

purpurascens]

T3577 Juncus acutus

T5942 Sida acuta

T6081 Spiraea japonica var. acuta
TCM Plant PIN YIN Name

DUAN SHE GU

SHE XIANG

AI SHI BAI MU

A SU JUE MING

DA YE MAO ZHU MU

MA ZHUANG QIE

A BI XI NI YA CI TONG

A BI XI NI YA NIU JIN GUO

A KA XI A LAN REN

CI HUA JIAO

CI YE SHI DA GONG LAO

ZHUI YE CHAI HU

SUAN GOU TENG

OU WUTOU

CU CI XIAO BO

CI DOU KOU

DING QIE

CI MA QIAN ZI

CI JI NU ZONG LV

JIAN JIAN AO YANG

XIAO GUO YE JIAO

SU DAN KE LE GUO

JIAN JIAN SUAN PAN ZI

CHANG WEI CU YE MU

CHAO FENG CAO

JIAN XING YAN CAO

JIAN ZI YU PAN

CU MAO YIN YANG HUO

SAN FEN SAN

JIAN RUI MAO CHA

JIAN ZI SU

JIAN ZI SU YE

JIAN DENG XIN CAO

HUANG HUA REN

JI JIAN XIU XIAN JU 
Acute Syngnathus*

Acuteangle Hedyotis

Acuteangulus Pencilwood*

Acuteleaf Ash Bark

Acute-leaf Pricklyash*

Acutelobed Angelica

Acutelobed Hornpoppy

Acutifoliate Podocarpium

Adder's Tongue

Adhesive Rehmannia Cocked Root

Adhesive Rehmannia Dried Root

Adhesive Rehmannia Fresh Root

Adlay

Adnate Groundsel*

Afghanistan Erysimum*

Afghanistan Lilac*

Africa Trumpetcreeper*

African Antiaris*

African Basil*

African Costus*

African Cucumber*

African Custard Apple*

African Erythrophleum

African frog

African Gambirplant*

African Holarrhena*

African Lily

African Mammey Apple

African Mitragyna*

African Myrsine

African Olive*

African Quassia*

African Rosewood

Afzel Poisonnut*

Afzeli Garcinia*

Aging Leek

Ailanthus-like Pricklyash

Ailanthus-like Pricklyash Bark

Ailanthus-like Pricklyash Root

Ainsliaefolious Cacalia

Air-plant

Airpotato Yam

Aizoon Stonecrop

Ajowan

Akee

Aleppo Gall (Galla Halepensis)

Alfalfa

Alfalfa Root

Alfred Stonecrop
T6257 Syngnathus acus

T3122 Hedyotis acutangula

T2306 Dysoxylum acutangulum

T2780 Fraxinus szaboana [Syn. Fraxinus chinensis var. acuminata]

T6859 Zanthoxylum acutifolium

T0474 Angelica acutiloba [Syn. Ligusticum acutilobum]

T2970 Glaucium oxylobum

T2133 Desmodium racemosum [Syn. Podocarpium podocarpum var.

oxyphyllum ]

T4506 Ophioglossum vulgatum

T5446 Rehmannia glutinosa [Syn. Rehmannia glutinosa f.

huechingensis]

T5445 Rehmannia glutinosa [Syn. Rehmannia glutinosa $\mathrm{f}$.

huechingensis]

T5447 Rehmannia glutinosa [Syn. Rehmannia glutinosa $\mathrm{f}$.

huechingensis]

T1613 Coix lacryma-jobi

T5875 Senecio adnatus

T2456 Erysimum perofskianum

T6258 Syringa afghanica

T4422 Newbouldia laevis

T0532 Antiaris africana

T4474 Ocimum kilimandscharicum

T1754 Costus afer

T1873 Cucumis africanus

T3237 Hexalobus crispiflorus

T2482 Erythrophleum africanum

T0207 African frog

T6605 Uncaria africana

T3262 Holarrhena africana

T0209 Agapanthus africanus

T4092 Mammea africana

T4255 Mitragyna africanus

T4361 Myrsine africana

T4486 Olea africana

T5369 Quassia africana

T2004 Dalbergia melanoxylon

T6164 Strychnos afzelii

T2849 Garcinia afzelii

T0320 Allium senescens

T6860 Zanthoxylum ailanthoides

T6862 Zanthoxylum ailanthoides

T6861 Zanthoxylum ailanthoides

T1097 Cacalia ainsliaeflora

T1043 Bryophyllum pinnatum

T2191 Dioscorea bulbifera

T5850 Sedum aizoon

T6488 Trachyspermum ammi

T0956 Blighia sapida

T5374 Quercus infectoria

T4148 Medicago sativa

T4149 Medicago sativa

T5851 Sedum alfredii [Syn. Sedum formosanum]
JIAN HAI LONG

JIN CAO

RUI JIAO JIAN MU

JIAN YE CEN

JIAN YE HUA JIAO

DONG DANG GUI

JIAN LIE HAI YING SU

SHAN MA HUANG

PING ER XIAO CAO

SHU DI HUANG

GAN DI HUANG

\section{XIAN DI HUANG}

\section{YI MI}

TIE SHENG QIAN LI GUANG

A FU HAN TANG JIE

A FU HAN DING XIANG

FEI ZHOU ZI WEI

FEI ZHOU JIAN XUE FENG HOU

FEI ZHOU LUO LE

FEI ZHOU BI QIAO JIANG

FEI ZHOU HUANG GUA

FEI ZHOU FAN LI ZHI

FEI ZHOU GE MU

FEI ZHOU WA

FEI ZHOU GOU TENG

FEI ZHOU ZHI XIE MU

FEI ZHOU BAI ZI LIAN

FEI ZHOU HUANG GUO MU

FEI ZHOU MAO ZHU MU

TIE ZI

FEI ZHOU GAN LAN

FEI ZHOU KU MU

FEI ZHOU HUANG TAN

A FU ZE ER MA QIAN ZI

A FU ZE LI SHAN ZHU ZI

SHAN JIU

CHU YE HUA JIAO

CHU YE HUA JIAO PI

CHU YE HUA JIAO GEN

TU ER FENG XIE JIA CAO

LUO DI SHENG GEN

HUANG YAO ZI

FEI CAI

A YU WEI

XI FEI LI ZHI GUO

MO SHI ZI

MU XU

MU XU GEN

DONG NAN JING TIAN 
Algarroba

Algerian Cottonthistle*

Algerian Iris

Algerian Statice

Algid Rhodiola*

Alkanet

Alliaceous Pencilwood*

Alligator Alternanthera

Allspice

Allugha Galangal*

Alpine Bog-swertia

Alpine Clubmoss*

Alpine Currant

Alpine Edelweiss

Alpine Gentian

Alpine Groundsel*

Alpine Larkspur

Alpine Meadowrue

Alpine Oleandra

Alpine Poppy

Alpine Pricklyash

Alpine Sowthistle

Alpine Totara

Alpine Yarrow

Alplne Thermopsis

Altai Anemone*

Altai Heteropappus

Alternateleaf Melaleuca*

Alternateleaf Thermopsis*

Alternate-leaved Golden-saxifrage

Amaranthinecolor Goosefoot*

Amazon lily

Amazonian Jewelvine*

Amazonian Poisonnut*

Ambiguous Consolida*

Amboina Pitch Tree

American Agave

American Aspen

American Avocado

American Bittersweet

American Coralbean*

American Cranberry

American Crotalaria*

American Eleutherine

American Elm

American Euphorbia

American Ginseng

American Ginseng Stem-leaf

American Green Alder

American Lotus

American Maidenhair Fern

American Pokeweed

American Ragweed*
T5210 Prosopis juliflora

T4496 Onopordum algeriense

T3473 Iris unguicularis

T3841 Limonium bonduellii

T5491 Rhodiola algida

T0447 Anchusa officinalis

T2307 Dysoxylum alliaceum

T0379 Alternanthera philoxeroides

T4894 Pimenta dioica

T0352 Alpinia allughas

T6230 Swertia perennis

T3964 Lycopodium alpinum [Syn. Diphasiastrum alpinum]

T5544 Ribes alpinum

T3749 Leontopodium alpinum

T2902 Gentiana algida

T5878 Senecio alpinus

T2074 Delphinium elatum

T6373 Thalictrum alpinum

T4488 Oleandra wallichii

T4621 Papaver alpinum

T6881 Zanthoxylum hamiltonianum

T1414 Cicerbita alpina

T5049 Podocarpus nivalis

T0060 Achillea alpina [Syn. Achillea sibirica]

T6424 Thermopsis alpina

T0463 Anemone altaica

T3233 Heteropappus altaicus

T4153 Melaleuca alternifolia

T6425 Thermopsis alternifolia

T1402 Chrysosplenium alternifolium

T1360 Chenopodium amaranticolor

T2524 Eucharis amazonica

T2117 Derris amazonica

T6165 Strychnos amazonica

T1646 Consolida ambigua

T0213 Agathis dammara

T0215 Agave americana

T5163 Populus tremuloides

T4731 Persea americana [Syn. Persea gratissima]

T1293 Celastrus scandens

T2458 Erythrina americana

T6671 Vaccinium macrocarpon

T1814 Crotalaria anagyroides

T2339 Eleutherine americana

T6594 Ulmus americana

T2600 Euphorbia maculata

T4610 Panax quinquefolium

T4611 Panax quinquefolium

T0325 Alnus crispa

T4396 Nelumbo lutea

T0175 Adiantum pedatum

T4861 Phytolacca americana [Syn. Phytolacca decandra]

T0397 Ambrosia artemisiaefolium)
MU DOU SHU

A ER JI ER DA CHI JI

A ER JI LI YA YUAN WEI

A ER JI LI YA BU XUE CAO

JI SHI HONG JING TIAN

YAO YONG NIU SHE CAO

CONG JIAN MU

KONG XIN XIAN

DUO XIANG GUO

A LU HA LIANG JIANG

SU GEN ZHANG YA CAI

GAO SHAN BIAN ZHI SHI SONG

GAO SHAN CHA BIAO

GAO SHAN HUO RONG CAO

BAI HUA LONG DAN

YA KE BEI QIAN LI GUANG

GAO FEI YAN CAO

GAO SHAN TANG SONG CAO

GAO SHAN TIAO JUE

GAO SHAN YING SU

GAO SHAN HUA JIAO

GAO SHAN YAN SHEN

GAO SHAN LUO HAN SONG

YI ZHI HAO

GAO SHAN HUANG HUA

A ER TAI YIN LIAN HUA

A ER TAI ZI WAN

HU SHENG YE BAI QIAN CENG

HU SHENG YE YE JUE MING

JIN YAO

XIAN SE LI

YA MA XUN BAI HE

YA MA XUN YU TENG

YA MA XUN MA QIAN ZI

LIANG SI FEI YAN CAO

BEI KE SHAN

FAN MA

CHAN YANG

E LI

MEI ZHOU NAN SHE TENG

MEI ZHOU CI TONG

MEI ZHOU SUAN GUO LUO

MEI ZHOU YE BAI HE

XIAO HONG SUAN

MEI ZHOU YU

BAN DI JIN

XI YANG SHEN

XI YANG SHEN JING YE

MEI ZHOU LU QI MU

JIN HUANG LIAN

TIE SI QI

MEI SHANG LU

MEI ZHOU TUN CAO 
American Spineginseng*

American Umbrellaleaf

American Vouacapoua*

Ammbergris

Ampelopsis

Amphibian Jujube*

Amphibious Knotweed

Amplexicaul Swertia

Amplexifolious Glorybower

Amur Adonis

Amur Barberry

Amur Corktree

Amur Corydalis

Amur Grape

Amur Jackinthepulpit

Amur Lilac

Amur Maackia

Amur Maple

Amur Poppy

Amur Valeriana

Amygdalate Apricot Seed

Anantmul

Anatomicum Fig*

Anattotree

Ancher St.John'swort*

Ancients Euphorbia

Ancistrocladus*

Andrographis*

Angelica

Angelin-tree

Angled Bittersweet

Angletwig Magnoliavine

Angola Alangium*

Angola Cucumber*

Angola Padauk*

Angola Uvaria*

Angostura-bark Tree

Angulate Groundsel*

Angulate Mistletoe*

Anhui Anemone*

Anhui Fritillary

Anise

Annual Adonis*

Annual Fleabane

Annual Mercury

Anomalus Mallotu

Ansu Apricot Seed

Antao Nutmeg

Antidysenteric Brucea*

Antifebrile Dichroa

Apisin

Appendiculate Cremastra

Apple
T4517 Oplopanax horridus

T2230 Diphylleia cymosa

T6804 Vouacapoua americana

T4855 Physeter catodon

T0423 Ampelopsis brevipedunculata

T6914 Ziziphus amphibia

T5097 Polygonum amphibium

T6221 Swertia franchetiana

T1569 Clerodendrum serratum var. amplexifolium

T0184 Adonis amurensis

T0897 Berberis amurensis

T4789 Phellodendron amurense

T1706 Corydalis ambigua var. amurensis [Syn. Corydalis ambigua]

T6794 Vitis amurensis

T0617 Arisaema amurense

T6259 Syringa amurensis [Syn. Syringa reticulata var. amurensis]

T4009 Maackia amurensis

T0049 Acer ginnala

T4630 Papaver nudicaule ssp. amurense

T6675 Valeriana amurensis

T5215 Prunus amygdalus

T3200 Hemidesmus indicus

T4199 Mesembryanthemum anatomicum

T0947 Bixa orellana

T3337 Hypericum ancherii

T2580 Euphorbia antiquorum

T0450 Ancistrocladus korupensis

T0459 Andrographis serpyllifolia

T0477 Angelica archangelica

T0452 Andira inermis

T1285 Celastrus angulatus

T5797 Schisandra propinqua

T0283 Alangium lamarckii

T1874 Cucumis angolensis

T5300 Pterocarpus angolensis

T6658 Uvaria angolensis

T2829 Galipea officinalis

T5880 Senecio angulatus

T6772 Viscum angulatum

T0464 Anemone anhuiensis

T2781 Fritillaria anhuiensis

T4895 Pimpinella anisum

T0185 Adonis annua

T2421 Erigeron annuus

T4196 Mercurialis annua

T4079 Mallotus anomalus

T5219 Prunus armeniaca var. ansu

T4354 Myristica simiarum

T1037 Brucea antidysenterica

T2158 Dichroa febrifuga

T0539 Apis cerana

T1787 Cremastra appendiculata

T4088 Malus pumila
MEI ZHOU CI SHEN

SHAN XI WO ER QI

MEI GUO KE YA SHU

LONG XIAN XIANG

SHE PU TAO

SHUI LU ZAO

LIANG QI LIAO

BAO JING ZHANG YA CAI

SAN TAI HUA

FU SHOU CAO

XIAO BO

HUANG BAI

DONG BEI YAN HU SUO

SHAN PU TAO

DONG BEI TIAN NAN XING

BAO MA ZI

CHAO XIAN HUAI

CHA TIAO QI

HEI SHUI YE YING SU

HEI SHUI XIE CAO

BA DAN XING REN

YIN DU BA QIA

MING SONG YE JU

HONG MU

AN SHI JIN SI TAO

HUO YANG LE

GOU ZHI TENG

BAI LI XIANG YE CHUN XIN LIAN

YUAN DANG GUI

WU CI KE YA SHU

DIAO GAN MA

HAN RUI WU WEI ZI

AN GE LA BA JIAO FENG

AN GE LA HUANG GUA

AN GE LA ZI TAN

GUAN ZI YU PAN

AN GU SI TU LA SHU

LENG JIAO QIAN LI GUANG

LENG ZHI HU JI SHENG

AN HUI YIN LIAN HUA

AN HUI BEI MU

HUI QIN

QIU FU SHOU CAO

YI NIAN PENG

YI NIAN SHENG SHAN DIAN

XIU MAO YE TONG

SHAN XING REN

FEI LV BIN ROU DOU KOU

KANG LI YA DAN ZI

CHANG SHAN

FENG DU

DU JUAN LAN

PING GUO 


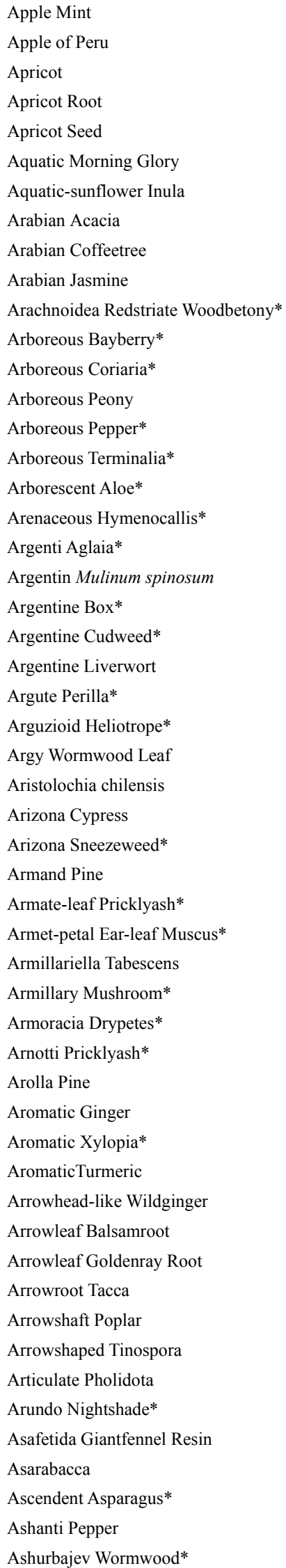

T4188 Mentha rotundifolia
T4423 Nicandra physaloides
T5218 Prunus armeniaca
T5217 Prunus armeniaca
T5216 Prunus armeniaca
T3444 Ipomoea aquatica [Syn. Convolvulus repens; Ipomoea reptans]
T3432 Inula helianthus-aquatica

T0016 Acacia arabica

T1608 Coffea arabica

T3557 Jasminum sambac

T4685 Pedicularis striata ssp. arachnoidea

T4342 Myrica arborea

T1689 Coriaria arborea

T4581 Paeonia arborea

T4932 Piper arboreum

T6343 Terminalia arborea

T0334 Aloe arborescens var. natalensis

T3319 Hymenocallis arenicola

T0231 Aglaia argentea

T4313 Mulinum spinosum

T1085 Buxus argentea

T3027 Gnaphalium gaudichaudianum

T4990 Plagiochasma rupestre

T4713 Perilla arguta

T3168 Heliotropium arguzioides

T0664 Artemisia argyi

T0623 Aristolochia chilensis

T1895 Cupressus arizonica

T3132 Helenium arizonicum

T4906 Pinus armandii

T6864 Zanthoxylum armatum

T2802 Frullania muscicola

T0646 Armillariella tabescens

T0644 Armillaria mellea

T2288 Drypetes armoracia

T6865 Zanthoxylum arnottianum

T4909 Pinus cembra

T6907 Zingiber aromaticum

T6850 Xylopia aromatica

T1903 Curcuma aromatica

T0730 Asarum sagittarioides

T0865 Balsamorhiza sagittata

T3812 Ligularia sagitta

T6275 Tacca chantrieri [Syn. Tacca minor; Tacca esquirolii]

T5156 Populus nigra var. thevestina

T6467 Tinospora sagittata

T4821 Pholidota articulata

T5990 Solanum arundo

T2695 Ferula assafoetida

T0725 Asarum europaeum

T0745 Asparagus adscendents

T4946 Piper guineense

T0665 Artemisia ashurbajevii
YU XIANG CAO

JIA SUAN JIANG

XING ZI

XING SHU GEN

XING REN

WENG CAI

SHUI CHAO YANG

A LA BO JIN HE HUAN

XIAO GUO KA FEI

MO LI HUA

ZHU SI HONG WEN MA XIAN HAO

QIAO MU ZHUANG YANG MEI

CAI SHI MU MA SANG

QIAO MU SHAO YAO

QIAO MU HU JIAO

QIAO MU ZHUANG LAN REN

WU GONG ZHANG

SHA SHENG SHUI GUI JIAO

YIN SE MI ZI LAN

DUO CI LUO CAO

YIN BAI HUANG YANG

A GEN TING SHU QU CAO

ZI BEI TAI

RUI ZI SU

A GU JI TIAN JIE CAI

AI YE

ZHI LI MA DOU LING

LV GAN BAI

YA LI SANG NA DUI XIN JU

HUA SHAN SONG

MAO ZHU YE HUA JIAO

KUI BAN ER YE TAI

LIANG JUN

MI HUAN JUN

LA GEN HE GUO MU

A NUO TI HUA JIAO

RUI SHI SHI SONG

FANG XIANG JIANG

FANG XIANG MU BAN SHU

YU JIN

SHAN CI GU

JIAN YE TOU WU GEN

JIAN GEN SHU

JIAN GAN YANG

QING NIU DAN

JIE JING SHI XIAN TAO

A LUN DUO QIE

A WEI

OU XI XIN

SHANG JU TIAN MEN DONG

JI NEI YA HU JIAO

A SHI HAO 
Asia Belltree

Asian Colubrina

Asian Cowparsnip*

Asian lichen

Asian Pigeonwings

Asian Tetracera

Asian White Birch Bark

Asiatic Cornelian Cherry

Asiatic Moonseed

Asiatic Moonseed Root

Asiatic Pennywort

Asiatic Plantain

Asiatic Yarrow

AsiaticToddalia

Asica Baneberry

Asidefield Galingale

Aspen

Assam Crotalaria Seed

Assam Horsechestnut

Assam Tea

Assuki Bean

Astrantia

Asymmetry Rhamnella*

Atlas Cedar

Atuntsuen Rhodiola

Aucher St.John'swort*

Aureate Mayweed*

Auricled Hedyotis

Auricledleaf Mosquitotrap

Auricula

Auriculate Acacia

Auriculate Millettia*

Auriculate Swallowwort

Auriculate Twayblade*

Auriform Plagiogyria

Austral Akebia

Austral Akebia Root

Austral Bird's Foot Trefoil

Austral Rhubarb

Australia Stephania*

Australia Yew

Australian Blackwood

Australian Cowplant

Australian Nightshade

Australian Tea-tree

Autumn Lycoris

Autumn Zephyrlily

Aweto (Chinese Caterpillar Fungus)

Avicenna's Pricklyash

Avrons

Axillary Southem Wildjujube

Ayapana Eupatorium*
T5402 Radermachera sinica

T1627 Colubrina asiatica

T3215 Heracleum lanatum var. asiaticum

T5342 Pyrenula japonica

T1577 Clitoria ternatea

T6353 Tetracera asiatica

T0935 Betula platyphylla var. japonica

T1698 Cornus officinalis [Syn. Macrocarpium officinale]

T4182 Menispermum dauricum

T4183 Menispermum dauricum

T1311 Centella asiatica

T5002 Plantago asiatica

T0061 Achillea asiatica

T6471 Toddalia asiatica [Syn. Toddalia aculeata; Paullinia asiatica]

T1418 Cimicifuga asiatica

T1975 Cyperus haspan

T5162 Populus tremula

T1815 Crotalaria assamica

T0198 Aesculus assamica

T1153 Camellia sinensis var. assamica

T6752 Vigna angularis [Syn. Dolichus angularis; Phaseolus

angularis]

T0810 Astrantia major

T5453 Rhamnella inaequilatera

T1281 Cedrus atlantica

T5492 Rhodiola atuntsuensis

T3341 Hypericum aucheri

T4123 Matricaria aurea

T3123 Hedyotis auricularia

T1958 Cynanchum otophyllum

T5197 Primula auricula

T0017 Acacia auriculaeformis

T4233 Millettia auriculata

T1952 Cynanchum auriculatum

T3861 Liparis auriculata

T5000 Plagiogyria stenoptera

T0279 Akebia trifoliata var. australis

T0280 Akebia trifoliata var. australis

T3927 Lotus australis

T5468 Rheum emodi [Syn. Rheum australe]

T6130 Stephania japonica var. australis

T0832 Austrotaxus spicata

T0025 Acacia melanoxylon

T3080 Gymnema sylvestre

T5991 Solanum aviculare [Syn. Solanum laciniatum]

T3767 Leptospermum polygalifolium ssp. polygalifolium

T3988 Lycoris squamigera

T6903 Zephyranthes candida

T1683 Cordyceps sinensis

T6866 Zanthoxylum avicennae

T5589 Rubus chamaemorus

T1381 Choerospondias axillaris

T2552 Eupatorium ayapana
CAI DOU SHU

SHE TENG

YA ZHOU DU HUO

RI BEN XIAO HE YI

HU DIE HUA DOU

XI YE TENG

HUA MU PI

SHAN ZHU YU

BIAN FU GE

BIAN FU GE GEN

JI XUE CAO

CHE QIAN

YA ZHOU SHI

FEI LONG ZHANG XUE

LEI YE SHENG MA

QI PAN SHA CAO

OU ZHOU SHAN YANG

ZI XIAO RONG ZI

CHANG BING QI YE SHU

PU ER CHA

CHI DOU

DA XING QIN

BU DUI CHENG MAO RU

BEI FEI XUE SONG

DE QIN HONG JING TIAN

AO SHI JIN SI TAO

JIN SE MU JU

ER CAO

QING YANG SHEN

ER ZHUANG BAO CHUN HUA

ER XING JIN HE HUAN

ER XING JI XUE TENG

ER YE NIU PI XIAO

ER XING YANG ER LAN

ER XING LIU ZU JUE

BAI MU TONG

BAI MU TONG GEN

AO ZHOU BAI MAI GEN

ZANG BIAN DA HUANG

AO DA LI YA QIAN JIN TENG

AO DA LI YA HONG DOU SHAN

HEI MU JIN HE HUAN

CHI GENG TENG

AO ZHOU QIE

YUAN ZHI YE AO ZHOU CHA

LU CONG

GAN FENG CAO

DONG CHONG XIA CAO

YING BU BO

XUAN GOU ZI

NAN SUAN ZAO

A YA PAN ZE LAN 
Aztec Dahlia

Aztec Marigold

Aztec Marigold Leaf

Aztec Tobacco

Azure Eupatorium*

Azure Monkshood

Babylon Weeping Willow Branch

Babylon Weeping Willow Root-bast

Bacciform Cipadessa

Badgersbane

Baikal Meadowrue

Baikal Skullcap

Bailai's Chrysanthemum

Bailan Flower

Baillon Veratrilla

Balansa Melodinus*

Bald Pyrrosia

Balearic Box

Balkan Toadflax

Ballander Magnoliavine

Balloonflower

Balloonvine Heartseed

Bally Aloe*

Balmleaf Metittis

Balsam Croton*

Balsam Fir

Balsam Poplar

Balsamiferous Blumea

Balsampear

Baluchistan Barberry

Bamboo Yellow*

Bambooleaf Pricklyash

Bambooleaf Pricklyash Root

Bambooleaf Seseli

Bambooleaf Thorowax

Bambusa Hypocrella*

Bank Pepper*

Barbados Cotton

Barbary Wolfberry Fruit

Barbary Wolfberry Root-bark*

Barbate Cyclea

Barbate Deadnettle

Barbate Filmy Fern

Barbed Skullcap

Barberry-like-dileaf Canthium

Barbey Larkspur*

Bark-less Puff-ball

Barley Germinating Fruit

Basil

Basil Fruit

Bastard Speedwell

Bastardtoadflaxlike Swallowwort

Bat Dung
T1996 Dahlia pinnata [Syn. Dahlia variabilis]

T6278 Tagetes erecta

T6279 Tagetes erecta

T4427 Nicotiana rustica

T2553 Eupatorium azureum

T0095 Aconitum fischeri

T5651 Salix babylonica

T5650 Salix babylonica

T1446 Cipadessa baccifera

T0114 Aconitum lycoctonum

T6375 Thalictrum baicalense

T5834 Scutellaria baicalensis

T0854 Baileya multiradiata

T4209 Michelia alba

T6691 Veratrilla baillonii

T4176 Melodinus balansae

T5353 Pyrrosia calvata

T1086 Buxus balearica

T3843 Linaria dalmatica

T5801 Schisandra sphaerandra

T5011 Platycodon grandiflorum

T1197 Cardiospermum halicacabum

T0335 Aloe ballyi

T4175 Melittis melissophyllum

T1838 Croton balsamifera

T0004 Abies balsamea

T5147 Populus balsamifera

T0957 Blumea balsamifera

T4263 Momordica charantia

T0898 Berberis baluchistanica

T5939 Shiraia bambusicola

T6887 Zanthoxylum planispinum

T6888 Zanthoxylum planispinum

T5934 Seseli meirei

T1066 Bupleurum marginatum

T3371 Hypocrella bambusae

T4935 Piper banksii

T3054 Gossypium barbadense]

T3955 Lycium barbarum

T3954 Lycium barbarum

T1931 Cyclea barbata

T3681 Lamium barbatum

T3324 Hymenophyllum barbatum

T5835 Scutellaria barbata [Syn. Scutellaria rivularis]

T1178 Canthium berberidifolium

T2061 Delphinium barbeyi

T3701 Lasiosphaera fenzlii

T3282 Hordeum vulgare

T4470 Ocimum basilicum

T4471 Ocimum basilicum

T6729 Veronica spuria

T1961 Cynanchum thesioides

T6732 Vespertilio superans
DA LI HUA

WAN SHOU JU

WAN SHOU JU YE

HUANG HUA YAN CAO

TIAN LAN ZE LAN

BO YE WU TOU

LIU ZHI

LIU BAI PI

YA LUO CHUN

LANG DU WU TOU

BEI JIA ER TANG SONG CAO

HUANG QIN

BAI LAI SHI JU

BAI LAN HUA

HUANG QIN JIAO

BEI SHI SHAN CHENG

GUANG SHI WEI

XI BAN YA HUANG YANG

DA ER MA WEI YA LIU CHUAN YU

QIU RUI WU WEI ZI

JIE GENG

JIA KU GUA

BEI LI LU HUI

OU ZHOU MI FENG HUA

XIANG BA DOU

XIANG ZHI LENG SHAN

ZHI YANG

AI NA XIANG

KU GUA

BI LU ZHI XIAO BO

ZHU XUANG

ZHU YE JIAO

ZHU YE JIAO GEN

SAN YE FANG FENG

ZHU YE CHAI HU

ZHU HONG JUN

BAN KE HU JIAO

HAI DAO MIAN

NING XIA GOU QI ZI

NING XIA GOU QI GEN PI

YIN BU HUAN

YE ZHI MA

MO JUE

BAN ZHI LIAN

SI XIAO BO SHUANG YE YU GU MU BA BI CUI QUE HUA

MA BO

MAI YA

LUO LE

LUO LE ZI

YI ZHI XIANG

DI SHAO GUA

YE MING SHA 


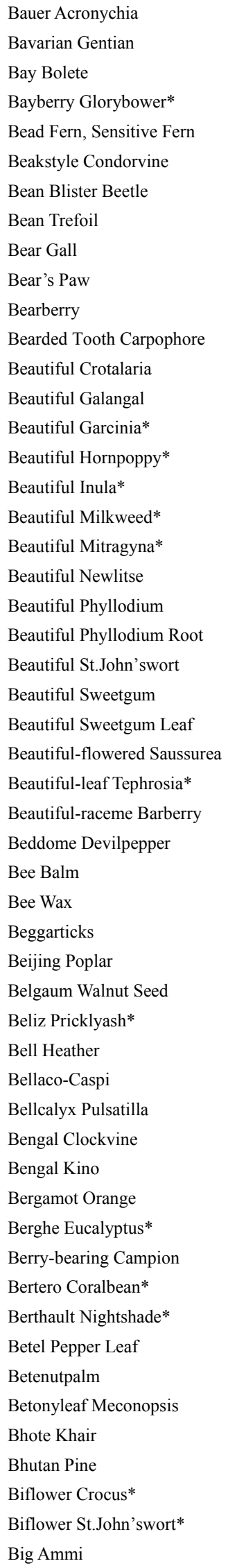

T0148 Acronychia baueri

T2903 Gentiana bavarica

T6848 Xerocomus badius

T1560 Clerodendron myricoides

T4492 Onoclea sensibilis

T4118 Marsdenia oreophila

T2384 Epicauta gorhami

T0441 Anagyris foetida

T5871 Selenarctos thibetanus; Ursus arctos

T5872 Selenarctos thibetanus; Ursus arctos

T0588 Arctostaphylos uva-ursi

T3229 Hericium erinaceus [Syn. Hydnum erinaceus]

T1834 Crotalaria spectabilis

T0363 Alpinia speciosa

T2875 Garcinia speciosa

T2971 Glaucium pulchrum

T3435 Inula magnifica

T0739 Asclepias speciosa

T4258 Mitragyna speciosa

T4405 Neolitsea pulchella

T2131 Desmodium pulchellum [Syn. Phyllodium pulchellum]

T2132 Desmodium pulchellum [Syn. Phyllodium pulchellum]

T3342 Hypericum bellum

T3867 Liquidambar formosana [Syn. Liquidambar taiwaniana]

T3866 Liquidambar formosana [Syn. Liquidambar taiwaniana]

T5767 Saussurea pulchella

T6332 Tephrosia calophylla

T0900 Berberis calliobotrys

T5429 Rauwolfia beddomei

T4174 Melissa officinalis

T0542 Apis cerana

T0938 Bidens bipinnata

T5148 Populus beijingensis

T0300 Aleurites moluccana

T6867 Zanthoxylum belizense

T2417 Erica cinerea

T3251 Himatanthus sucuuba

T5323 Pulsatilla campanella

T6444 Thunbergia grandiflora

T1083 Butea monosperma

T1470 Citrus bergamia

T2501 Eucalyptus berghei

T1871 Cucubalus baccifer

T2460 Erythrina berteroana

T5992 Solanum berthaultii

T4937 Piper betle

T0606 Areca catechu

T4141 Meconopsis betonicifolia

T2499 Eskemukerjea megacarpum

T4910 Pinus excelsa

T1807 Crocus chrysanthus-biflorus

T3353 Hypericum geminiflorum

T0410 Ammi majus
BAO RUI SHAN YOU GAN

BA FA LI YA LONG DAN

HE RONG GAI NIU GAN JUN

YANG MEI CHANG SHAN

BEI MEI QIU ZI JUE

HUI ZHU NIU NAI CAI

GE SHANG TING CHANG

CHOU WEI HONG DOU

XIONG DAN

XIONG ZHANG

XIONG GUO

HOU TOU JUN

MEI LI ZHU SHI DOU

DA CAO KOU

MEI LI TENG HUANG

MEI LI HAI YING SU

MEI LI XUAN FU HUA

MEI LI MA LI JIN

MEI LI MAO ZHU MU

MEI LI XIN MU JIANG ZI

PAI QIAN CAO

PAI QIAN CAO GEN

MEI LI JIN SI TAO

LU LU TONG

FENG XIANG SHU

MEI HUA FENG MAO JU

MEI LI YE HUI MAO DOU

MEI SUI XIAO BO

BI SHI LUO FU MU

XIANG FENG HUA

MI LA

GUI ZHEN CAO

BEI JING YANG

SHI LI ZI

BO LI ZI HUA JIAO

HUI SE OU SHI NAN

SU KU BA DOU HUA

ZHONG E BAI TOU WENG

DA HUA SHAN QIAN NIU

DAN ZI ZI MAO

XIANG NING MENG

BO SHI AN

BAI NIU XI

BO SHI CI TONG

BO SHI QIE

JU JIANG YE

BING LANG

HUO XIANG YE LV RONG HAO

QIAO GUI

SHUANG HUA FAN HONG HUA

SHUANG HUA JIN SI TAO

DA A MI 
Big Anise*

Big Anisetree*

Big Cord-grass

Big Goniothalamus*

Big Hogfennel*

Big Russula*

Big Sagebrush

Bigdentate Ampelopsis

Bigelov Sneezeweed*

Bigflower Cape Jasmine

Bigflower Cape Jasmine Leaf

Bigflower Carpesium*

Bigflower Goniothalamus

Bigflower Heartseed*

Bigflower Ladyslipper

Bigflower Magnoliavine*

Bigflower Pencilwood*

Bigflower Rhodiola

Big-flowered Javatea

Bigfruit Rosemyrtle*

Bigleaf Beautyberry

Bigleaf Breaketplant

Bigleaf Magnolia

Bigleaf Polyscias*

Bigleaf Poplar

Bigleaf Thorowax

Bignay Chinalaurel

Bignose Rhiancanthus

Bigseed Jointfir

Bigseed Swertia

Bigstreak Seseli*

Bigthyrse Rabdosia

Bimble Box

Biond Magnolia

Bipinnatifid Ginseng

Birchleaf Pear

Bird Rape

Bird-in-a-bush

Birdsfoot Trefoil

Bispore Mushroom*

Bistort

Bisulcate Milkvetch*

Biting Stonecrop

Bitter

Bitter Brucea*

Bitter Citrus

Bitter Cucumber*

Bitter Milkwort*

Bitter Nightshade Fruit

Bitter Russula

Bitter Vetch
T4899 Pimpinella magna

DA HUI QIN

T3403 Illicium majus

T6063 Spartina cynosuroides

T3046 Goniothalamus giganteus

T4757 Peucedanum grande

T5621 Russula lepida

T0702 Artemisia tridentata

T0426 Ampelopsis grossedentata [Syn. Ampelopsis cantoniesis var. grossedentata]

T3136 Helenium bigelovii

T2884 Gardenia jasminoides var. grandiflora

T2885 Gardenia jasminoides var. grandiflora

T1212 Carpesium eximum

T3047 Goniothalamus griffithii

T1196 Cardiospermum grandiflorum

T1984 Cypripedium macranthum [Syn. Cypripedium tibeticum]

T5792 Schisandra grandiflora

T2311 Dysoxylum macranthum

T5494 Rhodiola crenulata [Syn. Rhodiola euryphylla]

T4541 Orthosiphon stamineus [Syn. Orthosiphon aristatus;

Orthosiphon grandiflorus; Orthosiphon spicatus]

T5527 Rhodomyrtus macrocarpa

T1120 Callicarpa macrophylla

T1380 Chlorophytum malayense

T4048 Magnolia rostrata

T5130 Polyscias amplifolia

T5155 Populus lasiocarpa

T1065 Bupleurum longiradiatum

T0535 Antidesma bunius

T5483 Rhinacanthus nasutus

T3032 Gnetum montanum f. megalocarpum

T6225 Swertia macrosperma

T5930 Seseli grandivittatum

T3514 Isodon megathyrsus

T2516 Eucalyptus populnea

T4035 Magnolia biondii [Syn. Magnolia fargesii]

T4604 Panax japonicus var. bipinnatifidus

T5361 Pyrus betulaefolia

T1005 Brassica campestris [Syn. Brassica campestris var. oleifera]

T1708 Corydalis bulbosa [Syn. Corydalis solida]

T3928 Lotus corniculatus

T0210 Agaricus bisporus

T5099 Polygonum bistorta

T0787 Astragalus bisulcatus

T5849 Sedum acre

T3621 Kaempferia marginata

T1036 Brucea amarissima

T1469 Citrus aurantium var. amara

T1879 Cucumis sativus var. hanzil

T5070 Polygala amarella

T5998 Solanum dulcamara

T5624 Russula rosacea

T3705 Lathyrus montanus
DA BA JIAO

YAN DI HE

DA GE NA XIANG

DA QIAN HU

DA HONG GU

SAN CHI HAO

XIAN CHI SHE PU TAO

BI SHI DUI XIN JU

SHUI ZHI

SHUI ZHI YE

DA HUA JIN WA ER

DA HUA GE NA XIANG

DA HUA DAO DI LING

DA HUA SHAO LAN

DA HUA WU WEI ZI

DA HUA JIAN MU

DA HUA HONG JING TIAN

XIONG RUI ZHUANG ZHI GUAN

CAO

DA GUO TAO JIN NIANG

DA YE ZI ZHU

DA YE DIAO LAN

DA YE HOU PO

DA YE NAN YANG SHEN

DA YE YANG

DA YE CHAI HU

WU YUE CHA

BAI HE LING ZHI

DA ZI MAI MA TENG

DA ZI ZHANG YA CAI

DA TIAO WEN XIE HAO

DA ZHUI XIANG CHA CAI

YANG YE AN

WANG CHUN YU LAN

YU YE SAN QI

TANG LI

YUN TAI ZI

SHAN YAN HU SUO

DI YANG QUE

SHUANG BAO MO GU

QUAN SHEN

ER GOU HUANG QI

TAI JING TIAN

KU SHAN NAI

KU YA DAN ZI

DAI DAI HUA

KU HUANG GUA

KU WEI YUAN ZHI

QIAN NIAN BU LAN XIN

KU HONG GU

SHAN DI XIANG WAN DOU 
Bitter Willow Bast

Bitter Willow Branch-leaf

Bitterleaf

Bitterness Sneezeweed*

Bittersweet

Black Bryony

Black Bui*

Black Cottonwood

Black Currant

Black Dianella*

Black Elder

Black Falsehellebore

Black Hellebore

Black Henbane Leaf

Black Henbane Root

Black Henbane Seed

Black Honeysuckle

Black Indian Hemp

Black Locust Flower

Black Magnoliavine*

Black Mustard

Black Nightshade

Black Oak

Black Pepper

Black Rice Spermoderm*

Black Silkvine

Black Soyabean

Black Soyabean Leaf

Black Soyabean Spermoderm

Black Thorowax

Black Walnut

Black Wattle

Black White Quebracho*

Blackberrylily

Blacken Saussurea

Blackend Swallowwort

Blackflawer Rabdosia*

Blackflower Cassytha*

Blackfruit Greenbrier

Blackfruit Madder

Blackhair Denrdobium

Blackhaw

Black-powder Fungus in Corn

Black-purple Scurrula*

Black-rock Ophiorrhiza*

Black-spot Hornpoppy

Blackstick Coralbean*

Blacktiger Kadsura

Blasia*

Bleedingheart

Bleedingheart Glorybower

Blepharostoma*
T5653 Salix purpurea

T5654 Salix purpurea

T6712 Vernonia amygdalina

T3131 Helenium amarum

T6005 Solanum lyratum

T6291 Tamus communis

T0863 Ballota nigra

T5164 Populus trichocarpa

T5546 Ribes nigrum

T2140 Dianella nigra

T5704 Sambucus nigra

T6698 Veratrum nigrum

T3183 Helleborus niger

T3328 Hyoscyamus niger

T3327 Hyoscyamus niger

T3329 Hyoscyamus niger

T3916 Lonicera nigra

T0551 Apocynum cannabinum

T5552 Robinia pseudoacacia

T5796 Schisandra nigra

T1012 Brassica nigra

T6008 Solanum nigrum

T5381 Quercus tinctoria

T4957 Piper nigrum

T4547 Oryza sativa cv

T4728 Periploca nigrescens

T3000 Glycine max

T3002 Glycine max

T3001 Glycine max

T1074 Bupleurum smithii

T3566 Juglans nigra

T0027 Acacia mollissima

T0769 Aspidosperma nigricans

T0892 Belamcanda chinensis

T5762 Saussurea nigrescens

T1951 Cynanchum atratum

T3531 Isodon trichocarpus

T1253 Cassytha melantha

T5978 Smilax glauco-china

T5580 Rubia cordifolia var. pratensis

T2111 Dendrobium williamsonii

T6739 Viburnum prunifolium

T6656 Ustilago maydis

T5830 Scurrura atropurpurea

T4511 Ophiorrhiza kuroiwai

T2966 Glaucium corniculatum

T2470 Erythrina melanacantha

T3613 Kadsura coccinea [Syn. Kadsura chenensis; Kadsura

hainanensis]

T0949 Blasia pusilla

T2150 Dicentra formosa

T1570 Clerodendrum thomsonae

T0953 Blepharostoma trichophyllum
SHUI YANG MU BAI PI

SHUI YANG ZHI YE

BIAN TAO ZHUANG BAN JIU JU

KU WEI DUI XIN JU

BAI MAO TENG

JIANG GUO SHU YU

HEI BA LUO CAO

MAO GUO YANG

HEI CHA BIAO

HEI JIE GENG LAN

XI YANG JIE GU MU

LI LU

TI GEN CAO

LANG DANG YE

LANG DANG GEN

LANG DANG ZI

HEI REN DONG

JIA ZHU TAO MA

CI HUAI HUA

NEI FENG XIAO

HEI JIE

LONG KUI

ZHUO SE LI

HU JIAO

HEI SE MI PI KANG

HEI GANG LIU

HEI DA DOU

HEI DA DOU YE

HEI DA DOU PI

HEI CHAI HU

HEI HU TAO

ROU JIN HE HUAN

HEI BAI JIAN MU

SHE GAN

DUN BAO XUE LIAN

BAI WEI

HEI HUA YAN MING CAO

HEI HUA WU GEN TENG

HEI GUO BA QIA

HEI GUO QIAN CAO

HEI MAO SHI HU

YING YE JIA MI

YU MI HEI MEI

HEI ZI LI GUO JI SHENG

HEI YAN SHE GEN CAO

XIAO JIAO HAI YING SU

HEI CI CI TONG

LENG FAN TUAN[yn

HU BAO TAI

MEI LI HE BAO MU DAN

LONG TU ZHU

JIE MAO TAI 


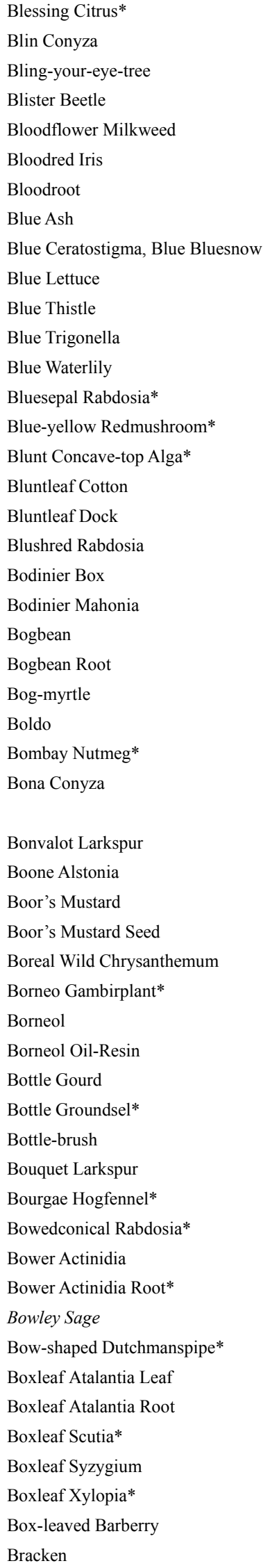

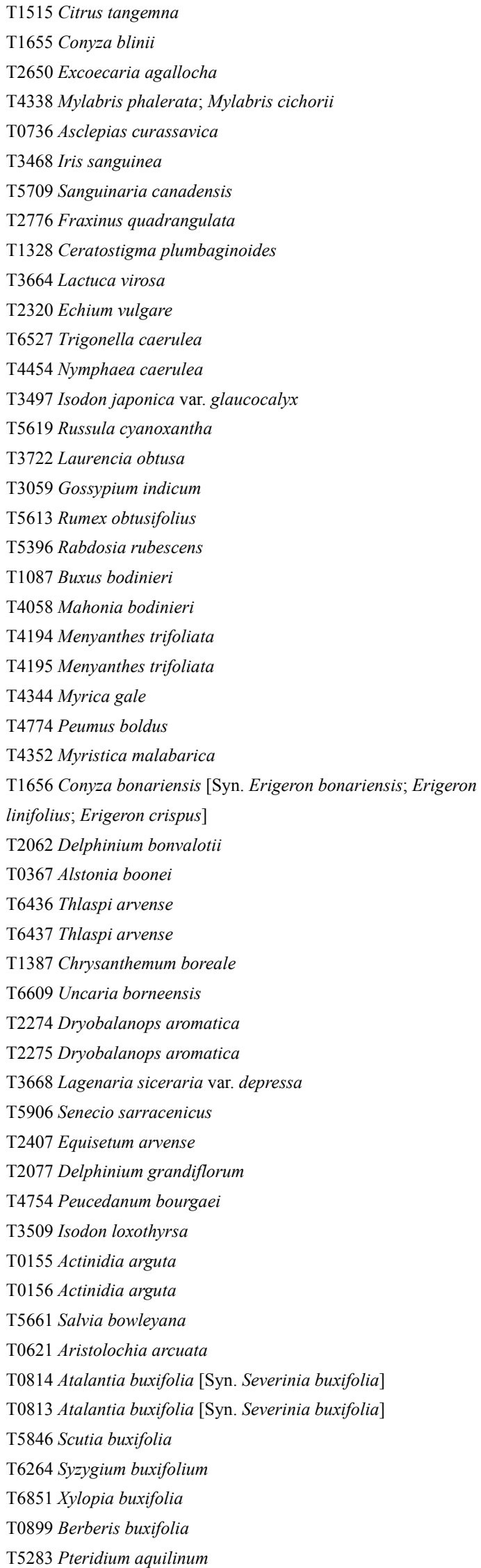

FU JU

KU HAO

HAI QI

BAN MAO

LIAN SHENG GUI ZI HUA

DOU CHI CAO

MEI ZHOU XUE GEN CAO

SI LENG LA SHU

JIAO ZHU HUA

DU WO JU

LAN JI

LAN HU LU BA

LAN SHUI LIAN

LAN E XIANG CHA CAI

LAN HUANG HONG GU

DUN XING AO DING ZAO

YIN DU MIAN

DUN YE SUAN MO

DONG LING CAO

QUE SHE HUANG YANG

XIAO GUO SHI DA GONG LAO

SHUI CAI

SHUI CAI GEN

XIANG YANG MEI

BO LU DU SHU

MENG MAI ROU DOU KOU

XIANG SI CAO

CHUAN QIAN CUI QUE HUA

GAN LAO JI GU CHANG SHAN

XI MING

XI MING ZI

BEI YE JU

PO LUO ZHOU GOU TENG

BING PIAN

LONG NAO GAO XIANG

HU GUA

PING QIAN LI GUANG

WEN JING

CUI QUE HUA

BO SHI QIAN HU

WAN ZHUI XIANG CHA CAI

MI HOU LI

MI HOU LI GEN

NAN DAN SHEN

GONG XING MA DOU LING

DONG FENG JU YE

DONG FENG JU GEN

HUANG YANG YE DUI CI TENG

CHI NAN

HUANG YANG YE MU BAN SHU

HUANG YANG XIAO BO

OU ZHOU JUE 


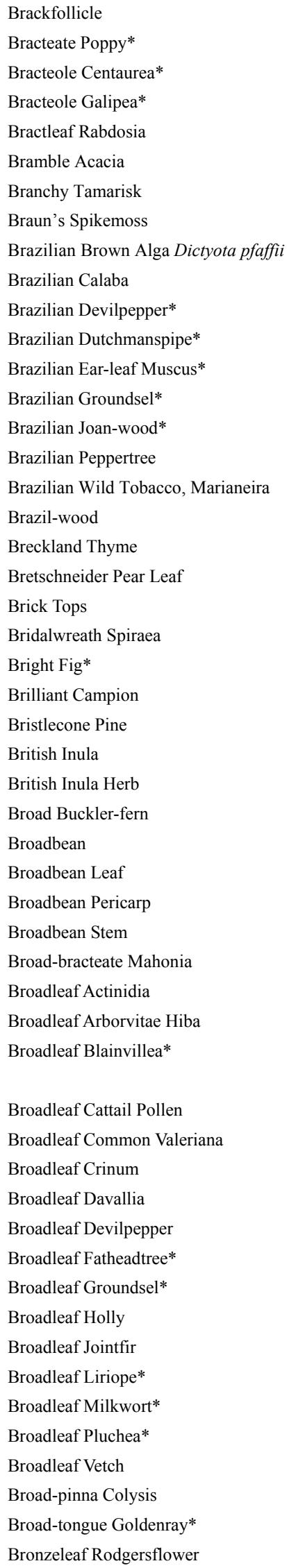

T6578 Tylophora atrofolliculata

T4623 Papaver bracteatum

T1304 Centaurea bracteata

T2827 Galipea bracteata

T3515 Isodon melissoides

T0034 Acacia victoria

T6290 Tamarix ramosissima

T5859 Selaginella braunii

T2171 Dictyota pfaffii

T1125 Calophyllum brasiliense

T5422 Rauvolfia bahiensis

T0622 Aristolochia chamissonis

T2801 Frullania brasiliensis

T5881 Senecio brasiliensis

T3562 Joannesia princeps

T5789 Schinus terebinthifolius

T0075 Acnistus arborescens

T1103 Caesalpinia echinata

T6452 Thymus serpyllum

T5362 Pyrus bretschneideri

T4367 Naematoloma sublateritium

T6084 Spiraea prunifolia

T2722 Ficus nitida

T3950 Lychnis fulgens

T4905 Pinus aristata

T3427 Inula britannica

T3426 Inula britannica

T2277 Dryopteris austriaca

T6744 Vicia faba

T6747 Vicia faba

T6745 Vicia faba

T6746 Vicia faba

T4061 Mahonia eurybracteata

T0163 Actinidia latifolia

T6443 Thujopsis dolobrata

T0948 Blainvillea acmella [Syn. Verbesina acmella; Eclipta latifolia;

Blainvillea latifolia

T6587 Typha latifolia

T6680 Valeriana officinalis var. latifolia

T1799 Crinum latifolium

T2054 Davallia solida

T5435 Rauwolfia latifrons

T4390 Nauclea latifolia

T5897 Senecio platyphyllus

T3393 Ilex latifolia

T3031 Gnetum latifolium

T3873 Liriope platyphylla

T5085 Polygala senega var. latifolia

T0848 Baccharis latifolia

T6742 Vicia amoena

T1629 Colysis pothifolia [Syn. Hemionitis pothifolia]

T3811 Ligularia platyglossa

T5556 Rodgersia podophylla
SAN FEN DAN

DA HONG YING SU

BAO PIAN SHI CHE JU

BAO PIAN TU LA SHU

BAO YE XIANG CHA CAI

WEI DUO LI YA JIN HE HUAN

DUO ZHI CHENG LIU

MAO ZHI JUAN BAI

BA XI ZONG ZAO

BA XI HU TONG

BA XI LUO FU MU

BA XI MA DOU LING

BA XI ER YE TAI

BA XI QIAN LI GUANG

BA XI QIAO AN MU

XIAO RU XIANG

BA XI YE YAN

JI YUN SHI

BAI LI XIANG

LI YE

ZHUAN HONG REN SAN

XIAO YE HUA

LIANG YE RONG

DA HUA JIAN QIU LUO

CI GUO SONG

XUAN FU HUA

DA HUA XUAN FU HUA CAO

AO DI LI LIN MAO JUE

CAN DOU

CAN DOU YE

CAN DOU JIA KE

CAN DOU JING

KUAN BAO SHI DA GONG LAO

KUO YE MI HOU TAO

LUO HAN BAI

YU LIN CAI

KUAN YE XIANG PU
KUO YE XIE CAO
XI NAN WEN SHU LAN
KUO YE GU SUI BU
KUO YE LUO FU MU
KUAN YE WU TAN
KUAN YE QIAN LI GUANG
DA YE DONG QING
KUAN YE MAI MA TENG
KUO YE SHAN MAI DONG
KUAN YE MEI YUAN ZHI
KUAN YE KUO BAO JU
SHAN YE WAN DOU
KUAN YU XIAN JUE
KUAN SHE TUO WU
RI BEN GUI DENG QING




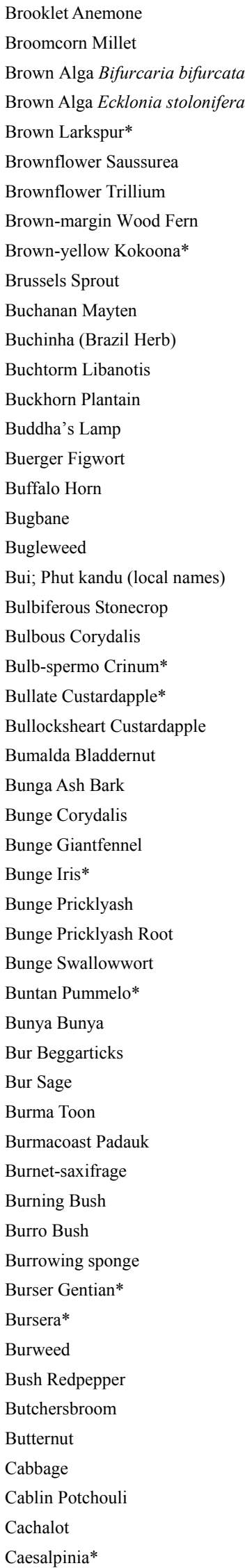

T0471 Anemone rivularis

T4618 Panicum miliaceum

T0943 Bifurcaria bifurcata

T2322 Ecklonia stolonifera

T2063 Delphinium brownii

T5765 Saussurea phaeantha

T6532 Trillium erectum

T2286 Dryopteris sacrosancta

T3638 Kokoona ochracea

T1018 Brassica oleracea var. gemmifera

T4128 Maytenus buchananii

T3936 Luffa operculata

T3796 Libanotis buchtormensis

T5006 Plantago lanceolata

T4335 Mussaenda pubescens

T5826 Scrophularia buergeriana

T1045 Bubalus bubalis

T1420 Cimicifuga foetida

T3982 Lycopus virginicus

T0862 Ballota limbata

T5852 Sedum bulbiferum

T1712 Corydalis cava

T1797 Crinum bulbispermum

T0504 Annona bullata

T0511 Annona reticulata

T6097 Staphylea bumalda

T2766 Fraxinus bungeana

T1709 Corydalis bungeana

T2697 Ferula borealis

T3454 Iris bungei

T6869 Zanthoxylum bungeanum

T6870 Zanthoxylum bungeanum

T1953 Cynanchum bungei

T1481 Citrus grandis f. buntan

T0578 Araucaria bidwillii

T0941 Bidens tripartita

T0395 Ambrosia acanthicarpa

T6474 Toona ciliata

T5301 Pterocarpus indicus

T4900 Pimpinella saxifraga

T2164 Dictamnus albus

T3322 Hymenoclea salsola

T1575 Cliona celata

T2905 Gentiana burseri

T1080 Bursera graveolens

T6837 Xanthium chinense

T1187 Capsicum frutescens

T5618 Ruscus aculeatus

T3563 Juglans cinerea

T1017 Brassica oleracea var. capitata

T5059 Pogostemon cablin [Syn. Mentha cablin]

T4856 Physeter catodon

T1101 Caesalpinia decapetala
HU ZHANG CAO

SHU MI

SHUANG CHA ZAO

ZONG ZAO

BAO SHI FEI YAN CAO

HE HUA XUE LIAN

HE HUA YAN LING CAO

RI BEN LIN MAO JUE

ZHE HUANG KAO GU NA

BAO ZI GAN LAN

BU CHANG NAN MEI DENG MU

NANG GAI SI GUA

YAN FENG

CHANG YE CHE QIAN

SHAN GAN CAO

BEI XUAN SHEN

SHUI NIU JIAO

SHENG MA

FU JI NI YA DI SUN

\section{XIAO JIAN CAO}

AO XIAN ZI JIN

LIN JING ZHONG ZI WEN SHU LAN

PAO ZHUANG FAN LI ZHI

NIU XIN FAN LI ZHI

SHENG GU YOU

XIAO YE CEN

KU DI DING

SHA QIAN HU

BENG GE YUAN WEI

HUA JIAO

HUA JIAO GEN

BAI SHOU WU

WEN DAN YOU

DA YE NAN YANG SHAN

LANG PA CAO

CI GUO TUN CAO

HONG CHUN

ZI TAN

HU ER CAO YE HUI QIN

BAI SE BAI XIAN

MEI GUO HAI MO JU

YIN JU CHUAN BEI HAI MIAN

BU SHI LONG DAN

LIE WEI LIE LAN

ZHONG GUO CANG ER

LA JIAO

JIA YE SHU

HUI HU TAO

GAN LAN

GUANG HUO XIANG

MO XIANG JING

YUN SHI YE 


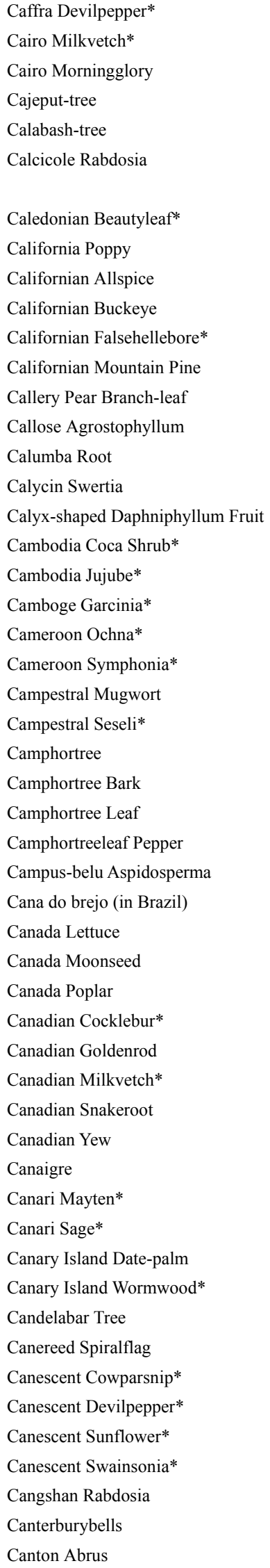

T5430 Rauwolfia caffra

T0797 Astragalus kahiricus

T3448 Ipomoea cairica [Syn. Ipomoea palmata]

T4154 Melaleuca leucadendra

T1791 Crescentia cujete

T3483 Isodon calcicola

T1126 Calophyllum caledonicum

T2495 Eschscholzia californica

T1140 Calycanthus occidentalis

T0199 Aesculus californica

T6694 Veratrum californicum

T4918 Pinus monticola

T5363 Pyrus calleryana

T0254 Agrostophyllum callosum

T3559 Jateorhiza palmata

T6213 Swertia calycina

T2032 Daphniphyllum calycinum

T2490 Erythroxylum cambodianum

T6915 Ziziphus cambodiana

T2851 Garcinia cambogia

T4464 Ochna calodendron

T6242 Symphonia globulifera

T0668 Artemisia campestris

T5928 Seseli campestre

T1435 Cinnamomum camphora

T1436 Cinnamomum camphora

T1437 Cinnamomum camphora

T4961 Piper polysyphorum

T0761 Aspidosperma campus-belus

T1757 Costus spicatus

T3658 Lactuca canadensis

T4181 Menispermum canadense

T5149 Populus canadensis

T6835 Xanthium canadense

T6022 Solidago altissima

T0789 Astragalus canadensis var. mortonii

T0723 Asarum canadense

T6309 Taxus canadensis

T5610 Rumex hymenosepalus

T4129 Maytenus canariensis

T5665 Salvia canariensis

T4819 Phoenix canariensis

T0671 Artemisia canariensis

T0577 Araucaria angustifolia

T1756 Costus speciosus

T3211 Heracleum canescens

T5431 Rauwolfia canescens

T3147 Helianthus canescens

T6207 Swainsonia canescens

T3482 Isodon bulleyana

T1156 Campanula medium

T0010 Abrus fruticulosus [Syn. Abrus cantoniensis]
KA FU LA LUO FU MU

KAI LUO HUANG QI

WU ZHAO LONG

BAI QIAN CENG

PAO DAN GUO

JIN WU MAO HUI YAN XIANG CHA

CAi

SU GE LAN HU TONG

HUA LING CAO

JIA ZHOU XIA LA MEI

JIA ZHOU QI YE SHU

JIA ZHOU LI LU

JIA ZHOU SHAN SONG

YE LI ZHI YE

YING PI HE YE LAN

FEI ZHOU FANG JI

BAO E ZHANG YA CAI

NIU ER FENG ZI

JIAN PU ZHAI GU KE

JIAN PU ZHAI ZAO

TENG HUANG SHAN ZHU ZI

KA MAI LONG JIN LIAN MU

KA MAI LONG XIN FO NI A

TIAN YE HAO

PING DI XI FENG QIN

ZHANG MU

ZHANG SHU PI

ZHANG SHU YE

ZHANG YE HU JIAO

BAI JIAN MU

SUI ZHUANG BI QIAO JIANG

JIA NA DA WO JU

MEI GUO BIAN FU GE

JIA YANG

JIA NA DA CANG ER

GAO YI ZHI HUANG HUA

JIA NA DA HUANG QI

JIA NA DA XI XIN

JIA NA DA HONG DOU SHAN

MO E SUAN MO

JIA NA LI MEI DENG MU

JIA NA LI SHU WEI CAO

ZHEN KUI

JIA NA LI HAO

ZHAI YE NAN YANG SHAN

ZHANG LIU TOU

HUI BAI DU HUO

HUI BAI MAO LUO FU MU

HUI BAI XIANG RI KUI

HUI BAI KU MA DOU

CANG SHAN XIANG CHA CAI

FENG LING CAO

JI GU CAO 


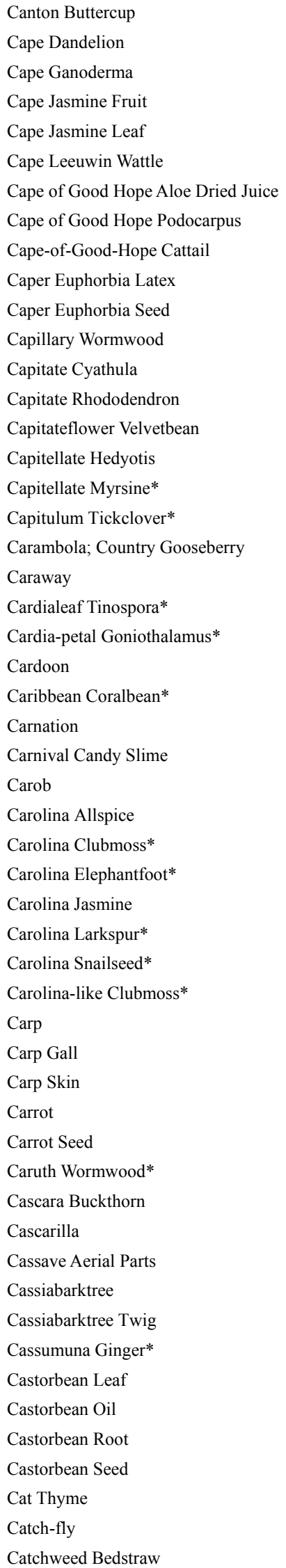

T5413 Ranunculus cantoniensis

T6687 Venidium decurrens

T2845 Ganoderma capense

T2882 Gardenia jasminoides [Syn. Gardenia florida]

T2883 Gardenia jasminoides [Syn. Gardenia florida]

T0294 Albizzia lophantha

T0338 Aloe ferox

T5037 Podocarpus elongatu

T6586 Typha capensis

T2598 Euphorbia lathyris

T2597 Euphorbia lathyris

T0672 Artemisia capillaris

T1923 Cyathula capitata

T5504 Rhododendron capitatum

T6147 Stizolobium capitatum

T3124 Hedyotis capitellata

T4362 Myrsine capitellata

T2129 Desmodium cephalotes

T0834 Averrhoa carambola

T1217 Carum carvi

T6464 Tinospora cordifolia

T3043 Goniothalamus cardiopetalus

T1965 Cynara cardunculus

T2462 Erythrina caribea

T2142 Dianthus caryophyllus

T0590 Arcyria denudata

T1326 Ceratonia siliqua

T1139 Calycanthus floridus

T3967 Lycopodium carolinianum

T2331 Elephantopus carolinianus

T2899 Gelsemium sempervirens

T2065 Delphinium carolinianum

T1584 Cocculus carolinus

T3968 Lycopodium carolinianum var. affine

T1980 Cyprinus carpio

T1981 Cyprinus carpio

T1982 Cyprinus carpio

T2050 Daucus carota var. sativa

T2051 Daucus carota var. sativa

T0673 Artemisia caruthii

T5463 Rhamnus purshiana

T1844 Croton eluteria

T4104 Manihot esculenta

T1439 Cinnamomum cassia [Syn. Cinnamomum aromaticum]

T1438 Cinnamomum cassia [Syn. Cinnamomum aromaticum]

T6908 Zingiber cassumunar

T5548 Ricinus communis

T5549 Ricinus communis

T5547 Ricinus communis

T5550 Ricinus communis

T6362 Teucrium marum

T5954 Silene fortunei

T2830 Galium aparine
ZI KOU CAO

NI JIN ZHAN JU

BAO GAI LING ZHI

ZHI ZI

ZHI ZI YE

YU ZHUANG HE HUAN

HAO WANG JIAO LU HUI

HAO WANG JIAO LUO HAN SONG

HAO WANG JIAO XIANG PU

XU SUI ZI JING ZHONG BAI ZHI

QIAN JIN ZI

YIN CHEN HAO

MA NIU XI

TOU HUA DU JUAN

LI DOU

XIAO TOU LIANG HOU CHA

XIAO TOU TIE ZI

JIA MU DOU

YANG TAO

GE LU ZI

XIN XING YE QING NIU DAN

XIN XING BAN GE NA XIANG

CI CAI JI

JIA LE BI CI TONG

SHE XIANG SHI ZHU

AN HONG TUAN WANG JUN

CHANG JIAO DOU

MEI GUO XIA LA MEI

KA LUO LAI NA SHI SONG

KA LUO LAI NA DI DAN CAO

CHANG LV GOU WEN

KA LUO LAI NA CUI QUE

MEI GUO QING TENG

SI KA LUO LAI NA SHI SONG

LIYU

LI YU DAN

LI YU PI

HU LUO BO

HU LUO BO ZI

KA SI HAO

BO XI SHU LI

KU XIANG SHU

MU SHU DI SHANG BU FEN

ROU GUI

GUI ZHI

YE JIANG

BI MA YE

BI MA YOU

BI MA GEN

BI MA ZI

MA SHI XIANG KE KE

YING ZI CAO

BA XIAN CAO 


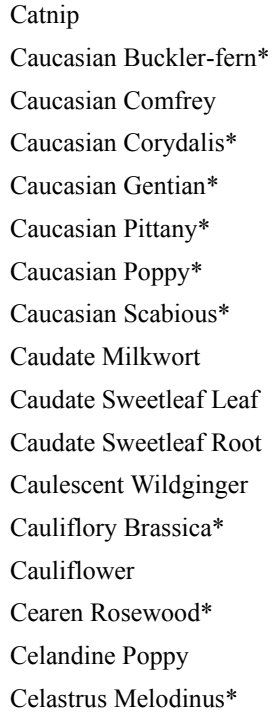

T4413 Nepeta cataria

T2278 Dryopteris caucasica

T6245 Symphytum caucasicum

T1711 Corydalis caucasica

T2907 Gentiana caucasa

T2166 Dictamnus caucasicus

T4624 Papaver caucasicum

T5774 Scabiosa caucasica

T5072 Polygala caudata

T6251 Symplocos caudata

T6250 Symplocos caudata

T0724 Asarum caulescens

T1015 Brassica oleracea var. botrytis subvar. cauliflora

T1014 Brassica oleracea var. botrytis

T1998 Dalbergia cearensis

T6195 Stylophorum diphyllum

T4177 Melodinus celastroides

T0662 Artemisia apiacea [Syn. Artemisia carvifolia; Artemisia

caruifolia ]

T4515 Opilia celtidifolia

T0710 Artocarpus champeden

T5817 Scolopendra subspinipes mutilans

T2913 Gentiana kaufmanniana

T3257 Hippophae rhamnoides subsp. turkestanica

T0451 Ancistrocladus likoko

T6176 Strychnos icaja

T5853 Sedum cepaea

T2420 Ericerus pela

T3970 Lycopodium cernuum

T3190 Helminthostachys zeylanica

T1445 Cinnamomum zeylanicum

T2493 Erythroxylum zeylanicum

T1971 Cynoglossum zeylanicum [Syn. Anchusa zeylanica;

Cynoglossum furcatum; Cynoglossum formosanum ]

T3292 Hunteria zeylanica

T2216 Diospyros ebenum

T5994 Solanum chacoense

T1471 Citrus chachiensis

T1472 Citrus chachiensis

T0425 Ampelopsis chaffanjonii

T1058 Bupleurum chaishoui

T6360 Teucrium chamaedrys

T0399 Ambrosia chamissonis

T1159 Campomanesia lineatifolia

T4210 Michelia champaca

T3742 Lentinus edodes

T0877 Bauhinia championii

T2279 Dryopteris championii

T3787 Liatris champmanii

T5201 Primula modesta

T5959 Sinapis arvensis

T6836 Xanthium chasei
JIA JING JIE

GAO JIA SUO LIN MAO JUE

XIN FEI CAO

GAO JIA SUO ZI JIN

GAO JIA SUO LONG DAN

GAO JIA SUO BAI XIAN

GAO JIA SUO YING SU

GAO JIA SUO LAN PEN HUA

SHUI HUANG YANG MU

SHAN FAN YE

SHAN FAN GEN

SHUANG YE XI XIN

JING HUA HUA YE CAI

HUA YE CAI

XI A LA HUANG TAN

ER YE BAO YING SU

NAN SHE TENG ZHUANG SHAN

CHENG

QING HAO

PU YE SHAN YOU ZI

YIN NI MIAN BAO GUO

WU GONG

ZHONG YA QIN JIAO

ZHONG YA SHA JI

ZHONG FEI GOU ZHI TENG

ZHONG FEI MA QIAN

XI PA JING TIAN

CHONG BAI LA

PU DI WU GONG

RU DI WU GONG

XI LAN ROU GUI

XI LAN GU KE

LIU LI CAO

ZI LAN SHU

WU MU XIE

CHA QIE

GAN

GAN PI

YU YE SHE PU TAO

CHAI SHOU

SHI CAN XIANG KE KE

CHA MI SEN TUN CAO

HUANG MIAN GUI

XIANG XUN

LONG XU TENG

MAO GUAN ZHONG

CHA SHI SHE BIAN JU

CHANG BAI SHAN BAO CHUN

YE OU BAI JIE

CAI SI CANG ER 


\begin{tabular}{|c|}
\hline Chaulmoogratree Seed \\
\hline Chaxiong Ligusticum \\
\hline Chayu St.John'swort \\
\hline Cheliensis Goniothalamus \\
\hline Chemens Phoebe* \\
\hline Chengkou Mahonia \\
\hline Chenille Plant, Red-hot Cat's-tail \\
\hline Cherimoya \\
\hline Cherokee Rose Leaf \\
\hline Cherokee Rose Seed \\
\hline Cherry Tomato* \\
\hline Chestnut Sage \\
\hline Chicken \\
\hline Chicken Brain \\
\hline Chicken's Gizzard Endothelium \\
\hline Chicken-feather Vegatable* \\
\hline Chile Calceolaria* \\
\hline Chile Podocarpus* \\
\hline China Alyxia \\
\hline China Amsonia \\
\hline China Douglas Fir \\
\hline China Ixeris \\
\hline China Paris \\
\hline China Weasel-snout \\
\hline China Winterberry Currant \\
\hline Chinaberry-tree \\
\hline Chinaberry-tree Bark \\
\hline Chinaberry-tree Flower \\
\hline Chinaberry-tree Fruit \\
\hline Chinaberry-tree Leaf* \\
\hline Chinaroot Greenbrier \\
\hline Chinese Alangium \\
\hline Chinese Aloe Dried Juice \\
\hline Chinese Angelica \\
\hline Chinese Arborvitae Branch \\
\hline Chinese Arborvitae Kernel* \\
\hline Chinese Arborvitae Leaf \\
\hline Chinese Artichoke \\
\hline Chinese Arundina \\
\hline Chinese Ash Bark \\
\hline Chinese Aspen \\
\hline Chinese Astilbe \\
\hline Chinese Atractylodes \\
\hline Chinese Atropanthe \\
\hline Chinese Aucuba \\
\hline Chinese Azalea \\
\hline Chinese Azalea Fruit \\
\hline Chinese Bastardtoadflax \\
\hline Chinese Bitterleaf* \\
\hline Chinese Box Juvenile Leaf \\
\hline Chinese Brake \\
\hline Chinese Brake \\
\hline Chinese Bretschneidera \\
\hline
\end{tabular}

T3300 Hydnocarpus anthelminticus

T3825 Ligusticum sinense $\mathrm{cv}$. chaxiong

T3369 Hypericum wightianum subsp. axillare

T3044 Goniothalamus cheliensis

T4815 Phoebe chemensii

T4072 Mahonia shenii

T0035 Acalypha hispida

T0505 Annona cherimolia

T5567 Rosa laevigata

T5568 Rosa laevigata

T3963 Lycopersicon esculentum var. cerasiforme

T5667 Salvia castanea

T2837 Gallus gallus domesticus

T2836 Gallus gallus domesticus

T2838 Gallus gallus domesticus

T5309 Pterocladia tenuis

T1110 Calceolaria inamoena

T5036 Podocarpus andina

T0383 Alyxia sinensis

T0434 Amsonia sinensis

T5261 Pseudotsuga sinensis

T3547 Ixeris chinensis

T4649 Paris polyphylla var. chinensis

T2825 Galeobdolon chinense [Syn. Lamium chinense]

T5545 Ribes fasciculatum var. chinense

T4155 Melia azadirachta

T4156 Melia azedarach

T4159 Melia azedarach

T4157 Melia azedarach

T4158 Melia azedarach

T5976 Smilax china [Syn. Smilax japonica]

T0281 Alangium chinense

T0348 Aloe vera var. chinensis

T0495 Angelica sinensis

T6439 Thuja orientalis [Syn. Platycladus orientalis; Biota orientalis]

T0944 Biota orientalis [Syn. Thuja orientalis; Platycladus orientalis]

T6440 Thuja orientalis [Syn. Platycladus orientalis; Biota orientalis]

T6094 Stachys tuberifera

T0721 Arundina chinensis

T2767 Fraxinus chinensis

T5144 Populus adenopoda

T0784 Astilbe chinensis

T0819 Atractylodes chinensis

T5822 Scopolia sinensis

T0827 Aucuba chinensis ssp. omeiensis

T5516 Rhododendron molle

T5517 Rhododendron molle

T6431 Thesium chinense

T6714 Vernonia chinense

T1092 Buxus microphylla var. sinica

T5295 Pteris multifida

T5298 Pteris vittata

T1025 Bretschneidera sinensis
DA FENG ZI

CHA XIONG

CHA YU BIAN DI JIN

GE NA XIANG

CHE SHI NAN

CHENG KOU SHI DA GONG LAO

CU YING MAO TIE XIAN CAI

MAO YE FAN LI ZHI

JIN YING YE

JIN YING ZI

YING TAO FAN QIE

LI SE SHU WEI CAO

JI ROU

JI NAO

JI NEI JIN

JI MAO CAI

BU MEI HE BAO HUA

ZHI LI LUO HAN SONG

LIAN ZHU TENG

SHUI GAN CAO

HUANG SHAN

SHAN KU MAI

HUA CHONG LOU

XIAO YE ZHI MA

HUA CHA BIAO

KU LIAN PI

LIAN HUA

KU LIAN SHI

KU LIAN YE

BA QIA

BA JIAO FENG

BAN WEN LU HUI

DANG GUI

CE BAI ZHI JIE

BAI ZI REN

CE BAI YE

KUAI JING SHUI SU

ZHU YE LAN

BAI LA SHU

XIANG YE YANG

LUO XIN FU

BEI CANG ZHU

TIAN PENG ZI

TIAN JIAO BAN

NAO YANG HUA

NAO YANG HUA ZI

BAI RUI CAO

ZHONG GUO BAN JIU JU

HUANG YANG MU YE

FENG WEI CAO

WU GONG CAO

BO LE SHU 


\begin{tabular}{|c|}
\hline Chinese Buckeye \\
\hline Chinese Cedar \\
\hline Chinese Cedrela* \\
\hline Chinese Chestnut \\
\hline Chinese Chestnut Bast \\
\hline Chinese Cinquefoil \\
\hline Chinese Clematis \\
\hline Chinese Clinopodium \\
\hline Chinese Common Jasminorange \\
\hline Chinese Coriaria \\
\hline Chinese Coriaria Leaf \\
\hline Chinese Corktree \\
\hline Chinese Corydalis \\
\hline Chinese Cricket \\
\hline Chinese Crinum \\
\hline Chinese Crossostephium Root \\
\hline Chinese Cryptocarya \\
\hline Chinese Cryptolepis \\
\hline Chinese Date \\
\hline Chinese Desmos \\
\hline Chinese Dodder Seed \\
\hline Chinese Dregea \\
\hline Chinese Dunnia \\
\hline Chinese Dwarf Cherry Seed \\
\hline Chinese Eaglewood \\
\hline Chinese Elm Bark \\
\hline Chinese Ephedra \\
\hline Chinese Ephedra Root \\
\hline Chinese Eupatorium \\
\hline Chinese Fevervine \\
\hline Chinese Floweringquince \\
\hline Chinese Forgetmenot \\
\hline Chinese Galangal \\
\hline Chinese Gambirplant \\
\hline Chinese Globeflower \\
\hline Chinese Golden Larch \\
\hline
\end{tabular}

Chinese Goldthread

Chinese Hawthorn

Chinese Hawthorn Flower

Chinese Hawthorn Leaf

Chinese Hibiscus Flower

Chinese Hibiscus Leaf

Chinese Holly Bark

Chinese Holly Leaf

Chinese Honeylocust

Chinese Honeylocust Leaf

Chinese Honeylocust Root-bark

Chinese Honeylocust Thorn

Chinese Hydrangea

Chinese Incarvillea

Chinese Inula

Chinese Iris
T0201 Aesculus chinensis

T1867 Cryptomeria fortunei

T1278 Cedrela sinensis

T1254 Castanea mollissima

T1256 Castanea mollissima

T5181 Potentilla chinensis

T1545 Clematis chinensis

T1573 Clinopodium chinense

T4318 Murraya exotica

T1692 Coriaria sinica [Syn. Coriaria nepalensis]

T1693 Coriaria sinica [Syn. Coriaria nepalensis]

T4791 Phellodendron chinense

T1713 Corydalis cheilanthifolia

T3066 Gryllulus chinensis

T1796 Crinum asiaticum var. sinicum

T1812 Crossostephium chinense

T1862 Cryptocarya chinensis

T1866 Cryptolepis sinensis

T6916 Ziziphus jujuba

T2136 Desmos cochinchinensis [Syn. Desmos chinensis]

T1912 Cuscuta chinensis

T2262 Dregea sinensis

T2294 Dunnia sinensis

T5223 Prunus humilis [Syn. Cerasus humilis]

T0555 Aquilaria sinensis

T6596 Ulmus parvifolia

T2380 Ephedra sinica

T2381 Ephedra sinica

T2555 Eupatorium chinense

T4576 Paederia chinensis

T1343 Chaenomeles sinensis

T1967 Cynoglossum amabile

T0354 Alpinia chinensis

T6633 Uncaria sinensis

T6552 Trollius chinensis [Syn. Trollius asiaticus var. chinensis]

T5256 Pseudolarix amabilis [Syn. Larix amabilis; Pseudolarix

kaempferi]

T1662 Coptis chinensis

T1775 Crataegus pinnatifida

T1776 Crataegus pinnatifida

T1777 Crataegus pinnatifida

T3242 Hibiscus rosa-sinensis

T3243 Hibiscus rosa-sinensis

T3389 Ilex cornuta

T3390 Ilex cornuta

T2978 Gleditsia sinensis [Syn. Gleditsia horrida]

T2981 Gleditsia sinensis [Syn. Gleditsia horrida]

T2980 Gleditsia sinensis [Syn. Gleditsia horrida]

T2979 Gleditsia sinensis [Syn. Gleditsia horrida]

T3303 Hydrangea chinensis

T3420 Incarvillea sinensis

T3428 Inula britannica var. chinensis

T3464 Iris lactea var. chinensis [Syn. Iris pallasii var. chinensis]
QI YE SHU

LIU SHAN

ZHONG GUO YANG CHUN

BAN LI

LI SHU PI

WEI LING CAI

WEI LING XIAN

FENG LUN CAI

ZHONG HUA JIU LI XIANG

MA SANG

MA SANG YE

HUANG PI SHU

HUA ZI JIN

XI SHUAI

WEN SHU LAN

FU RONG JU GEN

HOU KE GUI

BAI YE TENG

DA ZAO

JIA YING ZHAO

TU SI ZI

KU SHENG

XIU QIU QIAN CAO

OU LI REN

BAI MU XIANG

LANG YU PI

MA HUANG

MA HUANG GEN

HUA ZE LAN

ZHONG HUA JI SHI TENG

MU GUA

GOU SHI HUA

LIAN JIANG

HUA GOU TENG

JIN LIAN HUA

TU JING PI

HUANG LIAN

SHAN ZHA

SHAN ZHA HUA

SHAN ZHA YE

FU SANG HUA

FU SANG YE

GOU GU SHU PI

GOU GU YE

ZAO JIA

ZAO JIA YE

ZAO JIA GEN PI

ZAO JIA CI

ZHONG GUO XIU QIU

JIAO HAO

ZHONG GUO XUAN FU HUA

MA LIN ZI 


\begin{tabular}{|c|}
\hline Chinese Iris \\
\hline Chinese Ivy \\
\hline Chinese Ixora \\
\hline Chinese Juniper \\
\hline Chinese Juniper Leaf \\
\hline Chinese Knotweed \\
\hline Chinese Ligusticum \\
\hline Chinese Lizardtail \\
\hline Chinese Lobelia \\
\hline Chinese Loropetalum \\
\hline Chinese Lycoris \\
\hline Chinese Magnolia Flower \\
\hline Chinese Magnoliavine \\
\hline Chinese Mahonia \\
\hline Chinese Mahonia Leaf \\
\hline Chinese Milkvetch \\
\hline Chinese Milkvetch Seed \\
\hline Chinese Milkwort \\
\hline Chinese Morina \\
\hline Chinese Mulberry \\
\hline Chinese Narcissus Bulb \\
\hline Chinese Narcissus Flower \\
\hline Chinese Nardostachys \\
\hline Chinese Onion \\
\hline Chinese Orthodon \\
\hline Chinese Osbeckia \\
\hline Chinese Pearleaf Crabapple \\
\hline Chinese Peashrub Root \\
\hline Chinese Photinia \\
\hline Chinese Pink \\
\hline Chinese Pistache \\
\hline Chinese Plumyew Branch- \\
\hline
\end{tabular}

Chinese Plumyew Seed

Chinese Podocarpus Leaf

Chinese Podocarpus Seed

Chinese Pothos

Chinese Privet

Chinese Pulsatilla

Chinese Pyrola

Chinese Rose

Chinese Sage

Chinese Seabuckthorn*

Chinese Seriphidium

Chinese Silkvine Root-bark

Chinese Silvergrass

Chinese Siphonostegia

Chinese Soapberry Leaf

Chinese Soapberry Peel

Chinese Soapberry Seed

Chinese Spikemoss

Chinese Spiranthes*
T3465 Iris pallasii var. chinensis

T3113 Hedera nepalensis var. sinensis

T3549 Ixora chinensis

T5639 Sabina chinensis

T5987 Sobina chinensis

T5100 Polygonum chinense

T3824 Ligusticum sinense

T5750 Saururus chinensis

T3898 Lobelia chinensis [Syn. Lobelia radicans]

T3926 Loropetalum chinense

T3984 Lycoris chinensis

T4036 Magnolia coco

T5791 Schisandra chinensis

T4062 Mahonia fortunei

T4063 Mahonia fortunei

T0805 Astragalus sinicus

T0806 Astragalus sinicus

T5073 Polygala chinensis [Syn. Polygala glomerata]

T4279 Morina chinensis

T4293 Morus cathayana

T4384 Narcissus tazetta var. chinensis

T4385 Narcissus tazetta var. chinensis

T4386 Nardostachys chinensis

T0313 Allium chinense

T4304 Mosla chinensis [Syn. Orthodon chinensis]

T4549 Osbeckia chinensis

T4086 Malus asiatica

T1191 Caragana sinica

T4828 Photinia serrulata

T2143 Dianthus chinensis

T4979 Pistacia chinensis

T1323 Cephalotaxus sinensis [Syn. Cephalotaxus harringtonia var. sinensis]

T1324 Cephalotaxus sinensis [Syn. Cephalotaxus harringtonia var. sinensis]

T5045 Podocarpus macrophyllus var. maki

T5044 Podocarpus macrophyllus var. maki

T5189 Pothos chinensis

T3830 Ligustrum sinense

T5325 Pulsatilla chinensis

T5345 Pyrola calliantha [Syn. Pyrola rotundifolia ssp. chinensis]

T5562 Rosa chinensis

T5692 Salvia sinica

T3256 Hippophae rhamnoides subsp. sinensis

T5920 Seriphidium cinum [Syn. Artemisia cina]

T4729 Periploca sepium

T4254 Miscanthus sinensis

T5966 Siphonostegia chinensis

T5721 Sapindus mukorossi

T5720 Sapindus mukorossi

T5719 Sapindus mukorossi

T5867 Selaginella sinensis

T6087 Spiranthes sinensis
MA LIN

CHANG CHUN TENG

LONG CHUAN HUA

YUAN BAI

$\mathrm{HUI}^{(4)} \mathrm{YE}$

HUO TAN MU CAO

GAO BEN

SAN BAI CAO

BAN BIAN LIAN

JI MU

ZHONG GUO SHI SUAN

YE HE HUA

WU WEI ZI

XI YE GONG LAO MU

XI YE GONG LAO YE

ZI YUN YING

ZI YUN YING ZI

DA JIN NIU CAO

YUAN E CI XU DUAN

HUA SANG

SHUI XIAN GEN

SHUI XIAN HUA

GAN SONG

QIAO TOU

SHI XIANG RU

JIN JIN XIANG

LIN QIN

JIN QUE GEN

SHI NAN

SHI ZHU

HUANG LIAN YA

ZHONG GUO CU FEI ZHI YE

ZHONG GUO CU FEI ZI

DUAN YE LUO HAN SONG YE

DUAN YE LUO HAN SONG SHI

SHI GAN ZI

NV ZHEN XIAO LA SHU

BAI TOU WENG

LU XIAN CAO

YUE JI HUA

NI DAN SHEN

ZHONG GUO SHA JI

HUI HAO

XIANG JIA PI

MANG JING

YIN XING CAO

WU HUAN ZI YE

WU HUAN ZI PI

WU HUAN ZI

ZHONG HUA JUAN BAI

ZHONG GUO SHOU CAO 


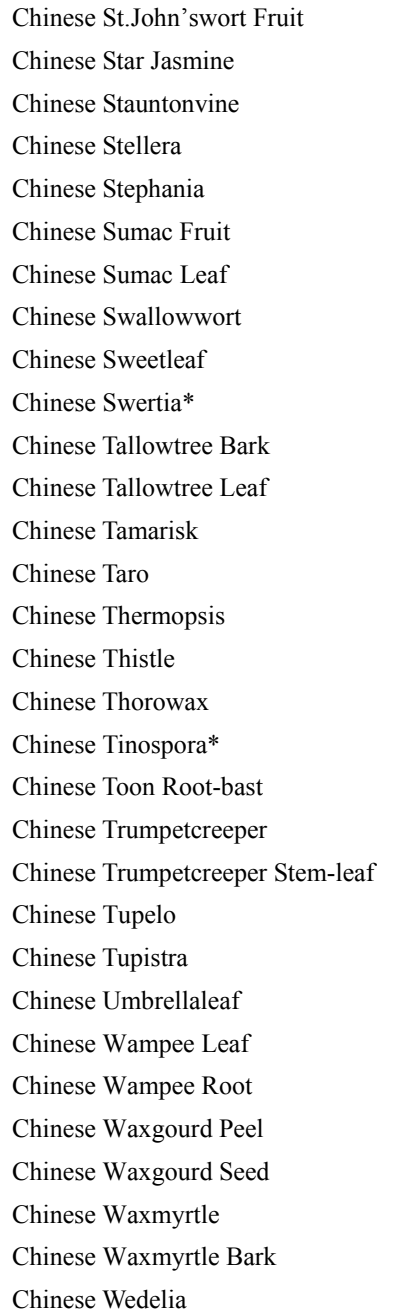

Chinese Weeping Cypress Leaf

Chinese White Poplar

Chinese Wingnut

Chinese Wisteria

Chinese Wisteria Seed

Chinese Wolfberry Fruit

Chinese Wolfberry Leaf

Chinese Wolfberry Root-bark

Chinese Yew

Chirata Swertia*

Chirita

Chittagong Chickrassy

Chive-like

Chloro-white Fibraurea*

Cholla

Chongming Many-flowered May-apple*

Christina Loosestrife

Chrusanthemum-like Groundsel

Chuandian Soapberry Seed

Chuanxiong (Wallich Ligusticum)

Chuchuhuasc Mayten*
T3344 Hypericum chinense

T6484 Trachelospermum jasminoides

T6099 Stauntonia chinensis

T6105 Stellera chamaejasme

T6133 Stephania sinica

T5531 Rhus chinensis [Syn. Rhus semialata]

T5530 Rhus chinensis [Syn. Rhus semialata]

T1954 Cynanchum chinense

T6252 Symplocos chinensis

T6214 Swertia chinensis

T5723 Sapium sebiferum

T5724 Sapium sebiferum

T6286 Tamarix chinensis

T0333 Alocasia cucullata [Syn. Arum cucullatum]

T6426 Thermopsis chinensis

T1448 Cirsium chinense

T1059 Bupleurum chinense

T6468 Tinospora sinensis

T6475 Toona sinensis

T1160 Campsis grandiflora

T1161 Campsis grandiflora

T4457 Nyssa sinensis

T6566 Tupistra chinensis

T2232 Diphylleia sinensis

T1538 Clausena lansium

T1537 Clausena lansium

T0894 Benincasa hispida

T0895 Benincasa hispida

T4348 Myrica rubra

T4349 Myrica rubra

T6814 Wedelia chinensis [Syn. Solidago chinensis; Wedelia

calendulacea $]$

T1896 Cupressus funebris

T5161 Populus tomentosa

T5308 Pterocarya stenoptera

T6826 Wisteria sinensis

T6827 Wisteria sinensis

T3958 Lycium chinense

T3957 Lycium chinense

T3956 Lycium chinense

T6310 Taxus chinensis

T6215 Swertia chirata

T1370 Chirita micronusa

T1410 Chukrasia tabularis

T0319 Allium schoenoprasum

T2714 Fibraurea chloroleuca

T4519 Opuntia dillenii

T2303 Dysosma subrosea

T3998 Lysimachia christinae

T5884 Senecio chrysanthemoides

T5717 Sapindus delavayi [Syn. Pancovia delavayi]

T3820 Ligusticum chuanxiong [Syn. Ligusticum wallichii]

T4130 Maytenus chuchuhuasca
JIN SI TAO GUO SHI

LUO SHI TENG

YE MU GUA

LANG DU

JIN BU HUAN

YAN FU ZI

YAN FU YE

E RONG TENG

HUA SHAN FAN

DANG YAO

WU JIU MU GEN PI

WU JIU YE

CHENG LIU

JIAN WEI YU

XIAO YE YE JUE MING

KU AO

CHAI HU

ZHONG HUA QING NIU DAN

CHUN BAI PI

ZI WEI

ZI WEI JING YE

ZI SHU

KAI KOU JIAN

WO ER QI

HUANG PI YE

HUANG PI GEN

DONG GUA PI

DONG GUA ZI

YANG MEI

YANG MEI SHU PI

PENG QI JU

BAI SHU YE

MAO BAI YANG

MA LIU YE

ZI TENG

ZI TENG ZI

GOU QI ZI

GOU QI YE

GOU QI GEN PI

HONG DOU SHAN

ZHAI RUI TA ZHANG YA CAI

CHUN ZHU JU TAI

MA LIAN

XI XIANG CONG

LV BAI TIAN XIAN TENG

XIAN REN ZHANG

CHONG MING BA JIAO LIAN

DA JIN QIAN CAO

TU SAN QI

PI SHAO ZI

CHUAN XIONG

QIU SHI MEI DENG MU 


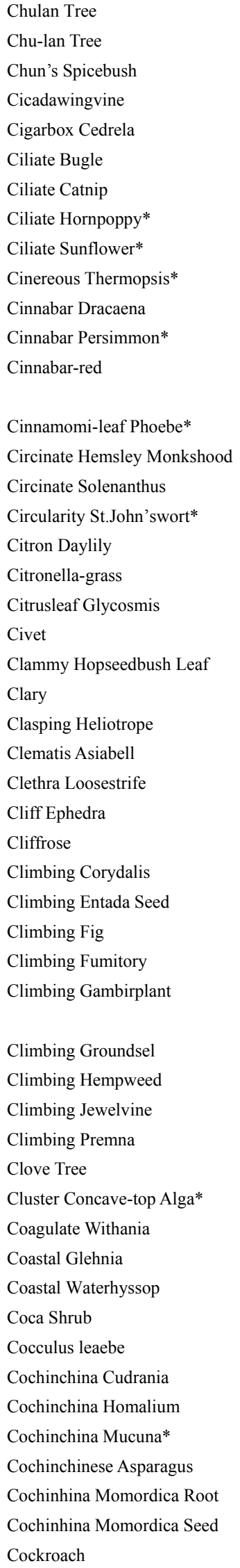

\begin{tabular}{|c|c|}
\hline T1374 Chloranthus spicatus & JIN SU LAN \\
\hline T0240 Aglaia odorata & MI ZI LAN \\
\hline T3849 Lindera chunii & DING HU DIAO ZHANG \\
\hline T5847 Securidaca inappendiculata & CHAN YI TENG \\
\hline T1277 Cedrela odorata & YAN YANG CHUN \\
\hline T0262 Ajuga ciliata & JIN GU CAO \\
\hline T4414 Nepeta ciliaris & YUAN MAO JING JIE \\
\hline T2968 Glaucium fimbrilligerum & HAI YING SU \\
\hline T3148 Helianthus ciliaris & YUAN MAO XIANG RI KUI \\
\hline T6427 Thermopsis cinerea & HUI HUANG HUA \\
\hline T2252 Dracaena cinnabari & ZHU HONG LONG XUE SHU \\
\hline T2215 Diospyros cinnabarina & ZHU HONG SHI \\
\hline \multicolumn{2}{|l|}{ cinnabarinus $]$} \\
\hline T4816 Phoebe cinnamomifolia & YU GUO XIAO YE NAN \\
\hline T0100 Aconitum hemsleyanium var. circinacum & QUAN JU GUA YE WU TOU \\
\hline T6021 Solenanthus circinatus & CHANG RUI LIU LI CAO \\
\hline T3339 Hypericum annulatum & HUAN ZHUANG JIN SI TAO \\
\hline T3192 Hemerocallis citrina & HUANG HUA CAI \\
\hline T1944 Cymbopogon nardus & JING XIANG MAO \\
\hline T3006 Glycosmis citrifolia & SHAN XIAO JU \\
\hline T6800 Viverra zibetha & LING MAO XIANG \\
\hline T2243 Dodonaea viscosa & CHE SANG ZI YE \\
\hline T5691 Salvia sclarea & NAN OU DAN SHEN \\
\hline T3167 Heliotropium amplexicaule & BAO JING TIAN JIE CAI \\
\hline T1597 Codonopsis clematidea & XIN JIANG DANG SHEN \\
\hline T3999 Lysimachia clethroides & ZHEN ZHU CAI \\
\hline T2379 Ephedra saxatilis & ZANG MA HUANG \\
\hline T1766 Cowania mexicana & XUAN YA MEI GUI \\
\hline T1714 Corydalis claviculata & BANG ZHUANG ZI JIN \\
\hline T2363 Entada phaseoloides [Syn. Lens phaseoloides] & KE TENG ZI \\
\hline T2723 Ficus pumila & BI LI \\
\hline T0183 Adlumia cirrhosa [Syn. Adlumia fungosa] & XUN ZHUANG SHAN YUAN CAO \\
\hline T6631 Uncaria scandens [Syn. Nauclea pilosa; Uruparia pilosa; & PAN ZHI GOU TENG \\
\hline \multicolumn{2}{|l|}{ Uncaria pilosa] } \\
\hline T5907 Senecio scandens [Syn. Senecio chinensis] & QIAN LI GUANG \\
\hline T4230 Mikania scandens & WEI GAN JU \\
\hline T2123 Derris scandens & PAN YUAN YU TENG \\
\hline T5195 Premna subscandens & PAN YUAN CHOU HUANG JING \\
\hline T6263 Syzygium aromaticum [Syn. Eugenia caryophyllata] & DING XIANG \\
\hline T3715 Laurencia caespitosa & CU SHENG AO DING ZAO \\
\hline T6828 Withania coagulans & NING GU SHUI QIE \\
\hline T2983 Glehnia littoralis & BEI SHA SHEN \\
\hline T0852 Bacopa monniera & JIA MA CHI XIAN \\
\hline T2491 Erythroxylum coca & GU KE \\
\hline \multicolumn{2}{|l|}{ T1587 Cocculus leaebe } \\
\hline T1884 Cudrania cochinchinensis & GOU JI \\
\hline T3275 Homalium cochinchinensis & TIAN LIAO MU \\
\hline T4309 Mucuna cochinchinensis & MAO DOU \\
\hline T0746 Asparagus cochinchinensis [Syn. Asparagus lucidus] & TIAN MEN DONG \\
\hline T4264 Momordica cochinchinensis & MU BIE GEN \\
\hline T4265 Momordica cochinchinensis & MU BIE ZI \\
\hline T0950 Blatta orientalis & ZHANG LANG \\
\hline
\end{tabular}




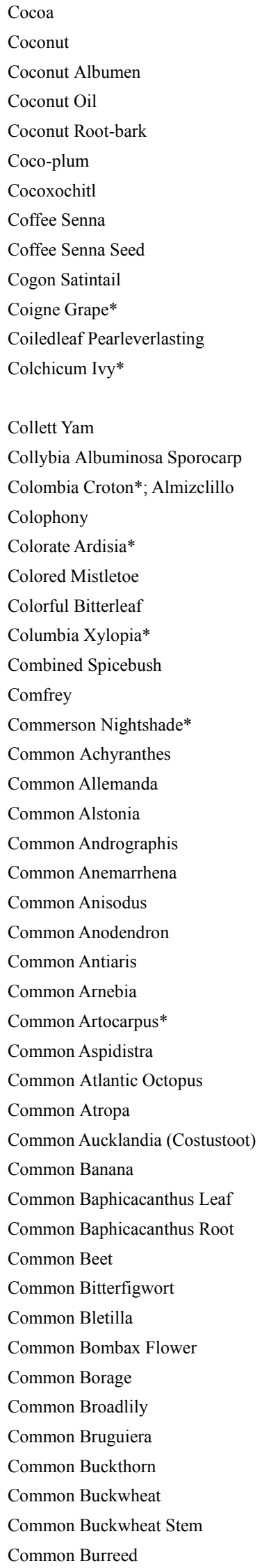

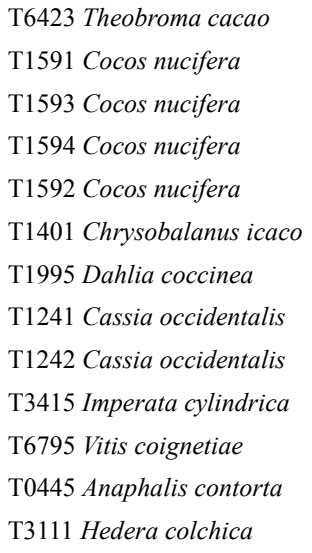

KE KE

YE ZI

YE ZI RANG

YE ZI YOU

YE ZI PI

YI KOU KE MEI

HONG DA LI HUA

WANG JIANG NAN

WANG JIANG NAN ZI

YIN DU BAI MAO

XIE PU TAO

XUAN YE XIANG QING

QIU SHUI XIAN CHANG CHUN

TENG

CHA RUI SHU YU

JI ZONG

GE LUN BI YA BA DOU

SONG XIANG

YOU SE ZI JIN NIU

HU JI SHENG

YOU SE BAN JIU JU

GE LUN BI YA MU BAN SHU

WU YAO

XI MEN FEI CAO

KE MO SEN QIE

TU NIU XI

RUAN ZHI HUANG CHAN

XIANG PI MU

CHUAN XIN LIAN

ZHI MU

SAI LANG DANG

SHAN TENG

JIAN XUE FENG HOU

JIA ZI CAO

MIAN BAO GUO

ZHI ZHU BAO DAN

ZHANG YU

DIAN QIE

MU XIANG

XIANG JIAO

MA LAN YE

MA LAN GEN

TIAN CAI

KU XUAN SHEN

BAI JI

MU MIAN HUA

LIU LI JU

LEI GONG QI

MU LAN

YAO SHU LI

QIAO MAI

QIAO MAI JIE

SAN LENG 
Common Butterbush

Common Camptotheac

Common Caper

Common Cardaria

Common Carpesium

Common Carpesium Fruit

Common Cashew Fruit

Common Cerberustree

Common Ceriops

Common Chamomile

Common Chicory

Common Cissampelos

Common Cnidium

Common Cocklebur*

Common Cockscomb Flower

Common Cockscomb Seed

Common Coelogyne

Common Coleostephus

Common Coltsfoot

Common Crapemyrtle Flower

Common Crapemyrtle Leaf

Common Crapemyrtle Root

Common Cruculigo

Common Cyanotis

Common Dayflower

Common Devilpepper

Common Devilpepper Stem and Leaf

Common Duckwood

Common Dysosma

Common Elsholtzia

Common Enhydra

Common Euscaphis

Common Evolvulus

Common Fenugreek

Common Fibraurea

Common Figwort

Common Flax

Common Flax Seed

Common Floweringquince

Common Fordia

Common Four-o'clock Leaf

Common Four-o'clock Root

Common Foxglove

Common Ginger Dried Rhizome

Common Goatsrue

Common Goldenrod

Common Gold-hair Moss*

Common Gorse

Common Gromwell

Common Groundsel

Common Hawthorn

Common Heron's Bill

Common Hoarhound
T1316 Cephalanthus occidentalis

T1162 Camptotheca acuminata

T1180 Capparis spinosa

T1195 Cardaria draba

T1210 Carpesium abrotanoides

T1211 Carpesium abrotanoides

T0438 Anacardium occidentale

T1330 Cerbera manghas

T1336 Ceriops tagal [Syn. Rhizophora tagal]

T0523 Anthemis nobilis

T1415 Cichorium intybus

T1452 Cissampelos pareira

T1582 Cnidium monnieri

T6838 Xanthium commune

T1299 Celosia cristata

T1297 Celosia cristada

T1606 Coelogyne ovalis

T1618 Coleostephus myconis

T6574 Tussilago farfara

T3671 Lagerstroemia indica

T3672 Lagerstroemia indica

T3670 Lagerstroemia indica

T1901 Curculigo orchioides

T1922 Cyanotis vaga

T1633 Commelina communis

T5423 Rauvolfia verticillata

T5424 Rauvolfia verticillata

T3738 Lemna minor

T2305 Dysosma versipellis [Syn. Podophyllum versipelle]

T2341 Elsholtzia ciliata

T2361 Enhydra fluctuans

T2636 Euscaphis japonica

T2649 Evolvulus alsinoides

T6528 Trigonella foenum-graecum

T2715 Fibraurea recisa

T5829 Scrophularia nodosa

T3859 Linum usitatissimum

T3860 Linum usitatissimum

T1342 Chaenomeles lagenaria [Syn. Chaenomeles speciosa]

T2752 Fordia cauliflora

T4253 Mirabilis jalapa

T4252 Mirabilis jalapa

T2177 Digitalis purpurea

T6909 Zingiber officinale

T2824 Galega officinalis

T6024 Solidago virgaurea var. leiocarpa [Syn. Solidago decurrens]

T5133 Polytrichum commune

T6590 Ulex europaeus

T3882 Lithospermum officinale

T5915 Senecio vulgaris

T1773 Crataegus monogyna

T2438 Erodium stephanianum

T4114 Marrubium vulgare
FENG XIANG SHU YE

XI SHU

LAO SHU GUA

QUN XIN CAI

TIAN MING JING

TIAN MING JING GUO

DU XIAN ZI

NIU XIN QIE ZI

JIAO GUO MU

GAO GUI CHUN HUANG JU

JU QU

XI SHENG TENG

SHE CHUANG ZI

PU TONG CANG ER

JI GUAN HUA

JI GUAN ZI

BEI MU LAN

QIAO GUAN JU

KUAN DONG HUA

ZI WEI HUA

ZI WEI YE

ZI WEI GEN

XIAN MAO

LU SHUI CAO

YA ZHI CAO

LUO FU MU

LUO FU MU JING YE

FU PING

GUI JIU

BAN BIAN SU

ZHAO JU

YE YA CHUN

TU DING GUI

HU LU BA

TIAN XIAN TENG

LIN SHENG XUAN SHEN

YA MA

YA MAZI

QIU MU GUA

GAN HUA DOU

ZI MO LI YE

ZI MO LI GEN

MAO DI HUANG

GAN JIANG

SHAN YANG DOU

YI ZHI HUANG HUA

JIN FA XIAN

JING DOU

BAI GUO ZI CAO

OU ZHOU QIAN LI GUANG

DAN ZI SHAN ZHA

MANG NIU ER MIAO

OU XIA ZHI CAO 


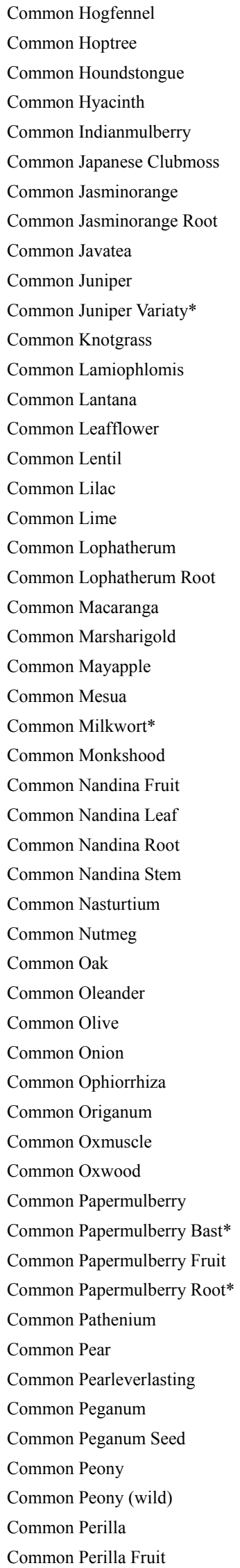

T0480 Angelica decursiva [Syn. Peucedanum decursivum]

T5282 Ptelea trifoliata

T1969 Cynoglossum officinale

T3297 Hyacinthus orientalis

T4286 Morinda umbellata

T3973 Lycopodium japonicum [Syn. Lycopodium clavatum]

T4323 Murraya paniculata [Syn. Chalcas paniculata]

T4324 Murraya paniculata [Syn. Chalcas paniculata]

T4542 Orthosiphon wulfenioides [Syn. Coleus wulfenioides]

T3583 Juniperus communis

T3584 Juniperus communis var. depressa

T5098 Polygonum aviculare

T3679 Lamiophlomis rotata [Syn. Phlomis rotata]

T3687 Lantana camara

T4843 Phyllanthus urinaria

T3741 Lens culinaris

T6262 Syringa vulgaris

T6461 Tilia vulgaris

T3920 Lophatherum gracile

T3921 Lophatherum gracile

T4013 Macaranga tanarius

T1137 Caltha palustris

T5058 Podophyllum peltatum

T4203 Mesua ferrea

T5089 Polygala vulgaris

T0084 Aconitum carmichaeli

T4371 Nandina domestica

T4370 Nandina domestica

T4368 Nandina domestica

T4369 Nandina domestica

T6555 Tropaeolum majus

T4351 Myristica fragrans

T5377 Quercus robur

T4420 Nerium oleander

T4487 Olea europaea

T0311 Allium cepa

T4513 Ophiorrhiza mungos

T4526 Origanum vulgare

T2157 Dichotomanthes tristaniaecarpa

T1784 Cratoxylum cochinchinense

T1032 Broussonetia papyrifera

T1033 Broussonetia papyrifera

T1035 Broussonetia papyrifera

T1034 Broussonetia papyrifera

T4660 Parthenium hysterophorus

T5364 Pyrus communis

T0446 Anaphalis margaritacea

T4687 Peganum harmala

T4688 Peganum harmala

T4580 Paeonia albiflora [Syn. Paeonia lactiflora]

T4584 Paeonia lactiflora wild

T4719 Perilla frutescens var. arguta

T4715 Perilla frutescens
QIAN HU

YU JU

YAO YONG DAO TI HU

FENG XIN ZI

YANG JIAO TENG

SHEN JIN CAO

JIU LI XIANG

JIU LI XIANG GEN

JI JIAO SHEN

OU ZHOU CI BAI

OU ZHOU CI BAI BIAN ZHONG

BIAN XU

DU YI WEI

WU SE MEI

YE XIA ZHU

BING DOU

OU DING XIANG

DUAN SHU

DAN ZHU YE

DAN ZHU YE GEN

XUE TONG

MA TI YE

DUN YE GUI JIU

TIE LI MU

PU TONG YUAN ZHI

WU TOU

NAN TIAN ZHU ZI

NAN TIAN ZHU YE

NAN TIAN ZHU GEN

NAN TIAN ZHU GENG

HAN LIAN HUA

ROU DOU KOU

OU ZHOU BAI LI

OU ZHOU JIA ZHU TAO

YOU GAN LAN

YANG CONG

SHE GEN CAO

TU XIANG RU

NIU JIN TIAO

HUANG NIU MU

GOU SHU

GOU SHU BAI PI

GOU SHU GUO

GOU SHU GEN

YIN JIAO JU

XI YANG LI

DA YE BAI TOU WENG

LUO TUO PENG

LUO TUO PENG ZI

BAI SHAO

CHI SHAO wild

ZI SU

BAI SU ZI 


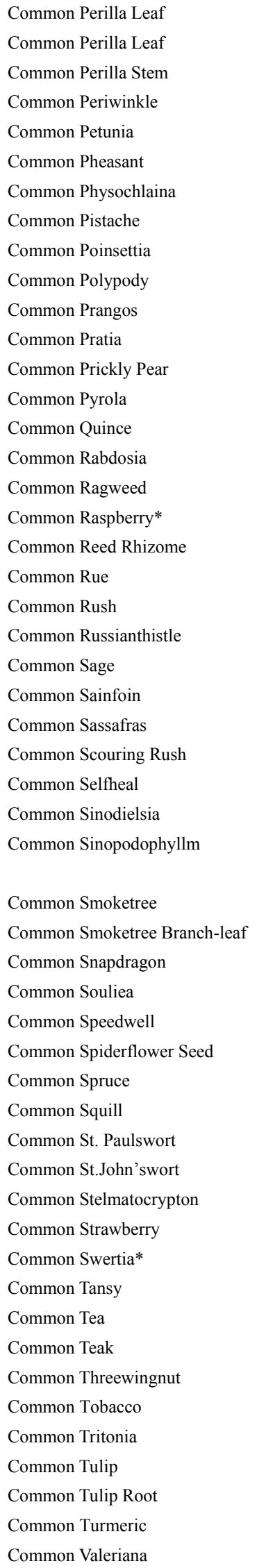

T4714 Perilla frutescens

T4721 Perilla frutescens var. arguta

T4720 Perilla frutescens var. arguta

T6761 Vinca minor

T4751 Petunia hybrida

T4786 Phasianus colchicus

T4859 Physochlaina physaloides

T4982 Pistacia vera

T2614 Euphorbia pulcherrima

T5127 Polypodium vulgare

T5191 Prangos pabularia

T5193 Pratia nummularia

T4521 Opuntia vulgaris

T5347 Pyrola decorata

T1937 Cydonia oblonga

T3479 Isodon amethystoides

T0398 Ambrosia artemisiifolia

T5588 Rubus allegheniensis

T4829 Phragmites communis

T5626 Ruta graveolens

T3578 Juncus effusus

T5657 Salsola collina

T5685 Salvia plebeia

T4491 Onobrychis viciifolia

T5746 Sassafras tzumu

T2408 Equisetum hiemale

T5214 Prunella vulgaris

T5963 Sinodielsia yunnanensis

T5057 Podophyllum emodii [Syn. Podophyllum emodii var. chinense;

Podophyllum sikkimenosis; Sinopodophyllum emodii]

T1758 Cotinus coggygria

T1759 Cotinus coggygria var. cinerea

T0537 Antirrhinum majus

T6060 Souliea vaginata

$\mathrm{T} 6723$ Veronica arvensis

T1550 Cleome gynandra [Syn. Gynandropsis gynandra]

T4869 Picea abies

T5813 Scilla scilloides

T5950 Siegesbeckia orientalis

T3361 Hypericum perforatum

T6106 Stelmatocrypton khasianum

T2763 Fragaria ananassa

T6239 Swertia swertiopsis

T6298 Tanacetum vulgare

T1152 Camellia sinensis [Syn. Thea sinensis]

T6325 Tectona grandis

T6542 Tripterygium wilfordii

T4428 Nicotiana tabacum

T6548 Tritonia crocosmaeflora

T6561 Tulipa gesneriana

T6562 Tulipa gesneriana

T1905 Curcuma longa

T6679 Valeriana officinalis
BAI SU YE

ZI SU YE

ZI SU GENG

MAN CHANG CHUN HUA

BI DONG QIE

ZHI

PAO NANG CAO

A YUE HUN ZI

YI PIN HONG

DUO ZU JUE

SHUAN CHI QIN

TONG CHUI YU DAI CAO

LV XIAN REN ZHANG

PU TONG LU TI CAO

WEN PO

XIANG CHA CAI

TUN CAO

PU TONG XUAN GOU ZI

LU GEN

CHOU CAO

DENG XIN CAO

ZHU MAO CAI

LI ZHI CAO

LV DOU

CHA SHU

MU ZEI

XIA KU CAO

DIAN QIN

TAO ER QI

HUANG LU

HUANG LU ZHI YE

JIN YU CAO

HUANG SAN QI

ZHI LI PO PO NA

BAI HUA CAI ZI

OU ZHOU YUN SHAN

MIAN ZAO ER

XI XIAN

GUAN YE LIAN QIAO

SHENG TENG

CAO MEI

PU TONG ZHANG YA CAI

JU HAO

CHA YE

YOU MU

LEI GONG TENG

YAN CAO

XIONG HUANG LAN

YU JIN XIANG

YU JIN XIANG GEN

JIANG HUANG

XIE CAO 
Common Wallflower

Common Waterhyacinth

Common Watershield

Common Wedgelet Fern

Common Vetch

Common White Jasmine

Common White Quebracho

Common Vladimiria

Common Woadwaxen

Common Wormwood

Common Xylopia

Common Yam

Common Yarrow

Common Yew

CommonGaultheria

Compact Wormwood*

Complanate Clubmoss

Compositeleaf Eupatorium*

Concentrated Beef Extract

Condurango

Conespike Flemingia

Confusable Larkspur*

Confusable Ochrosia

Confuse Myrrhtree*

Confused Mahonia

Congested-flower Rosewood*

Conglobate Ulva Frond*

Congo Antiaris*

Congo Holarrhena*

Conic Gymnadenia

Conicum Conocephalus*

Conifer Macaranga

Consolidated Larkspur*

Constricted Alstonia*

Contorted Tanglehead

Contracted Sawwort

Convolvulate Asiabell

Coprinus Sporocarp

Coral Ardisia

Coralhead Plant

Coralhead Plant Vine

Coral-tree

Cordate Gambirplant*

Cordate Syzygium*

Cordateleaf Sida

Cordate-oblong Beautyleaf*

Coriaceous Passionflower*

Coriaceousleaf Actinidia

Coriander Seed

Corinan Tree*

Corkywing Euonymus

Corn Gromwell

Corn Poppy
T1356 Cheiranthus cheiri

T2325 Eichhornia crassipes

T1004 Brasenia schreberi

T6116 Stenoloma chusanum

T6750 Vicia sativa

T3556 Jasminum officinale

T0772 Aspidosperma quebracho-blanco

T6802 Vladimiria souliei [Syn. Jurinea souliei]

T2901 Genista tinctoria

T0659 Artemisia absinthium

T6855 Xylopia vielana

T2190 Dioscorea batatas [Syn. Dioscorea opposita]

T0065 Achillea millefolium

T6307 Taxus baccata

T2893 Gaultheria leucocarpa var. cumingiana

T0674 Artemisia compacta

T3971 Lycopodium complanatum

T2556 Eupatorium compositifolium

T0983 Bos taurus domesticus

T4115 Marsdenia condurango

T2738 Flemingia strobilifera

T2067 Delphinium confusum

T4468 Ochrosia confusa

T1634 Commiphora confusa

T4060 Mahonia confusa

T2000 Dalbergia congestiflora

T6598 Ulva conglobata

T0534 Antiaris welwitschii

T3264 Holarrhena congolensis

T3077 Gymnadenia conopsea

T1644 Conocephalum conicum

T4012 Macaranga conifera

T2068 Delphinium consolida

T0368 Alstonia constricta

T3234 Heteropogon contortus

T5922 Serratula strangulata

T1598 Codonopsis convolvulacea

T1660 Coprinus atramentarius

T0594 Ardisia crenata

T0012 Abrus precatorius

T0011 Abrus precatorius

T2478 Erythrina variegata [Syn. Erythrina indica]

T6612 Uncaria cordata

T6265 Syzygium cordatum

T5943 Sida cordifolia

T1127 Calophyllum cordato-oblongum

T4664 Passiflora coriaceae

T0165 Actinidia rubricaulis var. coriacea

T1687 Coriandrum sativum

T1752 Corynanthe johimbe

T2545 Euonymus phellomana

T3880 Lithospermum arvense

T4625 Papaver commutatum [Syn. Papaver rhoeas]
GUI ZHU XIANG

SHUI HU LU

CHUN

DA YE JIN HUA CAO

DA CHAO CAI

SU FANG HUA

PU TONG BAI JIAN MU

CHUAN MU XIANG

RAN LIAO MU

ZHONG YA KU HAO

MU BAN SHU

SHAN YAO

YANG SHI CAO

JIANG GUO ZI SHAN

BAI ZHU SHU

MI HAO

GUO JIANG LONG

FU YE ZE LAN

XIA TIAN GAO

NAN MEI NIU NAI CAI

QIU SUI QIAN JIN BA

YI SI CUI QUE

YI SI MEI GUI SHU

HUN XIAO MO YAO

HU BEI SHI DA GONG LAO

JU HUA HUANG TAN

LI CAI

GANG GUO JIAN XUE FENG HOU

GANG GUO HE ZHI XIE MU

SHOU ZHANG SHEN

SHE TAI

ZHEN YE XUE TONG

QIANG GU FEI YAN CAO

SHU JI GU CHANG SHAN

DI JIN

YI BAO MA HUA TOU

JI DAN SHEN

GUI GAI

ZHU SHA GEN

XIANG SI ZI

XIANG SI TENG

CI TONG

XIN XING GOU TENG

XIN XING PU TAO

HUANG HUA ZI

CHANG YUAN XIN XING HU TONG

GE YANG XI FAN LIAN

GE YE MI HOU TAO

HU SUI ZI

KE NAN SHU

SHUAN CHI WEI MAO

MAI JIA GONG

LI CHUN HUA 
Cornflower

Corniculate Aegiceras

Corniculate Spurgentian

Cornish Heath

Cornuted Plantain*

Coromandel Lannea

Coronarious Gingerlily

Coronary Ervatamia*

Corsican Pine

Corydalisleaf Meadowrue

Corymb Rose-bay*

Corymbose Hedyotis

Cotton-leaf Leprous Tree*

Cottonrose Hibiscus Flower

Couminga Erythrophleum*

Country Monkshood

Country Stringbush*

Cow Milk

Cowage Velvet-bean

Cowberry Leaf

Cow-bezoar (Ox-gallstone)

Cowherb

Cowlily

Cowparsnipleaf Bugbane

Cow-pea

Crab-craw Orchis*

Crassleaf Tephrosia*

Creeping Acroptilon

Creeping Bugle

Creeping Ceratostigma, Creeping Bluesnow

Creeping Corydalis

Creeping Hypoestes*

Creeping Juniper

Creeping Lagotis*

Creeping Mahonia

Creeping Ninenode

Creeping Oxalis

Creeping Rockfoil

Creeping Rostellularia

Creeping Skyflower

Creeping Skyflower Leaf

Creeping Telosma

Crenate Quassiawood*

Creosote-bush

Crepinleaf Erysimum*

Crescent-shaped Euphorbia

Crest Iris

Crestedspike Ragweed

Cretan Brake

Crispate Crotalaria*

Crispateleaf Ardisia

Crisped Common Perilla

Crisped Common Perilla Stem
T1305 Centaurea cyanus

T0194 Aegiceras corniculatum

T3092 Halenia corniculata

T2419 Erica vagans

T5003 Plantago cornuti

T3685 Lannea grandis [Syn. Lannea coromandelica]

T3118 Hedychium coronarium

T2440 Ervatamia coronaria

T4914 Pinus laricio

T6395 Thalictrum isopyroides

T6272 Tabernaemontana corymbosa

T3126 Hedyotis corymbosa [Syn. Oldenlandia corymbosa]

T3561 Jatropha gossypifolia

T3241 Hibiscus mutabilis

T2484 Erythrophleum couminga

T0115 Aconitum monticola

T6822 Wikstroemia monticola

T0989 Bos taurus domesticus; Bubalus bubalis

T4310 Mucuna pruriens

T6673 Vaccinium vitis-idaea

T0987 Bos taurus domesticus; Bubalus bubalis

T6668 Vaccaria segetalis [Syn. Vaccaria pyramidata]

T4450 Nuphar pumilum

T1421 Cimicifuga heracleifolia

T6754 Vigna unguiculata

T5806 Schlumbergera truncata

T6333 Tephrosia crassifolia

T0154 Acroptilon repens

T0271 Ajuga reptans

T1327 Ceratostigma minus

T1735 Corydalis repens

T3375 Hypoestes serpens

T3589 Juniperus horizontalis

T3676 Lagotis stolonifera

T4071 Mahonia repens

T5279 Psychotria serpens

T4564 Oxalis corniculata [Syn. Oxalis repens]

T5773 Saxifraga stolonifera

T5576 Rostellularia procumbens [Syn. Justicia procumbens]

T2295 Duranta repens

T2296 Duranta repens

T6329 Telosma procumbens

T4879 Picrasma crenata

T3695 Larrea tridentata

T2453 Erysimum crepidifolium

T2599 Euphorbia lunulata

T3456 Iris cristata

T0406 Ambrosia psilostachya var. coronopifolia

T5287 Pteris cretica

T1818 Crotalaria crispata

T0595 Ardisia crispa

T4722 Perilla frutescens var. crispa

T4723 Perilla frutescens var. crispa
SHI CHE JU

LA ZHU GUO

HUA MAO

YING GUO OU SHI NAN

JIAO ZHUANG CHE QIAN

HOU PI SHU

TU QIANG HUO

GUAN ZHUANG GOU YA HUA

KE XI JIA SONG

ZI JIN YE TANG SONG CAO

SAN FANG HUA XU HONG YUE GUI

SHUI XIAN CAO

MIAN YE MA FENG SHU

MU FU RONG HUA

KAO MING GE MU

SHAN DI WU TOU

SHAN DI YAO HUA

NIU RU

CI YANG LI DOU

YUE JU YE

NIU HUANG

WANG BU LIU XING

PING PENG CAO

DA SAN YE SHENG MA

JIANG DOU

XIE ZHUA LAN

HOU YE HUI MAO DOU

DING YU JU

PU FU JIN GU CAO

XIAO JIAO ZHU HUA

QUAN YE YAN HU SUO

PU FU QIANG DAO YAO

PING PU YUAN BAI

PU FU JING TU ER CAO

PU FU SHI DA GONG LAO

MAN JIU JIE

ZUO JIANG CAO

HU ER CAO

JUE CHUANG

JIA LIAN QIAO

JIA LIAN QIAO YE

WO JING YE LAI XIANG

YUAN CHI KU MU

SAN CHI LA RUI A

HUAN YANG SHEN YE TANG JIE

MAO YAN CAO

SHI GUAN YUAN WEI

GUAN LUO SUI TUN CAO

DA YE JING KOU BIAN CAO

ZOU BO ZHUANG ZHU SHI DOU

BAI LIANG JIN

HUI HUI SU

HUI HUI SU GENG 


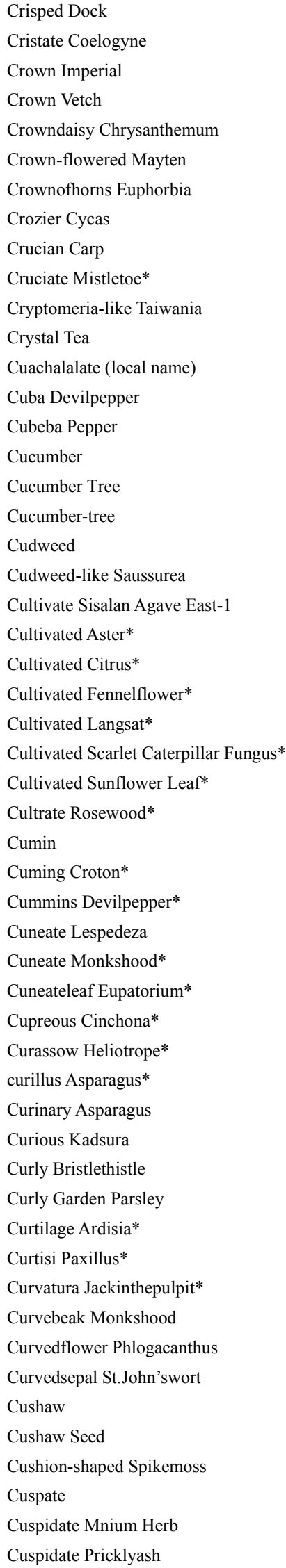

T5607 Rumex crispus

T1605 Coelogyne cristata

T2788 Fritillaria imperialis

T1702 Coronilla varia

T1390 Chrysanthemum coronarium

T4131 Maytenus confertiflorus

T2601 Euphorbia milii

T1926 Cycas circinalis

T1194 Carassius auratus

T6776 Viscum cruciatum

T6284 Taiwania cryptomerioides

T3735 Ledum palustre

T0431 Amphipterygium adstringens

T5432 Rauwolfia cubana

T4944 Piper cubeba

T1878 Cucumis sativus

T2112 Dendrosicyos socotrana

T4033 Magnolia acuminata

T3026 Gnaphalium affine [Syn. Gnaphalium multiceps]

T5753 Saussurea gnaphaloides

T0221 Agave east-one

T0779 Aster cultivars

T1473 Citrus cultivars

T4433 Nigella sativa

T3686 Lansium domesticum

T1681 Cordyceps militaris cv

T3146 Helianthus annuus cv

T2001 Dalbergia cultrata

T1889 Cuminum cyminum

T1842 Croton cumingii

T5433 Rauwolfia cumminsii

T3770 Lespedeza cuneata

T0134 Aconitum subcuneatum

T2557 Eupatorium cuneifolium

T1427 Cinchona cuprea

T3169 Heliotropium curassavicum

T0747 Asparagus curillus

T0751 Asparagus officinalis

T3614 Kadsura heteroclita [Syn. Uvaria heteroclita]

T1200 Carduus crispus

T4748 Petroselinum crispum

T0596 Ardisia hortorum

T4680 Paxillus curtisii

T0619 Arisaema curvatum

T0082 Aconitum campylorrhynchum

T4803 Phlogacanthus curviflorus

T3345 Hypericum curvisepalum

T1880 Cucurbita moschata

T1881 Cucurbita moschata

T5865 Selaginella pulvinata

T0762 Aspidosperma cuspa

T4259 Mnium cuspidatum

T6872 Zanthoxylum cuspidatum
NIU ER DA HUANG

MAO CHUN BEI MU LAN

XI BEI MU

DUO BIAN XIAO GUAN HUA

TONG HAO

MI HUA MEI DENG MU

TIE HAI TANG

QUAN YE SU TIE

JIN YU

SHI ZI XING FENG JI SHENG

TAI WAN SHAN

LA BA CHA

SHOU LIAN LIANG YI MU

GU BA LUO FU MU

BI CHENG QIE

HUANG GUA

JIAN JIAN MU LAN

SHU QU CAO

SHU QU FENG MAO JU

DONG YI HAO JIAN MA

ZAI PEI ZI WAN

ZAI PEI GAN JU

ZAI PEI HEI ZHONG CAO

ZAI ZHONG LANG SE MU

REN GONG YONG CHONG CAO

ZAI PEI XIANG RI KUI YE

XIAO DAO XING HUANG TAN

ZI RAN QIN

KA MING BA DOU

KE MING XI LUO FU MU

YE GUAN MEN

XIE XING WU TOU

XIE YE ZE LAN

TONG SE JI NA SHU

YAN TIAN JIE CAI

WAN QU TIAN MEN DONG

SHI DIAO BAI

YI XING NAN WU WEI ZI

FEI LIAN

ZHOU YE OU QIN

TING YUAN ZI JIN NIU

KE DI SI WANG ZHE JUN

WAN QU TIAN NAN XING

WAN ZHUO WU TOU

HUO YAN HUA

WAN E JIN SI TAO

NAN GUA

NAN GUA ZI

DIAN ZHUANG JUAN BAI

JIAN BAI JIAN MU

SHUI MU CAO

HUA JIAO LE 


\begin{tabular}{|c|}
\hline Custard Apple \\
\hline Cutechu \\
\hline Cut-leaf Balsamroot \\
\hline Cutleaf Coneflower \\
\hline Cutleaf Groundcherry \\
\hline Cyclamen \\
\hline Cylinder Immortelle \\
\hline Cymose Brassica* \\
\hline Dadah Artocarpus* \\
\hline Daffodil \\
\hline Daghestan Sweetclover \\
\hline Daghestan Sweetclover Root \\
\hline Dahuria Gentian \\
\hline Dahurian Angelica \\
\hline Dahurian Bugbane \\
\hline Dahurian Larch \\
\hline Dahurian Loosestrife \\
\hline Dahurian Patrinia \\
\hline Dahurian Pulsatilla* \\
\hline Dahurian Rhododendron \\
\hline Daimyo Oak Leaf \\
\hline Dalmatian Pyrethrum \\
\hline Damask Rose \\
\hline Danshen \\
\hline Dark-hair Gold-hair Moss* \\
\hline Darkhairy Milkvetch* \\
\hline Darkred Rhodiola \\
\hline Darksanguine Cinquefoil* \\
\hline Dark-yellow Corydalis \\
\hline Darling Pea \\
\hline Darwin Barberry \\
\hline Dateplum Persimmon \\
\hline David Epimedium \\
\hline David Falsepanax \\
\hline David Peach Bast \\
\hline David Peach Juvenile Branch \\
\hline David's Spikemoss \\
\hline Dawn Redwood \\
\hline Dawo Rabdosia \\
\hline Davurian Buckthorn \\
\hline Deadly Calabarbean \\
\hline Deadly Carrot \\
\hline Death Cap \\
\hline Decaline Nightshade* \\
\hline Decapistil Pokeweed* \\
\hline Decary Pricklyash* \\
\hline Deciduous Cypress \\
\hline Decumbent Bugle \\
\hline Decumbent Corydalis \\
\hline Decurrent Archangelica \\
\hline Deeplobed Larkspur* \\
\hline Deeplobed Merremia* \\
\hline Deep-lobed-leaf Incarvi \\
\hline
\end{tabular}

T0513 Annona squamosa

T0019 Acacia catechu

T0864 Balsamorhiza macrophylla

T5604 Rudbeckia laciniata

T4848 Physalis angulata

T1929 Cyclamen europaeum

T6847 Xeranthemum cylindraceum

T1016 Brassica oleracea var. botrytis subvar. cymosa

T0711 Artocarpus dadah

T4379 Narcissus pseudonarcissus

T4172 Melilotus suaveolens

T4173 Melilotus suaveolens

T2910 Gentiana dahurica

T0478 Angelica dahurica [Syn. Angelica porphyrocaulis]

T1419 Cimicifuga dahurica

T3690 Larix gmelini

T4001 Lysimachia davurica

T4672 Patrinia scabiosaefolia

T5326 Pulsatilla dahurica

T5508 Rhododendron dauricum

T5372 Quercus dentata

T1388 Chrysanthemum cinerariaefolium

T5564 Rosa damascena

T5680 Salvia miltiorrhiza

T5135 Polytrichum pollidisetum

T0786 Astragalus atropubescens

T5493 Rhodiola coccinea

T5180 Potentilla atrosanguinea

T1723 Corydalis lutea

T6208 Swainsonia galegifolia

T0901 Berberis darwinii

T2222 Diospyros lotus

T2392 Epimedium davidii

T4444 Nothopanax davidii

T5220 Prunus davidiana

T5221 Prunus davidiana

T5860 Selaginella davidii

T4208 Metasequoia glyptostroboides

T3484 Isodon dawoensis

T5457 Rhamnus davurica

T4860 Physostigma venenosum

T6419 Thapsia garganica

T0385 Amanita phalloides

T5996 Solanum decemlineata

T4863 Phytolacca dodecandra

T6873 Zanthoxylum decaryi

T6305 Taxodium distichum

T0263 Ajuga decumbens

T1715 Corydalis decumbens [Syn. Corydalis amabilis]

T0583 Archangelica decurrens

T2073 Delphinium dissectum

T4197 Merremia dissecta

T3419 Incarvillea dissectifoliola
FAN LI ZHI

HAI ER CHA

JIN GUANG JU

KU ZHI

OU ZHOU XIAN KE LAI

CHANG TONG HAN HUA

JU SAN HUA YE CAI

DA DA HE MIAN BAO GUO

HUANG SHUI XIAN

PI HAN CAO

PI HAN CAO GEN

DA WU LI QIN JIAO

BAI ZHI

XING AN SHENG MA

LUO YE SONG

HUANG LIAN HUA

HUANG HUA BAI JIANG

XING AN BAI TOU WENG

MAN SHAN HONG

HU YE

CHU CHONG JU

DA MA SHI GE QIANG WEI

DAN SHEN

CANG MAO JIN FA XUAN

ROU MAO HUANG QI

SHEN HONG HONG JING TIAN

AN HONG WEI LING CAI

SHEN HUANG ZI JIN

SHAN YANG DOU YE KU MA DOU

DA ER WEN XIAO BO

JUN QIAN ZI

CHUAN DIAN YIN YANG HUO

YI YE LIANG WANG CHA

SHAN TAO JING BAI PI

SHAN TAO ZHI

MAN SHENG JUAN BAI

SHUI SHAN

DAO FU XIANG CHA CAI

SHU LI

DU BIAN DOU

DU HU LUO BO

DU E GAO

SHI XIAN QIE

SHI ER RUI SHANG LU

DE KA RUI HUA JIAO

LUO YU SHAN

BAI MAO XIA KU CAO

XIA TIAN WU

XIA YAN GU DANG GUI

SHEN LIE CUI QUE HUA

SHEN LIE YU HUANG CAO

SHEN LIE YE JIAO HAO 
Deerbrush

Degen St.John'swort*

Delacay Honeylocust

Delavay Fritillary

Delavay Meadowrue

Delavay Peony

Delavay Stephania

Delavy Monkshood

Delicate Jew's Ear*

Delicious Actinidia

Delightful Dendrobium

Delightful Skullcap*

Delphinileaf Sopubia*

Deltoid Goldthread

Deltoid Yam

Dendrob Mycena

Denne Denrdobium

Dense Euphorbia*

Denseflower Dendrobium

Denseflower Lemongrass*

Denseflower Libanotis

Denseflower Loosestrife

Denseflower Ragweed*

Denseflower Tylophora*

Densefruit Cucumber*

Densefruit Pittany Root-bark

Densevein Schefflera

Denticulate Vladimiria

Denuded Larkspur*

Deodar Cedar

Depressed Orange

Depressed Plantain

Desert Rose

Desertliving Asparagus*

Desertliving Cistanche

Desertliving Groundsel*

Desfontainia spinosa

Dew Rabdosia

Devil's Clow

Devil-in-a-bush

Deviltree Alstonia

Diamondleaf Persimmon

Dicentrine Stephania*

Dichotoma Forked Fern

Dichotomous Ervatamia*

Dichotomous Fimbristylis

Diderrichi Fatheadtree*

Diels Millettia

Diels Stephania

Diels Trema

Difengpi Anisetree

Different-flowered Indigo
T1276 Ceanothus integerrimus

T3346 Hypericum degenii

T2975 Gleditsia delavayi

T2784 Fritillaria delavayi

T6378 Thalictrum delavayi

T4582 Paeonia delavayi

T6120 Stephania delavayi [Syn. Stephania epigaea]

T0090 Aconitum delavayi

T0831 Auricularia delicata

T0160 Actinidia deliciosa

T2095 Dendrobium amoenum

T5832 Scutellaria amabilis

T6050 Sopubia delphinifolia

T1664 Coptis deltoidea

T2195 Dioscorea deltoidea

T4336 Mycena dendrobii

T2096 Dendrobium aurantiacum var. denneanum

T2588 Euphorbia fortissima

T2100 Dendrobium densiflorum

T1940 Cymbopogon densiflorus

T3797 Libanotis condensata

T4000 Lysimachia congestiflora

T0400 Ambrosia confertiflora

T6579 Tylophora crebriflora

T1877 Cucumis myriocarpus

T2167 Dictamnus dasycarpus

T5785 Schefflera venulosa

T6801 Vladimiria denticulata

T2071 Delphinium denudatum

T1282 Cedrus deodara

T1475 Citrus depressa

T5004 Plantago depressa

T0168 Adenium obesum

T0750 Asparagus gobicus

T1455 Cistanche deserticola

T5886 Senecio eremophilus

T2126 Desfontainia spinosa

T3495 Isodon irrorata

T3106 Harpagophytum procumbens

T4430 Nigella arvensis

T0370 Alstonia macrophylla

T2226 Diospyros rhombifolia

T6121 Stephania dicentrinifera

T2161 Dicranopteris pedata [Syn. Polypodium pedatum;

Dicranopteris dichotoma]

T2441 Ervatamia dichotoma

T2730 Fimbristylis dichotoma

T4389 Nauclea diderrichii

T4234 Millettia dielsiana

T6122 Stephania dielsiana

T6494 Trema dielsiana

T3400 Illicium difengpi

T3422 Indigofera heteranthazha
QUAN YUAN YE MEI ZHOU CHA

DI GEN JIN SI TAO

YUN NAN ZAO JIA

LENG SHA BEI MU

PIAN CHI TANG SONG CAO

DIAN MU DAN

DI BU RONG

MA ER SHAN WU TOU

ZHOU MU ER

MEI WEI MI HOU TAO

KE AI SHI HU

KE AI HUANG QIN

CUI QUE YE DUAN GUAN CAO

SAN JIAO YE HUANG LIAN

SAN JIAO YE SHU YU

SHI HU XIAO GU

DIE QIAO SHI HU

NONG DA JI

MI HUA SHI HU

MI HUA XIANG MAO

MI HUA YAN FENG

JU HUA GUO LU HUANG

MI HUA TUN CAO

MI HUA WA ER TENG

MI GUO HUANG GUA

BAI XIAN PI

MI MAI E ZHANG CHAI

YUE XI MU XIANG

LU CUI QUE

XUE SONG

BIAN PING JU

PING CHE QIAN

SHA MO QIANG WEI

GE BI TIAN MEN

ROU CONG RONG

SHA SHENG QIAN LI GUANG

DUO CI DI SHI MU

LU ZHU XIANG CHA CAI

NAN FEI GOU MA

YE HEI ZHONG CAO

DA YE TANG JIAO SHU

LAO YA SHI

HE BAO DI BU RONG

MANG QI GU

ER QI GOU YA HUA

PIAO FU CAO

DI SHI WU TAN

KUN MING JI XUE TENG

XUE SAN SHU

SHAN YOU MA

DI FENG PI

YI HUA MU LAN 


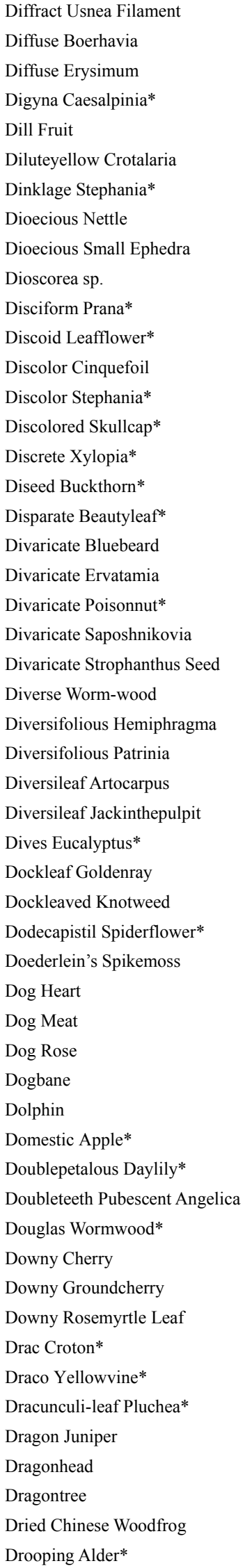

\begin{tabular}{|c|c|}
\hline T6653 Usnea diffracta & HUAN JIE SONG LUO \\
\hline T0969 Boerhavia diffusa & HUANG XI XIN \\
\hline T2454 Erysimum diffusum & TANG JIE \\
\hline T1102 Caesalpinia digyna & ER CI YUN SHI \\
\hline T0472 Anethum graveolens & SHI LUO ZI \\
\hline T1813 Crotalaria albida & HUANG HUA DI DING \\
\hline T6123 Stephania dinklagei & DING KE LA QIAN JIN TENG \\
\hline T6652 Urtica dioica & YI ZHU QIAN MA \\
\hline T2374 Ephedra minuta var. dioeca & YI ZHU AI MA HUANG \\
\hline \multicolumn{2}{|l|}{ T2209 Dioscorea sp. } \\
\hline T5190 Prana discifera & DA PENG TENG \\
\hline T4833 Phyllanthus discoides & PAN ZHUANG YE XIA ZHU \\
\hline T5182 Potentilla discolor & FAN BAI CAO \\
\hline T6124 Stephania discolor & CAI WEN QIAN JIN TENG \\
\hline T5836 Scutellaria discolor & ZI BEI HUANG QIN \\
\hline T6853 Xylopia discreta & XI SHU MU BAN SHU \\
\hline T5458 Rhamnus disperma & SHUANG ZHONG ZI SHU LI \\
\hline T1128 Calophyllum dispar & BU DENG HONG HOU KE \\
\hline T1221 Caryopteris divaricata & $\mathrm{YOU}^{(2)}$ \\
\hline T2442 Ervatamia divaricata & DAN BAN GOU YA HUA \\
\hline T6170 Strychnos divaricans & FEN CHA MA QIAN ZI \\
\hline T5727 Saposhnikovia divaricata [Syn. Ledebouriella seseloides] & FANG FENG \\
\hline T6155 Strophanthus divaricatus & YANG JIAO AO ZI \\
\hline T0661 Artemisia anomala & LIU JI NU \\
\hline T3201 Hemiphragma heterophyllum & BIAN DA XIU QIU \\
\hline T4670 Patrinia heterophylla & YI YE BAI JIANG \\
\hline T0713 Artocarpus heterophyllus & BO LUO MI \\
\hline T0620 Arisaema heterophyllum & YI YE TIAN NAN XING \\
\hline T2508 Eucalyptus dives & FU AN \\
\hline T3809 Ligularia lapathifolia & NIU BANG YE DU WU \\
\hline T5106 Polygonum lapathifolium & YU LIAO \\
\hline T5060 Polanisia dodecandra & SHI ER RUI CHOU SHI CAI \\
\hline T5861 Selaginella doederleinii & DA YE CAI \\
\hline T1171 Canis familiaris & GOU XIN \\
\hline T1170 Canis familiaris & GOU ROU \\
\hline T5561 Rosa canina & QUAN CHI QIANG WEI \\
\hline T0553 Apocynum venetum & LUO BU MA \\
\hline T2092 Delphinus delphis & HAI TUN YU \\
\hline T4087 Malus domestica & PING GUO HAI TANG \\
\hline T3194 Hemerocallis fulva var. kwanso & CHONG BAN XUAN CAO \\
\hline T0492 Angelica pubescens f. biserrata [Syn. Angelica pubescens] & DU HUO \\
\hline T0675 Artemisia douglasiana & DAO SHI HAO \\
\hline T5244 Prunus tomentosa & SHAN YING TAO \\
\hline T4854 Physalis pubescens & KU ZHI \\
\hline T5528 Rhodomyrtus tomentosa & SHAN REN YE \\
\hline T1843 Croton draconoide & DE LA KE BA DOU \\
\hline T1994 Daemonorops draco & QI LIN JIE \\
\hline T0844 Baccharis dracunculifolia & XIAO LONG YE KUO BAO JU \\
\hline T3582 Juniperus chinensis var. kaizuka & LONG BAI \\
\hline T2257 Dracocephalum moldavicum & XIANG QING LAN \\
\hline T2254 Dracaena draco & LONG XUE SHU \\
\hline T5409 Rana temporaria chensinensis; Rana amurensis & HA SHI MA \\
\hline T0330 Alnus pendula & CHUI QI MU \\
\hline
\end{tabular}




\begin{tabular}{l} 
Drooping Sedge* \\
Drooping Snailseed* \\
Droughtdysentery Holarrhena Bark \\
Drug Snowbell \\
Drug Sweetflag \\
Drummond St.John'swort \\
Drupaceous Plumyew* \\
Drupaceous Scurfpea* \\
Dry Silene \\
Dry-living Rabdosia \\
Dubious Barberry \\
Duhat \\
Dulcin Garcinia* \\
Dunn Wampee \\
Dunn's Plagiogyria \\
Dutchman's Breeches \\
Dutchmanspipe \\
Dwarf Chickling Pea \\
Dwarf Cornel \\
Dwarf Flowering Cherry Seed \\
Dwarf Gorse \\
Dwarf Many-flowered May-apple \\
Dwarf Marigold \\
Dwarf Nightshade* \\
Dwarf Ophiorrhiza \\
Dwarf Pomegranate \\
Dwarf Sunflower* \\
Dyebark Evodia \\
Dyed Morinda \\
Dye-maker's False Puffboll \\
\hline
\end{tabular}

Dyer's Alkanet

Dyers Woad

Dyetree Leaf

Dzungaria Hogfennel*

Dzungaria Monkshood

E.Chuan Swertia

Eaglewood

Early Lilac

Early Meadowrue

Earthworm

East African Sandalwood

East China Globethistle

East-African Poisonnut*

East-African Senna*

East-Anatolia Centaurea*

East-Asia Low Meadowrue

Easter Heraldtrumpet

Eastern Arborvitae

Eastern Bracken Fern

Eastern Groundsel

Eastern Monkshood
T1203 Carex pendula

T1588 Cocculus pendulus

T3263 Holarrhena antidysenterica

T6202 Styrax officinalis

T0142 Acorus calamus

T3347 Hypericum drummondii

T1317 Cephalotaxus drupacea

T5271 Psoralea drupacea

T5955 Silene jenisseensis

T3536 Isodon xerophilus

T0903 Berberis dubia

T6266 Syzygium cumini

T2853 Garcinia dulcis

T1534 Clausena dunniana

T4997 Plagiogyria dunnii

T2148 Dicentra cucullaria

T0638 Aristolochia sipho

T3703 Lathyrus cicera

T1699 Cornus suecica

T5224 Prunus japonica [Syn. Cerasus japonica]

T6592 Ulex nanus

T2298 Dysosma difformis

T5805 Schkuhria pinnata

T5997 Solanum demissum

T4514 Ophiorrhiza pumila

T5333 Punica granatum cv. nana

T3152 Helianthus pumilus

T2643 Evodia meliifolia

T4285 Morinda tinctoria

T4978 Pisolithus tinctorius [Syn. Lycoperdon capitatum; Scleroderma

tinctorium]

T0304 Alkanna tinctoria

T3477 Isatis tinctoria

T5010 Platycarya strobilacea

T4763 Peucedanum morisonii

T0131 Aconitum soongaricum

T6217 Swertia davidii

T0554 Aquilaria agallocha

T6260 Syringa oblata

T6379 Thalictrum dioicum

T4796 Pheretima aspergillum; Allolobophora caliginosa trapezoides

T4556 Osyris tenuifolia

T2316 Echinops grijsii

T6191 Strychnos usambarensis

T1246 Cassia singueana

T1307 Centaurea pseudoscabiosa ssp. pseudoscabiosa

T6414 Thalictrum thunbergii

T0886 Beaumontia grandiflora

T6438 Thuja occidentalis

T5284 Pteridium aquilinum var. latiusculum

T5914 Senecio vernalis

T0121 Aconitum orientale
XIA CHUI TAI CAO

CHUI MU FANG JI

ZHI XIE MU PI

YAO YONG AN XI XIANG

BAI CHANG

DE LA MENG DE JIN SI TAO

HE GUO CU FEI

HE GUO ZHUANG BU GU ZHI

HAN MAI PING CAO

HAN SHENG XIANG CHA CAI

ZHI YI XIAO BO

YANG SHI GUO

TIAN SHAN ZHU ZI

HEI GUO HUANG PI

DAO YE LIU ZU JUE

DOU ZHUANG HE BAO MU DAN

OU ZHOU MA DOU LING

BIAN JIA SHAN LI DOU

AI LAI MU

YU LI REN

AI JING DOU

XIAO BA JIAO LIAN

SHI KU JU

AI QIE

DUAN XIAO SHE GEN CAO

YUE JI SHI LIU

AI XIANG RI KUI

LIAN YE WU ZHU YU

RAN SE JI YAN TENG

DOU BAO JUN

OU ZI CAO

OU ZHOU SONG LAN

HUA XIANG SHU YE

ZHUN GE ER QIAN HU

ZHUN GE ER WU TOU

CHUAN DONG ZHANG YA CAI

CHEN XIANG

ZI DING XIANG

YI XING TANG SONG CAO

QIU YIN

XIAO HUA SHA ZHEN

HUA DONG LAN CI TOU

DONG FEI MA QIAN

DONG FEI JUE MING

DONG AN NA TUO LI YA SHI CHE

JU

YAN GUO CAO

QING MING HUA

BEI MEI YA BAI

JUE

CHUN QIAN LI GUANG

GAO JIA SUO WU TOU 
Eastern Wahoo

Easy-lobed Tyromyces*

Ebei Fritillary

Ebracteolate Euphorbia

Ecasto-leaf Rosewood*

Echinate Licorice*

Echiumlike Andrographis

Edible Abelmoschus

Edible Aglaia*

Edible Bamboo

Edible Bitterleaf

Edible Canna

Edible Casimiroa

Edible Eugenia*

Edible Kudzuvine

Edible Milkvetch*

Edible Oxystelma Variety*

Edible Tulip

Eelgrass

Egypt Cultivate Coralbean*

Egypt Planted Iris

Egyptian Carissa

Egyptian Clover*

Egyptian Cottonthistle*

Egyptian Groundsel

Egyptian Sage*

Eichwald Heliotrope*

Einkorn

Elder*

Elecampane Inula

Elegant Goldenray*

Elegant Sneezeweed*

Elegant Stephania

Elephant Bone

Elephant Gall

Elephant Meat

Elephant Tree

Ellgrass

Ellipse Navicula*

Elliptic Aglaia*

Elliptic Gambirplant*

Elliptic Gynura*

Elliptical Erycibe

Elliptical Ochrosia

Ellipticleaf Olibanum*

Ellipticleaf Pricklyash*

Ellisi Porous Agaric*

Elm-leaf Raspberry*

Elodia St.John'swort

Elongate Andrographis*

Elongate Barrenwort

Emarginate Amaranth

Emarginate Soapberry Seed
T2537 Euonymus atropurpureus

T6589 Tyromyces fissilis

T2785 Fritillaria ebeiensis

T2584 Euphorbia ebracteolata

T2002 Dalbergia ecastophyllum

T3012 Glycyrrhiza echinata

T0453 Andrographis echioides

T3240 Hibiscus esculentus

T0233 Aglaia edulis

T4844 Phyllostachys edulis

T6716 Vernonia esculenta

T1172 Canna edulis

T1226 Casimiroa edulis

T2532 Eugenia edulis

T5312 Pueraria edulis

T0792 Astragalus cibarius

T4566 Oxystelma esculentum var. alpini

T6560 Tulipa edulis

T6683 Vallisneria spiralis

T2469 Erythrina lysistemon

T3455 Iris carthaliniae

T1207 Carissa edulis

T6516 Trifolium alexandrinum

T4495 Onopordum alexandrinum

T5876 Senecio aegypticus

T5659 Salvia aegyptiaca

T3170 Heliotropium eichwaldii

T6545 Triticum monococcum

T5707 Sambucus williamsii

T3431 Inula helenium

T3804 Ligularia elegans

T3137 Helenium elegans

T6125 Stephania elegans

T2337 Elephas maximus

T2336 Elephas maximus

T2338 Elephas maximus

T1081 Bursera microphylla

T6925 Zostera marina

T4394 Navicula delognei f. elliptica

T0234 Aglaia elliptica

T6614 Uncaria elliptica

T3087 Gynura elliptica

T2447 Erycibe elliptilimba

T4469 Ochrosia elliptica

T0995 Boswellia ovalifoliolata

T6885 Zanthoxylum ovalifolium

T5128 Polyporus ellisii

T5602 Rubus ulmifolius

T3348 Hypericum elodeoides

T0454 Andrographis elongata

T2394 Epimedium elongatum

T0388 Amaranthus lividus

T5718 Sapindus emarginatus
ZI GUO WEI MAO

YI LYE GAN LAO JUN

E BEI BEI MU

YUE XIAN DA JI

YI KA TUO YE HUANG TAN

JI GAN CAO

LAN JI CHUAN XIN LIAN

KA FEI HUANG KUI

KE SHI MI ZI LAN

MENG ZONG ZHU

BAN JIU JU

JIAO YU

XIANG ROU GUO

KE SHI FAN YING TAO

SHI YONG GE

SHI YONG HUANG QI

BIAN ZHONG JIAN HUAI TENG

GUANG CI GU

KU CAO

AI JI ZAI PEI CI TONG

AI JI ZHONG ZHI YUAN WEI

AI JI JIA HU CI

AI JI CHE ZHOU CAO

AI JI DA CHI JI

AI JI QIAN LI GUANG

AI JI SHU WEI CAO

AI SHI TIAN JIE CAI

DAN LI XIAO MAI

JIE GU MU

TU MU XIANG

YA ZHI TUO WU

YA MEI DUI XIN JU

YA LI QIAN JIN TENG

XIANG GU

XIANG DAN

XIANG ROU

XIAO YE LIE LAN

HAI DAI

TUO YUAN ZHOU XING ZAO

TUE YUAN MI ZI LAN

TUO YUAN GOU TENG

TUO YUAN SAN QI CAO

AO MAI DING GONG TENG

GU CHENG MEI GUI SHU

TUO YUAN YE RU XIANG SHU

TUO YUAN YE HUA JIAO

AI LI SI DUO KONG JUN

YU YE MAO MEI

TING JING BIAN DI JIN

SHEN CHANG CHUAN XIN LIAN

CHUAN XI YIN YANG HUO

AO TOU XIAN

AO TOU WU HUAN ZI 
Emblic Leafflower

Emblic Leafflower Bark

Emblic Leafflower Leaf

Emblic Leafflower Root

Emei Larkspur

Emei Spikemoss*

Emitic Devilpepper

English Daisy

English Ivy

English Walnut Bark

English Walnut Exocarp

English Walnut Leaf

English Walnut Seed

Enormous Euphorbia

Entire Groundsel

Entire Micromelum

Entire Ochna

Entireleaf Gymnopetalum

Erect Fig

Erect Hypecoum

Erect Juniper*

Erect Periwinkle

Erect St.John'swort

Erect Stephania*

Ergot

Ermans Birch

Ernest Milkvetch*

Eschscholtz Falsehellebore*

Ethiopian Jujube*

Eucalyptus Leaf

Euchretaleaf Common Jasminorange

Eucommia

Eucommia Leaf

Eupatoriumlike Inula

Eurasia Greenbrier

European Alder

European Ash

European Barberry

European Beech

European Bladdernut, Pinnate Bladdernut

European Boxwood

European Bugleweed

European Columbine

European Cowlily

European Cowparsnip*

European Euonymus

European Goldenrod

European Grape

European Grape Stem and Leaf

European Gymnadenia

European Hop Female-flower

European Ladyslipper

European Leadwort*
T4834 Phyllanthus emblica

T4836 Phyllanthus emblica

T4837 Phyllanthus emblica

T4835 Phyllanthus emblica

T2081 Delphinium omeiense

T5864 Selaginella omeiensis

T5427 Rauvolfia vomitoria

T0893 Bellis perennis

T3112 Hedera helix

T3569 Juglans regia

T3567 Juglans regia

T3570 Juglans regia

T3568 Juglans regia

T2593 Euphorbia ingens

T5889 Senecio integerrimus

T4223 Micromelum integerrimum

T4465 Ochna integerrima

T3081 Gymnopetalum integrifolium

T2716 Ficus beecheyana [Syn. Ficus erecta var. beecheyana]

T3332 Hypecoum erectum

T3586 Juniperus erectopatens

T6759 Vinca erecta

T3349 Hypericum erectum

T6126 Stephania erecta

T1541 Claviceps purpurea

T0931 Betula ermanii

T0794 Astragalus ernestii

T6695 Veratrum eschscholtzii

T6921 Zizyphus abyssinica

T2510 Eucalyptus globulus

T4317 Murraya euchrestifolia [Syn. Clausena euchrestifolia]

T2530 Eucommia ulmoides

T2531 Eucommia ulmoides

T3429 Inula eupatorioides

T5974 Smilax aspera

T0326 Alnus glutinosa

T2768 Fraxinus excelsior

T0919 Berberis vulgaris

T2662 Fagus sylvatica

T6098 Staphylea pinnata

T1094 Buxus sempervirens

T3979 Lycopus europaeus

T0558 Aquilegia vulgaris

T4449 Nuphar luteum

T3220 Heracleum pyrenaicum

T2539 Euonymus europaeus

T6023 Solidago virgaurea

$\mathrm{T} 6798$ Vitis vinifera

T6799 Vitis vinifera

T3076 Gymnadenia albida

T3289 Humulus lupulus

T1983 Cypripedium calceolus

T5027 Plumbago europaea
AN MO LE

YOU GAN MU PI

YOU GAN YE

YOU GAN GEN

E MEI CUI QUE HUA

E MEI JUAN BAI

CUI TU LUO FU MU

CHU JU

YANG CHANG CHUN TENG

HU TAO SHU PI

HU TAO QING PI

HU TAO YE

HU TAO REN

JU DA JI

QUAN YUAN QIAN LI GUANG

XIAO YUN MU

JIN LIAN MU

FENG GUA

TIAN XIAN GUO

ZHI LI JIAO HUI XIANG

ZHI LI CI BAI

ZHI LI CHANG CHUN HUA

XIAO LIAN QIAO

ZHI LI QIAN JIN TENG

MAI JIAO

YUE HUA

SUO GUO HUANG QI

AI XI SHOU SHI LI LU

AI SAI E BI YA ZAO

AN YE

DOU YE JIU LI XIANG

DU ZHONG

DU ZHONG YE

ZE LAN YANG ER JU

SUI BA QIA

OU ZHOU QI MU

OU ZHOU BAI LA SHU

OU ZHOU XIAO BO

OU ZHOU SHUI QING GANG

OU ZHOU SHENG GU YOU

JIN SHU HUANG YANG

OU DI SUN

OU ZHOU LOU DOU CAI

OU ZHOU PING PENG CAO

OU ZHOU DU HUO

OU ZHOU WEI MAO

MAO GUO YI ZHI HUANG HUA

PU TAO

PU TAO TENG YE

BAI SHOU SHEN

PI JIU HUA

SHAO LAN

OU ZHOU LAN MO LI 
European Mountainash

European Privet

European Pyrola

European Scopolia

European Silver Fir

European Stickseed

European Strawberry

European Waterhemlock Root

European Verbena

Evergreen Dogwood

Evergreen Euonymus

Evergreen Mucuna

Evergreen Wood Fern

Everlasting Pea

Ewers Stonecrop*

Expanse Erycibe*

Expansum Fig*

Extensed Wingfruitvine

Extracts of Aloe spp.

Extreme-fragrant Machilus*

Extreme-fragrant Tailgrape*

Extreme-fragrant Yarrow*

Extreme-wide Coralbean*

Eyelid-leaf Sage*

Eyeshaped Dendrobium

Faber Galeola

Faber Groundsel

Faber Meadowrue

Faber's St.John'swort

Fairy Primrose

Falcate Micromelum

Falcate Milkvetch*

Falcon Monkshood*

Fallax Cladonia Lichen

Falsa Arnica (in Brazil)

False Chinese Swertia

False Goldenray*

False Iris*

False Narcissus*

False Simon Poplar

Falselittleconical Rabdosia

Falsenettleleaf Pepper

Falsesour Cherry

Fangchi

Fan-shaped Corallodiscus

Farges Aralia

Farges Epimedium

Farges Glorybower*

Farges Holboellia

Farges Meadowrue

Farges Paris

Fascicled Bushmint*

Fascicled Swertia*
T6053 Sorbus aucuparia

T3831 Ligustrum vulgare

T5351 Pyrola rotundifolia

T5820 Scopolia carniolica

T0003 Abies alba

T3688 Lappula echinata

T2764 Fragaria vesca

T1416 Cicuta virosa

T6709 Verbena officinalis

T1696 Cornus capitata [Syn. Dendrobenthamia capitata]

T2542 Euonymus japonicus

T4311 Mucuna sempervirens

T2283 Dryopteris marginalis

T3704 Lathyrus latifolius

T5854 Sedum ewersii

T2448 Erycibe expansa

T4201 Mesembryanthemum expansum

T4350 Myriopteron extensum

T0346 Aloe spp.

T4017 Machilus odoratissima

T0655 Artabotrys odoratissimus

T0062 Achillea fragrantissima

T2467 Erythrina latissima

T5660 Salvia blepharophylla

T2102 Dendrobium fimbriatum var. oculatum

T2826 Galeola faberi

T5887 Senecio faberi

T6381 Thalictrum faberi

T3350 Hypericum faberi

T5199 Primula malacoides

T4221 Micromelum falcatum

T0795 Astragalus falcatus

T0092 Aconitum falconeri

T1525 Cladonia fallax

T3952 Lychnophora ericoides

T6231 Swertia pseudochinensis

T3818 Ligulariopsis shichuana

T3470 Iris spuria

T4380 Narcissus pseudonarcissus ssp. pseudonarcissus

T5157 Populus pseudo-simonii

T5390 Rabdosia coetsoides

T4938 Piper boehmeriaefolium

T5236 Prunus pseudocerasus

T0628 Aristolochia fangchi

T1672 Corallodiscus flabellatus [Syn. Didissandra flabellat]

T0574 Aralia fargesii

T2395 Epimedium fargesii

T1564 Clerodendron trichotomum var. fargesii

T3270 Holboellia fargesii

T6382 Thalictrum fargesii

T4647 Paris fargesii

T3379 Hyptis fasciculata

T6220 Swertia fasciculata
OU ZHOU HUA QIU

OU ZHOU NV ZHEN

YUAN YE LU TI CAO

OU LANG DANG

OU ZHOU LENG SHAN

DONG BEI HE SHI

YE CAO MEI

DU QIN GEN

MA BIAN CAO

JI SU ZI

TIAO JING CAO

CHANG CHUN YOU MA TENG

BIAN BAO LIN MAO JUE

SU GEN XIANG WAN DOU

YI WO SI JING TIAN

GUANG BU DING GONG TENG

KUO ZHANG SONG YE JU

CHI GUO TENG

DUO ZHONG LU HUI TI QU WU

JI XIANG RUN NAN

JI XIANG YING ZHAO

JI XIANG SHI CAO

JI KUAN CI TONG

JIE MAO YE SHU WEI CAO

LIU SU SHI HU

SHAN HU LAN

MI SAN QIAN LI GUANG

DA YE TANG SONG CAO

YANG ZI XIAO LIAN QIAO

BAO CHUN HUA

XIAO GAN

LIAN XING HUANG QI

FA KANG WU TOU

JIN SHUA BA

ZHANG YA CAI

JIA TUO WU

JIA YUAN WEI

JIA SHUI XIAN

XIAO QING YANG

JIA XI ZHUI XIANG CHA CAI

ZHU YE JU

YING TAO

GUANG FANG JI

SHI DAN CAO

LONG YAN DU HUO

CHUAN E YIN YANG HUO

AI TONG ZI

WU YE GUA TENG

CHENG KOU TANG SONG CAO

QIU YAO GE CHONG LOU

CU SHENG SHAN XIANG

CU HUA ZHANG YA CAI 
Fasle Yellowflower Milkwort*

Fatraina (in Madagascar)

Faurie Crapemyrtle*

Faurie Shield Fern*

Faurie's Brake

Feather Cockscomb

Feathered Geranium

Featherleaf Rodgersflower

Febrifuge Holarrhena*

Female fall webworm moth

Fen Orchid

Fendler's Meadowrue

Fengqing Kadsura

Fennel Fruit

Fennel Root

Fennel Stem and Leaf

Fennelleaf Pondweed

Fernlike Asparagus

Ferruginous Rosewood*

Ferruginous Snowbell*

Fewcalycle Groundsel*

Feverfew

Fevervine

Fevervine Fruit

Fewflower Kopsia*

Fewflower Lysionotus

Few-flowered Fumitory

Fewradiate Bailai's Chrysanthemum*

Ffat-footed Clitocybe

Fiddle-leaf Resurrectionlily*

Field Bindweed

Field Goniothalamus*

Field Groundsel

Field Lacquertree

Field Marigold

Field Pepperwort

Field Sowthistle

Fig

Fig Leaf

Figuerleaved Brake

Figwortflower Picrorhiza

Fiji Fagara*

Fiji Garcinia*

Filamentary Meadowrue

Filiform Cassytha

Fimbriate Dendrobium*

Fimbriate Falsehellebore*

Findley Dendrobium*

Fineleaf Schizonepeta

Fine-leaved Fumitory

Fine-leaved Gay-feather*

Fine-leaved Sneezeweed

Fine-leaved Yarrow
T5075 Polygala fallax [Syn. Polygala aureocauda]

T5702 Samadera madagascariensis

T3669 Lagerstroemia fauriei

T5132 Polystichum fauriei

T5290 Pteris fauriei [Syn. Pteris fauriei var. minor]

T1296 Celosia argentea

T1362 Chenopodium botrys

T5555 Rodgersia pinnata

T3265 Holarrhena febrifuga

T3370 Hyphantria cunea

T3862 Liparis loeselii

T6383 Thalictrum fendleri

T3615 Kadsura interior

T2744 Foeniculum vulgare

T2745 Foeniculum vulgare

T2746 Foeniculum vulgare

T5177 Potamogeton pectinatus

T0749 Asparagus filicinus

T2003 Dalbergia ferruginea

T6198 Styrax ferrugineus

T5895 Senecio paucicazyculatus

T6294 Tanacetum parthenium

T4577 Paederia scandens

T4578 Paederia scandens

T3644 Kopsia pauciflora

T4005 Lysionotus pauciflorus

T2810 Fumaria vaillantii

T0855 Baileya pauciradiata

T1576 Clitocybe clavipes

T3622 Kaempferia pandurata

T1650 Convolvulus arvensis

T3042 Goniothalamus arvensis

T5894 Senecio oryzetorum

T6482 Toxicodendron succedaneum [Syn. Rhus succedanea]

T1112 Calendula arvensis

T3756 Lepidium campestre

T6025 Sonchus arvensis

T2717 Ficus carica

T2718 Ficus carica

T5289 Pteris dactylina

T4888 Picrorhiza scrophulariiflora

T2655 Fagara vitiensis

T2871 Garcinia pseudoguttifera

T6384 Thalictrum filamentosum

T1251 Cassytha filiformis

T2101 Dendrobium fimbriatum

T6696 Veratrum fimbriatum

T2103 Dendrobium findleyanum

T5804 Schizonepeta tenuifolia [Syn. Nepeta tenuifolia]

T2808 Fumaria parviflora

T3795 Liatris tenuifolia

T3141 Helenium tenuifolium

T0064 Achillea leptophylla
JIA HUANG HUA YUAN ZHI

MA DAO HUANG LIAN SHU

FU RUI ZI WEI

FU RUI ER JUE

JIN CHAI FENG WEI JUE

QIANG XIANG

XIANG LI

YU YE GUI DENG QING

TUI RE ZHI XIE MU

LUO XI YANG ER SUAN

FEN SHI TANG SONG CAO

NEI NAN WU WEI ZI

HUI XIANG

HUI XIANG GEN

HUI XIANG JING YE

BI CHI YAN ZI CAI

TU BAI BU

TIE XIU SE HUANG TAN

XIU SE AN XI XIANG

SHAO FU E QIAN LI GUANG

CHU AI JU

JI SHI TENG

JI SHI TENG GUO

SHAO HUA RUI MU

SHI DIAO LAN

WEI LAN QIU GUO ZI JIN

SHAO BIAN HUA BAI LAI SHI JU

BANG BING BEI SAN

TI QIN ZHUANG SHAN NAI

TIAN XUAN HUA

TIAN YE GE NA XIANG

DA BAI DING CAO

LIN BEI ZI

XIAO JIN ZHAN HUA

HUANG YE DU XING CAI

NIU SHE TOU

WU HUA GUO

WU HUA GUO YE

JIN JI WEI

XI ZANG HU HUANG LIAN

FEI JI AI JIAO

FEI JI TENG HUANG

HUA TANG SONG CAO

WU YE TENG

LIU SU JIN SHI HU

LIU SU LI LU

FEN LAI SHI HU

JING JIE

XIAO HUA QIU GUO ZI JIN

XI YE SHE BIAN JU

XI YE DUI XIN JU

XI YE SHI 
Fine-nerved Plagiogyria

Finet Monkshood

Fingerleaf Rodgersflower

First Wormwood

Fischer Euphorbia

Fish Pelargonium

Fisherman Leafflower*

Fishhook Cactus

Fistular Onion

Fiveangular Senna*

Fiveleaf Akebia

Fiveleaf Akebia Root

Fiveleaf Akebia Seed

Fiveleaf Carpetweed

Fiveleaf Crotalaria*

Fiveleaf Gynostemma

Five-leaf Merremia*

Fiveribbed Thyme

Five-room Honeysuckle*

Fivestyle Larkspur*

Flabellate Galangal*

Flaccid Anemone

Flaccid Knotweed

Flagelliform Liverwort*

Flamboyanttree

Flameray Gerbera

Flannel Mullein

Flatfruit Gynostemma

Flatshoot Mistletoe

Flatshort Mistletoe

Flatspine Pricklyash Bark

Flatspine Pricklyash Leaf

Flatspine Pricklyash Root

Flatstem Lilyturf*

Flatstem Milkvetch

Flatstiped Corydalis

Flavescent Crotalaria*

Flavescent Croton*

Flax Lily

Fleecy Milk-cap

Fleshfingered Citron

Fleshy Artocarpus*

Fleshy-flower Hemsleya

Flexedstem Rabdosia

Flexuose Climbing Fern

Flexuose Leafflower*

Flexuous Bitterleaf*

Flexuous Lemongrass*

Flexuous Milkvetch *

Flixweed Tansymustard Seed

Floatingleaf Pondweed
T4998 Plagiogyria euphlebia

T0094 Aconitum finetianum

T5554 Rodgersia aesculifolia

T0689 Artemisia princeps

T2587 Euphorbia fischeriana

T4691 Pelargonium hortorum

T4841 Phyllanthus piscatorum

T4097 Mammillaria microcarpa

T0314 Allium fistulosum

T1243 Cassia quinquangula

T0273 Akebia quinata

T0274 Akebia quinata

T0275 Akebia quinata

T4261 Mollugo pentaphylla

T1829 Crotalaria quinquefolia

T3085 Gynostemma pentaphyllum

T4198 Merremia quinquefolia

T6450 Thymus quinquecostatus

T3917 Lonicera quinquelocularis

T2083 Delphinium pentagynum

T0355 Alpinia flabellata

T0467 Anemone flaccida

T5105 Polygonum hydropiper var. flaccidum [Syn. Polygonum

flaccidum]

T0885 Bazzania trilobata

T2060 Delonix regia

T2951 Gerbera jamesonii

T6705 Verbascum thapsus

T3083 Gynostemma compressum

T6774 Viscum articulatum

T6773 Viscum articulactum

T6894 Zanthoxylum simulans

T6895 Zanthoxylum simulans

T6893 Zanthoxylum simulans

T4508 Ophiopogon planiscapus

T0793 Astragalus complanatus

T1727 Corydalis mucronifera

T1820 Crotalaria fulva

T1845 Croton flavens

T2141 Dianella tasmanica

T3657 Lactarius vellereus

T1501 Citrus medica var. sarcodactylis

T0709 Artocarpus altilis

T3204 Hemsleya carnosiflora

T3489 Isodon flexicaulis

T3989 Lygodium flexuosum [Syn. Lygodium pinnatifidum;

Ophioglossum flexuosum]

T4838 Phyllanthus flexuosus

T6717 Vernonia flexuosa

T1942 Cymbopogon flexuosus

T0796 Astragalus flexuosus

T2125 Descurainia sophia

T5176 Potamogeton natans
HUA ZHONG LIU ZU JUE

GAN WAN WU TOU

MU HE

KUI HAO

LANG DU DA JI

SHI LA HONG

YU FU YE XIA ZHU

XIAO GUO YIN MAO QIU

CONG BAI

WU LENG JUE MING

MU TONG

MU TONG GEN

YU ZHI ZI

SU MI CAO

WU YE ZHU SHI DOU

JIAO GU LAN

WU YE YU HUANG CAO

WU MAI BAI LI XIANG

WU SHI REN DONG

WU ZHU FEI YAN CAO

SHAN SHAN JIANG

E ZHANG CAO

LA LIAO

BIAN TAI

FENG HUANG MU

FU LANG HUA

MAO RUI HUA

BIAN GUO JIAO GU LAN

FENG XIANG JI SHENG

BIAN ZHI HU JI SHENG

YE HUA JIAO PI

YE HUA JIAO YE

YE HUA JIAO GEN

BIAN JING YAN JIE CAO

BIAN JING HUANG QI

BIAN BING HUANG JIN

AN HUANG ZHU SHI DOU

DAN HUANG BA DOU

TA SI MA NI YA JIE GENG LAN

RONG BAI RU GU

FO SHOU

FEI HOU MIAN BAO GUO

ROU HUA XUE DAN

ROU JING XIANG CHA CAI

QU ZHOU HAI JIN SHA

LUO E YE XIA ZHU

WAN YAN BAN JIU JU

WAN YAN XIANG MAO

WAN YAN HUANG QI

BO NIANG HAO

FU YE YAN ZI CAI 
Florida Waltheria

Florida Yellowtrumpet

Florists Chrysanthemum Flower

Florists Cyclamen

Flowering Ash

Flowering Fern

Flowery Stonebean-orchis

Fluticose Germander*

Foetid Giantfennel*

Folk Coralbean*

Folkers Coralbean*

Fomes Officinalis Sporocarp

Foochow Yam

Forbes Notopterygium

Forbes Wildginger

Fordi Lasianthus*

Fordi Phlegmariurus

Forest Gray Gum Leaf

Forest Horsetail

Forest Mint

Formosan False Cypress

Formosan Michelia*

Forrest Bugle

Forrest Gingerlily

Forrest Pyrola

Forrest Rabdosia*

Forrest Silkvine

Forrest's St.John'swort

Forskahl Coleus

Forskahl Coleus

Forster Ninenode*

Fortunate Wood Fern

Fortune Eupatorium

Fortune Japanese Spiraea

Fortune Meadowrue

Fortune Paulownia

Fortune Plumyew

Fortune Windmillpalm

Fortune's Drynaria Rhizome

Fortune's Holly Fern

Forture Euonymus

Fouquieria splendens

Four Sepals Gaultheria

Four-arris Eupatorium*

Fourfile Germander

Fourflower Litse

Foursplit Rhodiola

Fourstamen Stephania

Fourteech Schnabelia

Fourwing Eveningprimrose*

Fourwing Milkvetch*

Foveolate Aglaia*

Foxglove-like Sage
T6808 Waltheria americana

T6324 Tecoma stans

T1395 Chrysanthemum morifolium [Syn. Dendranthema morifolium]

T1930 Cyclamen persicum

T2773 Fraxinus ornus

T4553 Osmunda ragalis

T1053 Bulbophyllum odoratissimum [Syn. Stelis odoratissimum]

T6361 Teucrium fruticans

T2700 Ferula foetida

T2465 Erythrina folkersii

T2463 Erythrina crysragalli

T2749 Fomes officinalis

T2197 Dioscorea futschauensis

T4445 Notopterygium forbesii [Syn. Notopterygium franchetii]

T0726 Asarum forbesii

T3697 Lasianthus fordii

T4800 Phlegmariurus fordii

T2521 Eucalyptus tereticornis

T2412 Equisetum sylvaticum

T4192 Mentha sylvestris

T1347 Chamaecyparis formosensis

T4211 Michelia compressa var. formosana

T0264 Ajuga forrestii

T3119 Hedychium forrestii

T5348 Pyrola forrestiana

T3490 Isodon forrestii

T4726 Periploca forrestii

T3352 Hypericum forrestii

T1619 Coleus barbatus

T1620 Coleus forskahlii

T5275 Psychotria forsteriana

T2287 Dryopteris sublaeta

T2559 Eupatorium fortunei

T6082 Spiraea japonica var. fortunei

T6388 Thalictrum fortunei

T4678 Paulownia fortunei

T1318 Cephalotaxus fortunei

T6485 Trachycarpus fortunei

T2273 Drynaria fortunei

T1986 Cyrtomium fortunei

T2540 Euonymus fortunei

T2762 Fouquieria splendens

T2894 Gaultheria tetramera

T2568 Eupatorium quadrangularae

T6367 Teucrium quadrifarium

T3886 Litsea euosma

T5498 Rhodiola quadrifida

T6136 Stephania tetrandra

T5807 Schnabelia tetradonta

T4483 Oenothera tetraptera

T0808 Astragalus tetraplerus

T0236 Aglaia foveolata

T5671 Salvia digitaloides
HE TA CAO

HUANG ZHONG HUA

JU HUA

XIAN KE LAI

HUA BAI LA SHU

OU ZI QI

MI HUA SHI DOU LAN

GUAN CONG XIANG KE KE

CHOU A WEI

FU KE CI TONG

JI GUAN CI TONG

A LI HONG

FU ZHOU SHU YU

KUAN YE QIANG HUO

DU HENG

HUA NAN MA WEI SHAN

XI YE AN YE

LIN WEN JING

SEN LIN BO HE

HONG GUI

WU XIN SHI

LI ZHI HAO

YUAN BAN JIANG HUA

DA LI LU TI CAO

ZI E XIANG CHA CAI

XI NAN GANG LIU

CHUAN DIAN JIN SI TAO

RAN MAO QIAO RUI HUA

MAO HOU QIAO RUI HUA

FU SI TE JIU JIE

QIAN LIE LIN MAO JUE

PEI LAN

GUANG YE FEN HUA XIU XIAN JU

HUA DONG TANG SONG CAO

PAO TONG

SAN JIAN SHAN

ZONG LV PI

GU SUI BU

HUN TOU JI

FU FANG TENG

HUA LAI CI SHU

SI LIE BAI ZHU

SI LENG ZE LAN

TIE ZHOU CAO

QING XIANG MU JIANG ZI

SI LIE HONG JING TIAN

FANG JI

SI CHI SI LENG CAO

SI CHI YUE JIAN CAO

SI CHI HUANG QI

FENG CHAO MI ZI LAN

MAO DI HUANG SHU WEI CAO 
Foxtail Millet

Foxtail-like Galingale

Foxtail-like Sophora

Foxtail-like Sophora Root*

Fragile Codium Frond

Fragrant Albizia*

Fragrant Citrus

Fragrant Citrus Seed

Fragrant Datura*

Fragrant Erythrophleum

Fragrant Eupatorium

Fragrant Gananga

Fragrant Gaultheria

Fragrant Glorybower

Fragrant Gynocardia

Fragrant Sneezeweed*

Fragrant Snowbell

Fragrant Solomonseal

Fragrant Tailgrape*

Franchet Groundcherry

Franchet Groundcherry Root

Franchet Monkshood

Franchet Sophora

Frangipani

Free-flowering Barberry

French Broom

French Marigold

French Rose

French Tamarisk

Fresh Common Ginger

Fringecups

Fringed Iris

Froes Poisonnut*

Frog Orchid

Frutescent Cladostachys

Fruticose Cudrania

Fruticose Thorowax*

Fugacious Poppy*

Fukang Giantfennel Root

Fukien Wildginger

Funadoko Orange*

Fungus Coprinopsis episcopalis

Fungus Curvularia lunata

Fungus Gelasinospora santi-florii

Fungus Lasiodiplodia theobromae

Fungus Stachybotrys atra

Fungus Stachybotrys chartarum

Fungus Stachybotrys nephrospora

Fungus-infected Rice Spike

Funneled Physochlaina

Furcate Angelica*
T5938 Setaria italica

T1973 Cyperus alopecuroides

T6029 Sophora alopecuroides

T6028 Sophora alopecuroides

T1595 Codium fragile

T0295 Albizzia odoratissima

T1486 Citrus junos

T1487 Citrus junos

T2040 Datura metaloides

T2487 Erythrophleum suaveolens

T2567 Eupatorium odoratum

T1164 Cananga odorata

T2891 Gaultheria fragrantissima

T1556 Clerodendron fragrans

T3082 Gynocardia odorata

T3133 Helenium aromaticum

T6201 Styrax obassia

T5093 Polygonatum odoratum [Syn. Polygonatum officinale]

T0656 Artabotrys suaveolens

T4846 Physalis alkekengi var. franchetii

T4847 Physalis alkekengi var. franchetii

T0097 Aconitum franchetll

T6033 Sophora franchetiana

T5032 Plumeria rubra

T0904 Berberis floribunda

T1989 Cytisus monspessulanus

T6282 Tagetes patula

T5565 Rosa gallica

T6287 Tamarix gallica

T6910 Zingiber officinale

T6328 Tellima grandifolia

T3461 Iris japonica

T6173 Strychnos froesii

T1604 Coeloglossum viride [Syn. Coeloglossum viride var.

bracteatum ]

T2058 Deeringia amaranthoides [Syn. Cladostachys frutescens]

T1885 Cudrania fruticosa

T1061 Bupleurum fruticescens

T4628 Papaver fugax

T2701 Ferula fukanensis

T0727 Asarum fukienense

T1477 Citrus funadoko

T1659 Coprinopsis episcopalis

T1910 Curvularia lunata

T2896 Gelasinospora santi-florii

T3700 Lasiodiplodia theobromae

T6089 Stachybotrys atra

T6090 Stachybotrys chartarum

T6091 Stachybotrys nephrospora

T6655 Ustilaginoidea virens

T4858 Physochlaina infundibularis

T0481 Angelica furcijuga
SU MI

KAN MAI NIANG ZHUANG SHA

$\mathrm{CAO}$

KU DOU ZI

KU DOU GEN

SHUI SONG

XIANG HE HUAN

CHENG ZI

CHENG ZI HE

XIANG MAN TUO LUO

YE XIANG GE MU

FEI JI CAO

YI LAN

FANG XIANG BAI ZHU

CHOU MO LI

MA DAN GUO

FANG XIANG DUI XIN JU

YU LING HUA

YU ZHU

XIANG YING ZHAO

GUA JIN DENG

GUA JIN DENG GEN

DA DU WU TOU

MIN HUAI

HONG JI DAN HUA

DUO HUA XIAO BO

FA GUO JIN QUE ER

KONG QUE CAO

FA GUO QIANG WEI

FA GUO CHENG LIU

SHENG JIANG

XIN SHAO NA CAO

HU DIE HUA

FU SHI MA QIAN ZI

AO SHE LAN

JIANG GUO XIAN

ZHE TENG

MU CHAI HU

YI XIAN YING SU

FU KANG A WEI GEN

FU JIAN XI XIN

ZHOU CHANG JU

JING GU NU

LOU DOU PAO NANG CAO

FEN CHA DANG GUI 
Furcate Gloiopeltis Frond

Furfuraceous Many-flowered May-apple*

Furstamen Prismatomeris

Gabon Uvaria*

Galanga Galangal

Galanga Resurrectionlily

Galericulate Skullcap

Gambier Gambirplant

Gamboge Tree Resin

Gansu Greenbrier

Garabato; Unganangi; Cat's Claw

Garden Balsam

Garden Balsam Seed

Garden Balsum Flower

Garden Burnet

Garden Chervil

Garden Cress

Garden Eggplant

Garden Eggplant Leaf

Garden Euphorbia

Garden Lettuce

Garden Lovage

Garden Millingtonia

Garden Pansy

Garden Parsnip

Garden Pea

Garden Plum

Garden Radish

Garden Radish Seed

Garden Sorrel

Garden Sorrel Leaf

Garlic

Garlicsmell Germander*

Garou Bush

Garretti Senna*

Gaudicha Garcinia*

Gaumei Fringe-petal*

Gay-feather

Geckowood

Geniculate Monkshood

Genipa

Genonggang

Genuflex Angelica*

Gerard Ephedra

Gerard Linearstripe Rabdosia*

Gerbera

German Catchfly

German Gentian

German Iris

Gesnerialike Rabdosia

Ghostplant Wormwood

Giant Dogwood

Giant Granadilla
T2994 Gloiopeltis furcata

T2299 Dysosma furfuracea

T5207 Prismatomeris tetrandra

T6663 Uvaria klaineana

T0356 Alpinia galanga

T3620 Kaempferia galanga

T5838 Scutellaria galericulata

T6615 Uncaria gambir

T2866 Garcinia morella

T5980 Smilax menispermoidea

T6616 Uncaria guianensis

T3410 Impatiens balsamina

T3412 Impatiens balsamina

T3411 Impatiens balsamina

T5712 Sanguisorba officinalis

T0528 Anthriscus cerefolium

T3758 Lepidium sativum

T6007 Solanum melongena

T6006 Solanum melongena

T2590 Euphorbia hirta

T3662 Lactuca sativa

T3785 Levisticum officinale

T4247 Millingtonia hortensis

T6766 Viola tricolor

T4669 Pastinaca sativa

T4983 Pisum sativum

T5222 Prunus domestica

T5419 Raphanus sativus

T5420 Raphanus sativus

T5605 Rumex acetosa

T5606 Rumex acetosa

T0318 Allium sativum

T6368 Teucrium scordium

T2025 Daphne gnidium

T1233 Cassia garrettiana

T2855 Garcinia gaudichaudii

T1811 Crossopetalum gaumeri

T3793 Liatris spicata

T5748 Sauropus androgynus

T0098 Aconitum geniculatum

T2900 Genipa americana

T1783 Cratoxylum arborescens

T0482 Angelica genuflexa

T2368 Ephedra gerardiana

T3508 Isodon lophanthoides var. gerardiana

T2950 Gerbera anandria [Syn. Leibnitzia anandria]

T3951 Lychnis viscaria

T2911 Gentiana germanica

T3459 Iris germanica

T3491 Isodon gesneroides

T0680 Artemisia lactiflora

T1697 Cornus controversa [Syn. Bothrocaryum controversum]

T4667 Passiflora quadrangularis
LU JIAO CAI

BI LIN BA JIAO LIAN

NAN SHAN HUA

JIA PENG ZI YU PAN

DA LIANG JIANG

SHAN NAI

BING TOU CAO

ER CHA GOU TENG

TENG HUANG

FANG JI YE BA QIA

GUI YA NA GOU TENG

FENG XIAN

JI XING ZI

FENG XIAN HUA

DI YU

XUE WEI CAI

JIA DU XING CAI

QIE ZI

QIE YE

DA FEI YANG CAO

WO JU

OU DANG GUI

ZI MEI SHU

SAN SE JIN

OU FANG FENG

WAN DOU

YANG LI

LAI FU

LAI FU ZI

SUAN MO

SUAN MO YE

DA SUAN

SUAN WEI XIANG KE KE

JING YA MA YE RUI XIANG

JIA LEI JUE MING

GAO DI CHA SHAN ZHU ZI

GAO MEI YING BAN

SHE BIAN JU

TONG XU SHOU GONG MU

XI BAN WU TOU

JING NI PING

QIAO MU ZHUANG HUANG NIU MU

QU XI DANG GUI

SHAN LING MA HUANG

XIA JI XIAN WEN XIANG CHA CAI

DA DING CAO

YANG JIAN QIU LUO

DE GUO LONG DAN

DE GUO YUAN WEI

JU TAI XIANG CHA CAI

YA JIAO AI

DENG TAI SHU

DA GUO XI FAN LIAN 


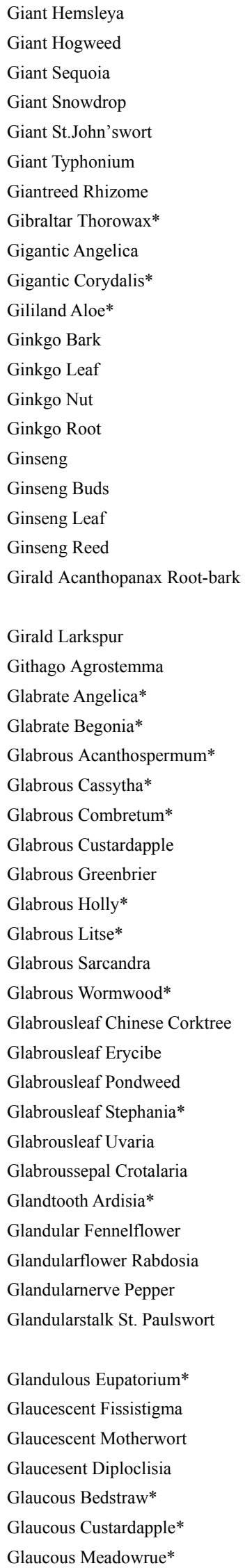

T3206 Hemsleya gigantha

T3216 Heracleum mantegazzianum

T5917 Sequoia gigantea

T2821 Galanthus elwelii

T3340 Hypericum ascyron

T6588 Typhoniun giganteum

T0722 Arundo donax

T1063 Bupleurum gibraltaricum

T0483 Angelica gigas

T1716 Corydalis gigantea

T0339 Aloe gililandii

T2963 Ginkgo biloba

T2964 Ginkgo biloba

T2961 Ginkgo biloba

T2962 Ginkgo biloba

T4599 Panax ginseng [Syn. Panax schinseng]

T4600 Panax ginseng [Syn. Panax schinseng]

T4602 Panax ginseng [Syn. Panax schinseng]

T4601 Panax ginseng [Syn. Panax schinseng]

T0036 Acanthopanax giraldii [Syn. Acanthopanax giraldii var.

inermis; Eleutherococcus giraldii]

T2076 Delphinium giraldii

T0252 Agrostemma githago

T0484 Angelica glabra

T0889 Begonia glabra

T0046 Acanthospermum glabratum

T1252 Cassytha glabella

T1631 Combretum imberbe

T0506 Annona glabra

T5977 Smilax glabra

T3397 Ilex pubescens var. glaber

T3888 Litsea glutinosa var. glabrara

T5730 Sarcandra glabra [Syn. Chloranthus glaber]

T0677 Artemisia glabella

T4792 Phellodendron chinense var. glabriusculum

T2450 Erycibe schmidtii

T5175 Potamogeton lucens

T6127 Stephania glabra

T6659 Uvaria boniana

T1837 Crotalaria usaramoensis

T0593 Ardisia cornudentata

T4432 Nigella glandulifera

T5388 Rabdosia adenantha

T4936 Piper bavinum

T5952 Siegesbeckia orientalis var. pubescens [Syn. Siegesbeckia pubescens]

T2560 Eupatorium glandulosum

T2734 Fissistigma glaucescens [Syn. Melodorum glaucescens]

T3751 Leonurus glaucescens

T2233 Diploclisia glaucescens

T2831 Galium glaucum

T0507 Annona glauca

T6390 Thalictrum glaucum
JU HUA XUE DAN

DA YE NIU FANG FENG

JU SHAN

DA XUE HUA LIAN

HUANG HAI TANG

YU BAI FU

LU ZHU GEN

ZHI BU LUO TUO CAI HU

CHAO XIAN DANG GUI

JU ZI JIN

JI SHI LU HUI

BAI GUO SHU PI

BAI GUO YE

BAI GUO

BAI GUO GEN

REN SHEN

REN SHEN HUA LEI

REN SHEN YE

REN SHEN LU

HONG MAO WU JIA PI

QIN LING CUI QUE HUA

MAI XIAN WENG

GUANG HUA DANG GUI

GUANG JIE QIU HAI TANG

GUANG CI BAO JU

WU MAO WU GEN TENG

WU MAO FENG CHE ZI

YUAN HUA FAN LI ZHI

TU FU LING

TU MAO DONG QING

WU MAO CHAN GAO SHU

JIU JIE CHA

WU MAO HAO

TU YE HUANG PI SHU

GUANG YE DING GONG TENG

GUANG YE YAN ZI CAI

GUANG YE DI BU RONG

GUANG YE ZI YU PAN

GUANG E ZHU SHI DOU

XIAN CHI ZI JIN NIU

XIAN MAO HEI ZHONG CAO

XIAN HUA XIANG CHA CAI

XIAN MAI JU

XIAN GENG XI XIAN

XIAN ZE LAN

BAI YE GUA FU MU

HUI BAI YI MU CAO

CANG BAI CHENG GOU FENG

FEN LU ZHU YANG YANG

ROU MAO FAN LI ZHI

LV TANG SONG CAO 
Glaucousback Honeysuckle Glaucousback Threewingnut Globe Artichoke Globe Candytuft Globe Cordia* Globeamaranth Globefish Globose Condorvine Globular Pepper Glomerate Blumea* Glomerule Sweetleaf Glory Bush Glory-of-the-snow Glossy Buckthorn Glossy Privet Fruit Gluey Litse Glutinose Bluebeard Gmelin Sealavender Herb Goat Hide

Goat Milk

Goat Pancreas

Goat Willow

Goering Lemongrass

Goldback Fern

Golden Adonis

Golden Buckwheat Root

Golden Corydalis

Golden Lycoris

Goldenchain Laburnum

Goldencoma Shield Fern

Goldenflower Dendrobium

Golden-seal

Goldenshower Senna Fruit

Goldenthread Cordyceps

Gold-enthread Meadowrue

Golden-wing Milkvetch*

Goldenyellow Jerusalemsage*

Goldenyellow Thelephore*

Goldenyellow Thorowax

Goldflower Actinidia

Goldhair Hedyotis

Goldmat Rhododendron

Goldon-belt*

Goldregen

Goldsaxifrage Herb

Gold-wire Brush*

Gongbo Monkshood

Goose Fat

Goose Tail-meat

Goose-bowel Vegatable*

Gordon Euryale Root

Gorgonian Gorgoniae suberogorgia

Gorgonian Junceella fragilis
T3911 Lonicera hypoglauca

T6540 Tripterygium hypoglaucum

T1966 Cynara scolymus

T3386 Iberis umbellata

T1677 Cordia globosa

T3040 Gomphrena globosa

T2805 Fugu ocellatus

T4116 Marsdenia globifera

T4956 Piper mullesua

T0958 Blumea glomerata

T6253 Symplocos glomerata

T6455 Tibouchina semidecandra

T1368 Chionodoxa luciliae

T5460 Rhamnus frangula [Syn. Frangula alnus]

T3828 Ligustrum lucidum

T3887 Litsea glutinosa

T1222 Caryopteris glutinosa

T3842 Limonium gmelinii

T1181 Capra hircus; Ovis aries

T1182 Capra hircus; Ovis aries

T1183 Capra hircus; Ovis aries

T5652 Salix caprea

T1943 Cymbopogon goeringii

T4988 Pityrogramma triangularis

T0186 Adonis chrysocyatha

T2657 Fagopyrum cymosum [Syn. Polygonum cymosum]

T1707 Corydalis aurea

T3983 Lycoris aurea

T3648 Laburnum anagyroides

T2280 Dryopteris chrysocoma

T2098 Dendrobium chrysanthum

T3309 Hydrastis canadensis

T1232 Cassia fistula

T1682 Cordyceps ophioglossoides

T6389 Thalictrum glandulosissimum

T0791 Astragalus chrysopterus

T4806 Phlomis aurea

T6420 Thelephora aurantiotincta

T1056 Bupleurum aureum

T0159 Actinidia chrysantha

T3125 Hedyotis chrysotricha [Syn. Oldenlandia chrysotricha]

T5505 Rhododendron chrysanthum

T3776 Lethariella zahlbruckneri

T1988 Cytisus laburnum

T1403 Chrysosplenium grayanum

T3775 Lethariella cladonioides

T0107 Aconitum kongboense

T0516 Anser cygnoides domestica

T0517 Anser cygnoides domestica

T2359 Endarachne binghamiae

T2632 Euryale ferox

T3053 Gorgoniae suberogorgia

T3574 Junceella fragilis
XIAN YE REN DONG

KUN MING SHAN HAI TANG

CAI JI

SAN XING QU QU HUA

QIU ZHUANG PO BU MU

QIAN RI HONG

HE TUN

QIU HUA NIU NAI CAI

DUAN JU

TUAN JI AI NA XIANG

TUAN HUA SHAN FAN

XUE GUANG HUA

OU SHU LI

NV ZHEN ZI

CHAN GAO MU JIANG ZI

NIAN YE YOU ${ }^{(2)}$

BU XUE CAO

YANG PI

YANG RU

YANG YI

HUANG HUA ER LIU

YE XIANG MAO

SAN JIAO FEN YE JUE

JIN HUANG CE JIN ZHAN HUA

TIAN QIAO MAI GEN

JIN HUANG JIN

DA YI ZHI JIAN

DU DOU

HUANG MAO LIN MAO JUE

SHU HUA SHI HU

BAI MAO GEN ${ }^{(4)}$

PO LUO MEN ZAO JIA

DA TUAN NANG CHONG CAO

JIN SI MA WEI LIAN

JIN YI HUANG QI

JIN HUANG CAO SU

JIN HUANG GE JUN

JIN HUANG CHAI HU

JIN HUA MI HOU TAO

JIN MAO ER CAO

NIU PI CHA

JIN YAO DAI

LIAN HUA JIN QUE ER

JIN QIAN KU YE CAO

JIN SI SHUA

GONG BU WU TOU

BAI E GAO

E CUI

E CHANG CAI

QIAN SHI GEN

LIU SHAN HU

CUI DENG XIN LIU SHAN HU 
Gorgonian Junceella gemmacea

Gorgonian Junceella juncea

Gorgonian Muricella sinensis

Gortschakov Corydalis*

Govan Corydalis

Graceful Jessamine

Gracile Eupatorium*

Gram Chickpea

Grand Aglaia*

Grand Crinum

Grand Globethistle*

Grand Litse*

Grapefruit

Grapeleaf Hibiscus*

Grass Vetchling

Grasshopper

Grassland Euphorbia*

Grassleaf Saussurea

Grassleaf Sweetflag

Grassleaf Sweetflag Leaf

Grassleaved Sweetflag

Gratiole

Graywhite Wormwood*

Great Burdock Fruit

Great Burdock Leaf*

Great Burdock Root

Great Maple

Great Willowherb (Firewood)

Greater Bird's-foot-trefoil

Greater Celandine

Greater Periwinkle

Greater Water-parsnip

Grecian Foxglove

Grecian Laurel Fruit

Grecian Laurel Leaf

Grecian Silkvine

Greek Silk-hair Yarrow*

Greek Yarrow

Green Alectoria Filament

Green Anemone

Green Falsehellebore

Green Hellebore*

Green Poplar

Greenish Lily

Greenleaf Piptanthus, Evergreen Laburnum

Greensnake vine

Greenspike Erythrophleum*

Greenstem Forsythia

Greenyellow Stephania

Gregg Sage*

Grey Arcyria*
T3575 Junceella gemmacea

T3576 Junceella juncea

T4315 Muricella sinensis

T1717 Corydalis gortschakovii

T1718 Corydalis govaniana

T2897 Gelsemium elegans

T2561 Eupatorium gracile

T1412 Cicer arietinum

T0237 Aglaia grandis

T1794 Crinum asiaticum

T2315 Echinops giganteus

T3890 Litsea grandis

T1503 Citrus paradisi

T3249 Hibiscus vitifolius

T3706 Lathyrus nissolia

T5559 Romalea microptera

T2620 Euphorbia stepposa

T5754 Saussurea graminea

T0144 Acorus gramineus

T0145 Acorus gramineus

T0146 Acorus tatarinowii

T3061 Gratiola officinalis

T0669 Artemisia cana

T0586 Arctium lappa

T0585 Arctium lappa

T0584 Arctium lappa

T0053 Acer pseudoplatanus

T1352 Chamaenerion angustifolium [Syn. Epilobium angustifolium]

T3930 Lotus pedunculatus

T1357 Chelidonium majus

T6760 Vinca herbacea [Syn. Vinca major]

T5969 Sium latifolium

T2175 Digitalis lanata

T3726 Laurus nobilis

T3725 Laurus nobilis

T4727 Periploca graeca

T0063 Achillea holosericea

T0058 Achillea ageratifolia

T0298 Alectoria vivens

T0526 Anthopleura stell

T6700 Veratrum viride

T3189 Helleborus viridis

T5150 Populus cathayana

T3832 Lilium brownii var. viridulum [Syn. Lilium brownii var.

colchesteri]

T4976 Piptanthus nepalensis

T4725 Periploca calophylla

T2483 Erythrophleum chlorostachyum

T2757 Forsythia viridissima

T6137 Stephania viridiflavens

T5674 Salvia greggii

T0589 Arcyria cinerea
LEI DENG XIN LIU SHAN HU

DENG XIN LIU SHAN HU

ZHONG HUA XIAO JIAN LIU SHAN

$\mathrm{HU}$

GE CAI KE SHI ZI JIN

KU MANG HUANG JIN

GOU WEN

XI ZE LAN

HUI HUI DOU

JU DA MI ZI LAN

YA ZHOU WEN SHU LAN

JU DA LAN CI TOU

DA MU JIANG ZI

PU TAO YOU

PU TAO YE MU JIN

HE CAO XIANG WAN DOU

HUANG CHONG

CAO YUAN DA JI

HE YE FENG MAO JU

JIN QIAN PU

JIN QIAN PU YE

SHI CHANG PU

YAO SHUI BA JIAO

QING AI

NIU BANG ZI

NIU BANG YE

NIU BANG GEN

OU YA QI

HONG KUAI ZI

HUA XU GENG BAI MAI GEN

BAI QU CAI

DA CHANG CHUN HUA

OU ZE QIN

MAO HUA MAO DI HUANG

YUE GUI ZI

YUE GUI YE

XI LA GANG LIU

XI LA SI MAO SHI

XI LA SHI CAO

JIN SI DAI

LV HAI KUI

LV LI LU

LV TI GEN CAO

QING YANG

BAI HE

HUANG HUA MU

QING SHE TENG

LU SUI GE MU

JIN ZHONG HUA

HUANG YE DI BU RONG

GE SHI SHU WEI CAO

HUI JIN SE TUAN WANG JUN 
Grey Basil

Grey Coralbean*

Grey Greenbrier*

Greyblue Pericampylus

Greyblue Spicebush

Greyblue Spicebush Leaf

Greyhair Asiabell

Greyish Gambirplant*

Greyish Nannoglottis*

Greyish-green Corydalis

Greyleaf Actinidia

Greyleaf Maesa

Greywhite Nightshade*

Greywhite Seseli*

Griffith Garcinia*

Griffith Mahonia

Grossnerve Pepper*

Grosvenor Siraitia

Groundfig Spurge

Guamachil Apea-earring

Guanabana

Guanguangtree

Guangxi Hogfennel

Guangxi Lycoris

Guangxi Many-flowered May-apple*

Guangxi Mayten*

Guara, Mestizo, or Guacharaco (in

Colombia)

Guarana

Guava Bark

Guava Leaf

Guava Seed

Guava Unripe Fruit

Guinea Bitterleaf*

Guinea Yam

Guizhou Arachniodes

Guizhou Evodia

Guizhou Swertia*

Gulfweed Sargassum micracanthum

Gulin Goldthread*

Gulin Hemsleya

Gum-arabic Tree

Gummy Atractylodes*

Gummy Gardenia*

Gummy St. Paulswort*

Gynura

Gypsophila

H.Collett's Rhododendron

Hada Mosla*

Haichow Elsholtzia

Hainan Amonum

Hainan Devilpepper

Hainan Goniothalamus
T4472 Ocimum canum

T2466 Erythrina glauca

T5973 Smilax aristolochiaefolia

T4710 Pericampylus glaucus

T3850 Lindera glauca

T3851 Lindera glauca

T1596 Codonopsis canescens

T6611 Uncaria canescens

T4372 Nannoglottis ravida

T1705 Corydalis adunca

T0162 Actinidia glaucophylla

T4027 Maesa chisia

T5999 Solanum incanum

T5931 Seseli incanum

T2856 Garcinia griffithii

T4065 Mahonia griffithii

T4943 Piper crassinervium

T5967 Siraitia grosvenorii [Syn. Momordica grosvenorii]

T2581 Euphorbia chamaesyce

T4985 Pithecolobium dulce

T0509 Annona muricata

T6556 Tsoongiodendron odorum

T4758 Peucedanum guangxiense

T3985 Lycoris guangxiensis

T2300 Dysosma guangxiensis

T4132 Maytenus guangsiensis

T1892 Cupania latifolia

T4677 Paullinia cupana

T5266 Psidium guajava

T5267 Psidium guajava

T5268 Psidium guajava

T5265 Psidium guajava

T6718 Vernonia guineensis

T2207 Dioscorea rotundata [Syn. Dioscorea cayenensis]

T0564 Arachniodes nipponica

T2645 Evodia rutaecarpa var. bodinieri

T6224 Swertia kouitchensis

T5738 Sargassum micracanthum

T1666 Coptis gulinensis

T3210 Hemsleya penxianensis var. gulinensis

T0028 Acacia nilotica

T0820 Atractylodes gummifera

T2881 Gardenia gummifera

T5949 Siegesbeckia gummifer

T3088 Gynura segetum [Syn. Gynura japonica]

T3089 Gypsophila acutifolia

T5507 Rhododendron collettianum

T4540 Orthodon hadai

T2343 Elsholtzia splendens

T0417 Amomum longiligulare

T5426 Rauvolfia verticillata var. hainanensis

T3048 Goniothalamus howii
ZHANG NAO LUO LE

HUI CI TONG

HUI BA QIA

XI YUAN TENG

SHAN HU JIAO

SHAN HU JIAO YE

HUI MAO DANG SHEN

QIAN HUI GOU TENG

QIAN HUI MAO GUAN JU

HUI LV YAN HU SUO

HUA NAN MI HOU TAO

HUI YE DU JING SHAN

HUI BAI QIE

HUI BAI XIE HAO

GE LI FEI SI TENG HUANG

GE LI FEI SI SHI DA GONG LAO

CU YE MAI HU JIAO

LUO HAN GUO

MAO GUO DI JIN

NIU TI DOU

CI GUO FAN LI ZHI

GUAN GUANG MU

GUANG XI QIAN HU

GUANG XI SHI SUAN

GUANG XI BA JIAO LIAN

GUANG XI MEI DENG MU

BA XI XIANG WU HUAN ZI

FAN SHI LIU PI

FAN SHI LIU YE

FAN SHI LIU ZI

FAN SHI LIU GAN

JI NEI YA BAN JIU JU

YUAN SHU YU

BO SHI WU ZHU YU

GUI ZHOU ZHANG YA CAI

GU LIN YE LIAN

GU LIN XUE DAN

A LA BO JIAO JIN HE HUAN

OU CANG ZHU

JIAO ZHI ZI

JIAO XI XIAN

SAN QI CAO

HUANG JIE GU DAN

A FU HAN DU JUAN HUA

HA DA SHI JI NING

XIANG RU

HAI NAN SHA REN

HAI NAN LUO FU MU

HAI NAN GE NA XIANG 


Hainan Jointfir
Hainan Pencilwood
Hainan Plumyew
Hainan Sarcococca
Hainan Tinospora
Hair Larkspur
Hair Vegetable*
Hairfruit Rhododendron*
Hairless Chinese Hawthorn
Hairnode Valeriana*
Hairspike Pepper*
Hairstalk Denrdobium
Hairstalk Narrowleaf Rabdosia*
Hairstalk St. Paulswort

Hairstyle Poisonnut

Hairy Antler

Hairy Bayberry

Hairy Campion

Hairy Clovereshrub

Hairy Datura Flower

Hairy Datura Leaf

Hairy Datura Root

Hairy Datura Seed

Hairy Heartseed*

Hairy Heliotrope*

Hairy Hellebore*

Hairy Micromelum*

Hairy Pepper

Hairy Peppertree*

Hairy Rhynchotechum

Hairy St.John'swort

Hairy Willowweed

Hairy Wormwood

Hairydtalk Loosestrife

Hairyflower Actinidia

Hairyflower Photinia*

Hairyfruit Heliotrope*

Hairyfruit Michelia

Hairyfruit Milkweed*

Hairyfruit Rabdosia*

Hairyfruit Ventilago

Hairyleaf Aralia

Hairyleaf Handelia

Hairyleaf South Ailanthus

Hairylobed Butterbur

Hairypod Fishvane

Hairypod Glochidion*

Hairysepal Rabdosia

Hairysepal Raspberry

Hairystalk Tinospora

Hairyvein Agrimonia

Halberd-like Eupatorium*
T3030 Gnetum hainanense

T2309 Dysoxylum hainanense

T1319 Cephalotaxus hainanensis [Syn. Cephalotaxus mannii]

T5733 Sarcococca vagans

T6466 Tinospora hainanensis

T2079 Delphinium kamaonense var. glabrescens

T4438 Nostoc flagelliforme

T5522 Rhododendron seniavinii

T1779 Crataegus pinnatifida var. psilosa

T6674 Valeriana alternifolia var. stolonifera

T4971 Piper trichostachyon

T2097 Dendrobium capillipes

T3481 Isodon angustifolius var. glabrescens

T5951 Siegesbeckia orientalis var. glabrescens [Syn. Siegesbeckia

glabrescens]

T6183 Strychnos nitida

T1337 Cervus nippon; Cervus elaphus

T4343 Myrica esculent

T3948 Lychnis coronaria

T1163 Campylotropis hirtella

T2037 Datura innoxia

T2038 Datura innoxia

T2036 Datura innoxia

T2039 Datura innoxia

T1198 Cardiospermum hirsutum

T3173 Heliotropium hirsutum

T3185 Helleborus orientalis var. hirsutus

T4222 Micromelum hirsutum

T4962 Piper puberulum

T5788 Schinus molle

T5543 Rhynchotechum vestitum

T3355 Hypericum hirsutum

T2387 Epilobium hirsutum

T0705 Artemisia vestita

T3997 Lysimachia capillipes

T0161 Actinidia eriantha

T4826 Photinia lactiflora

T3175 Heliotropium lasiocarpum

T4214 Michelia spaerantha

T0737 Asclepias eriocarpa

T3530 Isodon trichocarpa

T6688 Ventilago calyculata

T0570 Aralia dasyphylla

T3097 Handelia trichophylla

T0258 Ailanthus malabarica

T4745 Petasites tricholobus

T2119 Derris eriocarpa

T2988 Glochidion eriocarpum

T5391 Rabdosia eriocalyx

T5590 Rubus chroosepalus

T6463 Tinospora capillipes

T0248 Agrimonia pilosa

T2577 Eupatorium subhastatum
HAI NAN MAI MA TENG

HAI NAN JIAN MU

HAI NAN CU FEI

HAI NAN YE SHAN HUA

HAI NAN QING NIU DAN

ZHAN MAO CUI QUE HUA

FA CAI

MAO GUO DU JUAN

WU MAO SHAN ZHA

MAO JIE XIE CAO

MAO SUI HU JIAO

DUAN BANG SHI HU

MAO GENG XIA YE XIANG CHA CAI

MAO GENG XI XIAN

MAO ZHU MA QIAN

LU RONG

MAO YANG MEI

MAO JIAN QIU LUO

MAO HANG ZI SHAO

MAO MAN TUO LUO HUA

MAO MAN TUO LUO YE

MAO MAN TUO LUO GEN

MAO MAN TUO LUO ZI

MAO DAO DI LING

YING MAO TIAN JIE CAI

YING MAO TI GEN CAO

YIN MAO XIAO YUN MU

MAO JU

ROU MAO XIAO RU XIANG

MAO XIAN ZHU JU TAI

YING MAO JIN SI TAO

SHUI JIE GU DAN

MAO LIAN HAO

XI GENG XIANG CAO

MAO HUA MI HOU TAO

MAO HUA SHI NAN

MAO GUO TIAN JIE CAI

MAO GUO HAN XIAO

MAO GUO MA LI JIN

MAO GUO XIANG CHA CAI

MAO GUO YI HE GUO

TOU XU CONG MU

TIAN SHAN SHI

MA LA BA CHU

MAO LIE FENG DOU CAI

MAO GUO YU TENG

MAO GUO SUAN PAN ZI

MAO E XIANG CHA CAI

MAO E MEI

JIN GUO LAN

LONG YACAO

JIN JI ZE LAN 


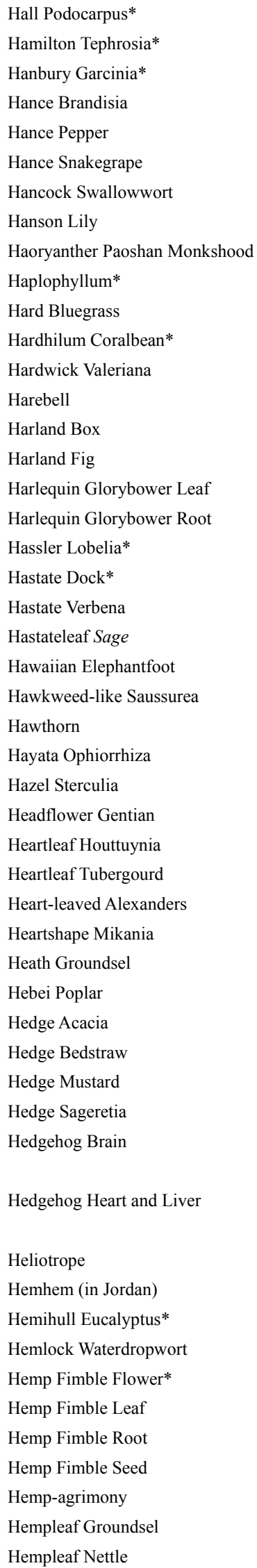

T5040 Podocarpus hallii

T6335 Tephrosia hamiltonii

T2857 Garcinia hanburyi

T1003 Brandisia hancei

T4948 Piper hancei

T0424 Ampelopsis brevipedunculata var. hancei

T1955 Cynanchum hancockianum

T3834 Lilium hansonii

T0117 Aconitum nagarum var. lasiandrum

T3100 Haplophyllum acutifolium

T5033 Poa sphondylodes

T2468 Erythrina lithosperma

T6676 Valeriana hardwickii

T1157 Campanula rotundifolia

T1088 Buxus harlandii

T2719 Ficus fistulosa [Syn. Ficus harlandii]

T1562 Clerodendron trichotomum

T1563 Clerodendron trichotomum

T3899 Lobelia hassleri

T5609 Rumex hastatus

T6707 Verbena hastata

T5664 Salvia bulleyana

T2333 Elephantopus mollis

T5770 Saussurea superba [Syn. Saussurea hieracioides]

T1774 Crataegus oxyacantha

T4509 Ophiorrhiza hayatana

T6138 Sterculia foetida

T2908 Gentiana cephalantha

T3284 Houttuynia cordata

T6435 Thladiantha cordifolia

T6913 Zizia aptera

T4228 Mikania cordata

T5913 Senecio sylvaticus

T5153 Populus hopeiensis

T3778 Leucaena glauca [Syn. Leucaena leucocephala]

T2832 Galium mollugo

T5968 Sisymbrium officinale

T5645 Sageretia theezans [Syn. Sageretia thea]

T2427 Erinaceus europaeus; Hemiechinus dauuricus; Hemiechinus auritus

T2428 Erinaceus europaeus; Hemiechinus dauuricus; Hemiechinus auritus

T3171 Heliotropium europaeum

T0448 Anchusa strigosa

T2512 Eucalyptus hemiphloia

T4480 Oenanthe crocata

T1175 Cannabis sativa

T1176 Cannabis sativa

T1174 Cannabis sativa

T1173 Cannabis sativa

T2554 Eupatorium cannabinum

T5883 Senecio cannabifolius

T6651 Urtica cannabina
HA SHI LUO HAN SONG

HAN MI ER DUN HUI YE

TENG HUANG SHU

LAI JIANG TENG

SHAN JU

GUANG YE SHE PU TAO

HUA BEI BAI QIAN

HUANG BAI HE

XUAN WEI WU TOU

JIAN YE YUN XIANG CAO

LONG XU CAO

YING HE CI TONG

CHANG XU XIE CAO

YUAN YE FENG LING CAO

XI YE HUANG YANG

SHUI TONG MU

CHOU WU TONG

CHOU WU TONG GEN

HA SHI SHAN GENG CAI

JI YE SUAN MO

JI YE MA BIAN CAO

JI YE SHU WEI CAO

ROU MAO DI DAN CAO

CHANG MAO FENG MAO JU

YING GUO SHAN ZHA

XIA YE SHE GEN CAO

JIA MA SHU

TOU HUA LONG DAN

YU XING CAO

XIN YE CHI BO

JI JI QIN

JIA ZE LAN

YE SHENG QIAN LI GUANG

HE BEI YANG

YIN HE HUAN

SU ZHU YANG YANG

ZUAN GUO SUAN JIE

QUE MEI TENG

WEI NAO

WEI XIN GAN

OU ZHOU TIAN JIE CAI

CU MAO NIU SHE CAO

BAN PI AN

ZANG HONG HUA SE SHUI QIN

MA HUA

MA YE

MA GEN

HUO MA REN

DA MA YE ZE LAN

MA YE QIAN LI GUANG

QIAN MA 


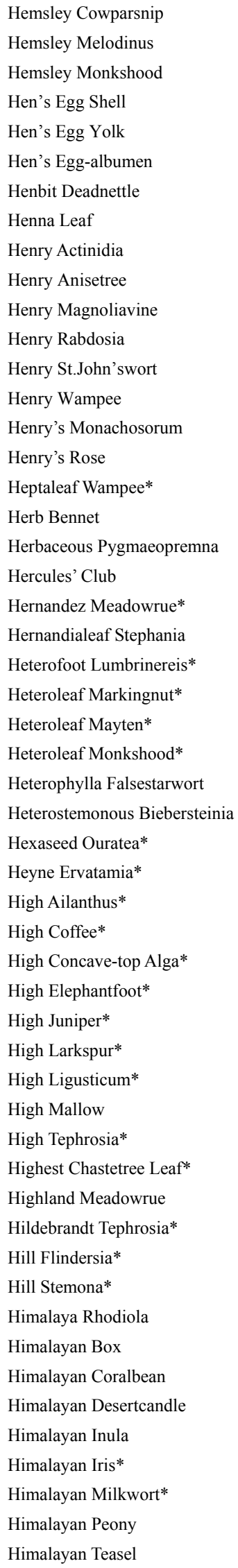

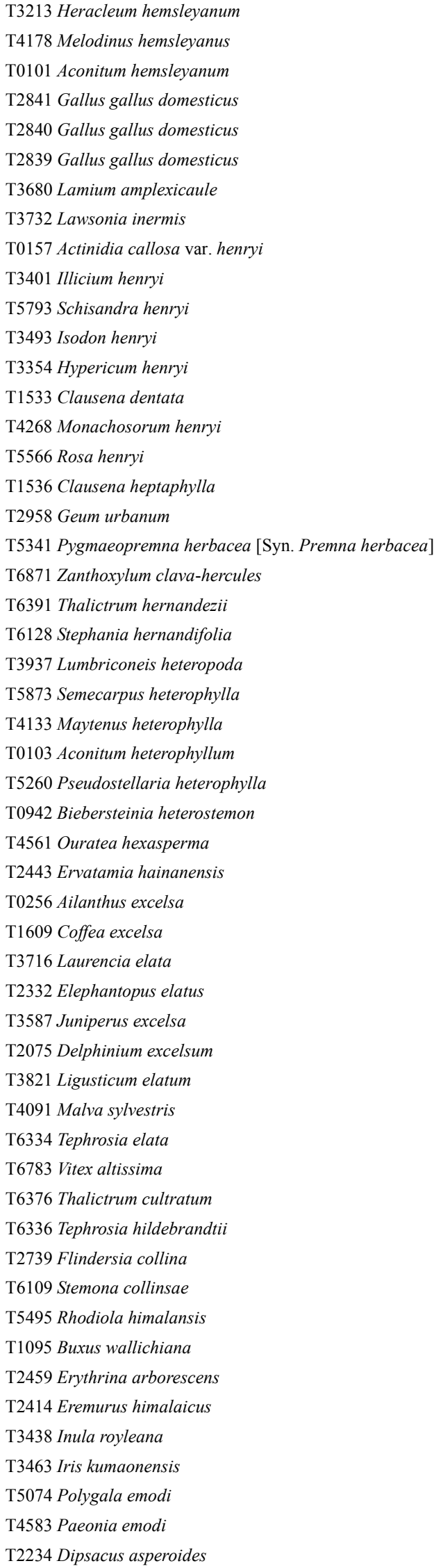

NIU WEI DU HUO

CHUAN SHAN CHENG

GUA YE WU TOU

JI ZI KE

JI ZI HUANG

JI ZI BAI

BAO GAI CAO

ZHI JIA HUA YE

JING LI MI HOU TAO

HONG HUI XIANG

YI GENG WU WEI ZI

E XI XIANG CHA CAI

HENG LI DI ER CAO

YE HUANG PI

XI ZI JUE

RUAN TIAO QI QIANG WEI

QI YE HUANG PI

XIANG CAO SHUI YANG MEI

QIAN JIE CAO

MEI GUO CI JIAO

HE SHI TANG SONG CAO

RU LAN

YI ZU SUO SHA CAN

YI YE ROU TUO GUO

YI YE MEI DENG MU

YI YE WU TOU

YI YE JIA FAN LV

XUN DAO NIU

LIU ZI SAI JIN LIAN MU

HAI NAN GOU YA HUA

GAO CHU

GAO KA FEI

GAO AO DING ZAO

GAO DI DAN CAO

GAO DA CI BAI

GAO DA CUI QUE HUA

GAO DANG GUI

OU JIN KUI

GAO HUI MAO DOU

ZUI GAO MU JING YE

GAO YUAN TANG SONG CAO

XI SHI HUI MAO DOU

SHAN QIU JU PAN MU

XIAO QIU BAI BU

XI MA HONG JING TIAN

WA LI XI HUANG YANG

QIAO MU CI TONG

XI MA DU WEI CAO

XI MA XUAN FU HUA

XI MA LA YA YUAN WEI

XI MA LA YA YUAN ZHI

DUO HUA SHAO YAO

CHUAN XU DUAN 


\begin{tabular}{|c|c|}
\hline Himalayan Yew & T6317 Taxus wallichiana \\
\hline Hindu Datura Flower & T2044 Datura metel \\
\hline Hindu Datura Leaf & T2042 Datura metel \\
\hline Hindu Datura Root & T2041 Datura metel \\
\hline Hindu Datura Seed & T2043 Datura metel \\
\hline Hindu Lotus Large Rhizome & T4402 Nelumbo nucifera \\
\hline Hindu Lotus Leaf & T4398 Nelumbo nucifera \\
\hline Hindu Lotus Leaf-base & T4399 Nelumbo nucifera \\
\hline Hindu Lotus Petiole & T4397 Nelumbo nucifera \\
\hline Hindu Lotus Plumule & T4401 Nelumbo nucifera \\
\hline Hindu Lotus Seed & T4400 Nelumbo nucifera \\
\hline Hinoki False Cypress & T1349 Chamaecyparis obtusa \\
\hline Hippomane Cupflower* & T4429 Nierembergia hippomanica \\
\hline Hirsute Bittersweet* & T1295 Celastrus strigillosus \\
\hline Hirsute Cissampelos* & T1453 Cissampelos pareira var. hirsute \\
\hline Hirsute Euchresta* & T2529 Euchresta strigillosa \\
\hline Hirsute Gambirplant* & T6617 Uncaria hirsuta \\
\hline Hirsute Licorice & T3011 Glycyrrhiza aspera \\
\hline Hirsute Respberry & T5592 Rubus hirsutus \\
\hline Hirsute Yam* & T2199 Dioscorea hirsuta \\
\hline Hirsutefruit White Quebracho* & T0763 Aspidosperma dasycarpon \\
\hline HirsuteMillettia & T4239 Millettia nitida var. hirsutissima \\
\hline Hisped Vineclethra & T1549 Clematoclethra scanden \\
\hline Hispid Arthraxon & T0707 Arthraxon hispidus \\
\hline Hispid Fig & T2727 Ficus simplicissima \\
\hline Hispid Onosma* & T4498 Onosma hispida \\
\hline Hispid Pepper* & T4949 Piper hispidum \\
\hline Hispid Ragweed* & T0402 Ambrosia hispida \\
\hline Hispid Sage & T5687 Salvia prionitis \\
\hline Hispid Yam & T2200 Dioscorea hispida \\
\hline Hispid 纤孔菌 & T3425 Inonotus hispidus \\
\hline Hispidus Girald Acanthopanax & T0037 Acanthopanax giraldii var. hispidus \\
\hline Hoary Pepperwort & T3757 Lepidium draba \\
\hline Hog's Fennel & T4764 Peucedanum officinale \\
\hline Hogweed & T3224 Heracleum sphondylium \\
\hline Holland Planted Saffron* & T1806 Crocus antalyensis cv \\
\hline Hollowed Wampee & T1535 Clausena excavata \\
\hline Hollyhock Flower & T0382 Althaea rosea \\
\hline Hollyhock-like Yam & T2189 Dioscorea althaeoides \\
\hline Hollyleaf Acanthus & T0048 Acanthus ilicifolius \\
\hline Homoloba Lespedeza* & T3772 Lespedeza homoloba \\
\hline Honan Meadowrue & T6392 Thalictrum honanenae \\
\hline Hondapara & T2181 Dillenia indica \\
\hline Honey & T0540 Apis cerana \\
\hline Honeyed Acacia & T0026 Acacia mellifera \\
\hline Honeylocust & T2982 Gleditsia triacanthos \\
\hline Hongkong Pencilwood & T2310 Dysoxylum hongkongense \\
\hline Hooked Dendrobium & T2094 Dendrobium aduncum \\
\hline Hooked Pepper* & T4928 Piper aduncum \\
\hline Hooked Spikemoss & T5870 Selaginella uncinata \\
\hline Hookedhair Madder & T5582 Rubia oncotricha \\
\hline Hookedhairypod Tickclover & T2130 Desmodium gangeticum \\
\hline
\end{tabular}

XI MA LA YA HONG DOU SHAN

YANG JIN HUA

MAN TUO LUO YE

MAN TUO LUO GEN

MAN TUO LUO ZI

OU

HE YE

HE YE DI

HE GENG

LIAN ZI XIN

LIAN ZI

RI BEN BIAN BAI

MA ZHUANG SAI YA MA

CU MAO NAN SHE TENG

YA HU NU

FU MAO SHAN DOU GEN

MAO GOU TENG

CU MAO GAN CAO

CI BO

CU MAO SHU YU

CU MAO GUO BAI JIAN MU

FENG CHENG JI XUE TENG

GANG MAO TENG SHAN LIU

JIN CAO

CU YE RONG

CU YING MAO DIAN ZI CAO

YING MAO HU JIAO

CU YING MAO TUN CAO

HONG GEN CAO

BAI SHU LANG

CU YING MAO XIAN KONG JUN

MAO GENG HONG MAO WU JIA

MAO DU XING CAI

YAO YONG QIAN HU

NIU FANG FENG

HE LAN ZHONG ZHI FAN HONG

HUA

SHAN HUANG PI

SHU KUI HUA

SHU KUI YE SHU YU

LAO SHU LE

TONG XING LIE PIAN HU ZHI ZI

HE NAN TANG SONG CAO

WU YA GUO

FENG MI

JU MI JIN HE HUAN

SAN CI ZAO JIA

XIANG GANG JIAN MU

GOU ZHUANG SHI HU

GOU ZHUANG HU JIAO

CUI YUN CAO

GOU MAO QIAN CAO

HONG MU JI CAO 
Hookedspine Bittersweet

Hooker Cucumber*

Hooker Onosma

Hop-shaped Dodder

Horse Bean

Horse Mint

Horsechestnut

Horseradish

Horsetail Beefwood

Horseweed Fleabane

Hosie Ormosia
Hottentot Fig
Howe
Huapei Scabious
Huaxi Fritillary
Hubei Landpick
Hubei Liriope
Human Hair
Human Placenta
Human Urine
Human Urine Sediment
Humifuse Euphorbia
Hupeh Anemone
Hupeh Fritillary
Hupeh Hawthorn
Hupeh Snakegourd
Hyacinth Dolichos Seed
Hyacinth Falselily
Hyberna Euphorbia
Hybrid Bluebeard*
Hybrid Clover*
Hybrid Lily*
Hybrid Pathenium*
Hypoglaucous Collett Yam

Hyrcanian Box*

Hyssop-leaved Boneset

Iberian Narcissus*

Iberian Oak*

Iceland Moss

Iceland Poppy

Icosandrous Pokeweed*

Ignat Poisonnut Seed

Ili Larkspur

Ilicis-leaf Mayten*

Illyrian Cottonthistle*

Imbricate Mosquito Fern

Imbricate Podocarpus

Immature Persimmon Fruit Juice

Incarnate Gloeostereum

Incarnate Milkweed*

Incised Corydalis
T1286 Celastrus flagellaris

T1874 Cucumis hookeri

T4499 Onosma hookeri

T1914 Cuscuta lupuliformis

T6748 Vicia faba var. equina

T4185 Mentha longifolia

T0202 Aesculus hippocastanum

T0647 Armoracia lapathifolia

T1258 Casuarina equisetifolia

T1657 Conyza canadensis [Syn. Erigeron canadensis]

T4530 Ormosia hosiei

T4200 Mesembryanthemum edule

T1272 Caulerpa sertularioides

T5778 Scabiosa tschiliensis

T2794 Fritillaria siechuanica

T5096 Polygonatum zanlanscianense

T3875 Liriope spicata var. prolifera

T3278 Homo sapiens

T3279 Homo sapiens

T3276 Homo sapiens

T3277 Homo sapiens

T2591 Euphorbia humifusa

T0468 Anemone hupehensis

T2787 Fritillaria hupehensis

T1770 Crataegus hupehensis

T6509 Trichosanthes hupehensis

T2246 Dolichos lablab

T4443 Notholirion hyacinthinum [Syn. Notholirion bulbuliferum]

T2592 Euphorbia hyberna

T1220 Caryopteris clandonensis

T6520 Trifolium hybridum

T3838 Lilium speciosum $\mathrm{x}$ L. nobilissimum

T4659 Parthenium argentatum $\times$ P. tomentosa

T2201 Dioscorea hypoglauca [Syn. Dioscorea collettii var.

hypoglauca]

T1089 Buxus hyrcana

T2562 Eupatorium hyssopifolium

T4374 Narcissus bujei

T5373 Quercus iberica

T1341 Cetraria islandica

T4629 Papaver nudicaule

T4865 Phytolacca icosandra

T6177 Strychnos ignatii

T2078 Delphinium iliense

T4135 Maytenus ilicifolia

T4497 Onopordum illyricum

T0838 Azolla imbricata [Syn. Salvinia imbricata]

T5041 Podocarpus imbricatus

T2219 Diospyros kaki

T2993 Gloeostereum incarnatum

T0738 Asclepias incarnata

T1719 Corydalis incisa
CI NAN SHE TENG

HU KE HUANG GUA

XI HUA DIAN ZI CAO

PI JIU HUA TU SI ZI

MA CAN DOU

OU BO HE

OU ZHOU QI YE SHU

LA GEN

MU MA HUANG

QI ZHOU YI ZHI HAO Conyza

canadeni Erigeron canadeni

HONG DOU

SHI YONG RI ZHONG HUA

BANG YE JUE ZAO

HUA BEI LAN PEN HUA

HUA XI BEI MU

HU BEI HUANG JING

HU BEI SHAN MAI DONG

XUE YU

ZI HE CHE

REN NIAO

REN ZHONG BAI

DI JIN CAO

DA PO WAN HUA HUA

HU BEI BEI MU

HU BEI SHAN ZHA

HU BEI GUA LOU

BIAN DOU

JIA BAI HE

HAI BO NA DA JI

ZA JIAO YOU ${ }^{(2)}$

ZA JIAO CHE ZHOU CAO

ZA JIAO BAI HEX

ZA JIAO YIN JIAO JU

BI XIE

HE KA NI YA HUANG YANG

SHEN XIANG CAO YE ZE LAN

YI BI LI YA SHUI XIAN

YI BI LI YA LI

BING DAO YI

YE YING SU

ER SHI RUI SHANG LU

LV SONG GUO

YI LI CUI QUE HUA

DONG QING YE MEI DENG MU

YI LI LI YA DA CHI JI

MAN JIANG HONG

JI MAO SONG

SHI QI

YU ER

ROU HONG MA LI JIN

ZI HUA YU DENG CAO 


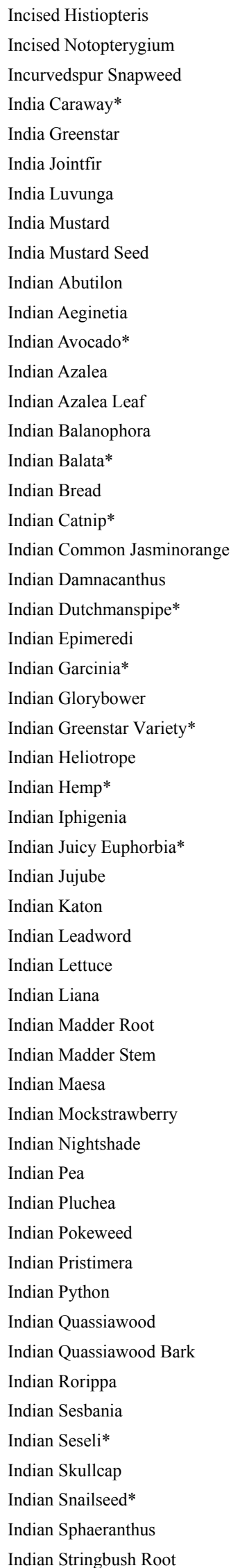

T3260 Histiopteris incisa

T4446 Notopterygium incisum

T3414 Impatiens siculifer

T1216 Carum ajowan

T5065 Polyalthia longifolia

T3035 Gnetum ula

T3946 Luvunga scandens

T1007 Brassica juncea

T1008 Brassica juncea

T0015 Abutilon indicum

T0195 Aeginetia indica

T4733 Persea indica

T5523 Rhododendron simsii

T5524 Rhododendron simsii

T0858 Balanophora indica [Syn. Langodorffia indica]

T4105 Manilkara indica

T5169 Poria cocos

T4415 Nepeta hindostana

T4319 Murraya koenigii

T2020 Damnacanthus indicus

T0630 Aristolochia indica

T0503 Anisomeles indica [Syn. Epimeredi indica]

T2859 Garcinia indica

T1557 Clerodendron indicum

T5066 Polyalthia longifolia var. pendula

T3174 Heliotropium indicum

T1177 Cannabis sativa var. indica

T3443 Iphigenia indica

T2603 Euphorbia nivulia

T6920 Ziziphus mauritiana

T5708 Sandoricum koetjape [Syn. Sandoricum indicum]

T5028 Plumbago indica

T3659 Lactuca indica

T0449 Ancistrocladus heyneanus

T5578 Rubia cordifolia

T5579 Rubia cordifolia

T4028 Maesa indica

T2290 Duchesnea indica

T6000 Solanum indicum

T3710 Lathyrus sativus

T0847 Baccharis indica [Syn. Pluchea indica]

T4864 Phytolacca esculenta [Syn. Phytolacca acinosa]

T5208 Pristimera indica

T5368 Python molurus bivittatus

T4881 Picrasma quassioides [Syn. Picrasma ailanthoides]

T4882 Picrasma quassioides [Syn. Picrasma ailanthoides]

T5560 Rorippa montana [Syn. Rorippa dubia; Sisymbrium dublium]

T5926 Sesbania sesban

T5932 Seseli indicum

T5840 Scutellaria indica

T1585 Cocculus indicus

T6071 Sphaeranthus indicus

T6820 Wikstroemia indica
LI JUE

QIANG HUO

HUANG JIN FENG

YIN DU ZANG HUI XIANG

BIAN ZHONG CHANG YE AN LUO

YIN DU MAI MA TENG

SAN YE TENG JU

JIE CAI

JIE ZI

MO PAN CAO

YE GU

YIN DU E LI

DU JUAN HUA

DU JUAN HUA YE

YIN DU SHE GU

IN DU TIE XIAN ZI

FU LING

YIN DU JIA JING JIE

YIN DU JIU LI XIANG

HU CI

YIN DU MA DOU LING

GUANG FANG FENG

YIN DU TENG HUANG

CHANG GUAN JIA MO LI

BIAN ZHONG CHANG YE AN LUO

DA WEI YAO

YIN DU DA MA

CAO BEI MU

YIN DU DUO ZHI DA JI

MIAN ZAO

YIN DU SHAN DAO LIAN YE

ZI XUE HUA

SHAN WO JU

HAI NI GOU ZHI TENG

QIAN CAO GEN

QIAN CAO TENG

LIANG MIAN QING

SHE MEI

TIAN QIE ZI

CAO XIANG WAN DOU

KUO BAO JU

SHANG LU

BIAN SHUO TENG

MANG SHE

KU MU

KU SHU PI

HAN CAI

AI JI TIAN JING

YIN DU XIE HAO

HAN XIN CAO

YIN DU MU FANG JI

RONG MAO DAI XING CAO

LIAO GE WANG GEN 


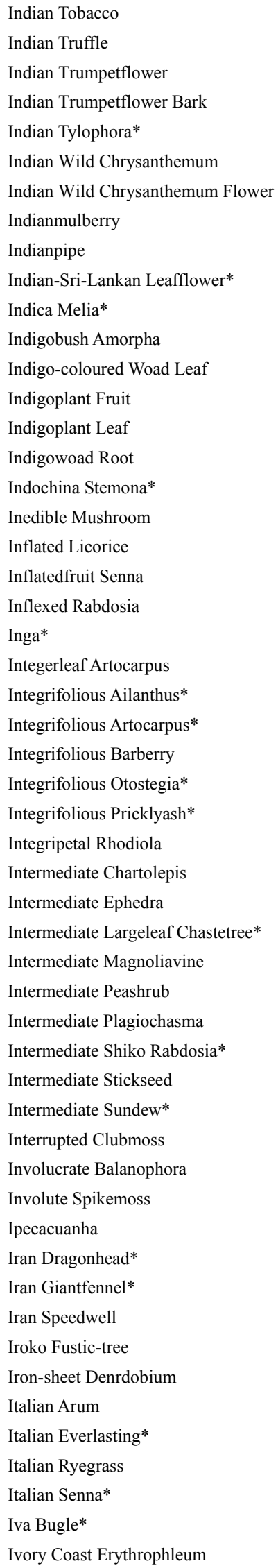

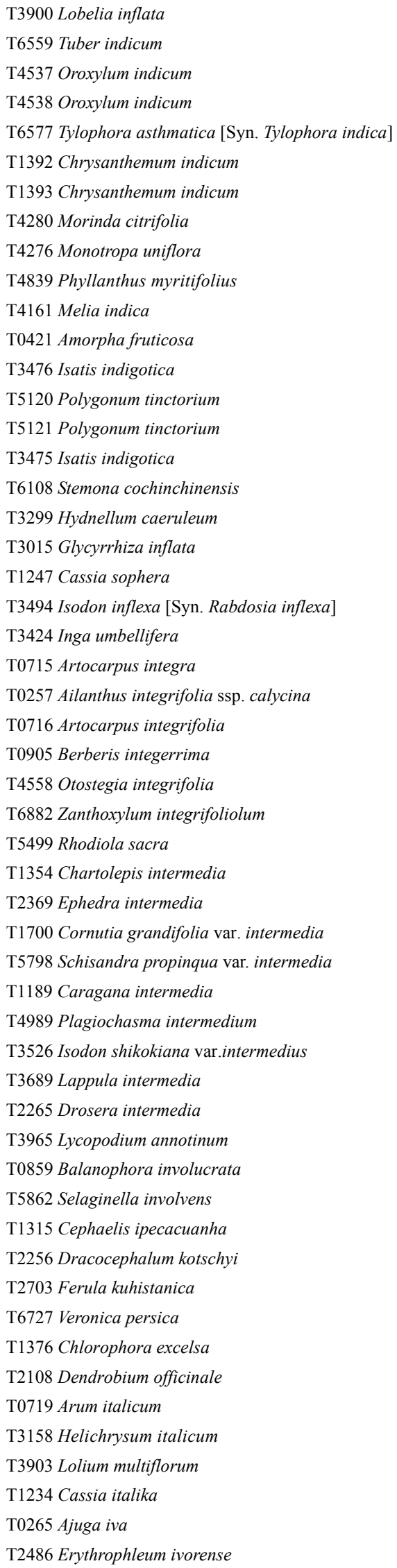

BEI MEI ZHOU SHAN GENG CAI

YIN DU KUAI JUN

MU HU DIE

MU HU DIE SHU PI

YIN DU WA ER TENG

YE JU

YE JU HUA

HAI BA JI

SHUI JING LAN

YIN DU SI LI LAN KA YE XIA ZHU

YIN JIAN

ZI SUI HUAI

DA QING YE

LIAO LAN GUO

LIAO LAN YE

BAN LAN GEN

YIN DU ZHI NA BAI BU

LAN SE YA CHI JUN

ZHANG GUO GAN CAO

JIANG MANG

NEI ZHE XIANG CHA CAI

SAN XING HUA XU YIN JIA

QUAN YUAN GUI MU

QUAN YUAN CHU

QUAN YUAN YE BO LUO MI

QUAN YUAN YE XIAO BO

QUAN YUAN YE AO TUO SI TE CAO

QUAN YUAN YE HUA JIAO

SHENG DI HONG JING TIAN

BO LIN JU

ZHONG MA HUANG

ZHONG JIAN DA YE KE NU CAO

ZHONG JIAN WU WEI ZI

ZHONG JIAN JIN JI ER

WU WEN ZI BEI TAI

JIAN XING SI GUO XIANG CHA CAI

ZHONG JIAN HE SHI

ZHONG JIAN MAO GAO CAI

DAN SUI SHI SONG

TONG QIAO SHE GU

YAN ZHOU JUAN BAI

TU GEN

YI LANG QING LAN

YI LANG A WEI

A LA BO PO PO NA

GAO HUANG LU SANG

TIE PI SHI HU

YI DA LI JIASNG NAN XING

YI DA LI LA JU

DUO HUA HEI MAI CAO

YI DA LI JUE MING ZI

AI WA JIN GU CAO

XIANG YA HAI AN GE MU 


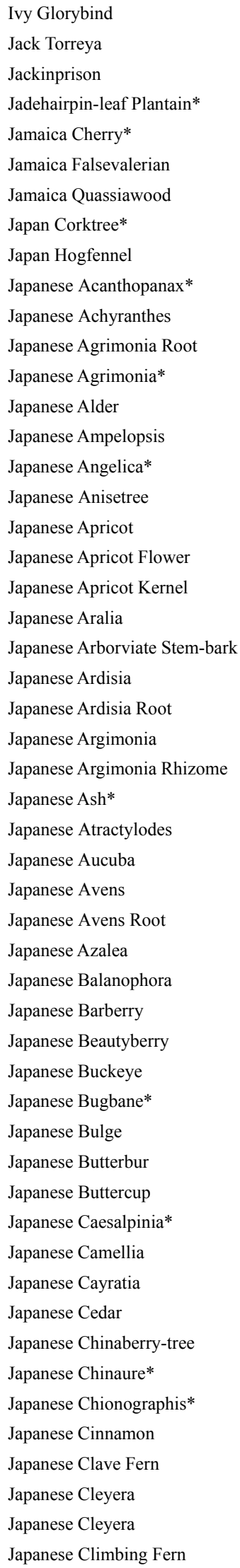

\begin{tabular}{|c|c|}
\hline T1143 Calystegia hederacea & MIAN GEN TENG \\
\hline T6478 Torreya jackii & CHANG YE FEI SHU \\
\hline T4431 Nigella damascena & HEI ZHONG CAO \\
\hline T5005 Plantago hostifolia & YU ZAN YE CHE QIAN \\
\hline T4314 Muntingia calabura & YA MAI JIA YING TAO \\
\hline T6095 Stachytarpheta jamaicensis & JIA MA BIAN \\
\hline T4880 Picrasma excelsa & YA MAI JIA KU MU \\
\hline T4793 Phellodendron japonicum & RI BEN HUANG BAI \\
\hline T4760 Peucedanum japonicum & BIN HAI QIAN HU \\
\hline T0039 Acanthopanax japonicus & RI BEN WU JIA \\
\hline T0074 Achyranthes fauriei & RI BEN NIU XI \\
\hline T0250 Agrimonia pilosa var. japonica & XIAN HE CAO GEN \\
\hline T0247 Agrimonia japonica & RI BEN LONG YA CAO \\
\hline T0328 Alnus japonica & CHI YANG \\
\hline T0427 Ampelopsis japonica [Syn. Paullinia japonica] & BAI LIAN \\
\hline T0485 Angelica japonica & RI BEN DANG GUI \\
\hline T3399 Illicium anisatum & RI BEN MANG CAO \\
\hline T5228 Prunus mume & WU MEI \\
\hline T5226 Prunus mume & BAI MEI HUA \\
\hline T5227 Prunus mume & MEI HE REN \\
\hline T0568 Aralia chinensis & CONG MU \\
\hline T6442 Thuja standishii & RI BEN XIANG BAI JING PI \\
\hline T0598 Ardisia japonica & ZI JIN NIU \\
\hline T0599 Ardisia japonica & ZI JIN NIU GEN \\
\hline T0249 Agrimonia pilosa var. japonica & XIAN HE CAO \\
\hline T0251 Agrimonia pilosa var. japonica & XIAN HE CAO GEN YA \\
\hline T2771 Fraxinus japonica & RI BEN BAI LA SHU \\
\hline T0821 Atractylodes japonica & GUAN CANG ZHU \\
\hline T0828 Aucuba japonica & DONG YING SHAN HU MU \\
\hline T2955 Geum japonicum & SHUI YANG MEI \\
\hline T2956 Geum japonicum & SHUI YANG MEI GEN \\
\hline T5511 Rhododendron japonicum & RI BEN DU JUAN HUA \\
\hline T0860 Balanophora japonica & GE XUN \\
\hline T0916 Berberis thunbergii & RI BEN XIAO BO \\
\hline T1119 Callicarpa japonica & RI BEN ZI ZHU \\
\hline T0204 Aesculus turbinata & RI BEN QI YE SHU \\
\hline T1422 Cimicifuga japonica & RI BEN SHENG MA \\
\hline T0267 Ajuga nipponensis & ZI BEI JIN PAN \\
\hline T4740 Petasites japonicus & FENG DOU CAI \\
\hline T5414 Ranunculus japonicus & MAO GEN \\
\hline T1104 Caesalpinia japonica & RI BEN SU MU \\
\hline T1145 Camellia japonica & SHAN CHA \\
\hline T1275 Cayratia japonica & WU LIAN MEI \\
\hline T1868 Cryptomeria japonica & RI BEN LIU SHAN \\
\hline T4160 Melia azedarach var. japonica & RI BEN KU LIAN \\
\hline T0966 Boenninghausenia japonica & RI BEN CHOU JIE CAO \\
\hline T1369 Chionographis japonica & RI BEN BAI SI CAO \\
\hline T1441 Cinnamomum japonicum & GUI PI \\
\hline T4502 Onychium japonicum [Syn. Tricomanes japonicum] & XIAO YE JI WEI \\
\hline T1571 Cleyera ochnacea $[$ Syn. Cleyera japonica $]$ & YANG TONG \\
\hline T6350 Ternstroemia japonica & RI BEN HOU PI XIANG \\
\hline T3990 Lygodium japonicum & HAI JIN SHA \\
\hline
\end{tabular}




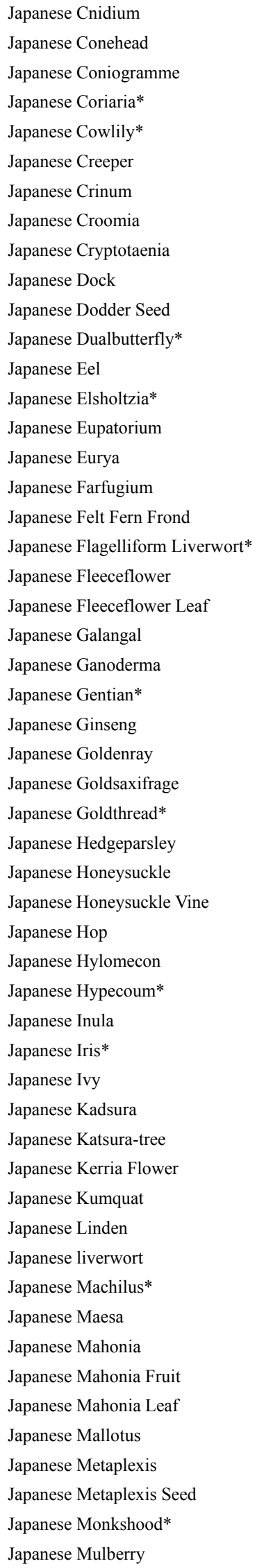

T1581 Cnidium japonicum

T6154 Strobilanthes japonicus [Syn. Championella japonica]

T1640 Coniogramme japonica [Syn. Hemionitis japonica]

T1690 Coriaria japonica

T4448 Nuphar japonicum

T4662 Parthenocissus tricuspidata

T1795 Crinum asiaticum var. japonicum

T1810 Croomia japonica

T1869 Cryptotaenia japonica

T5611 Rumex japonicus

T1913 Cuscuta japonica

T6537 Tripterospermum japonicum

T0500 Anguilla japonica

T2342 Elsholtzia nipponica

T2563 Eupatorium japonicum

T2631 Eurya japonica

T2691 Farfugium japonicum

T5357 Pyrrosia lingua

T0883 Bazzania japonica

T5101 Polygonum cuspidatum

T5102 Polygonum cuspidatum

T0357 Alpinia japonica

T2846 Ganoderma japonicum [Syn. Ganoderma sinense]

T2912 Gentiana japonica

T4607 Panax pseudo-ginseng var. japonicus

T3808 Ligularia japonica [Syn. Arnica japonica; Senecio japonica]

T1404 Chrysosplenium japonicum

T1667 Coptis japonica

T6476 Torilis japonica

T3912 Lonicera japonica

T3913 Lonicera japonica

T3288 Humulus japonicus [Syn. Humulus scandens]

T3316 Hylomecon japonica

T3333 Hypecoum japonicum

T3433 Inula japonica

T3462 Iris komonoensis

T3115 Hedera rhombea

T3616 Kadsura japonica

T1332 Cercidiphyllum japonicum

T3628 Kerria japonica

T2759 Fortunella japonica

T6457 Tilia japonica

T3550 Jackiella javanica

T4016 Machilus japonica

T4029 Maesa japonica

T4066 Mahonia japonica

T4068 Mahonia japonica

T4067 Mahonia japonica

T4082 Mallotus japonicus

T4206 Metaplexis japonica

T4207 Metaplexis japonica

T0104 Aconitum japonicum

T4291 Morus australis
BING SHE CHUANG

HONG ZE LAN

FENG YA JUE

RI BEN MA SANG

RI BEN PING PENG CAO

DI JIN

RI BEN WEN SHU LAN

JIN GANG DA

YA ER QIN

YANG TI

DA TU SI ZI

RI BEN SHUANG HU DIE

MAN LI YU

RI BEN XIANG RU

CHENG GAN CAO

LING MU

LIAN PENG CAO

SHI WEI

RI BEN BIAN TAI

HU ZHANG

HU ZHANG YE

TU SHA REN

ZI ZHI

RI BEN LONG DAN

ZHU JIE SAN QI

DA TOU TUO WU

RI BEN JIN YAO

RI BEN HUANG LIAN

HUA NAN HE SHI

JIN YIN HUA

REN DONG TENG

LV CAO

HE QING HUA

DI TANG CAO

JIN FEI CAO

RI BEN YUAN WEI

LING XING CHANG CHUN TENG

RI BEN NAN WU WEI ZI

RI BEN LIAN XIANG SHU

DI TANG HUA

JIN GAN

HUA DONG DUAN

ZHAO WA JIA KE TAI

RI BEN NAN

DU JING SHAN

HUA NAN GONG LAO MU

HUA NAN GONG LAO ZI

HUA NAN GONG LAO YE

YE WU TONG

LUO MO

LUO MO ZI

RI BEN WU TOU

AO DA LI YA SANG 
Japanese Mushroom*(Sheep Polypore)

Japanese Ophiorrhiza

Japanese Orixa

Japanese Osmunda Frond

Japanese Pachysandra

Japanese Pagodatree

Japanese Pagodatree Fruit

Japanese Pagodatree Root

Japanese Phtheirospermum

Japanese Pieris

Japanese Pittosporum

Japanese Plum

Japanese Plum Kernel

Japanese Plumyew

Japanese Polypody

Japanese Premna

Japanese Pricklyash

Japanese Privet

Japanese Privet

Japanese Pyrola

Japanese Rabdosia

Japanese Raisin Tree Root

Japanese Raisin Tree Seed

Japanese Raspberry

Japanese Rose Root

Japanese Sapium

Japanese Scopolia

Japanese Skimmia

Japanese Snailseed

Japanese Snailseed Stem

Japanese Snakegourd

Japanese Snakegourd Seed

Japanese Snowbell Stem-bark

Japanese Spicebush

Japanese Spiraea

Japanese Spiraea Leaf

Japanese St.John'swort

Japanese Staphania

Japanese Star Jasmine*

Japanese Stauntonvine

Japanese Stauntonvine Fruit

Japanese Stemona

Japanese Swallowwort*

Japanese Sweetflag*

Japanese Swertia*

Japanese Tachibana

Japanese Teasel

Japanese Thistle

Japanese Toadflax

Japanese Trileaf Ladybell*

Japanese Umbrellaleaf

Japanese White Birch Bark

Japanese White Chinaure*
T0288 Albatrellus ovinus

T4510 Ophiorrhiza japonica

T4527 Orixa japonica

T4552 Osmunda japonica

T4575 Pachysandra terminalis

T6034 Sophora japonica

T6036 Sophora japonica

T6035 Sophora japonica

T4831 Phtheirospermum japonicum [Syn. Geradia japonica]

T4890 Pieris japonica

T4987 Pittosporum tobira

T5239 Prunus salicina

T5238 Prunus salicina

T1320 Cephalotaxus harringtonia

T5124 Polypodium niponicum

T5194 Premna microphylla

T6886 Zanthoxylum piperitum

T3827 Ligustrum japonicum

T3829 Ligustrum robustum

T5350 Pyrola japonica

T3496 Isodon japonica [Syn. Rabdosia japonica]

T3285 Hovenia dulcis

T3286 Hovenia dulcis

T5595 Rubus parviforlius

T5569 Rosa multiflora

T5722 Sapium japonicum

T5821 Scopolia japonica

T5970 Skimmia japonica

T1589 Cocculus trilobus [Syn. Cocculus sarmentosus]

T1590 Cocculus trilobus [Syn. Cocculus sarmentosus]

T6507 Trichosanthes cucumeroides

T6508 Trichosanthes cucumeroides

T6200 Styrax japonica

T3853 Lindera obtusiloba

T6079 Spiraea japonica

T6080 Spiraea japonica

T3356 Hypericum japonicum

T6129 Stephania japonica

T6483 Trachelospermum asiaticum

T6100 Stauntonia hexaphylla

T6101 Stauntonia hexaphylla

T6111 Stemona japonica

T1956 Cynanchum japonicum

T0143 Acorus calamus var. angustatus

T6223 Swertia japonica

T1513 Citrus tachibana

T2235 Dipsacus japonicus

T1449 Cirsium japonicum

T3844 Linaria japonica

T0169 Adenophora triphylla var. japonica

T2231 Diphylleia grayi

T0933 Betula platyphylla

T0965 Boenninghausenia albiflora var. japonica
RE BEN MO GU

RI BEN SHE GEN CAO

CHOU SHAN YANG

ZI QI

XUE SHAN LIN

HUAI

HUAI JIAO

HUAI GEN

SONG HAO

RI BEN MA ZUI MU

HAI TONG

LI ZI

LI HE REN

RI BEN CU FEI

SHUI LONG GU

DOU FU CHAI

HU JIAO HUA JIAO

RI BEN NV ZHEN

CU ZHUANG NV ZHEN

RI BEN LU TI CAO

MAO YE XIANG CHA CAI

ZHI JU GEN

ZHI JU ZI

MAO MEI

QIANG WEI GEN

BAI MU WU JIU

DONG LANG DANG

XIANG YIN YU

MU FANG JI

QING TENG XIANG

WANG GUA

WANG GUA ZI

RI BEN AN XI XIANG JING PI

SAN ZUAN FENG

XIU XIAN JU

XIU XIAN JU YE

DI ER CAO

QIAN JIN TENG

RI BEN LUO SHI

NA TENG

NA TENG GUO

WAN SHENG BAI BU

RI BEN NIU PI XIAO

RI BEN CHANG PU

RI BEN ZHANG YA CAI

LI HUA JU

XU DUAN

DA JI

HAI BIN LIU CHUAN YU

RI BEN SAN YE SHA SEN

SHAN HE YE

HONG HUA PI

RI BEN BAI SONG FENG CAO 


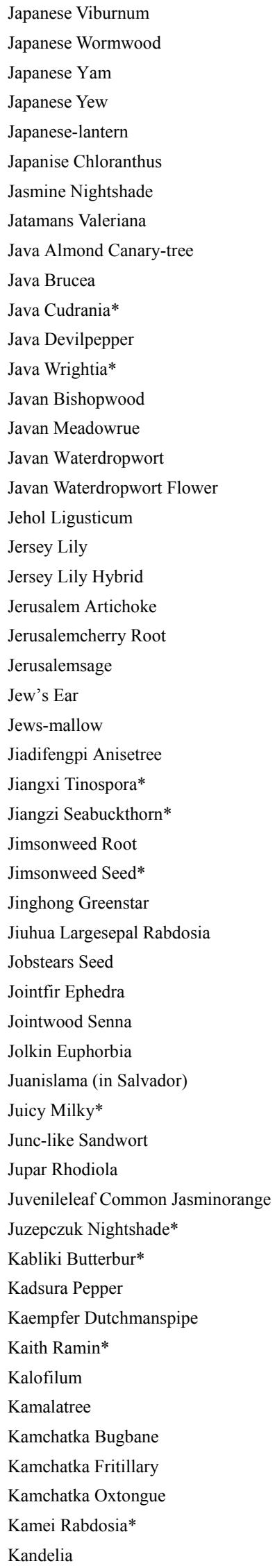

\author{
T6734 Viburnum awabuki \\ T0678 Artemisia japonica \\ T2202 Dioscorea japonica \\ T6311 Taxus cuspidata \\ T4845 Physalis alkekengi \\ T1371 Chloranthus japonicus \\ T6001 Solanum jasminoides \\ T6677 Valeriana jatamansii [Syn. Valeriana wallichii] \\ T1166 Canarium commune \\ T1038 Brucea javanica [Syn. Brucea sumatrana; Rhus javanica] \\ T1886 Cudrania javanensis \\ T5442 Rauwolfia serpentina \\ T6833 Wrightia javanica \\ T0945 Bischofia javanica [Syn. Bischofia trifoliata] \\ T6408 Thalictrum sessile \\ T4482 Oenanthe javanica \\ T4481 Oenanthe javanica \\ T3822 Ligusticum jeholense \\ T0390 Amaryllis belladonna \\ T0391 Amaryllis belladonna [hybrida] \\ T3155 Helianthus tuberosus \\ T6009 Solanum pseudo-capsicum \\ T4814 Phlomis umbrosa \\ T0830 Auricularia auricula \\ T1675 Corchorus olitorius \\ T3402 Illicium jiadifengpi \\ T6465 Tinospora craveniana \\ T3255 Hippophae rhamnoides subsp. gyantsensis \\ T2046 Datura stramonium \\ T2047 Datura stramonium \\ T5064 Polyalthia cheliensis \\ T3512 Isodon macrocalyx var. jiuhua \\ T1614 Coix lacryma-jobi var. ma-yuen \\ T2366 Ephedra distachya \\ T1239 Cassia nodosa \\ T2594 Euphorbia jolkini \\ T1111 Calea urticifolia \\ T3654 Lactarius rolemus \\ T0607 Arenaria juncea \\ T5496 Rhodiola juparensis \\ T4321 Murraya microphylla \\ T6002 Solanum juzepczukii \\ T4741 Petasites kablikianus \\ T4950 Piper kadsura [Syn. Piper futokadsura] \\ T0631 Aristolochia kaempferi \\ T3052 Gonystylus keithii \\ T1129 Calophyllum inophyllum \\ T4084 Mallotus philippinensis \\ T1425 Cimicifuga simplex \\ T2782 Fritillaria camtschatcensis \\ T4885 Picris kamtschatica \\ T3498 Isodon kameba \\ T3626 Kandelia candel
}

RI BEN JIA MI

MU HAO

RI BEN SHU YU

ZI SHAN

SUAN JIANG

YIN XIAN CAO

SU XIN YE BAI YING

ZHI ZHU XIANG

ZHAO WA GAN LAN

YA DAN ZI

ZHAO WA ZHE SHU

YIN DU LUO FU MU

ZHAO WA DAO DIAO BI

QIU FENG MU

WU BING TANG SONG CAO

SHUI QIN

QIN HUA

LIAO GAO BEN

GU TING HUA

GU TING HUA ZA JIAO ZHONG

JU YU

YU SHAN HU GEN

CAO SU

MU ER

CHANG SHUO HUANG MA

JIA DI FENG PI

JIANG XI QING NIU DAN

JIANG ZI SHA JI

OU MAN TUO LUO GEN

WAN TAO HUA ZI

JING HONG AN LUO

JIU HUA DA E XIANG CHA CAI

YI YI REN

SHUANG SUI MA HUANG

SHEN HUANG DOU

NAN DA JI

YOU KA MEI JU

DUO ZHI RU GU

LAO NIU JIN

YUAN CONG HONG JING TIAN

NEN YE JIU LI XIANG

JU SHI QIE

KA BU LI FENG DOU CAI

HAI FENG TENG

ZHU SHA LIAN

KAI TE LENG ZHU MU

HAI TANG GUO

LV SONG QIU MAO

YE SHENG MA

HEI BAI HE

KAN CHA JIA MAO LIAN CAI

KA MEI XIANG CHA CAI

QIU QIE SHU 
Kangting Larkspur

Kansas Gay-feather

Kansu Hawthorn

Kansu Sandwort

Kansui Euphorbia

Kapok Ceiba

Karaba Sage*

Karroo Acacia

Kashgar Tamarisk

Kashmir Larkspur

Katsumada Galangal

Katsura-tree

Kava Pepper

Kawakami Gambirplant*

Kayea assamica

Kazinoki Papermulberry

Keislce Angelica*

Kelp Thallus

Kenaf Hibiscus

Kenya Coralbean*

Kenya Crinum*

Kenya Embelia*

Kenya Myrrhtree*

Kenya Tephrosia*

Kerri Petrocosmea*

Kerri Stemona*

Khasi Nightshade Fruit

Khasya Pine

Khat

Khingan Fir

Kidney Bean

Kidney Vetch

Kidneyleaf Goldenray

Kidneyleaf Goldenray Variety*

King Orange

King Solomonseal

Kingsbloom

Kinokuni Citrus

Kirilow Groundsel Herb

Kirilow Rhodiola

Kirin Monkshood*

Kiushu Brake

Klein Cinquefoil

Kleini Senna*

Klotzsch Wormwood*

Knotteflower Phyla Herb

Ko Condorvine

Kobus Magnolia

Koch Gentian*

Kohl-rabi

Kola Garcinia*

Komarov Mosquitotrap
T2087 Delphinium tatsienense

T3790 Liatris pycnostachya

T1771 Crataegus kansuensis

T0608 Arenaria kansuensis [Syn. Arenaria kumaonensis]

T2596 Euphorbia kansui

T1284 Ceiba pentandra

T5676 Salvia karabachensis

T0022 Acacia karroo

T6288 Tamarix hispida

T2066 Delphinium cashmerianum

T0358 Alpinia katsumadai

T1333 Cercidiphyllum japonicum var. sinense

T4955 Piper methysticum

T6619 Uncaria kawakamii

T3627 Kayea assamica

T1031 Broussonetia kazinoki

T0487 Angelica keislcei

T3678 Laminaria japonica

T3238 Hibiscus cannabinus

T2461 Erythrina burttii

T1798 Crinum kirkii

T2350 Embelia schimperi

T1636 Commiphora kua var. gowlello

T6331 Tephrosia aequilata

T4747 Petrocosmea kerrii

T6112 Stemona kerrii

T6003 Solanum khasianum

T4911 Pinus kesiya

T1266 Catha edulis

T0006 Abies nephrolepis

T4785 Phaseolus vulgaris

T0531 Anthyllis vulneraria

T3805 Ligularia fischeri

T3806 Ligularia fischeri var. spiciformis

T1502 Citrus nobilis

T5092 Polygonatum kingianum

T4587 Paeonia officinalis

T1489 Citrus kinokuni

T6330 Tephroseris kirilowii [Syn. Senecio integrifolius var. fauriei]

T5497 Rhodiola kirilowii

T0106 Aconitum kirinense

T5292 Pteris kiuschiuensis

T5184 Potentilla kleiniana

T1235 Cassia kleinii

T0679 Artemisia klotzschiana

T3865 Lippia nodiflora

T4117 Marsdenia koi

T4040 Magnolia kobus

T2914 Gentiana kochiana

T1019 Brassica oleracea var. gongylodes

T2860 Garcinia kola

T1957 Cynanchum komarovii
KANG DING CUI QUE HUA

MI SUI HUA SHE BIAN JU

GAN SU SHAN ZHA

XUE LING ZHI

GAN SUI

JI BEI

KA LA BA DAN SHEN

KA LUO JIN HE HUAN

GANG MAO CHENG LIU

KE SHEN MI ER CUI QUE

CAO DOU KOU

LIAN XIANG SHU

KA WA HU JIAO

CHUAN SHANG LONG MI GOU

TENG

XIAO GOU SHU

KAI SHI DANG GUI

KUN BU

DA MA JIN

KEN NI YA CI TONG

KEN NI YA WEN SHU LAN

KEN NI YA XIAN SUAN QIANG

KEN NI YA MO YAO

KEN NI YA HUI YE

SHI HU DIE

DI TANG BAI BU

CI TIAN QIE

KA XI YA SONG

QIAO CHA

CHOU LENG SHAN

BAI FAN DOU

LIAO SHANG RONG MAO HUA

HU LU QI

HU LU QI BIAN ZHONG

CHUAN JU

DIAN HUANG JING

YAO YONG MU DAN

RU JU

GOU SHE CAO

XIA YE HONG JING TIAN

JI LIN WU TOU

PING YU FENG WEI JUE

SHE HAN WEI LING CAI

KE LEI NI JUE MING

KE SHI HAO

PENG LAI CAO

DA YE NIU NAI CAI

RI BEN XIN YI

KU HE LONG DAN

PIE LAN

KE LE TENG HUANG

NIU XIN PIAO ZI 
Kombe Strophanthus*

Kongu

Konish Chinafir

Konka Mountain Monkshood

Koraen Atractylodes

Korea Patrinia*

Korea Poplar

Korean Acanthopanax*

Korean Big Thyme*

Korean Epimedium

Korean Fir

Korean Forsythia*

Korean Monkshood

Korean Pine

Korean Pine Seed

Korean Pulsatilla

Korean Rhododendron

Korean Sophora

Korean Spruce

Kotake (in Japanese)

Krukov Mayten*

Kua Myrrhtree*

Kudincha Holly

Kulaso Aloe Dried Juice

Kunming Mosquitotrap

Kunming Rabdosia*

Kunming Thorowax*

Kuroo Gentian*

Kurz Alangium

Kusnezoff Monkshood

Kwangsi Jasminorange

Kwangsi Turmeric

Kweichou Uvaria

Laburnum Crotalaria*

Lac

Lacebark Pine

Laciniate Lettuce*

Laevigate Parameria

Lagopsis

Lakoocha Artocarpus*

Lalang Grass Leaf

Lalang Grass Rhizome

Lambert Barberry

Lambsquarters Juvenile

Lanatchead Saussurea

Lancaster's St.John'swort

Lance Coreopsis

Lanceleaf Actinodaphne*

Lanceleaf Dogbane*

Lanceleaf Eupatorium*

Lanceleaf Thermopsis

Lanceleaf Wingseedtree

Lance-leaved Gambirplant
T6157 Strophanthus kombe

T3280 Hopea parviflora

T1890 Cunninghamia konishii

T0112 Aconitum liljestrandii

T0822 Atractylodes koreana

T4671 Patrinia saniculaefolia

T5154 Populus koreana

T0040 Acanthopanax koreanum

T6448 Thymus magnus

T2398 Epimedium koreanum

T0005 Abies koreana

T2754 Forsythia koreana

T0088 Aconitum coreanum

T4913 Pinus koraiensis

T4912 Pinus koraiensis

T5324 Pulsatilla cernua

T5519 Rhododendron mucronulatum

T6037 Sophora koreensis

T4873 Picea koraiensis

T5734 Sarcodon aspratus

T4136 Maytenus krukovii

T1635 Commiphora kua

T3392 Ilex kudingcha

T0347 Aloe vera [Syn. Aloe barbadensis]

T1963 Cynanchum wallichii

T3499 Isodon kunmingensis

T1064 Bupleurum kunmingense

T2915 Gentiana kuroo

T0282 Alangium kurzii

T0108 Aconitum kusnezoffii

T4320 Murraya kwangsiensis

T1904 Curcuma kwangsiensis

T6664 Uvaria kweichowensis

T1824 Crotalaria laburnifolia

T3649 Laccifer lacca

T4908 Pinus bungeana

T3660 Lactuca laciniata

T4642 Parameria laevigata

T3675 Lagopsis supina

T0717 Artocarpus lakoocha

T3417 Imperata cylindrica var. major

T3416 Imperata cylindrica var. major

T0908 Berberis lambertii

T1359 Chenopodium album

T5757 Saussurea laniceps

T3357 Hypericum lancasteri

T1685 Coreopsis lanceolata

T0166 Actinodaphne lancifolia

T0552 Apocynum lancifolium

T2564 Eupatorium lancifolium

T6428 Thermopsis lanceolata

T5310 Pterospermum lanceaefolium

T6622 Uncaria lancifolia
KANG PI DU MAO XUAN HUA

XIAO HUA PO LEI

TAI WAN SHAN MU

GONG GA SHAN WU TOU

CHAO XIAN CANG ZHU

BIAN DOU CAI YE BAI JIANG

XIANG YANG

CHAO XIAN WU JIA

CHAO XIAN DA BAI LI XIANG

CHAO XIAN YIN YANG HUO

CHAO XIAN LENG SHAN

CHAO XIAN LIAN QIAO

HUANG HUA WU TOU

HONG SONG

HAI SONG ZI

CHAO XIAN BAI TOU WENG

YING SHAN HONG

CHAO XIAN LANG YA CI

HONG PI YUN SHAN

KE SHI MEI DENG MU

KU A MO YAO

KU DING CHA DONG QING

LU HUI

DUAN JIE SHEN

KUN MING XIANG CHA CAI

KUN MING CHAI HU

KU RUO LONG DAN

MAO BA JIAO FENG

BEI WU TOU

GUANG XI JIU LI XIANG

GUANG XI E SHU

LIU GUO ZI YU PAN

JIN LIAN HUA ZHU SHI DOU

ZI CAO RONG

BAI PI SONG

SUI BIAN WO JU

CHANG JIE ZHU

XIA ZHI CAO

LA KOU SHA MIAN BAO GUO

MAO CAO YE

BAI MAO GEN ${ }^{(1)}$

LAN BO TE XIAO BO

LI

MIAN TOU XUE LIAN

ZHAN E JIN SI TAO

XIAN YE JIN JI JU

PI ZHEN YE HUANG ROU NAN

HONG MA

ZHEN YE ZE LAN

MU MA DOU

ZHAI YE BAN FENG HE

PI ZHEN YE GOU TENG 


\author{
Lanceolate Kadsura* \\ Lanceolate Maesa* \\ Lanceolate Periwinkle* \\ Lanceolate Starwort \\ Lanceolate Stringbush* \\ Lanceolate Sumac* \\ Lancepod \\ Langpetal Globeflower \\ Lantern-tree \\ Lanyu Garcinia \\ Larch-leaf St.John'swort* \\ Large Caesalpinia* \\ Large Euphorbia Root \\ Largeflower Astilbe \\ Largeflower Euonymus \\ Largeflower Falsehellebore \\ Largeflower Jasmine* \\ Largeflower Jerusalemsage \\ Largeflower Purslane \\ Largeflower Uvaria \\ Largeflower Wildginger \\ Large-flowered Dendranthema* \\ Large-flowered Epimedium \\ Large-flowered Epimedium Variety
}

Largeflower-like Honeysuckle

Largefruit Euphorbia*

Largehead Atractylodes

Largeleaf Aglaia*

Largeleaf Ampelopsis

Largeleaf Chinese Ash Bark

Largeleaf Curculigo

Largeleaf Devilpepper*

Largeleaf Gambirplant

Largeleaf Gentian

Largeleaf Hydrangea

Largeleaf Inula

Largeleaf Japanese Ginseng

Largeleaf Pipper*

Largeleaf Rabdosia

Largeleaf Spicebush

Largeleaf Spicebush Branch-leaf

Largeleaf Spicebush Root-bark

Largeleaf Uvaria*

Largeseed Bugle

Largeseed Hemsleya

Largesepal Rabdosia

Largesepal Rabdosia

Largeserrate Mosla

Late-flower Boneset

Lateripening Bartsia
T3617 Kadsura lancilimba

T4030 Maesa lanceolata

T1267 Catharanthus lanceus

T6104 Stellaria dichotoma var. lanceolata

T6821 Wikstroemia lanceolata

T5533 Rhus lanceolata

T3904 Lonchocarpus atropurpureus

T6554 Trollius macropetalus

T1792 Crinodendron hookerianum

T2861 Garcinia linii

T3358 Hypericum laricifolium

T1105 Caesalpinia major

T2602 Euphorbia nematocypha

T0785 Astilbe macroflora

T2541 Euonymus grandiflorus

T6697 Veratrum grandiflorum

T3554 Jasminum grandiflorum

T4808 Phlomis grandiflora var. grandiflora

T5172 Portulaca grandiflora

T6662 Uvaria grandiflora

T0729 Asarum maximum

T2093 Dendranthema grandiflorum

T2396 Epimedium grandiflorum

T2397 Epimedium grandiflorum var. thumbergianum

T3914 Lonicera macranthoides

T2626 Euphorbia wallichii

T0824 Atractylodes macrocephala [Syn. Atractylis macrocephala]

T0235 Aglaia elliptifolia

T0428 Ampelopsis megalophylla

T2777 Fraxinus rhynchophylla [Syn. Fraxinus chinensis var.

rhynchophylla]

T1900 Curculigo capitulata [Syn. Leucojum capitulata]

T5437 Rauwolfia macrophylla

T6625 Uncaria macrophylla

T2919 Gentiana macrophylla

T3304 Hydrangea macrophylla

T3430 Inula grandis

T4605 Panax japonicus var. major

T4951 Piper laetispicum

T5394 Rabdosia macrophylla

T3852 Lindera megaphylla

T3857 Lindera umbellata [Syn. Lindera erythrocarpa]

T3856 Lindera umbellata [Syn. Lindera erythrocarpa]

T6665 Uvaria macrophylla

T0266 Ajuga macrosperma

T3208 Hemsleya macrosperma

T3511 Isodon macrocalyx

T3513 Isodon macrocalyx

T4306 Mosla grosseserrata

T2575 Eupatorium serotinum

T4479 Odontites serotina
PI ZHEN YE NAN WU WEI ZI

PI ZHEN DU JING SHAN

JIAN ZHUANG CHANG CHUN HUA

YIN CHAI HU

PI ZHEN XING YAO HUA

PI ZHEN QI SHU

CHANG BAN JIN LIAN HUA

HONG BAI HE MU

TAI WAN LV DAO TENG HUANG

LUO YE SONG YE JIN SI TAO

DA YUN SHI

DA LANG DU

DA HUA LUO XIN FU

YE DU ZHONG

MAO YE LI LU

SU XIN HUA

DA HUA CAO SU

DA HUA MA CHI XIAN

DA HUA ZI YU PAN

DA HUA XI XIN

DA HUA JU

DA HUA YIN YANG HUO

DA HUA YIN YANG HUO BIAN

ZHONG

HUI ZHAN MAO REN DONG

DA GUO DA JI

BAI ZHU

DA YE SHU LAN

DA YE SHE PU TAO

CEN PI

DA YE XIAN MAO

DA YE LUO FU MU

DA YE GOU TENG

QIN JIAO

BA XIAN HUA

DA YE TU MU XIANG

QIN LING ZHU ZI SHEN

DA YE JU

DA YE XIANG CHA CAI

HEI KE NAN

DIAO ZHANG ZHI YE

DIAO ZHANG GEN PI

DA YE ZI YU PAN

DA ZI JIN GU CAO

DA ZI XUE DAN

DA E XIANG CHA CAI

DA E BIAN XING XIANG CHA CAI

orm

JI NING

WAN HUA ZE LAN

CHI YE CAO 


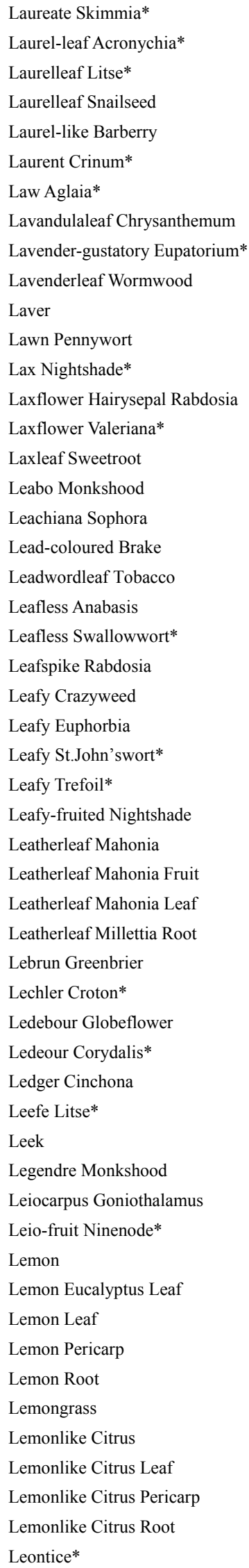

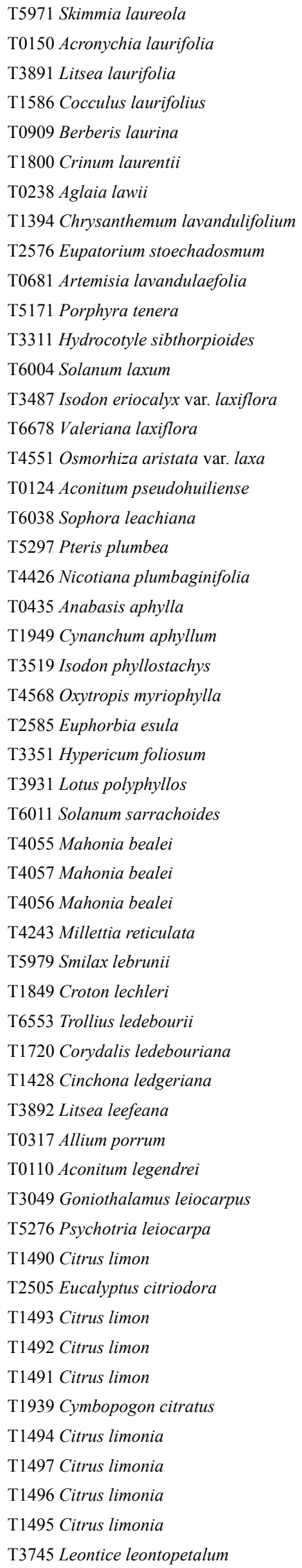

GUANG RONG YIN YU

YUE GUI YE SHAN YOU GAN

YUE GUI SHU YE MU JIANG ZI

HENG ZHOU WU YAO

YUE GUI XIAO BO

LAO SHI WEN SHU LAN

YAN XIANG JU

DUO XU GONG

YE AI HAO

ZI CAI

TIAN HU SUI

XI SHU QIE

SHU HUA MAO E XIANG CHA CAI

SHU HUA JIE CAO

XIANG GEN QIN

LEI BO WU TOU

LI QI HUAI

LI BING FENG WEI JUE

HUI YE YAN CAO

WU YE JIA MU ZEI

WU YE BAI QIAN

YE SUI XIANG CHA CAI

DUO YE JI DOU

JI CHANG LANG DU

DUO YE JIN SI TAO

DUO YE BAI MAI GEN

YE GUO QIE

SHI DA GONG LAO MU

SHI DA GONG LAO ZI

SHI DA GONG LAO YE

JI XUE TENG GEN

CU CAO BA QIA

LAI KE BA DOU

DUAN BAN JIN LIAN HUA

DUI YE YUAN HU

JIN JI LE

LI FEI MU JIANG ZI

JIU CONG

MIAN NING WU TOU

JIN PING GE NA XIANG

PING HUA GUO JIU JE

NING MENG

NING MENG AN YE

NING MENG YE

NING MENG PI

NING MENG GEN

XIANG MAO

LI MENG

LI MENG YE

LI MENG PI

LI MENG GEN

HUA BAN SHI ZU CAO 
Leopard Leather Mushroom*; Tengutake (in T0384 Amanita pantherina Japanese)

Leopard Plant

Leprieur Caloglossa Frond

Leprous Tree

Leptopeel Cucumber*

Leschenault Mahonia

Lesser Galangal

Lettuce Ulva Frond

Levant Cotton

Levant Cotton Oil

Levant Cotton Root

Liangshan Rabdosia

Liaodong Aralia

Liaodong Aralia Leaf*

Liberian Coffee

Licorice

Lidded Cleistocalyx

Light Larkspur*

Light-hoar Tickclover*

Lightyellow Snapweed

Lightyellow Sophora

Lightyellow Sophora Seed

Lignum-vitae

Lihsien Rabdosia

Likiang Ephedra

Likiang Hogfennel

Likiang Monkshood*

Likiang Skullcap

Lilac Chastetree

Lilac Daphne

Lilac Daphne Root

Lilac Pink

Lily Magnolia Bark

Lily Magnolia Buds

Lily of Valley

Limbate Otostegia

Lime

Limited Alstonia*

Limoncillo (in Costa Rica)

Limpricht Begonia

Linden Hibiscus

Linden Viburnum

Lindenleaf Tickclover*

Lindley Eupatorium

Lindley Pleurospermum

Linea Andrographis*

Linear Brake

Linear Croton*

Linear Koelpinia

Linear Stonecrop

Linearisepal Goldthread*

Linearleaf Gentian
T3801 Ligularia clivorum

T1122 Caloglossa leprieurii

T3560 Jatropha curcas

T1875 Cucumis leptodermus

T4069 Mahonia leschenaultii

T0359 Alpinia officinarum

T6599 Ulva lactuca

T3055 Gossypium herbaceum

T3057 Gossypium herbaceum

T3056 Gossypium herbaceum

T3504 Isodon liangshanica

T0572 Aralia elata

T0573 Aralia elata

T1610 Coffea liberica

T3013 Glycyrrhiza glabra

T1544 Cleistocalyx operculatus

T2069 Delphinium corumbosum

T2128 Desmodium canum

T3413 Impatiens nolitangere

T6031 Sophora flavescens [Syn. Sophora angustfolia]

T6032 Sophora flavescens [Syn. Sophora angustfolia]

T3067 Guajacum officinale

T3505 Isodon lihsienensis

T2372 Ephedra likiangensis

T4756 Peucedanum govanianum var. bicolo

T0096 Aconitum forrestii [Syn. Aconitum likiangense]

T5841 Scutellaria likiangensis

T6782 Vitex agnuscastus

T2023 Daphne genkwa

T2024 Daphne genkwa

T2145 Dianthus superbus

T4042 Magnolia liliflora

T4041 Magnolia liliflora

T1649 Convallaria keiskei [Syn. Convallaria majalis]

T4559 Otostegia limbata

T1466 Citrus aurantifolia

T0373 Alstonia restricta

T5965 Siparuna pauciflora

T0890 Begonia limprichtii

T3248 Hibiscus tiliaceus

T6735 Viburnum dilatatum

T2135 Desmodium tiliaefolium

T2565 Eupatorium lindleyanum

T5020 Pleurospermum lindleyanum

T0455 Andrographis lineata

T5293 Pteris linearis

T1850 Croton linearis

T3634 Koelpinia linearis

T5856 Sedum lineare [Syn. Sedum obtuso-lineare]

T1668 Coptis linearisepala

T2921 Gentiana manshurica
BAO BAN E GAO

SHAN GANG TUO WU

ZHE GU CAI

MA FENG SHU

BO PI HUANG GUA

LAI SHI NA TE SHI DA GONG LAO

GAO LIANG JIANG

SHI CHUN

MIAN HUA

MIAN ZI YOU

MIAN HUA GEN

LIANG SHAN XIANG CHA CAI

LIAO DONG CONG MU

LIAO DONG CONG MU YE

DA GUO KA FEI

GUANG GUO GAN CAO

SHUI RONG

GUANG FEI YAN CAO

DAN HUI BAI SHAN MA HUANG

SHUI JIN FENG

KU SHEN

KU SHEN SHI

YU CHUANG MU

LI XIAN XIANG CHA CAI

LI JIANG MA HUANG

LI JIANG QIAN HU

LI JIANG WU TOU

LI JIANG HUANG QIN

SUI HUA MU JING

YUAN HUA

YUAN HUA GEN

QU MAI

ZI YU LAN PI

XIN YI

LING LAN

YOU YAN AO TUO SI TE CAO

LAI MENG

YOU XIAN YA JIAO SHU

SHAO HUA XI PA MU

JI YE QIU HAI TANG

HUANG JIN

JIA MI

DUAN YE SHAN MA HUANG

CHENG GAN SHENG MA

TIAN SHAN LING ZI QIN

TIAO WEN CHUAN XIN LIAN

XIAN YU FENG WEI JUE

XIAN YE BA DOU

XIE WEI JU

FO JIA CAO

XIAN E HUANG LIAN

DONG BEI LONG DAN 


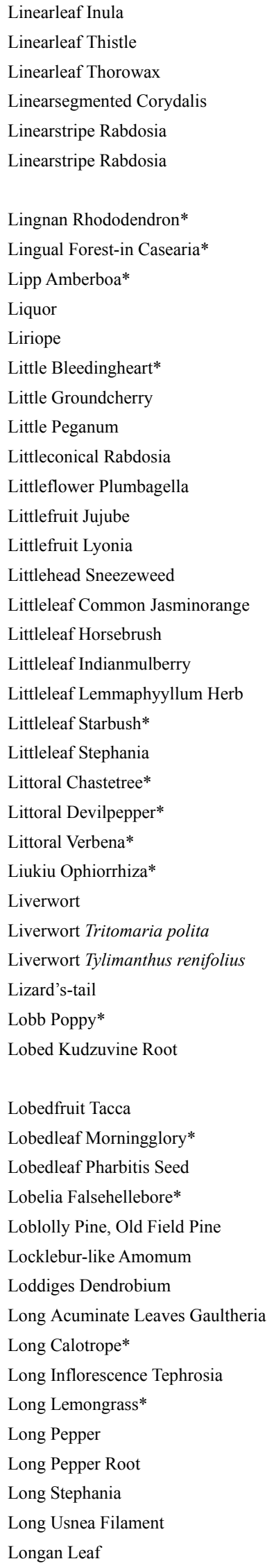

\begin{tabular}{|c|c|}
\hline T3434 Inula linariaefolia & XIAN YE XUAN FU HUA \\
\hline T1450 Cirsium lineare & TIAO YE JI \\
\hline T1055 Bupleurum angustissimum & XIAN YE CHAI HU \\
\hline T1721 Corydalis linearioides & TIAO LIE HUANG JIN \\
\hline T3506 Isodon lophanthoides & XIAN WEN XIANG CHA CAI \\
\hline T3507 Isodon lophanthoides [Syn. Rabdosia lophanthoides; Hyssopus & XI HUA XIAN WEN XIANG CHA CAI \\
\hline \multicolumn{2}{|l|}{ lophanthoides; Plectranthus striatus; Isodon striatus] } \\
\hline T5513 Rhododendron mariae & LING NAN DU JUAN \\
\hline T1225 Casearia sylvestris var. lingua & SHE XING LIN SHENG JIAO GU CUI \\
\hline T0392 Amberboa lippi & LI PU PO JU \\
\hline T3869 Liquor & JIU Liquor \\
\hline T4507 Ophiopogon japonicus & MAI DONG \\
\hline T2153 Dicentra pusilla & XI XIAO HE BAO MU DAN \\
\hline T4851 Physalis minima & TIAN PAO ZI \\
\hline T4689 Peganum nigellastrum & LUO TUO HAO \\
\hline T5389 Rabdosia coetsa & XI ZHUI XIANG CHA CAI \\
\hline T5026 Plumbagella micrantha & JI WA CAO \\
\hline T6923 Zizyphus oenoplia & XIAO GUO ZAO \\
\hline T3992 Lyonia ovalifolia var. elliptica & XIAO GUO NAN ZHU \\
\hline T3139 Helenium microcephalum & XIAO TOU DUI XIN JU \\
\hline T4325 Murraya paniculata var. exotica & XIAO YE JIU LI XIANG \\
\hline T6355 Tetradymia glabrata & GUANG SI SHI JU \\
\hline T4284 Morinda parvifolia & BAI YAN TENG \\
\hline T3736 Lemmaphyllum microphyllum & LUO YAN CAO \\
\hline T6571 Turraea parvifolia & XIAO YE DU LIAN \\
\hline T6135 Stephania succifera & XIAO YE DI BU RONG \\
\hline $\mathrm{T} 6784$ Vitex littoralis & BIN MU JING \\
\hline T5436 Rauwolfia littoralis & HAI BIN LUO FU MU \\
\hline T6708 Verbena littoralis & HAI BIAN MA BIAN CAO \\
\hline T4512 Ophiorrhiza liukiuensis & LIU QIU SHE GEN CAO \\
\hline \multicolumn{2}{|l|}{ T2819 Gackstroemia decipiens } \\
\hline \multicolumn{2}{|l|}{ T6547 Tritomaria polita } \\
\hline \multicolumn{2}{|l|}{ T6575 Tylimanthus renifolius } \\
\hline T5749 Saururus cernuus & MEI ZHOU SAN BAI CAO \\
\hline T2496 Eschscholzia lobbii & LUO BO HUA LING CAO \\
\hline $\begin{array}{l}\text { T5313 Pueraria lobata [Syn. Pueraria thunbergiana; Pueraria } \\
\text { pseudohirsuta] }\end{array}$ & GE GEN \\
\hline T6276 Tacca plantaginea [Syn. Schizocapsa plantaginea] & LIE GUO SHU \\
\hline T3449 Ipomoea hederacea & LIE YE QIAN NIU \\
\hline T4779 Pharbitis nil & QIAN NIU ZI \\
\hline T6693 Veratrum album var. lobelianum [Syn. Veratrum lobelianum] & BAN BIAN LIAN ZHUANG LI LU \\
\hline T4927 Pinus taeda & TAI DA SONG \\
\hline T0420 Amomum xanthioides & SUO SHA MI \\
\hline T2105 Dendrobium loddigesii & MEI HUA SHI HU \\
\hline T2892 Gaultheria griffithiana & WEI YE BAI ZHU \\
\hline T1136 Calotropis procera & CHANG NIU JIAO GUA \\
\hline T6337 Tephrosia noctiflora & YE HUA HUI MAO DOU \\
\hline T1946 Cymbopogon procerus & CHANG XIANG MAO \\
\hline T4953 Piper longum & BI BA \\
\hline T4954 Piper longum & BI BA GEN \\
\hline T6131 Stephania longa & FEN JI DU \\
\hline T6654 Usnea longissima & SONG LUO \\
\hline T2627 Euphoria longan [Syn. Dimocarpus longan] & LONG YAN YE \\
\hline
\end{tabular}


Longbeak Eucalyptus

Longbract Cattail Pollen

Longcalyx Pink*

Longeared Epigynum

Long-eared Pseudophegopteris

Longflower Bloomdaisy

Longflower Galipea*

Longflower Gambirplant*

Longflower Kopsia*

Longflower Laurentia

Longflower Lily

Longflower Valerian*

Longfruit Hemsleya

Longhair Milkwort

Longhairy Michelia*

Long-headed Poppy

Longleaf Carpesium

Longleaf Cryptocarya*

Longleaf Debregeasia

Longleaf Goniothalamus

Longleaf Groundsel*

Longleaf Mammey*

Longleaf Periwinkle*

Longleaf Podocarpus Leaf

Longleaf Podocarpus Seed

Longleaf Rabdosia

Longleaf Speedwell

Longleaf Tansy

Long-leaved Pine

Longlived Leaf

Longpedicel Chinese Buscherry Seed

Longpedicel Holly

Longpeduncle Kadsura

Longpetal Vouacapoua*

Longpetiole Juniper*

Longradiate Angelica*

Longroot Onion

Long-rostrate Eucalyptus*

Longshen Rabdosia

LongspikeCroton*

Longspur Corydalis*

longstalk Gynostemma

Longstalk Pepper*

Longstalk Plumyew*

Longstamen Onion

Longstyle Lindelofia

Longstyle Meadowrue*

Long-tail Mulberry*

Longthyrsus Poisonnut*

Longtube Daylily*

Longtube Ground Ivy

Longtube Rabdosia
T2502 Eucalyptus camaldulensis

T6584 Typha angustata

T2146 Dianthus superbus var. longicalycinus

T2386 Epigynum auritum

T5259 Pseudophegopteris subaurita

T4735 Pertya glabrescens

T2828 Galipea longiflora

T6624 Uncaria longiflora

T3642 Kopsia longiflora

T3541 Isotoma longiflora [Syn. Laurentia longiflora]

T3835 Lilium longiflorum

T1313 Centranthus longiflorus ssp. longiflorus

T3205 Hemsleya dolichocarpa

T5090 Polygala wattersii

T4213 Michelia lanuginosa

T4626 Papaver dubium

T1213 Carpesium longifolium

T1863 Cryptocarya longifolia

T2056 Debregeasia longifolia

T3045 Goniothalamus gardneri

T5891 Senecio longifolius

T4095 Mammea longifolia

T1268 Catharanthus longifolius

T5043 Podocarpus macrophyllus

T5042 Podocarpus macrophyllus

T5400 Rabdosia stracheyi

T6725 Veronica longifolia

T6292 Tanacetum longifolium

T4919 Pinus palustris

T6816 Welwitschia mirabilis

T5225 Prunus japonica var. nakaii

T3395 Ilex pedunculosa

T3619 Kadsura peltigera [Syn. Kadsura longipedunculata]

T6805 Vouacapoua macropetala

T3590 Juniperus macropoda

T0488 Angelica longeradiata

T0324 Allium victorialis

T2518 Eucalyptus rostrata

T3510 Isodon lungshengensis

T1851 Croton macrostachys

T1722 Corydalis longicalcarata

T3084 Gynostemma longipes

T4969 Piper sulvaticum

T1321 Cephalotaxus harringtonia var. drupacea

T0316 Allium macrostemon

T3846 Lindelofia stylosa

T6396 Thalictrum longistylum

T4297 Morus macroura

T6171 Strychnos dolichothyrsa

T3196 Hemerocallis longituba

T2973 Glechoma longituba

T5393 Rabdosia longituba
CHI AN

PU HUANG

CHANG E QU MAI

SI MAO TENG

GUANG NANG ZI BING JUE

JIN WU MAO SAO JU

CHANG HUA TU LA SHU

CHANG HUA GOU TENG

CHANG HUA RUI MU

TONG BAN CAO

SHE XIANG BAI HE

CHANG HUA XIE CAO

CHANG GUO XUE DAN

CHANG MAO ZI YUAN ZHI

CHANG MAO HAN XIAO

CHANG GUO YING SU

CHANG YE TIAN MING JING

CHANG YE HOU KE GUI

CHANG YE SHUI MA

CHANG YE GE NA XIANG

CHANG YE QIAN LI GUANG

CHANG YE MAN MI PING GUO

CHANG YE CHANG CHUN HUA

LUO HAN SONG YE

LUO HAN SONG SHI

CHANG YE XIANG CHA CAI

CHANG WEI PO PO NA

CHANG YE AI JU

CHANG YE SONG

BAI SUI YE

CHANG GENG YU LI REN

CHANG GENG DONG QING

CHANG GENG NAN WU WEI ZI

CHANG HUA BAN KE YA SHU

CHANG BING YUAN BAI

CHANG BIAN HUA DANG GUI

GE CONG

CHANG HUI AN

LONG SHENG XIANG CHA CAI

CHANG SUI BA DOU

CHANG JU YAN HU SUO

CHANG GENG JIAO GU LAN

CHANG BING HU JIAO

CHANG GENG CU FEI

XIE BAI

MING XIAN HUA ZHU CHANG ZHU

LIU LI CAO

CHANG ZHU TANG SONG CAO

NAI SANG

CHANG HUA XU MA QIAN ZI

CHANG GUAN XUAN CAO

JIN XIAN CAO

CHANG GUAN XIANG CHA CAI 


\begin{tabular}{|c|c|}
\hline Lopseed & T4830 Phryma leptostachya \\
\hline Loquat & T2431 Eriobotrya japonica \\
\hline Loquat Leaf & T2433 Eriobotrya japonica \\
\hline Loquat Seed & T2432 Eriobotrya japonica \\
\hline Lotusleaftung & T3231 Hernandia sonora $[$ Syn. Hernandia ovigera $]$ \\
\hline Louis Vepris* & T6690 Vepris louisii \\
\hline Loureir Cinnamon* & T1442 Cinnamomum loureirii \\
\hline Low Ardisia* & T0597 Ardisia humilis \\
\hline Low Bearberry* & T0587 Arctostaphylos pumila \\
\hline Low Lily & T3836 Lilium pumilum [Syn. Lilium tenuifolium] \\
\hline Low Meadowrue & T6399 Thalictrum minus \\
\hline Low Rhubarb* & T5471 Rheum nanum \\
\hline Low Sagebrush & T0663 Artemisia arbuscula \\
\hline Low Uvaria & T6660 Uvaria chamae \\
\hline Lowdaphne Stringbush & T6819 Wikstroemia chamaedaphne \\
\hline Love-lies-bleeding & T0387 Amaranthus caudatus \\
\hline Lovely Crinum* & T1793 Crinum amabile \\
\hline Lovely Gloriosa & T2995 Gloriosa superba \\
\hline Lovely Hemsleya & T3203 Hemsleya amabilis \\
\hline Lucid Ganoderma & T2848 Ganoderma lucidum \\
\hline Lucid Indianmulberry* & T4282 Morinda lucida \\
\hline Lucid Meadowrue* & T6397 Thalictrum lucidum \\
\hline Lucid Monkshood* & T0113 Aconitum lucidusculum \\
\hline Lucidum Onychium & T4503 Onychium lucidum \\
\hline Luederitz Acacia* & T0023 Acacia luederitzii \\
\hline Lunate Peltate Sundew & T2267 Drosera peltata var. lunata \\
\hline Lushan Rabdosia* & T3521 Isodon rubescens var. lushanensis \\
\hline Lushien Rabdosia* & T3522 Isodon rubescens var. lushiensis \\
\hline Luzon Viburnum* & T6737 Viburnum luzonicum \\
\hline Lychee & T3876 Litchi chinensis \\
\hline Lychee Seed & T3877 Litchi chinensis \\
\hline Lyrate Boesenbergia* & T0970 Boesenbergia pandurata \\
\hline Lyrate Hemistepta & $\begin{array}{l}\text { T3202 Hemistepta lyrata [Syn. Hemistepta carthamoides; Saussurea } \\
\text { carthamoides] }\end{array}$ \\
\hline Lyrate-like Croton* & T1857 Croton sublyratus \\
\hline MacLean Leek* & T0315 Allium macleanii \\
\hline Macradenous Milkwort* & T5077 Polygala macradenia \\
\hline Macroanther Crinum* & T1802 Crinum macrantherum \\
\hline Macrocalyx Ochna Bark* & T4466 Ochna macrocalyx \\
\hline Macropodous Daphniphyllum & T2033 Daphniphyllum macropodum \\
\hline Madagascar Evodia* & T2642 Evodia madagascariensis \\
\hline Madagascar Hani-wood* & T3110 Harungana madagascariensis \\
\hline Madagascar Periwinkle & T1271 Catharanthus roseus $[$ Syn. Vinca rosea; Lochera rosea] \\
\hline Madagascar Swartzia* & T6211 Swartzia madagascariensis \\
\hline Madder & T5584 Rubia tinctorum \\
\hline Madonna Lily & T3833 Lilium candidum \\
\hline Madrid Squill* & T5810 Scilla maderensis [Syn. Autonoë madeirensis] \\
\hline Madura Crotalaria* & T1825 Crotalaria madurensis \\
\hline Magnific Medinilla* & T4152 Medinilla magnifica \\
\hline Magnolia compressa & T4037 Magnolia compressa \\
\hline Maidenhair Meadowrue & T6385 Thalictrum flavum \\
\hline Maidenhair-like Meadowrue* & T6400 Thalictrum minus var. adiantifolium \\
\hline Mainge Tailgrape* & T0654 Artabotrys maingayi \\
\hline
\end{tabular}

LAO PO ZI ZHEN XIAN

PI PA

PI PA YE

PI PA HE

LIAN YE TONG

LU YI CI JU

MU GUI

AI ZI JIN NIU

AI SHENG XIONG GUO

XI YE BAI HE

XIAO TANG SONG CAO

AI DA HUANG

BEI MEI AI HAO

AN ZI YU PAN

HE SHUO YAO HUA

WEI SUI XIAN

SU MEN DA LA WEN SHU LAN

JIA LAN

LUO GUO DI

LING ZHI

GUANG ZE BA JI

TOU MING TANG SONG CAO

GUANG ZE WU TOU

LI BING JIN FEN JUE

LE SHI JIN HE HUAN

MAO GAO CAI

LU SHAN XIANG CHA CAI

LU SHI DONG LING CAO

LV SONG JIA MI

LI ZHI

LI ZHI HE

QIN ZHUANG AO CHUN JIANG NI HU CAI

JIN QIN ZHUANG BA DOU

MAI KE LIN JIU

DA XIAN YUAN ZHI

DA HUA YAO WEN SHU LAN

CHANG E JIN LIAN MU PI

JIAO RANG MU

MA DAO CHOU TAN

MA DAO HA NI MU

CHANG CHUN HUA

MA DAO SI WO CI DOU

YANG QIAN CAO

QING LIANG BAI HE

MA DE LI MIAN ZAO ER

MA DU LA ZHU SHI DOU

HONG WEI SUAN JIAO GAN

HUANG TANG SONG CAO

TIE XIAN JUE YE TANG SONG CAO MAN GE YING ZHAO 
Maire Alstonia

Maire Yew

Maize

Maize Bran

Maize Style

Malaba Bauhinia*

Malaba Pencilwood*

Malabanut

Malabar Randia; Sping Randia

Malabartree Euphorbia

Malacca Jewelvine*

Malay Blumea

Malay Bushbeech

Malay Glycosmis

Malaya Dieingtree*

Malaysia Kopsia*

Malaysian Box*

Malaytea Scurfpea

Male Fern Rhizome

Male-fern

Mamiaho (in Malagasy language,

Madagascar)

Mammee Apple

Manaplant Alhagi Sweet Secretion

Manchineel

Manchurian Ash

Manchurian Dutchmanspipe

Manchurian Monkshood

Manchurian Rhododendron

Manchurian Sage*

Manchurian Walnet

Manchurian Wildginger

Mango

Mango Bark

Mango Leaf

Mango Seed

Mangosteen

Mangrove Frult

Manio

Manipur Mahonia

Mann Garcinia*

Mansonia Heartwood

Manybracteole Bugle

Many-cleft Cinquefoil

Manyflower Ash

Manyflower Bayberry*

Manyflower Christmasbush*

Manyflower Dysosma*

Manyflower Fissistimga

Manyflower Garcinia

Manyflower Garcinia*

Manyflower Giantfennel*

Manyflower Glorybower Leaf
T0371 Alstonia mairei

T6312 Taxus mairei

T6902 Zea mays

T6900 Zea mays

T6901 Zea mays

T0878 Bauhinia malabarica

T2312 Dysoxylum malabaricum

T0170 Adhatoda vasica

T5411 Randia spinosa

T2624 Euphorbia tirucalli

T2120 Derris malaccensis

T0959 Blumea lacera

T3024 Gmelina arborea

T3008 Glycosmis pentaphylla

T5729 Saprosma scortechinii

T3641 Kopsia griffithii

T1090 Buxus malaiana

T5270 Psoralea corylifolia

T2281 Dryopteris crassirhizoma

T2282 Dryopteris filix-mas

T0876 Baseonema acuminatum

T4093 Mammea americana

T0302 Alhagi pseudalhagi

T3252 Hippomane mancinella

T2772 Fraxinus mandshurica

T0632 Aristolochia manshuriensis

T0139 Aconitum variegatum

T5515 Rhododendron micranthum

T5689 Salvia przewalskii var. mandarinorum

T3564 Juglans mandshurica

T0728 Asarum heterotropoides var. mandshuricum

T4099 Mangifera indica

T4101 Mangifera indica

T4102 Mangifera indica

T4100 Mangifera indica

T2863 Garcinia mangostana

T5488 Rhizophora mucronata

T5050 Podocarpus nubigenus

T4070 Mahonia manipurensis

T2864 Garcinia mannii

T4107 Mansonia gagei

T0270 Ajuga remota

T5185 Potentilla multifida

T2769 Fraxinus floribunda

T4345 Myrica multiflora

T0296 Alchornea floribunda

T2297 Dysosma aurantiocaulis

T2736 Fissistigma polyanthum

T2867 Garcinia multiflora

T2870 Garcinia polyantha

T2705 Ferula polyantha

T1554 Clerodendron cyrtophyllum
YANG JIAO MIAN

MEI LI HONG DOU SHAN

YU SHU SHU

YU MI FU

YU MI XU

MA LA BA YANG TI JIA

MA LA BA JIAN MU

DA BO GU

SHAN SHI LIU

LU YU SHU

MA LIU JIA YU TENG

HONG TOU CAO

YUN NAN SHI ZI

JIU BING YE

MA LAI BAN DAO RAN MU SHU

MA LAI XI YA RUI MU

MA LAI XI YA HUANG YANG

BU GU ZHI

GUAN ZHONG

MIAN MA

MEI ZHOU MAN MI PING GUO

LUO TUO CI

MA FENG MU

SHUI QU LIU

GUAN MU TONG

BAN HUA WU TOU

ZHAO SHAN BAI

ZI DAN SHEN

HU TAO QIU

LIAO XI XIN

MANG GUO

MANG GUO SHU PI

MANG GUO YE

MANG GUO HE

DAO NIAN ZI

HONG QIE DONG GUO

YUN WU LUO HAN SONG

MAN NI PU ER SHI DA GONG LAO

MAN TENG HUANG

MAN SUO NI YA XIN CAI

YUAN JU JIN GU CAO

DUO LIE WEI LING CAI

XI MA BAI LA SHU

DUO HUA YANG MEI

DUO HUA SHAN MA GAN

DUO HUA BA JIAO LIAN

HEI FENG TENG

SHAN ZHU ZI

DUO HUA TENG HUANG

DUO HUA A WEI

LU BIAN QING 
Manyflower Solomonseal

Manyflower Starbush*

Manyflower Tupelo*

Manyflower Tylophora

Manyflower Wormwood

Manyfruit Idesia

Manyleaf Meadowrue

Manyleaf Paris

Manynerve Embelia

Manypetal Marshmarigold

Manyprickle Acanthopanax

Manyprickle Acanthopanax Leaf

Manyprickle Acanthopanax Root-bark

Manyraceme Sweetvetch

Manyradiate Bailai's Chrysanthemum*

Manyroot Monkshood

Manyseed

Manysperma Bitterleaf*

Manyspike Cordia*

Manyspike Knotweed

Manyspike Podocarpus Seed

Manyspike Ragweed

Manyspike Tanoak Leaf

Manyspiny Pricklyash*

Manysplltted Monkshood

Manytoothed Groundsel*

Manyumbell Giantfennel

Many-veined Gambirplant*

Marginate Microlepia

Marginated Buckler-fern*

Marguerite

Marine Widgeonweed

Maritime Persimmon*

Marloth Aloe

Marschall Corydalis *

Marsh Horsetail

Marsh Parsley

Marsh Ramalina*

Marshmallow

Marshmarigold-leaved Beesia

Marshmarigold-like Goldenray*

Marshy Betony

Marshy Euphorbia*

Mary Arthromeris

Masaikai Caper

Mashan Hogfennel

Masson Pine Leaf:

Masters Elaeocarpus

Masters Pine

Masterwort

Mastic Africa Juniper*

Mastic Juniper*
T5091 Polygonatum cyrtonema [Syn. Polygonatum multiflorum]

T6569 Turraea floribunda

T4458 Nyssa sylvatica

T6580 Tylophora floribunda

T0687 Artemisia myriantha

T3387 Idesia polycarpa

T6387 Thalictrum foliolosum

T4648 Paris polyphylla

T2346 Embelia oblongifolia

T1138 Caltha polypetala

T0041 Acanthopanax senticosus [Syn. Eleutherococcus senticosus]

T0043 Acanthopanax senticosus [Syn. Eleutherococcus senticosus]

T0042 Acanthopanax senticosus [Syn. Eleutherococcus senticosus]

$\mathrm{T} 3129$ Hedysarum polybotrys

T0856 Baileya pleniradiata

T0105 Aconitum karakolicum

T5069 Polycarpon prostratum

T6720 Vernonia pogosperma

T1678 Cordia multispicata

T5114 Polygonum polystachyum

T5052 Podocarpus polystachyus

T0404 Ambrosia polystachya

T3878 Lithocarpus polystachyus

T6883 Zanthoxylum myriacanthum

T0123 Aconitum polyschistum

T5898 Senecio polyodon

T2698 Ferula ferulaeoides

T6626 Uncaria nervosa

T4219 Microlepia marginata

T2284 Dryopteris marginata

T1391 Chrysanthemum frutescens

T5616 Ruppia maritime

T2224 Diospyros maritima

T0341 Aloe marlothii

T1724 Corydalis marschalliana

T2409 Equisetum palustre

T4767 Peucedanum palustre

T5405 Ramalina paludosa

T0381 Althaea officinalis

T0888 Beesia calthaefolia

T3800 Ligularia calthaefolia

T6092 Stachys palustris

T2606 Euphorbia palustris

T0708 Arthromeris mairei [Syn. Polypodium mairei]

T1179 Capparis masaikai

T4762 Peucedanum mashanens

T4916 Pinus massoniana

T2329 Elaeocarpus mastersii

T4907 Pinus armandii var. mastersiana

T4766 Peucedanum ostruthium

T3602 Juniperus thurifera var. africana

T3601 Juniperus thurifera
DUO HUA HUANG JING

DUO HUA U LIAN

DUO HUA LAN GUO SHU

WA ER TENG

YI KUA

SHAN TONG ZI

MA WEI LIAN

ZAO XIU

MA GUI HUA

DUO BAN LV TI CAO

CI WU JIA

CI WU JIA YE

CI WU JIA PI

DUO XU YAN HUANG QI

DUO BIAN HUA BAI LAI SHI JU

DUO GEN WU TOU

DUO JIA CAO

BO GE BAN JIU JU

DUO SUI PO BU MU

DUO SUI LIAO

DUO SUI LUO HAN SONG SHI

DUO SUI TUN CAO

DUO SUI SHI KE YE

DA YE CHOU HUA JIAO

DUO LIE WU TOU

DUO CHI QIAN LI GUANG

DUO SAN A WEI

DUO MAI GOU TENG

BIAN YUAN LIN GAI JUE

BIAN YUAN LIN MAO JUE

MU TONG HAO

HAI SHENG CHUN MAN ZAO

HAI SHI

MA SHI LU HUI

MA CHANG LI ZI JIN

GU JIE CAO

ZHAO ZE QIAN HU

ZHAO ZE SHU HUA

YAO SHU KUI

TIE PO LUO

LV TI CAO YE TUO WU

GUANG YE SHUI SU

ZHAO SHENG DA JI

FENG WEI PA SHAN HU

MA BING LANG

MA SHAN QIAN HU

MA WEI SONG YE

MA SI TE SI DU YING

TAI WAN GUO SONG

OU QIAN HU

XIANG CI BAI FEI ZHOU BIAN

ZHONG

RU XIANG BAI 
Mastic-tree

Matai

Matarique (in Mexico)

Matico Pepper

Matteuccia Frond

Mature Winter-vegetable Spiced Juice

Maxima Dutchmanspipe*

Maxima Marigold*

Maximilian's Sunflower

Maximowicz Goldsaxifrage*

Maximowicz Hawthorn

Maximowicz Rhubarb*

May-apple

Mayweed

Meadaw Horsetail

Meadow Buttercup

Meadow Cranesbill

Meadow Eyebright

Meadow Gentian

Meadow Monkshood

Meadow Peavine

Meadow Saffron

Meadowruelike Isopyrum

Media Yew

Media Yew (hybrid)

Medicinal Angelica*

Medicinal Betonica

Medicinal Breynia Leaf*

Medicinal Changium

Medicinal Cinchona

Medicinal Citron

Medicinal Citron Leaf

Medicinal Ervatamia

Medicinal Evodia

Medicinal Fatheadtree

Medicinal Fumaria

Medicinal Hyssop

Medicinal Indianmulberry

Medicinal Kopsia

Medicinal Lavender*

Medicinal Ligusticum

Medicinal Padauk*

Medicinal Rhubarb

Medicinal Sage

Medicinal Sage Leaf

Medicine Terminalia

Medicine Terminalia Leaf

Mediinal Cyathula

Mediterranean Brown Alga Dilophus

ligulatus

Mediterranean Coriaria

Mediterranean Cypress
T4980 Pistacia lentiscus

T5055 Podocarpus spicatus

T5248 Psacalium peltatum

T4931 Piper angustifolium

T4127 Matteuccia struthiopteris

T1006 Brassica chinensis

T0633 Aristolochia maxima

T6280 Tagetes maxima

T3149 Helianthus maximiliani

T1405 Chrysosplenium maximowiczii

T1772 Crataegus maximowiczii

T5470 Rheum maximowiczii

T4666 Passiflora incarnata

T4124 Matricaria chamomilla [Syn. Matricaria recutita]

T2410 Equisetum pratense

T5412 Ranunculus acris

T2944 Geranium pratense

T2628 Euphrasia officinalis

T2906 Gentiana campestris

T0138 Aconitum umbrosum

T3709 Lathyrus pratensis

T1616 Colchicum autumnale

T3540 Isopyrum thalictroides

T6313 Taxus media

T6318 Taxus x media

T0489 Angelica officinalis

T0929 Betonica officinalis

T1026 Breynia officinalis

T1353 Changium smyrnioides

T1429 Cinchona officinalis

T1498 Citrus medica

T1499 Citrus medica

T2444 Ervatamia heyneana

T2644 Evodia rutaecarpa

T4391 Nauclea officinalis

T2807 Fumaria officinalis

T3384 Hyssopus officinalis

T4283 Morinda officinalis

T3643 Kopsia officinalis

T3728 Lavandula officinalis

T1583 Cnidium officinale [Syn. Ligusticum officinale]

T5303 Pterocarpus officinalis

T5472 Rheum officinale

T5682 Salvia officinalis

T5683 Salvia officinalis

T6346 Terminalia chebula

T6347 Terminalia chebula

T1924 Cyathula officinalis

T2183 Dilophus ligulatus

T1691 Coriaria myrtifolia

T1899 Cupressus sempervirens
XIANG HUANG LIAN MU

SUI HUA LUO HAN SONG

DUN ZHUANG LI JU

XIA YE HU JIAO

XIAO YE GUAN ZHONG

CHEN DONG CAI LU ZHI

DA MA DOU LING

ZUI DA WAN SHOU JU

MA SHI XIANG RI KUI

MA SHI JIN YAO

MAO SHAN ZHA

MA SHI DA HUANG

FEN HONG SE XI FAN LIAN

MU JU

CAO WEN JING

CAO DI MAO GEN

CAO YUAN LAO GUAN CAO

XIAO MI CAO

TIAN YE LONG DAN

CAO DI WU TOU

MU DI XIANG WAN DOU

QIU SHUI XIAN

TANG SONG CAO ZHUANG BIAN

GUO CAO

JIE ZHI HONG DOU SHAN

ZA JIAO JIE ZHI HONG DOU SHAN

YAO YONG DANG GUI

YAO SHUI SU

YAO YONG HEI MIAN SHEN YE

MING DANG SHEN

ZHENG JI NA SHU

JU YUAN

JU YUAN YE

HAI SHI GOU YA HUA

WU ZHU YU

DAN MU

YAO YONG QIU GUO ZI JIN

SHEN XIANG CAO

BA JI TIAN

YUN NAN RUI MU

YAO YONG XUN YI CAO

YAO YONG SHE CHUANG

YAO YONG ZI TAN

DA HUANG

YAO YONG DAN SHEN

YAO YONG DAN SHEN YE

HE ZI

HE ZI YE

CHUAN NIU XI

DI ZHONG HAI ZONG HAI ZAO

DI ZHONG HAI MA SANG

DI ZHONG HAI BAI MU 
Mediterranean Euphorbia; Mediterranean

Spurge

Mediterranean Mullein

Medlar Barberry*

Medusa Saussurea

Meiwa Kumquat

Mellea Armillaria Sporocarp

Melliferous Sage*

Membranous Casearia*

Membranous Milkvetch

Mengzi Peperomia*

Messania Sweetclover*

Metternich Rhododendron*

Mexican Ageratum

Mexican Aster

Mexican Cypress*

Mexican Pricklepoppy

Mexican Sunflower

Mexican Tea

Mexico Glorybind*

Mexico Sneezeweed*

Mexico Wormwood*

Mezereon

Micranthine Corydalis*

Microcap Sage*

Microwhite White Quebracho*

Mikanioid Eupatorium*

Mileen Swertia

Milkweed

Milk-white Russula

Milky Gentian*

Minima Micromelum*

Minus Hard-fern*

Minute Duckweed

Minwan Anisetree

Miquel Linden

Mirifica Kudzuvine

Miscol Rosewood*

Mist flower

Mistassini Primrose

Mitten Crab Chelae

Mittschelich Poisonnut*

Mocketprivet-like Oak

Moderate Asiabell

Moellendorff Spikemoss

Moliniform Dendrobium*

Molly Jewelvine*

Monarch-of-the-East

Mongolian Adonis*

Mongolian Ammopiptanthus

Mongolian Cymabria

Mongolian Dandelion

Mongolian Ephedra
T2582 Euphorbia characias

T6704 Verbascum sinuatum

T0921 Berberis zycium

T5759 Saussurea medusa

T2758 Fortunella crassifolia

T0645 Armillariella mellea

T5678 Salvia mellifera

T1224 Casearia membranacea

T0798 Astragalus membranaceus

T4704 Peperomia duclouxii

T4171 Melilotus messanensis

T5514 Rhododendron metternichii var. hondoese

T0230 Ageratum houstonianum

T1753 Cosmos bipinnata

T6306 Taxodium mucronatum

T0610 Argemone mexicana

T6470 Tithonia tagiliflora

T1361 Chenopodium ambrosioides

T5551 River corymbosa

T3138 Helenium mexicanum

T0684 Artemisia mexicana var. angustifolia

T2026 Daphne mezereum

T1725 Corydalis micrantha

T5679 Salvia microstegia

T0775 Aspidosperma subincanum

T2566 Eupatorium mikanioides

T6226 Swertia mileensis

T0740 Asclepias syriaca

T5620 Russula delica

T2916 Gentiana lactea

T4224 Micromelum minutum

T0951 Blechnum minus

T3739 Lemna perpusilla

T3405 Illicium minwanense

T6458 Tilia miqueliana

T5315 Pueraria mirifica

T2005 Dalbergia miscolobium

T2572 Eupatorium rugosum

T5200 Primula mistassinica

T2435 Eriocheir sinensis

T6180 Strychnos mittschelichii

T5376 Quercus phillyraeoides

T1600 Codonopsis pilosula var. modesta [Syn. Codonopsis modesta]

T5863 Selaginella moellendorffii

T2106 Dendrobium moniliforme

T2121 Derris mollis

T5747 Sauromatum guttatum

T0187 Adonis mongolica

T0414 Ammopiptanthus mongolicus [Syn. Piptanthus mongolicus]

T1938 Cymbaria mongolica

T6301 Taraxacum mongolicum

T2367 Ephedra equisetina
DI ZHONG HAI DA JI

DI ZHONG HAI MAO RUI HUA

GOU QI XIAO BO

SHUI MU XUE LIAN

JIN DAN

ZHEN MO

JU MI SHU WEI CAO

MO ZHI JIAO GU CUI

HUANG QI

MENG ZI CAO HU JIAO

XI XI LI CAO MU XI

MEI TE NI DU JUAN HUA

XIONG ER CAO

DA BO SI JU

MO XI GE LUO YU SHAN

JI YING SU

MO XI GE XIANG RI KUI

TU JING JIE

MO XI GE XUAN HUA

MO XI GE DUI XIN JU

MO XI GE HAO

OU YA RUI XIANG

XIAO HUA ZI JIN

XIAO GAI SHU WEI CAO

WEI BAI BAI JIAN MU

WEI GAN JU ZE LAN

QING YE DAN

XU LI YA MA LI JIN

MEI WEI HONG GU

RU BAI LONG DAN

JI XIAO XIAO YUN XIANG MU

XIAO WU MAO JUE

XI MAI FU PING

MIN WAN BA JIAO

PU TI SHU HUA

GUO YE GE

MI SI KE HUANG TAN

ZHOU YE ZE LAN

JIA NA DA BAO CHUN

XIE KE

MI SHI MA QIAN ZI

FEI LI GUI LI

SU HUA DANG SHEN

JIANG NAN JUAN BAI

XI JING SHI HU

MO LI YU TENG

KU BAO

MENG GU CE JIN ZHAN HUA

SHA DONG QING

GUANG YAO DA HUANG HUA

PU GONG YING

MU ZEI MA HUANG 
Mongolian Jerusalemsage

Mongolian Milkvetch

Mongolian Mulberry

Mongolian Oak

Mongolian Saussurea*

Mongolian Snakegourd

Mongolian Snakegourd Root

Mongolian Snakegourd Seed

Mongolian Spiraea

Mongolian Wormwood

Monochlamys Maidenhair

Monoleaf Atalantia*

Monoseed Honeylocust*

Monoseed Wormwood*

Montana Chamomile*

Montana Corydalis*

Montana Custardapple*

Montana Glycosmis*

Montana Rue*

Montane Baliospermum

Montane Germander*

Montane Larkspur

Montane Rabdosia

Montery Cypress

Montery Pine

Moon-carrot

Moor Sideritis*

Morrow Honeysuckle

Mother-of-pearl

Mountain Balsampear

Mountain Immortelle

Mountain Laurel

Mountain Parsley

Mountain Podocarpus*

Mountain Sneezeweed*

Mountain Spicy Tree

Mountain Tobacco

Mountain Yam

Mountainous Garcinia

Mountjasmine Rue*

Moupin Dutchmanspipe

Mozambique Mayten*

Muchlovable Denrdobium

Mucosa Rollinia*

Mucronate Glorybind*

Mucronated Jujube*

Mudpuppy

Mugwort

Muhul Myrrhtree*

Mukitake (in Japanese)

Mullein Nightbrier Leaf

Mulleinleaf Inula*

Multiflower White Quebracho*
T4810 Phlomis mongolica

T0800 Astragalus mongholicus

T4298 Morus mongolica

T5375 Quercus mongolica

T5760 Saussurea mongolica

T6510 Trichosanthes kirilowii

T6512 Trichosanthes kirilowii

T6511 Trichosanthes kirilowii

T6083 Spiraea mongolica

T0685 Artemisia mongolica

T0174 Adiantum monochlamys

T0815 Atalantia monophylla

T2977 Gleditsia monosperma

T0686 Artemisia monosperma

T0522 Anthemis cretica ssp. cretica [Syn. Anthemis montana]

T1726 Corydalis montana

T0508 Annona montana

T3007 Glycosmis montana

T5628 Ruta montana

T0861 Baliospermum montanum

T6363 Teucrium montanum

T2082 Delphinium oreophilum

T3516 Isodon oresbia

T1897 Cupressus macrocarpa

T4920 Pinus radiata

T5933 Seseli libanotis

T5945 Sideritis moorei

T3915 Lonicera morrowii

T1804 Cristaria plicata; Hyriopsis cumingii

T4266 Momordica dioica

T2471 Erythrina poeppigiana

T3623 Kalmia latifolia

T4765 Peucedanum oreoselinum

T5046 Podocarpus montanus

T3135 Helenium autumnale var. montanum

T3885 Litsea cubeba

T0651 Arnica montana

T2212 Dioscorea tokoro

T2858 Garcinia hombroniana

T5629 Ruta oreojasme

T0635 Aristolochia moupinensis

T4137 Maytenus mossambicensis

T2104 Dendrobium gratiosissimum

T5558 Rollinia mucosa

T1652 Convolvulus erinaceus

T6922 Zizyphus mucronata

T4395 Necturus maculosus

T0706 Artemisia vulgaris

T1637 Commiphora mukul

T4617 Panellus serotinus

T6018 Solanum verbascifolium

T3440 Inula verbascifolia

T0767 Aspidosperma multiflorum
MENG GU CAO SU

MENG GU HUANG QI

MENG SANG

MENG GU LI

MENG GU FENG MAO JU

GUA LOU

TIAN HUA FEN

GUA LOU ZI

MENG GU XIU XIAN JU

MENG GU HAO

DAN GAI TIE XIAN JUE

DAN YE DONG FENG JU

DAN ZHONG ZAO JIA

DAN ZI HAO

MENG DA NA CHUN HUANG JU

MENG DA NA ZI JIN

SHAN FAN LI ZHI

MENG DA NA SHAN XIAO JU

MENG DA NA YUN XIANG

BAN ZI MU

SHAN XIANG KE KE

XI SHAN CUI QUE

SHAN DI XIANG CHA CAI

DA GUO BAI MU

FU SHE SONG

LI BA NEN XIE HAO

MU ER DU MA CAO

MO LUO SHI REN DONG

ZHEN ZHU MU

SHAN KU GUA

SHAN DI CI TONG

KUAN YE SHAN YUE GUI

SHAN QIAN HU

SHAN DI LUO HAN SONG

SHAN DI DUI XIN JU

CHENG QIE ZI

SHAN JIN CHE

SHAN BI XIE

SHAN FENG GUO

SHAN MO LI YUN XIANG

HUAI TONG

MO SANG BI KE MEI DENG MU

BEI QIAO SHI HU

NIAN ZHI LUO LIN

JI XUAN HUA

JIAN YE ZAO

BAN YUAN

BEI AI

MU KU ER MO YAO

HOU SHU SHAN GU

YE YAN YE

MAO RUI HUA YE TU MU XIANG

DUO HUA BAI JIAN MU 
Multipistillate Spiderflower*

Multiradiate

Mung Bean Blister Beetle

Mural Goosefoot*

Murasakishimeji (in Japanese)

Muricate Amberboa*

Muriculate Eucheuma Frond

Murri Polyscias*

Muscicolous Woodbetony

Musengerra Podocarpus

Musenna Albizia

Mushroom

Musk-mallow

Muskmelon Fruit Pedicel

Muskroot

Musky Yarrow

Mussaenda*

Mussot Swertia

Mutable Magnolia*

Myrrh

Myrtleleaf Heimia

Nagai Podocarpus

Nagai Podocarpus Root

Nakai Podocarpus

Naked Leafyflower

Naked-caule Goldsaxifrage

Nakedstamen Monkshood

Nanchuan Bugbane*

Nandewa Cotton*

Nanling Hogfennel

Nanmu

Nantou Begonia*

Nar Uvaria*

Narrow Coriaria*

Narrow Gambirplant*

Narrow Rough Star Thistle*

Narrowbambooleaf Thorowax

Narrowbract Goldenray

Narrowflower Poisonnut

Narrowfruit Glycosmis Root*

Narrowfruit Hogfennel*

Narrowhead Goldenray

Narrowleaf Agave

Narrowleaf Alstonia*

Narrowleaf Angelica

Narrowleaf Cattail Pollen

Narrowleaf Dittary*

Narrowleaf Euphorbia

Narrowleaf Germander

Narrowleaf Kadsura*

Narrowleaf Lupin*

Narrowleaf Magnoliavine*

Narrowleaf Meadowrue
T1551 Cleome icosandra

T2423 Erigeron multiradiatus

T4008 Lytta caraganae

T1364 Chenopodium murale

T3766 Lepista nuda

T0393 Amberboa muricata

T2525 Eucheuma muricatum

T5131 Polyscias murrayi

T4682 Pedicularis muscicola

T5039 Podocarpus gracilior

T0291 Albizzia anthelmintica

T0211 Agaricus campestris

T0002 Abelmoschus moschatus [Syn. Hibiscus abelmoschus]

T1876 Cucumis melo

T0193 Adoxa moschatellina

T0066 Achillea moschata

T4334 Mussaenda hirsutissim

T6227 Swertia mussotii

T4043 Magnolia mutabilis

T1638 Commiphora myrrha [Syn. Commiphora molmol]

T3130 Heimia myrtifolia

T4346 Myrica nagi [Syn. Podocarpus nagi]

T4347 Myrica nagi [Syn. Podocarpus nagi]

T5047 Podocarpus nakaii

T0999 Bougainvillea glabra

T1406 Chrysosplenium nudicaule

T0099 Aconitum gymnandrum

T1423 Cimicifuga nanchuanensis

T3060 Gossypium sturtianum var. nandewarence

T4761 Peucedanum longshengens

T4818 Phoebe nanmu

T0891 Begonia nantoensis

T6666 Uvaria narum

T1688 Coriaria angustissima

T6606 Uncaria attenuata

T1302 Centaurea aspera subsp stenophylla

T1067 Bupleurum marginatum var. stenophyllum

T3807 Ligularia intermedia

T6166 Strychnos angustiflora

T3010 Glycosmis stenocarpa

T4772 Peucedanum stenocarpum

T3815 Ligularia stenocephala

T0217 Agave angustifolia

T0366 Alstonia angustifolia

T0476 Angelica anomala

T6585 Typha angustifolia

T2165 Dictamnus angustifolius

T2586 Euphorbia esula var. cyparissoides

T6366 Teucrium polium

T3612 Kadsura angustifolia

T3940 Lupinus angustifolius

T5794 Schisandra lancifolia

T6394 Thalictrum incidum
DUO RUI BAI HUA CAI

DUO SHE FEI PENG

QING NIANG ZI

BI SHENG LI

ZI DING XIANG MO

AN BEI JU

QI LIN CAI

MO LEI NAN YANG SHEN

XIAN SHENG MA XIAN HAO

XI LUO HAN SONG

QU CHONG HE HUAN

$\mathrm{MO} \mathrm{GU}$

HUANG KUI

GUA DI

WU FU HUA

SHE XIANG SHI CAO

YU YE JIN HUA

CHUAN XI ZHANG YA CAI

BIAN XING MU LAN

MO YAO

HUANG WEI

ZHU BAI

ZHU BAI GEN

TAI WAN LUO HAN SONG

GUANG YE ZI HUA

ZANG YAO LUO JING JIN YAO

LU RUI WU TOU

NAN CHUAN SHENG MA

NAN DE WA MIAN

NAN LING QIAN HU

NAN MU

NAN TOU QIU HAI TANG

NA ER ZI YU PAN

XIA MA SANG

XIA GOU TENG

XIA YE CU CAO SHI CHE JU ub ZHAI ZHU YE CHAI HU

XIA BAO TUO WU

NIU YAN MA QIAN

XIA GUO SHAN XIAO JU GEN

XIA GUO QIAN HU

ZHAI TOU TUO WU

DUAN YE LONG SHE LAN

XIA YE JI GU CHANG SHAN

XIA YE DANG GUI

XIA YE XIANG PU

XIA YE BAI XIAN

XI YE DA JI

HUI BAI SHI CAN

XIA XIE NAN WU WEI ZI

AI SAI E BI YA YU SHAN DOU

XIA YE WU WEI ZI

XIA YE TANG SONG CAO 
Narrowleaf Paris

Narrowleaf Rabdosia*

Narrowleaf Scabious

Narrowleaf Screwtree

Narrowleaf Senna Leaf

Narrowleaf Senna*

Narrowleaf Spicebush

Narrowleaf Swertia

Narrowleaf Vetch

Narrowraceme Meadowrue

Naudin Citrullus*

Needle-hear Fern*

Neem Tree

Negundo Chastetree Leaf

Negundo Chastetree Root

Negundo Chastetree Seed*

Nemoricolous Greenstar*

Nepal Camphortree

Nepal Cowparsnip

Nepal Cranesbill

Nepal Dock

Nepal Kobresia*

Nepal Meconopsis

Nepal Monkshood

Nepetaleaf Leontis

Nepeta-like Croton*

Nerrowleaf Peashrub

Nervate Twayblade

Nervous Brake

Netvein Goldenray

New Beauty Saussurea*

New Caledonian Guioa*

New Zealand Flax

New Zealand Kowhai

New Zealand liverwort

New Zealand liverwort Tylimanthus tenellus

New Zealand Podocarpus*

Newcaledonian Coelospermum*

Nguang-Chum; Duang-Sum (local names)

Nickernut Caesalpinia

Nicola Centaurea*

Nicotianflower Lobelia*

Niger Bean*

Nigerian Satinwood

Nightblooming Cestrum

Nightjasmine

Nigroline Garcinia*

Nikoo Maple

Nile Starbush*

Nilgiris Helicia

Nilotic Tamarisk*

Ningguo Fritillary

Ningpo Figwort
T4651 Paris polyphylla var. stenophylla

T3480 Isodon angustifolia

T5775 Scabiosa comosa

T3163 Helicteres angustifolia

T1230 Cassia angustifolia

T1237 Cassia leptophylla

T3847 Lindera angustifolia

T6212 Swertia angustifolia

T6743 Vicia angustifolia

T6374 Thalictrum atriplex

T1462 Citrullus naudinianus

T4026 Macrothelypteris oligophlebia

T0836 Azadiractica indica

T6788 Vitex negundo

T6787 Vitex negundo

T6789 Vitex negundo

T5067 Polyalthia nemoralis

T1440 Cinnamomum glanduliferum

T3219 Heracleum nepalense

T2943 Geranium nepalense

T5612 Rumex nepalensis

T3633 Kobresia nepalensis

T4144 Meconopsis nepaulensis

T0093 Aconitum ferox

T3744 Leonotis nepetaefolia

T1852 Croton nepetaefolius

T1192 Caragana stenophylla

T3863 Liparis nervosa

T5288 Pteris cretica var. nervosa [Syn. Pteris nervosa]

T3803 Ligularia dictyoneura [Syn. Senecio dictyoneurus]

T5761 Saussurea neopulchella

T3072 Guioa crenulata

T4825 Phormium tenax

T6044 Sophora tetraptera

T3760 Lepidolaena taylorii

T6576 Tylimanthus tenellus

T5038 Podocarpus ferrugineu

T1607 Coelospermum billardieri

T2954 Getonia floribunda

T1100 Caesalpinia crista

T1306 Centaurea nicolai

T3901 Lobelia nicotianaefolia

T3647 Lablab niger

T2239 Distemonanthus benthamianus

T1338 Cestrum nocturnum

T4453 Nyctanthes arbor-tristis

T2868 Garcinia nigrolineata

T0050 Acer nikoense

T6570 Turraea nilotica

T3162 Helicia nilagirica

T6289 Tamarix nilotica

T2789 Fritillaria ningguoensis

T5828 Scrophularia ningpoensis
XIA YE CHONG LOU

XIA YE XIANG CHA CAI

MENG GU SHAN LUO BO

SHAN ZHI MA

FAN XIE YE

XIA YE JUE MING

XIA YE SHAN HU JIAO

XIA YE ZHANG YA CAI

ZHAI YE YE WAN DOU

XIA XU TANG SONG CAO

NA SHI XI GUA

JIN JI WEI BA CAO GEN

YIN DU LIAN

HUANG JING YE

HUANG JING GEN

HUANG JING ZHONG ZI

LING SHUI AN LUO

YUN NAN ZHANG

NI BO ER DU HUO

NI BO ER LAO GUAN CAO

NI BO ER YANG TI

NI BO ER SONG CAO

NI BO ER LV RONG HAO

NI BO ER WU TOU

JING JIE YE SHI ER CAO

SI JING JIE BA DOU

XIA YE JIN JI ER

JIAN XUE QING

FENG WEI JUE

WANG MAI TOU WU

XIN MEI FENG MAO JU

XIN XI LAN MA

SI CHI HUAI

XIN XI LAN LUO HAN SONG

XIN SU GE LAN XUE GUO MU

CI GUO SU MU

NI GU LA SHI CHE JU

YAN CAO HUA SHAN GENG CAI

BIAN DOU

NI RI LI YA LIANG RUI SU MU

YE XIANG SHU

YE HUA

HEI XIAN TIAO TENG HUANG

MAO GUO QI

NI LUO HE JIN YIN LIAN

SHEN LU SHAN LONG YAN

NI LUO HE CHENG LIU

NING GUO BEI MU

XUAN SHEN 
Ningxia Fritillary

Nippon Hawthorn

Nippon Yam

Nirur Leafflower*

Nitidleaf Croton

Nitidleaf Senna*

Nitraria*

Niu-Chang chih; Jang Jy (in Taiwan)

Noble Arnebia*

Noble Artocarpus*

Noble Dendrobium

Node Elephantfoot*

Node Ferulago*

Non-cirrose Citrullus*

Non-stipe Eucalyptus*

North Sea Bryozoan

North Viet-Nam Gambirplant

Northeast Clubmos*

Northeast Dim-flower Carpesium*

North-east Polypody*

Northeast Seriphidium

Northeast Spineginseng*, Tall Oplopanax

Northeast Walnut*

Northern Angelica*

Northern Catalpa

Northern Dutchmanspipe

Northern Dutchmanspipe Root

Northern Monkshood*

Northern Pitch Pine

Northern Wolfberry Root-bark*

Northsea Angelica*

Norway Maple

Novel-Holland Pepper*

Nude Fern

Nude-stem Eargrass*

Nudicaulous Grounsel Herb

Nummulite Rabdosia*

Nutant Aussiepoplar*

Nutgrass Galingale

Nut-vomitive Poisonnut

Nyctaginiflower Petunia*

Oak-of-Cappadocia

Oat

Oblique Fuscoporia*

Oblique Pinna Brake

Oblong-leaf Borneol Oil-Resin*

Oblongleaf Milkvetch*

Oblong-leaved Barberry

Obovaleaf Dandelion Root

Obovate Peony

Obovateleaf Lemmaphyllum*

Obscure Devilpepper*
T2795 Fritillaria taipaiensis var. ningxiaensis

T1769 Crataegus cuneata

T2203 Dioscorea nipponica

T4840 Phyllanthus niruri

T1853 Croton oblongifolius [Syn. Croton laevigatus]

T1236 Cassia laevigata [Syn. Cassia floribunda]

T4436 Nitraria tangutorum

T0538 Antrodia camphorata

T0650 Arnebia nobilis

T0718 Artocarpus nobilis

T2107 Dendrobium nobile

T2335 Elephantus nudatus

T2713 Ferulago nodosa

T1461 Citrullus ecirrhosus

T2500 Eucalyptus apodophylla

T2742 Flustra foliacea

T6618 Uncaria homomalla [Syn. Uruparia homomalla; Uruparia

tonkinensis; Uruparia lanosa var. parvifora]

T3293 Huperzia miyoshiana

T1214 Carpesium triste var. manshuricum

T5126 Polypodium virginianum

T5921 Seriphidium finitum [Syn. Artemisia finita]

T4516 Oplopanax elatus

T3565 Juglans mandshurica var. sieboldiana

T0499 Angelica ursina

T1265 Catalpa speciosa

T0624 Aristolochia contorta

T0625 Aristolochia contorta

T0129 Aconitum septentrionale

T4922 Pinus rigida

T3959 Lycium chinense var. potaninii

T0475 Angelica acutiloba var. sugiyamae

T0052 Acer platanoides

T4958 Piper nove-hollandae

T5269 Psilotum nudum

T3127 Hedyotis nudicaulis

T5893 Senecio nudicaulis

T5014 Plectranthus nummularius

T3274 Homalanthus nutans

T1978 Cyperus rotundus

T6184 Strychnos nux-vomica

T4752 Petunia nyctaginiflora

T0403 Ambrosia maritima

T0833 Avena sativa

T2818 Fuscoporia obliqua

T5296 Pteris oshimensis

T2276 Dryobalanops oblongifolia

T0799 Astragalus miser var. oblongifolia

T0910 Berberis oblonga

T6302 Taraxacum obovatum

T4586 Paeonia obovata

T3737 Lemmaphyllum microphyllum var. obovatum

T5439 Rauwolfia obscura
NING XIA BEI MU

YE SHAN ZHA

CHUAN LONG SHU YU

ZHU ZI CAO

GUANG YE BA DOU

GUANG YE JUE MING

BAI CI

GAO GUI JIA ZI CAO

GAO GUI BO LUO MI

SHI HU

LUO DI DAN CAO

JIE JIE LEI A WEI

WU JUAN XU XI GUA

WU BING YE AN

BEI HAI XIAN TAI CHONG

BEI YUE GOU TENG

DONG BEI SHI SHAN

DONG BEI AN HUA JIN WA ER

DONG BEI DUO ZU JUE

DONG BEI HUI HAO

DONG BEI CI REN SHEN

DONG BEI HU TAO

BEI FANG DANG GUI

HUANG JIN SHU

BEI MA DOU LING

BEI MA DOU LING GEN

BEI FANG WU TOU

GANG SONG

BEI FANG GOU QI GEN PI

BEI HAI DANG GUI

NUO WEI QI

XIN HE LAN HU JIAO

SHI SHUA BA

LUO JING ER CAO

ZI BEI TIAN KUI CAO

YUAN BAN XIANG CHA CAI

XIA CHUI AO YANG

XIANG FU

MA QIAN ZI

ZI MO LI HUA BI DONG QIE

YAN HAI TUN CAO

YAN MAI

HUA HE KONG JUN

XIE YU FENG WEI JUE

JU YUAN YE LONG NAO XIANG

JU YUAN YE HUANG QI

CHANG YUAN YE XIAO BO

DAO LUAN YE PU GONG YING GEN

CAO SHAO YAO

DAO LUAN YE FU SHI JUE

GANG GUO LUO FU MU 
Obtuse Cryptolepis*

Obtuse Eucalyptus Leaf*

Obtuse Frangipani*

Obtuseleaf Achyranthes

Obtuseleaf Cassia Bark, Wild Cinnamon

Bark

Obtuseleaf Erycibe

Obtuseleaf Euphorbia Latex*

Obtuseleaf Euphorbia*

Obtuseleaf Senna*

Obtuselobed Anemone

Occidental Cocklebur*

Occidental Larkspur*

Ochotsk Corydalis

Octapistil Pokeweed*

Octet Wampee*

Odollam Cerberustree*

Odorate Rosewood

Odorous Hellebore*

Officinal Asparagus

Officinal Dandelion

Officinal Ervatamia*

Officinal Evodia

Officinal Magnolia

Officinal Primrose*

Oilpalm

Oiltea Camellia

Oiltea Camellia Root-bark

Oily Daphne*

Oily-leaf Milkvetch*

Okamoto Maple

Okinawan Softcoral Clavularia viridis

Oldham Bamboo Shoot

Oldham Fissistigma

Oldham Gypsophila

Oldworld Arrowhead Corm

Oleanderleaf Allemanda

Olga Gentian*

Olga Heliotrope*

Olibanum

Olive

Olive Ninenode*

Olive-green White Quebracho*

Oliver Plumyew

Oliver Rosewood*

Olivier Gentian*

Ombutree Pokeberry

Omei Kudzuvine

Omei Meadowrue

Omei Mountain Goldthread

Omoto Nipponlily Root

Omphalo-fruit Common Jasminorange*

Omphalo-fruit Jasminorange*
T1865 Cryptolepis obtusa

T2503 Eucalyptus camaldulensis var. obtusa

T5031 Plumeria obtusa

T0072 Achyranthes aspera var. indica

T1434 Cinnamomum bejolghota [Syn. Cinnamomum obtusifolium;

Laurus bejolghota]

T2449 Erycibe obtusifolia

T2604 Euphorbia obtusifoli

T2605 Euphorbia obtusifolia var. obtusifolia

T1240 Cassia obtusifolia

T0469 Anemone obtusiloba

T6839 Xanthium occidentale

T2080 Delphinium occidentale

T1728 Corydalis ochotensis

T4866 Phytolacca octandra

T1532 Clausena anisata

T1331 Cerbera odollam

T2008 Dalbergia odorifera

T3184 Helleborus odorus

T0752 Asparagus officinalis

T6303 Taraxacum officinale

T2445 Ervatamia officinalis

T2646 Evodia rutaecarpa var. officinalis

T4045 Magnolia officinalis

T5204 Primula veris [Syn. Primula officinalis]

T2328 Elaeis guineensis

T1146 Camellia oleifera

T1147 Camellia oleifera

T2029 Daphne oleoides

T0801 Astragalus oleifolius

T0051 Acer okamotoanum

T1542 Clavularia viridis

T5961 Sinocalamus oldhami

T2735 Fissistigma oldhamii [Syn. Melodorum oldhamii]

T3090 Gypsophila oldhamiana

T5647 Sagittaria sagittifolia

T0308 Allemanda neriifolia

T2923 Gentiana olgae

T3176 Heliotropium olgae

T0994 Boswellia carterii

T1165 Canarium album

T5277 Psychotria oleoides

T0770 Aspidosperma olivaceum

T1322 Cephalotaxus oliveri

T2009 Dalbergia oliveri

T2924 Gentiana olivieri

T4862 Phytolacca dioica

T5316 Pueraria omeiensis

T6402 Thalictrum omeiense

T1669 Coptis omeiensis

T5557 Rohdea japonica [Syn. Orontium japonicum]

T4322 Murraya omphalocarpa

T4326 Murraya paniculata var. omphalocarpa
DUN XING BAI YE TENG

DUN XING CHI AN YE

DUN XING JI DAN HUA

DUN YE TU NIU XI

DUN YE GUI PI

DING GONG TENG

DUN YE DA JI XIANG JIANG

DUN YE DA JI

DUN YE JUE MING

DUN LIE YIN LIAN HUA

XI FANG CANG ER

XI FANG CUI QUE

HUANG ZI JIN

AO ZHOU SHANG LU

BA JIAO HUANG PI

AO DAO LA MU HAI MANG GUO

JIANG ZHEN XIANG

XIANG TIE KUAI ZI

XIAO BAI BU

YAO YONG PU GONG YING

YAO YONG GOU YA HUA

SHI $\mathrm{HU}^{(3)}$

HOU PO

HUANG HUA JIU LUN CAO

YOU ZONG

CHA ZI XIN

YOU CHA GEN PI

YOU RUI XIANG

YOU YE HUANG QI

CHAO XIAN WU JIAO FENG

CHONG SHENG RUAN SHAN HU

LV SUN PIAN

GUA FU MU

XIA CAO

CI GU

HUANG CHAN

AO LIE GE LONG DAN

AO ER JIA TIAN JIE CAI

RU XIANG

QING GUO

YOU GAN LAN JIU JIE

HE LU BAI JIAN MU

BI ZI CU FEI

AO LI FO HUANG TAN

AO SHI LONG DAN

A GEN TING SHANG LU

E MEI GE

E MEI TANG SONG CAO

E MEI YE HUANG LIAN

WAN NIAN QING GEN

QI GUO JIU LI XIANG

QI GUO QIAN LI XIANG 
Omphalos Parmelia*

One Sided Racemes Leucothoe

Oneseed Ephedra

Onychium auratum

Oolong Tea

Opium

Opium Poppy

Opium Poppy Pericarp

Opposite Poisonnut*

Oppositeleaf Fig

Oppositeleaf Goldsaxifrage*

Oppositeleaf Rhodiola

Orange Daylily

Orange Lycoris

Orange Magnoliavine

Orange Monkeyflower*

Orange Mullein

Orange Poppy*

Orange Stonecrop

Orange-ball-tree

Orangeeye Butterflybush

Oregon Alder

Oregon Bleedingheart

Oregon-grape

Oreintal Consolida*

Oriental Bittersweet

Oriental Bittersweet Fruit

Oriental Bittersweet Leaf

Oriental Bittersweet Root

Oriental Blechnum Frond

Oriental Blueberry Fruit

Oriental Buckthorn

Oriental Buckthorn

Oriental Chain Fern

Oriental Cocklebur*

Oriental Ervatamia*

Oriental Fatheadtree*

Oriental Foxglove*

Oriental Gambirplant*

Oriental Germander

Oriental Henbane*

Oriental Ostrich Fern

Oriental Poppy

Oriental Sesame (black seed)

Oriental Sesame (white seed)

Oriental Sesame Leaf

Oriental Sesame Root

Oriental Stephania

Oriental Sweetgum Resin

Oriental Variegated Coralbean Bark

Oriental Waterplantain

Oriental Vismia*

Orientvine
T4657 Parmelia saxatilis var. omphalodes

T3784 Leucothoe grayana

T2375 Ephedra monosperma

T4501 Onychium auratum

T1155 Camellia sinensis var. viridis

T4635 Papaver somniferum

T4636 Papaver somniferum

T4637 Papaver somniferum

T6169 Strychnos decussata

T2720 Ficus hispida

T1407 Chrysosplenium oppositifolium

T5500 Rhodiola subopposita

T3193 Hemerocallis fulva

T3987 Lycoris sanguinea

T5802 Schisandra sphenanthera

T4251 Mimulus aurantiacus

T6702 Verbascum phlomoides

T4622 Papaver auranticum

T5855 Sedum kamtschaticum

T1047 Buddleja globosa

T1046 Buddleja davidii

T0329 Alnus oregana

T2151 Dicentra oregana

T4054 Mahonia aquifolium

T1647 Consolida orientalis

T1288 Celastrus orbiculatus [Syn. Celastrus articulatus]

T1291 Celastrus orbiculatus [Syn. Celastrus articulatus]

T1290 Celastrus orbiculatus [Syn. Celastrus articulatus]

T1289 Celastrus orbiculatus [Syn. Celastrus articulatus]

T0952 Blechnum orientale

T6670 Vaccinium bracteatum

T5455 Rhamnus crenata

T5456 Rhamnus crenatus

T6831 Woodwardia orientalis

T6840 Xanthium orientale

T2446 Ervatamia orientalis

T4392 Nauclea orientalis

T2176 Digitalis orientalis

T6627 Uncaria orientalis

T6364 Teucrium orientale

T3330 Hyoscyamus orientalis

T4126 Matteuccia orientalis

T4632 Papaver orientale

T5927 Sesamum indicum [Syn. Sesamum orientale]

T5925 Sesamum indicum [Syn. Sesamum orientale]

T5924 Sesamum indicum

T5923 Sesamum indicum

T6119 Stephania cepharantha

T3868 Liquidambar orientalis

T2479 Erythrina variegata var. orientalis

T0303 Alisma orientale [Syn. Alisma plantago-aquatica var. orientale]

$\mathrm{T} 6780$ Vismia orientalis

T5964 Sinomenium acutum
QI SHI HUA

MU LI LU

DAN ZI MA HUANG

WU LONG CHA

YA PIAN

YING SU

YING SU KE

DUI SHENG MA QIAN

DUI YE RONG

DUI YE JIN YAO

HU SHENG HONG JING TIAN

XUAN CAO GEN

TIE SE JIAN

HUA ZHONG WU WEI ZI

JU SE GOU SUAN JIANG

JU SE MAO RUI HUA

JU HUANG YING SU

HENG GEN FEI CAI

QIU HUA ZUI YU CAO

DA YE ZUI YU CAO

AO LEI TONG QI MU

E LE GANG HE BAO MU DAN

JIAN YE SHI DA GONG LAO

DONG FANG FEI YAN CAO

NAN SHE TENG

NAN SHE TENG GUO

NAN SHE TENG YE

NAN SHE TENG GEN

WU MAO JUE

NAN ZHU ZHI

LI LA GEN

HUANG YAO

DONG FANG GOU JI

DONG FANG CANG ER

DONG FANG GOU YA HUA

DONG FANG WU TAN

DONG FANG YANG DI HUANG

DONG FANG GOU TENG

DONG FANG XIANG KE KE

DONG FANG TIAN XIAN ZI

DONG FANG JIA GUO JUE

JIN DONG YING SU

HEI ZHI MA

BAI ZHI MA

HU MA YE

HU MA GEN

BAI YAO ZI

SU HE XIANG

HAI TONG PI

ZE XIE

DONG FANG WEI SI MU QING FENG TENG 
Orris

Osage Orange

Oshiroishimeji (in Japanese)

Osmarien Broom*

Osun Padauk*

Oswegotea

Otoba Nutmeg*

Oval Kumquat

Oval Kumquat Leaf

Oval Periwinkle*

Ovate Catalpa

Ovate Catalpa Bast

Ovate Catalpa Fruit

Ovate Catalpa Leaf

Ovateleaf Anemone

Ovateleaf Garcinia*

Ovateleaf Heliotrope*

Ovateleaf Holly

Ovateleaf Knotweed

Ovateleaf Mayten*

Ovateleaf Pepper

Ovateleaf Rhododendron*

Ovateleaf Tylophora

Ovate-leafThreevein Aster*

Ovatepetal Sandwort

Ox Blood

Ox Brain

Ox Gall

Ox Kidney

Ox Liver

Ox Lung

Ox Thyroid

Oxford Ragwort

Oxhide Gelatin

Oxlip

Oxtail Greenbrier

Oyama Magnolia

Oyster

Oyster Meat

Pacific Gypsophila

Pacific Wood Fern

Pacific Yew

Paene Milkwort*

Painted Fern

Pale Bittersweet

Pale Butterflybush

Pale Corydalis

Pale Persicaria

Pale Russula*

Pale Treebine*

Palmerston Pitch Tree*

Panamanian Albizia*
T3458 Iris florentina

T4022 Maclura pomifera

T3993 Lyophyllum connatum

T1990 Cytisus osmariensis

T5304 Pterocarpus osun

T4269 Monarda didyma

T4353 Myristica otoba

T2760 Fortunella margarita

T2761 Fortunella margarita

T1269 Catharanthus ovalis

T1262 Catalpa ovata

T1261 Catalpa ovata

T1263 Catalpa ovata

T1264 Catalpa ovata

T0465 Anemone begoniifolia

T2869 Garcinia ovalifolia

T3177 Heliotropium ovalifolium

T3398 Ilex rotunda

T5118 Polygonum suffultum

T4138 Maytenus ovatus

T4933 Piper attenuatum

T5520 Rhododendron ovatum [Syn. Rhododendron lamprophyllum;

Azalea ovata ]

T6582 Tylophora ovata

T0777 Aster ageratoides var. ovatus

T0609 Arenaria kansuensis var. ovatipeatala

T0991 Bos taurus domesticus; Bubalus bubalis

T0988 Bos taurus domesticus; Bubalus bubalis

T0984 Bos taurus domesticus; Bubalus bubalis

T0990 Bos taurus domesticus; Bubalus bubalis

T0986 Bos taurus domesticus; Bubalus bubalis

T0985 Bos taurus domesticus; Bubalus bubalis

T0992 Bos taurus domesticus; Bubalus bubalis

T5912 Senecio squalidus

T0982 Bos taurus domesticus

T5198 Primula elatior

T5981 Smilax riparia

T4050 Magnolia sieboldii

T1768 Crassostrea gigas

T4555 Ostrea rivularis; Ostrea talienwhanensis; Ostrea gigas

T3091 Gypsophila pacifica

T2285 Dryopteris pacifica

T6308 Taxus brevifolia

T5079 Polygala paenea

T0818 Athyrium filix-femina

T1287 Celastrus hypoleucus

T1048 Buddleja officinalis

$\mathrm{T} 1740$ Corydalis sempervirens

T5109 Polygonum nodosum

T5622 Russula ochroleuca

T1454 Cissus pallida

T0214 Agathis palmerstoni

T0290 Albizzia adinocephala
XI OU YUAN WEI

SANG CHENG

AO MA JIN QUE HUA

E SUN ZI TAN

MEI GUO BO HE

AO TUO ROU DOU KOU

JIN JU

JIN JU YE

LUAN YUAN CHANG CHUN HUA

ZI MU

ZI BAI PI

ZI SHI

ZI YE

LUAN YE YIN LIAN HUA

LUAN YE TENG HUANG

LUAN YE TIAN JIE CAI

JIU BI YING

HONG SAN QI

LUAN YE MEI DENG MU

LUAN YE HU JIAO

MA YIN HUA

LUAN YE WA ER TENG

LUAN YE SAN ZHE MAI ZI WAN

LUAN BAN ZAO ZHUI

NIU XUE

NIU NAO

NIU DAN

NIU SHEN

NIU GAN

NIU FEI

NIU YE

NIU JIN QIAN LI GUANG

HUANG MING JIAO

GAO BAO CHUN

NIU WEI CAI

TIAN NV MU LAN

MU LI

MU LI ROU

SHAN YIN CHAI HU

TAI PING YANG LIN MAO JUE

DUAN YE HONG DOU SHAN

PEI NI YUAN ZHI

TI GAI JUE

MIAN TENG

MI MENG HUA

CANG BAI ZI JIN

JIE LIAO

HUANG BAI HONG GU

CANG BAI FEN TENG

PA SHI BEI KE SHAN

BA NA MA HE HUAN 
Pangolin

Paniculate Bolbostemma

Paniculate Crotalaria*

Paniculate Goldraintree Flower

Paniculate Goldraintree Root-bark

Paniculate Hydrangea

Paniculate Microcos

Paniculate Onosma

Paniculate Ostodes*

Paniculate Spotflower

Paniculate Swallowwort

Paniculed Bittersweet Seed

Panniform Pyrrosia

Paohsing Euonymus

Paoshan Monkshood

Papaya Fruit

Papaya Leaf

Paper Reed

Paper-white Narcissus

Papillose Box*

Papua-New-Guinea Persimmon*

Para Rubbertree

Paradoxy Grape-hyacinth*

Paraguay Tea

Parasite Scurrula

Parchment-like Milky*

Pardleather-like Mushroom

Parisshape Loosestrife

Parker Raspberry

Parmelia Lichen

Parqui Cestrum

Parry Northern Mahonia

Parvivesiculose Gulfweed*

Pashi Pear Fruit

Pasific Mikania*

Paspalum Ergot*

Passionflower

Passionfruit

Pasture Gentian*

Patentflower Monkshood

Patience Dock

Paucivitat Cowparsnip

Pawpaw

Pax Ash

Peach

Peach Bast

Peach Flower

Peach Fritillary*

Peach Juvenile Branch

Peach Kernel

Peach Leaf
T4106 Manis pentadactyla

T0971 Bolbostemma paniculatum

T1828 Crotalaria paniculata

T3635 Koelreuteria paniculata

T3636 Koelreuteria paniculata

T3306 Hydrangea paniculata

T4217 Microcos paniculata [Syn. Grewia microcos]

T4500 Onosma paniculatum

T4554 Ostodes paniculata

T6075 Spilanthes acmella

T1959 Cynanchum paniculatum

T1292 Celastrus paniculatus

T5355 Pyrrosia drakeana

T2543 Euonymus mupinensis

T0080 Aconitum bullatifolium var. homotrichum [Syn. Aconitum

nagarum ]

T1205 Carica papaya

T1206 Carica papaya

T1977 Cyperus papyrus

T4377 Narcissus papyraceus

T1093 Buxus papillosa

T2223 Diospyros mafiensis

T3236 Hevea brasiliensis

T4333 Muscari paradoxum

T3394 Ilex paraguariensis

T3925 Loranthus parasiticus [Syn. Loranthus chinenis; Taxillus

chinensis]

T3652 Lactarius pergamenus

T3743 Lentinus lepideus

T4004 Lysimachia paridiformis

T5594 Rubus parkeri

T4656 Parmelia saxatilis

T1339 Cestrum parqui

T4059 Mahonia borealis

T5739 Sargassum parvivesiculosum

T5365 Pyrus pashia

T4229 Mikania mendocina

T1540 Claviceps paspali

T4663 Passiflora caerulea

T4665 Passiflora edulis

T2920 Gentiana makinoi

T0086 Aconitum chasmanthum

T5614 Rumex patientia

T3218 Heracleum moellendorffii var. paucivitatum

T0744 Asimina triloba

T2774 Fraxinus paxiana

T5229 Prunus persica

T5232 Prunus persica

T5231 Prunus persica

T2791 Fritillaria persica

T5235 Prunus persica

T5233 Prunus persica

T5234 Prunus persica
CHUAN SHAN JIA

JIA BEI MU

YUAN ZHUI ZHU SHI DOU

LUAN HUA

LUAN SHU

FEN TUAN HUA

PO BU YE

DIAN ZI CAO

YUAN ZHUI HUA YE LUN MU

TIAN WEN CAO

XU CHANG QING

DENG YOU TENG ZI

ZHAN MAO SHI WEI

BAO XING WEI MAO

BAO SHAN WU TOU

FAN MU GUA

FAN MU GUA YE

ZHI SHA CAO

BAI SHUI XIAN

DUO RU TOU HUANG YANG

BA BU YA XIN JI NEI YA SHI

XIANG JIAO SHU

QI YI PU TAO FENG XIN ZI

BA LA GUI CHA

SANG JI SHENG

SI YANG PI ZHI RU GU

BAO PI GU

CHONG LOU PAI CAO

WU PAO ZI

SHI HUA

PA KE YE XIANG SHU

PA LI BEI FANG SHI DA GONG LAO

XI NANG MA WEI ZAO

CHUAN LI GUO

TAI PING YANG JIA ZE LAN

QUE BAI MAI JIAO

XI FAN LIAN

JI DAN GUO

MU YE LONG DAN

ZHAN HUA WU TOU

NIU XI XI

ZOU MA QIN

PAO PAO SHU

QIN LING BAI LA SHU

TAO

TAO JING BAI PI

TAO HUA

TAO BEI MU

TAO ZHI

TAO REN

TAO YE 
Peach Root

Peachform Mango

Peachliking Pumpkin

Peanut

Peanut Branch-leaf

Peanut Oil

Pear Aglaia*

Pearleaf Microglossa Root

Pear-like Puff-ball

Pearlwort

Peashrub*

Pecan

Pectinat Bushmint*

Pectinate Speedwell*

Pedate Pinellia

Pedicellate Gentian

Pedunculate Acronychia

Peking Euphorbia

Peking Oak

Peking Pyrrosia Frond

Peltate Milkwort*

Peltate Sundew*

Peltate Yam

Peltateleaf Meadowrue

Peltateleaf Pepper*

Pendentseed Jointfir

Pendulous Eucalyptus Leaf

Pendulous Monkshood

Pengxian Hemsleya

Penicillium verruculosum

Pennsylvanian Cocklebur*

Pennyroyal Mint

Penta-androus Caltrap*

Pentagonous Ardisia

Pentagynous Dillenia

Pentland Mandevilla*

Pentopetia androsaernifolia

Pepper Mint

Pepper-tree

Peppertree Pricklyash

Pepperweed Seed

Peppery Milky

Perak Devilpepper

Pere L. F. Faurie's Rhododendron

Peregrin Bleedingheart*

Peregrine Hoarhound

Perennial Ragweed

Perfoliate Knotweed Root

Perforated Haplophyllum

Perforated Harrisonia

Pericarp

Perry Aloe*
T5230 Prunus persica

T4103 Mangifera persiciformis

T1883 Cucurbita pepo var. akoda

T0559 Arachis hypogaea

T0561 Arachis hypogaea

T0560 Arachis hypogaea

T0242 Aglaia pirifera

T4218 Microglossa pyrifolia

T3961 Lycoperdon pyriforme

T5646 Sagina japonica [Syn. Spergula japonica]

T1188 Caragana chamlagu

T1218 Carya illinoensis

T3380 Hyptis pectinata

T6726 Veronica pectinata var. glandulosa

T4903 Pinellia pedatisecta

T2925 Gentiana pedicellata

T0151 Acronychia pedunculata

T2608 Euphorbia pekinensis

T5371 Quercus aliena

T5354 Pyrrosia davidii

T5080 Polygala peltatum

T2266 Drosera peltata

T2213 Dioscorea zingiberensis

T6393 Thalictrum ichangense [Syn. Thalictrum tripeltatum;

Thalictrum multipeltatum]

T4960 Piper peltatum

T3034 Gnetum pendulum

T2504 Eucalyptus camaldulensis var. pendula

T0122 Aconitum pendulum

T3209 Hemsleya pengxianensis

T4701 Penicillium verruculosum

T6841 Xanthium pennsylvanicum

T4187 Mentha pulegium

T6496 Tribulus pentandrus

T0602 Ardisia quinquegona

T2182 Dillenia pentagyna

T4098 Mandevilla pentlandiana

T4702 Pentopetia androsaernifolia

T4186 Mentha piperita

T4025 Macropiper excelsum

T6892 Zanthoxylum schinifolium

T3755 Lepidium apetalum [Syn. Lepidium micranthum]

T3653 Lactarius piperatus [Syn. Agaricus piperatus]

T5441 Rauwolfia perakensis

T5509 Rhododendron fauriei

T2152 Dicentra peregrina

T4111 Marrubium peregrinum

T0405 Ambrosia psilostachya

T5111 Polygonum perfoliatum

T3104 Haplophyllum perforatum

T3109 Harrisonia perforata

T1488 Citrus junos

T0342 Aloe perryi
TAO GEN

BIAN TAO

TAO NAN GUA

LUO HUA SHENG

LUO HUA SHENG ZHI YE

LUO HUA SHENG YOU

LI MI ZI LAN

XIAO SHE JU GEN

LI XING MA BO

QI GU CAO

JIN JI ER

MEI GUO SHAN HE TAO

ZHI SHAN XIANG

SHU CHI PO PO NA

ZHANG YE BAN XIA

HUA GENG LONG DAN

SHA TANG MU

DA JI ${ }^{(3)}$

HU LI

BEI JING SHI WEI

ZU YE CAO

DUN ZHUANG MAO GAO CAI

DUN YE SHU YU

DUN YE TANG SONG CAO

DUN YE HU JIAO

CHUI ZI MAI MA TENG

CHUI ZHI CHI AN YE

TIE BANG CHUI

PENG XIAN XUE DAN

Mold

BIN XI FA NI YA CANG ER

CHUN E BO HE

WU XIONG RUI JI LI

LUO SAN SHU

XIAO HUA WU YA GUO

PENG TE MAN DE MU

PEN TUO PO TI CAO

LA BO HE

GAO DA HU JIAO

QING JIAO

TING LI ZI

LA RU GU

PI LI LUO FU MU

FU LEI SHI DU JUAN HUA

YI YANG HE BAO MU DAN

YANG OU XIA ZHI CAO

LUO SUI TUN CAO

GANG BAN GUI GEN

DA YE YUN XIANG CAO

NIU JIN GUO

CHENG ZI PI

PEI LI LU HUI 


\begin{tabular}{|c|}
\hline Persia Giantfennel Variety* \\
\hline Persia Motherwort* \\
\hline Persia Poppy \\
\hline Persimmon \\
\hline Persimmon Leaf \\
\hline Persimmon Persistent Caly \\
\hline Persimmon Root \\
\hline Personator Padritree* \\
\hline Pertusate Ulva Frond \\
\hline Peru Balmtree Resin \\
\hline Peru Coca Shrub \\
\hline Peru False Heath \\
\hline Peru Squill* \\
\hline Peruvian Groundcherry \\
\hline Petalformed Meadowrue \\
\hline Petioled Pyrrosia Frond \\
\hline Petrofracted Pepper \\
\hline Petty Euphorbia \\
\hline Peyote \\
\hline Phellandral Eucalyptus* \\
\hline Phellinus igniarius \\
\hline Philadelphia Fleabane \\
\hline Philadelphia Groundcherry* \\
\hline Philippine Bushbeech* \\
\hline Philippine Flemingia \\
\hline Philippine Flemingia \\
\hline Philippine Groundsel* \\
\hline Philippine Maidenhair* \\
\hline Philippine Mallotus* \\
\hline Philippine Nettletree \\
\hline Philippine Podocarpus \\
\hline Phoenix Date \\
\hline Phoenix Tree Bast \\
\hline Phoenix Tree Leaf \\
\hline Phoenix Tree Seed \\
\hline Picrorhiza \\
\hline Picture Houndstongue* \\
\hline Picture Kalopanax* \\
\hline Pig Gall \\
\hline Pigeon Vetch \\
\hline Pilose Asiabell \\
\hline Pilose Curculigo* \\
\hline Pilose Gerbera \\
\hline Pilose Germander \\
\hline Pilose Purslane* \\
\hline Piloseleaf Desmos Root \\
\hline Pilosity Peavine* \\
\hline Pilular Adina \\
\hline Pine Mushroom \\
\hline Pineapple \\
\hline Pineapple Guava \\
\hline Pineleaf Groundsel \\
\hline inewoods Coneflow \\
\hline
\end{tabular}

T2704 Ferula persica var. latisecta

T3753 Leonurus persicus

T4633 Papaver persicum

T2221 Diospyros kaki

T2220 Diospyros kaki

T2217 Diospyros kaki

T2218 Diospyros kaki

T6142 Stereospermum personatum

T6600 Ulva pertusa

T4358 Myroxylon pereirae

T2492 Erythroxylum novogranatense

T2652 Fabiana imbricata

T5812 Scilla peruviana

T4852 Physalis peruviana

T6403 Thalictrum petaloideum

T5358 Pyrrosia petiolosa

T4964 Piper retrofractum

T2609 Euphorbia peplus

T3923 Lophophora williamsii

T2515 Eucalyptus phellandra

T4787 Phellinus igniarius

T2424 Erigeron philadelphicus

T4853 Physalis philadelphica

T3025 Gmelina philippensis

T2737 Flemingia philippinensis [Syn. Moghania philippinensis]

T4260 Moghania philippinensis

T5896 Senecio phillipicus

T0173 Adiantum lunulatum

T4083 Mallotus philippinensis

T1298 Celtis philippinensis

T5051 Podocarpus philippinensis

T4820 Phoenix dactylifera

T2731 Firmiana simplex

T2732 Firmiana simplex

T2733 Firmiana simplex

T4887 Picrorhiza kurrooa

T1970 Cynoglossum pictum

T3624 Kalopanax pictum

T6206 Sus scrofa domestica

T6749 Vicia hirsuta

T1599 Codonopsis pilosula

T1902 Curculigo pilosa

T2952 Gerbera piloselloides

T6365 Teucrium pilosum [Syn. Teucrium japonicum var. pilosum]

T5174 Portulaca pilosa

T2137 Desmos dumosus

T3708 Lathyrus palustris var. pilosus

T0180 Adina pilulifera [Syn. Cephalanthus pilulifera]

T6504 Tricholoma matsutake [Syn. Armillaria matsutake]

T0444 Ananas comosus

T2693 Feijoa sellowiana

T5874 Senecio abrotanifolius

T5603 Rudbeckia bicolor
BO SI A WEI BIAN ZHONG

BO SI YI MU CAO

BO SI YING SU

SHI ZI

SHI YE

SHI DI

SHI GEN

JIA MIAN YU YE QIU

KONG SHI CHUN

BI LU XIANG JIAO

BI LU GU KE

PI QI QIE

BI LU MIAN ZAO ER

DENG LONG CAO

BAN RUI TANG SONG CAO

YOU BING SHI WEI

CHANG GUO BI BA

BO AI DA JI

AN LU LONG SHE LAN

SHUI HUI XIANG AN

SANG HUANG

FEI CHENG FEI PENG

FEI CHENG SUAN JIANG

FEI LV BIN SHI ZI

MAN XING QIAN JIN BA

FEI LV BIN QIAN JIN BA

FEI LV BIN QIAN LI GUANG

BAN YUE XING TIE XIAN JUE

CU KANG CHAI

FEI LV BIN PIAO SHU

FEI LV BIN LUO HAN SONG

WU LOU ZI

WU TONG BAI PI

WU TONG YE

WU TONG ZI

HU HUANG LIAN

ZHUO SE LIU LI CAO

ZHUO SE CI QIU

ZHU DAN

XIAO CHAO CAI

DANG SHEN

MAO XIAN MAO

MAO DA DING CAO

CHANG MAO XIANG KE KE

MAO MA CHI XIAN

MAO YE JIA YING ZHAO GEN

ROU MAO SHAN LI DOU

SHUI TUAN HUA

SONG XUN

FENG LI

FEI YUE GUO

SONG YE QIAN LI GUANG

SHUANG SE JIN GUANG JU 
Pink Plumepoppy

Pink Reineckea

Pink-fringed Milky

Pinkscale Gay-feather

Pinnan Galangal

Pinnate Rue*

Pinnateleaf Lilac

Pipewart

Piscidi Cuttongue tree*

Piscidia erythrina

Pittosporumlike Nothapodytes

Planeleaf Alangium

Planeleaf Alangium Variety*

Plantain Banana

Plantain Banana Cultivariaty*

Platyangle Pricklepoppy*

Plumleaf Cratoxylum

pobequini Fatheadtree*

Poets Narcissus

Poienician Juniper

Poiret Barberry

Poison Euphorbia*

Poison Ivy

Poisonhemlock

Poisonous Buttercup

Poisonous-shrub Anise*

Polyandrous Euptelea*

Polyandrous Pokeweed

Polyanthus Narcissus

Polybract Split-calyx Liverwort*

Polymorphic Angelica

Polymorphism Gold-hair Moss*

Polypore

Pomegranate

Pomegranate Heartwood

Pomegranate Leaf

Pomegranate Peel

Pomegranate Root

Pomegranate Seed

Pomelo

Pond Frog

Pond Frog Gall

Poongaoil Pongamia

Poplar-leaf Argyreia*

Poplar-leaf-like White Quebracho*

Popyporus Agaric

Porrect Wormwood*

Portiatree

Portlan Euphorbia*

Potanin Barberry

Potanin Iris

Potanin Larkspur
T4020 Macleaya cordata

T5449 Reineckea carnea

T3656 Lactarius torminosus

T3788 Liatris elegans

T0361 Alpinia pinnanensis

T5630 Ruta pinnata

T6261 Syringa pinnafolia

T2434 Eriocaulon buergerianum

T6806 Walsura piscidia

T4977 Piscidia erythrina

T4439 Nothapodytes pittosporoides

T0285 Alangium platanifolium

T0284 Alangium paltanifolium var. platanifolium

T4330 Musa paradisiaca

T4332 Musa x paradisiaca cultivar

T0611 Argemone platyceras

T1785 Cratoxylum prunifolium

T4393 Nauclea pobequinii

T4378 Narcissus poeticus

T3593 Juniperus phoenicea

T0912 Berberis poiretii

T2610 Euphorbia poisonii

T6481 Toxicodendron radicans

T1642 Conium maculatum

T5415 Ranunculus sceleratus

T3408 Illicium tsangii

T2630 Euptelea polyandra

T4867 Phytolacca polyandra

T4383 Narcissus tazetta

T1365 Chiloscyphus polyanthus

T0491 Angelica polymorpha

T5134 Polytrichum ohioense

T2751 Fomitopsis spraguei

T5332 Punica granatum

T5329 Punica granatum

T5330 Punica granatum

T5328 Punica granatum

T5327 Punica granatum

T5331 Punica granatum

T1474 Citrus decumana

T5407 Rana nigromaculata; Rana plancyi

T5408 Rana nigromaculata; Rana plancyi

T5143 Pongamia pinnata

T0615 Argyreia populifolia

T0771 Aspidosperma populifolium

T5129 Polyporus umbellatus

T0688 Artemisia porrecta

T6432 Thespesia populnea [Syn. Hibiscus populneus]

T2611 Euphorbia portlandica

T0913 Berberis potaninii

T3466 Iris potaninii

T2084 Delphinium potaninii
BO LUO HUI

JI XIANG CAO

MAO TOU RU GU

HUA LI SHE BIAN JU

ZHU SUI SHAN JIANG

YU ZHUANG YUN XIANG

YU YE DING XIANG

GU JING CAO

PI XI DI GE SHE SHU

YA MAI JIA DU YU DOU

MA BI MU

GUA MU

GUA MU BIAN ZHONG

FEN BA JIAO

FEN BA JIAO ZA JIAO ZHONG ZHI

BIAN ZHONG

KUO GUO JI YING SU

KU DING CHA

BO SHI WU TAN

HONG KOU SHUI XIAN

FEI NI JI CI BAI

XI YE XIAO BO

PO SEN DA JI

DU QI TENG

DU SHEN

SHI LONG RUI

DU AI BA JIAO

DUO XIONG RUI LING CHUN MU

DUO XIONG RUI SHANG LU

DUO HUA SHUI XIAN

LIE E TAI

GUAI QIN

DUO XING JIN FA XIAN

CENG KONG JUN

SUAN SHI LIU

SHI LIU XIN CAI

SHI LIU YE

SHI LIU PI

SHI LIU GEN

SHI LIU ZHONG ZI

ZHU LUAN

QING WA

QING WA DAN

SHUI LIU DOU

YANG YE YIN BEI TENG

SI YANG SHU YE BAI JIAN MU

ZHU LING

SHEN HAO

YANG YE XIAO JIN

BO TE LAN DA JI

SHAO CHI XIAO BO

JUAN QIAO YUAN WEI

HEI SHUI CUI QUE 
Potanin Larkspur Variety*

Potato

Potmarigold Calendula

praecox Stachyurus*

Prain Microtoena

Prairie Mimosa

Pratt Solomonseal

Prawn

Prepared Common Monkshood Daughter

Root

Prettiest Caesalpinia

Pretty Brake

Pretty Crocus

Prettyleaf Winchia

Prickly Juniper

Prickly Lettuce

Prickly Pear

Prickly Sowthistle

Pricklyash

Pricklyfruit Licorice

Primrose Denrdobium

Prince's-feather

Prinos-like Salacia

Procumbent Hypecoum*

Proliferous Euphorbia*

Prolongated Spleenwort

Propolis

Prostrate Pachysandra*

Prostrate Rice-flower*

Protozoon Ochromonas malhamensis

Provincialis Gayfeather*

Przewallsk Rhododendron*

Przewalsk Ephedra

Przewalsk Fritillary

Przewalsk Sage

Przewalsk Skullcap

Pseudobald Pyrrosia*

Pseudoiva Bugle*

Pseudomonas Locust*

Pseudo-racemose Glycosmis*

Pseud-oriental Groundsel*

Pseudoriental Poppy*

Pseudostapfia Monkshood

Puberulent Monkshood

Puberulent Sneezeweed*

Pubescence Epimedium

Pubescent Birch*

Pubescent Holly

Pubescent Nightshade

Pubescent Sunflower*
T2085 Delphinium potaninii var. jiufengshanense

T6017 Solanum tuberosum

T1113 Calendula officinalis

T6096 Stachyurus praecox

T4226 Microtoena prainiana

T2127 Desmanthus illinoensis

T5094 Polygonatum prattii

T4696 Penaeus orientalis

T0083 Aconitum carmichaeli

T1107 Caesalpinia pulcherrima

T5286 Pteris bella

T1809 Crocus speciosus

T6825 Winchia calophylla

T3592 Juniperus oxycedrus

T3663 Lactuca serriola

T4520 Opuntia ficus-indica

T6026 Sonchus asper [Syn. Sonchus oleraceus var. asper]

T6863 Zanthoxylum americanum [Syn. Xanthoxylum americanum]

T3018 Glycyrrhiza pallidiflora

T2109 Dendrobium primulinum

T5110 Polygonum orientale

T5648 Salacia prinoides [Syn. Salacia chinensis]

T3335 Hypecoum procumbens

T2612 Euphorbia prolifera

T0776 Asplenium prolongatum

T0543 Apis mellifera ligustica

T4574 Pachysandra procumbens

T4892 Pimelea prostrata

T4467 Ochromonas malhamensis

T3789 Liatris provincialis

T5521 Rhododendron przewalskii

T2377 Ephedra przewalskii

T2792 Fritillaria przewalskii

T5688 Salvia przewalskii

T5842 Scutellaria przewalskii

T5359 Pyrrosia pseudocalvata

T0269 Ajuga pseudoiva

T5553 Robinia pseudomonas

T3009 Glycosmis pseudoracemosa

T5899 Senecio pseudoorientalis

T4634 Papaver pseudorientale

T0125 Aconitum pseudostapfianum

T0078 Aconitum barbatum var. puberulum [Syn. Aconitum

ochranthum ]

T3140 Helenium puberulum

T2401 Epimedium pubescens

T0934 Betula pubescens

T3396 Ilex pubescens

T6010 Solanum pubescens

$\mathrm{T} 3150$ Helianthus mollis
HEI SHUI CUI QUE HUA BIAN

ZHONG

MA LING SHU

IIN ZHAN JU

JING JIE HUA

NAN CHUAN GUAN CHUN HUA

YI LI NUO HE HUAN CAO

KANG DING YU ZHU

HAI XIAKihinouye

FU ZI

JI MEI YUN SHI

CHANG BING FENG WEI JUE

MEI LI FAN HONG HUA

PEN JIA SHU

CI GUI

YE WO JU

LI GUO XIAN REN ZHANG

XU DUAN JU

MEI ZHOU HUA JIAO

CI GUO GAN CAO

BAO CHUN SHI HU

HONG CAO

SUO LA MU

PING ZHAN JIAO HUI XIANG

TU GUA LANG DU

CHANG SHENG TIE JIAO JUE

FENG JIAO

YANG WO BAN DENG GUO

PING WO DAO HUA

TU ER FENG

LONG SHU DU JUAN

MO GUO MA HUANG

GAN SU BEI MU

GAN XI SHU WEI CAO

AI YE HUANG QIN

NI GUANG SHI WEI

JIA DAN BAO JUN YANG HUAI

JIA ZONG ZHUANG HUA XU SHAN

XIAO JU

JIA DONG FANG QIAN LI GUANG

JIA JIN DONG YING SU

NI YU LONG WU TOU

NIU BIAN

WEI MAO DUI XIN JU ROU MAO YIN YANG HUO

MAO ZHI HUA

MAO DONG QING

ROU MAO QIE

ROU MAO XIANG RI KUI 


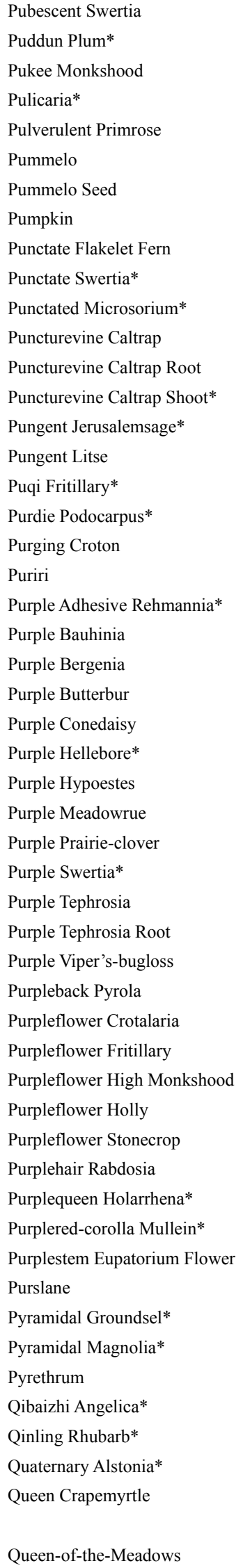

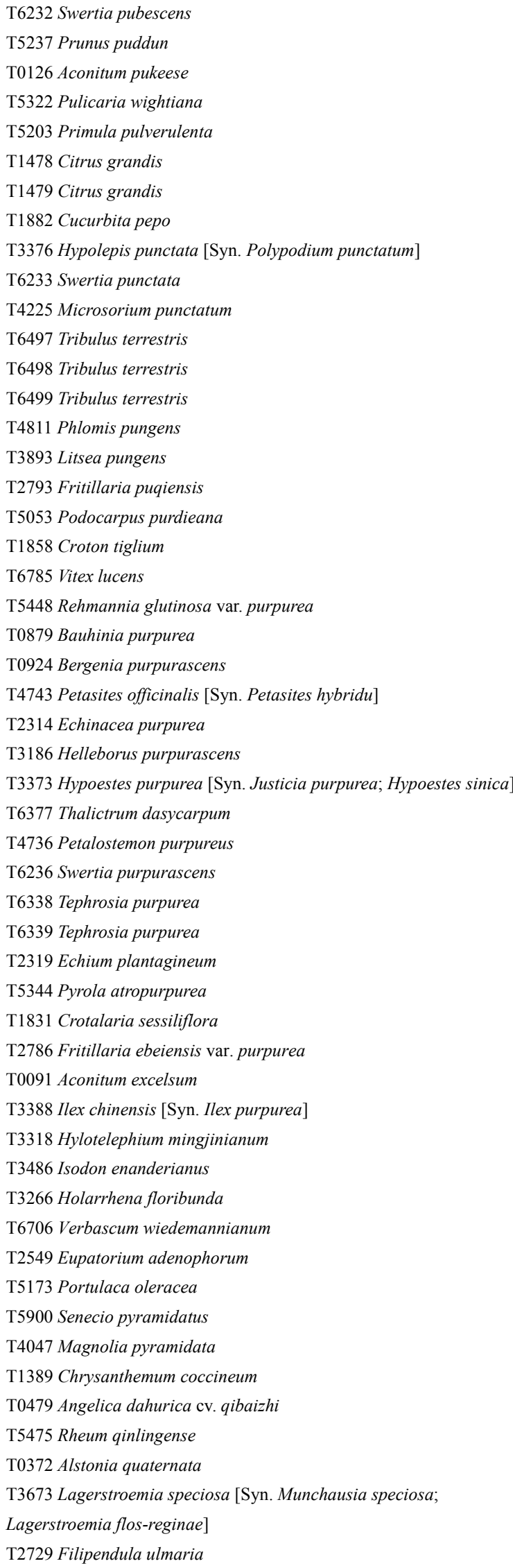

MAO ZHANG YA CAI

PU DUN LI

PU GE WU TOU

ZAO CAO

YIN FEN BAO CHUN

$\mathrm{YOU}^{(4)}$

YOU HE

XI HU LU

JI JUE

XI DIAN ZHANG YA CAI

XING JUE

CI JI LI

JI LI GEN

JI LI MIAO

CI CAO SU

ZHEN CAI

PU QI BEI MU

PU ER DI LUO HAN SONG

BA DOU

XIN XI LAN MU JING

ZI DI HUANG

ZI YANG TI JIA

YAN BAI CAI

ZI FENG DOU CAI

ZI HUA SONG GUO JU

ZI TI GEN CAO

QIANG DAO YAO

CU GUO TANG SONG CAO

ZI SE BAN RUI DOU

ZI SE ZHANG YA CAI

HUI YE

HUI YE GEN

CHE QIAN YE LAN JI

ZI BEI LU TI CAO

YE BAI HE

ZI HUA E BEI BEI MU

ZI HUA GAO WU TOU

SI JI QING

ZI HUA JING TIAN

ZI MAO XIANG CHA CAI

FAN HUA ZHI XIE MU

ZI HUA GUAN MAO RUI HUA

ZI JING ZE LAN HUA

MA CHI XIAN

JIN ZI TA XING QIAN LI GUANG

JIN ZI TA MU LAN

HONG HUA CHU CHONG JU

QI BAI ZHI

QIN LING DA HUANG

SI SHU JI GU CHANG SHAN

DA HUA ZI WEI

XUAN GUO WEN ZI CAO 


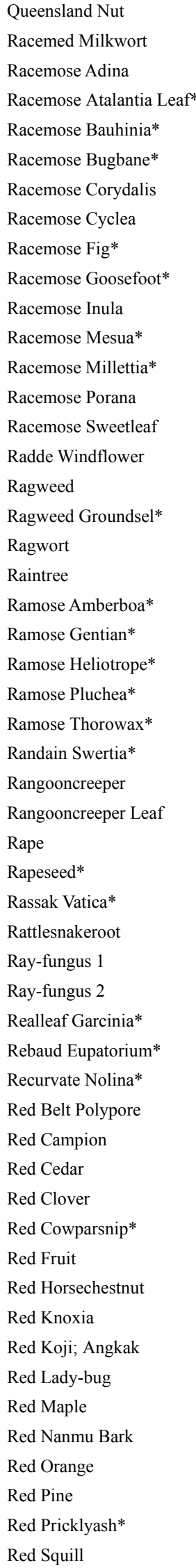

T4011 Macadamia ternifolia

T5081 Polygala polygama

T5960 Sinoadina racemosa [Syn. Adina racemosa]

T0816 Atalantia racemosa

T0880 Bauhinia racemosa

T1424 Cimicifuga racemosa

T1733 Corydalis racemosa

T1932 Cyclea racemosa

T2724 Ficus racemosa

T1363 Chenopodium championii

T3437 Inula racemosa

T4204 Mesua racemosa

T4242 Millettia racemosa

T5167 Porana racemosa

T6254 Symplocos racemosa

T0470 Anemone raddeana

T0396 Ambrosia ambrosioids

T5879 Senecio ambrosioides

T5890 Senecio jacobaea

T5703 Samanea saman

T0394 Amberboa ramosa

T2926 Gentiana ramosa

T3178 Heliotropium ramosissimum

T0849 Baccharis ramosissima

T1068 Bupleurum polyclonum

T6237 Swertia randainensis

T5384 Quisqualis indica

T5385 Quisqualis indica

T1009 Brassica napus

T1011 Brassica napus var. napus

T6686 Vatica rassak

T5196 Prenanthes acerifolia

T6152 Strepyomyes niveus

T6153 Strepyomyes spheroids

T2854 Garcinia engenifolia

T2569 Eupatorium rebaudianum

T4437 Nolina recurvata

T2750 Fomitopsis pinicola [Syn. Fomes pinicola; Polyporus pinicola]

T3949 Lychnis dioica

T3603 Juniperus virginiana

T6521 Trifolium pratense

T3212 Heracleum granatense

T1778 Crataegus pinnatifida var. major

T0200 Aesculus carnea

T3632 Knoxia valerianoides

T4270 Monascus kaoliang

T3287 Huechys sanguinea

T0054 Acer rubrum

T4018 Machilus thunbergii

T1476 Citrus erythrosa

T4921 Pinus resinosa

T6891 Zanthoxylum rubescens

T6649 Urginea maritima
AO ZHOU JIAN GUO

KU YUAN ZHI

JI ZI MU

ZONG ZHUANG DONG FENG JU YE

ZONG ZHUANG HUA YANG TI JIA

ZONG ZHUANG SHENG MA

XIAO HUA HUANG JIN

LUN HUAN TENG

JU GUO RONG

ZONG ZHUANG HUA LI

ZONG ZHUANG TU MU XIANG

ZONG ZHUANG TIE LI MU

ZONG ZHUANG JI XUE TENG

FEI E TENG

ZHU ZI SHU

DUO BEI YIN LIAN HUA

PU TONG TUN CAO

TUN CAO QIAN LI GUANG

CAO DIAN QIAN LI GUANG

YU SHU

FEN ZHI PO JU

DUO ZHI LONG DAN

DUO ZHI TIAN JIE CAI

DUO ZHI KUO BAO JU

DUO ZHI CHAI HU

LUAN DA SHAN ZHANG YA CAI

SHI JUN ZI

SHI JUN ZI YE

OW ZHOU YOU CAI

YOU CAI ZI

QING MEI

QI YE PAN GUO JU

ZHEN YE TENG HUANG

TIAN YE JU

XIA WAN NUO LIN

HONG YUAN CENG KONG JUN

HONG JIAN QIU LUO

BEI MEI YUAN BAI

HONG CHE ZHOU CAO

HONG DU HUO

SHAN LI HONG

HONG QI YE SHU

HONG YA DA JI

GAO LIANG HONG QU

HONG NIANG ZI

HONG HUA QI

HONG NAN PI

ZHU JU

DUO ZHI SONG

HONG HUA JIAO

HAI CONG 
Red Tasselflower*

Red Thorowax

Red Viburnum*

Red Vinespinach flower

Red-and-Yellow Garden Raspberry

Redback Christmashush

Redbark Cinchona

Redbracted Lysidice

Redcalyx Glotybower

Redcalyx Millettia*

Reddish Jackinthepulpit

Reddrop Barberry

Redflower Gentian

Redflower Kopsia

Redflower Magnoliavine

Redflower Meconopsis

Redflower Pyrola

Redflower Sweetvetch

Redflowered Swisscentaury*

Redfoot Wormwood

Redfruit Devilpepper

Redfruit Pencilwood

Redhaw Hawthorn

Red-knees

Red-knees Fruit

Red-milk Grass*

Red-Rock-Ears

Redroot Gromwell

Redspot Swertia

Redstriate Woodbetony

Red-water Tree

Redwood

Reed Canary-grass

Reeves Skimmia

Regel Ephedra

Regel Eyebright

Regel Threewingnut

Regnell Morningglory*

Rehder Skullcap;

Reindeer Moss

Reini Milkwort*

Religious Anisetree*

Remote Lemongrass

Renard Groundsel*

Reniform Pelargonium*

Reniform Rabdosia*

Repent Corydalis*

Resinoid Cistus*

Resinoid Euphorbia*

Resinoid Sumac*

Restharrow

Reticulate Leafflower*

Reticulatefruit Larkspur
T2356 Emilia coccinea

T1072 Bupleurum scorzonerifolium

T6736 Viburnum erubescens

T0875 Basella rubra

T5593 Rubus idaeus

T0297 Alchornea trewioides

T1433 Cinchona succirubra

T3995 Lysidice rhodostegia

T1555 Clerodendron fortunatum

T4235 Millettia erythrocalyx

T0618 Arisaema consanguineum

T0902 Berberis diaphana

T2927 Gentiana rhodantha

T3640 Kopsia fruticosa

T5799 Schisandra rubriflora

T4145 Meconopsis punicea

T5349 Pyrola incarnata

T3128 Hedysarum multijugum

T5465 Rhaponticum carthamoides

T0691 Artemisia rubripes

T5425 Rauvolfia verticillata f. rubrocarpa

T2308 Dysoxylum binectariferum

T1780 Crataegus sanguinea

T5104 Polygonum hydropiper

T5103 Polygonum hydropiper

T2623 Euphorbia makinoi

T6602 Umbilicaria hypococcinea

T3881 Lithospermum erythrorhizon

T6219 Swertia erythrosticta

T4684 Pedicularis striata

T2485 Erythrophleum guineense

T5918 Sequoia sempervirens

T4777 Phalaris arundinacea

T5972 Skimmia reevesiana

T2378 Ephedra regeliana

T2629 Euphrasia regelii

T6541 Tripterygium regelii

T3451 Ipomoea regnellii

T5843 Scutellaria rehderiana

T1526 Cladonia rangiferina

T5082 Polygala reinii

T3406 Illicium religiosum

T1941 Cymbopogon distans

T5901 Senecio renardii

T4692 Pelargonium reniforme

$\mathrm{T} 3502$ Isodon latifolia var. reniformis

T1736 Corydalis repens var. humosides

T1870 Csitus ladaniferus

T2616 Euphorbia resinifera

T5535 Rhus retinorrhoea

T4493 Ononis spinosa

T4842 Phyllanthus reticulatus

T2072 Delphinium dictyocarpum
FEI YI DIAN HONG

HONG CHAI HU

HONG JIA MI

LUO KUI HUA

FU PEN ZI

HONG BEI SHAN MA GAN

HONG SE JIN JI NA SHU

YI HUA

GUI DENG LONG

HONG E JI XUE TENG

TIAN NAN XING

XIAN HUANG XIAO BO

HONG HUA LONG DAN

HONG HUA RUI MU

HONG HUA WU WEI ZI

HONG HUA LV RONG HAO

HONG HUA LU TI CAO

HONG HUA YAN HUANG QI

LU CAO

HONG ZU HAO

HONG GUO LUO FU MU

HONG GUO JIAN MU

LIAO NING SHAN ZHA

SHUI LIAO

LIAO SHI

HONG RU CAO

HONG SHI ER

ZI CAO

HONG ZHI ZHANG YA CAI

HONG WEN MA XIAN HAO

JI NEI YA GE MU

BEI MEI HONG SHAN

YI CAO

YIN YU

XI ZI MA HUANG

DUAN XIAN XIAO MI CAO

HEI MAN

RUI SHI QIAN NIU

GAN SU HUANG QIN

SHI RUI

SHI YE CAO

DONG DU HUI

YUN XIANG CAO

LEI SHI QIAN LI GUANG

SHEN YE TIAN ZHU KUI

SHEN XING XIANG CHA CAI

TU YAN HU

SHU ZHI BAN RI HUA

SHU ZHI DA JI

SHU ZHI YAN FU MU

CI MANG BING HUA

LONG YAN JING

WANG GUO CUI QUE HUA 


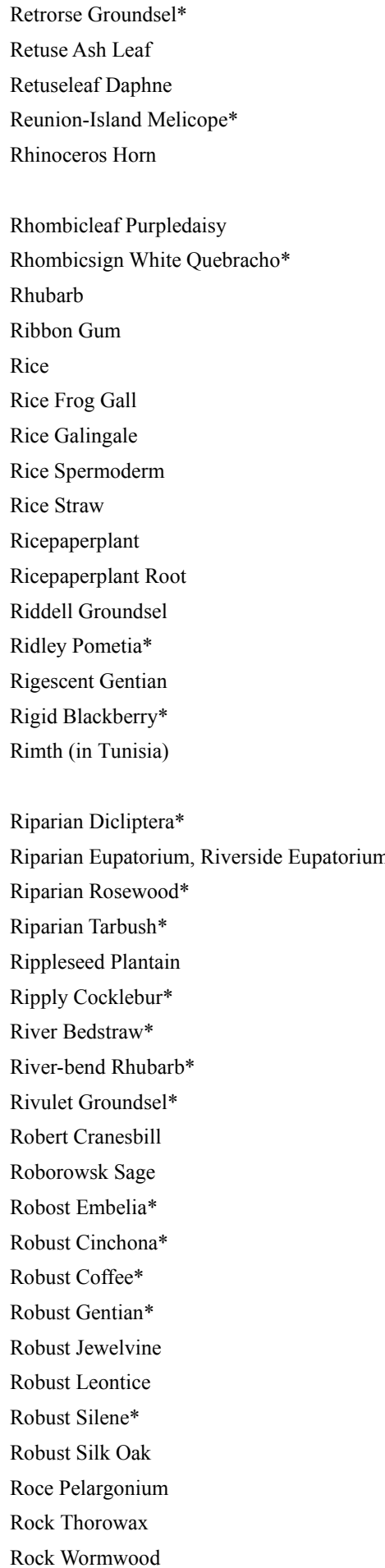

Rock-Ears*

Rocket Candytuft

Rocket Consolida

Rock-ginger Fern

Rockliving Corydalis

Roof Iris

Root-tuber Cremanthodium
T5902 Senecio retrorsus

T2770 Fraxinus insularis

T2030 Daphne retusa

T4164 Melicope coodeana

T5484 Rhinoceros unicornis; Rhinoceros sondaicus; Rhinoceros

sumatrensis

T4447 Notoseris rhombiformis

T0774 Aspidosperma rhombeosignatum

T5476 Rheum rhaponticum

T2522 Eucalyptus viminalis

T4545 Oryza sativa

T5406 Rana limnocharis

T1976 Cyperus iria

T4546 Oryza sativa

T4544 Oryza sativa

T6357 Tetrapanax papyriferus

T6356 Tetrapanax papyriferus

T5903 Senecio riddellii

T5136 Pometia ridleyi

T2928 Gentiana rigescens

T5596 Rubus rigidus

T3096 Hammada scoparia [Syn. Arthrophytum scoparium; Haloxylon

articulatum ssp. scoparium; Haloxylon scoparium]

T2160 Dicliptera riparia

T2570 Eupatorium riparium

T2011 Dalbergia riparia

T2741 Flourensia riparia

T5007 Plantago major

T6842 Xanthium riparium

T2833 Galium rivale

T5469 Rheum hotaoense

T5904 Senecio rivularis

T2946 Geranium robertianum

T5690 Salvia roborowskii

T2349 Embelia robusta

T1430 Cinchona robusta

T1611 Coffea robusta

T2929 Gentiana robusta

T2122 Derris robusta

T3746 Leontice robustum

T5953 Silene firma

T3063 Grevillea robusta

T4690 Pelargonium graveolens

T1070 Bupleurum rockii

T0692 Artemisia rupestris [Syn. Artemisia dentata; Artemisia viridis; Artemisia viridifolia]

T6601 Umbilicaria esculenta [Syn. Gyrophora esculenta]

T3385 Iberis amara

T1645 Consolida ajacis [Syn. Delphinium ajacis]

T5252 Pseudodrynaria coronans

T1747 Corydalis thalictrifolia

T3471 Iris tectorum

T1786 Cremanthodium ellisii
WAN QU QIAN LI GUANG

KU LI MU YE

AO YE RUI XIANG

RU NI WENG DAO MI ZHU YU

XI JIAO

LING YE ZI JU

LENG ZHUANG BAI JIAN MU

SHI YONG DA HUANG

DUO ZHI AN

JING MI

XIA MA DAN

SUI MI SHA CAO

MI PI KANG

DAO CAO

TONG TUO MU

TONG HUA GEN

RUI DE QIAN LI GUANG

LI DE LI FAN LONG YAN

DIAN LONG DAN

JIAN YING XUAN GOU ZI

HE AN GOU GAN CAI

HE AN ZE LAN

HE AN HUANG TAN

HE AN FU LAO JU

DA CHE QIAN

XIAO XI CANG ER

XI LIU ZHU YANG YANG

HE TAO DA HUANG

XI QIAN LI GUANG

XIAN XI LAO GUAN CAO

NIAN MAO SHU WEI CAO

CU ZHUANG SUAN TENG ZI

CU ZHUANG JIN JI NA

CU ZHUANG KA FEI

CU ZHUANG LONG DAN

CU ZHUANG YU TENG

HONG MAO QI

YING YE NV LOU CAI

YIN HUA

XIANG YE

LI JIAMH CHAI HU

XIN JIANG YI ZHI HAO

SHI ER

QU QU HUA

FEI YAN CAO

CHUAN SHI JIAN

YAN HUANG LIAN

YUAN WEI

KUAI GEN CHUI TOU JU 
Roquette

Rosary Ear-leaf Muscus*

Rose Corydalis*

Rose Glorybower (Clerodendrum)

Rose Hypoestes*

Rose Mallow

Roseapple

Rosemary

Rosemyrtle

Rose-of-Sharon

Rosepink Zephyrlily

Rosering Gaillardia

Rosmarin-leaf Groundsel*

Rosthorn Rabdosia

Rosthorn Snakegourd

Rosthorn Yam

Rostral Ninenode*

Rostratefruit Gynostemma

Rotate Hymenocallis*

Rough Comfrey

Rough Gentian

Rough Leucas

Rough Star Thistle

Roughfruit Rabdosia*

Roughleaf Raspberry

Rough-veined Rapanea*

Round Cardamom

Roundfruit Glochidion*

Roundfruit Licorice

Roundleaf Coca Shrub*

Roundleaf Corydalis*

Roundleaf Eupatorium*

Round-leaf Flytrap*

Roundleaf Heliotrope*

Roundleaf Pharbitis Seed

Roundleaf Schefflera*

Roundleaf Sundew

Roundleaf Thorowax*

Roundpod Jute Leaf

Roundpod Jute Seed

Roundwingfruit Cyclocarya

Roxburg Aglaia*

R oxburgh Engelhardtia Root

Roxburgh Peristrophe

Roxburgh Rose

Roxburgh Wormwood

Royal Jelly

Royal Paulownia

Royle Euphorbia Latex

Roylei Condorvine*

Rubi Pholidota*

Rueleaf Wormwood*

Rufous Turtle Dove
T2439 Eruca sativa

T2803 Frullania tamarisci ssp. moniliata [Syn. Frullania moniliata]

T1737 Corydalis rosea

T1565 Clerodendrum bungei

T3374 Hypoestes rosea

T6645 Urena lobata

T6267 Syzygium jambos

T5575 Rosmarinus officinalis

T5529 Rhodomyrtus tomentosa

T3343 Hypericum calycinum

T6904 Zephyranthes grandiflora [Syn. Zephyranthes carinata]

T2820 Gaillardia pulchella

T5905 Senecio rosmarinifolius

T3520 Isodon rosthornii

T6513 Trichosanthes rosthornii [Syn. Trichosanthes uniflora]

T2204 Dioscorea nipponica ssp. rosthornii

T5278 Psychotria rostrata

T3086 Gynostemma yixingense

T3321 Hymenocallis rotata

T6244 Symphytum asperum

T2930 Gentiana scabra

T3779 Leucas aspera

T1301 Centaurea aspera ssp. aspera

T3500 Isodon lasiocarpa

T5587 Rubus alceaefolius

T5416 Rapanea neurophylla

T0416 Amomum kravanh [Syn. Amomum cardamomum]

T2989 Glochidion sphaerogynum

T3021 Glycyrrhiza squamulosa

T2489 Erythroxylon rotundifolium

T1738 Corydalis rotundatour

T2571 Eupatorium rotundifolium

T2187 Dionaea rotundifolia

T3179 Heliotropium rotundifolium

T4780 Pharbitis purpurea

T5783 Schefflera rotundifolia

T2268 Drosera rotundifolia

T1071 Bupleurum rotundifolium

T1673 Corchorus capsularis

T1674 Corchorus capsularis

T1936 Cyclocarya paliurus

T0244 Aglaia roxburghiana

T2360 Engelhardia roxburghiana

T4730 Peristrophe roxburghiana

T5570 Rosa roxburghii

T0690 Artemisia roxbugiana

T0541 Apis cerana

T4679 Paulownia tomentosa

T2617 Euphorbia royleana

T4119 Marsdenia roylei

T4822 Pholidota rubra

T0693 Artemisia sativum

T6150 Streptopelia orientalis
ZHI MA CAI

CHUAN ZHU ER YE TAI

MEI GUI HONG JIN

CHOU MU DAN

DAN HONG QIANG DAO YAO

DI TAO HUA

$\mathrm{PU}^{(3)} \mathrm{TAO}$

MI DIE XIANG

TAO JIN NIANG

DA E JIN SI TAO

FENG YU HUA

TIAN REN JU

MI DIE XIANG YE QIAN LI GUANG

YING HUA XIANG CHA CAI

SHUANG BIAN GUA LOU

CHAI HUANG JIANG

HUI ZHUANG JIU JIE

HUI GUO JIAO GU LAN

FU ZHUANG SHUI GUI JIAO

CU XI MEN FEI CAO

LONG DAN

FENG CHAO CAO

CU CAO SHI CHE JU

CU GUO XIANG CHA CAI

CU YE XUAN GOU ZI

CU YE MAI MI HUA SHU

BAI DOU KOU

YUAN GUO SUAN PAN ZI

YUAN GUO GAN CAO

YUAN XING YE GU KE

YUAN YE SHAN WU GUI

YUAN YE ZE LAN

YUAN YE BU YING CAO

YUAN YE TIAN JIE CAI

YUAN YE QIAN NIU ZI

YUAN YE E ZHANG CHAI

YUAN YE MAO GAO CAI

YUAN YE CHAI HU

HUANG MA YE

HUANG MA ZI

QING QIAN LIU

LUO KE SI BAO MI ZI LAN

HUANG QI II

GUAN YIN CAO

CI LI

HUI BAO HAO

FENG RU

MAO PAO TONG

BA WANG BIAN

ROU LEI NIU NAI CAI

HONG SHI XIAN TAO

YUN XIANG YE HAO

BAN JIU 
Rugose Meadowrue*

Rugose Rabdosia*

Rugose Rose Flower

Runner Bean

Rupestrine Dragonhead

Ruspol Aloe*

Russian Boschniakia

Russian Comfrey

Russian Hogfennel*

Russianolive

Russianolive Bark

Rust-coloured Crotalaria

Rusty Foxglove

Rustyhair Taxillus

Rutaceae Diosma

Ruyuan Rhododendron

S. Sichuan Egretgrass

S. Yunnan Beautyleaf

Saba Aloe

Saccate Mullein*

Sachalin Eupatorium*

Sachalin Monkshood*

Sachaline Corktree*

Sacred Spindle-tree

Safflower

Saffron Crocus Stigma

Saghalin Spruce

Sagittate Epimedium

Sago Frond

Sago Seed

Saline Cistanche

Saline Saussurea

Saline Swainsonia

Salsify

Saltliving Anabasis

Salt-loving Iris

Salutary Croton*

Salvia pisidica

Salviaflower Coralbean*

Salwin Camellia

Samalanga Syzygium

Sampson St.John'swort

Sanchi

Sanchi Buds

Sanctity Blackberry*

Sand Pear Leaf

Sandal Beadtree

Sandalwood

Sandalwood Padauk

Sandbox-tree

Sandland Milkwort*
T6407 Thalictrum rugosum

T3523 Isodon rugosus [Syn. Rabdosia rugosa]

T5572 Rosa rugosa

T4782 Phaseolus multiflorus

T2258 Dracocephalum rupestre

T0343 Aloe ruspoliana

T0993 Boschniakia rossica

T6249 Symphytum $\mathrm{x}$ uplandicum

T4770 Peucedanum ruthenicum

T2326 Elaeagnus angustifolia

T2327 Elaeagnus angustifolia

T1819 Crotalaria ferruginea

T2174 Digitalis ferruginea

T6304 Taxillus levinei

T1617 Coleonema pulchellum

T5512 Rhododendron lingii

T2240 Diuranthera inarticulata

T1130 Calophyllum polyanthum

T0344 Aloe sabaea

T6703 Verbascum saccatum

T2573 Eupatorium sachalinense [Syn. Eupatorium glehni]

T0127 Aconitum sachalinense

T4794 Phellodendron sachalinense

T2546 Euonymus sacrosancta

T1215 Carthamus tinctorius

T1808 Crocus sativus

T4870 Picea glehnii

T2402 Epimedium sagittatum

T1928 Cycas revoluta

T1927 Cycas revoluta

T1456 Cistanche salsa

T5768 Saussurea salsa

T6210 Swainsonia salsula [Syn. Sphaerophysa salsula]

T6489 Tragopogon porrifolius

T0437 Anabasis salsa

T3460 Iris halophila

T1854 Croton salutaris

T5684 Salvia pisidica

T2473 Erythrina salviiflora

T1148 Camellia saluenensis

T6268 Syzygium samarangense

T3363 Hypericum sampsonii

T4608 Panax pseudo-ginseng var. notoginseng [Syn. Panax

notoginseng]

T4609 Panax pseudo-ginseng var. notoginseng [Syn. Panax

notoginseng]

T5597 Rubus sanctus

T5366 Pyrus pyrifolia

T0167 Adenanthera pavonina

T5715 Santalum album

T5305 Pterocarpus santalinus

T3296 Hura crepitans

T5083 Polygala sabulosa
ZOU WEN TANG SONG CAO

ZHOU YE XIANG CHA CAI

MEI GUI HUA

HONG HUA CAI DOU

YANG QING LAN

LA SHI LU HUI

CAO CONG RONG

E GUO XI MEN FEI CAO

E GUO QIAN HU

SHA ZAO

SHA ZAO SHU PI

XIANG LING CAO

XIU MAO DI HUANG

XIU MAO JI SHENG

MEI LI BU KU

RU YUAN DU JUAN

NAN CHUAN LU SI CAO

DIAN NAN HONG HOU KE

SA BA LU HUI

NANG ZHUANG MAO RUI HUA

KU YE DAO ZE LAN

KU YE WU TOU

KU YE DAO HUANG BAI

MAO YE WEI MAO

HONG HUA

ZANG HONG HUA

SA HA LIN YUN SHAN

JIAN YE YIN YANG HUO

SU TIE YE

SU TIE SHU GUO

YAN SHENG ROU CONG RONG

YAN DI FENG MAO JU

KU MA DOU

SUAN YE PO LUO MEN SHEN

YAN SHENG JIA MU ZEI

XI YAN YUAN WEI

YI KANG BA DOU

DU YU SHU WEI CAO

SHU WEI CAO HUA CI TONG

NU JIANG SHAN CHA

YANG PU TAO YE

YUAN BAO CAO

SAN QI

SAN QI HUA LEI

SHEN SHENG XUAN GOU ZI

SHA LI YE

HAI HONG DOU

TAN XIANG

SI ZI TAN

SHA HE SHU

SHA DI YUAN ZHI 
Sandliving Sophora

Sandwich Eugenia*

Sanguineous Spikemoss

Santonica

Sappan Caesalpinia

Sargent Cypress

Sargentgloryvine

Sariola Lettuce*

Sarmentose Pepper

Sarmentose Pepper Spike

Sasak Stephania*

Sasanqua Camellia

Sassafras

Satsuma

Sausagetree

Sawara False Cypress

Savin

Savin Juniper

Savoury Rhododendron

Savoy Cabbage

Sawtooth Clubmoss*

Scabrous Boulder Fern

Scabrous Cowparsnip

Scabrous Doellingeria

Scabrous Elephantfoot

Scabrous Gay-feather*

Scabrous Gentian*

Scabrous Mosla

Scabrous Patrinia

Scale Figwort*

Scale Licorice*

Scaly Blazing Star

Scalyseed Ephedra

Scammony Glorybind

Scandent Schefflera

Scarlet Caterpillar Fungus

Scarlet Kafirlily

Scarlet Pimpernel

Scarlet Runner Bean

Scarlet Sage

Scarlet Swertia

Scheffleri Uvaria*

Schischkin Foxglove*

Schleicher Fumitory

Schoenocoulon officinale

Schrenk Wormwood*

Scorpion

Scorzonera

Scotch Broom

Scotch Cottonthistle

Scotch Pine

Scots Lovage

Scouler Corydalis*
T6039 Sophora moorcroftiana

T2534 Eugenia sandwicensis

T5866 Selaginella sanguinolenta

T0683 Artemisia maritima

T1108 Caesalpinia sappan

T1898 Cupressus sargentii

T5741 Sargentodoxa cuneata

T3661 Lactuca sariola

T4965 Piper sarmentosum

T4966 Piper sarmentosum

T6132 Stephania sasakii

T1149 Camellia sasanqua

T5744 Sassafras albidum

T1518 Citrus unshiu

T3629 Kigelia pinnata

T1350 Chamaecyparis pisifera

T5640 Sabina vulgaris

T3595 Juniperus sabina

T5502 Rhododendron anthopogonoides

T1020 Brassica oleracea var. sabauda

T3977 Lycopodium serratum var. thunbergii

T2113 Dennstaedtia scabra [Syn. Dicksonia scabra]

T3222 Heracleum scabridum

T2244 Doellingeria scaber [Syn. Aster scaber]

T2334 Elephantopus scaber

T3791 Liatris scabra

T2931 Gentiana scabra var. buesgeri

T4307 Mosla scabra [Syn. Mosla punctata]

T4673 Patrinia scabra

T5827 Scrophularia lepidota

T3017 Glycyrrhiza lepidota

T3794 Liatris squarrosa

T2371 Ephedra lepidosperma

T1654 Convolvulus scammonia

T5781 Schefflera arboricola

T1680 Cordyceps militaris

T1578 Clivia miniata

T0440 Anagallis arvensis

T4781 Phaseolus coccineus

T5693 Salvia splendens

T6234 Swertia punicea

T6667 Uvaria scheffleri

T2178 Digitalis schischkinii

T2809 Fumaria schleicheri

T5808 Schoenocaulon officinale

T0694 Artemisia schrenkiana

T1084 Buthus martensi

T5824 Scorzonera hispanica

T1991 Cytisus scoparius [Syn. Spartium scoparium]

T4494 Onopordum acanthium

T4926 Pinus sylvestris

T3823 Ligusticum scoticum

T1739 Corydalis scouleri
SHA SHENG HUAI

SAN WEI ZHI FAN YING TAO

YUAN ZHI JUAN BAI

BIN HAO

SU MU

SA JIN TE BAI MU

DA XUE TENG

SA LI LA WO JU

JIA JU

JIA JU ZI

TAI WAN QIAN JIN TENG

CHA MEI

MEI ZHOU CHA MU

WU HE MI JU

DIAO DENG SHU

RI BEN HUA BAI

CHOU BAI

CHA ZI YUAN BAI

XIAO YE PI PA

YU YI GAN LAN

JU CHI SHI SONG

WAN JUE

DIAN BAI ZHI

DONG FENG CAI

KU DI DAN

CU CAO SHE BIAN JU

CU CAO LONG DAN

SHI JI NING

CAO YE BAI JIANG

LIN PIAN XUAN SHEN

MEI ZHOU GAN CAO

CU SHE BIAN JU

BAN ZI MA HUANG

SI GE MENG XUAN HAU

E ZHANG TENG

YONG CHONG CAO

JUN ZI LAN

LIU LI FAN LV

DUO HUA CAI DOU

XI YANG HONG

ZI HONG ZHANG YA CAI

XIE FEI ZI YU PAN

SI SHI MAO DI HUANG

YAN JIN

WEI JING BAI HE

XUE LING HAO

QUAN XIE

XI JUAN YA CONG

JIN QUE ER

DA CHI JI

OU ZHOU CHI SONG

SU GE LAN DANG GUI

SI KAO LE ZI JIN 
Scythian Lamb

Sea Euphorbia*

Seabuckthorn Fruit

Sea-cucunber Intestines

Seashore Angelica*

Sector Pluchea

Seibo Euonymus*

Selago-like Climbing Fern

Sello Basil*

Semi-frutex Mayweed*

Semiserration Eupatorium*

Senega Snakeroot

Senegal Coralbean*

Senegalese Strophanthus*

Senna Lemongrass*

Sensitive Plant

Sensitiveplant-like Senna

Seoul Siebold Wildginger

Sepiaria

Septemlobate Kalopanax Bark

Septic Fig*

Serbian Yarrow

Sericeous Cinquefoil

Sericeous Newlitse

Sericeous Pluchea*

Sericeous-leaf Rosewood

Serpent-head

Serrate Chloranthus

Serrate Chloranthus Stem-leaf

Serrate Clubmoss

Serrate Glorybower

Serrate Mulberry*

Serrate Rabdosia

Serrate-leaf Myrsine

Serrate-leaved Mayten

Sessile Neonauclea

Sessile Stemona

Sessileflower Acanthopanax Root-bark

Sessile-fruit Chinaure

Setose Abelmoschus

Setose Asparagus

Setose Thistle

Sevenlobed Yam

Sewerzow Corydalis*

Seville Orange Unripe Fruit

Seville Orange Young Fruit

Shackshack Crotalaria

Shady Broadleaf Rabdosia*

Shady Groundsel

Shady Rabdosia*

Shagbark Hickory
T1411 Cibotium barometz [Syn. Polypodium barometz]

T2607 Euphorbia paralias

T3254 Hippophae rhamnoides

T6145 Stichopus japonicus

T0486 Angelica keiskei

T0845 Baccharis flabellata

T2547 Euonymus sieboldianus

T3294 Huperzia selago [Syn. Lycopodium selago]

T4475 Ocimum selloi

T4125 Matricaria suffruticosa

T2574 Eupatorium semiserratum

T5084 Polygala senega

T2474 Erythrina senegalensis

T6159 Strophanthus sarmentosus var. senegambiae

T1947 Cymbopogon sennaarensis

T4248 Mimosa pudica

T1238 Cassia mimosoides

T0732 Asarum sieboldii var. seoulensis

T0197 Aegle marmelos

T3625 Kalopanax septemlobus

T2726 Ficus septica

T0059 Achillea alexandri-regis

T5186 Potentilla reptans var. sericophylla

T4406 Neolitsea sericea

T5025 Pluchea sericea

T2012 Dalbergia sericea

T4505 Ophiocephalus argus

T1372 Chloranthus serratus

T1373 Chloranthus serratus

T3295 Huperzia serrata [Syn. Lycopodium serratum]

T1561 Clerodendron serratum

T4299 Morus serrata

T5397 Rabdosia serra

T4364 Myrsine semiserrata

T4139 Maytenus serrata

T4408 Neonauclea sessilifolia [Syn. Nauclea sessilifolia; Adina

sessilifolia $]$

T6113 Stemona sessilifolia

T0044 Acanthopanax sessiliflorus

T0967 Boenninghausenia sessilicarpa

T0001 Abelmoschus manihot

T0753 Asparagus setaceus [Syn. Asparagus plumosus]

T1451 Cirsium setosum [Syn. Cerratula setosa; Cirsium segetum;

Cephalanoplos segetum]

T2208 Dioscorea septemloba

T1741 Corydalis sewerzowi

T1467 Citrus aurantium

T1468 Citrus aurantium

T1821 Crotalaria incana

T3532 Isodon umbrosa

T5892 Senecio nemorensis

T3533 Isodon umbrosa var. latifolia

T1219 Carya ovata
JIN MAO GOU

HAI YANG DA JI

CU LIU GUO

HAI SHEN CHANG

BIN HAI DANG GUI

SHAN XING KUO BAO JU

XI BO SHI WEI MAO

XIAO JIE JIN CAO

SAI LE LUO LE

BAN GUAN MU MU JU

BAN JU CHI ZHUANG ZE LAN

MEI YUAN ZHI

SAI NEI JIA ER CI TONG

XI FEI YANG JIAO AO

XIN NONG XIANG MAO

HAN XIU CAO

SHAN BIAN DOU ZI

HAN CHENG XI XIN

$\mathrm{MU}^{(4)} \mathrm{JU}$

CI QIU SHU PI

FU YE RONG

SAI ER WEI YA SHI CAO

JIN JIN BANG

ZHOU SHAN XIN MU JIANG ZI

JUAN MAO KUO BAO JU

JUAN MAO HUANG TAN

WU LI

JI JI

JI JI JING YE

QIAN CENG TA

SAN TAI HONG HUA

JU CHI SANG

XI HUANG CAO

CHI YE TIE ZI

CHI YE MEI DENG MU

WU BING XIN WU TAN

ZHI LI BAI BU

WU GENG WU JIA PI

SHI JIAO CAO

HUANG SHU KUI HUA

WEN ZHU

XIAO JI

MIAN BI XIE

XIE SHI ZI JIN

ZHI KE

ZHI SHI

GUANG YE ZHU SHI DOU

YIN DI KUAN YE XIANG CHA CAI

HUANG WAN

YIN DI XIANG CHA CAI

CU PI SHAN HE TAO 
Shagspine Peashrub

Shallot

Shandong Hypodematium

Shanyang Monkshood*

Sharpleaf Clubmoss*

Sharpleaf Galangal

Sharpleaf Gambirplant

Sharpleaf Leafflower*

Sharpleaf Mangrove

Sharpleaf Meadowrue

Sharpleaf Sabia

Sharpleaf Senna Leaf

Sharpspur Corydalis

Sharptooth Incarvillea

Shearer's Pyrrosia Frond

Sheathstipe Greenbrier

Shellfish Pricklyash

Shellfish Pricklyash Root

Shepherdspurse

Shepherdspurse Seed

Shield Floatingheart

Shiko Rabdosia*

Shining Adinandra*

Shining Clubmoss*

Shining Devilpepper*

Shining Gentianella*

Shining Milkwort*

Shining Rosewood*

Shiningleaf Birch Bark

Shiningleaf Millettia

Shiny Bugleweed

Shiny Bugleweed Root

Shiny Peperomia

Shinyleaf Pricklyash

Shinyleaf yellowhorn

Shkioki Angelica*

Shore Juniper

Shore Podgrass

Shortbractleaf Galingale*

Shortbraet Honeysuckle

Shortclustered Plantainlily

Shortflower Bottle Gourd*

Shortflower Crotalaria*

Shortfluff Consolida*

Shortfluff Dutchmanspipe*

Shortfluff Euphorbia*

Shortfluff Holarrhena*

Shorthair Cowparsnip

Shorthairy Antenoron

Shorthorned Epimedium

Shorthorned Epimedium Root

Shortleaf Agave
T1190 Caragana jubata

T0310 Allium ascalonicum

T3372 Hypodematium sinense

T0128 Aconitum sanyoense

T3966 Lycopodium annotinum var. acrifolium

T0360 Alpinia oxyphylla

T6629 Uncaria rhynchophylla [Syn. Nauclea rhynchophylla]

T4832 Phyllanthus acuminatus

T5487 Rhizophora apiculata

T6372 Thalictrum acutifolium

T5638 Sabia swinhoei

T1229 Cassia acutifolia

T1745 Corydalis suaveolens [Syn. Corydalis sheareri]

T3418 Incarvillea arguta

T5360 Pyrrosia sheareri

T5984 Smilax stans [Syn. Smilax vaginata var. stans]

T6876 Zanthoxylum dissitum

T6877 Zanthoxylum dissitum

T1184 Capsella bursa-pastoris

T1185 Capsella bursa-pastoris

T4456 Nymphoides peltatum

T5398 Rabdosia shikokiana

T0182 Adinandra nitida

T3974 Lycopodium lucidulum

T5438 Rauwolfia nitida

T2940 Gentianella nitida

T5078 Polygala nitida

T2006 Dalbergia nitidula

T0932 Betula luminifera

T4238 Millettia nitida

T3980 Lycopus lucidus

T3981 Lycopus lucidus

T4705 Peperomia pellucida

T6884 Zanthoxylum nitidum

T6845 Xanthoceras sorbifolia

T0494 Angelica shkiokiana

T3585 Juniperus conferta

T6526 Triglochin maritimum

T1974 Cyperus brevibracteatus

T3918 Lonicera similis

T3283 Hosta sieboldiana

T3667 Lagenaria breviflora

T1816 Crotalaria breviflora

T1648 Consolida pubescens

T0636 Aristolochia pubescens

T2613 Euphorbia pubescens

T3268 Holarrhena pubescens

T3217 Heracleum moellendorffii [Syn. Heracleum microcarpum;

Heracleum morifolium]

T0519 Antenoron neofiliforme

T2390 Epimedium brevicornum

T2391 Epimedium brevicornum

T0219 Agave cantala
GUI JIAN JIN JI ER

HU CONG

SHAN DONG ZHONG ZU JUE

SHAN YANG WU TOU

LIANG NIAN SHI SONG

YI ZHI REN

GOU TENG

JIAN YE YE XIA ZHU

HONG SHU

JIAN YE TANG SONG CAO

JIAN YE QING FENG TENG

JIAN YE FAN XIE YE

JIAN JU ZI JIN

MA TONG HUA

LU SHAN SHI WEI

QIAO BING BA QIA

DA YE HUA JIAO

DA YE HUA JIAO GEN

JI CAI

JI CAI ZI

XING CAI

SI GUO XIANG CHA CAI

GUANG LIANG YANG TONG

GUANG LIANG SHI SONG

GUANG LIANG LUO FU MU

GUANG LIANG JIA LONG DAN

GUANG LIANG YUAN ZHI

GUANG LIANG HUANG TAN

LIANG YE HUA PI

LIANG YE YAN DOU TENG

ZE LAN

ZE LAN GEN

CAO HU JIAO

RU DI JIN NIU

WEN GUAN MU

SHI SHI DANG GUI

AN CI BAI

HAI JIU CAI

DUAN BAO YE SHA CAO

XI ZHAN MAO REN DONG

DA YU BIAO HUA

DUAN HUA HU LU

DUAN HUA ZHU SHI DOU

DUAN ROU MAO FEI YAN CAO

DUAN ROU MAO MA DOU LING

DUAN ROU MAO DA JI

DUAN ROU MAO ZHI XIE MU

DUAN MAO DU HUO

DUAN MAO JIN XIAN CAO GEN

YIN YANG HUO

YIN YANG HUO GEN

XIA YE LONG SHE LAN 
Shortleaf Anabasis

Shortleaf Crotalaria*

Shortleaf Gentian*

Shortleaf Kyllinga

Shortleaf Wormwood*

Shortlobe Ligusticum

Shortpetalflower

Shortscape Fleabane

Shortsepal Goldthread

Shortspike Pepper*

Shortspine Pricklyash*

Shortstalk Bushclover

Shortstalk Monkshood

Shortstamen Stephania*

Shortstem Archangelica*

Short-stipe Agrostophyllum*

Short-stipe Liriope*

Shorttooth Milkvetch*

Shorttube Lycoris

Shoulang Yam

Showy Bleedingheart Root

Showy Mountainsah

Shrub Lespedeza

Shrub Thoroughwax (Shrubby Hare's-ear)

Shrubalthea Bark

Shrubalthea Flower

Shrubalthea Fruit

Shrubby Ajania

Shrubby Asparagus*

Shrubby Baeckea

Shrubby Clover*

Shrubby Crinum*'bush' or 'march lily'

Shrubby Devilpepper*

Shrubby Holly*

Shrubby Milkwort*

Shrubby Otostegia*

Shrubby Woodwardia

Siam Falsenettle

Siam Rosewood

Siamense Common Jasminorange

Siamese Senna

Siberia Thorowax

Siberian Adonis

Siberian Cocklebur

Siberian Corydalis

Siberian Cranesbill

Siberian Elm

Siberian Fir

Siberian Fritillary

Siberian Goldenray

Siberian Hazelnut
T0436 Anabasis brevifolia

T1817 Crotalaria brevifolia

T2904 Gentiana brachyphylla

T3646 Kyllinga brevifolia

T0667 Artemisia brevifolia

T3819 Ligusticum brachylobum

T1001 Brachystemma calycinum

T2422 Erigeron breviscapus

T1663 Coptis chinensis var. brevisepala

T4939 Piper brachystachyum

T6868 Zanthoxylum brachyacanthum

T3771 Lespedeza cyrtobotrya

T0079 Aconitum brachypodum

T6118 Stephania brachyandra

T0582 Archangelica brevicaulis [Syn. Angelicarpa brevicaulis;

Angelica brevicaulis]

T0253 Agrostophyllum brevipes

T3872 Liriope muscari

T0788 Astragalus canadensis var. brevidens

T3986 Lycoris radiata [Syn. Amaryllis radiata]

T2193 Dioscorea cirrhosa [Syn. Dioscorea pogonoides]

T2154 Dicentra spectabilis

T6054 Sorbus decora

T3769 Lespedeza bicolor

T1062 Bupleurum fruticosum

T3245 Hibiscus syriacus

T3244 Hibiscus syriacus

T3246 Hibiscus syriacus

T0260 Ajania fruticulosa

T0748 Asparagus dumosus

T0853 Baeckea frutescens

T6519 Trifolium fruticosa

T1801 Crinum macowanii

T5434 Rauwolfia fruticosa

T3391 Ilex dumosa

T5076 Polygala fruticosa

T4557 Otostegia fruticosa

T6830 Woodfordia fruticosa

T0963 Boehmeria siamensis

T1999 Dalbergia cochinchinensis

T4327 Murraya siamensis

T1244 Cassia siamea

T1073 Bupleurum sibiricum

T0188 Adonis sibirica

T6843 Xanthium sibiricum [Syn. Xanthium strumarium]

T1742 Corydalis sibirica

T2947 Geranium sibiricum

T6597 Ulmus pumila

T0007 Abies sibirica

T2790 Fritillaria pallidiflora

T3813 Ligularia sibirica

T1751 Corylus heterophylla
DUAN YE JIA MU ZEI

DUAN YE ZHU SHI DOU

DUAN YE LONG DAN

SHUI WU GONG

DUAN YE JUAN HAO

DUAN PIAN GAO BEN

DUAN BAN HUA

DENG ZHAN XI XIN

DUAN E HUANG LIAN

DUAN SUI HU JIAO

DUAN CI HUA JIAO

DUAN GENG HU ZHI ZI

XUE SHANG YI ZHI HAO

BAI XIAN SHU

DUA JING GU DANG GUI

DUAN BING HE YE LAN

DUAN TING SHAN MAI DONG

DUAN CHI HUANG QI

SHI SUAN

SHU LIANG

HE BAO MU DAN GEN

MEI LI HUA QIU

HU ZHI ZI

GUAN MU CHAI HU

MU JIN PI

MU JIN HUA

MU JIN ZI

GUAN MU YA JU

GUAN MU TIAN MEN DONG

GANG SONG

GUAN MU ZHUANG CHE ZHOU

CAO

GUAN MU WEN SHU LAN

GUAN MU LUO FU MU

GUAN CONG DONG QING

GUAN MU YUAN ZHI

GUAN MU AO TUO SI TE CAO

XIA ZI HUA

SHU XU ZHU MA

JIAO ZHI HUANG TAN

YUAN DONG JIU LI XIANG

TIE DAO MU

XING AN CHAI HU

BEI CE JIN ZHAN HUA

CANG ER

BEI ZI JIN

SHU ZHANG LAO GUAN CAO

YU SHU

XI BO LI YA LENG SHAN

YI BEI MU

XI BO LI YA TOU WU

ZHEN 
Siberian Knotweed*

Siberian Milkwort

Siberian Motherwort

Siberian Phlojodicarpus

Siberian Solomonseal

Siberian Spruce

Siberian Stone Pine

Siberian Tansy

Siberian Veronicastrum

Sichuna Rabdosia

Sickle Alfalfa

Sickle Senna Seed

Sickle-leaved Hare's-ear

Sieber Acacia*

Sieber Senna

Siebold Alder*

Siebold Elder

Siebold Greenbrier

Siebold Wildginger

Sieve Sedge

Sievers Wormwood

Sieversia Milkvetch

Sikkim Mahonia

Sikkim Microula

Siko Milkvetch*

Siko Stringbush*

Siliculose Onychium

Silk Cocoon

Silk Mulberry*

Silk-rubber Tree

Silktree Albizia Bark

Silkworm Egg

Silkworm Feculae

Silkworm King

Silkworm Larva

Silky Ant

Silky Rose

Silver Maple

Silver Ragwort

Silver Wattle

Silveredge Agave

Silverleaf Cotoneaster

Silverleaf Morningglory*

Silverweed Cinquefoil

Silvervine Actinidia

Silvery Aleuritopteris

Simon Poplar

Simons Mahonia

Simple Arachniodes

Simple Digenea Frond

Simple Pronephrium

Simple Rostellularia

Simpleleaf Shrub Chastetree
T5115 Polygonum sibiricum [Syn. Persicaria sibirica]

T5086 Polygala sibirica

T3754 Leonurus sibiricus

T4804 Phlojodicarpus sibiricus

T5095 Polygonatum sibiricum

T4875 Picea obovata

T4923 Pinus sibirica

T6295 Tanacetum sibiricum [Syn. Filifolium sibiricum]

T6731 Veronicastrum sibiricum

T3525 Isodon setschwanensis

T4147 Medicago falcata

T1250 Cassia tora

T1060 Bupleurum falcatum

T0029 Acacia sieberiana

T1245 Cassia sieberiana

T0331 Alnus sieboldiana

T5705 Sambucus sieboldiana

T5982 Smilax sieboldii

T0731 Asarum sieboldii

T1202 Carex kobomugi

T0696 Artemisia sieversiana

T0804 Astragalus sieversianus

T4073 Mahonia sikkimensis

T4227 Microula sikkimensis

T0803 Astragalus shikokianus

T6823 Wikstroemia sikokiana

T4504 Onychium siliculosum

T0976 Bombyx mori

T4292 Morus bombycis

T2811 Funtumia elastica

T0292 Albizzia julibrissin

T0979 Bombyx mori

T0978 Bombyx mori

T0977 Bombyx mori

T0975 Bombyx mori

T2753 Formica fusca

T5573 Rosa sericea

T0055 Acer saccharinum

T5885 Senecio cineraria

T0020 Acacia dealbata

T0218 Agave angustifolia var. marginata

T1761 Cotoneaster pannosus

T3445 Ipomoea argyrophylla

T5179 Potentilla anserina

T0164 Actinidia polygama

T0301 Aleuritopteris argentea

T5158 Populus simonii

T4074 Mahonia simonsii

T0565 Arachniodes simplicior

T2173 Digenea simplex

T5209 Pronephrium simplex [Syn. Meniscium simplex]

T3610 Justicia simplex

T6790 Vitex rotundifolia [Syn. Vitex trifollia var. simplicifolia]
XI BO LI YA LIAO[yn

XI BO LI YA YUAN ZHI

XI YE YI MU CAO

ZHANG GUO QIN

HUANG JING

XI BO LI YA YUN SHAN

XI BO LI YA HONG SONG

SI BO LI YAAI JU

ZHAN LONG JIAN

SI CHUAN XIANG CHA CAI

YE MU XU

JUE MING ZI

ZI HU

XI BO JIN HE HUAN

XI BO JUE MING

XI BO DE QI MU

LAN SHAI PIAO

NIAN YU XU

XI XIN

SHA ZUAN TAI CAO

BAI HAO

MIAN MAO HUANG QI

XI JIN SHI DA GONG LAO

WEI KONG CAO

SI GUO HUANG QI

SI GUO YAO HUA

JIN FEN JUE

CAN JIAN

CAN SANG

SI JIAO SHU

HE HUAN PI

YUAN CAN ZI

YUAN CAN SHA

YUAN CAN E

BAI JIANG CAN

HEI MA YI

JUAN MAO QIANG WEI

YIN BAI QI

YIN BAI QIAN LI GUANG

YIN BAI JIN HE HUAN

YIN BIAN LONG SHE LAN

ZHAN MAO XUN ZI

YIN YE SHU

E RONG WEI LING CAI

MU TIAN LIAO

TONG JING CAO

XIAO YE YANG

XI MENG SI SHI DA GONG LAO

CHANG WEI FU YE ER JUE

HAI REN CAO

DAN YE XIN YUE JUE

DAN JUE CHUANG

DAN YE MAN JING 
Simpleleaf Shrub Chastetree Fruit

Simplex Rice-flower*

Singharanut

Singkwa Towelgourd

Singleleaf Acronychia*

Sinkiang Fritillary

Sinkiang Globethistle

Sinkiang Poplar*

Sinkiang-Tibet Arnebia

Siris-acacia

Sisal Hemp-plant

Sisso Rosewood

Sitka Spruce

Sixangular Dysosma

Sixpetal Tailgrape

Skin-carp

Skunk Cabbage

Skyblue Broomrape

Slamm Black Poplar

Sleeping Lotusleaftung

Slender Dutchmanspipe

Slender Dutchmanspipe Root

Slender Maesa

Slender Periwinkle*

Slender Phlegmariurus

Slender-leaf Crotalaria

Slenderstalk Dicranostigma

Slenderstalk Litse*

Slenderstyle Acanthopanax Root-bark

Slim-top Meadowrue

Slimy Rabdosia

Slimy Tobacco*

Slimysheathed Waxy Cap 橄榄白蜡伞

Sloughy Litse*

Small Beefwood*

Small Bugbane

Small Burnet*

Small Centipeda

Small Ephedra

Small Gorse*

Small Ochotsk Corydalis

Small Piptanthus*

Small Rattle-box

Small Ungernia*

Small Yellow Daylily

Smallcalyx Woodbetony

Smaller Concave-top Alga*

Smallflower Acanthus*

Smallflower Beggarticks

Smallflower Bruguiera Fruit*

Smallflower Bugle*

Smallflower Embelia
T6791 Vitex rotundifolia [Syn. Vitex trifollia var. simplicifolia]

T4893 Pimelea simplex

T6492 Trapa bispinosa

T3933 Luffa acutangula

T0149 Acronychia haplophylla

T2800 Fritillaria walujewii

T2317 Echinops ritro

T5146 Populus alba var. pyramdalis

T0648 Arnebia euchroma

T0293 Albizzia lebbeck

T0226 Agave sisalana

T2013 Dalbergia sissoo

T4876 Picea sitchensis

T2302 Dysosma pleiantha [Syn. Podophyllum pleianthum]

T0653 Artabotrys hexapetalus [Syn. Annona hexapetalus]

T3199 Hemibarbus labeo

T3994 Lysichitum americanum

T4536 Orobanche coerulescens

T5166 Populus xiaohei

T3230 Hernandia nymphaeifolia

T0626 Aristolochia debilis [Syn. Aristolochia longa]

T0627 Aristolochia debilis [Syn. Aristolochia longa]

T4032 Maesa tenera

T1270 Catharanthus pusillus

T4801 Phlegmariurus phlegmaria [Syn. Lycopodium phlegmaria]

T1822 Crotalaria intermedia

T2162 Dicranostigma franchetianum [Syn. Dicranostigma

leptopodum]

T3889 Litsea gracilipes

T0038 Acanthopanax gracilistylus

T6409 Thalictrum simplex [Syn. Thalictrum simplex var. brevipes]

T3492 Isodon glutinosa

T4425 Nicotiana glutinosa

T3313 Hygrophorus olivaceoalbus

T3896 Litsea turfosa

T1259 Casuarina stricta

T1417 Cimicifuga acerina

T5711 Sanguisorba minor

T1312 Centipeda minima

T2373 Ephedra minuta

T6591 Ulex minor

T1729 Corydalis ochotensis var. raddeana

T4975 Piptanthus nanus

T1827 Crotalaria nana

T6641 Ungernia minor

T3197 Hemerocallis minor

T4681 Pedicularis decora

T3718 Laurencia majuscula

T0047 Acanthus ebracteatus

T0939 Bidens parviflora

T1040 Bruguiera parviflora

T0268 Ajuga parviflora

T2347 Embelia parviflora
DAN YE MAN JING ZI

DAN ZHI DAO HUA

LING

YUE SI GUA

DAN YE YOU GAN

XIN JIANG BEI MU

XIN JIANG LAN CI TOU

XIN JIANG YANG

XIN ZANG JIA ZI CAO

KUO JIA HE HUAN

JIAN MA

YIN DU HUANG TAN

XI TE KA YUN SHAN

LIU JIAO LIAN

YING ZHAO

CHONG CHUN YU

MEI ZHOU GUAN YIN LIAN

LIE DANG

XIAO HEI YANG

SHUI LIAN YE TONG

MA DOU LING

QING MU XIANG

RUAN RUO DU JING SHAN

XI XIAO CHANG CHUN HUA

MA WEI SHAN

XI YE ZHU SHI DOU

TU CHUANG HUA

XI BING MU JIANG ZI

WU JIA PI

YING SHUI HUANG LIAN

JIAO NIAN XIANG CHA CAI

JIAO YAN CAO

NI ZHAO MU JIANG ZI

XIAO MU MA HUANG

SAN MIAN DAO

XIAO DI YU

E BU SHI CAO

AI MA HUANG

XIAO JING DOU

XIAO HUANG ZI JIN

XIAO SHA DONG QING

XIAO ZHU SHI DOU

XIAO BO SI SHI SUAN

XIAO XUAN CAO GEN

MEI GUAN MA XIAN HAO

LUE DA AO DING ZAO

XIAO HUA LAO SHU LE.

XIAO HUA GUI ZHEN

XIAO HUA MU LAN GUO

XIAO HUA XIA KU CAO

XIAO HUA SUAN TENG ZI 
Smallflower Hemsleya

Smallflower Magnoliavine*

Smallflower Milkwort

Smallflower Mimosa*

Smallflower Pawpaw

Smallflower Saussurea*

Smallflower Xylopia*

Smallflower Yam

Smallfruit Fig

Smallfruit Loosestrife*

Smallfruit Meadowrue

Smallfruit Rose

Smallfruit Rue*

Small-grand Concave-top Alga*

Smallheartleaved Morningglory

Smallleaf Black Thorowax

Smallleaf Fritsch Spiraea*

Smallleaf Jointfir

Smallleaf Knema

Small-leaf Knema

Small-leaf Meadowrue

Small-leaf Photinia*

Smallleaf Rabdosia*

Small-leaf Raspberry

Small-leaf Tansy*

Small-leafElaeocarpus*

Smallleaf-hirsute Alder*

Small-leaved Box

Smallligulatecorolla Aster

Small-tree Ardisia*

Smilax bockii

Smirch Groundsel*

Smirnow Leontice*

Smith Meadowrue

Smoke Longan*

Smooth Butterbur*

Smooth Gambirplant

Smooth Mulberry*

Smoothbranched Supplejack

Smoothfruit Ventilago

Smoothstalk Madder

Snake Sansevieria

Snakebubble Raspberry

Snakefruit Corydalis

Snaw Gentian

Sneezeweed

Snow Azalea

Snow Lotus

Snowbellleaf Tickclover

Snowdrop

Snowwhite Sunflower*

Soapbark Tree

Soapwort
T3207 Hemsleya graciliflora [Syn. Alsomitra graciliflora]

T5795 Schisandra micrantha

T5087 Polygala telephioides

T4250 Mimosa tenuiflora

T0743 Asimina parviflora

T5763 Saussurea parviflora

T6854 Xylopia parviflora

T2206 Dioscorea parviflora

T2721 Ficus microcarpa

T4003 Lysimachia microcarpa

T6398 Thalictrum microgynum

T5563 Rosa cymosa

T5627 Ruta microcarpa

T3717 Laurencia glandulifera

T3450 Ipomoea obscura

T1075 Bupleurum smithii var. parvifolium

T6078 Spiraea fritschiana var. parvifolia

T3033 Gnetum parvifolium [Syn. Gnetum indicum]

T3630 Kleinhovia hospita

T3631 Knema globularia

T6380 Thalictrum elegans

T4827 Photinia parvifolia

T3517 Isodon parvifolia

T5601 Rubus taiwanicolus

T6293 Tanacetum microphyllum

T2330 Elaeocarpus parvifolius

T0327 Alnus hirsute var. microphylla

T1091 Buxus microphylla

T0778 Aster albescens

T0591 Ardisia arborescens

T5975 Smilax bockii

T5908 Senecio sceleratus

T3747 Leontice smirnowii

T6410 Thalictrum smithii

T2184 Dimocarpus fumatus

T4742 Petasites laevigatus

T6621 Uncaria laevigata

T4295 Morus laevigata

T0922 Berchemia polyphylla var. leioclada

T6689 Ventilago leiocarpa

T5583 Rubia schumannina

T5714 Sansevieria trifasciata

T5591 Rubus cochinchinensis

T1730 Corydalis ophiocarpa

T2922 Gentiana nivalis

T3134 Helenium autumnale

T5518 Rhododendron mucronatum

T5755 Saussurea involucrata

T2134 Desmodium styracifolium

T2822 Galanthus nivalis

T3151 Helianthus niveus

T5382 Quillaja saponaria

T5726 Saponaria officinalis
XI HUA XUE DAN

XIAO HUA WU WEI ZI

XIAO HUA YUAN ZHI

XI HUA HAN XIU CAO

XIAO HUA PAO PAO

XIAO HUA FENG MAO JU

XIAO HUA MU BAN SHU

XIAO HUA DUN YE SHU YU

RONG SHU

XIAO GUO XIANG CAO

XIAO GUO TANG SONG CAO

XIAO GUO QIANG WEI GEN

XIAO GUO YUN XIANG

XIAO XIAN AO DING ZAO

XIAO XIN YE SHU

XIAO YE HEI CHAI HU

XIAO YE HUA BEI XIU XIAN JU

XIAO YE MAI MA TENG

MIAN TOU YE

XIAO YE HONG GUANG SHU

XIAO YE TANG SONG CAO

XIAO YE SHI NAN

XIAO YE XIANG CHA CAI

XIAO YE XUAN GOU ZI

XIAO YE JU HAO

XIAO YE DU YING

XIAO YE YING MAO QI MU

XIAO YE HUANG YANG

XIAO SHE ZI WAN

XIAO QIAO MU ZI JIN NIU

XI NAN BA QIA

LA QIAN LI GUANG

SI MI SHI MU DAN CAO

BIAN ZHU TANG SONG CAO

YAN SE LONG YAN

PING HUA FENG DOU CAI

PING HUA FA LIANG GOU TENG

PING HUA SANG

GUANG ZHI GOU ER CHA

YI HE GUO

DA YE QIAN CAO

HU WEI LAN

SHE PAO JIN

SHE GUO HUANG JIN

XUE LONG DAN

DUI XIN JU

BAI HUA YING SHAN HONG

XUE LIAN

GUANG JIN QIAN CAO

XUE HUA LIAN

XUE BAI XIANG RI KUI

ZAO PI SHU

FEI ZAO CAO 
Soda-apple Nightshade

Sodome Nightshade*

Soft Argyreia*

Soft Comfrey

Soft Hawksbeard

Soft Persimmon*

Softcoral Lemnalia bournei

Softcoral Nephthea chabroli

Soft-hair Cowparsnip

Somniferous Withania

Soncoya

Songaria Cynomorium

Sorghum

Sorrel Rhubarb

South Asia Michelia

South China Evodia

South China Honeyloeust

South Chrysanthemum

South Dodder Seed

South Houndstongue*

South-Africa Tufted Everlasting*

South-African Eriosema*

Southern Catalpa

Southern Magnolia

Southern Maidenhair

Southern Marigold

Southern Red-cedar

Southern-caledonian Tulipwood*

Southern-Spain Hawksbeard*

South-India Andrographis*

South-India Sago Seed*

South-Japan Threewingnut*

South-western Honeysuckle

Sowthistle Tasselflower

Sowthistle-leaf Ixeris

Soybean Oil

Soybean Paste

Spanish Chestnut

Spanish Haplophyllum*

Spanish Heath

Spanish Oak

Sparseflower Croton*

Sparteine Crotalaria*

Spatulate Alstonia*

Spearmint

Spectacular Alstonia*

Spectacular Senna*

Spicate Centaurium*

Spicate Clerodendranthus

Spicate Pseudoelephantopus

Spicate Woodbetony

Spicebush
T6014 Solanum surattense

T6012 Solanum sodomeum [Syn. Solanum sodomaeum]

T0613 Argyreia mollis

T6247 Symphytum orientale

T1788 Crepis mollis

T2225 Diospyros mollis

T3740 Lemnalia bournei

T4417 Nephthea chabroli

T3214 Heracleum lanatum

T6829 Withania somnifera

T0510 Annona purpurea

T1972 Cynomorium songaricum

T6056 Sorghum vulgare

T5474 Rheum palmatum

T4212 Michelia doltsopa

T2638 Evodia austrosinensis

T2976 Gleditsia fera

T1397 Chrysanthemum segetum

T1911 Cuscuta australis

T1968 Cynoglossum australe

T3157 Helichrysum caespititium

T2436 Eriosema kraussianum

T1260 Catalpa bignonioides

T4039 Magnolia grandiflora

T0171 Adiantum capillus-veneris

T6281 Tagetes minuta

T3598 Juniperus silicicola

T3107 Harpullia austro-caledonica

T1790 Crepis tingitana

T0460 Andrographis viscosula

T1925 Cycas beddomei

T6539 Tripterygium doianum

T3908 Lonicera bournei

T2357 Emilia sonchifolia

T3548 Ixeris sonchifolia

T2999 Glycine max

T3003 Glycine max

T1255 Castanea sativa

T3102 Haplophyllum hispanicum

T2416 Erica australis

T5378 Quercus rubra

T1856 Croton sparsiflorus

T1833 Crotalaria spartioides

T0375 Alstonia spatulata

T4190 Mentha spicata

T0376 Alstonia spectabilis

T5916 Senna spectabilis

T1310 Centaurium spicatum

T1553 Clerodendranthus spicatus

T5253 Pseudoelephantopus spicatus

T4683 Pedicularis spicata

T3848 Lindera benzoin
YE DIAN QIE

SUO DUO MI QIE

RUAN YIN BEI TENG

DONG FANG XI MEN FEI CAO

ROU SE HUAN YANG SHEN

RUAN SHI

BO LUN LIN HUA RUAN SHAN HU

RUAN MAO DU HUO

CUI MIAN SHUI QIE

ZI FAN LI ZHI

SUO YANG

GAO LIANG

ZHANG YE DA HUANG

NAN YA HAN XIAO

HUA NAN WU ZHU YU

HUA NAN ZAO JIA

NAN TONG HAO

NAN FANG TU SI ZI

NAN FANG LIU LI CAO

NAN FEI CONG SHENG LA JU

NAN FEI JI TOU SHU

MEI GUO ZI

HE HUA YU LAN

ZHU ZONG CAO

WEI XIAO WAN SHOU JU

NAN MEI ZHOU GUI

NAN SU GE LAN JIA SHAN LUO

NAN XI BAN YA HUAN YANG SHEN

NAN YIN DU CHUAN XIN LIAN

NAN YIN DU SU TIE SHU GUO

NAN RI BEN LEI GONG TENG

XI NAN REN DONG

YI DIAN HONG

BAO JING KU MAI CAI

DOU YOU

JIANG

OU ZHOU LI

XI BAN YA YUN XIANG CAO

NAN FANG OU SHI NAN

HONG LI

SAN HUA BA DOU

YING ZHAO DOU ZHU SHI DOU

DAO ZHUANG JI GU CHANG SHAN

LIU LAN XIANG

ZHUANG GUAN JI GU CHANG

SHAN

ZHUANG GUAN FAN XIE

SUI ZHUANG BAI JIN HUA

MAO XU CAO

JIA DI DAN CAO

SUI HUA MA XIAN HAO

GUI PI DIAO ZHANG 
Spiderweb Blueeargrass, Pearl Cyanotis* Spiked Gingerlily

Spiked Loosestrife

Spiked Monkshood

Spinach Jointfir

Spinach-like Jointfir

Spine Aralia

Spine Date

Spine Date Seed

Spined Cordia*

Spined Custardapple*

Spineless Agave

Spineless Common Jujube

Spineless Mitragyna*

Spineless Powderpuff*

Spinish

Spiny Alsophila

Spiny Meconopsis

Spiny Sida*

Spiny Thorowax*

Spinyflower Alternanthera

Spinyflower Strophanthus

Spinyfruit Pricklyash

Spinyleaf Prickleyash

Spiral Acacia*

Splidleaf Poppy*

Sponge Amphimedon paraviridis

Sponge Haliclona variclona

Sponge Iotrochota baculifera

Sponge Petrosia strongylata

Sponge Psammaplysilla purpurea

Sponge Sphaeciospongia vesparia

Spoonleaf Nardostachys

Spotted Gum

Spottedleaf Euphorbia

Spread Gentian

Spreading Creosote-bush

Spreading Dogbane

Spreading Hedyitis

Spreading Meadowrue*

Spreading Rabdosia*

Spreading St.John'swort

Spreading Swertia*

Sprenger Magnolia

Spring Knotweed

Spring Larkspur

Spring Snowflake

Spruce Rosewood*

Spurless Barrenwort*

Spurless Columbine

Squirrel's Foot Fern

Squirting Cucumber
T1921 Cyanotis arachnoidea [Syn. Cyanotis bodinieri]

T3120 Hedychium spicatum

T4007 Lythrum salicaria

T0133 Aconitum spicatum

T3028 Gnetum gnemon

T3029 Gnetum gnemonoides

T0567 Aralia armata

T6918 Ziziphus jujuba var. spinosa

T6919 Ziziphus jujuba var. spinosa

T1679 Cordia spinescens

T0512 Annona spinescens

T0216 Agave americana var. marginata [Syn. Agave americana var.

variegata]

T6917 Ziziphus jujuba var. inermis

T4256 Mitragyna inermis

T1115 Calliandra inermis

T6076 Spinacia oleracea

T0364 Alsophila spinulosa

T4143 Meconopsis horridula

T5944 Sida spinosa

T1076 Bupleurum spinosum

T0380 Alternanthera repens

T6156 Strophanthus gratus

T6878 Zanthoxylum echinocarpum

T6874 Zanthoxylum dimorphophyllum var. spinifolum

T0031 Acacia spirorbis

T4631 Papaver nudicaule var. chinense

T0430 Amphimedon paraviridis

T3093 Haliclona variclona

T3442 Iotrochota baculifera

T4750 Petrosia strongylata

T5249 Psammaplysilla purpurea

T6070 Sphaeciospongia vesparia

T4387 Nardostachys jatamansi

T2514 Eucalyptus maculata

T2622 Euphorbia supina

T2917 Gentiana leptoclada

T3693 Larrea divaricata

T0550 Apocynum androsaemifolium

T4485 Oldenlandia diffusa [Syn. Hedyotis diffusa]

T6412 Thalictrum squarrosum

T3485 Isodon effusa

T3360 Hypericum patulum

T6229 Swertia patens

T4052 Magnolia sprengeri

T5113 Polygonum persicaria

T2088 Delphinium tricorne

T3782 Leucojum vernum

T2016 Dalbergia spruceana

T2393 Epimedium ecalcaratum

T0557 Aquilegia ecalcarata

T2053 Davallia mariesii

T2313 Ecballium elaterium
ZHEN ZHU LU SHUI CAO

TU LIANG JIANG

QIAN QU CAI

SUI ZHUANG WU TOU

XIAN ZHOU MAI MA TENG

MA LAI XI YA MAI MA TENG

HU CI CONG MU

SUAN ZAO

SUAN ZAO REN

YOU CI PO BU MU

CI ZHUANG FAN LI ZHI

WU CI FAN MA

WU CI ZAO

WU CI MAO ZHU MU

WU CI ZHU YING HUA

BO CAI

SUO LUO

DUO CI LV RONG HAO

DUO CI HUANG HUA REN

DUO CI CHAI HU

CI HUA LIAN ZI CAO

XUAN HUA YANG JIAO AO

CI KE HUA JIAO

CI YI YE HUA JIAO

LUO XUAN JIN HE HUAN

LIE YE YE YING SU

XIAO BANG XIOU QIOU HAI MIAN

ZI SHA ROU HAI MIAN

SHI YE GAN SONG

BAN WEN AN

BAN YE DI JIN

MAN ZHI LONG DAN

JI CHA KAI LA RUI A

DIAN DI MEI YE CHA YE HUA

BAI HUA SHE SHE CAO

ZHAN ZHI TANG SONG CAO

KAI ZHAN XIANG CHA CAI

JIN SI MEI

XIE JING ZHANG YA CAI

WU DANG MU LAN

TAO YE LIAO

SAN JU AI CUI QUE

XUE PIAN LIAN

QIAO HUANG TAN

WU JU YIN YANG HUO

WU JU LOU DOU CAI

HAI ZHOU GU SUI BU

PEN GUA 
Srilanka Glochidion

Sri-Lankan Bridelia

S-shape Coralbean*

St. Brigid

St. Johnswortleaf Skullcap

St. Marys

Stag's-horn Sumach

Stalkedfruit Meadowrue*

Stalkedfruit Pricklyash

Staminate Sage*

Star Anise

Staunton's Spikemoss

Stavisacre

Stellate Cladonia

Stellate hair Carpetweed

Stemless Carline Thistle

Steudner Heliotrope*

Stevenson Rosewood*

Sticky Clary

Sticky Rice

Stiffleaf Juniper Fruit

Stilted Pyrrosia

Stinking Polecat, Wood Witch

Stipefruit Mistletoe

Stipular Crotalaria*

Stipulate Dolichandrone

Stoat-osmyl Deadnettle*

Stomach Motherwort

Stone Mallotus*

Straight-raceme Barberry

Straw Mushroom

Strawberry Clover

Straw-coloured Gentian

Stria Agave*

Striate Kummerowia

Strict Conyza

Strict Meadowrue*

Strict Verbena*

Strictleaf Cryptocarya*

Strictleaf Dracaena Leaf

Strigose Microlepia

Stringbushlike Rabdosia

Stringy Stonecrop

Striped Crotalaria

Strongfragrant Loosestrife

Stuhlmann Millettia*

Stygian Euphorbia*

Stylose Mangrove

Suakwa Vegetablesponge

Suakwa Vegetablesponge Seed

Suave Holarrhena*
T2990 Glochidion zeylanicum

T1028 Bridelia retusa

T2475 Erythrina sigmoidea

T0466 Anemone coronaria

T5839 Scutellaria hypericifolia

T5957 Silybum marianum

T5540 Rhus typhina

T6404 Thalictrum podocarpum

T6889 Zanthoxylum podocarpum

T5695 Salvia staminea

T3409 Illicium verum

T5868 Selaginella stauntoniana

T2086 Delphinium staphisagria

T1528 Cladonia stellaris [Syn. Cladonia alpestris]

T2984 Glinus lotoides [Syn. Mollugo lotoides]

T1208 Carlina acaulis

T3181 Heliotropium steudneri

T2017 Dalbergia stevensonii

T5673 Salvia glutinosa

T4548 Oryza sativa var. glutinosa

T3594 Juniperus rigida

T5356 Pyrrosia gralla

T4778 Phallus impudicus

T6777 Viscum multinerve

T1835 Crotalaria stipularia

T2245 Dolichandrone stipulata

T3682 Lamium galeobdolon

T3750 Leonurus cardiaca

T4085 Mallotus repandus var. chrysocarpus [Syn. Mallotus

chrysocarpus; Mallotus repandus]

T0911 Berberis orthobotrys

T6803 Volvariella volvacea

T6518 Trifolium fragiferum

T2934 Gentiana straminea

T0227 Agave striata

T3645 Kummerowia striata

T1658 Conyza stricta

T6413 Thalictrum strictum

T6710 Verbena stricta

T1864 Cryptocarya strictifolia

T1684 Cordyline strcta

T4220 Microlepia strigosa [Syn. Trichomanes strigosa]

T3535 Isodon wikstroemioides

T5857 Sedum sarmentosum

T1826 Crotalaria mucronata

T4002 Lysimachia foenum-graecum

T4244 Millettia stuhlmannii

T2621 Euphorbia stygiana

T5489 Rhizophora stylosa

T3934 Luffa cylindrica

T3935 Luffa cylindrica

T3267 Holarrhena mitis
CHUI ZHU SUAN PAN ZI

SI LI LAN KA TU MI SHU

AI SI XING CI TONG

HUA GUAN YIN LIAN HUA

CHUAN HUANG QIN

SHUI FEI JI

LU JIAO QI SHU

BING GUO TANG SONG CAO

BING GUO HUA JIAO

XIONG RUI ZHUANG SHU WEI CAO

BA JIAO HUI XIANG

HAN SHENG JUAN BAI

SI TA WEI CUI QUE HUA

TAI BAI HUA

XING SU CAO

CHAO XIAN JI

SI SHI TIAN JIE CAI

SI TE WEN HUANG TAN

JIAO ZHI SHU WEI CAO

NUO DAO

DU SONG SHI

XI NAN SHI WEI

BAI GUI BI

BING GUO HU JI SHENG

TUO YE ZHU SHI DOU

XI NAN MAO WEI SHU

YOU CHOU YE ZHI MA

WEI YI MU CAO

SHI YAN FENG

ZHI ZONG ZHUANG HUA XU XIAO

BO

CAO GU

CAO MEI CHE ZHOU CAO

MA HUA JIAO

TIAO WEN LONG SHE LAN

JI YAN CAO

JIAN TENG BAI JIU CAO

BI ZHI TANG SONG CAO

JIAN TING MA BIAN CAO

ZHI LI YE HOU KE GUI

JIAN YE TIE SHU YE

CU MAO LIN GAI JUE

YAO HUA XIANG CHA CAI

SHI ZHI JIA

ZHU SHI DOU

LING XIANG CAO

SI TU JI XUE TENG

YOU AN DI JIN

HONG HAI LAN

SI GUA

SI GUA ZI

WEN ROU ZHI XIE MU 
Subcapitate Aralia

Suberect Spatholobus

Suberose Coralbean*

Suberous Greenstar

Subglobose Asiabell

Sublessing Wormwood*

Subsessile St.John'swort

Subshrubby Peony Bark

Subterranean Clover

Subtomentose Milky*

Subtriplinerved Mahonia

Suckling Clover

Sudach Citrus*

Suffrutescent Securinega

Sugar Bush

Sugar Maple

Sugarcane

Sulfur Tuft

Sulphur Maidenhair Fern*

Sultanate-Oman Myrrhtree*

Sumach

Sumatra Snowbell

Sumatran Yew

Summer Snowflake

Summer-hyacinth

Sumpweed

Sun Euphorbia

Sunflower Flower

Sunflower Leaf

Sunflower Seed

Sunflower Stem Pith

Sungpan Monkshood

Sunn Crotalaria

Supine Heliotrope*

Surinam Quassia

Surrounded Swertia

Swamp Cottonwood

Swamp Loosestrife

Swamp Mahogany Leaf

Swampy Gentianopsis

Swede Seed

Swedish Turnip

Sweet Blackberry*

Sweet Broomwort

Sweet Cicely

Sweet Marjoram

Sweet Orange

Sweet Osmanthus

Sweet Pea

Sweet Pepper

Sweet Potato

Sweet Tooth

Sweet Uvaria*
T0575 Aralia subcapitata

T6066 Spatholobus suberectus

T2477 Erythrina suberosa

T5068 Polyalthia suberosa

T1601 Codonopsis subglobosa

T0699 Artemisia sublessingiana

T3365 Hypericum subsessile

T4585 Paeonia moutan [Syn. Paeonia suffruticosa]

T6525 Trifolium subterraneum

T3655 Lactarius subvellereus

T4064 Mahonia gracilipes

T6517 Trifolium dubium

T1511 Citrus sudachii

T5848 Securinega suffruticosa

T5212 Protea mellifera

T0056 Acer saccharum

T5641 Saccharum officinarum

T4366 Naematoloma fasciculare

T0178 Adiantum sulphureum

T1639 Commiphora wightii

T5532 Rhus coriaria

T6197 Styrax benzoin

T6316 Taxus sumatrana

T3781 Leucojum aestivum

T2842 Galtonia candicans

T3543 Iva frutescens

T2589 Euphorbia helioscopia

T3142 Helianthus annuus

T3144 Helianthus annuus

T3145 Helianthus annuus

T3143 Helianthus annuus

T0135 Aconitum sungpanense

T1823 Crotalaria juncea

T3182 Heliotropium supinum

T5370 Quassia amara

T6216 Swertia cincta

T5152 Populus heterophylla

T2057 Decodon verticillatus

T2517 Eucalyptus robusta

T2941 Gentianopsis paludosa

T1010 Brassica napus var. napobrassica

T1022 Brassica rutabaga

T5600 Rubus suavissimus

T5818 Scoparia dulcis

T4360 Myrrhis odorata

T4524 Origanum majorana

T1508 Citrus sinensis

T4550 Osmanthus fragrans

T3707 Lathyrus odoratus

T1186 Capsicum annuum

T3447 Ipomoea batatas [Syn. Convolvulus batatas]

T3302 Hydnum repandum

T6661 Uvaria dulcis
AN HUI CONG MU

MI HUA DOU

SHUAN ZHUANG CI TONG

AN LUO

QIU HUA DANG SHEN

YA LIE XING HAO

JI WU BING JIN SI TAO

MU DAN PI

DI XIA CHE ZHOU CAO

YA RONG GAI RU GU

XI BING SHI DA GONG LAO

DUN YE CHE ZHOU CAO

SU DA QI GAN JU

YI YE QIU

MI PU LUO TI YA MU

TANG QI

YAO YONG GAN ZHE

CU SHENG HUANG REN SAN

LIU HUANG TIE XIAN JUE

A MAN SU DAN MO YAO

XI XI LI QI SHU

AN XI XIANG

SU MEN DA LA HONG DOU SHAN

XIA XUE PIAN LIAN

XIA FENG XIN ZI

YI WA JU

ZE QI

XIANG RI KUI HUA

XIANG RI KUI YE

XIANG RI KUI ZI

XIANG RI KUI JING SUI

SONG PAN WU TOU

SHU MA

YANG XIN TIAN JIE CAI

MEI ZHOU KU MU

XI NAN ZHANG YA CAI

YI YE YANG

DI KE DONG

DA YE AN YE

SHI SHENG BIAN LEI

WU JING GAN LAN

RUI DIAN GAN LAN

TIAN CHA

YE GAN CAO

OU ZHOU MO YAO

TIAN NIU ZHI

TIAN CHENG

GUI HUA

XIANG WAN DOU

HONG HAI JIAO

GAN SHU

MEI WEI CHI JUN

TIAN ZI YU PAN 
Sweet Viburnum*

Sweet Woodruff

Sweet Wormwood

Sweet-briar

Sweetcane Culm

Sweetroot Polypody*

Sweetscented Basil

Sweetscented Oleander

Sweet-tongue Lippia*

Sword Jackbean

Sword-bean

Swordleaf Dianella

Swordleaf Dracaena

Swordlike Atractylodes

Sylvatic Sapium*

Syneilesis*

Syria Origanum*

Syrian Rue*

Szechuan Epimedium

Szechwan Adonis

Szechwan Chinaberry Bark

Szechwan Chinaberry Fruit

Szechwan Cyclea

Szechwan Sabia

Szechwan Tangshen

Szechwan-Tibet Rabdosia

Taibaien Aralia

Tail-leaf Monachosorum

Taillikeleaf Rabdosia

Taishan Sumac Fruit

Taiton Wildginger

Taiuia Root*

Taiwan Agrimony

Taiwan Angelica

Taiwan Barberry

Taiwan Beautyberry

Taiwan Bletilla*

Taiwan Buckthorn*

Taiwan Bugle*

Taiwan Butterbur*

Taiwan Common Jasminorange

Taiwan Corktree*

Taiwan Dandelion*

Taiwan Davallia

Taiwan Dualbutterfly

Taiwan Euchresta

$\mathrm{T}$ aiwan Goniothalamus

Taiwan Hibiscus

Taiwan Hogfennel

Taiwan Incense Cedar

Taiwan Juniper

Taiwan Juniper
T6738 Viburnum odoratissimum

T0757 Asperula odorata

T0660 Artemisia annиa

T5571 Rosa rubiginosa

T5642 Saccharum sinensis

T5123 Polypodium glycyrrhiza

T4473 Ocimum gratissimum

T4418 Nerium indicum

T3864 Lippia dulcis

T1169 Canavalia gladiata

T1168 Canavalia ensiformis

T2139 Dianella ensifolia

T2253 Dracaena cochinchinensis

T0823 Atractylodes lancea

T6146 Stillingia sylvatica [Syn. Sapium sylvatica]

T6256 Syneilesis palmata

T4525 Origanum syriacum

T5625 Ruta chalepensis

T2404 Epimedium sutchuenense

T0190 Adonis sutchuenensis

T4162 Melia toosendan

T4163 Melia toosendan

T1933 Cyclea sutchuenensis

T5637 Sabia schumanniana

T1602 Codonopsis tangshen

T3518 Isodon pharicus

T0576 Aralia taibaiensis

T4267 Monachosorum flagellare

T5392 Rabdosia excisa

T5539 Rhus taishanensis

T0734 Asarum taitoense

T1274 Cayaponia tayuya

T2558 Eupatorium formosanum

T0498 Angelica taiwaniana

T0907 Berberis kawakamii

T1118 Callicarpa formosana

T0954 Bletilla formosana

T5459 Rhamnus formosana

T0272 Ajuga taiwanensis

T4739 Petasites formosanus

T4316 Murraya crenulata

T4790 Phellodendron amurense var. wilsonii

T6300 Taraxacum formosanum

T2052 Davallia divaricata [Syn. Davallia formosana; Davallia

orientalis]

T6538 Tripterospermum taiwanense

T2526 Euchresta formosana

T3041 Goniothalamus amuyon

$\mathrm{T} 3247$ Hibiscus taiwanensis

T4755 Peucedanum formosanum

T1121 Calocedrus macrolepis var. formosana

T3588 Juniperus formosana

T3600 Juniperus taiwaniana
XIANG QI JIA MI

XIANG CHE YE CAO

HUANG HUA HAO

XIU HONG QIANG WEI

GAN ZHE

TIAN GEN DUO ZU JUE

DING XIANG LUO LE

JIA ZHU TAO

TIAN SHE CAO

DAO DOU

YANG DAO DOU

SHAN MAO ER

JIAN YE LONG XUE SHU

CANG ZHU

CAO WU JIU

TU ER SAN

XU LI YA NIU ZHI

SUI ZHUANG YUN XIANG

SI CHUAN YIN YANG HUO

SHU CE JIN ZHAN HUA

CHUAN LIAN PI

CHUAN LIAN ZI

SI CHUAN LUN HUAN TENG

SI CHUAN QING FENG TENG

CHUAN DANG SHEN

CHUAN ZANG XIANG CHA CAI

TAI BAI CONG MU

WEI YE XI ZI JUE

WEI YE XIANG CHA CAI

TAI SHAN YAN FU ZI

TAI DONG XI XIN

TA YOU XIE GUA

TAI WAN ZE LAN

HANG BAI ZHI

TAI WAN XIAO BO

DU HONG HUA

LAN YU BAI JI

TAI WAN SHU LI

TAI WAN JIN GU CAO

TAI WAN FENG DOU CAI

TAI WAN JIU LI XIANG

TAI WAN HUANG BO

TAI WAN PU GONG YING

DA YE GU SUI BU

TAI WAN SHUANG HU DIE TAI WAN SHAN DOU GEN TAI WAN GE NA XIANG

TAI WAN FU RONG

TAI WAN QIAN HU

TAI WAN CUI BAI

CI BAI

SHAN CI BAI 
Taiwan Loquat

Taiwan Magnoliavine

Taiwan Malabar Randia

Taiwan Meadowrue

Taiwan Mosla

Taiwan Pepper*

Taiwan Pieris*

Taiwan Sassafras

Taiwan Snowbell

Taiwan Spiraea*

Taiwan Spruce

Taiwan Thistle*

Taiwan Tournefortia*

Taizhong Buckthorn*

Tala Monkshood*

Tali Corydalis

Tall Camomile

Tall Ephedra

Tall Eupatorium

Tall Gastrodia

Tall Hibiscus

Tall Hymenodictyon

Tall Meadowrue

Tall Monkshood

Tall Skullcap

Tamarind Fruit

Tamarisk-leaf Juniper*

Tamariskoid Spikemoss

Tangerine Pericarp

Tangerine Seed

Tangle Thallus

Tangut Anisodus

Tangut Clematis

Tangut Daphne

Tangut Monkshood

Tangut Przewalskia

Tangut Rhubarb

Tankan Citrus

Tankan Citrus Pericarp

Tankasché (in Mexico)

Tansy

Taproot Angelica*

Tarbush

Taro

Tarragon

Tartarian Buckwheat

Tatarion Aster

Taurine Wormwood*

Tea Flower

Tea Root

Tea Seed

Teat-shaped Ardisia

Tenacious Condorvine
T2430 Eriobotrya deflexa

T5790 Schisandra arisanensis

T5410 Randia formosa

T6415 Thalictrum urbainii

T4539 Orthodon formosanus

T4970 Piper taiwanense

T4889 Pieris formosa

T5745 Sassafras randainense

T6199 Styrax formosanus

T6077 Spiraea formosana

T4874 Picea morrisonicola

T1447 Cirisum japonica var. takaoense

T6480 Tournefortia sarmentosa

T5462 Rhamnus nakaharai

T0136 Aconitum talassicum

T1746 Corydalis taliensis

T0520 Anthemis altissima

T2376 Ephedra procera

T2550 Eupatorium altissimum

T2890 Gastrodia elata

T3239 Hibiscus elatus

T3323 Hymenodictyon excelsum

T6405 Thalictrum polygamum

T0130 Aconitum sinomontanum

T5831 Scutellaria altissima

T6285 Tamarindus indica

T3596 Juniperus sabina var. tamariscifolia

T5869 Selaginella tamariscina

T1506 Citrus reticulata

T1504 Citrus reticulata

T2321 Ecklonia kurome

T0502 Anisodus tanguticus [Syn. Scopolia tangutica]

T1546 Clematis tangutica

T2031 Daphne tangutica

T0137 Aconitum tanguticum

T5247 Przewalskia tangutica

T5481 Rheum tanguticum

T1516 Citrus tankan

T1517 Citrus tankan

T6102 Stauranthus perforatus

T1400 Chrysanthemum vulgare

T0493 Angelica radix

T2740 Flourensia cernua

T1624 Colocasia antiquorum

T0676 Artemisia dracunculus

T2661 Fagopyrum tataricum

T0781 Aster tataricus

T0701 Artemisia taurica

T1150 Camellia sinensis [Syn. Thea sinensis]

T1151 Camellia sinensis [Syn. Thea sinensis]

T1154 Camellia sinensis [Syn. Thea sinensis]

T0600 Ardisia mamillata [Syn. Tinus mamillata]

T4120 Marsdenia tenacissima
TAI WAN PI PA

A LI SHAN WU WEI ZI

BA NA MA SHAN SHI LIU

TAI WAN TANG SONG CAO

TAI WAN JI NING

TAI WAN HU JIAO

MEI LI MA ZUI MU

TAI WAN CHA MU

TAI WAN AN XI XIANG

TAI WAN XIU XIAN JU

TAI WAN YUN SHAN

TAI WAN JI

ZI DAN TENG

TAI ZHONG SHU LI

TA LA WU TOU

WU WEI CAO

GAO CHUN HUANG JU

SHU ZHUANG MA HUANG

GAO ZE LAN

TIAN MA

GAO HONG JIN

TU LIAN QIAO

ZA XING TANG SONG CAO

GAO WU TOU

GAO HUANG QIN

SUAN JIAO

CHENG LIU YE YUAN BAI

JUAN BAI

JU PI

JU HE

HEI KUN BU

ZANG QIE

GAN QING TIE XIAN LIAN

SHAN GAN RUI XIANG

GAN QING WU TOU

MA NIAO PAO

TANG GU TE DA HUANG

JIAO GAN

JIAO GAN PI

AI JU

ZHI GEN DANG GUI

FU CHUI FE LAO JU

YE YU

XIA YE QING HAO

KU QIAO MAI

ZI WAN

NIU HAO

CHA HUA

CHA SHU GEN

CHA ZI

HU SHE HONG

TONG GUANG TENG 


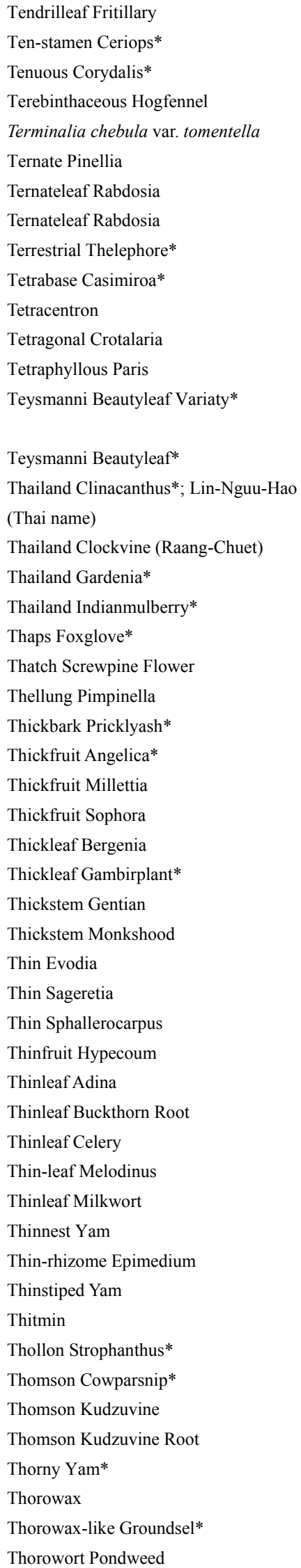

T2783 Fritillaria cirrhosa

T1335 Ceriops decandra

T1732 Corydalis pallida var. tenuis

T4773 Peucedanum terebinthaceum

T6348 Terminalia chebula var. tomentella

T4904 Pinellia ternata

T3528 Isodon ternifolia

T3529 Isodon ternifolius

T6421 Thelephora terrestris

T1227 Casimiroa tetrameria

T6352 Tetracentron sinense

T1836 Crotalaria tetragona

T4655 Paris tetraphylla

T1134 Calophyllum teysmannii var. inophylloide

T1133 Calophyllum teysmannii

T1572 Clinacanthus siamensis

T6445 Thunbergia laurifolia

T2887 Gardenia thailandica

T4281 Morinda coreia

T2180 Digitalis thapsii

T4616 Pandanus tectorius

T4902 Pimpinella thelungiana

T6879 Zanthoxylum elephantiasis

T0490 Angelica pachycarpa

T4240 Millettia pachycarpa

T6040 Sophora pachycarpa

T0923 Bergenia crassifolia

T6610 Uncaria callophylla

T2909 Gentiana crassicaulis

T0089 Aconitum crassicaule

T2641 Evodia lepta [Syn. Ilex lepta]

T5644 Sageretia gracilis

T6073 Sphallerocarpus gracilis

T3334 Hypecoum leptocarpum

T0181 Adina rubella

T5461 Rhamnus leptophylla

T0546 Apium leptophyllum

T4179 Melodinus tenuicaudatus

T5088 Polygala tenuifolia

T2198 Dioscorea gracillima

T2399 Epimedium leptorrhizum

T2211 Dioscorea tenuipes

T5048 Podocarpus neriifolius

T6162 Strophanthus thollonii

T3226 Heracleum thomsoni

T5314 Pueraria lobata var. thomsonii

T5320 Pueraria thomsonii

T2196 Dioscorea dumetorum

T1069 Bupleurum rigidum

T5882 Senecio bupleuroides

T5178 Potamogeton perfoliatus
CHUAN BEI MU

SHI XIONG RUI JIAO GUO MU

XI SHEN SHAN ZI JIN

SHI FANG FENG

WEI MAO HE ZI

BAN XIA

NIU WEI CAO XIANG CHA CAI

CHONG YA YAO

LU SHENG GE JUN

SI JI XIANG ROU GUO

SHUI QING SHU

HUA JIN DAN

WANG SUN

TE SI MAN NI HU TONG BIAN ZHONG

TE SI MAN NI HU TONG

TAI GUO NIU XU HUA

TAI GUO SHAN QIAN NIU

TAI GUO ZHI ZI

TAI GUO BA JI

SA SHI MAO DI HUANG

LU DOU LE HUA

YANG HONG SHAN

HOU PI HUA JIAO

HOU GUO DANG GUI

KU TAN ZI

GAN SU HUAI SHU

HOU YE YAN BAI CAI

HOU YE GOU TENG

CU JING QIN JIAO

CU JING WU TOU

SAN CHA KU

XI QUE MEI TENG

MO GUO QIN

XI GUO JIAO HUI XIANG

XI YE SHUI TUAN HUA

JIANG LI MU GEN

XIAN YE QIN

BO YE SHAN CHENG

YUAN ZHI

XIAN XI SHU YU

QIAN LING YIN YANG HUO

XI BING SHU YU

BAI RI QING

SE LUN YANG JIAO AO

TANG MU XUN DU HUO

FEN GE

GAN GE TENG GEN

JING JI SHU YU

JIAN YING CHAI HU

CHAI HU ZHUANG QIAN LI GUANG

SUAN SHUI CAO 


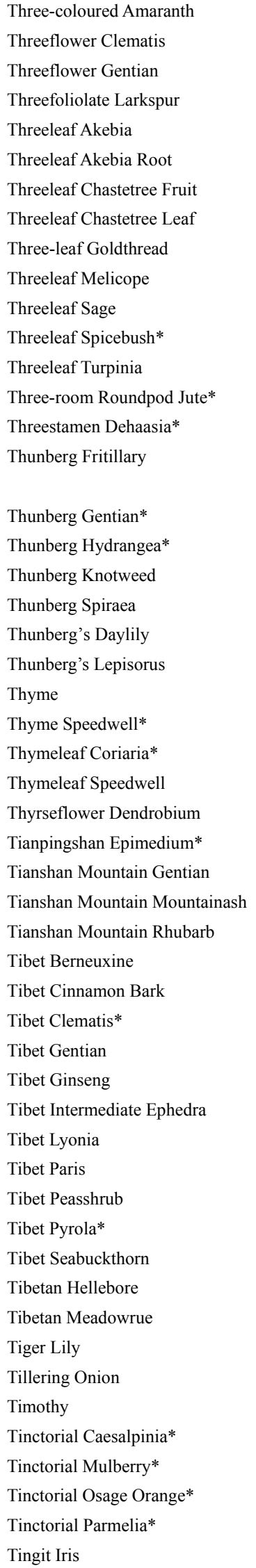

T0389 Amaranthus tricolor

T1547 Clematis terniflora [Syn. Clematis maximowicziana]

T2938 Gentiana triflora

T2089 Delphinium trifoliolatum

T0277 Akebia trifoliata

T0278 Akebia trifoliata

T6793 Vitex trifolia

T6792 Vitex trifolia

T1671 Coptis trifolia

T4169 Melicope triphylla

T5697 Salvia trijuga

T3855 Lindera triloba

T6568 Turpinia ternata

T1676 Corchorus trilocularis

T2059 Dehaasia triandra

T2798 Fritillaria verticillata var. thunbergii [Syn. Fritillaria

thunbergii]

T2935 Gentiana thunbergii

T3305 Hydrangea macrophylla var. thunbergii

T5119 Polygonum thunbergii

T6086 Spiraea thunbergii

T3198 Hemerocallis thunbergii

T3764 Lepisorus thunbergianus

T6454 Thymus vulgaris

T6730 Veronica thymoides ssp. pseudocinerea

T1695 Coriaria thymifolia

T6728 Veronica serpyllifolia

T2110 Dendrobium thyrsiflorum

T2400 Epimedium myrianthum

T2936 Gentiana tianschanica

T6055 Sorbus tianschanica

T5482 Rheum wittrocki

T0926 Berneuxia thibetica

T1444 Cinnamomum tamala

T1548 Clematis tibetana

T2937 Gentiana tibetica

T4606 Panax pseudo-ginseng

T2370 Ephedra intermedia var. tibetica

T3991 Lyonia ovalifolia

T4650 Paris polyphylla var. pseudothibetica

T1193 Caragana tibetica

T5346 Pyrola calliantha var. tibetana

T3259 Hippophae thibetana

T3187 Helleborus thibetanus

T6386 Thalictrum foetidum

T3839 Lilium tigrinum [Syn. Lilium lancifolium]

T0312 Allium cepa var. agrogatum

T4802 Phleum pratense

T1109 Caesalpinia tinctoria

T4302 Morus tinctoria

T4023 Maclura tinctoria

T4658 Parmelia tinctorum

$\mathrm{T} 3472$ Iris tingitana
YAN LAI HONG

BAI HUA TENG

SAN HUA LONG DAN

SAN XIAO YE CUI QUE HUA

SAN YE MU TONG

SAN YE MU TONG GEN

MAN JING ZI

MAN JING YE

SAN YE HUANG LIAN

SAN YE MI ZHU YU

SAN YE SHU WEI CAO

SAN YE DIAO ZHANG

SAN CHU SHAN XIANG YUAN

SAN SHI HUANG MA

SAN RUI LIAN GUI

ZHE BEI MU

\section{ZAN SHI LONG DAN}

SE BO GE XIU QIU

SHUI MA TIAO

ZHEN ZHU XIU XIAN JU

SHE XIANG XUAN

WA WEI

SHE XIANG CAO

JIA HUI SE JIU LI XIANG PO PO NA

BAI LI XIANG YE MA SANG

XIAO PO PO NA

JU HUA SHI HU

TIAN PING SHAN YIN YANG HUO

TIAN SHAN QIN JIAO

TIAN SHAN HUA QIU

TIAN SHAN DA HUANG

YAN JIN CAI

SAN TIAO JIN

XI ZANG TIE XIAN LIAN

XI ZANG QIN JIAO

ZANG SAN QI

XI ZANG ZHONG MA HUANG

LI MU

CHANG YAO GE CHONG LOU

MAO CI JIN JI ER

XI ZANG LU TI CAO

XI ZANG SHA JI

TIE KUAI ZI

XIANG TANG SONG CAO

JUAN DAN

FEN NIE CONG TOU

TI MU CAO

SE ZE YUN SHI

RAN SE SANG

ZHUO SE SANG CHENG

MEI YI

DAN JI ER YUAN WEI 
Tinileaf Eupatorium*

Tiny Ardisia

Tithymalus-like Pedilanthus

Toad

Toad Gall

Toad Skin

Toad Skin Secretion Cake

Tokyo Cherry

Tokyo Violet

Tomato

Tomentase Pummelo

Tomentose Aglaia*

Tomentose Bushmint*

Tomentose Calaba

Tomentose Caudate Croton

Tomentose Germander

Tomentose Glorybower

Tomentose Sage*

Tomentose Sophora

Tomentose Wrightia

Tongol Goldenray

Tongue-on-tree

Tonkin Bursera*

Tonkin Cyclea

Tonkin Snowbell

Tonkin Sophora Root

Toolur

Tooth Ammi

Toothedfruit Dock

Toothedpetal Corydalis

Toothleaf Goldenray

Toothwing Senna*

Top Primrose

Topeng Pygeum

Torreya*

Tortedfruit Screwtree

Tortile Acacia*

Tortuous Fig*

Tortuous Hellebore*

Tortuous Seseli*

Tosa Goldsaxifrage*

Tosa Swertia*

Toxic Poisonnut*

Toxic Tephrosia*

Treacle Erysimum

Treasure Maesa

Tree Beautyberry

Tree Clubmoss

Tree Falsespiraea

Tree Heath

Tree of Heaven Ailanthus Bast

Tree Phyllirea

Tree Wisteria
T2578 Eupatorium tinifolium

T0601 Ardisia pusilla

T4686 Pedilanthus tithymaloides

T1049 Bufo bufo gargarizans; Bufo melanostictus

T1050 Bufo bufo gargarizans; Bufo melanostictus

T1051 Bufo bufo gargarizans; Bufo melanostictus

T1052 Bufo bufo gargarizans; Bufo melanostictus

T5246 Prunus yedoensis

T6767 Viola yedoensis

T3962 Lycopersicon esculentum

T1483 Citrus grandis var. tomentosa

T0246 Aglaia tomentosa

T3382 Hyptis tomentosa

T1135 Calophyllum tomentosum

T1841 Croton caudatus var. tomentosus

T6370 Teucrium tomentosum

T1567 Clerodendrum mandarinorum

T5696 Salvia tomentosa

T6045 Sophora tomentosa

T6834 Wrightia tomentosa

T3816 Ligularia tongolensis

T2844 Ganoderma applanatum

T1082 Bursera tonkinensis

T1934 Cyclea tonkinensis

T6204 Styrax tonkinensis

T6043 Sophora subprostrata [Syn. Sophora tonkinensis]

T2511 Eucalyptus grandis

T0412 Ammi visnaga

T5608 Rumex dentatus

T1734 Corydalis remota [Syn. Corydalis bulbosa var. typica]

T3802 Ligularia dentata

T1231 Cassia dentata

T5202 Primula obconica

T5340 Pygeum topengii

T6477 Torreya grandis

$\mathrm{T} 3164$ Helicteres isora

T0033 Acacia tortilis ssp. raddiana

T4202 Mesembryanthemum tortuosum

T3188 Helleborus torquatus [Syn. Helleborus serbicus]

T5936 Seseli tortuosum

T1408 Chrysosplenium tosaense

T6240 Swertia tosaensis

T6188 Strychnos toxifera

T6341 Tephrosia toxicaria

T2452 Erysimum cheiranthoides

T4031 Maesa perlarius

T1116 Callicarpa arborea

T3976 Lycopodium obscurum

T6051 Sorbaria arborea

T2415 Erica arborea

T0255 Ailanthus altissima

T4797 Phillyrea latifolia

T0972 Bolusanthus speciosus
SI MIAN MAO JIA MI YE ZE LAN

CHUAN CHAN JIU JIE LONG

YU DAI GEN

CHAN CHU

CHAN CHU DAN

CHAN PI

CHAN SU

RI BEN YING HUA

ZI HUA DI DING

FAN QIE

HUA ZHOU YOU

RONG MAO MI ZI LAN

RONG MAO SHAN XIANG

RONG MAO HU TONG

MAO YE BA DOU

RONG MAO XIANG KE KE

HAI TONG

RONG MAO DAN SHEN

LING NAN HUAI SHU

YAN MU

DONG E LUO DU WU

SHU SHE

YUE NAN LIE LAN

NAN LUN HUAN TENG

YUE NAN AN XI XIANG

SHAN DOU GEN

JU AN

CHI A MI

NIU SHE CAO

CHI BAN YAN HU SUO

CHI YE TUO WU

CHI CHI JUE MING

E BAO CHUN

TUN XING GUO

FEI SHU

HUO SUO MA

NIU XUAN JIN HE HUAN

NIU QU SONG YE JU

NIU QU TI GEN CAO

XUAN NIU XIE HAO

SHANG ZUO ZHOU JIN YAO

SHANG ZUO ZHOU ZHANG YA CAI

DU MA QIAN

DU HUI MAO DOU

GUI ZHU TANG JIE

JI YU DAN

QIAO MU ZI ZHU

YU BAI SHI SONG

GAO CONG ZHEN ZHU MEI

OU SHI NAN

CHU BAI PI

KUO YE OU NV ZFEN 
Treelike Rhododendron

Triangular Dutchmanspipe*

Triangular-leaf St.John'swort*

Trichroism Morningglory*

Tricolor Leucopaxillus*

Tricuspid Cudrania

Tricuspidate Falsenettle

Trifoliate Acanthopanax

Trifoliate Euchresta

Trifoliate Jewelvine

Trifoliate-orange

Trifoliate-orange Leaf

Trifoliate-orange Root-bark

Trifoliate-orange Seed

Trifoliate-orange Unripe Fruit

Trifoliate-orange Young Fruit

Trifoliolate Verbena*

Tri-hard-tip Snakegourd*

Trileaf Wood*

Trilobate Macaranga*

Trilobedleaf Kudzuvine

Trinervure Poisonnut*

Trinidad Pepper*

Tripartite Nightshade*

Tripartite Wormwood*

Triplet Lily

Triplinervia Poisonnut*

Triquetrous Tadehagi

Trisphare Ungernia*

Trogopterus Dung

Trojan Milkvetch*

Tropic Ageratum

Tropical American Hymenocallis Leaf

tropical fungus Botryodiplodia theobromae

True Indigo

True Lacquer

True Lacquer Seed

Tschonosk Trillium

Tsihanim Pricklyash*

Tubaroot Jewelvine

Tubeflower Dutchmanspipe

Tubeflower Gentian

Tuber Fleeceflower

Tuber Fleeceflower Stem

Tuber Onion

Tuber Onion Seed

Tuber Stemona

Tuberculate Bredia

Tuberculate Pepper*

Tuberculate Rue*

Tuberculate Speranskia

Tuberose
T5503 Rhododendron arboreum

T0640 Aristolochia triangularis

T3366 Hypericum triquetrifolium

T3452 Ipomoea tricolor

T3783 Leucopaxillus tricolor

T1888 Cudrania tricuspidata

T0962 Boehmeria platanifolia [Syn. Boehmeria tricuspis]

T0045 Acanthopanax trifoliatus

T2527 Euchresta japonica

T2124 Derris trifoliata

T5137 Poncirus trifoliata

T5139 Poncirus trifoliata

T5142 Poncirus trifoliata

T5138 Poncirus trifoliata

T5140 Poncirus trifoliata

T5141 Poncirus trifoliata

T6711 Verbena triphylla [Syn. Lippia citriodora]

T6514 Trichosanthes tricuspidata

T6536 Triphyophyllum peltatum

T4014 Macaranga triloba

T5318 Pueraria phaseoloides

T6189 Strychnos trinervis

T4929 Piper aequale

T6016 Solanum tripartitum

T0704 Artemisia tripartita

T1029 Brodiaea california

T6190 Strychnos triplinervia

T6277 Tadehagi triquetrum

T6642 Ungernia trisphaera

T6551 Trogopterus xanthipes; Pteromys volans

T0809 Astragalus trojanus

T0229 Ageratum conyzoides

T3320 Hymenocallis littoralis [Syn. Hymenocallis americana;

Pancratium littoralis]

T0997 Botryodiplodia theobromae

T3423 Indigofera tinctoria

T5542 Rhus verniciflua [Syn. Toxicadendron verniciflum]

T5541 Rhus verniciflua [Syn. Toxicadendron verniciflum]

T6535 Trillium tschonoskii

T6898 Zanthoxylum tsihanimposa

T2118 Derris elliptica

T0642 Aristolochia tubiflora

T2932 Gentiana siphonantha

T5107 Polygonum multiflorum

T5108 Polygonum multiflorum

T0322 Allium tuberosum

T0323 Allium tuberosum

T6115 Stemona tuberosa

T1023 Bredia tuberculata

T4972 Piper tuberculatum

T5633 Ruta tuberculata [Syn. Haplophyllum tuberculatum]

T6069 Speranskia tuberculata

T5061 Polianthes tuberosa
SHU XING DU JUAN

SAN JIAO MA DOU LING

SAN LENG YE JIN SI TAO

SAN SE QIAN NIU

SAN SE BAI ZHUANG GU

ZHE SHU

CHI MA

CI SAN JIA

SAN XIAU YE SHAN DOU GEN

YU TENG

GOU JU

GOU JU YE

ZHI GEN PI

GOU JU HE

GOU JU ZHI KE

GOU JU ZHI SHI

SAN YE MA BIAN CAO

SAN YING JIAN GUA LOU

SAN YE MU

SAN LIE XUE TONG

SAN LIE YE GE

SAN YE MAI MA QIAN

TE LI NI DA HU JIAO

SAN LIE QIE

SAN LIE HAO

LI JI SAN CHU MAI MA QIAN

HU LU CHA

SAN QIU BO SI SHI SUAN

WU LING ZHI

TE LUO YI HUANG QI

SHENG HONG JI

SHUI GUI JIAO YE

MU LAN

SHENG QI

QI ZI

YAN LING CAO

QI HAN NING HUA JIAO

MAO YU TENG

GUAN HUA MA DOU LING

GUAN HUA QIN JIAO

HE SHOU WU

YE JIAO TENG

JIU CAI

JIU ZI

BAI BU

HONG MAO YE HAI TANG

LIU TU HU JIAO

LIU ZHUANG DAN YE YUN XIANG

TOU GU CAO

WAN XIANG YU 


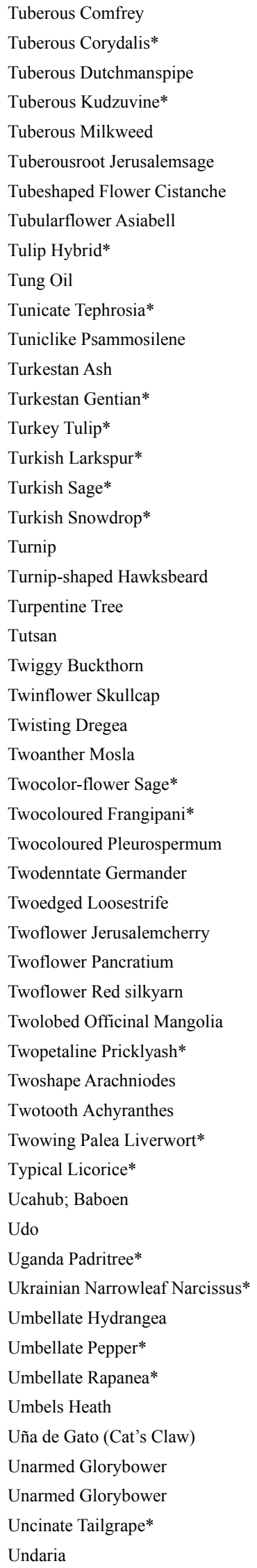

\begin{tabular}{|c|c|}
\hline T6248 Symphytum tuberosum & KUAI JING XI MEN FEI CAO \\
\hline T1748 Corydalis tuberosa & KUAI JING ZI JIN \\
\hline T0641 Aristolochia tuberosa & KUAI JING MA DOU LING \\
\hline T5321 Pueraria tuberosa & KUAI JING GE \\
\hline T0741 Asclepias tuberosa & KUAI JING MA LI JIN \\
\hline T4813 Phlomis tuberosa & KUAI JING CAO SU \\
\hline T1458 Cistanche tubulosa & GUAN HUA ROU CONG RONG \\
\hline T1603 Codonopsis tubulosa & GUAN HUA DANG SHEN \\
\hline T6563 Tulipa hybrida & YU JIN XIANG ZA JIAO ZHONG \\
\hline T0299 Aleurites cordata $[$ Syn. Aleurites fordii $]$ & TONG YOU \\
\hline T6342 Tephrosia tunicata & BAO MO HUI MAO DOU \\
\hline T5250 Psammosilene tunicoides & JIN TIE SUO \\
\hline T2775 Fraxinus potamophila & TU ER QI SI TAN BAI LA SHU \\
\hline T2939 Gentiana turkestanorum & TU ER QI SI TAN LONG DAN \\
\hline T6565 Tulipa turkestani & TU ER QI YU JIN XIANG \\
\hline T2070 Delphinium crispulum & TU ER QI CUI QUE HUA \\
\hline T5668 Salvia cilicica & TU ER QI SHU WEI CAO \\
\hline T2823 Galanthus plicatus ssp. byzantinus & TU ER QI XUE HUA LIAN \\
\hline T1021 Brassica rapa & WU QING \\
\hline T1789 Crepis napifera & YUAN JING HUAN YANG SHEN \\
\hline T4981 Pistacia terebinthus & RU DU XIANG \\
\hline T3338 Hypericum androsaemum & TU SAN JIN SI TAO \\
\hline T5464 Rhamnus virgata & ZHOU ZHI SHU LI \\
\hline T5844 Scutellaria scordifolia & BING TOU HUANG QIN \\
\hline T2263 Dregea volubilis & NAN SHAN TENG \\
\hline T4305 Mosla dianthera & DA YE XIANG RU \\
\hline T5670 Salvia dichroantha & ER SE HUA SHU WEI CAO \\
\hline T5030 Plumeria bicolor & SHUANG SE JI DAN HUA \\
\hline T5019 Pleurospermum govanianum var. bicolor & SHUANG SE SUO ZI QIN \\
\hline T6359 Teucrium bidentatum & ER CHI XIANG KE KE \\
\hline T4006 Lythrum anceps & RI BEN QIAN QU CAI \\
\hline T5993 Solanum capsicastrum & YE HAI JIAO \\
\hline T4612 Pancratium biflorum & QUAN NENG HUA \\
\hline T3953 Lycianthes biflora & HONG SI XIAN \\
\hline T4034 Magnolia biloba & AO YE HOU PO \\
\hline T6875 Zanthoxylum dipetalum & ER DUN ZHUANG HUA JIAO \\
\hline \multicolumn{2}{|l|}{ T0562 Arachniodes dimorphophylla } \\
\hline T0073 Achyranthes bidentata & NIU XI \\
\hline T4108 Marchantia paleacea var. diptera & ER YI TUO BAO DI QIAN \\
\hline T3014 Glycyrrhiza glabra var. typica & OU YA GAN CAO \\
\hline T6770 Virola surinamensis [Syn. Myristica surinamensis] & SU LI NAN ROU DOU KOU \\
\hline T0569 Aralia cordata & TU DANG GUI \\
\hline T6141 Stereospermum kunthianum & WU GAN DA YU YE QIU \\
\hline T4373 Narcissus angustifolius & WU KE LAN XIA YE SHUI XIAN \\
\hline T3308 Hydrangea umbellata & SAN XING XIU QIU \\
\hline T4973 Piper umbellatum & SAN XING HU JIAO \\
\hline T5418 Rapanea umbellata & SAN HUA MI HUA SHU \\
\hline T2418 Erica umbellata & SAN XING OU SHI NAN \\
\hline T6637 Uncaria tomentosa & BI LU GOU TENG \\
\hline $\mathrm{T} 1558$ Clerodendron inerme & SHUI HU MAN \\
\hline T1566 Clerodendrum inerme & KU LANG SHU \\
\hline T0657 Artabotrys uncinatus & YOU GOU YING ZHAO \\
\hline T6640 Undaria pinnatifida & QUN DAI CAI \\
\hline
\end{tabular}




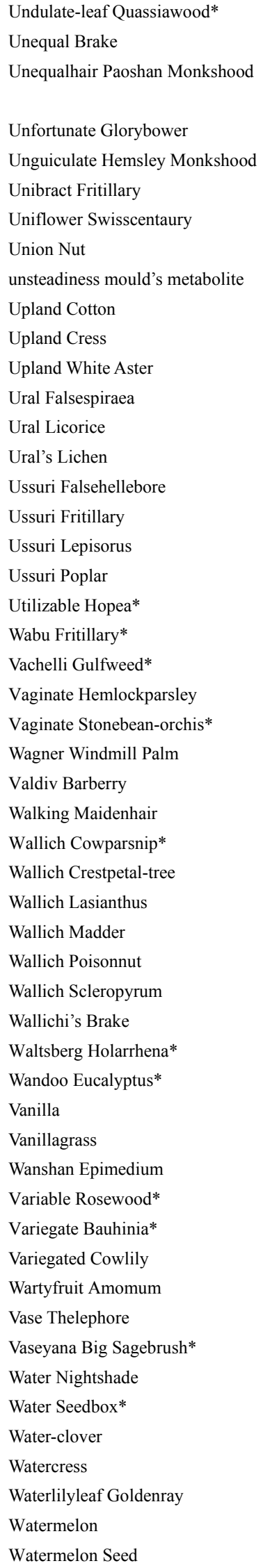

T3098 Hannoa undulata

T5291 Pteris inaequalis

T0116 Aconitum nagarum var. heterotrichum [Syn. Aconitum

bullatifolium ]

T1559 Clerodendron infortunatum

T0102 Aconitum hemsleyanum var. leueanthus

T2796 Fritillaria unibracteata

T5466 Rhaponticum uniflorum

T0998 Bouchardatia neurococca

T6643 unsteadiness mould's metabolite

T3058 Gossypium hirsutum [Syn. Gossypium mexicanum]

T0870 Barbarea vulgaris

T0780 Aster ptarmicoides

T6052 Sorbaria sorbifolia

T3022 Glycyrrhiza uralensis

T6603 Umbilicaria proboscidea

T6699 Veratrum nigrum var. ussuriense

T2797 Fritillaria ussuriensis

T3765 Lepisorus ussuriensis

T5165 Populus ussuriensis

T3281 Hopea utilis

T2799 Fritillaria wabuensia

T5740 Sargassum vachellianum

T1641 Conioselinum vaginatum

T1054 Bulbophyllum vaginatum

T6486 Trachycarpus wagnerianus

T0918 Berberis valdiviana

T0172 Adiantum caudatum

T3227 Heracleum wallichii

T3922 Lophopetalum wallichii

T3698 Lasianthus wallichii

T5585 Rubia wallichiana

T6193 Strychnos wallichiana

T5815 Scleropyrum wallichianum

T5299 Pteris wallichinan

T3269 Holarrhena waltsbergii

T2523 Eucalyptus wandoo

T6684 Vanilla planifolia

T3250 Hierochloe odorata

T2405 Epimedium wanshanense

T2018 Dalbergia variabilis

T0881 Bauhinia variegata

T4451 Nuphar variegatum

T0418 Amomum muricarpum

T6422 Thelephora vialis

T0703 Artemisia tridentata ssp. vaseyana

T6015 Solanum torvum

T3932 Ludwigia octovalvis

T4121 Marsilea quadrifolia

T4388 Nasturtium officinale

T3810 Ligularia nelumbifolia

T1464 Citrullus vulgaris [Syn. Citrullus lanatus]

T1465 Citrullus vulgaris [Syn. Citrullus lanatus]
BO YE KU MU

BIAN YI FENG WEI JUE

XIAO BAI CHENG

QIAN YU DA QING

ZHUA KUI GUA YE WU TOU

AN ZI BEI MU

LOU LU

LU DI MIAN

OU ZHOU SHAN JIE

TUN CAO ZI WAN

ZHEN ZHU MEI

GAN CAO

WU LA ER DI YI

WU SU LI LI LU

PING BEI MU

WU SU LI WA WEI

DA QING YANG

YOU YONG PO LEI

WA BU BEI MU

WA SHI MA WEI ZAO

XIN JIANG GAO BEN

QIAO SHI DOU LAN

WA SHI ZONG LV

WA SHI XIAO BO

BIAN YE TIE XIAN JUE

WA SHI DU HUO

WO LI HE GUAN BAN

XIE JI CU YE MU

GUANG JING QIAN CAO

CHANG ZI MA QIAN

YING HE

SAN CHA FENG WEI JUE

WO SHI ZHI XIE MU

WO SHI AN

XIANG ZI LAN

MAO XIANG HUA

WAN SHAN YIN YANG HUO

YI BIAN HUANG TAN

CAI BAN YANG TI JIA

BAN YE PING PENG CAO

YOU GUO DOU KOU

LIAN ZUO GE JUN

WA SI YA NA SAN CHI HAO

SHUI QIE

MAO CAO LONG

PING

DOU BAN CAI

LIAN YE TUO WU

XI GUA

XI GUA ZI REN 
Watery Speedwell

Watt Tupistra

Wattle

Waxy Litse*

Waxy Meadowrue

Wayaka Yambean

Wayaka Yambean Seed

Weaversbroom

Weeping Forsythia

Weeping Viburnum*

Veined Argyreia

Veined Inula

Veined Rabdosia

Veined Swertia*

Veinfruit Seabuckthorn

Weisi Rabdosia

Veitch Dysosma

Veitch Mahonia

Veitch Peony

Vellayim

Welsh Poppy

Welted Thistle

Velutinous Cinquefoil

Velutinous Gambirplant*

Velutinous Hoarhound*

Velvet Tamarind

Wenchuan Thorowax*

Venenous Alstonia*

Venenous Pricklyash*

Vengai Padauk

Wengujin Culcuma*

Venus Maidenhair

Venus Tailgrape*

Venus's Flytrap

Verecun Plum*

Vermeil-sulphureous Laetiporus*

Vermiculate Thamnolia Thallus

Vermilion Rhododendron

Verrucatespot Euonymus

Versicolorous Dutchmanspipe

Versicolorous Mosquitotrap

Versicolorous Pink

Verticillate Bushmint*

Verticillate Cladonia

Very-hard Agave*

Vesper Iris

West Africa Afrormosia

West Indian Mahogany

Vested Acronychia*

Western Arborvitae

Western Hemlock

Western Juniper
T6722 Veronica anagallis-aquatica

T6567 Tupistra wattii [Syn. Campylandra wattii]

T0024 Acacia mearnsii

T3895 Litsea sebifera

T6406 Thalictrum revolutum

T4573 Pachyrrhizus erosus

T4572 Pachyrhizus erosus

T6064 Spartium junceum

T2756 Forsythia suspensa

T6740 Viburnum suspensum

T0614 Argyreia nervosa

T3436 Inula nervosa

T5395 Rabdosia nervosa

T6228 Swertia nervosa

T3253 Hippophae neurocarpa

T3534 Isodon weisiensis

T2304 Dysosma veitchii

T4076 Mahonia veitchiorum

T4590 Paeonia veitchii

T3271 Holoptelea integrifolia

T4142 Meconopsis cambrica

T1199 Carduus acanthoides

T5183 Potentilla griffithii var. velutina

T6638 Uncaria veluntina

T4113 Marrubium velutinum

T2138 Dialium guineense

T1078 Bupleurum wenchuanense

T0377 Alstonia venenata

T6899 Zanthoxylum veneficium

T5302 Pterocarpus marsupium

T1907 Curcuma wengujin

T0179 Adiantum venustum

T0658 Artabotrys venustus

T2186 Dionaea muscipula

T5245 Prunus verecunda

T3666 Laetiporus sulphureus var. miniatus

T6416 Thamnolia vermicularis

T5506 Rhododendron cinnabarinum

T2548 Euonymus verrucosides

T0643 Aristolochia versicolar

T1962 Cynanchum versicolor

T2147 Dianthus versicolor

T3383 Hyptis verticillata

T1529 Cladonia verticillata

T0224 Agave rigidissima

T3457 Iris dichotoma

T0208 Afrormosia elata

T6241 Swietenia mahogany

T0153 Acronychia vestita

T6441 Thuja plicata

T6558 Tsuga heterophylla

T3591 Juniperus occidentalis
BEI SHUI KU MAI

WAN RUI KAI KOU JIAN

HEI JING SHU

LA ZHI MU JIANG ZI

WAI JUAN TANG SONG CAO

DOU SHU

DI GUA ZI

YING ZHAO DOU

LIAN QIAO

XUAN CHUI JIA MI

YE MAI YIN BEI TENG

XIAN MAI XUAN FU HUA

XIAN MAI XIANG CHA CAI

XIAN MAI ZHANG YA CAI

LEI GUO SHA JI

WEI XI XIANG CHA CAI

CHUAN BA JIAO LIAN

CHUAN DIAN SHI DA GONG LAO

CHUAN CHI SHAO

YIN MIAN YU

WEI ER SHI LV RONG HAO

JIE MAO FEI LIAN

CHANG ROU MAO WEI LING CAI

DUAN RONG MAO GOU TENG

DUAN RONG MAO OU XIA ZHI CAO

WEN CHUAN CHAI HU

YIN DU YA JIAO SHU

DU HUA JIAO

NANG ZHUANG ZI TAN

WEN YU JIN

XI YE TIE XIAN JUE

XIU LI YING ZHAO

BU YING CAO

WEI RUI LI

ZHU HONG LIU HUANG SE XUN

KONG JUN

XUE CHA

ZHU SHA DU JUAN

YOU DIAN WEI MAO

BIAN SE MA DOU LING

WAN SHENG BAI WEI

BIAN SE SHI ZHU

LUN SHENG SHAN XIANG

XIAO LA BA

JI JIAN LONG SHE LAN

BAI HUA SHE GAN

XI FEI HONG DOU SHU

TAO HUA XIN MU

BAO SHAN YOU GAN

BEI MEI XIANG BAI

YI YE TIE SHAN

XI FANG CI BAI 


\begin{tabular}{|c|}
\hline Western Red Cedar \\
\hline Western Sage \\
\hline West-Shiko Rabdosia* \\
\hline Vetchleaf Sophora \\
\hline Vetchleaf Sophora Leaf \\
\hline Vetchleaf Sophora Root \\
\hline Vetchleaf Sophora Seed \\
\hline Vetiver \\
\hline Wetland St.John'swort* \\
\hline Wheat \\
\hline Wheelstamentree \\
\hline Whiplash Star-of-Bethlehem \\
\hline White Ash \\
\hline White Aspen \\
\hline White Bryony \\
\hline White Bur Sage \\
\hline White Chinaure \\
\hline White Clover \\
\hline White Cowparsnip* \\
\hline White Dysosma* \\
\hline White Falsehellebore \\
\hline White Flax \\
\hline White Fuliga* \\
\hline White Gambirplant \\
\hline White Henna* \\
\hline White Linden* \\
\hline White Lotus, Egyptian Lotus \\
\hline White Lupin \\
\hline White Mangrove \\
\hline White Milkweed \\
\hline White Mistletoe \\
\hline White Mulberry Branch \\
\hline White Mulberry Fruit \\
\hline White Mulberry Leaf \\
\hline White Mulberry Root-bast \\
\hline White Mullein \\
\hline White Mustard Seed \\
\hline White Pearlweed \\
\hline White Poppy* \\
\hline White Pummelo* \\
\hline White Snakeroot \\
\hline White Swallow-wort \\
\hline White Sweetclover \\
\hline Whitebackleaf Mallotus \\
\hline Whiteflower Caesalpinia \\
\hline Whiteflower Danshen \\
\hline Whiteflower Embelia \\
\hline Whiteflower Hogfennel \\
\hline Whiteflower Lagopsis \\
\hline Whiteflower Leadword \\
\hline Whiteflower Mucuna \\
\hline Whiteflower Patrinia \\
\hline
\end{tabular}

T3597 Juniperus scopulorum

T0682 Artemisia ludoviciana

T3527 Isodon shikokiana var. occidentalis

T6046 Sophora viciifolia

T6048 Sophora viciifolia

T6047 Sophora viciifolia

T6049 Sophora viciifolia

T6733 Vetiveria zizanioides

T3367 Hypericum uliginosum

T6544 Triticum aestivum [Syn. Triticum vulgare]

T6549 Trochodendron aralioides

T4534 Ornithogalum caudatum

T2765 Fraxinus americana

T5145 Populus alba

T1042 Bryonia alba

T0401 Ambrosia dumosa

T0964 Boenninghausenia albiflora

T6522 Trifolium repens

T3221 Heracleum rapula

T2301 Dysosma majorensis [Syn. Podophyllum majorense; Dysosma

lichuanensis]

T6692 Veratrum album

T3858 Linum album

T2806 Fuligo candida

T6632 Uncaria sessilifructus [Syn. Nauclea sessilifructus]

T3731 Lawsonia alba

T6456 Tilia alburnum

T4455 Nymphaea lotus

T3939 Lupinus albus

T3677 Laguncularia racemosa

T0735 Asclepias albicans

T6771 Viscum album

T4290 Morus alba

T4288 Morus alba

T4289 Morus alba

T4287 Morus alba

T6701 Verbascum lychnites

T5958 Sinapis alba [Syn. Brassica alba; Brassica hirta]

T3996 Lysimachia candida

T4620 Papaver album

T1482 Citrus grandis f. hakunikuju

T2579 Eupatorium urticaefolium

T6763 Vincetoxicum officinale [Syn. Cynanchum vincetoxicum]

T4170 Melilotus albus

T4080 Mallotus apelta

T1106 Caesalpinia minax

T5681 Salvia miltiorrhiza f. alba

T2348 Embelia ribes

T4768 Peucedanum praeruptorum

T4112 Marrubium supinum [Syn. Lagopsis supina]

T5029 Plumbago zeylanica

T4308 Mucuna birdwoodiana

T4676 Patrinia villosa
LUO JI SHAN YUAN BAI

LU DE WEI HAO

XI SI GUO XIANG CHA CAI

BAI CI HUA

BAI CI HUA YE

BAI CI HUA GEN

BAI CI HUA ZI

YAN LAN CAO

SHI SHENG JIN SI TAO

XIAO MAI

KUN LAN SHU

HU YAN WAN NIAN QING

NEI GUO BAI CEN

YIN BAI YANG

BAI XIE GEN

BAI CI GUO TUN CAO

YAN JIAO CAO

SAN XIAO CAO

BAI YUN HUA

BAI BA JIAO LIAN

BAI LI LU

BAI YA MA

LIANG BAI MEI RONG JUN

BAI GOU TENG

BAI SAN MO HUA

BAI DUAN

CHI YE SHUI LIAN

BAI YU SHAN DOU

JIA HONG SHU

BIAN BAI MA LI JIN

LUAN YE HU JI SHENG

SANG ZHI

SANG SHI

SANG YE

SANG BAI PI

JIAN QIU LUO MAO RUI HUA

BAI JIE ZI

DAN TIAO CAO

BAI HUA YING SU

BAI YOU

QIAN MA YE ZE LAN

YAO YONG BAI QIAN

BAI XIANG CAO MU XI

BAI BEI YE

KU SHI LIAN

BAI HUA DAN SHEN

XIAN SUAN QIANG

BAI HUA QIAN HU

BAI HUA XIA ZHI CAO

BAI HUA DAN

BAI HUA YOU MA TENG

BAI JIANG 
Whiteflower Rostellularia

Whiteflower Trillium

Whiteflower Trillium*

White-flowerButterbur*

Whitehairy Beautyberry

Whiteleaf Aglaia*

Whiteleaf Japanese Magnolia

Whiteleaf Nepeta

Whiteleaf Rabdosia*

Whitepilose Rabdosia

White-punctated Combretum*

Whitespot Betony

Whitethorn*

Whitethroat Monkshood

Whittaker Sundew*

Whorlleaf Litse

Viatic Nightshade*

Widely distributed in nature

Wiedemann Sage*

Viet Nam Croton*; Kho Sam Cho La

Wight's St.John'swort

Wightiana Chaulmoogratree Seed*

Wild Angelica

Wild Bleedingheart

Wild Boar Gall

Wild Cabbage

Wild Carrot

Wild Carrot Fruit

Wild Gourd

Wild Gromwell*

Wild Honeysuckle

Wild Leek

Wild Mint

Wild Pea

Wild poplar

Wild Rum-cherry

Wild Spikenard

Wild Thermopsis

Wildcelery

Wildcelery Variety

Wildginger-leaf Morning Glory*

Wildhairdaisy

Wilford Cranesbill

Wilford Swallowwort

Willmott Ceratostigma

Villous Amomum

Villous Rangooncreeper*

Willowleaf Ash Bark

Willowleaf Magnolia*

Willowleaf Sarcococca

Willowleaf Swallowwort

Willowweedleaf Skullcap
T3609 Justicia procumbens var. leucantha

T6531 Trillium camtschaticum

T6533 Trillium kamtschaticum

T4738 Petasites albus

T1117 Callicarpa candicans

T0239 Aglaia leucophylla

T4044 Magnolia obovata

T4416 Nepeta leucophylla

T3503 Isodon leucophyllus

T3478 Isodon albopilosus

T1630 Combretum albopunctatum

T6093 Stachys sylvatica

T4435 Nitraria schoberi

T0111 Aconitum leucostomum

T2270 Drosera whittakeri

T3897 Litsea verticillata

T6019 Solanum viarum

T6817 widely distributed in nature

T5699 Salvia wagneriana

T1859 Croton tonkinensis

T3368 Hypericum wightianum

T3301 Hydnocarpus wightiana

T0497 Angelica sylvestris

T2149 Dicentra eximia

T6205 Sus scrofa

T1013 Brassica oleracea

T2048 Daucus carota

T2049 Daucus carota

T1460 Citrullus colocynthis

T3883 Lithospermum ruderale

T3909 Lonicera confusa

T0309 Allium ampeloprasum

T4184 Mentha haplocalyx [Syn. Mentha canadaensis; Mentha arvensis BO HE

var. haplocalyx; Mentha arvensis]

T3713 Lathyrus sylvestris

T5151 Populus davidiana

T5240 Prunus serotina

T3381 Hyptis suaveolens

T6429 Thermopsis lupinoides

T0544 Apium graveolens

T0545 Apium graveolens var. dulce

T3446 Ipomoea asarifolia

T5756 Saussurea japonica

T2949 Geranium wilfordii

T1964 Cynanchum wilfordii [Syn. Cynoctonum wilfordii]

T1329 Ceratostigma willmottianum

T0419 Amomum villosum

T5386 Quisqualis indica var. villosa

T2779 Fraxinus stylosa

T4049 Magnolia salicifolia

T5732 Sarcococca saligna

T1960 Cynanchum stauntonii

T5837 Scutellaria epilobifolia
BAI HUA JUE CHUANG

YU ER QI

JI LIN YAN LING CAO

BAI HUA FENG DOU CAI

BAI MAO ZI ZHU

BAI YE MI ZI LAN

RI BEN HOU PO

BAI YE JING JIE

BAI YE XIANG CHA CAI

BAI ROU MAO XIANG CHA CAI

BAI DIAN FENG CHE ZI

LIN DI SHUI SU

DONG QIANG

BAI HOU WU TOU

HUI TE KE MAO GAO CAI

DIE DA LAO

XIAO LU QIE

DONG JIN BA DOU

BIAN DI JIN

WEI SHI DA FENG ZI

LIN BAI ZHI

SUI MAO HE BAO MU DAN

YE ZHU DAN

YE JIE

HE SHI FENG

NAN HE SHI

YAO XI GUA

LU BIAN ZI CAO

HUA NAN REN DONG

DA TOU SUAN

LIN SHENG SHAN LI DOU

SHAN YANG

YE HEI YING

SHE BAI ZI

YE JUE MING

HAN QIN

HAN QIN BIAN ZHONG

XI XIN YE QIAN NIU

FENG MAO JU

LAO GUAN CAO

GE SHAN XIAO

ZI JIN LIAN

SHA REN

MAO SHI JUN ZI

LIU YE CEN

LIU YE MU LAN

YE SHAN HUA

LIU YE BAI QIAN

LIU YE CAI HUANG QIN 
Wilson Barberry

Wilson Buckeye Seed

Wilson Citron

Wilson Orange Young Fruit

Wilson Plumyew

Wilson Yarrow

Vinegar

Winged Euonymus

Winged Jurinea*

Winged Laggera

Winged Physochlaina*

Winged Yam

Wingfruit Milkvetch*

Winked Marshweed

Winter Daffodil

Winter Daphne Flower

Winter Daphne Root

Winter Jasmine

Winter Lemongrass*

Winterberry Euonymus

Wintergreen Barberry

Wintersweet Bud

Violet Monkshood*

Violet Morningglory*

Viper's-glass

Virescent Larkspur

Virgate Wormwood

Virginia Chain Fern

Virginia Pepperweed Seed

Virginia Witch Hazel

Viridian Common Perilla*

Viscid Primrose*

Viscid Wormwood*

Viscidhair Skullcap

Viscidhairy Knotweed

Viscose Cinquefoil*

Volcanic Peperomia*

Wolfs-milk Slime

Volga Adonis

Voluble Rosewood*

Wood Sage

Woodland Beakchervil

Woodland Monkshood

Woodland Sunflower

Woodland Wormwood

Woods Lcaquertree

Woods Lcaquertree Leaf

Wood-sorrel

Woody Fleabane

Woolly Gambirplant*

Woolly Lespedeza

Woolly Morning Glory

Woolly Philydrum

\author{
T0920 Berberis wilsonae \\ T0205 Aesculus wilsonii \\ T1520 Citrus wilsonii \\ T1521 Citrus wilsonii \\ T1325 Cephalotaxus wilsoniana \\ T0069 Achillea wilsoniana \\ T6764 Vinegar \\ T2536 Euonymus alatus \\ T3604 Jurinea alata \\ T3674 Laggera alata \\ T4857 Physochlaina alaica \\ T2188 Dioscorea alata \\ T0802 Astragalus pterocarpus \\ T3840 Limnophila rugosa \\ T6144 Sternbergia lutea \\ T2028 Daphne odora \\ T2027 Daphne odora \\ T3555 Jasminum nudiflorum \\ T1948 Cymbopogon winterianus \\ T2538 Euonymus bungeanus \\ T0906 Berberis julianae \\ T1366 Chimonanthus fragrans [Syn. Chimonanthus praecox] \\ T0140 Aconitum violaceum \\ T3453 Ipomoea violacea \\ T5825 Scorzonera humilis \\ T2090 Delphinium virescens \\ T0695 Artemisia scoparia [Syn. Artemisia capillaris var. scoparia] \\ T6832 Woodwardia virginica \\ T3759 Lepidium virginicum \\ T3095 Hamamelis virginiana \\ T4716 Perilla frutescens $\mathrm{f}$. viridis \\ T5205 Primula viscosa \\ T0670 Artemisia cana ssp. viscidula \\ T5845 Scutellaria viscidula \\ T5122 Polygonum viscosum \\ T5188 Potentilla viscosa \\ T4708 Peperomia vulcanica \\ T3960 Lycogala epidendrum \\ T0192 Adonis wolgensis \\ T2019 Dalbergia volubilis \\ T6369 Teucrium scorodonia \\ T0529 Anthriscus sylvestris \\ T0120 Aconitum nemorum \\ T3154 Helianthus strumosus \\ T0700 Artemisia sylvatica \\ T5538 Rhus sylvestris \\ T5537 Rhus sylvestris \\ T4562 Oxalis acetosella \\ T3441 Inula viscosa \\ T6623 Uncaria lanosa \\ T3774 Lespedeza tomentosa \\ T0616 Argyreia speciosa \\ T4799 Philydrum lanuginosum
}

JIN HUA XIAO BO

SUO LUO ZI

XIANG YUAN

XIANG YUAN ZHI SHI

TAI WAN CU FEI

YUN NAN SHI

CU Vinegar

GUI JIAN YU

YI CHI LING JU

LIU LENG JU

YI PAO NANG CAO

MAO SHU

CHI GUO HUANG QI

SHUI HUI XIANG

HUANG SI TAN BAO

RUI XIANG HUA

RUI XIANG GEN

YING CHUN HUA

WEN TE XIANG MAO

SI MIAN MU

TU HUANG LIAN

LA MEI HUA

ZI WU TOU

QING ZI QIAN NIU

AI SHENG YA CONG

DAN LV CUI QUE

HUANG HAO

FU JI NI YA GOU JI JUE

BEI MEI TING LI ZI

MEI ZHOU JIN LV MEI

QING ZI SU

NIAN BAO CHUN

NIAN HAO

ZHAN MAO HUANG QIN

ZHAN MAO LIAO

NIAN WEI LING CAI

HUO SHAN YAN CAO HU JIAO

FEN LIU JUN

FU ER JIA CE JIN ZHAN HUA

CHAN RAO HUANG TAN

LIN SHI CAN

E SHEN

LIN DI WU TOU

LIN DI XIANG RI KUI

LIN DI HAO

YE QI SHU ZI

YE QI SHU YE

SHAN ZUO JIANG CAO

NIAN XING TU MU XIANG

MIAN MAO GOU TENG

XIAO XUE REN SHEN

MEI LI YIN BEI TENG

TIAN CONG 
Woolly Seseli*

Woolly Tylophora

Woollyfruit Rabdosia*

Wooly Dutchmanspipe

Worm-killed Bitterleaf

Wormwood-like Motherwort

Wrinkled Gianthyssop

Wrinkledleaf Pyrola

Wrinkleleaf Magnolia*

Wru Croton*

Wushan Epimedium

Wych Elm

Xanthorrhiza Turmeric*

Yadong Monkshood

Yangtao Actinidia

Yanhusuo

Ya-Yaa (Thai name)

Yeast and other biological sources

Yeddo Spruce

Yeddo Spruce

Yellow Adonis

Yellow Barberry

Yellow Bedstraw

Yellow Bird's-nest

Yellow Bugle

Yellow Cedar

Yellow Cinnamon

Yellow Concave-top Alga*

Yellow Daylily

Yellow Everlasting

Yellow Gentian

Yellow Groundsel*

Yellow Hardpeel Puff-bal1*

Yellow Hornpoppy*

Yellow Licorice

Yellow Lupin

Yellow Monkshood

Yellow Oleander

Yellow Ouratea*

Yellow Oxytropis

Yellow Pitcherplant

Yellow Poplar

Yellow Prairie-clover

Yellow Toadflax

Yellow Yam

Yellowbow Dendrobium

Yellowflag Iris

Yellowflower Coleus

Yellowflower Corydalis

Yellowflower Hornpoppy*

Yellowflower Milkwort

Yellowflower Rabdosia
T5929 Seseli ericephalum

T6581 Tylophora mollissima

T3501 Isodon lasiocarpus

T0634 Aristolochia mollissima

T6713 Vernonia anthelmintica

T3752 Leonurus heterophyllus [Syn. Leonurus artemisia]

T0212 Agastache rugosus

T5352 Pyrola rugosa

T4046 Magnolia praecocissima

T1860 Croton urucurana

T2406 Epimedium wushanense

T6595 Ulmus glabra

T1908 Curcuma xanthorrhiza

T0077 Aconitum balfourii

T0158 Actinidia chinensis

T1750 Corydalis yanhusuo [Syn. Corydalis turtschaninovii $\mathrm{f}$.

yanhusuo]

T0812 Asystasia intrusa

T6857 Yeast and other biological sources

T4871 Picea jezoensis

T4872 Picea jezoensis var. jezoensis

T0191 Adonis vernalis

T0917 Berberis tschonoskiana

T2835 Galium verum

T4275 Monotropa hypopitys

T0261 Ajuga chamaepitys

T1348 Chamaecyparis nootkatensis

T1443 Cinnamomum parthenoxylum [Syn. Cinnamomum porrectum]

T3721 Laurencia nipponica

T3195 Hemerocallis lilio-asphodelus

T3156 Helichrysum arenarium

T2918 Gentiana lutea

T5888 Senecio flavus

T5814 Scleroderma citrinum

T2969 Glaucium flavum

T3016 Glycyrrhiza kansuensis

T3943 Lupinus luteus

T0076 Aconitum anthora

T6433 Thevetia neriifolia [Syn. Thevetia peruviana]

T4560 Ouratea flava

T4567 Oxytropis campestris

T5742 Sarracenia flava

T3871 Liriodendron tulipifera

T4737 Petalostemum purpureum

T3845 Linaria vulgaris

T2205 Dioscorea panthaica

T2099 Dendrobium chrysotoxum

T3467 Iris pseudacorus

T1621 Coleus xanthanthus

T1731 Corydalis pallida

T2967 Glaucium davum

T5071 Polygala arillata

T3524 Isodon sculponeata [Syn. Rabdosia sculponeata]
MIAN MAO XIE HAO

MIAN MAO WA ER TENG

MIAN MAO GUO XIANG CHA CAI

MIAN MAO MA DOU LING

QU CHONG BAN JIU JU

YI MU CAO

HUO XIANG

ZHOU YE LU TI CAO

ZHOU YE MU LAN

WULU BA DOU

WU SHAN YIN YANG HUO

SHAN YU

HUANG GEN JIANG HUANG

YA DONG WU TOU

MI HOU TAO

YAN HU SUO

CHA RU SHI WAN CUO

RI BEN YU LIN SONG

YU LIN YUN SHAN

CHUN FU SHOU CAO

HUANG XIAO BO

PENG ZI CAI

HUANG SHUI JING LAN

HUANG JIN GU CAO

HUANG BIAN BAI

XIANG ZHANG

HUANG SE AO DING CAO

BEI HUANG HUA CAI

SHA SHENG LA JU

HUANG LONG DAN

HUANG SE QIAN LI GUANG

HUANG YING PI MA BO

HUANG HAI YING SU

HUANG GAN CAO

HUANG YU SHAN DOU

HUANG WU TOU

HUANG HUA JIA ZHU TAO

HUANG SAI JIN LIAN MU

TIAN YE JI DOU

HUANG PING ZI CAO

BEI MEI E ZHANG QIU

HUANG SE BAN RUI DOU

LIU CHUAN YU

HUANG SHAN YAO

GU CHUI SHI HU

HUANG CHANG PU

HUANG QIAO RUI HUA

JU HUA HUANG LIAN

HUANG HUA HAI YING SU

HUANG HUA YUAN ZHI

HUANG HUA XIANG CHA CAI 


\begin{tabular}{|c|}
\hline Yellowflower Sage \\
\hline Yellowflower Spiderflower* \\
\hline Yellow-flowering Pea \\
\hline Yellowfruit Nightshade \\
\hline Yellowhair Aralia \\
\hline Yellowhair Honeysuckle \\
\hline Yellowhair Monkshood \\
\hline Yellow-hairy Calyx Kudzuvine \\
\hline Yellow-heart Pricklyash \\
\hline Yellowish Erysimum* \\
\hline Yellowish Rabdosia \\
\hline Yellowish Swainsonia* \\
\hline Yellowish Swertia* \\
\hline Yellowjuice Garcinia \\
\hline Yellowleaf Sophora \\
\hline Yellowmouth Dutchmanspipe \\
\hline Yellow-red Kopsia* \\
\hline Yellowroot \\
\hline Yerbadetajo \\
\hline Yinchow Thorowax \\
\hline Youth-and-old-age \\
\hline Yucatan Tithonia \\
\hline Yuko Citrus* \\
\hline Yulan Magnolia \\
\hline Yun Hogfennel \\
\hline Yungning Cowparsnip \\
\hline Yunnan Alstonia \\
\hline Yunnan Amentotaxus \\
\hline Yunnan Anisetree \\
\hline Yunnan Combretum* \\
\hline Yunnan Craibiodendron \\
\hline Yunnan Cuttongue tree \\
\hline Yunnan Devilpepper \\
\hline Yunnan Dodder \\
\hline Yunnan Galangal \\
\hline Yunnan Gambirplant \\
\hline Yunnan Garcinia \\
\hline Yunnan Gaultheria \\
\hline Yunnan Gingerlily \\
\hline Yunnan Goldthread \\
\hline Yunnan Hawthorn \\
\hline Yunnan Hemlock \\
\hline Yunnan Kudzuvine \\
\hline Yunnan Landflower Mushroon \\
\hline Yunnan Larkspur \\
\hline Yunnan Licorice \\
\hline Yunnan Madder \\
\hline Yunnan Manyleaf Paris \\
\hline Yunnan Mayten \\
\hline Yunnan Michelia \\
\hline Yunnan Parakmeria \\
\hline Yunnan Pholidota \\
\hline unnan Pleurospermum \\
\hline
\end{tabular}

T5672 Salvia flava

T1552 Cleome viscosa

T1830 Crotalaria retusa

T6020 Solanum xanthocarpum

T0571 Aralia decaisneaa

T3910 Lonicera fulvotomentosa

T0087 Aconitum chrysotrichum

T5311 Pueraria calycina

T6880 Zanthoxylum flavum

T2455 Erysimum ochroleucum

T3488 Isodon flavidus

T6209 Swainsonia luteola

T6235 Swertia punicea var. lutescens

T2880 Garcinia xanthochymus

T6030 Sophora chrysophylla

T0629 Aristolochia heterophylla

T3639 Kopsia flavida

T6846 Xanthorhiza simplicissima

T2323 Eclipta prostrata [Syn. Eclipta alba]

T1079 Bupleurum yinchowense

T6912 Zinnia elegans

T6469 Tithonia diversifolia

T1522 Citrus yuko

T4038 Magnolia denudata [Syn. Magnolia heptapata]

T4769 Peucedanum rubricaule

T3228 Heracleum yungningense

T0378 Alstonia yunnanensis

T0409 Amentotaxus yunnanensis

T3407 Illicium simonsii

T1632 Combretum yunnanensis

T1767 Craibiodendron yunnanese

T6807 Walsura yunnanensis

T5428 Rauvolfia yunnanensis

T1915 Cuscuta reflexa

T0353 Alpinia blepharocalyx

T6639 Uncaria yunnanensis

T2852 Garcinia cowa

T2895 Gaultheria yunnanensis

T3121 Hedychium yunnanense

T1670 Coptis teetoides [Syn. Coptis teeta]

T1781 Crataegus scabrifolia

T6557 Tsuga dumosa

T5317 Pueraria peduncularis

T0287 Albatrellus confluens

T2091 Delphinium yunnanense

T3023 Glycyrrhiza yunnanensis

T5586 Rubia yunnanensis

T4652 Paris polyphylla var. yunnanensis

T4134 Maytenus hookeri

T4215 Michelia yunnanensis

T4641 Parakmeria yunnanensis

T4823 Pholidota yunnanensis

T5021 Pleurospermum rivulorum
HUANG HUA SHU WEI CAO

HUANG HUA CAO

AO ZHU SHI DOU

HUANG GUO QIE

HUANG MAO CONG MU

HUANG HE MAO REN DONG

HUANG MAO WU TOU

HUANG MAO GE

HUANG XIN HUA JIAO

HUANG BAI TANG JIE

DAN HUANG XIANG CHA CAI

DAN HUANG KU MA DOU

DAN HUANG ZHANG YA CAI

DA YE TENG HUANG

HUANG YE HUAI

HAN FANG JI

HUANG HONG SE RUI MU

HUANG GEN SHU

MO HAN LIAN

YIN ZHOU CHAI HU

BAI RI CAO

ZHONG BIN JU

YU KE GAN JU

YULAN

YUN QIAN HU

YONG NING DU HUO

DIAN JI GU CHANG SHAN

YUN NAN SUI HUA SHAN

YUN NAN BA JIAO

YUN NAN FENG CHE ZI

JIN YE ZI

YUN NAN GE SHE SHU

YUN NAN LUO FU MU

YUN NAN TU SI ZI

YUN NAN CAO KOU

DIAN GOU TENG

YUN NAN SHAN ZHU ZI

DIAN BAI ZHU SHU

DIAN JIANG HUA

YUN NAN HUANG LIAN

YUN NAN SHAN ZHA

YUN NAN TIE SHAN

YUN NAN GE TENG

YUN NAN DI HUA JUN

XIAO CAO WU

YUN NAN GAN CAO

XIAO HONG SHEN

YUN NAN CHONG LOU

YUN NAN MEI DENG MU

YUN NAN HAN XIAO

YUN NAN NI DAN XING MU LAN

YUN NAN SHI XIAN TAO

YUN NAN QIANG HUO 
Yunnan Rabdosia

Yunnan Rhodiola

Yunnan Sage

Yunnan Sarcococca

Yunnan Seabuckthorn*

Yunnan Seseli

Yunnan Skullcap

Yunnan Torreya

Yunnan Yew

Yunnanwest Monkshood

Zambesi Croton*

Zedoary Turmeric

Zerumbet Ginger

Zhejiang Swertia*

Zhongba Monkshood Daughter Root*

Zonal Geranium

Zuiho Machilus
T5401 Rabdosia yuennanensis

T5501 Rhodiola yunnanesis

T5701 Salvia yunnanensis

T5731 Sarcococca coriacea [Syn. Sarcococca wallichii]

T3258 Hippophae rhamnoides subsp. yunnanensis

T5937 Seseli yunnanense

T5833 Scutellaria amoena

T6479 Torreya yunnanensis

T6319 Taxus yunnanensis

T0081 Aconitum bulleyanum

T1861 Croton zambesicus

T1909 Curcuma zedoaria [Syn. Curcuma aeruginosa]

T6911 Zingiber zerumbet

T6222 Swertia hickinii

T0085 Aconitum carmichaeli cv

T4694 Pelargonium zonale

T4019 Machilus zuihoensis
BU YU HONG

YUN NAN HONG JING TIAN

YUN NAN SHU WEI CAO

YUN NAN YE SHAN HUA

YUN NAN SHA JI

SONG YE FANG FENG

DIAN HUANG QIN

YUN NAN FEI SHU

YUN NAN HONG DOU SHAN

DIAN XI WU TOU

ZAN BI XI BA DOU

PING E SHU

HONG QIU JIANG

ZHE JIANG ZHANG YA CAI

ZHONG BA E ZHANG YE FU ZI

MA TI WEN TIAN ZHU KUI

TAI WAN RUI FANG RUN NAN

The asterisk (*) following a plant English name shows that the name is given by authors of the books. 


\section{TCM Plant PIN-YIN/Chinese Name Index}

\begin{tabular}{|c|c|}
\hline PIN YIN Name & Chinese Name \\
\hline A BI XI NI YA CI TONG & 阿比西尼亚刺桐 \\
\hline A BI XI NI YA NIU JIN GUO & 阿比西尼亚牛筋果* \\
\hline A ER JI ER DA CHI JI & 阿尔及尔大翅蓟 \\
\hline A ER JI LI YA BU XUE CAO & 阿尔及利亚补血草 \\
\hline A ER JI LI YA YUAN WEI & 阿尔及利亚荅尾 \\
\hline A ER TAI YIN LIAN HUA & 阿尔泰银莲花 \\
\hline A ER TAI ZI WAN & 阿尔泰紫苑 \\
\hline A FU HAN DING XIANG & 阿富汗丁香 \\
\hline A FU HAN DU JUAN HUA & 阿富汗杜鹃花 \\
\hline A FU HAN TANG JIE & 阿富汗糖芥 \\
\hline A FU ZE ER MA QIAN ZI & 阿弗泽尔马钱子 \\
\hline A FU ZE LI SHAN ZHU ZI & 阿夫泽里山竹子* \\
\hline A GEN TING SHANG LU & 阿根廷商陆 \\
\hline A GEN TING SHU QU CAO & 阿根廷鼠曲草 \\
\hline A GU JI TIAN JIE CAI & 阿古济天芥菜 \\
\hline A JIANG LAN REN & 阿江榄仁 \\
\hline A KA XI A LAN REN & 阿卡西阿榄仁 \\
\hline A LA BO JIAO JIN HE HUAN & 阿拉伯胶金合欢 \\
\hline A LA BO JIN HE HUAN & 阿拉伯金合欢 \\
\hline A LA BO PO PO NA & 阿拉伯婆婆纳 \\
\hline A LI HONG & 阿里红 \\
\hline A LI SHAN WU WEI ZI & 阿里山五味子 \\
\hline A LU HA LIANG JIANG & 阿陆哈良姜 \\
\hline A LUN DUO QIE & 阿伦多茄 \\
\hline A MAN SU DAN MO YAO & 阿曼苏丹没药 \\
\hline A NUO TI HUA JIAO & 阿诺提花椒 \\
\hline A SHI HAO & 阿氏蒿* \\
\hline A SU JUE MING & 阿苏决明 \\
\hline A WEI & 阿魏 \\
\hline A YA PAN ZE LAN & 阿亚潘泽兰 \\
\hline A YU WEI & 阿育魏 \\
\hline A YUE HUN ZI & 阿月浑子 \\
\hline AI DA HUANG & 矮大黄 \\
\hline AI JI CHE ZHOU CAO & 埃及车轴草 \\
\hline AI JI DA CHI JI & 埃及大翅蓟 \\
\hline AI JI JIA HU CI & 埃及假虎刺 \\
\hline AI JI QIAN LI GUANG & 埃及千里光* \\
\hline AI JI SHU WEI CAO & 埃及鼠尾草 \\
\hline AI JI TIAN JING & 埃及田菁 \\
\hline AI JI ZAI PEI CI TONG & 埃及栽培刺桐 \\
\hline AI JI ZHONG ZHI YUAN WEI & 埃及种植茑尾 \\
\hline AI JING DOU & 矮荆豆 \\
\hline
\end{tabular}

\section{PIN YIN Name}

A BI XI NI YA CI TONG

A BI XI NI YA NIU JIN GUO

A ER JI ER DA CHI JI

A ER JI LI YA BU XUE CAO

A ER JI LI YA YUAN WEI

A ER TAI YIN LIAN HUA

A ER TAI ZI WAN

A FU HAN DING XIANG

A FU HAN DU JUAN HUA

A FUHAN TANG JIE

A FU ZE ER MA QIAN Z

A FU ZE LI SHAN ZHU ZI

A GEN TING SHANG LU

A GEN TING SHU QU CAO

A GU JI TIAN JIE CAI

A JIANG LAN REN

A LA BO JIAO JIN HE HUAN

A LA BO JIN HE HUAN

A LA BO PO PO NA

A LI SHAN WU WEI ZI

A LU HA LIANG JIANG

A LUN DUO QIE

A MAN SUDAN MO YAO

A NUO TI HUA JIAO

A SU JUE MING

AWE

A YU WEI

A YUE HUN ZI

AI JI CHE ZHOU CAO

AI JI DA CHI JI

AI JI QIAN LI GUANG

AI JI ZHONG ZHI YUAN WEI

AI JING DOU
English Name

Abyssinia Coralbean*

Abyssinia Harrisonia*

Algerian Cottonthistle*

Algerian Statice

Algerian Iris

Altai Anemone*

Altai Heteropappus

Afghanistan Lilac*

H.Collett's Rhododendron

Afghanistan Erysimum*

Afzel Poisonnut*

Afzeli Garcinia*

Ombutree Pokeberry

Argentine Cudweed*

Arguzioid Heliotrope*

Acacia Terminalia*

Gum-arabic Tree

Arabian Acacia

Iran Speedwell

Fomes Officinalis Sporocarp

Taiwan Magnoliavine

Allugha Galangal*

Arundo Nightshade*

Sultanate-Oman Myrrhtree*

Arnotti Pricklyash*

Ashurbajev Wormwood*

Absus Senna*

Asafetida Giantfennel Resin

Ayapana Eupatorium*

Ajowan

Common Pistache

Low Rhubarb*

Egyptian Clover*

Egyptian Cottonthistle*

Egyptian Carissa

Egyptian Groundsel

Egyptian Sage*

Indian Sesbania

Egypt Cultivate Coralbean*

Egypt Planted Iris

Dwarf Gorse
Plant Code and Latin Name

T2457 Erythrina abyssinica

T3108 Harrisonia abyssinica

T4496 Onopordum algeriense

T3841 Limonium bonduellii

T3473 Iris unguicularis

T0463 Anemone altaica

T3233 Heteropappus altaicus

T6258 Syringa afghanica

T5507 Rhododendron collettianum

T2456 Erysimum perofskianum

T6164 Strychnos afzelii

T2849 Garcinia afzelii

T4862 Phytolacca dioica

T3027 Gnaphalium gaudichaudianum

T3168 Heliotropium arguzioides

T6344 Terminalia arjuna

T6349 Terminalia stuhlmannii

T0028 Acacia nilotica

T0016 Acacia arabica

T6727 Veronica persica

T2749 Fomes officinalis

T5790 Schisandra arisanensis

T0352 Alpinia allughas

T5990 Solanum arundo

T1639 Commiphora wightii

T6865 Zanthoxylum arnottianum

T0665 Artemisia ashurbajevii

T1228 Cassia absus

T2695 Ferula assafoetida

T2552 Eupatorium ayapana

T6488 Trachyspermum ammi

T4982 Pistacia vera

T5471 Rheum nanum

T6516 Trifolium alexandrinum

T4495 Onopordum alexandrinum

T1207 Carissa edulis

T5876 Senecio aegypticus

T5659 Salvia aegyptiaca

T5926 Sesbania sesban

T2469 Erythrina lysistemon

T3455 Iris carthaliniae

T6592 Ulex nanus 
AI JU

AI LAI MU

AI LI SI DUO KONG JUN

AI MA HUANG

AI NA XIANG

AI QIE

AI SAI E BI YA YU SHAN DOU

AI SAI E BI YA ZAO

AI SHENG BO LAN DI

AI SHENG XIONG GUO

AI SHENG YA CONG

AI SHI BAI MU

AI SHI TIAN JIE CAI

AI SI XING CI TONG

AI TONG ZI

AI WA JIN GU CAO

AI XI SHOU SHI LI LU

AI XIANG RI KUI

AI YE

AI YE HUANG QIN

AI ZI JIN NIU

AN BEI JU

AN CI BAI

AN GE LA BA JIAO FENG

AN GE LA HUANG GUA

AN GE LA ZI TAN

AN GU SI TU LA SHU

AN HONG TUAN WANG JUN

AN HONG WEI LING CAI

AN HUANG ZHU SHI DOU

AN HUI BEI MU

AN HUI CONG MU

AN HUI YIN LIAN HUA

AN LU LONG SHE LAN

AN LUO

AN MO LE

AN SHI JIN SI TAO

AN XI XIANG

AN YE

AN ZI BEI MU

AN ZI YU PAN

AN ZONG TAN TUAN JUN

AO DA LI YA HONG DOU SHAN

AO DA LI YA QIAN JIN TENG

AO DA LI YA SANG

AO DA LI YA YAN DIAN

AO DAO LA MU HAI MANG GUO

AO DI LI LIN MAO JUE

AO ER JIA TIAN JIE CAI

AO LEI TONG QI MU

AO LI FO HUANG TAN

AO LIE GE LONG DAN

AO MA JIN QUE HUA
艾菊

矮梾木

爱丽思多孔菌

矮麻黄

艾纳香

矮茄*

埃塞俄比亚羽扇豆

埃塞俄比亚柊

矮生博兰地*

矮生熊果*

矮生鸦葱

艾氏柏木*

艾氏天芥菜

爱思形刺桐*

矮桐子

艾娃筋骨草*

埃希首氏藜芦

矮向日葵

艾叶

艾叶黄芩

矮紫金牛

安倍菊

岸刺柏

安哥拉八角枫

安哥拉黄瓜*

安哥拉紫檀

安古斯图腊树

暗红团网菌

暗红委陵菜*

暗黄猪屏豆

安徽贝母

安徽淴木

安徽银莲花*

暗绿龙舌兰

暗罗

庵摩勒

安氏金丝桃

安息香

桉叶

暗紫贝母

暗紫玉盘

暗棕碳团菌*

澳大利亚红豆杉

澳大利亚千金藤

澳大利亚桑*

澳大利亚赝靛*

奥道拉姆海杧果

奥地利鳞毛硕

奥尔加天芥菜

奥雷同桤木

奥利佛黄檀*

奥列格龙胆*

奥马金雀花
Tansy

Dwarf Cornel

Ellisi Porous Agaric*

Small Ephedra

Balsamiferous Blumea

Dwarf Nightshade*

Narrowleaf Lupin*

Ethiopian Jujube*

Low Bearberry*

Viper's-glass

Abrams Cypress*

Eichwald Heliotrope*

S-shape Coralbean*

Farges Glorybower*

Iva Bugle*

Eschscholtz Falsehellebore*

Dwarf Sunflower*

Argy Wormwood Leaf

Przewalsk Skullcap

Low Ardisia*

Muricate Amberboa*

Shore Juniper

Angola Alangium*

Angola Cucumber*

Angola Padauk*

Angostura-bark Tree

Carnival Candy Slime

Darksanguine Cinquefoil*

Flavescent Crotalaria*

Anhui Fritillary

Subcapitate Aralia

Anhui Anemone*

Peyote

Suberous Greenstar

Emblic Leafflower

Ancher St.John'swort*

Sumatra Snowbell

Eucalyptus Leaf

Unibract Fritillary

Low Uvaria

Australia Yew

Australia Stephania*

Japanese Mulberry

Odollam Cerberustree*

Broad Buckler-fern

Olga Heliotrope*

Oregon Alder

Oliver Rosewood*

Olga Gentian*

Osmarien Broom*
T1400 Chrysanthemum vulgare

T1699 Cornus suecica

T5128 Polyporus ellisii

T2373 Ephedra minuta

T0957 Blumea balsamifera

T5997 Solanum demissum

T3940 Lupinus angustifolius

T6921 Zizyphus abyssinica

T0925 Berlandiera pumila

T0587 Arctostaphylos pumila

T5825 Scorzonera humilis

T1894 Cupressus abramsiana

T3170 Heliotropium eichwaldii

T2475 Erythrina sigmoidea

T1564 Clerodendron trichotomum var. fargesii

T0265 Ajuga iva

T6695 Veratrum eschscholtzii

T3152 Helianthus pumilus

T0664 Artemisia argyi

T5842 Scutellaria przewalskii

T0597 Ardisia humilis

T0393 Amberboa muricata

T3585 Juniperus conferta

T0283 Alangium lamarckii

T1874 Cucumis angolensis

T5300 Pterocarpus angolensis

T2829 Galipea officinalis

T0590 Arcyria denudata

T5180 Potentilla atrosanguinea

T1820 Crotalaria fulva

T2781 Fritillaria anhuiensis

T0575 Aralia subcapitata

T0464 Anemone anhuiensis

T3923 Lophophora williamsii

T5068 Polyalthia suberosa

T4834 Phyllanthus emblica

T3337 Hypericum ancherii

T6197 Styrax benzoin

T2510 Eucalyptus globulus

T2796 Fritillaria unibracteata

T6660 Uvaria chamae

T3378 Hypoxylon fuscum

T0832 Austrotaxus spicata

T6130 Stephania japonica var. australis

T4291 Morus australis

T0868 Baptisia australis

T1331 Cerbera odollam

T2277 Dryopteris austriaca

T3176 Heliotropium olgae

T0329 Alnus oregana

T2009 Dalbergia oliveri

T2923 Gentiana olgae

T1990 Cytisus osmariensis 


\begin{tabular}{|c|c|c|c|}
\hline AO MAI DING GONG TENG & 凹脉丁公藤 & Elliptical Erycibe & T2447 Erycibe elliptilimba \\
\hline AO PA CAO & 奥帕草 & & T4518 Oppopanax chironium \\
\hline \multirow[t]{2}{*}{ AO SHE LAN } & 凹舌兰 & Frog Orchid & T1604 Coeloglossum viride [Syn. \\
\hline & & & Coeloglossum viride var. bracteatum] \\
\hline AO SHI DAN NI SU MU & 奥氏丹尼苏木 & & T2022 Daniellia oliveri \\
\hline AO SHI JIN SI TAO & 奥氏金丝桃* & Aucher St.John'swort* & T3341 Hypericum aucheri \\
\hline AO SHI LONG DAN & 奥氏龙胆* & Olivier Gentian* & T2924 Gentiana olivieri \\
\hline AO TOU WU HUAN ZI & 凹头无患子* & Emarginate Soapberry Seed & T5718 Sapindus emarginatus \\
\hline AO TOU XIAN & 凹头苋 & Emarginate Amaranth & T0388 Amaranthus lividus \\
\hline AO TUO ROU DOU KOU & 奥托肉豆冦 & Otoba Nutmeg* & T4353 Myristica otoba \\
\hline AO XIAN ZI JIN & 凹陷紫堇 & Bulbous Corydalis & T1712 Corydalis cava \\
\hline AO YE HOU PO & 凹叶厚朴 & Twolobed Officinal Mangolia & T4034 Magnolia biloba \\
\hline AO YE RUI XIANG & 凹叶瑞香 & Retuseleaf Daphne & T2030 Daphne retusa \\
\hline AO ZHOU BAI MAI GEN & 澳洲百脉根 & Austral Bird's Foot Trefoil & T3927 Lotus australis \\
\hline AO ZHOU JIAN GUO & 澳洲坚果 & Queensland Nut & T4011 Macadamia ternifolia \\
\hline \multirow[t]{2}{*}{ AO ZHOU QIE } & 澳洲茄 & Australian Nightshade & T5991 Solanum aviculare [Syn. Solanum \\
\hline & & & laciniatum] \\
\hline AO ZHOU SHANG LU & 澳洲商陆 & Octapistil Pokeweed* & T4866 Phytolacca octandra \\
\hline AO ZHU SHI DOU & 凹猪屎豆 & Yellow-flowering Pea & T1830 Crotalaria retusa \\
\hline BA BEI SUAN TENG ZI & 巴贝酸藤子 & & T2344 Embelia barbeyana \\
\hline BA BI CUI QUE HUA & 巴比翠雀花 & Barbey Larkspur* & T2061 Delphinium barbeyi \\
\hline BA BU YA XIN JI NEI YA SHI & 巴布亚新几内亚柿 & Papua-New-Guinea Persimmon* & T2223 Diospyros mafiensis \\
\hline BA DAN XING REN & 巴旦杏仁 & Amygdalate Apricot Seed & T5215 Prunus amygdalus \\
\hline BA DOU & 巴豆 & Purging Croton & T1858 Croton tiglium \\
\hline BA FA LI YA LONG DAN & 巴伐利亚龙胆 & Bavarian Gentian & T2903 Gentiana bavarica \\
\hline BA JI TIAN & 巴戟天 & Medicinal Indianmulberry & T4283 Morinda officinalis \\
\hline BA JIAO FENG & 八角枫 & Chinese Alangium & T0281 Alangium chinense \\
\hline BA JIAO HUANG PI & 八角黄皮 & Octet Wampee* & T1532 Clausena anisata \\
\hline BA JIAO HUI XIANG & 八角茴香 & Star Anise & T3409 Illicium verum \\
\hline BA LA GUI CHA & 巴拉圭茶 & Paraguay Tea & T3394 Ilex paraguariensis \\
\hline BA NA MA HE HUAN & 巴拿马合欢 & Panamanian Albizia* & T0290 Albizzia adinocephala \\
\hline BA NA MA SHAN SHI LIU & 巴拿马山石榴* & Taiwan Malabar Randia & T5410 Randia formosa \\
\hline BA QIA & 茏葜 & Chinaroot Greenbrier & T5976 Smilax china $[$ Syn. Smilax japonica $]$ \\
\hline BA WANG BIAN & 霸王鞭 & Royle Euphorbia Latex & T2617 Euphorbia royleana \\
\hline BA XI ER YE TAI & 巴西耳叶苔 & Brazilian Ear-leaf Muscus* & T2801 Frullania brasiliensis \\
\hline BA XI HU TONG & 巴西胡桐 & Brazilian Calaba & T1125 Calophyllum brasiliense \\
\hline BA XI LUO FU MU & 巴西萝芙木 & Brazilian Devilpepper* & T5422 Rauvolfia bahiensis \\
\hline BA XI MA DOU LING & 巴西马䛜铃 & Brazilian Dutchmanspipe* & T0622 Aristolochia chamissonis \\
\hline BA XI QIAN LI GUANG & 巴西千里光 & Brazilian Groundsel* & T5881 Senecio brasiliensis \\
\hline BA XI QIAO AN MU & 巴西乔安木 & Brazilian Joan-wood* & T3562 Joannesia princeps \\
\hline BA XI REN SHEN & 巴西人参 & & T4775 Pfaffia paniculata \\
\hline BA XI XIANG WU HUAN ZI & 巴西香无患子 & Guarana & T4677 Paullinia cupana \\
\hline BA XI YE YAN & 巴西野烟 & Brazilian Wild Tobacco; Marianeira & T0075 Acnistus arborescens \\
\hline BA XI ZONG ZAO & 巴西棕藻* & Brazilian Brown Alga Dictyota pfaffii & T2171 Dictyota pfaffii \\
\hline BA XIAN CAO & 八仙草 & Catchweed Bedstraw & T2830 Galium aparine \\
\hline BA XIAN HUA & 八仙花 & Largeleaf Hydrangea & T3304 Hydrangea macrophylla \\
\hline BAI BA JIAO LIAN & 白八角莲 & White Dysosma* & T2301 Dysosma majorensis [Syn. \\
\hline & & & Podophyllum majorense; Dysosma \\
\hline & & & lichuanensis] \\
\hline BAI BEI YE & 白背叶 & Whitebackleaf Mallotus & T4080 Mallotus apelta \\
\hline BAI BU & 百部(对叶百部) & Tuber Stemona & T6115 Stemona tuberosa \\
\hline BAI CHANG & 白菖 & Drug Sweetflag & T0142 Acorus calamus \\
\hline BAI CI & 白刺 & Nitraria* & T4436 Nitraria tangutorum \\
\hline
\end{tabular}




\begin{tabular}{|c|c|c|c|}
\hline BAI CI GUO TUN CAO & 白刺果豚草 & White Bur Sage & T0401 Ambrosia dumosa \\
\hline BAI CI HUA & 白刺花 & Vetchleaf Sophora & T6046 Sophora viciifolia \\
\hline BAI CI HUA GEN & 白刺花根 & Vetchleaf Sophora Root & T6047 Sophora viciifolia \\
\hline BAI CI HUA YE & 白刺花叶 & Vetchleaf Sophora Leaf & T6048 Sophora viciifolia \\
\hline BAI CI HUA ZI & 白刺花籽 & Vetchleaf Sophora Seed & T6049 Sophora viciifolia \\
\hline BAI DIAN FENG CHE ZI & 白点风车子* & White-punctated Combretum* & T1630 Combretum albopunctatum \\
\hline BAI DOU KOU & 白豆蔻 & Round Cardamom & $\begin{array}{l}\text { T0416 Amomum kravanh }[\text { Syn. Amomum } \\
\text { cardamomum }]\end{array}$ \\
\hline BAI DUAN & 白椴 & White Linden* & T6456 Tilia alburnum \\
\hline BAI E GAO & 白鵝膏 & Goose Fat & T0516 Anser cygnoides domestica \\
\hline BAI FAN DOU & 白饭豆 & Kidney Bean & T4785 Phaseolus vulgaris \\
\hline BAI GOU TENG & 白钩藤 & White Gambirplant & $\begin{array}{l}\text { T6632 Uncaria sessilifructus [Syn. Nauclea } \\
\text { sessilifructus] }\end{array}$ \\
\hline BAI GUI BI & 白鬼笔 & Stinking Polecat, Wood Witch & T4778 Phallus impudicus \\
\hline BAI GUO & 白果 & Ginkgo Nut & T2961 Ginkgo biloba \\
\hline BAI GUO GEN & 白果根 & Ginkgo Root & T2962 Ginkgo biloba \\
\hline BAI GUO SHU PI & 白果树皮 & Ginkgo Bark & T2963 Ginkgo biloba \\
\hline BAI GUO YE & 白果叶(银杏叶) & Ginkgo Leaf & T2964 Ginkgo biloba \\
\hline BAI GUO ZI CAO & 白果紫草 & Common Gromwell & T3882 Lithospermum officinale \\
\hline BAI HAO & 白蒿 & Sievers Wormwood & T0696 Artemisia sieversiana \\
\hline BAI HE & 百合 & Greenish Lily & $\begin{array}{l}\text { T3832 Lilium brownii var. viridulum [Syn. } \\
\text { Lilium brownii var. colchesteri] }\end{array}$ \\
\hline BAI HE LING ZHI & 白鹤灵芝 & Bignose Rhiancanthus & T5483 Rhinacanthus nasutus \\
\hline BAI HOU WU TOU & 白喉乌头 & Whitethroat Monkshood & T0111 Aconitum leucostomum \\
\hline BAI HUA CAI ZI & 白花菜籽 & Common Spiderflower Seed & $\begin{array}{l}\text { T1550 Cleome gynandra }[\text { Syn. Gynandropsis } \\
\text { gynandra] }\end{array}$ \\
\hline BAI HUA DAN & 白花丹 & Whiteflower Leadword & T5029 Plumbago zeylanica \\
\hline BAI HUA DAN SHEN & 白花丹参 & Whiteflower Danshen & T5681 Salvia miltiorrhiza f. alba \\
\hline BAI HUA FENG DOU CAI & 白花蜂斗菜 & White-flowerButterbur* & T4738 Petasites albus \\
\hline BAI HUA JUE CHUANG & 白花爵床 & Whiteflower Rostellularia & T3609 Justicia procumbens var. leucantha \\
\hline BAI HUA LONG DAN & 白花龙胆 & Alpine Gentian & T2902 Gentiana algida \\
\hline BAI HUA QIAN HU & 白花前胡 & Whiteflower Hogfennel & T4768 Peucedanum praeruptorum \\
\hline BAI HUA SHE GAN & 白花射干 & Vesper Iris & T3457 Iris dichotoma \\
\hline BAI HUA SHE SHE CAO & 白花蛇舌草 & Spreading Hedyitis & $\begin{array}{l}\text { T4485 Oldenlandia diffusa }[\text { Syn. Hedyotis } \\
\text { diffusa] }\end{array}$ \\
\hline BAI HUA TENG & 白花藤 & Threeflower Clematis & $\begin{array}{l}\text { T1547 Clematis terniflora }[\text { Syn. Clematis } \\
\text { maximowicziana }]\end{array}$ \\
\hline BAI HUA XIA ZHI CAO & 白花夏至草 & Whiteflower Lagopsis & $\begin{array}{l}\text { T4112 Marrubium supinum }[\text { Syn. Lagopsis } \\
\text { supina] }\end{array}$ \\
\hline BAI HUA YING SHAN HONG & 白花映山红 & Snow Azalea & T5518 Rhododendron mucronatum \\
\hline BAI HUA YING SU & 白花罂粟 & White Poppy* & T4620 Papaver album \\
\hline BAI HUA YOU MA TENG & 白花油麻藤 & Whiteflower Mucuna & T4308 Mucuna birdwoodiana \\
\hline BAI JI & 白茂 & Common Bletilla & T0955 Bletilla striata \\
\hline BAI JIAN MU & 白坚木 & Campus-belu Aspidosperma & T0761 Aspidosperma campus-belus \\
\hline BAI JIANG & 败酱(白花败酱) & Whiteflower Patrinia & T4676 Patrinia villosa \\
\hline BAI JIANG CAN & 白僵蚕 & Silkworm Larva & T0975 Bombyx mori \\
\hline BAI JIANG JUN & 白僵菌 & & T0887 Beauveria bassiana \\
\hline BAI JIE ZI & 白芥子 & White Mustard Seed & $\begin{array}{l}\text { T5958 Sinapis alba }[\text { Syn. Brassica alba; } \\
\text { Brassica hirta }]\end{array}$ \\
\hline BAI LA SHU & 白蜡树 & Chinese Ash Bark & T2767 Fraxinus chinensis \\
\hline BAI LAI SHI JU & 白莱氏菊 & Bailai's Chrysanthemum & T0854 Baileya multiradiata \\
\hline BAI LAN HUA & 白兰花 & Bailan Flower & T4209 Michelia alba \\
\hline BAI LI LU & 白藜芦 & White Falsehellebore & T6692 Veratrum album \\
\hline
\end{tabular}




\begin{tabular}{|c|c|c|c|}
\hline BAI LI XIANG & 百里香 & Breckland Thyme & T6452 Thymus serpyllum \\
\hline BAI LI XIANG YE CHUN XIN LIAN & 百里香叶穿心莲* & Andrographis* & T0459 Andrographis serpyllifolia \\
\hline BAI LI XIANG YE MA SANG & 百里香叶马桑 & Thymeleaf Coriaria* & T1695 Coriaria thymifolia \\
\hline BAI LIAN & 白蔹 & Japanese Ampelopsis & $\begin{array}{l}\text { T0427 Ampelopsis japonica [Syn. Paullinia } \\
\text { japonica] }\end{array}$ \\
\hline BAI LIANG JIN & 百两金 & Crispateleaf Ardisia & T0595 Ardisia crispa \\
\hline BAI MAO GEN ${ }^{(1)}$ & 白茅根 & Lalang Grass Rhizome & T3416 Imperata cylindrica var. major \\
\hline BAI MAO GEN ${ }^{(4)}$ & 白毛茛 & Golden-seal & T3309 Hydrastis canadensis \\
\hline BAI MAO TENG & 白毛藤 & Bittersweet & T6005 Solanum lyratum \\
\hline BAI MAO XIA KU CAO & 白毛夏枯草 & Decumbent Bugle & T0263 Ajuga decumbens \\
\hline BAI MAO ZI ZHU & 白毛紫珠 & Whitehairy Beautyberry & T1117 Callicarpa candicans \\
\hline BAI MEI HUA & 白梅花 & Japanese Apricot Flower & T5226 Prunus mите \\
\hline BAI MU TONG & 白木通 & Austral Akebia & T0279 Akebia trifoliata var. australis \\
\hline BAI MU TONG GEN & 白木通根 & Austral Akebia Root & T0280 Akebia trifoliata var. australis \\
\hline BAI MU WU JIU & 白木乌柏 & Japanese Sapium & T5722 Sapium japonicum \\
\hline BAI MU XIANG & 白木香 & Chinese Eaglewood & T0555 Aquilaria sinensis \\
\hline BAI NIU XI & 白牛溙 & Berry-bearing Campion & T1871 Cucubalus baccifer \\
\hline BAI PI SONG & 白皮松 & Lacebark Pine & T4908 Pinus bungeana \\
\hline BAI QIAN CENG & 白千层 & Cajeput-tree & T4154 Melaleuca leucadendra \\
\hline BAI QU CAI & 白屈菜 & Greater Celandine & T1357 Chelidonium majus \\
\hline BAI RI CAO & 百日草 & Youth-and-old-age & T6912 Zinnia elegans \\
\hline BAI RI QING & 百日青 & Thitmin & T5048 Podocarpus neriifolius \\
\hline BAI ROU MAO XIANG CHA CAI & 白柔毛香茶菜 & Whitepilose Rabdosia & T3478 Isodon albopilosus \\
\hline BAI RUI CAO & 百荵草 & Chinese Bastardtoadflax & T6431 Thesium chinense \\
\hline BAI SAN MO HUA & 白散沫花* & White Henna* & T3731 Lawsonia alba \\
\hline BAI SE BAI XIAN & 白色白鲜* & Burning Bush & T2164 Dictamnus albus \\
\hline BAI SHAO & 白莐 & Common Peony & $\begin{array}{l}\text { T4580 Paeonia albiflora [Syn. Paeonia } \\
\text { lactiflora] }\end{array}$ \\
\hline BAI SHOU SHEN & 白手参* & European Gymnadenia & T3076 Gymnadenia albida \\
\hline BAI SHOU WU & 白首乌 & Bunge Swallowwort & T1953 Cynanchum bungei \\
\hline BAI SHU LANG & 白薯莨 & Hispid Yam & T2200 Dioscorea hispida \\
\hline BAI SHU YE & 柏树叶 & Chinese Weeping Cypress Leaf & T1896 Cupressus funebris \\
\hline BAI SHUI XIAN & 白水仙 & Paper-white Narcissus & T4377 Narcissus papyraceus \\
\hline BAI SU YE & 白苏叶 & Common Perilla Leaf & T4714 Perilla frutescens \\
\hline BAI SU ZI & 白苏子 & Common Perilla Fruit & T4715 Perilla frutescens \\
\hline BAI SUI YE & 百岁叶 & Longlived Leaf & T6816 Welwitschia mirabilis \\
\hline BAI TA GE & 白塔蛤 & & T5772 Saxidomus giganteus \\
\hline BAI TOU WENG & 白头翁 & Chinese Pulsatilla & T5325 Pulsatilla chinensis \\
\hline BAI WEI & 白薇 & Blackend Swallowwort & T1951 Cynanchum atratum \\
\hline BAI XIAN PI & 白鲜皮 & Densefruit Pittany Root-bark & T2167 Dictamnus dasycarpus \\
\hline BAI XIAN SHU & 白线薯 & Shortstamen Stephania* & T6118 Stephania brachyandra \\
\hline BAI XIANG CAO MU XI & 白香草木犀 & White Sweetclover & T4170 Melilotus albus \\
\hline BAI XIE GEN & 白泻根 & White Bryony & T1042 Bryonia alba \\
\hline BAI XUE GUO MU & 白雪果木 & & T1367 Chioccoca alba \\
\hline BAI YA MA & 白亚麻 & White Flax & T3858 Linum album \\
\hline BAI YAN TENG & 百眼藤 & Littleleaf Indianmulberry & T4284 Morinda parvifolia \\
\hline BAI YAO ZI & 白药子 & Oriental Stephania & T6119 Stephania cepharantha \\
\hline BAI YE GUA FU MU & 白叶瓜馥木 & Glaucescent Fissistigma & $\begin{array}{l}\text { T2734 Fissistigma glaucescens } \text { [Syn. } \\
\text { Melodorum glaucescens] }\end{array}$ \\
\hline BAI YE JING JIE & 白叶荆芥 & Whiteleaf Nepeta & T4416 Nepeta leucophylla \\
\hline BAI YE MI ZI LAN & 白叶米仔兰 & Whiteleaf Aglaia* & T0239 Aglaia leucophylla \\
\hline BAI YE TENG & 白叶藤 & Chinese Cryptolepis & T1866 Cryptolepis sinensis \\
\hline BAI YE XIANG CHA CAI & 白叶香茶菜 & Whiteleaf Rabdosia* & T3503 Isodon leucophyllus \\
\hline
\end{tabular}




\begin{tabular}{|c|c|c|c|}
\hline BAI YOU & 白柚 & White Pummelo* & T1482 Citrus grandis f. hakunikuju \\
\hline BAI YU SHAN DOU & 白羽扇豆 & White Lupin & T3939 Lupinus albus \\
\hline BAI YUN HUA & 白云花 & White Cowparsnip* & T3221 Heracleum rapula \\
\hline BAI ZHI & 白芷 & Dahurian Angelica & $\begin{array}{l}\text { T0478 Angelica dahurica }[\text { Syn. Angelica } \\
\text { porphyrocaulis }]\end{array}$ \\
\hline BAI ZHI MA & 白芝麻 & Oriental Sesame (white seed) & $\begin{array}{l}\text { T5925 Sesamum indicum [Syn. Sesamum } \\
\text { orientale] }\end{array}$ \\
\hline BAI ZHU & 白术 & Largehead Atractylodes & $\begin{array}{l}\text { T0824 Atractylodes macrocephala }[\text { Syn. } \\
\text { Atractylis macrocephala }]\end{array}$ \\
\hline BAI ZHU SHU & 白珠树 & CommonGaultheria & T2893 Gaultheria leucocarpa var. cumingiana \\
\hline BAI ZI REN & 柏子仁 & Chinese Arborvitae Kernel* & $\begin{array}{l}\text { T0944 Biota orientalis [Syn. Thuja orientalis; } \\
\text { Platycladus orientalis] }\end{array}$ \\
\hline BAN BIAN LIAN & 半边莲 & Chinese Lobelia & $\begin{array}{l}\text { T3898 Lobelia chinensis [Syn. Lobelia } \\
\text { radicans] }\end{array}$ \\
\hline BAN BIAN LIAN ZHUANG LI LU & 半边莲状藜芦 & Lobelia Falsehellebore* & $\begin{array}{l}\text { T6693 Veratrum album var. lobelianum }[\text { Syn. } \\
\text { Veratrum lobelianum }]\end{array}$ \\
\hline BAN BIAN SU & 半边苏 & Common Elsholtzia & T2341 Elsholtzia ciliata \\
\hline BAN DI JIN & 斑地锦 & American Euphorbia & T2600 Euphorbia maculata \\
\hline BAN DIAN XIAO QIU QIANG & 斑点小球腔菌* & & T3768 Leptosphaeria maculans \\
\hline JUN & & & \\
\hline BAN GUAN MU MU JU & 半灌木母菊 & Semi-frutex Mayweed* & T4125 Matricaria suffruticosa \\
\hline BAN HUA WU TOU & 斑花乌头 & Manchurian Monkshood & T0139 Aconitum variegatum \\
\hline BAN JIU & 斑加 & Rufous Turtle Dove & T6150 Streptopelia orientalis \\
\hline BAN JIU JU & 斑鸠菊 & Edible Bitterleaf & T6716 Vernonia esculenta \\
\hline BAN JU CHI ZHUANG ZE LAN & 半锯齿状泽兰 & Semiserration Eupatorium* & T2574 Eupatorium semiserratum \\
\hline BAN KE HU JIAO & 斑克胡椒 & Bank Pepper* & T4935 Piper banksii \\
\hline BAN LAN GEN & 板蓝根 & Indigowoad Root & T3475 Isatis indigotica \\
\hline BAN LI & 板栗 & Chinese Chestnut & T1254 Castanea mollissima \\
\hline BAN MAO & 斑螫 & Blister Beetle & T4338 Mylabris phalerata; Mylabris cichorii \\
\hline BAN PI AN & 半皮桉 & Hemihull Eucalyptus* & T2512 Eucalyptus hemiphloia \\
\hline BAN RUI TANG SONG CAO & 瓣荵唐松草 & Petalformed Meadowrue & T6403 Thalictrum petaloideum \\
\hline BAN WEN AN & 斑纹桉* & Spotted Gum & T2514 Eucalyptus maculata \\
\hline BAN WEN LU HUI & 斑纹芦荟 & Chinese Aloe Dried Juice & T0348 Aloe vera var. chinensis \\
\hline BAN XIA & 半夏 & Ternate Pinellia & T4904 Pinellia ternata \\
\hline BAN YE DI JIN & 斑叶地锦 & Spottedleaf Euphorbia & T2622 Euphorbia supina \\
\hline BAN YE PING PENG CAO & 斑叶萍蓬草 & Variegated Cowlily & T4451 Nuphar variegatum \\
\hline BAN YUAN & 班螈 & Mudpuppy & T4395 Necturus maculosus \\
\hline BAN YUE XING TIE XIAN JUE & 半月形铁线蕨 & Philippine Maidenhair* & T0173 Adiantum lunulatum \\
\hline BAN ZHEN ZHONG HUA SHU & 斑疹钟花树 & & T6270 Tabebuia impetiginosa \\
\hline BAN ZHI LIAN & 半枝莲 & Barbed Skullcap & $\begin{array}{l}\text { T5835 Scutellaria barbata [Syn. Scutellaria } \\
\text { rivularis] }\end{array}$ \\
\hline BAN ZI MA HUANG & 斑子麻黄 & Scalyseed Ephedra & T2371 Ephedra lepidosperma \\
\hline BAN ZI MU & 斑籽木 & Montane Baliospermum & T0861 Baliospermum montanum \\
\hline BANG BING BEI SAN & 棒柄杯伞 & Ffat-footed Clitocybe & T1576 Clitocybe clavipes \\
\hline BANG YE JUE ZAO & 棒叶蕨藻 & Howe & T1272 Caulerpa sertularioides \\
\hline BANG ZHUANG ZI JIN & 棒状紫堇 & Climbing Corydalis & T1714 Corydalis claviculata \\
\hline BAO BAN E GAO & 豹斑鹅膏 & $\begin{array}{l}\text { Leopard Leather Mushroom*; } \\
\text { Tengutake (in Japanese) }\end{array}$ & T0384 Amanita pantherina \\
\hline BAO CHUN HUA & 报春花 & Fairy Primrose & T5199 Primula malacoides \\
\hline BAO CHUN SHI HU & 报春石斛 & Primrose Denrdobium & T2109 Dendrobium primulinum \\
\hline BAO E ZHANG YA CAI & 苞萝獐牙菜 & Calycin Swertia & T6213 Swertia calycina \\
\hline BAO GAI CAO & 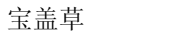 & Henbit Deadnettle & T3680 Lamium amplexicaule \\
\hline BAO GAI LING ZHI & 宝盖灵芝 & Cape Ganoderma & T2845 Ganoderma capense \\
\hline
\end{tabular}


BAO JING KU MAI CAI
BAO JING TIAN JIE CAI
BAO JING ZHANG YA CAI
BAO MA ZI

BAO MO HUI MAO DOU

BAO PI GU

BAO PIAN SHI CHE JU

BAO PIAN SHU WEI CAO

BAO PIAN TU LA SHU

BAO RUI SHAN YOU GAN

BAO SHAN WU TOU

\section{BAO SHAN YOU GAN}

BAO SHI FEI YAN CAO

BAO XING WEI MAO

BAO YE XIANG CHA CAI

BAO ZI GAN LAN

BEI AI

BEI CANG ZHU

BEI CE JIN ZHAN HUA

BEI FANG DANG GUI

BEI FANG GOU QI GEN PI

BEI FANG WU TOU

BEI FEI XUE SONG

BEI FEN NAI AO LE MU

BEI HAI DANG GUI

BEI HAI XIAN TAI CHONG

BEI HUANG HUA CAI

BEI JIA ER TANG SONG CAO

BEI JING SHI WEI

BEI JING YANG

BEI KE SHAN

BEI LI LU HUI

BEI MA DOU LING

BEI MA DOU LING GEN

BEI MEI AI HAO

BEI MEI E ZHANG QIU

BEI MEI HONG SHAN

BEI MEI QIU ZI JUE

BEI MEI TING LI ZI

BEI MEI XIANG BAI

BEI MEI YA BAI

BEI MEI YUAN BAI

BEI MEI ZHOU SHAN GENG CAI

BEI MU LAN

BEI QIAO SHI HU

BEI SHA SHEN

BEI SHI SHAN CHENG

BEI SHUI KU MAI

BEI WU TOU

BEI XUAN SHEN

BEI YE JU
抱茎苦荬菜

抱茎天芥菜*

抱茎獐牙菜

暴马子

\section{包膜灰毛豆* \\ 豹皮菇 \\ 苍片矢车菊* \\ 苞片鼠尾草 \\ 苞片图腊树 \\ 包瑞山油柑 \\ 保山乌头}

\section{包山油柑}

包氏飞燕草

宝兴卫矛

苞叶香茶菜

抱子甘蓝

北艾

北苍术

北侧金盏花

北方当归*

北方枸杞根皮

北方乌头

北非雪松

被粉奈奥勒姆

北海当归

北海蘚苔虫

北黄花菜

贝加尔唐松草

北京石韦

北京杨

贝壳杉

贝利芦荟*

北马盶铃

北马㝸铃根

北美矮蒿

北美鹅掌楸

北美红杉

北美球子偋

北美葶艻子

北美香柏

北美崖柏

北美圆柏

北美洲山梗菜

贝母兰

杯鞘石斛

北沙参

贝氏山橙*

北水苦荬

北乌头 (草乌)

北玄参

北野菊
Sowthistle-leaf Ixeris

Clasping Heliotrope

Amplexicaul Swertia

Amur Lilac

Tunicate Tephrosia*

Pardleather-like Mushroom

Bracteole Centaurea*

Bracteole Galipea*

Bauer Acronychia

Paoshan Monkshood

Vested Acronychia*

Brown Larkspur*

Paohsing Euonymus

Bractleaf Rabdosia

Brussels Sprout

Mugwort

Chinese Atractylodes

Siberian Adonis

Northern Angelica*

Northern Wolfberry Root-bark*

Northern Monkshood*

Atlas Cedar

Northsea Angelica*

North Sea Bryozoan

Yellow Daylily

Baikal Meadowrue

Peking Pyrrosia Frond

Beijing Poplar

Amboina Pitch Tree

Bally Aloe*

Northern Dutchmanspipe

Northern Dutchmanspipe Root

Low Sagebrush

Yellow Poplar

Redwood

Bead Fern, Sensitive Fern

Virginia Pepperweed Seed

Western Arborvitae

Eastern Arborvitae

Red Cedar

Indian Tobacco

Common Coelogyne

Muchlovable Denrdobium

Coastal Glehnia

Balansa Melodinus*

Watery Speedwell

Kusnezoff Monkshood

Buerger Figwort

Boreal Wild Chrysanthemum
T3548 Ixeris sonchifolia

T3167 Heliotropium amplexicaule

T6221 Swertia franchetiana

T6259 Syringa amurensis [Syn. Syringa

reticulata var. amurensis]

T6342 Tephrosia tunicata

T3743 Lentinus lepideus

T1304 Centaurea bracteata

T5662 Salvia bracteata

T2827 Galipea bracteata

T0148 Acronychia baueri

T0080 Aconitum bullatifolium var.

homotrichum [Syn. Aconitum nagarum]

T0153 Acronychia vestita

T2063 Delphinium brownii

T2543 Euonymus mupinensis

T3515 Isodon melissoides

T1018 Brassica oleracea var. gemmifera

T0706 Artemisia vulgaris

T0819 Atractylodes chinensis

T0188 Adonis sibirica

T0499 Angelica ursina

T3959 Lycium chinense var. potaninii

T0129 Aconitum septentrionale

T1281 Cedrus atlantica

T1579 Cneorum pulverulentum

T0475 Angelica acutiloba var. sugiyamae

T2742 Flustra foliacea

T3195 Hemerocallis lilio-asphodelus

T6375 Thalictrum baicalense

T5354 Pyrrosia davidii

T5148 Populus beijingensis

T0213 Agathis dammara

T0335 Aloe ballyi

T0624 Aristolochia contorta

T0625 Aristolochia contorta

T0663 Artemisia arbuscula

T3871 Liriodendron tulipifera

T5918 Sequoia sempervirens

T4492 Onoclea sensibilis

T3759 Lepidium virginicum

T6441 Thuja plicata

T6438 Thuja occidentalis

T3603 Juniperus virginiana

T3900 Lobelia inflata

T1606 Coelogyne ovalis

T2104 Dendrobium gratiosissimum

T2983 Glehnia littoralis

T4176 Melodinus balansae

T6722 Veronica anagallis-aquatica

T0108 Aconitum kusnezoffii

T5826 Scrophularia buergeriana

T1387 Chrysanthemum boreale 
BEI YUE GOU TENG

BEI ZI JIN

BENG GE YUAN WEI

BI BA

BI BA GEN

BI CHENG QIE

BI CHI YAN ZI CAI

BI CHUAN JIU JIE MU

BI DONG QIE

BI LI

BI LIN BA JIAO LIAN

\section{BI LU GOU TENG}

BI LU GU KE

BI LU MIAN ZAO ER

BI LU XIANG JIAO

BI LU ZHI XIAO BO

BI MA GEN

BI MA YE

BI MA YOU

BI MA ZI

BI SHENG LI

BI SHI DUI XIN JU

BI SHI LUO FU MU

BI XIE

BI ZHI TANG SONG CAO

BI ZI CU FEI

BIAN BAI MA LI JIN

BIAN BAO LIN MAO JUE

BIAN BING HUANG JIN

BIAN DA XIU QIU

BIAN DI JIN

BIAN DOU

BIAN DOU

BIAN DOU CAI YE BAI JIANG

BIAN FU GE

BIAN FU GE GEN

BIAN GUO JIAO GU LAN

BIAN JIA SHAN LI DOU

BIAN JING HUANG QI

BIAN JING YAN JIE CAO

BIAN PING JU

BIAN SE HE KE BAO

BIAN SE MA DOU LING

BIAN SE SHI ZHU

BIAN SHUO TENG

BIAN TAI

BIAN TAO

BIAN TAO ZHUANG BAN JIU JU

BIAN XING MU LAN
北越钩藤

North Viet-Nam Gambirplant

北紫堇

崩格岛尾*

荜苃

荜苃根

荜澄茄

菼齿眼子菜

比川九节木

碧冬茄

薜荔

秕鳞八角莲

秘鲁钩藤*

秘鲁古柯

秘鲁绵柊儿

秘鲁香胶

俾路支小檗

菼麻根

菼麻叶

菼麻油

菼麻子

壁生藜*

比氏堆心菊*

比氏萝芙木*

草薢粉草薢)

笔直唐松草*

篦子粗柶

变白马利筋

边孢鳞毛硕

扁柄黄堇

鞭打绣球

遍地金

扁豆

扁豆

变豆菜叶败酱*

蝙蝠葛

蝙蝠葛根

扁果绞股蓝

扁英山黧豆

扁茎黄花

扁茎沿阶草

扁平桔

变色裸壳孢*

变色马兒铃

变色石竹

扁蒴藤

鞭苔

扁桃

扁桃状斑鸭菊

变形木兰*
Siberian Corydalis

Bunge Iris*

Long Pepper

Long Pepper Root

Cubeba Pepper

Fennelleaf Pondweed

Common Petunia

Climbing Fig

Furfuraceous Many-flowered

May-apple*

Uña de Gato (Cat's Claw)

Peru Coca Shrub

Peru Squill*

Peru Balmtree Resin

Baluchistan Barberry

Castorbean Root

Castorbean Leaf

Castorbean Oil

Castorbean Seed

Mural Goosefoot*

Bigelov Sneezeweed*

Beddome Devilpepper

Hypoglaucous Collett Yam

Strict Meadowrue*

Oliver Plumyew

White Milkweed

Evergreen Wood Fern

Flatstiped Corydalis

Diversifolious Hemiphragma

Wight's St.John'swort

Hyacinth Dolichos Seed

Niger Bean*

Korea Patrinia*

Asiatic Moonseed

Asiatic Moonseed Root

Flatfruit Gynostemma

Dwarf Chickling Pea

Flatstem Milkvetch

Flatstem Lilyturf*

Depressed Orange

Versicolorous Dutchmanspipe

Versicolorous Pink

Indian Pristimera

Flagelliform Liverwort*

Peachform Mango

Bitterleaf

Mutable Magnolia*
T6618 Uncaria homomalla [Syn. Uruparia

homomalla; Uruparia tonkinensis; Uruparia

lanosa var. parvifora]

T1742 Corydalis sibirica

T3454 Iris bungei

T4953 Piper longum

T4954 Piper longum

T4944 Piper cubeba

T5177 Potamogeton pectinatus

T5274 Psychotria beccaroides

T4751 Petunia hybrida

T2723 Ficus pumila

T2299 Dysosma furfuracea

T6637 Uncaria tomentosa

T2492 Erythroxylum novogranatense

T5812 Scilla peruviana

T4358 Myroxylon pereirae

T0898 Berberis baluchistanica

T5547 Ricinus communis

T5548 Ricinus communis

T5549 Ricinus communis

T5550 Ricinus communis

T1364 Chenopodium murale

T3136 Helenium bigelovii

T5429 Rauwolfia beddomei

T2201 Dioscorea hypoglauca [Syn. Dioscorea

collettii var. hypoglauca]

T6413 Thalictrum strictum

T1322 Cephalotaxus oliveri

T0735 Asclepias albicans

T2283 Dryopteris marginalis

T1727 Corydalis mucronifera

T3201 Hemiphragma heterophyllum

T3368 Hypericum wightianum

T2246 Dolichos lablab

T3647 Lablab niger

T4671 Patrinia saniculaefolia

T4182 Menispermum dauricum

T4183 Menispermum dauricum

T3083 Gynostemma compressum

T3703 Lathyrus cicera

T0793 Astragalus complanatus

T4508 Ophiopogon planiscapus

T1475 Citrus depressa

T2354 Emericella variecolor

T0643 Aristolochia versicolar

T2147 Dianthus versicolor

T5208 Pristimera indica

T0885 Bazzania trilobata

T4103 Mangifera persiciformis

T6712 Vernonia amygdalina

T4043 Magnolia mutabilis 


BIAN XU
BIAN YE TIE XIAN JUE
BIAN YI FENG WEI JUE
BIAN YUAN BIAN E TAI
BIAN YUAN LIN GAI JUE
BIAN YUAN LIN MAO JUE
BIAN ZHI HU JI SHENG
BIAN ZHONG CHANG YE AN LUO
BIAN ZHONG JIAN HUAI TENG
BIAN ZHU TANG SONG CAO
BIN HAI DANG GUI
BIN HAI QIAN HU
BIN HAO
BIN MU JING
BIN XI FA NI YA CANG ER
BING CHI XIAN
BING DAO YI
BING DOU
BING GUO HU JI SHENG
BING GUO HUA JIAO
BING GUO TANG SONG CAO
BING HUA JU
BING LANG
BING PIAN
BING SHE CHUANG
BING TOU CAO
BING TOU HUANG QIN
BING YE SUO LUO
BO AI DA JI
BO BAN HE YE TAI
BO BAN JIU JU
BO
BOA

BO LE SHU
BO LI ZI HUA JIAO
BO LIN JU
BO LU DU SHU
BO LUN LIN HUA RUAN SHAN
HU
BO LUO HUI
BO LUO MI
BO LUO XIANG TENG
BO NIANG HAO
BO PI HUANG GUA
BO SHI AN
BO SHI CI TONG
BO SHI QIAN HU
BO SHI QIE
BO SHI WU TAN
BO SHI WU ZHU YU

\begin{tabular}{|c|c|}
\hline 萹蓄 & Common Knotgrass \\
\hline 鞭叶铁线蕨 & Walking Maidenhair \\
\hline 变异凤尾蔴 & Unequal Brake \\
\hline \multicolumn{2}{|l|}{ 边缘扁荳苔* } \\
\hline 边缘鳞盖蕨 & Marginate Microlepia \\
\hline 边缘鳞毛蕨 & Marginated Buckler-fern* \\
\hline 扁枝檞寄生 & Flatshort Mistletoe \\
\hline 变种长叶暗罗 & Indian Greenstar Variety* \\
\hline 变种尖槐藤* & Edible Oxystelma Variety* \\
\hline 鞭柱唐松草 & Smith Meadowrue \\
\hline 滨海当归 & Seashore Angelica* \\
\hline 滨海前胡 & Japan Hogfennel \\
\hline 滨蒿 & Santonica \\
\hline 滨牡荆 & Littoral Chastetree* \\
\hline $\begin{array}{l}\text { 宾夕法尼亚苍耳* } \\
\text { 并齿蘚 }\end{array}$ & Pennsylvanian Cocklebur* \\
\hline 冰岛衣 & Iceland Moss \\
\hline 兵豆 & Common Lentil \\
\hline 柄果桷寄生 & Stipefruit Mistletoe \\
\hline 柄果花椒 & Stalkedfruit Pricklyash \\
\hline 柄果唐松草 & Stalkedfruit Meadowrue* \\
\hline \multicolumn{2}{|l|}{ 柄花菊 } \\
\hline 槟榔 & Betenutpalm \\
\hline 冰片 & Borneol \\
\hline 滨蛇床 & Japanese Cnidium \\
\hline 并头草 & Galericulate Skullcap \\
\hline 并头黄芩 & Twinflower Skullcap \\
\hline \multicolumn{2}{|l|}{ 柄叶杪椤* } \\
\hline 孛艾大戟 & Petty Euphorbia \\
\hline \multicolumn{2}{|l|}{ 波瓣合叶苔 } \\
\hline 菠菜 & Spinish \\
\hline 波戈斑加菊 & Manysperma Bitterleaf* \\
\hline 薄荷 & Wild Mint \\
\hline
\end{tabular}

$\begin{array}{ll}\text { 伯乐树 } & \text { Chinese Bretschneidera } \\ \text { 伯利兹花椒 } & \text { Beliz Pricklyash* } \\ \text { 薄鳞菊 } & \text { Intermediate Chartolepis } \\ \text { 博路都树 } & \text { Boldo } \\ \text { 波伦鳞花软珊瑚 } & \text { Softcoral Lemnalia bournei }\end{array}$

博落回

波罗蜜

波罗香藤

播娘蒿

薄皮黄瓜*

伯氏桉*

伯氏刺桐*

博氏前胡*

博氏茄*

波氏乌檀

波氏吴荣英

Pink Plumepoppy
T5098 Polygonum aviculare

T0172 Adiantum caudatum

T5291 Pteris inaequalis

T5403 Radula marginata

T4219 Microlepia marginata

T2284 Dryopteris marginata

T6773 Viscum articulactum

T5066 Polyalthia longifolia var. pendula

T4566 Oxystelma esculentum var. alpini

T6410 Thalictrum smithii

T0486 Angelica keiskei

T4760 Peucedanum japonicum

T0683 Artemisia maritima

T6784 Vitex littoralis

T6841 Xanthium pennsylvanicum

T6358 Tetraplodon mnioides [Syn.

Tetraplodon bryoides; Splachnum mnioides]

T1341 Cetraria islandica

T3741 Lens culinaris

T6777 Viscum multinerve

T6889 Zanthoxylum podocarpum

T6404 Thalictrum podocarpum

T5035 Podanthus ovatifolius

T0606 Areca catechu

T2274 Dryobalanops aromatica

T1581 Cnidium japonicum

T5838 Scutellaria galericulata

T5844 Scutellaria scordifolia

T6856 Yathea podophylla

T2609 Euphorbia peplus

T5779 Scapania undulata

T6076 Spinacia oleracea

T6720 Vernonia pogosperma

T4184 Mentha haplocalyx [Syn. Mentha

canadaensis; Mentha arvensis var. haplocalyx;

Mentha arvensis]

T1025 Bretschneidera sinensis

T6867 Zanthoxylum belizense

T1354 Chartolepis intermedia

T4774 Peumus boldus

T3740 Lemnalia bournei

T4020 Macleaya cordata

T0713 Artocarpus heterophyllus

T3611 Kadsura ananosma

T2125 Descurainia sophia

T1875 Cucumis leptodermus

T2501 Eucalyptus berghei

T2460 Erythrina berteroana

T4754 Peucedanum bourgaei

T5992 Solanum berthaultii

T4393 Nauclea pobequinii

T2645 Evodia rutaecarpa var. bodinieri 


\begin{tabular}{|c|c|}
\hline BO SI A WEI BIAN ZHONG & 波斯阿魏变种* \\
\hline BO SI YI MU CAO & 波斯益母草* \\
\hline BO SI YING SU & 波斯篑粟 \\
\hline BO TE LAN DA JI & 波特兰大戟* \\
\hline BO XI SHU LI & 波希鼠李* \\
\hline BO YE KU MU & 波叶苦木 \\
\hline BO YE SHAN CHENG & 薄叶山橙 \\
\hline BO YE WANG YI ZAO & 薄叶网胰藻 \\
\hline BO YE WU TOU & 薄叶乌头 \\
\hline BU CHANG NAN MEI DENG MU & 布昌南美登木 \\
\hline BU DENG HONG HOU KE & 不等红厚壳* \\
\hline BU DUI CHENG MAO RU & 不对称猫乳* \\
\hline BU GU ZHI & 补骨脂 \\
\hline BU LANG WEI JI & 布郎维吉* \\
\hline BU MEI HE BAO HUA & 不美荷包花* \\
\hline BU SHI LONG DAN & 布氏龙胆 \\
\hline BU XUE CAO & 补血草 \\
\hline BU YING CAO & 捕蝇草 \\
\hline BU YU HONG & 不育红 \\
\hline CAI BAN YANG TI JIA & 彩斑羊蹄甲* \\
\hline CAI DOU SHU & 菜豆树 \\
\hline CAI JI & 菜薊 \\
\hline CAI SHI MU MA SANG & 采食木马桑 \\
\hline CAI SI CANG ER & 蔡斯苍耳* \\
\hline CAI WEN QIAN JIN TENG & 彩纹千金藤* \\
\hline CAN DOU & 虫豆 \\
\hline CAN DOU JIA KE & 虫豆荚壳 \\
\hline CAN DOU JING & 虫豆茎 \\
\hline CAN DOU YE & 虫豆叶 \\
\hline CAN JIAN & 虫茧 \\
\hline CAN SANG & 虫桑* \\
\hline CANG BAI BING ROU CHI JUN & 苍白柄肉齿菌* \\
\hline CANG BAI CHENG GOU FENG & 苍白秤钩风 \\
\hline CANG BAI FEN TENG & 苍白粉藤 \\
\hline CANG BAI QI SHE TAI & 苍白岐舌苔* \\
\hline CANG BAI ZI JIN & 苍白紫堇 \\
\hline CANG ER & 苍耳 \\
\hline CANG MAO JIN FA XUAN & 苍毛金发癬 \\
\hline CANG SHAN XIANG CHA CAI & 苍山香茶菜 \\
\hline CANG ZHU & 苍术(茅苍术) \\
\hline CAO BEI MU & 草贝母 \\
\hline CAO CONG RONG & 草苁蓉 \\
\hline CAO DI MAO GEN & 草地毛茛* \\
\hline CAO DI WU TOU & 草地乌头 \\
\hline CAO DIAN QIAN LI GUANG & 草甸千里光 \\
\hline CAO DOU KOU & 草豆冦 \\
\hline CAO GU & 草菇 \\
\hline CAO HU JIAO & 草胡椒 \\
\hline CAO MAO JIA DU JUAN & 踈毛假杜鹃* \\
\hline CAO MEI & 草莓 \\
\hline CAO MEI CHE ZHOU CAO & 草莓车轴草 \\
\hline CAO SHAO YAO & 草苟药 \\
\hline
\end{tabular}

Persia Giantfennel Variety*
Persia Motherwort*
Persia Poppy
Portlan Euphorbia*
Cascara Buckthorn
Undulate-leaf Quassiawood*
Thin-leaf Melodinus

Azure Monkshood

Buchanan Mayten

Disparate Beautyleaf*

Asymmetry Rhamnella*

Malaytea Scurfpea

Chile Calceolaria*

Burser Gentian*

Gmelin Sealavender Herb

Venus's Flytrap

Yunnan Rabdosia

Variegate Bauhinia*

Asia Belltree

Globe Artichoke

Arboreous Coriaria*

Chase Cocklebur*

Discolor Stephania*

Broadbean

Broadbean Pericarp

Broadbean Stem

Broadbean Leaf

Silk Cocoon

Silk Mulberry*

Glaucesent Diploclisia

Pale Treebine*

Pale Corydalis

Siberian Cocklebur

Dark-hair Gold-hair Moss*

Cangshan Rabdosia

Swordlike Atractylodes

Indian Iphigenia

Russian Boschniakia

Meadow Buttercup

Meadow Monkshood

Ragwort

Katsumada Galangal

Straw Mushroom

Shiny Peperomia

Common Strawberry

Strawberry Clover

Obovate Peony
T2704 Ferula persica var. latisecta

T3753 Leonurus persicus

T4633 Papaver persicum

T2611 Euphorbia portlandica

T5463 Rhamnus purshiana

T3098 Hannoa undulata

T4179 Melodinus tenuicaudatus

T3310 Hydroclathrus tenuis

T0095 Aconitum fischeri

T4128 Maytenus buchananii

T1128 Calophyllum dispar

T5453 Rhamnella inaequilatera

T5270 Psoralea corylifolia

T1041 Brunsvigia radulosa

T1110 Calceolaria inamoena

T2905 Gentiana burseri

T3842 Limonium gmelinii

T2186 Dionaea muscipula

T5401 Rabdosia yuennanensis

T0881 Bauhinia variegata

T5402 Radermachera sinica

T1966 Cynara scolymus

T1689 Coriaria arborea

T6836 Xanthium chasei

T6124 Stephania discolor

T6744 Vicia faba

T6745 Vicia faba

T6746 Vicia faba

T6747 Vicia faba

T0976 Bombyx mori

T4292 Morus bombycis

T5735 Sarcodon glaucopus

T2233 Diploclisia glaucescens

T1454 Cissus pallida

T5803 Schistochila glaucescens

T1740 Corydalis sempervirens

T6843 Xanthium sibiricum [Syn. Xanthium strumarium]

T5135 Polytrichum pollidisetum

T3482 Isodon bulleyana

T0823 Atractylodes lancea

T3443 Iphigenia indica

T0993 Boschniakia rossica

T5412 Ranunculus acris

T0138 Aconitum umbrosum

T5890 Senecio jacobaea

T0358 Alpinia katsumadai

T6803 Volvariella volvacea

T4705 Peperomia pellucida

T0872 Barleria strigosa

T2763 Fragaria ananassa

T6518 Trifolium fragiferum

T4586 Paeonia obovata 


\begin{tabular}{|c|c|c|c|}
\hline CAO SU & 糛苏 & Jerusalemsage & T4814 Phlomis umbrosa \\
\hline CAO WEN JING & 草问荆 & Meadaw Horsetail & T2410 Equisetum pratense \\
\hline CAO WU JIU & 草乌柏 & Sylvatic Sapium* & $\begin{array}{l}\text { T6146 Stillingia sylvatica }[\text { Syn. Sapium } \\
\text { sylvatica] }\end{array}$ \\
\hline CAO XIANG WAN DOU & 草香踠豆 & Indian Pea & T3710 Lathyrus sativus \\
\hline CAO YE BAI JIANG & 糙叶败奨 & Scabrous Patrinia & T4673 Patrinia scabra \\
\hline CAO YE YI WA JU & 踈叶依瓦菊* & & $\mathrm{T} 3542$ Iva asperifolia \\
\hline CAO YUAN DA JI & 草原大戟 & Grassland Euphorbia* & T2620 Euphorbia stepposa \\
\hline CAO YUAN LAO GUAN CAO & 草原老鸛草 & Meadow Cranesbill & T2944 Geranium pratense \\
\hline CE BAI YE & 侧柏叶 & Chinese Arborvitae Leaf & $\begin{array}{l}\text { T6440 Thuja orientalis }[\text { Syn. Platycladus } \\
\text { orientalis; Biota orientalis] }\end{array}$ \\
\hline CE BAI ZHI JIE & 侧柏枝节 & Chinese Arborvitae Branch & $\begin{array}{l}\text { T6439 Thuja orientalis }[\text { Syn. Platycladus } \\
\text { orientalis; Biota orientalis] }\end{array}$ \\
\hline CEN PI & 梣皮 & Largeleaf Chinese Ash Bark & $\begin{array}{l}\text { T2777 Fraxinus rhynchophylla [Syn. Fraxinus } \\
\text { chinensis var. rhynchophylla }]\end{array}$ \\
\hline CENG KONG JUN & 层孔菌* & Polypore & T2751 Fomitopsis spraguei \\
\hline CHA HUA & 茶花 & Tea Flower & T1150 Camellia sinensis [Syn. Thea sinensis] \\
\hline CHA MEI & 茶梅 & Sasanqua Camellia & T1149 Camellia sasanqua \\
\hline CHA MI SEN TUN CAO & 查米森豚草 & Chamisson Ragweed & T0399 Ambrosia chamissonis \\
\hline CHA QIE & 查茄 & Cha Nightshade* & T5994 Solanum chacoense \\
\hline CHA RU SHI WAN CUO & 插入十万错* & Ya-Yaa (Thai name) & T0812 Asystasia intrusa \\
\hline CHA RUI SHU YU & 叉苾薯蓣 & Collett Yam & T2194 Dioscorea collettii \\
\hline CHA SHI SHE BIAN JU & 查氏蛇鞭菊* & Champman Gay-feather* & T3787 Liatris champmanii \\
\hline CHA SHU & 檫树 & Common Sassafras & T5746 Sassafras tzumu \\
\hline CHA SHU GEN & 茶树根 & Tea Root & T1151 Camellia sinensis [Syn. Thea sinensis] \\
\hline CHA TIAO QI & 茶条棫 & Amur Maple & T0049 Acer ginnala \\
\hline CHA XIONG & 茶芦 & Chaxiong Ligusticum & T3825 Ligusticum sinense cv. chaxiong \\
\hline CHA YE & 茶叶 & Common Tea & T1152 Camellia sinensis [Syn. Thea sinensis] \\
\hline CHA YU BIAN DI JIN & 察隅遍地金 & Chayu St.John'swort & T3369 Hypericum wightianum subsp. axillare \\
\hline CHA ZI & 茶子 & Tea Seed & T1154 Camellia sinensis [Syn. Thea sinensis] \\
\hline CHA ZI XIN & 茶子心 & Oiltea Camellia & T1146 Camellia oleifera \\
\hline CHA ZI YUAN BAI & 叉子圆柏 & Savin Juniper & T3595 Juniperus sabina \\
\hline CHAI HU & 柴胡(北柴胡) & Chinese Thorowax & T1059 Bupleurum chinense \\
\hline CHAI HU ZHUANG QIAN LI & 柴胡状千里光 & Thorowax-like Groundsel* & T5882 Senecio bupleuroides \\
\hline GUANG & & & \\
\hline CHAI HUANG JIANG & 柴黄姜 & Rosthorn Yam & T2204 Dioscorea nipponica ssp. rosthornii \\
\hline CHAI SHOU & 柴首 & Chaishou Thorowax & T1058 Bupleurum chaishoui \\
\hline CHAN CHU & 蟾蜍 & Toad & $\begin{array}{l}\text { T1049 Bufo bufo gargarizans; Bufo } \\
\text { melanostictus }\end{array}$ \\
\hline CHAN CHU DAN & 蟾蜍胆 & Toad Gall & $\begin{array}{l}\text { T1050 Bufo bufo gargarizans; Bufo } \\
\text { melanostictus }\end{array}$ \\
\hline CHAN GAO MU JIANG ZI & 潺槁木姜子 & Gluey Litse & T3887 Litsea glutinosa \\
\hline CHAN PI & 蟾皮 & Toad Skin & $\begin{array}{l}\text { T1051 Bufo bufo gargarizans; Bufo } \\
\text { melanostictus }\end{array}$ \\
\hline CHAN RAO HUANG TAN & 缠绕黄檀 & Voluble Rosewood* & T2019 Dalbergia volubilis \\
\hline CHAN SU & 蟾酥 & Toad Skin Secretion Cake & $\begin{array}{l}\text { T1052 Bufo bufo gargarizans; Bufo } \\
\text { melanostictus }\end{array}$ \\
\hline CHAN YANG & 颤杨 & American Aspen & T5163 Populus tremuloides \\
\hline CHAN YI TENG & 蝉翼藤 & Cicadawingvine & T5847 Securidaca inappendiculata \\
\hline CHANG BAI SHAN BAO CHUN & 长白山报春 & Changpai Mountains Primrose & T5201 Primula modesta \\
\hline CHANG BAN JIN LIAN HUA & 长瓣金莲花 & Langpetal Globeflower & T6554 Trollius macropetalus \\
\hline CHANG BIAN HUA DANG GUI & 长边花当归* & Longradiate Angelica* & T0488 Angelica longeradiata \\
\hline CHANG BING FENG WEI JUE & 长柄凤尾蕨 & Pretty Brake & T5286 Pteris bella \\
\hline
\end{tabular}




\begin{tabular}{|c|c|c|c|}
\hline CHANG BING HU JIAO & 长柄胡椒 & Longstalk Pepper* & T4969 Piper sulvaticum \\
\hline CHANG BING QI YE SHU & 长柄七叶树 & Assam Horsechestnut & T0198 Aesculus assamica \\
\hline CHANG BING YUAN BAI & 长柄圆柏* & Longpetiole Juniper* & T3590 Juniperus macropoda \\
\hline CHANG CHUN HUA & 长春花 & Madagascar Periwinkle & $\begin{array}{l}\text { T1271 Catharanthus roseus }[\text { Syn. Vinca rosea } \\
\text { Lochera rosea }]\end{array}$ \\
\hline CHANG CHUN TENG & 常春藤 & Chinese Ivy & T3113 Hedera nepalensis var. sinensis \\
\hline CHANG CHUN YOU MA TENG & 常春油麻藤 & Evergreen Mucuna & T4311 Mucuna sempervirens \\
\hline CHANG E JIN LIAN MU PI & 长萼金莲木皮* & Macrocalyx Ochna Bark* & T4466 Ochna macrocalyx \\
\hline CHANG E QU MAI & 长萝㫿麦 & Longcalyx Pink* & T2146 Dianthus superbus var. longicalycinus \\
\hline CHANG GENG CU FEI & 长梗粗榧 & Longstalk Plumyew* & $\begin{array}{l}\text { T1321 Cephalotaxus harringtonia var. } \\
\text { drupacea }\end{array}$ \\
\hline CHANG GENG DONG QING & 长梗冬青 & Longpedicel Holly & T3395 Ilex pedunculosa \\
\hline CHANG GENG JIAO GU LAN & 长梗绞股蓝 & longstalk Gynostemma & T3084 Gynostemma longipes \\
\hline CHANG GENG NAN WU WEI ZI & 长梗南五味子 & Longpeduncle Kadsura & $\begin{array}{l}\text { T3619 Kadsura peltigera }[\text { Syn. Kadsura } \\
\text { longipedunculata }]\end{array}$ \\
\hline CHANG GENG YU LI REN & 长梗郁李仁 & Longpedicel Chinese Buscherry Seed & T5225 Prunus japonica var. nakaii \\
\hline CHANG GUAN JIA MO LI & 长管假茉莉 & Indian Glorybower & T1557 Clerodendron indicum \\
\hline CHANG GUAN XIANG CHA CAI & 长管香茶菜 & Longtube Rabdosia & T5393 Rabdosia longituba \\
\hline CHANG GUAN XUAN CAO & 长管萱草* & Longtube Daylily* & T3196 Hemerocallis longituba \\
\hline CHANG GUO BI BA & 长果荜苃 & Petrofracted Pepper & T4964 Piper retrofractum \\
\hline CHANG GUO XUE DAN & 长果雪胆 & Longfruit Hemsleya & T3205 Hemsleya dolichocarpa \\
\hline CHANG GUO YING SU & 长果罂粟 & Long-headed Poppy & T4626 Papaver dubium \\
\hline CHANG HU JIAO & 长胡椒 & & T4945 Piper elongatum \\
\hline CHANG HUA BAN KE YA SHU & 长花瓣柯桠树* & Longpetal Vouacapoua* & T6805 Vouacapoua macropetala \\
\hline CHANG HUA GOU TENG & 长花钩藤* & Longflower Gambirplant* & T6624 Uncaria longiflora \\
\hline CHANG HUA RUI MU & 长花荵木* & Longflower Kopsia* & T3642 Kopsia longiflora \\
\hline CHANG HUA TU LA SHU & 长花图腊树 & Longflower Galipea* & T2828 Galipea longiflora \\
\hline CHANG HUA XIE CAO & 长花顽草 & Longflower Valerian* & $\begin{array}{l}\text { T1313 Centranthus longiflorus ssp. } \\
\text { longiflorus }\end{array}$ \\
\hline CHANG HUA XU MA QIAN ZI & 长花序马钱子 & Longthyrsus Poisonnut* & T6171 Strychnos dolichothyrsa \\
\hline CHANG HUI AN & 长喙桉 & Long-rostrate Eucalyptus* & $\mathrm{T} 2518$ Eucalyptus rostrata \\
\hline CHANG JI HUANG & 长吉黄 & & T5477 Rheum sp. \\
\hline CHANG JIAO DOU & 长角豆 & Carob & T1326 Ceratonia siliqua \\
\hline CHANG JIE ZHU & 长节珠 & Laevigate Parameria & $\mathrm{T} 4642$ Parameria laevigata \\
\hline CHANG JU YAN HU SUO & 长矩延胡索 & Longspur Corydalis* & T1722 Corydalis longicalcarata \\
\hline CHANG LV GOU WEN & 常绿钩吻 & Carolina Jasmine & T2899 Gelsemium sempervirens \\
\hline CHANG MAO FENG MAO JU & 长毛凤毛菊 & Hawkweed-like Saussurea & $\begin{array}{l}\text { T5770 Saussurea superba }[\text { Syn. Saussurea } \\
\text { hieracioides }]\end{array}$ \\
\hline CHANG MAO HAN XIAO & 长毛含笑* & Longhairy Michelia* & T4213 Michelia lanuginosa \\
\hline CHANG MAO XIANG KE KE & 长毛香科科 & Pilose Germander & $\begin{array}{l}\text { T6365 Teucrium pilosum [Syn. Teucrium } \\
\text { japonicum var. pilosum] }\end{array}$ \\
\hline CHANG MAO ZI YUAN ZHI & 长毛籽远志 & Longhair Milkwort & T5090 Polygala wattersii \\
\hline CHANG NIU JIAO GUA & 长牛角瓜* & Long Calotrope* & T1136 Calotropis procera \\
\hline CHANG ROU MAO WEI LING & 长柔毛委陵菜 & Velutinous Cinquefoil & T5183 Potentilla griffithii var. velutina \\
\hline CAI & & & \\
\hline CHANG RUI LIU LI CAO & 长苾琉璃草 & Circinate Solenanthus & T6021 Solenanthus circinatus \\
\hline CHANG SHAN & 常山 & Antifebrile Dichroa & T2158 Dichroa febrifuga \\
\hline CHANG SHENG TIE JIAO JUE & 长生铁角蕨 & Prolongated Spleenwort & T0776 Asplenium prolongatum \\
\hline CHANG SHUO HUANG MA & 长龩黄麻 & Jews-mallow & T1675 Corchorus olitorius \\
\hline CHANG SUI BA DOU & 长穗巴豆 & LongspikeCroton* & T1851 Croton macrostachys \\
\hline CHANG TONG HAN HUA & 长筒旱花 & Cylinder Immortelle & T6847 Xeranthemum cylindraceum \\
\hline CHANG WEI CU YE MU & 长尾粗叶木 & Acuminate Lasianthus & T3696 Lasianthus acuminatissimus \\
\hline CHANG WEI FU YE ER JUE & 长尾复叶耳蕨 & Simple Arachniodes & T0565 Arachniodes simplicior \\
\hline
\end{tabular}




\begin{tabular}{|c|c|c|}
\hline CHANG WEI PO PO NA & 长尾婆婆纳 & Longleaf Speedwell \\
\hline CHANG XIANG MAO & 长香茅* & Long Lemongrass* \\
\hline CHANG XU XIE CAO & 长序㖪草 & Hardwick Valeriana \\
\hline CHANG YAO GE CHONG LOU & 长药隔重楼 & Tibet Paris \\
\hline CHANG YE AI JU & 长叶艾菊 & Longleaf Tansy \\
\hline CHANG YE AN LUO & 长叶暗罗 & India Greenstar \\
\hline CHANG YE CHANG CHUN HUA & 长叶长春花 & Longleaf Periwinkle* \\
\hline CHANG YE CHE QIAN & 长叶车前 & Buckhorn Plantain \\
\hline CHANG YE FEI SHU & 长叶榧树 & Jack Torreya \\
\hline CHANG YE GE NA XIANG & 长叶哥纳香 & Longleaf Goniothalamus \\
\hline CHANG YE HOU KE GUI & 长叶厚壳桂* & Longleaf Cryptocarya* \\
\hline CHANG YE KUAN MU & 长叶宽木 & \\
\hline CHANG YE MAN MI PING GUO & 长叶曼密苹果 & Longleaf Mammey* \\
\hline CHANG YE QIAN LI GUANG & 长叶千里光* & Longleaf Groundsel* \\
\hline CHANG YE SHUI MA & 长叶水麻 & Longleaf Debregeasia \\
\hline CHANG YE SONG & 长叶松 & Long-leaved Pine \\
\hline CHANG YE TIAN MING JING & 长叶天名精 & Longleaf Carpesium \\
\hline CHANG YE XIANG CHA CAI & 长叶香茶菜 & Longleaf Rabdosia \\
\hline CHANG YUAN XIN XING HU & 长圆心形胡桐* & Cordate-oblong Beautyleaf* \\
\hline \multicolumn{3}{|l|}{ TONG } \\
\hline CHANG YUAN YE XIAO BO & 长圆叶小檗 & Oblong-leaved Barberry \\
\hline CHANG ZHU TANG SONG CAO & 长柱唐松草 & Longstyle Meadowrue* \\
\hline CHANG ZI MA QIAN & 长籽马钱 & Wallich Poisonnut \\
\hline CHAO AO DING CAO & 巢凹顶藻 & \\
\hline CHAO FENG CAO & 潮风草 & Acuminate Swallowwort \\
\hline CHAO XIAN BAI TOU WENG & 朝鲜白头翁 & Korean Pulsatilla \\
\hline CHAO XIAN CANG ZHU & 朝鲜苍术 & Koraen Atractylodes \\
\hline CHAO XIAN DA BAI LI XIANG & 朝鲜大百里香* & Korean Big Thyme* \\
\hline CHAO XIAN DANG GUI & 朝鲜当归 & Gigantic Angelica \\
\hline CHAO XIAN HUAI & 朝鲜槐 & Amur Maackia \\
\hline CHAO XIAN JI & 朝鲜蓟 & Stemless Carline Thistle \\
\hline CHAO XIAN LANG YA CI & 朝鲜狼牙刺 & Korean Sophora \\
\hline CHAO XIAN LENG SHAN & 朝鲜冷杉* & Korean Fir \\
\hline CHAO XIAN LIAN QIAO & 朝鲜连尧* & Korean Forsythia* \\
\hline CHAO XIAN LUO WAN & 朝鲜裸苑* & \\
\hline CHAO XIAN WU JIA & 朝鲜五加 & Korean Acanthopanax* \\
\hline CHAO XIAN WU JIAO FENG & 朝鲜五角枫 & Okamoto Maple \\
\hline CHAO XIAN YIN YANG HUO & 朝鲜淫羊蕉 & Korean Epimedium \\
\hline CHE QIAN & 车前 & Asiatic Plantain \\
\hline CHE QIAN YE LAN JI & 车前叶蓝薊 & Purple Viper's-bugloss \\
\hline CHE SANG ZI YE & 车桑仔叶 & Clammy Hopseedbush Leaf \\
\hline CHE SHI NAN & 车氏楠* & Chemens Phoebe* \\
\hline CHEN DONG CAI LU ZHI & 陈冬菜卤汁 & Mature Winter-vegetable Spiced Juice \\
\hline CHEN XIANG & 沉香 & Eaglewood \\
\hline CHENG GAN CAO & 秤杆草 & Japanese Eupatorium \\
\hline CHENG GAN SHENG MA & 秤杆升麻 & Lindley Eupatorium \\
\hline CHENG KOU SHI DA GONG & 城口十大功劳 & Chengkou Mahonia \\
\hline \multicolumn{3}{|l|}{ LAO } \\
\hline CHENG KOU TANG SONG CAO & 城口唐松草 & Farges Meadowrue \\
\hline CHENG LIU & 柽柳 & Chinese Tamarisk \\
\hline CHENG LIU YE YUAN BAI & 柽柳叶圆柏* & Tamarisk-leaf Juniper* \\
\hline CHENG QIE ZI & 澄茄子 & Mountain Spicy Tree \\
\hline CHENG ZI & 橙子 & Fragrant Citrus \\
\hline
\end{tabular}

T6725 Veronica longifolia T1946 Cymbopogon procerus T6676 Valeriana hardwickii T4650 Paris polyphylla var. pseudothibetica T6292 Tanacetum longifolium T5065 Polyalthia longifolia T1268 Catharanthus longifolius T5006 Plantago lanceolata T6478 Torreya jackii T3045 Goniothalamus gardneri T1863 Cryptocarya longifolia T2634 Eurycoma longifolia T4095 Mammea longifolia T5891 Senecio longifolius T2056 Debregeasia longifolia T4919 Pinus palustris T1213 Carpesium longifolium T5400 Rabdosia stracheyi T1127 Calophyllum cordato-oblongum

T0910 Berberis oblonga

T6396 Thalictrum longistylum

T6193 Strychnos wallichiana

T3720 Laurencia nidifica

T1950 Cynanchum ascyrifolium

T5324 Pulsatilla cernua

T0822 Atractylodes koreana

T6448 Thymus magnus

T0483 Angelica gigas

T4009 Maackia amurensis

T1208 Carlina acaulis

T6037 Sophora koreensis

T0005 Abies koreana

T2754 Forsythia koreana

T3079 Gymnaster koraiensis

T0040 Acanthopanax koreanum

T0051 Acer okamotoanum

T2398 Epimedium koreanum

T5002 Plantago asiatica

T2319 Echium plantagineum

T2243 Dodonaea viscosa

T4815 Phoebe chemensii

T1006 Brassica chinensis

T0554 Aquilaria agallocha

T2563 Eupatorium japonicum

T2565 Eupatorium lindleyanum

T4072 Mahonia shenii

T6382 Thalictrum fargesii

T6286 Tamarix chinensis

T3596 Juniperus sabina var. tamariscifolia T3885 Litsea cubeba

T1486 Citrus junos 


\begin{tabular}{|c|c|c|c|}
\hline CHENG ZI HE & 橙子核 & Fragrant Citrus Seed & T1487 Citrus junos \\
\hline CHENG ZI PI & 橙子皮 & Pericarp & T1488 Citrus junos \\
\hline CHI A MI & 齿阿米 & Tooth Ammi & T0412 Ammi visnaga \\
\hline CHI AN & 赤桉 & Longbeak Eucalyptus & T2502 Eucalyptus camaldulensis \\
\hline CHI BAN YAN HU SUO & 齿瓣延胡索 & Toothedpetal Corydalis & $\begin{array}{l}\text { T1734 Corydalis remota }[\text { Syn. Corydalis } \\
\text { bulbosa var. typica] }\end{array}$ \\
\hline CHI CHI JUE MING & 齿翅决明* & Toothwing Senna* & T1231 Cassia dentata \\
\hline CHI DOU & 赤豆 & Assuki Bean & $\begin{array}{l}\text { T6752 Vigna angularis [Syn. Dolichus } \\
\text { angularis; Phaseolus angularis] }\end{array}$ \\
\hline CHI GENG TENG & 匙美藤 & Australian Cowplant & T3080 Gymnema sylvestre \\
\hline CHI GUO HUANG QI & 翅果黄芪* & Wingfruit Milkvetch* & T0802 Astragalus pterocarpus \\
\hline CHI GUO TENG & 翅果藤 & Extensed Wingfruitvine & T4350 Myriopteron extensum \\
\hline CHI MA & 赤麻 & Tricuspidate Falsenettle & $\begin{array}{l}\text { T0962 Boehmeria platanifolia [Syn. } \\
\text { Boehmeria tricuspis] }\end{array}$ \\
\hline CHI NAN & 赤楠 & Boxleaf Syzygium & T6264 Syzygium buxifolium \\
\hline CHI SHAO wild & 赤䓎 & Common Peony (wild) & T4584 Paeonia lactiflora wild \\
\hline CHI YANG & 赤杨 & Japanese Alder & T0328 Alnus japonica \\
\hline CHI YE CAO & 齿叶草 & Lateripening Bartsia & T4479 Odontites serotina \\
\hline CHI YE MEI DENG MU & 齿叶美登木 & Serrate-leaved Mayten & T4139 Maytenus serrata \\
\hline CHI YE SHUI LIAN & 齿叶睡莲 & White Lotus, Egyptian Lotus & T4455 Nymphaea lotus \\
\hline CHI YE TIE ZI & 齿叶铁仔 & Serrate-leaf Myrsine & T4364 Myrsine semiserrata \\
\hline CHI YE TUO WU & 齿叶囊吾 & Toothleaf Goldenray & T3802 Ligularia dentata \\
\hline CHONG BAI LA & 虫白蜡 & Cera Chinensis Wax & T2420 Ericerus pela \\
\hline CHONG BAN XUAN CAO & 重瓣萱草 & Doublepetalous Daylily* & T3194 Hemerocallis fulva var. kwanso \\
\hline CHONG CHUN YU & 重唇鱼 & Skin-carp & T3199 Hemibarbus labeo \\
\hline CHONG LOU PAI CAO & 重楼排草 & Parisshape Loosestrife & T4004 Lysimachia paridiformis \\
\hline CHONG MING BA JIAO LIAN & 崇明八角莲 & $\begin{array}{l}\text { Chongming Many-flowered } \\
\text { May-apple* }\end{array}$ & T2303 Dysosma subrosea \\
\hline $\begin{array}{l}\text { CHONG SHENG RUAN SHAN } \\
\text { HU }\end{array}$ & 冲绳软珊瑚* & $\begin{array}{l}\text { Okinawan Softcoral Clavularia } \\
\text { viridis }\end{array}$ & $\mathrm{T} 1542$ Clavularia viridis \\
\hline CHONG YA YAO & 虫牙药 & Ternateleaf Rabdosia & T3529 Isodon ternifolius \\
\hline CHOU A WEI & 臭阿魏 & Foetid Giantfennel* & T2700 Ferula foetida \\
\hline CHOU BAI & 臭柏 & Savin & T5640 Sabina vulgaris \\
\hline CHOU CAO & 臭草 & Common Rue & T5626 Ruta graveolens \\
\hline CHOU LENG SHAN & 臭冷杉 & Khingan Fir & T0006 Abies nephrolepis \\
\hline CHOU MO LI & 臭茉莉 & Fragrant Glorybower & T1556 Clerodendron fragrans \\
\hline CHOU MU DAN & 臭牡丹 & Rose Glorybower (Clerodendrum) & T1565 Clerodendrum bungei \\
\hline CHOU SHAN YANG & 臭山羊 & Japanese Orixa & T4527 Orixa japonica \\
\hline CHOU WEI HONG DOU & 臭味红豆 & Bean Trefoil & T0441 Anagyris foetida \\
\hline CHOU WU TONG & 臭梧桐 & Harlequin Glorybower Leaf & T1562 Clerodendron trichotomum \\
\hline CHOU WU TONG GEN & 臭梧桐根 & Harlequin Glorybower Root & T1563 Clerodendron trichotomum \\
\hline CHU AI JU & 雏艾菊 & Feverfew & T6294 Tanacetum parthenium \\
\hline CHU BAI PI & 㯪白皮 & Tree of Heaven Ailanthus Bast & T0255 Ailanthus altissima \\
\hline CHU CHONG JU & 除虫菊 & Dalmatian Pyrethrum & T1388 Chrysanthemum cinerariaefolium \\
\hline CHU JU & 雏菊 & English Daisy & T0893 Bellis perennis \\
\hline CHU TU HAI CONG & 出土海葱* & & T6647 Urginea epigea \\
\hline CHU YE HUA JIAO & 㯪叶花椒 & Ailanthus-like Pricklyash & T6860 Zanthoxylum ailanthoides \\
\hline CHU YE HUA JIAO GEN & 㯪叶花椒根 & Ailanthus-like Pricklyash Root & T6861 Zanthoxylum ailanthoides \\
\hline CHU YE HUA JIAO PI & 㯪叶花椒皮 & Ailanthus-like Pricklyash Bark & T6862 Zanthoxylum ailanthoides \\
\hline CHUAN BA JIAO LIAN & 川八角莲 & Veitch Dysosma & T2304 Dysosma veitchii \\
\hline CHUAN BEI MU & 川贝母 & Tendrilleaf Fritillary & T2783 Fritillaria cirrhosa \\
\hline CHUAN CHAN JIU JIE LONG & 川产九节龙 & Tiny Ardisia & T0601 Ardisia pusilla \\
\hline CHUAN CHI SHAO & 川赤䓎 & Veitch Peony & T4590 Paeonia veitchii \\
\hline
\end{tabular}




\begin{tabular}{|c|c|}
\hline CHUAN DANG SHEN & 川党参 \\
\hline CHUAN DIAN JIN SI TAO & 川滇金丝桃 \\
\hline CHUAN DIAN SHI DA GONG LAO & 川滇十大功劳 \\
\hline CHUAN DIAN YIN YANG HUO & 川滇淫羊蕉 \\
\hline CHUAN DONG ZHANG YA CAI & 川东獐牙菜 \\
\hline CHUAN E YIN YANG HUO & 川鄂淫羊藿 \\
\hline CHUAN HUANG QIN & 川黄芩 \\
\hline CHUAN JU & 川橘 \\
\hline CHUAN LI GUO & 川梨果 \\
\hline CHUAN LIAN PI & 川楝皮 \\
\hline CHUAN LIAN ZI & 川楝子 \\
\hline CHUAN LONG SHU YU & 穿龙薯蓣 \\
\hline CHUAN MU XIANG & 川木香 \\
\hline CHUAN NIU XI & 川牛膝 \\
\hline CHUAN QIAN CUI QUE HUA & 川黔翠雀花 \\
\hline CHUAN SHAN CHENG & 川山橙 \\
\hline CHUAN SHAN JIA & 穿山甲 \\
\hline CHUAN SHANG LONG MI GOU & 川上泷弥钩藤 \\
\hline TENG & \\
\hline CHUAN SHI JIAN & 穿石剑 \\
\hline CHUAN XI YIN YANG HUO & 川西淫羊藿 \\
\hline CHUAN XI ZHANG YA CAI & 川西獐牙菜 \\
\hline CHUAN XIN LIAN & 穿心莲 \\
\hline CHUAN XIONG & 川芦 \\
\hline CHUAN XU DUAN & 川续断 \\
\hline CHUAN ZANG XIANG CHA CAI & 川藏香茶菜 \\
\hline CHUAN ZHU ER YE TAI & 串珠耳叶苔 \\
\hline CHUI MU FANG JI & 垂木防己 \\
\hline CHUI QI MU & 垂桤木 \\
\hline CHUI ZHI CHI AN YE & 垂枝赤桉叶 \\
\hline CHUI ZHU SUAN PAN ZI & 枉柱算盘子 \\
\hline CHUI ZI MAI MA TENG & 垂子买麻藤 \\
\hline CHUN & 莼 \\
\hline CHUN BAI PI & 椿白皮 \\
\hline CHUN E BO HE & 唇䓯薄荷 \\
\hline CHUN FU SHOU CAO & 春福寿草 \\
\hline CHUN QIAN LI GUANG & 春千里光 \\
\hline CHUN ZHU JU TAI & 唇柱芭苔 \\
\hline CI BAI & 刺柏 \\
\hline $\mathrm{CI} \mathrm{BO}$ & 刺菠 \\
\hline CI CAI JI & 刺菜蓟 \\
\hline CI CAO SU & 刺糛苏 \\
\hline CI DOU KOU & 刺豆蔻 \\
\hline $\mathrm{CI} \mathrm{GU}$ & 慈菇 \\
\hline CI GUI & 刺桧 \\
\hline CI GUO FAN LI ZHI & 刺果番荔枝 \\
\hline CI GUO GAN CAO & 刺果甘草 \\
\hline CI GUO SONG & 刺果松 \\
\hline CI GUO SU MU & 刺果苏木 \\
\hline CI GUO TUN CAO & 刺果豚草 \\
\hline
\end{tabular}

Szechwan Tangshen

Forrest's St.John'swort

Veitch Mahonia

David Epimedium

E.Chuan Swertia

Farges Epimedium

St. Johnswortleaf Skullcap

King Orange

Pashi Pear Fruit

Szechwan Chinaberry Bark

Szechwan Chinaberry Fruit

Nippon Yam

Common Vladimiria

Mediinal Cyathula

Bonvalot Larkspur

Hemsley Melodinus

Pangolin

Kawakami Gambirplant*

Rock-ginger Fern

Elongate Barrenwort

Mussot Swertia

Common Andrographis

Chuanxiong (Wallich Ligusticum)

Himalayan Teasel

Szechwan-Tibet Rabdosia

Rosary Ear-leaf Muscus*

Drooping Snailseed*

Drooping Alder*

Pendulous Eucalyptus Leaf

Srilanka Glochidion

Pendentseed Jointfir

Common Watershield

Chinese Toon Root-bast

Pennyroyal Mint

Yellow Adonis

Eastern Groundsel

Chirita

Taiwan Juniper

Hirsute Respberry

Cardoon

Pungent Jerusalemsage*

Aculeate Amomum*

Oldworld Arrowhead Corm

Prickly Juniper

Guanabana

Pricklyfruit Licorice

Bristlecone Pine

Nickernut Caesalpinia

Bur Sage
T1602 Codonopsis tangshen

T3352 Hypericum forrestii

T4076 Mahonia veitchiorum

T2392 Epimedium davidii

T6217 Swertia davidii

T2395 Epimedium fargesii

T5839 Scutellaria hypericifolia

T1502 Citrus nobilis

T5365 Pyrus pashia

T4162 Melia toosendan

T4163 Melia toosendan

T2203 Dioscorea nipponica

T6802 Vladimiria souliei [Syn. Jurinea souliei]

T1924 Cyathula officinalis

T2062 Delphinium bonvalotii

T4178 Melodinus hemsleyanus

T4106 Manis pentadactyla

T6619 Uncaria kawakamii

T5252 Pseudodrynaria coronans

T2394 Epimedium elongatum

T6227 Swertia mussotii

T0457 Andrographis paniculata [Syn. Justicia paniculata $]$

T3820 Ligusticum chuanxiong [Syn.

Ligusticum wallichii]

T2234 Dipsacus asperoides

T3518 Isodon pharicus

T2803 Frullania tamarisci ssp. moniliata

[Syn. Frullania moniliata]

T1588 Cocculus pendulus

T0330 Alnus pendula

T2504 Eucalyptus camaldulensis var. pendula

T2990 Glochidion zeylanicum

T3034 Gnetum pendulum

T1004 Brasenia schreberi

T6475 Toona sinensis

T4187 Mentha pulegium

T0191 Adonis vernalis

T5914 Senecio vernalis

T1370 Chirita micronusa

T3588 Juniperus formosana

T5592 Rubus hirsutus

T1965 Cynara cardunculus

T4811 Phlomis pungens

T0415 Amomum aculeatum

T5647 Sagittaria sagittifolia

T3592 Juniperus oxycedrus

T0509 Annona muricata

T3018 Glycyrrhiza pallidiflora

T4905 Pinus aristata

T1100 Caesalpinia crista

T0395 Ambrosia acanthicarpa 


CI HUA JIAO
CI HUA LIAN ZI CAO
CI HUAI HUA
CI JI LI
CI JI NU ZONG LV
CI KE HUA JIAO
CI LI
CI MA QIAN ZI
CI MANG BING HUA
CI NAN SHE TENG
CI QIU SHU PI
CI SAN JIA
CI TIAN QIE
CI TONG
CI TOU FU YE ER JUE
CI WU JIA

CI WU JIA

CI WU JIA PI
CI WU JIA YE
CI YANG LI DOU
CI YE SHI DA GONG LAO
CI YI YE HUA JIAO

CI ZHUANG FAN LI ZHI

CONG BAI

CONG JIAN MU

CONG LI ZAO

CONG MU

CONG ZHU XUE LIAN

$\mathrm{CU}$

\section{CU CAO BA QIA}

CU CAO LONG DAN

CU CAO SHE BIAN JU

CU CAO SHI CHE JU

CU CI XIAO BO

CU GUO TANG SONG CAO

CU GUO XIANG CHA CAI

CU HUA ZHANG YA CAI

CU JING QIN JIAO

CU JING WU TOU

CU KANG CHAI

CU LIU GUO

CU MAO GAN CAO

CU MAO GUO BAI JIAN MU

CU MAO LIN GAI JUE

CU MAO NAN SHE TENG

CU MAO NIU SHE CAO

CU MAO SHU YU

CU MAO YIN YANG HUO

CU PI SHAN HE TAO
刺花椒

刺花莲子草

刺槐花

刺教藜

刺急怒棕㭣

刺壳花椒

刺梨

刺马钱子*

刺芒柄花

刺南蛇藤

刺楸树皮

刺三甲

刺天茄

刺桐

刺头复叶耳䓲

刺五加

刺五加皮

刺五加叶

刺痒黧豆

刺叶十大功劳

刺异叶花椒

刺状番荔枝

葱白

憼樫木*

从粒藻

淴木

从株雪莲

醋

粗踈茏荔

粗楉龙胆

粗糙蛇鞭菊

粗粘矢车菊

簇刺小檗

粗果唐松草

粗果香茶菜

簇花獐牙菜

粗茎秦艽

粗茎乌头

粗糠柴

醋柳果

粗毛甘草

粗毛果白坚木

粗毛鳞盖偋

粗毛南蛇藤

粗毛牛舌草

粗毛薯蓣*

粗毛淫羊藿

粗皮山核桃
Acanthoid Pricklyash*

Spinyflower Alternanthera

Black Locust Flower

Puncturevine Caltrap

Aculeate Ruffle Palm

Spinyfruit Pricklyash

Roxburgh Rose

Aculeate Poisonnut*

Restharrow

Hookedspine Bittersweet

Septemlobate Kalopanax Bark

Trifoliate Acanthopanax

Khasi Nightshade Fruit

Coral-tree

Manyprickle Acanthopanax

Manyprickle Acanthopanax Root-bark

Manyprickle Acanthopanax Leaf

Cowage Velvet-bean

Acanthus-leaved Mahonia

Spinyleaf Prickleyash

Spined Custardapple*

Fistular Onion

Alliaceous Pencilwood*

Japanese Aralia

Vinegar

Lebrun Greenbrier

Scabrous Gentian*

Scabrous Gay-feather*

Rough Star Thistle

Actino-spiny Barberry

Purple Meadowrue

Roughfruit Rabdosia*

Fascicled Swertia*

Thickstem Gentian

Thickstem Monkshood

Philippine Mallotus*

Seabuckthorn Fruit

Hirsute Licorice

Hirsutefruit White Quebracho*

Strigose Microlepia

Hirsute Bittersweet*

Hemhem (in Jordan)

Hirsute Yam*

Acuminatum Epimedium

Shagbark Hickory
T6858 Zanthoxylum acanthopodium

T0380 Alternanthera repens

T5552 Robinia pseudoacacia

T6497 Tribulus terrestris

T0259 Aiphanes aculeata

T6878 Zanthoxylum echinocarpum

T5570 Rosa roxburghii

T6163 Strychnos aculeata

T4493 Ononis spinosa

T1286 Celastrus flagellaris

T3625 Kalopanax septemlobus

T0045 Acanthopanax trifoliatus

T6003 Solanum khasianum

T2478 Erythrina variegata [Syn. Erythrina indica]

T0563 Arachniodes exilis

T0041 Acanthopanax senticosus [Syn.

Eleutherococcus senticosus]

T0042 Acanthopanax senticosus [Syn.

Eleutherococcus senticosus]

T0043 Acanthopanax senticosus [Syn.

Eleutherococcus senticosus]

T4310 Mucuna pruriens

T4053 Mahonia acanthifolia

T6874 Zanthoxylum dimorphophyllum var.

spinifolum

T0512 Annona spinescens

T0314 Allium fistulosum

T2307 Dysoxylum alliaceum

T0996 Botryococcus braunii

T0568 Aralia chinensis

T5771 Saussurea tridactyla var. maidugonla

T6764 Vinegar

T5979 Smilax lebrunii

T2931 Gentiana scabra var. buesgeri

T3791 Liatris scabra

T1301 Centaurea aspera ssp. aspera

T0896 Berberis actinacantha

T6377 Thalictrum dasycarpum

T3500 Isodon lasiocarpa

T6220 Swertia fasciculata

T2909 Gentiana crassicaulis

T0089 Aconitum crassicaule

T4083 Mallotus philippinensis

T3254 Hippophae rhamnoides

T3011 Glycyrrhiza aspera

T0763 Aspidosperma dasycarpon

T4220 Microlepia strigosa [Syn. Trichomanes

strigosa]

T1295 Celastrus strigillosus

T0448 Anchusa strigosa

T2199 Dioscorea hirsuta

T2389 Epimedium acuminatum

T1219 Carya ovata 


CU SHE BIAN JU
CU SHENG AO DING ZAO
CU SHENG HUA DUO GUO SHU
CU SHENG HUANG REN SAN
CU SHENG KA BU MU
CU SHENG SHAN XIANG
CU WEN HAI LONG
CU XI MEN FEI CAO
CU YE MAI HU JIAO
CU YE MAI MI HUA SHU
CU YE RONG
CU YE XUAN GOU ZI
CU YING MAO DIAN ZI CAO
CU YING MAO TIE XIAN CAI
CU YING MAO TUN CAO
CU YING MAO XIAN KONG JUN
CU ZHUANG JIN JI NA
CU ZHUANG KA FEI
CU ZHUANG LONG DAN
CU ZHUANG NV ZHEN
CU ZHUANG SUAN TENG ZI
CU ZHUANG YU TENG
CUI DENG XIN LIU SHAN HU
CUI MIAN SHUI QIE
CUI QUE HUA
CUI QUE YE DUAN GUAN CAO
CUI TU LUO FU MU
CUI YUN CAO
DA A MI
DA BA JIAO
DA BAI DING CAO
DA BO GU
DA BO SI JU
DA CAO KOU
DA CHANG CHUN HUA
DA CHAO CAI
DA CHE QIAN
DA JI
DAN BAO GUO
DHAO

DA DOU

DA DU WU TOU

DA E BIAN XING XIANG CHA

CAI

DA E JIN SI TAO
DA E XIANG CHA CAI
DA ER MA WEI YA LIU CHUAN YU
DA ER WEN XIAO BO
DA FEI YANG CAO
DA FENG ZI
DA GE NA XIANG
DA GUO BAI MU

粗蛇鞭菊

簇生凹顶藻

簇生花多果树*

簇生黄韧伞

簇生卡布木

簇生山香

粗吻海龙

粗西门肺草*

粗叶脉胡椒*

粗叶脉密花树*

粗叶榕

粗叶悬钩子

粗硬毛滇紫草*

粗硬毛铁苋菜*

粗硬毛豚草*

粗硬毛纤孔菌

粗壮金鸡纳*

粗壮咖啡

粗壮龙胆

粗壮女贞

粗壮酸藤子

粗壮鱼藤*

脆灯心柳珊瑚

催眠睡茄

翠雀花

翠雀叶短冠草

催吐萝芙木

翠云草

大阿米

大八角

大白顶草

大驳骨

大波斯菊

大草冦

大长春花*

大巢菜

大车前

大翅蓟

达达赫面包果*

大丁草

\section{大豆}

大渡乌头

大颌变型香茶菜

大萼金丝桃

大蓦香茶菜

达耳马威亚柳穿鱼

达尔文小檗

大飞扬草

大风子

大哥纳香

大果柏木
Scaly Blazing Star

Cluster Concave-top Alga*

Sulfur Tuft

Fascicled Bushmint*

Rough Comfrey

Grossnerve Pepper*

Rough-veined Rapanea*

Hispid Fig

Roughleaf Raspberry

Hispid Onosma*

Chenille Plant, Red-hot Cat's-tail

Hispid Ragweed*

Robust Cinchona*

Robust Coffee*

Robust Gentian*

Japanese Privet

Robost Embelia*

Robust Jewelvine

Gorgonian Junceella fragilis

Somniferous Withania

Bouquet Larkspur

Delphinileaf Sopubia*

Emitic Devilpepper

Hooked Spikemoss

Big Ammi

Big Anisetree*

Field Groundsel

Malabanut

Mexican Aster

Beautiful Galangal

Greater Periwinkle

Common Vetch

Rippleseed Plantain

Scotch Cottonthistle

Dadah Artocarpus*

Gerbera

Soybean

Franchet Monkshood

Largesepal Rabdosia

Rose-of-Sharon

Largesepal Rabdosia

Balkan Toadflax

Darwin Barberry

Garden Euphorbia

Chaulmoogratree Seed

Big Goniothalamus*

Montery Cypress
T3794 Liatris squarrosa

T3715 Laurencia caespitosa

T5016 Pleiocarpa pycnantha var. tubicina

T4366 Naematoloma fasciculare

T1096 Cabucala fasciculata

T3379 Hyptis fasciculata

T6487 Trachyrhamphus serratus

T6244 Symphytum asperum

T4943 Piper crassinervium

T5416 Rapanea neurophylla

T2727 Ficus simplicissima

T5587 Rubus alceaefolius

T4498 Onosma hispida

T0035 Acalypha hispida

T0402 Ambrosia hispida

T3425 Inonotus hispidus

T1430 Cinchona robusta

T1611 Coffea robusta

T2929 Gentiana robusta

T3829 Ligustrum robustum

T2349 Embelia robusta

T2122 Derris robusta

T3574 Junceella fragilis

T6829 Withania somnifera

T2077 Delphinium grandiflorum

T6050 Sopubia delphinifolia

T5427 Rauvolfia vomitoria

T5870 Selaginella uncinata

T0410 Ammi majus

T3403 Illicium majus

T5894 Senecio oryzetorum

T0170 Adhatoda vasica

T1753 Cosmos bipinnata

T0363 Alpinia speciosa

T6760 Vinca herbacea [Syn. Vinca major]

T6750 Vicia sativa

T5007 Plantago major

T4494 Onopordum acanthium

T0711 Artocarpus dadah

T2950 Gerbera anandria [Syn. Leibnitzia

anandria ]

T2998 Glycine max

T0097 Aconitum franchetll

T3513 Isodon macrocalyx

T3343 Hypericum calycinum

T3511 Isodon macrocalyx

T3843 Linaria dalmatica

T0901 Berberis darwinii

T2590 Euphorbia hirta

T3300 Hydnocarpus anthelminticus

T3046 Goniothalamus giganteus

T1897 Cupressus macrocarpa 


DA GUO DA JI
DA GUO KA FEI
DA GUO TAO JIN NIANG
DA GUO XI FAN LIAN
DA HONG GU
DA HONG YING SU
DA HUA CAO SU
DA HUA DAO DI LING
DA HUA DI AO DOU
DA HUA GE NA XIANG
DA HUA HONG JING TIAN
DA HUA JIAN MU
DA HUA JIAN QIU LUO
DA HUA JIN WA ER
DA HUA JU
DA HUA LUO XIN FU
DA HUA MA CHI XIAN
DA HUA SHAN QIAN NIU
DA HUA SHAO LAN
DA HUA WU WEI ZI
DA HUA XI XIN
DA HUA XUAN FU HUA CAO
DA HUA YAO WEN SHU LAN
DA HUA YIN YANG HUO
DA HUA YIN YANG HUO BIAN
ZHONG
DA HUA ZI WEI
DANA

大果大戟

大果咖啡

大果桃金娘

大果西番莲

大红菇

大红罂粟

大花糙苏*

大花倒地铃

大花迪奥豆

大花哥纳香

大花红景天

大花樫木*

大花剪秋罗

大花金挖耳

大花菊*

大花落新妇

大花马齿苋

大花山牵牛

大花杓兰

大花五味子

大花细辛

大花旋覆花草

大花药文殊兰*

大花淫羊蕉

大花淫羊嚾变种

大花紫薇

\section{大花紫玉盘 \\ 大黄(药用大黄) \\ 大茴芹* \\ 大蓟 \\ 大戟 \\ 大金牛草}

DA JIN QIAN CAO

DA LANG DU

DA LI HUA

DA LI LU TI CAO

DA LIANG JIANG

DA MA DOU LING

DA MA JIN

DA MA SHI GE QIANG WEI

DA MA YE ZE LAN

DA MU JIANG ZI

DA PENG TENG

DA PO WAN HUA HUA

DA QIAN HU

DA QING YANG

DA QING YE

大金钱草(金钱草)
大狼毒
大丽花
大理鹿蹄草
大良姜
大马淣铃*
大麻槿
大马士革蓄薇
大麻叶泽兰
大木姜子
搭棚藤
打破碗花花
大前胡
大青杨
大青叶

\author{
Largefruit Euphorbia* \\ Liberian Coffee \\ Bigfruit Rosemyrtle* \\ Giant Granadilla \\ Big Russula* \\ Bracteate Poppy* \\ Largeflower Jerusalemsage \\ Bigflower Heartseed*
}

Bigflower Goniothalamus

Bigflower Rhodiola

Bigflower Pencilwood*

Brilliant Campion

Bigflower Carpesium*

Large-flowered Dendranthema*

Largeflower Astilbe

Largeflower Purslane

Bengal Clockvine

Bigflower Ladyslipper

Bigflower Magnoliavine*

Largeflower Wildginger

British Inula Herb

Macroanther Crinum*

Large-flowered Epimedium

Large-flowered Epimedium Variety

Queen Crapemyrtle

Largeflower Uvaria

Medicinal Rhubarb

Big Anise*

Japanese Thistle

Peking Euphorbia

Chinese Milkwort

Christina Loosestrife

Large Euphorbia Root

Aztec Dahlia

Forrest Pyrola

Galanga Galangal

Maxima Dutchmanspipe*

Kenaf Hibiscus

Damask Rose

Hemp-agrimony

Grand Litse*

Disciform Prana*

Hupeh Anemone

Big Hogfennel*

Ussuri Poplar

Indigo-coloured Woad Leaf
T2626 Euphorbia wallichii

T1610 Coffea liberica

T5527 Rhodomyrtus macrocarpa

T4667 Passiflora quadrangularis

T5621 Russula lepida

T4623 Papaver bracteatum

T4808 Phlomis grandiflora var. grandiflora

T1196 Cardiospermum grandiflorum

T2185 Dioclea grandiflora

T3047 Goniothalamus griffithii

T5494 Rhodiola crenulata [Syn. Rhodiola

euryphylla]

T2311 Dysoxylum macranthum

T3950 Lychnis fulgens

T1212 Carpesium eximum

T2093 Dendranthema grandiflorum

T0785 Astilbe macroflora

T5172 Portulaca grandiflora

T6444 Thunbergia grandiflora

T1984 Cypripedium macranthum [Syn.

Cypripedium tibeticum]

T5792 Schisandra grandiflora

T0729 Asarum maximum

T3426 Inula britannica

T1802 Crinum macrantherum

T2396 Epimedium grandiflorum

T2397 Epimedium grandiflorum var.

thumbergianum

T3673 Lagerstroemia speciosa [Syn.

Munchausia speciosa; Lagerstroemia

flos-reginae]

T6662 Uvaria grandiflora

T5472 Rheum officinale

T4899 Pimpinella magna

T1449 Cirsium japonicum

T2608 Euphorbia pekinensis

T5073 Polygala chinensis [Syn. Polygala

glomerata]

T3998 Lysimachia christinae

T2602 Euphorbia nematocypha

T1996 Dahlia pinnata [Syn. Dahlia variabilis]

T5348 Pyrola forrestiana

T0356 Alpinia galanga

T0633 Aristolochia maxima

T3238 Hibiscus cannabinus

T5564 Rosa damascena

T2554 Eupatorium cannabinum

T3890 Litsea grandis

T5190 Prana discifera

T0468 Anemone hupehensis

T4757 Peucedanum grande

T5165 Populus ussuriensis

T3476 Isatis indigotica 


\begin{tabular}{|c|c|c|}
\hline DA SAN YE SHENG MA & 大三叶升麻 & Cowparsnipleaf Bugbane \\
\hline DA SUAN & 大蒜 & Garlic \\
\hline DA TIAO WEN XIE HAO & 大条纹邪蒿* & Bigstreak Seseli* \\
\hline DA TOU SUAN & 大头蒜 & Wild Leek \\
\hline DA TOU TUO WU & 大头㯻吾 & Japanese Goldenray \\
\hline DA TU SI ZI & 大菟丝子 & Japanese Dodder Seed \\
\hline DA TUAN NANG CHONG CAO & 大团囊虫草 & Goldenthread Cordyceps \\
\hline DA WEI YAO & 大尾摇 & Indian Heliotrope \\
\hline DA WU LI QIN JIAO & 达乌里秦艽 & Dahuria Gentian \\
\hline DA XIAN YUAN ZHI & 大腺远志 & Macradenous Milkwort* \\
\hline DA XING QIN & 大星芹 & Astrantia \\
\hline DA XUE HUA LIAN & 大雪花莲 & Giant Snowdrop \\
\hline DA XUE TENG & 大血藤 & Sargentgloryvine \\
\hline DA YE AN YE & 大叶桉叶 & Swamp Mahogany Leaf \\
\hline DA YE BAI TOU WENG & 大叶白头翁 & Common Pearleverlasting \\
\hline DA YE CAI & 大叶菜 & Doederlein’s Spikemoss \\
\hline DA YE CHAI HU & 大叶柴胡 & Bigleaf Thorowax \\
\hline DA YE CHOU HUA JIAO & 大叶臭花椒 & Manyspiny Pricklyash* \\
\hline DA YE DIAO LAN & 大叶吊兰 & Bigleaf Breaketplant \\
\hline DA YE DONG QING & 大叶冬青 & Broadleaf Holly \\
\hline DA YE GOU TENG & 大叶钩藤 & Largeleaf Gambirplant \\
\hline DA YE GU SUI BU & 大叶骨碎补 & Taiwan Davallia \\
\hline DA YE GUA TAI MU & 大叶瓜泰木 & \\
\hline DA YE HOU PO & 大叶厚朴 & Bigleaf Magnolia \\
\hline DA YE HUA JIAO & 大叶花椒 & Shellfish Pricklyash \\
\hline DA YE HUA JIAO GEN & 大叶花椒根 & Shellfish Pricklyash Root \\
\hline DA YE JIN HUA CAO & 大叶金花草 & Common Wedgelet Fern \\
\hline DA YE JING KOU BIAN CAO & 大叶井口边草 & Cretan Brake \\
\hline DA YE JU & 大叶蒟 & Largeleaf Pipper* \\
\hline DA YE KU NUO NI & 大叶库诺尼* & \\
\hline DA YE LUO FU MU & 大叶萝芙木 & Largeleaf Devilpepper* \\
\hline DA YE MAO ZHU MU & 大叶帽柱木 & Abura Mitragyna \\
\hline DA YE NAN YANG SHAN & 大叶南洋杉 & Bunya Bunya \\
\hline DA YE NAN YANG SHEN & 大叶南洋参* & Bigleaf Polyscias* \\
\hline DA YE NIU FANG FENG & 大叶牛防风 & Giant Hogweed \\
\hline DA YE NIU NAI CAI & 大叶牛奶菜 & Ko Condorvine \\
\hline DA YE QIAN CAO & 大叶茜草 & Smoothstalk Madder \\
\hline DA YE SHE PU TAO & 大叶蛇葡萄 & Largeleaf Ampelopsis \\
\hline DA YE SHU LAN & 大叶树兰 & Largeleaf Aglaia* \\
\hline DA YE TANG JIAO SHU & 大叶糖胶树 & Deviltree Alstonia \\
\hline DA YE TANG SONG CAO & 大叶唐松草 & Faber Meadowrue \\
\hline DA YE TENG HUANG & 大叶藤黄 & Yellowjuice Garcinia \\
\hline DA YE TU MU XIANG & 大叶土木香 & Largeleaf Inula \\
\hline DA YE XIAN MAO & 大叶仙茅 & Largeleaf Curculigo \\
\hline DA YE XIANG CHA CAI & 大叶香茶菜 & Largeleaf Rabdosia \\
\hline DA YE XIANG RU & 大叶香薷 & Twoanther Mosla \\
\hline DA YE YANG & 大叶杨 & Bigleaf Poplar \\
\hline DA YE YOU MU YUN XIANG & 大叶柚木芸香* & \\
\hline DA YE YUN XIANG CAO & 大叶芸香草 & Perforated Haplophyllum \\
\hline DA YE ZI YU PAN & 大叶紫玉盘 & Largeleaf Uvaria* \\
\hline
\end{tabular}

T1421 Cimicifuga heracleifolia

T0318 Allium sativum

T5930 Seseli grandivittatum

T0309 Allium ampeloprasum

T3808 Ligularia japonica [Syn. Arnica

japonica; Senecio japonica]

T1913 Cuscuta japonica

T1682 Cordyceps ophioglossoides

T3174 Heliotropium indicum

T2910 Gentiana dahurica

T5077 Polygala macradenia

T0810 Astrantia major

T2821 Galanthus elwelii

T5741 Sargentodoxa cuneata

T2517 Eucalyptus robusta

T0446 Anaphalis margaritacea

T5861 Selaginella doederleinii

T1065 Bupleurum longiradiatum

T6883 Zanthoxylum myriacanthum

T1380 Chlorophytum malayense

T3393 Ilex latifolia

T6625 Uncaria macrophylla

T2052 Davallia divaricata [Syn. Davallia

formosana; Davallia orientalis]

T3069 Guatteria amplifolia

T4048 Magnolia rostrata

T6876 Zanthoxylum dissitum

T6877 Zanthoxylum dissitum

T6116 Stenoloma chusanum

T5287 Pteris cretica

T4951 Piper laetispicum

T1891 Cunonia macrophylla

T5437 Rauwolfia macrophylla

T4257 Mitragyna macrophylla

T0578 Araucaria bidwillii

T5130 Polyscias amplifolia

T3216 Heracleum mantegazzianum

T4117 Marsdenia koi

T5583 Rubia schumannina

T0428 Ampelopsis megalophylla

T0235 Aglaia elliptifolia

T0370 Alstonia macrophylla

T6381 Thalictrum faberi

T2880 Garcinia xanthochymus

T3430 Inula grandis

T1900 Curculigo capitulata [Syn. Leucojum

capitulata]

T5394 Rabdosia macrophylla

T4305 Mosla dianthera

T5155 Populus lasiocarpa

T6320 Teclea grandifolia

T3104 Haplophyllum perforatum

T6665 Uvaria macrophylla 


DA YE ZI ZHU
DA YE ZUI YU CAO
DA YI ZHI JIAN
DA YU BIAO HUA
DA YUN SHI
DA ZAO
DA ZHUI XIANG CHA CAI
DA ZI JIN GU CAO
DA ZI MAI MA TENG
DA ZI XUE DAN
DA ZI ZHANG YA CAI
DAI DAI HUA
DAN BAN GOU YA HUA
DAN GAI TIE XIAN JUE
DAN HONG QIANG DAO YAO
DAN HUANG BA DOU
DAN HUANG KU MA DOU
DAN HUANG MU XI CAO
DAN HUANG XIANG CHA CAI
DAN HUANG ZHANG YA CAI
DAN HUI BAI SHAN MA HUANG
DAN JI ER YUAN WEI
DAN JUE CHUANG
DAN LI XIAO MAI
DAN LV CUI QUE
DAN MU
DAN SHEN
DAN SUI SHI SONG
DAN TIAO CAO
DAN YE DONG FENG JU
DAN YE MAN JING

DAN YE MAN JING ZI

DAN YE XIN YUE JUE

DAN YE YOU GAN

DAN ZHI DAO HUA

DAN ZHONG ZAO JIA

DAN ZHU YE

DAN ZHU YE GEN

DAN ZI HAO

DAN ZI MA HUANG

DAN ZI SHAN ZHA

DAN ZI ZI MAO

DANG GUI

DANG SHEN

DANG YAO

DAO CAO

DAO CHI MEI JUN

DAO DOU

DAO FU XIANG CHA CAI

DAO GEN MEI
大叶紫珠

大叶醉鱼草

大一枝箭

大鱼鳔花

大云实

大東

大雉香茶菜

大籽筋骨草

大籽买麻藤

大籽雪胆

大籽獐牙菜

玳玳花

单瓣狗牙花

单盖铁线蕣

淡红枪刀药*

淡黄巴豆*

淡黄苦马豆*

淡黄木犀草

淡黄香茶菜

淡黄獐牙菜

淡灰白山蚂蝗*

丹吉尔茑尾

单爵床

单粒小麦

淡绿翠雀*

胆木

丹参

单穗石松

单条草

单叶东风橘*

单叶蔓荆

单叶莫荆子

单叶新月鄀

单叶油柑*

单枝稻花

单种㿝荚*

淡竹叶

淡竹叶根

单籽蒿*

单子麻黄

单子山楂

单籽紫铆

当归

党参

当药

稻草

稻赤霉菌

刀豆

道孚香茶菜

稻根需*
Bigleaf Beautyberry

Orangeeye Butterflybush

Golden Lycoris

Shortclustered Plantainlily

Large Caesalpinia*

Chinese Date

Bigthyrse Rabdosia

Largeseed Bugle

Bigseed Jointfir

Largeseed Hemsleya

Bigseed Swertia

Bitter Citrus

Divaricate Ervatamia

Monochlamys Maidenhair

Rose Hypoestes*

Flavescent Croton*

Yellowish Swainsonia*

Yellowish Rabdosia

Yellowish Swertia*

Light-hoar Tickclover*

Tingit Iris

Simple Rostellularia

Einkorn

Virescent Larkspur

Medicinal Fatheadtree

Danshen

Interrupted Clubmoss

White Pearlweed

Monoleaf Atalantia*

Simpleleaf Shrub Chastetree

Simpleleaf Shrub Chastetree Fruit

Simple Pronephrium

Singleleaf Acronychia*

Simplex Rice-flower*

Monoseed Honeylocust*

Common Lophatherum

Common Lophatherum Root

Monoseed Wormwood*

Oneseed Ephedra

Common Hawthorn

Bengal Kino

Chinese Angelica

Pilose Asiabell

Chinese Swertia*

Rice Straw

Sword Jackbean

Dawo Rabdosia
T1120 Callicarpa macrophylla

T1046 Buddleja davidii

T3983 Lycoris aurea

T3283 Hosta sieboldiana

T1105 Caesalpinia major

T6916 Ziziphus jujuba

T3514 Isodon megathyrsus

T0266 Ajuga macrosperma

T3032 Gnetum montanum f. megalocarpum

T3208 Hemsleya macrosperma

T6225 Swertia macrosperma

T1469 Citrus aurantium var. amara

T2442 Ervatamia divaricata

T0174 Adiantum monochlamys

T3374 Hypoestes rosea

T1845 Croton flavens

T6209 Swainsonia luteola

T5452 Reseda luteola

T3488 Isodon flavidus

T6235 Swertia punicea var. lutescens

T2128 Desmodium canum

T3472 Iris tingitana

T3610 Justicia simplex

T6545 Triticum monococcum

T2090 Delphinium virescens

T4391 Nauclea officinalis

T5680 Salvia miltiorrhiza

T3965 Lycopodium annotinum

T3996 Lysimachia candida

T0815 Atalantia monophylla

T6790 Vitex rotundifolia [Syn. Vitex trifollia var. simplicifolia ]

T6791 Vitex rotundifolia [Syn. Vitex trifollia var. simplicifolia]

T5209 Pronephrium simplex [Syn. Meniscium simplex]

T0149 Acronychia haplophylla

T4893 Pimelea simplex

T2977 Gleditsia monosperma

T3920 Lophatherum gracile

T3921 Lophatherum gracile

T0686 Artemisia monosperma

T2375 Ephedra monosperma

T1773 Crataegus monogyna

T1083 Butea monosperma

T0495 Angelica sinensis

T1599 Codonopsis pilosula

T6214 Swertia chinensis

T4544 Oryza sativa

T2960 Gibberella zeae

T1169 Canavalia gladiata

T3484 Isodon dawoensis

T5490 Rhizopus oryzae 


\begin{tabular}{|c|c|c|c|}
\hline DAO LUAN YE FU SHI JUE & 倒卵叶伏石烣 & Obovateleaf Lemmaphyllum* & $\begin{array}{l}\text { T3737 Lemmaphyllum microphyllum var. } \\
\text { obovatum }\end{array}$ \\
\hline DAO LUAN YE PU GONG YING & 倒卵叶蒲公英根 & Obovaleaf Dandelion Root & T6302 Taraxacum obovatum \\
\hline \multicolumn{4}{|l|}{ GEN } \\
\hline DAO NIAN ZI & 倒捻子 & Mangosteen & T2863 Garcinia mangostana \\
\hline DAO SHI HAO & 道氏蒿 & Douglas Wormwood* & T0675 Artemisia douglasiana \\
\hline DAO YE LIU ZU JUE & 倒叶瘤足嵚 & Dunn's Plagiogyria & T4997 Plagiogyria dunnii \\
\hline DAO ZHUANG JI GU CHANG & 刀状鸡骨常山* & Spatulate Alstonia* & T0375 Alstonia spatulata \\
\hline \multicolumn{4}{|l|}{ SHAN } \\
\hline DE GUO LONG DAN & 德国龙胆 & German Gentian & T2911 Gentiana germanica \\
\hline DE GUO YUAN WEI & 德国茑尾 & German Iris & T3459 Iris germanica \\
\hline DE KA RUI HUA JIAO & 得卡瑞花椒 & Decary Pricklyash* & T6873 Zanthoxylum decaryi \\
\hline DE LA KE BA DOU & 得拉克巴豆 & Drac Croton* & T1843 Croton draconoide \\
\hline DE LA MENG DE JIN SI TAO & 德拉蒙德金丝桃* & Drummond St.John'swort & T3347 Hypericum drummondii \\
\hline DE QIN HONG JING TIAN & 德钦红景天 & Atuntsuen Rhodiola & T5492 Rhodiola atuntsuensis \\
\hline DENG LONG CAO & 灯笼草 & Peruvian Groundcherry & T4852 Physalis peruviana \\
\hline \multirow[t]{2}{*}{ DENG TAI SHU } & 灯台树 & Giant Dogwood & T1697 Cornus controversa $[$ Syn. \\
\hline & & & Bothrocaryum controversum] \\
\hline DENG XIN CAO & 灯芯草 & Common Rush & T3578 Juncus effusus \\
\hline DENG XIN DAI ER DOU & 灯心戴尔豆 & & T5273 Psorothamnus junceus \\
\hline DENG XIN LIU SHAN HU & 灯心柳珊瑚 & Gorgonian Junceella juncea & T3576 Junceella juncea \\
\hline DENG YOU TENG ZI & 灯油藤子 & Paniculed Bittersweet Seed & T1292 Celastrus paniculatus \\
\hline DENG ZHAN XI XIN & 灯盏细辛 & Shortscape Fleabane & T2422 Erigeron breviscapus \\
\hline DI BU RONG & 地不容 & Delavay Stephania & $\begin{array}{l}\text { T6120 Stephania delavayi [Syn. Stephania } \\
\text { epigaea] }\end{array}$ \\
\hline DI ER CAO & 地耳草 & Japanese St.John'swort & T3356 Hypericum japonicum \\
\hline DI FENG PI & 地枫皮 & Difengpi Anisetree & T3400 Illicium difengpi \\
\hline DI GEN JIN SI TAO & 迪根金丝桃* & Degen St.John'swort* & T3346 Hypericum degenii \\
\hline DI GUA ZI & 地瓜子 & Wayaka Yambean Seed & T4572 Pachyrhizus erosus \\
\hline DI JIN & 地筋 & Contorted Tanglehead & T3234 Heteropogon contortus \\
\hline DI JIN & 地锦 & Japanese Creeper & T4662 Parthenocissus tricuspidata \\
\hline DI JIN CAO & 地锦草 & Humifuse Euphorbia & T2591 Euphorbia humifusa \\
\hline DI KE DONG & 敌克冬 & Swamp Loosestrife & T2057 Decodon verticillatus \\
\hline DI SHAO GUA & 地梢瓜 & Bastardtoadflaxlike Swallowwort & T1961 Cynanchum thesioides \\
\hline DI SHI WU TAN & 迪氏乌檀 & Diderrichi Fatheadtree* & T4389 Nauclea diderrichii \\
\hline DI SUO LUO & 地梭罗 & & T4109 Marchantia polymorpha \\
\hline DI TANG BAI BU & 棣棠百部* & Kerri Stemona* & T6112 Stemona kerrii \\
\hline DI TANG CAO & 棣棠草 & Japanese Hypecoum* & T3333 Hypecoum japonicum \\
\hline DI TANG HUA & 棣棠花 & Japanese Kerria Flower & T3628 Kerria japonica \\
\hline DI TAO HUA & 地桃花 & Rose Mallow & T6645 Urena lobata \\
\hline DI TU YI & 地图衣 & & T5485 Rhizocarpon geographicum \\
\hline DI XIA CHE ZHOU CAO & 地下车轴草 & Subterranean Clover & T6525 Trifolium subterraneum \\
\hline DI YANG QUE & 地羊鹊 & Birdsfoot Trefoil & T3928 Lotus corniculatus \\
\hline DI YU & 地榆 & Garden Burnet & T5712 Sanguisorba officinalis \\
\hline DI ZHONG HAI BAI MU & 地中海柏木 & Mediterranean Cypress & T1899 Cupressus sempervirens \\
\hline DI ZHONG HAI DA JI & 地中海大戟* & Mediterranean Euphorbia; & $\mathrm{T} 2582$ Euphorbia characias \\
\hline & & Mediterranean Spurge & \\
\hline DI ZHONG HAI JU & 地中海菊 & & T1580 Cnicus benedictu \\
\hline DI ZHONG HAI MA SANG & 地中海马桑* & Mediterranean Coriaria & T1691 Coriaria myrtifolia \\
\hline DI ZHONG HAI MAO RUI HUA & 地中海毛莎花 & Mediterranean Mullein & T6704 Verbascum sinuatum \\
\hline DI ZHONG HAI ZONG HAI ZAO & 地中海棕海藻 & $\begin{array}{l}\text { Mediterranean Brown Alga Dilophus } \\
\text { ligulatus }\end{array}$ & T2183 Dilophus ligulatus \\
\hline DIAN BAI ZHI & 滇白鿊 & Scabrous Cowparsnip & T3222 Heracleum scabridum \\
\hline
\end{tabular}




DIAN BAI ZHU SHU
DIAN DI MEI YE CHA YE HUA
DIAN GOU TENG
DIAN HUANG JING
DIAN HUANG QIN
DIAN JI GU CHANG SHAN
DIAN JIANG HUA
DIAN LONG DAN
DIAN MU DAN
DIAN NAN HONG HOU KE
DIAN QIE
DIAN QIN
DIAN XI WU TOU
DIAN ZHUANG JUAN BAI
DIAN ZI CAO
DIAO DENG SHU
DIAO GAN MA
DIAO ZHANG GEN PI

DIAO ZHANG ZHI YE
DIE DA LAO
DIE QIAO SHI HU
DING GONG TENG
DING HU DIAO ZHANG
DING KE LA QIAN JIN TENG
DING QIE
DING XIANG

DING XIANG LUO LE
DING YU JU
DONG AN NA TUO LI YA SHI
CHE JU

DONG BEI AN HUA JIN WA ER DONG BEI CI REN SHEN DONG BEI DUO ZU JUE DONG BEI HE SHI DONG BEI HU TAO DONG BEI HUI HAO DONG BEI LONG DAN DONG BEI SHI SHAN DONG BEI TIAN NAN XING DONG BEI YAN HU SUO

DONG CHONG XIA CAO DONG DANG GUI

DONG DU HUI

DONG E LUO DU WU DONG FANG CANG ER DONG FANG FEI YAN CAO DONG FANG GOU JI
滇白珠树

点地梅叶茶叶花

滇钩藤

滇黄精

滇黄芩

滇鸡骨常山

滇姜花

滇龙胆

滇牡丹

滇南红厚壳

颠茄

滇芹

\section{滇西乌头}

\section{垫状卷柏}

滇紫草

吊灯树

吊干麻

钓樟根皮

钓樟枝叶

\section{跌打老}

叠鞘石斛

丁公藤
鼎湖钓樟
丁克拉千金藤
丁茄(野颠茄)
丁香

丁香罗勒

顶羽菊

东安纳托利亚矢车菊

东北暗花金挖耳*

东北刺人参

东北多足蓱

东北鹤禹

东北胡桃*

东北蛔蒿

东北龙胆

东北石杉

东北天南星

东北延胡索

冬虫夏草

东当归

东毒茴

东俄洛䍒吾

东方苍耳*

东方飞燕草*

东方狗脊
Yunnan Gaultheria

Spreading Dogbane

Yunnan Gambirplant

King Solomonseal

Yunnan Skullcap

Yunnan Alstonia

Yunnan Gingerlily

Rigescent Gentian

Delavay Peony

S. Yunnan Beautyleaf

Common Atropa

Common Sinodielsia

Yunnanwest Monkshood

Cushion-shaped Spikemoss

Paniculate Onosma

Sausagetree

Angled Bittersweet

Largeleaf Spicebush Root-bark

Largeleaf Spicebush Branch-leaf

Whorlleaf Litse

Denne Denrdobium

Obtuseleaf Erycibe

Chun's Spicebush

Dinklage Stephania*

Aculeate Nightshade*

Clove Tree

Sweetscented Basil

Creeping Acroptilon

East-Anatolia Centaurea*

Northeast Dim-flower Carpesium*

Northeast Spineginseng*, Tall Oplopanax

North-east Polypody*

European Stickseed

Northeast Walnut*

Northeast Seriphidium

Linearleaf Gentian

Northeast Clubmos*

Amur Jackinthepulpit

Amur Corydalis

Aweto (Chinese Caterpillar Fungus)

Acutelobed Angelica

Religious Anisetree*

Tongol Goldenray

Oriental Cocklebur*

Oreintal Consolida*

Oriental Chain Fern
T2895 Gaultheria yunnanensis

T0550 Apocynum androsaemifolium

T6639 Uncaria yunnanensis

T5092 Polygonatum kingianum

T5833 Scutellaria amoena

T0378 Alstonia yunnanensis

T3121 Hedychium yunnanense

T2928 Gentiana rigescens

T4582 Paeonia delavayi

T1130 Calophyllum polyanthum

T0825 Atropa belladonna

T5963 Sinodielsia yunnanensis

T0081 Aconitum bulleyanum

T5865 Selaginella pulvinata

T4500 Onosma paniculatum

T3629 Kigelia pinnata

T1285 Celastrus angulatus

T3856 Lindera umbellata [Syn. Lindera

erythrocarpa]

T3857 Lindera umbellata [Syn. Lindera

erythrocarpa]

T3897 Litsea verticillata

T2096 Dendrobium aurantiacum var.

denneanum

T2449 Erycibe obtusifolia

T3849 Lindera chunii

T6123 Stephania dinklagei

T5989 Solanum aculeatissimum

T6263 Syzygium aromaticum [Syn. Eugenia caryophyllata]

T4473 Ocimum gratissimum

T0154 Acroptilon repens

T1307 Centaurea pseudoscabiosa ssp.

pseudoscabiosa

T1214 Carpesium triste var. manshuricum

T4516 Oplopanax elatus

T5126 Polypodium virginianum

T3688 Lappula echinata

T3565 Juglans mandshurica var. sieboldiana

T5921 Seriphidium finitum [Syn. Artemisia finita]

T2921 Gentiana manshurica

T3293 Huperzia miyoshiana

T0617 Arisaema amurense

T1706 Corydalis ambigua var. amurensis

[Syn. Corydalis ambigua]

T1683 Cordyceps sinensis

T0474 Angelica acutiloba [Syn. Ligusticum

acutilobum]

T3406 Illicium religiosum

T3816 Ligularia tongolensis

T6840 Xanthium orientale

T1647 Consolida orientalis

T6831 Woodwardia orientalis 


\begin{tabular}{|c|c|c|c|}
\hline DONG FANG GOU TENG & 东方钩藤* & Oriental Gambirplant* & T6627 Uncaria orientalis \\
\hline DONG FANG GOU YA HUA & 东方狗牙花 & Oriental Ervatamia* & T2446 Ervatamia orientalis \\
\hline DONG FANG JIA GUO JUE & 东方荚果蕨 & Oriental Ostrich Fern & T4126 Matteuccia orientalis \\
\hline DONG FANG TIAN XIAN ZI & 东方天仙子 & Oriental Henbane* & T3330 Hyoscyamus orientalis \\
\hline DONG FANG WEI SI MU & 东方维斯木* & Oriental Vismia* & $\mathrm{T} 6780$ Vismia orientalis \\
\hline DONG FANG WU TAN & 东方乌檀 & Oriental Fatheadtree* & T4392 Nauclea orientalis \\
\hline DONG FANG XI MEN FEI CAO & 东方西门肺草* & Soft Comfrey & T6247 Symphytum orientale \\
\hline DONG FANG XIANG KE KE & 东方香科科 & Oriental Germander & T6364 Teucrium orientale \\
\hline DONG FANG YANG DI HUANG & 东方洋地黄 & Oriental Foxglove* & T2176 Digitalis orientalis \\
\hline DONG FEI JUE MING & 东非决明 & East-African Senna* & T1246 Cassia singueana \\
\hline DONG FEI MA QIAN & 东非马钱 & East-African Poisonnut* & T6191 Strychnos usambarensis \\
\hline DONG FENG CAI & 东风菜 & Scabrous Doellingeria & T2244 Doellingeria scaber [Syn. Aster scaber] \\
\hline DONG FENG JU GEN & 东风橘根 & Boxleaf Atalantia Root & $\begin{array}{l}\text { T0813 Atalantia buxifolia }[\text { Syn. Severinia } \\
\text { buxifolia }]\end{array}$ \\
\hline DONG FENG JU YE & 东风橘叶 & Boxleaf Atalantia Leaf & $\begin{array}{l}\text { T0814 Atalantia buxifolia }[\text { Syn. Severinia } \\
\text { buxifolia }]\end{array}$ \\
\hline DONG GUA PI & 冬瓜皮 & Chinese Waxgourd Peel & T0894 Benincasa hispida \\
\hline DONG GUA ZI & 冬瓜籽 & Chinese Waxgourd Seed & T0895 Benincasa hispida \\
\hline DONG JIN BA DOU & 东京巴豆* & Viet Nam Croton*; Kho Sam Cho La & T1859 Croton tonkinensis \\
\hline DONG LANG DANG & 东莨菪 & Japanese Scopolia & T5821 Scopolia japonica \\
\hline DONG LING CAO & 冬凌草(碎米桠) & Blushred Rabdosia & T5396 Rabdosia rubescens \\
\hline DONG NAN JING TIAN & 东南景天 & Alfred Stonecrop & $\begin{array}{l}\text { T5851 Sedum alfredii [Syn. Sedum } \\
\text { formosanum] }\end{array}$ \\
\hline DONG QIANG & 东店 & Whitethorn* & T4435 Nitraria schoberi \\
\hline DONG QING YE MEI DENG MU & 冬青叶美登木 & Ilicis-leaf Mayten* & T4135 Maytenus ilicifolia \\
\hline DONG YA TANG SONG CAO & 东亚唐松草 & & T6401 Thalictrum minus var. hypoleucum \\
\hline DONG YA ZHI YE TAI & 东亚指叶苔 & & T3761 Lepidozia fauriana \\
\hline DONG YA Zi JIN NIU & 东亚紫金牛 & & T0603 Ardisia sieboldii \\
\hline DONG YI HAO JIAN MA & 东一号剑麻 & Cultivate Sisalan Agave East-1 & T0221 Agave east-one \\
\hline DONG YING SHAN HU MU & 东赢珊瑚木 & Japanese Aucuba & T0828 Aucuba japonica \\
\hline DOU BAN CAI & 豆瓣菜 & Watercress & T4388 Nasturtium officinale \\
\hline DOU BAO JUN & 豆包菌 & Dye-maker's False Puffboll & $\begin{array}{l}\text { T4978 Pisolithus tinctorius [Syn. Lycoperdon } \\
\text { capitatum; Scleroderma tinctorium] }\end{array}$ \\
\hline DOU CHI CAO & 豆鼓草 & Bloodred Iris & T3468 Iris sanguinea \\
\hline DOU FU CHAI & 豆腐柴 & Japanese Premna & T5194 Premna microphylla \\
\hline DOU SHU & 豆薯 & Wayaka Yambean & T4573 Pachyrrhizus erosus \\
\hline DOU YE JIU LI XIANG & 豆叶九里香 & Euchretaleaf Common Jasminorange & $\begin{array}{l}\text { T4317 Murraya euchrestifolia [Syn. Clausena } \\
\text { euchrestifolia] }\end{array}$ \\
\hline DOU YOU & 豆油 & Soybean Oil & T2999 Glycine max \\
\hline DOU ZHUANG HE BAO MU & 兜状荷包牡丹 & Dutchman's Breeches & T2148 Dicentra cucullaria \\
\hline DAN & & & \\
\hline DU AI BA JIAO & 毒矮八角 & Poisonous-shrub Anise* & T3408 Illicium tsangii \\
\hline DU BIAN DOU & 毒扁豆 & Deadly Calabarbean & T4860 Physostigma venenosum \\
\hline DU DOU & 毒豆 & Goldenchain Laburnum & T3648 Laburnum anagyroides \\
\hline DU E GAO & 毒鹅膏 & Death Cap & T0385 Amanita phalloides \\
\hline DU HENG & 杜衡 & Forbes Wildginger & T0726 Asarum forbesii \\
\hline DU HONG HUA & 杜虹花 & Taiwan Beautyberry & T1118 Callicarpa formosana \\
\hline DU HU LUO BO & 毒胡萝卜 & Deadly Carrot & T6419 Thapsia garganica \\
\hline DU HUA JIAO & 毒花椒* & Venenous Pricklyash* & T6899 Zanthoxylum veneficium \\
\hline DU HUI MAO DOU & 毒灰毛豆 & Toxic Tephrosia* & T6341 Tephrosia toxicaria \\
\hline DU HUO & 独活 & Doubleteeth Pubescent Angelica & $\begin{array}{l}\text { T0492 Angelica pubescens f. biserrata [Syn. } \\
\text { Angelica pubescens] }\end{array}$ \\
\hline DU JING SHAN & 杜茎山 & Japanese Maesa & T4029 Maesa japonica \\
\hline
\end{tabular}




\begin{tabular}{|c|c|}
\hline DU JUAN HUA & 杜鹃花 \\
\hline DU JUAN HUA YE & 杜鹃花叶 \\
\hline DU JUAN LAN & 杜鹃兰 \\
\hline DU MA QIAN & 毒马钱 \\
\hline DU QI TENG & 毒漆藤 \\
\hline DU QIN GEN & 毒芹根 \\
\hline DU SHEN & 毒参 \\
\hline DU SONG SHI & 杜松实 \\
\hline DU WO JU & 毒莴草 \\
\hline DU XIAN ZI & 都咸子 \\
\hline DU YI WEI & 独一味 \\
\hline DU YU SHU WEI CAO & 毒鱼鼠尾草 \\
\hline DU ZHONG & 杜仲 \\
\hline DU ZHONG YE & 杜仲叶 \\
\hline DUA JING GU DANG GUI & 短茎古当归 \\
\hline DUAN BAN HUA & 短瓣花 \\
\hline DUAN BAN JIN LIAN HUA & 短瓣金莲花 \\
\hline DUAN BANG SHI HU & 短棒石斛 \\
\hline DUAN BAO YE SHA CAO & 短苞叶莎草 \\
\hline DUAN BING HE YE LAN & 短柄禾叶兰: \\
\hline DUAN CHI HUANG QI & 短齿黄芪* \\
\hline DUAN CI HUA JIAO & 短刺花椒* \\
\hline DUAN E HUANG LIAN & 短颌黄连 \\
\hline DUAN GENG HU ZHI ZI & 短梗胡枝子 \\
\hline DUAN HUA HU LU & 短花葫芦* \\
\hline DUAN HUA ZHU SHI DOU & 短花猪屎豆 \\
\hline DUAN JIE SHEN & 断节参 \\
\hline DUAN JU & 短蒟 \\
\hline DUAN MAO DU HUO & 短毛独活 \\
\hline
\end{tabular}

\begin{tabular}{|c|c|}
\hline DUAN MAO JIN XIAN CAO GEN & 短毛金线草根 \\
\hline DUAN PIAN GAO BEN & 短片㩰本 \\
\hline DUAN RONG MAO GOU TENG & 短线毛钩藤* \\
\hline DUAN RONG MAO OU XIA ZHI & 短线毛欧夏至草* \\
\hline \multicolumn{2}{|l|}{ CAO } \\
\hline DUAN ROU MAO DA JI & 短柔毛大戙* \\
\hline DUAN ROU MAO FEI YAN CAO & 短柔毛飞燕草* \\
\hline DUAN ROU MAO MA DOU & 短柔毛马兜铃* \\
\hline \multicolumn{2}{|l|}{ LING } \\
\hline DUAN ROU MAO ZHI XIE MU & 短柔毛止泻木* \\
\hline DUAN SHE GU & 短蛇菰* \\
\hline DUAN SHU & 根树* \\
\hline DUAN SUI HU JIAO & 短穗胡椒 \\
\hline DUAN TING SHAN MAI DONG & 短葶山麦冬 \\
\hline DUAN XIAN XIAO MI CAO & 短腺小米草 \\
\hline DUAN XIAO SHE GEN CAO & 短小蛇根草 \\
\hline DUAN YE HONG DOU SHAN & 短叶红豆杉 \\
\hline DUAN YE JIA MU ZEI & 短叶假木贼 \\
\hline DUAN YE JUAN HAO & 短叶绢蒿 \\
\hline DUAN YE & 短叶龙胆* \\
\hline
\end{tabular}

Indian Azalea

Indian Azalea Leaf

Appendiculate Cremastra

Toxic Poisonnut*

Poison Ivy

European Waterhemlock Root

Poisonhemlock

Stiffleaf Juniper Fruit

Blue Lettuce

Common Cashew Fruit

Common Lamiophlomis

Salvia pisidica

Eucommia

Eucommia Leaf

Shortstem Archangelica*

Shortpetalflower

Ledebour Globeflower

Hairstalk Denrdobium

Shortbractleaf Galingale*

Short-stipe Agrostophyllum*

Shorttooth Milkvetch*

Shortspine Pricklyash*

Shortsepal Goldthread

Shortstalk Bushclover

Shortflower Bottle Gourd*

Shortflower Crotalaria*

Kunming Mosquitotrap

Globular Pepper

Shorthair Cowparsnip

Shorthairy Antenoron

Shortlobe Ligusticum

Velutinous Gambirplant*

Velutinous Hoarhound*

Shortfluff Euphorbia*

Shortfluff Consolida*

Shortfluff Dutchmanspipe*

Shortfluff Holarrhena*

Abbreviate Balanophora*

Common Lime

Shortspike Pepper*

Short-stipe Liriope*

Regel Eyebright

Dwarf Ophiorrhiza

Pacific Yew

Shortleaf Anabasis

Shortleaf Wormwood*

Shortleaf Gentian*
T5523 Rhododendron simsii

T5524 Rhododendron simsii

T1787 Cremastra appendiculata

T6188 Strychnos toxifera

T6481 Toxicodendron radicans

T1416 Cicuta virosa

T1642 Conium maculatum

T3594 Juniperus rigida

T3664 Lactuca virosa

T0438 Anacardium occidentale

T3679 Lamiophlomis rotata [Syn. Phlomis

rotata]

T5684 Salvia pisidica

T2530 Eucommia ulmoides

T2531 Eucommia ulmoides

T0582 Archangelica brevicaulis [Syn.

Angelicarpa brevicaulis; Angelica brevicaulis]

T1001 Brachystemma calycinum

T6553 Trollius ledebourii

T2097 Dendrobium capillipes

T1974 Cyperus brevibracteatus

T0253 Agrostophyllum brevipes

T0788 Astragalus canadensis var. brevidens

T6868 Zanthoxylum brachyacanthum

T1663 Coptis chinensis var. brevisepala

T3771 Lespedeza cyrtobotrya

T3667 Lagenaria breviflora

T1816 Crotalaria breviflora

T1963 Cynanchum wallichii

T4956 Piper mullesua

T3217 Heracleum moellendorffii [Syn.

Heracleum microcarpum; Heracleum

morifolium]

T0519 Antenoron neofiliforme

T3819 Ligusticum brachylobum

T6638 Uncaria veluntina

T4113 Marrubium velutinum

T2613 Euphorbia pubescens

T1648 Consolida pubescens

T0636 Aristolochia pubescens

T3268 Holarrhena pubescens

T0857 Balanophora abbreviata

T6461 Tilia vulgaris

T4939 Piper brachystachyum

T3872 Liriope muscari

T2629 Euphrasia regelii

T4514 Ophiorrhiza pumila

T6308 Taxus brevifolia

T0436 Anabasis brevifolia

T0667 Artemisia brevifolia

T2904 Gentiana brachyphylla 


DUAN YE LONG SHE LAN
DUAN YE LUO HAN SONG SHI
DUAN YE LUO HAN SONG YE
DUAN YE PENG QI JU
DUAN YE SHAN MA HUANG
DUAN YE ZHU SHI DOU
DUI SHENG MA QIAN
DUI XIN JU
DUI YE JIN YAO
DUI YE RONG
DUI YE YUAN HU
DUN BAO XUE LIAN
DUN LIE YIN LIAN HUA
DUN XING AO DING ZAO
DUN XING BAI YE TENG
DUN XING CHI AN YE
DUN XING JI DAN HUA
DUN YE CHE ZHOU CAO
DUN YE DA JI
DUN YE DA JI XIANG JIANG
DUN YE GUI JIU
DUN YE GUI PI

DUN YE HU JIAO
DUN YE JUE MING
DUN YE SHU YU
DUN YE SUAN MO
DUN YE TANG SONG CAO

DUN YE TU NIU XI
DUN ZHUANG LI JU
DUN ZHUANG MAO GAO CAI
DUO BAN LV TI CAO
DUO BEI YIN LIAN HUA
DUO BIAN HUA BAI LAI SHI JU
DUO BIAN XIAO GUAN HUA
DUO CHI QIAN LI GUANG
DUO CI CHAI HU
DUO CI DI SHI MU
DUO CI HUANG HUA REN
DUO CI LUO CAO
DUO CI LV RONG HAO
DUO GEN WU TOU
DUO GUO SHU
DUO GUO YI NAN MU
DUO HUA A WEI
DUO HUA BA JIAO LIAN
DUO HUA BAI JIAN MU
DUO HUA CAI DOU
DUO HUA HEI MAI CAO
DUO HUA HUANG JING

短叶龙舌兰

短叶罗汉松实

短叶罗汉松叶

短叶蟛蜞菊*

椴叶山蚂蝗

短叶猪屎豆*

对生马钱

堆心菊

对叶金腰

对叶榕

对叶元胡

针苍雪莲

针裂银莲花

针形凹顶藻

针形白叶藤*

针形赤桉叶*

针形鸡蛋花*

针叶车轴草

针叶大戟*

钝叶大戟橡浆*

盾叶鬼臼

钝叶桂皮

盾叶胡椒*
钝叶决明
盾叶薯蓣
针叶酸模
盾叶唐松草

钝叶土牛滕
盾状粒菊
盾状茅膏菜*
多瓣驴蹄草
多被银莲花(两头尖)
多边花白莱氏菊*
多变小冠花
多齿千里光
多刺柴胡*
多刺迪氏木
多刺黄花稔
多刺骡草*
多刺绿线蒿
多根乌头
多果树
多果依南木*
多花阿魏*
多花八角莲
多花白坚木*
多花菜豆
多花黑麦草
多花黄精

Narrowleaf Agave
Chinese Podocarpus Seed
Chinese Podocarpus Leaf

Lindenleaf Tickclover*

Shortleaf Crotalaria*

Opposite Poisonnut*

Sneezeweed

Oppositeleaf Goldsaxifrage*

Oppositeleaf Fig

Ledeour Corydalis*

Blacken Saussurea

Obtuselobed Anemone

Blunt Concave-top Alga*

Obtuse Cryptolepis*

Obtuse Eucalyptus Leaf*

Obtuse Frangipani*

Suckling Clover

Obtuseleaf Euphorbia*

Obtuseleaf Euphorbia Latex*

Common Mayapple

Obtuseleaf Cassia Bark, Wild

Cinnamon Bark

Peltateleaf Pepper*

Obtuseleaf Senna*

Peltate Yam

Bluntleaf Dock

Peltateleaf Meadowrue

Obtuseleaf Achyranthes

Matarique (in Mexico)

Peltate Sundew*

Manypetal Marshmarigold

Radde Windflower

Manyradiate Bailai's Chrysanthemum*

Crown Vetch

Manytoothed Groundsel*

Spiny Thorowax*

Desfontainia spinosa

Spiny Sida*

Argentin Mulinum spinosum

Spiny Meconopsis

Manyroot Monkshood

Manyflower Giantfennel*

Manyflower Dysosma*

Multiflower White Quebracho*

Scarlet Runner Bean

Italian Ryegrass

Manyflower Solomonseal
T0217 Agave angustifolia

T5044 Podocarpus macrophyllus var. maki

T5045 Podocarpus macrophyllus var. maki T6905 Zexmenia brevifolia

T2135 Desmodium tiliaefolium

T1817 Crotalaria brevifolia

T6169 Strychnos decussata

T3134 Helenium autumnale

T1407 Chrysosplenium oppositifolium

T2720 Ficus hispida

T1720 Corydalis ledebouriana

T5762 Saussurea nigrescens

T0469 Anemone obtusiloba

T3722 Laurencia obtusa

T1865 Cryptolepis obtusa

T2503 Eucalyptus camaldulensis var. obtusa

T5031 Plumeria obtusa

T6517 Trifolium dubium

T2605 Euphorbia obtusifolia var. obtusifolia

T2604 Euphorbia obtusifoli

T5058 Podophyllum peltatum

T1434 Cinnamomum bejolghota [Syn.

Cinnamomum obtusifolium; Laurus

bejolghota]

T4960 Piper peltatum

T1240 Cassia obtusifolia

T2213 Dioscorea zingiberensis

T5613 Rumex obtusifolius

T6393 Thalictrum ichangense [Syn.

Thalictrum tripeltatum; Thalictrum

multipeltatum]

T0072 Achyranthes aspera var. indica

T5248 Psacalium peltatum

T2266 Drosera peltata

T1138 Caltha polypetala

T0470 Anemone raddeana

T0856 Baileya pleniradiata

T1702 Coronilla varia

T5898 Senecio polyodon

T1076 Bupleurum spinosum

T2126 Desfontainia spinosa

T5944 Sida spinosa

T4313 Mulinum spinosum

T4143 Meconopsis horridula

T0105 Aconitum karakolicum

T5015 Pleiocarpa mutica

T2358 Enantia polycarpa

T2705 Ferula polyantha

T2297 Dysosma aurantiocaulis

T0767 Aspidosperma multiflorum

T4781 Phaseolus coccineus

T3903 Lolium multiflorum

T5091 Polygonatum cyrtonema [Syn. 


\begin{tabular}{|c|c|c|c|}
\hline DUO HUA LAN GUO SHU & 多花蓝果树 & Manyflower Tupelo* & T4458 Nyssa sylvatica \\
\hline DUO HUA SHAN MA GAN & 多花山麻杆 & Manyflower Christmasbush* & T0296 Alchornea floribunda \\
\hline DUO HUA SHAO YAO & 多花苟药 & Himalayan Peony & T4583 Paeonia emodi \\
\hline DUO HUA SHUI XIAN & 多花水仙 & Polyanthus Narcissus & T4383 Narcissus tazetta \\
\hline DUO HUA TENG HUANG & 多花藤黄* & Manyflower Garcinia* & T2870 Garcinia polyantha \\
\hline DUO HUA U LIAN & 多花杜楝 & Manyflower Starbush* & T6569 Turraea floribunda \\
\hline DUO HUA XIAO BO & 多花小檗 & Free-flowering Barberry & T0904 Berberis floribunda \\
\hline DUO HUA YANG MEI & 多花杨梅 & Manyflower Bayberry* & T4345 Myrica multiflora \\
\hline DUO JIA CAO & 多荚草 & Manyseed & T5069 Polycarpon prostratum \\
\hline DUO LIE WEI LING CAI & 多裂委陵菜 & Many-cleft Cinquefoil & T5185 Potentilla multifida \\
\hline DUO LIE WU TOU & 多裂乌头 & Manysplltted Monkshood & T0123 Aconitum polyschistum \\
\hline DUO MAI GOU TENG & 多脉钩藤* & Many-veined Gambirplant* & T6626 Uncaria nervosa \\
\hline DUO MAI NAN MEI ROU DOU & 多脉南美肉豆冦* & & T6769 Virola multinervia \\
\hline \multicolumn{4}{|l|}{$\mathrm{KOU}$} \\
\hline DUO NI FEI SHA REN & 多尼非砂仁 & & T0206 Aframomum daniellin \\
\hline DUO RU TOU HUANG YANG & 多乳头黄杨* & Papillose Box* & T1093 Buxus papillosa \\
\hline DUO RUI BAI HUA CAI & 多䓌白花菜* & Multipistillate Spiderflower* & T1551 Cleome icosandra \\
\hline DUO SAN A WEI & 多伞阿魏 & Manyumbell Giantfennel & T2698 Ferula ferulaeoides \\
\hline DUO SHE FEI PENG & 多舌飞蓬 & Multiradiate & T2423 Erigeron multiradiatus \\
\hline DUO SUI LIAO & 多穗苶 & Manyspike Knotweed & T5114 Polygonum polystachyum \\
\hline DUO SUI LUO HAN SONG SHI & 多穗罗汉松实 & Manyspike Podocarpus Seed & T5052 Podocarpus polystachyus \\
\hline DUO SUI PO BU MU & 多穗破布木* & Manyspike Cordia* & T1678 Cordia multispicata \\
\hline DUO SUI SHI KE YE & 多穗石柯叶 & Manyspike Tanoak Leaf & T3878 Lithocarpus polystachyus \\
\hline DUO SUI SHU WEI CAO & 多穗鼠尾草 & & T5686 Salvia polystachya \\
\hline DUO SUI TUN CAO & 多穗豚草 & Manyspike Ragweed & T0404 Ambrosia polystachya \\
\hline DUO TOU GE NI DI MU & 多头格尼迪木* & & T3039 Gnidia polycephala \\
\hline DUO XIANG GUO & 多香果 & Allspice & T4894 Pimenta dioica \\
\hline DUO XING JIN FA XIAN & 多形金发藓 & Polymorphism Gold-hair Moss* & T5134 Polytrichum ohioense \\
\hline DUO XIONG RUI LING CHUN & 多雄蒿领春木* & Polyandrous Euptelea* & T2630 Euptelea polyandra \\
\hline \multicolumn{4}{|l|}{ MU } \\
\hline DUO XIONG RUI SHANG LU & 多雄荵商陆 & Polyandrous Pokeweed & T4867 Phytolacca polyandra \\
\hline DUO XU GONG & 多须公 & Lavender-gustatory Eupatorium* & T2576 Eupatorium stoechadosmum \\
\hline DUO XU YAN HUANG QI & 多序岩黄芪 & Manyraceme Sweetvetch & T3129 Hedysarum polybotrys \\
\hline DUO YE BAI MAI GEN & 多叶百脉根 & Leafy Trefoil* & T3931 Lotus polyphyllos \\
\hline DUO YE JI DOU & 多叶棘豆 & Leafy Crazyweed & T4568 Oxytropis myriophylla \\
\hline DUO YE JIN SI TAO & 多叶金丝桃* & Leafy St.John'swort* & T3351 Hypericum foliosum \\
\hline DUO ZHI AN & 多枝桉 & Ribbon Gum & T2522 Eucalyptus viminalis \\
\hline DUO ZHI CHAI HU & 多枝柴胡 & Ramose Thorowax* & T1068 Bupleurum polyclonum \\
\hline DUO ZHI CHENG LIU & 多枝柽柳 & Branchy Tamarisk & T6290 Tamarix ramosissima \\
\hline DUO ZHI KUO BAO JU & 多枝阔苞菊* & Ramose Pluchea* & T0849 Baccharis ramosissima \\
\hline DUO ZHI LONG DAN & 多枝龙胆* & Ramose Gentian* & T2926 Gentiana ramosa \\
\hline DUO ZHI LONG XUE SHU & 多枝龙血树* & & T2255 Dracaena surculosa \\
\hline DUO ZHI PU TE MU & 多疬普特木 & & T5336 Putterlickia verrucosa \\
\hline DUO ZHI RU GU & 多汁乳菇 & Juicy Milky* & T3654 Lactarius rolemus \\
\hline DUO ZHI SONG & 多脂松 & Red Pine & T4921 Pinus resinosa \\
\hline DUO ZHI TIAN JIE CAI & 多枝天芥菜 & Ramose Heliotrope* & T3178 Heliotropium ramosissimum \\
\hline DUO ZHI ZHI TENG HUANG & 多直枝藤黄* & & T2879 Garcinia virgata \\
\hline DUO ZHONG LU HUI TI QU WU & 多种芦荟提取物 & Extracts of Aloe spp. & T0346 Aloe spp. \\
\hline DUO ZU JUE & 多足应 & Common Polypody & T5127 Polypodium vulgare \\
\hline E BAO CHUN & 鄂报春 & Top Primrose & T5202 Primula obconica \\
\hline E BEI BEI MU & 鄂北贝母 & Ebei Fritillary & T2785 Fritillaria ebeiensis \\
\hline E BU SHI CAO & 鹅不食草 & Small Centipeda & T1312 Centipeda minima \\
\hline
\end{tabular}




E CHANG CAI
E CHI TENG
E CUI
E GUO QIAN HU
E GUO XI MEN FEI CAO
E LE GANG HE BAO MU DAN
E LI

E LI

E MEI CUI QUE HUA
E MEI GE
E MEI JUAN BAI
E MEI TANG SONG CAO
E MEI YE HUANG LIAN
E RONG TENG
E RONG WEI LING CAI
E SHEN
E SUN ZI TAN
E XI XIANG CHA CAI
E ZHANG CAO
E ZHANG TENG
EN GE MU
ER CAO
ER CHA GOU TENG
ER CHI XIANG KE KE
ER CI YUN SHI
ER DUN ZHUANG HUA JIAO
ER GOU HUANG QI
ER JIAO DUO JIA ZAO
ER QI GOU YA HUA
ER RUI HE LIAN DOU

\section{ER RUI ZI SU}

ER SE HUA SHU WEI CAO

ER SHI RUI SHANG LU

ER XING JI XUE TENG

ER XING JIN HE HUAN

ER XING LIU ZU JUE

ER XING YANG ER LAN

ER YE BAO YING SU

ER YE NIU PI XIAO

ER YI TUO BAO DI QIAN

ER ZHUANG BAO CHUN HUA

FA CAI

FA GUO CHENG LIU

FA GUO JIN QUE ER

FA GUO QIANG WEI

FA KANG WU TOU

FAN BAI CAO

FAN HUA ZHI XIE MU

FAN LI ZHI

FAN MA

FAN MU GUA

FAN MU GUA YE
鹅肠菜

萼翅藤

鹅膵

俄国前胡

俄国西门肺草*

俄勒冈荷包生丹

鳄梨

峨嵋翠雀花

峨嵋葛

峨眉卷柏

峨嵋唐松草

峨嵋野黄连

我鸟线藤

我线委陵菜

峨参

娥孙紫檀

鄂西香茶菜

我掌草

我掌藤

恩格木

耳草

儿茶钩藤

二齿香科科

二雌云实*

二盾状花椒

二沟黄芪

二角多甲藻

二岐狗牙花

二萝荷莲豆

二思紫苏

二色花鼠尾草

二十萝商陆

耳形鸡血藤

耳形金合欢

耳形瘤足痰

耳形羊耳兰

二叶苍罂粟

耳叶牛皮消

二翼托苍地钱

耳状报春花

发菜

法国柽柳

法国金雀儿

法国蓄薇

法康乌头

翻白草

繁花止泻木*

番荔枝

番麻

番木瓜

番木瓜叶
Goose-bowel Vegatable*

Goose Tail-meat

Russian Hogfennel*

Russian Comfrey

Oregon Bleedingheart

American Avocado

Emei Larkspur

Omei Kudzuvine

Emei Spikemoss*

Omei Meadowrue

Omei Mountain Goldthread

Chinese Swallowwort

Silverweed Cinquefoil

Woodland Beakchervil

Osun Padauk*

Henry Rabdosia

Flaccid Anemone

Scandent Schefflera

Auricled Hedyotis

Gambier Gambirplant

Twodenntate Germander

Digyna Caesalpinia*

Twopetaline Pricklyash*

Bisulcate Milkvetch*

Dichotomous Ervatamia*

Twocolor-flower Sage*

Icosandrous Pokeweed*

Auriculate Millettia*

Auriculate Acacia

Auriform Plagiogyria

Auriculate Twayblade*

Celandine Poppy

Auriculate Swallowwort

Twowing Palea Liverwort*

Auricula

Hair Vegetable*

French Tamarisk

French Broom

French Rose

Falcon Monkshood*

Discolor Cinquefoil

Purplequeen Holarrhena*

Custard Apple

American Agave

Papaya Fruit

Papaya Leaf
T2359 Endarachne binghamiae T1142 Calycopteris floribunda

T0517 Anser cygnoides domestica

T4770 Peucedanum ruthenicum

T6249 Symphytum x uplandicum

T2151 Dicentra oregana

T4731 Persea americana [Syn. Persea

gratissima ]

T2081 Delphinium omeiense

T5316 Pueraria omeiensis

T5864 Selaginella omeiensis

T6402 Thalictrum omeiense

T1669 Coptis omeiensis

T1954 Cynanchum chinense

T5179 Potentilla anserina

T0529 Anthriscus sylvestris

T5304 Pterocarpus osun

T3493 Isodon henryi

T0467 Anemone flaccida

T5781 Schefflera arboricola

T4489 Ongokea gore

T3123 Hedyotis auricularia

T6615 Uncaria gambir

T6359 Teucrium bidentatum

T1102 Caesalpinia digyna

T6875 Zanthoxylum dipetalum

T0787 Astragalus bisulcatus

T4712 Peridinium bipes

T2441 Ervatamia dichotoma

T2272 Drymaria diandra [Syn. Drymaria

cordata ssp. diandra]

T1622 Collinsonia canadensis

T5670 Salvia dichroantha

T4865 Phytolacca icosandra

T4233 Millettia auriculata

T0017 Acacia auriculaeformis

T5000 Plagiogyria stenoptera

T3861 Liparis auriculata

T6195 Stylophorum diphyllum

T1952 Cynanchum auriculatum

T4108 Marchantia paleacea var. diptera

T5197 Primula auricula

T4438 Nostoc flagelliforme

T6287 Tamarix gallica

T1989 Cytisus monspessulanus

T5565 Rosa gallica

T0092 Aconitum falconeri

T5182 Potentilla discolor

T3266 Holarrhena floribunda

T0513 Annona squamosa

T0215 Agave americana

T1205 Carica papaya

T1206 Carica papaya 
FAN QIE

FAN SHI LIU GAN

FAN SHI LIU PI

FAN SHI LIU YE

FAN SHI LIU ZI

FAN XIE YE

FANG FENG

FANG JI

FANG JI YE BA QIA

FANG XIANG BAI ZHU

FANG XIANG DUI XIN JU

FANG XIANG JIANG

FANG XIANG MU BAN SHU

FEI CAI

FEI CHENG FEI PENG

FEI CHENG SUAN JIANG

FEI E TENG

FEI HOU MIAN BAO GUO

FEI JI AI JIAO

FEI JI CAO

FEI JI TENG HUANG

FEI LI GUI LI

FEI LIAN

FEI LONG ZHANG XUE

FEI LV BIN LUO HAN SONG

FEI LV BIN PIAO SHU

FEI LV BIN QIAN JIN BA

FEI LV BIN QIAN LI GUANG

FEI LV BIN ROU DOU KOU

FEI LV BIN SHI ZI

FEI NI JI CI BAI

FEI SHU

FEI YAN CAO

FEI YI DIAN HONG

FEI YUE GUO

FEI ZAO CAO

FEI ZHOU BAI ZI LIAN

FEI ZHOU BI QIAO JIANG

FEI ZHOU FAN LI ZHI

FEI ZHOU FANG JI

FEI ZHOU GAN LAN

FEI ZHOU GE MU

FEI ZHOU GOU TENG

FEI ZHOU HUANG GUA

FEI ZHOU HUANG GUO MU

FEI ZHOU HUANG TAN

FEI ZHOU JIAN XUE FENG HOU

FEI ZHOU KU MU

FEI ZHOU LUO LE

FEI ZHOU MAO ZHU MU

FEI ZHOU WA
番茄

番石榴干

番石榴皮

番石榴叶

番石榴䊏

番泻叶

防风

防己粉防己)

防已叶菝荔

芳香白珠

芳香堆心菊

芳香姜

芳香木瓣树*

费菜

费城飞蓬

费城酸浆

飞蛾藤

肥厚面包果*

斐济崖椒

飞机草

斐济藤黄*

菲利桂标

飞廉

飞龙掌血.

菲律宾罗汉松

菲律宾朴树

菲律宾千斤拔*

菲律宾千里光*

菲律宾肉豆冦

菲律宾石梓*

腓尼基刺柏

榧树

飞燕草

绯一点红*

费约果

肥㿝草

非洲百子莲*

非洲闭鞘姜

非洲番荔枝

非洲防已

非洲橄榄

非洲格木

非洲钩藤*

非洲黄瓜*

非洲黄果木

非洲黄檀

非洲见血封喉

非洲苦木

非洲罗勒

非洲帽柱木*

非洲蛙
Tomato

Guava Unripe Fruit

Guava Bark

Guava Leaf

Guava Seed

Narrowleaf Senna Leaf

Divaricate Saposhnikovia

Fourstamen Stephania

Gansu Greenbrier

Fragrant Gaultheria

Fragrant Sneezeweed*

Aromatic Ginger

Aromatic Xylopia*

Aizoon Stonecrop

Philadelphia Fleabane

Philadelphia Groundcherry*

Racemose Porana

Fleshy Artocarpus*

Fiji Fagara*

Fragrant Eupatorium

Fiji Garcinia*

Mocketprivet-like Oak

Curly Bristlethistle

AsiaticToddalia

Philippine Podocarpus

Philippine Nettletree

Philippine Flemingia

Philippine Groundsel*

Antao Nutmeg

Philippine Bushbeech*

Poienician Juniper

Torreya*

Rocket Consolida

Red Tasselflower*

Pineapple Guava

Soapwort

African Lily

African Costus*

African Custard Apple*

Calumba Root

African Olive*

African Erythrophleum

African Gambirplant*

African Cucumber*

African Mammey Apple

African Rosewood

African Antiaris*

African Quassia*

African Basil*

African Mitragyna*

African frog
T3962 Lycopersicon esculentum

T5265 Psidium guajava

T5266 Psidium guajava

T5267 Psidium guajava

T5268 Psidium guajava

T1230 Cassia angustifolia

T5727 Saposhnikovia divaricata [Syn.

Ledebouriella seseloides]

T6136 Stephania tetrandra

T5980 Smilax menispermoidea

T2891 Gaultheria fragrantissima

T3133 Helenium aromaticum

T6907 Zingiber aromaticum

T6850 Xylopia aromatica

T5850 Sedum aizoon

T2424 Erigeron philadelphicus

T4853 Physalis philadelphica

T5167 Porana racemosa

T0709 Artocarpus altilis

T2655 Fagara vitiensis

T2567 Eupatorium odoratum

T2871 Garcinia pseudoguttifera

T5376 Quercus phillyraeoides

T1200 Carduus crispus

T6471 Toddalia asiatica [Syn. Toddalia

aculeata; Paullinia asiatica]

T5051 Podocarpus philippinensis

T1298 Celtis philippinensis

T4260 Moghania philippinensis

T5896 Senecio phillipicus

T4354 Myristica simiarum

T3025 Gmelina philippensis

T3593 Juniperus phoenicea

T6477 Torreya grandis

T1645 Consolida ajacis [Syn. Delphinium ajacis]

T2356 Emilia coccinea

T2693 Feijoa sellowiana

T5726 Saponaria officinalis

T0209 Agapanthus africanus

T1754 Costus afer

T3237 Hexalobus crispiflorus

T3559 Jateorhiza palmata

T4486 Olea africana

T2482 Erythrophleum africanum

T6605 Uncaria africana

T1873 Cucumis africanus

T4092 Mammea africana

T2004 Dalbergia melanoxylon

T0532 Antiaris africana

T5369 Quassia africana

T4474 Ocimum kilimandscharicum

T4255 Mitragyna africanus

T0207 African frog 


\begin{tabular}{|c|c|c|c|}
\hline FEI ZHOU ZHI XIE MU & 非洲止泻木* & African Holarrhena* & T3262 Holarrhena africana \\
\hline FEI ZHOU ZI WEI & 非州紫葳 & Africa Trumpetcreeper* & T4422 Newbouldia laevis \\
\hline FEN BA JIAO & 粉芭蕉 & Plantain Banana & $\mathrm{T} 4330$ Musa paradisiaca \\
\hline FEN BA JIAO ZA JIAO ZHONG & 粉芭蕉杂交种植变种* & Plantain Banana Cultivariaty* & T4332 Musa $\mathrm{x}$ paradisiaca cultivar \\
\hline \multicolumn{4}{|l|}{ ZHI BIAN ZHONG } \\
\hline FEN CHA DANG GUI & 分叉当归 & Furcate Angelica* & T0481 Angelica furcijuga \\
\hline FEN CHA MA QIAN ZI & 分叉马钱子* & Divaricate Poisonnut* & T6170 Strychnos divaricans \\
\hline FEN GE & 粉葛 & Thomson Kudzuvine & T5314 Pueraria lobata var. thomsonii \\
\hline FEN HONG SE XI FAN LIAN & 粉红色西番莲 & May-apple & T4666 Passiflora incarnata \\
\hline FEN JI DU & 粪箕笃 & Long Stephania & T6131 Stephania longa \\
\hline FEN LAI SHI HU & 芬来石斛 & Findley Dendrobium* & T2103 Dendrobium findleyanum \\
\hline FEN LIU JUN & 粉瘤菌 & Wolfs-milk Slime & T3960 Lycogala epidendrum \\
\hline FEN LU ZHU YANG YANG & 粉绿猪殊殊 & Glaucous Bedstraw* & T2831 Galium glaucum \\
\hline FEN NIE CONG TOU & 分莧葱头 & Tillering Onion & T0312 Allium cepa var. agrogatum \\
\hline FEN ROU CENG KONG JUN & 粉肉层孔菌 & & T2747 Fomes cajanderi \\
\hline FEN SHI TANG SONG CAO & 芬氏唐松草 & Fendler's Meadowrue & T6383 Thalictrum fendleri \\
\hline FEN TUAN HUA & 粉团花 & Paniculate Hydrangea & T3306 Hydrangea paniculata \\
\hline FEN ZHI PO JU & 分枝珀菊* & Ramose Amberboa* & T0394 Amberboa ramosa \\
\hline FENG CHAO CAO & 蜂巢草 & Rough Leucas & T3779 Leucas aspera \\
\hline FENG CHAO MI ZI LAN & 蜂巢米仔兰* & Foveolate Aglaia* & T0236 Aglaia foveolata \\
\hline FENG CHENG JI XUE TENG & 丰城鸡血藤 & HirsuteMillettia & T4239 Millettia nitida var. hirsutissima \\
\hline FENG DOU CAI & 蜂斗菜 & Japanese Butterbur & T4740 Petasites japonicus \\
\hline FENG DU & 蜂毒 & Apisin & T0539 Apis cerana \\
\hline FENG GUA & 凤瓜 & Entireleaf Gymnopetalum & T3081 Gymnopetalum integrifolium \\
\hline FENG HUANG MU & 凤凰木 & Flamboyanttree & T2060 Delonix regia \\
\hline FENG JIAO & 蜂胶 & Propolis & T0543 Apis mellifera ligustica \\
\hline FENG LI & 凤梨 & Pineapple & T0444 Ananas comosus \\
\hline FENG LING CAO & 风铃草 & Canterburybells & T1156 Campanula medium \\
\hline FENG LUN CAI & 风轮菜 & Chinese Clinopodium & T1573 Clinopodium chinense \\
\hline FENG MAO JU & 风毛菊 & Wildhairdaisy & T5756 Saussurea japonica \\
\hline FENG MI & 蜂蜜 & Honey & T0540 Apis cerana \\
\hline FENG RU & 蜂乳 & Royal Jelly & T0541 Apis cerana \\
\hline FENG WEI CAO & 凤尾草 & Chinese Brake & T5295 Pteris multifida \\
\hline FENG WEI CHA & 凤尾茶 & & T2340 Elsholtzia bodinieri \\
\hline FENG WEI JUE & 凤尾茨 & Nervous Brake & $\begin{array}{l}\text { T5288 Pteris cretica var. nervosa }[\text { Syn. Pteris } \\
\text { nervosa] }\end{array}$ \\
\hline FENG WEI PA SHAN HU & 凤尾爬山虎 & Mary Arthromeris & $\begin{array}{l}\text { T0708 Arthromeris mairei [Syn. Polypodium } \\
\text { mairei] }\end{array}$ \\
\hline FENG XIAN & 凤仙 & Garden Balsam & T3410 Impatiens balsamina \\
\hline FENG XIAN HUA & 凤仙花 & Garden Balsum Flower & T3411 Impatiens balsamina \\
\hline FENG XIANG JI SHENG & 枫香寄生 & Flatshoot Mistletoe & T6774 Viscum articulatum \\
\hline FENG XIANG SHU & 枫香树 & Beautiful Sweetgum Leaf & $\begin{array}{l}\text { T3866 Liquidambar formosana [Syn. } \\
\text { Liquidambar taiwaniana] }\end{array}$ \\
\hline FENG XIANG SHU YE & 风箱树叶 & Common Butterbush & T1316 Cephalanthus occidentalis \\
\hline FENG XIN ZI & 风信子 & Common Hyacinth & T3297 Hyacinthus orientalis \\
\hline FENG YA JUE & 风Y鄀 & Japanese Coniogramme & $\begin{array}{l}\text { T1640 Coniogramme japonica }[\text { Syn. } \\
\text { Hemionitis japonica }]\end{array}$ \\
\hline FENG YU HUA & 风雨花 & Rosepink Zephyrlily & $\begin{array}{l}\text { T6904 Zephyranthes grandiflora }[\text { Syn. } \\
\text { Zephyranthes carinata }]\end{array}$ \\
\hline FO JIA CAO & 佛甲草 & Linear Stonecrop & $\begin{array}{l}\text { T5856 Sedum lineare [Syn. Sedum } \\
\text { obtuso-lineare] }\end{array}$ \\
\hline FO SHOU & 佛手 & Fleshfingered Citron & T1501 Citrus medica var. sarcodactylis \\
\hline FU AN & 富桉* & Dives Eucalyptus* & T2508 Eucalyptus dives \\
\hline
\end{tabular}




\begin{tabular}{|c|c|}
\hline FU CHUI FE LAO JU & 俯垂弗劳菊 \\
\hline FU ER JIA CE JIN ZHAN HUA & 伏尔加侧金戞花 \\
\hline FU FANG TENG & 扶芳藤 \\
\hline FU JI NI YA DI SUN & 弗吉尼亚地笋* \\
\hline FU JI NI YA GOU JI JUE & 弗吉尼亚狗脊蒴 \\
\hline FU JIAN XI XIN & 福建细辛 \\
\hline FU JU & 福橘 \\
\hline FU KANG A WEI GEN & 阜康阿魏根 \\
\hline FU KE CI TONG & 福克刺桐 \\
\hline FU LANG HUA & 扶郎花 \\
\hline FU LEI SHI DU JUAN HUA & 福雷氏杜鹃花 \\
\hline FU LING & 获苓 \\
\hline FU MAO SHAN DOU GEN & 覆毛山豆根 \\
\hline FU MU & 福木 \\
\hline FU NING TENG & 富宁藤 \\
\hline FU PEN ZI & 覆盆子 \\
\hline FU PING & 浮萍 \\
\hline FU RONG JU GEN & 芙蓉菊根 \\
\hline FU RUI ER JUE & 福瑞耳蕨* \\
\hline FU RUI ZI WEI & 福瑞紫薇* \\
\hline FU SANG HUA & 扶桑花 \\
\hline FU SANG YE & 扶桑叶 \\
\hline FU SHE SONG & 辐射松 \\
\hline FU SHI MA QIAN ZI & 福氏马钱子* \\
\hline FU SHOU CAO & 福寿草 \\
\hline FU SI TE JIU JIE & 福斯特九节* \\
\hline FU YE RONG & 腐叶榕* \\
\hline FU YE YAN ZI CAI & 浮叶眼子菜 \\
\hline FU YE ZE LAN & 复叶泽兰 \\
\hline FU ZHOU SHU YU & 福州薯蓣 \\
\hline FU ZHUANG SHUI GUI JIAO & 辐状水鬼蕉* \\
\hline FU ZI & 附子 \\
\hline GAN & 柑 \\
\hline GAN CAO & 甘草 \\
\hline GAN DI HUANG & 干地黄 \\
\hline GAN FENG CAO & 肝风草 \\
\hline GAN GE TENG GEN & 甘葛藤根 \\
\hline GAN HUA DOU & 干花豆 \\
\hline GAN JIANG & 干姜 \\
\hline GAN LAN & 甘蓝 \\
\hline GAN LAO JI GU CHANG SHAN & 干酪鸡骨常山 \\
\hline GAN PI & 相皮 \\
\hline GAN QING TIE XIAN LIAN & 甘青铁线莲 \\
\hline GAN QING WU TOU & 甘青乌头 \\
\hline GAN SHU & 甘薯 \\
\hline GAN SONG & 甘松 \\
\hline GAN SU BEI MU & 甘肃贝母 \\
\hline GAN SU HUAI SHU & 甘肃槐树 \\
\hline GAN SU HUANG QIN & 甘肃黄芩 \\
\hline GAN SU SHAN ZHA & 甘 \\
\hline
\end{tabular}

Tarbush

Volga Adonis

Forture Euonymus

Bugleweed

Virginia Chain Fern

Fukien Wildginger

Blessing Citrus*

Fukang Giantfennel Root

Folk Coralbean*

Flameray Gerbera

Pere L. F. Faurie's Rhododendron Indian Bread

Hirsute Euchresta*

Red-and-Yellow Garden Raspberry

Common Duckwood

Chinese Crossostephium Root

Faurie Shield Fern*

Faurie Crapemyrtle*

Chinese Hibiscus Flower

Chinese Hibiscus Leaf

Montery Pine

Froes Poisonnut*

Amur Adonis

Forster Ninenode*

Septic Fig*

Floatingleaf Pondweed

Compositeleaf Eupatorium*

Foochow Yam

Rotate Hymenocallis*

Prepared Common Monkshood

Daughter Root

Chachi Citrus

Ural Licorice

Adhesive Rehmannia Dried Root

Autumn Zephyrlily

Thomson Kudzuvine Root

Common Fordia

Common Ginger Dried Rhizome

Cabbage

Boone Alstonia

Chachi Citrus Pericarp

Tangut Clematis

Tangut Monkshood

Sweet Potato

Chinese Nardostachys

Przewalsk Fritillary

Thickfruit Sophora

Rehder Skullcap;

Kansu Hawthorn
T2740 Flourensia cernua

T0192 Adonis wolgensis

T2540 Euonymus fortunei

T3982 Lycopus virginicus

T6832 Woodwardia virginica

T0727 Asarum fukienense

T1515 Citrus tangemna

T2701 Ferula fukanensis

T2465 Erythrina folkersii

T2951 Gerbera jamesonii

T5509 Rhododendron fauriei

T5169 Poria cocos

T2529 Euchresta strigillosa

T2877 Garcinia subelliptica

T4645 Parepigynum funingense

T5593 Rubus idaeus

T3738 Lemna minor

T1812 Crossostephium chinense

T5132 Polystichum fauriei

T3669 Lagerstroemia fauriei

T3242 Hibiscus rosa-sinensis

T3243 Hibiscus rosa-sinensis

T4920 Pinus radiata

T6173 Strychnos froesii

T0184 Adonis amurensis

T5275 Psychotria forsteriana

T2726 Ficus septica

T5176 Potamogeton natans

T2556 Eupatorium compositifolium

T2197 Dioscorea futschauensis

T3321 Hymenocallis rotata

T0083 Aconitum carmichaeli

T1471 Citrus chachiensis

T3022 Glycyrrhiza uralensis

T5445 Rehmannia glutinosa [Syn. Rehmannia

glutinosa f. huechingensis]

T6903 Zephyranthes candida

T5320 Pueraria thomsonii

T2752 Fordia cauliflora

T6909 Zingiber officinale

T1017 Brassica oleracea var. capitata

T0367 Alstonia boonei

T1472 Citrus chachiensis

T1546 Clematis tangutica

T0137 Aconitum tanguticum

T3447 Ipomoea batatas [Syn. Convolvulus

batatas]

T4386 Nardostachys chinensis

T2792 Fritillaria przewalskii

T6040 Sophora pachycarpa

T5843 Scutellaria rehderiana

T1771 Crataegus kansuensis 
GAN SUI

GAN WAN WU TOU

GAN XI SHU WEI CAO

GAN ZHE

GANG BAN GUI GEN

GANG GUO HE ZHI XIE MU

GANG GUO JIAN XUE FENG

HOU

GANG GUO LUO FU MU

GANG MAO CHENG LIU

GANG MAO TENG SHAN LIU

GANG SONG

GANG SONG

GAO AO DING ZAO

GAO BAO CHUN

GAO BEN

GAO CHU

GAO CHUN HUANG JU

GAO CONG ZHEN ZHU MEI

GAO DA CI BAI

GAO DA CUI QUE HUA

GAO DA HU JIAO

GAO DA SHAN LAN

GAO DANG GUI

GAO DI CHA SHAN ZHU ZI

GAO DI DAN CAO

GAO FEI YAN CAO

GAO GUI BO LUO MI

GAO GUI CHUN HUANG JU

GAO GUI JIA ZI CAO

GAO GUI YOU MU YUN XIANG

GAO HAI CONG

GAO HONG JIN

GAO HUANG LU SANG

GAO HUANG QIN

GAO HUI MAO DOU

GAO JIA SUO BAI XIAN

GAO JIA SUO LAN PEN HUA

GAO JIA SUO LIN MAO JUE

GAO JIA SUO LONG DAN

GAO JIA SUO WU TOU

GAO JIA SUO YING SU

GAO JIA SUO ZI JIN

GAO KA FEI

GAO LIANG

GAO LIANG HONG QU

GAO LIANG JIANG

GAO MEI YING BAN

GAO SHAN BIAN ZHI SHI SONG

GAO SHAN CHA BIAO

GAO SHAN HUA JIAO

GAO SHAN HUANG HUA
甘遂

贑皖乌头

甘西鼠尾草

甘蔗

杜板归根

刚果河止泻木*

刚果见血封喉

刚果萝芙木

刚毛柽柳

刚毛藤山柳

岗松

刚松

高凹顶藻

高报春

㩰本

高㯪

高春黄菊

高从珍珠梅

高大刺柏

高大翠雀花

高大胡椒

高大山榄*

高当归*

告地查山竹子*

高地胆草

高飞燕草

高贵波罗蜜*

高贵春黄菊*

高贵假紫草*

高贵柚木芸香*

高海葱

高红槿

高黄绿桑

高黄芩

高灰毛豆

高加索白鲜

高加索蓝盆花*

高加索鳞毛葓

高加索龙胆*

高加索乌头

高加索罂粟

高加索紫堇

高咖啡

高粱

高梁红曲*

高良姜

高梅缨瓣

高山扁枝石松

高山茶蔍*

高山花椒

高山黄华
Kansui Euphorbia

Finet Monkshood

Przewalsk Sage

Sweetcane Culm

Perfoliate Knotweed Root

Congo Holarrhena*

Congo Antiaris*

Obscure Devilpepper*

Kashgar Tamarisk

Hisped Vineclethra

Shrubby Baeckea

Northern Pitch Pine

High Concave-top Alga*

Oxlip

Chinese Ligusticum

High Ailanthus*

Tall Camomile

Tree Falsespiraea

High Juniper*

High Larkspur*

Pepper-tree

High Ligusticum*

Gaudicha Garcinia*

High Elephantfoot*

Alpine Larkspur

Noble Artocarpus*

Common Chamomile

Noble Arnebia*

Tall Hibiscus

Iroko Fustic-tree

Tall Skullcap

High Tephrosia*

Caucasian Pittany*

Caucasian Scabious*

Caucasian Buckler-fern*

Caucasian Gentian*

Eastern Monkshood

Caucasian Poppy*

Caucasian Corydalis*

High Coffee*

Sorghum

Red Koji; Angkak

Lesser Galangal

Gaumei Fringe-petal*

Alpine Clubmoss*

Alpine Currant

Alpine Pricklyash

Alplne Thermopsis
T2596 Euphorbia kansui

T0094 Aconitum finetianum

T5688 Salvia przewalskii

T5642 Saccharum sinensis

T5111 Polygonum perfoliatum

T3264 Holarrhena congolensis

T0534 Antiaris welwitschii

T5439 Rauwolfia obscura

T6288 Tamarix hispida

T1549 Clematoclethra scanden

T0853 Baeckea frutescens

T4922 Pinus rigida

T3716 Laurencia elata

T5198 Primula elatior

T3824 Ligusticum sinense

T0256 Ailanthus excelsa

T0520 Anthemis altissima

T6051 Sorbaria arborea

T3587 Juniperus excelsa

T2075 Delphinium excelsum

T4025 Macropiper excelsum

T5001 Planchonia grandis

T3821 Ligusticum elatum

T2855 Garcinia gaudichaudii

T2332 Elephantopus elatus

T2074 Delphinium elatum

T0718 Artocarpus nobilis

T0523 Anthemis nobilis

T0650 Arnebia nobilis

T6322 Teclea nobilis

T6646 Urginea altissima

T3239 Hibiscus elatus

T1376 Chlorophora excelsa

T5831 Scutellaria altissima

T6334 Tephrosia elata

T2166 Dictamnus caucasicus

T5774 Scabiosa caucasica

T2278 Dryopteris caucasica

T2907 Gentiana caucasa

T0121 Aconitum orientale

T4624 Papaver caucasicum

T1711 Corydalis caucasica

T1609 Coffea excelsa

T6056 Sorghum vulgare

T4270 Monascus kaoliang

T0359 Alpinia officinarum

T1811 Crossopetalum gaumeri

T3964 Lycopodium alpinum [Syn.

Diphasiastrum alpinum]

T5544 Ribes alpinum

T6881 Zanthoxylum hamiltonianum

T6424 Thermopsis alpina 


\begin{tabular}{|c|c|c|}
\hline GAO SHAN HUO RONG CAO & 高山火线草 & Alpine Edelweiss \\
\hline GAO SHAN LUO HAN SONG & 高山罗汉松 & Alpine Totara \\
\hline GAO SHAN TANG SONG CAO & 高山唐松草 & Alpine Meadowrue \\
\hline GAO SHAN TIAO JUE & 高山条蕨 & Alpine Oleandra \\
\hline GAO SHAN YAN SHEN & 高山岩参 & Alpine Sowthistle \\
\hline GAO SHAN YING SU & 高山罂粟 & Alpine Poppy \\
\hline GAO WU TOU & 高乌头 & Tall Monkshood \\
\hline GAO YI ZHI HUANG HUA & 高一枝黄花 & Canadian Goldenrod \\
\hline GAO YUAN TANG SONG CAO & 高原唐松草 & Highland Meadowrue \\
\hline GAO ZE LAN & 高泽兰 & Tall Eupatorium \\
\hline GE BI TIAN MEN & 戈壁天门冬 & Desertliving Asparagus* \\
\hline GE CAI KE SHI ZI JIN & 格蔡科氏紫堇 & Gortschakov Corydalis* \\
\hline GE CONG & 茖葱 & Longroot Onion \\
\hline GE GEN & 葛根 & Lobed Kudzuvine Root \\
\hline GE LI FEI SI SHI DA GONG LAO & 格里菲思十大功劳 & Griffith Mahonia \\
\hline GE LI FEI SI TENG HUANG & 格里菲思藤黄* & Griffith Garcinia* \\
\hline GE LU ZI & 葛缕子 & Caraway \\
\hline GE LUN BI YA BA DOU & 哥伦比亚巴豆 & Colombia Croton*; Almizclillo \\
\hline GE LUN BI YA MU BAN SHU & 哥仑比亚木瓣树* & Columbia Xylopia* \\
\hline GE NA XIANG & 哥纳香 & Cheliensis Goniothalamus \\
\hline GE SHAN XIAO & 隔山消 & Wilford Swallowwort \\
\hline GE SHANG TING CHANG & 葛上亭长 & Bean Blister Beetle \\
\hline GE SHI SHU WEI CAO & 格氏鼠尾草* & Gregg Sage* \\
\hline GE XUN & 葛荁 & Japanese Balanophora \\
\hline GE YANG XI FAN LIAN & 革样西番莲* & Coriaceous Passionflower* \\
\hline GE YE MI HOU TAO & 革叶猕猴桃 & Coriaceousleaf Actinidia \\
\hline GE ZHI HUA DI QIAN & 革质花地钱 & \\
\hline GONG BU WU TOU & 工布乌头 & Gongbo Monkshood \\
\hline GONG GA SHAN WU TOU & 贡嘎山乌头 & Konka Mountain Monkshood \\
\hline GONG XING MA DOU LING & 弓形马㹸铃 & Bow-shaped Dutchmanspipe* \\
\hline GOU GU SHU PI & 枸骨树皮 & Chinese Holly Bark \\
\hline GOU GU YE & 枸骨叶 & Chinese Holly Leaf \\
\hline GOU JI & 构棘 & Cochinchina Cudrania \\
\hline GOU JU & 枸橘 & Trifoliate-orange \\
\hline GOU JU HE & 枸橘核 & Trifoliate-orange Seed \\
\hline GOU JU YE & 枸橘叶 & Trifoliate-orange Leaf \\
\hline GOU JU ZHI KE & 枸橘枳壳 & Trifoliate-orange Unripe Fruit \\
\hline GOU JU ZHI SHI & 枸橘枳实 & Trifoliate-orange Young Fruit \\
\hline GOU MAO QIAN CAO & 钩毛茜草 & Hookedhair Madder \\
\hline GOU QI GEN PI & 枸杞根皮(地骨皮) & Chinese Wolfberry Root-bark \\
\hline GOU QI XIAO BO & 枸杞小檗 & Medlar Barberry* \\
\hline GOU QI YE & 枸杞叶 & Chinese Wolfberry Leaf \\
\hline GOU QI ZI & 枸杞子 & Chinese Wolfberry Fruit \\
\hline GOU ROU & 狗肉 & Dog Meat \\
\hline GOU SHE CAO & 狗舌草 & Kirilow Groundsel Herb \\
\hline GOU SHI HUA & 狗屎花 & Chinese Forgetmenot \\
\hline GOU SHU & 构树 & Common Papermulberry \\
\hline GOU SHU BAI PI & 构树白皮 & Common Papermulberry Bast* \\
\hline GOU SHU GEN & 构树根 & Common Papermulberry Root* \\
\hline GOU SHU GUO & 构树果 & Common Papermulberry Fruit \\
\hline
\end{tabular}

T3749 Leontopodium alpinum

T5049 Podocarpus nivalis

T6373 Thalictrum alpinum

T4488 Oleandra wallichii

T1414 Cicerbita alpina

T4621 Papaver alpinum

T0130 Aconitum sinomontanum

T6022 Solidago altissima

T6376 Thalictrum cultratum

T2550 Eupatorium altissimum

T0750 Asparagus gobicus

T1717 Corydalis gortschakovii

T0324 Allium victorialis

T5313 Pueraria lobata [Syn. Pueraria

thunbergiana; Pueraria pseudohirsuta]

T4065 Mahonia griffithii

T2856 Garcinia griffithii

T1217 Carum carvi

T1855 Croton schiedeanus

T6852 Xylopia columbiana

T3044 Goniothalamus cheliensis

T1964 Cynanchum wilfordii [Syn. Cynoctonum wilfordii]

T2384 Epicauta gorhami

T5674 Salvia greggii

T0860 Balanophora japonica

T4664 Passiflora coriaceae

T0165 Actinidia rubricaulis var. coriacea

T1703 Corsinia coriandrina

T0107 Aconitum kongboense

T0112 Aconitum liljestrandii

T0621 Aristolochia arcuata

T3389 Ilex cornuta

T3390 Ilex cornuta

T1884 Cudrania cochinchinensis

T5137 Poncirus trifoliata

T5138 Poncirus trifoliata

T5139 Poncirus trifoliata

T5140 Poncirus trifoliata

T5141 Poncirus trifoliata

T5582 Rubia oncotricha

T3956 Lycium chinense

T0921 Berberis zycium

T3957 Lycium chinense

T3958 Lycium chinense

T1170 Canis familiaris

T6330 Tephroseris kirilowii [Syn. Senecio integrifolius var. fauriei]

T1967 Cynoglossum amabile

T1032 Broussonetia papyrifera

T1033 Broussonetia papyrifera

T1034 Broussonetia papyrifera

T1035 Broussonetia papyrifera 


GOU TENG
GOU WEN
GOU XIN
GOU ZHI TENG
GOU ZHUANG HU JIAO
GOU ZHUANG SHI HU
GU BA LUO FU MU
GU CHENG MEI GUI SHU
GU CHUI SHI HU
GU JIE CAO
GU JING CAO
GU KE
GU LIN XUE DAN
GU LIN YE LIAN
GU SUI BU
GU TING HUA
GU TING HUA ZA JIAO ZHONG
GUA DI
GUA FU MU

GUA JIN DENG
GUA JIN DENG GEN
GUA LOU
GUA LOU ZI
GUA MU
GUA MU BIAN ZHONG

GUA YE WU TOU
GUAI QIN
GUAN CANG ZHU
GUAN CONG DONG QING
GUAN CONG XIANG KE KE
GUAN GUANG MU
GUAN HUA DANG SHEN
GUAN HUA MA DOU LING
GUAN HUA QIN JIAO
GUAN HUA ROU CONG RONG
GUAN LUO SUI TUN CAO

\section{GUAN MU AO TUO SI TE CAO} GUAN MU CHAI HU

\section{GUAN MU LUO FU MU} GUAN MU TIAN MEN DONG GUAN MU TONG GUAN MU WEN SHU LAN GUAN MU YA JU GUAN MU YUAN ZHI GUAN MU ZHUANG CHE ZHOU CAO GUAN SHANG ZHANG YA CAI GUAN YE LIAN QIAO

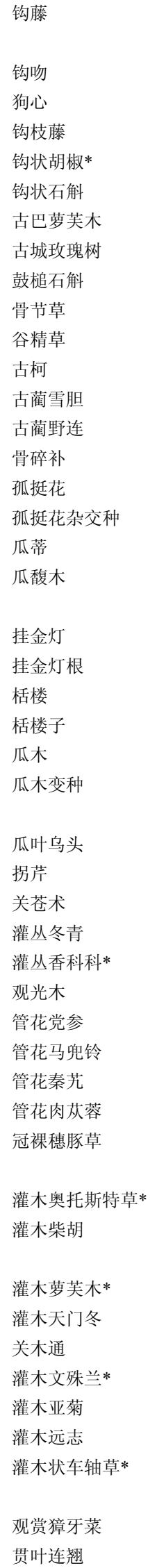

Sharpleaf Gambirplant

Graceful Jessamine

Dog Heart

Ancistrocladus*

Hooked Pepper*

Hooked Dendrobium

Cuba Devilpepper

Elliptical Ochrosia

Yellowbow Dendrobium

Marsh Horsetail

Pipewart

Coca Shrub

Gulin Hemsleya

Gulin Goldthread*

Fortune's Drynaria Rhizome

Jersey Lily

Jersey Lily Hybrid

Muskmelon Fruit Pedicel

Oldham Fissistigma

Franchet Groundcherry

Franchet Groundcherry Root

Mongolian Snakegourd

Mongolian Snakegourd Seed

Planeleaf Alangium

Planeleaf Alangium Variety*

Hemsley Monkshood

Polymorphic Angelica

Japanese Atractylodes

Shrubby Holly*

Fluticose Germander*

Guanguangtree

Tubularflower Asiabell

Tubeflower Dutchmanspipe

Tubeflower Gentian

Tubeshaped Flower Cistanche

Crestedspike Ragweed

Shrubby Otostegia*

Shrub Thoroughwax (Shrubby

Hare's-ear)

Shrubby Devilpepper*

Shrubby Asparagus*

Manchurian Dutchmanspipe

Shrubby Crinum* 'bush' or `march lily'

Shrubby Ajania

Shrubby Milkwort*

Shrubby Clover*

Common St.John'swort
T6629 Uncaria rhynchophylla [Syn. Nauclea rhynchophylla]

T2897 Gelsemium elegans

T1171 Canis familiaris

T0450 Ancistrocladus korupensis

T4928 Piper aduncum

T2094 Dendrobium aduncum

T5432 Rauwolfia cubana

T4469 Ochrosia elliptica

T2099 Dendrobium chrysotoxum

T2409 Equisetum palustre

T2434 Eriocaulon buergerianum

T2491 Erythroxylum coca

T3210 Hemsleya penxianensis var. gulinensis

T1666 Coptis gulinensis

T2273 Drynaria fortunei

T0390 Amaryllis belladonna

T0391 Amaryllis belladonna [hybrida]

T1876 Cucumis melo

T2735 Fissistigma oldhamii [Syn. Melodorum

oldhamii]

T4846 Physalis alkekengi var. franchetii

T4847 Physalis alkekengi var. franchetii

T6510 Trichosanthes kirilowii

T6511 Trichosanthes kirilowii

T0285 Alangium platanifolium

T0284 Alangium paltanifolium var.

platanifolium

T0101 Aconitum hemsleyanum

T0491 Angelica polymorpha

T0821 Atractylodes japonica

T3391 Ilex dumosa

T6361 Teucrium fruticans

T6556 Tsoongiodendron odorum

T1603 Codonopsis tubulosa

T0642 Aristolochia tubiflora

T2932 Gentiana siphonantha

T1458 Cistanche tubulosa

T0406 Ambrosia psilostachya var.

coronopifolia

T4557 Otostegia fruticosa

T1062 Bupleurum fruticosum

T5434 Rauwolfia fruticosa

T0748 Asparagus dumosus

T0632 Aristolochia manshuriensis

T1801 Crinum macowanii

T0260 Ajania fruticulosa

T5076 Polygala fruticosa

T6519 Trifolium fruticosa

T6218 Swertia decora

T3361 Hypericum perforatum 


\begin{tabular}{|c|c|c|}
\hline GUAN YIN CAO & 观音草 & Roxburgh Peristrophe \\
\hline GUAN ZHONG & 贯众 & Male Fern Rhizome \\
\hline GUAN ZHUANG GOU YA HUA & 冠状狗牙花 & Coronary Ervatamia* \\
\hline GUAN ZHUANG MEI YING SU & 灌状美罂粟 & \\
\hline GUAN ZI YU PAN & 管紫玉盘 & Angola Uvaria* \\
\hline GUANG BU DING GONG TENG & 广布丁公藤* & Expanse Erycibe* \\
\hline GUANG CI BAO JU & 光刺苍菊 & Glabrous Acanthospermum* \\
\hline GUANG CI GU & 光慈姑 & Edible Tulip \\
\hline GUANG E ZHU SHI DOU & 光萝猪屎豆 & Glabroussepal Crotalaria \\
\hline GUANG FANG FENG & 广防风 & Indian Epimeredi \\
\hline GUANG FANG JI & 广防己 & Fangchi \\
\hline GUANG FEI YAN CAO & 光飞燕草 & Light Larkspur* \\
\hline GUANG GUO GAN CAO & 光果甘草 & Licorice \\
\hline GUANG HUA DANG GUI & 光滑当归* & Glabrate Angelica* \\
\hline GUANG HUO XIANG & 广蕉香 & Cablin Potchouli \\
\hline GUANG JIE QIU HAI TANG & 光洁秋海棠* & Glabrate Begonia* \\
\hline GUANG JIN QIAN CAO & 广金钱草 & Snowbellleaf Tickclover \\
\hline GUANG JING QIAN CAO & 光茎茜草 & Wallich Madder \\
\hline GUANG LIANG HUANG TAN & 光亮黄檀* & Shining Rosewood* \\
\hline GUANG LIANG JIA LONG DAN & 光亮假龙胆* & Shining Gentianella* \\
\hline GUANG LIANG LUO FU MU & 光亮萝芙木 & Shining Devilpepper* \\
\hline GUANG LIANG SHI SONG & 光亮石松* & Shining Clubmoss* \\
\hline GUANG LIANG YANG TONG & 光亮杨桐 & Shining Adinandra* \\
\hline GUANG LIANG YUAN ZHI & 光亮远志 & Shining Milkwort* \\
\hline GUANG NANG ZI BING JUE & 光晒紫柄茨 & Long-eared Pseudophegopteris \\
\hline GUANG RONG YIN YU & 光荣茵芋 & Laureate Skimmia* \\
\hline GUANG SHI WEI & 光石韦 & Bald Pyrrosia \\
\hline GUANG SI SHI JU & 光四室菊 & Littleleaf Horsebrush \\
\hline GUANG XI BA JIAO LIAN & 广西八角莲 & Guangxi Many-flowered May-apple* \\
\hline GUANG XI E SHU & 广西莪术 & Kwangsi Turmeric \\
\hline GUANG XI JIU LI XIANG & 广西九里香 & Kwangsi Jasminorange \\
\hline GUANG XI MEI DENG MU & 广西美登木 & Guangxi Mayten* \\
\hline GUANG XI QIAN HU & 广西前胡 & Guangxi Hogfennel \\
\hline GUANG XI SHI SUAN & 广西石蒜 & Guangxi Lycoris \\
\hline GUANG YAO DA HUANG HUA & 光药大黄花 & Mongolian Cymabria \\
\hline GUANG YE BA DOU & 光叶巴豆 & Nitidleaf Croton \\
\hline GUANG YE DI BU RONG & 光叶地不容 & Glabrousleaf Stephania* \\
\hline GUANG YE DING GONG TENG & 光叶丁公藤 & Glabrousleaf Erycibe \\
\hline GUANG YE FEN HUA XIU XIAN & 光叶粉花绣线菊 & Fortune Japanese Spiraea \\
\hline $\mathrm{JU}$ & & \\
\hline GUANG YE JUE MING & 光叶决明 & Nitidleaf Senna* \\
\hline GUANG YE SHE PU TAO & 光叶蛇葡萄 & Hance Snakegrape \\
\hline GUANG YE SHUI SU & 光叶水苏 & Marshy Betony \\
\hline GUANG YE YAN ZI CAI & 光叶眼子菜 & Glabrousleaf Pondweed \\
\hline GUANG YE ZHU SHI DOU & 光叶猪屎豆 & Shackshack Crotalaria \\
\hline GUANG YE ZI HUA & 光叶子花 & Naked Leafyflower \\
\hline GUANG YE ZI YU PAN & 光叶紫玉盘 & Glabrousleaf Uvaria \\
\hline GUANG ZE BA JI & 光泽巴戟* & Lucid Indianmulberry* \\
\hline
\end{tabular}

T4730 Peristrophe roxburghiana T2281 Dryopteris crassirhizoma T2440 Ervatamia coronaria T0960 Bocconia frutescens T6658 Uvaria angolensis T2448 Erycibe expansa T0046 Acanthospermum glabratum T6560 Tulipa edulis T1837 Crotalaria usaramoensis T0503 Anisomeles indica [Syn. Epimeredi indica] T0628 Aristolochia fangchi T2069 Delphinium corumbosum T3013 Glycyrrhiza glabra T0484 Angelica glabra T5059 Pogostemon cablin [Syn. Mentha cablin]

T0889 Begonia glabra

T2134 Desmodium styracifolium

T5585 Rubia wallichiana

T2006 Dalbergia nitidula

T2940 Gentianella nitida

T5438 Rauwolfia nitida

T3974 Lycopodium lucidulum

T0182 Adinandra nitida

T5078 Polygala nitida

T5259 Pseudophegopteris subaurita

T5971 Skimmia laureola

T5353 Pyrrosia calvata

T6355 Tetradymia glabrata

T2300 Dysosma guangxiensis

T1904 Curcuma kwangsiensis

T4320 Murraya kwangsiensis

T4132 Maytenus guangsiensis

T4758 Peucedanum guangxiense

T3985 Lycoris guangxiensis

T1938 Cymbaria mongolica

T1853 Croton oblongifolius [Syn. Croton laevigatus]

T6127 Stephania glabra

T2450 Erycibe schmidtii

T6082 Spiraea japonica var. fortunei

T1236 Cassia laevigata [Syn. Cassia floribunda]

T0424 Ampelopsis brevipedunculata var. hancei

T6092 Stachys palustris

T5175 Potamogeton lucens

T1821 Crotalaria incana

T0999 Bougainvillea glabra

T6659 Uvaria boniana

T4282 Morinda lucida 


GUANG ZE WU TOU
GUANG ZHI GOU ER CHA
GUI DENG LONG
GUI GAI
GUI HUA
GUI JIAN JIN JI ER
GUI JIAN YU
GUI JIU
GUI PI
GUI PI DIAO ZHANG
GUI YA NA GOU TENG
GUI ZHEN CAO
GUI ZHI

GUI ZHOU ZHANG YA CAI
GUI ZHU TANG JIE
GUI ZHU XIANG
GUO JIANG LONG
GUO YE GE
HA DA SHI JI NING
HA SHI LUO HAN SONG
HA SHI MA

HA SHI SHAN GENG CAI HAI BA JI

HAI BIAN MA BIAN CAO HAI BIN LIU CHUAN YU HAI BIN LUO FU MU HAI BO NA DA JI HAI CONG HAI DAI HAI DAO MIAN HAI ER CHA HAI FENG TENG HAI HONG DOU HAI JIN BI XIE HAI JIN SHA HAI JIU CAI HAI NAN CU FEI

HAI NAN GE NA XIANG HAI NAN GOU YA HUA HAI NAN JIAN MU HAI NAN LUO FU MU HAI NAN MAI MA TENG HAI NAN QING NIU DAN HAI NAN SHA REN HAI NAN YE SHAN HUA HAI NI GOU ZHI TENG HAI QI HAI REN CAO HAI SHEN CHANG

\section{光泽乌头 \\ 光枝勾儿茶 鬼灯笼 \\ 鬼盖 \\ 桂花 \\ 鬼箭锦鸡儿 \\ 鬼箭羽 \\ 鬼臼 \\ 桂皮 \\ 桂皮钓樟 \\ 圭亚那钩藤 \\ 鬼针草 \\ 桂枝}

贵州獐牙菜

桂竹糖芥

桂竹香

过江龙

裹叶葛

哈达石荠苛

哈氏罗汉松

哈士蟆

哈氏山梗菜*

海巴戟

海边马鞭草*

海滨柳穿鱼

海滨萝芙木*

海博纳大戟

海苾

海带

海岛棉

孩儿茶

海风藤

海红豆

海锦草薢

海金沙

海非菜

海南粗榧

海南哥纳香

海南狗牙花

海南樫木

海南萝芙木

海南买麻藤

海南青牛胆

海南砂仁

海南野山花

海尼钩枝藤

海漆

海人草

海参肠
Lucid Monkshood*

Smoothbranched Supplejack

Redcalyx Glotybower

Coprinus Sporocarp

Sweet Osmanthus

Shagspine Peashrub

Winged Euonymus

Common Dysosma

Japanese Cinnamon

Spicebush

Garabato; Unganangi; Cat's Claw

Beggarticks

Cassiabarktree Twig

Guizhou Swertia*

Treacle Erysimum

Common Wallflower

Complanate Clubmoss

Mirifica Kudzuvine

Hada Mosla*

Hall Podocarpus*

Dried Chinese Woodfrog

Hassler Lobelia*

Indianmulberry

Littoral Verbena*

Japanese Toadflax

Littoral Devilpepper*

Hyberna Euphorbia

Red Squill

Ellgrass

Barbados Cotton

Cutechu

Kadsura Pepper

Sandal Beadtree

Japanese Climbing Fern

Shore Podgrass

Hainan Plumyew

Hainan Goniothalamus

Heyne Ervatamia*

Hainan Pencilwood

Hainan Devilpepper

Hainan Jointfir

Hainan Tinospora

Hainan Amonum

Hainan Sarcococca

Indian Liana

Bling-your-eye-tree

Simple Digenea Frond

Sea-cucunber Intestines
T0113 Aconitum lucidusculum

T0922 Berchemia polyphylla var. leioclada

T1555 Clerodendron fortunatum

T1660 Coprinus atramentarius

T4550 Osmanthus fragrans

T1190 Caragana jubata

T2536 Euonymus alatus

T2305 Dysosma versipellis [Syn. Podophyllum versipelle]

T1441 Cinnamomum japonicum

T3848 Lindera benzoin

T6616 Uncaria guianensis

T0938 Bidens bipinnata

T1438 Cinnamomum cassia [Syn.

Cinnamomum aromaticum]

T6224 Swertia kouitchensis

T2452 Erysimum cheiranthoides

T1356 Cheiranthus cheiri

T3971 Lycopodium complanatum

T5315 Pueraria mirifica

T4540 Orthodon hadai

T5040 Podocarpus hallii

T5409 Rana temporaria chensinensis; Rana

amurensis

T3899 Lobelia hassleri

T4280 Morinda citrifolia

T6708 Verbena littoralis

T3844 Linaria japonica

T5436 Rauwolfia littoralis

T2592 Euphorbia hyberna

T6649 Urginea maritima

T6925 Zostera marina

T3054 Gossypium barbadense]

T0019 Acacia catechu

T4950 Piper kadsura [Syn. Piper futokadsura]

T0167 Adenanthera pavonina

T2210 Dioscorea spongiosa

T3990 Lygodium japonicum

T6526 Triglochin maritimum

T1319 Cephalotaxus hainanensis [Syn.

Cephalotaxus mannii]

T3048 Goniothalamus howii

T2443 Ervatamia hainanensis

T2309 Dysoxylum hainanense

T5426 Rauvolfia verticillata var. hainanensis

T3030 Gnetum hainanense

T6466 Tinospora hainanensis

T0417 Amomum longiligulare

T5733 Sarcococca vagans

T0449 Ancistrocladus heyneanus

T2650 Excoecaria agallocha

T2173 Digenea simplex

T6145 Stichopus japonicus 


\begin{tabular}{|c|c|c|c|}
\hline HAI SHENG CHUN MAN ZAO & 海生川蔓藻* & Marine Widgeonweed & T5616 Ruppia maritime \\
\hline HAI SHI & 海柿* & Maritime Persimmon* & T2224 Diospyros maritima \\
\hline HAI SHI GOU YA HUA & 海氏狗牙花 & Medicinal Ervatamia & T2444 Ervatamia heyneana \\
\hline HAI SONG ZI & 海松子 & Korean Pine Seed & T4912 Pinus koraiensis \\
\hline HAI TANG GUO & 海棠果 & Kalofilum & T1129 Calophyllum inophyllum \\
\hline HAI TONG & 海通 & Tomentose Glorybower & T1567 Clerodendrum mandarinorum \\
\hline HAI TONG & 海桐 & Japanese Pittosporum & T4987 Pittosporum tobira \\
\hline HAI TONG PI & 海桐皮 & Oriental Variegated Coralbean Bark & T2479 Erythrina variegata var. orientalis \\
\hline HAI TUN YU & 海豘鱼 & Dolphin & T2092 Delphinus delphis \\
\hline HAI XIAKihinouye & 海虾 & Prawn & T4696 Penaeus orientalis \\
\hline HAI YAN & 海燕 & & T0782 Asterina pectinifera \\
\hline HAI YANG DA JI & 海洋大戟 & Sea Euphorbia* & T2607 Euphorbia paralias \\
\hline HAI YING SU & 海篥 & Ciliate Hornpoppy* & T2968 Glaucium fimbrilligerum \\
\hline HAI YUN & 海蕴 & & $\begin{array}{l}\text { T4403 Nemacystus decipiens [Syn. Mesogloea } \\
\text { decipiens; Cladosiphon decipiens] }\end{array}$ \\
\hline HAI ZHOU GU SUI BU & 海州骨碎补 & Squirrel's Foot Fern & T2053 Davallia mariesii \\
\hline HAN CAI & 蔊菜 & Indian Rorippa & $\begin{array}{l}\text { T5560 Rorippa montana [Syn. Rorippa dubia; } \\
\text { Sisymbrium dublium }]\end{array}$ \\
\hline HAN CHENG XI XIN & 汉城细辛 & Seoul Siebold Wildginger & T0732 Asarum sieboldii var. seoulensis \\
\hline HAN FANG JI & 汉防己 & Yellowmouth Dutchmanspipe & T0629 Aristolochia heterophylla \\
\hline HAN LIAN HUA & 旱莲花 & Common Nasturtium & T6555 Tropaeolum majus \\
\hline HAN MAI PING CAO & 旱麦瓶草 & Dry Silene & T5955 Silene jenisseensis \\
\hline HAN MI ER DUN HUI YE & 汉密尔顿灰叶 & Hamilton Tephrosia* & T6335 Tephrosia hamiltonii \\
\hline HAN QIN & 旱芹 & Wildcelery & T0544 Apium graveolens \\
\hline HAN QIN BIAN ZHONG & 旱芹变种 & Wildcelery Variety & T0545 Apium graveolens var. dulce \\
\hline HAN RUI WU WEI ZI & 含莈五味子 & Angletwig Magnoliavine & T5797 Schisandra propinqua \\
\hline HAN SHENG JUAN BAI & 旱生卷柏 & Staunton's Spikemoss & T5868 Selaginella stauntoniana \\
\hline HAN SHENG XIANG CHA CAI & 旱生香茶菜 & Dry-living Rabdosia & T3536 Isodon xerophilus \\
\hline HAN XIN CAO & 韩信草 & Indian Skullcap & T5840 Scutellaria indica \\
\hline HAN XIU CAO & 含羞草 & Sensitive Plant & T4248 Mimosa pudica \\
\hline HANG BAI ZHI & 杭白芷 & Taiwan Angelica & T0498 Angelica taiwaniana \\
\hline HAO WANG JIAO LU HUI & 好望角芦荟 & Cape of Good Hope Aloe Dried Juice & T0338 Aloe ferox \\
\hline HAO WANG JIAO LUO HAN & 好望角罗汉松 & Cape of Good Hope Podocarpus & T5037 Podocarpus elongatu \\
\hline SONG & & & \\
\hline HAO WANG JIAO XIANG PU & 好望角香蒲 & Cape-of-Good-Hope Cattail & T6586 Typha capensis \\
\hline HE AN FU LAO JU & 河岸弗劳菊* & Riparian Tarbush* & T2741 Flourensia riparia \\
\hline HE AN GOU GAN CAI & 河岸狗肝菜 & Riparian Dicliptera* & T2160 Dicliptera riparia \\
\hline HE AN HUANG TAN & 河岸黄檀 & Riparian Rosewood* & T2011 Dalbergia riparia \\
\hline HE AN ZE LAN & 河岸泽兰 & Riparian Eupatorium, Riverside & T2570 Eupatorium riparium \\
\hline & & Eupatorium & \\
\hline HE BAO DI BU RONG & 荷苞地不容 & Dicentrine Stephania* & T6121 Stephania dicentrinifera \\
\hline HE BAO MU DAN GEN & 荷包牡丹根 & Showy Bleedingheart Root & T2154 Dicentra spectabilis \\
\hline HE BEI YANG & 河北杨 & Hebei Poplar & T5153 Populus hopeiensis \\
\hline HE CAO XIANG WAN DOU & 禾草香踠豆 & Grass Vetchling & T3706 Lathyrus nissolia \\
\hline HE CAO YE JIA BEI FANG FENG & 禾草叶假北防风* & & T3734 Ledebouria graminifolia \\
\hline HE ER TI SHAN MA CHA & 赫尔梯山马茶 & & T6273 Tabernaemontana holstii \\
\hline HE GENG & 荷梗 & Hindu Lotus Petiole & T4397 Nelumbo nucifera \\
\hline HE GUO CU FEI & 核果粗榧 & Drupaceous Plumyew* & T1317 Cephalotaxus drupacea \\
\hline HE GUO ZHUANG BU GU ZHI & 核果状补骨脂 & Drupaceous Scurfpea* & T5271 Psoralea drupacea \\
\hline HE HUA XUE LIAN & 褐花雪莲 & Brownflower Saussurea & T5765 Saussurea phaeantha \\
\hline HE HUA YAN LING CAO & 褐花延龄草 & Brownflower Trillium & T6532 Trillium erectum \\
\hline HE HUA YU LAN & 荷花玉兰 & Southern Magnolia & T4039 Magnolia grandiflora \\
\hline HE HUAN PI & 合欢皮 & Silktree Albizia Bark & T0292 Albizzia julibrissin \\
\hline
\end{tabular}




\begin{tabular}{|c|c|c|c|}
\hline HE KA NI YA HUANG YANG & 赫卡尼亚黄杨* & Hyrcanian Box* & T1089 Buxus hyrcana \\
\hline HE LAN ZHONG ZHI FAN & 荷兰种植番红花 & Holland Planted Saffron* & T1806 Crocus antalyensis cv \\
\hline \multicolumn{4}{|l|}{ HONG HUA } \\
\hline HE LU BAI JIAN MU & 褐绿白坚木 & Olive-green White Quebracho* & T0770 Aspidosperma olivaceum \\
\hline HE NAN TANG SONG CAO & 河南唐松草 & Honan Meadowrue & T6392 Thalictrum honanenae \\
\hline HE QING HUA & 荷青花 & Japanese Hylomecon & T3316 Hylomecon japonica \\
\hline HE RONG GAI NIU GAN JUN & 褐线盖牛肝菌 & Bay Bolete & T6848 Xerocomus badius \\
\hline HE SE ZHONG HUA SHU & 褐色钟花树 & & T6269 Tabebuia avellanedae \\
\hline HE SHI FENG & 鹤虫风 & Wild Carrot & T2048 Daucus carota \\
\hline HE SHI TANG SONG CAO & 鹤氏唐松草 & Hernandez Meadowrue* & T6391 Thalictrum hernandezii \\
\hline HE SHOU WU & 何首乌 & Tuber Fleeceflower & T5107 Polygonum multiflorum \\
\hline HE SHUO YAO HUA & 河䍭荛花 & Lowdaphne Stringbush & T6819 Wikstroemia chamaedaphne \\
\hline HE TA CAO & 和他草 & Florida Waltheria & T6808 Waltheria americana \\
\hline HE TAO DA HUANG & 河套大黄 & River-bend Rhubarb* & T5469 Rheum hotaoense \\
\hline HE TUN & 河豚 & Globefish & T2805 Fugu ocellatus \\
\hline HE YE & 荷叶 & Hindu Lotus Leaf & T4398 Nelumbo nucifera \\
\hline HE YE DI & 荷叶蒂 & Hindu Lotus Leaf-base & T4399 Nelumbo nucifera \\
\hline HE YE FENG MAO JU & 禾叶凤毛菊 & Grassleaf Saussurea & T5754 Saussurea graminea \\
\hline HE ZI & 诃子 & Medicine Terminalia & T6346 Terminalia chebula \\
\hline HE ZI YE & 诃子叶 & Medicine Terminalia Leaf & T6347 Terminalia chebula \\
\hline HEI BA LUO CAO & 黑巴洛草 & Black Bui* & T0863 Ballota nigra \\
\hline HEI BAI HE & 黑百合 & Kamchatka Fritillary & T2782 Fritillaria camtschatcensis \\
\hline HEI BAI JIAN MU & 黑白坚木* & Black White Quebracho* & T0769 Aspidosperma nigricans \\
\hline HEI CHA BIAO & 黑茶蔍 & Black Currant & T5546 Ribes nigrum \\
\hline HEI CHAI HU & 黑柴胡 & Black Thorowax & T1074 Bupleurum smithii \\
\hline HEI CI CI TONG & 黑刺刺桐* & Blackstick Coralbean* & T2470 Erythrina melanacantha \\
\hline HEI DA DOU & 黑大豆 & Black Soyabean & T3000 Glycine max \\
\hline HEI DA DOU PI & 黑大豆皮 & Black Soyabean Spermoderm & T3001 Glycine max \\
\hline HEI DA DOU YE & 黑大豆叶 & Black Soyabean Leaf & T3002 Glycine max \\
\hline HEI FENG TENG & 黑风藤 & Manyflower Fissistimga & T2736 Fissistigma polyanthum \\
\hline HEI GANG LIU & 黑杜柳 & Black Silkvine & T4728 Periploca nigrescens \\
\hline HEI GUO BA QIA & 黑果菝葜 & Blackfruit Greenbrier & T5978 Smilax glauco-china \\
\hline HEI GUO HUANG PI & 黑果黄皮 & Dunn Wampee & T1534 Clausena dunniana \\
\hline HEI GUO QIAN CAO & 黑果茜草 & Blackfruit Madder & T5580 Rubia cordifolia var. pratensis \\
\hline HEI HU TAO & 黑胡桃 & Black Walnut & T3566 Juglans nigra \\
\hline HEI HUA WU GEN TENG & 黑花无根藤* & Blackflower Cassytha* & T1253 Cassytha melantha \\
\hline HEI HUA YAN MING CAO & 黑花延命草 & Blackflawer Rabdosia* & T3531 Isodon trichocarpus \\
\hline HEI JIE & 黑芥 & Black Mustard & T1012 Brassica nigra \\
\hline HEI JIE GENG LAN & 黑桔梗兰* & Black Dianella* & T2140 Dianella nigra \\
\hline HEI JING SHU & 黑荆树 & Wattle & T0024 Acacia mearnsii \\
\hline HEI KE NAN & 黑壳楠 & Largeleaf Spicebush & T3852 Lindera megaphylla \\
\hline HEI KUN BU & 黑昆布 & Tangle Thallus & T2321 Ecklonia kurome \\
\hline HEI MA YI & 黑蚂蚁 & Silky Ant & T2753 Formica fusca \\
\hline HEI MAN & 黑菖 & Regel Threewingnut & T6541 Tripterygium regelii \\
\hline HEI MAO SHI HU & 黑毛石斛 & Blackhair Denrdobium & T2111 Dendrobium williamsonii \\
\hline HEI MU JIN HE HUAN & 黑木金合欢 & Australian Blackwood & T0025 Acacia melanoxylon \\
\hline HEI REN DONG & 黑忍冬 & Black Honeysuckle & T3916 Lonicera nigra \\
\hline HEI SE MI PI KANG & 黑色米皮糠 & Black Rice Spermoderm* & T4547 Oryza sativa cv \\
\hline HEI SHI ER & 黑石耳 & & T2115 Dermatocarpon minitum \\
\hline HEI SHUI CUI QUE & 黑水翠雀 & Potanin Larkspur & T2084 Delphinium potaninii \\
\hline HEI SHUI CUI QUE HUA BIAN & 黑水翠雀花变种* & Potanin Larkspur Variety* & T2085 Delphinium potaninii var. \\
\hline ZHONG & & & jiufengshanense \\
\hline HEI SHUI XIE CAO & 黑水颎草 & Amur Valeriana & T6675 Valeriana amurensis \\
\hline
\end{tabular}




\begin{tabular}{|c|c|}
\hline HEI SHUI YE YING SU & 黑水野罂严 \\
\hline HEI XIAN TIAO TENG HUANG & 黑线条藤黄* \\
\hline HEI YAN SHE GEN CAO & 黑岩蛇根草 \\
\hline HEI ZHI MA & 黑芝麻 \\
\hline HEI ZHONG CAO & 黑种草 \\
\hline HEI ZI LI GUO JI SHENG & 黑紫梨果寄生* \\
\hline HENG GEN FEI CAI & 横根费菜 \\
\hline HENG LI DI ER CAO & 亨利地耳草 \\
\hline HENG ZHOU WU YAO & 衡州乌药 \\
\hline HONG A BU TA CAO & 红阿布塔草 \\
\hline HONG BAI HE MU & 红百合木 \\
\hline HONG BEI SHAN MA GAN & 红背山麻杆 \\
\hline HONG CAO & 荭草 \\
\hline HONG CHAI HU & 红柴胡 \\
\hline HONG CHE ZHOU CAO & 红车轴草 \\
\hline HONG CHUN & 红椿 \\
\hline HONG DA LI HUA & 红大丽花 \\
\hline HONG DOU & 红豆 \\
\hline HONG DOU SHAN & 红豆杉 \\
\hline HONG DU HUO & 红独活 \\
\hline HONG E JI XUE TENG & 红䓯鸡血藤 \\
\hline HONG GEN CAO & 红根草 \\
\hline HONG GUI & 红桧 \\
\hline HONG GUO JIAN MU & 红果樫木 \\
\hline HONG GUO LUO FU MU & 红果萝芙木 \\
\hline HONG HAI JIAO & 红海椒 \\
\hline HONG HAI LAN & 红海榄 \\
\hline HONG HUA & 红花 \\
\hline HONG HUA CAI DOU & 红花菜豆 \\
\hline HONG HUA CHU CHONG JU & 红花除虫菊 \\
\hline HONG HUA JIAO & 红花椒* \\
\hline HONG HUA LONG DAN & 红花龙胆 \\
\hline HONG HUA LU TI CAO & 红花鹿蹄草 \\
\hline HONG HUA LV RONG HAO & 红花绿线蒿 \\
\hline HONG HUA PI & 红桦皮 \\
\hline HONG HUA QI & 红花槭 \\
\hline HONG HUA RUI MU & 红花蒿木 \\
\hline HONG HUA WU WEI ZI & 红花五味子 Z1572 \\
\hline HONG HUA YAN HUANG QI & 红花岩黄芪 \\
\hline HONG HUI XIANG & 红茴香 \\
\hline HONG JI DAN HUA & 红鸡蛋花 \\
\hline HONG JIA MI & 红荚蒾 \\
\hline HONG JIAN QIU LUO & 红剪秋罗 \\
\hline HONG KOU SHUI XIAN & 红口水仙 \\
\hline HONG KUAI ZI & 红筷子 \\
\hline HONG LI & 红栋 \\
\hline HONG MA & 红麻 \\
\hline HONG MAO QI & 红毛七 \\
\hline HONG M & \\
\hline
\end{tabular}

Amur Poppy

Nigroline Garcinia*

Black-rock Ophiorrhiza*

Oriental Sesame (black seed)

Jackinprison

Black-purple Scurrula*

Orange Stonecrop

Henry St.John'swort

Laurelleaf Snailseed

Lantern-tree

Redback Christmashush

Prince's-feather

Red Thorowax

Red Clover

Burma Toon

Cocoxochitl

Hosie Ormosia

Chinese Yew

Red Cowparsnip*

Redcalyx Millettia*

Hispid Sage

Formosan False Cypress

Redfruit Pencilwood

Redfruit Devilpepper

Sweet Pepper

Stylose Mangrove

Safflower

Runner Bean

Pyrethrum

Red Pricklyash*

Redflower Gentian

Redflower Pyrola

Redflower Meconopsis

Japanese White Birch Bark

Red Maple

Redflower Kopsia

Redflower Magnoliavine

Redflower Sweetvetch

Henry Anisetree

Frangipani

Red Viburnum*

Red Campion

Poets Narcissus

Great Willowherb (Firewood)

Spanish Oak

Lanceleaf Dogbane*

Robust Leontice

Girald Acanthopanax Root-bark
T4630 Papaver nudicaule ssp. amurense T2868 Garcinia nigrolineata

T4511 Ophiorrhiza kuroiwai

T5927 Sesamum indicum [Syn. Sesamum orientale]

T4431 Nigella damascena

T5830 Scurrura atropurpurea

T5855 Sedum kamtschaticum

T3354 Hypericum henryi

T1586 Cocculus laurifolius

T0014 Abuta rufescens

T1792 Crinodendron hookerianum

T0297 Alchornea trewioides

T5110 Polygonum orientale

T1072 Bupleurum scorzonerifolium

T6521 Trifolium pratense

T6474 Toona ciliata

T1995 Dahlia coccinea

T4530 Ormosia hosiei

T6310 Taxus chinensis

T3212 Heracleum granatense

T4235 Millettia erythrocalyx

T5687 Salvia prionitis

T1347 Chamaecyparis formosensis

T2308 Dysoxylum binectariferum

T5425 Rauvolfia verticillata f. rubrocarpa

T1186 Capsicum annuum

T5489 Rhizophora stylosa

T1215 Carthamus tinctorius

T4782 Phaseolus multiflorus

T1389 Chrysanthemum coccineum

T6891 Zanthoxylum rubescens

T2927 Gentiana rhodantha

T5349 Pyrola incarnata

T4145 Meconopsis punicea

T0933 Betula platyphylla

T0054 Acer rubrum

T3640 Kopsia fruticosa

T5799 Schisandra rubriflora

T3128 Hedysarum multijugum

T3401 Illicium henryi

T5032 Plumeria rubra

T6736 Viburnum erubescens

T3949 Lychnis dioica

T4378 Narcissus poeticus

T1352 Chamaenerion angustifolium [Syn.

Epilobium angustifolium]

T5378 Quercus rubra

T0552 Apocynum lancifolium

T3746 Leontice robustum

T0036 Acanthopanax giraldii [Syn.

Acanthopanax giraldii var. inermis;

Eleutherococcus giraldii] 


\begin{tabular}{|c|c|c|c|}
\hline HONG MAO YE HAI TANG & 红毛野海棠 & Tuberculate Bredia & T1023 Bredia tuberculata \\
\hline HONG MU & 红木 & Anattotree & T0947 Bixa orellana \\
\hline HONG MU JI CAO & 红母鸡草 & Hookedhairypod Tickclover & T2130 Desmodium gangeticum \\
\hline HONG NAN PI & 红楠皮 & Red Nanmu Bark & T4018 Machilus thunbergii \\
\hline HONG NIANG ZI & 红娘子 & Red Lady-bug & T3287 Huechys sanguinea \\
\hline HONG PI YUN SHAN & 红皮云杉 & Korean Spruce & T4873 Picea koraiensis \\
\hline HONG QI YE SHU & 红七叶树 & Red Horsechestnut & T0200 Aesculus carnea \\
\hline HONG QIE DONG GUO & 红茄苳果 & Mangrove Frult & T5488 Rhizophora mucronata \\
\hline HONG QIU JIANG & 红球姜 & Zerumbet Ginger & T6911 Zingiber zerumbet \\
\hline HONG RU CAO & 红乳草 & Red-milk Grass* & T2623 Euphorbia makinoi \\
\hline HONG SAN QI & 红三七 & Ovateleaf Knotweed & T5118 Polygonum suffultum \\
\hline HONG SE JIN JI NA SHU & 红色金鸡纳树 & Redbark Cinchona & T1433 Cinchona succirubra \\
\hline HONG SHI ER & 红石耳 & Red-Rock-Ears & T6602 Umbilicaria hypococcinea \\
\hline HONG SHI XIAN TAO & 红石仙桃* & Rubi Pholidota* & T4822 Pholidota rubra \\
\hline HONG SHU & 红树 & Sharpleaf Mangrove & T5487 Rhizophora apiculata \\
\hline HONG SHUAN JUN & 红栓菌 & Cinnabar-red & $\begin{array}{l}\text { T6491 Trametes cinnabarina [Syn. Polyporus } \\
\text { cinnabarinus; Boletus cinnabarinus] }\end{array}$ \\
\hline HONG SI XIAN & 红丝线 & Twoflower Red silkyarn & T3953 Lycianthes biflora \\
\hline HONG SONG & 红松 & Korean Pine & T4913 Pinus koraiensis \\
\hline HONG TOU CAO & 红头草 & Malay Blumea & T0959 Blumea lacera \\
\hline HONG WEI SUAN JIAO GAN & 宏伟酸脚杆* & Magnific Medinilla* & T4152 Medinilla magnifica \\
\hline HONG WEN MA XIAN HAO & 红纹马先蒿 & Redstriate Woodbetony & T4684 Pedicularis striata \\
\hline HONG YA DA JI & 红芽大戟 & Red Knoxia & T3632 Knoxia valerianoides \\
\hline HONG YUAN CENG KONG JUN & 红缘层孔菌 & Red Belt Polypore & $\begin{array}{l}\text { T2750 Fomitopsis pinicola }[\text { Syn. Fomes } \\
\text { pinicola; Polyporus pinicola }]\end{array}$ \\
\hline HONG ZE LAN & 红泽兰 & Japanese Conehead & $\begin{array}{l}\text { T6154 Strobilanthes japonicus [Syn. } \\
\text { Championella japonica] }\end{array}$ \\
\hline HONG ZHI ZHANG YA CAI & 红直獐牙菜 & Redspot Swertia & T6219 Swertia erythrosticta \\
\hline HONG ZU HAO & 红足蒿 & Redfoot Wormwood & T0691 Artemisia rubripes \\
\hline HOU GUO DANG GUI & 厚果当归 & Thickfruit Angelica* & T0490 Angelica pachycarpa \\
\hline HOU KE GUI & 厚壳桂 & Chinese Cryptocarya & T1862 Cryptocarya chinensis \\
\hline HOU PI HUA JIAO & 厚皮花椒 & Thickbark Pricklyash* & T6879 Zanthoxylum elephantiasis \\
\hline HOU PI SHU & 厚皮树 & Coromandel Lannea & $\begin{array}{l}\text { T3685 Lannea grandis [Syn. Lannea } \\
\text { coromandelica] }\end{array}$ \\
\hline HOU PO & 厚朴 & Officinal Magnolia & T4045 Magnolia officinalis \\
\hline HOU SHU SHAN GU & 后熟扇菇* & Mukitake (in Japanese) & T4617 Panellus serotinus \\
\hline HOU TOU JUN & 猴头菌 & Bearded Tooth Carpophore & $\begin{array}{l}\text { T3229 Hericium erinaceus }[\text { Syn. Hydnum } \\
\text { erinaceus] }\end{array}$ \\
\hline HOU WANG ZAO & 厚网藻 & & T4570 Pachydictyon coriaceum \\
\hline HOU YE GOU TENG & 厚叶钩藤* & Thickleaf Gambirplant* & T6610 Uncaria callophylla \\
\hline HOU YE HUI MAO DOU & 厚叶灰毛豆* & Crassleaf Tephrosia* & T6333 Tephrosia crassifolia \\
\hline HOU YE YAN BAI CAI & 厚叶岩白菜 & Thickleaf Bergenia & T0923 Bergenia crassifolia \\
\hline HU BAO TAI & 恶苍苔 & Blasia* & T0949 Blasia pusilla \\
\hline HU BEI BEI MU & 湖北贝母 & Hupeh Fritillary & T2787 Fritillaria hupehensis \\
\hline HU BEI GUA LOU & 湖北栝楼 & Hupeh Snakegourd & T6509 Trichosanthes hupehensis \\
\hline HU BEI HUANG JING & 湖北黄精 & Hubei Landpick & T5096 Polygonatum zanlanscianense \\
\hline HU BEI SHAN MAI DONG & 湖北山麦冬 & Hubei Liriope & T3875 Liriope spicata var. prolifera \\
\hline HU BEI SHAN ZHA & 湖北山楂 & Hupeh Hawthorn & T1770 Crataegus hupehensis \\
\hline HU BEI SHI DA GONG LAO & 湖北十大功劳 & Confused Mahonia & T4060 Mahonia confusa \\
\hline $\mathrm{HU}$ CI & 虎刺 & Indian Damnacanthus & T2020 Damnacanthus indicus \\
\hline HU CI CONG MU & 虎刺淴木 & Spine Aralia & T0567 Aralia armata \\
\hline HU CONG & 胡葱 & Shallot & T0310 Allium ascalonicum \\
\hline HU DIE HUA & 蝴蝶花 & Fringed Iris & T3461 Iris japonica \\
\hline
\end{tabular}




\begin{tabular}{|c|c|}
\hline HU DIE HUA DOU & 蝴蝶花豆 \\
\hline HU ER CAO & 虎耳草 \\
\hline HU ER CAO YE HUI QIN & 虎耳草叶茴芹 \\
\hline HU GUA & 玈瓜 \\
\hline HU HUANG LIAN & 胡黄连 \\
\hline HU JI SHENG & 槲寄生 \\
\hline HU JIAO & 胡椒 \\
\hline HU JIAO HUA JIAO & 胡椒花椒 \\
\hline HU KE HUANG GUA & 虎克黄瓜* \\
\hline HU LI & 槲栋 \\
\hline HU LU BA & 胡卢巴 \\
\hline HU LU CHA & 葫芦茶 \\
\hline HU LU QI & 葫芦七 \\
\hline HU LU QI BIAN ZHONG & 葫芦七变种* \\
\hline HU LUO BO & 胡萝卜 \\
\hline HU LUO BO ZI & 胡萝卜子 \\
\hline HU MA GEN & 胡麻根 \\
\hline HU MA YE & 胡麻叶 \\
\hline HU SHE HONG & 虎舌红 \\
\hline HU SHENG HONG JING TIAN & 互生红景天 \\
\hline HU SHENG YE BAI QIAN CENG & 互生叶白千层 \\
\hline HU SHENG YE YE JUE MING & 互生叶野决明 \\
\hline HU SUI ZI & 胡荌子 \\
\hline HU TAO QING PI & 胡桃青皮 \\
\hline HU TAO QIU & 胡桃楸 \\
\hline HU TAO REN & 胡桃仁 \\
\hline HU TAO SHU PI & 胡桃树皮 \\
\hline HU TAO YE & 胡桃叶 \\
\hline HU WEI LAN & 虎尾兰 \\
\hline HU YAN WAN NIAN QING & 虎眼万年青 \\
\hline HU YE & 檞叶 \\
\hline HU ZHANG & 虎杖 \\
\hline HU ZHANG CAO & 虎掌草 \\
\hline HU ZHANG YE & 虎杖叶 \\
\hline HU ZHI ZI & 胡枝子 \\
\hline HUA BAI LA SHU & 花白蜡树 \\
\hline HUA BAN SHI ZU CAO & 花瓣狮足草 \\
\hline HUA BEI BAI QIAN & 华北白前 \\
\hline HUA BEI LAN PEN HUA & 华北蓝盆花 \\
\hline HUA CHA BIAO & 华茶蔍 \\
\hline HUA CHONG LOU & 华重楼 \\
\hline HUA DONG DUAN & 华东椴 \\
\hline HUA DONG LAN CI TOU & 华东蓝刺头 \\
\hline HUA DONG TANG SONG CAO & 华东唐松草 \\
\hline HUA GENG LONG DAN & 花梗龙胆 \\
\hline HUA GOU TENG & 华钩藤 \\
\hline HUA GUAN YIN LIAN HUA & 花冠银莲花 \\
\hline HUA HE KONG JUN & 桦褐孔菌 \\
\hline HUA JIAO & 花椒 \\
\hline HUA JIAO GEN & 花椒根 \\
\hline HUA JIAO LE & 花椒簕 \\
\hline HUA JIN DAN & 化金丹 \\
\hline
\end{tabular}

Asian Pigeonwings
Creeping Rockfoil
Burnet-saxifrage
Bottle Gourd
Picrorhiza
Colored Mistletoe
Black Pepper
Japanese Pricklyash
Hooker Cucumber*
Peking Oak
Common Fenugreek
Triquetrous Tadehagi
Kidneyleaf Goldenray
Kidneyleaf Goldenray Variety*
Carrot
Carrot Seed
Oriental Sesame Root
Oriental Sesame Leaf
Teat-shaped Ardisia

Oppositeleaf Rhodiola

Alternateleaf Melaleuca*

Alternateleaf Thermopsis*

Coriander Seed

English Walnut Exocarp

Manchurian Walnet

English Walnut Seed

English Walnut Bark

English Walnut Leaf

Snake Sansevieria

Whiplash Star-of-Bethlehem

Daimyo Oak Leaf

Japanese Fleeceflower

Brooklet Anemone

Japanese Fleeceflower Leaf

Shrub Lespedeza

Flowering Ash

Leontice*

Hancock Swallowwort

Huapei Scabious

China Winterberry Currant

China Paris

Japanese Linden

East China Globethistle

Fortune Meadowrue

Pedicellate Gentian

Chinese Gambirplant

St. Brigid

Oblique Fuscoporia*

Bunge Pricklyash

Bunge Pricklyash Root

Cuspidate Pricklyash

Tetragonal Crotalaria
T1577 Clitoria ternatea

T5773 Saxifraga stolonifera

T4900 Pimpinella saxifraga

T3668 Lagenaria siceraria var. depressa

T4887 Picrorhiza kurrooa

T6775 Viscum coloratum

T4957 Piper nigrum

T6886 Zanthoxylum piperitum

T1874 Cucumis hookeri

T5371 Quercus aliena

T6528 Trigonella foenum-graecum

T6277 Tadehagi triquetrum

T3805 Ligularia fischeri

T3806 Ligularia fischeri var. spiciformis

T2050 Daucus carota var. sativa

T2051 Daucus carota var. sativa

T5923 Sesamum indicum

T5924 Sesamum indicum

T0600 Ardisia mamillata [Syn. Tinus

mamillata]

T5500 Rhodiola subopposita

T4153 Melaleuca alternifolia

T6425 Thermopsis alternifolia

T1687 Coriandrum sativum

T3567 Juglans regia

T3564 Juglans mandshurica

T3568 Juglans regia

T3569 Juglans regia

T3570 Juglans regia

T5714 Sansevieria trifasciata

T4534 Ornithogalum caudatum

T5372 Quercus dentata

T5101 Polygonum cuspidatum

T0471 Anemone rivularis

T5102 Polygonum cuspidatum

T3769 Lespedeza bicolor

T2773 Fraxinus ornus

T3745 Leontice leontopetalum

T1955 Cynanchum hancockianum

T5778 Scabiosa tschiliensis

T5545 Ribes fasciculatum var. chinense

T4649 Paris polyphylla var. chinensis

T6457 Tilia japonica

T2316 Echinops grijsii

T6388 Thalictrum fortunei

T2925 Gentiana pedicellata

T6633 Uncaria sinensis

T0466 Anemone coronaria

T2818 Fuscoporia obliqua

T6869 Zanthoxylum bungeanum

T6870 Zanthoxylum bungeanum

T6872 Zanthoxylum cuspidatum

T1836 Crotalaria tetragona 


\begin{tabular}{|c|c|}
\hline HUA LAI CI SHU & 华来刺树 \\
\hline HUA LI SHE BIAN JU & 华丽蛇鞭菊 \\
\hline HUA LING CAO & 花菱草 \\
\hline HUA MAO & 花针 \\
\hline HUA MU PI & 桦木皮 \\
\hline HUA NAN GONG LAO MU & 华南功劳木 \\
\hline HUA NAN GONG LAO YE & 华南功劳叶 \\
\hline HUA NAN GONG LAO ZI & 华南功劳子 \\
\hline HUA NAN HE SHI & 华南鹤虫 \\
\hline HUA NAN MA WEI SHAN & 华南马尾杉 \\
\hline HUA NAN MI HOU TAO & 华南猕猴桃 \\
\hline HUA NAN REN DONG & 华南忍冬(山银花) \\
\hline HUA NAN WU ZHU YU & 华南吴茱英 \\
\hline HUA NAN ZAO JIA & 华南㿝荚 \\
\hline HUA SANG & 华桑 \\
\hline HUA SHAN FAN & 华山矾 \\
\hline HUA SHAN SONG & 华山松 \\
\hline HUA TANG SONG CAO & 花唐松草 \\
\hline HUA TAO SHU & 滑桃树 \\
\hline HUA XI BEI MU & 华西贝母 \\
\hline HUA XIANG SHU YE & 化香树叶 \\
\hline HUA XU GENG BAI MAI GEN & 花序梗百脉根* \\
\hline HUA YE CAI & 花椰菜 \\
\hline HUA YE JIA DU JUAN & 花叶假杜鹃 \\
\hline HUA ZE LAN & 华泽兰 \\
\hline HUA ZHONG LIU ZU JUE & 华中瘤足蕨 \\
\hline HUA ZHONG WU WEI ZI & 华中五味子 \\
\hline HUA ZHOU YOU & 化州柚 \\
\hline HUA ZI JIN & 华紫堇 \\
\hline HUAI & 槐 \\
\hline HUAI GEN & 槐根 \\
\hline HUAI JIAO & 槐角 \\
\hline HUAI TONG & 淮通 \\
\hline HUAN JIE SONG LUO & 环节松萝 \\
\hline HUAN YANG SHEN YE TANG & 还阳参叶糖芥 \\
\hline JIE & \\
\hline HUAN ZHUANG JIN SI TAO & 环状金丝桃 \\
\hline HUANG BAI & 黄柏(黄檗) \\
\hline HUANG BAI HE & 黄百合 \\
\hline HUANG BAI HONG GU & 黄白红菇 \\
\hline HUANG BAI TANG JIE & 黄白糖芥 \\
\hline HUANG BIAN BAI & 黄扁柏 \\
\hline HUANG CHAN & 黄蝉 \\
\hline HUANG CHANG PU & 黄菖蒲 \\
\hline HUANG CHONG & 蝗虫 \\
\hline HUANG GAN CAO & 黄甘草 \\
\hline HUANG GEN JIANG HUANG & 黄根姜黄* \\
\hline HUANG GEN SHU & 黄根树 \\
\hline HUANG GUA & 黄瓜 \\
\hline HUANG GUO QIE & 黄果茄 \\
\hline HUANG HAI TANG & 黄海棠 \\
\hline HUANG HAI YING SU & 黄海罂粟* \\
\hline HUANG HAO & 黄蒿 \\
\hline
\end{tabular}

Fouquieria splendens

Pinkscale Gay-feather

California Poppy

Corniculate Spurgentian

Asian White Birch Bark

Japanese Mahonia

Japanese Mahonia Leaf

Japanese Mahonia Fruit

Japanese Hedgeparsley

Fordi Phlegmariurus

Greyleaf Actinidia

Wild Honeysuckle

South China Evodia

South China Honeyloeust

Chinese Mulberry

Chinese Sweetleaf

Armand Pine

Filamentary Meadowrue

Huaxi Fritillary

Dyetree Leaf

Greater Bird's-foot-trefoil

Cauliflower

Chinese Eupatorium

Fine-nerved Plagiogyria

Orange Magnoliavine

Tomentase Pummelo

Chinese Corydalis

Japanese Pagodatree

Japanese Pagodatree Root

Japanese Pagodatree Fruit

Moupin Dutchmanspipe

Diffract Usnea Filament

Crepinleaf Erysimum*

Circularity St.John'swort*

Amur Corktree

Hanson Lily

Pale Russula*

Yellowish Erysimum*

Yellow Cedar

Oleanderleaf Allemanda

Yellowflag Iris

Grasshopper

Yellow Licorice

Xanthorrhiza Turmeric*

Yellowroot

Cucumber

Yellowfruit Nightshade

Giant St.John'swort

Yellow Hornpoppy*

Virgate Wormwood
T2762 Fouquieria splendens

T3788 Liatris elegans

T2495 Eschscholzia californica

T3092 Halenia corniculata

T0935 Betula platyphylla var. japonica

T4066 Mahonia japonica

T4067 Mahonia japonica

T4068 Mahonia japonica

T6476 Torilis japonica

T4800 Phlegmariurus fordii

T0162 Actinidia glaucophylla

T3909 Lonicera confusa

T2638 Evodia austrosinensis

T2976 Gleditsia fera

T4293 Morus cathayana

T6252 Symplocos chinensis

T4906 Pinus armandii

T6384 Thalictrum filamentosum

T6495 Trewin nudiflora

T2794 Fritillaria siechuanica

T5010 Platycarya strobilacea

T3930 Lotus pedunculatus

T1014 Brassica oleracea var. botrytis

T0871 Barleria lupulina

T2555 Eupatorium chinense

T4998 Plagiogyria euphlebia

T5802 Schisandra sphenanthera

T1483 Citrus grandis var. tomentosa

T1713 Corydalis cheilanthifolia

T6034 Sophora japonica

T6035 Sophora japonica

T6036 Sophora japonica

T0635 Aristolochia moupinensis

T6653 Usnea diffracta

T2453 Erysimum crepidifolium

T3339 Hypericum annulatum

T4789 Phellodendron amurense

T3834 Lilium hansonii

T5622 Russula ochroleuca

T2455 Erysimum ochroleucum

T1348 Chamaecyparis nootkatensis

T0308 Allemanda neriifolia

T3467 Iris pseudacorus

T5559 Romalea microptera

T3016 Glycyrrhiza kansuensis

T1908 Curcuma xanthorrhiza

T6846 Xanthorhiza simplicissima

T1878 Cucumis sativus

T6020 Solanum xanthocarpum

T3340 Hypericum ascyron

T2969 Glaucium flavum

T0695 Artemisia scoparia [Syn. Artemisia 


\begin{tabular}{|c|c|}
\hline HUANG HE MAO REN DONG & 黄褐毛忍冬 \\
\hline HUANG HONG SE RUI MU & 黄红色蕊木* \\
\hline HUANG HUA BAI JIANG & 黄花败酱 \\
\hline HUANG HUA CAI & 黄花菜 \\
\hline HUANG HUA CAO & 黄花草 \\
\hline HUANG HUA DI DING & 黄花地丁 \\
\hline HUANG HUA ER LIU & 黄花儿柳 \\
\hline HUANG HUA HAI YING SU & 黄花海罂粟 \\
\hline HUANG HUA HAO & 黄花蒿 \\
\hline HUANG HUA JIA ZHU TAO & 黄花夹竹桃 \\
\hline HUANG HUA JIU LUN CAO & 黄花九轮草 \\
\hline HUANG HUA MU & 黄花木 \\
\hline HUANG HUA REN & 黄花稔 \\
\hline HUANG HUA SHU WEI CAO & 黄花鼠尾草 \\
\hline HUANG HUA WU TOU & 黄花乌头关白阤 \\
\hline HUANG HUA XIANG CHA CAI & 黄花香茶菜 \\
\hline HUANG HUA YAN CAO & 黄花烟草 \\
\hline HUANG HUA YUAN ZHI & 黄花远志 \\
\hline HUANG HUA ZI & 黄花仔 \\
\hline HUANG JIE GU DAN & 黄接骨丹 \\
\hline HUANG JIN & 黄槿 \\
\hline HUANG JIN FENG & 黄金凤 \\
\hline HUANG JIN GU CAO & 黃筋骨草* \\
\hline HUANG JIN SHU & 黄金树 \\
\hline HUANG JING & 黄精 \\
\hline HUANG JING GEN & 黄荆根 \\
\hline HUANG JING YE & 黄荆叶 \\
\hline HUANG JING ZHONG ZI & 黄荆种子 \\
\hline HUANG KUI & 黄葵 \\
\hline HUANG LIAN & 黄连 \\
\hline HUANG LIAN HUA & 黄莲花 \\
\hline HUANG LIAN YA & 黄练芽 \\
\hline HUANG LONG DAN & 黄龙胆 \\
\hline HUANG LU & 黄栌 \\
\hline HUANG LU ZHI YE & 黄栌枝叶 \\
\hline HUANG MA YE & 黄麻叶 \\
\hline HUANG MA ZI & 黄麻子 \\
\hline HUANG MAO CONG MU & 黄毛淴木 \\
\hline HUANG MAO GE & 黄毛葛 \\
\hline HUANG MAO LIN MAO JUE & 黄毛鳞毛蕨 \\
\hline HUANG MAO WU TOU & 黄毛乌头 \\
\hline HUANG MIAN GUI & 黄缅桂 \\
\hline HUANG MING JIAO & 黄明胶 \\
\hline HUANG NIU MU & 黄牛木 \\
\hline HUANG PI GEN & 黄皮根 \\
\hline HUANG PI SHU & 黄皮树 \\
\hline HUANG PI YE & 黄皮叶 \\
\hline HUANG PING ZI CAO & 黄瓶子草 \\
\hline
\end{tabular}

Yellowhair Honeysuckle
Yellow-red Kopsia*
Dahurian Patrinia
Citron Daylily
Yellowflower Spiderflower*
Diluteyellow Crotalaria
Goat Willow
Yellowflower Hornpoppy*
Sweet Wormwood
Yellow Oleander
Officinal Primrose*
Greenleaf Piptanthus, Evergreen
Laburnum
Acute Sida
Yellowflower Sage
Korean Monkshood
Yellowflower Rabdosia

Aztec Tobacco

Yellowflower Milkwort

Cordateleaf Sida

Gypsophila

Linden Hibiscus

Incurvedspur Snapweed

Yellow Bugle

Northern Catalpa

Siberian Solomonseal

Negundo Chastetree Root

Negundo Chastetree Leaf

Negundo Chastetree Seed*

Musk-mallow

Chinese Goldthread
Dahurian Loosestrife
Chinese Pistache
Yellow Gentian
Common Smoketree
Common Smoketree Branch-leaf
Roundpod Jute Leaf
Roundpod Jute Seed
Yellowhair Aralia
Yellow-hairy Calyx Kudzuvine
Goldencoma Shield Fern
Yellowhair Monkshood
Champac Michelia
Oxhide Gelatin
Common Oxwood
Chinese Wampee Root
Chinese Corktree
Chinese Wampee Leaf
Yellow Pitcherplant

capillaris var. scoparia]

T3910 Lonicera fulvotomentosa

T3639 Kopsia flavida

T4672 Patrinia scabiosaefolia

T3192 Hemerocallis citrina

T1552 Cleome viscosa

T1813 Crotalaria albida

T5652 Salix caprea

T2967 Glaucium davum

T0660 Artemisia annua

T6433 Thevetia neriifolia [Syn. Thevetia peruviana]

T5204 Primula veris [Syn. Primula officinalis]

T4976 Piptanthus nepalensis

T5942 Sida acuta

T5672 Salvia flava

T0088 Aconitum coreanum

T3524 Isodon sculponeata [Syn. Rabdosia

sculponeata]

T4427 Nicotiana rustica

T5071 Polygala arillata

T5943 Sida cordifolia

T3089 Gypsophila acutifolia

T3248 Hibiscus tiliaceus

T3414 Impatiens siculifer

T0261 Ajuga chamaepitys

T1265 Catalpa speciosa

T5095 Polygonatum sibiricum

T6787 Vitex negundo

T6788 Vitex negundo

T6789 Vitex negundo

T0002 Abelmoschus moschatus [Syn. Hibiscus

abelmoschus]

T1662 Coptis chinensis

T4001 Lysimachia davurica

T4979 Pistacia chinensis

T2918 Gentiana lutea

T1758 Cotinus coggygria

T1759 Cotinus coggygria var. cinerea

T1673 Corchorus capsularis

T1674 Corchorus capsularis

T0571 Aralia decaisneaa

T5311 Pueraria calycina

T2280 Dryopteris chrysocoma

T0087 Aconitum chrysotrichum

T4210 Michelia champaca

T0982 Bos taurus domesticus

T1784 Cratoxylum cochinchinense

T1537 Clausena lansium

T4791 Phellodendron chinense

T1538 Clausena lansium

T5742 Sarracenia flava 


\begin{tabular}{|c|c|}
\hline HUANG QI & 黄芪(膜荚黄芪) \\
\hline HUANG QI II & 黄杞 \\
\hline HUANG QIAO RUI HUA & 黄鞘荵花 \\
\hline HUANG QIN & 黄芩 \\
\hline HUANG QIN JIAO & 黄秦艽 \\
\hline HUANG SAI JIN LIAN MU & 黄赛金莲木* \\
\hline HUANG SAN QI & 黄三七 \\
\hline HUANG SE AO DING CAO & 黄色凹顶藻 \\
\hline HUANG SE BAN RUI DOU & 黄色瓣芯豆 \\
\hline HUANG SE QIAN LI GUANG & 黄色千里光* \\
\hline HUANG SHAN & 黄杉 \\
\hline HUANG SHAN YAO & 黄山药 \\
\hline HUANG SHU KUI HUA & 黄蜀葵花 \\
\hline HUANG SHUI JING LAN & 黄水晶兰 \\
\hline HUANG SHUI XIAN & 黄水仙 \\
\hline HUANG SI TAN BAO & 黄斯坦堡 \\
\hline HUANG TANG SONG CAO & 黄唐松草 \\
\hline HUANG WAN & 黄苑 \\
\hline HUANG WEI & 黄薇 \\
\hline HUANG WU TOU & 黄乌头* \\
\hline HUANG XI XIN & 黄细心 \\
\hline HUANG XIAO BO & 黄小檗 \\
\hline HUANG XIN HUA JIAO & 黄心花椒 \\
\hline HUANG YAN MU & 黄颜木 \\
\hline HUANG YANG MU YE & 黄杨木叶 \\
\hline HUANG YANG XIAO BO & 黄杨小檗 \\
\hline HUANG YANG YE DUI CI TENG & 黄杨叶对刺藤* \\
\hline HUANG YANG YE MU BAN SHU & 黄杨叶木瓣树 \\
\hline HUANG YAO & 黄药 \\
\hline HUANG YAO ZI & 黄药子 \\
\hline HUANG YE DI BU RONG & 黄叶地不容 \\
\hline HUANG YE DU XING CAI & 荒野独行菜 \\
\hline HUANG YE HUAI & 黄叶槐 \\
\hline HUANG YING PI MA BO & 黄硬皮马勃 \\
\hline HUANG YU SHAN DOU & 黄羽扇豆 \\
\hline HUANG ZHONG HUA & 黄钟花 \\
\hline HUANG ZI JIN & 黄紫堇 \\
\hline HUI BA QIA & 灰菝荰 \\
\hline HUI BAI DU HUO & 灰白独活* \\
\hline HUI BAI KU MA DOU & 灰白苦马豆* \\
\hline HUI BAI MAO LUO FU MU & 灰白毛萝芙木 \\
\hline HUI BAI QIE & 灰白茄* \\
\hline HUI BAI SHI CAN & 灰白石蚕* \\
\hline HUI BAI XIANG RI KUI & 灰白向日葵* \\
\hline HUI BAI XIE HAO & 灰白邪槁* \\
\hline HUI BAI YI MU CAO & 灰白益母草 \\
\hline HUI BAO HAO & 灰苞蒿 \\
\hline HUI CI TONG & 灰刺桐 \\
\hline HUI GUO JIAO GU LAN & 㗒果绞股蓝 \\
\hline HUI HAO & 蛔蒿 \\
\hline HUI HU TAO & 灰胡桃 \\
\hline HUI HUANG HUA & 灰黄华* \\
\hline
\end{tabular}

\author{
Membranous Milkvetch \\ R oxburgh Engelhardtia Root \\ Yellowflower Coleus \\ Baikal Skullcap \\ Baillon Veratrilla \\ Yellow Ouratea* \\ Common Souliea \\ Yellow Concave-top Alga* \\ Yellow Prairie-clover \\ Yellow Groundsel* \\ China Douglas Fir \\ Yellow Yam \\ Setose Abelmoschus \\ Yellow Bird's-nest \\ Daffodil \\ Winter Daffodil \\ Maidenhair Meadowrue \\ Shady Groundsel \\ Myrtleleaf Heimia \\ Yellow Monkshood \\ Diffuse Boerhavia \\ Yellow Barberry \\ Yellow-heart Pricklyash
}

Chinese Box Juvenile Leaf

Box-leaved Barberry

Boxleaf Scutia*

Boxleaf Xylopia*

Oriental Buckthorn

Airpotato Yam

Greenyellow Stephania

Field Pepperwort

Yellowleaf Sophora

Yellow Hardpeel Puff-ball*

Yellow Lupin

Florida Yellowtrumpet

Ochotsk Corydalis

Grey Greenbrier*

Canescent Cowparsnip*

Canescent Swainsonia*

Canescent Devilpepper*

Greywhite Nightshade*

Narrowleaf Germander

Canescent Sunflower*

Greywhite Seseli*

Glaucescent Motherwort

Roxburgh Wormwood

Grey Coralbean*

Rostratefruit Gynostemma

Chinese Seriphidium

Butternut

Cinereous Thermopsis*
T0798 Astragalus membranaceus

T2360 Engelhardia roxburghiana

T1621 Coleus xanthanthus

T5834 Scutellaria baicalensis

T6691 Veratrilla baillonii

T4560 Ouratea flava

T6060 Souliea vaginata

T3721 Laurencia nipponica

T4737 Petalostemum purpureum

T5888 Senecio flavus

T5261 Pseudotsuga sinensis

T2205 Dioscorea panthaica

T0001 Abelmoschus manihot

T4275 Monotropa hypopitys

T4379 Narcissus pseudonarcissus

T6144 Sternbergia lutea

T6385 Thalictrum flavum

T5892 Senecio nemorensis

T3130 Heimia myrtifolia

T0076 Aconitum anthora

T0969 Boerhavia diffusa

T0917 Berberis tschonoskiana

T6880 Zanthoxylum flavum

T1378 Chlorophora tinctoria

T1092 Buxus microphylla var. sinica

T0899 Berberis buxifolia

T5846 Scutia buxifolia

T6851 Xylopia buxifolia

T5456 Rhamnus crenatus

T2191 Dioscorea bulbifera

T6137 Stephania viridiflavens

T3756 Lepidium campestre

T6030 Sophora chrysophylla

T5814 Scleroderma citrinum

T3943 Lupinus luteus

T6324 Tecoma stans

T1728 Corydalis ochotensis

T5973 Smilax aristolochiaefolia

T3211 Heracleum canescens

T6207 Swainsonia canescens

T5431 Rauwolfia canescens

T5999 Solanum incanum

T6366 Teucrium polium

T3147 Helianthus canescens

T5931 Seseli incanum

T3751 Leonurus glaucescens

T0690 Artemisia roxbugiana

T2466 Erythrina glauca

T3086 Gynostemma yixingense

T5920 Seriphidium cinum [Syn. Artemisia

cina]

T3563 Juglans cinerea

T6427 Thermopsis cinerea 


\begin{tabular}{|c|c|c|c|}
\hline HUI HUI DOU & 回回豆 & Gram Chickpea & T1412 Cicer arietinum \\
\hline HUI HUI SU & 回回苏 & Crisped Common Perilla & T4722 Perilla frutescens var. crispa \\
\hline HUI HUI SU GENG & 回回苏梗 & Crisped Common Perilla Stem & T4723 Perilla frutescens var. crispa \\
\hline HUI JIN SE TUAN WANG JUN & 灰烬色团网菌* & Grey Arcyria* & T0589 Arcyria cinerea \\
\hline HUI LV YAN HU SUO & 灰绿延胡索 & Greyish-green Corydalis & T1705 Corydalis adunca \\
\hline HUI MAO DANG SHEN & 灰毛党参 & Greyhair Asiabell & T1596 Codonopsis canescens \\
\hline HUI QIN & 茴芹 & Anise & T4895 Pimpinella anisum \\
\hline HUI SE OU SHI NAN & 灰色欧石南 & Bell Heather & T2417 Erica cinerea \\
\hline HUI TE KE MAO GAO CAI & 惠特克茅膏菜* & Whittaker Sundew* & T2270 Drosera whittakeri \\
\hline HUI XIANG & 茴香 & Fennel Fruit & T2744 Foeniculum vulgare \\
\hline HUI XIANG GEN & 茴香根 & Fennel Root & T2745 Foeniculum vulgare \\
\hline HUI XIANG JING YE & 茴香茎叶 & Fennel Stem and Leaf & T2746 Foeniculum vulgare \\
\hline $\mathrm{HUI}^{(4)} \mathrm{YE}$ & 桧叶 & Chinese Juniper Leaf & T5987 Sobina chinensis \\
\hline HUI YE & 灰叶 & Purple Tephrosia & T6338 Tephrosia purpurea \\
\hline HUI YE DU JING SHAN & 灰叶杜茎山 & Greyleaf Maesa & T4027 Maesa chisia \\
\hline HUI YE GEN & 灰叶根 & Purple Tephrosia Root & T6339 Tephrosia purpurea \\
\hline HUI YE YAN CAO & 灰叶烟草 & Leadwordleaf Tobacco & T4426 Nicotiana plumbaginifolia \\
\hline HUI ZHAN MAO REN DONG & 灰毡毛忍冬 & Largeflower-like Honeysuckle & T3914 Lonicera macranthoides \\
\hline HUI ZHU NIU NAI CAI & 嚎柱牛奶菜 & Beakstyle Condorvine & T4118 Marsdenia oreophila \\
\hline HUI ZHUANG JIU JIE & 喙状九节* & Rostral Ninenode* & T5278 Psychotria rostrata \\
\hline HUN TOU JI & 昏头鸡 & Fortune's Holly Fern & T1986 Cyrtomium fortunei \\
\hline HUN XIAO MO YAO & 混淆没药* & Confuse Myrrhtree* & T1634 Commiphora confusa \\
\hline HUO MA REN & 火麻仁 & Hemp Fimble Seed & T1173 Cannabis sativa \\
\hline HUO SHAN YAN CAO HU JIAO & 火山岩草胡椒 & Volcanic Peperomia* & T4708 Peperomia vulcanica \\
\hline HUO SUO MA & 火索麻 & Tortedfruit Screwtree & $\mathrm{T} 3164$ Helicteres isora \\
\hline HUO TAN MU CAO & 火炭母草 & Chinese Knotweed & T5100 Polygonum chinense \\
\hline HUO XIANG & 藿香 & Wrinkled Gianthyssop & T0212 Agastache rugosus \\
\hline HUO XIANG YE LV RONG HAO & 藿香叶绿绒蒿 & Betonyleaf Meconopsis & T4141 Meconopsis betonicifolia \\
\hline HUO YAN HUA & 火焰花 & Curvedflower Phlogacanthus & T4803 Phlogacanthus curviflorus \\
\hline HUO YANG LE & 火秧簕 & Ancients Euphorbia & T2580 Euphorbia antiquorum \\
\hline IN DU TIE XIAN ZI & & Indian Balata* & T4105 Manilkara indica \\
\hline JI BEI & 吉贝 & Kapok Ceiba & T1284 Ceiba pentandra \\
\hline JI CAI & 荠菜 & Shepherdspurse & T1184 Capsella bursa-pastoris \\
\hline JI CAI ZI & 荠菜子 & Shepherdspurse Seed & T1185 Capsella bursa-pastoris \\
\hline JI CHA KAI LA RUI A & 极叉开拉瑞阿 & Spreading Creosote-bush & T3693 Larrea divaricata \\
\hline JI CHANG LANG DU & 鸡肠狼毒 & Leafy Euphorbia & T2585 Euphorbia esula \\
\hline JI DAN GUO & 鸡蛋果 & Passionfruit & T4665 Passiflora edulis \\
\hline JI DAN SHEN & 鸡蛋参 & Convolvulate Asiabell & T1598 Codonopsis convolvulacea \\
\hline JI GAN CAO & 棘甘草* & Echinate Licorice* & T3012 Glycyrrhiza echinata \\
\hline JI GU CAO & 鸡骨草 & Canton Abrus & $\begin{array}{l}\text { T0010 Abrus fruticulosus [Syn. Abrus } \\
\text { cantoniensis] }\end{array}$ \\
\hline JI GUAN CI TONG & 鸡冠刺桐 & Folkers Coralbean* & T2463 Erythrina crysragalli \\
\hline JI GUAN HUA & 鸡冠花 & Common Cockscomb Flower & T1299 Celosia cristata \\
\hline JI GUAN ZI & 鸡冠子 & Common Cockscomb Seed & T1297 Celosia cristada \\
\hline JI JI & 及己 & Serrate Chloranthus & T1372 Chloranthus serratus \\
\hline JI JI JING YE & 及己茎叶 & Serrate Chloranthus Stem-leaf & T1373 Chloranthus serratus \\
\hline JI JI QIN & 菆夰芹 & Heart-leaved Alexanders & T6913 Zizia aptera \\
\hline JI JIAN DAN QING MEI & 极简单青霉* & & T4699 Penicillium simplicissimum \\
\hline JI JIAN LONG SHE LAN & 极坚龙舌兰* & Very-hard Agave* & T0224 Agave rigidissima \\
\hline JI JIAN XIU XIAN JU & 急尖绣线菊 & Acute Spiraea & T6081 Spiraea japonica var. acuta \\
\hline JI JIAO SHEN & 鸡脚参 & Common Javatea & $\begin{array}{l}\text { T4542 Orthosiphon wulfenioides [Syn. Coleus } \\
\text { wulfenioides] }\end{array}$ \\
\hline JI JUE & 姬㛶 & Punctate Flakelet Fern & T3376 Hypolepis punctata [Syn. Polypodium \\
\hline
\end{tabular}




\begin{tabular}{|c|c|c|}
\hline JI KUAN CI TONG & 极宽刺桐* & Extreme-wide Coralbean* \\
\hline JI LI GEN & 薮藜根 & Puncturevine Caltrap Root \\
\hline JI LI MIAO & 薮藜苗 & Puncturevine Caltrap Shoot* \\
\hline JI LIN WU TOU & 吉林乌头 & Kirin Monkshood* \\
\hline JI LIN YAN LING CAO & 吉林延龄草 & Whiteflower Trillium* \\
\hline JI MAO CAI & 鸡毛菜 & Chicken-feather Vegatable* \\
\hline JI MAO SONG & 鸡毛松 & Imbricate Podocarpus \\
\hline JI MEI YUN SHI & 极美云实* & Prettiest Caesalpinia \\
\hline JI MU & 松木 & Chinese Loropetalum \\
\hline JI NAO & 鸡脑 & Chicken Brain \\
\hline JI NEI JIN & 鸡内金 & Chicken's Gizzard Endothelium \\
\hline JI NEI YA BAN JIU JU & 几内亚斑加菊 & Guinea Bitterleaf* \\
\hline JI NEI YA GE MU & 几内亚格木 & Red-water Tree \\
\hline JI NEI YA HU JIAO & 几内亚胡椒 & Ashanti Pepper \\
\hline JI NING & 荠苧 & Largeserrate Mosla \\
\hline JI ROU & 鸡肉 & Chicken \\
\hline JI RUAN RONG TAI & 极软线苔* & \\
\hline JI SHI HONG JING TIAN & 吉氏红景天 & Algid Rhodiola* \\
\hline JI SHI LU HUI & 基氏芦荟* & Gililand Aloe* \\
\hline JI SHI TENG & 鸡屎藤 & Fevervine \\
\hline JI SHI TENG GUO & 鸡屎藤果 & Fevervine Fruit \\
\hline JI SU ZI & 鸡嗉子 & Evergreen Dogwood \\
\hline JI WA CAO & 鸡娃草 & Littleflower Plumbagella \\
\hline JI WU BING JIN SI TAO & 近无柄金丝桃 & Subsessile St.John'swort \\
\hline JI XIANG CAO & 吉祥草 & Pink Reineckea \\
\hline JI XIANG RUN NAN & 极香润楠* & Extreme-fragrant Machilus* \\
\hline JI XIANG SHI CAO & 极香萻草* & Extreme-fragrant Yarrow* \\
\hline JI XIANG YING ZHAO & 极香鹰爪* & Extreme-fragrant Tailgrape* \\
\hline JI XIAO XIAO YUN XIANG MU & 极小小芸香木* & Minima Micromelum* \\
\hline JI XING ZI & 急性子 & Garden Balsam Seed \\
\hline JI XUAN HUA & 棘旋花* & Mucronate Glorybind* \\
\hline JI XUE CAO & 积雪草 & Asiatic Pennywort \\
\hline JI XUE TENG GEN & 鸡血藤根 & Leatherleaf Millettia Root \\
\hline JI YAN CAO & 鸡眼草 & Striate Kummerowia \\
\hline JI YE MA BIAN CAO & 戟叶马鞭草 & Hastate Verbena \\
\hline JI YE QIU HAI TANG & 载叶秋海棠 & Limpricht Begonia \\
\hline JI YE SHU WEI CAO & 戟叶鼠尾草 & Hastateleaf Sage \\
\hline JI YE SUAN MO & 戟叶酸膜 & Hastate Dock* \\
\hline JI YING SU & 蓟罂粟 & Mexican Pricklepoppy \\
\hline JI YU DAN & 鲫鱼胆 & Treasure Maesa \\
\hline JI YUN SHI & 棘云实 & Brazil-wood \\
\hline JI ZI BAI & 鸡子白 & Hen's Egg-albumen \\
\hline JI ZI HUANG & 鸡子黄 & Hen's Egg Yolk \\
\hline JI ZI KE & 鸡子壳 & Hen's Egg Shell \\
\hline JI ZI MU & 鸡子木 & Racemose Adina \\
\hline JI ZONG & 鸡塔 & Collybia Albuminosa Sporocarp \\
\hline JIA BAI HE & 假百合 & Hyacinth Falselily \\
\hline JIA BEI MU & 假贝母 & Paniculate Bolbostemma \\
\hline JIA DAN BAO JUN YANG HUAI & 假单胞菌洋槐* & Pseudomonas Locust* \\
\hline JIA DI DAN CAO & 假地胆草 & Spicate Pseudoelephantopus \\
\hline
\end{tabular}

punctatum]

T2467 Erythrina latissima

T6498 Tribulus terrestris

T6499 Tribulus terrestris

T0106 Aconitum kirinense

T6533 Trillium kamtschaticum

T5309 Pterocladia tenuis

T5041 Podocarpus imbricatus

T1107 Caesalpinia pulcherrima

T3926 Loropetalum chinense

T2836 Gallus gallus domesticus

T2838 Gallus gallus domesticus

T6718 Vernonia guineensis

T2485 Erythrophleum guineense

T4946 Piper guineense

T4306 Mosla grosseserrata

T2837 Gallus gallus domesticus

T6502 Trichocolea mollissima

T5491 Rhodiola algida

T0339 Aloe gililandii

T4577 Paederia scandens

T4578 Paederia scandens

T1696 Cornus capitata [Syn.

Dendrobenthamia capitata]

T5026 Plumbagella micrantha

T3365 Hypericum subsessile

T5449 Reineckea carnea

T4017 Machilus odoratissima

T0062 Achillea fragrantissima

T0655 Artabotrys odoratissimus

T4224 Micromelum minutum

T3412 Impatiens balsamina

T1652 Convolvulus erinaceus

T1311 Centella asiatica

T4243 Millettia reticulata

T3645 Kummerowia striata

T6707 Verbena hastata

T0890 Begonia limprichtii

T5664 Salvia bulleyana

T5609 Rumex hastatus

T0610 Argemone mexicana

T4031 Maesa perlarius

T1103 Caesalpinia echinata

T2839 Gallus gallus domesticus

T2840 Gallus gallus domesticus

T2841 Gallus gallus domesticus

T5960 Sinoadina racemosa [Syn. Adina racemosa]

T1623 Collybia albuminosa

T4443 Notholirion hyacinthinum [Syn.

Notholirion bulbuliferum]

T0971 Bolbostemma paniculatum

T5553 Robinia pseudomonas

T5253 Pseudoelephantopus spicatus 


\begin{tabular}{|c|c|c|c|}
\hline JIA DI FENG PI & 假地枫皮 & Jiadifengpi Anisetree & T3402 Illicium jiadifengpi \\
\hline JIA DONG FANG QIAN LI GUANG & 假东方千里光 & Pseud-oriental Groundsel* & T5899 Senecio pseudoorientalis \\
\hline JIA DU XING CAI & 家独行菜 & Garden Cress & T3758 Lepidium sativum \\
\hline JIA HONG SHU & 假红树 & White Mangrove & T3677 Laguncularia racemosa \\
\hline JIA HUANG HUA YUAN ZHI & 假黄花远志 & Fasle Yellowflower Milkwort* & $\begin{array}{l}\text { T5075 Polygala fallax [Syn. Polygala } \\
\text { aureocauda }]\end{array}$ \\
\hline JIA HUI SE JIU LI XIANG PO PO & 假灰色九里香婆婆纳* & Thyme Speedwell* & T6730 Veronica thymoides ssp. pseudocinerea \\
\hline \multicolumn{4}{|l|}{ NA } \\
\hline JIA JIN DONG YING SU & 假近东罂粟 & Pseudoriental Poppy* & T4634 Papaver pseudorientale \\
\hline JIA JING JIE & 假荆芥 & Catnip & T4413 Nepeta cataria \\
\hline JIA JU & 假蒟 & Sarmentose Pepper & T4965 Piper sarmentosum \\
\hline JIA JU ZI & 假蒟子 & Sarmentose Pepper Spike & T4966 Piper sarmentosum \\
\hline JIA KU GUA & 假苦瓜 & Balloonvine Heartseed & T1197 Cardiospermum halicacabum \\
\hline JIA LAN & 嘉兰 & Lovely Gloriosa & T2995 Gloriosa superba \\
\hline JIA LE BI CI TONG & 加勒比刺桐* & Caribbean Coralbean* & T2462 Erythrina caribea \\
\hline JIA LEI JUE MING & 加雷决明 & Garretti Senna* & T1233 Cassia garrettiana \\
\hline JIA LIAN QIAO & 假连勊 & Creeping Skyflower & T2295 Duranta repens \\
\hline JIA LIAN QIAO YE & 假连趐叶 & Creeping Skyflower Leaf & T2296 Duranta repens \\
\hline JIA MA BIAN & 假马鞭 & Jamaica Falsevalerian & T6095 Stachytarpheta jamaicensis \\
\hline JIA MA CHI XIAN & 假马齿苋 & Coastal Waterhyssop & T0852 Bacopa monniera \\
\hline JIA MA SHU & 家麻树 & Hazel Sterculia & T6138 Sterculia foetida \\
\hline JIA MI & 荚䔎 & Linden Viburnum & T6735 Viburnum dilatatum \\
\hline JIA MIAN YU YE QIU & 假面羽叶楸* & Personator Padritree* & T6142 Stereospermum personatum \\
\hline JIA MU DOU & 假木豆 & Capitulum Tickclover* & T2129 Desmodium cephalotes \\
\hline JIA NA DA BAO CHUN & 加拿大报春* & Mistassini Primrose & T5200 Primula mistassinica \\
\hline JIA NA DA CANG ER & 加拿大苍耳 & Canadian Cocklebur* & T6835 Xanthium canadense \\
\hline JIA NA DA HONG DOU SHAN & 加拿大红豆杉 & Canadian Yew & T6309 Taxus canadensis \\
\hline JIA NA DA HUANG QI & 加拿大黄芪* & Canadian Milkvetch* & T0789 Astragalus canadensis var. mortonii \\
\hline JIA NA DA WO JU & 加拿大莴菅 & Canada Lettuce & T3658 Lactuca canadensis \\
\hline JIA NA DA XI XIN & 加拿大细辛 & Canadian Snakeroot & T0723 Asarum canadense \\
\hline JIA NA LI HAO & 加那利蒿* & Canary Island Wormwood* & T0671 Artemisia canariensis \\
\hline JIA NA LI MEI DENG MU & 加那利美登木 & Canari Mayten* & T4129 Maytenus canariensis \\
\hline JIA NA LI SHU WEI CAO & 加那利鼠尾草* & Canari Sage* & T5665 Salvia canariensis \\
\hline JIA PENG ZI YU PAN & 加蓬紫玉盘 & Gabon Uvaria* & T6663 Uvaria klaineana \\
\hline JIA SHAN HU JIAO & 假山胡椒 & & T4640 Parabenzoin trilobum \\
\hline JIA SHUI XIAN & 假水仙 & False Narcissus* & $\begin{array}{l}\text { T4380 Narcissus pseudonarcissus ssp. } \\
\text { pseudonarcissus }\end{array}$ \\
\hline JIA SUAN JIANG & 假酸浆 & Apple of Peru & T4423 Nicandra physaloides \\
\hline JIA TUO WU & 假㯱吾 & False Goldenray* & T3818 Ligulariopsis shichuana \\
\hline JIA XI ZHUI XIANG CHA CAI & 假细雉香茶菜 & Falselittleconical Rabdosia & T5390 Rabdosia coetsoides \\
\hline JIA YANG & 加杨 & Canada Poplar & T5149 Populus canadensis \\
\hline JIA YE SHU & 假叶树 & Butchersbroom & T5618 Ruscus aculeatus \\
\hline JIA YING ZHAO & 假鹰爪 & Chinese Desmos & $\begin{array}{l}\text { T2136 Desmos cochinchinensis [Syn. Desmos } \\
\text { chinensis] }\end{array}$ \\
\hline JIA YUAN WEI & 假怠尾 & False Iris* & $\mathrm{T} 3470$ Iris spuria \\
\hline JIA ZE LAN & 假泽兰 & Heartshape Mikania & T4228 Mikania cordata \\
\hline JIA ZHOU LI LU & 加州藜芦 & Californian Falsehellebore* & T6694 Veratrum californicum \\
\hline JIA ZHOU QI YE SHU & 加州七叶树 & Californian Buckeye & T0199 Aesculus californica \\
\hline JIA ZHOU SHAN SONG & 加州山松 & Californian Mountain Pine & T4918 Pinus monticola \\
\hline JIA ZHOU XIA LA MEI & 加州夏腊梅 & Californian Allspice & T1140 Calycanthus occidentalis \\
\hline JIA ZHU TAO & 夹竹桃 & Sweetscented Oleander & T4418 Nerium indicum \\
\hline JIA ZHU TAO MA & 夹竹桃麻 & Black Indian Hemp & T0551 Apocynum cannabinum \\
\hline JIA ZI CAO & 假紫草 & Common Arnebia & T0649 Arnebia guttata \\
\hline
\end{tabular}




\begin{tabular}{|c|c|c|c|}
\hline JIA ZONG ZHUANG HUA XU & 假总状花序山小橘* & Pseudo-racemose Glycosmis* & T3009 Glycosmis pseudoracemosa \\
\hline \multicolumn{4}{|l|}{ SHAN XIAO JU } \\
\hline JIAN BAI JIAN MU & 尖白坚木* & Cuspate & T0762 Aspidosperma cuspa \\
\hline JIAN CHI BU LI KE ER CAO & 尖齿布里克尔草 & & T1027 Brickellia arguta var. odontolepis \\
\hline JIAN DENG XIN CAO & 尖灯芯草 & Acute Rush* & T3577 Juncus acutus \\
\hline JIAN GAN YANG & 箭杆杨 & Arrowshaft Poplar & T5156 Populus nigra var. thevestina \\
\hline JIAN GEN SHU & 箭根薯 & Arrowroot Tacca & $\begin{array}{l}\text { T6275 Tacca chantrieri }[\text { Syn. Tacca minor; } \\
\text { Tacca esquirolii] }\end{array}$ \\
\hline JIAN HAI LONG & 尖海龙 & Acute Syngnathus* & T6257 Syngnathus acus \\
\hline JIAN JIAN AO YANG & 渐尖澳杨 & Acuminate Aussiepoplar* & T3273 Homalanthus acuminatus \\
\hline JIAN JIAN MU LAN & 渐尖木兰 & Cucumber-tree & T4033 Magnolia acuminata \\
\hline JIAN JIAN SUAN PAN ZI & 渐尖算盘子* & Acuminate Glochidion* & T2987 Glochidion acuminatum \\
\hline JIAN JU ZI JIN & 尖距紫堇 & Sharpspur Corydalis & $\begin{array}{l}\text { T1745 Corydalis suaveolens [Syn. Corydalis } \\
\text { sheareri] }\end{array}$ \\
\hline JIAN LIE HAI YING SU & 尖裂海罂粟 & Acutelobed Hornpoppy & T2970 Glaucium oxylobum \\
\hline JIAN MA & 剑麻 & Sisal Hemp-plant & T0226 Agave sisalana \\
\hline JIAN PU ZHAI GU KE & 柬埔寨古柯* & Cambodia Coca Shrub* & T2490 Erythroxylum cambodianum \\
\hline JIAN PU ZHAI ZAO & 柬埔寨杳* & Cambodia Jujube* & T6915 Ziziphus cambodiana \\
\hline JIAN QIU LUO MAO RUI HUA & 剪秋罗毛芯花 & White Mullein & T6701 Verbascum lychnites \\
\hline JIAN RUI MAO CHA & 尖锐毛茶* & Acute Antirhea* & T0536 Antirhea acutata \\
\hline JIAN TENG BAI JIU CAO & 坚挺白酒草* & Strict Conyza & $\mathrm{T} 1658$ Conyza stricta \\
\hline JIAN TING MA BIAN CAO & 坚挺马鞭草* & Strict Verbena* & T6710 Verbena stricta \\
\hline JIAN WEI YU & 尖尾芋 & Chinese Taro & T0333 Alocasia cucullata [Syn. Arum cucullatum] \\
\hline JIAN XING SI GUO XIANG CHA & 间型四国香茶菜 & Intermediate Shiko Rabdosia* & T3526 Isodon shikokiana var.intermedius \\
\hline \multicolumn{4}{|l|}{ CAI } \\
\hline JIAN XING YAN CAO & 尖形烟草* & Acuminate Tobacco* & T4424 Nicotiana acuminata \\
\hline JIAN XUE FENG HOU & 见血封喉 & Common Antiaris & $\begin{array}{l}\text { T0533 Antiaris toxicaria }[\text { Syn. Ambora } \\
\text { toxicaria }]\end{array}$ \\
\hline JIAN XUE QING & 见血清 & Nervate Twayblade & T3863 Liparis nervosa \\
\hline JIAN YE BAO SHI MU & 尖叶饱食木* & & T1030 Brosimum acutifolium \\
\hline JIAN YE CEN & 尖叶梣 & Acuteleaf Ash Bark & $\begin{array}{l}\text { T2780 Fraxinus szaboana [Syn. Fraxinus } \\
\text { chinensis var. acuminata] }\end{array}$ \\
\hline JIAN YE FAN XIE YE & 尖叶番泻叶 & Sharpleaf Senna Leaf & T1229 Cassia acutifolia \\
\hline JIAN YE HUA JIAO & 尖叶花椒 & Acute-leaf Pricklyash* & T6859 Zanthoxylum acutifolium \\
\hline JIAN YE LONG XUE SHU & 剑叶龙血树 & Swordleaf Dracaena & T2253 Dracaena cochinchinensis \\
\hline JIAN YE QING FENG TENG & 尖叶清风藤 & Sharpleaf Sabia & T5638 Sabia swinhoei \\
\hline JIAN YE SHI DA GONG LAO & 尖叶十大功劳* & Oregon-grape & T4054 Mahonia aquifolium \\
\hline JIAN YE TANG SONG CAO & 尖叶唐松草 & Sharpleaf Meadowrue & T6372 Thalictrum acutifolium \\
\hline JIAN YE TIE SHU YE & 剑叶铁树叶 & Strictleaf Dracaena Leaf & T1684 Cordyline strcta \\
\hline JIAN YE TOU WU GEN & 箭叶㯻吾根 & Arrowleaf Goldenray Root & T3812 Ligularia sagitta \\
\hline JIAN YE YE XIA ZHU & 尖叶叶下珠 & Sharpleaf Leafflower* & T4832 Phyllanthus acuminatus \\
\hline JIAN YE YIN YANG HUO & 箭叶淫羊藿 & Sagittate Epimedium & T2402 Epimedium sagittatum \\
\hline JIAN YE YUN XIANG CAO & 尖叶芸香草* & Haplophyllum* & T3100 Haplophyllum acutifolium \\
\hline JIAN YE ZAO & 尖叶菄 & Mucronated Jujube* & T6922 Zizyphus mucronata \\
\hline JIAN YING CHAI HU & 坚硬柴胡* & Thorowax & T1069 Bupleurum rigidum \\
\hline JIAN YING XUAN GOU ZI & 坚硬悬钩子* & Rigid Blackberry* & T5596 Rubus rigidus \\
\hline JIAN ZHUANG CHANG CHUN & 剑状长春花 & Lanceolate Periwinkle* & T1267 Catharanthus lanceus \\
\hline \multicolumn{4}{|l|}{ HUA } \\
\hline \multirow[t]{2}{*}{ JIAN ZI SU } & 尖紫苏 & Acute Common Perilla & T4717 Perilla frutescens var. acuta [Syn. \\
\hline & & & Perilla frutescens var. purpurascens] \\
\hline \multirow[t]{2}{*}{ JIAN ZI SU YE } & 尖紫苏叶 & Acute Common Perilla Leaf & T4718 Perilla frutescens var. acuta [Syn. \\
\hline & & & Perilla frutescens var. purpurascens] \\
\hline JIAN ZI YU PAN & 尖紫玉盘* & Acuminate Uvaria* & T6657 Uvaria acuminata \\
\hline
\end{tabular}


JIANG

JIANG CAN

JIANG DOU

JIANG GUO KU SHU

JIANG GUO SHU YU

JIANG GUO XIAN

JIANG GUO ZI SHAN

JIANG HUANG

JIANG LI MU GEN

JIANG MANG

JIANG NAN JUAN BAI

JIANG XI QING NIU DAN

JIANG ZHEN XIANG

JIANG ZI SHA JI

JIAO CHA CAI
JIAO GAN
JIAO GAN PI
JIAO GU LAN
JIAO GUO MU
JIAO HAO
JIAO MO

JIAO NIAN XIANG CHA CAI

JIAO RANG MU

JIAO XI XIAN

JIAO YAN CAO

JIAO YU

JIAO ZHI HUANG TAN

JIAO ZHI SHU WEI CAO

JIAO ZHI ZI

JIAO ZHU HUA

JIAO ZHUANG CHE QIAN

JIE CAI

JIE GENG

JIE GU MU

JIE JIE LEI A WEI

JIE JING SHI XIAN TAO

JIE LIAO

JIE MAO FEI LIAN

JIE MAO TAI

JIE MAO YE SHU WEI CAO

JIE XING YE TAI

JIE ZHI HONG DOU SHAN

JIE ZI

JIN BU HUAN

JIN CAO

JIN CAO

JIN CHAI FENG WEI JUE

JIN DAN

JIN DONG YING SU

JIN FA XIAN
酱

僵蚕

剅豆

浆果苦树

浆果薯蓣

浆果苋

酱果紫杉

姜黄

降梨木根

茫芒

江南卷柏

江西青牛胆

降真香(降香)

江孜沙棘

角叉菜

蕉相

蕉柑皮

绞股蓝

角果木

角蒿

礁膜

胶黏香茶菜

交让木

胶狶莶

胶烟草

蕉芋

交趾黄檀

胶质鼠尾草

胶桅子

角柱花

角状车前*

芥菜

桔梗

接骨木

结节类阿魏

节茎石仙桃

节蓼

节毛飞廉

睫毛苔

睫毛叶鼠尾草*

截形叶苔*

介质红豆杉

芥子

金不换

菍草

金草

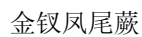

金弹

近东罂粟

金发蘚
Soybean Paste

Cow-pea

Black Bryony

Frutescent Cladostachys

Common Yew

Common Turmeric

Thinleaf Buckthorn Root

Inflatedfruit Senna

Moellendorff Spikemoss

Jiangxi Tinospora*

Odorate Rosewood

Jiangzi Seabuckthorn*

Tankan Citrus

Tankan Citrus Pericarp

Fiveleaf Gynostemma

Common Ceriops

Chinese Incarvillea

Slimy Rabdosia

Macropodous Daphniphyllum

Gummy St. Paulswort*

Slimy Tobacco*

Edible Canna

Siam Rosewood

Sticky Clary

Gummy Gardenia*

Blue Ceratostigma, Blue Bluesnow

Cornuted Plantain*

India Mustard

Balloonflower

Elder*

Node Ferulago*

Articulate Pholidota

Pale Persicaria

Welted Thistle

Blepharostoma*

Eyelid-leaf Sage*

Media Yew

India Mustard Seed

Chinese Stephania

Hispid Arthraxon

Acuteangle Hedyotis

Faurie's Brake

Meiwa Kumquat

Oriental Poppy

Common Gold-hair Moss*
T3003 Glycine max

T0974 Bombyx batryticatus

T6754 Vigna unguiculata

T4886 Picrodendron baccatum

T6291 Tamus communis

T2058 Deeringia amaranthoides [Syn.

Cladostachys frutescens]

T6307 Taxus baccata

T1905 Curcuma longa

T5461 Rhamnus leptophylla

T1247 Cassia sophera

T5863 Selaginella moellendorffii

T6465 Tinospora craveniana

T2008 Dalbergia odorifera

T3255 Hippophae rhamnoides subsp.

gyantsensis

T1384 Chondrus ocelladus

T1516 Citrus tankan

T1517 Citrus tankan

T3085 Gynostemma pentaphyllum

T1336 Ceriops tagal [Syn. Rhizophora tagal]

T3420 Incarvillea sinensis

T4274 Monostroma nitidum

T3492 Isodon glutinosa

T2033 Daphniphyllum macropodum

T5949 Siegesbeckia gummifer

T4425 Nicotiana glutinosa

T1172 Canna edulis

T1999 Dalbergia cochinchinensis

T5673 Salvia glutinosa

T2881 Gardenia gummifera

T1328 Ceratostigma plumbaginoides

T5003 Plantago cornuti

T1007 Brassica juncea

T5011 Platycodon grandiflorum

T5707 Sambucus williamsii

T2713 Ferulago nodosa

T4821 Pholidota articulata

T5109 Polygonum nodosum

T1199 Carduus acanthoides

T0953 Blepharostoma trichophyllum

T5660 Salvia blepharophylla

T3581 Jungermannia truncata

T6313 Taxus media

T1008 Brassica juncea

T6133 Stephania sinica

T0707 Arthraxon hispidus

T3122 Hedyotis acutangula

T5290 Pteris fauriei [Syn. Pteris fauriei var.

minor]

T2758 Fortunella crassifolia

T4632 Papaver orientale

T5133 Polytrichum commune 


\begin{tabular}{|c|c|c|}
\hline JIN FEI CAO & 金沸草 & Japanese Inula \\
\hline JIN FEN JUE & 金粉蕨 & Siliculose Onychium \\
\hline JIN GAN & 金柑 & Japanese Kumquat \\
\hline JIN GANG DA & 金刚大 & Japanese Croomia \\
\hline JIN GU CAO & 筋骨草 & Ciliate Bugle \\
\hline JIN GUANG JU & 金光菊 & Cutleaf Coneflower \\
\hline JIN GUO LAN & 金果榄 & Hairystalk Tinospora \\
\hline JIN HUA MI HOU TAO & 金花猕猴桃 & Goldflower Actinidia \\
\hline JIN HUA XIAO BO & 金花小檗 & Wilson Barberry \\
\hline JIN HUANG CAO SU & 金黄糙苏* & Goldenyellow Jerusalemsage* \\
\hline JIN HUANG CE JIN ZHAN HUA & 金黄侧金戞花 & Golden Adonis \\
\hline JIN HUANG CHAI HU & 金黄柴胡 & Goldenyellow Thorowax \\
\hline JIN HUANG GE JUN & 金黄革菌 & Goldenyellow Thelephore* \\
\hline JIN HUANG JIN & 金黄堇 & Golden Corydalis \\
\hline JIN HUANG LIAN & 金黄莲* & American Lotus \\
\hline JIN JI ER & 锦鸡儿 & Peashrub* \\
\hline JIN JI LE & 金鸡勒 & Ledger Cinchona \\
\hline JIN JI WEI & 金鸡尾 & Figuerleaved Brake \\
\hline JIN JI WEI BA CAO GEN & 金鸡尾巴草根 & Needle-hear Fern* \\
\hline JIN JI ZE LAN & 近戟泽兰 & Halberd-like Eupatorium* \\
\hline JIN JIN BANG & 金金棒 & Sericeous Cinquefoil \\
\hline JIN JIN XIANG & 金锦香 & Chinese Osbeckia \\
\hline JIN JU & 金橘 & Oval Kumquat \\
\hline JIN JU YE & 金橘叶 & Oval Kumquat Leaf \\
\hline JIN LIAN HUA & 金莲花 & Chinese Globeflower \\
\hline JIN LIAN HUA ZHU SHI DOU & 金链花猪屎豆 & Laburnum Crotalaria* \\
\hline JIN LIAN MU & 金莲木 & Entire Ochna \\
\hline JIN MAO ER CAO & 金毛耳草 & Goldhair Hedyotis \\
\hline JIN MAO GOU & 金毛狗 & Scythian Lamb \\
\hline JIN PING GE NA XIANG & 金屏哥纳香 & Leiocarpus Goniothalamus \\
\hline JIN QIAN KU YE CAO & 金钱苦叶草 & Goldsaxifrage Herb \\
\hline JIN QIAN PU & 金钱蒲 & Grassleaf Sweetflag \\
\hline JIN QIAN PU YE & 金钱蒲叶 & Grassleaf Sweetflag Leaf \\
\hline JIN QIN ZHUANG BA DOU & 近琴状巴豆 & Lyrate-like Croton* \\
\hline JIN QUE ER & 金雀儿 & Scotch Broom \\
\hline JIN QUE GEN & 金雀根 & Chinese Peashrub Root \\
\hline JIN SE MU JU & 金色母菊* & Aureate Mayweed* \\
\hline JIN SHU HUANG YANG & 锦熟黄杨 & European Boxwood \\
\hline JIN SHUA BA & 金刷把 & Fallax Cladonia Lichen \\
\hline JIN SI DAI & 金丝带 & Green Alectoria Filament \\
\hline JIN SI MA WEI LIAN & 金丝马尾连 & Gold-enthread Meadowrue \\
\hline JIN SI MEI & 金丝梅 & Spreading St.John'swort \\
\hline JIN SI SHUA & 金丝刷 & Gold-wire Brush* \\
\hline JIN SI TAO GUO SHI & 金丝桃果实 & Chinese St.John'swort Fruit \\
\hline JIN SU LAN & 金粟兰 & Chulan Tree \\
\hline JIN TIE SUO & 金铁锁 & Tuniclike Psammosilene \\
\hline JIN WU MAO HUI YAN XIANG & 近无毛灰岩香茶菜 & Calcicole Rabdosia \\
\hline \multicolumn{3}{|l|}{ CHA CAI } \\
\hline JIN WU MAO SAO JU & 近无毛掃菊* & Longflower Bloomdaisy \\
\hline
\end{tabular}

T3433 Inula japonica

T4504 Onychium siliculosum

T2759 Fortunella japonica

T1810 Croomia japonica

T0262 Ajuga ciliata

T5604 Rudbeckia laciniata

T6463 Tinospora capillipes

T0159 Actinidia chrysantha

T0920 Berberis wilsonae

T4806 Phlomis aurea

T0186 Adonis chrysocyatha

T1056 Bupleurum aureum

T6420 Thelephora aurantiotincta

T1707 Corydalis aurea

T4396 Nelumbo lutea

T1188 Caragana chamlagu

T1428 Cinchona ledgeriana

T5289 Pteris dactylina

T4026 Macrothelypteris oligophlebia

T2577 Eupatorium subhastatum

T5186 Potentilla reptans var. sericophylla

T4549 Osbeckia chinensis

T2760 Fortunella margarita

T2761 Fortunella margarita

T6552 Trollius chinensis [Syn. Trollius

asiaticus var. chinensis]

T1824 Crotalaria laburnifolia

T4465 Ochna integerrima

T3125 Hedyotis chrysotricha [Syn.

Oldenlandia chrysotricha]

T1411 Cibotium barometz [Syn. Polypodium barometz]

T3049 Goniothalamus leiocarpus

T1403 Chrysosplenium grayanum

T0144 Acorus gramineus

T0145 Acorus gramineus

T1857 Croton sublyratus

T1991 Cytisus scoparius [Syn. Spartium

scoparium ]

T1191 Caragana sinica

T4123 Matricaria aurea

T1094 Buxus sempervirens

T1525 Cladonia fallax

T0298 Alectoria vivens

T6389 Thalictrum glandulosissimum

T3360 Hypericum patulum

T3775 Lethariella cladonioides

T3344 Hypericum chinense

T1374 Chloranthus spicatus

T5250 Psammosilene tunicoides

T3483 Isodon calcicola

T4735 Pertya glabrescens 


\begin{tabular}{|c|c|c|c|}
\hline JIN XIAN CAO & 金线草 & Longtube Ground Ivy & T2973 Glechoma longituba \\
\hline JIN YAO & 金腰 & Alternate-leaved Golden-saxifrage & T1402 Chrysosplenium alternifolium \\
\hline JIN YAO DAI & 金腰带 & Goldon-belt* & T3776 Lethariella zahlbruckneri \\
\hline JIN YE ZI & 金叶子 & Yunnan Craibiodendron & T1767 Craibiodendron yunnanese \\
\hline JIN YI HUANG QI & 金翼黄芪 & Golden-wing Milkvetch* & T0791 Astragalus chrysopterus \\
\hline JIN YIN HUA & 金银花(忍冬) & Japanese Honeysuckle & T3912 Lonicera japonica \\
\hline JIN YING YE & 金樱叶 & Cherokee Rose Leaf & T5567 Rosa laevigata \\
\hline JIN YING ZI & 金樱子 & Cherokee Rose Seed & T5568 Rosa laevigata \\
\hline JIN YU & 金鱼 & Crucian Carp & T1194 Carassius auratus \\
\hline JIN YU CAO & 金鱼草 & Common Snapdragon & T0537 Antirrhinum majus \\
\hline JIN ZHAN JU & 金盏菊 & Potmarigold Calendula & T1113 Calendula officinalis \\
\hline JIN ZHONG HUA & 金钟花 & Greenstem Forsythia & T2757 Forsythia viridissima \\
\hline JIN ZI TA MU LAN & 金字塔木兰* & Pyramidal Magnolia* & T4047 Magnolia pyramidata \\
\hline JIN ZI TA XING QIAN LI GUANG & 金字塔形千里光 & Pyramidal Groundsel* & T5900 Senecio pyramidatus \\
\hline JING DIAN MEI & 茎点霉* & & T4824 Phoma lingam \\
\hline JING DOU & 荆豆 & Common Gorse & T6590 Ulex europaeus \\
\hline JING GU NU & 粳谷奴 & Fungus-infected Rice Spike & T6655 Ustilaginoidea virens \\
\hline JING HONG AN LUO & 景洪暗罗 & Jinghong Greenstar & T5064 Polyalthia cheliensis \\
\hline JING HUA HUA YE CAI & 茎花花梛菜* & Cauliflory Brassica* & $\begin{array}{l}\text { T1015 Brassica oleracea var. botrytis subvar. } \\
\text { cauliflora }\end{array}$ \\
\hline JING JI GUA TAI MU & 荆棘瓜泰木* & & T3071 Guatteria dumetorum \\
\hline JING JI SHU YU & 荆棘薯蓣* & Thorny Yam* & T2196 Dioscorea dumetorum \\
\hline JING JIE & 荆芥 & Fineleaf Schizonepeta & $\begin{array}{l}\text { T5804 Schizonepeta tenuifolia }[\text { Syn. Nepeta } \\
\text { tenuifolia }]\end{array}$ \\
\hline JING JIE HUA & 旌节花 & praecox Stachyurus* & T6096 Stachyurus praecox \\
\hline JING JIE YE SHI ER CAO & 荆芥叶狮耳草 & Nepetaleaf Leontis & T3744 Leonotis nepetaefolia \\
\hline JING LI MI HOU TAO & 京梨猕猴桃 & Henry Actinidia & T0157 Actinidia callosa var. henryi \\
\hline JING MI & 粳米 & Rice & T4545 Oryza sativa \\
\hline JING NI PING & 京尼平 & Genipa & T2900 Genipa americana \\
\hline JING SHENG HUA AI SUO LUO & 茎生花艾索罗那* & & T3537 Isolona cauliflora \\
\hline NA & & & \\
\hline JING XIANG MAO & 精香茅 & Citronella-grass & T1944 Cymbopogon nardus \\
\hline JING YA MA YE RUI XIANG & 茎亚麻叶瑞香 & Garou Bush & T2025 Daphne gnidium \\
\hline JIU & 酒 & Liquor & T3869 Liquor \\
\hline JIU BI YING & 救必应 & Ovateleaf Holly & T3398 Ilex rotunda \\
\hline JIU BING YE & 酒饼叶 & Malay Glycosmis & T3008 Glycosmis pentaphylla \\
\hline JIU CAI & 非菜 & Tuber Onion & T0322 Allium tuberosum \\
\hline JIU CENG FENG & 九层风 & & $\begin{array}{l}\text { T1530 Cladostachys amaranthoides [Syn. } \\
\text { Achyranthes amaranthoides; Cladostachys } \\
\text { frutescens; Deeringia amaranthoides] }\end{array}$ \\
\hline JIU CONG & 韭葱 & Leek & T0317 Allium porrum \\
\hline JIU HUA DA E XIANG CHA CAI & 九华大萼香茶菜 & Jiuhua Largesepal Rabdosia & T3512 Isodon macrocalyx var. jiuhua \\
\hline JIU JIE CHA & 九节茶 & Glabrous Sarcandra & $\begin{array}{l}\text { T5730 Sarcandra glabra }[\text { Syn. Chloranthus } \\
\text { glaber }]\end{array}$ \\
\hline JIU LI XIANG & 九里香 & Common Jasminorange & $\begin{array}{l}\text { T4323 Murraya paniculata }[\text { Syn. Chalcas } \\
\text { paniculata] }\end{array}$ \\
\hline JIU LI XIANG GEN & 九里香根 & Common Jasminorange Root & $\begin{array}{l}\text { T4324 Murraya paniculata }[\text { Syn. Chalcas } \\
\text { paniculata }]\end{array}$ \\
\hline JIU ZI & 非子 & Tuber Onion Seed & T0323 Allium tuberosum \\
\hline JU AN & 巨桉 & Toolur & T2511 Eucalyptus grandis \\
\hline JU CHI SANG & 锯齿桑* & Serrate Mulberry* & T4299 Morus serrata \\
\hline JU CHI SHI SONG & 锯齿石松 & Sawtooth Clubmoss* & T3977 Lycopodium serratum var. thunbergii \\
\hline JU DA JI & 巨大戟 & Enormous Euphorbia & T2593 Euphorbia ingens \\
\hline
\end{tabular}




JU DA LAN CI TOU
JU DA MI ZI LAN
JU GUO RONG
JU HAO
JU HE
JU HUA
JU HUA GUO LU HUANG
JU HUA HUANG LIAN
JU HUA HUANG TAN
JU HUA SHI HU
JU HUA XUE DAN
JU HUANG YING SU
JU JIANG YE
JU MAO LEI A WEI
JU MI JIN HE HUAN
JU MI SHU WEI CAO
JU PI
JU QU
JU REN ZHU
JU SAN HUA YE CAI

JU SE GOU SUAN JIANG

JU SE MAO RUI HUA

JU SHAN

JU SHI QIE

JU TAI XIANG CHA CAI

JU YU

JU YUAN

JU YUAN YE

JU YUAN YE HUANG QI

JU YUAN YE LONG NAO

XIANG

JU ZI JIN

JUAN BAI

JUAN DAN

JUAN MAO HUANG TAN

JUAN MAO KUO BAO JU

JUAN MAO QIANG WEI

JUAN QIAO YUAN WEI

JUE

JUE CHUANG

JUE MING ZI

JUN QIAN ZI

JUN ZI LAN

KA BU LI FENG DOU CAI

KA FEI HUANG KUI

KA FU LA LUO FU MU

KA LA BA DAN SHEN

KA LI YU RUI

KA LUO JIN HE HUAN

\section{巨大蓝刺头* \\ 巨大米仔兰* \\ 聚果榕 \\ 菊蒿 \\ 橘核 \\ 菊花}

\section{聚花过路黄}

菊花黄连

聚花黄檀*

聚花石斛*

巨花雪胆

桔黄罂粟*

蒟酱叶

具毛类阿魏*

具蜜金合欢

具蜜鼠尾草

橘皮(陈皮)

菊芭

巨人柱

\section{聚伞花楖菜}

橘色沟酸浆

橘色毛芯花*

巨杉

菊氏茄*

草苔香茶菜

菊芋

枸椽

枸椽叶

矩圆叶黄芪*

矩圆叶龙脑香*

\section{巨紫堇}

卷柏

卷丹

\section{绢毛黄檀 \\ 绢毛阔苍菊* \\ 绢毛蓄薇 \\ 卷鞘萝尾 \\ 屏}

爵床

决明子

君迁子

君子兰

卡布里蜂斗菜

咖啡黄葵

卡夫拉萝芙木

卡拉巴丹参

卡里玉芯

卡罗金合欢*
Grand Globethistle*

Grand Aglaia*

Racemose Fig*

Common Tansy

Tangerine Seed

Florists Chrysanthemum Flower

Denseflower Loosestrife

Yellowflower Corydalis

Congested-flower Rosewood*

Thyrseflower Dendrobium

Giant Hemsleya

Orange Poppy*

Betel Pepper Leaf

Honeyed Acacia

Melliferous Sage*

Tangerine Pericarp

Common Chicory

Cymose Brassica*

Orange Monkeyflower*

Orange Mullein

Giant Sequoia

Juzepczuk Nightshade*

Gesnerialike Rabdosia

Jerusalem Artichoke

Medicinal Citron

Medicinal Citron Leaf

Oblongleaf Milkvetch*

Oblong-leaf Borneol Oil-Resin*

Gigantic Corydalis*

Tamariskoid Spikemoss

Tiger Lily

Sericeous-leaf Rosewood

Sericeous Pluchea*

Silky Rose

Potanin Iris

Eastern Bracken Fern

Creeping Rostellularia

Sickle Senna Seed

Dateplum Persimmon

Scarlet Kafirlily

Kabliki Butterbur*

Edible Abelmoschus

Caffra Devilpepper*

Karaba Sage*

Karroo Acacia
T2315 Echinops giganteus

T0237 Aglaia grandis

T2724 Ficus racemosa

T6298 Tanacetum vulgare

T1504 Citrus reticulata

T1395 Chrysanthemum morifolium [Syn.

Dendranthema morifolium]

T4000 Lysimachia congestiflora

T1731 Corydalis pallida

T2000 Dalbergia congestiflora

T2110 Dendrobium thyrsiflorum

T3206 Hemsleya gigantha

T4622 Papaver auranticum

T4937 Piper betle

T2712 Ferulago capillaries

T0026 Acacia mellifera

T5678 Salvia mellifera

T1506 Citrus reticulata

T1415 Cichorium intybus

T1209 Carnegiea gigantea

T1016 Brassica oleracea var. botrytis subvar.

cymosa

T4251 Mimulus aurantiacus

T6702 Verbascum phlomoides

T5917 Sequoia gigantea

T6002 Solanum juzepczukii

T3491 Isodon gesneroides

T3155 Helianthus tuberosus

T1498 Citrus medica

T1499 Citrus medica

T0799 Astragalus miser var. oblongifolia

T2276 Dryobalanops oblongifolia

T1716 Corydalis gigantea

T5869 Selaginella tamariscina

T3839 Lilium tigrinum [Syn. Lilium

lancifolium]

T2012 Dalbergia sericea

T5025 Pluchea sericea

T5573 Rosa sericea

T3466 Iris potaninii

T5284 Pteridium aquilinum var. latiusculum

T5576 Rostellularia procumbens [Syn.

Justicia procumbens]

T1250 Cassia tora

T2222 Diospyros lotus

T1578 Clivia miniata

T4741 Petasites kablikianus

T3240 Hibiscus esculentus

T5430 Rauwolfia caffra

T5676 Salvia karabachensis

T1204 Careya arborea

T0022 Acacia karroo 


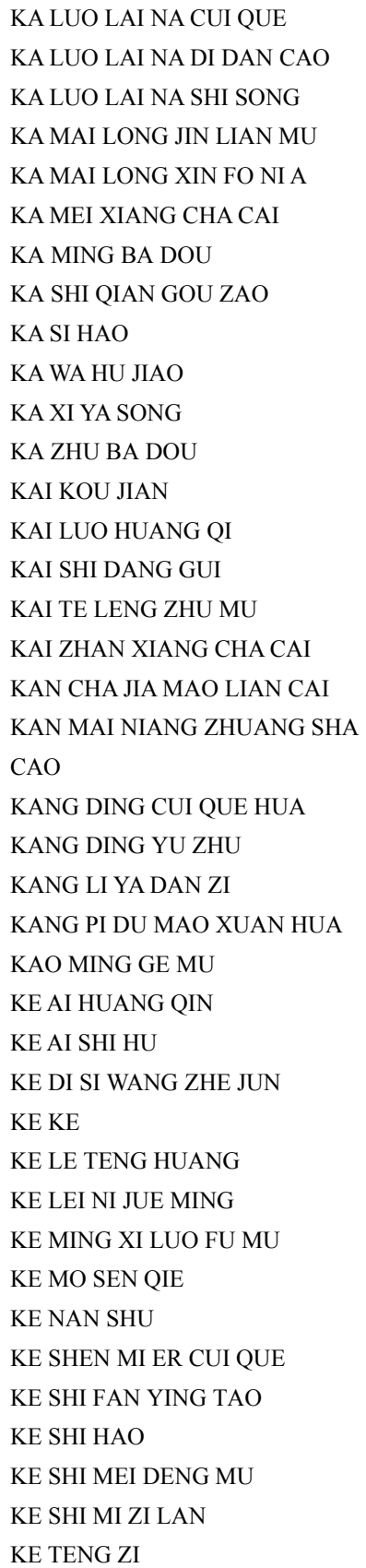

KE XI JIA SONG

KE YI YI SHOU SHEN

KEN NI YA CI TONG

KEN NI YA HUI YE

KEN NI YA MO YAO

KEN NI YA WEN SHU LAN

KEN NI YA XIAN SUAN QIANG

KONG QUE CAO

KONG SHI CHUN

KONG XIN XIAN

KU A MO YAO

KU AO

$\begin{array}{ll}\text { 卡罗来纳翠雀* } & \text { Carolina Larkspur* } \\ \text { 卡罗来纳地胆草 } & \text { Carolina Elephantfoot* } \\ \text { 卡罗来纳石松* } & \text { Carolina Clubmoss* } \\ \text { 喀麦隆金莲木 } & \text { Cameroon Ochn* } \\ \text { 喀麦隆辛佛尼阿* } & \text { Cameroon Symphonia* } \\ \text { 卡美香茶菜 } & \text { Kamei Rabdosia* } \\ \text { 卡明巴豆* } & \text { Cuming Croton* } \\ \text { 卡氏前沟藻 } & \\ \text { 卡斯蒿* } & \text { Caruth Wormwood* } \\ \text { 卡瓦胡椒 } & \text { Kava Pepper } \\ \text { 卡西亚松 } & \text { Khasya Pine } \\ \text { 卡朱巴豆 } & \\ \text { 开口箭 } & \text { Chinese Tupistra } \\ \text { 开罗黄芪 } & \text { 开罗 Milkvetch* } \\ \text { 凯氏当归* } & \text { Keislce Angelica* } \\ \text { 凯特棱柱木 } & \text { Kaith Ramin* } \\ \text { 开展香茶菜 } & \text { Spreading Rabdosia* } \\ \text { 堪察加毛莲菜 } & \text { Kamchatka Oxtongue } \\ \text { 看麦娘状莎草 } & \text { Foxtail-like Galingale } \\ & \end{array}$

康定翠雀花

康定玉竹

抗㾐鸦胆子

康毗毒毛旋花*

考明格木

可爱黄芩*

可爱石解

柯蒂斯网褶菌*

可可

可乐藤黄*

克雷尼决明

柯明西萝芙木

克默森茄

柯楠树

克什米尔翠雀

可食番樱桃*

克氏蒿*

克氏美登木*

可食米仔兰*

榼藤子

科西嘉松*
可疑翼手参
肯尼亚刺桐
肯尼亚灰叶
肯尼亚没药
肯尼亚文殊兰*
肯尼亚咸酸蔃
孔雀草
孔石莼
空心苋
库阿没药*
苦芙

Kangting Larkspur

Pratt Solomonseal Antidysenteric Brucea* Kombe Strophanthus* Delightful Skullcap* Delightful Dendrobium Curtisi Paxillus* Cocoa

Kola Garcinia*

Kleini Senna*

Cummins Devilpepper*

Corinan Tree*

Kashmir Larkspur

Edible Eugenia*

Klotzsch Wormwood*

Krukov Mayten*

Edible Aglaia*

Climbing Entada Seed

Corsican Pine

Kenya Coralbean*

Kenya Tephrosia*

Kenya Crinum*

Kenya Embelia*

French Marigold

Pertusate Ulva Frond

Alligator Alternanthera

Kua Myrrhtree*

Chinese Thistle
T2065 Delphinium carolinianum T2331 Elephantopus carolinianus T3967 Lycopodium carolinianum T4464 Ochna calodendron T6242 Symphonia globulifera T3498 Isodon kameba T1842 Croton cumingii T0429 Amphidinium carterae T0673 Artemisia caruthii T4955 Piper methysticum T4911 Pinus kesiya T1839 Croton cajucara T6566 Tupistra chinensis T0797 Astragalus kahiricus T0487 Angelica keislcei T3052 Gonystylus keithii T3485 Isodon effusa T4885 Picris kamtschatica T1973 Cyperus alopecuroides Couminga Erythrophleum*

Commerson Nightshade*

Kenya Myrrhtree*
T2087 Delphinium tatsienense

T5094 Polygonatum prattii

T1037 Brucea antidysenterica

T6157 Strophanthus kombe

T2484 Erythrophleum couminga

T5832 Scutellaria amabilis

T2095 Dendrobium amoenum

T4680 Paxillus curtisii

T6423 Theobroma cacao

T2860 Garcinia kola

T1235 Cassia kleinii

T5433 Rauwolfia cumminsii

T5995 Solanum commersonii

T1752 Corynanthe johimbe

T2066 Delphinium cashmerianum

T2532 Eugenia edulis

T0679 Artemisia klotzschiana

T4136 Maytenus krukovii

T0233 Aglaia edulis

T2363 Entada phaseoloides [Syn. Lens phaseoloides]

T4914 Pinus laricio

T1625 Colochirous anceps

T2461 Erythrina burttii

T6331 Tephrosia aequilata

T1636 Commiphora kua var. gowlello

T1798 Crinum kirkii

T2350 Embelia schimperi

T6282 Tagetes patula

T6600 Ulva pertusa

T0379 Alternanthera philoxeroides

T1635 Commiphora kua

T1448 Cirsium chinense 


KU BAO
KU CAO
KU DI DAN
KU DI DING
KU DING CHA
KU DING CHA DONG QIN
KU DOU GEN
KU DOU ZI
KU GUA
KU HAO
KU HE LONG DAN
KU HONG GU
KU HUANG GUA
KU LANG SHU
KU LI MU YE
KU LIAN PI
KU LIAN SHI
KU LIAN YE
KU MA DOU

KU MANG HUANG JIN KU MU

KU QIAO MAI
KU RUO LONG DAN
KU SHAN NAI
KU SHEN

KU SHEN SHI

KU SHENG

KU SHI LIAN

KU SHU PI

KU TAN ZI

KU WEI DUI XIN JU

KU WEI YUAN ZHI

KU XIANG SHU

KU XUAN SHEN

KU YA DAN ZI

KU YE DAO HUANG BAI

KU YE DAO ZE LAN

KU YE WU TOU

KU YUAN ZHI

KU ZHI

KU ZHI

KUAI GEN CHUI TOU JU KUAI JING CAO SU

KUAI JING GE

KUAI JING MA DOU LING

KUAI JING MA LI JIN

KUAI JING SHUI SU
枯苍

苦草

苦地胆

苦地丁

苦丁茶

苦丁茶冬青

苦豆根

苦豆子

苦瓜

苦蒿

库赫龙胆*

苦红菇

苦黄瓜

苦郎树

苦栃木叶

苦楝皮

苦楝实

苦楝叶

苦马豆

库芙黄堇

苦木

苦养麦

库若龙胆*

苦山柰

苦参

苦参实

苦绳

苦石莲

苦树皮

苦檀子

苦味堆心菊

苦味远志

苦香树

苦玄参

苦鸦胆子

库页岛黄柏

库页岛泽兰

库页乌头*

苦远志

苦蘵

毛酸浆

块根垂头菊

块茎糙苏

块茎葛*

块茎马兜铃

块茎马利筋

块茎水苏
Monarch-of-the-East

Eelgrass

Scabrous Elephantfoot

Bunge Corydalis

Plumleaf Cratoxylum

Kudincha Holly

Foxtail-like Sophora Root*

Foxtail-like Sophora

Balsampear

Blin Conyza

Koch Gentian*

Bitter Russula

Bitter Cucumber*

Unarmed Glorybower

Retuse Ash Leaf

Chinaberry-tree Bark

Chinaberry-tree Fruit

Chinaberry-tree Leaf*

Saline Swainsonia

Govan Corydalis

Indian Quassiawood

Tartarian Buckwheat

Kuroo Gentian*

Bitter

Lightyellow Sophora

Lightyellow Sophora Seed

Chinese Dregea

Whiteflower Caesalpinia

Indian Quassiawood Bark

Thickfruit Millettia

Bitterness Sneezeweed*

Bitter Milkwort*

Cascarilla

Common Bitterfigwort

Bitter Brucea*

Sachaline Corktree*

Sachalin Eupatorium*

Sachalin Monkshood*

Racemed Milkwort

Cutleaf Groundcherry

Downy Groundcherry

Root-tuber Cremanthodium

Tuberousroot Jerusalemsage

Tuberous Kudzuvine*

Tuberous Dutchmanspipe

Tuberous Milkweed

Chinese Artichoke
T5747 Sauromatum guttatum

T6683 Vallisneria spiralis

T2334 Elephantopus scaber

T1709 Corydalis bungeana

T1785 Cratoxylum prunifolium

T3392 Ilex kudingcha

T6028 Sophora alopecuroides

T6029 Sophora alopecuroides

T4263 Momordica charantia

T1655 Conyza blinii

T2914 Gentiana kochiana

T5624 Russula rosacea

T1879 Cucumis sativus var. hanzil

T1566 Clerodendrum inerme

T2770 Fraxinus insularis

T4156 Melia azedarach

T4157 Melia azedarach

T4158 Melia azedarach

T6210 Swainsonia salsula [Syn. Sphaerophysa

salsula]

T1718 Corydalis govaniana

T4881 Picrasma quassioides [Syn. Picrasma

ailanthoides]

T2661 Fagopyrum tataricum

T2915 Gentiana kuroo

T3621 Kaempferia marginata

T6031 Sophora flavescens [Syn. Sophora

angustfolia]

T6032 Sophora flavescens [Syn. Sophora

angustfolia]

T2262 Dregea sinensis

T1106 Caesalpinia minax

T4882 Picrasma quassioides [Syn. Picrasma

ailanthoides]

T4240 Millettia pachycarpa

T3131 Helenium amarum

T5070 Polygala amarella

T1844 Croton eluteria

T4884 Picria felterrae

T1036 Brucea amarissima

T4794 Phellodendron sachalinense

T2573 Eupatorium sachalinense [Syn.

Eupatorium glehni]

T0127 Aconitum sachalinense

T5081 Polygala polygama

T4848 Physalis angulata

T4854 Physalis pubescens

T1786 Cremanthodium ellisii

T4813 Phlomis tuberosa

T5321 Pueraria tuberosa

T0641 Aristolochia tuberosa

T0741 Asclepias tuberosa

T6094 Stachys tuberifera 


\begin{tabular}{|c|c|c|c|}
\hline KUAI JING XI MEN FEI CAO & 块茎西门肺草* & Tuberous Comfrey & T6248 Symphytum tuberosum \\
\hline KUAI JING ZI JIN & 块茎紫堇 & Tuberous Corydalis* & T1748 Corydalis tuberosa \\
\hline KUAN BAO SHI DA GONG LAO & 宽苞十大功劳 & Broad-bracteate Mahonia & T4061 Mahonia eurybracteata \\
\hline KUAN DONG HUA & 款冬花 & Common Coltsfoot & T6574 Tussilago farfara \\
\hline KUAN SHE TUO WU & 宽舌㯻吾 & Broad-tongue Goldenray* & T3811 Ligularia platyglossa \\
\hline KUAN YE GE NI DI MU & 宽叶格尼迪木 & & T3038 Gnidia latifolia \\
\hline KUAN YE KUO BAO JU & 宽叶阔苍菊* & Broadleaf Pluchea* & T0848 Baccharis latifolia \\
\hline KUAN YE MAI MA TENG & 宽叶买麻藤 & Broadleaf Jointfir & T3031 Gnetum latifolium \\
\hline KUAN YE MEI YUAN ZHI & 宽叶美远志 & Broadleaf Milkwort* & T5085 Polygala senega var. latifolia \\
\hline KUAN YE QIAN LI GUANG & 宽叶千里光 & Broadleaf Groundsel* & T5897 Senecio platyphyllus \\
\hline \multirow[t]{2}{*}{ KUAN YE QIANG HUO } & 宽叶㒸活 & Forbes Notopterygium & T4445 Notopterygium forbesii [Syn. \\
\hline & & & Notopterygium franchetii] \\
\hline KUAN YE SHAN YUE GUI & 宽叶山月桂 & Mountain Laurel & T3623 Kalmia latifolia \\
\hline KUAN YE WU TAN & 宽叶乌檀 & Broadleaf Fatheadtree* & T4390 Nauclea latifolia \\
\hline KUAN YE XIANG PU & 宽叶香蒲 & Broadleaf Cattail Pollen & T6587 Typha latifolia \\
\hline KUAN YU XIAN JUE & 宽羽线蕨 & Broad-pinna Colysis & $\begin{array}{l}\text { T1629 Colysis pothifolia }[\text { Syn. Hemionitis } \\
\text { pothifolia }]\end{array}$ \\
\hline KUI BAN ER YE TAI & 盔瓣耳叶苔 & Armet-petal Ear-leaf Muscus* & T2802 Frullania muscicola \\
\hline KUI HAO & 魁蒿 & First Wormwood & T0689 Artemisia princeps \\
\hline KUN BU & 昆布 & Kelp Thallus & T3678 Laminaria japonica \\
\hline KUN LAN SHU & 昆栏树 & Wheelstamentree & T6549 Trochodendron aralioides \\
\hline KUN MING CHAI HU & 昆明柴胡* & Kunming Thorowax* & T1064 Bupleurum kunmingense \\
\hline KUN MING JI XUE TENG & 昆明鸡血藤 & Diels Millettia & T4234 Millettia dielsiana \\
\hline KUN MING SHAN HAI TANG & 昆明山海棠 & Glaucousback Threewingnut & T6540 Tripterygium hypoglaucum \\
\hline KUN MING XIANG CHA CAI & 昆明香茶菜 & Kunming Rabdosia* & T3499 Isodon kunmingensis \\
\hline KUO BAO JU & 阔苞菊 & Indian Pluchea & T0847 Baccharis indica $[$ Syn. Pluchea indica $]$ \\
\hline KUO GUO JI YING SU & 阔果蓟罂粟 & Platyangle Pricklepoppy* & T0611 Argemone platyceras \\
\hline KUO JIA HE HUAN & 阔荚合欢 & Siris-acacia & T0293 Albizzia lebbeck \\
\hline KUO YE GU SUI BU & 阔叶骨碎补 & Broadleaf Davallia & T2054 Davallia solida \\
\hline KUO YE LUO FU MU & 阔叶萝芙木 & Broadleaf Devilpepper & T5435 Rauwolfia latifrons \\
\hline KUO YE MI HOU TAO & 阔叶猕猴桃 & Broadleaf Actinidia & T0163 Actinidia latifolia \\
\hline KUO YE OU NV ZFEN & 阔叶欧女贞 & Tree Phyllirea & T4797 Phillyrea latifolia \\
\hline KUO YE SHAN MAI DONG & 阔叶山麦冬 & Broadleaf Liriope* & T3873 Liriope platyphylla \\
\hline KUO YE XIE CAO & 阔叶缴草 & Broadleaf Common Valeriana & T6680 Valeriana officinalis var. latifolia \\
\hline KUO ZHANG SONG YE JU & 扩张松叶菊 & Expansum Fig* & T4201 Mesembryanthemum expansum \\
\hline LA BA CHA & 喇叭茶 & Crystal Tea & T3735 Ledum palustre \\
\hline LA BA FEN SHI RUI & 喇叭粉石芯 & & T1523 Cladonia chlorophaea \\
\hline LA BA ZHUANG DUO GUO SHU & 喇叭状多果树* & & T5017 Pleiocarpa tubicina \\
\hline LA BO HE & 辣薄荷 & Pepper Mint & T4186 Mentha piperita \\
\hline LA GEN & 辣根 & Horseradish & T0647 Armoracia lapathifolia \\
\hline LA GEN HE GUO MU & 辣根核果木* & Armoracia Drypetes* & T2288 Drypetes armoracia \\
\hline LA JIAO & 辣椒 & Bush Redpepper & T1187 Capsicum frutescens \\
\hline LA KOU SHA MIAN BAO GUO & 拉口沙面包果 & Lakoocha Artocarpus* & T0717 Artocarpus lakoocha \\
\hline LA LIAO & 辣䓹 & Flaccid Knotweed & $\begin{array}{l}\text { T5105 Polygonum hydropiper var. flaccidum } \\
\text { [Syn. Polygonum flaccidum }]\end{array}$ \\
\hline LA MEI HUA & 腊梅花 & Wintersweet Bud & T1366 Chimonanthus fragrans [Syn. \\
\hline & & & Chimonanthus praecox] \\
\hline LA QIAN LI GUANG & 辣千里光 & Smirch Groundsel* & T5908 Senecio sceleratus \\
\hline LA RU GU & 辣乳菇 & Peppery Milky & $\begin{array}{l}\text { T3653 Lactarius piperatus [Syn. Agaricus } \\
\text { piperatus] }\end{array}$ \\
\hline LA SHI LU HUI & 拉氏芦荟* & Ruspol Aloe* & T0343 Aloe ruspoliana \\
\hline LA ZHI MU JIANG ZI & 蜡质木姜子* & Waxy Litse* & T3895 Litsea sebifera \\
\hline LA ZHU GUO & 蜡烛果 & Corniculate Aegiceras & T0194 Aegiceras corniculatum \\
\hline
\end{tabular}




\begin{tabular}{|c|c|c|c|}
\hline LAI FU & 莱菔 & Garden Radish & T5419 Raphanus sativus \\
\hline LAI FU ZI & 莱菔子 & Garden Radish Seed & T5420 Raphanus sativus \\
\hline LAI JIANG TENG & 来江藤 & Hance Brandisia & T1003 Brandisia hancei \\
\hline LAI KE BA DOU & 莱克巴豆 & Lechler Croton* & T1849 Croton lechleri \\
\hline LAI MENG & 来檬 & Lime & T1466 Citrus aurantifolia \\
\hline LAI SHI NA TE SHI DA GONG & 莱施纳特十大功劳 & Leschenault Mahonia & T4069 Mahonia leschenaultii \\
\hline \multicolumn{4}{|l|}{ LAO } \\
\hline LAN BO TE XIAO BO & 兰伯特小檗 & Lambert Barberry & T0908 Berberis lambertii \\
\hline LAN E XIANG CHA CAI & 兰萝香茶菜 & Bluesepal Rabdosia* & T3497 Isodon japonica var. glaucocalyx \\
\hline LAN HU LU BA & 兰胡卢巴 & Blue Trigonella & T6527 Trigonella caerulea \\
\hline LAN HUANG HONG GU & 兰黄红菇 & Blue-yellow Redmushroom* & T5619 Russula cyanoxantha \\
\hline LAN JI & 蓝薊 & Blue Thistle & T2320 Echium vulgare \\
\hline LAN JI CHUAN XIN LIAN & 蓝薊穿心莲 & Echiumlike Andrographis & T0453 Andrographis echioides \\
\hline LAN SE YA CHI JUN & 蓝色亚齿菌* & Inedible Mushroom & T3299 Hydnellum caeruleum \\
\hline LAN SHAI PIAO & 蓝筛朴 & Siebold Elder & T5705 Sambucus sieboldiana \\
\hline LAN SHUI LIAN & 兰睡莲 & Blue Waterlily & T4454 Nymphaea caerulea \\
\hline LAN YU BAI JI & 兰屿白芨 & Taiwan Bletilla* & T0954 Bletilla formosana \\
\hline LAN YU LUO YE RONG & 兰屿落叶榕 & & T2725 Ficus ruficaulis var. antaoensis \\
\hline LANG DANG GEN & 莨宕根 & Black Henbane Root & T3327 Hyoscyamus niger \\
\hline LANG DANG YE & 莨宕叶 & Black Henbane Leaf & T3328 Hyoscyamus niger \\
\hline LANG DANG ZI & 莨宕子 & Black Henbane Seed & T3329 Hyoscyamus niger \\
\hline LANG DU & 狼毒 & Chinese Stellera & T6105 Stellera chamaejasme \\
\hline LANG DU DA JI & 狼毒大戟 & Fischer Euphorbia & T2587 Euphorbia fischeriana \\
\hline LANG DU WU TOU & 狼毒乌头 & Badgersbane & T0114 Aconitum lycoctonum \\
\hline LANG PA CAO & 狼杷草 & Bur Beggarticks & T0941 Bidens tripartita \\
\hline LANG YU PI & 榔榆皮 & Chinese Elm Bark & T6596 Ulmus parvifolia \\
\hline LAO GUAN CAO & 老鹳草 & Wilford Cranesbill & T2949 Geranium wilfordii \\
\hline LAO NIU JIN & 老牛筋 & Junc-like Sandwort & T0607 Arenaria juncea \\
\hline LAO PO ZI ZHEN XIAN & 老婆子针线 & Lopseed & T4830 Phryma leptostachya \\
\hline LAO SHI WEN SHU LAN & 劳氏文殊兰* & Laurent Crinum* & T1800 Crinum laurentii \\
\hline LAO SHU GUA & 老鼠瓜 & Common Caper & T1180 Capparis spinosa \\
\hline LAO SHU LE & 老鼠簕 & Hollyleaf Acanthus & T0048 Acanthus ilicifolius \\
\hline LAO YA SHI & 老鸦柿 & Diamondleaf Persimmon & T2226 Diospyros rhombifolia \\
\hline LE SHI JIN HE HUAN & 勒氏金合欢* & Luederitz Acacia* & T0023 Acacia luederitzii \\
\hline LEI A WEI & 短裂片类阿魏* & & T2711 Ferulago brachyloba \\
\hline LEI BO WU TOU & 雷波乌头 & Leabo Monkshood & T0124 Aconitum pseudohuiliense \\
\hline LEI DENG XIN LIU SHAN HU & 蕾灯心柳珊瑚 & Gorgonian Junceella gemmacea & T3575 Junceella gemmacea \\
\hline LEI GONG QI & 雷公七 & Common Broadlily & T1574 Clintonia alpina \\
\hline LEI GONG TENG & 雷公藤 & Common Threewingnut & T6542 Tripterygium wilfordii \\
\hline LEI GUO SHA JI & 肋果沙棘 & Veinfruit Seabuckthorn & T3253 Hippophae neurocarpa \\
\hline LEI SHI QIAN LI GUANG & 雷氏千里光* & Renard Groundsel* & T5901 Senecio renardii \\
\hline LEI YE SHENG MA & 类叶升麻 & Asica Baneberry & T1418 Cimicifuga asiatica \\
\hline LENG FAN TUAN[yn & 冷饭团 & Blacktiger Kadsura & $\begin{array}{l}\text { T3613 Kadsura coccinea [Syn. Kadsura } \\
\text { chenensis; Kadsura hainanensis] }\end{array}$ \\
\hline LENG JIAO QIAN LI GUANG & 棱角千里光 & Angulate Groundsel* & T5880 Senecio angulatus \\
\hline LENG SHA BEI MU & 棱砂贝母 & Delavay Fritillary & T2784 Fritillaria delavayi \\
\hline LENG ZHI HU JI SHENG & 棱核檞寄生 & Angulate Mistletoe* & T6772 Viscum angulatum \\
\hline LENG ZHUANG BAI JIAN MU & 棱状白坚木* & Rhombicsign White Quebracho* & T0774 Aspidosperma rhombeosignatum \\
\hline LI & 藜 & Lambsquarters Juvenile & T1359 Chenopodium album \\
\hline LI BA NEN XIE HAO & 黎巴嫩邪蒿* & Moon-carrot & T5933 Seseli libanotis \\
\hline LI BIAN ZHI YI & 栋扁枝衣 & & T2637 Evernia prunastri \\
\hline LI BING FENG WEI JUE & 栗柄凤尾蕨 & Lead-coloured Brake & T5297 Pteris plumbea \\
\hline LI BING JIN FEN JUE & 栗柄金粉偋 & Lucidum Onychium & T4503 Onychium lucidum \\
\hline
\end{tabular}




\begin{tabular}{|c|c|c|}
\hline LI CAI & 蚛菜 & Conglobate Ulva Frond* \\
\hline LI CHUN HUA & 丽春花 & Corn Poppy \\
\hline LI DE LI FAN LONG YAN & 里德利番龙眼* & Ridley Pometia* \\
\hline LI DOU & 黎豆 & Capitateflower Velvetbean \\
\hline LI FEI MU JIANG ZI & 里菲木姜子 & Leefe Litse* \\
\hline LI GUO XIAN REN ZHANG & 梨果仙人掌 & Prickly Pear \\
\hline LI HE REN & 李核仁 & Japanese Plum Kernel \\
\hline LI HUA JU & 立花橘 & Japanese Tachibana \\
\hline LI JI SAN CHU MAI MA QIAN & 离基三出脉马钱* & Triplinervia Poisonnut* \\
\hline LI JIAMH CHAI HU & 丽江柴胡 & Rock Thorowax \\
\hline LI JIANG HUANG QIN & 丽江黄芩 & Likiang Skullcap \\
\hline LI JIANG MA HUANG & 丽江麻黄 & Likiang Ephedra \\
\hline LI JIANG QIAN HU & 漓江前胡 & Likiang Hogfennel \\
\hline LI JIANG WU TOU & 丽江乌头 & Likiang Monkshood* \\
\hline LI JUE & 栗蕨 & Incised Histiopteris \\
\hline LI LA GEN & 黎辣根 & Oriental Buckthorn \\
\hline LI LU & 藜芦 & Black Falsehellebore \\
\hline LI MENG & 黎檬 & Lemonlike Citrus \\
\hline LI MENG GEN & 黎檬根 & Lemonlike Citrus Root \\
\hline LI MENG PI & 黎檬皮 & Lemonlike Citrus Pericarp \\
\hline LI MENG YE & 黎檬叶 & Lemonlike Citrus Leaf \\
\hline LI MI ZI LAN & 梨米仔兰* & Pear Aglaia* \\
\hline LI MU & 悷木 & Tibet Lyonia \\
\hline LI PU PO JU & 利普珀菊* & Lipp Amberboa* \\
\hline LI QI HUAI & 利奇槐 & Leachiana Sophora \\
\hline LI SE SHU WEI CAO & 栗色鼠尾草 & Chestnut Sage \\
\hline LI SHU PI & 栗树皮 & Chinese Chestnut Bast \\
\hline LI SHUO ZHU XIAN & 梨蒴珠藓 & \\
\hline LI XIAN XIANG CHA CAI & 理县香茶菜 & Lihsien Rabdosia \\
\hline LI XING MA BO & 梨形马勃 & Pear-like Puff-ball \\
\hline LI YE & 梨叶 & Bretschneider Pear Leaf \\
\hline LI YU & 鲤鱼 & Carp \\
\hline LI YU DAN & 鲤鱼胆 & Carp Gall \\
\hline LI YU PI & 鲤鱼皮 & Carp Skin \\
\hline LI ZHI & 荔枝 & Lychee \\
\hline LI ZHI CAO & 荔枝草 & Common Sage \\
\hline LI ZHI HAO & 痢止蒿 & Forrest Bugle \\
\hline LI ZHI HE & 荔枝核 & Lychee Seed \\
\hline LI ZI & 李子 & Japanese Plum \\
\hline LIAN HUA & 楝花 & Chinaberry-tree Flower \\
\hline LIAN HUA JIN QUE ER & 链花金雀儿* & Goldregen \\
\hline LIAN JIANG & 廉姜 & Chinese Galangal \\
\hline LIAN PENG CAO & 莲蓬草 & Japanese Farfugium \\
\hline LIAN QIAN CAO & 连钱草 & \\
\hline LIAN QIAO & 连翅 & Weeping Forsythia \\
\hline LIAN SHENG GUI ZI HUA & 莲生桂子花 & Bloodflower Milkweed \\
\hline LIAN XIANG SHU & 连香树 & Katsura-tree \\
\hline LIAN XING HUANG QI & 镰形黄芪* & Falcate Milkvetch* \\
\hline LIAN YE TONG & 莲叶桐 & Lotusleaftung \\
\hline
\end{tabular}

T6598 Ulva conglobata

T4625 Papaver commutatum [Syn. Papaver rhoeas]

T5136 Pometia ridleyi

T6147 Stizolobium capitatum

T3892 Litsea leefeana

T4520 Opuntia ficus-indica

T5238 Prunus salicina

T1513 Citrus tachibana

T6190 Strychnos triplinervia

T1070 Bupleurum rockii

T5841 Scutellaria likiangensis

T2372 Ephedra likiangensis

T4756 Peucedanum govanianum var. bicolo T0096 Aconitum forrestii [Syn. Aconitum

likiangense]

T3260 Histiopteris incisa

T5455 Rhamnus crenata

T6698 Veratrum nigrum

T1494 Citrus limonia

T1495 Citrus limonia

T1496 Citrus limonia

T1497 Citrus limonia

T0242 Aglaia pirifera

T3991 Lyonia ovalifolia

T0392 Amberboa lippi

T6038 Sophora leachiana

T5667 Salvia castanea

T1256 Castanea mollissima

T0874 Bartramia pomiformis

T3505 Isodon lihsienensis

T3961 Lycoperdon pyriforme

T5362 Pyrus bretschneideri

T1980 Cyprinus carpio

T1981 Cyprinus carpio

T1982 Cyprinus carpio

T3876 Litchi chinensis

T5685 Salvia plebeia

T0264 Ajuga forrestii

T3877 Litchi chinensis

T5239 Prunus salicina

T4159 Melia azedarach

T1988 Cytisus laburnum

T0354 Alpinia chinensis

T2691 Farfugium japonicum

T2974 Glechoma lungituba

T2756 Forsythia suspensa

T0736 Asclepias curassavica

T1333 Cercidiphyllum japonicum var. sinense T0795 Astragalus falcatus

T3231 Hernandia sonora [Syn. Hernandia ovigera]

T3810 Ligularia nelumbifolia 


\begin{tabular}{|c|c|c|c|}
\hline LIAN YE WU ZHU YU & 楝叶吴荣英 & Dyebark Evodia & T2643 Evodia meliifolia \\
\hline LIAN ZHU TENG & 链珠藤 & China Alyxia & T0383 Alyxia sinensis \\
\hline LIAN ZI & 莲子 & Hindu Lotus Seed & T4400 Nelumbo nucifera \\
\hline LIAN ZI XIN & 莲子心 & Hindu Lotus Plumule & T4401 Nelumbo nucifera \\
\hline LIAN ZUO GE JUN & 莲座革菌 & Vase Thelephore & T6422 Thelephora vialis \\
\hline LIANG BAI MEI RONG JUN & 亮白煤绒菌* & White Fuliga* & T2806 Fuligo candida \\
\hline LIANG HUA GE NI DI MU & 亮花格尼迪木 & & T3037 Gnidia lamprantha \\
\hline LIANG JUN & 亮菌 & Armillariella Tabescens & T0646 Armillariella tabescens \\
\hline LIANG MIAN QING & 两面青 & Indian Maesa & T4028 Maesa indica \\
\hline LIANG NIAN SHI SONG & 两年石松 & Sharpleaf Clubmoss* & T3966 Lycopodium annotinum var. acrifolium \\
\hline LIANG QI LIAO & 两栖苶 & Amphibious Knotweed & T5097 Polygonum amphibium \\
\hline LIANG SHAN DU JUAN & 凉山杜鹃 & & T5510 Rhododendron huianum \\
\hline LIANG SHAN XIANG CHA CAI & 凉山香茶菜 & Liangshan Rabdosia & T3504 Isodon liangshanica \\
\hline LIANG SI FEI YAN CAO & 两似飞燕草* & Ambiguous Consolida* & T1646 Consolida ambigua \\
\hline LIANG YE HUA PI & 亮叶桦皮 & Shiningleaf Birch Bark & T0932 Betula luminifera \\
\hline LIANG YE RONG & 亮叶榕 & Bright Fig* & T2722 Ficus nitida \\
\hline LIANG YE YAN DOU TENG & 亮叶岩豆藤 & Shiningleaf Millettia & T4238 Millettia nitida \\
\hline LIAO DONG CONG MU & 辽东淴木 & Liaodong Aralia & T0572 Aralia elata \\
\hline LIAO DONG CONG MU YE & 辽东淴木叶 & Liaodong Aralia Leaf* & T0573 Aralia elata \\
\hline LIAO GAO BEN & 辽莕本 & Jehol Ligusticum & T3822 Ligusticum jeholense \\
\hline LIAO GE WANG GEN & 了哥王根 & Indian Stringbush Root & T6820 Wikstroemia indica \\
\hline LIAO LAN GUO & 蓼蓝果 & Indigoplant Fruit & T5120 Polygonum tinctorium \\
\hline LIAO LAN YE & 蓼蓝叶 & Indigoplant Leaf & T5121 Polygonum tinctorium \\
\hline LIAO NING SHAN ZHA & 辽宁山楂 & Redhaw Hawthorn & T1780 Crataegus sanguinea \\
\hline LIAO SHANG RONG MAO HUA & 疗伤线毛花 & Kidney Vetch & T0531 Anthyllis vulneraria \\
\hline LIAO SHI & 蓼实 & Red-knees Fruit & T5103 Polygonum hydropiper \\
\hline LIAO XI XIN & 辽细辛 & Manchurian Wildginger & $\begin{array}{l}\text { T0728 Asarum heterotropoides var. } \\
\text { mandshuricum }\end{array}$ \\
\hline LIE DANG & 列当 & Skyblue Broomrape & T4536 Orobanche coerulescens \\
\hline LIE E TAI & 裂罡苔 & Polybract Split-calyx Liverwort* & T1365 Chiloscyphus polyanthus \\
\hline LIE GUO SHU & 裂果薯 & Lobedfruit Tacca & $\begin{array}{l}\text { T6276 Tacca plantaginea }[\text { Syn. Schizocapsa } \\
\text { plantaginea }]\end{array}$ \\
\hline LIE TI MU CENG KONG JUN & 裂蹄木层孔菌 & & T4788 Phellinus linteus \\
\hline LIE WEI LIE LAN & 烈味裂榄 & Bursera* & T1080 Bursera graveolens \\
\hline LIE YE QIAN NIU & 裂叶牵牛 & Lobedleaf Morningglory* & T3449 Ipomoea hederacea \\
\hline LIE YE YE YING SU & 裂叶野罂粟 & Splidleaf Poppy* & T4631 Papaver nudicaule var. chinense \\
\hline LIN BAI ZHI & 林白芷 & Wild Angelica & T0497 Angelica sylvestris \\
\hline LIN BEI ZI & 林背子 & Field Lacquertree & $\begin{array}{l}\text { T6482 Toxicodendron succedaneum }[\text { Syn. } \\
\text { Rhus succedanea }]\end{array}$ \\
\hline LIN DI HAO & 林地蒿* & Woodland Wormwood & T0700 Artemisia sylvatica \\
\hline LIN DI SHUI SU & 林地水苏 & Whitespot Betony & T6093 Stachys sylvatica \\
\hline LIN DI WU TOU & 林地乌头 & Woodland Monkshood & T0120 Aconitum nemorum \\
\hline LIN DI XIANG RI KUI & 林地向日葵* & Woodland Sunflower & T3154 Helianthus strumosus \\
\hline LIN JING ZHONG ZI WEN SHU & 鳞茎种子文殊兰* & Bulb-spermo Crinum* & T1797 Crinum bulbispermum \\
\hline LAN & & & \\
\hline LIN PIAN XUAN SHEN & 鳞片玄参* & Scale Figwort* & T5827 Scrophularia lepidota \\
\hline LIN QIN & 林檎 & Chinese Pearleaf Crabapple & T4086 Malus asiatica \\
\hline LIN SHENG SHAN LI DOU & 林生山黧豆 & Wild Pea & T3713 Lathyrus sylvestris \\
\hline LIN SHENG XUAN SHEN & 林生玄参 & Common Figwort & T5829 Scrophularia nodosa \\
\hline LIN SHI CAN & 林石蚕 & Wood Sage & T6369 Teucrium scorodonia \\
\hline LIN WEN JING & 林问荆 & Forest Horsetail & T2412 Equisetum sylvaticum \\
\hline LING & 菱 & Singharanut & T6492 Trapa bispinosa \\
\hline LING LAN & 铃兰 & Lily of Valley & T1649 Convallaria keiskei [Syn. Convallaria \\
\hline
\end{tabular}




LING MAO XIANG
LING MU
LING NAN DU JUAN
LING NAN HUAI SHU
LING SHUI AN LUO
LING XIANG CAO
LING XING CHANG CHUN TENG
LING YE ZI JU
LING ZHI
LIU BAI PI
LIU CHUAN YU
LIU GUO ZI YU PAN
LIU HUANG TIE XIAN JUE
LIU JI NU
LIU JIAO LIAN
LIU LAN XIANG
LIU LENG JU
LIU LI CAO

LIU LI FAN LV
LIU LI JU
LIU QIU SHE GEN CAO
LIU SHAN
LIU SHAN HU

LIU SU JIN SHI HU
LIU SU LI LU
LIU SU SHI HU
LIU TU HU JIAO
LIU YE BAI QIAN
LIU YE CAI HUANG QIN
LIU YE CEN
LIU YE MU LAN
LIU ZHI

LIU ZHUANG DAN YE YUN

XIANG

LIU ZI SAI JIN LIAN MU

LONG BAI

LONG CHUAN HUA

LONG DAN

LONG KUI

LONG NAO GAO XIANG

LONG SHENG XIANG CHA CAI

LONG SHU DU JUAN

LONG TU ZHU

LONG XIAN XIANG

LONG XU CAO

LONG XU TENG

LONG XUE SHU

LONG YA CAO

\section{灵猫香 \\ 柃木 \\ 岭南杜鹃 \\ 岭南槐树 \\ 陵水暗罗 \\ 灵香草 \\ 菱形常春藤 \\ 菱叶紫菊 \\ 灵芝(赤芝) \\ 柳白皮 \\ 柳穿鱼 \\ 瘤果紫玉盘 \\ 硫磺铁线藓* \\ 刘寄奴 \\ 六角莲 \\ 留兰香 \\ 六棱菊 \\ 琉璃草}

\begin{tabular}{l} 
琉璃繁缕 \\
琉璃草 \\
硫球蛇根草 \\
柳杉 \\
柳珊瑚 Gorgoniae \\
suberogorgia \\
流苏金石斛 \\
流苏蘩芦 \\
流苏石斛 \\
瘤突胡椒 \\
柳叶白前 \\
柳叶菜黄芩* \\
柳叶梣 \\
柳叶木兰 \\
柳枝 \\
瘤状单叶芸香 \\
\\
六籽赛金莲木* \\
龙柏 \\
龙船花 \\
龙胆 \\
龙葵 \\
龙脑膏香 \\
龙胜香茶菜 \\
陇蜀杜鹃 \\
龙吐珠 \\
龙涎香 \\
龙须草 \\
龙须藤 \\
龙血树 \\
龙芽草 \\
\hline
\end{tabular}

majalis]

T6800 Viverra zibetha

T2631 Eurya japonica

T5513 Rhododendron mariae

T6045 Sophora tomentosa

T5067 Polyalthia nemoralis

T4002 Lysimachia foenum-graecum

T3115 Hedera rhombea

T4447 Notoseris rhombiformis

T2848 Ganoderma lucidum

T5650 Salix babylonica

T3845 Linaria vulgaris

T6664 Uvaria kweichowensis

T0178 Adiantum sulphureum

T0661 Artemisia anomala

T2302 Dysosma pleiantha [Syn. Podophyllum

pleianthum]

T4190 Mentha spicata

T3674 Laggera alata

T1971 Cynoglossum zeylanicum [Syn.

Anchusa zeylanica; Cynoglossum furcatum;

Cynoglossum formosanum ]

T0440 Anagallis arvensis

T0981 Borago officinalis

T4512 Ophiorrhiza liukiuensis

T1867 Cryptomeria fortunei

T3053 Gorgoniae suberogorgia

Fimbriate Dendrobium*

Fimbriate Falsehellebore*

Eyeshaped Dendrobium

Tuberculate Pepper*

Willowleaf Swallowwort

Willowweedleaf Skullcap

Willowleaf Ash Bark

Willowleaf Magnolia*

Babylon Weeping Willow Branch

Tuberculate Rue*

Hexaseed Ouratea*
Dragon Juniper
Chinese Ixora
Rough Gentian
Black Nightshade
Borneol Oil-Resin
Longshen Rabdosia
Przewallsk Rhododendron*
Bleedingheart Glorybower
Ammbergris
Hard Bluegrass
Champion Bauhinia
Dragontree
Hairyvein Agrimonia

T2101 Dendrobium fimbriatum T6696 Veratrum fimbriatum

T2102 Dendrobium fimbriatum var. oculatum T4972 Piper tuberculatum

T1960 Cynanchum stauntonii

T5837 Scutellaria epilobifolia

T2779 Fraxinus stylosa

T4049 Magnolia salicifolia

T5651 Salix babylonica

T5633 Ruta tuberculata [Syn. Haplophyllum tuberculatum]

T4561 Ouratea hexasperma

T3582 Juniperus chinensis var. kaizuka

T3549 Ixora chinensis

T2930 Gentiana scabra

T6008 Solanum nigrum

T2275 Dryobalanops aromatica

T3510 Isodon lungshengensis

T5521 Rhododendron przewalskii

T1570 Clerodendrum thomsonae

T4855 Physeter catodon

T5033 Poa sphondylodes

T0877 Bauhinia championii

T2254 Dracaena draco

T0248 Agrimonia pilosa 


\begin{tabular}{|c|c|}
\hline LONG YAN DU HUO & 龙眼独活 \\
\hline LONG YAN JING & 龙眼睛 \\
\hline LONG YAN YE & 龙眼叶 \\
\hline LOU DI QING MEI & 娄地青霉 \\
\hline LOU DOU PAO NANG CAO & 漏斗泡囊草 \\
\hline LOU LU & 漏芦 \\
\hline LU BEI GUI HUA & 绿背桂花 \\
\hline LU BIAN QING & 路边青 \\
\hline LU BIAN ZI CAO & 路边紫草 \\
\hline LU CAO & 鹿草 \\
\hline LU CONG & 鹿葱 \\
\hline LU CUI QUE & 露翠雀 \\
\hline LU DE WEI HAO & 陆得威蒿 \\
\hline LU DI MIAN & 陆地棉 \\
\hline LU DOU LE HUA & 露兜簕花 \\
\hline LU GEN & 芦根 \\
\hline LU HUI & 芦荟(库拉索芦荟) \\
\hline LU JIAO CAI & 鹿角菜 \\
\hline LU JIAO QI SHU & 鹿角漆树 \\
\hline LU LU TONG & 路路通 \\
\hline LU RONG & 鹿茸 \\
\hline LU RUI WU TOU & 露芯乌头 \\
\hline LU SHAN SHI WEI & 庐山石韦 \\
\hline LU SHAN XIANG CHA CAI & 鲁山香茶菜 \\
\hline LU SHENG GE JUN & 陆生革菌* \\
\hline LU SHI DONG LING CAO & 卢氏冬凌草 \\
\hline LU SHUI CAO & 露水草 \\
\hline LU SUI GE MU & 绿穗格木 \\
\hline LU XIAN CAO & 鹿衔草 \\
\hline LU YI CI JU & 路易刺橘 \\
\hline LU YU SHU & 绿玉树 \\
\hline LU ZHU GEN & 芦竹根 \\
\hline LU ZHU XIANG CHA CAI & 露珠香茶菜 \\
\hline LUAN BAN ZAO ZHUI & 卵瓣蚤缀 \\
\hline LUAN DA SHAN ZHANG YA CAI & 峦大山獐芽菜* \\
\hline LUAN HUA & 森华 \\
\hline LUAN SHU & 奕树 \\
\hline LUAN YE HU JI SHENG & 卵叶檞寄生 \\
\hline LUAN YE HU JIAO & 卵叶胡椒 \\
\hline LUAN YE MEI DENG MU & 卵叶美登木 \\
\hline LUAN YE SAN ZHE MAI ZI & 卵叶三褶脉紫苑 \\
\hline \multicolumn{2}{|l|}{ WAN } \\
\hline LUAN YE TENG HUANG & 卵叶藤黄* \\
\hline LUAN YE TIAN JIE CAI & 卵叶天芥菜 \\
\hline LUAN YE WA ER TENG & 卵叶娃儿藤* \\
\hline LUAN YE YIN LIAN HUA & 卵叶银莲花 \\
\hline LUAN YUAN CHANG CHUN HUA & 卵圆长春花 \\
\hline LUE DAAO DING ZAO & 略大凹顶藻 \\
\hline LUN HUAN TENG & 轮环藤 \\
\hline
\end{tabular}

Farges Aralia
Reticulate Leafflower*
Longan Leaf

Funneled Physochlaina

Uniflower Swisscentaury

Manyflower Glorybower Leaf

Wild Gromwel1*

Redflowered Swisscentaury*

Autumn Lycoris

Denuded Larkspur*

Western Sage

Upland Cotton

Thatch Screwpine Flower

Common Reed Rhizome

Kulaso Aloe Dried Juice

Furcate Gloiopeltis Frond

Stag's-horn Sumach

Beautiful Sweetgum

Hairy Antler

Nakedstamen Monkshood

Shearer's Pyrrosia Frond

Lushan Rabdosia*

Terrestrial Thelephore*

Lushien Rabdosia*

Common Cyanotis

Greenspike Erythrophleum*

Chinese Pyrola

Louis Vepris*

Malabartree Euphorbia

Giantreed Rhizome

Dew Rabdosia

Ovatepetal Sandwort

Randain Swertia*

Paniculate Goldraintree Flower

Paniculate Goldraintree Root-bark

White Mistletoe

Ovateleaf Pepper

Ovateleaf Mayten*

Ovate-leafThreevein Aster*

Ovateleaf Garcinia*

Ovateleaf Heliotrope*

Ovateleaf Tylophora

Ovateleaf Anemone

Oval Periwinkle*

Smaller Concave-top Alga*

Racemose Cyclea
T0574 Aralia fargesii

T4842 Phyllanthus reticulatus

T2627 Euphoria longan [Syn. Dimocarpus

longan]

T4698 Penicillium roqueforti

T4858 Physochlaina infundibularis

T5466 Rhaponticum uniflorum

T2651 Excoecaria cochinchinensis var. viridis

T1554 Clerodendron cyrtophyllum

T3883 Lithospermum ruderale

T5465 Rhaponticum carthamoides

T3988 Lycoris squamigera

T2071 Delphinium denudatum

T0682 Artemisia ludoviciana

T3058 Gossypium hirsutum [Syn. Gossypium

mexicanum]

T4616 Pandanus tectorius

T4829 Phragmites communis

T0347 Aloe vera [Syn. Aloe barbadensis]

T2994 Gloiopeltis furcata

T5540 Rhus typhina

T3867 Liquidambar formosana [Syn.

Liquidambar taiwaniana ]

T1337 Cervus nippon; Cervus elaphus

T0099 Aconitum gymnandrum

T5360 Pyrrosia sheareri

T3521 Isodon rubescens var. lushanensis

T6421 Thelephora terrestris

$\mathrm{T} 3522$ Isodon rubescens var. lushiensis

T1922 Cyanotis vaga

T2483 Erythrophleum chlorostachyum

T5345 Pyrola calliantha [Syn. Pyrola

rotundifolia ssp. chinensis]

T6690 Vepris louisii

T2624 Euphorbia tirucalli

T0722 Arundo donax

T3495 Isodon irrorata

T0609 Arenaria kansuensis var. ovatipeatala

T6237 Swertia randainensis

T3635 Koelreuteria paniculata

T3636 Koelreuteria paniculata

T6771 Viscum album

T4933 Piper attenuatum

T4138 Maytenus ovatus

T0777 Aster ageratoides var. ovatus

T2869 Garcinia ovalifolia

T3177 Heliotropium ovalifolium

T6582 Tylophora ovata

T0465 Anemone begoniifolia

T1269 Catharanthus ovalis

T3718 Laurencia majuscula

T1932 Cyclea racemosa 


LUN SHENG SHAN XIANG
LUO BO HUA LING CAO
LUO BU MA
LUO DI DAN CAO
LUO DI SHENG GEN
LUO E YE XIA ZHU
LUO FU MU
LUO FU MU JING YE
LUO GUO DI
LUO HAN BAI
LUO HAN GUO
LUO HAN SONG SHI
LUO HAN SONG YE
LUO HUA NAN MEI ROU DOU
KOU
LUO HUA SHENG
LUO HUA SHENG YOU
LUO HUA SHENG ZHI YE
LUO JI SHAN YUAN BAI
LUO JING ER CAO
LUO KE SI BAO MI ZI LAN
LUO KUI HUA
LUO KUI SHU

轮生山香*

罗勃花菱草

罗布麻

裸地胆草

落地生根

落萼叶下珠

萝芙木

萝芙木茎叶

罗锅底

罗汉柏

罗汉果

罗汉松实

罗汉松叶

落花南美肉豆冦*

$\begin{array}{ll}\text { 落花生 } & \text { Peanut } \\ \text { 落花生油 } & \text { Peanut Oil } \\ \text { 落花生枝叶 } & \text { Peanut Branch-leaf } \\ \text { 落矶山圆柏 } & \text { Western Red Cedar } \\ \text { 裸茎耳草 } & \text { Nude-stem Eargrass* } \\ \text { 罗克斯堡米仔兰 } & \text { Roxburg Aglaia* } \\ \text { 落葵花 } & \text { Red Vinespinach flower }\end{array}$

落葵薯

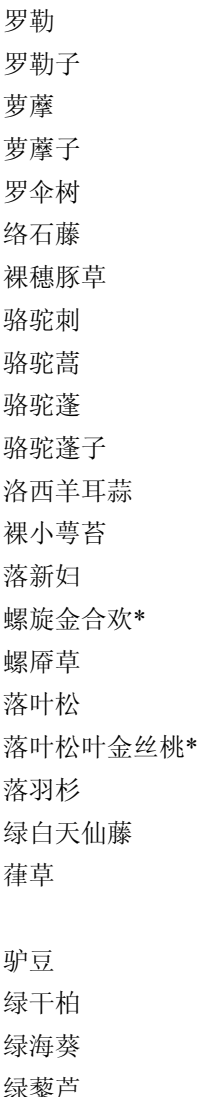

Verticillate Bushmint*
Lobb Poppy*
Dogbane
Node Elephantfoot*
Air-plant
Flexuose Leafflower*
Common Devilpepper
Common Devilpepper Stem and Leaf
Lovely Hemsleya
Broadleaf Arborvitae Hiba
Grosvenor Siraitia

Longleaf Podocarpus Seed

Longleaf Podocarpus Leaf

LUO LE
LUO LE ZI
LUO MO
LUO MO ZI
LUO SAN SHU
LUO SHI TENG
LUO SUI TUN CAO
LUO TUO CI
LUO TUO HAO
LUO TUO PENG
LUO TUO PENG ZI
LUO XI YANG ER SUAN
LUO XIAO E TAI
LUO XIN FU
LUO XUAN JIN HE HUAN
LUO YAN CAO
LUO YE SONG
LUO YE SONG YE JIN SI TAO
LUO YU SHAN
LV BAI TIAN XIAN TENG
LV CAO

LV DOU

LV GAN BAI

LV HAI KUI

LV LI LU
Basil

Basil Fruit

Japanese Metaplexis

Japanese Metaplexis Seed

Pentagonous Ardisia

Chinese Star Jasmine

Perennial Ragweed

Manaplant Alhagi Sweet Secretion

Little Peganum

Common Peganum

Common Peganum Seed

Fen Orchid

Chinese Astilbe

Spiral Acacia*

Littleleaf Lemmaphyyllum Herb

Dahurian Larch

Larch-leaf St.John'swort*

Deciduous Cypress

Chloro-white Fibraurea*

Japanese Hop

Common Sainfoin

Arizona Cypress

Green Anemone

Green Falsehellebore
T3383 Hyptis verticillata

T2496 Eschscholzia lobbii

T0553 Apocynum venetum

T2335 Elephantus nudatus

T1043 Bryophyllum pinnatum

T4838 Phyllanthus flexuosus

T5423 Rauvolfia verticillata

T5424 Rauvolfia verticillata

T3203 Hemsleya amabilis

T6443 Thujopsis dolobrata

T5967 Siraitia grosvenorii [Syn. Momordica grosvenorii]

T5042 Podocarpus macrophyllus

T5043 Podocarpus macrophyllus

T6768 Virola caducifolia

T0559 Arachis hypogaed

T0560 Arachis hypogaea

T0561 Arachis hypogaea

T3597 Juniperus scopulorum

T3127 Hedyotis nudicaulis

T0244 Aglaia roxburghiana

T0875 Basella rubra

T0515 Anredera cordifolia [Syn.

Baussingaultia cordifolia; Baussingaultia

gracilis f. pseudobaselloides; Baussingaultia

gracilis var. pseudobaselloides]

T4470 Ocimum basilicum

T4471 Ocimum basilicum

T4206 Metaplexis japonica

T4207 Metaplexis japonica

T0602 Ardisia quinquegona

T6484 Trachelospermum jasminoides

T0405 Ambrosia psilostachya

T0302 Alhagi pseudalhagi

T4689 Peganum nigellastrum

T4687 Peganum harmala

T4688 Peganum harmala

T3862 Liparis loeselii

T4339 Mylia nuda

T0784 Astilbe chinensis

T0031 Acacia spirorbis

T3736 Lemmaphyllum microphyllum

T3690 Larix gmelini

T3358 Hypericum laricifolium

T6305 Taxodium distichum

T2714 Fibraurea chloroleuca

T3288 Humulus japonicus [Syn. Humulus

scandens]

T4491 Onobrychis viciifolia

T1895 Cupressus arizonica

T0526 Anthopleura stell

T6700 Veratrum viride 


\begin{tabular}{|c|c|c|}
\hline LV SE MU MEI & 绿色木霉 & \\
\hline LV SONG GUO & 吕宋果 & Ignat Poisonnut Seed \\
\hline LV SONG JIA MI & 吕宋荚蒾 & Luzon Viburnum* \\
\hline LV SONG QIU MAO & 吕宋楸毛 & Kamalatree \\
\hline LV SUN PIAN & 绿笋片 & Oldham Bamboo Shoot \\
\hline LV TANG SONG CAO & 绿唐松草 & Glaucous Meadowrue* \\
\hline LV TI CAO YE TUO WU & 驴蹄草叶㯻吾 & Marshmarigold-like Goldenray* \\
\hline LV TI GEN CAO & 绿德根草 & Green Hellebore* \\
\hline LV XIAN REN ZHANG & 绿仙人掌 & Common Prickly Pear \\
\hline LV ZAO JI GEN YING MAO ZAO & 绿藻基根硬毛藻 & \\
\hline LV ZI SHAN XIAO JU & 绿籽山小橘* & \\
\hline MA BI MU & 马比木 & Pittosporumlike Nothapodytes \\
\hline MA BIAN CAO & 马鞭草 & European Verbena \\
\hline MA BING LANG & 马槟榔 & Masaikai Caper \\
\hline MA BO & 马勃 & Bark-less Puff-ball \\
\hline MA CAN DOU & 马虫豆 & Horse Bean \\
\hline MA CHANG LI ZI JIN & 马长里紫堇 & Marschall Corydalis * \\
\hline MA CHI XIAN & 马齿苋 & Purslane \\
\hline MA DAN GUO & 马蛋果 & Fragrant Gynocardia \\
\hline MA DAO CHOU TAN & 马岛臭檀 & Madagascar Evodia* \\
\hline MA DAO HA NI MU & 马岛哈尼木 & Madagascar Hani-wood* \\
\hline MA DAO HUANG LIAN SHU & 马岛黄楝树* & Fatraina (in Madagascar) \\
\hline MA DAO SI WO CI DOU & 马岛斯沃茨豆 & Madagascar Swartzia* \\
\hline MA DE LI MIAN ZAO ER & 马德里绵柊儿* & Madrid Squill* \\
\hline MA DOU LING & 马兒铃 & Slender Dutchmanspipe \\
\hline MA DU LA ZHU SHI DOU & 马都拉猪屎豆 & Madura Crotalaria* \\
\hline MA ER SHAN WU TOU & 马耳山乌头 & Delavy Monkshood \\
\hline MA FENG MU & 马疯木 & Manchineel \\
\hline MA FENG SHU & 麻风树 & Leprous Tree \\
\hline MA GEN & 麻根 & Hemp Fimble Root \\
\hline MA GUI HUA & 马桂花 & Manynerve Embelia \\
\hline MA HUA & 麻花 & Hemp Fimble Flower* \\
\hline MA HUA JIAO & 麻花艽 & Straw-coloured Gentian \\
\hline MA HUANG & 麻黄(草麻黄) & Chinese Ephedra \\
\hline MA HUANG GEN & 麻黄根 & Chinese Ephedra Root \\
\hline MA LA BA CHU & 马拉巴㯪 & Hairyleaf South Ailanthus \\
\hline MA LA BA JIAN MU & 马拉巴樫木* & Malaba Pencilwood* \\
\hline MA LA BA YANG TI JIA & 马拉巴羊蹄甲* & Malaba Bauhinia* \\
\hline MA LAI BAN DAO RAN MU & 马来半岛染木树* & Malaya Dieingtree* \\
\hline \multicolumn{3}{|l|}{ SHU } \\
\hline MA LAI XI YA HUANG YANG & 马来西亚黄杨* & Malaysian Box* \\
\hline MA LAI XI YA MAI MA TENG & 马来西亚买麻藤 & Spinach-like Jointfir \\
\hline MA LAI XI YA RUI MU & 马来西亚蒿木 & Malaysia Kopsia* \\
\hline MA LAN GEN & 马蓝根 & Common Baphicacanthus Root \\
\hline MA LAN YE & 马蓝叶 & Common Baphicacanthus Leaf \\
\hline MA LIAN & 麻楝 & Chittagong Chickrassy \\
\hline MA LIN & 马萄 & Chinese Iris \\
\hline MA LIN ZI & 马萄子 & Chinese Iris \\
\hline
\end{tabular}

T6503 Trichoderma virida

T6177 Strychnos ignatii

T6737 Viburnum luzonicum

T4084 Mallotus philippinensis

T5961 Sinocalamus oldhami

T6390 Thalictrum glaucum

T3800 Ligularia calthaefolia

T3189 Helleborus viridis

T4521 Opuntia vulgaris

T1346 Chaetomorpha basiretorsa

T3005 Glycosmis chlorosperma

T4439 Nothapodytes pittosporoides

T6709 Verbena officinalis

T1179 Capparis masaikai

T3701 Lasiosphaera fenzlii

T6748 Vicia faba var. equina

T1724 Corydalis marschalliana

T5173 Portulaca oleracea

T3082 Gynocardia odorata

T2642 Evodia madagascariensis

T3110 Harungana madagascariensis

T5702 Samadera madagascariensis

T6211 Swartzia madagascariensis

T5810 Scilla maderensis [Syn. Autonoë

madeirensis]

T0626 Aristolochia debilis [Syn. Aristolochia

longa]

T1825 Crotalaria madurensis

T0090 Aconitum delavayi

T3252 Hippomane mancinella

T3560 Jatropha curcas

T1174 Cannabis sativa

T2346 Embelia oblongifolia

T1175 Cannabis sativa

T2934 Gentiana straminea

T2380 Ephedra sinica

T2381 Ephedra sinica

T0258 Ailanthus malabarica

T2312 Dysoxylum malabaricum

T0878 Bauhinia malabarica

T5729 Saprosma scortechinii

T1090 Buxus malaiana

T3029 Gnetum gnemonoides

T3641 Kopsia griffithii

T0866 Baphicacanthus cusia [Syn.

Strobilanthes cusia]

T0867 Baphicacanthus cusia [Syn.

Strobilanthes cusia]

T1410 Chukrasia tabularis

T3465 Iris pallasii var. chinensis

T3464 Iris lactea var. chinensis [Syn. Iris

pallasii var. chinensis] 
MA LING SHU

MA LIU JIA YU TENG

MA LIU YE

MA NIAO PAO

MA NIU XI

MA QIAN ZI

MA SANG

MA SANG YE

MA SHAN QIAN HU

MA SHI DA HUANG

MA SHI JIN YAO

MA SHI LU HUI

MA SHI XIANG KE KE

MA SHI XIANG RI KUI

MA SI TE SI DU YING

MA TI WEN TIAN ZHU KUI

MA TI YE

MA TONG HUA

MA WEI LIAN

MA WEI SHAN

MA WEI SONG YE

MA YE

MA YE QIAN LI GUANG

MA YIN HUA

MA ZHUANG QIE

MA ZHUANG SAI YA MA

MAI DONG

MAI JIA GONG

MAI JIAO

MAI KE LIN JIU

MAI XIAN WENG

MAI YA

MAN CHANG CHUN HUA

MAN GE YING ZHAO

MAN HUO XIANG

MAN JIANG HONG

MAN JING YE

MAN JING ZI

MAN JIU JIE

MAN LI YU

MAN NI DUO TAN CAO

MAN NI PU ER SHI DA GONG

LAO

MAN SHAN HONG

MAN SHENG JUAN BAI

MAN SUO NI YA XIN CAI

MAN TENG HUANG

MAN TUO LUO GEN

MAN TUO LUO YE

MAN TUO LUO ZI
马铃薯

马六甲鱼藤

麻柳叶

马尿泡

麻牛膝

马钱子

马桑

马桑叶

马山前胡

马氏大黄*

马氏金腰*

马氏芦荟

马氏香科科

马氏向日葵*

马斯特斯杜英

马蹄纹天竺葵

马蹄叶

马桶花

马尾连

马尾杉

\section{马尾松叶}

麻叶

麻叶千里光

马银花

麻状茄*

马状赛亚麻

麦冬

麦家公

麦角菌

麦克林韭

麦仙翁

麦芽

蔓长春花

曼戈鹰爪

蔓蕉香

满江红

蔓荆叶

蔓荆子

蔓九节

鳗鳎鱼

曼尼多坦草*

曼尼普尔十大功劳

满山红(兴安杜鹃)

蔓生卷柏

曼索尼亚心材

曼藤黄*

曼陀罗根

曼陀罗叶

曼陀罗子
Potato

Malacca Jewelvine*

Chinese Wingnut

Tangut Przewalskia

Capitate Cyathula

Nut-vomitive Poisonnut

Chinese Coriaria

Chinese Coriaria Leaf

Mashan Hogfennel

Maximowicz Rhubarb*

Maximowicz Goldsaxifrage*

Marloth Aloe

Cat Thyme

Maximilian's Sunflower

Masters Elaeocarpus

Zonal Geranium

Common Marsharigold

Sharptooth Incarvillea

Manyleaf Meadowrue

Slender Phlegmariurus

Masson Pine Leaf:

Hemp Fimble Leaf

Hempleaf Groundsel

Ovateleaf Rhododendron*

Abutilon Nightshade*

Hippomane Cupflower*

Liriope

Corn Gromwell

Ergot

MacLean Leek*

Githago Agrostemma

Barley Germinating Fruit

Common Periwinkle

Mainge Tailgrape*

Imbricate Mosquito Fern

Threeleaf Chastetree Leaf

Threeleaf Chastetree Fruit

Creeping Ninenode

Japanese Eel

Manipur Mahonia

Dahurian Rhododendron

David's Spikemoss

Mansonia Heartwood

Mann Garcinia*

Hindu Datura Root

Hindu Datura Leaf

Hindu Datura Seed
T6017 Solanum tuberosum

T2120 Derris malaccensis

T5308 Pterocarya stenoptera

T5247 Przewalskia tangutica

T1923 Cyathula capitata

T6184 Strychnos nux-vomica

T1692 Coriaria sinica [Syn. Coriaria nepalensis]

T1693 Coriaria sinica [Syn. Coriaria nepalensis]

T4762 Peucedanum mashanens

T5470 Rheum maximowiczii

T1405 Chrysosplenium maximowiczii

T0341 Aloe marlothii

T6362 Teucrium marum

T3149 Helianthus maximiliani

T2329 Elaeocarpus mastersii

T4694 Pelargonium zonale

T1137 Caltha palustris

T3418 Incarvillea arguta

T6387 Thalictrum foliolosum

T4801 Phlegmariurus phlegmaria [Syn.

Lycopodium phlegmaria]

T4916 Pinus massoniana

T1176 Cannabis sativa

T5883 Senecio cannabifolius

T5520 Rhododendron ovatum [Syn.

Rhododendron lamprophyllum; Azalea ovata]

T5988 Solanum abutiloides

T4429 Nierembergia hippomanica

T4507 Ophiopogon japonicus

T3880 Lithospermum arvense

T1541 Claviceps purpurea

T0315 Allium macleanii

T0252 Agrostemma githago

T3282 Hordeum vulgare

T6761 Vinca minor

T0654 Artabotrys maingayi

T6371 Teucrium viscidum var. miquelianum

T0838 Azolla imbricata [Syn. Salvinia

imbricata]

T6792 Vitex trifolia

T6793 Vitex trifolia

T5279 Psychotria serpens

T0500 Anguilla japonica

T2250 Dorstenia mannii

T4070 Mahonia manipurensis

T5508 Rhododendron dauricum

T5860 Selaginella davidii

T4107 Mansonia gagei

T2864 Garcinia mannii

T2041 Datura metel

T2042 Datura metel

T2043 Datura metel 


\begin{tabular}{|c|c|c|c|}
\hline MAN XING QIAN JIN BA & 蔓性千斤拔 & Philippine Flemingia & $\begin{array}{l}\text { T2737 Flemingia philippinensis [Syn. } \\
\text { Moghania philippinensis] }\end{array}$ \\
\hline MAN ZHI LONG DAN & 蔓枝龙胆 & Spread Gentian & T2917 Gentiana leptoclada \\
\hline MANG GUO & 杧果 & Mango & T4099 Mangifera indica \\
\hline MANG GUO HE & 杧果核 & Mango Seed & T4100 Mangifera indica \\
\hline MANG GUO SHU PI & 杧果树皮 & Mango Bark & T4101 Mangifera indica \\
\hline MANG GUO YE & 杧果叶 & Mango Leaf & T4102 Mangifera indica \\
\hline MANG JING & 芒茎 & Chinese Silvergrass & T4254 Miscanthus sinensis \\
\hline MANG NIU ER MIAO & 陇牛儿苗 & Common Heron's Bill & T2438 Erodium stephanianum \\
\hline MANG QI GU & 芒萁骨 & Dichotoma Forked Fern & $\begin{array}{l}\text { T2161 Dicranopteris pedata }[\text { Syn. } \\
\text { Polypodium pedatum; Dicranopteris } \\
\text { dichotoma }]\end{array}$ \\
\hline MANG SHE & 蟒蛇 & Indian Python & T5368 Python molurus bivittatus \\
\hline MAO BA JIAO FENG & 毛八角枫 & Kurz Alangium & T0282 Alangium kurzii \\
\hline MAO BAI YANG & 毛白杨 & Chinese White Poplar & T5161 Populus tomentosa \\
\hline MAO CAO LONG & 毛草龙 & Water Seedbox* & T3932 Ludwigia octovalvis \\
\hline MAO CAO YE & 茅草叶 & Lalang Grass Leaf & T3417 Imperata cylindrica var. major \\
\hline MAO CHUN BEI MU LAN & 毛唇贝母兰 & Cristate Coelogyne & T1605 Coelogyne cristata \\
\hline MAO CI JIN JI ER & 毛刺锦鸡儿 & Tibet Peasshrub & T1193 Caragana tibetica \\
\hline MAO DA DING CAO & 毛大丁草 & Pilose Gerbera & T2952 Gerbera piloselloides \\
\hline MAO DAO DI LING & 毛倒地铃 & Hairy Heartseed* & T1198 Cardiospermum hirsutum \\
\hline MAO DI HUANG & 毛地黄(紫花洋地黄) & Common Foxglove & T2177 Digitalis purpurea \\
\hline MAO DI HUANG SHU WEI CAO & 毛地黄鼠尾草 & Foxglove-like Sage & T5671 Salvia digitaloides \\
\hline MAO DI QIAN & 毛地钱 & & T2293 Dumortiera hirsuta \\
\hline MAO DONG QING & 毛冬青 & Pubescent Holly & T3396 Ilex pubescens \\
\hline MAO DOU & 猫豆 & Cochinchina Mucuna* & T4309 Mucuna cochinchinensis \\
\hline MAO DU XING CAI & 毛独行菜 & Hoary Pepperwort & T3757 Lepidium draba \\
\hline MAO E MEI & 毛萼梅 & Hairysepal Raspberry & T5590 Rubus chroosepalus \\
\hline MAO E XIANG CHA CAI & 毛萼香茶菜 & Hairysepal Rabdosia & T5391 Rabdosia eriocalyx \\
\hline MAO GAO CAI & 茅膏菜 & Lunate Peltate Sundew & T2267 Drosera peltata var. lunata \\
\hline MAO GEN & 毛茛 & Japanese Buttercup & T5414 Ranunculus japonicus \\
\hline MAO GENG HONG MAO WU JIA & 毛梗红毛五加 & Hispidus Girald Acanthopanax & T0037 Acanthopanax giraldii var. hispidus \\
\hline MAO GENG XI XIAN & 毛梗豨莶 & Hairstalk St. Paulswort & $\begin{array}{l}\text { T5951 Siegesbeckia orientalis var. glabrescens } \\
\text { [Syn. Siegesbeckia glabrescens] }\end{array}$ \\
\hline $\begin{array}{l}\text { MAO GENG XIA YE XIANG } \\
\text { CHA CAI }\end{array}$ & 毛梗狭叶香茶菜 & Hairstalk Narrowleaf Rabdosia* & T3481 Isodon angustifolius var. glabrescens \\
\hline MAO GOU TENG & 毛钩藤 & Hirsute Gambirplant* & T6617 Uncaria hirsuta \\
\hline MAO GUAN ZHONG & 毛贯众 & Champion Wood Fern & T2279 Dryopteris championii \\
\hline MAO GUO DI JIN & 毛果地锦 & Groundfig Spurge & T2581 Euphorbia chamaesyce \\
\hline MAO GUO DU JUAN & 毛果杜鹃 & Hairfruit Rhododendron* & T5522 Rhododendron seniavinii \\
\hline MAO GUO HAN XIAO & 毛果含笑 & Hairyfruit Michelia & T4214 Michelia spaerantha \\
\hline MAO GUO MA LI JIN & 毛果马利筋* & Hairyfruit Milkweed* & T0737 Asclepias eriocarpa \\
\hline MAO GUO QI & 毛果槭 & Nikoo Maple & T0050 Acer nikoense \\
\hline MAO GUO SUAN PAN ZI & 毛果算盘子 & Hairypod Glochidion* & T2988 Glochidion eriocarpum \\
\hline MAO GUO TIAN JIE CAI & 毛果天芥菜 & Hairyfruit Heliotrope* & T3175 Heliotropium lasiocarpum \\
\hline MAO GUO XIANG CHA CAI & 毛果香茶菜 & Hairyfruit Rabdosia* & T3530 Isodon trichocarpa \\
\hline MAO GUO YANG & 毛果杨 & Black Cottonwood & T5164 Populus trichocarpa \\
\hline MAO GUO YI HE GUO & 毛果翼核果 & Hairyfruit Ventilago & T6688 Ventilago calyculata \\
\hline MAO GUO YI ZHI HUANG HUA & 毛果一枝黄花 & European Goldenrod & T6023 Solidago virgaurea \\
\hline MAO GUO YU TENG & 毛果鱼藤 & Hairypod Fishvane & T2119 Derris eriocarpa \\
\hline MAO GUO YUN XIANG & 毛果芸香 & & T4891 Pilocarpus jaborandi \\
\hline MAO HANG ZI SHAO & 毛杭子梢 & Hairy Clovereshrub & T1163 Campylotropis hirtella \\
\hline MAO HOU QIAO RUI HUA & 毛喉鞘芯花 & Forskahl Coleus & T1620 Coleus forskahlii \\
\hline
\end{tabular}




\begin{tabular}{|c|c|c|}
\hline MAO HUA MAO DI HUANG & 毛花毛地黄 & Grecian Foxglove \\
\hline MAO HUA MI HOU TAO & 毛花猕猴桃 & Hairyflower Actinidia \\
\hline MAO HUA SHI NAN & 毛花石楠 & Hairyflower Photinia* \\
\hline MAO JIAN QIU LUO & 毛剪秋罗 & Hairy Campion \\
\hline MAO JIE XIE CAO & 毛节缴草 & Hairnode Valeriana* \\
\hline MAO JU & 毛蒟 & Hairy Pepper \\
\hline MAO LIAN HAO & 毛莲蒿 & Hairy Wormwood \\
\hline MAO LIE FENG DOU CAI & 毛裂蜂斗菜* & Hairylobed Butterbur \\
\hline MAO MA CHI XIAN & 毛马齿苋 & Pilose Purslane* \\
\hline MAO MAI LIAO & 毛脉苶 & \\
\hline MAO MAN TUO LUO GEN & 毛曼陀罗根 & Hairy Datura Root \\
\hline MAO MAN TUO LUO HUA & 毛曼陀罗花 & Hairy Datura Flower \\
\hline MAO MAN TUO LUO YE & 毛曼陀罗叶 & Hairy Datura Leaf \\
\hline MAO MAN TUO LUO ZI & 毛曼陀罗子 & Hairy Datura Seed \\
\hline MAO MEI & 茅莓 & Japanese Raspberry \\
\hline MAO PAO TONG & 毛泡桐 & Royal Paulownia \\
\hline MAO REN GE JUN & 毛㓞革菌 & \\
\hline MAO RUI HUA & 毛芯花 & Flannel Mullein \\
\hline MAO RUI HUA YE TU MU & 毛蒿花叶土木香 & Mulleinleaf Inula* \\
\hline \multicolumn{3}{|l|}{ XIANG } \\
\hline MAO SHAN ZHA & 毛山楂 & Maximowicz Hawthorn \\
\hline MAO SHI JUN ZI & 毛使君子 & Villous Rangooncreeper* \\
\hline MAO SHU & 毛薯 & Winged Yam \\
\hline MAO SHU MEI & 毛束霉 & \\
\hline MAO SUI HU JIAO & 毛穗胡椒 & Hairspike Pepper* \\
\hline MAO TOU RU GU & 毛头乳菇 & Pink-fringed Milky \\
\hline MAO XIAN MAO & 毛仙茅* & Pilose Curculigo* \\
\hline MAO XIAN ZHU JU TAI & 毛线柱草苔 & Hairy Rhynchotechum \\
\hline MAO XIANG HUA & 茅香花 & Vanillagrass \\
\hline MAO XU CAO & 猫须草 & Spicate Clerodendranthus \\
\hline MAO YAN CAO & 猫眼草 & Crescent-shaped Euphorbia \\
\hline MAO YANG MEI & 毛杨梅 & Hairy Bayberry \\
\hline MAO YE BA DOU & 毛叶巴豆 & Tomentose Caudate Croton \\
\hline MAO YE FAN LI ZHI & 毛叶番荔枝 & Cherimoya \\
\hline MAO YE JIA YING ZHAO GEN & 毛叶假鹰爪根 & Piloseleaf Desmos Root \\
\hline MAO YE LI LU & 毛叶藜芦 & Largeflower Falsehellebore \\
\hline MAO YE WEI MAO & 毛叶卫矛 & Sacred Spindle-tree \\
\hline MAO YE XIANG CHA CAI & 毛叶香茶菜 & Japanese Rabdosia \\
\hline MAO YU TENG & 毛鱼藤 & Tubaroot Jewelvine \\
\hline MAO ZHANG YA CAI & 毛獐牙菜 & Pubescent Swertia \\
\hline MAO ZHI HUA & 毛枝桦 & Pubescent Birch* \\
\hline MAO ZHI JUAN BAI & 毛枝卷柏 & Braun's Spikemoss \\
\hline MAO ZHU MA QIAN & 毛竹马钱 & Hairstyle Poisonnut \\
\hline MAO ZHU YE HUA JIAO & 毛竹叶花椒 & Armate-leaf Pricklyash* \\
\hline MEI GUAN MA XIAN HAO & 美观马先蒿 & Smallcalyx Woodbetony \\
\hline MEI GUI HONG JIN & 玫瑰红堇 & Rose Corydalis* \\
\hline MEI GUI HUA & 玫瑰花 & Rugose Rose Flower \\
\hline MEI GUO BIAN FU GE & 美国蝙蝠葛 & Canada Moonseed \\
\hline MEI GUO BO HE & 美国薄荷 & Oswegotea \\
\hline MEI GUO CI JIAO & 美国刺椒 & Hercules' Club \\
\hline MEI GUO HAI MO JU & 美国海墨菊 & Burro Bush \\
\hline MEI GUO KE YA SHU & 美国柯桠树* & American Vouacapoua* \\
\hline
\end{tabular}

T2175 Digitalis lanata

T0161 Actinidia eriantha T4826 Photinia lactiflora T3948 Lychnis coronaria T6674 Valeriana alternifolia var. stolonifera T4962 Piper puberulum T0705 Artemisia vestita T4745 Petasites tricholobus T5174 Portulaca pilosa T5018 Pleuropterus ciliinervis T2036 Datura innoxia T2037 Datura innoxia T2038 Datura innoxia T2039 Datura innoxia T5595 Rubus parviforlius T4679 Paulownia tomentosa T6143 Stereum hirsutum T6705 Verbascum thapsus T3440 Inula verbascifolia

T1772 Crataegus maximowiczii T5386 Quisqualis indica var. villosa T2188 Dioscorea alata T6515 Trichurus terrophilus T4971 Piper trichostachyon T3656 Lactarius torminosus T1902 Curculigo pilosa T5543 Rhynchotechum vestitum T3250 Hierochloe odorata T1553 Clerodendranthus spicatus T2599 Euphorbia lunulata T4343 Myrica esculent T1841 Croton caudatus var. tomentosus T0505 Annona cherimolia

T2137 Desmos dumosus T6697 Veratrum grandiflorum T2546 Euonymus sacrosancta T3496 Isodon japonica [Syn. Rabdosia japonica ]

T2118 Derris elliptica T6232 Swertia pubescens T0934 Betula pubescens T5859 Selaginella braunii T6183 Strychnos nitida T6864 Zanthoxylum armatum T4681 Pedicularis decora T1737 Corydalis rosea T5572 Rosa rugosa T4181 Menispermum canadense T4269 Monarda didyma T6871 Zanthoxylum clava-hercules T3322 Hymenoclea salsola T6804 Vouacapoua americana 


\begin{tabular}{|c|c|}
\hline MEI GUO QING TENG & 美国青藤 \\
\hline MEI GUO SHAN HE TAO & 美国山核桃 \\
\hline MEI GUO XIA LA MEI & 美国夏腊梅 \\
\hline MEI GUO ZI & 美国梓 \\
\hline MEI HE REN & 梅核仁 \\
\hline MEI HUA FENG MAO JU & 美花风毛菊 \\
\hline MEI HUA SHI HU & 美花石斛 \\
\hline MEI LI BU KU & 美丽布枯* \\
\hline MEI LI FAN HONG HUA & 美丽番红花 \\
\hline MEI LI HAI YING SU & 美丽海罂粟* \\
\hline MEI LI HE BAO MU DAN & 美丽荷包牡丹 \\
\hline MEI LI HONG DOU SHAN & 美丽红豆杉 \\
\hline MEI LI HUA QIU & 美丽花楸 \\
\hline MEI LI JIN SI TAO & 美丽金丝桃 \\
\hline MEI LI MA LI JIN & 美丽马利筋 \\
\hline MEI LI MA ZUI MU & 美丽马醉木 \\
\hline MEI LI MAO ZHU MU & 美丽帽柱木 \\
\hline MEI LI TE LE JU & 美丽特勒菊 \\
\hline MEI LI TENG HUANG & 美丽藤黄* \\
\hline MEI LI XIN MU JIANG ZI & 美丽新木姜子 \\
\hline MEI LI XUAN FU HUA & 美丽旋覆花* \\
\hline MEI LI YE HUI MAO DOU & 美丽叶灰毛豆* \\
\hline MEI LI YIN BEI TENG & 美丽银背藤* \\
\hline MEI LI ZHU SHI DOU & 美丽猪屎豆 \\
\hline MEI SHANG LU & 美商陆 \\
\hline MEI SUI XIAO BO & 美穗小檗 \\
\hline MEI TE NI DU JUAN HUA & 梅特尼杜鹃花 \\
\hline MEI WEI CHI JUN & 美味齿菌 \\
\hline MEI WEI HONG GU & 美味红菇 \\
\hline MEI WEI MI HOU TAO & 美味猕猴桃 \\
\hline MEI YI & 梅衣 \\
\hline MEI YUAN ZHI & 美远志 \\
\hline MEI ZHOU CHA MU & 美洲檫木 \\
\hline MEI ZHOU CI SHEN & 美州刺参 \\
\hline MEI ZHOU CI TONG & 美洲刺桐 \\
\hline MEI ZHOU GAN CAO & 美洲甘草 \\
\hline MEI ZHOU GUAN YIN LIAN & 美洲观音莲 \\
\hline MEI ZHOU HUA JIAO & 美洲花椒 \\
\hline MEI ZHOU JIN LV MEI & 美洲金缕梅 \\
\hline MEI ZHOU KU MU & 美洲苦木 \\
\hline MEI ZHOU LU QI MU & 美洲绿桤木 \\
\hline MEI ZHOU MAN MI PING GUO & 美洲曼密苹果 \\
\hline MEI ZHOU NAN SHE TENG & 美洲南蛇藤 \\
\hline MEI ZHOU SAN BAI CAO & 美洲三白草 \\
\hline MEI ZHOU SUAN GUO LUO & 美洲酸果萝 \\
\hline MEI ZHOU TUN CAO & 美洲豚草 \\
\hline MEI ZHOU XUE GEN CAO & 美洲血根草 \\
\hline MEI ZHOU YE BAI HE & 美洲野百合 \\
\hline MEI ZHOU YU & 美洲榆 \\
\hline MENG DA NA CHUN HUANG JU & 蒙大拿春黄菊 \\
\hline
\end{tabular}

\author{
Carolina Snailseed* \\ Pecan \\ Carolina Allspice \\ Southern Catalpa \\ Japanese Apricot Kernel \\ Beautiful-flowered Saussurea \\ Loddiges Dendrobium \\ Rutaceae Diosma \\ Pretty Crocus \\ Beautiful Hornpoppy* \\ Bleedingheart \\ Maire Yew \\ Showy Mountainsah \\ Beautiful St.John'swort \\ Beautiful Milkweed* \\ Taiwan Pieris* \\ Beautiful Mitragyna* \\ Beautiful Garcinia* \\ Beautiful Newlitse \\ Beautiful Inula* \\ Beautiful-leaf Tephrosia* \\ Woolly Morning Glory \\ Beautiful Crotalaria \\ American Pokeweed \\ Beautiful-raceme Barberry \\ Metternich Rhododendron*
}

Sweet Tooth

Milk-white Russula

Delicious Actinidia

Tinctorial Parmelia*

Senega Snakeroot

Sassafras

American Spineginseng*

American Coralbean*

Scale Licorice*

Skunk Cabbage

Pricklyash

Virginia Witch Hazel

Surinam Quassia

American Green Alder

Mammee Apple

American Bittersweet

Lizard's-tail

American Cranberry

American Ragweed*

Bloodroot

American Crotalaria*

American Elm

Montana Chamomile*
T1584 Cocculus carolinus

T1218 Carya illinoensis

T1139 Calycanthus floridus

T1260 Catalpa bignonioides

T5227 Prunus mume

T5767 Saussurea pulchella

T2105 Dendrobium loddigesii

T1617 Coleonema pulchellum

T1809 Crocus speciosus

T2971 Glaucium pulchrum

T2150 Dicentra formosa

T6312 Taxus mairei

T6054 Sorbus decora

T3342 Hypericum bellum

T0739 Asclepias speciosa

T4889 Pieris formosa

T4258 Mitragyna speciosa

T6326 Telekia speciosa

T2875 Garcinia speciosa

T4405 Neolitsea pulchella

T3435 Inula magnifica

T6332 Tephrosia calophylla

T0616 Argyreia speciosa

T1834 Crotalaria spectabilis

T4861 Phytolacca americana [Syn.

Phytolacca decandra]

T0900 Berberis calliobotrys

T5514 Rhododendron metternichii var.

hondoese

T3302 Hydnum repandum

T5620 Russula delica

T0160 Actinidia deliciosa

T4658 Parmelia tinctorum

T5084 Polygala senega

T5744 Sassafras albidum

T4517 Oplopanax horridus

T2458 Erythrina americana

T3017 Glycyrrhiza lepidota

T3994 Lysichitum americanum

T6863 Zanthoxylum americanum [Syn.

Xanthoxylum americanum]

T3095 Hamamelis virginiana

T5370 Quassia amara

T0325 Alnus crispa

T4093 Mammea americana

T1293 Celastrus scandens

T5749 Saururus cernuus

T6671 Vaccinium macrocarpon

T0397 Ambrosia artemisiaefolium)

T5709 Sanguinaria canadensis

T1814 Crotalaria anagyroides

T6594 Ulmus americana

T0522 Anthemis cretica ssp. cretica [Syn. 


\begin{tabular}{|c|c|c|}
\hline MENG DA NA SHAN XIAO JU & 蒙大拿山小橘* & Montana Glycosmis* \\
\hline MENG DA NA YUN XIANG & 蒙大拿芸香 & Montana Rue* \\
\hline MENG DA NA ZI JIN & 蒙大拿紫堇* & Montana Corydalis* \\
\hline MENG DI TENG & 蒙迪藤 & \\
\hline MENG GU CAO SU & 蒙古糙苏 & Mongolian Jerusalemsage \\
\hline MENG GU CE JIN ZHAN HUA & 蒙古侧金戞花 & Mongolian Adonis* \\
\hline MENG GU FENG MAO JU & 蒙古风毛菊 & Mongolian Saussurea* \\
\hline MENG GU HAO & 蒙古蒿 & Mongolian Wormwood \\
\hline MENG GU HUANG QI & 蒙古黄芪 & Mongolian Milkvetch \\
\hline MENG GU LI & 蒙古栋 & Mongolian Oak \\
\hline MENG GU SHAN LUO BO & 蒙古山萝卜 & Narrowleaf Scabious \\
\hline MENG GU XIU XIAN JU & 蒙古绣线菊 & Mongolian Spiraea \\
\hline MENG MAI ROU DOU KOU & 孟买肉豆蔻 & Bombay Nutmeg* \\
\hline MENG SANG & 蒙桑 & Mongolian Mulberry \\
\hline MENG ZI CAO HU JIAO & 蒙自草胡椒 & Mengzi Peperomia* \\
\hline MENG ZONG ZHU & 孟宗竹 & Edible Bamboo \\
\hline MI DIE XIANG & 迷迭香 & Rosemary \\
\hline MI DIE XIANG YE QIAN LI & 迷迭香叶千里光* & Rosmarin-leaf Groundsel* \\
\hline \multicolumn{3}{|l|}{ GUANG } \\
\hline MI GUO HUANG GUA & 密果黄瓜* & Densefruit Cucumber* \\
\hline MI HAO & 密蒿 & Compact Wormwood* \\
\hline MI HOU LI & 猕猴梨 & Bower Actinidia \\
\hline MI HOU LI GEN & 猕猴梨根 & Bower Actinidia Root* \\
\hline MI HOU TAO & 猕猴桃 & Yangtao Actinidia \\
\hline MI HUA DOU & 密花豆 & Suberect Spatholobus \\
\hline MI HUA MEI DENG MU & 密花美登木 & Crown-flowered Mayten \\
\hline MI HUA SHI DOU LAN & 密花石豆兰 & Flowery Stonebean-orchis \\
\hline MI HUA SHI HU & 密花石斛 & Denseflower Dendrobium \\
\hline MI HUA TUN CAO & 密花豚草 & Denseflower Ragweed* \\
\hline MI HUA WA ER TENG & 密花娃儿藤 & Denseflower Tylophora* \\
\hline MI HUA XIANG MAO & 密花香茅 & Denseflower Lemongrass* \\
\hline MI HUA YAN FENG & 密花岩凤 & Denseflower Libanotis \\
\hline MI HUAN JUN & 蜜环菌 & Armillary Mushroom* \\
\hline MI HUO DA JI & 迷惑大戟* & \\
\hline MI LA & 蜜蜡 & Bee Wax \\
\hline MI MAI E ZHANG CHAI & 密脉找掌柴 & Densevein Schefflera \\
\hline MI MENG HUA & 密蒙花 & Pale Butterflybush \\
\hline MI PI KANG & 米皮糠 & Rice Spermoderm \\
\hline MI PU LUO TI YA MU & 蜜普罗梯亚木 & Sugar Bush \\
\hline MI SAN QIAN LI GUANG & 密伞千里光 & Faber Groundsel \\
\hline MI SHI MA QIAN ZI & 米氏马钱子* & Mittschelich Poisonnut* \\
\hline MI SI KE HUANG TAN & 米斯科黄檀 & Miscol Rosewood* \\
\hline MI SUI HUA SHE BIAN JU & 密穗花蛇鞭菊* & Kansas Gay-feather \\
\hline MI TI BING HUA JU & 米梯柄花菊 & \\
\hline MI XIAO YING QIN & 密小鹰芹* & \\
\hline MI ZI LAN & 米仔兰 & Chu-lan Tree \\
\hline MIAN BAO GUO & 面包果 & Common Artocarpus* \\
\hline MIAN BI XIE & 绵草薢 & Sevenlobed Yam \\
\hline MIAN GEN TENG & 面根藤 & Ivy Glorybind \\
\hline MIAN HUA & 棉花 & Levant Cotton \\
\hline
\end{tabular}

Anthemis montana]

T3007 Glycosmis montana

T5628 Ruta montana

T1726 Corydalis montana

T4271 Mondia whitei

T4810 Phlomis mongolica

T0187 Adonis mongolica

T5760 Saussurea mongolica

T0685 Artemisia mongolica

T0800 Astragalus mongholicus

T5375 Quercus mongolica

T5775 Scabiosa comosa

T6083 Spiraea mongolica

T4352 Myristica malabarica

T4298 Morus mongolica

T4704 Peperomia duclouxii

T4844 Phyllostachys edulis

T5575 Rosmarinus officinalis

T5905 Senecio rosmarinifolius

T1877 Cucumis myriocarpus

T0674 Artemisia compacta

T0155 Actinidia arguta

T0156 Actinidia arguta

T0158 Actinidia chinensis

T6066 Spatholobus suberectus

T4131 Maytenus confertiflorus

T1053 Bulbophyllum odoratissimum [Syn.

Stelis odoratissimum ]

T2100 Dendrobium densiflorum

T0400 Ambrosia confertiflora

T6579 Tylophora crebriflora

T1940 Cymbopogon densiflorus

T3797 Libanotis condensata

T0644 Armillaria mellea

T2583 Euphorbia decipiens

T0542 Apis cerana

T5785 Schefflera venulosa

T1048 Buddleja officinalis

T4546 Oryza sativa

T5212 Protea mellifera

T5887 Senecio faberi

T6180 Strychnos mittschelichii

T2005 Dalbergia miscolobium

T3790 Liatris pycnostachya

T5034 Podanthus mitiqui

T0839 Azorella compacta

T0240 Aglaia odorata

T0714 Artocarpus incisa [Syn. Artocarpus

communis]

T2208 Dioscorea septemloba

T1143 Calystegia hederacea

T3055 Gossypium herbaceum 


\begin{tabular}{|c|c|c|c|}
\hline MIAN HUA GEN & 棉花根 & Levant Cotton Root & T3056 Gossypium herbaceum \\
\hline MIAN MA & 绵马 & Male-fern & T2282 Dryopteris filix-mas \\
\hline MIAN MAO GOU TENG & 绵毛钩藤* & Woolly Gambirplant* & T6623 Uncaria lanosa \\
\hline MIAN MAO GUO XIANG CHA & 绵毛果香茶菜 & Woollyfruit Rabdosia* & T3501 Isodon lasiocarpus \\
\hline \multicolumn{4}{|l|}{ CAI } \\
\hline MIAN MAO HUANG QI & 绵毛黄芪 & Sieversia Milkvetch & T0804 Astragalus sieversianus \\
\hline MIAN MAO MA DOU LING & 绵毛马癿铃 & Wooly Dutchmanspipe & T0634 Aristolochia mollissima \\
\hline MIAN MAO WA ER TENG & 绵毛娃儿藤 & Woolly Tylophora & T6581 Tylophora mollissima \\
\hline MIAN MAO XIE HAO & 绵毛邪蒿 & Woolly Seseli* & T5929 Seseli ericephalum \\
\hline MIAN NING WU TOU & 冕宁乌头 & Legendre Monkshood & T0110 Aconitum legendrei \\
\hline MIAN TENGWarb & 绵藤 & Pale Bittersweet & T1287 Celastrus hypoleucus \\
\hline MIAN TOU XUE LIAN & 绵头雪莲 & Lanatchead Saussurea & T5757 Saussurea laniceps \\
\hline MIAN TOU YE & 面头叶 & Smallleaf Knema & T3630 Kleinhovia hospita \\
\hline MIAN YE MA FENG SHU & 棉叶麻风树 & Cotton-leaf Leprous Tree* & T3561 Jatropha gossypifolia \\
\hline MIAN ZAO & 缅查 & Indian Jujube & T6920 Ziziphus mauritiana \\
\hline MIAN ZAO ER & 绵柊儿 & Common Squill & T5813 Scilla scilloides \\
\hline MIAN ZI YOU & 棉籽油 & Levant Cotton Oil & T3057 Gossypium herbaceum \\
\hline MIN HUAI & 闽槐 & Franchet Sophora & T6033 Sophora franchetiana \\
\hline MIN WAN BA JIAO & 闽皖八角 & Minwan Anisetree & T3405 Illicium minwanense \\
\hline MING DANG SHEN & 明党参 & Medicinal Changium & T1353 Changium smyrnioides \\
\hline MING SONG YE JU & 明松叶菊 & Anatomicum Fig* & T4199 Mesembryanthemum anatomicum \\
\hline MING XIAN HUA ZHU CHANG & 明显花柱长柱琉璃草* & Longstyle Lindelofia & T3846 Lindelofia stylosa \\
\hline \multicolumn{4}{|l|}{ ZHU LIU LI CAO } \\
\hline MO E SUAN MO & 膜萝酸模 & Canaigre & T5610 Rumex hymenosepalus \\
\hline MO GU & 蘑菇 & Mushroom & T0211 Agaricus campestris \\
\hline MO GUO MA HUANG & 膜果麻黄 & Przewalsk Ephedra & T2377 Ephedra przewalskii \\
\hline MO GUO QIN & 迷果芹 & Thin Sphallerocarpus & T6073 Sphallerocarpus gracilis \\
\hline MO HAN LIAN & 墨早莲 & Yerbadetajo & T2323 Eclipta prostrata $[$ Syn. Eclipta alba] \\
\hline MO JUE & 膜蕨 & Barbate Filmy Fern & T3324 Hymenophyllum barbatum \\
\hline MO LEI NAN YANG SHEN & 墨累南洋参* & Murri Polyscias* & T5131 Polyscias murrayi \\
\hline MO LI HUA & 茉莉花 & Arabian Jasmine & T3557 Jasminum sambac \\
\hline MO LI YU TENG & 莫里鱼藤* & Molly Jewelvine* & T2121 Derris mollis \\
\hline MO LUO SHI REN DONG & 莫罗氏忍冬 & Morrow Honeysuckle & T3915 Lonicera morrowii \\
\hline MO PAN CAO & 磨盘草 & Indian Abutilon & T0015 Abutilon indicum \\
\hline MO SANG BI KE MEI DENG MU & 莫桑比克美登木* & Mozambique Mayten* & T4137 Maytenus mossambicensis \\
\hline MO SHI ZI & 没食子 & Aleppo Gall (Galla Halepensis) & T5374 Quercus infectoria \\
\hline MO XI GE DUI XIN JU & 墨西哥堆心菊 & Mexico Sneezeweed* & T3138 Helenium mexicanum \\
\hline MO XI GE HAO & 墨西哥蒿 & Mexico Wormwood* & T0684 Artemisia mexicana var. angustifolia \\
\hline MO XI GE LUO YU SHAN & 墨西哥落羽杉 & Mexican Cypress* & T6306 Taxodium mucronatum \\
\hline MO XI GE XIANG RI KUI & 墨西哥向日葵 & Mexican Sunflower & T6470 Tithonia tagiliflora \\
\hline MO XI GE XUAN HUA & 墨西哥旋花 & Mexico Glorybind* & T5551 River corymbosa \\
\hline MO XIANG JING & 抹香鲸 & Cachalot & T4856 Physeter catodon \\
\hline \multirow[t]{2}{*}{ MO YAO } & 没药 & Myrrh & T1638 Commiphora myrrha [Syn. \\
\hline & & & Commiphora molmol] \\
\hline MO ZHI JIAO GU CUI & 膜质脚骨脆* & Membranous Casearia* & T1224 Casearia membranacea \\
\hline MO ZHI JU & 膜质菊 & & T3325 Hymenoxys grandiflora \\
\hline MU BAN SHU & 木瓣树 & Common Xylopia & T6855 Xylopia vielana \\
\hline MU BIE GEN & 木鳖根 & Cochinhina Momordica Root & T4264 Momordica cochinchinensis \\
\hline MU BIE ZI & 木鳖子 & Cochinhina Momordica Seed & T4265 Momordica cochinchinensis \\
\hline MU CHAI HU & 木柴胡 & Fruticose Thorowax* & T1061 Bupleurum fruticescens \\
\hline MU DAN PI & 牡丹皮 & Subshrubby Peony Bark & T4585 Paeonia moutan [Syn. Paeonia suffruticosa] \\
\hline MU DI XIANG WAN DOU & 牧地香踠豆 & Meadow Peavine & T3709 Lathyrus pratensis \\
\hline MU DOU SHU & 牧豆树 & Algarroba & T5210 Prosopis juliflora \\
\hline
\end{tabular}




\begin{tabular}{|c|c|c|c|}
\hline MU ER & 木耳 & Jew's Ear & T0830 Auricularia auricula \\
\hline MU ER DU MA CAO & 穆尔毒马草* & Moor Sideritis* & T5945 Sideritis moorei \\
\hline MU FANG JI & 木防己 & Japanese Snailseed & $\begin{array}{l}\text { T1589 Cocculus trilobus [Syn. Cocculus } \\
\text { sarmentosus] }\end{array}$ \\
\hline MU FU RONG HUA & 木芙蓉花 & Cottonrose Hibiscus Flower & T3241 Hibiscus mutabilis \\
\hline MU GUA & 木瓜 & Chinese Floweringquince & T1343 Chaenomeles sinensis \\
\hline MU GUI & 牡桂 & Loureir Cinnamon* & T1442 Cinnamomum loureirii \\
\hline MU HAO & 牡蒿 & Japanese Wormwood & T0678 Artemisia japonica \\
\hline MU HE & 慕荷 & Fingerleaf Rodgersflower & T5554 Rodgersia aesculifolia \\
\hline MU HU DIE & 木蝴蝶 & Indian Trumpetflower & T4537 Oroxylum indicum \\
\hline MU HU DIE SHU PI & 木蝴蝶树皮 & Indian Trumpetflower Bark & T4538 Oroxylum indicum \\
\hline MU JIN HUA & 木槿花 & Shrubalthea Flower & T3244 Hibiscus syriacus \\
\hline MU JIN PI & 木槿皮 & Shrubalthea Bark & T3245 Hibiscus syriacus \\
\hline MU JIN ZI & 木槿子 & Shrubalthea Fruit & T3246 Hibiscus syriacus \\
\hline $\mathrm{MU}^{(3)} \mathrm{JU}$ & 母菊 & Mayweed & $\begin{array}{l}\text { T4124 Matricaria chamomilla [Syn. } \\
\text { Matricaria recutita] }\end{array}$ \\
\hline $\mathrm{MU}^{(4)} \mathrm{JU}$ & 木橘 & Sepiaria & T0197 Aegle marmelos \\
\hline MU KU ER MO YAO & 穆库尔没药 & Muhul Myrrhtree* & T1637 Commiphora mukul \\
\hline MU LAN ${ }^{(3)}$ & 木榄 & Common Bruguiera & T1039 Bruguiera gymnorrhiza \\
\hline MU LAN ${ }^{(2)}$ & 木蓝 & True Indigo & T3423 Indigofera tinctoria \\
\hline MU LI & 牡蚛 & Oyster & T1768 Crassostrea gigas \\
\hline MU LI LU & 木藜芦 & One Sided Racemes Leucothoe & T3784 Leucothoe grayana \\
\hline MU LI ROU & 牡蚛肉 & Oyster Meat & $\begin{array}{l}\text { T4555 Ostrea rivularis; Ostrea } \\
\text { talienwhanensis; Ostrea gigas }\end{array}$ \\
\hline MU MA DOU & 牧马豆 & Lanceleaf Thermopsis & T6428 Thermopsis lanceolata \\
\hline MU MA HUANG & 木麻黄 & Horsetail Beefwood & T1258 Casuarina equisetifolia \\
\hline MU MIAN HUA & 木棉花 & Common Bombax Flower & $\begin{array}{l}\text { T0973 Bombax malabaricum [Syn. } \\
\text { Gossampinus malabarica] }\end{array}$ \\
\hline MU SHU DI SHANG BU FEN & 木薯地上部分 & Cassave Aerial Parts & T4104 Manihot esculenta \\
\hline MU TI CENG KONG JUN & 木蹄层孔菌 & & $\begin{array}{l}\text { T2748 Fomes fomentarius [Syn. } \\
\text { Pyropolyporus fomentarius; Boletus } \\
\text { fomentarius; Polyporus fomentarius] }\end{array}$ \\
\hline MU TIAN LIAO & 木天苶 & Silvervine Actinidia & T0164 Actinidia polygama \\
\hline MU TONG & 木通 & Fiveleaf Akebia & T0273 Akebia quinata \\
\hline MU TONG GEN & 木通根 & Fiveleaf Akebia Root & T0274 Akebia quinata \\
\hline MU TONG HAO & 木茼蒿 & Marguerite & T1391 Chrysanthemum frutescens \\
\hline MU XIANG & 木香 & Common Aucklandia (Costustoot) & T5758 Saussurea lappa [Syn. Aucklandia lappa $]$ \\
\hline MU XU & 苜宿 & Alfalfa & T4148 Medicago sativa \\
\hline MU XU GEN & 苜宿根 & Alfalfa Root & T4149 Medicago sativa \\
\hline MU YE LONG DAN & 牧野龙胆 & Pasture Gentian* & T2920 Gentiana makinoi \\
\hline MU ZEI & 木贼 & Common Scouring Rush & T2408 Equisetum hiemale \\
\hline MU ZEI MA HUANG & 木贼麻黄 & Mongolian Ephedra & T2367 Ephedra equisetina \\
\hline NA ER ZI YU PAN & 那耳紫玉盘 & Nar Uvaria* & T6666 Uvaria narum \\
\hline NA SHI XI GUA & 纳氏西瓜* & Naudin Citrullus* & T1462 Citrullus naudinianus \\
\hline NA TENG & 那藤 & Japanese Stauntonvine & T6100 Stauntonia hexaphylla \\
\hline NA TENG GUO & 那藤果 & Japanese Stauntonvine Fruit & T6101 Stauntonia hexaphylla \\
\hline NAI SANG & 奶桑 & Long-tail Mulberry* & T4297 Morus macroura \\
\hline NAN CHUAN GUAN CHUN HUA & 南川冠唇花 & Prain Microtoena & T4226 Microtoena prainiana \\
\hline NAN CHUAN LU SI CAO & 南川鹭鹋草 & S. Sichuan Egretgrass & T2240 Diuranthera inarticulata \\
\hline NAN CHUAN SHENG MA & 南川升麻 & Nanchuan Bugbane* & T1423 Cimicifuga nanchuanensis \\
\hline NAN DA JI & 南大戟 & Jolkin Euphorbia & T2594 Euphorbia jolkini \\
\hline NAN DAN SHEN & 南丹参 & Bowley Sage & T5661 Salvia bowleyana \\
\hline NAN DE WA MIAN & 南德瓦棉 & Nandewa Cotton* & T3060 Gossypium sturtianum var. \\
\hline
\end{tabular}




NAN FANG LIU LI CAO
NAN FANG OU SHI NAN
NAN FANG TU SI ZI
NAN FEI CONG SHENG LA JU
NAN FEI GOU MA
NAN FEI JI TOU SHU
NAN FEI ZHANG GUI
NAN GUA
NAN GUA ZI
NAN HE SHI
NAN LING QIAN HU
NAN LUN HUAN TENG
NAN MEI FANG JI
NAN MEI NIU NAI CAI
NAN MEI ZHOU GUI
NAN MU
NAN OU DAN SHEN
NAN RI BEN LEI GONG TENG
NAN SHAN HUA
NAN SHAN TENG
NAN SHE TENG

NAN SHE TENG YE

NAN SHE TENG GUO

NAN SHE TENG ZHUANG

SHAN CHENG

NAN SU GE LAN JIA SHAN LUO

NAN SUAN ZAO

NAN TIAN ZHU GEN

NAN TIAN ZHU GENG

NAN TIAN ZHU YE

NAN TIAN ZHU ZI

NAN TONG HAO

NAN TOU QIU HAI TANG

NAN XI BAN YA HUAN YANG SHEN

NAN YA HAN XIAO

NAN YIN DU CHUAN XIN LIAN

NAN YIN DU SU TIE SHU GUO

NAN ZHU ZHI

NANG GAI SI GUA

NANG ZHUANG MAO RUI HUA

NANG ZHUANG ZI TAN

NAO YANG HUA

NAO YANG HUA ZI

NEI FENG XIAO

NEI GUO BAI CEN

NEI HUA YI WA JU

\section{南方琉璃草* \\ 南方欧石南* \\ 南方菟丝子 \\ 南非丛生蜡菊* \\ 南非钩麻 \\ 南非鸡头薯 \\ 南非樟桂* \\ 南瓜 \\ 南瓜子 \\ 南鹤䖝 \\ 南岭前胡 \\ 南轮环藤 \\ 南美防己 \\ 南美牛奶菜 \\ 南美洲桧 \\ 楠木 \\ 南欧丹参 \\ 南日本雷公藤 \\ 南山花 \\ 南山藤 \\ 南蛇藤}

南蛇藤根

南蛇藤叶

南蛇藤果

南蛇藤状山橙

南苏格兰假山萝*

南酸李

南天竹根

南天竹梗

南天竹叶

南天竹子

南茼高

南投秋海棠

南西班牙还阳参

南亚含笑

南印度穿心莲

南印度苏铁树果

南烛子

囊盖丝瓜*

囊状毛荵花*

囊状紫檀

闹羊花

闹羊花子

内风消五味子

美国白梣

内华依瓦菊
South Houndstongue*

Spanish Heath

South Dodder Seed

South-Africa Tufted Everlasting*

Devil's Clow

South-African Eriosema*

Cushaw

Cushaw Seed

Wild Carrot Fruit

Nanling Hogfennel

Tonkin Cyclea

Condurango

Southern Red-cedar

Nanmu

Clary

South-Japan Threewingnut*

Furstamen Prismatomeris

Twisting Dregea

Oriental Bittersweet

Oriental Bittersweet Root

Oriental Bittersweet Leaf

Oriental Bittersweet Fruit

Celastrus Melodinus*

Southern-caledonian Tulipwood* Axillary Southem Wildjujube

Common Nandina Root

Common Nandina Stem

Common Nandina Leaf

Common Nandina Fruit

South Chrysanthemum

Nantou Begonia*

Southern-Spain Hawksbeard*

South Asia Michelia

South-India Andrographis*

South-India Sago Seed*

Oriental Blueberry Fruit

Buchinha (Brazil Herb)

Saccate Mullein*

Vengai Padauk

Chinese Azalea

Chinese Azalea Fruit

Black Magnoliavine*

White Ash nandewarence

T1968 Cynoglossum australe

T2416 Erica australis

T1911 Cuscuta australis

T3157 Helichrysum caespititium

T3106 Harpagophytum procumbens

T2436 Eriosema kraussianum

T4477 Ocotea bullata

T1880 Cucurbita moschata

T1881 Cucurbita moschata

T2049 Daucus carota

T4761 Peucedanum longshengens

T1934 Cyclea tonkinensis

T1383 Chondrodendron tomentosum

T4115 Marsdenia condurango

T3598 Juniperus silicicola

T4818 Phoebe nanmu

T5691 Salvia sclarea

T6539 Tripterygium doianum

T5207 Prismatomeris tetrandra

T2263 Dregea volubilis

T1288 Celastrus orbiculatus [Syn. Celastrus

articulatus]

T1289 Celastrus orbiculatus [Syn. Celastrus

articulatus]

T1290 Celastrus orbiculatus [Syn. Celastrus

articulatus]

T1291 Celastrus orbiculatus [Syn. Celastrus

articulatus]

T4177 Melodinus celastroides

T3107 Harpullia austro-caledonica

T1381 Choerospondias axillaris

T4368 Nandina domestica

T4369 Nandina domestica

T4370 Nandina domestica

T4371 Nandina domestica

T1397 Chrysanthemum segetum

T0891 Begonia nantoensis

T1790 Crepis tingitana

T4212 Michelia doltsopa

T0460 Andrographis viscosula

T1925 Cycas beddomei

T6670 Vaccinium bracteatum

T3936 Luffa operculata

T6703 Verbascum saccatum

T5302 Pterocarpus marsupium

T5516 Rhododendron molle

T5517 Rhododendron molle

T5796 Schisandra nigra

T2765 Fraxinus americana

T3545 Iva nevadensis 


NEI NAN WU WEI ZI
NEI ZHE XIANG CHA CAI
NEN YE JIU LI XIANG
NI A LUO
NI BO ER DU HUO
NI BO ER LAO GUAN CAO
NI BO ER LV RONG HAO
NI BO ER SONG CAO
NI BO ER WU TOU
NI BO ER YANG TI
NI DAN SHEN
NI DONG FENG LUO
NI GU LA SHI CHE JU
NI GUANG SHI WEI
NI HU CAI
NI JIN ZHAN JU
NI LUO HE CHENG LIU
NI LUO HE JIN YIN LIAN
NI RI LI YA LIANG RUI SU MU
NI TAN XIAN

NI TAN XIAN

NI YU LONG WU TOU

NI ZHAO MU JIANG ZI

NIAN BAO CHUN

NIAN HAO

NIAN MAO SHU WEI CAO

NIAN WEI LING CAI

NIAN XING AI LEI JU

NIAN XING GUO SHI SUAN

JIANG

NIAN XING TU MU XIANG

NIAN YE YOU ${ }^{(2)}$

NIAN YU

NIAN YU XU

NIAN ZHI JIN ZHI JU

NIAN ZHI LUO LIN

NING BIAN E TAI

NING GU SHUI QIE

NING GUO BEI MU

NING MENG

NING MENG AN YE

NING MENG GEN

NING MENG PI

NING MENG YE

NING XIA BEI MU

NING XIA GOU QI GEN PI

NING XIA GOU QI ZI

NIU BANG GEN

NIU BANG YE

NIU BANG YE DU WU

NIU BANG ZI

NIU BIAN
内南五味子

内折香茶菜

嫩叶九里香

逆阿落

尼泊尔独活

尼泊尔老鹳草

尼泊尔绿线蒿

尼泊尔嵩草

尼泊尔乌头

尼泊尔羊蹄

拟丹参

泥东风螺*

尼古拉矢车菊*

拟光石韦

泥胡菜

\section{拟金戞菊 \\ 尼罗河柽柳* \\ 尼罗河金银楝 \\ 尼日利亚两聴苏木 \\ 泥炭蘚}

$\begin{array}{ll}\text { 拟玉龙乌头 } & \text { Pseudostapfia Monkshood } \\ \text { 泥沼木姜子 } & \text { Sloughy Litse* } \\ \text { 黏报春* } & \text { Viscid Primrose* } \\ \text { 黏蒿* } & \text { Viscid Wormwood* } \\ \text { 黏毛鼠尾草 } & \text { Roborowsk Sage } \\ \text { 黏委陵菜 } & \text { Viscose Cinquefoil* }\end{array}$

黏性埃勒菊

黏性果实酸浆*

黏性土木香*

黏叶获

鲇鱼

鲇鱼须

黏质金枝菊*

黏质罗林

宁扁萼苔

凝固睡茄

宁国贝母

柠檬

柠檬桉叶

柠檬根

柠檬皮

柠檬叶

宁夏贝母

宁夏枸杞根皮

宁夏枸杞子

牛蒡根

牛蒡叶

牛蒡叶㯻吾

牛蒡子

牛扁
Fengqing Kadsura

Inflexed Rabdosia

Juvenileleaf Common Jasminorange

Nepal Cowparsnip

Nepal Cranesbill

Nepal Meconopsis

Nepal Kobresia*

Nepal Monkshood

Nepal Dock

Chinese Sage

Nicola Centaurea*

Pseudobald Pyrrosia*

Lyrate Hemistepta

Cape Dandelion

Nilotic Tamarisk*

Nile Starbush*

Nigerian Satinwood

Viscose Cinquefoil*

Woody Fleabane

Glutinose Bluebeard

Siebold Greenbrier

Mucosa Rollinia*

Coagulate Withania

Ningguo Fritillary

Lemon

Lemon Eucalyptus Leaf

Lemon Root

Lemon Pericarp

Lemon Leaf

Ningxia Fritillary

Barbary Wolfberry Root-bark*

Barbary Wolfberry Fruit

Great Burdock Root

Great Burdock Leaf*

Dockleaf Goldenray

Great Burdock Fruit

Puberulent Monkshood
T3615 Kadsura interior

T3494 Isodon inflexa [Syn. Rabdosia inflexa]

T4321 Murraya microphylla

T5112 Polygonum periginatoris

T3219 Heracleum nepalense

T2943 Geranium nepalense

T4144 Meconopsis nepaulensis

T3633 Kobresia nepalensis

T0093 Aconitum ferox

T5612 Rumex nepalensis

T5692 Salvia sinica

T0843 Babylonia lutosa

T1306 Centaurea nicolai

T5359 Pyrrosia pseudocalvata

T3202 Hemistepta lyrata [Syn. Hemistepta

carthamoides; Saussurea carthamoides]

T6687 Venidium decurrens

T6289 Tamarix nilotica

T6570 Turraea nilotica

T2239 Distemonanthus benthamianus

T6072 Sphagnum palustre [Syn. Sphagnum

obtusifolium; Sphagnum cymbifolium]

T0125 Aconitum pseudostapfianum

T3896 Litsea turfosa

T5205 Primula viscosa

T0670 Artemisia cana ssp. viscidula

T5690 Salvia roborowskii

T5188 Potentilla viscosa

T2324 Egletes viscosa

T4850 Physalis ixocarpa

T3441 Inula viscosa

T1222 Caryopteris glutinosa

T4644 Parasilurus asotus

T5982 Smilax sieboldii

T1409 Chrysothamnus viscidiflorus

T5558 Rollinia mucosa

T5404 Radula perrottetii

T6828 Withania coagulans

T2789 Fritillaria ningguoensis

T1490 Citrus limon

T2505 Eucalyptus citriodora

T1491 Citrus limon

T1492 Citrus limon

T1493 Citrus limon

T2795 Fritillaria taipaiensis var. ningxiaensis

T3954 Lycium barbarum

T3955 Lycium barbarum

T0584 Arctium lappa

T0585 Arctium lappa

T3809 Ligularia lapathifolia

T0586 Arctium lappa

T0078 Aconitum barbatum var. puberulum 


\begin{tabular}{|c|c|c|c|}
\hline & & & [Syn. Aconitum ochranthum] \\
\hline NIU DAN & 牛胆 & Ox Gall & T0984 Bos taurus domesticus; Bubalus bubalis \\
\hline NIU ER DA HUANG & 牛耳大黄 & Crisped Dock & T5607 Rumex crispus \\
\hline NIU ER FENG ZI & 牛耳枫子 & Calyx-shaped Daphniphyllum Fruit & T2032 Daphniphyllum calycinum \\
\hline NIU FANG FENG & 牛防风 & Hogweed & T3224 Heracleum sphondylium \\
\hline NIU FEI & 牛肺 & Ox Lung & T0985 Bos taurus domesticus; Bubalus bubalis \\
\hline NIU GAN & 牛肝 & Ox Liver & T0986 Bos taurus domesticus; Bubalus bubalis \\
\hline NIU HAO & 牛蒿 & Taurine Wormwood* & T0701 Artemisia taurica \\
\hline NIU HUANG & 牛黄 & Cow-bezoar (Ox-gallstone) & T0987 Bos taurus domesticus; Bubalus bubalis \\
\hline NIU JIN GUO & 牛筋果 & Perforated Harrisonia & T3109 Harrisonia perforata \\
\hline NIU JIN QIAN LI GUANG & 牛津千里光* & Oxford Ragwort & T5912 Senecio squalidus \\
\hline NIU JIN TIAO & 牛筋条 & Common Oxmuscle & T2157 Dichotomanthes tristaniaecarpa \\
\hline NIU NAO & 牛脑 & Ox Brain & T0988 Bos taurus domesticus; Bubalus bubalis \\
\hline NIU PI CHA & 牛皮茶 & Goldmat Rhododendron & T5505 Rhododendron chrysanthum \\
\hline NIU QU SONG YE JU & 扭曲松叶菊 & Tortuous Fig* & T4202 Mesembryanthemum tortuosum \\
\hline NIU QU TI GEN CAO & 扭曲德根草* & Tortuous Hellebore* & $\begin{array}{l}\text { T3188 Helleborus torquatus [Syn. Helleborus } \\
\text { serbicus] }\end{array}$ \\
\hline NIU RU & 牛乳 & Cow Milk & T0989 Bos taurus domesticus; Bubalus bubalis \\
\hline NIU SHE CAO & 牛舌草 & Toothedfruit Dock & T5608 Rumex dentatus \\
\hline NIU SHE TOU & 牛舌头 & Field Sowthistle & T6025 Sonchus arvensis \\
\hline NIU SHEN & 牛肾 & Ox Kidney & T0990 Bos taurus domesticus; Bubalus bubalis \\
\hline NIU TI DOU & 牛蹄豆 & Guamachil Apea-earring & T4985 Pithecolobium dulce \\
\hline NIU WEI CAI & 牛尾菜 & Oxtail Greenbrier & T5981 Smilax riparia \\
\hline NIU WEI CAO XIANG CHA CAI & 牛尾草 & Ternateleaf Rabdosia & T3528 Isodon ternifolia \\
\hline NIU WEI DU HUO & 牛尾独活 & Hemsley Cowparsnip & T3213 Heracleum hemsleyanum \\
\hline NIU XI & 牛溙 & Twotooth Achyranthes & T0073 Achyranthes bidentata \\
\hline NIU XI XI & 牛西西 & Patience Dock & T5614 Rumex patientia \\
\hline NIU XIN FAN LI ZHI & 牛心番荔枝 & Bullocksheart Custardapple & T0511 Annona reticulata \\
\hline NIU XIN PIAO ZI & 牛心朴子 & Komarov Mosquitotrap & T1957 Cynanchum komarovii \\
\hline NIU XIN QIE ZI & 牛心茄子 & Common Cerberustree & T1330 Cerbera manghas \\
\hline NIU XUAN JIN HE HUAN & 扭旋金合欢* & Tortile Acacia* & T0033 Acacia tortilis ssp. raddiana \\
\hline NIU XUE & 牛血. & Ox Blood & T0991 Bos taurus domesticus; Bubalus bubalis \\
\hline NIU YAN MA QIAN & 牛眼马钱 & Narrowflower Poisonnut & T6166 Strychnos angustiflora \\
\hline NIU YAN PENG QI JU & 牛眼蟛蜞菊 & & T6906 Zexmenia buphthalmiflora \\
\hline NIU YE & 牛庿 & Ox Thyroid & T0992 Bos taurus domesticus; Bubalus bubalis \\
\hline NONG DA JI & 浓大戟* & Dense Euphorbia* & T2588 Euphorbia fortissima \\
\hline NU JIANG SHAN CHA & 怒江山茶 & Salwin Camellia & T1148 Camellia saluenensis \\
\hline NUO DAO & 糯稻 & Sticky Rice & T4548 Oryza sativa var. glutinosa \\
\hline NUO WEI QI & 挪威棫 & Norway Maple & T0052 Acer platanoides \\
\hline NV ZHEN XIAO LA SHU & 女贞小蜡树 & Chinese Privet & T3830 Ligustrum sinense \\
\hline NV ZHEN ZI & 女贞子 & Glossy Privet Fruit & T3828 Ligustrum lucidum \\
\hline $\mathrm{OU}$ & 藕 & Hindu Lotus Large Rhizome & T4402 Nelumbo nucifera \\
\hline OU BO HE & 欧薄荷 & Horse Mint & T4185 Mentha longifolia \\
\hline OU CANG ZHU & 欧苍术 & Gummy Atractylodes* & T0820 Atractylodes gummifera \\
\hline OU DANG GUI & 欧当归 & Garden Lovage & T3785 Levisticum officinale \\
\hline OU DI SUN & 欧地笋 & European Bugleweed & T3979 Lycopus europaeus \\
\hline OU DING XIANG & 欧丁香 & Common Lilac & T6262 Syringa vulgaris \\
\hline OU FANG FENG & 欧防风 & Garden Parsnip & T4669 Pastinaca sativa \\
\hline OU JIN KUI & 欧锦葵 & High Mallow & T4091 Malva sylvestris \\
\hline OU LANG DANG & 欧莨宕 & European Scopolia & T5820 Scopolia carniolica \\
\hline OU LI REN & 欧李仁 & Chinese Dwarf Cherry Seed & T5223 Prunus humilis [Syn. Cerasus humilis] \\
\hline OU MAN TUO LUO GEN & 欧曼陀罗根 & Jimsonweed Root & T2046 Datura stramonium \\
\hline OU QIAN HU & 欧前胡 & Masterwort & T4766 Peucedanum ostruthium \\
\hline
\end{tabular}




\begin{tabular}{|c|c|c|c|}
\hline OU SHI NAN & 欧石南 & Tree Heath & T2415 Erica arborea \\
\hline OU SHU LI & 欧鼠李 & Glossy Buckthorn & T5460 Rhamnus frangula $[$ Syn. Frangula alnus $]$ \\
\hline OU WUTOU & 欧乌头 & Aconite & T0118 Aconitum napellus \\
\hline OU XI XIN & 欧细辛 & Asarabacca & T0725 Asarum europaeum \\
\hline OU XIA ZHI CAO & 欧夏至草 & Common Hoarhound & T4114 Marrubium vulgare \\
\hline OU YA GAN CAO & 欧亚甘草 & Typical Licorice* & T3014 Glycyrrhiza glabra var. typica \\
\hline OU YA QI & 欧亚械 & Great Maple & T0053 Acer pseudoplatanus \\
\hline OU YA RUI XIANG & 欧亚瑞香 & Mezereon & T2026 Daphne mezereum \\
\hline OU ZE QIN & 欧泽芹 & Greater Water-parsnip & T5969 Sium latifolium \\
\hline OU ZHOU BAI LA SHU & 欧洲白蜡树 & European Ash & T2768 Fraxinus excelsior \\
\hline OU ZHOU BAI LI & 欧洲白栋 & Common Oak & T5377 Quercus robur \\
\hline OU ZHOU CHI SONG & 欧洲赤松 & Scotch Pine & T4926 Pinus sylvestris \\
\hline OU ZHOU CI BAI & 欧洲刺柏 & Common Juniper & T3583 Juniperus communis \\
\hline OU ZHOU CI BAI BIAN ZHONG & 欧洲刺柏变种 & Common Juniper Variaty* & T3584 Juniperus communis var. depressa \\
\hline OU ZHOU DU HUO & 欧洲独活 & European Cowparsnip* & T3220 Heracleum pyrenaicum \\
\hline OU ZHOU HUA QIU & 欧洲花楸 & European Mountainash & T6053 Sorbus aucuparia \\
\hline OU ZHOU JIA ZHU TAO & 欧洲夹竹桃 & Common Oleander & T4420 Nerium oleander \\
\hline OU ZHOU JUE & 欧洲蕨 & Bracken & T5283 Pteridium aquilinum \\
\hline OU ZHOU LAN MO LI & 欧洲蓝茉莉 & European Leadwort* & T5027 Plumbago europaea \\
\hline OU ZHOU LENG SHAN & 欧洲冷杉 & European Silver Fir & T0003 Abies alba \\
\hline OU ZHOU LI & 欧洲栗 & Spanish Chestnut & T1255 Castanea sativa \\
\hline OU ZHOU LOU DOU CAI & 欧洲耧斗菜 & European Columbine & T0558 Aquilegia vulgaris \\
\hline OU ZHOU MA DOU LING & 欧洲马㕷铃 & Dutchmanspipe & T0638 Aristolochia sipho \\
\hline OU ZHOU MI FENG HUA & 欧洲蜜蜂花 & Balmleaf Metittis & T4175 Melittis melissophyllum \\
\hline OU ZHOU MO YAO & 欧洲没药 & Sweet Cicely & T4360 Myrrhis odorata \\
\hline OU ZHOU NV ZHEN & 欧洲女贞 & European Privet & T3831 Ligustrum vulgare \\
\hline OU ZHOU PING PENG CAO & 欧洲萍蓬草 & European Cowlily & T4449 Nuphar luteum \\
\hline OU ZHOU QI MU & 欧洲桤木 & European Alder & T0326 Alnus glutinosa \\
\hline OU ZHOU QI YE SHU & 欧洲七叶树 & Horsechestnut & T0202 Aesculus hippocastanum \\
\hline OU ZHOU QIAN LI GUANG & 欧洲千里光 & Common Groundsel & T5915 Senecio vulgaris \\
\hline OU ZHOU SHAN JIE & 欧州山芥 & Upland Cress & T0870 Barbarea vulgaris \\
\hline OU ZHOU SHAN YANG & 欧洲山杨 & Aspen & T5162 Populus tremula \\
\hline OU ZHOU SHENG GU YOU & 欧洲省沽油 & European Bladdernut, Pinnate Bladdernut & T6098 Staphylea pinnata \\
\hline OU ZHOU SHUI QING GANG & 欧洲水青冈 & European Beech & T2662 Fagus sylvatica \\
\hline OU ZHOU SONG LAN & 欧州菘蓝 & Dyers Woad & T3477 Isatis tinctoria \\
\hline OU ZHOU TIAN JIE CAI & 欧洲天芥菜* & Heliotrope & T3171 Heliotropium europaeum \\
\hline OU ZHOU WEI MAO & 欧洲卫矛 & European Euonymus & T2539 Euonymus europaeus \\
\hline OU ZHOU XIAN KE LAI & 欧洲仙客来 & Cyclamen & T1929 Cyclamen europaeum \\
\hline OU ZHOU XIAO BO & 欧洲小檗 & European Barberry & T0919 Berberis vulgaris \\
\hline OU ZHOU YUN SHAN & 欧洲云杉 & Common Spruce & T4869 Picea abies \\
\hline OU ZI CAO & 欧紫草 & Dyer's Alkanet & T0304 Alkanna tinctoria \\
\hline OU ZI QI & 欧紫萁 & Flowering Fern & T4553 Osmunda ragalis \\
\hline OW ZHOU YOU CAI & 欧洲油菜 & Rape & T1009 Brassica napus \\
\hline PA KE YE XIANG SHU & 帕克夜香树 & Parqui Cestrum & T1339 Cestrum parqui \\
\hline PA LI BEI FANG SHI DA GONG & 帕里北方十大功劳 & Parry Northern Mahonia & T4059 Mahonia borealis \\
\hline \multicolumn{4}{|l|}{ LAO } \\
\hline PA SHI BEI KE SHAN & 帕氏贝壳杉 & Palmerston Pitch Tree* & T0214 Agathis palmerstoni \\
\hline \multirow[t]{2}{*}{ PAI QIAN CAO } & 排钱草 & Beautiful Phyllodium & T2131 Desmodium pulchellum [Syn. \\
\hline & & & Phyllodium pulchellum] \\
\hline \multirow[t]{2}{*}{ PAI QIAN CAO GEN } & 排钱草根 & Beautiful Phyllodium Root & T2132 Desmodium pulchellum [Syn. \\
\hline & & & Phyllodium pulchellum ] \\
\hline PAN YUAN CHOU HUANG JING & 攀援臭黄荆 & Climbing Premna & T5195 Premna subscandens \\
\hline I YUAN YU TENG & 援鱼藤 & Climbing Jewelvine & T2123 Derris scandens \\
\hline
\end{tabular}




\begin{tabular}{|c|c|c|c|}
\hline PAN ZHI GOU TENG & 攀枝钩藤 & Climbing Gambirplant & $\begin{array}{l}\text { T6631 Uncaria scandens [Syn. Nauclea } \\
\text { pilosa; Uruparia pilosa; Uncaria pilosa] }\end{array}$ \\
\hline PAN ZHUANG YE XIA ZHU & 盘状叶下珠* & Discoid Leafflower* & T4833 Phyllanthus discoides \\
\hline PANG DA HAI & 胖大海 & & T6139 Sterculia lychnophora \\
\hline PAO DAN GUO & 炮弹果 & Calabash-tree & T1791 Crescentia cujete \\
\hline PAO NANG CAO & 泡囊草 & Common Physochlaina & T4859 Physochlaina physaloides \\
\hline PAO PAO SHU & 泡泡树 & Pawpaw & T0744 Asimina triloba \\
\hline PAO TONG & 泡桐 & Fortune Paulownia & T4678 Paulownia fortunei \\
\hline PAO ZHUANG FAN LI ZHI & 泡状番荔枝 & Bullate Custardapple* & T0504 Annona bullata \\
\hline PEI LAN & 佩兰 & Fortune Eupatorium & T2559 Eupatorium fortunei \\
\hline PEI LI LU HUI & 佩里芦荟* & Perry Aloe* & T0342 Aloe perryi \\
\hline PEI NI YUAN ZHI & 佩尼远志 & Paene Milkwort* & T5079 Polygala paenea \\
\hline PEN GUA & 喷瓜 & Squirting Cucumber & T2313 Ecballium elaterium \\
\hline PEN JIA SHU & 盆架树 & Prettyleaf Winchia & T6825 Winchia calophylla \\
\hline PEN TUO PO TI CAO & 盆托坡梯草 & Pentopetia androsaernifolia & T4702 Pentopetia androsaernifolia \\
\hline PENG LAI CAO & 蓬莱草 & Knotteflower Phyla Herb & T3865 Lippia nodiflora \\
\hline PENG QI JU & 蟛蜞菊 & Chinese Wedelia & $\begin{array}{l}\text { T6814 Wedelia chinensis }[\text { Syn. Solidago } \\
\text { chinensis; Wedelia calendulacea] }\end{array}$ \\
\hline PENG TE MAN DE MU & 彭特曼得木 & Pentland Mandevilla* & T4098 Mandevilla pentlandiana \\
\hline PENG XIAN XUE DAN & 彭县雪胆 & Pengxian Hemsleya & T3209 Hemsleya pengxianensis \\
\hline PENG ZI CAI & 蓬子菜 & Yellow Bedstraw & T2835 Galium verum \\
\hline PI HAN CAO & 辟汗草 & Daghestan Sweetclover & T4172 Melilotus suaveolens \\
\hline PI HAN CAO GEN & 辟汗草根 & Daghestan Sweetclover Root & T4173 Melilotus suaveolens \\
\hline PI JIU HUA & 啤酒花 & European Hop Female-flower & T3289 Humulus lupulus \\
\hline PI JIU HUA TU SI ZI & 啤酒花菟丝子 & Hop-shaped Dodder & T1914 Cuscuta lupuliformis \\
\hline PI LI LUO FU MU & 霹䨌萝芙木 & Perak Devilpepper & T5441 Rauwolfia perakensis \\
\hline PI PA & 枇杷 & Loquat & T2431 Eriobotrya japonica \\
\hline PI PA HE & 枇杷核 & Loquat Seed & T2432 Eriobotrya japonica \\
\hline PI PA YE & 枇杷叶 & Loquat Leaf & T2433 Eriobotrya japonica \\
\hline PI QI QIE & 皮契茄 & Peru False Heath & T2652 Fabiana imbricata \\
\hline PI SHAO ZI & 皮哨子 & Chuandian Soapberry Seed & $\begin{array}{l}\text { T5717 Sapindus delavayi }[\text { Syn. Pancovia } \\
\text { delavayi] }\end{array}$ \\
\hline PI XI DI GE SHE SHU & 匹西狄割舌树* & Piscidi Cuttongue tree* & T6806 Walsura piscidia \\
\hline PI ZHEN DU JING SHAN & 披针杜茎山 & Lanceolate Maesa* & T4030 Maesa lanceolata \\
\hline PI ZHEN QI SHU & 披针漆树* & Lanceolate Sumac* & T5533 Rhus lanceolata \\
\hline PI ZHEN XING YAO HUA & 披针形荛花* & Lanceolate Stringbush* & T6821 Wikstroemia lanceolata \\
\hline PI ZHEN YE GOU TENG & 披针叶钩藤* & Lance-leaved Gambirplant & T6622 Uncaria lancifolia \\
\hline PI ZHEN YE HUANG ROU NAN & 披针叶黄肉楠 & Lanceleaf Actinodaphne* & T0166 Actinodaphne lancifolia \\
\hline PI ZHEN YE NAN WU WEI ZI & 披针叶南五味子 & Lanceolate Kadsura* & T3617 Kadsura lancilimba \\
\hline PIAN CHI TANG SONG CAO & 偏翅唐松草 & Delavay Meadowrue & T6378 Thalictrum delavayi \\
\hline PIAO FU CAO & 飘拂草 & Dichotomous Fimbristylis & T2730 Fimbristylis dichotoma \\
\hline PIE LAN & 僌蓝. & Kohl-rabi & T1019 Brassica oleracea var. gongylodes \\
\hline PING & 苹 & Water-clover & T4121 Marsilea quadrifolia \\
\hline PING BEI MU & 平贝母 & Ussuri Fritillary & T2797 Fritillaria ussuriensis \\
\hline PING CHE QIAN & 平车前 & Depressed Plantain & T5004 Plantago depressa \\
\hline PING DI XI FENG QIN & 平地西风芹* & Campestral Seseli* & T5928 Seseli campestre \\
\hline PING E SHU & 平莪术 & Zedoary Turmeric & $\begin{array}{l}\text { T1909 Curcuma zedoaria [Syn. Curcuma } \\
\text { aeruginosa] }\end{array}$ \\
\hline PING ER XIAO CAO & 瓶尔小草 & Adder's Tongue & T4506 Ophioglossum vulgatum \\
\hline PING GUO & 苹果 & Apple & T4088 Malus pumila \\
\hline PING GUO HAI TANG & 苹果海棠* & Domestic Apple* & T4087 Malus domestica \\
\hline PING HUA FA LIANG GOU TENG & 平滑发亮钩藤* & Smooth Gambirplant & T6621 Uncaria laevigata \\
\hline PING HUA FENG DOU CAI & 平滑蜂斗菜* & Smooth Butterbur* & T4742 Petasites laevigatus \\
\hline
\end{tabular}




PING HUA GUO JIU JE
PING HUA SANG
PING PENG CAO
PING PU YUAN BAI
PING QIAN LI GUANG
PING WO DAO HUA
PING YU FENG WEI JUE
PING YUAN BA DOU
PING ZHAN JIAO HUI XIANG
PO BU YE
PO LUO MEN ZAO JIA
PO LUO ZHOU GOU TENG
PO SEN DA JI
PU DI WU GONG
PU DUN LI
PU ER CHA
PU ER DI LUO HAN SONG
PU FU JIN GU CAO
PU FU JING TU ER CAO
PU FU QIANG DAO YAO
PU FU SHI DA GONG LAO
PU GE WU TOU
PU GONG YING
PU HUANG
PU QI BEI MU
PU SUO MU
PU TAO
PU TAO TENG YE
PU TAO YE MU JIN
PU TAO YOU
PU(3) TAO
PU TAO YOU DA HONG JU ZA
JIAO ZHONG
PU TI SHU HUA
PU TONG BAI JIAN MU
PU TONG CANG ER
PU TONG LU TI CAO
PU TONG TUN CAO
PU TONG XUAN GOU ZI
PU TONG YUAN ZHI
PU TONG ZHANG YA CAI
PU YE SHAN YOU ZI
QI BAI ZHI
QI GU CAO
QI GUO JIU LI XIANG

QI GUO QIAN LI XIANG

QI HAN NING HUA JIAO

QI LIN CAI

QI LIN JIE

QI PAN SHA CAO

QI SHI HUA

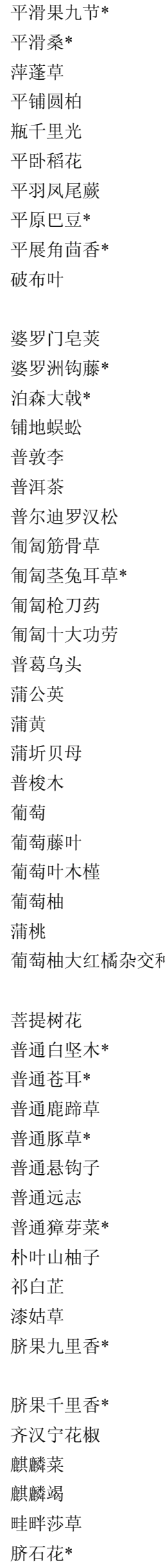

\section{Leio-fruit Ninenode* \\ Smooth Mulberry* \\ Cowlily \\ Creeping Juniper \\ Bottle Groundsel* \\ Prostrate Rice-flower* \\ Kiushu Brake}

Procumbent Hypecoum*

Paniculate Microcos

Goldenshower Senna Fruit

Borneo Gambirplant*

Poison Euphorbia*

Cernuous Clubmoss

Puddun Plum*

Assam Tea

Purdie Podocarpus*

Creeping Bugle

Creeping Lagotis*

Creeping Hypoestes*

Creeping Mahonia

Pukee Monkshood

Mongolian Dandelion

Longbract Cattail Pollen

Puqi Fritillary*

European Grape

European Grape Stem and Leaf

Grapeleaf Hibiscus*

Grapefruit

Roseapple

Miquel Linden
Common White Quebracho
Common Cocklebur*
Common Pyrola
Ragweed
Common Raspberry*
Common Milkwort*
Common Swertia*
Celtis-leaf Opilia*
Qibaizhi Angelica*
Pearlwort
Omphalo-fruit Common
Jasminorange*
Omphalo-fruit Jasminorange*
Tsihanim Pricklyash*
Muriculate Eucheuma Frond
Draco Yellowvine*
Asidefield Galingale
Omphalos Parmelia*

T5276 Psychotria leiocarpa

T4295 Morus laevigata

T4450 Nuphar pumilum

T3589 Juniperus horizontalis

T5906 Senecio sarracenicus

T4892 Pimelea prostrata

T5292 Pteris kiuschiuensis

T1840 Croton campestris

T3335 Hypecoum procumbens

T4217 Microcos paniculata [Syn. Grewia

microcos]

T1232 Cassia fistula

T6609 Uncaria borneensis

T2610 Euphorbia poisonii

T3970 Lycopodium cernuum

T5237 Prunus puddun

T1153 Camellia sinensis var. assamica

T5053 Podocarpus purdieana

T0271 Ajuga reptans

T3676 Lagotis stolonifera

T3375 Hypoestes serpens

T4071 Mahonia repens

T0126 Aconitum pukeese

T6301 Taraxacum mongolicum

T6584 Typha angustata

T2793 Fritillaria puqiensis

T5272 Psorospermum febrifugum

T6798 Vitis vinifera

T6799 Vitis vinifera

T3249 Hibiscus vitifolius

T1503 Citrus paradisi

T6267 Syzygium jambos

T1505 Citrus paradisi $\mathrm{x}$ Citrus tangerina

T6458 Tilia miqueliana

T0772 Aspidosperma quebracho-blanco

T6838 Xanthium commune

T5347 Pyrola decorata

T0396 Ambrosia ambrosioids

T5588 Rubus allegheniensis

T5089 Polygala vulgaris

T6239 Swertia swertiopsis

T4515 Opilia celtidifolia

T0479 Angelica dahurica cv. qibaizhi

T5646 Sagina japonica [Syn. Spergula japonica]

T4322 Murraya omphalocarpa

T4326 Murraya paniculata var. omphalocarpa T6898 Zanthoxylum tsihanimposa

T2525 Eucheuma muricatum

T1994 Daemonorops draco

T1975 Cyperus haspan

T4657 Parmelia saxatilis var. omphalodes 


\begin{tabular}{|c|c|c|}
\hline QI YE HUANG PI & 七叶黄皮* & Heptaleaf Wampee* \\
\hline QI YE PAN GUO JU & 槭叶盘果菊 & Rattlesnakeroot \\
\hline QI YE SHU & 七叶树 & Chinese Buckeye \\
\hline QI YI PU TAO FENG XIN ZI & 奇异葡萄风信子* & Paradoxy Grape-hyacinth* \\
\hline QI ZHOU YI ZHI HAO & 祁州一支蒿 & Horseweed Fleabane \\
\hline QI ZI & 漆子 & True Lacquer Seed \\
\hline QIAN CAO GEN & 茜草根 & Indian Madder Root \\
\hline QIAN CAO TENG & 茜草藤 & Indian Madder Stem \\
\hline QIAN CENG TA & 千层塔(蛇足石杉) & Serrate Clubmoss \\
\hline QIAN HU & 前胡 & Common Hogfennel \\
\hline QIAN HUI GOU TENG & 浅灰钩藤* & Greyish Gambirplant* \\
\hline QIAN HUI MAO GUAN JU & 浅灰毛冠菊* & Greyish Nannoglottis* \\
\hline QIAN JIE CAO & 千解草 & Herbaceous Pygmaeopremna \\
\hline QIAN JIN TENG & 千金藤 & Japanese Staphania \\
\hline QIAN JIN ZI & 千金子 & Caper Euphorbia Seed \\
\hline QIAN LI GUANG & 千里光 & Climbing Groundsel \\
\hline QIAN LIE LIN MAO JUE & 浅裂鳞毛蕨 & Fortunate Wood Fern \\
\hline QIAN LING YIN YANG HUO & 黔岭淫羊藿 & Thin-rhizome Epimedium \\
\hline QIAN MA & 寽麻 & Hempleaf Nettle \\
\hline QIAN MA YE ZE LAN & 寽麻叶泽兰 & White Snakeroot \\
\hline QIAN NIAN BU LAN XIN & 千年不烂心 & Bitter Nightshade Fruit \\
\hline QIAN NIU ZI & 牵牛子 & Lobedleaf Pharbitis Seed \\
\hline QIAN QU CAI & 千屈菜 & Spiked Loosestrife \\
\hline QIAN RI HONG & 千日红 & Globeamaranth \\
\hline QIAN SHI GEN & 苂实根 & Gordon Euryale Root \\
\hline QIAN YU DA QING & 欠愉大青 & Unfortunate Glorybower \\
\hline QIANG DAO YAO & 枪刀药 & Purple Hypoestes \\
\hline QIANG GU FEI YAN CAO & 强固飞燕草* & Consolidated Larkspur* \\
\hline QIANG HUO & 㒸活 & Incised Notopterygium \\
\hline QIANG WEI GEN & 蓄薇根 & Japanese Rose Root \\
\hline QIANG XIANG & 青葙 & Feather Cockscomb \\
\hline QIAO BING BA QIA & 鞘柄菝葜 & Sheathstipe Greenbrier \\
\hline QIAO CHA & 巧茶 & Khat \\
\hline QIAO GUAN JU & 鞘冠菊 & Common Coleostephus \\
\hline QIAO GUI & 乔桧 & Bhutan Pine \\
\hline QIAO HUANG TAN & 俏黄檀* & Spruce Rosewood* \\
\hline QIAO MAI & 养麦 & Common Buckwheat \\
\hline QIAO MAI JIE & 养麦秸 & Common Buckwheat Stem \\
\hline QIAO MU CI TONG & 乔木刺桐 & Himalayan Coralbean \\
\hline QIAO MU HU JIAO & 乔木胡椒 & Arboreous Pepper* \\
\hline QIAO MU SHAO YAO & 乔木苟药* & Arboreous Peony \\
\hline QIAO MU ZHUANG HUANG & 乔木状黄牛木* & Genonggang \\
\hline \multicolumn{3}{|l|}{ NIU MU } \\
\hline QIAO MU ZHUANG LAN REN & 乔木状榄仁 & Arboreous Terminalia* \\
\hline QIAO MU ZHUANG YANG MEI & 乔木状杨梅* & Arboreous Bayberry* \\
\hline QIAO MU ZI ZHU & 乔木紫珠 & Tree Beautyberry \\
\hline
\end{tabular}

T1536 Clausena heptaphylla

T5196 Prenanthes acerifolia

T0201 Aesculus chinensis

T4333 Muscari paradoxum

T1657 Conyza canadensis [Syn. Erigeron

canadensis]

T5541 Rhus verniciflua [Syn. Toxicadendron

verniciflum]

T5578 Rubia cordifolia

T5579 Rubia cordifolia

T3295 Huperzia serrata [Syn. Lycopodium

serratum]

T0480 Angelica decursiva [Syn. Peucedanum

decursivum]

T6611 Uncaria canescens

T4372 Nannoglottis ravida

T5341 Pygmaeopremna herbacea [Syn.

Premna herbacea]

T6129 Stephania japonica

T2597 Euphorbia lathyris

T5907 Senecio scandens [Syn. Senecio chinensis]

T2287 Dryopteris sublaeta

T2399 Epimedium leptorrhizum

T6651 Urtica cannabina

T2579 Eupatorium urticaefolium

T5998 Solanum dulcamara

T4779 Pharbitis nil

T4007 Lythrum salicaria

T3040 Gomphrena globosa

T2632 Euryale ferox

T1559 Clerodendron infortunatum

T3373 Hypoestes purpurea [Syn. Justicia

purpurea; Hypoestes sinica]

T2068 Delphinium consolida

T4446 Notopterygium incisum

T5569 Rosa multiflora

T1296 Celosia argentea

T5984 Smilax stans [Syn. Smilax vaginata var. stans]

T1266 Catha edulis

T1618 Coleostephus myconis

T4910 Pinus excelsa

T2016 Dalbergia spruceana

T2658 Fagopyrum esculentum

T2659 Fagopyrum esculentum

T2459 Erythrina arborescens

T4932 Piper arboreum

T4581 Paeonia arborea

T1783 Cratoxylum arborescens

T6343 Terminalia arborea

T4342 Myrica arborea

T1116 Callicarpa arborea 


\begin{tabular}{|c|c|c|}
\hline QIAO SHI DOU LAN & 鞘石豆兰 & Vaginate Stonebean-orchis* \\
\hline QIAO TOU & 菾头 & Chinese Onion \\
\hline QIE YE & 茄叶 & Garden Eggplant Leaf \\
\hline QIE ZI & 茄子 & Garden Eggplant \\
\hline QIN HUA & 芹花 & Javan Waterdropwort Flower \\
\hline QIN JIAO & 秦艽 & Largeleaf Gentian \\
\hline QIN LING BAI LA SHU & 秦岭白蜡树 & Pax Ash \\
\hline QIN LING CUI QUE HUA & 秦岭翠雀花 & Girald Larkspur \\
\hline QIN LING DA HUANG & 秦岭大黄 & Qinling Rhubarb* \\
\hline QIN LING ZHU ZI SHEN & 秦岭珠子参 & Largeleaf Japanese Ginseng \\
\hline QIN ZHUANG AO CHUN JIANG & 琴状凹唇姜 & Lyrate Boesenbergia* \\
\hline QING AI & 清艾 & Graywhite Wormwood* \\
\hline QING FENG TENG & 青风藤 & Orientvine \\
\hline QING GUO & 青果 & Olive \\
\hline QING HAO & 青蒿 & Celery Wormwood \\
\hline QING JIAO & 青椒 & Peppertree Pricklyash \\
\hline QING LIANG BAI HE & 清亮百合* & Madonna Lily \\
\hline QING MEI & 青梅 & Rassak Vatica* \\
\hline QING MING HUA & 清明花 & Easter Heraldtrumpet \\
\hline QING MU XIANG & 青木香(马兒铃根) & Slender Dutchmanspipe Root \\
\hline QING NIANG ZI & 青娘子 & Mung Bean Blister Beetle \\
\hline QING NIU DAN & 青牛胆 & Arrowshaped Tinospora \\
\hline QING QIAN LIU & 青钱柳 & Roundwingfruit Cyclocarya \\
\hline QING SHE TENG & 青蛇藤 & Greensnake vine \\
\hline QING TENG XIANG & 青藤香 & Japanese Snailseed Stem \\
\hline QING WA & 青蛙 & Pond Frog \\
\hline QING WA DAN & 青蛙胆 & Pond Frog Gall \\
\hline QING XIANG MU JIANG ZI & 清香木姜子 & Fourflower Litse \\
\hline QING YANG & 青杨 & Green Poplar \\
\hline QING YANG SHEN & 青羊参 & Auricledleaf Mosquitotrap \\
\hline QING YE DAN & 青叶胆 & Mileen Swertia \\
\hline QING ZI QIAN NIU & 青紫牵牛 & Violet Morningglory* \\
\hline QING ZI SU & 青紫苏 & Viridian Common Perilla* \\
\hline QIU FENG MU & 秋枫木 & Javan Bishopwood \\
\hline QIU FU SHOU CAO & 秋福寿草 & Annual Adonis* \\
\hline QIU HUA DANG SHEN & 球花党参 & Subglobose Asiabell \\
\hline QIU HUA NIU NAI CAI & 球花牛奶菜 & Globose Condorvine \\
\hline QIU HUA ZUI YU CAO & 球花醉鱼草 & Orange-ball-tree \\
\hline QIU MU GUA & 秋木瓜 & Common Floweringquince \\
\hline QIU QIE SHU & 秋茄树 & Kandelia \\
\hline QIU RUI WU WEI ZI & 球萝五味子 & Ballander Magnoliavine \\
\hline QIU SHENG BI HUA MU & 丘生闭花木 & \\
\hline QIU SHI MEI DENG MU & 丘氏美登木 & Chuchuhuasc Mayten* \\
\hline QIU SHUI XIAN & 秋水仙 & Meadow Saffron \\
\hline QIU SHUI XIAN CHANG CHUN & 秋水仙常春藤* & Colchicum Ivy* \\
\hline \multicolumn{3}{|l|}{ TENG } \\
\hline QIU SUI QIAN JIN BA & 球穗千斤拔 & Conespike Flemingia \\
\hline QIU YAO GE CHONG LOU & 球药隔重楼 & Farges Paris \\
\hline
\end{tabular}

T1054 Bulbophyllum vaginatum

T0313 Allium chinense

T6006 Solanum melongena

T6007 Solanum melongena

T4481 Oenanthe javanica

T2919 Gentiana macrophylla

T2774 Fraxinus paxiana

T2076 Delphinium giraldii

T5475 Rheum qinlingense

T4605 Panax japonicus var. major

T0970 Boesenbergia pandurata

T0669 Artemisia cana

T5964 Sinomenium acutum

T1165 Canarium album

T0662 Artemisia apiacea [Syn. Artemisia

carvifolia; Artemisia caruifolia ]

T6892 Zanthoxylum schinifolium

T3833 Lilium candidum

T6686 Vatica rassak

T0886 Beaumontia grandiflora

T0627 Aristolochia debilis [Syn. Aristolochia

longa]

T4008 Lytta caraganae

T6467 Tinospora sagittata

T1936 Cyclocarya paliurus

T4725 Periploca calophylla

T1590 Cocculus trilobus [Syn. Cocculus sarmentosus]

T5407 Rana nigromaculata; Rana plancyi

T5408 Rana nigromaculata; Rana plancyi

T3886 Litsea euosma

T5150 Populus cathayana

T1958 Cynanchum otophyllum

T6226 Swertia mileensis

T3453 Ipomoea violacea

T4716 Perilla frutescens $\mathrm{f}$. viridis

T0945 Bischofia javanica [Syn. Bischofia

trifoliata ]

T0185 Adonis annua

T1601 Codonopsis subglobosa

T4116 Marsdenia globifera

T1047 Buddleja globosa

T1342 Chaenomeles lagenaria [Syn.

Chaenomeles speciosa]

T3626 Kandelia candel

T5801 Schisandra sphaerandra

T1543 Cleistanthus collinus

T4130 Maytenus chuchuhuasca

T1616 Colchicum autumnale

T3111 Hedera colchica

T2738 Flemingia strobilifera

T4647 Paris fargesii 


\begin{tabular}{|c|c|c|c|}
\hline QIU YIN & 虾蚓 & Earthworm & $\begin{array}{l}\text { T4796 Pheretima aspergillum; Allolobophora } \\
\text { caliginosa trapezoides }\end{array}$ \\
\hline QIU YUAN YE TAI & 秋圆叶苔 & & T3552 Jamesoniella autumnalis \\
\hline QIU ZHUANG PO BU MU & 球状破布木* & Globe Cordia* & T1677 Cordia globosa \\
\hline QU CHONG BAN JIU JU & 驱虫斑加菊 & Worm-killed Bitterleaf & T6713 Vernonia anthelmintica \\
\hline QU CHONG CAO & 驱虫草 & & T6074 Spigelia anthelmia \\
\hline QU CHONG HE HUAN & 驱虫合欢* & Musenna Albizia & T0291 Albizzia anthelmintica \\
\hline QU MAI & 㫿麦 & Lilac Pink & T2145 Dianthus superbus \\
\hline QU QU HUA & 屈曲花 & Rocket Candytuft & T3385 Iberis amara \\
\hline QU XI DANG GUI & 曲膝当归* & Genuflex Angelica* & T0482 Angelica genuflexa \\
\hline QU YU CAO DI LAO GUAN CAO & 区域草地老鹳草 & & T2945 Geranium pratense ssp. funitimum \\
\hline QU ZHOU HAI JIN SHA & 曲轴海金沙 & Flexuose Climbing Fern & $\begin{array}{l}\text { T3989 Lygodium flexuosum [Syn. Lygodium } \\
\text { pinnatifidum; Ophioglossum flexuosum] }\end{array}$ \\
\hline QUAN CHI QIANG WEI & 犬齿蓄薇 & Dog Rose & T5561 Rosa canina \\
\hline QUAN JU GUA YE WU TOU & 拳距瓜叶乌头 & Circinate Hemsley Monkshood & $\begin{array}{l}\text { T0100 Aconitum hemsleyanium var. } \\
\text { circinacum }\end{array}$ \\
\hline QUAN NENG HUA & 全能花 & Twoflower Pancratium & T4612 Pancratium biflorum \\
\hline QUAN SHEN & 拳参 & Bistort & T5099 Polygonum bistorta \\
\hline QUAN XIE & 全蝎 & Scorpion & T1084 Buthus martensi \\
\hline QUAN YE SU TIE & 拳叶苏铁 & Crozier Cycas & T1926 Cycas circinalis \\
\hline QUAN YE YAN HU SUO & 全叶延胡索 & Creeping Corydalis & T1735 Corydalis repens \\
\hline QUAN YUAN CHU & 全缘㯪 & Integrifolious Ailanthus* & T0257 Ailanthus integrifolia ssp. calycina \\
\hline QUAN YUAN GUI MU & 全缘桂木 & Integerleaf Artocarpus & T0715 Artocarpus integra \\
\hline QUAN YUAN QIAN LI GUANG & 全缘千里光 & Entire Groundsel & T5889 Senecio integerrimus \\
\hline $\begin{array}{l}\text { QUAN YUAN YE AO TUO SI TE } \\
\text { CAO }\end{array}$ & 全缘叶奥托斯特草* & Integrifolious Otostegia* & T4558 Otostegia integrifolia \\
\hline QUAN YUAN YE BO LUO MI & 全缘叶波罗蜜* & Integrifolious Artocarpus* & T0716 Artocarpus integrifolia \\
\hline QUAN YUAN YE HUA JIAO & 全缘叶花椒 & Integrifolious Pricklyash* & T6882 Zanthoxylum integrifoliolum \\
\hline $\begin{array}{l}\text { QUAN YUAN YE MEI ZHOU } \\
\text { CHA }\end{array}$ & 全缘叶美洲茶 & Deerbrush & T1276 Ceanothus integerrimus \\
\hline QUAN YUAN YE TE SA JU & 全缘叶特萨菊 & & T6351 Tessaria integriforia \\
\hline QUAN YUAN YE XIAO BO & 全缘叶小檗 & Integrifolious Barberry & T0905 Berberis integerrima \\
\hline QUE BAI MAI JIAO & 雀稗麦角 & Paspalum Ergot* & T1540 Claviceps paspali \\
\hline QUE MEI TENG & 雀梅藤 & Hedge Sageretia & $\begin{array}{l}\text { T5645 Sageretia theezans }[\text { Syn. Sageretia } \\
\text { thea] }\end{array}$ \\
\hline QUE SHE HUANG YANG & 雀舌黄杨 & Bodinier Box & T1087 Buxus bodinieri \\
\hline QUN DAI CAI & 裙带菜 & Undaria & T6640 Undaria pinnatifida \\
\hline QUN XIN CAI & 群心菜 & Common Cardaria & T1195 Cardaria draba \\
\hline RAN LIAO MU & 染料木 & Common Woadwaxen & T2901 Genista tinctoria \\
\hline RAN LIAO SI SHI MU & 染料斯氏木 & & T5940 Sickingia tinctoria \\
\hline RAN MAO QIAO RUI HUA & 髼毛鞘荵花 & Forskahl Coleus & T1619 Coleus barbatus \\
\hline RAN SE JI YAN TENG & 染色鸡眼藤 & Dyed Morinda & T4285 Morinda tinctoria \\
\hline RAN SE SANG & 染色桑 & Tinctorial Mulberry* & T4302 Morus tinctoria \\
\hline RE BEN MO GU & 日本蘑菇 & $\begin{array}{l}\text { Japanese Mushroom*(Sheep } \\
\text { Polypore) }\end{array}$ & T0288 Albatrellus ovinus \\
\hline REN DONG TENG & 忍冬藤 & Japanese Honeysuckle Vine & T3913 Lonicera japonica \\
\hline REN GONG YONG CHONG CAO & 人工蛹虫草 & Cultivated Scarlet Caterpillar Fungus* & T1681 Cordyceps militaris cv \\
\hline REN NIAO & 人尿 & Human Urine & T3276 Homo sapiens \\
\hline REN SHEN & 人参 & Ginseng & T4599 Panax ginseng [Syn. Panax schinseng] \\
\hline REN SHEN HUA LEI & 人参花蕾 & Ginseng Buds & T4600 Panax ginseng [Syn. Panax schinseng] \\
\hline REN SHEN LU & 人参芦 & Ginseng Reed & T4601 Panax ginseng [Syn. Panax schinseng] \\
\hline REN SHEN XI YANG SHEN ZA & 人参西洋参杂交种 & & T4603 Panax ginseng x P. quinquefolium \\
\hline
\end{tabular}




\begin{tabular}{|c|c|}
\hline REN SHEN YE & 人参叶 \\
\hline REN ZHONG BAI & 人中白 \\
\hline RI BEN AN XI XIANG JING PI & 日本安息香茎皮 \\
\hline RI BEN BAI LA SHU & 日本白蜡树 \\
\hline RI BEN BAI SI CAO & 日本白丝草 \\
\hline RI BEN BAI SONG FENG CAO & 日本白松风草 \\
\hline RI BEN BIAN BAI & 日本扁柏 \\
\hline RI BEN BIAN TAI & 日本鞭苔 \\
\hline RI BEN CE ER & 日本侧耳 \\
\hline RI BEN CHANG PU & 日本菖蒲 \\
\hline RI BEN CHOU JIE CAO & 日本臭节草* \\
\hline RI BEN CU FEI & 日本粗榧 \\
\hline RI BEN DANG GUI & 日本当归 \\
\hline RI BEN DONG FENG LUO & 日本东风螺* \\
\hline RI BEN DU JUAN HUA & 日本杜鹃花 \\
\hline RI BEN DUO CI YU TAI & 日本多刺羽苔* \\
\hline RI BEN GUI DENG QING & 日本鬼灯擎 \\
\hline RI BEN HOU PI XIANG & 日本厚皮香 \\
\hline RI BEN HOU PO & 日本厚朴 \\
\hline RI BEN HUA BAI & 日本花柏 \\
\hline RI BEN HUANG BAI & 日本黄柏 \\
\hline RI BEN HUANG LIAN & 日本黄连 \\
\hline RI BEN JIA MI & 日本荚蒾 \\
\hline RI BEN JIN YAO & 日本金腰 \\
\hline RI BEN KU LIAN & 日本苦楝 \\
\hline RI BEN LIAN XIANG SHU & 日本连香树* \\
\hline RI BEN LIN MAO JUE & 日本鳞毛蕨 \\
\hline RI BEN LIU SHAN & 日本柳杉 \\
\hline RI BEN LONG DAN & 日本龙胆* \\
\hline RI BEN LONG YA CAO & 日本龙芽草* \\
\hline RI BEN LU TI CAO & 日本鹿蹄草 \\
\hline RI BEN LUO SHI & 日本络石 \\
\hline RI BEN MA SANG & 日本马桑 \\
\hline RI BEN MA ZUI MU & 日本马醉木 \\
\hline RI BEN MANG CAO & 日本䒭草 \\
\hline RI BEN NAN & 日本楠 \\
\hline RI BEN NAN WU WEI ZI & 日本南五味子 \\
\hline RI BEN NIU PI XIAO & 日本牛皮消 \\
\hline RI BEN NIU XI & 日本牛膝 \\
\hline RI BEN NV ZHEN & 日本女贞 \\
\hline RI BEN PING PENG CAO & 日本萍蓬草 \\
\hline RI BEN QI YE SHU & 日本七叶树 \\
\hline RI BEN QIAN QU CAI & 日本千屈菜 \\
\hline RI BEN SAN YE SHA SEN & 日本三叶沙参 \\
\hline RI BEN SHE GEN CAO & 日本蛇根草 \\
\hline RI BEN SHENG MA & 日本升麻 \\
\hline RI BEN SHU YU & 日本薯蓣 \\
\hline RI BEN SHUANG HU DIE & 日本双蝴蝶* \\
\hline RI BEN SU MU & 日本苏木 \\
\hline RI BEN WEN SHU LAN & 日本文殊兰 \\
\hline RI BEN WU JIA & 日本五加 \\
\hline RI BEN WU TOU & 日本乌头 \\
\hline RI BEN XIANG BAI JING PI & 日本香柏茎皮 \\
\hline
\end{tabular}

Ginseng Leaf

Human Urine Sediment

Japanese Snowbell Stem-bark

Japanese Ash*

Japanese Chionographis*

Japanese White Chinaure*

Hinoki False Cypress

Japanese Flagelliform Liverwort*

Japanese Sweetflag*

Japanese Chinaure*

Japanese Plumyew

Japanese Angelica*

Japanese Azalea

Bronzeleaf Rodgersflower

Japanese Cleyera

Whiteleaf Japanese Magnolia

Sawara False Cypress

Japan Corktree*

Japanese Goldthread*

Japanese Viburnum

Japanese Goldsaxifrage

Japanese Chinaberry-tree

Japanese Katsura-tree

Brown-margin Wood Fern

Japanese Cedar

Japanese Gentian*

Japanese Agrimonia*

Japanese Pyrola

Japanese Star Jasmine*

Japanese Coriaria*

Japanese Pieris

Japanese Anisetree

Japanese Machilus*

Japanese Kadsura

Japanese Swallowwort*

Japanese Achyranthes

Japanese Privet

Japanese Cowlily*

Japanese Buckeye

Twoedged Loosestrife

Japanese Trileaf Ladybel1*

Japanese Ophiorrhiza

Japanese Bugbane*

Japanese Yam

Japanese Dualbutterfly*

Japanese Caesalpinia*

Japanese Crinum

Japanese Acanthopanax*

Japanese Monkshood*

Japanese Arborviate Stem-bark
T4602 Panax ginseng [Syn. Panax schinseng]

T3277 Homo sapiens

T6200 Styrax japonica

T2771 Fraxinus japonica

T1369 Chionographis japonica

T0965 Boenninghausenia albiflora var. japonica

T1349 Chamaecyparis obtusa

T0883 Bazzania japonica

T3684 Lamptreomyces japonicus

T0143 Acorus calamus var. angustatus

T0966 Boenninghausenia japonica

T1320 Cephalotaxus harringtonia

T0485 Angelica japonica

T0842 Babylonia japonica

T5511 Rhododendron japonicum

T4991 Plagiochila acanthophylla ssp. japonica

T5556 Rodgersia podophylla

T6350 Ternstroemia japonica

T4044 Magnolia obovata

T1350 Chamaecyparis pisifera

T4793 Phellodendron japonicum

T1667 Coptis japonica

T6734 Viburnum awabuki

T1404 Chrysosplenium japonicum

T4160 Melia azedarach var. japonica

T1332 Cercidiphyllum japonicum

T2286 Dryopteris sacrosancta

T1868 Cryptomeria japonica

T2912 Gentiana japonica

T0247 Agrimonia japonica

T5350 Pyrola japonica

T6483 Trachelospermum asiaticum

T1690 Coriaria japonica

T4890 Pieris japonica

T3399 Illicium anisatum

T4016 Machilus japonica

T3616 Kadsura japonica

T1956 Cynanchum japonicum

T0074 Achyranthes fauriei

T3827 Ligustrum japonicum

T4448 Nuphar japonicum

T0204 Aesculus turbinata

T4006 Lythrum anceps

T0169 Adenophora triphylla var. japonica T4510 Ophiorrhiza japonica

T1422 Cimicifuga japonica

T2202 Dioscorea japonica

T6537 Tripterospermum japonicum

T1104 Caesalpinia japonica

T1795 Crinum asiaticum var. japonicum

T0039 Acanthopanax japonicus

T0104 Aconitum japonicum

T6442 Thuja standishii 


\begin{tabular}{|c|c|c|}
\hline RI BEN XIANG RU & 日本香蔐* & Japanese Elsholtzia* \\
\hline RI BEN XIAO BO & 日本小檗 & Japanese Barberry \\
\hline RI BEN XIAO HE YI & 日本小核衣* & Asian lichen \\
\hline RI BEN XIN YI & 日本辛夷 & Kobus Magnolia \\
\hline RI BEN YING HUA & 日本樱花 & Tokyo Cherry \\
\hline RI BEN YU LIN SONG & 日本鱼鳞松 & Yeddo Spruce \\
\hline RI BEN YUAN WEI & 日本禹尾 & Japanese Iris* \\
\hline RI BEN ZHANG YA CAI & 日本獐牙菜 & Japanese Swertia* \\
\hline RI BEN ZI ZHU & 日本紫珠 & Japanese Beautyberry \\
\hline RONG BAI RU GU & 线白乳菇 & Fleecy Milk-cap \\
\hline RONG MAO DAI XING CAO & 线毛戴星草 & Indian Sphaeranthus \\
\hline RONG MAO DAN SHEN & 线毛丹参 & Tomentose Sage* \\
\hline RONG MAO HU TONG & 咠毛胡桐 & Tomentose Calaba \\
\hline RONG MAO MI ZI LAN & 线毛米仔兰* & Tomentose Aglaia* \\
\hline RONG MAO SHAN XIANG & 茸毛山香 & Tomentose Bushmint* \\
\hline RONG MAO XIANG KE KE & 线毛香科科 & Tomentose Germander \\
\hline RONG SHU & 榕树 & Smallfruit Fig \\
\hline ROU CONG RONG & 肉苁蓉 & Desertliving Cistanche \\
\hline ROU DOU KOU & 肉豆冦 & Common Nutmeg \\
\hline ROU GUI & 肉桂 & Cassiabarktree \\
\hline ROU HONG MA LI JIN & 肉红马利筋 & Incarnate Milkweed* \\
\hline ROU HUA XUE DAN & 肉花雪胆 & Fleshy-flower Hemsleya \\
\hline ROU JIN HE HUAN & 柔金合欢 & Black Wattle \\
\hline ROU JING XIANG CHA CAI & 柔茎香茶菜 & Flexedstem Rabdosia \\
\hline ROU LEI NIU NAI CAI & 柔雷牛奶菜 & Roylei Condorvine* \\
\hline ROU MAO DI DAN CAO & 菜毛地胆草 & Hawaiian Elephantfoot \\
\hline ROU MAO FAN LI ZHI & 柔毛番荔枝 & Glaucous Custardapple* \\
\hline ROU MAO HUANG QI & 柔毛黄芪* & Darkhairy Milkvetch* \\
\hline ROU MAO QIE & 柔毛茄 & Pubescent Nightshade \\
\hline ROU MAO SHAN LI DOU & 柔毛山黧豆 & Pilosity Peavine* \\
\hline ROU MAO XIANG RI KUI & 柔毛向日葵 & Pubescent Sunflower* \\
\hline ROU MAO XIAO RU XIANG & 柔毛肖乳香 & Hairy Peppertree* \\
\hline ROU MAO YIN YANG HUO & 柔毛淫羊藿 & Pubescence Epimedium \\
\hline ROU SE HUAN YANG SHEN & 柔色还阳参 & Soft Hawksbeard \\
\hline RU BAI LONG DAN & 乳白龙胆* & Milky Gentian* \\
\hline RU DI JIN NIU & 入地金牛(两面针) & Shinyleaf Pricklyash \\
\hline RU DI WU GONG & 入地蜈蚣 & Ceylan Helminthostachys \\
\hline RU DU XIANG & 萗笃香 & Turpentine Tree \\
\hline RU JU & 乳桔 & Kinokuni Citrus \\
\hline RU LAN & 汝兰 & Hernandialeaf Stephania \\
\hline RU NI WENG DAO MI ZHU YU & 儒尼翁岛蜜茱英 & Reunion-Island Melicope* \\
\hline RU XIANG & 乳香 & Olibanum \\
\hline RU XIANG BAI & 乳香柏 & Mastic Juniper* \\
\hline RU YUAN DU JUAN & 乳源杜鹃 & Ruyuan Rhododendron \\
\hline RUAN GU HAI TOU HONG & 软骨海头红 & \\
\hline RUAN GU ZAO & 软骨藻 & \\
\hline RUAN MAO DU HUO & 软毛独活 & Soft-hair Cowparsnip \\
\hline RUAN RUO DU JING SHAN & 软弱杜茎山 & Slender Maesa \\
\hline RUAN SHI & 软柿 & Soft Persimmon* \\
\hline RUAN TIAO QI QIANG WEI & 软条七蓄薇 & Henry's Rose \\
\hline RUAN YIN BEI TENG & 软银背藤 & Soft Argyreia* \\
\hline RUAN ZHI HUANG CHAN & 软枝黄蝉 & Common Allemanda \\
\hline
\end{tabular}

T2342 Elsholtzia nipponica T0916 Berberis thunbergii T5342 Pyrenula japonica T4040 Magnolia kobus T5246 Prunus yedoensis T4871 Picea jezoensis T3462 Iris komonoensis T6223 Swertia japonica T1119 Callicarpa japonica T3657 Lactarius vellereus T6071 Sphaeranthus indicus T5696 Salvia tomentosa T1135 Calophyllum tomentosum T0246 Aglaia tomentosa T3382 Hyptis tomentosa T6370 Teucrium tomentosum T2721 Ficus microcarpa T1455 Cistanche deserticola T4351 Myristica fragrans T1439 Cinnamomum cassia [Syn. Cinnamomum aromaticum ] T0738 Asclepias incarnata T3204 Hemsleya carnosiflora T0027 Acacia mollissima T3489 Isodon flexicaulis T4119 Marsdenia roylei T2333 Elephantopus mollis T0507 Annona glauca T0786 Astragalus atropubescens T6010 Solanum pubescens T3708 Lathyrus palustris var. pilosus T3150 Helianthus mollis T5788 Schinus molle T2401 Epimedium pubescens T1788 Crepis mollis T2916 Gentiana lactea T6884 Zanthoxylum nitidum T3190 Helminthostachys zeylanica T4981 Pistacia terebinthus T1489 Citrus kinokuni T6128 Stephania hernandifolia T4164 Melicope coodeana T0994 Boswellia carterii T3601 Juniperus thurifera T5512 Rhododendron lingii T5023 Plocamium cartilagineum T1382 Chondria armata [Syn. Lophura armata] T3214 Heracleum lanatum T4032 Maesa tenera T2225 Diospyros mollis T5566 Rosa henryi T0613 Argyreia mollis T0307 Allemanda cathartica 


\begin{tabular}{|c|c|c|c|}
\hline RUI DE QIAN LI GUANG & 瑞德千里光* & Riddell Groundsel & T5903 Senecio riddellii \\
\hline RUI DIAN GAN LAN & 瑞典甘蓝* & Swedish Turnip & T1022 Brassica rutabaga \\
\hline RUI JIAO JIAN MU & 锐角樫木* & Acuteangulus Pencilwood* & T2306 Dysoxylum acutangulum \\
\hline RUI SHI QIAN NIU & 瑞氏汼牛 & Regnell Morningglory* & T3451 Ipomoea regnellii \\
\hline RUI SHI SHI SONG & 瑞士石松 & Arolla Pine & T4909 Pinus cembra \\
\hline RUI XIANG GEN & 瑞香根 & Winter Daphne Root & T2027 Daphne odora \\
\hline RUI XIANG HUA & 瑞香花 & Winter Daphne Flower & T2028 Daphne odora \\
\hline RUI ZI SU & 锐紫苏 & Argute Perilla* & T4713 Perilla arguta \\
\hline SA BA LU HUI & 萨芭芦荟 & Saba Aloe & T0344 Aloe sabaea \\
\hline SA HA LIN YUN SHAN & 萨哈林云杉 & Saghalin Spruce & T4870 Picea glehnii \\
\hline SA JIN TE BAI MU & 萨金特柏木 & Sargent Cypress & T1898 Cupressus sargentii \\
\hline SA LI LA WO JU & 萨利拉莴菅 & Sariola Lettuce* & T3661 Lactuca sariola \\
\hline SA SHI MAO DI HUANG & 萨氏毛地黄* & Thaps Foxglove* & T2180 Digitalis thapsii \\
\hline SAI ER WEI YA SHI CAO & 塞尔维亚著草 & Serbian Yarrow & T0059 Achillea alexandri-regis \\
\hline SAI LA LI ANG SHUI XIAN & 塞拉利昂水仙 & & T4375 Narcissus leonensis \\
\hline SAI LANG DANG & 赛莨宕 & Common Anisodus & T0501 Anisodus luridus \\
\hline SAI LE LUO LE & 塞勒罗勒 & Sello Basil* & T4475 Ocimum selloi \\
\hline SAI NEI JIA ER CI TONG & 塞内加尔刺桐* & Senegal Coralbean* & T2474 Erythrina senegalensis \\
\hline Salvia spp. & 鼠尾草属 & & T5694 Salvia spp. \\
\hline SAN BAI CAO & 三白草 & Chinese Lizardtail & T5750 Saururus chinensis \\
\hline SAN CHA FENG WEI JUE & 三叉凤尾蕨 & Wallichi's Brake & T5299 Pteris wallichinan \\
\hline SAN CHA KU & 三叉苦 & Thin Evodia & T2641 Evodia lepta $[$ Syn. Ilex lepta $]$ \\
\hline SAN CHI HAO & 三齿蒿 & Big Sagebrush & T0702 Artemisia tridentata \\
\hline SAN CHI LA RUI A & 三齿拉瑞阿 & Creosote-bush & T3695 Larrea tridentata \\
\hline SAN CHU SHAN XIANG YUAN & 三出山香圆* & Threeleaf Turpinia & T6568 Turpinia ternata \\
\hline SAN CI ZAO JIA & 三刺㿝荚 & Honeylocust & T2982 Gleditsia triacanthos \\
\hline SAN FANG HUA XU HONG YUE & 伞房花序红月桂* & Corymb Rose-bay* & T6272 Tabernaemontana corymbosa \\
\hline \multicolumn{4}{|l|}{ GUI } \\
\hline SAN FEN DAN & 三分丹 & Brackfollicle & T6578 Tylophora atrofolliculata \\
\hline SAN FEN SAN & 三分三 & Acutangular Scopolia & $\begin{array}{l}\text { T5819 Scopolia acutangula [Syn. Anisodus } \\
\text { acutangulus] }\end{array}$ \\
\hline SAN HUA BA DOU & 散花巴豆 & Sparseflower Croton* & T1856 Croton sparsiflorus \\
\hline SAN HUA LONG DAN & 三花龙胆 & Threeflower Gentian & T2938 Gentiana triflora \\
\hline SAN HUA MI HUA SHU & 伞花密花树* & Umbellate Rapanea* & T5418 Rapanea umbellata \\
\hline SAN JIAN SHAN & 三尖杉 & Fortune Plumyew & T1318 Cephalotaxus fortunei \\
\hline SAN JIAO FEN YE JUE & 三角粉叶硕* & Goldback Fern & T4988 Pityrogramma triangularis \\
\hline SAN JIAO MA DOU LING & 三角马䅐铃* & Triangular Dutchmanspipe* & T0640 Aristolochia triangularis \\
\hline SAN JIAO YE HUANG LIAN & 三角叶黄连 & Deltoid Goldthread & T1664 Coptis deltoidea \\
\hline SAN JIAO YE SHU YU & 三角叶薯蓣 & Deltoid Yam & T2195 Dioscorea deltoidea \\
\hline SAN JU AI CUI QUE & 三距矮翠雀 & Spring Larkspur & T2088 Delphinium tricorne \\
\hline SAN LENG & 三棱 & Common Burreed & T6062 Sparganium stoloniferum \\
\hline SAN LENG YE JIN SI TAO & 三棱叶金丝桃* & Triangular-leaf St.John'swort* & T3366 Hypericum triquetrifolium \\
\hline SAN LIE HAO & 三裂蒿* & Tripartite Wormwood* & T0704 Artemisia tripartita \\
\hline SAN LIE QIE & 三裂茄 & Tripartite Nightshade* & T6016 Solanum tripartitum \\
\hline SAN LIE XUE TONG & 三裂血桐* & Trilobate Macaranga* & T4014 Macaranga triloba \\
\hline SAN LIE YE GE & 三裂叶葛 & Trilobedleaf Kudzuvine & T5318 Pueraria phaseoloides \\
\hline SAN MIAN DAO & 三面刀 & Small Bugbane & T1417 Cimicifuga acerina \\
\hline SAN QI & 三七 & Sanchi & $\begin{array}{l}\text { T4608 Panax pseudo-ginseng var. notoginseng } \\
\text { [Syn. Panax notoginseng] }\end{array}$ \\
\hline SAN QI CAO & 三七草 & Gynura & T3088 Gynura segetum [Syn. Gynura japonica] \\
\hline SAN QI HUA LEI & 三七花蕾 & Sanchi Buds & $\begin{array}{l}\text { T4609 Panax pseudo-ginseng var. notoginseng } \\
\text { [Syn. Panax notoginseng] }\end{array}$ \\
\hline SAN QIU BO SI SHI SUAN & 三球波斯石蒜 & Trisphare Ungernia* & T6642 Ungernia trisphaera \\
\hline
\end{tabular}




SAN RUI LIAN GUI
SAN SE BAI ZHUANG GU
SAN SE JIN
SAN SE QIAN NIU
SAN SHI HUANG MA
SAN TAI HONG HUA
SAN TAI HUA
SAN TIAO JIN
SAN WEI ZHI FAN YING TAO
SAN XIAO CAO
SAN XIAO YE CUI QUE HUA
SAN XIAU YE SHAN DOU GEN
SAN XING HU JIAO
SAN XING HUA XU YIN JIA
SAN XING OU SHI NAN
SAN XING QU QU HUA
SAN XING XIU QIU
SAN YE DIAO ZHANG
SAN YE FANG FENG
SAN YE HUANG LIAN
SAN YE MA BIAN CAO
SAN YE MAI MA QIAN
SAN YE MI ZHU YU
SAN YE MU
SAN YE MU TONG
SAN YE MU TONG GEN
SAN YE SHU WEI CAO
SAN YE TENG JU
SAN YING JIAN GUA LOU
SAN ZHONG JU ZA JIAO
ZHONG [XX
SAN ZUAN FENG
SANG BAI PI
SANG CHENG
SANG DAO BU SHI MU
SANG HANG
SANG
SAN
SAN
SAN

SANG SHI
SANG YE
SANG ZHI
SE BO GE XIU QIU
SE LUN YANG JIAO AO
SE ZE YUN SHI
SEN LIN BO HE
SHA DI YUAN ZHI
SHA DONG QING

SHA HE SHU

SHA LI YE

SHA MO QIANG WEI

SHA QIAN HU

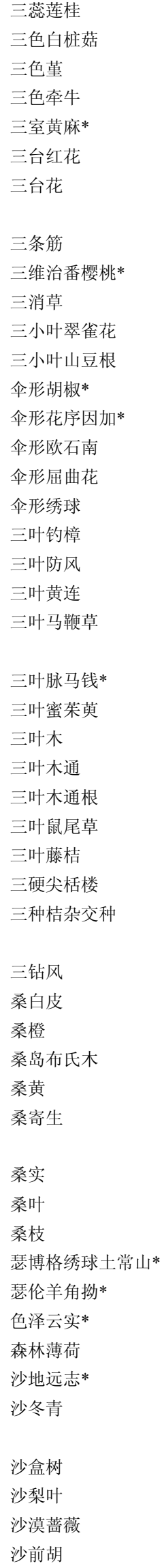

Threestamen Dehaasia*

Tricolor Leucopaxillus*

Garden Pansy

Trichroism Morningglory*

Three-room Roundpod Jute*

Serrate Glorybower

Amplexifolious Glorybower

Tibet Cinnamon Bark

Sandwich Eugenia*

White Clover

Threefoliolate Larkspur

Trifoliate Euchresta

Umbellate Pepper*

Inga*

Umbels Heath

Globe Candytuft

Umbellate Hydrangea

Threeleaf Spicebush*

Bambooleaf Seseli

Three-leaf Goldthread

Trifoliolate Verbena*

Trinervure Poisonnut*

Threeleaf Melicope

Trileaf Wood*

Threeleaf Akebia

Threeleaf Akebia Root

Threeleaf Sage

India Luvunga

Tri-hard-tip Snakegourd*

Japanese Spicebush

White Mulberry Root-bast

Osage Orange

Phellinus igniarius

Parasite Scurrula

White Mulberry Fruit

White Mulberry Leaf

White Mulberry Branch

Thunberg Hydrangea*

Thollon Strophanthus*

Tinctorial Caesalpinia*

Forest Mint

Sandland Milkwort*

Mongolian Ammopiptanthus

Sandbox-tree

Sand Pear Leaf

Desert Rose

Bunge Giantfennel
T2059 Dehaasia triandra

T3783 Leucopaxillus tricolor

T6766 Viola tricolor

T3452 Ipomoea tricolor

T1676 Corchorus trilocularis

T1561 Clerodendron serratum

T1569 Clerodendrum serratum var.

amplexifolium

T1444 Cinnamomum tamala

T2534 Eugenia sandwicensis

T6522 Trifolium repens

T2089 Delphinium trifoliolatum

T2527 Euchresta japonica

T4973 Piper umbellatum

T3424 Inga umbellifera

T2418 Erica umbellata

T3386 Iberis umbellata

T3308 Hydrangea umbellata

T3855 Lindera triloba

T5934 Seseli meirei

T1671 Coptis trifolia

T6711 Verbena triphylla [Syn. Lippia

citriodora]

T6189 Strychnos trinervis

T4169 Melicope triphylla

T6536 Triphyophyllum peltatum

T0277 Akebia trifoliata

T0278 Akebia trifoliata

T5697 Salvia trijuga

T3946 Luvunga scandens

T6514 Trichosanthes tricuspidata

$\mathrm{T} 1519$ [Citrus unshiu $\mathrm{x}$ Citrus sinensis] $\mathrm{x}$

Citrus iyo

T3853 Lindera obtusiloba

T4287 Morus alba

T4022 Maclura pomifera

T1002 Brackenridgea zanguebarica

T4787 Phellinus igniarius

T3925 Loranthus parasiticus [Syn. Loranthus

chinenis; Taxillus chinensis]

T4289 Morus alba

T4289 Morus alba

T4290 Morus alba

T3305 Hydrangea macrophylla var. thunbergii

T6162 Strophanthus thollonii

T1109 Caesalpinia tinctoria

T4192 Mentha sylvestris

T5083 Polygala sabulosa

T0414 Ammopiptanthus mongolicus [Syn.

Piptanthus mongolicus]

T3296 Hura crepitans

T5366 Pyrus pyrifolia

T0168 Adenium obesum

T2697 Ferula borealis 
SHA REN

SHA SHENG HUAI

SHA SHENG LA JU

SHA SHENG QIAN LI GUANG

SHA SHENG SHUI GUI JIAO

SHA TANG MU

SHA ZAO

SHA ZAO SHU PI

SHA ZUAN TAI CAO

SHAN BI XIE

SHAN BIAN DOU ZI

SHAN CHA

SHAN CI BAI

SHAN CI GU

SHAN DI CI TONG

SHAN DI DUI XIN JU

SHAN DI LUO HAN SONG

SHAN DI WU TOU

SHAN DI XIANG CHA CAI

SHAN DI XIANG WAN DOU

SHAN DI YAO HUA

SHAN DONG ZHONG ZU JUE

SHAN DOU GEN

\section{SHAN FAN GEN}

SHAN FAN LI ZHI

SHAN FAN YE

SHAN FENG GUO

SHAN GAN CAO

SHAN GAN RUI XIANG

SHAN GANG TUO WU

SHAN HE YE

SHAN HU GEN HAI TOU HONG

SHAN HU JIAO

SHAN HU JIAO YE

SHAN HU LAN

SHAN HUANG PI

SHAN JIN CHE

SHAN JIU

SHAN JU

SHAN KU GUA

SHAN KU MAI

SHAN LI HONG

SHAN LING MA HUANG

SHAN MA HUANG

SHAN MAI DONG

SHAN MAO ER

SHAN MO LI YUN XIANG

SHAN NAI

SHAN PU TAO

SHAN QIAN HU

SHAN QIU JU PAN MU
砂仁(阳春砂)

砂生槐

沙生蜡菊

沙生千里光*

砂生水鬼蕉*

沙塘木

沙查

沙菄树皮

砂钻苔草

山草薢

山扁豆子

山茶

山刺柏

山慈菇

山地刺桐

山地堆心菊*

山地罗汉松*

山地乌头

山地香茶菜

山地香踠豆

山地荛花*

山东肿足痰

山豆根

山矾根

山番荔枝

山矾叶

山风果

山甘草

陕甘瑞香

山冈㯻吾

山荷叶

珊瑚根海头红*

山胡椒

山胡椒叶

珊瑚兰

山黄皮

山金车

山非

山蔔

山苦瓜

山苦荬

山里红

山岭麻黄

山蚂蝗

山麦冬

山猫儿

山茉莉芸香

山柰

山葡萄

山前胡*

山丘巨盘木*
Villous Amomum

Sandliving Sophora

Yellow Everlasting

Desertliving Groundsel*

Arenaceous Hymenocallis*

Pedunculate Acronychia

Russianolive

Russianolive Bark

Sieve Sedge

Mountain Yam

Sensitiveplant-like Senna

Japanese Camellia

Taiwan Juniper

Arrowhead-like Wildginger

Mountain Immortelle

Mountain Sneezeweed*

Mountain Podocarpus*

Country Monkshood

Montane Rabdosia

Bitter Vetch

Country Stringbush*

Shandong Hypodematium

Tonkin Sophora Root

Caudate Sweetleaf Root

Montana Custardapple*

Caudate Sweetleaf Leaf

Mountainous Garcinia

Buddha's Lamp

Tangut Daphne

Leopard Plant

Japanese Umbrellaleaf

Greyblue Spicebush

Greyblue Spicebush Leaf

Faber Galeola

Hollowed Wampee

Mountain Tobacco

Aging Leek

Hance Pepper

Mountain Balsampear

China Ixeris

Red Fruit

Gerard Ephedra

Acutifoliate Podocarpium

Swordleaf Dianella

Mountjasmine Rue*

Galanga Resurrectionlily

Amur Grape

Mountain Parsley

Hill Flindersia*
T0419 Amomum villosum

T6039 Sophora moorcroftiana

T3156 Helichrysum arenarium

T5886 Senecio eremophilus

T3319 Hymenocallis arenicola

T0151 Acronychia pedunculata

T2326 Elaeagnus angustifolia

T2327 Elaeagnus angustifolia

T1202 Carex kobomugi

T2212 Dioscorea tokoro

T1238 Cassia mimosoides

T1145 Camellia japonica

T3600 Juniperus taiwaniana

T0730 Asarum sagittarioides

T2471 Erythrina poeppigiana

T3135 Helenium autumnale var. montanum

T5046 Podocarpus montanus

T0115 Aconitum monticola

T3516 Isodon oresbia

T3705 Lathyrus montanus

T6822 Wikstroemia monticola

T3372 Hypodematium sinense

T6043 Sophora subprostrata [Syn. Sophora

tonkinensis]

T6250 Symplocos caudata

T0508 Annona montana

T6251 Symplocos caudata

T2858 Garcinia hombroniana

T4335 Mussaenda pubescens

T2031 Daphne tangutica

T3801 Ligularia clivorum

T2231 Diphylleia grayi

T5024 Plocamium corallorrhiza

T3850 Lindera glauca

T3851 Lindera glauca

T2826 Galeola faberi

T1535 Clausena excavata

T0651 Arnica montana

T0320 Allium senescens

T4948 Piper hancei

T4266 Momordica dioica

T3547 Ixeris chinensis

T1778 Crataegus pinnatifida var. major

T2368 Ephedra gerardiana

T2133 Desmodium racemosum [Syn.

Podocarpium podocarpum var. oxyphyllum]

T3874 Liriope spicata

T2139 Dianella ensifolia

T5629 Ruta oreojasme

T3620 Kaempferia galanga

T6794 Vitis amurensis

T4765 Peucedanum oreoselinum

T2739 Flindersia collina 


\begin{tabular}{|c|c|c|c|}
\hline SHAN REN YE & 山稔叶 & Downy Rosemyrtle Leaf & T5528 Rhodomyrtus tomentosa \\
\hline SHAN SHAN JIANG & 扇山姜 & Flabellate Galangal* & T0355 Alpinia flabellata \\
\hline SHAN SHI LIU & 山石榴 & Malabar Randia; Sping Randia & T5411 Randia spinosa \\
\hline SHAN TAO JING BAI PI & 山桃茎白皮 & David Peach Bast & T5220 Prunus davidiana \\
\hline SHAN TAO ZHI & 山桃枝 & David Peach Juvenile Branch & T5221 Prunus davidiana \\
\hline SHAN TENG & 鳝藤 & Common Anodendron & T0514 Anodendron affine \\
\hline SHAN TONG ZI & 山桐子 & Manyfruit Idesia & T3387 Idesia polycarpa \\
\hline SHAN WO JU & 山莴苢 & Indian Lettuce & T3659 Lactuca indica \\
\hline SHAN XI WO ER QI & 陕西窝儿七 & American Umbrellaleaf & T2230 Diphylleia cymosa \\
\hline SHAN XIANG KE KE & 山香科科 & Montane Germander* & T6363 Teucrium montanum \\
\hline SHAN XIAO JU & 山小橘 & Citrusleaf Glycosmis & T3006 Glycosmis citrifolia \\
\hline SHAN XING KUO BAO JU & 扇形阔苞菊 & Sector Pluchea & T0845 Baccharis flabellata \\
\hline SHAN XING REN & 山杏仁 & Ansu Apricot Seed & T5219 Prunus armeniaca var. ansu \\
\hline SHAN YAN HU SUO & 山延胡索 & Bird-in-a-bush & $\begin{array}{l}\text { T1708 Corydalis bulbosa }[\text { Syn. Corydalis } \\
\text { solida }]\end{array}$ \\
\hline SHAN YANG & 山杨 & Wild poplar & T5151 Populus davidiana \\
\hline SHAN YANG DOU & 山羊豆 & Common Goatsrue & T2824 Galega officinalis \\
\hline $\begin{array}{l}\text { SHAN YANG DOU YE KU MA } \\
\text { DOU }\end{array}$ & 山羊豆叶苦马豆 & Darling Pea & T6208 Swainsonia galegifolia \\
\hline SHAN YANG HUANG QI & 山羊黄芪* & & T0790 Astragalus caprinus \\
\hline SHAN YANG WU TOU & 山阳乌头 & Shanyang Monkshood* & T0128 Aconitum sanyoense \\
\hline SHAN YAO & 山药 & Common Yam & $\begin{array}{l}\text { T2190 Dioscorea batatas [Syn. Dioscorea } \\
\text { opposita] }\end{array}$ \\
\hline SHAN YE WAN DOU & 山野踠豆 & Broadleaf Vetch & T6742 Vicia amoena \\
\hline SHAN YIN CHAI HU & 山银柴胡 & Pacific Gypsophila & T3091 Gypsophila pacifica \\
\hline SHAN YING TAO & 山樱桃 & Downy Cherry & T5244 Prunus tomentosa \\
\hline SHAN YOU MA & 山油麻 & Diels Trema & T6494 Trema dielsiana \\
\hline SHAN YU & 山榆 & Wych Elm & T6595 Ulmus glabra \\
\hline SHAN ZHA & 山楂 & Chinese Hawthorn & T1775 Crataegus pinnatifida \\
\hline SHAN ZHA HUA & 山楂花 & Chinese Hawthorn Flower & T1776 Crataegus pinnatifida \\
\hline SHAN ZHA YE & 山楂叶 & Chinese Hawthorn Leaf & T1777 Crataegus pinnatifida \\
\hline SHAN ZHI MA & 山芝麻 & Narrowleaf Screwtree & T3163 Helicteres angustifolia \\
\hline SHAN ZHU YU & 山茱英 & Asiatic Cornelian Cherry & $\begin{array}{l}\text { T1698 Cornus officinalis [Syn. Macrocarpium } \\
\text { officinale] }\end{array}$ \\
\hline SHAN ZHU ZI & 山竹子 & Manyflower Garcinia & T2867 Garcinia multiflora \\
\hline SHAN ZHUANG AO DING ZAO & 栅状凹顶藻 & & T3723 Laurencia palisada \\
\hline SHAN ZUO JIANG CAO & 山酢浆草 & Wood-sorrel & T4562 Oxalis acetosella \\
\hline SHANG JU TIAN MEN DONG & 上举天门冬* & Ascendent Asparagus* & T0745 Asparagus adscendents \\
\hline SHANG LU & 商陆 & Indian Pokeweed & $\begin{array}{l}\text { T4864 Phytolacca esculenta } \text { [Syn. Phytolacca } \\
\text { acinosa] }\end{array}$ \\
\hline $\begin{array}{l}\text { SHANG ZUO JIAN YE GUANG E } \\
\text { TAI }\end{array}$ & 上佐尖叶光䓵苔* & & T5168 Porella acutifolia ssp. tosana \\
\hline SHANG ZUO ZHOU JIN YAO & 上佐州金腰* & Tosa Goldsaxifrage* & T1408 Chrysosplenium tosaense \\
\hline $\begin{array}{l}\text { SHANG ZUO ZHOU ZHANG YA } \\
\text { CAI }\end{array}$ & 上佐州獐芽菜* & Tosa Swertia* & T6240 Swertia tosaensis \\
\hline $\begin{array}{l}\text { SHAO BIAN HUA BAI LAI SHI } \\
\text { JU }\end{array}$ & 少边花白莱氏菊* & Fewradiate Bailai's Chrysanthemum* & T0855 Baileya pauciradiata \\
\hline SHAO CHI XIAO BO & 少齿小檗 & Potanin Barberry & T0913 Berberis potaninii \\
\hline SHAO FU E QIAN LI GUANG & 少副蕓千里光 & Fewcalycle Groundsel* & T5895 Senecio paucicazyculatus \\
\hline SHAO HUA RUI MU & 少花芯木 & Fewflower Kopsia* & T3644 Kopsia pauciflora \\
\hline SHAO HUA XI PA MU & 少花西帕木 & Limoncillo (in Costa Rica) & T5965 Siparuna pauciflora \\
\hline SHAO LAN & 杓兰 & European Ladyslipper & T1983 Cypripedium calceolus \\
\hline SHE BAI ZI & 蛇百子 & Wild Spikenard & T3381 Hyptis suaveolens \\
\hline
\end{tabular}




\begin{tabular}{|c|c|c|c|}
\hline SHE BIAN JU & 蛇鞭菊 & Gay-feather & T3793 Liatris spicata \\
\hline SHE CHUANG ZI & 蛇床子 & Common Cnidium & T1582 Cnidium monnieri \\
\hline SHE GAN & 射干 & Blackberrylily & T0892 Belamcanda chinensis \\
\hline SHE GEN CAO & 蛇根草 & Common Ophiorrhiza & T4513 Ophiorrhiza mungos \\
\hline SHE GUO HUANG JIN & 蛇果黄堇 & Snakefruit Corydalis & T1730 Corydalis ophiocarpa \\
\hline SHE HAN WEI LING CAI & 蛇含委陵菜 & Klein Cinquefoil & T5184 Potentilla kleiniana \\
\hline SHE MEI & 蛇莓 & Indian Mockstrawberry & T2290 Duchesnea indica \\
\hline SHE PAO JIN & 蛇泡筋 & Snakebubble Raspberry & T5591 Rubus cochinchinensis \\
\hline SHE PU TAO & 蛇葡萄 & Ampelopsis & T0423 Ampelopsis brevipedunculata \\
\hline SHE TAI & 蛇苔 & Conicum Conocephalus* & T1644 Conocephalum conicum \\
\hline SHE TENG & 蛇藤 & Asian Colubrina & T1627 Colubrina asiatica \\
\hline SHE XIANG & 㯍香 & Abelmusk & $\begin{array}{l}\text { T4303 Moschus moschiferus; Moschus } \\
\text { berezovskii; Moschus sifanicus }\end{array}$ \\
\hline SHE XIANG BAI HE & 膺香百合 & Longflower Lily & T3835 Lilium longiflorum \\
\hline SHE XIANG CAO & 㯍香草 & Thyme & T6454 Thymus vulgaris \\
\hline SHE XIANG MANG ZI & 鹰香芒籽 & & T0817 Atherosperma moschatum \\
\hline SHE XIANG SHI CAO & 貨香著草 & Musky Yarrow & T0066 Achillea moschata \\
\hline SHE XIANG SHI ZHU & 瘪香石竹 & Carnation & T2142 Dianthus caryophyllus \\
\hline SHE XIANG XUAN & 瘪香萱 & Thunberg's Daylily & T3198 Hemerocallis thunbergii \\
\hline $\begin{array}{l}\text { SHE XING LIN SHENG JIAO GU } \\
\text { CUI }\end{array}$ & 舌形林生脚骨脆* & Lingual Forest-in Casearia* & T1225 Casearia sylvestris var. lingua \\
\hline SHEN CHANG CHUAN XIN & 伸长穿心莲* & Elongate Andrographis* & T0454 Andrographis elongata \\
\hline LIAN & & & \\
\hline SHEN HAO & 伸蒿* & Porrect Wormwood* & T0688 Artemisia porrecta \\
\hline SHEN HONG HONG JING TIAN & 深红红景天 & Darkred Rhodiola & T5493 Rhodiola coccinea \\
\hline SHEN HUANG DOU & 神黄豆 & Jointwood Senna & T1239 Cassia nodosa \\
\hline SHEN HUANG ZI JIN & 深黄紫堇* & Dark-yellow Corydalis & T1723 Corydalis lutea \\
\hline SHEN JIN CAO & 伸筋草 & Common Japanese Clubmoss & T3973 Lycopodium japonicum [Syn. \\
\hline & & & Lycopodium clavatum] \\
\hline SHEN LIE CUI QUE HUA & 深裂翠雀花 & Deeplobed Larkspur* & T2073 Delphinium dissectum \\
\hline SHEN LIE YE JIAO HAO & 深裂叶角蒿* & Deep-lobed-leaf Incarvillea* & T3419 Incarvillea dissectifoliola \\
\hline SHEN LIE YU HUANG CAO & 深裂鱼黄草* & Deeplobed Merremia* & T4197 Merremia dissecta \\
\hline SHEN LU SHAN LONG YAN & 深绿山龙眼 & Nilgiris Helicia & T3162 Helicia nilagirica \\
\hline SHEN SHENG XUAN GOU ZI & 神圣悬钩子* & Sanctity Blackberry* & T5597 Rubus sanctus \\
\hline SHEN XIANG CAO & 神香草 & Medicinal Hyssop & T3384 Hyssopus officinalis \\
\hline SHEN XIANG CAO YE ZE LAN & 神香草叶泽兰 & Hyssop-leaved Boneset & T2562 Eupatorium hyssopifolium \\
\hline SHEN XING XIANG CHA CAI & 肾形香茶菜 & Reniform Rabdosia* & T3502 Isodon latifolia var. reniformis \\
\hline SHEN YE TIAN ZHU KUI & 肾叶天答葵 & Reniform Pelargonium* & T4692 Pelargonium reniforme \\
\hline SHENG DI HONG JING TIAN & 圣地红景天 & Integripetal Rhodiola & T5499 Rhodiola sacra \\
\hline SHENG GU YOU & 省沽油 & Bumalda Bladdernut & T6097 Staphylea bumalda \\
\hline SHENG HONG JI & 胜红蓟 & Tropic Ageratum & T0229 Ageratum conyzoides \\
\hline SHENG JIANG & 生姜 & Fresh Common Ginger & T6910 Zingiber officinale \\
\hline SHENG MA & 升麻 & Bugbane & T1420 Cimicifuga foetida \\
\hline SHENG MU & 升木 & & T3884 Lithraea caustica \\
\hline SHENG QI & 生漆 & True Lacquer & $\begin{array}{l}\text { T5542 Rhus verniciflua [Syn. Toxicadendron } \\
\text { verniciflum] }\end{array}$ \\
\hline SHENG TENG & 生藤 & Common Stelmatocrypton & T6106 Stelmatocrypton khasianum \\
\hline SHENG ZAO & 绳藻 & & T1385 Chorda filum \\
\hline SHI CAN XIANG KE KE & 石蚕香科科 & Chamaedrys Germander & T6360 Teucrium chamaedrys \\
\hline SHI CHANG PU & 石菖蒲 & Grassleaved Sweetflag & T0146 Acorus tatarinowii \\
\hline SHI CHE JU & 矢车菊 & Cornflower & T1305 Centaurea cyanus \\
\hline SHI CHUN & 石莼 & Lettuce Ulva Frond & T6599 Ulva lactuca \\
\hline SHI DA GONG LAO MU & 十大功劳木 & Leatherleaf Mahonia & T4055 Mahonia bealei \\
\hline
\end{tabular}




\begin{tabular}{|c|c|c|c|}
\hline SHI DA GONG LAO YE & 十大功劳叶 & Leatherleaf Mahonia Leaf & T4056 Mahonia bealei \\
\hline SHI DA GONG LAO ZI & 十大功劳子 & Leatherleaf Mahonia Fruit & T4057 Mahonia bealei \\
\hline \multirow[t]{2}{*}{ SHI DAN CAO } & 石胆草 & Fan-shaped Corallodiscus & T1672 Corallodiscus flabellatus [Syn. \\
\hline & & & Didissandra flabellat] \\
\hline SHI DI & 柿蒂 & Persimmon Persistent Calyx & T2217 Diospyros kaki \\
\hline SHI DI QIAN & 石地钱 & & T5444 Reboulia hemisphaerica \\
\hline SHI DIAO BAI & 石入柏 & Curinary Asparagus & T0751 Asparagus officinalis \\
\hline SHI DIAO LAN & 石吊兰 & Fewflower Lysionotus & T4005 Lysionotus pauciflorus \\
\hline SHI ER & 石耳 & Rock-Ears* & $\begin{array}{l}\text { T6601 Umbilicaria esculenta [Syn. Gyrophora } \\
\text { esculenta] }\end{array}$ \\
\hline SHI ER RUI CHOU SHI CAI & 十二萝臭矢菜* & Dodecapistil Spiderflower* & T5060 Polanisia dodecandra \\
\hline SHI ER RUI SHANG LU & 十二莈商陆 & Decapistil Pokeweed* & T4863 Phytolacca dodecandra \\
\hline SHI FANG FENG & 石防风 & Terebinthaceous Hogfennel & T4773 Peucedanum terebinthaceum \\
\hline SHI GAN ZI & 石相子 & Chinese Pothos & T5189 Pothos chinensis \\
\hline SHI GEN & 柿根 & Persimmon Root & T2218 Diospyros kaki \\
\hline SHI GUAN YUAN WEI & 饰冠茑尾 & Crest Iris & T3456 Iris cristata \\
\hline SHI HU & 石斛 & Noble Dendrobium & T2107 Dendrobium nobile \\
\hline SHI HU DIE & 石蝴蝶 & Kerri Petrocosmea* & T4747 Petrocosmea kerrii \\
\hline SHI HU XIAO GU & 石斛小菇 & Dendrob Mycena & T4336 Mycena dendrobii \\
\hline SHI HU ${ }^{(3)}$ & 石虎 & Officinal Evodia & T2646 Evodia rutaecarpa var. officinalis \\
\hline SHI HUA & 石花 & Parmelia Lichen & T4656 Parmelia saxatilis \\
\hline SHI JI NING & 石荠苧 & Scabrous Mosla & T4307 Mosla scabra [Syn. Mosla punctata $]$ \\
\hline SHI JIAO CAO & 石椒草 & Sessile-fruit Chinaure & T0967 Boenninghausenia sessilicarpa \\
\hline SHI JUN ZI & 使君子 & Rangooncreeper & T5384 Quisqualis indica \\
\hline SHI JUN ZI YE & 使君子叶 & Rangooncreeper Leaf & T5385 Quisqualis indica \\
\hline SHI KU JU & 史库菊 & Dwarf Marigold & T5805 Schkuhria pinnata \\
\hline SHI LA HONG & 石腊红 & Fish Pelargonium & T4691 Pelargonium hortorum \\
\hline SHI LI ZI & 石栗子 & Belgaum Walnut Seed & T0300 Aleurites moluccana \\
\hline SHI LIU GEN & 石榴根 & Pomegranate Root & T5327 Punica granatum \\
\hline SHI LIU PI & 石榴皮 & Pomegranate Peel & T5328 Punica granatum \\
\hline SHI LIU XIN CAI & 石榴心材 & Pomegranate Heartwood & T5329 Punica granatum \\
\hline SHI LIU YE & 石榴叶 & Pomegranate Leaf & T5330 Punica granatum \\
\hline SHI LIU ZHONG ZI & 石榴种子 & Pomegranate Seed & T5331 Punica granatum \\
\hline SHI LONG RUI & 石龙芮 & Poisonous Buttercup & T5415 Ranunculus sceleratus \\
\hline SHI LUO ZI & 莳萝子 & Dill Fruit & T0472 Anethum graveolens \\
\hline SHI NAN & 石楠 & Chinese Photinia & T4828 Photinia serrulata \\
\hline SHI NAN TENG & 石南藤 & & $\begin{array}{l}\text { T4974 Piper wallichii [Syn. Piper wallichii } \\
\text { var. hupehense }]\end{array}$ \\
\hline SHI QI & 柿漆 & Immature Persimmon Fruit Juice & T2219 Diospyros kaki \\
\hline SHI RUI & 石苾 & Reindeer Moss & T1526 Cladonia rangiferina \\
\hline SHI SHENG BIAN LEI & 湿生扁蕾 & Swampy Gentianopsis & T2941 Gentianopsis paludosa \\
\hline SHI SHENG JIN SI TAO & 湿生金丝桃 & Wetland St.John'swort* & T3367 Hypericum uliginosum \\
\hline SHI SHI DANG GUI & 施氏当归* & Shkioki Angelica* & T0494 Angelica shkiokiana \\
\hline SHI SHUA BA & 石刷把 & Nude Fern & T5269 Psilotum nudum \\
\hline SHI SUAN & 石蒜 & Shorttube Lycoris & T3986 Lycoris radiata [Syn. Amaryllis radiata] \\
\hline SHI WEI & 石韦 & Japanese Felt Fern Frond & T5357 Pyrrosia lingua \\
\hline SHI XIAN QIE & 十线茄* & Decaline Nightshade* & T5996 Solanum decemlineata \\
\hline SHI XIANG RU & 石香薷 & Chinese Orthodon & T4304 Mosla chinensis [Syn. Orthodon chinensis] \\
\hline SHI XIONG RUI JIAO GUO MU & 十雄荵角果木 & Ten-stamen Ceriops* & T1335 Ceriops decandra \\
\hline SHI YAN FENG & 石岩枫 & Stone Mallotus* & T4085 Mallotus repandus var. chrysocarpus \\
\hline & & & [Syn. Mallotus chrysocarpus; Mallotus \\
\hline & & & repandus] \\
\hline SHI YE & 柿叶 & Persimmon Leaf & T2220 Diospyros kaki \\
\hline
\end{tabular}




\begin{tabular}{|c|c|}
\hline SHI YE CAO & 柿叶草 \\
\hline SHI YE GAN SONG & 匙叶甘松 \\
\hline SHI YONG DA HUANG & 食用大黄 \\
\hline SHI YONG GE & 食用葛 \\
\hline SHI YONG HUANG QI & 食用黄芪 \\
\hline SHI YONG RI ZHONG HUA & 食用日中花 \\
\hline SHI ZHI JIA & 石指甲 \\
\hline SHI ZHU & 石竹 \\
\hline SHI ZI & 柿子 \\
\hline SHI ZI XING FENG JI SHENG & 十字形椡寄生* \\
\hline SHOU LIAN LIANG YI MU & 收敛两翼木 \\
\hline SHOU ZHANG SHEN & 手掌参 \\
\hline SHU CE JIN ZHAN HUA & 蜀侧金戞花 \\
\hline SHU CHI PO PO NA & 梳齿婆婆纳* \\
\hline SHU DI HUANG & 熟地黄 \\
\hline SHU HUA JIE CAO & 疏花颟草* \\
\hline SHU HUA MAO E XIANG CHA & 疏花毛䓫香茶菜 \\
\hline CAI & \\
\hline SHU HUA SHI HU & 束花石斛 \\
\hline SHU JI GU CHANG SHAN & 束鸡骨常山* \\
\hline SHU KUI HUA & 蜀葵花 \\
\hline SHU KUI YE SHU YU & 蜀葵叶薯蓣 \\
\hline SHU LI & 鼠李 \\
\hline SHU LIANG & 薯莨 \\
\hline SHU MA & 菽麻 \\
\hline SHU MI & 㯟米 \\
\hline SHU QU CAO & 鼠曲草 \\
\hline SHU QU FENG MAO JU & 鼠麳凤毛菊 \\
\hline SHU SHE & 树舌 \\
\hline SHU WEI CAO HUA CI TONG & 鼠尾草花刺桐 \\
\hline SHU XING DU JUAN & 树形杜鹃 \\
\hline SHU XU ZHU MA & 束序茾麻 \\
\hline SHU ZHANG LAO GUAN CAO & 鼠掌老鹳草 \\
\hline SHU ZHI BAN RI HUA & 树脂半日花 \\
\hline SHU ZHI DA JI & 树脂大戟 \\
\hline SHU ZHI YAN FU MU & 树脂盐肤木* \\
\hline SHU ZHUANG MA HUANG & 树状麻黄 \\
\hline SHUAN CHI QIN & 栓翅芹 \\
\hline SHUAN CHI WEI MAO & 栓翅卫矛 \\
\hline SHUAN ZHUANG CI TONG & 拴状刺桐* \\
\hline SHUANG BAO MO GU & 双狍蘑菇 \\
\hline SHUANG BIAN GUA LOU & 双边栝楼 \\
\hline SHUANG CHA ZAO & 双叉藻 \\
\hline SHUANG HUA FAN HONG HUA & 双花番红花* \\
\hline SHUANG HUA JIN SI TAO & 双花金丝桃* \\
\hline SHUANG SE JI DAN HUA & 双色鸡蛋花* \\
\hline SHUANG SE JIN GUANG JU & 双色金光菊* \\
\hline SHUANG SE SUO ZI QIN & 双色梭子芹 \\
\hline
\end{tabular}

Reini Milkwort*

Spoonleaf Nardostachys

Rhubarb

Edible Kudzuvine

Edible Milkvetch*

Hottentot Fig

Stringy Stonecrop

Chinese Pink

Persimmon

Cruciate Mistletoe*

Cuachalalate (local name)

Conic Gymnadenia

Szechwan Adonis

Pectinate Speedwell*

Adhesive Rehmannia Cocked Root

Laxflower Valeriana*

Laxflower Hairysepal Rabdosia

Goldenflower Dendrobium

Constricted Alstonia*

Hollyhock Flower

Hollyhock-like Yam

Davurian Buckthorn

Shoulang Yam

Sunn Crotalaria

Broomcorn Millet

Cudweed

Cudweed-like Saussurea
Tongue-on-tree
Salviaflower Coralbean*
Treelike Rhododendron
Siam Falsenettle
Siberian Cranesbill
Resinoid Cistus*
Resinoid Euphorbia*
Resinoid Sumac*
Tall Ephedra
Common Prangos
Corkywing Euonymus
Suberose Coralbean*
Bispore Mushroom*
Rosthorn Snakegourd
Brown Alga Bifurcaria bifurcata
Biflower Crocus*
Biflower St.John'swort*
Twocoloured Frangipani*
Pinewoods Coneflower
Twocoloured Pleurospermum

Cudweed-like Saussurea

Treelike Rhododendron

Resinoid Cistus*

Resinoid Euphorbia

Common Prangos

Corkywing Euonymus

Bispore Mushroom*

Rosthorn Snakegourd

Twocoloured Pleurospermum
T5082 Polygala reinii

T4387 Nardostachys jatamansi

T5476 Rheum rhaponticum

T5312 Pueraria edulis

T0792 Astragalus cibarius

T4200 Mesembryanthemum edule

T5857 Sedum sarmentosum

T2143 Dianthus chinensis

T2221 Diospyros kaki

T6776 Viscum cruciatum

T0431 Amphipterygium adstringens

T3077 Gymnadenia conopsea

T0190 Adonis sutchuenensis

T6726 Veronica pectinata var. glandulosa

T5446 Rehmannia glutinosa [Syn. Rehmannia

glutinosa f. huechingensis]

T6678 Valeriana laxiflora

T3487 Isodon eriocalyx var. laxiflora

T2098 Dendrobium chrysanthum

T0368 Alstonia constricta

T0382 Althaea rosea

T2189 Dioscorea althaeoides

T5457 Rhamnus davurica

T2193 Dioscorea cirrhosa [Syn. Dioscorea pogonoides]

T1823 Crotalaria juncea

T4618 Panicum miliaceum

T3026 Gnaphalium affine [Syn. Gnaphalium

multiceps]

T5753 Saussurea gnaphaloides

T2844 Ganoderma applanatum

T2473 Erythrina salviiflora

T5503 Rhododendron arboreum

T0963 Boehmeria siamensis

T2947 Geranium sibiricum

T1870 Csitus ladaniferus

T2616 Euphorbia resinifera

T5535 Rhus retinorrhoea

T2376 Ephedra procera

T5191 Prangos pabularia

T2545 Euonymus phellomana

T2477 Erythrina suberosa

T0210 Agaricus bisporus

T6513 Trichosanthes rosthornii [Syn.

Trichosanthes uniflora]

T0943 Bifurcaria bifurcata

T1807 Crocus chrysanthus-biflorus

T3353 Hypericum geminiflorum

T5030 Plumeria bicolor

T5603 Rudbeckia bicolor

T5019 Pleurospermum govanianum var.

bicolor 


SHUANG SUI MA HUANG
SHUANG YE XI XIN
SHUANG ZHONG ZI SHU LI
SHUI CAI
SHUI CAI GEN
SHUI CHAO YANG
SHUI FEI JI
SHUI GAN CAO
SHUI GUI JIAO YE

双穗麻黄
双叶细辛
双种子鼠李*
睡菜
睡菜根
水朝阳
水飞蓟
水甘草
水鬼蕉叶

\section{SHUI HU LU}

SHUI HU MAN

SHUI HUANG YANG MU

SHUI HUI XIANG

SHUI HUI XIANG AN

SHUI JIE GU DAN

SHUI JIN FENG

SHUI JING LAN

SHUI LIAN YE TONG

SHUI LIAO

SHUI LIU DOU

SHUI LONG GU

SHUI LU ZAO

SHUI MA TIAO

SHUI MU CAO

SHUI MU XUE LIAN

SHUI NIU JIAO

SHUI QIE

SHUI QIN

SHUI QING SHU

SHUI QU LIU

SHUI RONG

SHUI SHAN

SHUI SONG

SHUI TONG MU

SHUI TUAN HUA

SHUI WU GONG

SHUI XIAN CAO

SHUI XIAN GEN

SHUI XIAN HUA

SHUI YANG MEI

SHUI YANG MEI GEN

SHUI YANG MU BAI PI

SHUI YANG ZHI YE

SHUI ZHI

SHUI ZHI YE

SI BO LI YAAI JU

SI CHI HUAI

SI CHI HUANG QI

\begin{tabular}{|c|c|}
\hline 水葫芦 & Common Waterhyacinth \\
\hline 水胡满 & Unarmed Glorybower \\
\hline 水黄杨木 & Caudate Milkwort \\
\hline 水茴香 & Winked Marshweed \\
\hline 水茴香桉 & Phellandral Eucalyptus* \\
\hline 水接骨丹 & Hairy Willowweed \\
\hline 水金风 & Lightyellow Snapweed \\
\hline 水晶兰 & Indianpipe \\
\hline 睡莲叶桐* & Sleeping Lotusleaftung \\
\hline 水蓼 & Red-knees \\
\hline 水流豆 & Poongaoil Pongamia \\
\hline 水龙骨 & Japanese Polypody \\
\hline 水陆杳 & Amphibian Jujube* \\
\hline 水麻节 & Thunberg Knotweed \\
\hline 水木草 & Cuspidate Mnium Herb \\
\hline 水母雪莲 & Medusa Saussurea \\
\hline 水牛角 & Buffalo Horn \\
\hline 水茄 & Water Nightshade \\
\hline 水芹 & Javan Waterdropwort \\
\hline 水青树 & Tetracentron \\
\hline 水曲柳 & Manchurian Ash \\
\hline 水榕 & Lidded Cleistocalyx \\
\hline 水杉 & Dawn Redwood \\
\hline 水松 & Fragile Codium Frond \\
\hline 水桐木 & Harland Fig \\
\hline 水团花 & Pilular Adina \\
\hline 水蜈蚣 & Shortleaf Kyllinga \\
\hline 水线草 & Corymbose Hedyotis \\
\hline 水仙根 & Chinese Narcissus Bulb \\
\hline 水仙花 & Chinese Narcissus Flower \\
\hline 水杨梅 & Japanese Avens \\
\hline 水杨梅根 & Japanese Avens Root \\
\hline 水杨木白皮 & Bitter Willow Bast \\
\hline 水杨枝叶 & Bitter Willow Branch-leaf \\
\hline 水桅 & Bigflower Cape Jasmine \\
\hline 水桅叶 & Bigflower Cape Jasmine Leaf \\
\hline 西伯利亚艾菊 & Siberian Tansy \\
\hline 四翅槐 & New Zealand Kowhai \\
\hline 四翅黄芪* & Fourwing Milkvetch* \\
\hline
\end{tabular}

Jointfir Ephedra

Caulescent Wildginger

Diseed Buckthorn*

Bogbean

Bogbean Root

Aquatic-sunflower Inula

St. Marys

China Amsonia

Tropical American Hymenocallis

Leaf

Common Waterhyacinth

Glorybowe

Winked Marshweed

Lightyellow Snapweed

Sleeping Lotusleaftung

Amphibian Jujube*

rg Knotweed

Medusa Saussurea

Javan Waterdropwort

Lidded Cleistocalyx

Fourwing Milkvetch*
T2366 Ephedra distachya

T0724 Asarum caulescens

T5458 Rhamnus disperma

T4194 Menyanthes trifoliata

T4195 Menyanthes trifoliata

T3432 Inula helianthus-aquatica

T5957 Silybum marianum

T0434 Amsonia sinensis

T3320 Hymenocallis littoralis [Syn.

Hymenocallis americana; Pancratium

littoralis]

T2325 Eichhornia crassipes

T1558 Clerodendron inerme

T5072 Polygala caudata

T3840 Limnophila rugosa

T2515 Eucalyptus phellandra

T2387 Epilobium hirsutum

T3413 Impatiens nolitangere

T4276 Monotropa uniflora

T3230 Hernandia nymphaeifolia

T5104 Polygonum hydropiper

T5143 Pongamia pinnata

T5124 Polypodium niponicum

T6914 Ziziphus amphibia

T5119 Polygonum thunbergii

T4259 Mnium cuspidatum

T5759 Saussurea medusa

T1045 Bubalus bubalis

T6015 Solanum torvum

T4482 Oenanthe javanica

T6352 Tetracentron sinense

T2772 Fraxinus mandshurica

T1544 Cleistocalyx operculatus

T4208 Metasequoia glyptostroboides

T1595 Codium fragile

T2719 Ficus fistulosa [Syn. Ficus harlandii]

T0180 Adina pilulifera [Syn. Cephalanthus

pilulifera]

T3646 Kyllinga brevifolia

T3126 Hedyotis corymbosa [Syn. Oldenlandia

corymbosa]

T4384 Narcissus tazetta var. chinensis

T4385 Narcissus tazetta var. chinensis

T2955 Geum japonicum

T2956 Geum japonicum

T5653 Salix purpurea

T5654 Salix purpurea

T2884 Gardenia jasminoides var. grandiflora

T2885 Gardenia jasminoides var. grandiflora T6295 Tanacetum sibiricum [Syn. Filifolium sibiricum]

T6044 Sophora tetraptera

T0808 Astragalus tetraplerus 


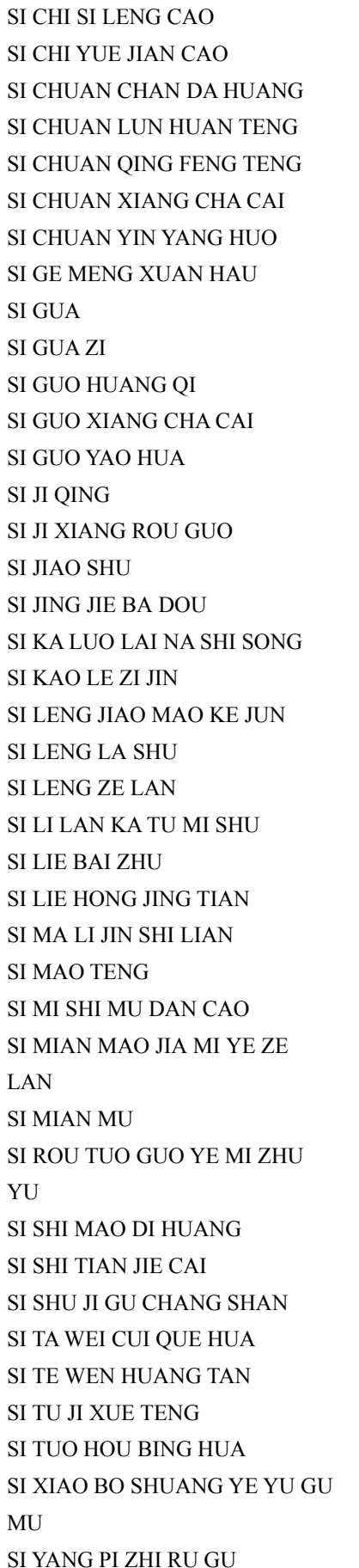

SI YANG PI ZHI RU GU
SI YANG SHU YE BAI JIAN MU

SI ZI TAN

SONG HAO

SONG LUO

SONG PAN WU TOU

SONG XIANG

SONG XUN

SONG YE FANG FENG
四齿四棱草

四翅月见草

四川产大黄

四川轮环藤

四川清风藤

四川香茶菜

四川淫羊蕉

司格蒙旋花*

丝瓜

丝瓜子

四国黄花*

四国香茶菜

四国荛花*

四季青

四基香肉果

丝胶树

似荆芥巴豆*

似卡罗来纳石松*

斯考勒紫革

四棱角毛壳菌*

四棱蜡树

四棱泽兰

斯里兰卡土密树

四裂白珠

四裂红景天

似马利筋石莲*

思茅藤

斯密氏牡丹草

似绵毛荚蒾叶泽兰*

丝棉木

似肉托果叶蜜茱英*

斯氏毛地黄

斯氏天芥菜*

四数鸡骨常山*

斯塔维翠雀花

斯特文黄檀

斯图鸡血藤*

斯托厚柄花

似小檗双叶鱼骨木

\section{似羊皮纸乳菇*}

似杨树叶白坚木*

似紫檀*

松蒿

松萝

松潘乌头

松香

松䓥

松叶防风
Fourteech Schnabelia

Fourwing Eveningprimrose*

Szechwan Cyclea

Szechwan Sabia

Sichuna Rabdosia

Szechuan Epimedium

Scammony Glorybind

Suakwa Vegetablesponge

Suakwa Vegetablesponge Seed

Siko Milkvetch*

Shiko Rabdosia*

Siko Stringbush*

Purpleflower Holly

Tetrabase Casimiroa*

Silk-rubber Tree

Nepeta-like Croton*

Carolina-like Clubmoss*

Scouler Corydalis*

Blue Ash

Four-arris Eupatorium*

Sri-Lankan Bridelia

Four Sepals Gaultheria

Foursplit Rhodiola

Longeared Epigynum

Smirnow Leontice*

Tinileaf Eupatorium*

Winterberry Euonymus

Schischkin Foxglove*

Steudner Heliotrope*

Quaternary Alstonia*

Stavisacre

Stevenson Rosewood*

Stuhlmann Millettia*

Barberry-like-dileaf Canthium

Parchment-like Milky*

Poplar-leaf-like White Quebracho*

Sandalwood Padauk

Japanese Phtheirospermum

Long Usnea Filament

Sungpan Monkshood

Colophony

Pine Mushroom

Yunnan Seseli
T5807 Schnabelia tetradonta

T4483 Oenothera tetraptera

T5479 Rheum sp.

T1933 Cyclea sutchuenensis

T5637 Sabia schumanniana

T3525 Isodon setschwanensis

T2404 Epimedium sutchuenense

T1654 Convolvulus scammonia

T3934 Luffa cylindrica

T3935 Luffa cylindrica

T0803 Astragalus shikokianus

T5398 Rabdosia shikokiana

T6823 Wikstroemia sikokiana

T3388 Ilex chinensis [Syn. Ilex purpurea]

T1227 Casimiroa tetrameria

T2811 Funtumia elastica

T1852 Croton nepetaefolius

T3968 Lycopodium carolinianum var. affine

T1739 Corydalis scouleri

T1344 Chaetomium quadrangulatum

T2776 Fraxinus quadrangulata

T2568 Eupatorium quadrangularae

T1028 Bridelia retusa

T2894 Gaultheria tetramera

T5498 Rhodiola quadrifida

T5962 Sinocrassula asclepiadea

T2386 Epigynum auritum

T3747 Leontice smirnowii

T2578 Eupatorium tinifolium

T2538 Euonymus bungeanus

T4166 Melicope semecarpifolia

T2178 Digitalis schischkinii

T3181 Heliotropium steudneri

T0372 Alstonia quaternata

T2086 Delphinium staphisagria

T2017 Dalbergia stevensonii

T4244 Millettia stuhlmannii

T4571 Pachypodanthium staudii

T1178 Canthium berberidifolium

T3652 Lactarius pergamenus

T0771 Aspidosperma populifolium

T5305 Pterocarpus santalinus

T4831 Phtheirospermum japonicum [Syn.

Geradia japonica]

T6654 Usnea longissima

T0135 Aconitum sungpanense

T4917 Pinus massoniana

T6504 Tricholoma matsutake [Syn. Armillaria

matsutake]

T5937 Seseli yunnanense 


\begin{tabular}{|c|c|c|c|}
\hline SONG YE QIAN LI GUANG & 松叶千里光 & Pineleaf Groundsel & T5874 Senecio abrotanifolius \\
\hline SU DA QI GAN JU & 苏打其柑桔 & Sudach Citrus* & T1511 Citrus sudachii \\
\hline SU DAN KE LE GUO & 苏丹可乐果 & Acuminate Colanut & T1615 Cola acuminata \\
\hline SU FANG HUA & 素方花 & Common White Jasmine & T3556 Jasminum officinale \\
\hline SU GE LAN DANG GUI & 苏格兰当归 & Scots Lovage & T3823 Ligusticum scoticum \\
\hline SU GE LAN HU TONG & 苏格兰胡桐* & Caledonian Beautyleaf* & T1126 Calophyllum caledonicum \\
\hline SU GEN XIANG WAN DOU & 宿根香踠豆 & Everlasting Pea & T3704 Lathyrus latifolius \\
\hline SU GEN ZHANG YA CAI & 宿根獐芽菜 & Alpine Bog-swertia & T6230 Swertia perennis \\
\hline SU HE XIANG & 苏合香 & Oriental Sweetgum Resin & T3868 Liquidambar orientalis \\
\hline SU HUA DANG SHEN & 素花党参 & Moderate Asiabell & $\begin{array}{l}\text { T1600 Codonopsis pilosula var. modesta }[\text { Syn. } \\
\text { Codonopsis modesta] }\end{array}$ \\
\hline SU KU BA DOU HUA & 苏库巴斗花 & Bellaco-Caspi & T3251 Himatanthus sucuuba \\
\hline SU LI NAN ROU DOU KOU & 苏里南肉豆蔻 & Ucahub; Baboen & $\begin{array}{l}\text { T6770 Virola surinamensis [Syn. Myristica } \\
\text { surinamensis] }\end{array}$ \\
\hline SU MEN BAI JIU CAO & 苏门白酒草 & & T2426 Erigeron sumatrensis \\
\hline SU MEN DA LA HONG DOU & 苏门答腊红豆杉 & Sumatran Yew & T6316 Taxus sumatrana \\
\hline \multicolumn{4}{|l|}{ SHAN } \\
\hline SU MEN DA LA WEN SHU LAN & 苏门答腊文殊兰 & Lovely Crinum* & T1793 Crinum amabile \\
\hline SU MI & 粟米 & Foxtail Millet & T5938 Setaria italica \\
\hline SU MI CAO & 粟米草 & Fiveleaf Carpetweed & T4261 Mollugo pentaphylla \\
\hline SU MU & 苏木 & Sappan Caesalpinia & T1108 Caesalpinia sappan \\
\hline SU TIE SHU GUO & 苏铁树果 & Sago Seed & T1927 Cycas revoluta \\
\hline SU TIE YE & 苏铁叶 & Sago Frond & T1928 Cycas revoluta \\
\hline SU XIN HUA & 素馨花 & Largeflower Jasmine* & T3554 Jasminum grandiflorum \\
\hline SU XIN YE BAI YING & 素馨叶白英 & Jasmine Nightshade & T6001 Solanum jasminoides \\
\hline SU ZHU YANG YANG & 粟猪殊殊 & Hedge Bedstraw & T2832 Galium mollugo \\
\hline SUAN CHOU MU JI CAO & 蒜臭母鸡草 & & T4746 Petiveria alliacea \\
\hline SUAN GOU TENG & 酸钩藤* & Acid Gambirplant* & T6604 Uncaria acida \\
\hline SUAN JIANG & 酸浆 & Japanese-lantern & T4845 Physalis alkekengi \\
\hline SUAN JIAO & 酸角 & Tamarind Fruit & T6285 Tamarindus indica \\
\hline SUAN MO & 酸模 & Garden Sorrel & T5605 Rumex acetosa \\
\hline SUAN MO YE & 酸模叶 & Garden Sorrel Leaf & T5606 Rumex acetosa \\
\hline SUAN SHI LIU & 酸石榴 & Pomegranate & T5332 Punica granatum \\
\hline SUAN SHUI CAO & 酸水草 & Thorowort Pondweed & T5178 Potamogeton perfoliatus \\
\hline SUAN WEI XIANG KE KE & 蒜味香科科 & Garlicsmell Germander* & T6368 Teucrium scordium \\
\hline SUAN YE PO LUO MEN SHEN & 蒜叶婆罗门参 & Salsify & T6489 Tragopogon porrifolius \\
\hline SUAN ZAO & 酸本 & Spine Date & T6918 Ziziphus jujuba var. spinosa \\
\hline SUAN ZAO REN & 酸柊仁 & Spine Date Seed & T6919 Ziziphus jujuba var. spinosa \\
\hline SUI BA QIA & 穗茏葜 & Eurasia Greenbrier & T5974 Smilax aspera \\
\hline SUI BIAN WO JU & 穗变莴芭* & Laciniate Lettuce* & T3660 Lactuca laciniata \\
\hline SUI HUA LUO HAN SONG & 穗花罗汉松 & Matai & T5055 Podocarpus spicatus \\
\hline SUI HUA MA XIAN HAO & 穗花马先蒿 & Spicate Woodbetony & T4683 Pedicularis spicata \\
\hline SUI HUA MU JING & 穗花牡荆 & Lilac Chastetree & T6782 Vitex agnuscastus \\
\hline SUI MAO HE BAO MU DAN & 繸毛荷包牡丹 & Wild Bleedingheart & T2149 Dicentra eximia \\
\hline SUI MI SHA CAO & 碎米莎草 & Rice Galingale & T1976 Cyperus iria \\
\hline SUI ZHUANG BAI JIN HUA & 穗状百金花 & Spicate Centaurium* & T1310 Centaurium spicatum \\
\hline SUI ZHUANG BI QIAO JIANG & 穗状闭鞘姜* & Cana do brejo (in Brazil) & T1757 Costus spicatus \\
\hline SUI ZHUANG WU TOU & 穗状乌头* & Spiked Monkshood & T0133 Aconitum spicatum \\
\hline SUI ZHUANG YUN XIANG & 繸状芸香 & Syrian Rue* & T5625 Ruta chalepensis \\
\hline SUO DUO MI QIE & 索多米茄 & Sodome Nightshade* & $\begin{array}{l}\text { T6012 Solanum sodomeum }[\text { Syn. Solanum } \\
\text { sodomaeum }]\end{array}$ \\
\hline SUO GUO HUANG QI & 梭果黄芪 & Ernest Milkvetch* & T0794 Astragalus ernestii \\
\hline SUO LA MU & 椒拉木 & Prinos-like Salacia & T5648 Salacia prinoides [Syn. Salacia chinensis] \\
\hline
\end{tabular}




\begin{tabular}{|c|c|c|}
\hline SUO LUO & 岿椤 & Spiny Alsophila \\
\hline SUO LUO ZI & 娑罗子 & Wilson Buckeye Seed \\
\hline SUO SHA MI & 缩砂密 & Locklebur-like Amomum \\
\hline SUO YANG & 锁阳 & Songaria Cynomorium \\
\hline TA HUA BAI LI XIANG & 塔花百里香* & \\
\hline TA LA WU TOU & 塔拉乌头 & Tala Monkshood* \\
\hline TA SI MA NI YA JIE GENG LAN & 塔斯马尼亚桔梗兰 & Flax Lily \\
\hline TA YOU XIE GUA & 塔尤泻瓜 & Taiuia Root* \\
\hline TAI BAI CONG MU & 太白淴木 & Taibaien Aralia \\
\hline TAI BAI HUA & 太白花 & Stellate Cladonia \\
\hline TAI DA SONG & 台大松 & Loblolly Pine, Old Field Pine \\
\hline TAI DONG XI XIN & 台东细辛 & Taiton Wildginger \\
\hline TAI GUO BA JI & 泰国巴戟 & Thailand Indianmulberry* \\
\hline TAI GUO NIU XU HUA & 泰国扭序花 & Thailand Clinacanthus*; \\
\hline & & Lin-Nguu-Hao (Thai name) \\
\hline TAI GUO SHAN QIAN NIU & 泰国山牵牛 & Thailand Clockvine (Raang-Chuet) \\
\hline TAI GUO ZHI ZI & 泰国杤子* & Thailand Gardenia* \\
\hline TAI JING TIAN & 苔景天 & Biting Stonecrop \\
\hline TAI PING YANG JIA ZE LAN & 太平洋假泽兰 & Pasific Mikania* \\
\hline TAI PING YANG LIN MAO JUE & 太平洋鳞毛蕨 & Pacific Wood Fern \\
\hline TAI SHAN YAN FU ZI & 泰山盐麸子 & Taishan Sumac Fruit \\
\hline TAI WAN AN XI XIANG & 台湾安息香 & Taiwan Snowbell \\
\hline TAI WAN CHA MU & 台湾檫木 & Taiwan Sassafras \\
\hline TAI WAN CU FEI & 台湾粗榧 & Wilson Plumyew \\
\hline TAI WAN CUI BAI & 台湾翠柏 & Taiwan Incense Cedar \\
\hline TAI WAN FENG DOU CAI & 台湾蜂斗菜* & Taiwan Butterbur* \\
\hline TAI WAN FU RONG & 台湾芙蓉 & Taiwan Hibiscus \\
\hline TAI WAN GE NA XIANG & 台湾哥纳香 & T aiwan Goniothalamus \\
\hline TAI WAN GUO SONG & 台湾果松 & Masters Pine \\
\hline TAI WAN HU JIAO & 台湾胡椒* & Taiwan Pepper* \\
\hline TAI WAN HUANG BO & 台湾黄檗 & Taiwan Corktree* \\
\hline TAI WAN JI & 台湾蓟 & Taiwan Thistle* \\
\hline TAI WAN JI NING & 台湾荠艼 & Taiwan Mosla \\
\hline TAI WAN JIN GU CAO & 台湾筋骨草 & Taiwan Bugle* \\
\hline TAI WAN JIU LI XIANG & 台湾九里香 & Taiwan Common Jasminorange \\
\hline TAI WAN LUO HAN SONG & 台湾罗汉松 & Nakai Podocarpus \\
\hline TAI WAN LV DAO TENG HUANG & 台湾绿岛藤黄* & Lanyu Garcinia \\
\hline TAI WAN PI PA & 台湾枇杷 & Taiwan Loquat \\
\hline TAI WAN PU GONG YING & 台湾蒲公英 & Taiwan Dandelion* \\
\hline TAI WAN QIAN HU & 台湾前胡 & Taiwan Hogfennel \\
\hline TAI WAN QIAN JIN TENG & 台湾千金藤 & Sasak Stephania* \\
\hline TAI WAN RUI FANG RUN NAN & 台湾瑞芳润楠* & Zuiho Machilus \\
\hline TAI WAN SHAN & 台湾杉 & Cryptomeria-like Taiwania \\
\hline TAI WAN SHAN DOU GEN & 台湾山豆根 & Taiwan Euchresta \\
\hline TAI WAN SHAN MU & 台湾杉木 & Konish Chinafir \\
\hline TAI WAN SHU LI & 台湾鼠李 & Taiwan Buckthorn* \\
\hline TAI WAN SHUANG HU DIE & 台湾双蝴蝶 & Taiwan Dualbutterfly \\
\hline TAI WAN TANG SONG CAO & 台湾唐松草 & Taiwan Meadowrue \\
\hline TAI WAN XIAO BO & 台湾小檗 & Taiwan Barberry \\
\hline TAI WAN XIU XIAN JU & 台湾绣线菊 & Taiwan Spiraea* \\
\hline TAI WAN YUN SHAN & 台湾云杉 & Taiwan Spruce \\
\hline TAI WAN ZE LAN & 台湾泽兰 & Taiwan Agrimony \\
\hline
\end{tabular}

T0364 Alsophila spinulosa

T0205 Aesculus wilsonii

T0420 Amomum xanthioides

T1972 Cynomorium songaricum

T6451 Thymus satureioide

T0136 Aconitum talassicum

T2141 Dianella tasmanica

T1274 Cayaponia tayuya

T0576 Aralia taibaiensis

T1528 Cladonia stellaris [Syn. Cladonia

alpestris]

T4927 Pinus taeda

T0734 Asarum taitoense

T4281 Morinda coreia

T1572 Clinacanthus siamensis

T6445 Thunbergia laurifolia

T2887 Gardenia thailandica

T5849 Sedum acre

T4229 Mikania mendocina

T2285 Dryopteris pacifica

T5539 Rhus taishanensis

T6199 Styrax formosanus

T5745 Sassafras randainense

T1325 Cephalotaxus wilsoniana

T1121 Calocedrus macrolepis var. formosana

T4739 Petasites formosanus

T3247 Hibiscus taiwanensis

T3041 Goniothalamus amuyon

T4907 Pinus armandii var. mastersiana

T4970 Piper taiwanense

T4790 Phellodendron amurense var. wilsonii

T1447 Cirisum japonica var. takaoense

T4539 Orthodon formosanus

T0272 Ajuga taiwanensis

T4316 Murraya crenulata

T5047 Podocarpus nakaii

T2861 Garcinia linii

T2430 Eriobotrya deflexa

T6300 Taraxacum formosanum

T4755 Peucedanum formosanum

T6132 Stephania sasakii

T4019 Machilus zuihoensis

T6284 Taiwania cryptomerioides

T2526 Euchresta formosana

T1890 Cunninghamia konishii

T5459 Rhamnus formosana

T6538 Tripterospermum taiwanense

T6415 Thalictrum urbainii

T0907 Berberis kawakamii

T6077 Spiraea formosana

T4874 Picea morrisonicola

T2558 Eupatorium formosanum 


$\begin{array}{lll}\text { TAI ZHONG SHU LI } & \text { 台中鼠李 } & \text { Taizhong Buckthorn* } \\ \text { TAN MANG GUO } & \text { 坦杧果 } & \\ \text { TAN XIANG } & \text { 檀香 } & \text { Sandalwood } \\ \text { TANG GU TE DA HUANG } & \text { 唐古特大黄 } & \text { Tangut Rhubarb } \\ \text { TANG JIE } & \text { 糖芥 } & \text { Diffuse Erysimum } \\ \text { TANG LI } & \text { 棠梨 } & \text { Birchleaf Pear } \\ \text { TANG MU XUN DU HUO } & \text { 汤姆逊独活 } & \text { Thomson Cowparsnip* } \\ \text { TANG QI } & \text { 糖械 } & \text { Sugar Maple } \\ \text { TANG SONG CAO ZHUANG } & \text { 唐松草状扁果草 } & \text { Meadowruelike Isopyrum } \\ \text { BIAN GUO CAO } & & \\ \text { TAO } & \text { 桃 } & \text { Peach } \\ \text { TAO BEI MU } & \text { 桃贝母 } & \text { Peach Fritillary* } \\ \text { TAO ER QI } & \text { 桃儿七 } & \text { Common Sinopodophyllm }\end{array}$

TAO GEN
TAO HUA
TAO HUA XIN MU
TAO JIN NIANG
TAO JING BAI PI
TAO NAN GUA
TAO REN
TAO YE
TAO YE LIAO
TAO ZHI
TE LI NI DA HU JIAO
TE LUO YI HUANG QI
TE SI MAN NI HU TONG
TE SI MAN NI HU TONG BIAN
ZHONG
TENG CANG CHI MEI

TENG CANG CHI MEI

TENG HUANG

TENG HUANG SHAN ZHU ZI

TENG HUANG SHU

TENG SHI SONG

TI GAI JUE

TI GEN CAO

TI MU CAO

TI QIN ZHUANG SHAN NAI

TIAN CAI

TIAN CHA

TIAN CHENG

TIAN CONG

TIAN GEN DUO ZU JUE

TIAN HU SUI

TIAN HUA FEN

TIAN JIAO BAN

TIAN LAN ZE LAN

TIAN LIAO MU

TIAN MA

TIAN MEN DONG

$\begin{array}{ll}\text { 桃根 } & \text { Peach Root } \\ \text { 桃花 } & \text { Peach Flower } \\ \text { 桃花心木 } & \text { West Indian Mahogany } \\ \text { 桃金娘 } & \text { Rosemyrtle } \\ \text { 桃茎白皮 } & \text { Peach Bast } \\ \text { 桃南瓜 } & \text { Peachliking Pumpkin } \\ \text { 桃仁 } & \text { Peach Kernel } \\ \text { 桃叶 } & \text { Peach Leaf } \\ \text { 桃叶蓼 } & \text { Spring Knotweed } \\ \text { 桃枝 } & \text { Peach Juvenile Branch } \\ \text { 特立尼达胡椒 } & \text { Trinidad Pepper* } \\ \text { 特洛伊黄花 } & \text { Trojan Milkvetch* } \\ \text { 特思曼尼胡桐 } & \text { Teysmanni Beautyleaf* } \\ \text { 特思曼尼胡桐变种* } & \text { Teysmanni Beautyleaf Variaty* }\end{array}$

藤仓赤需

藤黄

藤黄山竹子*

藤黄树

藤石松

蹄盖蕨

德根草

梯牧草

提琴状山奈*

甜菜

甜茶

甜橙

田葱

甜根多足蓱

天胡荽

天花粉

天脚板

天蓝泽兰

天料木

天麻

天门冬

Gamboge Tree Resin
Camboge Garcinia*
Hanbury Garcinia*
Painted Fern
Black Hellebore
Timothy
Fiddle-leaf Resurrectionlily*
Common Beet
Sweet Blackberry*
Sweet Orange
Woolly Philydrum
Sweetroot Polypody*
Lawn Pennywort
Mongolian Snakegourd Root
Chinese Aucuba
Azure Eupatorium*
Cochinchina Homalium
Tall Gastrodia
Cochinchinese Asparagus

\begin{abstract}
T5462 Rhamnus nakaharai
T6299 Tanghinia venenifera

T5715 Santalum album

T5481 Rheum tanguticum

T2454 Erysimum diffusum

T5361 Pyrus betulaefolia

T3226 Heracleum thomsoni

T0056 Acer saccharum
\end{abstract}

T3540 Isopyrum thalictroides

T5229 Prunus persica

T2791 Fritillaria persica

T5057 Podophyllum emodii [Syn.

Podophyllum emodii var. chinense;

Podophyllum sikkimenosis; Sinopodophyllum

emodii]

T5230 Prunus persica

T5231 Prunus persica

T6241 Swietenia mahogany

T5529 Rhodomyrtus tomentosa

T5232 Prunus persica

T1883 Cucurbita pepo var. akoda

T5233 Prunus persica

T5234 Prunus persica

T5113 Polygonum persicaria

T5235 Prunus persica

T4929 Piper aequale

T0809 Astragalus trojanus

T1133 Calophyllum teysmannii

T1134 Calophyllum teysmannii var.

inophylloide

T2959 Gibberella fujikuroi

T2866 Garcinia morella

T2851 Garcinia cambogia

T2857 Garcinia hanburyi

T3969 Lycopodium casuarinoides

T0818 Athyrium filix-femina

T3183 Helleborus niger

T4802 Phleum pratense

T3622 Kaempferia pandurata

T0928 Beta vulgaris

T5600 Rubus suavissimus

T1508 Citrus sinensis

T4799 Philydrum lanuginosum

T5123 Polypodium glycyrrhiza

T3311 Hydrocotyle sibthorpioides

T6512 Trichosanthes kirilowii

T0827 Aucuba chinensis ssp. omeiensis

T2553 Eupatorium azureum

T3275 Homalium cochinchinensis

T2890 Gastrodia elata

T0746 Asparagus cochinchinensis [Syn.

Asparagus lucidus] 


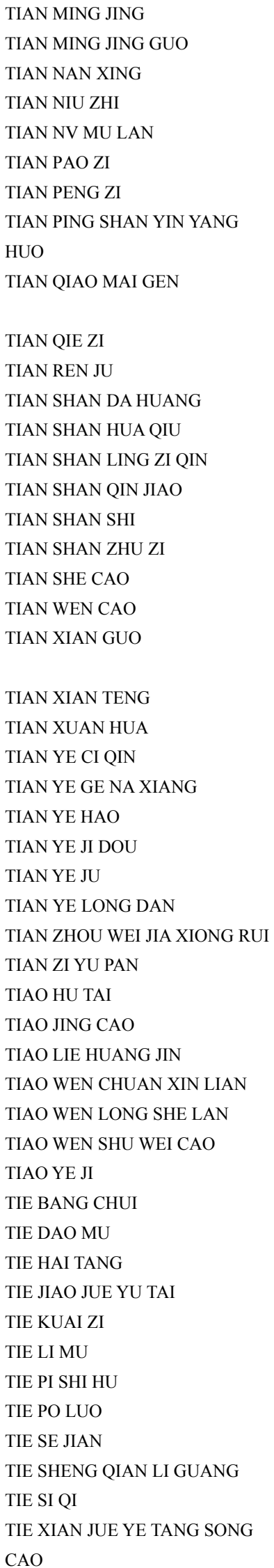

$\begin{array}{lll}\text { 天名精 } & \text { Common Carpesium } & \text { T1210 Carpesium abrotanoides } \\ \text { 天名精果 } & \text { Common Carpesium Fruit } & \text { T1211 Carpesium abrotanoides } \\ \text { 天南星 } & \text { Reddish Jackinthepulpit } & \text { T0618 Arisaema consanguineum } \\ \text { 甜牛至* } & \text { Sweet Marjoram } & \text { T4524 Origanum majorana } \\ \text { 天女木兰 } & \text { Oyama Magnolia } & \text { T4050 Magnolia sieboldii } \\ \text { 天泡子 } & \text { Little Groundcherry } & \text { T4851 Physalis minima } \\ \text { 天蓬子 } & \text { Chinese Atropanthe } & \text { T5822 Scopolia sinensis } \\ \text { 天平山淫羊蕉 } & \text { Tianpingshan Epimedium* } & \text { T2400 Epimedium myrianthum }\end{array}$

\section{天菾麦根}

天茄子

天人菊

天山大黄

天山花楸

天山棱子芹

天山秦艽

天山蓄

甜山竹子*

甜舌草

天文草

天仙果

天仙藤

田旋花

田野刺芹

田野哥纳香*

田野蒿*

田野棘豆

甜叶菊

田野龙胆

甜周围假雄荵

甜紫玉盘*

条滸苔

调经草

条裂黄堇

条纹穿心莲*

条纹龙舌兰

条纹鼠尾草*

条叶蓟

铁棒锤

铁刀木

铁海棠

铁角偋羽苔*

铁筷子

铁力木

铁皮石斛

铁破锣

铁色箭

贴生千里光*

铁丝七

铁线蕨叶唐松草*
Common Carpesium
Common Carpesium Fruit
Reddish Jackinthepulpit
Sweet Marjoram
Oyama Magnolia
Little Groundcherry
Chinese Atropanthe
Tianpingshan Epimedium*

Golden Buckwheat Root

Indian Nightshade

Rosering Gaillardia

Tianshan Mountain Rhubarb

Tianshan Mountain Mountainash

Lindley Pleurospermum

Tianshan Mountain Gentian

Hairyleaf Handelia

Dulcin Garcinia*

Sweet-tongue Lippia*

Paniculate Spotflower

Erect Fig

Common Fibraurea

Field Bindweed

Field Goniothalamus*

Campestral Mugwort

Yellow Oxytropis

Rebaud Eupatorium*

Meadow Gentian

Sweet Uvaria*

Evergreen Euonymus

Linearsegmented Corydalis

Linea Andrographis*

Stria Agave*

Linearleaf Thistle

Pendulous Monkshood

Siamese Senna

Crownofhorns Euphorbia

Tibetan Hellebore

Common Mesua

Iron-sheet Denrdobium

Marshmarigold-leaved Beesia

Orange Lycoris

Adnate Groundsel*

American Maidenhair Fern

Maidenhair-like Meadowrue*
T2657 Fagopyrum cymosum [Syn. Polygonum cymosum]

T6000 Solanum indicum

T2820 Gaillardia pulchella

T5482 Rheum wittrocki

T6055 Sorbus tianschanica

T5020 Pleurospermum lindleyanum

T2936 Gentiana tianschanica

T3097 Handelia trichophylla

T2853 Garcinia dulcis

T3864 Lippia dulcis

T6075 Spilanthes acmella

T2716 Ficus beecheyana [Syn. Ficus erecta

var. beecheyana]

T2715 Fibraurea recisa

T1650 Convolvulus arvensis

T2451 Eryngium campestre

T3042 Goniothalamus arvensis

T0668 Artemisia campestris

T4567 Oxytropis campestris

T2569 Eupatorium rebaudianum

T2906 Gentiana campestris

T4709 Periandra dulcis

T6661 Uvaria dulcis

T2365 Enteromorpha clathrata

T2542 Euonymus japonicus

T1721 Corydalis linearioides

T0455 Andrographis lineata

T0227 Agave striata

T5677 Salvia lineata

T1450 Cirsium lineare

T0122 Aconitum pendulum

T1244 Cassia siamea

T2601 Euphorbia milii

T4992 Plagiochila asplenioides

T3187 Helleborus thibetanus

T4203 Mesua ferrea

T2108 Dendrobium officinale

T0888 Beesia calthaefolia

T3987 Lycoris sanguinea

T5875 Senecio adnatus

T0175 Adiantum pedatum

T6400 Thalictrum minus var. adiantifolium 


\begin{tabular}{|c|c|c|}
\hline TIE XIU SE HUANG TAN & 铁锈色黄檀 & Ferruginous Rosewood* \\
\hline TIE ZHOU CAO & 铁轴草 & Fourfile Germander \\
\hline TIE ZI & 铁仔 & African Myrsine \\
\hline TING JING BIAN DI JIN & 挺茎遍地金 & Elodia St.John'swort \\
\hline TING LI ZI & 葶芳子 & Pepperweed Seed \\
\hline TING YUAN ZI JIN NIU & 庭园紫金牛* & Curtilage Ardisia* \\
\hline TONG BAN CAO & 同瓣草 & Longflower Laurentia \\
\hline TONG CHUI YU DAI CAO & 铜锤玉带草 & Common Pratia \\
\hline TONG GUANG TENG & 通光藤 & Tenacious Condorvine \\
\hline TONG HAO & 茼蒿 & Crowndaisy Chrysanthemum \\
\hline TONG HUA GEN & 通花根 & Ricepaperplant Root \\
\hline TONG JING CAO & 通经草 & Silvery Aleuritopteris \\
\hline TONG LUO HAN & 铜罗汉 & \\
\hline TONG QIAO SHE GU & 筒鞘蛇菰 & Involucrate Balanophora \\
\hline TONG SE JI NA SHU & 铜色鸡纳树* & Cupreous Cinchona* \\
\hline TONG TUO MU & 通脱木 & Ricepaperplant \\
\hline TONG XING LIE PIAN HU ZHI & 同形裂片胡枝子* & Homoloba Lespedeza* \\
\hline ZI & & \\
\hline TONG XU SHOU GONG MU & 同序守宫木* & Geckowood \\
\hline TONG YOU & 桐油 & Tung Oil \\
\hline TOU GU CAO & 透骨草 & Tuberculate Speranskia \\
\hline TOU HUA DU JUAN & 头花杜鹃 & Capitate Rhododendron \\
\hline TOU HUA LONG DAN & 头花龙胆 & Headflower Gentian \\
\hline TOU MING TANG SONG CAO & 透明唐松草* & Lucid Meadowrue* \\
\hline TOU XU CONG MU & 头序淴木 & Hairyleaf Aralia \\
\hline TU BAI BU & 土百部 & Fernlike Asparagus \\
\hline TU CHUANG HUA & 秃疮花 & Slenderstalk Dicranostigma \\
\hline TU DANG GUI & 土当归 & Udo \\
\hline TU DING GUI & 土丁桂 & Common Evolvulus \\
\hline TU ER FENG & 兔耳风 & Provincialis Gayfeather* \\
\hline TU ER FENG XIE JIA CAO & 兔儿风蟹甲草 & Ainsliaefolious Cacalia \\
\hline TU ER QI CUI QUE HUA & 土耳其翠雀花 & Turkish Larkspur* \\
\hline TU ER QI SHU WEI CAO & 土耳其鼠尾草 & Turkish Sage* \\
\hline TU ER QI SI TAN BAI LA SHU & 土耳其斯坦白蜡树 & Turkestan Ash \\
\hline TU ER QI SI TAN LONG DAN & 土耳其斯坦龙胆* & Turkestan Gentian* \\
\hline TU ER QI XUE HUA LIAN & 土耳其雪花莲 & Turkish Snowdrop* \\
\hline TU ER QI YU JIN XIANG & 土耳其郁金香* & Turkey Tulip* \\
\hline TU ER SAN & 兔儿半 & Syneilesis* \\
\hline TU FU LING & 土获苓 & Glabrous Greenbrier \\
\hline TU GEN & 吐根 & Ipecacuanha \\
\hline TU GUA LANG DU & 土瓜狼毒 & Proliferous Euphorbia* \\
\hline TU HUANG LIAN & 土黄连 & Wintergreen Barberry \\
\hline TU JING JIE & 土荆芥 & Mexican Tea \\
\hline TU JING PI & 土荆皮 & Chinese Golden Larch \\
\hline TU LIAN QIAO & 土连翅 & Tall Hymenodictyon \\
\hline TU LIANG JIANG & 土良姜 & Spiked Gingerlily \\
\hline TU MAO DONG QING & 秃毛冬青 & Glabrous Holly* \\
\hline TU MU XIANG & 土木香 & Elecampane Inula \\
\hline TU NIU XI & 土牛膝 & Common Achyranthes \\
\hline
\end{tabular}

T2003 Dalbergia ferruginea

T6367 Teucrium quadrifarium

T4361 Myrsine africana

T3348 Hypericum elodeoides

T3755 Lepidium apetalum [Syn. Lepidium

micranthum]

T0596 Ardisia hortorum

T3541 Isotoma longiflora [Syn. Laurentia

longiflora]

T5193 Pratia nummularia

T4120 Marsdenia tenacissima

T1390 Chrysanthemum coronarium

T6356 Tetrapanax papyriferus

T0301 Aleuritopteris argentea

T4231 Milingtonia hortensis

T0859 Balanophora involucrata

T1427 Cinchona cuprea

T6357 Tetrapanax papyriferus

T3772 Lespedeza homoloba

T5748 Sauropus androgynus

T0299 Aleurites cordata [Syn. Aleurites fordii]

T6069 Speranskia tuberculata

T5504 Rhododendron capitatum

T2908 Gentiana cephalantha

T6397 Thalictrum lucidum

T0570 Aralia dasyphylla

T0749 Asparagus filicinus

T2162 Dicranostigma franchetianum [Syn.

Dicranostigma leptopodum]

T0569 Aralia cordata

T2649 Evolvulus alsinoides

T3789 Liatris provincialis

T1097 Cacalia ainsliaeflora

T2070 Delphinium crispulum

T5668 Salvia cilicica

T2775 Fraxinus potamophila

T2939 Gentiana turkestanorum

T2823 Galanthus plicatus ssp. byzantinus

T6565 Tulipa turkestani

T6256 Syneilesis palmata

T5977 Smilax glabra

T1315 Cephaelis ipecacuanha

T2612 Euphorbia prolifera

T0906 Berberis julianae

T1361 Chenopodium ambrosioides

T5256 Pseudolarix amabilis [Syn. Larix

amabilis; Pseudolarix kaempferi]

T3323 Hymenodictyon excelsum

T3120 Hedychium spicatum

T3397 Ilex pubescens var. glaber

T3431 Inula helenium

T0071 Achyranthes aspera 


\begin{tabular}{|c|c|}
\hline TU QIANG HUO & 土芫活 \\
\hline TU SAN JIN SI TAO & 土三金丝桃 \\
\hline TU SAN QI & 土三七 \\
\hline TU SHA REN & 土砂仁 \\
\hline TU SI ZI & 菟丝子 \\
\hline TU XIANG RU & 土香薷 \\
\hline TU YAN HU & 土延胡 \\
\hline TU YE HUANG PI SHU & 秃叶黄皮树 \\
\hline TUAN HUA SHAN FAN & 团花山矾 \\
\hline TUAN JI AI NA XIANG & 团集艾纳香* \\
\hline TUE YUAN MI ZI LAN & 椭圆米仔兰* \\
\hline TUI RE ZHI XIE MU & 退热止泻木 \\
\hline TUN CAO & 豚草 \\
\hline TUN CAO QIAN LI GUANG & 豚草千里光* \\
\hline TUN CAO ZI WAN & 豚草紫苑 \\
\hline TUN XING GUO & 眤形果 \\
\hline TUO YE ZHU SHI DOU & 托叶猪屎豆* \\
\hline TUO YUAN DUO TAN CAO & 椭圆多坦草 \\
\hline TUO YUAN GOU TENG & 椭圆钩藤 \\
\hline TUO YUAN SAN QI CAO & 椭圆三七草* \\
\hline TUO YUAN YE HUA JIAO & 椭圆叶花椒 \\
\hline TUO YUAN YE RU XIANG SHU & 椭圆叶乳香树* \\
\hline TUO YUAN ZHOU XING ZAO & 椭圆舟形藻 \\
\hline WA BU BEI MU & 瓦布贝母 \\
\hline WA ER TENG & 娃儿藤 \\
\hline WA LI XI HUANG YANG & 瓦利希黄杨 \\
\hline WA SHI DU HUO & 瓦氏独活 \\
\hline WA SHI MA WEI ZAO & 瓦氏马尾藻 \\
\hline WA SHI XIAO BO & 瓦氏小檗* \\
\hline WA SHI ZONG LV & 瓦氏棕㭣 \\
\hline WA SI YA NA SAN CHI HAO & 瓦斯亚那三齿蒿 \\
\hline WA WEI & 瓦韦 \\
\hline WAI JUAN TANG SONG CAO & 外卷唐松草 \\
\hline WAI LAI CAI ZONG & 外来菜棕* \\
\hline WAN DOU & 踠豆 \\
\hline WAN E JIN SI TAO & 弯蔩金丝桃 \\
\hline WAN HUA ZE LAN & 晚花泽兰* \\
\hline WAN JUE & 碗㓹 \\
\hline WAN NIAN QING GEN & 万年青根 \\
\hline WAN QU QIAN LI GUANG & 弯曲千里光* \\
\hline WAN QU TIAN MEN DONG & 弯曲天门冬* \\
\hline WAN QU TIAN NAN XING & 弯曲天南星 \\
\hline WAN QU ZHI YE TAI & 弯曲指叶苔* \\
\hline WAN RUI KAI KOU JIAN & 弯芯开口箭 \\
\hline WAN SHAN YIN YANG HUO & 万山淫羊藿 \\
\hline WAN SHENG BAI BU & 蔓生百部 \\
\hline WAN SHENG BAI WEI & 蔓生白薇 \\
\hline WAN SHOU JU & 万寿菊 \\
\hline WAN SHOU JU YE & 万寿菊叶 \\
\hline N TAO HUA ZI & 万桃花子 \\
\hline
\end{tabular}

Coronarious Gingerlily
Tutsan
Chrusanthemum-like Groundsel
Japanese Galangal
Chinese Dodder Seed
Common Origanum
Repent Corydalis*
Glabrousleaf Chinese Corktree

Glomerule Sweetleaf

Glomerate Blumea*

Elliptic Aglaia*

Febrifuge Holarrhena*

Common Ragweed

Ragweed Groundsel*

Upland White Aster

Topeng Pygeum

Stipular Crotalaria*

Elliptic Gambirplant*

Elliptic Gynura*

Ellipticleaf Pricklyash*

Ellipticleaf Olibanum*

Ellipse Navicula*

Wabu Fritillary*

Manyflower Tylophora

Himalayan Box

Wallich Cowparsnip*

Vachelli Gulfweed*

Valdiv Barberry

Wagner Windmill Palm

Vaseyana Big Sagebrush*

Thunberg's Lepisorus

Waxy Meadowrue

Garden Pea

Curvedsepal St.John'swort

Late-flower Boneset

Scabrous Boulder Fern

Omoto Nipponlily Root

Retrorse Groundsel*

curillus Asparagus*

Curvatura Jackinthepulpit*

Watt Tupistra

Wanshan Epimedium

Japanese Stemona

Versicolorous Mosquitotrap

Aztec Marigold

Aztec Marigold Leaf

Jimsonweed Seed*
T3118 Hedychium coronarium

T3338 Hypericum androsaemum

T5884 Senecio chrysanthemoides

T0357 Alpinia japonica

T1912 Cuscuta chinensis

T4526 Origanum vulgare

T1736 Corydalis repens var. humosides

T4792 Phellodendron chinense var.

glabriusculum

T6253 Symplocos glomerata

T0958 Blumea glomerata

T0234 Aglaia elliptica

T3265 Holarrhena febrifuga

T0398 Ambrosia artemisiifolia

T5879 Senecio ambrosioides

T0780 Aster ptarmicoides

T5340 Pygeum topengii

T1835 Crotalaria stipularia

T2248 Dorstenia elliptica

T6614 Uncaria elliptica

T3087 Gynura elliptica

T6885 Zanthoxylum ovalifolium

T0995 Boswellia ovalifoliolata

T4394 Navicula delognei f. elliptica

T2799 Fritillaria wabuensia

T6580 Tylophora floribunda

T1095 Buxus wallichiana

T3227 Heracleum wallichii

T5740 Sargassum vachellianum

T0918 Berberis valdiviana

T6486 Trachycarpus wagnerianus

T0703 Artemisia tridentata ssp. vaseyana

T3764 Lepisorus thunbergianus

T6406 Thalictrum revolutum

T5636 Sabal peregrina

T4983 Pisum sativum

T3345 Hypericum curvisepalum

T2575 Eupatorium serotinum

T2113 Dennstaedtia scabra [Syn. Dicksonia

scabra]

T5557 Rohdea japonica [Syn. Orontium

japonicum ]

T5902 Senecio retrorsus

T0747 Asparagus curillus

T0619 Arisaema curvatum

T3762 Lepidozia incurvata

T6567 Tupistra wattii [Syn. Campylandra wattii]

T2405 Epimedium wanshanense

T6111 Stemona japonica

T1962 Cynanchum versicolor

T6278 Tagetes erecta

T6279 Tagetes erecta

T2047 Datura stramonium 


\begin{tabular}{|c|c|}
\hline WAN XIANG YU & 晚香玉 \\
\hline WAN YAN BAN JIU JU & 蜿蜒斑加菊 \\
\hline WAN YAN HUANG QI & 蜿蜒黄芪* \\
\hline WAN YAN XIANG MAO & 蜿蜒香茅 \\
\hline WAN ZHUI XIANG CHA CAI & 弯雉香茶菜 \\
\hline WAN ZHUO WU TOU & 万豚乌头 \\
\hline WANG BU LIU XING & 王不留行 \\
\hline WANG CHUN YU LAN & 望春玉兰 \\
\hline WANG DI ZAO & 网地藻 \\
\hline WANG GUA & 王瓜 \\
\hline WANG GUA ZI & 王瓜子 \\
\hline WANG GUO CUI QUE HUA & 网果翠雀花 \\
\hline WANG JIANG NAN & 望江南 \\
\hline WANG JIANG NAN ZI & 望江南子 \\
\hline WANG MAI TOU WU & 网脉㯻吾 \\
\hline WANG SUN & 王孙 \\
\hline WEI BAI BAI JIAN MU & 微白白坚木* \\
\hline WEI DUO LI YA JIN HE HUAN & 维多利亚金合欢* \\
\hline WEI ER SHI LV RONG HAO & 威尔士绿绒蒿 \\
\hline WEI GAN JU & 薇甘菊 \\
\hline WEI GAN JU ZE LAN & 薇甘菊泽兰 \\
\hline WEI JING BAI HE & 苇茎百合 \\
\hline WEI KONG CAO & 微孔草 \\
\hline WEI LAN QIU GUO ZI JIN & 维兰球果紫堇 \\
\hline WEI LIAN SI SHI MU & 威廉斯氏木 \\
\hline WEI LING CAI & 委陵菜 \\
\hline WEI LING XIAN & 威灵仙 \\
\hline WEI MAO DUI XIN JU & 微毛堆心菊* \\
\hline WEI MAO HE ZI & 微毛诃子 \\
\hline WEI NAO & 猬脑 \\
\hline WEI RUI LI & 味瑞李 \\
\hline WEI SHI DA FENG ZI & 韦氏大风子 \\
\hline WEI SHI SHU WEI CAO & 威氏鼠尾草 \\
\hline WEI SUI XIAN & 尾穗苋 \\
\hline WEI XI XIANG CHA CAI & 维西香茶菜 \\
\hline WEI XIAO WAN SHOU JU & 微小万寿菊* \\
\hline WEI XIN GAN & 猬心肝 \\
\hline WEI YE BAI ZHU & 尾叶白珠 \\
\hline WEI YE XI ZI JUE & 尾叶稀子偋 \\
\hline WEI YE XIANG CHA CAI & 尾叶香茶菜 \\
\hline WEI YI MU CAO & 胃益母草 \\
\hline WEN CHUAN CHAI HU & 汶川柴胡 \\
\hline WEN DAN YOU & 文旦柚 \\
\hline WEN GUAN MU & 文冠木 \\
\hline WEN JING & 问荆 \\
\hline WEN PO & 榅桲 \\
\hline WEN ROU ZHI XIE MU & 温柔止泻木 \\
\hline WEN SHU LAN & 文殊兰 \\
\hline
\end{tabular}

Tuberose

Flexuous Bitterleaf*

Flexuous Milkvetch *

Flexuous Lemongrass*

Bowedconical Rabdosia*

Curvebeak Monkshood

Cowherb

Biond Magnolia

Japanese Snakegourd

Japanese Snakegourd Seed

Reticulatefruit Larkspur

Coffee Senna

Coffee Senna Seed

Netvein Goldenray

Tetraphyllous Paris

Microwhite White Quebracho*

Bramble Acacia

Welsh Poppy

Climbing Hempweed

Mikanioid Eupatorium*

Schoenocoulon officinale

Sikkim Microula

Few-flowered Fumitory

Chinese Cinquefoil

Chinese Clematis

Puberulent Sneezeweed*

Terminalia chebula var. tomentella

Hedgehog Brain

Verecun Plum*

Wightiana Chaulmoogratree Seed*

Love-lies-bleeding

Weisi Rabdosia

Southern Marigold

Hedgehog Heart and Liver

Long Acuminate Leaves Gaultheria

Tail-leaf Monachosorum

Taillikeleaf Rabdosia

Stomach Motherwort

Wenchuan Thorowax*

Buntan Pummelo*

Shinyleaf yellowhorn

Bottle-brush

Common Quince

Suave Holarrhena*

Chinese Crinum
T5061 Polianthes tuberosa

T6717 Vernonia flexuosa

T0796 Astragalus flexuosus

T1942 Cymbopogon flexuosus

T3509 Isodon loxothyrsa

T0082 Aconitum campylorrhynchum

T6668 Vaccaria segetalis [Syn. Vaccaria pyramidata]

T4035 Magnolia biondii [Syn. Magnolia

fargesii]

T2169 Dictyota dichotoma

T6507 Trichosanthes cucumeroides

T6508 Trichosanthes cucumeroides

T2072 Delphinium dictyocarpum

T1241 Cassia occidentalis

T1242 Cassia occidentalis

T3803 Ligularia dictyoneura [Syn. Senecio

dictyoneurus]

T4655 Paris tetraphylla

T0775 Aspidosperma subincanum

T0034 Acacia victoria

T4142 Meconopsis cambrica

T4230 Mikania scandens

T2566 Eupatorium mikanioides

T5808 Schoenocaulon officinale

T4227 Microula sikkimensis

T2810 Fumaria vaillantii

T5941 Sickingia williamsii

T5181 Potentilla chinensis

T1545 Clematis chinensis

T3140 Helenium puberulum

T6348 Terminalia chebula var. tomentella

T2427 Erinaceus europaeus; Hemiechinus

dauuricus; Hemiechinus auritus

T5245 Prunus verecunda

T3301 Hydnocarpus wightiana

T5700 Salvia wiedemannii

T0387 Amaranthus caudatus

T3534 Isodon weisiensis

T6281 Tagetes minuta

T2428 Erinaceus europaeus; Hemiechinus

dauuricus; Hemiechinus auritus

T2892 Gaultheria griffithiana

T4267 Monachosorum flagellare

T5392 Rabdosia excisa

T3750 Leonurus cardiaca

T1078 Bupleurum wenchuanense

T1481 Citrus grandis f. buntan

T6845 Xanthoceras sorbifolia

T2407 Equisetum arvense

T1937 Cydonia oblonga

T3267 Holarrhena mitis

T1796 Crinum asiaticum var. sinicum 


\begin{tabular}{|c|c|}
\hline WEN TE XIANG MAO & 文特香茅 \\
\hline WEN YU JIN & 温郁金(片姜黄) \\
\hline WEN ZHU & 文竹 \\
\hline WENG CAI & 䔨菜 \\
\hline WO ER QI & 窝儿七 \\
\hline WO JING YE LAI XIANG & 卧茎夜来香 \\
\hline WO JU & 莫苣 \\
\hline WO LI HE GUAN BAN & 沃利赫冠瓣 \\
\hline WO SHI AN & 沃氏桉* \\
\hline WO SHI ZHI XIE MU & 沃氏止泻木* \\
\hline WU BING TANG SONG CAO & 无柄唐松草* \\
\hline WU BING XIN WU TAN & 无柄新乌檀 \\
\hline WU BING YE AN & 无柄叶桉* \\
\hline WU CI FAN MA & 无刺番麻 \\
\hline WU CI KE YA SHU & 无刺柯桠树 \\
\hline WU CI MAO ZHU MU & 无刺帽柱木 \\
\hline WU CI ZAO & 无刺杳 \\
\hline WU CI ZHU YING HUA & 无刺朱缨花* \\
\hline WU DANG MU LAN & 武当木兰 \\
\hline WU FU HUA & 五福花 \\
\hline WU GAN DA YU YE QIU & 乌干达羽叶楸 \\
\hline WU GENG WU JIA PI & 无梗五加皮 \\
\hline WU GONG & 蜈蚣 \\
\hline WU GONG CAO & 蜈蚣草 \\
\hline WU GONG ZHANG & 蜈蚣掌 \\
\hline WU HE MI JU & 无核蜜桔 \\
\hline WU HUA GUO & 无花果 \\
\hline WU HUA GUO YE & 无花果叶 \\
\hline WU HUAN ZI & 无患子 \\
\hline WU HUAN ZI PI & 无患子皮 \\
\hline WU HUAN ZI YE & 无患子叶 \\
\hline WU JIA PI & 五加皮 \\
\hline WU JIA QIAN HU & 五加前胡 \\
\hline WU JING GAN LAN & 芜菁甘蓝 \\
\hline WU JIU MU GEN PI & 乌柏木根皮 \\
\hline WU JIU YE & 乌柏叶 \\
\hline WU JU LOU DOU CAI & 无距耧斗菜 \\
\hline WU JU YIN YANG HUO & 无距淫羊嚾 \\
\hline WU JUAN XU XI GUA & 无卷须西瓜 \\
\hline WU KE LAN XIA YE SHUI XIAN & 乌克兰狭叶水仙 \\
\hline WU LA ER DI YI & 乌拉尔地衣 \\
\hline WU LENG JUE MING & 五棱决明 \\
\hline WU LI & 乌鳢 \\
\hline WU LIAN MEI & 乌蔹莓 \\
\hline WU LING ZHI & 五灵脂 \\
\hline WU LONG CHA & 乌龙茶 \\
\hline WU LOU ZI & 无漏子 \\
\hline WU MAI BAI LI XIANG & 五脉百里香 \\
\hline WU MAO CHAN GAO SHU & 无毛漽槁树 \\
\hline
\end{tabular}

Winter Lemongrass*

Wengujin Culcuma*

Setose Asparagus

Aquatic Morning Glory

Chinese Umbrellaleaf

Creeping Telosma

Garden Lettuce

Wallich Crestpetal-tree

Wandoo Eucalyptus*

Waltsberg Holarrhena*

Javan Meadowrue

Sessile Neonauclea

Non-stipe Eucalyptus*

Spineless Agave

Angelin-tree

Spineless Mitragyna*

Spineless Common Jujube

Spineless Powderpuff*

Sprenger Magnolia

Muskroot

Uganda Padritree*

Sessileflower Acanthopanax Root-bark

Centipede

Chinese Brake

Arborescent Aloe*

Satsuma

Fig

Fig Leaf

Chinese Soapberry Seed

Chinese Soapberry Peel

Chinese Soapberry Leaf

Slenderstyle Acanthopanax Root-bark

Swede Seed

Chinese Tallowtree Bark

Chinese Tallowtree Leaf

Spurless Columbine

Spurless Barrenwort*

Non-cirrose Citrullus*

Ukrainian Narrowleaf Narcissus*

Ural's Lichen

Fiveangular Senna*

Serpent-head

Japanese Cayratia

Trogopterus Dung

Oolong Tea

Phoenix Date

Fiveribbed Thyme

Glabrous Litse*
T1948 Cymbopogon winterianus

T1907 Curcuma wengujin

T0753 Asparagus setaceus [Syn. Asparagus plumosus]

T3444 Ipomoea aquatica [Syn. Convolvulus repens; Ipomoea reptans]

T2232 Diphylleia sinensis

T6329 Telosma procumbens

T3662 Lactuca sativa

T3922 Lophopetalum wallichii

T2523 Eucalyptus wandoo

T3269 Holarrhena waltsbergii

T6408 Thalictrum sessile

T4408 Neonauclea sessilifolia [Syn. Nauclea sessilifolia; Adina sessilifolia]

T2500 Eucalyptus apodophylla

T0216 Agave americana var. marginata [Syn.

Agave americana var. variegata]

T0452 Andira inermis

T4256 Mitragyna inermis

T6917 Ziziphus jujuba var. inermis

T1115 Calliandra inermis

T4052 Magnolia sprengeri

T0193 Adoxa moschatellina

T6141 Stereospermum kunthianum

T0044 Acanthopanax sessiliflorus

T5817 Scolopendra subspinipes mutilans

T5298 Pteris vittata

T0334 Aloe arborescens var. natalensis

T1518 Citrus unshiu

T2717 Ficus carica

T2718 Ficus carica

T5719 Sapindus mukorossi

T5720 Sapindus mukorossi

T5721 Sapindus mukorossi

T0038 Acanthopanax gracilistylus

T6103 Steganotaenia araliacea

T1010 Brassica napus var. napobrassica

T5723 Sapium sebiferum

T5724 Sapium sebiferum

T0557 Aquilegia ecalcarata

T2393 Epimedium ecalcaratum

T1461 Citrullus ecirrhosus

T4373 Narcissus angustifolius

T6603 Umbilicaria proboscidea

T1243 Cassia quinquangula

T4505 Ophiocephalus argus

T1275 Cayratia japonica

T6551 Trogopterus xanthipes; Pteromys volans

T1155 Camellia sinensis var. viridis

T4820 Phoenix dactylifera

T6450 Thymus quinquecostatus

T3888 Litsea glutinosa var. glabrara 


\begin{tabular}{|c|c|c|c|}
\hline WU MAO FENG CHE ZI & 无毛风车子 & Glabrous Combretum* & T1631 Combretum imberbe \\
\hline WU MAO HAO & 无毛蒿 & Glabrous Wormwood* & T0677 Artemisia glabella \\
\hline WU MAO JUE & 乌毛蕨 & Oriental Blechnum Frond & T0952 Blechnum orientale \\
\hline WU MAO SHAN ZHA & 无毛山楂 & Hairless Chinese Hawthorn & T1779 Crataegus pinnatifida var. psilosa \\
\hline WU MAO WU GEN TENG & 无毛无根藤* & Glabrous Cassytha* & T1252 Cassytha glabella \\
\hline WU MEI & 乌梅 & Japanese Apricot & T5228 Prunus mume \\
\hline WU MO & 乌墨 & & $\begin{array}{l}\text { T2533 Eugenia jambolana [Syn. Syzygium } \\
\text { cumin; } \text { Myrtus cumini }]\end{array}$ \\
\hline WU MU XIE & 乌木屑 & Ceylon Persimmon Sawdust & T2216 Diospyros ebenum \\
\hline WU PAO ZI & 乌泡子 & Parker Raspberry & T5594 Rubus parkeri \\
\hline WU QING & 芜青 & Turnip & T1021 Brassica rapa \\
\hline WU SE MEI & 五色梅 & Common Lantana & T3687 Lantana camara \\
\hline WU SHAN YIN YANG HUO & 巫山淫羊蕉 & Wushan Epimedium & T2406 Epimedium wushanense \\
\hline WU SHI REN DONG & 五室忍冬* & Five-room Honeysuckle* & T3917 Lonicera quinquelocularis \\
\hline WU SU LI LI LU & 乌苏里藜芦 & Ussuri Falsehellebore & T6699 Veratrum nigrum var. ussuriense \\
\hline WU SU LI WA WEI & 乌苏里瓦韦 & Ussuri Lepisorus & T3765 Lepisorus ussuriensis \\
\hline WU TONG BAI PI & 梧桐白皮 & Phoenix Tree Bast & T2731 Firmiana simplex \\
\hline WU TONG YE & 梧桐叶 & Phoenix Tree Leaf & T2732 Firmiana simplex \\
\hline WU TONG ZI & 梧桐子 & Phoenix Tree Seed & T2733 Firmiana simplex \\
\hline WU TOU & 乌头(川乌) & Common Monkshood & T0084 Aconitum carmichaeli \\
\hline WU WEI CAO & 五味草 & Tali Corydalis & T1746 Corydalis taliensis \\
\hline WU WEI ZI & 五味子(北五味子) & Chinese Magnoliavine & T5791 Schisandra chinensis \\
\hline WU WEN ZI BEI TAI & 无纹紫背苔 & Intermediate Plagiochasma & T4989 Plagiochasma intermedium \\
\hline WU XIN SHI & 乌心石 & Formosan Michelia* & T4211 Michelia compressa var. formosana \\
\hline WU XIONG RUI JI LI & 五雄荵蔟藜* & Penta-androus Caltrap* & T6496 Tribulus pentandrus \\
\hline WU YA GUO & 五桠果 & Hondapara & T2181 Dillenia indica \\
\hline WU YAO & 乌药 & Combined Spicebush & $\begin{array}{l}\text { T3854 Lindera strychnifolia }[\text { Syn. Lindera } \\
\text { aggregata }]\end{array}$ \\
\hline WU YE BAI QIAN & 无叶白前* & Leafless Swallowwort* & T1949 Cynanchum aphyllum \\
\hline WU YE GUA TENG & 五叶瓜藤 & Farges Holboellia & T3270 Holboellia fargesii \\
\hline WU YE JIA MU ZEI & 无叶假木贼 & Leafless Anabasis & T0435 Anabasis aphylla \\
\hline WU YE TENG & 无爷藤 & Filiform Cassytha & T1251 Cassytha filiformis \\
\hline WU YE YU HUANG CAO & 五叶鱼黄草* & Five-leaf Merremia* & T4198 Merremia quinquefolia \\
\hline WU YE ZHU SHI DOU & 五叶猪屎豆* & Fiveleaf Crotalaria* & T1829 Crotalaria quinquefolia \\
\hline WU YUE CHA & 五月茶 & Bignay Chinalaurel & T0535 Antidesma bunius \\
\hline WU ZHAO LONG & 五爪龙 & Cairo Morningglory & T3448 Ipomoea cairica $[$ Syn. Ipomoea palmata $]$ \\
\hline WU ZHU FEI YAN CAO & 五柱飞燕草 & Fivestyle Larkspur* & T2083 Delphinium pentagynum \\
\hline WU ZHU MAI DA JI & 五主脉大戟* & & T2615 Euphorbia quinquecostata \\
\hline WU ZHU YU & 吴茱英 & Medicinal Evodia & T2644 Evodia rutaecarpa \\
\hline WULU BA DOU & 乌鲁巴豆 & Wru Croton* & T1860 Croton urucurana \\
\hline XI A LA HUANG TAN & 西阿拉黄檀 & Cearen Rosewood* & T1998 Dalbergia cearensis \\
\hline XI BAN WU TOU & 膝瓣乌头 & Geniculate Monkshood & T0098 Aconitum geniculatum \\
\hline XI BAN YA HUANG YANG & 西班牙黄杨 & Balearic Box & T1086 Buxus balearica \\
\hline XI BAN YA YUN XIANG CAO & 西班牙芸香草 & Spanish Haplophyllum* & T3102 Haplophyllum hispanicum \\
\hline XI BEI MU & 西贝母 & Crown Imperial & T2788 Fritillaria imperialis \\
\hline XI BING MU JIANG ZI & 细柄木姜子* & Slenderstalk Litse* & T3889 Litsea gracilipes \\
\hline XI BING SHI DA GONG LAO & 细柄十大功劳 & Subtriplinerved Mahonia & T4064 Mahonia gracilipes \\
\hline XI BING SHU YU & 细柄薯蓣 & Thinstiped Yam & T2211 Dioscorea tenuipes \\
\hline XI BO DE QI MU & 西博德桤木* & Siebold Alder* & T0331 Alnus sieboldiana \\
\hline XI BO JIN HE HUAN & 西博金合欢* & Sieber Acacia* & T0029 Acacia sieberiana \\
\hline XI BO JUE MING & 西博决明 & Sieber Senna & T1245 Cassia sieberiana \\
\hline XI BO LI YA HONG SONG & 西伯利亚红松 & Siberian Stone Pine & T4923 Pinus sibirica \\
\hline XI BO LI YA LENG SHAN & 西伯利亚冷杉 & Siberian Fir & T0007 Abies sibirica \\
\hline
\end{tabular}




XI BO LI YA LIAO[yn
XI BO LI YA TOU WU
XI BO LI YA YUAN ZHI
XI BO LI YA YUN SHAN
XI BO SHI WEI MAO
XI CHANG NAN MEI DOU
XI DIAN ZHANG YA CAI
XI FAN LIAN
XI FANG CANG ER
XI FANG CI BAI
XI FANG CUI QUE
XI FEI HONG DOU SHU
XI FEI LI ZHI GUO
XI FEI YANG JIAO AO
XI GENG XIANG CAO
XI GUA
XI GUA ZI REN
XI GUO JIAO HUI XIANG
XI HU LU
XI HUA DIAN ZI CAO
XI HUA HAN XIU CAO XIAN WEN XIANG CHA
CAI

CAI

XI HUA XUE DAN
XI HUANG CAO
XI JIAO
XI JIN SHI DA GONG LAO
XI JING SHI HU
XI JUAN YA CONG
XI LA GANG LIU
XI LA SHI CAO
XI LA SI MAO SHI
XI LAN GU KE
XI LAN ROU GUI
XI LIU ZHU YANG YANG
XI LUO HAN SONG
XI MA BAI LA SHU
XI MA DU WEI CAO
XI MA HONG JING TIAN
XI MA LA YA HONG DOU SHAN
XI MA LA YA YUAN WEI
XI MA LA YA YUAN ZHI
XI MA XUAN FU HUA
XI MAI FU PING
XI MEN FEI CAO
XI MENG SI SHI DA GONG LAO

\section{西伯利亚䓹}

西伯利亚革吾

西伯利亚远志

西伯利亚云杉

西博氏卫矛

细长南美豆

细点獐牙菜

西番莲

西方苍耳*

西方刺柏*.

西方翠雀

西非红豆树

西非荔枝果

西非羊角拗

细梗香草

西瓜

西瓜䊏仁

细果角茴香

西葫芦

细花滇紫草

细花含羞草

细花线纹香茶菜

\section{细花雪胆}

溪黄草

犀角

锡金十大功劳

细茎石斛

细卷鸦葱

希腊杜柳

希腊蓄草

希腊丝毛蓄

锡兰古柯*

锡兰肉桂

溪流猪种种*

西罗汉松

喜马白蜡树

喜马独尾草

喜马红景天

喜马拉雅红豆杉

喜马拉雅总尾

喜马拉雅远志

喜马旋覆花

稀脉浮萍

西门肺草

西蒙斯十大功劳
Siberian Knotweed*

Siberian Goldenray

Siberian Milkwort

Siberian Spruce

Seibo Euonymus*

Punctate Swertia*

Passionflower

Occidental Cocklebur*

Western Juniper

Occidental Larkspur*

West Africa Afrormosia

Akee

Senegalese Strophanthus*

Hairydtalk Loosestrife

Watermelon

Watermelon Seed

Thinfruit Hypecoum

Pumpkin

Hooker Onosma

Smallflower Mimosa*

Linearstripe Rabdosia

Smallflower Hemsleya

Serrate Rabdosia

Rhinoceros Horn

Sikkim Mahonia

Moliniform Dendrobium*

Scorzonera

Grecian Silkvine

Greek Yarrow

Greek Silk-hair Yarrow*

Ceylon Coca Shrub*

Ceylon Cinnamon

River Bedstraw*

Musengerra Podocarpus

Manyflower Ash

Himalayan Desertcandle

Himalaya Rhodiola

Himalayan Yew

Himalayan Iris*

Himalayan Milkwort*

Himalayan Inula

Minute Duckweed

Comfrey

Simons Mahonia
T5115 Polygonum sibiricum [Syn. Persicaria sibirica $]$

T3813 Ligularia sibirica

T5086 Polygala sibirica

T4875 Picea obovata

T2547 Euonymus sieboldianus

T0439 Anadenanthera colubrine

T6233 Swertia punctata

T4663 Passiflora caerulea

T6839 Xanthium occidentale

T3591 Juniperus occidentalis

T2080 Delphinium occidentale

T0208 Afrormosia elata

T0956 Blighia sapida

T6159 Strophanthus sarmentosus var. senegambiae

T3997 Lysimachia capillipes

T1464 Citrullus vulgaris [Syn. Citrullus lanatus]

T1465 Citrullus vulgaris [Syn. Citrullus lanatus]

T3334 Hypecoum leptocarpum

T1882 Cucurbita pepo

T4499 Onosma hookeri

T4250 Mimosa tenuiflora

T3507 Isodon lophanthoides [Syn. Rabdosia lophanthoides; Hyssopus lophanthoides;

Plectranthus striatus; Isodon striatus]

T3207 Hemsleya graciliflora [Syn. Alsomitra graciliflora]

T5397 Rabdosia serra

T5484 Rhinoceros unicornis; Rhinoceros

sondaicus; Rhinoceros sumatrensis

T4073 Mahonia sikkimensis

T2106 Dendrobium moniliforme

T5824 Scorzonera hispanica

T4727 Periploca graeca

T0058 Achillea ageratifolia

T0063 Achillea holosericea

T2493 Erythroxylum zeylanicum

T1445 Cinnamomum zeylanicum

T2833 Galium rivale

T5039 Podocarpus gracilior

T2769 Fraxinus floribunda

T2414 Eremurus himalaicus

T5495 Rhodiola himalansis

T6317 Taxus wallichiana

T3463 Iris kumaonensis

T5074 Polygala emodi

T3438 Inula royleana

T3739 Lemna perpusilla

T6246 Symphytum officinale

T4074 Mahonia simonsii 


\begin{tabular}{|c|c|c|c|}
\hline XI MING & 菥冥 & Boor's Mustard & T6436 Thlaspi arvense \\
\hline XI MING ZI & 菥冥子 & Boor's Mustard Seed & T6437 Thlaspi arvense \\
\hline XI NAN BA QIA & 西南菝葜 & Smilax bockii & T5975 Smilax bockii \\
\hline XI NAN GANG LIU & 西南杜柳 & Forrest Silkvine & T4726 Periploca forrestii \\
\hline XI NAN MAO WEI SHU & 西南猫尾树 & Stipulate Dolichandrone & T2245 Dolichandrone stipulata \\
\hline XI NAN REN DONG & 西南忍冬 & South-western Honeysuckle & T3908 Lonicera bournei \\
\hline XI NAN SHI WEI & 西南石韦 & Stilted Pyrrosia & T5356 Pyrrosia gralla \\
\hline XI NAN WEN SHU LAN & 西南文殊兰 & Broadleaf Crinum & T1799 Crinum latifolium \\
\hline XI NAN ZHANG YA CAI & 西南獐牙菜 & Surrounded Swertia & T6216 Swertia cincta \\
\hline XI NANG MA WEI ZAO & 细囊马尾藻 & Parvivesiculose Gulfweed* & T5739 Sargassum parvivesiculosum \\
\hline XI OU YUAN WEI & 西欧苪尾 & Orris & T3458 Iris florentina \\
\hline XI PA JING TIAN & 西帕景天* & Cepaea Stonecrop* & T5853 Sedum cepaea \\
\hline XI QIAN LI GUANG & 溪千里光 & Rivulet Groundsel* & T5904 Senecio rivularis \\
\hline XI QUE MEI TENG & 细雀梅藤 & Thin Sageretia & T5644 Sageretia gracilis \\
\hline XI SHAN CUI QUE & 喜山翠雀* & Montane Larkspur & T2082 Delphinium oreophilum \\
\hline XI SHEN SHAN ZI JIN & 细深山紫堇 & Tenuous Corydalis* & T1732 Corydalis pallida var. tenuis \\
\hline XI SHENG TENG & 锡生藤 & Common Cissampelos & T1452 Cissampelos pareira \\
\hline XI SHI HUI MAO DOU & 希氏灰毛豆* & Hildebrandt Tephrosia* & T6336 Tephrosia hildebrandtii \\
\hline XI SHU & 喜树 & Common Camptotheac & T1162 Camptotheca acuminata \\
\hline XI SHU MU BAN SHU & 稀疏木瓣树 & Discrete Xylopia* & T6853 Xylopia discreta \\
\hline XI SHU QIE & 稀疏茄* & Lax Nightshade* & T6004 Solanum laxum \\
\hline XI SHUAI & 蟋蟀 & Chinese Cricket & T3066 Gryllulus chinensis \\
\hline XI SI GUO XIANG CHA CAI & 西四国香茶菜 & West-Shiko Rabdosia* & T3527 Isodon shikokiana var. occidentalis \\
\hline XI TAI & 溪苔 & & T4695 Pellia epiphylla \\
\hline XI TE KA YUN SHAN & 西特喀云杉 & Sitka Spruce & T4876 Picea sitchensis \\
\hline XI XI LI CAO MU XI & 西西里草木犀 & Messania Sweetclover* & T4171 Melilotus messanensis \\
\hline XI XI LI QI SHU & 西西里漆树 & Sumach & T5532 Rhus coriaria \\
\hline XI XIAN & 豨莶 & Common St. Paulswort & T5950 Siegesbeckia orientalis \\
\hline XI XIANG CONG & 细香葱 & Chive-like & T0319 Allium schoenoprasum \\
\hline XI XIAO CHANG CHUN HUA & 细小长春花 & Slender Periwinkle* & T1270 Catharanthus pusillus \\
\hline XI XIAO HE BAO MU DAN & 细小荷包牡丹 & Little Bleedingheart* & T2153 Dicentra pusilla \\
\hline XI XIAO LUO ZAO & 细小裸藻 & & T2535 Euglena gracilis \\
\hline XI XIN & 细辛 & Siebold Wildginger & T0731 Asarum sieboldii \\
\hline$X I X I N$ YE $Q I A N N I U$ & 细辛叶牵牛* & Wildginger-leaf Morning Glory* & T3446 Ipomoea asarifolia \\
\hline XI YAN YUAN WEI & 喜盐茓尾 & Salt-loving Iris & T3460 Iris halophila \\
\hline XI YANG HONG & 西洋红 & Scarlet Sage & T5693 Salvia splendens \\
\hline XI YANG JIE GU MU & 西洋接骨木 & Black Elder & T5704 Sambucus nigra \\
\hline XI YANG LI & 西洋梨 & Common Pear & T5364 Pyrus communis \\
\hline XI YANG SHEN & 西洋参 & American Ginseng & T4610 Panax quinquefolium \\
\hline XI YANG SHEN JING YE & 西洋参茎叶 & American Ginseng Stem-leaf & T4611 Panax quinquefolium \\
\hline XI YE AN YE & 细叶桉叶 & Forest Gray Gum Leaf & T2521 Eucalyptus tereticornis \\
\hline XI YE BAI HE & 细叶百合 & Low Lily & T3836 Lilium pumilum [Syn. Lilium tenuifolium] \\
\hline XI YE DA JI & 细叶大戟 & Narrowleaf Euphorbia & T2586 Euphorbia esula var. cyparissoides \\
\hline XI YE DUI XIN JU & 细叶堆心菊 & Fine-leaved Sneezeweed & T3141 Helenium tenuifolium \\
\hline XI YE GONG LAO MU & 细叶功劳木 & Chinese Mahonia & T4062 Mahonia fortunei \\
\hline XI YE GONG LAO YE & 细叶功劳叶 & Chinese Mahonia Leaf & T4063 Mahonia fortunei \\
\hline XI YE HUANG YANG & 细叶黄杨 & Harland Box & T1088 Buxus harlandii \\
\hline XI YE SHE BIAN JU & 细叶蛇鞭菊 & Fine-leaved Gay-feather* & T3795 Liatris tenuifolia \\
\hline XI YE SHI & 细叶蓄 & Fine-leaved Yarrow & T0064 Achillea leptophylla \\
\hline XI YE SHUI TUAN HUA & 细叶水团花 & Thinleaf Adina & T0181 Adina rubella \\
\hline XI YE TENG & 锡叶藤 & Asian Tetracera & T6353 Tetracera asiatica \\
\hline XI YE TIE XIAN JUE & 细叶铁线蕨 & Venus Maidenhair & T0179 Adiantum venustum \\
\hline XI YE XIAO BO & 细叶小檗 & Poiret Barberry & T0912 Berberis poiretii \\
\hline
\end{tabular}




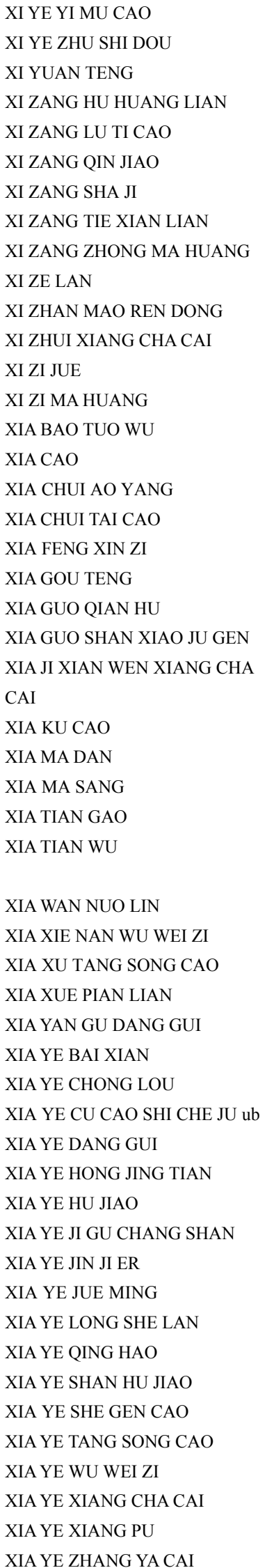

细叶益母草

细叶猪康豆*

细圆藤

西藏胡黄连

西藏鹿蹄草

西藏秦艽

西藏沙棘

西藏铁线莲*

西藏中麻黄

细泽兰*

细毡毛忍冬

细雉香茶菜

稀子荻

细子麻黄

狭苍㯻吾

霞草

下垂澳杨

下垂苔草*

夏风信子

狭钩藤

狭果前胡

狭果山小橘根*

狭基线纹香茶菜

\section{夏枯草 \\ 虾蟆胆 \\ 狭马桑* \\ 霞天膏 \\ 夏天无}

下弯诺林*

狭叶南五味子

狭序唐松草

夏雪片莲

下延古当归

狭叶白鲜

狭叶重楼

狭叶粗粘矢车菊*

狭叶当归(川白芷)

狭叶红景天

狭叶胡椒

狭叶鸡骨常山

狭叶锦鸡儿

狭叶决明

狭叶龙舌兰

狭叶青蒿

狭叶山胡椒

狭叶蛇根草

狭叶唐松草

狭叶五味子

狭叶香茶菜

狭叶香蒲

狭叶獐牙菜
Siberian Motherwort

Slender-leaf Crotalaria

Greyblue Pericampylus

Figwortflower Picrorhiza

Tibet Pyrola*

Tibet Gentian

Tibet Seabuckthorn

Tibet Clematis*

Tibet Intermediate Ephedra

Gracile Eupatorium*

Shortbraet Honeysuckle

Littleconical Rabdosia

Henry's Monachosorum

Regel Ephedra

Narrowbract Goldenray

Oldham Gypsophila

Nutant Aussiepoplar*

Drooping Sedge*

Summer-hyacinth

Narrow Gambirplant*

Narrowfruit Hogfennel*

Narrowfruit Glycosmis Root*

Gerard Linearstripe Rabdosia*

Common Selfheal

Rice Frog Gall

Narrow Coriaria*

Concentrated Beef Extract

Decumbent Corydalis

Recurvate Nolina*

Narrowleaf Kadsura*

Narrowraceme Meadowrue

Summer Snowflake

Decurrent Archangelica

Narrowleaf Dittary*

Narrowleaf Paris

Narrow Rough Star Thistle*

Narrowleaf Angelica

Kirilow Rhodiola

Matico Pepper

Narrowleaf Alstonia*

Nerrowleaf Peashrub

Narrowleaf Senna*

Shortleaf Agave

Tarragon

Narrowleaf Spicebush

Hayata Ophiorrhiza

Narrowleaf Meadowrue

Narrowleaf Magnoliavine*

Narrowleaf Rabdosia*

Narrowleaf Cattail Pollen

Narrowleaf Swertia
T3754 Leonurus sibiricus

T1822 Crotalaria intermedia

T4710 Pericampylus glaucus

T4888 Picrorhiza scrophulariflora

T5346 Pyrola calliantha var. tibetana

T2937 Gentiana tibetica

T3259 Hippophae thibetana

T1548 Clematis tibetana

T2370 Ephedra intermedia var. tibetica

T2561 Eupatorium gracile

T3918 Lonicera similis

T5389 Rabdosia coetsa

T4268 Monachosorum henryi

T2378 Ephedra regeliana

T3807 Ligularia intermedia

T3090 Gypsophila oldhamiana

T3274 Homalanthus nutans

T1203 Carex pendula

T2842 Galtonia candicans

T6606 Uncaria attenuata

T4772 Peucedanum stenocarpum

T3010 Glycosmis stenocarpa

T3508 Isodon lophanthoides var. gerardiana

T5214 Prunella vulgaris

T5406 Rana limnocharis

T1688 Coriaria angustissima

T0983 Bos taurus domesticus

T1715 Corydalis decumbens [Syn. Corydalis amabilis]

T4437 Nolina recurvata

T3612 Kadsura angustifolia

T6374 Thalictrum atriplex

T3781 Leucojum aestivum

T0583 Archangelica decurrens

T2165 Dictamnus angustifolius

T4651 Paris polyphylla var. stenophylla

T1302 Centaurea aspera subsp stenophylla

T0476 Angelica anomala

T5497 Rhodiola kirilowii

T4931 Piper angustifolium

T0366 Alstonia angustifolia

T1192 Caragana stenophylla

T1237 Cassia leptophylla

T0219 Agave cantala

T0676 Artemisia dracunculus

T3847 Lindera angustifolia

T4509 Ophiorrhiza hayatana

T6394 Thalictrum incidum

T5794 Schisandra lancifolia

T3480 Isodon angustifolia

T6585 Typha angustifolia

T6212 Swertia angustifolia 


\begin{tabular}{|c|c|c|}
\hline XIA ZHI CAO & 夏至草 & Lagopsis \\
\hline XIA ZI HUA & 虾子花 & Shrubby Woodwardia \\
\hline XIAN CHI SHE PU TAO & 显齿蛇葡萄 & Bigdentate Ampelopsis \\
\hline XIAN CHI ZI JIN NIU & 腺齿紫金牛 & Glandtooth Ardisia* \\
\hline XIAN DI HUANG & 鲜地黄(生地) & Adhesive Rehmannia Fresh Ro \\
\hline XIAN E HUANG LIAN & 线苧黄连 & Linearisepal Goldthread* \\
\hline XIAN GENG XI XIAN & 腺梗豨莶 & Glandularstalk St. Paulswort \\
\hline XIAN HE CAO & 仙鹤草 & Japanese Argimonia \\
\hline XIAN HE CAO GEN & 仙鹤草根 & Japanese Agrimonia Root \\
\hline XIAN HE CAO GEN YA & 仙鹤草根芽 & Japanese Argimonia Rhizome \\
\hline XIAN HUA XIANG CHA CAI & 腺花香茶菜 & Glandularflower Rabdosia \\
\hline XIAN HUANG XIAO BO & 鲜黄小檗 & Reddrop Barberry \\
\hline XIAN KE LAI & 仙客来 & Florists Cyclamen \\
\hline XIAN MAI JU & 腺脉蒟 & Glandularnerve Pepper \\
\hline XIAN MAI XIANG CHA CAI & 显脉香茶菜 & Veined Rabdosia \\
\hline XIAN MAI XUAN FU HUA & 显脉旋覆花 & Veined Inula \\
\hline XIAN MAI ZHANG YA CAI & 显脉獐牙菜 & Veined Swertia* \\
\hline XIAN MAO & 仙茅 & Common Cruculigo \\
\hline XIAN MAO HEI ZHONG CAO & 腺毛黑种草 & Glandular Fennelflower \\
\hline XIAN REN ZHANG & 仙人掌 & Cholla \\
\hline XIAN SE LI & 苋色藜 & Amaranthinecolor Goosefoot* \\
\hline XIAN SHENG MA XIAN HAO & 藓生马先蒿 & Muscicolous Woodbetony \\
\hline XIAN SUAN QIANG & 咸酸蔃 & Whiteflower Embelia \\
\hline XIAN WEN XIANG CHA CAI & 线纹香茶菜 & Linearstripe Rabdosia \\
\hline XIAN XI LAO GUAN CAO & 纤细老鹳草 & Robert Cranesbill \\
\hline XIAN XI SHU YU & 纤细薯蓣 & Thinnest Yam \\
\hline XIAN YE BA DOU & 线叶巴豆 & Linear Croton* \\
\hline XIAN YE CHAI HU & 线叶柴胡 & Linearleaf Thorowax \\
\hline XIAN YE JIN JI JU & 线叶金鸡菊 & Lance Coreopsis \\
\hline XIAN YE QIN & 纤叶芹 & Thinleaf Celery \\
\hline XIAN YE REN DONG & 腺叶忍冬 & Glaucousback Honeysuckle \\
\hline XIAN YE XUAN FU HUA & 线叶旋覆花 & Linearleaf Inula \\
\hline XIAN YU FENG WEI JUE & 线羽凤尾薮 & Linear Brake \\
\hline XIAN ZE LAN & 腺泽兰* & Glandulous Eupatorium* \\
\hline XIAN ZHOU MAI MA TENG & 显轴买麻藤 & Spinach Jointfir \\
\hline XIAN ZHUANG WANG DI ZAO & 线状网地藻 & \\
\hline XIANG BA DOU & 香巴豆* & Balsam Croton* \\
\hline XIANG CAO SHUI YANG MEI & 香草水杨梅* & Herb Bennet \\
\hline XIANG CHA CAI & 香茶菜 & Common Rabdosia \\
\hline XIANG CHE YE CAO & 香车叶草 & Sweet Woodruff \\
\hline XIANG CI BAI FEI ZHOU BIAN & 香刺柏非洲变种* & Mastic Africa Juniper* \\
\hline \multicolumn{3}{|l|}{ ZHONG } \\
\hline XIANG DAN & 象胆 & Elephant Gall \\
\hline XIANG DOU & 香豆 & \\
\hline XIANG FENG HUA & 香蜂花 & Bee Balm \\
\hline XIANG FU & 香附 & Nutgrass Galingale \\
\hline XIANG GANG JIAN MU & 香港樫木 & Hongkong Pencilwood \\
\hline XIANG GEN QIN & 香根芹 & Laxleaf Sweetroot \\
\hline XIANG GU & 象骨 & Elephant Bone \\
\hline XIANG HE HUAN & 香合欢 & Fragrant Albizia* \\
\hline
\end{tabular}

T3675 Lagopsis supina

T6830 Woodfordia fruticosa

T0426 Ampelopsis grossedentata [Syn.

Ampelopsis cantoniesis var. grossedentata]

T0593 Ardisia cornudentata

T5447 Rehmannia glutinosa [Syn. Rehmannia

glutinosa f. huechingensis]

T1668 Coptis linearisepala

T5952 Siegesbeckia orientalis var. pubescens

[Syn. Siegesbeckia pubescens]

T0249 Agrimonia pilosa var. japonica

T0250 Agrimonia pilosa var. japonica

T0251 Agrimonia pilosa var. japonica

T5388 Rabdosia adenantha

T0902 Berberis diaphana

T1930 Cyclamen persicum

T4936 Piper bavinum

T5395 Rabdosia nervosa

T3436 Inula nervosa

T6228 Swertia nervosa

T1901 Curculigo orchioides

T4432 Nigella glandulifera

T4519 Opuntia dillenii

T1360 Chenopodium amaranticolor

T4682 Pedicularis muscicola

T2348 Embelia ribes

T3506 Isodon lophanthoides

T2946 Geranium robertianum

T2198 Dioscorea gracillima

$\mathrm{T} 1850$ Croton linearis

T1055 Bupleurum angustissimum

T1685 Coreopsis lanceolata

T0546 Apium leptophyllum

T3911 Lonicera hypoglauca

T3434 Inula linariaefolia

T5293 Pteris linearis

T2560 Eupatorium glandulosum

T3028 Gnetum gnemon

T2170 Dictyota linearis

T1838 Croton balsamifera

T2958 Geum urbanum

T3479 Isodon amethystoides

T0757 Asperula odorata

T3602 Juniperus thurifera var. africana

T2336 Elephas maximus

T2237 Dipteryx odorata

T4174 Melissa officinalis

T1978 Cyperus rotundus

T2310 Dysoxylum hongkongense

T4551 Osmorhiza aristata var. laxa

T2337 Elephas maximus

T0295 Albizzia odoratissima 


\begin{tabular}{|c|c|c|c|}
\hline XIANG HUANG LIAN MU & 香黄连木 & Mastic-tree & T4980 Pistacia lentiscus \\
\hline XIANG JIA PI & 香加皮 & Chinese Silkvine Root-bark & T4729 Periploca sepium \\
\hline \multirow[t]{2}{*}{ XIANG JIAO } & 香蕉 & Common Banana & T4331 Musa paradisiaca var. sapientum [Syn. \\
\hline & & & Musa sapientum] \\
\hline XIANG JIAO SHU & 橡胶树 & Para Rubbertree & T3236 Hevea brasiliensis \\
\hline XIANG LI & 香藜 & Feathered Geranium & T1362 Chenopodium botrys \\
\hline XIANG LING CAO & 响铃草 & Rust-coloured Crotalaria & T1819 Crotalaria ferruginea \\
\hline XIANG MAN TUO LUO & 香曼陀罗 & Fragrant Datura* & T2040 Datura metaloides \\
\hline XIANG MAO & 香茅 & Lemongrass & T1939 Cymbopogon citratus \\
\hline XIANG MO ZHI JU & 香膜质菊 & & T3326 Hymenoxys odorata \\
\hline XIANG NING MENG & 香柠檬 & Bergamot Orange & T1470 Citrus bergamia \\
\hline XIANG PI MU & 象皮木 & Common Alstonia & T0374 Alstonia scholaris \\
\hline XIANG QI JIA MI & 香气荚蒾* & Sweet Viburnum* & T6738 Viburnum odoratissimum \\
\hline XIANG QING LAN & 香青兰 & Dragonhead & T2257 Dracocephalum moldavicum \\
\hline XIANG RI KUI HUA & 向日葵花 & Sunflower Flower & T3142 Helianthus annuus \\
\hline XIANG RI KUI JING SUI & 向日葵茎髓 & Sunflower Stem Pith & T3143 Helianthus annuus \\
\hline XIANG RI KUI YE & 向日葵叶 & Sunflower Leaf & T3144 Helianthus annuus \\
\hline XIANG RI KUI ZI & 向日葵籽 & Sunflower Seed & T3145 Helianthus annuus \\
\hline XIANG ROU & 象肉 & Elephant Meat & T2338 Elephas maximus \\
\hline XIANG ROU GUO & 香肉果 & Edible Casimiroa & T1226 Casimiroa edulis \\
\hline XIANG RU & 香薷 & Haichow Elsholtzia & T2343 Elsholtzia splendens \\
\hline \multirow[t]{3}{*}{ XIANG SI CAO } & 香丝草 & Bona Conyza & T1656 Conyza bonariensis [Syn. Erigeron \\
\hline & & & bonariensis; Erigeron linifolius; Erigeron \\
\hline & & & crispus] \\
\hline XIANG SI TENG & 相思藤 & Coralhead Plant Vine & T0011 Abrus precatorius \\
\hline XIANG SI ZI & 相思子 & Coralhead Plant & T0012 Abrus precatorius \\
\hline XIANG TANG SONG CAO & 香唐松草 & Tibetan Meadowrue & T6386 Thalictrum foetidum \\
\hline XIANG TIE KUAI ZI & 香铁筷子 & Odorous Hellebore* & T3184 Helleborus odorus \\
\hline XIANG WAN DOU & 香踠豆 & Sweet Pea & T3707 Lathyrus odoratus \\
\hline XIANG XUN & 香草 & Champignon & T3742 Lentinus edodes \\
\hline XIANG YA HAI AN GE MU & 象牙海岸格木 & Ivory Coast Erythrophleum & T2486 Erythrophleum ivorense \\
\hline XIANG YANG & 香杨 & Korea Poplar & T5154 Populus koreana \\
\hline XIANG YANG MEI & 香杨梅 & Bog-myrtle & T4344 Myrica gale \\
\hline XIANG YE & 香叶 & Roce Pelargonium & T4690 Pelargonium graveolens \\
\hline XIANG YE YANG & 响叶杨 & Chinese Aspen & T5144 Populus adenopoda \\
\hline XIANG YIN YU & 香茵芋 & Japanese Skimmia & T5970 Skimmia japonica \\
\hline XIANG YING ZHAO & 香鹰爪 & Fragrant Tailgrape* & T0656 Artabotrys suaveolens \\
\hline XIANG YUAN & 香侬 & Wilson Citron & T1520 Citrus wilsonii \\
\hline XIANG YUAN ZHI SHI & 香侬枳实 & Wilson Orange Young Fruit & T1521 Citrus wilsonii \\
\hline \multirow[t]{2}{*}{ XIANG ZHANG } & 香樟 & Yellow Cinnamon & T1443 Cinnamomum parthenoxylum [Syn. \\
\hline & & & Cinnamomum porrectum] \\
\hline XIANG ZHI LENG SHAN & 香脂冷杉 & Balsam Fir & T0004 Abies balsamea \\
\hline XIANG ZI LAN & 香子兰 & Vanilla & T6684 Vanilla planifolia \\
\hline XIAO BA JIAO LIAN & 小八角莲 & Dwarf Many-flowered May-apple & T2298 Dysosma difformis \\
\hline XIAO BAI BU & 小百部 & Officinal Asparagus & T0752 Asparagus officinalis \\
\hline \multirow[t]{2}{*}{ XIAO BAI CHENG } & 小白撑 & Unequalhair Paoshan Monkshood & T0116 Aconitum nagarum var. heterotrichum \\
\hline & & & [Syn. Aconitum bullatifolium $]$ \\
\hline XIAO BANG XIOU QIOU HAI & 小棒绣球海绵 & Sponge Iotrochota baculifera & T3442 Iotrochota baculifera \\
\hline \multicolumn{4}{|l|}{ MIAN } \\
\hline XIAO BO & 小檗 & Amur Barberry & T0897 Berberis amurensis \\
\hline XIAO BO SI SHI SUAN & 小波斯石蒜 & Small Ungernia* & T6641 Ungernia minor \\
\hline XIAO CAO WU & 小草乌 & Yunnan Larkspur & T2091 Delphinium yunnanense \\
\hline XIAO CHAO CAI & 小巢菜 & Pigeon Vetch & T6749 Vicia hirsuta \\
\hline
\end{tabular}




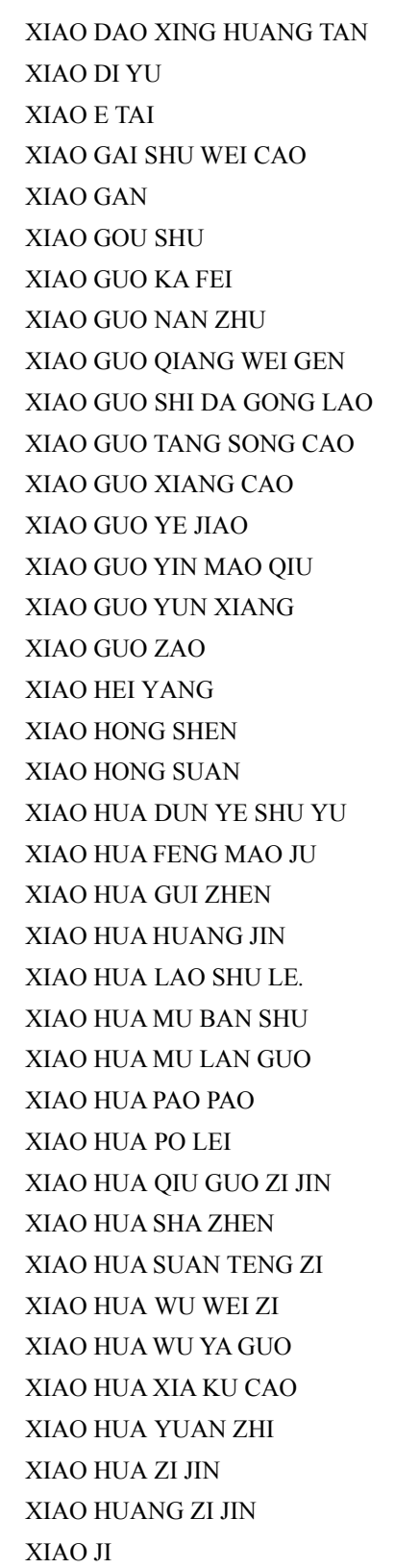

Cultrate Rosewood*

Small Burnet*

Microcap Sage*

Falcate Micromelum

Kazinoki Papermulberry

Arabian Coffeetree

Littlefruit Lyonia

Smallfruit Rose

Bodinier Mahonia

Smallfruit Meadowrue

Smallfruit Loosestrife*

Acuminate Banana

Fishhook Cactus

Smallfruit Rue*

Littlefruit Jujube

Slamm Black Poplar

Yunnan Madder

American Eleutherine

Smallflower Yam

Smallflower Saussurea*

Smallflower Beggarticks

Racemose Corydalis

Smallflower Acanthus*

Smallflower Xylopia*

Smallflower Bruguiera Fruit*

Smallflower Pawpaw

Kongu

Fine-leaved Fumitory

East African Sandalwood

Smallflower Embelia

Smallflower Magnoliavine*

Pentagynous Dillenia

Smallflower Bugle*

Smallflower Milkwort

Micranthine Corydalis*

Small Ochotsk Corydalis

Setose Thistle

Bulbiferous Stonecrop

Black-spot Hornpoppy

Creeping Ceratostigma, Creeping

Bluesnow

Selago-like Climbing Fern

Field Marigold

Small Gorse*

Verticillate Cladonia

Erect St.John'swort

Dracunculi-leaf Pluchea*

Viatic Nightshade*

Wheat
T2001 Dalbergia cultrata

T5711 Sanguisorba minor

T4340 Mylia taylorii

T5679 Salvia microstegia

T4221 Micromelum falcatum

T1031 Broussonetia kazinoki

T1608 Coffea arabica

T3992 Lyonia ovalifolia var. elliptica

T5563 Rosa cymosa

T4058 Mahonia bodinieri

T6398 Thalictrum microgynum

T4003 Lysimachia microcarpa

T4329 Musa acuminata

T4097 Mammillaria microcarpa

T5627 Ruta microcarpa

T6923 Zizyphus oenoplia

T5166 Populus xiaohei

T5586 Rubia yunnanensis

T2339 Eleutherine americana

T2206 Dioscorea parviflora

T5763 Saussurea parviflora

T0939 Bidens parviflora

T1733 Corydalis racemosa

T0047 Acanthus ebracteatus

T6854 Xylopia parviflora

T1040 Bruguiera parviflora

T0743 Asimina parviflora

T3280 Hopea parviflora

T2808 Fumaria parviflora

T4556 Osyris tenuifolia

T2347 Embelia parviflora

T5795 Schisandra micrantha

T2182 Dillenia pentagyna

T0268 Ajuga parviflora

T5087 Polygala telephioides

T1725 Corydalis micrantha

T1729 Corydalis ochotensis var. raddeana

T1451 Cirsium setosum [Syn. Cerratula

setosa; Cirsium segetum; Cephalanoplos

segetum]

T5852 Sedum bulbiferum

T2966 Glaucium corniculatum

T1327 Ceratostigma minus

T3294 Huperzia selago [Syn. Lycopodium selago]

T1112 Calendula arvensis

T6591 Ulex minor

T1529 Cladonia verticillata

T3349 Hypericum erectum

T0844 Baccharis dracunculifolia

T6019 Solanum viarum

T6544 Triticum aestivum [Syn. Triticum vulgare]

T5986 Smyrnium olusatrum 


\begin{tabular}{|c|c|}
\hline XIAO MI CAO & 小米草 \\
\hline XIAO MU MA HUANG & 小木麻黄 \\
\hline XIAO PO PO NA & 小婆婆纳 \\
\hline XIAO QIAO MU ZI JIN NIU & 小乔木紫金牛 \\
\hline XIAO QING YANG & 小青杨 \\
\hline XIAO QIU BAI BU & 小丘百部 \\
\hline XIAO RU XIANG & 肖乳香 \\
\hline XIAO SHA DONG QING & 小沙冬青 \\
\hline XIAO SHE JU GEN & 小舌菊根 \\
\hline XIAO SHE ZI WAN & 小舌紫苑 \\
\hline XIAO TANG SONG CAO & 小唐松草 \\
\hline XIAO TOU DUI XIN JU & 小头堆心菊 \\
\hline XIAO TOU LIANG HOU CHA & 小头凉喉茶 \\
\hline XIAO TOU TIE ZI & 小头铁仔* \\
\hline XIAO WU MAO JUE & 小乌毛蕨* \\
\hline XIAO XI CANG ER & 小溪苍耳* \\
\hline XIAO XIAN AO DING ZAO & 小腺凹顶藻 \\
\hline XIAO XIN YE SHU & 小心叶薯 \\
\hline XIAO XING HUA YAN QIANG & 小形花岩蓄薇* \\
\hline WEI & \\
\hline XIAO XUAN CAO GEN & 小萱草根 \\
\hline XIAO XUE REN SHEN & 小雪人参 \\
\hline XIAO YE CEN & 小叶梣 \\
\hline XIAO YE DI BU RONG & 小叶地不容 \\
\hline XIAO YE DU LIAN & 小叶杜楝 \\
\hline XIAO YE DU YING & 小叶杜英* \\
\hline XIAO YE GUAN ZHONG & 小叶贯众 \\
\hline XIAO YE HEI CHAI HU & 小叶黑柴胡 \\
\hline XIAO YE HONG GUANG SHU & 小叶红光树 \\
\hline XIAO YE HUA & 笑愿花 \\
\hline XIAO YE HUA BEI XIU XIAN JU & 小叶华北绣线菊 \\
\hline XIAO YE HUANG YANG & 小叶黄杨 \\
\hline XIAO YE JI WEI & 小野鸡尾 \\
\hline XIAO YE JIU LI XIANG & 小叶九里香 \\
\hline XIAO YE JU HAO & 小叶菊蒿 \\
\hline XIAO YE LIE LAN & 小叶裂榄 \\
\hline XIAO YE MAI MA TENG & 小叶买麻藤 \\
\hline XIAO YE PI PA & 小叶枇杷 \\
\hline XIAO YE SHI NAN & 小叶石楠 \\
\hline XIAO YE TANG SONG CAO & 小叶唐松草 \\
\hline XIAO YE XIANG CHA CAI & 小叶香茶菜 \\
\hline XIAO YE XUAN GOU ZI & 小叶悬钩子 \\
\hline XIAO YE YANG & 小叶杨 \\
\hline XIAO YE YE JUE MING & 小叶野决明 \\
\hline XIAO YE YING MAO QI MU & 小叶硬毛桤木 \\
\hline XIAO YE ZHI MA & 小野芝麻 \\
\hline XIAO YUN MU & 小芸木 \\
\hline XIAO ZHU SHI DOU & 小猪屎豆 \\
\hline XIE BAI & 茢白 \\
\hline IE CAO & 顺草 \\
\hline
\end{tabular}

Meadow Eyebright

Small Beefwood*

Thymeleaf Speedwell

Small-tree Ardisia*

False Simon Poplar

Hill Stemona*

Brazilian Peppertree

Small Piptanthus*

Pearleaf Microglossa Root

Smallligulatecorolla Aster

Low Meadowrue

Littlehead Sneezeweed

Capitellate Hedyotis

Capitellate Myrsine*

Minus Hard-fern*

Ripply Cocklebur*

Small-grand Concave-top Alga*

Smallheartleaved Morningglory

Small Yellow Daylily

Woolly Lespedeza

Bunga Ash Bark

Littleleaf Stephania

Littleleaf Starbush*

Small-leafElaeocarpus*

Matteuccia Frond

Smallleaf Black Thorowax

Small-leaf Knema

Bridalwreath Spiraea

Smallleaf Fritsch Spiraea*

Small-leaved Box

Japanese Clave Fern

Littleleaf Common Jasminorange

Small-leaf Tansy*

Elephant Tree

Smallleaf Jointfir

Savoury Rhododendron

Small-leaf Photinia*

Small-leaf Meadowrue

Smallleaf Rabdosia*

Small-leaf Raspberry

Simon Poplar

Chinese Thermopsis

Smallleaf-hirsute Alder*

China Weasel-snout

Entire Micromelum

Small Rattle-box

Longstamen Onion

Common Valeriana
T2628 Euphrasia officinalis

T1259 Casuarina stricta

T6728 Veronica serpyllifolia

T0591 Ardisia arborescens

T5157 Populus pseudo-simonii

T6109 Stemona collinsae

T5789 Schinus terebinthifolius

T4975 Piptanthus nanus

T4218 Microglossa pyrifolia

T0778 Aster albescens

T6399 Thalictrum minus

T3139 Helenium microcephalum

T3124 Hedyotis capitellata

T4362 Myrsine capitellata

T0951 Blechnum minus

T6842 Xanthium riparium

T3717 Laurencia glandulifera

T3450 Ipomoea obscura

T1459 Cistus parviflorus

T3197 Hemerocallis minor

T3774 Lespedeza tomentosa

T2766 Fraxinus bungeana

T6135 Stephania succifera

T6571 Turraea parvifolia

T2330 Elaeocarpus parvifolius

T4127 Matteuccia struthiopteris

T1075 Bupleurum smithii var. parvifolium

T3631 Knema globularia

T6084 Spiraea prunifolia

T6078 Spiraea fritschiana var. parvifolia

T1091 Buxus microphylla

T4502 Onychium japonicum [Syn. Tricomanes

japonicum]

T4325 Murraya paniculata var. exotica

T6293 Tanacetum microphyllum

T1081 Bursera microphylla

T3033 Gnetum parvifolium [Syn. Gnetum

indicum]

T5502 Rhododendron anthopogonoides

T4827 Photinia parvifolia

T6380 Thalictrum elegans

T3517 Isodon parvifolia

T5601 Rubus taiwanicolus

T5158 Populus simonii

T6426 Thermopsis chinensis

T0327 Alnus hirsute var. microphylla

T2825 Galeobdolon chinense [Syn. Lamium

chinense]

T4223 Micromelum integerrimum

T1827 Crotalaria nana

T0316 Allium macrostemon

T6679 Valeriana officinalis 


\begin{tabular}{|c|c|}
\hline XIE FEI ZI YU PAN & 谢飞紫玉盘* \\
\hline XIE JI CU YE MU & 斜基粗叶木 \\
\hline XIE JING ZHANG YA CAI & 斜茎獐牙菜 \\
\hline XIE KE & 蟹壳 \\
\hline XIE PU TAO & 楔葡萄 \\
\hline XIE SHI ZI JIN & 谢氏紫堇 \\
\hline XIE WEI JU & 蝎尾菊 \\
\hline XIE XING WU TOU & 楔形乌头* \\
\hline XIE YE ZE LAN & 楔叶泽兰 \\
\hline XIE YU FENG WEI JUE & 斜羽凤尾蕨 \\
\hline XIE ZHUA LAN & 蟹爪兰 \\
\hline XIN FEI CAO & 辛菲草 \\
\hline XIN HE LAN HU JIAO & 新荷兰胡椒* \\
\hline XIN JIANG BEI MU & 新疆贝母 \\
\hline XIN JIANG DANG SHEN & 新疆党参 \\
\hline XIN JIANG GAO BEN & 新疆䕁本 \\
\hline XIN JIANG LAN CI TOU & 新疆蓝刺头 \\
\hline XIN JIANG YANG & 新疆杨 \\
\hline XIN JIANG YI ZHI HAO & 新疆一支蒿 \\
\hline XIN MEI FENG MAO JU & 新美风毛菊* \\
\hline XIN NONG XIANG MAO & 信浓香茅 \\
\hline XIN SHAO NA CAO & 新哨纳草 \\
\hline XIN SU GE LAN XUE GUO MU & 新苏格兰穴果木 \\
\hline XIN XI LAN LUO HAN SONG & 新西兰罗汉松 \\
\hline XIN XI LAN MA & 新西兰麻 \\
\hline XIN XI LAN MU JING & 新西兰牡荆 \\
\hline XIN XI LAN YE TAI & 新西兰叶苔* \\
\hline XIN XING BAN GE NA XIANG & 心形瓣哥纳香 \\
\hline XIN XING GOU TENG & 心形钩藤* \\
\hline XIN XING PU TAO & 心形蒲桃* \\
\hline XIN XING SHEN YE YE TAI & 心形伸叶叶苔* \\
\hline XIN XING YE QING NIU DAN & 心形叶青牛胆* \\
\hline XIN YE CHI BO & 心叶赤爮 \\
\hline XIN YI & 辛夷 \\
\hline XIN YUE XING YE CAO SU & 新月型叶糙苏* \\
\hline XIN ZANG JIA ZI CAO & 新藏假紫草 \\
\hline XING AN BAI TOU WENG & 兴安白头翁 \\
\hline XING AN CHAI HU & 兴安柴胡 \\
\hline XING AN SHENG MA & 兴安升麻 \\
\hline XING CAI & 苦菜 \\
\hline XING JUE & 星蕨 \\
\hline XING REN & 杏仁 \\
\hline XING SHU GEN & 杏树根 \\
\hline XING SU CAO & 星粟草 \\
\hline XING ZI & 杏子 \\
\hline XIONG DAN & 熊胆 \\
\hline XIONG ER CAO & 熊耳草 \\
\hline XIONG GUO & 熊果 \\
\hline XIONG HUANG LAN & 雄黄兰 \\
\hline XIONG RUI ZHUANG SHU WEI & 雄蒿状鼠尾草* \\
\hline $\mathrm{CAO}$ & \\
\hline XIONG RUI ZHUANG ZHI & 雄芯状直管草 \\
\hline
\end{tabular}

Scheffleri Uvaria*

Wallich Lasianthus

Spreading Swertia*

Mitten Crab Chelae

Coigne Grape*

Sewerzow Corydalis*

Linear Koelpinia

Cuneate Monkshood*

Cuneateleaf Eupatorium*

Oblique Pinna Brake

Crab-craw Orchis*

Caucasian Comfrey

Novel-Holland Pepper*

Sinkiang Fritillary

Clematis Asiabell

Vaginate Hemlockparsley

Sinkiang Globethistle

Sinkiang Poplar*

Rock Wormwood

New Beauty Saussurea*

Senna Lemongrass*

Fringecups

Newcaledonian Coelospermum*

New Zealand Podocarpus*

New Zealand Flax

Puriri

Cardia-petal Goniothalamus*

Cordate Gambirplant*

Cordate Syzygium*

Cardialeaf Tinospora*

Heartleaf Tubergourd

Lily Magnolia Buds

Sinkiang-Tibet Arnebia

Dahurian Pulsatilla*

Siberia Thorowax

Dahurian Bugbane

Shield Floatingheart

Punctated Microsorium*

Apricot Seed

Apricot Root

Stellate hair Carpetweed

Apricot

Bear Gall

Mexican Ageratum

Bearberry

Common Tritonia

Staminate Sage*

Big-flowered Javatea
T6667 Uvaria scheffleri

T3698 Lasianthus wallichii

T6229 Swertia patens

T2435 Eriocheir sinensis

T6795 Vitis coignetiae

T1741 Corydalis sewerzowi

T3634 Koelpinia linearis

T0134 Aconitum subcuneatum

T2557 Eupatorium cuneifolium

T5296 Pteris oshimensis

T5806 Schlumbergera truncata

T6245 Symphytum caucasicum

T4958 Piper nove-hollandae

T2800 Fritillaria walujewii

T1597 Codonopsis clematidea

T1641 Conioselinum vaginatum

T2317 Echinops ritro

T5146 Populus alba var. pyramdalis

T0692 Artemisia rupestris [Syn. Artemisia dentata;

Artemisia viridis; Artemisia viridifolia]

T5761 Saussurea neopulchella

T1947 Cymbopogon sennaarensis

T6328 Tellima grandifolia

T1607 Coelospermum billardieri

T5038 Podocarpus ferrugineu

T4825 Phormium tenax

$\mathrm{T} 6785$ Vitex lucens

T3580 Jungermannia sp.

T3043 Goniothalamus cardiopetalus

T6612 Uncaria cordata

T6265 Syzygium cordatum

T3579 Jungermannia exsertifolia ssp. cordifolia

T6464 Tinospora cordifolia

T6435 Thladiantha cordifolia

T4041 Magnolia liliflora

T4809 Phlomis lunariifolia

T0648 Arnebia euchroma

T5326 Pulsatilla dahurica

T1073 Bupleurum sibiricum

T1419 Cimicifuga dahurica

T4456 Nymphoides peltatum

T4225 Microsorium punctatum

T5216 Prunus armeniaca

T5217 Prunus armeniaca

T2984 Glinus lotoides [Syn. Mollugo lotoides]

T5218 Prunus armeniaca

T5871 Selenarctos thibetanus; Ursus arctos

T0230 Ageratum houstonianum

T0588 Arctostaphylos uva-ursi

T6548 Tritonia crocosmaeflora

T5695 Salvia staminea

T4541 Orthosiphon stamineus [Syn. 
GUAN CAO

\begin{tabular}{|c|c|}
\hline XIONG ZHANG & 熊掌 \\
\hline XIU HONG QIANG WEI & 锈红蓄薇* \\
\hline XIU LI YING ZHAO & 秀丽鹰爪* \\
\hline XIU MAO DI HUANG & 锈毛地黄 \\
\hline XIU MAO JI SHENG & 锈毛寄生 \\
\hline XIU MAO YE TONG & 锈毛野桐 \\
\hline XIU QIU QIAN CAO & 绣球茜草 \\
\hline XIU QIU SHU WEI CAO & 绣球鼠尾草* \\
\hline XIU SE AN XI XIANG & 锈色安息香 \\
\hline XIU XIAN JU & 绣线菊 \\
\hline XIU XIAN JU YE & 绣线菊叶 \\
\hline XU CHANG QING & 徐长卿 \\
\hline XU DUAN & 续断 \\
\hline XU DUAN JU & 续断菊 \\
\hline XU LI YA MA LI JIN & 叙利亚马利筋 \\
\hline XU LI YA NIU ZHI & 叙利亚牛至* \\
\hline XU SUI ZI JING ZHONG BAI ZHI & 续随子茎中白汁 \\
\hline XUAN CAO GEN & 萱草根 \\
\hline XUAN CHUI GEN NAI LA CAO & 悬垂根乃拉草* \\
\hline XUAN CHUI JIA MI & 悬垂荚蒾 \\
\hline XUAN FU HUA & 旋覆花 \\
\hline XUAN GOU ZI & 悬钩子 \\
\hline XUAN GUO WEN ZI CAO & 旋果蚊子草 \\
\hline XUAN HUA YANG JIAO AO & 旋花羊角拗 \\
\hline XUAN NIU XIE HAO & 旋扭邪蒿 \\
\hline XUAN SHEN & 玄参 \\
\hline XUAN WEI WU TOU & 宣威乌头 \\
\hline XUAN YA MEI GUI & 悬崖玫瑰* \\
\hline XUAN YE XIANG QING & 旋叶香青 \\
\hline XUE BAI XIANG RI KUI & 雪白向日葵* \\
\hline XUE CHA & 雪茶 \\
\hline XUE GUANG HUA & 雪光花 \\
\hline XUE HONG SHUAN JUN & 血红栓菌 \\
\hline XUE HUA LIAN & 雪花莲 \\
\hline XUE LIAN & 雪莲 \\
\hline XUE LING HAO & 雪岭蒿 \\
\hline XUE LING ZHI & 雪灵芝 \\
\hline XUE LONG DAN & 雪龙胆 \\
\hline XUE PIAN LIAN & 雪片莲 \\
\hline XUE SAN SHU & 血散薯 \\
\hline XUE SHAN LIN & 雪山林 \\
\hline XUE SHANG YI ZHI HAO & 雪上一支蒿 \\
\hline XUE SHENG SHUI XIAN & 雪生水仙 \\
\hline XUE SONG & 雪松 \\
\hline XUE TONG & 血桐 \\
\hline XUE WEI CAI & 雪维菜 \\
\hline XUE YU & 血余 \\
\hline XUN DAO NIU & 熏倒牛 \\
\hline XUN ZHUANG SHAN YL & 荁状山缘草 \\
\hline
\end{tabular}

Orthosiphon aristatus; Orthosiphon grandiflorus; Orthosiphon spicatus] T5872 Selenarctos thibetanus; Ursus arctos T5571 Rosa rubiginosa T0658 Artabotrys venustus T2174 Digitalis ferruginea T6304 Taxillus levinei T4079 Mallotus anomalus T2294 Dunnia sinensis T5675 Salvia hydrangea T6198 Styrax ferrugineus T6079 Spiraea japonica T6080 Spiraea japonica T1959 Cynanchum paniculatum T2235 Dipsacus japonicus T6026 Sonchus asper [Syn. Sonchus oleraceus var. asper]

T0740 Asclepias syriaca

T4525 Origanum syriacum

T2598 Euphorbia lathyris

T3193 Hemerocallis fulva T3073 Gunnera perpensa

Weeping Viburnum*

British Inula

Avrons

Queen-of-the-Meadows

Spinyflower Strophanthus

Tortuous Seseli*

Ningpo Figwort

Haoryanther Paoshan Monkshood

Cliffrose

Coiledleaf Pearleverlasting

Snowwhite Sunflower*

Vermiculate Thamnolia Thallus

Glory-of-the-snow

Snowdrop
Snow Lotus
Schrenk Wormwood*
Kansu Sandwort
Snaw Gentian
Spring Snowflake
Diels Stephania
Japanese Pachysandra
Shortstalk Monkshood
Deodar Cedar
Common Macaranga
Garden Chervil
Human Hair
Heterostemonous Biebersteinia
Climbing Fumitory

T6740 Viburnum suspensum

T3427 Inula britannica

T5589 Rubus chamaemorus

T2729 Filipendula ulmaria

T6156 Strophanthus gratus

T5936 Seseli tortuosum

T5828 Scrophularia ningpoensis

T0117 Aconitum nagarum var. lasiandrum

T1766 Cowania mexicana

T0445 Anaphalis contorta

T3151 Helianthus niveus

T6416 Thamnolia vermicularis

T1368 Chionodoxa luciliae

T5339 Pycnoporus sanguineus

T2822 Galanthus nivalis

T5755 Saussurea involucrata

T0694 Artemisia schrenkiana

T0608 Arenaria kansuensis [Syn. Arenaria

kumaonensis]

T2922 Gentiana nivalis

T3782 Leucojum vernum

T6122 Stephania dielsiana

T4575 Pachysandra terminalis

T0079 Aconitum brachypodum

T4376 Narcissus nivalis

T1282 Cedrus deodara

T4013 Macaranga tanarius

T0528 Anthriscus cerefolium

T3278 Homo sapiens

T0942 Biebersteinia heterostemon

T0183 Adlumia cirrhosa [Syn. Adlumia fungosa] 


\begin{tabular}{|c|c|}
\hline YA DAN ZI & 鸦胆子 \\
\hline YA DONG WU TOU & 亚东乌头 \\
\hline YA ER QIN & 鸭儿芹 \\
\hline YA HU NU & 亚乎奴 \\
\hline YA JIAO AI & 鸭脚艾 \\
\hline YA KE BEI QIAN LI GUANG & 牙克贝千里光 \\
\hline YA LI QIAN JIN TENG & 雅丽千金藤 \\
\hline YA LI SANG NA DUI XIN JU & 亚利桑那堆心菊* \\
\hline YA LIE XING HAO & 亚列兴蒿 \\
\hline YA LUO CHUN & 亚罗椿 \\
\hline YA MA & 亚麻 \\
\hline YA MA XUN BAI HE & 亚马逊百合* \\
\hline YA MA XUN MA QIAN ZI & 亚马逊马钱子 \\
\hline YA MA XUN YU TENG & 亚马逊鱼藤* \\
\hline YA MA ZI & 亚麻子 \\
\hline YA MAI JIA DU YU DOU & 牙买加毒鱼豆 \\
\hline YA MAI JIA KU MU & 牙买加苦木 \\
\hline YA MAI JIA YING TAO & 牙买加樱桃 \\
\hline YA MEI DUI XIN JU & 雅美堆心菊* \\
\hline YA PIAN & 鸦片 \\
\hline YA RONG GAI RU GU & 亚线盖乳菇 \\
\hline YA ZHI CAO & 鸭跖草 \\
\hline YA ZHI TUO WU & 雅致㯻吾* \\
\hline YA ZHOU DU HUO & 亚洲独活* \\
\hline YA ZHOU SHI & 亚洲萻 \\
\hline YA ZHOU WEN SHU LAN & 亚洲文殊兰* \\
\hline YAN BAI CAI & 岩白菜 \\
\hline YAN CAO & 烟草 \\
\hline YAN CAO HUA SHAN GENG CAI & 烟草花山梗菜* \\
\hline YAN DI FENG MAO JU & 盐地风毛菊 \\
\hline YAN DI HE & 盐地禾 \\
\hline YAN FENG & 岩凤 \\
\hline YAN FU YE & 盐麸叶 \\
\hline YAN FU ZI & 盐麸子 \\
\hline YAN GUO CAO & 烟锅草 \\
\hline YAN HAI TUN CAO & 沿海豚草 \\
\hline YAN HU SUO & 延胡索(元胡) \\
\hline YAN HUANG LIAN & 岩黄连 \\
\hline YAN JIAO CAO & 岩椒草 \\
\hline YAN JIAO CAO SUO SUO & 盐角草梭梭* \\
\hline YAN JIN & 烟堇 \\
\hline YAN JIN CAI & 岩筋菜 \\
\hline YAN LAI HONG & 雁来红 \\
\hline YAN LAN CAO & 岩兰草 \\
\hline YAN LING CAO & 延龄草 \\
\hline YAN MAI & 燕麦 \\
\hline YAN MU & 胭木 \\
\hline YAN QU MEI & 烟曲霉 \\
\hline YAN SE LONG YAN & 烟色龙眼 \\
\hline YAN SHENG JIA MU ZEI & 盐生假木贼 \\
\hline YAN SHENG ROU CONG & 盐生肉苁蓉 \\
\hline
\end{tabular}

Java Brucea

Yadong Monkshood

Japanese Cryptotaenia

Hirsute Cissampelos*

Ghostplant Wormwood

Alpine Groundsel*

Elegant Stephania

Arizona Sneezeweed*

Sublessing Wormwood*

Bacciform Cipadessa

Common Flax

Amazon lily

Amazonian Poisonnut*

Amazonian Jewelvine*

Common Flax Seed

Piscidia erythrina

Jamaica Quassiawood

Jamaica Cherry*

Elegant Sneezeweed*

Opium

Subtomentose Milky*

Common Dayflower

Elegant Goldenray*

Asian Cowparsnip*

Asiatic Yarrow

Grand Crinum

Purple Bergenia

Common Tobacco

Nicotianflower Lobelia*

Saline Saussurea

Big Cord-grass

Buchtorm Libanotis

Chinese Sumac Leaf

Chinese Sumac Fruit

East-Asia Low Meadowrue

Oak-of-Cappadocia

Yanhusuo

Rockliving Corydalis

White Chinaure

Schleicher Fumitory

Tibet Berneuxine

Three-coloured Amaranth

Vetiver

Tschonosk Trillium

Oat

Tomentose Wrightia

Smoke Longan*

Saltliving Anabasis

Saline Cistanche
T1038 Brucea javanica [Syn. Brucea

sumatrana; Rhus javanica]

T0077 Aconitum balfourii

T1869 Cryptotaenia japonica

T1453 Cissampelos pareira var. hirsute

T0680 Artemisia lactiflora

T5878 Senecio alpinus

T6125 Stephania elegans

T3132 Helenium arizonicum

T0699 Artemisia sublessingiana

T1446 Cipadessa baccifera

T3859 Linum usitatissimum

T2524 Eucharis amazonica

T6165 Strychnos amazonica

T2117 Derris amazonica

T3860 Linum usitatissimum

T4977 Piscidia erythrina

T4880 Picrasma excelsa

T4314 Muntingia calabura

T3137 Helenium elegans

T4635 Papaver somniferum

T3655 Lactarius subvellereus

T1633 Commelina communis

T3804 Ligularia elegans

T3215 Heracleum lanatum var. asiaticum

T0061 Achillea asiatica

T1794 Crinum asiaticum

T0924 Bergenia purpurascens

T4428 Nicotiana tabacum

T3901 Lobelia nicotianaefolia

T5768 Saussurea salsa

T6063 Spartina cynosuroides

T3796 Libanotis buchtormensis

T5530 Rhus chinensis [Syn. Rhus semialata]

T5531 Rhus chinensis [Syn. Rhus semialata]

T6414 Thalictrum thunbergii

T0403 Ambrosia maritima

T1750 Corydalis yanhusuo [Syn. Corydalis

turtschaninovii f. yanhusuo]

T1747 Corydalis thalictrifolia

T0964 Boenninghausenia albiflora

T3094 Haloxylon salicornicum

T2809 Fumaria schleicheri

T0926 Berneuxia thibetica

T0389 Amaranthus tricolor

T6733 Vetiveria zizanioides

T6535 Trillium tschonoskii

T0833 Avena sativa

T6834 Wrightia tomentosa

T0755 Aspergillus fumigatus

T2184 Dimocarpus fumatus

T0437 Anabasis salsa

T1456 Cistanche salsa 


\begin{tabular}{|c|c|}
\hline YAN TIAN JIE CAI & 盐天芥菜 \\
\hline YAN XIANG JU & 岩香菊 \\
\hline YAN YANG CHUN & 烟洋椿 \\
\hline YAN ZHOU JUAN BAI & 充州卷柏 \\
\hline YANG CHANG CHUN TENG & 洋常春藤 \\
\hline YANG CONG & 洋葱 \\
\hline YANG DAO DOU & 洋刀豆 \\
\hline YANG HONG SHAN & 羊红膻 \\
\hline YANG JIAN QIU LUO & 洋剪秋罗 \\
\hline YANG JIAO AO ZI & 羊角拗子 \\
\hline YANG JIAO MIAN & 羊角棉 \\
\hline YANG JIAO TENG & 羊角藤 \\
\hline YANG JIN HUA & 洋金花 \\
\hline YANG LI & 洋李 \\
\hline YANG MEI & 杨梅 \\
\hline YANG MEI CHANG SHAN & 杨梅常山 \\
\hline YANG MEI SHU PI & 杨梅树皮 \\
\hline YANG OU XIA ZHI CAO & 洋欧夏至草 \\
\hline YANG PI & 羊皮 \\
\hline YANG PU TAO YE & 洋蒲桃叶 \\
\hline YANG QIAN CAO & 洋茜草 \\
\hline YANG QING LAN & 岩青兰 \\
\hline YANG RU & 羊乳 \\
\hline YANG SHI CAO & 洋蓄草 \\
\hline YANG SHI GUO & 羊屎果 \\
\hline YANG TAO & 洋桃 \\
\hline YANG TI & 羊蹄 \\
\hline YANG TONG & 杨桐 \\
\hline YANG WO BAN DENG GUO & 仰卧板発果* \\
\hline YANG XIN TIAN JIE CAI & 仰心天芥菜* \\
\hline YANG YE AN & 杨叶桉 \\
\hline YANG YE XIAO JIN & 杨叶肖槿 \\
\hline YANG YE YIN BEI TENG & 杨叶银背藤 \\
\hline YANG YI & 羊胰 \\
\hline YANG ZI XIAO LIAN QIAO & 扬子小连趐 \\
\hline YAO HUA XIANG CHA CAI & 芫花香茶菜 \\
\hline YAO SHU KUI & 药蜀葵 \\
\hline YAO SHU LI & 药鼠李 \\
\hline YAO SHUI BA JIAO & 药水八角 \\
\hline YAO SHUI SU & 药水苏 \\
\hline YAO SHUI SU JUE CHUANG & 药水苏爵床* \\
\hline YAO XI GUA & 药西瓜 \\
\hline YAO YONG AN XI XIANG & 药用安息香 \\
\hline YAO YONG BAI QIAN & 药用白前 \\
\hline YAO YONG DAN SHEN & 药用丹参* \\
\hline YAO YONG DAN SHEN YE & 药用丹参叶* \\
\hline YAO YONG DANG GUI & 药用当归* \\
\hline YAO YONG DAO TI HU & 药用倒提壶 \\
\hline YAO YONG GAN ZHE & 药用甘蔗* \\
\hline YAO YONG GOU YA HUA & 药用狗牙花 \\
\hline YAO YONG HEI MIAN SHEN YE & 黑面神叶 \\
\hline
\end{tabular}

Curassow Heliotrope*

Lavandulaleaf Chrysanthemum

Cigarbox Cedrela

Involute Spikemoss

English Ivy

Common Onion

Sword-bean

Thellung Pimpinella

German Catchfly

Divaricate Strophanthus Seed

Maire Alstonia

Common Indianmulberry

Hindu Datura Flower

Garden Plum

Chinese Waxmyrtle

Bayberry Glorybower*

Chinese Waxmyrtle Bark

Peregrine Hoarhound

Goat Hide

Samalanga Syzygium

Madder

Rupestrine Dragonhead

Goat Milk

Common Yarrow

Duhat

Carambola; Country Gooseberry

Japanese Dock

Japanese Cleyera

Prostrate Pachysandra*

Supine Heliotrope*

Bimble Box

Portiatree

Poplar-leaf Argyreia

Goat Pancreas

Faber's St.John'swort

Stringbushlike Rabdosia

Marshmallow

Common Buckthorn

Gratiole

Medicinal Betonica

Wild Gourd

Drug Snowbell

White Swallow-wort

Medicinal Sage

Medicinal Sage Leaf

Medicinal Angelica*

Common Houndstongue

Sugarcane

Officinal Ervatamia*

Medicinal Breynia Leaf*
T3169 Heliotropium curassavicum

T1394 Chrysanthemum lavandulifolium

T1277 Cedrela odorata

T5862 Selaginella involvens

T3112 Hedera helix

T0311 Allium cepa

T1168 Canavalia ensiformis

T4902 Pimpinella thelungiana

T3951 Lychnis viscaria

T6155 Strophanthus divaricatus

T0371 Alstonia mairei

T4286 Morinda umbellata

T2044 Datura metel

T5222 Prunus domestica

T4348 Myrica rubra

T1560 Clerodendron myricoides

T4349 Myrica rubra

T4111 Marrubium peregrinum

T1181 Capra hircus; Ovis aries

T6268 Syzygium samarangense

T5584 Rubia tinctorum

T2258 Dracocephalum rupestre

T1182 Capra hircus; Ovis aries

T0065 Achillea millefolium

T6266 Syzygium cumini

T0834 Averrhoa carambola

T5611 Rumex japonicus

T1571 Cleyera ochnacea [Syn. Cleyera japonica]

T4574 Pachysandra procumbens

T3182 Heliotropium supinum

T2516 Eucalyptus populnea

T6432 Thespesia populnea [Syn. Hibiscus populneus]

T0615 Argyreia populifolia

T1183 Capra hircus; Ovis aries

T3350 Hypericum faberi

T3535 Isodon wikstroemioides

T0381 Althaea officinalis

T5454 Rhamnus cathartica

T3061 Gratiola officinalis

T0929 Betonica officinalis

T3605 Justicia betonica

T1460 Citrullus colocynthis

T6202 Styrax officinalis

T6763 Vincetoxicum officinale [Syn.

Cynanchum vincetoxicum]

T5682 Salvia officinalis

T5683 Salvia officinalis

T0489 Angelica officinalis

T1969 Cynoglossum officinale

T5641 Saccharum officinarum

T2445 Ervatamia officinalis

T1026 Breynia officinalis 


\begin{tabular}{|c|c|c|}
\hline YAO YONG MU DAN & 药用牡丹 & Kingsbloom \\
\hline YAO YONG NIU SHE CAO & 药用牛舌草 & Alkanet \\
\hline YAO YONG PU GONG YING & 药用蒲公英 & Officinal Dandelion \\
\hline YAO YONG QIAN HU & 药用前胡 & Hog's Fennel \\
\hline YAO YONG QIU GUO ZI JIN & 药用球果紫堇 & Medicinal Fumaria \\
\hline YAO YONG SHE CHUANG & 药用蛇床 & Medicinal Ligusticum \\
\hline YAO YONG XUN YI CAO & 药用薰衣草 & Medicinal Lavender* \\
\hline YAO YONG ZI TAN & 药用紫檀* & Medicinal Padauk* \\
\hline YE AI HAO & 野艾蒿 & Lavenderleaf Wormwood \\
\hline YE BAI HE & 野百合 & Purpleflower Crotalaria \\
\hline YE CAO MEI & 野草莓 & European Strawberry \\
\hline YE DIAN QIE & 野巔茄 & Soda-apple Nightshade \\
\hline YE DU ZHONG & 野杜仲 & Largeflower Euonymus \\
\hline YE GAN CAO & 野甘草 & Sweet Broomwort \\
\hline YE GU & 野菰 & Indian Aeginetia \\
\hline YE GUAN MEN & 夜关门 & Cuneate Lespedeza \\
\hline YE GUO QIE & 叶果茄* & Leafy-fruited Nightshade \\
\hline YE HAI JIAO & 野海椒 & Twoflower Jerusalemcherry \\
\hline YE HE HUA & 夜合花 & Chinese Magnolia Flower \\
\hline YE HEI YING & 野黑樱 & Wild Rum-cherry \\
\hline YE HEI ZHONG CAO & 野黑种草 & Devil-in-a-bush \\
\hline YE HUA & 夜花 & Nightjasmine \\
\hline YE HUA HUI MAO DOU & 夜花灰毛豆 & Long Inflorescence Tephrosia \\
\hline YE HUA JIAO GEN & 野花椒根 & Flatspine Pricklyash Root \\
\hline YE HUA JIAO PI & 野花椒皮 & Flatspine Pricklyash Bark \\
\hline YE HUA JIAO YE & 野花椒叶 & Flatspine Pricklyash Leaf \\
\hline YE HUANG PI & 野黄皮 & Henry Wampee \\
\hline YE JIANG & 野姜 & Cassumuna Ginger* \\
\hline YE JIAO TENG & 夜交藤 & Tuber Fleeceflower Stem \\
\hline YE JIE & 野芥* & Wild Cabbage \\
\hline YE JU & 野菊 & Indian Wild Chrysanthemum \\
\hline YE JU HUA & 野菊花 & Indian Wild Chrysanthemum Flower \\
\hline YE JUE MING & 野决明 & Wild Thermopsis \\
\hline YE LI ZHI YE & 野梨枝叶 & Callery Pear Branch-leaf \\
\hline YE MAI YIN BEI TENG & 叶脉银背藤* & Veined Argyreia \\
\hline YE MING SHA & 夜明砂 & Bat Dung \\
\hline YE MU GUA & 野木瓜 & Chinese Stauntonvine \\
\hline YE MU XU & 野苜倹 & Sickle Alfalfa \\
\hline YE OU BAI JIE & 野欧白芥 & Charlock \\
\hline YE QI SHU YE & 野漆树叶 & Woods Lcaquertree Leaf \\
\hline YE QI SHU ZI & 野漆树子 & Woods Lcaquertree \\
\hline YE SHAN HUA & 野扇花 & Willowleaf Sarcococca \\
\hline YE SHAN ZHA & 野山楂 & Nippon Hawthorn \\
\hline YE SHENG MA & 野升麻 & Kamchatka Bugbane \\
\hline YE SHENG QIAN LI GUANG & 野生千里光* & Heath Groundsel \\
\hline YE SHENG SHAN YING TAO & 野生山樱桃* & \\
\hline YE SUI XIANG CHA CAI & 叶穗香茶菜 & Leafspike Rabdosia \\
\hline YE TAI & 叶苔 & \\
\hline YE WO JU & 野莴节 & Prickly Lettuce \\
\hline YE WU TONG & 野梧桐 & Japanese Mallotus \\
\hline YE XIA ZHU & 叶下珠 & Common Leafflower \\
\hline YE XIANG GE MU & 叶香格木 & Fragrant Erythrophleum \\
\hline
\end{tabular}

T4587 Paeonia officinalis

T0447 Anchusa officinalis

T6303 Taraxacum officinale

T4764 Peucedanum officinale

T2807 Fumaria officinalis

T1583 Cnidium officinale [Syn. Ligusticum

officinale]

T3728 Lavandula officinalis

T5303 Pterocarpus officinalis

T0681 Artemisia lavandulaefolia

T1831 Crotalaria sessiliflora

T2764 Fragaria vesca

T6014 Solanum surattense

T2541 Euonymus grandiflorus

T5818 Scoparia dulcis

T0195 Aeginetia indica

T3770 Lespedeza cuneata

T6011 Solanum sarrachoides

T5993 Solanum capsicastrum

T4036 Magnolia coco

T5240 Prunus serotina

T4430 Nigella arvensis

T4453 Nyctanthes arbor-tristis

T6337 Tephrosia noctiflora

T6893 Zanthoxylum simulans

T6894 Zanthoxylum simulans

T6895 Zanthoxylum simulans

T1533 Clausena dentata

T6908 Zingiber cassumunar

T5108 Polygonum multiflorum

T1013 Brassica oleracea

T1392 Chrysanthemum indicum

T1393 Chrysanthemum indicum

T6429 Thermopsis lupinoides

T5363 Pyrus calleryana

T0614 Argyreia nervosa

T6732 Vespertilio superans

T6099 Stauntonia chinensis

T4147 Medicago falcata

T5959 Sinapis arvensis

T5537 Rhus sylvestris

T5538 Rhus sylvestris

T5732 Sarcococca saligna

T1769 Crataegus cuneata

T1425 Cimicifuga simplex

T5913 Senecio sylvaticus

T5241 Prunus serrulata var. spontanea

T3519 Isodon phyllostachys

T6550 Trocholejeunea sandvicensis

T3663 Lactuca serriola

T4082 Mallotus japonicus

T4843 Phyllanthus urinaria

T2487 Erythrophleum suaveolens 


\begin{tabular}{|c|c|c|}
\hline YE XIANG MAO & 野香茅 & Goering Lemongrass \\
\hline YE XIANG SHU & 夜香树 & Nightblooming Cestrum \\
\hline YE YA CHUN & 野鸦椿 & Common Euscaphis \\
\hline YE YAN YE & 野烟叶 & Mullein Nightbrier Leaf \\
\hline YE YING SU & 野篑粟 & Iceland Poppy \\
\hline YE YU & 野芋 & Taro \\
\hline YE ZHI MA & 野芝麻 & Barbate Deadnettle \\
\hline YE ZHU DAN & 野猪胆 & Wild Boar Gall \\
\hline YE ZI & 椰子 & Coconut \\
\hline YE ZI PI & 椰子皮 & Coconut Root-bark \\
\hline YE ZI RANG & 梆子襄瓜 & Coconut Albumen \\
\hline YE ZI YOU & 椰子油 & Coconut Oil \\
\hline YI BAO MA HUA TOU & 缢苍麻花头 & Contracted Sawwort \\
\hline YI BEI MU & 伊贝母 & Siberian Fritillary \\
\hline YI BI LI YA LI & 伊比利亚栋 & Iberian Oak* \\
\hline YI BI LI YA SHUI XIAN & 伊比利亚水仙 & Iberian Narcissus* \\
\hline YI BIAN HE SHE ZAO & 易变褐舌藻* & \\
\hline YI BIAN HUANG TAN & 易变黄檀 & Variable Rosewood* \\
\hline YI CAO & 曧草 & Reed Canary-grass \\
\hline YI CHI LING JU & 翼翅苓菊* & Winged Jurinea* \\
\hline YI DA LI JIASNG NAN XING & 意大利疆南星 & Italian Arum \\
\hline YI DA LI JUE MING ZI & 意大利决明子 & Italian Senna* \\
\hline YI DA LI LA JU & 意大利蜡菊* & Italian Everlasting* \\
\hline YI DIAN HONG & 一点红 & Sowthistle Tasselflower \\
\hline YI GENG WU WEI ZI & 翼梗五味子 & Henry Magnoliavine \\
\hline YI HE GUO & 翼核果 & Smoothfruit Ventilago \\
\hline YI HUA & 仪花 & Redbracted Lysidice \\
\hline YI HUA MU LAN & 异花木兰 & Different-flowered Indigo \\
\hline YI HUA WU ZHU YU & 异花吴茱英 & \\
\hline YI KA TUO YE HUANG TAN & 伊卡托叶黄檀 & Ecasto-leaf Rosewood* \\
\hline YI KANG BA DOU & 益康巴豆* & Salutary Croton* \\
\hline YI KOU KE MEI & 一口可梅 & Coco-plum \\
\hline YI KUA & 伊夸 & Manyflower Wormwood \\
\hline YI LAN & 依兰 & Fragrant Gananga \\
\hline YI LANG A WEI & 伊朗阿魏 & Iran Giantfennel* \\
\hline YI LANG QING LAN & 伊朗青兰* & Iran Dragonhead* \\
\hline YI LI CUI QUE HUA & 伊犁翠雀花 & Ili Larkspur \\
\hline YI LI LI YA DA CHI JI & 伊利里亚大翅蓟* & Illyrian Cottonthistle* \\
\hline YI LI NUO HE HUAN CAO & 伊利诺合欢草 & Prairie Mimosa \\
\hline YI LYE GAN LAO JUN & 易裂干酪菌* & Easy-lobed Tyromyces* \\
\hline YI MEI NI A BU TA CAO & 依美尼阿布塔草 & \\
\hline YI MI & 薏米 & Adlay \\
\hline YI MU CAO & 益母草 & Wormwood-like Motherwor \\
\hline YI NIAN PENG & 一年蓬 & Annual Fleabane \\
\hline YI NIAN SHENG SHAN DIAN & 一年生山靛 & Annual Mercury \\
\hline YI PAO NANG CAO & 翼泡囊草 & Winged Physochlaina* \\
\hline YI PIN HONG & 一品红 & Common Poinsettia \\
\hline YI SI CUI QUE & 疑似翠雀* & Confusable Larkspur* \\
\hline YI SI MEI GUI SHU & 疑似玫瑰树 & Confusable Ochrosia \\
\hline YI WA JU & 依瓦菊 & Sumpweed \\
\hline YI WO SI JING TIAN & 伊沃斯景天 & Ewers Stonecrop* \\
\hline YI XIAN HAI CONG & 一现海苟* & \\
\hline
\end{tabular}

T1943 Cymbopogon goeringii T1338 Cestrum nocturnum T2636 Euscaphis japonica T6018 Solanum verbascifolium T4629 Papaver nudicaule T1624 Colocasia antiquorum T3681 Lamium barbatum T6205 Sus scrofa T1591 Cocos nucifera T1592 Cocos nucifera T1593 Cocos nucifera T1594 Cocos nucifera T5922 Serratula strangulata T2790 Fritillaria pallidiflora T5373 Quercus iberica T4374 Narcissus bujei T6067 Spatoglossum variabile T2018 Dalbergia variabilis T4777 Phalaris arundinacea T3604 Jurinea alata T0719 Arum italicum T1234 Cassia italika T3158 Helichrysum italicum T2357 Emilia sonchifolia T5793 Schisandra henryi T6689 Ventilago leiocarpa T3995 Lysidice rhodostegia T3422 Indigofera heteranthazha T2639 Evodia baberi T2002 Dalbergia ecastophyllum T1854 Croton salutaris

T1401 Chrysobalanus icaco T0687 Artemisia myriantha T1164 Cananga odorata T2703 Ferula kuhistanica T2256 Dracocephalum kotschyi T2078 Delphinium iliense T4497 Onopordum illyricum T2127 Desmanthus illinoensis T6589 Tyromyces fissilis T0013 Abuta imene T1613 Coix lacryma-jobi T3752 Leonurus heterophyllus [Syn. Leonurus artemisia]

T2421 Erigeron aпnииs T4196 Mercurialis annua T4857 Physochlaina alaica T2614 Euphorbia pulcherrima T2067 Delphinium confusum T4468 Ochrosia confusa T3543 Iva frutescens T5854 Sedum ewersii T6648 Urginea fugax 
YI XIAN YING SU
YI XING NAN WU WEI Z

YI XING TANG SONG CAO

YI YANG HE BAO MU DAN

YI YE BAI JIANG

YI YE JIA FAN LV

YI YE LIANG WANG CHA

YI YE MEI DENG MU

YI YE QIU

YI YE ROU TUO GUO

YI YE TIAN NAN XING

YI YE TIE SHAN

YI YE WU TOU

YI YE YANG

YI YI REN

YI ZHI HAO

YI ZHI HUANG HUA

YI ZHI REN

YI ZHI XIANG

YI ZHU AI MA HUANG

YI ZHU QIAN MA

YI ZU SUO SHA CAN

YIN BAI HUANG YANG

YIN BAI JIN HE HUAN

YIN BAI QI

YIN BAI QIAN LI GUANG

YIN BAI YANG

YIN BIAN LONG SHE LAN

YIN BU HUAN

YIN CHAI HU

YIN CHEN HAO

YIN DI KUAN YE XIANG CHA

CAI

YIN DI XIANG CHA CAI

YIN DU BA QIA

YIN DU BAI MAO

YIN DU DA MA

YIN DU DUO ZHI DA JI

YIN DU E LI

YIN DU FANG JI

YIN DU HUANG TAN

YIN DU JIA JING JIE

YIN DU JIU LI XIANG

YIN DU KUAI JUN

YIN DU LIAN

YIN DU LUO FU MU

YIN DU MA DOU LING

YIN DU MAI MA TENG

YIN DU MIAN

YIN DU MU FANG JI

YIN DU SHAN DAO LIAN YE

一现罍粟*
异型南五味子
异性唐松草
异样荷包牡丹*
异叶败酱(墓头回)
异叶假繁缕(太子参)
异叶梁王茶
异叶美登木
一叶萩
异叶肉托果
异叶天南星
异叶铁杉
异叶乌头
异叶杨
薏蔌仁
一支蒿
一枝黄花

益智仁

一支香

异株矮麻黄

异株菖麻

异足索沙蚕

银白黄杨*

银白金合欢*

银白槭

银白千里光*

银白杨

银边龙舌兰

银不换

银柴胡

茵陈蒿

阴地宽叶香茶菜

阴地香茶菜

印度菝葜

印度白茅

印度大麻*

印度多汁大戟

印度鳄梨*

印度防已

印度黄檀

印度假荆芥

印度九里香

印度块菌

印度楝

印度萝芙木

印度马悓铃*

印度买麻藤

印度棉*

印度木防己*

印度山道楝叶
Fugacious Poppy*

Curious Kadsura

Early Meadowrue

Peregrin Bleedingheart*

Diversifolious Patrinia

Heterophylla Falsestarwort

David Falsepanax

Heteroleaf Mayten*

Suffrutescent Securinega

Heteroleaf Markingnut*

Diversileaf Jackinthepulpit

Western Hemlock

Heteroleaf Monkshood*

Swamp Cottonwood

Jobstears Seed

Alpine Yarrow

Common Goldenrod

Sharpleaf Galangal

Bastard Speedwell

Dioecious Small Ephedra

Dioecious Nettle

Heterofoot Lumbrinereis*

Argentine Box*

Silver Wattle

Silver Maple

Silver Ragwort

White Aspen

Silveredge Agave

Barbate Cyclea

Lanceolate Starwort

Capillary Wormwood

Shady Broadleaf Rabdosia*

Shady Rabdosia*

Anantmul

Cogon Satintail

Indian Hemp*

Indian Juicy Euphorbia*

Indian Avocado*

Sisso Rosewood

Indian Catnip*

Indian Common Jasminorange

Indian Truffle

Neem Tree

Java Devilpepper

Indian Dutchmanspipe*

India Jointfir

Bluntleaf Cotton

Indian Snailseed*

Indian Katon
T4628 Papaver fugax

T3614 Kadsura heteroclita [Syn. Uvaria

heteroclita]

T6379 Thalictrum dioicum

T2152 Dicentra peregrina

T4670 Patrinia heterophylla

T5260 Pseudostellaria heterophylla

T4444 Nothopanax davidii

T4133 Maytenus heterophylla

T5848 Securinega suffruticosa

T5873 Semecarpus heterophylla

T0620 Arisaema heterophyllum

T6558 Tsuga heterophylla

T0103 Aconitum heterophyllum

T5152 Populus heterophylla

T1614 Coix lacryma-jobi var. ma-yuen

T0060 Achillea alpina [Syn. Achillea sibirica]

T6024 Solidago virgaurea var. leiocarpa [Syn.

Solidago decurrens]

T0360 Alpinia oxyphylla

T6729 Veronica spuria

T2374 Ephedra minuta var. dioeca

T6652 Urtica dioica

T3937 Lumbriconeis heteropoda

T1085 Buxus argentea

T0020 Acacia dealbata

T0055 Acer saccharinum

T5885 Senecio cineraria

T5145 Populus alba

T0218 Agave angustifolia var. marginata

T1931 Cyclea barbata

T6104 Stellaria dichotoma var. lanceolata

T0672 Artemisia capillaris

T3532 Isodon umbrosa

T3533 Isodon umbrosa var. latifolia

T3200 Hemidesmus indicus

T3415 Imperata cylindrica

T1177 Cannabis sativa var. indica

T2603 Euphorbia nivulia

T4733 Persea indica

T0443 Anamirta paniculata

T2013 Dalbergia sissoo

T4415 Nepeta hindostana

T4319 Murraya koenigii

T6559 Tuber indicum

T0836 Azadiractica indica

T5442 Rauwolfia serpentina

T0630 Aristolochia indica

T3035 Gnetum ula

T3059 Gossypium indicum

T1585 Cocculus indicus

T5708 Sandoricum koetjape [Syn. Sandoricum indicum] 


\begin{tabular}{|c|c|c|}
\hline YIN DU SHE GU & 印度蛇菇 & Indian Balanophora \\
\hline YIN DU SI LI LAN KA YE XIA & 印度斯里兰卡叶下珠* & Indian-Sri-Lankan Leafflower* \\
\hline \multicolumn{3}{|l|}{ ZHU } \\
\hline YIN DU TENG HUANG & 印度藤黄* & Indian Garcinia* \\
\hline YIN DU WA ER TENG & 印度娃儿藤 & Indian Tylophora* \\
\hline YIN DU XIE HAO & 印度邪蒿 & Indian Seseli* \\
\hline YIN DU YA JIAO SHU & 印度鸭脚树 & Venenous Alstonia* \\
\hline YIN DU ZANG HUI XIANG & 印度藏茴香 & India Caraway* \\
\hline YIN DU ZHI NA BAI BU & 印度支那百部* & Indochina Stemona* \\
\hline YIN FEN BAO CHUN & 银粉报春 & Pulverulent Primrose \\
\hline YIN HE HUAN & 银合欢 & Hedge Acacia \\
\hline YIN HUA & 银桦 & Robust Silk Oak \\
\hline YIN HUA YAO XIAO YING QIN & 隐花药小鹰芹* & \\
\hline YIN JIAN & 印楝 & Indica Melia* \\
\hline YIN JIAO JU & 银胶菊 & Common Pathenium \\
\hline YIN JU CHUAN BEI HAI MIAN & 隐居穿贝海绵 & Burrowing sponge \\
\hline YIN MAO XIAO YUN MU & 硬毛小芸木* & Hairy Micromelum* \\
\hline YIN MIAN YU & 印缅榆 & Vellayim \\
\hline YIN NI MIAN BAO GUO & 印尼面包果 & Cempedak \\
\hline YIN SE MI ZI LAN & 银色米仔兰 & Argenti Aglaia* \\
\hline YIN XIAN CAO & 银线草 & Japanise Chloranthus \\
\hline YIN XING CAO & 阴行草 & Chinese Siphonostegia \\
\hline YIN YANG HUO & 渓羊藿 & Shorthorned Epimedium \\
\hline YIN YANG HUO GEN & 淫羊藿根 & Shorthorned Epimedium Root \\
\hline YIN YE SHU & 银叶薯* & Silverleaf Morningglory* \\
\hline YIN YU & 茵芋 & Reeves Skimmia \\
\hline YIN ZHOU CHAI HU & 银洲柴胡 & Yinchow Thorowax \\
\hline YING BU BO & 鹰不泊 & Avicenna's Pricklyash \\
\hline YING CHUN HUA & 迎春花 & Winter Jasmine \\
\hline YING GUO OU SHI NAN & 英国欧石南 & Cornish Heath \\
\hline YING GUO SHAN ZHA & 英国山督 & Hawthorn \\
\hline YING HE & 硬核 & Wallich Scleropyrum \\
\hline YING HE CI TONG & 硬核刺桐 & Hardhilum Coralbean* \\
\hline YING HUA XIANG CHA CAI & 㾉花香茶菜 & Rosthorn Rabdosia \\
\hline YING MAO HU JIAO & 硬毛胡椒 & Hispid Pepper* \\
\hline YING MAO JIN SI TAO & 硬毛金丝桃 & Hairy St.John'swort \\
\hline YING MAO TI GEN CAO & 硬毛德根草 & Hairy Hellebore* \\
\hline YING MAO TIAN JIE CAI & 硬毛天芥菜* & Hairy Heliotrope* \\
\hline YING PI HE YE LAN & 硬皮禾叶兰 & Callose Agrostophyllum \\
\hline YING SHAN HONG & 迎山红 & Korean Rhododendron \\
\hline YING SHUI HUANG LIAN & 硬水黄连 & Slim-top Meadowrue \\
\hline YING SU & 罂粟 & Opium Poppy \\
\hline YING SU KE & 罂粟壳 & Opium Poppy Pericarp \\
\hline YING TAO & 樱桃 & Falsesour Cherry \\
\hline YING TAO FAN QIE & 樱桃番茄 & Cherry Tomato* \\
\hline YING YE JIA MI & 樱叶荚蒾 & Blackhaw \\
\hline YING YE NV LOU CAI & 硬叶女娄菜 & Robust Silene* \\
\hline YING ZHAO & 鹰爪 & Sixpetal Tailgrape \\
\hline
\end{tabular}

T0858 Balanophora indica [Syn. Langodorffia indica]

T4839 Phyllanthus myritifolius

T2859 Garcinia indica

T6577 Tylophora asthmatica [Syn. Tylophora indica]

T5932 Seseli indicum

T0377 Alstonia venenata

T1216 Carum ajowan

T6108 Stemona cochinchinensis

T5203 Primula pulverulenta

T3778 Leucaena glauca [Syn. Leucaena

leucocephala]

T3063 Grevillea robusta

T0840 Azorella cryptantha

T4161 Melia indica

T4660 Parthenium hysterophorus

T1575 Cliona celata

T4222 Micromelum hirsutum

T3271 Holoptelea integrifolia

T0710 Artocarpus champeden

T0231 Aglaia argentea

T1371 Chloranthus japonicus

T5966 Siphonostegia chinensis

T2390 Epimedium brevicornum

T2391 Epimedium brevicornum

T3445 Ipomoea argyrophylla

T5972 Skimmia reevesiana

T1079 Bupleurum yinchowense

T6866 Zanthoxylum avicennae

T3555 Jasminum nudiflorum

T2419 Erica vagans

T1774 Crataegus oxyacantha

T5815 Scleropyrum wallichianum

T2468 Erythrina lithosperma

T3520 Isodon rosthornii

T4949 Piper hispidum

T3355 Hypericum hirsutum

T3185 Helleborus orientalis var. hirsutus

T3173 Heliotropium hirsutum

T0254 Agrostophyllum callosum

T5519 Rhododendron mucronulatum

T6409 Thalictrum simplex [Syn. Thalictrum

simplex var. brevipes]

T4636 Papaver somniferum

T4637 Papaver somniferum

T5236 Prunus pseudocerasus

T3963 Lycopersicon esculentum var. cerasiforme T6739 Viburnum prunifolium

T5953 Silene firma

T0653 Artabotrys hexapetalus [Syn. Annona hexapetalus] 


\begin{tabular}{|c|c|c|c|}
\hline YING ZHAO DOU & 鹰爪豆 & Weaversbroom & T6064 Spartium junceum \\
\hline YING ZHAO DOU ZHU SHI DOU & 鹰爪豆猪屎豆* & Sparteine Crotalaria* & T1833 Crotalaria spartioides \\
\hline YING ZHI YE TAI & 硬指叶苔 & & T3763 Lepidozia vitrea \\
\hline YING ZI CAO & 蝇子草 & Catch-fly & T5954 Silene fortunei \\
\hline YONG CHONG CAO & 蛹虫草 & Scarlet Caterpillar Fungus & T1680 Cordyceps militaris \\
\hline YONG NING DU HUO & 永宁独活 & Yungning Cowparsnip & T3228 Heracleum yungningense \\
\hline YOU AN DI JIN & 幽暗地锦* & Stygian Euphorbia* & T2621 Euphorbia stygiana \\
\hline YOU BING SHI WEI & 有柄石韦 & Petioled Pyrrosia Frond & T5358 Pyrrosia petiolosa \\
\hline YOU CAI ZI & 油菜籽 & Rapeseed* & T1011 Brassica napus var. napus \\
\hline YOU CHA GEN PI & 油茶根皮 & Oiltea Camellia Root-bark & T1147 Camellia oleifera \\
\hline YOU CHOU YE ZHI MA & 鼠臭野芝麻* & Stoat-osmyl Deadnettle* & T3682 Lamium galeobdolon \\
\hline YOU CI PO BU MU & 有刺破布木 & Spined Cordia* & T1679 Cordia spinescens \\
\hline YOU DIAN WEI MAO & 疮点卫矛 & Verrucatespot Euonymus & T2548 Euonymus verrucosides \\
\hline YOU GAN GEN & 油相根 & Emblic Leafflower Root & T4835 Phyllanthus emblica \\
\hline YOU GAN LAN & 油橄榄 & Common Olive & T4487 Olea europaea \\
\hline YOU GAN LAN JIU JIE & 油橄榄九节 & Olive Ninenode* & T5277 Psychotria oleoides \\
\hline YOU GAN MU PI & 油柑木皮 & Emblic Leafflower Bark & T4836 Phyllanthus emblica \\
\hline YOU GAN YE & 油柑叶 & Emblic Leafflower Leaf & T4837 Phyllanthus emblica \\
\hline YOU GOU YING ZHAO & 有钩鹰爪* & Uncinate Tailgrape* & T0657 Artabotrys uncinatus \\
\hline YOU GUO DOU KOU & 疮果豆蔻 & Wartyfruit Amomum & T0418 Amотит muricarpum \\
\hline YOU HE & 柚核 & Pummelo Seed & T1479 Citrus grandis \\
\hline YOU KA MEI JU & 尤卡美菊 & Juanislama (in Salvador) & T1111 Calea urticifolia \\
\hline YOU MU & 柚木 & Common Teak & T6325 Tectona grandis \\
\hline YOU PU TAO YOU ZA JIAO & 柚葡萄柚杂交种 & & T1480 Citrus grandis cv. x Citrus paradisi \\
\hline \multicolumn{4}{|l|}{ ZHONG } \\
\hline YOU RUI XIANG & 油瑞香 & Oily Daphne* & T2029 Daphne oleoides \\
\hline YOU SE BAN JIU JU & 有色斑鸠菊 & Colorful Bitterleaf & T6715 Vernonia colorata \\
\hline YOU SE ZI JIN NIU & 有色紫金牛* & Colorate Ardisia* & T0592 Ardisia colorata \\
\hline YOU XIAN YA JIAO SHU & 有限鸭脚树 & Limited Alstonia* & T0373 Alstonia restricta \\
\hline YOU YAN AO TUO SI TE CAO & 有檐奥托斯特草 & Limbate Otostegia & T4559 Otostegia limbata \\
\hline YOU YE HUANG QI & 油叶黄芪* & Oily-leaf Milkvetch* & T0801 Astragalus oleifolius \\
\hline YOU YONG PO LEI & 有用坡垒* & Utilizable Hopea* & T3281 Hopea utilis \\
\hline YOU ZONG & 油棕 & Oilpalm & T2328 Elaeis guineensis \\
\hline $\mathrm{YOU}^{(2)}$ & 获 & Divaricate Bluebeard & T1221 Caryopteris divaricata \\
\hline $\mathrm{YOU}^{(4)}$ & 柚 & Pummelo & T1478 Citrus grandis \\
\hline YU BAI FU & 禹白附 & Giant Typhonium & T6588 Typhoniun giganteum \\
\hline YU BAI SHI SONG & 玉柏石松 & Tree Clubmoss & T3976 Lycopodium obscurum \\
\hline YU CHUANG MU & 愈疮木 & Lignum-vitae & T3067 Guajacum officinale \\
\hline YU DA HUANG & 芋大黄 & & T5478 Rheum sp. \\
\hline YU DAI GEN & 玉带根 & Tithymalus-like Pedilanthus & T4686 Pedilanthus tithymaloides \\
\hline YU ER & 榆耳 & Incarnate Gloeostereum & T2993 Gloeostereum incarnatum \\
\hline YU ER QI & 鱼儿七 & Whiteflower Trillium & T6531 Trillium camtschaticum \\
\hline YU FU YE XIA ZHU & 渔夫叶下珠 & Fisherman Leafflower* & T4841 Phyllanthus piscatorum \\
\hline YU GUO XIAO YE NAN & 玉桂小叶楠 & Cinnamomi-leaf Phoebe* & T4816 Phoebe cinnamomifolia \\
\hline YU JIN & 郁金 & AromaticTurmeric & T1903 Curcuma aromatica \\
\hline YU JIN XIANG & 郁金香 & Common Tulip & T6561 Tulipa gesneriana \\
\hline YU JIN XIANG GEN & 郁金香根 & Common Tulip Root & T6562 Tulipa gesneriana \\
\hline YU JIN XIANG ZA JIAO ZHONG & 郁金香杂交种 & Tulip Hybrid* & T6563 Tulipa hybrida \\
\hline YU JU & 渝橘 & Common Hoptree & T5282 Ptelea trifoliata \\
\hline YU KE GAN JU & 玉克柑桔 & Yuko Citrus* & T1522 Citrus yuko \\
\hline YU LAN & 玉兰 & Yulan Magnolia & $\begin{array}{l}\text { T4038 Magnolia denudata [Syn. Magnolia } \\
\text { heptapata] }\end{array}$ \\
\hline YU LI REN & 郁李仁 & Dwarf Flowering Cherry Seed & T5224 Prunus japonica $[$ Syn. Cerasus japonica $]$ \\
\hline
\end{tabular}




\begin{tabular}{|c|c|c|c|}
\hline YU LIAO & 鱼苶 & Dockleaved Knotweed & T5106 Polygonum lapathifolium \\
\hline YU LIN CAI & 鱼鳞菜 & Broadleaf Blainvillea* & $\begin{array}{l}\text { T0948 Blainvillea acmella }[\text { Syn. Verbesina } \\
\text { acmella; Eclipta latifolia } ; \text { Blainvillea latifolia }]\end{array}$ \\
\hline YU LIN YUN SHAN & 鱼鳞云杉 & Yeddo Spruce & T4872 Picea jezoensis var. jezoensis \\
\hline YU LING HUA & 玉铃花 & Fragrant Snowbell & T6201 Styrax obassia \\
\hline YU MI FU & 玉米麸 & Maize Bran & T6900 Zea mays \\
\hline YU MI HEI MEI & 玉米黑霉 & Black-powder Fungus in Corn & T6656 Ustilago maydis \\
\hline YU MI XU & 玉米须 & Maize Style & T6901 Zea mays \\
\hline YU SHAN HU GEN & 玉珊瑚根 & Jerusalemcherry Root & T6009 Solanum pseudo-capsicum \\
\hline YU SHU & 雨树 & Raintree & T5703 Samanea saman \\
\hline YU SHU & 榆树 & Siberian Elm & T6597 Ulmus pumila \\
\hline YU SHU SHU & 玉蜀㯟 & Maize & T6902 Zea mays \\
\hline YU TENG & 鱼藤 & Trifoliate Jewelvine & T2124 Derris trifoliata \\
\hline YU XIANG CAO & 鱼香草 & Apple Mint & T4188 Mentha rotundifolia \\
\hline YU XING CAO & 鱼腥草 & Heartleaf Houttuynia & T3284 Houttuynia cordata \\
\hline YU YE DING XIANG & 羽叶丁香 & Pinnateleaf Lilac & T6261 Syringa pinnafolia \\
\hline YU YE GUI DENG QING & 羽叶鬼灯檠 & Featherleaf Rodgersflower & T5555 Rodgersia pinnata \\
\hline YU YE JIN HUA & 玉叶金花 & Mussaenda* & T4334 Mussaenda hirsutissim \\
\hline YU YE MAO MEI & 榆叶茅莓 & Elm-leaf Raspberry* & T5602 Rubus ulmifolius \\
\hline YU YE SAN QI & 羽叶三七 & Bipinnatifid Ginseng & T4604 Panax japonicus var. bipinnatifidus \\
\hline YU YE SHE PU TAO & 羽叶蛇葡萄 & Chaffanjon Ampelopsis & T0425 Ampelopsis chaffanjonii \\
\hline YU YI GAN LAN & 羽衣甘蓝 & Savoy Cabbage & T1020 Brassica oleracea var. sabauda \\
\hline YU ZAN YE CHE QIAN & 玉穊叶车前* & Jadehairpin-leaf Plantain* & T5005 Plantago hostifolia \\
\hline YU ZHI ZI & 预知子 & Fiveleaf Akebia Seed & T0275 Akebia quinata \\
\hline \multirow[t]{2}{*}{ YU ZHU } & 玉竹 & Fragrant Solomonseal & T5093 Polygonatum odoratum [Syn. \\
\hline & & & Polygonatum officinale] \\
\hline YU ZHUANG HE HUAN & 羽状合欢 & Cape Leeuwin Wattle & T0294 Albizzia lophantha \\
\hline YU ZHUANG YUN XIANG & 羽状芸香 & Pinnate Rue* & T5630 Ruta pinnata \\
\hline YUAN BAI & 圆柏 & Chinese Juniper & T5639 Sabina chinensis \\
\hline YUAN BAN JIANG HUA & 圆瓣姜花 & Forrest Gingerlily & T3119 Hedychium forrestii \\
\hline YUAN BAN XIANG CHA CAI & 圆板香茶菜* & Nummulite Rabdosia* & T5014 Plectranthus nummularius \\
\hline YUAN BAO CAO & 元宝草 & Sampson St.John'swort & T3363 Hypericum sampsonii \\
\hline YUAN CAN E & 原蚕蛾 & Silkworm King & T0977 Bombyx mori \\
\hline YUAN CAN SHA & 原虫沙 & Silkworm Feculae & T0978 Bombyx mori \\
\hline YUAN CAN ZI & 原虫子 & Silkworm Egg & T0979 Bombyx mori \\
\hline YUAN CHI KU MU & 圆齿苦木 & Crenate Quassiawood* & T4879 Picrasma crenata \\
\hline YUAN CONG HONG JING TIAN & 园从红景天 & Jupar Rhodiola & T5496 Rhodiola juparensis \\
\hline YUAN DANG GUI & 圆当归 & Angelica & T0477 Angelica archangelica \\
\hline YUAN DONG JIU LI XIANG & 远东九里香 & Siamense Common Jasminorange & T4327 Murraya siamensis \\
\hline YUAN E CI XU DUAN & 圆䓯刺续断 & Chinese Morina & T4279 Morina chinensis \\
\hline YUAN GUO GAN CAO & 圆果甘草 & Roundfruit Licorice & T3021 Glycyrrhiza squamulosa \\
\hline YUAN GUO SUAN PAN ZI & 圆果算盘子 & Roundfruit Glochidion* & T2989 Glochidion sphaerogynum \\
\hline YUAN HUA & 芫花 & Lilac Daphne & T2023 Daphne genkwa \\
\hline YUAN HUA FAN LI ZHI & 圆滑番荔枝 & Glabrous Custardapple & T0506 Annona glabra \\
\hline YUAN HUA GEN & 芫花根 & Lilac Daphne Root & T2024 Daphne genkwa \\
\hline YUAN JING HUAN YANG SHEN & 芫菁还阳参 & Turnip-shaped Hawksbeard & T1789 Crepis napifera \\
\hline YUAN JU JIN GU CAO & 远距筋骨草* & Manybracteole Bugle & T0270 Ajuga remota \\
\hline YUAN MAO JING JIE & 缘毛荆芥 & Ciliate Catnip & T4414 Nepeta ciliaris \\
\hline YUAN MAO XIANG RI KUI & 缘毛向日葵 & Ciliate Sunflower* & T3148 Helianthus ciliaris \\
\hline YUAN SHU YU & 圆薯蓣 & Guinea Yam & $\begin{array}{l}\text { T2207 Dioscorea rotundata [Syn. Dioscorea } \\
\text { cayenensis] }\end{array}$ \\
\hline YUAN WEI & 䲸尾(川射干) & Roof Iris & T3471 Iris tectorum \\
\hline YUAN XING YE GU KE & 圆形叶古柯* & Roundleaf Coca Shrub* & T2489 Erythroxylon rotundifolium \\
\hline
\end{tabular}




\begin{tabular}{|c|c|}
\hline YUAN YE BU YING CAO & 圆叶捕蝇草 \\
\hline YUAN YE CHAI HU & 圆叶柴胡 \\
\hline YUAN YE E ZHANG CHAI & 圆叶鹅掌柴* \\
\hline YUAN YE FENG LING CAO & 圆叶风铃草 \\
\hline YUAN YE LU TI CAO & 圆叶鹿蹄草 \\
\hline YUAN YE MAO GAO CAI & 圆叶茅膏菜 \\
\hline YUAN YE QIAN NIU ZI & 圆叶牵牛子 \\
\hline YUAN YE SHAN WU GUI & 圆叶山乌龟 \\
\hline YUAN YE TAI & 园叶苔 \\
\hline YUAN YE TIAN JIE CAI & 圆叶天芥菜* \\
\hline YUAN YE ZE LAN & 圆叶泽兰 \\
\hline YUAN ZHI & 远志 \\
\hline YUAN ZHI JUAN BAI & 圆枝卷柏 \\
\hline YUAN ZHI YE AO ZHOU CHA & 远志叶澳州茶* \\
\hline YUAN ZHUI HUA YE LUN MU & 圆雉花叶轮木 \\
\hline YUAN ZHUI ZHU SHI DOU & 圆雉猪屎豆 \\
\hline YUE GUI SHU YE MU JIANG ZI & 月桂树叶木姜子* \\
\hline YUE GUI XIAO BO & 月桂小檗 \\
\hline YUE GUI YE & 月桂叶 \\
\hline YUE GUI YE SHAN YOU GAN & 月桂叶山油柑* \\
\hline YUE GUI ZI & 月桂子 \\
\hline YUE HAN SI TONG SHAN MACHA & 约翰司通山马茶 \\
\hline YUE HUA & 岳桦 \\
\hline YUE JI HUA & 月季花 \\
\hline YUE JI SHI LIU & 月季石榴 \\
\hline YUE JU YE & 越橘叶 \\
\hline YUE NAN AN XI XIANG & 越南安息香 \\
\hline YUE NAN LIE LAN & 越南裂榄* \\
\hline YUE SI GUA & 粤丝瓜 \\
\hline YUE XI MU XIANG & 越西木香 \\
\hline YUE XIAN DA JI & 月腺大戟 \\
\hline YUN NAN BA JIAO & 云南八角 \\
\hline YUN NAN CAO KOU & 云南草冦 \\
\hline YUN NAN CHONG LOU & 云南重楼 \\
\hline YUN NAN DI HUA JUN & 云南地花菌 \\
\hline YUN NAN FEI SHU & 云南榧树 \\
\hline YUN NAN FENG CHE ZI & 云南风车子 \\
\hline YUN NAN GAN CAO & 云南甘草 \\
\hline YUN NAN GE SHE SHU & 云南割舌树 \\
\hline YUN NAN GE TENG & 云南葛藤 \\
\hline YUN NAN HAN XIAO & 云南含笑 \\
\hline YUN NAN HONG DOU SHAN & 云南红豆杉 \\
\hline YUN NAN HONG JING TIAN & 云南红景天 \\
\hline YUN NAN HUANG LIAN & 云南黄连 \\
\hline YUN NAN LUO FU MU & 云南萝芙木 \\
\hline YUN NAN MEI DENG MU & 云南美登木 \\
\hline YUN NAN NI DAN XING MU & 云南拟单性木兰 \\
\hline \multicolumn{2}{|l|}{ LAN } \\
\hline YUN NAN QIANG HUO & 云南㒸活 \\
\hline YUN NAN RUI MU & 云南荵木 \\
\hline YUN NAN SHA JI & 云南沙棘 \\
\hline
\end{tabular}

Round-leaf Flytrap*

Roundleaf Thorowax*

Roundleaf Schefflera*

Harebell

European Pyrola

Roundleaf Sundew

Roundleaf Pharbitis Seed

Roundleaf Corydalis*

Roundleaf Heliotrope*

Roundleaf Eupatorium*

Thinleaf Milkwort

Sanguineous Spikemoss

Australian Tea-tree

Paniculate Ostodes*

Paniculate Crotalaria*

Laurelleaf Litse*

Laurel-like Barberry

Grecian Laurel Leaf

Laurel-leaf Acronychia*

Grecian Laurel Fruit

Ermans Birch

Chinese Rose

Dwarf Pomegranate

Cowberry Leaf

Tonkin Snowbell

Tonkin Bursera*

Singkwa Towelgourd

Denticulate Vladimiria

Ebracteolate Euphorbia

Yunnan Anisetree

Yunnan Galangal

Yunnan Manyleaf Paris

Yunnan Landflower Mushroom*

Yunnan Torreya

Yunnan Combretum*

Yunnan Licorice

Yunnan Cuttongue tree

Yunnan Kudzuvine

Yunnan Michelia

Yunnan Yew

Yunnan Rhodiola

Yunnan Goldthread

Yunnan Devilpepper

Yunnan Mayten

Yunnan Parakmeria

Yunnan Pleurospermum

Medicinal Kopsia

Yunnan Seabuckthorn*
T2187 Dionaea rotundifolia

T1071 Bupleurum rotundifolium

T5783 Schefflera rotundifolia

T1157 Campanula rotundifolia

T5351 Pyrola rotundifolia

T2268 Drosera rotundifolia

T4780 Pharbitis purpurea

T1738 Corydalis rotundatour

T3553 Jamesoniella colorata

T3179 Heliotropium rotundifolium

T2571 Eupatorium rotundifolium

T5088 Polygala tenuifolia

T5866 Selaginella sanguinolenta

T3767 Leptospermum polygalifolium ssp.

polygalifolium

T4554 Ostodes paniculata

T1828 Crotalaria paniculata

T3891 Litsea laurifolia

T0909 Berberis laurina

T3725 Laurus nobilis

T0150 Acronychia laurifolia

T3726 Laurus nobilis

T6274 Tabernaemontana johnstonii

T0931 Betula ermanii

T5562 Rosa chinensis

T5333 Punica granatum cv. nana

T6673 Vaccinium vitis-idaea

T6204 Styrax tonkinensis

T1082 Bursera tonkinensis

T3933 Luffa acutangula

T6801 Vladimiria denticulata

T2584 Euphorbia ebracteolata

T3407 Illicium simonsii

T0353 Alpinia blepharocalyx

T4652 Paris polyphylla var. yunnanensis

T0287 Albatrellus confluens

T6479 Torreya yunnanensis

T1632 Combretum yunnanensis

T3023 Glycyrrhiza yunnanensis

T6807 Walsura yunnanensis

T5317 Pueraria peduncularis

T4215 Michelia yunnanensis

T6319 Taxus yunnanensis

T5501 Rhodiola yunnanesis

T1670 Coptis teetoides [Syn. Coptis teeta]

T5428 Rauvolfia yunnanensis

T4134 Maytenus hookeri

T4641 Parakmeria yunnanensis

T5021 Pleurospermum rivulorum

T3643 Kopsia officinalis

T3258 Hippophae rhamnoides subsp.

yunnanensis 


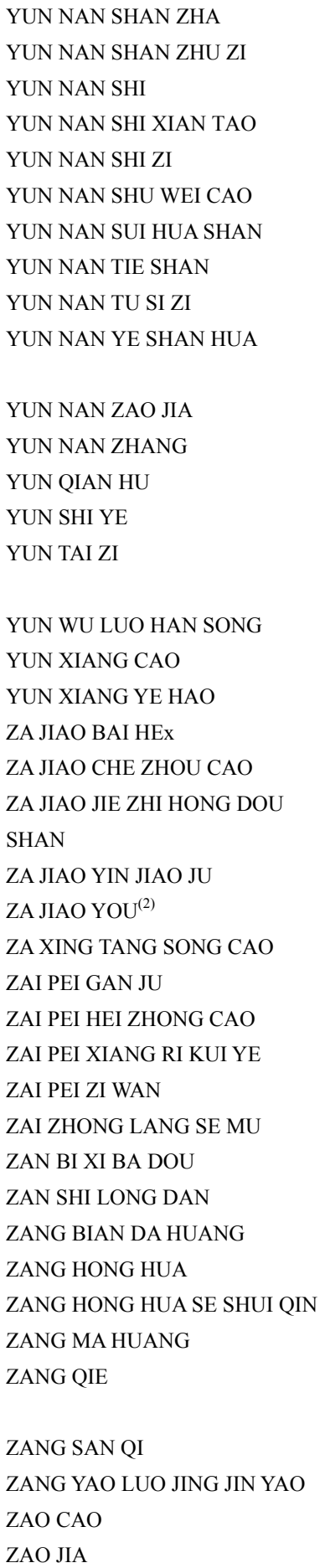

ZAO JIA CI

ZAO JIA GEN PI

ZAO JIA YE

ZAO PI SHU

ZAO WEI KOU MO

\section{云南山楂 \\ 云南山竹子 \\ 云南蓄 \\ 云南石仙桃 \\ 云南石梓 \\ 云南鼠尾草 \\ 云南穗花杉 \\ 云南铁杉 \\ 云南菟丝子 \\ 云南野扇花 \\ 云南㿝荚 \\ 云南樟 \\ 云前胡 \\ 云实叶 \\ 芸䔔子 \\ 云雾罗汉松 \\ 芸香草 \\ 芸香叶蒿 \\ 杂交百合 \\ 杂交车轴草 \\ 杂交介质红豆杉 \\ 杂交银胶菊 \\ 杂交获* \\ 杂性唐松草 \\ 栽培柑桔* \\ 栽培黑种草 \\ 栽培向日葵叶* \\ 栽培紫苑 \\ 栽种榔色木 \\ 赞比西巴豆 \\ 赞氏龙胆 \\ 藏边大黄 \\ 藏红花 \\ 藏红花色水芹 \\ 藏麻黄 \\ 藏茄(山莨若) \\ 藏三七 \\ 藏药裸茎金腰 \\ 蚤草 \\ 㿝荚 \\ 㿝荚刺}

皇荚根皮

㿝英叶

皇皮树*

皇味口蘑
Yunnan Hawthorn

Yunnan Garcinia

Wilson Yarrow

Yunnan Pholidota

Malay Bushbeech

Yunnan Sage

Yunnan Amentotaxus

Yunnan Hemlock

Yunnan Dodder

Yunnan Sarcococca

Delacay Honeylocust

Nepal Camphortree

Yun Hogfennel

Caesalpinia*

Bird Rape

Manio

Remote Lemongrass

Rueleaf Wormwood*

Hybrid Lily*

Hybrid Clover*

Media Yew (hybrid)

Hybrid Pathenium*

Hybrid Bluebeard*

Tall Meadowrue

Cultivated Citrus*

Cultivated Fennelflower*

Cultivated Sunflower Leaf*

Cultivated Aster*

Cultivated Langsat*

Zambesi Croton*

Thunberg Gentian*

Austral Rhubarb

Saffron Crocus Stigma

Hemlock Waterdropwort

Cliff Ephedra

Tangut Anisodus

Tibet Ginseng

Naked-caule Goldsaxifrage

Pulicaria*

Chinese Honeylocust

Chinese Honeylocust Thorn

Chinese Honeylocust Root-bark

Chinese Honeylocust Leaf

Soapbark Tree
T1781 Crataegus scabrifolia

T2852 Garcinia cowa

T0069 Achillea wilsoniana

T4823 Pholidota yunnanensis

T3024 Gmelina arborea

T5701 Salvia yunnanensis

T0409 Amentotaxus yunnanensis

T6557 Tsuga dumosa

T1915 Cuscuta reflexa

T5731 Sarcococca coriacea [Syn. Sarcococca

wallichii]

T2975 Gleditsia delavayi

T1440 Cinnamomum glanduliferum

T4769 Peucedanum rubricaule

T1101 Caesalpinia decapetala

T1005 Brassica campestris [Syn. Brassica

campestris var. oleifera]

T5050 Podocarpus nubigenus

T1941 Cymbopogon distans

T0693 Artemisia sativum

T3838 Lilium speciosum $\mathrm{x}$ L. nobilissimum

T6520 Trifolium hybridum

T6318 Taxus x media

T4659 Parthenium argentatum x P. tomentosa

T1220 Caryopteris clandonensis

T6405 Thalictrum polygamum

T1473 Citrus cultivars

T4433 Nigella sativa

T3146 Helianthus annuus cv

T0779 Aster cultivars

T3686 Lansium domesticum

T1861 Croton zambesicus

T2935 Gentiana thunbergii

T5468 Rheum emodi [Syn. Rheum australe]

T1808 Crocus sativus

T4480 Oenanthe crocata

T2379 Ephedra saxatilis

T0502 Anisodus tanguticus [Syn. Scopolia

tangutica]

T4606 Panax pseudo-ginseng

T1406 Chrysosplenium nudicaule

T5322 Pulicaria wightiana

T2978 Gleditsia sinensis [Syn. Gleditsia

horrida]

T2979 Gleditsia sinensis [Syn. Gleditsia

horrida ]

T2980 Gleditsia sinensis [Syn. Gleditsia

horrida ]

T2981 Gleditsia sinensis [Syn. Gleditsia

horrida]

T5382 Quillaja saponaria

T6506 Tricholoma saponaceum 


\begin{tabular}{|c|c|c|}
\hline ZAO XIU & 蚤休(七叶一枝花) & Manyleaf Paris \\
\hline ZE LAN & 泽兰 & Shiny Bugleweed \\
\hline ZE LAN GEN & 泽兰根 & Shiny Bugleweed Root \\
\hline ZE LAN YANG ER JU & 泽兰羊耳菊 & Eupatoriumlike Inula \\
\hline ZE QI & 泽漆 & Sun Euphorbia \\
\hline ZE WU TOU & 泽乌头 & \\
\hline ZE XIAN & 泽蘚 & \\
\hline ZE XIE & 泽泻 & Oriental Waterplantain \\
\hline ZHAI RUI TA ZHANG YA CAI & 其瑞塔獐牙菜 & Chirata Swertia* \\
\hline ZHAI TOU TUO WU & 窄头㯻吾 & Narrowhead Goldenray \\
\hline ZHAI YE BAN FENG HE & 窄叶半枫荷 & Lanceleaf Wingseedtree \\
\hline ZHAI YE NAN YANG SHAN & 窄叶南洋杉 & Candelabar Tree \\
\hline ZHAI YE YE WAN DOU & 窄叶野踠豆 & Narrowleaf Vetch \\
\hline ZHAI ZHU YE CHAI HU & 窄竹叶柴胡 & Narrowbambooleaf Thorowax \\
\hline ZHAN E JIN SI TAO & 展蕓金丝桃 & Lancaster's St.John'swort \\
\hline ZHAN HUA WU TOU & 展花乌头 & Patentflower Monkshood \\
\hline ZHAN LONG JIAN & 斩龙剑 & Siberian Veronicastrum \\
\hline ZHAN MAO CUI QUE HUA & 展毛翠雀花 & Hair Larkspur \\
\hline ZHAN MAO HUANG QIN & 黏毛黄芩 & Viscidhair Skullcap \\
\hline ZHAN MAO LIAO & 粘毛蓼 & Viscidhairy Knotweed \\
\hline ZHAN MAO SHI WEI & 毡毛石韦 & Panniform Pyrrosia \\
\hline ZHAN MAO XUN ZI & 毡毛栒子 & Silverleaf Cotoneaster \\
\hline ZHAN ZHI TANG SONG CAO & 展枝唐松草 & Spreading Meadowrue* \\
\hline ZHANG GUO GAN CAO & 胀果甘草 & Inflated Licorice \\
\hline ZHANG GUO QIN & 胀果芹 & Siberian Phlojodicarpus \\
\hline ZHANG LANG & 蟑螂 & Cockroach \\
\hline ZHANG LIU TOU & 樟柳头 & Canereed Spiralflag \\
\hline ZHANG MU & 樟木 & Camphortree \\
\hline ZHANG NAO LUO LE & 樟脑罗勒 & Grey Basil \\
\hline ZHANG SHU PI & 樟树皮 & Camphortree Bark \\
\hline ZHANG SHU YE & 樟树叶 & Camphortree Leaf \\
\hline ZHANG YA CAI & 獐牙菜 & False Chinese Swertia \\
\hline ZHANG YE BAN XIA & 掌叶半夏 & Pedate Pinellia \\
\hline ZHANG YE DA HUANG & 掌叶大黄 & Sorrel Rhubarb \\
\hline ZHANG YE HU JIAO & 掌叶胡椒 & Camphortreeleaf Pepper \\
\hline ZHANG YU & 章鱼 & Common Atlantic Octopus \\
\hline ZHAO JU & 沼菊 & Common Enhydra \\
\hline ZHAO SHAN BAI & 照山白 & Manchurian Rhododendron \\
\hline ZHAO SHENG DA JI & 沼生大戟 & Marshy Euphorbia* \\
\hline ZHAO WA DAO DIAO BI & 爪哇倒吊笔* & Java Wrightia* \\
\hline ZHAO WA GAN LAN & 爪哇橄榄 & Java Almond Canary-tree \\
\hline ZHAO WA JIA KE TAI & 爪哇甲克苔 & Japanese liverwort \\
\hline ZHAO WA ZHE SHU & 爪哇柘树* & Java Cudrania* \\
\hline ZHAO ZE QIAN HU & 沼泽前胡* & Marsh Parsley \\
\hline ZHAO ZE SHU HUA & 沼泽树花 & Marsh Ramalina* \\
\hline ZHE BEI MU & 浙贝母 & Thunberg Fritillary \\
\hline ZHE GU CAI & 府鸪菜 & Leprieur Caloglossa Frond \\
\hline ZHE HUANG KAO GU NA & 赫黄栲古那 & Brown-yellow Kokoona* \\
\hline ZHE JIANG ZHANG YA CAI & 浙江獐牙菜 & Zhejiang Swertia* \\
\hline
\end{tabular}

T4648 Paris polyphylla T3980 Lycopus lucidus T3981 Lycopus lucidus T3429 Inula eupatorioides T2589 Euphorbia helioscopia T0141 Aconitum zeravschanicum T4798 Philonotis fontana T0303 Alisma orientale [Syn. Alisma plantago-aquatica var. orientale] T6215 Swertia chirata T3815 Ligularia stenocephala T5310 Pterospermum lanceaefolium T0577 Araucaria angustifolia T6743 Vicia angustifolia T1067 Bupleurum marginatum var. stenophyllum

T3357 Hypericum lancasteri T0086 Aconitum chasmanthum T6731 Veronicastrum sibiricum T2079 Delphinium kamaonense var. glabrescens

T5845 Scutellaria viscidula T5122 Polygonum viscosum T5355 Pyrrosia drakeana T1761 Cotoneaster pannosus T6412 Thalictrum squarrosum T3015 Glycyrrhiza inflata T4804 Phlojodicarpus sibiricus T0950 Blatta orientalis T1756 Costus speciosus T1435 Cinnamomum camphora T4472 Ocimum canum T1436 Cinnamomum camphora T1437 Cinnamomum camphora T6231 Swertia pseudochinensis T4903 Pinellia pedatisecta T5474 Rheum palmatum T4961 Piper polysyphorum T4478 Octopus vulgaris T2361 Enhydra fluctuans T5515 Rhododendron micranthum T2606 Euphorbia palustris T6833 Wrightia javanica T1166 Canarium commune T3550 Jackiella javanica T1886 Cudrania javanensis T4767 Peucedanum palustre T5405 Ramalina paludosa T2798 Fritillaria verticillata var. thunbergii [Syn. Fritillaria thunbergii] T1122 Caloglossa leprieurii T3638 Kokoona ochracea T6222 Swertia hickinii 


\begin{tabular}{|c|c|c|c|}
\hline ZHE SHU & 柘树 & Tricuspid Cudrania & T1888 Cudrania tricuspidata \\
\hline ZHE TENG & 柘藤 & Fruticose Cudrania & T1885 Cudrania fruticosa \\
\hline ZHEN & 榛 & Siberian Hazelnut & T1751 Corylus heterophylla \\
\hline ZHEN CAI & 枕材 & Pungent Litse & T3893 Litsea pungens \\
\hline ZHEN KUI & 针葵 & Canary Island Date-palm & T4819 Phoenix canariensis \\
\hline ZHEN MO & 榛蘑 & Mellea Armillaria Sporocarp & T0645 Armillariella mellea \\
\hline ZHEN XIAN & 真蘚 & & T1044 Bryum argenteum \\
\hline ZHEN YE TENG HUANG & 真叶藤黄* & Realleaf Garcinia* & T2854 Garcinia eugenifolia \\
\hline ZHEN YE XUE TONG & 针叶血桐 & Conifer Macaranga & T4012 Macaranga conifera \\
\hline ZHEN YE ZE LAN & 针叶泽兰* & Lanceleaf Eupatorium* & T2564 Eupatorium lancifolium \\
\hline ZHEN ZHU CAI & 珍珠菜 & Clethra Loosestrife & T3999 Lysimachia clethroides \\
\hline \multirow[t]{2}{*}{ ZHEN ZHU LU SHUI CAO } & 珍珠露水草 & Spiderweb Blueeargrass, Pearl & T1921 Cyanotis arachnoidea [Syn. Cyanotis \\
\hline & & Cyanotis* & bodinieri] \\
\hline ZHEN ZHU MEI & 珍珠梅 & Ural Falsespiraea & T6052 Sorbaria sorbifolia \\
\hline ZHEN ZHU MU & 珍珠母 & Mother-of-pearl & T1804 Cristaria plicata; Hyriopsis cumingii \\
\hline ZHEN ZHU XIU XIAN JU & 珍珠绣线菊 & Thunberg Spiraea & T6086 Spiraea thunbergii \\
\hline ZHENG JI NA SHU & 正鸡纳树 & Medicinal Cinchona & T1429 Cinchona officinalis \\
\hline ZHI & 雉 & Common Pheasant & T4786 Phasianus colchicus \\
\hline ZHI BU LUO TUO CAI HU & 直布罗陀柴胡 & Gibraltar Thorowax* & T1063 Bupleurum gibraltaricum \\
\hline ZHI GEN DANG GUI & 直根当归 & Taproot Angelica* & T0493 Angelica radix \\
\hline ZHI GEN PI & 枳根皮 & Trifoliate-orange Root-bark & T5142 Poncirus trifoliata \\
\hline ZHI JIA HUA YE & 指甲花叶 & Henna Leaf & T3732 Lawsonia inermis \\
\hline ZHI JU GEN & 枳椇根 & Japanese Raisin Tree Root & T3285 Hovenia dulcis \\
\hline ZHI JU ZI & 枳椇子 & Japanese Raisin Tree Seed & T3286 Hovenia dulcis \\
\hline ZHI KE & 枳壳 & Seville Orange Unripe Fruit & T1467 Citrus aurantium \\
\hline ZHI LI BAI BU & 直立百部 & Sessile Stemona & T6113 Stemona sessilifolia \\
\hline ZHI LI CHANG CHUN HUA & 直立长春花 & Erect Periwinkle & T6759 Vinca erecta \\
\hline ZHI LI CI BAI & 直立刺柏 & Erect Juniper* & T3586 Juniperus erectopatens \\
\hline ZHI LI DIAN LAN & 直立靛兰 & & T3421 Indigofera arrecta \\
\hline ZHI LI JIAO HUI XIANG & 直立角茴香 & Erect Hypecoum & T3332 Hypecoum erectum \\
\hline ZHI LI LUO HAN SONG & 智利罗汉松 & Chile Podocarpus* & T5036 Podocarpus andina \\
\hline ZHI LI MA DOU LING & 智利马兒铃 & Aristolochia chilensis & T0623 Aristolochia chilensis \\
\hline ZHI LI PO PO NA & 直立婆婆纳 & Common Speedwell & T6723 Veronica arvensis \\
\hline ZHI LI QIAN JIN TENG & 直立千金藤 & Erect Stephania* & T6126 Stephania erecta \\
\hline ZHI LI YE HOU KE GUI & 直立叶厚壳桂* & Strictleaf Cryptocarya* & T1864 Cryptocarya strictifolia \\
\hline ZHI LI ZI JIN & 直立紫堇 & & T1744 Corydalis stricta \\
\hline ZHI MA CAI & 芝麻菜 & Roquette & T2439 Eruca sativa \\
\hline ZHI MU & 知母 & Common Anemarrhena & T0462 Anemarrhena asphodeloides \\
\hline ZHI SHA CAO & 纸莎草 & Paper Reed & T1977 Cyperus papyrus \\
\hline ZHI SHAN XIANG & 栉山香 & Pectinat Bushmint* & T3380 Hyptis pectinata \\
\hline ZHI SHI & 枳实 & Seville Orange Young Fruit & T1468 Citrus aurantium \\
\hline ZHI XIE MU PI & 止泻木皮 & Droughtdysentery Holarrhena Bark & T3263 Holarrhena antidysenterica \\
\hline ZHI YANG & 脂杨 & Balsam Poplar & T5147 Populus balsamifera \\
\hline ZHI YI XIAO BO & 置疑小檗 & Dubious Barberry & T0903 Berberis dubia \\
\hline ZHI ZHI SHAN MA CHA & 纸质山马茶 & & T6271 Tabernaemontana chartacea \\
\hline ZHI ZHU BAO DAN & 蜘蛛抱蛋 & Common Aspidistra & T0759 Aspidistra elatior \\
\hline ZHI ZHU XIANG & 蜘蛛香 & Jatamans Valeriana & $\begin{array}{l}\text { T6677 Valeriana jatamansii [Syn. Valeriana } \\
\text { wallichii] }\end{array}$ \\
\hline ZHI ZHUANG E AN & 枝状萝桉* & & T2506 Eucalyptus cladocalyx \\
\hline ZHI ZI & 栃子 & Cape Jasmine Fruit & $\begin{array}{l}\text { T2882 Gardenia jasminoides [Syn. Gardenia } \\
\text { florida] }\end{array}$ \\
\hline ZHI ZI YE & 栃子叶 & Cape Jasmine Leaf & $\begin{array}{l}\text { T2883 Gardenia jasminoides [Syn. Gardenia } \\
\text { florida] }\end{array}$ \\
\hline
\end{tabular}




ZHI ZONG ZHUANG HUA XU
XIAO BO
ZHONG BA E ZHANG YE FU ZI
ZHONG BIN JU
ZHONG E BAI TOU WENG
ZHONG FEI GOU ZHI TENG
ZHONG FEI MA QIAN
ZHONG GUO BAN JIU JU
ZHONG GUO CANG ER
ZHONG GUO CU FEI ZHI YE

ZHONG GUO CU FEI ZI

ZHONG GUO SHA JI
ZHONG GUO SHI SUAN
ZHONG GUO SHOU CAO
ZHONG GUO XIU QIU
ZHONG GUO XUAN FU HUA
ZHONG GUO YANG CHUN
ZHONG HUA JI SHI TENG
ZHONG HUA JIU LI XIANG
ZHONG HUA JUAN BAI
ZHONG HUA QING NIU DAN
ZHONG HUA XIAO JIAN LIU
SHAN HU
ZHONG JIANDA YE KE NUCAO

ZHONG JIAN DA YE KE NU CAO

ZHONG JIAN HE SHI

ZHONG JIAN JIN JI ER

ZHONG JIAN MAO GAO CAI

ZHONG JIAN WU WEI ZI

ZHONG MA HUANG

ZHONG YA KU HAO

ZHONG YA QIN JIAO

ZHONG YA SHA JI

ZHOU CHANG JU

\section{ZHOU MU ER}

ZHOU SHAN XIN MU JIANG ZI

ZHOU SHUO XIAN

ZHOU YE LU TI CAO

ZHOU YE MU LAN

ZHOU YE OU QIN

ZHOU YE XIANG CHA CAI

ZHOU YE ZE LAN

ZHOU ZHI SHU LI

\section{ZHU BAI}

ZHU BAI GEN

ZHU DAN

ZHU HONG JUN

ZHU HONG LIU HUANG SE

XUN KONG JUN

ZHU HONG LONG XUE SHU

ZHU HONG SHI

ZHU HONG SHU WEI CAO

\section{直总状花序小檗}

中坝鹅掌叶附子

肿柄菊

钟萼白头翁

中非钩枝藤

中非马钱

中国斑鸭菊

中国苍耳*

中国粗榧枝叶

中国粗榧子

中国沙棘

中国石蒜

中国绶草*

中国绣球

中国旋覆花

中国洋椿*

中华鸡屎藤*

中华九里香

中华卷柏

中华青牛胆

中华小尖柳珊瑚

中间大叶科努草*

中间鹤禹

中间锦鸡儿

中间茅膏菜*

中间五味子

中麻黄

中亚苦蒿

中亚秦艽

中亚沙棘

舟常桔

皱木耳

舟山新木姜子

皱蒴藓

皱叶鹿蹄草

皱叶木兰

皱叶欧芹

皱叶香茶菜

皱叶泽兰

帚枝鼠李

竹柏

竹柏根

猪胆

竹红菌

朱红硫黄色绚孔菌*

朱红龙血树

朱红柿*

朱红鼠尾草
Straight-raceme Barberry

Zhongba Monkshood Daughter Root*

Yucatan Tithonia

Bellcalyx Pulsatilla

Central-African Ancistrocladus*

Central-African Poisonnut*

Chinese Bitterleaf*

Burweed

Chinese Plumyew Branch-leaf

Chinese Plumyew Seed

Chinese Seabuckthorn*

Chinese Lycoris

Chinese Spiranthes*

Chinese Hydrangea

Chinese Inula

Chinese Cedrela*

Chinese Fevervine

Chinese Common Jasminorange

Chinese Spikemoss

Chinese Tinospora*

Gorgonian Muricella sinensis

Intermediate Largeleaf Chastetree*

Intermediate Stickseed

Intermediate Peashrub

Intermediate Sundew*

Intermediate Magnoliavine

Intermediate Ephedra

Common Wormwood

Central Asia Gentian

Central Asia Seabuckthorn*

Funadoko Orange*

Delicate Jew's Ear*

Sericeous Newlitse

Wrinkledleaf Pyrola

Wrinkleleaf Magnolia*

Curly Garden Parsley

Rugose Rabdosia*

Mist flower

Twiggy Buckthorn

Nagai Podocarpus

Nagai Podocarpus Root

Pig Gall

Bambusa Hypocrella*

Vermeil-sulphureous Laetiporus*

Cinnabar Dracaena

Cinnabar Persimmon*
T0911 Berberis orthobotrys

T0085 Aconitum carmichaeli $\mathrm{cv}$

T6469 Tithonia diversifolia

T5323 Pulsatilla campanella

T0451 Ancistrocladus likoko

T6176 Strychnos icaja

T6714 Vernonia chinense

T6837 Xanthium chinense

T1323 Cephalotaxus sinensis [Syn.

Cephalotaxus harringtonia var. sinensis]

T1324 Cephalotaxus sinensis [Syn.

Cephalotaxus harringtonia var. sinensis]

T3256 Hippophae rhamnoides subsp. sinensis

T3984 Lycoris chinensis

T6087 Spiranthes sinensis

T3303 Hydrangea chinensis

T3428 Inula britannica var. chinensis

T1278 Cedrela sinensis

T4576 Paederia chinensis

T4318 Murraya exotica

T5867 Selaginella sinensis

T6468 Tinospora sinensis

T4315 Muricella sinensis

T1700 Cornutia grandifolia var. intermedia

T3689 Lappula intermedia

T1189 Caragana intermedia

T2265 Drosera intermedia

T5798 Schisandra propinqua var. intermedia

T2369 Ephedra intermedia

T0659 Artemisia absinthium

T2913 Gentiana kaufmanniana

T3257 Hippophae rhamnoides subsp. turkestanica

T1477 Citrus funadoko

T0831 Auricularia delicata

T4406 Neolitsea sericea

T0829 Aulacomnium androgynum

T5352 Pyrola rugosa

T4046 Magnolia praecocissima

T4748 Petroselinum crispum

T3523 Isodon rugosus [Syn. Rabdosia rugosa]

T2572 Eupatorium rugosum

T5464 Rhamnus virgata

T4346 Myrica nagi [Syn. Podocarpus nagi]

T4347 Myrica nagi [Syn. Podocarpus nagi]

T6206 Sus scrofa domestica

T3371 Hypocrella bambusae

T3666 Laetiporus sulphureus var. miniatus

T2252 Dracaena cinnabari

T2215 Diospyros cinnabarina

T5669 Salvia cinnabarina 


\begin{tabular}{|c|c|c|c|}
\hline ZHU JIE SAN QI & 竹节三七 & Japanese Ginseng & T4607 Panax pseudo-ginseng var. japonicus \\
\hline ZHU JU & 朱橘 & Red Orange & T1476 Citrus erythrosa \\
\hline ZHU LING & 猪苓 & Popyporus Agaric & T5129 Polyporus umbellatus \\
\hline ZHU LUAN & 朱奕 & Pomelo & T1474 Citrus decumana \\
\hline ZHU MAO CAI & 猪毛菜 & Common Russianthistle & T5657 Salsola collina \\
\hline ZHU SHA DU JUAN & 硃砂杜鹃 & Vermilion Rhododendron & T5506 Rhododendron cinnabarinum \\
\hline ZHU SHA GEN & 硃砂根 & Coral Ardisia & T0594 Ardisia crenata \\
\hline ZHU SHA LIAN & 朱砂莲 & Kaempfer Dutchmanspipe & T0631 Aristolochia kaempferi \\
\hline ZHU SHI DOU & 猪屎豆 & Striped Crotalaria & T1826 Crotalaria mucronata \\
\hline ZHU SI HONG WEN MA XIAN & 蛛丝红纹马先蒿 & Arachnoidea Redstriate Woodbetony* & T4685 Pedicularis striata ssp. arachnoidea \\
\hline \multicolumn{4}{|l|}{ HAO } \\
\hline ZHU SUI SHAN JIANG & 柱穗山姜 & Pinnan Galangal & T0361 Alpinia pinnanensis \\
\hline ZHU TAI SHU WEI CAO & 烛台鼠尾草* & & T5666 Salvia candelabrum \\
\hline ZHU XUANG & 竹黄 & Bamboo Yellow* & T5939 Shiraia bambusicola \\
\hline ZHU YE CHAI HU & 竹叶柴胡 & Bambooleaf Thorowax & T1066 Bupleurum marginatum \\
\hline ZHU YE JIAO & 竹叶椒 & Bambooleaf Pricklyash & T6887 Zanthoxylum planispinum \\
\hline ZHU YE JIAO GEN & 竹叶椒根 & Bambooleaf Pricklyash Root & T6888 Zanthoxylum planispinum \\
\hline ZHU YE JU & 草叶蒟 & Falsenettleleaf Pepper & T4938 Piper boehmeriaefolium \\
\hline ZHU YE LAN & 竹叶兰 & Chinese Arundina & T0721 Arundina chinensis \\
\hline ZHU ZI CAO & 珠子草 & Nirur Leafflower* & T4840 Phyllanthus niruri \\
\hline ZHU ZI SHU & 珠仔树 & Racemose Sweetleaf & T6254 Symplocos racemosa \\
\hline ZHU ZONG CAO & 猪鬃草 & Southern Maidenhair & T0171 Adiantum capillus-veneris \\
\hline ZHUA KUI GUA YE WU TOU & 爪盔瓜叶乌头 & Unguiculate Hemsley Monkshood & T0102 Aconitum hemsleyanum var. leueanthus \\
\hline ZHUAN HONG REN SAN & 砖红韧伞 & Brick Tops & T4367 Naematoloma sublateritium \\
\hline ZHUANG GUAN FAN XIE & 壮观番泻* & Spectacular Senna* & T5916 Senna spectabilis \\
\hline ZHUANG GUAN JI GU CHANG & 壮观鸡骨常山* & Spectacular Alstonia* & T0376 Alstonia spectabilis \\
\hline \multicolumn{4}{|l|}{ SHAN } \\
\hline ZHUI YE CHAI HU & 雉叶柴胡 & Acicular Thorowax & T1057 Bupleurum bicaule \\
\hline ZHUN GE ER LAN PEN HUA & 准噶尔兰盆花 & & T5776 Scabiosa soongorica \\
\hline ZHUN GE ER QIAN HU & 准噶尔前胡 & Dzungaria Hogfennel* & T4763 Peucedanum morisonii \\
\hline ZHUN GE ER WU TOU & 准葛尔乌头 & Dzungaria Monkshood & T0131 Aconitum soongaricum \\
\hline ZHUO SE CI QIU & 着色刺楸* & Picture Kalopanax* & T3624 Kalopanax pictum \\
\hline ZHUO SE LI & 着色栋* & Black Oak & T5381 Quercus tinctoria \\
\hline ZHUO SE LIU LI CAO & 着色琉璃草* & Picture Houndstongue* & T1970 Cynoglossum pictum \\
\hline ZHUO SE SANG CHENG & 着色桑橙* & Tinctorial Osage Orange* & T4023 Maclura tinctoria \\
\hline ZI BAI PI & 梓白皮 & Ovate Catalpa Bast & T1261 Catalpa ovata \\
\hline ZI BEI HUANG QIN & 紫背黄芩 & Discolored Skullcap* & T5836 Scutellaria discolor \\
\hline ZI BEI JIN PAN & 紫背金盘 & Japanese Bulge & T0267 Ajuga nipponensis \\
\hline ZI BEI LU TI CAO & 紫背鹿蹄草 & Purpleback Pyrola & T5344 Pyrola atropurpurea \\
\hline ZI BEI TAI & 紫背苔 & Argentine Liverwort & T4990 Plagiochasma rupestre \\
\hline ZI BEI TIAN KUI CAO & 紫背天葵草 & Nudicaulous Grounsel Herb & T5893 Senecio nudicaulis \\
\hline ZI CAI & 紫菜 & Laver & T5171 Porphyra tenera \\
\hline ZI CAO & 紫草 & Redroot Gromwell & T3881 Lithospermum erythrorhizon \\
\hline ZI CAO RONG & 紫草茸 & Lac & T3649 Laccifer lacca \\
\hline ZI DAN SHEN & 紫丹参 & Manchurian Sage* & T5689 Salvia przewalskii var. mandarinorum \\
\hline ZI DAN TENG & 紫丹藤 & Taiwan Tournefortia* & T6480 Tournefortia sarmentosa \\
\hline ZI DI HUANG & 紫地黄 & Purple Adhesive Rehmannia* & T5448 Rehmannia glutinosa var. purpurea \\
\hline ZI DING XIANG & 紫丁香 & Early Lilac & T6260 Syringa oblata \\
\hline ZI DING XIANG MO & 紫丁香蘑 & Murasakishimeji (in Japanese) & T3766 Lepista nuda \\
\hline ZI E XIANG CHA CAI & 紫萝香茶菜 & Forrest Rabdosia* & T3490 Isodon forrestii \\
\hline ZI FAN LI ZHI & 紫番荔枝 & Soncoya & T0510 Annona purpurea \\
\hline ZI FENG DOU CAI & 紫蜂斗菜 & Purple Butterbur & T4743 Petasites officinalis [Syn. Petasites hybridu] \\
\hline ZI GUO WEI MAO & 紫果卫矛 & Eastern Wahoo & T2537 Euonymus atropurpureus \\
\hline
\end{tabular}




\begin{tabular}{|c|c|c|c|}
\hline ZI HE CHE & 紫河车 & Human Placenta & T3279 Homo sapiens \\
\hline ZI HONG ZHANG YA CAI & 紫红獐牙菜 & Scarlet Swertia & T6234 Swertia punicea \\
\hline ZI HU & 紫胡 & Sickle-leaved Hare's-ear & T1060 Bupleurum falcatum \\
\hline ZI HUA DI DING & 紫花地丁 & Tokyo Violet & $\mathrm{T} 6767$ Viola yedoensis \\
\hline ZI HUA E BEI BEI MU & 紫花鄂北贝母 & Purpleflower Fritillary & T2786 Fritillaria ebeiensis var. purpurea \\
\hline ZI HUA GAO WU TOU & 紫花高乌头 & Purpleflower High Monkshood & T0091 Aconitum excelsum \\
\hline ZI HUA GUAN MAO RUI HUA & 紫花冠毛蒿花* & Purplered-corolla Mullein* & T6706 Verbascum wiedemannianum \\
\hline ZI HUA JING TIAN & 紫花景天 & Purpleflower Stonecrop & T3318 Hylotelephium mingjinianum \\
\hline ZI HUA SONG GUO JU & 紫花松果菊 & Purple Conedaisy & T2314 Echinacea purpurea \\
\hline ZI HUA YU DENG CAO & 紫花鱼灯草 & Incised Corydalis & T1719 Corydalis incisa \\
\hline ZI JIN LIAN & 紫金莲 & Willmott Ceratostigma & T1329 Ceratostigma willmottianum \\
\hline ZI JIN NIU & 紫金牛 & Japanese Ardisia & T0598 Ardisia japonica \\
\hline ZI JIN NIU GEN & 紫金牛根 & Japanese Ardisia Root & T0599 Ardisia japonica \\
\hline ZI JIN YE TANG SONG CAO & 紫堇叶唐松草 & Corydalisleaf Meadowrue & T6395 Thalictrum isopyroides \\
\hline ZI JING ZE LAN HUA & 紫茎泽兰花 & Purplestem Eupatorium Flower & T2549 Eupatorium adenophorum \\
\hline ZI KOU CAO & 自扣草 & Canton Buttercup & T5413 Ranunculus cantoniensis \\
\hline ZI LAN SHU & 仔榄树 & Ceylon Hunteria & T3292 Hunteria zeylanica \\
\hline ZI LUO KE BAO & 紫裸壳胞 & & T2352 Emericella purpurea \\
\hline ZI MAO XIANG CHA CAI & 紫毛香茶菜 & Purplehair Rabdosia & T3486 Isodon enanderianus \\
\hline ZI MEI SHU & 姊妹树 & Garden Millingtonia & T4247 Millingtonia hortensis \\
\hline ZI MO LI GEN & 紫茉莉根 & Common Four-o'clock Root & T4252 Mirabilis jalapa \\
\hline ZI MO LI HUA BI DONG QIE & 紫茉莉花碧冬茄* & Nyctaginiflower Petunia* & T4752 Petunia nyctaginiflora \\
\hline ZI MO LI YE & 紫茉莉叶 & Common Four-o'clock Leaf & T4253 Mirabilis jalapa \\
\hline ZI MU & 梓木 & Ovate Catalpa & T1262 Catalpa ovata \\
\hline ZI QI & 紫萁 & Japanese Osmunda Frond & T4552 Osmunda japonica \\
\hline ZI RAN QIN & 孜然芹 & Cumin & T1889 Cuminum cyminum \\
\hline ZI SE BAN RUI DOU & 紫色瓣葶豆 & Purple Prairie-clover & T4736 Petalostemon purpureus \\
\hline ZI SE ZHANG YA CAI & 紫色獐芽菜* & Purple Swertia* & T6236 Swertia purpurascens \\
\hline ZI SHA ROU HAI MIAN & 紫沙肉海绵* & Sponge Psammaplysilla purpurea & T5249 Psammaplysilla purpurea \\
\hline ZI SHAN & 紫杉 & Japanese Yew & T6311 Taxus cuspidata \\
\hline ZI SHI & 梓实 & Ovate Catalpa Fruit & T1263 Catalpa ovata \\
\hline ZI SHU & 紫树 & Chinese Tupelo & T4457 Nyssa sinensis \\
\hline ZI SU & 紫苏 & Common Perilla & T4719 Perilla frutescens var. arguta \\
\hline ZI SU GENG & 紫苏梗 & Common Perilla Stem & T4720 Perilla frutescens var. arguta \\
\hline ZI SU YE & 紫苏叶 & Common Perilla Leaf & T4721 Perilla frutescens var. arguta \\
\hline ZI SUI HUAI & 紫穗槐 & Indigobush Amorpha & T0421 Amorpha fruticosa \\
\hline ZI TAN & 紫檀 & Burmacoast Padauk & T5301 Pterocarpus indicus \\
\hline ZI TENG & 紫藤 & Chinese Wisteria & T6826 Wisteria sinensis \\
\hline ZI TENG ZI & 紫藤子 & Chinese Wisteria Seed & T6827 Wisteria sinensis \\
\hline ZI TI GEN CAO & 紫德根草 & Purple Hellebore* & T3186 Helleborus purpurascens \\
\hline ZI WAN & 紫苑 & Tatarion Aster & T0781 Aster tataricus \\
\hline ZI WEI & 紫蒇 & Chinese Trumpetcreeper & T1160 Campsis grandiflora \\
\hline ZI WEI GEN & 紫薇根 & Common Crapemyrtle Root & T3670 Lagerstroemia indica \\
\hline ZI WEI HUA & 紫薇花 & Common Crapemyrtle Flower & T3671 Lagerstroemia indica \\
\hline ZI WEI JING YE & 紫葳茎叶 & Chinese Trumpetcreeper Stem-leaf & T1161 Campsis grandiflora \\
\hline ZI WEI YE & 紫薇叶 & Common Crapemyrtle Leaf & T3672 Lagerstroemia indica \\
\hline ZI WU TOU & 紫乌头* & Violet Monkshood* & T0140 Aconitum violaceum \\
\hline ZI XIAO RONG ZI & 自消容子 & Assam Crotalaria Seed & T1815 Crotalaria assamica \\
\hline ZI XUE HUA & 紫雪花 & Indian Leadword & T5028 Plumbago indica \\
\hline ZI YANG TI JIA & 紫羊蹄甲 & Purple Bauhinia & T0879 Bauhinia purpurea \\
\hline ZI YE & 梓叶 & Ovate Catalpa Leaf & T1264 Catalpa ovata \\
\hline ZI YU LAN PI & 紫玉兰皮 & Lily Magnolia Bark & T4042 Magnolia liliflora \\
\hline ZI YUN YING & 紫云英 & Chinese Milkvetch & T0805 Astragalus sinicus \\
\hline
\end{tabular}




\begin{tabular}{|c|c|c|}
\hline ZI YUN YING ZI & 紫云英子 & Chinese Milkvetch Seed \\
\hline ZI ZHI & 紫芝 & Japanese Ganoderma \\
\hline ZONG BAO GE NI DI MU & 总苞格尼迪木* & \\
\hline ZONG JUAN SHI RUI & 纵卷石荵* & \\
\hline ZONG KUI CAO SU & 棕盔踈苏* & \\
\hline ZONG LV PI & 棕㭣皮 & Fortune Windmillpalm \\
\hline ZONG ZAO & 棕藻 Ecklonia stolonifera & Brown Alga Ecklonia stolonifera \\
\hline ZONG ZHUANG DONG FENG JU & 总状东风橘叶* & Racemose Atalantia Leaf* \\
\hline \multicolumn{3}{|l|}{ YE } \\
\hline ZONG ZHUANG HUA LI & 总状花藜 & Racemose Goosefoot* \\
\hline ZONG ZHUANG HUA YANG TI JIA & 总状花羊蹄甲 & Racemose Bauhinia* \\
\hline ZONG ZHUANG JI XUE TENG & 总状鸡血藤* & Racemose Millettia* \\
\hline ZONG ZHUANG JIA RUI XIANG & 总状假瑞香 & \\
\hline ZONG ZHUANG SHENG MA & 总状升麻 & Racemose Bugbane* \\
\hline ZONG ZHUANG TIE LI MU & 总状铁力木 & Racemose Mesua* \\
\hline ZONG ZHUANG TU MU XIANG & 总状土木香 & Racemose Inula \\
\hline ZOU BO ZHUANG ZHU SHI & 皱波状猪屎豆 & Crispate Crotalaria* \\
\hline \multicolumn{3}{|l|}{ DOU } \\
\hline ZOU MA QIN & 走马芹 & Paucivitat Cowparsnip \\
\hline ZOU WEN TANG SONG CAO & 绉纹唐松草 & Rugose Meadowrue* \\
\hline ZU YE CAO & 足叶草 & Peltate Milkwort* \\
\hline ZUAN GUO SUAN JIE & 钻果蒜芥 & Hedge Mustard \\
\hline ZUI DA WAN SHOU JU & 最大万寿菊* & Maxima Marigold* \\
\hline ZUI GAO MU JING YE & 最高牡荆叶* & Highest Chastetree Leaf* \\
\hline ZUO JIANG CAO & 酢浆草 & Creeping Oxalis \\
\hline
\end{tabular}

T0806 Astragalus sinicus

T2846 Ganoderma japonicum [Syn.

Ganoderma sinense]

T3036 Gnidia involucrata

T1524 Cladonia convoluta

T4807 Phlomis brunneogaleata

T6485 Trachycarpus fortunei

T2322 Ecklonia stolonifera

T0816 Atalantia racemosa

T1363 Chenopodium championii

T0880 Bauhinia racemosa

T4242 Millettia racemosa

T2034 Daphnopsis racemosa

T1424 Cimicifuga racemosa

T4204 Mesua racemosa

T3437 Inula racemosa

T1818 Crotalaria crispata

T3218 Heracleum moellendorffii var. paucivitatum

T6407 Thalictrum rugosum

T5080 Polygala peltatum

T5968 Sisymbrium officinale

T6280 Tagetes maxima

T6783 Vitex altissima

T4564 Oxalis corniculata [Syn. Oxalis repens]

The asterisk $(*)$ following a plant name shows that the name is given by authors of the books. 


\section{TCM Plant Traditional Effect Index}

This index lists all 1311 normalized TCM traditional effects terms in English appeared in the encyclopedia in alphabetical order and the related plant code number (from T0001 to T6926) and Latin name follow the bold term immediately.

abate fever T0947 Bixa orellana, T3295 Huperzia serrata [Syn. Lycopodium serratum], T4516 Oplopanax elatus, T5626 Ruta graveolens.

abate jaundice T0672 Artemisia capillaris, T0695 Artemisia scoparia [Syn. Artemisia capillaris var. scoparia], T4538 Oroxylum indicum, T5414 Ranunculus japonicus, T6227 Swertia mussotii.

abate swelling T5740 Sargassum vachellianum.

accept $q \boldsymbol{i}$ and calm asthma T0554 Aquilaria agallocha, T0555 Aquilaria sinensis, T5270 Psoralea corylifolia.

allay thirst T0158 Actinidia chinensis, T0423 Ampelopsis brevipedunculata, T0976 Bombyx mori, T0988 Bos taurus domesticus; Bubalus bubalis, T1266 Catha edulis, T2222 Diospyros lotus, T2431 Eriobotrya japonica, T2763 Fragaria ananassa, T4102 Mangifera indica, T5332 Punica granatum, T5419 Raphanus sativus, T6347 Terminalia chebula, T6544 Triticum aestivum [Syn. Triticum vulgare].

allay vexation and thirst T3829 Ligustrum robustum.

anesthetize T0533 Antiaris toxicaria [Syn. Ambora toxicaria], T1674 Corchorus capsularis.

anesthetize and settle pain T0502 Anisodus tanguticus [Syn. Scopolia tangutica], T4323 Murraya paniculata [Syn. Chalcas paniculata], T4325 Murraya paniculata var. exotica, T5819 Scopolia acutangula [Syn. Anisodus acutangulus].

antiallergic T3936 Luffa operculata.

anticancer T0830 Auricularia auricula, T0831 Auricularia delicata, T1162 Camptotheca acuminata, T1271 Catharanthus roseus [Syn. Vinca rosea; Lochera rosea], T1318 Cephalotaxus fortunei, T1319 Cephalotaxus hainanensis [Syn. Cephalotaxus mannii], T1322 Cephalotaxus oliveri, T1323 Cephalotaxus sinensis [Syn. Cephalotaxus harringtonia var. sinensis], T1904 Curcuma kwangsiensis, T1909 Curcuma zedoaria [Syn. Curcuma aeruginosa], T2143 Dianthus chinensis, T2145 Dianthus superbus, T2147 Dianthus versicolor, T2748 Fomes fomentarius [Syn. Pyropolyporus fomentarius; Boletus fomentarius; Polyporus fomentarius], T2844 Ganoderma applanatum, T2897 Gelsemium elegans, T3229 Hericium erinaceus [Syn. Hydnum erinaceus], T3231 Hernandia sonora [Syn. Hernandia ovigera], T3742 Lentinus edodes, T4120 Marsdenia tenacissima, T4131 Maytenus confertiflorus, T4134 Maytenus hookeri, T5129 Polyporus umbellatus, T5396 Rabdosia rubescens, T6311 Taxus cuspidata, T6478 Torreya jackii.

anticarcinoma T0165 Actinidia rubricaulis var. coriacea, T3102 Haplophyllum hispanicum, T3325 Hymenoxys grandiflora, T3326 Hymenoxys odorata, T4130 Maytenus chuchuhuasca, T4366 Naematoloma fasciculare, T4367 Naematoloma sublateritium, T4457 Nyssa sinensis, T5830 Scurrura atropurpurea, T6109 Stemona collinsae. arouse liquor T0860 Balanophora japonica, T1471 Citrus chachiensis, T1472 Citrus chachiensis, T1473 Citrus cultivars, T1474 Citrus decumana, T1478 Citrus grandis, T1516 Citrus tankan, T1517 Citrus tankan, T1518 Citrus unshiu, T2758 Fortunella crassifolia, T2759 Fortunella japonica, T2760 Fortunella margarita, T4088 Malus pumila. arouse spirit T1608 Coffea arabica, T1610 Coffea liberica, T6416 Thamnolia vermicularis.

arrest diarrhea T3348 Hypericum elodeoides, T3368 Hypericum wightianum, T5268 Psidium guajava, T5517 Rhododendron molle.

astringe intestines T4635 Papaver somniferum, T4637 Papaver somniferum, T5327 Punica granatum, T5329 Punica granatum, T5332 Punica granatum, T5361 Pyrus betulaefolia, T6266 Syzygium cumini. astringe intestines and check diarrhea T3162 Helicia nilagirica, T3569 Juglans regia, T4343 Myrica esculent, T4351 Myristica fragrans, T4629 Papaver nudicaule, T4630 Papaver nudicaule ssp. amurense, T4631 Papaver nudicaule var. chinense, T5228 Prunus mume, T5328 Punica granatum, T5568 Rosa laevigata.

astringe intestines and check dysentery T5112 Polygonum periginatoris. astringe intestines and secure essence T5529 Rhodomyrtus tomentosa. astringe intestines and stanch bleeding T0255 Ailanthus altissima, T6475 Toona sinensis. 
attack gastrointestinal accumulation T2585 Euphorbia esula. attack toxin T2650 Excoecaria agallocha, T2857 Garcinia hanburyi,

T3287 Huechys sanguinea, T3327 Hyoscyamus niger.

attack toxin and cure sores T4265 Momordica cochinchinensis, T4338

Mylabris phalerata; Mylabris cichorii.

attack toxin and dissipate binds $\mathrm{T} 1084$ Buthus martensi.

attack toxin and expel stasis T4008 Lytta caraganae.

attack toxin and kill worms $\mathrm{T} 3300$ Hydnocarpus anthelminticus.

attack toxin and relieve pain T4240 Millettia pachycarpa.

boost essence T3279 Homo sapiens, T4658 Parmelia tinctorum.

boost essence and blood T1337 Cervus nippon; Cervus elaphus, T1455

Cistanche deserticola, T1456 Cistanche salsa, T1952 Cynanchum

auriculatum, T1953 Cynanchum bungei, T1972 Cynomorium

songaricum, T5927 Sesamum indicum [Syn. Sesamum orientale].

boost essence and replenish marrow T5446 Rehmannia glutinosa [Syn.

Rehmannia glutinosa f. huechingensis].

boost heart and liver T3743 Lentinus lepideus.

boost intestines and stomach $\mathrm{T} 1660$ Coprinus atramentarius.

boost kidney T5938 Setaria italica, T6073 Sphallerocarpus gracilis, T6544

Triticum aestivum [Syn. Triticum vulgare], T6750 Vicia sativa.

boost kidney and fortify spleen T1958 Cynanchum otophyllum.

boost kidney and nourish liver T5498 Rhodiola quadrifida.

boost kidney and quiet spirit T5697 Salvia trijuga.

boost kidney and secure essence T4400 Nelumbo nucifera.

boost kidney and settle asthma T6264 Syzygium buxifolium.

boost kidney and strengthen lumbus T2540 Euonymus fortunei.

boost kidney and strengthen sinews T5848 Securinega suffruticosa.

boost kidney and supplement vacuity T1017 Brassica oleracea var.

capitata.

boost liver T0541 Apis cerana.

boost liver and brighten eyes T0793 Astragalus complanatus.

boost liver and kidney T3389 Ilex cornuta, T3390 Ilex cornuta, T4828

Photinia serrulata.

boost lung T0985 Bos taurus domesticus; Bubalus bubalis.

boost lung and kidney T2195 Dioscorea deltoidea.

boost lung and quiet heart T6902 Zea mays.

boost lung and relieve cough T4350 Myriopteron extensum.

boost lung and stomach T0989 Bos taurus domesticus; Bubalus bubalis.

boost qi T1961 Cynanchum thesioides, T2837 Gallus gallus domesticus,

T5244 Prunus tomentosa, T5924 Sesamum indicum, T6150 Streptopelia orientalis.

boost $q i$ and disinhibit water T3981 Lycopus lucidus.

boost $q \boldsymbol{i}$ and engender liquid $\mathrm{T} 1596$ Codonopsis canescens, T1597

Codonopsis clematidea, T1599 Codonopsis pilosula, T1600 Codonopsis

pilosula var. modesta [Syn. Codonopsis modesta], T1601 Codonopsis

subglobosa, T1602 Codonopsis tangshen, T1603 Codonopsis tubulosa,

T3447 Ipomoea batatas [Syn. Convolvulus batatas], T5791 Schisandra

chinensis, T5802 Schisandra sphenanthera.

boost $q i$ and fortify spleen T0041 Acanthopanax senticosus [Syn.

Eleutherococcus senticosus], T0824 Atractylodes macrocephala [Syn.

Atractylis macrocephala], T1254 Castanea mollissima, T1591 Cocos

nucifera, T1593 Cocos nucifera, T4681 Pedicularis decora, T4902

Pimpinella thelungiana, T5260 Pseudostellaria heterophylla, T5465
Rhaponticum carthamoides, T6492 Trapa bispinosa.

boost $q i$ and nourish yin T0163 Actinidia latifolia, T6087 Spiranthes sinensis.

boost $q \boldsymbol{i}$ and secure exterior T0791 Astragalus chrysopterus, T0794 Astragalus ernestii, T0798 Astragalus membranaceus, T0800 Astragalus mongholicus, T3128 Hedysarum multijugum.

boost $q i$ and supplement vacuity T2962 Ginkgo biloba, T2963 Ginkgo biloba, T4820 Phoenix dactylifera.

boost qi-blood T2846 Ganoderma japonicum [Syn. Ganoderma sinense], T2848 Ganoderma lucidum.

boost stomach T4088 Malus pumila, T4099 Mangifera indica, T4103 Mangifera persiciformis.

boost stomach and engender liquid T2094 Dendrobium aduncum, T2096 Dendrobium aurantiacum var. denneanum, T2098 Dendrobium chrysanthum, T2100 Dendrobium densiflorum, T2102 Dendrobium fimbriatum var. oculatum, T2105 Dendrobium loddigesii, T2106 Dendrobium moniliforme, T2107 Dendrobium nobile, T2108 Dendrobium officinale, T2983 Glehnia littoralis.

boost wits and nourish heart $\mathrm{T} 5499$ Rhodiola sacra.

break accumulation T2584 Euphorbia ebracteolata, T2587 Euphorbia fischeriana.

break accumulation and free stool T2596 Euphorbia kansui.

break accumulation and kill worms T6105 Stellera chamaejasme, T6820 Wikstroemia indica.

break blood T3306 Hydrangea paniculata, T6062 Sparganium stoloniferum.

break blood and dispel stasis T1904 Curcuma kwangsiensis, T1909 Curcuma zedoaria [Syn. Curcuma aeruginosa].

break blood and disperse concretion T2597 Euphorbia lathyris, T4338 Mylabris phalerata; Mylabris cichorii.

break blood and disperse swelling T6267 Syzygium jambos.

break blood and free menstruation T2143 Dianthus chinensis, T2145 Dianthus superbus, T2147 Dianthus versicolor, T2536 Euonymus alatus, T5028 Plumbago indica.

break blood and move qi T1903 Curcuma aromatica, T1905 Curcuma longa.

break blood and soften hard T3412 Impatiens balsamina.

break foul T2008 Dalbergia odorifera, T2013 Dalbergia sissoo, T3868 Liquidambar orientalis.

break phlegm and disinhibit orifices T1008 Brassica juncea.

break phlegm and open orifices T2975 Gleditsia delavayi, T2976 Gleditsia fera.

break phlegm and repel foulness T6197 Styrax benzoin, T6204 Styrax tonkinensis.

break $q i$ and dissipate binds $\mathrm{T} 5137$ Poncirus trifoliata.

break $q i$ and dissipate glomus T1468 Citrus aurantium, T1521 Citrus wilsonii, T5141 Poncirus trifoliata.

break qi and move phlegm T1467 Citrus aurantium, T5140 Poncirus trifoliata.

break stasis T1210 Carpesium abrotanoides, T3287 Huechys sanguinea.

break stasis and dissipate binds T5103 Polygonum hydropiper.

break stasis and free menstruation T0661 Artemisia anomala.

brighten eyes T0819 Atractylodes chinensis, T0821 Atractylodes japonica, 
T0823 Atractylodes lancea, T0986 Bos taurus domesticus; Bubalus bubalis, T1032 Broussonetia papyrifera, T1911 Cuscuta australis, T1912 Cuscuta chinensis, T1913 Cuscuta japonica, T2244 Doellingeria scaber [Syn. Aster scaber], T2407 Equisetum arvense, T2766 Fraxinus bungeana, T2767 Fraxinus chinensis, T2774 Fraxinus paxiana, T2777 Fraxinus rhynchophylla [Syn. Fraxinus chinensis var. rhynchophylla], T2779 Fraxinus stylosa, T2780 Fraxinus szaboana [Syn. Fraxinus chinensis var. acuminata], T2803 Frullania tamarisci ssp. moniliata [Syn. Frullania moniliata], T2841 Gallus gallus domesticus, T3779 Leucas aspera, T3955 Lycium barbarum, T3958 Lycium chinense, T3969 Lycopodium casuarinoides, T4263 Momordica charantia, T4471 Ocimum basilicum, T4551 Osmorhiza aristata var. laxa, T4656 Parmelia saxatilis, T4658 Parmelia tinctorum, T5214 Prunella vulgaris, T5227 Prunus mume, T5593 Rubus idaeus, T5690 Salvia roborowskii, T6150 Streptopelia orientalis, T6437 Thlaspi arvense, T6552 Trollius chinensis [Syn. Trollius asiaticus var. chinensis], T6553 Trollius ledebourii.

brighten eyes and blacken hair T3828 Ligustrum lucidum.

brighten eyes and eliminate eye screens T1296 Celosia argentea, T2434 Eriocaulon buergerianum.

brighten eyes and transform damp T1753 Cosmos bipinnata.

brighten eyes; flower: disinhibit urine T1305 Centaurea cyanus.

calm T0184 Adonis amurensis, T0554 Aquilaria agallocha, T0555 Aquilaria sinensis, T1084 Buthus martensi, T1525 Cladonia fallax, T4243 Millettia reticulata, T4423 Nicandra physaloides, T5817 Scolopendra subspinipes mutilans, T6457 Tilia japonica, T6458 Tilia miqueliana, T6533 Trillium kamtschaticum, T6535 Trillium tschonoskii, T6562 Tulipa gesneriana.

calm and lower blood pressure T4001 Lysimachia davurica.

calm and quiet spirit T6358 Tetraplodon mnioides [Syn. Tetraplodon bryoides; Splachnum mnioides].

calm and relieve pain T6135 Stephania succifera.

calm asthma T0078 Aconitum barbatum var. puberulum [Syn. Aconitum ochranthum], T1241 Cassia occidentalis, T2039 Datura innoxia, T2043 Datura metel, T2047 Datura stramonium, T2268 Drosera rotundifolia, T2279 Dryopteris championii, T3850 Lindera glauca, T3885 Litsea cubeba, T4042 Magnolia liliflora, T4371 Nandina domestica, T4715 Perilla frutescens, T4717 Perilla frutescens var. acuta [Syn. Perilla frutescens var. purpurascens], T4719 Perilla frutescens var. arguta, T4722 Perilla frutescens var. crispa, T4796 Pheretima aspergillum; Allolobophora caliginosa trapezoides, T4908 Pinus bungeana, T5502 Rhododendron anthopogonoides, T5522 Rhododendron seniavinii, T6729 Veronica spuria.

calm liver T0544 Apium graveolens, T2219 Diospyros kaki, T3142 Helianthus annuus, T3775 Lethariella cladonioides, T3859 Linum usitatissimum, T4796 Pheretima aspergillum; Allolobophora caliginosa trapezoides, T6076 Spinacia oleracea.

calm liver and brighten eyes T1184 Capsella bursa-pastoris, T1395 Chrysanthemum morifolium [Syn. Dendranthema morifolium], T1528 Cladonia stellaris [Syn. Cladonia alpestris], T5100 Polygonum chinense, T5871 Selenarctos thibetanus; Ursus arctos.

calm liver and extinguish wind T0644 Armillaria mellea, T0645 Armillariella mellea, T6618 Uncaria homomalla [Syn. Uruparia homomalla; Uruparia tonkinensis; Uruparia lanosa var. parvifora],
T6903 Zephyranthes candida.

calm liver and lower blood pressure T0428 Ampelopsis megalophylla, T1927 Cycas revoluta, T2729 Filipendula ulmaria, T4609 Panax pseudo-ginseng var. notoginseng [Syn. Panax notoginseng].

calm liver and quiet spirit T3372 Hypodematium sinense.

calm liver and raise spirit T0210 Agaricus bisporus, T0211 Agaricus campestris.

calm liver and relieve pain T0625 Aristolochia contorta, T0627 Aristolochia debilis [Syn. Aristolochia longa], T4580 Paeonia albiflora [Syn. Paeonia lactiflora].

calm liver and settle fright T6651 Urtica cannabina, T6652 Urtica dioica. calm liver and subdue yang T1804 Cristaria plicata; Hyriopsis cumingii, T2060 Delonix regia, T2890 Gastrodia elata, T5657 Salsola collina.

check diarrhea T0360 Alpinia oxyphylla, T1299 Celosia cristata, T1493 Citrus limon, T1497 Citrus limonia, T1911 Cuscuta australis, T1912 Cuscuta chinensis, T1913 Cuscuta japonica, T1937 Cydonia oblonga, T1939 Cymbopogon citratus, T1943 Cymbopogon goeringii, T2346 Embelia oblongifolia, T3567 Juglans regia, T4625 Papaver commutatum [Syn. Papaver rhoeas], T4787 Phellinus igniarius, T5266 Psidium guajava, T5570 Rosa roxburghii, T6476 Torilis japonica, T6746 Vicia faba.

check discharge T1183 Capra hircus; Ovis aries, T1299 Celosia cristata, T2632 Euryale ferox, T4036 Magnolia coco, T5327 Punica granatum, T5329 Punica granatum, T5567 Rosa laevigata.

check dysentery T0248 Agrimonia pilosa, T0249 Agrimonia pilosa var. japonica, T0444 Ananas comosus, T0542 Apis cerana, T2032 Daphniphyllum calycinum, T2079 Delphinium kamaonense var. glabrescens, T2268 Drosera rotundifolia, T3145 Helianthus annuus, T5531 Rhus chinensis [Syn. Rhus semialata], T6347 Terminalia chebula, T6374 Thalictrum atriplex.

check dysentery and diarrhea T0197 Aegle marmelos, T2438 Erodium stephanianum, T2943 Geranium nepalense, T2944 Geranium pratense, T2947 Geranium sibiricum, T2949 Geranium wilfordii, T4545 Oryza sativa, T6670 Vaccinium bracteatum.

check dysentery and promote astriction T5573 Rosa sericea.

check flooding and spotting T2234 Dipsacus asperoides, T2235 Dipsacus japonicus.

check hiccups T1168 Canavalia ensiformis, T1169 Canavalia gladiata .

check hyperactivity T0553 Apocynum venetum.

check sweating T0824 Atractylodes macrocephala [Syn. Atractylis macrocephala], T2381 Ephedra sinica.

check tetany T0979 Bombyx mori, T1332 Cercidiphyllum japonicum.

check turbid vaginal discharge T2961 Ginkgo biloba.

check vomiting T0012 Abrus precatorius, T0554 Aquilaria agallocha, T0555 Aquilaria sinensis, T1488 Citrus junos, T2644 Evodia rutaecarpa T2646 Evodia rutaecarpa var. officinalis, T3250 Hierochloe odorata, T3286 Hovenia dulcis, T3427 Inula britannica, T3428 Inula britannica var. chinensis, T3434 Inula linariaefolia, T4099 Mangifera indica, T4103 Mangifera persiciformis, T4829 Phragmites communis.

check vomiting and diarrhea T4818 Phoebe nanmu.

check vomiting and quiet fetus T0418 Amomum muricarpum. check vomiting and stanch bleeding T0924 Bergenia purpurascens. clean heat T0687 Artemisia myriantha. 
clear and discharge large intestines T0624 Aristolochia contorta, T0626 Aristolochia debilis [Syn. Aristolochia longa], T0630 Aristolochia indica, T0633 Aristolochia maxima, T0640 Aristolochia triangularis.

clear damp heat T0902 Berberis diaphana, T1672 Corallodiscus flabellatus [Syn. Didissandra flabellat], T1759 Cotinus coggygria var. cinerea, T1966 Cynara scolymus, T2718 Ficus carica, T2830 Galium aparine, T2909 Gentiana crassicaulis, T2910 Gentiana dahurica, T2913 Gentiana kaufmanniana, T2919 Gentiana macrophylla, T2932 Gentiana siphonantha, T2934 Gentiana straminea, T2936 Gentiana tianschanica, T2937 Gentiana tibetica, T3342 Hypericum bellum, T4369 Nandina domestica, T5520 Rhododendron ovatum [Syn. Rhododendron lamprophyllum; Azalea ovata], T5604 Rudbeckia laciniata, T5942 Sida acuta.

clear fire T5214 Prunella vulgaris.

clear fire and engender liquid T4610 Panax quinquefolium.

clear head and eyes T1152 Camellia sinensis [Syn. Thea sinensis], T3392 Ilex kudingcha, T3393 Ilex latifolia, T3829 Ligustrum robustum, T4184 Mentha haplocalyx [Syn. Mentha canadaensis; Mentha arvensis var. haplocalyx; Mentha arvensis], T6791 Vitex rotundifolia [Syn. Vitex trifollia var. simplicifolia], T6793 Vitex trifolia.

clear heart T2803 Frullania tamarisci ssp. moniliata [Syn. Frullania moniliata].

clear heart and cool blood T1907 Curcuma wengujin.

clear heart and cool liver T0987 Bos taurus domesticus; Bubalus bubalis. clear heart and downbear fire T3578 Juncus effusus.

clear heart and quiet spirit T3832 Lilium brownii var. viridulum [Syn. Lilium brownii var. colchesteri], T3835 Lilium longiflorum, T3836 Lilium pumilum [Syn. Lilium tenuifolium], T3839 Lilium tigrinum [Syn. Lilium lancifolium], T4385 Narcissus tazetta var. chinensis.

clear heart fire T0632 Aristolochia manshuriensis, T4401 Nelumbo nucifera.

clear heat T0157 Actinidia callosa var. henryi, T0423 Ampelopsis brevipedunculata, T0544 Apium graveolens, T0660 Artemisia annua, T0662 Artemisia apiacea [Syn. Artemisia carvifolia; Artemisia caruifolia], T0678 Artemisia japonica, T0705 Artemisia vestita, T0784 Astilbe chinensis, T0834 Averrhoa carambola, T0888 Beesia calthaefolia, T0973 Bombax malabaricum [Syn. Gossampinus malabarica], T1045 Bubalus bubalis, T1161 Campsis grandiflora, T1165 Canarium album, T1172 Canna edulis, T1194 Carassius auratus, T1199 Carduus acanthoides, T1200 Carduus crispus, T1210 Carpesium abrotanoides, T1266 Catha edulis, T1526 Cladonia rangiferina, T1746 Corydalis taliensis, T1831 Crotalaria sessiliflora, T1878 Cucumis sativus, T2101 Dendrobium fimbriatum, T2221 Diospyros kaki, T2222 Diospyros lotus, T2263 Dregea volubilis, T2293 Dumortiera hirsuta, T2334 Elephantopus scaber, T2445 Ervatamia officinalis, T2495 Eschscholzia californica, T2649 Evolvulus alsinoides, T2994 Gloiopeltis furcata, T3085 Gynostemma pentaphyllum, T3125 Hedyotis chrysotricha [Syn. Oldenlandia chrysotricha], T3143 Helianthus annuus, T3277 Homo sapiens, T3528 Isodon ternifolia, T3529 Isodon ternifolius, T3555 Jasminum nudiflorum, T3649 Laccifer lacca, T3815 Ligularia stenocephala, T3935 Luffa cylindrica, T4055 Mahonia bealei, T4062 Mahonia fortunei, T4066 Mahonia japonica, T4072 Mahonia shenii, T4080 Mallotus apelta, T4178 Melodinus hemsleyanus, T4331 Musa paradisiaca var. sapientum [Syn. Musa sapientum], T4368 Nandina domestica, T4471 Ocimum basilicum, T4500 Onosma paniculatum, T4616 Pandanus tectorius, T4796 Pheretima aspergillum; Allolobophora caliginosa trapezoides, T4840 Phyllanthus niruri, T4848 Physalis angulata, T5120 Polygonum tinctorium, T5126 Polypodium virginianum, T5127 Polypodium vulgare, T5239 Prunus salicina, T5423 Rauvolfia verticillata, T5425 Rauvolfia verticillata f. rubrocarpa, T5426 Rauvolfia verticillata var. hainanensis, T5427 Rauvolfia vomitoria, T5435 Rauwolfia latifrons, T5441 Rauwolfia perakensis, T5484 Rhinoceros unicornis; Rhinoceros sondaicus; Rhinoceros sumatrensis, T5719 Sapindus mukorossi, T5767 Saussurea pulchella, T5857 Sedum sarmentosum, T5863 Selaginella moellendorffii, T5978 Smilax glauco-china, T6330 Tephroseris kirilowii [Syn. Senecio integrifolius var. fauriei], T6372 Thalictrum acutifolium, T6381 Thalictrum faberi, T6388 Thalictrum fortunei, T6403 Thalictrum petaloideum, T6431 Thesium chinense, T6522 Trifolium repens, T6723 Veronica arvensis, T6752 Vigna angularis [Syn. Dolichus angularis; Phaseolus angularis], T6820 Wikstroemia indica, T6912 Zinnia elegans.

clear heat and allay thirst T3234 Heteropogon contortus, T3957 Lycium chinense, T6416 Thamnolia vermicularis.

clear heat and boost yin T1950 Cynanchum ascyrifolium, T1951 Cynanchum atratum, T1962 Cynanchum versicolor, T2952 Gerbera piloselloides, T6582 Tylophora ovata.

clear heat and brighten eyes T1981 Cyprinus carpio, T5762 Saussurea nigrescens, T6072 Sphagnum palustre [Syn. Sphagnum obtusifolium; Sphagnum cymbifolium], T6426 Thermopsis chinensis, T6732 Vespertilio superans.

clear heat and calm liver T0553 Apocynum venetum, T1271 Catharanthus roseus [Syn. Vinca rosea; Lochera rosea], T1697 Cornus controversa [Syn. Bothrocaryum controversum], T2731 Firmiana simplex, T2732 Firmiana simplex, T2733 Firmiana simplex, T4843 Phyllanthus urinaria, T5428 Rauvolfia yunnanensis, T6625 Uncaria macrophylla, T6629 Uncaria rhynchophylla [Syn. Nauclea rhynchophylla], T6633 Uncaria sinensis.

clear heat and check dysentery T0511 Annona reticulata, T5299 Pteris wallichinan, T5375 Quercus mongolica.

clear heat and cool blood T0266 Ajuga macrosperma, T0379 Alternanthera philoxeroides, T0852 Bacopa monniera, T0964 Boenninghausenia albiflora, T1160 Campsis grandiflora, T2231 Diphylleia grayi, T2232 Diphylleia sinensis, T3155 Helianthus tuberosus, T3387 Idesia polycarpa, T3549 Ixora chinensis, T4148 Medicago sativa, T4506 Ophioglossum vulgatum, T4582 Paeonia delavayi, T4584 Paeonia lactiflora wild, T4585 Paeonia moutan [Syn. Paeonia suffruticosa], T4586 Paeonia obovata, T4590 Paeonia veitchii, T5447 Rehmannia glutinosa [Syn. Rehmannia glutinosa f. huechingensis], T5826 Scrophularia buergeriana, T5828 Scrophularia ningpoensis, T5851 Sedum alfredii [Syn. Sedum formosanum].

clear heat and disinhibit damp T0010 Abrus fruticulosus [Syn. Abrus cantoniensis], T0156 Actinidia arguta, T0161 Actinidia eriantha, T0180 Adina pilulifera [Syn. Cephalanthus pilulifera], T0297 Alchornea trewioides, T0424 Ampelopsis brevipedunculata var. hancei, T0428 Ampelopsis megalophylla, T0468 Anemone hupehensis, T0573 Aralia elata, T0601 Ardisia pusilla, T0672 Artemisia capillaris, T0695 
Artemisia scoparia [Syn. Artemisia capillaris var. scoparia], T0696 Artemisia sieversiana, T0751 Asparagus officinalis, T0859 Balanophora involucrata, T0906 Berberis julianae, T0933 Betula platyphylla, T0935 Betula platyphylla var. japonica, T1017 Brassica oleracea var. capitata, T1034 Broussonetia papyrifera, T1163 Campylotropis hirtella, T1184 Capsella bursa-pastoris, T1251 Cassytha filiformis, T1261 Catalpa ovata, T1275 Cayratia japonica, T1282 Cedrus deodara, T1311 Centella asiatica, T1402 Chrysosplenium alternifolium, T1561 Clerodendron serratum, T1569 Clerodendrum serratum var. amplexifolium, T1657 Conyza canadensis [Syn. Erigeron canadensis], T1733 Corydalis racemosa, T1813 Crotalaria albida, T1826 Crotalaria mucronata, T1967 Cynoglossum amabile, T1969 Cynoglossum officinale, T2056 Debregeasia longifolia, T2272 Drymaria diandra [Syn. Drymaria cordata ssp. diandra], T2348 Embelia ribes, T2719 Ficus fistulosa [Syn. Ficus harlandii], T2927 Gentiana rhodantha, T2941 Gentianopsis paludosa, T2950 Gerbera anandria [Syn. Leibnitzia anandria], T2993 Gloeostereum incarnatum, T3192 Hemerocallis citrina, T3193 Hemerocallis fulva, T3195 Hemerocallis lilio-asphodelus, T3197 Hemerocallis minor, T3243 Hibiscus rosa-sinensis, T3244 Hibiscus syriacus, T3245 Hibiscus syriacus, T3270 Holboellia fargesii, T3311 Hydrocotyle sibthorpioides, T3356 Hypericum japonicum, T3479 Isodon amethystoides, T3646 Kyllinga brevifolia, T3670 Lagerstroemia indica, T3675 Lagopsis supina, T3774 Lespedeza tomentosa, T3830 Ligustrum sinense, T3932 Ludwigia octovalvis, T3989 Lygodium flexuosum [Syn. Lygodium pinnatifidum; Ophioglossum flexuosum], T4028 Maesa indica, T4109 Marchantia polymorpha, T4149 Medicago sativa, T4217 Microcos paniculata [Syn. Grewia microcos], T4220 Microlepia strigosa [Syn. Trichomanes strigosa], T4225 Microsorium punctatum, T4248 Mimosa pudica, T4252 Mirabilis jalapa, T4361 Myrsine africana, T4370 Nandina domestica, T4538 Oroxylum indicum, T4564 Oxalis corniculata [Syn. Oxalis repens], T4823 Pholidota yunnanensis, T4831 Phtheirospermum japonicum [Syn. Geradia japonica], T4835 Phyllanthus emblica, T4836 Phyllanthus emblica, T4882 Picrasma quassioides [Syn. Picrasma ailanthoides], T5072 Polygala caudata, T5097 Polygonum amphibium, T5100 Polygonum chinense, T5101 Polygonum cuspidatum, T5124 Polypodium niponicum, T5151 Populus davidiana, T5158 Populus simonii, T5161 Populus tomentosa, T5174 Portulaca pilosa, T5220 Prunus davidiana, T5230 Prunus persica, T5232 Prunus persica, T5284 Pteridium aquilinum var. latiusculum, T5288 Pteris cretica var. nervosa [Syn. Pteris nervosa], T5290 Pteris fauriei [Syn. Pteris fauriei var. minor], T5295 Pteris multifida, T5297 Pteris plumbea, T5400 Rabdosia stracheyi, T5587 Rubus alceaefolius, T5754 Saussurea graminea, T5844 Scutellaria scordifolia, T5859 Selaginella braunii, T5860 Selaginella davidii, T5862 Selaginella involvens, T5866 Selaginella sanguinolenta, T5867 Selaginella sinensis, T5870 Selaginella uncinata, T5943 Sida cordifolia, T5954 Silene fortunei, T5957 Silybum marianum, T5966 Siphonostegia chinensis, T6005 Solanum lyratum, T6020 Solanum xanthocarpum, T6095 Stachytarpheta jamaicensis, T6250 Symplocos caudata, T6282 Tagetes patula, T6508 Trichosanthes cucumeroides.

clear heat and disinhibit $q i \mathrm{~T} 3063$ Grevillea robusta.

clear heat and disinhibit throat T0595 Ardisia crispa, T6046 Sophora viciifolia, T6047 Sophora viciifolia. clear heat and disinhibit urine T0273 Akebia quinata, T0277 Akebia trifoliata, T0279 Akebia trifoliata var. australis, T1148 Camellia saluenensis, T2161 Dicranopteris pedata [Syn. Polypodium pedatum; Dicranopteris dichotoma], T2412 Equisetum sylvaticum, T2882 Gardenia jasminoides [Syn. Gardenia florida], T3303 Hydrangea chinensis, T3921 Lophatherum gracile, T3950 Lychnis fulgens, T4194 Menyanthes trifoliata, T4636 Papaver somniferum, T5002 Plantago asiatica, T5004 Plantago depressa, T5007 Plantago major, T5171 Porphyra tenera, T5750 Saururus chinensis, T6071 Sphaeranthus indicus, T6356 Tetrapanax papyriferus, T6794 Vitis amurensis.

clear heat and disinhibit water T0628 Aristolochia fangchi, T0629 Aristolochia heterophylla, T0894 Benincasa hispida, T1197 Cardiospermum halicacabum, T2132 Desmodium pulchellum [Syn. Phyllodium pulchellum], T4274 Monostroma nitidum, T4482 Oenanthe javanica, T4847 Physalis alkekengi var. franchetii, T6357 Tetrapanax papyriferus.

clear heat and dispel damp T1359 Chenopodium album, T1553 Clerodendranthus spicatus, T3460 Iris halophila, T3464 Iris lactea var. chinensis [Syn. Iris pallasii var. chinensis], T4159 Melia azedarach.

clear heat and disperse phlegm T2525 Eucheuma muricatum.

clear heat and disperse stagnation T6339 Tephrosia purpurea.

clear heat and disperse swelling T1338 Cestrum nocturnum, T1451 Cirsium setosum [Syn. Cerratula setosa; Cirsium segetum; Cephalanoplos segetum].

clear heat and downbear fire T0328 Alnus japonica, T3233 Heteropappus altaicus.

clear heat and drain fire T0446 Anaphalis margaritacea, T0462 Anemarrhena asphodeloides, T0722 Arundo donax, T0959 Blumea lacera, T2294 Dunnia sinensis, T5309 Pterocladia tenuis, T5775 Scabiosa comosa, T6373 Thalictrum alpinum.

clear heat and dry damp T0659 Artemisia absinthium, T0897 Berberis amurensis, T0907 Berberis kawakamii, T0912 Berberis poiretii, T0916 Berberis thunbergii, T0920 Berberis wilsonae, T1038 Brucea javanica [Syn. Brucea sumatrana; Rhus javanica], T1662 Coptis chinensis, T1663 Coptis chinensis var. brevisepala, T1664 Coptis deltoidea, T1669 Coptis omeiensis, T1670 Coptis teetoides [Syn. Coptis teeta], T2079 Delphinium kamaonense var. glabrescens, T2165 Dictamnus angustifolius, T2167 Dictamnus dasycarpus, T2766 Fraxinus bungeana, T2767 Fraxinus chinensis, T2772 Fraxinus mandshurica, T2774 Fraxinus paxiana, T2777 Fraxinus rhynchophylla [Syn. Fraxinus chinensis var. rhynchophylla], T2779 Fraxinus stylosa, T2780 Fraxinus szaboana [Syn. Fraxinus chinensis var. acuminata], T2908 Gentiana cephalantha, T2921 Gentiana manshurica, T2928 Gentiana rigescens, T2930 Gentiana scabra, T2938 Gentiana triflora, T3564 Juglans mandshurica, T4060 Mahonia confusa, T4064 Mahonia gracilipes, T4158 Melia azedarach, T4479 Odontites serotina, T4887 Picrorhiza kurrooa, T4888 Picrorhiza scrophularififlora, T5833 Scutellaria amoena, T5834 Scutellaria baicalensis, T5839 Scutellaria hypericifolia, T5841 Scutellaria likiangensis, T5843 Scutellaria rehderiana, T5845 Scutellaria viscidula, T6029 Sophora alopecuroides, T6031 Sophora flavescens [Syn. Sophora angustfolia], T6039 Sophora moorcroftiana, T6375 Thalictrum baicalense, T6376 Thalictrum cultratum, T6378 Thalictrum delavayi, T6385 Thalictrum flavum, T6386 Thalictrum 
foetidum, T6387 Thalictrum foliolosum, T6389 Thalictrum glandulosissimum, T6410 Thalictrum smithii, T6474 Toona ciliata.

clear heat and eliminate damp T0635 Aristolochia moupinensis, T2134 Desmodium styracifolium, T2738 Flemingia strobilifera, T5118 Polygonum suffultum.

clear heat and eliminate vexation T3920 Lophatherum gracile, T6060 Souliea vaginata.

clear heat and engender liquid T2716 Ficus beecheyana [Syn. Ficus erecta var. beecheyana], T2717 Ficus carica, T2867 Garcinia multiflora, T3416 Imperata cylindrica var. major, T4402 Nelumbo nucifera, T4609 Panax pseudo-ginseng var. notoginseng [Syn. Panax notoginseng], T4829 Phragmites communis, T5642 Saccharum sinensis, T6512 Trichosanthes kirilowii, T6526 Triglochin maritimum.

clear heat and expel pus T1614 Coix lacryma-jobi var. ma-yuen.

clear heat and free stool T1232 Cassia fistula, T5611 Rumex japonicus, T5612 Rumex nepalensis.

clear heat and interrupt malaria T3109 Harrisonia perforata.

clear heat and lower blood pressure T2442 Ervatamia divaricata, T2444 Ervatamia heyneana.

clear heat and moisten lung T2783 Fritillaria cirrhosa, T2784 Fritillaria delavayi, T2792 Fritillaria przewalskii, T2796 Fritillaria unibracteata, T2797 Fritillaria ussuriensis, T3769 Lespedeza bicolor, T5483 Rhinacanthus nasutus, T5967 Siraitia grosvenorii [Syn. Momordica grosvenorii].

clear heat and move $q \boldsymbol{i}$ T4852 Physalis peruviana.

clear heat and nourish liver T1048 Buddleja officinalis.

clear heat and nourish yin T4605 Panax japonicus var. major.

clear heat and percolate damp T2243 Dodonaea viscosa.

clear heat and promote contraction T3732 Lawsonia inermis, T6251 Symplocos caudata.

clear heat and quicken blood T5183 Potentilla griffithii var. velutina, T6007 Solanum melongena.

clear heat and rectify damp T3360 Hypericum patulum.

clear heat and relieve cough T0608 Arenaria kansuensis [Syn. Arenaria kumaonensis], T2327 Elaeagnus angustifolia, T3926 Loropetalum chinense, T4284 Morinda parvifolia, T4760 Peucedanum japonicum, T5501 Rhodiola yunnanesis, T6521 Trifolium pratense.

clear heat and relieve pain T4143 Meconopsis horridula.

clear heat and remove damp T0776 Asplenium prolongatum, T0963 Boehmeria siamensis, T1406 Chrysosplenium nudicaule, T1884 Cudrania cochinchinensis.

clear heat and resolve exterior $\mathrm{T} 1415$ Cichorium intybus, T2113 Dennstaedtia scabra [Syn. Dicksonia scabra], T2697 Ferula borealis, T3163 Helicteres angustifolia, T4527 Orixa japonica, T6788 Vitex negundo.

clear heat and resolve phlegm T0174 Adiantum monochlamys, T0971 Bolbostemma paniculatum, T2359 Endarachne binghamiae, T2798 Fritillaria verticillata var. thunbergii [Syn. Fritillaria thunbergii], T3933 Luffa acutangula, T3934 Luffa cylindrica, T5145 Populus alba, T5720 Sapindus mukorossi, T6510 Trichosanthes kirilowii, T6513 Trichosanthes rosthornii [Syn. Trichosanthes uniflora], T6615 Uncaria gambir, T6925 Zostera marina.

clear heat and resolve summerheat T1366 Chimonanthus fragrans [Syn.
Chimonanthus praecox], T1464 Citrullus vulgaris [Syn. Citrullus lanatus], T1785 Cratoxylum prunifolium, T4398 Nelumbo nucifera, T4602 Panax ginseng [Syn. Panax schinseng], T6803 Volvariella volvacea.

clear heat and resolve toxin T0002 Abelmoschus moschatus [Syn. Hibiscus abelmoschus], T0011 Abrus precatorius, T0012 Abrus precatorius, T0045 Acanthopanax trifoliatus, T0048 Acanthus ilicifolius, T0071 Achyranthes aspera, T0072 Achyranthes aspera var. indica, T0137 Aconitum tanguticum, T0171 Adiantum capillus-veneris, T0172 Adiantum caudatum, T0175 Adiantum pedatum, T0181 Adina rubella, T0190 Adonis sutchuenensis, T0195 Aeginetia indica, T0229 Ageratum conyzoides, T0262 Ajuga ciliata, T0263 Ajuga decumbens, T0264 Ajuga forrestii, T0267 Ajuga nipponensis, T0295 Albizzia odoratissima, T0299 Aleurites cordata [Syn. Aleurites fordii], T0302 Alhagi pseudalhagi, T0333 Alocasia cucullata [Syn. Arum cucullatum], T0364 Alsophila spinulosa, T0374 Alstonia scholaris, T0378 Alstonia yunnanensis, T0388 Amaranthus lividus, T0389 Amaranthus tricolor, T0421 Amorpha fruticosa, T0426 Ampelopsis grossedentata [Syn. Ampelopsis cantoniesis var. grossedentata], T0427 Ampelopsis japonica [Syn. Paullinia japonica], T0434 Amsonia sinensis, T0457 Andrographis paniculata [Syn. Justicia paniculata], T0471 Anemone rivularis, T0513 Annona squamosa, T0561 Arachis hypogaea, T0563 Arachniodes exilis, T0565 Arachniodes simplicior, T0594 Ardisia crenata, T0600 Ardisia mamillata [Syn. Tinus mamillata], T0602 Ardisia quinquegona, T0610 Argemone mexicana, T0631 Aristolochia kaempferi, T0641 Aristolochia tuberosa, T0642 Aristolochia tubiflora, T0643 Aristolochia versicolar, T0721 Arundina chinensis, T0736 Asclepias curassavica, T0805 Astragalus sinicus, T0827 Aucuba chinensis ssp. omeiensis, T0853 Baeckea frutescens, T0860 Balanophora japonica, T0866 Baphicacanthus cusia [Syn. Strobilanthes cusia], T0867 Baphicacanthus cusia [Syn. Strobilanthes cusia], T0879 Bauhinia purpurea, T0892 Belamcanda chinensis, T0931 Betula ermanii, T0938 Bidens bipinnata, T0941 Bidens tripartita, T0952 Blechnum orientale, T0962 Boehmeria platanifolia [Syn. Boehmeria tricuspis], T0967 Boenninghausenia sessilicarpa, T0987 Bos taurus domesticus; Bubalus bubalis, T1003 Brandisia hancei, T1004 Brasenia schreberi, T1043 Bryophyllum pinnatum, T1044 Bryum argenteum, T1051 Bufo bufo gargarizans; Bufo melanostictus, T1087 Buxus bodinieri, T1092 Buxus microphylla var. sinica, T1101 Caesalpinia decapetala, T1118 Callicarpa formosana, T1119 Callicarpa japonica, T1151 Camellia sinensis [Syn. Thea sinensis], T1162 Camptotheca acuminata, T1179 Capparis masaikai, T1190 Caragana jubata, T1222 Caryopteris glutinosa, T1238 Cassia mimosoides, T1264 Catalpa ovata, T1305 Centaurea cyanus, T1316 Cephalanthus occidentalis, T1384 Chondrus ocelladus, T1387 Chrysanthemum boreale, T1392 Chrysanthemum indicum, T1393 Chrysanthemum indicum, T1394 Chrysanthemum lavandulifolium, T1412 Cicer arietinum, T1417 Cimicifuga acerina, T1419 Cimicifuga dahurica, T1420 Cimicifuga foetida, T1421 Cimicifuga heracleifolia, T1423 Cimicifuga nanchuanensis, T1425 Cimicifuga simplex, T1448 Cirsium chinense, T1530 Cladostachys amaranthoides [Syn. Achyranthes amaranthoides; Cladostachys frutescens; Deeringia amaranthoides], T1544 Cleistocalyx operculatus, T1554 Clerodendron cyrtophyllum, T1555 Clerodendron fortunatum, T1624 Colocasia antiquorum, T1633 Commelina communis, 
T1640 Coniogramme japonica [Syn. Hemionitis japonica], T1644 Conocephalum conicum, T1655 Conyza blinii, T1656 Conyza bonariensis [Syn. Erigeron bonariensis; Erigeron linifolius; Erigeron crispus], T1692 Coriaria sinica [Syn. Coriaria nepalensis], T1693 Coriaria sinica [Syn. Coriaria nepalensis], T1696 Cornus capitata [Syn. Dendrobenthamia capitata], T1727 Corydalis mucronifera, T1728 Corydalis ochotensis, T1731 Corydalis pallida, T1745 Corydalis suaveolens [Syn. Corydalis sheareri], T1747 Corydalis thalictrifolia, T1753 Cosmos bipinnata, T1784 Cratoxylum cochinchinense, T1787 Cremastra appendiculata, T1796 Crinum asiaticum var. sinicum, T1799 Crinum latifolium, T1810 Croomia japonica, T1866 Cryptolepis sinensis, T1931 Cyclea barbata, T1933 Cyclea sutchuenensis, T1934 Cyclea tonkinensis, T1954 Cynanchum chinense, T1971 Cynoglossum zeylanicum [Syn. Anchusa zeylanica; Cynoglossum furcatum; Cynoglossum formosanum], T1986 Cyrtomium fortunei, T1996 Dahlia pinnata [Syn. Dahlia variabilis], T2033 Daphniphyllum macropodum, T2050 Daucus carota var. sativa, T2058 Deeringia amaranthoides [Syn. Cladostachys frutescens], T2131 Desmodium pulchellum [Syn. Phyllodium pulchellum], T2157 Dichotomanthes tristaniaecarpa, T2162 Dicranostigma franchetianum [Syn. Dicranostigma leptopodum], T2191 Dioscorea bulbifera, T2193 Dioscorea cirrhosa [Syn. Dioscorea pogonoides], T2194 Dioscorea collettii, T2200 Dioscorea hispida, T2206 Dioscorea parviflora, T2218 Diospyros kaki, T2233 Diploclisia glaucescens, T2244 Doellingeria scaber [Syn. Aster scaber], T2279 Dryopteris championii, T2280 Dryopteris chrysocoma, T2281 Dryopteris crassirhizoma, T2282 Dryopteris filix-mas, T2290 Duchesnea indica, T2298 Dysosma difformis, T2302 Dysosma pleiantha [Syn. Podophyllum pleianthum], T2304 Dysosma veitchii, T2305 Dysosma versipellis [Syn. Podophyllum versipelle], T2316 Echinops grijsii, T2317 Echinops ritro, T2357 Emilia sonchifolia, T2387 Epilobium hirsutum, T2421 Erigeron annuus, T2510 Eucalyptus globulus, T2517 Eucalyptus robusta, T2529 Euchresta strigillosa, T2555 Eupatorium chinense, T2590 Euphorbia hirta, T2591 Euphorbia humifusa, T2628 Euphrasia officinalis, T2641 Evodia lepta [Syn. Ilex lepta], T2657 Fagopyrum cymosum [Syn. Polygonum cymosum], T2659 Fagopyrum esculentum, T2691 Farfugium japonicum, T2715 Fibraurea recisa, T2730 Fimbristylis dichotoma, T2732 Firmiana simplex, T2756 Forsythia suspensa, T2757 Forsythia viridissima, T2826 Galeola faberi, T2835 Galium verum, T2839 Gallus gallus domesticus, T2852 Garcinia cowa, T2884 Gardenia jasminoides var. grandiflora, T2973 Glechoma longituba, T2974 Glechoma lungituba, T2988 Glochidion eriocarpum, T2989 Glochidion sphaerogynum, T3092 Halenia corniculata, T3122 Hedyotis acutangula, T3123 Hedyotis auricularia, T3126 Hedyotis corymbosa [Syn. Oldenlandia corymbosa], T3174 Heliotropium indicum, T3187 Helleborus thibetanus, T3200 Hemidesmus indicus, T3201 Hemiphragma heterophyllum, T3202 Hemistepta lyrata [Syn. Hemistepta carthamoides; Saussurea carthamoides], T3203 Hemsleya amabilis, T3208 Hemsleya macrosperma, T3241 Hibiscus mutabilis, T3283 Hosta sieboldiana, T3284 Houttuynia cordata, T3288 Humulus japonicus [Syn. Humulus scandens], T3292 Hunteria zeylanica, T3295 Huperzia serrata [Syn. Lycopodium serratum], T3308 Hydrangea umbellata, T3318 Hylotelephium mingjinianum, T3323 Hymenodictyon excelsum, T3332 Hypecoum erectum, T3334 Hypecoum leptocarpum,
T3340 Hypericum ascyron, T3348 Hypericum elodeoides, T3361 Hypericum perforatum, T3363 Hypericum sampsonii, T3368 Hypericum wightianum, T3376 Hypolepis punctata [Syn. Polypodium punctatum], T3388 Ilex chinensis [Syn. Ilex purpurea], T3394 Ilex paraguariensis, T3396 Ilex pubescens, T3397 Ilex pubescens var. glaber, T3398 Ilex rotunda, T3414 Impatiens siculifer, T3423 Indigofera tinctoria, T3448 Ipomoea cairica [Syn. Ipomoea palmata], T3457 Iris dichotoma, T3461 Iris japonica, T3466 Iris potaninii, T3471 Iris tectorum, T3475 Isatis indigotica, T3476 Isatis indigotica, T3479 Isodon amethystoides, T3495 Isodon irrorata, T3496 Isodon japonica [Syn. Rabdosia japonica], T3507 Isodon lophanthoides [Syn. Rabdosia lophanthoides; Hyssopus lophanthoides; Plectranthus striatus; Isodon striatus], T3547 Ixeris chinensis, T3548 Ixeris sonchifolia, T3588 Juniperus formosana, T3645 Kummerowia striata, T3659 Lactuca indica, T3662 Lactuca sativa, T3671 Lagerstroemia indica, T3672 Lagerstroemia indica, T3736 Lemmaphyllum microphyllum, 13737 Lemmaphyllum microphyllum var. obovatum, T3738 Lemna minor, T3764 Lepisorus thunbergianus, T3765 Lepisorus ussuriensis, T3770 Lespedeza cuneata, T3845 Linaria vulgaris, T3863 Liparis nervosa, T3878 Lithocarpus polystachyus, T3898 Lobelia chinensis [Syn. Lobelia radicans], T3908 Lonicera bournei, T3909 Lonicera confusa, T3910 Lonicera fulvotomentosa, T3911 Lonicera hypoglauca, T3912 Lonicera japonica, T3913 Lonicera japonica, T3914 Lonicera macranthoides, T3918 Lonicera similis, T3928 Lotus corniculatus, T3953 Lycianthes biflora, T3990 Lygodium japonicum, T3996 Lysimachia candida, T3998 Lysimachia christinae, T4006 Lythrum anceps, T4007 Lythrum salicaria, T4009 Maackia amurensis, T4026 Macrothelypteris oligophlebia, T4082 Mallotus japonicus, T4091 Malva sylvestris, T4120 Marsdenia tenacissima, T4121 Marsilea quadrifolia, T4124 Matricaria chamomilla [Syn. Matricaria recutita], T4127 Matteuccia struthiopteris, T4144 Meconopsis nepaulensis, T4145 Meconopsis punicea, T4170 Melilotus albus, T4173 Melilotus suaveolens, T4182 Menispermum dauricum, T4183 Menispermum dauricum, T4208 Metasequoia glyptostroboides, T4215 Michelia yunnanensis, T4219 Microlepia marginata, T4227 Microula sikkimensis, T4253 Mirabilis jalapa, T4280 Morinda citrifolia, T4384 Narcissus tazetta var. chinensis, T4391 Nauclea officinalis, T4423 Nicandra physaloides, T4456 Nymphoides peltatum, T4485 Oldenlandia diffusa [Syn. Hedyotis diffusa], T4502 Onychium japonicum [Syn. Tricomanes japonicum], T4503 Onychium lucidum, T4514 Ophiorrhiza pumila, T4530 Ormosia hosiei, T4552 Osmunda japonica, T4562 Oxalis acetosella, T4568 Oxytropis myriophylla, T4648 Paris polyphylla, T4649 Paris polyphylla var. chinensis, T4650 Paris polyphylla var. pseudothibetica, T4651 Paris polyphylla var. stenophylla, T4652 Paris polyphylla var. yunnanensis, T4672 Patrinia scabiosaefolia, T4676 Patrinia villosa, T4686 Pedilanthus tithymaloides, T4691 Pelargonium hortorum, T4705 Peperomia pellucida, T4710 Pericampylus glaucus, T4730 Peristrophe roxburghiana, T4740 Petasites japonicus, T4798 Philonotis fontana, T4800 Phlegmariurus fordii, T4801 Phlegmariurus phlegmaria [Syn. Lycopodium phlegmaria], T4803 Phlogacanthus curviflorus, T4827 Photinia parvifolia, T4837 Phyllanthus emblica, T4838 Phyllanthus flexuosus, T4845 Physalis alkekengi, T4846 Physalis alkekengi var. franchetii, T4854 Physalis pubescens, T4859 Physochlaina physaloides, T4881 Picrasma quassioides [Syn. Picrasma 
ailanthoides], T4884 Picria felterrae, T4998 Plagiogyria euphlebia, T5000 Plagiogyria stenoptera, T5018 Pleuropterus ciliinervis, T5033 Poa sphondylodes, T5061 Polianthes tuberosa, T5099 Polygonum bistorta, T5109 Polygonum nodosum, T5110 Polygonum orientale, T5114 Polygonum polystachyum, T5121 Polygonum tinctorium, T5149 Populus canadensis, T5150 Populus cathayana, T5172 Portulaca grandiflora, T5173 Portulaca oleracea, T5176 Potamogeton natans, T5177 Potamogeton pectinatus, T5181 Potentilla chinensis, T5182 Potentilla discolor, T5188 Potentilla viscosa, T5194 Premna microphylla, T5199 Primula malacoides, T5209 Pronephrium simplex [Syn. Meniscium simplex], T5267 Psidium guajava, T5289 Pteris dactylina, T5323 Pulsatilla campanella, T5324 Pulsatilla cernua, T5325 Pulsatilla chinensis, T5326 Pulsatilla dahurica, T5363 Pyrus calleryana, T5388 Rabdosia adenantha, T5393 Rabdosia longituba, T5396 Rabdosia rubescens, T5397 Rabdosia serra, T5407 Rana nigromaculata; Rana plancyi, T5408 Rana nigromaculata; Rana plancyi, T5415 Ranunculus sceleratus, T5424 Rauvolfia verticillata, T5444 Reboulia hemisphaerica, T5455 Rhamnus crenata, T5457 Rhamnus davurica, T5466 Rhaponticum uniflorum, T5468 Rheum emodi [Syn. Rheum australe], T5497 Rhodiola kirilowii, T5524 Rhododendron simsii, T5557 Rohdea japonica [Syn. Orontium japonicum], T5567 Rosa laevigata, T5569 Rosa multiflora, T5576 Rostellularia procumbens [Syn. Justicia procumbens], T5592 Rubus hirsutus, T5595 Rubus parviforlius, T5607 Rumex crispus, T5608 Rumex dentatus, T5613 Rumex obtusifolius, T5614 Rumex patientia, T5654 Salix purpurea, T5685 Salvia plebeia, T5712 Sanguisorba officinalis, T5714 Sansevieria trifasciata, T5730 Sarcandra glabra [Syn. Chloranthus glaber], T5741 Sargentodoxa cuneata, T5797 Schisandra propinqua, T5798 Schisandra propinqua var. intermedia, T5818 Scoparia dulcis, T5835 Scutellaria barbata [Syn. Scutellaria rivularis], T5836 Scutellaria discolor, T5838 Scutellaria galericulata, T5840 Scutellaria indica, T5852 Sedum bulbiferum, T5856 Sedum lineare [Syn. Sedum obtuso-lineare], T5861 Selaginella doederleinii, T5871 Selenarctos thibetanus; Ursus arctos, T5884 Senecio chrysanthemoides, T5892 Senecio nemorensis, T5894 Senecio oryzetorum, T5907 Senecio scandens [Syn. Senecio chinensis], T5950 Siegesbeckia orientalis, T5951 Siegesbeckia orientalis var. glabrescens [Syn. Siegesbeckia glabrescens], T5952 Siegesbeckia orientalis var. pubescens [Syn. Siegesbeckia pubescens], T5953 Silene firma, T5998 Solanum dulcamara, T6003 Solanum khasianum, T6008 Solanum nigrum, T6025 Sonchus arvensis, T6026 Sonchus asper [Syn. Sonchus oleraceus var. asper], T6032 Sophora flavescens [Syn. Sophora angustfolia], T6043 Sophora subprostrata [Syn. Sophora tonkinensis], T6087 Spiranthes sinensis, T6092 Stachys palustris, T6116 Stenoloma chusanum, T6118 Stephania brachyandra, T6119 Stephania cepharantha, T6121 Stephania dicentrinifera, T6122 Stephania dielsiana, T6125 Stephania elegans, T6128 Stephania hernandifolia, T6129 Stephania japonica, T6131 Stephania longa, T6133 Stephania sinica, T6135 Stephania succifera, T6137 Stephania viridiflavens, T6139 Sterculia lychnophora, T6205 Sus scrofa, T6206 Sus scrofa domestica, T6214 Swertia chinensis, T6217 Swertia davidii, T6219 Swertia erythrosticta, T6224 Swertia kouitchensis, T6226 Swertia mileensis, T6228 Swertia nervosa, T6234 Swertia punicea, T6252 Symplocos chinensis, T6260 Syringa oblata, T6275 Tacca chantrieri [Syn. Tacca minor; Tacca esquirolii], T6277 Tadehagi triquetrum, T6278 Tagetes erecta, T6279 Tagetes erecta, T6285 Tamarindus indica, T6295 Tanacetum sibiricum [Syn. Filifolium sibiricum], T6301 Taraxacum mongolicum, T6365 Teucrium pilosum [Syn. Teucrium japonicum var. pilosum], T6374 Thalictrum atriplex, T6393 Thalictrum ichangense [Syn. Thalictrum tripeltatum; Thalictrum multipeltatum], T6398 Thalictrum microgynum, T6402 Thalictrum omeiense, T6409 Thalictrum simplex [Syn. Thalictrum simplex var. brevipes], T6412 Thalictrum squarrosum, T6414 Thalictrum thunbergii, T6432 Thespesia populnea [Syn. Hibiscus populneus], T6435 Thladiantha cordifolia, T6436 Thlaspi arvense, T6463 Tinospora capillipes, T6467 Tinospora sagittata, T6469 Tithonia diversifolia, T6537 Tripterospermum japonicum, T6538 Tripterospermum taiwanense, T6552 Trollius chinensis [Syn. Trollius asiaticus var. chinensis], T6553 Trollius ledebourii, T6554 Trollius macropetalus, T6555 Tropaeolum majus, T6560 Tulipa edulis, T6566 Tupistra chinensis, T6567 Tupistra wattii [Syn. Campylandra wattii], T6598 Ulva conglobata, T6599 Ulva lactuca, T6600 Ulva pertusa, T6601 Umbilicaria esculenta [Syn. Gyrophora esculenta], T6645 Urena lobata, T6653 Usnea diffracta, T6654 Usnea longissima, T6655 Ustilaginoidea virens, T6684 Vanilla planifolia, T6691 Veratrilla baillonii, T6705 Verbascum thapsus, T6716 Vernonia esculenta, T6722 Veronica anagallis-aquatica, T6731 Veronicastrum sibiricum, T6735 Viburnum dilatatum, T6767 Viola yedoensis, T6814 Wedelia chinensis [Syn. Solidago chinensis; Wedelia calendulacea].

clear heat and settle fright T0942 Biebersteinia heterostemon, T5184 Potentilla kleiniana.

clear heat and stanch bleeding T1112 Calendula arvensis, T5354 Pyrrosia davidii, T5355 Pyrrosia drakeana, T5356 Pyrrosia gralla, T5357 Pyrrosia lingua' T5358 Pyrrosia petiolosa, T5360 Pyrrosia sheareri.

clear heat and transform damp T1106 Caesalpinia minax, T2984 Glinus lotoides [Syn. Mollugo lotoides], T4261 Mollugo pentaphylla, T4799 Philydrum lanuginosum, T5554 Rodgersia aesculifolia, T6049 Sophora viciifolia.

clear heat toxin T1709 Corydalis bungeana.

clear intestines and dry damp T6028 Sophora alopecuroides.

clear liver T1241 Cassia occidentalis, T1242 Cassia occidentalis, T5690 Salvia roborowskii.

clear liver and brighten eyes T0049 Acer ginnala, T0984 Bos taurus domesticus; Bubalus bubalis, T1035 Broussonetia papyrifera, T1236 Cassia laevigata [Syn. Cassia floribunda], T1240 Cassia obtusifolia, T1250 Cassia tora, T1746 Corydalis taliensis, T1804 Cristaria plicata; Hyriopsis cumingii, T2336 Elephas maximus, T2772 Fraxinus mandshurica, T3040 Gomphrena globosa, T3635 Koelreuteria paniculata, T3636 Koelreuteria paniculata, T4289 Morus alba, T4291 Morus australis, T4293 Morus cathayana, T4298 Morus mongolica, T4976 Piptanthus nepalensis, T5413 Ranunculus cantoniensis, T6034 Sophora japonica, T6036 Sophora japonica.

clear liver and disinhibit gallbladder T1705 Corydalis adunca, T6227 Swertia mussotii, T6234 Swertia punicea, T6901 Zea mays.

clear liver and relieve constipation T0338 Aloe ferox, T0347 Aloe vera [Syn. Aloe barbadensis], T0348 Aloe vera var. chinensis.

clear liver fire T1296 Celosia argentea, T3827 Ligustrum japonicum, T4401 Nelumbo nucifera. 
clear lung T1241 Cassia occidentalis, T2790 Fritillaria pallidiflora, T2800 Fritillaria walujewii, T3242 Hibiscus rosa-sinensis, T4388 Nasturtium officinale, T5521 Rhododendron przewalskii.

clear lung and boost qi T5494 Rhodiola crenulata [Syn. Rhodiola euryphylla].

clear lung and calm liver T1150 Camellia sinensis [Syn. Thea sinensis]. clear lung and disinhibit throat T3701 Lasiosphaera fenzlii, T3961

Lycoperdon pyriforme, T4537 Oroxylum indicum, T6139 Sterculia lychnophora.

clear lung and downbear fire T0746 Asparagus cochinchinensis [Syn. Asparagus lucidus].

clear lung and downbear $q \boldsymbol{i}$ T0624 Aristolochia contorta, T0626 Aristolochia debilis [Syn. Aristolochia longa], T0630 Aristolochia indica, T0633 Aristolochia maxima, T0640 Aristolochia triangularis.

clear lung and engender liquid T4573 Pachyrrhizus erosus.

clear lung and moisten dryness T2301 Dysosma majorensis [Syn. Podophyllum majorense; Dysosma lichuanensis], T4289 Morus alba, T4291 Morus australis, T4293 Morus cathayana, T4298 Morus mongolica, T4665 Passiflora edulis.

clear lung and relieve cough T0173 Adiantum lunulatum, T0759 Aspidistra elatior, T1705 Corydalis adunca, T1789 Crepis napifera, T2213 Dioscorea zingiberensis, T2433 Eriobotrya japonica, T2565 Eupatorium lindleyanum, T3247 Hibiscus taiwanensis, T3248 Hibiscus tiliaceus, T3373 Hypoestes purpurea [Syn. Justicia purpurea; Hypoestes sinica], T3736 Lemmaphyllum microphyllum, T3737 Lemmaphyllum microphyllum var. obovatum, T4520 Opuntia ficus-indica, T5449 Reineckea carnea, T6304 Taxillus levinei.

clear lung and suppress cough T6055 Sorbus tianschanica.

clear lung and transform phlegm T0895 Benincasa hispida, T1006 Brassica chinensis, T1465 Citrullus vulgaris [Syn. Citrullus lanatus], T1967 Cynoglossum amabile, T3190 Helminthostachys zeylanica, T3246 Hibiscus syriacus, T6511 Trichosanthes kirilowii.

clear lung heat T6581 Tylophora mollissima.

clear summerheat T2554 Eupatorium cannabinum, T4335 Mussaenda pubescens, T4526 Origanum vulgare, T4979 Pistacia chinensis, T5227 Prunus mume.

clear summerheat and eliminate damp T4307 Mosla scabra [Syn. Mosla punctata], T4399 Nelumbo nucifera.

clear summerheat and resolve toxin T0537 Antirrhinum majus, T1595 Codium fragile, T5402 Radermachera sinica.

clear summerheat and transform damp T4172 Melilotus suaveolens. clear summerheat heat T4101 Mangifera indica, T6492 Trapa bispinosa. clear vacuity fire T1961 Cynanchum thesioides.

clear vacuity heat T0607 Arenaria juncea, T3090 Gypsophila oldhamiana, T3091 Gypsophila pacifica, T3390 Ilex cornuta, T4056 Mahonia bealei, T4057 Mahonia bealei, T4063 Mahonia fortunei, T4067 Mahonia japonica, T4068 Mahonia japonica, T5955 Silene jenisseensis, T6104 Stellaria dichotoma var. lanceolata.

close sores T0563 Arachniodes exilis, T1336 Ceriops tagal [Syn. Rhizophora tagal], T1594 Cocos nucifera, T1777 Crataegus pinnatifida, T2598 Euphorbia lathyris, T3673 Lagerstroemia speciosa [Syn. Munchausia speciosa; Lagerstroemia flos-reginae], T3775 Lethariella cladonioides, T5228 Prunus mume, T5266 Psidium guajava, T6745 Vicia faba, T6747 Vicia faba.

close sores and engender flesh T0019 Acacia catechu, T0427 Ampelopsis japonica [Syn. Paullinia japonica], T0791 Astragalus chrysopterus, T0794 Astragalus ernestii, T0798 Astragalus membranaceus, T0800 Astragalus mongholicus, T1896 Cupressus funebris, T1994 Daemonorops draco, T2253 Dracaena cochinchinensis, T3128 Hedysarum multijugum, T3388 Ilex chinensis [Syn. Ilex purpurea].

close sores and stanch bleeding $\mathrm{T} 3857$ Lindera umbellata [Syn. Lindera erythrocarpa], T5290 Pteris fauriei [Syn. Pteris fauriei var. minor].

constrain blood T5142 Poncirus trifoliata.

constrain lung T4637 Papaver somniferum, T5361 Pyrus betulaefolia, T5374 Quercus infectoria.

constrain lung and astringe intestines T2964 Ginkgo biloba, T6346 Terminalia chebula, T6348 Terminalia chebula var. tomentella.

constrain lung and relieve cough T2454 Erysimum diffusum, T4371 Nandina domestica, T4629 Papaver nudicaule, T4630 Papaver nudicaule ssp. amurense, T4631 Papaver nudicaule var. chinense, T5228 Prunus mume.

constrain lung and settle asthma T2961 Ginkgo biloba, T6266 Syzygium cumini.

constrain sweat T0944 Biota orientalis [Syn. Thuja orientalis; Platycladus orientalis], T4548 Oryza sativa var. glutinosa, T5531 Rhus chinensis [Syn. Rhus semialata], T6918 Ziziphus jujuba var. spinosa, T6919 Ziziphus jujuba var. spinosa.

constrain sweat and astringe intestines T5821 Scopoliajaponica. constrain sweat and dry damp T4670 Patrinia heterophylla, T4673 Patrinia scabra.

constrain sweat and secure exterior T3129 Hedysarum polybotrys. constrain yin and check sweating T4580 Paeonia albiflora [Syn. Paeonia lactiflora].

consume sore and kill worms $\mathrm{T} 1858$ Croton tiglium.

contract damp T0019 Acacia catechu.

contract damp and close sores T6615 Uncaria gambir.

contract damp and kill worms T0299 Aleurites cordata [Syn. Aleurites fordii].

contract damp and relieve itch T1316 Cephalanthus occidentalis, T6339 Tephrosia purpurea, T6476 Torilis japonica.

contract uterus and stanch bleeding T1541 Claviceps purpurea.

cool blood T0607 Arenaria juncea, T0678 Artemisia japonica, T1045 Bubalus bubalis, T1161 Campsis grandiflora, T2334 Elephantopus scaber, T3090 Gypsophila oldhamiana, T3091 Gypsophila pacifica, T3242 Hibiscus rosa-sinensis, T3334 Hypecoum leptocarpum, T3649 Laccifer lacca, T4388 Nasturtium officinale, T4402 Nelumbo nucifera, T4500 Onosma paniculatum, T5002 Plantago asiatica, T5004 Plantago depressa, T5007 Plantago major, T5018 Pleuropterus ciliinervis, T5120 Polygonum tinctorium, T5484 Rhinoceros unicornis; Rhinoceros sondaicus; Rhinoceros sumatrensis, T5955 Silene jenisseensis, T6048 Sophora viciifolia, T6522 Trifolium repens.

cool blood and check diarrhea T2993 Gloeostereum incarnatum, T5323 Pulsatilla campanella, T5324 Pulsatilla cernua, T5325 Pulsatilla chinensis, T5326 Pulsatilla dahurica.

cool blood and check dysentery T5181 Potentilla chinensis.

cool blood and clear heat T3444 Ipomoea aquatica [Syn. Convolvulus 
repens; Ipomoea reptans], T5545 Ribes fasciculatum var. chinense.

cool blood and disinhibit throat T3475 Isatis indigotica.

cool blood and disperse macula T3476 Isatis indigotica, T5121

Polygonum tinctorium.

cool blood and disperse stasis T6276 Tacca plantaginea [Syn. Schizocapsa plantaginea].

cool blood and disperse swelling T0262 Ajuga ciliata, T0866 Baphicacanthus cusia [Syn. Strobilanthes cusia], T3123 Hedyotis auricularia, T6046 Sophora viciifolia, T6047 Sophora viciifolia, T6484 Trachelospermum jasminoides, T6767 Viola yedoensis.

cool blood and disperse welling abscess T5680 Salvia miltiorrhiza, T5681 Salvia miltiorrhiza f. alba, T5688 Salvia przewalskii.

cool blood and dissipate blood T0263 Ajuga decumbens.

cool blood and dissipate stasis T0267 Ajuga nipponensis, T1034 Broussonetia papyrifera, T4564 Oxalis corniculata [Syn. Oxalis repens], T5685 Salvia plebeia, T6814 Wedelia chinensis [Syn. Solidago chinensis; Wedelia calendulacea].

cool blood and extinguish wind T1986 Cyrtomium fortunei, T4730 Peristrophe roxburghiana.

cool blood and quicken blood T0648 Arnebia euchroma, T0649 Arnebia guttata, T1448 Cirsium chinense, T3881 Lithospermum erythrorhizon, T5647 Sagittaria sagittifolia, T6092 Stachys palustris.

cool blood and relieve pain T4479 Odontites serotina.

cool blood and resolve toxin T0297 Alchornea trewioides, T0753 Asparagus setaceus [Syn. Asparagus plumosus], T0875 Basella rubra, T1197 Cardiospermum halicacabum, T1251 Cassytha filiformis, T2927 Gentiana rhodantha, T3002 Glycine max, T3192 Hemerocallis citrina, T3244 Hibiscus syriacus, T3247 Hibiscus taiwanensis, T3933 Luffa acutangula, T3934 Luffa cylindrica, T4248 Mimosa pudica, T4520 Opuntia ficus-indica, T4658 Parmelia tinctorum, T4836 Phyllanthus emblica, T5100 Polygonum chinense, T5156 Populus nigra var. thevestina, T5472 Rheum officinale, T5474 Rheum palmatum, T5481 Rheum tanguticum, T5646 Sagina japonica [Syn. Spergula japonica], T5773 Saxifraga stolonifera, T6367 Teucrium quadrifarium.

cool blood and stanch bleeding T0226 Agave sisalana, T0687 Artemisia myriantha, T0696 Artemisia sieversiana, T0805 Astragalus sinicus, T0859 Balanophora involucrata, T0867 Baphicacanthus cusia [Syn. Strobilanthes cusia], T0890 Begonia limprichtii, T1043 Bryophyllum pinnatum, T1116 Callicarpa arborea, T1113 Calendula officinalis, T1145 Camellia japonica, T1199 Carduus acanthoides, T1200 Carduus crispus, T1212 Carpesium eximum, T1299 Celosia cristata, T1449 Cirsium japonicum, T1451 Cirsium setosum [Syn. Cerratula setosa; Cirsium segetum; Cephalanoplos segetum], T1529 Cladonia verticillata, T1547 Clematis terniflora [Syn. Clematis maximowicziana], T1554 Clerodendron cyrtophyllum, T1684 Cordyline strcta, T1896 Cupressus funebris, T2191 Dioscorea bulbifera, T2218 Diospyros kaki, T2281 Dryopteris crassirhizoma, T2282 Dryopteris filix-mas, T2290 Duchesnea indica, T2323 Eclipta prostrata [Syn. Eclipta alba], T2412 Equisetum sylvaticum, T2591 Euphorbia humifusa, T2691 Farfugium japonicum, T3092 Halenia corniculata, T3193 Hemerocallis fulva, T3195 Hemerocallis lilio-asphodelus, T3197 Hemerocallis minor, T3241 Hibiscus mutabilis, T3340 Hypericum ascyron, T3363 Hypericum sampsonii, T3373 Hypoestes purpurea [Syn. Justicia purpurea;
Hypoestes sinica], T3416 Imperata cylindrica var. major, T3423 Indigofera tinctoria, T3547 Ixeris chinensis, T3681 Lamium barbatum, T3736 Lemmaphyllum microphyllum, T3737 Lemmaphyllum microphyllum var. obovatum, T3863 Liparis nervosa, T4009 Maackia amurensis, T4259 Mnium cuspidatum, T4398 Nelumbo nucifera, T4519 Opuntia dillenii, T4521 Opuntia vulgaris, T5133 Polytrichum commune, T5173 Portulaca oleracea, T5182 Potentilla discolor, T5207 Prismatomeris tetrandra, T5295 Pteris multifida, T5449 Reineckea carnea, T5557 Rohdea japonica [Syn. Orontium japonicum], T5578 Rubia cordifolia, T5582 Rubia oncotricha, T5583 Rubia schumannina, T5584 Rubia tinctorum, T5585 Rubia wallichiana, T5605 Rumex acetosa, T5607 Rumex crispus, T5611 Rumex japonicus, T5612 Rumex nepalensis, T5613 Rumex obtusifolius, T5701 Salvia yunnanensis, T5712 Sanguisorba officinalis, T5754 Saussurea graminea, T5852 Sedum bulbiferum, T5868 Selaginella stauntoniana, T6034 Sophora japonica, T6036 Sophora japonica, T6119 Stephania cepharantha, T6250 Symplocos caudata, T6440 Thuja orientalis [Syn. Platycladus orientalis; Biota orientalis], T6508 Trichosanthes cucumeroides, T6584 Typha angustata, T6585 Typha angustifolia, T6587 Typha latifolia, T6601 Umbilicaria esculenta [Syn. Gyrophora esculenta], T6904 Zephyranthes grandiflora [Syn. Zephyranthes carinata].

cool blood and supplement blood T5445 Rehmannia glutinosa [Syn. Rehmannia glutinosa f. huechingensis].

cool liver T1526 Cladonia rangiferina, T6374 Thalictrum atriplex.

cool liver and resolve toxin T2258 Dracocephalum rupestre.

cool liver and settle fright T4648 Paris polyphylla, T4649 Paris polyphylla var. chinensis, T4650 Paris polyphylla var. pseudothibetica, T4651 Paris polyphylla var. stenophylla, T4652 Paris polyphylla var. yunnanensis.

cool liver and stanch bleeding T1184 Capsella bursa-pastoris, T2257 Dracocephalum moldavicum, T5606 Rumex acetosa.

course liver and disinhibit gallbladder T1907 Curcuma wengujin, T1966 Cynara scolymus, T5957 Silybum marianum.

course liver and free network vessels T3360 Hypericum patulum.

course liver and harmonize stomach T0275 Akebia quinata, T4537

Oroxylum indicum.

course liver and quicken blood T2555 Eupatorium chinense.

course liver and rectify $q i \mathrm{~T} 1479$ Citrus grandis.

course liver and resolve depression T5137 Poncirus trifoliata.

course wind T1675 Corchorus olitorius, T6889 Zanthoxylum podocarpum. course wind and clear heat T0015 Abutilon indicum, T0167 Adenanthera pavonina, T0948 Blainvillea acmella [Syn. Verbesina acmella; Eclipta latifolia; Blainvillea latifolia], T1395 Chrysanthemum morifolium [Syn. Dendranthema morifolium], T1410 Chukrasia tabularis, T1573 Clinopodium chinense, T2257 Dracocephalum moldavicum, T2258 Dracocephalum rupestre, T2720 Ficus hispida, T3749 Leontopodium alpinum, T4413 Nepeta cataria, T5115 Polygonum sibiricum [Syn. Persicaria sibirica], T5687 Salvia prionitis, T6023 Solidago virgaurea. course wind and diffuse lung T4714 Perilla frutescens. course wind and dispel damp T2189 Dioscorea althaeoides. course wind and dissipate cold T4937 Piper betle.

course wind and dissipate heat T0480 Angelica decursiva [Syn. Peucedanum decursivum], T0586 Arctium lappa, T3819 Ligusticum brachylobum, T4186 Mentha piperita, T4289 Morus alba, T4291 Morus 
australis, T4293 Morus cathayana, T4298 Morus mongolica, T4761 Peucedanum longshengens, T4768 Peucedanum praeruptorum, T4769 Peucedanum rubricaule.

course wind and outthrust papules T1417 Cimicifuga acerina, T1423

Cimicifuga nanchuanensis.

course wind and overcome damp T3520 Isodon rosthornii.

course wind and resolve exterior T0967 Boenninghausenia sessilicarpa,

T1446 Cipadessa baccifera, T1535 Clausena excavata, T1538 Clausena

lansium, T3646 Kyllinga brevifolia, T4223 Micromelum integerrimum,

T4307 Mosla scabra [Syn. Mosla punctata], T4317 Murraya euchrestifolia [Syn. Clausena euchrestifolia], T4320 Murraya kwangsiensis, T4470 Ocimum basilicum, T6286 Tamarix chinensis, T6290 Tamarix ramosissima, T6735 Viburnum dilatatum.

decay wart and mole T5026 Plumbagella micrantha.

diffuse damp and arouse spleen T0463 Anemone altaica.

diffuse lung T5011 Platycodon grandiflorum.

diffuse lung and calm asthma T2367 Ephedra equisetina, T2368 Ephedra gerardiana, T2369 Ephedra intermedia, T2372 Ephedra likiangensis, T2373 Ephedra minuta, T2375 Ephedra monosperma, T2379 Ephedra saxatilis, T2380 Ephedra sinica.

diffuse lung and calm panting T0591 Ardisia arborescens.

diffuse lung and effuse exterior T2521 Eucalyptus tereticornis.

diffuse lung and outthrust papules T0586 Arctium lappa.

diffuse lung and rectify $q \boldsymbol{i} \mathrm{T} 3803$ Ligularia dictyoneura [Syn. Senecio dictyoneurus].

diffuse lung and resolve exterior T4003 Lysimachia microcarpa.

diffuse lung and suppress cough T0676 Artemisia dracunculus, T1258

Casuarina equisetifolia, T1479 Citrus grandis, T4689 Peganum nigellastrum.

diffuse lung and transform phlegm T4718 Perilla frutescens var. acuta

[Syn. Perilla frutescens var. purpurascens], T4721 Perilla frutescens var. arguta, T6259 Syringa amurensis [Syn. Syringa reticulata var. amurensis].

diffuse lung qi T4687 Peganum harmala.

disinhibit damp T0137 Aconitum tanguticum, T0157 Actinidia callosa var. henryi, T0567 Aralia armata, T0895 Benincasa hispida, T0941 Bidens tripartita, T0973 Bombax malabaricum [Syn. Gossampinus malabarica], T1143 Calystegia hederacea, T1172 Canna edulis, T1199 Carduus acanthoides, T1200 Carduus crispus, T1258 Casuarina equisetifolia, T1526 Cladonia rangiferina, T1675 Corchorus olitorius, T1747 Corydalis thalictrifolia, T1831 Crotalaria sessiliflora, T2334 Elephantopus scaber, T2649 Evolvulus alsinoides, T2715 Fibraurea recisa, T2902 Gentiana algida, T3126 Hedyotis corymbosa [Syn. Oldenlandia corymbosa], T3142 Helianthus annuus, T3361 Hypericum perforatum, T3524 Isodon sculponeata [Syn. Rabdosia sculponeata], T3528 Isodon ternifolia, T3529 Isodon ternifolius, T3555 Jasminum nudiflorum, T4144 Meconopsis nepaulensis, T4145 Meconopsis punicea, T4183 Menispermum dauricum, T4335 Mussaenda pubescens, T4485 Oldenlandia diffusa [Syn. Hedyotis diffusa], T4502 Onychium japonicum [Syn. Tricomanes japonicum], T4526 Origanum vulgare, T4840 Phyllanthus niruri, T4979 Pistacia chinensis, T5687 Salvia prionitis, T5856 Sedum lineare [Syn. Sedum obtuso-lineare], T5863 Selaginella moellendorffii, T5939 Shiraia bambusicola, T5978 Smilax glauco-china,
T6116 Stenoloma chusanum, T6217 Swertia davidii, T6224 Swertia kouitchensis, T6231 Swertia pseudochinensis, T6234 Swertia punicea, T6359 Teucrium bidentatum, T6398 Thalictrum microgynum, T6431 Thesium chinense, T6912 Zinnia elegans.

disinhibit damp and abate jaundice T0608 Arenaria kansuensis [Syn. Arenaria kumaonensis], T3507 Isodon lophanthoides [Syn. Rabdosia lophanthoides; Hyssopus lophanthoides; Plectranthus striatus; Isodon striatus], T4148 Medicago sativa, T5207 Prismatomeris tetrandra, T5397 Rabdosia serra, T5560 Rorippa montana [Syn. Rorippa dubia; Sisymbrium dublium], T6219 Swertia erythrosticta, T6226 Swertia mileensis, T6260 Syringa oblata, T6409 Thalictrum simplex [Syn. Thalictrum simplex var. brevipes].

disinhibit damp and check diarrhea T2387 Epilobium hirsutum, T5528 Rhodomyrtus tomentosa.

disinhibit damp and disperse glomus T3928 Lotus corniculatus.

disinhibit damp and disperse stagnation T5576 Rostellularia procumbens [Syn. Justicia procumbens].

disinhibit damp and disperse swelling T0426 Ampelopsis grossedentata [Syn. Ampelopsis cantoniesis var. grossedentata], T1921 Cyanotis arachnoidea [Syn. Cyanotis bodinieri], T2363 Entada phaseoloides [Syn. Lens phaseoloides], T3628 Kerria japonica, T3681 Lamium barbatum, T3996 Lysimachia candida, T4837 Phyllanthus emblica, T6131 Stephania longa, T6367 Teucrium quadrifarium.

disinhibit damp and eliminate impediment T4814 Phlomis umbrosa. disinhibit damp and eliminate turbidity T2208 Dioscorea septemloba. disinhibit damp and fortify stomach $\mathbf{T 6 2 1 4}$ Swertia chinensis. disinhibit damp and free milk T4120 Marsdenia tenacissima. disinhibit damp and free strangury T2213 Dioscorea zingiberensis, T2973 Glechoma longituba, T2974 Glechoma lungituba.

disinhibit damp and harmonize center T4305 Mosla dianthera. disinhibit damp and harmonize stomach T5395 Rabdosia nervosa. disinhibit damp and quicken blood T4842 Phyllanthus reticulatus. disinhibit damp and relieve itch T4154 Melaleuca leucadendra. disinhibit damp and relieve pain T2327 Elaeagnus angustifolia, T3398 Ilex rotunda.

disinhibit damp and resolve toxin T3444 Ipomoea aquatica [Syn. Convolvulus repens; Ipomoea reptans], T5671 Salvia digitaloides.

disinhibit damp and stanch bleeding T3672 Lagerstroemia indica.

disinhibit damp and transform turbidity T6504 Tricholoma matsutake [Syn. Armillaria matsutake].

disinhibit damp and turbidity T2197 Dioscorea futschauensis, T2198 Dioscorea gracillima, T2201 Dioscorea hypoglauca [Syn. Dioscorea collettii var. hypoglauca], T2210 Dioscorea spongiosa, T2212 Dioscorea tokoro.

disinhibit damp heat T5185 Potentilla multifida.

disinhibit diaphragm T1488 Citrus junos, T5993 Solanum capsicastrum.

disinhibit gallbladder T0647 Armoracia lapathifolia, T1315 Cephaelis ipecacuanha.

disinhibit gallbladder and free intestines T0984 Bos taurus domesticus; Bubalus bubalis.

disinhibit joints T1411 Cibotium barometz [Syn. Polypodium barometz], T4290 Morus alba, T4445 Notopterygium forbesii [Syn. Notopterygium franchetii], T4446 Notopterygium incisum. 
disinhibit liver and gallbladder T6656 Ustilago maydis. disinhibit lung and transform phlegm T1007 Brassica juncea.

disinhibit pharynx and larynx T4184 Mentha haplocalyx [Syn. Mentha canadaensis; Mentha arvensis var. haplocalyx; Mentha arvensis], T4845 Physalis alkekengi.

disinhibit qi T1750 Corydalis yanhusuo [Syn. Corydalis turtschaninovii $\mathrm{f}$. yanhusuo].

disinhibit sinews and bones T0467 Anemone flaccida.

disinhibit throat T0892 Belamcanda chinensis, T1165 Canarium album, T1981 Cyprinus carpio, T2244 Doellingeria scaber [Syn. Aster scaber], T3240 Hibiscus esculentus, T4210 Michelia champaca, T5011 Platycodon grandiflorum, T6463 Tinospora capillipes, T6467 Tinospora sagittata, T6655 Ustilaginoidea virens.

disinhibit throat and disperse goiter T0992 Bos taurus domesticus; Bubalus bubalis.

disinhibit throat and disperse swelling T0197 Aegle marmelos, T4538 Oroxylum indicum, T6043 Sophora subprostrata [Syn. Sophora tonkinensis], T6084 Spiraea prunifolia, T6086 Spiraea thunbergii.

disinhibit throat and relieve cough T2257 Dracocephalum moldavicum. disinhibit throat and restore voice T5406 Rana limnocharis.

disinhibit urine T0011 Abrus precatorius, T0184 Adonis amurensis, T0274 Akebia quinata, T0275 Akebia quinata, T0278 Akebia trifoliata, T0280 Akebia trifoliata var. australis, T0379 Alternanthera philoxeroides, T0388 Amaranthus lividus, T0423 Ampelopsis brevipedunculata, T0598 Ardisia japonica, T0632 Aristolochia manshuriensis, T0647 Armoracia lapathifolia, T0708 Arthromeris mairei [Syn. Polypodium mairei], T0722 Arundo donax, T0834 Averrhoa carambola, T1032 Broussonetia papyrifera, T1152 Camellia sinensis [Syn. Thea sinensis], T1174 Cannabis sativa, T1241 Cassia occidentalis, T1385 Chorda filum, T1464 Citrullus vulgaris [Syn. Citrullus lanatus], T1471 Citrus chachiensis, T1473 Citrus cultivars, T1474 Citrus decumana, T1516 Citrus tankan, T1518 Citrus unshiu, T1608 Coffea arabica, T1610 Coffea liberica, T1922 Cyanotis vaga, T2407 Equisetum arvense, T2410 Equisetum pratense, T2612 Euphorbia prolifera, T2628 Euphrasia officinalis, T2730 Fimbristylis dichotoma, T2749 Fomes officinalis, T3066 Gryllulus chinensis, T3122 Hedyotis acutangula, T3128 Hedysarum multijugum, T3143 Helianthus annuus, T3174 Heliotropium indicum, T3394 Ilex paraguariensis, T3594 Juniperus rigida, T3662 Lactuca sativa, T3758 Lepidium sativum, T3765 Lepisorus ussuriensis, T3785 Levisticum officinale, T3815 Ligularia stenocephala, T3885 Litsea cubeba, T3920 Lophatherum gracile, T3988 Lycoris squamigera, T4254 Miscanthus sinensis, T4290 Morus alba, T4388 Nasturtium officinale, T4438 Nostoc flagelliforme, T4644 Parasilurus asotus, T4785 Phaseolus vulgaris, T4829 Phragmites communis, T4846 Physalis alkekengi var. franchetii, T4848 Physalis angulata, T4854 Physalis pubescens, T5419 Raphanus sativus, T5452 Reseda luteola, T5605 Rumex acetosa, T5606 Rumex acetosa, T5626 Ruta graveolens, T5651 Salix babylonica, T5726 Saponaria officinalis, T5953 Silene firma, T5963 Sinodielsia yunnanensis, T6210 Swainsonia salsula [Syn. Sphaerophysa salsula], T6423 Theobroma cacao, T6598 Ulva conglobata, T6673 Vaccinium vitis-idaea, T6798 Vitis vinifera, T6820 Wikstroemia indica, T6862 Zanthoxylum ailanthoides, T6902 Zea mays.

disinhibit urine T4797 Phillyrea latifolia. disinhibit urine and abate jaundice T4147 Medicago falcata.

disinhibit urine and disperse edema T1415 Cichorium intybus, T1984 Cypripedium macranthum [Syn. Cypripedium tibeticum], T2479 Erythrina variegata var. orientalis, T3200 Hemidesmus indicus, T3752 Leonurus heterophyllus [Syn. Leonurus artemisia], T3754 Leonurus sibiricus, T3898 Lobelia chinensis [Syn. Lobelia radicans], T4050 Magnolia sieboldii, T5818 Scoparia dulcis, T5835 Scutellaria barbata [Syn. Scutellaria rivularis], T6099 Stauntonia chinensis, T6100 Stauntonia hexaphylla, T6433 Thevetia neriifolia [Syn. Thevetia peruviana], T6901 Zea mays.

disinhibit urine and draw toxin T0791 Astragalus chrysopterus, T0794 Astragalus ernestii, T0798 Astragalus membranaceus, T0800 Astragalus mongholicus.

disinhibit urine and free channels T6311 Taxus cuspidata.

disinhibit urine and free milk T4573 Pachyrrhizus erosus.

disinhibit urine and free stool T0389 Amaranthus tricolor, T2580 Euphorbia antiquorum, T3286 Hovenia dulcis, T4091 Malva sylvestris, T4845 Physalis alkekengi, T4861 Phytolacca americana [Syn. Phytolacca decandra], T4864 Phytolacca esculenta [Syn. Phytolacca acinosa].

disinhibit urine and free strangury T0001 Abelmoschus manihot, T0073 Achyranthes bidentata, T0753 Asparagus setaceus [Syn. Asparagus plumosus], T0759 Aspidistra elatior, T0853 Baeckea frutescens, T1931 Cyclea barbata, T1933 Cyclea sutchuenensis, T1950 Cynanchum ascyrifolium, T1951 Cynanchum atratum, T1962 Cynanchum versicolor, T2119 Derris eriocarpa, T2134 Desmodium styracifolium, T2143 Dianthus chinensis, T2145 Dianthus superbus, T2147 Dianthus versicolor, T2952 Gerbera piloselloides, T3288 Humulus japonicus [Syn. Humulus scandens], T3416 Imperata cylindrica var. major, T3764 Lepisorus thunbergianus, T3769 Lespedeza bicolor, T4456 Nymphoides peltatum, T4562 Oxalis acetosella, T5033 Poa sphondylodes, T5098 Polygonum aviculare, T5354 Pyrrosia davidii, T5355 Pyrrosia drakeana T5356 Pyrrosia gralla, T5357 Pyrrosia lingua' T5358 Pyrrosia petiolosa T5360 Pyrrosia sheareri, T6301 Taraxacum mongolicum, T6582 Tylophora ovata, T6597 Ulmus pumila.

disinhibit urine and quiet spirit T3289 Humulus lupulus.

disinhibit urine and remove stone T4432 Nigella glandulifera.

disinhibit urine and resolve toxin $\mathrm{T} 1357$ Chelidonium majus, T1823 Crotalaria juncea, T4760 Peucedanum japonicum, T6286 Tamarix chinensis, T6290 Tamarix ramosissima.

disinhibit water T0544 Apium graveolens, T1033 Broussonetia papyrifera, T1194 Carassius auratus, T1229 Cassia acutifolia, T1230 Cassia angustifolia, T1878 Cucumis sativus, T2115 Dermatocarpon minitum, T2321 Ecklonia kurome, T2585 Euphorbia esula, T3668 Lagenaria siceraria var. depressa, T3678 Laminaria japonica, T3867 Liquidambar formosana [Syn. Liquidambar taiwaniana], T3935 Luffa cylindrica, T4616 Pandanus tectorius, T5223 Prunus humilis [Syn. Cerasus humilis], T5224 Prunus japonica [Syn. Cerasus japonica], T5225 Prunus japonica var. nakaii, T5238 Prunus salicina, T5740 Sargassum vachellianum, T6259 Syringa amurensis [Syn. Syringa reticulata var. amurensis], T6330 Tephroseris kirilowii [Syn. Senecio integrifolius var. fauriei], T6640 Undaria pinnatifida, T6750 Vicia sativa, T6752 Vigna angularis [Syn. Dolichus angularis; Phaseolus angularis], T6826 
Wisteria sinensis, T6925 Zostera marina.

disinhibit water and disperse distention T1051 Bufo bufo gargarizans; Bufo melanostictus, T6602 Umbilicaria hypococcinea.

disinhibit water and disperse edema T0172 Adiantum caudatum, T0553 Apocynum venetum, T1004 Brasenia schreberi, T1263 Catalpa ovata, T1590 Cocculus trilobus [Syn. Cocculus sarmentosus], T1595 Codium fragile, T1633 Commelina communis, T1696 Cornus capitata [Syn. Dendrobenthamia capitata], T1881 Cucurbita moschata, T1954 Cynanchum chinense, T2282 Dryopteris filix-mas, T2367 Ephedra equisetina, T2368 Ephedra gerardiana, T2369 Ephedra intermedia, T2372 Ephedra likiangensis, T2373 Ephedra minuta, T2375 Ephedra monosperma, T2379 Ephedra saxatilis, T2380 Ephedra sinica, T2589 Euphorbia helioscopia, T3738 Lemna minor, T3999 Lysimachia clethroides, T4026 Macrothelypteris oligophlebia, T4121 Marsilea quadrifolia, T4287 Morus alba, T4306 Mosla grosseserrata, T4505 Ophiocephalus argus, T4818 Phoebe nanmu, T5115 Polygonum sibiricum [Syn. Persicaria sibirica], T5407 Rana nigromaculata; Rana plancyi, T5409 Rana temporaria chensinensis; Rana amurensis, T5685 Salvia plebeia, T6129 Stephania japonica, T6136 Stephania tetrandra, T6227 Swertia mussotii, T6436 Thlaspi arvense, T6541 Tripterygium regelii, T6599 Ulva lactuca, T6600 Ulva pertusa, T6709 Verbena officinalis, T6799 Vitis vinifera, T6861 Zanthoxylum ailanthoides.

disinhibit water and free stool T1240 Cassia obtusifolia, T1250 Cassia tora, T5231 Prunus persica.

disinhibit water and free strangury T0171 Adiantum capillus-veneris, T0173 Adiantum lunulatum, T0175 Adiantum pedatum, T0264 Ajuga forrestii, T3002 Glycine max, T3448 Ipomoea cairica [Syn. Ipomoea palmata], T3578 Juncus effusus, T3990 Lygodium japonicum, T3998 Lysimachia christinae, T4855 Physeter catodon, T5838 Scutellaria galericulata, T6596 Ulmus parvifolia.

disinhibit water and harmonize center T0568 Aralia chinensis, T0570 Aralia dasyphylla.

disinhibit water and kill worms T2051 Daucus carota var. sativa.

disinhibit water and percolate damp T0303 Alisma orientale [Syn. Alisma plantago-aquatica var. orientale], T1352 Chamaenerion angustifolium [Syn. Epilobium angustifolium], T5129 Polyporus umbellatus, T5169 Poria cocos.

disinhibit water and precipitate $q i \mathrm{~T} 1980$ Cyprinus carpio.

disinhibit water and resolve toxin T4843 Phyllanthus urinaria, T5362 Pyrus bretschneideri, T5366 Pyrus pyrifolia.

disinhibit water and transform damp T5289 Pteris dactylina.

dispel cold T2644 Evodia rutaecarpa, T2646 Evodia rutaecarpa var. officinalis, T3877 Litchi chinensis, T6528 Trigonella foenum-graecum, T6889 Zanthoxylum podocarpum.

dispel cold and relieve pain T1441 Cinnamomum japonicum, T4964 Piper retrofractum.

dispel damp T0301 Aleuritopteris argentea, T4080 Mallotus apelta. dispel damp and check diarrhea T6895 Zanthoxylum simulans.

dispel damp and disinhibit urine T0071 Achyranthes aspera, T0072 Achyranthes aspera var. indica.

dispel damp and disinhibit water T5176 Potamogeton natans. dispel damp and disperse swelling T0421 Amorpha fruticosa. dispel damp and dissipate cold T0932 Betula luminifera, T1901 Curculigo orchioides.

dispel damp and fortify spleen T3766 Lepista nuda.

dispel damp and fortify stomach T0142 Acorus calamus.

dispel damp and free network vessels T1205 Carica papaya, T4938 Piper

boehmeriaefolium, T6653 Usnea diffracta, T6654 Usnea longissima.

dispel damp and kill worms T4859 Physochlaina physaloides, T6869

Zanthoxylum bungeanum, T6892 Zanthoxylum schinifolium.

dispel damp and relieve itch T2988 Glochidion eriocarpum.

dispel damp and relieve pain T0006 Abies nephrolepis, T0138 Aconitum umbrosum, T0478 Angelica dahurica [Syn. Angelica porphyrocaulis], T0572 Aralia elata, T2805 Fugu ocellatus, T5964 Sinomenium acutum.

dispel damp and resolve toxin T1932 Cyclea racemosa, T4689 Peganum nigellastrum, T4789 Phellodendron amurense, T4790 Phellodendron amurense var. wilsonii, T4791 Phellodendron chinense, T4792 Phellodendron chinense var. glabriusculum.

dispel damp and strengthen spleen T0358 Alpinia katsumadai, T0819 Atractylodes chinensis, T0821 Atractylodes japonica, T0823 Atractylodes lancea.

dispel damp and transform stasis T1533 Clausena dentata, T1534 Clausena dunniana.

dispel phlegm T0012 Abrus precatorius, T0598 Ardisia japonica, T1315 Cephaelis ipecacuanha, T1385 Chorda filum, T2268 Drosera rotundifolia, T2321 Ecklonia kurome, T3678 Laminaria japonica, T3802 Ligularia dentata, T3805 Ligularia fischeri, T3807 Ligularia intermedia, T3813 Ligularia sibirica, T3988 Lycoris squamigera, T4423 Nicandra physaloides, T4518 Oppopanax chironium, T4814 Phlomis umbrosa, T4858 Physochlaina infundibularis, T4908 Pinus bungeana, T5011 Platycodon grandiflorum, T5073 Polygala chinensis [Syn. Polygala glomerata], T5087 Polygala telephioides, T5502 Rhododendron anthopogonoides, T5508 Rhododendron dauricum, T5522 Rhododendron seniavinii, T5594 Rubus parkeri, T5719 Sapindus mukorossi, T5726 Saponaria officinalis, T6640 Undaria pinnatifida.

dispel phlegm and allay thirst $\mathrm{T} 1247$ Cassia sophera.

dispel phlegm and disinhibit damp T0595 Ardisia crispa.

dispel phlegm and disinhibit throat T1858 Croton tiglium.

dispel phlegm and dissipate binds T3308 Hydrangea umbellata.

dispel phlegm and eliminate damp T5071 Polygala arillata.

dispel phlegm and interrupt malaria T0363 Alpinia speciosa, T2158 Dichroa febrifuga.

dispel phlegm and open orifices T5086 Polygala sibirica, T5088 Polygala tenuifolia.

dispel phlegm and promote vomiting T3984 Lycoris chinensis, T3986 Lycoris radiata [Syn. Amaryllis radiata], T6429 Thermopsis lupinoides.

dispel phlegm and relieve cough T3709 Lathyrus pratensis, T5513 Rhododendron mariae, T5981 Smilax riparia.

dispel phlegm and settle asthma T2439 Eruca sativa, T4418 Nerium indicum.

dispel phlegm and suppress cough T2697 Ferula borealis, T5560 Rorippa montana [Syn. Rorippa dubia; Sisymbrium dublium].

dispel stasis T1181 Capra hircus; Ovis aries, T1212 Carpesium eximum, T1416 Cicuta virosa, T1558 Clerodendron inerme, T4418 Nerium indicum, T5238 Prunus salicina, T5587 Rubus alceaefolius, T6507 Trichosanthes cucumeroides. 
dispel stasis and disperse swelling T1449 Cirsium japonicum, T1784 Cratoxylum cochinchinense, T2328 Elaeis guineensis, T4670 Patrinia heterophylla, T4673 Patrinia scabra, T4740 Petasites japonicus, T5411 Randia spinosa, T5537 Rhus sylvestris, T5613 Rumex obtusifolius.

dispel stasis and engender flesh T5586 Rubia yunnanensis, T5697 Salvia trijuga.

dispel stasis and harmonize construction T5301 Pterocarpus indicus.

dispel stasis and regulate menstruation T2130 Desmodium gangeticum, T4005 Lysionotus pauciflorus.

dispel stasis and relieve pain T0813 Atalantia buxifolia [Syn. Severinia buxifolia], T0890 Begonia limprichtii, T1101 Caesalpinia decapetala, T1129 Calophyllum inophyllum, T1491 Citrus limon, T1495 Citrus limonia, T1796 Crinum asiaticum var. sinicum, T2298 Dysosma difformis, T2302 Dysosma pleiantha [Syn. Podophyllum pleianthum], T2304 Dysosma veitchii, T2305 Dysosma versipellis [Syn. Podophyllum versipelle], T3360 Hypericum patulum, T3479 Isodon amethystoides, T4131 Maytenus confertiflorus, T5365 Pyrus pashia.

dispel stasis and stanch bleeding T1566 Clerodendrum inerme, T2280 Dryopteris chrysocoma, T3278 Homo sapiens, T4399 Nelumbo nucifera, T4549 Osbeckia chinensis, T4552 Osmunda japonica.

dispel summerheat T0687 Artemisia myriantha, T1490 Citrus limon, T1494 Citrus limonia, T4263 Momordica charantia, T4397 Nelumbo nucifera.

dispel summerheat and effuse exterior T2563 Eupatorium japonicum. dispel summerheat and engender liquid T1544 Cleistocalyx operculatus. dispel summerheat and resolve exterior T0212 Agastache rugosus. dispel summerheat and transform damp T1278 Cedrela sinensis.

dispel wind T0065 Achillea millefolium, T0359 Alpinia officinarum, T0544 Apium graveolens, T0567 Aralia armata, T0750 Asparagus gobicus, T0784 Astilbe chinensis, T0819 Atractylodes chinensis, T0821 Atractylodes japonica, T0823 Atractylodes lancea, T0888 Beesia calthaefolia, T1032 Broussonetia papyrifera, T1171 Canis familiaris, T1175 Cannabis sativa, T1180 Capparis spinosa, T1199 Carduus acanthoides, T1200 Carduus crispus, T1437 Cinnamomum camphora, T1582 Cnidium monnieri, T1650 Convolvulus arvensis, T1746 Corydalis taliensis, T2039 Datura innoxia, T2043 Datura metel, T2047 Datura stramonium, T2113 Dennstaedtia scabra [Syn. Dicksonia scabra], T2154 Dicentra spectabilis, T2263 Dregea volubilis, T3082 Gynocardia odorata, T3142 Helianthus annuus, T3594 Juniperus rigida, T3674 Laggera alata, T3810 Ligularia nelumbifolia, T3847 Lindera angustifolia, T3878 Lithocarpus polystachyus, T4020 Macleaya cordata, T4029 Maesa japonica, T4126 Matteuccia orientalis, T4188 Mentha rotundifolia, T4662 Parthenocissus tricuspidata, T4842 Phyllanthus reticulatus, T4912 Pinus koraiensis, T5107 Polygonum multiflorum, T5626 Ruta graveolens, T5651 Salix babylonica, T5741 Sargentodoxa cuneata, T5767 Saussurea pulchella, T5804 Schizonepeta tenuifolia [Syn. Nepeta tenuifolia], T5963 Sinodielsia yunnanensis, T5978 Smilax glauco-china, T6359 Teucrium bidentatum, T6499 Tribulus terrestris, T6578 Tylophora atrofolliculata, T6843 Xanthium sibiricum [Syn. Xanthium strumarium].

dispel wind and attack toxin T2897 Gelsemium elegans.

dispel wind and brighten eyes T0805 Astragalus sinicus, T0806 Astragalus sinicus, T1185 Capsella bursa-pastoris, T3957 Lycium chinense.

dispel wind and check tetany T0617 Arisaema amurense, T0618 Arisaema consanguineum, T0620 Arisaema heterophyllum, T1084 Buthus martensi, T4903 Pinellia pedatisecta.

dispel wind and clear heat T0979 Bombyx mori, T3865 Lippia nodiflora, T5119 Polygonum thunbergii, T5234 Prunus persica, T5773 Saxifraga stolonifera, T6079 Spiraea japonica, T6082 Spiraea japonica var. fortunei.

dispel wind and disinhibit damp T0045 Acanthopanax trifoliatus, T0600 Ardisia mamillata [Syn. Tinus mamillata], T0721 Arundina chinensis, T1003 Brandisia hancei, T1924 Cyathula officinalis, T2020 Damnacanthus indicus, T2136 Desmos cochinchinensis [Syn. Desmos chinensis], T2194 Dioscorea collettii, T2723 Ficus pumila, T3113 Hedera nepalensis var. sinensis, T3115 Hedera rhombea, T3471 Iris tectorum, T4439 Nothapodytes pittosporoides, T4527 Orixa japonica, T4542 Orthosiphon wulfenioides [Syn. Coleus wulfenioides], T5114 Polygonum polystachyum, T5178 Potamogeton perfoliatus, T5401 Rabdosia yuennanensis, T5645 Sageretia theezans [Syn. Sageretia thea], T5650 Salix babylonica, T5704 Sambucus nigra, T5707 Sambucus williamsii, T5795 Schisandra micrantha, T5976 Smilax china [Syn. Smilax japonica], T5982 Smilax sieboldii, T5984 Smilax stans [Syn. Smilax vaginata var. stans], T6645 Urena lobata, T6676 Valeriana hardwickii.

dispel wind and disperse macula T0583 Archangelica decurrens.

dispel wind and dissipate cold T0098 Aconitum geniculatum, T0138 Aconitum umbrosum, T0440 Anagallis arvensis, T0676 Artemisia dracunculus, T0724 Asarum caulescens, T0726 Asarum forbesii, T0727 Asarum fukienense, T0728 Asarum heterotropoides var. mandshuricum, T0729 Asarum maximum, T0730 Asarum sagittarioides, T0731 Asarum sieboldii, T0732 Asarum sieboldii var. seoulensis, T0926 Berneuxia thibetica, T1046 Buddleja davidii, T1434 Cinnamomum bejolghota [Syn. Cinnamomum obtusifolium; Laurus bejolghota], T1435 Cinnamomum camphora, T1440 Cinnamomum glanduliferum, T1443 Cinnamomum parthenoxylum [Syn. Cinnamomum porrectum], T1550 Cleome gynandra [Syn. Gynandropsis gynandra], T2636 Euscaphis japonica, T3026 Gnaphalium affine [Syn. Gnaphalium multiceps], T3118 Hedychium coronarium, T3213 Heracleum hemsleyanum, T3217 Heracleum moellendorffii [Syn. Heracleum microcarpum; Heracleum morifolium], T3228 Heracleum yungningense, T3653 Lactarius piperatus [Syn. Agaricus piperatus], T4000 Lysimachia congestiflora, T4039 Magnolia grandiflora, T4763 Peucedanum morisonii, T4938 Piper boehmeriaefolium, T4965 Piper sarmentosum, T5639 Sabina chinensis, T6106 Stelmatocrypton khasianum, T6422 Thelephora vialis, T6874 Zanthoxylum dimorphophyllum var. spinifolum, T6877 Zanthoxylum dissitum, T6895 Zanthoxylum simulans.

dispel wind and dissipate cold and eliminate damp T4962 Piper puberulum.

dispel wind and dissipate heat T2434 Eriocaulon buergerianum.

dispel wind and dissipate stasis T4444 Nothopanax davidii, T6100 Stauntonia hexaphylla.

dispel wind and downbear fire T3432 Inula helianthus-aquatica.

dispel wind and dry damp T3300 Hydnocarpus anthelminticus, T4906 Pinus armandii, T4916 Pinus massoniana, T4917 Pinus massoniana, 
T5517 Rhododendron molle, T6073 Sphallerocarpus gracilis.

dispel wind and effuse exterior T0491 Angelica polymorpha, T6365

Teucrium pilosum [Syn. Teucrium japonicum var. pilosum].

dispel wind and effuse sweat T3222 Heracleum scabridum.

dispel wind and eliminate damp T0069 Achillea wilsoniana, T0077

Aconitum balfourii, T0079 Aconitum brachypodum, T0084 Aconitum carmichaeli, T0100 Aconitum hemsleyanium var. circinacum, T0101 Aconitum hemsleyanum, T0105 Aconitum karakolicum, T0106 Aconitum kirinense, T0107 Aconitum kongboense, T0116 Aconitum nagarum var. heterotrichum [Syn. Aconitum bullatifolium], T0117 Aconitum nagarum var. lasiandrum, T0122 Aconitum pendulum, T0130 Aconitum sinomontanum, T0274 Akebia quinata, T0278 Akebia trifoliata, T0280 Akebia trifoliata var. australis, T0281 Alangium chinense, T0285 Alangium platanifolium, T0364 Alsophila spinulosa, T0383 Alyxia sinensis, T0414 Ammopiptanthus mongolicus [Syn. Piptanthus mongolicus], T0476 Angelica anomala, T0479 Angelica dahurica cv. qibaizhi, T0492 Angelica pubescens f. biserrata [Syn. Angelica pubescens], T0498 Angelica taiwaniana, T0500 Anguilla japonica, T0539 Apis cerana, T0568 Aralia chinensis, T0569 Aralia cordata, T0570 Aralia dasyphylla, T0571 Aralia decaisneaa, T0574 Aralia fargesii, T0582 Archangelica brevicaulis [Syn. Angelicarpa brevicaulis; Angelica brevicaulis], T0634 Aristolochia mollissima, T0847 Baccharis indica [Syn. Pluchea indica], T0877 Bauhinia championii, T0886 Beaumontia grandiflora, T0922 Berchemia polyphylla var. leioclada, T0938 Bidens bipinnata, T0945 Bischofia javanica [Syn. Bischofia trifoliata], T0957 Blumea balsamifera, T0978 Bombyx mori, T1023 Bredia tuberculata, T1031 Broussonetia kazinoki, T1285 Celastrus angulatus, T1286 Celastrus flagellaris, T1288 Celastrus orbiculatus [Syn. Celastrus articulatus], T1290 Celastrus orbiculatus [Syn. Celastrus articulatus], T1361 Chenopodium ambrosioides, T1371 Chloranthus japonicus, T1436 Cinnamomum camphora, T1545 Clematis chinensis, T1547 Clematis terniflora [Syn. Clematis maximowicziana], T1556 Clerodendron fragrans, T1589 Cocculus trilobus [Syn. Cocculus sarmentosus], T1590 Cocculus trilobus [Syn. Cocculus sarmentosus], T1640 Coniogramme japonica [Syn. Hemionitis japonica], T1641 Conioselinum vaginatum, T1692 Coriaria sinica [Syn. Coriaria nepalensis], T1721 Corydalis linearioides, T1815 Crotalaria assamica, T1900 Curculigo capitulata [Syn. Leucojum capitulata], T1943 Cymbopogon goeringii, T1976 Cyperus iria, T2058 Deeringia amaranthoides [Syn. Cladostachys frutescens], T2062 Delphinium bonvalotii, T2081 Delphinium omeiense, T2084 Delphinium potaninii, T2085 Delphinium potaninii var. jiufengshanense, T2133 Desmodium racemosum [Syn. Podocarpium podocarpum var. oxyphyllum], T2203 Dioscorea nipponica, T2204 Dioscorea nipponica ssp. rosthornii, T2233 Diploclisia glaucescens, T2262 Dregea sinensis, T2391 Epimedium brevicornum, T2422 Erigeron breviscapus, T2459 Erythrina arborescens, T2478 Erythrina variegata [Syn. Erythrina indica], T2541 Euonymus grandiflorus, T2631 Eurya japonica, T2641 Evodia lepta [Syn. Ilex lepta], T2657 Fagopyrum cymosum [Syn. Polygonum cymosum], T2731 Firmiana simplex, T2732 Firmiana simplex, T2735 Fissistigma oldhamii [Syn. Melodorum oldhamii], T2737 Flemingia philippinensis [Syn. Moghania philippinensis], T2749 Fomes officinalis, T2750 Fomitopsis pinicola [Syn. Fomes pinicola; Polyporus pinicola], T2895 Gaultheria yunnanensis, T2946 Geranium robertianum, T3033 Gnetum parvifolium [Syn. Gnetum indicum], T3201 Hemiphragma heterophyllum, T3214 Heracleum lanatum, T3221 Heracleum rapula, T3234 Heteropogon contortus, T3294 Huperzia selago [Syn. Lycopodium selago], T3371 Hypocrella bambusae, T3395 Ilex pedunculosa, T3400 Illicium difengpi, T3401 Illicium henryi, T3403 Illicium majus, T3413 Impatiens nolitangere, T3414 Impatiens siculifer, T3417 Imperata cylindrica var. major, T3614 Kadsura heteroclita [Syn. Uvaria heteroclita], T3624 Kalopanax pictum, T3625 Kalopanax septemlobus, T3746 Leontice robustum, T3776 Lethariella zahlbruckneri, T3796 Libanotis buchtormensis, T3822 Ligusticum jeholense, T3824 Ligusticum sinense, T3849 Lindera chunii, T3852 Lindera megaphylla, T3856 Lindera umbellata [Syn. Lindera erythrocarpa], T3871 Liriodendron tulipifera, T3965 Lycopodium annotinum, T3969 Lycopodium casuarinoides, T3970 Lycopodium cernuum, T3971 Lycopodium complanatum, T3973 Lycopodium japonicum [Syn. Lycopodium clavatum], T3976 Lycopodium obscurum, T3997 Lysimachia capillipes, T4004 Lysimachia paridiformis, T4005 Lysionotus pauciflorus, T4085 Mallotus repandus var. chrysocarpus [Syn. Mallotus chrysocarpus; Mallotus repandus], T4260 Moghania philippinensis, T4267 Monachosorum flagellare, T4268 Monachosorum henryi, T4324 Murraya paniculata [Syn. Chalcas paniculata], T4347 Myrica nagi [Syn. Podocarpus nagi], T4445 Notopterygium forbesii [Syn. Notopterygium franchetii], T4446 Notopterygium incisum, T4453 Nyctanthes arbor-tristis, T4503 Onychium lucidum, T4577 Paederia scandens, T4663 Passiflora caerulea, T4690 Pelargonium graveolens, T4704 Peperomia duclouxii, T4725 Periploca calophylla, T4726 Periploca forrestii, T4778 Phallus impudicus, T4801 Phlegmariurus phlegmaria [Syn. Lycopodium phlegmaria], T4810 Phlomis mongolica, T4838 Phyllanthus flexuosus, T4948 Piper hancei, T4975 Piptanthus nanus, T5021 Pleurospermum rivulorum, T5029 Plumbago zeylanica, T5057 Podophyllum emodii [Syn. Podophyllum emodii var. chinense; Podophyllum sikkimenosis; Sinopodophyllum emodii], T5110 Polygonum orientale, T5143 Pongamia pinnata, T5156 Populus nigra var. thevestina, T5193 Pratia nummularia, T5256 Pseudolarix amabilis [Syn. Larix amabilis; Pseudolarix kaempferi], T5269 Psilotum nudum, T5279 Psychotria serpens, T5298 Pteris vittata, T5341 Pygmaeopremna herbacea [Syn. Premna herbacea], T5391 Rabdosia eriocalyx, T5516 Rhododendron molle, T5555 Rodgersia pinnata, T5569 Rosa multiflora, T5591 Rubus cochinchinensis, T5609 Rumex hastatus, T5640 Sabina vulgaris, T5726 Saponaria officinalis, T5730 Sarcandra glabra [Syn. Chloranthus glaber], T5746 Sassafras tzumu, T5756 Saussurea japonica, T5793 Schisandra henryi, T5799 Schisandra rubriflora, T5847 Securidaca inappendiculata, T5861 Selaginella doederleinii, T5975 Smilax bockii, T6069 Speranskia tuberculata, T6439 Thuja orientalis [Syn. Platycladus orientalis; Biota orientalis], T6477 Torreya grandis, T6540 Tripterygium hypoglaucum, T6541 Tripterygium regelii, T6542 Tripterygium wilfordii, T6566 Tupistra chinensis, T6727 Veronica persica, T6731 Veronicastrum sibiricum, T6737 Viburnum luzonicum, T6738 Viburnum odoratissimum, T6742 Vicia amoena, T6773 Viscum articulactum, T6774 Viscum articulatum, T6787 Vitex negundo, T6799 Vitis vinifera, T6845 Xanthoceras sorbifolia, T6861 Zanthoxylum ailanthoides, T6862 Zanthoxylum ailanthoides, T6893 Zanthoxylum simulans, T6894 
Zanthoxylum simulans.

dispel wind and eliminate evil T6000 Solanum indicum.

dispel wind and eliminate impediment T0144 Acorus gramineus, T0146 Acorus tatarinowii, T0156 Actinidia arguta, T5872 Selenarctos thibetanus; Ursus arctos.

dispel wind and free impediment T2208 Dioscorea septemloba.

dispel wind and free network vessels T0644 Armillaria mellea, T1567 Clerodendrum mandarinorum, T1629 Colysis pothifolia [Syn. Hemionitis pothifolia], T1884 Cudrania cochinchinensis, T1939 Cymbopogon citratus, T2030 Daphne retusa, T2031 Daphne tangutica, T2738 Flemingia strobilifera, T2890 Gastrodia elata, T3316 Hylomecon japonica, T3363 Hypericum sampsonii, T3797 Libanotis condensata, T3897 Litsea verticillata, T4800 Phlegmariurus fordii, T5101 Polygonum cuspidatum, T5108 Polygonum multiflorum, T5515 Rhododendron micranthum, T5964 Sinomenium acutum, T6484 Trachelospermum jasminoides, T6618 Uncaria homomalla [Syn. Uruparia homomalla; Uruparia tonkinensis; Uruparia lanosa var. parvifora], T6651 Urtica cannabina, T6652 Urtica dioica, T6884 Zanthoxylum nitidum.

dispel wind and kill worms T2980 Gleditsia sinensis [Syn. Gleditsia horrida], T3857 Lindera umbellata [Syn. Lindera erythrocarpa], T5028 Plumbago indica, T6031 Sophora flavescens [Syn. Sophora angustfolia]. dispel wind and move $q i \mathrm{~T} 3886$ Litsea euosma.

dispel wind and move water T2131 Desmodium pulchellum [Syn. Phyllodium pulchellum], T6127 Stephania glabra.

dispel wind and open orifices T2275 Dryobalanops aromatica. dispel wind and outthrust papules $\mathrm{T} 3742$ Lentinus edodes.

dispel wind and overcome damp T0080 Aconitum bullatifolium var. homotrichum [Syn. Aconitum nagarum], T0086 Aconitum chasmanthum, T0108 Aconitum kusnezoffii, T0123 Aconitum polyschistum, T0135 Aconitum sungpanense, T0838 Azolla imbricata [Syn. Salvinia imbricata], T1289 Celastrus orbiculatus [Syn. Celastrus articulatus], T5755 Saussurea involucrata, T5934 Seseli meirei, T5937 Seseli yunnanense, T5972 Skimmia reevesiana.

dispel wind and percolate damp T4253 Mirabilis jalapa.

dispel wind and quicken blood T0572 Aralia elata, T1191 Caragana sinica, T1649 Convallaria keiskei [Syn. Convallaria majalis], T1767 Craibiodendron yunnanese, T3411 Impatiens balsamina, T5151 Populus davidiana, T5158 Populus simonii, T5637 Sabia schumanniana, T5848 Securinega suffruticosa, T6713 Vernonia anthelmintica, T6888 Zanthoxylum planispinum.

dispel wind and quicken network vessels T0645 Armillariella mellea, T0708 Arthromeris mairei [Syn. Polypodium mairei], T3285 Hovenia dulcis, T3643 Kopsia officinalis, T4219 Microlepia marginata, T5368 Python molurus bivittatus, T6099 Stauntonia chinensis, T6131 Stephania longa, T6183 Strychnos nitida.

dispel wind and relieve cough T2056 Debregeasia longifolia, T4443 Notholirion hyacinthinum [Syn. Notholirion bulbuliferum].

dispel wind and relieve itch T0963 Boehmeria siamensis, T1160 Campsis grandiflora, T1730 Corydalis ophiocarpa, T1936 Cyclocarya paliurus, T2165 Dictamnus angustifolius, T2167 Dictamnus dasycarpus, T3687 Lantana camara, T4231 Milingtonia hortensis, T4247 Millingtonia hortensis, T5104 Polygonum hydropiper, T6325 Tectona grandis. dispel wind and relieve pain T0060 Achillea alpina [Syn. Achillea sibirica], T0078 Aconitum barbatum var. puberulum [Syn. Aconitum ochranthum], T0094 Aconitum finetianum, T0628 Aristolochia fangchi, T0629 Aristolochia heterophylla, T0635 Aristolochia moupinensis, T1291 Celastrus paniculatus, T1372 Chloranthus serratus, T1552 Cleome viscosa, T1563 Clerodendron trichotomum, T1583 Cnidium officinale [Syn. Ligusticum officinale], T1586 Cocculus laurifolius, T2052 Davallia divaricata [Syn. Davallia formosana; Davallia orientalis], T2053 Davallia mariesii, T2267 Drosera peltata var. lunata, T2412 Equisetum sylvaticum, T2447 Erycibe elliptilimba, T2883 Gardenia jasminoides [Syn. Gardenia florida], T3080 Gymnema sylvestre, T3820 Ligusticum chuanxiong [Syn. Ligusticum wallichii], T3851 Lindera glauca, T4361 Myrsine africana, T5144 Populus adenopoda, T5308 Pterocarya stenoptera, T5781 Schefflera arboricola, T5785 Schefflera venulosa, T5969 Sium latifolium, T6084 Spiraea prunifolia, T6086 Spiraea thunbergii, T6118 Stephania brachyandra, T6119 Stephania cepharantha, T6125 Stephania elegans, T6129 Stephania japonica, T6136 Stephania tetrandra, T6250 Symplocos caudata, T6468 Tinospora sinensis, T6471 Toddalia asiatica [Syn. Toddalia aculeata; Paullinia asiatica].

dispel wind and remove damp T1530 Cladostachys amaranthoides [Syn. Achyranthes amaranthoides; Cladostachys frutescens; Deeringia amaranthoides].

dispel wind and resolve exterior T0478 Angelica dahurica [Syn. Angelica porphyrocaulis], T0692 Artemisia rupestris [Syn. Artemisia dentata; Artemisia viridis; Artemisia viridifolia], T0814 Atalantia buxifolia [Syn. Severinia buxifolia], T3006 Glycosmis citrifolia, T4154 Melaleuca leucadendra, T5727 Saposhnikovia divaricata [Syn. Ledebouriella seseloides], T6789 Vitex negundo.

dispel wind and resolve summerheat T6367 Teucrium quadrifarium.

dispel wind and resolve tetany T0975 Bombyx mori, T5547 Ricinus communis.

dispel wind and resolve toxin T2617 Euphorbia royleana, T2981 Gleditsia sinensis [Syn. Gleditsia horrida], T3000 Glycine max, T3001 Glycine max, T3991 Lyonia ovalifolia, T6005 Solanum lyratum.

dispel wind and settle fright T1332 Cercidiphyllum japonicum, T5290 Pteris fauriei [Syn. Pteris fauriei var. minor].

dispel wind and soothe liver T6531 Trillium camtschaticum.

dispel wind and transform damp T1959 Cynanchum paniculatum, T6866 Zanthoxylum avicennae.

dispel wind and transform phlegm T0088 Aconitum coreanum, T4814 Phlomis umbrosa, T6580 Tylophora floribunda, T6588 Typhoniun giganteum, T6697 Veratrum grandiflorum, T6698 Veratrum nigrum.

dispel wind quicken blood T1373 Chloranthus serratus.

dispel wind-damp T0036 Acanthopanax giraldii [Syn. Acanthopanax giraldii var. inermis; Eleutherococcus giraldii], T0038 Acanthopanax gracilistylus, T0042 Acanthopanax senticosus [Syn. Eleutherococcus senticosus], T0044 Acanthopanax sessiliflorus, T0090 Aconitum delavayi, T0096 Aconitum forrestii [Syn. Aconitum likiangense], T0099 Aconitum gymnandrum, T0125 Aconitum pseudostapfianum, T0154 Acroptilon repens, T0164 Actinidia polygama, T0240 Aglaia odorata, T0467 Anemone flaccida, T0470 Anemone raddeana, T0503 Anisomeles indica [Syn. Epimeredi indica], T1327 Ceratostigma minus, T1343 
Chaenomeles sinensis, T1374 Chloranthus spicatus, T1411 Cibotium barometz [Syn. Polypodium barometz], T1557 Clerodendron indicum, T1562 Clerodendron trichotomum, T1565 Clerodendrum bungei, T1812 Crossostephium chinense, T1922 Cyanotis vaga, T1923 Cyathula capitata, T1958 Cynanchum otophyllum, T2077 Delphinium grandiflorum, T2091 Delphinium yunnanense, T2197 Dioscorea futschauensis, T2198 Dioscorea gracillima, T2201 Dioscorea hypoglauca [Syn. Dioscorea collettii var. hypoglauca], T2210 Dioscorea spongiosa, T2211 Dioscorea tenuipes, T2212 Dioscorea tokoro, T2231 Diphylleia grayi, T2232 Diphylleia sinensis, T2389 Epimedium acuminatum, T2390 Epimedium brevicornum, T2392 Epimedium davidii, T2394 Epimedium elongatum, T2398 Epimedium koreanum, T2401 Epimedium pubescens, T2402 Epimedium sagittatum, T2404 Epimedium sutchuenense, T2406 Epimedium wushanense, T2438 Erodium stephanianum, T2479 Erythrina variegata var. orientalis, T2542 Euonymus japonicus, T2721 Ficus microcarpa, T2734 Fissistigma glaucescens [Syn. Melodorum glaucescens], T2736 Fissistigma polyanthum, T2909 Gentiana crassicaulis, T2910 Gentiana dahurica, T2913 Gentiana kaufmanniana, T2919 Gentiana macrophylla, T2932 Gentiana siphonantha, T2934 Gentiana straminea, T2936 Gentiana tianschanica, T2937 Gentiana tibetica, T2943 Geranium nepalense, T2944 Geranium pratense, T2947 Geranium sibiricum, T2949 Geranium wilfordii, T3199 Hemibarbus labeo, T3390 Ilex cornuta, T3410 Impatiens balsamina, T3420 Incarvillea sinensis, T3436 Inula nervosa, T3726 Laurus nobilis, T3925 Loranthus parasiticus [Syn. Loranthus chinenis; Taxillus chinensis], T4124 Matricaria chamomilla [Syn. Matricaria recutita], T4210 Michelia champaca, T4279 Morina chinensis, T4286 Morinda umbellata, T4290 Morus alba, T4575 Pachysandra terminalis, T4687 Peganum harmala, T4688 Peganum harmala, T4710 Pericampylus glaucus, T4729 Periploca sepium, T4828 Photinia serrulata, T4950 Piper kadsura [Syn. Piper futokadsura], T4974 Piper wallichii [Syn. Piper wallichii var. hupehense], T5102 Polygonum cuspidatum, T5189 Pothos chinensis, T5344 Pyrola atropurpurea, T5345 Pyrola calliantha [Syn. Pyrola rotundifolia ssp. chinensis], T5346 Pyrola calliantha var. tibetana, T5347 Pyrola decorata, T5349 Pyrola incarnata, T5350 Pyrola japonica, T5351 Pyrola rotundifolia, T5523 Rhododendron simsii, T5950 Siegesbeckia orientalis, T5951 Siegesbeckia orientalis var. glabrescens [Syn. Siegesbeckia glabrescens], T5952 Siegesbeckia orientalis var. pubescens [Syn. Siegesbeckia pubescens], T5981 Smilax riparia, T6120 Stephania delavayi [Syn. Stephania epigaea], T6128 Stephania hernandifolia, T6304 Taxillus levinei, T6437 Thlaspi arvense, T6440 Thuja orientalis [Syn. Platycladus orientalis; Biota orientalis], T6675 Valeriana amurensis, T6679 Valeriana officinalis, T6680 Valeriana officinalis var. latifolia, T6771 Viscum album, T6772 Viscum angulatum, T6775 Viscum coloratum, T6777 Viscum multinerve, T6831 Woodwardia orientalis, T6883 Zanthoxylum myriacanthum.

dispel wind-heat T0584 Arctium lappa, T1296 Celosia argentea, T2325 Eichhornia crassipes, T6791 Vitex rotundifolia [Syn. Vitex trifollia var. simplicifolia], T6793 Vitex trifolia.

dispel wind-phlegm T0599 Ardisia japonica.

disperse accumulation T0606 Areca catechu, T1381 Choerospondias axillaris, T1831 Crotalaria sessiliflora, T2049 Daucus carota, T2422
Erigeron breviscapus, T2748 Fomes fomentarius [Syn. Pyropolyporus fomentarius; Boletus fomentarius; Polyporus fomentarius], T3412 Impatiens balsamina, T5073 Polygala chinensis [Syn. Polygala glomerata], T5189 Pothos chinensis, T5229 Prunus persica, T5239 Prunus salicina, T5719 Sapindus mukorossi, T5720 Sapindus mukorossi, T5993 Solanum capsicastrum, T6062 Sparganium stoloniferum, T6312 Taxus mairei.

disperse accumulation and disinhibit damp T6277 Tadehagi triquetrum. disperse accumulation and disinhibit water T1049 Bufo bufo gargarizans; Bufo melanostictus.

disperse accumulation and expel stone T4000 Lysimachia congestiflora. disperse accumulation and fortify stomach T0692 Artemisia rupestris

[Syn. Artemisia dentata; Artemisia viridis; Artemisia viridifolia], T1954 Cynanchum chinense.

disperse accumulation and free stool T0708 Arthromeris mairei [Syn.

Polypodium mairei], T6651 Urtica cannabina, T6652 Urtica dioica.

disperse accumulation and gan T2502 Eucalyptus camaldulensis.

disperse accumulation and kill worms T1696 Cornus capitata [Syn.

Dendrobenthamia capitata], T4240 Millettia pachycarpa, T4779

Pharbitis nil, T4780 Pharbitis purpurea.

disperse accumulation and move water T3468 Iris sanguinea.

disperse accumulation and relieve pain T3436 Inula nervosa, T6049 Sophora viciifolia.

disperse accumulation and resolve toxin T6551 Trogopterus xanthipes; Pteromys volans.

disperse accumulation and transform phlegm T2720 Ficus hispida.

disperse accumulation and transform stagnation T5469 Rheum hotaoense.

disperse concretion and conglomeration T0164 Actinidia polygama.

disperse concretion and transform stone T2838 Gallus gallus domesticus. disperse damp T3306 Hydrangea paniculata.

disperse distention T1508 Citrus sinensis, T4264 Momordica cochinchinensis.

disperse edema T0157 Actinidia callosa var. henryi.

disperse edema and abate jaundice T6752 Vigna angularis [Syn. Dolichus angularis; Phaseolus angularis].

disperse food T1152 Camellia sinensis [Syn. Thea sinensis], T1478 Citrus grandis, T1483 Citrus grandis var. tomentosa, T1488 Citrus junos, T1937 Cydonia oblonga, T2115 Dermatocarpon minitum, T2994 Gloiopeltis furcata, T3308 Hydrangea umbellata, T3620 Kaempferia galanga, T4544 Oryza sativa, T5113 Polygonum persicaria, T5361 Pyrus betulaefolia, T5419 Raphanus sativus, T5570 Rosa roxburghii.

disperse food and assisting movement T6878 Zanthoxylum echinocarpum. disperse food and check diarrhea T2421 Erigeron annuus.

disperse food and harmonize center T0647 Armoracia lapathifolia.

disperse food and precipitate $q \boldsymbol{i} \mathrm{T} 1021$ Brassica rapa.

disperse food and promote lactation T1205 Carica papaya.

disperse food and rectify $\boldsymbol{q} i \mathrm{~T} 2387$ Epilobium hirsutum.

disperse food and relieve pain T0354 Alpinia chinensis.

disperse food and transform accumulation T0661 Artemisia anomala, T3282 Hordeum vulgare, T4577 Paederia scandens, T5365 Pyrus pashia.

disperse food and transform phlegm T2758 Fortunella crassifolia, T2759 
Fortunella japonica, T2760 Fortunella margarita, T4820 Phoenix dactylifera.

disperse food and transform stagnation T5461 Rhamnus leptophylla. disperse food distention T5420 Raphanus sativus.

disperse gan T0387 Amaranthus caudatus, T0969 Boerhavia diffusa, T1591

Cocos nucifera, T1593 Cocos nucifera.

disperse glomus T0988 Bos taurus domesticus; Bubalus bubalis.

disperse glomus and dissipate binds T4904 Pinellia ternata.

disperse phlegm T0892 Belamcanda chinensis, T1960 Cynanchum

stauntonii, T3426 Inula britannica, T3427 Inula britannica, T3428 Inula

britannica var. chinensis, T3433 Inula japonica, T3434 Inula

linariaefolia, T4957 Piper nigrum, T5740 Sargassum vachellianum.

disperse phlegm and allay thirst T1343 Chaenomeles sinensis.

disperse phlegm and disinhibit water T4403 Nemacystus decipiens [Syn.

Mesogloea decipiens; Cladosiphon decipiens].

disperse phlegm and move water T0726 Asarum forbesii.

disperse phlegm and relieve cough T3953 Lycianthes biflora.

disperse phlegm and transform accumulation T1468 Citrus aurantium,

T1521 Citrus wilsonii, T5141 Poncirus trifoliata.

disperse phlegm rheum T1390 Chrysanthemum coronarium, T1397

Chrysanthemum segetum.

disperse qi T2048 Daucus carota.

disperse sore toxin T0503 Anisomeles indica [Syn. Epimeredi indica].

disperse stagnation and disinhibit damp T1544 Cleistocalyx operculatus.

disperse stagnation and harmonize center T0932 Betula luminifera.

disperse stasis T3277 Homo sapiens, T3295 Huperzia serrata [Syn.

Lycopodium serratum].

disperse stasis and relieve pain T1931 Cyclea barbata, T6020 Solanum xanthocarpum.

disperse stasis and settle pain T0180 Adina pilulifera [Syn. Cephalanthus pilulifera].

disperse swelling T0471 Anemone rivularis, T0529 Anthriscus sylvestris, T0894 Benincasa hispida, T1181 Capra hircus; Ovis aries, T1558 Clerodendron inerme, T2048 Daucus carota, T2200 Dioscorea hispida, T2546 Euonymus sacrosancta, T2585 Euphorbia esula, T2830 Galium aparine, T2857 Garcinia hanburyi, T2885 Gardenia jasminoides var. grandiflora, T3155 Helianthus tuberosus, T3570 Juglans regia, T3668 Lagenaria siceraria var. depressa, T3674 Laggera alata, T4029 Maesa japonica, T4785 Phaseolus vulgaris, T4814 Phlomis umbrosa, T4848 Physalis angulata, T4951 Piper laetispicum, T4965 Piper sarmentosum, T5099 Polygonum bistorta, T5214 Prunella vulgaris, T5247 Przewalskia tangutica, T5563 Rosa cymosa, T5626 Ruta graveolens, T5651 Salix babylonica, T5857 Sedum sarmentosum, T6177 Strychnos ignatii, T6210 Swainsonia salsula [Syn. Sphaerophysa salsula], T6548 Tritonia crocosmaeflora, T6435 Thladiantha cordifolia, T6552 Trollius chinensis [Syn. Trollius asiaticus var. chinensis], T6553 Trollius ledebourii, T6772 Viscum angulatum.

disperse swelling and close sores T5712 Sanguisorba officinalis. disperse swelling and dispel damp T4985 Pithecolobium dulce.

disperse swelling and dissipate binds T1007 Brassica juncea, T1787 Cremastra appendiculata.

disperse swelling and draw out toxin T5550 Ricinus communis. disperse swelling and engender flesh T0955 Bletilla striata, T0994
Boswellia carterii, T1638 Commiphora myrrha [Syn. Commiphora molmol].

disperse swelling and expel pus T0479 Angelica dahurica cv. qibaizhi, T0498 Angelica taiwaniana, T3241 Hibiscus mutabilis, T3547 Ixeris chinensis, T6512 Trichosanthes kirilowii.

disperse swelling and free network vessels T6866 Zanthoxylum avicennae.

disperse swelling and outthrust pus T2979 Gleditsia sinensis [Syn. Gleditsia horrida].

disperse swelling and relieve pain T0108 Aconitum kusnezoffii, T0122 Aconitum pendulum, T0144 Acorus gramineus, T0146 Acorus tatarinowii, T0267 Ajuga nipponensis, T0293 Albizzia lebbeck, T0302 Alhagi pseudalhagi, T0476 Angelica anomala, T0643 Aristolochia versicolar, T0736 Asclepias curassavica, T0827 Aucuba chinensis ssp. omeiensis, T1108 Caesalpinia sappan, T1644 Conocephalum conicum, T1693 Coriaria sinica [Syn. Coriaria nepalensis], T1697 Cornus controversa [Syn. Bothrocaryum controversum], T1731 Corydalis pallida, T2162 Dicranostigma franchetianum [Syn. Dicranostigma leptopodum], T2444 Ervatamia heyneana, T2445 Ervatamia officinalis, T2449 Erycibe obtusifolia, T2450 Erycibe schmidtii, T2631 Eurya japonica, T2718 Ficus carica, T2749 Fomes officinalis, T2752 Fordia cauliflora, T2884 Gardenia jasminoides var. grandiflora, T2897 Gelsemium elegans, T3088 Gynura segetum [Syn. Gynura japonica], T3187 Helleborus thibetanus, T3203 Hemsleya amabilis, T3208 Hemsleya macrosperma, T3294 Huperzia selago [Syn. Lycopodium selago], T3320 Hymenocallis littoralis [Syn. Hymenocallis americana; Pancratium littoralis], T3411 Impatiens balsamina, T3461 Iris japonica, T3471 Iris tectorum, T3679 Lamiophlomis rotata [Syn. Phlomis rotata], T3796 Libanotis buchtormensis, T3852 Lindera megaphylla, T3880 Lithospermum arvense, T3887 Litsea glutinosa, T4018 Machilus thunbergii, T4182 Menispermum dauricum, T4183 Menispermum dauricum, T4303 Moschus moschiferus; Moschus berezovskii; Moschus sifanicus, T4317 Murraya euchrestifolia [Syn. Clausena euchrestifolia], T4391 Nauclea officinalis, T4605 Panax japonicus var. major, T4607 Panax pseudo-ginseng var. japonicus, T4648 Paris polyphylla, T4649 Paris polyphylla var. chinensis, T4650 Paris polyphylla var. pseudothibetica, T4651 Paris polyphylla var. stenophylla, T4652 Paris polyphylla var. yunnanensis, T4800 Phlegmariurus fordii, T4852 Physalis peruviana, T4884 Picria felterrae, T5028 Plumbago indica, T5279 Psychotria serpens, T5393 Rabdosia longituba, T5513 Rhododendron mariae, T5592 Rubus hirsutus, T5650 Salix babylonica, T5731 Sarcococca coriacea [Syn. Sarcococca wallichii], T5794 Schisandra lancifolia, T5797 Schisandra propinqua, T5798 Schisandra propinqua var. intermedia, T6007 Solanum melongena, T6051 Sorbaria arborea, T6052 Sorbaria sorbifolia, T6075 Spilanthes acmella, T6166 Strychnos angustiflora, T6353 Tetracera asiatica, T6432 Thespesia populnea [Syn. Hibiscus populneus], T6542 Tripterygium wilfordii, T6567 Tupistra wattii [Syn. Campylandra wattii], T6792 Vitex trifolia, T6845 Xanthoceras sorbifolia, T6884 Zanthoxylum nitidum.

eliminate inflammation and disperse swelling T6432 Thespesia populnea [Syn. Hibiscus populneus],

disperse swelling and resolve toxin T2062 Delphinium bonvalotii, T2081 Delphinium omeiense, T2084 Delphinium potaninii, T2085 Delphinium 
potaninii var. jiufengshanense, T2243 Dodonaea viscosa, T2866 Garcinia morella, T2883 Gardenia jasminoides [Syn. Gardenia florida]. disperse swelling and settle pain T1589 Cocculus trilobus [Syn. Cocculus sarmentosus], T4604 Panax japonicus var. bipinnatifidus, T4606 Panax pseudo-ginseng, T4608 Panax pseudo-ginseng var. notoginseng [Syn. Panax notoginseng].

disperse swelling and stanch bleeding T3995 Lysidice rhodostegia, T4568 Oxytropis myriophylla, T5444 Reboulia hemisphaerica.

disperse swelling and transform stasis T3936 Luffa operculata.

disperse swelling toxin T0584 Arctium lappa, T2197 Dioscorea futschauensis, T2210 Dioscorea spongiosa, T4687 Peganum harmala, T6018 Solanum verbascifolium, T6120 Stephania delavayi [Syn. Stephania epigaea].

disperse swollen welling abscess T0470 Anemone raddeana, T0516 Anser cygnoides domestica, T1685 Coreopsis lanceolata, T1709 Corydalis bungeana, T4279 Morina chinensis, T5230 Prunus persica.

disperse welling abscess T4106 Manis pentadactyla, T6499 Tribulus terrestris.

disperse welling abscess and dissipate binds T0750 Asparagus gobicus. disperse welling abscess and expel pus T0895 Benincasa hispida, T5250 Psammosilene tunicoides.

disperse welling abscess and promote lactation T0173 Adiantum lunulatum.

dissipate binds T2024 Daphne genkwa, T2599 Euphorbia lunulata, T2757 Forsythia viridissima, T2790 Fritillaria pallidiflora, T2800 Fritillaria walujewii, T3287 Huechys sanguinea, T3668 Lagenaria siceraria var. depressa, T3815 Ligularia stenocephala, T5214 Prunella vulgaris.

dissipate binds and disperse concretion T1162 Camptotheca acuminata. dissipate binds and disperse goiter T2191 Dioscorea bulbifera.

dissipate binds and disperse swelling T1005 Brassica campestris [Syn. Brassica campestris var. oleifera], T1092 Buxus microphylla var. sinica, T2608 Euphorbia pekinensis, T2636 Euscaphis japonica, T2691 Farfugium japonicum, T2756 Forsythia suspensa, T2798 Fritillaria verticillata var. thunbergii [Syn. Fritillaria thunbergii], T3202 Hemistepta lyrata [Syn. Hemistepta carthamoides; Saussurea carthamoides], T4265 Momordica cochinchinensis, T5139 Poncirus trifoliata, T5415 Ranunculus sceleratus, T5723 Sapium sebiferum, T5958 Sinapis alba [Syn. Brassica alba; Brassica hirta], T6184 Strychnos nux-vomica, T6193 Strychnos wallichiana, T6257 Syngnathus acus, T6301 Taraxacum mongolicum, T6521 Trifolium pratense, T6560 Tulipa edulis.

dissipate binds and draw out toxin T0971 Bolbostemma paniculatum. dissipate binds and relieve pain T0333 Alocasia cucullata [Syn. Arum cucullatum], T0427 Ampelopsis japonica [Syn. Paullinia japonica], T1017 Brassica oleracea var. capitata, T2632 Euryale ferox, T3443 Iphigenia indica.

dissipate blood T4254 Miscanthus sinensis.

dissipate blood and disperse swelling T6006 Solanum melongena.

dissipate blood and eliminate accumulation T6732 Vespertilio superans.

dissipate blood and relieve pain $\mathrm{T} 1640$ Coniogramme japonica [Syn. Hemionitis japonica].

dissipate cold T0356 Alpinia galanga, T0359 Alpinia officinarum, T0472 Anethum graveolens, T1180 Capparis spinosa, T3120 Hedychium spicatum, T3869 Liquor, T6864 Zanthoxylum armatum, T6887 Zanthoxylum planispinum.

dissipate cold and dry damp T0363 Alpinia speciosa, T2051 Daucus carota var. sativa, T3222 Heracleum scabridum.

dissipate cold and effuse exterior T4551 Osmorhiza aristata var. laxa.

dissipate cold and interrupt malaria T3124 Hedyotis capitellata.

dissipate cold and relieve cough T3871 Liriodendron tulipifera.

dissipate cold and relieve pain T0077 Aconitum balfourii, T0084 Aconitum carmichaeli, T0088 Aconitum coreanum, T0105 Aconitum karakolicum, T0106 Aconitum kirinense, T0123 Aconitum polyschistum, T0135 Aconitum sungpanense, T0470 Anemone raddeana, T0492 Angelica pubescens f. biserrata [Syn. Angelica pubescens], T0664 Artemisia argyi, T0681 Artemisia lavandulaefolia, T0685 Artemisia mongolica, T0689 Artemisia princeps, T0691 Artemisia rubripes, T0706 Artemisia vulgaris, T1217 Carum carvi, T1439 Cinnamomum cassia [Syn. Cinnamomum aromaticum], T1641 Conioselinum vaginatum, T1889 Cuminum cyminum, T2895 Gaultheria yunnanensis, T3214 Heracleum lanatum, T4550 Osmanthus fragrans, T4954 Piper longum, T6488 Trachyspermum ammi, T6876 Zanthoxylum dissitum, T6888 Zanthoxylum planispinum, T6893 Zanthoxylum simulans, T6894 Zanthoxylum simulans.

dissipate cold and resolve exterior T1438 Cinnamomum cassia [Syn. Cinnamomum aromaticum], T2422 Erigeron breviscapus, T3822 Ligusticum jeholense, T3824 Ligusticum sinense, T4445 Notopterygium forbesii [Syn. Notopterygium franchetii], T4446 Notopterygium incisum, T4718 Perilla frutescens var. acuta [Syn. Perilla frutescens var. purpurascens], T4721 Perilla frutescens var. arguta, T5560 Rorippa montana [Syn. Rorippa dubia; Sisymbrium dublium], T6910 Zingiber officinale.

dissipate depression and open binds T1808 Crocus sativus.

dissipate exopathogen T2340 Elsholtzia bodinieri.

dissipate heat T6843 Xanthium sibiricum [Syn. Xanthium strumarium].

dissipate heat and relieve pain $\mathrm{T} 2274$ Dryobalanops aromatica.

dissipate lung $q i$ T2761 Fortunella margarita.

dissipate screen and disperse swelling T1981 Cyprinus carpio.

dissipate stasis T0322 Allium tuberosum, T0324 Allium victorialis, T0567 Aralia armata, T0950 Blatta orientalis, T1097 Cacalia ainsliaeflora, T1161 Campsis grandiflora, T1174 Cannabis sativa, T2612 Euphorbia prolifera, T2830 Galium aparine, T3088 Gynura segetum [Syn. Gynura japonica], T3674 Laggera alata, T4020 Macleaya cordata, T5073 Polygala chinensis [Syn. Polygala glomerata], T5087 Polygala telephioides, T5090 Polygala wattersii, T5105 Polygonum hydropiper var. flaccidum [Syn. Polygonum flaccidum], T5518 Rhododendron mucronatum, T5563 Rosa cymosa, T5850 Sedum aizoon, T5855 Sedum kamtschaticum, T5883 Senecio cannabifolius, T6872 Zanthoxylum cuspidatum.

dissipate stasis and disperse swelling T0135 Aconitum sungpanense, T0240 Aglaia odorata, T0468 Anemone hupehensis, T0515 Anredera cordifolia [Syn. Baussingaultia cordifolia; Baussingaultia gracilis $\mathrm{f}$. pseudobaselloides; Baussingaultia gracilis var. pseudobaselloides], T1031 Broussonetia kazinoki, T1120 Callicarpa macrophylla, T1145 Camellia japonica, T1147 Camellia oleifera, T1441 Cinnamomum japonicum, T1552 Cleome viscosa, T1624 Colocasia antiquorum, T1657 
Conyza canadensis [Syn. Erigeron canadensis], T1684 Cordyline strcta, T2290 Duchesnea indica, T2357 Emilia sonchifolia, T2973 Glechoma longituba, T2974 Glechoma lungituba, T3006 Glycosmis citrifolia, T3089 Gypsophila acutifolia, T3316 Hylomecon japonica, T3356 Hypericum japonicum, T3387 Idesia polycarpa, T3507 Isodon lophanthoides [Syn. Rabdosia lophanthoides; Hyssopus lophanthoides; Plectranthus striatus; Isodon striatus], T3560 Jatropha curcas, T3845 Linaria vulgaris, T3998 Lysimachia christinae, T4223 Micromelum integerrimum, T4384 Narcissus tazetta var. chinensis, T4686 Pedilanthus tithymaloides, T4730 Peristrophe roxburghiana, T4821 Pholidota articulata, T5397 Rabdosia serra, T5402 Radermachera sinica, T5499 Rhodiola sacra, T5724 Sapium sebiferum, T5884 Senecio chrysanthemoides, T6035 Sophora japonica.

dissipate stasis and quicken blood T3809 Ligularia lapathifolia.

dissipate stasis and relieve pain T0010 Abrus fruticulosus [Syn. Abrus cantoniensis], T0048 Acanthus ilicifolius, T0069 Achillea wilsoniana, T0264 Ajuga forrestii, T0266 Ajuga macrosperma, T0281 Alangium chinense, T0282 Alangium kurzii, T0285 Alangium platanifolium, T0602 Ardisia quinquegona, T0721 Arundina chinensis, T1215 Carthamus tinctorius, T1561 Clerodendron serratum, T1569 Clerodendrum serratum var. amplexifolium, T1574 Clintonia alpina, T1629 Colysis pothifolia [Syn. Hemionitis pothifolia], T1866 Cryptolepis sinensis, T1933 Cyclea sutchuenensis, T1996 Dahlia pinnata [Syn. Dahlia variabilis], T2030 Daphne retusa, T2031 Daphne tangutica, T2124 Derris trifoliata, T2139 Dianella ensifolia, T2545 Euonymus phellomana, T3221 Heracleum rapula, T3349 Hypericum erectum, T3520 Isodon rosthornii, T3549 Ixora chinensis, T3849 Lindera chunii, T3897 Litsea verticillata, T4221 Micromelum falcatum, T4284 Morinda parvifolia, T4323 Murraya paniculata [Syn. Chalcas paniculata], T4325 Murraya paniculata var. exotica, T4642 Parameria laevigata, T4705 Peperomia pellucida, T4823 Pholidota yunnanensis, T4956 Piper mullesua, T5517 Rhododendron molle, T5595 Rubus parviforlius, T5756 Saussurea japonica, T5847 Securidaca inappendiculata, T5868 Selaginella stauntoniana, T5989 Solanum aculeatissimum, T6014 Solanum surattense, T6015 Solanum torvum, T6121 Stephania dicentrinifera, T6122 Stephania dielsiana, T6133 Stephania sinica, T6137 Stephania viridiflavens, T6566 Tupistra chinensis.

dissipate stasis and resolve toxin T2296 Duranta repens, T3190 Helminthostachys zeylanica, T3373 Hypoestes purpurea [Syn. Justicia purpurea; Hypoestes sinica], T5189 Pothos chinensis.

dissipate stasis and settle pain T1994 Daemonorops draco, T2253 Dracaena cochinchinensis, T5250 Psammosilene tunicoides, T5516 Rhododendron molle, T6874 Zanthoxylum dimorphophyllum var. spinifolum.

dissipate stasis and stanch bleeding T1971 Cynoglossum zeylanicum [Syn. Anchusa zeylanica; Cynoglossum furcatum; Cynoglossum formosanum], T3395 Ilex pedunculosa, T5501 Rhodiola yunnanesis, T6567 Tupistra wattii [Syn. Campylandra wattii].

dissipate stasis and strengthen sinews T5207 Prismatomeris tetrandra. dissipate stasis blood T5722 Sapium japonicum.

dissipate toxin swelling T6440 Thuja orientalis [Syn. Platycladus orientalis; Biota orientalis].

dissipate wind and brighten eyes T6497 Tribulus terrestris. dissipate wind and clear heat T4773 Peucedanum terebinthaceum. dissipate wind and eliminate damp T2505 Eucalyptus citriodora.

dissipate wind and relieve itch T4305 Mosla dianthera.

dissipate wind-cold T4035 Magnolia biondii [Syn. Magnolia fargesii], T4038 Magnolia denudata [Syn. Magnolia heptapata], T4041 Magnolia liliflora, T4052 Magnolia sprengeri, T5822 Scopolia sinensis.

dissipate wind-heat T2408 Equisetum hiemale, T2721 Ficus microcarpa, T3392 Ilex kudingcha, T3393 Ilex latifolia, T3829 Ligustrum robustum, T4184 Mentha haplocalyx [Syn. Mentha canadaensis; Mentha arvensis var. haplocalyx; Mentha arvensis].

downbear counterflow and check vomiting T1202 Carex kobomugi, T1261 Catalpa ovata, T2433 Eriobotrya japonica, T4904 Pinellia ternata, T6910 Zingiber officinale.

downbear counterflow and disperse food T4954 Piper longum.

downbear counterflow and precipitate $q \boldsymbol{i} \mathrm{T} 2217$ Diospyros kaki.

downbear counterflow $q i$ T4369 Nandina domestica.

downbear fire T0708 Arthromeris mairei [Syn. Polypodium mairei], T1154 Camellia sinensis [Syn. Thea sinensis], T3277 Homo sapiens.

downbear fire and disinhibit throat T6346 Terminalia chebula, T6348 Terminalia chebula var. tomentella.

downbear fire and transform phlegm T5531 Rhus chinensis [Syn. Rhus semialata].

downbear qi T1960 Cynanchum stauntonii, T2008 Dalbergia odorifera, T2013 Dalbergia sissoo, T3426 Inula britannica, T3427 Inula britannica, T3428 Inula britannica var. chinensis, T3433 Inula japonica, T3434 Inula linariaefolia, T4717 Perilla frutescens var. acuta [Syn. Perilla frutescens var. purpurascens], T4719 Perilla frutescens var. arguta, T4722 Perilla frutescens var. crispa, T6261 Syringa pinnafolia.

downbear $q \boldsymbol{i}$ and disperse phlegm T4715 Perilla frutescens, T4773 Peucedanum terebinthaceum.

downbear $q \boldsymbol{i}$ and transform phlegm T0480 Angelica decursiva [Syn. Peucedanum decursivum], T3819 Ligusticum brachylobum, T4761 Peucedanum longshengens, T4768 Peucedanum praeruptorum, T4769 Peucedanum rubricaule, T5216 Prunus armeniaca, T5219 Prunus armeniaca var. ansu, T5284 Pteridium aquilinum var. latiusculum, T5420 Raphanus sativus, T5645 Sageretia theezans [Syn. Sageretia thea].

drain fire T0457 Andrographis paniculata [Syn. Justicia paniculata], T0907 Berberis kawakamii, T4370 Nandina domestica, T4789 Phellodendron amurense, T4790 Phellodendron amurense var. wilsonii, T4791 Phellodendron chinense, T4792 Phellodendron chinense var. glabriusculum, T6372 Thalictrum acutifolium, T6381 Thalictrum faberi, T6388 Thalictrum fortunei.

drain fire and relieve dysphoria T2882 Gardenia jasminoides [Syn. Gardenia florida].

drain fire and resolve toxin T0334 Aloe arborescens var. natalensis, T0906 Berberis julianae, T0912 Berberis poiretii, T0916 Berberis thunbergii, T0920 Berberis wilsonae, T1662 Coptis chinensis, T1663 Coptis chinensis var. brevisepala, T1664 Coptis deltoidea, T1669 Coptis omeiensis, T1670 Coptis teetoides [Syn. Coptis teeta], T2902 Gentiana algida, T3011 Glycyrrhiza aspera, T3013 Glycyrrhiza glabra, T3015 Glycyrrhiza inflata, T3016 Glycyrrhiza kansuensis, T3021 Glycyrrhiza squamulosa, T3022 Glycyrrhiza uralensis, T3023 Glycyrrhiza 
yunnanensis, T4060 Mahonia confusa, T4064 Mahonia gracilipes, T4887 Picrorhiza kurrooa, T4888 Picrorhiza scrophulariiflora, T5833 Scutellaria amoena, T5834 Scutellaria baicalensis, T5839 Scutellaria hypericifolia, T5841 Scutellaria likiangensis, T5843 Scutellaria rehderiana, T5845 Scutellaria viscidula, T6231 Swertia pseudochinensis, T6268 Syzygium samarangense, T6375 Thalictrum baicalense, T6376 Thalictrum cultratum, T6378 Thalictrum delavayi, T6385 Thalictrum flavum, T6387 Thalictrum foliolosum, T6389 Thalictrum glandulosissimum.

drain fire and stanch bleeding T1655 Conyza blinii.

drain heat $\mathrm{T} 5606$ Rumex acetosa.

drain heat and free intestines T5472 Rheum officinale, T5474 Rheum palmatum, $\mathrm{T} 5481$ Rheum tanguticum.

drain heat and free stool T5605 Rumex acetosa.

drain heat and free strangury T0303 Alisma orientale [Syn. Alisma plantago-aquatica var. orientale].

drain heat and move stagnation T1229 Cassia acutifolia, T1230 Cassia angustifolia.

drain liver and brighten eyes T3564 Juglans mandshurica.

drain liver and settle fright T2908 Gentiana cephalantha, T2921 Gentiana manshurica, T2928 Gentiana rigescens, T2930 Gentiana scabra, T2938 Gentiana triflora.

drain lung and calm asthma T2125 Descurainia sophia, T3755 Lepidium apetalum [Syn. Lepidium micranthum], T3759 Lepidium virginicum, T4287 Morus alba.

drain lung and disperse phlegm T1813 Crotalaria albida.

drain precipitation T0533 Antiaris toxicaria [Syn. Ambora toxicaria], T1645 Consolida ajacis [Syn. Delphinium ajacis], T2231 Diphylleia grayi, T2232 Diphylleia sinensis, T2650 Excoecaria agallocha.

drain precipitation and cold accumulation $\mathrm{T} 1858$ Croton tiglium.

drain precipitation and expel water T5723 Sapium sebiferum, T5724

Sapium sebiferum, T6819 Wikstroemia chamaedaphne.

drain precipitation and free stagnation T5550 Ricinus communis.

drain precipitation and free stool T3231 Hernandia sonora [Syn. Hernandia ovigera].

drain precipitation and kill worms T5457 Rhamnus davurica.

drain water and expel rheum T2023 Daphne genkwa, T2596 Euphorbia kansui, T2608 Euphorbia pekinensis, T3632 Knoxia valerianoides.

drain water and free stool T4779 Pharbitis nil, T4780 Pharbitis purpurea.

drain water retension T5770 Saussurea superba [Syn. Saussurea hieracioides].

draw out pus T2036 Datura innoxia, T2041 Datura metel, T2046 Datura stramonium.

draw out pus and relieve itch T5548 Ricinus communis.

draw out toxin T1416 Cicuta virosa, T2293 Dumortiera hirsuta, T2584 Euphorbia ebracteolata, T2587 Euphorbia fischeriana, T2599 Euphorbia lunulata.

draw out toxin and disperse swelling T2139 Dianella ensifolia.

draw out toxin and engender flesh T0557 Aquilegia ecalcarata, T3887 Litsea glutinosa.

draw out toxin and remove putrid T2580 Euphorbia antiquorum.

draw sore toxin T1337 Cervus nippon; Cervus elaphus.

draw toxin and close sores T3129 Hedysarum polybotrys. draw toxin and expel pus T3128 Hedysarum multijugum.

dry damp T0190 Adonis sutchuenensis, T0353 Alpinia blepharocalyx, T0446 Anaphalis margaritacea, T0457 Andrographis paniculata [Syn. Justicia paniculata], T1582 Cnidium monnieri, T2627 Euphoria longan [Syn. Dimocarpus longan], T2644 Evodia rutaecarpa, T2646 Evodia rutaecarpa var. officinalis, T3120 Hedychium spicatum, T4055 Mahonia bealei, T4056 Mahonia bealei, T4057 Mahonia bealei, T4062 Mahonia fortunei, T4063 Mahonia fortunei, T4066 Mahonia japonica, T4067 Mahonia japonica, T4068 Mahonia japonica, T4072 Mahonia shenii, T6393 Thalictrum ichangense [Syn. Thalictrum tripeltatum; Thalictrum multipeltatum], T6402 Thalictrum omeiense, T6403 Thalictrum petaloideum, T6414 Thalictrum thunbergii, T6860 Zanthoxylum ailanthoides.

dry damp and check discharge T6683 Vallisneria spiralis.

dry damp and disinhibit water T0824 Atractylodes macrocephala [Syn. Atractylis macrocephala].

dry damp and disperse phlegm T4034 Magnolia biloba, T4045 Magnolia officinalis.

dry damp and fortify spleen T5267 Psidium guajava.

dry damp and kill worms T1566 Clerodendrum inerme, T4881 Picrasma quassioides [Syn. Picrasma ailanthoides], T5323 Pulsatilla campanella, T5324 Pulsatilla cernua, T5325 Pulsatilla chinensis, T5326 Pulsatilla dahurica.

dry damp and relieve itch T3588 Juniperus formosana.

dry damp and resolve itch $\mathrm{T} 0167$ Adenanthera pavonina, T3630 Kleinhovia hospita, T4937 Piper betle.

dry damp and resolve toxin T5234 Prunus persica, T6268 Syzygium samarangense, T6338 Tephrosia purpurea.

dry damp and transform phlegm T1483 Citrus grandis var. tomentosa, T1506 Citrus reticulata.

effuse exterior T0314 Allium fistulosum, T5963 Sinodielsia yunnanensis.

effuse exterior and clear heat T2627 Euphoria longan [Syn. Dimocarpus longan].

effuse exterior and dispel summerheat T4305 Mosla dianthera.

effuse exterior and dissipate cold T3796 Libanotis buchtormensis.

effuse exterior and dissipate wind T5389 Rabdosia coetsa.

effuse exterior and outthrust papules T1419 Cimicifuga dahurica, T1420 Cimicifuga foetida, T1421 Cimicifuga heracleifolia, T1425 Cimicifuga simplex.

effuse exterior and relieve cough T5000 Plagiogyria stenoptera.

effuse exterior and resolve summerheat T5059 Pogostemon cablin [Syn. Mentha cablin].

effuse sweat T1315 Cephaelis ipecacuanha, T5452 Reseda luteola, T5575 Rosmarinus officinalis.

effuse sweat and abate fever T6457 Tilia japonica, T6458 Tilia miqueliana.

effuse sweat and disinhibit water T0610 Argemone mexicana. effuse sweat and eliminate summerheat T2343 Elsholtzia splendens. effuse sweat and outthrust papules T4456 Nymphoides peltatum. effuse sweat and remove damp T5113 Polygonum persicaria. effuse sweat and resolve exterior T2367 Ephedra equisetina, T2368 Ephedra gerardiana, T2369 Ephedra intermedia, T2372 Ephedra likiangensis, T2373 Ephedra minuta, T2375 Ephedra monosperma, 
T2379 Ephedra saxatilis, T2380 Ephedra sinica, T3118 Hedychium coronarium, $\mathrm{T} 3738$ Lemna minor, 55609 Rumex hastatus.

effuse sweat and resolve summerheat T2341 Elsholtzia ciliata, T4304 Mosla chinensis [Syn. Orthodon chinensis].

eliminate damp T1180 Capparis spinosa, T1437 Cinnamomum camphora, T1558 Clerodendron inerme, T1876 Cucumis melo, T1941 Cymbopogon distans, T2263 Dregea volubilis, T2584 Euphorbia ebracteolata, T2587 Euphorbia fischeriana, T3082 Gynocardia odorata, T3125 Hedyotis chrysotricha [Syn. Oldenlandia chrysotricha], T3295 Huperzia serrata [Syn. Lycopodium serratum], T3594 Juniperus rigida, T3674 Laggera alata, T3847 Lindera angustifolia, T4349 Myrica rubra, T4368 Nandina domestica, T5105 Polygonum hydropiper var. flaccidum [Syn. Polygonum flaccidum], T5106 Polygonum lapathifolium, T5767 Saussurea pulchella, T5977 Smilax glabra, T5980 Smilax menispermoidea, T6499 Tribulus terrestris, T6843 Xanthium sibiricum [Syn. Xanthium strumarium].

eliminate damp and relieve pain T1656 Conyza bonariensis [Syn. Erigeron bonariensis; Erigeron linifolius; Erigeron crispus].

eliminate damp and resolve cold phlegm T4904 Pinellia ternata.

eliminate food stagnation T5384 Quisqualis indica.

eliminate gan fever T4887 Picrorhiza kurrooa, T4888 Picrorhiza scrophulariiflora, T6104 Stellaria dichotoma var. lanceolata.

eliminate heat T3003 Glycine max, T5938 Setaria italica, T6507 Trichosanthes cucumeroides, T6544 Triticum aestivum [Syn. Triticum vulgare].

eliminate heat and dry damp T0255 Ailanthus altissima, T6475 Toona sinensis.

eliminate heat in blood T2882 Gardenia jasminoides [Syn. Gardenia florida], T3954 Lycium barbarum, T3956 Lycium chinense.

eliminate heat vexation T1758 Cotinus coggygria.

eliminate impediment T6826 Wisteria sinensis.

eliminate impediment and check diarrhea T1614 Coix lacryma-jobi var. ma-yuen.

eliminate inflammation T0423 Ampelopsis brevipedunculata, T0646 Armillariella tabescens, T0964 Boenninghausenia albiflora, T1955 Cynanchum hancockianum, T2844 Ganoderma applanatum, T3106 Harpagophytum procumbens, T3289 Humulus lupulus, T3394 Ilex paraguariensis, T4503 Onychium lucidum, T6139 Sterculia lychnophora.

eliminate inflammation and disinhibit urine $\mathrm{T} 1557$ Clerodendron indicum.

eliminate inflammation and disperse swelling T6432 Thespesia populnea [Syn. Hibiscus populneus].

eliminate inflammation and engender flesh T6920 Ziziphus mauritiana.

eliminate inflammation and move water T3432 Inula helianthus-aquatica.

eliminate inflammation and relieve pain T0543 Apis mellifera ligustica, T3643 Kopsia officinalis, T3960 Lycogala epidendrum, T4208 Metasequoia glyptostroboides, T6276 Tacca plantaginea [Syn. Schizocapsa plantaginea].

eliminate macula and resolve toxin T2598 Euphorbia lathyris.

eliminate phlegm and suppress cough T3803 Ligularia dictyoneura [Syn. Senecio dictyoneurus].

eliminate putridity T2584 Euphorbia ebracteolata, T2587 Euphorbia fischeriana.

eliminate putridity and close sores T2729 Filipendula ulmaria, T2857 Garcinia hanburyi.

eliminate putridity and engender flesh T4031 Maesa perlarius, T6080 Spiraea japonica.

eliminate screen T2408 Equisetum hiemale, T5414 Ranunculus japonicus. eliminate screen and brighten eyes T1048 Buddleja officinalis, T2274 Dryobalanops aromatica, T5907 Senecio scandens [Syn. Senecio chinensis], T6079 Spiraea japonica, T6082 Spiraea japonica var. fortunei.

eliminate screens T4471 Ocimum basilicum.

eliminate steam T0660 Artemisia annua, T0662 Artemisia apiacea [Syn.

Artemisia carvifolia; Artemisia caruifolia], T0705 Artemisia vestita.

eliminate welling abscess T6596 Ulmus parvifolia.

eliminate vexation T4088 Malus pumila, T4829 Phragmites communis, T5227 Prunus mume.

eliminate vexation and allay thirst T0155 Actinidia arguta, T0438 Anacardium occidentale, T0462 Anemarrhena asphodeloides, T1152 Camellia sinensis [Syn. Thea sinensis], T3286 Hovenia dulcis, T3392 Ilex kudingcha, T3393 Ilex latifolia, T4545 Oryza sativa, T4618 Panicum miliaceum, T6492 Trapa bispinosa.

eliminate vexation and heat T1006 Brassica chinensis.

eliminate vexation and quiet spirit T4121 Marsilea quadrifolia.

eliminate wind-damp T0298 Alectoria vivens, T4283 Morinda officinalis, T5638 Sabia swinhoei.

engender blood and harmonize blood T3615 Kadsura interior.

engender flesh T0542 Apis cerana, T1452 Cissampelos pareira, T1453

Cissampelos pareira var. hirsute, T2092 Delphinus delphis, T2293

Dumortiera hirsuta, T2420 Ericerus pela, T4478 Octopus vulgaris.

engender flesh and close sores T6716 Vernonia esculenta.

engender flesh and cure sores T1552 Cleome viscosa.

engender flesh and kill worms T3407 Illicium simonsii.

engender flesh and relieve pain T3089 Gypsophila acutifolia, T4917 Pinus massoniana.

engender flesh and stanch bleeding T0180 Adina pilulifera [Syn. Cephalanthus pilulifera], T4207 Metaplexis japonica, T4686 Pedilanthus tithymaloides, T5528 Rhodomyrtus tomentosa, T5554 Rodgersia aesculifolia, T6145 Stichopus japonicus.

engender hairs T1175 Cannabis sativa, T2981 Gleditsia sinensis [Syn. Gleditsia horrida].

engender liquid T0535 Antidesma bunius, T0834 Averrhoa carambola, T1165 Canarium album, T1961 Cynanchum thesioides, T2221 Diospyros kaki, T4088 Malus pumila, T4099 Mangifera indica, T4103 Mangifera persiciformis, T4288 Morus alba, T4599 Panax ginseng [Syn. Panax schinseng], T4979 Pistacia chinensis, T5228 Prunus mume, T5229 Prunus persica, T5239 Prunus salicina, T5312 Pueraria edulis, T5313 Pueraria lobata [Syn. Pueraria thunbergiana; Pueraria pseudohirsuta], T5316 Pueraria omeiensis, T5318 Pueraria phaseoloides, T5320 Pueraria thomsonii, T6266 Syzygium cumini, T6507 Trichosanthes cucumeroides, T6919 Ziziphus jujuba var. spinosa.

engender liquid and allay thirst T1006 Brassica chinensis, T1153 Camellia sinensis var. assamica, T1179 Capparis masaikai, T1471 Citrus chachiensis, T1473 Citrus cultivars, T1474 Citrus decumana, 
T1490 Citrus limon, T1494 Citrus limonia, T1516 Citrus tankan, T1518 Citrus unshiu, T2220 Diospyros kaki, T2569 Eupatorium rebaudianum, T3962 Lycopersicon esculentum, T4086 Malus asiatica, T4348 Myrica rubra, T4600 Panax ginseng [Syn. Panax schinseng], T4602 Panax ginseng [Syn. Panax schinseng], T4731 Persea americana [Syn. Persea gratissima], T4786 Phasianus colchicus, T4834 Phyllanthus emblica, T5093 Polygonatum odoratum [Syn. Polygonatum officinale], T5094 Polygonatum prattii, T5179 Potentilla anserina, T5186 Potentilla reptans var. sericophylla, $\mathrm{T} 5218$ Prunus armeniaca.

engender liquid and boost blood T3876 Litchi chinensis.

engender liquid and boost lung T2190 Dioscorea batatas [Syn. Dioscorea opposita], T2202 Dioscorea japonica.

engender liquid and eliminate vexation T0713 Artocarpus heterophyllus, T0722 Arundo donax.

engender liquid and moisten dryness T0989 Bos taurus domesticus; Bubalus bubalis, T5447 Rehmannia glutinosa [Syn. Rehmannia glutinosa f. huechingensis].

engender liquid and quiet spirit T4682 Pedicularis muscicola.

enrich and invigorate T0541 Apis cerana.

enrich and nourish T4785 Phaseolus vulgaris.

enrich and nourish blood vessel T1888 Cudrania tricuspidata.

enrich and supplement T0541 Apis cerana, T0924 Bergenia purpurascens,

T3200 Hemidesmus indicus, T6432 Thespesia populnea [Syn. Hibiscus populneus].

enrich kidney T3955 Lycium barbarum, T3958 Lycium chinense.

enrich kidney and replenish essence T5091 Polygonatum cyrtonema [Syn.

Polygonatum multiflorum], T5092 Polygonatum kingianum, T5095

Polygonatum sibiricum.

enrich liver and nourish kidney T1819 Crotalaria ferruginea .

enrich yin and boost qi T4821 Pholidota articulata.

enrich yin and clear heat T0155 Actinidia arguta, T2094 Dendrobium aduncum, T2096 Dendrobium aurantiacum var. denneanum, T2098 Dendrobium chrysanthum, T2100 Dendrobium densiflorum, T2102 Dendrobium fimbriatum var. oculatum, T2105 Dendrobium loddigesii, T2106 Dendrobium moniliforme, T2107 Dendrobium nobile, T2108 Dendrobium officinale, T5133 Polytrichum commune, T5445 Rehmannia glutinosa [Syn. Rehmannia glutinosa f. huechingensis].

enrich yin and downbear fire T3276 Homo sapiens, T5826 Scrophularia buergeriana, T5828 Scrophularia ningpoensis.

enrich yin and moisten dryness T0462 Anemarrhena asphodeloides, T0746 Asparagus cochinchinensis [Syn. Asparagus lucidus], T0982 Bos taurus domesticus, T2840 Gallus gallus domesticus.

enrich yin and nourish blood T5107 Polygonum multiflorum. enrich yin and remove heat T5186 Potentilla reptans var. sericophylla. enrich yin and supplement blood T1189 Caragana intermedia.

enrich yin and supplement kidney T1035 Broussonetia papyrifera, T2301

Dysosma majorensis [Syn. Podophyllum majorense; Dysosma

lichuanensis], T4681 Pedicularis decora.

enrich yin and supplement vacuity T4644 Parasilurus asotus.

enrish yin and nourish blood T4288 Morus alba.

expel phlegm and precipitate $q \boldsymbol{i} \mathrm{T} 1153$ Camellia sinensis var. assamica.

expel pus T0791 Astragalus chrysopterus, T0794 Astragalus ernestii,

T0798 Astragalus membranaceus, T0800 Astragalus mongholicus,
T2601 Euphorbia milii, T5011 Platycodon grandiflorum.

expel pus and break stasis T4672 Patrinia scabiosaefolia, T4676 Patrinia villosa.

expel pus and disinhibit urine $\mathrm{T} 3284$ Houttuynia cordata.

expel pus and draw out toxin T4917 Pinus massoniana.

expel pus and engender flesh T1673 Corchorus capsularis.

expel pus and relieve cough T3233 Heteropappus altaicus.

expel roundworm T0659 Artemisia absinthium, T1122 Caloglossa

leprieurii, T1176 Cannabis sativa, T2173 Digenea simplex, T4002

Lysimachia foenum-graecum, T4231 Milingtonia hortensis, T6312 Taxus

mairei, T6864 Zanthoxylum armatum, T6887 Zanthoxylum planispinum.

expel roundworms $\mathrm{T} 3342$ Hypericum bellum.

expel roundworms T3380 Hyptis pectinata.

expel stasis and break accumulation T2384 Epicauta gorhami.

expel stasis and free menstruation T5472 Rheum officinale, T5474 Rheum

palmatum, T5481 Rheum tanguticum.

expel stasis blood T1923 Cyathula capitata.

expel stone and disinhibit water T1553 Clerodendranthus spicatus.

expel tapeworm T0251 Agrimonia pilosa var. japonica.

expel water T2024 Daphne genkwa, T2599 Euphorbia lunulata, T2601

Euphorbia milii.

expel water and dispel phlegm T6105 Stellera chamaejasme.

expel water and disperse swelling T1858 Croton tiglium, T2597

Euphorbia lathyris, T4861 Phytolacca americana [Syn. Phytolacca

decandra], T4864 Phytolacca esculenta [Syn. Phytolacca acinosa].

expel wind T1137 Caltha palustris, T2746 Foeniculum vulgare.

expel wind and dissipate cold T4956 Piper mullesua, T5987 Sobina

chinensis.

expel wind and eliminate damp T2449 Erycibe obtusifolia, T2450 Erycibe

schmidtii, T2538 Euonymus bungeanus, T3867 Liquidambar formosana

[Syn. Liquidambar taiwaniana], T4678 Paulownia fortunei, T4679

Paulownia tomentosa, T4873 Picea koraiensis, T5648 Salacia prinoides

[Syn. Salacia chinensis], T5998 Solanum dulcamara, T6808 Waltheria americana.

expel wind and quicken blood T6264 Syzygium buxifolium.

expel wind and relieve pain T6003 Solanum khasianum.

expel worms T0264 Ajuga forrestii, T0446 Anaphalis margaritacea, T0511 Annona reticulata, T0606 Areca catechu, T0952 Blechnum orientale, T1101 Caesalpinia decapetala, T1382 Chondria armata [Syn. Lophura armata], T1595 Codium fragile, T1881 Cucurbita moschata, T1986 Cyrtomium fortunei, T2277 Dryopteris austriaca, T2279 Dryopteris championii, T2282 Dryopteris filix-mas, T2346 Embelia oblongifolia, T2410 Equisetum pratense, T2852 Garcinia cowa, T3466 Iris potaninii, T3688 Lappula echinata, T3935 Luffa cylindrica, T4163 Melia toosendan, T4364 Myrsine semiserrata, T5151 Populus davidiana, T5328 Punica granatum, T5329 Punica granatum, T5921 Seriphidium finitum [Syn. Artemisia finita], T6201 Styrax obassia, T6653 Usnea diffracta, T6654 Usnea longissima.

expel worms and relieve itch T4085 Mallotus repandus var. chrysocarpus [Syn. Mallotus chrysocarpus; Mallotus repandus].

expel worms and treat lichen T4156 Melia azedarach, T4162 Melia toosendan.

extinguish wind and check tetany T2836 Gallus gallus domesticus, T2890 
Gastrodia elata, T4710 Pericampylus glaucus, T5817 Scolopendra subspinipes mutilans, T6625 Uncaria macrophylla, T6629 Uncaria rhynchophylla [Syn. Nauclea rhynchophylla], T6633 Uncaria sinensis.

extinguish wind and harmonize blood T0483 Angelica gigas. extinguish wind and settle fright T6424 Thermopsis alpina. fortify and move spleen T5573 Rosa sericea.

fortify spleen T0353 Alpinia blepharocalyx, T0387 Amaranthus caudatus, T0535 Antidesma bunius, T0541 Apis cerana, T1143 Calystegia hederacea, T2246 Dolichos lablab, T3950 Lychnis fulgens, T4178 Melodinus hemsleyanus, T5244 Prunus tomentosa, T5575 Rosmarinus officinalis, T6231 Swertia pseudochinensis.

fortify spleen and assisting movement T4436 Nitraria tangutorum.

fortify spleen and boost kidney T1188 Caragana chamlagu, T1604 Coeloglossum viride [Syn. Coeloglossum viride var. bracteatum], T3000 Glycine max, T3077 Gymnadenia conopsea.

fortify spleen and boost lung T4450 Nuphar pumilum.

fortify spleen and boost $q \boldsymbol{i}$ T1202 Carex kobomugi, T6489 Tragopogon porrifolius.

fortify spleen and boost stomach T5067 Polyalthia nemoralis.

fortify spleen and check diarrhea T2188 Dioscorea alata, T4548 Oryza sativa var. glutinosa, T6056 Sorghum vulgare.

fortify spleen and disinhibit damp T1238 Cassia mimosoides, T3418 Incarvillea arguta, T3645 Kummerowia striata, T3770 Lespedeza cuneata, T3840 Limnophila rugosa, T3886 Litsea euosma, T6264 Syzygium buxifolium, T6754 Vigna unguiculata.

fortify spleen and disinhibit water T1035 Broussonetia papyrifera, T2998 Glycine max, T6744 Vicia faba.

fortify spleen and disperse accumulation T2658 Fagopyrum esculentum, T6229 Swertia patens, T6676 Valeriana hardwickii.

fortify spleen and disperse food T1769 Crataegus cuneata, T1771 Crataegus kansuensis, T1772 Crataegus maximowiczii, T1780 Crataegus sanguinea, T1781 Crataegus scabrifolia, T1952 Cynanchum auriculatum, T1953 Cynanchum bungei, T2189 Dioscorea althaeoides, T2838 Gallus gallus domesticus, T4194 Menyanthes trifoliata, T5341 Pygmaeopremna herbacea [Syn. Premna herbacea], T5758 Saussurea lappa [Syn. Aucklandia lappa].

fortify spleen and eliminate damp T0881 Bauhinia variegata, T2720 Ficus hispida.

fortify spleen and harmonize center T2050 Daucus carota var. sativa.

fortify spleen and harmonize stomach T1623 Collybia albuminosa, T1751 Corylus heterophylla, T1980 Cyprinus carpio, T2136 Desmos cochinchinensis [Syn. Desmos chinensis], T3431 Inula helenium, T3437 Inula racemosa.

fortify spleen and nourish stomach T0559 Arachis hypogaea, T2190 Dioscorea batatas [Syn. Dioscorea opposita], T2202 Dioscorea japonica, T3229 Hericium erinaceus [Syn. Hydnum erinaceus].

fortify spleen and percolate damp T1614 Coix lacryma-jobi var. ma-yuen. fortify spleen and promote digestion T0210 Agaricus bisporus, T0211 Agaricus campestris, T2716 Ficus beecheyana [Syn. Ficus erecta var. beecheyana], T2717 Ficus carica, T3742 Lentinus edodes, T4636 Papaver somniferum, T4644 Parasilurus asotus.

fortify spleen and quiet heart T5169 Poria cocos.

fortify spleen and regulate menstruation T2326 Elaeagnus angustifolia. fortify spleen and stomach T0298 Alectoria vivens, T0983 Bos taurus domesticus, T1964 Cynanchum wilfordii [Syn. Cynoctonum wilfordii], T2846 Ganoderma japonicum [Syn. Ganoderma sinense], T2848 Ganoderma lucidum, T5179 Potentilla anserina, T5872 Selenarctos thibetanus; Ursus arctos, T6656 Ustilago maydis.

fortify spleen and supplement center T0991 Bos taurus domesticus; Bubalus bubalis.

fortify spleen and supplement lung T0500 Anguilla japonica, T1596 Codonopsis canescens, T1597 Codonopsis clematidea, T1599 Codonopsis pilosula, T1600 Codonopsis pilosula var. modesta [Syn. Codonopsis modesta], T1601 Codonopsis subglobosa, T1602 Codonopsis tangshen, T1603 Codonopsis tubulosa, T2727 Ficus simplicissima.

fortify spleen and supplement vacuity $\mathrm{T} 3774$ Lespedeza tomentosa, T4147 Medicago falcata, T5071 Polygala arillata.

fortify spleen and suppress cough T0151 Acronychia pedunculata.

fortify spleen and warm stomach T0363 Alpinia speciosa, T6267 Syzygium jambos.

fortify stomach T0158 Actinidia chinensis, T0357 Alpinia japonica, T0659 Artemisia absinthium, T1242 Cassia occidentalis, T1608 Coffea arabica, T1610 Coffea liberica, T1687 Coriandrum sativum, T4551 Osmorhiza aristata var. laxa, T5570 Rosa roxburghii, T6889 Zanthoxylum podocarpum.

fortify stomach and disperse accumulation T1546 Clematis tangutica.

fortify stomach and disperse food T0418 Amomum muricarpum, T1775 Crataegus pinnatifida, T1778 Crataegus pinnatifida var. major, T2763 Fragaria ananassa, T3254 Hippophae rhamnoides, T3256 Hippophae rhamnoides subsp. sinensis, T3258 Hippophae rhamnoides subsp. yunnanensis, T3289 Humulus lupulus, T3962 Lycopersicon esculentum, T4100 Mangifera indica, T5122 Polygonum viscosum, T6071 Sphaeranthus indicus.

fortify stomach and disperse stagnation T4217 Microcos paniculata [Syn. Grewia microcos].

fortify stomach and expel worms T6895 Zanthoxylum simulans.

fortify stomach and harmonize center $\mathrm{T} 3480$ Isodon angustifolia, T4172 Melilotus suaveolens.

fortify stomach and rectify $\boldsymbol{q} i \mathrm{~T} 0311$ Allium cepa, T3725 Laurus nobilis.

fortify stomach and relieve pain T1492 Citrus limon, T1496 Citrus limonia, T2505 Eucalyptus citriodora, T6435 Thladiantha cordifolia.

fortify stomach and resolve phlegm T1501 Citrus medica var. sarcodactylis.

free bowels and drain heat T5469 Rheum hotaoense.

free channels and network vessels T0871 Barleria lupulina, T1327 Ceratostigma minus, T2438 Erodium stephanianum, T2943 Geranium nepalense, T2944 Geranium pratense, T2947 Geranium sibiricum, T2949 Geranium wilfordii, T3436 Inula nervosa, T4950 Piper kadsura [Syn. Piper futokadsura], T5950 Siegesbeckia orientalis, T5951 Siegesbeckia orientalis var. glabrescens [Syn. Siegesbeckia glabrescens], T5952 Siegesbeckia orientalis var. pubescens [Syn. Siegesbeckia pubescens], T5981 Smilax riparia, T6588 Typhoniun giganteum.

free channels and quicken blood T1853 Croton oblongifolius [Syn. Croton laevigatus], T5648 Salacia prinoides [Syn. Salacia chinensis].

free channels and quicken network vessels T1589 Cocculus trilobus [Syn. 
Cocculus sarmentosus], T2753 Formica fusca, T4308 Mucuna birdwoodiana, T6166 Strychnos angustiflora, T6738 Viburnum odoratissimum

free channels and relieve pain T1943 Cymbopogon goeringii. free intestines and stomach T1006 Brassica chinensis. free joints T5977 Smilax glabra, T5980 Smilax menispermoidea. free medicinal strength T3869 Liquor.

free menstruation T0941 Bidens tripartita, T1356 Cheiranthus cheiri, T2546 Euonymus sacrosancta, T3779 Leucas aspera.

free menstruation and check discharge T4575 Pachysandra terminalis. free menstruation and milk T0632 Aristolochia manshuriensis, T4106 Manis pentadactyla.

free menstruation and promote lactation T4178 Melodinus hemsleyanus. free menstruation and quicken blood T2734 Fissistigma glaucescens [Syn. Melodorum glaucescens].

free menstruation and relieve pain T0994 Boswellia carterii, T1288 Celastrus orbiculatus [Syn. Celastrus articulatus], T1903 Curcuma aromatica, T1905 Curcuma longa, T3056 Gossypium herbaceum.

free milk T1508 Citrus sinensis, T1980 Cyprinus carpio, T2590 Euphorbia hirta, T3662 Lactuca sativa, T4206 Metaplexis japonica, T6357 Tetrapanax papyriferus.

free milk and disinhibit water T4983 Pisum sativum.

free nasal orifices T1312 Centipeda minima.

free network vessels T2426 Erigeron sumatrensis, T3913 Lonicera japonica, T4516 Oplopanax elatus, T4796 Pheretima aspergillum; Allolobophora caliginosa trapezoides, T6066 Spatholobus suberectus.

free network vessels and disperse swelling T1008 Brassica juncea.

free network vessels and dissipate stasis T3613 Kadsura coccinea [Syn. Kadsura chenensis; Kadsura hainanensis], T4324 Murraya paniculata [Syn. Chalcas paniculata], T5075 Polygala fallax [Syn. Polygala aureocauda].

free network vessels and relieve pain T0080 Aconitum bullatifolium var. homotrichum [Syn. Aconitum nagarum], T1053 Bulbophyllum odoratissimum [Syn. Stelis odoratissimum], T1084 Buthus martensi, T1545 Clematis chinensis, T1715 Corydalis decumbens [Syn. Corydalis amabilis], T1767 Craibiodendron yunnanese, T1799 Crinum latifolium, T1921 Cyanotis arachnoidea [Syn. Cyanotis bodinieri], T2062 Delphinium bonvalotii, T2081 Delphinium omeiense, T2084 Delphinium potaninii, T2085 Delphinium potaninii var. jiufengshanense, T2194 Dioscorea collettii, T2213 Dioscorea zingiberensis, T3737 Lemmaphyllum microphyllum var. obovatum, T4118 Marsdenia oreophila, T5021 Pleurospermum rivulorum, T5817 Scolopendra subspinipes mutilans, T6184 Strychnos nux-vomica, T6193 Strychnos wallichiana, T6252 Symplocos chinensis, T6862 Zanthoxylum ailanthoides.

free orifices and relieve pain T0479 Angelica dahurica cv. qibaizhi, T0498 Angelica taiwaniana, T0728 Asarum heterotropoides var. mandshuricum, T0731 Asarum sieboldii, T0732 Asarum sieboldii var. seoulensis.

free $q \boldsymbol{i}$ and effuse sweat T0319 Allium schoenoprasum.

free stool T0334 Aloe arborescens var. natalensis, T1101 Caesalpinia decapetala, T1173 Cannabis sativa, T1229 Cassia acutifolia, T1230 Cassia angustifolia, T1236 Cassia laevigata [Syn. Cassia floribunda], T1238 Cassia mimosoides, T1241 Cassia occidentalis, T1242 Cassia occidentalis, T1291 Celastrus paniculatus, T2612 Euphorbia prolifera, T3394 Ilex paraguariensis, T3447 Ipomoea batatas [Syn. Convolvulus batatas], T3935 Luffa cylindrica, T5613 Rumex obtusifolius, T5272 Psorospermum febrifugum, T6032 Sophora flavescens [Syn. Sophora angustfolia].

free stool and kill worms T5607 Rumex crispus, T5614 Rumex patientia. free strangury T0155 Actinidia arguta, T0158 Actinidia chinensis, T3240 Hibiscus esculentus, T3668 Lagenaria siceraria var. depressa, T5372 Quercus dentata.

free strangury and expel stone T4149 Medicago sativa.

free strangury and transform turbid T1001 Brachystemma calycinum.

free yang T0310 Allium ascalonicum, T0314 Allium fistulosum.

harmonize blood T4720 Perilla frutescens var. arguta, T4723 Perilla frutescens var. crispa, T5518 Rhododendron mucronatum, T5523 Rhododendron simsii, T5572 Rosa rugosa.

harmonize blood and check dysentery T4665 Passiflora edulis. harmonize blood and dispel wind T1188 Caragana chamlagu.

harmonize blood and relieve pain T0569 Aralia cordata, T0574 Aralia fargesii, T1292 Celastrus orbiculatus [Syn. Celastrus articulatus].

harmonize blood and resolve toxin T3113 Hedera nepalensis var. sinensis, T3115 Hedera rhombea.

harmonize blood and stanch bleeding T0382 Althaea rosea. harmonize blood and vanquish toxin T0094 Aconitum finetianum. harmonize center T4190 Mentha spicata, T5938 Setaria italica. harmonize center and check vomiting T6325 Tectona grandis. harmonize center and downbear counterflow T4818 Phoebe nanmu. harmonize center and moisten intestines T1465 Citrullus vulgaris [Syn. Citrullus lanatus].

harmonize center and precipitate qi T4983 Pisum sativum.

harmonize center and promote digestion T1486 Citrus junos.

harmonize center and relieve pain T4086 Malus asiatica.

harmonize exterior and interior T1055 Bupleurum angustissimum, T1056 Bupleurum aureum, T1058 Bupleurum chaishoui, T1059 Bupleurum chinense, T1065 Bupleurum longiradiatum, T1066 Bupleurum marginatum, T1067 Bupleurum marginatum var. stenophyllum, T1072 Bupleurum scorzonerifolium, T1073 Bupleurum sibiricum, T1075 Bupleurum smithii var. parvifolium, T1078 Bupleurum wenchuanense, T1079 Bupleurum yinchowense.

harmonize spleen and stomach T1390 Chrysanthemum coronarium, T1397 Chrysanthemum segetum, T1888 Cudrania tricuspidata.

harmonize stomach T1182 Capra hircus; Ovis aries, T1469 Citrus aurantium var. amara, T2263 Dregea volubilis, T4188 Mentha rotundifolia, T6145 Stichopus japonicus.

harmonize stomach and arouse spleen T0417 Amomum longiligulare, T0419 Amomum villosum, T0420 Amomum xanthioides, T4386 Nardostachys chinensis, T4387 Nardostachys jatamansi.

harmonize stomach and check vomiting T3372 Hypodematium sinense.

harmonize stomach and disperse accumulation T1467 Citrus aurantium, T5140 Poncirus trifoliata, T6285 Tamarindus indica.

harmonize stomach and disperse food T2452 Erysimum cheiranthoides, T2733 Firmiana simplex, T4348 Myrica rubra.

harmonize stomach and fortify center T6017 Solanum tuberosum. harmonize stomach and free stool T1384 Chondrus ocelladus. 
harmonize stomach and inhibit acid T4306 Mosla grosseserrata. harmonize stomach and soothe sinews T1343 Chaenomeles sinensis. harmonize stomach and transform damp T0212 Agastache rugosus, T1342 Chaenomeles lagenaria [Syn. Chaenomeles speciosa], T4170 Melilotus albus, T4284 Morinda parvifolia.

harmonize stomach and transform phlegm T5226 Prunus mume. harmonize stomach and transform turbidity T0978 Bombyx mori. hasten delivery $\mathrm{T} 3758$ Lepidium sativum. hasten delivery or stop delivery $\mathrm{T} 1179$ Capparis masaikai. increase appetite T0041 Acanthopanax senticosus [Syn. Eleutherococcus senticosus], T0472 Anethum graveolens, T1341 Cetraria islandica, T4546 Oryza sativa, T4547 Oryza sativa cv.

increase appetite and check vomiting T5059 Pogostemon cablin [Syn. Mentha cablin].

increase appetite and disperse food T0416 Amomum kravanh [Syn. Amomum cardamomum].

induce sweat T3394 Ilex paraguariensis.

inhibit acid T2841 Gallus gallus domesticus, T6412 Thalictrum squarrosum.

inhibit secretion T0825 Atropa belladonna.

interrupt malaria T0249 Agrimonia pilosa var. japonica, T0378 Alstonia yunnanensis, T0606 Areca catechu, T0653 Artabotrys hexapetalus [Syn. Annona hexapetalus], T0660 Artemisia annua, T0662 Artemisia apiacea [Syn. Artemisia carvifolia; Artemisia caruifolia], T0947 Bixa orellana, T1176 Cannabis sativa, T1446 Cipadessa baccifera, T1535 Clausena excavata, T1561 Clerodendron serratum, T1562 Clerodendron trichotomum, T1569 Clerodendrum serratum var. amplexifolium, T1853 Croton oblongifolius [Syn. Croton laevigatus], T2295 Duranta repens, T2310 Dysoxylum hongkongense, T2421 Erigeron annuus, T2695 Ferula assafoetida, T3144 Helianthus annuus, T3146 Helianthus annuus cv, T3303 Hydrangea chinensis, T3304 Hydrangea macrophylla, T3308 Hydrangea umbellata, T3327 Hyoscyamus niger, T4803 Phlogacanthus curviflorus, T5107 Polygonum multiflorum, T5110 Polygonum orientale, T5184 Potentilla kleiniana, T5413 Ranunculus cantoniensis, T5414 Ranunculus japonicus, T5415 Ranunculus sceleratus, T5852 Sedum bulbiferum, T6402 Thalictrum omeiense, T6709 Verbena officinalis, T6723 Veronica arvensis, T6727 Veronica persica.

interrupt malaria and abate fever T1428 Cinchona ledgeriana, T1429 Cinchona officinalis, T1433 Cinchona succirubra.

interrupt malaria and kill worms T2136 Desmos cochinchinensis [Syn.

Desmos chinensis].

interrupt malaria and settle pain T1841 Croton caudatus var. tomentosus. invigorate yang T6073 Sphallerocarpus gracilis.

invigorate yang and secure essence T0323 Allium tuberosum.

joint bones T1206 Carica papaya, T2234 Dipsacus asperoides, T2235 Dipsacus japonicus, T3685 Lannea grandis [Syn. Lannea coromandelica], T5733 Sarcococca vagans.

joint bones and disperse swelling T4031 Maesa perlarius.

joint bones and engender flesh T1871 Cucubalus baccifer.

joint sinews and bones T0170 Adhatoda vasica, T0871 Barleria lupulina, T6138 Sterculia foetida.

kill snails T0398 Ambrosia artemisiifolia.

kill worms T0012 Abrus precatorius, T0215 Agave americana, T0248
Agrimonia pilosa, T0255 Ailanthus altissima, T0314 Allium fistulosum, T0318 Allium sativum, T0364 Alsophila spinulosa, T0435 Anabasis aphylla, T0513 Annona squamosa, T0707 Arthraxon hispidus, T0750 Asparagus gobicus, T0752 Asparagus officinalis, T1097 Cacalia ainsliaeflora, T1210 Carpesium abrotanoides, T1211 Carpesium abrotanoides, T1278 Cedrela sinensis, T1374 Chloranthus spicatus, T1388 Chrysanthemum cinerariaefolium, T1389 Chrysanthemum coccineum, T1437 Cinnamomum camphora, T1535 Clausena excavata, T1558 Clerodendron inerme, T1582 Cnidium monnieri, T1591 Cocos nucifera, T1593 Cocos nucifera, T1645 Consolida ajacis [Syn. Delphinium ajacis], T1693 Coriaria sinica [Syn. Coriaria nepalensis], T1815 Crotalaria assamica, T1867 Cryptomeria fortunei, T2048 Daucus carota, T2049 Daucus carota, T2162 Dicranostigma franchetianum [Syn. Dicranostigma leptopodum], T2281 Dryopteris crassirhizoma, T2533 Eugenia jambolana [Syn. Syzygium cumin; Myrtus cumini], T2536 Euonymus alatus, T2546 Euonymus sacrosancta, T2567 Eupatorium odoratum, T2584 Euphorbia ebracteolata, T2585 Euphorbia esula, T2587 Euphorbia fischeriana, T2599 Euphorbia lunulata, T2612 Euphorbia prolifera, T2624 Euphorbia tirucalli, T2695 Ferula assafoetida, T2857 Garcinia hanburyi, T2979 Gleditsia sinensis [Syn. Gleditsia horrida], T3263 Holarrhena antidysenterica, T3327 Hyoscyamus niger, T3420 Incarvillea sinensis, T3567 Juglans regia, T3570 Juglans regia, T3630 Kleinhovia hospita, T3726 Laurus nobilis, T4020 Macleaya cordata, T4127 Matteuccia struthiopteris, T4157 Melia azedarach, T4552 Osmunda japonica, T4690 Pelargonium graveolens, T4890 Pieris japonica, T5098 Polygonum aviculare, T5113 Polygonum persicaria, T5185 Potentilla multifida, T5220 Prunus davidiana, T5221 Prunus davidiana, T5232 Prunus persica, T5234 Prunus persica, T5235 Prunus persica, T5317 Pueraria peduncularis, T5327 Punica granatum, T5516 Rhododendron molle, T5537 Rhus sylvestris, T5542 Rhus verniciflua [Syn. Toxicadendron verniciflum], T5595 Rubus parviforlius, T5605 Rumex acetosa, T5613 Rumex obtusifolius, T5719 Sapindus mukorossi, T5726 Saponaria officinalis, T5920 Seriphidium cinum [Syn. Artemisia cina], T6032 Sophora flavescens [Syn. Sophora angustfolia], T6035 Sophora japonica, T6048 Sophora viciifolia, T6177 Strychnos ignatii, T6219 Swertia erythrosticta, T6277 Tadehagi triquetrum, T6330 Tephroseris kirilowii [Syn. Senecio integrifolius var. fauriei], T6474 Toona ciliata, T6475 Toona sinensis, T6476 Torilis japonica, T6691 Veratrilla baillonii, T6697 Veratrum grandiflorum, T6698 Veratrum nigrum, T6826 Wisteria sinensis, T6827 Wisteria sinensis, T6831 Woodwardia orientalis, T6860 Zanthoxylum ailanthoides, T6870 Zanthoxylum bungeanum.

kill worms and cure sores T1436 Cinnamomum camphora.

kill worms and disinhibit damp T5455 Rhamnus crenata.

kill worms and disperse accumulation T1632 Combretum yunnanensis, T4542 Orthosiphon wulfenioides [Syn. Coleus wulfenioides].

kill worms and disperse gan $\mathrm{T} 1049$ Bufo bufo gargarizans; Bufo melanostictus.

kill worms and dissipate binds T2978 Gleditsia sinensis [Syn. Gleditsia horrida].

kill worms and ejection T6693 Veratrum album var. lobelianum [Syn. Veratrum lobelianum].

kill worms and relieve diarrhea T4083 Mallotus philippinensis, T4084 
Mallotus philippinensis.

kill worms and relieve itch T0142 Acorus calamus, T0297 Alchornea trewioides, T0749 Asparagus filicinus, T0853 Baeckea frutescens, T1261 Catalpa ovata, T1264 Catalpa ovata, T1359 Chenopodium album, T1361 Chenopodium ambrosioides, T1594 Cocos nucifera, T2077 Delphinium grandiflorum, T2124 Derris trifoliata, T2459 Erythrina arborescens, T2478 Erythrina variegata [Syn. Erythrina indica], T2510 Eucalyptus globulus, T2517 Eucalyptus robusta, T2580 Euphorbia antiquorum, T2602 Euphorbia nematocypha, T2617 Euphorbia royleana, T2651 Excoecaria cochinchinensis var. viridis, T3245 Hibiscus syriacus, T3560 Jatropha curcas, T3624 Kalopanax pictum, T3625 Kalopanax septemlobus, T4158 Melia azedarach, T4159 Melia azedarach, T4572 Pachyrhizus erosus, T4906 Pinus armandii, T4916 Pinus massoniana, T5010 Platycarya strobilacea, T5026 Plumbagella micrantha, T5256 Pseudolarix amabilis [Syn. Larix amabilis; Pseudolarix kaempferi], T5308 Pterocarya stenoptera, T5368 Python molurus bivittatus, T5483 Rhinacanthus nasutus, T5608 Rumex dentatus, T5611 Rumex japonicus, T5612 Rumex nepalensis, T5646 Sagina japonica [Syn. Spergula japonica], T5907 Senecio scandens [Syn. Senecio chinensis], T6155 Strophanthus divaricatus, T6713 Vernonia anthelmintica.

kill worms and relieve pain T0099 Aconitum gymnandrum, T6029 Sophora alopecuroides, T6101 Stauntonia hexaphylla, T6858 Zanthoxylum acanthopodium.

loosen center T0201 Aesculus chinensis, T0202 Aesculus hippocastanum, T0205 Aesculus wilsonii, T4544 Oryza sativa.

loosen center and induce stagnation T2998 Glycine max.

loosen center and rectify $\boldsymbol{q} i \mathrm{~T} 4720$ Perilla frutescens var. arguta, T4723 Perilla frutescens var. crispa.

loosen chest and disinhibit qi T1486 Citrus junos, T1786 Cremanthodium ellisii.

loosen chest and dissipate binds T6510 Trichosanthes kirilowii, T6513 Trichosanthes rosthornii [Syn. Trichosanthes uniflora].

loosen chest and resolve depression $\mathrm{T} 3192$ Hemerocallis citrina.

loosen intestines and stomach T3447 Ipomoea batatas [Syn. Convolvulus batatas].

lower blood pressure T0544 Apium graveolens, T0553 Apocynum venetum, T0561 Arachis hypogaea, T0625 Aristolochia contorta, T0627 Aristolochia debilis [Syn. Aristolochia longa], T0631 Aristolochia kaempferi, T0830 Auricularia auricula, T0831 Auricularia delicata, T0987 Bos taurus domesticus; Bubalus bubalis, T1190 Caragana jubata, T1241 Cassia occidentalis, T1311 Centella asiatica, T1385 Chorda filum, T1393 Chrysanthemum indicum, T1417 Cimicifuga acerina, T1562 Clerodendron trichotomum, T1565 Clerodendrum bungei, T1662 Coptis chinensis, T1663 Coptis chinensis var. brevisepala, T1664 Coptis deltoidea, T1669 Coptis omeiensis, T1670 Coptis teetoides [Syn. Coptis teeta], T1776 Crataegus pinnatifida, T1777 Crataegus pinnatifida, T2115 Dermatocarpon minitum, T2445 Ervatamia officinalis, T2530 Eucommia ulmoides, T2531 Eucommia ulmoides, T2565 Eupatorium lindleyanum, T2569 Eupatorium rebaudianum, T2659 Fagopyrum esculentum, T2908 Gentiana cephalantha, T2921 Gentiana manshurica, T2928 Gentiana rigescens, T2930 Gentiana scabra, T2938 Gentiana triflora, T2973 Glechoma longituba, T3144 Helianthus annuus, T3146 Helianthus annuus cv, T3878 Lithocarpus polystachyus, T3898 Lobelia chinensis [Syn. Lobelia radicans], T3955 Lycium barbarum, T3958 Lycium chinense, T4028 Maesa indica, T4034 Magnolia biloba, T4045 Magnolia officinalis, T4195 Menyanthes trifoliata, T4290 Morus alba, T4438 Nostoc flagelliforme, T4487 Olea europaea, T4796 Pheretima aspergillum; Allolobophora caliginosa trapezoides, T5423 Rauvolfia verticillata, T5424 Rauvolfia verticillata, T5425 Rauvolfia verticillata $\mathrm{f}$. rubrocarpa, T5426 Rauvolfia verticillata var. hainanensis, T5427 Rauvolfia vomitoria, T5435 Rauwolfia latifrons, T5441 Rauwolfia perakensis, T5442 Rauwolfia serpentina, T5657 Salsola collina, T5723 Sapium sebiferum, T5817 Scolopendra subspinipes mutilans, T5969 Sium latifolium, T6478 Torreya jackii, T6697 Veratrum grandiflorum, T6698 Veratrum nigrum, T6772 Viscum angulatum, T6803 Volvariella volvacea.

lower blood sugar levels T0303 Alisma orientale [Syn. Alisma plantago-aquatica var. orientale], T3085 Gynostemma pentaphyllum, T4599 Panax ginseng [Syn. Panax schinseng], T5445 Rehmannia glutinosa [Syn. Rehmannia glutinosa f. huechingensis], T5447 Rehmannia glutinosa [Syn. Rehmannia glutinosa f. huechingensis].

lower blood-fat T0311 Allium cepa, T4487 Olea europaea.

lower cholesterol T0303 Alisma orientale [Syn. Alisma plantago-aquatica var. orientale], T1775 Crataegus pinnatifida, T1778 Crataegus pinnatifida var. major, T2731 Firmiana simplex, T2732 Firmiana simplex, T2733 Firmiana simplex, T2846 Ganoderma japonicum [Syn. Ganoderma sinense], T2848 Ganoderma lucidum, T5101 Polygonum cuspidatum, T5107 Polygonum multiflorum, T6528 Trigonella foenum-graecum, T6584 Typha angustata, T6585 Typha angustifolia, T6587 Typha latifolia.

lower vacuity heat T3954 Lycium barbarum, T3956 Lycium chinense, T4887 Picrorhiza kurrooa, T4888 Picrorhiza scrophulariiflora.

lubricate intestines T1173 Cannabis sativa, T4331 Musa paradisiaca var. sapientum [Syn. Musa sapientum], T5549 Ricinus communis.

lubricate intestines and free stool T5967 Siraitia grosvenorii [Syn. Momordica grosvenorii], T6511 Trichosanthes kirilowii.

lubricate intestines and precipitate accumulation T0560 Arachis hypogaea.

moisten dryness T0843 Babylonia lutosa, T1173 Cannabis sativa, T1182 Capra hircus; Ovis aries, T1526 Cladonia rangiferina, T4912 Pinus koraiensis, T6076 Spinacia oleracea, T6206 Sus scrofa domestica.

moisten dryness and free stool T3860 Linum usitatissimum.

moisten dryness and harmonize center T5642 Saccharum sinensis.

moisten dryness and lubricate intestines T0474 Angelica acutiloba [Syn. Ligusticum acutilobum], T5223 Prunus humilis [Syn. Cerasus humilis], T5224 Prunus japonica [Syn. Cerasus japonica], T5225 Prunus japonica var. nakaii, T6510 Trichosanthes kirilowii, T6513 Trichosanthes rosthornii [Syn. Trichosanthes uniflora].

moisten dryness and transform phlegm T0983 Bos taurus domesticus.

moisten intestines T0300 Aleurites moluccana, T4288 Morus alba, T4536 Orobanche coerulescens, T4715 Perilla frutescens, T4717 Perilla frutescens var. acuta [Syn. Perilla frutescens var. purpurascens], T4719 Perilla frutescens var. arguta, T4722 Perilla frutescens var. crispa, T5229 Prunus persica, T5238 Prunus salicina, T5927 Sesamum indicum [Syn. Sesamum orientale].

moisten intestines and free stool T0540 Apis cerana, T0944 Biota 
orientalis [Syn. Thuja orientalis; Platycladus orientalis], T0993 Boschniakia rossica, T1005 Brassica campestris [Syn. Brassica campestris var. oleifera], T1356 Cheiranthus cheiri, T1455 Cistanche deserticola, T1456 Cistanche salsa, T1972 Cynomorium songaricum, T2999 Glycine max, T3568 Juglans regia, T4487 Olea europaea, T4976 Piptanthus nepalensis, T5107 Polygonum multiflorum, T5216 Prunus armeniaca, T5219 Prunus armeniaca var. ansu, T5233 Prunus persica, T5454 Rhamnus cathartica, T5460 Rhamnus frangula [Syn. Frangula alnus], T5657 Salsola collina, T5925 Sesamum indicum [Syn. Sesamum orientale], T6139 Sterculia lychnophora, T6428 Thermopsis lanceolata.

moisten intestines and relieve constipation T0495 Angelica sinensis, T4804 Phlojodicarpus sibiricus.

moisten lung T0216 Agave americana var. marginata [Syn. Agave americana var. variegata], T2101 Dendrobium fimbriatum, T2221 Diospyros kaki, T3955 Lycium barbarum, T3958 Lycium chinense, T4331 Musa paradisiaca var. sapientum [Syn. Musa sapientum], T6111 Stemona japonica, T6113 Stemona sessilifolia, T6115 Stemona tuberosa.

moisten lung and clear heart T3875 Liriope spicata var. prolifera, T4507 Ophiopogon japonicus.

moisten lung and disinhibit throat T2839 Gallus gallus domesticus. moisten lung and engender liquid T1476 Citrus erythrosa, T1515 Citrus tangemna, T1598 Codonopsis convolvulacea, T5260 Pseudostellaria heterophylla, T5531 Rhus chinensis [Syn. Rhus semialata].

moisten lung and precipitate $q \boldsymbol{i}$ T0781 Aster tataricus, T2431 Eriobotrya japonica, T6574 Tussilago farfara.

moisten lung and relieve cough T0540 Apis cerana, T0749 Asparagus filicinus, T0830 Auricularia auricula, T0831 Auricularia delicata, T0859 Balanophora involucrata, T1183 Capra hircus; Ovis aries, T1751 Corylus heterophylla, T3011 Glycyrrhiza aspera, T3013 Glycyrrhiza glabra, T3015 Glycyrrhiza inflata, T3016 Glycyrrhiza kansuensis, T3021 Glycyrrhiza squamulosa, T3022 Glycyrrhiza uralensis, T3023 Glycyrrhiza yunnanensis, T3344 Hypericum chinense, T3983 Lycoris aurea, T4050 Magnolia sieboldii, T4823 Pholidota yunnanensis, T5363 Pyrus calleryana, T6097 Staphylea bumalda.

moisten lung and settle asthma T5218 Prunus armeniaca.

moisten lung and suppress cough T4195 Menyanthes trifoliata, T5215 Prunus amygdalus.

moisten lung and transform phlegm T0438 Anacardium occidentale, T0559 Arachis hypogaea, T1053 Bulbophyllum odoratissimum [Syn. Stelis odoratissimum], T1353 Changium smyrnioides.

moisten skin T0167 Adenanthera pavonina, T0516 Anser cygnoides domestica, T1183 Capra hircus; Ovis aries, T4188 Mentha rotundifolia, T5549 Ricinus communis.

moisten skin and engender flesh T0540 Apis cerana, T0543 Apis mellifera ligustica.

move blood and quicken network vessels T2053 Davallia mariesii. move blood and relieve itch T2835 Galium verum.

move qi T0322 Allium tuberosum, T0357 Alpinia japonica, T0359 Alpinia officinarum, T0472 Anethum graveolens, T1328 Ceratostigma plumbaginoides, T1329 Ceratostigma willmottianum, T1508 Citrus sinensis, T2479 Erythrina variegata var. orientalis, T2612 Euphorbia prolifera, T3619 Kadsura peltigera [Syn. Kadsura longipedunculata], T4002 Lysimachia foenum-graecum, T4163 Melia toosendan, T4209
Michelia alba, T5167 Porana racemosa, T6800 Viverra zibetha. move $q i$ and check diarrhea T3263 Holarrhena antidysenterica. move $q i$ and disinhibit damp T2727 Ficus simplicissima.

move $q \boldsymbol{i}$ and disperse accumulation T4048 Magnolia rostrata, T5717

Sapindus delavayi [Syn. Pancovia delavayi].

move $q i$ and disperse food T1563 Clerodendron trichotomum.

move $q i$ and dissipate binds T4855 Physeter catodon.

move $q i$ and dissipate blood T1289 Celastrus orbiculatus [Syn. Celastrus articulatus].

move $q i$ and dissipate cold $\mathrm{T} 3847$ Lindera angustifolia.

move $q \boldsymbol{i}$ and dissipate stasis $\mathrm{T} 1775$ Crataegus pinnatifida, T1778

Crataegus pinnatifida var. major, T2720 Ficus hispida, T3381 Hyptis

suaveolens, T4036 Magnolia coco.

move $q \boldsymbol{i}$ and fortify stomach T0859 Balanophora involucrata, T5363

Pyrus calleryana.

move $q \boldsymbol{i}$ and free network vessels T6106 Stelmatocrypton khasianum.

move $q \boldsymbol{i}$ and harmonize center T4718 Perilla frutescens var. acuta [Syn.

Perilla frutescens var. purpurascens], T4721 Perilla frutescens var. arguta.

move $q \boldsymbol{i}$ and loosen center T3849 Lindera chunii.

move $\boldsymbol{q} i$ and quicken blood T0151 Acronychia pedunculata, T0274 Akebia quinata, T0278 Akebia trifoliata, T0280 Akebia trifoliata var. australis, T0814 Atalantia buxifolia [Syn. Severinia buxifolia], T0877 Bauhinia championii, T0967 Boenninghausenia sessilicarpa, T0994 Boswellia carterii, T1381 Choerospondias axillaris, T1113 Calendula officinalis, T1443 Cinnamomum parthenoxylum [Syn. Cinnamomum porrectum], T1583 Cnidium officinale [Syn. Ligusticum officinale], T1715 Corydalis decumbens [Syn. Corydalis amabilis], T3418 Incarvillea arguta, T3820 Ligusticum chuanxiong [Syn. Ligusticum wallichii], T3885 Litsea cubeba, T4323 Murraya paniculata [Syn. Chalcas paniculata], T4325 Murraya paniculata var. exotica, T4470 Ocimum basilicum, T4519 Opuntia dillenii, T4521 Opuntia vulgaris, T5029 Plumbago zeylanica, T6118 Stephania brachyandra, T6197 Styrax benzoin, T6204 Styrax tonkinensis, T6683 Vallisneria spiralis.

move $q i$ and quicken blood and relieve pain T4962 Piper puberulum. move $q i$ and regulate center T0417 Amomum longiligulare, T0419 Amomum villosum, T0420 Amomum xanthioides.

move qi and relieve depression T1907 Curcuma wengujin, T1978 Cyperus rotundus, T5572 Rosa rugosa.

move $q \boldsymbol{i}$ and relieve pain T0554 Aquilaria agallocha, T0555 Aquilaria sinensis, T0631 Aristolochia kaempferi, T0642 Aristolochia tubiflora, T0942 Biebersteinia heterostemon, T1258 Casuarina equisetifolia, T1440 Cinnamomum glanduliferum, T1444 Cinnamomum tamala, T1535 Clausena excavata, T1537 Clausena lansium, T1734 Corydalis remota [Syn. Corydalis bulbosa var. typica], T1904 Curcuma kwangsiensis, T1909 Curcuma zedoaria [Syn. Curcuma aeruginosa], T2363 Entada phaseoloides [Syn. Lens phaseoloides], T2636 Euscaphis japonica, T2643 Evodia meliifolia, T2745 Foeniculum vulgare, T3164 Helicteres isora, T3270 Holboellia fargesii, T3400 Illicium difengpi, T3403 Illicium majus, T3554 Jasminum grandiflorum, T3613 Kadsura coccinea [Syn. Kadsura chenensis; Kadsura hainanensis], T3614 Kadsura heteroclita [Syn. Uvaria heteroclita], T3746 Leontice robustum, T3850 Lindera glauca, T3856 Lindera umbellata [Syn. Lindera 
erythrocarpa], T3866 Liquidambar formosana [Syn. Liquidambar taiwaniana], T3997 Lysimachia capillipes, T4039 Magnolia grandiflora, T4157 Melia azedarach, T4158 Melia azedarach, T4324 Murraya paniculata [Syn. Chalcas paniculata], T4428 Nicotiana tabacum, T4527 Orixa japonica, T4690 Pelargonium graveolens, T4938 Piper boehmeriaefolium, T4944 Piper cubeba, T4948 Piper hancei, T4965 Piper sarmentosum, T4966 Piper sarmentosum, T5042 Podocarpus macrophyllus, T5044 Podocarpus macrophyllus var. maki, T5189 Pothos chinensis, T5591 Rubus cochinchinensis, T5758 Saussurea lappa [Syn. Aucklandia lappa], T5793 Schisandra henryi, T6062 Sparganium stoloniferum, T6339 Tephrosia purpurea, T6802 Vladimiria souliei [Syn. Jurinea souliei], T6878 Zanthoxylum echinocarpum.

move $q \boldsymbol{i}$ and transform phlegm T1538 Clausena lansium, T4100 Mangifera indica, T4937 Piper betle.

move qi-blood T6018 Solanum verbascifolium, T6675 Valeriana amurensis,

T6679 Valeriana officinalis, T6680 Valeriana officinalis var. latifolia.

move stagnation and transform damp T5104 Polygonum hydropiper.

move stasis T5579 Rubia cordifolia.

move water T0606 Areca catechu, T3426 Inula britannica, T3427 Inula

britannica, T3428 Inula britannica var. chinensis, T3433 Inula japonica,

T3434 Inula linariaefolia.

move water and disperse swelling T1756 Costus speciosus, T2125

Descurainia sophia, T3755 Lepidium apetalum [Syn. Lepidium micranthum], T3759 Lepidium virginicum, T3980 Lycopus lucidus, T4304 Mosla chinensis [Syn. Orthodon chinensis].

move water and dissipate damp T2343 Elsholtzia splendens.

move water and free stool T5461 Rhamnus leptophylla.

normalize qi T2733 Firmiana simplex, T2746 Foeniculum vulgare.

normalize $q \boldsymbol{i}$ and loosen chest $\mathrm{T} 1586$ Cocculus laurifolius.

normalize $q i$ and relieve pain $\mathrm{T} 3854$ Lindera strychnifolia [Syn. Lindera aggregata].

normalize $\boldsymbol{q} \boldsymbol{i}$ and transform phlegm T0813 Atalantia buxifolia [Syn. Severinia buxifolia].

nourish blood T0986 Bos taurus domesticus; Bubalus bubalis, T0989 Bos taurus domesticus; Bubalus bubalis, T3279 Homo sapiens, T4912 Pinus koraiensis, T6076 Spinacia oleracea.

nourish blood and boost essence T5925 Sesamum indicum [Syn. Sesamum orientale].

nourish blood and dispel wind $\mathrm{T} 3860$ Linum usitatissimum.

nourish blood and extinguish wind T2840 Gallus gallus domesticus.

nourish blood and free milk T4478 Octopus vulgaris.

nourish blood and free network vessels T3124 Hedyotis capitellata.

nourish blood and quicken blood T0991 Bos taurus domesticus; Bubalus bubalis.

nourish blood and quiet spirit T4555 Ostrea rivularis; Ostrea talienwhanensis; Ostrea gigas, T5680 Salvia miltiorrhiza, T5681 Salvia miltiorrhiza f. alba, T5688 Salvia przewalskii, T6916 Ziziphus jujuba, T6917 Ziziphus jujuba var. inermis.

nourish blood and regulate menstruation T0495 Angelica sinensis, T4580 Paeonia albiflora [Syn. Paeonia lactiflora], T4804 Phlojodicarpus sibiricus.

nourish blood and stanch bleeding T0982 Bos taurus domesticus, T5529 Rhodomyrtus tomentosa. nourish body and quicken blood T3675 Lagopsis supina.

nourish heart T6544 Triticum aestivum [Syn. Triticum vulgare].

nourish heart and quiet spirit T0944 Biota orientalis [Syn. Thuja orientalis; Platycladus orientalis], T1292 Celastrus orbiculatus [Syn. Celastrus articulatus], T1381 Choerospondias axillaris, T4194 Menyanthes trifoliata, T4400 Nelumbo nucifera, T4902 Pimpinella thelungiana, T5108 Polygonum multiflorum, T5497 Rhodiola kirilowii, T5701 Salvia yunnanensis.

nourish liver T3955 Lycium barbarum, T3958 Lycium chinense, T6918 Ziziphus jujuba var. spinosa.

nourish liver and boost kidney T2326 Elaeagnus angustifolia.

nourish liver and brighten eyes T1789 Crepis napifera.

nourish lung and enrich kidney T2188 Dioscorea alata.

nourish qi blood T5586 Rubia yunnanensis.

nourish yin and calm liver T3001 Glycine max.

nourish yin and clear lung T2983 Glehnia littoralis.

nourish yin and engender liquid T3873 Liriope platyphylla, T3874 Liriope spicata, T3875 Liriope spicata var. prolifera, T4507 Ophiopogon japonicus.

nourish yin and harmonize stomach T1353 Changium smyrnioides.

nourish yin and moisten dryness T5093 Polygonatum odoratum [Syn. Polygonatum officinale], T5094 Polygonatum prattii.

nourish yin and moisten lung T3832 Lilium brownii var. viridulum [Syn. Lilium brownii var. colchesteri], T3835 Lilium longiflorum, T3836 Lilium pumilum [Syn. Lilium tenuifolium], T3839 Lilium tigrinum [Syn. Lilium lancifolium], T5091 Polygonatum cyrtonema [Syn. Polygonatum multiflorum], T5092 Polygonatum kingianum, T5095 Polygonatum sibiricum, T6601 Umbilicaria esculenta [Syn. Gyrophora esculenta].

open orifices T2978 Gleditsia sinensis [Syn. Gleditsia horrida], T3868 Liquidambar orientalis.

open orifices and arouse spirit $\mathrm{T} 1052$ Bufo bufo gargarizans; Bufo melanostictus, T4303 Moschus moschiferus; Moschus berezovskii; Moschus sifanicus, T6197 Styrax benzoin, T6204 Styrax tonkinensis.

open orifices and free spirit $\mathrm{T} 2274$ Dryobalanops aromatica.

open stomach and transform phlegm T4696 Penaeus orientalis.

open stomach $q i$ T2761 Fortunella margarita.

outthrust papules T1687 Coriandrum sativum, T3145 Helianthus annuus, T4184 Mentha haplocalyx [Syn. Mentha canadaensis; Mentha arvensis var. haplocalyx; Mentha arvensis], T5312 Pueraria edulis, T5313 Pueraria lobata [Syn. Pueraria thunbergiana; Pueraria pseudohirsuta], T5316 Pueraria omeiensis, T5318 Pueraria phaseoloides, T5320 Pueraria thomsonii, T5804 Schizonepeta tenuifolia [Syn. Nepeta tenuifolia].

outthrust papules and relieve itch T3738 Lemna minor.

outthrust welling abscess and pus T3145 Helianthus annuus.

overcome damp and relieve pain T3213 Heracleum hemsleyanum, T3217

Heracleum moellendorffii [Syn. Heracleum microcarpum; Heracleum morifolium], T3228 Heracleum yungningense, T5727 Saposhnikovia divaricata [Syn. Ledebouriella seseloides].

percolate damp and kill worms T4851 Physalis minima. percolate damp and relieve itch $\mathrm{T} 2590$ Euphorbia hirta. precipitate heat toxin T5993 Solanum capsicastrum. precipitate qi T0606 Areca catechu, T1168 Canavalia ensiformis, T1169 
Canavalia gladiata, T1508 Citrus sinensis, T2644 Evodia rutaecarpa, T2646 Evodia rutaecarpa var. officinalis, T4544 Oryza sativa, T4546 Oryza sativa, T4547 Oryza sativa cv, T4957 Piper nigrum, T5223 Prunus humilis [Syn. Cerasus humilis], T5224 Prunus japonica [Syn. Cerasus japonica], T5225 Prunus japonica var. nakaii, T5419 Raphanus sativus.

precipitate $q \boldsymbol{i}$ and disperse accumulation T2659 Fagopyrum esculentum. precipitate $q i$ and disperse food T1186 Capsicum annuum, T1187 Capsicum frutescens.

precipitate $q \boldsymbol{i}$ and disperse phlegm $\mathrm{T} 6347$ Terminalia chebula. precipitate $q \boldsymbol{i}$ and eliminate fullness T4034 Magnolia biloba, T4045 Magnolia officinalis.

precipitate $q \boldsymbol{i}$ and loosen chest T4086 Malus asiatica.

precipitate $q i$ and loosen intestines T2658 Fagopyrum esculentum.

precipitate $q i$ and move blood T6497 Tribulus terrestris.

precipitate $q i$ and move water T2439 Eruca sativa.

precipitate $q i$ and regulate center T1472 Citrus chachiensis, T1517 Citrus tankan.

precipitate $q i$ and relieve pain T4953 Piper longum.

promote astriction T2181 Dillenia indica, T2766 Fraxinus bungeana, T2767 Fraxinus chinensis, T2774 Fraxinus paxiana, T2777 Fraxinus rhynchophylla [Syn. Fraxinus chinensis var. rhynchophylla], T2779 Fraxinus stylosa, T2780 Fraxinus szaboana [Syn. Fraxinus chinensis var. acuminata], T2841 Gallus gallus domesticus, T5266 Psidium guajava, T6474 Toona ciliata.

promote astriction and check drain T0293 Albizzia lebbeck.

promote astriction and secure astriction T1927 Cycas revoluta.

promote astriction and stanch bleeding T0248 Agrimonia pilosa, T0249 Agrimonia pilosa var. japonica, T0955 Bletilla striata, T0962 Boehmeria platanifolia [Syn. Boehmeria tricuspis], T1256 Castanea mollissima, T3361 Hypericum perforatum.

promote astriction and stem desertion T2826 Galeola faberi. promote contraction T3394 Ilex paraguariensis, T4797 Phillyrea latifolia. promote contraction and check discharge T3570 Juglans regia. promote contraction and check drain T5265 Psidium guajava, T5330 Punica granatum.

promote contraction and resolve toxin T5530 Rhus chinensis [Syn. Rhus semialata].

promote contraction and secure astriction T5791 Schisandra chinensis, T5802 Schisandra sphenanthera.

promote contraction and stanch bleeding T1118 Callicarpa formosana, T1119 Callicarpa japonica, T3376 Hypolepis punctata [Syn. Polypodium punctatum], T3926 Loropetalum chinense, T4006 Lythrum anceps, T4007 Lythrum salicaria, T4082 Mallotus japonicus, T4361 Myrsine africana, T5183 Potentilla griffithii var. velutina, T5499 Rhodiola sacra, T5555 Rodgersia pinnata, T5673 Salvia glutinosa, T6485 Trachycarpus fortunei.

promote contraction and stem desertion T5188 Potentilla viscosa, T6353 Tetracera asiatica.

promote lactation T0533 Antiaris toxicaria [Syn. Ambora toxicaria], T1881 Cucurbita moschata, T1961 Cynanchum thesioides, T2624 Euphorbia tirucalli, T3018 Glycyrrhiza pallidiflora, T3240 Hibiscus esculentus, T4436 Nitraria tangutorum, T4644 Parasilurus asotus,
T6507 Trichosanthes cucumeroides, T6771 Viscum album, T6777 Viscum multinerve.

promote lactation and disperse edema T6668 Vaccaria segetalis [Syn. Vaccaria pyramidata].

promote lactation and free stool T0002 Abelmoschus moschatus [Syn. Hibiscus abelmoschus].

promote vomiting T0533 Antiaris toxicaria [Syn. Ambora toxicaria], T1262 Catalpa ovata, T1291 Celastrus paniculatus, T1315 Cephaelis ipecacuanha, T1645 Consolida ajacis [Syn. Delphinium ajacis], T1876 Cucumis melo, T3988 Lycoris squamigera, T6132 Stephania sasakii, T6697 Veratrum grandiflorum, T6698 Veratrum nigrum.

promote vomiting and expel phlegm drool T0299 Aleurites cordata [Syn. Aleurites fordii].

promote yang and dissipate binds T0313 Allium chinense, T0316 Allium macrostemon.

promote yang and transform qi T1438 Cinnamomum cassia [Syn. Cinnamomum aromaticum].

protect hepatic function T3085 Gynostemma pentaphyllum.

quicken blood T0060 Achillea alpina [Syn. Achillea sibirica], T0065 Achillea millefolium, T0300 Aleurites moluccana, T0495 Angelica sinensis, T0535 Antidesma bunius, T0598 Ardisia japonica, T0886 Beaumontia grandiflora, T1175 Cannabis sativa, T1189 Caragana intermedia, T1328 Ceratostigma plumbaginoides, T1329 Ceratostigma willmottianum, T1682 Cordyceps ophioglossoides, T1750 Corydalis yanhusuo [Syn. Corydalis turtschaninovii f. yanhusuo], T1955 Cynanchum hancockianum, T2154 Dicentra spectabilis, T2267 Drosera peltata var. lunata, T2347 Embelia parviflora, T2410 Equisetum pratense, T2601 Euphorbia milii, T3619 Kadsura peltigera [Syn. Kadsura longipedunculata], T3775 Lethariella cladonioides, T3859 Linum usitatissimum, T3964 Lycopodium alpinum [Syn. Diphasiastrum alpinum], T4227 Microula sikkimensis, T4253 Mirabilis jalapa, T4335 Mussaenda pubescens, T4500 Onosma paniculatum, T4662 Parthenocissus tricuspidata, T4787 Phellinus igniarius, T4804 Phlojodicarpus sibiricus, T4951 Piper laetispicum, T5018 Pleuropterus ciliinervis, T5106 Polygonum lapathifolium, T5110 Polygonum orientale, T5167 Porana racemosa, T5193 Pratia nummularia, T5229 Prunus persica, T5626 Ruta graveolens, T5704 Sambucus nigra, T5707 Sambucus williamsii, T5741 Sargentodoxa cuneata, T6015 Solanum torvum, T6066 Spatholobus suberectus, T6533 Trillium kamtschaticum, T6535 Trillium tschonoskii, T6578 Tylophora atrofolliculata, T6735 Viburnum dilatatum, T6737 Viburnum luzonicum, T6800 Viverra zibetha, T6872 Zanthoxylum cuspidatum.

quicken blood and disinhibit water T0036 Acanthopanax giraldii [Syn. Acanthopanax giraldii var. inermis; Eleutherococcus giraldii], T3000 Glycine max.

quicken blood and dispel stasis T0122 Aconitum pendulum, T1108 Caesalpinia sappan, T1799 Crinum latifolium, T1924 Cyathula officinalis, T4584 Paeonia lactiflora wild, T4586 Paeonia obovata, T4590 Paeonia veitchii, T5233 Prunus persica, T5671 Salvia digitaloides, T5680 Salvia miltiorrhiza, T5681 Salvia miltiorrhiza f. alba, T5688 Salvia przewalskii, T5701 Salvia yunnanensis, T5794 Schisandra lancifolia, T5966 Siphonostegia chinensis, T5975 Smilax bockii, T6051 Sorbaria arborea, T6052 Sorbaria sorbifolia, T6584 Typha angustata, 
T6585 Typha angustifolia, T6587 Typha latifolia.

quicken blood and dispel wind T1025 Bretschneidera sinensis, T5939

Shiraia bambusicola.

quicken blood and disperse swelling T0130 Aconitum sinomontanum, T0537 Antirrhinum majus, T0601 Ardisia pusilla, T0938 Bidens bipinnata, T0982 Bos taurus domesticus, T1137 Caltha palustris, T1199 Carduus acanthoides, T1200 Carduus crispus, T1254 Castanea mollissima, T1305 Centaurea cyanus, T1361 Chenopodium ambrosioides, T1556 Clerodendron fragrans, T1557 Clerodendron indicum, T1869 Cryptotaenia japonica, T2020 Damnacanthus indicus, T2131 Desmodium pulchellum [Syn. Phyllodium pulchellum], T2189 Dioscorea althaeoides, T2206 Dioscorea parviflora, T3414 Impatiens siculifer, T3457 Iris dichotoma, T3496 Isodon japonica [Syn. Rabdosia japonica], T4320 Murraya kwangsiensis, T4948 Piper hancei, T5424 Rauvolfia verticillata, T5547 Ricinus communis, T5714 Sansevieria trifasciata, T5781 Schefflera arboricola, T5785 Schefflera venulosa, T6008 Solanum nigrum, T6155 Strophanthus divaricatus, T6645 Urena lobata.

quicken blood and disperse welling abscess T0292 Albizzia julibrissin, T2657 Fagopyrum cymosum [Syn. Polygonum cymosum], T4726 Periploca forrestii.

quicken blood and dissipate binds T0751 Asparagus officinalis, T4303 Moschus moschiferus; Moschus berezovskii; Moschus sifanicus.

quicken blood and dissipate stasis T0071 Achyranthes aspera, T0072 Achyranthes aspera var. indica, T0591 Ardisia arborescens, T0680 Artemisia lactiflora, T0692 Artemisia rupestris [Syn. Artemisia dentata; Artemisia viridis; Artemisia viridifolia], T0969 Boerhavia diffusa, T1372 Chloranthus serratus, T1450 Cirsium lineare, T1706 Corydalis ambigua var. amurensis [Syn. Corydalis ambigua], T1734 Corydalis remota [Syn. Corydalis bulbosa var. typica], T1735 Corydalis repens, T2008 Dalbergia odorifera, T2013 Dalbergia sissoo, T3187 Helleborus thibetanus, T3254 Hippophae rhamnoides, T3256 Hippophae rhamnoides subsp. sinensis, T3258 Hippophae rhamnoides subsp. yunnanensis, T3479 Isodon amethystoides, T3746 Leontice robustum, T3853 Lindera obtusiloba, T4106 Manis pentadactyla, T4317 Murraya euchrestifolia [Syn. Clausena euchrestifolia], T4582 Paeonia delavayi, T4585 Paeonia moutan [Syn. Paeonia suffruticosa], T5101 Polygonum cuspidatum, T5109 Polygonum nodosum, T5400 Rabdosia stracheyi, T5730 Sarcandra glabra [Syn. Chloranthus glaber], T5746 Sassafras tzumu, T5838 Scutellaria galericulata, T6709 Verbena officinalis, T6728 Veronica serpyllifolia, T6861 Zanthoxylum ailanthoides.

quicken blood and free menstruation T0571 Aralia decaisneaa, T0978 Bombyx mori, T1215 Carthamus tinctorius, T1439 Cinnamomum cassia [Syn. Cinnamomum aromaticum], T2541 Euonymus grandiflorus, T4432 Nigella glandulifera, T5401 Rabdosia yuennanensis, T5755 Saussurea involucrata, T6154 Strobilanthes japonicus [Syn. Championella japonica], T5865 Selaginella pulvinata, T5869 Selaginella tamariscina, T6668 Vaccaria segetalis [Syn. Vaccaria pyramidata], T6895 Zanthoxylum simulans.

quicken blood and free network vessels T0364 Alsophila spinulosa, T0428 Ampelopsis megalophylla, T0634 Aristolochia mollissima, T0926 Berneuxia thibetica, T1435 Cinnamomum camphora, T2538 Euonymus bungeanus, T2723 Ficus pumila, T2752 Fordia cauliflora, T2895
Gaultheria yunnanensis, T3396 Ilex pubescens, T3397 Ilex pubescens var. glaber, T3480 Isodon angustifolia, T3680 Lamium amplexicaule, T3990 Lygodium japonicum, T4085 Mallotus repandus var. chrysocarpus [Syn. Mallotus chrysocarpus; Mallotus repandus], T4234 Millettia dielsiana, T4542 Orthosiphon wulfenioides [Syn. Coleus wulfenioides], T5124 Polypodium niponicum, T5144 Populus adenopoda, T5221 Prunus davidiana, T5235 Prunus persica, T5982 Smilax sieboldii, T5984 Smilax stans [Syn. Smilax vaginata var. stans], T6542 Tripterygium wilfordii, T6827 Wisteria sinensis.

quicken blood and free vessels T0273 Akebia quinata, T0277 Akebia trifoliata, T0279 Akebia trifoliata var. australis, T3270 Holboellia fargesii, T3869 Liquor.

quicken blood and joint bones T1147 Camellia oleifera, T2387 Epilobium hirsutum.

quicken blood and move $q \boldsymbol{i}$ T4221 Micromelum falcatum, T5731 Sarcococca coriacea [Syn. Sarcococca wallichii].

quicken blood and move stasis T1371 Chloranthus japonicus.

quicken blood and nourish heart T2964 Ginkgo biloba.

quicken blood and promote milk T2316 Echinops grijsii, T2317 Echinops ritro, T5466 Rhaponticum uniflorum, T6356 Tetrapanax papyriferus.

quicken blood and quiet spirit T4906 Pinus armandii, T4916 Pinus massoniana.

quicken blood and regulate menstruation T0073 Achyranthes bidentata, T0298 Alectoria vivens, T0963 Boehmeria siamensis, T0999 Bougainvillea glabra, T1023 Bredia tuberculata, T1151 Camellia sinensis [Syn. Thea sinensis], T1163 Campylotropis hirtella, T1285 Celastrus angulatus, T1352 Chamaenerion angustifolium [Syn. Epilobium angustifolium], T1900 Curculigo capitulata [Syn. Leucojum capitulata], T1976 Cyperus iria, T2348 Embelia ribes, T2542 Euonymus japonicus, T2545 Euonymus phellomana, T3340 Hypericum ascyron, T3363 Hypericum sampsonii, T3413 Impatiens nolitangere, T3752 Leonurus heterophyllus [Syn. Leonurus artemisia], T3754 Leonurus sibiricus, T3774 Lespedeza tomentosa, T3776 Lethariella zahlbruckneri, T3785 Levisticum officinale, T3999 Lysimachia clethroides, T4311 Mucuna sempervirens, T4450 Nuphar pumilum, T4510 Ophiorrhiza japonica, T4513 Ophiorrhiza mungos, T4813 Phlomis tuberosa, T5118 Polygonum suffultum, T5555 Rodgersia pinnata, T5562 Rosa chinensis, T5566 Rosa henryi, T5569 Rosa multiflora, T5672 Salvia flava, T5697 Salvia trijuga, T5762 Saussurea nigrescens, T5893 Senecio nudicaulis, T6228 Swertia nervosa, T6676 Valeriana hardwickii, T6677 Valeriana jatamansii [Syn. Valeriana wallichii].

quicken blood and relieve pain T0079 Aconitum brachypodum, T0098 Aconitum geniculatum, T0100 Aconitum hemsleyanium var. circinacum, T0101 Aconitum hemsleyanum, T0116 Aconitum nagarum var. heterotrichum [Syn. Aconitum bullatifolium], T0117 Aconitum nagarum var. lasiandrum, T0170 Adhatoda vasica, T0275 Akebia quinata, T0295 Albizzia odoratissima, T0383 Alyxia sinensis, T0594 Ardisia crenata, T0726 Asarum forbesii, T0759 Aspidistra elatior, T1046 Buddleja davidii, T1100 Caesalpinia crista, T1286 Celastrus flagellaris, T1290 Celastrus orbiculatus [Syn. Celastrus articulatus], T1374 Chloranthus spicatus, T1417 Cimicifuga acerina, T1550 Cleome gynandra [Syn. Gynandropsis gynandra], T1638 Commiphora myrrha [Syn. Commiphora molmol], T1672 Corallodiscus flabellatus [Syn. 
Didissandra flabellat], T1721 Corydalis linearioides, T1730 Corydalis ophiocarpa, T1745 Corydalis suaveolens [Syn. Corydalis sheareri], T1810 Croomia japonica, T1907 Curcuma wengujin, T1934 Cyclea tonkinensis, T1984 Cypripedium macranthum [Syn. Cypripedium tibeticum], T2027 Daphne odora, T2028 Daphne odora, T2203 Dioscorea nipponica, T2204 Dioscorea nipponica ssp. rosthornii, T2231 Diphylleia grayi, T2232 Diphylleia sinensis, T2295 Duranta repens, T2409 Equisetum palustre, T2719 Ficus fistulosa [Syn. Ficus harlandii], T2721 Ficus microcarpa, T2731 Firmiana simplex, T2735 Fissistigma oldhamii [Syn. Melodorum oldhamii], T2736 Fissistigma polyanthum, T3063 Grevillea robusta, T3201 Hemiphragma heterophyllum, T3292 Hunteria zeylanica, T3303 Hydrangea chinensis, T3401 Illicium henryi, T3410 Impatiens balsamina, T3624 Kalopanax pictum, T3625 Kalopanax septemlobus, T3681 Lamium barbatum, T3991 Lyonia ovalifolia, T3995 Lysidice rhodostegia, T3996 Lysimachia candida, T4004 Lysimachia paridiformis, T4444 Nothopanax davidii, T4577 Paederia scandens, T4663 Passiflora caerulea, T4689 Peganum nigellastrum, T4725 Periploca calophylla, T4778 Phallus impudicus, T4810 Phlomis mongolica, T4827 Photinia parvifolia, T4974 Piper wallichii [Syn. Piper wallichii var. hupehense], T5057 Podophyllum emodii [Syn. Podophyllum emodii var. chinense; Podophyllum sikkimenosis; Sinopodophyllum emodii], T5119 Polygonum thunbergii, T5230 Prunus persica, T5341 Pygmaeopremna herbacea [Syn. Premna herbacea], T5396 Rabdosia rubescens, T5576 Rostellularia procumbens [Syn. Justicia procumbens], T5640 Sabina vulgaris, T5793 Schisandra henryi, T5799 Schisandra rubriflora, T5813 Scilla scilloides, T5840 Scutellaria indica, T5942 Sida acuta, T6099 Stauntonia chinensis, T6138 Sterculia foetida, T6444 Thunbergia grandiflora, T6551 Trogopterus xanthipes; Pteromys volans, T6580 Tylophora floribunda, T6742 Vicia amoena, T6777 Viscum multinerve.

quicken blood and resolve toxin T0440 Anagallis arvensis, T0568 Aralia chinensis, T0570 Aralia dasyphylla, T0595 Ardisia crispa, T0729 Asarum maximum, T0957 Blumea balsamifera, T1288 Celastrus orbiculatus [Syn. Celastrus articulatus], T2133 Desmodium racemosum [Syn. Podocarpium podocarpum var. oxyphyllum], T2262 Dregea sinensis, T2272 Drymaria diandra [Syn. Drymaria cordata ssp. diandra], T2737 Flemingia philippinensis [Syn. Moghania philippinensis], T2955 Geum japonicum, T2956 Geum japonicum, T3646 Kyllinga brevifolia, T3925 Loranthus parasiticus [Syn. Loranthus chinenis; Taxillus chinensis], T4252 Mirabilis jalapa, T4260 Moghania philippinensis, T5184 Potentilla kleiniana, T5560 Rorippa montana [Syn. Rorippa dubia; Sisymbrium dublium], T5639 Sabina chinensis, T5954 Silene fortunei, T5987 Sobina chinensis.

quicken blood and settle pain T1871 Cucubalus baccifer, T2614 Euphorbia pulcherrima.

quicken blood and soothe sinews T0471 Anemone rivularis, T5586 Rubia yunnanensis, T5866 Selaginella sanguinolenta.

quicken blood and stanch bleeding T0001 Abelmoschus manihot, T0600 Ardisia mamillata [Syn. Tinus mamillata], T0736 Asclepias curassavica, T0952 Blechnum orientale, T2193 Dioscorea cirrhosa [Syn. Dioscorea pogonoides], T2220 Diospyros kaki, T3318 Hylotelephium mingjinianum, T3388 Ilex chinensis [Syn. Ilex purpurea], T3645 Kummerowia striata, T3659 Lactuca indica, T3670 Lagerstroemia indica, T3671
Lagerstroemia indica, T4413 Nepeta cataria, T5269 Psilotum nudum, T5297 Pteris plumbea, T5310 Pterospermum lanceaefolium, T5541 Rhus verniciflua [Syn. Toxicadendron verniciflum], T5567 Rosa laevigata, T6531 Trillium camtschaticum, T6540 Tripterygium hypoglaucum, T6722 Veronica anagallis-aquatica, T6830 Woodfordia fruticosa.

quicken blood and strengthen sinews T3992 Lyonia ovalifolia var. elliptica.

quicken blood and transform stasis T1001 Brachystemma calycinum, T1005 Brassica campestris [Syn. Brassica campestris var. oleifera], T1769 Crataegus cuneata, T1771 Crataegus kansuensis, T1772 Crataegus maximowiczii, T1780 Crataegus sanguinea, T1781 Crataegus scabrifolia, T1808 Crocus sativus, T2052 Davallia divaricata [Syn. Davallia formosana; Davallia orientalis], T3679 Lamiophlomis rotata [Syn. Phlomis rotata], T3980 Lycopus lucidus, T4143 Meconopsis horridula, T4562 Oxalis acetosella, T5231 Prunus persica, T5497 Rhodiola kirilowii, T5578 Rubia cordifolia, T5582 Rubia oncotricha, T5583 Rubia schumannina, T5584 Rubia tinctorum, T5585 Rubia wallichiana, T5638 Sabia swinhoei, T5661 Salvia bowleyana.

quicken blood and vessels T0038 Acanthopanax gracilistylus, T0042 Acanthopanax senticosus [Syn. Eleutherococcus senticosus], T0044 Acanthopanax sessiliflorus.

quicken network vessels T4965 Piper sarmentosum.

quicken network vessels and relieve pain T2422 Erigeron breviscapus, T5822 Scopolia sinensis, T6691 Veratrilla baillonii.

quiet fetus T0824 Atractylodes macrocephala [Syn. Atractylis macrocephala], T1490 Citrus limon, T1494 Citrus limonia, T1911 Cuscuta australis, T1912 Cuscuta chinensis, T1913 Cuscuta japonica, T1980 Cyprinus carpio, T1982 Cyprinus carpio, T2530 Eucommia ulmoides, T4399 Nelumbo nucifera, T4718 Perilla frutescens var. acuta [Syn. Perilla frutescens var. purpurascens], T4720 Perilla frutescens var. arguta, T4721 Perilla frutescens var. arguta, T4723 Perilla frutescens var. crispa, T5687 Salvia prionitis, T5833 Scutellaria amoena, T5834 Scutellaria baicalensis, T5839 Scutellaria hypericifolia, T5841 Scutellaria likiangensis, T5843 Scutellaria rehderiana, T5845 Scutellaria viscidula, T6775 Viscum coloratum, T6777 Viscum multinerve.

quiet heart T6522 Trifolium repens, T6919 Ziziphus jujuba var. spinosa.

quiet heart and quicken blood T5071 Polygala arillata.

quiet heart and settle epilepsy $\mathrm{T} 3329$ Hyoscyamus niger.

quiet heart and spirit T0561 Arachis hypogaea, T1390 Chrysanthemum coronarium, T1397 Chrysanthemum segetum, T2846 Ganoderma japonicum [Syn. Ganoderma sinense], T2848 Ganoderma lucidum, T5086 Polygala sibirica, T5088 Polygala tenuifolia, T5791 Schisandra chinensis, T5802 Schisandra sphenanthera, T5850 Sedum aizoon, T5855 Sedum kamtschaticum, T6675 Valeriana amurensis, T6679 Valeriana officinalis, T6680 Valeriana officinalis var. latifolia, T6918 Ziziphus jujuba var. spinosa.

quiet roundworm T5228 Prunus mume.

quiet spirit T0295 Albizzia odoratissima, T0463 Anemone altaica, T1171 Canis familiaris, T3229 Hericium erinaceus [Syn. Hydnum erinaceus], T3775 Lethariella cladonioides, T3950 Lychnis fulgens, T4243 Millettia reticulata, T4436 Nitraria tangutorum, T4599 Panax ginseng [Syn. Panax schinseng], T5423 Rauvolfia verticillata, T5425 Rauvolfia 
verticillata f. rubrocarpa, T5426 Rauvolfia verticillata var. hainanensis, T5427 Rauvolfia vomitoria, T5435 Rauwolfia latifrons, T5441 Rauwolfia perakensis, T5465 Rhaponticum carthamoides, T5575 Rosmarinus officinalis, T6295 Tanacetum sibiricum [Syn. Filifolium sibiricum], T6656 Ustilago maydis, T6800 Viverra zibetha.

quiet spirit and boost wits T4600 Panax ginseng [Syn. Panax schinseng]. quiet spirit and calm T0298 Alectoria vivens, T4248 Mimosa pudica. quiet spirit and relieve pain T4665 Passiflora edulis. quiet spirit and resolve depression T0292 Albizzia julibrissin. quiet spirit and settle asthma T4859 Physochlaina physaloides. quiet spirit and settle fright $\mathrm{T} 1804$ Cristaria plicata; Hyriopsis cumingii. raise spirit T1266 Catha edulis, T1615 Cola acuminata, T6423 Theobroma cacao.

rectify qi T0201 Aesculus chinensis, T0202 Aesculus hippocastanum, T0205 Aesculus wilsonii, T1483 Citrus grandis var. tomentosa, T3283 Hosta sieboldiana, T3524 Isodon sculponeata [Syn. Rabdosia sculponeata], T3877 Litchi chinensis, T4190 Mentha spicata, T4526 Origanum vulgare, T6801 Vladimiria denticulata.

rectify $q i$ and dispel wind $\mathrm{T} 5139$ Poncirus trifoliata.

rectify $\boldsymbol{q} \boldsymbol{i}$ and disperse accumulation $\mathrm{T} 3006$ Glycosmis citrifolia.

rectify $q \boldsymbol{i}$ and disperse cold T6677 Valeriana jatamansii [Syn. Valeriana wallichii].

rectify $\boldsymbol{q} i$ and disperse food T4714 Perilla frutescens, T5183 Potentilla griffithii var. velutina, T6356 Tetrapanax papyriferus.

rectify $\boldsymbol{q} \boldsymbol{i}$ and disperse food with relieving pain T6789 Vitex negundo. rectify $\boldsymbol{q} \boldsymbol{i}$ and disperse swelling T1352 Chamaenerion angustifolium [Syn.

Epilobium angustifolium].

rectify $q i$ and dissipate cold T4439 Nothapodytes pittosporoides.

rectify $q \boldsymbol{i}$ and dissipate stasis T4349 Myrica rubra.

rectify $q \boldsymbol{i}$ and downbear counterflow T1498 Citrus medica, T1506 Citrus reticulata, T1520 Citrus wilsonii.

rectify $q \boldsymbol{i}$ and eliminate damp T4042 Magnolia liliflora.

rectify $q i$ and fortify spleen T5385 Quisqualis indica.

rectify $q i$ and harmonize blood $\mathrm{T} 1604$ Coeloglossum viride [Syn.

Coeloglossum viride var. bracteatum], T3077 Gymnadenia conopsea.

rectify $\boldsymbol{q} i$ and harmonize stomach T1476 Citrus erythrosa, T1493 Citrus

limon, T1497 Citrus limonia, T1515 Citrus tangemna, T2340 Elsholtzia

bodinieri, T2744 Foeniculum vulgare, T4443 Notholirion hyacinthinum

[Syn. Notholirion bulbuliferum], T5715 Santalum album.

rectify $q \boldsymbol{i}$ and loosen chest T0313 Allium chinense, T0316 Allium macrostemon.

rectify $q \boldsymbol{i}$ and open depression T1366 Chimonanthus fragrans [Syn. Chimonanthus praecox].

rectify $q i$ and promote digestion T1217 Carum carvi, T6602 Umbilicaria hypococcinea.

rectify $q \boldsymbol{i}$ and quicken blood T2521 Eucalyptus tereticornis, T2563 Eupatorium japonicum, T3802 Ligularia dentata, T3805 Ligularia fischeri, T3807 Ligularia intermedia, T3813 Ligularia sibirica, T4530 Ormosia hosiei, T5795 Schisandra micrantha, T6877 Zanthoxylum dissitum.

rectify $\boldsymbol{q} i$ and regulate center T1889 Cuminum cyminum.

rectify $\boldsymbol{q} \boldsymbol{i}$ and regulate menstruation $\mathrm{T} 4385$ Narcissus tazetta var. chinensis. rectify $\boldsymbol{q} i$ and relieve pain $\mathrm{T} 0130$ Aconitum sinomontanum, T0491 Angelica polymorpha, T0641 Aristolochia tuberosa, T1163 Campylotropis hirtella, T1487 Citrus junos, T1501 Citrus medica var. sarcodactylis, T1504 Citrus reticulata, T1706 Corydalis ambigua var. amurensis [Syn. Corydalis ambigua], T1735 Corydalis repens, T1928 Cycas revoluta, T1932 Cyclea racemosa, T2193 Dioscorea cirrhosa [Syn. Dioscorea pogonoides], T2205 Dioscorea panthaica, T2529 Euchresta strigillosa, T3409 Illicium verum, T3557 Jasminum sambac, T3876 Litchi chinensis, T4386 Nardostachys chinensis, T4387 Nardostachys jatamansi, T4763 Peucedanum morisonii, T6120 Stephania delavayi [Syn. Stephania epigaea], T6275 Tacca chantrieri [Syn. Tacca minor; Tacca esquirolii], T6527 Trigonella caerulea, T6787 Vitex negundo.

rectify $q i$ and remove damp T5122 Polygonum viscosum.

rectify $\boldsymbol{q} \boldsymbol{i}$ and resolve depression T2758 Fortunella crassifolia, T2759

Fortunella japonica, T2760 Fortunella margarita.

rectify $q \boldsymbol{i}$ and stanch bleeding T1673 Corchorus capsularis.

rectify $q \boldsymbol{i}$ and transform damp T0680 Artemisia lactiflora, T4397 Nelumbo nucifera.

rectify $\boldsymbol{q} \boldsymbol{i}$ and transform phlegm $\mathrm{T} 1660$ Coprinus atramentarius, T2510

Eucalyptus globulus, T3742 Lentinus edodes, T3840 Limnophila rugosa,

T6504 Tricholoma matsutake [Syn. Armillaria matsutake].

reduce phlegm T6819 Wikstroemia chamaedaphne.

reduce urine T0360 Alpinia oxyphylla, T2961 Ginkgo biloba, T4548 Oryza sativa var. glutinosa.

regulate center T0357 Alpinia japonica, T1247 Cassia sophera.

regulate center and increase appetite T1506 Citrus reticulata, T6902 Zea mays.

regulate digestive system T1341 Cetraria islandica.

regulate function among herbs T3011 Glycyrrhiza aspera, T3013 Glycyrrhiza glabra, T3015 Glycyrrhiza inflata, T3016 Glycyrrhiza kansuensis, Т3021 Glycyrrhiza squamulosa, T3022 Glycyrrhiza uralensis, T3023 Glycyrrhiza yunnanensis.

regulate menstruation T0301 Aleuritopteris argentea, T0969 Boerhavia diffusa, T1143 Calystegia hederacea, T1682 Cordyceps ophioglossoides, T2736 Fissistigma polyanthum, T3240 Hibiscus esculentus, T3997 Lysimachia capillipes, T5523 Rhododendron simsii, T5545 Ribes fasciculatum var. chinense, T5594 Rubus parkeri, T5953 Silene firma, T6295 Tanacetum sibiricum [Syn. Filifolium sibiricum], T6876 Zanthoxylum dissitum.

regulate menstruation and check discharge T4777 Phalaris arundinacea. regulate menstruation and free milk T3361 Hypericum perforatum. regulate menstruation and promote pregnancy T3615 Kadsura interior. regulate menstruation and quicken blood T5498 Rhodiola quadrifida. regulate menstruation and relieve pain T0474 Angelica acutiloba [Syn. Ligusticum acutilobum], T1978 Cyperus rotundus, T5515 Rhododendron micranthum, T5661 Salvia bowleyana, T5680 Salvia miltiorrhiza, T5681 Salvia miltiorrhiza f. alba, T5688 Salvia przewalskii.

regulate menstruation and stanch bleeding T1528 Cladonia stellaris [Syn. Cladonia alpestris], T2614 Euphorbia pulcherrima, T2826 Galeola faberi, T3349 Hypericum erectum, T5753 Saussurea gnaphaloides, T5757 Saussurea laniceps, T5759 Saussurea medusa, T6653 Usnea diffracta, T6654 Usnea longissima, T6749 Vicia hirsuta. 
regulate $q i \mathrm{~T} 1469$ Citrus aurantium var. amara.

regulate $q \boldsymbol{i}$ and relieve pain $\mathrm{T} 1590$ Cocculus trilobus [Syn. Cocculus sarmentosus], T2536 Euonymus alatus.

regulate $q i$ and resolve depression $\mathrm{T} 3431$ Inula helenium, T3437 Inula racemosa.

regulate thoroughfare and controlling vessels T1337 Cervus nippon; Cervus elaphus.

relax tension and relieve pain $\mathrm{T} 0540$ Apis cerana, T3011 Glycyrrhiza aspera, T3013 Glycyrrhiza glabra, T3015 Glycyrrhiza inflata, T3016 Glycyrrhiza kansuensis, T3021 Glycyrrhiza squamulosa, T3022 Glycyrrhiza uralensis, T3023 Glycyrrhiza yunnanensis.

relieve constipation T5606 Rumex acetosa.

relieve cough T0444 Ananas comosus, T0948 Blainvillea acmella [Syn. Verbesina acmella; Eclipta latifolia; Blainvillea latifolia], T1312 Centipeda minima, T1675 Corchorus olitorius, T1960 Cynanchum stauntonii, T1969 Cynoglossum officinale, T2101 Dendrobium fimbriatum, T3143 Helianthus annuus, T3380 Hyptis pectinata, T3457 Iris dichotoma, T3567 Juglans regia, T3765 Lepisorus ussuriensis, T3779 Leucas aspera, T3802 Ligularia dentata, T3805 Ligularia fischeri, T3807 Ligularia intermedia, T3813 Ligularia sibirica, T3970 Lycopodium cernuum, T3973 Lycopodium japonicum [Syn. Lycopodium clavatum], T4004 Lysimachia paridiformis, T4036 Magnolia coco, T4099 Mangifera indica, T4103 Mangifera persiciformis, T4209 Michelia alba, T4368 Nandina domestica, T4908 Pinus bungeana, T5087 Polygala telephioides, T5375 Quercus mongolica, T5502 Rhododendron anthopogonoides, T5508 Rhododendron dauricum, T5518 Rhododendron mucronatum, T5522 Rhododendron seniavinii, T5523 Rhododendron simsii, T5594 Rubus parkeri, T5609 Rumex hastatus, T5836 Scutellaria discolor, T5859 Selaginella braunii, T5862 Selaginella involvens, T5943 Sida cordifolia, T6750 Vicia sativa, T6772 Viscum angulatum, T6787 Vitex negundo.

relieve cough and asthma T0707 Arthraxon hispidus, T0985 Bos taurus domesticus; Bubalus bubalis, T6581 Tylophora mollissima.

relieve cough and calm asthma T0364 Alsophila spinulosa, T0624 Aristolochia contorta, T0626 Aristolochia debilis [Syn. Aristolochia longa], T0630 Aristolochia indica, T0633 Aristolochia maxima, T0640 Aristolochia triangularis, T0926 Berneuxia thibetica, T1604 Coeloglossum viride [Syn. Coeloglossum viride var. bracteatum], T1819 Crotalaria ferruginea, T1941 Cymbopogon distans, T1943 Cymbopogon goeringii, T2749 Fomes officinalis, T3056 Gossypium herbaceum, T3077 Gymnadenia conopsea, T3221 Heracleum rapula, T3928 Lotus corniculatus, T4003 Lysimachia microcarpa, T4120 Marsdenia tenacissima, T4124 Matricaria chamomilla [Syn. Matricaria recutita], T4688 Peganum harmala, T4858 Physochlaina infundibularis, T4974 Piper wallichii [Syn. Piper wallichii var. hupehense], T5145 Populus alba, T5216 Prunus armeniaca, T5219 Prunus armeniaca var. ansu, T5548 Ricinus communis, T6259 Syringa amurensis [Syn. Syringa reticulata var. amurensis], T6789 Vitex negundo, T6825 Winchia calophylla.

relieve cough and dispel phlegm T0374 Alstonia scholaris, T0729 Asarum maximum, T0781 Aster tataricus, T0933 Betula platyphylla, T0935 Betula platyphylla var. japonica, T1786 Cremanthodium ellisii, T2023 Daphne genkwa, T2203 Dioscorea nipponica, T2204 Dioscorea nipponica ssp. rosthornii, T2517 Eucalyptus robusta, T2978 Gleditsia sinensis [Syn. Gleditsia horrida], T3758 Lepidium sativum, T3770 Lespedeza cuneata, T4203 Mesua ferrea, T4358 Myroxylon pereirae, T4510 Ophiorrhiza japonica, T4513 Ophiorrhiza mungos, T4607 Panax pseudo-ginseng var. japonicus, T4902 Pimpinella thelungiana, T5057 Podophyllum emodii [Syn. Podophyllum emodii var. chinense; Podophyllum sikkimenosis; Sinopodophyllum emodii], T5457 Rhamnus davurica, T5504 Rhododendron capitatum, T6428 Thermopsis lanceolata, T6440 Thuja orientalis [Syn. Platycladus orientalis; Biota orientalis], T6653 Usnea diffracta, T6654 Usnea longissima.

relieve cough and free strangury T5647 Sagittaria sagittifolia.

relieve cough and kill worms T6111 Stemona japonica, T6113 Stemona sessilifolia, T6115 Stemona tuberosa.

relieve cough and settle asthma T0923 Bergenia crassifolia, T2220 Diospyros kaki.

relieve cough and transform phlegm T0015 Abutilon indicum, T0078 Aconitum barbatum var. puberulum [Syn. Aconitum ochranthum], T0263 Ajuga decumbens, T0814 Atalantia buxifolia [Syn. Severinia buxifolia], T1493 Citrus limon, T1497 Citrus limonia, T2050 Daucus carota var. sativa, T2262 Dregea sinensis, T2432 Eriobotrya japonica, T2589 Euphorbia helioscopia, T2738 Flemingia strobilifera, T2783 Fritillaria cirrhosa, T2784 Fritillaria delavayi, T2787 Fritillaria hupehensis, T2792 Fritillaria przewalskii, T2796 Fritillaria unibracteata, T2797 Fritillaria ussuriensis, T2895 Gaultheria yunnanensis, T3033 Gnetum parvifolium [Syn. Gnetum indicum], T3254 Hippophae rhamnoides, T3256 Hippophae rhamnoides subsp. sinensis, T3258 Hippophae rhamnoides subsp. yunnanensis, T3628 Kerria japonica, T3749 Leontopodium alpinum, T3810 Ligularia nelumbifolia, T4000 Lysimachia congestiflora, T4005 Lysionotus pauciflorus, T4438 Nostoc flagelliforme, T4834 Phyllanthus emblica, T5072 Polygala caudata, T5161 Populus tomentosa, T5184 Potentilla kleiniana, T5515 Rhododendron micranthum, T5519 Rhododendron mucronulatum, T5521 Rhododendron przewalskii, T5530 Rhus chinensis [Syn. Rhus semialata], T5637 Sabia schumanniana, T6278 Tagetes erecta, T6574 Tussilago farfara, T6773 Viscum articulactum, T6774 Viscum articulatum, T6910 Zingiber officinale.

relieve headache $\mathrm{T} 3246$ Hibiscus syriacus.

relieve itch T0750 Asparagus gobicus, T1650 Convolvulus arvensis, T1777 Crataegus pinnatifida, T1867 Cryptomeria fortunei, T2049 Daucus carota, T2533 Eugenia jambolana [Syn. Syzygium cumin; Myrtus cumini], T2584 Euphorbia ebracteolata, T2587 Euphorbia fischeriana, T3342 Hypericum bellum, T3569 Juglans regia, T3851 Lindera glauca, T4102 Mangifera indica, T4828 Photinia serrulata, T5098 Polygonum aviculare, T5727 Saposhnikovia divaricata [Syn. Ledebouriella seseloides], T6072 Sphagnum palustre [Syn. Sphagnum obtusifolium; Sphagnum cymbifolium], T6365 Teucrium pilosum [Syn. Teucrium japonicum var. pilosum], T6499 Tribulus terrestris.

relieve pain T0065 Achillea millefolium, T0086 Aconitum chasmanthum, T0090 Aconitum delavayi, T0107 Aconitum kongboense, T0125 Aconitum pseudostapfianum, T0157 Actinidia callosa var. henryi, T0201 Aesculus chinensis, T0202 Aesculus hippocastanum, T0205 Aesculus wilsonii, T0229 Ageratum conyzoides, T0356 Alpinia galanga, T0359 Alpinia officinarum, T0471 Anemone rivularis, T0495 Angelica sinensis, 
T0539 Apis cerana, T0582 Archangelica brevicaulis [Syn. Angelicarpa brevicaulis; Angelica brevicaulis], T0634 Aristolochia mollissima, T0708 Arthromeris mairei [Syn. Polypodium mairei], T0724 Asarum caulescens, T0727 Asarum fukienense, T0729 Asarum maximum, T0814 Atalantia buxifolia [Syn. Severinia buxifolia], T0886 Beaumontia grandiflora, T1052 Bufo bufo gargarizans; Bufo melanostictus, T1262 Catalpa ovata, T1327 Ceratostigma minus, T1416 Cicuta virosa, T1434 Cinnamomum bejolghota [Syn. Cinnamomum obtusifolium; Laurus bejolghota], T1452 Cissampelos pareira, T1453 Cissampelos pareira var. hirsute, T1508 Citrus sinensis, T1525 Cladonia fallax, T1541 Claviceps purpurea, T1577 Clitoria ternatea, T1592 Cocos nucifera, T1604 Coeloglossum viride [Syn. Coeloglossum viride var. bracteatum], T1650 Convolvulus arvensis, T1705 Corydalis adunca, T1719 Corydalis incisa, T1727 Corydalis mucronifera, T1746 Corydalis taliensis, T1750 Corydalis yanhusuo [Syn. Corydalis turtschaninovii f. yanhusuo], T1955 Cynanchum hancockianum, T2036 Datura innoxia, T2039 Datura innoxia, T2041 Datura metel, T2043 Datura metel, T2046 Datura stramonium, T2047 Datura stramonium, T2077 Delphinium grandiflorum, T2091 Delphinium yunnanense, T2130 Desmodium gangeticum, T2263 Dregea volubilis, T2546 Euonymus sacrosancta, T2644 Evodia rutaecarpa, T2646 Evodia rutaecarpa var. officinalis, T2746 Foeniculum vulgare, T3077 Gymnadenia conopsea, T3356 Hypericum japonicum, T3457 Iris dichotoma, T3560 Jatropha curcas, T3567 Juglans regia, T3619 Kadsura peltigera [Syn. Kadsura longipedunculata], T3620 Kaempferia galanga, T3670 Lagerstroemia indica, T3797 Libanotis condensata, T3802 Ligularia dentata, T3805 Ligularia fischeri, T3807 Ligularia intermedia, T3809 Ligularia lapathifolia, T3813 Ligularia sibirica, T3822 Ligusticum jeholense, T3824 Ligusticum sinense, T3868 Liquidambar orientalis, T3877 Litchi chinensis, T3964 Lycopodium alpinum [Syn. Diphasiastrum alpinum], T4002 Lysimachia foenum-graecum, T4020 Macleaya cordata, T4145 Meconopsis punicea, T4163 Melia toosendan, T4264 Momordica cochinchinensis, T4267 Monachosorum flagellare, T4268 Monachosorum henryi, T4343 Myrica esculent, T4349 Myrica rubra, T4445 Notopterygium forbesii [Syn. Notopterygium franchetii], T4446 Notopterygium incisum, T4551 Osmorhiza aristata var. laxa, T4635 Papaver somniferum, T4637 Papaver somniferum, T4662 Parthenocissus tricuspidata, T4804 Phlojodicarpus sibiricus, T4951 Piper laetispicum, T5142 Poncirus trifoliata, T5268 Psidium guajava, T5415 Ranunculus sceleratus, T5572 Rosa rugosa, T5575 Rosmarinus officinalis, T5651 Salix babylonica, T5690 Salvia roborowskii, T5720 Sapindus mukorossi, T5767 Saussurea pulchella, T5883 Senecio cannabifolius, T5963 Sinodielsia yunnanensis, T6009 Solanum pseudo-capsicum, T6018 Solanum verbascifolium, T6069 Speranskia tuberculata, T6100 Stauntonia hexaphylla, T6128 Stephania hernandifolia, T6177 Strychnos ignatii, T6197 Styrax benzoin, T6204 Styrax tonkinensis, T6463 Tinospora capillipes, T6467 Tinospora sagittata, T6494 Trema dielsiana, T6528 Trigonella foenum-graecum, T6533 Trillium kamtschaticum, T6535 Trillium tschonoskii, T6548 Tritonia crocosmaeflora, T6578 Tylophora atrofolliculata, T6675 Valeriana amurensis, T6679 Valeriana officinalis, T6680 Valeriana officinalis var. latifolia, T6693 Veratrum album var. lobelianum [Syn. Veratrum lobelianum], T6731 Veronicastrum sibiricum, T6800 Viverra zibetha, T6801 Vladimiria denticulata, T6860 Zanthoxylum ailanthoides, T6864 Zanthoxylum armatum, T6872 Zanthoxylum cuspidatum, T6887 Zanthoxylum planispinum.

Relieve pain T3106 Harpagophytum procumbens.

relieve pain and check tetany T5934 Seseli meirei, T5937 Seseli yunnanense.

relieve pain and disperse swelling T3548 Ixeris sonchifolia.

relieve pain and draw out pus T2038 Datura innoxia, T2042 Datura metel.

relieve pain and itch T0610 Argemone mexicana, T1959 Cynanchum paniculatum.

relieve pain and quiet fetus T3431 Inula helenium, T3437 Inula racemosa. relieve pain and settle tetany T2037 Datura innoxia, T2044 Datura metel. relieve pain due to impediment T0608 Arenaria kansuensis [Syn. Arenaria kumaonensis], T0990 Bos taurus domesticus; Bubalus bubalis, T2909 Gentiana crassicaulis, T2910 Gentiana dahurica, T2913 Gentiana kaufmanniana, T2919 Gentiana macrophylla, T2932 Gentiana siphonantha, T2934 Gentiana straminea, T2936 Gentiana tianschanica, T2937 Gentiana tibetica, T4950 Piper kadsura [Syn. Piper futokadsura], T5638 Sabia swinhoei.

relieve stuffed nose T4035 Magnolia biondii [Syn. Magnolia fargesii], T4038 Magnolia denudata [Syn. Magnolia heptapata], T4041 Magnolia liliflora, T4052 Magnolia sprengeri.

repel foulness T2554 Eupatorium cannabinum.

repel foulness and harmonize center T2558 Eupatorium formosanum, T2559 Eupatorium fortunei.

repel foulness and open depression T3557 Jasminum sambac.

replenish essence T1170 Canis familiaris.

resolve binds $\mathrm{T} 1504$ Citrus reticulata, $\mathrm{T} 3877$ Litchi chinensis.

resolve depression T4184 Mentha haplocalyx [Syn. Mentha canadaensis; Mentha arvensis var. haplocalyx; Mentha arvensis], T4688 Peganum harmala.

resolve depression and quiet heart $\mathrm{T} 3778$ Leucaena glauca [Syn. Leucaena leucocephala].

resolve dog toxin T1958 Cynanchum otophyllum.

resolve epidemic toxin T4029 Maesa japonica.

resolve exterior T0310 Allium ascalonicum, T0319 Allium schoenoprasum, T1941 Cymbopogon distans, T3779 Leucas aspera, T4002 Lysimachia foenum-graecum, T4190 Mentha spicata, T4335 Mussaenda pubescens, T4526 Origanum vulgare, T5167 Porana racemosa, T5519 Rhododendron mucronulatum, T5804 Schizonepeta tenuifolia [Syn. Nepeta tenuifolia], T6787 Vitex negundo.

resolve exterior and abate fever T0557 Aquilegia ecalcarata, T5836 Scutellaria discolor.

resolve exterior and allay fever T0591 Ardisia arborescens.

resolve exterior and clear heat T6338 Tephrosia purpurea.

resolve exterior and disinhibit damp T3381 Hyptis suaveolens, T6749 Vicia hirsuta.

resolve exterior and outthrust papules T0838 Azolla imbricata [Syn. Salvinia imbricata].

resolve heat T0158 Actinidia chinensis, T0533 Antiaris toxicaria [Syn. Ambora toxicaria], T3106 Harpagophytum procumbens, T4785 Phaseolus vulgaris, T3380 Hyptis pectinata, T5272 Psorospermum 
febrifugum.

resolve heat toxin T0154 Acroptilon repens, T0902 Berberis diaphana, T1685 Coreopsis lanceolata, T3827 Ligustrum japonicum, T4254 Miscanthus sinensis, T5102 Polygonum cuspidatum.

resolve liquor T1486 Citrus junos, T1937 Cydonia oblonga, T3285 Hovenia dulcis.

resolve liquor and arouse spleen T0713 Artocarpus heterophyllus, T1428 Cinchona ledgeriana, T1429 Cinchona officinalis, T1433 Cinchona succirubra.

resolve liquor jaundice $\mathrm{T} 1758$ Cotinus coggygria.

resolve liquor toxin T3286 Hovenia dulcis, T4573 Pachyrrhizus erosus, T5202 Primula obconica.

resolve snake toxin T1958 Cynanchum otophyllum, T2749 Fomes officinalis, T5723 Sapium sebiferum.

resolve sore toxin $\mathrm{T} 1672$ Corallodiscus flabellatus [Syn. Didissandra flabellat], T2718 Ficus carica, T4101 Mangifera indica, T5520 Rhododendron ovatum [Syn. Rhododendron lamprophyllum; Azalea ovata], T5523 Rhododendron simsii.

resolve spasm T4518 Oppopanax chironium, T5727 Saposhnikovia divaricata [Syn. Ledebouriella seseloides].

resolve spasm and relieve pain T0501 Anisodus luridus, T0825 Atropa belladonna, T3329 Hyoscyamus niger, T5247 Przewalskia tangutica.

resolve summerheat T0660 Artemisia annua, T0662 Artemisia apiacea [Syn. Artemisia carvifolia; Artemisia caruifolia], T1137 Caltha palustris.

resolve summerheat and engender liquid T1464 Citrullus vulgaris [Syn. Citrullus lanatus].

resolve summerheat and transform damp $\mathrm{T} 2558$ Eupatorium formosanum, T2559 Eupatorium fortunei.

resolve tetany and disperse swelling T0502 Anisodus tanguticus [Syn. Scopolia tangutica].

resolve tetany and settle pain T5821 Scopolia japonica.

resolve toxin T0060 Achillea alpina [Syn. Achillea sibirica], T0065 Achillea millefolium, T0174 Adiantum monochlamys, T0249 Agrimonia pilosa var. japonica, T0274 Akebia quinata, T0278 Akebia trifoliata, T0280 Akebia trifoliata var. australis, T0310 Allium ascalonicum, T0314 Allium fistulosum, T0318 Allium sativum, T0322 Allium tuberosum, T0324 Allium victorialis, T0371 Alstonia mairei, T0379 Alternanthera philoxeroides, T0444 Ananas comosus, T0463 Anemone altaica, T0535 Antidesma bunius, T0540 Apis cerana, T0542 Apis cerana, T0544 Apium graveolens, T0567 Aralia armata, T0598 Ardisia japonica, T0678 Artemisia japonica, T0705 Artemisia vestita, T0726 Asarum forbesii, T0834 Averrhoa carambola, T0838 Azolla imbricata [Syn. Salvinia imbricata], T0888 Beesia calthaefolia, T0897 Berberis amurensis, T0932 Betula luminifera, T0947 Bixa orellana, T0950 Blatta orientalis, T0973 Bombax malabaricum [Syn. Gossampinus malabarica], T0982 Bos taurus domesticus, T0989 Bos taurus domesticus; Bubalus bubalis, T1045 Bubalus bubalis, T1052 Bufo bufo gargarizans; Bufo melanostictus, T1097 Cacalia ainsliaeflora, T1152 Camellia sinensis [Syn. Thea sinensis], T1165 Canarium album, T1171 Canis familiaris, T1172 Canna edulis, T1182 Capra hircus; Ovis aries, T1188 Caragana chamlagu, T1194 Carassius auratus, T1206 Carica papaya, T1210 Carpesium abrotanoides, T1239 Cassia nodosa, T1242 Cassia occidentalis, T1266 Catha edulis, T1271 Catharanthus roseus [Syn.
Vinca rosea; Lochera rosea], T1278 Cedrela sinensis, T1336 Ceriops tagal [Syn. Rhizophora tagal], T1353 Changium smyrnioides, T1371 Chloranthus japonicus, T1381 Choerospondias axillaris, T1437 Cinnamomum camphora, T1570 Clerodendrum thomsonae, T1589 Cocculus trilobus [Syn. Cocculus sarmentosus], T1831 Crotalaria sessiliflora, T1867 Cryptomeria fortunei, T1878 Cucumis sativus, T1963 Cynanchum wallichii, T1964 Cynanchum wilfordii [Syn. Cynoctonum wilfordii], T2024 Daphne genkwa, T2027 Daphne odora, T2092 Delphinus delphis, T2130 Desmodium gangeticum, T2165 Dictamnus angustifolius, T2167 Dictamnus dasycarpus, T2181 Dillenia indica, T2216 Diospyros ebenum, T2221 Diospyros kaki, T2267 Drosera peltata var. lunata, T2327 Elaeagnus angustifolia, T2334 Elephantopus scaber, T2533 Eugenia jambolana [Syn. Syzygium cumin; Myrtus cumini], T2563 Eupatorium japonicum, T2601 Euphorbia milii, T2612 Euphorbia prolifera, T2624 Euphorbia tirucalli, T2627 Euphoria longan [Syn. Dimocarpus longan], T2649 Evolvulus alsinoides, T2830 Galium aparine, T2941 Gentianopsis paludosa, T3003 Glycine max, T3040 Gomphrena globosa, T3082 Gynocardia odorata, T3085 Gynostemma pentaphyllum, T3144 Helianthus annuus, T3146 Helianthus annuus cv, T3162 Helicia nilagirica, T3242 Hibiscus rosa-sinensis, T3243 Hibiscus rosa-sinensis, T3246 Hibiscus syriacus, T3356 Hypericum japonicum, T3360 Hypericum patulum, T3410 Impatiens balsamina, T3420 Incarvillea sinensis, T3432 Inula helianthus-aquatica, T3524 Isodon sculponeata [Syn. Rabdosia sculponeata], T3528 Isodon ternifolia, T3529 Isodon ternifolius, T3555 Jasminum nudiflorum, T3567 Juglans regia, T3569 Juglans regia, T3628 Kerria japonica, T3649 Laccifer lacca, T3673 Lagerstroemia speciosa [Syn. Munchausia speciosa; Lagerstroemia flos-reginae], T3674 Laggera alata, T3685 Lannea grandis [Syn. Lannea coromandelica], T3726 Laurus nobilis, T3742 Lentinus edodes, T3815 Ligularia stenocephala, T3866 Liquidambar formosana [Syn. Liquidambar taiwaniana], T3886 Litsea euosma, T3969 Lycopodium casuarinoides, T3970 Lycopodium cernuum, T3973 Lycopodium japonicum [Syn. Lycopodium clavatum], T3988 Lycoris squamigera, T3997 Lysimachia capillipes, T4004 Lysimachia paridiformis, T4020 Macleaya cordata, T4055 Mahonia bealei, T4056 Mahonia bealei, T4062 Mahonia fortunei, T4063 Mahonia fortunei, T4066 Mahonia japonica, T4067 Mahonia japonica, T4072 Mahonia shenii, T4080 Mallotus apelta, T4178 Melodinus hemsleyanus, T4188 Mentha rotundifolia, T4206 Metaplexis japonica, T4225 Microsorium punctatum, T4263 Momordica charantia, T4264 Momordica cochinchinensis, T4331 Musa paradisiaca var. sapientum [Syn. Musa sapientum], T4335 Mussaenda pubescens, T4368 Nandina domestica, T4370 Nandina domestica, T4388 Nasturtium officinale, T4478 Octopus vulgaris, T4500 Onosma paniculatum, T4544 Oryza sativa, T4548 Oryza sativa var. glutinosa, T4618 Panicum miliaceum, T4799 Philydrum lanuginosum, T4814 Phlomis umbrosa, T4831 Phtheirospermum japonicum [Syn. Geradia japonica], T4834 Phyllanthus emblica, T4848 Physalis angulata, T4979 Pistacia chinensis, T4983 Pisum sativum, T5002 Plantago asiatica, T5004 Plantago depressa, T5007 Plantago major, T5073 Polygala chinensis [Syn. Polygala glomerata], T5087 Polygala telephioides, T5090 Polygala wattersii, T5097 Polygonum amphibium, T5101 Polygonum cuspidatum, T5103 Polygonum hydropiper, T5104 Polygonum hydropiper, T5105 Polygonum 
hydropiper var. flaccidum [Syn. Polygonum flaccidum], T5106 Polygonum lapathifolium, T5107 Polygonum multiflorum, T5120 Polygonum tinctorium, T5157 Populus pseudo-simonii, T5167 Porana racemosa, T5174 Portulaca pilosa, T5193 Pratia nummularia, T5217 Prunus armeniaca, T5220 Prunus davidiana, T5221 Prunus davidiana, T5232 Prunus persica, T5235 Prunus persica, T5272 Psorospermum febrifugum, T5411 Randia spinosa, T5484 Rhinoceros unicornis; Rhinoceros sondaicus; Rhinoceros sumatrensis, T5537 Rhus sylvestris, T5563 Rosa cymosa, T5606 Rumex acetosa, T5626 Ruta graveolens, T5642 Saccharum sinensis, T5721 Sapindus mukorossi, T5850 Sedum aizoon, T5855 Sedum kamtschaticum, T5857 Sedum sarmentosum, T5862 Selaginella involvens, T5870 Selaginella uncinata, T5938 Setaria italica, T5977 Smilax glabra, T5978 Smilax glauco-china, T5980 Smilax menispermoidea, T6039 Sophora moorcroftiana, T6048 Sophora viciifolia, T6177 Strychnos ignatii, T6359 Teucrium bidentatum, T6372 Thalictrum acutifolium, T6373 Thalictrum alpinum, T6381 Thalictrum faberi, T6386 Thalictrum foetidum, T6388 Thalictrum fortunei, T6403 Thalictrum petaloideum, T6410 Thalictrum smithii, T6431 Thesium chinense, T6482 Toxicodendron succedaneum [Syn. Rhus succedanea], T6492 Trapa bispinosa, T6548 Tritonia crocosmaeflora, T6651 Urtica cannabina, T6652 Urtica dioica, T6673 Vaccinium vitis-idaea, T6709 Verbena officinalis, T6713 Vernonia anthelmintica, T6728 Veronica serpyllifolia, T6799 Vitis vinifera, T6820 Wikstroemia indica, T6827 Wisteria sinensis, T6831 Woodwardia orientalis, T6843 Xanthium sibiricum [Syn. Xanthium strumarium], T6893 Zanthoxylum simulans, T6894 Zanthoxylum simulans, T6912 Zinnia elegans.

resolve toxin and close sores T2188 Dioscorea alata, T2658 Fagopyrum esculentum, T4109 Marchantia polymorpha, T4487 Olea europaea, T5308 Pterocarya stenoptera, T5395 Rabdosia nervosa, T6746 Vicia faba.

resolve toxin and cure sores T0976 Bombyx mori, T1950 Cynanchum ascyrifolium, T1951 Cynanchum atratum, T1952 Cynanchum auriculatum, T1953 Cynanchum bungei, T1962 Cynanchum versicolor, T2428 Erinaceus europaeus; Hemiechinus daunicus; Hemiechinus auritus, T2952 Gerbera piloselloides, T3851 Lindera glauca, T5010 Platycarya strobilacea, T6439 Thuja orientalis [Syn. Platycladus orientalis; Biota orientalis], T6582 Tylophora ovata.

resolve toxin and disinhibit damp T1819 Crotalaria ferruginea, T4000 Lysimachia congestiflora, T4656 Parmelia saxatilis, T5126 Polypodium virginianum, T5127 Polypodium vulgare, T6075 Spilanthes acmella, T6488 Trachyspermum ammi, T6526 Triglochin maritimum, T6788 Vitex negundo.

resolve toxin and disinhibit throat T0586 Arctium lappa, T0975 Bombyx mori, T5449 Reineckea carnea.

resolve toxin and disperse swelling T0001 Abelmoschus manihot, T0015 Abutilon indicum, T0047 Acanthus ebracteatus, T0069 Achillea wilsoniana, T0098 Aconitum geniculatum, T0156 Actinidia arguta, T0161 Actinidia eriantha, T0226 Agave sisalana, T0424 Ampelopsis brevipedunculata var. hancei, T0571 Aralia decaisneaa, T0625 Aristolochia contorta, T0627 Aristolochia debilis [Syn. Aristolochia longa], T0852 Bacopa monniera, T0871 Barleria lupulina, T0922 Berchemia polyphylla var. leioclada, T0933 Betula platyphylla, T0935 Betula platyphylla var. japonica, T0959 Blumea lacera, T0977 Bombyx mori, T0984 Bos taurus domesticus; Bubalus bubalis, T1001 Brachystemma calycinum, T1021 Brassica rapa, T1100 Caesalpinia crista, T1241 Cassia occidentalis, T1256 Castanea mollissima, T1275 Cayratia japonica, T1286 Celastrus flagellaris, T1289 Celastrus orbiculatus [Syn. Celastrus articulatus], T1290 Celastrus orbiculatus [Syn. Celastrus articulatus], T1311 Centella asiatica, T1359 Chenopodium album, T1395 Chrysanthemum morifolium [Syn. Dendranthema morifolium], T1450 Cirsium lineare, T1547 Clematis terniflora [Syn. Clematis maximowicziana], T1561 Clerodendron serratum, T1565 Clerodendrum bungei, T1569 Clerodendrum serratum var. amplexifolium, T1573 Clinopodium chinense, T1660 Coprinus atramentarius, T1813 Crotalaria albida, T1880 Cucurbita moschata, T1884 Cudrania cochinchinensis, T1928 Cycas revoluta, T2161 Dicranopteris pedata [Syn. Polypodium pedatum; Dicranopteris dichotoma], T2205 Dioscorea panthaica, T2213 Dioscorea zingiberensis, T2301 Dysosma majorensis [Syn. Podophyllum majorense; Dysosma lichuanensis], T2336 Elephas maximus, T2348 Embelia ribes, T2365 Enteromorpha clathrata, T2435 Eriocheir sinensis, T2442 Ervatamia divaricata, T2536 Euonymus alatus, T2586 Euphorbia esula var. cyparissoides, T2716 Ficus beecheyana [Syn. Ficus erecta var. beecheyana], T2717 Ficus carica, T2723 Ficus pumila, T2753 Formica fusca, T2946 Geranium robertianum, T2950 Gerbera anandria [Syn. Leibnitzia anandria], T2984 Glinus lotoides [Syn. Mollugo lotoides], T2998 Glycine max, T3080 Gymnema sylvestre, T3163 Helicteres angustifolia, T3193 Hemerocallis fulva, T3195 Hemerocallis lilio-asphodelus, T3197 Hemerocallis minor, T3248 Hibiscus tiliaceus, T3311 Hydrocotyle sibthorpioides, T3349 Hypericum erectum, T3479 Isodon amethystoides, T3680 Lamium amplexicaule, T3687 Lantana camara, T3778 Leucaena glauca [Syn. Leucaena leucocephala], T3808 Ligularia japonica [Syn. Arnica japonica; Senecio japonica], T3830 Ligustrum sinense, T3847 Lindera angustifolia, T3865 Lippia nodiflora, T3882 Lithospermum officinale, T3932 Ludwigia octovalvis, T3983 Lycoris aurea, T4085 Mallotus repandus var. chrysocarpus [Syn. Mallotus chrysocarpus; Mallotus repandus], T4104 Manihot esculenta, T4131 Maytenus confertiflorus, T4203 Mesua ferrea, T4261 Mollugo pentaphylla, T4323 Murraya paniculata [Syn. Chalcas paniculata], T4325 Murraya paniculata var. exotica, T4470 Ocimum basilicum, T4519 Opuntia dillenii, T4521 Opuntia vulgaris, T4549 Osbeckia chinensis, T4564 Oxalis corniculata [Syn. Oxalis repens], T4577 Paederia scandens, T4678 Paulownia fortunei, T4679 Paulownia tomentosa, T4704 Peperomia duclouxii, T4813 Phlomis tuberosa, T4937 Piper betle, T4956 Piper mullesua, T4978 Pisolithus tinctorius [Syn. Lycoperdon capitatum; Scleroderma tinctorium], T5029 Plumbago zeylanica, T5086 Polygala sibirica, T5088 Polygala tenuifolia, T5111 Polygonum perfoliatum, T5288 Pteris cretica var. nervosa [Syn. Pteris nervosa], T5295 Pteris multifida, T5375 Quercus mongolica, T5401 Rabdosia yuennanensis, T5562 Rosa chinensis, T5701 Salvia yunnanensis, T5750 Saururus chinensis, T5806 Schlumbergera truncata, T5813 Scilla scilloides, T5844 Scutellaria scordifolia, T5851 Sedum alfredii [Syn. Sedum formosanum], T5942 Sida acuta, T6017 Solanum tuberosum, T6023 Solidago virgaurea, T6024 Solidago virgaurea var. leiocarpa [Syn. Solidago decurrens], T6060 Souliea vaginata, T6080 Spiraea japonica, T6095 Stachytarpheta jamaicensis, T6101 Stauntonia 
hexaphylla, T6127 Stephania glabra, T6183 Strychnos nitida, T6264 Syzygium buxifolium, T6429 Thermopsis lupinoides, T6444 Thunbergia grandiflora, T6580 Tylophora floribunda, T6744 Vicia faba, T6834 Wrightia tomentosa, T6883 Zanthoxylum myriacanthum, T6904 Zephyranthes grandiflora [Syn. Zephyranthes carinata].

resolve toxin and disperse welling abscess T6752 Vigna angularis [Syn. Dolichus angularis; Phaseolus angularis].

resolve toxin and dissipate binds T0382 Althaea rosea, T1049 Bufo bufo gargarizans; Bufo melanostictus, T1050 Bufo bufo gargarizans; Bufo melanostictus, T1826 Crotalaria mucronata, T2028 Daphne odora, T2787 Fritillaria hupehensis, T2980 Gleditsia sinensis [Syn. Gleditsia horrida], T3632 Knoxia valerianoides, T3984 Lycoris chinensis, T3986 Lycoris radiata [Syn. Amaryllis radiata], T4186 Mentha piperita, T4835 Phyllanthus emblica, T4861 Phytolacca americana [Syn. Phytolacca decandra], T4864 Phytolacca esculenta [Syn. Phytolacca acinosa], T5647 Sagittaria sagittifolia, T5817 Scolopendra subspinipes mutilans, T5826 Scrophularia buergeriana, T5828 Scrophularia ningpoensis, T5975 Smilax bockii, T5982 Smilax sieboldii, T5984 Smilax stans [Syn. Smilax vaginata var. stans].

resolve toxin and draw out pus T0215 Agave americana.

resolve toxin and eliminate damp T2325 Eichhornia crassipes, T5339 Pycnoporus sanguineus, T5413 Ranunculus cantoniensis, T6491 Trametes cinnabarina [Syn. Polyporus cinnabarinus; Boletus cinnabarinus].

resolve toxin and eliminate inflammation T1869 Cryptotaenia japonica, T6808 Waltheria americana.

resolve toxin and eliminate welling abscess T3980 Lycopus lucidus, T5604 Rudbeckia laciniata, T5943 Sida cordifolia, T5976 Smilax china [Syn. Smilax japonica].

resolve toxin and engender flesh T2337 Elephas maximus, T4578 Paederia scandens.

resolve toxin and interrupt malaria T1039 Bruguiera gymnorrhiza. resolve toxin and kill worms T0250 Agrimonia pilosa var. japonica, T0311 Allium cepa, T0468 Anemone hupehensis, T0500 Anguilla japonica, T1038 Brucea javanica [Syn. Brucea sumatrana; Rhus javanica], T1046 Buddleja davidii, T1205 Carica papaya, T1285 Celastrus angulatus, T1372 Chloranthus serratus, T1719 Corydalis incisa, T1733 Corydalis racemosa, T2023 Daphne genkwa, T2521 Eucalyptus tereticornis, T2589 Euphorbia helioscopia, T2975 Gleditsia delavayi, T2976 Gleditsia fera, T2999 Glycine max, T3057 Gossypium herbaceum, T3460 Iris halophila, T3464 Iris lactea var. chinensis [Syn. Iris pallasii var. chinensis], T4247 Millingtonia hortensis, T4358 Myroxylon pereirae, T4428 Nicotiana tabacum, T4830 Phryma leptostachya, T4882 Picrasma quassioides [Syn. Picrasma ailanthoides], T5143 Pongamia pinnata, T5298 Pteris vittata, T5330 Punica granatum, T5384 Quisqualis indica, T5385 Quisqualis indica, T5391 Rabdosia eriocalyx, T5428 Rauvolfia yunnanensis, T5717 Sapindus delavayi [Syn. Pancovia delavayi], T5724 Sapium sebiferum, T6540 Tripterygium hypoglaucum, T6541 Tripterygium regelii, T T6542 Tripterygium wilfordii, 6764 Vinegar.

resolve toxin and outthrust papules T0648 Arnebia euchroma, T0649 Arnebia guttata, T3881 Lithospermum erythrorhizon, T6145 Stichopus japonicus.

resolve toxin and relieve itch $\mathrm{T} 1373$ Chloranthus serratus, T2505
Eucalyptus citriodora, T3371 Hypocrella bambusae, T4307 Mosla scabra [Syn. Mosla punctata].

resolve toxin and relieve pain T0730 Asarum sagittarioides, T2538

Euonymus bungeanus.

resolve toxin and repel foulness T4385 Narcissus tazetta var. chinensis. resolve toxin and settle pain T4506 Ophioglossum vulgatum, T6588

Typhoniun giganteum.

resolve toxin and transform damp T1546 Clematis tangutica.

resolve toxin of fish and crab T1486 Citrus junos, T4714 Perilla frutescens, T4718 Perilla frutescens var. acuta [Syn. Perilla frutescens var. purpurascens], T4721 Perilla frutescens var. arguta.

resolve toxin of fish and meat T0472 Anethum graveolens.

restore pulse and stem desertion T4599 Panax ginseng [Syn. Panax schinseng].

return fire to its source T1439 Cinnamomum cassia [Syn. Cinnamomum aromaticum].

return yang and free vessels T6909 Zingiber officinale.

return yang and treat collapse T0083 Aconitum carmichaeli, T0085

Aconitum carmichaeli $\mathrm{cv}$.

rough essence T0977 Bombyx mori.

rough essence and arrest emission T2838 Gallus gallus domesticus.

rough essence and stem desertion T1698 Cornus officinalis [Syn.

Macrocarpium officinale].

secure essence T0360 Alpinia oxyphylla, T4401 Nelumbo nucifera, T5244

Prunus tomentosa.

secure essence 'nd reduce urine T5569 Rosa multiflora.

secure essence and reduce urine T1911 Cuscuta australis, T1912 Cuscuta chinensis, T1913 Cuscuta japonica, T5568 Rosa laevigata, T5593 Rubus idaeus.

secure essence $q i \quad$ T6670 Vaccinium bracteatum.

secure $q \boldsymbol{i}$ and rough essence T5374 Quercus infectoria.

secure teeth T6498 Tribulus terrestris.

settle asthma T1176 Cannabis sativa, T5414 Ranunculus japonicus, T5517 Rhododendron molle.

settle fright T1045 Bubalus bubalis, T2091 Delphinium yunnanense, T5289 Pteris dactylina, T5299 Pteris wallichinan, T5484 Rhinoceros unicornis; Rhinoceros sondaicus; Rhinoceros sumatrensis, T6145 Stichopus japonicus.

settle fright epilepsy T0088 Aconitum coreanum.

settle pain T0096 Aconitum forrestii [Syn. Aconitum likiangense], T0183 Adlumia cirrhosa [Syn. Adlumia fungosa], T0542 Apis cerana, T1328 Ceratostigma plumbaginoides, T1329 Ceratostigma willmottianum, T2092 Delphinus delphis, T2154 Dicentra spectabilis, T2420 Ericerus pela, T2495 Eschscholzia californica, T3594 Juniperus rigida, T4418 Nerium indicum, T4625 Papaver commutatum [Syn. Papaver rhoeas], T4629 Papaver nudicaule, T4630 Papaver nudicaule ssp. amurense, T4631 Papaver nudicaule var. chinense, T5202 Primula obconica, T5414 Ranunculus japonicus, T6028 Sophora alopecuroides, T6132 Stephania sasakii, T6889 Zanthoxylum podocarpum.

settle pain and calm T1716 Corydalis gigantea.

settle pain and relieve cough $\mathrm{T} 1357$ Chelidonium majus. settle pain and resolve tetany $\mathrm{T} 3328$ Hyoscyamus niger. settle pain and suppress cough T2968 Glaucium fimbrilligerum. 
settle tetany T6457 Tilia japonica, T6458 Tilia miqueliana.

soften hardness T1385 Chorda filum, T5740 Sargassum vachellianum.

soften hardness and disperse swelling T4555 Ostrea rivularis; Ostrea

talienwhanensis; Ostrea gigas.

soften hardness and dissipate binds T0275 Akebia quinata, T0847

Baccharis indica [Syn. Pluchea indica], T2321 Ecklonia kurome, T2359

Endarachne binghamiae, T2365 Enteromorpha clathrata, T3678

Laminaria japonica, T4403 Nemacystus decipiens [Syn. Mesogloea

decipiens; Cladosiphon decipiens], T6640 Undaria pinnatifida, T6925

Zostera marina.

soften hardness and transform phlegm T5309 Pterocladia tenuis.

soothe channels and network vessels T6689 Ventilago leiocarpa.

soothe channels and quicken blood T4018 Machilus thunbergii.

soothe channels and quicken network vessels T4238 Millettia nitida,

T6504 Tricholoma matsutake [Syn. Armillaria matsutake].

soothe depressed liver $\boldsymbol{q} i$ T2761 Fortunella margarita.

soothe liver T0201 Aesculus chinensis, T0202 Aesculus hippocastanum,

T0205 Aesculus wilsonii, T1469 Citrus aurantium var. amara, T2644

Evodia rutaecarpa, T2646 Evodia rutaecarpa var. officinalis, T4163

Melia toosendan, T5226 Prunus mume.

soothe liver and disinhibit gallbladder T1406 Chrysosplenium nudicaule. soothe liver and harmonize stomach T5362 Pyrus bretschneideri, T5366 Pyrus pyrifolia.

soothe liver and move $q i \quad \mathrm{~T} 2432$ Eriobotrya japonica.

soothe liver and quicken network vessels T3867 Liquidambar formosana [Syn. Liquidambar taiwaniana].

soothe liver and resolve depression T3554 Jasminum grandiflorum.

soothe liver and upbear yang T1055 Bupleurum angustissimum, T1056

Bupleurum aureum, T1058 Bupleurum chaishoui, T1059 Bupleurum

chinense, T1065 Bupleurum longiradiatum, T1066 Bupleurum

marginatum, T1067 Bupleurum marginatum var. stenophyllum, T1072

Bupleurum scorzonerifolium, T1073 Bupleurum sibiricum, T1075

Bupleurum smithii var. parvifolium, T1078 Bupleurum wenchuanense,

T1079 Bupleurum yinchowense.

soothe sinews and free network vessels T3976 Lycopodium obscurum, T3989 Lygodium flexuosum [Syn. Lygodium pinnatifidum; Ophioglossum flexuosum].

soothe sinews and joint bones T6540 Tripterygium hypoglaucum.

soothe sinews and quicken blood T0045 Acanthopanax trifoliatus, T0282 Alangium kurzii, T0414 Ammopiptanthus mongolicus [Syn. Piptanthus mongolicus], T0964 Boenninghausenia albiflora, T1841 Croton caudatus var. tomentosus, T3125 Hedyotis chrysotricha [Syn. Oldenlandia chrysotricha], T3320 Hymenocallis littoralis [Syn. Hymenocallis americana; Pancratium littoralis], T3808 Ligularia japonica [Syn. Arnica japonica; Senecio japonica], T3965 Lycopodium annotinum, T3969 Lycopodium casuarinoides, T3970 Lycopodium cernuum, T3971 Lycopodium complanatum, T3973 Lycopodium japonicum [Syn. Lycopodium clavatum], T4575 Pachysandra terminalis, T4975 Piptanthus nanus, T5100 Polygonum chinense, T5797 Schisandra propinqua, T5798 Schisandra propinqua var. intermedia, T6069 Speranskia tuberculata, T6773 Viscum articulactum, T6774 Viscum articulatum.

soothe sinews and quicken network vessels T0281 Alangium chinense,
T0285 Alangium platanifolium, T0569 Aralia cordata, T0574 Aralia fargesii, T1342 Chaenomeles lagenaria [Syn. Chaenomeles speciosa], T1922 Cyanotis vaga, T2211 Dioscorea tenuipes, T2459 Erythrina arborescens, T2478 Erythrina variegata [Syn. Erythrina indica], T2540 Euonymus fortunei, T2727 Ficus simplicissima, T3614 Kadsura heteroclita [Syn. Uvaria heteroclita], T3653 Lactarius piperatus [Syn. Agaricus piperatus], T3657 Lactarius vellereus, T4147 Medicago falcata, T4239 Millettia nitida var. hirsutissima, T5279 Psychotria serpens, T5298 Pteris vittata, T5638 Sabia swinhoei, T5860 Selaginella davidii, T6422 Thelephora vialis, T6468 Tinospora sinensis, T6830 Woodfordia fruticosa.

soothe sinews and relieve pain T2273 Drynaria fortunei, T5252 Pseudodrynaria coronans.

soothe stagnation and move $\boldsymbol{q} i \mathrm{~T} 1146$ Camellia oleifera, T1492 Citrus limon, T1496 Citrus limonia.

soothe wind and brighten eyes $\mathrm{T} 2409$ Equisetum palustre.

soothe wind and drain heat T6024 Solidago virgaurea var. leiocarpa [Syn. Solidago decurrens].

soothe wind and rectify $q \boldsymbol{i} \mathrm{T} 1533$ Clausena dentata, T1534 Clausena dunniana.

soothe wind and resolve exterior T2510 Eucalyptus globulus, T2517 Eucalyptus robusta.

stanch bleeding T0156 Actinidia arguta, T0170 Adhatoda vasica, T0215 Agave americana, T0229 Ageratum conyzoides, T0324 Allium victorialis, T0328 Alnus japonica, T0371 Alstonia mairei, T0542 Apis cerana, T0544 Apium graveolens, T0830 Auricularia auricula, T0831 Auricularia delicata, T0843 Babylonia lutosa, T0881 Bauhinia variegata, T0973 Bombax malabaricum [Syn. Gossampinus malabarica], T0976 Bombyx mori, T0977 Bombyx mori, T0993 Boschniakia rossica, T1033 Broussonetia papyrifera, T1044 Bryum argenteum, T1087 Buxus bodinieri, T1171 Canis familiaris, T1174 Cannabis sativa, T1210 Carpesium abrotanoides, T1254 Castanea mollissima, T1336 Ceriops tagal [Syn. Rhizophora tagal], T1452 Cissampelos pareira, T1453 Cissampelos pareira var. hirsute, T1592 Cocos nucifera, T1656 Conyza bonariensis [Syn. Erigeron bonariensis; Erigeron linifolius; Erigeron crispus], T1682 Cordyceps ophioglossoides, T1866 Cryptolepis sinensis, T1969 Cynoglossum officinale, T1982 Cyprinus carpio, T1994 Daemonorops draco, T2157 Dichotomanthes tristaniaecarpa, T2253 Dracaena cochinchinensis, T2327 Elaeagnus angustifolia, T2407 Equisetum arvense, T2420 Ericerus pela, T2426 Erigeron sumatrensis, T2567 Eupatorium odoratum, T2659 Fagopyrum esculentum, T2734 Fissistigma glaucescens [Syn. Melodorum glaucescens], T2825 Galeobdolon chinense [Syn. Lamium chinense], T2841 Gallus gallus domesticus, T2857 Garcinia hanburyi, T2882 Gardenia jasminoides [Syn. Gardenia florida], T3054 Gossypium barbadense], T3055 Gossypium herbaceum, T3058 Gossypium hirsutum [Syn. Gossypium mexicanum], T3088 Gynura segetum [Syn. Gynura japonica], T3285 Hovenia dulcis, T3294 Huperzia selago [Syn. Lycopodium selago], T3295 Huperzia serrata [Syn. Lycopodium serratum], T3324 Hymenophyllum barbatum, T3528 Isodon ternifolia, T3529 Isodon ternifolius, T3560 Jatropha curcas, T3701 Lasiosphaera fenzlii, T3764 Lepisorus thunbergianus, T3765 Lepisorus ussuriensis, T3769 Lespedeza bicolor, T3851 Lindera glauca, T3866 Liquidambar formosana [Syn. 
Liquidambar taiwaniana], T3887 Litsea glutinosa, T3961 Lycoperdon pyriforme, T3989 Lygodium flexuosum [Syn. Lygodium pinnatifidum; Ophioglossum flexuosum], T4080 Mallotus apelta, T4101 Mangifera indica, T4121 Marsilea quadrifolia, T4126 Matteuccia orientalis, T4127 Matteuccia struthiopteris, T4178 Melodinus hemsleyanus, T4343 Myrica esculent, T4401 Nelumbo nucifera, T4494 Onopordum acanthium, T4502 Onychium japonicum [Syn. Tricomanes japonicum], T4656 Parmelia saxatilis, T4705 Peperomia pellucida, T4787 Phellinus igniarius, T4978 Pisolithus tinctorius [Syn. Lycoperdon capitatum; Scleroderma tinctorium], T5043 Podocarpus macrophyllus, T5045 Podocarpus macrophyllus var. maki, T5099 Polygonum bistorta, T5105 Polygonum hydropiper var. flaccidum [Syn. Polygonum flaccidum], T5109 Polygonum nodosum, T5138 Poncirus trifoliata, T5185 Potentilla multifida, T5228 Prunus mume, T5250 Psammosilene tunicoides, T5265 Psidium guajava, T5284 Pteridium aquilinum var. latiusculum, T5299 Pteris wallichinan, T5328 Punica granatum, T5332 Punica granatum, T5339 Pycnoporus sanguineus, T5344 Pyrola atropurpurea, T5345 Pyrola calliantha [Syn. Pyrola rotundifolia ssp. chinensis], T5346 Pyrola calliantha var. tibetana, T5347 Pyrola decorata, T5349 Pyrola incarnata, T5350 Pyrola japonica, T5351 Pyrola rotundifolia, T5372 Quercus dentata, T5374 Quercus infectoria, T5389 Rabdosia coetsa, T5411 Randia spinosa, T5419 Raphanus sativus, T5497 Rhodiola kirilowii, T5524 Rhododendron simsii, T5552 Robinia pseudoacacia, T5563 Rosa cymosa, T5579 Rubia cordifolia, T5587 Rubus alceaefolius, T5592 Rubus hirsutus, T5594 Rubus parkeri, T5609 Rumex hastatus, T5687 Salvia prionitis, T5704 Sambucus nigra, T5707 Sambucus williamsii, T5746 Sassafras tzumu, T5804 Schizonepeta tenuifolia [Syn. Nepeta tenuifolia], T5833 Scutellaria amoena, T5834 Scutellaria baicalensis, T5839 Scutellaria hypericifolia, T5841 Scutellaria likiangensis, T5843 Scutellaria rehderiana, T5845 Scutellaria viscidula, T5850 Sedum aizoon, T5855 Sedum kamtschaticum, T5856 Sedum lineare [Syn. Sedum obtuso-lineare], T5862 Selaginella involvens, T5863 Selaginella moellendorffii, T5866 Selaginella sanguinolenta, T5867 Selaginella sinensis, T5870 Selaginella uncinata, T5883 Senecio cannabifolius, T6026 Sonchus asper [Syn. Sonchus oleraceus var. asper], T6076 Spinacia oleracea, T6116 Stenoloma chusanum, T6491 Trametes cinnabarina [Syn. Polyporus cinnabarinus; Boletus cinnabarinus], T6494 Trema dielsiana, T6533 Trillium kamtschaticum, T6535 Trillium tschonoskii, T6728 Veronica serpyllifolia, T6745 Vicia faba, T6746 Vicia faba, T6747 Vicia faba, T6750 Vicia sativa, T6773 Viscum articulactum, T6774 Viscum articulatum.

stanch bleeding and close sores T2279 Dryopteris championii.

stanch bleeding and disperse stasis T2540 Euonymus fortunei, T3276 Homo sapiens.

stanch bleeding and disperse swelling T0374 Alstonia scholaris, T0378 Alstonia yunnanensis, T0661 Artemisia anomala, T1815 Crotalaria assamica, T4305 Mosla dianthera, T5614 Rumex patientia, T5840 Scutellaria indica.

stanch bleeding and dissipate stasis T1282 Cedrus deodara, T1928 Cycas revoluta, T1967 Cynoglossum amabile, T1986 Cyrtomium fortunei, T2435 Eriocheir sinensis, T3033 Gnetum parvifolium [Syn. Gnetum indicum], T3842 Limonium gmelinii, T4118 Marsdenia oreophila, T4402 Nelumbo nucifera, T4604 Panax japonicus var. bipinnatifidus, T4605
Panax japonicus var. major, T4607 Panax pseudo-ginseng var. japonicus, T4608 Panax pseudo-ginseng var. notoginseng [Syn. Panax notoginseng], T5104 Polygonum hydropiper, T5172 Portulaca grandiflora, T5835 Scutellaria barbata [Syn. Scutellaria rivularis], T6471 Toddalia asiatica [Syn. Toddalia aculeata; Paullinia asiatica], T6482 Toxicodendron succedaneum [Syn. Rhus succedanea], T6705 Verbascum thapsus, T6764 Vinegar.

stanch bleeding and engender flesh T5288 Pteris cretica var. nervosa [Syn. Pteris nervosa], T5468 Rheum emodi [Syn. Rheum australe], T6874 Zanthoxylum dimorphophyllum var. spinifolum.

stanch bleeding and joint bones T4346 Myrica nagi [Syn. Podocarpus nagi].

stanch bleeding and kill worms T2866 Garcinia morella, T4026 Macrothelypteris oligophlebia, T5871 Selenarctos thibetanus; Ursus arctos.

stanch bleeding and relieve pain T0298 Alectoria vivens, T1120 Callicarpa macrophylla, T1747 Corydalis thalictrifolia, T5118 Polygonum suffultum, T6883 Zanthoxylum myriacanthum.

stanch bleeding and settle pain T0922 Berchemia polyphylla var. leioclada, T2008 Dalbergia odorifera, T2013 Dalbergia sissoo, T3460 Iris halophila, T3464 Iris lactea var. chinensis [Syn. Iris pallasii var. chinensis], T3776 Lethariella zahlbruckneri, T5301 Pterocarpus indicus, T6615 Uncaria gambir.

stanch bleeding and transform phlegm T1683 Cordyceps sinensis.

strengthen bones T2841 Gallus gallus domesticus.

strengthen essence and enrich kidney T0572 Aralia elata.

strengthen heart T0184 Adonis amurensis, T0533 Antiaris toxicaria [Syn. Ambora toxicaria], T1674 Corchorus capsularis, T6155 Strophanthus divaricatus, T6433 Thevetia neriifolia [Syn. Thevetia peruviana].

strengthen heart and calm T0190 Adonis sutchuenensis.

strengthen heart and disinhibit urine T1151 Camellia sinensis [Syn. Thea sinensis], T1991 Cytisus scoparius [Syn. Spartium scoparium], T2175 Digitalis lanata, T2177 Digitalis purpurea, T2452 Erysimum cheiranthoides, T4418 Nerium indicum, T5557 Rohdea japonica [Syn. Orontium japonicum].

strengthen heart and lower blood pressure T4682 Pedicularis muscicola. strengthen lumbus T6727 Veronica persica.

strengthen lumbus and knees T0990 Bos taurus domesticus; Bubalus bubalis, T1411 Cibotium barometz [Syn. Polypodium barometz], T2347 Embelia parviflora, T4974 Piper wallichii [Syn. Piper wallichii var. hupehense], T5722 Sapium japonicum, T6831 Woodwardia orientalis.

strengthen lumbus and legs T3389 Ilex cornuta.

strengthen sinews and bones T0036 Acanthopanax giraldii [Syn. Acanthopanax giraldii var. inermis; Eleutherococcus giraldii], T0038 Acanthopanax gracilistylus, T0042 Acanthopanax senticosus [Syn. Eleutherococcus senticosus], T0044 Acanthopanax sessiliflorus, T0073 Achyranthes bidentata, T0644 Armillaria mellea, T0645 Armillariella mellea, T0969 Boerhavia diffusa, T1337 Cervus nippon; Cervus elaphus, T1901 Curculigo orchioides, T1952 Cynanchum auriculatum, T1953 Cynanchum bungei, T1963 Cynanchum wallichii, T1964 Cynanchum wilfordii [Syn. Cynoctonum wilfordii], T2234 Dipsacus asperoides, T2235 Dipsacus japonicus, T2389 Epimedium acuminatum, T2390 Epimedium brevicornum, T2392 Epimedium davidii, T2394 Epimedium 
elongatum, T2398 Epimedium koreanum, T2401 Epimedium pubescens, T2402 Epimedium sagittatum, T2404 Epimedium sutchuenense, T2406 Epimedium wushanense, T2530 Eucommia ulmoides, T2531 Eucommia ulmoides, T2736 Fissistigma polyanthum, T2737 Flemingia philippinensis [Syn. Moghania philippinensis], T3199 Hemibarbus labeo, T3925 Loranthus parasiticus [Syn. Loranthus chinenis; Taxillus chinensis], T4260 Moghania philippinensis, T4283 Morinda officinalis, T4536 Orobanche coerulescens, T4729 Periploca sepium, T4828 Photinia serrulata, T5344 Pyrola atropurpurea, T5345 Pyrola calliantha [Syn. Pyrola rotundifolia ssp. chinensis], T5346 Pyrola calliantha var. tibetana, T5347 Pyrola decorata, T5349 Pyrola incarnata, T5350 Pyrola japonica, T5351 Pyrola rotundifolia, T5924 Sesamum indicum, T6670 Vaccinium bracteatum, T6689 Ventilago leiocarpa, T6771 Viscum album, T6772 Viscum angulatum, T6775 Viscum coloratum, T6798 Vitis vinifera.

strengthen sinews and lumbus T2386 Epigynum auritum.

strengthen spleen T1498 Citrus medica, T1520 Citrus wilsonii.

supplement blood T2347 Embelia parviflora, T4178 Melodinus hemsleyanus, T4438 Nostoc flagelliforme, T6066 Spatholobus suberectus.

supplement blood and enrich yin T5446 Rehmannia glutinosa [Syn. Rehmannia glutinosa f. huechingensis].

supplement blood and quicken blood T0474 Angelica acutiloba [Syn. Ligusticum acutilobum], T4238 Millettia nitida, T4239 Millettia nitida var. hirsutissima, T4308 Mucuna birdwoodiana.

supplement blood and soothe sinews T4311 Mucuna sempervirens.

supplement blood and stanch bleeding T4234 Millettia dielsiana.

supplement brain and dispel wind T0988 Bos taurus domesticus; Bubalus bubalis.

supplement brain and marrow T5924 Sesamum indicum.

supplement center and boost $\boldsymbol{q i}$ T3011 Glycyrrhiza aspera, T3013 Glycyrrhiza glabra, T3015 Glycyrrhiza inflata, T3016 Glycyrrhiza kansuensis, Т3021 Glycyrrhiza squamulosa, Т3022 Glycyrrhiza uralensis, T3023 Glycyrrhiza yunnanensis, T4548 Oryza sativa var. glutinosa, T4618 Panicum miliaceum, T4786 Phasianus colchicus, T6916 Ziziphus jujuba, T6917 Ziziphus jujuba var. inermis.

supplement center and harmonize blood T3447 Ipomoea batatas [Syn. Convolvulus batatas].

supplement essence and boost $q \boldsymbol{i}$ T4206 Metaplexis japonica.

supplement essence and replenish marrow T2837 Gallus gallus domesticus.

supplement fire and reinforce yang T0083 Aconitum carmichaeli, T0085 Aconitum carmichaeli cv, T1439 Cinnamomum cassia [Syn. Cinnamomum aromaticum].

supplement kidney T0322 Allium tuberosum, T2803 Frullania tamarisci ssp. moniliata [Syn. Frullania moniliata], T4057 Mahonia bealei, T4068 Mahonia japonica, T6150 Streptopelia orientalis.

supplement kidney and boost essence T0990 Bos taurus domesticus; Bubalus bubalis, T2753 Formica fusca, T3568 Juglans regia, T4207 Metaplexis japonica.

supplement kidney and disinhibit water T6527 Trigonella caerulea. supplement kidney and fortify brain T4432 Nigella glandulifera. supplement kidney and invigorate yang T0977 Bombyx mori, T0993
Boschniakia rossica, T1337 Cervus nippon; Cervus elaphus, T1455 Cistanche deserticola, T1456 Cistanche salsa, T1900 Curculigo capitulata [Syn. Leucojum capitulata], T1901 Curculigo orchioides, T1972 Cynomorium songaricum, T2389 Epimedium acuminatum, T2390 Epimedium brevicornum, T2391 Epimedium brevicornum, T2392 Epimedium davidii, T2394 Epimedium elongatum, T2398 Epimedium koreanum, T2401 Epimedium pubescens, T2402 Epimedium sagittatum, T2404 Epimedium sutchuenense, T2406 Epimedium wushanense, T4283 Morinda officinalis, T4536 Orobanche coerulescens, T4696 Penaeus orientalis, T4974 Piper wallichii [Syn. Piper wallichii var. hupehense], T5270 Psoralea corylifolia, T6257 Syngnathus acus.

supplement kidney and quiet spirit T0041 Acanthopanax senticosus [Syn. Eleutherococcus senticosus].

supplement kidney and rough essence T2190 Dioscorea batatas [Syn. Dioscorea opposita], T2202 Dioscorea japonica, T3770 Lespedeza cuneata, T6754 Vigna unguiculata.

supplement kidney and secure essence T0793 Astragalus complanatus, T5067 Polyalthia nemoralis.

supplement kidney and strengthen bones T2052 Davallia divaricata [Syn. Davallia formosana; Davallia orientalis], T2053 Davallia mariesii, T2273 Drynaria fortunei, T5252 Pseudodrynaria coronans.

supplement kidney and strengthen lumbus T0515 Anredera cordifolia [Syn. Baussingaultia cordifolia; Baussingaultia gracilis $\mathrm{f}$. pseudobaselloides; Baussingaultia gracilis var. pseudobaselloides], T1963 Cynanchum wallichii, T4642 Parameria laevigata.

supplement kidney and strengthen sinews T1254 Castanea mollissima. supplement liver T0986 Bos taurus domesticus; Bubalus bubalis, T6919 Ziziphus jujuba var. spinosa.

supplement liver and boost kidney T4656 Parmelia saxatilis, T5593 Rubus idaeus, T5925 Sesamum indicum [Syn. Sesamum orientale].

supplement liver and kidney T0038 Acanthopanax gracilistylus, T0042 Acanthopanax senticosus [Syn. Eleutherococcus senticosus], T0044 Acanthopanax sessiliflorus, T0073 Achyranthes bidentata, T0323 Allium tuberosum, T1698 Cornus officinalis [Syn. Macrocarpium officinale], T1911 Cuscuta australis, T1912 Cuscuta chinensis, T1913 Cuscuta japonica, T1964 Cynanchum wilfordii [Syn. Cynoctonum wilfordii], T2234 Dipsacus asperoides, T2235 Dipsacus japonicus, T2323 Eclipta prostrata [Syn. Eclipta alba], T2530 Eucommia ulmoides, T2531 Eucommia ulmoides, T2805 Fugu ocellatus, T3828 Ligustrum lucidum, T4279 Morina chinensis, T5927 Sesamum indicum [Syn. Sesamum orientale], T6670 Vaccinium bracteatum, T6775 Viscum coloratum, T6777 Viscum multinerve, T6831 Woodwardia orientalis.

supplement lung and boost kidney T1680 Cordyceps militaris, T1681 Cordyceps militaris cv, T1683 Cordyceps sinensis, T1952 Cynanchum auriculatum, T1953 Cynanchum bungei, T5409 Rana temporaria chensinensis; Rana amurensis, T5501 Rhodiola yunnanesis.

supplement lung and boost spleen T1191 Caragana sinica.

supplement lung and relieve cough T4276 Monotropa uniflora, T6267 Syzygium jambos.

supplement original $q \boldsymbol{i}$ greatly T4599 Panax ginseng [Syn. Panax schinseng], T4600 Panax ginseng [Syn. Panax schinseng], T4682 Pedicularis muscicola.

supplement qi T3279 Homo sapiens. 
supplement $q i$ and blood T0983 Bos taurus domesticus, T3743 Lentinus lepideus, T5179 Potentilla anserina, T5872 Selenarctos thibetanus; Ursus arctos, T6689 Ventilago leiocarpa, T6798 Vitis vinifera, T6803 Volvariella volvacea.

supplement $q i$ and clear lung T5499 Rhodiola sacra.

supplement $q i$ and disinhibit water T3129 Hedysarum polybotrys, T3199 Hemibarbus labeo.

supplement $q i$ and fortify spleen T4545 Oryza sativa.

supplement $q \boldsymbol{i}$ and nourish blood T0830 Auricularia auricula, T0831 Auricularia delicata, T1598 Codonopsis convolvulacea.

supplement qi and nourish yin T4610 Panax quinquefolium.

supplement $q i$ and quiet spirit T0572 Aralia elata.

supplement $q i$ and reinforce yang T4516 Oplopanax elatus.

supplement spleen and boost kidney T3992 Lyonia ovalifolia var. elliptica, T5236 Prunus pseudocerasus.

supplement spleen and boost lung T4599 Panax ginseng [Syn. Panax schinseng], T4600 Panax ginseng [Syn. Panax schinseng].

supplement spleen and boost $q \boldsymbol{i}$ T0529 Anthriscus sylvestris, T5091 Polygonatum cyrtonema [Syn. Polygonatum multiflorum], T5092 Polygonatum kingianum, T5095 Polygonatum sibiricum.

supplement spleen and check diarrhea T4400 Nelumbo nucifera. supplement spleen and engender liquid T6055 Sorbus tianschanica. supplement spleen and fortify stomach T4505 Ophiocephalus argus. supplement spleen and warm stomach $\mathrm{T} 1170$ Canis familiaris.

supplement stomach and spleen T0513 Annona squamosa, T0540 Apis cerana, T2195 Dioscorea deltoidea.

supplement vacuity T1181 Capra hircus; Ovis aries, T1182 Capra hircus; Ovis aries, T3085 Gynostemma pentaphyllum, T4275 Monotropa hypopitys, T4607 Panax pseudo-ginseng var. japonicus, T5407 Rana nigromaculata; Rana plancyi.

supplement vacuity and boost essence T3957 Lycium chinense.

supplement vacuity and boost kidney T2955 Geum japonicum, T2956 Geum japonicum.

supplement vacuity and fortify spleen T5075 Polygala fallax [Syn. Polygala aureocauda].

supplement vacuity and stanch bleeding T0923 Bergenia crassifolia. supplement vacuity detriment T0989 Bos taurus domesticus; Bubalus bubalis.

supplement yin T3389 Ilex cornuta.

suppress cough T0216 Agave americana var. marginata [Syn. Agave americana var. variegata], T0301 Aleuritopteris argentea, T0598 Ardisia japonica, T0752 Asparagus officinalis, T0784 Astilbe chinensis, T1087 Buxus bodinieri, T2036 Datura innoxia, T2041 Datura metel, T2046 Datura stramonium, T2157 Dichotomanthes tristaniaecarpa, T2268 Drosera rotundifolia, T2902 Gentiana algida, T4195 Menyanthes trifoliata, T4275 Monotropa hypopitys, T4516 Oplopanax elatus, T4625 Papaver commutatum [Syn. Papaver rhoeas], T4635 Papaver somniferum, T5721 Sapindus mukorossi, T6282 Tagetes patula, T6537 Tripterospermum japonicum, T6538 Tripterospermum taiwanense, T6729 Veronica spuria, T6766 Viola tricolor.

suppress cough and calm asthma T2037 Datura innoxia, T2038 Datura innoxia, T2042 Datura metel, T2044 Datura metel, T3040 Gomphrena globosa, T5989 Solanum aculeatissimum, T6014 Solanum surattense. suppress cough and dispel phlegm T1050 Bufo bufo gargarizans; Bufo melanostictus, T1927 Cycas revoluta, T2599 Euphorbia lunulata, T3394 Ilex paraguariensis.

suppress cough and dispel wind T1555 Clerodendron fortunatum, T6454 Thymus vulgaris.

suppress cough and interrupt malaria T3323 Hymenodictyon excelsum. suppress cough and relieve pain T3332 Hypecoum erectum. suppress cough and settle asthma T6075 Spilanthes acmella. suppress cough and transform phlegm T3026 Gnaphalium affine [Syn. Gnaphalium multiceps], T4247 Millingtonia hortensis.

terminate lactation T3282 Hordeum vulgare.

track wind T2979 Gleditsia sinensis [Syn. Gleditsia horrida].

track wind and dissipate cold T3657 Lactarius vellereus.

transform accumulation T0950 Blatta orientalis.

transform concretion and disperse accumulation T2695 Ferula assafoetida.

transform damp T2246 Dolichos lablab, T2554 Eupatorium cannabinum, T3242 Hibiscus rosa-sinensis, T4209 Michelia alba.

transform damp and check discharge T0999 Bougainvillea glabra.

transform damp and check dysentery T5149 Populus canadensis, T5150 Populus cathayana.

transform damp and disinhibit urine T2341 Elsholtzia ciliata. transform damp and disinhibit water T5103 Polygonum hydropiper.

transform damp and disperse stagnation T1784 Cratoxylum cochinchinense, T1785 Cratoxylum prunifolium.

transform damp and disperse swelling T4966 Piper sarmentosum.

transform damp and harmonize center T2563 Eupatorium japonicum, T4304 Mosla chinensis [Syn. Orthodon chinensis], T4470 Ocimum basilicum, T5389 Rabdosia coetsa.

transform damp and move $q \boldsymbol{i} \mathrm{T} 0144$ Acorus gramineus, T0146 Acorus tatarinowii, T0416 Amomum kravanh [Syn. Amomum cardamomum].

transform damp and repel foulness T6561 Tulipa gesneriana.

transform damp aromaticly T5059 Pogostemon cablin [Syn. Mentha cablin].

transform phlegm T0216 Agave americana var. marginata [Syn. Agave americana var. variegata], T1152 Camellia sinensis [Syn. Thea sinensis], T1210 Carpesium abrotanoides, T1472 Citrus chachiensis, T1478 Citrus grandis, T1488 Citrus junos, T1498 Citrus medica, T1517 Citrus tankan, T1520 Citrus wilsonii, T1526 Cladonia rangiferina, T2048 Daucus carota, T2426 Erigeron sumatrensis, T2790 Fritillaria pallidiflora, T2800 Fritillaria walujewii, T2994 Gloiopeltis furcata, T3878 Lithocarpus polystachyus, T4717 Perilla frutescens var. acuta [Syn. Perilla frutescens var. purpurascens], T4719 Perilla frutescens var. arguta, T4722 Perilla frutescens var. crispa, T5419 Raphanus sativus, T6729 Veronica spuria.

transform phlegm and calm asthma T1154 Camellia sinensis [Syn. Thea sinensis], T2565 Eupatorium lindleyanum, T4855 Physeter catodon, T5961 Sinocalamus oldhami.

transform phlegm and disinhibit damp T0048 Acanthus ilicifolius, T4549 Osbeckia chinensis.

transform phlegm and disperse accumulation T2365 Enteromorpha clathrata.

transform phlegm and dissipate binds T0617 Arisaema amurense, T0618 
Arisaema consanguineum, T0620 Arisaema heterophyllum, T0975 Bombyx mori, T2298 Dysosma difformis, T2302 Dysosma pleiantha [Syn. Podophyllum pleianthum], T2304 Dysosma veitchii, T2305 Dysosma versipellis [Syn. Podophyllum versipelle], T4903 Pinellia pedatisecta.

transform phlegm and expel rheum T5958 Sinapis alba [Syn. Brassica alba; Brassica hirta].

transform phlegm and interrupt malaria T6252 Symplocos chinensis. transform phlegm and open orifices T0142 Acorus calamus, T0144 Acorus gramineus, T0146 Acorus tatarinowii, T0463 Anemone altaica. transform phlegm and precipitate $q \boldsymbol{i}$ T5215 Prunus amygdalus. transform phlegm and quiet spirit T6056 Sorghum vulgare. transform phlegm and relieve cough T2119 Derris eriocarpa, T2752 Fordia cauliflora, T3006 Glycosmis citrifolia, T3520 Isodon rosthornii, T4231 Milingtonia hortensis, T4274 Monostroma nitidum, T5939 Shiraia bambusicola.

transform phlegm and resolve toxin T4840 Phyllanthus niruri.

transform phlegm and soften hardness T5171 Porphyra tenera, T6599 Ulva lactuca, T6600 Ulva pertusa.

transform rheum T4787 Phellinus igniarius.

transform stagnation T3674 Laggera alata, T4102 Mangifera indica.

transform stagnation and relieve pain T1232 Cassia fistula, T1836

Crotalaria tetragona, T4948 Piper hancei.

transform stasis T2748 Fomes fomentarius [Syn. Pyropolyporus fomentarius;

Boletus fomentarius; Polyporus fomentarius].

transform stasis and disperse accumulation T0945 Bischofia javanica [Syn.

Bischofia trifoliata].

transform stasis and disperse concretion T2132 Desmodium pulchellum

[Syn. Phyllodium pulchellum], T4134 Maytenus hookeri.

transform stasis and disperse swelling T1287 Celastrus hypoleucus.

transform stasis and dissipate binds T1160 Campsis grandiflora, T2541

Euonymus grandiflorus, T4704 Peperomia duclouxii.

transform stasis and move water T6154 Strobilanthes japonicus [Syn.

Championella japonica].

transform stasis and relieve pain T0853 Baeckea frutescens, T2136 Desmos cochinchinensis [Syn. Desmos chinensis], T5672 Salvia flava.

transform stasis and stanch bleeding T0776 Asplenium prolongatum,

T2161 Dicranopteris pedata [Syn. Polypodium pedatum; Dicranopteris

dichotoma], T2602 Euphorbia nematocypha, T3981 Lycopus lucidus,

T4606 Panax pseudo-ginseng, T5566 Rosa henryi, T5865 Selaginella

pulvinata, T5869 Selaginella tamariscina, T6551 Trogopterus xanthipes;

Pteromys volans.

treat lichen T3630 Kleinhovia hospita.

upbear yang T4398 Nelumbo nucifera.

upbear yang and check diarrhea T5312 Pueraria edulis, T5313 Pueraria

lobata [Syn. Pueraria thunbergiana; Pueraria pseudohirsuta], T5316

Pueraria omeiensis, T5318 Pueraria phaseoloides, T5320 Pueraria thomsonii.

upbear yang and effuse exterior T1991 Cytisus scoparius [Syn. Spartium scoparium].

upbear yang and raise fall T1419 Cimicifuga dahurica, T1420 Cimicifuga foetida, T1421 Cimicifuga heracleifolia, T1423 Cimicifuga nanchuanensis, T4601 Panax ginseng [Syn. Panax schinseng].

upbear yang and resolve exterior T5317 Pueraria peduncularis. warm center T0322 Allium tuberosum, T1168 Canavalia ensiformis, T1169 Canavalia gladiata, T2837 Gallus gallus domesticus, T3758 Lepidium sativum, T6261 Syringa pinnafolia, T6860 Zanthoxylum ailanthoides.

warm center and boost $q i$ T6147 Stizolobium capitatum.

warm center and check diarrhea T0957 Blumea balsamifera, T1258 Casuarina equisetifolia.

warm center and check vomiting T0416 Amomum kravanh [Syn. Amomum cardamomum].

warm center and dissipate cold T0099 Aconitum gymnandrum, T0491 Angelica polymorpha, T1008 Brassica juncea, T1186 Capsicum annuum, T1187 Capsicum frutescens, T2643 Evodia meliifolia, T3850 Lindera glauca, T4944 Piper cubeba, T4953 Piper longum, T4957 Piper nigrum, T4966 Piper sarmentosum, T6858 Zanthoxylum acanthopodium, T6909 Zingiber officinale.

warm center and downbear counterflow T0554 Aquilaria agallocha, T0555 Aquilaria sinensis, T6263 Syzygium aromaticum [Syn. Eugenia caryophyllata].

warm center and fortify stomach T4964 Piper retrofractum.

warm center and move $\boldsymbol{q} i \mathrm{~T} 3620$ Kaempferia galanga, T3852 Lindera megaphylla, T3853 Lindera obtusiloba, T3880 Lithospermum arvense, T4223 Micromelum integerrimum, T4351 Myristica fragrans, T4954 Piper longum.

warm center and move stagnation T0318 Allium sativum.

warm center and normalize $q i \quad \mathrm{~T} 4018$ Machilus thunbergii.

warm center and precipitate $q \boldsymbol{i} \mathrm{T} 1937$ Cydonia oblonga.

warm center and rectify $q \boldsymbol{i} \mathrm{T} 1435$ Cinnamomum camphora.

warm center and relieve pain T1443 Cinnamomum parthenoxylum [Syn. Cinnamomum porrectum], T1812 Crossostephium chinense, T1939 Cymbopogon citratus, T2087 Delphinium tatsienense, T3885 Litsea cubeba, T6229 Swertia patens, T6869 Zanthoxylum bungeanum, T6892 Zanthoxylum schinifolium.

warm center and supplement blood T5042 Podocarpus macrophyllus, T5044 Podocarpus macrophyllus var. maki.

warm center and supplement vacuity T4859 Physochlaina physaloides.

warm center and transform damp T0418 Amomum muricarpum, T4048 Magnolia rostrata.

warm channels T0077 Aconitum balfourii, T0084 Aconitum carmichaeli, T0105 Aconitum karakolicum.

warm channels and dissipate cold T0108 Aconitum kusnezoffii.

warm channels and free network vessels T1438 Cinnamomum cassia [Syn. Cinnamomum aromaticum], T1444 Cinnamomum tamala.

warm channels and relieve pain T0164 Actinidia polygama, T5541 Rhus verniciflua [Syn. Toxicadendron verniciflum].

warm kidney T0360 Alpinia oxyphylla, T4944 Piper cubeba, T4982 Pistacia vera, T6261 Syringa pinnafolia, T6528 Trigonella foenum-graecum.

warm kidney and disperse cold T2744 Foeniculum vulgare, T3854 Lindera strychnifolia [Syn. Lindera aggregata].

warm kidney and harmonize center T2745 Foeniculum vulgare. warm kidney and invigorate yang T1170 Canis familiaris, T1582 Cnidium monnieri, T5753 Saussurea gnaphaloides, T5755 Saussurea involucrata, T5757 Saussurea laniceps, T5759 Saussurea medusa, T6263 Syzygium aromaticum [Syn. Eugenia caryophyllata]. 
warm liver and kidney T1441 Cinnamomum japonicum.

warm lung T0752 Asparagus officinalis.

warm lung and settle asthma T3568 Juglans regia.

warm lung and transform rheum T0724 Asarum caulescens, T0727

Asarum fukienense, T0728 Asarum heterotropoides var. mandshuricum,

T0731 Asarum sieboldii, T0732 Asarum sieboldii var. seoulensis, T4550

Osmanthus fragrans, T6909 Zingiber officinale.

warm menstruation and quicken blood T1434 Cinnamomum bejolghota

[Syn. Cinnamomum obtusifolium; Laurus bejolghota].

warm menstruation and stanch bleeding T0664 Artemisia argyi, T0681

Artemisia lavandulaefolia, T0685 Artemisia mongolica, T0689 Artemisia

princeps, T0691 Artemisia rubripes, T0706 Artemisia vulgaris.

warm spleen T0360 Alpinia oxyphylla, T4982 Pistacia vera.

warm spleen and check diarrhea T5270 Psoralea corylifolia.

warm spleen and kidney T0472 Anethum graveolens.

warm spleen and stomach T1441 Cinnamomum japonicum.

warm stomach T0353 Alpinia blepharocalyx, T0356 Alpinia galanga,

T0359 Alpinia officinarum, T3120 Hedychium spicatum, T3250

Hierochloe odorata.

warm stomach and center $\mathrm{T} 3856$ Lindera umbellata [Syn. Lindera

erythrocarpa].

warm stomach and check vomiting T0358 Alpinia katsumadai.

warm stomach and dissipate cold T0354 Alpinia chinensis.

warm stomach and eliminate accumulation T0847 Baccharis indica [Syn.

Pluchea indica].

warm stomach and harmonize center T1436 Cinnamomum camphora.

warm stomach and regulate center T2343 Elsholtzia splendens.

warm stomach and relieve pain T5504 Rhododendron capitatum.

warm yang T6423 Theobroma cacao.

warm yang and disinhibit water T1649 Convallaria keiskei [Syn.

Convallaria majalis].

warm yang and dissipate cold T3409 Illicium verum. 


\section{TCM Plant Traditional Indication Index}

This index lists all 2528 normalized TCM traditional indications terms in English appeared in the encyclopedia in alphabetical order and the related plant code number (from T0001 to T6926) and Latin name follow the bold term immediately.

abdominal distention T0819 Atractylodes chinensis, T0821 Atractylodes japonica, T0823 Atractylodes lancea, T1493 Citrus limon, T1497 Citrus limonia, T2115 Dermatocarpon minitum, T2529 Euchresta strigillosa, T3289 Humulus lupulus, T3381 Hyptis suaveolens, T3758 Lepidium sativum, T4184 Mentha haplocalyx [Syn. Mentha canadaensis; Mentha arvensis var. haplocalyx; Mentha arvensis], T4190 Mentha spicata, T4852 Physalis peruviana, T6339 Tephrosia purpurea.

abdominal distention and constipation T4034 Magnolia biloba, T4045 Magnolia officinalis, T5223 Prunus humilis [Syn. Cerasus humilis], T5224 Prunus japonica [Syn. Cerasus japonica], T5225 Prunus japonica var. nakaii, T5457 Rhamnus davurica.

abdominal distention and diarrhea T3282 Hordeum vulgare, T4983 Pisum sativum.

abdominal distention and emaciation T4887 Picrorhiza kurrooa, T4888 Picrorhiza scrophulariiflora.

abdominal distention and pain T0357 Alpinia japonica, T0942 Biebersteinia heterostemon, T3886 Litsea euosma.

abdominal distention and torpid intake T2998 Glycine max.

abdominal distention with edema T5409 Rana temporaria chensinensis; Rana amurensis, T5966 Siphonostegia chinensis.

abdominal fullness with glomus and congestion T1546 Clematis tangutica.

abdominal lump glomus T0060 Achillea alpina [Syn. Achillea sibirica], T4662 Parthenocissus tricuspidata.

abdominal pain T0060 Achillea alpina [Syn. Achillea sibirica], T0197 Aegle marmelos, T0302 Alhagi pseudalhagi, T0631 Aristolochia kaempferi, T0963 Boehmeria siamensis, T1100 Caesalpinia crista, T1147 Camellia oleifera, T1258 Casuarina equisetifolia, T1357 Chelidonium majus, T1359 Chenopodium album, T1434 Cinnamomum bejolghota [Syn. Cinnamomum obtusifolium; Laurus bejolghota], T1535 Clausena excavata, T1537 Clausena lansium, T1555
Clerodendron fortunatum, T1586 Cocculus laurifolius, T1590 Cocculus trilobus [Syn. Cocculus sarmentosus], T1673 Corchorus capsularis, T1836 Crotalaria tetragona, T1931 Cyclea barbata, T1943 Cymbopogon goeringii, T2343 Elsholtzia splendens, T2444 Ervatamia heyneana, T2445 Ervatamia officinalis, T2529 Euchresta strigillosa, T2745 Foeniculum vulgare, T3120 Hedychium spicatum, T3221 Heracleum rapula, T3241 Hibiscus mutabilis, T3468 Iris sanguinea, T3614 Kadsura heteroclita [Syn. Uvaria heteroclita], T3619 Kadsura peltigera [Syn. Kadsura longipedunculata], T4348 Myrica rubra, T4544 Oryza sativa, T4580 Paeonia albiflora [Syn. Paeonia lactiflora], T4625 Papaver commutatum [Syn. Papaver rhoeas], T4636 Papaver somniferum, T4789 Phellodendron amurense, T4790 Phellodendron amurense var. wilsonii, T4791 Phellodendron chinense, T4792 Phellodendron chinense var. glabriusculum, T5268 Psidium guajava, T5460 Rhamnus frangula [Syn. Frangula alnus], T5604 Rudbeckia laciniata, T5865 Selaginella pulvinata, T5869 Selaginella tamariscina, T6049 Sophora viciifolia, T6787 Vitex negundo.

abdominal pain and clouded spirit T1052 Bufo bufo gargarizans; Bufo melanostictus.

abdominal pain and cramp T5363 Pyrus calleryana.

abdominal pain and diarrhea T0354 Alpinia chinensis, T0635 Aristolochia moupinensis, T1546 Clematis tangutica, T1745 Corydalis suaveolens [Syn. Corydalis sheareri], T1747 Corydalis thalictrifolia, T1775 Crataegus pinnatifida, T1778 Crataegus pinnatifida var. major, T1932 Cyclea racemosa, T2517 Eucalyptus robusta, T3554 Jasminum grandiflorum, T4154 Melaleuca leucadendra, T4261 Mollugo pentaphylla, T4718 Perilla frutescens var. acuta [Syn. Perilla frutescens var. purpurascens], T4721 Perilla frutescens var. arguta, T4957 Piper nigrum, T5469 Rheum hotaoense, T5727 Saposhnikovia divaricata [Syn. Ledebouriella seseloides], T6119 Stephania cepharantha, T6402 Thalictrum omeiense, T6410 Thalictrum smithii, T6861 Zanthoxylum ailanthoides, T6862 Zanthoxylum ailanthoides. 
abdominal pain and vomiting due to roundworm T6869 Zanthoxylum bungeanum, T6892 Zanthoxylum schinifolium.

abdominal pain due to ascariasis T3342 Hypericum bellum, T4127 Matteuccia struthiopteris.

abdominal pain due to stagnation T5541 Rhus verniciflua [Syn. Toxicadendron verniciflum], T5613 Rumex obtusifolius.

abdominal pain due to static blood T1640 Coniogramme japonica [Syn. Hemionitis japonica].

abdominal pain due to summerheat damage T3866 Liquidambar formosana [Syn. Liquidambar taiwaniana].

abdominal pain due to worm accumulation T0314 Allium fistulosum, T1632 Combretum yunnanensis, T2049 Daucus carota, T2546 Euonymus sacrosancta, T4156 Melia azedarach, T4157 Melia azedarach, T4162 Melia toosendan, T4163 Melia toosendan, T4542 Orthosiphon wulfenioides [Syn. Coleus wulfenioides], T4779 Pharbitis nil, T4780 Pharbitis purpurea, T5328 Punica granatum, T6476 Torilis japonica, T6858 Zanthoxylum acanthopodium.

abdominal pain glomus distention T0417 Amomum longiligulare, T0419 Amomum villosum, T0420 Amomum xanthioides.

aberratio mensium T1001 Brachystemma calycinum, T4607 Panax pseudo-ginseng var. japonicus.

abnormal increase of lipoxygenase T3106 Harpagophytum procumbens. abscess T0945 Bischofia javanica [Syn. Bischofia trifoliata], T5730 Sarcandra glabra [Syn. Chloranthus glaber].

absence of sweating T2343 Elsholtzia splendens, T2367 Ephedra equisetina, T2368 Ephedra gerardiana, T2369 Ephedra intermedia, T2372 Ephedra likiangensis, T2373 Ephedra minuta, T2375 Ephedra monosperma, T2379 Ephedra saxatilis, T2380 Ephedra sinica.

abundant and sticky phlegm T5521 Rhododendron przewalskii.

abundant phlegm T3427 Inula britannica, T3428 Inula britannica var. chinensis, T3434 Inula linariaefolia, T3758 Lepidium sativum, T4518 Oppopanax chironium, T4908 Pinus bungeana, T5502 Rhododendron anthopogonoides, T5519 Rhododendron mucronulatum.

abundant phlegm and asthma T3802 Ligularia dentata, T3805 Ligularia fischeri, T3807 Ligularia intermedia, T3813 Ligularia sibirica.

abundant phlegm and shortness of breath T6504 Tricholoma matsutake [Syn. Armillaria matsutake].

accumulated fire toxin T6043 Sophora subprostrata [Syn. Sophora tonkinensis].

accumulation and diarrhea T5420 Raphanus sativus.

accumulation and distention-fullness T2597 Euphorbia lathyris.

accumulation from phlegm T6105 Stellera chamaejasme.

accumulation with abdominal distention T1544 Cleistocalyx operculatus, T5573 Rosa sericea.

accumulation with abdominal pain T5472 Rheum officinale, T5474 Rheum palmatum, T5481 Rheum tanguticum.

accumulation-gathering T2384 Epicauta gorhami, T5229 Prunus persica.

accumulation-gathering and distention-fullness T3893 Litsea pungens. aching and weightiness of limbs T1342 Chaenomeles lagenaria [Syn. Chaenomeles speciosa].

aching cold in lumbus and knees T1441 Cinnamomum japonicum. aching in lumbus and knees T0041 Acanthopanax senticosus [Syn. Eleutherococcus senticosus], T0073 Achyranthes bidentata, T0569 Aralia cordata, T0574 Aralia fargesii, T1683 Cordyceps sinensis, T1698 Cornus officinalis [Syn. Macrocarpium officinale], T1952 Cynanchum auriculatum, T1953 Cynanchum bungei, T2540 Euonymus fortunei, T3199 Hemibarbus labeo, T3925 Loranthus parasiticus [Syn. Loranthus chinenis; Taxillus chinensis], T4279 Morina chinensis, T4283 Morinda officinalis, T4974 Piper wallichii [Syn. Piper wallichii var. hupehense], T5722 Sapium japonicum, T6484 Trachelospermum jasminoides.

aching in lumbus and legs T0045 Acanthopanax trifoliatus, T0568 Aralia chinensis, T0570 Aralia dasyphylla, T0571 Aralia decaisneaa, T2347 Embelia parviflora, T4308 Mucuna birdwoodiana, T6772 Viscum angulatum.

aching lumbus and tinnitus T4288 Morus alba.

aching pain in back and shoulder T1550 Cleome gynandra [Syn. Gynandropsis gynandra].

aching pain in joints T0470 Anemone raddeana, T1697 Cornus controversa [Syn. Bothrocaryum controversum], T2909 Gentiana crassicaulis, T2910 Gentiana dahurica, T2913 Gentiana kaufmanniana, T2919 Gentiana macrophylla, T2932 Gentiana siphonantha, T2934 Gentiana straminea, T2936 Gentiana tianschanica, T2937 Gentiana tibetica, T3970 Lycopodium cernuum, T3973 Lycopodium japonicum [Syn. Lycopodium clavatum], T4290 Morus alba, T4688 Peganum harmala, T5727 Saposhnikovia divaricata [Syn. Ledebouriella seseloides].

aching pain in limbs T0819 Atractylodes chinensis, T0821 Atractylodes japonica, T0823 Atractylodes lancea, T4655 Paris tetraphylla.

aching pain in lumbus and back T1337 Cervus nippon; Cervus elaphus, $\mathrm{T} 6831$ Woodwardia orientalis.

aching pain in lumbus muscle T0539 Apis cerana.

aching sinews and bones T0598 Ardisia japonica, T2438 Erodium stephanianum, T2657 Fagopyrum cymosum [Syn. Polygonum cymosum], T2943 Geranium nepalense, T2944 Geranium pratense, T2947 Geranium sibiricum, T2949 Geranium wilfordii, T3479 Isodon amethystoides.

aching sinews in limb joints T4950 Piper kadsura [Syn. Piper futokadsura].

acid vomiting T2841 Gallus gallus domesticus.

acne T1641 Conioselinum vaginatum.

acute appendicitis T1709 Corydalis bungeana, T3908 Lonicera bournei, T3909 Lonicera confusa, T3910 Lonicera fulvotomentosa, T3911 Lonicera hypoglauca, T3912 Lonicera japonica, T3914 Lonicera macranthoides, T3918 Lonicera similis, T5472 Rheum officinale, T5474 Rheum palmatum, T5481 Rheum tanguticum, T5730 Sarcandra glabra [Syn. Chloranthus glaber], T6554 Trollius macropetalus.

acute bacillary dysentery $\mathrm{T} 0130$ Aconitum sinomontanum, T5018 Pleuropterus ciliinervis, T5173 Portulaca oleracea, T5907 Senecio scandens [Syn. Senecio chinensis], T6032 Sophora flavescens [Syn. Sophora angustfolia], T6234 Swertia punicea.

acute bronchitis $\mathrm{T} 1943$ Cymbopogon goeringii, T3646 Kyllinga brevifolia, T5508 Rhododendron dauricum, T6454 Thymus vulgaris.

acute cholecystitis T1055 Bupleurum angustissimum, T1056 Bupleurum 
aureum, T1058 Bupleurum chaishoui, T1059 Bupleurum chinense, T1065 Bupleurum longiradiatum, T1066 Bupleurum marginatum, T1067 Bupleurum marginatum var. stenophyllum, T1072 Bupleurum scorzonerifolium, T1073 Bupleurum sibiricum, T1075 Bupleurum smithii var. parvifolium, T1078 Bupleurum wenchuanense, T1079 Bupleurum yinchowense, T1573 Clinopodium chinense, T5400 Rabdosia stracheyi.

acute conjunctivitis T1573 Clinopodium chinense, T1662 Coptis chinensis, T1663 Coptis chinensis var. brevisepala, T1664 Coptis deltoidea, T1669 Coptis omeiensis, T1670 Coptis teetoides [Syn. Coptis teeta], T1692 Coriaria sinica [Syn. Coriaria nepalensis], T2908 Gentiana cephalantha, T2921 Gentiana manshurica, T2928 Gentiana rigescens, T2930 Gentiana scabra, T2938 Gentiana triflora, T3432 Inula helianthus-aquatica, T3827 Ligustrum japonicum, T4121 Marsilea quadrifolia, T4789 Phellodendron amurense, T4790 Phellodendron amurense var. wilsonii, T4791 Phellodendron chinense, T4792 Phellodendron chinense var. glabriusculum, T6031 Sophora flavescens [Syn. Sophora angustfolia], T6095 Stachytarpheta jamaicensis, T6226 Swertia mileensis, T6231 Swertia pseudochinensis, T6260 Syringa oblata, T6412 Thalictrum squarrosum, T6554 Trollius macropetalus.

acute conjunctivitis nephelium T1747 Corydalis thalictrifolia, T4843 Phyllanthus urinaria.

acute dermitis T6414 Thalictrum thunbergii.

acute diarrhea T6260 Syringa oblata.

acute dysentery T3828 Ligustrum lucidum, T5344 Pyrola atropurpurea, T5345 Pyrola calliantha [Syn. Pyrola rotundifolia ssp. chinensis], T5346 Pyrola calliantha var. tibetana, T5347 Pyrola decorata, T5349 Pyrola incarnata, T5350 Pyrola japonica, T5351 Pyrola rotundifolia, T6554 Trollius macropetalus.

acute eczema T0916 Berberis thunbergii.

acute endometritis T2715 Fibraurea recisa.

acute enteritis T0060 Achillea alpina [Syn. Achillea sibirica], T0130 Aconitum sinomontanum, T1554 Clerodendron cyrtophyllum, T2590 Euphorbia hirta, T5018 Pleuropterus ciliinervis, T6599 Ulva lactuca, T6600 Ulva pertusa.

acute febrile diseases T5447 Rehmannia glutinosa [Syn. Rehmannia glutinosa f. huechingensis], T5484 Rhinoceros unicornis; Rhinoceros sondaicus; Rhinoceros sumatrensis.

acute fright wind T1210 Carpesium abrotanoides, T2890 Gastrodia elata, T5817 Scolopendra subspinipes mutilans.

acute gastritis T1101 Caesalpinia decapetala, T2422 Erigeron breviscapus, T6226 Swertia mileensis.

acute gastroenteritis T0501 Anisodus luridus, T0502 Anisodus tanguticus [Syn. Scopolia tangutica], T0881 Bauhinia variegata, T1785 Cratoxylum prunifolium, T2988 Glochidion eriocarpum, T3476 Isatis indigotica, T3613 Kadsura coccinea [Syn. Kadsura chenensis; Kadsura hainanensis], T4881 Picrasma quassioides [Syn. Picrasma ailanthoides], T5247 Przewalskia tangutica, T5847 Securidaca inappendiculata, T6135 Stephania succifera, T6469 Tithonia diversifolia.

acute glomerulonephritis T3416 Imperata cylindrica var. major. acute heart pain T0542 Apis cerana. acute hepatitis T0048 Acanthus ilicifolius, T0897 Berberis amurensis, T3416 Imperata cylindrica var. major, T5395 Rabdosia nervosa, T5607 Rumex crispus, T5957 Silybum marianum, T6119 Stephania cepharantha.

acute icterohepatitis T0468 Anemone hupehensis, T1545 Clematis chinensis, T1583 Cnidium officinale [Syn. Ligusticum officinale], T2882 Gardenia jasminoides [Syn. Gardenia florida], T2908 Gentiana cephalantha, T2921 Gentiana manshurica, T2928 Gentiana rigescens, T2930 Gentiana scabra, T2938 Gentiana triflora, T3820 Ligusticum chuanxiong [Syn. Ligusticum wallichii], T5400 Rabdosia stracheyi, T5472 Rheum officinale, T5474 Rheum palmatum, T5481 Rheum tanguticum, T5857 Sedum sarmentosum, T6227 Swertia mussotii, T6231 Swertia pseudochinensis, T6234 Swertia punicea.

acute infant fright wind T0987 Bos taurus domesticus; Bubalus bubalis. acute infection of upper respiratory tract T0975 Bombyx mori.

acute infectious hepatitis T6540 Tripterygium hypoglaucum.

acute jaundice T6031 Sophora flavescens [Syn. Sophora angustfolia].

acute laryngitis T3908 Lonicera bournei, T3909 Lonicera confusa, T3910 Lonicera fulvotomentosa, T3911 Lonicera hypoglauca, T3912 Lonicera japonica, T3914 Lonicera macranthoides, T3918 Lonicera similis.

acute leukemia T1162 Camptotheca acuminata.

acute lymphangitis T6552 Trollius chinensis [Syn. Trollius asiaticus var. chinensis], T6553 Trollius ledebourii, T6554 Trollius macropetalus.

acute mastitis T0478 Angelica dahurica [Syn. Angelica porphyrocaulis], T0933 Betula platyphylla, T0935 Betula platyphylla var. japonica, T6510 Trichosanthes kirilowii, T6513 Trichosanthes rosthornii [Syn. Trichosanthes uniflora].

acute myocardial infarction T3396 Ilex pubescens, T3397 Ilex pubescens var. glaber.

acute nephritis T0598 Ardisia japonica, T1553 Clerodendranthus spicatus, T2244 Doellingeria scaber [Syn. Aster scaber], T2327 Elaeagnus angustifolia, T5704 Sambucus nigra, T5707 Sambucus williamsii, T6008 Solanum nigrum.

acute nephritis with edema T3416 Imperata cylindrica var. major, T3752 Leonurus heterophyllus [Syn. Leonurus artemisia], T3754 Leonurus sibiricus.

acute or chronic tonsillitis T6554 Trollius macropetalus.

acute otitis media T6251 Symplocos caudata, T6554 Trollius macropetalus.

acute pancreatitis T1055 Bupleurum angustissimum, T1056 Bupleurum aureum, T1058 Bupleurum chaishoui, T1059 Bupleurum chinense, T1065 Bupleurum longiradiatum, T1066 Bupleurum marginatum, T1067 Bupleurum marginatum var. stenophyllum, T1072 Bupleurum scorzonerifolium, T1073 Bupleurum sibiricum, T1075 Bupleurum smithii var. parvifolium, T1078 Bupleurum wenchuanense, T1079 Bupleurum yinchowense.

acute parotitis $\mathrm{T} 3476$ Isatis indigotica.

acute pelvic inflammation T2715 Fibraurea recisa.

acute periostitis T6552 Trollius chinensis [Syn. Trollius asiaticus var. chinensis], T6553 Trollius ledebourii.

acute peritonitis T5472 Rheum officinale, T5474 Rheum palmatum, T5481 Rheum tanguticum. 
acute pharyngolaryngitis T3423 Indigofera tinctoria, T5833 Scutellaria amoena, T5834 Scutellaria baicalensis, T5839 Scutellaria hypericifolia, T5841 Scutellaria likiangensis, T5843 Scutellaria rehderiana, T5845 Scutellaria viscidula, T6226 Swertia mileensis.

acute rheumatic arthritis T1180 Capparis spinosa.

acute spasm in limbs T3011 Glycyrrhiza aspera, T3013 Glycyrrhiza glabra, T3015 Glycyrrhiza inflata, T3016 Glycyrrhiza kansuensis, T3021 Glycyrrhiza squamulosa, T3022 Glycyrrhiza uralensis, T3023 Glycyrrhiza yunnanensis.

acute spinal cord inflammation T0502 Anisodus tanguticus [Syn. Scopolia tangutica].

acute surgical infection T1662 Coptis chinensis, T1663 Coptis chinensis var. brevisepala, T1664 Coptis deltoidea, T1669 Coptis omeiensis, T1670 Coptis teetoides [Syn. Coptis teeta], T5680 Salvia miltiorrhiza, T5681 Salvia miltiorrhiza f. alba, T5688 Salvia przewalskii.

acute throat wind T2027 Daphne odora.

acute tonsillitis T0933 Betula platyphylla, T0935 Betula platyphylla var. japonica, T2715 Fibraurea recisa, T4391 Nauclea officinalis, T4845 Physalis alkekengi, T5445 Rehmannia glutinosa [Syn. Rehmannia glutinosa f. huechingensis], T5447 Rehmannia glutinosa [Syn. Rehmannia glutinosa f. huechingensis], T5833 Scutellaria amoena, T5834 Scutellaria baicalensis, T5839 Scutellaria hypericifolia, T5841 Scutellaria likiangensis, T5843 Scutellaria rehderiana, T5845 Scutellaria viscidula, T6043 Sophora subprostrata [Syn. Sophora tonkinensis], T6226 Swertia mileensis, T6251 Symplocos caudata.

acute urethritis T0273 Akebia quinata, T0277 Akebia trifoliata, T0279 Akebia trifoliata var. australis.

AD syndrome T2961 Ginkgo biloba.

adjuvant in anesthesia T0281 Alangium chinense, T0285 Alangium platanifolium, T6136 Stephania tetrandra.

agkistrodon bite T5393 Rabdosia longituba.

allergic conjunctivitis) T3936 Luffa operculata.

allergic dermatitis T1573 Clinopodium chinense, T4838 Phyllanthus flexuosus, T5847 Securidaca inappendiculata, T6226 Swertia mileensis.

allergic rhinitis T2367 Ephedra equisetina, T2368 Ephedra gerardiana, T2369 Ephedra intermedia, T2372 Ephedra likiangensis, T2373 Ephedra minuta, T2375 Ephedra monosperma, T2379 Ephedra saxatilis, T2380 Ephedra sinica, T3936 Luffa operculata, T4582 Paeonia delavayi, T4585 Paeonia moutan [Syn. Paeonia suffruticosa], T6843 Xanthium sibiricum [Syn. Xanthium strumarium].

alopecia areata T2048 Daucus carota, T2273 Drynaria fortunei, T5252 Pseudodrynaria coronans, T5270 Psoralea corylifolia.

altitude stress T5499 Rhodiola sacra.

amebic dysentery T0318 Allium sativum, T1038 Brucea javanica [Syn. Brucea sumatrana; Rhus javanica], T4034 Magnolia biloba, T4045 Magnolia officinalis, T5323 Pulsatilla campanella, T5324 Pulsatilla cernua, T5325 Pulsatilla chinensis, T5326 Pulsatilla dahurica, T6820 Wikstroemia indica.

amenorrhea T0071 Achyranthes aspera, T0072 Achyranthes aspera var. indica, T0250 Agrimonia pilosa var. japonica, T0273 Akebia quinata, T0274 Akebia quinata, T0277 Akebia trifoliata, T0278 Akebia trifoliata, T0279 Akebia trifoliata var. australis, T0280 Akebia trifoliata var. australis, T0300 Aleurites moluccana, T0474 Angelica acutiloba [Syn. Ligusticum acutilobum], T0680 Artemisia lactiflora, T0736 Asclepias curassavica, T0941 Bidens tripartita, T0978 Bombyx mori, T0991 Bos taurus domesticus; Bubalus bubalis, T0994 Boswellia carterii, T1163 Campylotropis hirtella, T1175 Cannabis sativa, T1215 Carthamus tinctorius, T1285 Celastrus angulatus, T1286 Celastrus flagellaris, T1289 Celastrus orbiculatus [Syn. Celastrus articulatus], T1356 Cheiranthus cheiri, T1361 Chenopodium ambrosioides, T1372 Chloranthus serratus, T1439 Cinnamomum cassia [Syn. Cinnamomum aromaticum], T1450 Cirsium lineare, T1589 Cocculus trilobus [Syn. Cocculus sarmentosus], T1638 Commiphora myrrha [Syn. Commiphora molmol], T1808 Crocus sativus, T1903 Curcuma aromatica, T1905 Curcuma longa, T1928 Cycas revoluta, T1976 Cyperus iria, T2020 Damnacanthus indicus, T2130 Desmodium gangeticum, T2132 Desmodium pulchellum [Syn. Phyllodium pulchellum], T2143 Dianthus chinensis, T2145 Dianthus superbus, T2147 Dianthus versicolor, T2347 Embelia parviflora, T2348 Embelia ribes, T2384 Epicauta gorhami, T2387 Epilobium hirsutum, T2536 Euonymus alatus, T2546 Euonymus sacrosancta, T2723 Ficus pumila, T3254 Hippophae rhamnoides, T3256 Hippophae rhamnoides subsp. sinensis, T3258 Hippophae rhamnoides subsp. yunnanensis, T3410 Impatiens balsamina, T3412 Impatiens balsamina, T3496 Isodon japonica [Syn. Rabdosia japonica], T3549 Ixora chinensis, T3752 Leonurus heterophyllus [Syn. Leonurus artemisia], T3754 Leonurus sibiricus, T3774 Lespedeza tomentosa, T3785 Levisticum officinale, T3867 Liquidambar formosana [Syn. Liquidambar taiwaniana], T3980 Lycopus lucidus, T4234 Millettia dielsiana, T4311 Mucuna sempervirens, T4584 Paeonia lactiflora wild, T4586 Paeonia obovata, T4590 Paeonia veitchii, T4604 Panax japonicus var. bipinnatifidus, T4787 Phellinus igniarius, T5028 Plumbago indica, T5101 Polygonum cuspidatum, T5229 Prunus persica, T5230 Prunus persica, T5231 Prunus persica, T5233 Prunus persica, T5269 Psilotum nudum, T5541 Rhus verniciflua [Syn. Toxicadendron verniciflum], T5560 Rorippa montana [Syn. Rorippa dubia; Sisymbrium dublium], T5567 Rosa laevigata, T5626 Ruta graveolens, T5680 Salvia miltiorrhiza, T5681 Salvia miltiorrhiza f. alba, T5688 Salvia przewalskii, T5741 Sargentodoxa cuneata, T5755 Saussurea involucrata, T5793 Schisandra henryi, T5865 Selaginella pulvinata, T5869 Selaginella tamariscina, T6062 Sparganium stoloniferum, T6154 Strobilanthes japonicus [Syn. Championella japonica], T6278 Tagetes erecta, T6357 Tetrapanax papyriferus, T6471 Toddalia asiatica [Syn. Toddalia aculeata; Paullinia asiatica], T6507 Trichosanthes cucumeroides, T6551 Trogopterus xanthipes; Pteromys volans, T6668 Vaccaria segetalis [Syn. Vaccaria pyramidata], T6675 Valeriana amurensis, T6676 Valeriana hardwickii, T6679 Valeriana officinalis, T6680 Valeriana officinalis var. latifolia, T6895 Zanthoxylum simulans.

amenorrhea and dysmenorrhea T0069 Achillea wilsoniana, T0275 Akebia quinata, T0495 Angelica sinensis, T0759 Aspidistra elatior, T1108 Caesalpinia sappan, T1438 Cinnamomum cassia [Syn. Cinnamomum aromaticum], T1583 Cnidium officinale [Syn. Ligusticum officinale], T1750 Corydalis yanhusuo [Syn. Corydalis turtschaninovii f. yanhusuo], T1904 Curcuma kwangsiensis, T1909 Curcuma zedoaria [Syn. Curcuma aeruginosa], T1934 Cyclea 
tonkinensis, T1978 Cyperus rotundus, T3221 Heracleum rapula, T3411 Impatiens balsamina, T3820 Ligusticum chuanxiong [Syn. Ligusticum wallichii], T4550 Osmanthus fragrans, T4804 Phlojodicarpus sibiricus, T6709 Verbena officinalis.

amenorrhea and scant milk T0632 Aristolochia manshuriensis.

amenorrhea due to blood stasis T0060 Achillea alpina [Syn. Achillea sibirica], T0065 Achillea millefolium, T0073 Achyranthes bidentata, T1775 Crataegus pinnatifida, T1778 Crataegus pinnatifida var. major, T1923 Cyathula capitata, T2541 Euonymus grandiflorus, T2597 Euphorbia lathyris, T3287 Huechys sanguinea, T4106 Manis pentadactyla, T4303 Moschus moschiferus; Moschus berezovskii; Moschus sifanicus, T4418 Nerium indicum, T4530 Ormosia hosiei, T4582 Paeonia delavayi, T4585 Paeonia moutan [Syn. Paeonia suffruticosa], T4607 Panax pseudo-ginseng var. japonicus, T5029 Plumbago zeylanica, T5104 Polygonum hydropiper, T5472 Rheum officinale, T5474 Rheum palmatum, T5481 Rheum tanguticum, T5578 Rubia cordifolia, T5582 Rubia oncotricha, T5583 Rubia schumannina, T5584 Rubia tinctorum, T5585 Rubia wallichiana.

amenorrhea with concretion and conglomeration T1924 Cyathula officinalis, T2838 Gallus gallus domesticus.

amnesia T0144 Acorus gramineus, T0146 Acorus tatarinowii, T5086 Polygala sibirica, T5088 Polygala tenuifolia, T5701 Salvia yunnanensis. amnesia and heavy dreams T1292 Celastrus orbiculatus [Syn. Celastrus articulatus].

anaphylactic diseases (hay fever, pollinosis, allergic rhinitis, allergic conjunctivitis) T3936 Luffa operculata.

ancylostomiasis T0318 Allium sativum, T1211 Carpesium abrotanoides, T1361 Chenopodium ambrosioides, T1881 Cucurbita moschata, T2049 Daucus carota, T2279 Dryopteris championii, T2281 Dryopteris crassirhizoma, T2510 Eucalyptus globulus, T5173 Portulaca oleracea, T5537 Rhus sylvestris, T6277 Tadehagi triquetrum.

anemia T1353 Changium smyrnioides, T5207 Prismatomeris tetrandra, T5586 Rubia yunnanensis.

anemia and amenorrhea T4478 Octopus vulgaris, T6689 Ventilago leiocarpa.

anemia and constipation T0989 Bos taurus domesticus; Bubalus bubalis. anemia and generalized pain T5108 Polygonum multiflorum. anemia and scant milk T4178 Melodinus hemsleyanus. anemia due to bleeding T6066 Spatholobus suberectus.

anemia due to folic acid deficiency T0495 Angelica sinensis, T4804 Phlojodicarpus sibiricus.

anemia due to malnutrition T6066 Spatholobus suberectus.

anemia with yellow complexion T0986 Bos taurus domesticus; Bubalus bubalis, T4580 Paeonia albiflora [Syn. Paeonia lactiflora], T4600 Panax ginseng [Syn. Panax schinseng], T5445 Rehmannia glutinosa [Syn. Rehmannia glutinosa f. huechingensis], T6066 Spatholobus suberectus.

anesthesia T0108 Aconitum kusnezoffii, T1330 Cerbera manghas, T2037 Datura innoxia, T2044 Datura metel.

angiitis T0264 Ajuga forrestii, T3092 Halenia corniculata.

angina pectoris T1215 Carthamus tinctorius, T1395 Chrysanthemum morifolium [Syn. Dendranthema morifolium], T1775 Crataegus pinnatifida, T1778 Crataegus pinnatifida var. major, T2274
Dryobalanops aromatica, T2389 Epimedium acuminatum, T2390 Epimedium brevicornum, T2392 Epimedium davidii, T2394 Epimedium elongatum, T2398 Epimedium koreanum, T2401 Epimedium pubescens, T2402 Epimedium sagittatum, T2404 Epimedium sutchuenense, T2406 Epimedium wushanense, T2846 Ganoderma japonicum [Syn. Ganoderma sinense], T2848 Ganoderma lucidum, T2964 Ginkgo biloba, T3388 Ilex chinensis [Syn. Ilex purpurea], T3396 Ilex pubescens, T3397 Ilex pubescens var. glaber, T3875 Liriope spicata var. prolifera, T3925 Loranthus parasiticus [Syn. Loranthus chinenis; Taxillus chinensis], T3926 Loropetalum chinense, T4303 Moschus moschiferus; Moschus berezovskii; Moschus sifanicus, T4507 Ophiopogon japonicus, T4608 Panax pseudo-ginseng var. notoginseng [Syn. Panax notoginseng], T4953 Piper longum, T5312 Pueraria edulis, T5313 Pueraria lobata [Syn. Pueraria thunbergiana; Pueraria pseudohirsuta], T5316 Pueraria omeiensis, T5318 Pueraria phaseoloides, T5320 Pueraria thomsonii, T5680 Salvia miltiorrhiza, T5681 Salvia miltiorrhiza f. alba, T5688 Salvia przewalskii, T6510 Trichosanthes kirilowii, T6513 Trichosanthes rosthornii [Syn. Trichosanthes uniflora], T6584 Typha angustata, T6585 Typha angustifolia, T6587 Typha latifolia.

angitis T1327 Ceratostigma minus, T6676 Valeriana hardwickii.

animal and insect bites T4254 Miscanthus sinensis, T5950 Siegesbeckia orientalis, T5951 Siegesbeckia orientalis var. glabrescens [Syn. Siegesbeckia glabrescens], T5952 Siegesbeckia orientalis var. pubescens [Syn. Siegesbeckia pubescens].

annexitis T6217 Swertia davidii.

anorexy for greasy T1705 Corydalis adunca.

ant-fistula T2428 Erinaceus europaeus; Hemiechinus daunicus; Hemiechinus auritus.

anthrax T5821 Scopolia japonica.

anxiety and depression T1808 Crocus sativus.

aphonia T2839 Gallus gallus domesticus, T3701 Lasiosphaera fenzlii, T3961 Lycoperdon pyriforme, T4537 Oroxylum indicum, T5719 Sapindus mukorossi.

aphonia due to lung heat T0161 Actinidia eriantha.

aphonia due to throat pain T4609 Panax pseudo-ginseng var. notoginseng [Syn. Panax notoginseng], T5967 Siraitia grosvenorii [Syn. Momordica grosvenorii], T6139 Sterculia lychnophora, T6346 Terminalia chebula, T6348 Terminalia chebula var. tomentella.

appendicitis T0646 Armillariella tabescens, T3548 Ixeris sonchifolia, T4582 Paeonia delavayi, T4585 Paeonia moutan [Syn. Paeonia suffruticosa], T5101 Polygonum cuspidatum, T5173 Portulaca oleracea, T5844 Scutellaria scordifolia, T5907 Senecio scandens [Syn. Senecio chinensis], T6025 Sonchus arvensis, T6716 Vernonia esculenta.

ardent fever T0987 Bos taurus domesticus; Bubalus bubalis, T3323 Hymenodictyon excelsum, T4145 Meconopsis punicea.

ardent fever and convulsion T5295 Pteris multifida.

ardent fever and manic agitation T4796 Pheretima aspergillum; Allolobophora caliginosa trapezoides.

ardent fever incessant T0002 Abelmoschus moschatus [Syn. Hibiscus abelmoschus], T1633 Commelina communis.

ardent fever with clouded spirit T1045 Bubalus bubalis, T3476 Isatis 
indigotica, T5447 Rehmannia glutinosa [Syn. Rehmannia glutinosa f. huechingensis].

ardent fever with fright wind T5184 Potentilla kleiniana.

ardent fever with headache T0866 Baphicacanthus cusia [Syn. Strobilanthes cusia], T0867 Baphicacanthus cusia [Syn. Strobilanthes cusia], T2510 Eucalyptus globulus, T2517 Eucalyptus robusta, T3475 Isatis indigotica.

ardent fever with vexation and thirst T0462 Anemarrhena asphodeloides, T5833 Scutellaria amoena, T5834 Scutellaria baicalensis, T5839 Scutellaria hypericifolia, T5841 Scutellaria likiangensis, T5843 Scutellaria rehderiana, T5845 Scutellaria viscidula.

ardent fever (hyperpyrexia) T6295 Tanacetum sibiricum [Syn. Filifolium sibiricum].

armpit welling abscess T6099 Stauntonia chinensis.

arrhythmia T1991 Cytisus scoparius [Syn. Spartium scoparium], T3925 Loranthus parasiticus [Syn. Loranthus chinenis; Taxillus chinensis], T5557 Rohdea japonica [Syn. Orontium japonicum], T6031 Sophora flavescens [Syn. Sophora angustfolia], T6919 Ziziphus jujuba var. spinosa.

arsenic poisoning T4503 Onychium lucidum.

arthralgia T5661 Salvia bowleyana, T5701 Salvia yunnanensis.

arthritis T0495 Angelica sinensis, T0647 Armoracia lapathifolia, T3882 Lithospermum officinale, T3970 Lycopodium cernuum, T3973 Lycopodium japonicum [Syn. Lycopodium clavatum], T4689 Peganum nigellastrum, T4804 Phlojodicarpus sibiricus.

ascariasis T0255 Ailanthus altissima, T0264 Ajuga forrestii, T0338 Aloe ferox, T0347 Aloe vera [Syn. Aloe barbadensis], T0348 Aloe vera var. chinensis, T0364 Alsophila spinulosa, T0446 Anaphalis margaritacea, T0468 Anemone hupehensis, T0659 Artemisia absinthium, T1122 Caloglossa leprieurii, T1176 Cannabis sativa, T1211 Carpesium abrotanoides, T1361 Chenopodium ambrosioides, T1382 Chondria armata [Syn. Lophura armata], T1595 Codium fragile, T1696 Cornus capitata [Syn. Dendrobenthamia capitata], T1881 Cucurbita moschata, T2049 Daucus carota, T2173 Digenea simplex, T2281 Dryopteris crassirhizoma, T3466 Iris potaninii, T3688 Lappula echinata, T3935 Luffa cylindrica, T4002 Lysimachia foenum-graecum, T4026 Macrothelypteris oligophlebia, T4083 Mallotus philippinensis, T4084 Mallotus philippinensis, T4156 Melia azedarach, T4162 Melia toosendan, T4231 Milingtonia hortensis, T4247 Millingtonia hortensis, T4779 Pharbitis nil, T4780 Pharbitis purpurea, T5098 Polygonum aviculare, T5151 Populus davidiana, T5158 Populus simonii, T5173 Portulaca oleracea, T5176 Potamogeton natans, T5298 Pteris vittata, T5327 Punica granatum, T5329 Punica granatum, T5384 Quisqualis indica, T5717 Sapindus delavayi [Syn. Pancovia delavayi], T5920 Seriphidium cinum [Syn. Artemisia cina], T5921 Seriphidium finitum [Syn. Artemisia finita], T6032 Sophora flavescens [Syn. Sophora angustfolia], T6035 Sophora japonica, T6101 Stauntonia hexaphylla, T6111 Stemona japonica, T6113 Stemona sessilifolia, T6115 Stemona tuberosa, T6312 Taxus mairei, T6474 Toona ciliata, T6475 Toona sinensis, T6691 Veratrilla baillonii, T6713 Vernonia anthelmintica.

ascaridosis in biliary tract T0502 Anisodus tanguticus [Syn. Scopolia tangutica]. ascites T1049 Bufo bufo gargarizans; Bufo melanostictus, T1263 Catalpa ovata, T1357 Chelidonium majus, T1633 Commelina communis, T1756 Costus speciosus, T2023 Daphne genkwa, T2131 Desmodium pulchellum [Syn. Phyllodium pulchellum], T2132 Desmodium pulchellum [Syn. Phyllodium pulchellum], T2439 Eruca sativa, T2580 Euphorbia antiquorum, T2585 Euphorbia esula, T2596 Euphorbia kansui, T2631 Eurya japonica, T3066 Gryllulus chinensis, T3432 Inula helianthus-aquatica, T3668 Lagenaria siceraria var. depressa, T3774 Lespedeza tomentosa, T5129 Polyporus umbellatus, T5238 Prunus salicina, T5407 Rana nigromaculata; Rana plancyi, T5461 Rhamnus leptophylla, T5557 Rohdea japonica [Syn. Orontium japonicum], T5723 Sapium sebiferum, T5835 Scutellaria barbata [Syn. Scutellaria rivularis], T5862 Selaginella involvens, T6210 Swainsonia salsula [Syn. Sphaerophysa salsula].

ascites (hydroperitoneum) 75770 Saussurea superba [Syn. Saussurea hieracioides], T5115 Polygonum sibiricum [Syn. Persicaria sibirica].

ascites ulcer T1194 Carassius auratus, T2601 Euphorbia milii.

ashen nail T3411 Impatiens balsamina.

asthma T0216 Agave americana var. marginata [Syn. Agave americana var. variegata], T0364 Alsophila spinulosa, T0539 Apis cerana, T0923 Bergenia crassifolia, T0926 Berneuxia thibetica, T1176 Cannabis sativa, T1452 Cissampelos pareira, T1533 Clausena dentata, T1534 Clausena dunniana, T1684 Cordyline strcta, T1819 Crotalaria ferruginea, T1880 Cucurbita moschata, T2101 Dendrobium fimbriatum, T2268 Drosera rotundifolia, T2279 Dryopteris championii, T2505 Eucalyptus citriodora, T2738 Flemingia strobilifera, T2749 Fomes officinalis, T3026 Gnaphalium affine [Syn. Gnaphalium multiceps], T3040 Gomphrena globosa, T3056 Gossypium herbaceum, T3190 Helminthostachys zeylanica, T3758 Lepidium sativum, T3765 Lepisorus ussuriensis, T3779 Leucas aspera, T3850 Lindera glauca, T3885 Litsea cubeba, T3953 Lycianthes biflora, T4003 Lysimachia microcarpa, T4371 Nandina domestica, T4549 Osbeckia chinensis, T4796 Pheretima aspergillum; Allolobophora caliginosa trapezoides, T4908 Pinus bungeana, T4937 Piper betle, T5502 Rhododendron anthopogonoides, T5513 Rhododendron mariae, T5645 Sageretia theezans [Syn. Sageretia thea], T5943 Sida cordifolia, T5989 Solanum aculeatissimum, T6014 Solanum surattense, T6043 Sophora subprostrata [Syn. Sophora tonkinensis], T6075 Spilanthes acmella, T6105 Stellera chamaejasme, T6206 Sus scrofa domestica, T6259 Syringa amurensis [Syn. Syringa reticulata var. amurensis], T6264 Syzygium buxifolium, T6581 Tylophora mollissima, T6789 Vitex negundo.

asthma (for women) T2391 Epimedium brevicornum.

asthma (raw) T5865 Selaginella pulvinata, T5869 Selaginella tamariscina.

asthma and abundant phlegm T0591 Ardisia arborescens.

asthma and cough T0078 Aconitum barbatum var. puberulum [Syn. Aconitum ochranthum], T5517 Rhododendron molle.

asthma and fullness due to external contraction cough T5216 Prunus armeniaca, T5219 Prunus armeniaca var. ansu.

atherosclerosis T2410 Equisetum pratense. 
atrial fibrillation T0184 Adonis amurensis.

aversion of cold T1337 Cervus nippon; Cervus elaphus, T2343 Elsholtzia splendens.

aversion of cold during convalescence T5499 Rhodiola sacra.

aversion to light T5871 Selenarctos thibetanus; Ursus arctos.

bacillary dysentery T0318 Allium sativum, T0457 Andrographis paniculata [Syn. Justicia paniculata], T0643 Aristolochia versicolar, T0897 Berberis amurensis, T0912 Berberis poiretii, T0920 Berberis wilsonae, T1044 Bryum argenteum, T1785 Cratoxylum prunifolium, T2357 Emilia sonchifolia, T2589 Euphorbia helioscopia, T2590 Euphorbia hirta, T2657 Fagopyrum cymosum [Syn. Polygonum cymosum], T3203 Hemsleya amabilis, T3208 Hemsleya macrosperma, T4391 Nauclea officinalis, T4882 Picrasma quassioides [Syn. Picrasma ailanthoides], T5375 Quercus mongolica, T5730 Sarcandra glabra [Syn. Chloranthus glaber], T6043 Sophora subprostrata [Syn. Sophora tonkinensis], T6119 Stephania cepharantha, T6135 Stephania succifera, T6277 Tadehagi triquetrum.

backache T1455 Cistanche deserticola, T1456 Cistanche salsa, T1683 Cordyceps sinensis, T1928 Cycas revoluta, T5107 Polygonum multiflorum.

bacteriogenic dysentery T5149 Populus canadensis, T5150 Populus cathayana.

bad breath T1152 Camellia sinensis [Syn. Thea sinensis], T2336 Elephas maximus, T2558 Eupatorium formosanum, T2559 Eupatorium fortunei, T4170 Melilotus albus, T4172 Melilotus suaveolens, T4470 Ocimum basilicum, T4550 Osmanthus fragrans.

bad breath and slimy tongue fur T6561 Tulipa gesneriana.

bald sores T1955 Cynanchum hancockianum, T2162 Dicranostigma franchetianum [Syn. Dicranostigma leptopodum], T2338 Elephas maximus, T3567 Juglans regia, T4265 Momordica cochinchinensis, T4520 Opuntia ficus-indica, T5607 Rumex crispus, T5646 Sagina japonica [Syn. Spergula japonica].

bald white scalp sore T1278 Cedrela sinensis, T4917 Pinus massoniana, T5611 Rumex japonicus, T5612 Rumex nepalensis.

bedsore T0297 Alchornea trewioides.

bee sting T1624 Colocasia antiquorum, T2206 Dioscorea parviflora, T2213 Dioscorea zingiberensis, T3003 Glycine max, T5120 Polygonum tinctorium, T6367 Teucrium quadrifarium.

bee-fistula T2428 Erinaceus europaeus; Hemiechinus daunicus; Hemiechinus auritus.

behcet's syndrome T6542 Tripterygium wilfordii.

belching T0353 Alpinia blepharocalyx.

belching and low food intake T1498 Citrus medica, T1520 Citrus wilsonii.

beriberi T0038 Acanthopanax gracilistylus, T0042 Acanthopanax senticosus [Syn. Eleutherococcus senticosus], T0044 Acanthopanax sessiliflorus, T0559 Arachis hypogaea, T0606 Areca catechu, T0680 Artemisia lactiflora, T0721 Arundina chinensis, T0853 Baeckea frutescens, T0895 Benincasa hispida, T1258 Casuarina equisetifolia, T1342 Chaenomeles lagenaria [Syn. Chaenomeles speciosa], T1435 Cinnamomum camphora, T1556 Clerodendron fragrans, T1614 Coix lacryma-jobi var. ma-yuen, T1980 Cyprinus carpio, T2038 Datura innoxia, T2042 Datura metel, T2343 Elsholtzia splendens, T2744
Foeniculum vulgare, T3436 Inula nervosa, T3856 Lindera umbellata [Syn. Lindera erythrocarpa], T3893 Litsea pungens, T4287 Morus alba, T4386 Nardostachys chinensis, T4387 Nardostachys jatamansi, T4505 Ophiocephalus argus, T4546 Oryza sativa, T4785 Phaseolus vulgaris, T4799 Philydrum lanuginosum, T4906 Pinus armandii, T4916 Pinus massoniana, T5071 Polygala arillata, T5110 Polygonum orientale, T5151 Populus davidiana, T5171 Porphyra tenera, T5189 Pothos chinensis, T5223 Prunus humilis [Syn. Cerasus humilis], T5224 Prunus japonica [Syn. Cerasus japonica], T5225 Prunus japonica var. nakaii, T5231 Prunus persica, T5238 Prunus salicina, T5244 Prunus tomentosa, T5548 Ricinus communis, T5750 Saururus chinensis, T6069 Speranskia tuberculata, T6528 Trigonella foenum-graecum, T6870 Zanthoxylum bungeanum, T6925 Zostera marina.

beriberi with edema T0500 Anguilla japonica, T0629 Aristolochia heterophylla, T1343 Chaenomeles sinensis, T2119 Derris eriocarpa, T2644 Evodia rutaecarpa, T2646 Evodia rutaecarpa var. officinalis, T3996 Lysimachia candida, T4720 Perilla frutescens var. arguta, T4723 Perilla frutescens var. crispa, T4983 Pisum sativum, T6129 Stephania japonica, T6136 Stephania tetrandra, T6677 Valeriana jatamansii [Syn. Valeriana wallichii].

beriberi with general edema T5818 Scoparia dulcis.

bilharziosis T1881 Cucurbita moschata, T2131 Desmodium pulchellum [Syn. Phyllodium pulchellum], T5308 Pterocarya stenoptera, T6709 Verbena officinalis.

binding depression of liver $\boldsymbol{q} \boldsymbol{i}$ T5137 Poncirus trifoliata.

bitter taste T2908 Gentiana cephalantha, T2921 Gentiana manshurica, T2928 Gentiana rigescens, T2930 Gentiana scabra, T2938 Gentiana triflora, T6228 Swertia nervosa.

bitter taste and slimy tongue fur T2554 Eupatorium cannabinum.

bitter taste and tidal fever T6224 Swertia kouitchensis.

bladder qi pain $\mathrm{T} 1504$ Citrus reticulata.

bladder vacuity cold T3854 Lindera strychnifolia [Syn. Lindera aggregata].

bleeding T1299 Celosia cristata, T1402 Chrysosplenium alternifolium, T2321 Ecklonia kurome, T3678 Laminaria japonica, T4009 Maackia amurensis, T4234 Millettia dielsiana, T5173 Portulaca oleracea, T5354 Pyrrosia davidii, T5355 Pyrrosia drakeana, T5356 Pyrrosia gralla, T5357 Pyrrosia lingua T5358 Pyrrosia petiolosa, T5360 Pyrrosia sheareri, T6119 Stephania cepharantha, T6584 Typha angustata, T6585 Typha angustifolia, T6587 Typha latifolia, T6640 Undaria pinnatifida.

bleeding due to blood heat T5578 Rubia cordifolia, T5582 Rubia oncotricha, T5583 Rubia schumannina, T5584 Rubia tinctorum, T5585 Rubia wallichiana, 55852 Sedum bulbiferum.

bleeding due to external injury T0019 Acacia catechu, T0156 Actinidia arguta, T0180 Adina pilulifera [Syn. Cephalanthus pilulifera], T0181 Adina rubella, T0229 Ageratum conyzoides, T0248 Agrimonia pilosa, T0264 Ajuga forrestii, T0267 Ajuga nipponensis, T0295 Albizzia odoratissima, T0298 Alectoria vivens, T0328 Alnus japonica, T0371 Alstonia mairei, T0374 Alstonia scholaris, T0378 Alstonia yunnanensis, T0423 Ampelopsis brevipedunculata, T0427 Ampelopsis japonica [Syn. Paullinia japonica], T0572 Aralia elata, T0634 Aristolochia mollissima, T0736 Asclepias curassavica, T0776 Asplenium 
prolongatum, T0805 Astragalus sinicus, T0859 Balanophora involucrata, T0871 Barleria lupulina, T0955 Bletilla striata, T1043 Bryophyllum pinnatum, T1116 Callicarpa arborea, T1118 Callicarpa formosana, T1119 Callicarpa japonica, T1120 Callicarpa macrophylla, T1129 Calophyllum inophyllum, T1163 Campylotropis hirtella, T1210 Carpesium abrotanoides, T1212 Carpesium eximum, T1251 Cassytha filiformis, T1336 Ceriops tagal [Syn. Rhizophora tagal], T1434 Cinnamomum bejolghota [Syn. Cinnamomum obtusifolium; Laurus bejolghota], T1449 Cirsium japonicum, T1451 Cirsium setosum [Syn. Cerratula setosa; Cirsium segetum; Cephalanoplos segetum], T1529 Cladonia verticillata, T1554 Clerodendron cyrtophyllum, T1566 Clerodendrum inerme, T1655 Conyza blinii, T1656 Conyza bonariensis [Syn. Erigeron bonariensis; Erigeron linifolius; Erigeron crispus], T1657 Conyza canadensis [Syn. Erigeron canadensis], T1672 Corallodiscus flabellatus [Syn. Didissandra flabellat], T1684 Cordyline strcta, T1815 Crotalaria assamica, T1928 Cycas revoluta, T1932 Cyclea racemosa, T1969 Cynoglossum officinale, T1971 Cynoglossum zeylanicum [Syn. Anchusa zeylanica; Cynoglossum furcatum; Cynoglossum formosanum], T2008 Dalbergia odorifera, T2008 Dalbergia odorifera, T2013 Dalbergia sissoo, T2013 Dalbergia sissoo, T2161 Dicranopteris pedata [Syn. Polypodium pedatum; Dicranopteris dichotoma], T2193 Dioscorea cirrhosa [Syn. Dioscorea pogonoides], T2323 Eclipta prostrata [Syn. Eclipta alba], T2327 Elaeagnus angustifolia, T2348 Embelia ribes, T2407 Equisetum arvense, T2510 Eucalyptus globulus, T2540 Euonymus fortunei, T2602 Euphorbia nematocypha, T2614 Euphorbia pulcherrima, T2631 Eurya japonica, T2732 Firmiana simplex, T2734 Fissistigma glaucescens [Syn. Melodorum glaucescens], T2825 Galeobdolon chinense [Syn. Lamium chinense], T2857 Garcinia hanburyi, T2866 Garcinia morella, T2950 Gerbera anandria [Syn. Leibnitzia anandria], T2998 Glycine max, T3054 Gossypium barbadense], T3055 Gossypium herbaceum, T3058 Gossypium hirsutum [Syn. Gossypium mexicanum], T3088 Gynura segetum [Syn. Gynura japonica], T3089 Gypsophila acutifolia, T3092 Halenia corniculata, T3202 Hemistepta lyrata [Syn. Hemistepta carthamoides; Saussurea carthamoides], T3294 Huperzia selago [Syn. Lycopodium selago], T3324 Hymenophyllum barbatum, T3340 Hypericum ascyron, T3349 Hypericum erectum, T3361 Hypericum perforatum, T3376 Hypolepis punctata [Syn. Polypodium punctatum], T3387 Idesia polycarpa, T3388 Ilex chinensis [Syn. Ilex purpurea], T3395 Ilex pedunculosa, T3528 Isodon ternifolia, T3529 Isodon ternifolius, T3672 Lagerstroemia indica, T3701 Lasiosphaera fenzlii, T3732 Lawsonia inermis, T3851 Lindera glauca, T3856 Lindera umbellata [Syn. Lindera erythrocarpa], T3857 Lindera umbellata [Syn. Lindera erythrocarpa], T3863 Liparis nervosa, T3866 Liquidambar formosana [Syn. Liquidambar taiwaniana], T3876 Litchi chinensis, T3887 Litsea glutinosa, T3953 Lycianthes biflora, T3961 Lycoperdon pyriforme, T3989 Lygodium flexuosum [Syn. Lygodium pinnatifidum; Ophioglossum flexuosum], T3990 Lygodium japonicum, T3995 Lysidice rhodostegia, T4006 Lythrum anceps, T4007 Lythrum salicaria, T4026 Macrothelypteris oligophlebia, T4029 Maesa japonica, T4080 Mallotus apelta, T4082 Mallotus japonicus, T4118 Marsdenia oreophila, T4126 Matteuccia orientalis, T4305 Mosla dianthera, T4346 Myrica nagi [Syn. Podocarpus nagi], T4413 Nepeta cataria, T4503 Onychium lucidum, T4604 Panax japonicus var. bipinnatifidus, T4605 Panax japonicus var. major, T4607 Panax pseudo-ginseng var. japonicus, T4608 Panax pseudo-ginseng var. notoginseng [Syn. Panax notoginseng], T4658 Parmelia tinctorum, T4686 Pedilanthus tithymaloides, T4705 Peperomia pellucida, T4730 Peristrophe roxburghiana, T4836 Phyllanthus emblica, T4978 Pisolithus tinctorius [Syn. Lycoperdon capitatum; Scleroderma tinctorium], T5104 Polygonum hydropiper, T5109 Polygonum nodosum, T5118 Polygonum suffultum, T5172 Portulaca grandiflora, T5184 Potentilla kleiniana, T5185 Potentilla multifida, T5194 Premna microphylla, T5250 Psammosilene tunicoides, T5267 Psidium guajava, T5290 Pteris fauriei [Syn. Pteris fauriei var. minor], T5295 Pteris multifida, T5299 Pteris wallichinan, T5310 Pterospermum lanceaefolium, T5339 Pycnoporus sanguineus, T5374 Quercus infectoria, T5411 Randia spinosa, T5444 Reboulia hemisphaerica, T5468 Rheum emodi [Syn. Rheum australe], T5501 Rhodiola yunnanesis, T5524 Rhododendron simsii, T5528 Rhodomyrtus tomentosa, T5529 Rhodomyrtus tomentosa, T5537 Rhus sylvestris, T5555 Rodgersia pinnata, T5563 Rosa cymosa, T5566 Rosa henryi, T5587 Rubus alceaefolius, T5595 Rubus parviforlius, T5704 Sambucus nigra, T5707 Sambucus williamsii, T5746 Sassafras tzumu, T5753 Saussurea gnaphaloides, T5757 Saussurea laniceps, T5759 Saussurea medusa, T5785 Schefflera venulosa, T5821 Scopolia japonica, T5840 Scutellaria indica, T5850 Sedum aizoon, T5852 Sedum bulbiferum, T5855 Sedum kamtschaticum, T5856 Sedum lineare [Syn. Sedum obtuso-lineare], T5860 Selaginella davidii, T5861 Selaginella doederleinii, T5862 Selaginella involvens, T5863 Selaginella moellendorffii, T5866 Selaginella sanguinolenta, T5867 Selaginella sinensis, T5870 Selaginella uncinata, T5883 Senecio cannabifolius, T5942 Sida acuta, T5998 Solanum dulcamara, T6003 Solanum khasianum, T6116 Stenoloma chusanum, T6119 Stephania cepharantha, T6145 Stichopus japonicus, T6177 Strychnos ignatii, T6276 Tacca plantaginea [Syn. Schizocapsa plantaginea], T6367 Teucrium quadrifarium, T6482 Toxicodendron succedaneum [Syn. Rhus succedanea], T6485 Trachycarpus fortunei, T6491 Trametes cinnabarina [Syn. Polyporus cinnabarinus; Boletus cinnabarinus], T6531 Trillium camtschaticum, T6533 Trillium kamtschaticum, T6535 Trillium tschonoskii, T6544 Triticum aestivum [Syn. Triticum vulgare], T6548 Tritonia crocosmaeflora, T6567 Tupistra wattii [Syn. Campylandra wattii], T6584 Typha angustata, T6585 Typha angustifolia, T6587 Typha latifolia, T6615 Uncaria gambir, T6618 Uncaria homomalla [Syn. Uruparia homomalla; Uruparia tonkinensis; Uruparia lanosa var. parvifora], T6653 Usnea diffracta, T6654 Usnea longissima, T6705 Verbascum thapsus, T6728 Veronica serpyllifolia, T6731 Veronicastrum sibiricum, T6747 Vicia faba, T6861 Zanthoxylum ailanthoides, T6874 Zanthoxylum dimorphophyllum var. spinifolum, T6883 Zanthoxylum myriacanthum.

bleeding due to internal damage T5866 Selaginella sanguinolenta.

bleeding due to tympanites T1181 Capra hircus; Ovis aries.

bleeding during operation T3863 Liparis nervosa.

bleeding from dental extraction T1120 Callicarpa macrophylla.

bleeding from hemorrhoids T0060 Achillea alpina [Syn. Achillea sibirica], T0065 Achillea millefolium, T0830 Auricularia auricula, 
T0831 Auricularia delicata, T1112 Calendula arvensis, T1145 Camellia japonica, T1662 Coptis chinensis, T1663 Coptis chinensis var. brevisepala, T1664 Coptis deltoidea, T1669 Coptis omeiensis, T1670 Coptis teetoides [Syn. Coptis teeta], T1747 Corydalis thalictrifolia, T3123 Hedyotis auricularia, T3244 Hibiscus syriacus, T3295 Huperzia serrata [Syn. Lycopodium serratum], T3659 Lactuca indica, T3933 Luffa acutangula, T3934 Luffa cylindrica, T4178 Melodinus hemsleyanus, T4203 Mesua ferrea, T4305 Mosla dianthera, T4307 Mosla scabra [Syn. Mosla punctata], T4502 Onychium japonicum [Syn. Tricomanes japonicum], T4505 Ophiocephalus argus, T4519 Opuntia dillenii, T4520 Opuntia ficus-indica, T4521 Opuntia vulgaris, T5099 Polygonum bistorta, T5173 Portulaca oleracea, T5176 Potamogeton natans, T5323 Pulsatilla campanella, T5324 Pulsatilla cernua, T5325 Pulsatilla chinensis, T5326 Pulsatilla dahurica, T5372 Quercus dentata, T5569 Rosa multiflora, T5611 Rumex japonicus, T5612 Rumex nepalensis, T5685 Salvia plebeia, T5712 Sanguisorba officinalis, T6034 Sophora japonica, T6036 Sophora japonica, T6522 Trifolium repens.

bleeding from wounds T3363 Hypericum sampsonii, T3560 Jatropha curcas, T5567 Rosa laevigata.

bleeding hemorrhoids T0267 Ajuga nipponensis, T5181 Potentilla chinensis, T5863 Selaginella moellendorffii.

bleeding knife wound T3373 Hypoestes purpurea [Syn. Justicia purpurea; Hypoestes sinica], T3555 Jasminum nudiflorum, T3765 Lepisorus ussuriensis, T5288 Pteris cretica var. nervosa [Syn. Pteris nervosa], T5297 Pteris plumbea, T5389 Rabdosia coetsa.

bleeding of digestive tract T0060 Achillea alpina [Syn. Achillea sibirica], T0922 Berchemia polyphylla var. leioclada, T1116 Callicarpa arborea, T5472 Rheum officinale, T5474 Rheum palmatum, T5481 Rheum tanguticum, T6745 Vicia faba, T6747 Vicia faba.

bleeding of skin T2323 Eclipta prostrata [Syn. Eclipta alba].

blood amassment yellowing T2435 Eriocheir sinensis.

blood and liquid depletion $\mathrm{T} 1173$ Cannabis sativa.

blood conglomeration T1210 Carpesium abrotanoides.

blood deficiency syndrome T0495 Angelica sinensis, T4804 Phlojodicarpus sibiricus.

blood depletion and amenorrhea T5445 Rehmannia glutinosa [Syn. Rehmannia glutinosa f. huechingensis].

blood dizziness T3276 Homo sapiens.

blood dysentery T0249 Agrimonia pilosa var. japonica, T0302 Alhagi pseudalhagi, T0427 Ampelopsis japonica [Syn. Paullinia japonica], T0601 Ardisia pusilla, T0830 Auricularia auricula, T0831 Auricularia delicata, T0991 Bos taurus domesticus; Bubalus bubalis, T1005 Brassica campestris [Syn. Brassica campestris var. oleifera], T1441 Cinnamomum japonicum, T1451 Cirsium setosum [Syn. Cerratula setosa; Cirsium segetum; Cephalanoplos segetum], T1896 Cupressus funebris, T2218 Diospyros kaki, T2281 Dryopteris crassirhizoma, T2323 Eclipta prostrata [Syn. Eclipta alba], T2363 Entada phaseoloides [Syn. Lens phaseoloides], T3145 Helianthus annuus, T3278 Homo sapiens, T4399 Nelumbo nucifera, T4604 Panax japonicus var. bipinnatifidus, T4606 Panax pseudo-ginseng, T5372 Quercus dentata, T5530 Rhus chinensis [Syn. Rhus semialata], T5712 Sanguisorba officinalis, T5966 Siphonostegia chinensis, T6006
Solanum melongena, T6034 Sophora japonica, T6036 Sophora japonica, T6440 Thuja orientalis [Syn. Platycladus orientalis; Biota orientalis].

blood ejection T0001 Abelmoschus manihot, T0216 Agave americana var. marginata [Syn. Agave americana var. variegata], T0248 Agrimonia pilosa, T0249 Agrimonia pilosa var. japonica, T0266 Ajuga macrosperma, T0322 Allium tuberosum, T0382 Althaea rosea, T0446 Anaphalis margaritacea, T0568 Aralia chinensis, T0570 Aralia dasyphylla, T0598 Ardisia japonica, T0600 Ardisia mamillata [Syn. Tinus mamillata], T0648 Arnebia euchroma, T0649 Arnebia guttata, T0664 Artemisia argyi, T0681 Artemisia lavandulaefolia, T0685 Artemisia mongolica, T0687 Artemisia myriantha, T0689 Artemisia princeps, T0691 Artemisia rubripes, T0706 Artemisia vulgaris, T0722 Arundo donax, T0776 Asplenium prolongatum, T0860 Balanophora japonica, T0867 Baphicacanthus cusia [Syn. Strobilanthes cusia], T0890 Begonia limprichtii, T0923 Bergenia crassifolia, T0924 Bergenia purpurascens, T0952 Blechnum orientale, T0955 Bletilla striata, T0973 Bombax malabaricum [Syn. Gossampinus malabarica], T0982 Bos taurus domesticus, T1043 Bryophyllum pinnatum, T1045 Bubalus bubalis, T1145 Camellia japonica, T1184 Capsella bursa-pastoris, T1199 Carduus acanthoides, T1200 Carduus crispus, T1210 Carpesium abrotanoides, T1254 Castanea mollissima, T1311 Centella asiatica, T1449 Cirsium japonicum, T1451 Cirsium setosum [Syn. Cerratula setosa; Cirsium segetum; Cephalanoplos segetum], T1547 Clematis terniflora [Syn. Clematis maximowicziana], T1662 Coptis chinensis, T1663 Coptis chinensis var. brevisepala, T1664 Coptis deltoidea, T1669 Coptis omeiensis, T1670 Coptis teetoides [Syn. Coptis teeta], T1808 Crocus sativus, T1896 Cupressus funebris, T1907 Curcuma wengujin, T1928 Cycas revoluta, T1967 Cynoglossum amabile, T1969 Cynoglossum officinale, T1986 Cyrtomium fortunei, T2191 Dioscorea bulbifera, T2193 Dioscorea cirrhosa [Syn. Dioscorea pogonoides], T2221 Diospyros kaki, T2257 Dracocephalum moldavicum, T2281 Dryopteris crassirhizoma, T2290 Duchesnea indica, T2323 Eclipta prostrata [Syn. Eclipta alba], T2407 Equisetum arvense, T2540 Euonymus fortunei, T2882 Gardenia jasminoides [Syn. Gardenia florida], T3054 Gossypium barbadense], T3055 Gossypium herbaceum, T3058 Gossypium hirsutum [Syn. Gossypium mexicanum], T3088 Gynura segetum [Syn. Gynura japonica], T3241 Hibiscus mutabilis, T3276 Homo sapiens, T3277 Homo sapiens, T3278 Homo sapiens, T3318 Hylotelephium mingjinianum, T3334 Hypecoum leptocarpum, T3349 Hypericum erectum, T3361 Hypericum perforatum, T3363 Hypericum sampsonii, T3373 Hypoestes purpurea [Syn. Justicia purpurea; Hypoestes sinica], T3387 Idesia polycarpa, T3423 Indigofera tinctoria, T3460 Iris halophila, T3464 Iris lactea var. chinensis [Syn. Iris pallasii var. chinensis], T3547 Ixeris chinensis, T3736 Lemmaphyllum microphyllum, T3737 Lemmaphyllum microphyllum var. obovatum, T3769 Lespedeza bicolor, T3866 Liquidambar formosana [Syn. Liquidambar taiwaniana], T3881 Lithospermum erythrorhizon, T3954 Lycium barbarum, T3956 Lycium chinense, T3981 Lycopus lucidus, T4006 Lythrum anceps, T4007 Lythrum salicaria, T4121 Marsilea quadrifolia, T4127 Matteuccia struthiopteris, T4259 Mnium cuspidatum, T4398 Nelumbo nucifera, T4402 Nelumbo nucifera, T4413 Nepeta cataria, T4502 Onychium 
japonicum [Syn. Tricomanes japonicum], T4519 Opuntia dillenii, T4521 Opuntia vulgaris, T4549 Osbeckia chinensis, T4552 Osmunda japonica, T4564 Oxalis corniculata [Syn. Oxalis repens], T4582 Paeonia delavayi, T4584 Paeonia lactiflora wild, T4585 Paeonia moutan [Syn. Paeonia suffruticosa], T4586 Paeonia obovata, T4590 Paeonia veitchii, T4604 Panax japonicus var. bipinnatifidus, T4605 Panax japonicus var. major, T4606 Panax pseudo-ginseng, T4607 Panax pseudo-ginseng var. japonicus, T4608 Panax pseudo-ginseng var. notoginseng [Syn. Panax notoginseng], T4656 Parmelia saxatilis, T4720 Perilla frutescens var. arguta, T4723 Perilla frutescens var. crispa, T4730 Peristrophe roxburghiana, T5043 Podocarpus macrophyllus, T5045 Podocarpus macrophyllus var. maki, T5099 Polygonum bistorta, T5118 Polygonum suffultum, T5120 Polygonum tinctorium, T5121 Polygonum tinctorium, T5182 Potentilla discolor, T5230 Prunus persica, T5269 Psilotum nudum, T5295 Pteris multifida, T5354 Pyrrosia davidii, T5355 Pyrrosia drakeana, T5356 Pyrrosia gralla, T5357 Pyrrosia lingua T5358 Pyrrosia petiolosa, T5360 Pyrrosia sheareri, T5372 Quercus dentata, T5419 Raphanus sativus, T5449 Reineckea carnea, T5484 Rhinoceros unicornis; Rhinoceros sondaicus; Rhinoceros sumatrensis, T5497 Rhodiola kirilowii, T5518 Rhododendron mucronatum, T5523 Rhododendron simsii, T5529 Rhodomyrtus tomentosa, T5554 Rodgersia aesculifolia, T5557 Rohdea japonica [Syn. Orontium japonicum], T5579 Rubia cordifolia, T5605 Rumex acetosa, T5607 Rumex crispus, T5611 Rumex japonicus, T5612 Rumex nepalensis, T5613 Rumex obtusifolius, T5614 Rumex patientia, T5672 Salvia flava, T5673 Salvia glutinosa, T5685 Salvia plebeia, T5687 Salvia prionitis, T5701 Salvia yunnanensis, T5712 Sanguisorba officinalis, T5754 Saussurea graminea, T5804 Schizonepeta tenuifolia [Syn. Nepeta tenuifolia], T5833 Scutellaria amoena, T5834 Scutellaria baicalensis, T5835 Scutellaria barbata [Syn. Scutellaria rivularis], T5839 Scutellaria hypericifolia, T5840 Scutellaria indica, T5841 Scutellaria likiangensis, T5843 Scutellaria rehderiana, T5845 Scutellaria viscidula, T5850 Sedum aizoon, T5855 Sedum kamtschaticum, T5862 Selaginella involvens, T5863 Selaginella moellendorffii, T5865 Selaginella pulvinata, T5869 Selaginella tamariscina, T5870 Selaginella uncinata, T5924 Sesamum indicum, T6034 Sophora japonica, T6036 Sophora japonica, T6116 Stenoloma chusanum, T6119 Stephania cepharantha, T6367 Teucrium quadrifarium, T6440 Thuja orientalis [Syn. Platycladus orientalis; Biota orientalis], T6471 Toddalia asiatica [Syn. Toddalia aculeata; Paullinia asiatica], T6482 Toxicodendron succedaneum [Syn. Rhus succedanea], T6485 Trachycarpus fortunei, T6584 Typha angustata, T6585 Typha angustifolia, T6587 Typha latifolia, T6601 Umbilicaria esculenta [Syn. Gyrophora esculenta], T6615 Uncaria gambir, T6764 Vinegar, T6895 Zanthoxylum simulans, T6904 Zephyranthes grandiflora [Syn. Zephyranthes carinata].

blood ejection due to blood heat $\mathrm{T} 3340$ Hypericum ascyron, T3416 Imperata cylindrica var. major, T4401 Nelumbo nucifera, T5133 Polytrichum commune, T5445 Rehmannia glutinosa [Syn. Rehmannia glutinosa f. huechingensis], T5447 Rehmannia glutinosa [Syn. Rehmannia glutinosa f. huechingensis], T5851 Sedum alfredii [Syn. Sedum formosanum].

blood ejection due to internal damage $\mathrm{T} 1566$ Clerodendrum inerme. blood ejection from knocks and falls T0529 Anthriscus sylvestris, T1448 Cirsium chinense.

blood heat T4398 Nelumbo nucifera, T5833 Scutellaria amoena, T5834 Scutellaria baicalensis, T5839 Scutellaria hypericifolia, T5841 Scutellaria likiangensis, T5843 Scutellaria rehderiana, T5845 Scutellaria viscidula, T6036 Sophora japonica.

blood loss T1604 Coeloglossum viride [Syn. Coeloglossum viride var. bracteatum], T3077 Gymnadenia conopsea.

blood qi pain T2835 Galium verum.

blood stagnation and menstrual block T1160 Campsis grandiflora.

blood stasis T2435 Eriocheir sinensis.

blood stasis and abdominal pain T1005 Brassica campestris [Syn. Brassica campestris var. oleifera], T1492 Citrus limon, T1496 Citrus limonia, T6278 Tagetes erecta.

blood stasis and dysmenorrhea T1001 Brachystemma calycinum, T1561 Clerodendron serratum, T1569 Clerodendrum serratum var. amplexifolium, T4450 Nuphar pumilum.

blood stasis and menstrual block T0383 Alyxia sinensis, T0999 Bougainvillea glabra.

blood stasis and $\boldsymbol{q} i$ stagnation T1903 Curcuma aromatica, T1905 Curcuma longa.

blood stasis lumbago T5648 Salacia prinoides [Syn. Salacia chinensis].

blood stasis swelling and pain T0108 Aconitum kusnezoffii, T0170 Adhatoda vasica, T0324 Allium victorialis, T1566 Clerodendrum inerme, T3853 Lindera obtusiloba, T5238 Prunus salicina, T5562 Rosa chinensis.

blood strangury T0073 Achyranthes bidentata, T0544 Apium graveolens, T0977 Bombyx mori, T1001 Brachystemma calycinum, T1145 Camellia japonica, T1210 Carpesium abrotanoides, T1311 Centella asiatica, T1451 Cirsium setosum [Syn. Cerratula setosa; Cirsium segetum; Cephalanoplos segetum], T1554 Clerodendron cyrtophyllum, T1907 Curcuma wengujin, T1924 Cyathula officinalis, T1950 Cynanchum ascyrifolium, T1951 Cynanchum atratum, T1962 Cynanchum versicolor, T2143 Dianthus chinensis, T2145 Dianthus superbus, T2147 Dianthus versicolor, T2952 Gerbera piloselloides, T3002 Glycine max, T3143 Helianthus annuus, T3278 Homo sapiens, T3363 Hypericum sampsonii, T3645 Kummerowia striata, T3681 Lamium barbatum, T3737 Lemmaphyllum microphyllum var. obovatum, T3933 Luffa acutangula, T3934 Luffa cylindrica, T3990 Lygodium japonicum, T4787 Phellinus igniarius, T5354 Pyrrosia davidii, T5355 Pyrrosia drakeana, T5356 Pyrrosia gralla, T5357 Pyrrosia lingua T5358 Pyrrosia petiolosa, T5360 Pyrrosia sheareri, T5835 Scutellaria barbata [Syn. Scutellaria rivularis], T5838 Scutellaria galericulata, T5966 Siphonostegia chinensis, T6006 Solanum melongena, T6034 Sophora japonica, T6036 Sophora japonica, T6582 Tylophora ovata, T6722 Veronica anagallis-aquatica, T6870 Zanthoxylum bungeanum.

blood strangury with inhibited pain T6584 Typha angustata, T6585 Typha angustifolia, T6587 Typha latifolia.

blood vacuity $\mathrm{T} 3742$ Lentinus edodes, T4238 Millettia nitida, T4308 Mucuna birdwoodiana, T4311 Mucuna sempervirens, T5042 Podocarpus macrophyllus, T5044 Podocarpus macrophyllus var. maki, T6066 Spatholobus suberectus.

blood vacuity and general weakness T4234 Millettia dielsiana, T4239 
Millettia nitida var. hirsutissima, T5529 Rhodomyrtus tomentosa.

blood vacuity and numbness in limbs T5701 Salvia yunnanensis.

blood vacuity during convalescence T5179 Potentilla anserina.

blood vacuity of women T4438 Nostoc flagelliforme.

blood vacuity with yellow complexion T3129 Hedysarum polybotrys, T5446 Rehmannia glutinosa [Syn. Rehmannia glutinosa f. huechingensis].

blood vaculty T0474 Angelica acutiloba [Syn. Ligusticum acutilobum], T0495 Angelica sinensis, T1598 Codonopsis convolvulacea, T1680 Cordyceps militaris, T1681 Cordyceps militaris cv, T2347 Embelia parviflora, T4804 Phlojodicarpus sibiricus.

bloody stool T0600 Ardisia mamillata [Syn. Tinus mamillata], T1118 Callicarpa formosana, T1119 Callicarpa japonica, T1254 Castanea mollissima, T2927 Gentiana rhodantha, T5182 Potentilla discolor, T5754 Saussurea graminea, T5863 Selaginella moellendorffii, T5868 Selaginella stauntoniana.

bloody urine T1118 Callicarpa formosana, T1119 Callicarpa japonica, T3765 Lepisorus ussuriensis, T5868 Selaginella stauntoniana.

bloody urine (hematuria) T5002 Plantago asiatica, T5004 Plantago depressa, T5007 Plantago major.

blurred vision T2803 Frullania tamarisci ssp. moniliata [Syn. Frullania moniliata], T4656 Parmelia saxatilis, T5227 Prunus mume.

body lichen T0750 Asparagus gobicus, T5821 Scopolia japonica.

boil T4005 Lysionotus pauciflorus, T4253 Mirabilis jalapa, T4789 Phellodendron amurense, T4790 Phellodendron amurense var. wilsonii, T4791 Phellodendron chinense, T4792 Phellodendron chinense var. glabriusculum, T6494 Trema dielsiana.

boil and lichen T2036 Datura innoxia, T2041 Datura metel, T2046 Datura stramonium.

bone marrow infection T5680 Salvia miltiorrhiza, T5681 Salvia miltiorrhiza f. alba, T5688 Salvia przewalskii.

bone stuck in throat T1545 Clematis chinensis, T1982 Cyprinus carpio, T5569 Rosa multiflora.

bone tuberculosis $\mathrm{T} 1550$ Cleome gynandra [Syn. Gynandropsis gynandra], T3564 Juglans mandshurica, T4005 Lysionotus pauciflorus, T5279 Psychotria serpens, T6105 Stellera chamaejasme, T6540 Tripterygium hypoglaucum.

brain leak T0988 Bos taurus domesticus; Bubalus bubalis, T4215 Michelia yunnanensis.

brandy nose T1160 Campsis grandiflora, T4020 Macleaya cordata.

breast milk stoppage $\mathrm{T} 0156$ Actinidia arguta, T0173 Adiantum lunulatum, T2720 Ficus hispida, T3270 Holboellia fargesii, T4120 Marsdenia tenacissima, T6777 Viscum multinerve.

bronchial asthma $\mathrm{T} 1941$ Cymbopogon distans, T2367 Ephedra equisetina, T2368 Ephedra gerardiana, T2369 Ephedra intermedia, T2372 Ephedra likiangensis, T2373 Ephedra minuta, T2375 Ephedra monosperma, T2379 Ephedra saxatilis, T2380 Ephedra sinica, T3106 Harpagophytum procumbens, T3885 Litsea cubeba, T4796 Pheretima aspergillum; Allolobophora caliginosa trapezoides, T6820 Wikstroemia indica.

bronchitis T0151 Acronychia pedunculata, T0967 Boenninghausenia sessilicarpa, T1381 Choerospondias axillaris, T1943 Cymbopogon goeringii, T2565 Eupatorium lindleyanum, T2720 Ficus hispida,
T3011 Glycyrrhiza aspera, T3013 Glycyrrhiza glabra, T3015 Glycyrrhiza inflata, T3016 Glycyrrhiza kansuensis, T3021 Glycyrrhiza squamulosa, T3022 Glycyrrhiza uralensis, T3023 Glycyrrhiza yunnanensis, T3056 Gossypium herbaceum, T3246 Hibiscus syriacus, T3568 Juglans regia, T3709 Lathyrus pratensis, T3749 Leontopodium alpinum, T4248 Mimosa pudica, T4403 Nemacystus decipiens [Syn. Mesogloea decipiens; Cladosiphon decipiens], T4689 Peganum nigellastrum, T5072 Polygala caudata, T5907 Senecio scandens [Syn. Senecio chinensis], T6132 Stephania sasakii.

bubo T2601 Euphorbia milii.

bulging mounting T5740 Sargassum vachellianum.

burns T1084 Buthus martensi, T1118 Callicarpa formosana, T1119 Callicarpa japonica, T2988 Glochidion eriocarpum, T4789 Phellodendron amurense, T4790 Phellodendron amurense var. wilsonii, T4791 Phellodendron chinense, T4792 Phellodendron chinense var. glabriusculum, T5018 Pleuropterus ciliinervis, T5549 Ricinus communis, T6440 Thuja orientalis [Syn. Platycladus orientalis; Biota orientalis], T6521 Trifolium pratense, T6691 Veratrilla baillonii.

burns and scalds T0001 Abelmoschus manihot, T0002 Abelmoschus moschatus [Syn. Hibiscus abelmoschus], T0267 Ajuga nipponensis, T0299 Aleurites cordata [Syn. Aleurites fordii], T0382 Althaea rosea, T0421 Amorpha fruticosa, T0427 Ampelopsis japonica [Syn. Paullinia japonica], T0540 Apis cerana, T0563 Arachniodes exilis, T0680 Artemisia lactiflora, T0776 Asplenium prolongatum, T0827 Aucuba chinensis ssp. omeiensis, T0838 Azolla imbricata [Syn. Salvinia imbricata], T0853 Baeckea frutescens, T0879 Bauhinia purpurea, T0955 Bletilla striata, T1044 Bryum argenteum, T1137 Caltha palustris, T1146 Camellia oleifera, T1147 Camellia oleifera, T1151 Camellia sinensis [Syn. Thea sinensis], T1152 Camellia sinensis [Syn. Thea sinensis], T1163 Campylotropis hirtella, T1251 Cassytha filiformis, T1289 Celastrus orbiculatus [Syn. Celastrus articulatus], T1366 Chimonanthus fragrans [Syn. Chimonanthus praecox], T1381 Choerospondias axillaris, T1437 Cinnamomum camphora, T1644 Conocephalum conicum, T1692 Coriaria sinica [Syn. Coriaria nepalensis], T1758 Cotinus coggygria, T1759 Cotinus coggygria var. cinerea, T1878 Cucumis sativus, T2092 Delphinus delphis, T2161 Dicranopteris pedata [Syn. Polypodium pedatum; Dicranopteris dichotoma], T2188 Dioscorea alata, T2243 Dodonaea viscosa, T2290 Duchesnea indica, T2293 Dumortiera hirsuta, T2327 Elaeagnus angustifolia, T2505 Eucalyptus citriodora, T2510 Eucalyptus globulus, T2536 Euonymus alatus, T2601 Euphorbia milii, T2658 Fagopyrum esculentum, T2715 Fibraurea recisa, T2839 Gallus gallus domesticus, T2852 Garcinia cowa, T2866 Garcinia morella, T2927 Gentiana rhodantha, T3241 Hibiscus mutabilis, T3361 Hypericum perforatum, T3376 Hypolepis punctata [Syn. Polypodium punctatum], T3387 Idesia polycarpa, T3388 Ilex chinensis [Syn. Ilex purpurea], T3396 Ilex pubescens, T3397 Ilex pubescens var. glaber, T3414 Impatiens siculifer, T3479 Isodon amethystoides, T3775 Lethariella cladonioides, T3827 Ligustrum japonicum, T3845 Linaria vulgaris, T3932 Ludwigia octovalvis, T3969 Lycopodium casuarinoides, T3983 Lycoris aurea, T3984 Lycoris chinensis, T3986 Lycoris radiata [Syn. Amaryllis radiata], T3990 Lygodium japonicum, T4026 Macrothelypteris oligophlebia, T4072 Mahonia shenii, T4109 Marchantia polymorpha, 
T4261 Mollugo pentaphylla, T4349 Myrica rubra, T4487 Olea europaea, T4502 Onychium japonicum [Syn. Tricomanes japonicum], T4520 Opuntia ficus-indica, T4562 Oxalis acetosella, T4564 Oxalis corniculata [Syn. Oxalis repens], T4705 Peperomia pellucida, T4798 Philonotis fontana, T4881 Picrasma quassioides [Syn. Picrasma ailanthoides], T4937 Piper betle, T5101 Polygonum cuspidatum, T5156 Populus nigra var. thevestina, T5172 Portulaca grandiflora, T5288 Pteris cretica var. nervosa [Syn. Pteris nervosa], T5290 Pteris fauriei [Syn. Pteris fauriei var. minor], T5395 Rabdosia nervosa, T5444 Reboulia hemisphaerica, T5472 Rheum officinale, T5474 Rheum palmatum, T5481 Rheum tanguticum, T5499 Rhodiola sacra, T5614 Rumex patientia, T5650 Salix babylonica, T5712 Sanguisorba officinalis, T5850 Sedum aizoon, T5855 Sedum kamtschaticum, T5856 Sedum lineare [Syn. Sedum obtuso-lineare], T5857 Sedum sarmentosum, T5859 Selaginella braunii, T5863 Selaginella moellendorffii, T5866 Selaginella sanguinolenta, T5867 Selaginella sinensis, T5870 Selaginella uncinata, T5925 Sesamum indicum [Syn. Sesamum orientale], T5976 Smilax china [Syn. Smilax japonica], T6025 Sonchus arvensis, T6087 Spiranthes sinensis, T6116 Stenoloma chusanum, T6234 Swertia punicea, T6264 Syzygium buxifolium, T6716 Vernonia esculenta, T6728 Veronica serpyllifolia, T6883 Zanthoxylum myriacanthum, T6884 Zanthoxylum nitidum, T6920 Ziziphus mauritiana.

calculus of urinary system T2838 Gallus gallus domesticus, T3989 Lygodium flexuosum [Syn. Lygodium pinnatifidum; Ophioglossum flexuosum], T3998 Lysimachia christinae, T4248 Mimosa pudica.

cancer of esophagus T2263 Dregea volubilis.

candida vaginitis T5101 Polygonum cuspidatum.

carcinoma T0275 Akebia quinata, T0543 Apis mellifera ligustica, T1319

Cephalotaxus hainanensis [Syn. Cephalotaxus mannii], T1322 Cephalotaxus oliveri, T1323 Cephalotaxus sinensis [Syn. Cephalotaxus harringtonia var. sinensis], T2191 Dioscorea bulbifera, T2846 Ganoderma japonicum [Syn. Ganoderma sinense], T2848 Ganoderma lucidum, T2857 Garcinia hanburyi, T3126 Hedyotis corymbosa [Syn. Oldenlandia corymbosa], T3480 Isodon angustifolia, T3742 Lentinus edodes, T4120 Marsdenia tenacissima, T4131 Maytenus confertiflorus, T4134 Maytenus hookeri, T4303 Moschus moschiferus; Moschus berezovskii; Moschus sifanicus, T4485 Oldenlandia diffusa [Syn. Hedyotis diffusa], T5835 Scutellaria barbata [Syn. Scutellaria rivularis], T5838 Scutellaria galericulata.

carcinoma in digestive tract $\mathrm{T} 0156$ Actinidia arguta, T3229 Hericium erinaceus [Syn. Hydnum erinaceus].

carcinoma of cardia T1162 Camptotheca acuminata.

carcinoma of esophagus T0161 Actinidia eriantha, T1162 Camptotheca acuminata, T1318 Cephalotaxus fortunei, T1831 Crotalaria sessiliflora, T2143 Dianthus chinensis, T2145 Dianthus superbus, T2147 Dianthus versicolor, T2748 Fomes fomentarius [Syn. Pyropolyporus fomentarius; Boletus fomentarius; Polyporus fomentarius], T2844 Ganoderma applanatum, $\mathrm{T} 5129$ Polyporus umbellatus, T5396 Rabdosia rubescens.

carcinoma of intestine T1162 Camptotheca acuminata.

carcinoma of liver T1162 Camptotheca acuminata, T1831 Crotalaria sessiliflora.

carcinoma of lung T1318 Cephalotaxus fortunei, T1831 Crotalaria sessiliflora, T1904 Curcuma kwangsiensis, T1909 Curcuma zedoaria [Syn. Curcuma aeruginosa], T5129 Polyporus umbellatus.

carcinoma of mammary glands T3443 Iphigenia indica, T5396 Rabdosia rubescens, T6311 Taxus cuspidata.

carcinoma of ovary T1904 Curcuma kwangsiensis, T1909 Curcuma zedoaria [Syn. Curcuma aeruginosa], T6311 Taxus cuspidata.

carcinoma of rectum T1318 Cephalotaxus fortunei, T2143 Dianthus chinensis, T2145 Dianthus superbus, T2147 Dianthus versicolor.

carcinoma of salivary gland T3443 Iphigenia indica.

carcinoma of skin T1904 Curcuma kwangsiensis, T1909 Curcuma zedoaria [Syn. Curcuma aeruginosa].

carcinoma of stomach T0161 Actinidia eriantha, T1162 Camptotheca acuminata, T1318 Cephalotaxus fortunei, T1831 Crotalaria sessiliflora, T1904 Curcuma kwangsiensis, T1909 Curcuma zedoaria [Syn. Curcuma aeruginosa], T2263 Dregea volubilis, T2748 Fomes fomentarius [Syn. Pyropolyporus fomentarius; Boletus fomentarius; Polyporus fomentarius].

carcinoma of thyroid T1904 Curcuma kwangsiensis, T1909 Curcuma zedoaria [Syn. Curcuma aeruginosa], T4020 Macleaya cordata.

carcinoma of uterine cervix T0617 Arisaema amurense, T0618 Arisaema consanguineum, T0620 Arisaema heterophyllum, T0830 Auricularia auricula, T0831 Auricularia delicata, T1831 Crotalaria sessiliflora, T3842 Limonium gmelinii, T4020 Macleaya cordata, T4903 Pinellia pedatisecta.

carcinoma of vagina T0830 Auricularia auricula, T0831 Auricularia delicata.

cardiac failure T0553 Apocynum venetum, T1468 Citrus aurantium, T1521 Citrus wilsonii, T2175 Digitalis lanata, T2177 Digitalis purpurea, T2452 Erysimum cheiranthoides, T4599 Panax ginseng [Syn. Panax schinseng], T5141 Poncirus trifoliata, T5557 Rohdea japonica [Syn. Orontium japonicum], T6155 Strophanthus divaricatus, T6433 Thevetia neriifolia [Syn. Thevetia peruviana].

cardiac insufficiency T0184 Adonis amurensis.

cardiovascular diseases T3231 Hernandia sonora [Syn. Hernandia ovigera].

cassava poisoning T3248 Hibiscus tiliaceus, T3685 Lannea grandis [Syn. Lannea coromandelica], T4503 Onychium lucidum.

center qi fall T0791 Astragalus chrysopterus, T0794 Astragalus ernestii, T0798 Astragalus membranaceus, T0800 Astragalus mongholicus, T1419 Cimicifuga dahurica, T1420 Cimicifuga foetida, T1421 Cimicifuga heracleifolia, T1423 Cimicifuga nanchuanensis.

center vacuity with stomachache T5067 Polyalthia nemoralis.

centipede bite T3400 Illicium difengpi, T3403 Illicium majus, T4217 Microcos paniculata [Syn. Grewia microcos], T4835 Phyllanthus emblica, T6177 Strychnos ignatii, T6551 Trogopterus xanthipes; Pteromys volans.

central angiospastic retinitis $\mathrm{T} 3396$ Ilex pubescens, T3397 Ilex pubescens var. glaber.

central retinitis $\mathrm{T} 3396$ Ilex pubescens, $\mathrm{T} 3397$ Ilex pubescens var. glaber. cerebral and cardiovascular diseases T6775 Viscum coloratum.

cerebral atherosclerosis T5680 Salvia miltiorrhiza, T5681 Salvia miltiorrhiza f. alba, T5688 Salvia przewalskii.

cerebral ischemia T1583 Cnidium officinale [Syn. Ligusticum officinale], 
T3820 Ligusticum chuanxiong [Syn. Ligusticum wallichii].

cerebral thrombosis T0502 Anisodus tanguticus [Syn. Scopolia tangutica], T1215 Carthamus tinctorius, T3396 Ilex pubescens, T3397 Ilex pubescens var. glaber, T4303 Moschus moschiferus; Moschus berezovskii; Moschus sifanicus.

cerebrovascular insufficiency diseases T2961 Ginkgo biloba.

cervical erosion T0543 Apis mellifera ligustica.

cervicitis T6008 Solanum nigrum.

chancre T2200 Dioscorea hispida, T2883 Gardenia jasminoides [Syn. Gardenia florida].

chest and rib-side pain T0491 Angelica polymorpha, T1750 Corydalis yanhusuo [Syn. Corydalis turtschaninovii f. yanhusuo], T2008 Dalbergia odorifera, T2013 Dalbergia sissoo, T2555 Eupatorium chinense, T4163 Melia toosendan, T4604 Panax japonicus var. bipinnatifidus, T4823 Pholidota yunnanensis.

chest and rib-side stabbing pain T1583 Cnidium officinale [Syn. Ligusticum officinale], T3820 Ligusticum chuanxiong [Syn. Ligusticum wallichii].

chest impediment T0313 Allium chinense, T0316 Allium macrostemon, T0322 Allium tuberosum, T1438 Cinnamomum cassia [Syn. Cinnamomum aromaticum], T2546 Euonymus sacrosancta, T3869 Liquor, T4221 Micromelum falcatum, T6510 Trichosanthes kirilowii, T6513 Trichosanthes rosthornii [Syn. Trichosanthes uniflora].

chest impediment and angina T5661 Salvia bowleyana, T5671 Salvia digitaloides, T5701 Salvia yunnanensis.

chest impediment and heart pain T2964 Ginkgo biloba, T3396 Ilex pubescens, $\mathrm{T} 3397$ Ilex pubescens var. glaber.

chest oppression and pain T5497 Rhodiola kirilowii.

chest oppression with cough asthma T2697 Ferula borealis, T3432 Inula helianthus-aquatica.

chest oppression with retching T5833 Scutellaria amoena, T5834 Scutellaria baicalensis, T5839 Scutellaria hypericifolia, T5841 Scutellaria likiangensis, T5843 Scutellaria rehderiana, T5845 Scutellaria viscidula.

chest pain T1381 Choerospondias axillaris, T3797 Libanotis condensata. chest pain due to cough T0045 Acanthopanax trifoliatus.

child aphonia T1050 Bufo bufo gargarizans; Bufo melanostictus, T5406 Rana limnocharis.

child convulsion T2890 Gastrodia elata.

child epilepsy T1660 Coprinus atramentarius.

child food accumulation T0847 Baccharis indica [Syn. Pluchea indica], T1954 Cynanchum chinense.

child fright epilepsy T2836 Gallus gallus domesticus, T6197 Styrax benzoin, T6204 Styrax tonkinensis.

child gan accumulation T0338 Aloe ferox, T0347 Aloe vera [Syn. Aloe barbadensis], T0348 Aloe vera var. chinensis, T0387 Amaranthus caudatus, T0500 Anguilla japonica, T0941 Bidens tripartita, T0950 Blatta orientalis, T0969 Boerhavia diffusa, T1049 Bufo bufo gargarizans; Bufo melanostictus, T1143 Calystegia hederacea, T1188 Caragana chamlagu, T1211 Carpesium abrotanoides, T1238 Cassia mimosoides, T1247 Cassia sophera, T1563 Clerodendron trichotomum, T1684 Cordyline strcta, T1815 Crotalaria assamica, T1826 Crotalaria mucronata, T1831 Crotalaria sessiliflora, T1954 Cynanchum chinense,
T1958 Cynanchum otophyllum, T2020 Damnacanthus indicus, T2049 Daucus carota, T2267 Drosera peltata var. lunata, T2272 Drymaria diandra [Syn. Drymaria cordata ssp. diandra], T2422 Erigeron breviscapus, T2502 Eucalyptus camaldulensis, T2838 Gallus gallus domesticus, T3770 Lespedeza cuneata, T3950 Lychnis fulgens, T3999 Lysimachia clethroides, T4000 Lysimachia congestiflora, T4240 Millettia pachycarpa, T4526 Origanum vulgare, T4549 Osbeckia chinensis, T4843 Phyllanthus urinaria, T4887 Picrorhiza kurrooa, T4888 Picrorhiza scrophulariiflora, T5071 Polygala arillata, T5073 Polygala chinensis [Syn. Polygala glomerata], T5075 Polygala fallax [Syn. Polygala aureocauda], T5104 Polygonum hydropiper, T5122 Polygonum viscosum, T5183 Potentilla griffithii var. velutina, T5189 Pothos chinensis, T5384 Quisqualis indica, T5385 Quisqualis indica, T5409 Rana temporaria chensinensis; Rana amurensis, T5717 Sapindus delavayi [Syn. Pancovia delavayi], T5848 Securinega suffruticosa, T6229 Swertia patens, T6285 Tamarindus indica, T6489 Tragopogon porrifolius, $\mathrm{T} 6831$ Woodwardia orientalis.

child gan accumulation with fever T2909 Gentiana crassicaulis, T2910 Gentiana dahurica, T2913 Gentiana kaufmanniana, T2919 Gentiana macrophylla, T2932 Gentiana siphonantha, T2934 Gentiana straminea, T2936 Gentiana tianschanica, T2937 Gentiana tibetica, T3954 Lycium barbarum, T3956 Lycium chinense.

child gan fever T0607 Arenaria juncea, T0678 Artemisia japonica, T3090 Gypsophila oldhamiana, T3091 Gypsophila pacifica, T3932 Ludwigia octovalvis, T5955 Silene jenisseensis, T6104 Stellaria dichotoma var. lanceolata.

child gan sore T1952 Cynanchum auriculatum, T1953 Cynanchum bungei.

child head sore T2348 Embelia ribes.

child heat sore T5407 Rana nigromaculata; Rana plancyi.

child indigestion T2715 Fibraurea recisa, T2840 Gallus gallus domesticus, T5114 Polygonum polystachyum, T5375 Quercus mongolica, T6145 Stichopus japonicus.

child jaundice T5101 Polygonum cuspidatum.

child measles T1366 Chimonanthus fragrans [Syn. Chimonanthus praecox], T1425 Cimicifuga simplex, T5804 Schizonepeta tenuifolia [Syn. Nepeta tenuifolia], T5818 Scoparia dulcis.

child milk accumulation T3840 Limnophila rugosa.

child mounting qi T5362 Pyrus bretschneideri, T5366 Pyrus pyrifolia.

child oxyuria disease T6827 Wisteria sinensis.

child wind papules T0382 Althaea rosea.

children wind-heat T0434 Amsonia sinensis.

cholangitis T5957 Silybum marianum.

cholecystalgia T0501 Anisodus luridus, T0825 Atropa belladonna.

cholecystitis T0137 Aconitum tanguticum, T0646 Armillariella tabescens, T0647 Armoracia lapathifolia, T0912 Berberis poiretii, T0920 Berberis wilsonae, T1406 Chrysosplenium nudicaule, T1657 Conyza canadensis [Syn. Erigeron canadensis], T2233 Diploclisia glaucescens, T2941 Gentianopsis paludosa, T3156 Helichrysum arenarium, T3332 Hypecoum erectum, T3334 Hypecoum leptocarpum, T3507 Isodon lophanthoides [Syn. Rabdosia lophanthoides; Hyssopus lophanthoides; Plectranthus striatus; Isodon striatus], T3547 Ixeris chinensis, T4194 Menyanthes trifoliata, T4391 Nauclea officinalis, T5247 Przewalskia 
tangutica, T5397 Rabdosia serra, T5472 Rheum officinale, T5474 Rheum palmatum, T5481 Rheum tanguticum, T5730 Sarcandra glabra [Syn. Chloranthus glaber], T5867 Selaginella sinensis, T6095 Stachytarpheta jamaicensis, T6227 Swertia mussotii, T6234 Swertia punicea, T6789 Vitex negundo, T6901 Zea mays.

cholera T0318 Allium sativum, T1153 Camellia sinensis var. assamica, T1262 Catalpa ovata, T4086 Malus asiatica, T6056 Sorghum vulgare.

cholera cramp T4983 Pisum sativum, T6439 Thuja orientalis [Syn. Platycladus orientalis; Biota orientalis].

cholera with vomiting T2216 Diospyros ebenum.

cholera with vomiting and diarrhea T4190 Mentha spicata, T5362 Pyrus bretschneideri, T5366 Pyrus pyrifolia.

cholera with vomiting of sour matter T4548 Oryza sativa var. glutinosa. chorioblastoma T6043 Sophora subprostrata [Syn. Sophora tonkinensis]. chorionitis T2753 Formica fusca.

chronic anemia T6184 Strychnos nux-vomica, T6193 Strychnos wallichiana.

chronic appendicitis T6705 Verbascum thapsus.

chronic bacillary dysentery T0130 Aconitum sinomontanum.

chronic bone marrow infection T0625 Aristolochia contorta, T0627 Aristolochia debilis [Syn. Aristolochia longa].

chronic bronchitis T0553 Apocynum venetum, T0625 Aristolochia contorta, T0627 Aristolochia debilis [Syn. Aristolochia longa], T1357 Chelidonium majus, T1943 Cymbopogon goeringii, T2023 Daphne genkwa, T2203 Dioscorea nipponica, T2204 Dioscorea nipponica ssp. rosthornii, T2389 Epimedium acuminatum, T2390 Epimedium brevicornum, T2392 Epimedium davidii, T2394 Epimedium elongatum, T2398 Epimedium koreanum, T2401 Epimedium pubescens, T2402 Epimedium sagittatum, T2404 Epimedium sutchuenense, T2406 Epimedium wushanense, T2766 Fraxinus bungeana, T2767 Fraxinus chinensis, T2774 Fraxinus paxiana, T2777 Fraxinus rhynchophylla [Syn. Fraxinus chinensis var. rhynchophylla], T2779 Fraxinus stylosa, T2780 Fraxinus szaboana [Syn. Fraxinus chinensis var. acuminata], T2783 Fritillaria cirrhosa, T2784 Fritillaria delavayi, T2792 Fritillaria przewalskii, T2796 Fritillaria unibracteata, T2798 Fritillaria verticillata var. thunbergii [Syn. Fritillaria thunbergii], T2846 Ganoderma japonicum [Syn. Ganoderma sinense], T2848 Ganoderma lucidum, T3284 Houttuynia cordata, T3828 Ligustrum lucidum, T3885 Litsea cubeba, T4005 Lysionotus pauciflorus, T4796 Pheretima aspergillum; Allolobophora caliginosa trapezoides, T5508 Rhododendron dauricum, T5989 Solanum aculeatissimum, T6008 Solanum nigrum, T6014 Solanum surattense, T6043 Sophora subprostrata [Syn. Sophora tonkinensis], T6259 Syringa amurensis [Syn. Syringa reticulata var. amurensis], T6440 Thuja orientalis [Syn. Platycladus orientalis; Biota orientalis], T6574 Tussilago farfara.

chronic cervicitis T6043 Sophora subprostrata [Syn. Sophora tonkinensis].

chronic cholecystitis T0897 Berberis amurensis, T6691 Veratrilla baillonii.

chronic conjunctivitis T6031 Sophora flavescens [Syn. Sophora angustfolia].

chronic constipation T4976 Piptanthus nepalensis, T5309 Pterocladia tenuis. chronic cough and asthma T1049 Bufo bufo gargarizans; Bufo melanostictus, T2584 Euphorbia ebracteolata, T2587 Euphorbia fischeriana, T4851 Physalis minima, T5637 Sabia schumanniana.

chronic diarrhea T0197 Aegle marmelos, T0255 Ailanthus altissima, T0542 Apis cerana, T0791 Astragalus chrysopterus, T0794 Astragalus ernestii, T0798 Astragalus membranaceus, T0800 Astragalus mongholicus, T1425 Cimicifuga simplex, T1604 Coeloglossum viride [Syn. Coeloglossum viride var. bracteatum], T1826 Crotalaria mucronata, T2053 Davallia mariesii, T3077 Gymnadenia conopsea, T4400 Nelumbo nucifera, T4635 Papaver somniferum, T4637 Papaver somniferum, T5169 Poria cocos, T5270 Psoralea corylifolia, T5327 Punica granatum, T5328 Punica granatum, T5472 Rheum officinale, T5474 Rheum palmatum, T5481 Rheum tanguticum, T5554 Rodgersia aesculifolia, T6347 Terminalia chebula, T6474 Toona ciliata, T6475 Toona sinensis, T6670 Vaccinium bracteatum.

chronic diarrhea and dysentery T3567 Juglans regia, T5228 Prunus mume, T5568 Rosa laevigata, T6266 Syzygium cumini, T6346 Terminalia chebula, T6348 Terminalia chebula var. tomentella, T6670 Vaccinium bracteatum.

chronic dysentery T0255 Ailanthus altissima, T1419 Cimicifuga dahurica, T1420 Cimicifuga foetida, T1421 Cimicifuga heracleifolia, T2032 Daphniphyllum calycinum, T2050 Daucus carota var. sativa, T2051 Daucus carota var. sativa, T4635 Papaver somniferum, T5265 Psidium guajava, T5327 Punica granatum, T5328 Punica granatum, T5332 Punica granatum, T5569 Rosa multiflora, T5654 Salix purpurea, T6251 Symplocos caudata, T6267 Syzygium jambos, T6347 Terminalia chebula, T6474 Toona ciliata, T6475 Toona sinensis.

chronic eczema T2651 Excoecaria cochinchinensis var. viridis.

chronic endometritis T2715 Fibraurea recisa.

chronic enteritis T0130 Aconitum sinomontanum, T6599 Ulva lactuca, T6600 Ulva pertusa.

chronic fright wind T1210 Carpesium abrotanoides, T2037 Datura innoxia, T2044 Datura metel, T2890 Gastrodia elata, T5817 Scolopendra subspinipes mutilans.

chronic gastritis T0791 Astragalus chrysopterus, T0794 Astragalus ernestii, T0798 Astragalus membranaceus, T0800 Astragalus mongholicus, T1101 Caesalpinia decapetala, T3164 Helicteres isora, T3229 Hericium erinaceus [Syn. Hydnum erinaceus], T3613 Kadsura coccinea [Syn. Kadsura chenensis; Kadsura hainanensis].

chronic gastroenteritis T0501 Anisodus luridus, T0502 Anisodus tanguticus [Syn. Scopolia tangutica], T3085 Gynostemma pentaphyllum, T5247 Przewalskia tangutica.

chronic glomerulonephritis T0791 Astragalus chrysopterus, T0794 Astragalus ernestii, T0798 Astragalus membranaceus, T0800 Astragalus mongholicus, T3416 Imperata cylindrica var. major.

chronic hepatitis T0048 Acanthus ilicifolius, T0541 Apis cerana, T0680 Artemisia lactiflora, T0897 Berberis amurensis, T1604 Coeloglossum viride [Syn. Coeloglossum viride var. bracteatum], T1876 Cucumis melo, T1927 Cycas revoluta, T3077 Gymnadenia conopsea, T3742 Lentinus edodes, T5396 Rabdosia rubescens, T5607 Rumex crispus, T5957 Silybum marianum.

chronic ischiatitis T5848 Securinega suffruticosa.

chronic leukemia T1162 Camptotheca acuminata. 
chronic liver diseases T6231 Swertia pseudochinensis.

chronic medullitis T1556 Clerodendron fragrans.

chronic nephritis T0423 Ampelopsis brevipedunculata, T0495 Angelica sinensis, T0598 Ardisia japonica, T1553 Clerodendranthus spicatus, T2327 Elaeagnus angustifolia, T3128 Hedysarum multijugum, T4804 Phlojodicarpus sibiricus, T4906 Pinus armandii, T4916 Pinus massoniana, T5704 Sambucus nigra, T5707 Sambucus williamsii, T6540 Tripterygium hypoglaucum.

chronic osteomyelitis T0333 Alocasia cucullata [Syn. Arum cucullatum]. chronic pain in lumbus and legs T3614 Kadsura heteroclita [Syn. Uvaria heteroclita].

chronic pharyngitis T1053 Bulbophyllum odoratissimum [Syn. Stelis odoratissimum].

chronic pharyngolaryngitis T6507 Trichosanthes cucumeroides.

chronic rheumatic arthritis T1180 Capparis spinosa, T2749 Fomes officinalis.

chronic scab T1903 Curcuma aromatica, T1905 Curcuma longa.

chronic sinus infections T6843 Xanthium sibiricum [Syn. Xanthium strumarium].

chronic sores T6132 Stephania sasakii.

chronic trachitis T0598 Ardisia japonica, T0933 Betula platyphylla, T0935 Betula platyphylla var. japonica, T1051 Bufo bufo gargarizans; Bufo melanostictus, T1053 Bulbophyllum odoratissimum [Syn. Stelis odoratissimum], T1655 Conyza blinii, T2203 Dioscorea nipponica, T2204 Dioscorea nipponica ssp. rosthornii, T2327 Elaeagnus angustifolia, T3033 Gnetum parvifolium [Syn. Gnetum indicum], T3085 Gynostemma pentaphyllum, T4908 Pinus bungeana, T5522 Rhododendron seniavinii, T5607 Rumex crispus, T6008 Solanum nigrum, T6105 Stellera chamaejasme, T6729 Veronica spuria, T6787 Vitex negundo.

chronic tympanitis T1570 Clerodendrum thomsonae.

chronic ulcer T1903 Curcuma aromatica, T1905 Curcuma longa.

chronic urticaria T4184 Mentha haplocalyx [Syn. Mentha canadaensis;

Mentha arvensis var. haplocalyx; Mentha arvensis].

chronic uterine cervical infection $\mathrm{T} 3284$ Houttuynia cordata .

chronic wound ulcer T0791 Astragalus chrysopterus, T0794 Astragalus ernestii, T0798 Astragalus membranaceus, T0800 Astragalus mongholicus.

chyluria T1184 Capsella bursa-pastoris, T1199 Carduus acanthoides, T1200 Carduus crispus, T3125 Hedyotis chrysotricha [Syn. Oldenlandia chrysotricha], T3143 Helianthus annuus, T3646 Kyllinga brevifolia, T5098 Polygonum aviculare.

cinnabar toxin T0838 Azolla imbricata [Syn. Salvinia imbricata].

cirrhosis T0853 Baeckea frutescens, T1876 Cucumis melo, T5957 Silybum marianum.

cirrhosis with ascites T0553 Apocynum venetum, T1240 Cassia obtusifolia, T1250 Cassia tora, T2727 Ficus simplicissima, T5844 Scutellaria scordifolia, T6436 Thlaspi arvense.

clavus T0318 Allium sativum, T0543 Apis mellifera ligustica.

clear-eye blindness T0986 Bos taurus domesticus; Bubalus bubalis, T1185 Capsella bursa-pastoris, T1240 Cassia obtusifolia, T1250 Cassia tora, T6732 Vespertilio superans.

clear-eye blindness with internal obstruction T1981 Cyprinus carpio. clenched jaw T3000 Glycine max.

climacteric hypertension T2389 Epimedium acuminatum, T2390 Epimedium brevicornum, T2392 Epimedium davidii, T2394 Epimedium elongatum, T2398 Epimedium koreanum, T2401 Epimedium pubescens, T2402 Epimedium sagittatum, T2404 Epimedium sutchuenense, T2406 Epimedium wushanense.

clouded flowery vision T0049 Acer ginnala, T0793 Astragalus complanatus.

clouded spirit T4855 Physeter catodon.

clouded spirit with delirious speech T1662 Coptis chinensis, T1663 Coptis chinensis var. brevisepala, T1664 Coptis deltoidea, T1669 Coptis omeiensis, T1670 Coptis teetoides [Syn. Coptis teeta], T4401 Nelumbo nucifera.

clouded spirit with loss of speech T2978 Gleditsia sinensis [Syn. Gleditsia horrida].

clove boil $\mathrm{T} 1372$ Chloranthus serratus, T2243 Dodonaea viscosa.

clove boil and swollen welling abscess T3113 Hedera nepalensis var. sinensis, T3115 Hedera rhombea.

clove sore T0195 Aeginetia indica, T0595 Ardisia crispa, T0600 Ardisia mamillata [Syn. Tinus mamillata], T0805 Astragalus sinicus, T1049 Bufo bufo gargarizans; Bufo melanostictus, T1197 Cardiospermum halicacabum, T1275 Cayratia japonica, T1387 Chrysanthemum boreale, T1392 Chrysanthemum indicum, T1394 Chrysanthemum lavandulifolium, T1403 Chrysosplenium grayanum, T1448 Cirsium chinense, T1565 Clerodendrum bungei, T2298 Dysosma difformis, T2302 Dysosma pleiantha [Syn. Podophyllum pleianthum], T2304 Dysosma veitchii, T2305 Dysosma versipellis [Syn. Podophyllum versipelle], T2580 Euphorbia antiquorum, T2590 Euphorbia hirta, T2649 Evolvulus alsinoides, T2897 Gelsemium elegans, T3144 Helianthus annuus, T3146 Helianthus annuus cv, T3411 Impatiens balsamina, T3928 Lotus corniculatus, T3997 Lysimachia capillipes, T4206 Metaplexis japonica, T4428 Nicotiana tabacum, T4530 Ormosia hosiei, T4836 Phyllanthus emblica, T4837 Phyllanthus emblica, T4846 Physalis alkekengi var. franchetii, T4848 Physalis angulata, T4854 Physalis pubescens, T4917 Pinus massoniana, T5097 Polygonum amphibium, T5194 Premna microphylla, T5557 Rohdea japonica [Syn. Orontium japonicum], T5651 Salix babylonica, T5750 Saururus chinensis, T5856 Sedum lineare [Syn. Sedum obtuso-lineare], T5894 Senecio oryzetorum, T5993 Solanum capsicastrum, T6005 Solanum lyratum, T6008 Solanum nigrum, T6015 Solanum torvum, T6205 Sus scrofa, T6279 Tagetes erecta, T6843 Xanthium sibiricum [Syn. Xanthium strumarium].

clove sore and eczema T2691 Farfugium japonicum.

clove sore and swollen boil T1199 Carduus acanthoides, T1200 Carduus crispus, T1831 Crotalaria sessiliflora, T2835 Galium verum, T4004 Lysimachia paridiformis.

clove sore and swollen welling abscess T1709 Corydalis bungeana, T1884 Cudrania cochinchinensis.

clove sore of nose T1050 Bufo bufo gargarizans; Bufo melanostictus. clove sore running yellow $\mathrm{T} 1662$ Coptis chinensis, $\mathrm{T} 1663$ Coptis chinensis var. brevisepala, T1664 Coptis deltoidea, T1669 Coptis omeiensis, T1670 Coptis teetoides [Syn. Coptis teeta].

clove sore scrofula T5975 Smilax bockii. 
clove sore tubercle T3983 Lycoris aurea.

clove sore with fever T6735 Viburnum dilatatum.

clove toxin sores T1353 Changium smyrnioides.

cns depression T0184 Adonis amurensis.

cold asthma T6261 Syringa pinnafolia.

cold damage febrile disease T1179 Capparis masaikai.

cold damage mania T1808 Crocus sativus.

cold dysentery T0417 Amomum longiligulare, T0419 Amomum villosum,

T0420 Amomum xanthioides, T4714 Perilla frutescens, T6860

Zanthoxylum ailanthoides.

cold impediment in lumbus and knees T1901 Curculigo orchioides.

cold limbs and faint pulse T0083 Aconitum carmichaeli, T0085

Aconitum carmichaeli cv, T4599 Panax ginseng [Syn. Panax

schinseng].

cold malaria T1170 Canis familiaris.

cold mounting T0472 Anethum graveolens, T1439 Cinnamomum cassia

[Syn. Cinnamomum aromaticum], T2744 Foeniculum vulgare, T2745

Foeniculum vulgare, T4954 Piper longum, T6488 Trachyspermum ammi.

cold mounting with abdominal pain T1441 Cinnamomum japonicum, T1889 Cuminum cyminum, T1978 Cyperus rotundus, T2636 Euscaphis japonica, T3409 Illicium verum, T3885 Litsea cubeba, T4550 Osmanthus fragrans, T4944 Piper cubeba, T6877 Zanthoxylum dissitum.

cold mounting with pain T0077 Aconitum balfourii, T0084 Aconitum carmichaeli, T0105 Aconitum karakolicum, T0108 Aconitum kusnezoffii.

cold pain in abdomen T0322 Allium tuberosum, T0360 Alpinia oxyphylla, T0472 Anethum graveolens, T0664 Artemisia argyi, T0681 Artemisia lavandulaefolia, T0685 Artemisia mongolica, T0689 Artemisia princeps, T0691 Artemisia rubripes, T0706 Artemisia vulgaris, T2087 Delphinium tatsienense, T4283 Morinda officinalis.

cold pain in chest and abdomen T3868 Liquidambar orientalis.

cold pain in heart and abdomen T0077 Aconitum balfourii, T0083 Aconitum carmichaeli, T0084 Aconitum carmichaeli, T0085 Aconitum carmichaeli cv, T0105 Aconitum karakolicum, T0106 Aconitum kirinense, T0108 Aconitum kusnezoffii, T0138 Aconitum umbrosum, T0363 Alpinia speciosa, T1021 Brassica rapa, T1439 Cinnamomum cassia [Syn. Cinnamomum aromaticum], T2037 Datura innoxia, T2044 Datura metel, T2695 Ferula assafoetida, T3250 Hierochloe odorata, T3869 Liquor, T6860 Zanthoxylum ailanthoides.

cold pain in lesser-abdomen T2744 Foeniculum vulgare, T6528 Trigonella foenum-graecum.

cold pain in lumbus and knees T0582 Archangelica brevicaulis [Syn. Angelicarpa brevicaulis; Angelica brevicaulis], T0993 Boschniakia rossica, T1439 Cinnamomum cassia [Syn. Cinnamomum aromaticum], T4324 Murraya paniculata [Syn. Chalcas paniculata], T4536 Orobanche coerulescens, T5270 Psoralea corylifolia.

cold pain in stomach duct T0418 Amomum muricarpum, T1434 Cinnamomum bejolghota [Syn. Cinnamomum obtusifolium; Laurus bejolghota], T1812 Crossostephium chinense.

cold pain in stomach duct and abdomen T0318 Allium sativum, T0359 Alpinia officinarum, T0554 Aquilaria agallocha, T0555 Aquilaria sinensis, T1113 Calendula officinalis, T1217 Carum carvi, T1441 Cinnamomum japonicum, T1889 Cuminum cyminum, T1939 Cymbopogon citratus, T2644 Evodia rutaecarpa, T2646 Evodia rutaecarpa var. officinalis, T3222 Heracleum scabridum, T3409 Illicium verum, T3436 Inula nervosa, T3620 Kaempferia galanga, T3746 Leontice robustum, T3850 Lindera glauca, T3852 Lindera megaphylla, T3885 Litsea cubeba, T4550 Osmanthus fragrans, T4944 Piper cubeba, T4953 Piper longum, T4956 Piper mullesua, T6261 Syringa pinnafolia, T6263 Syzygium aromaticum [Syn. Eugenia caryophyllata], T6488 Trachyspermum ammi, T6858 Zanthoxylum acanthopodium, T6869 Zanthoxylum bungeanum, T6892 Zanthoxylum schinifolium, T6893 Zanthoxylum simulans, T6894 Zanthoxylum simulans, T6909 Zingiber officinale.

cold pain of joints T0354 Alpinia chinensis.

cold qi retching T4954 Piper longum.

cold rheum T6860 Zanthoxylum ailanthoides.

cold rheum asthma cough T6909 Zingiber officinale.

cold rheum cough T1007 Brassica juncea, T5755 Saussurea involucrata. cold stagnation lumbago T1217 Carum carvi.

cold stomachache T5715 Santalum album.

cold suppurative sore T5989 Solanum aculeatissimum, T6014 Solanum surattense.

cold-damp abdominal pain T1641 Conioselinum vaginatum.

cold-damp accumulation and obstruction T0363 Alpinia speciosa.

cold-damp ascites T3295 Huperzia serrata [Syn. Lycopodium serratum].

cold-damp diarrhea T0680 Artemisia lactiflora, T2644 Evodia

rutaecarpa, T2646 Evodia rutaecarpa var. officinalis, T4966 Piper sarmentosum.

cold-damp diarrhea and dysentery T0957 Blumea balsamifera. cold-damp dribbling urination and turbid urine T4944 Piper cubeba. cold-damp impediment pain T1438 Cinnamomum cassia [Syn. Cinnamomum aromaticum], T1441 Cinnamomum japonicum, T1444 Cinnamomum tamala, T3885 Litsea cubeba, T6909 Zingiber officinale. cold-damp red swelling T0319 Allium schoenoprasum.

cold-damp vaginal discharge T3222 Heracleum scabridum.

cold-damp vomiting and diarrhea T0353 Alpinia blepharocalyx, T1435 Cinnamomum camphora.

cold-damp water drum distention T3885 Litsea cubeba.

cold-heat headache T0212 Agastache rugosus, T1419 Cimicifuga dahurica, T1420 Cimicifuga foetida, T1421 Cimicifuga heracleifolia .

cold-heat in turn T1055 Bupleurum angustissimum, T1056 Bupleurum aureum, T1058 Bupleurum chaishoui, T1059 Bupleurum chinense, T1065 Bupleurum longiradiatum, T1066 Bupleurum marginatum, T1067 Bupleurum marginatum var. stenophyllum, T1072 Bupleurum scorzonerifolium, T1073 Bupleurum sibiricum, T1075 Bupleurum smithii var. parvifolium, T1078 Bupleurum wenchuanense, T1079 Bupleurum yinchowense.

cold-heat warm malaria T5323 Pulsatilla campanella, T5324 Pulsatilla cernua, T5325 Pulsatilla chinensis, T5326 Pulsatilla dahurica.

colic of gastrointestinal tract T0825 Atropa belladonna.

coma T0987 Bos taurus domesticus; Bubalus bubalis, T2274 Dryobalanops aromatica.

common cold T0011 Abrus precatorius, T0015 Abutilon indicum, T0099 
Aconitum gymnandrum, T0151 Acronychia pedunculata, T0318 Allium sativum, T0730 Asarum sagittarioides, T0791 Astragalus chrysopterus, T0794 Astragalus ernestii, T0798 Astragalus membranaceus, T0800 Astragalus mongholicus, T0813 Atalantia buxifolia [Syn. Severinia buxifolia], T0932 Betula luminifera, T0952 Blechnum orientale, T0964 Boenninghausenia albiflora, T0967 Boenninghausenia sessilicarpa, T1055 Bupleurum angustissimum, T1056 Bupleurum aureum, T1058 Bupleurum chaishoui, T1059 Bupleurum chinense, T1065 Bupleurum longiradiatum, T1066 Bupleurum marginatum, T1067 Bupleurum marginatum var. stenophyllum, T1072 Bupleurum scorzonerifolium, T1073 Bupleurum sibiricum, T1075 Bupleurum smithii var. parvifolium, T1078 Bupleurum wenchuanense, T1079 Bupleurum yinchowense, T1152 Camellia sinensis [Syn. Thea sinensis], T1373 Chloranthus serratus, T1387 Chrysanthemum boreale, T1392 Chrysanthemum indicum, T1393 Chrysanthemum indicum, T1394 Chrysanthemum lavandulifolium, T1395 Chrysanthemum morifolium [Syn. Dendranthema morifolium], T1446 Cipadessa baccifera, T1533 Clausena dentata, T1534 Clausena dunniana, T1545 Clematis chinensis, T1555 Clerodendron fortunatum, T1573 Clinopodium chinense, T1633 Commelina communis, T1656 Conyza bonariensis [Syn. Erigeron bonariensis; Erigeron linifolius; Erigeron crispus], T1784 Cratoxylum cochinchinense, T1785 Cratoxylum prunifolium, T1986 Cyrtomium fortunei, T2263 Dregea volubilis, T2267 Drosera peltata var. lunata, T2279 Dryopteris championii, T2290 Duchesnea indica, T2334 Elephantopus scaber, T2340 Elsholtzia bodinieri, T2510 Eucalyptus globulus, T2517 Eucalyptus robusta, T2521 Eucalyptus tereticornis, T2563 Eupatorium japonicum, T2627 Euphoria longan [Syn. Dimocarpus longan], T2691 Farfugium japonicum, T2697 Ferula borealis, T3213 Heracleum hemsleyanum, T3217 Heracleum moellendorffii [Syn. Heracleum microcarpum; Heracleum morifolium], T3228 Heracleum yungningense, T3323 Hymenodictyon excelsum, T3360 Hypericum patulum, T3381 Hyptis suaveolens, T3495 Isodon irrorata, T3528 Isodon ternifolia, T3529 Isodon ternifolius, T3779 Leucas aspera, T3803 Ligularia dictyoneura [Syn. Senecio dictyoneurus], T4003 Lysimachia microcarpa, T4121 Marsilea quadrifolia, T4188 Mentha rotundifolia, T4190 Mentha spicata, T4248 Mimosa pudica, T4317 Murraya euchrestifolia [Syn. Clausena euchrestifolia], T4320 Murraya kwangsiensis, T4335 Mussaenda pubescens, T4423 Nicandra physaloides, T4503 Onychium lucidum, T4773 Peucedanum terebinthaceum, T4798 Philonotis fontana, T4810 Phlomis mongolica, T4814 Phlomis umbrosa, T4848 Physalis angulata, T4852 Physalis peruviana, T4854 Physalis pubescens, T5298 Pteris vittata, T5401 Rabdosia yuennanensis, T5519 Rhododendron mucronulatum, T5592 Rubus hirsutus, T5609 Rumex hastatus, T5714 Sansevieria trifasciata, T5727 Saposhnikovia divaricata [Syn. Ledebouriella seseloides], T5934 Seseli meirei, T5937 Seseli yunnanense, T5963 Sinodielsia yunnanensis, T5967 Siraitia grosvenorii [Syn. Momordica grosvenorii], T5969 Sium latifolium, T6075 Spilanthes acmella, T6106 Stelmatocrypton khasianum, T6133 Stephania sinica, T6286 Tamarix chinensis, T6290 Tamarix ramosissima, T6359 Teucrium bidentatum, T6521 Trifolium pratense, T6645 Urena lobata, T6722 Veronica anagallis-aquatica, T6731 Veronicastrum sibiricum, T6738 Viburnum odoratissimum, 66787 Vitex negundo, T6788 Vitex negundo, T6843 Xanthium sibiricum [Syn. Xanthium strumarium].

common cold (root) T5317 Pueraria peduncularis.

common cold due to wind-cold T4714 Perilla frutescens, T4938 Piper boehmeriaefolium, T5167 Porana racemosa.

common cold in early stage T2756 Forsythia suspensa.

common cold with cough T0814 Atalantia buxifolia [Syn. Severinia buxifolia], T0838 Azolla imbricata [Syn. Salvinia imbricata], T1199 Carduus acanthoides, T1200 Carduus crispus, T1675 Corchorus olitorius, T2157 Dichotomanthes tristaniaecarpa, T2984 Glinus lotoides [Syn. Mollugo lotoides], T3006 Glycosmis citrifolia, T3674 Laggera alata, T3997 Lysimachia capillipes, T4223 Micromelum integerrimum, T4261 Mollugo pentaphylla, T4284 Morinda parvifolia, T4616 Pandanus tectorius.

common cold with fever T0045 Acanthopanax trifoliatus, T0060 Achillea alpina [Syn. Achillea sibirica], T0137 Aconitum tanguticum, T0171 Adiantum capillus-veneris, T0174 Adiantum monochlamys, T0229 Ageratum conyzoides, T0374 Alstonia scholaris, T0378 Alstonia yunnanensis, T0379 Alternanthera philoxeroides, T0426 Ampelopsis grossedentata [Syn. Ampelopsis cantoniesis var. grossedentata], T0503 Anisomeles indica [Syn. Epimeredi indica], T0591 Ardisia arborescens, T0942 Biebersteinia heterostemon, T0948 Blainvillea acmella [Syn. Verbesina acmella; Eclipta latifolia; Blainvillea latifolia], T1236 Cassia laevigata [Syn. Cassia floribunda], T1251 Cassytha filiformis, T1258 Casuarina equisetifolia, T1410 Chukrasia tabularis, T2131 Desmodium pulchellum [Syn. Phyllodium pulchellum], T2257 Dracocephalum moldavicum, T2282 Dryopteris filix-mas, T2720 Ficus hispida, T2757 Forsythia viridissima, T2902 Gentiana algida, T2941 Gentianopsis paludosa, T2989 Glochidion sphaerogynum, T3123 Hedyotis auricularia, T3163 Helicteres angustifolia, T3332 Hypecoum erectum, T3334 Hypecoum leptocarpum, T3398 Ilex rotunda, T3555 Jasminum nudiflorum, T3645 Kummerowia striata, T3769 Lespedeza bicolor, T3830 Ligustrum sinense, T3932 Ludwigia octovalvis, T3990 Lygodium japonicum, T4124 Matricaria chamomilla [Syn. Matricaria recutita], T4154 Melaleuca leucadendra, T4217 Microcos paniculata [Syn. Grewia microcos], T4514 Ophiorrhiza pumila, T4526 Origanum vulgare, T4834 Phyllanthus emblica, T5284 Pteridium aquilinum var. latiusculum, T5423 Rauvolfia verticillata, T5425 Rauvolfia verticillata f. rubrocarpa, T5426 Rauvolfia verticillata var. hainanensis, T5427 Rauvolfia vomitoria, T5435 Rauwolfia latifrons, T5441 Rauwolfia perakensis, T5560 Rorippa montana [Syn. Rorippa dubia; Sisymbrium dublium], T5576 Rostellularia procumbens [Syn. Justicia procumbens], T5595 Rubus parviforlius, T5626 Ruta graveolens, T5685 Salvia plebeia, T5687 Salvia prionitis, T5754 Saussurea graminea, T5767 Saussurea pulchella, T5804 Schizonepeta tenuifolia [Syn. Nepeta tenuifolia], T5836 Scutellaria discolor, T6116 Stenoloma chusanum, T6252 Symplocos chinensis, T6277 Tadehagi triquetrum, T6375 Thalictrum baicalense, T6376 Thalictrum cultratum, T6385 Thalictrum flavum, T6387 Thalictrum foliolosum, T6389 Thalictrum glandulosissimum, T6552 Trollius chinensis [Syn. Trollius asiaticus var. chinensis], T6553 Trollius ledebourii, T6581 Tylophora mollissima, T6814 Wedelia chinensis [Syn. Solidago chinensis; Wedelia calendulacea]. 
common cold with fever and chills T1384 Chondrus ocelladus.

common cold with headache T0479 Angelica dahurica cv. qibaizhi, T0498 Angelica taiwaniana, T0557 Aquilegia ecalcarata, T1939 Cymbopogon citratus, T2113 Dennstaedtia scabra [Syn. Dicksonia scabra], T2189 Dioscorea althaeoides, T2258 Dracocephalum rupestre, T3432 Inula helianthus-aquatica, T4002 Lysimachia foenum-graecum, T4307 Mosla scabra [Syn. Mosla punctata], T4470 Ocimum basilicum, T4663 Passiflora caerulea, T5000 Plagiogyria stenoptera, T5391 Rabdosia eriocalyx, T5396 Rabdosia rubescens, T6888 Zanthoxylum planispinum.

common cold with headache and fever T3646 Kyllinga brevifolia. common cold with headache and nasal congestion T2422 Erigeron breviscapus.

common cold without sweating T0610 Argemone mexicana.

common wart T2162 Dicranostigma franchetianum [Syn. Dicranostigma leptopodum].

concretion and accumulation $\mathrm{T} 1049$ Bufo bufo gargarizans; Bufo melanostictus.

concretion and accumulation with glomus distention T5103 Polygonum hydropiper.

concretion and conglomeration T0073 Achyranthes bidentata, T0584 Arctium lappa, T0661 Artemisia anomala, T1160 Campsis grandiflora, T1215 Carthamus tinctorius, T1406 Chrysosplenium nudicaule, T1438 Cinnamomum cassia [Syn. Cinnamomum aromaticum], T1583 Cnidium officinale [Syn. Ligusticum officinale], T1638 Commiphora myrrha [Syn. Commiphora molmol], T1706 Corydalis ambigua var. amurensis [Syn. Corydalis ambigua], T1735 Corydalis repens, T1858 Croton tiglium, T2132 Desmodium pulchellum [Syn. Phyllodium pulchellum], T2384 Epicauta gorhami, T2536 Euonymus alatus, T2546 Euonymus sacrosancta, T2584 Euphorbia ebracteolata, T2587 Euphorbia fischeriana, T2695 Ferula assafoetida, T3412 Impatiens balsamina, T3671 Lagerstroemia indica, T3820 Ligusticum chuanxiong [Syn. Ligusticum wallichii], T3980 Lycopus lucidus, T4036 Magnolia coco, T4106 Manis pentadactyla, T4338 Mylabris phalerata; Mylabris cichorii, T4582 Paeonia delavayi, T4585 Paeonia moutan [Syn. Paeonia suffruticosa], T4607 Panax pseudo-ginseng var. japonicus, T5101 Polygonum cuspidatum, T5231 Prunus persica, T5233 Prunus persica, T5594 Rubus parkeri, T5701 Salvia yunnanensis, T5865 Selaginella pulvinata, T5869 Selaginella tamariscina, T6154 Strobilanthes japonicus [Syn. Championella japonica], T6497 Tribulus terrestris, T6764 Vinegar, T6831 Woodwardia orientalis.

concretion and conglomeration (root) T1113 Calendula officinalis.

concretion and conglomeration with mass T1001 Brachystemma calycinum, T1907 Curcuma wengujin.

concretion conglomeration accumulation and gathering T0164 Actinidia polygama, T0950 Blatta orientalis, T1904 Curcuma kwangsiensis, T1909 Curcuma zedoaria [Syn. Curcuma aeruginosa], T2596 Euphorbia kansui, T4303 Moschus moschiferus; Moschus berezovskii; Moschus sifanicus, T4855 Physeter catodon, T5680 Salvia miltiorrhiza, T5681 Salvia miltiorrhiza f. alba, T5688 Salvia przewalskii, T5723 Sapium sebiferum, T5966 Siphonostegia chinensis, T6062 Sparganium stoloniferum, T6257 Syngnathus acus, T6709
Verbena officinalis.

congesting lung T2367 Ephedra equisetina, T2368 Ephedra gerardiana, T2369 Ephedra intermedia, T2372 Ephedra likiangensis, T2373 Ephedra minuta, T2375 Ephedra monosperma, T2379 Ephedra saxatilis, T2380 Ephedra sinica.

congestive cardiac failure T0184 Adonis amurensis, T1649 Convallaria keiskei [Syn. Convallaria majalis], T4418 Nerium indicum.

conjunctivitis T1655 Conyza blinii, T1709 Corydalis bungeana, T2334 Elephantopus scaber, T2715 Fibraurea recisa, T2720 Ficus hispida, T2721 Ficus microcarpa, T3969 Lycopodium casuarinoides, T4215 Michelia yunnanensis, T4248 Mimosa pudica, T4976 Piptanthus nepalensis, T5804 Schizonepeta tenuifolia [Syn. Nepeta tenuifolia], T5892 Senecio nemorensis, T5907 Senecio scandens [Syn. Senecio chinensis], T6060 Souliea vaginata, T6214 Swertia chinensis, T6217 Swertia davidii, T6278 Tagetes erecta, T6374 Thalictrum atriplex.

constipation T0002 Abelmoschus moschatus [Syn. Hibiscus abelmoschus], T0338 Aloe ferox, T0347 Aloe vera [Syn. Aloe barbadensis], T0348 Aloe vera var. chinensis, T0462 Anemarrhena asphodeloides, T0495 Angelica sinensis, T0572 Aralia elata, T0708 Arthromeris mairei [Syn. Polypodium mairei], T0746 Asparagus cochinchinensis [Syn. Asparagus lucidus], T0984 Bos taurus domesticus; Bubalus bubalis, T1005 Brassica campestris [Syn. Brassica campestris var. oleifera], T1101 Caesalpinia decapetala, T1236 Cassia laevigata [Syn. Cassia floribunda], T1241 Cassia occidentalis, T1242 Cassia occidentalis, T1291 Celastrus paniculatus, T1356 Cheiranthus cheiri, T1465 Citrullus vulgaris [Syn. Citrullus lanatus], T2612 Euphorbia prolifera, T2716 Ficus beecheyana [Syn. Ficus erecta var. beecheyana], T2999 Glycine max, T3231 Hernandia sonora [Syn. Hernandia ovigera], T3444 Ipomoea aquatica [Syn. Convolvulus repens; Ipomoea reptans], T3447 Ipomoea batatas [Syn. Convolvulus batatas], T3845 Linaria vulgaris, T3935 Luffa cylindrica, T4331 Musa paradisiaca var. sapientum [Syn. Musa sapientum], T4665 Passiflora edulis, T4804 Phlojodicarpus sibiricus, T5137 Poncirus trifoliata, T5231 Prunus persica, T5244 Prunus tomentosa, T5272 Psorospermum febrifugum, T5419 Raphanus sativus, T5420 Raphanus sativus, T5446 Rehmannia glutinosa [Syn. Rehmannia glutinosa f. huechingensis], T5461 Rhamnus leptophylla, T5605 Rumex acetosa, T5611 Rumex japonicus, T5612 Rumex nepalensis, T5613 Rumex obtusifolius, T5614 Rumex patientia, T6032 Sophora flavescens [Syn. Sophora angustfolia], T6206 Sus scrofa domestica, T6285 Tamarindus indica, T6510 Trichosanthes kirilowii, T6513 Trichosanthes rosthornii [Syn. Trichosanthes uniflora].

constipation with abdominal pain T1229 Cassia acutifolia, T1230 Cassia angustifolia.

constrictive aortitis T0495 Angelica sinensis, T4804 Phlojodicarpus sibiricus.

consumption cough and hemoptysis T0781 Aster tataricus, T2790 Fritillaria pallidiflora, T2800 Fritillaria walujewii, T6574 Tussilago farfara.

consumption cough with phlegm and blood T2927 Gentiana rhodantha. consumption damage and cough T6677 Valeriana jatamansii [Syn. Valeriana wallichii].

contracture of muscles and joints T6069 Speranskia tuberculata. 
contracture pain in stomach duct and abdomen T0502 Anisodus tanguticus [Syn. Scopolia tangutica].

contusion from knocks and falls T6866 Zanthoxylum avicennae. contusion in chest and rib-side T3431 Inula helenium, T3437 Inula racemosa.

contusion in joints T3560 Jatropha curcas.

convulsion T0987 Bos taurus domesticus; Bubalus bubalis, T1084 Buthus martensi, T1841 Croton caudatus var. tomentosus, T2274 Dryobalanops aromatica, T2890 Gastrodia elata, T4957 Piper nigrum, T5817 Scolopendra subspinipes mutilans, T6497 Tribulus terrestris.

convulsion due to high fever T2995 Gloriosa superba.

convulsion due to liver heat and liver wind T5871 Selenarctos thibetanus; Ursus arctos.

convulsion of hands and feet T4696 Penaeus orientalis.

convulsion of limbs $\mathrm{T} 3653$ Lactarius piperatus [Syn. Agaricus piperatus].

convulsion with cold limbs T1332 Cercidiphyllum japonicum.

cootie T6111 Stemona japonica, T6113 Stemona sessilifolia, T6115 Stemona tuberosa.

copper coin lichen T2963 Ginkgo biloba, T4358 Myroxylon pereirae.

cor pulmonale T3295 Huperzia serrata [Syn. Lycopodium serratum].

corn and common wart T1038 Brucea javanica [Syn. Brucea sumatrana; Rhus javanica].

corneal nephelium T5100 Polygonum chinense.

coronary heart disease T0644 Armillaria mellea, T2846 Ganoderma japonicum [Syn. Ganoderma sinense], T2848 Ganoderma lucidum, T2964 Ginkgo biloba, T3388 Ilex chinensis [Syn. Ilex purpurea], T4487 Olea europaea, T4953 Piper longum.

coronary heart disease with pattern of $q i$ stagnation and blood stasis T5715 Santalum album.

cough T0015 Abutilon indicum, T0151 Acronychia pedunculata, T0301 Aleuritopteris argentea, T0364 Alsophila spinulosa, T0444 Ananas comosus, T0471 Anemone rivularis, T0480 Angelica decursiva [Syn. Peucedanum decursivum], T0571 Aralia decaisneaa, T0584 Arctium lappa, T0753 Asparagus setaceus [Syn. Asparagus plumosus], T0784 Astilbe chinensis, T0813 Atalantia buxifolia [Syn. Severinia buxifolia], T0923 Bergenia crassifolia, T0926 Berneuxia thibetica, T1021 Brassica rapa, T1087 Buxus bodinieri, T1165 Canarium album, T1194 Carassius auratus, T1258 Casuarina equisetifolia, T1357 Chelidonium majus, T1359 Chenopodium album, T1373 Chloranthus serratus, T1440 Cinnamomum glanduliferum, T1529 Cladonia verticillata, T1555 Clerodendron fortunatum, T1751 Corylus heterophylla, T1796 Crinum asiaticum var. sinicum, T1933 Cyclea sutchuenensis, T1967 Cynoglossum amabile, T2050 Daucus carota var. sativa, T2056 Debregeasia longifolia, T2101 Dendrobium fimbriatum, T2119 Derris eriocarpa, T2221 Diospyros kaki, T2258 Dracocephalum rupestre, T2268 Drosera rotundifolia, T2302 Dysosma pleiantha [Syn. Podophyllum pleianthum], T2304 Dysosma veitchii, T2305 Dysosma versipellis [Syn. Podophyllum versipelle], T2367 Ephedra equisetina, T2368 Ephedra gerardiana, T2369 Ephedra intermedia, T2372 Ephedra likiangensis, T2373 Ephedra minuta, T2375 Ephedra monosperma, T2379 Ephedra saxatilis, T2380 Ephedra sinica, T2521 Eucalyptus tereticornis, T2563 Eupatorium japonicum, T2749 Fomes officinalis, T2752 Fordia cauliflora, T2994 Gloiopeltis furcata, T3040 Gomphrena globosa, T3056 Gossypium herbaceum, T3190 Helminthostachys zeylanica, T3234 Heteropogon contortus, T3289 Humulus lupulus, T3332 Hypecoum erectum, T3628 Kerria japonica, T3701 Lasiosphaera fenzlii, T3758 Lepidium sativum, T3802 Ligularia dentata, T3803 Ligularia dictyoneura [Syn. Senecio dictyoneurus], T3805 Ligularia fischeri, T3807 Ligularia intermedia, T3813 Ligularia sibirica, T3819 Ligusticum brachylobum, T3953 Lycianthes biflora, T3961 Lycoperdon pyriforme, T3970 Lycopodium cernuum, T3973 Lycopodium japonicum [Syn. Lycopodium clavatum], T4003 Lysimachia microcarpa, T4004 Lysimachia paridiformis, T4099 Mangifera indica, T4100 Mangifera indica, T4103 Mangifera persiciformis, T4190 Mentha spicata, T4195 Menyanthes trifoliata, T4209 Michelia alba, T4307 Mosla scabra [Syn. Mosla punctata], T4317 Murraya euchrestifolia [Syn. Clausena euchrestifolia], T4335 Mussaenda pubescens, T4350 Myriopteron extensum, T4502 Onychium japonicum [Syn. Tricomanes japonicum], T4510 Ophiorrhiza japonica, T4513 Ophiorrhiza mungos, T4514 Ophiorrhiza pumila, T4527 Orixa japonica, T4549 Osbeckia chinensis, T4600 Panax ginseng [Syn. Panax schinseng], T4618 Panicum miliaceum, T4625 Papaver commutatum [Syn. Papaver rhoeas], T4665 Passiflora edulis, T4761 Peucedanum longshengens, T4763 Peucedanum morisonii, T4768 Peucedanum praeruptorum, T4769 Peucedanum rubricaule, T4773 Peucedanum terebinthaceum, T4798 Philonotis fontana, T4834 Phyllanthus emblica, T4843 Phyllanthus urinaria, T4852 Physalis peruviana, T4902 Pimpinella thelungiana, T5000 Plagiogyria stenoptera, T5057 Podophyllum emodii [Syn. Podophyllum emodii var. chinense; Podophyllum sikkimenosis; Sinopodophyllum emodii], T5087 Polygala telephioides, T5119 Polygonum thunbergii, T5171 Porphyra tenera, T5216 Prunus armeniaca, T5219 Prunus armeniaca var. ansu, T5361 Pyrus betulaefolia, T5374 Quercus infectoria, T5502 Rhododendron anthopogonoides, T5518 Rhododendron mucronatum, T5523 Rhododendron simsii, T5560 Rorippa montana [Syn. Rorippa dubia; Sisymbrium dublium], T5576 Rostellularia procumbens [Syn. Justicia procumbens], T5640 Sabina vulgaris, T5645 Sageretia theezans [Syn. Sageretia thea], T5726 Saponaria officinalis, T5804 Schizonepeta tenuifolia [Syn. Nepeta tenuifolia], T5963 Sinodielsia yunnanensis, T6046 Sophora viciifolia, T6075 Spilanthes acmella, T6079 Spiraea japonica, T6082 Spiraea japonica var. fortunei, T6105 Stellera chamaejasme, T6106 Stelmatocrypton khasianum, T6116 Stenoloma chusanum, T6282 Tagetes patula, T6574 Tussilago farfara, T6766 Viola tricolor, T6772 Viscum angulatum, T6789 Vitex negundo, T6888 Zanthoxylum planispinum.

cough and abundant phlegm T0776 Asplenium prolongatum, T1343 Chaenomeles sinensis, T2426 Erigeron sumatrensis, T3520 Isodon rosthornii, T4274 Monostroma nitidum, T4814 Phlomis umbrosa, T5594 Rubus parkeri, T5939 Shiraia bambusicola.

cough and asthma T0462 Anemarrhena asphodeloides, T0676 Artemisia dracunculus, T0729 Asarum maximum, T0892 Belamcanda chinensis, T1241 Cassia occidentalis, T1493 Citrus limon, T1497 Citrus limonia, T1538 Clausena lansium, T1831 Crotalaria sessiliflora, T1941 Cymbopogon distans, T2036 Datura innoxia, T2038 Datura innoxia, 
T2039 Datura innoxia, T2041 Datura metel, T2042 Datura metel, T2043 Datura metel, T2046 Datura stramonium, T2047 Datura stramonium, T2220 Diospyros kaki, T2257 Dracocephalum moldavicum, T2367 Ephedra equisetina, T2368 Ephedra gerardiana, T2369 Ephedra intermedia, T2372 Ephedra likiangensis, T2373 Ephedra minuta, T2375 Ephedra monosperma, T2379 Ephedra saxatilis, T2380 Ephedra sinica, T2596 Euphorbia kansui, T3222 Heracleum scabridum, T3279 Homo sapiens, T3328 Hyoscyamus niger, T3427 Inula britannica, T3428 Inula britannica var. chinensis, T3434 Inula linariaefolia, T3770 Lespedeza cuneata, T3840 Limnophila rugosa, T3990 Lygodium japonicum, T4369 Nandina domestica, T4418 Nerium indicum, T4432 Nigella glandulifera, T4688 Peganum harmala, T4714 Perilla frutescens, T4715 Perilla frutescens, T4717 Perilla frutescens var. acuta [Syn. Perilla frutescens var. purpurascens], T4719 Perilla frutescens var. arguta, T4722 Perilla frutescens var. crispa, T4800 Phlegmariurus fordii, T4956 Piper mullesua, T4974 Piper wallichii [Syn. Piper wallichii var. hupehense], T5145 Populus alba, T5308 Pterocarya stenoptera, T5519 Rhododendron mucronulatum, T5521 Rhododendron przewalskii, T5609 Rumex hastatus, T5981 Smilax riparia, T6055 Sorbus tianschanica, T6286 Tamarix chinensis, T6290 Tamarix ramosissima, T6521 Trifolium pratense, T6825 Winchia calophylla.

cough and asthma due to lung heat T2927 Gentiana rhodantha, T5182 Potentilla discolor.

cough and asthma due to lung vacuity T0529 Anthriscus sylvestris, T1596 Codonopsis canescens, T1597 Codonopsis clematidea, T1599 Codonopsis pilosula, T1600 Codonopsis pilosula var. modesta [Syn. Codonopsis modesta], T1601 Codonopsis subglobosa, T1602 Codonopsis tangshen, T1603 Codonopsis tubulosa, T1604 Coeloglossum viride [Syn. Coeloglossum viride var. bracteatum], T2190 Dioscorea batatas [Syn. Dioscorea opposita], T2202 Dioscorea japonica, T3077 Gymnadenia conopsea, T4599 Panax ginseng [Syn. Panax schinseng], T6346 Terminalia chebula, T6348 Terminalia chebula var. tomentella.

cough and asthma with abundant phlegm T0480 Angelica decursiva [Syn. Peucedanum decursivum], T0781 Aster tataricus, T1813 Crotalaria albida, T2125 Descurainia sophia, T2439 Eruca sativa, T2565 Eupatorium lindleyanum, T3426 Inula britannica, T3433 Inula japonica, T3755 Lepidium apetalum [Syn. Lepidium micranthum], T3759 Lepidium virginicum, T3819 Ligusticum brachylobum, T4005 Lysionotus pauciflorus, T4231 Milingtonia hortensis, T4761 Peucedanum longshengens, T4768 Peucedanum praeruptorum, T4769 Peucedanum rubricaule, T4858 Physochlaina infundibularis, T4904 Pinellia ternata, T5504 Rhododendron capitatum, T5515 Rhododendron micranthum, T5958 Sinapis alba [Syn. Brassica alba; Brassica hirta], T5961 Sinocalamus oldhami, T6574 Tussilago farfara, T6580 Tylophora floribunda.

cough and asthma with phlegm-blood T4610 Panax quinquefolium. cough and asthma without phlegm T2037 Datura innoxia, T2044 Datura metel.

cough and counterflow T0438 Anacardium occidentale, T0985 Bos taurus domesticus; Bubalus bubalis, T2839 Gallus gallus domesticus, T6497 Tribulus terrestris. cough and counterflow with asthma and fullness T6819 Wikstroemia chamaedaphne.

cough and heavy head (flower) T0240 Aglaia odorata.

cough and hemoptysis T0881 Bauhinia variegata, T0922 Berchemia polyphylla var. leioclada, T0982 Bos taurus domesticus, T2691 Farfugium japonicum, T3390 Ilex cornuta, T3764 Lepisorus thunbergianus.

cough and hemoptysis or dyspnea T3954 Lycium barbarum, T3956 Lycium chinense.

cough and $q \boldsymbol{i}$ counterflow T1980 Cyprinus carpio.

cough and shortness of breath T4687 Peganum harmala, T4908 Pinus bungeana.

cough and sore pharynx T5073 Polygala chinensis [Syn. Polygala glomerata], T6567 Tupistra wattii [Syn. Campylandra wattii].

cough asthma qith phlegm-drool and atrophicexuberant T2975 Gleditsia delavayi, T2976 Gleditsia fera.

cough harmful to lung T1674 Corchorus capsularis.

cough of phlegm asthma T1786 Cremanthodium ellisii, T2262 Dregea sinensis, T2964 Ginkgo biloba, T2978 Gleditsia sinensis [Syn. Gleditsia horrida], T3246 Hibiscus syriacus, T3933 Luffa acutangula, T3934 Luffa cylindrica, T3988 Lycoris squamigera, T4247 Millingtonia hortensis, T4859 Physochlaina physaloides, T5161 Populus tomentosa, T5457 Rhamnus davurica, T5548 Ricinus communis, T5560 Rorippa montana [Syn. Rorippa dubia; Sisymbrium dublium], T6428 Thermopsis lanceolata.

cough of phlegm-rheum T2020 Damnacanthus indicus, T3893 Litsea pungens.

cough of profuse phlegm $\mathrm{T} 3873$ Liriope platyphylla, T3874 Liriope spicata, T5513 Rhododendron mariae.

cough with blood ejection T0746 Asparagus cochinchinensis [Syn. Asparagus lucidus], T1034 Broussonetia papyrifera, T1684 Cordyline strcta, T2955 Geum japonicum, T5773 Saxifraga stolonifera, T6087 Spiranthes sinensis.

cough with inhibited phlegm T0595 Ardisia crispa, T0749 Asparagus filicinus, T5087 Polygala telephioides.

cough with phlegm T1660 Coprinus atramentarius, T4820 Phoenix dactylifera.

cough with phlegm and blood T2783 Fritillaria cirrhosa, T2784 Fritillaria delavayi, T2792 Fritillaria przewalskii, T2796 Fritillaria unibracteata, T2797 Fritillaria ussuriensis, T5595 Rubus parviforlius, T5647 Sagittaria sagittifolia.

cough with profuse phlegm T0586 Arctium lappa, T0617 Arisaema amurense, T0618 Arisaema consanguineum, T0620 Arisaema heterophyllum, T0984 Bos taurus domesticus; Bubalus bubalis, T1312 Centipeda minima, T1390 Chrysanthemum coronarium, T1397 Chrysanthemum segetum, T1488 Citrus junos, T1498 Citrus medica, T1506 Citrus reticulata, T1520 Citrus wilsonii, T1927 Cycas revoluta, T1960 Cynanchum stauntonii, T2023 Daphne genkwa, T2432 Eriobotrya japonica, T2787 Fritillaria hupehensis, T2895 Gaultheria yunnanensis, T2961 Ginkgo biloba, T3011 Glycyrrhiza aspera, T3013 Glycyrrhiza glabra, T3015 Glycyrrhiza inflata, T3016 Glycyrrhiza kansuensis, T3021 Glycyrrhiza squamulosa, T3022 Glycyrrhiza uralensis, T3023 Glycyrrhiza yunnanensis, T3026 Gnaphalium affine 
[Syn. Gnaphalium multiceps], T3254 Hippophae rhamnoides, T3256 Hippophae rhamnoides subsp. sinensis, T3258 Hippophae rhamnoides subsp. yunnanensis, T3323 Hymenodictyon excelsum, T3528 Isodon ternifolia, T3529 Isodon ternifolius, T4000 Lysimachia congestiflora, T4048 Magnolia rostrata, T4203 Mesua ferrea, T4438 Nostoc flagelliforme, T4718 Perilla frutescens var. acuta [Syn. Perilla frutescens var. purpurascens], T4721 Perilla frutescens var. arguta, T4903 Pinellia pedatisecta, T5011 Platycodon grandiflorum, T5071 Polygala arillata, T5086 Polygala sibirica, T5088 Polygala tenuifolia, T5101 Polygonum cuspidatum, T6440 Thuja orientalis [Syn. Platycladus orientalis; Biota orientalis], T6653 Usnea diffracta, T6654 Usnea longissima.

coughing of blood T1044 Bryum argenteum, T1087 Buxus bodinieri, T1451 Cirsium setosum [Syn. Cerratula setosa; Cirsium segetum; Cephalanoplos segetum], T1971 Cynoglossum zeylanicum [Syn. Anchusa zeylanica; Cynoglossum furcatum; Cynoglossum formosanum ], T2193 Dioscorea cirrhosa [Syn. Dioscorea pogonoides], T3645 Kummerowia striata, T3737 Lemmaphyllum microphyllum var. obovatum, T4502 Onychium japonicum [Syn. Tricomanes japonicum], T5288 Pteris cretica var. nervosa [Syn. Pteris nervosa], T5870 Selaginella uncinata, T6830 Woodfordia fruticosa.

courbature T6731 Veronicastrum sibiricum.

cracked nipple T0875 Basella rubra.

cracking T0516 Anser cygnoides domestica, T3388 Ilex chinensis [Syn. Ilex purpurea], T4188 Mentha rotundifolia, T4190 Mentha spicata.

cracking from frostbite T0299 Aleurites cordata [Syn. Aleurites fordii].

cracking of hands and feet T0540 Apis cerana, T0955 Bletilla striata,

T3857 Lindera umbellata [Syn. Lindera erythrocarpa].

cracking of skin T3395 Ilex pedunculosa.

cramp and swelling of feet T4018 Machilus thunbergii.

crane's knee wind T0440 Anagallis arvensis, T0569 Aralia cordata, T0574 Aralia fargesii, T3033 Gnetum parvifolium [Syn. Gnetum indicum], T3213 Heracleum hemsleyanum, T3217 Heracleum moellendorffii [Syn. Heracleum microcarpum; Heracleum morifolium], T3228 Heracleum yungningense, T5414 Ranunculus japonicus, T5645 Sageretia theezans [Syn. Sageretia thea].

crimson tongue T5826 Scrophularia buergeriana, T5828 Scrophularia ningpoensis.

crippling wilt T0819 Atractylodes chinensis, T0821 Atractylodes japonica, T0823 Atractylodes lancea.

cystitis T1553 Clerodendranthus spicatus, T1557 Clerodendron indicum, T2056 Debregeasia longifolia, T2119 Derris eriocarpa, T3187 Helleborus thibetanus, T3233 Heteropappus altaicus, T3284 Houttuynia cordata, T3288 Humulus japonicus [Syn. Humulus scandens], T3289 Humulus lupulus, T3471 Iris tectorum, T6023 Solidago virgaurea, $\mathrm{T} 6731$ Veronicastrum sibiricum.

damp beriberi $\mathrm{T} 0383$ Alyxia sinensis.

damp edema T0819 Atractylodes chinensis, T0821 Atractylodes japonica, T0823 Atractylodes lancea.

damp evil brew T2554 Eupatorium cannabinum.

damp impediment T0819 Atractylodes chinensis, T0821 Atractylodes japonica, T0823 Atractylodes lancea, T0824 Atractylodes macrocephala [Syn. Atractylis macrocephala], T4505 Ophiocephalus argus, T6860 Zanthoxylum ailanthoides.

damp impediment and hypertonicity T1614 Coix lacryma-jobi var. ma-yuen, T2316 Echinops grijsii, T2317 Echinops ritro, T5466 Rhaponticum uniflorum.

damp impediment pain T0990 Bos taurus domesticus; Bubalus bubalis.

damp itchy in genitals T0038 Acanthopanax gracilistylus, T0042 Acanthopanax senticosus [Syn. Eleutherococcus senticosus], T0044 Acanthopanax sessiliflorus, T4042 Magnolia liliflora, T5924 Sesamum indicum.

damp itchy in sore and papules T2048 Daucus carota.

damp itchy skin T0088 Aconitum coreanum, T3736 Lemmaphyllum Microphyllum, T3737 Lemmaphyllum microphyllum var. obovatum T5115 Polygonum sibiricum [Syn. Persicaria sibirica].

damp lichen T5907 Senecio scandens [Syn. Senecio chinensis].

damp phlegm streaming sore T5958 Sinapis alba [Syn. Brassica alba; Brassica hirta].

damp sore T0427 Ampelopsis japonica [Syn. Paullinia japonica], T0672 Artemisia capillaris, T0695 Artemisia scoparia [Syn. Artemisia capillaris var. scoparia], T1546 Clematis tangutica, T1582 Cnidium monnieri, T2197 Dioscorea futschauensis, T2210 Dioscorea spongiosa, T2325 Eichhornia crassipes, T2857 Garcinia hanburyi, T4172 Melilotus suaveolens, T4851 Physalis minima, T4906 Pinus armandii, T4916 Pinus massoniana, T5220 Prunus davidiana, T5232 Prunus persica, T5950 Siegesbeckia orientalis, T5951 Siegesbeckia orientalis var. glabrescens [Syn. Siegesbeckia glabrescens], T5952 Siegesbeckia orientalis var. pubescens [Syn. Siegesbeckia pubescens], T6864 Zanthoxylum armatum, T6870 Zanthoxylum bungeanum, T6887 Zanthoxylum planispinum.

damp sore with swelling toxin $\mathrm{T} 2194$ Dioscorea collettii.

damp toxin $\mathrm{T} 3001$ Glycine max, $\mathrm{T} 3687$ Lantana camara.

damp toxin of scab sore T5266 Psidium guajava.

damp toxin sore T6031 Sophora flavescens [Syn. Sophora angustfolia].

damp toxin with pruritus T3674 Laggera alata.

damp ulceration on skin T2165 Dictamnus angustifolius, T2167 Dictamnus dasycarpus.

damp warmth T0660 Artemisia annua, T0662 Artemisia apiacea [Syn. Artemisia carvifolia; Artemisia caruifolia].

damp-erosion of sores T6268 Syzygium samarangense.

damp-heat diarrhea T0171 Adiantum capillus-veneris, T0573 Aralia elata, T1705 Corydalis adunca, T1733 Corydalis racemosa, T1826 Crotalaria mucronata, T2718 Ficus carica, T2984 Glinus lotoides [Syn. Mollugo lotoides], T4057 Mahonia bealei, T4068 Mahonia japonica, T4564 Oxalis corniculata [Syn. Oxalis repens], T5767 Saussurea pulchella, T6031 Sophora flavescens [Syn. Sophora angustfolia], T6060 Souliea vaginata.

damp-heat diarrhea dysentery T0867 Baphicacanthus cusia [Syn. Strobilanthes cusia], T0906 Berberis julianae, T0916 Berberis thunbergii, T0920 Berberis wilsonae, T1662 Coptis chinensis, T1663 Coptis chinensis var. brevisepala, T1664 Coptis deltoidea, T1669 Coptis omeiensis, T1670 Coptis teetoides [Syn. Coptis teeta], T1813 Crotalaria albida, T1933 Cyclea sutchuenensis, T1966 Cynara scolymus, T2772 Fraxinus mandshurica, T2950 Gerbera anandria [Syn. Leibnitzia anandria], T3245 Hibiscus syriacus, T3288 Humulus 
japonicus [Syn. Humulus scandens], T3878 Lithocarpus polystachyus, T3932 Ludwigia octovalvis, T5576 Rostellularia procumbens [Syn. Justicia procumbens], T5942 Sida acuta, T6226 Swertia mileensis, T6375 Thalictrum baicalense, T6376 Thalictrum cultratum, T6378 Thalictrum delavayi, T6385 Thalictrum flavum, T6387 Thalictrum foliolosum, T6389 Thalictrum glandulosissimum, T6403 Thalictrum petaloideum, T6767 Viola yedoensis.

damp-heat diarrhea, T0907 Berberis kawakamii.

damp-heat disease T6357 Tetrapanax papyriferus.

damp-heat dysentery T0161 Actinidia eriantha, T0446 Anaphalis margaritacea, T0641 Aristolochia tuberosa, T0902 Berberis diaphana, T0912 Berberis poiretii, T1004 Brasenia schreberi, T1561 Clerodendron serratum, T1569 Clerodendrum serratum var. amplexifolium, T1931 Cyclea barbata, T2079 Delphinium kamaonense var. glabrescens, T2387 Epilobium hirsutum, T3564 Juglans mandshurica, T3765 Lepisorus ussuriensis, T4060 Mahonia confusa, T4064 Mahonia gracilipes, T4072 Mahonia shenii, T4789 Phellodendron amurense, T4790 Phellodendron amurense var. wilsonii, T4791 Phellodendron chinense, T4792 Phellodendron chinense var. glabriusculum, T5118 Polygonum suffultum, T5174 Portulaca pilosa, T5554 Rodgersia aesculifolia, T5856 Sedum lineare [Syn. Sedum obtuso-lineare], T5866 Selaginella sanguinolenta, T6028 Sophora alopecuroides, T6386 Thalictrum foetidum, T6393 Thalictrum ichangense [Syn. Thalictrum tripeltatum; Thalictrum multipeltatum], T6912 Zinnia elegans.

damp-heat edema T0157 Actinidia callosa var. henryi, T0635 Aristolochia moupinensis, T2730 Fimbristylis dichotoma, T4144 Meconopsis nepaulensis, T4145 Meconopsis punicea.

damp-heat generalized pain T0628 Aristolochia fangchi.

damp-heat glomus in chest T1662 Coptis chinensis, T1663 Coptis chinensis var. brevisepala, T1664 Coptis deltoidea, T1669 Coptis omeiensis, T1670 Coptis teetoides [Syn. Coptis teeta].

damp-heat impediment T2132 Desmodium pulchellum [Syn. Phyllodium pulchellum], T2165 Dictamnus angustifolius, T2167 Dictamnus dasycarpus.

damp-heat impediment pain T0632 Aristolochia manshuriensis, T1672 Corallodiscus flabellatus [Syn. Didissandra flabellat].

damp-heat inhibited urination T2719 Ficus fistulosa [Syn. Ficus harlandii].

damp-heat itchy sore T5993 Solanum capsicastrum.

damp-heat jaundice T0158 Actinidia chinensis, T0424 Ampelopsis brevipedunculata var. hancei, T0457 Andrographis paniculata [Syn. Justicia paniculata], T0471 Anemone rivularis, T0567 Aralia armata, T0571 Aralia decaisneaa, T0595 Ardisia crispa, T1017 Brassica oleracea var. capitata, T1251 Cassytha filiformis, T1261 Catalpa ovata, T1311 Centella asiatica, T1415 Cichorium intybus, T1876 Cucumis melo, T2165 Dictamnus angustifolius, T2167 Dictamnus dasycarpus, T2194 Dioscorea collettii, T2826 Galeola faberi, T2908 Gentiana cephalantha, T2921 Gentiana manshurica, T2928 Gentiana rigescens, T2930 Gentiana scabra, T2938 Gentiana triflora, T2973 Glechoma longituba, T2974 Glechoma lungituba, T3126 Hedyotis corymbosa [Syn. Oldenlandia corymbosa], T3356 Hypericum japonicum, T3479 Isodon amethystoides, T3507 Isodon lophanthoides [Syn. Rabdosia lophanthoides; Hyssopus lophanthoides; Plectranthus striatus; Isodon striatus], T3578 Juncus effusus, T3830 Ligustrum sinense, T3998 Lysimachia christinae, T4056 Mahonia bealei, T4063 Mahonia fortunei, T4067 Mahonia japonica, T4109 Marchantia polymorpha, T4120 Marsdenia tenacissima, T4144 Meconopsis nepaulensis, T4194 Menyanthes trifoliata, T4368 Nandina domestica, T4369 Nandina domestica, T4485 Oldenlandia diffusa [Syn. Hedyotis diffusa], T4502 Onychium japonicum [Syn. Tricomanes japonicum], T4848 Physalis angulata, T4854 Physalis pubescens, T5072 Polygala caudata, T5101 Polygonum cuspidatum, T5397 Rabdosia serra, T5472 Rheum officinale, T5474 Rheum palmatum, T5481 Rheum tanguticum, T5754 Saussurea graminea, T5775 Scabiosa comosa, T5861 Selaginella doederleinii, T5862 Selaginella involvens, T5863 Selaginella moellendorffii, T5943 Sida cordifolia, T6039 Sophora moorcroftiana, T6217 Swertia davidii, T6224 Swertia kouitchensis, T6226 Swertia mileensis, T6231 Swertia pseudochinensis, T6301 Taraxacum mongolicum, T6372 Thalictrum acutifolium, T6374 Thalictrum atriplex, T6381 Thalictrum faberi, T6388 Thalictrum fortunei, T6393 Thalictrum ichangense [Syn. Thalictrum tripeltatum; Thalictrum multipeltatum], T6402 Thalictrum omeiense.

damp-heat pain in limbs T0629 Aristolochia heterophylla.

damp-heat sore and papules T2165 Dictamnus angustifolius, T2167 Dictamnus dasycarpus.

damp-heat sore toxin T2208 Dioscorea septemloba, T4127 Matteuccia struthiopteris.

damp-heat strangury $\mathrm{T} 3360$ Hypericum patulum.

damp-heat strangury pain T2161 Dicranopteris pedata [Syn. Polypodium pedatum; Dicranopteris dichotoma], T2213 Dioscorea zingiberensis, T4760 Peucedanum japonicum, T5126 Polypodium virginianum, $\mathrm{T} 5127$ Polypodium vulgare.

damp-heat vaginal discharge T2908 Gentiana cephalantha, T2921 Gentiana manshurica, T2928 Gentiana rigescens, T2930 Gentiana scabra, T2938 Gentiana triflora, T5520 Rhododendron ovatum [Syn. Rhododendron lamprophyllum; Azalea ovata], T6116 Stenoloma chusanum.

damp-heat vomiting diarrhea T5604 Rudbeckia laciniata.

damp-heat with strangury turbidity T5977 Smilax glabra, T5980 Smilax menispermoidea.

damp-sore of skin T4470 Ocimum basilicum.

damp-sore with flowing water T6615 Uncaria gambir.

damp-stagnation obstructing internally $\mathrm{T} 5104$ Polygonum hydropiper.

damp-swelling in scrotum T3413 Impatiens nolitangere.

deadlimb T0617 Arisaema amurense, T0618 Arisaema consanguineum, T0620 Arisaema heterophyllum, T3971 Lycopodium complanatum, T4903 Pinellia pedatisecta, T5279 Psychotria serpens, T6166 Strychnos angustiflora.

deafness T0015 Abutilon indicum, T0144 Acorus gramineus, T0146 Acorus tatarinowii, T0516 Anser cygnoides domestica, T0517 Anser cygnoides domestica, T1084 Buthus martensi, T2275 Dryobalanops aromatica, T2908 Gentiana cephalantha, T2921 Gentiana manshurica, T2928 Gentiana rigescens, T2930 Gentiana scabra, T2938 Gentiana triflora.

deafness in early stage T5312 Pueraria edulis, T5313 Pueraria lobata 
[Syn. Pueraria thunbergiana; Pueraria pseudohirsuta], T5316 Pueraria omeiensis, T5318 Pueraria phaseoloides, T5320 Pueraria thomsonii.

decayed teeth T1996 Dahlia pinnata [Syn. Dahlia variabilis], T5646 Sagina japonica [Syn. Spergula japonica].

decayed toothache T0297 Alchornea trewioides, T1359 Chenopodium album, T4020 Macleaya cordata, T5144 Populus adenopoda, T5457 Rhamnus davurica.

decayed toothache due to wind T3329 Hyoscyamus niger.

deep pus ulcer T0568 Aralia chinensis, T0570 Aralia dasyphylla.

deep-lying heat sore pharynx T2839 Gallus gallus domesticus.

deep-source nasal congestion T0212 Agastache rugosus, T0476 Angelica anomala, T0479 Angelica dahurica cv. qibaizhi, T0498 Angelica taiwaniana, T0728 Asarum heterotropoides var. mandshuricum, T0731 Asarum sieboldii, T0732 Asarum sieboldii var. seoulensis, T2434 Eriocaulon buergerianum, T3222 Heracleum scabridum, T3680 Lamium amplexicaule, T4953 Piper longum, T5646 Sagina japonica [Syn. Spergula japonica], T6000 Solanum indicum, T6843 Xanthium sibiricum [Syn. Xanthium strumarium].

deep-source nasal congestion and headache T5059 Pogostemon cablin [Syn. Mentha cablin].

deep-source nasal congestion and runny nose T1312 Centipeda minima.

delacrimation T6791 Vitex rotundifolia [Syn. Vitex trifollia var. simplicifolia], T6793 Vitex trifolia.

delacrimation and photophobia T1048 Buddleja officinalis, T1240 Cassia obtusifolia, T1250 Cassia tora, T2434 Eriocaulon buergerianum.

depressed liver-gallbladder heat T4479 Odontites serotina.

depression T0292 Albizzia julibrissin, T5848 Securinega suffruticosa.

depression in heart-chest T2798 Fritillaria verticillata var. thunbergii [Syn. Fritillaria thunbergii].

depression of $\boldsymbol{q} \boldsymbol{i}$ and blood T0945 Bischofia javanica [Syn. Bischofia trifoliata].

dermatitis T0495 Angelica sinensis, T2165 Dictamnus angustifolius, T2167 Dictamnus dasycarpus, T2641 Evodia lepta [Syn. Ilex lepta], T3381 Hyptis suaveolens, T3830 Ligustrum sinense, T4391 Nauclea officinalis, T4804 Phlojodicarpus sibiricus, T4837 Phyllanthus emblica, T5445 Rehmannia glutinosa [Syn. Rehmannia glutinosa f. huechingensis], T5447 Rehmannia glutinosa [Syn. Rehmannia glutinosa f. huechingensis], T6018 Solanum verbascifolium, T6338 Tephrosia purpurea, T6339 Tephrosia purpurea.

dermatitis $^{\text {[5509] }}$ T6690 Vepris louisii.

dermatomyositis T2753 Formica fusca.

desiccation of liquid and intestine dryness T5223 Prunus humilis [Syn. Cerasus humilis], T5224 Prunus japonica [Syn. Cerasus japonica], T5225 Prunus japonica var. nakaii.

desire to sleep T1152 Camellia sinensis [Syn. Thea sinensis].

deviated eyes and mouth T0088 Aconitum coreanum, T0617 Arisaema amurense, T0618 Arisaema consanguineum, T0620 Arisaema heterophyllum, T3113 Hedera nepalensis var. sinensis, T3115 Hedera rhombea, T4085 Mallotus repandus var. chrysocarpus [Syn. Mallotus chrysocarpus; Mallotus repandus], T4903 Pinellia pedatisecta, T6588
Typhoniun giganteum.

diabetes mellitus T0158 Actinidia chinensis, T0462 Anemarrhena asphodeloides, T0541 Apis cerana, T0568 Aralia chinensis, T0570 Aralia dasyphylla, T0572 Aralia elata, T0746 Asparagus cochinchinensis [Syn. Asparagus lucidus], T0975 Bombyx mori, T0976 Bombyx mori, T0988 Bos taurus domesticus; Bubalus bubalis, T0989 Bos taurus domesticus; Bubalus bubalis, T1182 Capra hircus; Ovis aries, T1412 Cicer arietinum, T1476 Citrus erythrosa, T1515 Citrus tangemna, T2188 Dioscorea alata, T2195 Dioscorea deltoidea, T2222 Diospyros lotus, T2569 Eupatorium rebaudianum, T2837 Gallus gallus domesticus, T3668 Lagenaria siceraria var. depressa, T3954 Lycium barbarum, T3955 Lycium barbarum, T3956 Lycium chinense, T3958 Lycium chinense, T4086 Malus asiatica, T4102 Mangifera indica, T4121 Marsilea quadrifolia, T4263 Momordica charantia, T4288 Morus alba, T4516 Oplopanax elatus, T4544 Oryza sativa, T4573 Pachyrrhizus erosus, T4600 Panax ginseng [Syn. Panax schinseng], T4602 Panax ginseng [Syn. Panax schinseng], T4731 Persea americana [Syn. Persea gratissima], T4786 Phasianus colchicus, T4983 Pisum sativum, T5091 Polygonatum cyrtonema [Syn. Polygonatum multiflorum], T5092 Polygonatum kingianum, T5095 Polygonatum sibiricum, T5239 Prunus salicina, T5312 Pueraria edulis, T5313 Pueraria lobata [Syn. Pueraria thunbergiana; Pueraria pseudohirsuta], T5316 Pueraria omeiensis, T5318 Pueraria phaseoloides, T5320 Pueraria thomsonii, T5419 Raphanus sativus, T5445 Rehmannia glutinosa [Syn. Rehmannia glutinosa f. huechingensis], T5446 Rehmannia glutinosa [Syn. Rehmannia glutinosa f. huechingensis], T5447 Rehmannia glutinosa [Syn. Rehmannia glutinosa f. huechingensis], T5642 Saccharum sinensis, T5938 Setaria italica, T6076 Spinacia oleracea, T6087 Spiranthes sinensis, T6147 Stizolobium capitatum, T6311 Taxus cuspidata, T6492 Trapa bispinosa, T6507 Trichosanthes cucumeroides, T6510 Trichosanthes kirilowii, T6513 Trichosanthes rosthornii [Syn. Trichosanthes uniflora], T6544 Triticum aestivum [Syn. Triticum vulgare], T6754 Vigna unguiculata, T6901 Zea mays.

diabetes mellitus due to internal heat T0791 Astragalus chrysopterus, T0794 Astragalus ernestii, T0798 Astragalus membranaceus, T0800 Astragalus mongholicus, T1698 Cornus officinalis [Syn. Macrocarpium officinale], T3234 Heteropogon contortus, T3875 Liriope spicata var. prolifera, T4507 Ophiopogon japonicus, T4599 Panax ginseng [Syn. Panax schinseng], T4610 Panax quinquefolium, T5093 Polygonatum odoratum [Syn. Polygonatum officinale], T5094 Polygonatum prattii, T6512 Trichosanthes kirilowii.

diabetes mellitus with profuse urination T4548 Oryza sativa var. glutinosa.

diabetes $^{[5509]}$ T3394 Ilex paraguariensis.

diaphragm food T6744 Vicia faba.

diarrhea T0015 Abutilon indicum, T0293 Albizzia lebbeck, T0302 Alhagi pseudalhagi, T0303 Alisma orientale [Syn. Alisma plantago-aquatica var. orientale], T0318 Allium sativum, T0357 Alpinia japonica, T0358 Alpinia katsumadai, T0359 Alpinia officinarum, T0383 Alyxia sinensis, T0388 Amaranthus lividus, T0418 Amomum muricarpum, T0428 Ampelopsis megalophylla, T0457 Andrographis paniculata [Syn. Justicia paniculata], T0468 Anemone hupehensis, T0641 Aristolochia 
tuberosa, T0642 Aristolochia tubiflora, T0894 Benincasa hispida, T0912 Berberis poiretii, T0933 Betula platyphylla, T0935 Betula platyphylla var. japonica, T0938 Bidens bipinnata, T0963 Boehmeria siamensis, T0973 Bombax malabaricum [Syn. Gossampinus malabarica], T1003 Brandisia hancei, T1031 Broussonetia kazinoki, T1152 Camellia sinensis [Syn. Thea sinensis], T1172 Canna edulis, T1186 Capsicum annuum, T1187 Capsicum frutescens, T1251 Cassytha filiformis, T1258 Casuarina equisetifolia, T1278 Cedrela sinensis, T1299 Celosia cristata, T1343 Chaenomeles sinensis, T1352 Chamaenerion angustifolium [Syn. Epilobium angustifolium], T1359 Chenopodium album, T1381 Choerospondias axillaris, T1423 Cimicifuga nanchuanensis, T1440 Cinnamomum glanduliferum, T1443 Cinnamomum parthenoxylum [Syn. Cinnamomum porrectum], T1493 Citrus limon, T1497 Citrus limonia, T1530 Cladostachys amaranthoides [Syn. Achyranthes amaranthoides; Cladostachys frutescens; Deeringia amaranthoides], T1641 Conioselinum vaginatum, T1784 Cratoxylum cochinchinense, T1928 Cycas revoluta, T1939 Cymbopogon citratus, T1943 Cymbopogon goeringii, T1964 Cynanchum wilfordii [Syn. Cynoctonum wilfordii], T1980 Cyprinus carpio, T2058 Deeringia amaranthoides [Syn. Cladostachys frutescens], T2136 Desmos cochinchinensis [Syn. Desmos chinensis], T2181 Dillenia indica, T2341 Elsholtzia ciliata, T2346 Embelia oblongifolia, T2348 Embelia ribes, T2510 Eucalyptus globulus, T2580 Euphorbia antiquorum, T2636 Euscaphis japonica, T2658 Fagopyrum esculentum, T2717 Ficus carica, T2719 Ficus fistulosa [Syn. Ficus harlandii], T2723 Ficus pumila, T2766 Fraxinus bungeana, T2767 Fraxinus chinensis, T2774 Fraxinus paxiana, T2777 Fraxinus rhynchophylla [Syn. Fraxinus chinensis var. rhynchophylla], T2779 Fraxinus stylosa, T2780 Fraxinus szaboana [Syn. Fraxinus chinensis var. acuminata], T2994 Gloiopeltis furcata, T3125 Hedyotis chrysotricha [Syn. Oldenlandia chrysotricha], T3241 Hibiscus mutabilis, T3340 Hypericum ascyron, T3348 Hypericum elodeoides, T3356 Hypericum japonicum, T3368 Hypericum wightianum, T3373 Hypoestes purpurea [Syn. Justicia purpurea; Hypoestes sinica], T3381 Hyptis suaveolens, T3388 Ilex chinensis [Syn. Ilex purpurea], T3418 Incarvillea arguta, T3507 Isodon lophanthoides [Syn. Rabdosia lophanthoides; Hyssopus lophanthoides; Plectranthus striatus; Isodon striatus], T3569 Juglans regia, T3674 Laggera alata, T3758 Lepidium sativum, T3770 Lespedeza cuneata, T3866 Liquidambar formosana [Syn. Liquidambar taiwaniana], T3885 Litsea cubeba, T3926 Loropetalum chinense, T3990 Lygodium japonicum, T4006 Lythrum anceps, T4007 Lythrum salicaria, T4028 Maesa indica, T4048 Magnolia rostrata, T4055 Mahonia bealei, T4060 Mahonia confusa, T4062 Mahonia fortunei, T4064 Mahonia gracilipes, T4066 Mahonia japonica, T4072 Mahonia shenii, T4183 Menispermum dauricum, T4217 Microcos paniculata [Syn. Grewia microcos], T4343 Myrica esculent, T4361 Myrsine africana, T4368 Nandina domestica, T4369 Nandina domestica, T4397 Nelumbo nucifera, T4502 Onychium japonicum [Syn. Tricomanes japonicum], T4544 Oryza sativa, T4545 Oryza sativa, T4577 Paederia scandens, T4600 Panax ginseng [Syn. Panax schinseng], T4629 Papaver nudicaule, T4630 Papaver nudicaule ssp. amurense, T4636 Papaver somniferum, T4789 Phellodendron amurense, T4790 Phellodendron amurense var. wilsonii,
T4791 Phellodendron chinense, T4792 Phellodendron chinense var. glabriusculum, T4802 Phleum pratense, T4834 Phyllanthus emblica, T4835 Phyllanthus emblica, T4840 Phyllanthus niruri, T4953 Piper longum, T5100 Polygonum chinense, T5104 Polygonum hydropiper, T5105 Polygonum hydropiper var. flaccidum [Syn. Polygonum flaccidum], T5106 Polygonum lapathifolium, T5112 Polygonum periginatoris, T5114 Polygonum polystachyum, T5124 Polypodium niponicum, T5129 Polyporus umbellatus, T5171 Porphyra tenera, T5194 Premna microphylla, T5268 Psidium guajava, T5288 Pteris cretica var. nervosa [Syn. Pteris nervosa], T5289 Pteris dactylina, T5290 Pteris fauriei [Syn. Pteris fauriei var. minor], T5295 Pteris multifida, T5312 Pueraria edulis, T5313 Pueraria lobata [Syn. Pueraria thunbergiana; Pueraria pseudohirsuta], T5316 Pueraria omeiensis, T5318 Pueraria phaseoloides, T5320 Pueraria thomsonii, T5330 Punica granatum, T5332 Punica granatum, T5341 Pygmaeopremna herbacea [Syn. Premna herbacea], T5361 Pyrus betulaefolia, T5365 Pyrus pashia, T5374 Quercus infectoria, T5388 Rabdosia adenantha, T5389 Rabdosia coetsa, T5397 Rabdosia serra, T5419 Raphanus sativus, T5497 Rhodiola kirilowii, T5499 Rhodiola sacra, T5528 Rhodomyrtus tomentosa, T5563 Rosa cymosa, T5573 Rosa sericea, T5626 Ruta graveolens, T5687 Salvia prionitis, T5754 Saussurea graminea, T5833 Scutellaria amoena, T5834 Scutellaria baicalensis, T5839 Scutellaria hypericifolia, T5841 Scutellaria likiangensis, T5843 Scutellaria rehderiana, T5845 Scutellaria viscidula, T5860 Selaginella davidii, T5870 Selaginella uncinata, T5934 Seseli meirei, T5937 Seseli yunnanense, T5938 Setaria italica, T5943 Sida cordifolia, T5976 Smilax china [Syn. Smilax japonica], T6024 Solidago virgaurea var. leiocarpa [Syn. Solidago decurrens], T6075 Spilanthes acmella, T6133 Stephania sinica, T6177 Strychnos ignatii, T6206 Sus scrofa domestica, T6250 Symplocos caudata, T6372 Thalictrum acutifolium, T6373 Thalictrum alpinum, T6381 Thalictrum faberi, T6388 Thalictrum fortunei, T6436 Thlaspi arvense, T6463 Tinospora capillipes, T6467 Tinospora sagittata, T6488 Trachyspermum ammi, T6544 Triticum aestivum [Syn. Triticum vulgare], T6645 Urena lobata, T6801 Vladimiria denticulata, T6860 Zanthoxylum ailanthoides, T6869 Zanthoxylum bungeanum, T6892 Zanthoxylum schinifolium, T6909 Zingiber officinale, T6910 Zingiber officinale, T6920 Ziziphus mauritiana.

diarrhea and abdomen pain T5391 Rabdosia eriocalyx.

diarrhea and dysentery T0045 Acanthopanax trifoliatus, T0142 Acorus calamus, T0661 Artemisia anomala, T1858 Croton tiglium, T2438 Erodium stephanianum, T2521 Eucalyptus tereticornis, T2837 Gallus gallus domesticus, T2838 Gallus gallus domesticus, T2943 Geranium nepalense, T2944 Geranium pratense, T2947 Geranium sibiricum, T2949 Geranium wilfordii, T2956 Geum japonicum, T2964 Ginkgo biloba, T4182 Menispermum dauricum, T4549 Osbeckia chinensis, T4618 Panicum miliaceum, T4884 Picria felterrae, T4965 Piper sarmentosum, T5517 Rhododendron molle, T6131 Stephania longa, T6252 Symplocos chinensis, T6476 Torilis japonica, T6754 Vigna unguiculata.

diarrhea and dysentery due to cold $q i$ T4306 Mosla grosseserrata. diarrhea and tenesmus T0313 Allium chinense, T0316 Allium macrostemon, T0606 Areca catechu, T5758 Saussurea lappa [Syn. 
Aucklandia lappa].

diarrhea due to spleen-kidney vacuity T1911 Cuscuta australis, T1912

Cuscuta chinensis, T1913 Cuscuta japonica.

diarrhea due to spleen-stamach vacuity cold T4548 Oryza sativa var. glutinosa.

diarrhea dysentery T5182 Potentilla discolor.

diarrhea in pregnancy T0212 Agastache rugosus.

diarrhea with hematochezia T0982 Bos taurus domesticus.

diarrhea with pus blood T2337 Elephas maximus.

diarrhea, T4663 Passiflora caerulea.

dietary imbalance after illness T1472 Citrus chachiensis, T1517 Citrus tankan.

difficult delivery T0979 Bombyx mori, T1174 Cannabis sativa, T2836 Gallus gallus domesticus.

difficult lactation T6497 Tribulus terrestris.

diffusive intravascular clotting T5680 Salvia miltiorrhiza, T5681 Salvia miltiorrhiza f. alba, T5688 Salvia przewalskii.

dim vision T1035 Broussonetia papyrifera, T1048 Buddleja officinalis, T1152 Camellia sinensis [Syn. Thea sinensis], T1240 Cassia obtusifolia, T1250 Cassia tora, T2094 Dendrobium aduncum, T2096 Dendrobium aurantiacum var. denneanum, T2098 Dendrobium chrysanthum, T2100 Dendrobium densiflorum, T2102 Dendrobium fimbriatum var. oculatum, T2105 Dendrobium loddigesii, T2106 Dendrobium moniliforme, T2107 Dendrobium nobile, T2108 Dendrobium officinale, T3828 Ligustrum lucidum, T4658 Parmelia tinctorum, T6150 Streptopelia orientalis.

dim vision and eye screen T1526 Cladonia rangiferina.

diphtheria T1001 Brachystemma calycinum, T1662 Coptis chinensis, T1663 Coptis chinensis var. brevisepala, T1664 Coptis deltoidea, T1669 Coptis omeiensis, T1670 Coptis teetoides [Syn. Coptis teeta], T3875 Liriope spicata var. prolifera, T4507 Ophiopogon japonicus, T4834 Phyllanthus emblica, T5100 Polygonum chinense, T5445 Rehmannia glutinosa [Syn. Rehmannia glutinosa f. huechingensis], T5447 Rehmannia glutinosa [Syn. Rehmannia glutinosa f. huechingensis], T5557 Rohdea japonica [Syn. Orontium japonicum], T6039 Sophora moorcroftiana, T6566 Tupistra chinensis, T6580 Tylophora floribunda, T6709 Verbena officinalis, T6814 Wedelia chinensis [Syn. Solidago chinensis; Wedelia calendulacea].

dislocation T1533 Clausena dentata, T1534 Clausena dunniana.

disquieted heart spirit T0292 Albizzia julibrissin, T4555 Ostrea rivularis; Ostrea talienwhanensis; Ostrea gigas, T5086 Polygala sibirica, T5088 Polygala tenuifolia, T5169 Poria cocos, T6675 Valeriana amurensis, T6679 Valeriana officinalis, T6680 Valeriana officinalis var. latifolia.

disquieted heart spirit (root) T4859 Physochlaina physaloides.

disquieted spirit-mind T6358 Tetraplodon mnioides [Syn. Tetraplodon bryoides; Splachnum mnioides].

disseminated swelling of welling abscess and sores T3213 Heracleum hemsleyanum, T3217 Heracleum moellendorffii [Syn. Heracleum microcarpum; Heracleum morifolium], T3228 Heracleum yungningense.

distended head and headache T4172 Melilotus suaveolens.

distended head and headache due to liver-gallbladder repletion fire T2908 Gentiana cephalantha, T2921 Gentiana manshurica, T2928
Gentiana rigescens, T2930 Gentiana scabra, T2938 Gentiana triflora. distended head and oppression in chest T2558 Eupatorium formosanum, T2559 Eupatorium fortunei.

distended red eyes and screen T2434 Eriocaulon buergerianum. distending pain in breast T0201 Aesculus chinensis, T0202 Aesculus hippocastanum, T0205 Aesculus wilsonii, T3282 Hordeum vulgare.

distending pain in chest and abdomen T0625 Aristolochia contorta, T0627 Aristolochia debilis [Syn. Aristolochia longa], T3854 Lindera strychnifolia [Syn. Lindera aggregata].

distending pain in chest and rib-side T0201 Aesculus chinensis, T0202 Aesculus hippocastanum, T0205 Aesculus wilsonii, T1966 Cynara scolymus, T1978 Cyperus rotundus, T3431 Inula helenium, T3437 Inula racemosa.

distending pain in chest and stomach duct T1932 Cyclea racemosa, T5758 Saussurea lappa [Syn. Aucklandia lappa].

distending pain in rib-side T0275 Akebia quinata, T6062 Sparganium stoloniferum.

distending pain in stomach duct T0275 Akebia quinata, T1186 Capsicum annuum, T1187 Capsicum frutescens, T1415 Cichorium intybus, T1440 Cinnamomum glanduliferum, T1498 Citrus medica, T1520 Citrus wilsonii, T1769 Crataegus cuneata, T1771 Crataegus kansuensis, T1772 Crataegus maximowiczii, T1780 Crataegus sanguinea, T1781 Crataegus scabrifolia, T1876 Cucumis melo, T1978 Cyperus rotundus, T2136 Desmos cochinchinensis [Syn. Desmos chinensis], T2387 Epilobium hirsutum, T3431 Inula helenium, T3437 Inula racemosa, T4039 Magnolia grandiflora, T4163 Melia toosendan, T4351 Myristica fragrans, T4937 Piper betle, T4966 Piper sarmentosum, T5420 Raphanus sativus, T5934 Seseli meirei, T5937 Seseli yunnanense, T6106 Stelmatocrypton khasianum, T6602 Umbilicaria hypococcinea, T6675 Valeriana amurensis, T6679 Valeriana officinalis, T6680 Valeriana officinalis var. latifolia, T6802 Vladimiria souliei [Syn. Jurinea souliei].

distending pain in stomach duct and abdomen T0144 Acorus gramineus, T0146 Acorus tatarinowii, T0606 Areca catechu, T0692 Artemisia rupestris [Syn. Artemisia dentata; Artemisia viridis; Artemisia viridifolia], T1469 Citrus aurantium var. amara, T1483 Citrus grandis var. tomentosa, T1904 Curcuma kwangsiensis, T1909 Curcuma zedoaria [Syn. Curcuma aeruginosa], T1958 Cynanchum otophyllum, T2193 Dioscorea cirrhosa [Syn. Dioscorea pogonoides], T3725 Laurus nobilis, T3849 Lindera chunii, T3997 Lysimachia capillipes, T4217 Microcos paniculata [Syn. Grewia microcos], T4443 Notholirion hyacinthinum [Syn. Notholirion bulbuliferum].

distention and oppression in stomach duct and abdomen T2365 Enteromorpha clathrata, T4714 Perilla frutescens.

distention fullness T4944 Piper cubeba, T6910 Zingiber officinale.

distention fullness and pain in stomach duct and abdomen T0358 Alpinia katsumadai, T4470 Ocimum basilicum.

distention fullness and rapid asthma T4287 Morus alba.

distention fullness and sudden pain in chest and abdomen T1858 Croton tiglium, T4048 Magnolia rostrata.

distention fullness in chest and abdomen T0130 Aconitum sinomontanum, T0363 Alpinia speciosa, T1468 Citrus aurantium, T1521 Citrus wilsonii, T3840 Limnophila rugosa, T4002 Lysimachia 
foenum-graecum, T4386 Nardostachys chinensis, T4387 Nardostachys jatamansi, T4786 Phasianus colchicus, T5141 Poncirus trifoliata.

distention fullness in chest and diaphragm T0240 Aglaia odorata, T3620 Kaempferia galanga, T4526 Origanum vulgare.

distention fullness in chest and rib-side T2125 Descurainia sophia, T3755 Lepidium apetalum [Syn. Lepidium micranthum], T3759 Lepidium virginicum.

distention fullness in chest and stomach duct T4718 Perilla frutescens var. acuta [Syn. Perilla frutescens var. purpurascens], T4721 Perilla frutescens var. arguta.

distention fullness in heart and abdomen T4954 Piper longum.

distention fullness in stomach duct T0353 Alpinia blepharocalyx, T1775 Crataegus pinnatifida, T1778 Crataegus pinnatifida var. major, T1964 Cynanchum wilfordii [Syn. Cynoctonum wilfordii], T4505 Ophiocephalus argus, T4964 Piper retrofractum, T4965 Piper sarmentosum, T6819 Wikstroemia chamaedaphne.

distention fullness in stomach duct and abdomen T1170 Canis familiaris, T1623 Collybia albuminosa, T2048 Daucus carota, T5137 Poncirus trifoliata, T5385 Quisqualis indica, T5469 Rheum hotaoense.

distention in rib-side T1467 Citrus aurantium, T1501 Citrus medica var. sarcodactylis, T5140 Poncirus trifoliata.

distention oppression in stomach duct T0274 Akebia quinata, T0278 Akebia trifoliata, T0280 Akebia trifoliata var. australis.

dizziness T0334 Aloe arborescens var. natalensis, T0553 Apocynum venetum, T0645 Armillariella mellea, T0859 Balanophora involucrata, T0923 Bergenia crassifolia, T1337 Cervus nippon; Cervus elaphus, T1353 Changium smyrnioides, T1366 Chimonanthus fragrans [Syn. Chimonanthus praecox], T1395 Chrysanthemum morifolium [Syn. Dendranthema morifolium], T1697 Cornus controversa [Syn. Bothrocaryum controversum], T2060 Delonix regia, T2244 Doellingeria scaber [Syn. Aster scaber], T2805 Fugu ocellatus, T2846 Ganoderma japonicum [Syn. Ganoderma sinense], T2848 Ganoderma lucidum, T2890 Gastrodia elata, T3001 Glycine max, T3142 Helianthus annuus, T3372 Hypodematium sinense, T3775 Lethariella cladonioides, T3776 Lethariella zahlbruckneri, T3785 Levisticum officinale, T3845 Linaria vulgaris, T4238 Millettia nitida, T4279 Morina chinensis, T4821 Pholidota articulata, T4904 Pinellia ternata, T5226 Prunus mume, T5423 Rauvolfia verticillata, T5425 Rauvolfia verticillata f. rubrocarpa, T5426 Rauvolfia verticillata var. hainanensis, T5427 Rauvolfia vomitoria, T5428 Rauvolfia yunnanensis, T5435 Rauwolfia latifrons, T5441 Rauwolfia perakensis, T5497 Rhodiola kirilowii, T5657 Salsola collina, T5927 Sesamum indicum [Syn. Sesamum orientale], T6278 Tagetes erecta, T6410 Thalictrum smithii, T6843 Xanthium sibiricum [Syn. Xanthium strumarium].

dizziness (for men) T2391 Epimedium brevicornum.

dizziness and dim vision T0298 Alectoria vivens, T1296 Celosia argentea, T1525 Cladonia fallax, T1528 Cladonia stellaris [Syn. Cladonia alpestris], T1819 Crotalaria ferruginea, T2955 Geum japonicum, T3827 Ligustrum japonicum, T3955 Lycium barbarum, T3958 Lycium chinense, T5593 Rubus idaeus, T6036 Sophora japonica, T6076 Spinacia oleracea.

dizziness and headache T0625 Aristolochia contorta, T0627 Aristolochia debilis [Syn. Aristolochia longa], T1055 Bupleurum angustissimum,
T1056 Bupleurum aureum, T1058 Bupleurum chaishoui, T1059 Bupleurum chinense, T1065 Bupleurum longiradiatum, T1066 Bupleurum marginatum, T1067 Bupleurum marginatum var. stenophyllum, T1072 Bupleurum scorzonerifolium, T1073 Bupleurum sibiricum, T1075 Bupleurum smithii var. parvifolium, T1078 Bupleurum wenchuanense, T1079 Bupleurum yinchowense, T1240 Cassia obtusifolia, T1250 Cassia tora, T3557 Jasminum sambac, T4289 Morus alba, T4291 Morus australis, T4293 Morus cathayana, T4298 Morus mongolica, T6531 Trillium camtschaticum, T6533 Trillium kamtschaticum, T6535 Trillium tschonoskii, T6791 Vitex rotundifolia [Syn. Vitex trifollia var. simplicifolia], T6793 Vitex trifolia.

dizziness and insomnia T1961 Cynanchum thesioides.

dizziness and palpitation T5446 Rehmannia glutinosa [Syn. Rehmannia glutinosa f. huechingensis].

dizziness and tinnitus T0793 Astragalus complanatus, T1698 Cornus officinalis [Syn. Macrocarpium officinale], T1952 Cynanchum auriculatum, T1953 Cynanchum bungei, T2323 Eclipta prostrata [Syn. Eclipta alba], T3828 Ligustrum lucidum, T4056 Mahonia bealei, T4057 Mahonia bealei, T4063 Mahonia fortunei, T4067 Mahonia japonica, T4068 Mahonia japonica, T4432 Nigella glandulifera, T5100 Polygonum chinense.

dizziness due to anemia T3774 Lespedeza tomentosa, T5107 Polygonum multiflorum.

dizziness syndrome T0644 Armillaria mellea.

dizzy and distended head T1242 Cassia occidentalis, T1247 Cassia sophera.

dizzy head T0644 Armillaria mellea, T4060 Mahonia confusa, T4064 Mahonia gracilipes, $\mathrm{T} 6087$ Spiranthes sinensis.

dizzy head and distention eyes T0428 Ampelopsis megalophylla.

dizzy head and insomnia T5586 Rubia yunnanensis.

dizzy head and tinnitus T1188 Caragana chamlagu.

dizzy head and vision T1964 Cynanchum wilfordii [Syn. Cynoctonum wilfordii], T3390 Ilex cornuta, T5446 Rehmannia glutinosa [Syn. Rehmannia glutinosa f. huechingensis], T5498 Rhodiola quadrifida.

dizzy head and vision due to insufficiency of liver-kidney and blood vacuity essence depletion T4288 Morus alba.

dizzy head due to blood vacuity T3675 Lagopsis supina.

dog bite T1547 Clematis terniflora [Syn. Clematis maximowicziana], T1932 Cyclea racemosa, T1958 Cynanchum otophyllum, T4428 Nicotiana tabacum.

dormant papules with pruritus T3738 Lemna minor, T4184 Mentha haplocalyx [Syn. Mentha canadaensis; Mentha arvensis var. haplocalyx; Mentha arvensis], T4470 Ocimum basilicum.

double tongue T4042 Magnolia liliflora, T4050 Magnolia sieboldii.

dream emission T5791 Schisandra chinensis, T5802 Schisandra sphenanthera, T6670 Vaccinium bracteatum.

dribbling and inhibited voidings of urination T0172 Adiantum caudatum, T0175 Adiantum pedatum, T1933 Cyclea sutchuenensis, T3240 Hibiscus esculentus, T3764 Lepisorus thunbergianus, T5033 Poa sphondylodes, T5978 Smilax glauco-china, T6131 Stephania longa.

dribbling pain of urination T0173 Adiantum lunulatum, T0428 Ampelopsis megalophylla, T0595 Ardisia crispa, T1589 Cocculus 
trilobus [Syn. Cocculus sarmentosus], T1813 Crotalaria albida, T1823 Crotalaria juncea, T1931 Cyclea barbata, T1941 Cymbopogon distans, T2902 Gentiana algida, T2908 Gentiana cephalantha, T2921 Gentiana manshurica, T2928 Gentiana rigescens, T2930 Gentiana scabra, T2938 Gentiana triflora, T3303 Hydrangea chinensis, T3555 Jasminum nudiflorum, T3765 Lepisorus ussuriensis, T3920 Lophatherum gracile, T4502 Onychium japonicum [Syn. Tricomanes japonicum], T4730 Peristrophe roxburghiana, T4802 Phleum pratense, T5071 Polygala arillata, T5301 Pterocarpus indicus, T5372 Quercus dentata, T6526 Triglochin maritimum.

dribbling urinary block T2243 Dodonaea viscosa, T3738 Lemna minor, T5011 Platycodon grandiflorum.

dribbling urination T0753 Asparagus setaceus [Syn. Asparagus plumosus], T0993 Boschniakia rossica, T1241 Cassia occidentalis, T1826 Crotalaria mucronata, T1911 Cuscuta australis, T1912 Cuscuta chinensis, T1913 Cuscuta japonica, T2243 Dodonaea viscosa, T3142 Helianthus annuus, T4845 Physalis alkekengi, T5151 Populus davidiana, T5158 Populus simonii, T6901 Zea mays.

dripping with inhibited pain T2143 Dianthus chinensis, T2145 Dianthus superbus, T2147 Dianthus versicolor, T5098 Polygonum aviculare, T5354 Pyrrosia davidii, T5355 Pyrrosia drakeana, T5356 Pyrrosia gralla, T5357 Pyrrosia lingua T5358 Pyrrosia petiolosa, T5360 Pyrrosia sheareri.

drooling T0360 Alpinia oxyphylla, T0825 Atropa belladonna, T2558 Eupatorium formosanum, T2559 Eupatorium fortunei.

drug poisoning T0516 Anser cygnoides domestica, T3000 Glycine max.

drunkenness T1428 Cinchona ledgeriana, T1429 Cinchona officinalis, T1433 Cinchona succirubra, T1471 Citrus chachiensis, T1473 Citrus cultivars, T1474 Citrus decumana, T1478 Citrus grandis, T1486 Citrus junos, T1488 Citrus junos, T1516 Citrus tankan, T1518 Citrus unshiu, T3286 Hovenia dulcis, T5194 Premna microphylla, T6492 Trapa bispinosa.

dry blood tisis (consumptive disease due to blood disorders) T5498 Rhodiola quadrifida.

dry cough T0318 Allium sativum, T0462 Anemarrhena asphodeloides, T2783 Fritillaria cirrhosa, T2784 Fritillaria delavayi, T2792 Fritillaria przewalskii, T2796 Fritillaria unibracteata, T2797 Fritillaria ussuriensis, T3124 Hedyotis capitellata, T5093 Polygonatum odoratum [Syn. Polygonatum officinale], T5094 Polygonatum prattii, T6097 Staphylea bumalda.

dry cough due to lung dryness T0158 Actinidia chinensis, T2797 Fritillaria ussuriensis, T2983 Glehnia littoralis, T3875 Liriope spicata var. prolifera, T4507 Ophiopogon japonicus, T4602 Panax ginseng [Syn. Panax schinseng], T4912 Pinus koraiensis, T5260 Pseudostellaria heterophylla.

dry cough lesser phlegm T6139 Sterculia lychnophora.

dry cough with hoarseness $\mathrm{T} 2717$ Ficus carica.

dry cough without phlegm T4635 Papaver somniferum.

dry cracked skin T3860 Linum usitatissimum, T5925 Sesamum indicum [Syn. Sesamum orientale].

dry eyes with clouded flowery vision T5826 Scrophularia buergeriana, T5828 Scrophularia ningpoensis.

dry lichen T4265 Momordica cochinchinensis, T5907 Senecio scandens
[Syn. Senecio chinensis].

dry mouth and throat $\mathrm{T} 3873$ Liriope platyphylla, T3874 Liriope spicata. dry mouth with vexation and thirst T2094 Dendrobium aduncum, T2096 Dendrobium aurantiacum var. denneanum, T2098 Dendrobium chrysanthum, T2100 Dendrobium densiflorum, T2102 Dendrobium fimbriatum var. oculatum, T2105 Dendrobium loddigesii, T2106 Dendrobium moniliforme, T2107 Dendrobium nobile, T2108 Dendrobium officinale.

dry stool T0559 Arachis hypogaea, T0843 Babylonia lutosa, T5549 Ricinus communis, T5550 Ricinus communis, T5642 Saccharum sinensis, T6428 Thermopsis lanceolata.

dry throat T4665 Passiflora edulis, T5093 Polygonatum odoratum [Syn. Polygonatum officinale], T5094 Polygonatum prattii.

dry throat and phlegm node T1526 Cladonia rangiferina.

dry-itchy skin T3878 Lithocarpus polystachyus.

dryness in eyes and mouth T6542 Tripterygium wilfordii.

duodenal bleeding T2323 Eclipta prostrata [Syn. Eclipta alba], T3926 Loropetalum chinense, T6440 Thuja orientalis [Syn. Platycladus orientalis; Biota orientalis].

duodenal ulcer T0255 Ailanthus altissima, T0541 Apis cerana, T0825 Atropa belladonna, T1884 Cudrania cochinchinensis, T1954 Cynanchum chinense, T2783 Fritillaria cirrhosa, T2784 Fritillaria delavayi, T2792 Fritillaria przewalskii, T2796 Fritillaria unibracteata, T2798 Fritillaria verticillata var. thunbergii [Syn. Fritillaria thunbergii], T3229 Hericium erinaceus [Syn. Hydnum erinaceus], T3613 Kadsura coccinea [Syn. Kadsura chenensis; Kadsura hainanensis], T4082 Mallotus japonicus, T5183 Potentilla griffithii var. velutina, T5712 Sanguisorba officinalis, T6145 Stichopus japonicus, T6275 Tacca chantrieri [Syn. Tacca minor; Tacca esquirolii].

dysentery T0002 Abelmoschus moschatus [Syn. Hibiscus abelmoschus], T0071 Achyranthes aspera, T0072 Achyranthes aspera var. indica, T0171 Adiantum capillus-veneris, T0172 Adiantum caudatum, T0175 Adiantum pedatum, T0180 Adina pilulifera [Syn. Cephalanthus pilulifera], T0190 Adonis sutchuenensis, T0197 Aegle marmelos, T0226 Agave sisalana, T0248 Agrimonia pilosa, T0263 Ajuga decumbens, T0264 Ajuga forrestii, T0275 Akebia quinata, T0297 Alchornea trewioides, T0314 Allium fistulosum, T0318 Allium sativum, T0322 Allium tuberosum, T0388 Amaranthus lividus, T0389 Amaranthus tricolor, T0424 Ampelopsis brevipedunculata var. hancei, T0428 Ampelopsis megalophylla, T0444 Ananas comosus, T0468 Anemone hupehensis, T0495 Angelica sinensis, T0500 Anguilla japonica, T0563 Arachniodes exilis, T0567 Aralia armata, T0573 Aralia elata, T0594 Ardisia crenata, T0598 Ardisia japonica, T0600 Ardisia mamillata [Syn. Tinus mamillata], T0852 Bacopa monniera, T0877 Bauhinia championii, T0890 Begonia limprichtii, T0897 Berberis amurensis, T0907 Berberis kawakamii, T0933 Betula platyphylla, T0935 Betula platyphylla var. japonica, T0938 Bidens bipinnata, T0947 Bixa orellana, T0973 Bombax malabaricum [Syn. Gossampinus malabarica], T1031 Broussonetia kazinoki, T1101 Caesalpinia decapetala, T1106 Caesalpinia minax, T1153 Camellia sinensis var. assamica, T1163 Campylotropis hirtella, T1172 Canna edulis, T1205 Carica papaya, T1242 Cassia occidentalis, T1247 Cassia sophera, T1251 Cassytha filiformis, T1258 Casuarina 
equisetifolia, T1275 Cayratia japonica, T1278 Cedrela sinensis, T1282 Cedrus deodara, T1286 Celastrus flagellaris, T1288 Celastrus orbiculatus [Syn. Celastrus articulatus], T1289 Celastrus orbiculatus [Syn. Celastrus articulatus], T1299 Celosia cristata, T1311 Centella asiatica, T1315 Cephaelis ipecacuanha, T1316 Cephalanthus occidentalis, T1343 Chaenomeles sinensis, T1357 Chelidonium majus, T1359 Chenopodium album, T1387 Chrysanthemum boreale, T1392 Chrysanthemum indicum, T1394 Chrysanthemum lavandulifolium, T1443 Cinnamomum parthenoxylum [Syn. Cinnamomum porrectum], T1530 Cladostachys amaranthoides [Syn. Achyranthes amaranthoides; Cladostachys frutescens; Deeringia amaranthoides], T1552 Cleome viscosa, T1562 Clerodendron trichotomum, T1573 Clinopodium chinense, T1657 Conyza canadensis [Syn. Erigeron canadensis], T1662 Coptis chinensis, T1663 Coptis chinensis var. brevisepala, T1664 Coptis deltoidea, T1669 Coptis omeiensis, T1670 Coptis teetoides [Syn. Coptis teeta], T1673 Corchorus capsularis, T1675 Corchorus olitorius, T1684 Cordyline strcta, T1687 Coriandrum sativum, T1709 Corydalis bungeana, T1727 Corydalis mucronifera, T1728 Corydalis ochotensis, T1733 Corydalis racemosa, T1747 Corydalis thalictrifolia, T1826 Crotalaria mucronata, T1831 Crotalaria sessiliflora, T1927 Cycas revoluta, T1928 Cycas revoluta, T1967 Cynoglossum amabile, T1969 Cynoglossum officinale, T2039 Datura innoxia, T2043 Datura metel, T2047 Datura stramonium, T2058 Deeringia amaranthoides [Syn. Cladostachys frutescens], T2115 Dermatocarpon minitum, T2181 Dillenia indica, T2233 Diploclisia glaucescens, T2257 Dracocephalum moldavicum, T2258 Dracocephalum rupestre, T2268 Drosera rotundifolia, T2280 Dryopteris chrysocoma, T2290 Duchesnea indica, T2348 Embelia ribes, T2505 Eucalyptus citriodora, T2510 Eucalyptus globulus, T2533 Eugenia jambolana [Syn. Syzygium cumin; Myrtus cumini], T2580 Euphorbia antiquorum, T2591 Euphorbia humifusa, T2636 Euscaphis japonica, T2644 Evodia rutaecarpa, T2646 Evodia rutaecarpa var. officinalis, T2649 Evolvulus alsinoides, T2658 Fagopyrum esculentum, T2659 Fagopyrum esculentum, T2695 Ferula assafoetida, T2715 Fibraurea recisa, T2716 Ficus beecheyana [Syn. Ficus erecta var. beecheyana], T2717 Ficus carica, T2720 Ficus hispida, T2839 Gallus gallus domesticus, T2840 Gallus gallus domesticus, T2927 Gentiana rhodantha, T2988 Glochidion eriocarpum, T2994 Gloiopeltis furcata, T3040 Gomphrena globosa, T3123 Hedyotis auricularia, T3125 Hedyotis chrysotricha [Syn. Oldenlandia chrysotricha], T3126 Hedyotis corymbosa [Syn. Oldenlandia corymbosa], T3163 Helicteres angustifolia, T3242 Hibiscus rosa-sinensis, T3263 Holarrhena antidysenterica, T3284 Houttuynia cordata, T3311 Hydrocotyle sibthorpioides, T3332 Hypecoum erectum, T3340 Hypericum ascyron, T3342 Hypericum bellum, T3356 Hypericum japonicum, T3363 Hypericum sampsonii, T3381 Hyptis suaveolens, T3388 Ilex chinensis [Syn. Ilex purpurea], T3392 Ilex kudingcha, T3393 Ilex latifolia, $\mathrm{T} 3398$ Ilex rotunda, T3418 Incarvillea arguta, $\mathrm{T} 3507$ Isodon lophanthoides [Syn. Rabdosia lophanthoides; Hyssopus lophanthoides; Plectranthus striatus; Isodon striatus], T3528 Isodon ternifolia, T3529 Isodon ternifolius, $\mathrm{T} 3547$ Ixeris chinensis, Т3557 Jasminum sambac, T3569 Juglans regia, T3645 Kummerowia striata, T3646 Kyllinga brevifolia, T3670 Lagerstroemia indica, T3672 Lagerstroemia indica,
T3736 Lemmaphyllum microphyllum, T3737 Lemmaphyllum microphyllum var. obovatum, T3758 Lepidium sativum, T3770 Lespedeza cuneata, T3774 Lespedeza tomentosa, T3830 Ligustrum sinense, T3847 Lindera angustifolia, T3866 Liquidambar formosana [Syn. Liquidambar taiwaniana], T3926 Loropetalum chinense, T3928 Lotus corniculatus, T3953 Lycianthes biflora, T3989 Lygodium flexuosum [Syn. Lygodium pinnatifidum; Ophioglossum flexuosum], T3990 Lygodium japonicum, T3999 Lysimachia clethroides, T4006 Lythrum anceps, T4007 Lythrum salicaria, T4055 Mahonia bealei, T4056 Mahonia bealei, T4062 Mahonia fortunei, T4063 Mahonia fortunei, T4066 Mahonia japonica, T4067 Mahonia japonica, T4086 Malus asiatica, T4148 Medicago sativa, T4170 Melilotus albus, T4172 Melilotus suaveolens, T4225 Microsorium punctatum, T4261 Mollugo pentaphylla, T4263 Momordica charantia, T4280 Morinda citrifolia, T4307 Mosla scabra [Syn. Mosla punctata], T4335 Mussaenda pubescens, T4343 Myrica esculent, T4348 Myrica rubra, T4349 Myrica rubra, T4361 Myrsine africana, T4385 Narcissus tazetta var. chinensis, T4397 Nelumbo nucifera, T4485 Oldenlandia diffusa [Syn. Hedyotis diffusa], T4502 Onychium japonicum [Syn. Tricomanes japonicum], T4519 Opuntia dillenii, T4520 Opuntia ficus-indica, T4521 Opuntia vulgaris, T4527 Orixa japonica, T4552 Osmunda japonica, T4564 Oxalis corniculata [Syn. Oxalis repens], T4577 Paederia scandens, T4582 Paeonia delavayi, T4585 Paeonia moutan [Syn. Paeonia suffruticosa], T4625 Papaver commutatum [Syn. Papaver rhoeas], T4665 Passiflora edulis, T4672 Patrinia scabiosaefolia, T4676 Patrinia villosa, T4802 Phleum pratense, T4804 Phlojodicarpus sibiricus, T4835 Phyllanthus emblica, T4840 Phyllanthus niruri, T4842 Phyllanthus reticulatus, T4843 Phyllanthus urinaria, T4845 Physalis alkekengi, T4846 Physalis alkekengi var. franchetii, T4848 Physalis angulata, T4854 Physalis pubescens, T4881 Picrasma quassioides [Syn. Picrasma ailanthoides], T4887 Picrorhiza kurrooa, T4888 Picrorhiza scrophulariiflora, T5011 Platycodon grandiflorum, T5097 Polygonum amphibium, T5098 Polygonum aviculare, T5100 Polygonum chinense, T5104 Polygonum hydropiper, T5105 Polygonum hydropiper var. flaccidum [Syn. Polygonum flaccidum], T5106 Polygonum lapathifolium, T5109 Polygonum nodosum, T5112 Polygonum periginatoris, T5114 Polygonum polystachyum, T5119 Polygonum thunbergii, T5151 Populus davidiana, T5156 Populus nigra var. thevestina, T5158 Populus simonii, T5161 Populus tomentosa, T5183 Potentilla griffithii var. velutina, T5184 Potentilla kleiniana, T5188 Potentilla viscosa, T5194 Premna microphylla, T5209 Pronephrium simplex [Syn. Meniscium simplex], T5267 Psidium guajava, T5284 Pteridium aquilinum var. latiusculum, T5288 Pteris cretica var. nervosa [Syn. Pteris nervosa], T5289 Pteris dactylina, T5290 Pteris fauriei [Syn. Pteris fauriei var. minor], T5295 Pteris multifida, T5297 Pteris plumbea, T5298 Pteris vittata, T5299 Pteris wallichinan, T5339 Pycnoporus sanguineus, T5361 Pyrus betulaefolia, T5388 Rabdosia adenantha, T5397 Rabdosia serra, T5401 Rabdosia yuennanensis, T5407 Rana nigromaculata; Rana plancyi, T5419 Raphanus sativus, T5497 Rhodiola kirilowii, T5518 Rhododendron mucronatum, T5529 Rhodomyrtus tomentosa, T5531 Rhus chinensis [Syn. Rhus semialata], T5555 Rodgersia pinnata, T5557 Rohdea japonica [Syn. Orontium 
japonicum], T5563 Rosa cymosa, T5567 Rosa laevigata, T5587 Rubus alceaefolius, T5595 Rubus parviforlius, T5607 Rumex crispus, T5614 Rumex patientia, T5685 Salvia plebeia, T5687 Salvia prionitis, T5859 Selaginella braunii, T5862 Selaginella involvens, T5867 Selaginella sinensis, T5870 Selaginella uncinata, T5892 Senecio nemorensis, T5943 Sida cordifolia, T5954 Silene fortunei, T5976 Smilax china [Syn. Smilax japonica], T6008 Solanum nigrum, T6025 Sonchus arvensis, T6029 Sophora alopecuroides, T6046 Sophora viciifolia, T6047 Sophora viciifolia, T6060 Souliea vaginata, T6092 Stachys palustris, T6116 Stenoloma chusanum, T6121 Stephania dicentrinifera, T6128 Stephania hernandifolia, T6133 Stephania sinica, T6137 Stephania viridiflavens, T6177 Strychnos ignatii, T6206 Sus scrofa domestica, T6214 Swertia chinensis, T6217 Swertia davidii, T6231 Swertia pseudochinensis, T6250 Symplocos caudata, T6264 Syzygium buxifolium, T6275 Tacca chantrieri [Syn. Tacca minor; Tacca esquirolii], T6282 Tagetes patula, T6353 Tetracera asiatica, T6359 Teucrium bidentatum, T6365 Teucrium pilosum [Syn. Teucrium japonicum var. pilosum], T6372 Thalictrum acutifolium, T6373 Thalictrum alpinum, T6374 Thalictrum atriplex, T6381 Thalictrum faberi, T6388 Thalictrum fortunei, T6409 Thalictrum simplex [Syn. Thalictrum simplex var. brevipes], T6432 Thespesia populnea [Syn. Hibiscus populneus], T6436 Thlaspi arvense, T6463 Tinospora capillipes, T6467 Tinospora sagittata, T6491 Trametes cinnabarina [Syn. Polyporus cinnabarinus; Boletus cinnabarinus], T6492 Trapa bispinosa, T6508 Trichosanthes cucumeroides, T6602 Umbilicaria hypococcinea, T6645 Urena lobata, T6691 Veratrilla baillonii, T6709 Verbena officinalis, T6722 Veronica anagallis-aquatica, T6788 Vitex negundo, T6801 Vladimiria denticulata, T6814 Wedelia chinensis [Syn. Solidago chinensis; Wedelia calendulacea], T6830 Woodfordia fruticosa, T6831 Woodwardia orientalis, T6843 Xanthium sibiricum [Syn. Xanthium strumarium], T6920 Ziziphus mauritiana.

dysentery (seed) T0533 Antiaris toxicaria [Syn. Ambora toxicaria].

dysentery abdominal pain T3524 Isodon sculponeata [Syn. Rabdosia sculponeata].

dysentery with ardent fever T0181 Adina rubella.

dysentery with hematochezia and hematuria T2882 Gardenia jasminoides [Syn. Gardenia florida].

dysentery with pus and blood T0542 Apis cerana, T1038 Brucea javanica [Syn. Brucea sumatrana; Rhus javanica], T2826 Galeola faberi.

dysentery, T6412 Thalictrum squarrosum.

dysfunctional uterine bleeding T0541 Apis cerana, T1199 Carduus acanthoides, T1200 Carduus crispus.

dysmenorrhea T0071 Achyranthes aspera, T0072 Achyranthes aspera var. indica, T0073 Achyranthes bidentata, T0201 Aesculus chinensis, T0202 Aesculus hippocastanum, T0205 Aesculus wilsonii, T0273 Akebia quinata, T0277 Akebia trifoliata, T0279 Akebia trifoliata var. australis, T0474 Angelica acutiloba [Syn. Ligusticum acutilobum], T0601 Ardisia pusilla, T0642 Aristolochia tubiflora, T0661 Artemisia anomala, T0680 Artemisia lactiflora, T0994 Boswellia carterii, T1005 Brassica campestris [Syn. Brassica campestris var. oleifera], T1129 Calophyllum inophyllum, T1151 Camellia sinensis [Syn. Thea sinensis], T1160 Campsis grandiflora, T1163 Campylotropis hirtella, T1215
Carthamus tinctorius, T1288 Celastrus orbiculatus [Syn. Celastrus articulatus], T1356 Cheiranthus cheiri, T1361 Chenopodium ambrosioides, T1434 Cinnamomum bejolghota [Syn. Cinnamomum obtusifolium; Laurus bejolghota], T1439 Cinnamomum cassia [Syn. Cinnamomum aromaticum], T1441 Cinnamomum japonicum, T1444 Cinnamomum tamala, T1450 Cirsium lineare, T1537 Clausena lansium, T1638 Commiphora myrrha [Syn. Commiphora molmol], T1698 Cornus officinalis [Syn. Macrocarpium officinale], T1706 Corydalis ambigua var. amurensis [Syn. Corydalis ambigua], T1734 Corydalis remota [Syn. Corydalis bulbosa var. typica], T1735 Corydalis repens, T1903 Curcuma aromatica, T1905 Curcuma longa, T1907 Curcuma wengujin, T1959 Cynanchum paniculatum, T1976 Cyperus iria, T1994 Daemonorops draco, T2193 Dioscorea cirrhosa [Syn. Dioscorea pogonoides], T2253 Dracaena cochinchinensis, T2536 Euonymus alatus, T2541 Euonymus grandiflorus, T2542 Euonymus japonicus, T2644 Evodia rutaecarpa, T2646 Evodia rutaecarpa var. officinalis, T3088 Gynura segetum [Syn. Gynura japonica], T3222 Heracleum scabridum, T3340 Hypericum ascyron, T3410 Impatiens balsamina, T3413 Impatiens nolitangere, T3567 Juglans regia, T3613 Kadsura coccinea [Syn. Kadsura chenensis; Kadsura hainanensis], T3614 Kadsura heteroclita [Syn. Uvaria heteroclita], T3619 Kadsura peltigera [Syn. Kadsura longipedunculata], T3679 Lamiophlomis rotata [Syn. Phlomis rotata], T3746 Leontice robustum, T3752 Leonurus heterophyllus [Syn. Leonurus artemisia], T3754 Leonurus sibiricus, T3774 Lespedeza tomentosa, T3785 Levisticum officinale, T3854 Lindera strychnifolia [Syn. Lindera aggregata], T3897 Litsea verticillata, T3970 Lycopodium cernuum, T3973 Lycopodium japonicum [Syn. Lycopodium clavatum], T4005 Lysionotus pauciflorus, T4144 Meconopsis nepaulensis, T4145 Meconopsis punicea, T4190 Mentha spicata, T4311 Mucuna sempervirens, T4510 Ophiorrhiza japonica, T4513 Ophiorrhiza mungos, T4549 Osbeckia chinensis, T4582 Paeonia delavayi, T4584 Paeonia lactiflora wild, T4585 Paeonia moutan [Syn. Paeonia suffruticosa], T4586 Paeonia obovata, T4590 Paeonia veitchii, T4629 Papaver nudicaule, T4630 Papaver nudicaule ssp. amurense, T4663 Passiflora caerulea, T4665 Passiflora edulis, T4948 Piper hancei, T4956 Piper mullesua, T4974 Piper wallichii [Syn. Piper wallichii var. hupehense], T5028 Plumbago indica, T5057 Podophyllum emodii [Syn. Podophyllum emodii var. chinense; Podophyllum sikkimenosis; Sinopodophyllum emodii], T5075 Polygala fallax [Syn. Polygala aureocauda], T5104 Polygonum hydropiper, T5233 Prunus persica, T5365 Pyrus pashia, T5401 Rabdosia yuennanensis, T5515 Rhododendron micranthum, T5545 Ribes fasciculatum var. chinense, T5555 Rodgersia pinnata, T5562 Rosa chinensis, T5569 Rosa multiflora, T5680 Salvia miltiorrhiza, T5681 Salvia miltiorrhiza f. alba, T5688 Salvia przewalskii, T5697 Salvia trijuga, T5701 Salvia yunnanensis, T5730 Sarcandra glabra [Syn. Chloranthus glaber], T5741 Sargentodoxa cuneata, T5781 Schefflera arboricola, T6099 Stauntonia chinensis, T6118 Stephania brachyandra, T6154 Strobilanthes japonicus [Syn. Championella japonica], T6471 Toddalia asiatica [Syn. Toddalia aculeata; Paullinia asiatica], T6488 Trachyspermum ammi, T6540 Tripterygium hypoglaucum, T6584 Typha angustata, T6585 Typha angustifolia, T6587 Typha latifolia, 
T6668 Vaccaria segetalis [Syn. Vaccaria pyramidata], T6675 Valeriana amurensis, T6676 Valeriana hardwickii, T6679 Valeriana officinalis, T6680 Valeriana officinalis var. latifolia, T6830 Woodfordia fruticosa.

dysmenorrhea due to anemia T0495 Angelica sinensis, T4804 Phlojodicarpus sibiricus.

dysphagia-occlusion $\quad \mathrm{T} 0240$ Aglaia odorata, T0353 Alpinia blepharocalyx, T0417 Amomum longiligulare, T0419 Amomum villosum, T0420 Amomum xanthioides, T1501 Citrus medica var. sarcodactylis, T2659 Fagopyrum esculentum, T2761 Fortunella margarita, T3412 Impatiens balsamina, T3436 Inula nervosa, T4544 Oryza sativa, T4546 Oryza sativa, T4547 Oryza sativa cv, T5139 Poncirus trifoliata, T5284 Pteridium aquilinum var. latiusculum.

dysphagia-occlusion and stomach reflux T0322 Allium tuberosum, T0354 Alpinia chinensis, T0516 Anser cygnoides domestica, T6507 Trichosanthes cucumeroides.

ear sore T3420 Incarvillea sinensis.

early stage of sores and welling abscess toxin T0333 Alocasia cucullata [Syn. Arum cucullatum].

early stage of toxin swelling T1640 Coniogramme japonica [Syn. Hemionitis japonica].

early stage of welling abscess and flat abscess T0116 Aconitum nagarum var. heterotrichum [Syn. Aconitum bullatifolium].

eczema T0019 Acacia catechu, T0142 Acorus calamus, T0180 Adina pilulifera [Syn. Cephalanthus pilulifera], T0181 Adina rubella, T0297 Alchornea trewioides, T0379 Alternanthera philoxeroides, T0421 Amorpha fruticosa, T0457 Andrographis paniculata [Syn. Justicia paniculata], T0495 Angelica sinensis, T0635 Aristolochia moupinensis, T0643 Aristolochia versicolar, T0736 Asclepias curassavica, T0853 Baeckea frutescens, T0897 Berberis amurensis, T0906 Berberis julianae, T0912 Berberis poiretii, T0941 Bidens tripartita, T0963 Boehmeria siamensis, T0973 Bombax malabaricum [Syn. Gossampinus malabarica], T1051 Bufo bufo gargarizans; Bufo melanostictus, T1261 Catalpa ovata, T1290 Celastrus orbiculatus [Syn. Celastrus articulatus], T1359 Chenopodium album, T1387 Chrysanthemum boreale, T1392 Chrysanthemum indicum, T1394 Chrysanthemum lavandulifolium, T1558 Clerodendron inerme, T1565 Clerodendrum bungei, T1582 Cnidium monnieri, T1589 Cocculus trilobus [Syn. Cocculus sarmentosus], T1662 Coptis chinensis, T1663 Coptis chinensis var. brevisepala, T1664 Coptis deltoidea, T1669 Coptis omeiensis, T1670 Coptis teetoides [Syn. Coptis teeta], T1675 Corchorus olitorius, T1719 Corydalis incisa, T1921 Cyanotis arachnoidea [Syn. Cyanotis bodinieri], T1922 Cyanotis vaga, T1959 Cynanchum paniculatum, T2194 Dioscorea collettii, T2267 Drosera peltata var. lunata, T2334 Elephantopus scaber, T2341 Elsholtzia ciliata, T2357 Emilia sonchifolia, T2459 Erythrina arborescens, T2478 Erythrina variegata [Syn. Erythrina indica], T2479 Erythrina variegata var. orientalis, T2505 Eucalyptus citriodora, T2510 Eucalyptus globulus, T2517 Eucalyptus robusta, T2590 Euphorbia hirta, T2627 Euphoria longan [Syn. Dimocarpus longan], T2641 Evodia lepta [Syn. Ilex lepta], T2721 Ficus microcarpa, T2852 Garcinia cowa, T2897 Gelsemium elegans, T2988 Glochidion eriocarpum, T2989 Glochidion sphaerogynum, Т3080 Gymnema sylvestre, T3123 Hedyotis auricularia, T3163 Helicteres angustifolia, T3246 Hibiscus syriacus, T3340 Hypericum ascyron, T3381 Hyptis suaveolens, T3388 Ilex chinensis [Syn. Ilex purpurea], T3398 Ilex rotunda, T3420 Incarvillea sinensis, T3560 Jatropha curcas, T3588 Juniperus formosana, T3628 Kerria japonica, T3672 Lagerstroemia indica, T3808 Ligularia japonica [Syn. Arnica japonica; Senecio japonica], T3830 Ligustrum sinense, T3866 Liquidambar formosana [Syn. Liquidambar taiwaniana], T3867 Liquidambar formosana [Syn. Liquidambar taiwaniana], T3928 Lotus corniculatus, T4020 Macleaya cordata, T4055 Mahonia bealei, T4062 Mahonia fortunei, T4066 Mahonia japonica, T4080 Mallotus apelta, T4085 Mallotus repandus var. chrysocarpus [Syn. Mallotus chrysocarpus; Mallotus repandus], T4154 Melaleuca leucadendra, T4247 Millingtonia hortensis, T4284 Morinda parvifolia, T4305 Mosla dianthera, T4307 Mosla scabra [Syn. Mosla punctata], T4324 Murraya paniculata [Syn. Chalcas paniculata], T4391 Nauclea officinalis, T4500 Onosma paniculatum, T4564 Oxalis corniculata [Syn. Oxalis repens], T4789 Phellodendron amurense, T4790 Phellodendron amurense var. wilsonii, T4791 Phellodendron chinense, T4792 Phellodendron chinense var. glabriusculum, T4804 Phlojodicarpus sibiricus, T4834 Phyllanthus emblica, T4837 Phyllanthus emblica, T4845 Physalis alkekengi, T4881 Picrasma quassioides [Syn. Picrasma ailanthoides], T4882 Picrasma quassioides [Syn. Picrasma ailanthoides], T4884 Picria felterrae, T5098 Polygonum aviculare, T5100 Polygonum chinense, T5104 Polygonum hydropiper, T5105 Polygonum hydropiper var. flaccidum [Syn. Polygonum flaccidum], T5106 Polygonum lapathifolium, T5172 Portulaca grandiflora, T5173 Portulaca oleracea, T5178 Potamogeton perfoliatus, T5234 Prunus persica, T5256 Pseudolarix amabilis [Syn. Larix amabilis; Pseudolarix kaempferi], T5308 Pterocarya stenoptera, T5323 Pulsatilla campanella, T5324 Pulsatilla cernua, T5325 Pulsatilla chinensis, T5326 Pulsatilla dahurica, T5395 Rabdosia nervosa, T5455 Rhamnus crenata, T5483 Rhinacanthus nasutus, T5576 Rostellularia procumbens [Syn. Justicia procumbens], T5605 Rumex acetosa, T5613 Rumex obtusifolius, T5626 Ruta graveolens, T5646 Sagina japonica [Syn. Spergula japonica], T5712 Sanguisorba officinalis, T5723 Sapium sebiferum, T5724 Sapium sebiferum, T5773 Saxifraga stolonifera, T5818 Scoparia dulcis, T5907 Senecio scandens [Syn. Senecio chinensis], T5934 Seseli meirei, T5937 Seseli yunnanense, T6017 Solanum tuberosum, T6018 Solanum verbascifolium, T6028 Sophora alopecuroides, T6029 Sophora alopecuroides, T6031 Sophora flavescens [Syn. Sophora angustfolia], T6111 Stemona japonica, T6113 Stemona sessilifolia, T6115 Stemona tuberosa, T6118 Stephania brachyandra, T6136 Stephania tetrandra, T6338 Tephrosia purpurea, T6339 Tephrosia purpurea, T6359 Teucrium bidentatum, T6365 Teucrium pilosum [Syn. Teucrium japonicum var. pilosum], T6367 Teucrium quadrifarium, T6414 Thalictrum thunbergii, T6542 Tripterygium wilfordii, T6843 Xanthium sibiricum [Syn. Xanthium strumarium], T6862 Zanthoxylum ailanthoides.

eczema leukoplakia T6359 Teucrium bidentatum.

eczema of lower limb T5867 Selaginella sinensis.

eczema of skin T0503 Anisomeles indica [Syn. Epimeredi indica], T1361 Chenopodium ambrosioides, T2323 Eclipta prostrata [Syn. Eclipta 
alba], T4835 Phyllanthus emblica, T6116 Stenoloma chusanum.

eczema shank sore T5267 Psidium guajava.

eczema titillation T0229 Ageratum conyzoides, T0659 Artemisia absinthium, T2533 Eugenia jambolana [Syn. Syzygium cumin; Myrtus cumini], T4102 Mangifera indica, T4158 Melia azedarach, T4937 Piper betle, T5389 Rabdosia coetsa, T5685 Salvia plebeia.

eczema with pruritus T1566 Clerodendrum inerme.

edema T0038 Acanthopanax gracilistylus, T0042 Acanthopanax senticosus [Syn. Eleutherococcus senticosus], T0044 Acanthopanax sessiliflorus, T0071 Achyranthes aspera, T0072 Achyranthes aspera var. indica, T0172 Adiantum caudatum, T0180 Adina pilulifera [Syn. Cephalanthus pilulifera], T0264 Ajuga forrestii, T0273 Akebia quinata, T0277 Akebia trifoliata, T0279 Akebia trifoliata var. australis, T0318 Allium sativum, T0388 Amaranthus lividus, T0553 Apocynum venetum, T0567 Aralia armata, T0571 Aralia decaisneaa, T0573 Aralia elata, T0606 Areca catechu, T0610 Argemone mexicana, T0624 Aristolochia contorta, T0626 Aristolochia debilis [Syn. Aristolochia longa], T0629 Aristolochia heterophylla, T0630 Aristolochia indica, T0632 Aristolochia manshuriensis, T0633 Aristolochia maxima, T0640 Aristolochia triangularis, T0721 Arundina chinensis, T0726 Asarum forbesii, T0824 Atractylodes macrocephala [Syn. Atractylis macrocephala], T0838 Azolla imbricata [Syn. Salvinia imbricata], T0894 Benincasa hispida, T0895 Benincasa hispida, T1003 Brandisia hancei, T1004 Brasenia schreberi, T1031 Broussonetia kazinoki, T1034 Broussonetia papyrifera, T1035 Broussonetia papyrifera, T1049 Bufo bufo gargarizans; Bufo melanostictus, T1170 Canis familiaris, T1238 Cassia mimosoides, T1263 Catalpa ovata, T1282 Cedrus deodara, T1352 Chamaenerion angustifolium [Syn. Epilobium angustifolium], T1357 Chelidonium majus, T1533 Clausena dentata, T1534 Clausena dunniana, T1556 Clerodendron fragrans, T1589 Cocculus trilobus [Syn. Cocculus sarmentosus], T1590 Cocculus trilobus [Syn. Cocculus sarmentosus], T1595 Codium fragile, T1614 Coix lacryma-jobi var. ma-yuen, T1633 Commelina communis, T1649 Convallaria keiskei [Syn. Convallaria majalis], T1696 Cornus capitata [Syn. Dendrobenthamia capitata], T1826 Crotalaria mucronata, T1921 Cyanotis arachnoidea [Syn. Cyanotis bodinieri], T1922 Cyanotis vaga, T2020 Damnacanthus indicus, T2023 Daphne genkwa, T2024 Daphne genkwa, T2048 Daucus carota, T2131 Desmodium pulchellum [Syn. Phyllodium pulchellum], T2136 Desmos cochinchinensis [Syn. Desmos chinensis], T2272 Drymaria diandra [Syn. Drymaria cordata ssp. diandra], T2282 Dryopteris filix-mas, T2325 Eichhornia crassipes, T2341 Elsholtzia ciliata, T2432 Eriobotrya japonica, T2439 Eruca sativa, T2452 Erysimum cheiranthoides, T2580 Euphorbia antiquorum, T2585 Euphorbia esula, T2596 Euphorbia kansui, T2597 Euphorbia lathyris, T2599 Euphorbia lunulata, T2608 Euphorbia pekinensis, T2612 Euphorbia prolifera, T2617 Euphorbia royleana, T2723 Ficus pumila, T2727 Ficus simplicissima, T2749 Fomes officinalis, T2837 Gallus gallus domesticus, T3066 Gryllulus chinensis, T3125 Hedyotis chrysotricha [Syn. Oldenlandia chrysotricha], T3126 Hedyotis corymbosa [Syn. Oldenlandia corymbosa], T3193 Hemerocallis fulva, T3195 Hemerocallis lilio-asphodelus, T3197 Hemerocallis minor, T3199 Hemibarbus labeo, T3270 Holboellia fargesii, T3288 Humulus japonicus [Syn. Humulus scandens], T3289 Humulus lupulus, T3311
Hydrocotyle sibthorpioides, T3416 Imperata cylindrica var. major, T3448 Ipomoea cairica [Syn. Ipomoea palmata], T3479 Isodon amethystoides, T3528 Isodon ternifolia, T3529 Isodon ternifolius, T3578 Juncus effusus, T3594 Juniperus rigida, T3628 Kerria japonica, T3668 Lagenaria siceraria var. depressa, T3675 Lagopsis supina, $\mathrm{T} 3738$ Lemna minor, T3742 Lentinus edodes, T3752 Leonurus heterophyllus [Syn. Leonurus artemisia], T3754 Leonurus sibiricus, T3758 Lepidium sativum, T3765 Lepisorus ussuriensis, T3770 Lespedeza cuneata, T3774 Lespedeza tomentosa, T3785 Levisticum officinale, T3815 Ligularia stenocephala, T3840 Limnophila rugosa, T3856 Lindera umbellata [Syn. Lindera erythrocarpa], T3886 Litsea euosma, T3898 Lobelia chinensis [Syn. Lobelia radicans], T3932 Ludwigia octovalvis, T3933 Luffa acutangula, T3934 Luffa cylindrica, T3935 Luffa cylindrica, T3981 Lycopus lucidus, T3988 Lycoris squamigera, T3989 Lygodium flexuosum [Syn. Lygodium pinnatifidum; Ophioglossum flexuosum], T3990 Lygodium japonicum, T3999 Lysimachia clethroides, T4026 Macrothelypteris oligophlebia, T4029 Maesa japonica, T4042 Magnolia liliflora, T4121 Marsilea quadrifolia, T4147 Medicago falcata, T4148 Medicago sativa, T4194 Menyanthes trifoliata, T4195 Menyanthes trifoliata, T4248 Mimosa pudica, T4252 Mirabilis jalapa, T4274 Monostroma nitidum, T4287 Morus alba, T4304 Mosla chinensis [Syn. Orthodon chinensis], T4305 Mosla dianthera, T4432 Nigella glandulifera, T4439 Nothapodytes pittosporoides, T4445 Notopterygium forbesii [Syn. Notopterygium franchetii], T4446 Notopterygium incisum, T4456 Nymphoides peltatum, T4482 Oenanthe javanica, T4485 Oldenlandia diffusa [Syn. Hedyotis diffusa], T4505 Ophiocephalus argus, T4526 Origanum vulgare, T4542 Orthosiphon wulfenioides [Syn. Coleus wulfenioides], T4644 Parasilurus asotus, T4785 Phaseolus vulgaris, T4799 Philydrum lanuginosum, T4801 Phlegmariurus phlegmaria [Syn. Lycopodium phlegmaria], T4818 Phoebe nanmu, T4831 Phtheirospermum japonicum [Syn. Geradia japonica], T4837 Phyllanthus emblica, T4840 Phyllanthus niruri, T4845 Physalis alkekengi, T4846 Physalis alkekengi var. franchetii, T4848 Physalis angulata, T4854 Physalis pubescens, T4937 Piper betle, T4966 Piper sarmentosum, T5071 Polygala arillata, T5103 Polygonum hydropiper, T5115 Polygonum sibiricum [Syn. Persicaria sibirica], T5129 Polyporus umbellatus, T5169 Poria cocos, T5171 Porphyra tenera, T5176 Potamogeton natans, T5179 Potentilla anserina, T5220 Prunus davidiana, T5223 Prunus humilis [Syn. Cerasus humilis], T5224 Prunus japonica [Syn. Cerasus japonica], T5225 Prunus japonica var. nakaii, T5231 Prunus persica, T5232 Prunus persica, T5288 Pteris cretica var. nervosa [Syn. Pteris nervosa], T5289 Pteris dactylina, T5362 Pyrus bretschneideri, T5366 Pyrus pyrifolia, T5400 Rabdosia stracheyi, T5407 Rana nigromaculata; Rana plancyi, T5461 Rhamnus leptophylla, T5557 Rohdea japonica [Syn. Orontium japonicum], T5560 Rorippa montana [Syn. Rorippa dubia; Sisymbrium dublium], T5576 Rostellularia procumbens [Syn. Justicia procumbens], T5645 Sageretia theezans [Syn. Sageretia thea], T5723 Sapium sebiferum, T5750 Saururus chinensis, T5770 Saussurea superba [Syn. Saussurea hieracioides], T5835 Scutellaria barbata [Syn. Scutellaria rivularis], T5862 Selaginella involvens, T5863 Selaginella moellendorffii, T5870 Selaginella uncinata, T6005 Solanum lyratum, T6031 Sophora 
flavescens [Syn. Sophora angustfolia], T6046 Sophora viciifolia, T6047 Sophora viciifolia, T6100 Stauntonia hexaphylla, T6127 Stephania glabra, T6131 Stephania longa, T6210 Swainsonia salsula [Syn. Sphaerophysa salsula], T6227 Swertia mussotii, T6264 Syzygium buxifolium, T6356 Tetrapanax papyriferus, T6357 Tetrapanax papyriferus, T6367 Teucrium quadrifarium, T6527 Trigonella caerulea, T6598 Ulva conglobata, T6599 Ulva lactuca, T6600 Ulva pertusa, T6709 Verbena officinalis, T6744 Vicia faba, T6750 Vicia sativa, T6752 Vigna angularis [Syn. Dolichus angularis; Phaseolus angularis], T6798 Vitis vinifera, T6799 Vitis vinifera, T6819 Wikstroemia chamaedaphne, T6820 Wikstroemia indica, T6826 Wisteria sinensis, T6901 Zea mays, T6902 Zea mays, T6925 Zostera marina.

edema and enlarged abdomen T1858 Croton tiglium, T3898 Lobelia chinensis [Syn. Lobelia radicans].

edema and inhibited urination T2343 Elsholtzia splendens.

edema and qi fullness T2589 Euphorbia helioscopia.

edema distention fullness T0303 Alisma orientale [Syn. Alisma plantago-aquatica var. orientale], T1033 Broussonetia papyrifera, T1229 Cassia acutifolia, T1230 Cassia angustifolia, T3000 Glycine max, T3632 Knoxia valerianoides, T3867 Liquidambar formosana [Syn. Liquidambar taiwaniana], T4779 Pharbitis nil, T4780 Pharbitis purpurea, T4861 Phytolacca americana [Syn. Phytolacca decandra], T4864 Phytolacca esculenta [Syn. Phytolacca acinosa], T5457 Rhamnus davurica, T6105 Stellera chamaejasme.

edema due to heart disease T0184 Adonis amurensis, T1151 Camellia sinensis [Syn. Thea sinensis], T1589 Cocculus trilobus [Syn. Cocculus sarmentosus], T1991 Cytisus scoparius [Syn. Spartium scoparium], T2175 Digitalis lanata, T2177 Digitalis purpurea, T5813 Scilla scilloides, T6259 Syringa amurensis [Syn. Syringa reticulata var. amurensis].

edema in body and face T3980 Lycopus lucidus.

edema in chest and abdomen T2125 Descurainia sophia, T3755 Lepidium apetalum [Syn. Lepidium micranthum], T3759 Lepidium virginicum.

edema in face and foot T3898 Lobelia chinensis [Syn. Lobelia radicans]. edema in lower limb T0628 Aristolochia fangchi, T1984 Cypripedium macranthum [Syn. Cypripedium tibeticum], T4729 Periploca sepium, T5097 Polygonum amphibium.

edema in pregnancy T0553 Apocynum venetum, T1980 Cyprinus carpio, T4505 Ophiocephalus argus.

effusion in thorax T5770 Saussurea superba [Syn. Saussurea hieracioides].

effusion of back T1049 Bufo bufo gargarizans; Bufo melanostictus, T1565 Clerodendrum bungei, T3479 Isodon amethystoides.

effusion of back from welling abscess and flat abscess T0542 Apis cerana, T2316 Echinops grijsii, T2317 Echinops ritro, T2658 Fagopyrum esculentum, T5086 Polygala sibirica, T5088 Polygala tenuifolia, T5466 Rhaponticum uniflorum.

effusion of vaginal coat T2744 Foeniculum vulgare.

elephantiasis T0852 Bacopa monniera, T3082 Gynocardia odorata, T3570 Juglans regia.

elephantiasis of scrotum T2744 Foeniculum vulgare.

emission T0255 Ailanthus altissima, T0303 Alisma orientale [Syn. Alisma plantago-aquatica var. orientale], T0323 Allium tuberosum, T0360 Alpinia oxyphylla, T0542 Apis cerana, T0859 Balanophora involucrata, T0993 Boschniakia rossica, T1411 Cibotium barometz [Syn. Polypodium barometz], T1819 Crotalaria ferruginea, T1927 Cycas revoluta, T1964 Cynanchum wilfordii [Syn. Cynoctonum wilfordii], T2838 Gallus gallus domesticus, T2955 Geum japonicum, T2962 Ginkgo biloba, T2964 Ginkgo biloba, T3279 Homo sapiens, T3568 Juglans regia, T3615 Kadsura interior, T3770 Lespedeza cuneata, T3955 Lycium barbarum, T3958 Lycium chinense, T4206 Metaplexis japonica, T4207 Metaplexis japonica, T4400 Nelumbo nucifera, T4470 Ocimum basilicum, T4536 Orobanche coerulescens, T4629 Papaver nudicaule, T4630 Papaver nudicaule ssp. amurense, T4789 Phellodendron amurense, T4790 Phellodendron amurense var. wilsonii, T4791 Phellodendron chinense, T4792 Phellodendron chinense var. glabriusculum, T4821 Pholidota articulata, T5107 Polygonum multiflorum, T5244 Prunus tomentosa, T5374 Quercus infectoria, T5529 Rhodomyrtus tomentosa, T5568 Rosa laevigata, T5593 Rubus idaeus, T6025 Sonchus arvensis, T6087 Spiranthes sinensis, T6257 Syngnathus acus, T6353 Tetracera asiatica, T6431 Thesium chinense, T6474 Toona ciliata, T6475 Toona sinensis, T6750 Vicia sativa, T6754 Vigna unguiculata.

emission and premature ejaculation T0793 Astragalus complanatus, T1972 Cynomorium songaricum.

encephalitis T2756 Forsythia suspensa, T3476 Isatis indigotica.

encephalitis b T0379 Alternanthera philoxeroides, T2282 Dryopteris filix-mas, T3423 Indigofera tinctoria.

encephalitis of early stage T0379 Alternanthera philoxeroides. endometrorrhagia T5868 Selaginella stauntoniana.

enduring cough T1465 Citrullus vulgaris [Syn. Citrullus lanatus], T3567 Juglans regia, T4371 Nandina domestica, T4637 Papaver somniferum, T5228 Prunus mume, T5344 Pyrola atropurpurea, T5345 Pyrola calliantha [Syn. Pyrola rotundifolia ssp. chinensis], T5346 Pyrola calliantha var. tibetana, T5347 Pyrola decorata, T5349 Pyrola incarnata, T5350 Pyrola japonica, T5351 Pyrola rotundifolia.

enduring cough and aphonia T6347 Terminalia chebula.

enduring cough and asthma T0707 Arthraxon hispidus, T2846 Ganoderma japonicum [Syn. Ganoderma sinense], T2848 Ganoderma lucidum, T3568 Juglans regia, T4629 Papaver nudicaule, T4630 Papaver nudicaule ssp. amurense, T4631 Papaver nudicaule var. chinense, T6825 Winchia calophylla.

enduring cough and vacuity asthma T1683 Cordyceps sinensis, T5791 Schisandra chinensis, T5802 Schisandra sphenanthera.

enduring cough with bloody phlegm T1819 Crotalaria ferruginea.

enduring diarrhea T3128 Hedysarum multijugum, T4601 Panax ginseng [Syn. Panax schinseng], T4631 Papaver nudicaule var. chinense, T5329 Punica granatum.

enduring diarrhea due to damp T0479 Angelica dahurica cv. qibaizhi, T0498 Angelica taiwaniana.

enduring dysentery T2721 Ficus microcarpa, T3348 Hypericum elodeoides, T3368 Hypericum wightianum, T4631 Papaver nudicaule var. chinense, T5181 Potentilla chinensis, T5329 Punica granatum, T5573 Rosa sericea.

enduring illness $q \boldsymbol{i}$ vacuity T4599 Panax ginseng [Syn. Panax schinseng], 
T6150 Streptopelia orientalis

enduring illness vacuity T0163 Actinidia latifolia.

enduring low fever T3600 Juniperus taiwaniana.

enduring malaria T0607 Arenaria juncea, T3090 Gypsophila

oldhamiana, T3091 Gypsophila pacifica, T5107 Polygonum

multiflorum, T5955 Silene jenisseensis, T6727 Veronica persica.

enduring sores T0019 Acacia catechu, T0180 Adina pilulifera [Syn.

Cephalanthus pilulifera], T0540 Apis cerana, T0542 Apis cerana,

T0994 Boswellia carterii, T1777 Crataegus pinnatifida, T2293

Dumortiera hirsuta, T2420 Ericerus pela, T3295 Huperzia serrata

[Syn. Lycopodium serratum], T3407 Illicium simonsii, T4478 Octopus

vulgaris, T4696 Penaeus orientalis, T5308 Pterocarya stenoptera,

T5374 Quercus infectoria, T6615 Uncaria gambir.

enduring vanquished sore $\mathrm{T} 1170$ Canis familiaris.

enduring welling abscess and flat abscess T3129 Hedysarum polybotrys.

enduring yin flat abscess T1337 Cervus nippon; Cervus elaphus.

enlargement of testes T6099 Stauntonia chinensis.

enteritis T0180 Adina pilulifera [Syn. Cephalanthus pilulifera], T0424

Ampelopsis brevipedunculata var. hancei, T0897 Berberis amurensis,

T1316 Cephalanthus occidentalis, T1357 Chelidonium majus, T1573

Clinopodium chinense, T1657 Conyza canadensis [Syn. Erigeron

canadensis], T1709 Corydalis bungeana, T2327 Elaeagnus

angustifolia, T2357 Emilia sonchifolia, T2591 Euphorbia humifusa,

T2716 Ficus beecheyana [Syn. Ficus erecta var. beecheyana], T3123

Hedyotis auricularia, T3126 Hedyotis corymbosa [Syn. Oldenlandia

corymbosa], T3163 Helicteres angustifolia, T3203 Hemsleya amabilis,

T3208 Hemsleya macrosperma, T3254 Hippophae rhamnoides, T3256

Hippophae rhamnoides subsp. sinensis, T3258 Hippophae rhamnoides

subsp. yunnanensis, T3363 Hypericum sampsonii, T3528 Isodon ternifolia, T3529 Isodon ternifolius, T3547 Ixeris chinensis, T3548 Ixeris sonchifolia, T3847 Lindera angustifolia, T4148 Medicago sativa, T4220 Microlepia strigosa [Syn. Trichomanes strigosa], T4248 Mimosa pudica, T4307 Mosla scabra [Syn. Mosla punctata], T4391 Nauclea officinalis, T4485 Oldenlandia diffusa [Syn. Hedyotis diffusa], T4834 Phyllanthus emblica, T4842 Phyllanthus reticulatus, T4843 Phyllanthus urinaria, T5104 Polygonum hydropiper, T5149 Populus canadensis, T5150 Populus cathayana, T5173 Portulaca oleracea, T5188 Potentilla viscosa, T5555 Rodgersia pinnata, T5587 Rubus alceaefolius, T5607 Rumex crispus, T5892 Senecio nemorensis, T5907 Senecio scandens [Syn. Senecio chinensis], T5954 Silene fortunei, T6043 Sophora subprostrata [Syn. Sophora tonkinensis], T6075 Spilanthes acmella, T6116 Stenoloma chusanum, T6276 Tacca plantaginea [Syn. Schizocapsa plantaginea], T6277 Tadehagi triquetrum, T6353 Tetracera asiatica.

enteritis and diarrhea T0643 Aristolochia versicolar, T2079 Delphinium kamaonense var. glabrescens, T2529 Euchresta strigillosa, T3162 Helicia nilagirica, T3164 Helicteres isora, T5570 Rosa roxburghii, T6028 Sophora alopecuroides.

enteritis and dysentery T0642 Aristolochia tubiflora, T0776 Asplenium prolongatum.

enuresis T2805 Fugu ocellatus, T2838 Gallus gallus domesticus, T2962 Ginkgo biloba, T3000 Glycine max, T3568 Juglans regia, T3770 Lespedeza cuneata, T5270 Psoralea corylifolia, T5569 Rosa multiflora.

enuresis and frequent urination T1698 Cornus officinalis [Syn. Macrocarpium officinale], T1911 Cuscuta australis, T1912 Cuscuta chinensis, T1913 Cuscuta japonica, T2961 Ginkgo biloba, T3854 Lindera strychnifolia [Syn. Lindera aggregata], T5568 Rosa laevigata.

enuresis due to kidney vacuity T0360 Alpinia oxyphylla.

epidemic encephalitis T0318 Allium sativum, T3475 Isatis indigotica, T4552 Osmunda japonica.

epidemic febrile diseases $\mathrm{T} 5484$ Rhinoceros unicornis; Rhinoceros sondaicus; Rhinoceros sumatrensis.

epidemic hemorrhagic conjunctivitis T0379 Alternanthera philoxeroides.

epidemic meningitis T4789 Phellodendron amurense, T4790 Phellodendron amurense var. wilsonii, T4791 Phellodendron chinense, T4792 Phellodendron chinense var. glabriusculum.

epidemic parotitis T0045 Acanthopanax trifoliatus, T0379 Alternanthera philoxeroides, T0586 Arctium lappa, T0866 Baphicacanthus cusia [Syn. Strobilanthes cusia], T0867 Baphicacanthus cusia [Syn. Strobilanthes cusia], T0906 Berberis julianae, T0959 Blumea lacera, T0975 Bombyx mori, T1275 Cayratia japonica, T2282 Dryopteris filix-mas, T2290 Duchesnea indica, T3163 Helicteres angustifolia, T3457 Iris dichotoma, T3475 Isatis indigotica, T3476 Isatis indigotica, T4183 Menispermum dauricum, T4186 Mentha piperita, T4482 Oenanthe javanica, T4519 Opuntia dillenii, T4520 Opuntia ficus-indica, T4521 Opuntia vulgaris, T4796 Pheretima aspergillum; Allolobophora caliginosa trapezoides, T4852 Physalis peruviana, T5121 Polygonum tinctorium, T5613 Rumex obtusifolius, T6003 Solanum khasianum, T6017 Solanum tuberosum, T6116 Stenoloma chusanum, T6128 Stephania hernandifolia, T6301 Taraxacum mongolicum, T6440 Thuja orientalis [Syn. Platycladus orientalis; Biota orientalis], T6548 Tritonia crocosmaeflora, T6820 Wikstroemia indica.

epididymis tubercle T6105 Stellera chamaejasme, T6540 Tripterygium hypoglaucum.

epilepsy T0088 Aconitum coreanum, T0142 Acorus calamus, T0190 Adonis sutchuenensis, T0298 Alectoria vivens, T0463 Anemone altaica, T0617 Arisaema amurense, T0618 Arisaema consanguineum, T0620 Arisaema heterophyllum, T0644 Armillaria mellea, T0645 Armillariella mellea, T0975 Bombyx mori, T1152 Camellia sinensis [Syn. Thea sinensis], T1525 Cladonia fallax, T1876 Cucumis melo, T2037 Datura innoxia, T2044 Datura metel, T2091 Delphinium yunnanense, T2412 Equisetum sylvaticum, T2596 Euphorbia kansui, T2975 Gleditsia delavayi, T2976 Gleditsia fera, T2978 Gleditsia sinensis [Syn. Gleditsia horrida], T3775 Lethariella cladonioides, T4418 Nerium indicum, T4423 Nicandra physaloides, T4903 Pinellia pedatisecta, T5547 Ricinus communis, T5871 Selenarctos thibetanus; Ursus arctos, T6145 Stichopus japonicus, T6358 Tetraplodon mnioides [Syn. Tetraplodon bryoides; Splachnum mnioides], T6416 Thamnolia vermicularis, T6522 Trifolium repens, T6697 Veratrum grandiflorum, T6698 Veratrum nigrum, T6819 Wikstroemia chamaedaphne, T6903 Zephyranthes candida.

epilepsy and profuse phlegm T4957 Piper nigrum.

epilepsy of pregnancy T6625 Uncaria macrophylla, T6629 Uncaria rhynchophylla [Syn. Nauclea rhynchophylla], T6633 Uncaria sinensis. 
eruptive dermatitis T1662 Coptis chinensis, T1663 Coptis chinensis var. brevisepala, T1664 Coptis deltoidea, T1669 Coptis omeiensis, T1670 Coptis teetoides [Syn. Coptis teeta].

erysipelas T0434 Amsonia sinensis, T0457 Andrographis paniculata [Syn. Justicia paniculata], T0586 Arctium lappa, T0678 Artemisia japonica, T0866 Baphicacanthus cusia [Syn. Strobilanthes cusia], T0897 Berberis amurensis, T0906 Berberis julianae, T0947 Bixa orellana, T1005 Brassica campestris [Syn. Brassica campestris var. oleifera], T1043 Bryophyllum pinnatum, T1256 Castanea mollissima, T1275 Cayratia japonica, T1565 Clerodendrum bungei, T2517 Eucalyptus robusta, T2521 Eucalyptus tereticornis, T2658 Fagopyrum esculentum, T2731 Firmiana simplex, T2757 Forsythia viridissima, T3396 Ilex pubescens, T3397 Ilex pubescens var. glaber, T3410 Impatiens balsamina, T3423 Indigofera tinctoria, T3475 Isatis indigotica, T3476 Isatis indigotica, T3738 Lemna minor, T3990 Lygodium japonicum, T4206 Metaplexis japonica, T4564 Oxalis corniculata [Syn. Oxalis repens], T4678 Paulownia fortunei, T4679 Paulownia tomentosa, T4845 Physalis alkekengi, T4846 Physalis alkekengi var. franchetii, T5121 Polygonum tinctorium, T5173 Portulaca oleracea, T5194 Premna microphylla, T5557 Rohdea japonica [Syn. Orontium japonicum], T5646 Sagina japonica [Syn. Spergula japonica], T5651 Salix babylonica, T5680 Salvia miltiorrhiza, T5681 Salvia miltiorrhiza f. alba, T5688 Salvia przewalskii, T5773 Saxifraga stolonifera, T5818 Scoparia dulcis, T5856 Sedum lineare [Syn. Sedum obtuso-lineare], T5907 Senecio scandens [Syn. Senecio chinensis], T6005 Solanum lyratum, T6008 Solanum nigrum, T6440 Thuja orientalis [Syn. Platycladus orientalis; Biota orientalis], T6767 Viola yedoensis.

erysipelas wandering wind T6393 Thalictrum ichangense [Syn. Thalictrum tripeltatum; Thalictrum multipeltatum].

erythematous lupus T0334 Aloe arborescens var. natalensis, T2753 Formica fusca, T6540 Tripterygium hypoglaucum, T6542 Tripterygium wilfordii.

erythra itch-pain T3630 Kleinhovia hospita.

excessive leukorrhea T0478 Angelica dahurica [Syn. Angelica porphyrocaulis], T1411 Cibotium barometz [Syn. Polypodium barometz], T2208 Dioscorea septemloba, T2246 Dolichos lablab, T4400 Nelumbo nucifera, T5569 Rosa multiflora, T6029 Sophora alopecuroides.

exterior damp T0819 Atractylodes chinensis, T0821 Atractylodes japonica, T0823 Atractylodes lancea.

exterior vacuity and common cold T2955 Geum japonicum.

externally contracted ardent fever T1428 Cinchona ledgeriana, T1429 Cinchona officinalis, T1433 Cinchona succirubra.

externally contracted wind evil T5234 Prunus persica.

externally contracted wind-cold T4039 Magnolia grandiflora, T4445 Notopterygium forbesii [Syn. Notopterygium franchetii], T4446 Notopterygium incisum, T4956 Piper mullesua, T5727 Saposhnikovia divaricata [Syn. Ledebouriella seseloides].

externally contracted wind-heat T0480 Angelica decursiva [Syn. Peucedanum decursivum], T1395 Chrysanthemum morifolium [Syn. Dendranthema morifolium], T1933 Cyclea sutchuenensis, T2798 Fritillaria verticillata var. thunbergii [Syn. Fritillaria thunbergii], T3819 Ligusticum brachylobum, T4413 Nepeta cataria, T4761
Peucedanum longshengens, T4768 Peucedanum praeruptorum, T4769 Peucedanum rubricaule, T6791 Vitex rotundifolia [Syn. Vitex trifollia var. simplicifolia], T6793 Vitex trifolia.

externally contracted wind-heat cough T2787 Fritillaria hupehensis. exuberance of fire with tidal fever T4789 Phellodendron amurense, T4790 Phellodendron amurense var. wilsonii, T4791 Phellodendron chinense, T4792 Phellodendron chinense var. glabriusculum.

exuberant heat and vexation thirst T1554 Clerodendron cyrtophyllum. exuberant heat fluid damage T1464 Citrullus vulgaris [Syn. Citrullus lanatus], T6526 Triglochin maritimum.

exuberant heat stirring wind T6625 Uncaria macrophylla, T6629 Uncaria rhynchophylla [Syn. Nauclea rhynchophylla], T6633 Uncaria sinensis.

exudative dermatitis T6403 Thalictrum petaloideum.

exudative eczema T6584 Typha angustata, T6585 Typha angustifolia, T6587 Typha latifolia.

eye diseases T1528 Cladonia stellaris [Syn. Cladonia alpestris], T4188 Mentha rotundifolia, T6416 Thamnolia vermicularis.

eye pain T1185 Capsella bursa-pastoris, T3635 Koelreuteria paniculata. eye pain and tearing T3636 Koelreuteria paniculata.

eye screen T1035 Broussonetia papyrifera, T1185 Capsella bursa-pastoris, T1236 Cassia laevigata [Syn. Cassia floribunda], T1296 Celosia argentea, T1746 Corydalis taliensis, T1804 Cristaria plicata; Hyriopsis cumingii, T2267 Drosera peltata var. lunata, T2272 Drymaria diandra [Syn. Drymaria cordata ssp. diandra], T2336 Elephas maximus, T2408 Equisetum hiemale, T2517 Eucalyptus robusta, T2766 Fraxinus bungeana, T2767 Fraxinus chinensis, T2772 Fraxinus mandshurica, T2774 Fraxinus paxiana, T2777 Fraxinus rhynchophylla [Syn. Fraxinus chinensis var. rhynchophylla], T2779 Fraxinus stylosa, T2780 Fraxinus szaboana [Syn. Fraxinus chinensis var. acuminata], T2841 Gallus gallus domesticus, T3311 Hydrocotyle sibthorpioides, T3363 Hypericum sampsonii, T3957 Lycium chinense, T4349 Myrica rubra, T4471 Ocimum basilicum, T5413 Ranunculus cantoniensis, T5690 Salvia roborowskii, T5871 Selenarctos thibetanus; Ursus arctos, T5907 Senecio scandens [Syn. Senecio chinensis], T6072 Sphagnum palustre [Syn. Sphagnum obtusifolium; Sphagnum cymbifolium], T6092 Stachys palustris, T6843 Xanthium sibiricum [Syn. Xanthium strumarium].

eye swelling T3635 Koelreuteria paniculata.

eyeball night pain T5214 Prunella vulgaris.

eyebrow bone pain T0479 Angelica dahurica cv. qibaizhi, T0498 Angelica taiwaniana.

eyeground hemorrhage T0830 Auricularia auricula, T0831 Auricularia delicata, T1184 Capsella bursa-pastoris.

eyelid laceration T0610 Argemone mexicana.

facial muscle spasm T2890 Gastrodia elata.

facial paralysis $\mathrm{T} 0975$ Bombyx mori, $\mathrm{T} 3680$ Lamium amplexicaule, T5183 Potentilla griffithii var. velutina, T5848 Securinega suffruticosa.

fasciolopsiasis T1591 Cocos nucifera, T1593 Cocos nucifera.

fatigue hypodynamia T0819 Atractylodes chinensis, T0821 Atractylodes japonica, T0823 Atractylodes lancea, T0824 Atractylodes macrocephala [Syn. Atractylis macrocephala], T2846 Ganoderma japonicum [Syn. Ganoderma sinense], T2848 Ganoderma lucidum, 
T3011 Glycyrrhiza aspera, T3013 Glycyrrhiza glabra, T3015 Glycyrrhiza inflata, T3016 Glycyrrhiza kansuensis, T3021 Glycyrrhiza squamulosa, T3022 Glycyrrhiza uralensis, T3023 Glycyrrhiza yunnanensis, T3128 Hedysarum multijugum, T3743 Lentinus lepideus, T4545 Oryza sativa, T5465 Rhaponticum carthamoides, T6150 Streptopelia orientalis.

fatigue hypodynamia due to spleen vacuity T5091 Polygonatum cyrtonema [Syn. Polygonatum multiflorum], T5092 Polygonatum kingianum, T5095 Polygonatum sibiricum.

$\begin{array}{lllll}\text { fatigue hypodynamia due to spleen-stomach vacuity } & \text { T0387 }\end{array}$ Amaranthus caudatus.

fatigued spirit T1337 Cervus nippon; Cervus elaphus, T1608 Coffea arabica, T1610 Coffea liberica, T1623 Collybia albuminosa.

fatigued spirit and amnesia T1961 Cynanchum thesioides.

fatigued spirit and desire to sleep T0210 Agaricus bisporus, T0211 Agaricus campestris.

fatigued spirit and dizziness T4385 Narcissus tazetta var. chinensis.

fatigued spirit and hypodynamia $\mathrm{T} 3742$ Lentinus edodes.

fatigued spirit and lassitude T4516 Oplopanax elatus.

fatty liver T5957 Silybum marianum.

fearful throbbing T5701 Salvia yunnanensis.

febrile diseases T1986 Cyrtomium fortunei, T2290 Duchesnea indica, T2882 Gardenia jasminoides [Syn. Gardenia florida], T3155 Helianthus tuberosus, T3200 Hemidesmus indicus, T3233 Heteropappus altaicus.

febrile diseases clouded spirit T0144 Acorus gramineus, T0146 Acorus tatarinowii, T0463 Anemone altaica, T0987 Bos taurus domesticus; Bubalus bubalis, T1907 Curcuma wengujin, T4303 Moschus moschiferus; Moschus berezovskii; Moschus sifanicus.

febrile diseases due to external contraction T1554 Clerodendron cyrtophyllum.

febrile diseases fluid damage T0155 Actinidia arguta, T4602 Panax ginseng [Syn. Panax schinseng], T5186 Potentilla reptans var. sericophylla.

febrile diseases macular eruption T4127 Matteuccia struthiopteris, T4500 Onosma paniculatum.

febrile diseases tetanic reversal T2840 Gallus gallus domesticus.

febrile diseases thirst T1878 Cucumis sativus, T2628 Euphrasia officinalis, T6512 Trichosanthes kirilowii.

febrile diseases with vexation and agitation T6060 Souliea vaginata.

febrile diseases with vexation and fullness $\mathrm{T} 4148$ Medicago sativa, T4149 Medicago sativa.

febrile diseases with vexation and thirst T0722 Arundo donax, T3392 Ilex kudingcha, T3393 Ilex latifolia, T3416 Imperata cylindrica var. major, T3920 Lophatherum gracile, T3933 Luffa acutangula, T3934 Luffa cylindrica, T4331 Musa paradisiaca var. sapientum [Syn. Musa sapientum], T4402 Nelumbo nucifera, T4605 Panax japonicus var. major, T4829 Phragmites communis, T5445 Rehmannia glutinosa [Syn. Rehmannia glutinosa f. huechingensis], T6206 Sus scrofa domestica.

febrile infectious diseases T1662 Coptis chinensis, T1663 Coptis chinensis var. brevisepala, T1664 Coptis deltoidea, T1669 Coptis omeiensis, T1670 Coptis teetoides [Syn. Coptis teeta], T4029 Maesa japonica, T4479 Odontites serotina. fecal stoppage T1858 Croton tiglium, T4845 Physalis alkekengi, T6076 Spinacia oleracea, T6651 Urtica cannabina, T6652 Urtica dioica.

fetal bleeding T2234 Dipsacus asperoides, T2235 Dipsacus japonicus.

fetal spotting T0664 Artemisia argyi, T0681 Artemisia lavandulaefolia, T0685 Artemisia mongolica, T0689 Artemisia princeps, T0691 Artemisia rubripes, T0706 Artemisia vulgaris, T1982 Cyprinus carpio.

fetal spotting (precipitation of blood in pregnancy) T5687 Salvia prionitis.

fetal spotting due to kidney vacuity T1911 Cuscuta australis, T1912 Cuscuta chinensis, T1913 Cuscuta japonica.

fetus qi disharmony T4720 Perilla frutescens var. arguta, T4723 Perilla frutescens var. crispa.

fever T0015 Abutilon indicum, T0457 Andrographis paniculata [Syn. Justicia paniculata], T0947 Bixa orellana, T1359 Chenopodium album, T1705 Corydalis adunca, T2343 Elsholtzia splendens, T2367 Ephedra equisetina, T2368 Ephedra gerardiana, T2369 Ephedra intermedia, T2372 Ephedra likiangensis, T2373 Ephedra minuta, T2375 Ephedra monosperma, T2379 Ephedra saxatilis, T2380 Ephedra sinica, T2697 Ferula borealis, T2882 Gardenia jasminoides [Syn. Gardenia florida], T3921 Lophatherum gracile, T4335 Mussaenda pubescens, T4789 Phellodendron amurense, T4790 Phellodendron amurense var. wilsonii, T4791 Phellodendron chinense, T4792 Phellodendron chinense var. glabriusculum, T4887 Picrorhiza kurrooa, T4888 Picrorhiza scrophulariiflora, T5863 Selaginella moellendorffii.

fever and aversion to wind T2367 Ephedra equisetina, T2368 Ephedra gerardiana, T2369 Ephedra intermedia, T2372 Ephedra likiangensis, T2373 Ephedra minuta, T2375 Ephedra monosperma, T2379 Ephedra saxatilis, T2380 Ephedra sinica, T6910 Zingiber officinale.

fever and chest pain T1705 Corydalis adunca.

fever and cough T4470 Ocimum basilicum, T5943 Sida cordifolia.

fever and dry mouth T2631 Eurya japonica.

fever and headache T1395 Chrysanthemum morifolium [Syn. Dendranthema morifolium], T2554 Eupatorium cannabinum, T2563 Eupatorium japonicum.

fever and headache due to external contraction T1544 Cleistocalyx operculatus.

fever and sore pharynx T4801 Phlegmariurus phlegmaria [Syn. Lycopodium phlegmaria].

fever due to external contraction T0071 Achyranthes aspera, T0072 Achyranthes aspera var. indica, T0608 Arenaria kansuensis [Syn. Arenaria kumaonensis], T1055 Bupleurum angustissimum, T1056 Bupleurum aureum, T1058 Bupleurum chaishoui, T1059 Bupleurum chinense, T1065 Bupleurum longiradiatum, T1066 Bupleurum marginatum, T1067 Bupleurum marginatum var. stenophyllum, T1072 Bupleurum scorzonerifolium, T1073 Bupleurum sibiricum, T1075 Bupleurum smithii var. parvifolium, T1078 Bupleurum wenchuanense, T1079 Bupleurum yinchowense, T3749 Leontopodium alpinum.

fever due to summerheat damage T5402 Radermachera sinica. fever in children T6224 Swertia kouitchensis, T6375 Thalictrum baicalense, T6376 Thalictrum cultratum, T6385 Thalictrum flavum, T6387 Thalictrum foliolosum, T6389 Thalictrum glandulosissimum.

filariasis T2510 Eucalyptus globulus, T2517 Eucalyptus robusta. fire eye T3989 Lygodium flexuosum [Syn. Lygodium pinnatifidum; 
Ophioglossum flexuosum], T5414 Ranunculus japonicus, T6224 Swertia kouitchensis.

fire flow T0594 Ardisia crenata.

fish bone stuck in throat T4384 Narcissus tazetta var. chinensis.

fish mouth sore toxin T1964 Cynanchum wilfordii [Syn. Cynoctonum wilfordii].

fistula T2384 Epicauta gorhami, T4786 Phasianus colchicus, T4917 Pinus massoniana, T6080 Spiraea japonica.

flat abscess T6267 Syzygium jambos.

flat abscess (leaf) T0240 Aglaia odorata.

flat wart T1614 Coix lacryma-jobi var. ma-yuen, T5856 Sedum lineare [Syn. Sedum obtuso-lineare]

flooding T0215 Agave americana, T0860 Balanophora japonica, T0952 Blechnum orientale, T0973 Bombax malabaricum [Syn. Gossampinus malabarica], T0976 Bombyx mori, T1145 Camellia japonica, T1174 Cannabis sativa, T1191 Caragana sinica, T1419 Cimicifuga dahurica, T1420 Cimicifuga foetida, T1421 Cimicifuga heracleifolia, T1673 Corchorus capsularis, T1674 Corchorus capsularis, T1682 Cordyceps ophioglossoides, T1888 Cudrania tricuspidata, T1986 Cyrtomium fortunei, T2133 Desmodium racemosum [Syn. Podocarpium podocarpum var. oxyphyllum], T2161 Dicranopteris pedata [Syn. Polypodium pedatum; Dicranopteris dichotoma], T2218 Diospyros kaki, T2540 Euonymus fortunei, T3054 Gossypium barbadense], T3055 Gossypium herbaceum, T3058 Gossypium hirsutum [Syn. Gossypium mexicanum], T3547 Ixeris chinensis, T3671 Lagerstroemia indica, T3957 Lycium chinense, T4006 Lythrum anceps, T4007 Lythrum salicaria, T4307 Mosla scabra [Syn. Mosla punctata], T4361 Myrsine africana, T4606 Panax pseudo-ginseng, T4670 Patrinia heterophylla, T4673 Patrinia scabra, T4787 Phellinus igniarius, T5552 Robinia pseudoacacia, T5579 Rubia cordifolia, T5594 Rubus parkeri, T5697 Salvia trijuga, T5924 Sesamum indicum, T6018 Solanum verbascifolium, T6250 Symplocos caudata, T6485 Trachycarpus fortunei, T6551 Trogopterus xanthipes; Pteromys volans, T6615 Uncaria gambir, T6830 Woodfordia fruticosa.

flooding and spotting T0170 Adhatoda vasica, T0229 Ageratum conyzoides, T0249 Agrimonia pilosa var. japonica, T0255 Ailanthus altissima, T0664 Artemisia argyi, T0678 Artemisia japonica, T0681 Artemisia lavandulaefolia, T0685 Artemisia mongolica, T0689 Artemisia princeps, T0691 Artemisia rubripes, T0706 Artemisia vulgaris, T0736 Asclepias curassavica, T0791 Astragalus chrysopterus, T0794 Astragalus ernestii, T0798 Astragalus membranaceus, T0800 Astragalus mongholicus, T0830 Auricularia auricula, T0831 Auricularia delicata, T0859 Balanophora involucrata, T0867 Baphicacanthus cusia [Syn. Strobilanthes cusia], T0962 Boehmeria platanifolia [Syn. Boehmeria tricuspis], T0978 Bombyx mori, T0982 Bos taurus domesticus, T0993 Boschniakia rossica, T1116 Callicarpa arborea, T1118 Callicarpa formosana, T1119 Callicarpa japonica, T1160 Campsis grandiflora, T1184 Capsella bursa-pastoris, T1425 Cimicifuga simplex, T1448 Cirsium chinense, T1449 Cirsium japonicum, T1451 Cirsium setosum [Syn. Cerratula setosa; Cirsium segetum; Cephalanoplos segetum], T1547 Clematis terniflora [Syn. Clematis maximowicziana], T1655 Conyza blinii, T1706 Corydalis ambigua var. amurensis [Syn. Corydalis ambigua], T1735 Corydalis repens, T1900 Curculigo capitulata [Syn. Leucojum capitulata], T1971 Cynoglossum zeylanicum [Syn. Anchusa zeylanica; Cynoglossum furcatum; Cynoglossum formosanum], T2234 Dipsacus asperoides, T2235 Dipsacus japonicus, T2281 Dryopteris crassirhizoma, T2282 Dryopteris filix-mas, T2290 Duchesnea indica, T2323 Eclipta prostrata [Syn. Eclipta alba], T2407 Equisetum arvense, T2435 Eriocheir sinensis, T2591 Euphorbia humifusa, T2826 Galeola faberi, T2837 Gallus gallus domesticus, T3056 Gossypium herbaceum, T3088 Gynura segetum [Syn. Gynura japonica], T3123 Hedyotis auricularia, T3193 Hemerocallis fulva, T3195 Hemerocallis lilio-asphodelus, T3197 Hemerocallis minor, T3241 Hibiscus mutabilis, T3242 Hibiscus rosa-sinensis, T3278 Homo sapiens, T3340 Hypericum ascyron, T3349 Hypericum erectum, T3361 Hypericum perforatum, T3373 Hypoestes purpurea [Syn. Justicia purpurea; Hypoestes sinica], T3659 Lactuca indica, T3679 Lamiophlomis rotata [Syn. Phlomis rotata], T3736 Lemmaphyllum microphyllum, T3842 Limonium gmelinii, T3863 Liparis nervosa, T3926 Loropetalum chinense, T3933 Luffa acutangula, T3934 Luffa cylindrica, T4259 Mnium cuspidatum, T4343 Myrica esculent, T4398 Nelumbo nucifera, T4399 Nelumbo nucifera, T4400 Nelumbo nucifera, T4552 Osmunda japonica, T4604 Panax japonicus var. bipinnatifidus, T4605 Panax japonicus var. major, T4607 Panax pseudo-ginseng var. japonicus, T4608 Panax pseudo-ginseng var. notoginseng [Syn. Panax notoginseng], T4656 Parmelia saxatilis, T4658 Parmelia tinctorum, T5104 Polygonum hydropiper, T5118 Polygonum suffultum, T5133 Polytrichum commune, T5173 Portulaca oleracea, T5228 Prunus mume, T5265 Psidium guajava, T5323 Pulsatilla campanella, T5324 Pulsatilla cernua, T5325 Pulsatilla chinensis, T5326 Pulsatilla dahurica, T5328 Punica granatum, T5332 Punica granatum, T5354 Pyrrosia davidii, T5355 Pyrrosia drakeana, T5356 Pyrrosia gralla, T5357 Pyrrosia lingua T5358 Pyrrosia petiolosa, T5360 Pyrrosia sheareri, T5497 Rhodiola kirilowii, T5518 Rhododendron mucronatum, T5523 Rhododendron simsii, T5529 Rhodomyrtus tomentosa, T5541 Rhus verniciflua [Syn. Toxicadendron verniciflum], T5554 Rodgersia aesculifolia, T5567 Rosa laevigata, T5607 Rumex crispus, T5609 Rumex hastatus, T5611 Rumex japonicus, T5612 Rumex nepalensis, T5614 Rumex patientia, T5661 Salvia bowleyana, T5671 Salvia digitaloides, T5672 Salvia flava, T5685 Salvia plebeia, T5701 Salvia yunnanensis, T5712 Sanguisorba officinalis, T5773 Saxifraga stolonifera, T5804 Schizonepeta tenuifolia [Syn. Nepeta tenuifolia], T5850 Sedum aizoon, T5855 Sedum kamtschaticum, T5856 Sedum lineare [Syn. Sedum obtuso-lineare], T5862 Selaginella involvens, T6034 Sophora japonica, T6036 Sophora japonica, T6474 Toona ciliata, T6475 Toona sinensis, T6584 Typha angustata, T6585 Typha angustifolia, T6587 Typha latifolia, T6653 Usnea diffracta, T6654 Usnea longissima, T6742 Vicia amoena, T6831 Woodwardia orientalis, T6904 Zephyranthes grandiflora [Syn. Zephyranthes carinata].

flooding and spotting [ = metrorrhagia and metrostaxis] T0001 Abelmoschus manihot, T1033 Broussonetia papyrifera, T1034 Broussonetia papyrifera, T2193 Dioscorea cirrhosa [Syn. Dioscorea pogonoides], T3129 Hedysarum polybotrys, T3460 Iris halophila, T3464 Iris lactea var. chinensis [Syn. Iris pallasii var. chinensis], T4127 Matteuccia struthiopteris, T4549 Osbeckia chinensis, T5105 
Polygonum hydropiper var. flaccidum [Syn. Polygonum flaccidum], T5182 Potentilla discolor, T5185 Potentilla multifida, T5401 Rabdosia yuennanensis, T5573 Rosa sericea, T6533 Trillium kamtschaticum, T6535 Trillium tschonoskii, T6601 Umbilicaria esculenta [Syn. Gyrophora esculenta].

flooding and spotting due to blood heat T5445 Rehmannia glutinosa [Syn. Rehmannia glutinosa f. huechingensis], T5447 Rehmannia glutinosa [Syn. Rehmannia glutinosa f. huechingensis].

flooding and spotting with vaginal discharge T0248 Agrimonia pilosa, T0297 Alchornea trewioides, T0500 Anguilla japonica, T0890 Begonia limprichtii, T1337 Cervus nippon; Cervus elaphus, T1698 Cornus officinalis [Syn. Macrocarpium officinale], T2280 Dryopteris chrysocoma, T5568 Rosa laevigata, T6773 Viscum articulactum, T6774 Viscum articulatum.

flooding and vaginal discharge with strangury-turbidity T4584 Paeonia lactiflora wild, T4586 Paeonia obovata, T4590 Paeonia veitchii.

flooding spotting and vaginal discharge T4121 Marsilea quadrifolia.

flowing phlegm T1008 Brassica juncea, T2584 Euphorbia ebracteolata, T2587 Euphorbia fischeriana.

flowing rheum in chest T2596 Euphorbia kansui.

fluid damage and constipation T5826 Scrophularia buergeriana, T5828 Scrophularia ningpoensis.

fluid damage and thirst T0535 Antidesma bunius, T2101 Dendrobium fimbriatum, T2983 Glehnia littoralis, T3875 Liriope spicata var. prolifera, T4288 Morus alba, T4507 Ophiopogon japonicus, T4599 Panax ginseng [Syn. Panax schinseng], T4600 Panax ginseng [Syn. Panax schinseng], T4609 Panax pseudo-ginseng var. notoginseng [Syn. Panax notoginseng], T5218 Prunus armeniaca, T5791 Schisandra chinensis, T5802 Schisandra sphenanthera, T6266 Syzygium cumini, T6919 Ziziphus jujuba var. spinosa.

fluid damage with vexation and thirst T5447 Rehmannia glutinosa [Syn. Rehmannia glutinosa f. huechingensis].

food accumulation T1152 Camellia sinensis [Syn. Thea sinensis], T1467 Citrus aurantium, T1563 Clerodendron trichotomum, T1687 Coriandrum sativum, T1696 Cornus capitata [Syn. Dendrobenthamia capitata], T1904 Curcuma kwangsiensis, T1909 Curcuma zedoaria [Syn. Curcuma aeruginosa], T1958 Cynanchum otophyllum, T2365 Enteromorpha clathrata, T2387 Epilobium hirsutum, T2505 Eucalyptus citriodora, T2580 Euphorbia antiquorum, T2612 Euphorbia prolifera, T2748 Fomes fomentarius [Syn. Pyropolyporus fomentarius; Boletus fomentarius; Polyporus fomentarius], T3282 Hordeum vulgare, T4954 Piper longum, T5140 Poncirus trifoliata, T5239 Prunus salicina, T5361 Pyrus betulaefolia, T5401 Rabdosia yuennanensis, T5469 Rheum hotaoense, T6049 Sophora viciifolia, T6105 Stellera chamaejasme, T6312 Taxus mairei.

food accumulation abdominal distention T0680 Artemisia lactiflora, T0708 Arthromeris mairei [Syn. Polypodium mairei], T3308 Hydrangea umbellata, T3436 Inula nervosa, T4542 Orthosiphon wulfenioides [Syn. Coleus wulfenioides], T5267 Psidium guajava, T5461 Rhamnus leptophylla.

food accumulation abdominal pain T0142 Acorus calamus, T0661 Artemisia anomala, T3006 Glycosmis citrifolia, T3254
Hippophae rhamnoides, T3256 Hippophae rhamnoides subsp. sinensis, T3258 Hippophae rhamnoides subsp. yunnanensis.

food accumulation and distending pain in stomach duct T1291 Celastrus paniculatus.

food accumulation and qi distention T3885 Litsea cubeba.

food accumulation and $\boldsymbol{q} \boldsymbol{i}$ stagnation T0692 Artemisia rupestris [Syn. Artemisia dentata; Artemisia viridis; Artemisia viridifolia], T4034 Magnolia biloba, T4045 Magnolia officinalis, T4048 Magnolia rostrata, T5223 Prunus humilis [Syn. Cerasus humilis], T5224 Prunus japonica [Syn. Cerasus japonica], T5225 Prunus japonica var. nakaii.

food accumulation diarrhea T2998 Glycine max, T6789 Vitex negundo.

food accumulation distention and fullness T0932 Betula luminifera, T1352 Chamaenerion angustifolium [Syn. Epilobium angustifolium], T2189 Dioscorea althaeoides, T5189 Pothos chinensis, T5419 Raphanus sativus, T5570 Rosa roxburghii, T6356 Tetrapanax papyriferus.

food accumulation stomachache T5183 Potentilla griffithii var. velutina.

food damage T2733 Firmiana simplex.

food damage diarrhea T5113 Polygonum persicaria.

food damage vomiting and diarrhea T0356 Alpinia galanga.

food poisoning T2715 Fibraurea recisa, T3000 Glycine max, T3162 Helicia nilagirica, T3984 Lycoris chinensis, T3986 Lycoris radiata [Syn. Amaryllis radiata], T3988 Lycoris squamigera.

food retention and abdominal distention T4088 Malus pumila.

food stagnation T0606 Areca catechu, T1478 Citrus grandis, T1769 Crataegus cuneata, T1771 Crataegus kansuensis, T1772 Crataegus maximowiczii, T1780 Crataegus sanguinea, T1781 Crataegus scabrifolia, T2838 Gallus gallus domesticus, T4100 Mangifera indica, T4428 Nicotiana tabacum, T4544 Oryza sativa, T5420 Raphanus sativus, T5719 Sapindus mukorossi, T5720 Sapindus mukorossi.

food stagnation abdominal distention T5167 Porana racemosa.

food stagnation and abdominal fullness T1381 Choerospondias axillaris.

food stagnation and diarrhea T5244 Prunus tomentosa.

food stagnation distention and fullness T1179 Capparis masaikai.

food stagnation in torpid stomach T0417 Amomum longiligulare, T0419 Amomum villosum, T0420 Amomum xanthioides, T2758 Fortunella crassifolia, T2759 Fortunella japonica, T2760 Fortunella margarita.

food stagnation with torpid intake T3006 Glycosmis citrifolia.

food-denying dysentery T0144 Acorus gramineus, T0146 Acorus tatarinowii.

foot lichen T1046 Buddleja davidii, T2590 Euphorbia hirta, T3524 Isodon sculponeata [Syn. Rabdosia sculponeata], T3560 Jatropha curcas, T4307 Mosla scabra [Syn. Mosla punctata], T5026 Plumbagella micrantha, T5104 Polygonum hydropiper, T5105 Polygonum hydropiper var. flaccidum [Syn. Polygonum flaccidum], T5724 Sapium sebiferum.

foot rot T0180 Adina pilulifera [Syn. Cephalanthus pilulifera], T1205 Carica papaya, T1316 Cephalanthus occidentalis, T2136 Desmos cochinchinensis [Syn. Desmos chinensis], T4799 Philydrum lanuginosum.

forking qi with pain $\mathrm{T} 3431$ Inula helenium, T3437 Inula racemosa. 
fracture T0002 Abelmoschus moschatus [Syn. Hibiscus abelmoschus], T0036 Acanthopanax giraldii [Syn. Acanthopanax giraldii var. inermis; Eleutherococcus giraldii], T0038 Acanthopanax gracilistylus, T0042 Acanthopanax senticosus [Syn. Eleutherococcus senticosus], T0044 Acanthopanax sessiliflorus, T0122 Aconitum pendulum, T0282 Alangium kurzii, T0502 Anisodus tanguticus [Syn. Scopolia tangutica], T0515 Anredera cordifolia [Syn. Baussingaultia cordifolia; Baussingaultia gracilis f. pseudobaselloides; Baussingaultia gracilis var. pseudobaselloides], T0568 Aralia chinensis, T0569 Aralia cordata, T0570 Aralia dasyphylla, T0574 Aralia fargesii, T0708 Arthromeris mairei [Syn. Polypodium mairei], T0813 Atalantia buxifolia [Syn. Severinia buxifolia], T0871 Barleria lupulina, T1147 Camellia oleifera, T1206 Carica papaya, T1327 Ceratostigma minus, T1328 Ceratostigma plumbaginoides, T1329 Ceratostigma willmottianum, T1372 Chloranthus serratus, T1533 Clausena dentata, T1534 Clausena dunniana, T1535 Clausena excavata, T1644 Conocephalum conicum, T1799 Crinum latifolium, T1841 Croton caudatus var. tomentosus, T1853 Croton oblongifolius [Syn. Croton laevigatus], T1871 Cucubalus baccifer, T1963 Cynanchum wallichii, T1967 Cynoglossum amabile, T2612 Euphorbia prolifera, T2614 Euphorbia pulcherrima, T2734 Fissistigma glaucescens [Syn. Melodorum glaucescens], T2955 Geum japonicum, T3089 Gypsophila acutifolia, T3202 Hemistepta lyrata [Syn. Hemistepta carthamoides; Saussurea carthamoides], T3303 Hydrangea chinensis, T3373 Hypoestes purpurea [Syn. Justicia purpurea; Hypoestes sinica], T3387 Idesia polycarpa, T3418 Incarvillea arguta, T3444 Ipomoea aquatica [Syn. Convolvulus repens; Ipomoea reptans], T3613 Kadsura coccinea [Syn. Kadsura chenensis; Kadsura hainanensis], T3624 Kalopanax pictum, T3625 Kalopanax septemlobus, T3685 Lannea grandis [Syn. Lannea coromandelica], T3778 Leucaena glauca [Syn. Leucaena leucocephala], T3880 Lithospermum arvense, T3991 Lyonia ovalifolia, T3995 Lysidice rhodostegia, T4109 Marchantia polymorpha, T4118 Marsdenia oreophila, T4143 Meconopsis horridula, T4221 Micromelum falcatum, T4223 Micromelum integerrimum, T4320 Murraya kwangsiensis, T4346 Myrica nagi [Syn. Podocarpus nagi], T4444 Nothopanax davidii, T4542 Orthosiphon wulfenioides [Syn. Coleus wulfenioides], T4678 Paulownia fortunei, T4679 Paulownia tomentosa, T4686 Pedilanthus tithymaloides, T4726 Periploca forrestii, T4938 Piper boehmeriaefolium, T5189 Pothos chinensis, T5270 Psoralea corylifolia, T5279 Psychotria serpens, T5402 Radermachera sinica, T5515 Rhododendron micranthum, T5555 Rodgersia pinnata, T5730 Sarcandra glabra [Syn. Chloranthus glaber], T5733 Sarcococca vagans, T5781 Schefflera arboricola, T5785 Schefflera venulosa, T5794 Schisandra lancifolia, T5819 Scopolia acutangula [Syn. Anisodus acutangulus], T5942 Sida acuta, T6051 Sorbaria arborea, T6052 Sorbaria sorbifolia, T6444 Thunbergia grandiflora, T6531 Trillium camtschaticum, T6540 Tripterygium hypoglaucum, T6580 Tylophora floribunda, T6618 Uncaria homomalla [Syn. Uruparia homomalla; Uruparia tonkinensis; Uruparia lanosa var. parvifora], T6738 Viburnum odoratissimum.

fracture and pain T3560 Jatropha curcas.

fracture due to knocks and falls T0045 Acanthopanax trifoliatus, T0295 Albizzia odoratissima, T2262 Dregea sinensis, T2347 Embelia parviflora, T2387 Epilobium hirsutum, T2540 Euonymus fortunei, T3996 Lysimachia candida, T4031 Maesa perlarius, T6567 Tupistra wattii [Syn. Campylandra wattii], T6735 Viburnum dilatatum, T6883 Zanthoxylum myriacanthum.

fracture with damage $\mathrm{T} 3124$ Hedyotis capitellata.

fracture with wound sinew T4821 Pholidota articulata.

frequent dreaming T5108 Polygonum multiflorum, T5697 Salvia trijuga, T6919 Ziziphus jujuba var. spinosa.

frequent urination T0323 Allium tuberosum, T0360 Alpinia oxyphylla, T0793 Astragalus complanatus, T1411 Cibotium barometz [Syn. Polypodium barometz], T1604 Coeloglossum viride [Syn. Coeloglossum viride var. bracteatum], T2188 Dioscorea alata, T2190 Dioscorea batatas [Syn. Dioscorea opposita], T2202 Dioscorea japonica, T2837 Gallus gallus domesticus, T2838 Gallus gallus domesticus, T3077 Gymnadenia conopsea, T3568 Juglans regia, T3770 Lespedeza cuneata, T4279 Morina chinensis, T4600 Panax ginseng [Syn. Panax schinseng], T4786 Phasianus colchicus, T5270 Psoralea corylifolia, T5569 Rosa multiflora, T6754 Vigna unguiculata.

frequent urination and enuresis T5593 Rubus idaeus, T5791 Schisandra chinensis, T5802 Schisandra sphenanthera.

frequent urination at night T2962 Ginkgo biloba.

frequent urination in children T1826 Crotalaria mucronata.

fright epilepsy T0427 Ampelopsis japonica [Syn. Paullinia japonica], T0986 Bos taurus domesticus; Bubalus bubalis, T0987 Bos taurus domesticus; Bubalus bubalis, T1804 Cristaria plicata; Hyriopsis cumingii, T1841 Croton caudatus var. tomentosus, T1907 Curcuma wengujin, T2039 Datura innoxia, T2043 Datura metel, T2047 Datura stramonium, T2290 Duchesnea indica, T3329 Hyoscyamus niger, T3868 Liquidambar orientalis, T5086 Polygala sibirica, T5088 Polygala tenuifolia, T6457 Tilia japonica, T6458 Tilia miqueliana.

fright palpitation T1808 Crocus sativus, T4400 Nelumbo nucifera, T5701 Salvia yunnanensis, T6919 Ziziphus jujuba var. spinosa.

fright palpitation and amnesia T0142 Acorus calamus, T4600 Panax ginseng [Syn. Panax schinseng].

fright palpitation and fearful throbbing T0944 Biota orientalis [Syn. Thuja orientalis; Platycladus orientalis], T6918 Ziziphus jujuba var. spinosa.

fright palpitation and insomnia T4599 Panax ginseng [Syn. Panax schinseng], T5086 Polygala sibirica, T5088 Polygala tenuifolia, T5169 Poria cocos.

fright palpitation and vacuity vexation $\mathrm{T} 3832$ Lilium brownii var. viridulum [Syn. Lilium brownii var. colchesteri], T3835 Lilium longiflorum, T3836 Lilium pumilum [Syn. Lilium tenuifolium], T3839 Lilium tigrinum [Syn. Lilium lancifolium].

fright palpitation due to heart heat T3304 Hydrangea macrophylla.

fright wind T0338 Aloe ferox, T0347 Aloe vera [Syn. Aloe barbadensis], T0348 Aloe vera var. chinensis, T0617 Arisaema amurense, T0618 Arisaema consanguineum, T0620 Arisaema heterophyllum, T2272 Drymaria diandra $[$ Syn. Drymaria cordata ssp. diandra], T3764 Lepisorus thunbergianus, T4903 Pinellia pedatisecta, T5871 Selenarctos thibetanus; Ursus arctos, T5934 Seseli meirei, T5937 Seseli yunnanense.

fright wind and convulsion T0975 Bombyx mori, T2908 Gentiana 
cephalantha, T2921 Gentiana manshurica, T2928 Gentiana rigescens, T2930 Gentiana scabra, T2938 Gentiana triflora, T4710 Pericampylus glaucus, T4796 Pheretima aspergillum; Allolobophora caliginosa trapezoides.

frostbite [ = kibe] $\mathrm{T} 0122$ Aconitum pendulum, T0414 Ammopiptanthus mongolicus [Syn. Piptanthus mongolicus], T0827 Aucuba chinensis ssp. omeiensis, T0977 Bombyx mori, T1007 Brassica juncea, T1186 Capsicum annuum, T1187 Capsicum frutescens, T1594 Cocos nucifera, T1769 Crataegus cuneata, T1771 Crataegus kansuensis, T1772 Crataegus maximowiczii, T1780 Crataegus sanguinea, T1781 Crataegus scabrifolia, T3388 Ilex chinensis [Syn. Ilex purpurea], T3675 Lagopsis supina, T3925 Loranthus parasiticus [Syn. Loranthus chinenis; Taxillus chinensis], T4157 Melia azedarach. T4519 Opuntia dillenii, T4521 Opuntia vulgaris, T4578 Paederia scandens, T4975 Piptanthus nanus, T5419 Raphanus sativus, T6006 Solanum melongena.

frostbite flowing water and pus T4978 Pisolithus tinctorius [Syn. Lycoperdon capitatum; Scleroderma tinctorium].

fullness and oppression in chest and diaphragm T0480 Angelica decursiva [Syn. Peucedanum decursivum], T1007 Brassica juncea, T3819 Ligusticum brachylobum, T4761 Peucedanum longshengens, T4768 Peucedanum praeruptorum, T4769 Peucedanum rubricaule.

fullness and oppression in chest and stomach duct T6561 Tulipa gesneriana.

fullness and oppression in heart and abdomen 75215 Prunus amygdalus.

fullness in chest T6497 Tribulus terrestris.

fullness in chest and rapid asthma T1960 Cynanchum stauntonii.

fullness in chest and rib-side pain T5011 Platycodon grandiflorum, T5958 Sinapis alba [Syn. Brassica alba; Brassica hirta].

fulminant fever with vexation and thirst T4482 Oenanthe javanica.

furunculosis T5330 Punica granatum.

galactostasis T0273 Akebia quinata, T0273 Akebia quinata, T0277 Akebia trifoliata, T0277 Akebia trifoliata, T0279 Akebia trifoliata var. australis, T0279 Akebia trifoliata var. australis, T1352 Chamaenerion angustifolium [Syn. Epilobium angustifolium], T1508 Citrus sinensis, T2262 Dregea sinensis, T2316 Echinops grijsii, T2317 Echinops ritro, T3193 Hemerocallis fulva, T3195 Hemerocallis lilio-asphodelus, T3197 Hemerocallis minor, T3282 Hordeum vulgare, T3340 Hypericum ascyron, T3361 Hypericum perforatum, T3662 Lactuca sativa, T3867 Liquidambar formosana [Syn. Liquidambar taiwaniana], T3933 Luffa acutangula, T3934 Luffa cylindrica, T4106 Manis pentadactyla, T4178 Melodinus hemsleyanus, T5466 Rhaponticum uniflorum, T6356 Tetrapanax papyriferus, T6668 Vaccaria segetalis [Syn. Vaccaria pyramidata], T6901 Zea mays.

galloping gan of teeth and gum T2435 Eriocheir sinensis, T4471 Ocimum basilicum.

gallstones T0502 Anisodus tanguticus [Syn. Scopolia tangutica], T1406 Chrysosplenium nudicaule, T1903 Curcuma aromatica, T1905 Curcuma longa, T2838 Gallus gallus domesticus, T3156 Helichrysum arenarium, T3998 Lysimachia christinae, T4000 Lysimachia congestiflora, T5472 Rheum officinale, T5474 Rheum palmatum, T5481 Rheum tanguticum, T5957 Silybum marianum, T6789 Vitex negundo, T6901 Zea mays.

gan accumulation T0541 Apis cerana, T0877 Bauhinia championii, T1051 Bufo bufo gargarizans; Bufo melanostictus, T1591 Cocos nucifera, T1593 Cocos nucifera, T2048 Daucus carota, T2115 Dermatocarpon minitum, T2336 Elephas maximus, T2927 Gentiana rhodantha, T4102 Mangifera indica, T5106 Polygonum lapathifolium, T5179 Potentilla anserina, T5423 Rauvolfia verticillata, T5425 Rauvolfia verticillata f. rubrocarpa, T5426 Rauvolfia verticillata var. hainanensis, T5427 Rauvolfia vomitoria, T5435 Rauwolfia latifrons, T5441 Rauwolfia perakensis, T5528 Rhodomyrtus tomentosa, T5576 Rostellularia procumbens [Syn. Justicia procumbens], T5719 Sapindus mukorossi, T6474 Toona ciliata, T6475 Toona sinensis, T6599 Ulva lactuca, T6600 Ulva pertusa, T6602 Umbilicaria hypococcinea, T6656 Ustilago maydis, T6732 Vespertilio superans.

gan disease T0976 Bombyx mori, T3645 Kummerowia striata, T4851 Physalis minima, T5407 Rana nigromaculata; Rana plancyi, T6279 Tagetes erecta.

gan of nose T1150 Camellia sinensis [Syn. Thea sinensis], T6409 Thalictrum simplex [Syn. Thalictrum simplex var. brevipes].

gan of teeth and gum T2131 Desmodium pulchellum [Syn. Phyllodium pulchellum], T2838 Gallus gallus domesticus, T2866 Garcinia morella, T3277 Homo sapiens, T3865 Lippia nodiflora, T4303 Moschus moschiferus; Moschus berezovskii; Moschus sifanicus, T6615 Uncaria gambir.

gan sore pox toxin T2841 Gallus gallus domesticus.

gastric ulcer T0543 Apis mellifera ligustica, T0825 Atropa belladonna, T1101 Caesalpinia decapetala, T1884 Cudrania cochinchinensis, T2783 Fritillaria cirrhosa, T2784 Fritillaria delavayi, T2792 Fritillaria przewalskii, T2796 Fritillaria unibracteata, T2798 Fritillaria verticillata var. thunbergii [Syn. Fritillaria thunbergii], T3011 Glycyrrhiza aspera, T3013 Glycyrrhiza glabra, T3015 Glycyrrhiza inflata, T3016 Glycyrrhiza kansuensis, T3021 Glycyrrhiza squamulosa, T3022 Glycyrrhiza uralensis, T3023 Glycyrrhiza yunnanensis, T3164 Helicteres isora, T3229 Hericium erinaceus [Syn. Hydnum erinaceus], T3613 Kadsura coccinea [Syn. Kadsura chenensis; Kadsura hainanensis], T4082 Mallotus japonicus, T5183 Potentilla griffithii var. velutina, T6145 Stichopus japonicus, T6275 Tacca chantrieri [Syn. Tacca minor; Tacca esquirolii].

gastritis T1055 Bupleurum angustissimum, T1056 Bupleurum aureum, T1058 Bupleurum chaishoui, T1059 Bupleurum chinense, T1065 Bupleurum longiradiatum, T1066 Bupleurum marginatum, T1067 Bupleurum marginatum var. stenophyllum, T1072 Bupleurum scorzonerifolium, T1073 Bupleurum sibiricum, T1075 Bupleurum smithii var. parvifolium, T1078 Bupleurum wenchuanense, T1079 Bupleurum yinchowense, T1727 Corydalis mucronifera, T1954 Cynanchum chinense, T3496 Isodon japonica [Syn. Rabdosia japonica], T4194 Menyanthes trifoliata, T4248 Mimosa pudica, T6224 Swertia kouitchensis, T6231 Swertia pseudochinensis, T6339 Tephrosia purpurea, T6691 Veratrilla baillonii.

gastritis with edema T1954 Cynanchum chinense.

gastroenteritis T0137 Aconitum tanguticum, T2421 Erigeron annuus, T2563 Eupatorium japonicum, T2715 Fibraurea recisa, T3092 Halenia corniculata, T4144 Meconopsis nepaulensis, T6122 Stephania 
dielsiana, T6275 Tacca chantrieri [Syn. Tacca minor; Tacca esquirolii]. gastrointestinal accumulation T2658 Fagopyrum esculentum. gastrointestinal damp-heat diarrhea T4887 Picrorhiza kurrooa, T4888

Picrorhiza scrophulariiflora.

gastrointestinal flatulence T3263 Holarrhena antidysenterica. gastrointestinal spasm $\mathrm{T} 1750$ Corydalis yanhusuo [Syn. Corydalis turtschaninovii f. yanhusuo].

gastrointestinal ulcer T0060 Achillea alpina [Syn. Achillea sibirica]. gastroptosis T1055 Bupleurum angustissimum, T1056 Bupleurum aureum, T1058 Bupleurum chaishoui, T1059 Bupleurum chinense, T1065 Bupleurum longiradiatum, T1066 Bupleurum marginatum, T1067 Bupleurum marginatum var. stenophyllum, T1072 Bupleurum scorzonerifolium, T1073 Bupleurum sibiricum, T1075 Bupleurum smithii var. parvifolium, T1078 Bupleurum wenchuanense, T1079 Bupleurum yinchowense.

gastrospasm T0572 Aralia elata, T0625 Aristolochia contorta, T0627 Aristolochia debilis [Syn. Aristolochia longa].

ge syndrome T4369 Nandina domestica.

general arthralgia T2995 Gloriosa superba.

generalized fever T5826 Scrophularia buergeriana, T5828 Scrophularia ningpoensis.

generalized fever and aversion to cold T4101 Mangifera indica. generalized fever with macule T1950 Cynanchum ascyrifolium, T1951 Cynanchum atratum, T1962 Cynanchum versicolor, T2952 Gerbera piloselloides, T6582 Tylophora ovata.

generalized pain T3118 Hedychium coronarium, T3674 Laggera alata, T4029 Maesa japonica, T4551 Osmorhiza aristata var. laxa.

genital carcinoma T1904 Curcuma kwangsiensis, T1909 Curcuma zedoaria [Syn. Curcuma aeruginosa].

genital sweating T4954 Piper longum, T5374 Quercus infectoria.

genital swelling T1662 Coptis chinensis, T1663 Coptis chinensis var. brevisepala, T1664 Coptis deltoidea, T1669 Coptis omeiensis, T1670 Coptis teetoides [Syn. Coptis teeta], T4714 Perilla frutescens.

genital swelling and itch T2908 Gentiana cephalantha, T2921 Gentiana manshurica, T2928 Gentiana rigescens, T2930 Gentiana scabra, T2938 Gentiana triflora.

gingiva painful swelling T0584 Arctium lappa, T3201 Hemiphragma heterophyllum, T5267 Psidium guajava, T6043 Sophora subprostrata [Syn. Sophora tonkinensis].

gingiva ulcerating $\mathrm{T} 3420$ Incarvillea sinensis. gingivitis T6095 Stachytarpheta jamaicensis.

globus hystericus T1366 Chimonanthus fragrans [Syn. Chimonanthus praecox], T5139 Poncirus trifoliata, T5226 Prunus mume.

glomerulonephritis T6542 Tripterygium wilfordii.

glomus T0357 Alpinia japonica.

glomus accumulation T6541 Tripterygium regelii.

glomus and congestion in chest and diaphragm T4086 Malus asiatica. glomus and oppression in chest and diaphragm T1808 Crocus sativus. glomus and oppression in chest and stomach duct T0212 Agastache rugosus, T1978 Cyperus rotundus.

glomus distention in stomach duct and abdomen T1490 Citrus limon, T1494 Citrus limonia, T1937 Cydonia oblonga.

glomus fullness T0472 Anethum graveolens, T3850 Lindera glauca,
T3928 Lotus corniculatus, T4720 Perilla frutescens var. arguta, T4723 Perilla frutescens var. crispa.

glomus fullness in chest and diaphragm T3426 Inula britannica, T3427 Inula britannica, T3428 Inula britannica var. chinensis, T3433 Inula japonica, T3434 Inula linariaefolia.

glomus in chest T0819 Atractylodes chinensis, T0821 Atractylodes japonica, T0823 Atractylodes lancea, T1467 Citrus aurantium, T4904 Pinellia ternata, T5140 Poncirus trifoliata.

glomus qi T0988 Bos taurus domesticus; Bubalus bubalis.

goiter and carcinoma of neck T0847 Baccharis indica [Syn. Pluchea indica], T1385 Chorda filum, T2365 Enteromorpha clathrata, T4403 Nemacystus decipiens [Syn. Mesogloea decipiens; Cladosiphon decipiens], T6257 Syngnathus acus, T6599 Ulva lactuca, T6600 Ulva pertusa.

goiter and carcinomas of neck T5740 Sargassum vachellianum.

goiter and tuberculosis T0275 Akebia quinata, T1486 Citrus junos, T2191 Dioscorea bulbifera, T2302 Dysosma pleiantha [Syn. Podophyllum pleianthum], T2304 Dysosma veitchii, T2305 Dysosma versipellis [Syn. Podophyllum versipelle], T2321 Ecklonia kurome, T2525 Eucheuma muricatum, T2756 Forsythia suspensa, T2994 Gloiopeltis furcata, T3308 Hydrangea umbellata, T3678 Laminaria japonica, T5171 Porphyra tenera, T5214 Prunella vulgaris, T6640 Undaria pinnatifida, T6925 Zostera marina.

goiters with phlegm node T4904 Pinellia ternata.

goose-foot wind $\mathrm{T} 1867$ Cryptomeria fortunei, $\mathrm{T} 3410$ Impatiens balsamina, T3411 Impatiens balsamina, T5026 Plumbagella micrantha, T5548 Ricinus communis.

goose-mouth sore T4080 Mallotus apelta, T4618 Panicum miliaceum, T6268 Syzygium samarangense, T6393 Thalictrum ichangense [Syn. Thalictrum tripeltatum; Thalictrum multipeltatum].

great wind sore T0145 Acorus gramineus.

gum erosion T0293 Albizzia lebbeck.

gum hemorrhage T0155 Actinidia arguta, T0805 Astragalus sinicus, T0867 Baphicacanthus cusia [Syn. Strobilanthes cusia], T1118 Callicarpa formosana, T1119 Callicarpa japonica, T3278 Homo sapiens, T4796 Pheretima aspergillum; Allolobophora caliginosa trapezoides, T5207 Prismatomeris tetrandra, T5606 Rumex acetosa, T6552 Trollius chinensis [Syn. Trollius asiaticus var. chinensis], T6553 Trollius ledebourii.

gum swelling T0906 Berberis julianae, T4854 Physalis pubescens, T5651 Salix babylonica.

gum swelling and pain T0476 Angelica anomala, T0571 Aralia decaisneaa, T1662 Coptis chinensis, T1663 Coptis chinensis var. brevisepala, T1664 Coptis deltoidea, T1669 Coptis omeiensis, T1670 Coptis teetoides [Syn. Coptis teeta], T3396 Ilex pubescens, T3397 Ilex pubescens var. glaber, T3457 Iris dichotoma, T4848 Physalis angulata, T5199 Primula malacoides, T5852 Sedum bulbiferum, T6439 Thuja orientalis [Syn. Platycladus orientalis; Biota orientalis], T6552 Trollius chinensis [Syn. Trollius asiaticus var. chinensis], T6553 Trollius ledebourii, T6791 Vitex rotundifolia [Syn. Vitex trifollia var. simplicifolia], T6793 Vitex trifolia.

gum swelling erosion T1007 Brassica juncea. habitual abortion T4505 Ophiocephalus argus. 
habitual constipation T1238 Cassia mimosoides, T1240 Cassia obtusifolia, T1247 Cassia sophera, T1250 Cassia tora, T5454 Rhamnus cathartica, T5460 Rhamnus frangula [Syn. Frangula alnus].

hacking of blood T0267 Ajuga nipponensis, T0600 Ardisia mamillata [Syn. Tinus mamillata], T5182 Potentilla discolor, T5701 Salvia yunnanensis.

hacking of pus blood T3233 Heteropappus altaicus.

hair loss T3860 Linum usitatissimum.

hair loss during convalescence T5927 Sesamum indicum [Syn. Sesamum orientale].

hard glomus below heart $\mathrm{T} 3427$ Inula britannica, $\mathrm{T} 3428$ Inula britannica var. chinensis, T3434 Inula linariaefolia.

hard swelling T6521 Trifolium pratense, T6522 Trifolium repens.

hasten delivery $\mathrm{T} 1595$ Codium fragile.

hasty asthma T4600 Panax ginseng [Syn. Panax schinseng].

head louse T1361 Chenopodium ambrosioides, T1645 Consolida ajacis [Syn. Delphinium ajacis], T3630 Kleinhovia hospita.

head sore T4843 Phyllanthus urinaria.

head wind T0569 Aralia cordata, T0574 Aralia fargesii, T2657 Fagopyrum cymosum [Syn. Polygonum cymosum], T5234 Prunus persica, T6792 Vitex trifolia, T6843 Xanthium sibiricum [Syn. Xanthium strumarium].

head wind dizziness T0988 Bos taurus domesticus; Bubalus bubalis, T4773 Peucedanum terebinthaceum.

head wind headache T0060 Achillea alpina [Syn. Achillea sibirica], T0077 Aconitum balfourii, T0084 Aconitum carmichaeli, T0105 Aconitum karakolicum, T0108 Aconitum kusnezoffii, T0957 Blumea balsamifera, T4828 Photinia serrulata.

head wind white scaling T5531 Rhus chinensis [Syn. Rhus semialata].

headache T0045 Acanthopanax trifoliatus, T0080 Aconitum bullatifolium var. homotrichum [Syn. Aconitum nagarum], T0088 Aconitum coreanum, T0302 Alhagi pseudalhagi, T0319 Allium schoenoprasum, T0492 Angelica pubescens f. biserrata [Syn. Angelica pubescens], T0553 Apocynum venetum, T0567 Aralia armata, T0569 Aralia cordata, T0574 Aralia fargesii, T0582 Archangelica brevicaulis [Syn. Angelicarpa brevicaulis; Angelica brevicaulis], T0584 Arctium lappa, T0644 Armillaria mellea, T0645 Armillariella mellea, T0708 Arthromeris mairei [Syn. Polypodium mairei], T0724 Asarum caulescens, T0726 Asarum forbesii, T0727 Asarum fukienense, T0728 Asarum heterotropoides var. mandshuricum, T0729 Asarum maximum, T0731 Asarum sieboldii, T0732 Asarum sieboldii var. seoulensis, T0952 Blechnum orientale, T1084 Buthus martensi, T1152 Camellia sinensis [Syn. Thea sinensis], T1288 Celastrus orbiculatus [Syn. Celastrus articulatus], T1289 Celastrus orbiculatus [Syn. Celastrus articulatus], T1552 Cleome viscosa, T1583 Cnidium officinale [Syn. Ligusticum officinale], T1586 Cocculus laurifolius, T1641 Conioselinum vaginatum, T1697 Cornus controversa [Syn. Bothrocaryum controversum], T1943 Cymbopogon goeringii, T1986 Cyrtomium fortunei, T2028 Daphne odora, T2030 Daphne retusa, T2031 Daphne tangutica, T2130 Desmodium gangeticum, T2257 Dracocephalum moldavicum, T2343 Elsholtzia splendens, T2434 Eriocaulon buergerianum, T2628 Euphrasia officinalis, T2643 Evodia meliifolia, T2697 Ferula borealis, T2890 Gastrodia elata, T3001
Glycine max, T3040 Gomphrena globosa, T3118 Hedychium coronarium, T3163 Helicteres angustifolia, T3213 Heracleum hemsleyanum, T3214 Heracleum lanatum, T3217 Heracleum moellendorffii [Syn. Heracleum microcarpum; Heracleum morifolium], T3222 Heracleum scabridum, T3228 Heracleum yungningense, T3246 Hibiscus syriacus, T3283 Hosta sieboldiana, T3303 Hydrangea chinensis, T3334 Hypecoum leptocarpum, T3392 Ilex kudingcha, T3393 Ilex latifolia, T3548 Ixeris sonchifolia, T3779 Leucas aspera, T3785 Levisticum officinale, T3796 Libanotis buchtormensis, T3820 Ligusticum chuanxiong [Syn. Ligusticum wallichii], T3829 Ligustrum robustum, T3845 Linaria vulgaris, T3847 Lindera angustifolia, T3950 Lychnis fulgens, T4001 Lysimachia davurica, T4035 Magnolia biondii [Syn. Magnolia fargesii], T4038 Magnolia denudata [Syn. Magnolia heptapata], T4041 Magnolia liliflora, T4052 Magnolia sprengeri, T4144 Meconopsis nepaulensis, T4145 Meconopsis punicea, T4170 Melilotus albus, T4186 Mentha piperita, T4190 Mentha spicata, T4317 Murraya euchrestifolia [Syn. Clausena euchrestifolia], T4386 Nardostachys chinensis, T4387 Nardostachys jatamansi, T4629 Papaver nudicaule, T4630 Papaver nudicaule ssp. amurense, T4631 Papaver nudicaule var. chinense, T4689 Peganum nigellastrum, T4821 Pholidota articulata, T4904 Pinellia ternata, T4953 Piper longum, T5021 Pleurospermum rivulorum, T5234 Prunus persica, T5301 Pterocarpus indicus, T5428 Rauvolfia yunnanensis, T5528 Rhodomyrtus tomentosa, T5575 Rosmarinus officinalis, T5592 Rubus hirsutus, T5609 Rumex hastatus, T5640 Sabina vulgaris, T5657 Salsola collina, T5781 Schefflera arboricola, T5804 Schizonepeta tenuifolia [Syn. Nepeta tenuifolia], T5934 Seseli meirei, T5937 Seseli yunnanense, T5963 Sinodielsia yunnanensis, T5969 Sium latifolium, T6000 Solanum indicum, T6024 Solidago virgaurea var. leiocarpa [Syn. Solidago decurrens], T6076 Spinacia oleracea, T6079 Spiraea japonica, T6082 Spiraea japonica var. fortunei, T6128 Stephania hernandifolia, T6139 Sterculia lychnophora, T6250 Symplocos caudata, T6359 Teucrium bidentatum, T6497 Tribulus terrestris, T6653 Usnea diffracta, T6654 Usnea longissima.

headache (especially in forehead and superciliary region) T0478 Angelica dahurica [Syn. Angelica porphyrocaulis].

headache (herb) T4859 Physochlaina physaloides.

headache ${ }^{[5509]}$ T4797 Phillyrea latifolia.

headache and dizziness T0544 Apium graveolens, T1199 Carduus acanthoides, T1200 Carduus crispus, T1393 Chrysanthemum indicum, T1804 Cristaria plicata; Hyriopsis cumingii, T4580 Paeonia albiflora [Syn. Paeonia lactiflora], T5214 Prunella vulgaris.

headache and generalized pain T0784 Astilbe chinensis, T2367 Ephedra equisetina, T2368 Ephedra gerardiana, T2369 Ephedra intermedia, T2372 Ephedra likiangensis, T2373 Ephedra minuta, T2375 Ephedra monosperma, T2379 Ephedra saxatilis, T2380 Ephedra sinica, T5423 Rauvolfia verticillata, T5425 Rauvolfia verticillata f. rubrocarpa, T5426 Rauvolfia verticillata var. hainanensis, T5427 Rauvolfia vomitoria, T5435 Rauwolfia latifrons, T5441 Rauwolfia perakensis, T5727 Saposhnikovia divaricata [Syn. Ledebouriella seseloides], T6457 Tilia japonica, T6458 Tilia miqueliana.

headache and nasal congestion T0476 Angelica anomala, T4039 Magnolia grandiflora, T6910 Zingiber officinale. 
headache and red eyes T1241 Cassia occidentalis, T4184 Mentha haplocalyx [Syn. Mentha canadaensis; Mentha arvensis var. haplocalyx; Mentha arvensis], T6373 Thalictrum alpinum.

headache and sore pharynx T4413 Nepeta cataria.

headache due to externally contracted wind-cold T3822 Ligusticum jeholense, $\mathrm{T} 3824$ Ligusticum sinense.

headache due to externally contracted wind-heat T0049 Acer ginnala, T4976 Piptanthus nepalensis, T5119 Polygonum thunbergii, T5312 Pueraria edulis, T5313 Pueraria lobata [Syn. Pueraria thunbergiana; Pueraria pseudohirsuta], T5316 Pueraria omeiensis, T5318 Pueraria phaseoloides, T5320 Pueraria thomsonii.

headache due to febrile disease T1045 Bubalus bubalis.

headache without sweating T2367 Ephedra equisetina, T2368 Ephedra gerardiana, T2369 Ephedra intermedia, T2372 Ephedra likiangensis, T2373 Ephedra minuta, T2375 Ephedra monosperma, T2379 Ephedra saxatilis, T2380 Ephedra sinica, T4304 Mosla chinensis [Syn. Orthodon chinensis], T4445 Notopterygium forbesii [Syn. Notopterygium franchetii], T4446 Notopterygium incisum.

heart disease T1452 Cissampelos pareira, T1453 Cissampelos pareira var. hirsute, T3394 Ilex paraguariensis.

heart palpitation T5661 Salvia bowleyana.

heartache T1182 Capra hircus; Ovis aries.

heart-spleen vacuity $\mathrm{T} 3743$ Lentinus lepideus.

heat bind and accumulation T1229 Cassia acutifolia, T1230 Cassia angustifolia.

heat bind constipation T5469 Rheum hotaoense, T5607 Rumex crispus, T6139 Sterculia lychnophora.

heat bind in bladder T5002 Plantago asiatica, T5004 Plantago depressa, T5007 Plantago major.

heat blood strangury T0979 Bombyx mori.

heat cough T4846 Physalis alkekengi var. franchetii.

heat diarrhea T0853 Baeckea frutescens, T4616 Pandanus tectorius, T5099 Polygonum bistorta.

heat dysentery T0696 Artemisia sieversiana, T2221 Diospyros kaki, T2766 Fraxinus bungeana, T2767 Fraxinus chinensis, T2774 Fraxinus paxiana, T2777 Fraxinus rhynchophylla [Syn. Fraxinus chinensis var. rhynchophylla], T2779 Fraxinus stylosa, T2780 Fraxinus szaboana [Syn. Fraxinus chinensis var. acuminata], T3476 Isatis indigotica, T3865 Lippia nodiflora, T5121 Polygonum tinctorium, T5312 Pueraria edulis, T5313 Pueraria lobata [Syn. Pueraria thunbergiana; Pueraria pseudohirsuta], T5316 Pueraria omeiensis, T5318 Pueraria phaseoloides, T5320 Pueraria thomsonii, T5605 Rumex acetosa.

heat entering construction-blood T5680 Salvia miltiorrhiza, T5681 Salvia miltiorrhiza f. alba, T5688 Salvia przewalskii, T5826 Scrophularia buergeriana, T5828 Scrophularia ningpoensis.

heat entering pericardium T4401 Nelumbo nucifera.

heat impediment T4799 Philydrum lanuginosum.

heat impediment swelling and pain T4124 Matricaria chamomilla [Syn. Matricaria recutita], T5680 Salvia miltiorrhiza, T5681 Salvia miltiorrhiza f. alba, T5688 Salvia przewalskii.

heat inpediment T2056 Debregeasia longifolia.

heat pain in stomach duct and abdomen T6463 Tinospora capillipes, T6467 Tinospora sagittata. heat sore $\mathrm{T} 2840$ Gallus gallus domesticus.

heat strangury T0073 Achyranthes bidentata, T0264 Ajuga forrestii, T0297 Alchornea trewioides, T0721 Arundina chinensis, T0722 Arundo donax, T0736 Asclepias curassavica, T0853 Baeckea frutescens, T0906 Berberis julianae, T1001 Brachystemma calycinum, T1251 Cassytha filiformis, T1526 Cladonia rangiferina, T1831 Crotalaria sessiliflora, T1950 Cynanchum ascyrifolium, T1951 Cynanchum atratum, T1962 Cynanchum versicolor, T2134 Desmodium styracifolium, T2143 Dianthus chinensis, T2145 Dianthus superbus, T2147 Dianthus versicolor, T2325 Eichhornia crassipes, T2628 Euphrasia officinalis, T2950 Gerbera anandria [Syn. Leibnitzia anandria], T2952 Gerbera piloselloides, T2973 Glechoma longituba, T2974 Glechoma lungituba, T3284 Houttuynia cordata, T3288 Humulus japonicus [Syn. Humulus scandens], T3528 Isodon ternifolia, T3529 Isodon ternifolius, T3953 Lycianthes biflora, T3990 Lygodium japonicum, T3998 Lysimachia christinae, T4121 Marsilea quadrifolia, T4252 Mirabilis jalapa, T4369 Nandina domestica, T4370 Nandina domestica, T4456 Nymphoides peltatum, T4800 Phlegmariurus fordii, T4840 Phyllanthus niruri, T4848 Physalis angulata, T4854 Physalis pubescens, T5098 Polygonum aviculare, T5354 Pyrrosia davidii, T5355 Pyrrosia drakeana, T5356 Pyrrosia gralla, T5357 Pyrrosia lingua' T5358 Pyrrosia petiolosa, T5360 Pyrrosia sheareri, T5838 Scutellaria galericulata, T5857 Sedum sarmentosum, T5954 Silene fortunei, T6024 Solidago virgaurea var. leiocarpa [Syn. Solidago decurrens], T6095 Stachytarpheta jamaicensis, T6582 Tylophora ovata, T6709 Verbena officinalis.

heat strangury with inhibited pain T0303 Alisma orientale [Syn. Alisma plantago-aquatica var. orientale], T0632 Aristolochia manshuriensis, T1633 Commelina communis, T2756 Forsythia suspensa, T3126 Hedyotis corymbosa [Syn. Oldenlandia corymbosa], T3416 Imperata cylindrica var. major, T4485 Oldenlandia diffusa [Syn. Hedyotis diffusa], T4829 Phragmites communis, T6226 Swertia mileensis, T6301 Taraxacum mongolicum.

heat toxin T2884 Gardenia jasminoides var. grandiflora.

heat toxin and phlegm-fire stasis T0892 Belamcanda chinensis.

heat toxin blood dysentery T2193 Dioscorea cirrhosa [Syn. Dioscorea pogonoides], T3908 Lonicera bournei, T3909 Lonicera confusa, T3910 Lonicera fulvotomentosa, T3911 Lonicera hypoglauca, T3912 Lonicera japonica, T3913 Lonicera japonica, T3914 Lonicera macranthoides, T3918 Lonicera similis, T5173 Portulaca oleracea.

heat toxin diarrhea dysentery T1544 Cleistocalyx operculatus.

heat toxin dysentery T0511 Annona reticulata, T1554 Clerodendron cyrtophyllum, T6367 Teucrium quadrifarium.

heat toxin macula T1425 Cimicifuga simplex, T2280 Dryopteris chrysocoma.

heat toxin scab and lai T1538 Clausena lansium.

heat toxin sore and welling abscess T2293 Dumortiera hirsuta, T5836 Scutellaria discolor.

heat toxin sores T0906 Berberis julianae, T1662 Coptis chinensis, T1663 Coptis chinensis var. brevisepala, T1664 Coptis deltoidea, T1669 Coptis omeiensis, T1670 Coptis teetoides [Syn. Coptis teeta], T3284 Houttuynia cordata, T3288 Humulus japonicus [Syn. Humulus scandens], T3957 Lycium chinense, T5018 Pleuropterus ciliinervis, 
T5388 Rabdosia adenantha, T5626 Ruta graveolens.

heat toxin swollen welling abscess T0161 Actinidia eriantha, T0902 Berberis diaphana, T0920 Berberis wilsonae, T1004 Brasenia schreberi, T3388 Ilex chinensis [Syn. Ilex purpurea], T4803 Phlogacanthus curviflorus, T5835 Scutellaria barbata [Syn. Scutellaria rivularis], T5851 Sedum alfredii [Syn. Sedum formosanum], T5852 Sedum bulbiferum, T5856 Sedum lineare [Syn. Sedum obtuso-lineare], T6007 Solanum melongena, T6119 Stephania cepharantha.

heat vexation T0158 Actinidia chinensis, T2222 Diospyros lotus, T5227 Prunus mume, T5642 Saccharum sinensis, T6544 Triticum aestivum [Syn. Triticum vulgare].

heat vexation and disquiet T1390 Chrysanthemum coronarium, T1397 Chrysanthemum segetum, $\mathrm{T} 1526$ Cladonia rangiferina.

heat vexation and insomnia T4555 Ostrea rivularis; Ostrea talienwhanensis; Ostrea gigas.

heat vexation and thirst T2221 Diospyros kaki, T2433 Eriobotrya japonica, T4834 Phyllanthus emblica, T6794 Vitis amurensis.

heat vexation in chest T0273 Akebia quinata, T0277 Akebia trifoliata, T0279 Akebia trifoliata var. australis, T1471 Citrus chachiensis, T1473 Citrus cultivars, T1474 Citrus decumana, T1516 Citrus tankan, T1518 Citrus unshiu.

heat wind in heart and diaphragm T1232 Cassia fistula.

heaven-borne sore T1316 Cephalanthus occidentalis, T4848 Physalis angulata, T4851 Physalis minima, T4852 Physalis peruviana, T4854 Physalis pubescens, T6745 Vicia faba.

heavy dreams and amnesia T0463 Anemone altaica.

heavy head and generalized pain T0819 Atractylodes chinensis, T0821 Atractylodes japonica, T0823 Atractylodes lancea.

\section{heavy head and low food intake T4577 Paederia scandens.}

hematochezia T0226 Agave sisalana, T0248 Agrimonia pilosa, T0661 Artemisia anomala, T0678 Artemisia japonica, T0791 Astragalus chrysopterus, T0794 Astragalus ernestii, T0798 Astragalus membranaceus, T0800 Astragalus mongholicus, T0955 Bletilla striata, T0962 Boehmeria platanifolia [Syn. Boehmeria tricuspis], T0976 Bombyx mori, T0991 Bos taurus domesticus; Bubalus bubalis, T1033 Broussonetia papyrifera, T1145 Camellia japonica, T1449 Cirsium japonicum, T1451 Cirsium setosum [Syn. Cerratula setosa; Cirsium segetum; Cephalanoplos segetum], T1655 Conyza blinii, T1684 Cordyline strcta, T1928 Cycas revoluta, T2193 Dioscorea cirrhosa [Syn. Dioscorea pogonoides], T2221 Diospyros kaki, T2279 Dryopteris championii, T2281 Dryopteris crassirhizoma, T2363 Entada phaseoloides [Syn. Lens phaseoloides], T2407 Equisetum arvense, T2420 Ericerus pela, T2591 Euphorbia humifusa, T2691 Farfugium japonicum, T3054 Gossypium barbadense], T3055 Gossypium herbaceum, T3058 Gossypium hirsutum [Syn. Gossypium mexicanum], T3088 Gynura segetum [Syn. Gynura japonica], T3129 Hedysarum polybotrys, T3193 Hemerocallis fulva, T3195 Hemerocallis lilio-asphodelus, T3197 Hemerocallis minor, T3334 Hypecoum leptocarpum, T3340 Hypericum ascyron, T3349 Hypericum erectum, T3444 Ipomoea aquatica [Syn. Convolvulus repens; Ipomoea reptans], T3460 Iris halophila, T3461 Iris japonica, T3464 Iris lactea var. chinensis [Syn. Iris pallasii var. chinensis], T3736 Lemmaphyllum microphyllum, T3769 Lespedeza bicolor, T3926 Loropetalum chinense, T4006 Lythrum anceps, T4007 Lythrum salicaria, T4259 Mnium cuspidatum, T4361 Myrsine africana, T4398 Nelumbo nucifera, T4502 Onychium japonicum [Syn. Tricomanes japonicum], T4510 Ophiorrhiza japonica, T4513 Ophiorrhiza mungos, T4549 Osbeckia chinensis, T4552 Osmunda japonica, T4604 Panax japonicus var. bipinnatifidus, T4605 Panax japonicus var. major, T4607 Panax pseudo-ginseng var. japonicus, T4608 Panax pseudo-ginseng var. notoginseng [Syn. Panax notoginseng], T4629 Papaver nudicaule, T4630 Papaver nudicaule ssp. amurense, T5104 Polygonum hydropiper, T5118 Polygonum suffultum, T5133 Polytrichum commune, T5142 Poncirus trifoliata, T5173 Portulaca oleracea, T5228 Prunus mume, T5288 Pteris cretica var. nervosa [Syn. Pteris nervosa], T5295 Pteris multifida, T5328 Punica granatum, T5372 Quercus dentata, T5374 Quercus infectoria, T5419 Raphanus sativus, T5449 Reineckea carnea, T5468 Rheum emodi [Syn. Rheum australe], T5518 Rhododendron mucronatum, T5529 Rhodomyrtus tomentosa, T5530 Rhus chinensis [Syn. Rhus semialata], T5541 Rhus verniciflua [Syn. Toxicadendron verniciflum], T5554 Rodgersia aesculifolia, T5594 Rubus parkeri, T5605 Rumex acetosa, T5607 Rumex crispus, T5614 Rumex patientia, T5673 Salvia glutinosa, T5712 Sanguisorba officinalis, T5804 Schizonepeta tenuifolia [Syn. Nepeta tenuifolia], T5840 Scutellaria indica, T5850 Sedum aizoon, T5855 Sedum kamtschaticum, T5856 Sedum lineare [Syn. Sedum obtuso-lineare], T5862 Selaginella involvens, T5865 Selaginella pulvinata, T5869 Selaginella tamariscina, T5870 Selaginella uncinata, T6034 Sophora japonica, T6046 Sophora viciifolia, T6047 Sophora viciifolia, T6048 Sophora viciifolia, T6076 Spinacia oleracea, T6116 Stenoloma chusanum, T6251 Symplocos caudata, T6346 Terminalia chebula, T6348 Terminalia chebula var. tomentella, T6367 Teucrium quadrifarium, T6440 Thuja orientalis [Syn. Platycladus orientalis; Biota orientalis], T6485 Trachycarpus fortunei, T6584 Typha angustata, T6585 Typha angustifolia, T6587 Typha latifolia, T6615 Uncaria gambir, T6752 Vigna angularis [Syn. Dolichus angularis; Phaseolus angularis], T6764 Vinegar, T6904 Zephyranthes grandiflora [Syn. Zephyranthes carinata].

hematochezia due to blood heat T5445 Rehmannia glutinosa [Syn. Rehmannia glutinosa f. huechingensis], T5447 Rehmannia glutinosa [Syn. Rehmannia glutinosa f. huechingensis].

hematochezia from hemorrhoids T3192 Hemerocallis citrina, T3928 Lotus corniculatus, T4148 Medicago sativa.

hematosepsis T5907 Senecio scandens [Syn. Senecio chinensis].

hematuria T0248 Agrimonia pilosa, T0297 Alchornea trewioides, T0322 Allium tuberosum, T0379 Alternanthera philoxeroides, T0635 Aristolochia moupinensis, T0648 Arnebia euchroma, T0649 Arnebia guttata, T0661 Artemisia anomala, T0962 Boehmeria platanifolia [Syn. Boehmeria tricuspis], T0976 Bombyx mori, T0993 Boschniakia rossica, T1184 Capsella bursa-pastoris, T1199 Carduus acanthoides, T1200 Carduus crispus, T1275 Cayratia japonica, T1449 Cirsium japonicum, T1451 Cirsium setosum [Syn. Cerratula setosa; Cirsium segetum; Cephalanoplos segetum], T1684 Cordyline strcta, T1924 Cyathula officinalis, T2193 Dioscorea cirrhosa [Syn. Dioscorea pogonoides], T2323 Eclipta prostrata [Syn. Eclipta alba], T2412 Equisetum 
sylvaticum, T2420 Ericerus pela, T2590 Euphorbia hirta, T2591 Euphorbia humifusa, T2691 Farfugium japonicum, T2830 Galium aparine, T3295 Huperzia serrata [Syn. Lycopodium serratum], T3340 Hypericum ascyron, T3416 Imperata cylindrica var. major, T3444 Ipomoea aquatica [Syn. Convolvulus repens; Ipomoea reptans], T3662 Lactuca sativa, T3736 Lemmaphyllum microphyllum, T3737 Lemmaphyllum microphyllum var. obovatum, T3764 Lepisorus thunbergianus, T3769 Lespedeza bicolor, T3881 Lithospermum erythrorhizon, T4082 Mallotus japonicus, T4121 Marsilea quadrifolia, T4127 Matteuccia struthiopteris, T4248 Mimosa pudica, T4370 Nandina domestica, T4502 Onychium japonicum [Syn. Tricomanes japonicum], T4564 Oxalis corniculata [Syn. Oxalis repens], T4604 Panax japonicus var. bipinnatifidus, T4605 Panax japonicus var. major, T4607 Panax pseudo-ginseng var. japonicus, T5097 Polygonum amphibium, T5228 Prunus mume, T5288 Pteris cretica var. nervosa [Syn. Pteris nervosa], T5295 Pteris multifida, T5354 Pyrrosia davidii, T5355 Pyrrosia drakeana, T5356 Pyrrosia gralla, T5357 Pyrrosia lingua T5358 Pyrrosia petiolosa, T5360 Pyrrosia sheareri, T5541 Rhus verniciflua [Syn. Toxicadendron verniciflum], T5685 Salvia plebeia, T5712 Sanguisorba officinalis, T5850 Sedum aizoon, T5855 Sedum kamtschaticum, T5865 Selaginella pulvinata, T5869 Selaginella tamariscina, T6034 Sophora japonica, T6046 Sophora viciifolia, T6047 Sophora viciifolia, T6116 Stenoloma chusanum, T6440 Thuja orientalis [Syn. Platycladus orientalis; Biota orientalis], T6485 Trachycarpus fortunei, T6584 Typha angustata, T6585 Typha angustifolia, T6587 Typha latifolia, T6615 Uncaria gambir, T6745 Vicia faba, T6814 Wedelia chinensis [Syn. Solidago chinensis; Wedelia calendulacea].

hematuria due to blood heat T5445 Rehmannia glutinosa [Syn. Rehmannia glutinosa f. huechingensis].

hemicrania [ = hemilateral headache] T6618 Uncaria homomalla [Syn. Uruparia homomalla; Uruparia tonkinensis; Uruparia lanosa var. parvifora].

hemiplegia T0077 Aconitum balfourii, T0084 Aconitum carmichaeli, T0105 Aconitum karakolicum, T0298 Alectoria vivens, T0617 Arisaema amurense, T0618 Arisaema consanguineum, T0620 Arisaema heterophyllum, T0877 Bauhinia championii, T1084 Buthus martensi, T1191 Caragana sinica, T1562 Clerodendron trichotomum, T1567 Clerodendrum mandarinorum, T1715 Corydalis decumbens [Syn. Corydalis amabilis], T1767 Craibiodendron yunnanese, T2449 Erycibe obtusifolia, T2450 Erycibe schmidtii, T2540 Euonymus fortunei, T2890 Gastrodia elata, T2995 Gloriosa superba, T3657 Lactarius vellereus, T3675 Lagopsis supina, T3680 Lamium amplexicaule, T4903 Pinellia pedatisecta, T5298 Pteris vittata, T5401 Rabdosia yuennanensis, T5746 Sassafras tzumu, T5950 Siegesbeckia orientalis, T5951 Siegesbeckia orientalis var. glabrescens [Syn. Siegesbeckia glabrescens], T5952 Siegesbeckia orientalis var. pubescens [Syn. Siegesbeckia pubescens], T6166 Strychnos angustiflora, T6184 Strychnos nux-vomica, T6193 Strychnos wallichiana, T6540 Tripterygium hypoglaucum.

hemoptysis T0229 Ageratum conyzoides, T0248 Agrimonia pilosa, T0249 Agrimonia pilosa var. japonica, T0379 Alternanthera philoxeroides, T0678 Artemisia japonica, T0753 Asparagus setaceus [Syn. Asparagus plumosus], T0830 Auricularia auricula, T0831 Auricularia delicata, T0859 Balanophora involucrata, T0923 Bergenia crassifolia, T0924 Bergenia purpurascens, T0941 Bidens tripartita, T0948 Blainvillea acmella [Syn. Verbesina acmella; Eclipta latifolia; Blainvillea latifolia], T0955 Bletilla striata, T0962 Boehmeria platanifolia [Syn. Boehmeria tricuspis], T0964 Boenninghausenia albiflora, T0973 Bombax malabaricum [Syn. Gossampinus malabarica], T1118 Callicarpa formosana, T1119 Callicarpa japonica, T1120 Callicarpa macrophylla, T1145 Camellia japonica, T1184 Capsella bursa-pastoris, T1251 Cassytha filiformis, T1465 Citrullus vulgaris [Syn. Citrullus lanatus], T1547 Clematis terniflora [Syn. Clematis maximowicziana], T1969 Cynoglossum officinale, T2191 Dioscorea bulbifera, T2193 Dioscorea cirrhosa [Syn. Dioscorea pogonoides], T2281 Dryopteris crassirhizoma, T2323 Eclipta prostrata [Syn. Eclipta alba], T2407 Equisetum arvense, T2412 Equisetum sylvaticum, T2540 Euonymus fortunei, T2591 Euphorbia humifusa, T2659 Fagopyrum esculentum, T3088 Gynura segetum [Syn. Gynura japonica], T3242 Hibiscus rosa-sinensis, T3244 Hibiscus syriacus, T3279 Homo sapiens, T3340 Hypericum ascyron, T3349 Hypericum erectum, T3361 Hypericum perforatum, T3363 Hypericum sampsonii, T3373 Hypoestes purpurea [Syn. Justicia purpurea; Hypoestes sinica], T3736 Lemmaphyllum microphyllum, T3829 Ligustrum robustum, T3866 Liquidambar formosana [Syn. Liquidambar taiwaniana], T3926 Loropetalum chinense, T3983 Lycoris aurea, T4060 Mahonia confusa, T4064 Mahonia gracilipes, T4549 Osbeckia chinensis, T4604 Panax japonicus var. bipinnatifidus, T4605 Panax japonicus var. major, T4607 Panax pseudo-ginseng var. japonicus, T4608 Panax pseudo-ginseng var. notoginseng [Syn. Panax notoginseng], T4720 Perilla frutescens var. arguta, T4723 Perilla frutescens var. crispa, T5043 Podocarpus macrophyllus, T5045 Podocarpus macrophyllus var. maki, T5133 Polytrichum commune, T5374 Quercus infectoria, T5419 Raphanus sativus, T5449 Reineckea carnea, T5494 Rhodiola crenulata [Syn. Rhodiola euryphylla], T5499 Rhodiola sacra, T5552 Robinia pseudoacacia, T5557 Rohdea japonica [Syn. Orontium japonicum], T5673 Salvia glutinosa, T5685 Salvia plebeia, T5712 Sanguisorba officinalis, T5840 Scutellaria indica, T5850 Sedum aizoon, T5855 Sedum kamtschaticum, T5862 Selaginella involvens, T6092 Stachys palustris, T6440 Thuja orientalis [Syn. Platycladus orientalis; Biota orientalis], T6482 Toxicodendron succedaneum [Syn. Rhus succedanea], T6584 Typha angustata, T6585 Typha angustifolia, T6587 Typha latifolia, T6615 Uncaria gambir, T6745 Vicia faba, T6772 Viscum angulatum, T6895 Zanthoxylum simulans.

hemorrhagic fever T0379 Alternanthera philoxeroides.

hemorrhoids T0158 Actinidia chinensis, T0226 Agave sisalana, T0293 Albizzia lebbeck, T0322 Allium tuberosum, T0584 Arctium lappa, T0805 Astragalus sinicus, T0827 Aucuba chinensis ssp. omeiensis, T0860 Balanophora japonica, T0942 Biebersteinia heterostemon, T0984 Bos taurus domesticus; Bubalus bubalis, T1448 Cirsium chinense, T1556 Clerodendron fragrans, T1562 Clerodendron trichotomum, T1565 Clerodendrum bungei, T1623 Collybia albuminosa, T1687 Coriandrum sativum, T1799 Crinum latifolium, T1881 Cucurbita moschata, T1896 Cupressus funebris, T1994 Daemonorops draco, T2024 Daphne genkwa, T2062 Delphinium 
bonvalotii, T2081 Delphinium omeiense, T2084 Delphinium potaninii, T2085 Delphinium potaninii var. juufengshanense, T2218 Diospyros kaki, T2253 Dracaena cochinchinensis, T2363 Entada phaseoloides [Syn. Lens phaseoloides], T2525 Eucheuma muricatum, T2627 Euphoria longan [Syn. Dimocarpus longan], T2716 Ficus beecheyana [Syn. Ficus erecta var. beecheyana], T2718 Ficus carica, T2731 Firmiana simplex, T2732 Firmiana simplex, T2883 Gardenia jasminoides [Syn. Gardenia florida], T2994 Gloiopeltis furcata, T3163 Helicteres angustifolia, T3245 Hibiscus syriacus, T3320 Hymenocallis littoralis [Syn. Hymenocallis americana; Pancratium littoralis], T3444 Ipomoea aquatica [Syn. Convolvulus repens; Ipomoea reptans], T3460 Iris halophila, T3464 Iris lactea var. chinensis [Syn. Iris pallasii var. chinensis], T3479 Isodon amethystoides, T3845 Linaria vulgaris, T3932 Ludwigia octovalvis, T4020 Macleaya cordata, T4331 Musa paradisiaca var. sapientum [Syn. Musa sapientum], T4544 Oryza sativa, T4548 Oryza sativa var. glutinosa, T4564 Oxalis corniculata [Syn. Oxalis repens], T4607 Panax pseudo-ginseng var. japonicus, T4789 Phellodendron amurense, T4790 Phellodendron amurense var. wilsonii, T4791 Phellodendron chinense, T4792 Phellodendron chinense var. glabriusculum, T4836 Phyllanthus emblica, T4884 Picria felterrae, T4887 Picrorhiza kurrooa, T4888 Picrorhiza scrophulariiflora, T4917 Pinus massoniana, T5107 Polygonum multiflorum, T5142 Poncirus trifoliata, T5230 Prunus persica, T5375 Quercus mongolica, T5563 Rosa cymosa, T5606 Rumex acetosa, T5773 Saxifraga stolonifera, T5862 Selaginella involvens, T5925 Sesamum indicum [Syn. Sesamum orientale], T5942 Sida acuta, T6025 Sonchus arvensis, T6035 Sophora japonica, T6076 Spinacia oleracea, T6095 Stachytarpheta jamaicensis, T6264 Syzygium buxifolium, T6432 Thespesia populnea [Syn. Hibiscus populneus], T6432 Thespesia populnea [Syn. Hibiscus populneus], T6814 Wedelia chinensis [Syn. Solidago chinensis; Wedelia calendulacea], T6843 Xanthium sibiricum [Syn. Xanthium strumarium].

hemorrhoids and fistulas T0427 Ampelopsis japonica [Syn. Paullinia japonica], T0500 Anguilla japonica, T1210 Carpesium abrotanoides, T1550 Cleome gynandra [Syn. Gynandropsis gynandra], T2092 Delphinus delphis, T2161 Dicranopteris pedata [Syn. Polypodium pedatum; Dicranopteris dichotoma], T3202 Hemistepta lyrata [Syn. Hemistepta carthamoides; Saussurea carthamoides], T3935 Luffa cylindrica, T3984 Lycoris chinensis, T3986 Lycoris radiata [Syn. Amaryllis radiata], T4265 Momordica cochinchinensis, T5111 Polygonum perfoliatum, T5870 Selaginella uncinata, T6105 Stellera chamaejasme, T6601 Umbilicaria esculenta [Syn. Gyrophora esculenta].

hemorrhoids and fistulas ${ }^{[509]}$ T6139 Sterculia lychnophora.

hemorrhoids and swollen welling abscess T6615 Uncaria gambir.

hepatic disease and anemia (leaf) T3110 Harungana madagascariensis.

hepatitis T0011 Abrus precatorius, T0137 Aconitum tanguticum, T0155 Actinidia arguta, T0423 Ampelopsis brevipedunculata, T0495 Angelica sinensis, T0572 Aralia elata, T0598 Ardisia japonica, T0646 Armillariella tabescens, T0672 Artemisia capillaris, T0695 Artemisia scoparia [Syn. Artemisia capillaris var. scoparia], T0707 Arthraxon hispidus, T0751 Asparagus officinalis, T0881 Bauhinia variegata, T0964 Boenninghausenia albiflora, T1055 Bupleurum angustissimum,
T1056 Bupleurum aureum, T1058 Bupleurum chaishoui, T1059 Bupleurum chinense, T1065 Bupleurum longiradiatum, T1066 Bupleurum marginatum, T1067 Bupleurum marginatum var. stenophyllum, T1072 Bupleurum scorzonerifolium, T1073 Bupleurum sibiricum, T1075 Bupleurum smithii var. parvifolium, T1078 Bupleurum wenchuanense, T1079 Bupleurum yinchowense, T1151 Camellia sinensis [Syn. Thea sinensis], T1387 Chrysanthemum boreale, T1392 Chrysanthemum indicum, T1394 Chrysanthemum lavandulifolium, T1573 Clinopodium chinense, T1657 Conyza canadensis [Syn. Erigeron canadensis], T1662 Coptis chinensis, T1663 Coptis chinensis var. brevisepala, T1664 Coptis deltoidea, T1669 Coptis omeiensis, T1670 Coptis teetoides [Syn. Coptis teeta], T1696 Cornus capitata [Syn. Dendrobenthamia capitata], T1746 Corydalis taliensis, T1747 Corydalis thalictrifolia, T1967 Cynoglossum amabile, T1986 Cyrtomium fortunei, T2340 Elsholtzia bodinieri, T2601 Euphorbia milii, T2657 Fagopyrum cymosum [Syn. Polygonum cymosum], T2727 Ficus simplicissima, T2756 Forsythia suspensa, T2835 Galium verum, T2840 Gallus gallus domesticus, T2846 Ganoderma japonicum [Syn. Ganoderma sinense], T2848 Ganoderma lucidum, T2941 Gentianopsis paludosa, T3092 Halenia corniculata, T3113 Hedera nepalensis var. sinensis, T3115 Hedera rhombea, T3122 Hedyotis acutangula, T3203 Hemsleya amabilis, T3208 Hemsleya macrosperma, T3332 Hypecoum erectum, T3334 Hypecoum leptocarpum, T3340 Hypericum ascyron, T3342 Hypericum bellum, T3360 Hypericum patulum, T3457 Iris dichotoma, T3461 Iris japonica, T3471 Iris tectorum, T3475 Isatis indigotica, T3476 Isatis indigotica, T3496 Isodon japonica [Syn. Rabdosia japonica], T3990 Lygodium japonicum, T4028 Maesa indica, T4082 Mallotus japonicus, T4145 Meconopsis punicea, T4248 Mimosa pudica, T4804 Phlojodicarpus sibiricus, T4842 Phyllanthus reticulatus, T5071 Polygala arillata, T5100 Polygonum chinense, T5101 Polygonum cuspidatum, T5156 Populus nigra var. thevestina, T5161 Populus tomentosa, T5185 Potentilla multifida, T5207 Prismatomeris tetrandra, T5587 Rubus alceaefolius, T5614 Rumex patientia, T5680 Salvia miltiorrhiza, T5681 Salvia miltiorrhiza f. alba, T5688 Salvia przewalskii, T5690 Salvia roborowskii, T5838 Scutellaria galericulata, T5844 Scutellaria scordifolia, T5860 Selaginella davidii, T5892 Senecio nemorensis, T6046 Sophora viciifolia, T6047 Sophora viciifolia, T6116 Stenoloma chusanum, T6214 Swertia chinensis, T6227 Swertia mussotii, T6264 Syzygium buxifolium, T6275 Tacca chantrieri [Syn. Tacca minor; Tacca esquirolii], T6301 Taraxacum mongolicum, T6656 Ustilago maydis.

hepatitis a T3011 Glycyrrhiza aspera, T3013 Glycyrrhiza glabra, T3015 Glycyrrhiza inflata, T3016 Glycyrrhiza kansuensis, T3021 Glycyrrhiza squamulosa, T3022 Glycyrrhiza uralensis, T3023 Glycyrrhiza yunnanensis, T5543 Rhynchotechum vestitum.

hepatitis b T5543 Rhynchotechum vestitum.

hepatitis with rib-side pain T5687 Salvia prionitis.

herpes zoster T5856 Sedum lineare [Syn. Sedum obtuso-lineare].

hiccough T2217 Diospyros kaki, T3758 Lepidium sativum, T3876 Litchi chinensis, T6150 Streptopelia orientalis, T6263 Syzygium aromaticum [Syn. Eugenia caryophyllata].

hoarseness T4665 Passiflora edulis. 
hordeolum T1633 Commelina communis.

hydatid moles T6043 Sophora subprostrata [Syn. Sophora tonkinensis]. hydrothorax T2023 Daphne genkwa.

hyperchlorhydria T2644 Evodia rutaecarpa, T2646 Evodia rutaecarpa var. officinalis, T3880 Lithospermum arvense, T4306 Mosla grosseserrata, T5419 Raphanus sativus.

hypercholesterolemia T0544 Apium graveolens, T2964 Ginkgo biloba.

hyperlipemia T0303 Alisma orientale [Syn. Alisma plantago-aquatica var. orientale], T0311 Allium cepa, T0672 Artemisia capillaris, T0695 Artemisia scoparia [Syn. Artemisia capillaris var. scoparia], T0751 Asparagus officinalis, T1775 Crataegus pinnatifida, T1778 Crataegus pinnatifida var. major, T2731 Firmiana simplex, T2732 Firmiana simplex, T2733 Firmiana simplex, T2846 Ganoderma japonicum [Syn. Ganoderma sinense], T2848 Ganoderma lucidum, T3085 Gynostemma pentaphyllum, T3742 Lentinus edodes, T5101 Polygonum cuspidatum, T5107 Polygonum multiflorum, T6528 Trigonella foenum-graecum, T6584 Typha angustata, T6585 Typha angustifolia, T6587 Typha latifolia.

hyperplasia of mammary glands T0751 Asparagus officinalis, T5214 Prunella vulgaris.

hypertension T0210 Agaricus bisporus, T0211 Agaricus campestris, T0428 Ampelopsis megalophylla, T0539 Apis cerana, T0541 Apis cerana, T0544 Apium graveolens, T0553 Apocynum venetum, T0598 Ardisia japonica, T0608 Arenaria kansuensis [Syn. Arenaria kumaonensis], T0625 Aristolochia contorta, T0627 Aristolochia debilis [Syn. Aristolochia longa], T0631 Aristolochia kaempferi, T0644 Armillaria mellea, T0830 Auricularia auricula, T0831 Auricularia delicata, T1084 Buthus martensi, T1150 Camellia sinensis [Syn. Thea sinensis], T1184 Capsella bursa-pastoris, T1190 Caragana jubata, T1191 Caragana sinica, T1241 Cassia occidentalis, T1271 Catharanthus roseus [Syn. Vinca rosea; Lochera rosea], T1296 Celosia argentea, T1311 Centella asiatica, T1385 Chorda filum, T1387 Chrysanthemum boreale, T1392 Chrysanthemum indicum, T1393 Chrysanthemum indicum, T1394 Chrysanthemum lavandulifolium, T1395 Chrysanthemum morifolium [Syn. Dendranthema morifolium], T1417 Cimicifuga acerina, T1562 Clerodendron trichotomum, T1563 Clerodendron trichotomum, T1565 Clerodendrum bungei, T1586 Cocculus laurifolius, T1662 Coptis chinensis, T1663 Coptis chinensis var. brevisepala, T1664 Coptis deltoidea, T1669 Coptis omeiensis, T1670 Coptis teetoides [Syn. Coptis teeta], T1715 Corydalis decumbens [Syn. Corydalis amabilis], T1776 Crataegus pinnatifida, T1777 Crataegus pinnatifida, T1927 Cycas revoluta, T2115 Dermatocarpon minitum, T2219 Diospyros kaki, T2442 Ervatamia divaricata, T2444 Ervatamia heyneana, T2445 Ervatamia officinalis, T2530 Eucommia ulmoides, T2531 Eucommia ulmoides, T2565 Eupatorium lindleyanum, T2569 Eupatorium rebaudianum, T2659 Fagopyrum esculentum, T2729 Filipendula ulmaria, T2731 Firmiana simplex, T2732 Firmiana simplex, T2732 Firmiana simplex, T2890 Gastrodia elata, T3144 Helianthus annuus, T3146 Helianthus annuus cv, T3549 Ixora chinensis, T3742 Lentinus edodes, T3878 Lithocarpus polystachyus, T3925 Loranthus parasiticus [Syn. Loranthus chinenis; Taxillus chinensis], T3932 Ludwigia octovalvis, T4001 Lysimachia davurica, T4028 Maesa indica, T4039 Magnolia grandiflora, T4145
Meconopsis punicea, T4195 Menyanthes trifoliata, T4290 Morus alba, T4401 Nelumbo nucifera, T4438 Nostoc flagelliforme, T4516 Oplopanax elatus, T4582 Paeonia delavayi, T4585 Paeonia moutan [Syn. Paeonia suffruticosa], T4609 Panax pseudo-ginseng var. notoginseng [Syn. Panax notoginseng], T4796 Pheretima aspergillum; Allolobophora caliginosa trapezoides, T4837 Phyllanthus emblica, T4906 Pinus armandii, T4916 Pinus massoniana, T4976 Piptanthus nepalensis, T5214 Prunella vulgaris, T5312 Pueraria edulis, T5313 Pueraria lobata [Syn. Pueraria thunbergiana; Pueraria pseudohirsuta], T5316 Pueraria omeiensis, T5318 Pueraria phaseoloides, T5320 Pueraria thomsonii, T5365 Pyrus pashia, T5423 Rauvolfia verticillata, T5424 Rauvolfia verticillata, T5425 Rauvolfia verticillata $\mathrm{f}$. rubrocarpa, T5426 Rauvolfia verticillata var. hainanensis, T5427 Rauvolfia vomitoria, T5435 Rauwolfia latifrons, T5441 Rauwolfia perakensis, T5442 Rauwolfia serpentina, T5516 Rhododendron molle, T5657 Salsola collina, T5723 Sapium sebiferum, T5817 Scolopendra subspinipes mutilans, T5950 Siegesbeckia orientalis, T5951 Siegesbeckia orientalis var. glabrescens [Syn. Siegesbeckia glabrescens], T5952 Siegesbeckia orientalis var. pubescens [Syn. Siegesbeckia pubescens], T5969 Sium latifolium, T6136 Stephania tetrandra, T6478 Torreya jackii, T6531 Trillium camtschaticum, T6533 Trillium kamtschaticum, T6535 Trillium tschonoskii, T6599 Ulva lactuca, T6600 Ulva pertusa, T6618 Uncaria homomalla [Syn. Uruparia homomalla; Uruparia tonkinensis; Uruparia lanosa var. parvifora], T6651 Urtica cannabina, T6652 Urtica dioica, T6772 Viscum angulatum, T6775 Viscum coloratum, T6777 Viscum multinerve, T6803 Volvariella volvacea, T6901 Zea mays.

hypertension (bark) T3110 Harungana madagascariensis.

hypertension due to ascendant liver yang T5428 Rauvolfia yunnanensis. hypertension due to liver heat T2060 Delonix regia.

hypertonicity T6183 Strychnos nitida.

hypertonicity and numbness T2194 Dioscorea collettii, T2389 Epimedium acuminatum, T2390 Epimedium brevicornum, T2392 Epimedium davidii, T2394 Epimedium elongatum, T2398 Epimedium koreanum, T2401 Epimedium pubescens, T2402 Epimedium sagittatum, T2404 Epimedium sutchuenense, T2406 Epimedium wushanense, T2438 Erodium stephanianum, T2943 Geranium nepalense, T2944 Geranium pratense, T2947 Geranium sibiricum, T2949 Geranium wilfordii, T6843 Xanthium sibiricum [Syn. Xanthium strumarium].

hypertonicity and pain T0036 Acanthopanax giraldii [Syn. Acanthopanax giraldii var. inermis; Eleutherococcus giraldii].

hypertonicity in limb joints T2459 Erythrina arborescens, T2478 Erythrina variegata [Syn. Erythrina indica].

hypertonicity of limbs T0106 Aconitum kirinense, T0123 Aconitum polyschistum, T0138 Aconitum umbrosum, T0470 Anemone raddeana, T3867 Liquidambar formosana [Syn. Liquidambar taiwaniana], T5972 Skimmia reevesiana, T6791 Vitex rotundifolia [Syn. Vitex trifollia var. simplicifolia], T6793 Vitex trifolia.

hypertonicity of sinews and bones T0045 Acanthopanax trifoliatus, T0634 Aristolochia mollissima, T3653 Lactarius piperatus [Syn. Agaricus piperatus].

hypertonicity of sinews and vessels T1001 Brachystemma calycinum, T1342 Chaenomeles lagenaria [Syn. Chaenomeles speciosa], T1545 
Clematis chinensis, T2211 Dioscorea tenuipes, T2909 Gentiana crassicaulis, T2910 Gentiana dahurica, T2913 Gentiana kaufmanniana, T2919 Gentiana macrophylla, T2932 Gentiana siphonantha, T2934 Gentiana straminea, T2936 Gentiana tianschanica, T2937 Gentiana tibetica, T3869 Liquor, T4950 Piper kadsura [Syn. Piper futokadsura], T5021 Pleurospermum rivulorum, T6422 Thelephora vialis, T6484 Trachelospermum jasminoides, T6504 Tricholoma matsutake [Syn. Armillaria matsutake], T6742 Vicia amoena.

hypertrophic spinitis T6184 Strychnos nux-vomica, T6193 Strychnos wallichiana.

hypocorticosteroidism [ = Addison's disease] T3011 Glycyrrhiza aspera, T3013 Glycyrrhiza glabra, T3015 Glycyrrhiza inflata, T3016 Glycyrrhiza kansuensis, T3021 Glycyrrhiza squamulosa, T3022 Glycyrrhiza uralensis, T3023 Glycyrrhiza yunnanensis.

hypotension T4682 Pedicularis muscicola.

hypovitaminosis T6055 Sorbus tianschanica.

hypovitaminosis c T0155 Actinidia arguta.

hysteria T4386 Nardostachys chinensis, T4387 Nardostachys jatamansi.

icterohepatitis T0010 Abrus fruticulosus [Syn. Abrus cantoniensis], T0374 Alstonia scholaris, T0379 Alternanthera philoxeroides, T0426 Ampelopsis grossedentata [Syn. Ampelopsis cantoniesis var. grossedentata], T0678 Artemisia japonica, T1655 Conyza blinii, T1758 Cotinus coggygria, T2165 Dictamnus angustifolius, T2167 Dictamnus dasycarpus, T2327 Elaeagnus angustifolia, T2641 Evodia lepta [Syn. Ilex lepta], T6260 Syringa oblata, T6277 Tadehagi triquetrum.

ileitis T3106 Harpagophytum procumbens.

ileus T2999 Glycine max, T3164 Helicteres isora, T5472 Rheum officinale, T5474 Rheum palmatum, T5481 Rheum tanguticum.

ileus due to roundworm T0560 Arachis hypogaea.

impediment T4655 Paris tetraphylla, T4917 Pinus massoniana.

impediment pain T1008 Brassica juncea, T1046 Buddleja davidii, T1767 Craibiodendron yunnanese, T2038 Datura innoxia, T2042 Datura metel.

impediment pain in joints T1924 Cyathula officinalis, T3479 Isodon amethystoides, T3964 Lycopodium alpinum [Syn. Diphasiastrum alpinum], T5101 Polygonum cuspidatum, T5578 Rubia cordifolia, T5582 Rubia oncotricha, T5583 Rubia schumannina, T5584 Rubia tinctorum, T5585 Rubia wallichiana.

impediment pain in lumbus and knees T0515 Anredera cordifolia [Syn. Baussingaultia cordifolia; Baussingaultia gracilis f. pseudobaselloides; Baussingaultia gracilis var. pseudobaselloides], T2208 Dioscorea septemloba.

impediment pain in sinew and bone T5870 Selaginella uncinata.

impediment pain into network vessels T1001 Brachystemma calycinum.

impediment pain numbness T3852 Lindera megaphylla, T4303 Moschus moschiferus; Moschus berezovskii; Moschus sifanicus.

impotence T0083 Aconitum carmichaeli, T0085 Aconitum carmichaeli cv, T0298 Alectoria vivens, T0500 Anguilla japonica, T0990 Bos taurus domesticus; Bubalus bubalis, T1035 Broussonetia papyrifera, $\mathrm{T} 1170$
Canis familiaris, T1439 Cinnamomum cassia [Syn. Cinnamomum aromaticum], T1455 Cistanche deserticola, T1456 Cistanche salsa, T1582 Cnidium monnieri, T1604 Coeloglossum viride [Syn. Coeloglossum viride var. bracteatum], T1964 Cynanchum wilfordii [Syn. Cynoctonum wilfordii], T2301 Dysosma majorensis [Syn. Podophyllum majorense; Dysosma lichuanensis], T2805 Fugu ocellatus, T2955 Geum japonicum, T3077 Gymnadenia conopsea, T3279 Homo sapiens, T3568 Juglans regia, T4206 Metaplexis japonica, T4207 Metaplexis japonica, T4516 Oplopanax elatus, T4599 Panax ginseng [Syn. Panax schinseng], T4974 Piper wallichii [Syn. Piper wallichii var. hupehense], T4982 Pistacia vera, T5317 Pueraria peduncularis, T5697 Salvia trijuga, T5753 Saussurea gnaphaloides, T5755 Saussurea involucrata, T5757 Saussurea laniceps, T5759 Saussurea medusa, T5848 Securinega suffruticosa, T6184 Strychnos nux-vomica, T6193 Strychnos wallichiana, T6257 Syngnathus acus.

impotence and emission T0977 Bombyx mori, T1337 Cervus nippon; Cervus elaphus, T1683 Cordyceps sinensis, T1698 Cornus officinalis [Syn. Macrocarpium officinale], T1900 Curculigo capitulata [Syn. Leucojum capitulata], T1911 Cuscuta australis, T1912 Cuscuta chinensis, T1913 Cuscuta japonica, T1952 Cynanchum auriculatum, T1953 Cynanchum bungei, T2389 Epimedium acuminatum, T2390 Epimedium brevicornum, T2392 Epimedium davidii, T2394 Epimedium elongatum, T2398 Epimedium koreanum, T2401 Epimedium pubescens, T2402 Epimedium sagittatum, T2404 Epimedium sutchuenense, T2406 Epimedium wushanense, T2753 Formica fusca, T4283 Morinda officinalis, T5091 Polygonatum cyrtonema [Syn. Polygonatum multiflorum], T5092 Polygonatum kingianum, T5095 Polygonatum sibiricum, T5270 Psoralea corylifolia, T5446 Rehmannia glutinosa [Syn. Rehmannia glutinosa f. huechingensis].

impotence seminal cool T1901 Curculigo orchioides.

impotence with emission T4828 Photinia serrulata.

impotence with premature ejaculation T5593 Rubus idaeus.

improving cognitive function in elderly dementia patients T2961 Ginkgo biloba.

inability of legs and knees T0036 Acanthopanax giraldii [Syn. Acanthopanax giraldii var. inermis; Eleutherococcus giraldii], T1411 Cibotium barome [Syn. Polypodium barometz], T1963 Cynanchum wallichii.

inappetence T0041 Acanthopanax senticosus [Syn. Eleutherococcus senticosus], T0463 Anemone altaica, T0659 Artemisia absinthium, T0819 Atractylodes chinensis, T0821 Atractylodes japonica, T0823 Atractylodes lancea, T1278 Cedrela sinensis, T1415 Cichorium intybus, T1478 Citrus grandis, T1490 Citrus limon, T1494 Citrus limonia, T1608 Coffea arabica, T1610 Coffea liberica, T1660 Coprinus atramentarius, T1687 Coriandrum sativum, T1751 Corylus heterophylla, T1952 Cynanchum auriculatum, T1953 Cynanchum bungei, T1964 Cynanchum wilfordii [Syn. Cynoctonum wilfordii], T2717 Ficus carica, T2763 Fragaria ananassa, T3282 Hordeum vulgare, T3962 Lycopersicon esculentum, T4217 Microcos paniculata [Syn. Grewia microcos], T4400 Nelumbo nucifera, T4600 Panax ginseng [Syn. Panax schinseng], T4607 Panax pseudo-ginseng var. japonicus, T4957 Piper nigrum, T5067 Polyalthia nemoralis, T5071 Polygala arillata, T5226 Prunus mume, T5260 Pseudostellaria 
heterophylla, T5465 Rhaponticum carthamoides, T6285 Tamarindus indica, T6902 Zea mays.

inappetence due to glomus in stomach duct T2554 Eupatorium cannabinum.

incarcerated hernia of intestine T2744 Foeniculum vulgare.

incessant asthma and cough T3329 Hyoscyamus niger.

incessant bleeding T1994 Daemonorops draco, T2253 Dracaena cochinchinensis, T6540 Tripterygium hypoglaucum.

incessant chronic diarrhea T2190 Dioscorea batatas [Syn. Dioscorea opposita], T2202 Dioscorea japonica, T4351 Myristica fragrans, T5791 Schisandra chinensis, T5802 Schisandra sphenanthera.

incessant cough T2867 Garcinia multiflora, T6346 Terminalia chebula, T6348 Terminalia chebula var. tomentella.

incessant flooding and spotting T5446 Rehmannia glutinosa [Syn. Rehmannia glutinosa f. huechingensis], T6440 Thuja orientalis [Syn. Platycladus orientalis; Biota orientalis].

incessant intestinal wind bleeding T5138 Poncirus trifoliata.

incessant nosebleed T0328 Alnus japonica.

incessant postpartum bleeding T5183 Potentilla griffithii var. velutina.

incessant red and white vaginal discharge T6551 Trogopterus xanthipes; Pteromys volans.

incessant urinary turbidity T6504 Tricholoma matsutake [Syn. Armillaria matsutake].

incessant vaginal discharge T6670 Vaccinium bracteatum.

incessant vomiting and diarrhea T4018 Machilus thunbergii, T5363 Pyrus calleryana.

incised wound T0991 Bos taurus domesticus; Bubalus bubalis, T2154 Dicentra spectabilis, T2280 Dryopteris chrysocoma, T3980 Lycopus lucidus, T4917 Pinus massoniana, T5554 Rodgersia aesculifolia, T5653 Salix purpurea.

incised wound and bleeding T0661 Artemisia anomala, T0973 Bombax malabaricum [Syn. Gossampinus malabarica], T0977 Bombyx mori, T2420 Ericerus pela, T3776 Lethariella zahlbruckneri, T4207 Metaplexis japonica, T5301 Pterocarpus indicus.

indigestion T0156 Actinidia arguta, T0158 Actinidia chinensis, T0363 Alpinia speciosa, T0500 Anguilla japonica, T0647 Armoracia lapathifolia, T0881 Bauhinia variegata, T1101 Caesalpinia decapetala, T1217 Carum carvi, T1242 Cassia occidentalis, T1468 Citrus aurantium, T1506 Citrus reticulata, T1521 Citrus wilsonii, T1623 Collybia albuminosa, T1889 Cuminum cyminum, T1904 Curcuma kwangsiensis, T1909 Curcuma zedoaria [Syn. Curcuma aeruginosa], T1978 Cyperus rotundus, T2050 Daucus carota var. sativa, T2115 Dermatocarpon minitum, T2136 Desmos cochinchinensis [Syn. Desmos chinensis], T2189 Dioscorea althaeoides, T2203 Dioscorea nipponica, T2204 Dioscorea nipponica ssp. rosthornii, T2326 Elaeagnus angustifolia, T2340 Elsholtzia bodinieri, T2421 Erigeron annuus, T2563 Eupatorium japonicum, T2659 Fagopyrum esculentum, T2717 Ficus carica, T2720 Ficus hispida, T2763 Fragaria ananassa, T2838 Gallus gallus domesticus, T3120 Hedychium spicatum, T3229 Hericium erinaceus [Syn. Hydnum erinaceus], T3254 Hippophae rhamnoides, T3256 Hippophae rhamnoides subsp. sinensis, T3258 Hippophae rhamnoides subsp. yunnanensis, T3289 Humulus lupulus,
T3318 Hylotelephium mingjinianum, T3480 Isodon angustifolia, T3628 Kerria japonica, T3742 Lentinus edodes, T3779 Leucas aspera, T3926 Loropetalum chinense, T4147 Medicago falcata, T4194 Menyanthes trifoliata, T4217 Microcos paniculata [Syn. Grewia microcos], T4284 Morinda parvifolia, T4436 Nitraria tangutorum, T4644 Parasilurus asotus, T4802 Phleum pratense, T4887 Picrorhiza kurrooa, T4888 Picrorhiza scrophulariiflora, T5122 Polygonum viscosum, T5141 Poncirus trifoliata, T5341 Pygmaeopremna herbacea [Syn. Premna herbacea], T5365 Pyrus pashia, T5395 Rabdosia nervosa, T5419 Raphanus sativus, T5472 Rheum officinale, T5474 Rheum palmatum, T5481 Rheum tanguticum, T6056 Sorghum vulgare, T6071 Sphaeranthus indicus, T6214 Swertia chinensis, T6224 Swertia kouitchensis, T6229 Swertia patens, T6231 Swertia pseudochinensis, T6234 Swertia punicea, T6275 Tacca chantrieri [Syn. Tacca minor; Tacca esquirolii], T6339 Tephrosia purpurea, T6436 Thlaspi arvense, T6488 Trachyspermum ammi, T6602 Umbilicaria hypococcinea, T6651 Urtica cannabina, T6652 Urtica dioica, T6656 Ustilago maydis, T6789 Vitex negundo, T6801 Vladimiria denticulata.

indigestion of overnight food T1021 Brassica rapa, T1876 Cucumis melo.

induce abortion T2023 Daphne genkwa, T6512 Trichosanthes kirilowii.

infant ardent fever T1264 Catalpa ovata, T3764 Lepisorus thunbergianus, T4248 Mimosa pudica, T4506 Ophioglossum vulgatum.

infant ardent fever convulsion T0942 Biebersteinia heterostemon, T5592 Rubus hirsutus.

infant common cold T6229 Swertia patens.

infant diarrhea T2161 Dicranopteris pedata [Syn. Polypodium pedatum; Dicranopteris dichotoma], T2941 Gentianopsis paludosa, T5344 Pyrola atropurpurea, T5345 Pyrola calliantha [Syn. Pyrola rotundifolia ssp. chinensis], T5346 Pyrola calliantha var. tibetana, T5347 Pyrola decorata, T5349 Pyrola incarnata, T5350 Pyrola japonica, T5351 Pyrola rotundifolia.

infant fetal toxin T2730 Fimbristylis dichotoma.

infant fright wind T0088 Aconitum coreanum, T0984 Bos taurus domesticus; Bubalus bubalis, T1045 Bubalus bubalis, T1084 Buthus martensi, T1288 Celastrus orbiculatus [Syn. Celastrus articulatus], T1332 Cercidiphyllum japonicum, T2091 Delphinium yunnanense, T2540 Euonymus fortunei, T2927 Gentiana rhodantha, T4730 Peristrophe roxburghiana, T5183 Potentilla griffithii var. velutina, T5289 Pteris dactylina, T5290 Pteris fauriei [Syn. Pteris fauriei var. minor], T5299 Pteris wallichinan, T5626 Ruta graveolens, T5863 Selaginella moellendorffii, T5939 Shiraia bambusicola, T6278 Tagetes erecta, T6393 Thalictrum ichangense [Syn. Thalictrum tripeltatum; Thalictrum multipeltatum], T6580 Tylophora floribunda, T6618 Uncaria homomalla [Syn. Uruparia homomalla; Uruparia tonkinensis; Uruparia lanosa var. parvifora], T6651 Urtica cannabina, T6652 Urtica dioica, T6903 Zephyranthes candida.

infant fright wind and night crying T6625 Uncaria macrophylla, T6629 Uncaria rhynchophylla [Syn. Nauclea rhynchophylla], T6633 Uncaria sinensis.

infant mouth sore T0920 Berberis wilsonae, T2733 Firmiana simplex, T3989 Lygodium flexuosum [Syn. Lygodium pinnatifidum; Ophioglossum flexuosum], T4562 Oxalis acetosella. 
infant night crying T2836 Gallus gallus domesticus, T3040 Gomphrena globosa, T3578 Juncus effusus.

infant pneumonia T3348 Hypericum elodeoides, T3368 Hypericum wightianum.

infant retardation of walking T0038 Acanthopanax gracilistylus, T0042 Acanthopanax senticosus [Syn. Eleutherococcus senticosus], T0044 Acanthopanax sessiliflorus.

infantile cough asthma T6026 Sonchus asper [Syn. Sonchus oleraceus var. asper].

infantile night crying T4838 Phyllanthus flexuosus.

infection due to foot lichen T4391 Nauclea officinalis.

infection from wounds T0060 Achillea alpina [Syn. Achillea sibirica].

infection of biliary tract T3388 Ilex chinensis [Syn. Ilex purpurea], T4881 Picrasma quassioides [Syn. Picrasma ailanthoides], T4882 Picrasma quassioides [Syn. Picrasma ailanthoides].

infection of skin T0625 Aristolochia contorta, T0627 Aristolochia debilis [Syn. Aristolochia longa], T1959 Cynanchum paniculatum, T2023 Daphne genkwa, T3926 Loropetalum chinense, T6008 Solanum nigrum.

infection of upper respiratory tract T0457 Andrographis paniculata [Syn. Justicia paniculata], T0897 Berberis amurensis, T0912 Berberis poiretii, T0920 Berberis wilsonae, T1535 Clausena excavata, T1662 Coptis chinensis, T1663 Coptis chinensis var. brevisepala, T1664 Coptis deltoidea, T1669 Coptis omeiensis, T1670 Coptis teetoides [Syn. Coptis teeta], T1709 Corydalis bungeana, T2357 Emilia sonchifolia, T2715 Fibraurea recisa, T2783 Fritillaria cirrhosa, T2784 Fritillaria delavayi, T2792 Fritillaria przewalskii, T2796 Fritillaria unibracteata, T2798 Fritillaria verticillata var. thunbergii [Syn. Fritillaria thunbergii], T3476 Isatis indigotica, T3908 Lonicera bournei, T3909 Lonicera confusa, T3910 Lonicera fulvotomentosa, T3911 Lonicera hypoglauca, T3912 Lonicera japonica, T3914 Lonicera macranthoides, T3918 Lonicera similis, T4184 Mentha haplocalyx [Syn. Mentha canadaensis; Mentha arvensis var. haplocalyx; Mentha arvensis], T4881 Picrasma quassioides [Syn. Picrasma ailanthoides], T5018 Pleuropterus ciliinervis, T5833 Scutellaria amoena, T5834 Scutellaria baicalensis, T5839 Scutellaria hypericifolia, T5841 Scutellaria likiangensis, T5843 Scutellaria rehderiana, T5845 Scutellaria viscidula, T6122 Stephania dielsiana, T6135 Stephania succifera, T6278 Tagetes erecta, T6301 Taraxacum mongolicum, T6554 Trollius macropetalus, T6574 Tussilago farfara, T6766 Viola tricolor.

infection of urinary system T1199 Carduus acanthoides, T1200 Carduus crispus, T4388 Nasturtium officinale, T5018 Pleuropterus ciliinervis, T6071 Sphaeranthus indicus, T6234 Swertia punicea.

infections T0478 Angelica dahurica [Syn. Angelica porphyrocaulis], T0625 Aristolochia contorta, T0627 Aristolochia debilis [Syn. Aristolochia longa], T5110 Polygonum orientale.

infective cholecystitis T0672 Artemisia capillaris, T0695 Artemisia scoparia [Syn. Artemisia capillaris var. scoparia].

infective dermatitis T1959 Cynanchum paniculatum.

infective fever due to external injury T3092 Halenia corniculata.

infective hepatitis T1709 Corydalis bungeana, T4538 Oroxylum indicum, T4843 Phyllanthus urinaria, T5651 Salix babylonica, T6412 Thalictrum squarrosum, T6814 Wedelia chinensis [Syn. Solidago chinensis; Wedelia calendulacea], T6819 Wikstroemia chamaedaphne. infertility T0541 Apis cerana, T1455 Cistanche deserticola, T1456 Cistanche salsa, T3279 Homo sapiens, T3287 Huechys sanguinea, T5566 Rosa henryi.

infertility due to uterus cold T0664 Artemisia argyi, T0681 Artemisia lavandulaefolia, T0685 Artemisia mongolica, T0689 Artemisia princeps, T0691 Artemisia rubripes, T0706 Artemisia vulgaris, T0993 Boschniakia rossica, T1337 Cervus nippon; Cervus elaphus, T1582 Cnidium monnieri, T1900 Curculigo capitulata [Syn. Leucojum capitulata], T4536 Orobanche coerulescens, T4954 Piper longum, T5593 Rubus idaeus.

influenza T0099 Aconitum gymnandrum, T0333 Alocasia cucullata [Syn. Arum cucullatum], T0553 Apocynum venetum, T0728 Asarum heterotropoides var. mandshuricum, T0731 Asarum sieboldii, T0732 Asarum sieboldii var. seoulensis, T0791 Astragalus chrysopterus, T0794 Astragalus ernestii, T0798 Astragalus membranaceus, T0800 Astragalus mongholicus, T0866 Baphicacanthus cusia [Syn. Strobilanthes cusia], T1055 Bupleurum angustissimum, T1056 Bupleurum aureum, T1058 Bupleurum chaishoui, T1059 Bupleurum chinense, T1065 Bupleurum longiradiatum, T1066 Bupleurum marginatum, T1067 Bupleurum marginatum var. stenophyllum, T1072 Bupleurum scorzonerifolium, T1073 Bupleurum sibiricum, T1075 Bupleurum smithii var. parvifolium, T1078 Bupleurum wenchuanense, T1079 Bupleurum yinchowense, T1393 Chrysanthemum indicum, T1535 Clausena excavata, T1633 Commelina communis, T1709 Corydalis bungeana, T1727 Corydalis mucronifera, T2721 Ficus microcarpa, T3475 Isatis indigotica, T3528 Isodon ternifolia, T3529 Isodon ternifolius, T3908 Lonicera bournei, T3909 Lonicera confusa, T3910 Lonicera fulvotomentosa, T3911 Lonicera hypoglauca, T3912 Lonicera japonica, T3914 Lonicera macranthoides, T3918 Lonicera similis, T4220 Microlepia strigosa [Syn. Trichomanes strigosa], T4223 Micromelum integerrimum, T4552 Osmunda japonica, T4568 Oxytropis myriophylla, T4998 Plagiogyria euphlebia, T5592 Rubus hirsutus, T5797 Schisandra propinqua, T5798 Schisandra propinqua var. intermedia, T5907 Senecio scandens [Syn. Senecio chinensis], T6226 Swertia mileensis, T6375 Thalictrum baicalense, T6376 Thalictrum cultratum, T6385 Thalictrum flavum, T6387 Thalictrum foliolosum, T6389 Thalictrum glandulosissimum.

influenza in early stage T2756 Forsythia suspensa.

ingrown eyelash T4471 Ocimum basilicum.

inhibited urination T0274 Akebia quinata, T0278 Akebia trifoliata, T0280 Akebia trifoliata var. australis, T0303 Alisma orientale [Syn. Alisma plantago-aquatica var. orientale], T0628 Aristolochia fangchi, T0629 Aristolochia heterophylla, T0647 Armoracia lapathifolia, T0672 Artemisia capillaris, T0695 Artemisia scoparia [Syn. Artemisia capillaris var. scoparia], T0708 Arthromeris mairei [Syn. Polypodium mairei], T0722 Arundo donax, T0759 Aspidistra elatior, T0824 Atractylodes macrocephala [Syn. Atractylis macrocephala], T0838 Azolla imbricata [Syn. Salvinia imbricata], T0853 Baeckea frutescens, T1004 Brasenia schreberi, T1152 Camellia sinensis [Syn. Thea sinensis], T1238 Cassia mimosoides, T1240 Cassia obtusifolia, T1250 Cassia tora, T1258 Casuarina equisetifolia, T1263 Catalpa ovata, T1438 Cinnamomum cassia [Syn. Cinnamomum aromaticum], T1464 
Citrullus vulgaris [Syn. Citrullus lanatus], T1471 Citrus chachiensis, T1473 Citrus cultivars, T1474 Citrus decumana, T1516 Citrus tankan, T1518 Citrus unshiu, T1538 Clausena lansium, T1595 Codium fragile, T1614 Coix lacryma-jobi var. ma-yuen, T1819 Crotalaria ferruginea, T1980 Cyprinus carpio, T2125 Descurainia sophia, T2282 Dryopteris filix-mas, T2341 Elsholtzia ciliata, T2367 Ephedra equisetina, T2368 Ephedra gerardiana, T2369 Ephedra intermedia, T2372 Ephedra likiangensis, T2373 Ephedra minuta, T2375 Ephedra monosperma, T2379 Ephedra saxatilis, T2380 Ephedra sinica, T2628 Euphrasia officinalis, T2730 Fimbristylis dichotoma, T2927 Gentiana rhodantha, T3143 Helianthus annuus, T3199 Hemibarbus labeo, T3288 Humulus japonicus [Syn. Humulus scandens], T3448 Ipomoea cairica [Syn. Ipomoea palmata], T3578 Juncus effusus, T3628 Kerria japonica, T3662 Lactuca sativa, T3675 Lagopsis supina, T3755 Lepidium apetalum [Syn. Lepidium micranthum], T3758 Lepidium sativum, T3759 Lepidium virginicum, T3765 Lepisorus ussuriensis, T3885 Litsea cubeba, T3921 Lophatherum gracile, T3950 Lychnis fulgens, T3983 Lycoris aurea, T3988 Lycoris squamigera, T4194 Menyanthes trifoliata, T4225 Microsorium punctatum, T4254 Miscanthus sinensis, T4275 Monotropa hypopitys, T4287 Morus alba, T4304 Mosla chinensis [Syn. Orthodon chinensis], T4456 Nymphoides peltatum, T4616 Pandanus tectorius, T4688 Peganum harmala, T4851 Physalis minima, T5129 Polyporus umbellatus, T5223 Prunus humilis [Syn. Cerasus humilis], T5224 Prunus japonica [Syn. Cerasus japonica], T5225 Prunus japonica var. nakaii, T5231 Prunus persica, T5362 Pyrus bretschneideri, T5366 Pyrus pyrifolia, T5614 Rumex patientia, T5626 Ruta graveolens, T5953 Silene firma, T5966 Siphonostegia chinensis, T6031 Sophora flavescens [Syn. Sophora angustfolia], T6100 Stauntonia hexaphylla, T6136 Stephania tetrandra, T6210 Swainsonia salsula [Syn. Sphaerophysa salsula], T6357 Tetrapanax papyriferus, T6598 Ulva conglobata, T6676 Valeriana hardwickii, T6794 Vitis amurensis, T6799 Vitis vinifera, T6862 Zanthoxylum ailanthoides, $\mathrm{T} 6902$ Zea mays.

inhibited urination [= dysuria] T0388 Amaranthus lividus, T0894 Benincasa hispida, T1032 Broussonetia papyrifera, T1033 Broussonetia papyrifera, T3460 Iris halophila, T3464 Iris lactea var. chinensis [Syn. Iris pallasii var. chinensis], T3990 Lygodium japonicum, T4120 Marsdenia tenacissima, T4121 Marsilea quadrifolia, T4274 Monostroma nitidum, T4573 Pachyrrhizus erosus, T4644 Parasilurus asotus, T5002 Plantago asiatica, T5004 Plantago depressa, T5007 Plantago major, T5103 Polygonum hydropiper, T6861 Zanthoxylum ailanthoides.

inhibited urine and stool T0314 Allium fistulosum, T1205 Carica papaya, T2608 Euphorbia pekinensis, T3286 Hovenia dulcis, T4779 Pharbitis nil, T4780 Pharbitis purpurea.

inhibited voidings of reddish urine T0635 Aristolochia moupinensis, T3920 Lophatherum gracile, T4194 Menyanthes trifoliata.

innominate toxin swelling T0002 Abelmoschus moschatus [Syn. Hibiscus abelmoschus], T0100 Aconitum hemsleyanium var. circinacum, T0101 Aconitum hemsleyanum, T0122 Aconitum pendulum, T0123 Aconitum polyschistum, T0424 Ampelopsis brevipedunculata var. hancei, T0567 Aralia armata, T0595 Ardisia crispa, T0897 Berberis amurensis, T0962 Boehmeria platanifolia [Syn. Boehmeria tricuspis], T1097
Cacalia ainsliaeflora, T1731 Corydalis pallida, T1996 Dahlia pinnata [Syn. Dahlia variabilis], T2056 Debregeasia longifolia, T2301 Dysosma majorensis [Syn. Podophyllum majorense; Dysosma lichuanensis], T2599 Euphorbia lunulata, T2632 Euryale ferox, T2980 Gleditsia sinensis [Syn. Gleditsia horrida], T3080 Gymnema sylvestre, T3125 Hedyotis chrysotricha [Syn. Oldenlandia chrysotricha], T3495 Isodon irrorata, T3619 Kadsura peltigera [Syn. Kadsura longipedunculata], T3808 Ligularia japonica [Syn. Arnica japonica; Senecio japonica], T3827 Ligustrum japonicum, T3886 Litsea euosma, T3928 Lotus corniculatus, T3933 Luffa acutangula, T3934 Luffa cylindrica, T4264 Momordica cochinchinensis, T4527 Orixa japonica, T4530 Ormosia hosiei, T4562 Oxalis acetosella, T4577 Paederia scandens, T4687 Peganum harmala, T4843 Phyllanthus urinaria, T4979 Pistacia chinensis, T5090 Polygala wattersii, T5097 Polygonum amphibium, T5120 Polygonum tinctorium, T5167 Porana racemosa, T5193 Pratia nummularia, T5247 Przewalskia tangutica, T5646 Sagina japonica [Syn. Spergula japonica], T5720 Sapindus mukorossi, T5731 Sarcococca coriacea [Syn. Sarcococca wallichii], T6279 Tagetes erecta, T6367 Teucrium quadrifarium, T6742 Vicia amoena.

insect bites T2206 Dioscorea parviflora, T2213 Dioscorea zingiberensis, T3003 Glycine max, T4384 Narcissus tazetta var. chinensis, T5120 Polygonum tinctorium, T5857 Sedum sarmentosum.

insect bites with itching T6072 Sphagnum palustre [Syn. Sphagnum obtusifolium; Sphagnum cymbifolium].

insomnia T0292 Albizzia julibrissin, T0295 Albizzia odoratissima, T0553 Apocynum venetum, T0645 Armillariella mellea, T1381 Choerospondias axillaris, T1393 Chrysanthemum indicum, T1826 Crotalaria mucronata, T2846 Ganoderma japonicum [Syn. Ganoderma sinense], T2848 Ganoderma lucidum, T3229 Hericium erinaceus [Syn. Hydnum erinaceus], T3289 Humulus lupulus, T3372 Hypodematium sinense, T3775 Lethariella cladonioides, T3950 Lychnis fulgens, T4001 Lysimachia davurica, T4248 Mimosa pudica, T4400 Nelumbo nucifera, T4663 Passiflora caerulea, T4665 Passiflora edulis, T5107 Polygonum multiflorum, T5108 Polygonum multiflorum, T5169 Poria cocos, T5423 Rauvolfia verticillata, T5425 Rauvolfia verticillata f. rubrocarpa, T5426 Rauvolfia verticillata var. hainanensis, T5427 Rauvolfia vomitoria, T5435 Rauwolfia latifrons, T5441 Rauwolfia perakensis, T5657 Salsola collina, T5697 Salvia trijuga, T5850 Sedum aizoon, T5855 Sedum kamtschaticum, T6416 Thamnolia vermicularis, T6656 Ustilago maydis.

insomnia and emission T4401 Nelumbo nucifera.

insomnia and frequent dreaming T0041 Acanthopanax senticosus [Syn. Eleutherococcus senticosus], T2753 Formica fusca, T3832 Lilium brownii var. viridulum [Syn. Lilium brownii var. colchesteri], T3835 Lilium longiflorum, T3836 Lilium pumilum [Syn. Lilium tenuifolium], T3839 Lilium tigrinum [Syn. Lilium lancifolium], T4288 Morus alba, T4600 Panax ginseng [Syn. Panax schinseng], T5071 Polygala arillata, T6056 Sorghum vulgare.

insomnia and vexation T1813 Crotalaria albida, T2840 Gallus gallus domesticus, T3578 Juncus effusus, T3778 Leucaena glauca [Syn. Leucaena leucocephala], T3875 Liriope spicata var. prolifera, T4507 Ophiopogon japonicus, T5680 Salvia miltiorrhiza, T5681 Salvia miltiorrhiza f. alba, T5688 Salvia przewalskii. 
insomnia and vexation due to effulgent fire T1662 Coptis chinensis, T1663 Coptis chinensis var. brevisepala, T1664 Coptis deltoidea, T1669 Coptis omeiensis, T1670 Coptis teetoides [Syn. Coptis teeta].

intercostal neuralgia T0116 Aconitum nagarum var. heterotrichum [Syn. Aconitum bullatifolium].

internal bleeding T2220 Diospyros kaki, T6746 Vicia faba.

internal damage due to knocks and falls T5646 Sagina japonica [Syn. Spergula japonica].

internal damage stasis pain T1994 Daemonorops draco, T2253

Dracaena cochinchinensis.

internal heat and abdominal pain T0565 Arachniodes simplicior. internal or external obstruction and screen $\quad$ T6732 Vespertilio superans. intervallic fever T1101 Caesalpinia decapetala.

intestinal dry and constipation T0300 Aleurites moluccana, T0474 Angelica acutiloba [Syn. Ligusticum acutilobum], T0540 Apis cerana, T0944 Biota orientalis [Syn. Thuja orientalis; Platycladus orientalis], T0993 Boschniakia rossica, T1173 Cannabis sativa, T1384 Chondrus ocelladus, T1455 Cistanche deserticola, T1456 Cistanche salsa, T3568 Juglans regia, T3860 Linum usitatissimum, T3873 Liriope platyphylla, T3874 Liriope spicata, T3875 Liriope spicata var. prolifera, T4288 Morus alba, T4487 Olea europaea, T4507 Ophiopogon japonicus, T4536 Orobanche coerulescens, T4715 Perilla frutescens, T4717 Perilla frutescens var. acuta [Syn. Perilla frutescens var. purpurascens], T4719 Perilla frutescens var. arguta, T4722 Perilla frutescens var. crispa, T5107 Polygonum multiflorum, T5216 Prunus armeniaca, T5219 Prunus armeniaca var. ansu, T5229 Prunus persica, T5233 Prunus persica, T5238 Prunus salicina, T5454 Rhamnus cathartica, T5657 Salsola collina, T5925 Sesamum indicum [Syn. Sesamum orientale], T5927 Sesamum indicum [Syn. Sesamum orientale], T5967 Siraitia grosvenorii [Syn. Momordica grosvenorii], T6511 Trichosanthes kirilowii.

intestinal heat and constipation T2717 Ficus carica.

intestinal heat bleeding $\mathrm{T} 3155$ Helianthus tuberosus.

intestinal heat bleeding from hemorrhoids T0624 Aristolochia contorta, T0626 Aristolochia debilis [Syn. Aristolochia longa], T0630 Aristolochia indica, T0633 Aristolochia maxima, T0640 Aristolochia triangularis.

intestinal infection T5344 Pyrola atropurpurea, T5345 Pyrola calliantha [Syn. Pyrola rotundifolia ssp. chinensis], T5346 Pyrola calliantha var. tibetana, T5347 Pyrola decorata, T5349 Pyrola incarnata, T5350 Pyrola japonica, T5351 Pyrola rotundifolia.

intestinal pain due to qi disorder T0364 Alsophila spinulosa.

intestinal parasitic disease T0099 Aconitum gymnandrum, T0500 Anguilla japonica, T0511 Annona reticulata, T0513 Annona squamosa, T0952 Blechnum orientale, T2282 Dryopteris filix-mas, T2410 Equisetum pratense, T4552 Osmunda japonica, T5113 Polygonum persicaria, T6826 Wisteria sinensis.

intestinal welling abscess T0157 Actinidia callosa var. henryi, T0267 Ajuga nipponensis, T0318 Allium sativum, T0867 Baphicacanthus cusia [Syn. Strobilanthes cusia], T0895 Benincasa hispida, T0938 Bidens bipinnata, T0994 Boswellia carterii, T1614 Coix lacryma-jobi var. ma-yuen, T1638 Commiphora myrrha [Syn. Commiphora molmol], T2830 Galium aparine, T3126 Hedyotis corymbosa [Syn. Oldenlandia corymbosa], T3356 Hypericum japonicum, T3460 Iris halophila, T3464 Iris lactea var. chinensis [Syn. Iris pallasii var. chinensis], T3466 Iris potaninii, T3547 Ixeris chinensis, T3659 Lactuca indica, T4485 Oldenlandia diffusa [Syn. Hedyotis diffusa], T4577 Paederia scandens, T4672 Patrinia scabiosaefolia, T4676 Patrinia villosa, T5835 Scutellaria barbata [Syn. Scutellaria rivularis], T5838 Scutellaria galericulata, T5840 Scutellaria indica, T6767 Viola yedoensis.

intestinal welling abscess and abdominal pain T5472 Rheum officinale, T5474 Rheum palmatum, T5481 Rheum tanguticum, T5741 Sargentodoxa cuneata.

intestinal wind T0427 Ampelopsis japonica [Syn. Paullinia japonica], T0500 Anguilla japonica, T1441 Cinnamomum japonicum, T4505 Ophiocephalus argus.

intestinal wind bleeding T0255 Ailanthus altissima, T1005 Brassica campestris [Syn. Brassica campestris var. oleifera], T1112 Calendula arvensis, T1282 Cedrus deodara, T1289 Celastrus orbiculatus [Syn. Celastrus articulatus], T3244 Hibiscus syriacus, T3245 Hibiscus syriacus, T3361 Hypericum perforatum, T3863 Liparis nervosa, T3933 Luffa acutangula, T3934 Luffa cylindrica, T3935 Luffa cylindrica, T4584 Paeonia lactiflora wild, T4586 Paeonia obovata, T4590 Paeonia veitchii, T4678 Paulownia fortunei, T4679 Paulownia tomentosa, T5284 Pteridium aquilinum var. latiusculum, T5611 Rumex japonicus, T5612 Rumex nepalensis, T5727 Saposhnikovia divaricata [Syn. Ledebouriella seseloides], T6006 Solanum melongena, T6007 Solanum melongena, T6031 Sophora flavescens [Syn. Sophora angustfolia], T6034 Sophora japonica, T6036 Sophora japonica, T6474 Toona ciliata, T6475 Toona sinensis, T6508 Trichosanthes cucumeroides, T6601 Umbilicaria esculenta [Syn. Gyrophora esculenta], T6830 Woodfordia fruticosa.

intestinal wind bleeding (flower) T1113 Calendula officinalis.

intestinal wind blood dysentery T4550 Osmanthus fragrans.

intestinal worm accumulation $\mathrm{T} 1101$ Caesalpinia decapetala.

intestine gripping sand T2658 Fagopyrum esculentum.

intractable headache with pulling sensation T5817 Scolopendra subspinipes mutilans.

intractable impediment T2597 Euphorbia lathyris.

intractable lichen T0736 Asclepias curassavica, T1084 Buthus martensi, T1374 Chloranthus spicatus, T1719 Corydalis incisa, T2505 Eucalyptus citriodora, T2584 Euphorbia ebracteolata, T2587 Euphorbia fischeriana, T2857 Garcinia hanburyi, T2866 Garcinia morella, T3567 Juglans regia, T3984 Lycoris chinensis, T3986 Lycoris radiata [Syn. Amaryllis radiata], T4020 Macleaya cordata, T4085 Mallotus repandus var. chrysocarpus [Syn. Mallotus chrysocarpus; Mallotus repandus], T4338 Mylabris phalerata; Mylabris cichorii, T5010 Platycarya strobilacea, T5455 Rhamnus crenata, T5516 Rhododendron molle, T5531 Rhus chinensis [Syn. Rhus semialata], T5976 Smilax china [Syn. Smilax japonica], T6028 Sophora alopecuroides, T6029 Sophora alopecuroides, T6542 Tripterygium wilfordii.

invisible worm sores T5221 Prunus davidiana, T5235 Prunus persica. iron or wood intake $\mathrm{T} 1836$ Crotalaria tetragona. itching in genital region T6764 Vinegar. 
itching sore of skin T1316 Cephalanthus occidentalis.

itch-pain in sore and papules T1352 Chamaenerion angustifolium [Syn. Epilobium angustifolium].

itchy body T1161 Campsis grandiflora, T1175 Cannabis sativa, T6497 Tribulus terrestris.

itchy eye T5804 Schizonepeta tenuifolia [Syn. Nepeta tenuifolia].

itchy of skin T0838 Azolla imbricata [Syn. Salvinia imbricata], T3342 Hypericum bellum.

itchy papules T0933 Betula platyphylla, T0935 Betula platyphylla var. japonica, T1210 Carpesium abrotanoides, T1556 Clerodendron fragrans, T5339 Pycnoporus sanguineus, T6491 Trametes cinnabarina [Syn. Polyporus cinnabarinus; Boletus cinnabarinus].

itchy skin T0664 Artemisia argyi, T0672 Artemisia capillaris, T0681 Artemisia lavandulaefolia, T0685 Artemisia mongolica, T0689 Artemisia princeps, T0691 Artemisia rubripes, T0695 Artemisia scoparia [Syn. Artemisia capillaris var. scoparia], T0706 Artemisia vulgaris, T0853 Baeckea frutescens, T0963 Boehmeria siamensis, T0975 Bombyx mori, T1146 Camellia oleifera, T1147 Camellia oleifera, T1261 Catalpa ovata, T1264 Catalpa ovata, T1372 Chloranthus serratus, T1437 Cinnamomum camphora, T1446 Cipadessa baccifera, T1721 Corydalis linearioides, T1730 Corydalis ophiocarpa, T1869 Cryptotaenia japonica, T2165 Dictamnus angustifolius, T2167 Dictamnus dasycarpus, T2348 Embelia ribes, T2585 Euphorbia esula, T2590 Euphorbia hirta, T2805 Fugu ocellatus, T2988 Glochidion eriocarpum, T3288 Humulus japonicus [Syn. Humulus scandens], T3471 Iris tectorum, T3569 Juglans regia, T3646 Kyllinga brevifolia, T3675 Lagopsis supina, T3851 Lindera glauca, T3860 Linum usitatissimum, T3990 Lygodium japonicum, T4388 Nasturtium officinale, T4526 Origanum vulgare, T4572 Pachyrhizus erosus, T4687 Peganum harmala, T4917 Pinus massoniana, T5104 Polygonum hydropiper, T5178 Potamogeton perfoliatus, T5395 Rabdosia nervosa, T5640 Sabina vulgaris, T5840 Scutellaria indica, T6031 Sophora flavescens [Syn. Sophora angustfolia], T6541 Tripterygium regelii, T6843 Xanthium sibiricum [Syn. Xanthium strumarium], T6843 Xanthium sibiricum [Syn. Xanthium strumarium].

jaundice T0045 Acanthopanax trifoliatus, T0048 Acanthus ilicifolius, T0156 Actinidia arguta, T0175 Adiantum pedatum, T0264 Ajuga forrestii, T0379 Alternanthera philoxeroides, T0594 Ardisia crenata, T0600 Ardisia mamillata [Syn. Tinus mamillata], T0601 Ardisia pusilla, T0608 Arenaria kansuensis [Syn. Arenaria kumaonensis], T0610 Argemone mexicana, T0648 Arnebia euchroma, T0649 Arnebia guttata, T0660 Artemisia annua, T0662 Artemisia apiacea [Syn. Artemisia carvifolia; Artemisia caruifolia], T0672 Artemisia capillaris, T0695 Artemisia scoparia [Syn. Artemisia capillaris var. scoparia], T0696 Artemisia sieversiana, T0721 Arundina chinensis, T0859 Balanophora involucrata, T0867 Baphicacanthus cusia [Syn. Strobilanthes cusia], T0902 Berberis diaphana, T0912 Berberis poiretii, T0920 Berberis wilsonae, T0933 Betula platyphylla, T0935 Betula platyphylla var. japonica, T0938 Bidens bipinnata, T0941 Bidens tripartita, T0947 Bixa orellana, T0984 Bos taurus domesticus; Bubalus bubalis, T0989 Bos taurus domesticus; Bubalus bubalis, T1003 Brandisia hancei, T1004 Brasenia schreberi, T1031 Broussonetia kazinoki, T1044 Bryum argenteum, T1172 Canna edulis, T1194 Carassius auratus, T1197
Cardiospermum halicacabum, T1199 Carduus acanthoides, T1200 Carduus crispus, T1238 Cassia mimosoides, T1275 Cayratia japonica, T1357 Chelidonium majus, T1402 Chrysosplenium alternifolium, T1406 Chrysosplenium nudicaule, T1526 Cladonia rangiferina, T1554 Clerodendron cyrtophyllum, T1705 Corydalis adunca, T1733 Corydalis racemosa, T1759 Cotinus coggygria var. cinerea, T1784 Cratoxylum cochinchinense, T1785 Cratoxylum prunifolium, T1813 Crotalaria albida, T1884 Cudrania cochinchinensis, T1903 Curcuma aromatica, T1905 Curcuma longa, T1907 Curcuma wengujin, T1966 Cynara scolymus, T1980 Cyprinus carpio, T2020 Damnacanthus indicus, T2048 Daucus carota, T2132 Desmodium pulchellum [Syn. Phyllodium pulchellum], T2257 Dracocephalum moldavicum, T2258 Dracocephalum rupestre, T2272 Drymaria diandra [Syn. Drymaria cordata ssp. diandra], T2290 Duchesnea indica, T2334 Elephantopus scaber, T2363 Entada phaseoloides [Syn. Lens phaseoloides], T2649 Evolvulus alsinoides, T2715 Fibraurea recisa, T2738 Flemingia strobilifera, T2839 Gallus gallus domesticus, T2882 Gardenia jasminoides [Syn. Gardenia florida], T2884 Gardenia jasminoides var. grandiflora, T2927 Gentiana rhodantha, T3125 Hedyotis chrysotricha [Syn. Oldenlandia chrysotricha], T3192 Hemerocallis citrina, T3193 Hemerocallis fulva, T3195 Hemerocallis lilio-asphodelus, T3197 Hemerocallis minor, T3311 Hydrocotyle sibthorpioides, T3361 Hypericum perforatum, T3373 Hypoestes purpurea [Syn. Justicia purpurea; Hypoestes sinica], T3398 Ilex rotunda, T3416 Imperata cylindrica var. major, T3460 Iris halophila, T3464 Iris lactea var. chinensis [Syn. Iris pallasii var. chinensis], T3476 Isatis indigotica, T3528 Isodon ternifolia, T3529 Isodon ternifolius, T3645 Kummerowia striata, T3646 Kyllinga brevifolia, T3668 Lagenaria siceraria var. depressa, T3680 Lamium amplexicaule, T3845 Linaria vulgaris, T3881 Lithospermum erythrorhizon, T3970 Lycopodium cernuum, T3973 Lycopodium japonicum [Syn. Lycopodium clavatum], T3981 Lycopus lucidus, T4000 Lysimachia congestiflora, T4055 Mahonia bealei, T4060 Mahonia confusa, T4062 Mahonia fortunei, T4064 Mahonia gracilipes, T4066 Mahonia japonica, T4121 Marsilea quadrifolia, T4147 Medicago falcata, T4148 Medicago sativa, T4149 Medicago sativa, T4183 Menispermum dauricum, T4217 Microcos paniculata [Syn. Grewia microcos], T4482 Oenanthe javanica, T4526 Origanum vulgare, T4544 Oryza sativa, T4564 Oxalis corniculata [Syn. Oxalis repens], T4656 Parmelia saxatilis, T4789 Phellodendron amurense, T4790 Phellodendron amurense var. wilsonii, T4791 Phellodendron chinense, T4792 Phellodendron chinense var. glabriusculum, T4831 Phtheirospermum japonicum [Syn. Geradia japonica], T4835 Phyllanthus emblica, T4840 Phyllanthus niruri, T4845 Physalis alkekengi, T4846 Physalis alkekengi var. franchetii, T4847 Physalis alkekengi var. franchetii, T4851 Physalis minima, T5121 Polygonum tinctorium, T5176 Potamogeton natans, T5230 Prunus persica, T5284 Pteridium aquilinum var. latiusculum, T5288 Pteris cretica var. nervosa [Syn. Pteris nervosa], T5290 Pteris fauriei [Syn. Pteris fauriei var. minor], T5295 Pteris multifida, T5401 Rabdosia yuennanensis, T5407 Rana nigromaculata; Rana plancyi, T5413 Ranunculus cantoniensis, T5414 Ranunculus japonicus, T5531 Rhus chinensis [Syn. Rhus semialata], T5560 Rorippa montana [Syn. Rorippa dubia; Sisymbrium dublium], T5576 Rostellularia procumbens 
[Syn. Justicia procumbens], T5650 Salix babylonica, T5750 Saururus chinensis, T5833 Scutellaria amoena, T5834 Scutellaria baicalensis, T5839 Scutellaria hypericifolia, T5841 Scutellaria likiangensis, T5843 Scutellaria rehderiana, T5845 Scutellaria viscidula, T5856 Sedum lineare [Syn. Sedum obtuso-lineare], T5859 Selaginella braunii, T5870 Selaginella uncinata, T5907 Senecio scandens [Syn. Senecio chinensis], T5950 Siegesbeckia orientalis, T5951 Siegesbeckia orientalis var. glabrescens [Syn. Siegesbeckia glabrescens], T5952 Siegesbeckia orientalis var. pubescens [Syn. Siegesbeckia pubescens], T5966 Siphonostegia chinensis, T6005 Solanum lyratum, T6024 Solidago virgaurea var. leiocarpa [Syn. Solidago decurrens], T6028 Sophora alopecuroides, T6131 Stephania longa, T6206 Sus scrofa domestica, T6250 Symplocos caudata, T6357 Tetrapanax papyriferus, T6375 Thalictrum baicalense, T6376 Thalictrum cultratum, T6378 Thalictrum delavayi, T6385 Thalictrum flavum, T6386 Thalictrum foetidum, T6387 Thalictrum foliolosum, T6389 Thalictrum glandulosissimum, T6403 Thalictrum petaloideum, T6409 Thalictrum simplex [Syn. Thalictrum simplex var. brevipes], T6431 Thesium chinense, T6507 Trichosanthes cucumeroides, T6508 Trichosanthes cucumeroides, T6537 Tripterospermum japonicum, T6538 Tripterospermum taiwanense, T6541 Tripterygium regelii, T6749 Vicia hirsuta, T6750 Vicia sativa, T6752 Vigna angularis [Syn. Dolichus angularis; Phaseolus angularis], T6764 Vinegar, T6767 Viola yedoensis, T6788 Vitex negundo, T6901 Zea mays.

jaundice [ = icterus, ICT] T4072 Mahonia shenii, T4827 Photinia Parvifolia, T6219 Swertia erythrosticta, T6228 Swertia nervosa.

jaundice edema T3000 Glycine max.

jaundice hepatitis T5867 Selaginella sinensis.

jaundice with reddish urine T2134 Desmodium styracifolium.

joint running impediment pain T2536 Euonymus alatus.

joint running swelling pain $\mathrm{T} 5517$ Rhododendron molle.

joint running wind T6439 Thuja orientalis [Syn. Platycladus orientalis; Biota orientalis].

joints wind T4912 Pinus koraiensis.

keratitis T1055 Bupleurum angustissimum, T1056 Bupleurum aureum, T1058 Bupleurum chaishoui, T1059 Bupleurum chinense, T1065 Bupleurum longiradiatum, T1066 Bupleurum marginatum, T1067 Bupleurum marginatum var. stenophyllum, T1072 Bupleurum scorzonerifolium, T1073 Bupleurum sibiricum, T1075 Bupleurum smithii var. parvifolium, T1078 Bupleurum wenchuanense, T1079 Bupleurum yinchowense, T4320 Murraya kwangsiensis.

kersan disease T4902 Pimpinella thelungiana.

kersan disease with hypertonicity and pain $\mathrm{T} 3480$ Isodon angustifolia. kidney disease T6311 Taxus cuspidata.

kidney $q \boldsymbol{i}$ insecurity T5270 Psoralea corylifolia.

kidney vacuity $\mathrm{T} 1604$ Coeloglossum viride $[\mathrm{Syn}$. Coeloglossum viride var. bracteatum], T2540 Euonymus fortunei, T3077 Gymnadenia conopsea, $\mathrm{T} 3770$ Lespedeza cuneata.

kidney vacuity and edema T0568 Aralia chinensis, T0570 Aralia dasyphylla.

kidney vacuity and emission T2188 Dioscorea alata, T2190 Dioscorea batatas [Syn. Dioscorea opposita], T2195 Dioscorea deltoidea, T2202 Dioscorea japonica, T5067 Polyalthia nemoralis, T5236 Prunus pseudocerasus.

kidney vacuity asthma T1439 Cinnamomum cassia [Syn. Cinnamomum aromaticum], T6257 Syngnathus acus.

kidney vacuity cold T6528 Trigonella foenum-graecum.

kidney vacuity cough asthma T1900 Curculigo capitulata [Syn. Leucojum capitulata].

kidney vacuity dizziness T2956 Geum japonicum.

kidney vacuity dizziness and tinnitus T2753 Formica fusca.

kidney vacuity hasty asthma T5446 Rehmannia glutinosa [Syn. Rehmannia glutinosa f. huechingensis].

kidney vacuity impotence T0322 Allium tuberosum, T0323 Allium tuberosum, T0993 Boschniakia rossica, T1972 Cynomorium songaricum, T4536 Orobanche coerulescens, T4600 Panax ginseng [Syn. Panax schinseng], T4696 Penaeus orientalis, T6263 Syzygium aromaticum [Syn. Eugenia caryophyllata].

kidney vacuity lumbago T0364 Alsophila spinulosa, T1958 Cynanchum otophyllum, T1963 Cynanchum wallichii, T2052 Davallia divaricata [Syn. Davallia formosana; Davallia orientalis], T2273 Drynaria fortunei, T2326 Elaeagnus angustifolia, T2530 Eucommia ulmoides, T2744 Foeniculum vulgare, T3000 Glycine max, T3409 Illicium verum, T4286 Morinda umbellata, T4642 Parameria laevigata, T5252 Pseudodrynaria coronans, T5697 Salvia trijuga, T6527 Trigonella caerulea, T6727 Veronica persica, T6750 Vicia sativa, T6754 Vigna unguiculata.

kidney vacuity lumbar cold T4982 Pistacia vera.

kidney vacuity lumbar pain T4828 Photinia serrulata, T5501 Rhodiola yunnanesis.

kidney vacuity lumbar pain and back rigidity T1411 Cibotium barometz [Syn. Polypodium barometz].

kidney yang vacuity $\mathrm{T} 5270$ Psoralea corylifolia.

kindey vacuity toothache T2053 Davallia mariesii.

knee joint pain T5308 Pterocarya stenoptera.

knee pain and legs weakness T6831 Woodwardia orientalis.

knife wound T1866 Cryptolepis sinensis, T1896 Cupressus funebris, T1927 Cycas revoluta, T1967 Cynoglossum amabile, T2301 Dysosma majorensis [Syn. Podophyllum majorense; Dysosma lichuanensis], T4031 Maesa perlarius, T4109 Marchantia polymorpha, T5391 Rabdosia eriocalyx, T6471 Toddalia asiatica [Syn. Toddalia aculeata; Paullinia asiatica], T6601 Umbilicaria esculenta [Syn. Gyrophora esculenta].

knocks and falls T0015 Abutilon indicum, T0036 Acanthopanax giraldii [Syn. Acanthopanax giraldii var. inermis; Eleutherococcus giraldii], T0038 Acanthopanax gracilistylus, T0042 Acanthopanax senticosus [Syn. Eleutherococcus senticosus], T0044 Acanthopanax sessiliflorus, T0060 Achillea alpina [Syn. Achillea sibirica], T0065 Achillea millefolium, T0071 Achyranthes aspera, T0072 Achyranthes aspera var. indica, T0073 Achyranthes bidentata, T0079 Aconitum brachypodum, T0094 Aconitum finetianum, T0096 Aconitum forrestii [Syn. Aconitum likiangense], T0098 Aconitum geniculatum, T0100 Aconitum hemsleyanium var. circinacum, T0101 Aconitum hemsleyanum, T0107 Aconitum kongboense, T0108 Aconitum kusnezoffii, T0117 Aconitum nagarum var. lasiandrum, T0122 Aconitum pendulum, T0123 Aconitum polyschistum, T0130 Aconitum sinomontanum, T0135 Aconitum 
sungpanense, T0138 Aconitum umbrosum, T0144 Acorus gramineus, T0146 Acorus tatarinowii, T0156 Actinidia arguta, T0161 Actinidia eriantha, T0229 Ageratum conyzoides, T0240 Aglaia odorata, T0263 Ajuga decumbens, T0264 Ajuga forrestii, T0274 Akebia quinata, T0278 Akebia trifoliata, T0280 Akebia trifoliata var. australis, T0281 Alangium chinense, T0285 Alangium platanifolium, T0292 Albizzia julibrissin, T0322 Allium tuberosum, T0324 Allium victorialis, T0364 Alsophila spinulosa, T0378 Alstonia yunnanensis, T0383 Alyxia sinensis, T0424 Ampelopsis brevipedunculata var. hancei, T0427 Ampelopsis japonica [Syn. Paullinia japonica], T0428 Ampelopsis megalophylla, T0467 Anemone flaccida, T0468 Anemone hupehensis, T0471 Anemone rivularis, T0491 Angelica polymorpha, T0502 Anisodus tanguticus [Syn. Scopolia tangutica], T0515 Anredera cordifolia [Syn. Baussingaultia cordifolia; Baussingaultia gracilis $\mathrm{f}$. pseudobaselloides; Baussingaultia gracilis var. pseudobaselloides], T0535 Antidesma bunius, T0537 Antirrhinum majus, T0561 Arachis hypogaea, T0567 Aralia armata, T0568 Aralia chinensis, T0570 Aralia dasyphylla, T0594 Ardisia crenata, T0595 Ardisia crispa, T0600 Ardisia mamillata [Syn. Tinus mamillata], T0601 Ardisia pusilla, T0602 Ardisia quinquegona, T0617 Arisaema amurense, T0618 Arisaema consanguineum, T0620 Arisaema heterophyllum, T0642 Aristolochia tubiflora, T0661 Artemisia anomala, T0680 Artemisia lactiflora, T0721 Arundina chinensis, T0726 Asarum forbesii, T0729 Asarum maximum, T0730 Asarum sagittarioides, T0759 Aspidistra elatior, T0776 Asplenium prolongatum, T0827 Aucuba chinensis ssp. omeiensis, T0859 Balanophora involucrata, T0877 Bauhinia championii, T0881 Bauhinia variegata, T0886 Beaumontia grandiflora, T0922 Berchemia polyphylla var. leioclada, T0926 Berneuxia thibetica, T0938 Bidens bipinnata, T0952 Blechnum orientale, T0962 Boehmeria platanifolia [Syn. Boehmeria tricuspis], T0964 Boenninghausenia albiflora, T0967 Boenninghausenia sessilicarpa, T0969 Boerhavia diffusa, T0982 Bos taurus domesticus, T1001 Brachystemma calycinum, T1008 Brassica juncea, T1031 Broussonetia kazinoki, T1034 Broussonetia papyrifera, T1043 Bryophyllum pinnatum, T1046 Buddleja davidii, T1053 Bulbophyllum odoratissimum [Syn. Stelis odoratissimum], T1106 Caesalpinia minax, T1129 Calophyllum inophyllum, T1137 Caltha palustris, T1145 Camellia japonica, T1174 Cannabis sativa, T1188 Caragana chamlagu, T1191 Caragana sinica, T1199 Carduus acanthoides, T1200 Carduus crispus, T1212 Carpesium eximum, T1215 Carthamus tinctorius, T1251 Cassytha filiformis, T1256 Castanea mollissima, T1286 Celastrus flagellaris, T1290 Celastrus orbiculatus [Syn. Celastrus articulatus], T1311 Centella asiatica, T1316 Cephalanthus occidentalis, T1327 Ceratostigma minus, T1328 Ceratostigma plumbaginoides, T1329 Ceratostigma willmottianum, T1361 Chenopodium ambrosioides, T1371 Chloranthus japonicus, T1372 Chloranthus serratus, T1373 Chloranthus serratus, T1374 Chloranthus spicatus, T1384 Chondrus ocelladus, T1417 Cimicifuga acerina, T1423 Cimicifuga nanchuanensis, T1436 Cinnamomum camphora, T1443 Cinnamomum parthenoxylum [Syn. Cinnamomum porrectum], T1444 Cinnamomum tamala, T1450 Cirsium lineare, T1452 Cissampelos pareira, T1453 Cissampelos pareira var. hirsute, T1491 Citrus limon, T1495 Citrus limonia, T1535 Clausena excavata, T1555 Clerodendron fortunatum,
T1561 Clerodendron serratum, T1563 Clerodendron trichotomum, T1566 Clerodendrum inerme, T1569 Clerodendrum serratum var. amplexifolium, T1570 Clerodendrum thomsonae, T1574 Clintonia alpina, T1589 Cocculus trilobus [Syn. Cocculus sarmentosus], T1590 Cocculus trilobus [Syn. Cocculus sarmentosus], T1604 Coeloglossum viride [Syn. Coeloglossum viride var. bracteatum], T1624 Colocasia antiquorum, T1629 Colysis pothifolia [Syn. Hemionitis pothifolia], T1638 Commiphora myrrha [Syn. Commiphora molmol], T1640 Coniogramme japonica [Syn. Hemionitis japonica], T1645 Consolida ajacis [Syn. Delphinium ajacis], T1657 Conyza canadensis [Syn. Erigeron canadensis], T1672 Corallodiscus flabellatus [Syn. Didissandra flabellat], T1684 Cordyline strcta, T1692 Coriaria sinica [Syn. Coriaria nepalensis], T1706 Corydalis ambigua var. amurensis [Syn. Corydalis ambigua], T1715 Corydalis decumbens [Syn. Corydalis amabilis], T1721 Corydalis linearioides, T1730 Corydalis ophiocarpa, T1735 Corydalis repens, T1745 Corydalis suaveolens [Syn. Corydalis sheareri], T1767 Craibiodendron yunnanese, T1784 Cratoxylum cochinchinense, T1796 Crinum asiaticum var. sinicum, T1810 Croomia japonica, T1815 Crotalaria assamica, T1823 Crotalaria juncea, T1841 Croton caudatus var. tomentosus, T1866 Cryptolepis sinensis, T1871 Cucubalus baccifer, T1884 Cudrania cochinchinensis, T1900 Curculigo capitulata [Syn. Leucojum capitulata], T1903 Curcuma aromatica, T1905 Curcuma longa, T1922 Cyanotis vaga, T1924 Cyathula officinalis, T1927 Cycas revoluta, T1928 Cycas revoluta, T1939 Cymbopogon citratus, T1943 Cymbopogon goeringii, T1959 Cynanchum paniculatum, T1963 Cynanchum wallichii, T1976 Cyperus iria, T1984 Cypripedium macranthum [Syn. Cypripedium tibeticum], T1986 Cyrtomium fortunei, T1991 Cytisus scoparius [Syn. Spartium scoparium], T1994 Daemonorops draco, T2008 Dalbergia odorifera, T2013 Dalbergia sissoo, T2020 Damnacanthus indicus, T2027 Daphne odora, T2030 Daphne retusa, T2031 Daphne tangutica, T2037 Datura innoxia, T2039 Datura innoxia, T2043 Datura metel, T2044 Datura metel, T2047 Datura stramonium, T2052 Davallia divaricata [Syn. Davallia formosana; Davallia orientalis], T2053 Davallia mariesii, T2091 Delphinium yunnanense, T2130 Desmodium gangeticum, T2131 Desmodium pulchellum [Syn. Phyllodium pulchellum], T2132 Desmodium pulchellum [Syn. Phyllodium pulchellum], T2133 Desmodium racemosum [Syn. Podocarpium podocarpum var. oxyphyllum], T2136 Desmos cochinchinensis [Syn. Desmos chinensis], T2139 Dianella ensifolia, T2189 Dioscorea althaeoides, T2194 Dioscorea collettii, T2211 Dioscorea tenuipes, T2231 Diphylleia grayi, T2232 Diphylleia sinensis, T2234 Dipsacus asperoides, T2235 Dipsacus japonicus, T2244 Doellingeria scaber [Syn. Aster scaber], T2253 Dracaena cochinchinensis, T2267 Drosera peltata var. lunata, T2298 Dysosma difformis, T2302 Dysosma pleiantha [Syn. Podophyllum pleianthum], T2304 Dysosma veitchii, T2305 Dysosma versipellis [Syn. Podophyllum versipelle], T2348 Embelia ribes, T2357 Emilia sonchifolia, T2409 Equisetum palustre, T2422 Erigeron breviscapus, T2442 Ervatamia divaricata, T2459 Erythrina arborescens, T2478 Erythrina variegata [Syn. Erythrina indica], T2521 Eucalyptus tereticornis, T2545 Euonymus phellomana, T2546 Euonymus sacrosancta, T2555 Eupatorium chinense, T2563 
Eupatorium japonicum, T2601 Euphorbia milii, T2612 Euphorbia prolifera, T2691 Farfugium japonicum, T2721 Ficus microcarpa, T2723 Ficus pumila, T2727 Ficus simplicissima, T2731 Firmiana simplex, T2734 Fissistigma glaucescens [Syn. Melodorum glaucescens], T2735 Fissistigma oldhamii [Syn. Melodorum oldhamii], T2737 Flemingia philippinensis [Syn. Moghania philippinensis], T2752 Fordia cauliflora, T2830 Galium aparine, T2835 Galium verum, T2883 Gardenia jasminoides [Syn. Gardenia florida], T2885 Gardenia jasminoides var. grandiflora, T2895 Gaultheria yunnanensis, T2897 Gelsemium elegans, T2973 Glechoma longituba, T2974 Glechoma lungituba, T3033 Gnetum parvifolium [Syn. Gnetum indicum], T3063 Grevillea robusta, T3077 Gymnadenia conopsea, T3088 Gynura segetum [Syn. Gynura japonica], T3089 Gypsophila acutifolia, T3113 Hedera nepalensis var. sinensis, T3115 Hedera rhombea, T3118 Hedychium coronarium, T3123 Hedyotis auricularia, T3125 Hedyotis chrysotricha [Syn. Oldenlandia chrysotricha], T3155 Helianthus tuberosus, T3187 Helleborus thibetanus, T3201 Hemiphragma heterophyllum, T3241 Hibiscus mutabilis, T3270 Holboellia fargesii, T3276 Homo sapiens, T3294 Huperzia selago [Syn. Lycopodium selago], T3295 Huperzia serrata [Syn. Lycopodium serratum], T3303 Hydrangea chinensis, T3311 Hydrocotyle sibthorpioides, T3316 Hylomecon japonica, T3340 Hypericum ascyron, T3349 Hypericum erectum, T3356 Hypericum japonicum, T3360 Hypericum patulum, T3363 Hypericum sampsonii, T3381 Hyptis suaveolens, T3395 Ilex pedunculosa, T3398 Ilex rotunda, T3401 Illicium henryi, T3411 Impatiens balsamina, T3413 Impatiens nolitangere, T3414 Impatiens siculifer, T3420 Incarvillea sinensis, T3457 Iris dichotoma, T3479 Isodon amethystoides, T3479 Isodon amethystoides, T3496 Isodon japonica [Syn. Rabdosia japonica], T3547 Ixeris chinensis, T3549 Ixora chinensis, T3555 Jasminum nudiflorum, T3613 Kadsura coccinea [Syn. Kadsura chenensis; Kadsura hainanensis], T3614 Kadsura heteroclita [Syn. Uvaria heteroclita], T3619 Kadsura peltigera [Syn. Kadsura longipedunculata], T3624 Kalopanax pictum, T3625 Kalopanax septemlobus, T3645 Kummerowia striata, T3646 Kyllinga brevifolia, T3674 Laggera alata, T3675 Lagopsis supina, T3679 Lamiophlomis rotata [Syn. Phlomis rotata], T3680 Lamium amplexicaule, T3681 Lamium barbatum, T3725 Laurus nobilis, T3746 Leontice robustum, T3765 Lepisorus ussuriensis, T3770 Lespedeza cuneata, T3775 Lethariella cladonioides, T3778 Leucaena glauca [Syn. Leucaena leucocephala], T3802 Ligularia dentata, T3805 Ligularia fischeri, T3807 Ligularia intermedia, T3808 Ligularia japonica [Syn. Arnica japonica; Senecio japonica], T3809 Ligularia lapathifolia, T3813 Ligularia sibirica, T3830 Ligustrum sinense, T3847 Lindera angustifolia, T3851 Lindera glauca, T3853 Lindera obtusiloba, T3859 Linum usitatissimum, T3863 Liparis nervosa, T3885 Litsea cubeba, T3887 Litsea glutinosa, T3925 Loranthus parasiticus [Syn. Loranthus chinenis; Taxillus chinensis], T3964 Lycopodium alpinum [Syn. Diphasiastrum alpinum], T3965 Lycopodium annotinum, T3969 Lycopodium casuarinoides, T3970 Lycopodium cernuum, T3971 Lycopodium complanatum, T3973 Lycopodium japonicum [Syn. Lycopodium clavatum], T3980 Lycopus lucidus, T3984 Lycoris chinensis, T3986 Lycoris radiata [Syn. Amaryllis radiata], T3989 Lygodium flexuosum [Syn. Lygodium pinnatifidum; Ophioglossum flexuosum], T3991 Lyonia ovalifolia, T3992 Lyonia ovalifolia var. elliptica, T3995 Lysidice rhodostegia, T3998 Lysimachia christinae, T3999 Lysimachia clethroides, T4004 Lysimachia paridiformis, T4005 Lysionotus pauciflorus, T4036 Magnolia coco, T4080 Mallotus apelta, T4085 Mallotus repandus var. chrysocarpus [Syn. Mallotus chrysocarpus; Mallotus repandus], T4131 Maytenus confertiflorus, T4143 Meconopsis horridula, T4219 Microlepia marginata, T4225 Microsorium punctatum, T4234 Millettia dielsiana, T4239 Millettia nitida var. hirsutissima, T4248 Mimosa pudica, T4252 Mirabilis jalapa, T4260 Moghania philippinensis, T4284 Morinda parvifolia, T4303 Moschus moschiferus; Moschus berezovskii; Moschus sifanicus, T4311 Mucuna sempervirens, T4317 Murraya euchrestifolia [Syn. Clausena euchrestifolia], T4320 Murraya kwangsiensis, T4324 Murraya paniculata [Syn. Chalcas paniculata], T4335 Mussaenda pubescens, T4349 Myrica rubra, T4444 Nothopanax davidii, T4450 Nuphar pumilum, T4470 Ocimum basilicum, T4502 Onychium japonicum [Syn. Tricomanes japonicum], T4526 Origanum vulgare, T4542 Orthosiphon wulfenioides [Syn. Coleus wulfenioides], T4562 Oxalis acetosella, T4564 Oxalis corniculata [Syn. Oxalis repens], T4568 Oxytropis myriophylla, T4577 Paederia scandens, T4584 Paeonia lactiflora wild, T4586 Paeonia obovata, T4590 Paeonia veitchii, T4606 Panax pseudo-ginseng, T4607 Panax pseudo-ginseng var. japonicus, T4631 Papaver nudicaule var. chinense, T4642 Parameria laevigata, T4670 Patrinia heterophylla, T4673 Patrinia scabra, T4704 Peperomia duclouxii, T4705 Peperomia pellucida, T4710 Pericampylus glaucus, T4725 Periploca calophylla, T4726 Periploca forrestii, T4740 Petasites japonicus, T4800 Phlegmariurus fordii, T4801 Phlegmariurus phlegmaria [Syn. Lycopodium phlegmaria], T4810 Phlomis mongolica, T4814 Phlomis umbrosa, T4821 Pholidota articulata, T4837 Phyllanthus emblica, T4842 Phyllanthus reticulatus, T4884 Picria felterrae, T4903 Pinellia pedatisecta, T4906 Pinus armandii, T4916 Pinus massoniana, T4938 Piper boehmeriaefolium, T4948 Piper hancei, T4951 Piper laetispicum, T4956 Piper mullesua, T4965 Piper sarmentosum, T5018 Pleuropterus ciliinervis, T5057 Podophyllum emodii [Syn. Podophyllum emodii var. chinense; Podophyllum sikkimenosis; Sinopodophyllum emodii], T5071 Polygala arillata, T5073 Polygala chinensis [Syn. Polygala glomerata], T5075 Polygala fallax [Syn. Polygala aureocauda], T5087 Polygala telephioides, T5090 Polygala wattersii, T5100 Polygonum chinense, T5101 Polygonum cuspidatum, T5104 Polygonum hydropiper, T5106 Polygonum lapathifolium, T5109 Polygonum nodosum, T5114 Polygonum polystachyum, T5124 Polypodium niponicum, T5126 Polypodium virginianum, T5127 Polypodium vulgare, T5184 Potentilla kleiniana, T5189 Pothos chinensis, T5193 Pratia nummularia, T5207 Prismatomeris tetrandra, T5233 Prunus persica, T5238 Prunus salicina, T5250 Psammosilene tunicoides, T5269 Psilotum nudum, T5279 Psychotria serpens, T5297 Pteris plumbea, T5298 Pteris vittata, T5330 Punica granatum, T5339 Pycnoporus sanguineus, T5341 Pygmaeopremna herbacea [Syn. Premna herbacea], T5401 Rabdosia yuennanensis, T5455 Rhamnus crenata, T5472 Rheum officinale, T5474 Rheum palmatum, T5481 Rheum tanguticum, T5497 Rhodiola kirilowii, T5499 Rhodiola sacra, T5513 Rhododendron mariae, T5516 Rhododendron molle, $\mathrm{T} 5517$ Rhododendron molle, $\mathrm{T} 5518$ 
Rhododendron mucronatum, T5537 Rhus sylvestris, T5547 Ricinus communis, T5555 Rodgersia pinnata, T5560 Rorippa montana [Syn. Rorippa dubia; Sisymbrium dublium], T5562 Rosa chinensis, T5563 Rosa cymosa, T5576 Rostellularia procumbens [Syn. Justicia procumbens], T5578 Rubia cordifolia, T5579 Rubia cordifolia, T5582 Rubia oncotricha, T5583 Rubia schumannina, T5584 Rubia tinctorum, T5585 Rubia wallichiana, T5586 Rubia yunnanensis, T5587 Rubus alceaefolius, T5595 Rubus parviforlius, T5609 Rumex hastatus, T5611 Rumex japonicus, T5612 Rumex nepalensis, T5613 Rumex obtusifolius, T5614 Rumex patientia, T5626 Ruta graveolens, T5637 Sabia schumanniana, T5680 Salvia miltiorrhiza, T5681 Salvia miltiorrhiza $\mathrm{f}$. alba, T5685 Salvia plebeia, T5688 Salvia przewalskii, T5697 Salvia trijuga, T5704 Sambucus nigra, T5707 Sambucus williamsii, T5714 Sansevieria trifasciata, T5730 Sarcandra glabra [Syn. Chloranthus glaber], T5731 Sarcococca coriacea [Syn. Sarcococca wallichii], T5746 Sassafras tzumu, T5756 Saussurea japonica, T5793 Schisandra henryi, T5794 Schisandra lancifolia, T5795 Schisandra micrantha, T5797 Schisandra propinqua, T5798 Schisandra propinqua var. intermedia, T5813 Scilla scilloides, T5819 Scopolia acutangula [Syn. Anisodus acutangulus], T5835 Scutellaria barbata [Syn. Scutellaria rivularis], T5838 Scutellaria galericulata, T5840 Scutellaria indica, T5844 Scutellaria scordifolia, T5847 Securidaca inappendiculata, T5850 Sedum aizoon, T5855 Sedum kamtschaticum, T5865 Selaginella pulvinata, T5866 Selaginella sanguinolenta, T5868 Selaginella stauntoniana, T5869 Selaginella tamariscina, T5883 Senecio cannabifolius, T5893 Senecio nudicaulis, T5939 Shiraia bambusicola, T5942 Sida acuta, T5954 Silene fortunei, T5978 Smilax glauco-china, T5981 Smilax riparia, T5989 Solanum aculeatissimum, T6014 Solanum surattense, T6023 Solidago virgaurea, T6051 Sorbaria arborea, T6052 Sorbaria sorbifolia, T6062 Sparganium stoloniferum, T6075 Spilanthes acmella, T6118 Stephania brachyandra, T6121 Stephania dicentrinifera, T6122 Stephania dielsiana, T6133 Stephania sinica, T6137 Stephania viridiflavens, T6154 Strobilanthes japonicus [Syn. Championella japonica], T6155 Strophanthus divaricatus, T6166 Strychnos angustiflora, T6184 Strychnos nux-vomica, T6193 Strychnos wallichiana, T6252 Symplocos chinensis, T6257 Syngnathus acus, T6276 Tacca plantaginea [Syn. Schizocapsa plantaginea], T6353 Tetracera asiatica, T6367 Teucrium quadrifarium, T6393 Thalictrum ichangense [Syn. Thalictrum tripeltatum; Thalictrum multipeltatum], T6398 Thalictrum microgynum, T6444 Thunbergia grandiflora, T6468 Tinospora sinensis, T6471 Toddalia asiatica [Syn. Toddalia aculeata; Paullinia asiatica], T6484 Trachelospermum jasminoides, T6491 Trametes cinnabarina [Syn. Polyporus cinnabarinus; Boletus cinnabarinus], T6531 Trillium camtschaticum, T6533 Trillium kamtschaticum, T6535 Trillium tschonoskii, T6541 Tripterygium regelii, T6566 Tupistra chinensis, T6580 Tylophora floribunda, T6618 Uncaria homomalla [Syn. Uruparia homomalla; Uruparia tonkinensis; Uruparia lanosa var. parvifora], T6651 Urtica cannabina, T6652 Urtica dioica, T6675 Valeriana amurensis, T6679 Valeriana officinalis, T6680 Valeriana officinalis var. latifolia, T6689 Ventilago leiocarpa, T6691 Veratrilla baillonii, T6693 Veratrum album var. lobelianum [Syn. Veratrum lobelianum], T6722 Veronica anagallis-aquatica, T6728 Veronica serpyllifolia, T6737 Viburnum luzonicum, T6742 Vicia amoena, T6777 Viscum multinerve, T6792 Vitex trifolia, T6820 Wikstroemia indica, T6830 Woodfordia fruticosa, T6858 Zanthoxylum acanthopodium, T6861 Zanthoxylum ailanthoides, T6862 Zanthoxylum ailanthoides, T6872 Zanthoxylum cuspidatum, T6874 Zanthoxylum dimorphophyllum var. spinifolum, T6877 Zanthoxylum dissitum, T6888 Zanthoxylum planispinum, T6889 Zanthoxylum podocarpum, T6895 Zanthoxylum simulans.

lack of strength and sloppy stool T6916 Ziziphus jujuba, T6917 Ziziphus jujuba var. inermis.

lacquer sore T0322 Allium tuberosum, T0568 Aralia chinensis, T0570 Aralia dasyphylla, T1007 Brassica juncea, T1182 Capra hircus; Ovis aries, T1256 Castanea mollissima, T1758 Cotinus coggygria, T1759 Cotinus coggygria var. cinerea, T1769 Crataegus cuneata, T1771 Crataegus kansuensis, T1772 Crataegus maximowiczii, T1777 Crataegus pinnatifida, T1780 Crataegus sanguinea, T1781 Crataegus scabrifolia, T2723 Ficus pumila, T2988 Glochidion eriocarpum, T3124 Hedyotis capitellata, T5102 Polygonum cuspidatum, T5560 Rorippa montana [Syn. Rorippa dubia; Sisymbrium dublium], T5646 Sagina japonica [Syn. Spergula japonica], T6365 Teucrium pilosum [Syn. Teucrium japonicum var. pilosum].

lai T1256 Castanea mollissima, T1896 Cupressus funebris, T3671 Lagerstroemia indica, T4042 Magnolia liliflora.

lai lichen T1097 Cacalia ainsliaeflora.

lai sore T3413 Impatiens nolitangere, T5330 Punica granatum.

laryngeal carcinoma T0318 Allium sativum.

laryngeal infection in children T4845 Physalis alkekengi.

laryngitis T1179 Capparis masaikai, T1545 Clematis chinensis, T4274 Monostroma nitidum, T4403 Nemacystus decipiens [Syn. Mesogloea decipiens; Cladosiphon decipiens], T5309 Pterocladia tenuis, T5818 Scoparia dulcis, T5967 Siraitia grosvenorii [Syn. Momordica grosvenorii], T6043 Sophora subprostrata [Syn. Sophora tonkinensis], T6301 Taraxacum mongolicum, T6599 Ulva lactuca, T6600 Ulva pertusa.

lassitude in limbs T0529 Anthriscus sylvestris, T1596 Codonopsis canescens, T1597 Codonopsis clematidea, T1599 Codonopsis pilosula, T1600 Codonopsis pilosula var. modesta [Syn. Codonopsis modesta], T1601 Codonopsis subglobosa, T1602 Codonopsis tangshen, T1603 Codonopsis tubulosa, T2955 Geum japonicum.

lassitude in lumbar and knees T3992 Lyonia ovalifolia var. elliptica, T5925 Sesamum indicum [Syn. Sesamum orientale].

lassitude in lumbus and knees T2738 Flemingia strobilifera, T3955 Lycium barbarum, T3958 Lycium chinense, T5344 Pyrola atropurpurea, T5345 Pyrola calliantha [Syn. Pyrola rotundifolia ssp. chinensis], T5346 Pyrola calliantha var. tibetana, T5347 Pyrola decorata, T5349 Pyrola incarnata, T5350 Pyrola japonica, T5351 Pyrola rotundifolia, $\mathrm{T} 5950$ Siegesbeckia orientalis, T5951 Siegesbeckia orientalis var. glabrescens [Syn. Siegesbeckia glabrescens], T5952 Siegesbeckia orientalis var. pubescens [Syn. Siegesbeckia pubescens].

late afternoon tidal fever T2909 Gentiana crassicaulis, T2910 Gentiana dahurica, T2913 Gentiana kaufmanniana, T2919 Gentiana macrophylla, T2932 Gentiana siphonantha, T2934 Gentiana straminea, T2936 Gentiana tianschanica, T2937 Gentiana tibetica. 
leech in nose T2852 Garcinia cowa.

leg $q \boldsymbol{i}$ [ = beriberi] $\mathrm{T} 3766$ Lepista nuda, $\mathrm{T} 3937$ Lumbriconeis heteropoda, T4547 Oryza sativa cv., T5158 Populus simonii, T5391 Rabdosia eriocalyx, T6752 Vigna angularis [Syn. Dolichus angularis; Phaseolus angularis].

leg qi damp-erosion T5389 Rabdosia coetsa.

leg $q \boldsymbol{i}$ due to wind-damp T2272 Drymaria diandra [Syn. Drymaria cordata ssp. diandra].

leg $q \boldsymbol{i}$ puffy swelling T5740 Sargassum vachellianum.

leg qi swelling and pain T1436 Cinnamomum camphora, T3413 Impatiens nolitangere.

leg $q \boldsymbol{i}$ with edema T5156 Populus nigra var. thevestina.

leg $q i$ with swelling toxin T4714 Perilla frutescens.

leprosy T0099 Aconitum gymnandrum, T1046 Buddleja davidii, T1282 Cedrus deodara, T1624 Colocasia antiquorum, T2979 Gleditsia sinensis [Syn. Gleditsia horrida], T3082 Gynocardia odorata, T3289 Humulus lupulus, T3569 Juglans regia, T3860 Linum usitatissimum, T4562 Oxalis acetosella, T4917 Pinus massoniana, T5368 Python molurus bivittatus, T6031 Sophora flavescens [Syn. Sophora angustfolia], T6542 Tripterygium wilfordii, T6820 Wikstroemia indica.

leprosy (leprosy) T3560 Jatropha curcas.

leptochroa T4247 Millingtonia hortensis.

leptospirosis T0333 Alocasia cucullata [Syn. Arum cucullatum], T2517 Eucalyptus robusta, T2521 Eucalyptus tereticornis, T3284 Houttuynia cordata.

leukaemia T1318 Cephalotaxus fortunei.

leukaemia in early stage and carcinoma of uterine cervix in early stage T1903 Curcuma aromatica, T1905 Curcuma longa.

leukopenic complications of carcinoma T0897 Berberis amurensis, T0912 Berberis poiretii, T0920 Berberis wilsonae.

leukoplakia of vulva T3371 Hypocrella bambusae.

leukorrhea T0427 Ampelopsis japonica [Syn. Paullinia japonica], T0479 Angelica dahurica cv. qibaizhi, T0498 Angelica taiwaniana, T0567 Aralia armata, T0632 Aristolochia manshuriensis, T1001 Brachystemma calycinum, T1353 Changium smyrnioides, T1528 Cladonia stellaris [Syn. Cladonia alpestris], T1984 Cypripedium macranthum [Syn. Cypripedium tibeticum], T2161 Dicranopteris pedata [Syn. Polypodium pedatum; Dicranopteris dichotoma], T2194 Dioscorea collettii, T2197 Dioscorea futschauensis, T2210 Dioscorea spongiosa, T2632 Euryale ferox, T2721 Ficus microcarpa, T3221 Heracleum rapula, Т3363 Hypericum sampsonii, T3776 Lethariella zahlbruckneri, T4144 Meconopsis nepaulensis, T4145 Meconopsis punicea, T4821 Pholidota articulata, T4917 Pinus massoniana, T5289 Pteris dactylina, T5328 Punica granatum, T5499 Rhodiola sacra, T6008 Solanum nigrum, T6095 Stachytarpheta jamaicensis, T6378 Thalictrum delavayi, T6436 Thlaspi arvense, T6754 Vigna unguiculata, $\mathrm{T} 6831$ Woodwardia orientalis.

lichen T0338 Aloe ferox, T0347 Aloe vera [Syn. Aloe barbadensis], T0348 Aloe vera var. chinensis, T2124 Derris trifoliata, T2479 Erythrina variegata var. orientalis, T2521 Eucalyptus tereticornis, T2717 Ficus carica, T3630 Kleinhovia hospita, T3989 Lygodium flexuosum [Syn. Lygodium pinnatifidum; Ophioglossum flexuosum], T4253 Mirabilis jalapa, T4906 Pinus armandii, T4916 Pinus massoniana, T5143
Pongamia pinnata.

lichen and scab sore T3327 Hyoscyamus niger.

lichen lai T2062 Delphinium bonvalotii, T2081 Delphinium omeiense, T2084 Delphinium potaninii, T2085 Delphinium potaninii var. jiufengshanense.

lichen papules T6752 Vigna angularis [Syn. Dolichus angularis; Phaseolus angularis].

lichen sore T0100 Aconitum hemsleyanium var. circinacum, T0101 Aconitum hemsleyanum, T0318 Allium sativum, T0941 Bidens tripartita, T0957 Blumea balsamifera, T1867 Cryptomeria fortunei, T2624 Euphorbia tirucalli, T3287 Huechys sanguinea, T3413 Impatiens nolitangere, T3588 Juniperus formosana, T3852 Lindera megaphylla, T3991 Lyonia ovalifolia, T4208 Metasequoia glyptostroboides, T5234 Prunus persica.

limp aching in legs and knees T1254 Castanea mollissima.

limp aching inability of legs and knees T2531 Eucommia ulmoides.

limp aching lumbus and knees T0323 Allium tuberosum, T0990 Bos taurus domesticus; Bubalus bubalis, T1188 Caragana chamlagu, T1455 Cistanche deserticola, T1456 Cistanche salsa, T1604 Coeloglossum viride [Syn. Coeloglossum viride var. bracteatum], T1900 Curculigo capitulata [Syn. Leucojum capitulata], T1911 Cuscuta australis, T1912 Cuscuta chinensis, T1913 Cuscuta japonica, T1964 Cynanchum wilfordii [Syn. Cynoctonum wilfordii], T2234 Dipsacus asperoides, T2235 Dipsacus japonicus, T2323 Eclipta prostrata [Syn. Eclipta alba], T2805 Fugu ocellatus, T3077 Gymnadenia conopsea, T3390 Ilex cornuta, T4056 Mahonia bealei, T4057 Mahonia bealei, T4063 Mahonia fortunei, T4067 Mahonia japonica, T4068 Mahonia japonica, T4729 Periploca sepium, T5446 Rehmannia glutinosa [Syn. Rehmannia glutinosa f. huechingensis], T5753 Saussurea gnaphaloides, T5757 Saussurea laniceps, T5759 Saussurea medusa, T6773 Viscum articulactum, T6774 Viscum articulatum, T6775 Viscum coloratum.

limp aching lumbus and knees due to kidney vacuity T1035 Broussonetia papyrifera.

limp aching lumbus and knees due to kidney yin vacuity T5091 Polygonatum cyrtonema [Syn. Polygonatum multiflorum], T5092 Polygonatum kingianum, T5095 Polygonatum sibiricum.

limp aching lumbus and knees due to liver kidney yin vacuity T5107 Polygonum multiflorum.

limp aching numbness in limbs T1161 Campsis grandiflora.

limp wilting of lower limb T1972 Cynomorium songaricum.

limp wilting sinew and bone T0036 Acanthopanax giraldii [Syn. Acanthopanax giraldii var. inermis; Eleutherococcus giraldii], T0038 Acanthopanax gracilistylus, T0042 Acanthopanax senticosus [Syn. Eleutherococcus senticosus], T0044 Acanthopanax sessiliflorus, T1337 Cervus nippon; Cervus elaphus, T1901 Curculigo orchioides, T2389 Epimedium acuminatum, T2390 Epimedium brevicornum, T2392 Epimedium davidii, T2394 Epimedium elongatum, T2398 Epimedium koreanum, T2401 Epimedium pubescens, T2402 Epimedium sagittatum, T2404 Epimedium sutchuenense, T2406 Epimedium wushanense, T6771 Viscum album.

lip sore $\mathrm{T} 3829$ Ligustrum robustum.

liquor damage with thirst $\mathrm{T} 1472$ Citrus chachiensis, T1517 Citrus 
tankan, T2758 Fortunella crassifolia, T2759 Fortunella japonica, T2760 Fortunella margarita.

liquor jaundice T4042 Magnolia liliflora.

liquor jaundice accumulation T4050 Magnolia sieboldii.

lithiasis T5098 Polygonum aviculare.

liver depression and qi pain T1978 Cyperus rotundus, T4036 Magnolia coco.

liver depression and rib-side pain T1055 Bupleurum angustissimum, T1056 Bupleurum aureum, T1058 Bupleurum chaishoui, T1059 Bupleurum chinense, T1065 Bupleurum longiradiatum, T1066 Bupleurum marginatum, T1067 Bupleurum marginatum var. stenophyllum, T1072 Bupleurum scorzonerifolium, T1073 Bupleurum sibiricum, T1075 Bupleurum smithii var. parvifolium, T1078 Bupleurum wenchuanense, T1079 Bupleurum yinchowense, T4184 Mentha haplocalyx [Syn. Mentha canadaensis; Mentha arvensis var. haplocalyx; Mentha arvensis].

liver enlargement T3461 Iris japonica, T3471 Iris tectorum.

liver fire and red eyes T0049 Acer ginnala, T0334 Aloe arborescens var. natalensis, T3332 Hypecoum erectum, T5199 Primula malacoides, T6036 Sophora japonica.

liver fire headache T5775 Scabiosa comosa, T6034 Sophora japonica, T6625 Uncaria macrophylla, T6629 Uncaria rhynchophylla [Syn. Nauclea rhynchophylla], T6633 Uncaria sinensis.

liver gallbladder effulgent fire T3233 Heteropappus altaicus.

liver gallbladder pain T6802 Vladimiria souliei [Syn. Jurinea souliei].

liver heat and convulsion T4648 Paris polyphylla, T4649 Paris polyphylla var. chinensis, T4650 Paris polyphylla var. pseudothibetica, T4651 Paris polyphylla var. stenophylla, T4652 Paris polyphylla var. yunnanensis.

liver heat and dizziness $\mathrm{T} 3040$ Gomphrena globosa.

liver heat and red eyes T1236 Cassia laevigata [Syn. Cassia floribunda], T1804 Cristaria plicata; Hyriopsis cumingii, T2772 Fraxinus mandshurica, T5762 Saussurea nigrescens.

liver kidney vacuity T0793 Astragalus complanatus, T1964 Cynanchum wilfordii [Syn. Cynoctonum wilfordii], T3389 Ilex cornuta, T6670 Vaccinium bracteatum.

liver spleen enlargement T0048 Acanthus ilicifolius, T0680 Artemisia lactiflora, T2131 Desmodium pulchellum [Syn. Phyllodium pulchellum], T3457 Iris dichotoma, T4577 Paederia scandens, T5029 Plumbago zeylanica, T5587 Rubus alceaefolius, T5661 Salvia bowleyana, T6580 Tylophora floribunda.

liver stomach $q \boldsymbol{i}$ pain T1928 Cycas revoluta, T4537 Oroxylum indicum, T5226 Prunus mume, T5572 Rosa rugosa.

liver stomach $q \boldsymbol{i}$ stagnation T0275 Akebia quinata.

liver vacuity dim vision T1048 Buddleja officinalis, T2326 Elaeagnus angustifolia.

liver welling abscess T0867 Baphicacanthus cusia [Syn. Strobilanthes cusia].

liver wind headache T3859 Linum usitatissimum.

liver yang dizziness T1986 Cyrtomium fortunei, T6625 Uncaria macrophylla, T6629 Uncaria rhynchophylla [Syn. Nauclea rhynchophylla], T6633 Uncaria sinensis.

liver-gallbladder damp-heat T1705 Corydalis adunca. liver-kidney yin depletion T5446 Rehmannia glutinosa [Syn. Rehmannia glutinosa f. huechingensis].

lobar pneumonia T5907 Senecio scandens [Syn. Senecio chinensis].

local anesthesia T0728 Asarum heterotropoides var. mandshuricum, T0731 Asarum sieboldii, T0732 Asarum sieboldii var. seoulensis, T1052 Bufo bufo gargarizans; Bufo melanostictus, T4323 Murraya paniculata [Syn. Chalcas paniculata], T4325 Murraya paniculata var. exotica.

lumbago T0164 Actinidia polygama, T0170 Adhatoda vasica, T0275 Akebia quinata, T0495 Angelica sinensis, T0759 Aspidistra elatior, T0964 Boenninghausenia albiflora, T0967 Boenninghausenia sessilicarpa, T1289 Celastrus orbiculatus [Syn. Celastrus articulatus], T1434 Cinnamomum bejolghota [Syn. Cinnamomum obtusifolium; Laurus bejolghota], T1487 Citrus junos, T1504 Citrus reticulata, T1550 Cleome gynandra [Syn. Gynandropsis gynandra], T1680 Cordyceps militaris, T1681 Cordyceps militaris cv, T1959 Cynanchum paniculatum, T2030 Daphne retusa, T2031 Daphne tangutica, T2053 Davallia mariesii, T2263 Dregea volubilis, T2538 Euonymus bungeanus, T2541 Euonymus grandiflorus, T2735 Fissistigma oldhamii [Syn. Melodorum oldhamii], T3033 Gnetum parvifolium [Syn. Gnetum indicum], T3221 Heracleum rapula, T3287 Huechys sanguinea, T4131 Maytenus confertiflorus, T4182 Menispermum dauricum, T4725 Periploca calophylla, T4804 Phlojodicarpus sibiricus, T5075 Polygala fallax [Syn. Polygala aureocauda], T5230 Prunus persica, T5298 Pteris vittata, T5515 Rhododendron micranthum, T5579 Rubia cordifolia, T5637 Sabia schumanniana, T6431 Thesium chinense.

lumbago and limp aching T6087 Spiranthes sinensis.

lumbago and limp leg T0793 Astragalus complanatus, T1170 Canis familiaris, T3568 Juglans regia, T6771 Viscum album.

lumbar and back pain T2531 Eucommia ulmoides, T4118 Marsdenia oreophila.

lumbar and rib-side pain T3411 Impatiens balsamina.

lumbus kidney cold T4954 Piper longum.

lump glomus T0963 Boehmeria siamensis, T1215 Carthamus tinctorius, T1692 Coriaria sinica [Syn. Coriaria nepalensis], T2020 Damnacanthus indicus, T2580 Euphorbia antiquorum, T2695 Ferula assafoetida, T3412 Impatiens balsamina, T4519 Opuntia dillenii, T4521 Opuntia vulgaris, T5233 Prunus persica, T6356 Tetrapanax papyriferus.

lung abscess T3295 Huperzia serrata [Syn. Lycopodium serratum], T3476 Isatis indigotica.

lung cold cough T1008 Brassica juncea, T1479 Citrus grandis.

lung disease T1555 Clerodendron fortunatum, T3344 Hypericum chinense.

lung disease with blood ejection T2244 Doellingeria scaber [Syn. Aster scaber].

lung disease with cough T3955 Lycium barbarum, T3958 Lycium chinense.

lung heat abundant phlegm dry cough T5309 Pterocladia tenuis.

lung heat asthma and oppression T5220 Prunus davidiana, T5232 Prunus persica.

lung heat cough T0002 Abelmoschus moschatus [Syn. Hibiscus 
abelmoschus], T0011 Abrus precatorius, T0171 Adiantum capillus-veneris, T0173 Adiantum lunulatum, T0174 Adiantum monochlamys, T0175 Adiantum pedatum, T0263 Ajuga decumbens, T0264 Ajuga forrestii, T0266 Ajuga macrosperma, T0267 Ajuga nipponensis, T0378 Alstonia yunnanensis, T0457 Andrographis paniculata [Syn. Justicia paniculata], T0608 Arenaria kansuensis [Syn. Arenaria kumaonensis], T0736 Asclepias curassavica, T0759 Aspidistra elatior, T0859 Balanophora involucrata, T0860 Balanophora japonica, T0867 Baphicacanthus cusia [Syn. Strobilanthes cusia], T0920 Berberis wilsonae, T0941 Bidens tripartita, T0959 Blumea lacera, T1043 Bryophyllum pinnatum, T1353 Changium smyrnioides, T1696 Cornus capitata [Syn. Dendrobenthamia capitata], T1705 Corydalis adunca, T1746 Corydalis taliensis, T1789 Crepis napifera, T1950 Cynanchum ascyrifolium, T1951 Cynanchum atratum, T1962 Cynanchum versicolor, T2191 Dioscorea bulbifera, T2213 Dioscorea zingiberensis, T2433 Eriobotrya japonica, T2628 Euphrasia officinalis, T2783 Fritillaria cirrhosa, T2784 Fritillaria delavayi, T2790 Fritillaria pallidiflora, T2792 Fritillaria przewalskii, T2796 Fritillaria unibracteata, T2800 Fritillaria walujewii, T2902 Gentiana algida, T2950 Gerbera anandria [Syn. Leibnitzia anandria], T2952 Gerbera piloselloides, T3123 Hedyotis auricularia, T3241 Hibiscus mutabilis, T3242 Hibiscus rosa-sinensis, T3244 Hibiscus syriacus, T3247 Hibiscus taiwanensis, T3248 Hibiscus tiliaceus, T3288 Humulus japonicus [Syn. Humulus scandens], T3373 Hypoestes purpurea [Syn. Justicia purpurea; Hypoestes sinica], T3388 Ilex chinensis [Syn. Ilex purpurea], T3448 Ipomoea cairica [Syn. Ipomoea palmata], T3457 Iris dichotoma, T3547 Ixeris chinensis, $\mathrm{T} 3736$ Lemmaphyllum microphyllum, T3749 Leontopodium alpinum, T3769 Lespedeza bicolor, T3830 Ligustrum sinense, T3878 Lithocarpus polystachyus, T3926 Loropetalum chinense, T3935 Luffa cylindrica, T3983 Lycoris aurea, T4055 Mahonia bealei, T4062 Mahonia fortunei, T4066 Mahonia japonica, T4144 Meconopsis nepaulensis, T4183 Menispermum dauricum, T4368 Nandina domestica, T4370 Nandina domestica, T4506 Ophioglossum vulgatum, T4519 Opuntia dillenii, T4520 Opuntia ficus-indica, T4521 Opuntia vulgaris, T4537 Oroxylum indicum, T4573 Pachyrrhizus erosus, T4730 Peristrophe roxburghiana, T4760 Peucedanum japonicum, T4823 Pholidota yunnanensis, T4829 Phragmites communis, T4845 Physalis alkekengi, T4848 Physalis angulata, T4854 Physalis pubescens, T5099 Polygonum bistorta, T5100 Polygonum chinense, T5151 Populus davidiana, T5158 Populus simonii, T5177 Potamogeton pectinatus, T5184 Potentilla kleiniana, T5199 Primula malacoides, T5449 Reineckea carnea, T5468 Rheum emodi [Syn. Rheum australe], T5499 Rhodiola sacra, T5685 Salvia plebeia, T5714 Sansevieria trifasciata, T5719 Sapindus mukorossi, T5818 Scoparia dulcis, T5833 Scutellaria amoena, T5834 Scutellaria baicalensis, T5839 Scutellaria hypericifolia, T5841 Scutellaria likiangensis, T5843 Scutellaria rehderiana, T5845 Scutellaria viscidula, T5859 Selaginella braunii, T5861 Selaginella doederleinii, T6024 Solidago virgaurea var. leiocarpa [Syn. Solidago decurrens], T6047 Sophora viciifolia, T6217 Swertia davidii, T6304 Taxillus levinei, T6403 Thalictrum petaloideum, T6409 Thalictrum simplex [Syn. Thalictrum simplex var. brevipes], T6416 Thamnolia vermicularis, T6510 Trichosanthes kirilowii, T6513 Trichosanthes rosthornii [Syn. Trichosanthes uniflora], T6581 Tylophora mollissima, T6582 Tylophora ovata, T6691 Veratrilla baillonii.

lung heat cough asthma T0334 Aloe arborescens var. natalensis, T0374 Alstonia scholaris, T0624 Aristolochia contorta, T0626 Aristolochia debilis [Syn. Aristolochia longa], T0630 Aristolochia indica, T0633 Aristolochia maxima, T0640 Aristolochia triangularis, T2431 Eriobotrya japonica, T2510 Eucalyptus globulus, T2517 Eucalyptus robusta, T3126 Hedyotis corymbosa [Syn. Oldenlandia corymbosa], T3396 Ilex pubescens, T3397 Ilex pubescens var. glaber, T4120 Marsdenia tenacissima, T4124 Matricaria chamomilla [Syn. Matricaria recutita], T4287 Morus alba, T4485 Oldenlandia diffusa [Syn. Hedyotis diffusa], T5354 Pyrrosia davidii, T5355 Pyrrosia drakeana, T5356 Pyrrosia gralla, T5357 Pyrrosia lingua T5358 Pyrrosia petiolosa, T5360 Pyrrosia sheareri, T5775 Scabiosa comosa, T5840 Scutellaria indica, T6367 Teucrium quadrifarium.

lung heat cough due to yin vacuity T4605 Panax japonicus var. major.

lung heat dry cough T0540 Apis cerana, T0559 Arachis hypogaea, T1490 Citrus limon, T1494 Citrus limonia, T4289 Morus alba, T4291 Morus australis, T4293 Morus cathayana, T4298 Morus mongolica, T4331 Musa paradisiaca var. sapientum [Syn. Musa sapientum], T4388 Nasturtium officinale, T5091 Polygonatum cyrtonema [Syn. Polygonatum multiflorum], T5092 Polygonatum kingianum, T5095 Polygonatum sibiricum, T5218 Prunus armeniaca, T5967 Siraitia grosvenorii [Syn. Momordica grosvenorii], T6512 Trichosanthes kirilowii.

lung heat enduring cough T1183 Capra hircus; Ovis aries.

lung heat hemoptysis T0262 Ajuga ciliata, T1866 Cryptolepis sinensis, T3681 Lamium barbatum, T3863 Liparis nervosa, T5863 Selaginella moellendorffii.

lung heat phlegm cough T3284 Houttuynia cordata.

lung heat phlegm depression T0480 Angelica decursiva [Syn. Peucedanum decursivum], T3819 Ligusticum brachylobum, T4761 Peucedanum longshengens, T4768 Peucedanum praeruptorum, T4769 Peucedanum rubricaule.

lung heat $q \boldsymbol{i}$ counterflow T2867 Garcinia multiflora.

lung $q$ i block T1506 Citrus reticulata.

lung qi distention T2220 Diospyros kaki.

lung stomach yin damage T5093 Polygonatum odoratum [Syn. Polygonatum officinale], T5094 Polygonatum prattii.

lung vacuity $\mathrm{T} 0985$ Bos taurus domesticus; Bubalus bubalis.

lung vacuity and shortness of breath T4600 Panax ginseng [Syn. Panax schinseng].

lung vacuity cold cough T6267 Syzygium jambos.

lung vacuity consumption cough T0948 Blainvillea acmella [Syn. Verbesina acmella; Eclipta latifolia; Blainvillea latifolia].

lung vacuity cough T1598 Codonopsis convolvulacea, T4050 Magnolia sieboldii, T4276 Monotropa uniflora, T4821 Pholidota articulata, T6798 Vitis vinifera.

lung vacuity dry cough T6511 Trichosanthes kirilowii.

lung vacuity enduring cough T0624 Aristolochia contorta, T0626 Aristolochia debilis [Syn. Aristolochia longa], T0630 Aristolochia indica, T0633 Aristolochia maxima, T0640 Aristolochia triangularis, 
T0830 Auricularia auricula, T0831 Auricularia delicata, T1191 Caragana sinica, T2195 Dioscorea deltoidea.

lung vacuity taxation cough T6601 Umbilicaria esculenta [Syn. Gyrophora esculenta].

lung wilting T0746 Asparagus cochinchinensis [Syn. Asparagus lucidus], T3277 Homo sapiens, T6508 Trichosanthes cucumeroides.

lymphatic sarcoma T1318 Cephalotaxus fortunei.

lymphnoditis T3202 Hemistepta lyrata [Syn. Hemistepta carthamoides; Saussurea carthamoides], T5289 Pteris dactylina, T6567 Tupistra wattii [Syn. Campylandra wattii].

lymphrnoditis T0707 Arthraxon hispidus, T0867 Baphicacanthus cusia [Syn. Strobilanthes cusia], T2359 Endarachne binghamiae, T3243 Hibiscus rosa-sinensis, T3423 Indigofera tinctoria, T4264 Momordica cochinchinensis.

macula T0648 Arnebia euchroma, T0649 Arnebia guttata, T1419 Cimicifuga dahurica, T1420 Cimicifuga foetida, T1421 Cimicifuga heracleifolia, T3881 Lithospermum erythrorhizon, T5447 Rehmannia glutinosa [Syn. Rehmannia glutinosa f. huechingensis].

macular eruption T0867 Baphicacanthus cusia [Syn. Strobilanthes cusia], T4582 Paeonia delavayi, T4585 Paeonia moutan [Syn. Paeonia suffruticosa], T5120 Polygonum tinctorium, T5826 Scrophularia buergeriana, T5828 Scrophularia ningpoensis.

macular eruption and papules T1045 Bubalus bubalis, T3476 Isatis indigotica, T5121 Polygonum tinctorium.

maculopapular eruption T1986 Cyrtomium fortunei, T3475 Isatis indigotica.

malaria T0071 Achyranthes aspera, T0072 Achyranthes aspera var. indica, T0248 Agrimonia pilosa, T0249 Agrimonia pilosa var. japonica, T0318 Allium sativum, T0374 Alstonia scholaris, T0378 Alstonia yunnanensis, T0382 Althaea rosea, T0468 Anemone hupehensis, T0471 Anemone rivularis, T0500 Anguilla japonica, T0568 Aralia chinensis, T0570 Aralia dasyphylla, T0606 Areca catechu, T0653 Artabotrys hexapetalus [Syn. Annona hexapetalus], T0660 Artemisia annua, T0662 Artemisia apiacea [Syn. Artemisia carvifolia; Artemisia caruifolia], T0813 Atalantia buxifolia [Syn. Severinia buxifolia], T0814 Atalantia buxifolia [Syn. Severinia buxifolia], T0947 Bixa orellana, T1038 Brucea javanica [Syn. Brucea sumatrana; Rhus javanica], T1039 Bruguiera gymnorrhiza, T1048 Buddleja officinalis, T1055 Bupleurum angustissimum, T1056 Bupleurum aureum, T1058 Bupleurum chaishoui, T1059 Bupleurum chinense, T1065 Bupleurum longiradiatum, T1066 Bupleurum marginatum, T1067 Bupleurum marginatum var. stenophyllum, T1072 Bupleurum scorzonerifolium, T1073 Bupleurum sibiricum, T1075 Bupleurum smithii var. parvifolium, T1078 Bupleurum wenchuanense, T1079 Bupleurum yinchowense, T1176 Cannabis sativa, T1247 Cassia sophera, T1428 Cinchona ledgeriana, T1429 Cinchona officinalis, T1433 Cinchona succirubra, T1446 Cipadessa baccifera, T1535 Clausena excavata, T1538 Clausena lansium, T1561 Clerodendron serratum, T1562 Clerodendron trichotomum, T1563 Clerodendron trichotomum, T1569 Clerodendrum serratum var. amplexifolium, T1656 Conyza bonariensis [Syn. Erigeron bonariensis; Erigeron linifolius; Erigeron crispus], T1853 Croton oblongifolius [Syn. Croton laevigatus], T1888 Cudrania tricuspidata, T1931 Cyclea barbata, T1986 Cyrtomium fortunei, T2023
Daphne genkwa, T2131 Desmodium pulchellum [Syn. Phyllodium pulchellum], T2136 Desmos cochinchinensis [Syn. Desmos chinensis], T2158 Dichroa febrifuga, T2203 Dioscorea nipponica, T2204 Dioscorea nipponica ssp. rosthornii, T2263 Dregea volubilis, T2267 Drosera peltata var. lunata, T2272 Drymaria diandra [Syn. Drymaria cordata ssp. diandra], T2295 Duranta repens, T2310 Dysoxylum hongkongense, T2421 Erigeron annuus, T2505 Eucalyptus citriodora, T2517 Eucalyptus robusta, T2589 Euphorbia helioscopia, T2627 Euphoria longan [Syn. Dimocarpus longan], T2641 Evodia lepta [Syn. Ilex lepta], T2695 Ferula assafoetida, T2723 Ficus pumila, T3109 Harrisonia perforata, T3120 Hedychium spicatum, T3124 Hedyotis capitellata, T3144 Helianthus annuus, T3146 Helianthus annuиs cv, T3303 Hydrangea chinensis, T3304 Hydrangea macrophylla, T3308 Hydrangea umbellata, T3323 Hymenodictyon excelsum, T3327 Hyoscyamus niger, T3340 Hypericum ascyron, T3460 Iris halophila, T3464 Iris lactea var. chinensis [Syn. Iris pallasii var. chinensis], T3646 Kyllinga brevifolia, T3954 Lycium barbarum, T3956 Lycium chinense, T4170 Melilotus albus, T4172 Melilotus suaveolens, T4803 Phlogacanthus curviflorus, T4847 Physalis alkekengi var. franchetii, T5105 Polygonum hydropiper var. flaccidum [Syn. Polygonum flaccidum], T5110 Polygonum orientale, T5182 Potentilla discolor, T5184 Potentilla kleiniana, T5194 Premna microphylla, T5234 Prunus persica, T5413 Ranunculus cantoniensis, T5414 Ranunculus japonicus, T5415 Ranunculus sceleratus, T5576 Rostellularia procumbens [Syn. Justicia procumbens], T5852 Sedum bulbiferum, T5950 Siegesbeckia orientalis, T5951 Siegesbeckia orientalis var. glabrescens [Syn. Siegesbeckia glabrescens], T5952 Siegesbeckia orientalis var. pubescens [Syn. Siegesbeckia pubescens], T6005 Solanum lyratum, T6075 Spilanthes acmella, T6132 Stephania sasakii, T6135 Stephania succifera, T6177 Strychnos ignatii, T6252 Symplocos chinensis, T6375 Thalictrum baicalense, T6376 Thalictrum cultratum, T6385 Thalictrum flavum, T6387 Thalictrum foliolosum, T6389 Thalictrum glandulosissimum, T6653 Usnea diffracta, T6654 Usnea longissima, T6697 Veratrum grandiflorum, T6698 Veratrum nigrum, T6709 Verbena officinalis, T6723 Veronica arvensis, T6732 Vespertilio superans, T6749 Vicia hirsuta, T6750 Vicia sativa, T6788 Vitex negundo, T6866 Zanthoxylum avicennae.

malaria with ardent fever T1841 Croton caudatus var. tomentosus.

malaria with chills and fever T6402 Thalictrum omeiense.

malaria with fever T6226 Swertia mileensis.

malaria with splenomegaly T0834 Averrhoa carambola.

malign malaria T3323 Hymenodictyon excelsum.

malign obstruction in pregnancy T0418 Amomum muricarpum, T4718 Perilla frutescens var. acuta [Syn. Perilla frutescens var. purpurascens], T4721 Perilla frutescens var. arguta.

malign scab and lai sore T4349 Myrica rubra.

malign sore T1049 Bufo bufo gargarizans; Bufo melanostictus, T1660 Coprinus atramentarius, T1756 Costus speciosus, T2036 Datura innoxia, T2041 Datura metel, T2046 Datura stramonium, T2428 Erinaceus europaeus; Hemiechinus dauuricus; Hemiechinus auritus, T2866 Garcinia morella, T2975 Gleditsia delavayi, T2976 Gleditsia fera, T2980 Gleditsia sinensis [Syn. Gleditsia horrida], T3057 Gossypium herbaceum, T4338 Mylabris phalerata; Mylabris cichorii, 
T5368 Python molurus bivittatus, T5414 Ranunculus japonicus, T5605 Rumex acetosa, T5998 Solanum dulcamara, T6429 Thermopsis lupinoides, T6555 Tropaeolum majus, T6693 Veratrum album var. lobelianum [Syn. Veratrum lobelianum], T6697 Veratrum grandiflorum, T6698 Veratrum nigrum.

malign sore and scab lichen T1858 Croton tiglium.

malign sore and swelling toxin T0513 Annona squamosa, T1799 Crinum latifolium, T3984 Lycoris chinensis, T3986 Lycoris radiata [Syn. Amaryllis radiata], T5671 Salvia digitaloides.

malign sore with welling abscess and flat abscess T4303 Moschus moschiferus; Moschus berezovskii; Moschus sifanicus.

malign sores with welling abscess and flat abscess T1787 Cremastra appendiculata, T4917 Pinus massoniana.

malignant lymphoma T1904 Curcuma kwangsiensis, T1909 Curcuma zedoaria [Syn. Curcuma aeruginosa].

malignant tumor (therioma) T3231 Hernandia sonora [Syn. Hernandia ovigera].

malignity stroke stupor T4303 Moschus moschiferus; Moschus berezovskii; Moschus sifanicus.

mammary consumption T5137 Poncirus trifoliata.

mammary distention T1055 Bupleurum angustissimum, T1056 Bupleurum aureum, T1058 Bupleurum chaishoui, T1059 Bupleurum chinense, T1065 Bupleurum longiradiatum, T1066 Bupleurum marginatum, T1067 Bupleurum marginatum var. stenophyllum, T1072 Bupleurum scorzonerifolium, T1073 Bupleurum sibiricum, T1075 Bupleurum smithii var. parvifolium, T1078 Bupleurum wenchuanense, T1079 Bupleurum yinchowense.

mammary rock T1043 Bryophyllum pinnatum.

mammary sore T4696 Penaeus orientalis, T5400 Rabdosia stracheyi.

mammary welling abscess T0010 Abrus fruticulosus [Syn. Abrus cantoniensis], T0011 Abrus precatorius, T0045 Acanthopanax trifoliatus, T0161 Actinidia eriantha, T0171 Adiantum capillus-veneris, T0172 Adiantum caudatum, T0267 Ajuga nipponensis, T0446 Anaphalis margaritacea, T0567 Aralia armata, T0600 Ardisia mamillata [Syn. Tinus mamillata], T0634 Aristolochia mollissima, T0643 Aristolochia versicolar, T0932 Betula luminifera, T0971 Bolbostemma paniculatum, T1005 Brassica campestris [Syn. Brassica campestris var. oleifera], T1007 Brassica juncea, T1043 Bryophyllum pinnatum, T1188 Caragana chamlagu, T1190 Caragana jubata, T1338 Cestrum nocturnum, T1504 Citrus reticulata, T1506 Citrus reticulata, T1565 Clerodendrum bungei, T1624 Colocasia antiquorum, T1640 Coniogramme japonica [Syn. Hemionitis japonica], T1813 Crotalaria albida, T1986 Cyrtomium fortunei, T2024 Daphne genkwa, T2028 Daphne odora, T2133 Desmodium racemosum [Syn. Podocarpium podocarpum var. oxyphyllum], T2316 Echinops grijsii, T2317 Echinops ritro, T2517 Eucalyptus robusta, T2521 Eucalyptus tereticornis, T2590 Euphorbia hirta, T2787 Fritillaria hupehensis, T2798 Fritillaria verticillata var. thunbergii [Syn. Fritillaria thunbergii], T3080 Gymnema sylvestre, T3193 Hemerocallis fulva, T3195 Hemerocallis lilio-asphodelus, T3197 Hemerocallis minor, T3202 Hemistepta lyrata [Syn. Hemistepta carthamoides; Saussurea carthamoides], T3348 Hypericum elodeoides, T3363 Hypericum sampsonii, T3368 Hypericum wightianum, T3432 Inula helianthus-aquatica, T3457 Iris dichotoma, T3479 Isodon amethystoides, T3815 Ligularia stenocephala, T3932 Ludwigia octovalvis, T3990 Lygodium japonicum, T3996 Lysimachia candida, T3999 Lysimachia clethroides, T4252 Mirabilis jalapa, T4265 Momordica cochinchinensis, T4519 Opuntia dillenii, T4520 Opuntia ficus-indica, T4521 Opuntia vulgaris, T4648 Paris polyphylla, T4649 Paris polyphylla var. chinensis, T4650 Paris polyphylla var. pseudothibetica, T4651 Paris polyphylla var. stenophylla, T4652 Paris polyphylla var. yunnanensis, T4726 Periploca forrestii, T4827 Photinia parvifolia, T5090 Polygala wattersii, T5193 Pratia nummularia, T5214 Prunella vulgaris, T5298 Pteris vittata, T5466 Rhaponticum uniflorum, T5650 Salix babylonica, T5672 Salvia flava, T5701 Salvia yunnanensis, T5813 Scilla scilloides, T5862 Selaginella involvens, T5942 Sida acuta, T6092 Stachys palustris, T6301 Taraxacum mongolicum, T6367 Teucrium quadrifarium, T6431 Thesium chinense, T6645 Urena lobata, T6668 Vaccaria segetalis [Syn. Vaccaria pyramidata], T6767 Viola yedoensis, T6912 Zinnia elegans.

mammary welling abscess with swelling and pain T0173 Adiantum lunulatum.

mania and withdrawal T1804 Cristaria plicata; Hyriopsis cumingii, T1907 Curcuma wengujin, T3329 Hyoscyamus niger, T5231 Prunus persica, T6675 Valeriana amurensis, T6679 Valeriana officinalis, T6680 Valeriana officinalis var. latifolia.

mania and withdrawal with congesting phlegm T6693 Veratrum album var. lobelianum [Syn. Veratrum lobelianum].

manic agitation schizophrenia T4243 Millettia reticulata.

march hematoglobinuria T5587 Rubus alceaefolius.

marked emaciation T1337 Cervus nippon; Cervus elaphus, T3279 Homo sapiens.

massive head scourge T0866 Baphicacanthus cusia [Syn. Strobilanthes cusia], T3475 Isatis indigotica, T3495 Isodon irrorata.

mastitis T0264 Ajuga forrestii, T0594 Ardisia crenata, T0707 Arthraxon hispidus, T0776 Asplenium prolongatum, T1450 Cirsium lineare, T1573 Clinopodium chinense, T1826 Crotalaria mucronata, T1959 Cynanchum paniculatum, T2357 Emilia sonchifolia, T2691 Farfugium japonicum, T2988 Glochidion eriocarpum, T3123 Hedyotis auricularia, T3125 Hedyotis chrysotricha [Syn. Oldenlandia chrysotricha], T3243 Hibiscus rosa-sinensis, T3284 Houttuynia cordata, T3288 Humulus japonicus [Syn. Humulus scandens], T3496 Isodon japonica [Syn. Rabdosia japonica], T3908 Lonicera bournei, T3909 Lonicera confusa, T3910 Lonicera fulvotomentosa, T3911 Lonicera hypoglauca, T3912 Lonicera japonica, T3914 Lonicera macranthoides, T3918 Lonicera similis, T4121 Marsilea quadrifolia, T4391 Nauclea officinalis, T5295 Pteris multifida, T5393 Rabdosia longituba, T5587 Rubus alceaefolius, T5680 Salvia miltiorrhiza, T5681 Salvia miltiorrhiza f. alba, T5688 Salvia przewalskii, T5844 Scutellaria scordifolia, T5857 Sedum sarmentosum, T5861 Selaginella doederleinii, T6003 Solanum khasianum, T6008 Solanum nigrum, T6025 Sonchus arvensis, T6301 Taraxacum mongolicum, T6691 Veratrilla baillonii, T6808 Waltheria americana.

mastocarcinoma T0161 Actinidia eriantha.

maybe cure prostatauxe T5340 Pygeum topengii.

mazischesis T0560 Arachis hypogaea. 
measles papules T0379 Alternanthera philoxeroides, T0586 Arctium lappa, T0648 Arnebia euchroma, T0649 Arnebia guttata, T0867 Baphicacanthus cusia [Syn. Strobilanthes cusia], T1533 Clausena dentata, T1534 Clausena dunniana, T2282 Dryopteris filix-mas, T2517 Eucalyptus robusta, T2946 Geranium robertianum, T3163 Helicteres angustifolia, T3303 Hydrangea chinensis, T3416 Imperata cylindrica var. major, T3649 Laccifer lacca, T3881 Lithospermum erythrorhizon, T4028 Maesa indica, T4320 Murraya kwangsiensis, T4500 Onosma paniculatum, T4526 Origanum vulgare, T4552 Osmunda japonica, T4564 Oxalis corniculata [Syn. Oxalis repens], T5401 Rabdosia yuennanensis, T5804 Schizonepeta tenuifolia [Syn. Nepeta tenuifolia], T6145 Stichopus japonicus, T6375 Thalictrum baicalense, T6376 Thalictrum cultratum, T6385 Thalictrum flavum, T6387 Thalictrum foliolosum, T6389 Thalictrum glandulosissimum.

measles papules swelling toxin T1179 Capparis masaikai.

measles papules with ardent fever T3588 Juniperus formosana.

measles papules with complicated pneumonia T5408 Rana nigromaculata; Rana plancyi.

measles papulis $\mathrm{T} 3475$ Isatis indigotica.

meat-type food accumulation T1769 Crataegus cuneata, T1771 Crataegus kansuensis, T1772 Crataegus maximowiczii, T1775 Crataegus pinnatifida, T1778 Crataegus pinnatifida var. major, T1780 Crataegus sanguinea, T1781 Crataegus scabrifolia, T2695 Ferula assafoetida, T5365 Pyrus pashia.

medullitis T0195 Aeginetia indica, T0568 Aralia chinensis, T0570 Aralia dasyphylla, T1003 Brandisia hancei, T1416 Cicuta virosa, T2589 Euphorbia helioscopia, T6214 Swertia chinensis, T6540 Tripterygium hypoglaucum.

melancholia T2882 Gardenia jasminoides [Syn. Gardenia florida].

meningitis T1393 Chrysanthemum indicum, T6432 Thespesia populnea [Syn. Hibiscus populneus].

menstrual block T0571 Aralia decaisneaa, T0600 Ardisia mamillata [Syn. Tinus mamillata], T0661 Artemisia anomala, T0963 Boehmeria siamensis, T1001 Brachystemma calycinum, T1288 Celastrus orbiculatus [Syn. Celastrus articulatus], T1434 Cinnamomum bejolghota [Syn. Cinnamomum obtusifolium; Laurus bejolghota], T1640 Coniogramme japonica [Syn. Hemionitis japonica], T1884 Cudrania cochinchinensis, T1907 Curcuma wengujin, T2193 Dioscorea cirrhosa [Syn. Dioscorea pogonoides], T3413 Impatiens nolitangere, T3479 Isodon amethystoides, T3674 Laggera alata, T3779 Leucas aspera, T4432 Nigella glandulifera, T4549 Osbeckia chinensis, T4726 Periploca forrestii, T5401 Rabdosia yuennanensis, T5586 Rubia yunnanensis, T5594 Rubus parkeri, T5661 Salvia bowleyana, T5672 Salvia flava, T5697 Salvia trijuga, T5701 Salvia yunnanensis, T6830 Woodfordia fruticosa.

menstrual block and abdominal pain T3201 Hemiphragma heterophyllum.

menstrual disorder T0071 Achyranthes aspera, T0072 Achyranthes aspera var. indica, T0298 Alectoria vivens, T0301 Aleuritopteris argentea, T0474 Angelica acutiloba [Syn. Ligusticum acutilobum], T0495 Angelica sinensis, T0591 Ardisia arborescens, T0805 Astragalus sinicus, T0859 Balanophora involucrata, T0941 Bidens tripartita, T0999 Bougainvillea glabra, T1053 Bulbophyllum odoratissimum [Syn. Stelis odoratissimum], T1055 Bupleurum angustissimum, T1056 Bupleurum aureum, T1058 Bupleurum chaishoui, T1059 Bupleurum chinense, T1065 Bupleurum longiradiatum, T1066 Bupleurum marginatum, T1067 Bupleurum marginatum var. stenophyllum, T1072 Bupleurum scorzonerifolium, T1073 Bupleurum sibiricum, T1075 Bupleurum smithii var. parvifolium, T1078 Bupleurum wenchuanense, T1079 Bupleurum yinchowense, T1143 Calystegia hederacea, T1163 Campylotropis hirtella, T1352 Chamaenerion angustifolium [Syn. Epilobium angustifolium], T1356 Cheiranthus cheiri, T1443 Cinnamomum parthenoxylum [Syn. Cinnamomum porrectum], T1450 Cirsium lineare, T1492 Citrus limon, T1496 Citrus limonia, T1528 Cladonia stellaris [Syn. Cladonia alpestris], T1583 Cnidium officinale [Syn. Ligusticum officinale], T1706 Corydalis ambigua var. amurensis [Syn. Corydalis ambigua], T1735 Corydalis repens, T1871 Cucubalus baccifer, T1889 Cuminum cyminum, T1900 Curculigo capitulata [Syn. Leucojum capitulata], T1976 Cyperus iria, T1978 Cyperus rotundus, T2132 Desmodium pulchellum [Syn. Phyllodium pulchellum], T2231 Diphylleia grayi, T2232 Diphylleia sinensis, T2290 Duchesnea indica, T2326 Elaeagnus angustifolia, T2334 Elephantopus scaber, T2347 Embelia parviflora, T2387 Epilobium hirsutum, T2391 Epimedium brevicornum, T2540 Euonymus fortunei, T2542 Euonymus japonicus, T2545 Euonymus phellomana, T2546 Euonymus sacrosancta, T2563 Eupatorium japonicum, T2636 Euscaphis japonica, T2691 Farfugium japonicum, T2731 Firmiana simplex, T2736 Fissistigma polyanthum, T2826 Galeola faberi, T2955 Geum japonicum, T3056 Gossypium herbaceum, T3113 Hedera nepalensis var. sinensis, T3115 Hedera rhombea, T3124 Hedyotis capitellata, T3240 Hibiscus esculentus, T3340 Hypericum ascyron, T3349 Hypericum erectum, T3361 Hypericum perforatum, T3363 Hypericum sampsonii, T3418 Incarvillea arguta, T3549 Ixora chinensis, T3615 Kadsura interior, T3675 Lagopsis supina, T3681 Lamium barbatum, T3746 Leontice robustum, T3752 Leonurus heterophyllus [Syn. Leonurus artemisia], T3754 Leonurus sibiricus, T3765 Lepisorus ussuriensis, T3776 Lethariella zahlbruckneri, T3820 Ligusticum chuanxiong [Syn. Ligusticum wallichii], T3965 Lycopodium annotinum, T3969 Lycopodium casuarinoides, T3971 Lycopodium complanatum, T3997 Lysimachia capillipes, T3999 Lysimachia clethroides, T4005 Lysionotus pauciflorus, T4178 Melodinus hemsleyanus, T4234 Millettia dielsiana, T4238 Millettia nitida, T4308 Mucuna birdwoodiana, T4311 Mucuna sempervirens, T4385 Narcissus tazetta var. chinensis, T4432 Nigella glandulifera, T4444 Nothopanax davidii, T4450 Nuphar pumilum, T4470 Ocimum basilicum, T4510 Ophiorrhiza japonica, T4513 Ophiorrhiza mungos, T4575 Pachysandra terminalis, T4580 Paeonia albiflora [Syn. Paeonia lactiflora], T4604 Panax japonicus var. bipinnatifidus, T4605 Panax japonicus var. major, T4689 Peganum nigellastrum, T4777 Phalaris arundinacea, T4804 Phlojodicarpus sibiricus, T4813 Phlomis tuberosa, T4956 Piper mullesua, T5018 Pleuropterus ciliinervis, T5057 Podophyllum emodii [Syn. Podophyllum emodii var. chinense; Podophyllum sikkimenosis; Sinopodophyllum emodii], T5071 Polygala arillata, T5075 Polygala fallax [Syn. Polygala aureocauda], T5087 Polygala telephioides, T5106 Polygonum lapathifolium, T5184 Potentilla kleiniana, T5497 Rhodiola kirilowii, T5515 Rhododendron 
micranthum, T5523 Rhododendron simsii, T5562 Rosa chinensis, T5569 Rosa multiflora, T5572 Rosa rugosa, T5594 Rubus parkeri, T5671 Salvia digitaloides, T5672 Salvia flava, T5680 Salvia miltiorrhiza, T5681 Salvia miltiorrhiza f. alba, T5688 Salvia przewalskii, T5697 Salvia trijuga, T5701 Salvia yunnanensis, T5753 Saussurea gnaphaloides, T5755 Saussurea involucrata, T5757 Saussurea laniceps, T5759 Saussurea medusa, T5762 Saussurea nigrescens, T5793 Schisandra henryi, T5795 Schisandra micrantha, T5797 Schisandra propinqua, T5798 Schisandra propinqua var. intermedia, T5893 Senecio nudicaulis, T5966 Siphonostegia chinensis, T6066 Spatholobus suberectus, T6154 Strobilanthes japonicus [Syn. Championella japonica], T6295 Tanacetum sibiricum [Syn. Filifolium sibiricum], T6356 Tetrapanax papyriferus, T6645 Urena lobata, T6677 Valeriana jatamansii [Syn. Valeriana wallichii], T6689 Ventilago leiocarpa, T6728 Veronica serpyllifolia, T6749 Vicia hirsuta, T6750 Vicia sativa.

menstrual disorder $[=$ menoxenia] $\quad$ T0969 Boerhavia diffusa, T1189 Caragana intermedia, T1373 Chloranthus serratus, T1682 Cordyceps ophioglossoides, T2193 Dioscorea cirrhosa [Syn. Dioscorea pogonoides], T2734 Fissistigma glaucescens [Syn. Melodorum glaucescens], T3413 Impatiens nolitangere, T3779 Leucas aspera, T4239 Millettia nitida var. hirsutissima, T4564 Oxalis corniculata [Syn. Oxalis repens], T4938 Piper boehmeriaefolium, T5193 Pratia nummularia, T5446 Rehmannia glutinosa [Syn. Rehmannia glutinosa f. huechingensis], T5498 Rhodiola quadrifida, T5545 Ribes fasciculatum var. chinense, T5555 Rodgersia pinnata, T5563 Rosa cymosa, T5566 Rosa henryi, T5586 Rubia yunnanensis, T5661 Salvia bowleyana, T6533 Trillium kamtschaticum, T6535 Trillium tschonoskii, T6676 Valeriana hardwickii, T6722 Veronica anagallis-aquatica.

menstrual disorder ${ }^{[5509]}$ T4518 Oppopanax chironium.

menstrual disorder due to cold T0664 Artemisia argyi, T0681 Artemisia lavandulaefolia, T0685 Artemisia mongolica, T0689 Artemisia princeps, T0691 Artemisia rubripes, T0706 Artemisia vulgaris.

menstrual pain T3363 Hypericum sampsonii, T4962 Piper puberulum, T5661 Salvia bowleyana, T5671 Salvia digitaloides, T5672 Salvia flava.

menstruant's morbidity T5993 Solanum capsicastrum.

mental disease T0298 Alectoria vivens, T4423 Nicandra physaloides.

mental manic agitation T5821 Scopolia japonica.

mercurial poisoning T5977 Smilax glabra, $\mathrm{T} 5980$ Smilax menispermoidea.

miasmic malaria T2092 Delphinus delphis.

migraine T0088 Aconitum coreanum, T1084 Buthus martensi, T1374 Chloranthus spicatus, T1528 Cladonia stellaris [Syn. Cladonia alpestris], T1541 Claviceps purpurea, T1562 Clerodendron trichotomum, T3822 Ligusticum jeholense, T3824 Ligusticum sinense, T4625 Papaver commutatum [Syn. Papaver rhoeas], T4662 Parthenocissus tricuspidata, T5414 Ranunculus japonicus, T5727 Saposhnikovia divaricata [Syn. Ledebouriella seseloides], T6588 Typhoniun giganteum, T6791 Vitex rotundifolia [Syn. Vitex trifollia var. simplicifolia], T6793 Vitex trifolia.

mough and tongue sores T3830 Ligustrum sinense, T3932 Ludwigia octovalvis, T4730 Peristrophe roxburghiana, T6268 Syzygium samarangense.

mounting pain T1537 Clausena lansium, T4157 Melia azedarach, T4987 Pittosporum tobira.

mounting qi T0164 Actinidia polygama, T0274 Akebia quinata, T0275 Akebia quinata, T0278 Akebia trifoliata, T0280 Akebia trifoliata var. australis, T0598 Ardisia japonica, T0610 Argemone mexicana, T0680 Artemisia lactiflora, T0721 Arundina chinensis, T0813 Atalantia buxifolia [Syn. Severinia buxifolia], T0814 Atalantia buxifolia [Syn. Severinia buxifolia], T1092 Buxus microphylla var. sinica, T1113 Calendula officinalis, T1217 Carum carvi, T1258 Casuarina equisetifolia, T1289 Celastrus orbiculatus [Syn. Celastrus articulatus], T1359 Chenopodium album, T1479 Citrus grandis, T1487 Citrus junos, T1504 Citrus reticulata, T1586 Cocculus laurifolius, T1598 Codonopsis convolvulacea, T1775 Crataegus pinnatifida, T1778 Crataegus pinnatifida var. major, T1869 Cryptotaenia japonica, T2432 Eriobotrya japonica, T2536 Euonymus alatus, T2632 Euryale ferox, T2644 Evodia rutaecarpa, T2646 Evodia rutaecarpa var. officinalis, T2733 Firmiana simplex, T2746 Foeniculum vulgare, T3221 Heracleum rapula, T3852 Lindera megaphylla, T3854 Lindera strychnifolia [Syn. Lindera aggregata], T4100 Mangifera indica, T4163 Melia toosendan, T4268 Monachosorum henryi, T4530 Ormosia hosiei, T4616 Pandanus tectorius, T4663 Passiflora caerulea, T4690 Pelargonium graveolens, T4847 Physalis alkekengi var. franchetii, T4852 Physalis peruviana, T4948 Piper hancei, T4966 Piper sarmentosum, T5110 Polygonum orientale, T5137 Poncirus trifoliata, T5139 Poncirus trifoliata, T5189 Pothos chinensis, T5626 Ruta graveolens, T5720 Sapindus mukorossi, T6101 Stauntonia hexaphylla, T6528 Trigonella foenum-graecum, T6540 Tripterygium hypoglaucum, T6800 Viverra zibetha.

mounting $\boldsymbol{q} \boldsymbol{i}[\mathbf{=}$ hernia] $\mathrm{T} 1381$ Choerospondias axillaris, T1552 Cleome viscosa, T3006 Glycosmis citrifolia, T3270 Holboellia fargesii, T3613 Kadsura coccinea [Syn. Kadsura chenensis; Kadsura hainanensis], T4158 Melia azedarach, T4439 Nothapodytes pittosporoides, T4962 Piper puberulum, T5661 Salvia bowleyana, T5701 Salvia yunnanensis, T5717 Sapindus delavayi [Syn. Pancovia delavayi], T6527 Trigonella caerulea, T6876 Zanthoxylum dissitum.

mounting $q \boldsymbol{i}$ [ = hernia] T0036 Acanthopanax giraldii [Syn. Acanthopanax giraldii var. inermis; Eleutherococcus giraldii], T1288 Celastrus orbiculatus [Syn. Celastrus articulatus], T3460 Iris halophila, T3464 Iris lactea var. chinensis [Syn. Iris pallasii var. chinensis], T5391 Rabdosia eriocalyx, T6789 Vitex negundo.

mounting qi pain T1053 Bulbophyllum odoratissimum [Syn. Stelis odoratissimum], T3877 Litchi chinensis.

mounting $\boldsymbol{q} \boldsymbol{i}$ with unilateral sagging of one testicle T3360 Hypericum patulum.

mounting-conglomeration T6925 Zostera marina.

mounting-conglomeration accumulation-gathering T4584 Paeonia lactiflora wild, T4586 Paeonia obovata, T4590 Paeonia veitchii.

mouth and tongue sores T0378 Alstonia yunnanensis, T0959 Blumea lacera, T0977 Bombyx mori, T0987 Bos taurus domesticus; Bubalus bubalis, T1045 Bubalus bubalis, T1361 Chenopodium ambrosioides, T5447 Rehmannia glutinosa [Syn. Rehmannia glutinosa f. huechingensis]. 
mouth sore T0019 Acacia catechu, T0273 Akebia quinata, T0277 Akebia trifoliata, T0279 Akebia trifoliata var. australis, T0540 Apis cerana, T0632 Aristolochia manshuriensis, T0867 Baphicacanthus cusia [Syn. Strobilanthes cusia], T0897 Berberis amurensis, T0907 Berberis kawakamii, T0912 Berberis poiretii, T0916 Berberis thunbergii, T1151 Camellia sinensis [Syn. Thea sinensis], T1182 Capra hircus; Ovis aries, T1256 Castanea mollissima, T1419 Cimicifuga dahurica, T1420 Cimicifuga foetida, T1421 Cimicifuga heracleifolia, T1464 Citrullus vulgaris [Syn. Citrullus lanatus], T1554 Clerodendron cyrtophyllum, T1662 Coptis chinensis, T1663 Coptis chinensis var. brevisepala, T1664 Coptis deltoidea, T1669 Coptis omeiensis, T1670 Coptis teetoides [Syn. Coptis teeta], T2221 Diospyros kaki, T2274 Dryobalanops aromatica, T2290 Duchesnea indica, T2628 Euphrasia officinalis, T2838 Gallus gallus domesticus, T3277 Homo sapiens, T3342 Hypericum bellum, T3356 Hypericum japonicum, T3361 Hypericum perforatum, T3363 Hypericum sampsonii, T3420 Incarvillea sinensis, T3423 Indigofera tinctoria, T3578 Juncus effusus, T3624 Kalopanax pictum, T3625 Kalopanax septemlobus, T3920 Lophatherum gracile, T3990 Lygodium japonicum, T4172 Melilotus suaveolens, T4178 Melodinus hemsleyanus, T4303 Moschus moschiferus; Moschus berezovskii; Moschus sifanicus, T4789 Phellodendron amurense, T4790 Phellodendron amurense var. wilsonii, T4791 Phellodendron chinense, T4792 Phellodendron chinense var. glabriusculum, T4831 Phtheirospermum japonicum [Syn. Geradia japonica], T4836 Phyllanthus emblica, T4837 Phyllanthus emblica, T4843 Phyllanthus urinaria, T5099 Polygonum bistorta, T5121 Polygonum tinctorium, T5151 Populus davidiana, T5158 Populus simonii, T5457 Rhamnus davurica, T5569 Rosa multiflora, T6116 Stenoloma chusanum, T6224 Swertia kouitchensis, T6231 Swertia pseudochinensis, T6552 Trollius chinensis [Syn. Trollius asiaticus var. chinensis], T6553 Trollius ledebourii, T6615 Uncaria gambir, T6728 Veronica serpyllifolia.

mouth-level nape sore T4616 Pandanus tectorius, T5111 Polygonum perfoliatum.

mouth-lever nape sore (nuchal phlegmon) T5513 Rhododendron mariae.

multi-infarct dementia T2961 Ginkgo biloba.

multiple swollen welling abscess T5279 Psychotria serpens.

mumps T0048 Acanthus ilicifolius, T0471 Anemone rivularis, T1384 Chondrus ocelladus, T3990 Lygodium japonicum, T4884 Picria felterrae, T5289 Pteris dactylina, T5717 Sapindus delavayi [Syn. Pancovia delavayi], T6365 Teucrium pilosum [Syn. Teucrium japonicum var. pilosum], $\mathrm{T} 6767$ Viola yedoensis.

muscle numbness T2367 Ephedra equisetina, T2368 Ephedra gerardiana, T2369 Ephedra intermedia, T2372 Ephedra likiangensis, T2373 Ephedra minuta, T2375 Ephedra monosperma, T2379 Ephedra saxatilis, T2380 Ephedra sinica, T5108 Polygonum multiflorum.

muscle weakness T3984 Lycoris chinensis, T3986 Lycoris radiata [Syn. Amaryllis radiata], T6184 Strychnos nux-vomica, T6193 Strychnos wallichiana.

myocardiac ischemia T1775 Crataegus pinnatifida, T1778 Crataegus pinnatifida var. major.

nasal congestion T0478 Angelica dahurica $[$ Syn. Angelica porphyrocaulis], T0479 Angelica dahurica cv. qibaizhi, T0498 Angelica taiwaniana, T0728 Asarum heterotropoides var. mandshuricum, T0731 Asarum sieboldii, T0732 Asarum sieboldii var. seoulensis, T1312 Centipeda minima, T2367 Ephedra equisetina, T2368 Ephedra gerardiana, T2369 Ephedra intermedia, T2372 Ephedra likiangensis, T2373 Ephedra minuta, T2375 Ephedra monosperma, T2379 Ephedra saxatilis, T2380 Ephedra sinica, T6359 Teucrium bidentatum.

nasal congestion and runny nose T4035 Magnolia biondii [Syn. Magnolia fargesii], T4038 Magnolia denudata [Syn. Magnolia heptapata], T4041 Magnolia liliflora, T4052 Magnolia sprengeri, T4663 Passiflora caerulea, T6499 Tribulus terrestris.

nasitis T0707 Arthraxon hispidus, T4035 Magnolia biondii [Syn. Magnolia fargesii], T4038 Magnolia denudata [Syn. Magnolia heptapata], T4041 Magnolia liliflora, T4052 Magnolia sprengeri, T4215 Michelia yunnanensis, T4831 Phtheirospermum japonicum [Syn. Geradia japonica].

nasopharyngeal carcinoma T2844 Ganoderma applanatum, T3443 Iphigenia indica.

nasosinusitis T1044 Bryum argenteum, T4035 Magnolia biondii [Syn. Magnolia fargesii], T4038 Magnolia denudata [Syn. Magnolia heptapata], T4041 Magnolia liliflora, T4052 Magnolia sprengeri, T4859 Physochlaina physaloides, T5189 Pothos chinensis.

nausea T3372 Hypodematium sinense, T4305 Mosla dianthera.

nausea and vomiting T1278 Cedrela sinensis, T1486 Citrus junos, T1488 Citrus junos, T2558 Eupatorium formosanum, T2559 Eupatorium fortunei, T3282 Hordeum vulgare, T4718 Perilla frutescens var. acuta [Syn. Perilla frutescens var. purpurascens], T4721 Perilla frutescens var. arguta, T6325 Tectona grandis.

nephritis T0933 Betula platyphylla, T0935 Betula platyphylla var. japonica, T1655 Conyza blinii, T1709 Corydalis bungeana, T1819 Crotalaria ferruginea, T2119 Derris eriocarpa, T3594 Juniperus rigida, T4842 Phyllanthus reticulatus, T5687 Salvia prionitis, T5795 Schisandra micrantha, T5867 Selaginella sinensis, T6023 Solidago virgaurea, T6277 Tadehagi triquetrum, T6691 Veratrilla baillonii.

nephritis with edema T0273 Akebia quinata, T0277 Akebia trifoliata, T0279 Akebia trifoliata var. australis, T1184 Capsella bursa-pastoris, T1415 Cichorium intybus, T2334 Elephantopus scaber, T2884 Gardenia jasminoides var. grandiflora, T3998 Lysimachia christinae, T4843 Phyllanthus urinaria, T5685 Salvia plebeia, T6129 Stephania japonica, T6330 Tephroseris kirilowii [Syn. Senecio integrifolius var. fauriei], T6436 Thlaspi arvense.

nephropathy syndrome T6542 Tripterygium wilfordii.

nephropyelitis T0967 Boenninghausenia sessilicarpa, T2941 Gentianopsis paludosa.

nervous headache T6003 Solanum khasianum, T6843 Xanthium sibiricum [Syn. Xanthium strumarium].

nervous system diseases T3231 Hernandia sonora [Syn. Hernandia ovigera].

neuralgia T0135 Aconitum sungpanense, T0138 Aconitum umbrosum, T0495 Angelica sinensis, T0539 Apis cerana, T0617 Arisaema amurense, T0618 Arisaema consanguineum, T0620 Arisaema heterophyllum, T1589 Cocculus trilobus [Syn. Cocculus sarmentosus], 
T1715 Corydalis decumbens [Syn. Corydalis amabilis], T1750 Corydalis yanhusuo [Syn. Corydalis turtschaninovii f. yanhusuo], T2897 Gelsemium elegans, T4663 Passiflora caerulea, T4804 Phlojodicarpus sibiricus, T4903 Pinellia pedatisecta, T5848 Securinega suffruticosa, T6100 Stauntonia hexaphylla, T6122 Stephania dielsiana, T6133 Stephania sinica, T6135 Stephania succifera.

neurasthenia T0572 Aralia elata, T1381 Choerospondias axillaris, T1525 Cladonia fallax, T1598 Codonopsis convolvulacea, T1604 Coeloglossum viride [Syn. Coeloglossum viride var. bracteatum], T1683 Cordyceps sinensis, T1826 Crotalaria mucronata, T2389 Epimedium acuminatum, T2390 Epimedium brevicornum, T2392 Epimedium davidii, T2394 Epimedium elongatum, T2398 Epimedium koreanum, T2401 Epimedium pubescens, T2402 Epimedium sagittatum, T2404 Epimedium sutchuenense, T2406 Epimedium wushanense, T3077 Gymnadenia conopsea, T4248 Mimosa pudica, T4283 Morinda officinalis, T4436 Nitraria tangutorum, T4906 Pinus armandii, T4916 Pinus massoniana, T5107 Polygonum multiflorum, T5169 Poria cocos, T5465 Rhaponticum carthamoides, T5848 Securinega suffruticosa, T6533 Trillium kamtschaticum, T6535 Trillium tschonoskii, T6919 Ziziphus jujuba var. spinosa.

neuritis T1055 Bupleurum angustissimum, T1056 Bupleurum aureum, T1058 Bupleurum chaishoui, T1059 Bupleurum chinense, T1065 Bupleurum longiradiatum, T1066 Bupleurum marginatum, T1067 Bupleurum marginatum var. stenophyllum, T1072 Bupleurum scorzonerifolium, T1073 Bupleurum sibiricum, T1075 Bupleurum smithii var. parvifolium, T1078 Bupleurum wenchuanense, T1079 Bupleurum yinchowense.

neurodermatitis T0495 Angelica sinensis, T0750 Asparagus gobicus, T1215 Carthamus tinctorius, T1650 Convolvulus arvensis, T1959 Cynanchum paniculatum, T2023 Daphne genkwa, T4804 Phlojodicarpus sibiricus, T5026 Plumbagella micrantha, T5256 Pseudolarix amabilis [Syn. Larix amabilis; Pseudolarix kaempferi], T5323 Pulsatilla campanella, T5324 Pulsatilla cernua, T5325 Pulsatilla chinensis, T5326 Pulsatilla dahurica, T5680 Salvia miltiorrhiza, T5681 Salvia miltiorrhiza f. alba, T5688 Salvia przewalskii, T6119 Stephania cepharantha, T6540 Tripterygium hypoglaucum.

neurosis T4248 Mimosa pudica, T5107 Polygonum multiflorum, T5499 Rhodiola sacra.

night blindness T0819 Atractylodes chinensis, T0821 Atractylodes japonica, T0823 Atractylodes lancea, T0986 Bos taurus domesticus; Bubalus bubalis, T1240 Cassia obtusifolia, T1250 Cassia tora, T1789 Crepis napifera, T2434 Eriocaulon buergerianum, T3779 Leucas aspera, T3957 Lycium chinense, T3969 Lycopodium casuarinoides, T6076 Spinacia oleracea, T6732 Vespertilio superans.

night sweating T0607 Arenaria juncea, T0825 Atropa belladonna, T0944 Biota orientalis [Syn. Thuja orientalis; Platycladus orientalis], T1680 Cordyceps militaris, T1681 Cordyceps militaris cv, T2658 Fagopyrum esculentum, T2727 Ficus simplicissima, T3001 Glycine max, T3090 Gypsophila oldhamiana, T3091 Gypsophila pacifica, T3279 Homo sapiens, T3742 Lentinus edodes, T3950 Lychnis fulgens, T3969 Lycopodium casuarinoides, T4450 Nuphar pumilum, T4580 Paeonia albiflora [Syn. Paeonia lactiflora], T4887 Picrorhiza kurrooa, T4888 Picrorhiza scrophulariiflora, T5133 Polytrichum commune, T5530 Rhus chinensis [Syn. Rhus semialata], T5531 Rhus chinensis [Syn. Rhus semialata], T5955 Silene jenisseensis.

nipple moth T0950 Blatta orientalis, T1001 Brachystemma calycinum, T1210 Carpesium abrotanoides, T3356 Hypericum japonicum, T3396 Ilex pubescens, T3397 Ilex pubescens var. glaber, T3984 Lycoris chinensis, T3986 Lycoris radiata [Syn. Amaryllis radiata], T5105 Polygonum hydropiper var. flaccidum [Syn. Polygonum flaccidum], T5717 Sapindus delavayi [Syn. Pancovia delavayi], T6039 Sophora moorcroftiana, T6431 Thesium chinense.

no thought of food and drink T1492 Citrus limon, T1496 Citrus limonia, T4470 Ocimum basilicum, T4681 Pedicularis decora, T5758 Saussurea lappa [Syn. Aucklandia lappa].

node swelling T4954 Piper longum.

non-diffusion of lung $\boldsymbol{q} i \mathrm{~T} 2367$ Ephedra equisetina, T2368 Ephedra gerardiana, T2369 Ephedra intermedia, T2372 Ephedra likiangensis, T2373 Ephedra minuta, T2375 Ephedra monosperma, T2379 Ephedra saxatilis, T2380 Ephedra sinica.

non-digestion of food accumulation T0210 Agaricus bisporus, T0211 Agaricus campestris, T0275 Akebia quinata, T1483 Citrus grandis var. tomentosa, T1488 Citrus junos, T1546 Clematis tangutica, T1937 Cydonia oblonga, T2452 Erysimum cheiranthoides, T3620 Kaempferia galanga, T4470 Ocimum basilicum, T4714 Perilla frutescens, T5758 Saussurea lappa [Syn. Aucklandia lappa].

non-eruption of macula T1417 Cimicifuga acerina, T1423 Cimicifuga nanchuanensis, T1991 Cytisus scoparius [Syn. Spartium scoparium].

non-eruption of measles T0838 Azolla imbricata [Syn. Salvinia imbricata], T2721 Ficus microcarpa, T3649 Laccifer lacca, T3738 Lemna minor, T3742 Lentinus edodes, T4184 Mentha haplocalyx [Syn. Mentha canadaensis; Mentha arvensis var. haplocalyx; Mentha arvensis], T4413 Nepeta cataria, T4456 Nymphoides peltatum, T5312 Pueraria edulis, T5313 Pueraria lobata [Syn. Pueraria thunbergiana; Pueraria pseudohirsuta], T5316 Pueraria omeiensis, T5318 Pueraria phaseoloides, T5320 Pueraria thomsonii, T5560 Rorippa montana [Syn. Rorippa dubia; Sisymbrium dublium], T6286 Tamarix chinensis, T6290 Tamarix ramosissima.

noninfectious fever T1633 Commelina communis.

non-interaction of heart and kidney T4401 Nelumbo nucifera.

non-opened welling abscess and flat abscess T4338 Mylabris phalerata; Mylabris cichorii.

nose carcinoma T2897 Gelsemium elegans.

nose sores T3361 Hypericum perforatum.

nosebleed T5323 Pulsatilla campanella, T5324 Pulsatilla cernua, T5325 Pulsatilla chinensis, T5326 Pulsatilla dahurica, T6139 Sterculia lychnophora.

nosebleed (epistaxis) T0843 Babylonia lutosa, T0952 Blechnum orientale, T1171 Canis familiaris, T1251 Cassytha filiformis, T1296 Celosia argentea, T1528 Cladonia stellaris [Syn. Cladonia alpestris], T1592 Cocos nucifera, T1655 Conyza blinii, T1684 Cordyline strcta, T2157 Dichotomanthes tristaniaecarpa, T2323 Eclipta prostrata [Syn. Eclipta alba], T2365 Enteromorpha clathrata, T2884 Gardenia jasminoides var. grandiflora, T3242 Hibiscus rosa-sinensis, T3278 Homo sapiens, 
T3444 Ipomoea aquatica [Syn. Convolvulus repens; Ipomoea reptans], T3701 Lasiosphaera fenzlii, T3961 Lycoperdon pyriforme, T4190 Mentha spicata, T4248 Mimosa pudica, T4259 Mnium cuspidatum, T5354 Pyrrosia davidii, T5355 Pyrrosia drakeana, T5356 Pyrrosia gralla, T5357 Pyrrosia lingua T5358 Pyrrosia petiolosa, T5360 Pyrrosia sheareri, T5529 Rhodomyrtus tomentosa, T5569 Rosa multiflora, T6046 Sophora viciifolia, T6742 Vicia amoena, T6745 Vicia faba, T6749 Vicia hirsuta, T6750 Vicia sativa, T6814 Wedelia chinensis [Syn. Solidago chinensis; Wedelia calendulacea], T6830 Woodfordia fruticosa.

numbing wind $\mathrm{T} 3300$ Hydnocarpus anthelminticus.

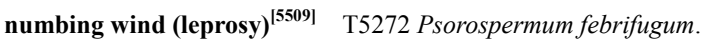

numbness and paralysis T2732 Firmiana simplex, T4308 Mucuna birdwoodiana, T6066 Spatholobus suberectus, T6184 Strychnos nux-vomica, T6193 Strychnos wallichiana.

numbness in joints T4290 Morus alba.

numbness in limbs T0077 Aconitum balfourii, T0084 Aconitum carmichaeli, T0105 Aconitum karakolicum, T0281 Alangium chinense, T0285 Alangium platanifolium, T0634 Aristolochia mollissima, T0644 Armillaria mellea, T0645 Armillariella mellea, T1175 Cannabis sativa, T1286 Celastrus flagellaris, T1288 Celastrus orbiculatus [Syn. Celastrus articulatus], T1545 Clematis chinensis, T1562 Clerodendron trichotomum, T1921 Cyanotis arachnoidea [Syn. Cyanotis bodinieri], T2030 Daphne retusa, T2031 Daphne tangutica, T2211 Dioscorea tenuipes, T2753 Formica fusca, T2890 Gastrodia elata, T3624 Kalopanax pictum, T3625 Kalopanax septemlobus, T3643 Kopsia officinalis, T3653 Lactarius piperatus [Syn. Agaricus piperatus], T3657 Lactarius vellereus, T3680 Lamium amplexicaule, T3785 Levisticum officinale, T3847 Lindera angustifolia, T3867 Liquidambar formosana [Syn. Liquidambar taiwaniana], T3897 Litsea verticillata, T3965 Lycopodium annotinum, T4190 Mentha spicata, T4238 Millettia nitida, T4311 Mucuna sempervirens, T4688 Peganum harmala, T4800 Phlegmariurus fordii, T4956 Piper mullesua, T5236 Prunus pseudocerasus, T5298 Pteris vittata, T5730 Sarcandra glabra [Syn. Chloranthus glaber], T5848 Securinega suffruticosa, T5958 Sinapis alba [Syn. Brassica alba; Brassica hirta], T6183 Strychnos nitida, T6504 Tricholoma matsutake [Syn. Armillaria matsutake].

numbness in lumbus and leg T1292 Celastrus orbiculatus [Syn. Celastrus articulatus].

numbness of limbs T3976 Lycopodium obscurum, T3989 Lygodium flexuosum [Syn. Lygodium pinnatifidum; Ophioglossum flexuosum], T4725 Periploca calophylla.

odynuria T1967 Cynoglossum amabile, T3284 Houttuynia cordata.

opacity of urine T1823 Crotalaria juncea, T3885 Litsea cubeba, T5124 Polypodium niponicum.

open pus sore of lung T6330 Tephroseris kirilowii [Syn. Senecio integrifolius var. fauriei].

ophthalmia T0293 Albizzia lebbeck.

oppression and depression in chest T2758 Fortunella crassifolia, T2759 Fortunella japonica, T2760 Fortunella margarita.

oppression in chest T1498 Citrus medica, T1520 Citrus wilsonii, T2246 Dolichos lablab, T5059 Pogostemon cablin [Syn. Mentha cablin].

oppression in chest and abdomen distention T0463 Anemone altaica,
T1486 Citrus junos, T2563 Eupatorium japonicum, T4209 Michelia alba.

oppression in chest and $q i$ bind T4855 Physeter catodon.

oppression in chest and vexation in heart T3192 Hemerocallis citrina.

oppression in chest due to glomus T1366 Chimonanthus fragrans [Syn.

Chimonanthus praecox], T1469 Citrus aurantium var. amara.

optic nerve atrophy T5312 Pueraria edulis, T5313 Pueraria lobata [Syn.

Pueraria thunbergiana; Pueraria pseudohirsuta], T5316 Pueraria

omeiensis, T5318 Pueraria phaseoloides, T5320 Pueraria thomsonii.

oral gan T3827 Ligustrum japonicum.

oral putrescence T0834 Averrhoa carambola.

oral ulcer T0172 Adiantum caudatum, T0543 Apis mellifera ligustica,

T1247 Cassia sophera, T1747 Corydalis thalictrifolia, T2357 Emilia sonchifolia, T3174 Heliotropium indicum, T4979 Pistacia chinensis, T5199 Primula malacoides.

osteoarthritis T0138 Aconitum umbrosum, T5704 Sambucus nigra, T5707 Sambucus williamsii.

osteomalacia T5270 Psoralea corylifolia.

osteoporosis T5270 Psoralea corylifolia.

otitis media T0015 Abutilon indicum, T0516 Anser cygnoides domestica, T0517 Anser cygnoides domestica, T0646 Armillariella tabescens, T1655 Conyza blinii, T1662 Coptis chinensis, T1663 Coptis chinensis var. brevisepala, T1664 Coptis deltoidea, T1669 Coptis omeiensis, T1670 Coptis teetoides [Syn. Coptis teeta], T1733 Corydalis racemosa, T1922 Cyanotis vaga, T2830 Galium aparine, T3284 Houttuynia cordata, T3392 Ilex kudingcha, T3393 Ilex latifolia, T4303 Moschus moschiferus; Moschus berezovskii; Moschus sifanicus, T4691 Pelargonium hortorum, T4859 Physochlaina physaloides, T5100 Polygonum chinense, T5189 Pothos chinensis, T5680 Salvia miltiorrhiza, T5681 Salvia miltiorrhiza f. alba, T5688 Salvia przewalskii, T5773 Saxifraga stolonifera, T5836 Scutellaria discolor, T5892 Senecio nemorensis, T5953 Silene firma, T6031 Sophora flavescens [Syn. Sophora angustfolia], T6206 Sus scrofa domestica, T6843 Xanthium sibiricum [Syn. Xanthium strumarium].

outcrop T2272 Drymaria diandra [Syn. Drymaria cordata ssp. diandra]. outcrop of sore and welling abscess T5228 Prunus mume.

ovarian carcinoma T2748 Fomes fomentarius [Syn. Pyropolyporus fomentarius; Boletus fomentarius; Polyporus fomentarius].

oxhide lichen T2772 Fraxinus mandshurica.

oxyuria T3466 Iris potaninii.

oxyuria disease T0318 Allium sativum, T0364 Alsophila spinulosa, T1211 Carpesium abrotanoides, T1361 Chenopodium ambrosioides, T1382 Chondria armata [Syn. Lophura armata], T1881 Cucurbita moschata, T2049 Daucus carota, T2281 Dryopteris crassirhizoma, T3688 Lappula echinata, T4083 Mallotus philippinensis, T4084 Mallotus philippinensis, T4127 Matteuccia struthiopteris, T4156 Melia azedarach, T4162 Melia toosendan, T5185 Potentilla multifida, T5384 Quisqualis indica, T5920 Seriphidium cinum [Syn. Artemisia cina], T5921 Seriphidium finitum [Syn. Artemisia finita], T6111 Stemona japonica, T6113 Stemona sessilifolia, T6115 Stemona tuberosa, T6201 Styrax obassia, T6713 Vernonia anthelmintica.

paddy field dermatitis T2835 Galium verum.

paddy field dermatitis (water ulcer) T2988 Glochidion eriocarpum. 
paddy-field dermatitis T3996 Lysimachia candida. pain after operation T1959 Cynanchum paniculatum.

pain from arthritis T1589 Cocculus trilobus [Syn. Cocculus sarmentosus], T3410 Impatiens balsamina, T5964 Sinomenium acutum.

pain from carcinoma T0079 Aconitum brachypodum, T0117 Aconitum nagarum var. lasiandrum.

pain from swollen welling abscess T2718 Ficus carica.

pain from ulcer in digestive tract T1017 Brassica oleracea var. capitata, T1357 Chelidonium majus.

pain in chest and abdomen T0201 Aesculus chinensis, T0202 Aesculus hippocastanum, T0205 Aesculus wilsonii, T5715 Santalum album.

pain in chest and abdomen and rib-side T1903 Curcuma aromatica, T1905 Curcuma longa, T1907 Curcuma wengujin.

pain in chest and back T4143 Meconopsis horridula.

pain in deep tissues T1715 Corydalis decumbens [Syn. Corydalis amabilis].

pain in heart and abdomen T0036 Acanthopanax giraldii [Syn. Acanthopanax giraldii var. inermis; Eleutherococcus giraldii], T0994 Boswellia carterii, T1008 Brassica juncea, T1706 Corydalis ambigua var. amurensis [Syn. Corydalis ambigua], T1734 Corydalis remota [Syn. Corydalis bulbosa var. typica], T1735 Corydalis repens, T2536 Euonymus alatus, T3853 Lindera obtusiloba, T4631 Papaver nudicaule var. chinense, T4635 Papaver somniferum, T4855 Physeter catodon, T5221 Prunus davidiana, T5235 Prunus persica, T5301 Pterocarpus indicus, T5680 Salvia miltiorrhiza, T5681 Salvia miltiorrhiza f. alba, T5688 Salvia przewalskii, T6062 Sparganium stoloniferum, T6105 Stellera chamaejasme, T6197 Styrax benzoin, T6204 Styrax tonkinensis, T6584 Typha angustata, T6585 Typha angustifolia, T6587 Typha latifolia.

pain in heart and stomach T5042 Podocarpus macrophyllus, T5044 Podocarpus macrophyllus var. maki.

pain in hepatic zone T3461 Iris japonica.

pain in joints T0077 Aconitum balfourii, T0079 Aconitum brachypodum, T0084 Aconitum carmichaeli, T0086 Aconitum chasmanthum, T0090 Aconitum delavayi, T0099 Aconitum gymnandrum, T0105 Aconitum karakolicum, T0108 Aconitum kusnezoffii, T0117 Aconitum nagarum var. lasiandrum, T0135 Aconitum sungpanense, T0483 Angelica gigas, T0881 Bauhinia variegata, T1043 Bryophyllum pinnatum, T1577 Clitoria ternatea, T1799 Crinum latifolium, T1955 Cynanchum hancockianum, T2193 Dioscorea cirrhosa [Syn. Dioscorea pogonoides], T2203 Dioscorea nipponica, T2204 Dioscorea nipponica ssp. rosthornii, T2302 Dysosma pleiantha [Syn. Podophyllum pleianthum], T2304 Dysosma veitchii, T2305 Dysosma versipellis [Syn. Podophyllum versipelle], T2750 Fomitopsis pinicola [Syn. Fomes pinicola; Polyporus pinicola], T3332 Hypecoum erectum, T3334 Hypecoum leptocarpum, T3496 Isodon japonica [Syn. Rabdosia japonica], T3886 Litsea euosma, T4665 Passiflora edulis, T4681 Pedicularis decora, T4796 Pheretima aspergillum; Allolobophora caliginosa trapezoides, T5569 Rosa multiflora, T6826 Wisteria sinensis.

pain in joints due to rheumatalgia T0071 Achyranthes aspera, T0072 Achyranthes aspera var. indica, T0568 Aralia chinensis, T0570 Aralia dasyphylla, T0631 Aristolochia kaempferi, T0642 Aristolochia tubiflora, T1005 Brassica campestris [Syn. Brassica campestris var. oleifera], T2745 Foeniculum vulgare, T2950 Gerbera anandria [Syn. Leibnitzia anandria], T3320 Hymenocallis littoralis [Syn. Hymenocallis americana; Pancratium littoralis], T3349 Hypericum erectum, T3400 Illicium difengpi, T3403 Illicium majus, T3797 Libanotis condensata, T3984 Lycoris chinensis, T3986 Lycoris radiata [Syn. Amaryllis radiata], T4020 Macleaya cordata, T4527 Orixa japonica, T4607 Panax pseudo-ginseng var. japonicus, T4663 Passiflora caerulea, T4842 Phyllanthus reticulatus, T5279 Psychotria serpens, T5396 Rabdosia rubescens, T5414 Ranunculus japonicus, T5609 Rumex hastatus, T5638 Sabia swinhoei, T5639 Sabina chinensis, T5987 Sobina chinensis, T6005 Solanum lyratum, T6888 Zanthoxylum planispinum.

pain in limbs T0079 Aconitum brachypodum, T0117 Aconitum nagarum var. lasiandrum, T0138 Aconitum umbrosum, T3822 Ligusticum jeholense, T3824 Ligusticum sinense, T6843 Xanthium sibiricum [Syn. Xanthium strumarium].

pain in lumbus and knees T0038 Acanthopanax gracilistylus, T0042 Acanthopanax senticosus [Syn. Eleutherococcus senticosus], T0044 Acanthopanax sessiliflorus, T0492 Angelica pubescens f. biserrata [Syn. Angelica pubescens], T1706 Corydalis ambigua var. amurensis [Syn. Corydalis ambigua], T1734 Corydalis remota [Syn. Corydalis bulbosa var. typica], T1735 Corydalis repens, T1923 Cyathula capitata, T2479 Erythrina variegata var. orientalis, T3213 Heracleum hemsleyanum, T3214 Heracleum lanatum, T3217 Heracleum moellendorffii [Syn. Heracleum microcarpum; Heracleum morifolium], T3228 Heracleum yungningense, T4656 Parmelia saxatilis, T6862 Zanthoxylum ailanthoides.

pain in lumbus and leg T3363 Hypericum sampsonii, T5591 Rubus cochinchinensis.

pain in lumbus and legs T0006 Abies nephrolepis, T0078 Aconitum barbatum var. puberulum [Syn. Aconitum ochranthum], T0100 Aconitum hemsleyanium var. circinacum, T0101 Aconitum hemsleyanum, T0125 Aconitum pseudostapfianum, T0644 Armillaria mellea, T0645 Armillariella mellea, T1417 Cimicifuga acerina, T1921 Cyanotis arachnoidea [Syn. Cyanotis bodinieri], T2231 Diphylleia grayi, T2232 Diphylleia sinensis, T3401 Illicium henryi, T3653 Lactarius piperatus [Syn. Agaricus piperatus], T3802 Ligularia dentata, T3805 Ligularia fischeri, T3807 Ligularia intermedia, T3813 Ligularia sibirica, T3976 Lycopodium obscurum, T4085 Mallotus repandus var. chrysocarpus [Syn. Mallotus chrysocarpus; Mallotus repandus], T5236 Prunus pseudocerasus, T5978 Smilax glauco-china, T6504 Tricholoma matsutake [Syn. Armillaria matsutake], T6531 Trillium camtschaticum, T6533 Trillium kamtschaticum, T6535 Trillium tschonoskii, T6777 Viscum multinerve.

pain in rib-side T0045 Acanthopanax trifoliatus, T1406 Chrysosplenium nudicaule, T1498 Citrus medica, T1520 Citrus wilsonii, T1705 Corydalis adunca, T2132 Desmodium pulchellum [Syn. Phyllodium pulchellum], T2908 Gentiana cephalantha, T2921 Gentiana manshurica, T2928 Gentiana rigescens, T2930 Gentiana scabra, T2938 Gentiana triflora, T3418 Incarvillea arguta, T4580 Paeonia albiflora [Syn. Paeonia lactiflora]. 
pain in rib-side due to stagnation T4584 Paeonia lactiflora wild, T4586

Paeonia obovata, T4590 Paeonia veitchii.

pain in sensory nerve endings $\mathrm{T} 1357$ Chelidonium majus.

pain in sinews and bones T0969 Boerhavia diffusa, T1025

Bretschneidera sinensis, T1292 Celastrus orbiculatus [Syn. Celastrus articulatus], T3026 Gnaphalium affine [Syn. Gnaphalium multiceps], T3360 Hypericum patulum, T3619 Kadsura peltigera [Syn. Kadsura longipedunculata], T3679 Lamiophlomis rotata [Syn. Phlomis rotata], T3680 Lamium amplexicaule, T4510 Ophiorrhiza japonica, T4513 Ophiorrhiza mungos, T4642 Parameria laevigata, T5576 Rostellularia procumbens [Syn. Justicia procumbens], T5813 Scilla scilloides, T5840 Scutellaria indica, T6252 Symplocos chinensis, T6548 Tritonia crocosmaeflora, T6827 Wisteria sinensis, T6845 Xanthoceras sorbifolia.

pain in stomach duct T0045 Acanthopanax trifoliatus, T0201 Aesculus chinensis, T0202 Aesculus hippocastanum, T0205 Aesculus wilsonii, T0567 Aralia armata, T0568 Aralia chinensis, T0570 Aralia dasyphylla, T0571 Aralia decaisneaa, T0642 Aristolochia tubiflora, T0877 Bauhinia championii, T1163 Campylotropis hirtella, T1384 Chondrus ocelladus, T1436 Cinnamomum camphora, T2027 Daphne odora, T3866 Liquidambar formosana [Syn. Liquidambar taiwaniana], T4305 Mosla dianthera, T4323 Murraya paniculata [Syn. Chalcas paniculata], T4325 Murraya paniculata var. exotica, T4604 Panax japonicus var. bipinnatifidus, T5137 Poncirus trifoliata, T5341 Pygmaeopremna herbacea [Syn. Premna herbacea], T5401 Rabdosia yuennanensis, T5731 Sarcococca coriacea [Syn. Sarcococca wallichii], T5785 Schefflera venulosa, T6548 Tritonia crocosmaeflora.

pain in stomach duct, abdomen and rib-side T1327 Ceratostigma minus. pain in stomach duct and abdomen T0229 Ageratum conyzoides, T0491 Angelica polymorpha, T0540 Apis cerana, T0634 Aristolochia mollissima, T0641 Aristolochia tuberosa, T0859 Balanophora involucrata, T1444 Cinnamomum tamala, T1750 Corydalis yanhusuo [Syn. Corydalis turtschaninovii f. yanhusuo], T1934 Cyclea tonkinensis, T2643 Evodia meliifolia, T3011 Glycyrrhiza aspera, T3013 Glycyrrhiza glabra, T3015 Glycyrrhiza inflata, T3016 Glycyrrhiza kansuensis, T3021 Glycyrrhiza squamulosa, T3022 Glycyrrhiza uralensis, T3023 Glycyrrhiza yunnanensis, T3328 Hyoscyamus niger, T3329 Hyoscyamus niger, T3567 Juglans regia, T3867 Liquidambar formosana [Syn. Liquidambar taiwaniana], T4004 Lysimachia paridiformis, T4304 Mosla chinensis [Syn. Orthodon chinensis], T4577 Paederia scandens, T4637 Papaver somniferum, T4962 Piper puberulum, T5021 Pleurospermum rivulorum, T5057 Podophyllum emodii [Syn. Podophyllum emodii var. chinense; Podophyllum sikkimenosis; Sinopodophyllum emodii], T5661 Salvia bowleyana, T5781 Schefflera arboricola, T6120 Stephania delavayi [Syn. Stephania epigaea].

pain in stomach duct and rib-side T4157 Melia azedarach, T6801 Vladimiria denticulata.

pain in stomach duct and rib-side due to liver $\boldsymbol{q} i$ depression T3554 Jasminum grandiflorum.

pain in throat T0805 Astragalus sinicus, T2162 Dicranostigma franchetianum [Syn. Dicranostigma leptopodum], T5501 Rhodiola yunnanesis. pain in tooth and cheek T3822 Ligusticum jeholense, T3824 Ligusticum sinense.

pain of blood stasis T3276 Homo sapiens, T4479 Odontites serotina, T6895 Zanthoxylum simulans.

pain of hot urine T0423 Ampelopsis brevipedunculata, T2134 Desmodium styracifolium.

pain of hot urine and inhibited urination T2410 Equisetum pratense.

pain wind T0319 Allium schoenoprasum, T1188 Caragana chamlagu, T1191 Caragana sinica, T1416 Cicuta virosa, T2020 Damnacanthus indicus, T2412 Equisetum sylvaticum, T3443 Iphigenia indica, T3594 Juniperus rigida, T4267 Monachosorum flagellare, T4324 Murraya paniculata [Syn. Chalcas paniculata], T5704 Sambucus nigra, T5707 Sambucus williamsii, T6018 Solanum verbascifolium, T6673 Vaccinium vitis-idaea.

pain wind in joints T6895 Zanthoxylum simulans.

pain wind in limbs T1262 Catalpa ovata.

painful bind in lesser-abdomen T2231 Diphylleia grayi, T2232 Diphylleia sinensis.

painful joints due to rheumatalgia T0096 Aconitum forrestii [Syn. Aconitum likiangense], T0100 Aconitum hemsleyanium var. circinacum, T0101 Aconitum hemsleyanum, T0107 Aconitum kongboense, T1288 Celastrus orbiculatus [Syn. Celastrus articulatus], T1640 Coniogramme japonica [Syn. Hemionitis japonica], T2263 Dregea volubilis, T3674 Laggera alata, T5102 Polygonum cuspidatum.

painful red eyes T3203 Hemsleya amabilis, T3208 Hemsleya macrosperma, T4263 Momordica charantia, T5323 Pulsatilla campanella, T5324 Pulsatilla cernua, T5325 Pulsatilla chinensis, T5326 Pulsatilla dahurica.

painful swelling from clove sore T3426 Inula britannica, T3433 Inula japonica, T4388 Nasturtium officinale, T5723 Sapium sebiferum.

painful swelling from knocks and falls T0262 Ajuga ciliata, T0266 Ajuga macrosperma, T0267 Ajuga nipponensis, T0293 Albizzia lebbeck, T0310 Allium ascalonicum, T0374 Alstonia scholaris, T0571 Aralia decaisneaa, T0813 Atalantia buxifolia [Syn. Severinia buxifolia], T0814 Atalantia buxifolia [Syn. Severinia buxifolia], T0871 Barleria lupulina, T1120 Callicarpa macrophylla, T1181 Capra hircus; Ovis aries, T1289 Celastrus orbiculatus [Syn. Celastrus articulatus], T1434 Cinnamomum bejolghota [Syn. Cinnamomum obtusifolium; Laurus bejolghota], T1441 Cinnamomum japonicum, T1552 Cleome viscosa, T1583 Cnidium officinale [Syn. Ligusticum officinale], T1697 Cornus controversa [Syn. Bothrocaryum controversum], T1734 Corydalis remota [Syn. Corydalis bulbosa var. typica], T1750 Corydalis yanhusuo [Syn. Corydalis turtschaninovii f. yanhusuo], T1808 Crocus sativus, T1971 Cynoglossum zeylanicum [Syn. Anchusa zeylanica; Cynoglossum furcatum; Cynoglossum formosanum], T2062 Delphinium bonvalotii, T2081 Delphinium omeiense, T2084 Delphinium potaninii, T2085 Delphinium potaninii var. jiufengshanense, T2124 Derris trifoliata, T2193 Dioscorea cirrhosa [Syn. Dioscorea pogonoides], T2200 Dioscorea hispida, T2290 Duchesnea indica, T2449 Erycibe obtusifolia, T2450 Erycibe schmidtii, T2602 Euphorbia nematocypha, T2614 Euphorbia pulcherrima, T2631 Eurya japonica, T2719 Ficus fistulosa [Syn. Ficus harlandii], T2720 Ficus hispida, T2736 Fissistigma polyanthum, T2857 Garcinia 
hanburyi, T3006 Glycosmis citrifolia, T3190 Helminthostachys zeylanica, T3292 Hunteria zeylanica, T3320 Hymenocallis littoralis [Syn. Hymenocallis americana; Pancratium littoralis], T3410 Impatiens balsamina, T3471 Iris tectorum, T3820 Ligusticum chuanxiong [Syn. Ligusticum wallichii], T3880 Lithospermum arvense, T3897 Litsea verticillata, T4020 Macleaya cordata, T4029 Maesa japonica, T4158 Melia azedarach, T4190 Mentha spicata, T4221 Micromelum falcatum, T4223 Micromelum integerrimum, T4323 Murraya paniculata [Syn. Chalcas paniculata], T4325 Murraya paniculata var. exotica, T4413 Nepeta cataria, T4418 Nerium indicum, T4503 Onychium lucidum, T4506 Ophioglossum vulgatum, T4604 Panax japonicus var. bipinnatifidus, T4608 Panax pseudo-ginseng var. notoginseng [Syn. Panax notoginseng], T4686 Pedilanthus tithymaloides, T4730 Peristrophe roxburghiana, T4962 Piper puberulum, T4974 Piper wallichii [Syn. Piper wallichii var. hupehense], T5105 Polygonum hydropiper var. flaccidum [Syn. Polygonum flaccidum], T5158 Populus simonii, T5172 Portulaca grandiflora, T5267 Psidium guajava, T5288 Pteris cretica var. nervosa [Syn. Pteris nervosa], T5444 Reboulia hemisphaerica, T5501 Rhodiola yunnanesis, T5741 Sargentodoxa cuneata, T5781 Schefflera arboricola, T5884 Senecio chrysanthemoides, T5975 Smilax bockii, T6018 Solanum verbascifolium, T6092 Stachys palustris, T6095 Stachytarpheta jamaicensis, T6138 Sterculia foetida, T6264 Syzygium buxifolium, T6578 Tylophora atrofolliculata, T6645 Urena lobata, T6738 Viburnum odoratissimum, T6788 Vitex negundo.

\section{painful swelling throat moth T2835 Galium verum.}

painful swollen breast T1978 Cyperus rotundus, T5086 Polygala sibirica, T5088 Polygala tenuifolia.

painful swollen testes T0274 Akebia quinata, T0278 Akebia trifoliata, T0280 Akebia trifoliata var. australis, T1504 Citrus reticulata, T2321 Ecklonia kurome, T2636 Euscaphis japonica, T3678 Laminaria japonica, T3877 Litchi chinensis, T4324 Murraya paniculata [Syn. Chalcas paniculata], T6020 Solanum xanthocarpum, T6432 Thespesia populnea [Syn. Hibiscus populneus], T6527 Trigonella caerulea, T6640 Undaria pinnatifida.

painful wound from knocks and falls T0019 Acacia catechu, T0634 Aristolochia mollissima, T0830 Auricularia auricula, T0831 Auricularia delicata, T0957 Blumea balsamifera, T1092 Buxus microphylla var. sinica, T1292 Celastrus orbiculatus [Syn. Celastrus articulatus], T1343 Chaenomeles sinensis, T1435 Cinnamomum camphora, T1931 Cyclea barbata, T1933 Cyclea sutchuenensis, T1996 Dahlia pinnata [Syn. Dahlia variabilis], T2295 Duranta repens, T2536 Euonymus alatus, T3329 Hyoscyamus niger, T3507 Isodon lophanthoides [Syn. Rabdosia lophanthoides; Hyssopus lophanthoides; Plectranthus striatus; Isodon striatus], T3548 Ixeris sonchifolia, T3849 Lindera chunii, T4268 Monachosorum henryi, T4582 Paeonia delavayi, T4585 Paeonia moutan [Syn. Paeonia suffruticosa], T4648 Paris polyphylla, T4649 Paris polyphylla var. chinensis, T4650 Paris polyphylla var. pseudothibetica, T4651 Paris polyphylla var. stenophylla, T4652 Paris polyphylla var. yunnanensis, T5118 Polygonum suffultum, T5119 Polygonum thunbergii, T5393 Rabdosia longituba, T5397 Rabdosia serra, T5447 Rehmannia glutinosa [Syn. Rehmannia glutinosa f. huechingensis], T5572 Rosa rugosa, T5591
Rubus cochinchinensis, T5606 Rumex acetosa, T5822 Scopolia sinensis, T6015 Solanum torvum, T6100 Stauntonia hexaphylla, T6548 Tritonia crocosmaeflora, T6676 Valeriana hardwickii, T6773 Viscum articulactum, T6774 Viscum articulatum.

palmus and breathe hard T1381 Choerospondias axillaris.

palpitation T0553 Apocynum venetum, T1438 Cinnamomum cassia [Syn. Cinnamomum aromaticum], T2452 Erysimum cheiranthoides, T2846 Ganoderma japonicum [Syn. Ganoderma sinense], T2848 Ganoderma lucidum, T5107 Polygonum multiflorum, T5516 Rhododendron molle, T5850 Sedum aizoon, T5855 Sedum kamtschaticum, T6295 Tanacetum sibiricum [Syn. Filifolium sibiricum], T6358 Tetraplodon mnioides [Syn. Tetraplodon bryoides; Splachnum mnioides], T6750 Vicia sativa.

palpitation and dizziness T5445 Rehmannia glutinosa [Syn. Rehmannia glutinosa $\mathrm{f}$. huechingensis].

palpitation and fearful throbbing T3778 Leucaena glauca [Syn. Leucaena leucocephala], T6060 Souliea vaginata.

palpitation and insomnia T0190 Adonis sutchuenensis, T1292 Celastrus orbiculatus [Syn. Celastrus articulatus], T1804 Cristaria plicata; Hyriopsis cumingii, T1952 Cynanchum auriculatum, T1953 Cynanchum bungei, T3743 Lentinus lepideus, T4194 Menyanthes trifoliata, T5497 Rhodiola kirilowii, T5791 Schisandra chinensis, T5802 Schisandra sphenanthera, T6675 Valeriana amurensis, T6679 Valeriana officinalis, T6680 Valeriana officinalis var. latifolia.

palpitation and night sweating T6798 Vitis vinifera.

palpitation and shortness of breath T3011 Glycyrrhiza aspera, T3013 Glycyrrhiza glabra, T3015 Glycyrrhiza inflata, T3016 Glycyrrhiza kansuensis, T3021 Glycyrrhiza squamulosa, T3022 Glycyrrhiza uralensis, T3023 Glycyrrhiza yunnanensis, T4729 Periploca sepium, T4902 Pimpinella thelungiana.

papules T1239 Cassia nodosa, T5818 Scoparia dulcis.

paralysis in limbs T3615 Kadsura interior.

paralysis T0978 Bombyx mori, T1288 Celastrus orbiculatus [Syn. Celastrus articulatus], T1976 Cyperus iria, T2422 Erigeron breviscapus, T3113 Hedera nepalensis var. sinensis, T3115 Hedera rhombea, T5236 Prunus pseudocerasus, T5368 Python molurus bivittatus, T5822 Scopolia sinensis, T5934 Seseli meirei, T5937 Seseli yunnanense, T6183 Strychnos nitida.

paralysis in limbs T5144 Populus adenopoda.

paralytic and pruritus T5964 Sinomenium acutum.

parkinson's disease T2964 Ginkgo biloba.

parotitis T0012 Abrus precatorius, T0015 Abutilon indicum, T0643 Aristolochia versicolar, T0952 Blechnum orientale, T1327 Ceratostigma minus, T1573 Clinopodium chinense, T1709 Corydalis bungeana, T1819 Crotalaria ferruginea, T1884 Cudrania cochinchinensis, T1996 Dahlia pinnata [Syn. Dahlia variabilis], T2298 Dysosma difformis, T3243 Hibiscus rosa-sinensis, T3423 Indigofera tinctoria, T3475 Isatis indigotica, T3736 Lemmaphyllum microphyllum, T4127 Matteuccia struthiopteris, T4552 Osmunda japonica, T5098 Polygonum aviculare, T5295 Pteris multifida, T5806 Schlumbergera truncata, T6119 Stephania cepharantha, T6814 Wedelia chinensis [Syn. Solidago chinensis; Wedelia calendulacea], T6843 Xanthium sibiricum [Syn. Xanthium strumarium].

paroxysmal fibrillation T6433 Thevetia neriifolia [Syn. Thevetia 
peruviana]

paroxysmal supraventricular tachycardia T6433 Thevetia neriifolia [Syn. Thevetia peruviana].

paroxysmal tachycardia T1649 Convallaria keiskei [Syn. Convallaria majalis].

pelvic inflamation $\mathrm{T} 3547$ Ixeris chinensis, T6217 Swertia davidii.

pelvic inflammation T0215 Agave americana, T1826 Crotalaria mucronata, T5169 Poria cocos.

pemphigus T5395 Rabdosia nervosa.

peptic ulcer T3011 Glycyrrhiza aspera, T3013 Glycyrrhiza glabra, T3015 Glycyrrhiza inflata, T3016 Glycyrrhiza kansuensis, T3021 Glycyrrhiza squamulosa, T3022 Glycyrrhiza uralensis, T3023 Glycyrrhiza yunnanensis, T6276 Tacca plantaginea [Syn. Schizocapsa plantaginea].

periodontitis T0933 Betula platyphylla, T0935 Betula platyphylla var. japonica, T2749 Fomes officinalis, T2852 Garcinia cowa.

periostitis T1003 Brandisia hancei.

peripheral arterial diseases T2964 Ginkgo biloba.

pernicious anemia T0495 Angelica sinensis, T4804 Phlojodicarpus sibiricus.

persistent flow of lochia T0661 Artemisia anomala, T3752 Leonurus heterophyllus [Syn. Leonurus artemisia], T3754 Leonurus sibiricus, T4234 Millettia dielsiana, T5301 Pterocarpus indicus.

persistent flow with abdominal pain T5701 Salvia yunnanensis.

pertussis T0318 Allium sativum, T0374 Alstonia scholaris, T0457 Andrographis paniculata [Syn. Justicia paniculata], T0752 Asparagus officinalis, T1357 Chelidonium majus, T1366 Chimonanthus fragrans [Syn. Chimonanthus praecox], T1789 Crepis napifera, T1881 Cucurbita moschata, T2191 Dioscorea bulbifera, T2268 Drosera rotundifolia, T2334 Elephantopus scaber, T2510 Eucalyptus globulus, T2721 Ficus microcarpa, T2738 Flemingia strobilifera, T3040 Gomphrena globosa, T3344 Hypericum chinense, T3646 Kyllinga brevifolia, T3769 Lespedeza bicolor, T3779 Leucas aspera, T3802 Ligularia dentata, T3805 Ligularia fischeri, T3807 Ligularia intermedia, T3813 Ligularia sibirica, T4284 Morinda parvifolia, T4370 Nandina domestica, T4371 Nandina domestica, T4937 Piper betle, T5100 Polygonum chinense, T5173 Portulaca oleracea, T5184 Potentilla kleiniana, T5721 Sapindus mukorossi, T5939 Shiraia bambusicola, T6075 Spilanthes acmella, T6092 Stachys palustris, T6111 Stemona japonica, T6113 Stemona sessilifolia, T6115 Stemona tuberosa, T6132 Stephania sasakii, T6206 Sus scrofa domestica, T6276 Tacca plantaginea [Syn. Schizocapsa plantaginea], T6278 Tagetes erecta, T6414 Thalictrum thunbergii, T6454 Thymus vulgaris, T6814 Wedelia chinensis [Syn. Solidago chinensis; Wedelia calendulacea], T6820 Wikstroemia indica.

pharyngitis T0015 Abutilon indicum, T5086 Polygala sibirica, T5088 Polygala tenuifolia, T6060 Souliea vaginata, T6122 Stephania dielsiana.

pharyngolaryngitis T0137 Aconitum tanguticum, T0707 Arthraxon hispidus, T0964 Boenninghausenia albiflora, T1655 Conyza blinii, T2133 Desmodium racemosum [Syn. Podocarpium podocarpum var. oxyphyllum], T2334 Elephantopus scaber, T2563 Eupatorium japonicum, T2715 Fibraurea recisa, T2844 Ganoderma applanatum,
T4215 Michelia yunnanensis, T4391 Nauclea officinalis, T6095 Stachytarpheta jamaicensis, T6214 Swertia chinensis, T6454 Thymus vulgaris, T6814 Wedelia chinensis [Syn. Solidago chinensis; Wedelia calendulacea].

phlegm aggregation T1468 Citrus aurantium, T1521 Citrus wilsonii, T5141 Poncirus trifoliata.

phlegm asthma T1152 Camellia sinensis [Syn. Thea sinensis], T2051 Daucus carota var. sativa, T4773 Peucedanum terebinthaceum, T4786 Phasianus colchicus.

phlegm congestion and hasty qi T0624 Aristolochia contorta, T0626 Aristolochia debilis [Syn. Aristolochia longa], T0630 Aristolochia indica, T0633 Aristolochia maxima, T0640 Aristolochia triangularis.

phlegm congestion and $\boldsymbol{q} \boldsymbol{i}$ counterflow T3426 Inula britannica, T3433 Inula japonica, T4715 Perilla frutescens, T4717 Perilla frutescens var. acuta [Syn. Perilla frutescens var. purpurascens], T4719 Perilla frutescens var. arguta, T4722 Perilla frutescens var. crispa.

phlegm congestion cough asthma T5420 Raphanus sativus.

phlegm containing blood T0749 Asparagus filicinus, T1680 Cordyceps militaris, T1681 Cordyceps militaris cv, T3832 Lilium brownii var. viridulum [Syn. Lilium brownii var. colchesteri], T3835 Lilium longiflorum, T3836 Lilium pumilum [Syn. Lilium tenuifolium], T3839 Lilium tigrinum [Syn. Lilium lancifolium], T4050 Magnolia sieboldii.

phlegm cough T4840 Phyllanthus niruri, T5530 Rhus chinensis [Syn. Rhus semialata], T5531 Rhus chinensis [Syn. Rhus semialata].

phlegm fire cough T1006 Brassica chinensis, T2798 Fritillaria verticillata var. thunbergii [Syn. Fritillaria thunbergii].

phlegm node T0847 Baccharis indica [Syn. Pluchea indica].

phlegm reversal T0144 Acorus gramineus, T0146 Acorus tatarinowii, T2274 Dryobalanops aromatica.

phlegm reversal with stupor T2975 Gleditsia delavayi, T2976 Gleditsia fera.

phlegm stagnation and qi counterflow T1007 Brassica juncea.

phlegm stagnation in chest and diaphragm T1467 Citrus aurantium, T5140 Poncirus trifoliata.

phlegm-damp congestion T1498 Citrus medica, T1520 Citrus wilsonii.

phlegm-damp cough T5862 Selaginella involvens, T6056 Sorghum vulgare.

phlegm-drool and congesting lung T2125 Descurainia sophia, T2978 Gleditsia sinensis [Syn. Gleditsia horrida], T3755 Lepidium apetalum [Syn. Lepidium micranthum], T3759 Lepidium virginicum, T3984 Lycoris chinensis, T3986 Lycoris radiata [Syn. Amaryllis radiata].

phlegm-heat cough T0895 Benincasa hispida, T1154 Camellia sinensis [Syn. Thea sinensis], T2525 Eucheuma muricatum, T2738 Flemingia strobilifera, T2787 Fritillaria hupehensis, T5419 Raphanus sativus, T6511 Trichosanthes kirilowii, T6615 Uncaria gambir.

phlegm-heat cough asthma T0048 Acanthus ilicifolius.

phlegmon T0333 Alocasia cucullata [Syn. Arum cucullatum], T3779 Leucas aspera, T4080 Mallotus apelta.

phlegm-rheum T0819 Atractylodes chinensis, T0821 Atractylodes japonica, T0823 Atractylodes lancea, T1438 Cinnamomum cassia [Syn. Cinnamomum aromaticum], T1692 Coriaria sinica [Syn. Coriaria nepalensis], T2597 Euphorbia lathyris, T2608 Euphorbia pekinensis, T5231 Prunus persica, T6819 Wikstroemia chamaedaphne. 
phlegm-rheum and accumulation-gathering T4779 Pharbitis nil, T4780 Pharbitis purpurea.

phlegm-rheum and edema T2321 Ecklonia kurome, T3678 Laminaria japonica, T6640 Undaria pinnatifida.

phlegm-rheum and food retention T4086 Malus asiatica.

phlegm-rheum and glomus accumulation T0983 Bos taurus domesticus. phlegm-rheum asthma fullness T1858 Croton tiglium.

phlegm-rheum cough asthma T0313 Allium chinense, T0316 Allium macrostemon, T0724 Asarum caulescens, T0726 Asarum forbesii, T0727 Asarum fukienense, T0728 Asarum heterotropoides var. mandshuricum, T0731 Asarum sieboldii, T0732 Asarum sieboldii var. seoulensis, T1501 Citrus medica var. sarcodactylis, T2589 Euphorbia helioscopia, T2599 Euphorbia lunulata, T4034 Magnolia biloba, T4045 Magnolia officinalis, T4550 Osmanthus fragrans, T6910 Zingiber officinale.

phlegm-rheum dizziness T0303 Alisma orientale [Syn. Alisma plantago-aquatica var. orientale], T0824 Atractylodes macrocephala [Syn. Atractylis macrocephala], T5169 Poria cocos.

phlegm-rheum rapid asthma T3632 Knoxia valerianoides.

phthisis T1884 Cudrania cochinchinensis, T3810 Ligularia nelumbifolia, T4350 Myriopteron extensum.

pineapple poisoning T3685 Lannea grandis [Syn. Lannea coromandelica].

pleuritis T1055 Bupleurum angustissimum, T1056 Bupleurum aureum, T1058 Bupleurum chaishoui, T1059 Bupleurum chinense, T1065 Bupleurum longiradiatum, T1066 Bupleurum marginatum, T1067 Bupleurum marginatum var. stenophyllum, T1072 Bupleurum scorzonerifolium, T1073 Bupleurum sibiricum, T1075 Bupleurum smithii var. parvifolium, T1078 Bupleurum wenchuanense, T1079 Bupleurum yinchowense, T2101 Dendrobium fimbriatum.

pneumonia T0137 Aconitum tanguticum, T0457 Andrographis paniculata [Syn. Justicia paniculata], T0866 Baphicacanthus cusia [Syn. Strobilanthes cusia], T0933 Betula platyphylla, T0935 Betula platyphylla var. japonica, T0967 Boenninghausenia sessilicarpa, T1869 Cryptotaenia japonica, T2298 Dysosma difformis, T2357 Emilia sonchifolia, T2697 Ferula borealis, T3174 Heliotropium indicum, T3284 Houttuynia cordata, T3295 Huperzia serrata [Syn. Lycopodium serratum], T3334 Hypecoum leptocarpum, T3709 Lathyrus pratensis, T3908 Lonicera bournei, T3909 Lonicera confusa, T3910 Lonicera fulvotomentosa, T3911 Lonicera hypoglanca, T3912 Lonicera japonica, T3914 Lonicera macranthoides, T3918 Lonicera similis, T4145 Meconopsis punicea, T4500 Onosma paniculatum, T4881 Picrasma quassioides [Syn. Picrasma ailanthoides], T5188 Potentilla viscosa, T5730 Sarcandra glabra [Syn. Chloranthus glaber], T5907 Senecio scandens [Syn. Senecio chinensis], T6705 Verbascum thapsus, T6814 Wedelia chinensis [Syn. Solidago chinensis; Wedelia calendulacea], T6820 Wikstroemia indica.

pneumonia with cough and asthma T5687 Salvia prionitis.

pneumonia, bronchitis T5494 Rhodiola crenulata [Syn. Rhodiola euryphylla].

poison from smoke T2048 Daucus carota.

poisoning from almonds T5217 Prunus armeniaca.

poisoning of fish or crab $\mathrm{T} 1165$ Canarium album, T4714 Perilla frutescens, T4718 Perilla frutescens var. acuta [Syn. Perilla frutescens var. purpurascens], T4721 Perilla frutescens var. arguta.

poisoning of mushrooms T3162 Helicia nilagirica, T5362 Pyrus bretschneideri, T5366 Pyrus pyrifolia.

poisonous bee stings T0333 Alocasia cucullata [Syn. Arum cucullatum], T1880 Cucurbita moschata.

poisonous insect stings T0107 Aconitum kongboense, T1359 Chenopodium album, T1436 Cinnamomum camphora, T1437 Cinnamomum camphora, T2131 Desmodium pulchellum [Syn. Phyllodium pulchellum], T2161 Dicranopteris pedata [Syn. Polypodium pedatum; Dicranopteris dichotoma], T2435 Eriocheir sinensis, T3241 Hibiscus mutabilis, T3770 Lespedeza cuneata, T3863 Liparis nervosa, T4578 Paederia scandens, T4881 Picrasma quassioides [Syn. Picrasma ailanthoides], T5402 Radermachera sinica, T5838 Scutellaria galericulata, T5850 Sedum aizoon, T5855 Sedum kamtschaticum, T6731 Veronicastrum sibiricum.

poisonous snake and rabid dog bite T6116 Stenoloma chusanum.

poisonous snake bite T0122 Aconitum pendulum, T0171 Adiantum capillus-veneris, T0172 Adiantum caudatum, T0263 Ajuga decumbens, T0379 Alternanthera philoxeroides, T0457 Andrographis paniculata [Syn. Justicia paniculata], T0617 Arisaema amurense, T0618 Arisaema consanguineum, T0620 Arisaema heterophyllum, T0631 Aristolochia kaempferi, T0635 Aristolochia moupinensis, T0641 Aristolochia tuberosa, T0642 Aristolochia tubiflora, T0678 Artemisia japonica, T0721 Arundina chinensis, T0890 Begonia limprichtii, T0941 Bidens tripartita, T0947 Bixa orellana, T0957 Blumea balsamifera, T1106 Caesalpinia minax, T1210 Carpesium abrotanoides, T1238 Cassia mimosoides, T1286 Celastrus flagellaris, T1289 Celastrus orbiculatus [Syn. Celastrus articulatus], T1372 Chloranthus serratus, T1589 Cocculus trilobus [Syn. Cocculus sarmentosus], T1644 Conocephalum conicum, T1719 Corydalis incisa, T1733 Corydalis racemosa, T1810 Croomia japonica, T1831 Crotalaria sessiliflora, T1896 Cupressus funebris, T1932 Cyclea racemosa, T1950 Cynanchum ascyrifolium, T1951 Cynanchum atratum, T1952 Cynanchum auriculatum, T1953 Cynanchum bungei, T1962 Cynanchum versicolor, T2027 Daphne odora, T2133 Desmodium racemosum [Syn. Podocarpium podocarpum var. oxyphyllum], T2191 Dioscorea bulbifera, T2194 Dioscorea collettii, T2205 Dioscorea panthaica, T2233 Diploclisia glaucescens, T2290 Duchesnea indica, T2298 Dysosma difformis, T2302 Dysosma pleiantha [Syn. Podophyllum pleianthum], T2304 Dysosma veitchii, T2305 Dysosma versipellis [Syn. Podophyllum versipelle], T2348 Embelia ribes, T2421 Erigeron annuus, T2536 Euonymus alatus, T2749 Fomes officinalis, T2753 Formica fusca, T2826 Galeola faberi, T2952 Gerbera piloselloides, T3080 Gymnema sylvestre, T3123 Hedyotis auricularia, T3126 Hedyotis corymbosa [Syn. Oldenlandia corymbosa], T3190 Helminthostachys zeylanica, T3318 Hylotelephium mingjinianum, T3340 Hypericum ascyron, T3349 Hypericum erectum, T3356 Hypericum japonicum, T3479 Isodon amethystoides, T3646 Kyllinga brevifolia, T3764 Lepisorus thunbergianus, T3808 Ligularia japonica [Syn. Arnica japonica; Senecio japonica], T3998 Lysimachia christinae, T4000 Lysimachia congestiflora, T4004 Lysimachia paridiformis, T4109 Marchantia polymorpha, T4203 Mesua ferrea, 
T4261 Mollugo pentaphylla, T4413 Nepeta cataria, T4456 Nymphoides peltatum, T4485 Oldenlandia diffusa [Syn. Hedyotis diffusa], T4607 Panax pseudo-ginseng var. japonicus, T4710 Pericampylus glaucus, T4740 Petasites japonicus, T4800 Phlegmariurus fordii, T4837 Phyllanthus emblica, T4840 Phyllanthus niruri, T4903 Pinellia pedatisecta, T5029 Plumbago zeylanica, T5073 Polygala chinensis [Syn. Polygala glomerata], T5099 Polygonum bistorta, T5104 Polygonum hydropiper, T5109 Polygonum nodosum, T5279 Psychotria serpens, T5424 Rauvolfia verticillata, T5537 Rhus sylvestris, T5714 Sansevieria trifasciata, T5723 Sapium sebiferum, T5797 Schisandra propinqua, T5798 Schisandra propinqua var. intermedia, T5835 Scutellaria barbata [Syn. Scutellaria rivularis], T5840 Scutellaria indica, T5852 Sedum bulbiferum, T6024 Solidago virgaurea var. leiocarpa [Syn. Solidago decurrens], T6075 Spilanthes acmella, T6120 Stephania delavayi [Syn. Stephania epigaea], T6121 Stephania dicentrinifera, T6129 Stephania japonica, T6132 Stephania sasakii, T6137 Stephania viridiflavens, T6367 Teucrium quadrifarium, T6482 Toxicodendron succedaneum [Syn. Rhus succedanea], T6541 Tripterygium regelii, T6580 Tylophora floribunda, T6582 Tylophora ovata, T6588 Typhoniun giganteum, T6645 Urena lobata, T6653 Usnea diffracta, T6654 Usnea longissima, T6731 Veronicastrum sibiricum, T6883 Zanthoxylum myriacanthum, T6904 Zephyranthes grandiflora [Syn. Zephyranthes carinata].

poisonous snake bites T0267 Ajuga nipponensis, T0333 Alocasia cucullata [Syn. Arum cucullatum], T0388 Amaranthus lividus, T0440 Anagallis arvensis, T0479 Angelica dahurica cv. qibaizhi, T0498 Angelica taiwaniana, T0871 Barleria lupulina, T0888 Beesia calthaefolia, T1118 Callicarpa formosana, T1119 Callicarpa japonica, T1371 Chloranthus japonicus, T1971 Cynoglossum zeylanicum [Syn. Anchusa zeylanica; Cynoglossum furcatum; Cynoglossum formosanum], T3292 Hunteria zeylanica, T3348 Hypericum elodeoides, T3368 Hypericum wightianum, T3479 Isodon amethystoides, T3932 Ludwigia octovalvis, T3996 Lysimachia candida, T4121 Marsilea quadrifolia, T4221 Micromelum falcatum, T4502 Onychium japonicum [Syn. Tricomanes japonicum], T4514 Ophiorrhiza pumila, T4549 Osbeckia chinensis, T4730 Peristrophe roxburghiana, T4884 Picria felterrae, T5087 Polygala telephioides, T5105 Polygonum hydropiper var. flaccidum [Syn. Polygonum flaccidum], T5209 Pronephrium simplex [Syn. Meniscium simplex], T5395 Rabdosia nervosa, T5401 Rabdosia yuennanensis, T5646 Sagina japonica [Syn. Spergula japonica], T5856 Sedum lineare [Syn. Sedum obtuso-lineare], T5954 Silene fortunei, T6087 Spiranthes sinensis, T6131 Stephania longa, T6135 Stephania succifera, T6214 Swertia chinensis, T6224 Swertia kouitchensis, T6252 Symplocos chinensis, T6566 Tupistra chinensis, T6601 Umbilicaria esculenta [Syn. Gyrophora esculenta], T6684 Vanilla planifolia, T6767 Viola yedoensis, T6861 Zanthoxylum ailanthoides, T6893 Zanthoxylum simulans, T6894 Zanthoxylum simulans.

poliomyelitis T2389 Epimedium acuminatum, T2390 Epimedium brevicornum, T2392 Epimedium davidii, T2394 Epimedium elongatum, T2398 Epimedium koreanum, T2401 Epimedium pubescens, T2402 Epimedium sagittatum, T2404 Epimedium sutchuenense, T2406 Epimedium wushanense, T3984 Lycoris chinensis, T3986 Lycoris radiata [Syn. Amaryllis radiata], T4239 Millettia nitida var. hirsutissima.

pollinosis T3936 Luffa operculata.

postauricular sore T3726 Laurus nobilis.

postlithotripsy urinary tract infection T0457 Andrographis paniculata

[Syn. Justicia paniculata].

postoperative pain $\mathrm{T} 3548$ Ixeris sonchifolia.

postpartum abdominal pain T0474 Angelica acutiloba [Syn. Ligusticum acutilobum], T2193 Dioscorea cirrhosa [Syn. Dioscorea pogonoides], T3614 Kadsura heteroclita [Syn. Uvaria heteroclita], T3877 Litchi chinensis, T3981 Lycopus lucidus, T4234 Millettia dielsiana, T4956 Piper mullesua, T5595 Rubus parviforlius, T5781 Schefflera arboricola, T5893 Senecio nudicaulis, T5966 Siphonostegia chinensis, T6154 Strobilanthes japonicus [Syn. Championella japonica], T6540 Tripterygium hypoglaucum, T6584 Typha angustata, T6585 Typha angustifolia, T6587 Typha latifolia.

postpartum abdominal pain due to stasis obstruction T2545 Euonymus phellomana, T3675 Lagopsis supina.

postpartum anemia T4311 Mucuna sempervirens.

postpartum arthralgia T5586 Rubia yunnanensis.

postpartum bleeding T1541 Claviceps purpurea, T3752 Leonurus heterophyllus [Syn. Leonurus artemisia], T3754 Leonurus sibiricus, T5173 Portulaca oleracea, T6584 Typha angustata, T6585 Typha angustifolia, T6587 Typha latifolia.

postpartum blood dizziness T3649 Laccifer lacca, T5804 Schizonepeta tenuifolia [Syn. Nepeta tenuifolia], T6197 Styrax benzoin, T6204 Styrax tonkinensis, T6764 Vinegar.

postpartum blood $q i$ distending pain T2280 Dryopteris chrysocoma.

postpartum blood qi vacuity T6773 Viscum articulactum, T6774 Viscum articulatum.

postpartum blood stasis T1108 Caesalpinia sappan, T3276 Homo sapiens, T3411 Impatiens balsamina, T4662 Parthenocissus tricuspidata, T5647 Sagittaria sagittifolia, T6097 Staphylea bumalda.

postpartum blood stasis abdominal pain T0073 Achyranthes bidentata, T1769 Crataegus cuneata, T1771 Crataegus kansuensis, T1772 Crataegus maximowiczii, T1780 Crataegus sanguinea, T1781 Crataegus scabrifolia, T1808 Crocus sativus, T1994 Daemonorops draco, T2253 Dracaena cochinchinensis, T2723 Ficus pumila, T3613 Kadsura coccinea [Syn. Kadsura chenensis; Kadsura hainanensis], T3659 Lactuca indica, T3746 Leontice robustum, T3980 Lycopus lucidus, T4604 Panax japonicus var. bipinnatifidus, T4672 Patrinia scabiosaefolia, T4676 Patrinia villosa, T5365 Pyrus pashia, T5730 Sarcandra glabra [Syn. Chloranthus glaber], T6062 Sparganium stoloniferum, T6436 Thlaspi arvense, T6551 Trogopterus xanthipes; Pteromys volans.

postpartum edema T1881 Cucurbita moschata.

postpartum fever T2927 Gentiana rhodantha.

postpartum flooding T3671 Lagerstroemia indica.

postpartum foot edema T4965 Piper sarmentosum.

postpartum galactostasis T0002 Abelmoschus moschatus [Syn. Hibiscus abelmoschus], T3124 Hedyotis capitellata, T3349 Hypericum erectum.

postpartum pain T1583 Cnidium officinale [Syn. Ligusticum officinale], T3820 Ligusticum chuanxiong [Syn. Ligusticum wallichii].

postpartum persistent flow of lochia T0591 Ardisia arborescens, T0600 
Ardisia mamillata [Syn. Tinus mamillata], T1005 Brassica campestris [Syn. Brassica campestris var. oleifera], T1923 Cyathula capitata, T4606 Panax pseudo-ginseng, T6683 Vallisneria spiralis.

postpartum scant milk T1881 Cucurbita moschata, T1952 Cynanchum auriculatum, T1953 Cynanchum bungei, T1964 Cynanchum wilfordii [Syn. Cynoctonum wilfordii], T1980 Cyprinus carpio, T2624 Euphorbia tirucalli, T2727 Ficus simplicissima, T2837 Gallus gallus domesticus, T2979 Gleditsia sinensis [Syn. Gleditsia horrida], T3018 Glycyrrhiza pallidiflora, T3240 Hibiscus esculentus, T4478 Octopus vulgaris, T4505 Ophiocephalus argus, T4644 Parasilurus asotus, T6357 Tetrapanax papyriferus, T6771 Viscum album.

postpartum stasis stagnation T6560 Tulipa edulis.

postpartum stasis stagnation abdominal pain T0661 Artemisia anomala, T0680 Artemisia lactiflora, T1706 Corydalis ambigua var. amurensis [Syn. Corydalis ambigua], T1734 Corydalis remota [Syn. Corydalis bulbosa var. typica], T1735 Corydalis repens, T1750 Corydalis yanhusuo [Syn. Corydalis turtschaninovii f. yanhusuo], T1775 Crataegus pinnatifida, T1778 Crataegus pinnatifida var. major, T1903 Curcuma aromatica, T1905 Curcuma longa, T2536 Euonymus alatus, T2546 Euonymus sacrosancta, T3088 Gynura segetum [Syn. Gynura japonica], T4549 Osbeckia chinensis, T4607 Panax pseudo-ginseng var. japonicus, T5661 Salvia bowleyana, T5680 Salvia miltiorrhiza, T5681 Salvia miltiorrhiza f. alba, T5688 Salvia przewalskii.

postpartum vacuity taxation T3628 Kerria japonica.

postpartum vacuity vexation T1950 Cynanchum ascyrifolium, T1951 Cynanchum atratum, T1962 Cynanchum versicolor, T2952 Gerbera piloselloides, T6582 Tylophora ovata.

postpartum vacuity weakness T1961 Cynanchum thesioides, T2347 Embelia parviflora, T4238 Millettia nitida, T5071 Polygala arillata, T5407 Rana nigromaculata; Rana plancyi.

postpartum wind pain T0571 Aralia decaisneaa.

postpartum wind tetany $\mathrm{T} 3000$ Glycine max, T6651 Urtica cannabina, T6652 Urtica dioica, T6735 Viburnum dilatatum.

pox T1239 Cassia nodosa.

pox without coming out T1188 Caragana chamlagu.

precipitate blood T4402 Nelumbo nucifera.

precipitate blood of large intestine T5552 Robinia pseudoacacia.

premature graying in beard and hair T1964 Cynanchum wilfordii [Syn. Cynoctonum wilfordii], T2323 Eclipta prostrata [Syn. Eclipta alba], T3828 Ligustrum lucidum, T4288 Morus alba, T4432 Nigella glandulifera, T5091 Polygonatum cyrtonema [Syn. Polygonatum multiflorum], T5092 Polygonatum kingianum, T5095 Polygonatum sibiricum, T5107 Polygonum multiflorum, T5446 Rehmannia glutinosa [Syn. Rehmannia glutinosa f. huechingensis], T5593 Rubus idaeus, T5925 Sesamum indicum [Syn. Sesamum orientale], T5927 Sesamum indicum [Syn. Sesamum orientale], T6670 Vaccinium bracteatum.

premenstrual abdominal pain $\mathrm{T} 3877$ Litchi chinensis.

prevention of cataracts T2321 Ecklonia kurome, T3678 Laminaria japonica, T6640 Undaria pinnatifida.

prevention of hair loss in early stage T5575 Rosmarinus officinalis.

prevention of influenza and encephalitis b T4906 Pinus armandii, T4916 Pinus massoniana.

prevention of ptosis T1419 Cimicifuga dahurica, T1420 Cimicifuga foetida, T1421 Cimicifuga heracleifolia.

prickly heat T1878 Cucumis sativus, T4159 Melia azedarach, T4307 Mosla scabra [Syn. Mosla punctata], T5818 Scoparia dulcis.

primary degenerative dementia of alzheimer disease T2961 Ginkgo biloba.

primary hepatoma T1904 Curcuma kwangsiensis, T1909 Curcuma zedoaria [Syn. Curcuma aeruginosa].

profuse menstruation T0170 Adhatoda vasica, T0382 Althaea rosea, T1199 Carduus acanthoides, T1200 Carduus crispus, T1819 Crotalaria ferruginea, T2234 Dipsacus asperoides, T2235 Dipsacus japonicus, T2614 Euphorbia pulcherrima, T4121 Marsilea quadrifolia, T4580 Paeonia albiflora [Syn. Paeonia lactiflora], T5344 Pyrola atropurpurea, T5345 Pyrola calliantha [Syn. Pyrola rotundifolia ssp. chinensis], T5346 Pyrola calliantha var. tibetana, T5347 Pyrola decorata, T5349 Pyrola incarnata, T5350 Pyrola japonica, T5351 Pyrola rotundifolia, T5573 Rosa sericea, T5605 Rumex acetosa, T6540 Tripterygium hypoglaucum, T6551 Trogopterus xanthipes; Pteromys volans, T6876 Zanthoxylum dissitum.

profuse pus T2165 Dictamnus angustifolius, T2167 Dictamnus dasycarpus.

prolapse of rectum T0584 Arctium lappa, T0791 Astragalus chrysopterus, T0794 Astragalus ernestii, T0798 Astragalus membranaceus, T0800 Astragalus mongholicus, T1425 Cimicifuga simplex, T1556 Clerodendron fragrans, T2038 Datura innoxia, T2039 Datura innoxia, T2042 Datura metel, T2043 Datura metel, T2047 Datura stramonium, T2130 Desmodium gangeticum, T2363 Entada phaseoloides [Syn. Lens phaseoloides], T2636 Euscaphis japonica, T2826 Galeola faberi, T3128 Hedysarum multijugum, T3245 Hibiscus syriacus, T4549 Osbeckia chinensis, T4601 Panax ginseng [Syn. Panax schinseng], T4629 Papaver nudicaule, T4630 Papaver nudicaule ssp. amurense, T4636 Papaver somniferum, T4637 Papaver somniferum, T5137 Poncirus trifoliata, T5328 Punica granatum, T5529 Rhodomyrtus tomentosa, T5547 Ricinus communis, T6261 Syringa pinnafolia, T6346 Terminalia chebula, T6348 Terminalia chebula var. tomentella, T6353 Tetracera asiatica, T6601 Umbilicaria esculenta [Syn. Gyrophora esculenta], T6618 Uncaria homomalla [Syn. Uruparia homomalla; Uruparia tonkinensis; Uruparia lanosa var. parvifora].

prolapse of rectum (scorch-fry) T5865 Selaginella pulvinata, T5869 Selaginella tamariscina.

prolapse of rectum due to diarrhea T3329 Hyoscyamus niger.

prolapse of rectum due to enduring diarrhea T3129 Hedysarum polybotrys.

prolapse of rectum due to $\boldsymbol{q} \boldsymbol{i}$ vacuity fall $\mathrm{T} 1055$ Bupleurum angustissimum, T1056 Bupleurum aureum, T1058 Bupleurum chaishoui, T1059 Bupleurum chinense, T1065 Bupleurum longiradiatum, T1066 Bupleurum marginatum, T1067 Bupleurum marginatum var. stenophyllum, T1072 Bupleurum scorzonerifolium, T1073 Bupleurum sibiricum, T1075 Bupleurum smithii var. parvifolium, T1078 Bupleurum wenchuanense, T1079 Bupleurum yinchowense.

prolapse of rectum with bleeding $\mathrm{T} 4787$ Phellinus igniarius.

prolapse of uterus T0298 Alectoria vivens, T1055 Bupleurum angustissimum, T1056 Bupleurum aureum, T1058 Bupleurum chaishoui, T1059 Bupleurum chinense, T1065 Bupleurum 
longiradiatum, T1066 Bupleurum marginatum, T1067 Bupleurum marginatum var. stenophyllum, T1072 Bupleurum scorzonerifolium, T1073 Bupleurum sibiricum, T1075 Bupleurum smithii var. parvifolium, T1078 Bupleurum wenchuanense, T1079 Bupleurum yinchowense, T1900 Curculigo capitulata [Syn. Leucojum capitulata], T2130 Desmodium gangeticum, T2540 Euonymus fortunei, T2636 Euscaphis japonica, T2826 Galeola faberi, T2946 Geranium robertianum, T3128 Hedysarum multijugum, T3270 Holboellia fargesii, T4821 Pholidota articulata, T5075 Polygala fallax [Syn. Polygala aureocauda], T5137 Poncirus trifoliata, T5188 Potentilla viscosa, T5547 Ricinus communis, T5563 Rosa cymosa, T5569 Rosa multiflora, T6261 Syringa pinnafolia, T6264 Syzygium buxifolium.

prostatitis T4209 Michelia alba.

provention of influenza T0364 Alsophila spinulosa.

pruritus of vulva T3555 Jasminum nudiflorum.

psoriasis T0495 Angelica sinensis, T0543 Apis mellifera ligustica, T0750 Asparagus gobicus, T0751 Asparagus officinalis, T1151 Camellia sinensis [Syn. Thea sinensis], T1162 Camptotheca acuminata, T1657 Conyza canadensis [Syn. Erigeron canadensis], T1799 Crinum latifolium, T2130 Desmodium gangeticum, T2165 Dictamnus angustifolius, T2167 Dictamnus dasycarpus, T2651 Excoecaria cochinchinensis var. viridis, T2963 Ginkgo biloba, T4804 Phlojodicarpus sibiricus, T5026 Plumbagella micrantha, T5270 Psoralea corylifolia, T5680 Salvia miltiorrhiza, T5681 Salvia miltiorrhiza f. alba, T5688 Salvia przewalskii, T6540 Tripterygium hypoglaucum, $\mathrm{T} 6542$ Tripterygium wilfordii.

ptosis of anus or uterus T1468 Citrus aurantium, T1521 Citrus wilsonii, T5141 Poncirus trifoliata.

pudendal itch T0249 Agrimonia pilosa var. japonica, T0853 Baeckea frutescens, T1285 Celastrus angulatus, T1582 Cnidium monnieri, T2049 Daucus carota, T2721 Ficus microcarpa, T4305 Mosla dianthera, T5098 Polygonum aviculare, T5323 Pulsatilla campanella, T5324 Pulsatilla cernua, T5325 Pulsatilla chinensis, T5326 Pulsatilla dahurica, T5712 Sanguisorba officinalis, T6031 Sophora flavescens [Syn. Sophora angustfolia], T6268 Syzygium samarangense.

pudendal itch and vaginal discharge T6476 Torilis japonica.

pudendal itch of women T1046 Buddleja davidii.

pulmonary abscess $\mathrm{T} 3548$ Ixeris sonchifolia.

pulmonary edema T1589 Cocculus trilobus [Syn. Cocculus sarmentosus].

pulmonary infection T5344 Pyrola atropurpurea, T5345 Pyrola calliantha [Syn. Pyrola rotundifolia ssp. chinensis], T5346 Pyrola calliantha var. tibetana, T5347 Pyrola decorata, T5349 Pyrola incarnata, T5350 Pyrola japonica, T5351 Pyrola rotundifolia.

pulmonary welling abscess T0263 Ajuga decumbens, T0457 Andrographis paniculata [Syn. Justicia paniculata], T0746 Asparagus cochinchinensis [Syn. Asparagus lucidus], T0895 Benincasa hispida, T1614 Coix lacryma-jobi var. ma-yuen, T1869 Cryptotaenia japonica, T1880 Cucurbita moschata, T2020 Damnacanthus indicus, T2538 Euonymus bungeanus, T2590 Euphorbia hirta, T2657 Fagopyrum cymosum [Syn. Polygonum cymosum], T2787 Fritillaria hupehensis, T2798 Fritillaria verticillata var. thunbergii [Syn. Fritillaria thunbergii], T3233 Heteropappus altaicus, T3254 Hippophae rhamnoides, T3256 Hippophae rhamnoides subsp. sinensis, T3258 Hippophae rhamnoides subsp. yunnanensis, T3288 Humulus japonicus [Syn. Humulus scandens], T3356 Hypericum japonicum, T3547 Ixeris chinensis, T3709 Lathyrus pratensis, T3736 Lemmaphyllum microphyllum, T3737 Lemmaphyllum microphyllum var. obovatum, T3778 Leucaena glauca [Syn. Leucaena leucocephala], T4506 Ophioglossum vulgatum, T4573 Pachyrrhizus erosus, T4829 Phragmites communis, T5613 Rumex obtusifolius, T5773 Saxifraga stolonifera, T5835 Scutellaria barbata [Syn. Scutellaria rivularis], T5840 Scutellaria indica, T6092 Stachys palustris, T6301 Taraxacum mongolicum, T6365 Teucrium pilosum [Syn. Teucrium japonicum var. pilosum], T6367 Teucrium quadrifarium, T6431 Thesium chinense, T6436 Thlaspi arvense.

pulmonary welling abscess with hacking of pus and blood T3284 Houttuynia cordata, T5011 Platycodon grandiflorum.

purpura T0648 Arnebia euchroma, T0649 Arnebia guttata, T2659 Fagopyrum esculentum, T3881 Lithospermum erythrorhizon, T5614 Rumex patientia.

purpuric dermatosis T1118 Callicarpa formosana, T1119 Callicarpa japonica.

purulence due to bone welling abscess T5010 Platycarya strobilacea.

purulence in ear top $\mathrm{T} 1552$ Cleome viscosa.

putrefying sore T0557 Aquilegia ecalcarata.

pyothorax T3174 Heliotropium indicum.

$q \boldsymbol{i}$ and yin vacuity T1961 Cynanchum thesioides, T5260 Pseudostellaria heterophylla.

$q i$ ascent with vexation and fullness T1472 Citrus chachiensis, T1517 Citrus tankan.

$q i$ bind in chest and diaphragm T1476 Citrus erythrosa, T1515 Citrus tangemna.

qi bind pain T4428 Nicotiana tabacum.

$q i$ blood vacuity depletion T3615 Kadsura interior.

qi counterflow with asthma T0554 Aquilaria agallocha, T0555 Aquilaria sinensis.

qi counterflow with cough and asthma T4779 Pharbitis nil, T4780 Pharbitis purpurea, T4855 Physeter catodon.

qi counterflow with rapid asthma T2433 Eriobotrya japonica, T3854 Lindera strychnifolia [Syn. Lindera aggregata].

qi depression T1171 Canis familiaris.

$q i$ depression and fulminant reversal T4303 Moschus moschiferus; Moschus berezovskii; Moschus sifanicus.

qi distention abdominal pain T1538 Clausena lansium, T2521 Eucalyptus tereticornis.

$q i$ distention in chest and abdomen T1092 Buxus microphylla var. sinica.

qi dysentery T0164 Actinidia polygama.

qi goiter T0992 Bos taurus domesticus; Bubalus bubalis.

qi pain T3120 Hedychium spicatum.

qi pain in heart and stomach T0151 Acronychia pedunculata, T0356 Alpinia galanga, T1943 Cymbopogon goeringii, T4530 Ormosia hosiei, T5189 Pothos chinensis, T5793 Schisandra henryi.

qi stagnation T5137 Poncirus trifoliata.

qi stagnation abdominal distention T3619 Kadsura peltigera [Syn. 
Kadsura longipedunculata].

$q i$ stagnation and blood stasis T1381 Choerospondias axillaris.

$q i$ stagnation and distention in stomach duct T0631 Aristolochia kaempferi.

qi stagnation and food accumulation T6120 Stephania delavayi [Syn. Stephania epigaea].

$q i$ stagnation and pain in stomach duct T6877 Zanthoxylum dissitum, T6878 Zanthoxylum echinocarpum.

$q i$ stagnation in chest and diaphragm T1488 Citrus junos.

qi stagnation stomachache T1537 Clausena lansium, T2194 Dioscorea collettii, T2205 Dioscorea panthaica, T2505 Eucalyptus citriodora.

$q i$ stagnation with abdominal pain and diarrhea T1146 Camellia oleifera.

qi strangury T0554 Aquilaria agallocha, T0555 Aquilaria sinensis, T6597 Ulmus pumila.

qi vacuity T0824 Atractylodes macrocephala [Syn. Atractylis macrocephala], T1188 Caragana chamlagu, T4610 Panax quinquefolium.

$q i$ vacuity and blood depletion T0830 Auricularia auricula, T0831 Auricularia delicata.

qi vacuity and dysentery T0989 Bos taurus domesticus; Bubalus bubalis.

$q i$ vacuity and general weakness T4516 Oplopanax elatus, T5497 Rhodiola kirilowii, T5499 Rhodiola sacra.

qi vacuity and hypodynamia T0791 Astragalus chrysopterus, T0794 Astragalus ernestii, T0798 Astragalus membranaceus, T0800 Astragalus mongholicus, T3129 Hedysarum polybotrys, T3981 Lycopus lucidus.

$q i$ vacuity and verging on desertion T4599 Panax ginseng [Syn. Panax schinseng], T4600 Panax ginseng [Syn. Panax schinseng].

qi vacuity edema T0791 Astragalus chrysopterus, T0794 Astragalus ernestii, T0798 Astragalus membranaceus, T0800 Astragalus mongholicus, T3129 Hedysarum polybotrys, T4577 Paederia scandens. qi-block deafness T0463 Anemone altaica.

qi-blood depletion T1596 Codonopsis canescens, T1597 Codonopsis clematidea, T1599 Codonopsis pilosula, T1600 Codonopsis pilosula var. modesta [Syn. Codonopsis modesta], T1601 Codonopsis subglobosa, T1602 Codonopsis tangshen, T1603 Codonopsis tubulosa, T3279 Homo sapiens, T3743 Lentinus lepideus, T4682 Pedicularis muscicola, T6689 Ventilago leiocarpa.

qi-blood fluids vacuity depletion T4600 Panax ginseng [Syn. Panax schinseng].

qi-blood stagnation T6062 Sparganium stoloniferum.

rabid dog bite T0440 Anagallis arvensis, T1491 Citrus limon, T1495 Citrus limonia, T1963 Cynanchum wallichii, T2036 Datura innoxia, T2041 Datura metel, T2046 Datura stramonium, T3287 Huechys sanguinea, T3953 Lycianthes biflora, T4008 Lytta caraganae, T4423 Nicandra physaloides, T5289 Pteris dactylina, T5401 Rabdosia yuennanensis, T5797 Schisandra propinqua, T5798 Schisandra propinqua var. intermedia, T6424 Thermopsis alpina, T6566 Tupistra chinensis.

rachitis in children T2841 Gallus gallus domesticus, T4536 Orobanche coerulescens.

raynaud's disease T0495 Angelica sinensis, T4804 Phlojodicarpus sibiricus.

recurrent neuritis T5848 Securinega suffruticosa.

red and white dysentery T0250 Agrimonia pilosa var. japonica, T0941 Bidens tripartita, T1145 Camellia japonica, T1184 Capsella bursa-pastoris, T2267 Drosera peltata var. lunata, T2993 Gloeostereum incarnatum, T3244 Hibiscus syriacus, T4655 Paris tetraphylla.

red and white dysentery (root) T0945 Bischofia javanica [Syn. Bischofia trifoliata].

red and white turbidity T3242 Hibiscus rosa-sinensis.

red and white vaginal discharge T0301 Aleuritopteris argentea, T0382 Althaea rosea, T0999 Bougainvillea glabra, T1672 Corallodiscus flabellatus [Syn. Didissandra flabellat], T2766 Fraxinus bungeana, T2767 Fraxinus chinensis, T2774 Fraxinus paxiana, T2777 Fraxinus rhynchophylla [Syn. Fraxinus chinensis var. rhynchophylla], T2779 Fraxinus stylosa, T2780 Fraxinus szaboana [Syn. Fraxinus chinensis var. acuminata], T3245 Hibiscus syriacus, T3615 Kadsura interior, T3645 Kummerowia striata, T4252 Mirabilis jalapa, T4662 Parthenocissus tricuspidata, T4670 Patrinia heterophylla, T4672 Patrinia scabiosaefolia, T4673 Patrinia scabra, T4676 Patrinia villosa, T4777 Phalaris arundinacea, T5118 Polygonum suffultum, T5327 Punica granatum, T5329 Punica granatum, T5614 Rumex patientia, T5671 Salvia digitaloides, T5712 Sanguisorba officinalis, T6226 Swertia mileensis, T6860 Zanthoxylum ailanthoides.

red bayberry sore $\mathrm{T} 3300$ Hydnocarpus anthelminticus.

red dysentery T0266 Ajuga macrosperma, T5099 Polygonum bistorta. red dysentery and abdominal pain T5181 Potentilla chinensis.

red dysentery and bloody stool T4127 Matteuccia struthiopteris.

red eyes T0540 Apis cerana, T0708 Arthromeris mairei [Syn. Polypodium mairei], T0897 Berberis amurensis, T0912 Berberis poiretii, T1152 Camellia sinensis [Syn. Thea sinensis], T1311 Centella asiatica, T1395 Chrysanthemum morifolium [Syn. Dendranthema morifolium], T1746 Corydalis taliensis, T1758 Cotinus coggygria, T2290 Duchesnea indica, T2517 Eucalyptus robusta, T2839 Gallus gallus domesticus, T2902 Gentiana algida, T3392 Ilex kudingcha, T3393 Ilex latifolia, T3423 Indigofera tinctoria, T3557 Jasminum sambac, T3829 Ligustrum robustum, T4186 Mentha piperita, T4190 Mentha spicata, T4796 Pheretima aspergillum; Allolobophora caliginosa trapezoides, T5413 Ranunculus cantoniensis, T5472 Rheum officinale, T5474 Rheum palmatum, T5481 Rheum tanguticum, T5605 Rumex acetosa, T5775 Scabiosa comosa, T6076 Spinacia oleracea, T6139 Sterculia lychnophora, T6206 Sus scrofa domestica, T6301 Taraxacum mongolicum, T6580 Tylophora floribunda, T6653 Usnea diffracta, T6654 Usnea longissima, T6799 Vitis vinifera, T6843 Xanthium sibiricum [Syn. Xanthium strumarium].

red eyes and clouded vision T4289 Morus alba, T4291 Morus australis, T4293 Morus cathayana, T4298 Morus mongolica.

red eyes and distending pain T1387 Chrysanthemum boreale, T1392 Chrysanthemum indicum, T1394 Chrysanthemum lavandulifolium, T1981 Cyprinus carpio.

red eyes and eye screen T2407 Equisetum arvense, T2409 Equisetum palustre, T5449 Reineckea carnea, T6079 Spiraea japonica, T6082 Spiraea japonica var. fortunei, T6497 Tribulus terrestris. 
red eyes and itch-pain $\mathrm{T} 1552$ Cleome viscosa.

red eyes and profuse eye discharge T4471 Ocimum basilicum.

red eyes and sore pharynx T1439 Cinnamomum cassia [Syn.

Cinnamomum aromaticum].

red eyes and tearing T3635 Koelreuteria paniculata.

red eyes with gall T0190 Adonis sutchuenensis, T0263 Ajuga decumbens, T0426 Ampelopsis grossedentata [Syn. Ampelopsis cantoniesis var. grossedentata], T0805 Astragalus sinicus, T0806 Astragalus sinicus, T0852 Bacopa monniera, T0888 Beesia calthaefolia, T0906 Berberis julianae, T0916 Berberis thunbergii, T0920 Berberis wilsonae, T1032 Broussonetia papyrifera, T1048 Buddleja officinalis, T1100 Caesalpinia crista, T1184 Capsella bursa-pastoris, T1240 Cassia obtusifolia, T1242 Cassia occidentalis, T1247 Cassia sophera, T1250 Cassia tora, T1296 Celosia argentea, T1393 Chrysanthemum indicum, T1395 Chrysanthemum morifolium [Syn. Dendranthema morifolium], T1638 Commiphora myrrha [Syn. Commiphora molmol], T1640 Coniogramme japonica [Syn. Hemionitis japonica], T1733 Corydalis racemosa, T1745 Corydalis suaveolens [Syn. Corydalis sheareri], T2161 Dicranopteris pedata [Syn. Polypodium pedatum; Dicranopteris dichotoma], T2244 Doellingeria scaber [Syn. Aster scaber], T2279 Dryopteris championii, T2340 Elsholtzia bodinieri, T2757 Forsythia viridissima, T2766 Fraxinus bungeana, T2767 Fraxinus chinensis, T2774 Fraxinus paxiana, T2777 Fraxinus rhynchophylla [Syn. Fraxinus chinensis var. rhynchophylla], T2779 Fraxinus stylosa, T2780 Fraxinus szaboana [Syn. Fraxinus chinensis var. acuminata], T2803 Frullania tamarisci ssp. moniliata [Syn. Frullania moniliata], T2908 Gentiana cephalantha, T2921 Gentiana manshurica, T2928 Gentiana rigescens, T2930 Gentiana scabra, T2938 Gentiana triflora, T2941 Gentianopsis paludosa, T2984 Glinus lotoides [Syn. Mollugo lotoides], T3040 Gomphrena globosa, T3122 Hedyotis acutangula, T3241 Hibiscus mutabilis, T3334 Hypecoum leptocarpum, T3356 Hypericum japonicum, T3361 Hypericum perforatum, T3564 Juglans mandshurica, T3636 Koelreuteria paniculata, T3675 Lagopsis supina, T3770 Lespedeza cuneata, T3990 Lygodium japonicum, T4055 Mahonia bealei, T4056 Mahonia bealei, T4060 Mahonia confusa, T4062 Mahonia fortunei, T4063 Mahonia fortunei, T4064 Mahonia gracilipes, T4066 Mahonia japonica, T4067 Mahonia japonica, T4072 Mahonia shenii, T4261 Mollugo pentaphylla, T4369 Nandina domestica, T4370 Nandina domestica, T4506 Ophioglossum vulgatum, T4584 Paeonia lactiflora wild, T4586 Paeonia obovata, T4590 Paeonia veitchii, T4672 Patrinia scabiosaefolia, T4676 Patrinia villosa, T4840 Phyllanthus niruri, T5115 Polygonum sibiricum [Syn. Persicaria sibirica], T5176 Potamogeton natans, T5184 Potentilla kleiniana, T5193 Pratia nummularia, T5214 Prunella vulgaris, T5576 Rostellularia procumbens [Syn. Justicia procumbens], T5690 Salvia roborowskii, T5856 Sedum lineare [Syn. Sedum obtuso-lineare], T5861 Selaginella doederleinii, T6034 Sophora japonica, T6372 Thalictrum acutifolium, T6375 Thalictrum baicalense, T6376 Thalictrum cultratum, T6378 Thalictrum delavayi, T6381 Thalictrum faberi, T6385 Thalictrum flavum, T6386 Thalictrum foetidum, T6387 Thalictrum foliolosum, T6388 Thalictrum fortunei, T6389 Thalictrum glandulosissimum, T6393 Thalictrum ichangense [Syn. Thalictrum tripeltatum; Thalictrum multipeltatum], T6402 Thalictrum omeiense,
T6403 Thalictrum petaloideum, T6409 Thalictrum simplex [Syn. Thalictrum simplex var. brevipes], T6426 Thermopsis chinensis, T6436 Thlaspi arvense, T6552 Trollius chinensis [Syn. Trollius asiaticus var. chinensis], T6553 Trollius ledebourii, T6555 Tropaeolum majus, T6767 Viola yedoensis, T6791 Vitex rotundifolia [Syn. Vitex trifollia var. simplicifolia], T6793 Vitex trifolia.

red eyes with pain due to liver fire T1662 Coptis chinensis, T1663 Coptis chinensis var. brevisepala, T1664 Coptis deltoidea, T1669 Coptis omeiensis, T1670 Coptis teetoides [Syn. Coptis teeta].

red face and eyes T0544 Apium graveolens.

red swelling of clove sore T3953 Lycianthes biflora.

red swollen breast T5608 Rumex dentatus.

red swollen due to knocks and falls T6904 Zephyranthes grandiflora [Syn. Zephyranthes carinata].

red swollen in throat T2244 Doellingeria scaber [Syn. Aster scaber], T2340 Elsholtzia bodinieri, T5199 Primula malacoides.

red swollen of throat T4730 Peristrophe roxburghiana.

red swollen sore and welling abscess T4760 Peucedanum japonicum, T6904 Zephyranthes grandiflora [Syn. Zephyranthes carinata].

reduced food intake T0387 Amaranthus caudatus, T1469 Citrus aurantium var. amara, T2837 Gallus gallus domesticus, T4099 Mangifera indica, T4103 Mangifera persiciformis, T4545 Oryza sativa. reduced food intake and diarrhea T0535 Antidesma bunius, T6263 Syzygium aromaticum [Syn. Eugenia caryophyllata].

reduced food intake and sloppy stool T0791 Astragalus chrysopterus, T0794 Astragalus ernestii, T0798 Astragalus membranaceus, T0800 Astragalus mongholicus, T1596 Codonopsis canescens, T1597 Codonopsis clematidea, T1599 Codonopsis pilosula, T1600 Codonopsis pilosula var. modesta [Syn. Codonopsis modesta], T1601 Codonopsis subglobosa, T1602 Codonopsis tangshen, T1603 Codonopsis tubulosa, T2246 Dolichos lablab, T3129 Hedysarum polybotrys.

reduced food intake due to spleen vacuity T2190 Dioscorea batatas [Syn. Dioscorea opposita], T2202 Dioscorea japonica, T4436 Nitraria tangutorum, T4450 Nuphar pumilum, T4599 Panax ginseng [Syn. Panax schinseng], T5169 Poria cocos, T6916 Ziziphus jujuba, T6917 Ziziphus jujuba var. inermis.

reduced food intake with abdominal distention T0311 Allium cepa, T0824 Atractylodes macrocephala [Syn. Atractylis macrocephala], T5938 Setaria italica, T6878 Zanthoxylum echinocarpum.

reduced food intake with dry mouth T1353 Changium smyrnioides, T5091 Polygonatum cyrtonema [Syn. Polygonatum multiflorum], T5092 Polygonatum kingianum, T5095 Polygonatum sibiricum.

reduced food intake with dry retching T2094 Dendrobium aduncum, T2096 Dendrobium aurantiacum var. denneanum, T2098 Dendrobium chrysanthum, T2100 Dendrobium densiflorum, T2102 Dendrobium fimbriatum var. oculatum, T2105 Dendrobium loddigesii, T2106 Dendrobium moniliforme, $\mathrm{T} 2107$ Dendrobium nobile, T2108 Dendrobium officinale.

reduced food intake with fatigue T2727 Ficus simplicissima. reduced food intake with nausea and vomiting T5572 Rosa rugosa. reduced food intake with vomiting T4351 Myristica fragrans. reduced sleep $\mathrm{T} 3192$ Hemerocallis citrina. 
renal colic T0825 Atropa belladonna.

replete body and constipation T2650 Excoecaria agallocha.

repletion heat constipation T0334 Aloe arborescens var. natalensis,

T5472 Rheum officinale, T5474 Rheum palmatum, T5481 Rheum

tanguticum.

resolve liquor toxin T0834 Averrhoa carambola, T4088 Malus pumila.

restlessness T2882 Gardenia jasminoides [Syn. Gardenia florida], T4400

Nelumbo nucifera.

retching blood T1118 Callicarpa formosana, T1119 Callicarpa japonica. retching counterflow T1106 Caesalpinia minax, T1217 Carum carvi,

T1467 Citrus aurantium, T1476 Citrus erythrosa, T1515 Citrus tangemna, T2840 Gallus gallus domesticus, T5140 Poncirus trifoliata.

retching counterflow and abdominal pain T6561 Tulipa gesneriana.

retching counterflow and reduced food intake T0480 Angelica decursiva [Syn. Peucedanum decursivum], T3819 Ligusticum brachylobum, T4761 Peucedanum longshengens, T4768 Peucedanum praeruptorum, 44769 Peucedanum rubricaule.

retching with counterflow T4618 Panicum miliaceum.

retention of afterbirth T0001 Abelmoschus manihot, T2979 Gleditsia sinensis [Syn. Gleditsia horrida].

retention of lochia T1215 Carthamus tinctorius, T2536 Euonymus alatus. retention of placenta T0073 Achyranthes bidentata, T1174 Cannabis sativa, T1924 Cyathula officinalis, T5647 Sagittaria sagittifolia.

retinal insufficiency syndrome T2964 Ginkgo biloba.

retinitis T5312 Pueraria edulis, T5313 Pueraria lobata [Syn. Pueraria thunbergiana; Pueraria pseudohirsuta], T5316 Pueraria omeiensis,

T5318 Pueraria phaseoloides, T5320 Pueraria thomsonii.

reversal cold of limbs T0098 Aconitum geniculatum, T1438 Cinnamomum cassia [Syn. Cinnamomum aromaticum].

reverting yin headache T2644 Evodia rutaecarpa, T2646 Evodia rutaecarpa var. officinalis.

rheumatic arthritis T0154 Acroptilon repens, T0281 Alangium chinense, T0285 Alangium platanifolium, T0295 Albizzia odoratissima, T0539 Apis cerana, T0541 Apis cerana, T0572 Aralia elata, T1545 Clematis chinensis, T1553 Clerodendranthus spicatus, T1656 Conyza bonariensis [Syn. Erigeron bonariensis; Erigeron linifolius; Erigeron crispus], T1715 Corydalis decumbens [Syn. Corydalis amabilis], T1812 Crossostephium chinense, T1943 Cymbopogon goeringii, T1959 Cynanchum paniculatum, T2231 Diphylleia grayi, T2232 Diphylleia sinensis, T2538 Euonymus bungeanus, T2736 Fissistigma polyanthum, T2766 Fraxinus bungeana, T2767 Fraxinus chinensis, T2774 Fraxinus paxiana, T2777 Fraxinus rhynchophylla [Syn. Fraxinus chinensis var. rhynchophylla], T2779 Fraxinus stylosa, T2780 Fraxinus szaboana [Syn. Fraxinus chinensis var. acuminata], T3371 Hypocrella bambusae, T3594 Juniperus rigida, T3646 Kyllinga brevifolia, T3984 Lycoris chinensis, T3986 Lycoris radiata [Syn. Amaryllis radiata], T4810 Phlomis mongolica, T5110 Polygonum orientale, T5207 Prismatomeris tetrandra, T5413 Ranunculus cantoniensis, T5767 Saussurea pulchella, T5799 Schisandra rubriflora, T5860 Selaginella davidii, T6075 Spilanthes acmella, T6129 Stephania japonica, T6155 Strophanthus divaricatus, T6540 Tripterygium hypoglaucum, T6542 Tripterygium wilfordii, T6618 Uncaria homomalla [Syn. Uruparia homomalla; Uruparia tonkinensis; Uruparia lanosa var. parvifora], T6820
Wikstroemia indica, T6843 Xanthium sibiricum [Syn. Xanthium strumarium].

rheumatic endocarditis T2203 Dioscorea nipponica, T2204 Dioscorea nipponica ssp. rosthornii.

rheumatic heart disease T1649 Convallaria keiskei [Syn. Convallaria majalis].

rheumatic pain in joints T0414 Ammopiptanthus mongolicus [Syn. Piptanthus mongolicus], T0629 Aristolochia heterophylla, T4975 Piptanthus nanus.

rheumatic pain in legs T1586 Cocculus laurifolius.

rheumatis T3106 Harpagophytum procumbens, T3394 Ilex paraguariensis.

rheumatism T0354 Alpinia chinensis, T1533 Clausena dentata, T1534 Clausena dunniana, T1555 Clerodendron fortunatum, T4690 Pelargonium graveolens, T5445 Rehmannia glutinosa [Syn. Rehmannia glutinosa f. huechingensis], T5447 Rehmannia glutinosa [Syn. Rehmannia glutinosa f. huechingensis], T6788 Vitex negundo.

rheumatism numbness T0099 Aconitum gymnandrum, T1692 Coriaria sinica [Syn. Coriaria nepalensis], T3411 Impatiens balsamina, T3414 Impatiens siculifer, T5184 Potentilla kleiniana, T5797 Schisandra propinqua, T5798 Schisandra propinqua var. intermedia, T5993 Solanum capsicastrum.

rheumatoid arthritis $\quad$ T2736 Fissistigma polyanthum, T5727 Saposhnikovia divaricata [Syn. Ledebouriella seseloides], T6184 Strychnos nux-vomica, T6193 Strychnos wallichiana, T6541 Tripterygium regelii, $\mathrm{T} 6542$ Tripterygium wilfordii.

rickets $\mathrm{T} 3742$ Lentinus edodes.

rigidity of neck T5312 Pueraria edulis, T5313 Pueraria lobata [Syn. Pueraria thunbergiana; Pueraria pseudohirsuta], T5316 Pueraria omeiensis, T5318 Pueraria phaseoloides, T5320 Pueraria thomsonii.

ringing in head T1154 Camellia sinensis [Syn. Thea sinensis].

roundworm reversal with abdominal pain T5228 Prunus mume, T5719 Sapindus mukorossi.

rumbling intestines and diarrhea T4944 Piper cubeba, T6802 Vladimiria souliei [Syn. Jurinea souliei].

running piglet T1439 Cinnamomum cassia [Syn. Cinnamomum aromaticum], T3856 Lindera umbellata [Syn. Lindera erythrocarpa].

salpingitis T2715 Fibraurea recisa.

sand T1288 Celastrus orbiculatus [Syn. Celastrus articulatus], T5119 Polygonum thunbergii, T6015 Solanum torvum.

sand and painful distention in stomach duct and abdomen T6677 Valeriana jatamansii [Syn. Valeriana wallichii].

sand distention and abdominal pain T2193 Dioscorea cirrhosa [Syn. Dioscorea pogonoides].

sand distention and vomiting diarrhea T2505 Eucalyptus citriodora.

sand foulness T1986 Cyrtomium fortunei.

sand foulness retching T0472 Anethum graveolens.

sand qi T2746 Foeniculum vulgare, T6787 Vitex negundo.

sand $q \boldsymbol{i}$ abdominal pain $\mathrm{T} 0726$ Asarum forbesii, T1153 Camellia sinensis var. assamica, T1311 Centella asiatica, T2721 Ficus microcarpa, T4240 Millettia pachycarpa, T5220 Prunus davidiana, T5230 Prunus persica, T5232 Prunus persica.

sand strangury $\mathrm{T} 0155$ Actinidia arguta, T0759 Aspidistra elatior, T1007 
Brassica juncea, T1311 Centella asiatica, T1907 Curcuma wengujin, T2134 Desmodium styracifolium, T5231 Prunus persica.

sarcoma T4184 Mentha haplocalyx [Syn. Mentha canadaensis; Mentha arvensis var. haplocalyx; Mentha arvensis].

scab and lai T0610 Argemone mexicana, T0696 Artemisia sieversiana, T1197 Cardiospermum halicacabum, T1251 Cassytha filiformis, T1693 Coriaria sinica [Syn. Coriaria nepalensis], T1719 Corydalis incisa, T2897 Gelsemium elegans, T3687 Lantana camara, T4937 Piper betle, T5143 Pongamia pinnata, T5401 Rabdosia yuennanensis, T5717 Sapindus delavayi [Syn. Pancovia delavayi].

scab and lichen T0012 Abrus precatorius, T0078 Aconitum barbatum var. puberulum [Syn. Aconitum ochranthum], T0215 Agave americana, T0295 Albizzia odoratissima, T0297 Alchornea trewioides, T0299 Aleurites cordata [Syn. Aleurites fordii], T0364 Alsophila spinulosa, T0463 Anemone altaica, T0516 Anser cygnoides domestica, T0752 Asparagus officinalis, T0805 Astragalus sinicus, T0853 Baeckea frutescens, T1311 Centella asiatica, T1359 Chenopodium album, T1361 Chenopodium ambrosioides, T1372 Chloranthus serratus, T1388 Chrysanthemum cinerariaefolium, T1389 Chrysanthemum coccineum, T1437 Cinnamomum camphora, T1582 Cnidium monnieri, T1594 Cocos nucifera, T1624 Colocasia antiquorum, T1641 Conioselinum vaginatum, T1733 Corydalis racemosa, T1823 Crotalaria juncea, T2136 Desmos cochinchinensis [Syn. Desmos chinensis], T2162 Dicranostigma franchetianum [Syn. Dicranostigma leptopodum], T2459 Erythrina arborescens, T2478 Erythrina variegata [Syn. Erythrina indica], T2510 Eucalyptus globulus, T2517 Eucalyptus robusta, T2580 Euphorbia antiquorum, T2599 Euphorbia lunulata, T2602 Euphorbia nematocypha, T2612 Euphorbia prolifera, T2716 Ficus beecheyana [Syn. Ficus erecta var. beecheyana], T2978 Gleditsia sinensis [Syn. Gleditsia horrida], T2980 Gleditsia sinensis [Syn. Gleditsia horrida], T2999 Glycine max, T3057 Gossypium herbaceum, T3245 Hibiscus syriacus, T3300 Hydnocarpus anthelminticus, T3420 Incarvillea sinensis, T3423 Indigofera tinctoria, T3560 Jatropha curcas, T3671 Lagerstroemia indica, T3709 Lathyrus pratensis, T3725 Laurus nobilis, T3726 Laurus nobilis, T3758 Lepidium sativum, T3856 Lindera umbellata $[\mathrm{Syn} . \quad$ Lindera erythrocarpa], T4104 Manihot esculenta, T4172 Melilotus suaveolens, T4240 Millettia pachycarpa, T4324 Murraya paniculata [Syn. Chalcas paniculata], T4428 Nicotiana tabacum, T4505 Ophiocephalus argus, T4520 Opuntia ficus-indica, T4562 Oxalis acetosella, T4564 Oxalis corniculata [Syn. Oxalis repens], T4572 Pachyrhizus erosus, T4656 Parmelia saxatilis, T4672 Patrinia scabiosaefolia, T4676 Patrinia villosa, T4690 Pelargonium graveolens, T4799 Philydrum lanuginosum, T4881 Picrasma quassioides [Syn. Picrasma ailanthoides], T4882 Picrasma quassioides [Syn. Picrasma ailanthoides], T4917 Pinus massoniana, T5028 Plumbago indica, T5256 Pseudolarix amabilis [Syn. Larix amabilis; Pseudolarix kaempferi], T5308 Pterocarya stenoptera, T5368 Python molurus bivittatus, T5414 Ranunculus japonicus, T5428 Rauvolfia yunnanensis, T5457 Rhamnus davurica, T5483 Rhinacanthus nasutus, T5537 Rhus sylvestris, T5605 Rumex acetosa, T5607 Rumex crispus, T5611 Rumex japonicus, T5612 Rumex nepalensis, T5613 Rumex obtusifolius, T5614 Rumex patientia, T5723 Sapium sebiferum, T5977 Smilax glabra,
T5980 Smilax menispermoidea, T6031 Sophora flavescens [Syn. Sophora angustfolia], T6048 Sophora viciifolia, T6105 Stellera chamaejasme, T6111 Stemona japonica, T6113 Stemona sessilifolia, T6115 Stemona tuberosa, T6155 Strophanthus divaricatus, T6219 Swertia erythrosticta, T6365 Teucrium pilosum [Syn. Teucrium japonicum var. pilosum], T6429 Thermopsis lupinoides, T6499 Tribulus terrestris, T6693 Veratrum album var. lobelianum [Syn. Veratrum lobelianum], T6697 Veratrum grandiflorum, T6698 Veratrum nigrum, T6788 Vitex negundo, T6843 Xanthium sibiricum [Syn. Xanthium strumarium], T6862 Zanthoxylum ailanthoides.

scab and lichen with bald sores T5156 Populus nigra var. thevestina. scab and lichen with itching T0749 Asparagus filicinus, T1435 Cinnamomum camphora, T4156 Melia azedarach, T4162 Melia toosendan, T5029 Plumbago zeylanica.

scab and lichen with itching sores $\mathrm{T} 3857$ Lindera umbellata [Syn. Lindera erythrocarpa].

scab and lichen with sore toxin T1436 Cinnamomum camphora, T6217 Swertia davidii.

scab and lichen with swelling of sores T1357 Chelidonium majus.

scab and lichen with welling abscess and flat abscess T0144 Acorus gramineus, 0146 Acorus tatarinowii.

scab and lichen; bark: dysentery T6432 Thespesia populnea [Syn. Hibiscus populneus].

scab sore T0142 Acorus calamus, T0145 Acorus gramineus, T1264 Catalpa ovata, T1556 Clerodendron fragrans, T1562 Clerodendron trichotomum, T1645 Consolida ajacis [Syn. Delphinium ajacis], T2024 Daphne genkwa, T2267 Drosera peltata var. lunata, T2387 Epilobium hirsutum, T2479 Erythrina variegata var. orientalis, T2521 Eucalyptus tereticornis, T2584 Euphorbia ebracteolata, T2587 Euphorbia fischeriana, T2649 Evolvulus alsinoides, T3407 Illicium simonsii, T3570 Juglans regia, T3630 Kleinhovia hospita, T3778 Leucaena glauca [Syn. Leucaena leucocephala], T4253 Mirabilis jalapa, T4358 Myroxylon pereirae, T4432 Nigella glandulifera, T4830 Phryma leptostachya, T4890 Pieris japonica, T5298 Pteris vittata, T5455 Rhamnus crenata, T5528 Rhodomyrtus tomentosa, T5595 Rubus parviforlius, T5998 Solanum dulcamara, T6330 Tephroseris kirilowii [Syn. Senecio integrifolius var. fauriei], T6542 Tripterygium wilfordii. scabies T1278 Cedrela sinensis.

scalds T0175 Adiantum pedatum, T0560 Arachis hypogaea, T0648 Arnebia euchroma, T0649 Arnebia guttata, T0661 Artemisia anomala, T0916 Berberis thunbergii, T0922 Berchemia polyphylla var. leioclada, T0933 Betula platyphylla, T0935 Betula platyphylla var. japonica, T0982 Bos taurus domesticus, T1043 Bryophyllum pinnatum, T1145 Camellia japonica, T1199 Carduus acanthoides, T1200 Carduus crispus, T1271 Catharanthus roseus [Syn. Vinca rosea; Lochera rosea], T1529 Cladonia verticillata, T1662 Coptis chinensis, T1663 Coptis chinensis var. brevisepala, T1664 Coptis deltoidea, T1669 Coptis omeiensis, T1670 Coptis teetoides [Syn. Coptis teeta], T1693 Coriaria sinica [Syn. Coriaria nepalensis], T1867 Cryptomeria fortunei, T1880 Cucurbita moschata, T1896 Cupressus funebris, T2279 Dryopteris championii, T2387 Epilobium hirsutum, T2517 Eucalyptus robusta, T2659 Fagopyrum esculentum, T2840 Gallus gallus domesticus, T2857 Garcinia hanburyi, T3244 Hibiscus syriacus, T3295 Huperzia 
serrata [Syn. Lycopodium serratum], T3318 Hylotelephium mingjinianum, T3340 Hypericum ascyron, T3363 Hypericum sampsonii, T3460 Iris halophila, T3464 Iris lactea var. chinensis [Syn. Iris pallasii var. chinensis], T3738 Lemna minor, T3830 Ligustrum sinense, T3881 Lithospermum erythrorhizon, T3970 Lycopodium cernuum, T3973 Lycopodium japonicum [Syn. Lycopodium clavatum], T4055 Mahonia bealei, T4062 Mahonia fortunei, T4066 Mahonia japonica, T4203 Mesua ferrea, T4500 Onosma paniculatum, T4519 Opuntia dillenii, T4521 Opuntia vulgaris, T4544 Oryza sativa, T4618 Panicum miliaceum, T4789 Phellodendron amurense, T4790 Phellodendron amurense var. wilsonii, T4791 Phellodendron chinense, T4792 Phellodendron chinense var. glabriusculum, T4956 Piper mullesua, T5308 Pterocarya stenoptera, T5419 Raphanus sativus, T5529 Rhodomyrtus tomentosa, T5557 Rohdea japonica [Syn. Orontium japonicum], T5562 Rosa chinensis, T5567 Rosa laevigata, T5569 Rosa multiflora, T5613 Rumex obtusifolius, T5714 Sansevieria trifasciata, T5860 Selaginella davidii, T5862 Selaginella involvens, T5907 Senecio scandens [Syn. Senecio chinensis], T5938 Setaria italica, T6017 Solanum tuberosum, T6028 Sophora alopecuroides, T6048 Sophora viciifolia, T6205 Sus scrofa, T6440 Thuja orientalis [Syn. Platycladus orientalis; Biota orientalis], T6544 Triticum aestivum [Syn. Triticum vulgare], T6601 Umbilicaria esculenta [Syn. Gyrophora esculenta], T6745 Vicia faba, T6746 Vicia faba.

scalp infection T2023 Daphne genkwa.

scant breast milk T0210 Agaricus bisporus, T0211 Agaricus campestris, T0559 Arachis hypogaea, T1191 Caragana sinica, T1598 Codonopsis convolvulacea, T1604 Coeloglossum viride [Syn. Coeloglossum viride var. bracteatum], T1961 Cynanchum thesioides, T2717 Ficus carica, T3077 Gymnadenia conopsea, T3279 Homo sapiens, T3925 Loranthus parasiticus [Syn. Loranthus chinenis; Taxillus chinensis], T4206 Metaplexis japonica, T4432 Nigella glandulifera, T4436 Nitraria tangutorum, T4573 Pachyrrhizus erosus, T4983 Pisum sativum, T5925 Sesamum indicum [Syn. Sesamum orientale], T6507 Trichosanthes cucumeroides.

scant fluid and thirst T4088 Malus pumila, T5229 Prunus persica. scant urine T1035 Broussonetia papyrifera.

scant urine in children T6008 Solanum nigrum.

scant urine with edema T0553 Apocynum venetum, T1633 Commelina communis, T1878 Cucumis sativus, T2134 Desmodium styracifolium, T3752 Leonurus heterophyllus [Syn. Leonurus artemisia], T3754 Leonurus sibiricus, T5169 Poria cocos.

scar T3395 Ilex pedunculosa.

scarlatina T0867 Baphicacanthus cusia [Syn. Strobilanthes cusia], T1662 Coptis chinensis, T1663 Coptis chinensis var. brevisepala, T1664 Coptis deltoidea, T1669 Coptis omeiensis, T1670 Coptis teetoides [Syn. Coptis teeta].

schizophrenia T1525 Cladonia fallax, T5848 Securinega suffruticosa. sciatica T0708 Arthromeris mairei [Syn. Polypodium mairei], T1715 Corydalis decumbens [Syn. Corydalis amabilis], T1727 Corydalis mucronifera, T2203 Dioscorea nipponica, T2204 Dioscorea nipponica ssp. rosthornii, T2723 Ficus pumila, T5279 Psychotria serpens, T6129 Stephania japonica, T6618 Uncaria homomalla [Syn. Uruparia homomalla; Uruparia tonkinensis; Uruparia lanosa var. parvifora],
T6843 Xanthium sibiricum [Syn. Xanthium strumarium].

scorpion sting T0382 Althaea rosea, T6551 Trogopterus xanthipes; Pteromys volans.

scourge epidemic with fever T0705 Artemisia vestita.

scratch T0827 Aucuba chinensis ssp. omeiensis.

scrofula T0048 Acanthus ilicifolius, T0078 Aconitum barbatum var. puberulum [Syn. Aconitum ochranthum], T0122 Aconitum pendulum, T0130 Aconitum sinomontanum, T0171 Adiantum capillus-veneris, T0175 Adiantum pedatum, T0275 Akebia quinata, T0333 Alocasia cucullata [Syn. Arum cucullatum], T0427 Ampelopsis japonica [Syn. Paullinia japonica], T0446 Anaphalis margaritacea, T0468 Anemone hupehensis, T0471 Anemone rivularis, T0567 Aralia armata, T0617 Arisaema amurense, T0618 Arisaema consanguineum, T0620 Arisaema heterophyllum, T0643 Aristolochia versicolar, T0726 Asarum forbesii, T0941 Bidens tripartita, T1049 Bufo bufo gargarizans; Bufo melanostictus, T1051 Bufo bufo gargarizans; Bufo melanostictus, T1254 Castanea mollissima, T1385 Chorda filum, T1387 Chrysanthemum boreale, T1392 Chrysanthemum indicum, T1394 Chrysanthemum lavandulifolium, T1692 Coriaria sinica [Syn. Coriaria nepalensis], T1709 Corydalis bungeana, T1787 Cremastra appendiculata, T1871 Cucubalus baccifer, T1967 Cynoglossum amabile, T1986 Cyrtomium fortunei, T1994 Daemonorops draco, T2024 Daphne genkwa, T2139 Dianella ensifolia, T2158 Dichroa febrifuga, T2162 Dicranostigma franchetianum [Syn. Dicranostigma leptopodum], T2253 Dracaena cochinchinensis, T2267 Drosera peltata var. lunata, T2298 Dysosma difformis, T2302 Dysosma pleiantha [Syn. Podophyllum pleianthum], T2304 Dysosma veitchii, T2305 Dysosma versipellis [Syn. Podophyllum versipelle], T2316 Echinops grijsii, T2317 Echinops ritro, T2321 Ecklonia kurome, T2365 Enteromorpha clathrata, T2428 Erinaceus europaeus; Hemiechinus daunricus; Hemiechinus auritus, T2432 Eriobotrya japonica, T2525 Eucheuma muricatum, T2584 Euphorbia ebracteolata, T2585 Euphorbia esula, T2587 Euphorbia fischeriana, T2589 Euphorbia helioscopia, T2599 Euphorbia lunulata, T2602 Euphorbia nematocypha, T2608 Euphorbia pekinensis, T2657 Fagopyrum cymosum [Syn. Polygonum cymosum], T2658 Fagopyrum esculentum, T2691 Farfugium japonicum, T2697 Ferula borealis, T2718 Ficus carica, T2756 Forsythia suspensa, T2757 Forsythia viridissima, T2761 Fortunella margarita, T2787 Fritillaria hupehensis, T2790 Fritillaria pallidiflora, T2798 Fritillaria verticillata var. thunbergii [Syn. Fritillaria thunbergii], T2800 Fritillaria walujewii, T2897 Gelsemium elegans, T2979 Gleditsia sinensis [Syn. Gleditsia horrida], T2980 Gleditsia sinensis [Syn. Gleditsia horrida], T3080 Gymnema sylvestre, T3163 Helicteres angustifolia, T3193 Hemerocallis fulva, T3195 Hemerocallis lilio-asphodelus, T3197 Hemerocallis minor, T3201 Hemiphragma heterophyllum, T3287 Huechys sanguinea, T3443 Iphigenia indica, T3460 Iris halophila, T3464 Iris lactea var. chinensis [Syn. Iris pallasii var. chinensis], T3674 Laggera alata, T3678 Laminaria japonica, T3680 Lamium amplexicaule, T3736 Lemmaphyllum microphyllum, T3815 Ligularia stenocephala, T3847 Lindera angustifolia, T3876 Litchi chinensis, T3988 Lycoris squamigera, T4005 Lysionotus pauciflorus, T4008 Lytta caraganae, T4091 Malva sylvestris, T4106 Manis pentadactyla, T4121 Marsilea quadrifolia, T4172 Melilotus 
suaveolens, T4173 Melilotus suaveolens, T4182 Menispermum dauricum, T4206 Metaplexis japonica, T4265 Momordica cochinchinensis, T4338 Mylabris phalerata; Mylabris cichorii, T4368 Nandina domestica, T4370 Nandina domestica, T4482 Oenanthe javanica, T4555 Ostrea rivularis; Ostrea talienwhanensis; Ostrea gigas, T4577 Paederia scandens, T4730 Peristrophe roxburghiana, T4796 Pheretima aspergillum; Allolobophora caliginosa trapezoides, T4835 Phyllanthus emblica, T4851 Physalis minima, T4903 Pinellia pedatisecta, T4917 Pinus massoniana, T5073 Polygala chinensis [Syn. Polygala glomerata], T5099 Polygonum bistorta, T5103 Polygonum hydropiper, T5105 Polygonum hydropiper var. flaccidum [Syn. Polygonum flaccidum], T5106 Polygonum lapathifolium, T5107 Polygonum multiflorum, T5109 Polygonum nodosum, T5182 Potentilla discolor, T5214 Prunella vulgaris, T5214 Prunella vulgaris, T5220 Prunus davidiana, T5226 Prunus mume, T5232 Prunus persica, T5295 Pteris multifida, T5323 Pulsatilla campanella, T5324 Pulsatilla cernua, T5325 Pulsatilla chinensis, T5326 Pulsatilla dahurica, T5401 Rabdosia yuennanensis, T5415 Ranunculus sceleratus, T5457 Rhamnus davurica, T5466 Rhaponticum uniflorum, T5550 Ricinus communis, T5562 Rosa chinensis, T5592 Rubus hirsutus, T5646 Sagina japonica [Syn. Spergula japonica], T5740 Sargassum vachellianum, T5835 Scutellaria barbata [Syn. Scutellaria rivularis], T5840 Scutellaria indica, T5862 Selaginella involvens, T5925 Sesamum indicum [Syn. Sesamum orientale], T5977 Smilax glabra, T5978 Smilax glauco-china, T5980 Smilax menispermoidea, T5982 Smilax sieboldii, T5984 Smilax stans [Syn. Smilax vaginata var. stans], T5989 Solanum aculeatissimum, T6014 Solanum surattense, T6018 Solanum verbascifolium, T6105 Stellera chamaejasme, T6257 Syngnathus acus, T6301 Taraxacum mongolicum, T6431 Thesium chinense, T6497 Tribulus terrestris, $\mathrm{T} 6541$ Tripterygium regelii, T6560 Tulipa edulis, T6588 Typhoniun giganteum, T6640 Undaria pinnatifida, T6653 Usnea diffracta, T6654 Usnea longissima, T6732 Vespertilio superans, T6766 Viola tricolor, T6767 Viola yedoensis, T6820 Wikstroemia indica, T6884 Zanthoxylum nitidum.

scrofula (juice) T0533 Antiaris toxicaria [Syn. Ambora toxicaria].

scrofula in early stage T1050 Bufo bufo gargarizans; Bufo melanostictus.

scrofula with phlegm node T0971 Bolbostemma paniculatum, T1084

Buthus martensi, T5826 Scrophularia buergeriana, T5828

Scrophularia ningpoensis.

scrofula with ulceration T5817 Scolopendra subspinipes mutilans.

scrotal damp itch T1582 Cnidium monnieri, T2584 Euphorbia ebracteolata, T2587 Euphorbia fischeriana.

scrotal eczema T2902 Gentiana algida, T3245 Hibiscus syriacus, T3547 Ixeris chinensis, T4690 Pelargonium graveolens, T4836 Phyllanthus emblica, T5010 Platycarya strobilacea, T6742 Vicia amoena.

scrotal enlargement T1352 Chamaenerion angustifolium [Syn. Epilobium angustifolium].

scrotal wind $\mathrm{T} 3306$ Hydrangea paniculata, T3569 Juglans regia.

scurvy T3394 Ilex paraguariensis, T4388 Nasturtium officinale.

seasonal diaphragm $q i$ T0599 Ardisia japonica.

seasonal epidemic fire toxin T1419 Cimicifuga dahurica, T1420

Cimicifuga foetida, T1421 Cimicifuga heracleifolia.

seeper in chest and abdomen T2608 Euphorbia pekinensis, T3984
Lycoris chinensis, $\mathrm{T} 3986$ Lycoris radiata [Syn. Amaryllis radiata]. seminal efflux T1604 Coeloglossum viride [Syn. Coeloglossum viride var. bracteatum], T3077 Gymnadenia conopsea.

senile bronchitis T3926 Loropetalum chinense.

senile cough and asthma T0752 Asparagus officinalis, T6111 Stemona

japonica, T6113 Stemona sessilifolia, T6115 Stemona tuberosa.

senile dementia T5848 Securinega suffruticosa.

senile nocturia T0529 Anthriscus sylvestris.

senile vacuity weakness T0541 Apis cerana.

senile vacuity weakness headache $\mathrm{T} 1786$ Cremanthodium ellisii.

septicemia T0541 Apis cerana, T1337 Cervus nippon; Cervus elaphus,

T1662 Coptis chinensis, T1663 Coptis chinensis var. brevisepala,

T1664 Coptis deltoidea, T1669 Coptis omeiensis, T1670 Coptis teetoides [Syn. Coptis teeta], T2846 Ganoderma japonicum [Syn. Ganoderma sinense], T2848 Ganoderma lucidum, T3085 Gynostemma pentaphyllum, T3409 Illicium verum, T3828 Ligustrum lucidum, T4308 Mucuna birdwoodiana, T6031 Sophora flavescens [Syn. Sophora angustfolia], T6043 Sophora subprostrata [Syn. Sophora tonkinensis], T6066 Spatholobus suberectus, T6119 Stephania cepharantha.

sequel of poliomyelitis T1567 Clerodendrum mandarinorum, T1715 Corydalis decumbens [Syn. Corydalis amabilis], T2736 Fissistigma polyanthum, T3976 Lycopodium obscurum, T5848 Securinega suffruticosa, T6184 Strychnos nux-vomica, T6193 Strychnos wallichiana, T6651 Urtica cannabina, T6652 Urtica dioica.

shank sore T0299 Aleurites cordata [Syn. Aleurites fordii], T1437 Cinnamomum camphora, T2732 Firmiana simplex, T6747 Vicia faba.

shcizophrenia T6819 Wikstroemia chamaedaphne.

shedding of eyebrow and hair T1175 Cannabis sativa.

shock T0502 Anisodus tanguticus [Syn. Scopolia tangutica], T1468 Citrus aurantium, T1521 Citrus wilsonii, T5141 Poncirus trifoliata.

shock due to acute infectious diseases T0502 Anisodus tanguticus [Syn. Scopolia tangutica].

short voidings of reddish urine T0273 Akebia quinata, T0277 Akebia trifoliata, T0279 Akebia trifoliata var. australis, T0932 Betula luminifera, T1878 Cucumis sativus, T2882 Gardenia jasminoides [Syn. Gardenia florida], T3192 Hemerocallis citrina, T3270 Holboellia fargesii, T5098 Polygonum aviculare, T6357 Tetrapanax papyriferus.

shortness of breath and hypodynamia T5497 Rhodiola kirilowii, T5499 Rhodiola sacra.

shortness of breath and palpitation T3128 Hedysarum multijugum.

shortness of breath and spontaneous sweating T1596 Codonopsis canescens, T1597 Codonopsis clematidea, T1599 Codonopsis pilosula, T1600 Codonopsis pilosula var. modesta [Syn. Codonopsis modesta], T1601 Codonopsis subglobosa, T1602 Codonopsis tangshen, T1603 Codonopsis tubulosa.

shoulder swelling T2243 Dodonaea viscosa.

silicosis T2846 Ganoderma japonicum [Syn. Ganoderma sinense], T2848 Ganoderma lucidum.

sinew and bone numbness T3796 Libanotis buchtormensis, T6893 Zanthoxylum simulans, T6894 Zanthoxylum simulans.

sinew and bone wound T0170 Adhatoda vasica, T2273 Drynaria fortunei, T5252 Pseudodrynaria coronans, T6138 Sterculia foetida.

sinew and bone wound with swelling pain T1254 Castanea mollissima. 
skin cancer T4130 Maytenus chuchuhuasca.

skin chap and pain T0543 Apis mellifera ligustica.

skin diseases T0495 Angelica sinensis, T3082 Gynocardia odorata,

T3845 Linaria vulgaris, T4804 Phlojodicarpus sibiricus, T5726 Saponaria officinalis, T6072 Sphagnum palustre [Syn. Sphagnum obtusifolium; Sphagnum cymbifolium], T6432 Thespesia populnea [Syn. Hibiscus populneus], T6713 Vernonia anthelmintica.

skin heat papules T4261 Mollugo pentaphylla.

skin intractable ulcer T2650 Excoecaria agallocha.

skin lichen T1240 Cassia obtusifolia, T1250 Cassia tora, T1936 Cyclocarya paliurus, T2617 Euphorbia royleana, T3600 Juniperus taiwaniana, T4519 Opuntia dillenii, T4521 Opuntia vulgaris, T5719 Sapindus mukorossi.

skin lichen lai T3308 Hydrangea umbellata.

skin numbness T3970 Lycopodium cernuum, T3973 Lycopodium japonicum [Syn. Lycopodium clavatum].

skin scabies T5411 Randia spinosa.

skin sores T4170 Melilotus albus, T6893 Zanthoxylum simulans, T6894 Zanthoxylum simulans.

skin suppurations T3908 Lonicera bournei, T3909 Lonicera confusa, T3910 Lonicera fulvotomentosa, T3911 Lonicera hypoglauca, T3912 Lonicera japonica, T3914 Lonicera macranthoides, T3918 Lonicera similis.

sleepless T0644 Armillaria mellea, T5701 Salvia yunnanensis, T6295 Tanacetum sibiricum [Syn. Filifolium sibiricum], T6358 Tetraplodon mnioides [Syn. Tetraplodon bryoides; Splachnum mnioides].

sleepless and amnesia T0944 Biota orientalis [Syn. Thuja orientalis; Platycladus orientalis], T1964 Cynanchum wilfordii [Syn. Cynoctonum wilfordii].

sleepless and vexation T4121 Marsilea quadrifolia.

sloppy stool T0824 Atractylodes macrocephala [Syn. Atractylis macrocephala].

sloppy stool and diarrhea T5169 Poria cocos.

slowness to work T3199 Hemibarbus labeo.

snake bite T0060 Achillea alpina [Syn. Achillea sibirica], T0595 Ardisia crispa, T0601 Ardisia pusilla, T0692 Artemisia rupestris [Syn. Artemisia dentata; Artemisia viridis; Artemisia viridifolia], T0726 Asarum forbesii, T0730 Asarum sagittarioides, T0977 Bombyx mori, T1084 Buthus martensi, T1197 Cardiospermum halicacabum, T1434 Cinnamomum bejolghota [Syn. Cinnamomum obtusifolium; Laurus bejolghota], T1450 Cirsium lineare, T1547 Clematis terniflora [Syn. Clematis maximowicziana], T1958 Cynanchum otophyllum, T2244 Doellingeria scaber [Syn. Aster scaber], T2323 Eclipta prostrata [Syn. Eclipta alba], T2555 Eupatorium chinense, T2563 Eupatorium japonicum, T2598 Euphorbia lathyris, T2691 Farfugium japonicum, T2927 Gentiana rhodantha, T3002 Glycine max, T3363 Hypericum sampsonii, T3460 Iris halophila, T3464 Iris lactea var. chinensis [Syn. Iris pallasii var. chinensis], T3984 Lycoris chinensis, T3986 Lycoris radiata [Syn. Amaryllis radiata], T3997 Lysimachia capillipes, T4178 Melodinus hemsleyanus, T4335 Mussaenda pubescens, T4428 Nicotiana tabacum, T4642 Parameria laevigata, T4838 Phyllanthus flexuosus, T4882 Picrasma quassioides [Syn. Picrasma ailanthoides], T5102 Polygonum cuspidatum, T5428 Rauvolfia yunnanensis, T5557
Rohdea japonica [Syn. Orontium japonicum], T5721 Sapindus mukorossi, T5724 Sapium sebiferum, T5857 Sedum sarmentosum, T5870 Selaginella uncinata, T6444 Thunbergia grandiflora, T6551 Trogopterus xanthipes; Pteromys volans, T6831 Woodwardia orientalis, T6834 Wrightia tomentosa, T6889 Zanthoxylum podocarpum.

snake bite or scorpion sting ${ }^{[5509]}$ T3200 Hemidesmus indicus.

snake or dog bite T4085 Mallotus repandus var. chrysocarpus [Syn. Mallotus chrysocarpus; Mallotus repandus].

snake or insect bites T0045 Acanthopanax trifoliatus, T0069 Achillea wilsoniana, T0274 Akebia quinata, T0278 Akebia trifoliata, T0280 Akebia trifoliata var. australis, T0318 Allium sativum, T0389 Amaranthus tricolor, T0503 Anisomeles indica [Syn. Epimeredi indica], T0625 Aristolochia contorta, T0627 Aristolochia debilis [Syn. Aristolochia longa], T0853 Baeckea frutescens, T0867 Baphicacanthus cusia [Syn. Strobilanthes cusia], T0938 Bidens bipinnata, T0950 Blatta orientalis, T0971 Bolbostemma paniculatum, T1241 Cassia occidentalis, T1290 Celastrus orbiculatus [Syn. Celastrus articulatus], T1357 Chelidonium majus, T1361 Chenopodium ambrosioides, T1787 Cremastra appendiculata, T1866 Cryptolepis sinensis, T2334 Elephantopus scaber, T2950 Gerbera anandria [Syn. Leibnitzia anandria], T3088 Gynura segetum [Syn. Gynura japonica], T3113 Hedera nepalensis var. sinensis, T3115 Hedera rhombea, T3410 Impatiens balsamina, T3423 Indigofera tinctoria, T3444 Ipomoea aquatica [Syn. Convolvulus repens; Ipomoea reptans], T3496 Isodon japonica [Syn. Rabdosia japonica], T3662 Lactuca sativa, T3851 Lindera glauca, T3898 Lobelia chinensis [Syn. Lobelia radicans], T4020 Macleaya cordata, T4158 Melia azedarach, T4183 Menispermum dauricum, T4206 Metaplexis japonica, T4305 Mosla dianthera, T4307 Mosla scabra [Syn. Mosla punctata], T4323 Murraya paniculata [Syn. Chalcas paniculata], T4325 Murraya paniculata var. exotica, T4470 Ocimum basilicum, T4519 Opuntia dillenii, T4520 Opuntia ficus-indica, T4521 Opuntia vulgaris, T4564 Oxalis corniculata [Syn. Oxalis repens], T4648 Paris polyphylla, T4649 Paris polyphylla var. chinensis, T4650 Paris polyphylla var. pseudothibetica, T4651 Paris polyphylla var. stenophylla, T4652 Paris polyphylla var. yunnanensis, T4714 Perilla frutescens, T4956 Piper mullesua, T5173 Portulaca oleracea, T5184 Potentilla kleiniana, T5194 Premna microphylla, T5267 Psidium guajava, T5295 Pteris multifida, T5298 Pteris vittata, T5396 Rabdosia rubescens, T5685 Salvia plebeia, T5712 Sanguisorba officinalis, T5844 Scutellaria scordifolia, T6651 Urtica cannabina, T6652 Urtica dioica.

soft tissue sprain T2206 Dioscorea parviflora.

sore T0319 Allium schoenoprasum, T0500 Anguilla japonica, T4368 Nandina domestica, T4789 Phellodendron amurense, T4790 Phellodendron amurense var. wilsonii, T4791 Phellodendron chinense, T4792 Phellodendron chinense var. glabriusculum, T4796 Pheretima aspergillum; Allolobophora caliginosa trapezoides, T5530 Rhus chinensis [Syn. Rhus semialata], T6279 Tagetes erecta.

sore (leaf) T0945 Bischofia javanica [Syn. Bischofia trifoliata].

sore and boil T0011 Abrus precatorius, T0130 Aconitum sinomontanum, T0293 Albizzia lebbeck, T0379 Alternanthera philoxeroides, T0468 Anemone hupehensis, T0867 Baphicacanthus cusia [Syn. Strobilanthes cusia], T0879 Bauhinia purpurea, T0888 Beesia calthaefolia, T1003 
Brandisia hancei, T1046 Buddleja davidii, T1785 Cratoxylum prunifolium, T2092 Delphinus delphis, T2193 Dioscorea cirrhosa [Syn. Dioscorea pogonoides], T2334 Elephantopus scaber, T2357 Emilia sonchifolia, T2365 Enteromorpha clathrata, T2505 Eucalyptus citriodora, T2591 Euphorbia humifusa, T2715 Fibraurea recisa, T2946 Geranium robertianum, T2984 Glinus lotoides [Syn. Mollugo lotoides], T3040 Gomphrena globosa, T3080 Gymnema sylvestre, T3233 Heteropappus altaicus, T3241 Hibiscus mutabilis, T3244 Hibiscus syriacus, T3398 Ilex rotunda, T3423 Indigofera tinctoria, T3471 Iris tectorum, T3709 Lathyrus pratensis, T3840 Limnophila rugosa, T3887 Litsea glutinosa, T3998 Lysimachia christinae, T4026 Macrothelypteris oligophlebia, T4120 Marsdenia tenacissima, T4188 Mentha rotundifolia, T4190 Mentha spicata, T4305 Mosla dianthera, T4423 Nicandra physaloides, T4881 Picrasma quassioides [Syn. Picrasma ailanthoides], T5126 Polypodium virginianum, T5127 Polypodium vulgare, T5172 Portulaca grandiflora, T5174 Portulaca pilosa, T5177 Potamogeton pectinatus, T5199 Primula malacoides, T5449 Reineckea carnea, T5548 Ricinus communis, T5850 Sedum aizoon, T5855 Sedum kamtschaticum, T5982 Smilax sieboldii, T5984 Smilax stans [Syn. Smilax vaginata var. stans], T6024 Solidago virgaurea var. leiocarpa [Syn. Solidago decurrens], T6029 Sophora alopecuroides, T6121 Stephania dicentrinifera, T6129 Stephania japonica, T6137 Stephania viridiflavens, T6145 Stichopus japonicus, T6275 Tacca chantrieri [Syn. Tacca minor; Tacca esquirolii], T6304 Taxillus levinei, T6386 Thalictrum foetidum, T6444 Thunbergia grandiflora, T6580 Tylophora floribunda, T6599 Ulva lactuca, T6600 Ulva pertusa, T6645 Urena lobata, T6716 Vernonia esculenta, T6772 Viscum angulatum.

sore and boil in children T5266 Psidium guajava.

sore and boil with swelling of clove T0047 Acanthus ebracteatus.

sore and lichen T1106 Caesalpinia minax, T1296 Celosia argentea, T2139 Dianella ensifolia, T3624 Kalopanax pictum, T3625 Kalopanax septemlobus, T3738 Lemna minor, T6474 Toona ciliata, T6475 Toona sinensis.

sore and papules T4979 Pistacia chinensis, T5231 Prunus persica.

sore and papules with intractable lichen T2979 Gleditsia sinensis [Syn. Gleditsia horrida].

sore and scab T1261 Catalpa ovata, T1558 Clerodendron inerme, T5549 Ricinus communis, T5724 Sapium sebiferum, T5804 Schizonepeta tenuifolia [Syn. Nepeta tenuifolia].

sore and scab in children T3893 Litsea pungens.

sore and welling abscess T0457 Andrographis paniculata [Syn. Justicia paniculata], T1673 Corchorus capsularis, T3192 Hemerocallis citrina, T4323 Murraya paniculata [Syn. Chalcas paniculata], T4325 Murraya paniculata var. exotica, T4370 Nandina domestica, T4618 Panicum miliaceum, T5107 Polygonum multiflorum, T5288 Pteris cretica var. nervosa [Syn. Pteris nervosa], T5457 Rhamnus davurica, T6101 Stauntonia hexaphylla, T6122 Stephania dielsiana, T6788 Vitex negundo.

sore and welling abscess with scab and lichen T6214 Swertia chinensis. sore in lower body $\mathrm{T} 1171$ Canis familiaris.

sore lai T4240 Millettia pachycarpa.

sore lichen and scab lai T1566 Clerodendrum inerme, T4158 Melia azedarach.

sore pharynx T0045 Acanthopanax trifoliatus, T0071 Achyranthes aspera, T0072 Achyranthes aspera var. indica, T0834 Averrhoa carambola, T0912 Berberis poiretii, T0947 Bixa orellana, T1417 Cimicifuga acerina, T1419 Cimicifuga dahurica, T1420 Cimicifuga foetida, T1421 Cimicifuga heracleifolia, T2290 Duchesnea indica, T2902 Gentiana algida, T3174 Heliotropium indicum, T3190 Helminthostachys zeylanica, T3201 Hemiphragma heterophyllum, T3396 Ilex pubescens, T3397 Ilex pubescens var. glaber, T3829 Ligustrum robustum, T4186 Mentha piperita, T4190 Mentha spicata, T4502 Onychium japonicum [Syn. Tricomanes japonicum], T5840 Scutellaria indica, T6028 Sophora alopecuroides, T6121 Stephania dicentrinifera, T6137 Stephania viridiflavens, T6276 Tacca plantaginea [Syn. Schizocapsa plantaginea], T6301 Taraxacum mongolicum, T6722 Veronica anagallis-aquatica.

sore red eyes and clouded vision $\mathrm{T} 3957$ Lycium chinense.

sore red swollen eyes and tearing T6437 Thlaspi arvense.

sore swollen throat T0267 Ajuga nipponensis, T3240 Hibiscus esculentus, T3548 Ixeris sonchifolia, T3765 Lepisorus ussuriensis, T3830 Ligustrum sinense, T6125 Stephania elegans, T6219 Swertia erythrosticta, T6224 Swertia kouitchensis, T6365 Teucrium pilosum [Syn. Teucrium japonicum var. pilosum].

sore toxin T0098 Aconitum geniculatum, T0389 Amaranthus tricolor, T0423 Ampelopsis brevipedunculata, T0434 Amsonia sinensis, T0561 Arachis hypogaea, T0932 Betula luminifera, T0973 Bombax malabaricum [Syn. Gossampinus malabarica], T1733 Corydalis racemosa, T1866 Cryptolepis sinensis, T2154 Dicentra spectabilis, T2612 Euphorbia prolifera, T2617 Euphorbia royleana, T2798 Fritillaria verticillata var. thunbergii [Syn. Fritillaria thunbergii], T3163 Helicteres angustifolia, T3283 Hosta sieboldiana, T3557 Jasminum sambac, T3687 Lantana camara, T3853 Lindera obtusiloba, T4502 Onychium japonicum [Syn. Tricomanes japonicum], T4830 Phryma leptostachya, T5298 Pteris vittata, T5537 Rhus sylvestris, T5554 Rodgersia aesculifolia, T6125 Stephania elegans, T6136 Stephania tetrandra, T6540 Tripterygium hypoglaucum, T6555 Tropaeolum majus, T6705 Verbascum thapsus, T6744 Vicia faba.

sore toxin and intractable lichen T4658 Parmelia tinctorum.

sore toxin of sore and boil T0659 Artemisia absinthium.

sore toxin of welling abscess and boil T5523 Rhododendron simsii, T6128 Stephania hernandifolia.

sore toxin of welling abscess and flat abscess T0982 Bos taurus domesticus, T2442 Ervatamia divaricata, T4009 Maackia amurensis, T5826 Scrophularia buergeriana, T5828 Scrophularia ningpoensis.

sore welling abscess and lichen lai T2077 Delphinium grandiflorum.

sores T0295 Albizzia odoratissima, T0478 Angelica dahurica [Syn. Angelica porphyrocaulis], T0495 Angelica sinensis, T0838 Azolla imbricata [Syn. Salvinia imbricata], T0912 Berberis poiretii, T0947 Bixa orellana, T0962 Boehmeria platanifolia [Syn. Boehmeria tricuspis], T1100 Caesalpinia crista, T1655 Conyza blinii, T2198 Dioscorea gracillima, T2201 Dioscorea hypoglauca [Syn. Dioscorea collettii var. hypoglauca], T2212 Dioscorea tokoro, T2274 Dryobalanops aromatica, T2521 Eucalyptus tereticornis, T2641 Evodia lepta [Syn. Ilex lepta], T2657 Fagopyrum cymosum [Syn. 
Polygonum cymosum], T3970 Lycopodium cernuum, T3973 Lycopodium japonicum [Syn. Lycopodium clavatum], T4055 Mahonia bealei, T4062 Mahonia fortunei, T4066 Mahonia japonica, T4082 Mallotus japonicus, T4172 Melilotus suaveolens, T4217 Microcos paniculata [Syn. Grewia microcos], T4604 Panax japonicus var. bipinnatifidus, T4804 Phlojodicarpus sibiricus, T5401 Rabdosia yuennanensis, T5419 Raphanus sativus, T5594 Rubus parkeri, T5727 Saposhnikovia divaricata [Syn. Ledebouriella seseloides], T6260 Syringa oblata, T6373 Thalictrum alpinum, T6548 Tritonia crocosmaeflora.

sores and scrofula T3568 Juglans regia.

sores clove boil T4519 Opuntia dillenii, T4521 Opuntia vulgaris.

sores eczema T3860 Linum usitatissimum.

sores scab and lichen T0088 Aconitum coreanum, T0707 Arthraxon hispidus.

sores with pus blood T2729 Filipendula ulmaria.

sores with welling abscess and flat abscess T0215 Agave americana,

T0479 Angelica dahurica cv. qibaizhi, T0498 Angelica taiwaniana,

T3878 Lithocarpus polystachyus, T3933 Luffa acutangula, T3934 Luffa cylindrica.

sparse vaginal discharge T5075 Polygala fallax [Syn. Polygala aureocauda].

spasm T0617 Arisaema amurense, T0618 Arisaema consanguineum, T0620 Arisaema heterophyllum, T1084 Buthus martensi, T4518 Oppopanax chironium, T4903 Pinellia pedatisecta, T5817 Scolopendra subspinipes mutilans.

spasm in limbs T2890 Gastrodia elata, T4580 Paeonia albiflora [Syn. Paeonia lactiflora].

spasm of biliary ducts or duodenum T0502 Anisodus tanguticus [Syn. Scopolia tangutica].

spasmodic abdominal pain T6229 Swertia patens.

spasmodic abdominal pain of gastrointestinal tract T6527 Trigonella caerulea.

spasmodic cough T4275 Monotropa hypopitys.

spider bite T1182 Capra hircus; Ovis aries.

spill pulse T4481 Oenanthe javanica.

spleen qi vacuity T0824 Atractylodes macrocephala [Syn. Atractylis macrocephala].

spleen vacuity and abdominal distention T4147 Medicago falcata.

spleen vacuity and fatigued body T4600 Panax ginseng [Syn. Panax schinseng], T5260 Pseudostellaria heterophylla.

spleen vacuity and food distention T0529 Anthriscus sylvestris.

spleen vacuity and functional weakness T1964 Cynanchum wilfordii [Syn. Cynoctonum wilfordii].

spleen vacuity and marked emaciation T0991 Bos taurus domesticus; Bubalus bubalis.

spleen vacuity and vaginal discharge T2720 Ficus hispida.

spleen vacuity cold dysentery T4982 Pistacia vera.

spleen vacuity diarrhea T1254 Castanea mollissima, T1419 Cimicifuga dahurica, T1420 Cimicifuga foetida, T1421 Cimicifuga heracleifolia, T1614 Coix lacryma-jobi var. ma-yuen, T1751 Corylus heterophylla, T2188 Dioscorea alata, T2195 Dioscorea deltoidea, T2326 Elaeagnus angustifolia, T3992 Lyonia ovalifolia var. elliptica, T4088 Malus pumila, T4398 Nelumbo nucifera, T4786 Phasianus colchicus, T5179 Potentilla anserina, T5236 Prunus pseudocerasus, T5606 Rumex acetosa, T6056 Sorghum vulgare, T6267 Syzygium jambos, T6492 Trapa bispinosa.

spleen vacuity edema T2727 Ficus simplicissima, T2998 Glycine max, T3447 Ipomoea batatas [Syn. Convolvulus batatas], T5075 Polygala fallax [Syn. Polygala aureocauda].

spleen vacuity $q \boldsymbol{i}$ fall T4601 Panax ginseng [Syn. Panax schinseng].

spleen vacuity with damp T2246 Dolichos lablab.

spleen weakness and functional weakness T6878 Zanthoxylum echinocarpum.

spleen-kidney vacuity T4681 Pedicularis decora, T5270 Psoralea corylifolia.

spleen-kidney yang vacuity T0041 Acanthopanax senticosus [Syn. Eleutherococcus senticosus].

spleen-stomach cold-damp obstructing T0358 Alpinia katsumadai.

spleen-stomach damp turbidity T0819 Atractylodes chinensis, T0821 Atractylodes japonica, T0823 Atractylodes lancea, T6561 Tulipa gesneriana.

spleen-stomach disharmony T1390 Chrysanthemum coronarium, T1397 Chrysanthemum segetum, T2452 Erysimum cheiranthoides.

spleen-stomach $q \boldsymbol{i}$ stagnation T4720 Perilla frutescens var. arguta, T4723 Perilla frutescens var. crispa.

spleen-stomach $q \boldsymbol{i}$ stagnation and damp obstruction T1506 Citrus reticulata.

spleen-stomach $q i$ stagnation due to damp obstructing middle-jiao T0416 Amomum kravanh [Syn. Amomum cardamomum].

spleen-stomach vacuity T1202 Carex kobomugi, T1596 Codonopsis canescens, T1597 Codonopsis clematidea, T1599 Codonopsis pilosula, T1600 Codonopsis pilosula var. modesta [Syn. Codonopsis modesta], T1601 Codonopsis subglobosa, T1602 Codonopsis tangshen, T1603 Codonopsis tubulosa, T3011 Glycyrrhiza aspera, T3013 Glycyrrhiza glabra, T3015 Glycyrrhiza inflata, T3016 Glycyrrhiza kansuensis, T3021 Glycyrrhiza squamulosa, T3022 Glycyrrhiza uralensis, T3023 Glycyrrhiza yunnanensis, T4178 Melodinus hemsleyanus, T4545 Oryza sativa, T4644 Parasilurus asotus, T5872 Selenarctos thibetanus; Ursus arctos, T6754 Vigna unguiculata.

spleen-stomach vacuity cold T0360 Alpinia oxyphylla, T4351 Myristica fragrans, T6869 Zanthoxylum bungeanum, T6892 Zanthoxylum schinifolium.

spleen-stomach vacuity heat T5938 Setaria italica.

splitting of anus T0955 Bletilla striata.

spontaneous external bleeding T0001 Abelmoschus manihot, T0226 Agave sisalana, T0229 Ageratum conyzoides, T0266 Ajuga macrosperma, T0322 Allium tuberosum, T0324 Allium victorialis, T0382 Althaea rosea, T0568 Aralia chinensis, T0570 Aralia dasyphylla, T0648 Arnebia euchroma, T0649 Arnebia guttata, T0664 Artemisia argyi, T0678 Artemisia japonica, T0681 Artemisia lavandulaefolia, T0685 Artemisia mongolica, T0687 Artemisia myriantha, T0689 Artemisia princeps, T0691 Artemisia rubripes, T0706 Artemisia vulgaris, T0830 Auricularia auricula, T0831 Auricularia delicata, T0867 Baphicacanthus cusia [Syn. Strobilanthes cusia], T0955 Bletilla striata, T0962 Boehmeria platanifolia [Syn. 
Boehmeria tricuspis], T0964 Boenninghausenia albiflora, T0982 Bos taurus domesticus, T1044 Bryum argenteum, T1045 Bubalus bubalis, T1116 Callicarpa arborea, T1118 Callicarpa formosana, T1119 Callicarpa japonica, T1120 Callicarpa macrophylla, T1145 Camellia japonica, T1184 Capsella bursa-pastoris, T1199 Carduus acanthoides, T1200 Carduus crispus, T1210 Carpesium abrotanoides, T1254 Castanea mollissima, T1311 Centella asiatica, T1449 Cirsium japonicum, T1451 Cirsium setosum [Syn. Cerratula setosa; Cirsium segetum; Cephalanoplos segetum], T1554 Clerodendron cyrtophyllum, T1662 Coptis chinensis, T1663 Coptis chinensis var. brevisepala, T1664 Coptis deltoidea, T1669 Coptis omeiensis, T1670 Coptis teetoides [Syn. Coptis teeta], T1907 Curcuma wengujin, T1969 Cynoglossum officinale, T2191 Dioscorea bulbifera, T2193 Dioscorea cirrhosa [Syn. Dioscorea pogonoides], T2257 Dracocephalum moldavicum, T2280 Dryopteris chrysocoma, T2281 Dryopteris crassirhizoma, T2407 Equisetum arvense, T2538 Euonymus bungeanus, T2882 Gardenia jasminoides [Syn. Gardenia florida], T3088 Gynura segetum [Syn. Gynura japonica], T3113 Hedera nepalensis var. sinensis, T3115 Hedera rhombea, T3193 Hemerocallis fulva, T3195 Hemerocallis lilio-asphodelus, T3197 Hemerocallis minor, T3276 Homo sapiens, T3277 Homo sapiens, T3334 Hypecoum leptocarpum, T3349 Hypericum erectum, T3363 Hypericum sampsonii, T3373 Hypoestes purpurea [Syn. Justicia purpurea; Hypoestes sinica], T3416 Imperata cylindrica var. major, T3460 Iris halophila, T3464 Iris lactea var. chinensis [Syn. Iris pallasii var. chinensis], T3547 Ixeris chinensis, T3645 Kummerowia striata, T3736 Lemmaphyllum microphyllum, T3737 Lemmaphyllum microphyllum var. obovatum, T3769 Lespedeza bicolor, T3881 Lithospermum erythrorhizon, T3926 Loropetalum chinense, T3954 Lycium barbarum, T3956 Lycium chinense, T3981 Lycopus lucidus, T4006 Lythrum anceps, T4007 Lythrum salicaria, T4121 Marsilea quadrifolia, T4127 Matteuccia struthiopteris, T4305 Mosla dianthera, T4398 Nelumbo nucifera, T4402 Nelumbo nucifera, T4413 Nepeta cataria, T4549 Osbeckia chinensis, T4552 Osmunda japonica, T4564 Oxalis corniculata [Syn. Oxalis repens], T4582 Paeonia delavayi, T4584 Paeonia lactiflora wild, T4585 Paeonia moutan [Syn. Paeonia suffruticosa], T4586 Paeonia obovata, T4590 Paeonia veitchii, T4604 Panax japonicus var. bipinnatifidus, T4605 Panax japonicus var. major, T4606 Panax pseudo-ginseng, T4607 Panax pseudo-ginseng var. japonicus, T4608 Panax pseudo-ginseng var. notoginseng [Syn. Panax notoginseng], T4720 Perilla frutescens var. arguta, T4723 Perilla frutescens var. crispa, T5002 Plantago asiatica, T5004 Plantago depressa, T5007 Plantago major, T5099 Polygonum bistorta, T5120 Polygonum tinctorium, T5121 Polygonum tinctorium, T5133 Polytrichum commune, T5230 Prunus persica, T5295 Pteris multifida, T5372 Quercus dentata, T5419 Raphanus sativus, T5449 Reineckea carnea, T5484 Rhinoceros unicornis; Rhinoceros sondaicus; Rhinoceros sumatrensis, T5523 Rhododendron simsii, T5554 Rodgersia aesculifolia, T5594 Rubus parkeri, T5607 Rumex crispus, T5611 Rumex japonicus, T5612 Rumex nepalensis, T5614 Rumex patientia, T5701 Salvia yunnanensis, T5712 Sanguisorba officinalis, T5804 Schizonepeta tenuifolia [Syn. Nepeta tenuifolia], T5833 Scutellaria amoena, T5834 Scutellaria baicalensis, T5835 Scutellaria barbata [Syn. Scutellaria rivularis], T5839
Scutellaria hypericifolia, T5841 Scutellaria likiangensis, T5843 Scutellaria rehderiana, T5845 Scutellaria viscidula, T5850 Sedum aizoon, T5851 Sedum alfredii [Syn. Sedum formosanum], T5855 Sedum kamtschaticum, T5863 Selaginella moellendorffii, T6034 Sophora japonica, T6036 Sophora japonica, T6047 Sophora viciifolia, T6048 Sophora viciifolia, T6076 Spinacia oleracea, T6119 Stephania cepharantha, T6440 Thuja orientalis [Syn. Platycladus orientalis; Biota orientalis], T6471 Toddalia asiatica [Syn. Toddalia aculeata; Paullinia asiatica], T6485 Trachycarpus fortunei, T6584 Typha angustata, T6585 Typha angustifolia, T6587 Typha latifolia, T6601 Umbilicaria esculenta [Syn. Gyrophora esculenta], T6764 Vinegar.

spontaneous external bleeding due to blood heat T5445 Rehmannia glutinosa [Syn. Rehmannia glutinosa f. huechingensis], T5447 Rehmannia glutinosa [Syn. Rehmannia glutinosa f. huechingensis].

spontaneous sweating T0824 Atractylodes macrocephala [Syn. Atractylis macrocephala], T1598 Codonopsis convolvulacea, T2658 Fagopyrum esculentum, T4548 Oryza sativa var. glutinosa, T4580 Paeonia albiflora [Syn. Paeonia lactiflora].

spontaneous sweating and night sweating T2381 Ephedra sinica, T3128 Hedysarum multijugum, T5791 Schisandra chinensis, T5802 Schisandra sphenanthera.

spontaneous sweating and night sweating due to vacuity T6918 Ziziphus jujuba var. spinosa.

spontaneous sweating due to exterior vacuity T0791 Astragalus chrysopterus, T0794 Astragalus ernestii, T0798 Astragalus membranaceus, T0800 Astragalus mongholicus, T3129 Hedysarum polybotrys.

sprain T0170 Adhatoda vasica, T3318 Hylotelephium mingjinianum, T4221 Micromelum falcatum, T4917 Pinus massoniana.

sprain and contusion T0948 Blainvillea acmella [Syn. Verbesina acmella; Eclipta latifolia; Blainvillea latifolia], T1147 Camellia oleifera, T1533 Clausena dentata, T1534 Clausena dunniana, T1931 Cyclea barbata, T2884 Gardenia jasminoides var. grandiflora, T2946 Geranium robertianum, T4018 Machilus thunbergii, T4510 Ophiorrhiza japonica, T4513 Ophiorrhiza mungos.

sprain from knocks and falls T1288 Celastrus orbiculatus [Syn. Celastrus articulatus], T1557 Clerodendron indicum, T2213 Dioscorea zingiberensis, T3976 Lycopodium obscurum, T5028 Plumbago indica, T5029 Plumbago zeylanica, T6008 Solanum nigrum, T6705 Verbascum thapsus.

sprain in joints T0116 Aconitum nagarum var. heterotrichum [Syn. Aconitum bullatifolium].

sprain of hands and feet T0569 Aralia cordata, T0574 Aralia fargesii.

squamous carcinoma of skin T1831 Crotalaria sessiliflora.

stabbing pain in chest and abdomen T1108 Caesalpinia sappan, T4608 Panax pseudo-ginseng var. notoginseng [Syn. Panax notoginseng].

stabbing pain in heart and abdomen $\mathrm{T} 1775$ Crataegus pinnatifida, T1778 Crataegus pinnatifida var. major.

stable intermittent claudication T2961 Ginkgo biloba.

stagnation of damp-heat in liver and gall T2882 Gardenia jasminoides [Syn. Gardenia florida].

stasis heat yellowing T1045 Bubalus bubalis.

stasis of lung $\boldsymbol{q} i \quad \mathrm{~T} 1960$ Cynanchum stauntonii. 
stasis pain from knocks and falls T0010 Abrus fruticulosus [Syn. Abrus cantoniensis], T0077 Aconitum balfourii, T0084 Aconitum carmichaeli, T0105 Aconitum karakolicum, T0151 Acronychia pedunculata, T0890 Begonia limprichtii, T0994 Boswellia carterii, T5400 Rabdosia stracheyi, T5966 Siphonostegia chinensis.

stasis pain in chest and abdomen T1638 Commiphora myrrha [Syn. Commiphora molmol].

stasis stagnation pain in stomach duct and abdomen T6872

Zanthoxylum cuspidatum.

stasis swelling due to injury T5419 Raphanus sativus.

stasis swelling from knocks and falls T0069 Achillea wilsoniana, T0282 Alangium kurzii, T0692 Artemisia rupestris [Syn. Artemisia dentata; Artemisia viridis; Artemisia viridifolia], T0853 Baeckea frutescens, T0879 Bauhinia purpurea, T1558 Clerodendron inerme, T2296 Duranta repens, T3221 Heracleum rapula, T3254 Hippophae rhamnoides, T3256 Hippophae rhamnoides subsp. sinensis, T3258 Hippophae rhamnoides subsp. yunnanensis, T3373 Hypoestes purpurea [Syn. Justicia purpurea; Hypoestes sinica], T3520 Isodon rosthornii, T3560 Jatropha curcas, T5411 Randia spinosa, T5424 Rauvolfia verticillata, T5701 Salvia yunnanensis.

stasis swelling pain T3809 Ligularia lapathifolia.

static blood T3842 Limonium gmelinii.

static blood from knocks and falls T5151 Populus davidiana.

static blood pain T5868 Selaginella stauntoniana.

static blood pain due to injury T5144 Populus adenopoda.

static blood swelling and distention T4568 Oxytropis myriophylla.

static blood swelling pain T4951 Piper laetispicum, T5883 Senecio cannabifolius, T6874 Zanthoxylum dimorphophyllum var. spinifolum.

steaming bone T2994 Gloiopeltis furcata.

steaming bone fever and chills T1232 Cassia fistula.

steaming bone taxation cough T5826 Scrophularia buergeriana, T5828 Scrophularia ningpoensis.

steaming bone taxation fever T0705 Artemisia vestita, T2231 Diphylleia grayi, T2232 Diphylleia sinensis, T3279 Homo sapiens, T5445 Rehmannia glutinosa [Syn. Rehmannia glutinosa f. huechingensis], T5498 Rhodiola quadrifida, T5762 Saussurea nigrescens, T6104 Stellaria dichotoma var. lanceolata.

steaming bone tidal fever T0462 Anemarrhena asphodeloides, T0607 Arenaria juncea, T0687 Artemisia myriantha, T1950 Cynanchum ascyrifolium, T1951 Cynanchum atratum, T1962 Cynanchum versicolor, T2952 Gerbera piloselloides, T3090 Gypsophila oldhamiana, T3091 Gypsophila pacifica, T4056 Mahonia bealei, T4057 Mahonia bealei, T4063 Mahonia fortunei, T4067 Mahonia japonica, T4068 Mahonia japonica, T4681 Pedicularis decora, T5955 Silene jenisseensis, T6060 Souliea vaginata, T6139 Sterculia lychnophora, T6582 Tylophora ovata.

steaming bone tidal fever due to yin vacuity T4582 Paeonia delavayi, T4585 Paeonia moutan [Syn. Paeonia suffruticosa], T4887 Picrorhiza kurrooa, T4888 Picrorhiza scrophulariiflora, T5133 Polytrichum commune.

steaming bone vexation thirst $\mathrm{T} 0302$ Alhagi pseudalhagi.

sterility T6257 Syngnathus acus.

sterility and infertility T5446 Rehmannia glutinosa [Syn. Rehmannia glutinosa $\mathrm{f}$. huechingensis].

sticky phlegm T0480 Angelica decursiva [Syn. Peucedanum decursivum],

T3819 Ligusticum brachylobum, T4761 Peucedanum longshengens,

T4768 Peucedanum praeruptorum, T4769 Peucedanum rubricaule.

sticky phlegm and oppression in chest T2790 Fritillaria pallidiflora, T2800 Fritillaria walujewii.

stiffness in joints T1017 Brassica oleracea var. capitata.

stings T0388 Amaranthus lividus.

stirring fetus disquieted T0418 Amomum muricarpum, T6772 Viscum angulatum, T6777 Viscum multinerve.

stirring fetus in pregnancy T0417 Amomum longiligulare, T0419 Amomum villosum, T0420 Amomum xanthioides, T0824 Atractylodes macrocephala [Syn. Atractylis macrocephala], T1911 Cuscuta australis, T1912 Cuscuta chinensis, T1913 Cuscuta japonica, T1980 Cyprinus carpio, T1982 Cyprinus carpio, T2530 Eucommia ulmoides, T3431 Inula helenium, T3437 Inula racemosa, T4399 Nelumbo nucifera, T5833 Scutellaria amoena, T5834 Scutellaria baicalensis, T5839 Scutellaria hypericifolia, T5841 Scutellaria likiangensis, T5843 Scutellaria rehderiana, T5845 Scutellaria viscidula, T6775 Viscum coloratum.

stirring fetus with bleeding T0542 Apis cerana.

stomach and esophagus hemorrhage T4978 Pisolithus tinctorius [Syn. Lycoperdon capitatum; Scleroderma tinctorium].

stomach cold T0356 Alpinia galanga, T6864 Zanthoxylum armatum, T6887 Zanthoxylum planispinum.

stomach cold abdominal pain T0353 Alpinia blepharocalyx, T1443 Cinnamomum parthenoxylum [Syn. Cinnamomum porrectum], T5504 Rhododendron capitatum.

stomach cold and hiccough T6267 Syzygium jambos.

stomach cold and $\boldsymbol{q} \boldsymbol{i}$ stagnation T1186 Capsicum annuum, T1187 Capsicum frutescens.

stomach cold distending pain T1435 Cinnamomum camphora, T3880 Lithospermum arvense, T4763 Peucedanum morisonii.

stomach cold pain T2895 Gaultheria yunnanensis, T3120 Hedychium spicatum, T4964 Piper retrofractum.

stomach cold with retching counterflow T0554 Aquilaria agallocha, T0555 Aquilaria sinensis, T2745 Foeniculum vulgare, T4944 Piper cubeba, T6263 Syzygium aromaticum [Syn. Eugenia caryophyllata].

stomach disease T3394 Ilex paraguariensis.

stomach disease and acid vomiting T6412 Thalictrum squarrosum.

stomach duck pain T1100 Caesalpinia crista, T2841 Gallus gallus domesticus.

stomach duct pain and abdominal distention T2555 Eupatorium chinense.

stomach fire toothache T0446 Anaphalis margaritacea, T0959 Blumea lacera.

stomach heat blood ejection T3863 Liparis nervosa.

stomach heat fluid damage T1490 Citrus limon, T1494 Citrus limonia, T2867 Garcinia multiflora.

stomach heat pain T0916 Berberis thunbergii.

stomach heat vexation thirst T6526 Triglochin maritimum.

stomach heat vomiting T0423 Ampelopsis brevipedunculata, T1662 Coptis chinensis, T1663 Coptis chinensis var. brevisepala, T1664 
Coptis deltoidea, T1669 Coptis omeiensis, T1670 Coptis teetoides [Syn. Coptis teeta], T2337 Elephas maximus, T2433 Eriobotrya japonica, T4829 Phragmites communis.

stomach hemorrhage T1866 Cryptolepis sinensis.

stomach qi pain T5122 Polygonum viscosum.

stomach reflux T0353 Alpinia blepharocalyx, T0559 Arachis hypogaea, T0976 Bombyx mori, T1182 Capra hircus; Ovis aries, T2837 Gallus gallus domesticus, T2841 Gallus gallus domesticus, T4544 Oryza sativa, T4547 Oryza sativa cv, T4636 Papaver somniferum, T5139 Poncirus trifoliata.

stomach reflux and dysphagia-occlusion T0989 Bos taurus domesticus; Bubalus bubalis.

stomach reflux vomiting T1254 Castanea mollissima, T3885 Litsea cubeba, T5363 Pyrus calleryana, T5938 Setaria italica.

stomach yin insufficiency T2983 Glehnia littoralis, T3873 Liriope platyphylla, T3874 Liriope spicata, T4602 Panax ginseng [Syn. Panax schinseng].

stomachache T0010 Abrus fruticulosus [Syn. Abrus cantoniensis], T0048 Acanthus ilicifolius, T0069 Achillea wilsoniana, T0099 Aconitum gymnandrum, T0130 Aconitum sinomontanum, T0374 Alstonia scholaris, T0383 Alyxia sinensis, T0471 Anemone rivularis, T0478 Angelica dahurica [Syn. Angelica porphyrocaulis], T0501 Anisodus luridus, T0529 Anthriscus sylvestris, T0625 Aristolochia contorta, T0627 Aristolochia debilis [Syn. Aristolochia longa], T0631 Aristolochia kaempferi, T0726 Asarum forbesii, T0730 Asarum sagittarioides, T0813 Atalantia buxifolia [Syn. Severinia buxifolia], T0814 Atalantia buxifolia [Syn. Severinia buxifolia], T1043 Bryophyllum pinnatum, T1205 Carica papaya, T1242 Cassia occidentalis, T1357 Chelidonium majus, T1437 Cinnamomum camphora, T1501 Citrus medica var. sarcodactylis, T1533 Clausena dentata, T1534 Clausena dunniana, T1555 Clerodendron fortunatum, T1590 Cocculus trilobus [Syn. Cocculus sarmentosus], T1592 Cocos nucifera, T1745 Corydalis suaveolens [Syn. Corydalis sheareri], T1931 Cyclea barbata, T1980 Cyprinus carpio, T2030 Daphne retusa, T2031 Daphne tangutica, T2062 Delphinium bonvalotii, T2081 Delphinium omeiense, T2084 Delphinium potaninii, T2085 Delphinium potaninii var. jiufengshanense, T2091 Delphinium yunnanense, T2154 Dicentra spectabilis, T2267 Drosera peltata var. lunata, T2301 Dysosma majorensis [Syn. Podophyllum majorense; Dysosma lichuanensis], T2327 Elaeagnus angustifolia, T2529 Euchresta strigillosa, T2612 Euphorbia prolifera, T2636 Euscaphis japonica, T2657 Fagopyrum cymosum [Syn. Polygonum cymosum], T2721 Ficus microcarpa, T2733 Firmiana simplex, T2735 Fissistigma oldhamii [Syn. Melodorum oldhamii], T2744 Foeniculum vulgare, T2749 Fomes officinalis, T3120 Hedychium spicatum, T3203 Hemsleya amabilis, T3208 Hemsleya macrosperma, T3221 Heracleum rapula, T3254 Hippophae rhamnoides, T3256 Hippophae rhamnoides subsp. sinensis, T3258 Hippophae rhamnoides subsp. yunnanensis, T3398 Ilex rotunda, T3418 Incarvillea arguta, T3457 Iris dichotoma, T3461 Iris japonica, T3468 Iris sanguinea, T3548 Ixeris sonchifolia, T3614 Kadsura heteroclita [Syn. Uvaria heteroclita], T3619 Kadsura peltigera [Syn. Kadsura longipedunculata], T3840 Limnophila rugosa, T3876 Litchi chinensis, T3897 Litsea verticillata, T3925 Loranthus parasiticus [Syn.
Loranthus chinenis; Taxillus chinensis], T4188 Mentha rotundifolia, T4190 Mentha spicata, T4223 Micromelum integerrimum, T4343 Myrica esculent, T4386 Nardostachys chinensis, T4387 Nardostachys jatamansi, T4503 Onychium lucidum, T4506 Ophioglossum vulgatum, T4519 Opuntia dillenii, T4521 Opuntia vulgaris, T4527 Orixa japonica, T4605 Panax japonicus var. major, T4618 Panicum miliaceum, T4629 Papaver nudicaule, T4630 Papaver nudicaule ssp. amurense, T4631 Papaver nudicaule var. chinense, T4823 Pholidota yunnanensis, T4938 Piper boehmeriaefolium, T4948 Piper hancei, T5247 Przewalskia tangutica, T5250 Psammosilene tunicoides, T5414 Ranunculus japonicus, T5645 Sageretia theezans [Syn. Sageretia thea], T5720 Sapindus mukorossi, T5795 Schisandra micrantha, T5819 Scopolia acutangula [Syn. Anisodus acutangulus], T5939 Shiraia bambusicola, T5989 Solanum aculeatissimum, T6003 Solanum khasianum, T6014 Solanum surattense, T6015 Solanum torvum, T6017 Solanum tuberosum, T6029 Sophora alopecuroides, T6049 Sophora viciifolia, T6055 Sorbus tianschanica, T6118 Stephania brachyandra, T6121 Stephania dicentrinifera, T6122 Stephania dielsiana, T6128 Stephania hernandifolia, T6129 Stephania japonica, T6133 Stephania sinica, T6135 Stephania succifera, T6137 Stephania viridiflavens, T6177 Strychnos ignatii, T6339 Tephrosia purpurea, T6471 Toddalia asiatica [Syn. Toddalia aculeata; Paullinia asiatica], T6566 Tupistra chinensis, T6772 Viscum angulatum, T6787 Vitex negundo, T6884 Zanthoxylum nitidum.

stomachache and abdominal distention T0967 Boenninghausenia sessilicarpa.

stomachache blood ejection T6567 Tupistra wattii [Syn. Campylandra wattii].

stomachache due to roundworm T6864 Zanthoxylum armatum, T6887 Zanthoxylum planispinum.

stomachache with acid regurgitation T6789 Vitex negundo.

stomachache with distention and oppression T0354 Alpinia chinensis.

stomatitis T1655 Conyza blinii, T2340 Elsholtzia bodinieri, T2852 Garcinia cowa, T2989 Glochidion sphaerogynum, T3348 Hypericum elodeoides, T3368 Hypericum wightianum, T5587 Rubus alceaefolius, T5730 Sarcandra glabra [Syn. Chloranthus glaber], T6060 Souliea vaginata, T6278 Tagetes erecta, T6580 Tylophora floribunda.

stone strangury T0045 Acanthopanax trifoliatus, T0155 Actinidia arguta, T0158 Actinidia chinensis, T0297 Alchornea trewioides, T0834 Averrhoa carambola, T1007 Brassica juncea, T1251 Cassytha filiformis, T2134 Desmodium styracifolium, T2143 Dianthus chinensis, T2145 Dianthus superbus, T2147 Dianthus versicolor, T2962 Ginkgo biloba, T2973 Glechoma longituba, T2974 Glechoma lungituba, T3568 Juglans regia, T3935 Luffa cylindrica, T3990 Lygodium japonicum, T4840 Phyllanthus niruri, T5231 Prunus persica, T5354 Pyrrosia davidii, T5355 Pyrrosia drakeana, T5356 Pyrrosia gralla, T5357 Pyrrosia lingua' T5358 Pyrrosia petiolosa, T5360 Pyrrosia sheareri, T6095 Stachytarpheta jamaicensis.

strangury T0071 Achyranthes aspera, T0072 Achyranthes aspera var. indica, T0610 Argemone mexicana, T1143 Calystegia hederacea, T1197 Cardiospermum halicacabum, T1487 Citrus junos, T1869 Cryptotaenia japonica, T1984 Cypripedium macranthum [Syn. Cypripedium tibeticum], T2143 Dianthus chinensis, T2145 Dianthus 
superbus, T2147 Dianthus versicolor, T2412 Equisetum sylvaticum, T2590 Euphorbia hirta, T2730 Fimbristylis dichotoma, T3200 Hemidesmus indicus, T3311 Hydrocotyle sibthorpioides, T3448 Ipomoea cairica [Syn. Ipomoea palmata], T3479 Isodon amethystoides, T3578 Juncus effusus, T3668 Lagenaria siceraria var. depressa, T3865 Lippia nodiflora, T4170 Melilotus albus, T4225 Microsorium punctatum, T4397 Nelumbo nucifera, T4482 Oenanthe javanica, T4542 Orthosiphon wulfenioides [Syn. Coleus wulfenioides], T4678 Paulownia fortunei, T4679 Paulownia tomentosa, T5124 Polypodium niponicum, T5171 Porphyra tenera, T5647 Sagittaria sagittifolia, T5651 Salix babylonica, T5863 Selaginella moellendorffii, T5870 Selaginella uncinata, T5943 Sida cordifolia, T6005 Solanum lyratum, T6752 Vigna angularis [Syn. Dolichus angularis; Phaseolus angularis], T6798 Vitis vinifera.

strangury syndrome T0001 Abelmoschus manihot, T0457 Andrographis paniculata [Syn. Justicia paniculata], T0895 Benincasa hispida, T1174 Cannabis sativa, T1402 Chrysosplenium alternifolium, T1561 Clerodendron serratum, T1569 Clerodendrum serratum var. amplexifolium, T1590 Cocculus trilobus [Syn. Cocculus sarmentosus], T3243 Hibiscus rosa-sinensis, T3769 Lespedeza bicolor, T4172 Melilotus suaveolens, T4564 Oxalis corniculata [Syn. Oxalis repens], T4855 Physeter catodon, T4979 Pistacia chinensis, T5288 Pteris cretica var. nervosa [Syn. Pteris nervosa], T5862 Selaginella involvens, T6046 Sophora viciifolia, T6047 Sophora viciifolia, T6356 Tetrapanax papyriferus, T6431 Thesium chinense, T6645 Urena lobata, T6673 Vaccinium vitis-idaea, T6912 Zinnia elegans.

strangury with pain T0266 Ajuga macrosperma, T2194 Dioscorea collettii, T2197 Dioscorea futschauensis, T2210 Dioscorea spongiosa, T3932 Ludwigia octovalvis, T6357 Tetrapanax papyriferus.

strangury with white turbidity T2208 Dioscorea septemloba.

strangury-turbidity T0171 Adiantum capillus-veneris, T0273 Akebia quinata, T0277 Akebia trifoliata, T0279 Akebia trifoliata var. australis, T0379 Alternanthera philoxeroides, T0462 Anemarrhena asphodeloides, T0567 Aralia armata, T0571 Aralia decaisneaa, T0608 Arenaria kansuensis [Syn. Arenaria kumaonensis], T0708 Arthromeris mairei [Syn. Polypodium mairei], T0924 Bergenia purpurascens, T1106 Caesalpinia minax, T1884 Cudrania cochinchinensis, T2649 Evolvulus alsinoides, T2830 Galium aparine, T3193 Hemerocallis fulva, T3195 Hemerocallis lilio-asphodelus, T3197 Hemerocallis minor, T3270 Holboellia fargesii, T3444 Ipomoea aquatica [Syn. Convolvulus repens; Ipomoea reptans], T3460 Iris halophila, T3464 Iris lactea var. chinensis [Syn. Iris pallasii var. chinensis], T4057 Mahonia bealei, T4068 Mahonia japonica, T4562 Oxalis acetosella, T4616 Pandanus tectorius, T5129 Polyporus umbellatus, T5161 Populus tomentosa, T5295 Pteris multifida, T5419 Raphanus sativus, T5605 Rumex acetosa, T5650 Salix babylonica, T5750 Saururus chinensis, T5976 Smilax china [Syn. Smilax japonica], T6264 Syzygium buxifolium, T6601 Umbilicaria esculenta [Syn. Gyrophora esculenta].

strangury-turbidity and vaginal discharge T0161 Actinidia eriantha, T1552 Cleome viscosa, T5002 Plantago asiatica, T5004 Plantago depressa, T5007 Plantago major, T6087 Spiranthes sinensis.

stream toxin $\mathrm{T} 1595$ Codium fragile. stubborn lichen with sore toxin T5157 Populus pseudo-simonii. stupor due to phlegm reversal T0142 Acorus calamus.

sty T3564 Juglans mandshurica.

subcutaneous static blood T0964 Boenninghausenia albiflora.

sudden clouding collapse T3868 Liquidambar orientalis.

sudden pain in heart and abdomen T4303 Moschus moschiferus;

Moschus berezovskii; Moschus sifanicus, T6800 Viverra zibetha.

sudden stroke and fulminant reversal T6197 Styrax benzoin, T6204

Styrax tonkinensis.

summer common cold T0212 Agastache rugosus, T0678 Artemisia japonica, T0687 Artemisia myriantha, T2341 Elsholtzia ciliata.

summer externally contracted wind-cold T4304 Mosla chinensis [Syn. Orthodon chinensis].

summer unacclimation in child T4121 Marsilea quadrifolia.

summerhea-damp and damage center T1278 Cedrela sinensis.

summerheat damage T1941 Cymbopogon distans, T2554 Eupatorium cannabinum, T4101 Mangifera indica.

summerheat stroke T1573 Clinopodium chinense, T2341 Elsholtzia ciliata, T4209 Michelia alba, T4261 Mollugo pentaphylla, T4307 Mosla scabra [Syn. Mosla punctata], T4335 Mussaenda pubescens, T4470 Ocimum basilicum, T4526 Origanum vulgare, T5109 Polygonum nodosum, T5400 Rabdosia stracheyi, T6285 Tamarindus indica, T6416 Thamnolia vermicularis, T6431 Thesium chinense, T6598 Ulva conglobata, T6788 Vitex negundo, T6889 Zanthoxylum podocarpum.

summerheat stroke and anidrosis T6367 Teucrium quadrifarium.

summerheat stroke with abdominal pain T5393 Rabdosia longituba. summerheat stroke with fever T0687 Artemisia myriantha, T1784 Cratoxylum cochinchinense, T1785 Cratoxylum prunifolium.

summerheat stroke with sand T1137 Caltha palustris.

summerheat stroke with vexation and thirst T1490 Citrus limon, T1494 Citrus limonia, T4573 Pachyrrhizus erosus.

summerheat stroke with vomiting and diarrhea $\mathrm{T} 1052$ Bufo bufo gargarizans; Bufo melanostictus, T3885 Litsea cubeba.

summerheat-damp T0660 Artemisia annua, T0662 Artemisia apiacea [Syn. Artemisia carvifolia; Artemisia caruifolia].

summerheat-damp and oppression in chest T4172 Melilotus suaveolens, T4397 Nelumbo nucifera.

summerheat-damp damage center T6499 Tribulus terrestris.

summerheat-damp diarrhea T0631 Aristolochia kaempferi, T3398 Ilex rotunda, T4335 Mussaenda pubescens, T4398 Nelumbo nucifera, T4399 Nelumbo nucifera, T5002 Plantago asiatica, T5004 Plantago depressa, T5007 Plantago major.

summerheat-damp exterior syndrome T2558 Eupatorium formosanum, T2559 Eupatorium fortunei.

summerheat-damp lassitude T5059 Pogostemon cablin [Syn. Mentha cablin].

summerheat-damp vomiting and diarrhea T2246 Dolichos lablab, T3645 Kummerowia striata, T3886 Litsea euosma.

summerheat-heat T0660 Artemisia annua, T0662 Artemisia apiacea [Syn. Artemisia carvifolia; Artemisia caruifolia].

summerheat-heat and cholera T5227 Prunus mume. summerheat-heat and oppression in chest T4170 Melilotus albus. 
summerheat-heat and thirst T1179 Capparis masaikai, T2989 Glochidion sphaerogynum, T4602 Panax ginseng [Syn. Panax schinseng].

summerheat-heat and vexation and thirst T1544 Cleistocalyx operculatus, T3829 Ligustrum robustum.

summerheat-heat and vomiting diarrhea T1238 Cassia mimosoides.

summerheat-heat diarrhea T1311 Centella asiatica, T5818 Scoparia dulcis.

summerheat-heat thirst T4979 Pistacia chinensis.

summerheat-heat vexation and thirst T1366 Chimonanthus fragrans [Syn. Chimonanthus praecox], T1464 Citrullus vulgaris [Syn. Citrullus lanatus], T4263 Momordica charantia, T4398 Nelumbo nucifera, T4785 Phaseolus vulgaris, T6492 Trapa bispinosa, T6803 Volvariella volvacea.

suppurative hematochezia T4789 Phellodendron amurense, T4790 Phellodendron amurense var. wilsonii, T4791 Phellodendron chinense, T4792 Phellodendron chinense var. glabriusculum.

suppurative infection T0019 Acacia catechu, T0634 Aristolochia mollissima, T4882 Picrasma quassioides [Syn. Picrasma ailanthoides].

suppurative nest sore T6031 Sophora flavescens [Syn. Sophora angustfolia].

suppurative osteomyelitis T6217 Swertia davidii.

suppurative sore T5143 Pongamia pinnata.

suppurative tympanitis T4080 Mallotus apelta.

sweat macule T1878 Cucumis sativus.

sweet-greasy in mouth T2558 Eupatorium formosanum, T2559 Eupatorium fortunei.

swelling and pain of sore and welling abscess T2974 Glechoma lungituba, T4279 Morina chinensis.

swelling and pus of sores T1656 Conyza bonariensis [Syn. Erigeron bonariensis; Erigeron linifolius; Erigeron crispus], T4335 Mussaenda pubescens, T6820 Wikstroemia indica.

swelling and toxin of sore and welling abscess T4814 Phlomis umbrosa, T6219 Swertia erythrosticta, T6374 Thalictrum atriplex.

swelling hemorrhoids T1007 Brassica juncea, T3624 Kalopanax pictum, T3625 Kalopanax septemlobus, T6125 Stephania elegans.

swelling in ear T2908 Gentiana cephalantha, T2921 Gentiana manshurica, T2928 Gentiana rigescens, T2930 Gentiana scabra, T2938 Gentiana triflora.

swelling in joints T5964 Sinomenium acutum.

swelling of clove T1660 Coprinus atramentarius, T2627 Euphoria longan [Syn. Dimocarpus longan], T3203 Hemsleya amabilis, T3208 Hemsleya macrosperma, T3876 Litchi chinensis, T4031 Maesa perlarius, T5579 Rubia cordifolia.

swelling of skin T3443 Iphigenia indica.

swelling of sore welling abscess and boil T0602 Ardisia quinquegona, T4203 Mesua ferrea.

swelling of sores T0476 Angelica anomala, T0866 Baphicacanthus cusia [Syn. Strobilanthes cusia], T0894 Benincasa hispida, T1162 Camptotheca acuminata, T2336 Elephas maximus, T2387 Epilobium hirsutum, T2533 Eugenia jambolana [Syn. Syzygium cumin; Myrtus cumini], T2536 Euonymus alatus, T2586 Euphorbia esula var. cyparissoides, T2631 Eurya japonica, T2955 Geum japonicum, T3460
Iris halophila, T3464 Iris lactea var. chinensis [Syn. Iris pallasii var. chinensis], T3507 Isodon lophanthoides [Syn. Rabdosia lophanthoides; Hyssopus lophanthoides; Plectranthus striatus; Isodon striatus], T4124 Matricaria chamomilla [Syn. Matricaria recutita], T4385 Narcissus tazetta var. chinensis, T4813 Phlomis tuberosa, T5109 Polygonum nodosum, T5110 Polygonum orientale, T5397 Rabdosia serra, T5661 Salvia bowleyana, T5672 Salvia flava, T5701 Salvia yunnanensis, T5781 Schefflera arboricola, T5934 Seseli meirei, T5937 Seseli yunnanense, T6062 Sparganium stoloniferum, T6155 Strophanthus divaricatus, T6560 Tulipa edulis, T6722 Veronica anagallis-aquatica.

swelling of sores and boils T0642 Aristolochia tubiflora, T1152 Camellia sinensis [Syn. Thea sinensis], T1290 Celastrus orbiculatus [Syn. Celastrus articulatus], T3126 Hedyotis corymbosa [Syn. Oldenlandia corymbosa], T3549 Ixora chinensis, T4485 Oldenlandia diffusa [Syn. Hedyotis diffusa], T6252 Symplocos chinensis.

swelling of sores and damp toxin T3201 Hemiphragma heterophyllum.

swelling of welling abscess T3624 Kalopanax pictum, T3625 Kalopanax septemlobus, T5642 Saccharum sinensis, T6764 Vinegar.

swelling of welling abscess and boil T0631 Aristolochia kaempferi.

swelling pain due to external injury T1108 Caesalpinia sappan, T1550 Cleome gynandra [Syn. Gynandropsis gynandra], T1853 Croton oblongifolius [Syn. Croton laevigatus], T4678 Paulownia fortunei, T4679 Paulownia tomentosa.

swelling pain due to stasis accumulation T2328 Elaeis guineensis.

swelling pain from fracture T0886 Beaumontia grandiflora, T1285 Celastrus angulatus, T1715 Corydalis decumbens [Syn. Corydalis amabilis], T3155 Helianthus tuberosus, T5704 Sambucus nigra, T5707 Sambucus williamsii.

swelling pain from hemorrhoids T0624 Aristolochia contorta, T0626 Aristolochia debilis [Syn. Aristolochia longa], T0630 Aristolochia indica, T0633 Aristolochia maxima, T0640 Aristolochia triangularis, T0852 Bacopa monniera, T0859 Balanophora involucrata, T4182 Menispermum dauricum, T4183 Menispermum dauricum, T5871 Selenarctos thibetanus; Ursus arctos.

swelling pain in joints T0130 Aconitum sinomontanum, T0659 Artemisia absinthium, T2546 Euonymus sacrosancta, T2624 Euphorbia tirucalli, T3679 Lamiophlomis rotata [Syn. Phlomis rotata], T4143 Meconopsis horridula, T4252 Mirabilis jalapa, T4286 Morinda umbellata, T4800 Phlegmariurus fordii, T5958 Sinapis alba [Syn. Brassica alba; Brassica hirta].

swelling pain in knees and feet T4789 Phellodendron amurense, T4790 Phellodendron amurense var. wilsonii, T4791 Phellodendron chinense, T4792 Phellodendron chinense var. glabriusculum.

swelling pain in perineum T2243 Dodonaea viscosa.

swelling pain in throat T0011 Abrus precatorius, T0073 Achyranthes bidentata, T0195 Aeginetia indica, T0197 Aegle marmelos, T0229 Ageratum conyzoides, T0262 Ajuga ciliata, T0263 Ajuga decumbens, T0264 Ajuga forrestii, T0378 Alstonia yunnanensis, T0426 Ampelopsis grossedentata [Syn. Ampelopsis cantoniesis var. grossedentata], T0457 Andrographis paniculata [Syn. Justicia paniculata], T0471 Anemone rivularis, T0567 Aralia armata, T0571 Aralia decaisneaa, T0584 Arctium lappa, T0586 Arctium lappa, T0594 Ardisia crenata, T0595 Ardisia crispa, T0602 Ardisia quinquegona, T0641 Aristolochia 
tuberosa, T0643 Aristolochia versicolar, T0736 Asclepias curassavica, T0746 Asparagus cochinchinensis [Syn. Asparagus lucidus], T0867 Baphicacanthus cusia [Syn. Strobilanthes cusia], T0892 Belamcanda chinensis, T0906 Berberis julianae, T0916 Berberis thunbergii, T0938 Bidens bipinnata, T0941 Bidens tripartita, T0959 Blumea lacera, T0975 Bombyx mori, T0977 Bombyx mori, T0987 Bos taurus domesticus; Bubalus bubalis, T1006 Brassica chinensis, T1043 Bryophyllum pinnatum, T1045 Bubalus bubalis, T1052 Bufo bufo gargarizans; Bufo melanostictus, T1161 Campsis grandiflora, T1165 Canarium album, T1361 Chenopodium ambrosioides, T1366 Chimonanthus fragrans [Syn. Chimonanthus praecox], T1384 Chondrus ocelladus, T1423 Cimicifuga nanchuanensis, T1425 Cimicifuga simplex, T1554 Clerodendron cyrtophyllum, T1561 Clerodendron serratum, T1569 Clerodendrum serratum var. amplexifolium, T1589 Cocculus trilobus [Syn. Cocculus sarmentosus], T1633 Commelina communis, T1672 Corallodiscus flabellatus [Syn. Didissandra flabellat], T1697 Cornus controversa [Syn. Bothrocaryum controversum], T1810 Croomia japonica, T1933 Cyclea sutchuenensis, T1950 Cynanchum ascyrifolium, T1951 Cynanchum atratum, T1962 Cynanchum versicolor, T2028 Daphne odora, T2131 Desmodium pulchellum [Syn. Phyllodium pulchellum], T2157 Dichotomanthes tristaniaecarpa, T2233 Diploclisia glaucescens, T2257 Dracocephalum moldavicum, T2258 Dracocephalum rupestre, T2267 Drosera peltata var. lunata, T2274 Dryobalanops aromatica, T2298 Dysosma difformis, T2302 Dysosma pleiantha [Syn. Podophyllum pleianthum], T2304 Dysosma veitchii, T2305 Dysosma versipellis [Syn. Podophyllum versipelle], T2442 Ervatamia divaricata, T2444 Ervatamia heyneana, T2445 Ervatamia officinalis, T2517 Eucalyptus robusta, T2529 Euchresta strigillosa, T2628 Euphrasia officinalis, T2641 Evodia lepta [Syn. Ilex lepta], T2657 Fagopyrum cymosum [Syn. Polygonum cymosum], T2691 Farfugium japonicum, T2717 Ficus carica, T2723 Ficus pumila, T2737 Flemingia philippinensis [Syn. Moghania philippinensis], T2749 Fomes officinalis, T2952 Gerbera piloselloides, T3080 Gymnema sylvestre, T3113 Hedera nepalensis var. sinensis, T3115 Hedera rhombea, T3122 Hedyotis acutangula, T3123 Hedyotis auricularia, T3126 Hedyotis corymbosa [Syn. Oldenlandia corymbosa], T3203 Hemsleya amabilis, T3208 Hemsleya macrosperma, T3308 Hydrangea umbellata, T3332 Hypecoum erectum, T3388 Ilex chinensis [Syn. Ilex purpurea], T3398 Ilex rotunda, T3457 Iris dichotoma, T3461 Iris japonica, T3471 Iris tectorum, T3479 Isodon amethystoides, T3528 Isodon ternifolia, T3529 Isodon ternifolius, T3643 Kopsia officinalis, T3659 Lactuca indica, T3736 Lemmaphyllum microphyllum, T3764 Lepisorus thunbergianus, T3779 Leucas aspera, T3852 Lindera megaphylla, T3928 Lotus corniculatus, T3932 Ludwigia octovalvis, T3984 Lycoris chinensis, T3986 Lycoris radiata [Syn. Amaryllis radiata], T3988 Lycoris squamigera, T3990 Lygodium japonicum, T3996 Lysimachia candida, T4000 Lysimachia congestiflora, T4002 Lysimachia foenum-graecum, T4091 Malva sylvestris, T4120 Marsdenia tenacissima, T4121 Marsilea quadrifolia, T4124 Matricaria chamomilla [Syn. Matricaria recutita], T4182 Menispermum dauricum, T4183 Menispermum dauricum, T4184 Mentha haplocalyx [Syn. Mentha canadaensis; Mentha arvensis var. haplocalyx; Mentha arvensis], T4210 Michelia champaca, T4260 Moghania philippinensis, T4335 Mussaenda pubescens, T4485 Oldenlandia diffusa [Syn. Hedyotis diffusa], T4538 Oroxylum indicum, T4564 Oxalis corniculata [Syn. Oxalis repens], T4568 Oxytropis myriophylla, T4605 Panax japonicus var. major, T4710 Pericampylus glaucus, T4740 Petasites japonicus, T4798 Philonotis fontana, T4845 Physalis alkekengi, T4848 Physalis angulata, T4854 Physalis pubescens, T4859 Physochlaina physaloides, T4884 Picria felterrae, T4979 Pistacia chinensis, T5011 Platycodon grandiflorum, T5072 Polygala caudata, T5100 Polygonum chinense, T5120 Polygonum tinctorium, T5171 Porphyra tenera, T5172 Portulaca grandiflora, T5184 Potentilla kleiniana, T5209 Pronephrium simplex [Syn. Meniscium simplex], T5339 Pycnoporus sanguineus, T5396 Rabdosia rubescens, T5423 Rauvolfia verticillata, T5424 Rauvolfia verticillata, T5425 Rauvolfia verticillata f. rubrocarpa, T5426 Rauvolfia verticillata var. hainanensis, T5427 Rauvolfia vomitoria, T5435 Rauwolfia latifrons, T5441 Rauwolfia perakensis, T5447 Rehmannia glutinosa [Syn. Rehmannia glutinosa $\mathrm{f}$. huechingensis], T5449 Reineckea carnea, T5468 Rheum emodi [Syn. Rheum australe], T5557 Rohdea japonica [Syn. Orontium japonicum], T5560 Rorippa montana [Syn. Rorippa dubia; Sisymbrium dublium], T5576 Rostellularia procumbens [Syn. Justicia procumbens], T5592 Rubus hirsutus, T5606 Rumex acetosa, T5685 Salvia plebeia, T5687 Salvia prionitis, T5804 Schizonepeta tenuifolia [Syn. Nepeta tenuifolia], T5826 Scrophularia buergeriana, T5828 Scrophularia ningpoensis, T5836 Scutellaria discolor, T5856 Sedum lineare [Syn. Sedum obtuso-lineare], T5857 Sedum sarmentosum, T5861 Selaginella doederleinii, T5953 Silene firma, T5954 Silene fortunei, T6023 Solidago virgaurea, T6024 Solidago virgaurea var. leiocarpa [Syn. Solidago decurrens], T6043 Sophora subprostrata [Syn. Sophora tonkinensis], T6046 Sophora viciifolia, T6047 Sophora viciifolia, T6084 Spiraea prunifolia, T6086 Spiraea thunbergii, T6087 Spiranthes sinensis, T6092 Stachys palustris, T6116 Stenoloma chusanum, T6119 Stephania cepharantha, T6128 Stephania hernandifolia, T6129 Stephania japonica, T6183 Strychnos nitida, T6217 Swertia davidii, T6234 Swertia punicea, T6275 Tacca chantrieri [Syn. Tacca minor; Tacca esquirolii], T6277 Tadehagi triquetrum, T6463 Tinospora capillipes, T6467 Tinospora sagittata, T6491 Trametes cinnabarina [Syn. Polyporus cinnabarinus; Boletus cinnabarinus], T6552 Trollius chinensis [Syn. Trollius asiaticus var. chinensis], T6553 Trollius ledebourii, T6560 Tulipa edulis, T6566 Tupistra chinensis, T6582 Tylophora ovata, T6655 Ustilaginoidea virens, T6866 Zanthoxylum avicennae.

swelling pain of scrotum T5548 Ricinus communis.

swelling pain of sore and boil T1657 Conyza canadensis [Syn. Erigeron canadensis], T1727 Corydalis mucronifera, T3248 Hibiscus tiliaceus, T3659 Lactuca indica, T4956 Piper mullesua, T6488 Trachyspermum ammi.

swelling pain of sores T1359 Chenopodium album, T4526 Origanum vulgare, T5106 Polygonum lapathifolium, T6231 Swertia pseudochinensis.

swelling pain of welling abscess and flat abscess T6184 Strychnos nux-vomica, T6193 Strychnos wallichiana.

swelling pain of welling abscess and sore T0314 Allium fistulosum, 
T0324 Allium victorialis, T0334 Aloe arborescens var. natalensis, T0470 Anemone raddeana, T2752 Fordia cauliflora, T2973 Glechoma longituba, T4109 Marchantia polymorpha, T4605 Panax japonicus var. major, T5712 Sanguisorba officinalis, T5813 Scilla scilloides, T5871 Selenarctos thibetanus; Ursus arctos.

swelling toxin malign sore T3555 Jasminum nudiflorum.

swelling toxin of clove sore T0388 Amaranthus lividus, T0938 Bidens bipinnata, T1210 Carpesium abrotanoides, T1395 Chrysanthemum morifolium [Syn. Dendranthema morifolium], T1547 Clematis terniflora [Syn. Clematis maximowicziana], T1573 Clinopodium chinense, T1819 Crotalaria ferruginea, T2538 Euonymus bungeanus, T3243 Hibiscus rosa-sinensis, T3932 Ludwigia octovalvis, T5267 Psidium guajava, T5295 Pteris multifida, T6301 Taraxacum mongolicum, T6552 Trollius chinensis [Syn. Trollius asiaticus var. chinensis], T6553 Trollius ledebourii, T6814 Wedelia chinensis [Syn. Solidago chinensis; Wedelia calendulacea].

swelling toxin of clove welling abscess T1311 Centella asiatica.

swelling toxin of flat abscess T0077 Aconitum balfourii, T0084 Aconitum carmichaeli, T0105 Aconitum karakolicum, T0108 Aconitum kusnezoffii, T5639 Sabina chinensis, T5958 Sinapis alba [Syn. Brassica alba; Brassica hirta].

swelling toxin of incised wound T5793 Schisandra henryi.

swelling toxin of limbs T2650 Excoecaria agallocha.

swelling toxin of sore and boil T0267 Ajuga nipponensis, T1190 Caragana jubata, T2033 Daphniphyllum macropodum, T2941 Gentianopsis paludosa, T3187 Helleborus thibetanus, T3308 Hydrangea umbellata, T3349 Hypericum erectum, T3479 Isodon amethystoides, T3547 Ixeris chinensis, T4261 Mollugo pentaphylla, T4831 Phtheirospermum japonicum [Syn. Geradia japonica], T5444 Reboulia hemisphaerica, T6025 Sonchus arvensis, T6075 Spilanthes acmella.

swelling toxin of sore and lichen T6069 Speranskia tuberculata.

swelling toxin of sore and scab T3649 Laccifer lacca.

swelling toxin of sore and welling abscess T0015 Abutilon indicum, T0427 Ampelopsis japonica [Syn. Paullinia japonica], T0721 Arundina chinensis, T1950 Cynanchum ascyrifolium, T1951 Cynanchum atratum, T1952 Cynanchum auriculatum, T1953 Cynanchum bungei, T1962 Cynanchum versicolor, T2732 Firmiana simplex, T2826 Galeola faberi, T2839 Gallus gallus domesticus, T2952 Gerbera piloselloides, T2998 Glycine max, T3475 Isatis indigotica, T3670 Lagerstroemia indica, T3913 Lonicera japonica, T3988 Lycoris squamigera, T3989 Lygodium flexuosum [Syn. Lygodium pinnatifidum; Ophioglossum flexuosum], T4263 Momordica charantia, T4384 Narcissus tazetta var. chinensis, T4859 Physochlaina physaloides, T5010 Platycarya strobilacea, T5176 Potamogeton natans, T5181 Potentilla chinensis, T5409 Rana temporaria chensinensis; Rana amurensis, T5569 Rosa multiflora, T5654 Salix purpurea, T5821 Scopolia japonica, T6060 Souliea vaginata, T6131 Stephania longa, T6582 Tylophora ovata.

swelling toxin of welling abscess and boil T0318 Allium sativum, T2206 Dioscorea parviflora, T2691 Farfugium japonicum, T2950 Gerbera anandria [Syn. Leibnitzia anandria], T3123 Hedyotis auricularia, T3356 Hypericum japonicum, T3361 Hypericum perforatum, T5415
Ranunculus sceleratus, T5907 Senecio scandens [Syn. Senecio chinensis], T6118 Stephania brachyandra.

swelling toxin of welling abscess and flat abscess T0310 Allium ascalonicum, T0471 Anemone rivularis, T0502 Anisodus tanguticus [Syn. Scopolia tangutica], T0827 Aucuba chinensis ssp. omeiensis, T0994 Boswellia carterii, T1118 Callicarpa formosana, T1119 Callicarpa japonica, T1278 Cedrela sinensis, T1289 Celastrus orbiculatus [Syn. Celastrus articulatus], T1451 Cirsium setosum [Syn. Cerratula setosa; Cirsium segetum; Cephalanoplos segetum], T1554 Clerodendron cyrtophyllum, T1561 Clerodendron serratum, T1569 Clerodendrum serratum var. amplexifolium, T1932 Cyclea racemosa, T2200 Dioscorea hispida, T2857 Garcinia hanburyi, T2866 Garcinia morella, T2979 Gleditsia sinensis [Syn. Gleditsia horrida], T3628 Kerria japonica, T3865 Lippia nodiflora, T4514 Ophiorrhiza pumila, T4904 Pinellia ternata, T5517 Rhododendron molle, T5550 Ricinus communis, T5607 Rumex crispus, T6133 Stephania sinica, T6166 Strychnos angustiflora.

swelling toxin of welling abscess and sore T0060 Achillea alpina [Syn. Achillea sibirica], T0065 Achillea millefolium, T0069 Achillea wilsoniana, T0106 Aconitum kirinense, T0135 Aconitum sungpanense, T0154 Acroptilon repens, T0157 Actinidia callosa var. henryi, T0180 Adina pilulifera [Syn. Cephalanthus pilulifera], T0190 Adonis sutchuenensis, T0229 Ageratum conyzoides, T0249 Agrimonia pilosa var. japonica, T0322 Allium tuberosum, T0371 Alstonia mairei, T0378 Alstonia yunnanensis, T0503 Anisomeles indica [Syn. Epimeredi indica], T0535 Antidesma bunius, T0601 Ardisia pusilla, T0661 Artemisia anomala, T0736 Asclepias curassavica, T0931 Betula ermanii, T0950 Blatta orientalis, T0955 Bletilla striata, T0959 Blumea lacera, T0977 Bombyx mori, T1001 Brachystemma calycinum, T1044 Bryum argenteum, T1084 Buthus martensi, T1172 Canna edulis, T1241 Cassia occidentalis, T1271 Catharanthus roseus [Syn. Vinca rosea; Lochera rosea], T1419 Cimicifuga dahurica, T1420 Cimicifuga foetida, T1421 Cimicifuga heracleifolia, T1449 Cirsium japonicum, T1644 Conocephalum conicum, T1745 Corydalis suaveolens [Syn. Corydalis sheareri], T1813 Crotalaria albida, T2191 Dioscorea bulbifera, T2435 Eriocheir sinensis, T2510 Eucalyptus globulus, T2601 Euphorbia milii, T2723 Ficus pumila, T3000 Glycine max, T3011 Glycyrrhiza aspera, T3013 Glycyrrhiza glabra, T3015 Glycyrrhiza inflata, T3016 Glycyrrhiza kansuensis, T3021 Glycyrrhiza squamulosa, T3022 Glycyrrhiza uralensis, T3023 Glycyrrhiza yunnanensis, T3242 Hibiscus rosa-sinensis, T3311 Hydrocotyle sibthorpioides, T3329 Hyoscyamus niger, T3567 Juglans regia, T3632 Knoxia valerianoides, T3673 Lagerstroemia speciosa [Syn. Munchausia speciosa; Lagerstroemia flos-reginae], T3736 Lemmaphyllum microphyllum, T3770 Lespedeza cuneata, T3830 Ligustrum sinense, T3983 Lycoris aurea, T3996 Lysimachia candida, T4178 Melodinus hemsleyanus, T4208 Metasequoia glyptostroboides, T4252 Mirabilis jalapa, T4520 Opuntia ficus-indica, T4552 Osmunda japonica, T4568 Oxytropis myriophylla, T4582 Paeonia delavayi, T4585 Paeonia moutan [Syn. Paeonia suffruticosa], T4648 Paris polyphylla, T4649 Paris polyphylla var. chinensis, T4650 Paris polyphylla var. pseudothibetica, T4651 Paris polyphylla var. stenophylla, T4652 Paris polyphylla var. yunnanensis, T4705 Peperomia pellucida, T4821 Pholidota articulata, 
T4823 Pholidota yunnanensis, T4861 Phytolacca americana [Syn. Phytolacca decandra], T4864 Phytolacca esculenta [Syn. Phytolacca acinosa], T5002 Plantago asiatica, T5004 Plantago depressa, T5007 Plantago major, T5028 Plumbago indica, T5061 Polianthes tuberosa, T5087 Polygala telephioides, T5101 Polygonum cuspidatum, T5182 Potentilla discolor, T5391 Rabdosia eriocalyx, T5468 Rheum emodi [Syn. Rheum australe], T5611 Rumex japonicus, T5612 Rumex nepalensis, T5613 Rumex obtusifolius, T5614 Rumex patientia, T5680 Salvia miltiorrhiza, T5681 Salvia miltiorrhiza f. alba, T5685 Salvia plebeia, T5688 Salvia przewalskii, T5797 Schisandra propinqua, T5798 Schisandra propinqua var. intermedia, T5833 Scutellaria amoena, T5834 Scutellaria baicalensis, T5839 Scutellaria hypericifolia, T5841 Scutellaria likiangensis, T5843 Scutellaria rehderiana, T5845 Scutellaria viscidula, T5943 Sida cordifolia, T5950 Siegesbeckia orientalis, T5951 Siegesbeckia orientalis var. glabrescens [Syn. Siegesbeckia glabrescens], T5952 Siegesbeckia orientalis var. pubescens [Syn. Siegesbeckia pubescens], T5976 Smilax china [Syn. Smilax japonica], T5978 Smilax glauco-china, T6116 Stenoloma chusanum, T6120 Stephania delavayi [Syn. Stephania epigaea], T6183 Strychnos nitida, T6278 Tagetes erecta, T6414 Thalictrum thunbergii, T6436 Thlaspi arvense, T6510 Trichosanthes kirilowii, T6511 Trichosanthes kirilowii, T6513 Trichosanthes rosthornii [Syn. Trichosanthes uniflora], T6691 Veratrilla baillonii.

swelling toxin sores T1371 Chloranthus japonicus, T6752 Vigna angularis [Syn. Dolichus angularis; Phaseolus angularis].

swelling welling abscess and sore and boil T1784 Cratoxylum cochinchinense, T1971 Cynoglossum zeylanicum [Syn. Anchusa zeylanica; Cynoglossum furcatum; Cynoglossum formosanum], T3548 Ixeris sonchifolia, T4730 Peristrophe roxburghiana, T4798 Philonotis fontana, T6403 Thalictrum petaloideum.

swift digestion with rapid hungering T1662 Coptis chinensis, T1663 Coptis chinensis var. brevisepala, T1664 Coptis deltoidea, T1669 Coptis omeiensis, T1670 Coptis teetoides [Syn. Coptis teeta].

swollen and painful eyes T2274 Dryobalanops aromatica.

swollen boil T0933 Betula platyphylla, T0935 Betula platyphylla var. japonica, T1101 Caesalpinia decapetala, T1417 Cimicifuga acerina, T1555 Clerodendron fortunatum, T2290 Duchesnea indica, T2325 Eichhornia crassipes, T2830 Galium aparine, T4884 Picria felterrae, T5120 Polygonum tinctorium, T5592 Rubus hirsutus, T5595 Rubus parviforlius, T6330 Tephroseris kirilowii [Syn. Senecio integrifolius var. fauriei], T6431 Thesium chinense, T6912 Zinnia elegans.

swollen boil and malign sore $\mathrm{T} 3737$ Lemmaphyllum microphyllum var. obovatum.

swollen boil with pus and ulcer T4391 Nauclea officinalis.

swollen hard breast T2028 Daphne odora.

swollen liver and spleen due to bilharziosis T1162 Camptotheca acuminata, 55189 Pothos chinensis.

swollen pain due to bleeding T1215 Carthamus tinctorius.

swollen pain in red eyes due to liver heat T5871 Selenarctos thibetanus; Ursus arctos.

swollen pain of sore toxin $\mathrm{T} 1728$ Corydalis ochotensis.

swollen sore of welling abscess and boil T0264 Ajuga forrestii, T0426 Ampelopsis grossedentata [Syn. Ampelopsis cantoniesis var. grossedentata], T2262 Dregea sinensis, T4219 Microlepia marginata. swollen throat T1311 Centella asiatica, T5472 Rheum officinale, T5474 Rheum palmatum, T5481 Rheum tanguticum.

swollen welling abscess T0175 Adiantum pedatum, T0379 Alternanthera philoxeroides, T0468 Anemone hupehensis, T0516 Anser cygnoides domestica, T0544 Apium graveolens, T0617 Arisaema amurense, T0618 Arisaema consanguineum, T0620 Arisaema heterophyllum, T0641 Aristolochia tuberosa, T0952 Blechnum orientale, T0976 Bombyx mori, T0984 Bos taurus domesticus; Bubalus bubalis, T1005 Brassica campestris [Syn. Brassica campestris var. oleifera], T1046 Buddleja davidii, T1106 Caesalpinia minax, T1275 Cayratia japonica, T1387 Chrysanthemum boreale, T1392 Chrysanthemum indicum, T1394 Chrysanthemum lavandulifolium, T1756 Costus speciosus, T1866 Cryptolepis sinensis, T1880 Cucurbita moschata, T1903 Curcuma aromatica, T1905 Curcuma longa, T2203 Dioscorea nipponica, T2204 Dioscorea nipponica ssp. rosthornii, T2231 Diphylleia grayi, T2232 Diphylleia sinensis, T2296 Duranta repens, T2298 Dysosma difformis, T2302 Dysosma pleiantha [Syn. Podophyllum pleianthum], T2304 Dysosma veitchii, T2305 Dysosma versipellis [Syn. Podophyllum versipelle], T2357 Emilia sonchifolia, T2365 Enteromorpha clathrata, T2517 Eucalyptus robusta, T2563 Eupatorium japonicum, T2591 Euphorbia humifusa, T2608 Euphorbia pekinensis, T2628 Euphrasia officinalis, T2659 Fagopyrum esculentum, T2717 Ficus carica, T2746 Foeniculum vulgare, T2787 Fritillaria hupehensis, T2790 Fritillaria pallidiflora, T2800 Fritillaria walujewii, T2946 Geranium robertianum, T2978 Gleditsia sinensis [Syn. Gleditsia horrida], T3163 Helicteres angustifolia, T3174 Heliotropium indicum, T3241 Hibiscus mutabilis, T3244 Hibiscus syriacus, T3410 Impatiens balsamina, T3418 Incarvillea arguta, T3423 Indigofera tinctoria, T3444 Ipomoea aquatica [Syn. Convolvulus repens; Ipomoea reptans], T3476 Isatis indigotica, T3668 Lagenaria siceraria var. depressa, T3687 Lantana camara, T3778 Leucaena glauca [Syn. Leucaena leucocephala], T3887 Litsea glutinosa, T3998 Lysimachia christinae, T4050 Magnolia sieboldii, T4106 Manis pentadactyla, T4265 Momordica cochinchinensis, T4572 Pachyrhizus erosus, T4607 Panax pseudo-ginseng var. japonicus, T4672 Patrinia scabiosaefolia, T4676 Patrinia villosa, T4903 Pinellia pedatisecta, T4983 Pisum sativum, T5073 Polygala chinensis [Syn. Polygala glomerata], T5099 Polygonum bistorta, T5100 Polygonum chinense, T5104 Polygonum hydropiper, T5121 Polygonum tinctorium, T5126 Polypodium virginianum, T5127 Polypodium vulgare, T5194 Premna microphylla, T5199 Primula malacoides, T5230 Prunus persica, T5375 Quercus mongolica, T5402 Radermachera sinica, T5413 Ranunculus cantoniensis, T5414 Ranunculus japonicus, T5447 Rehmannia glutinosa [Syn. Rehmannia glutinosa f. huechingensis], T5449 Reineckea carnea, T5562 Rosa chinensis, T5750 Saururus chinensis, T5804 Schizonepeta tenuifolia [Syn. Nepeta tenuifolia], T5850 Sedum aizoon, T5855 Sedum kamtschaticum, T5857 Sedum sarmentosum, T5977 Smilax glabra, T5980 Smilax menispermoidea, T5998 Solanum dulcamara, T6006 Solanum melongena, T6008 Solanum nigrum, T6015 Solanum torvum, T6017 Solanum tuberosum, T6024 Solidago virgaurea var. leiocarpa [Syn. Solidago decurrens], T6121 Stephania dicentrinifera, $\mathrm{T} 6129$ Stephania japonica, T6137 Stephania 
viridiflavens, T6154 Strobilanthes japonicus [Syn. Championella japonica], T6264 Syzygium buxifolium, T6276 Tacca plantaginea [Syn. Schizocapsa plantaginea], T6386 Thalictrum foetidum, T6484 Trachelospermum jasminoides, T6499 Tribulus terrestris, T6507 Trichosanthes cucumeroides, T6544 Triticum aestivum [Syn. Triticum vulgare], T6580 Tylophora floribunda, T6588 Typhoniun giganteum, T6653 Usnea diffracta, T6654 Usnea longissima, T6799 Vitis vinifera.

swollen welling abscess and clove sores T0071 Achyranthes aspera, T0072 Achyranthes aspera var. indica, T0263 Ajuga decumbens, T0625 Aristolochia contorta, T0627 Aristolochia debilis [Syn. Aristolochia longa], T1043 Bryophyllum pinnatum, T1393 Chrysanthemum indicum, T2244 Doellingeria scaber [Syn. Aster scaber], T2753 Formica fusca, T3202 Hemistepta lyrata [Syn. Hemistepta carthamoides; Saussurea carthamoides], T3859 Linum usitatissimum, T3898 Lobelia chinensis [Syn. Lobelia radicans], T3908 Lonicera bournei, T3909 Lonicera confusa, T3910 Lonicera fulvotomentosa, T3911 Lonicera hypoglauca, T3912 Lonicera japonica, T3914 Lonicera macranthoides, T3918 Lonicera similis, T4020 Macleaya cordata, T4506 Ophioglossum vulgatum, T4564 Oxalis corniculata [Syn. Oxalis repens], T5105 Polygonum hydropiper var. flaccidum [Syn. Polygonum flaccidum], T5173 Portulaca oleracea, T5472 Rheum officinale, T5474 Rheum palmatum, T5481 Rheum tanguticum, T5520 Rhododendron ovatum [Syn. Rhododendron lamprophyllum; Azalea ovata], T5524 Rhododendron simsii, T5560 Rorippa montana [Syn. Rorippa dubia; Sisymbrium dublium], T5567 Rosa laevigata, T5724 Sapium sebiferum, T6048 Sophora viciifolia, T6206 Sus scrofa domestica, T6257 Syngnathus acus.

swollen welling abscess and malign sore T0073 Achyranthes bidentata, T0122 Aconitum pendulum, T0635 Aristolochia moupinensis, T2213 Dioscorea zingiberensis.

swollen welling abscess and scrofula T5029 Plumbago zeylanica, T5547 Ricinus communis.

swollen welling abscess and sore toxin T0001 Abelmoschus manihot, T0586 Arctium lappa, T0871 Barleria lupulina, T2555 Eupatorium chinense, T4121 Marsilea quadrifolia, T4704 Peperomia duclouxii, T5604 Rudbeckia laciniata, T6135 Stephania succifera, T6566 Tupistra chinensis.

swollen welling abscess and sores T0374 Alstonia scholaris, T1425 Cimicifuga simplex, T1638 Commiphora myrrha [Syn. Commiphora molmol], T3764 Lepisorus thunbergianus, T3866 Liquidambar formosana [Syn. Liquidambar taiwaniana], T4056 Mahonia bealei, T4060 Mahonia confusa, T4063 Mahonia fortunei, T4064 Mahonia gracilipes, T4067 Mahonia japonica, T4085 Mallotus repandus var. chrysocarpus [Syn. Mallotus chrysocarpus; Mallotus repandus], T4584 Paeonia lactiflora wild, T4586 Paeonia obovata, T4590 Paeonia veitchii, T5103 Polygonum hydropiper, T5234 Prunus persica, T6034 Sophora japonica, T6087 Spiranthes sinensis, T6295 Tanacetum sibiricum [Syn. Filifolium sibiricum].

swollen welling abscess and toxin of clove T0174 Adiantum monochlamys, T1021 Brassica rapa, T1242 Cassia occidentalis, T1633 Commelina communis, T3448 Ipomoea cairica [Syn. Ipomoea palmata], T4740 Petasites japonicus, T5840 Scutellaria indica, T6023 Solidago virgaurea. swollen welling abscess and toxin of clove sore T3363 Hypericum sampsonii.

swollen welling abscess of sore and boil T0750 Asparagus gobicus.

syphilis T0610 Argemone mexicana, T2200 Dioscorea hispida, T3200 Hemidesmus indicus, T3737 Lemmaphyllum microphyllum var. obovatum, T4813 Phlomis tuberosa, T5401 Rabdosia yuennanensis, T5977 Smilax glabra, T5980 Smilax menispermoidea, T6219 Swertia erythrosticta.

systremma T4575 Pachysandra terminalis.

tachycardia T5516 Rhododendron molle.

taenia infection T0318 Allium sativum.

taeniasis T0250 Agrimonia pilosa var. japonica, T0251 Agrimonia pilosa var. japonica, T0957 Blumea balsamifera, T1211 Carpesium abrotanoides, T1881 Cucurbita moschata, T2049 Daucus carota, T2277 Dryopteris austriaca, T2346 Embelia oblongifolia, T3688 Lappula echinata, T4083 Mallotus philippinensis, T4084 Mallotus philippinensis, T4085 Mallotus repandus var. chrysocarpus [Syn. Mallotus chrysocarpus; Mallotus repandus], T4364 Myrsine semiserrata, T4779 Pharbitis nil, T4780 Pharbitis purpurea, T5327 Punica granatum, T5329 Punica granatum.

taxation cough T5483 Rhinacanthus nasutus.

taxation damage T1417 Cimicifuga acerina, T1423 Cimicifuga nanchuanensis, T1786 Cremanthodium ellisii, T3802 Ligularia dentata, T3805 Ligularia fischeri, T3807 Ligularia intermedia, T3813 Ligularia sibirica, T4859 Physochlaina physaloides, T4954 Piper longum, T5722 Sapium japonicum, T6367 Teucrium quadrifarium.

taxation damage and blood ejection T1003 Brandisia hancei, T3295 Huperzia serrata [Syn. Lycopodium serratum], T4510 Ophiorrhiza japonica, T4513 Ophiorrhiza mungos, T5793 Schisandra henryi.

taxation damage and coughing of blood T1884 Cudrania cochinchinensis.

taxation damage and lumbago T1552 Cleome viscosa, T4444 Nothopanax davidii, T4604 Panax japonicus var. bipinnatifidus, T5981 Smilax riparia, T6009 Solanum pseudo-capsicum.

taxation damage and pain T4562 Oxalis acetosella, T6128 Stephania hernandifolia.

taxation damage and sinew bone pain T2087 Delphinium tatsienense, T2301 Dysosma majorensis [Syn. Podophyllum majorense; Dysosma lichuanensis], T4234 Millettia dielsiana.

taxation damage and strength desertion T0598 Ardisia japonica.

taxation damage cough T0924 Bergenia purpurascens, T6266 Syzygium cumini, T6773 Viscum articulactum, T6774 Viscum articulatum.

taxation damage due to knocks and falls T5230 Prunus persica.

taxation damage fever T5954 Silene fortunei.

taxation damage hemoptysis $\mathrm{T} 1683$ Cordyceps sinensis, T3276 Homo sapiens, T4248 Mimosa pudica, T5529 Rhodomyrtus tomentosa, T6722 Veronica anagallis-aquatica.

taxation damage hypodynamia T2211 Dioscorea tenuipes.

taxation damage in lumbar muscle T0048 Acanthus ilicifolius, T0116 Aconitum nagarum var. heterotrichum [Syn. Aconitum bullatifolium], T0569 Aralia cordata, T0574 Aralia fargesii, T0886 Beaumontia grandiflora, T1958 Cynanchum otophyllum, T2267 Drosera peltata var. lunata, T2737 Flemingia philippinensis [Syn. Moghania 
philippinensis], T3318 Hylotelephium mingjinianum, T3400 Illicium difengpi, T3403 Illicium majus, T3969 Lycopodium casuarinoides, T4260 Moghania philippinensis, T4284 Morinda parvifolia, T5279 Psychotria serpens, T5746 Sassafras tzumu, T6015 Solanum torvum, T6468 Tinospora sinensis, T6689 Ventilago leiocarpa, T6830 Woodfordia fruticosa.

taxation damage pain in lumbus and legs T0298 Alectoria vivens, T3776 Lethariella zahlbruckneri.

taxation detriment and sprain T2203 Dioscorea nipponica, T2204 Dioscorea nipponica ssp. rosthornii.

taxation detriment due to knocks and falls T2205 Dioscorea panthaica.

taxation fatigue and hypodynamia T5075 Polygala fallax [Syn. Polygala aureocauda].

taxation fever T2994 Gloiopeltis furcata, T3277 Homo sapiens, T5407 Rana nigromaculata; Rana plancyi.

taxation fever cough T5447 Rehmannia glutinosa [Syn. Rehmannia glutinosa f. huechingensis].

taxation fever steaming bone T4060 Mahonia confusa, T4064 Mahonia gracilipes.

tearing in wind T2408 Equisetum hiemale, T2409 Equisetum palustre.

tearing with wind $\mathrm{T} 3564$ Juglans mandshurica.

temporomandibular lymphrnoditis T0975 Bombyx mori.

tenesmus T1038 Brucea javanica [Syn. Brucea sumatrana; Rhus javanica], T4789 Phellodendron amurense, T4790 Phellodendron amurense var. wilsonii, T4791 Phellodendron chinense, T4792 Phellodendron chinense var. glabriusculum, T6802 Vladimiria souliei [Syn. Jurinea souliei].

terrene leech bite T2567 Eupatorium odoratum.

testitis T0594 Ardisia crenata, T2723 Ficus pumila, T3270 Holboellia fargesii, T4100 Mangifera indica, T6264 Syzygium buxifolium.

tetanus T0088 Aconitum coreanum, T0617 Arisaema amurense, T0618 Arisaema consanguineum, T0620 Arisaema heterophyllum, T0979 Bombyx mori, T1049 Bufo bufo gargarizans; Bufo melanostictus, T1084 Buthus martensi, T2890 Gastrodia elata, T4903 Pinellia pedatisecta, T5547 Ricinus communis, T5727 Saposhnikovia divaricata [Syn. Ledebouriella seseloides], T5817 Scolopendra subspinipes mutilans, T5822 Scopolia sinensis, T5934 Seseli meirei, T5937 Seseli yunnanense, T5998 Solanum dulcamara, T6588 Typhoniun giganteum, T6903 Zephyranthes candida.

tetrodon poisoning T3685 Lannea grandis [Syn. Lannea coromandelica], T3726 Laurus nobilis, T3815 Ligularia stenocephala.

thirst T2763 Fragaria ananassa, T2867 Garcinia multiflora, T3163 Helicteres angustifolia, T3921 Lophatherum gracile, T3962 Lycopersicon esculentum, T4029 Maesa japonica, T4099 Mangifera indica, T4103 Mangifera persiciformis, T5312 Pueraria edulis, T5313 Pueraria lobata [Syn. Pueraria thunbergiana; Pueraria pseudohirsuta], T5316 Pueraria omeiensis, T5318 Pueraria phaseoloides, T5320 Pueraria thomsonii.

thirst due to spontaneous sweating T5260 Pseudostellaria heterophylla. thirst with dry throat T4610 Panax quinquefolium, T5186 Potentilla reptans var. sericophylla.

three worms T1232 Cassia fistula. throat erosion T5408 Rana nigromaculata; Rana plancyi.

throat impediment T0318 Allium sativum, T0950 Blatta orientalis, T0992 Bos taurus domesticus; Bubalus bubalis, T1008 Brassica juncea, T1210 Carpesium abrotanoides, T1464 Citrullus vulgaris [Syn. Citrullus lanatus], T1547 Clematis terniflora [Syn. Clematis maximowicziana], T1858 Croton tiglium, T1876 Cucumis melo, T1981 Cyprinus carpio, T2191 Dioscorea bulbifera, T2363 Entada phaseoloides [Syn. Lens phaseoloides], T2434 Eriocaulon buergerianum, T2756 Forsythia suspensa, T2978 Gleditsia sinensis [Syn. Gleditsia horrida], T3277 Homo sapiens, T3460 Iris halophila, T3464 Iris lactea var. chinensis [Syn. Iris pallasii var. chinensis], T3476 Isatis indigotica, T3578 Juncus effusus, T3908 Lonicera bournei, T3909 Lonicera confusa, T3910 Lonicera fulvotomentosa, T3911 Lonicera hypoglauca, T3912 Lonicera japonica, T3914 Lonicera macranthoides, T3918 Lonicera similis, T4303 Moschus moschiferus; Moschus berezovskii; Moschus sifanicus, T4537 Oroxylum indicum, T4544 Oryza sativa, T4648 Paris polyphylla, T4649 Paris polyphylla var. chinensis, T4650 Paris polyphylla var. pseudothibetica, T4651 Paris polyphylla var. stenophylla, T4652 Paris polyphylla var. yunnanensis, T4796 Pheretima aspergillum; Allolobophora caliginosa trapezoides, T5121 Polygonum tinctorium, T5531 Rhus chinensis [Syn. Rhus semialata], T5550 Ricinus communis, T5840 Scutellaria indica, T6035 Sophora japonica, T6131 Stephania longa, T6206 Sus scrofa domestica, T6484 Trachelospermum jasminoides, T6645 Urena lobata, T6655 Ustilaginoidea virens, T6709 Verbena officinalis, T6884 Zanthoxylum nitidum.

throat impediment sore with gall T5719 Sapindus mukorossi, T5720 Sapindus mukorossi.

throat impediment with nipple moth T2838 Gallus gallus domesticus, T5295 Pteris multifida.

throat moth T3865 Lippia nodiflora.

throat pain T0273 Akebia quinata, T0277 Akebia trifoliata, T0279 Akebia trifoliata var. australis, T0888 Beesia calthaefolia, T1179 Capparis masaikai, T1555 Clerodendron fortunatum, T1796 Crinum asiaticum var. sinicum, T1931 Cyclea barbata, T1932 Cyclea racemosa, T1961 Cynanchum thesioides, T2716 Ficus beecheyana [Syn. Ficus erecta var. beecheyana], T3334 Hypecoum leptocarpum, T3361 Hypericum perforatum, Т3475 Isatis indigotica, Т3999 Lysimachia clethroides, T4221 Micromelum falcatum, T4519 Opuntia dillenii, T4521 Opuntia vulgaris, T4527 Orixa japonica, T4834 Phyllanthus emblica, T4846 Physalis alkekengi var. franchetii, T4852 Physalis peruviana, T5804 Schizonepeta tenuifolia [Syn. Nepeta tenuifolia], T5835 Scutellaria barbata [Syn. Scutellaria rivularis], T5967 Siraitia grosvenorii [Syn. Momordica grosvenorii], T6133 Stephania sinica, T6889 Zanthoxylum podocarpum, T6920 Ziziphus mauritiana.

throat pain and swollen tongue T1934 Cyclea tonkinensis.

throat pain and throat impediment T1787 Cremastra appendiculata. throat swelling T1152 Camellia sinensis [Syn. Thea sinensis], T3311 Hydrocotyle sibthorpioides.

throat wind T1858 Croton tiglium, T3984 Lycoris chinensis, T3986 Lycoris radiata [Syn. Amaryllis radiata].

thromboangiitis obliterans T0967 Boenninghausenia sessilicarpa. 
thromboangiitis obliterans (buerger's disease) T2538 Euonymus bungeanus, T3388 Ilex chinensis [Syn. Ilex purpurea], T3396 Ilex pubescens, T3397 Ilex pubescens var. glaber, T4917 Pinus massoniana.

thrombocytopenia T1337 Cervus nippon; Cervus elaphus.

thrombocytopenic purpura T0805 Astragalus sinicus.

thrombophlebitis T0495 Angelica sinensis, T3388 Ilex chinensis [Syn. Ilex purpurea], T3396 Ilex pubescens, T3397 Ilex pubescens var. glaber, T4804 Phlojodicarpus sibiricus, T5680 Salvia miltiorrhiza, T5681 Salvia miltiorrhiza f. alba, T5688 Salvia przewalskii.

thyroid enlargement T2359 Endarachne binghamiae, T4403 Nemacystus decipiens [Syn. Mesogloea decipiens; Cladosiphon decipiens], T5214 Prunella vulgaris, T6598 Ulva conglobata.

tidal fever T5097 Polygonum amphibium, T6228 Swertia nervosa.

tidal fever with night sweat T3954 Lycium barbarum, T3956 Lycium chinense, T4789 Phellodendron amurense, T4790 Phellodendron amurense var. wilsonii, T4791 Phellodendron chinense, T4792 Phellodendron chinense var. glabriusculum, T5446 Rehmannia glutinosa [Syn. Rehmannia glutinosa $\mathrm{f}$. huechingensis].

tinea capitis T1285 Celastrus angulatus, T2023 Daphne genkwa, T2092 Delphinus delphis, T3363 Hypericum sampsonii, T3560 Jatropha curcas, T4157 Melia azedarach, T5010 Platycarya strobilacea, T5026 Plumbagella micrantha, T5455 Rhamnus crenata, T6489 Tragopogon porrifolius, $\mathrm{T} 6541$ Tripterygium regelii.

tinnitus T0144 Acorus gramineus, T0146 Acorus tatarinowii, T1819 Crotalaria ferruginea, T3142 Helianthus annuus, T3829 Ligustrum robustum, T5107 Polygonum multiflorum.

tinnitus and deafness T0142 Acorus calamus, T1337 Cervus nippon; Cervus elaphus, T2273 Drynaria fortunei, T5252 Pseudodrynaria coronans, T5446 Rehmannia glutinosa [Syn. Rehmannia glutinosa $\mathrm{f}$. huechingensis], T5927 Sesamum indicum [Syn. Sesamum orientale], T6234 Swertia punicea.

tinnitus and dim vision T1911 Cuscuta australis, T1912 Cuscuta chinensis, T1913 Cuscuta japonica, T5091 Polygonatum cyrtonema [Syn. Polygonatum multiflorum], T5092 Polygonatum kingianum, T5095 Polygonatum sibiricum.

toad head scourge T5407 Rana nigromaculata; Rana plancyi.

tongue sores T0273 Akebia quinata, T0277 Akebia trifoliata, T0279 Akebia trifoliata var. australis, T0632 Aristolochia manshuriensis, T1662 Coptis chinensis, T1663 Coptis chinensis var. brevisepala, T1664 Coptis deltoidea, T1669 Coptis omeiensis, T1670 Coptis teetoides [Syn. Coptis teeta], T3277 Homo sapiens, T3920 Lophatherum gracile, T4178 Melodinus hemsleyanus, T5099 Polygonum bistorta.

tonsillitis T0457 Andrographis paniculata [Syn. Justicia paniculata], T0967 Boenninghausenia sessilicarpa, T1545 Clematis chinensis, T1655 Conyza blinii, T1709 Corydalis bungeana, T1819 Crotalaria ferruginea, T2334 Elephantopus scaber, T2563 Eupatorium japonicum, T2697 Ferula borealis, T2721 Ficus microcarpa, T3288 Humulus japonicus [Syn. Humulus scandens], T3360 Hypericum patulum, T3908 Lonicera bournei, T3909 Lonicera confusa, T3910 Lonicera fulvotomentosa, T3911 Lonicera hypoglauca, T3912 Lonicera japonica, T3914 Lonicera macranthoides, T3918 Lonicera similis, T5018
Pleuropterus ciliinervis, T5680 Salvia miltiorrhiza, T5681 Salvia miltiorrhiza f. alba, T5688 Salvia przewalskii, T5907 Senecio scandens [Syn. Senecio chinensis], T6214 Swertia chinensis, T6301 Taraxacum mongolicum, T6567 Tupistra wattii [Syn. Campylandra wattii], T6691 Veratrilla baillonii, T6814 Wedelia chinensis [Syn. Solidago chinensis; Wedelia calendulacea].

tooth decay T3827 Ligustrum japonicum, T5651 Salix babylonica.

tooth mobilizing T2273 Drynaria fortunei, T2323 Eclipta prostrata [Syn. Eclipta alba], T5252 Pseudodrynaria coronans.

toothache T0060 Achillea alpina [Syn. Achillea sibirica], T0069 Achillea wilsoniana, T0079 Aconitum brachypodum, T0106 Aconitum kirinense, T0117 Aconitum nagarum var. lasiandrum, T0135 Aconitum sungpanense, T0471 Anemone rivularis, T0478 Angelica dahurica [Syn. Angelica porphyrocaulis], T0479 Angelica dahurica cv. qibaizhi, T0492 Angelica pubescens f. biserrata [Syn. Angelica pubescens], T0498 Angelica taiwaniana, T0569 Aralia cordata, T0574 Aralia fargesii, T0582 Archangelica brevicaulis [Syn. Angelicarpa brevicaulis; Angelica brevicaulis], T0708 Arthromeris mairei [Syn. Polypodium mairei], T0724 Asarum caulescens, T0726 Asarum forbesii, T0727 Asarum fukienense, T0728 Asarum heterotropoides var. mandshuricum, T0730 Asarum sagittarioides, T0731 Asarum sieboldii, T0732 Asarum sieboldii var. seoulensis, T0834 Averrhoa carambola, T1084 Buthus martensi, T1092 Buxus microphylla var. sinica, T1210 Carpesium abrotanoides, T1288 Celastrus orbiculatus [Syn. Celastrus articulatus], T1292 Celastrus orbiculatus [Syn. Celastrus articulatus], T1316 Cephalanthus occidentalis, T1650 Convolvulus arvensis, T1655 Conyza blinii, T1746 Corydalis taliensis, T1796 Crinum asiaticum var. sinicum, T1799 Crinum latifolium, T1931 Cyclea barbata, T1933 Cyclea sutchuenensis, T1955 Cynanchum hancockianum, T1959 Cynanchum paniculatum, T2023 Daphne genkwa, T2028 Daphne odora, T2056 Debregeasia longifolia, T2130 Desmodium gangeticum, T2162 Dicranostigma franchetianum [Syn. Dicranostigma leptopodum], T2340 Elsholtzia bodinieri, T2387 Epilobium hirsutum, T2434 Eriocaulon buergerianum, T3203 Hemsleya amabilis, T3208 Hemsleya macrosperma, T3213 Heracleum hemsleyanum, T3214 Heracleum lanatum, T3217 Heracleum moellendorffii [Syn. Heracleum microcarpum; Heracleum morifolium], T3221 Heracleum rapula, T3228 Heracleum yungningense, T3328 Hyoscyamus niger, T3392 Ilex kudingcha, T3393 Ilex latifolia, T3460 Iris halophila, T3464 Iris lactea var. chinensis [Syn. Iris pallasii var. chinensis], T3528 Isodon ternifolia, T3529 Isodon ternifolius, T3548 Ixeris sonchifolia, T3670 Lagerstroemia indica, T3675 Lagopsis supina, T3737 Lemmaphyllum microphyllum var. obovatum, T3779 Leucas aspera, T3796 Libanotis buchtormensis, T3829 Ligustrum robustum, T3876 Litchi chinensis, T3885 Litsea cubeba, T4002 Lysimachia foenum-graecum, T4349 Myrica rubra, T4361 Myrsine africana, T4470 Ocimum basilicum, T4527 Orixa japonica, T4549 Osbeckia chinensis, T4550 Osmanthus fragrans, T4827 Photinia parvifolia, T4953 Piper longum, T4956 Piper mullesua, T4966 Piper sarmentosum, T5109 Polygonum nodosum, T5142 Poncirus trifoliata, T5151 Populus davidiana, T5158 Populus simonii, T5266 Psidium guajava, T5308 Pterocarya stenoptera, T5374 Quercus infectoria, T5414 Ranunculus japonicus, T5592 Rubus hirsutus, T5650 Salix babylonica, T5690 Salvia 
roborowskii, T5781 Schefflera arboricola, T5840 Scutellaria indica, T5934 Seseli meirei, T5937 Seseli yunnanense, T6000 Solanum indicum, T6003 Solanum khasianum, T6018 Solanum verbascifolium, T6020 Solanum xanthocarpum, T6028 Sophora alopecuroides, T6075 Spilanthes acmella, T6079 Spiraea japonica, T6082 Spiraea japonica var. fortunei, T6122 Stephania dielsiana, T6133 Stephania sinica, T6135 Stephania succifera, T6139 Sterculia lychnophora, T6217 Swertia davidii, T6224 Swertia kouitchensis, T6231 Swertia pseudochinensis, T6276 Tacca plantaginea [Syn. Schizocapsa plantaginea], T6278 Tagetes erecta, T6414 Thalictrum thunbergii, T6498 Tribulus terrestris, T6860 Zanthoxylum ailanthoides, T6862 Zanthoxylum ailanthoides, T6864 Zanthoxylum armatum, T6877 Zanthoxylum dissitum, T6884 Zanthoxylum nitidum, T6887 Zanthoxylum planispinum, T6888 Zanthoxylum planispinum, T6893 Zanthoxylum simulans, T6894 Zanthoxylum simulans.

torpid intake T0210 Agaricus bisporus, T0211 Agaricus campestris, T3742 Lentinus edodes.

toxemia T5907 Senecio scandens [Syn. Senecio chinensis].

toxic hepatitis T0379 Alternanthera philoxeroides.

toxic jaundice T5101 Polygonum cuspidatum.

toxin of clove sore T2883 Gardenia jasminoides [Syn. Gardenia florida], T5892 Senecio nemorensis.

toxin of sore welling abscess and boils T2272 Drymaria diandra [Syn. Drymaria cordata ssp. diandra].

toxin swelling T0250 Agrimonia pilosa var. japonica, T0293 Albizzia lebbeck, T0598 Ardisia japonica, T0726 Asarum forbesii, T0924 Bergenia purpurascens, T1051 Bufo bufo gargarizans; Bufo melanostictus, T1240 Cassia obtusifolia, T1250 Cassia tora, T1624 Colocasia antiquorum, T1693 Coriaria sinica [Syn. Coriaria nepalensis], T1928 Cycas revoluta, T2590 Euphorbia hirta, T2756 Forsythia suspensa, T3295 Huperzia serrata [Syn. Lycopodium serratum], T3662 Lactuca sativa, T3680 Lamium amplexicaule, T3681 Lamium barbatum, T5411 Randia spinosa, T5719 Sapindus mukorossi, T5982 Smilax sieboldii, T5984 Smilax stans [Syn. Smilax vaginata var. stans], T5987 Sobina chinensis, T6205 Sus scrofa.

toxin swelling from hemorrhoids T1199 Carduus acanthoides, T1200 Carduus crispus, T4678 Paulownia fortunei, T4679 Paulownia tomentosa.

toxin swelling of sores T0045 Acanthopanax trifoliatus, T0079 Aconitum brachypodum, T0117 Aconitum nagarum var. lasiandrum, T0537 Antirrhinum majus, T0907 Berberis kawakamii, T0971 Bolbostemma paniculatum, T1087 Buxus bodinieri, T1151 Camellia sinensis [Syn. Thea sinensis], T1206 Carica papaya, T1437 Cinnamomum camphora, T1589 Cocculus trilobus [Syn. Cocculus sarmentosus], T1672 Corallodiscus flabellatus [Syn. Didissandra flabellat], T1685 Coreopsis lanceolata, T1719 Corydalis incisa, T2205 Dioscorea panthaica, T2927 Gentiana rhodantha, T3222 Heracleum scabridum, T3247 Hibiscus taiwanensis, T3447 Ipomoea batatas [Syn. Convolvulus batatas], T3646 Kyllinga brevifolia, T3765 Lepisorus ussuriensis, T3847 Lindera angustifolia, T3851 Lindera glauca, T3863 Liparis nervosa, T3885 Litsea cubeba, T3925 Loranthus parasiticus [Syn. Loranthus chinenis; Taxillus chinensis], T3969 Lycopodium casuarinoides, T4104 Manihot esculenta, T4248 Mimosa pudica,
T4445 Notopterygium forbesii [Syn. Notopterygium franchetii], T4446 Notopterygium incisum, T4456 Nymphoides peltatum, T4660 Parthenium hysterophorus, T4686 Pedilanthus tithymaloides, T4710 Pericampylus glaucus, T4799 Philydrum lanuginosum, T4937 Piper betle, T5184 Potentilla kleiniana, T5341 Pygmaeopremna herbacea [Syn. Premna herbacea], T5714 Sansevieria trifasciata, T5806 Schlumbergera truncata, T5838 Scutellaria galericulata, T5884 Senecio chrysanthemoides, T5942 Sida acuta, T6026 Sonchus asper [Syn. Sonchus oleraceus var. asper], T6127 Stephania glabra, T6275 Tacca chantrieri [Syn. Tacca minor; Tacca esquirolii], T6378 Thalictrum delavayi, T6469 Tithonia diversifolia, T6512 Trichosanthes kirilowii, T6541 Tripterygium regelii, T6750 Vicia sativa, T6820 Wikstroemia indica.

toxin swelling of sores and open sores T5817 Scolopendra subspinipes mutilans.

trachitis T1050 Bufo bufo gargarizans; Bufo melanostictus, T1387 Chrysanthemum boreale, T1392 Chrysanthemum indicum, T1394 Chrysanthemum lavandulifolium, T2697 Ferula borealis, T4275 Monotropa hypopitys, T5396 Rabdosia rubescens, T6814 Wedelia chinensis [Syn. Solidago chinensis; Wedelia calendulacea].

trachoma T4789 Phellodendron amurense, T4790 Phellodendron amurense var. wilsonii, T4791 Phellodendron chinense, T4792 Phellodendron chinense var. glabriusculum.

trance T3832 Lilium brownii var. viridulum [Syn. Lilium brownii var. colchesteri], T3835 Lilium longiflorum, T3836 Lilium pumilum [Syn. Lilium tenuifolium], T3839 Lilium tigrinum [Syn. Lilium lancifolium].

trichomonal vaginitis T4158 Melia azedarach.

trichomonas vaginalis $\mathrm{T} 3420$ Incarvillea sinensis, $\mathrm{T} 3560$ Jatropha curcas.

trichomoniasis T0248 Agrimonia pilosa, T0311 Allium cepa, T0318 Allium sativum, T1582 Cnidium monnieri, T1662 Coptis chinensis, T1663 Coptis chinensis var. brevisepala, T1664 Coptis deltoidea, T1669 Coptis omeiensis, T1670 Coptis teetoides [Syn. Coptis teeta], T3245 Hibiscus syriacus, T4020 Macleaya cordata, T5234 Prunus persica, T5308 Pterocarya stenoptera, T5719 Sapindus mukorossi, T5907 Senecio scandens [Syn. Senecio chinensis], T6031 Sophora flavescens [Syn. Sophora angustfolia], T6048 Sophora viciifolia.

trichuriasis T6101 Stauntonia hexaphylla.

trigeminal neuralgia T2890 Gastrodia elata.

try to using for carcinoma of stomach and carcinoma of esophagus T4704 Peperomia duclouxii.

tuberculosis T0163 Actinidia latifolia, T0318 Allium sativum, T0457 Andrographis paniculata [Syn. Justicia paniculata], T0641 Aristolochia tuberosa, T0752 Asparagus officinalis, T1680 Cordyceps militaris, T1681 Cordyceps militaris cv, T2101 Dendrobium fimbriatum, T2359 Endarachne binghamiae, T2584 Euphorbia ebracteolata, T2587 Euphorbia fischeriana, T2756 Forsythia suspensa, T2783 Fritillaria cirrhosa, T2784 Fritillaria delavayi, T2792 Fritillaria przewalskii, T2796 Fritillaria unibracteata, T2798 Fritillaria verticillata var. thunbergii [Syn. Fritillaria thunbergii], T3011 Glycyrrhiza aspera, T3013 Glycyrrhiza glabra, T3015 Glycyrrhiza inflata, T3016 Glycyrrhiza kansuensis, T3021 Glycyrrhiza squamulosa, T3022 Glycyrrhiza uralensis, T3023 Glycyrrhiza 
yunnanensis, T3288 Humulus japonicus [Syn. Humulus scandens], T3289 Humulus lupulus, T3709 Lathyrus pratensis, T4005 Lysionotus pauciflorus, T4039 Magnolia grandiflora, T4145 Meconopsis punicea, T4280 Morinda citrifolia, T4789 Phellodendron amurense, T4790 Phellodendron amurense var. wilsonii, T4791 Phellodendron chinense, T4792 Phellodendron chinense var. glabriusculum, T5071 Polygala arillata, T6055 Sorbus tianschanica, T6075 Spilanthes acmella, T6111 Stemona japonica, T6113 Stemona sessilifolia, T6115 Stemona tuberosa, T6132 Stephania sasakii, T6276 Tacca plantaginea [Syn. Schizocapsa plantaginea], T6440 Thuja orientalis [Syn. Platycladus orientalis; Biota orientalis], T6574 Tussilago farfara, T6731 Veronicastrum sibiricum.

tuberculosis and coughing of blood T1746 Corydalis taliensis, T5586 Rubia yunnanensis, T6026 Sonchus asper [Syn. Sonchus oleraceus var. asper].

tuberculosis and hacking of blood T4730 Peristrophe roxburghiana.

tuberculosis and hemoptysis T0226 Agave sisalana, T0598 Ardisia japonica, T1053 Bulbophyllum odoratissimum [Syn. Stelis odoratissimum], T1728 Corydalis ochotensis, T1866 Cryptolepis sinensis, T4056 Mahonia bealei, T4063 Mahonia fortunei, T4067 Mahonia japonica, T4361 Myrsine africana, T4519 Opuntia dillenii, T4520 Opuntia ficus-indica, T4521 Opuntia vulgaris, T4823 Pholidota yunnanensis, T5119 Polygonum thunbergii, T5284 Pteridium aquilinum var. latiusculum, T5613 Rumex obtusifolius, T6251 Symplocos caudata, T6747 Vicia faba, T6814 Wedelia chinensis [Syn. Solidago chinensis; Wedelia calendulacea].

tuberculosis and vacuity T4505 Ophiocephalus argus.

tuberculosis tidal fever T0678 Artemisia japonica.

tuberculosis with blood ejection T4506 Ophioglossum vulgatum.

tuberculosis with cough T0500 Anguilla japonica, T0749 Asparagus filicinus, T2727 Ficus simplicissima, T5133 Polytrichum commune,

T5494 Rhodiola crenulata [Syn. Rhodiola euryphylla].

tuberculosis fistula T2589 Euphorbia helioscopia.

tumor T0165 Actinidia rubricaulis var. coriacea.

turbid vaginal discharge T5186 Potentilla reptans var. sericophylla.

tympanites T1884 Cudrania cochinchinensis, T2092 Delphinus delphis,

T6548 Tritonia crocosmaeflora.

tympanitis T6131 Stephania longa.

typhoid fever T1662 Coptis chinensis, T1663 Coptis chinensis var. brevisepala, T1664 Coptis deltoidea, T1669 Coptis omeiensis, T1670 Coptis teetoides [Syn. Coptis teeta], T5907 Senecio scandens [Syn. Senecio chinensis].

ulcer T0311 Allium cepa, T1043 Bryophyllum pinnatum, T1727 Corydalis mucronifera, T2274 Dryobalanops aromatica, T2857 Garcinia hanburyi, T4789 Phellodendron amurense, T4790 Phellodendron amurense var. wilsonii, T4791 Phellodendron chinense, T4792 Phellodendron chinense var. glabriusculum.

ulcer in gastrointestinal tract T0495 Angelica sinensis, T4804 Phlojodicarpus sibiricus, T6656 Ustilago maydis.

ulcer of lower limb T0446 Anaphalis margaritacea, T1994 Daemonorops draco, T2253 Dracaena cochinchinensis, T2337 Elephas maximus, T2950 Gerbera anandria [Syn. Leibnitzia anandria], T3388 Ilex chinensis [Syn. Ilex purpurea], T3560 Jatropha curcas, T4020
Macleaya cordata, T4391 Nauclea officinalis, T5978 Smilax glauco-china.

ulcer of uterine cervix T4020 Macleaya cordata, T6043 Sophora subprostrata [Syn. Sophora tonkinensis].

ulcerating sore and boil T5385 Quisqualis indica.

ulcerating sore of leg T5415 Ranunculus sceleratus.

ulcerating sore toxin T2279 Dryopteris championii.

ulcerating sores T1251 Cassytha filiformis, T1285 Celastrus angulatus, T1336 Ceriops tagal [Syn. Rhizophora tagal], T1552 Cleome viscosa, T2188 Dioscorea alata, T2989 Glochidion sphaerogynum, T4006 Lythrum anceps, T4007 Lythrum salicaria, T4500 Onosma paniculatum, T6476 Torilis japonica.

ulcerating welling abscess and sore T2852 Garcinia cowa.

ulcerative bleeding T3033 Gnetum parvifolium [Syn. Gnetum indicum].

ulcerative colitis T3106 Harpagophytum procumbens.

unctuous strangury T2198 Dioscorea gracillima, T2201 Dioscorea hypoglauca [Syn. Dioscorea collettii var. hypoglauca], T2212 Dioscorea tokoro.

upflaming vacuity fire T1961 Cynanchum thesioides.

urethral stone T1553 Clerodendranthus spicatus, T1823 Crotalaria juncea, T2134 Desmodium styracifolium, T2325 Eichhornia crassipes, T2749 Fomes officinalis, T3143 Helianthus annuus, T4000 Lysimachia congestiflora, T4148 Medicago sativa, T4149 Medicago sativa, T4432 Nigella glandulifera, T5354 Pyrrosia davidii, T5355 Pyrrosia drakeana, T5356 Pyrrosia gralla, T5357 Pyrrosia lingua T5358 Pyrrosia petiolosa, T5360 Pyrrosia sheareri, T6264 Syzygium buxifolium, T6488 Trachyspermum ammi, T6902 Zea mays.

urethritis T2119 Derris eriocarpa, T3187 Helleborus thibetanus, T5098 Polygonum aviculare.

urinary and fecal stoppage T0382 Althaea rosea, T0389 Amaranthus tricolor, T1390 Chrysanthemum coronarium, T1397 Chrysanthemum segetum, T2596 Euphorbia kansui, T2597 Euphorbia lathyris, T2978 Gleditsia sinensis [Syn. Gleditsia horrida], T4091 Malva sylvestris, T4861 Phytolacca americana [Syn. Phytolacca decandra], T4864 Phytolacca esculenta [Syn. Phytolacca acinosa], T5723 Sapium sebiferum.

urinary incontinence T3742 Lentinus edodes, T4283 Morinda officinalis. urinary stoppage T0310 Allium ascalonicum, T2143 Dianthus chinensis, T2145 Dianthus superbus, T2147 Dianthus versicolor, T3066 Gryllulus chinensis, T4562 Oxalis acetosella, T4796 Pheretima aspergillum; Allolobophora caliginosa trapezoides, T5354 Pyrrosia davidii, T5355 Pyrrosia drakeana, T5356 Pyrrosia gralla, T5357 Pyrrosia lingua' T5358 Pyrrosia petiolosa, T5360 Pyrrosia sheareri, T5605 Rumex acetosa, T5651 Salix babylonica.

urinary strangury T2723 Ficus pumila.

urinary tract infection T0015 Abutilon indicum, T0195 Aeginetia indica, T0776 Asplenium prolongatum, T0897 Berberis amurensis, T0912 Berberis poiretii, T0920 Berberis wilsonae, T0933 Betula platyphylla, T0935 Betula platyphylla var. japonica, T1450 Cirsium lineare, T1557 Clerodendron indicum, T1969 Cynoglossum officinale, T2143 Dianthus chinensis, T2145 Dianthus superbus, T2147 Dianthus versicolor, T2233 Diploclisia glaucescens, T2357 Emilia sonchifolia, T2908 Gentiana cephalantha, T2921 Gentiana manshurica, T2928 
Gentiana rigescens, T2930 Gentiana scabra, T2938 Gentiana triflora, T3122 Hedyotis acutangula, T3203 Hemsleya amabilis, T3208 Hemsleya macrosperma, T3284 Houttuynia cordata, T3361 Hypericum perforatum, T3388 Ilex chinensis [Syn. Ilex purpurea], T3594 Juniperus rigida, T3989 Lygodium flexuosum [Syn. Lygodium pinnatifidum; Ophioglossum flexuosum], T4391 Nauclea officinalis, T4789 Phellodendron amurense, T4790 Phellodendron amurense var. wilsonii, T4791 Phellodendron chinense, T4792 Phellodendron chinense var. glabriusculum, T4843 Phyllanthus urinaria, T5207 Prismatomeris tetrandra, T5344 Pyrola atropurpurea, T5345 Pyrola calliantha [Syn. Pyrola rotundifolia ssp. chinensis], T5346 Pyrola calliantha var. tibetana, T5347 Pyrola decorata, T5349 Pyrola incarnata, T5350 Pyrola japonica, T5351 Pyrola rotundifolia, T5354 Pyrrosia davidii, T5355 Pyrrosia drakeana, T5356 Pyrrosia gralla, T5357 Pyrrosia lingua T5358 Pyrrosia petiolosa, T5360 Pyrrosia sheareri, T5393 Rabdosia longituba, T5639 Sabina chinensis, T6129 Stephania japonica, T6217 Swertia davidii, T6794 Vitis amurensis.

urinary turbidity T0323 Allium tuberosum.

urticari with sore and scab T1160 Campsis grandiflora.

urticaria T0539 Apis cerana, T0838 Azolla imbricata [Syn. Salvinia imbricata], T1561 Clerodendron serratum, T1569 Clerodendrum serratum var. amplexifolium, T1959 Cynanchum paniculatum, T2020 Damnacanthus indicus, T2835 Galium verum, T2988 Glochidion eriocarpum, T3294 Huperzia selago [Syn. Lycopodium selago], T3628 Kerria japonica, T3742 Lentinus edodes, T3847 Lindera angustifolia, T4247 Millingtonia hortensis, T4801 Phlegmariurus phlegmaria [Syn. Lycopodium phlegmaria], T5455 Rhamnus crenata, T5524 Rhododendron simsii, T5639 Sabina chinensis, T5987 Sobina chinensis, T6651 Urtica cannabina, T6652 Urtica dioica.

uterine bleeding T2426 Erigeron sumatrensis, T3926 Loropetalum chinense.

uterus cold T0083 Aconitum carmichaeli, T0085 Aconitum carmichaeli cv, T1439 Cinnamomum cassia [Syn. Cinnamomum aromaticum], T4599 Panax ginseng [Syn. Panax schinseng].

uterus cold and abdominal pain T5755 Saussurea involucrata. vacuity and edema T0036 Acanthopanax giraldii [Syn. Acanthopanax giraldii var. inermis; Eleutherococcus giraldii], T3128 Hedysarum multijugum.

vacuity and fever T4810 Phlomis mongolica.

vacuity and hypodynamia T0041 Acanthopanax senticosus [Syn. Eleutherococcus senticosus], T3085 Gynostemma pentaphyllum, T3229 Hericium erinaceus [Syn. Hydnum erinaceus], T6803 Volvariella volvacea.

vacuity and marked emaciation T0038 Acanthopanax gracilistylus, T0042 Acanthopanax senticosus [Syn. Eleutherococcus senticosus], T0044 Acanthopanax sessiliflorus, T5091 Polygonatum cyrtonema [Syn. Polygonatum multiflorum], T5092 Polygonatum kingianum, T5095 Polygonatum sibiricum.

vacuity and profuse sweating T1698 Cornus officinalis [Syn. Macrocarpium officinale], T4600 Panax ginseng [Syn. Panax schinseng], T5097 Polygonum amphibium, T6919 Ziziphus jujuba var. spinosa.

vacuity asthma T5186 Potentilla reptans var. sericophylla, T5270
Psoralea corylifolia, T6266 Syzygium cumini. vacuity cold and abdominal pain T2955 Geum japonicum. vacuity cold and hiccough T1168 Canavalia ensiformis, T1169 Canavalia gladiata.

vacuity cold chronic diarrhea and dysentery T0083 Aconitum carmichaeli, T0085 Aconitum carmichaeli $\mathrm{cv}$.

vacuity cold cough asthma T3221 Heracleum rapula.

vacuity cold diarrhea T1434 Cinnamomum bejolghota [Syn. Cinnamomum obtusifolium; Laurus bejolghota], T4859 Physochlaina physaloides.

vacuity cold in lumbus and knees T0554 Aquilaria agallocha, T0555 Aquilaria sinensis, T6263 Syzygium aromaticum [Syn. Eugenia caryophyllata].

vacuity cold of kidney and bladder T6870 Zanthoxylum bungeanum. vacuity cold of uterus T4283 Morinda officinalis.

vacuity cold vomiting and diarrhea T1439 Cinnamomum cassia [Syn. Cinnamomum aromaticum].

vacuity constipation T0554 Aquilaria agallocha, T0555 Aquilaria sinensis, T1972 Cynomorium songaricum, T4912 Pinus koraiensis.

vacuity consumption with cough T3875 Liriope spicata var. prolifera, T4507 Ophiopogon japonicus.

vacuity cough T4516 Oplopanax elatus.

vacuity desertion due to great sweating T1698 Cornus officinalis [Syn. Macrocarpium officinale].

vacuity desertion failure T4682 Pedicularis muscicola.

vacuity detriment $\mathrm{T} 1017$ Brassica oleracea var. capitata, T1888 Cudrania tricuspidata, T3279 Homo sapiens, T4820 Phoenix dactylifera, T5872 Selenarctos thibetanus; Ursus arctos.

vacuity detriment and emaciation weakness T4644 Parasilurus asotus. vacuity detriment and taxation damage T4206 Metaplexis japonica. vacuity detriment sterility $\mathrm{T} 3615$ Kadsura interior.

vacuity fever in children T3681 Lamium barbatum.

vacuity heat and diabetes mellitus T2190 Dioscorea batatas [Syn. Dioscorea opposita], T2202 Dioscorea japonica.

vacuity heat and hypodynamia T5545 Ribes fasciculatum var. chinense. vacuity heat and vexation fatigue T4610 Panax quinquefolium.

vacuity heat cough T3344 Hypericum chinense, T5642 Saccharum sinensis.

vacuity heat during convalescence T2094 Dendrobium aduncum, T2096 Dendrobium aurantiacum var. denneanum, T2098 Dendrobium chrysanthum, T2100 Dendrobium densiflorum, T2102 Dendrobium fimbriatum var. oculatum, T2105 Dendrobium loddigesii, T2106 Dendrobium moniliforme, T2107 Dendrobium nobile, T2108 Dendrobium officinale.

vacuity heat with vexation and thirst $\mathrm{T} 3288$ Humulus japonicus [Syn. Humulus scandens], T5228 Prunus mume. vacuity of $q \boldsymbol{i}$ and blood T6798 Vitis vinifera. vacuity strangury T2391 Epimedium brevicornum.

vacuity taxation T1191 Caragana sinica, T2846 Ganoderma japonicum [Syn. Ganoderma sinense], T2848 Ganoderma lucidum, T3774 Lespedeza tomentosa, T4207 Metaplexis japonica.

vacuity taxation blood ejection T2840 Gallus gallus domesticus, T3285 Hovenia dulcis. 
vacuity taxation cough T0216 Agave americana var. marginata [Syn. Agave americana var. variegata], T1188 Caragana chamlagu, T2188 Dioscorea alata, T2454 Erysimum diffusum, T4607 Panax pseudo-ginseng var. japonicus, T5215 Prunus amygdalus, T5409 Rana temporaria chensinensis; Rana amurensis, T5836 Scutellaria discolor.

vacuity taxation detriment T0989 Bos taurus domesticus; Bubalus bubalis.

vacuity taxation hemoptysis T2983 Glehnia littoralis.

vacuity taxation lung wilting T0982 Bos taurus domesticus.

vacuity taxation with cough T5501 Rhodiola yunnanesis.

vacuity taxation with emaciation T1604 Coeloglossum viride [Syn. Coeloglossum viride var. bracteatum], T3077 Gymnadenia conopsea.

vacuity taxation with emaciation and weakness T1181 Capra hircus; Ovis aries, T1182 Capra hircus; Ovis aries.

vacuity taxation with fever T3957 Lycium chinense, T5186 Potentilla reptans var. sericophylla.

vacuity taxation with kidney depletion T0990 Bos taurus domesticus; Bubalus bubalis.

vacuity taxation with marked emaciation T0983 Bos taurus domesticus, T0986 Bos taurus domesticus; Bubalus bubalis, T2837 Gallus gallus domesticus.

vacuity taxation with profuse sweating T4682 Pedicularis muscicola. vacuity taxation with steaming bone T0722 Arundo donax, T5239 Prunus salicina.

vacuity vexation and egersis T6918 Ziziphus jujuba var. spinosa. vacuity vexation and insomnia T0462 Anemarrhena asphodeloides, T5826 Scrophularia buergeriana, T5828 Scrophularia ningpoensis, T6919 Ziziphus jujuba var. spinosa.

vacuity vexation and thirst T1961 Cynanchum thesioides.

vacuity weakness T4275 Monotropa hypopitys.

vacuity with dizziness T0924 Bergenia purpurascens.

vacuity-fire toothache T4602 Panax ginseng [Syn. Panax schinseng].

vaginal discharge T0045 Acanthopanax trifoliatus, T0171 Adiantum capillus-veneris, T0249 Agrimonia pilosa var. japonica, T0255 Ailanthus altissima, T0274 Akebia quinata, T0278 Akebia trifoliata, T0280 Akebia trifoliata var. australis, T0298 Alectoria vivens, T0323 Allium tuberosum, T0476 Angelica anomala, T0542 Apis cerana, T0571 Aralia decaisneaa, T0678 Artemisia japonica, T0736 Asclepias curassavica, T0805 Astragalus sinicus, T0895 Benincasa hispida, T0902 Berberis diaphana, T0924 Bergenia purpurascens, T0952 Blechnum orientale, T0993 Boschniakia rossica, T1143 Calystegia hederacea, T1145 Camellia japonica, T1163 Campylotropis hirtella, T1174 Cannabis sativa, T1183 Capra hircus; Ovis aries, T1188 Caragana chamlagu, T1191 Caragana sinica, T1199 Carduus acanthoides, T1200 Carduus crispus, T1299 Celosia cristata, T1419 Cimicifuga dahurica, T1420 Cimicifuga foetida, T1421 Cimicifuga heracleifolia, T1425 Cimicifuga simplex, T1582 Cnidium monnieri, T1604 Coeloglossum viride [Syn. Coeloglossum viride var. bracteatum], T1819 Crotalaria ferruginea, T1927 Cycas revoluta, T1967 Cynoglossum amabile, T1969 Cynoglossum officinale, T1986 Cyrtomium fortunei, T2133 Desmodium racemosum [Syn. Podocarpium podocarpum var. oxyphyllum], T2188 Dioscorea alata, T2190 Dioscorea batatas [Syn. Dioscorea opposita], T2198 Dioscorea gracillima, T2201 Dioscorea hypoglauca [Syn. Dioscorea collettii var. hypoglauca], T2202 Dioscorea japonica, T2212 Dioscorea tokoro, T2281 Dryopteris crassirhizoma, T2326 Elaeagnus angustifolia, T2327 Elaeagnus angustifolia, T2334 Elephantopus scaber, T2387 Epilobium hirsutum, T2391 Epimedium brevicornum, T2649 Evolvulus alsinoides, T2657 Fagopyrum cymosum [Syn. Polygonum cymosum], T2658 Fagopyrum esculentum, T2659 Fagopyrum esculentum, T2718 Ficus carica, T2727 Ficus simplicissima, T2772 Fraxinus mandshurica, T2837 Gallus gallus domesticus, T2961 Ginkgo biloba, T2962 Ginkgo biloba, T2964 Ginkgo biloba, T3026 Gnaphalium affine [Syn. Gnaphalium multiceps], T3077 Gymnadenia conopsea, T3193 Hemerocallis fulva, T3195 Hemerocallis lilio-asphodelus, T3197 Hemerocallis minor, T3241 Hibiscus mutabilis, T3242 Hibiscus rosa-sinensis, T3243 Hibiscus rosa-sinensis, T3244 Hibiscus syriacus, T3295 Huperzia serrata [Syn. Lycopodium serratum], T3570 Juglans regia, T3649 Laccifer lacca, T3671 Lagerstroemia indica, T3681 Lamium barbatum, T3770 Lespedeza cuneata, T3932 Ludwigia octovalvis, T3957 Lycium chinense, T3981 Lycopus lucidus, T3990 Lygodium japonicum, T3999 Lysimachia clethroides, T4036 Magnolia coco, T4056 Mahonia bealei, T4057 Mahonia bealei, T4063 Mahonia fortunei, T4067 Mahonia japonica, T4068 Mahonia japonica, T4082 Mallotus japonicus, T4091 Malva sylvestris, T4172 Melilotus suaveolens, T4206 Metaplexis japonica, T4209 Michelia alba, T4397 Nelumbo nucifera, T4482 Oenanthe javanica, T4526 Origanum vulgare, T4552 Osmunda japonica, T4562 Oxalis acetosella, T4564 Oxalis corniculata [Syn. Oxalis repens], T4575 Pachysandra terminalis, T4629 Papaver nudicaule, T4630 Papaver nudicaule ssp. amurense, T4787 Phellinus igniarius, T5098 Polygonum aviculare, T5100 Polygonum chinense, T5129 Polyporus umbellatus, T5284 Pteridium aquilinum var. latiusculum, T5295 Pteris multifida, T5323 Pulsatilla campanella, T5324 Pulsatilla cernua, T5325 Pulsatilla chinensis, T5326 Pulsatilla dahurica, T5332 Punica granatum, T5529 Rhodomyrtus tomentosa, T5554 Rodgersia aesculifolia, T5567 Rosa laevigata, T5586 Rubia yunnanensis, T5750 Saururus chinensis, T5753 Saussurea gnaphaloides, T5757 Saussurea laniceps, T5759 Saussurea medusa, T5939 Shiraia bambusicola, T5954 Silene fortunei, T5966 Siphonostegia chinensis, T5976 Smilax china [Syn. Smilax japonica], T5977 Smilax glabra, T5980 Smilax menispermoidea, T6031 Sophora flavescens [Syn. Sophora angustfolia], T6357 Tetrapanax papyriferus, T6474 Toona ciliata, T6475 Toona sinensis, T6601 Umbilicaria esculenta [Syn. Gyrophora esculenta], T6645 Urena lobata, T6653 Usnea diffracta, T6654 Usnea longissima, T6683 Vallisneria spiralis, T6749 Vicia hirsuta, T6808 Waltheria americana.

vaginal discharge and pudendal itch T0318 Allium sativum.

vaginal protrusion (prolapse of uterus) T5671 Salvia digitaloides.

vaginitis T2715 Fibraurea recisa, T5724 Sapium sebiferum.

varicella T4552 Osmunda japonica.

variola T4548 Oryza sativa var. glutinosa.

various hemorrhage T2841 Gallus gallus domesticus, T4568 Oxytropis myriophylla, T5018 Pleuropterus ciliinervis.

various pain T0183 Adlumia cirrhosa [Syn. Adlumia fungosa], T6135 Stephania succifera.

various pains $\mathrm{T} 1716$ Corydalis gigantea, $\mathrm{T} 5821$ Scopolia japonica. 
various pains due to blood stasis and rheumatic impediment T0495

Angelica sinensis, T4804 Phlojodicarpus sibiricus.

vascular headache T0644 Armillaria mellea.

vascular migraine T4303 Moschus moschiferus; Moschus berezovskii;

Moschus sifanicus.

vertex headache T3822 Ligusticum jeholense, T3824 Ligusticum sinense,

T4551 Osmorhiza aristata var. laxa.

vesical calculus T3174 Heliotropium indicum.

vesicle sore T1197 Cardiospermum halicacabum.

vexation T2060 Delonix regia, T2882 Gardenia jasminoides [Syn.

Gardenia florida], T3921 Lophatherum gracile, T5661 Salvia

bowleyana.

vexation and agitation T1662 Coptis chinensis, T1663 Coptis chinensis var. brevisepala, T1664 Coptis deltoidea, T1669 Coptis omeiensis, T1670 Coptis teetoides [Syn. Coptis teeta], T3304 Hydrangea macrophylla, T4029 Maesa japonica, T5680 Salvia miltiorrhiza, T5681 Salvia miltiorrhiza f. alba, T5688 Salvia przewalskii.

vexation and agitation in night T4904 Pinellia ternata.

vexation and agitation with insomnia T5428 Rauvolfia yunnanensis.

vexation and fullness T2839 Gallus gallus domesticus.

vexation and oppression due to summerheat-heat T1448 Cirsium chinense.

vexation and reddish urine T0632 Aristolochia manshuriensis.

vexation and thirst T0438 Anacardium occidentale, T0834 Averrhoa carambola, T1152 Camellia sinensis [Syn. Thea sinensis], T1165 Canarium album, T2246 Dolichos lablab, T2431 Eriobotrya japonica, T3286 Hovenia dulcis, T3876 Litchi chinensis, T3957 Lycium chinense, T4348 Myrica rubra, T4545 Oryza sativa, T4618 Panicum miliaceum, T5826 Scrophularia buergeriana, T5828 Scrophularia ningpoensis, T6416 Thamnolia vermicularis.

vexation due to febrile disease $\mathrm{T} 2803$ Frullania tamarisci ssp. moniliata [Syn. Frullania moniliata].

viral conjunctivitis T3908 Lonicera bournei, T3909 Lonicera confusa, T3910 Lonicera fulvotomentosa, T3911 Lonicera hypoglauca, T3912 Lonicera japonica, T3914 Lonicera macranthoides, T3918 Lonicera similis.

viral infection T0866 Baphicacanthus cusia [Syn. Strobilanthes cusia], T3085 Gynostemma pentaphyllum.

viral hepatitis T0379 Alternanthera philoxeroides.

visceral agitation T6544 Triticum aestivum [Syn. Triticum vulgare], T6562 Tulipa gesneriana, T6675 Valeriana amurensis, T6679 Valeriana officinalis, T6680 Valeriana officinalis var. latifolia, T6916 Ziziphus jujuba, T6917 Ziziphus jujuba var. inermis.

vomiting T0012 Abrus precatorius, T0156 Actinidia arguta, T0357 Alpinia japonica, T0358 Alpinia katsumadai, T0359 Alpinia officinarum, T0417 Amomum longiligulare, T0418 Amomum muricarpum, T0419 Amomum villosum, T0420 Amomum xanthioides, T1168 Canavalia ensiformis, T1169 Canavalia gladiata, T1182 Capra hircus; Ovis aries, T1186 Capsicum annuum, T1187 Capsicum frutescens, T1469 Citrus aurantium var. amara, T1501 Citrus medica var. sarcodactylis, T1506 Citrus reticulata, T2643 Evodia meliifolia, T2744 Foeniculum vulgare, T2867 Garcinia multiflora, T3286 Hovenia dulcis, T3372 Hypodematium sinense, T4048 Magnolia rostrata, T4099 Mangifera indica, T4103 Mangifera persiciformis, T4443 Notholirion hyacinthinum [Syn. Notholirion bulbuliferum], T4600 Panax ginseng [Syn. Panax schinseng], T4953 Piper longum, T5139 Poncirus trifoliata, T5389 Rabdosia coetsa, T5419 Raphanus sativus, T5754 Saussurea graminea, T6869 Zanthoxylum bungeanum, T6892 Zanthoxylum schinifolium, T6909 Zingiber officinale, T6910 Zingiber officinale.

vomiting and diarrhea T0190 Adonis sutchuenensis, T0212 Agastache rugosus, T0360 Alpinia oxyphylla, T0363 Alpinia speciosa, T0374 Alstonia scholaris, T0819 Atractylodes chinensis, T0821 Atractylodes japonica, T0823 Atractylodes lancea, T1436 Cinnamomum camphora, T1441 Cinnamomum japonicum, T1592 Cocos nucifera, T2343 Elsholtzia splendens, T3234 Heteropogon contortus, T3431 Inula helenium, T3437 Inula racemosa, T4034 Magnolia biloba, T4039 Magnolia grandiflora, T4045 Magnolia officinalis, T4304 Mosla chinensis [Syn. Orthodon chinensis], T4348 Myrica rubra, T4470 Ocimum basilicum, T4714 Perilla frutescens, T6499 Tribulus terrestris, T6677 Valeriana jatamansii [Syn. Valeriana wallichii], T6788 Vitex negundo, T6888 Zanthoxylum planispinum, T6893 Zanthoxylum simulans, T6894 Zanthoxylum simulans.

vomiting and diarrhea with cramp T0978 Bombyx mori, T1342 Chaenomeles lagenaria [Syn. Chaenomeles speciosa], T1343 Chaenomeles sinensis, T4818 Phoebe nanmu.

vomiting and dysentery T4979 Pistacia chinensis.

vomiting and eructation T3427 Inula britannica, T3428 Inula britannica var. chinensis, T3434 Inula linariaefolia.

vomiting and hiccough T1202 Carex kobomugi, T1483 Citrus grandis var. tomentosa.

vomiting diarrhea with abdominal pain T1941 Cymbopogon distans, T2205 Dioscorea panthaica, T4086 Malus asiatica, T4526 Origanum vulgare, T5059 Pogostemon cablin [Syn. Mentha cablin], T5103 Polygonum hydropiper, T5836 Scutellaria discolor, T6827 Wisteria sinensis.

vomiting due to liver stomach qi stagnation T1498 Citrus medica, T1520 Citrus wilsonii.

vomiting in pregnancy T1490 Citrus limon, T1494 Citrus limonia, T2263 Dregea volubilis, T6277 Tadehagi triquetrum, T6285 Tamarindus indica.

vomiting nausea T0825 Atropa belladonna, T1353 Changium smyrnioides, T2838 Gallus gallus domesticus, T4904 Pinellia ternata, T5642 Saccharum sinensis, T6488 Trachyspermum ammi.

vomiting of milk in infants with stomach cold T0416 Amomum kravanh [Syn. Amomum cardamomum].

vomiting of sour matter T1937 Cydonia oblonga, T2431 Eriobotrya japonica, T4983 Pisum sativum, T6754 Vigna unguiculata.

vomiting of water due to congealing cold $q \boldsymbol{i}$ stagnation T5715 Santalum album.

vomiting with stomach cold T0416 Amomum kravanh [Syn. Amomum cardamomum], T1008 Brassica juncea, T3409 Illicium verum, T4957 Piper nigrum.

wandering wind of head and face T0167 Adenanthera pavonina. warm disease T2756 Forsythia suspensa, T3868 Liquidambar orientalis. warm disease ardent fever T5120 Polygonum tinctorium. 
warm disease fever T0457 Andrographis paniculata [Syn. Justicia paniculata], T1538 Clausena lansium, T1727 Corydalis mucronifera, T3908 Lonicera bournei, T3909 Lonicera confusa, T3910 Lonicera fulvotomentosa, T3911 Lonicera hypoglauca, T3912 Lonicera japonica, T3913 Lonicera japonica, T3914 Lonicera macranthoides, T3918 Lonicera similis, T5121 Polygonum tinctorium.

warm disease macular eruption T5445 Rehmannia glutinosa [Syn.

Rehmannia glutinosa f. huechingensis].

warm evil in construction T3476 Isatis indigotica.

warm heat disease T0462 Anemarrhena asphodeloides, T0867

Baphicacanthus cusia [Syn. Strobilanthes cusia].

warm heat disease with fever T0942 Biebersteinia heterostemon, T1950

Cynanchum ascyrifolium, T1951 Cynanchum atratum, T1962

Cynanchum versicolor, T2952 Gerbera piloselloides, T4582 Paeonia

delavayi, T4585 Paeonia moutan [Syn. Paeonia suffruticosa], T6582

Tylophora ovata.

warm heat macular eruption T2281 Dryopteris crassirhizoma.

warm malaria T0427 Ampelopsis japonica [Syn. Paullinia japonica],

T4670 Patrinia heterophylla, T4673 Patrinia scabra.

warm toxin macular eruption T0866 Baphicacanthus cusia [Syn.

Strobilanthes cusia], T3475 Isatis indigotica, T4584 Paeonia lactiflora

wild, T4586 Paeonia obovata, T4590 Paeonia veitchii.

wart T0971 Bolbostemma paniculatum, T1954 Cynanchum chinense,

T2597 Euphorbia lathyris, T3659 Lactuca indica, T4102 Mangifera

indica, T4338 Mylabris phalerata; Mylabris cichorii, T5026

Plumbagella micrantha.

water diarrhea T0328 Alnus japonica, T1937 Cydonia oblonga, T1943

Cymbopogon goeringii, T2193 Dioscorea cirrhosa [Syn. Dioscorea

pogonoides], T6746 Vicia faba.

water gu T5542 Rhus verniciflua [Syn. Toxicadendron verniciflum].

water-damp fullness T1980 Cyprinus carpio.

water-rheum collecting internally T0824 Atractylodes macrocephala

[Syn. Atractylis macrocephala].

water-rheum collecting lung T4287 Morus alba.

weakness during convalescence T0515 Anredera cordifolia [Syn.

Baussingaultia cordifolia; Baussingaultia gracilis f. pseudobaselloides;

Baussingaultia gracilis var. pseudobaselloides], T0541 Apis cerana,

T1751 Corylus heterophylla, T2837 Gallus gallus domesticus, T4607

Panax pseudo-ginseng var. japonicus, T5260 Pseudostellaria heterophylla, T6087 Spiranthes sinensis, T6489 Tragopogon porrifolius.

weakness in limbs T3970 Lycopodium cernuum, T3973 Lycopodium japonicum [Syn. Lycopodium clavatum].

weakness in lumbus and knees T3389 Ilex cornuta, T5755 Saussurea involucrata.

weakness in sinews and bones T1455 Cistanche deserticola, T1456 Cistanche salsa, T2530 Eucommia ulmoides, T4536 Orobanche coerulescens, 55638 Sabia swinhoei, T6670 Vaccinium bracteatum.

weakness of legs $\mathrm{T} 5972$ Skimmia reevesiana.

weakness of rigth $q i \quad \mathrm{~T} 3742$ Lentinus edodes.

weekness in legs and knees T4828 Photinia serrulata.

welling abscess $\mathrm{T} 0012$ Abrus precatorius, T0226 Agave sisalana, T0421

Amorpha fruticosa, T0495 Angelica sinensis, T2341 Elsholtzia ciliata,
T2756 Forsythia suspensa, T3001 Glycine max, T3190 Helminthostachys zeylanica, T3908 Lonicera bournei, T3909 Lonicera confusa, T3910 Lonicera fulvotomentosa, T3911 Lonicera hypoglauca, T3912 Lonicera japonica, T3914 Lonicera macranthoides, T3918 Lonicera similis, T4253 Mirabilis jalapa, T4804 Phlojodicarpus sibiricus, T5118 Polygonum suffultum, T5323 Pulsatilla campanella, T5324 Pulsatilla cernua, T5325 Pulsatilla chinensis, T5326 Pulsatilla dahurica, T5531 Rhus chinensis [Syn. Rhus semialata], T6018 Solanum verbascifolium, T6279 Tagetes erecta.

welling abscess and boil T0584 Arctium lappa, T1031 Broussonetia kazinoki, T1373 Chloranthus serratus, T1450 Cirsium lineare, T1731 Corydalis pallida, T2162 Dicranostigma franchetianum [Syn. Dicranostigma leptopodum], T3808 Ligularia japonica [Syn. Arnica japonica; Senecio japonica], T5250 Psammosilene tunicoides, T5339 Pycnoporus sanguineus, T5892 Senecio nemorensis, T6020 Solanum xanthocarpum, T6095 Stachytarpheta jamaicensis, T6491 Trametes cinnabarina [Syn. Polyporus cinnabarinus; Boletus cinnabarinus], T6808 Waltheria americana.

welling abscess and clove sore T1869 Cryptotaenia japonica, T3674 Laggera alata.

welling abscess and eczema T5925 Sesamum indicum [Syn. Sesamum orientale].

welling abscess and flat abscess T0463 Anemone altaica, T0648 Arnebia euchroma, T0649 Arnebia guttata, T1049 Bufo bufo gargarizans; Bufo melanostictus, T1051 Bufo bufo gargarizans; Bufo melanostictus, T1286 Celastrus flagellaris, T1562 Clerodendron trichotomum, T1565 Clerodendrum bungei, T1693 Coriaria sinica [Syn. Coriaria nepalensis], T1858 Croton tiglium, T1871 Cucubalus baccifer, T2139 Dianella ensifolia, T2580 Euphorbia antiquorum, T2584 Euphorbia ebracteolata, T2587 Euphorbia fischeriana, T2756 Forsythia suspensa, T3128 Hedysarum multijugum, T3396 Ilex pubescens, T3397 Ilex pubescens var. glaber, T3411 Impatiens balsamina, T3881 Lithospermum erythrorhizon, T4042 Magnolia liliflora, T4428 Nicotiana tabacum, T5220 Prunus davidiana, T5232 Prunus persica, T6497 Tribulus terrestris, T6560 Tulipa edulis.

welling abscess and flat abscess with clove sore T0138 Aconitum umbrosum, T0987 Bos taurus domesticus; Bubalus bubalis, T2132 Desmodium pulchellum [Syn. Phyllodium pulchellum], T3088 Gynura segetum [Syn. Gynura japonica], T4000 Lysimachia congestiflora, T5576 Rostellularia procumbens [Syn. Justicia procumbens], T6463 Tinospora capillipes, T6467 Tinospora sagittata, T6767 Viola yedoensis.

welling abscess and flat abscess with clove sores T0922 Berchemia polyphylla var. leioclada, T1052 Bufo bufo gargarizans; Bufo melanostictus.

welling abscess and flat abscess with swelling sore T3624 Kalopanax pictum, T3625 Kalopanax septemlobus, T5642 Saccharum sinensis, T6764 Vinegar.

welling abscess and flat abscess with swollen boil T0382 Althaea rosea. welling abscess and open sore T3026 Gnaphalium affine [Syn. Gnaphalium multiceps].

welling abscess and sores T0292 Albizzia julibrissin, T1338 Cestrum nocturnum, T1546 Clematis tangutica, T2062 Delphinium bonvalotii, 
T2081 Delphinium omeiense, T2084 Delphinium potaninii, T2085 Delphinium potaninii var. jiufengshanense, T2757 Forsythia viridissima, T3675 Lagopsis supina.

welling abscess and sores with sore and boil T0156 Actinidia arguta. welling abscess and toxin of clove T4264 Momordica cochinchinensis. welling abscess boil and clove sores $\mathrm{T} 3645$ Kummerowia striata.

welling abscess of ear T2517 Eucalyptus robusta.

welling abscess toxin T5579 Rubia cordifolia.

welling abscess with pus swelling T0002 Abelmoschus moschatus [Syn.

Hibiscus abelmoschus], T3145 Helianthus annuus.

welling abscessand sores T2716 Ficus beecheyana [Syn. Ficus erecta var. beecheyana].

white dysentery T4305 Mosla dianthera.

white mouth sore in children T3348 Hypericum elodeoides, T3368

Hypericum wightianum, T5894 Senecio oryzetorum.

white patch wind T0583 Archangelica decurrens, T1359 Chenopodium album, T1911 Cuscuta australis, T1912 Cuscuta chinensis, T1913 Cuscuta japonica, T2273 Drynaria fortunei, T2598 Euphorbia lathyris, T3390 Ilex cornuta, T3567 Juglans regia, T4432 Nigella glandulifera, T5252 Pseudodrynaria coronans, T5270 Psoralea corylifolia, T6713 Vernonia anthelmintica.

white turbidity T0048 Acanthus ilicifolius, T0255 Ailanthus altissima, T0360 Alpinia oxyphylla, T0895 Benincasa hispida, T0977 Bombyx mori, T1001 Brachystemma calycinum, T1275 Cayratia japonica, T1756 Costus speciosus, T2194 Dioscorea collettii, T2197 Dioscorea futschauensis, T2198 Dioscorea gracillima, T2201 Dioscorea hypoglauca [Syn. Dioscorea collettii var. hypoglauca], T2210 Dioscorea spongiosa, T2212 Dioscorea tokoro, T2391 Epimedium brevicornum, T2658 Fagopyrum esculentum, T3615 Kadsura interior, T3770 Lespedeza cuneata, T3932 Ludwigia octovalvis, T3990 Lygodium japonicum, T4252 Mirabilis jalapa, T5554 Rodgersia aesculifolia, T5651 Salix babylonica, T5685 Salvia plebeia, T6025 Sonchus arvensis, T6095 Stachytarpheta jamaicensis, T6474 Toona ciliata, T6475 Toona sinensis, T6754 Vigna unguiculata.

white turbidity vaginal discharge T1900 Curculigo capitulata [Syn. Leucojum capitulata].

whole body swelling T2995 Gloriosa superba.

wilting-impediment of limbs T0569 Aralia cordata, T0574 Aralia fargesii.

wilting-weakness in limbs T2737 Flemingia philippinensis [Syn. Moghania philippinensis], T4260 Moghania philippinensis.

wind damage and common cold T2056 Debregeasia longifolia, T3520 Isodon rosthornii, $\mathrm{T} 6789$ Vitex negundo.

wind damage common cold $\mathrm{T} 1137$ Caltha palustris.

wind due to blood heat T1161 Campsis grandiflora.

wind eye with ulceration of eyelid rim T6251 Symplocos caudata.

wind impediment T1171 Canis familiaris, T1205 Carica papaya, T3001

Glycine max, T4912 Pinus koraiensis, T5151 Populus davidiana,

T5234 Prunus persica, T5368 Python molurus bivittatus, T5579 Rubia

cordifolia, T6439 Thuja orientalis [Syn. Platycladus orientalis; Biota orientalis].

wind impediment and hypertonicity of sinews T3000 Glycine max.

wind impediment and lumbago T6197 Styrax benzoin, T6204 Styrax tonkinensis.

wind lai T6843 Xanthium sibiricum [Syn. Xanthium strumarium].

wind lai lichen T5091 Polygonatum cyrtonema [Syn. Polygonatum multiflorum], T5092 Polygonatum kingianum, T5095 Polygonatum sibiricum.

wind papule itching T0540 Apis cerana, T0978 Bombyx mori, T2257 Dracocephalum moldavicum, T2367 Ephedra equisetina, T2368 Ephedra gerardiana, T2369 Ephedra intermedia, T2372 Ephedra likiangensis, T2373 Ephedra minuta, T2375 Ephedra monosperma, T2379 Ephedra saxatilis, T2380 Ephedra sinica, T2434 Eriocaulon buergerianum, T3202 Hemistepta lyrata [Syn. Hemistepta carthamoides; Saussurea carthamoides], T3222 Heracleum scabridum, T4231 Milingtonia hortensis, T4906 Pinus armandii, T4916 Pinus massoniana, T5107 Polygonum multiflorum, T5108 Polygonum multiflorum, T5126 Polypodium virginianum, T5127 Polypodium vulgare, T5727 Saposhnikovia divaricata [Syn. Ledebouriella seseloides], T6286 Tamarix chinensis, T6290 Tamarix ramosissima, T6325 Tectona grandis.

wind papule itching due to blood heat T1160 Campsis grandiflora.

wind papule itching of skin T6499 Tribulus terrestris.

wind papules T0297 Alchornea trewioides, T0423 Ampelopsis brevipedunculata, T0586 Arctium lappa, T0692 Artemisia rupestris [Syn. Artemisia dentata; Artemisia viridis; Artemisia viridifolia], T0932 Betula luminifera, T1032 Broussonetia papyrifera, T1084 Buthus martensi, T1161 Campsis grandiflora, T1311 Centella asiatica, T2136 Desmos cochinchinensis [Syn. Desmos chinensis], T2194 Dioscorea collettii, T2325 Eichhornia crassipes, T2505 Eucalyptus citriodora, T2517 Eucalyptus robusta, T2984 Glinus lotoides [Syn. Mollugo lotoides], T3417 Imperata cylindrica var. major, T3737 Lemmaphyllum microphyllum var. obovatum, T4154 Melaleuca leucadendra, T4307 Mosla scabra [Syn. Mosla punctata], T5104 Polygonum hydropiper, T5269 Psilotum nudum, T5401 Rabdosia yuennanensis, T5704 Sambucus nigra, T5707 Sambucus williamsii, T5773 Saxifraga stolonifera, T5804 Schizonepeta tenuifolia [Syn. Nepeta tenuifolia], T5934 Seseli meirei, T5937 Seseli yunnanense, T5950 Siegesbeckia orientalis, T5951 Siegesbeckia orientalis var. glabrescens [Syn. Siegesbeckia glabrescens], T5952 Siegesbeckia orientalis var. pubescens [Syn. Siegesbeckia pubescens], T6367 Teucrium quadrifarium.

wind papules $[=$ rubella] T4828 Photinia serrulata, T6365 Teucrium pilosum [Syn. Teucrium japonicum var. pilosum].

wind sore T2981 Gleditsia sinensis [Syn. Gleditsia horrida].

wind stroke T0142 Acorus calamus, T1876 Cucumis melo, T2274 Dryobalanops aromatica, T2975 Gleditsia delavayi, T6183 Strychnos nitida.

wind stroke (apoplexy) T2976 Gleditsia fera.

wind stroke clenched jaw T2978 Gleditsia sinensis [Syn. Gleditsia horrida].

wind stroke paralysis T0116 Aconitum nagarum var. heterotrichum [Syn. Aconitum bullatifolium].

wind stroke with congesting phlegm T0088 Aconitum coreanum, T0617 Arisaema amurense, T0618 Arisaema consanguineum, T0620 Arisaema heterophyllum, T4903 Pinellia pedatisecta, T6588 Typhoniun 
giganteum, T6697 Veratrum grandiflorum, T6698 Veratrum nigrum. wind stroke with deviated eyes and mouth T1084 Buthus martensi. wind stroke with hemiplegia T0108 Aconitum kusnezoffii, T0164 Actinidia polygama, T0983 Bos taurus domesticus, T2753 Formica fusca, T3396 Ilex pubescens, T3397 Ilex pubescens var. glaber, T4696 Penaeus orientalis, T4796 Pheretima aspergillum; Allolobophora caliginosa trapezoides.

wind stroke with loss of speech T6358 Tetraplodon mnioides [Syn.

Tetraplodon bryoides; Splachnum mnioides].

wind stroke with orifice block T0987 Bos taurus domesticus; Bubalus bubalis.

wind stroke with phlegm reversal T3868 Liquidambar orientalis, T4303

Moschus moschiferus; Moschus berezovskii; Moschus sifanicus.

wind swelling T5651 Salix babylonica.

wind swelling and pruritus T5650 Salix babylonica.

wind toxin and beriberi $\mathrm{T} 3000$ Glycine max, T4937 Piper betle.

wind toxin and swollen welling abscess T5301 Pterocarpus indicus.

wind toxin swollen face T0584 Arctium lappa.

wind warmth T1395 Chrysanthemum morifolium [Syn. Dendranthema morifolium].

wind water edema T2367 Ephedra equisetina, T2368 Ephedra gerardiana, T2369 Ephedra intermedia, T2372 Ephedra likiangensis, T2373 Ephedra minuta, T2375 Ephedra monosperma, T2379 Ephedra saxatilis, T2380 Ephedra sinica, T5963 Sinodielsia yunnanensis.

wind yang harassing upper body T1528 Cladonia stellaris [Syn. Cladonia alpestris].

wind-cold common cold T0310 Allium ascalonicum, T0314 Allium fistulosum, T0476 Angelica anomala, T0478 Angelica dahurica [Syn. Angelica porphyrocaulis], T0676 Artemisia dracunculus, T0692 Artemisia rupestris [Syn. Artemisia dentata; Artemisia viridis; Artemisia viridifolia], T0724 Asarum caulescens, T0726 Asarum forbesii, T0727 Asarum fukienense, T0728 Asarum heterotropoides var. mandshuricum, T0729 Asarum maximum, T0731 Asarum sieboldii, T0732 Asarum sieboldii var. seoulensis, T0752 Asparagus officinalis, T0926 Berneuxia thibetica, T0957 Blumea balsamifera, T1312 Centipeda minima, T1371 Chloranthus japonicus, T1435 Cinnamomum camphora, T1440 Cinnamomum glanduliferum, T1443 Cinnamomum parthenoxylum [Syn. Cinnamomum porrectum], T1641 Conioselinum vaginatum, T1941 Cymbopogon distans, T2505 Eucalyptus citriodora, T2956 Geum japonicum, T3026 Gnaphalium affine [Syn. Gnaphalium multiceps], T3124 Hedyotis capitellata, T3221 Heracleum rapula, T3222 Heracleum scabridum, T3796 Libanotis buchtormensis, T3847 Lindera angustifolia, T4000 Lysimachia congestiflora, T4035 Magnolia biondii [Syn. Magnolia fargesii], T4038 Magnolia denudata [Syn. Magnolia heptapata], T4041 Magnolia liliflora, T4052 Magnolia sprengeri, T4305 Mosla dianthera, T4551 Osmorhiza aristata var. laxa, T4763 Peucedanum morisonii, T5021 Pleurospermum rivulorum, T5113 Polygonum persicaria, T5389 Rabdosia coetsa, T5639 Sabina chinensis, T5987 Sobina chinensis, T6457 Tilia japonica, T6458 Tilia miqueliana, T6677 Valeriana jatamansii [Syn. Valeriana wallichii], T6883 Zanthoxylum myriacanthum, T6910 Zingiber officinale.

wind-cold cough T1046 Buddleja davidii, T3426 Inula britannica, T3427
Inula britannica, T3428 Inula britannica var. chinensis, T3433 Inula japonica, T3434 Inula linariaefolia, T3810 Ligularia nelumbifolia, T3871 Liriodendron tulipifera, T4443 Notholirion hyacinthinum [Syn. Notholirion bulbuliferum], T4937 Piper betle, T4948 Piper hancei, T4965 Piper sarmentosum, T6111 Stemona japonica, T6113 Stemona sessilifolia, T6115 Stemona tuberosa, T6874 Zanthoxylum dimorphophyllum var. spinifolum.

wind-cold cough and asthma with abundant phlegm T1483 Citrus grandis var. tomentosa.

wind-cold exterior repletion syndrome T2367 Ephedra equisetina, T2368 Ephedra gerardiana, T2369 Ephedra intermedia, T2372 Ephedra likiangensis, T2373 Ephedra minuta, T2375 Ephedra monosperma, T2379 Ephedra saxatilis, T2380 Ephedra sinica.

wind-cold exterior syndrome T0491 Angelica polymorpha, T1438 Cinnamomum cassia [Syn. Cinnamomum aromaticum], T4718 Perilla frutescens var. acuta [Syn. Perilla frutescens var. purpurascens], T4721 Perilla frutescens var. arguta.

wind-cold headache T4962 Piper puberulum.

wind-cold impediment pain T1434 Cinnamomum bejolghota [Syn. Cinnamomum obtusifolium; Laurus bejolghota], T1939 Cymbopogon citratus, T3869 Liquor.

wind-cold sinew and bone numbness T1550 Cleome gynandra [Syn. Gynandropsis gynandra].

wind-cold-damp impediment T0036 Acanthopanax giraldii [Syn. Acanthopanax giraldii var. inermis; Eleutherococcus giraldii], T0038 Acanthopanax gracilistylus, T0042 Acanthopanax senticosus [Syn. Eleutherococcus senticosus], T0044 Acanthopanax sessiliflorus, T0077 Aconitum balfourii, T0080 Aconitum bullatifolium var. homotrichum [Syn. Aconitum nagarum], T0083 Aconitum carmichaeli, T0084 Aconitum carmichaeli, T0085 Aconitum carmichaeli cv, T0086 Aconitum chasmanthum, T0090 Aconitum delavayi, T0098 Aconitum geniculatum, T0105 Aconitum karakolicum, T0106 Aconitum kirinense, T0108 Aconitum kusnezoffii, T0123 Aconitum polyschistum, T0135 Aconitum sungpanense, T0138 Aconitum umbrosum, T0164 Actinidia polygama, T0470 Anemone raddeana, T0582 Archangelica brevicaulis [Syn. Angelicarpa brevicaulis; Angelica brevicaulis], T0696 Artemisia sieversiana, T0726 Asarum forbesii, T1641 Conioselinum vaginatum, T1923 Cyathula capitata, T2039 Datura innoxia, T2043 Datura metel, T2047 Datura stramonium, T2091 Delphinium yunnanense, T2203 Dioscorea nipponica, T2204 Dioscorea nipponica ssp. rosthornii, T2750 Fomitopsis pinicola [Syn. Fomes pinicola; Polyporus pinicola], T3213 Heracleum hemsleyanum, T3217 Heracleum moellendorffii [Syn. Heracleum microcarpum; Heracleum morifolium], T3221 Heracleum rapula, T3222 Heracleum scabridum, T3228 Heracleum yungningense, T3401 Illicium henryi, T3822 Ligusticum jeholense, T3824 Ligusticum sinense, T3970 Lycopodium cernuum, T3973 Lycopodium japonicum [Syn. Lycopodium clavatum], T4283 Morinda officinalis, T4439 Nothapodytes pittosporoides, T4445 Notopterygium forbesii [Syn. Notopterygium franchetii], T4446 Notopterygium incisum, T4516 Oplopanax elatus, T4725 Periploca calophylla, T4948 Piper hancei, T4950 Piper kadsura [Syn. Piper futokadsura], T4974 Piper wallichii [Syn. Piper wallichii var. hupehense], T5021 Pleurospermum rivulorum, T5113 Polygonum persicaria, T5517 
Rhododendron molle, T5822 Scopolia sinensis, T5924 Sesamum indicum, T6106 Stelmatocrypton khasianum, T6858 Zanthoxylum acanthopodium, T6862 Zanthoxylum ailanthoides, T6874 Zanthoxylum dimorphophyllum var. spinifolum, T6877 Zanthoxylum dissitum, T6893 Zanthoxylum simulans, T6894 Zanthoxylum simulans.

wind-cold-damp impediment (especially in lower part of body) T0492 Angelica pubescens f. biserrata [Syn. Angelica pubescens], T3214 Heracleum lanatum.

wind-damp T4838 Phyllanthus flexuosus, T6155 Strophanthus divaricatus, T6326 Telekia speciosa.

wind-damp (rheumatis) ${ }^{[5509]}$ T5248 Psacalium peltatum.

wind-damp bone pain T0010 Abrus fruticulosus [Syn. Abrus cantoniensis], T0012 Abrus precatorius, T0079 Aconitum brachypodum, T0117 Aconitum nagarum var. lasiandrum, T0500 Anguilla japonica, T0847 Baccharis indica [Syn. Pluchea indica], T0888 Beesia calthaefolia, T0922 Berchemia polyphylla var. leioclada, T1120 Callicarpa macrophylla, T1191 Caragana sinica, T1537 Clausena lansium, T1556 Clerodendron fragrans, T1557 Clerodendron indicum, T1657 Conyza canadensis [Syn. Erigeron canadensis], T1815 Crotalaria assamica, T1871 Cucubalus baccifer, T2052 Davallia divaricata [Syn. Davallia formosana; Davallia orientalis], T2233 Diploclisia glaucescens, T2505 Eucalyptus citriodora, T2641 Evodia lepta [Syn. Ilex lepta], T2721 Ficus microcarpa, T2980 Gleditsia sinensis [Syn. Gleditsia horrida], T3414 Impatiens siculifer, T3736 Lemmaphyllum microphyllum, T3849 Lindera chunii, T4154 Melaleuca leucadendra, T4268 Monachosorum henryi, T4317 Murraya euchrestifolia [Syn. Clausena euchrestifolia], T4937 Piper betle, T5401 Rabdosia yuennanensis, T5587 Rubus alceaefolius, T5626 Ruta graveolens, T5650 Salix babylonica, T5672 Salvia flava, T5795 Schisandra micrantha, T5847 Securidaca inappendiculata, T6095 Stachytarpheta jamaicensis, T6286 Tamarix chinensis, T6290 Tamarix ramosissima, T6866 Zanthoxylum avicennae, T6884 Zanthoxylum nitidum.

wind-damp bone pain (root cortex branchlet-leaf) T0945 Bischofia javanica [Syn. Bischofia trifoliata].

wind-damp edema T1097 Cacalia ainsliaeflora.

wind-damp impediment T1084 Buthus martensi, T1562 Clerodendron trichotomum, T2479 Erythrina variegata var. orientalis, T4085 Mallotus repandus var. chrysocarpus [Syn. Mallotus chrysocarpus; Mallotus repandus], T5648 Salacia prinoides [Syn. Salacia chinensis], T5753 Saussurea gnaphaloides, T5757 Saussurea laniceps, T5759 Saussurea medusa, T5975 Smilax bockii, T5978 Smilax glauco-china, T5981 Smilax riparia.

wind-damp impediment pain T0045 Acanthopanax trifoliatus, T0060 Achillea alpina [Syn. Achillea sibirica], T0065 Achillea millefolium, T0088 Aconitum coreanum, T0094 Aconitum finetianum, T0125 Aconitum pseudostapfianum, T0130 Aconitum sinomontanum, T0144 Acorus gramineus, T0146 Acorus tatarinowii, T0156 Actinidia arguta, T0161 Actinidia eriantha, T0170 Adhatoda vasica, T0229 Ageratum conyzoides, T0266 Ajuga macrosperma, T0273 Akebia quinata, T0274 Akebia quinata, T0277 Akebia trifoliata, T0278 Akebia trifoliata, T0279 Akebia trifoliata var. australis, T0280 Akebia trifoliata var. australis, T0281 Alangium chinense, T0285 Alangium platanifolium,
T0364 Alsophila spinulosa, T0383 Alyxia sinensis, T0463 Anemone altaica, T0491 Angelica polymorpha, T0503 Anisomeles indica [Syn. Epimeredi indica], T0567 Aralia armata, T0571 Aralia decaisneaa, T0584 Arctium lappa, T0595 Ardisia crispa, T0600 Ardisia mamillata [Syn. Tinus mamillata], T0601 Ardisia pusilla, T0602 Ardisia quinquegona, T0608 Arenaria kansuensis [Syn. Arenaria kumaonensis], T0628 Aristolochia fangchi, T0634 Aristolochia mollissima, T0661 Artemisia anomala, T0721 Arundina chinensis, T0724 Asarum caulescens, T0727 Asarum fukienense, T0728 Asarum heterotropoides var. mandshuricum, T0731 Asarum sieboldii, T0732 Asarum sieboldii var. seoulensis, T0759 Aspidistra elatior, T0776 Asplenium prolongatum, T0814 Atalantia buxifolia [Syn. Severinia buxifolia], T0877 Bauhinia championii, T0886 Beaumontia grandiflora, T0932 Betula luminifera, T0938 Bidens bipinnata, T0957 Blumea balsamifera, T0963 Boehmeria siamensis, T0978 Bombyx mori, T0994 Boswellia carterii, T1031 Broussonetia kazinoki, T1053 Bulbophyllum odoratissimum [Syn. Stelis odoratissimum], T1092 Buxus microphylla var. sinica, T1282 Cedrus deodara, T1285 Celastrus angulatus, T1286 Celastrus flagellaris, T1289 Celastrus orbiculatus [Syn. Celastrus articulatus], T1290 Celastrus orbiculatus [Syn. Celastrus articulatus], T1291 Celastrus paniculatus, T1342 Chaenomeles lagenaria [Syn. Chaenomeles speciosa], T1343 Chaenomeles sinensis, T1361 Chenopodium ambrosioides, T1371 Chloranthus japonicus, T1372 Chloranthus serratus, T1411 Cibotium barometz [Syn. Polypodium barometz], T1435 Cinnamomum camphora, T1436 Cinnamomum camphora, T1437 Cinnamomum camphora, T1440 Cinnamomum glanduliferum, T1443 Cinnamomum parthenoxylum [Syn. Cinnamomum porrectum], T1530 Cladostachys amaranthoides [Syn. Achyranthes amaranthoides; Cladostachys frutescens; Deeringia amaranthoides], T1545 Clematis chinensis, T1547 Clematis terniflora [Syn. Clematis maximowicziana], T1563 Clerodendron trichotomum, T1565 Clerodendrum bungei, T1582 Cnidium monnieri, T1583 Cnidium officinale [Syn. Ligusticum officinale], T1589 Cocculus trilobus [Syn. Cocculus sarmentosus], T1590 Cocculus trilobus [Syn. Cocculus sarmentosus], T1650 Convolvulus arvensis, T1721 Corydalis linearioides, T1831 Crotalaria sessiliflora, T1841 Croton caudatus var. tomentosus, T1884 Cudrania cochinchinensis, T1900 Curculigo capitulata [Syn. Leucojum capitulata], T1921 Cyanotis arachnoidea [Syn. Cyanotis bodinieri], T1922 Cyanotis vaga, T1934 Cyclea tonkinensis, T1941 Cymbopogon distans, T1958 Cynanchum otophyllum, T1959 Cynanchum paniculatum, T1984 Cypripedium macranthum [Syn. Cypripedium tibeticum], T2020 Damnacanthus indicus, T2030 Daphne retusa, T2031 Daphne tangutica, T2036 Datura innoxia, T2037 Datura innoxia, T2041 Datura metel, T2044 Datura metel, T2046 Datura stramonium, T2053 Davallia mariesii, T2058 Deeringia amaranthoides [Syn. Cladostachys frutescens], T2077 Delphinium grandiflorum, T2113 Dennstaedtia scabra [Syn. Dicksonia scabra], T2131 Desmodium pulchellum [Syn. Phyllodium pulchellum], T2133 Desmodium racemosum [Syn. Podocarpium podocarpum var. oxyphyllum], T2136 Desmos cochinchinensis [Syn. Desmos chinensis], T2189 Dioscorea althaeoides, T2194 Dioscorea collettii, T2197 Dioscorea futschauensis, T2198 Dioscorea gracillima, T2201 Dioscorea hypoglauca [Syn. Dioscorea collettii var. 
hypoglauca], T2210 Dioscorea spongiosa, T2211 Dioscorea tenuipes, T2212 Dioscorea tokoro, T2234 Dipsacus asperoides, T2235 Dipsacus japonicus, T2262 Dregea sinensis, T2267 Drosera peltata var. lunata, T2367 Ephedra equisetina, T2368 Ephedra gerardiana, T2369 Ephedra intermedia, T2372 Ephedra likiangensis, T2373 Ephedra minuta, T2375 Ephedra monosperma, T2379 Ephedra saxatilis, T2380 Ephedra sinica, T2389 Epimedium acuminatum, T2390 Epimedium brevicornum, T2392 Epimedium davidii, T2394 Epimedium elongatum, T2398 Epimedium koreanum, T2401 Epimedium pubescens, T2402 Epimedium sagittatum, T2404 Epimedium sutchuenense, T2406 Epimedium wushanense, T2422 Erigeron breviscapus, T2426 Erigeron sumatrensis, T2438 Erodium stephanianum, T2449 Erycibe obtusifolia, T2450 Erycibe schmidtii, T2459 Erythrina arborescens, T2478 Erythrina variegata [Syn. Erythrina indica], T2517 Eucalyptus robusta, T2540 Euonymus fortunei, T2542 Euonymus japonicus, T2545 Euonymus phellomana, T2631 Eurya japonica, T2720 Ficus hispida, T2723 Ficus pumila, T2727 Ficus simplicissima, T2731 Firmiana simplex, T2734 Fissistigma glaucescens [Syn. Melodorum glaucescens], T2735 Fissistigma oldhamii [Syn. Melodorum oldhamii], T2737 Flemingia philippinensis [Syn. Moghania philippinensis], T2738 Flemingia strobilifera, T2752 Fordia cauliflora, T2753 Formica fusca, T2805 Fugu ocellatus, T2890 Gastrodia elata, T2895 Gaultheria yunnanensis, T2897 Gelsemium elegans, T2909 Gentiana crassicaulis, T2910 Gentiana dahurica, T2913 Gentiana kaufmanniana, T2919 Gentiana macrophylla, T2932 Gentiana siphonantha, T2934 Gentiana straminea, T2936 Gentiana tianschanica, T2937 Gentiana tibetica, T2943 Geranium nepalense, T2944 Geranium pratense, T2946 Geranium robertianum, T2947 Geranium sibiricum, T2949 Geranium wilfordii, T2994 Gloiopeltis furcata, T3033 Gnetum parvifolium [Syn. Gnetum indicum], T3080 Gymnema sylvestre, T3113 Hedera nepalensis var. sinensis, T3115 Hedera rhombea, T3201 Hemiphragma heterophyllum, T3234 Heteropogon contortus, T3270 Holboellia fargesii, T3294 Huperzia selago [Syn. Lycopodium selago], T3316 Hylomecon japonica, T3329 Hyoscyamus niger, T3363 Hypericum sampsonii, T3381 Hyptis suaveolens, T3390 Ilex cornuta, T3395 Ilex pedunculosa, T3398 Ilex rotunda, T3410 Impatiens balsamina, T3413 Impatiens nolitangere, T3417 Imperata cylindrica var. major, T3420 Incarvillea sinensis, T3520 Isodon rosthornii, T3613 Kadsura coccinea [Syn. Kadsura chenensis; Kadsura hainanensis], T3614 Kadsura heteroclita [Syn. Uvaria heteroclita], T3615 Kadsura interior, T3624 Kalopanax pictum, T3625 Kalopanax septemlobus, T3628 Kerria japonica, T3643 Kopsia officinalis, T3726 Laurus nobilis, T3746 Leontice robustum, T3796 Libanotis buchtormensis, $\mathrm{T} 3809$ Ligularia lapathifolia, T3820 Ligusticum chuanxiong [Syn. Ligusticum wallichii], T3847 Lindera angustifolia, T3851 Lindera glauca, T3852 Lindera megaphylla, T3867 Liquidambar formosana [Syn. Liquidambar taiwaniana], T3871 Liriodendron tulipifera, T3897 Litsea verticillata, T3925 Loranthus parasiticus [Syn. Loranthus chinenis; Taxillus chinensis], T3965 Lycopodium annotinum, T3969 Lycopodium casuarinoides, T3971 Lycopodium complanatum, T3976 Lycopodium obscurum, T3990 Lygodium japonicum, T3995 Lysidice rhodostegia, T3996 Lysimachia candida, T3997 Lysimachia capillipes, T4005 Lysionotus pauciflorus,
T4106 Manis pentadactyla, T4126 Matteuccia orientalis, T4147 Medicago falcata, T4183 Menispermum dauricum, T4210 Michelia champaca, T4219 Microlepia marginata, T4221 Micromelum falcatum, T4223 Micromelum integerrimum, T4234 Millettia dielsiana, T4238 Millettia nitida, T4239 Millettia nitida var. hirsutissima, T4260 Moghania philippinensis, T4267 Monachosorum flagellare, T4279 Morina chinensis, T4311 Mucuna sempervirens, T4323 Murraya paniculata [Syn. Chalcas paniculata], T4324 Murraya paniculata [Syn. Chalcas paniculata], T4325 Murraya paniculata var. exotica, T4347 Myrica nagi [Syn. Podocarpus nagi], T4361 Myrsine africana, T4368 Nandina domestica, T4444 Nothopanax davidii, T4470 Ocimum basilicum, T4542 Orthosiphon wulfenioides [Syn. Coleus wulfenioides], T4549 Osbeckia chinensis, T4605 Panax japonicus var. major, T4631 Papaver nudicaule var. chinense, T4687 Peganum harmala, T4704 Peperomia duclouxii, T4710 Pericampylus glaucus, T4726 Periploca forrestii, T4729 Periploca sepium, T4801 Phlegmariurus phlegmaria [Syn. Lycopodium phlegmaria], T4814 Phlomis umbrosa, T4828 Photinia serrulata, T4873 Picea koraiensis, T4906 Pinus armandii, T4916 Pinus massoniana, T4938 Piper boehmeriaefolium, T4956 Piper mullesua, T4962 Piper puberulum, T4965 Piper sarmentosum, T4966 Piper sarmentosum, T5018 Pleuropterus ciliinervis, T5028 Plumbago indica, T5029 Plumbago zeylanica, T5057 Podophyllum emodii [Syn. Podophyllum emodii var. chinense; Podophyllum sikkimenosis; Sinopodophyllum emodii], T5071 Polygala arillata, T5075 Polygala fallax [Syn. Polygala aureocauda], T5104 Polygonum hydropiper, T5105 Polygonum hydropiper var. flaccidum [Syn. Polygonum flaccidum], T5106 Polygonum lapathifolium, T5108 Polygonum multiflorum, T5114 Polygonum polystachyum, T5124 Polypodium niponicum, T5144 Populus adenopoda, T5156 Populus nigra var. thevestina, T5179 Potentilla anserina, T5189 Pothos chinensis, T5230 Prunus persica, T5267 Psidium guajava, T5269 Psilotum nudum, T5284 Pteridium aquilinum var. latiusculum, T5308 Pterocarya stenoptera, T5341 Pygmaeopremna herbacea [Syn. Premna herbacea], T5344 Pyrola atropurpurea, T5345 Pyrola calliantha [Syn. Pyrola rotundifolia ssp. chinensis], T5346 Pyrola calliantha var. tibetana, T5347 Pyrola decorata, T5349 Pyrola incarnata, T5350 Pyrola japonica, T5351 Pyrola rotundifolia, T5391 Rabdosia eriocalyx, T5515 Rhododendron micranthum, T5516 Rhododendron molle, T5523 Rhododendron simsii, T5547 Ricinus communis, T5560 Rorippa montana [Syn. Rorippa dubia; Sisymbrium dublium], T5591 Rubus cochinchinensis, T5637 Sabia schumanniana, T5640 Sabina vulgaris, T5651 Salix babylonica, T5704 Sambucus nigra, T5707 Sambucus williamsii, T5727 Saposhnikovia divaricata [Syn. Ledebouriella seseloides], T5730 Sarcandra glabra [Syn. Chloranthus glaber], T5741 Sargentodoxa cuneata, T5746 Sassafras tzumu, T5755 Saussurea involucrata, T5756 Saussurea japonica, T5781 Schefflera arboricola, T5785 Schefflera venulosa, T5793 Schisandra henryi, T5817 Scolopendra subspinipes mutilans, T5861 Selaginella doederleinii, T5934 Seseli meirei, T5937 Seseli yunnanense, T5939 Shiraia bambusicola, T5950 Siegesbeckia orientalis, T5951 Siegesbeckia orientalis var. glabrescens [Syn. Siegesbeckia glabrescens], T5952 Siegesbeckia orientalis var. pubescens [Syn. Siegesbeckia pubescens], T5963 Sinodielsia yunnanensis, T5964 
Sinomenium acutum, T5972 Skimmia reevesiana, T5976 Smilax china [Syn. Smilax japonica], T5982 Smilax sieboldii, T5984 Smilax stans [Syn. Smilax vaginata var. stans], T6020 Solanum xanthocarpum, T6051 Sorbaria arborea, T6052 Sorbaria sorbifolia, T6066 Spatholobus suberectus, T6069 Speranskia tuberculata, T6084 Spiraea prunifolia, T6086 Spiraea thunbergii, T6100 Stauntonia hexaphylla, T6118 Stephania brachyandra, T6119 Stephania cepharantha, T6120 Stephania delavayi [Syn. Stephania epigaea], T6125 Stephania elegans, T6127 Stephania glabra, T6128 Stephania hernandifolia, T6129 Stephania japonica, T6131 Stephania longa, T6133 Stephania sinica, T6136 Stephania tetrandra, T6166 Strychnos angustiflora, T6250 Symplocos caudata, T6356 Tetrapanax papyriferus, T6422 Thelephora vialis, T6437 Thlaspi arvense, T6440 Thuja orientalis [Syn. Platycladus orientalis; Biota orientalis], T6468 Tinospora sinensis, T6484 Trachelospermum jasminoides, T6540 Tripterygium hypoglaucum, T6566 Tupistra chinensis, T6580 Tylophora floribunda, T6645 Urena lobata, T6651 Urtica cannabina, T6652 Urtica dioica, T6675 Valeriana amurensis, T6676 Valeriana hardwickii, T6679 Valeriana officinalis, T6680 Valeriana officinalis var. latifolia, T6693 Veratrum album var. lobelianum [Syn. Veratrum lobelianum], T6727 Veronica persica, T6737 Viburnum luzonicum, T6738 Viburnum odoratissimum, T6771 Viscum album, T6772 Viscum angulatum, T6773 Viscum articulactum, T6774 Viscum articulatum, T6775 Viscum coloratum, T6777 Viscum multinerve, T6787 Vitex negundo, T6791 Vitex rotundifolia [Syn. Vitex trifollia var. simplicifolia], T6793 Vitex trifolia, T6798 Vitis vinifera, T6799 Vitis vinifera, T6830 Woodfordia fruticosa, T6843 Xanthium sibiricum [Syn. Xanthium strumarium], T6861 Zanthoxylum ailanthoides, T6883 Zanthoxylum myriacanthum.

wind-damp impediment pattern T5158 Populus simonii.

wind-damp intractable impediment T6184 Strychnos nux-vomica, T6193 Strychnos wallichiana.

wind-damp lumbago T0122 Aconitum pendulum, T1453 Cissampelos pareira var. hirsute, T1629 Colysis pothifolia [Syn. Hemionitis pothifolia], T2213 Dioscorea zingiberensis, T5794 Schisandra lancifolia, T5848 Securinega suffruticosa.

wind-damp numbness T1327 Ceratostigma minus.

wind-damp numbness pain T5389 Rabdosia coetsa

wind-damp pain T0069 Achillea wilsoniana, T0116 Aconitum nagarum var. heterotrichum [Syn. Aconitum bullatifolium], T0142 Acorus calamus, T0467 Anemone flaccida, T0471 Anemone rivularis, T0569 Aralia cordata, T0574 Aralia fargesii, T0729 Asarum maximum, T0813 Atalantia buxifolia [Syn. Severinia buxifolia], T0838 Azolla imbricata [Syn. Salvinia imbricata], T1129 Calophyllum inophyllum, T1186 Capsicum annuum, T1187 Capsicum frutescens, T1275 Cayratia japonica, T1373 Chloranthus serratus, T1932 Cyclea racemosa, T2028 Daphne odora, T2409 Equisetum palustre, T2412 Equisetum sylvaticum, T2510 Eucalyptus globulus, T2732 Firmiana simplex, T3088 Gynura segetum [Syn. Gynura japonica], T3418 Incarvillea arguta, T3436 Inula nervosa, T3460 Iris halophila, T3464 Iris lactea var. chinensis [Syn. Iris pallasii var. chinensis], T3471 Iris tectorum, T3737 Lemmaphyllum microphyllum var. obovatum, T3765 Lepisorus ussuriensis, T3989 Lygodium flexuosum [Syn. Lygodium pinnatifidum; Ophioglossum flexuosum], T4004 Lysimachia paridiformis, T4195
Menyanthes trifoliata, T4423 Nicandra physaloides, T4503 Onychium lucidum, T4577 Paederia scandens, T4778 Phallus impudicus, T4823 Pholidota yunnanensis, T5122 Polygonum viscosum, T5193 Pratia nummularia, T5250 Psammosilene tunicoides, T5555 Rodgersia pinnata, T5563 Rosa cymosa, T5586 Rubia yunnanensis, T5720 Sapindus mukorossi, T5819 Scopolia acutangula [Syn. Anisodus acutangulus], T5989 Solanum aculeatissimum, T5998 Solanum dulcamara, T6003 Solanum khasianum, T6014 Solanum surattense, T6264 Syzygium buxifolium, T6367 Teucrium quadrifarium, T6471 Toddalia asiatica [Syn. Toddalia aculeata; Paullinia asiatica], T6578 Tylophora atrofolliculata, T6689 Ventilago leiocarpa, T6742 Vicia amoena, T6895 Zanthoxylum simulans.

wind-damp pain (external use with high toxicity) T1416 Cicuta virosa. wind-damp pain in lumbus and legs T0151 Acronychia pedunculata, T6304 Taxillus levinei, T6731 Veronicastrum sibiricum.

wind-damp pain in sinew and bone T0708 Arthromeris mairei [Syn. Polypodium mairei], T1003 Brandisia hancei, T1976 Cyperus iria, T2062 Delphinium bonvalotii, T2081 Delphinium omeiense, T2084 Delphinium potaninii, T2085 Delphinium potaninii var. jiufengshanense, T3118 Hedychium coronarium, T3285 Hovenia dulcis, T4662 Parthenocissus tricuspidata, T5298 Pteris vittata, T5592 Rubus hirsutus, T6889 Zanthoxylum podocarpum.

wind-damp pain in sinews and bones $\mathrm{T} 1746$ Corydalis taliensis.

wind-damp papules T6476 Torilis japonica.

wind-damp sore toxin T6477 Torreya grandis.

wind-damp with painful swollen joints T0078 Aconitum barbatum var. puberulum [Syn. Aconitum ochranthum].

wind-damp-heat impediment T0594 Ardisia crenata, T0635 Aristolochia moupinensis, T1561 Clerodendron serratum, T1569 Clerodendrum serratum var. amplexifolium, T3913 Lonicera japonica, T4060 Mahonia confusa, T4064 Mahonia gracilipes, T4575 Pachysandra terminalis, T4582 Paeonia delavayi, T4585 Paeonia moutan [Syn. Paeonia suffruticosa], T4678 Paulownia fortunei, T4679 Paulownia tomentosa, T5126 Polypodium virginianum, T5127 Polypodium vulgare, T6386 Thalictrum foetidum, T6845 Xanthoceras sorbifolia.

wind-fire eye T1251 Cassytha filiformis, T5907 Senecio scandens [Syn. Senecio chinensis].

wind-fire toothache T0181 Adina rubella, T0364 Alsophila spinulosa, T0722 Arundo donax, T0922 Berchemia polyphylla var. leioclada, T1692 Coriaria sinica [Syn. Coriaria nepalensis], T1869 Cryptotaenia japonica, T3432 Inula helianthus-aquatica, T3624 Kalopanax pictum, T3625 Kalopanax septemlobus, T3736 Lemmaphyllum microphyllum, T4605 Panax japonicus var. major, T5184 Potentilla kleiniana, T6234 Swertia punicea, T6250 Symplocos caudata, T6378 Thalictrum delavayi.

wind-heat common cold T0319 Allium schoenoprasum, T0457 Andrographis paniculata [Syn. Justicia paniculata], T0584 Arctium lappa, T0586 Arctium lappa, T0784 Astilbe chinensis, T0888 Beesia calthaefolia, T1106 Caesalpinia minax, T1425 Cimicifuga simplex, T1633 Commelina communis, T1746 Corydalis taliensis, T1931 Cyclea barbata, T2244 Doellingeria scaber [Syn. Aster scaber], T2281 Dryopteris crassirhizoma, T2325 Eichhornia crassipes, T2555 Eupatorium chinense, T2756 Forsythia suspensa, T3340 Hypericum 
ascyron, T3396 Ilex pubescens, T3397 Ilex pubescens var. glaber, T4056 Mahonia bealei, T4063 Mahonia fortunei, T4067 Mahonia japonica, T4186 Mentha piperita, T4289 Morus alba, T4291 Morus australis, T4293 Morus cathayana, T4298 Morus mongolica, T4502 Onychium japonicum [Syn. Tricomanes japonicum], T4527 Orixa japonica, T4831 Phtheirospermum japonicum [Syn. Geradia japonica], T4884 Picria felterrae, T6023 Solidago virgaurea, T6024 Solidago virgaurea var. leiocarpa [Syn. Solidago decurrens], T6234 Swertia punicea, T6338 Tephrosia purpurea, T6365 Teucrium pilosum [Syn. Teucrium japonicum var. pilosum], T6367 Teucrium quadrifarium, T6431 Thesium chinense, T6567 Tupistra wattii [Syn. Campylandra wattii], T6735 Viburnum dilatatum.

wind-heat common cold and headache T0571 Aralia decaisneaa.

wind-heat cough T0834 Averrhoa carambola, T3928 Lotus corniculatus, T6219 Swertia erythrosticta, T6537 Tripterospermum japonicum, T6538 Tripterospermum taiwanense.

wind-heat exterior syndrome T3738 Lemna minor, T4184 Mentha haplocalyx [Syn. Mentha canadaensis; Mentha arvensis var. haplocalyx; Mentha arvensis].

wind-heat headache T4143 Meconopsis horridula, T4796 Pheretima aspergillum; Allolobophora caliginosa trapezoides.

wind-heat impediment pain T1199 Carduus acanthoides, T1200 Carduus crispus, T1903 Curcuma aromatica, T1905 Curcuma longa.

wind-heat itching of skin T1296 Celosia argentea.

wind-heat macular eruption T0860 Balanophora japonica.

wind-heat red eye T0984 Bos taurus domesticus; Bubalus bubalis, T2408 Equisetum hiemale.

wind-heat toothache T2077 Delphinium grandiflorum.

wind-phlegm cough T0805 Astragalus sinicus.

wind-phlegm dizziness T0088 Aconitum coreanum, T0617 Arisaema amurense, T0618 Arisaema consanguineum, T0620 Arisaema heterophyllum, T4903 Pinellia pedatisecta.

withered-yellow facial complexion T5042 Podocarpus macrophyllus, T5044 Podocarpus macrophyllus var. maki.

wolf-fistula T2427 Erinaceus europaeus; Hemiechinus daunicus; Hemiechinus auritus.

women's diseases T0495 Angelica sinensis, T1978 Cyperus rotundus, T4804 Phlojodicarpus sibiricus.

women's hormone dysfunction diseases T1419 Cimicifuga dahurica, T1420 Cimicifuga foetida, T1421 Cimicifuga heracleifolia.

worm accumulation T0606 Areca catechu, T1210 Carpesium abrotanoides, T2048 Daucus carota, T2365 Enteromorpha clathrata, T2695 Ferula assafoetida, T3460 Iris halophila, T3464 Iris lactea var. chinensis [Syn. Iris pallasii var. chinensis], T5385 Quisqualis indica, T5542 Rhus verniciflua [Syn. Toxicadendron verniciflum], T5720 Sapindus mukorossi, T6105 Stellera chamaejasme, T6177 Strychnos ignatii.

wound T0311 Allium cepa, T0543 Apis mellifera ligustica, T2293 Dumortiera hirsuta, T3388 Ilex chinensis [Syn. Ilex purpurea], T3578 Juncus effusus, T4253 Mirabilis jalapa, T4303 Moschus moschiferus; Moschus berezovskii; Moschus sifanicus, T5308 Pterocarya stenoptera. wound swelling from knocks and falls T1287 Celastrus hypoleucus, T1799 Crinum latifolium, T2161 Dicranopteris pedata [Syn.
Polypodium pedatum; Dicranopteris dichotoma], T2538 Euonymus bungeanus, T3796 Libanotis buchtormensis, T3990 Lygodium japonicum, T4549 Osbeckia chinensis, T4605 Panax japonicus var. major, T4937 Piper betle, T5785 Schefflera venulosa.

wrenching and contusion T0483 Angelica gigas.

wrenching and contusion from knocks and falls T1958 Cynanchum otophyllum, T2273 Drynaria fortunei, T5252 Pseudodrynaria coronans.

yang brightness headache T1425 Cimicifuga simplex.

yang vacuity cold diarrhea T1901 Curculigo orchioides.

yang vacuity dizziness T1439 Cinnamomum cassia [Syn. Cinnamomum aromaticum].

yang vacuity external contraction T0083 Aconitum carmichaeli, T0085 Aconitum carmichaeli cv.

yang-collapse reversal flow T6909 Zingiber officinale.

yang-collapse vacuity desertion T0083 Aconitum carmichaeli, T0085 Aconitum carmichaeli cv.

yellow eyes T6398 Thalictrum microgynum.

yellow sweating T6764 Vinegar.

yellow swelling T1538 Clausena lansium, T6018 Solanum verbascifolium, T6866 Zanthoxylum avicennae.

yellow swelling in whole body T6398 Thalictrum microgynum.

yellow thick foul leukorrhagia T4789 Phellodendron amurense, T4790 Phellodendron amurense var. wilsonii, T4791 Phellodendron chinense, T4792 Phellodendron chinense var. glabriusculum.

yellow thick vaginal discharge T3564 Juglans mandshurica.

yellow-water sore T0557 Aquilegia ecalcarata, T1163 Campylotropis hirtella, T1693 Coriaria sinica [Syn. Coriaria nepalensis], T2165 Dictamnus angustifolius, T2167 Dictamnus dasycarpus, T3246 Hibiscus syriacus, T3340 Hypericum ascyron, T3348 Hypericum elodeoides, T3368 Hypericum wightianum, T3680 Lamium amplexicaule, T4830 Phryma leptostachya, T4845 Physalis alkekengi, T5033 Poa sphondylodes, T5717 Sapindus delavayi [Syn. Pancovia delavayi].

yin cold abdominal pain T0310 Allium ascalonicum, T0314 Allium fistulosum.

yin cold edema T0083 Aconitum carmichaeli, T0085 Aconitum carmichaeli cv.

yin damage liquid depletion T2094 Dendrobium aduncum, T2096 Dendrobium aurantiacum var. denneanum, T2098 Dendrobium chrysanthum, T2100 Dendrobium densiflorum, T2102 Dendrobium fimbriatum var. oculatum, T2105 Dendrobium loddigesii, T2106 Dendrobium moniliforme, $\mathrm{T} 2107$ Dendrobium nobile, T2108 Dendrobium officinale.

yin depletion and effulgent fire T4610 Panax quinquefolium.

yin flat abscess T5109 Polygonum nodosum, T6263 Syzygium aromaticum [Syn. Eugenia caryophyllata].

yin flat abscess and phlegm node T2367 Ephedra equisetina, T2368 Ephedra gerardiana, T2369 Ephedra intermedia, T2372 Ephedra likiangensis, T2373 Ephedra minuta, T2375 Ephedra monosperma, T2379 Ephedra saxatilis, T2380 Ephedra sinica.

yin flat abscess and sores T0083 Aconitum carmichaeli, T0085 Aconitum carmichaeli $\mathrm{cv}$. 
yin summerheat and headache T4305 Mosla dianthera.

yin syndrome with sores T0440 Anagallis arvensis.

yin vacuity and heat vexation $\mathrm{T} 3001$ Glycine max.

yin vacuity blood heat T2323 Eclipta prostrata [Syn. Eclipta alba].

yin vacuity consumption fever $\mathrm{T} 3983$ Lycoris aurea.

yin vacuity cough T1969 Cynoglossum officinale, T4450 Nuphar

pumilum.

yin vacuity enduring cough $\mathrm{T} 3832$ Lilium brownii var. viridulum [Syn.

Lilium brownii var. colchesteri], T3835 Lilium longiflorum, T3836

Lilium pumilum [Syn. Lilium tenuifolium], T3839 Lilium tigrinum [Syn.

Lilium lancifolium].

yin vacuity fever T0660 Artemisia annua, T0662 Artemisia apiacea [Syn.

Artemisia carvifolia; Artemisia caruifolia], T0746 Asparagus

cochinchinensis [Syn. Asparagus lucidus], T3276 Homo sapiens,

T6104 Stellaria dichotoma var. lanceolata.

yin vacuity internal heat $\mathrm{T} 6087$ Spiranthes sinensis.

yin vacuity lung dryness T0749 Asparagus filicinus, T0753 Asparagus setaceus [Syn. Asparagus plumosus], T3873 Liriope platyphylla, T3874 Liriope spicata.

yin vacuity taxation cough $\mathrm{T} 2783$ Fritillaria cirrhosa, T2784 Fritillaria delavayi, T2792 Fritillaria przewalskii, T2796 Fritillaria unibracteata, T2797 Fritillaria ussuriensis, T5091 Polygonatum cyrtonema [Syn. Polygonatum multiflorum], T5092 Polygonatum kingianum, T5095 Polygonatum sibiricum.

yin vacuity taxation fever T3390 Ilex cornuta.

yin vacuity tidal fever T6416 Thamnolia vermicularis.

yin vacuity tuberculosis T0607 Arenaria juncea, T3090 Gypsophila oldhamiana, T3091 Gypsophila pacifica, T5955 Silene jenisseensis.

zoster T0543 Apis mellifera ligustica, T0805 Astragalus sinicus, T0866 Baphicacanthus cusia [Syn. Strobilanthes cusia], T1151 Camellia sinensis [Syn. Thea sinensis], T1288 Celastrus orbiculatus [Syn. Celastrus articulatus], T1290 Celastrus orbiculatus [Syn. Celastrus articulatus], T1799 Crinum latifolium, T1869 Cryptotaenia japonica, T1959 Cynanchum paniculatum, T2193 Dioscorea cirrhosa [Syn. Dioscorea pogonoides], T2658 Fagopyrum esculentum, T3233 Heteropappus altaicus, T3311 Hydrocotyle sibthorpioides, T3318 Hylotelephium mingjinianum, T3865 Lippia nodiflora, T3970 Lycopodium cernuum, T3973 Lycopodium japonicum [Syn. Lycopodium clavatum], T3990 Lygodium japonicum, T4248 Mimosa pudica, T5184 Potentilla kleiniana, T5680 Salvia miltiorrhiza, T5681 Salvia miltiorrhiza f. alba, T5688 Salvia przewalskii, T6092 Stachys palustris, T6217 Swertia davidii. 